NATL INST OF STANDARDS \& TECH RIC

I || || || ||

A11 102145848

Moore, Charlotte 102145848

NSRDS-NBS 40

QC100. U573 V40;1972 C. 1 NBltplet table o

\title{
NBS
}

PUBLICATIONS

U.S. DEPARTMENT OF COMMERCE National Bureau

\section{A Multiplet Table of Astrophysical Interest}

1945 Edition

Part I-Table of Multiplets

Part II-Finding List of All Lines in the Table of Multiplets 


\section{NATIONAL BUREAU OF STANDARDS}

The National Bureau of Standards ${ }^{1}$ was established by an act of Congress March 3 , 1901. The Bureau's overall goal is to strengthen and advance the Nation's science and technology and facilitate their effective application for public benefit. To this end, the Bureau conducts research and provides: (1) a basis for the Nation's physical measurement system, (2) scientific and technological services for industry and government, (3) a technical basis for equity in trade, and (4) technical services to promote public safety. The Bureau consists of the Institute for Basic Standards, the Institute for Materials Research, the Institute for Applied Technology, the Center for Computer Sciences and Technology, and the Office for Information Programs.

THE INSTITUTE FOR BASIC STANDARDS provides the central basis within the United States of a complete and consistent system of physical measurement; coordinates that system with measurement systems of other nations; and furnishes essential services leading to accurate and uniform physical measurements throughout the Nation's scientific community, industry, and commerce. The Institute consists of a Center for Radiation Research, an Office of Measurement Services and the following divisions:

Applied Mathematics-Electricity-Heat-Mechanics-Optical Physics-Linac Radiation ${ }^{2}$ - Nuclear Radiation ${ }^{2}$ - Applied Radiation²-Quantum Electronics ${ }^{3}$ Electromagnetics ${ }^{3}$ - Time and Frequency ${ }^{3}$ - Laboratory Astrophysics ${ }^{3}-$ Cryo- $^{3}$ genics 3 .

THE INSTITUTE FOR MATERIALS RESEARCH conducts materials research leading to improved methods of measurement, standards, and data on the properties of well-characterized materials needed by industry, commerce, educational institutions, and Government; provides advisory and research services to other Government agencies: and develops, produces, and distributes standard reference materials. The Institute consists of the Office of Standard Reference Materials and the following divisions:

Analytical Chemistry-Polymers-Metallurgy-Inorganic Materials-Reactor Radiation-Physical Chemistry.

THE INSTITUTE FOR APPLIED TECHNOLOGY provides technical services to promote the use of available technology and to facilitate technological innovation in industry and Government; cooperates with public and private organizations leading to the development of technological standards (including mandatory safety standards), codes and methods of test; and provides technical advice and services to Government agencies upon request. The Institute also monitors NBS engineering standards activities and provides liaison between NBS and national and international engineering standards bodies. The Institute consists of the following divisions and offices:

Engineering Standards Services-Weights and Measures-Invention and Innovation-Product Evaluation Technology-Building Research-Electronic Technology-Technical Analysis-Measurement Engineering-Office of Fire Programs.

THE CENTER FOR COMPUTER SCIENCES AND TECHNOLOGY conducts research and provides technical services designed to aid Government agencies in improving cost effectiveness in the conduct of their programs through the selection, acquisition, and effective utilization of automatic data processing equipment; and serves as the principal focus within the executive branch for the development of Federal standards for automatic data processing equipment, techniques, and computer languages. The Center consists of the following offices and divisions:

Information Processing Standards-Computer Information-Computer Services -Systems Development-Information Processing Technology.

THE OFFICE FOR INFORMATION PROGRAMS promotes optimum dissemination and accessibility of scientific information generated within NBS and other agencies of the Federal Government; promotes the development of the National Standard Reference Data System and a system of information analysis centers dealing with the broader aspects of the National Measurement System; provides appropriate services to ensure that the NBS staff has optimum accessibility to the scientific information of the world, and directs the public information activities of the Bureau. The Office consists of the following organizational units:

Office of Standard Reference Data-Office of Technical Information anc Publications-Library-Office of International Relations.

\footnotetext{
1 Headquarters and Laboratories at Gaithersburg, Maryland, unless otherwise noted; mailing address Washing. ton, D.C. 20234.

2 Part of the Center for Radiation Research.

3 Located at Boulder, Colorado 80302.
} 
UNITED STATES DEPARTMENT OF COMMERCE - Maurice H. Stans, Secretary

U.5. NATIONAL BUREAU OF STANDARDS - Lewis M. Branscomb, Director

\title{
A Multiplet Table of Astrophysical Interest
}

\author{
Revised Edition
}

\section{Part I-Table of Multiplets}

Part II-Finding List of All Lines in the Table of Multiplets

\author{
Charlotte E. Moore \\ Office of Standard Reference Data \\ National Bureau of Standards \\ Washington, D.C. 20234 \\ Contributions from the Princeton University Observatory \\ No. 20, 1945 \\ Reprinted by permission of the Director \\ of the Princeton University Observatory
}

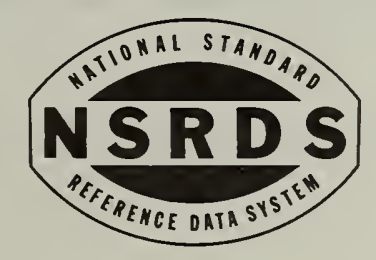

t. NSRDS-NBS 40

Nat. Stand. Ref. Data Ser., Nat. Bur. Stand. (U.S.), 40, 253 pages (Feb. 1972)

\section{CODEN : NSRDAP}

Reprint of NBS Technical Note 36 (PB151395). See author's note, pp. V-VI.

Issued February 1972 


\section{FOREWORD}

The National Standard Reference Data System provides effective access to the quantitative data of physical science, critically evaluated and compiled for convenience, and readily accessible through a variety of distribution channels. The System was established in 1963 by action of the President's Office of Science and Technology and the Federal Council for Science and Technology, with responsibility to administer it assigned to the National Bureau of Standards.

The System now comprises a complex of data centers and other activities, carried on in academic institutions and other laboratories both in and out of government. The independent operational status of existing critical data projects is maintained and encouraged. Data centers that are components of the NSRDS produce compilations of critically evaluated data, critical reviews of the state of quantitative knowledge in specialized areas, and computations of useful functions derived from standard reference data. In addition, the centers and projects establish criteria for evaluation and compilation of data and make recommendations on needed improvements in experimental techniques. They are normally closely associated with active research in the relevant field.

The technical scope of the NSRDS is indicated by the principal categories of data compilation projects now active or being planned; nuclear properties, atomic and molecular properties, solid state properties, thermodynamic and transport properties, chemical kinetics, and colloid and surface properties and mechanical properties.

The NSRDS receives advice and planning assistance from the National Research Council of the National Academy of Sciences-National Academy of Engineering. An overall Review Committee considers the program as a whole and makes recommendations on policy, long-term planning, and international collaboration. Advisory Panels, each concerned with a single technical area, meet regularly to examine major portions of the program, assign relative priorities, and identify specific key problems in need of further attention. For selected specific topics, the Advisory Panels sponsor subpanels which make detailed studies of users' needs, the present siate of knowledge, and existing data resources, as a basis for recommending one or more data compilation activities. This assembly of advisory services contributes greatly to the guidance of NSRDS activities.

The NSRDS-NBS series of publications is intended primarily to include evaluated reference data and critical reviews of long-term interest to the scientific and technical community.

Lewis M. Branscomb, Director 


\title{
AUTHOR'S FOREWORD
}

The present Multiplet Table was first published in 1945 by the Princeton University Observatory; it has not yet been superseded. In 1959 it was reprinted as Technical Note 36 of the National Bureau of Standards. This issue is now out of print and is being reprinted as NSRDS-NBS 40.

The format is not being changed. In the present publication a special note has been added in the heading for those spectra included in "Selected Tables of Atomic Spectra," NSRDS-NBS 3, to indicate the existence of a Revised Multiplet Table.

August 2, 1971

\begin{abstract}
Pending the completion of a current edition, the 1945 Multiplet Table is being reprinted here to meet continuing demands. The leading lines in 196 atomic spectra of 85 chemical elements are listed in related groups called multiplets. Estimated intensities, excitation potentials and multiplet designations are given for the individual lines, and each multiplet is assigned a number. An extensive bibliography covers the source material used for the compilation.

The Table is presented in two parts:

Part I includes the multiplets, with the spectra of each element being given in order of increasing ionization, and the elements in order of increasing atomic number.

Part II is a Finding List in which all the lines in Part I are entered in order of increasing wavelength, with their multiplet numbers.

The range of the Table is from $2951 \AA$ to $13164 \AA$ A. A supplementary table of "Forbidden Lines" extends from $2972 \AA$ to $12645 \AA$.
\end{abstract}

Key words: Atomic spectra, multiplet table; finding list, atomic spectra; multiplet table; spectra, atomic. 


\section{Editorial Note-Spectra in Technical Note 36 (PB151395), for which revised data are given in NSRDS-NBS $3 *$}

\begin{tabular}{|c|c|}
\hline Page & Spectrum \\
\hline 2 & $\mathrm{C}_{\mathrm{I}}$ \\
\hline 2 & $\mathrm{C}_{\text {II }}$ \\
\hline 3 & $C_{\text {III }}$ \\
\hline 3 & C IV \\
\hline New & $\mathrm{C} v$ \\
\hline 6 & $\mathrm{~N}$ Iv \\
\hline 6 & $\mathrm{Nv}$ \\
\hline New & $\mathrm{N}$ vi \\
\hline New & $\mathrm{N}$ vI \\
\hline 15 & Si I \\
\hline 16 & Si I \\
\hline 16 & Si I \\
\hline 17 & Si I \\
\hline
\end{tabular}

Reference

SEE REVISION IN NSRDS-NBS 3, Section 3, November 1970.

SEE

Section 3, November 1970.

SEE REVISION IN NSRDS-NBS 3, Section 4, August 1971.

SEE

Section 4, August 1971.

SEE REVISION IN NSRDS-NBS 3, Section 2, November 1967.

SEE REVISION IN NSRDS-NBS 3, Section 1, June 1965.

\section{Correction}

Part I 2 He II

Part II $76 \quad$ He II $\lambda 6570.0$ has been corrected to $\lambda 6527.10$.

$\lambda 6570.0$ Ref. A has been corrected to $\lambda 6527.10$ Ref. P.

Part II $76 \quad$ He II $\lambda 6570.0$ has been corrected to $\lambda 6527.10$.

* See List of Publications in the National Standard Reference Data Series at the back of this book for information about obtaining these publications. 


\section{Author's Note on the Reprinting of the 1945 Princeton Multiplet Table: U.S. Department of Commerce, N.B.S. Tech. Note 36, (PB151395), 1959}

The Multiplet Table that first appeared as Contributions from the Princeton University Observatory No. 20, 1945, is still a standard reference source used by astrophysicists, physicists, chemists, and many others. To date it has not been superseded and it continues to be in steady demand, although it is seriously in need of revision.

In 1959 this table was reprinted as U.S. Department of Commerce, National Bureau of Standards Technical Note 36 (PB151395). This issue is now out of print.

In view of the continuing requests, the Office of Standard Reference Data has decided to reprint Technical Note 36 as National Standard Reference Data Series-National Bureau of Standards, NSRDS-NBS 40, 1971, Parts I and II.

Similarly, Volumes I, II, and III of "Atomic Energy Levels," Circular of the National Bureau of Standards 467 are being reprinted in the same series, NSRDS-NBS 35, Parts I, II, III.

The present rapid technological advances by the astrophysicist in observing celestial spectra have created an urgent need for a current Multiplet Table of Astrophysical Interest. The correct interpretation of these spectra depends directly on the laboratory analyses of optical spectra. A critical compilation of spectroscopic data that provides the leading lines of individual atomic and ionic spectra of the more abundant elements, over the range from the x-ray to the microwave region is essential. In preparing such a table an effort should be made to envisage future developments in observing celestial spectra over this range and to design laboratory programs that will provide the requisite data.

Many gaps exist in our knowledge of atomic and ionic spectra. Sources that will produce clearly separated spectra in all stages of ionization for the elements $\mathrm{H}$ to $\mathrm{Ni}$ will be needed. Some of the less complex spectia can be traced along isoelectronic sequences, while more complex spectra have line lists containing thousands of lines. Encouraging progress is being made in the laboratory, where excellent spectrographs and carefully controlled sources can produce spectra that far outweigh the observations quoted in 1945.

Although it is not yet possible to provide a complete revision of this 1945 edition, current Multiplet Tables together with corresponding revised tables of Atomic Energy Levels are available for selected spectra. They are being published by the National Bureau of Standards under the title "Selected Tables of Atomic Spectra, A tomic Energy Levels and Multiplet Tables," as Sections of NSRDS. NBS 3. Section 1 contains these data for the spectra Si II, Si III, Si rv; Section 2 for Si I; Section 3 for C I, C II, C III, C rv, C v, C vi; Section 4 for N rv, N v, N vi, N vir. Similar tables for N I, N II, N III are in course of preparation as Section 5 . A number of additional spectra are partially completed for inclusion in this series. These new Multiplet Tables cover the entire observed range of individual spectra, and, therefore, supersede not only the 1945 Multiplet Table, but also the Ultraviolet Multiplet Table which appeared as Circular of the National Bureau of Standards 488, Section 1, 1950; Section 2, 1952; Sections 3, 4, 5, 1962.

In the present reprinted issue of the Princeton Table, the individual spectra that have been revised are clearly indicated. Readers are urged to use the revised data for the spectra thus marked and to note further such revisions of selected spectra as they appear in this series.

This work advances slowly, although a number of revised analyses of spectra have been published that supersede the 1945 data. A bibliography in the National Bureau of Standards Special Publication 306, Sections 1, 2, 3, 4, 1968-1969, provides reference material on individual spectra to about July 1968, continuing from the reference listings given in the Volumes on Atomic Energy Levels.

The most serious need for revision is perhaps in the infrared data. Current references to work on the spectra $\mathrm{HI}$ to $\mathrm{Ni}$ I in the range $>7000 \AA$ may be found in a forthcoming publication of the Proceedings of the Seventeenth International Astrophysical Symposium, on "Astronomical Spectra in the Infrared and Microwave Regions," held at the Institut d'Astrophysique, Université de Liège, Cointe-Ougrée, Belgium, June 28-30, 1971. 

Part I-Table of Multiplets 

I Introduction

1 Need for spectrum analysis. . . . . . . . . . . . . . .

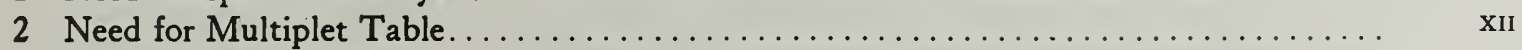

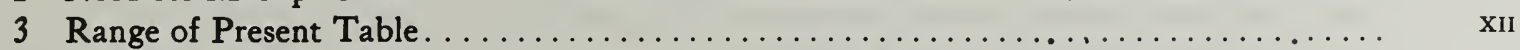

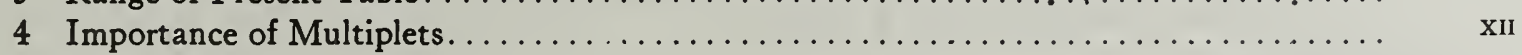

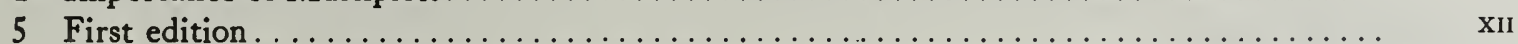

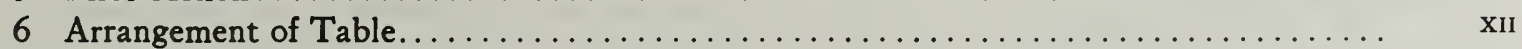

II The Multiplet Table-General Considerations

7 Astronomical Spectra........................................ XII

8 Astrophysical importance of spectra, $H-Z_{n} \ldots \ldots \ldots \ldots \ldots \ldots \ldots \ldots \ldots \ldots \ldots \ldots \ldots \ldots \ldots$ xIII

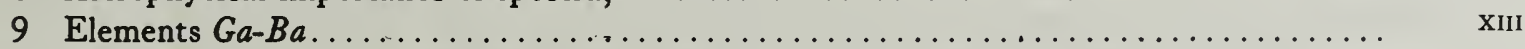

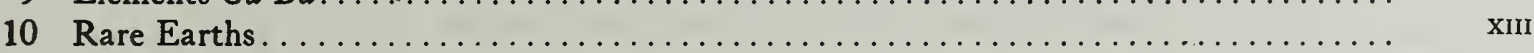

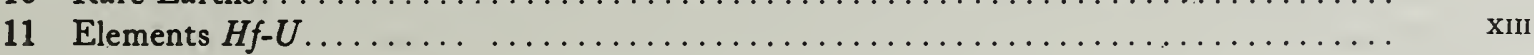

III Basis of Selection

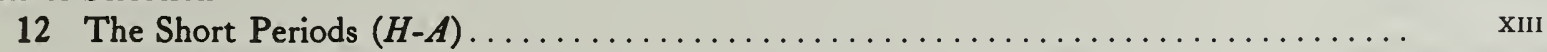

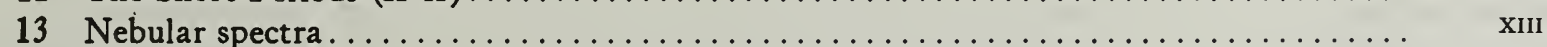

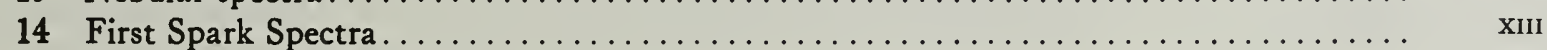

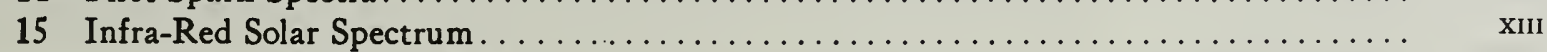

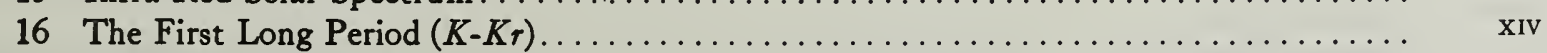

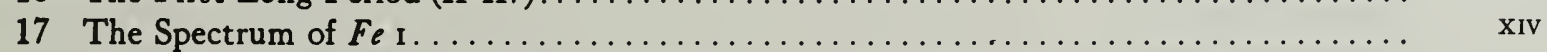

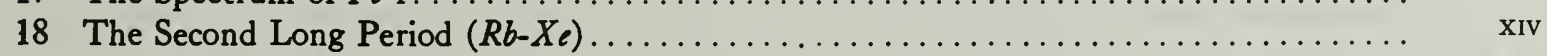

19 Forbidden Lines.................

IV General Arrangement of the Multiplet Table

20 Size of Present Revised Multiplet Table $\ldots \ldots \ldots \ldots \ldots \ldots \ldots \ldots \ldots \ldots \ldots \ldots \ldots \ldots \ldots$ XIV

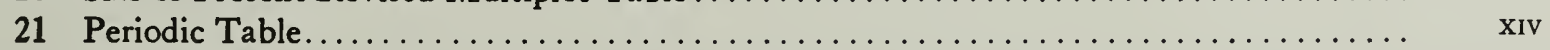

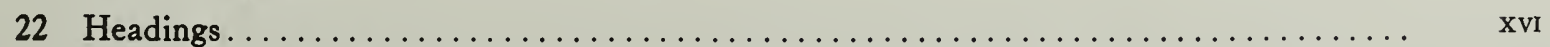

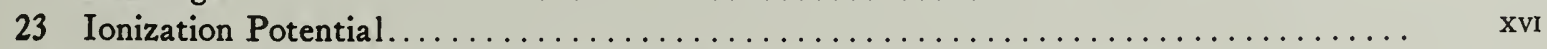

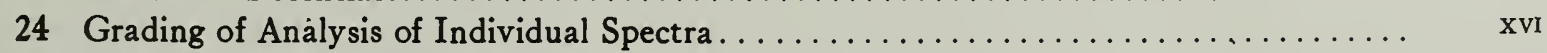

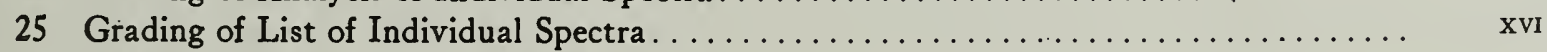

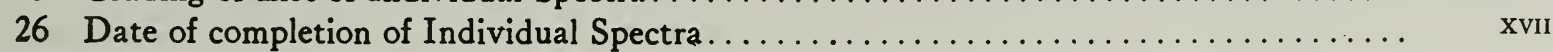

V Arrangement of the Multiplets of Each Spectrum

27 Spectrum Analysis-general arrangement of multıplets..................... xviI

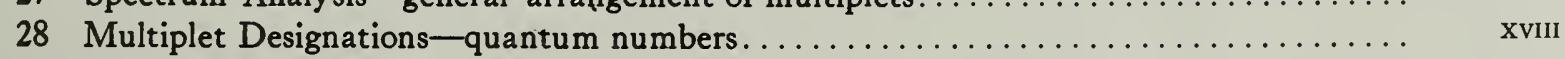

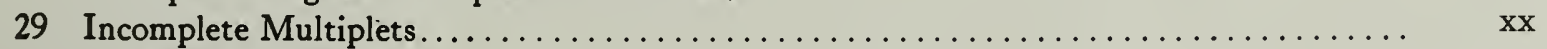

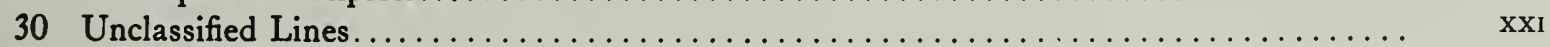

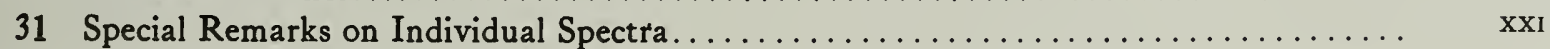

VI Columns of the Table of Multiplets

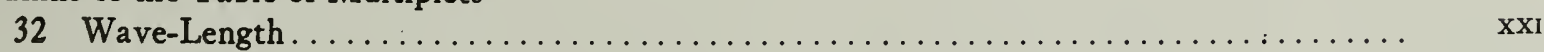

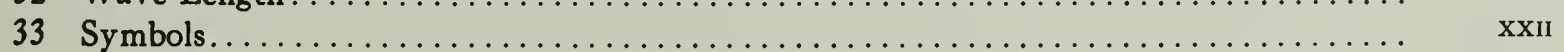

34 Intensity . . . .

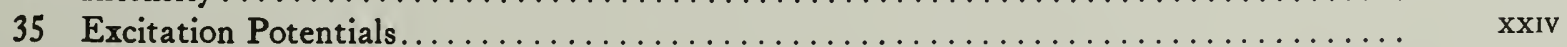

VII Spectroscopic Notation

A Series Spectra

36 Series Notation and Limits . . . . . . . . . . . . . . . . . . . . . . . . . . . . . . . . . .

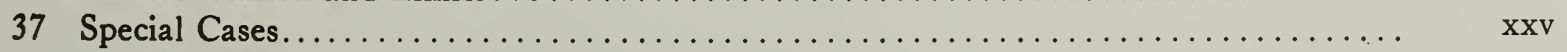

B Complex Spectra

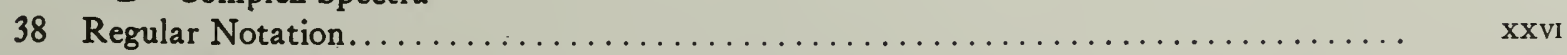

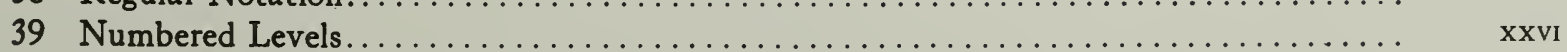

VIII Special Notes on Individual Spectra

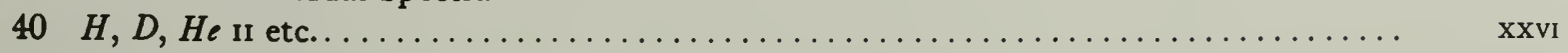

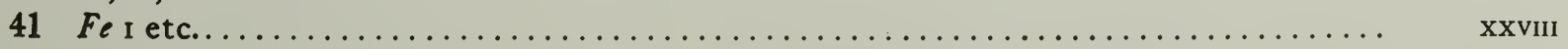


IX Spectra Omitted from the R M T

42 Spectra of Astrophysical Interest-No Analysis $\ldots \ldots \ldots \ldots \ldots \ldots \ldots \ldots \ldots \ldots \ldots \ldots \ldots \ldots \ldots$ xxvII

43 Analyzed Spectra without Astrophysical Importance $\ldots \ldots \ldots \ldots \ldots \ldots \ldots \ldots \ldots \ldots \ldots \ldots \ldots$

44 Spectra for which nothing is known $\ldots \ldots \ldots \ldots \ldots \ldots \ldots \ldots \ldots \ldots \ldots \ldots \ldots \ldots \ldots \ldots \ldots \ldots \ldots$

$\mathrm{X}$ Forbidden Lines

45 Basis of Selection . . . . . . . . .

XI Details of Publication

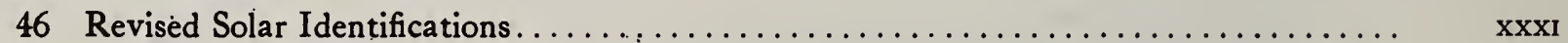

XII Bibliography-Description

47 References used for Wave-Length, Intensity and Analysis.................

XIII Acknowledgments

Bibliography

XXXIX

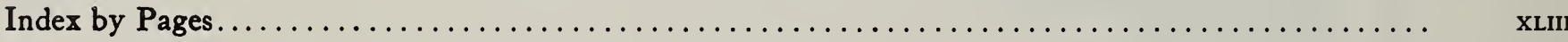

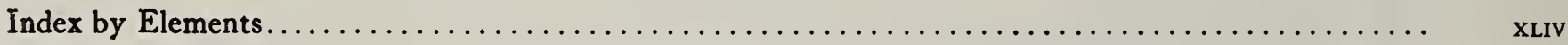

Revised Multiplet Table

Body of Table.

Forbidden Lines. 
The preparation of a Multiplet Table that will meet the needs of all astrophysicists both now and in the future is an almost overwhelming undertaking. The most eminent workers would have to exercise careful judgment in handling the spectroscopic literature today. The writer has been bold enough to attempt it, only because of the many requests for a revision of the earlier Table and the enthusiasm with which it was received in spite of its many faults. Admittedly the present work is far from ideal. With all its limitations, however, it could never have been published without a vast amount of collaboration. The generosity and encouragement of spectroscopists and astrophysicists both at home and abroad has been the inspiration for this book. No two people would present the same choice of material, and the writer feels that her judgment has been far from adequate for this task. Whatever usefulness the volume may have is due to the many workers who have stood by, ready to supply material, to discuss puzzling questions and to offer the most valuable suggestions.

Since 1932 work on spectrum analysis has progressed so speedily that the selection of data useful to the astrophysicist has been one of the major problems. Requests for an entirely complete Multiplet Table have been received, but the purpose of this work has been to provide a book whose scope is limited-one that contains astrophysically useful data but is not unwieldy because of the inclusion of other material from the vast storehouse of spectroscopic literature now accessible. The bibliography should be consulted by those who desire more complete Tables of Multiplets.

More work of astrophysical importance remains to be done, chiefly on the spectra of the rare-earths and on the second spark spectra in general. It is hoped that a supplement can be prepared to cover these spectra and that a large part of the present work will prove to be definitive.

This book has been brought to a conclusion during the second world war. Consequently, restrictions of all kinds have been imposed and assistance has been limited. A very careful attempt has been made to prepare the manuscript accurately. The writer believes, however, that errors have inevitably been made in the compiling and editing of more than 25,750 spectral lines, for the work has been done with the minimum amount of clerical aid. She relies upon the users to detect serious errors and report them to her so that a list of errata may be published. Suggestions will be welcome.

Mention has been made of the cordial cooperation experienced from the beginning of this work. It could not now have been brought to a conclusion without the hearty and enthusiastic support of Henry Norris Russell, the author of the first list of multiplets of astrophysical importance. He has generously offered his valuable collection of data on spectra and has been ever ready to help in spite of the many complications that have arisen in carrying out such an extensive program.

Charlotte E. Moore

Princeton University Observatory

Princeton, New Jersey

April 3, 1945 


\section{TABLE OF MULTIPLETS}

\section{INTRODUCTION}

1. The detailed interpretation of stellar spectra demands of the laboratory investigators an ever increasing amount of careful work on spectrum analysis. With the impetus provided by Hund's theory, remarkably rapid strides have been made. Additional encouragement, particularly in handling complex spectra, has resulted from the great development of mechanical devices to decrease the enormous labor of measuring and reducing spectrograms. The valuable machine developed by Harrison at the Massachusetts Institute of Technology for this purpose has already proven its worth and promises much more in the future.

2. From an astrophysical point of view there is a definite need for a compendium of multiplets. The manuscript lists prepared for the present work have been almost continuously on loan to various investigators.

Spectrum analysis has not been carried far enough to compile a completely satisfactory Table. So many spectra have been analyzed, however, that to wait for perfection is to retard scientific progress. For many spectra "prediction" may be invoked to extend the existing lists of observed laboratory lines, and this has been done throughout the work. Three general classes of lines are tabulated: those observed in the laboratory; predicted "permitted" lines calculated from combinations among spectroscopic term values; and predicted "forbidden" lines.

3. A complete multiplet table would be welcomed by many scientists. It is not the purpose of the present work to furnish this. The range of wave-lengths is roughly from $\lambda 2950$ to $\lambda 13000$. The violet limit is imposed by the ozone in our atmosphere, which cuts off stellar observations beyond this region. In the infra-red the scarcity and inaccuracy of laboratory wave-lengths have made it necessary to predict many lines.

Even within these limits, only the lines thought to be useful in the interpretation of astronomical spectra are listed. These are selected from the elements sufficiently abundant to appear in stellar spectra, and from only those stages of ionization and types of excitation which are to be expected.

4. The importance of handling the various laboratory spectra by multiplets was stressed in 1925 by Russell, ${ }^{1}$ who published the original multiplet table under the title "A List of Ultimate and Penultimate Lines of Astrophysical Interest." Useful as it was, this soon proved to be incomplete, not only because it was intentionally limited, but also because more data were becoming available. Work on spectrum analysis was proceeding so rapidly that an extension of his list was imperative.

5. When the writer was at the Mount Wilson Observatory in 1931 she prepared a solar multiplet table for private use in revising and extending the identification of lines in the solar and sun-spot spectra. This manuscript was constantly used by astronomers. In response to requests for copies, the laboratory data for light elements present in early type stars were added and a limited edition was printed in 1933.2 This edition was out of date and out of print almost immediately-the demand for it had not been anticipated. To meet the situation the present book was planned; it is the first book designed from the start as a multiplet table for astrophysicists. For this reason, solar wave-lengths and intensities are excluded. It is essentially a manual of laboratory data needed by astronomers.

6. In the Multiplet Table (Part I) the elements are arranged in order of increasing atomic number. For each spectrum of each element the multiplets are listed in order of increasing energy level (see $\$ 27$ for details), and are numbered for reference. While such an arrangement is useful in studying stellar spectra, it introduces one serious disadvantage. The search for a particular line is laborious. This has been a widespread and an entirely justified criticism of the earlier Table. A Finding List has, therefore, been prepared and forms Part II of this Contribution. Here every line in the Revised Multiplet Table (hereinafter referred to as the R M T), is entered in order of wavelength, listing the spectrum to which the line belongs, and the number of the multiplet containing it.

\section{THE MULTIPLET TABLE-General Considerations}

7. The astronomical spectra forming the basis of selection of the elements, spectra and lines included, fall into several general classes. The sun receives first consideration. The observed solar spectrum now extends from $\lambda 2914 *$ to $\lambda 13495$, which accounts for the range covered in this Table. In addition, the spot and chromospheric spectra, stellar spectra of every type from Wolf-Rayet stars down through M-stars, including giants and dwarfs, spectra of novae and nebulae, and of the corona must be taken into account.

\footnotetext{
${ }^{1}$ Mt. Wilson Contr. No. 286; Ap. J. 61, 223, 1925.

A Multiplet Table of Astrophysical Interest, Princeton 1933.

* Accurate measures have not been made to the violet of $\lambda 2949$.
} 
8. The astrophysical importance of a spectrum depends upon the abundance of the element in the most favorable celestial sources, and the number and excitation potentials of the lines in the visible region. Almost all of the elements of atomic number 1-30 $(H-Z n)$ have, on this account, preference over those that follow. The analyses of their arc spectra are almost all adequate for astrophysical purposes.

For the first spark spectra, which on the whole are more important, the analyses are fairly complete (except for $M n$ II and Co II) The lists for these spectra in the $\mathrm{R} \mathrm{M} \mathrm{T} \mathrm{include} \mathrm{all} \mathrm{but} \mathrm{the} \mathrm{weakest} \mathrm{observed} \mathrm{lines} \mathrm{except} \mathrm{for} \mathrm{a}$ few elements of low abundance.

The second spark spectra are less completely analyzed in the two short periods. In the first long period $F e$ III is complete and only a beginning has been made for any of the rest.

Detailed knowledge of spectra of more highly ionized atoms is confined to a few of the lighter elements.

9. The spectra of the elements from $G a$ to $B a$ are on the average considerably richer, and much less completely analyzed; but these elements are decidedly less abundant and the existing data are usually, though not always, fairly adequate for astrophysical purposes.

10. The rare earths, which are ro rarer than neighboring elements in cosmical abundance, usually have rich spectra, which adds to their significance. The arc spectra rarely appear. The first spark spectra are important in many stars, and lines of the second spark spectra of several of them have recently been identified. ${ }^{1}$ Analysis of the third spectra is barely begun; for the second spectra it is well advanced for six of these elements and well begun for four more.

The lists given here for the rare earths are approximately definitive for $L a$ II, $E u$ II and $L u$ II. It is hoped that greatly improved data for the others will be available in the near future. Extended tables for rare earths are likely to form the larger part of a supplement to the R M T.

11. The elements from $H f$ onward are of low abundance, and the data for them, though incomplete, meet most astrophysical needs tolerably.

\section{BASIS OF SELECTION}

\section{The Short Periods $(H-A)$.}

These spectra are so important in the hotter stars that the lists are entirely or almost complete for all degrees of ionization included, except for a few elements of low cosmic abundance.

The spectra of Wolf-Rayet stars, ${ }^{2}{ }^{3}$ novae and nebulae contain many "predicted" lines of these elements, not yet observed in the laboratory. For many light elements more predicted lines could probably have been included to advantage. More accurate values of predicted wave-lengths could also have been given, particularly in the spark spectra of $C, N$, and $O$. The precedent set by Edlén in his work on $W$ olf-Rayet $^{3}$ stars was followed. In many cases the term separations are known with sufficient accuracy to justify predictions to $0.1 \mathrm{~A}$, although he uses no decimals. Use of the photographic method of reproduction for this book has prevented all but the most necessary alterations of the original manuscript. Changes later realized to be improvements have been omitted because of this restriction.

13. Bowen's " work naturally forms the guide for selecting material related to nebular spectra. The leading nebular lines are due to forbidden lines of the light elements. In anticipation of future needs, the lists of forbidden lines have, however, been greatly extended throughout the first long period.

14. No particular type of stellar spectrum has influenced the choice of lines from the first spark spectra of light elements. The lists have not been restricted to include only those lines known to be present in the stars. The abundance of the element has been the chief factor considered in omitting lines. For elements known to be fairly abundant, favorable predicted lines have been added. The lists are as extensive as the present state of analysis permits.

15. For some years Mr. H. D. Babcock at Mount Wilson, has been preparing for publication a monograph on the Infra-Red Solar Spectrum. His work now covers the interval $\lambda \lambda 6600-13495$ and includes approximately 7300 lines. The leading accessible lines of the arc spectra of most of the light elements lie in this interval. For example, important solar lines are unquestionably due to $H, C_{\mathrm{I}}, N_{\mathrm{I}}, O_{\mathrm{I}}, M g_{\mathrm{I}}, S_{i} \mathrm{I}, P_{\mathrm{I}}$, and $S \mathrm{I}$. In fact, the presence of phosphorus could not be detected until the solar observations were extended to the infra-red. The present Table has been compiled with Mr. Babcock's work especially in mind.

For unblended lines the solar wave-lengths in this region are far more accurate than many laboratory measures. $S i$ affords an excellent illustration. The lines are sharp in the sun and the term separations among solar wave-

\footnotetext{
's Swings, Ap. J. 100, 132, 1944.

Payne, Zeit. fur Ap. 7, 1, 1933.

Edlén, Zeit. fur Ap. 7, 378, 1933.

Reo. Mod. Phys. 8, 55 (No. 2), 1936.
} 
numbers are so consistent that accurate solar term values can be calculated. These term values have been very useful in predicting wave-lengths. Similarly, the triplet and singlet "F" series of $M g$ I were extended with the aid of solar data. ${ }^{1}$ The constancy of the term separations proves beyond doubt the correctness of the identifications.

16. The First Long Period $(K-K r)$.

The elements in the first long period from $K$ through $N i$ constitute by far the major portion of this book (pp. 23-77), on account of the complexity of their spectra. Generally speaking, the arc and first spark spectra are well analyzed except for those mentioned in § 8. Many lines of these spectra (as far as $C u$ ) are present throughout the entire range of the solar and sun-spot spectra, the flash spectrum, stellar spectra like those of $\gamma$ Cygni and a Persei, and later type stars.

The only second spark spectrum in this group that can be given completely is that of $F e$ III. Astronomers eagerly await the definitive analysis of the rest.

17. The spectrum of $F_{e} \mathrm{I}$ deserves special mention. Although the importance of the analysis has long been realized, a complete monograph of this spectrum has only recently been published. ${ }^{2}$ Practically every known line of $F_{\ell} \mathrm{I}$ is present in the sun. An amazing number of predicted lines agree well with solar wave-lengths. A statistical study of these coincidences indicates that most of them are real. For the statistical work the predicted lines were graded as "good," "fair," or "poor." The grades were based on the behavior of all the lines of each multiplet in the solar spectrum, the agreement in wave-length, and other factors. Only the "good" and "fair" lines have been published to date. Since the grading was severe, and since predicted wave-lengths are much in demand, many of the lines graded "poor," but considered useful to other workers, have been retained in the R M T.

18. The Second Long Period $\left(R b-X_{\ell}\right)$.

These elements are observed chiefly in the solar and sun-spot spectra and later dwarf stars. Except for $Y$ II and $\mathrm{Zr}$ II the lists are restricted to the lines from low atomic energy levels. They are, however, more extensive than in the earlier Multiplet Table and slightly longer than are necessary to meet present needs.

19. Forbidden lines are assuming more and more significance in astronomical sources. A special section of the R M T (pp. 100-110) and one of the Finding List (pp. 87-96) are devoted to them. It is extremely difficult to predict what the future needs will be.

To list the array of possible predicted lines even among only abundant elements would be prohibitive. The present selection has been based largely on suggestions made by Dr. P. Swings. He was planning to publish a paper on this subject, but this was unknown to the writer when she was confronted with the problem of including them in the R M T. He generously suggested that they be given here instead of in a separate paper, and has examined the manuscript carefully. The author is extremely fortunate to have had the benefit of his extensive knowledge of both the theoretical and astrophysical aspects of forbidden lines while preparing this section of the Table. Details are discussed later in $\S 44$.

\section{GENERAL ARRANGEMENT OF THE MULTIPLET TABLE}

20. The foregoing remarks serve only as the most general guide to the scope of the material presented here. The book is colored throughout by individual judgment in the editing of spectroscopic literature. A serious attempt has been made to limit it in such a manner that it will be a useful astrophysical handbook. Even so, it is now more than twice the size of the earlier edition.

21. The elements in the $\mathrm{R} M \mathrm{~T}$ are discussed in order of increasing atomic number, and the spectra of each element in order of increasing ionization.

Table 1 gives a convenient arrangement of the Periodic Table of the elements. This Table is self-explanatory. The atomic number and chemical symbol of each element are given and elements with similar spectra in the short and long periods are connected by diagonal lines.

\footnotetext{
1 Russell, Babcock and Moore, Phys. Reo. (2) 46, 826 (No. 9), 1934. Babcock and Moore, Ap. J. 101, $374,1945$.

2 Russell, Moore and Weeks, Trans. Am. Phil. Soc. 34, 111 (Part 2), 1944.
} 


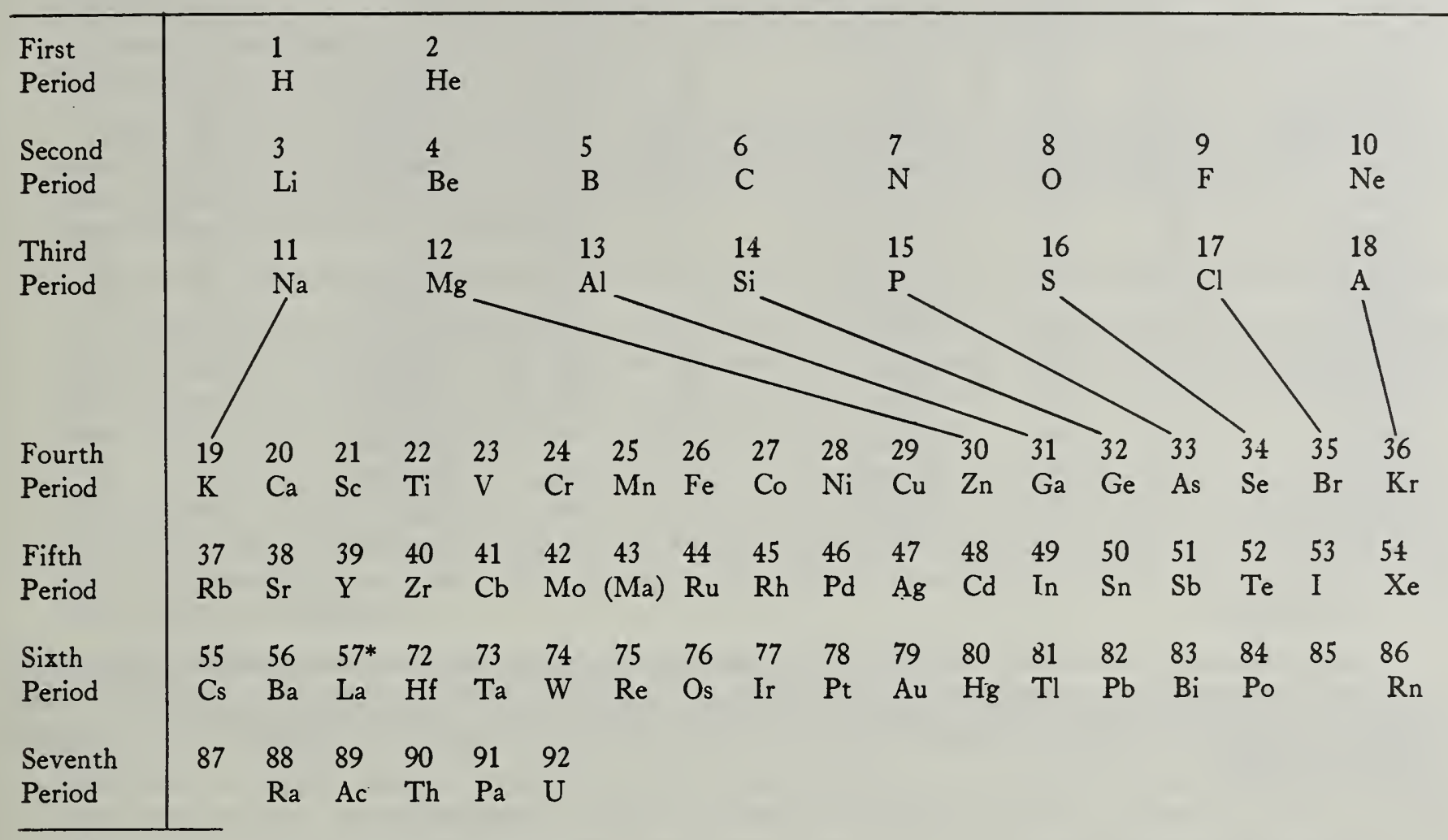

* Atomic numbers 58-71-Rare Earths. See below.

$\begin{array}{lllllllllllllll}\text { Rare } & 58 & 59 & 60 & 61 & 62 & 63 & 64 & 65 & 66 & 67 & 68 & 69 & 70 & 71 \\ \text { Earths } & \mathrm{Ce} & \mathrm{Pr} & \mathrm{Nd} & (\mathrm{Il}) & \mathrm{Sm} & \mathrm{Eu} & \mathrm{Gd} & \mathrm{Tb} & \mathrm{Dy} & \mathrm{Ho} & \mathrm{Er} & \mathrm{Tm} & \mathrm{Yb} & \mathrm{Lu}\end{array}$

In Table 2 the elements are listed in the alphabetical order of their names. The successive columns contain, respectively, the name, the chemical symbol, and the atomic number of each element.

TABLE 2

Alphabetical List of Elements

\begin{tabular}{lcc|lcc|lcc}
\hline \multicolumn{1}{c|}{ Name } & Symbol & $\begin{array}{c}\text { Atomic } \\
\text { No. }\end{array}$ & Name & $\begin{array}{c}\text { Symbol } \\
\text { Atomic } \\
\text { No. }\end{array}$ & Name & $\begin{array}{c}\text { Symbol } \\
\text { Atomic } \\
\text { No. }\end{array}$ \\
\hline Actinium & $\mathrm{Ac}$ & 89 & Chlorine & $\mathrm{Cl}$ & 17 & Holmium & $\mathrm{Ho}$ & 67 \\
Aluminium & $\mathrm{Al}$ & 13 & Chromium & $\mathrm{Cr}$ & 24 & Hydrogen & $\mathrm{H}$ & 1 \\
Antimony & $\mathrm{Sb}$ & 51 & Cobalt & $\mathrm{Co}$ & 27 & (Illinium & $\mathrm{Il}$ & 61 ) \\
Argon & $\mathrm{A}$ & 18 & Columbium & $\mathrm{Cb}$ & 41 & Indium & $\mathrm{In}$ & 49 \\
Arsenic & $\mathrm{As}$ & 33 & Copper & $\mathrm{Cu}$ & 29 & Iodine & $\mathrm{I}$ & 53 \\
Barium & $\mathrm{Ba}$ & 56 & Dysprosium & $\mathrm{Dy}$ & 66 & Iridium & $\mathrm{Ir}$ & 77 \\
Beryllium & $\mathrm{Be}$ & 4 & Erbium & $\mathrm{Er}$ & 68 & Iron & $\mathrm{Fe}$ & 26 \\
Bismuth & $\mathrm{Bi}$ & 83 & Europium & $\mathrm{Eu}$ & 63 & Krypton & $\mathrm{Kr}$ & 36 \\
Boron & $\mathrm{B}$ & 5 & Fluorine & $\mathrm{F}$ & 9 & Lanthanum & $\mathrm{La}$ & 57 \\
Bromine & $\mathrm{Br}$ & 35 & Gadolinium & $\mathrm{Gd}$ & 64 & Lead & $\mathrm{Pb}$ & 82 \\
Cadmium & $\mathrm{Cd}$ & 48 & Gallium & $\mathrm{Ga}$ & 31 & Lithium & $\mathrm{Li}$ & 3 \\
Caesium & $\mathrm{Cs}$ & 55 & Germanium & $\mathrm{Ge}$ & 32 & Lutecium & $\mathrm{Lu}$ & 71 \\
Calcium & $\mathrm{Ca}$ & 20 & Gold & $\mathrm{Au}$ & 79 & Magnesium & $\mathrm{Mg}$ & 12 \\
Carbon & $\mathrm{C}$ & 6 & Hafnium & $\mathrm{Hf}$ & 72 & Manganese & $\mathrm{Mn}$ & 25 \\
Cerium & $\mathrm{Ce}$ & 58 & Helium & $\mathrm{He}$ & 2 & (Masurium & $\mathrm{Ma}$ & $43) \dagger$ \\
& & & & & & & &
\end{tabular}

+ Not isolated.

International Chemical Symbols-1941. 
TABLE 2-Continued

\begin{tabular}{|c|c|c|c|c|c|c|c|c|}
\hline Name & Symbol & $\begin{array}{c}\text { Atomic } \\
\text { No. }\end{array}$ & Name & Symbol & $\begin{array}{c}\text { Atomic } \\
\text { No. }\end{array}$ & Name & Symbol & $\begin{array}{c}\text { Atomic } \\
\text { No. }\end{array}$ \\
\hline Mercury & $\mathrm{Hg}$ & 80 & Radium & $\mathrm{Ra}$ & 88 & Tellurium & $\overline{\mathrm{Te}}$ & 52 \\
\hline Molybdenum & Mo & 42 & Radon & $\mathrm{Rn}$ & 86 & Terbium & $\mathrm{Tb}$ & 65 \\
\hline Neodymium & $\mathrm{Nd}$ & 60 & Rhenium & $\operatorname{Re}$ & 75 & Thallium & $\mathrm{Tl}$ & 81 \\
\hline Neon & $\mathrm{Ne}$ & 10 & Rhodium & $\mathrm{Rh}$ & 45 & Thorium & Th & 90 \\
\hline Nickel & $\mathrm{Ni}$ & 28 & Rubidium & $\mathrm{Rb}$ & 37 & Thulium & $\mathrm{Tm}$ & 69 \\
\hline Nitrogen & $\mathrm{N}$ & 7 & Ruthenium & $\mathrm{Ru}$ & 44 & Tin & Sn & 50 \\
\hline Osmium & Os & 76 & Samarium & $\mathrm{Sm}$ & 62 & Titanium & $\mathrm{Ti}$ & 22 \\
\hline Oxygen & 0 & 8 & Scandium & $\mathrm{Sc}$ & 21 & Tungsten & W & 74 \\
\hline Palladium & $\mathrm{Pd}$ & 46 & Selenium & $\mathrm{Se}$ & 34 & Uranium & $\mathrm{U}$ & 92 \\
\hline Phosphorus & $\mathrm{P}$ & 15 & Silicon & $\mathrm{Si}$ & 14 & Vanadium & V & 23 \\
\hline Platinum & $\mathrm{Pt}$ & 78 & Silver & $\mathrm{Ag}$ & 47 & Xenon & $\mathrm{Xe}$ & 54 \\
\hline Polonium & Po & 84 & Sodium & $\mathrm{Na}$ & 11 & Ytterbium & $\mathrm{Yb}$ & 70 \\
\hline Potassium & $\mathrm{K}$ & 19 & Strontium & $\mathrm{Sr}$ & 38 & Yttrium & $\mathrm{Y}$ & 39 \\
\hline Praseodymium & $\operatorname{Pr}$ & 59 & Sulphur & S & 16 & Zinc & $\mathrm{Zn}$ & 30 \\
\hline Protoactinium & $\mathrm{Pa}$ & 91 & Tantalum & $\mathrm{Ta}$ & 73 & Zirconium & $\mathrm{Z}_{\mathrm{r}}$ & 40 \\
\hline
\end{tabular}

\section{Headings.}

Each spectrum of each element for which multiplets are given, begins with a heading containing four entries: the ionization potential, an astrophysical grade of the analysis, a grade of the list, expressing the fraction of classified lines listed, and finally, the date of completion of the manuscript of that spectrum. For example, $\operatorname{Cr} I$ (p. 37) starts with the heading

\section{Cr I I P 6.74 Anal A List B March 1941.}

\section{The Ionization Potential.}

For arc spectra many of these have been taken from the list published by Meggers in $1941 .^{1}$ For the first sparik spectra he has kindly furnished a similar list ${ }^{2}$ which has been extensively used. The monograph by Edlén ${ }^{3}$ has furnished many more, but the values have been recalculated using the factor 0.00012345 instead of the one he used (see § 35). Edlén's unpublished values are quoted ${ }^{4}$ for $N e$. For many other elements the I P has been obtained from the limits published in the papers on analysis, as for Edlén's results. The list by Boyce ${ }^{5}$ is frequently quoted, particularly in the section dealing with Forbidden Lines. Those interested in the source are advised to consult the part of the bibliography pertaining to analysis (Tables 9 and 10), or one of the above mentioned general lists.

24. The completeness of analysis from the standpoint of the astrophysicist ( $\$ \$ 8-11)$ is indicated by four grades. "Anal A" signifies that practically all the important lines of wave-length $>2950$ are classified, "Anal B" that only a small fraction remain unclassified, "Anal C" that a considerable proportion are unclassified and "Anal D" that the analysis is seriously incomplete.

This rating necessarily involves a large amount of opinion and should not be given too much weight. No two appraisements would agree completely. Its purpose is to indicate the present state of analysis with regard to the needs of the astronomer.

From the-viewpoint of the physicist, the state of the term analysis of the various spectra has been similarly summarized elsewhere by means of grades A, B, C etc. With the aid of Hund's theory the physicist can compare the number of predicted and observed terms and assign a grade accordingly, whether or not most of the leading lines occur in a given region of the spectrum. Both Boyce ${ }^{5}$ and Shenstone ${ }^{6}$ have published extensive surveys.

On account of the different viewpoints, the two grades are often not identical for the same spectrum.

25. A similar grading "List A, B, C, or D" is introduced to indicate the percentage of classified lines of a given spectrum included in the R M T. Since all lines of each spectrum considered are not equally useful to the astrophysicist, the omissions have been much more drastic in some cases than in others. For example, practically every

1 Journ. Opt. Soc. Am. 31, 39 (No. 1), 1941.

2 Unpublished material, April 1941.

'Nova Acta Regice Societatis Scientiarum Upsaliensis (IV) 9, No. 6, 1933.

- Communicated by Swings in a letter, March 1945.

- Reo. Mod. Phys. 13, 1 (No. 1), 1941.

- Reports on Progress in Physics 5, 210, 1939. 
line of $F e \mathrm{r}$ observed in the laboratory is present or accounted for in the solar spectrum. ${ }^{1}$ This applies to weak as well as strong lines. Hence, all classified lines of $F e \mathrm{I}$ to the red of $\lambda 2950$ are entered and the list entry in the heading is "List A". Most of the spark spectra of the first long period are in this class, unless the element is scarce in stars. For many spark spectra most of the observed lines are in the violet and ultra-violet. In such cases the list may be very short, although graded "List A". This means that only a small fraction of the total number of observed classified lines lie in the region considered in this book.

When all but the weakest classified lines are given, the list is graded " $\mathrm{B}$ ". The spectra of $\mathrm{Na}$ I and $M g$ I illustrate "List B", the higher series members having been omitted as unimportant. In anticipation of requests for more material, the general policy has been to include slightly more than is necessary. Since all classified lines are not given, however, the list cannot be graded " $A$ ".

"List C" denotes that most of the strong lines are entered: "List D", that only the leading strong lines are given.

In grading the lists, unclassified lines have not been given consideration (although the stronger ones are listed after the multiplets of a spectrum). The purpose of this grading is to enable the reader to judge how many classified lines have been omitted, regardless of whether or not the analysis is complete. Thus for Co II few lines are classified, but all these are listed. Hence this element is in the class "Anal C, List A."

26. The last entry at the head of each spectrum gives the month and year in which that section of manuscript was completed. This work has extended over such a long period that the date of publication does not apply even approximately to the date at which some spectra were last examined. It is hoped that the lists are up to date, but if important references have been missed, or if existing unpublished material should replace that included here, the writer invites such suggestions.

\section{ARRANGEMENT OF THE MULTIPLETS OF EACH SPECTRUM}

27. Reference must be made to some details of spectrum analysis in order to discuss the plan of presentation adopted here. In brief, the atoms of a gas, when excited by radiation, absorb certain wave-lengths corresponding to transitions of their outer electrons from low er energy levels to higher ones. From differences in the wave numbers of the observed lines, energy levels can be worked out, each line being produced by a transition between two such levels. Related levels are grouped accordingly to well known rules to form spectroscopic terms. Transitions between terms give rise to groups of related lines called multiplets.

In the RMT the terms of each spectrum have been arranged in order of increasing value of the component of lowest energy. This defines the relative level of the term, starting with the lowest term zero. The excitation potentials (columns 4 and 5) express in electron volts the values of the energy levels of those term components involved in the production of each line (see \$35).

To illustrate, the lowest term of $F_{\ell} \mathrm{I}$ is $\mathrm{a}^{5} \mathrm{D}$. This term is made up of five energy levels whose $E$ P's are respectively $0.00,0.05,0.09,0.11$ and 0.12 . The next term is $a^{5} \mathrm{~F}$. Here the components have E P's $0.86,0.91,0.95$, 0.99 and 1.01. For the purposes of this book the terms are considered in order of the lowest level of each, i.e. $\mathrm{a}^{5} \mathrm{D}$ $0.00, a^{5} \mathrm{~F} 0.86$ etc. This is to avoid confusion in spectra whose term values overlap seriously.

In each spectrum all multiplets with the same low term are in one group. The various groups are listed in the order of increasing value of the low terms. Within a group (which represents the combinations of a given low term with higher terms) the multiplets follow the order of increasing high term values.

For example, all combinations from $\mathrm{a}^{5} \mathrm{D}$ of $\mathrm{Fe}_{\mathrm{I}}$ (Multiplets 1-11) form the first group. These multiplets are listed in order of increasing high E P, 2.39, 2.82, 2.93, 3.20 etc. The next low term is ${ }^{5} \mathrm{~F}$. The combinations from this term form the next group (Multiplets 12-31) etc.

In certain multiplets, the lowest components of one or bcth of the terms involved are not represented. This does not alter the arrangement.

Whenever the low level changes, the break in the continuity is indicated by three long dashes between the groups. For $F e I$ the first of these occurs between multiplets 11 and 12.

When terms are widely separated this arrangement results in listing the multiplets from a given low term in the order of decreasing wave-length of the leading line of each multiplet, since increasing energy of the high terms automatically results in increasing wave number, or decreasing wave-length.

The wave-length criterion alone was used for part of the R M T until the overlapping of terms in some complez spectra of the first long period introduced serious complications of arrangement. Then the more rigorous procedure

1 Rusell, Moore and Weeks, Trans. Am. Phil. Soc. 34 (Part 2), 111, 1944. 
described above was adopted. Some spectra had been typed before the strictly orderly listing was put into effect. Owing to the excessive amount of labor involved in making such minor changes, slight irregularities of arrangement have not been corrected. For the greater part of the Table, however, the multiplets are in orderly array.

28. The energy levels that are grouped to form spectroscopic terms are defined by inner quantum numbers, commonly known as J-values. The terms have multiplicities (which are either all odd or all even in a given spectrum), and are further defined by azimuthal quantum numbers $L$ which have the values $1,2,3$ etc. for terms labeled $\mathrm{S}, \mathrm{P}, \mathrm{D}, \mathrm{F}, \mathrm{G}, \mathrm{H}, \mathrm{I}, \mathrm{K}$. The complete multiplet designation of any line includes all of these quantities for both the lower and upper energy level involved in the production of the line.

In the R M T a simplified plan has been adopted. The inner quantum numbers are listed separately from the rest of the designation, in column six, under the heading J. The J-value belonging to the lower term comes first and is followed by that of the higher term. In the next column, headed "Multiplet Desig" the spectroscopic designation of the lower term is always stated first, followed by a dash, then that of the higher term. For example, in multiplet No. 5 of $F e$ I the first line, 3719.935 has J-values $4-5$. The rest of the designation is $a^{5} \mathrm{D}-\mathrm{z}^{5} \mathrm{~F}^{\circ}$. In the complete notation the " 4 " appears as the subscript of $\mathrm{a}^{5} \mathrm{D}$ and 5 as that of $z^{5} \mathrm{~F}^{\circ}$, i.e. $\mathrm{a}^{5} \mathrm{D}_{4}-\mathrm{z}^{5} \mathrm{~F}^{\circ}{ }_{5}$. The complete designation of the second line $\lambda 3737.133$, is $\mathrm{a}^{5} \mathrm{D}_{3}-\mathrm{z}^{5} \mathrm{~F}_{4}^{\circ}$ etc. The " $\mathrm{a}$ " and " $\mathrm{z}$ " merely distinguish these ${ }^{5} \mathrm{D}$ and ${ }^{5} \mathrm{~F}^{\circ}$ terms from others of the same type. This notation is discussed later in $\S 38$. For both terms the superscript 5 denotes the multiplicity.

"Permitted" lines occur among combinations between two sets of terms, one "odd" and the other "even". The supersciipt "o" attached to "F tells that this is the odd term. When both terms belong to the same set (odd or even), the lines are commonly called "Forbidden".

Within a multiplet the arrangement of the individual lines is governed by the J-values. Each multiplet is entered as if it were written in multiplet array, i. e. the lines on the main diagonal come first, then the strongest satellite lines, then the next strongest etc. This is best illustrated by considering the inner quantum numbers, $\mathrm{J}$, of each type of spectroscopic term. For convenience the J-values of all terms from $\mathrm{S}$ through I, of multiplicities 1-11 and 2-10 are given in Table 3, which applies equally to odd or even terms.

Even multiplicites are on the left and odd on the right half of the table. The types of terms (in order of increasing $L$ value) $S, P, D, F, G, H, I$ are in the vertical column on the left.

To arrange any multiplet in standard array, such as Multiplet No. 5 of $F e \mathrm{I}, \mathrm{a}^{5} \mathrm{D}-\mathrm{z}^{5} \mathrm{~F}^{\circ}$, find the J-values of the two types of terms (odd or even) from Table 3. The term ${ }^{5} \mathrm{D}$ is listed under multiplicity 5 , and entry $\mathrm{D}$ on the left (J-values are $4,3,2,1,0)$. The term ${ }^{5} \mathrm{~F}^{\circ}$ has $\mathrm{J}$-values $5,4,3,2,1$. Write these arrays as follows, with the low term horizontally arranged, and the high one vertically arranged:

\begin{tabular}{l|ccccc}
\hline & ${ }^{5} \mathrm{D}_{4}$ & ${ }^{5} \mathrm{D}_{3}$ & ${ }^{5} \mathrm{D}_{2}$ & ${ }^{5} \mathrm{D}_{1}$ & ${ }^{5} \mathrm{D}_{0}$ \\
\hline $\mathrm{z}^{6} \mathrm{~F}^{\circ} \mathrm{D}$ & $\mathrm{x}_{1}$ & & & & \\
\hline${ }^{5} \mathrm{~F}_{5}^{\circ}$ & $\mathrm{y}_{1}$ & $\mathrm{x}_{2}$ & & & \\
${ }^{6} \mathrm{~F}^{\circ}{ }_{5}$ & $\mathrm{z}_{1}$ & $\mathrm{y}_{2}$ & $\mathrm{x}_{8}$ & & \\
${ }^{6} \mathrm{~F}_{8}^{\circ}$ & & $\mathrm{z}_{2}$ & $\mathrm{y}_{3}$ & $\mathrm{x}_{4}$ & \\
${ }^{6} \mathrm{~F}^{\circ}{ }_{2}$ & & & $\mathrm{z}_{3}$ & $\mathrm{y}_{4}$ & $\mathrm{x}_{5}$ \\
${ }^{5} \mathrm{~F}^{\circ}{ }_{1}$ & & & & \\
\hline
\end{tabular}


TABLE 3

J-Values of Spectroscopic Terms

\begin{tabular}{|c|c|c|c|c|c|c|c|c|c|c|c|c|}
\hline \multicolumn{2}{|c|}{ Multiplicity } & 2 & 4 & 6 & 8 & 10 & 1 & 3 & 5 & 7 & 9 & 11 \\
\hline $\mathrm{L}$ & Term & & & & & & & & & & & \\
\hline 1 & S & $1 / 2$ & $11 / 2$ & $21 / 2$ & $31 / 2$ & $41 / 2$ & 0 & 1 & 2 & 3 & 4 & 5 \\
\hline 2 & $P$ & $\begin{array}{r}11 / 2 \\
1 / 2\end{array}$ & $\begin{array}{r}21 / 2 \\
11 / 2 \\
1 / 2\end{array}$ & $\begin{array}{l}31 / 2 \\
21 / 2 \\
11 / 2\end{array}$ & $\begin{array}{l}41 / 2 \\
31 / 2 \\
21 / 2\end{array}$ & $\begin{array}{l}51 / 2 \\
41 / 2 \\
31 / 2\end{array}$ & 1 & $\begin{array}{l}2 \\
1 \\
0\end{array}$ & $\begin{array}{l}3 \\
2 \\
1\end{array}$ & $\begin{array}{l}4 \\
3 \\
2\end{array}$ & $\begin{array}{l}5 \\
4 \\
3\end{array}$ & $\begin{array}{l}6 \\
5 \\
4\end{array}$ \\
\hline 3 & $\mathrm{D}$ & $\begin{array}{l}21 / 2 \\
11 / 2\end{array}$ & $\begin{array}{r}31 / 2 \\
21 / 2 \\
11 / 2 \\
1 / 2\end{array}$ & $\begin{array}{r}41 / 2 \\
31 / 2 \\
21 / 2 \\
11 / 2 \\
1 / 2\end{array}$ & $\begin{array}{l}51 / 2 \\
41 / 2 \\
31 / 2 \\
21 / 2 \\
11 / 2\end{array}$ & $\begin{array}{l}61 / 2 \\
51 / 2 \\
41 / 2 \\
31 / 2 \\
21 / 2\end{array}$ & 2 & $\begin{array}{l}3 \\
2 \\
1\end{array}$ & $\begin{array}{l}4 \\
3 \\
2 \\
1 \\
0\end{array}$ & $\begin{array}{l}5 \\
4 \\
3 \\
2 \\
1\end{array}$ & $\begin{array}{l}6 \\
5 \\
4 \\
3 \\
2\end{array}$ & $\begin{array}{l}7 \\
6 \\
5 \\
4 \\
3\end{array}$ \\
\hline 4 & F & $\begin{array}{l}31 / 2 \\
21 / 2\end{array}$ & $\begin{array}{l}41 / 2 \\
31 / 2 \\
21 / 2 \\
11 / 2\end{array}$ & $\begin{array}{r}51 / 2 \\
41 / 2 \\
31 / 2 \\
21 / 2 \\
11 / 2 \\
1 / 2\end{array}$ & $\begin{array}{c}61 / 2 \\
51 / 2 \\
41 / 2 \\
31 / 2 \\
21 / 2 \\
11 / 2 \\
1 / 2\end{array}$ & $\begin{array}{l}71 / 2 \\
61 / 2 \\
51 / 2 \\
41 / 2 \\
31 / 2 \\
21 / 2 \\
11 / 2\end{array}$ & 3 & $\begin{array}{l}4 \\
3 \\
2\end{array}$ & $\begin{array}{l}5 \\
4 \\
3 \\
2 \\
1\end{array}$ & $\begin{array}{l}6 \\
5 \\
4 \\
3 \\
2 \\
1 \\
0\end{array}$ & $\begin{array}{l}7 \\
6 \\
5 \\
4 \\
3 \\
2 \\
1\end{array}$ & $\begin{array}{l}8 \\
7 \\
6 \\
5 \\
4 \\
3 \\
2\end{array}$ \\
\hline 5 & G & $\begin{array}{l}41 / 2 \\
31 / 2\end{array}$ & $\begin{array}{l}51 / 2 \\
41 / 2 \\
31 / 2 \\
21 / 2\end{array}$ & $\begin{array}{l}61 / 2 \\
51 / 2 \\
41 / 2 \\
31 / 2 \\
21 / 2 \\
11 / 2\end{array}$ & $\begin{array}{r}71 / 2 \\
61 / 2 \\
51 / 2 \\
41 / 2 \\
31 / 2 \\
21 / 2 \\
11 / 2 \\
11 / 2\end{array}$ & $\begin{array}{r}81 / 2 \\
71 / 2 \\
61 / 2 \\
51 / 2 \\
41 / 2 \\
31 / 2 \\
21 / 2 \\
11 / 2 \\
1 / 2\end{array}$ & 4 & $\begin{array}{l}5 \\
4 \\
3\end{array}$ & $\begin{array}{l}6 \\
5 \\
4 \\
3 \\
2\end{array}$ & $\begin{array}{l}7 \\
6 \\
5 \\
4 \\
3 \\
2 \\
1\end{array}$ & $\begin{array}{l}8 \\
7 \\
6 \\
5 \\
4 \\
3 \\
2 \\
1 \\
0\end{array}$ & $\begin{array}{l}9 \\
8 \\
7 \\
6 \\
5 \\
4 \\
3 \\
2 \\
1\end{array}$ \\
\hline 6 & $\mathrm{H}$ & $\begin{array}{l}51 / 2 \\
41 / 2\end{array}$ & $\begin{array}{l}61 / 2 \\
51 / 2 \\
41 / 2 \\
31 / 2\end{array}$ & $\begin{array}{l}71 / 2 \\
61 / 2 \\
51 / 2 \\
41 / 2 \\
31 / 2 \\
21 / 2\end{array}$ & $\begin{array}{l}81 / 2 \\
71 / 2 \\
61 / 2 \\
51 / 2 \\
41 / 2 \\
31 / 2 \\
21 / 2 \\
11 / 2\end{array}$ & $\begin{array}{l}91 / 2 \\
81 / 2 \\
71 / 2 \\
61 / 2 \\
51 / 2 \\
41 / 2 \\
31 / 2 \\
21 / 2 \\
11 / 2 \\
1 / 2\end{array}$ & 5 & $\begin{array}{l}6 \\
5 \\
4\end{array}$ & $\begin{array}{l}7 \\
6 \\
5 \\
4 \\
3\end{array}$ & $\begin{array}{l}8 \\
7 \\
6 \\
5 \\
4 \\
3 \\
2\end{array}$ & $\begin{array}{l}9 \\
8 \\
7 \\
6 \\
5 \\
4 \\
3 \\
2 \\
1\end{array}$ & $\begin{array}{r}10 \\
9 \\
8 \\
7 \\
6 \\
5 \\
4 \\
3 \\
2 \\
1 \\
0\end{array}$ \\
\hline 7 & I & $\begin{array}{l}61 / 2 \\
51 / 2\end{array}$ & $\begin{array}{l}71 / 2 \\
61 / 2 \\
51 / 2 \\
41 / 2\end{array}$ & $\begin{array}{l}81 / 2 \\
71 / 2 \\
61 / 2 \\
51 / 2 \\
41 / 2 \\
31 / 2\end{array}$ & $\begin{array}{l}91 / 2 \\
81 / 2 \\
71 / 2 \\
61 / 2 \\
51 / 2 \\
41 / 2 \\
31 / 2 \\
21 / 2\end{array}$ & $\begin{array}{c}101 / 2 \\
91 / 2 \\
81 / 2 \\
71 / 2 \\
61 / 2 \\
51 / 2 \\
41 / 2 \\
31 / 2 \\
21 / 2 \\
11 / 2\end{array}$ & 6 & $\begin{array}{l}7 \\
6 \\
5\end{array}$ & $\begin{array}{l}8 \\
7 \\
6 \\
5 \\
4\end{array}$ & $\begin{array}{l}9 \\
8 \\
7 \\
6 \\
5 \\
4 \\
3\end{array}$ & $\begin{array}{r}10 \\
9 \\
8 \\
7 \\
6 \\
5 \\
4 \\
3 \\
2\end{array}$ & $\begin{array}{r}11 \\
10 \\
9 \\
8 \\
7 \\
6 \\
5 \\
4 \\
3 \\
2 \\
1\end{array}$ \\
\hline
\end{tabular}


Only those combinations between the low and high terms, for which $J$ changes by $O$ or \pm 1 are "permitted". This rule restricts the number of lines to be expectea to those denoted by $\mathbf{x}, \mathbf{y}$, and $\mathbf{z}$, where the subscripts $1,2,3$ represent decreasing J-values. The main diagonal lines are $x_{1}-x_{6}$. The first satellites are $y_{1}-y_{4}$ and the second satellites, $\mathbf{z}_{1}-\mathbf{z}_{3}$.

In the R M T, the lines on the main diagonal are listed first, in order of decreasing J-values. From the example, the first lines entered are those in the positions $\mathbf{x}_{1}, \mathbf{x}_{2}, \mathbf{x}_{3}, \mathbf{x}_{4}, \mathbf{x}_{5}$ in the Multiplet. The line at $\mathbf{x}_{1}$ has the designation $a^{5} D_{4}-z^{5} F_{5}^{\circ}$, at $x_{2} a^{5} D_{3}-z^{5} F_{4}^{\circ}$ etc. In the $R M T$ the lines of this multiplet appear in the following order:'

\begin{tabular}{|c|c|c|c|c|}
\hline & $\lambda$ & $\mathrm{J}$ & Desig & \\
\hline$x_{1}$ & 3719.935 & $4-5$ & $\left.a^{5} D-z^{5} F^{\circ}\right)$ & \\
\hline$x_{2}$ & 3737.133 & $3-4$ & “ & \\
\hline$x_{3}$ & 3745.561 & $2-3$ & “6 & Main Diagonal \\
\hline$x_{4}$ & 3748.264 & $1-2$ & "6 & \\
\hline$x_{5}$ & 3745.901 & $0-1$ & " & \\
\hline$y_{1}$ & 3679.915 & 4-4 & "6 & \\
\hline$y_{2}$ & 3705.567 & $3-3$ & “6 & First Satellites \\
\hline$y_{3}$ & 3722.564 & $2-2$ & "6 & \\
\hline$y_{4}$ & 3733.319 & $1-1$ & “" & \\
\hline${ }^{4}$ & 3649.304 & $4-3$ & 66 & \\
\hline $\mathrm{z}_{2}$ & 3683.054 & $3-2$ & " & Second Satellites \\
\hline 3 & 3707.828 & $2-1$ & “ & \\
\hline
\end{tabular}

An example of a symmetrical multiplet should also be given. Multiplet No. 12 of $C_{r}$ II (p. 43) has the designation $a^{4} \mathrm{P}-\mathrm{z}^{4} \mathrm{P}^{\circ}$. Since the multiplicity (4) and type of term $(\mathrm{P})$ are identical for both terms, the J-values are also identical. From Table 3 the J-values for a ${ }^{4} \mathrm{P}$ term are $21 / 2,11 / 2,1 / 2$.

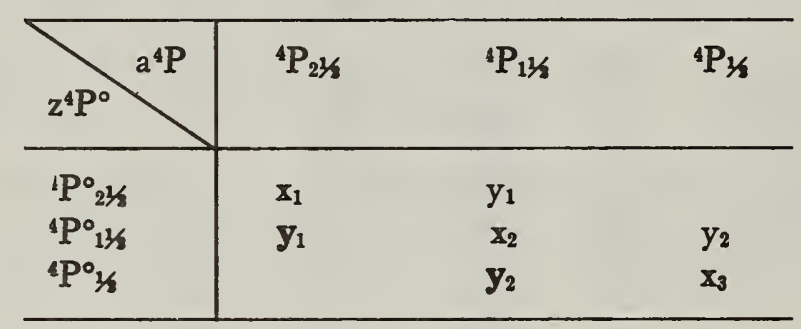

Here both sets of satellites involve the same J-values, $1 \frac{1}{2}-21 / 2,1 / 2-11 / 2$ and $21 / 2-11 / 2,11 / 2-1 / 2$. Throughout the $\mathrm{R}$ M T for such cases, combinations in which J-values read from larger $\mathrm{J}$ to smaller $\mathrm{J}$ are entered first. Here, for example the pair $21 / 2-11 / 2,11 / 2-1 / 2\left(y_{1}\right.$ and $y_{2}$ in bold face type above), precede the pair with $J$-values $11 / 2-21 / 2,1 / 2-11 / 2$ respectively.

According to elementary theory the leading line of the principal diagonal is the strongest in the multiplet, and first satellites are stronger than the second, while the two sets of satellites in a symmetrical multiplet are comparable. ${ }^{1}$

In the majority of spectra intersystem combinations occur, i.e. those in which the multiplicities of the terms differ by 2 or even 4 , as for example multiplets 1 and 332 of $F e \mathrm{I}, \mathrm{a}^{5} \mathrm{D}-\mathrm{z}^{7} \mathrm{D}^{\circ}$ and $\mathrm{z}^{7} \mathrm{~F}^{\circ}-\mathrm{e}^{3} \mathrm{G}$ respectively. These multiplets often include strong lines, particularly for the heavier elements. The rule $\Delta J=0$ or \pm 1 is strictly observed but there are no known formulae for the prediction of intensities, which are often apparently erratic. When intersystem lines are strong, intensities in regular multiplets often deviate from the formulae. The intersystem multiplets are arranged in the R M T on the "diagonal" basis described above, so far as irregularities permit.

29. For all types of multiplets the reader must bear in mind that the arrays described above, and the J-values in Table 3 give all the possible permitted theoretical transitions.

In many cases the $\mathrm{R} \mathrm{M} \mathrm{T} \mathrm{does} \mathrm{not} \mathrm{give} \mathrm{theoretically} \mathrm{complete} \mathrm{multiplets.} \mathrm{Reasons} \mathrm{for} \mathrm{this} \mathrm{are:}$

1. When the strongest. lines of a multiplet are likely to be very weak in astrophysical sources, the weaker ones have been deliberately omitted even though they may have been observed in the laboratory. Omissions are indicated by a " $f$ " following the "Multiplet Designation".

2. Individual lines in a multiplet are sometimes much fainter than theoretically expected and have never been observed. Sequences along the diagonals are thus broken. For such lines predicted positions are given only when it is believed that they may be observable astrophysically.

- For details see Russell, Mt. Wilson Contr. No. 537; Ap. J. 83, 129, 1936. 
3. In some cases one or more components of a spectroscopic term have not yet been indentified.

Such cases may be detected by comparing the column headed J for a given multiplet with the theoretical array of permitted lines, just described.

30. For the more important spectra, limited lists of the leading unclassified lines follow the multiplets. The $\mathrm{R} \mathrm{M} \mathrm{T} \mathrm{is} \mathrm{not} \mathrm{designed} \mathrm{as} \mathrm{a} \mathrm{source} \mathrm{for} \mathrm{the} \mathrm{investigator} \mathrm{who} \mathrm{is} \mathrm{interested} \mathrm{primarily} \mathrm{in} \mathrm{unclassified} \mathrm{lines.}$

Three general factors have controlled the selection: the abundance of the element in astronomical sources, the grade of the analysis and the accuracy of the laboratory material. Under "Anal A" more lines will be unclassified for a complex spectrum like $F \ell$ I than for a simpler spectrum, but the percentage of strong lines will be small. Under "Anal B" there will be more and stronger unclassified lines than if the grade were A, etc.

If A.S. King has made a temperature classification of the spectrum the leading unclassified lines can be readily chosen from his lists. In such cases his temperature class follows the intensity in the $\mathrm{R} \mathrm{M} \mathrm{T}$

Among arc spectra the lists of unclassified lines are longest for $N$ I, $T i$ I, $C r$ I, $M n$ I, $F e$ I, $N i$ I, $T m$ I. Only a few lines are listed for $S i$ I, $S$ I, $C a$ I, $S c$ I, $V$ I, $C_{0}$ I, $Y$ I, $L u$ I, and none for any other elements.

For first spark spectra the lists of unclassified lines may be summarized as follows:

Limited

$$
\text { Si II, } A \text { II, } F e \text { II, } C b \text { II, } L a \text { II, } N d \text { II, } S m \text { II, } G d \text { II, } T m \text { II. }
$$

Very limited

$$
O \text { II, } S \text { II, } C l \text { II, } T i \text { II, } V \text { II, } C_{r} \text { II, } Y \text { II, } Z r \text { II, } C e \text { II, } P r \text { II, } E u \text { II, } H f \text { II. }
$$

Measures inadequate $M n$ II, $C_{0}$ II, $N i$ II.

Lists are given for only five "third" spectra: $C$ III (where a dubious classification has been suggested for the lines) $N$ III, $S i$ III, $S$ III and $F e$ III. The only one of any length is that of $F_{e}$ III.

For some spark spectra, notably $L a$. II and $F e$ III it is not certain that the separation of the lines in different states of ionization is definitive. This is mentioned in the $\mathrm{R} \mathrm{M} \mathrm{T} \mathrm{under} \mathrm{these} \mathrm{spectra.} \mathrm{For} \mathrm{many} \mathrm{spectra} \mathrm{the}$ separation is so uncertain that no unclassified lines have been included. For no spectrum is a complete list given.

31. A few notes are appennded to the list of multiplets for certain spectra. These fall into two classes: those dealing with notation (see \$37), and those dealing with fine structure. This book does not discuss fine structure or isotope effects in any detail. Those spectra in which the fine structure or isotope effects should be called especially to the attention of the astrophysicist have this fact mentioned, as follows:

$$
\begin{array}{ll}
L i \text { II } & \text { Very wide fine structure } \\
M g \text { I, } A l \text { II } & \text { Fine structure } \\
H g \text { I, } T l_{\text {I }} & \text { Many lines show fine structure } \\
H e \text { II, } B i_{\text {I }} & \text { Wide fine structure. }
\end{array}
$$

\section{COLUMNS OF THE TABLE OF MULTIPLETS}

32. Wave-length.

The data for each spectrum are given in seven columns. The first contains the laboratory wave-length in I A units. In the earlier edition solar wave-lengths were listed for all but the lighter elements (see $\$ 5$ ). Since any solar line may be a blend, it was decided to replace this entry by laboratory material. An effort has been made to select the best available wave-length for each line. The individual lists are far from homogeneous-there is often an enormous range in accuracy among the lines of a given spectrum.

The reference from which each line has been taken is indicated in column two, by the letters A, B, C etc. Table 7 (p. zxiv), contains the number of the reference in the bibliography to which the letters refer. The letter " $P$ " in this column denotes throughout that the wave-length is predicted from the laboratory term values, which may be found in the references to analysis, Table 9 (p. xxvii).

The order of the letters represents roughly the estimated precision of the measures, but this must not be interpreted too literally because some investigators have measured only limited regions of a spectrum. Consequently, several accurate sources may be used within a multiplet. Furthermore, the letters A, B, etc. denote very different degrees of accuracy for-different spectra. While the earlier letters of the alphabet are the more favored choices, it would be erroneous to conclude that the letters are arranged strictly according to the writer's rating of the accuracy of the measures. 


\section{Symbols in the R M T.}

Since most of the symbols occur in column one, they will all be described here.

Wave-length column:

// This symbol follows the wave-length of the "Raie Ultime" as determined from observations in the laboratory. High transition probabilities as well as low energy levels are involved, and they do not always come from the lowest levels. When the known Raies Ulitmes are to the violet of $\lambda 2950$ they are not included in the R M T. With the exception of $\mathrm{Eu} \mathrm{II}^{1}$ they are ali quoted from Meggers ${ }^{2}$, who generously furnished the data on first spark spectra ${ }^{3}$ in advance of publication.

* An asterisk precedes the wave-length throughout the $\mathrm{R} \mathrm{M} \mathrm{T} \mathrm{to} \mathrm{denote} \mathrm{a} \mathrm{blend.} \mathrm{If} \mathrm{no} \mathrm{symbol} \mathrm{follows} \mathrm{the}$ wave-length, the line is blended with another line in the same spectrum. For example, the line *2970.106 appears in multiplets 10 and 11 of $F e \mathrm{I}$.

An “*” is also used to denote blended intensities. For symbols in the intensity column see $\$ 34 \mathrm{G}$.

$\$$ This symbol follows the wave-length (an “*”" always preceding) to indicate a blend of an arc and spark line; or of two spark lines of the same element in spectra of different degrees of ionization. When this pair of symbols appears with an arc line it denotes that the arc line is blended with a line in the first spark spectrum of the element. Similarly, if it appears with a line in a first spark spectrum, the spark line is blended with an arc line of that element. Examples:

Fe I Multiplet No. $28 * 3116.6338-B l e n d$ of $F_{e}$ I and $F_{e}$ II.

$C_{r}$ II Multiplet No. $4 \quad * 3349.34 \$-B l e n d$ of $C_{r}$ II and $C_{r}$ I.

All uses of pairs of symbols not covered by the general cases of blends of arc lines with those in the first spark spectra or vice-versa, as described above, are summarized in Table 4, where another pair of symbols "* and $8 \delta^{\prime}$ " is also introduced.

TABLE 4

Symbols Denoting Blends-Special Cases

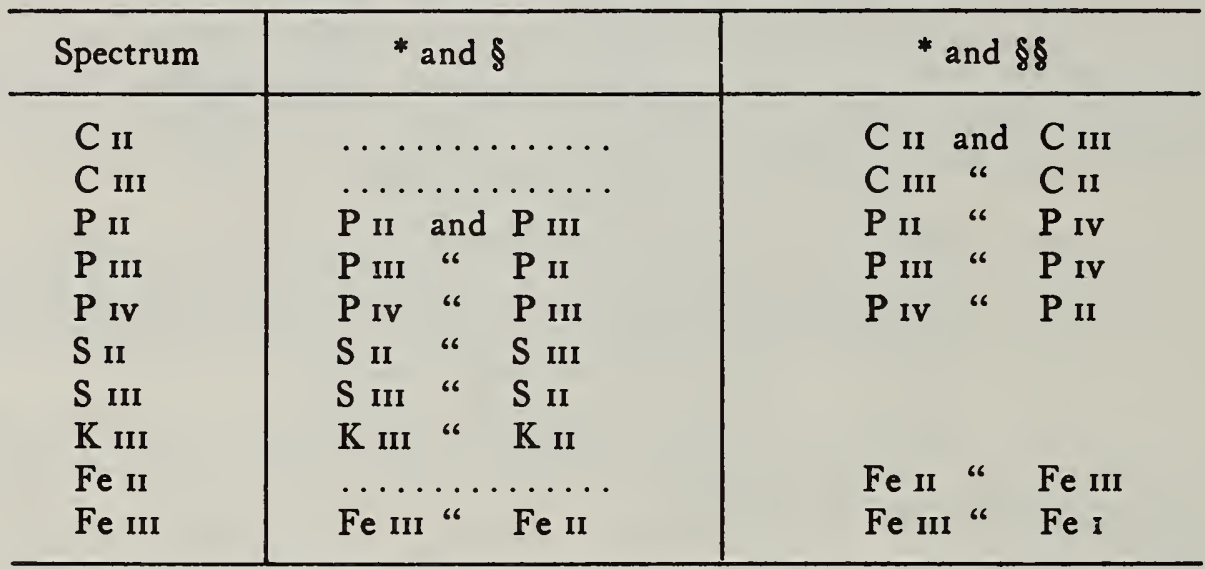

Column one of Table 4 indicates the spectrum in which the symbols are found. Columns two and three of the Table contain the pairs of symbols used and the meaning of each. For example:

$C$ II Multiplet No. $45{ }^{*} 4368.1488-B l e n d$ of $C$ II and $C$ iII.

$S$ II Multiplet No. $50 * 3860.648-$ Blend of $S$ II and $S$ inr.

The symbols mentioned above apply to blended lines which have come to the attention of the writer, but doubtless many more blends exist than are thus noted. A careful examination of the Finding List should reveal any important blends, but this list was prepared after the $\mathrm{R} \mathrm{M} \mathrm{T} \mathrm{was} \mathrm{typed,} \mathrm{and} \mathrm{consequently} \mathrm{could} \mathrm{not} \mathrm{be} \mathrm{used} \mathrm{to}$ check the thoroughness with which the blended lines are marked.

$\mathrm{m}$ An " $\mathrm{m}$ " preceding the wave-length indicates that the line is masked (see $\$ 34 \mathrm{~F}$ ).

$\dagger$ This symbol follows the Multiplet Designation to call attention to the fact that not all the lines observed in the multiplet are listed in the $\mathrm{R} \mathrm{M} \mathrm{T.} \mathrm{The} \mathrm{violet} \mathrm{limit} \lambda 2950$ explains the omission of some strong lines. Most of the omitted lines are too faint to be of astrophysical importance.

1 Russell, Unpublished material.

Meggers, Journ. Opt. Soc. Am. 31, 39 (No. 1), 1941.

April 1941. 


\section{Intensity.}

Column three contains the estimated laboratory intensity. It has been included only because of persistent requests. The intensities must be used with great caution not only because of the glaring lack of homogeneity in the estimates, but also on account of the difference in the intensity scales used by various investigators.

For each spectrum the writer has tried to adopt the best existing set of estimates made by a good observer who has covered a long range of wave-length. In the red, the intensities by Meggers and Kiess have been the first choice. In the visible, the arc intensities by A. S. King are given for arc spectra and for first spark spectra of the rare earths. For other spark spectra, spark intensities have been used.

When the intensities of the lines of a spectrum are taken from one or two main references they are not given in parentheses. If only a few intensities are from one source, or if the listed ones are probably on a very different scale from the majority used for a spectrum, parentheses are used. In general, the parentheses denote that the intensity is not the first or second choice. Table 8, Page zxvi, gives the references from which the intensities have been taken. The reader is warned not to assume that the wave-length and intensity come from the same reference. This may be the case, but frequently it is not.

The intensity column contans several types of notes discussed below under entries $A$ to $G$ :

A The letters used to describe the intensities are as follows:

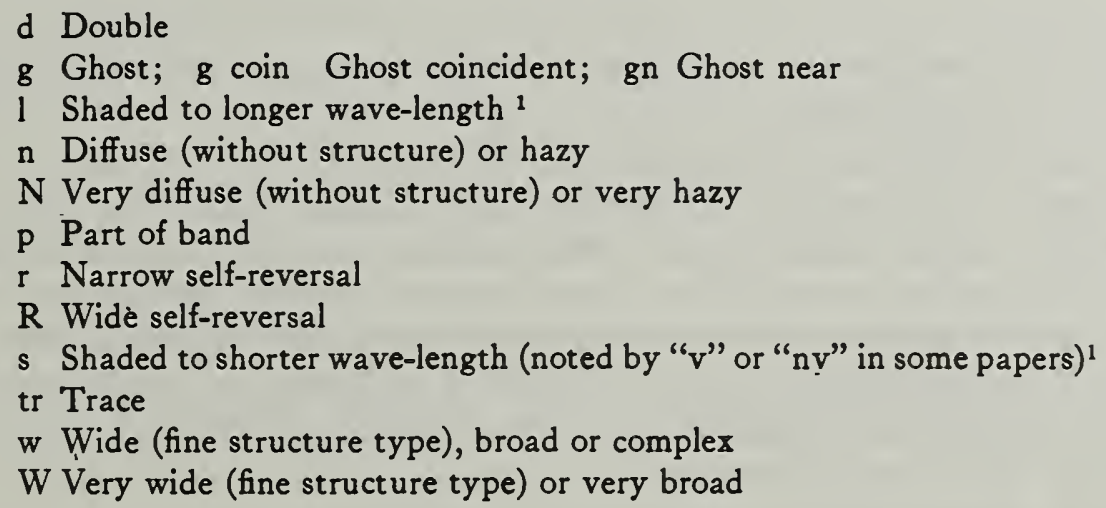

B The intensity column is often blank for predicted lines because most of them have not been observed in the laboratory. If the predicted position is assumed to be more accurate than the measured one, the laboratory intensity is given with a predicted wave-length.

$\mathrm{C}$ A dash indicates that the line is so faint that no laboratory intensity has been assigned, except for $H, D$ and He II, where no intensities are listed.

$D$ "Forb" indicates that the line is forbidden but has been observed in the laboratory. Lines due to Stark Effect are thus marked.

E Familiar "names" of selected lines are included:

Series Names:

$$
\begin{aligned}
& \mathrm{H} \quad \mathrm{Ha}, \mathrm{H} \beta \text { etc. } \\
& \text { D } \mathrm{D} a, \mathrm{D} \beta \text { “ }
\end{aligned}
$$

Fraunhofer Names:

$$
\begin{array}{ll}
\mathrm{He}_{\mathrm{I}} & \mathrm{D}_{8} \\
\mathrm{Na} & \mathrm{D}_{1} \text { and } \mathrm{D}_{2} \\
\mathrm{C} \mathrm{I}_{\text {II }} & \mathrm{H} \text { and } \mathrm{K}
\end{array}
$$

F When an important line is masked, " $m$ " precedes the wave-length, the predicted position of the masked line is given, and the spectrum to which the masking line belongs, is noted by the chemical symbol in the intensity column. The Roman numerals have been omitted except for masked lines of $F e$ iII. If for example " $F e$ " or " $T i$ " appears in the intensity column, the line in question is masked by $F_{\ell} \mathrm{I}$ or $T i_{i}$ t the "I" being omitted because of the limited space in this column. Similarly, a " + " is mostly" used for first spark spectra in place of "II," although the conventional use of Roman numerals is fully recognized.

\footnotetext{
${ }^{1}$ Recommended by the International Astronomical Union-Trans. Intern. Astr. Union 6, 100, 1938.
} 
G Symbols in the intensity column:

* Blended Intensity

$\odot$ Predicted line of $F e$ I present in the solar spectrum; $\odot$ ? denotes that the solar identification as $F e \mathrm{I}$ is subject to some question.

35. Columns four and five give the low and high excitation potentials (E P) of the levels involved in the production of the line (see $\$ 27$ ). Some E P's are given in parentheses in the R M T to denote that they are not accurately known.

In analyzing a spectrum it often happens that two or more sets of terms of different multiplicity exist that are unconnected, since no intersystem combinations have beèn detected. If. long series, whose correctness is unquestionable, can be found, the limits furnish a farrly accurate determination of the relative positions of the different types of terms. This is the case for $B e \mathrm{I}, B \mathrm{II}, C \mathrm{II}, O \mathrm{III}$, and $O \mathrm{Iv}$, but no symbol has been introduced to indicate that intersystem combinations have not been observed.

The limits are less accurate for $N$ II, $N$ IV, $O$ II, $O$ v and $F$ II. The E P's affected by this are in parentheses.

For $C_{l}$ II, two sets of terms are well known but they are unconnected. For this reason the lines are listed in two Groups, I and II. Within each group the relative values of the E P's are correct, but the terms in Group I are believed to be lower than those in Group II by about 0.6 volt. ${ }^{1}$ In Group II all values are, therefore, enclosed in parentheses.

For $C e$ III parentheses are used because the lowest level may not have been found.

For $S i$ II $P$ III and $M n$ II some terms are established by their internal separations, but are entirely unconnected with the rest of the terms. Here the $\mathrm{E} P$ columns contain question marks.

In the earlier Multiplet Table all E P's were obtained by multiplying the term values in $\mathrm{cm}^{-1}$ by the factor 0.00012345 . An improved value of this factor, 0.00012336 , was published by Birge ${ }^{2}$ in 1929 . Since then he has announced that 0.00012395 is more nearly correct ${ }^{3}$. This last change deserves serious consideration-it involves a change in " $e$ ", the charge on the electron, which will doubtless be carefully checked experimentally in the near future. The change is surprisingly large and affects many calculations of an astrophysical nature.

Although it is wrong, in principle, to perpetuate the use of an incorrect value of a fundamental physical constant, the old value 0.00012345 has been used throughout the R M T in calculating the E P's (and I P's for which the limits were known. See $\$ 23$ ). The reason is threefold:

1. The errors in stellar temperatures and other quantities based on observational data far exceed those introduced by the change in this factor.

2. Until a definitive value of the constant is available it has seemed an unjustifiable expenditure of time and money to revise the extensive calculations, many of which had already been done with the oldest value.

3. The change in the value of " $\mathrm{e}$ " enters into so many calculations, that to recalculate the E P's and I P's is far from sufficient. As soon as the new value is confirmed without likelihood of further change, it should be used in all calculations of astrophysical importance.

The last two columns contain J-values and Multiplet Designations. These have already been discussed in $\$ 28$, but a few comments'are in order. When levels of a term are so close that they are unresolved, all the J-values for the term should be listed. This is impossible because of limited space, and consequently the column headed $\mathrm{J}$ is frequently blank or has the J-value of only one level entered.

The multiplet numbers which appear in parentheses under the Multiplet Designation are reference numbers to be used in locating any line. (See \$6). In each spectrum the numbers start with "1." All lines in a multiplet have the same multiplet number. These numbers are entered in the Finding List.

\section{SPECTROSCOPIC NOTATION}

The notation used in the column headed "Multiplet Designation" differs for spectra which contain conspicuous series and for the complex spectra which do not.

\section{A. Series Spectra}

36. For many elements the spectra become more complex as the degree of ionization decreases. The terms of each spectrum are the parent terms or "limits" of the terms in the spectrum of the next lower degree of ionization. The addition of $s, \mathrm{p}, \mathrm{d}, \mathrm{f}$, etc. electrons to each limit produces arrays of terms accurately predictable from theory.

1 Harrison, Albertson and Hosford, Journ. Opt. Soc. Am. 31, 439 (No. 6), 1941.

2 Phys. Reo. Suppl. 1, 62 (No. 1), 1929.
'Rev. Mod. Phys. 13, 237 (No. 4), 1941;. Reports on Progress in Physics 8, 131, 1941. 
The simplest case is illustrated by $O$ vi. Here the lowest term of $O$ vir, $1 \mathrm{~s}^{2}{ }^{1} \mathrm{~S}$, is so much lower than any other that no other limit need be considered. The addition of a "running" s, p, d, f . . electron to this state produces series of doublet $\mathrm{S}, \mathrm{P}^{\circ}, \mathrm{D}, \mathrm{F}^{\circ} \ldots$ terms in $O$ vi. In this case the electron and the terms are of the same type. For example, the ground term of $O \mathrm{vI}$ is $1 \mathrm{~s}^{2} 2 \mathrm{~s}{ }^{2} \mathrm{~S}$, and the next term $1 \mathrm{~s}^{2} 2 \mathrm{p}^{2} \mathrm{P}^{\circ}$. The term type and total quantum number of the running electron suffice to define the configuration. In the $\mathrm{R} \mathrm{M} \mathrm{T} \mathrm{the} \mathrm{notation}{ }^{2} \mathrm{~S}, 2^{2} \mathrm{P}^{\circ}$ etc. is used in spectra of this type. To illustrate, Multiplet No. 1 of $L i$ I has the designation ${ }^{2}{ }^{2} \mathrm{~S}-2{ }^{2} \mathrm{P} \circ$. (Other features of the notation are discussed in $\$ 28$ and in Table 3).

The case of $O \mathrm{v}$ is more complicated because $2^{2} \mathrm{P}^{\circ}$ of $O \mathrm{vI}$ is not much higher than ${ }^{2} \mathrm{~S}$ and terms from both limits are important. The addition of a running electron to these limits gives the following terms:

\begin{tabular}{|c|c|c|c|c|c|c|c|c|c|c|c|}
\hline $\mathrm{O} \mathrm{vI}$ & Limit & $1 s^{2} 2 s$ & & & $1 s^{2} 2 p$ & & & & & & \\
\hline \multirow[t]{4}{*}{$\mathrm{Ov}$} & $\begin{array}{c}\text { Added } \\
\text { Electron }\end{array}$ & Config & \multicolumn{2}{|c|}{ Terms } & Config & \multicolumn{3}{|c|}{ Terms } & & & \\
\hline & $3 \mathrm{~s}$ & $1 s^{2} 2 s^{2} s$ & ${ }^{1} \mathrm{~S}$ & ${ }^{3} S$ & $1 s^{2} 2 p 3 s$ & ${ }^{1} \mathrm{P}^{\circ}$ & & & ${ }^{3} \mathrm{P}^{\circ}$ & & \\
\hline & $3 p$ & $1 s^{2} 2 s^{3} p$ & ${ }^{\prime} \mathrm{P}^{\circ}$ & ${ }^{8} \mathrm{P}^{\circ}$ & $1 s^{2} 2 p^{3} p$ & ${ }^{1} \mathrm{~S}$ & ${ }^{1} \mathrm{P}$ & ${ }^{1} \mathrm{D}$ & ${ }^{3} \mathrm{~S}$ & ${ }^{3} \mathrm{P}$ & ${ }^{3} \mathrm{D}$ \\
\hline & $3 d$ & $1 \mathrm{~s}^{2} 2 \mathrm{~s}^{2} \mathrm{~d}$ & ${ }^{1} \mathrm{D}$ & ${ }^{3} \mathrm{D}$ & $1 s^{2} 2 p^{3 d}$ & ${ }^{1} \mathrm{P}^{\circ}$ & ${ }^{1} \mathrm{D}^{\circ}$ & ${ }^{1} \mathrm{~F}^{\circ}$, & ${ }^{3} \mathrm{P}^{\circ}$ & ${ }^{3} \mathrm{D}^{\circ}$ & ${ }^{3} \mathrm{~F}^{\circ}$ \\
\hline
\end{tabular}

The configuration is $1 s^{2} 2 s^{2}$ gives only ${ }^{1} S$; and $1 s^{2} 2 p^{2}$ only ${ }^{1} S^{1} D{ }^{3} P$.

It appears from this array that if the terms having the limit $2^{2} \mathrm{~S}$ in $O^{\prime}$ vi are labeled ${ }^{1} \mathrm{~S}, 3^{3} \mathrm{~S}, 3^{1} \mathrm{P}^{\circ}, 3^{3} \mathrm{P}^{\circ}, 3^{1} \mathrm{D}, 3^{3} \mathrm{D}$, and those from $2^{2} \mathrm{P}^{\circ}$ are labeled $3 \mathrm{~s}^{1} \mathrm{P}^{\circ}, 3 \mathrm{~s}^{3} \mathrm{P}^{\circ}, 3 \mathrm{p}^{1} \mathrm{~S} \ldots \ldots 3 \mathrm{~d}^{1} \mathrm{P}^{\circ}$ no ambiguity occurs. This notation has been adopted in the R M T for a number of spectra in which two limits, one odd and one even, had to be considered.

When two or more of the effective limits are all even or all odd an addition to this notation is necessary. For terms derived from the lowest of such a group of limits, the running electron is given as before; for those derived from the next higher limit a prime is affixed; and for those from the limit above this a double prime. Where the lowest limit is an $\mathrm{S}$ term, the type of the electron and of the term itself are the same, and the former is omitted. For example, the limiting terms in $O$ II are ${ }^{4} \mathrm{~S}^{\circ},{ }^{2} \mathrm{D}^{\circ}$ and ${ }^{2} \mathrm{P}^{\circ}$ in order. The addition of a $3 \mathrm{p}$ electron to these gives (among others) the terms in $O \mathrm{I}$ here called $3^{5} \mathrm{P}$ from ${ }^{4} \mathrm{~S}^{\circ}, 3 \mathrm{p}^{\prime}{ }^{3} \mathrm{D}$ from ${ }^{2} \mathrm{D}^{\circ}$ and $3 \mathrm{p}^{\prime \prime}{ }^{3} \mathrm{D}$ from ${ }^{2} \mathrm{P}^{\circ}$.

In several spectra there remain terms which cannot be described by this scheme: but it has been found possible to give a special abbreviated form of the configuration notation, etc. which make their nature intelligible to one versed in the theory of spectral structure.

There is at present no general agreement regarding the use of abbreviated notation of this sort. The notation here adopted has been largely influenced by the limitations of the photographic process-and is not presented as an ideal system-but it illustrates the glaring need for the preparation and general adoption of a better one.

\section{Special Cases.}

The notation used in the R M T for $N e$ I, $N a$ II, $A$ I, $K$ II and $C a$ III deserves special mention. Paschen's notation formerly used for spectra of this type defined the total quantum number and the type of electron, but introduced subscripts that were not inner quantum numbers. A revised notation which is given in detail by Bacher and Goudsmit ${ }^{1}$ is adopted here. The levels with " $s$ " electrons were called by Paschen $s_{2}, s_{3}$, $s_{4}$ and $s_{5}$; those with " $p$ " electrons were $p_{1}$ to $p_{10}$ etc. In this book the subscripts used by Paschen have been omitted but the rest of his notation is retained with numbers assigned to the levels, in order of increasing values for the lowest group of levels of each type. All the members of a series have the same number, but with this arrangement homologous levels which have the same J-values for different elements are not always assigned the same index number. Ner illustrates the changes:

\section{Ne i Notation}

\begin{tabular}{cc|cc}
\hline Paschen & Revised & Paschen & Revised \\
\hline & & $\ldots \ldots$ & $\ldots \ldots$ \\
$3 s_{6}$ & $3 \mathrm{~s}^{\circ} 1^{\circ}$ & $\ldots \ldots$ & $\ldots \ldots$ \\
$3 \mathrm{~s}_{4}$ & $3 \mathrm{~s}^{\circ}$ & $4 \mathrm{~d}_{6}$ & $4 \mathrm{~d} 1^{\circ}$ \\
$\ldots \ldots$ & $\ldots \ldots$ & $4 \mathrm{~d}_{6}$ & $4 \mathrm{~d} 2^{\circ}$ \\
$3 \mathrm{p}_{10}$ & $3 \mathrm{p} 1$ & $4 \mathrm{~d}_{4}$ & $4 \mathrm{~d} 3^{\circ}$ \\
$3 \mathrm{p}_{8}$ & $3 \mathrm{p} 2$ & $4 \mathrm{~d}_{4}$ & $4 \mathrm{~d} 4^{\circ}$ \\
$3 \mathrm{p}_{8}$ & $3 \mathrm{p} 3$ & $\ldots \ldots$ & $\ldots \ldots$ \\
\hline
\end{tabular}

${ }^{1}$ Atomic Energy States, MeGraw Hill, New York, London, 1932. 
Most of the levels for spectra of this type are not grouped into terms and consequently multiplets in the ordinary sense cannot be listed. Arbitrary groups of lines have been formed and numbered to facilitate the search for a given line. In $\mathrm{Ne}$ I for example, all important lines from the level $3 \mathrm{~s} 1^{\circ}$ combining with " $3 \mathrm{p}$ " levels have multiplet number 1 ; those from $3 \mathrm{~s} 1^{\circ}$ combining with $4 \mathrm{p}$ levels have multiplet number 2 etc.

\section{B. Complex Spectra}

38. In the majority of complex spectra the terms are so numerous that it is impracticable to designate them by their configurations. For these spectra the prefixes $a, b, c, d$ are assigned to the low terms of each type and $z, y, x$ etc. to those which combine with them. In $F e \mathrm{I}$, for example, the lowest ${ }^{3} \mathrm{~F}$ term is ${ }^{3} \mathrm{~F}$, the next higher one $b^{3} \mathrm{~F}$ etc. There are ten ${ }^{3} \mathrm{G}^{\circ}$ terms. They are labeled $\mathrm{z}^{3} \mathrm{G}^{\circ}, \mathrm{y}^{3} \mathrm{G}^{\circ} \ldots \ldots \mathrm{q}^{3} \mathrm{G}^{\circ}$. In Multiplet No. 449 the designation is $b^{8} G-t^{3} G^{\circ}$. Here the low term is the second ${ }^{3} \mathrm{G}$ term as indicated by the prefix " $b$ ". The high term is the seventh odd " $\mathrm{G}$ term, as indicated by " $\mathrm{t}$ ".

39. In many complex spectra it is impossible to group all known levels into spectroscopic terms. These miscellaneous levels are assigned numbers, and the superscript "o" if they belong to the odd set. Many combinations between terms and miscellaneous levels are given in the R M T and assigned multiplet numbers. For example, the designation of multiplet number 450 of $F e \mathrm{I}$ is $\mathrm{b}^{3} \mathrm{G}-12^{\circ}$.

Numbered levels are numerous in spectra of the rare earths. The arrangement is similar to that described in $\$ 27$, i.e. the lines from a given low term are listed in order of increasing $E \mathrm{P}$ of the numbered levels.

In $S m$ II only the low levels have been grouped into terms. All high levels are numbered odd levels. In the R M T the combinations of the separate components of the low terms with arbitrarily grouped odd levels are assigned multiplet numbers. For example, the combinations of $a^{8} \mathrm{~F}_{3 / 6}$ with the levels labeled $1^{\circ}, 2^{\circ}, 5^{\circ}, 23^{\circ}, 35^{\circ}, 37^{\circ}$ have multiplet number 1 . The E P's increase for the various groups similarly to those in spectra with regular terms, as discussed in $\$ 27$.

\section{SPECIAL NOTES ON INDIVIDUAL SPECTRA}

40. $H$ The wave-lengths listed for these spectra have been calculated for the center of gravity of the lines, $D$ taking into account the fine structure, and using the values of $R_{H}, R_{D}$ and $R_{H e}$ respectively, given He II by Birge in $1941^{1}$. These computations were made by Dr. J. E. Mack for inclusion here. The writer is deeply indebted to Dr. Mack for his cordial cooperation in furnishing this unpublished material.

No intensities have been included for these spectra.

$O$ II Improved term values are needed. The writer has constructed the multiplets from Edlén's term list. Measures by different investigators are discordant, and considerable editing has been done, especially in the interpretation of blends.

For the sextet terms the configuration in abbreviated form is used to indicate that the terms are from the high limit $\mathrm{sp}^{3}{ }^{5} \mathrm{~S}^{\circ}$ in $O$ III, namely: $\mathrm{sp}^{3} 3 \mathrm{p}{ }^{6} \mathrm{P}, \mathrm{sp}^{3} 3 \mathrm{~d}^{6} \mathrm{D}^{\circ}, \mathrm{sp}^{3} 4 \mathrm{~s}^{\circ} \mathrm{S}^{\circ}$.

$\mathrm{Na}$ I The fine structure components of $\mathrm{D}_{1}$ and $\mathrm{D}_{2}$ have been measured with the interferometer by Meissner and Luft ${ }^{2}$, as follows:

\begin{tabular}{|c|c|c|}
\hline & $\begin{array}{c}D_{1} \\
5895.9316 \\
5895.9103\end{array}$ & $\begin{array}{c}\mathrm{D}_{2} \\
5889.9579 \\
5889.9380\end{array}$ \\
\hline $\begin{array}{l}\text { Center of } \\
\text { Gravity }\end{array}$ & $\overline{5895.9236}$ & 5889.9504 \\
\hline
\end{tabular}

The measures listed in the R M T are taken from a source where the lines appeared as impurities, since it was thought that for astrophysical purposes these measures might be preferable to those of the fine structure components.

The two lines $\lambda 11403$ and $\lambda 11381$ were also measured as impurities.

Improved laboratory intensities are needed for $\mathrm{Na}$ I.

1 Rev. Mod. Phys. 13, 233 (No. 4), 1941.

2 Ann. der Phys. (5) 29, 698, 1937. 
$\mathrm{Na}$ II The changes made in the Paschen notation for $\mathrm{Na}$ II have been discussed in $\S 37$. Some terms are also known in this spectrum, and two types of notation appear. The lines are listed in order of increasing low level and these levels combine with the terms. Although no complete multiplets are listed, multiplet numbers have been assigned as usual. For example, multiplet No. 17 is $3 p 9-4 s^{3} P^{\circ}$. In spectra of this type no attempt has been made to indicate omitted lines by the use of a " $\nmid$ ". The "List $D$ " indicates that only the leading lines are listed.

$M g$ I Two sets of series, $3^{1} \mathrm{D}-1 \mathrm{~F}^{\circ}$ and $3^{3} \mathrm{D}-{ }^{3} \mathrm{~F}^{\circ}$ have been extended by the use of infra-red solar wave-lengths from Babcock's Table (see $\S 15)^{1}$. This has been done on the assumption that the ${ }^{1} \mathrm{~F}^{\circ}$ and ${ }^{3} \mathrm{~F}^{\circ}$ terms are coincident, as Paschen suggested for the first members of the series. The predicted wave-lengths in the R M T are obtained from solar term values. The series appear to be so well confirmed that the solar wave-lengths are preferable to the predicted ones, but for uniformity, no exception has been made for these series lines of $M g$ I.

In Multiplets 7, 8 and 9 the J-values and designation apply to all three lines entered. In each case singlet combinations are involved. Normally one one line is observed in a combination of this type, but the fine structure components of each line are listed.

Al II The $\mathrm{G}$ and $\mathrm{H}$ terms given by Paschen and Ritschl ${ }^{2}$ are in both cases assumed to be coincident singlet and triplet terms. When combinations of these terms with singlet terms are listed in the R M T, ${ }^{1} \mathrm{G}$ or ${ }^{1} \mathrm{H}^{\circ}$ has been used in place of ${ }^{1,3} \mathrm{G}$ or ${ }^{1,3} \mathrm{H}^{\circ}$. Similarly, the last three entries are given as singlet combinations, but in reality they are probably singlet and triplet combinations. Double multiplicities for unresolved terms have not been used in the R M T.

Si II Owing to the use of the photographic method of publication, it has been impossible to add lines without retyping one or more pages. One predicted multiplet of $S i$ II has been omitted which should possibly have been inserted.

\begin{tabular}{cccccc}
\hline \multirow{2}{*}{ I A } & \multirow{2}{*}{ Ref } & \multicolumn{2}{c}{ E P } & J & $\begin{array}{c}\text { Multiplet } \\
\text { Desig }\end{array}$ \\
\hline 4075.81 & P & 9.80 & 12.82 & $21 / 2-11 / 2$ & $3^{2} \mathrm{D}-5^{2} \mathrm{P}^{\circ}$ \\
4077.09 & $\mathrm{P}$ & 9.79 & 12.82 & $11 / 2-1 / 2$ & \\
4073.05 & $\mathrm{P}$ & 9.79 & 12.82 & $11 / 2-11 / 2$ & \\
\hline
\end{tabular}

$P$ III The multiplets are listed slightly out of order, but it was thought unnecessary to retype the page on this account.

$S$ II The measures by different observers are very discordant. This spectrum needs thorough observation. Accurate wave-lengths, intensity estimates and term values, and further analysis are desirable.

$A$ II This spectrum is fairly well analyzed but needs careful editing before a definitive analysis can be published. Rosenthal ${ }^{3}$ has measured many lines and from his measures alone a consistent set of term values could probably be calculated. The lists of classified lines are not homogeneous and a larger residual in the observed minus calculated wave number must be permitted than for most spectra. The multiplets listed in the R M T appear to be fairly satisfactory in spite of the inaccurate term values.

One term, labeled $a^{2} \mathrm{P}$ by de Bruin is puzzling because it has no configuration assignment. It has been retained, but needs to be checked carefully when the analysis is carried further. This is the only case where both the running electron notation and the prefix "a" appear in a given spectrum.

$\mathrm{Ca}$ I Although the analyses of these spectra are almost completed, the spectra require further laboratory

$\mathrm{Ca}$ II observation. Accurate wave-lengths, especially of the fainter lines are urgently needed. It is surprisingly difficult to obtain accordant term values. The interferometer measures made at Allegheny furnish an excellent starting point, but these spectra still invite the attention of the laboratory investigator, from the violet through the infra-red.

1 Babcock and Moore, Ap. J. 101, 374, 1945.

- Ann. der Phys. (5) 18, 867, 1933.

- Ann. der Phys. (5) 4, 49, 1930. 
Sc II Multiplet No. 9. Enter intensity 2 for $\lambda 3923.503$.

41. Fe I The rigorous arrangement of multiplets described in $\$ 27$ applies only approximately to $F e \mathrm{I}$. In this spectrum the multiplet numbers reach 1352 but this figure is not definitive. Owing to an extension of the analysis which altered some term assignments, a number of multiplets were rearranged after the lines and multiplet numbers had been entered and checked in the Finding List.

All the revisions were entered in the R M T. For unchanged multiplets the original multiplet numbers were retained. The revised multiplets were inserted as nearly as possible in the correct place and assigned the available numbers, or to avoid duplication, a number followed by " $a$ ". As a result of these changes the multiplets do not always have consecutive numbers and some numbers are omitted. The renumbering of all the multiplets entailed so many changes in the Finding List that it was not undertaken.

In three multiplets of $F e$, Nos. 3, 7 and 81 , an "R" is entered under the multiplet number. A line has been inadvertently omitted from each of these multiplets. The omitted lines are listed on page 65 at the end of the $F e$ I multiplets, and preceding the list of unclassified lines.

In multiplet No. 78, columns one and two, $\lambda 3497.137 \mathrm{~V}$ should read 3497.15 P.

Multiplet No. 1151 should be rejected; $\lambda 4618.568$ is erroneous.

$N i$ I Attention has been called to the fact that the intensities in Multiplet No. 62 are not so abnormal as indicated here. It has been impossible to insert revised estimates.

$R h$ II The use of the symbol " $\nmid$ " to denote omitted lines has not been checked owing to the lack of a complete line list. It has been assumed from the term lists that the fainter members of the multiplets thus marked have been observed.

Ce II The lack of connection between Groups I and II has been mentioned ( $\$ 35$ ). It is assumed that the terms in Group I are the lower set.

The prefixes a, b and c etc. have been assigned to the low set of terms of each Group. There can be no ambiguity because in Group I the low set is even, while in Group II it is odd.

$W$ II All the miscellaneous levels published by Laun ${ }^{1}$ have been numbered in order. These numbers are used in the R M T in place of Laun's notation.

\section{SPECTRA OMITTED FROM THE R M T.}

These may be grouped in several general classes.

42. Spectra of probable astrophysical importance for which there is no analysis to date.

These spectra are mentioned in the R M T in the appropriate place with the remark "No Analysis" and the date. If A. S. King has assigned a Temperature Class to the lines, this fact is noted. For example: page 86, Ce I No Analysis May 1942 (Temperature Class). The spectra in this class are listed in Table 5.

TABLE 5

Spectra Omitted from R M T

No ANALYsis

\begin{tabular}{lc|cc}
\hline Spectrum & $\begin{array}{c}\text { Ref. to } \\
\text { Temp. Class }\end{array}$ & Spectrum & $\begin{array}{c}\text { Ref. to } \\
\text { Temp. Class }\end{array}$ \\
\hline Ce I & 215 & Dy I & 217 \\
Pr I & 215 & Dy II & 217,229 \\
Nd I & 219 & Ho I & 217 \\
Tb I & 217 & Ho II & 217 \\
Tb II & 217 & Th I & 123 \\
\hline
\end{tabular}

All but $T h$ I have been observed by A.S. King. The bibliography numbers of the references to the work on temperature classification are entered in column two.

'Bur. St. Journ. Res. 21, 207 (RP 1125), 1938. 
43. Analyzed spectra having lines observed in the visible region, but omitted from the $\mathrm{R} M \mathrm{~T}$ as unimportant astrophysically.

These spectra are mentioned in the R M T with the remark "See Introduction". They are listed in Table 6 with numbers from the bibliography referring to the papers on analysis.

TABLE 6

Spectra Omitted from R M T

Not of Astrophysical Interest

\begin{tabular}{|c|c|c|c|c|c|}
\hline Spectrum & $\begin{array}{l}\text { Ref. to } \\
\text { Analysis }\end{array}$ & Spectrum & $\begin{array}{l}\text { Ref. to } \\
\text { Analysis }\end{array}$ & Spectrum & $\begin{array}{l}\text { Ref. to } \\
\text { Analysis }\end{array}$ \\
\hline $\mathrm{B}_{\mathrm{I}}{ }^{1}$ & 16,89 & $\mathrm{Rb}_{\text {II }}$ & 238 & Cs II & 309,405 \\
\hline$F_{\text {IV }}$ & 84 & $\mathrm{Pd}$ II & 384,24 & Ta II & 192 \\
\hline F vI & 83,87 & $\mathrm{Ag}$ II & 383,24 & Pt II & 387 \\
\hline $\mathrm{Cl}_{\text {IV }}$ & 31,32 & $\mathrm{Cd}$ II & 372,401 & $\mathrm{Au}$ II & 324 \\
\hline $\mathrm{Ga}$ II & 376 & In II & 317 & $\mathrm{Hg}$ II & 313 \\
\hline Se II & 244 & Sb II & 236 & $\mathrm{Tl}$ II & 106 \\
\hline $\mathrm{Br} \mathrm{I}$ & 194 & I I & $107,69,325$ & $\mathrm{~Pb}$ II & 80 \\
\hline $\mathrm{Br}$ II & 232 & I II & 232 & $\mathrm{Bi}$ II & 64,115 \\
\hline $\mathrm{Kr}_{\mathrm{I}}$ & $274,276,165$ & $\mathrm{Xe}_{\mathrm{I}}$ & 156 & $\mathrm{R}_{\mathbf{I}}$ & 329 \\
\hline $\mathrm{Kr}_{\text {II }}$ & 53 & $\mathrm{Xe}$ II & 155 & Th III & 54 \\
\hline
\end{tabular}

The low abundance of these elements in celestial sources, and the high E P of the lines in the visible region have been the determining factors for omission.

44. There are three types of spectra for which little or nothing is known:

$E_{r}, U$. Lines have been observed in spectra of these elements, but the spectra of various degrees of ionization have not been separated.

Te II, Re II, Os II, Ir II, $P o, A c, P a$. The writer has found no references to work on these spectra.

$M a, I l, 85,87$. There is nothing known about these elements. It appears doubtful whether they have been successfully isolated.

No reference is made in the body of the R M T to those spectra whose leading lines are in the region to the violet of $\lambda 2950$, since this is a book designed for astrophysical use. Selected spectra of this type are included in the section dealing with Forbidden Lines (see $\S 45$ and pp. 100-110).

\section{FORBIDDEN LINES}

45. The author of a "Multiplet Table of Astrophysical Interest" published in 1945 is obliged to consider the probable importance of the forbidden lines of all abundant elements. This is indicated by the work of Bowen on nebular lines, of Edlén on coronal lines, and of Swings, Merrill and others on various astronomical spectra.

Following the body of the R M T is a Table of Forbidden Lines of Astrophysical Interest (pp. 100-110). This Table is arranged in detail similarly to the $\mathrm{R} \mathrm{MT}$. The lines in a multiplet are listed by diagonals and the multipl ets are listed in the order described in $\S 27$. In order to avoid duplication, all multiplets of forbidden lines have an " $F$ " following the multiplet number, $1 \mathrm{~F}, 2 \mathrm{~F}$ etc. Unlike the $\mathrm{R} \mathrm{M} \mathrm{T}$, the headings for each spectrum contain only the name of spectrum and the I P. No grading of analysis or list has been attempted and no date of completion of the manuscript is given. All of this section has been written between January and May 1945.

In preparing this manuscript the writer has been most cordially assisted by Dr. Swings. He has edited the lists and offered many valuable suggestions concerning the limitations of the Table. No explicit statement can be made as to the principles of selection adopted, but severe restrictions have been necessary in complex spectra because of the great array of possible forbidden transitions. For simple spectra only a limited number of transitions occurs, but as the complexity increases the number increases rapidly. The general principles followed are:

A Only transitions from metastable states are forbidden. Consequently only the lowest terms in a spectrum are considered.

1 Lines of $\mathrm{B}_{1}$ have not been observed in the visible, but should exist. 
B The lists are restricted to multiplets involving likely combinations as regards mu! -inlicity and azimuthal quantum numbers, except for those in which the lowest terms are involved. In $F e$ II, for example, many more combinations and more unlikely combinations from the lowest term, $a^{6} \mathrm{D}$ are listed than from higher terms. portant.

C Transitions involving $\Delta \mathrm{J}= \pm 2$ as well as $\Delta \mathrm{J}=\mathrm{O}$ or \pm 1 are listed for the multiplets most likely to be im-

$\mathrm{D}$ The high $\mathrm{E} \mathrm{P}$ is limited to about 4.0 for the most abundant elements and to about 3.5 for arc spectra of these elements.

$\mathrm{E}$ The lists have been extended to include lines that may be important in the red and infra-red. Forbidden lines of neutral atoms are included only for the most abundant elements.

The multiplets listed must be interpreted with caution, because of these restrictions. If complete multiplet arrays are written up from Table 3 , lines omitted from any multiplet among the forbidden lines can be detected. Those interested in longer lists must construct them from the term lists given in the papers on the analysis of each spectrum (Table 10 p. xxix).

The great majority of forbidden lines are predicted from the term values. If accurate measures have been obtained, they are entered with a letter indicating the source, as follows:

$$
\begin{array}{lll}
\text { N } & \text { Nebular } & N \text { II, } O \text { II, } O \text { III, Ne III, Ne V, S II } \\
\text { L } & \text { Laboratory } & O_{\text {I }} \\
\text { A } & \text { Auroral } & O_{\text {I }} \\
\text { C } & \text { Coronal } & \text { Entered under the predicted positions of lines of highly ionized } F e \text { and } N i
\end{array}
$$

When term values permit, the wave-lengths of predicted lines have been calculated to two decimal places. For some spectra the term values are not accurately known, but the internal separations are well established. For these the position is given to $0.1 \mathrm{~A}$. For the most inaccurate wave-lengths no decimals are recorded and in very dubious

\begin{tabular}{|c|c|c|c|c|c|}
\hline \multicolumn{4}{|c|}{ Term } & \multicolumn{2}{|c|}{ I P } \\
\hline$S p$ & Term & $\mathrm{Sp}$ & Term & $\mathrm{Sp}$ & $\mathrm{Sp}$ \\
\hline $\begin{array}{l}\mathrm{Cl} \text { II } \\
\mathrm{S} \text { xII } \\
\mathrm{A} \text { III } \\
\mathrm{A} \text { xI } \\
\mathrm{A} \text { xIV } \\
\mathrm{K} \text { v } \\
\mathrm{K} \text { vI }\end{array}$ & $\begin{array}{l}{ }^{1} \mathrm{~S} \\
{ }^{2} \mathrm{P} \\
{ }^{1} \mathrm{~S} \\
{ }^{3} \mathrm{P} \\
{ }^{2} \mathrm{P}^{\circ} \\
{ }^{2} \mathrm{P}^{\circ}{ }^{2} \mathrm{D}^{\circ} \\
{ }^{1} \mathrm{~S}\end{array}$ & $\begin{array}{l}\mathrm{Ca} \text { vII } \\
\mathrm{Ca} \mathrm{xv} \\
\mathrm{Fe} \times \mathbf{x} \\
\mathrm{Ni} \mathbf{x I I} \\
\mathrm{Ni} \mathbf{x I I} \\
\mathrm{Ni} \mathbf{x v} \\
\mathrm{Ni} \mathbf{x v I}\end{array}$ & $\begin{array}{l}{ }^{1} \mathrm{~S} \\
{ }^{3} \mathrm{P} \\
{ }^{3} \mathrm{P}^{\circ} \\
{ }^{2} \mathrm{P}^{\circ} \\
{ }^{3} \mathrm{P},{ }^{1} \mathrm{D} \\
{ }^{8} \mathrm{P} \\
{ }^{2} \mathrm{P}^{\circ}\end{array}$ & $\begin{array}{l}\mathrm{Ca} \mathbf{v} \\
\mathrm{Sc} \text { vI } \\
\mathrm{V} \text { viII } \\
\mathrm{Cr} \text { vIII } \\
\mathrm{Cr} \mathbf{I x} \\
\mathrm{Mn} \mathbf{I X} \\
\mathrm{Mn} \mathbf{x}\end{array}$ & 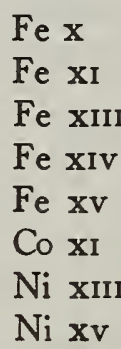 \\
\hline
\end{tabular}
cases a "?" follows the wave-length.

Some I P's and some predicted wave-lengths have been obtained by interpolation or extrapolation along the isoelectronic sequences. These are:

As in the body of the R M T, E P's in parentheses denote that the terms involved do not have observed connections with the rest of the terms of the spectrum.

Dr. Swings has pointed out that forbidden lines are essentially emission lines, and therefore, astrophysically the high $\mathrm{E} \mathrm{P}$ is the important one. For this reason the multiplets of a spectrum should be listed by high $\mathrm{E} P$ rather than by low E P ( $\$ 27)$. It is fully recognized that emission lines are better handled in this order and it is hoped that all multiplets having the same high term can be readily selected in any spectrum. The arrangement by low terms has been adopted merely for the sake of uniformity.

Another highly significant comment has been made by Dr. Swings ${ }^{1}$, namely, that "certain forbidden transitions that are not directly observable may play a role in astronomy, for example, by flourescence excitation, ionization or dissociation."

\footnotetext{
1 Letter, May 1945.
} 
The importance of lines in the extreme violet such as $\lambda 303.7$ of $\mathrm{He} \mathrm{II}, \lambda 303.7$ and $\lambda 374.4$ of $O \mathrm{III}$ and the pair at $\lambda 374.4$ of $N \mathrm{III}$, in producing the nebular lines has been fully discussed by Bowen ${ }^{1}$. The violet limit, $\lambda 2950$, imposed in this book has excluded both permitted and forbidden lines in the violet that are extremely important in the interpretation of forbidden lines observed in astronomical spectra. Readers are, therefore, urged to consult the individual papers on this subject, as it has been regarded as beyond the scope of the present work.

\section{DETAILS OF PUBLICATION}

The preparation of the manuscript of this book has covered such a long period of time that the typing has been done as various spectra were finished, which is not in the order of increasing atomic number. It has been practically impossible to terminate every section of the manuscript at the end of a typed page. Some important insertions have also broken the continuity of typing. Consequently, the pages are frequently unequal in length and some have large gaps. No serious effort has been made to avoid irregularities of this kind, for two reasons: first, the retyping and rechecking of these large pages in order to adjust spacing has seemed an unjustifiable procedure, particularly since there is always the chance of introducing new errors in handling so much tabular data; second, the blank spaces may prove to be useful for notes.

Doubtless there are more serious irregularities, namely inconsistencies in notation of similar spectra. During the course of the work the manuscript has been widely distributed to interested investigators. To date it has never all been assembled in one place. The writer has been unable to remember all the details connected with each spectrum, but has proceeded on the assumption that minor irregularities would not impair the value of the R M T so seriously as the delays required to correct all of them.

46. One of the purposes of this book has been to provide adequate material for fairly definitive identifications of solar lines. Mention has been made of the forthcoming publication by Babcock and others on the Infra-Red Solar Spectrum $\lambda \lambda 6600-13495$ ( $\$ \S 7,15)$. A similar program covering the violet solar spectrum is being carried on at Mount Wilson by Babcock. The writer has been working on the identifications of the solar lines throughout the entire solar spectrum, with the aid of the manuscript of the R M T. The publication of the results to the violet of $\lambda 6600$ has been postponed in order to complete the present book. It is planned to publish them as soon as possible.

\section{BIBLIOGRAPHY}

Following the text is a Bibliography in which all references used in the preparation of this book are listed in the alphabetical order of the names of the authors. Each reference is assigned a number for purposes of cross reference.

47. In the $\mathrm{R} \mathrm{M} \mathrm{T} \mathrm{(excluding} \mathrm{the} \mathrm{section} \mathrm{on} \mathrm{Forbidden} \mathrm{Lines)} \mathrm{each} \mathrm{spectrum} \mathrm{has} \mathrm{three} \mathrm{sets} \mathrm{of} \mathrm{references:} \mathrm{one}$ giving the sources from which the wave-lengths have been taken-Table 7; one giving the first, second, etc. choices of references for intensity estimates-Table 8; and one referring to papers on analysis-Table 9.

The Tables are arranged similarly. In each, the first column gives the chemical symbol of the element and the spectrum ( $I=\operatorname{arc}$, II first spark etc.), the second the number with which to enter the Bibliography. In Table 7 the letters $\mathrm{A}, \mathrm{B}, \mathrm{C}$, etc. are taken from column 2 of the $\mathrm{R} \mathrm{M} \mathrm{T} \mathrm{for} \mathrm{each} \mathrm{spectrum.} \mathrm{In} \mathrm{Table} 8$ the first choice for intensity is indicated in column one, the second in column two etc. In general, reference numbers are in italics when the intensities from the reference are in parentheses in the R M T (see $\$ 34$ ). Table 9 does not list choices. It contains references to papers on analysis that were used in compiling the $R \mathrm{M} T$.

Table 10 gives the sources used for analysis of spectra contained in the Table of Forbidden Lines. It is arranged similarly to Table 9.

Following the Bibliography are an index by pages, and one by elements arranged in the alphabetical order of the chemical symbols.

'Ap. J. 81, 1, 1935. 
TABLE 7

References-Wave-Length

\begin{tabular}{|c|c|c|c|c|c|c|c|c|c|c|c|c|c|c|c|c|c|c|c|}
\hline $\mathrm{Sp}$ & $\mathrm{A}$ & B & $\mathrm{C}$ & $\mathrm{D}$ & $\mathrm{E}$ & $\mathrm{F}$ & Sp & A & B & C & $\mathrm{D}$ & $\mathrm{E}$ & $\mathrm{F}$ & G & $\mathrm{H}$ & I & $\mathrm{J}$ & $\mathrm{K}$ & $\mathrm{L}$ \\
\hline $\mathrm{H}$ & 243 & & & & & & $\mathrm{Cl}$ II & 195 & & & & & & & & & & & \\
\hline D & 243 & & & & & & $\mathrm{Cl}$ ıI & 31 & 27 & & & & & & & & & & \\
\hline $\mathrm{He} \mathrm{I}$ & 263 & 299 & 319 & 174 & 275 & & $\mathrm{~A}_{\mathrm{I}}$ & 277 & 154 & 263 & & & & & & & & & \\
\hline $\mathrm{He} \mathrm{II}$ & 243 & & & & & & $A$ II & 340 & 18 & 48 & 47 & & & & & & & & \\
\hline $\mathrm{Li}_{I}$ & 206 & 175 & 149 & & & & A III & 49 & 51 & & & & & & & & & & \\
\hline Li II & 378 & 404 & & & & & A IV & 50 & & & & & & & & & & & \\
\hline $\mathrm{Be} I$ & 318 & 315 & 149 & & & 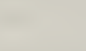 & $\mathrm{K}_{\mathrm{I}}$ & 149 & 403 & 263 & 259 & 88 & 116 & 108 & 380 & & & & \\
\hline Be II & 318 & & & & & & $\mathrm{~K}$ II & 46 & & & & & & & & & & & \\
\hline B II & 81 & & & 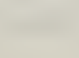 & & & $\mathrm{K}$ III & $47 a$ & $51 a$ & & & & & & & & & & \\
\hline B III & 81 & & & & & & $\mathrm{Ca} \mathrm{I}$ & 403 & 66 & 259 & 369 & 373 & 241 & 374 & & & & & \\
\hline$C_{I}$ & 185 & 300 & 169 & 160 & 370 & & $\begin{array}{l}\mathrm{Ca} \text { II } \\
\mathrm{Ca} \text { III }\end{array}$ & $\begin{array}{r}168 \\
11\end{array}$ & 403 & 66 & 375 & 390 & & & & & & & \\
\hline $\mathrm{C}_{\text {II }}$ & 122 & 81 & 89 & & & & Sc I & 245 & 253 & 349 & & & & & & & & & \\
\hline C III & 81 & 121 & & & & & Sc II & 245 & 253 & & & & & & & & & & \\
\hline$C_{\text {IV }}$ & 81 & & & & & & Sc $1 I I$ & 172 & & & & & & & & & & & \\
\hline $\mathrm{N}_{\mathrm{I}}$ & 93 & 78 & 160 & 370 & & & $\mathrm{Ti}_{\mathrm{I}}$ & 178 & 65 & 185 & 282 & 245 & 201 & 212 & 348 & 199 & 172 & 177 & 21 \\
\hline$N_{\text {II }}$ & 20 & 125 & 120 & & & & Ti II & 178 & 65 & 245 & 201 & 347 & 172 & & & & & & \\
\hline $\mathrm{N}$ III & 124 & 81 & & & & & Ti IV & 364 & & & & & & & & & & & \\
\hline $\mathrm{N}_{\text {IV }}$ & 126 & 81 & & & & & $\mathrm{~V}_{\mathrm{I}}$ & 264 & 368 & 245 & 242 & 266 & 213 & 109 & & & & & \\
\hline $\mathrm{O}_{\mathrm{I}}$ & 98 & 263 & 127 & 116 & 316 & 128 & $\mathrm{~V}_{\text {II }}$ & 266 & & & & & & & & & & & \\
\hline O II & 20 & 118 & 301 & & & & & & & & & (204) & & & & & & & \\
\hline O III & 119 & 86 & 302 & & & & Cr I & 183 & 184 & 245 & 142 & $\left\{\begin{array}{l}213 \\
\text {. }\end{array}\right.$ & $\int 173$ & & & & & & \\
\hline O IV & 81 & 126 & 86 & & & & $\mathrm{Cr}$ II & 184 & 245 & 142 & 110 & & & & & & & & \\
\hline $\mathrm{Ov}$ & 81 & & & & & & $\mathrm{Mn}$ I & 260 & 245 & 246 & 131 & & & & & & & & \\
\hline O vi & 81 & & & & & & $\mathrm{Mn}$ II & $\left\{\begin{array}{l}67 \\
60\end{array}\right\}$ & 245 & 108 & & & & & & & & & \\
\hline$F_{\text {I }}$ & 88 & & & & & & F & $(68)$ & 2.0 & 100 & & & & & & & & & \\
\hline F II & 73 & & & & & & $\mathrm{Fe} \mathrm{I*}$ & & & & & & & & & & & & \\
\hline F III & 72 & 85 & & & & & Fe II & 76 & 222 & 55 & & & & & & & & & \\
\hline $\mathrm{Ne} I$ & 162 & 277 & 154 & 276 & 311 & 263 & $\mathrm{Fe}$ III & 103 & 102 & 222 & & & & & & & & & \\
\hline $\mathrm{Ne}$ II & 52 & & & & & & Co I & 56 & 282 & 245 & 280 & 71 & 230 & 62 & 147 & & & & \\
\hline $\mathrm{Na} \mathrm{I}$ & 149 & 116 & 263 & 298 & 259 & 338 & Co II & 255 & & & & & & & & & & & \\
\hline & & & & & & & $\mathrm{Ni} I$ & 282 & 245 & 178 & 143 & 109 & 146 & 280 & 398 & & & & \\
\hline $\mathrm{Na}$ II & 130 & 393 & & & & & $\mathrm{Ni}$ II & 252 & 245 & 111 & & & & & & & & & \\
\hline $\mathrm{Mg} I$ & 295 & 314 & 141 & 261 & 116 & & $\mathrm{Cu}_{\mathrm{I}}$ & 58 & & & & & & & & & & & \\
\hline $\mathrm{Mg}$ II & 116 & & & & & & $\mathrm{Cu}$ II & 137 & & & & & & & & & & & \\
\hline $\mathrm{Al} \mathrm{I}$ & 403 & 315 & 116 & 319 & 310 & & $\mathrm{Zn}_{\mathrm{I}}$ & 149 & & & & & & & & & & & \\
\hline $\mathrm{Al}$ II & 319 & 377 & & & & & $\mathrm{Zn}$ II & 149 & 319 & & & & & & & & & & \\
\hline $\mathrm{Al} \mathrm{III}$ & 312 & & & & & & $\mathrm{Ga} I$ & 402 & & & & & & & & & & & \\
\hline Si I & 186 & & & & & & Ge I & 188 & 245 & & & & & & & & & & \\
\hline Si II & 117 & & & & & & $\mathrm{Ge}$ II & 188 & 235 & & & & & & & & & & \\
\hline Si III & 117 & & & & & & As I & 273 & & & & & & & & & & & \\
\hline Si IV & 117 & 101 & & & & & As Ií & 328 & & & & & & & & & & & \\
\hline$P_{\text {I }}$ & 180 & & & & & & $\mathrm{Se}_{\mathrm{I}}$ & 189 & & & & & & & & & & & \\
\hline P II & 133 & 181 & 70 & & & & $\mathrm{Rb}_{\mathrm{I}}$ & 149 & 333 & & & & & & & & & & \\
\hline P III & 133 & & & & & & Sr I & 399 & 245 & 116 & & & & & & & & & \\
\hline$P_{\text {IV }}$ & 133 & & & & & & Sr II & 399 & & & & & & & & & & & \\
\hline $\mathrm{P} v$ & 133 & & & & & & $Y_{\text {I }}$ & 254 & 245 & & & & & & & & & & \\
\hline$S_{I}$ & 297 & 129 & & & & & $Y_{\text {II }}$ & 254 & 351 & 245 & & & & & & & & & \\
\hline$S_{\text {II }}$ & 157 & 19 & 158 & 25 & 136 & & $\mathrm{Zr} \mathbf{I}$ & 197 & 282 & & & & & & & & & & \\
\hline S III & 157 & 159 & & & & & $\mathrm{Zr}$ II & 196 & & & & & & & & & & & \\
\hline S IV & 303 & & & & & & $\mathrm{Cb}$ I & 283 & & & & & & & & & & & \\
\hline $\mathrm{Cl}_{\mathrm{I}}$ & 182 & & & & & & $\mathrm{Cb}$ II & 283 & & & & & & & & & & & \\
\hline
\end{tabular}

- See references for Fe 1 at end of Table 7. 
TABLE 7-Continued

REFERENCES-WAVE-LENGTH

\begin{tabular}{|c|c|c|c|c|c|c|c|c|c|c|c|c|c|c|c|c|c|}
\hline $\mathrm{Sp}$ & A & B & C & $\mathrm{D}$ & $\mathrm{E}$ & Sp & A & B & C & $\mathrm{D}$ & $\mathrm{E}$ & Sp & A & B & $\mathrm{C}$ & $\mathrm{D}$ & $\mathrm{E}$ \\
\hline Mo I & 326 & 176 & 113 & & & Ce II & 144 & 9 & 245 & & & $\mathrm{Ta} \mathrm{I}$ & 200 & & & & \\
\hline Mo II & 245 & 113 & & & & $\mathrm{Ce}$ III & 362 & & & & & $\mathrm{~W}_{\mathrm{I}}$ & 22 & & & & \\
\hline $\mathrm{Ru} I$ & 245 & & & & & Pr II & 339 & 245 & & & & W II & 239 & & & & \\
\hline $\mathrm{Ru}_{\mathrm{II}}$ & 272 & & & & & $\mathrm{Nd}$ II & 10 & 245 & 219 & & & $\operatorname{Re} I$ & 257 & & & & \\
\hline $\mathrm{Rh}_{\mathrm{I}}$ & 304 & & & & & $\mathrm{Sm}$ & $\{221\}$ & $\{221\}$ & & & & Os I & 245 & & & & \\
\hline $\mathrm{Rh}_{\text {II }}$ & 150 & & & & & Sm I & $\left\{\begin{array}{l}4 \\
4\end{array}\right\}$ & $\{2\}$ & & & & Ir I $_{I}$ & 6 & & & & \\
\hline $\mathrm{Pd} \mathrm{I}$ & 256 & 109 & & & & Sm II & 221 & & & & & $P t_{I}$ & 109 & & & & \\
\hline $\mathrm{Ag} I$ & 149 & & & & & $\mathrm{Eu}_{\mathrm{I}}$ & 223 & & & & & $\mathrm{Au} \mathrm{I}$ & 324 & & & & \\
\hline $\mathrm{Cd} \mathrm{I}$ & 149 & 163 & & & & Eu II & 223 & & & & & $\mathrm{Hg} \mathrm{I}$ & 396 & 166 & & & \\
\hline In I & 402 & & & & & Gd I & 225 & & & & & $\mathrm{Tl} \mathrm{I}_{\mathrm{I}}$ & 307 & 109 & & & \\
\hline Sn I & 265 & & & & & Gd II & 225 & & & & & $\mathrm{~Pb}_{\mathrm{I}}$ & 149 & 135 & & & \\
\hline Sn II & 248 & & & & & $\operatorname{Tm} \mathrm{I}$ & 268 & & & & & $\mathrm{Bi}$ & 214 & 17 & & & \\
\hline $\mathrm{Sb}_{\mathrm{I}}$ & 279 & & & & & $\mathrm{Tm}$ & 269\{ & $\{268$ & & & & $\mathrm{Ra} I$ & 332 & & & & \\
\hline Te I & 18,9 & & & & & 1011 & $203\}$ & 224 & & & & $\mathrm{Ra}$ II & 331 & & & & \\
\hline Cs I & 259 & 149 & & & & $\mathrm{Yb} \mathrm{I}$ & 291 & & & & & Th II & 250 & & & & \\
\hline $\mathrm{Ba}$ I & 400 & & & & & Yb II & 291 & & & & & & & & & & \\
\hline $\mathrm{Ba}$ II & 400 & 245 & 2593 & 330 & & Lu I & 288 & & & & & & & & & & \\
\hline $\mathrm{La} I$ & 258 & & & & & Lu II & 288 & & & & & & & & & & \\
\hline La II & 258 & & & & & Hf I & 287 & & & & & & & & & & \\
\hline La III & 258 & & & & & Hf II & 289 & & & & & & & & & & \\
\hline
\end{tabular}

$\begin{array}{lllllllllllllllllllllllllll}\mathrm{Sp} & & \text { A } & \text { B } & \text { C } & \text { D } & \text { E } & \text { F } & \text { G } & \text { H } & \text { I } & \text { J } & \text { K } & \text { L } & \text { M } & \text { N } & \text { O } & \text { Q } & \text { R } & \text { S } & \text { T } & \text { U } & \text { V } & \text { W } & \text { X } & \text { Y }\end{array}$

Fe I $\left.\quad \begin{array}{llllllllllllll}164 & 161 & 278 & 185 & 262 & 282 & 59 & 167 & 14 & 371 & 57 & 281 & 220 & \left\{\begin{array}{l}139 \\ 138\end{array}\right.\end{array}\right\} \dagger^{2} 28015227 \quad 77391247 \quad 55171379 \uparrow 74$

† These references have been used for lines to the violet of the range covered in the RMT, but are included for completeness. 
TABLE 8

REFERENCES-INTENSITY

\begin{tabular}{|c|c|c|c|c|c|}
\hline $\mathrm{Sp}$ & Reference Numbers & $\mathrm{Sp}$ & Reference Numbers & $\mathrm{Sp}$ & Reference Numbers \\
\hline He I & 263275174116319 & $A_{\text {II }}$ & 340 & $\mathrm{Ru} \mathrm{II}$ & 272 \\
\hline $\mathrm{Li} I$ & 113175 & A III & $49 \quad 51$ & $\mathrm{Rh}_{\mathrm{I}}$ & $10 s$ \\
\hline $\mathrm{Li}_{\text {II }}$ & 378404 & A IV & 50 & $\mathrm{Rh}_{\text {II }}$ & 150 \\
\hline $\mathrm{Be} \mathrm{I}$ & 315318 & $\mathrm{~K}_{\mathrm{I}}$ & $259116 \quad 88108$ & $\mathrm{Pd} L$ & 256 \\
\hline Be II & 318 & $\mathrm{~K}_{\text {II }}$ & 41046 & $\mathrm{Ag} \mathrm{I}$ & 389 \\
\hline B II & 81 & $\mathrm{~K}$ III & $47 a 51 a$ & $\mathrm{Cd}_{\mathrm{I}}$ & 173 \\
\hline B III & 81 & $\mathrm{Ca} \mathrm{I}$ & 207259369241374 & $\operatorname{In}_{I}$ & 402 \\
\hline $\mathrm{C}_{\mathrm{I}}$ & 185300169160370 & Ca II & $259207375390 \quad 66$ & $\mathrm{Sn}_{\mathrm{I}}$ & 265 \\
\hline $\mathrm{C}_{\text {II }}$ & 81122 & $\mathrm{Ca}$ III & 11 & Sn II & 248 \\
\hline $\mathrm{C}_{\text {III }}$ & 81 & Sc I & 210253349367 & $\mathrm{Sb}_{\mathrm{I}}$ & 279 \\
\hline Civ & 81 & Sc II & 253365 & $\mathrm{Te}_{\mathrm{I}}$ & 189 \\
\hline $\mathrm{N}_{\mathbf{I}}$ & $93 \quad 78 \quad 160370$ & Sc III & 172 & Cs I & 259245 \\
\hline$N$ II & 20125120 & $\operatorname{Ti}_{\mathbf{I}}$ & 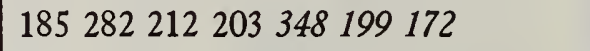 & $\mathrm{Ba} \mathrm{I}$ & 207259 \\
\hline $\mathrm{N}$ iII & 12481 & Ti II & 347 & $\mathrm{Ba}$ II & 207259330 \\
\hline $\mathrm{N}$ iv & 81 & Ti Iv & 364 & La I & 228258 \\
\hline $\mathrm{O}_{\mathrm{I}}$ & 98263127116128 & $V_{\text {I }}$ & 264213204 & La II & 258 \\
\hline O II & 20118301 & $V_{\text {II }}$ & 266 & La III & 258 \\
\hline $\mathrm{O}_{\text {III }}$ & 11986302 & Cr I & 183184204213 & $\mathrm{Ce}$ II & $215144 \quad 9245$ \\
\hline $\mathrm{O}_{\text {IV }}$ & 81126 & Cr II & 184 & Ce III & 362 \\
\hline $\mathrm{Ov}$ & 81 & $\mathrm{Mn}$ I & 26020913161113 & Pr II & 215339 \\
\hline $\mathrm{O}$ vi & 81 & Mn II & 6768108 & Nd in & 21910 \\
\hline $\mathrm{F}_{\text {I }}$ & 88 & $\mathrm{Fe}_{\mathrm{I}}$ & 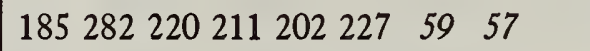 & Sm I & 221 \\
\hline $\mathrm{F}_{\text {II }}$ & 73 & & $\begin{array}{llllllll}281 & 280 & 55 & 15 & 77 & 391 & 171 & 74\end{array}$ & Sm II & 221 \\
\hline F III & 7285 & $\mathrm{Fe}$ II & 7622255 & $\mathrm{Eu} \mathbf{I}$ & 223 \\
\hline $\mathrm{Ne} \mathbf{I}$ & 263276311 & Fe III & 103102 & Eu II & 223 \\
\hline $\mathrm{Ne}$ II & 52 & Co $\mathrm{I}$ & $\begin{array}{lllllllll}205 & 208 & 282 & 280 & 71 & 56 & 230 & 62 & 147\end{array}$ & $\mathrm{Gd} \mathrm{I}$ & 225 \\
\hline $\mathrm{Na}$ & 259172116 & Co II & 255 & Gd II & 225 \\
\hline $\mathrm{Na}$ II & 130393 & $\mathrm{Ni} \mathrm{I}$ & $208205282 \quad 143109146280398245$ & $\operatorname{Tm} \mathrm{I}$ & 268 \\
\hline $\mathrm{Mg} \mathrm{I}$ & 261207295314170 & $\mathrm{Ni}$ II & 252382 & Tm II & 269268224 \\
\hline $\mathrm{Mg}$ iI & 116 & $\mathrm{Cu}_{\mathrm{I}}$ & 58 & $\mathrm{Yb}_{\mathrm{I}}$ & 291 \\
\hline $\mathrm{Al}$ & 315116319310 & $\mathrm{Cu}$ II & 137 & $\mathrm{Yb}_{\text {II }}$ & 291 \\
\hline $\mathrm{Al}$ iI & 319377 & $\mathrm{Zn}_{\mathrm{I}}$ & 149 & $\mathrm{Lu}$ I & 288 \\
\hline $\mathrm{Al}$ III & 312 & $Z_{\mathrm{n} \text { II }}$ & 372 & Lu II & 288 \\
\hline $\mathrm{Si}_{\mathrm{I}}$ & 186 & $\mathrm{Ga} I$ & 402174 & Hf I & 216 \\
\hline Si II & 117 & $\mathrm{Ge}_{\mathrm{I}}$ & 188132 & Hf II & 289 \\
\hline Si III & 117 & Ge II & 188235 & $\mathrm{Ta} \mathrm{I}$ & 200 \\
\hline Si IJ & 117101 & As I & 273 & $W_{I}$ & $218 \quad 22$ \\
\hline $\mathrm{P}_{\mathrm{I}}$ & 180 & As II & 328 & W II & 239 \\
\hline$P_{\text {II }}$ & 133181 & Se I & 189 & $\operatorname{Re} I$ & 257 \\
\hline$P_{\text {III }}$ & 133 & $\mathrm{Rb}_{\mathrm{I}}$ & 172 & Os I & 245 \\
\hline$P_{\text {iv }}$ & 133 & Sr $\mathrm{t}$ & 207259 & $\operatorname{Ir} \mathbf{I}$ & 6 \\
\hline $\mathrm{Pv}$ & 133 & Sr II & 207259 & Pt I & 109 \\
\hline$S_{\text {I }}$ & 297129 & $\mathrm{Y}_{\mathrm{I}}$ & 228 & $\mathrm{Au}_{\mathrm{I}}$ & 324 \\
\hline$S_{\text {II }}$ & $25 \quad 19136$ & $Y_{\text {II }}$ & 254351 & $\mathrm{Hg}$ I & 112108 \\
\hline$S_{\text {III }}$ & 157159 & $\mathrm{Zr}$ & 228282197 & $\mathrm{Tl} \mathrm{I}_{\mathrm{I}}$ & 109 \\
\hline S IV $_{\text {IV }}$ & 303 & $\mathrm{Zr}$ in & 196 & $\mathrm{~Pb}_{\mathrm{I}}$ & 109271 \\
\hline $\mathrm{Cl}_{\mathrm{I}}$ & 182 & $\mathrm{Cb}_{\mathrm{I}}$ & 283 & $\mathrm{Bi}$ & $\begin{array}{ll}308 \quad 17\end{array}$ \\
\hline $\mathrm{Cl}_{\text {II }}$ & 195 & $\mathrm{Cb}_{\text {II }}$ & 283 & $\mathrm{Ra}_{\mathrm{I}}$ & 332 \\
\hline $\mathrm{Cl}_{\text {III }}$ & $31 \quad 27$ & Mo I & 326176113 & $\mathrm{Ra}$ II & 331 \\
\hline$A_{\text {I }}$ & 263276293294 & $\begin{array}{l}\text { Mo II } \\
\text { Ru I }\end{array}$ & $\begin{array}{l}113 \\
251109\end{array}$ & Th II & 226 \\
\hline
\end{tabular}


TABLE 9

REFERENCES-ANALYSIS

\begin{tabular}{|c|c|c|c|}
\hline $\mathrm{Sp}$ & Reference Numbers & $\mathrm{Sp}$ & Reference Numbers \\
\hline $\mathrm{H}$ & 24323116 & $\mathrm{~S}_{\text {II }}$ & $\begin{array}{lll}157 & 19158 & 25136\end{array}$ \\
\hline $\mathrm{D}$ & 24323 & $\mathrm{~S}$ III & 157159136337 \\
\hline $\mathrm{He} \mathrm{I}$ & 1645319263275134 & $\mathrm{~S}$ iv & $\begin{array}{lll}303 & 27 & 29\end{array}$ \\
\hline He II & 24323 & $\mathrm{Cl}$ I & $182193 \quad 92$ \\
\hline $\mathrm{Li}$ I & 11616206 & $\mathrm{Cl}$ II & 19596 \\
\hline $\mathrm{Li}$ II & 1681 & $\mathrm{Cl}$ III & 3127 \\
\hline $\mathrm{Be} I$ & 318315 & $\mathrm{Cl}$ iv & 3132 \\
\hline Be II & 318 & $\mathrm{~A}_{\mathrm{I}}$ & $154276 \quad 42 \quad 16293 \quad 294$ \\
\hline B I & 1689 & A II & $\begin{array}{llll}92 & 42 & 48 & 47\end{array}$ \\
\hline B II & 8189 & $A$ III & $\begin{array}{lllll}49 & 51 & 42 & 44 & 96\end{array}$ \\
\hline B III & 81 & A IV & 4250 \\
\hline $\mathrm{C}_{\mathrm{I}}$ & 81300 & $\mathrm{~K}$ I & 11688 \\
\hline $\mathrm{C}_{\text {II }}$ & 8189 & $\mathrm{~K}$ II & $46 \quad 28105$ \\
\hline $\mathrm{C}_{\text {III }}$ & $\begin{array}{lll}81 & 89 & 82\end{array}$ & $\mathrm{~K}$ iII & 47a 92 \\
\hline Civ & 8182 & $\mathrm{Ca} \mathrm{I}$ & 373369259116 \\
\hline $\mathrm{N}_{\mathrm{I}}$ & 10478160 & Ca II & 375346390 \\
\hline$N_{\text {II }}$ & 8981125120 & Ca III & $28 \quad 16$ \\
\hline $\mathrm{N}$ III & 8981 & Sc I & 365 \\
\hline $\mathrm{N}_{\text {IV }}$ & $\begin{array}{lll}89 & 81 & 82\end{array}$ & Sc II & 365285 \\
\hline $\mathrm{N} v$ & 8182 & Sc III & 364392 \\
\hline $\mathrm{O}_{\mathrm{I}}$ & 98 & Ti I & 348355282185178 \\
\hline $\mathrm{O}$ II & $86 \quad 81350118$ & Ti II & 347 \\
\hline $\mathrm{O}$ III & $\begin{array}{lllll}95 & 81 & 119 & 86 & 302\end{array}$ & Ti iv & 364 \\
\hline O IV & 8182 & VI & 286305 \\
\hline $\mathrm{Ov}$ & 8182 & V II & 284 \\
\hline O vi & 8182 & $\mathrm{Cr}$ & 179183187346 \\
\hline $\mathrm{F}_{\mathrm{I}}$ & 88 & Cr II & 184 \\
\hline F II & 8573 & $\mathrm{Mn} \mathrm{I}$ & 6179260346 \\
\hline $\mathrm{F}$ III & $\begin{array}{lll}85 & 30 & 72\end{array}$ & Mn II & $67 \quad 68$ \\
\hline$F_{\text {IV }}$ & 84 & $\mathrm{Fe} I$ & $363 a$ \\
\hline F vI & 8387 & $\mathrm{Fe}$ II & $\begin{array}{llll}75 & 76 & 94 & 139\end{array}$ \\
\hline $\mathrm{Ne} I$ & $\begin{array}{llll}154 & 41276 & 16311\end{array}$ & $\mathrm{Fe}$ III & 103102 \\
\hline $\mathrm{Ne}$ II & 4152 & Co I & 363 \\
\hline $\mathrm{Na} I$ & 298338116 & Co II & 114 \\
\hline $\mathrm{Na}$ II & 13039316 & $\mathrm{Ni}$ I & 352 \\
\hline $\mathrm{Mg}$ I & 295296314345 & $\mathrm{Ni}$ II & 382233 \\
\hline $\mathrm{Mg}$ II & 11616 & $\mathrm{Cu}$ & 381 \\
\hline $\mathrm{Al}$ I & 315319 & $\mathrm{Cu}$ II & 386 \\
\hline $\mathrm{Al}$ II & 319377 & $\operatorname{Zn} \mathrm{I}$ & 149116 \\
\hline $\mathrm{Al}$ III & 31216 & $\mathrm{Zn} \mathrm{II}$ & 319372 \\
\hline $\mathrm{Si}$ I & 186 & $\mathrm{Ga} I$ & 116 \\
\hline Si II & $\begin{array}{lll}117 & 29 & 16\end{array}$ & Ga II & 376 \\
\hline Si III & $117 \quad 29$ & Ge I & 188327 \\
\hline Si Iv & 117101 & Ge II & 188234 \\
\hline $\mathrm{P}_{\mathrm{I}}$ & 180335 & As I & 273 \\
\hline$P_{\text {II }}$ & 26335336 & As II & 140328 \\
\hline$P_{\text {III }}$ & 29303336 & Se I & 343 \\
\hline$P_{\text {IV }}$ & 29336 & Se II & 244 \\
\hline P v & 33640 & $\mathrm{Br} I$ & 194 \\
\hline$S_{I}$ & 297342 & Br II & 232 \\
\hline
\end{tabular}


TABLE 9-Continued

RefERENCES-ANALYsis

\begin{tabular}{|c|c|c|c|}
\hline $\mathrm{Sp}$ & Reference Numbers & $\mathrm{Sp}$ & Reference Numbers \\
\hline $\begin{array}{l}\mathrm{Kr}_{\text {I }} \\
\mathrm{Kr}_{\text {II }} \\
\mathrm{Rb}_{\text {I }} \\
\mathrm{Rb}_{\text {II }} \\
\mathrm{Sr}_{\mathrm{I}} \\
\mathrm{Sr}_{\text {II }} \\
\mathrm{Y}_{\text {I }} \\
\mathrm{Y}_{\text {II }} \\
\mathrm{Zr}_{\text {r }} \\
\mathrm{Zr}_{\text {II }}\end{array}$ & $\begin{array}{lll}274 & 276 & 165 \\
53 & & \\
116 & & \\
238 & & \\
116 & 369 & 399 \\
116 & 259 & 399 \\
285 & & \\
285 & & \\
197 & 282 \\
196 & & \end{array}$ & $\begin{array}{l}\text { Gd I } \\
\text { Gd II } \\
\text { Tm I } \\
\text { Tm II } \\
\text { Yb I } \\
\text { Yb II } \\
\text { Lu I } \\
\text { Lu II } \\
\text { Hf I } \\
\text { Hf II }\end{array}$ & $\begin{array}{l}2357358 \\
\quad 8358 \\
270 \\
270269 \\
291359 \\
291359 \\
288267 \\
288 \\
287 \\
289\end{array}$ \\
\hline 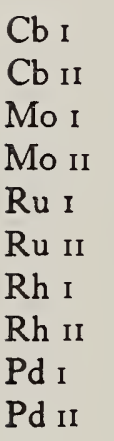 & $\begin{array}{l}290 \\
290 \\
63191 \\
190 \\
394145 \\
272292 \\
395 \quad 304 \\
150 \\
385 \\
384 \quad 24\end{array}$ & $\begin{array}{l}\text { Ta I } \\
\text { Ta II } \\
W_{\text {I }} \\
W_{\text {II }} \\
\text { Re I } \\
\text { Os I } \\
\text { Ir I } \\
\text { Pt I } \\
\text { Pt II } \\
\text { Au I }\end{array}$ & $\begin{array}{lr}192 & \\
192 & \\
237 & \\
239 & \\
257 & \\
1 & 5 \\
6 & \\
240 & 148 \\
387 & \\
324 & 306\end{array}$ \\
\hline $\begin{array}{l}\mathrm{Ag} \text { I } \\
\mathrm{Ag} \text { II } \\
\mathrm{Cd}_{\mathrm{I}} \\
\mathrm{Cd} \text { II } \\
\text { In I } \\
\text { In II } \\
\text { Sn I } \\
\text { Sn II } \\
\text { Sb I } \\
\text { Sb II }\end{array}$ & $\begin{array}{ll}388 & \\
383 & 24 \\
116 & 16 \\
372 & 401 \\
116 & \\
317 & \\
265 & \\
248 & \\
279 & \\
236 & \end{array}$ & $\begin{array}{l}\mathrm{Au} \text { II } \\
\mathrm{Hg} \text { I } \\
\mathrm{Hg} \text { II } \\
\mathrm{Tl}_{\text {I }} \\
\mathrm{Tl} \text { II } \\
\mathrm{Pb}_{\text {I }} \\
\mathrm{Pb}_{\text {II }} \\
\mathrm{Bi}_{\mathrm{I}} \\
\mathrm{Bi} \text { II } \\
\mathrm{Rn}_{\mathrm{I}}\end{array}$ & $\begin{array}{c}324 \\
16 \\
313 \\
116 \\
106 \\
16 \\
80 \\
16 \\
64115 \\
329\end{array}$ \\
\hline $\begin{array}{l}\text { Te I } \\
\text { I I } \\
\text { I II } \\
\text { Xe I } \\
\text { Xe II } \\
\text { Cs I } \\
\text { Cs II } \\
\text { Ba I } \\
\text { Ba II } \\
\text { La I }\end{array}$ & $\begin{array}{l}341 \\
107 \quad 69325 \\
232 \\
156 \\
155 \\
116 \\
309405 \\
400344353 \\
330400 \\
366\end{array}$ & $\begin{array}{l}\mathrm{Ra}_{\mathrm{I}} \\
\mathrm{Ra}_{\mathrm{II}} \\
\mathrm{Th} \text { II } \\
\text { Th III }\end{array}$ & $\begin{array}{l}332354 \\
331 \\
249250 \\
54\end{array}$ \\
\hline $\begin{array}{l}\text { La II } \\
\text { La III } \\
\text { Ce II } \\
\text { Ce III } \\
\text { Pr II } \\
\text { Nd II } \\
\text { Sm I } \\
\text { Sm II } \\
\text { Eu I } \\
\text { Eu II }\end{array}$ & $\begin{array}{rrrr}366 & & \\
366 & & \\
144 & 7 & 9 \\
362 & & \\
339 & 356 & \\
10 & & \\
2 & 4 & \\
3 & & \\
361 & & \\
360 & & \end{array}$ & & \\
\hline
\end{tabular}


TABLE 10

REFERENCES-FoRBIDDEN LINES

\begin{tabular}{|c|c|c|c|c|c|}
\hline $\mathrm{Sp}_{\mathrm{p}}$ & Reference Numbers & $\mathrm{Sp}$ & Reference Numbers & $\mathrm{Sp}$ & Reference Numbers \\
\hline $\mathrm{Be} \mathrm{I}$ & $318 \cdot 315$ & Ca vi & 33 & Fe xiv & 97 \\
\hline $\mathrm{C}_{1}$ & 81 & Ca viI & 337 & $\mathrm{Fe} \mathrm{xv}$ & 90 \\
\hline $\mathrm{N}_{\text {I }}$ & 104 & $\mathrm{Ca}$ XII & 97 & Co II & 114 \\
\hline$N$ II & $81 \quad 43409$ & Ca XIII & 97 & Co vi & 37 \\
\hline $\mathrm{O}_{\mathrm{I}}$ & $98 \quad 13 \quad 151$ & $\mathrm{Caxv}$ & 97 & Co vil & 12 \\
\hline $\mathrm{O}_{\text {II }}$ & 81409 & Sc II & 365 & Co viII & 60 \\
\hline $\mathrm{O}_{\mathrm{III}}$ & 81409 & Sc III & 364 & Co xI & 92 \\
\hline$F_{\text {II }}$ & 85 & Sc vi & 91231 & $\mathrm{Ni}_{\mathrm{I}}$ & 352 \\
\hline F III & 85 & Sc vil & 231 & $\mathrm{Ni}$ II & 382233 \\
\hline F IV & 84 & Ti 1 & 348 & $\mathrm{Ni}$ viI & 322 \\
\hline $\mathrm{Ne}$ III & $41 \quad 43409$ & Tin & 347 & $\mathrm{Ni}$ vilI & 12 \\
\hline $\mathrm{Ne}$ IV & 320 & Ti III & 364 & $\mathrm{Ni} \mathrm{Ix}$ & 60 \\
\hline $\mathrm{Ne} v$ & $100320 \quad 35397$ & Ti vII & 23191 & $\mathrm{Ni}$ xII & 97 \\
\hline $\mathrm{Na}$ IV & 393 & Ti vilI & 231 & Ni xiII & 97 \\
\hline $\mathrm{Na} v$ & 393 & $\mathrm{~V}_{\text {II }}$ & 284 & $\mathrm{Ni} \mathrm{xv}$ & 97 \\
\hline Mg vi & 393 & V III & 407 & $\mathrm{Ni} x v i$ & 97 \\
\hline $\mathrm{Al}$ vII & 393 & V IV & 406 & $\mathrm{Cu}_{\text {II }}$ & 386 \\
\hline Si I & 186 & V viII & 91 & $\mathrm{Kr}$ III & 152 \\
\hline$P_{1}$ & 180335 & $\mathrm{Cr}_{\mathrm{I}}$ & 179183187 & Sr II & 116259399 \\
\hline$P_{\text {II }}$ & 26335336 & $\mathrm{Cr}$ II & 184 & $Y_{\text {II }}$ & 285 \\
\hline$S_{\text {I }}$ & 9634233 & Cr III & 36 & $\mathrm{Yv}$ & 321 \\
\hline S II & 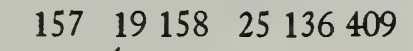 & Cr Iv & 3638 & $\mathrm{Zr}$ II & 196 \\
\hline S III & 157159136337 & Cr v & 406 & $Z_{\text {r III }}$ & 198 \\
\hline S vilI & 97337 & Cr viII & 92 & $Z_{r}$ vi & 321 \\
\hline S XII & 97 & Cr Ix & 91 & $\mathrm{Xe}$ II & 155 \\
\hline $\mathrm{Cl}$ II & 96195 & Mn II & 6768 & $\mathrm{Xe}$ III & 99153 \\
\hline $\mathrm{Cl}$ III & 3127 & Mn IV & 36 & La II & 366 \\
\hline $\mathrm{Cl}$ Iv & 3132 & $\mathrm{Mn} v$ & $34 \quad 38$ & La III & 366 \\
\hline A III & 96 & Mn vi & 60 & Eu II & 360 \\
\hline A IV & 4250 & Mn Ix & 92 & & \\
\hline $\mathrm{Av}$ & 323334 & $\operatorname{Mn} x$ & 91 & & \\
\hline$A x$ & 97 & $\mathrm{Fe}_{\mathrm{I}}$ & $363 a$ & & \\
\hline A XI & 97 & $\mathrm{Fe}$ II & $\begin{array}{llll}75 & 76 & 94 & 139\end{array}$ & & \\
\hline A XIV & 97 & $\mathrm{Fe}$ III & 103102 & & \\
\hline $\mathrm{K}$ IV & 9633 & $\mathrm{Fe} \mathrm{v}$ & 36 & & \\
\hline $\mathrm{K} v$ & 33 & $\mathrm{Fe}$ vi & $34 \quad 38$ & & \\
\hline K vI & 337408334 & $\mathrm{Fe}$ viI & 39 & & \\
\hline $\mathrm{CaI}$ & 373369259116 & $\mathrm{Fe} x$ & $92 \quad 97$ & & \\
\hline Ca II & 375 & $\mathrm{Fe} \mathrm{XI}$ & 9197 & & \\
\hline $\mathrm{Cav}$ & $\begin{array}{lll}96 & 33 & 91\end{array}$ & Fe XIII & 97 & & \\
\hline
\end{tabular}




\section{ACKNOWLEDGMENTS}

This book is the result of an enormous amount of cooperation for which the writer cannot express adequate appreciation. She is extremely grateful to all who have generously furnished material, offered valuable suggestions, and assisted in many other ways.

In spite of war conditions both M. A. Catalán of Madrid and B. Edlén of Lund have been active collaborators. At home, physicists, astronomers, librarians and many others have likewise contributed. To each of these the writer extends most hearty thanks.

At the Mount Wilson Observatory, W. S. Adams has willingly assembled interested members of his staff for consultation concerning the form, content and scope of the book. H. D. Babcock's unpublished solar material has been of inestimable value. A. S. King has obligingly settled many puzzling questions about intensities. P. W. Merrill has urged the publication of the book from the start and supported the work enthusiastically. A. H. Joy has furnished detailed material on stellar spectra observed at Mount Wilson.

The writer has also been in constant communication with the Bureau of Standards. W. F. Meggers and C. C. Kiess have furnished more material on analysis in advance of publication than any other contributors. Special mention should be made of the valuable work on $C r$ I and $C r$ I by Kiess. The multiplet lists of these spectra have already been in constant demand. . Many intricate details have been referred to Meggers. His continued interest and work on behalf of this book will not be forgotten.

In addition to the help with the forbidden lines, P. Swings together with O. Struve at the Yerkes Observatory have given most helpful assistance in the handling of the spectra of light elements from an astrophysical point of view.

Mention has already been made of the computations generously furnished by J. E. Mack.

The writer has been privileged to use the facilities of various institutions not directly connected with this program. At Wesleyan University the late Professor Slocum welcomed her most cordially to the Van Vleck Observatory. W. G. Cady, Chairman of the Department of Physics, was equally generous. Without the library privileges extended by these friends, the program would have been seriously impaired.

A welcome no less cordial has been extended in Cambridge, Massachusetts. J. C. Boyce, Harlow Shapley and Mrs. C. P. Gaposchkin have taken a most personal interest in the work. It has also been an enormous advantage to have free access to the libraries at the Cruft Laboratory, at the Massachusetts Institute of Technology and at the Harvard Observatory.

At Princeton this research program has been most enthusiastically supported by Henry Norris Russell over the long period of years since the publication of the first edition in 1933. The writer has had the great benefit of his wide experience in analyzing spectra, of his detailed knowledge of spectra and above all of his keen interest-all of which are recorded in the pages of the book itself. She expresses to him her gratitude with the hope that the readers will find the R M T worthy of all he has contributed to it.

Miss Margaret C. Shields of Fine Hall library in Princeton has cooperated so extensively that it is inadequate to express the personal gratitude of the writer in a single sentence. The same is true of Mrs. Jay Murray who has patiently and efficiently brought to a successful conclusion the appalling task of typing the Multiplet Table for photographic reproduction. Her painstaking care and skill are largely responsible for the completion of the work at this time.

Miss Marion Daly at Princeton and Miss Ada Spaterna of Washington, D. C., have spared no effort in typing the Finding List carefully and accurately.

The personal interest and help of President John A. Eckert of the Columbia Planograph Company have been one of the greatest sources of encouragement in completing the manuscript for publication.

In conclusion the writer wishes to record her appreciation of the cordial cooperation of her husband, Bancroft W. Sitterly. 
Albertion, Phyrs. Rev. (3) $45,304,1934$.

Albertson, Phys. Rev. (3) 47, 370, 1935.

3 Albertson, Yt. Filson Contr. No. 546; ㅅ․ J. 84, 36, 1936.

Albertson, Phys. Rev. (3) $53,644,1937$.

Albertson, Phys. Rev. (3) $\underline{53}, 940,1938$.

Albertson, Phys. Rev. (3) 54, 183, 1938.

Albertson, Undublished ater1al, Nov. 1941.

Albertson, Bruynes and Hanau, Phys. Rev. (a) $57,393,1940$.

Albertson and Harrison, Phys. Rev. (3) 53, 1309, 1937.

Albertson, Harr1son and Mclially, Jr., Phys. Rev. (3) 61, 167, 1943.

Anderson, J. A., Ap. J. 59, 76, 1934 .

Anderson, E. E. and Mack, Phys. Rev. (3) $\underline{59}, 717,1941$.

Babcock, Mt. W11son Contr. No. 359; AD. J. $\underline{57}, 309,1933$.

Babcock, Mt. F118on Contr. No. 343 ; AD. J. 66, $356,1937$.

Babcock, Unpublished materlal.

See Bacher and Goudmm1t, Atom1c Energy States, McGram-H111, N. I. and London, 1933.

Back und Goudsm1t, ze1t. F. Phys. 47, 174, 1938.

8 Bely, de Bruin, Bloch, L., Bloch, E., meas. quoted by Rosenthal, Ann. der Phyg. (5) $4.49,1930$.

9 Bartelt und Eckste1n, Ze1t. 1. Phys. 86, 77, 1933.

Beals, Publ. Dom. Ap. Obs. $6,17,1931$.

Behner, Ze1t. 1. 118s. Ptg. 33, 325, 1925.

Belke, Ze1t. 1. H1ss. Ptg. 17, 132 and 145, 1918.

B1rge, Rev. Lod. Phys. 13, 333 (Ho. 4), 1941.

Bla1r, Phrs. Rev. (a) 36, 173, 1930.

Bloch, L. et Bloch, E., Annsles de Physlaue (10) 12, 5, 1929.

Bowen, Phys. Rev. (3) $29,510,1927$.

Bowen, Phrs. Rev. (2) 31, 34, 1938.

Bowen, Phys. Rev. (3) 31, 497, 1938.

Bowen, Phrs. Rer. (3) $39,8,1932$.

Bowen, Phys. Rev. (3) $45,83,1934$.

Bowen, Phys. Rev. (3) $45,401,1934$.

Bowen, Phys. Rer. (2) 46, 377, 1934.

Bowen, Phys. Rev. (3) 46, 791, 1934.

Bowen, Phys. Rev. (2) 47, 934, 1935.

Bowen, Rev. Mod. Phys. 8, 55 (No. 2), 1936.

Bowen, Phys. Rev. (3) $53,1153,1937$.

Bowen, Phrs. B.ev. (2) $53,889,1938$.

Bowen, see Pasternack, Ap. J. $93,140,1940$.

Bowen and Edlén, Nature 143, 374, 1939.

Bowen and M1likkan, Phys. Rev. (2) 25, 295, 1935.

1 Boyco, Phys. Rer. (3) 46, 378, 1934.

Boyce, Phys. Rev. (3) 48, 396, 1935.

3 Boyce, Uon. Hot. Royal Astr. Soc. 96, 690 (No. 7), 1936.

4 Boyce, phre. Rev. (a) $49,351,1936$.

5 Boyce and Robinson, Journ. Oot. Soc. Am. 36, 143, 1936.

6 de Bruin, Ze1t. 1. Phys. 38, 94, 1926.

47 de Bruln, ze1t. 1. Phys. 51, 108, 1938.

47 de Bruln, ze1t. 1. Phy $.53,658,1939$.

48 de Bruln, Ze1t. 1. Phys. 61, 307, 1930.

49 de Bruln, Proc. Amsterdam Aoad. 36, 734 (No. 7), 1933.

50 de Bruin, Phrales 3,809 (No. 8), 1936.

51 de Bruin, Proc. Amsterdam Acad. 40, 340 (No. 4), 1937.

51 de Bruin, see Edlén, ze1t. 1. Physe. 104, 407, 1937.

53 de Bruln und Bakker, 2e1t. 1. Physe. 69, 19, 1931.

53 do Bruin, Fumphreys and Yeggers, Bur. St. Journ. Req.11, 409 (RP 599) 1933.

54 do Bruin, Rlinkenberg und Schuurmans, ze1t. f. Phys. 118, 58, 1941.
55 Burns, L1ck Bul1. 8,37 (No. 347), 1913.

56 Burns, Unpublished moterial. For interferometer meas, see ReP. No. 363.

57 Burns and Malters, Jr., Publ. Allegheny Obs. 6, 159 (No. 11), 1939

58 Burne and Nalters, Jr., Publ. Allegheny Obs 8,27 (No. 3), 1930.

59 Burns and Falters, Jr., Publ. Allegheny Obs. $\underline{8}, 39$ (Ho. 4), 1931.

60 Cady, N11loughby, Phys. Rev. (3) $43,333,1933$.

61 Catalán, Ph1l. Trans. Royal Soc. A 333, 137, 1933.

63 Catslán y Antunes, Ann. de 1a Soc. Esp. de F181ca y Qu1m1ca 34, 207, 1936

(6ome measures by Exner and Haschek included here)

63 Catalán y Ladar1aga, Ann.de la Soc. Egp. de F1s1ca y Qu1m1ca 31, 707, 1933

64 Crawford and YcLay, Proc. Royal Soc. A $143,540,1934$.

65 Crew, Ap. J. 60, 108, 1924.

66 Crew and McCauley, Ap. J. 39, 29, 1914.

67 Curt18, Phys. Rev. (a) $53,474,1938$.

68 Curt18, Unpublished mater1al, July 1941.

69 Dob, Proc. Royal Soc. A 139, 380, 1933.

70 Déjard1n, Cansa1an Journ. Res. ?, 556, 1932.

71 Dhe1n, Ze1t. P. W168. Ptg. 19, 389, 1930.

73 Dingle, Proc. Royal Soc. A 123, 144, 1939.

73 Dingle, Proc. Royal Soc. A $128,600,1930$.

74 Dingle, Mon. Not. Royel Astr. Soc. 94, 866, 1934.

75 Dobble, Phys. Rev. (3) $\underline{45}, 76,1934$.

76 Dobble, Ann. Solar Phys. Obs. 5, 1 (Part I), 1938.

77 Dobble, Unpublished mater1al.

78 Duffendack and Wolfe, Phys. Rev. (3) 34, 409, 1939.

79 Dunham, T. Jr., Unpublished mater1al, 1926.

80 Earls and Sawyer, Phys. Rev. (3) 47, 115, 1935.

81 Edlen, Nova Acta Reglae Soc1etat1s Sc1ent1arum Upsallens18, Ser. IV, $\underline{9}$ (No. 6), 1933.

83 Ealén, Ze1t. 1. Ap. ?, 378, 1933.

83 Edlén, Ze1t. 1. Phys. 89, 179, 1934.

84 Edlén, ze1t. 1. Phys. 93, 19, 1934.

85 Edlén, Ze1t. 1. Phys. 93, 433, 1935.

86 Edlén, Ze1t. 1. Phys. 93, 726, 1935.

87 Edlén, ze1t. 1. Phys. 94, 47, 1935.

88 Edlén, zelt. f. Phys. 98, 445, 1936.

89 Edlén, Ze1t. 1. Phys. 98, 561, 1936.

90 Edlén, ze1t. 1. Phys. 103, 536, 1936.

91 Edlén, ze1t. 1. Phys. 104, 188, 1937.

92 Edlén, zelt. 1. Phys. 104, 407, 1937.

93 Edlén, Festskr1ft T1llägned, Östen Bergstrand p. 135, 1938.

94 Edlén, Unpublished materlal, Feb. 1940.

95 Edlén, Baturw188. 30, 379, 1942.

96 Edzén, Phys. Rev. (3) 63, $434,1943$.

97 Edlén, ze1t. 1. Ap. 23, 30, 1942; see Swings, AD. J. 98, 116, 1943.

98 Edlén, Kungl. Svenska Vetenskapsakademlens Handl1ngar, Tredje Serien, 20 (No. 10), 1943.

99 Edlen, Phys. Rev. (3) $65,348,1944$.

100 Edlén, Unpublished material.

101 Edlén und Söderqvist, Ze1t. \&. Pays. 87, 317, 1933.

103 Edlén and Swings, Unpublished mater1al, Dec. 1941.

103 Edlén and Sw1ngs, Contr. LCDonald Obs. No. 49; Ap. J. 95, 533, 1943.

104 Ekefors, ze1t. 1. Phys. 63, 437, 1930.

105 Ekefors, ze1t. 1. Phys. 71, 53, 1931.

106 Ell18 and Sawyer, Phys. Rev. (3) 49, 145, 1936.

107 Evans, Proc. Royal Soc. A 133, 417, 1931.

108 Exner und Haschek, see Kayser, Handbuch der Sp. ‥ 1910.

109 Exner und Haschek, see Rayser, Handbuch der Sp. 6, 1913. 
110 Exner und Haschek, ses Kayser und Konen, Handbuch der Sp. Z Part 1, 1934.

111 Exner and Haschek, soe Shsnstone, Phys. Rev. (a) 30, 255, 1927.

112 Exner und Haschek, ses Kayser und Konen, Handbuch der Sp. I Part 2, 1930.

113 Exner und Haschek, see Kayser und Konen, Handbuch der 8p. ?, Part 3, 1934.

114 Findlay, Phys. Rev. (a) $36,5,1930$.

115 F1sher and Goudem1t, Phys. Rev. (2) 37, 1057, 1931.

116 See Fowler, Report on Ser1es in Line Spectra, Fleetway Pres8, London, 1933.

117 Fowler, Ph1l. Trans. Royal Soo. A $\underline{225}, 1,1925$.

118 Fowler, Proc. Royal Soc. A $110,476,1936$.

119 Fowler, Proc. Royal Soc. A 117, 317, 1928.

130 Fowler and Freeman, Proc. Royal Soc. A 114, 663, 1927.

131 Fowler and Selwyn, Proc. Royal Soc. A 120, 313, 1928.

123 See Fowler and Belmyn, Proo. Royal soc. A $120,313,1938$.

133 Fred, AD. J. 87, 176, 1938.

124 Freeman, Proc. Royal Soc. A 121, 318, 1938.

125 Freeman, Proc. Royal Soc. A 124, 654, 1939.

136 See Freeman, Proc. Royal Soc. A.127, 330, 1930.

127 Frer1chs, Phys. Rev. (a) $34,1239,1929$.

128 Frer1chs, Naturw188. 21, 849, 1933.

129 Frer1chs, Ze1t. P. Phys. 80, 150, 1933.

130 Fr1sch, ze1t. 1. Phys. 70, 498, 1931.

131 Fuchs, see Kayser und Konen, Handbuch der Sp. I, Part 3, 1934.

132 Gartle1n, Phys. Rev. (2) $31,782,1938$.

133 Geuter, ze1t. P. W188. Ptg. $\underline{5}, 1$, 1907. (See Kayser, Handbuch der Sp. $\underline{6}, 1913$.

134 G1bbs and Kruger, Phys. Rev. (a) 37, 1559, 1931.

135 G1eseler und Grotrian, Ze1t. 1. Phyg. 34, 374, 1925.

136 G1lles, Annales de Physique (10) 15, 267, 1931.

137 Green, L.C., See Shenstone, Ph11. Trans. Royal Soc. A 235, 195 (No. 751), 1936.

138 Green, L.C., Unpublished mater1al, 1937.

139 Green, L.C., Phys. Rev. (2) 55, 1209, 1939.

140 Green, J.B. and Barrows, Jr., Phys. Rev. (2) 47, 131, 1935.

141 Gremmer, see Paschen, S1tz. der Prouss. Akad. dor M188., Phys.-Math. Klasse 32, 709, 1931.

142 Hall, see Kayser und Konen, Handbuch der Sp. I, Part 1, 1924.

143 Hamm, Ze1t. P. .188. Ptg. 13, 105, 1913.

144 Harrison, Albertson and Hosford, Journ. Opt. Soc. Am. 31, 430, 1941.

145 Harrison and McNally, Jr., Phys. Rev. (2) $\underline{58}, 703,1940$.

146 Hasselberg, see Kayser, Handbuch der $\mathrm{gp} . \underline{6}, 1912$.

147 Hasselberg or Exner und Haschek, ses Kayser, Handbuch der Sp. $\underline{\mathbf{5}}, 1910$.

148 Haussmann, Ap. J. 66, 333, 1927.

149 Hetzler, Boreman and Burns, Phys. Rev. (2) $48,656,1935$.

150 H1tchcock, W. J., Unpublished materlal, Nov. 1943.

151 Hoppleld, Phys. Rev. (2) 37, 160, 1931.

152 Humphreys, Phys. Rev. (2) 4 ?7, 712, 1935.

153 Humphreys, Bur. St. Journ. Res. 16, 639 (RP 898) 1936.

154 Hurphreys, Bur. St. Journ. Res. 20, 17 (RP 1061) 1938.

155 Humphreys, Bur. St. Journ. Re日. 22,19 (RP 1164) 1939.

156 Humphreys and Meggers, Bur. St. Journ. Res. 10, 139 (RP 521), 1933.

157 Hunter, Ph1l. Trans. Royal Soc. A $233,303,1934$.

158 Ingram, Phys. Rev. (2) $32,172,1928$.

159 Ingram, Phys. Rev. (3) 33, 907, 1929.

160 Ingram, Phys. Rev. (2) 34, 421, 1929.

161 International Standard, see Trans. Intern. Astr. Union $3,86,1928$.

162 International standard, see Trans. Intern. Astr. Union $5,85,1935$.

163 International Standard, see Trans. Intern. Astr. Un1on $6,79,1938$.

164 International Standard, se Trans. Intern. Astr. Union $6,80,1938$.

165 International standard, see Trans. Intern. Astr. Union $6,89,1938$.
166 Jackson, Proc. Royal Soc. A 130, 395, 1931.

167 Jackson, Proc. Royal Soc. A 133, 553, 1931.

168 Jackson, Mon. Not. Royal Astr. Soc. 93, 98 (No. 1), 1932.

169 Johnson or Merton and Johnson, see Kayser und Konen, Handbuch der Sp. 8 ,

Part 1, 1932.

170 See Kayser, Handbuch der sp. $5,1910$.

171 Kayser, Handbuch der $\mathrm{sp} .6,893,1912$.

172 See Kayser, Handbuch der sp. $6,1912$.

173 Soo Kayser und Konen, Handbuch der Sp. I, Part 1, 1924.

174 Sae Kayser und Konen, Handbuch der Sp. 7, Part 2, 1930.

175 See Kayser und Konen, Handbuch der Sp. ?, Part 3, 1934.

176 K1ess, Sc1. Papers Bur. 8t. 19, 113 (No. 474), 1923.

177 K1e88, Unpublished mater1al, 1934?.

178 K1e88, Bur: 8t. Journ. Res. 1, 75 (RP 4), 1938.

179 K1es8, Bur. St. Journ. Res. 5, 775 (RP 229), 1930.

$180 \mathrm{K1088}$, Bur. St. Journ. Res. 8, 393 (RP 425), 1932.

181 K1e88, Unpublished mater1al, May 1933.

$182 \mathrm{~K} 1088$, Bur. St. Journ. Res. 10, 827 (RP 570), 1933.

$183 \mathrm{~K} 1 \mathrm{es8}$, Bur. St. Journ. Res: 15, 79 (RP 812) 1935.

184 K1e88, Unpublished mater1al, Jan. 1936, June 1940, Mar. 1941, Apr. 1941.

185 K1088, Bur. St. Journ. Res. 220, 33 (RP 1062), 1938.

186 K1es8, Bur. 8t. Journ. Res. 31, 185 (RP 1124), 1938.

187 K1ess, Unpublished mater1al, Feb. 1939, June 1940, Feb. 1941.

188 K1e88, Bur. St. Journ. Res. 34, 1 (AP 1366), 1940.

189 X1088, Letter, June 1942.

$190 \mathrm{~K} 1088$, Unpublished mater1al, Aug. 1943.

191 K1e88, Unpublished material, Oot. 1942.

192 K1e88, Unpublished mater1al, Dec. 1942.

193 K1ess and de Bruin, Bur. St. Journ. Res. ‥ 1117 (RP 73), 1939.

194 K1ess and de Bruin, Bur. 8t. Journ. Res. 4, 667 (RP 173), 1930.

195 K1888 and de Bruin, Bur. St. Journ. Reg. 23, 443 (RP 1244), 1939.

$196 \mathrm{~K} 1 \mathrm{es8}, \mathrm{C} . \mathrm{C}$. and K1e88, H. K. , Bur. 8t. Journ. ReB. 5, 1205 (RP 255) 1830.

$197 \mathrm{~K} 1088, \mathrm{C}$. C. and K1e88, H. K., Bur. St. Journ. Res. 6, 621 (RP 296) 1931.

$198 \mathrm{~K} 1088$ and Lang, Bur. St. Journ. Reg. 5, 305 (RP 203), 1930.

$199 \mathrm{~K} 1888$ and Meggers, S01. Papers Bur. St. 16, 54 (No. 372), 1920.

200 K1e日s and Stowell, Bur. St. Journ. Res. 12, 459 (RP 671), 1934.

201 K1lby, Ap. J. 30, 243, 1909.

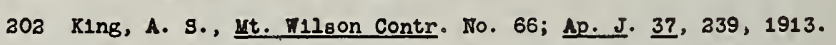

203 King, A. S., Mt. N118on Contr. No. 76; Ap. J. 39, 139, 1914.

204 King, A. S., 丝. Filson Contr. No. 94; Ap. J. 41, 86, 1915.

205 K1ng, A. S., ㅆt. F11son Contr. No. 108; Ap.J. 르, 347, 1915.

306 King, A. S., Mt. W1lioon Contr. No. 123; Ap. J. 44, 169, 1916.

307 K1ng, A. S., ㄴ. F1lion Contr. No. 150; Ap. J. 48, 13, 1918.

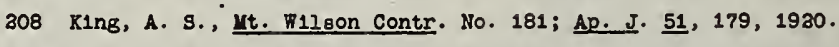

209 King, A. S., 省. W118on Contr. No. 198; Ap. J. 53, 133, 1931.

210 King, A. S., Lt. Filson Contr. No. 211; Ap. J. 54, 28, 1921.

311 King, A. S., Mt. M1lison Contr. No. 247; Ap. J. 56, 318, 1923.

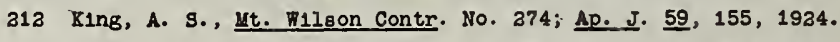

213 King, A. S., Mt. N1lion Contr. No. 283; Ap. J. 60, 282, 1924.

214 King, A. S., Unpublished mater1al, 19279.

215 King, A. S., Mt. \$1lson Contr. No. 368; Ap. J. 68, 194, 1928.

216 King, A. S., y. M1180n Contr. No. 384; Ap. J. 70, 105, 1939.

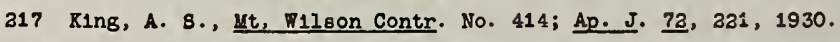

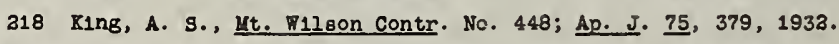

219 King, A. s., Mt. M1lson Contr. No. 470; Ap. J. 78, 9, 1933.

230 King, A. S., 贸. T1lion Contr. No. 496; Ap. J. 80, 124, 1934.

331 K1ng, A. S., 丝. F1lion Contr. No. 533; Ap. J. 83, 140, 1935.

238 King, A. 8., Yt. T1180n Contr. No. 584; Ap. I. 87, 109, 1938. 


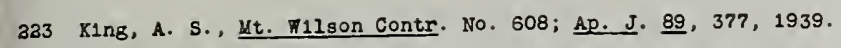

224 King, A. S., 帒. Tilison Contr. No. 651; Ap. J. 94, 336, 1941.

225 King, A. S., Mt. W118on Contr. No. 678; Ap. J. 97, 323, 1943.

$226 \mathrm{~K} 1 \mathrm{ng}, \mathrm{A} . \mathrm{S}$, , see S1tter1y and K1ng, Proc. Am. Ph1l. Soc. 86,339

$$
\text { (No. 3), } 1943 .
$$

227 King, A. S., Unpublished material.

228 King, A. S. and Carter, ㅆt. W1lson Contr. No. 326; Ap. J. 65, 86, 1927.

229 K1ng, A. S. and Moore, ‥ W11son Contr. No. 681; Ap. J. 98, 33, 1943.

230 Krebs, Ze1t. 1. M188. Ptg. 16, 293, 1917.

231 Kruger and Pattin, Phys. Rev. (2) $\underline{53}, 621,1937$.

232 Lacroute, Annales de Physique (11) 3 , 1, 1935.

233 Lang, Phys. Rev. (3) $31,773,1938$.

234 Lang, Proc. Nat. Acad. Sc1. 14, 33, 1938.

235 Lang, Phys. Rev. (3) $34,697,1939$.

236 Lang and Vestine, Phys. Rav. (2) $42,233,1932$.

237 Laporte and Mack, Phys. Rev. (2) $63,246,1943$.

238 Laporte, M1ller and Sawyer, Phys. Rev. (a) 38, 843, 1931.

239 Laun, Bur. St. Journ. Res. 21,207 (RP 1125), 1938.

240 L1vingood, Phys. Rev. (a) $34,185,1939$.

241 Lorenser, see Kayser und Konen, Handbuch der Sp. ?, Part 1, 1924.

242 Ludw1g, Ze1t. 1. H1s8. Ptg. 16, 157, 1917.

243 Mack, Unpubllahed materlal, June 1942.

344 Mart1n, Phys. Rev. (2) $48,938,1935$.

345 Mass. Inst. Tech., Wave Length Tables, W1ley, New York, 1939.

346 Us8s. Inst. Tech., Unpubl1shed mater1al, May 1941.

247 Nass. Inst. Tech., Unpubl1shed mater18l, June 1943.

248 McCormlck and Sanjer, Phys. Rev. (a) 54, 71, 1938.

249 Mclally, Jr., Unpublished materlal, Kay 1944.

250 MeNally, Jr., Harrison and Park, Journ. Opt. Soc. Am. 33, 334, 1943.

251 Messers, Sc1. Papers Bur. St. 20, 19 (No. 499), 1925.

252 Leggers (Bureau of Standards), see Shenstone, Phrs. Rev. 30, 355, 1927.

253 Megzers, Sc1. Papers_Bur. St. 223, 61 (No. 549), 1927.

254 Meggers, see Leggers and Russell, Bur. St. Journ. Res. 3 , 733 (RP 55), 1929.

255 Yeggers, see Findlay, Phys. Rev. (a) $36,5,1930$.

256 Megsers, see Shenstone, Phys. Rev. (3) 36, 669, 1930.

257 Leggers, Bur. St. Journ. Res. ․, 1027 (RP 32a), 1931.

258 Meggers, gee Russell and Meggers, Bur. St. Journ. Res. 9,635 (RP 497), 1933.

259 Yeggers, Bur. St. Journ. R8s. 10, 669 (RP 558), 1933.

260 Meggers, Bur. St. Journ. Res. 10, 75P (RP 564), 1933.

261 Meggers, Unpubl1shed mater1al, Feb. 1934.

268 Mesgers, Bur. St. Journ. RBs. 14, 33 (RP 755), 1935.

363 Meggers, Bur. St. Journ. Res. 14, 487 (RP 781) 1935.

264 Meggers, see Megzers and Russell, Bur. St. Journ. Res. 17, 125 (RP 906), 1936.

265 Meggers, Bur. St. Journ. Res. 24, 153 (RP 1375), 1940.

266 Meggers, see Leggers and Loore, Bur. St. Journ. Res. 25,83 (RP 1317), 1940.

367 Meggers, Journ. Opt. 8oc. Am. 31, 39, 1941.

368 Meggers, see King, A.S., yt. N118on Contr. No. 651; Ap. J. 94, 236, 1941.

369 Meggers, Unpublished mater1al, Nov. 1941

270 Meggers, Rev. Mod. Phjs. 14, 96, 1942.

371 Megsers, Unpublished mater1al, Jan. 1943.

273 Meggers, Unpublished materlal.

273 Meggers and de Bruin, Bur. St. Journ. Res. 3, 765 (RP 116), 1939.

374 Meggers, de Bruin and Humphreys, Bur. St. Journ Rsg. ?, 843 (RP 364), 1931.

375 Megsers and D1eks, Bur. 8t. Journ. Reg. 9, 131 (RP 483), 1932.
276 Meggers and Humphreys, Bur. St. Journ. Res. 10, 427 (RP 540), 1933

277 Yeggers and Humphreys, Bur. St. Journ. Res. 13, 293 (RP 710), 1934

278 Megsers and Humphreys, Bur. St. Journ. Res. 18, 543 (RP 993), 1937.

279 Msggers and Humphrejs, Bur. St. Journ. Res. 28, 463 (RP 1464), 1943.

380 Meggers and K1es8, Sc1. Papers Bur. St. 14, 637 (No. 334), 1918.

281 Meggers and K1ese, So1. Papers Bur. St. 19, 373 (No. 479), 1934.

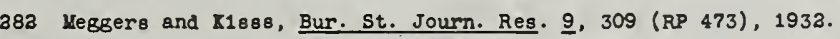

383 Meggers and Xing, Bur. St. Journ. Res. 16, 385 (RP 881), 1936.

284 Leggers and Loore, Bur. St. Journ. Res. 25, 83 (RP 1317), 1940.

385 Meggers and Russell, Bur. St. Journ. Res. ㄱ, 733 (RP 55), 1939.

286 Yegsers and Russell, Bur. St. Journ. Res. 17, 125 (RP 906), 1936.

387 Keggers and Scribner, Bur. St. Journ. Res. 4, 169 (RP 139), 1930

288 Leggers and Scribner, Bur. St. Journ. Res. 5,73 (RP 187), 1930.

389 Yegsers and Scr1bner, Bur. St. Journ. Res. 13, 625 (RP 732), 1934.

390 Keggers and Scribner, Bur. 8t. Journ. Res. 14, 629 (RP 793), 1935.

391 Meggers and Scribner, Bur. St. Journ. Res. 19, 651 (RP 1053), 1937.

293 Meggers and Shenstone, Phys. Rev. (3) $35,868,1930$.

293 Me1sener, Ze1t. 1. Phys. 39, 172, 1936.

294 Me1sener, Ze1t. 1. Phys. 40, 839, 1937.

295 Me1sener, Ann. der Phys. (5) $31,505,1938$.

296 Le1sener, Ann. der Phys. (5) 31, 518, 1938.

297 Me1sener, Bartelt und Eckste1n, Ze1t. 1. Phys. 86, 54, 1933.

298 Melsener und Luft, Ann. der Phys. (5) 29, 698, 1937.

399 Kerr1ll, Bull. Bur. St. 14, 159, 1918.

300 Merton and Johnson, see Fomler and Selmyn, Proc. Roysi Soc. A 118 , 34,1938 .

301 Mihul, Annales de Physique (10) 9, 294, 1938.

303 Mhul, Annales ds Physlque (10) $9,301,1928$ :

303 Mill1kan and Bowen, Phys. Rer. (3) $25,600,1925$.

304 Molnar and H1tchcock, Journ. Opt. Soc. Ám. 30, 523, 1940.

305 Loore, C. E., Phys. Rev. (a) $\underline{55}, 710,1939$.

308 Loore, C. E. and King, A. S., Publ. Astr. Soc. Pac111c 55, 27 (No. 333), 1943.

307 Narajan, Koda1kanal Obs. Bull. 4, 311 (No. 99), 1933.

308 Offermann, see Kayser und Konen, Handbuch der Sp. T, Part 1, 1924.

309 Olthoff and Sanyer, Phys. Rev. (3) $42,766,1932$.

310 Paschen, Ann. der Phys. (4) 29, 642, 1909

311 Paschen, Ann. der Phys. (4) 60, 405, 1919.

313 Paschen, Ann. der Phys. (4) 1ㅗ. 142, 1933.

313 Paschen, S1tz. der Prouss. Akad. der W188., Phys.-Nath. Klosse, $33,536,1938$.

314 Paschen, 81tz. dsr Preuss. Akad. der W18s., Phys.-Uath. Klasse, $33,709,1931$

315 Paschen, Ann. der Phys. (5) 12, 509, 1932.

316 Pasohen und Baok, Ann. der Phys. (4) 39, 897, 1912.

317 Paschen und Campbell, Ann. der Phys. (5) 31, 29, 1938.

318 Psschen und Zruger, Ann. der Phys. (5) $\underline{8}, 1005,1931$.

319 Paschen und R1techl, Ann. der Phyre. (5) 18, 867, 1933.

330 Paul and Polster, Phys. Rev. (3) $59,424,1941$.

331 Paul and Rense, Phys. Rev. (3) 56, 1110, 1939.

323 Ph1ll1ps and kruger, Phys. Rev. (3) 54, 839, 1938.

383 Ph1ll1ps and Parker, Phys. Rev. (2) 60, 301, 1941.

334 Platt and 8anyer, Phys. Rev. (a) $60,866,1941$.

325 Prlce, Phys. Rey. (a) 48, 477, 1935.

336 Puhlmann, 2e1t. 1. M188. Ptg. 17, 97, 1917.

337 Rao, Proc. Roral 800. A 134, 485, 1939.

338 Rao, Ind1an Journ. Phas. I, 581, 1933.

339 Rasmussen, Ze1t. 1. Phys. 80, 736, 1933. 
330 Rasmussen, ze1t. I. Phys. 83, 404, 1933.

331 Rarmussen, Ze1t. 1. Phys. 86, 24, 1933.

332 Rasmusssn, Ze1t. 1. Phys. 87, 607, 1934.

333 Re1nhe1mmer, Ann. der Phys. (4) 71, 162, 1923.

334 Rob1nson, Nature 137, 992, 1936.

335 Roblnson, Phys. Rev. (2) $49,297,1936$.

336 Robinson, Phys. Rev. (2) $\underline{51}, 736,1937$.

337 Roblnson, Phys. Rev. (2) $\underline{52}, 724,1937$.

338 Rood and Sawyer, Ap. J. 87, 68, 1938.

339 Rosen, Harr1son and McNally, Jr. , Phys. Rsy. (2) 60, 722, 1941.

340 Rosenthal, Ann. der Phys. (5) $4,49,1930$.

341 Ruedy, Phys. Rev. (3) $41,588,1932$.

343 Ruedy, Phys. Rev. (2) 44, 757, 1933.

343 Ruedy and G1bbs, Phys. Rev. (3) $46,880,1934$.

344 Russell, Mt. W11son Contr. No. 286; Ap. J. 61, 223, 1925.

345 Russell, Publ. Astr. Soc. Pac1e1c 38, 236, 1926.

346 Russell, Mt. T11son Contr. No. 342; Ap. J. 66, 233, 1927.

347 Russell, Mt. W1lson Contr. No. 344 ; Ap. J. 66, $383,1927$.

348 Russell, Mt. T1lson Contr. No. 345 ; Ap. J. 66, 347, 1927.

349 Russell, see Russell and Leggers, Sc1. Papers Bur. St. $\underline{22}, 329$

(No. 558), 1927.

350 Russell, Phys. Rev. (2) 31, 27, 1928.

351 Russell, see Meggers and Russell, Bur. St. Journ. Res. a. 733 (RP 55), 1929.

352 Russell, Phys. Rev. (2) $34,821,1929$.

353 Russell, see Meggers, Bur. St. Journ. Res. 10, 676, 684 (RP 558), 1933.

354 Russell, Phys. Rev. (2) $46,989,1934$.

355 Russell, Unpublished mater1al, Kay 1940.

356 Russell, Unpubllshed mater1al, Nov. 1941.

357 Russell, Mt. W1lson Contr. No. 663; Ap. J. 96, 11, 1942.

358 Russell, Unpublished materlal, 1943.

359 Russell, Unpublished materlal.

360 Russell, Albertson and Dav1s, phys. Rev. (2) 60, 641, 1941.

361 Russell and K1ng, A. S., 贸. W1lson Contr. No. 611; Ap. J. 90, 155, 1939.

362 Russell, K1ng, R. B. and Lang, Phys. Rev. (2) $\underline{52}, 456,1937$.

363 Russell, King, R. B. and Moore, Phys. Rsv. (2) $\underline{58}, 407,1940$.

363a Russell, Moore and Wesks, Trans. Am. Ph1l. Soc. 34, 111 (Part 2), 1944.

364 Russell and Lang, Mt. W1lson Contr. No. 337; Ap. J. 66, 13, 1927.

365 Russell and Meggers, Sc1. Papers Bur. St. $\underline{23}, 329$ (No. 558), $19 a 7$.

366 Russell and Neggers, Bur. St. Journ. Res. 9, 625 (RP 497), 1932.

367 Russell and Meggers, Unpublished matsrial.

368 Russell or Moore, see Meggers and Russell, Bur. St. Journ. Res. 17, 125 (RP 906) 1936.
369 Russell and Saunders, Ap. J. $61,38,1925$.

370 Ryde, Proc. Royal Soc. A 117, 164, 1927.

371 St. John and Babcock, Mt. W11son Contr. Ho. 202; Ap. J. 53, 260, 1921.

373 von Sal1s, Ann. der Phys. (4) 76, 145, 1925.

373 Saunders, Ap. J. $\underline{52}, 265,1220$.

374 Saunders, Unpublishsd material.

375 Sounders and Russell, Ap. J. 62, 1, 1925.

376 Sawyer and Lang, Phys. Rev. (2) 34, 712, 1929.

377 Sawyer und Paschen, Ann. der Phys. (4) 84, 1, 1927.

378 schüler, ze1t. 1 . Phys. $42,487,1927$.

379 schumacher, Ze1t. 1. W158. Ptg. 19, 149, 1919.

380 Segrs und Bakker, ze1t. 1. Phys. 72, 734, 1931.

381 Shenstone, Phys. Rev. (2) 28, 449, 1926.

382 Shenstone, Phys. Rev. (2) $30,255,1927$.

383 Shenstone, Phys. Rev. (2) $31,317,1928$.

384 Shenstone, Phys. Rev. (3) $32,30,1928$.

385 Shenstone, Phys. Rev. (3) 36, 669, 1930.

386 Shenstone, Phil. Trans. Royal Soc. A 235, 195 (Mo. 251), 1936.

387 Shenstone, Ph1l. Trans. Royal Soc. A $237,453,1938$.

388 Shenstone, Phys. Rev. (3) $\underline{57}, 894,1940$.

389 See Shenstone, Phys. Rey. (2) 57, 894, 1940.

390 Shenstone, Unpublished material.

391 Smith, Sincla1r, Unpublished material.

392 Smlth, Stanley, Proc. Wat. Acad. Sc1. 13, 65, 1927.

393 Södsrqvist, Nova Acta Reglae Soc1etat1s Sc1ent1arum Upsaliensis (IV) g (No. 7), 1934.

394 Sommer, Ze1t. P. Phys. 37, 1, 1936.

395 Sommer, Ze1t. 1. Phys. 45, 147, 1927.

396 See Stiles, Ap. J. 30, 48, 1909.

397 Stoy, L1ck Bull. 17, 179 (No. 480), 1935.

398 Stliting, see Kayser, Handbuch der Sp. ㅌ, 1912.

399 Sullivan, Un1v. P1ttsburgh Bull. 35, 1 (No. 1), 1938.

400 Sullivan and Burns, Sclence Studies 9, 7 (No. 3), 1941.

401 Takahash1, Ann. der Phys. (5) $3,27,1929$.

402 Uhler and Tanch, Ap. J. 55, 291, 1923.

403 Tagman, Un1v. P1ttaburgh Bu1l. 34, 1 (No. 1), 1937.

404 Werner, see Kayser und Konen, Handbuch der Sp. 7, Part 3, 1934.

405 Theatley and Sawyer, Phys. Rev. (2) 61, 591, 1942.

406 Wh1te, Phys. Rev. (2) $33,538,1929$.

407 White, Phys. Rev. (2) $33,672,1929$.

408 Th1tford, Phys. Rev. (2) 46, 793, 1934.

409 Wr1ght, Publ. L1ck Obs. 13, 193, 1918.

410 Zeeman und D1k, see de Bru1n, Zs1t. \&. Phys. 38, 94, 1936. 
INDEX-By Pagea

\begin{tabular}{|c|c|c|c|}
\hline Page & Sp & $\frac{1401 t}{\text { No }} \mathrm{sp}$ & $\frac{\text { Mult }}{\text { No }}$ \\
\hline 1 & $H$ & 1 - He II & 5 \\
\hline 3 & He II & 6 - C II & a1 \\
\hline 3 & C II & $23-N I$ & 6 \\
\hline 4 & N I & $7-: \because I$ & 24 \\
\hline 5 & N I & $25-N$ II & 68 \\
\hline 6 & N II & $69-0 I$ & 11 \\
\hline 7 & OI & $13-0 I$ & 44 \\
\hline 8 & $0 I$ & 45 - $0 I I$ & 63 \\
\hline 9 & 0 II & 63 - 0 III & 35 \\
\hline 10 & O III & $36-F$ II & 4 \\
\hline 11 & $F$ II & $5-\mathrm{Ne} I$ & 33 \\
\hline 13 & Ne I & 33 - Ne II & 70 \\
\hline 13 & Ne II & 71 - 绠 II & 9 \\
\hline 14 & Ls II & 10 - AI II & 65 \\
\hline 15 & Al II & $66-31 I$ & 38 \\
\hline 16 & 31 I & $29-51$ III & 8 \\
\hline 17 & S1 III & $9-P V$ & 1 \\
\hline 18 & S I & 1 - S II & 48 \\
\hline 19 & S II & 49 - Cl II & 8 \\
\hline 30 & Cl II & $9-C I$ III & 8 \\
\hline 31 & Cl III & $9-A I I$ & 43 \\
\hline 33 & A II & $44-A I I$ & 123 \\
\hline 23 & A II & $124-\mathrm{Ce} I$ & 13 \\
\hline 24 & $\mathrm{Ca} I$ & $14-C_{a}$ III & 4 \\
\hline 25 & So I & 1 - Sc III & 1 \\
\hline 26 & I1 I & 1 - T1 I & 54 \\
\hline 37 & T1 I & $55-21 I$ & 140 \\
\hline 28 & I1 I & $141-T 1 I$ & 192 \\
\hline 29 & T1 I & 193 - T1 I & 268 \\
\hline 30 & TI I & 269 - TI II & 32 \\
\hline 31 & T1 II & $23-\mathrm{T} 1 \mathrm{II}$ & 93 \\
\hline 32 & II II & $94-V I$ & 31 \\
\hline 33 & $V I$ & $33-V I$ & 78 \\
\hline 34 & $\nabla I$ & \multicolumn{2}{|c|}{79 - $\nabla$ I Uncloss. } \\
\hline 35 & V II & $1-\nabla I I$ & 61 \\
\hline 36 & VII & $68-V$ II & 163 \\
\hline 37 & $\nabla I I$ & $163-C r I$ & 18 \\
\hline 38 & Cr I & $19-\operatorname{Cr} I$ & 52 \\
\hline 39 & Cr I & $53-C r I$ & 114 \\
\hline
\end{tabular}

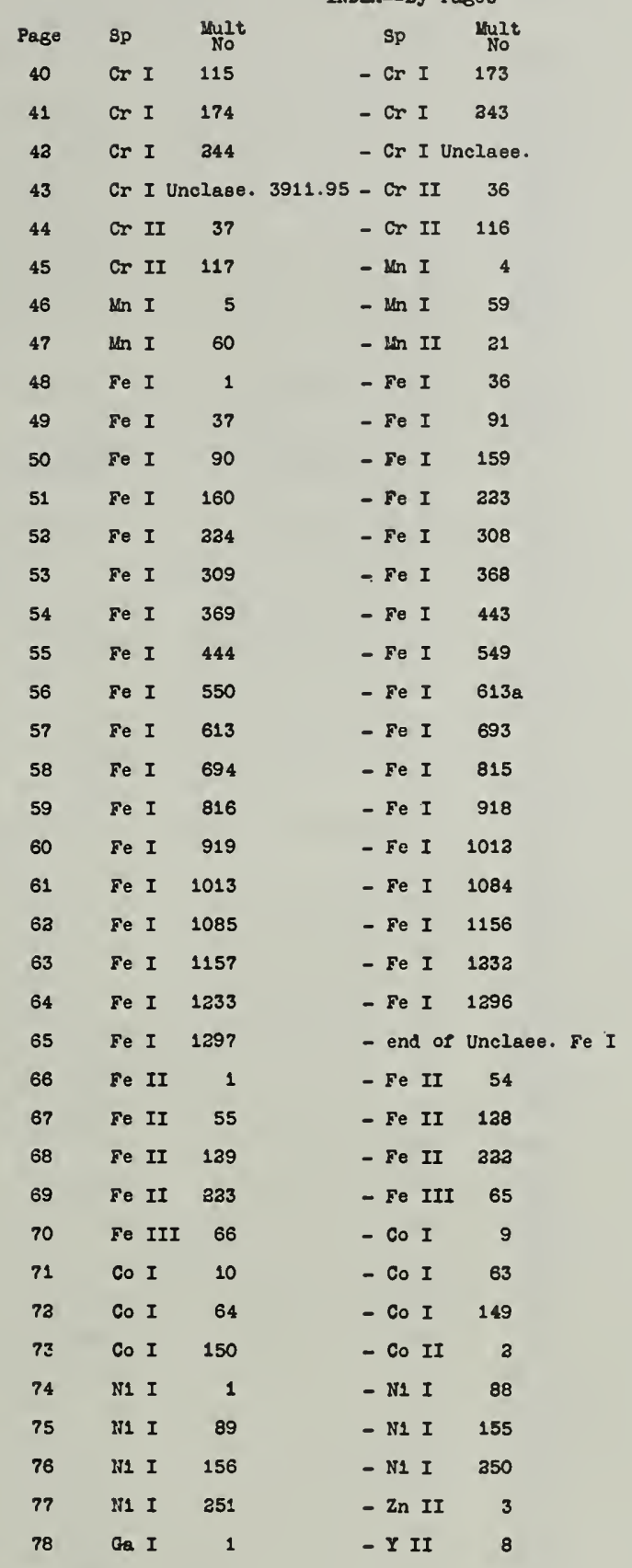

\begin{tabular}{|c|c|c|c|c|}
\hline Page & Sp & $\underset{\text { No }}{\text { Kult }} \mathrm{Sp}$ & & $\underset{\text { No }}{\operatorname{Mu} 2 t}$ \\
\hline 79 & Y II & $9-2 r$ & I & 17 \\
\hline 80 & $\mathrm{Zr} I$ & $18-\mathrm{Zr}$ & II & 23 \\
\hline 81 & $2 r$ II & $34-\mathrm{Zr}$ & II & 108 \\
\hline 83 & $2 r$ II & 109 - & II & 2 \\
\hline 83 & Mo II & $3-A B$ & II & \\
\hline 84 & Cd I & $1-\mathrm{La}$ & II & 4 \\
\hline 85 & La II & 5 - La & II & 86 \\
\hline 86 & La II & $87-\mathrm{Ce}$ & I & \\
\hline 87 & Ce II & $1-\mathrm{Ce}$ & II & 99 \\
\hline 88 & $\mathrm{Ce}$ II & $100-\mathrm{Ce}$ & II & 233 \\
\hline 89 & Ce II & $234-N a$ & II & $?$ \\
\hline 90 & Na II & $8-S m$ & I & 3 \\
\hline 91 & Sm II & $1-S m$ & II & 58 \\
\hline 93 & Sm II & $59-\mathrm{Gd}$ & II & 1 \\
\hline 93 & Gd II & $2-G d$ & II & 49 \\
\hline 94 & od II & $50-G d$ & II & 98 \\
\hline 95 & Gd II & $99-\mathrm{Tb}$ & II & \\
\hline 96 & Dy I & - Hr & II & 17 \\
\hline 97 & He II & $18-\mathrm{Ta}$ & I & 3 \\
\hline 98 & $\mathrm{Ta} I$ & $3-T 1$ & II & \\
\hline 99 & Po I & $-U$ & & \\
\hline
\end{tabular}

Forbldaen Linee

$\begin{array}{llcr}100 & \text { Be I } & 1 F-\text { Cl IV } & 3 F \\ 101 & \text { A III } & 1 F-\text { TI I } & 15 F \\ 103 & \text { TI I } & 16 F-\text { TI II } & 37 F \\ 103 & \text { T1 II } & 38 F-\text { V VIII } & 1 F \\ 104 & \text { Cr I } & 1 F-\text { Cr II } & 26 F \\ 105 & \text { Cr II } & 37 F-F e \text { I } & 3 F \\ 106 & \text { Fe I } & 4 F-F e \text { I } & 41 F \\ 107 & \text { Fe II } & 1 F-F e \text { II } & 38 F \\ 108 & \text { Fe II } & 39 F-F e X V & 1 F \\ 109 & \text { Co II } & 1 F-\text { N1 XII } & 1 F \\ 110 & \text { N1 XIII } & 1 F-\text { Eu II } & 3 F\end{array}$


INDEX--By Elements

\begin{tabular}{|c|c|c|c|c|c|c|c|c|c|c|c|}
\hline $8 p$ & Page & $\mathrm{Sp}$ & Page & $\mathrm{Sp}$ & Page & sp & Page & Sp & Page & Sp & Page \\
\hline A I & 21 & Dy II & 96 & $N$ III & 6 & S1 I & 15,16 & A XIV & 101 & Un $V$ & 105 \\
\hline A II & 21,23 & Er & 96 & N IV & 6 & S1 II & 16 & $\boldsymbol{N}$ VII & 100 & $\operatorname{lin} V I$ & 105 \\
\hline A III & 23 & Eu I & 92 & $\mathrm{~N} \mathrm{~V}$ & 6 & S1 III & 16,17 & $\mathrm{Be} I$ & 100 & $\operatorname{Ln} I X$ & 105 \\
\hline A IV & 23 & Eu II & 92 & $\mathrm{Na} I$ & 13 & S1 IV & 17 & C I & 100 & $\operatorname{Ln} x$ & 105 \\
\hline$A B I$ & 83 & $F I$ & 10 & $\mathrm{Na} I I$ & 13 & Sm I & 90 & $\mathrm{Ca} I$ & 101 & N I & 100 \\
\hline$A B I I$ & 83 & $F I I_{1}$ & 10,11 & Nd I & 89 & Sm II & 91,92 & $\mathrm{Ca}$ II & 101 & N II & 100 \\
\hline$A 1$ I & 14 & $F I I I$ & 11 & Na II & 89,90 & Sn. I & 84 & $\mathrm{Ca} \mathrm{V}$ & 101 & $\mathrm{Na} I V$ & 100 \\
\hline AI II & 14,15 & F IV & 11 & $\mathrm{Ne} I$ & 11,12 & Sn II & 84 & $\mathrm{Ca}$ VI & 101 & $\mathrm{Na} \mathrm{V}$ & 100 \\
\hline AI III & 15 & F VI & 11 & Ne II & 12,13 & Sr I & 78 & $\mathrm{Ca}$ VII & 101 & Ne III & 100 \\
\hline As $I$ & 78 & Fe I & $48-65$ & N1 I & $74-77$ & Sr II & 78 & $\mathrm{Ca} X I I$ & 101 & $\mathrm{Ne} I V$ & 100 \\
\hline As II & 78 & Fe II & $66-69$ & $\mathrm{N1}$ II & 77 & $T a I$ & 97,98 & $\mathrm{Ca}$ XIII & 101 & $\mathrm{Ne} \mathrm{V}$ & 100 \\
\hline Au I & 98 & Fe III & 69,70 & $O I$ & $6-8$ & TQ II & 98 & $\mathrm{Ca} X V$ & 101 & $I 11$ & 109 \\
\hline $\mathrm{Au}$ II & 98 & $\mathrm{Ga} \mathrm{I}$ & 78 & $0 I I$ & 8,9 & To I & 95 & Cl II & 100 & $\mathrm{N1}$ II & 109 \\
\hline B I & 2 & $\mathrm{Ga}$ II & 78 & 0 III & 9,10 & Tb II & 95 & CI III & 100 & N1 VII & 109 \\
\hline B II & 2 & Gd I & 92 & $0 \mathrm{IV}$ & 10 & Te I & 84 & $\mathrm{Cl}$ IV & 100 & II VIII & 109 \\
\hline B III & 2 & Gd II & $92-95$ & $0 \mathrm{~V}$ & 10 & Th I & 99 & Co II & 109 & N1 IX & 109 \\
\hline$B a I$ & 84 & $\mathrm{Ge} I$ & 78 & O VI & 10 & Th II & 99 & Co VI & 109 & N1 XII & 109 \\
\hline $\mathrm{Ba} I I$ & 84 & $\mathrm{Ge} I I$ & 78 & Os I & 98 & Th III & 99 & Co VII & 109 & N1 XIII & 110 \\
\hline $\mathrm{Be} I$ & 2 & $\mathrm{H}$ & 1 & $P I$ & 17 & TI I & $26-30$ & Co VIII & 109 & $\mathrm{~N} 1 \mathrm{XV}$ & 110 \\
\hline Be II & 2 & $\mathrm{He} I$ & 1 & P II & 17 & T1 II & $30-32$ & Co XI & 109 & N1 XVI & 110 \\
\hline B1 I & 99 & He II & 1,2 & P III & 17 & T1 IV & 32 & Cr I & 104 & $0 I$ & 100 \\
\hline B1 II & 99 & HP I & 96 & P IV & 17 & II I & 98 & Cr II & 104,105 & $0 \mathrm{II}$ & 100 \\
\hline Br I & 78 & HP II & 96,97 & $P V$ & 17 & II II & 98 & Cr III & 105 & $0 \mathrm{III}$ & 100 \\
\hline Br II & 78 & Hg I & 98 & $\mathrm{~Pb} I$ & 99 & $T m I$ & 96 & Cr IV & 105 & PI & 100 \\
\hline$c I$ & 2 & $\mathrm{Hg}$ II & 98 & $\mathrm{~Pb} I I$ & 99 & $\operatorname{Im} I I$ & 96 & $\mathrm{Cr} V$ & 105 & P II & 100 \\
\hline C II & 2,3 & H० I & 96 & $P d I$ & 83 & 0 & 99 & Cr VIII & 105 & S I & 100 \\
\hline C III & 3 & Ho II & 96 & $\mathrm{Pd} I I$ & 83 & $V I$ & $32-34$ & Cr IX & 105 & S II & 100 \\
\hline$C I V$ & 3 & I I & 84 & $\operatorname{Pr} I$ & 89 & V II & $35-37$ & $\mathrm{Cu} I I$ & 110 & S III & 100 \\
\hline Ca I & 23,24 & I II & 84 & Pr II & 89 & TI & 98 & Eu II & 110 & s VIII & 100 \\
\hline $\mathrm{Ca}$ II & 24 & In I & 84 & Pt I & 98 & VII & 98 & $F I I$ & 100 & S XII & 100 \\
\hline $\mathrm{Ce}$ III & 24 & In II & 84 & Pt II & 98 & $X \in I$ & 84 & $F I I I$ & 100 & Sc II & 101 \\
\hline $\mathrm{Cb} \mathrm{I}$ & 82 & Ir I & 98 & $\mathrm{Ra} I$ & 99 & Xe II & 84 & $F I V$ & 100 & Sc III & 101 \\
\hline $\mathrm{Cb} I I$ & 82 & $X I$ & 23 & Ra II & 99 & Y I & 78 & $\mathrm{Fe} I$ & 105,106 & Sc VI & 101 \\
\hline $\mathrm{Cd} \cdot \mathrm{I}$ & 84 & K II & 23 & $\mathrm{Rb} I$ & 78 & I II & 78,79 & $\mathrm{Fe} I I$ & 107,108 & Sc VII & 101 \\
\hline Cd II & 84 & $\mathrm{~K}$ III & 23 & $\mathrm{Rb} I I$ & 78 & Yo I & 96 & Fe III & 108 & S1 I & 100 \\
\hline $\mathrm{Ce} \mathrm{I}$ & 86 & $K r I$ & 78 & $\operatorname{Re} I$ & 98 & Ib II & 96 & $\mathrm{Fe} V$ & 108 & Sr II & 110 \\
\hline $\mathrm{Ce}$ II & $87-89$ & III II & 78 & Rh I & 83 & $\mathrm{Zn} I$ & 77 & Fe VI & 108 & T1 I & 101,10 \\
\hline $\mathrm{Ce}$ III & 89 & La I & 84 & Rh II & 83 & $\mathrm{Zn} I I$ & 77 & Fe VII & 108 & T1 II & 102,10 \\
\hline CI I & 19 & Lo II & $84-86$ & $\operatorname{Rn} I$ & 99 & $\mathrm{Zr} I$ & 79,80 & Fe $x$ & 108 & T1 III & 103 \\
\hline CI II & 19,20 & La III & 86 & Ru I & 83 & Zr II & $80-82$ & Fe XI & 108 & TI VII & 103 \\
\hline CI III & 20,21 & LI I & 2 & Ru II & 83 & & & Fe XIII & 108 & T1 VIII & 103 \\
\hline Cl IV & 21 & L1 II & 2 & $S I$ & 18 & & & Fe XIV & 108 & V II & 103 \\
\hline Co I & $70-73$ & $\mathrm{Lu} \mathrm{I}$ & 96 & S II & 18,19 & & & Fe XV & 108 & V III & 103 \\
\hline Co II & 73 & Lu II & 96 & S III & 19 & & & K IV & 101 & V IV & 103 \\
\hline Cr I & $37-43$ & Mg I & 13 & S IV & 19 & & & $\mathrm{~K} \mathrm{~V}$ & 101 & V VIII & 103 \\
\hline Cr II & $43-45$ & $M B I I$ & 13,14 & $\mathrm{Sb} I$ & 84 & & & K VI & 101 & Xe II & 110 \\
\hline Cs I & 84 & Mn I & $45-47$ & $\mathrm{Sb} I I$ & 84 & Forb1dden & Lines & $\mathrm{Kr}$ III & 110 & Xe III & 110 \\
\hline Cs II & 84 & Mn II & 47 & Sc I & 25 & A III & 101 & Lo II & 110 & Y II & 110 \\
\hline $\mathrm{Cu} \mathrm{I}$ & 77 & Mo I & 82 & Sc II & 25 & A IV & 101 & La III & 110 & $Y V$ & 110 \\
\hline $\mathrm{Cu}$ II & 77 & Mo II & 82,83 & Sc III & 25 & $A V$ & 101 & MS VI & 100 & $\mathrm{Zr}$ II & 110 \\
\hline D & 1 & N I & $3-5$ & Se I & 78 & $A X$ & 101 & Mn II & 105 & $\mathrm{Zr}$ III & 110 \\
\hline Dy I & 96 & $\mathrm{~N}$ II & 5,6 & $\mathrm{Se}$ II & 78 & $\mathrm{~A} X I$ & 101 & Un IV & 105 & $\mathrm{ZrVI}$ & 110 \\
\hline
\end{tabular}




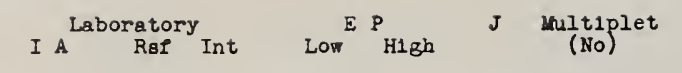
Hs II continued

\begin{tabular}{|c|c|c|c|}
\hline $\begin{array}{r}11626.40 \\
9344.93 \\
8236.77 \\
7592.74 \\
7177.50\end{array}$ & $\begin{array}{l}A \\
A \\
A \\
A \\
A\end{array}$ & $\begin{array}{l}\bar{z} \\
\bar{z}\end{array}$ & $\begin{array}{ll}53.02 & 53.08 \\
52.02 & 53.34 \\
52.02 & 53.51 \\
52.02 & 53.64 \\
53.02 & 53.74\end{array}$ \\
\hline $\begin{array}{l}6890.88 \\
6683.2 \\
6527.10 \\
6406.3 \\
6310.8 \\
6233.8\end{array}$ & $\begin{array}{l}\text { A } \\
\text { A } \\
\text { P } \\
\text { A } \\
\text { A } \\
\text { A }\end{array}$ & $\begin{array}{l}= \\
\bar{z}\end{array}$ & $\begin{array}{ll}52.02 & 53.81 \\
52.03 & 53.86 \\
52.02 & 53.91 \\
52.02 & 53.94 \\
52.02 & 53.97 \\
53.02 & 54.00\end{array}$ \\
\hline $\begin{array}{l}6170.6 \\
6118.3 \\
6074.1 \\
6036.7 \\
5694.46\end{array}$ & $\begin{array}{l}\mathrm{A} \\
\mathrm{A} \\
\mathrm{A} \\
\mathrm{A} \\
\mathrm{A}\end{array}$ & $\bar{z}$ & $\begin{array}{ll}52.03 & 54.02 \\
52.02 & 54.03 \\
53.02 & 54.05 \\
52.02 & 54.06 \\
52.02 & 54.18\end{array}$ \\
\hline
\end{tabular}

W1ds Fins Structure

$-\quad 5^{2} \mathrm{G}-7{ }^{2} \mathrm{H}^{\circ}$ etc
$=\quad(6) 8_{\mathrm{H}^{\circ}}^{2}$ etc
$-\quad 9^{2} \mathrm{H}^{\circ}$ etc
$-\quad 10^{2} \mathrm{H}^{\circ}$ etc $5^{3} \mathrm{a}-13^{2} \mathrm{H}^{\circ}$ etc (7) $13 \mathrm{H}^{\circ}$ etc $16^{2} \mathrm{H}^{\circ}$ etc 8) $18^{2} \mathrm{H}_{\mathrm{H}^{\circ}}$ etc
$30_{\mathrm{H}^{\circ}}^{2}$ stc
3 stc $30^{2} \mathrm{H}^{\circ}$ stc Lim1t

\section{REVISED $M$ U L T I P LET T A B L E}

I A Laboratory $\quad$ Rer Int Low $P_{\text {High }} J \quad$ (No) $^{\text {(Niplet }}$

I A Laboratory Int Low ${ }_{\text {H1gh }}^{\text {Ref }}$

$\underset{\text { Noultiplet }}{(\text { No })}$

BII I P 25.02 Anal B L1st $D$ Fsb 1943

CI continued

$3451.41 \quad A \quad 10 \quad 9.06 \quad 12.64 \quad 1-3 \quad 2^{1} P^{\circ}-2 p^{2}{ }^{1} D \quad 13164.1 \quad D \quad(100)$

$8.73 \quad 9.67 \quad 1-1 \quad 3 p^{3} s-4 s^{1} p^{0}$

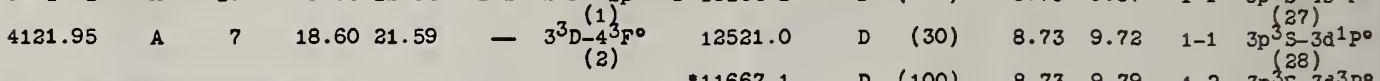

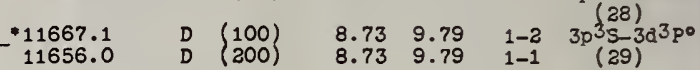
$5^{2} \mathrm{G}-18^{2} \mathrm{H}^{\circ}$ etc 4487.46

L1 I I P 5.37 Anal A L1st B Jan 1943

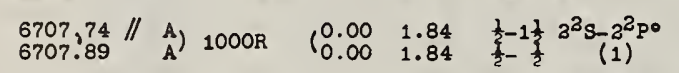

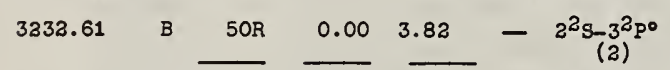

8136.52 B $\overline{(500)} \overline{1.84} 3.36-3^{2} \mathrm{po}^{0} 3^{3} \mathrm{~S}$

6103.642 C $500 \mathrm{R} \quad 1.843 .86 \quad-2^{2} \mathrm{P}^{0}-3^{3} \mathrm{D}$

$4971.92 \quad$ B $\quad 10 \mathrm{r} \quad 1.84 \quad 4.32 \quad-3^{2} \mathrm{P}_{-4}^{(4)} \mathrm{C}_{\mathrm{S}}$

\begin{tabular}{lllllll}
$4602.99 \quad$ B & $100 \mathrm{R}$ & 1.84 & 4.53 & $-2^{2} \mathrm{P}^{\circ}{ }_{-4}^{(5)} \mathrm{D}$ \\
\hline
\end{tabular}

L1 II I P 75.31 Anal A L1st $D$ Jan 1943

5483.55 to $)_{A} \quad 10 \quad 58.7761 .03 \quad-\quad 3^{3} \mathrm{~S}-2^{3} \mathrm{P}^{0}$

$3684.1 \quad B \quad \frac{3}{68.4871 .83}-3^{3} \mathrm{~s}_{(2)} 4^{3} \mathrm{P}^{\circ}$

4156.3 B $0.5 \overline{68.98} 71.95 \quad 0-1 \quad 3^{1} \mathrm{~s}_{(3)}{ }^{1} \mathrm{P}^{0}$

4881.3 B $2.5 \overline{69.07} 71.60-3^{3} \mathrm{p}^{0}-4^{3} \mathrm{~s}$

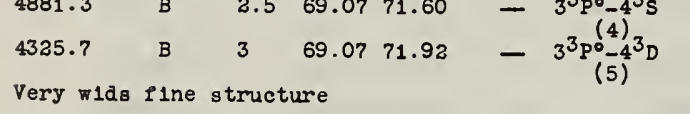

Be I I P 9.28 Anal A L1st C Feb 1943

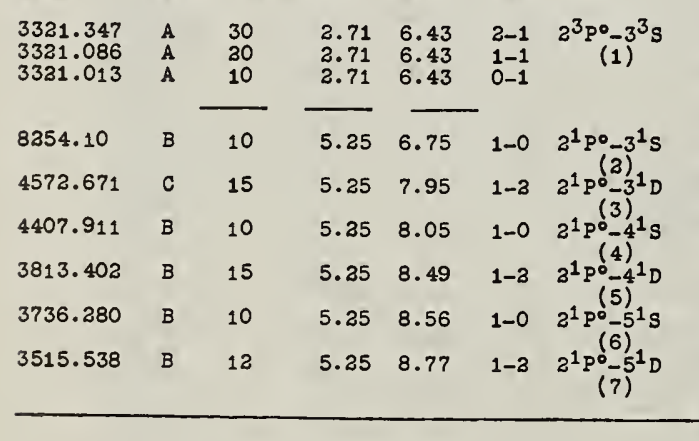

Be II I P 18.13 Anal A L1st $D$ Feb 1943

$\begin{array}{lllllll}3130.416 / / & A & 50 & 0.00 & 3.94 & \frac{1}{2}-1 \frac{1}{3} 2^{2} \mathrm{~S}-2^{2} \mathrm{P}^{\circ} \\ 3131.064 & \mathrm{~A} & 30 & 0.00 & 3.94 & \frac{1}{2}-\frac{1}{2} & (1)\end{array}$

3274.640 A $10 \quad-10.8914 .66-3^{2} \mathrm{~S}_{-} 4^{2} \mathrm{po}^{\circ}$

$\begin{array}{lllllll}5270.843 & \mathrm{~A} & 12 & & 11.91 & 14.25 & 1 \frac{1}{2} \\ 5270.322 & \mathrm{~A} & 10 & 3^{2} \mathrm{P}^{0}-4^{3} \mathrm{~S}\end{array}$

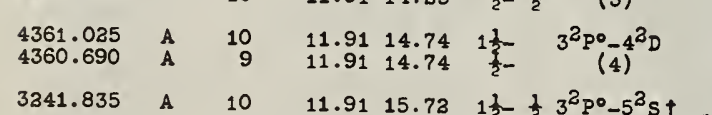

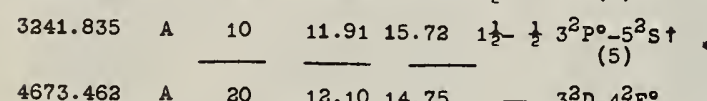
$14 \mathrm{H}^{\circ}$ etc BIII I P 37.77 Anal A L1st B Feb 1943

4497.58

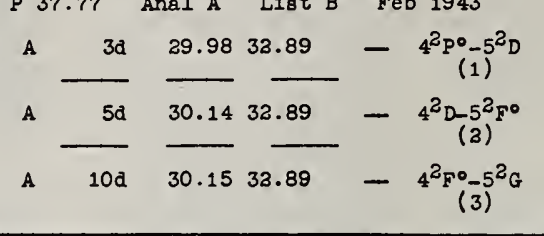

REVISFD

See NSRDS-NBS 3, Section 3, 1970 C I I P 11.20 Anal B L1st A Karch 1943

10691.36
10683.18
10685.44
107299.59
10707.44
10754.09
9658.49
9650.49
9603.09
9094.89
9078.32
9111.85
9088.57
9061.48
9063.53
5041.66

.5039 .05

4836.73

4817.33
4812.84

4771.72

4766.62
4775.87

4770.00

.4763 .41

4065.1
4064.2

4064.2

$$
\begin{array}{lr}
\text { A } & 50 \\
\text { A } & 25 \\
\text { A } & 10 \\
\text { A } & 8 \\
\text { A } & 8 \\
\text { P } & \\
\text { A } & 2 \\
\text { A } & 1 \\
\text { A } & 0 \\
\text { A } & 25 \\
\text { A } & 6 \\
\text { A } & 10 \\
\text { A } & 8 \\
\text { A } & 15 \\
\text { A } & 10 \\
\text { B } & 3 \\
\hline &
\end{array}
$$

$\begin{array}{rrr}50 & 7.46 & 8.61 \\ 25 & 7.45 & 8.61 \\ 10 & 7.45 & 8.60 \\ 8 & 7.46 & 8.61 \\ 8 & 7.45 & 8.60 \\ & 7.46 & 8.60\end{array}$

$\begin{array}{ll}7.46 & 8.73 \\ 7.45 & 8.73\end{array}$

$\begin{array}{ll}7.45 & 8.73 \\ 7.45 & 8.73\end{array}$

$7.46 \quad 8.81$
7.45

$\begin{array}{ll}7.46 & 8.81 \\ 7.45 & 8.81 \\ 7.46 & 8.81\end{array}$

$\begin{array}{ll}7.46 & 8.81 \\ 7.45 & 8.81\end{array}$

$\begin{array}{ll}7.45 & 8.81 \\ 7.45 & 8.81\end{array}$

7.458 .81

$7.46 \quad 9.90$

$\begin{array}{ll}7.45 & 9.90 \\ 7.45 & 9.90\end{array}$

$\begin{array}{lll}1 & 7.46 & 10.01 \\ 1 & 7.45 & 10.01 \\ \text { (1) } & 7.45 & 10.01\end{array}$

$2-3 \quad 3 s^{3} p^{0}-3 p^{3} D$

11656.0

12614.8
12565.0

12603.6

12565.0

12551.0

8018

7850

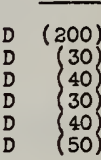

$8.81 \quad 9.79 \quad 2-2 \quad 3 p^{3} p-3 a^{3} p^{\circ}$

$\begin{array}{llll}8.81 & 9.79 & 1-1 & (30)\end{array}$

$\begin{array}{lll}8.81 & 9.79 & 2-1 \\ 8.81 & 9.79 & 1-0\end{array}$

$\begin{array}{lll}8.81 & 9.79 & 1-2 \\ 8.81 & 9.79 & 0-1\end{array}$

(1a) $8.8110 .35-3 p^{3} p-4 a^{3} D^{0}$

(1d) $8.8110 .38-3 p^{3} p-4 d^{3} p^{\circ}$
See NSRDS-NBS 3 , Section 3, 1970 C II I P 24.38 Anal A L18t A Feb 1943

$0-1$
$2-2$
$2-1$

$1-1$

6578.03

$0_{-1}^{1-1} \quad(5)$

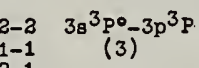

$2-1$
$1-0$
$1-2$
$0-1$

$2-3 \quad 3 \mathrm{~s}^{3} \mathrm{po}^{-4 \mathrm{p}^{3} \mathrm{D}}$

$\begin{array}{ll}2-3 & 3 \mathrm{~s}^{3} \mathrm{po}^{\circ}-4 \mathrm{p}^{3} \mathrm{D} \\ 1-2 & (4)^{-1}\end{array}$

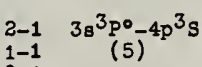

$\begin{array}{llll}7.46 & 10.04 & 2-2 & 3 \mathrm{~B}^{3} \mathrm{po}^{\circ}-4 \mathrm{p}^{3} \mathrm{p} \\ 7.45 & 10.04 & 1-1 & (6)\end{array}$

7.4610 .04

7.4510 .04

7.45
7.45
70.04

$1-1$
$2-1$
$1-0$

$1-0$
$1-2$
$0-1$

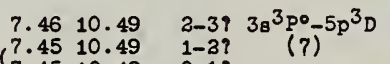

$i_{7.45}^{7.40 .49}$

$\begin{array}{lll}7.45 & 10.49 \quad 0-19\end{array}$

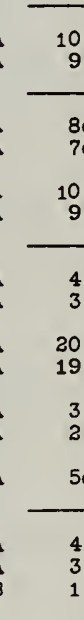

(10)

Odd 30.

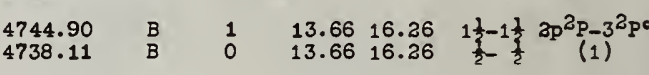

$2-1 \quad 3 \mathrm{~s}^{3} \mathrm{po}^{-}-3 \mathrm{p}^{3} \mathrm{~s}$

$\begin{array}{ccc}9405.77 & \text { A } & 20\end{array}$

$7.65 \quad 8.81 \quad 1-1 \quad 38^{1} P^{0}-3 p^{3} P$

7.658 .96

$\begin{array}{ll}1-1 & 3 g^{1} p^{\circ}-3 p^{3} p \\ 1-3 & 3 g^{1} p^{\circ}-3 p^{1} D\end{array}$

8335.19 A 10

5380.243 B 8

5052.122 B 6

4932.00 B 5

4371.33

4352.1

4268.99

4231.35

5793.51 5801.17
5805.76

11330.36 A 1

$7.65 \quad 9.13$ 1-0 $3 s^{1}{ }^{(9)}-3 p^{1} s$

10548.0 E (60)

6587.75

- 11894.9

11880.4
11849.3

11849.3
11863.0

.11754 .0

11747.5
11801.8

1166?.1

$11631.59 ?$
$11609.91 ?$

11609.91?

11638.609

11619.0
11603.948

7118.5
$7.65 \quad 9.95$

$7.65 \quad 10.09$

$7.65 \quad 10.15$

$\begin{array}{lll}7.65 & 10.47\end{array}$

7.6510 .49

$$
\text { 1-1 3s (10) }
$$$$
\text { 1-3 }{ }_{3 s^{3}}(11)^{0}-4 p^{1} D
$$$$
\text { 1-0 } 3 s^{(12)}\left(1 P^{\circ}-4 p^{1} s\right.
$$$$
\text { 1-1 } 3 \mathrm{~B}^{1}{ }^{\circ}-5 \mathrm{p}^{1} \mathrm{P}
$$$$
\text { 1-2? } 3 \mathrm{~s}^{(14)} \mathrm{P}^{(14)}-5 \mathrm{p}^{3} \mathrm{D}
$$$$
1-2 \quad 38 \mathrm{P}^{\circ}-5 \mathrm{p}^{1} \mathrm{D}
$$

$7.65 \quad 10.54$

$7.65 \quad 10.57$

$-0$

$(16)$

$7.91 \quad 10.04$

$\begin{array}{ll}7.91 & 10.04 \\ 7.91 & 10.04\end{array}$

$8.50 \quad 9.59 \quad 1-3 \quad 3 p^{1} P-3 a^{1} D^{\circ}$

$8.50 \quad 9.67 \quad 1-1 \quad 3 p^{1} p_{-4 g^{1}} p^{\circ}$

$8.5010 .31 \quad 1-2 \quad 3 p\left(\begin{array}{lll}20) \\ P-4 a^{1} D^{\circ}\end{array}\right.$

$\begin{array}{llll}8.50 & 10.37 & 1-1 & 3 p \\ & & (21) & (22) \\ & & & (22)\end{array}$

(300)

8.619 .65

$\begin{array}{rr}8.61 & 9.65 \\ 8.61 & 9.64 \\ 8.60 & 9.64 \\ 8.61 & 9.65\end{array}$

$\begin{array}{cc}3-2 & 3 p^{3} D-4 a^{3} p \\ 3-1 & (23)\end{array}$

$D$
$D$$\quad\left\{\begin{array}{l}15 \\ 10 \\ 15\end{array}\right\}$

8.619 .64

$\begin{array}{ll}8.61 & 9.66\end{array}$

\begin{tabular}{rrr}
8.61 & 9.66 \\
\hline & 8.60 & 9.65
\end{tabular}

$\begin{array}{ll}8.60 & 9.65 \\ 8.61 & 9.66\end{array}$

(100)

$8.61 \quad 9.67$

$8.61 \quad 9.67$

$\begin{array}{ll}8.60 & 9.67 \\ 8.61 & 9.67\end{array}$

(30)

$\begin{array}{ll}8.61 & 9.67 \\ 8.61 & 9.67\end{array}$

$1-0 ?$
$2-2$
$1-1$

1- $3-$

$\begin{array}{ll}3 p^{3} D-3 a^{3} F^{\circ} \\ -3 & (24)\end{array}$

$-3$

$3-3$
$2-2$
$1-1$
$3-2$
$3-1$
$2-3$

(25)

-1
-3
-2

(6a) $\begin{array}{ll}8.60 & 9.67\end{array}$
$3-2$
$2-1$
$1-0$

$3-3 \quad 3 p^{3} D-3 a^{3} D^{0}$
7236
7231
39

58
58
42
4267
336
3361

7236

3920.677

3920.677
3918.977

5889.97

4267.27

4267.02

3361.75

2992.63

3165.51
3167.95

3165.99

5536.0

5336.7

5121.69

3832.12

6783.75

6780.27

6800.50
6791.30

6787.09

6812.19
6798.04

5662.51

5648.08

5640.50

5145.16

5139.21
5137.26

5151.08

5143.49
5133.29

5133.39
5132.96

5033.2
5037.0

3871.63
3868.84

6115.31

- 7119. 45

7115.15

7133.52

7135.49
$* 7119.45$

6750.22

6738.36
6730.79

.6736 .84

6742.05

-6733.56

.6726 .84

\begin{tabular}{l} 
B \\
A \\
B \\
B \\
\hline A \\
A \\
A \\
A \\
A \\
A \\
A \\
A \\
A \\
A \\
A \\
A \\
A \\
A \\
A \\
A
\end{tabular}

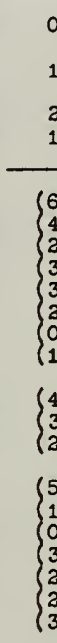

$$
\begin{aligned}
& 30.06 \quad 32.38 \quad 1 \frac{1}{2}-\frac{1}{2} 4^{2} \mathrm{P}^{0}-6^{2} \mathrm{~S} \\
& 20.06 \quad 22.47 \quad 1 \frac{1}{2}-1 \frac{1}{2} 4^{2} p^{(11)}-3 p^{3} p \\
& 20.0623 .28 \quad 1 \frac{1}{3}-2 \frac{1}{3} 4^{2} \mathrm{p}^{(13)}-3 \mathrm{p}^{2} \mathrm{D} \\
& 20.0623 .28 \quad \frac{1}{2}-1 \frac{1}{2} \quad \text { (13) }
\end{aligned}
$$

$1 \mathrm{~d} \quad 20.83 \quad 23.38 \quad 1 \frac{1}{3}-2 \frac{1}{3} \quad 2 p^{3} 2 p^{0}-3 p^{2} \mathrm{D}$

$3 \quad 20.8324 .03 \quad 1 \frac{1}{2}-\frac{1}{2} 2 p^{3} 2 p^{\circ}-3 p^{2} S$

\begin{tabular}{llllll} 
B & 2 & 20.83 & 24.03 & $1 \frac{1}{2}-\frac{1}{2}$ & $2 \mathrm{p}$ \\
B & 1 & 20.83 & 24.02 & $\frac{1}{2}-\frac{1}{2}$ & $(18)$ \\
\hline
\end{tabular}

- $\quad \overline{22.00} 24.02 \quad 1 \frac{1}{2}-\frac{1}{2} 3 \mathrm{~s}^{2} \mathrm{p}^{0}-3 \mathrm{p}^{2} \mathrm{~S}$

(2) $22.44 \quad 34.17 \quad 3 \frac{1}{2}-4 \frac{1}{3} \quad 3 p^{4} D-3 a^{4} F^{\circ}$

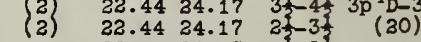

(1) $\left(22.4324 .17 \quad 1 \frac{1}{22}-2\right.$
$-3 p^{3} D-4 d^{3} F^{\circ}$ 


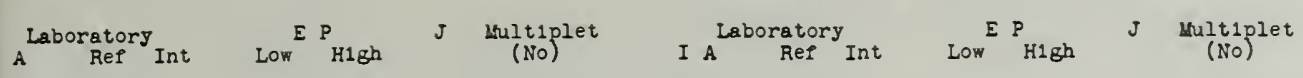
CII continued

5856.09 5856.09
5836.31
583.13 5823.13 5827.80 5817.87 3589.67 3590.87 3584.98 3587.68 3581.80 3581.80
3585.83

6098.62 6095.37 6102.5 4964.90 4959.52

7063.4

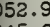

7045.8

4009.90

4021.13

4317.42

4321.95
.4325 .88

4313.50
4318.92

3039.67

5257.36

.5259.6

5249.43
5253.5588

3949.45

3947.60
$\cdot 2946.35$

3952.08

3948.15
-3946.35

3945.1

3876.188 -1
-13876.409

4076.00 4074.53

3980.35 3973.8 3978.87 3972.44 3970.20
3977.30

4411.52
4411.20 4410.06 4292.00 4296.1 3049.44

\section{$\left\{\begin{array}{l}3 \\ 3\end{array}\right.$} (2) $\begin{array}{llll}22.44 & 24.55 & 3 \frac{1}{2}-2 \frac{1}{2} & 3 \mathrm{p}^{4} \mathrm{D}-3 \mathrm{~d}^{4} \mathrm{po} \\ 22.44 & 24.55 & 2 \frac{1}{3}-1 \frac{1}{3} & (22)\end{array}$ $\begin{array}{lll}22.43 & 24.55 & 1 \frac{1}{3} \\ 22.44 & 24.55 & 23\end{array}$ $\begin{array}{lll}22.43 & 44.55 & 1 \frac{2}{1}-1 \\ 32.43 & 24.55 & \frac{2}{2}-\end{array}$

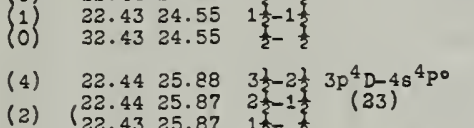

(1) $\begin{array}{lll}22.44 & 25.88 & 2 \frac{1}{2}-2\end{array}$

(2) $\begin{array}{llll}22.43 & 25.87 & 1 \frac{2}{2}-1 \\ 1 & 22.43 & 25.87 & \end{array}$

$22.47 \quad 24.50 \quad 1 \frac{1}{2}-2 \frac{1}{2} \quad 3 \mathrm{p}^{2} \mathrm{P}-3 \mathrm{~d}^{2} \mathrm{D}^{\circ}$ $\begin{array}{llll}22.47 & 24.50 & 1 \frac{1}{3}-1 \frac{2}{3} & (24) \\ 22.47 & 24.50 & 1 \frac{1}{2}-1 \frac{1}{2}\end{array}$

$22.4724 .96 \quad 1 \frac{1}{2}-1 \frac{1}{2} \quad 3 \mathrm{p}^{2} \mathrm{P}-3 \mathrm{~d}^{2} \mathrm{po}$ 22.4724 .96 (3)

Od $\quad 22.4724 .96 \quad \frac{1}{2}-1 \frac{1}{2}$

(1n) $22.80 \quad 24.55 \quad 1 \frac{1}{2}-2 \frac{1}{2} \quad 3 p^{4} s-3 d^{4} p^{0}$

(2) $22.8025 .88 \quad 1 \frac{1}{2}-2 \frac{1}{3} \quad 3 p^{4} s-4 s^{4} p 0$

(1) \begin{tabular}{lll}
22.80 & 25.87 & $1 \frac{1}{2}-1$ \\
22.80 & 25.87 & $1 \frac{2}{2}-$ \\
\hline
\end{tabular}

$23.02 \quad 25.88 \quad 2 \frac{1}{3}-2 \frac{1}{3} \quad 3 p^{4} p-4 s^{4} p_{0}$ $23.02 \quad 25.87 \quad 1 \frac{1}{13} \quad$ (28) (23.02 $25.87 \quad 2 \frac{1}{3}-1 \frac{1}{3}$

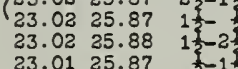

$$
\text { - }
$$

$\overline{23.28} 27.34-3 \mathrm{p}^{2} \mathrm{D}-4 \mathrm{~d}^{2} \mathrm{~F}^{\circ}$

(2)

$24.1726 .52 \quad 4 \frac{1}{3}-3 \frac{1}{2} \quad 3 \mathrm{~d}^{4} \mathrm{~F}^{\circ}-4 \mathrm{p}^{4} \mathrm{D}$

(3) $\left\{\begin{array}{lll}24.17 & 26.52 & 31 \\ 24.17 & 26.51 & 23 \\ 24.17 & 26.51 & 1 \\ 24.17 & 26.52 & 3\end{array}\right.$

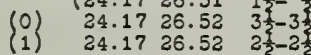

$24.1727 .29 \quad 3 \frac{1}{2}-3 \frac{1}{2} 3 d^{4} F^{\circ}-4 I^{2} F$

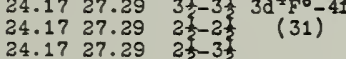
$\begin{array}{llll}24.17 & 27.30 & 4 \frac{1}{3}-4 \frac{1}{3} & 3 \mathrm{~d}^{4} \mathrm{~F}^{\circ}-4 \mathrm{I}^{4} F \\ 24.17 & 27.30 & 3 \frac{3}{2}-3 \frac{3}{2} & (32)^{2}\end{array}$

$\begin{array}{lll}24.17 & 27.30 & -2 \\ 24.17 & 27.30 & 2 \frac{1}{2}-3 \frac{1}{2}\end{array}$

$\begin{array}{llll}24.17 & 27.36 & 4 \frac{1}{2}-5 \frac{1}{2} & 3 \mathrm{~d}^{4} \mathrm{~F}^{0}-4 \mathrm{P}^{4} \mathrm{G} \\ 24.17 & 27.35 & 3 \frac{2}{2}-4 \frac{3}{2} & (33)^{-}\end{array}$ $\begin{array}{lll}24.17 & 27.35 & 3 \frac{1}{2}-4 \\ 24.17 & 27.35 & 2 \frac{1}{2}-3\end{array}$

$\begin{array}{lll}24.17 & 27.35 & 12 \\ 2 & -2 & \end{array}$

$\begin{array}{lll}24.17 & 27.35 & 4 \frac{1}{2}-4 \\ 24.17 & 27.35 & 3 \frac{1}{2}-3\end{array}$

$24.17 \quad 27.35 \quad 2 \frac{2}{2}-2 \frac{1}{2}$

(0) $24.2726 .52 \quad 3 \frac{1}{2}-3 \frac{1}{2} 3 d^{4} D^{0}-4 p^{4} D$ $24.2726 .71 \quad 3 \frac{1}{2}-2 \frac{1}{3} \quad 3 \mathrm{~d}^{4} \mathrm{D}^{\circ}-4 \mathrm{p}^{4} \mathrm{p}$ $24.27 \quad 26.71 \quad 2 \frac{1}{2}-1 \frac{1}{2} \quad(35)$ $\begin{array}{llll}24.27 & 27.30 & 3 \frac{1}{2}-4 \frac{1}{1} & 3 \mathrm{~d}^{4} D^{0}-4 \mathrm{P}^{4} \mathrm{~F} \\ 24.27 & 27.30 & 2 \frac{1}{2}-3 \frac{3}{2} & (36)\end{array}$ $\begin{array}{lll}24.27 & 27.30 & 2 \frac{1}{2}-3 \\ 24.27 & 27.30\end{array}$

$24.27 \quad 27.37 \quad 3 \frac{1}{3}-3 \frac{1}{2} 3 d^{4} D^{\circ}-4 I^{4} D$ $24.27 \quad 27.37 \quad 2 \frac{1}{2}-2 \frac{3}{3} \quad$ (37) $\begin{array}{lll}24.27 & 27.37 & 1 \frac{1}{3}-1 \\ 24.27 & 27.37 & 2 \frac{1}{2}-3\end{array}$

$\begin{array}{lll}24.27 & 27.37 & 1 \frac{1}{3}-2 \frac{1}{2} \\ 24.26 & 27.37 & \frac{1}{2}-1 \frac{1}{2}\end{array}$

$24.27 \quad 27.38 \quad 2 \frac{1}{3}-1 \frac{1}{3} \quad 3 \mathrm{~d}^{4} \mathrm{D}^{0}-4 \mathrm{I}^{2} \mathrm{D}$ 24.50 $27.29 \quad 2 \frac{1}{3}-3 \frac{1}{2} 3 \mathrm{~d}^{2} \mathrm{D}^{0}-4 \mathrm{P}^{2} \mathrm{~F}$ $24.50 \quad 27.29 \quad 1 \frac{1}{2}-2 \frac{3}{2} \quad(39)$ $24.5027 .30 \quad 2 \frac{1}{2}-3 \frac{1}{2} \quad 3 \mathrm{~d}^{2} \mathrm{D}^{\circ}-4 \mathrm{4}^{4} \mathrm{~F}$ $24.50 \quad 27.37 \quad 2 \frac{1}{2}-2 \frac{1}{2} \quad 3 d^{2} D^{\circ}-42^{4} \mathrm{D}$ $\begin{array}{llll}24.50 & 27.37 & 2 \frac{1}{2}-2 \frac{1}{3} & 3 \mathrm{~d}^{2} \mathrm{D}^{\circ}-4 \mathrm{I}^{2} \mathrm{D}\end{array}$

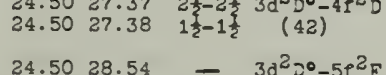
$24.5028 .54-3 \mathrm{~d}^{2} 2^{0}-5$ \begin{tabular}{llll}
0 & 22.43 & 25.88 & $1 \frac{3}{1}-2 \frac{1}{3}$ \\
(2) & 22.43 & 25.87 & $\frac{1}{2}-1 \frac{1}{2}$ \\
\hline
\end{tabular}

(1n) $22.80 \quad 24.55 \quad 1 \frac{1}{2}$ (n) $22.8024 .55 \quad 1$ (26)
C II cont1nued

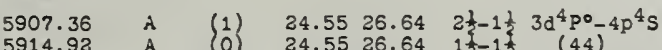

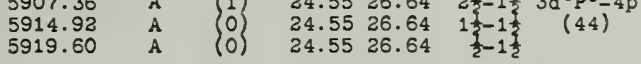

$\begin{array}{lllllll}4374.28 & \text { A } & 5 & 24.55 & 27.37 & 2 \frac{1}{3}-3 \frac{1}{3} & 3 \mathrm{~d}^{4} \mathrm{PO}-4 \mathrm{I}^{4} \mathrm{D}\end{array}$ 4371.59
-4368.1488

4376.78

3059.24

4734.75
4727.21

4630.52
4625.71

4618.85

\begin{tabular}{lll}
5119.55 & A & 2 \\
5114.07 & A & 2 \\
\hline
\end{tabular}

$\begin{array}{llll}24.55 & 27.37 & 1 \frac{1}{3}-2 \frac{3}{2} & (45) \\ 24.55 & 27.37 & \frac{1}{2}-1 \frac{3}{2} & \end{array}$

$\begin{array}{lll}24.55 & 27.37 & \frac{1}{2}-1 \\ 24.55 & 27.37\end{array}$

$24.55 \quad 27.37 \quad 1 \frac{1}{2}-2 \frac{1}{2} \quad 3 d^{4} \mathrm{PO}^{\circ}-4 \mathrm{P}^{2} \mathrm{D}$

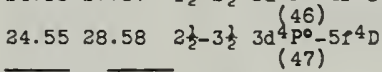

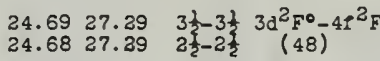

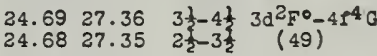
$24.6927 .36-3 \mathrm{~d}^{2} F^{0}-42^{2} G$

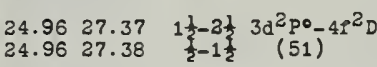
REVISED

See NSRDS-NBS 3, Section 3, 1970 C III I P 47.67 Anal B L1st A Feb 1943 $\begin{array}{lllllll}4647.40 & \text { A } & 20 & 29.39 & 32.05 & 1-2 & 3^{3} \mathrm{~s}-3^{3} \mathrm{po}^{\circ} \\ 4650.16 & \text { A } & 19 & 29.39 & 32.05 & 1-1 & (1) \\ 4651.35 & \text { A } & 18 & 39.39 & 32.05 & 1-0 & \end{array}$

\begin{tabular}{|c|c|c|}
\hline 5696.0 & A & 8 \\
\hline $\begin{array}{l}6744.2 \\
6730.7 \\
6727.1\end{array}$ & P & \\
\hline $\begin{array}{c}5272.56 \\
\cdot 5253.5588 \\
5244.5\end{array}$ & ${ }_{A}$ & $\begin{array}{l}2 \\
1 \\
0\end{array}$ \\
\hline
\end{tabular}

$31.97 \quad 34.13 \quad 1-2 \quad 3^{1} \mathrm{po}_{-} 3^{1} \mathrm{D}$

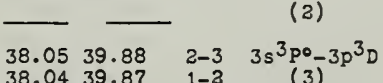
$\begin{array}{lll}38.04 & 39.87 & 1-3 \\ 38.04 & 39.87 & 0-1\end{array}$

$(3)^{-3 p^{3}}$

$38.05 \quad 40.39 \quad 2-1 \quad 3 s^{3} \mathrm{po}^{0}-3 \mathrm{p}^{3} \mathrm{~s}$ $\begin{array}{lll}38.04 & 40.39 & 1- \\ 38.04 & 40.39 & 0-1\end{array}$

4665.90 4663.53

\subsection{3}

3259.44
3257.90

4325.70

A

38.0540 .59

$\begin{array}{llll}38.19 & 41.97 & 1-2 & 4^{3} \mathrm{~s}-3 \mathrm{~d}^{3} \mathrm{po}\end{array}$

$\begin{array}{lll}38.19 & 41.98 & 1-1 \\ 38.19 & 41.98 & 1-0\end{array}$

3170.16

4516.93

4516.02

3609.61
3608.96

4247.56

3703.52

6156.

6155.

4388.24

4383.24
4379.97

3889.18
3885.99

3885.99
3883.80

4070.30

4068.97
4067.87

4122.05

4187.05

6857.3

$38.27 \quad 41.12 \quad 1-2 \quad 3 s^{1} \mathrm{po}^{\circ}-3 \mathrm{p}^{1} \mathrm{D}$

$38.48 \quad 42.37 \quad 0-1 \quad 4^{1} \mathrm{~S}-5^{1} \mathrm{po}$

$\begin{array}{cccc}39.22 & 41.95 & 2-1 & 4^{3} \mathrm{po}^{\mathrm{O}}-5^{3} \mathrm{~S} \\ 39.22 & 41.95 & 1,0-1 & (9)^{2}\end{array}$

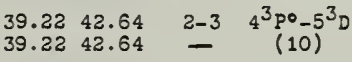

$39.47 \quad 42.37 \quad 1-1 \quad 3 p^{1} \mathrm{P}_{-5}^{1} \mathrm{po}$

$39.47 \quad 42.80 \quad 1-1 \quad \mathrm{pp}^{3 \mathrm{p}^{2}-3 \mathrm{~d}^{1} \mathrm{po}}$

$39.67 \quad 41.67 \quad 3-3 \quad 4^{3} D-3 d^{3} D^{\circ}$

$\begin{array}{llll}39.67 & 41.67 & 2-2 & \text { (13) } \\ 39.66 & 41.67 & 1-1 & \end{array}$

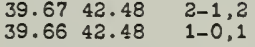

$39.67 \quad 42.84 \quad 3-4 \quad 4^{3} \mathrm{D}-5^{3} \mathrm{~F}^{\circ}$

$\begin{array}{lll}39.67 & 42.84 & 2- \\ 39.66 & 42.84 & 1-2\end{array}$

(15)

39.74 $42.77 \quad 4-5 \quad 4^{3} F^{\circ}-5^{3}$

$\begin{array}{rrrrrc}\text { B } & 10 & 39.74 & 42.77 & 4-5 & 4^{3} F^{0}-5^{3} G \\ \text { B } & 9 & 39.74 & 42.77 & 3-4 & (16) \\ \text { A } & 9 & 39.74 & 42.77 & 2-3 & \end{array}$

$\frac{39.74}{39.80} \overline{27}^{2-3}$

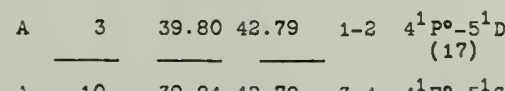

$39.84 \quad 42.78 \quad 3-4 \quad 4^{1} F^{\circ}-5^{1} G$

$39.88 \quad 41.67 \quad 3-3 \quad 3 \mathrm{p}^{3} \mathrm{D}-3 \mathrm{~d}^{3} \mathrm{D}^{\circ}$

$\begin{array}{lll}39.87 & 41.67 & 1-1\end{array}$
$6871 . ?$ $\begin{array}{ll}38.05 & 40.59 \\ 38.05 & 40.69 \\ 38.05 & 40.69\end{array}$

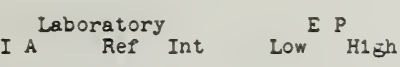

J vultiplet

C III continued $\begin{array}{llllll}5894.1 & \mathrm{P} & 39.8841 .97 & 3-2 & 3 \mathrm{p}^{3} \mathrm{D}-3 \mathrm{~d}^{3} \mathrm{P} \\ 5871.6 & \mathrm{P} & 39.8741 .98 & 2-1 & (20) \\ 5857.9 & \mathrm{P} & 39.87 & 41.98 & 1-0 & \end{array}$

$\begin{array}{lllllll}4162.80 & \mathrm{~A} & 5 & 39.88 & 42.84 & 3-4 & 3 \mathrm{p}^{3} \mathrm{D}-5^{3} \mathrm{~F}^{\circ} \\ 4156.50 & \mathrm{~A} & 4 & 39.87 & 42.84 & 2-3 & (21)\end{array}$ $\begin{array}{lllllll}4156.50 & \mathrm{~A} & 4 & 39.87 & 42.84 & 2-3 & \text { (21) } \\ 4152.43 & \mathrm{~A} & 3 & 39.87 & 42.84 & 1-2 & \end{array}$ $4152.43 \quad A \quad 39.87 \quad 42.84 \quad 1-2$

$\begin{array}{lllllll}5827.1 & A & 1 & 40.02 & 42.14 & 2-3 & 4^{1} \mathrm{D}-3 \mathrm{~d}^{2} \mathrm{~F}^{\circ}\end{array}$ $\begin{array}{llllllll}5249.6 \quad A & 0 & 40.02 & 42.37 & 2-1 & 4^{1} D^{(22)} 5^{1} \mathrm{po}\end{array}$

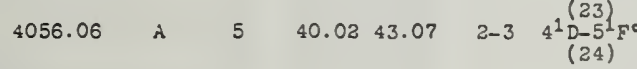

Lines attributed to C III--Classirication dublous $\begin{array}{lll}4593.47 & A & 2 d\end{array}$ $\begin{array}{lll}4361.85 & \text { A } & 4 \mathrm{~d} \\ 4001.56 & \mathrm{~A} & 0 \mathrm{~d} \\ & & \end{array}$ $3999.92 \quad A \quad$ Od

See NSRDS-NBS 3, Section 3, 1970 C IV I P 64.22 Anal A L1st A Feb 1943

$\begin{array}{llllll}5801.51 & A & 4 & 37.39 & 39.51 & 1 \\ 5 & -1 \frac{1}{2} & 3^{2} S-3^{2} \mathrm{PO}\end{array}$

\begin{tabular}{|c|c|c|c|c|c|c|}
\hline 3936 & $P$ & & 54.98 & 58.12 & $\frac{1}{2}-$ & $5^{2} S-6^{2} p o$ \\
\hline $\begin{array}{l}5023 \\
5021\end{array}$ & $\begin{array}{l}P \\
P\end{array}$ & & $\begin{array}{l}55.41 \\
55.41\end{array}$ & $\begin{array}{l}57.87 \\
57.87\end{array}$ & $\frac{1 \frac{1}{3}-\frac{1}{2}}{\frac{1}{2}}-\frac{3}{2}$ & $\frac{5^{2} \mathrm{p}^{0}-6^{2} \mathrm{~s}}{(3)}$ \\
\hline 4441.81 & A & Od & 55.41 & 58.19 & - & $\begin{array}{c}5^{2} P^{0}-6^{2} D \\
(4)\end{array}$ \\
\hline 4789 & $P$ & & 55.54 & 58.12 & - & $5^{2} \mathrm{D}-6^{2} \mathrm{po}$ \\
\hline 4647 & $P$ & & 55.54 & 58.19 & - & $\begin{array}{c}5^{2} D-6^{2} F^{\circ} \\
(6)\end{array}$ \\
\hline 4665 & $P$ & & 55.54 & 58.19 & - & $5^{2} F^{\circ}-6^{2} D$ \\
\hline 4658.64 & A & $5 d$ & 55.54 & 58.20 & - & $\begin{array}{c}5^{2} F^{0}-6^{2} G \\
(8)\end{array}$ \\
\hline 4660 & $P$ & & 55.55 & 58.19 & - & $\begin{array}{c}5^{2} G-6^{2} F^{\circ} \\
(9)\end{array}$ \\
\hline 6592 & $P$ & & 57.87 & 59.74 & $\frac{1}{2}-$ & $6^{2} s-7^{2} p$ \\
\hline 4217 & $P$ & & 57.87 & 60.80 & $\frac{1}{2}-$ & $\begin{array}{c}6^{2} S_{-8}^{2} p_{0} \\
(11)\end{array}$ \\
\hline
\end{tabular}

$39.67 \quad 42.48 \quad 3-2 \quad 4^{3} \mathrm{D}-5^{3} \mathrm{Po}^{\circ}$

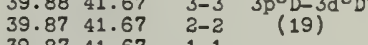

\begin{tabular}{|c|c|c|c|c|}
\hline $\begin{array}{l}8680.24 \\
8683.38 \\
8686.13 \\
8718.82 \\
8711.69 \\
8703.24 \\
8747.35 \\
8728.88\end{array}$ & $\begin{array}{l}\hat{A} \\
\hat{A} \\
\hat{A} \\
\hat{A} \\
\hat{A} \\
\hat{A} \\
\hat{A}\end{array}$ & $\begin{array}{r}10 \\
8 \\
7 \\
6 \\
7 \\
6 \\
0 \\
1\end{array}$ & $\begin{array}{ll}10.29 & 11.71 \\
10.29 & 11.71 \\
10.28 & 11.70 \\
10.29 & 11.71 \\
10.29 & 11.70 \\
10.28 & 11.70 \\
10.29 & 11.70 \\
10.29 & 11.70\end{array}$ & 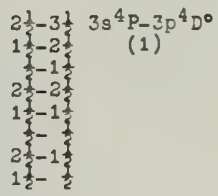 \\
\hline $\begin{array}{l}8216.28 \\
8210.64 \\
8200.31 \\
8242.34 \\
8223.07 \\
8184.80 \\
8187.95\end{array}$ & $\begin{array}{l}\hat{A} \\
\hat{A} \\
\hat{A} \\
\hat{A} \\
\hat{A}\end{array}$ & $\begin{array}{l}6 \\
2 \\
1 \\
4 \\
4 \\
4 \\
4\end{array}$ & $\begin{array}{ll}10.29 & 11.79 \\
10.29 & 11.79 \\
10.28 & 11.79 \\
10.29 & 11.79 \\
10.29 & 11.79 \\
10.29 & 11.79 \\
10.28 & 11.79\end{array}$ & $\begin{array}{ll}2 \frac{1}{2}-2 \frac{1}{3} & 3 s^{4} p-3 p^{4} p^{0} \\
1 & \left(2-1 \frac{2}{2}\right.\end{array}$ \\
\hline $\begin{array}{l}7468.29 \\
7442.28 \\
7423.63\end{array}$ & $\begin{array}{l}A \\
\hat{A}\end{array}$ & $\begin{array}{r}10 \\
8 \\
7\end{array}$ & $\begin{array}{ll}10.29 & 11.94 \\
10.29 & 11.94 \\
10.28 & 11.94\end{array}$ & $\begin{array}{cc}2 \frac{1}{2}-1 \frac{1}{3} & 3 s^{4} P-3 p^{4} s^{0} \\
1 \frac{1}{3}-1 \frac{3}{1} & (3) \\
\frac{1}{2}-1 \frac{1}{2} & \end{array}$ \\
\hline $\begin{aligned} & 4253.28 \\
- & 4254.7\end{aligned}$ & $\begin{array}{l}B \\
D\end{array}$ & $\begin{array}{l}4 \\
4\end{array}$ & $\begin{array}{rr}10.29 & 13.19 \\
10.29 & 13.19 \\
10.28 & 13.18\end{array}$ & $\begin{array}{ll}2 \frac{1}{2}-3 \frac{1}{2} & 3 \mathrm{~s}^{4} \mathrm{P}-4 \mathrm{p}^{4} \mathrm{D}^{0} \\
1 \frac{1}{2}-2 \frac{2}{2} & (4) \\
\frac{1}{2}-1 \frac{1}{2} & \end{array}$ \\
\hline $\begin{array}{l}4223.04 \\
4230.35 \\
4224.74 \\
4214.73 \\
4215.92\end{array}$ & $\begin{array}{l}\text { B } \\
\text { E } \\
B \\
B \\
B\end{array}$ & $\begin{array}{l}5 \\
4 \\
4 \\
5 \\
2\end{array}$ & $\begin{array}{ll}10.29 & 13.21 \\
10.29 & 13.21 \\
10.29 & 13.21 \\
10.29 & 13.21 \\
10.28 & 13.21\end{array}$ & $\begin{array}{c}2 \frac{1}{3} 8^{4} p-4 p^{4} p^{0} \\
(5)\end{array}$ \\
\hline $\begin{array}{l}4151.46 \\
4143.42 \\
4137.63\end{array}$ & $\begin{array}{l}\mathrm{B} \\
\mathrm{P} \\
\mathrm{B}\end{array}$ & $\begin{array}{r}12 \\
7\end{array}$ & $\begin{array}{ll}10.29 & 13.26 \\
10.29 & 13.26 \\
10.28 & 13.26\end{array}$ & $\begin{array}{l}2 \frac{1}{2}-1 \frac{1}{3} \\
1 \frac{1}{2}=1 \frac{1}{2} p-4 p^{4} s^{0} \\
\frac{1}{2}=1 \frac{1}{2}\end{array}$ \\
\hline
\end{tabular}

$58.1960 .80-6^{2} D-8^{2} P$ 
REVISED UULTIPLET TABLE 5372.66 11.71

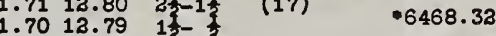
6437.0 5623.20

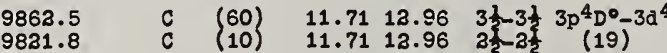

$\underset{\text { Lom }}{E P}$ H1gh $\quad$ Vultiplet

$11.71 \quad 13.57 \quad 3 \frac{1}{3}-2 \frac{1}{3} \quad 3 p^{4} p^{0}-5 s^{4} p$

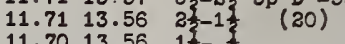

11.7013 .56

11.7013 .56

11.7013 .56

$\begin{array}{ll}11.71 & 13.62 \\ 11.71 & 13.61\end{array}$

11.7013 .61

11.7013 .60

11.7113 .61

11.7113 .61

$1 \frac{1}{2}-1 \frac{2}{2}$

(11.71 $13.62 \quad 3 \frac{1}{3}-3 \frac{1}{3} 3 p^{4} D^{0}-4 d^{4} D 1$

$\begin{array}{rrrr}11.71 & 13.62 & 3 \frac{2}{2}-2 \frac{1}{3} & (32) \\ 11.70 & 13.61 & 1 & 1\end{array}$

$11.71 \quad 13.63 \quad 3 \frac{1}{2}-2 \frac{1}{3} 3 p^{4} p^{0}-4 d^{4} p$

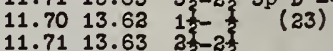

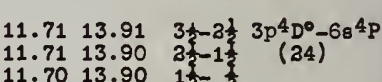




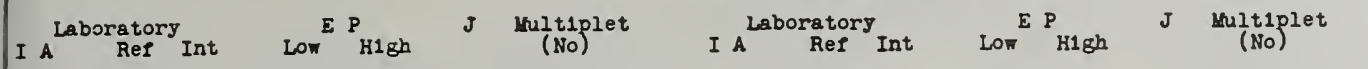 I cont1nued \\ NII oont1nued}

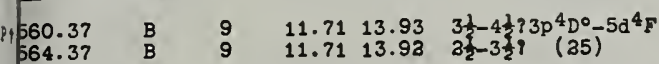

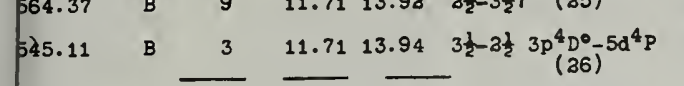

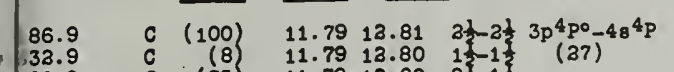

388.8

138.8

303.

539.0

506.5
548.0

$\left.\begin{array}{ll}c & \left(\begin{array}{l}75 \\ c \\ c\end{array}\right. \\ 30 \\ 75\end{array}\right\}$

$\begin{array}{lll}11.79 & 12.80 & 3 \\ 11.79 & 12.81 & 1 \\ 11.79 & 13.80 & \end{array}$

c (135) $11.79 \quad 13.96 \quad 3 \frac{1}{3}-3 \frac{1}{2} \quad 3 \mathrm{p}^{4} \mathrm{PO}^{\circ}-3 \mathrm{~d}^{4} \mathrm{D}$ $\left(\begin{array}{l}70 \\ 60\end{array}\right)$

$\begin{array}{lll}11.79 & 13.96 & 31-3 \\ 11.79 & 13.96 & 13 \\ 11.79 & 13.96 & 31\end{array}$

$11.79 \quad 13.57 \quad 3 \frac{1}{2}-2 \frac{1}{2} 3 p^{4} p^{0}-58^{4} p+$ $\begin{array}{lll}11.79 & 13.56 & 3 \\ 11.79 & 13.57 & 1 \frac{1}{2}-3\end{array}$

11.7913 .63 2t $3 \frac{1}{2} 3 p^{4} P^{\circ}-4 a^{4} D$

$11.7913 .63 \quad 1 \frac{1}{2}-3 \frac{1}{2} \quad(30)$

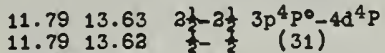
$\begin{array}{lll}11.79 & 13.63 & 1 \frac{1}{3}-3 \frac{2}{2} \\ 11.79 & 13.63 & 1 \frac{1}{2}\end{array}$

11.7913 .91 at $-313 p^{4} p^{0}-68^{4} p+$ $11.7913 .90 \quad 13-1$ (33) $\quad 4488.15$ $\begin{array}{lll}11.79 & 13.90 & 3 \\ 11.79 & 13.91 & 1 \frac{1}{2}-2\end{array}$

$11.7913 .942 \frac{1}{2}-2 \frac{1}{2} 3 p^{4} p^{0}-5 \mathrm{~d}^{4} \mathrm{p}$

3338.79

3331.32

3318.14

C (100) $11.94 \quad 12.96 \quad 1 \frac{1}{3}-3 \frac{1}{4} 93 \mathrm{p}^{4} 8^{\circ}-3^{4} \mathrm{P}$

$\begin{array}{ll}\text { C } & (75) \\ \text { C } & (150)\end{array}$

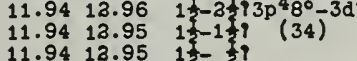

$11.9414 .09 \quad 1 \frac{1}{2}-2 \frac{1}{2} 3 p^{4} 8^{\circ}-78^{4} p$

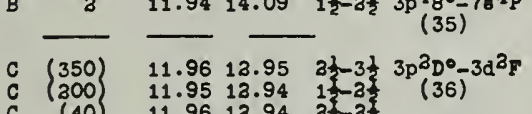

11.9613 .94

(60) $\quad 11.96 \quad 13.98 \quad 2 \frac{1}{2}-2 \frac{1}{2} \cdot 3 \mathrm{p}^{2} \mathrm{p}^{\circ}-3 \mathrm{~d}^{2} \mathrm{D}$

C $\begin{array}{llll}30 & 11.95 & 12.98 & 13 \\ 11.96 & 13.98 & 3 \frac{1}{2}-1 \frac{1}{2}\end{array}$

(37)

3593.60
3609.09
3615.88

1997.5

$\begin{array}{lllllll}4564.78 & C & 1 & 30.33 & 23.03 & 1-3 & 3 \mathrm{p}^{1} \mathrm{P}-3 \mathrm{~d}^{3} \mathrm{~F}^{\circ}\end{array}$

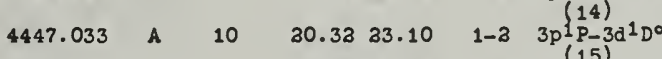

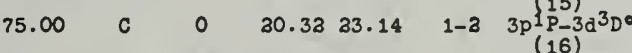
$20.32 \quad 23.47 \quad 1-1 \quad 3 p^{1} p_{-3 d^{1}} p^{\circ}$

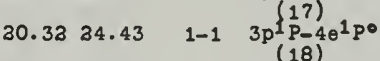
30.58 $23.04 \quad 3-4 \quad 3 p^{3} p-3 d^{3} F^{\circ}$ $30.56 \quad 23.03$
$30.56 \quad 33.03$ 30.56
30.53
33.03 30.58
30.56
33.03 30.56
30.58
33.03

$30.56 \quad 33.14$

30.5633 .15

$2-3$
$1-3$
$3-3$
$3-3$
$3-3$

(19)

5383.83

5007.316

4994.358
4987.377

709.45

$6065.5 \quad c \quad 0$

5941.67

5931.79

5953.39

5960.93

5495.70

5463.63

5454.26

5478.13
5453.13

3838.39 3847.38 3856.07
3855.08 3855.08
3829.80

N II I P 39.49 Anal B List A Oct 1943

3839.80
3843.20

4895. 3

6379.6

6365.

5679.

5876.02

5710.7
5686.3

$5730.6 ?$

5045.098

5010.620
5003.693

4630.537

4843.086

4621.393

4601.478
4607.153

3955.851

3408.138

6483.07

5747.35
5767.43

5073.60

4654.5

4667.38
4674.98

3994.996

3437.163 $1 D^{\circ}-3 p$
$(1)$

\section{$\frac{4}{5}$}

18.3930 .32

$\begin{array}{cccccc}c & 10 & 18.40 & 20.58 & 2-3 & 38^{3} \mathrm{Po}^{\circ}-3 \mathrm{p}^{3} \mathrm{D}\end{array}$

18.39
18.38
30.56

$18.39 \quad 20.56 \quad 1-1$

18.4030 .85

18.40
18.39
30.85

$3-$

$-1 \quad 3 s^{3} p 0-3 p^{3} s$

$8.38 \quad 30.85$
0.318

18.4031 .07
18.3921 .06
18.4021 .06

$\begin{array}{ll}18.40 & 21.06 \\ 18.39 & 21.06\end{array}$

$18.3931 .06 \quad 1-0$

$\begin{array}{lll}18.39 & 31.07 & 1-2 \\ 18.38 & 31.06 & 0-1\end{array}$

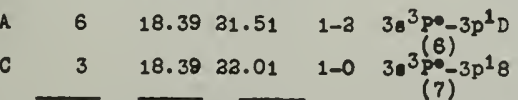

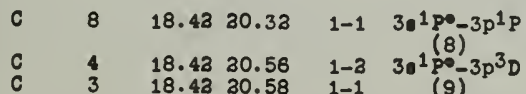

C $3 \quad 18.4320 .85 \quad 1-1 \quad 38^{1} p_{0}-3 p^{3} \mathrm{~g}$

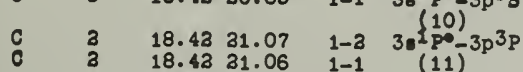

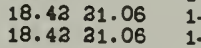

$18.42 \quad 21.51 \quad 1-3 \quad 38^{1} \mathrm{P}^{\bullet}-3 \mathrm{p}^{1} \mathrm{D}$

$18.4322 .01 \quad 1-0{ }_{38}^{\left(130^{2}-3 \mathrm{p}^{1} \mathrm{~g}\right.}$ $\begin{array}{lllllll}6610.58 & C & 6 & 31.51 & 33.37 & 2-3 & 3 \mathrm{p}^{1} \mathrm{D}-3 \mathrm{~d}^{1} \mathrm{~F}^{\circ}\end{array}$

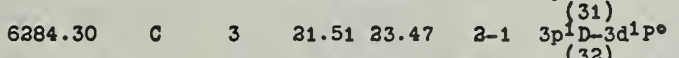

4337.749

5104.45

3033.80

6167.88

6173.40

8136.9
6150.9

6150.9
6114.8

4087.35

4095.92

4082.85
4076.83
4083.380

4073.055

4041.331

4043.537
4035.087

4057.00
4044.75

4038.080

6630.5

4176.164

4171.608

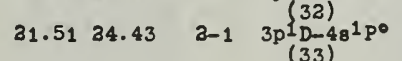

$-1$

$\begin{array}{ll}33.04 & 25.04 \\ 23.03 & \mathbf{2 5} .03\end{array}$

$\begin{array}{ll}33.03 & 35.03 \\ 33.02 & 25.03\end{array}$

$33.03 \quad 35.04$

23.02
33.03
35.03

$33.03 \quad 36.05$

33.0436 .08

$\infty \quad 33.0336 .05$

33.02
33.03
36.08

33.0338 .05

23.0438 .10

33.0338 .08

23.03
33.0438 .08
38.08

33.0338 .08

$4-3$
$3-2$
$2-1$
$3-3$
$2-2$
$3-3$

(35)

$33.03 \quad 36.10 \quad 3-4 \quad 38^{3} F^{\circ}-4 I^{1} \sigma$

$3-3$
$3-3$
$3-4$

$3-3$

$4-5 \quad 3 d^{3} r^{0}-4 r^{3} G$

$3-4$
$3-3$

$3-3$
$4-4$
$3-3$

$23.1034 .96 \quad 2-1 \quad 3 d^{1} D^{\bullet}-4 p^{1} p$

23.1026 .05 2-3 $3 \mathrm{~d}^{(41)}\left(\mathrm{D}^{\circ}-4 \mathrm{I}^{1} \mathrm{~F}\right.$
$30.58 \quad 23.15$

$30.56 \quad 33.14$
30.5833 .14

30.5833 .14
30.5633 .14

20.5623 .14

$30.58 \quad 33.31$

30.5633 .32

$30.56 \quad 33.32$

$30.56 \quad 33.31$
$30.56 \quad 33.32$

$30.58 \quad 34.38$

$30.56 \quad 34.37$

30.5634 .26

30.5634 .38

30.5624 .27

30.8533 .14

3-3 $3 p^{3} p-3 d^{3} D^{\circ}$

$1-1$
$3-2$

$3-3$

$3-2 \quad 3 p^{3} p-3 d^{3} p^{0}$

$2-1$
$1-0$
$3-3$

$1-1$

3-2 $3 p^{3} D-48^{3} p^{0}$

$3-1$
$1-0$
$3-3$

$3-2$
$1-1$

$\begin{array}{lll}1-3 & 3 p^{3} S-3 d^{3} D^{0} & 0\end{array}$

30.8523 .31

$30.85 \quad 33.32$

$20.85 \quad 33.47$

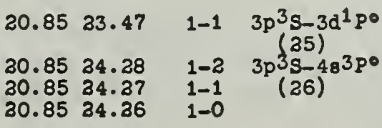

$21.06 \quad 33.10 \quad 1-3 \quad 3 p^{3} P-3 d^{1} D^{\circ}$

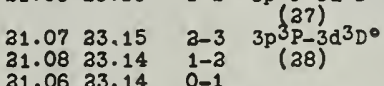

31.0733 .14

31.06
31.0733 .14
33.14

$21.07 \quad 33.31$

$\begin{array}{lll}21.06 & 33.33 \\ 31.07 & 33.32\end{array}$

31.0633 .32

$31.06 \quad 33.31$
$31.06 \quad 33.32$

21.0734 .38

$\begin{array}{ll}31.06 & 34.28 \\ 31.07 & 34.27\end{array}$

31.0734 .37
31.0834 .36

31.0634 .38

$31.08 \quad 34.37$

$1-3$
$0-1$
$2-3$

$1-1$
$3-1$

a-3 $3 \mathrm{p}^{3} \mathrm{p}-3 \mathrm{~d}^{3} \mathrm{po}$

$1-1$
$3-1$
$1-0$

$1-2$
$0-1$

$\begin{array}{cc}3-3 & 3 p^{3} p-48^{3} p^{\circ} \\ (30) & 0\end{array}$

$1-1$
$3-1$
$1-0$

$1-0$
$1-2$

32.01 $34.43 \quad 0-1 \quad 3 p^{1} s-48^{1} p^{\circ}$

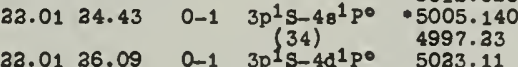

5033.11

5011.24

$3-3 \quad 3 d^{3} F^{0}-4 I^{1} F \quad \cdot 5179.50$

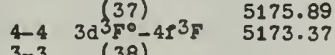

$23.1036 .05 \quad 2-3 \quad 3 d^{(42)}(43)^{\circ}-4 I^{3} F$

-4341.787

4181.17

4179.667
4173.51

$\because 4156.8$

- 4160.8

7139.8

7217.0
7259.3

6942.9

6976.8

7015.3

6813.36
6836.3

4432.739
4441.99

4433.48
4431.83

4427.21

6343.53

4694.55

.5535 .39

5526.26

5543.49

5565.30

5552.54
5540.16

5013.036

.4994 .358

4145.764

4133.669

4134.081

- 5173.32

5190.48

5180.34

5199.50

4860.35

4709.45

4731.59

4713.13
4704.33

4698.62

4706.41
4700.13

4700.12
4695.91
A Laboratory Ref Int Low H1gh

$\underset{(\text { No })}{\text { Multiplet }}$

N II continued

$4110.00 \quad C \quad$ On $\quad 23.10 \quad 36.10 \quad 3-2 \quad 3 d^{1} D^{\circ}-41^{3} D$

6504.9
6533.0

6493.0

6340.67

6357.0

6347.1

336.030 A

4553.536

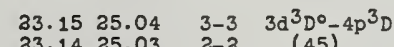


W J Multiplet J J J $P$ J Multiplet N II continued

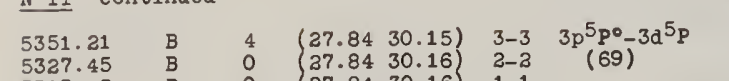
5313.43 5340.20 5320.96
5338.66 .5320 .96

.5179 .50 5171.46 5168.24 5183.21 5174.46
5170.08 5170.08
5186.17

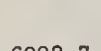

6888.7
6870.8 6870.8
6857.6

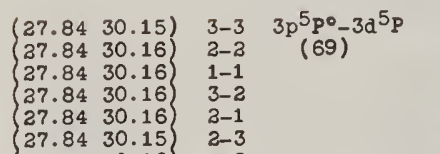

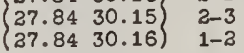

$\left(\begin{array}{llll}37.84 & 30.23) & 3-4 & 3 p^{5} p^{0}-3 d^{5} \mathrm{D}\end{array}\right.$ $\left\{\begin{array}{ll|l}37.84 & 30.23 & 2-3 \\ 27.84 & 30.22 & 1-2\end{array}\right.$ $\left\{\begin{array}{lll}27.84 & 30.23 & 3-3 \\ 27.84 & 30.22 & 3-2 \\ 27.84 & 30.22 & 1-1\end{array}\right.$ $\left\{\begin{array}{lll}27.84 & 30.22 & 2-2 \\ 27.84 & 30.22) & 1-1\end{array}\right.$ $\begin{array}{lll}27.84 & 30.22 & 1-1 \\ 27.84 & 30.32) & 3-2\end{array}$

$(28.36 \quad 30.15) \quad 3-3 \quad 3 p^{5} \mathrm{~s}^{0}-3 \mathrm{~d}^{5} \mathrm{p}$

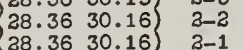

Laboratory
ReS Int Low ${ }_{\text {H1gh }}^{\text {E }}$ J Muitiplet
(No) N III continued

4544.80 4535.11
4527.86

6466.86

6453.95

6478.69

6463.03
6450.78

6487.55
6468.77

5314.45

5260.91

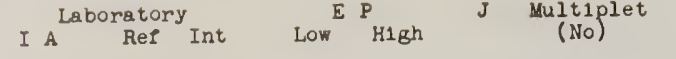
45
45
45
6
66
66
6
6
6
6
6
5
5
5
5

$4546.36 \quad \mathrm{~A} \quad 3 \quad 38.7941 .51 \quad 1 \frac{1}{2}-3 \frac{1}{4} 3 \mathrm{p}^{4} \mathrm{~S}-3 \mathrm{~d}^{4} \mathrm{po}$

5298.93
5272.60

5297.86
5270.59

NIII I P 47.34 Anal A L18t A Apr 1943

\begin{tabular}{|c|c|c|}
\hline $\begin{array}{l}4097.31 \\
4103.37\end{array}$ & $\begin{array}{l}A \\
A\end{array}$ & $\begin{array}{r}10 \\
9\end{array}$ \\
\hline $\begin{array}{l}4640.64 \\
4634.16 \\
4641.90\end{array}$ & $\begin{array}{l}A \\
A \\
A\end{array}$ & $\left.\begin{array}{r}(10) \\
9 \\
7\end{array}\right)$ \\
\hline 4514.89 & A & 7 \\
\hline 4510.92 & A & 6 \\
\hline $\begin{array}{l}4534.57 \\
4523.60 \\
4518.18 \\
4547.34 \\
4530.84\end{array}$ & $\begin{array}{l}\mathrm{A} \\
\mathrm{A} \\
\mathrm{A} \\
\mathrm{A} \\
\mathrm{A}\end{array}$ & 3 \\
\hline $\begin{array}{l}3771.08 \\
3754.62 \\
3745.83\end{array}$ & A & 4 \\
\hline $\begin{array}{l}367.36 \\
361.90 \\
358.72 \\
374.06 \\
365.79 \\
354.29 \\
353.78\end{array}$ & $\begin{array}{l}\hat{A} \\
\mathrm{~A} \\
\mathrm{~A} \\
\mathrm{~A} \\
\mathrm{~A}\end{array}$ & 4 \\
\hline
\end{tabular}

$4200.02 \quad \mathrm{~A}=\frac{6}{36.70} 39.64-1 \frac{1}{3}-2 \frac{1}{3} 38^{2} \mathrm{po}^{\circ}-3 \mathrm{p}^{2} \mathrm{D}$

4195.70

3355.47

3355.47
3342.77

3938.52

3934.41
3942.78

.4867 .18

4861.33
4858.88

4858.74

4884.14

4867.18

4896.71
4881.81

4348.36

4335.53
4328.15

4328.15
-4323.93

4353.66

4339.52
4330.44

4330.14

4333.93
4331.37

3792.87

3771.45

3779.23

3762.62
3752.65

3770.37
3757.60 $\begin{array}{llll}27.32 & 30.33 & \frac{1}{3}-1 \frac{1}{3} & 3^{2} \mathrm{~s}-3^{3} \mathrm{po} \\ 27.32 & 30.33 & \frac{1}{2}-\frac{3}{2} & (1)\end{array}$

30. 33 32. $39 \quad 1 \frac{1}{2}-2 \frac{1}{2} 3^{2} \mathrm{P}^{\mathrm{O}}-3^{2} \mathrm{D}$

$\begin{array}{llll}30.33 & 32.99 & 1 \frac{1}{2}-2 \frac{1}{3} & 3^{2} \mathrm{P}^{0}-3^{2} \\ 30.33 & 32.99 & \frac{1}{3} & (\text { a } \\ 30.33 & 32.99 & 1 \frac{1}{2}-1 \frac{3}{2} & \end{array}$

$\begin{array}{llll}35.52 & 38.25 & 2 \frac{1}{2}-3 \frac{1}{2} & 38^{4} \mathrm{po}^{\circ}-3 \mathrm{p}^{4} \mathrm{D} \\ 35.50 & 38.24 & 1 \frac{1}{3}-2 \frac{1}{3} & (3)\end{array}$

$\begin{array}{lll}35.50 & 38.23 & \\ 35.52 & 38.24 & 2\end{array}$

$\begin{array}{lll}35.52 & 38.24 & 2 \frac{1}{2} \\ 35.50 & 38.23 & 1 \\ 3 & 1\end{array}$

$\begin{array}{lll}35.50 & 38.23 & \frac{3}{3} \\ 35.52 & 38.23 & 2 \frac{1}{3} \\ 35.50 & \frac{3}{2}\end{array}$

$35.5038 .23 \quad 1 \frac{1}{2}-\frac{1}{2}$

$35.5238 .79 \quad 3 \frac{1}{2}-1 \frac{1}{3} \quad 38^{4} \mathrm{po}^{\circ}-3 \mathrm{p}^{4} \mathrm{~s}$ $\begin{array}{llll}35.50 & 38.79 & 1 \frac{1}{1}-1 \frac{3}{2} & (4)\end{array}$

$35.52 \quad 39.18 \quad 2 \frac{1}{3}-2 \frac{1}{3} \quad 38^{4} \mathrm{po}^{\circ}-3 \mathrm{p}^{4} \mathrm{P}$ $35.5039 .181^{1 \frac{2}{2}-1 \frac{1}{2}}(5)^{-3 p}$ 35. 5039.17 2

$35.50 \quad 39.17$ (1)

$\begin{array}{lll}35.50 & 39.18 & 1 \frac{2}{2}-2 \frac{3}{3} \\ 35.50 & 39.18 & \end{array}$

4003.64

3998.69

4379.09

Unclassified Lines of N III

$\begin{array}{lll}4294.76 & \text { A } & \text { On } \\ 4290.80 & \text { A } & 3 n \\ 4290.55 & \text { A } & 1 n \\ 4288.72 & \text { A } & 1 n \\ 4288.21 & \text { A } & \text { On } \\ 4284.51 & \text { A } & 1 \mathrm{n} \\ 3172.97 & \text { A } & 3 \\ 3171.14 & \text { A } & 1\end{array}$

See NSRDS-NBS 3 , SEVISED

See NSRDS-NBS 3, Section 4,
N IV I P 77.09 Anal B L18t A Feb 1943

$\begin{array}{llllll}3478.69 & A & ? & (46.57 & 50.11 \\ 3482 & \text { A } & 5 & 1-3 & 3^{3} \mathrm{~s}-3^{3} \mathrm{po} & 0 \\ 1-1 & & (1)\end{array}$ 3484

$\begin{array}{lll}36.68 & 39.62 & \\ 36.70 & 39.62 & 1 \frac{1}{2}-1 \frac{1}{2}\end{array}$

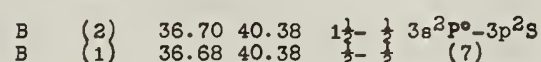

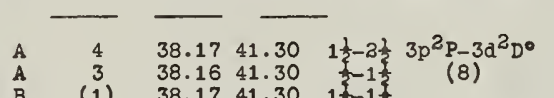

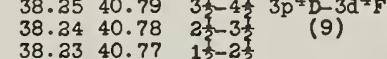

$\begin{array}{lll}38.23 & 40.77 & 1 \\ 38.25 & 40.78 & 3 \frac{1}{3}\end{array}$

$\begin{array}{lll}38.25 & 40.78 & 3 \frac{1}{3}-3 \frac{3}{3} \\ 38.24 & 40.77 & 3 \frac{3}{3}-2 \frac{1}{3}\end{array}$

$\begin{array}{lll}38.23 & 40.77 & 1 \frac{3}{3}-1 \frac{2}{2} \\ 38.25 & 40.77 & 3 \frac{3}{3} \\ 38.24 & 40.77 & 2 \frac{3}{2}-1 \frac{3}{2}\end{array}$

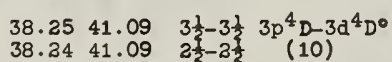

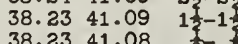

$\begin{array}{llll}38.23 & 41.08 & \\ 38.25 & 41.09 & 3 & 2\end{array}$

$\begin{array}{lll}38.24 & 41.09 & 25 \\ 38.23 & 41.08 & 1\end{array}$

38.3341 .08

38.2441 .09

$\begin{array}{rrrr}38.23 & 41.09 & 1 \frac{1}{2}-2 \frac{2}{3} \\ 38.23 & 41.09 & \frac{1}{2}-1 \frac{2}{2}\end{array}$

$\begin{array}{llll}38.25 & 41.51 & 3 \frac{1}{2}-2 \frac{1}{3} & 3 \mathrm{p}^{4} \mathrm{D}-3 \mathrm{a}^{4} \mathrm{p}^{\circ} \\ 38.24 & 41.51 & 2 \frac{2}{2}-1 \frac{3}{2} & (11)\end{array}$

$\begin{array}{lll}38.23 & 41.52 & 1 \% \\ 38.24 & 41.51 & 2\end{array}$

38.2341 .51

38.2341 .52

$\begin{array}{rrr}38.33 & 41.51 & 1 \frac{1}{3}-2 \frac{1}{3} \\ 38.23 & 41.51 & \frac{1}{2}-1 \frac{1}{2}\end{array}$
38.25 $40.79 \quad 3 \frac{1}{2}-4 \frac{1}{3} 3 p^{4} D-3 d^{4} F^{0}$
6383

\begin{tabular}{|c|c|c|c|c|c|c|}
\hline 6383 & P & & 48.00 & 49.94 & $0-1$ & $\begin{array}{c}3^{1} s-3^{1} p^{\circ} \\
\text { (a) }\end{array}$ \\
\hline 4057.80 & B & 3 & 49.98 & 52.98 & $1-2$ & $\begin{array}{c}3^{1} P^{0}-3^{1} D \\
(3)\end{array}$ \\
\hline $\begin{array}{l}7123.10 \\
7109.48 \\
7103.28 \\
7127.21 \\
7111.28 \\
7129\end{array}$ & $\begin{array}{l}A \\
A \\
A \\
A \\
A \\
\text { P }\end{array}$ & $\begin{array}{l}5 \\
3 \\
1 \\
1 \\
1\end{array}$ & $\left\{\begin{array}{l}50.11 \\
50.11 \\
50.11 \\
50.11 \\
50.11 \\
50.11\end{array}\right.$ & $\begin{array}{l}51.85 \\
51.85 \\
51.85 \\
51.85 \\
51.85 \\
51.85)\end{array}$ & $\begin{array}{l}2-3 \\
1-2 \\
0-1 \\
2-2 \\
1-1 \\
3-1\end{array}$ & $\begin{array}{c}3^{3} P^{\circ}-3^{3} D \\
(4)\end{array}$ \\
\hline $\begin{array}{l}5245 \\
5236 \\
5281\end{array}$ & $\begin{array}{l}P \\
P \\
P\end{array}$ & & $\left\{\begin{array}{l}57.46 \\
57.44 \\
57.46\end{array}\right.$ & $\begin{array}{l}59.81 \\
59.80 \\
59.80\end{array}$ & $\begin{array}{l}2-3 \\
1-2 \\
2-2\end{array}$ & $\begin{array}{c}38^{3} p^{0}-3 p^{3} D \\
(5)\end{array}$ \\
\hline $\begin{array}{l}4528 \\
4495 \\
4479\end{array}$ & $\begin{array}{l}P \\
P \\
P\end{array}$ & & $\left\{\begin{array}{l}57.46 \\
57.44 \\
57.43\end{array}\right.$ & $\begin{array}{l}60.19 \\
60.19 \\
60.19\end{array}$ & $\begin{array}{l}2-1 \\
1-1 \\
0-1\end{array}$ & $\begin{array}{c}3 s^{3} p^{\circ}-3 p^{3} s \\
(6)\end{array}$ \\
\hline $\begin{array}{l}3463.36 \\
3454 \\
3474.56 \\
3461.34 \\
3443 \\
3445\end{array}$ & $\begin{array}{l}B \\
P \\
B \\
B \\
P \\
P\end{array}$ & $\begin{array}{l}1 \\
0 \\
0\end{array}$ & $\left\{\begin{array}{l}57.46 \\
57.44 \\
57.46 \\
57.44 \\
57.44 \\
57.43\end{array}\right.$ & $\begin{array}{l}61.03 \\
61.01 \\
61.01 \\
61.01 \\
61.03 \\
61.01\end{array}$ & $\begin{array}{l}2-3 \\
1-1 \\
3-1 \\
1-0 \\
1-2 \\
0-1\end{array}$ & $\begin{array}{c}3 s^{3} p^{0}-3 p^{3} p \\
(7)\end{array}$ \\
\hline 3747.66 & B & 0 & 58.44 & 61.69 & $1-2$ & $\begin{array}{c}3 s^{1} p^{\circ}-3 p^{1} D \\
(8)\end{array}$ \\
\hline 5734 & $P$ & & 59.36 & 61.53 & $1-3$ & $3 p^{1} P-3 a^{1} D^{\circ}$ \\
\hline 3824 & $P$ & & 59.36 & 63.59 & $1-1$ & $\begin{array}{c}3 p^{1} p_{-4}^{(9)} p^{1} \\
(10)\end{array}$ \\
\hline $\begin{array}{l}4753 \\
4733 \\
4762 \\
4740 \\
4723\end{array}$ & $\begin{array}{l}P \\
P \\
P \\
P \\
P \\
P\end{array}$ & & $\left\{\begin{array}{l}59.81 \\
59.80 \\
59.81 \\
59.80 \\
59.80\end{array}\right.$ & $\begin{array}{l}62.41 \\
62.41 \\
62.41 \\
62.40 \\
62.41\end{array}$ & $\begin{array}{l}3-3 \\
2-2 \\
3-2 \\
2-1 \\
2-3\end{array}$ & $\begin{array}{c}3 p^{3} D-3 d^{3} D^{0} \\
(11)\end{array}$ \\
\hline $\begin{array}{l}3714 \\
3689 \\
3696\end{array}$ & $\begin{array}{l}\mathrm{P} \\
\mathrm{P} \\
\mathrm{P}\end{array}$ & & $\left\{\begin{array}{l}59.81 \\
59.80 \\
59.80\end{array}\right.$ & $\left.\begin{array}{l}63.14 \\
63.14 \\
63.14\end{array}\right\}$ & $\begin{array}{l}3-2 \\
2-1 \\
2-2\end{array}$ & $\begin{array}{c}3 p^{3} D-3 d^{3} p^{0} \\
(12)\end{array}$ \\
\hline
\end{tabular}

I A Raboratory Rnt

E P

J Kultipler

N IV continued

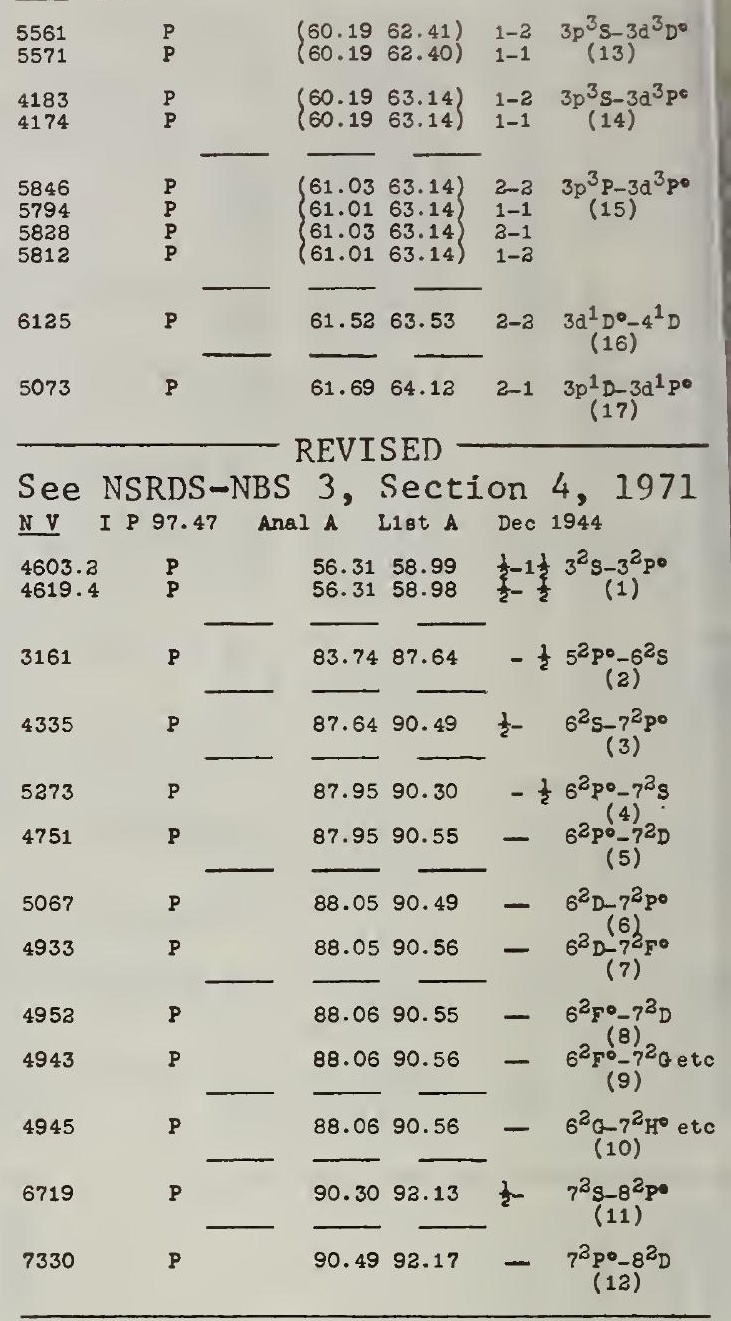

O I I P 13.56 Ansl A L18t B Apr 1944

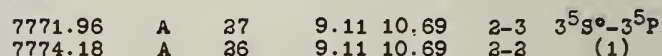

$\begin{array}{lllllll}7774.18 & \text { A } & 36 & 9.11 & 10.69 & 3-2 & (1) \\ 7775.40 & \text { A } & 25 & 9.11 & 10.69 & 3-1 & \end{array}$

$\begin{array}{ccccccc}6726.25 & A & 5 & 9.11 & 10.94 & 2-2 & 3^{5} \mathrm{~s}^{\circ}-3^{3} \mathrm{P} \\ 6726.50 & A & 2 & 9.11 & 10.94 & 2-1 & (3)\end{array}$

$\begin{array}{ccccccc}3947.301 & \mathrm{E} & 10 & 9.11 & 12.33 & 2-3 & 3^{5} 8^{\circ}-4^{5} \mathrm{P} \\ 3947 & 7 & 9.11 & 12.23 & 2-2 & (3)\end{array}$

$\begin{array}{rrrrrrr}3947.489 & E & 7 & 9.11 & 12.23 & 2-2 & (3) \\ 3947.594 & E & 4 & 9.11 & 13.33 & 2-1 & \end{array}$

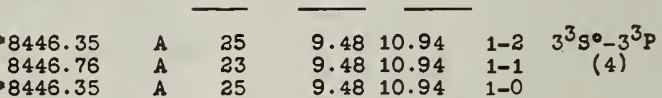

$4368.30 \quad D \quad(10) \quad 9.4813 .31 \quad 1-3^{3} s^{\circ}-4^{3} \mathrm{p}$

$3693.44 \quad D \quad(7)$

$9.4812 .821-\quad 3^{3} \mathrm{~S}^{0}-5^{3} \mathrm{P}$

$\begin{array}{lllllll}11302.32 & \text { B } & 15 & 10.69 & 11.79 & 3-3 & 3^{5} \mathrm{P}-4^{5} \mathrm{~S}^{\circ}\end{array}$

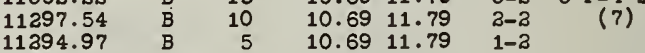

$\begin{array}{lllllll}9265.99 \quad A \quad & 16 & 10.69 & 13.03 & 3- & 3^{5} \mathrm{P}-3^{5} \mathrm{D}^{\circ}\end{array}$

$\begin{array}{llllll}9262.73 & \text { A } & 15 & 10.69 & 12.03 & 2- \\ 9260.88 & \text { A } & 14 & 10.69 & 12.03 & 1-\end{array}$

$\begin{array}{lllllll}6456.01 \quad A & 17 & 10.69 & 12.61 & 3-3 & 3^{5} \mathrm{P}-5^{5} \mathrm{~S}^{\circ}\end{array}$

$\begin{array}{lllllll}6454.48 & \mathrm{~A} & 16 & 10.69 & 12.61 & 3-2 & \text { (9) } \\ 6453.64 & \mathrm{~A} & 15 & 10.69 & 12.61 & 1-2 & \end{array}$

$\begin{array}{lllllll}6158.19 \quad A \quad 18 & 10.69 & 12.70 & 3- & 3^{5} \mathrm{P}-4^{5} \mathrm{D}^{\circ}\end{array}$

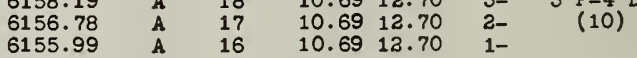

$\begin{array}{lllllll}5436.83 \quad D & (8) & 10.69 & 12.96 & 3-2 & 3^{5} \mathrm{P}-6^{5} \mathrm{~S}^{\circ}\end{array}$

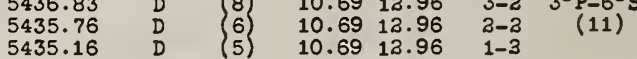




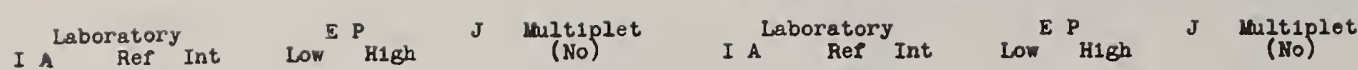

OI contiuued

$9522.01 \quad A$

9499.39

9599.3

.9498 .0

9487.49
9498.04

6266.8

6264.5

. 6261.55

6256.84
.6261 .55

.5410 .76

.5410 .76

5408.5
5404.8

8426.326

8428.342
8429.128

8429.128
8420.968

8434.730

7476.45
7479.06

7479.06
7480.66

7473.23

7477.21
7471.36

9760.65

9741.4

9741.4

6374.3

6366.33

6351.17

5492.8

5486 .

7886.3

7886.31
6653.78

OII continued

$14.0415 .34 \quad 4-53 p^{\prime} 3^{T}-3 d^{\prime} 3 g^{\circ}+3864.13$

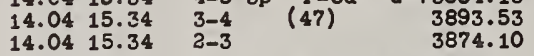

$\begin{array}{llllll}14.04 & 16.01 & 4-4 & 3 p^{\prime} & 3 F-4 d^{\prime} & 3 F^{\circ} 3883.197 \\ (48) & 3864.45\end{array}$

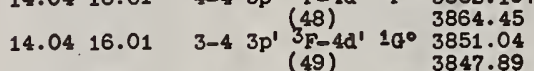

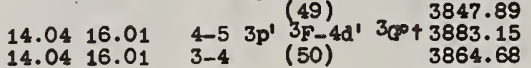

$\begin{array}{lllll}14.04 & 16.01 & 3-4 & (50) & 3864.68 \\ 14.04 & 16.01 & 3-3 & & 3856.16\end{array}$

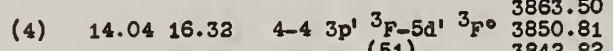

(4) $\quad 14.04 \quad 16.33 \quad 3-4 \quad 3 p^{\prime} 3 F_{-5 d^{\prime}} 1 q^{\circ} 3843.82$

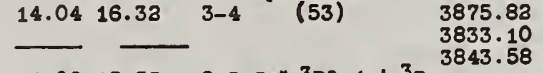

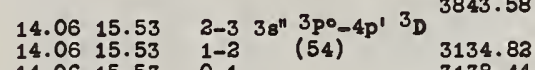

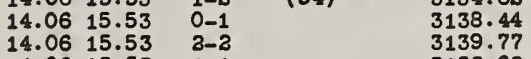

$\begin{array}{llll}14.06 & 15.53 & 3-2 & 3122.62 \\ 14.06 & 15.53 & 1-1 & 3139.44\end{array}$

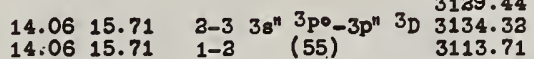

$\begin{array}{lllll}14.06 & 15.71 & 1-3 & (55) & 3113.71 \\ 14.06 & 15.71 & 0-1 & & 3134.03\end{array}$

$\begin{array}{lll}14.06 & 15.71 & 0-1 \\ 14.06 & 15.71 & 3-3\end{array}$

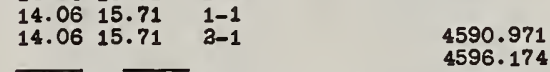

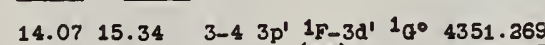

$\begin{array}{llll}14.07 & 15.34 & 3-43 p^{\prime} 1 F-3 a^{\prime} 3 j^{\circ} & 4347.425\end{array}$

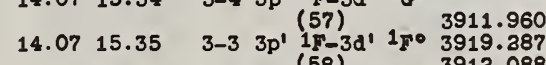

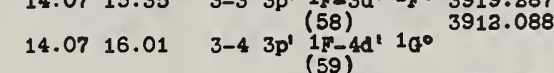

$\begin{array}{llll}14.07 & 16.01 & 3-4 \quad 3 p^{\prime} & (59) \\ F^{\prime}-4 d^{\prime} & 3 G^{\circ} & 4357.25\end{array}$

$14.0716 .02 \quad 3-33 \mathrm{p}^{\prime}{ }^{1 \mathrm{~F}-4 \mathrm{~d}^{\prime}} 1 \mathrm{~F}^{\circ} 4169.230$

(3) $\quad \begin{array}{llllll}14.07 & 16.32 & 3-4 & 3 p^{\prime} & (61) & 1\end{array}$

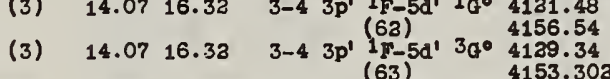

$4 \quad \overline{14.31} 15.38$ 1-3 38" 1po-3p" 1D 4133.806

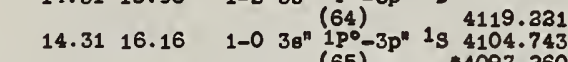

$\begin{array}{lll}35.53 & 38.71 & 1 \\ 35.54 & 38.70 & 35\end{array}$

$25.53 \quad 38.71$

\begin{tabular}{ll}
25.52 & 38.71 \\
25.53 & 38.70 \\
\hline
\end{tabular}

$\begin{array}{lll}25.53 & 38.70 & 1 \frac{3}{3}-2 \frac{2}{2} \\ 35.53 & 38.71 & \frac{3}{2}-1 \frac{2}{2}\end{array}$

$\begin{array}{llll}35.54 & 28.73 & 3 \frac{1}{2}-2 \frac{1}{4} \\ 25.53 & 38.73 & 1 \frac{1}{2}-1 \frac{1}{3}\end{array}$

35.5228 .73

25.5428 .73

25.5338 .73

35

$\begin{array}{lll}25.53 & 38.73 & 1 \frac{1}{\frac{1}{2}}-3 \frac{1}{3} \\ 25.53 & 38.73 & \frac{1}{2}-1 \frac{1}{2}\end{array}$

$\begin{array}{llll}35.55 & 28.76 & 3 \frac{1}{2}-3 \frac{1}{1} & 3 \mathrm{p}^{4} \mathrm{D}^{0}-3 \mathrm{~d}^{2} \\ 35.54 & 88.74 & 31-2 \frac{1}{2} & (13)\end{array}$

35.5538 .74

$\begin{array}{ll}35.54 & 38.76 \\ 35.53 & 38.74\end{array}$

$\begin{array}{lllll}81 & 35.54 & 39.47 & 32 & 1\end{array} \quad$ (14)

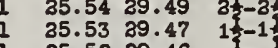

25.5329 .46

\begin{tabular}{rrr}
35.53 & 39.49 & $1 \frac{3}{3}-3 \frac{3}{2}$ \\
35.52 & 29.47 & $\frac{3}{2}-1 \frac{3}{2}$ \\
\hline
\end{tabular}

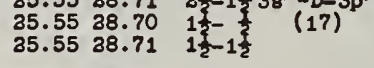

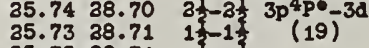

$\begin{array}{lll}35.73 & 38.71 & 5 \\ 35.74 & 38.71 & 3\end{array}$

35.73
35.71
35

$\begin{array}{lll}35.73 & 38.70 & 1 \frac{1}{2}-3 \frac{1}{2} \\ 25.73 & 38.71 & \frac{1}{2}-1\end{array}$

$35.73 \quad 38.73$ 13 $3 \frac{20}{30}$
$14.0415 .33 \quad 4-43 p^{\prime} 3 \bar{F}-3 d^{\prime} 3 F^{\circ} 3926.58$

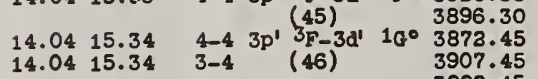

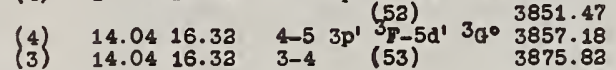

$(65)^{-3 p}+4097.260$ 4130.279

4105.000
41030.017

4103.017
4110.795

OIf I P 35.00 Anal A L1st A Mar $1944 \quad 4110.795$

\begin{tabular}{|c|c|c|c|c|c|}
\hline \multirow{3}{*}{$\begin{array}{l}4649.139 \\
4641.811 \\
4638.854 \\
4676.234 \\
4661.535 \\
4650.841 \\
4696.36 \\
4673.75\end{array}$} & $\begin{array}{l}A \\
A \\
A\end{array}$ & $\begin{array}{r}10 \\
9 \\
6\end{array}$ & 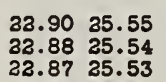 & 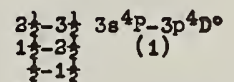 & \multirow{2}{*}{$\begin{array}{l}4084.66 \\
4096.543 \\
4112.029 \\
3967.441 \\
3985.46\end{array}$} \\
\hline & $\begin{array}{l}\mathrm{A} \\
\mathrm{A} \\
\mathrm{A} \\
\mathrm{B}\end{array}$ & $\begin{array}{l}8 \\
9 \\
6 \\
3\end{array}$ & $\begin{array}{ll}32.90 & 25.54 \\
32.88 & 25.53 \\
32.87 & 25.52 \\
22.90 & 35.53\end{array}$ & $\begin{array}{l}31 \\
13 \\
13\end{array}$ & \\
\hline & 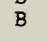 & & & & \\
\hline $\begin{array}{l}4349.426 \\
4330.865 \\
4325.77 \\
4366.896 \\
4345.562 \\
4319.531 \\
4317.139\end{array}$ & $\begin{array}{l}A \\
A \\
B \\
A \\
A \\
A\end{array}$ & $\begin{array}{l}8 \\
6 \\
3 \\
?\end{array}$ & 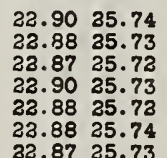 & $\begin{array}{c}3 \frac{1}{2} 3 \theta^{4} p-3 p^{4} p 0 \\
1(3)\end{array}$ & $\begin{array}{l}3301.56 \\
3305.15 \\
3306.60 \\
3377.59 \\
3390.13\end{array}$ \\
\hline
\end{tabular}

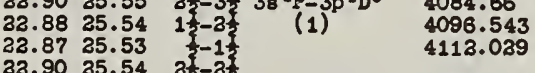

$33.8825 .53 \quad 11$

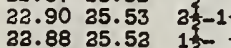

3749.49

3727.33

0721.35

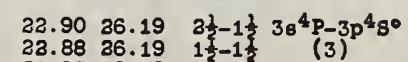

\begin{tabular}{lll}
23.88 & 36.19 & $1 \frac{1}{2}-1$ \\
32.87 & 36.19 & $\frac{1}{2}-1 \frac{1}{2}$ \\
\hline
\end{tabular}

4751.34
4710.04
4753.70

4705.355

$\begin{array}{llll}23.34 & 25.18 & 1 \frac{1}{2}-\frac{1}{2} & 38^{2} \mathrm{P}-3 \mathrm{p}^{2} \mathrm{~S}^{\circ} \\ 23.32 & 25.18 & (4) & \end{array}$

4699.21
4741.71

6640.90

4414.909

4416.975
4452.377

3273.263

3954.372

3945.048

3496.27

3488.18

3474.94

3390.25
3377.2

4075.868

4069.897

4069.636

4085.124

4078.862

4106.03
4094.18 $\begin{array}{llll}23.34 & 26.14 & 1 \frac{1}{2}-2 \frac{1}{4} & 3 \mathrm{~s}^{2} \mathrm{P}-3 \mathrm{p}^{2} \mathrm{p}^{\circ} \\ 23.33 & 26.11 & \frac{3}{2}-1 \frac{2}{2} & (5) \\ 33.34 & 36.11 & 1 \frac{1}{2}-1 \frac{3}{2} & \end{array}$

$\begin{array}{llll}23.34 & 26.45 & 1 \frac{1}{2}-1 \frac{1}{2} & 3 \mathrm{~B}^{2} \mathrm{P}-3 \mathrm{p}^{2} \mathrm{po} \\ 33.32 & 26.44 & (6)\end{array}$

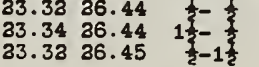

$25.1838 .71 \quad \frac{1}{3}-1 \frac{1}{3} 3 p^{3} g^{\circ}-3 d^{4} P$

$25.1838 .73 \frac{1}{2}-\frac{1}{2} 3 \mathrm{p}^{2} \mathrm{~g}^{\circ}-3 \mathrm{~d}^{4} \mathrm{D}$

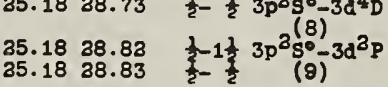

$25.5528 .58 \quad 3 \frac{1}{2}-4 \frac{1}{2} \quad 3 \mathrm{p}^{4} \mathrm{p}^{0}-3 \mathrm{~d}^{4} \mathrm{~F}$

25.5438 .57 ? $35-3 \frac{3}{3}(10)$

$25.5528 .57 \quad 3 \frac{1}{3}-3$

$25.5428 .56 \quad 31-3 \frac{1}{2}$

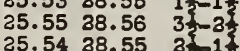

$25.53 \quad 38.56 \quad 1 \frac{1}{5}-3$

4395.95

4369.38
4406.03

4359.38

3470.81
3470.43

3470.42
3447.98

4924.60

4906.88
4890.93

4856.49

4856.7
4864.95

4845.01

3739.92

3762.63
3777.60

5306.73

5160.03

5176.00
5190.56

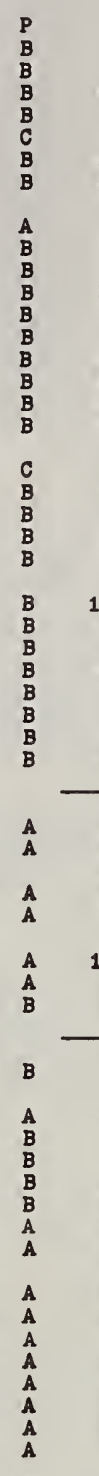

$\begin{array}{cccc}25.55 & 38.70 & 3 \frac{1}{2}-2 \frac{1}{3} & 3 p^{4} D^{0}-3 d^{4} P \\ 35.54 & 38.71 & 2 \frac{1}{2}-1 \frac{1}{2} & (11)\end{array}$

$\begin{array}{llll}25.55 & 28.73 & 3 \frac{1}{3}-3 \frac{1}{3} & 3 p^{4} D^{0}-3 d^{4} D \\ 35.54 & 28.73 & 3 \frac{1}{3}-2 \frac{5}{5} & (12)\end{array}$

$35.5528 .76 \quad 3 \frac{1}{3}-3 \frac{1}{2} 3 p^{4} p^{\circ}-3 d^{3} \bar{c}$

$101 \quad 25.5529 .49 \quad 3 \frac{1}{2}-3 \frac{1}{3} \quad 3 p^{4} D^{0}-48^{4} p$

$\begin{array}{llll}41 & 25.53 & 29.46 & 1\end{array}$

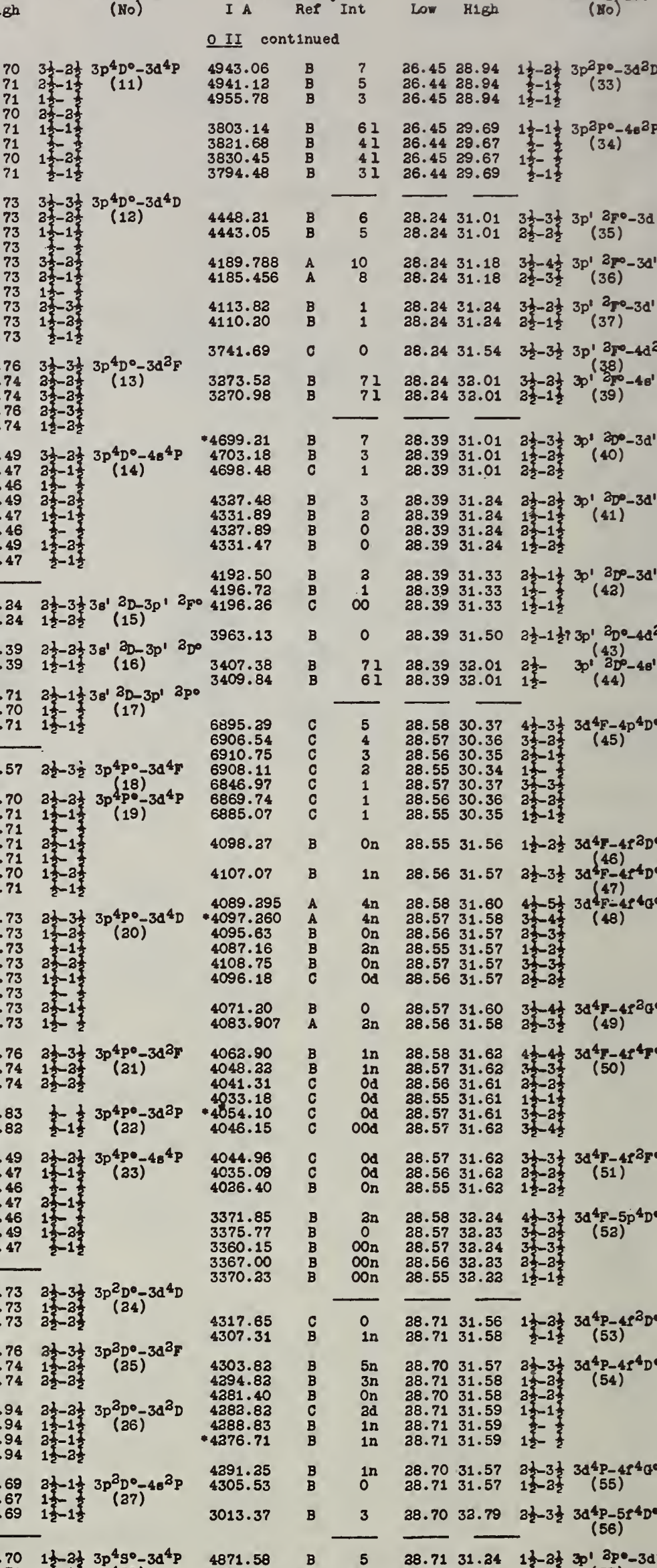

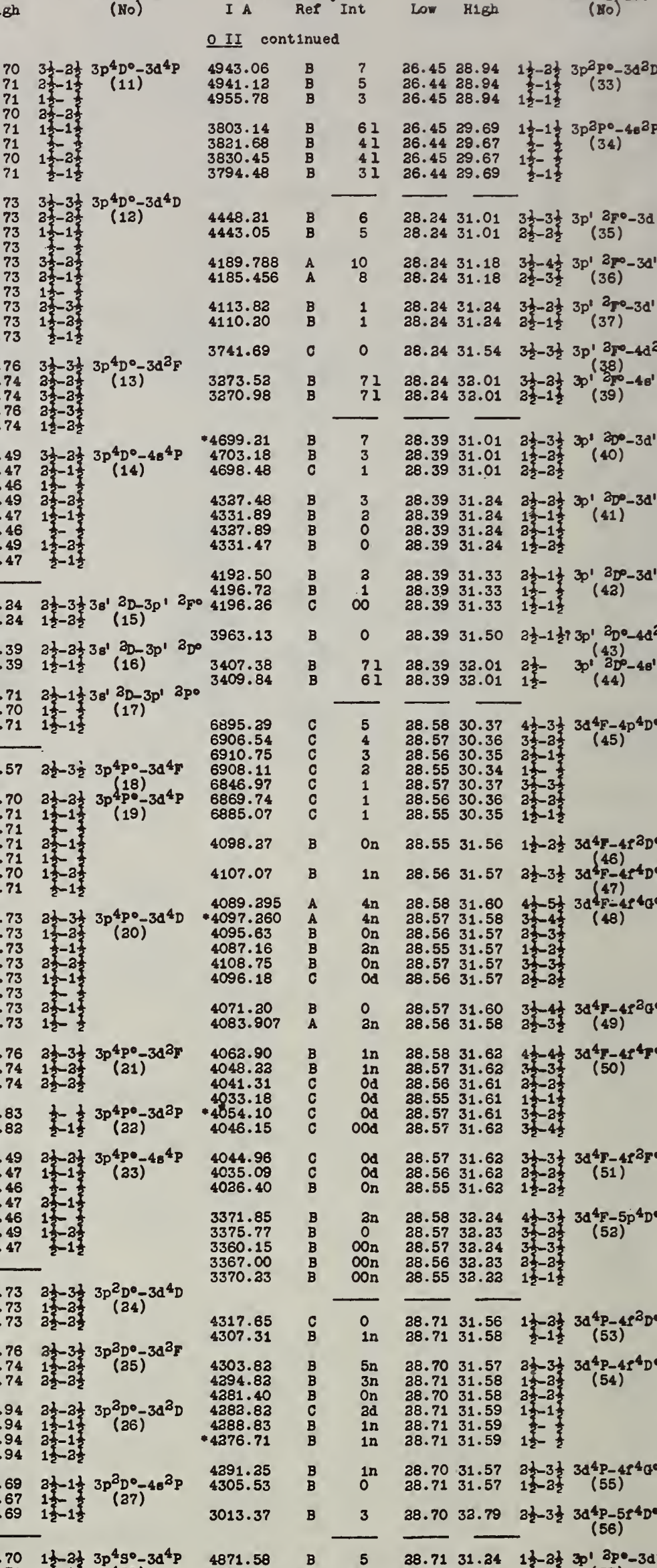

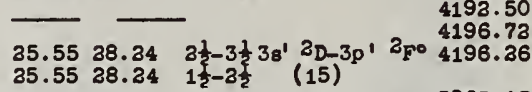

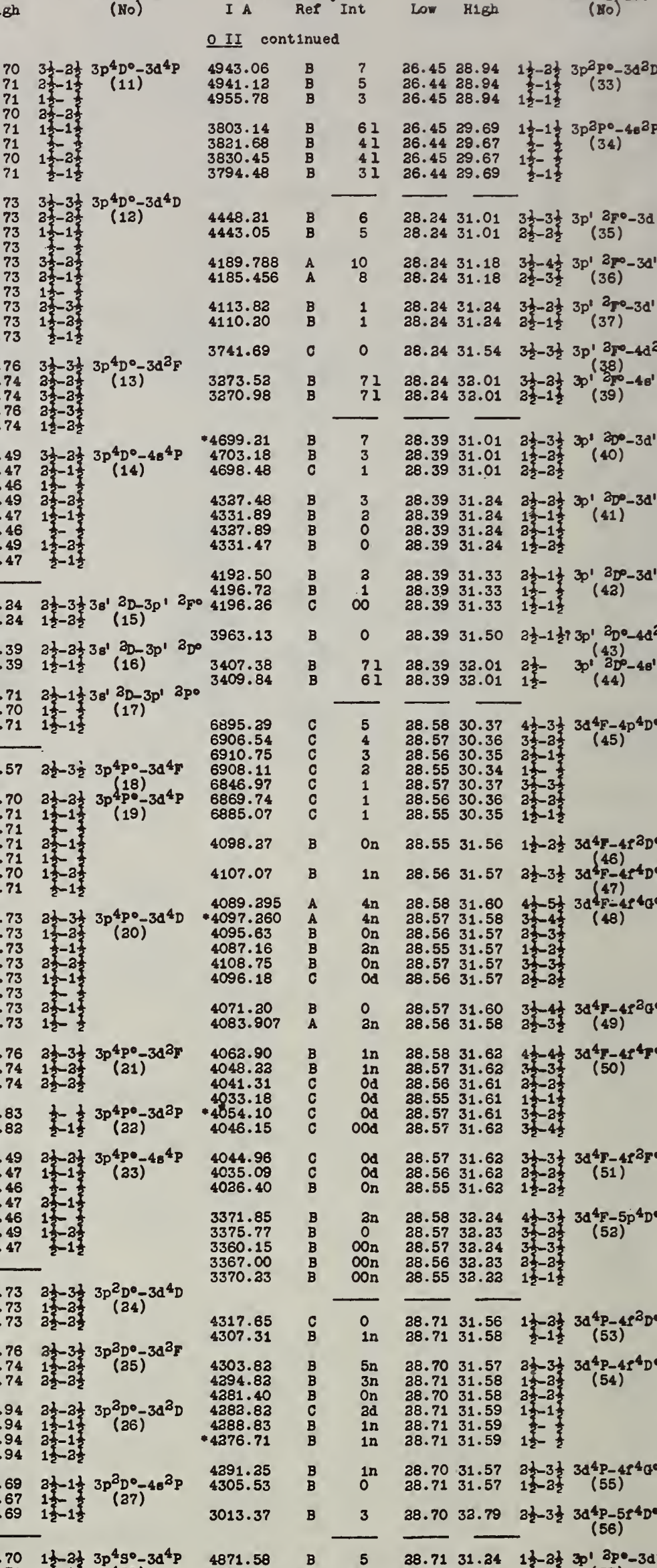

$35.5528 .392 \frac{1}{3}-2 \frac{1}{3} 3 \mathrm{~s}^{\prime}$ 2p-3p' $2 \mathrm{p}^{\circ}$

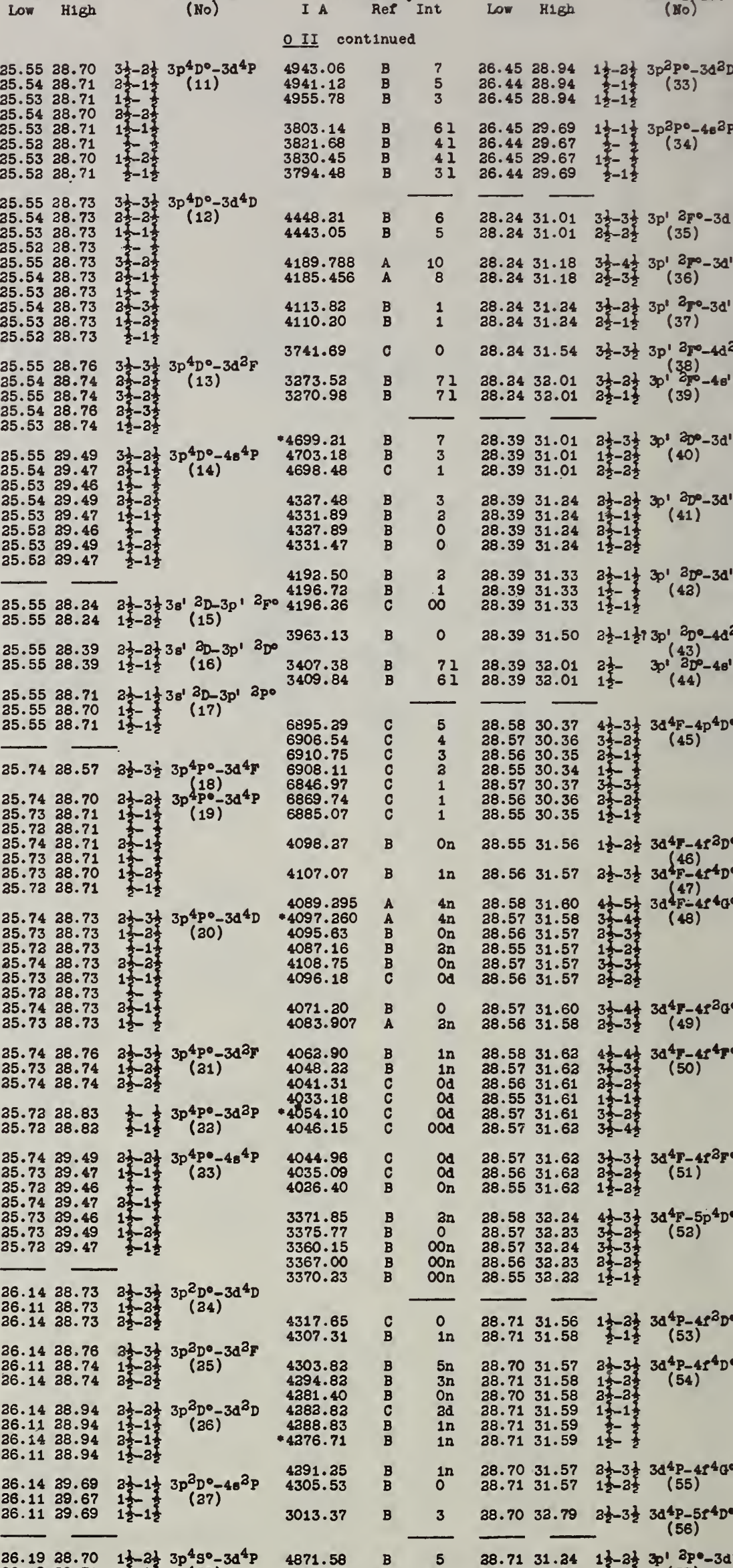

$25.5528 .712 \frac{1}{2}-1 \frac{1}{3} 3 g^{\prime} 3 D_{-3} 3 p^{\prime} 3 p \circ$

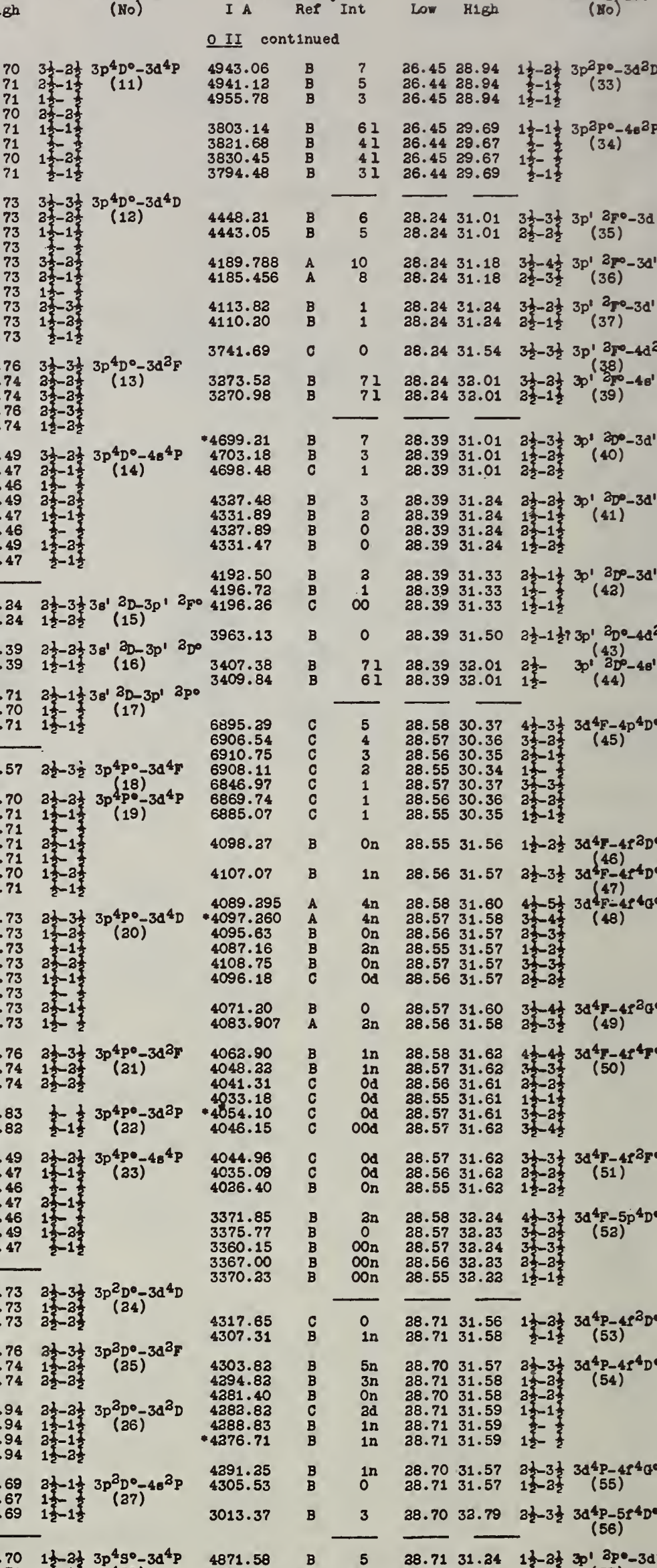

$35.7428 .57 \quad 2 \frac{1}{2}-3 \frac{1}{2} 3 p^{4} p^{\circ}-3 d^{4} \mathrm{r}$

$35.74 \quad 38.70 \quad 2 \frac{1}{2}-2 \frac{1}{2} 3 \mathrm{p}^{(18)}-3 \mathrm{P}^{4} \mathrm{P}$

25.7428 .73 3h-31 $3 p^{4 p 0}-3 \mathrm{~d}^{4} \mathrm{D}$

35.73 38.73 1 -

$\begin{array}{lll}35.74 & 38.73 & 31 \\ 35.73 & 38.73 & 1\end{array}$

$35.73 \quad 38.73$

$\begin{array}{lll}35.74 & 38.73 & 2 \frac{2}{2}-1 \frac{2}{2} \\ 25.73 & 38.73 & 1 \frac{1}{2}\end{array}$

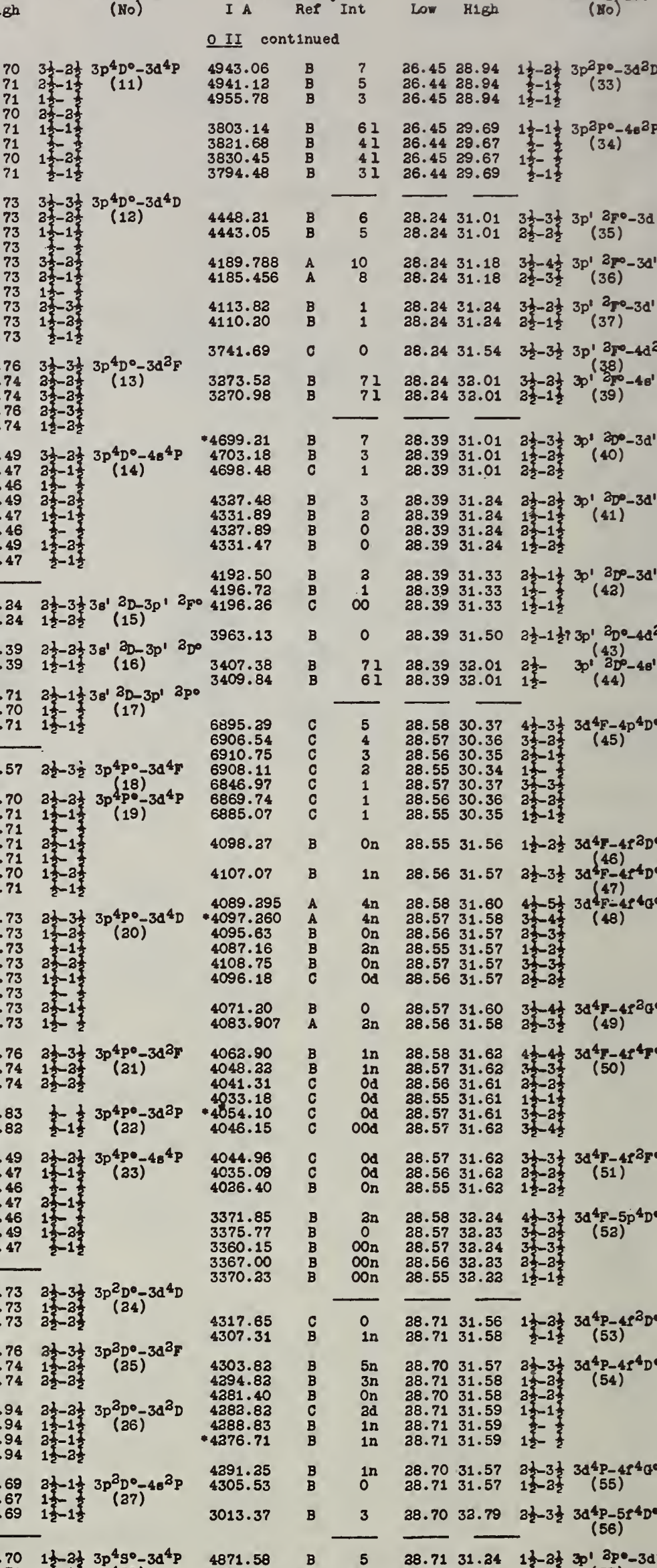

$25.74 \quad 38.76 \quad 3 \frac{1}{2}-3 \frac{1}{2} 3 p^{4} p^{0}-3 d^{2 y}$

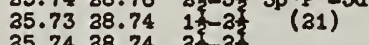

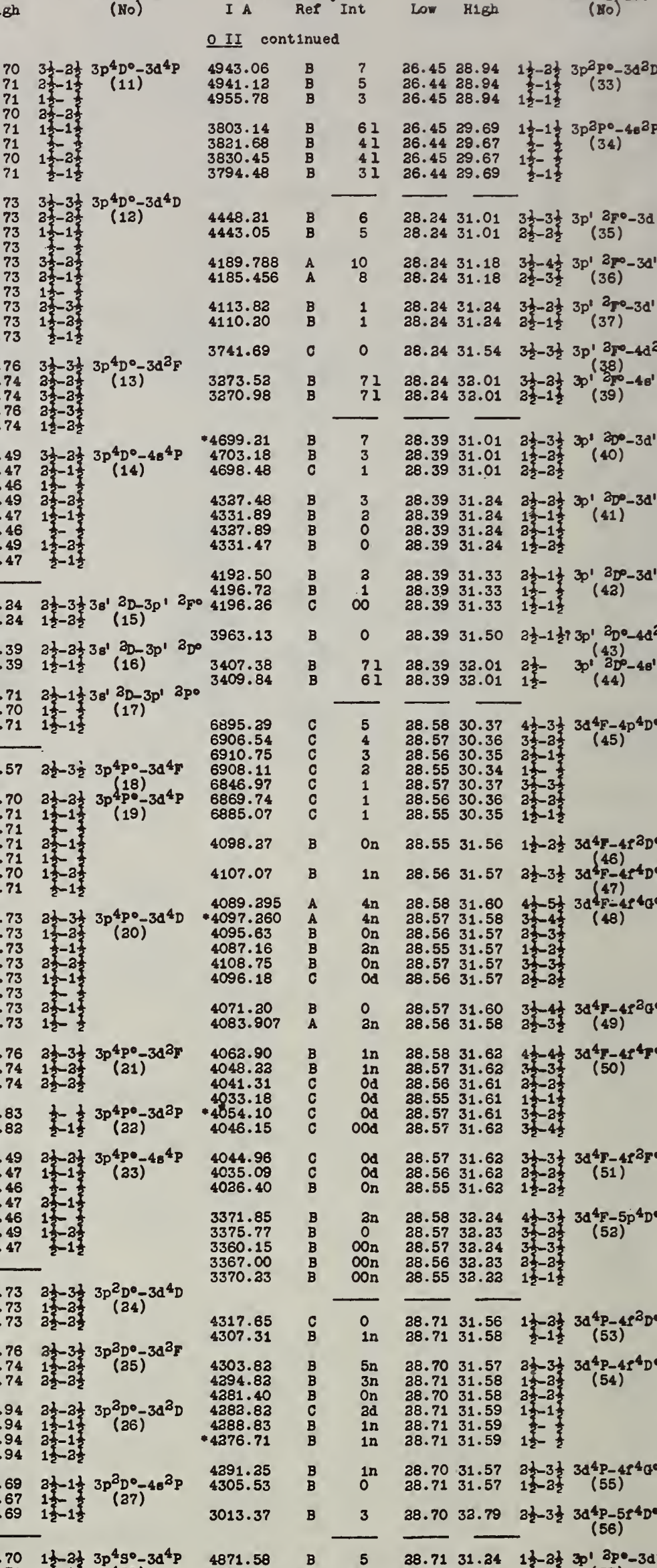

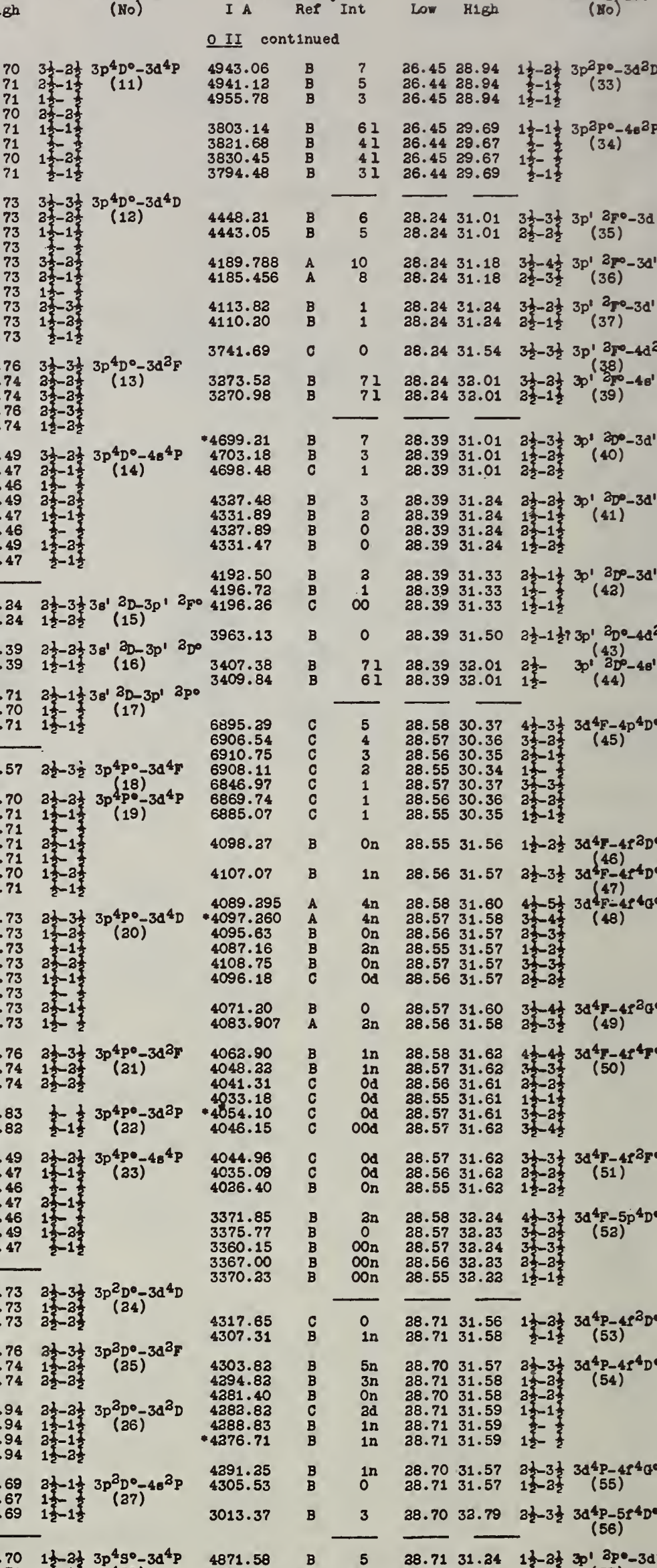

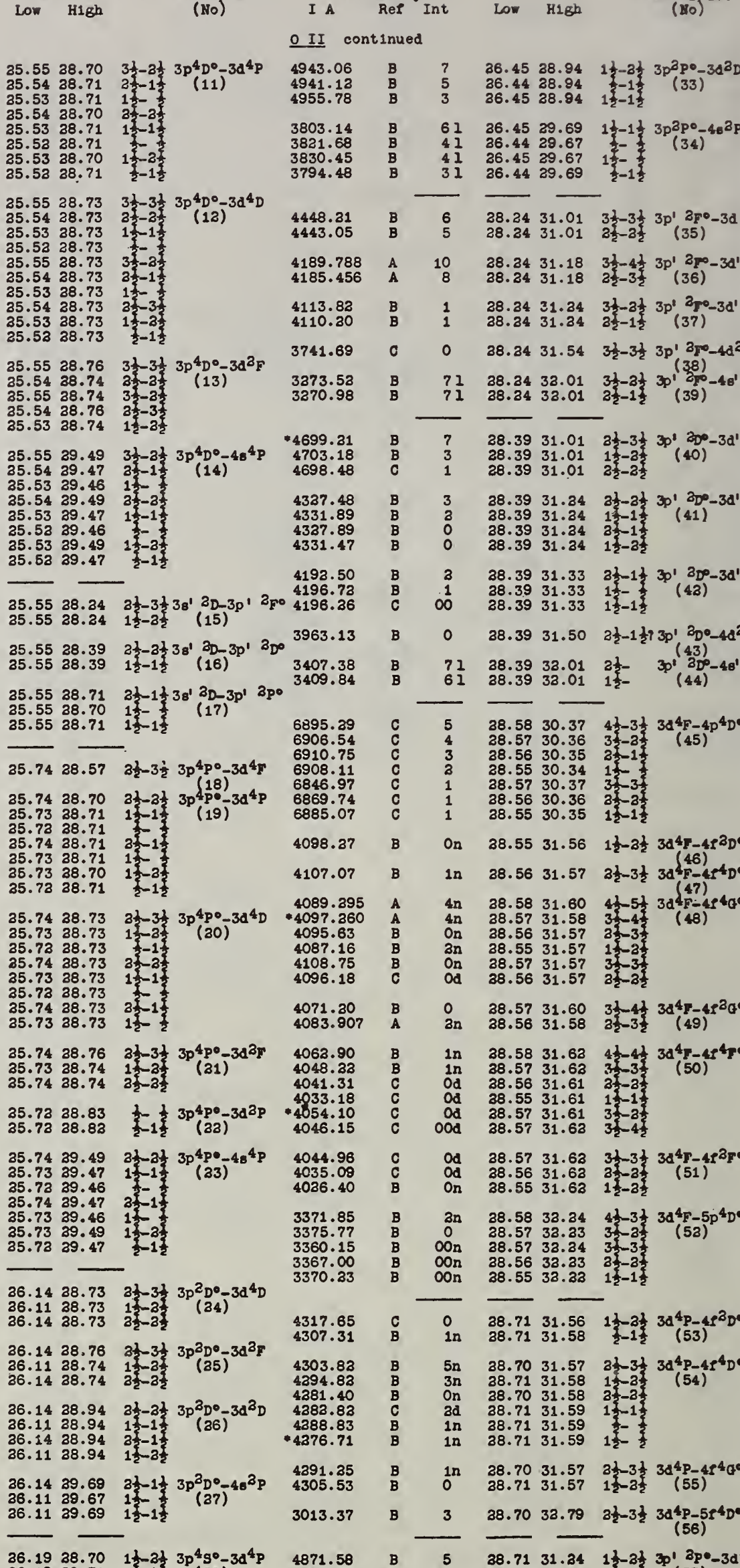

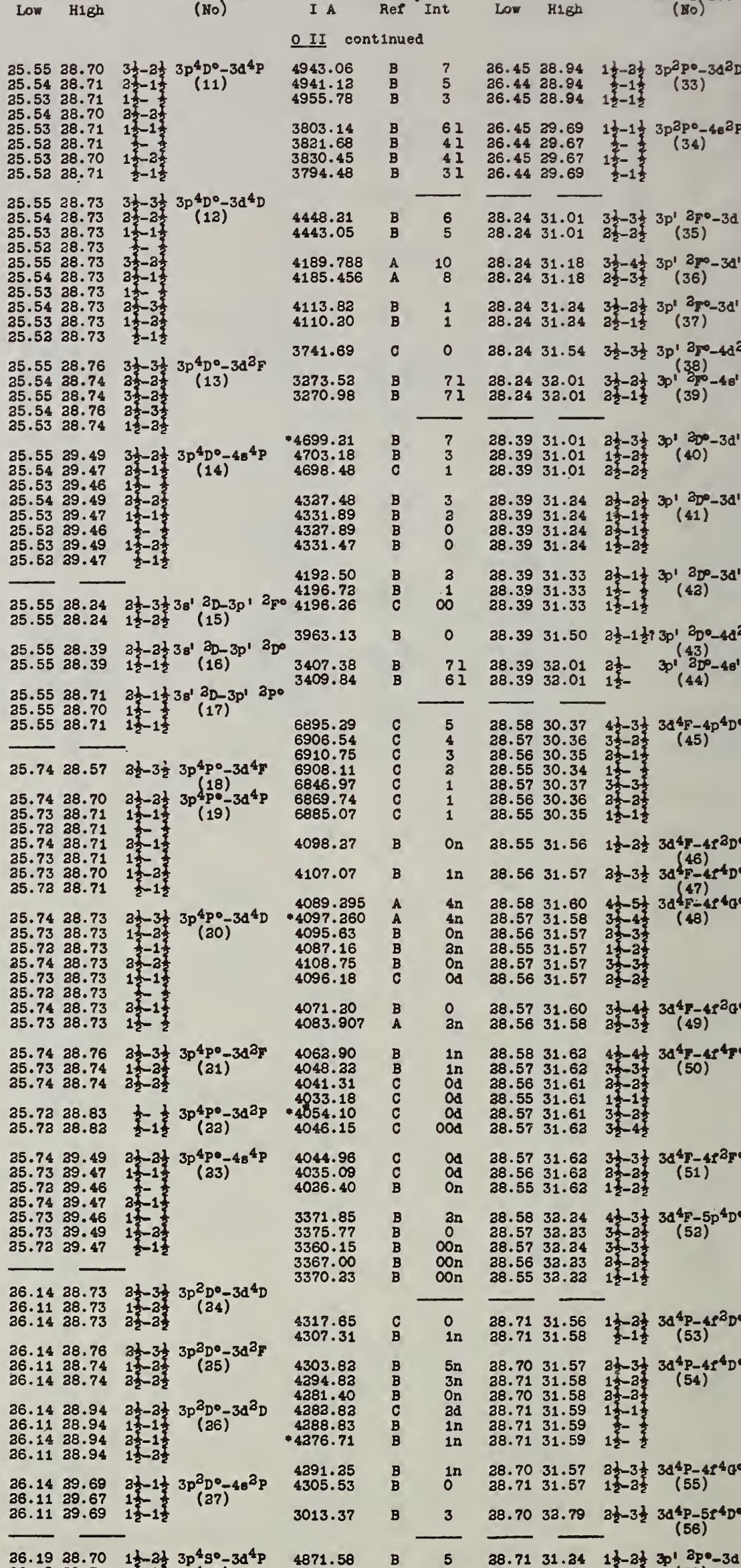

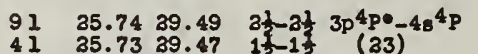

31 35.73 39.46

$\begin{aligned} & 35.74 \\ & 29.47\end{aligned} 25-1$

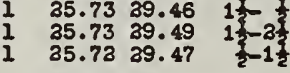

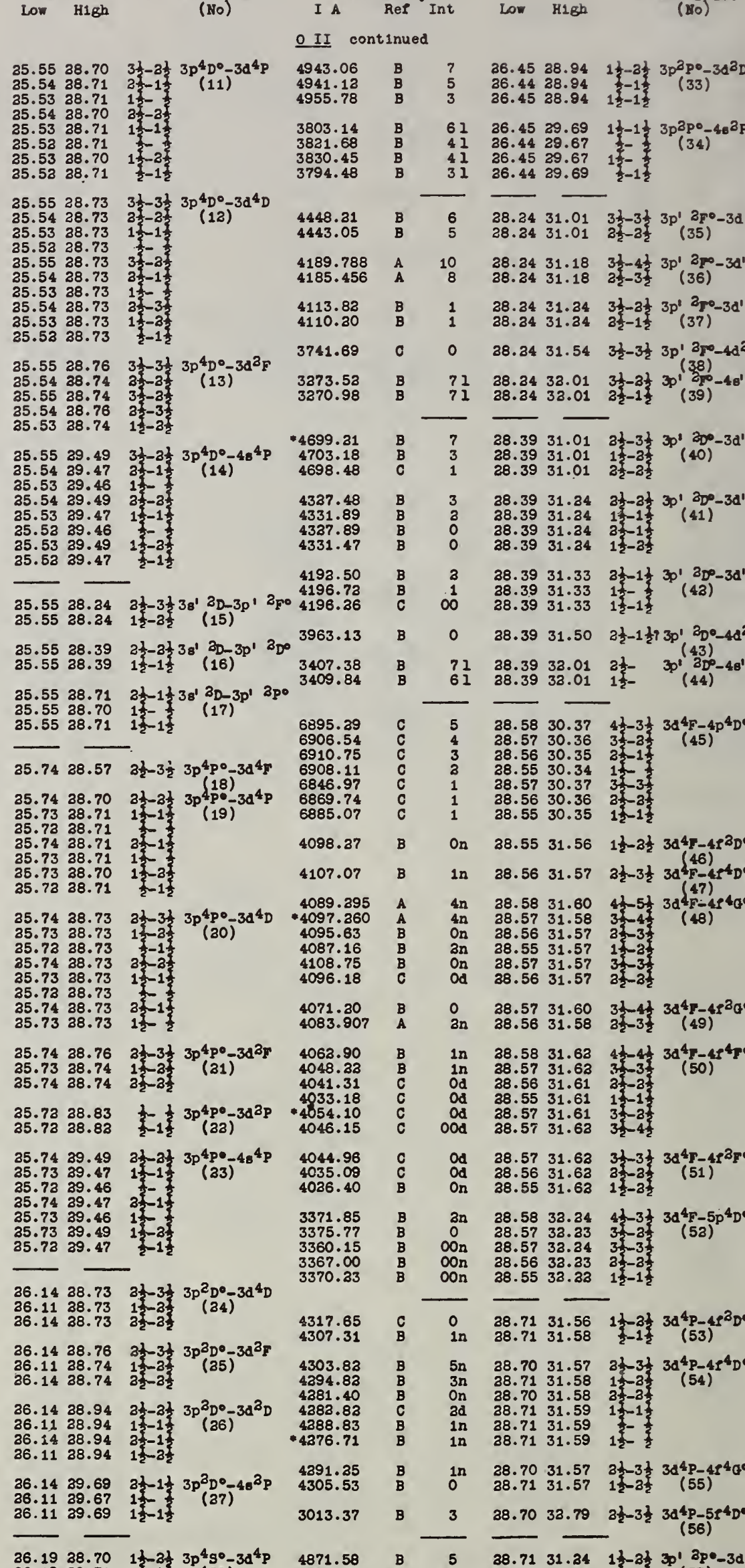

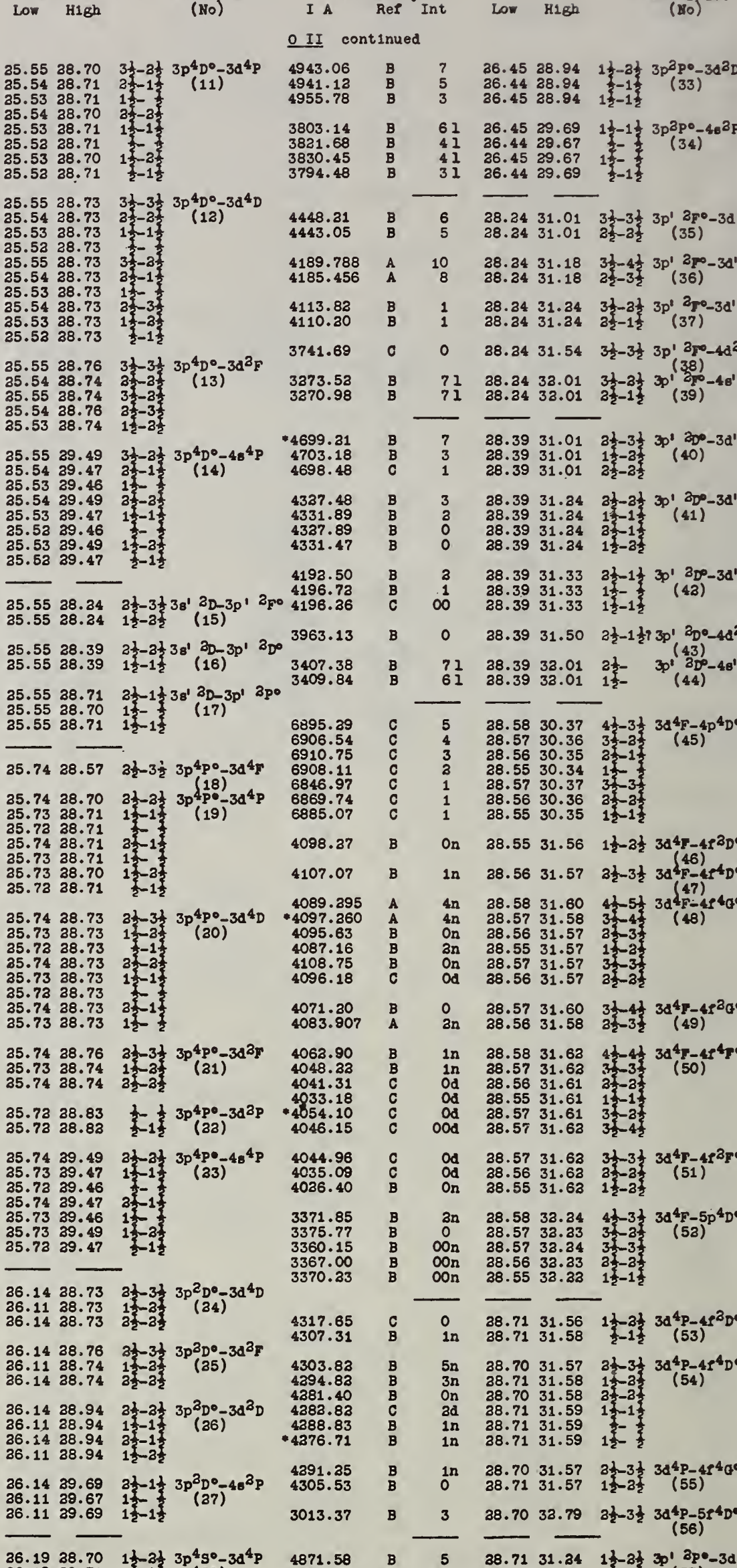

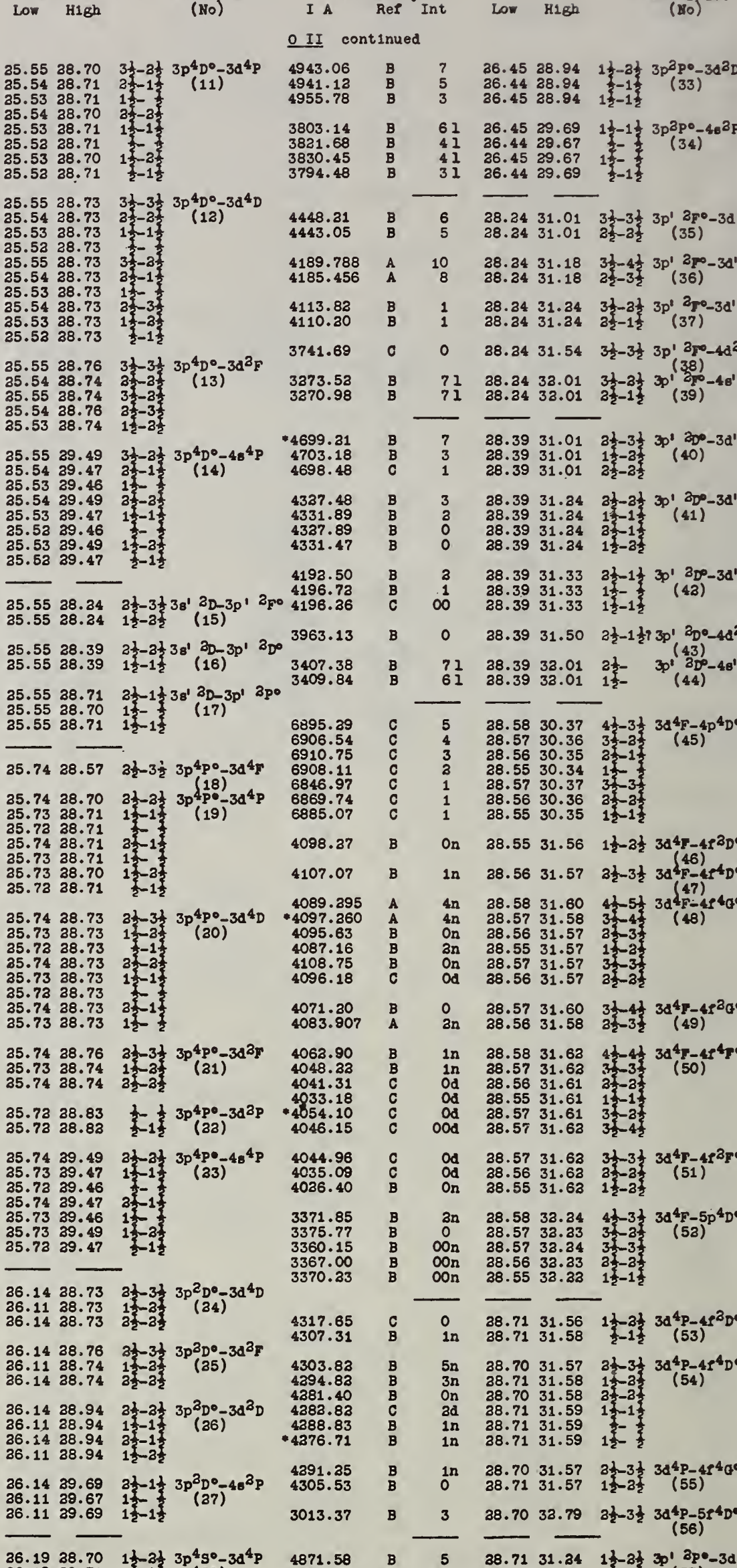

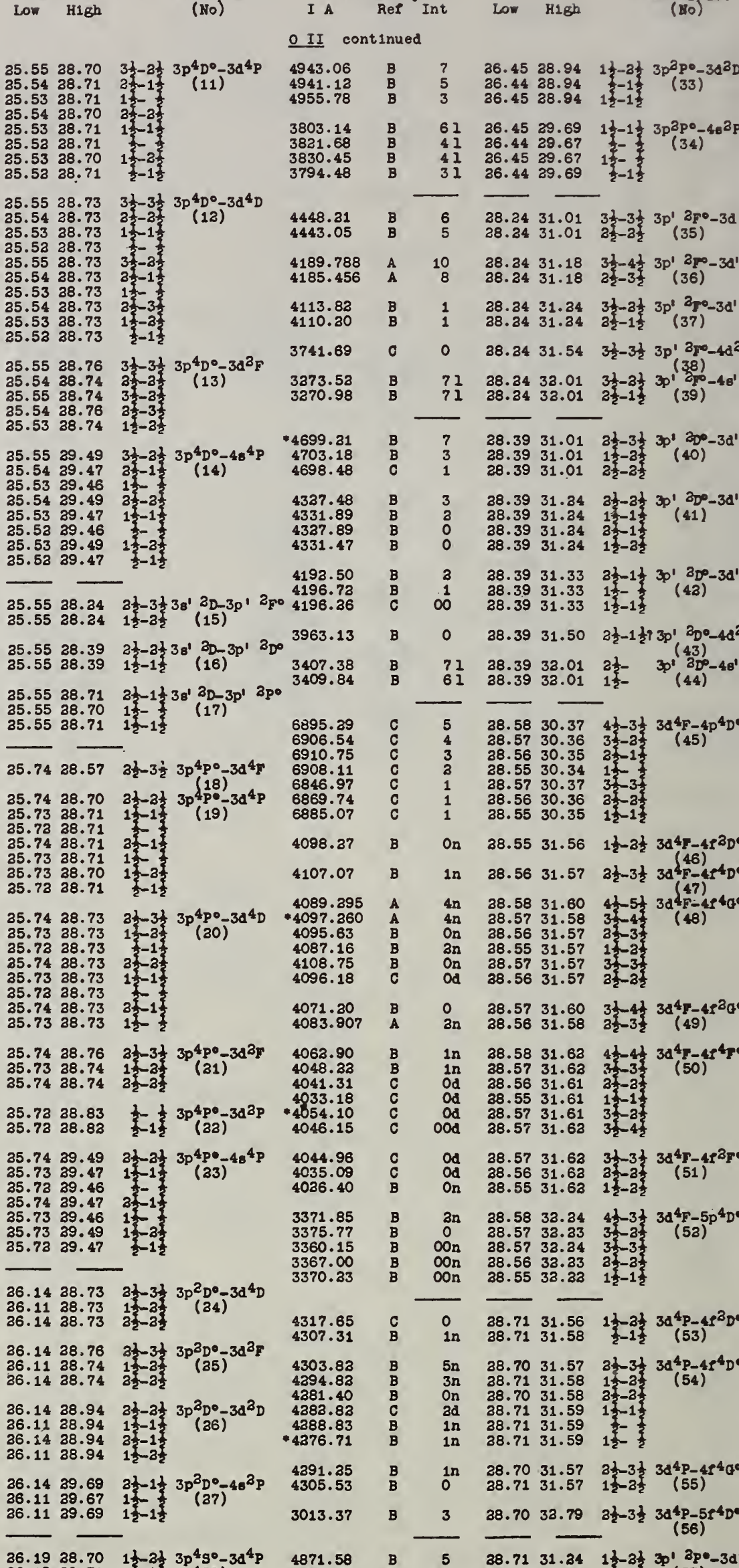

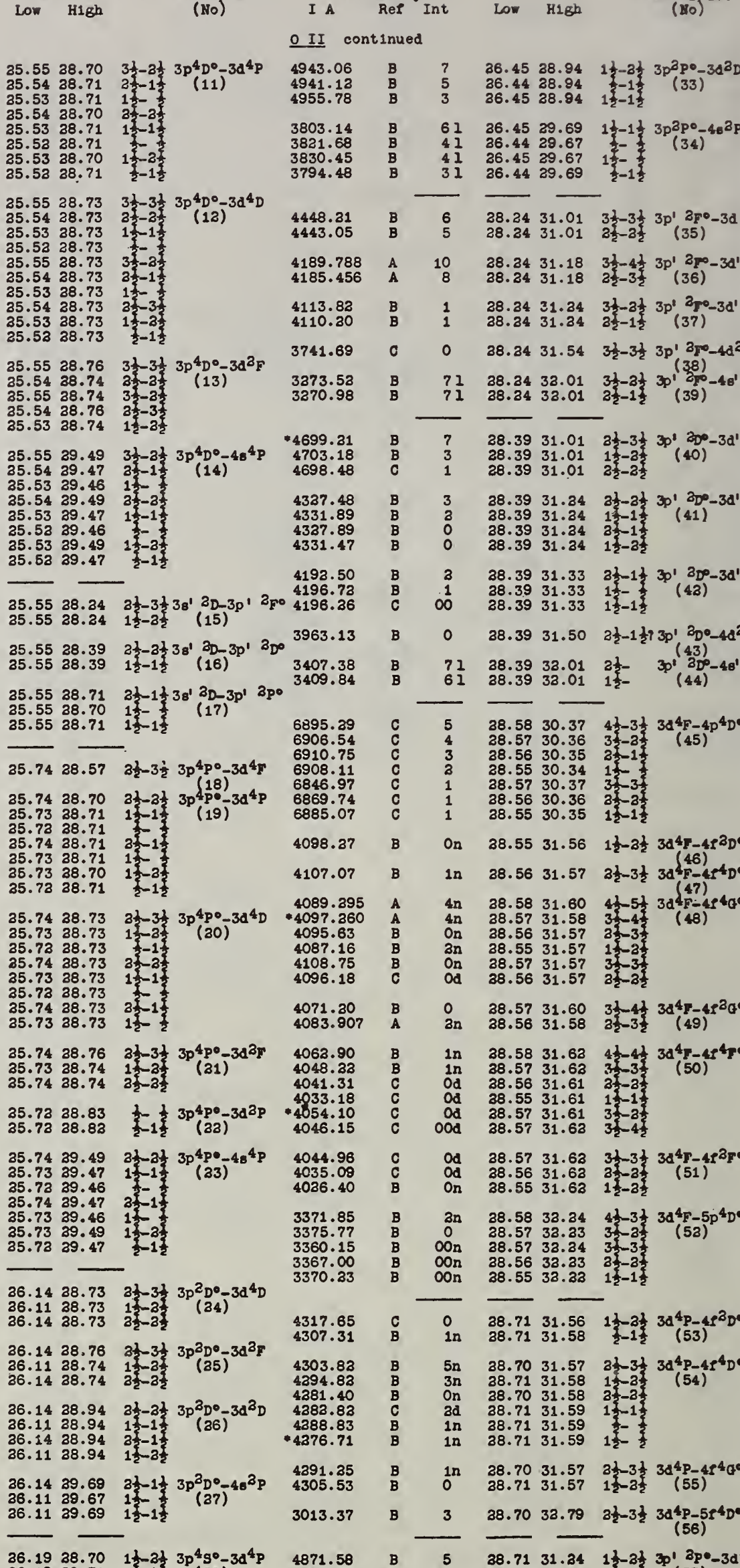

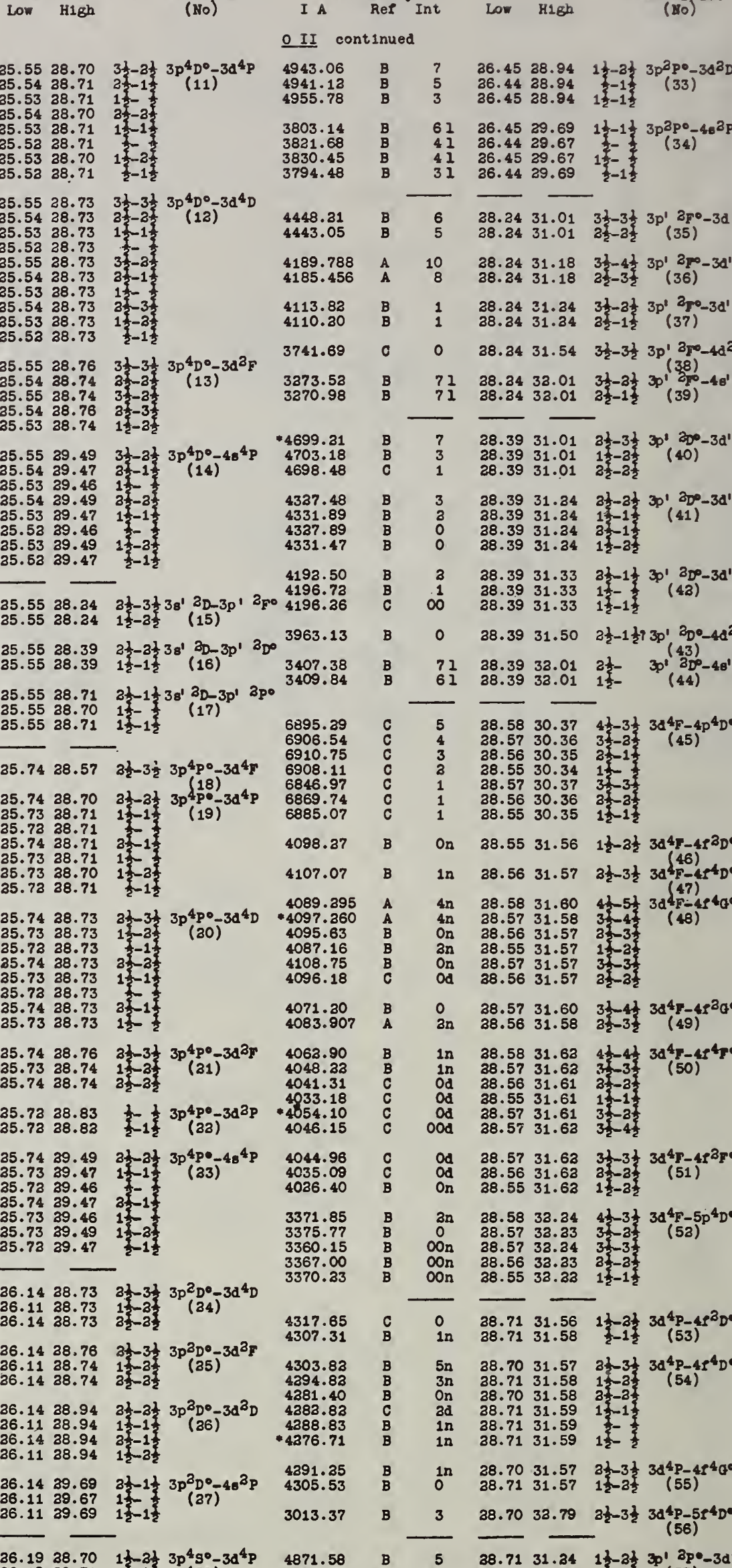

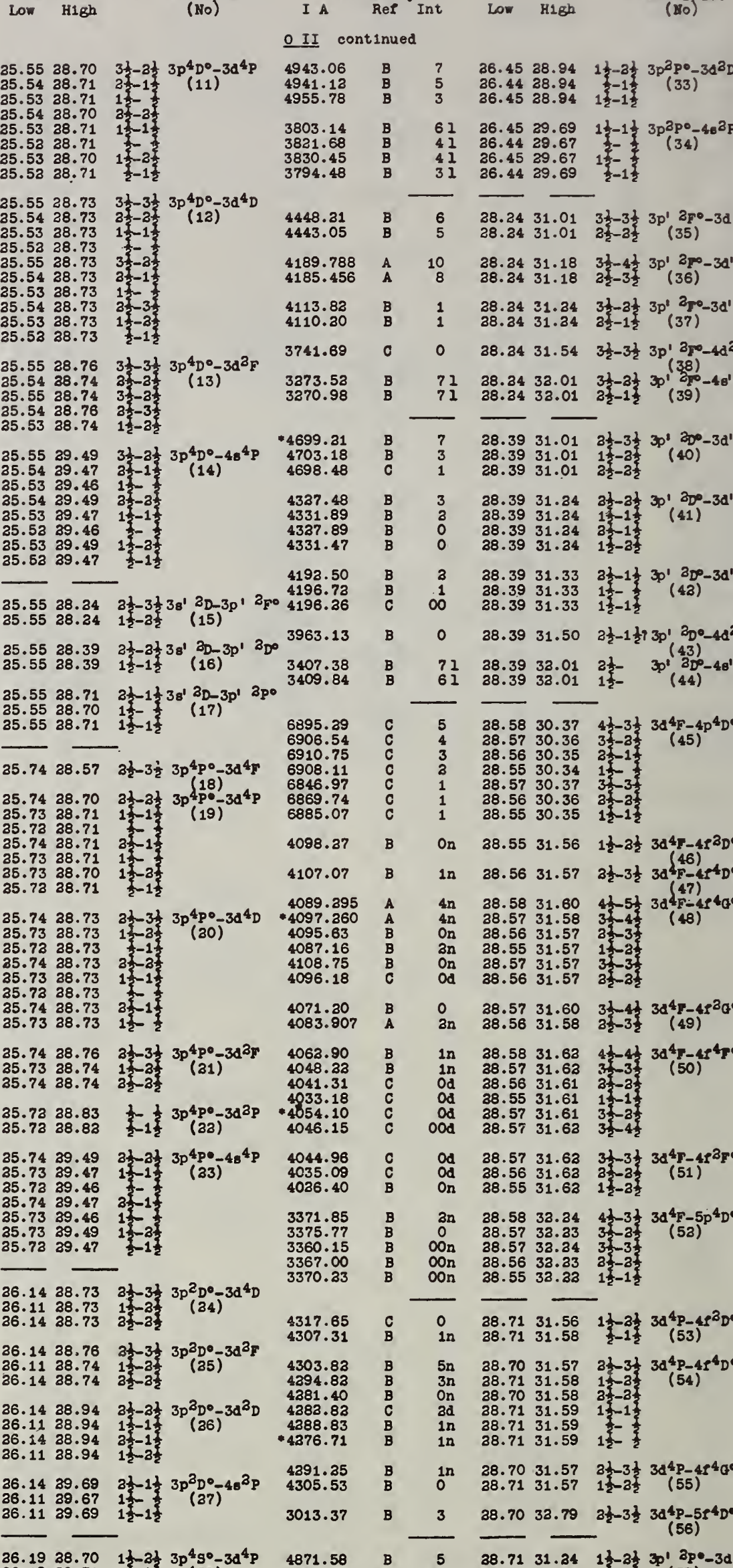

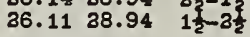

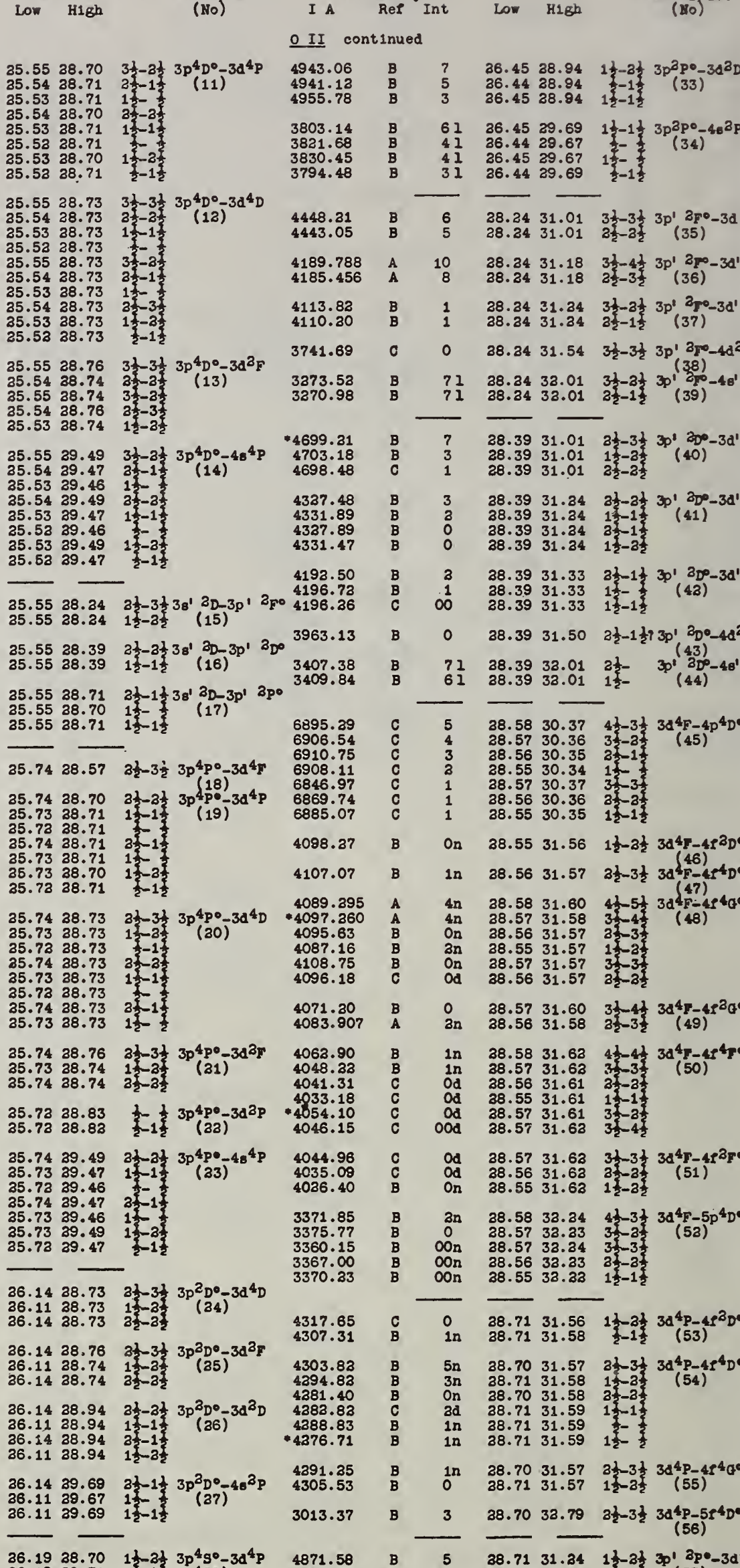

$\begin{array}{lll}36.11 & 39.67 & 13 \\ 36.11 & 29.69 & 1\end{array}$

$26.1928 .70 \quad 1 \frac{1}{2}-2 \frac{1}{3} \quad 3 p^{4} 5^{0}-3 d^{4} p$

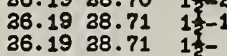

36.1938 .73 11-21 $3 p^{4} 8^{0}-3 d^{4} D$

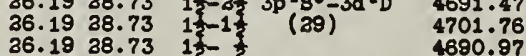

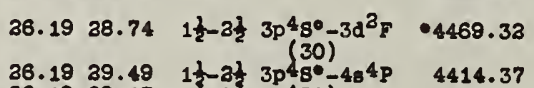

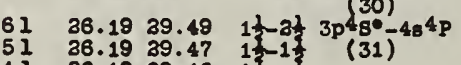

36.1939 .46 1:

$26.4538 .83 \quad 1 \frac{1}{2}-1 \frac{1}{2} 3 \mathrm{p}^{2} \mathrm{p}-3 \mathrm{~d}^{2} \mathrm{p}$

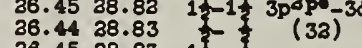

36.45 .88 .83
38.44 .38 .83

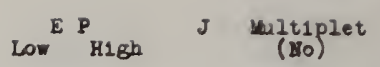

$36.4538 .94 \quad 1 \frac{1}{2}-3 \frac{1}{2} 3 p^{2} p^{\circ}-3 d^{2} \mathrm{D}$

$\begin{array}{llll}36.44 & 28.94 & 1 & -1 \\ 36.45 & 28.94 & 1 & 1\end{array}$

$36.4529 .69 \quad 1 \frac{1}{2}-1 \frac{1}{3} 3 \mathrm{p}^{3} \mathrm{po}^{-48^{2} \mathrm{P}}$

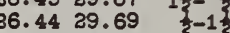

$38.2431 .01 \quad 3 \frac{3}{3}-3 \frac{1}{3} 3 p^{\prime} 3 p^{\circ}-3 d^{\prime}$ a $38.2431 .183 \frac{1}{3}-4 \frac{1}{2} 3 p^{\prime} 2 p^{\circ}-3 d^{\prime} 3 q$

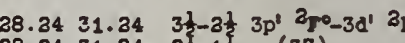

$38.2431 .54 \quad 3 \frac{1}{2}-3 \frac{1}{2} 3 p^{1} 3 p^{\circ}-4 d^{2} y$

28.3931 .01 3t $3 t^{\prime} 3 \mathrm{p}^{\prime} 200^{\circ}-3 \mathrm{~d}^{\prime} \quad \mathrm{a}_{\mathbf{T}}$

38.3931 .34 (1) $3 p^{\circ}-3 d^{\prime} 3 \mathrm{D}$ $\begin{array}{lll}38.39 & 31.34 & 13-1 \\ 38.39 & 31.34 & 3 \frac{1}{3} \\ 38.39 & 31.34 & 1 \frac{1}{2}-3\end{array}$

38.3931 .33 3t-1 30' 300-30' 3p $.33 \quad 1 \frac{1}{2}-1 \frac{2}{2}$

$d^{2} p$




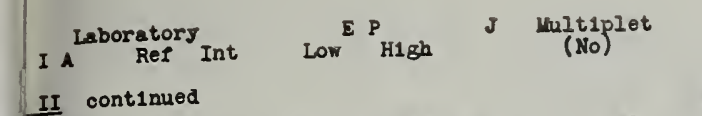

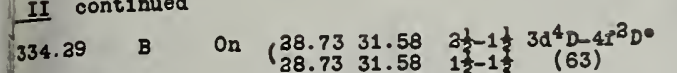
357.35

358.40
334.39
3308.98 308.98
315.35 357.35 315.35 333.78 344.43 (331.13 1375.52 1378.71 1377.40 1377.80
1383.13 1383.75 4274.13 4877.40 4373.17 3518.93 3495.4
3508.0 3494.86
3501.67 3453.31 3039.76
3039.51 3038.83 3007.08
3007.74 3007.74
3008.28

.4343 .3
14331.1 4371.6
4353.6 4342.0 4340.3 4285. 4315.8 4313.10 3500.5

3457.9

3047.

3032.

3085 .

6637.6

6878.19
6866.94

491.25
489.

4

4468.3

4477.8

4707.

$4689.33 \quad C \quad 0 \quad 38.9431 .58 \quad 1 \frac{1}{2}-3 \frac{1}{2} \quad 38^{2} D-41^{4} D^{\circ}$

$4677.00 \quad C \quad 0 \quad 38.9431 .58 \quad 3 \frac{1}{2}-3 \frac{1}{2} \quad 3 \mathrm{~d}^{3} \mathrm{C}-4 \mathrm{I}^{2} \mathrm{G}^{\circ}$

$4613.8^{\circ}$

4610.

4609.

4803.

4465.
4467.
.489

3785.

3374.77

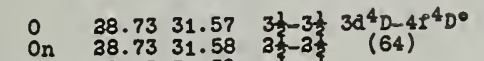
in $38.73 \quad 31.59$ od 38.7331 .59 1

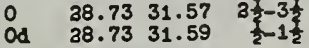

1 n $38.73 \quad 31.58 \quad 3 \frac{1}{2}-4 \frac{1}{3} 3 \mathrm{~d}^{4} D-42^{4} G^{\circ}$

od $38.73 \quad 31.58 \quad 3 \frac{1}{2}-3 \frac{1}{2} 3 d^{4} D-41^{3} a^{\circ}$

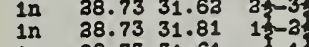

38.7331 .61 s

$38.73 \quad 31.61 \quad 1 \frac{1}{2}-1 \frac{1}{2}$

ood $\quad 38.73 \quad 32.63 \quad 3 \frac{1}{2}-3 \frac{1}{2} \quad 3 d^{4} D-4 r^{2} y_{0}$

$\begin{array}{lll}38.73 & 31.62 & 31 \\ 38.73 & 31.63 & 31 \\ 38.73 & 31.63 & 32\end{array}$

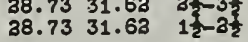

on $38.73 \quad 33.36 \quad 3 \frac{1}{2}-3 \frac{1}{2} 3 d^{4} \mathrm{p}-5 \mathrm{p}^{4} \mathrm{po}$

$\begin{array}{llll}\text { On } & 28.73 & 33.35 & 35 \\ \text { on } & 38.73 & 33.66 & 31 \\ \text { on } & 38.73 & 38.25 & \frac{1}{2}-1 \frac{1}{2}\end{array}$

On $\quad 38.73 \quad 33.31 \quad 3 \frac{1}{2}-1 \frac{1}{2} 3 d^{4} D-5 p^{3} D^{0}$

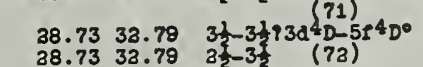

$38.73 \quad 33.813 \frac{1}{2}-4 \frac{1}{2} 3 d^{4} D-51^{4} a^{\circ}$

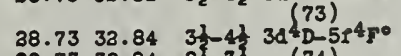

\begin{tabular}{lllll} 
C & 3 dd & 38.73 & 32.84 & 32 \\
B & 1 & 38.73 & 33.84 & 32 \\
\hline
\end{tabular}

an $38.7631 .58 \quad 3 \frac{1}{2}-4 \frac{1}{3 \mathrm{~d}^{3}} \bar{F}-45^{4} \sigma^{\circ}$

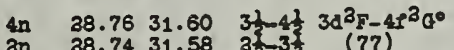

in $38.78 \quad 31.63 \quad 31-4 \frac{1}{3} 3 d^{3} \mathrm{~F}-42^{4} \mathrm{yo}^{\circ}$

$\begin{array}{cccc}3 \mathrm{n} & 38.74 & 31.62 & 31 \\ \infty & 38.78 & 31.62 & 31 \\ \text { On } & 38.74 & 31.61 & 31\end{array}$

On $\quad 38.7831 .63 \quad 31-313 d^{3} y^{2}-49^{3} F^{\circ}$

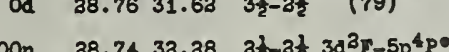

In $38.7632 .33 \quad 31-2 \frac{1}{3} 3 \mathrm{~d}^{320}-5 \mathrm{p}^{2} \mathrm{p}^{\circ}$

Odd $38.78 \quad 32.81 \quad 3 \frac{1}{2}-4 \frac{1}{2} 3 d^{3} \mathrm{~F}-52^{4} \mathrm{G}^{\circ}$ $38.78 \quad 33.83 \quad 3 \frac{1}{2}-4 \frac{1}{3} 3 \mathrm{~d}^{38} \mathrm{~F}_{-55^{3} \mathrm{G}^{\circ}}$

$28.83 \quad 30.68 \quad 13-1 \frac{1}{3} \quad 3 \mathrm{~d}^{2} \mathrm{p}-4 \mathrm{p}^{3} \mathrm{p} 0$ $\begin{array}{lll}38.83 & 30.68 & 1 \\ 38.83 & 30.67 & 1\end{array}$

$38.8331 .56 \quad 1 \frac{1}{3} 3 a^{3} \mathrm{P}-4 \mathrm{I}^{2} \mathrm{D}^{\circ}$

n

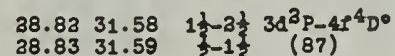

$38.83 \quad 31.57 \quad 1 \frac{1}{2}-2 \frac{1}{2} 3 \mathrm{~d}^{3} \mathrm{P}-4 \mathrm{I}^{4} \mathrm{G}^{\circ}$

- — (88)

38.9431 .83

$\begin{array}{lll}38.94 & 31.63 & 13 \\ 38.94 & 31.62 & 31 \\ \end{array}$

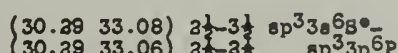

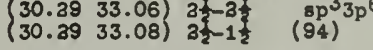

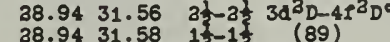

EVISED UULTIPLT T T B

I Aaboratory $A_{\text {Ref Int Iow }}^{\text {E P High J Lultiplet }}$

I ${ }_{\text {Laboratory }}^{\text {Lef }}$ Int

Low ${ }^{\text {E1gh }}$

J $\underset{(\text { No })}{(10 t}$

O II continued

OIII continued

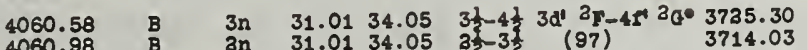
$\bullet$

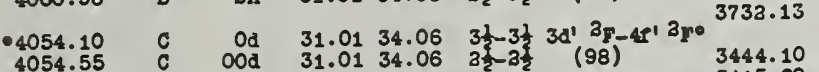

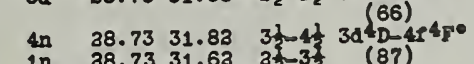

$38.73 \quad 31.81$ 3

On $38.73 \quad 33.24 \quad 3 \frac{1}{3}-3 \frac{1}{2} 3 d^{4} D-5 p^{4} D^{0}$

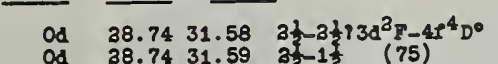
$38.7633 .84 \quad 3 \frac{1}{2}-4 \frac{1}{2} 3 d^{3} 7-5 t^{4} y^{\circ}$

4034.04

B 1 in

$31.0134 .08-3 d^{\prime} 3 y^{3}-4 s^{\prime} 20 \times 3430.60$

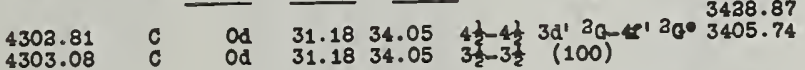

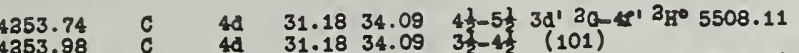

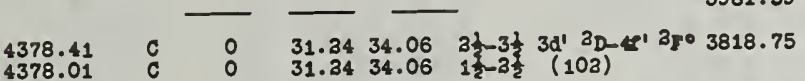

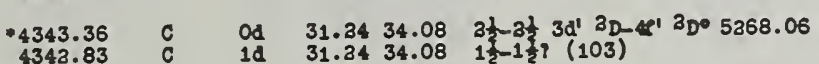

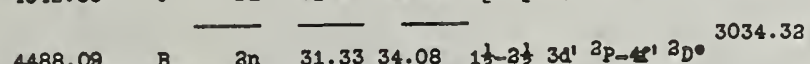

$\begin{array}{llllllll}4488.09 & B & \text { an } & 31.33 & 34.08 & 1 & 1 & \\ 4487.72 & B & \text { On } & 31.33 & 34.08 & \frac{1}{2}-1 \frac{1}{2} & (104) & 3703.37\end{array}$

$4843.38 \quad B \quad$ in $\overline{31.58} 34.10 \quad 1 \frac{1}{2} \quad 3 \mathrm{~d}^{\prime} 3 \mathrm{~g}-4 \mathrm{p}^{\prime} 3 \mathrm{po} 3695.70$

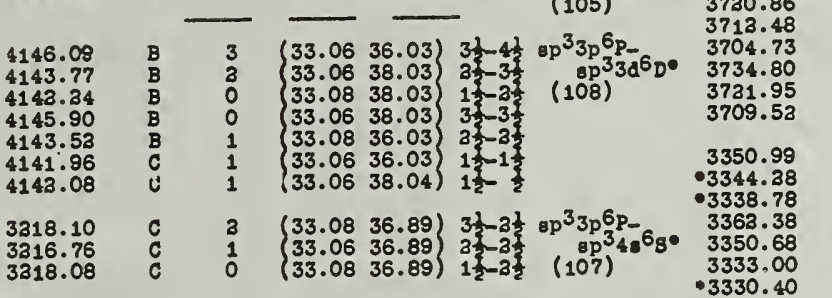

Strongest Unolassifled Lines Attributed to 0 II

$\begin{array}{lll}4506.50 & B & 3 n \\ 3440.61 & B & 3 n \\ 3419.87 & B & 3 n\end{array}$

$\begin{array}{lll}3419.87 & B & 3 n \\ 3081.46 & B & \text { an }\end{array}$

$\begin{array}{lll}3006.83 & \text { B } & 3 \\ 3008.04 & \text { B } & 3 \\ 3 & & 3\end{array}$

\begin{tabular}{lll}
3008.04 & B & 3 \\
3005.62 & B & 3 \\
\hline
\end{tabular}

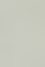

OIII I P 54.71 Anal B L16t A Sept 1943

$\begin{array}{llllllll}4339.5 \quad A \quad 00 & 33.0135 .93 & 1-1 & 38^{3} \mathrm{po}^{\circ}-3 \mathrm{p}^{1 \mathrm{p}} & 3454.90 \\ 3451.33\end{array}$

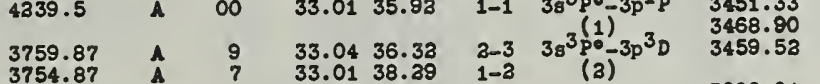

3757.21

3774.00
3810.96

A $\quad 5 \quad 33.0138 .29$

$\begin{array}{lllllll}3340.74 & A & 6 & 33.04 & 36.73 & 2-1 & 3 \mathrm{~s}^{3} \mathrm{po}^{-3 \mathrm{p}^{3} \mathrm{~s}} \\ 3312.30 & \text { A } & 5 & 33.01 & 38.73 & 1-1 & (3)\end{array}$

3312.30
3399.36

3047.13

3035.43

3043.02

3023.45
3034.57

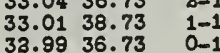

33.0437 .09

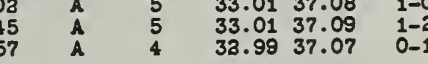

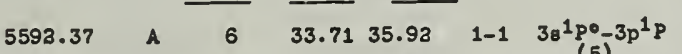

3983.78$$
33
$$

2983.66

3385.48

3260.98

3267.31
3384.57

3381.94
3305.77

3253.9

3338.57

3017.63
3004.35

3004.35
3996.51

3996.51
3024.36
3008.79

3997.71

3993.11

3363.83

3389.40

$A$
$\hat{A}$
$\hat{A}$
$\hat{A}$
$\hat{A}$

35

$\begin{array}{llll}A & 5 & 36.38 & 40.09 \\ A & 5 & 36.33 & 40.41\end{array}$

$\begin{array}{ll}36.32 & 40.40 \\ 36.39 & 40.40\end{array}$

3132.86

3121.71

3115.73

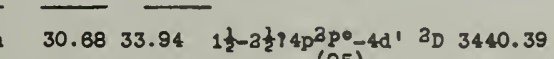

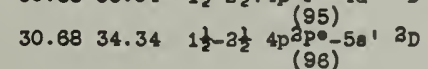

3715.08
4081.10
4073.90

3556.93

3455.13 3450.94
3448.05 m3448.05

3447.32

3468.15

$2-3$
$1-2$
$0-1$
$2-2$

33.0438 .29

33.0136 .38
33.0438 .38

$1-2$
$0-1$
$2-3$
$1-1$
$2-1$

33.0137 .07

$2-3 \quad 3 \mathrm{~s}^{3} \mathrm{po}^{2}-3 \mathrm{p}^{3} \mathrm{p}$

3088.04

$\overline{35.93} 40.05 \quad 1-3 \quad 3 \mathrm{p}^{1} \mathrm{p}-3 \mathrm{~d}^{3} \mathrm{~g}^{\circ}$

$\begin{array}{cccc}36.33 & 40.10 & 3-4 & 3 p^{3} p-3 d^{3} y^{\circ} \\ 36.29 & 40.08 & 3-3 & (8)\end{array}$

$\begin{array}{lll}36.29 & 40.08 & 2-3 \\ 38.28 & 40.05 & 1-3 \\ 36.32 & 40.08 & 3-3\end{array}$

36.3940 .05

36.29
36.33
36.05

38.3940 .09

$3-3$
$3-2$
$3-3$

36.3840 .09

$-2 \quad 3 p^{3} p-3 d^{1} p^{0}$

$\begin{array}{ll}36.39 & 40.40 \\ 36.38 & 40.40\end{array}$

36.3840 .40

36. 39 (3)

$\begin{array}{lll}36.39 & 40.41 & 3-3 \\ 36.38 & 40.40 & 1-3\end{array}$

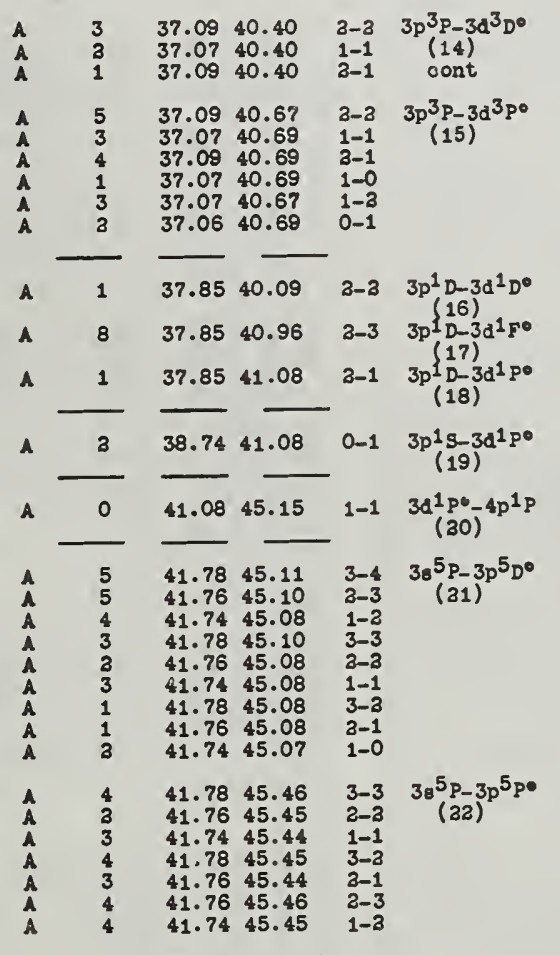

43.24 $46.37 \quad 2-3 \quad 38^{3} p-3 p^{3} D^{0}$

$43.24 \quad 46.71 \quad 3-3 \quad 38^{3} \mathrm{p}-3 \mathrm{r}^{3} \mathrm{p}$

$\begin{array}{llll}45.11 & 48.68 & 4-5 & 3 p^{5} p^{0}-3 d^{5} F \\ 45.10 & 48.67 & 3-4 & (25)^{2}\end{array}$

$\begin{array}{lll}45.10 & 48.67 & 3-4 \\ 45.08 & 48.66 & 2-3\end{array}$

$45.0848 .66 \quad 1-2$

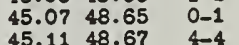

$45.10 \quad 48.66 \quad 3-3$

$\begin{array}{lll}45.08 & 48.86 & 2-3 \\ 45.08 & 48.65 & 1-1\end{array}$

$\begin{array}{lll}45.10 & 48.68 & 3-3 \\ 45.08 & 48.65 & 3-1\end{array}$

$\begin{array}{llll}45.11 & 49.11 & 4-4 & 3 p^{5} D^{\circ}-3 d^{5} D \\ 45.10 & 49.10 & 3-3 & (36)\end{array}$

45.0849 .10

$\begin{array}{ll}45.11 & 49.10 \\ 45.10 & 49.10\end{array}$

45.0849 .10

45.0849 .10

45.0849 .10

45.0749 .10

$45.46 \quad 49.11 \quad 3-4 \quad 3 p^{5 p 0}-3 d^{5 p}$

$45.4549 .10 \quad 3-3$

$45.4649 .10 \quad 3-3$

(1) $\quad \begin{array}{llll}25.45 & 49.10 & 3-3 \\ & 45.44 & 49.10 & 1-1\end{array}$

$45.46 \quad 49.14 \quad 3-3 \quad 3 p^{5} p^{0}-3 d^{5} p$

$\begin{array}{lll}45.45 & 49.15 & 3-2\end{array}$

$45.44 \quad 49.15 \quad 1-1$

$\begin{array}{lll}45.46 & 49.15 & 3-3 \\ 45.45 & 49.15 & 3-1\end{array}$

$\begin{array}{lll}45.45 & 49.14 & 2-3 \\ 45.44 & 49.15 & 1-3\end{array}$

(1) $46.05 \quad 49.82 \quad 0-1 \quad 4 p^{1} s-5 d^{1} p^{0}$

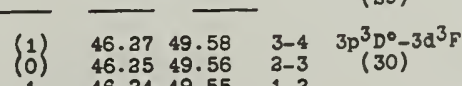

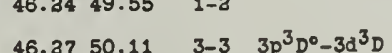

$\begin{array}{llll}46.35 & 50.10 & 3-3 & (31)\end{array}$

$\begin{array}{lll}46.24 & 50.10 & 1-1\end{array}$

$46.37 \quad 49.10 \quad 2-3 \quad 3 p^{5} g^{0}-3 d^{5} \mathrm{D}$

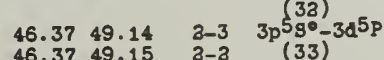

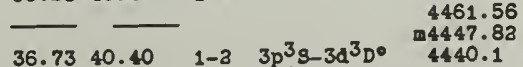

4539.7
4461.56
$\mathbf{m} 4447.82$
4440.1

$\begin{array}{lll}46.37 & 49.15 & 3-1\end{array}$

$46.71 \quad 49.43 \quad 3-3 \quad 3 \mathrm{p}^{3} \mathrm{po}^{20}-3 \mathrm{~d}^{3} \mathrm{p}$

$46.7150 .11 \quad 2-3 \quad 3 \mathrm{p}^{3 \mathrm{po}^{\circ}-3 \mathrm{~d}^{3} \mathrm{D}}$

48.7350 .10

$1-3$
$0-1$

$46.7350 .10 \quad 1-1$

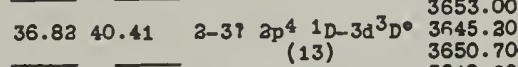

$\begin{array}{lllc}37.09 & 40.41 & 2-3 & 3 p^{3} P-3 d^{3} D^{\circ} \\ 37.07 & 40.40 & 1-2 & (14)\end{array}$

$\begin{array}{lll}37.07 & 40.40 & 1-3 \\ 37.08 & 40.40 & 0-1\end{array}$
45.1049 .11

46. $7150.10 \quad 3-3$ 
10

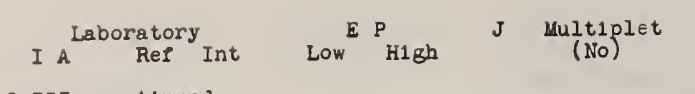

\section{O III continued}

$4569.50 \quad A \quad$ in $52.6355 .33 \quad 2-3$ ? $3 \mathrm{p}^{1}, \mathrm{D}^{0}-3 \mathrm{~d}^{1}, \mathrm{~s}$ $4474.95 \quad A \quad$ in $52.6355 .39 \quad 2-393 p_{(37)}^{(36)} D^{\circ}-3 d^{1} D$

OIV I P 77.08 Anal B L18t A Mar 1943

$\begin{array}{lllll}3063.46 & \text { B } & 6 & 44.1548 .18 & \frac{1}{3}-1 \frac{1}{3} 3^{2} S-3^{2} p_{0} \\ 3071.66 & \text { B } & 5 & 44.1548 .17\end{array}$

\begin{tabular}{|c|c|c|c|c|c|}
\hline 71.66 & $D$ & 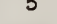 & 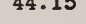 & .12 & टर \\
\hline $\begin{array}{l}3411.76 \\
3403.58 \\
3413.71\end{array}$ & $\begin{array}{l}\text { B } \\
\text { B } \\
\text { A }\end{array}$ & $\begin{array}{l}4 \\
3 \\
1\end{array}$ & $\begin{array}{l}48.18 \\
48.17 \\
48.18\end{array}$ & $\begin{array}{l}51.79 \\
51.79 \\
51.79\end{array}$ & $\begin{array}{cc}1 \frac{1}{2}-2 \frac{1}{2} & 3^{3} \mathrm{P}^{0}-3^{2} \mathrm{D} \\
\frac{1}{2}-1 \frac{1}{2} & (2) \\
1 \frac{2}{2}-1 \frac{2}{2} & \end{array}$ \\
\hline $\begin{array}{l}3385.55 \\
3381.28 \\
3381.33 \\
3409.75 \\
3396.83 \\
3390.37 \\
3425.57 \\
3405.97\end{array}$ & $\begin{array}{l}\text { B } \\
\text { B } \\
P \\
B \\
B \\
\text { P } \\
B \\
B\end{array}$ & $\begin{array}{l}\left\{\begin{array}{l}6 \\
4\end{array}\right) \\
\left\{\begin{array}{l}2 \\
2\end{array}\right) \\
(0)\end{array}$ & $\begin{array}{l}54.19 \\
54.16 \\
54.14 \\
54.19 \\
54.16 \\
54.14 \\
54.19 \\
54.16\end{array}$ & $\begin{array}{l}57.84 \\
57.81 \\
57.79 \\
57.81 \\
57.79 \\
57.78 \\
57.79 \\
57.78\end{array}$ & 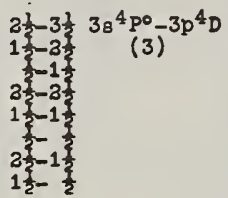 \\
\hline
\end{tabular}

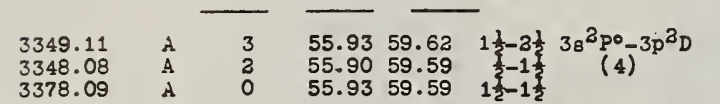

3052.5

.3736 .78

3729.03

$-3725.81$

3758.45

3744.73
3736.78

3736.78
3774.38

3774.38
3755.82

3209.64

3194.75
3185.72

3185.72
3180.72

3180.72
3216.31

3199.53

3188.65
3188.17

3180.98
3177.80

3375.50

3375.50
3362.63
3354.31

4789.25

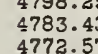

titis

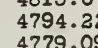

4823.93

3995.17

3995.17
3956.82
3930.63

3930.63
3977.10

3945.29

3974.66
3942.14

5362.4
5305.

$59.5961 .91{ }_{1 \frac{1}{2}-1 \frac{1}{2}}^{5}(11)$

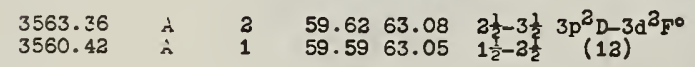

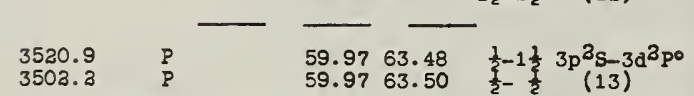

(4) $57.8461 .14 \quad 3 \frac{1}{2}-4 \frac{1}{3} \quad 3 p^{4} p-3 d^{4} F^{0}$

(3) $\quad \begin{array}{r}57.79 \\ 57.78\end{array} 61.11 \quad 1 \frac{1}{2}-3$

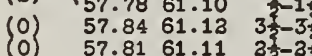

(0) $\quad 57.8161 .11 \quad 3726$

$\begin{array}{lll}57.84 & 61.11 & 3 \frac{2}{3}-3 \frac{1}{3} \\ 57.81 & 61.10 & 3 \frac{1}{2}-1 \frac{1}{2}\end{array}$

(3) $57.8461 .68 \quad 3 \frac{1}{2}-3 \frac{1}{3} \quad 3 p^{4} D-3 a^{4} D^{\circ}$

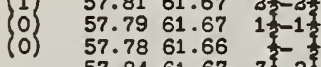

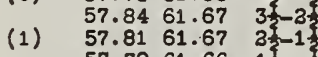

$\begin{array}{lll}57.79 & 61.66 & 1 \\ 57.81 & 61.68 & 3\end{array}$

(0) \begin{tabular}{lll}
57.79 & 61.67 & $1 \frac{1}{3}-3 \frac{1}{3}$ \\
\hline
\end{tabular}

(3) $58.54 \quad 62.20 \quad 1 \frac{1}{3}-2 \frac{1}{2} \quad 3 p^{4} s-3 d^{4} p^{\circ}$

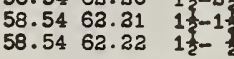

(5) $59.1161 .68 \quad 2 \frac{1}{2}-3 \frac{1}{7} \quad 3 p^{4} P-3 a^{4} D^{0}$

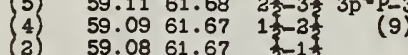

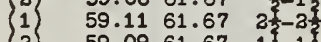

(2) $\begin{aligned} & 59.0961 .67 \\ & 2\end{aligned} \quad \begin{array}{ll}59.08 & 61.66\end{array}$

$\begin{array}{lll}59.11 & 61.67 & 23 \\ 59.09 & 61.66 & 13\end{array}$

(2) $59.1162 .30 \quad 2 \frac{1}{2}-3 \frac{1}{2} 3 \mathrm{p}^{4} \mathrm{P}-3 \mathrm{~d}^{4} \mathrm{p}^{\mathrm{P}}$

59.0962 .21 1 $1 \frac{1}{2}$ (10)

(1) $59.1162 .21 \quad 2 \frac{2}{2}-1$

59.0962 .22
59.0962 .20

(0) $59.0862 .21 \quad \frac{1}{2}-1 \frac{1}{2}$

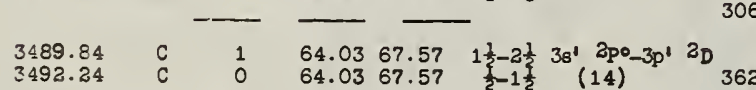

$4568 \quad P \quad-\frac{5^{2} 5^{\circ}-6^{2} \mathrm{D}}{(15)}$
REVISED YULTIPLET TABLE

I A Laboratory ${\text { Ref Int Low }{ }_{\text {HIgh }} \mathrm{J} \text { Multiplet }}_{(\text {No) }}$ OV I P 113.38 Anal B L1st A Feb 1943

\begin{tabular}{|c|c|c|c|c|c|c|}
\hline 5114 & $\mathbf{P}$ & & 69.39 & 71.70 & $0-1$ & $\begin{array}{c}3^{1} \mathrm{~S}-3^{1} \mathrm{P}^{\circ} \\
\text { (1) }\end{array}$ \\
\hline 3144.58 & A & 1 & 71.70 & 75.63 & $1-3$ & $\begin{array}{c}3^{1} P^{0}-3^{1} D \\
(3)\end{array}$ \\
\hline $\begin{array}{l}5600 \\
5582 \\
5573 \\
5606 \\
5584 \\
5608\end{array}$ & $\begin{array}{l}P \\
P \\
P \\
P \\
P \\
P\end{array}$ & & $\left\{\begin{array}{l}71.93 \\
71.91 \\
71.91 \\
71.92 \\
71.91 \\
71.93\end{array}\right.$ & $\left.\begin{array}{l}74.13 \\
74.13 \\
74.13 \\
74.13 \\
74.12 \\
74.13\end{array}\right\}$ & $\begin{array}{l}2-3 \\
1-3 \\
0-1 \\
2-3 \\
1-1 \\
2-1\end{array}$ & $\frac{3^{3} \mathrm{P}^{0}-3^{3} \mathrm{D}}{(3)}$ \\
\hline
\end{tabular}
$\begin{array}{lllll}4123.90 & A & 3 \\ 4120 & P\end{array} \quad\left\{\begin{array}{cccc}80.63 & 83.62 \\ 80.58 & 83.58 & 2-3 & 38^{3} \mathrm{P}^{\circ}-3 \mathrm{p}^{3} \mathrm{D} \\ 1-2 & (4)\end{array}\right.$ $\begin{array}{ll}80.56 & 83.56 \\ 80.63 & 83.58\end{array} \quad 0-1$ $\begin{array}{ll}80.63 & 83.58 \\ 80.58 & 83.56\end{array}\left\{\begin{array}{l}2-3 \\ 1-1\end{array}\right.$ 4123
4179
4151 4151
4211

3275.67 3239
3222

3058.68

4554.38

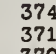

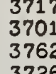

3762
3726
3703

3703
3693

3298
3249

3249
3232
3364

3230
3245

4925
4940

4158.76

4135.9
4131.7

6830
6790

6790
6767
6878

6878
6819

6909

5473
5376

5376
5432
5352
5417

5417
5343

6339

4522

3702

7438

$\begin{array}{ccc}\mathrm{P} & & \\ \mathrm{P} & & \\ \mathrm{P} & \\ \mathrm{P} & \\ \mathrm{P} & \\ \mathrm{A} & 0 \\ \mathrm{P} & & \\ \mathrm{P} & \end{array}$

$\left.\begin{array}{lll}80.58 & 83.56 \\ 80.63 & 83.56\end{array}\right) \quad \begin{aligned} & 1-1 \\ & 2-1\end{aligned}$

$(80.6384 .39) \quad 2-1 \quad 38^{3} \mathrm{p}^{0}-3 \mathrm{p}^{3} \mathrm{~s}$

$\left\{\begin{array}{lll}80.58 & 84.39 \\ 80.56 & 84.39\end{array}\right) \quad \begin{aligned} & 1-1 \\ & 0-1\end{aligned}$

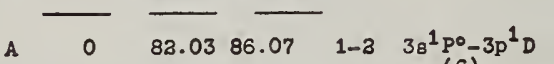

A $\quad-\quad \frac{0}{83.04} 85.75 \quad 1-2 \quad 3 p^{1} P-3 d^{1} \div D^{\circ}$

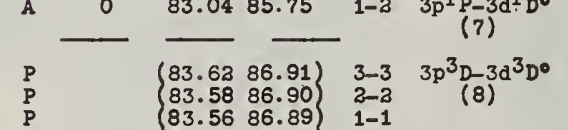

$\begin{array}{ll}83.58 & 86.90 \\ 83.56 & 86.89 \\ 83.63 & 86.90 \\ 8-1 & 3-1\end{array}$

$\begin{array}{lll}83.62 & 86.90 & 3-2 \\ 83.58 & 86.89 & 2-1\end{array}$

$\begin{array}{lll}83.58 & 86.91 & 2-1 \\ 8-3\end{array}$

$83.5686 .90) \quad 1-3$

83.63 87.36) 3-a $3 p^{3} \mathrm{D}-3 \mathrm{~d}^{3} \mathrm{p}^{\circ}$

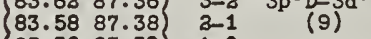

$\begin{array}{lll}83.58 & 87.38 & 2-1 \\ 83.56 & 87.39 & 1-0\end{array}$

$\begin{array}{lll}83.58 & 87.36 & 1-0 \\ 83 & -3\end{array}$

$\begin{array}{lll}83.58 & 87.36 & 2-2 \\ 83.56 & 87.38 & 1-1 \\ 83.56 & 87.36 & 1-3\end{array}$

P

$\left[\begin{array}{lll}84.39 & 86.90 \\ 84.39 & 86.89\end{array}\right) \quad 1-3 \quad 3 p^{3} \mathrm{~S}-3 \mathrm{~d}^{3} \mathrm{D}^{\circ}$

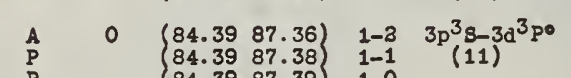

\section{P} 87.38 1-1 (11)

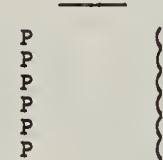

$\left(\begin{array}{lll}85.11 & 86.91\end{array}\right) \quad 2-3 \quad 3 p^{3} P-3 d^{3} D^{\circ}$ $\begin{array}{llll}85.08 & 86.90 \\ 1-3 & \text { (13) }\end{array}$

\begin{tabular}{ll|l}
85.07 & 86.89 & $0-1$ \\
85.11 & 86.90 & $2-3$
\end{tabular}

$\left\{\begin{array}{ll}85.08 & 86.89 \\ 85.11 & 86.89\end{array}\right\} \quad \begin{aligned} & 1-1 \\ & 2-1\end{aligned}$

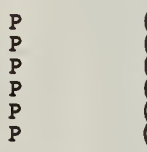

$(85.1187 .36) \quad 2-2 \quad 3 p^{3} \mathrm{p}-3 \mathrm{~d}^{3} \mathrm{p}^{\circ}$

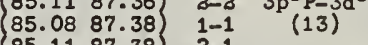

$\begin{array}{lll}85.11 & 87.38 & 2-1 \\ 85.08 & 87.39 & 1-0\end{array}$

$\left\{\begin{array}{ll}85.08 & 87.36 \\ 85.07 & 87.38\end{array}\right) \quad \begin{aligned} & 1-2 \\ & 0-1\end{aligned}$

$P \quad \overline{86.07} 88.03 \quad 2-3 \quad 3 p^{1} \mathrm{D}-3 \mathrm{~d}^{1} \mathrm{~F}^{\circ}$

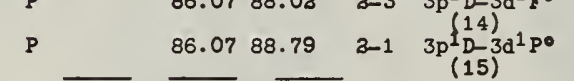

$P \quad \overline{88.79} 93.13 \quad 1-3 \quad 3 \mathrm{~d}_{(16)}^{1 \mathrm{P}^{\circ}-4^{1} \mathrm{D}}$

$P \quad \overline{(89.15} 90.81)-4^{3} \mathrm{~S}-4^{3} \mathrm{Po}^{\circ}$

OVI I P 137.53 Ansl A L1st A Feb 1943 $\begin{array}{lllllll}3811.35 & \text { A } & 3 & 79.01 & 82.25 & \frac{1}{2}-1 \frac{1}{2} & 3^{2} \mathrm{~S}-3^{2} \mathrm{P} \text { o } \\ 3834.24 & \text { A } & 1 & 79.01 & 82.23 & \frac{1}{2}-\frac{1}{2} & (1)\end{array}$ $3068 \quad \mathrm{P}+123.46127 .49$ है $6^{2} \mathrm{~S}-7^{2} \mathrm{P}^{\circ}$ 3623

$\mathrm{P} \quad \overline{123.84} 137.25-6^{2} \mathrm{P}^{\circ}-7^{2} \mathrm{~S}$ 3314

$P \quad \begin{array}{r}123.84137 .57-6^{2} P^{0}-7^{2} \mathrm{D} \\ \hline\end{array}$
I A Laboratory Ref Int Low $_{\text {High }}$ J (Ro) $^{\text {Litiplet }}$

OVI continued

$3509 \quad P \quad 123.97127 .49-6^{2} D_{-12} P^{\circ}$

$3426 \quad P \quad 123.97127 .57-6^{2} D^{(5)}(6)$

$3438 \quad P \quad 123.98137 .57-6^{2} \mathrm{~F}^{0}-7^{3} \mathrm{D}$

$3433 \quad P \quad 123.98127 .57-186^{2} \mathrm{Fo}^{(7)} ?^{2} \mathrm{G}$

$3434 \quad P \quad \overline{123.98} 127.57-6^{2} \mathrm{G}-7^{2} \mathrm{H}^{\circ}$ etc

$4751 \quad \mathrm{P}=\overline{137.25} 129.85$ t $-7^{2} \mathrm{~S}-8^{2} \mathrm{PO}^{\circ}$

$5602 \quad P \quad 127.49129 .69 \quad-\frac{1}{2} \quad 7^{2} p^{\circ}-8^{2} 8$

$5112 \quad P \quad 127.49129 .90-7^{2} \mathrm{P}^{(11)}-8^{2} \mathrm{D}$

$5410 \quad \mathrm{P}=\overline{127.57} 129.85-7^{2} \mathrm{D}-8^{2} \mathrm{po}$

$\begin{array}{lll}5279 & P & 127.57139 .90-7^{2} \mathrm{D}-8^{2} \mathrm{~F}^{\circ}\end{array}$

$5298 \quad P \quad 127.57139 .90-7^{2} F^{\circ}-8^{2} \mathrm{D}$

5289
$P$$\quad \begin{aligned} & 137.57129 .90-7^{2} F^{\circ}-8^{2} 6 \\ & (16)\end{aligned}$

$5293 \quad P=\overline{137.57} 139.90-7^{2} Q-8^{2} F^{\circ}$

$\begin{array}{lll}5391 & P & 137.57129 .90-7^{2} \mathrm{G}-82 \mathrm{~K}\end{array}$

\begin{tabular}{|c|c|c|c|c|}
\hline$I$ & 7.35 & & L1st $D$ & Uay 1944 \\
\hline $\begin{array}{l}398.68 \\
482.72 \\
514.93 \\
331.95 \\
425.64 \\
553.34 \\
573.41\end{array}$ & $\begin{array}{l}\mathbf{A} \\
\mathbf{A} \\
\mathbf{A} \\
\mathbf{A} \\
\mathbf{A} \\
\mathbf{A} \\
\mathbf{A}\end{array}$ & $\begin{array}{r}17 \\
11 \\
9 \\
15 \\
14 \\
14 \\
14\end{array}$ & $\begin{array}{ll}13.64 & 14.31 \\
12.68 & 14.33 \\
13.70 & 14.34 \\
13.64 & 14.33 \\
13.68 & 14.34 \\
12.68 & 14.31 \\
13.70 & 14.33\end{array}$ & $\begin{array}{ll}-3 \frac{1}{2} & 3 s^{4} p-3 p^{4} p^{0} \\
-1 \frac{1}{2} & (1)^{2} \\
-1 \frac{1}{2} & \\
-3 \frac{1}{2} & \end{array}$ \\
\hline $\begin{array}{l}6856.03 \\
6903.46 \\
6909.83 \\
6773.97 \\
6834.36 \\
6870.32 \\
6708.37 \\
6795.53\end{array}$ & $\begin{array}{l}\mathbf{A} \\
\mathbf{A} \\
\mathbf{A} \\
\mathbf{A} \\
\mathbf{A} \\
\mathbf{A} \\
\mathbf{A} \\
\mathbf{A}\end{array}$ & $\begin{array}{r}16 \\
15 \\
13 \\
11 \\
13 \\
13 \\
7 \\
9\end{array}$ & $\begin{array}{ll}12.64 & 14.44 \\
13.68 & 14.46 \\
12.70 & 14.48 \\
12.64 & 14.46 \\
12.68 & 14.48 \\
12.70 & 14.49 \\
12.64 & 14.48 \\
12.68 & 14.49\end{array}$ & 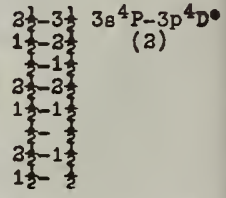 \\
\hline $\begin{array}{l}6339.64 \\
6348.50 \\
6413.66\end{array}$ & $\begin{array}{l}\mathbf{A} \\
\mathbf{A} \\
\mathbf{A}\end{array}$ & $\begin{array}{l}16 \\
15 \\
14\end{array}$ & $\begin{array}{ll}12.64 & 14.63 \\
13.68 & 14.62 \\
12.70 & 14.62\end{array}$ & $\begin{array}{c}3 \frac{1}{2}-1 \frac{1}{2} \\
1 \frac{1}{2}-1 \frac{1}{2}{ }^{4} \mathrm{P}-3 \mathrm{p}^{4} \mathrm{~s} \\
\frac{1}{2}-1 \frac{1}{2}\end{array}$ \\
\hline $\begin{array}{l}7754.70 \\
7800.32 \\
7607.17\end{array}$ & $\begin{array}{l}\mathbf{A} \\
\hat{A} \\
\mathbf{A}\end{array}$ & $\begin{array}{l}19 \\
18 \\
15\end{array}$ & $\begin{array}{ll}12.93 & 14.53 \\
12.97 & 14.55 \\
12.93 & 14.55\end{array}$ & $\begin{array}{ll}2 \frac{1}{2} & 3 s^{2} P-3 p^{2} D \\
1 \frac{1}{2} & (4) \\
1 \frac{1}{2} & \end{array}$ \\
\hline $\begin{array}{l}7311.02 \\
7489.14\end{array}$ & $\begin{array}{l}\mathbf{A} \\
\mathbf{A}\end{array}$ & $\begin{array}{r}13 \\
8\end{array}$ & $\begin{array}{ll}12.93 & 14.63 \\
13.97 & 14.62\end{array}$ & $\frac{\frac{1}{2}}{\frac{1}{2}}{ }^{3 \mathrm{~s}^{2} \mathrm{P}-3 \mathrm{p}^{2} \mathrm{~S}^{\circ}} \frac{(5)}{(5)}$ \\
\hline $\begin{array}{l}7037.45 \\
7127.88 \\
6966.35 \\
7202.37\end{array}$ & $\begin{array}{l}\mathbf{A} \\
\mathrm{A} \\
\mathrm{A} \\
\mathrm{A}\end{array}$ & $\begin{array}{l}15 \\
14 \\
10 \\
13\end{array}$ & $\begin{array}{ll}12.93 & 14.68 \\
12.97 & 14.70 \\
12.93 & 14.70 \\
12.97 & 14.68\end{array}$ & $\begin{array}{c}\frac{1}{2} 3 \mathrm{~s}^{2} \mathrm{P}-3 \mathrm{p}^{2} \mathrm{I} \\
(6)\end{array}$ \\
\hline
\end{tabular}

F II I P 34.84 Anal B L1st D Kay 1944

$\begin{array}{lll}3847.086 & \text { A } & 20 \\ 3849.987 & \text { A } & 15\end{array} \quad\left\{\begin{array}{ccc}31.81 & 25.02 \\ 31.81 & 35.01 \\ 31.81 & 25.01\end{array}\right\} \begin{array}{ccc}2-3 & 38^{5} \mathrm{~S}^{\circ}-3 \mathrm{p}^{5} \mathrm{P} \\ 2-3 & (1)\end{array}$

$\begin{array}{lll}3847.086 & \text { A } & 20 \\ 3849.987 & \text { A } & 15 \\ 3851.667 & \text { A } & 10\end{array} \quad\left\{\begin{array}{lll}21.81 & 25.02 \\ 31.81 & 35.01 \\ 31.81 & 25.01\end{array}\right\} \begin{aligned} & 2-3 \\ & 2-3 \\ & 2-1\end{aligned}$

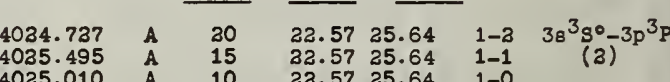

$\begin{array}{llllll}4025.010 & \text { A } & 10 & 22.57 & 25.64 & 1-0\end{array}$

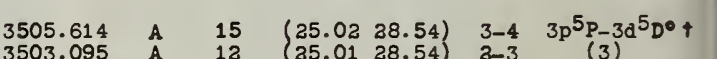

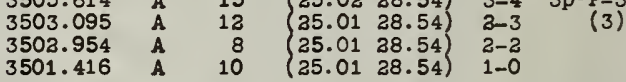

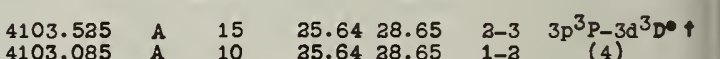

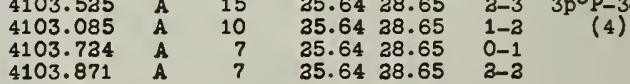




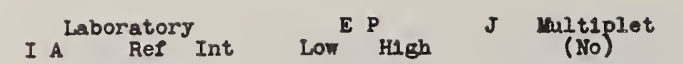
Ne I continued

$\begin{array}{llr}9373.28 & D & 300 \\ 9313.98 & D & 300 \\ 9300.85 & D & 600 \\ 9221.59 & D & 200 \\ 9220.05 & D & 400 \\ 8654.51 & D & 400 \\ 8654.3835 & B & 1500 \\ 8647.05 & D & 300\end{array}$

$\begin{array}{lll}5145.011 & E & E\end{array} \quad\left\{\begin{array}{l}10 \\ 10\end{array}\right)$

-4884.915 B (10)

$\begin{array}{lll}9425.38 & D & 500 \\ 9326.53 & D & 600\end{array}$

8679.491 B 500

$9547.40 \quad D \quad 300$

$\begin{array}{lll}9534.17 & D & 500 \\ 9459.21 & D & 300\end{array}$

$\begin{array}{llr}8783.755 & B & 1000 \\ 8771.70 & D & 400\end{array}$

5965.474 B (10)

$10562.43 \quad F \quad \overline{200}$

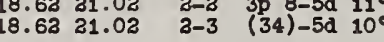

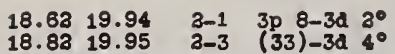

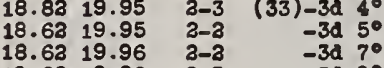
18.6219 .96 18.6230 .05 $\begin{array}{lll}18.62 & 30.05 \\ 18.62 & 30.05\end{array}$ $18.62 \quad 20.57 \quad 2-1 \quad 3 p 8-584^{\circ}$ $18.6320 .05 \quad 0-1 \quad 3 p$ 6-3a $13^{\circ}$ $18.65 \quad 19.94 \quad 1-0 \quad 3 p$ 9-3d $1^{\circ}$

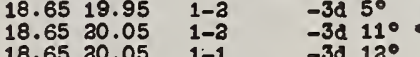

$\begin{array}{lllll}18.65 & 30.71 \quad 1-2 \quad 3 p & 9-4 a & 11^{\circ} & 3554.39\end{array}$

$(40)^{10-3 d} 12^{\circ}$

3208.99 3188.74

3244.15
3314.38

3243.34

3348.15
3269.86
3363.43

3363.43

3118.02
3169.30

3151.16

3178.61

3209.38

3039.65
3035.98

3030.85

3059.16

3044.16
.3072 .68

$\begin{array}{lll}18.88 & 20.05 \quad 0-1\end{array}$

3367.20

3388.46
3330.78
$18.63 \quad 31.02 \quad 2-3 \quad 3 p 8-5 d 11^{\circ}$ $\begin{array}{lllll}18.63 & 19.94 & 0-1 & 3 p 6-3 d & 2^{\circ} \\ 18.63 & 19.95 & 0-1 & (36)-3 d & 6^{\circ}\end{array}$ $\begin{array}{llll}18.65 & 19.94 & 1-1 & (38)-3 d \\ 3^{\circ}\end{array}$

I A Reboratory Int

For changes in Paschen's notation see text $\$ 37$

\begin{tabular}{|c|c|c|c|c|c|}
\hline \multirow[b]{2}{*}{$\begin{array}{l}\text { NeII } \\
3694.22 \\
3734.94 \\
3751.26 \\
3664.09 \\
3709.64 \\
3766.29 \\
3777.16\end{array}$} & \multicolumn{2}{|c|}{ I P 40.91} & \multirow{2}{*}{\multicolumn{2}{|c|}{$\begin{array}{cc}\text { Anal A } & \text { L1st A } \\
37.05 & 30.39 \\
37.13 & 30.42 \\
37.15 & 30.44 \\
37.05 & 30.42 \\
37.12 & 30.44 \\
37.12 & 30.39 \\
37.15 & 30.42\end{array}$}} & \multirow{2}{*}{$\begin{array}{l}\text { A June } 1944 \\
3 \frac{1}{3}-38^{4} p-3 p^{4} p 0 \\
1 \frac{1}{3}-1 \frac{1}{(1)}\end{array}$} \\
\hline & $\begin{array}{l}\mathbf{A} \\
\mathbf{A} \\
\mathbf{A} \\
\mathbf{A} \\
\mathbf{A} \\
\mathbf{A} \\
\mathbf{A}\end{array}$ & $\begin{array}{r}10 \\
7 \\
5 \\
9 \\
7 \\
8 \\
8 \\
8\end{array}$ & & & \\
\hline $\begin{array}{l}3334.87 \\
3355.05 \\
3360.63 \\
3297.74 \\
3337.16 \\
3344.43 \\
3270.79 \\
3311.30\end{array}$ & $\begin{array}{l}\mathbf{A} \\
\mathbf{A} \\
\mathbf{A} \\
\mathbf{A} \\
\mathbf{A} \\
\mathbf{A} \\
\mathbf{A} \\
\mathbf{A}\end{array}$ & $\begin{array}{r}10 \\
? \\
5 \\
7 \\
5 \\
5 \\
2 \\
3\end{array}$ & $\begin{array}{l}37.05 \\
37.12 \\
27.15 \\
37.05 \\
37.12 \\
37.15 \\
37.05 \\
27.12\end{array}$ & $\begin{array}{l}30.75 \\
30.79 \\
30.83 \\
30.79 \\
30.83 \\
30.84 \\
30.83 \\
30.84\end{array}$ & $\frac{38^{4} P-3 p^{4} D^{0}}{(3)^{3}}$ \\
\hline $\begin{array}{l}3135.82 \\
3187.60\end{array}$ & ${ }_{\mathbf{A}}^{\mathbf{A}}$ & $\frac{1}{3}$ & $\begin{array}{l}27.05 \\
27.12\end{array}$ & $\begin{array}{l}30.99 \\
30.99\end{array}$ & $\begin{array}{l}2 \frac{1}{2}-2 \frac{1}{12} 3 B^{4} P-3 p^{2} D^{0} \\
1 \frac{1}{2}-2 \frac{1}{2} \\
(3)\end{array}$ \\
\hline $\begin{array}{l}3001.65 \\
3028.84\end{array}$ & A & $\begin{array}{l}6 \\
4\end{array}$ & $\begin{array}{l}37.12 \\
37.15\end{array}$ & $\begin{array}{l}31.23 \\
31.23\end{array}$ & 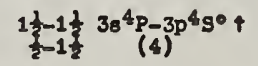 \\
\hline $\begin{array}{l}3713.09 \\
3727.08 \\
3643.89\end{array}$ & $\begin{array}{l}\mathbf{A} \\
\mathbf{A} \\
\mathbf{A}\end{array}$ & $\begin{array}{r}10 \\
9 \\
5\end{array}$ & $\begin{array}{l}37.66 \\
27.74 \\
37.66\end{array}$ & $\begin{array}{l}30.99 \\
31.05 \\
31.05\end{array}$ & $\begin{array}{c}1 \frac{1}{2}-2 \frac{1}{2} 38^{3} p-3 p^{2} D^{0} \\
1 \frac{1}{2}-1 \frac{2}{2}\end{array}$ \\
\hline $\begin{array}{l}3481.96 \\
3557.84\end{array}$ & ${ }_{A}^{A}$ & $\begin{array}{l}6 \\
4\end{array}$ & $\begin{array}{l}27.66 \\
27.74\end{array}$ & $\begin{array}{l}31.21 \\
31.31\end{array}$ & $\frac{1}{3} \mathrm{~s}^{2} \mathrm{P}-3 \mathrm{p}^{2} \mathrm{~g}^{\circ}$ \\
\hline $\begin{array}{l}3333.75 \\
3378.28 \\
3309.78 \\
3392.78\end{array}$ & $\begin{array}{l}\mathbf{A} \\
\mathbf{A} \\
\mathbf{A} \\
\mathbf{A}\end{array}$ & $\begin{array}{l}7 \\
5 \\
3 \\
5\end{array}$ & $\begin{array}{l}27.66 \\
27.74 \\
27.66 \\
37.74\end{array}$ & $\begin{array}{l}31.38 \\
31.39 \\
31.39 \\
31.38\end{array}$ & $\begin{array}{c}1 \frac{1}{2} 38^{3} p-3 p^{3} p 0 \\
(7)^{2}\end{array}$ \\
\hline $\begin{array}{l}3034.48 \\
3047.57 \\
3054.69 \\
3027.04 \\
3037.73 \\
3045.58 \\
3017.34\end{array}$ & $\begin{array}{l}\mathbf{A} \\
\mathbf{A} \\
\mathbf{A} \\
\mathbf{A} \\
\mathbf{A} \\
\mathbf{A} \\
\mathbf{A}\end{array}$ & $\begin{array}{l}5 \\
6 \\
5 \\
4 \\
4 \\
4 \\
3\end{array}$ & $\begin{array}{l}30.39 \\
30.42 \\
30.44 \\
30.39 \\
30.42 \\
30.44 \\
30.39\end{array}$ & $\begin{array}{l}34.46 \\
34.47 \\
34.48 \\
34.47 \\
34.48 \\
34.50 \\
34.48\end{array}$ & ${ }^{3 \frac{1}{4} p^{0}-3 d^{4} D}$ \\
\hline $\begin{array}{l}3568.53 \\
3574.64 \\
3574.23\end{array}$ & $\stackrel{A}{A}$ & $\begin{array}{l}6 \\
5 \\
0\end{array}$ & $\begin{array}{l}30.42 \\
30.48 \\
30.43\end{array}$ & $\begin{array}{l}33.88 \\
33.87 \\
33.87\end{array}$ & 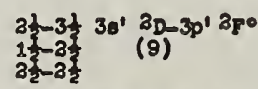 \\
\hline $\begin{array}{r}3345.49 \\
3319.75 \\
\cdot 3345.88\end{array}$ & $\begin{array}{l}A \\
A \\
A\end{array}$ & $\begin{array}{l}3 \\
3 \\
1\end{array}$ & $\begin{array}{l}30.43 \\
30.42 \\
30.42\end{array}$ & $\begin{array}{l}34.11 \\
34.14 \\
34.11\end{array}$ & 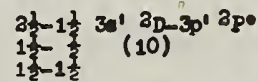 \\
\hline $\begin{array}{l}3230.16 \\
3232.38 \\
3231.97\end{array}$ & $\begin{array}{l}\hat{A} \\
\hat{A} \\
\mathbf{A}\end{array}$ & $\begin{array}{l}5 \\
3 \\
0\end{array}$ & $\begin{array}{l}30.42 \\
30.42 \\
30.42\end{array}$ & $\begin{array}{l}34.34 \\
34.24 \\
34.24\end{array}$ & 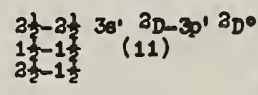 \\
\hline $\begin{array}{l}3329.30 \\
3357.90 \\
3374.10 \\
3379.39 \\
3320.29 \\
3345.88 \\
3362.89 \\
3367.05 \\
3386.24 \\
3390.56\end{array}$ & $\begin{array}{l}A \\
A \\
A \\
A \\
A \\
A \\
A \\
A \\
A \\
P \\
A \\
A\end{array}$ & $\begin{array}{l}4 \\
3 \\
3 \\
1 \\
2 \\
1 \\
1 \\
3\end{array}$ & $\begin{array}{l}30.75 \\
30.79 \\
30.83 \\
30.84 \\
30.75 \\
30.79 \\
30.83 \\
30.79 \\
30.83 \\
30.84\end{array}$ & $\begin{array}{l}34.46 \\
34.47 \\
34.48 \\
34.50 \\
34.47 \\
34.48 \\
34.50 \\
34.46 \\
34.47 \\
34.48\end{array}$ & 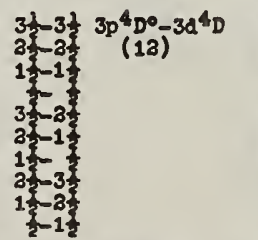 \\
\hline $\begin{array}{l}3318.21 \\
3198.63 \\
3190.86 \\
3213.70 \\
3164.46 \\
3165.70 \\
3198.88 \\
3132.22 \\
3173.58\end{array}$ & $\begin{array}{l}\mathbf{A} \\
\mathbf{A} \\
\mathbf{A} \\
\mathbf{A} \\
\mathbf{A} \\
\mathbf{A} \\
\mathbf{P} \\
\mathbf{A} \\
\mathbf{A}\end{array}$ & $\begin{array}{l}8 \\
5 \\
2 \\
3 \\
3 \\
4\end{array}$ & $\begin{array}{l}30.75 \\
30.79 \\
30.83 \\
30.84 \\
30.75 \\
30.79 \\
30.83 \\
30.75 \\
30.79\end{array}$ & $\begin{array}{l}34.59 \\
34.65 \\
34.69 \\
34.68 \\
34.65 \\
34.69 \\
34.68 \\
34.69 \\
34.68\end{array}$ & 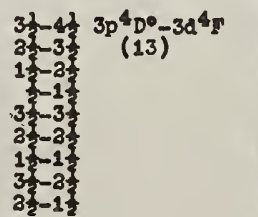 \\
\hline
\end{tabular}

3417.71

3356.35

3416.87

3453.10

3314.60

3355.39

3353.63

3094.08

3088.23
3143.74

3551.5

3613.35

3546.28

3456.68

3503.81
3375.20

3806.30 3790.96

3561.23
3571.2

3590.47

3659.93
3632.75

3543.90

3565.84

3475.25

3448.12

3397.90

3721.88

3753.83

3829.77

3818.44
3800.02

3701.81

3838.08

3697.09

3644.88

3438.78

3443.70

3339.50

3224.82

3097.15

3098.91

3411.38

3440.80
3413.13
3438.97

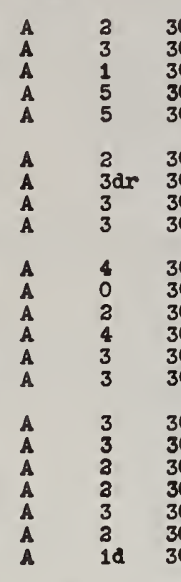

$30.75 \quad 34.60 \quad 3 \frac{1}{3}-3 \frac{1}{4} 3 \mathrm{p}^{4} \mathrm{D}^{\circ}-3 \mathrm{~d}^{2} \mathrm{~F}$

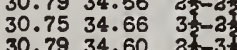

$\begin{array}{lll}30.79 & 34.60 & 2 \frac{1}{3}-3 \frac{1}{2} \\ 30.83 & 34.66 & 1 \frac{3}{2}-2 \frac{1}{2}\end{array}$

$30.7934 .60 \quad 3 \frac{1}{3}-2 \frac{1}{3} 3 \mathrm{p}^{4} \mathrm{D}^{0}-3 \mathrm{~d}^{2} \mathrm{D}$ $30.83 \quad 34.62$ 1 1 (15) $\begin{array}{lll}30.83 & 34.60 & 1 \frac{1}{2}-2 \frac{1}{2} \\ 30.84 & 34.62 & \frac{1}{2}-1 \frac{1}{2}\end{array}$

$30.7534 .71 \quad 3 \frac{1}{2}-2 \frac{1}{2} \quad 3 p^{4} p^{0}-3 d^{4} p$ 30.7934 .69 3 3 (16)

$\begin{array}{lll}30.79 & 34.71 & 34-3 \\ 30.83 & 34.69 & 17\end{array}$

$30.83 \quad 34.71 \quad 1 \frac{1}{3}-2$

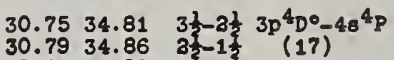

$30.83 \quad 34.90 \quad 18$

$\begin{array}{lll}30.79 & 34.81 & 34-2 \\ 30.83 & 34.86 & 13-1\end{array}$

$\begin{array}{llll}30.84 & 34.90 & 1 \\ 30.84 & 34.86 & \frac{1}{2}-1 \frac{1}{2}\end{array}$

$30.9934 .46 \quad 3 \frac{1}{2}-3 \frac{1}{2} 3 \mathrm{p}^{2} \mathrm{D}^{0}-3 \mathrm{~d}^{4} \mathrm{D}$

30.9934 .65 3t-3t $3 \mathrm{p}^{2 D^{\circ}-3 d^{4} F}$

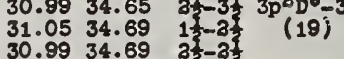

$30.9934 .60 \quad 3 \frac{2}{3}-3 \frac{1}{2} 3 \mathrm{p}^{2} \mathrm{D}^{\circ}-3 \mathrm{a}^{2} \mathrm{~F}$

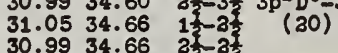

$30.99 \quad 34.60 \quad 2 \frac{1}{2}-2 \frac{1}{2} 3 \mathrm{p}^{2} \mathrm{D}^{\circ}-3 \mathrm{~d}^{2} \mathrm{D}$ $\begin{array}{llll}31.05 & 34.62 & 1 \frac{1}{3}-1 \frac{1}{3} & \text { (21) } \\ 31.05 & 34.60 & 1 \frac{1}{2}-2 \frac{1}{2} & \end{array}$

$30.9934 .71 \quad 2 \frac{1}{3}-2 \frac{1}{3} 3 \mathrm{p}^{2} \mathrm{p}^{0}-3 \mathrm{~d}^{4} \mathrm{p}$ $31.05 \quad 34.71 \quad 1 \frac{1}{2}-2 \frac{1}{2} \quad$ (28)

$30.99 \quad 34.78 \quad 3 \frac{1}{2}-1 \frac{1}{2} 3 p^{2} D^{0}-3 d^{2} p$ $\begin{array}{lll}31.05 & 34.73 & 1 \\ 31.05 & 34.78 & 1\end{array}$

$30.99 \quad 34.98 \quad 2 \frac{1}{2}-1 \frac{1}{2} 3 p^{2} p^{0}-48^{2} p$

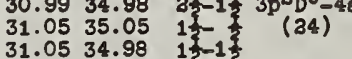

$31.3134 .68 \quad \frac{1}{2-1 \frac{1}{2}} 3 \mathrm{p}^{2} \mathrm{~g}^{\circ}-3 \mathrm{~d}^{4} \mathrm{~F}$

$31.3134 .62 \quad \frac{1}{2}-1 \frac{1}{2} 3 \mathrm{p}^{0.99^{\circ}-3 \mathrm{~d}^{2} \mathrm{D}}$

$31.3134 .69 \quad \frac{1}{2}-1 \frac{1}{2} 3 \mathrm{p}^{d} \mathrm{~g}^{\circ}-3 \mathrm{~d}^{4} \mathrm{P}$

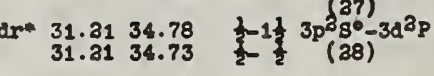

$31.2334 .47 \quad 12-313 p^{4} g^{\circ}-3 a^{4} D$ $\begin{array}{llll}31.23 & 34.48 & 1 \frac{1}{2}-1 \frac{1}{2} & 3 p^{4} \\ (30)\end{array}$

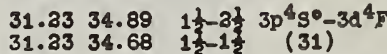
$31.23 \quad 34.66 \quad 1 \frac{1}{2}-2 \frac{1}{2} 3 p^{4} S^{0}-3 d^{3} \bar{F}$

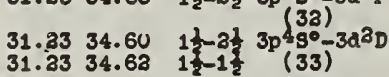

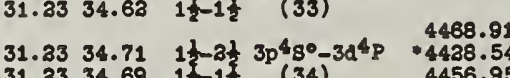
$\begin{array}{lll}31.23 & 34.69 & 1 \frac{1}{3}-1 \frac{1}{2} \\ 31.23 & 34.88 & 1 \frac{1}{2}-\frac{1}{2}\end{array}$

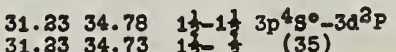

$31.2334 .81 \quad 12-213 p^{4} s 0-48^{4} p$

31.38 $34.68 \quad 1 \frac{1}{2}-2 \frac{1}{2} 3 \mathrm{p}^{3 \mathrm{po}-3 \mathrm{~d}^{4} \mathrm{~F}}$ $31.38 \quad 34.68 \quad 1 \frac{1}{2}-2 \frac{1}{2} 3 \mathrm{p}^{3} \mathrm{3P}$ - $3 \mathrm{~d}^{2} \mathrm{~F}$ $31.38 \quad 34.60 \quad 1 \frac{1}{2}-2 \frac{1}{2} 3 \mathrm{p}^{6 \mathrm{PO}^{\circ}}-3 \mathrm{~d}^{3} \mathrm{D}$ $\begin{array}{llll}31.39 & 34.82 & 1 & 1 \\ 31.38 & 34.62 & 1 \frac{1}{2}-1 \frac{1}{2} & (39)\end{array}$

$31.38 \quad 34.71 \quad 1 \frac{1}{3}-2 \frac{1}{3} 3 \mathrm{p}^{2} \mathrm{p} 0-3 \mathrm{~d}^{4} \mathrm{p}$ $31.3834 .78 \quad 1 \frac{1}{2}-1 \frac{1}{4} 3 \mathrm{p}^{2} \mathrm{po}^{\circ}-3 \mathrm{~d}^{2} \mathrm{p}$ 31.3934 .73 t 31.3934 .78 安-1章

$31.38 \quad 34.98 \quad 1 \frac{1}{4}-1 \frac{1}{2} 3 \mathrm{p}^{2} \mathrm{po}_{-48^{2} \mathrm{P}}$

$\begin{array}{lll}31.38 & 34.98 & 1 \\ 31.39 & 35.05 & 1 \\ 31.39 & 34.98 & (48)\end{array}$

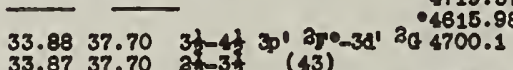
4634.73
4719.37
.4615 .98 4439.95 4244.17 4332.66

4615.98 4574.49 4582.05 1600.11

4471.53 .4413 .20 4439.30
4473.22 4431.38 33.8737 .70 aर $=3 \frac{3}{2}$ (43) 4538.66

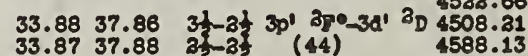

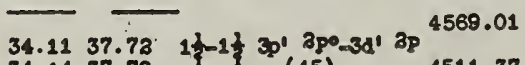

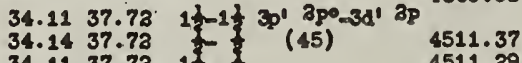

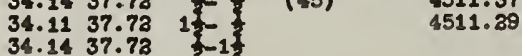

Laboratory Int

E P

tortiplot

Ne II continued

34.11 $37.81 \quad 1 \frac{1}{2}-1 \frac{1}{2} 3 p^{\prime} 3 p^{\circ}-3 d^{\prime} 2_{D}$ $34.1138 .04 \quad 1 \frac{1}{2}-2 \frac{1}{2} 3 \mathrm{p}^{(46)}(3 \mathrm{p})$

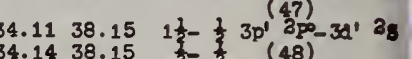
1438.15 रे $\frac{1}{2}(48)$

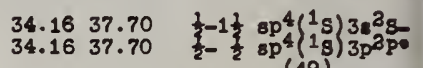
- - (49)

34.34 37.72 $3 \frac{1}{2}-1 \frac{1}{2} 3 p^{\prime} 3 p^{\circ}-3 d^{\prime}$ ap $34.24 \quad 37.72 \quad 1 \frac{1}{2}-1 \frac{3}{2}$

$34.2437 .862 \frac{1}{2}-2 \frac{1}{2} 3 p^{\prime} 300-3 d^{\prime} 30$ $34.34 \quad 37.81$ 11 1 (51) \begin{tabular}{lll}
34.24 & 37.81 & $3 \frac{1}{3}$ \\
34.24 & 37.86 & $1 \frac{1}{2}-2 \frac{1}{2}$ \\
\hline
\end{tabular}

$34.4637 .38 \quad 3 \frac{1}{3}-3 \frac{1}{2} 3 d^{4} D-41^{4} D^{\circ}$ $\begin{array}{lllll}34.47 & 37.39 & 23 & -23 & (53)\end{array}$ $\begin{aligned} & 34.48 \\ & 34.50\end{aligned} 37.39 \quad 13-1$

$34.46 \quad 37.39 \quad 3 \frac{5}{2}$

34.4737 .39 33 1

34.4837 .40

34.5037 .39 (5)

$34.4637 .39 \quad 3 \frac{1}{1}-4 \frac{1}{7} 3 \mathrm{~d}^{4} \mathrm{D}-41^{4} \mathrm{~F}^{\circ}$

$34.48 \quad 37.51$ 1

$\begin{array}{lll}34.48 & 37.51 \\ 34.50 & 37.47\end{array}$

$34.4637 .47 \quad 3=-1$

34.4737 .51 25

$34.48 \quad 37.48 \quad 11-21 \quad 3 d^{4} D-4 f^{4} G^{\circ}$ $\begin{array}{llll}34.47 & 37.48 & 32 & 21 \\ 34.46 & 37.48 & 3 & \end{array}$

$34.5937 .38 \quad 4 \frac{1}{3}-3 \frac{1}{2} 3 \mathrm{~d}^{4} F-45^{4} \mathrm{D}^{\circ}$

$\begin{array}{llll}34.65 & 37.39 & 3 \frac{1}{2}-2 \frac{1}{3} & \text { (55) }\end{array}$

$34.68 \quad 37.40 \quad 13-$

$\begin{array}{llll}34.68 & 37.39 & 12-1 \\ 34.68 & 37.39 & 1 & 1\end{array}$

34.5937 .39 4t $-4 \frac{1}{3} 3 \mathrm{~d}^{4} \mathrm{~F}-4 \mathrm{~F}^{4} \mathrm{~F}^{\circ}$

$34.65 \quad 37.47 \quad 35-3 \frac{30}{(56)}$

$34.69 \quad 37.51 \quad 31-2$

$34.6937 .47 \quad 35$

$34.65 \quad 37.39 \quad 33-4$

$\begin{array}{llll}34.69 & 37.47 & 32-3 \\ 34.68 & 37.51 & 1\end{array}$

$34.5937 .46 \quad 4 \frac{1}{3}-5 \frac{1}{3} \quad 3 \mathrm{~d}^{4} \mathrm{~F}-42^{400}$

$34.8537 .46 \quad 37-47$ (57)

$34.8937 .49 \quad 23-3$

$34.68 \quad 37.48 \quad 13-2$

$\begin{array}{lll}34.69 & 37.48 & 3 \\ 34.65 & 37.48 & 312\end{array}$

$34.6637 .392 \frac{1}{2}-2 \frac{1}{2} 3 a^{2} F-4 t^{4} L^{\circ}$

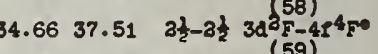

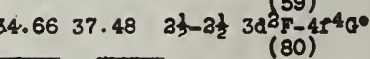

$34.8837 .39 \quad 1 \frac{1}{2}-2 \frac{1}{2} 3 d^{3} D-4 t^{4} D^{\circ}$ $34.60 \quad 37.39$ 32-27 (81) $\begin{array}{llll}34.62 & 37.39 & 1 & 1 \\ 34.60 & 37.39 & 3 & 1 \\ 34.62 & 37.40 & 1 & 1\end{array}$

$34.6037 .51 \quad 32-2 \frac{1}{3} 3 d^{2} p-48^{4}$.p

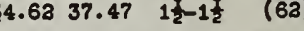
$34.6237 .481 \frac{1}{2}-2 \frac{1}{2} 3 d^{2} D-42^{4} \cdot 6 \bullet$

34.7137 .38 at $-3 \frac{1}{2} 3 a^{4} P-48^{4} D^{\circ}$ 34.6937 .39 19

$34.71 \quad 37.39$ 3 31

$34.66 \quad 37.40$ 15

$\begin{array}{lll}34.66 & 37.70 & 37.39 \\ 34.7 & 37\end{array}$

$34.7137 .47 \quad 2 \frac{1}{2}-3 \frac{1}{2} 3 a^{4} P-47^{4} \cdot 0$ 34.8937 .51 1 30 (85) 34.7137 .51 2t-27

$34.71 \quad 37.47 \quad 3 \frac{1}{2}-1$

$34.8937 .48 \quad 1 \frac{1}{2}-3 \frac{1}{2} 3 d^{4} P-4 P^{4} g^{4}$

34.78 37.391 1 -at $3 \mathrm{~d}^{2} \mathrm{P}-4 \mathrm{~s}^{4} \mathrm{D}$

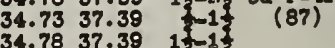

34.7337 .40
34.7837 .40

$34.78 \quad 37.51 \quad 1 \frac{1}{3}-2 \frac{1}{3} 3 \mathrm{~d}^{2} \mathrm{P}-4 \mathrm{r}^{4} \mathrm{~F}$

$\begin{array}{llll}34.73 & 37.47 & 15 & 1 \\ 34.78 & 37.47 & (88)\end{array}$

$34.7837 .48 \quad 1 \frac{1}{2}-2 \frac{1}{2} 3 d^{2} p-44^{6}$

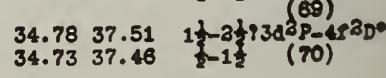


$I_{\text {A Laboratory }}$ ReI Int Low $_{\text {H1gh }}^{\text {P I tultiplet }}$

쏘 II continued

\begin{tabular}{|c|c|c|c|c|c|c|}
\hline $\begin{array}{l}4390.585 \\
4384.643\end{array}$ & $\hat{A}$ & $\begin{array}{l}0 \\
8\end{array}$ & $\begin{array}{l}9.96 \\
9.95\end{array}$ & $\begin{array}{l}13.77 \\
13.77\end{array}$ & $1 \frac{1}{\frac{1}{2}}-1 \frac{1}{2}$ & $\begin{array}{c}4^{2} \mathrm{P}^{\circ}-5^{3} \mathrm{D} \\
(10)\end{array}$ \\
\hline $\begin{array}{l}3553.51 \\
3549.61\end{array}$ & $\hat{A}$ & $\begin{array}{l}5 \\
4 \\
\end{array}$ & $\begin{array}{l}9.96 \\
9.95\end{array}$ & $\begin{array}{l}13.43 \\
13.43\end{array}$ & $1 \frac{1}{2}$ & $\begin{array}{c}4^{2} \mathrm{p}^{\circ}-7^{2} \mathrm{~s} \\
(11)^{-3}\end{array}$ \\
\hline $\begin{array}{l}3538.86 \\
3535.04\end{array}$ & $\hat{A}$ & 5 & $\begin{array}{l}9.96 \\
9.95\end{array}$ & $\begin{array}{l}13.44 \\
13.44\end{array}$ & $1 \frac{1}{2}$ & $\begin{array}{c}4^{2} p \circ-6^{2} p \\
(13)\end{array}$ \\
\hline $\begin{array}{l}3175.84 \\
3173.79\end{array}$ & $\hat{A}$ & $\begin{array}{l}3 \\
1\end{array}$ & $\begin{array}{l}9.96 \\
9.95\end{array}$ & $\begin{array}{l}13.84 \\
13.84\end{array}$ & & $\begin{array}{c}4^{3} \mathrm{po}^{\circ}-8^{2} \mathrm{~s} \\
(13)\end{array}$ \\
\hline $\begin{array}{l}3168.98 \\
3165.94\end{array}$ & $\hat{A}$ & $\begin{array}{l}3 \\
3\end{array}$ & $\begin{array}{l}9.96 \\
9.95\end{array}$ & $\begin{array}{l}13.85 \\
13.85\end{array}$ & $1 \frac{1}{2}-1 \frac{1}{2}$ & $4^{4}{ }^{30}-7^{3} \mathrm{D}$ \\
\hline 9633.0 & $\mathbf{P}$ & & 11.53 & 13.80 & & \\
\hline 6346.67 & $\boldsymbol{A}$ & 5 & 11.53 & 13.46 & & \\
\hline 5264.14 & $\mathbf{A}$ & 5 & 11.53 & 13.86 & & \\
\hline 4739.59 & $\mathbf{A}$ & 5 & 11.53 & 14.12 & & \\
\hline 4436.48 & $A$ & 5 & 11.53 & 14.30 & - & \\
\hline 4343.47 & $\mathbf{A}$ & 4 & 11.53 & 14.43 & - & \\
\hline 4109.54 & A & 3 & 11.53 & 14.53 & & \\
\hline 4013.80 & $\mathbf{A}$ & 3 & 11.53 & 14.59 & & $\begin{array}{l}a_{D-1} a^{2} F^{\circ} \\
(2 a)\end{array}$ \\
\hline 6545.80 & A & 5 & 11.58 & 13.47 & & \\
\hline 5401.05 & $A$ & 5 & 11.58 & 13.86 & & \\
\hline 4851.10 & A & 5 & 11.58 & 14.12 & & \\
\hline 4534.26 & A & 4 & 11.58 & 14.30 & - & \\
\hline 4331.93 & $\boldsymbol{A}$ & 3 & 11.58 & 14.43 & - & \\
\hline 4193.44 & A & 3 & 11.58 & 14.53 & . & ${ }_{1}{ }^{G}$ \\
\hline 4093.90 & A & 1 & 11.58 & 14.59 & & $\begin{array}{c}4^{2} F^{0}-12^{2} G \\
(29)\end{array}$ \\
\hline
\end{tabular}

A1 I I P 5.96 Anol A L18t B July 1944

$\begin{array}{lllllll}3961.523 / / & \mathrm{A} & 10 \mathrm{R} & 0.01 & 3.13 & 1 \frac{1}{2} & 3^{2} \mathrm{po}_{-} 4^{2} \mathrm{~S}\end{array}$

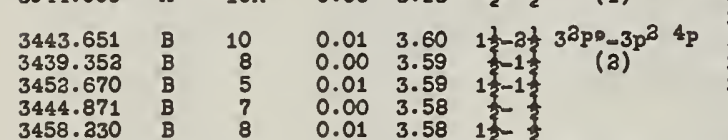

$\begin{array}{lllllll}3458.330 & \mathrm{~B} & 8 & 0.01 & 3.58 & 1 \frac{3}{2} & \frac{3}{2}\end{array}$

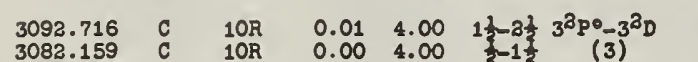

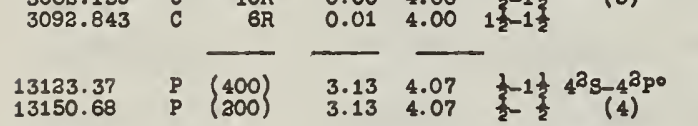

6695.97
6698.63

5557.0

5557.95

3057.155

3059.047
3066.158

3064.302

3054.694

$P$ (300)

$\begin{array}{ll}3.13 & 4.07 \\ 3.13 & 4.97\end{array}$

$4^{2}{ }^{2}-5^{2} p$

6343.36
6331.78

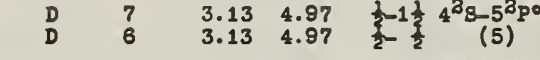

$\begin{array}{llllll}\mathrm{C} & \text { 1n } & 3.13 & 5.35 & \frac{1}{3}-1 \frac{1}{2} & 4^{2} \mathrm{~S}-6^{2} \mathrm{p}_{0} \\ \mathrm{C} & 1 \mathrm{n} & 3.13 & 5.35 & \frac{1}{2}-\frac{1}{2} & (6)\end{array}$

\begin{tabular}{rr} 
B & 10 \\
B & 4 \\
B & 5 \\
B & 5 \\
B & 9 \\
B & 6 \\
\hline
\end{tabular}

$3.60 \quad 7.63 \quad 3 \frac{1}{2}-2 \frac{1}{2} \quad 3 p^{2} 4 p-4$

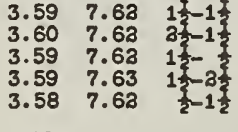

$(7)$

$\left.\begin{array}{llllll}11255.69 & P \\ 11253.81 & P\end{array}\right)(300) \quad\left(\begin{array}{lllll}4.00 & 5.10 & 3 \frac{1}{3} & 3^{2} D-44^{3} F^{\circ} \\ 4.00 & 5.10 & 1 \frac{2}{2}- & (8)\end{array}\right.$

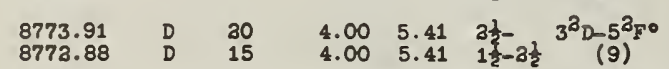

$\begin{array}{llrllll}7836.15 & D & 10 & 4.00 & 5.58 & 21 & 3^{3} D-6^{2} F 0 \\ 7835.33 & D & 9 & 4.00 & 5.58 & 1 \frac{1}{2}-2 \frac{1}{2} & (10)\end{array}$

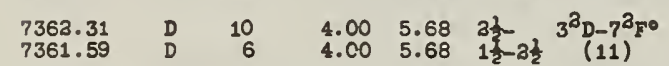

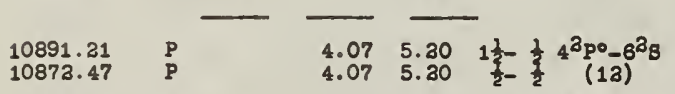

10782.1

10768.39
10786.78

$\begin{array}{llll}4.07 & 5.20 & \frac{1}{2}-\frac{1}{2} & (13) \\ 4.07 & 5.31 & 1 \frac{1}{3}-8 \frac{1}{2} & 4^{3} \mathrm{po}^{\circ}-5^{3} \mathrm{D}\end{array}$

$\begin{array}{llll}4.07 & 5.21 & 1 & 1 \\ 4.07 & 5.31 & 1 \frac{1}{2}-1 \frac{1}{2} & (13)\end{array}$

8923.56

$\begin{array}{llll}4.07 & 5.45 & 1 \frac{1}{3}-2 \frac{1}{2} & 4^{2} \mathrm{po}^{\circ}-8^{2} \mathrm{D} \\ 4.07 & 5.45 & \frac{1}{2}-1 \frac{1}{2} & (14)\end{array}$

$8841.36 \quad D \quad 3 \quad 4.07 \quad 5.47 \quad 1 \frac{1}{3}-2 \frac{1}{2} 4^{3} \mathrm{po}^{\circ}-7^{3} \mathrm{~s}$

$\begin{array}{lllllll}8838.91 & D & 1 & 4.07 & 5.47 & \text { 2-1 } & (15)\end{array}$

$\begin{array}{lllllll}8075.37 & D & 4 & 4.07 & 5.60 & 1 \frac{1}{3}-2 \frac{1}{3} & 4^{2} \mathrm{po}-7^{2} \mathrm{D} \\ 8065.99 & D & 3 & 4.07 & 5.60 & \frac{1}{2}-1 \frac{1}{2} & (16)\end{array}$

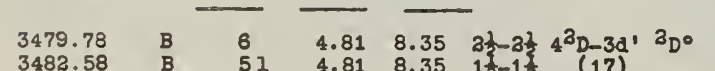

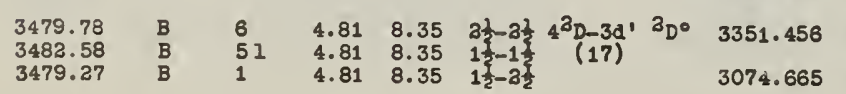

I A Laboratory ${ }_{\text {ReI }}$ Int Low ${ }_{\text {H1gh }}$ J Hultiplet Al I continued

\begin{tabular}{lllllll}
3931.97 & $B$ & 5 & 5.21 & 8.35 & $21-21$ & $5^{2} D-3 d^{\prime}{ }^{2} D^{\circ}$ \\
3935.77 & $B$ & 4 & 5.31 & 8.35 & $12-1$ & \\
\hline
\end{tabular} I A Rof Int Low ${ }_{\text {H1gh }}^{P}$

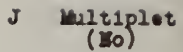
1I II continued

6231.78
6236.18

3738.003

3738.003
3733.910
3731.950

AI II I P 18.75 Anal A L1st A JuIy 1944

$3900.680 \quad B \quad 10 \quad 7.39 \quad 10.55 \quad 1-3 \quad 3^{1} \mathrm{PO}^{\circ}-3^{1} \mathrm{D}$

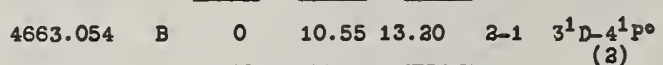

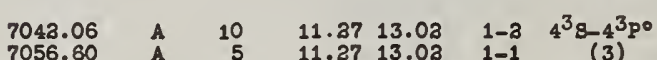

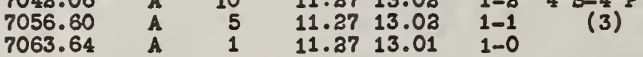

$8640.70 \quad A \quad \frac{1}{11.77} 13.30 \quad 0-1 \quad 4^{1} \mathrm{~s}-4^{1} \mathrm{po}$

$\begin{array}{llllllllllll}3275.776 & \text { B } & 4 & 11.77 & 15.54 & 0-1 & 4^{1} \mathrm{~s}-51 \mathrm{po} \\ (5)\end{array}$

$\begin{array}{lllllll}10076.29 & A & 6 & 11.80 & 13.02 & 3-3 & 3^{3} \mathrm{D}-4^{3} \mathrm{po} \\ 10107.19 & A & 4 & 11.80 & 13.02 & 2-1 & (6) \\ 10123.50 & A & 0.5 & 11.80 & 13.01 & 1-0 & (6)\end{array}$

10122.50

10077.32

10108.01
10108.37

3586.557
3587.068
3587.450
3586.912
3586.936
3587.309
3587.342
3587.185
3587.195
3586.708
3586.811
35

3587.195

-1
-1

3314.981

315.516

6837.14

883.48

3731.950

3654.995
3651.096

3651.065

3649.184
+3649.332
30.86

3026.776

3026.781

3024.098

3024.114

3998.158

2998.163

$\begin{array}{llllll}2995.530 & \mathrm{p} & 1.5 & 13.02 & 17.14 & 1- \\ 3995.546 & \mathrm{p} & & & \\ 3994.259 & \mathrm{P} & 1 & 13.01 & 17.14 & \mathrm{O}_{1}\end{array}$

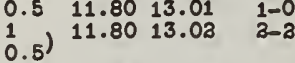

$\begin{array}{lll}0.5 & \\ 0.2 & 11.8013 .03 \quad 1-1\end{array}$

$\begin{array}{rrrrr}10 & 11.80 & 15.24 & 3-4 & 3^{3} D-4^{3} F^{\circ} \\ 9 & 11.80 & 15.24 & 2-3 & (7)\end{array}$

\begin{tabular}{llll}
9 & 11.80 & 15.24 & $2-3$ \\
8 & 11.80 & 15.34 & $1-3$ \\
\hline & 11.80 & 15.34 & $3-3$
\end{tabular}

3) $11.8015 .34 \quad 3-3$

3.5 , $11.8015 .34 \quad 3-3$

0.5 , $11.8015 .34 \quad 3-38$

$\begin{array}{llll}\text { A } & 1.55 \text { orb } 11.80 & 15.24 & 2-4 \\ \text { A } & \text {.25orb } 11.80 & 15.34 & 1-4\end{array}$

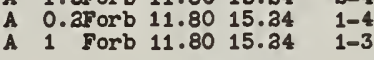

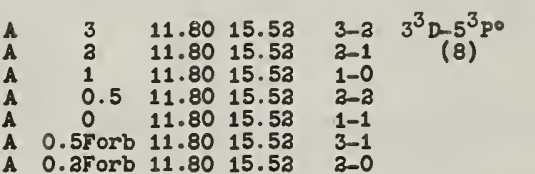

A $0.3 \mathrm{~F}$ orb $11.8015 .53 \quad 3-0$

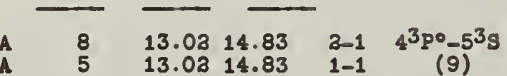

$\begin{array}{lllll}\mathbf{A} & 5 & 13.02 & 14.83 & 1-1 \\ \mathrm{~A} & \mathbf{1} & 13.01 & 14.83 & 0-1 \\ \mathrm{~A} & & & \end{array}$

$\begin{array}{cccccc}A & 10 & 13.02 & 15.00 & 2-3 & 4^{3} \mathrm{po}^{\circ}-4^{3} \mathrm{D} \\ \mathrm{A} & 9 & 13.02 & 15.00 & 1-2 & (10)\end{array}$

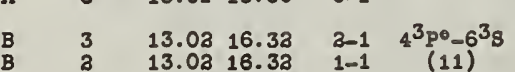

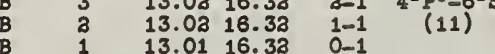

\begin{tabular}{lllll} 
A & $(8)$ \\
A & 13.03 & 16.40 & $2-3$ & $4^{3} \mathrm{PO}_{-5} 5^{3} \mathrm{D}$ \\
\hline & 53.02 & 16.40 & $1-2$ & $(12)$
\end{tabular}

A 7 Forb 13.0216 .40

A 1.5 Forb $13.0116 .40 \quad 0-3$

P) $\quad 1.5 \quad 13.03 \quad 17.10 \quad 2-1 \quad 4^{3} \mathrm{po}^{\mathrm{p}}-7^{3} \mathrm{~s}$

$\begin{array}{llll}\text { P) } & 1 & 13.03 \quad 17.10 \quad 1-1\end{array}$

(13)

$\begin{array}{llll}0.5 & 13.01 & 17.10 \quad 0-1\end{array}$

P) $\quad$ a $\quad 13.03 \quad 17.14 \quad 2-4^{3} \mathrm{P}^{\circ}-6^{3} \mathrm{D}$

$6919.98 \quad$ B $\quad 0.5 \quad 13.30 \quad 14.98 \quad 1-0 \quad 4^{1 \mathrm{pO}^{\circ}}-5^{1} \mathrm{~s}$

$5593.23 \quad B \quad 10 \quad 13.2015 .41 \quad 1-3 \quad 4^{1 \mathrm{Po}^{-}-5^{1} \mathrm{D}}$

$\begin{array}{llllllll}3866.160 & \text { B } & \text { a } & 13.20 & 16.39 & 1-0 & 4^{1} \mathrm{P}^{-} 0^{1} \mathrm{~S}\end{array}$

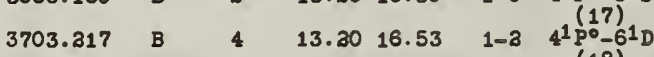

$\begin{array}{llllllll}3135.875 & B & 3 & 13.30 & 17.13 & 1-0 & 4^{1} \mathrm{Po}_{-71} \mathrm{G}\end{array}$

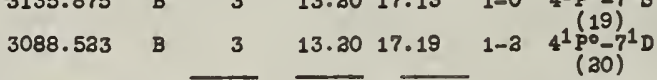

$7471.41 \quad A \quad 8 \quad \overline{13.59} 15.34$ 3-3 $4^{1} \mathrm{D}-4^{1} \mathrm{~F}$

$6335.74 \quad A \quad 10 \quad 13.59 \quad 15.54 \quad 2-1 \quad{ }_{4}^{1} D_{-5}\left(\begin{array}{llll}\text { po } \\ \text { P }\end{array}\right.$

$4237.57 \quad P \quad 13.5916 .50 \quad 3-1 \quad 4^{1}{ }_{D-5}(3)$

$\left.4036.5 \quad B \quad 5 \quad 13.59 \quad 16.66 \quad 2-1 \quad 4^{1} \frac{23}{D-6}\right)_{p o}$

$\left.3438.916 \quad B \quad 6 \quad 13.5917 .19 \quad 2-3 \quad 4^{1} \frac{1}{D-6}\right)_{F}$

$\begin{array}{llllllll}3351.456 & \text { B } & 3 & 13.59 & 17.37 & 2-1 & \left.4^{1} \mathrm{D}-7\right)_{\mathrm{Po}}\end{array}$

$\begin{array}{llllllll}3074.665 & B & 6 & 13.59 & 17.60 & 2-3 & 4^{1} \mathrm{D}-7 \mathrm{~F}^{\circ} & (36)\end{array}$

$3041.878 \quad$ B $\quad 6 \quad 13.5917 .65 \quad 2-1 \quad 4^{1}{ }^{1}-81_{P 0}$ $\begin{array}{lllllll}6696.39 & \text { B } & 0.5 & 14.83 & 16.67 & 1-3 & 5^{3} \mathrm{~s}_{-} 6^{3} \mathrm{pt}\end{array}$

$\begin{array}{llll}4998.43 \quad P & 14.83 \quad 17.29\end{array}$

$-5^{3} 8-7^{3} p$

4998.43

$\left.2^{3} 8^{2}-8^{3}\right)^{20}$

$5^{3}$ S- $\left.^{(31)}\right)_{00}$ ${ }^{3} \mathrm{~s}^{2}(32)$ 3774.3

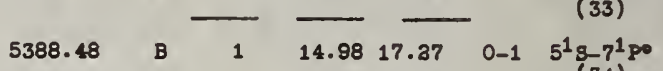
$\begin{array}{llllllll}4629.7 & B & 1 & 14.98 & 17.65 & 0-1 & 51 \mathrm{~g} & (34) \mathrm{po}\end{array}$

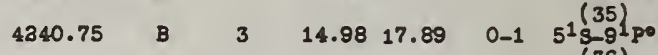
$4009.58 \quad B \quad 1 \quad 14.9818 .06 \quad 0-1 \quad 5^{1} \mathrm{~g}_{-10^{1}} \mathrm{pe}$ $\begin{array}{lllllll}3859.33 \quad \text { B } & 3 & 14.98 & 18.18 & 0-1 & 5^{1} \mathrm{~S}_{11}^{1}{ }^{1} \mathrm{po}\end{array}$ 3753.10 8354.35
8359.57 8359.57 8363.52
8359.23

8363.30

5853.62

5867.81

5371.84

5085.02

5093.65

5100.3

4609.7

4585.820

4588.194

4588.082

4336.827

.4323 .509

4327.899

4237.509

4327.430
4327.945

4237.875

4226.918
4227.545

3995.860

3998.159

3996.381

3996.075

3996.323

-3983. 7

3842.037

3842.313
3842.317

3842.317

3734.567

3734.715
3734.805

3656.319

3597.50

3552.00

3516.05

3463.63

A

1
10
9
8
1
1

$14.98 \quad 18.37$

15.0016 .47 $0-1 \quad 5^{1} 8-1 a^{1} p^{2}$ 15.0016 .47 15.0016 .47
15.0016 .47 15.0016 .47
15.0016 .47 $\begin{array}{ll}3-4 & 4^{3} p-5^{3} r^{\circ} \\ 3-3 & (40)\end{array}$

$15.00 \quad 17.11 \quad 3-4 \quad 4^{3} p-6^{3} F^{0}$

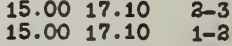
$15.0017 .29 \quad 3,2$

$4^{3} p-7^{3} p$ $15.00 \quad 17.43 \quad 3-4 \quad 4^{3} D_{-7}^{(3)} 7^{\circ 0}$

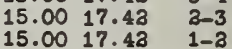
(43)$$
15.00
$$

$15.00 \quad 17.67$

$\begin{array}{lll}6 & 15.00 & 17.69 \\ 5 & 15.00 & 17.69\end{array}$

$4 \quad 15.0017 .69$

$\begin{array}{lll}0.5 & 15.00 & 17.69 \\ 1 & 15.00 & 17.69\end{array}$

$\begin{array}{lc}- & 4^{3} p-8^{3} p o \\ 3-4 & (44) \\ 3-4 & 4-8 p^{30} \\ 2-3 & (45) \\ 1-3 & \\ 3-3 & \\ 2-3 & \end{array}$

$\begin{array}{llll}8 & 15.00 & 17.93\end{array}$

\begin{tabular}{lll}
4 & 15.00 & 17.92 \\
3 & 15.00 & 17.92 \\
\hline & 15.00 & 17.93
\end{tabular}

4) 15.0017 .92

$0.5 \quad 15.00 \quad 17.93$

$\begin{array}{ccc}0 & 15.00 & 17.92 \\ 0 \text { OForb } & 15.00 & 17.92\end{array}$

OForb 15.0017 .92
0.5 Forb 15.0017 .93

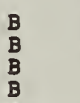

$\begin{array}{llll}5 & 15.00 & 18.09 \\ 4 & 15.00 & 18.09\end{array}$

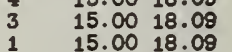

$\begin{array}{lll}1 & 15.00 & 18.09 \\ 0.5 & 15.00 & 18.09\end{array}$

B 0.5 Forb 15.0018 .09

$\begin{array}{lll}0.5 & 15.0018 .09\end{array}$$$
\begin{aligned}
& 3 \\
& 3 \\
& 1
\end{aligned}
$$

15.0018 .21
15.0018 .31

1.

$-4^{3} \mathrm{p}-10^{3} \mathrm{po}$ $\begin{array}{cc}3-4 & 4^{3} p-113 F^{\circ} \\ 2-3 & (49)\end{array}$

$1 \quad 15.0018 .30$

$\begin{array}{lll}0.5 & 15.00 & 18.30 \\ 0 & 15.00 & 18.30\end{array}$

$1-3$

$4^{3} p-9^{3} F^{\circ}$

$1-3$
$3-3$

$2-3$
$3-3$

$-3$

$\begin{array}{cc}-4 & 4^{3} p-10^{3}=0 \\ -3 & (47)\end{array}$

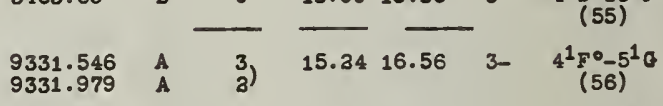

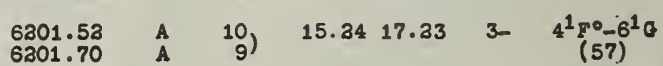

$5158.187 \quad B \quad$ 1d $\quad 15.34 \quad 17.63 \quad 3-\quad 4^{1} F^{0}-7^{10} G$

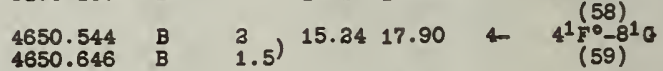

$\begin{array}{lllllll}4356.711 & B & 3 \\ 4356.807 & B & 1.5) & 15.34 & 18.07 & 3- & 4^{1} F^{\circ}-99^{2} G \\ (60)\end{array}$

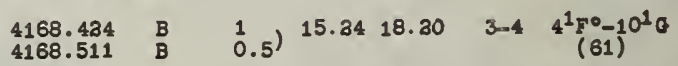

$\begin{array}{llllllll}4039.397 & B & 0 & 15.34 & 18.30 & 3-4 & 4^{1} F^{\circ}-11^{10 G} \\ 4039.302 & B & 0.5\end{array}$

$3946.406 \quad B \quad 0.5 A \quad 15.34 \quad 18.37 \quad 3-\quad 4^{1} \mathrm{~F}^{\circ}-12^{1 / G}$

\begin{tabular}{|c|c|c|c|c|c|c|}
\hline $\begin{array}{r}9290.649 \\
9290.747 \\
9388.145 \\
9388.550 \\
9386.578 \\
9386.794\end{array}$ & $\begin{array}{l}\hat{A} \\
\hat{A} \\
\hat{A} \\
\hat{A} \\
\hat{A}\end{array}$ & $\begin{array}{l}6 \\
5 \\
3 \\
3 \\
3 \\
1 \\
3 \\
3\end{array}$ & $\begin{array}{l}15.34 \\
15.34 \\
15.34\end{array}$ & $\begin{array}{l}16.56 \\
16.56 \\
16.56\end{array}$ & $\begin{array}{l}4- \\
3- \\
3-\end{array}$ & $\begin{array}{c}4^{3} F^{0}-5^{3} a \\
(64)\end{array}$ \\
\hline 6495.45 & B & 0.5 & 15.24 & 17.14 & 4 & $\begin{array}{c}4^{3} F^{0}-6^{3} D \\
(65)\end{array}$ \\
\hline
\end{tabular}




\section{A ${ }^{\text {Laboratory }}$ II continued}

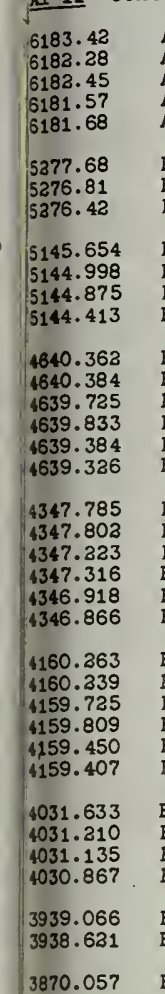

Laboratory Int Low ${ }_{\text {Hef }}$ J Multiplet Al II continued

$\begin{array}{lllllll}5324.61 & B & 4 & 15.54 & 17.86 & 1-0 & 5^{1} \mathrm{p}^{\circ}-9^{1} \mathrm{~S}\end{array}$ $\begin{array}{llllllll}5285.85 & B & 6 & 15.54 & 17.87 & 1-3 & { }_{5}^{(101)} \\ P^{\circ}-9^{1} \mathrm{D} & \end{array}$ $4918.98 \quad$ B $\quad 3 \quad 15.5418 .05 \quad 1-0 \quad{ }_{5}^{(102)} \frac{1 \mathrm{P}_{-10^{1} \mathrm{~S}}}{10}$ $4898.76 \quad B \quad 5 \quad \quad 15.54 \quad 18.06 \quad 1-2 \quad 5_{1}^{(103)} \mathrm{P}^{\circ}-10^{1} \mathrm{D}$ $4666.8 \quad$ B $\quad 11 \quad 15.5418 .18 \quad 1-0 \quad 5_{5}^{(104)} \mathrm{P}_{0}^{0} 1_{11}^{1} \mathrm{~S}$ $4655.05 \quad$ B $\quad 0.5 \quad 15.5418 .19 \quad 1-2 \quad 5 \quad \begin{array}{llll}105) & \left.1 \mathrm{P}^{\circ}\right)^{1} \mathrm{D}\end{array}$

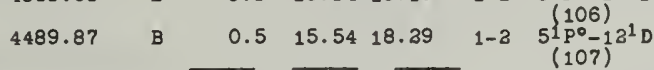
9124.27 A $0.5 \quad \overline{16.33} 17.67 \quad 1-2 \quad 6^{3} \mathrm{~S}-8^{3} \mathrm{po}^{0}$ 6001.18 B $1 \quad \overline{16.39} 18.45 \quad 0-1 \quad 6^{1} \mathrm{~s}-15^{1} \mathrm{po}$ $\left.\begin{array}{l}3.5 \\ 1.5\end{array}\right) 15.24 \quad 17.90 \quad 3-$ $\left.\begin{array}{l}1.5 \\ 0.5\end{array}\right) 15.24 \quad 17.90 \quad 2$ $4.5) 15.2418 .0744^{3} \mathrm{~F}^{\circ}-9^{3} \mathrm{O}$ $\left.\begin{array}{lll}3.5 \\ 3.0 \\ 1.5\end{array}\right) 15.34 \quad 18.07 \quad 3$ $\left.\begin{array}{l}1.5 \\ 1.0 \\ 0.5\end{array}\right) 15.2418 .07$

$3.5) 15.24 \quad 18.30 \quad 4$ $1.5,15.3418 .20 \quad 3-$ $\left.\begin{array}{l}1 \\ 0.5\end{array}\right) 15.34 \quad 18.30 \quad 3-$

$\begin{array}{lllll}0.5 d & 15.24 & 18.30 & 4- & 4^{3} \mathrm{~F}^{0}-11^{3} \mathrm{G}\end{array}$ $\begin{array}{llll}0.5) & & & \\ \text { On } & 15.34 & 18.30 & 2\end{array}$

$\begin{array}{lllll}0.5 d & 15.24 & 18.37 & 4 & 4^{3} F^{0}-12^{3} a \\ 0.5 d & 15.24 & 18.37 & 3- & (73)\end{array}$ $\begin{array}{lll}0.5 & 15.2418 .434 & 4 \\ 4 & 4^{3} F^{\circ}-13^{3} G \\ (74)\end{array}$

$1 \quad 15.41 \quad 17.19 \quad 3-3 \quad 5^{1} D-6^{1} F^{\circ}$ $\begin{array}{llllll}0.5 & 15.41 & 17.27 \quad 3-1 & \left.5^{1} \mathrm{D}-7\right)_{\mathrm{p}}\end{array}$ $\begin{array}{lllll}3 & 15.41 & 17.60 \quad 2-3 & 5^{1} D_{-7} F^{\circ}\end{array}$ $\begin{array}{lllll}3 & 15.41 & 17.65 & 3-1 & 5^{1} \mathrm{D}-8 \mathrm{P}_{\mathrm{PO}}\end{array}$ $\begin{array}{lllll}3 & 15.41 & 17.87 & 2-3 & 5^{1} D_{-} 8^{7} F^{\circ}\end{array}$ $\begin{array}{lllll}3 & 15.41 & 17.89 & 2-1 & 5^{1}(79) P_{\text {Po }}\end{array}$ $\begin{array}{lllll}0.5 & 15.41 & 18.06 & 2-3 & 5^{1} \mathrm{D}^{(80} \mathrm{F}^{\circ}\end{array}$ $1 \quad 15.41 \quad 18.06 \quad 2-1 \quad 5^{1}{ }^{(81)}\left(0^{1} \mathrm{P}^{\circ}\right.$ $3 \quad 15.41 \quad 18.18 \quad 3-1 \quad 5^{1} \mathrm{D}-11^{1} \mathrm{po}$ $\begin{array}{lllll}0.5 & 15.41 & 18.19 & 3-3 & 5^{1} \mathrm{D}-10^{1} \mathrm{~F}^{\circ}\end{array}$ $3 \quad 15.41 \quad 18.27 \quad 3-1 \quad 5^{1} D_{-13}^{(84)}$ po $0.5 \quad 15.41 \quad 18.29 \quad 2-3 \quad 5^{1} D_{-11^{1}} F^{\circ}$

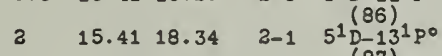
$\begin{array}{lllll}0.5 & 15.41 & 18.45 & 3-1 & 5^{1}(87) \\ & \left(85^{1} p_{0}\right.\end{array}$

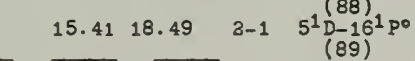

$\begin{array}{lllll}3 & 15.52 & 17.10 & 2-1 & 5^{3} \mathrm{P}^{0}-7^{3} \mathrm{~S}\end{array}$ $\begin{array}{lllll}1 & 15.52 & 17.10 & 1-1 & (90)\end{array}$

$2 \quad 15.5217 .14 \quad 2-\quad 5^{3} \mathrm{P}^{\circ}-6^{3} \mathrm{D}$ $\begin{array}{lll}1 & 15.52 & 17.14 \\ 0.5 & 15.52 & 17.14\end{array}$

$\begin{array}{llllc}10 & 15.52 & 17.55 & 2-1 & 5^{3} \mathrm{p}^{0}-8^{3} \mathrm{~s} \\ \text { (8) } & 15.52 & 17.55 & 1-1 & (92)\end{array}$

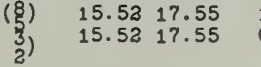

$\begin{array}{llllc}10 & 15.52 & 17.57 & 2- & 5^{3} P^{0}-7^{3} D \\ \text { (q) } & 15.52 & 17.57 & 1- & (93)^{2}\end{array}$ 3) $15.52 \quad 17.57$

$2 p_{0}-g_{3} s$ $\begin{array}{ccccc}7 & 15.52 & 17.84 & 2-1 & 5^{3} \mathrm{p}^{0}-9^{3} \mathrm{~s} \\ 5 & 15.52 & 17.84 & 1-1 & (94)\end{array}$ $8 \quad 15.5217 .86 \quad 2-5^{3} \mathrm{P}^{0}-8^{3} \mathrm{D}$ $\begin{array}{lllll}6 & 15.52 & 17.86 & 1- & (95) \\ 3 & 15.52 & 17.86 & 0-1 & \end{array}$ $\begin{array}{lllll}5 & 15.52 & 18.04 & 2-1 & 5^{3} \mathrm{po}^{\circ}-10^{3} \mathrm{~s} \\ 3 & 15.52 & 18.04 & 1-1 & (96)\end{array}$ $\begin{array}{lllll}3 & 15.52 & 18.04 & 1-1 & (96) \\ 2 & 15.52 & 18.04 & 0-1 & \end{array}$ $\begin{array}{lllll}1 & 15.52 & 18.18 & 2- & 5^{3} \mathrm{P}^{0}-10^{3} \mathrm{D} \\ 0.5 & 15.52 & 18.18 & 1- & (97)\end{array}$ $\begin{array}{llll}0.5 & 15.52 & 18.18 & 1- \\ 0.5 & 15.52 & 18.18 & 0-\end{array}$ $\begin{array}{lllll}15.54 & 17.19 & 1-2 & 5^{1} \mathrm{P}^{0}-7^{1} \mathrm{D}\end{array}$ $\begin{array}{llll}15.54 & 17.57 \quad 1-0 & 51 \mathrm{PO}^{-8} \mathrm{~s}\end{array}$ $\begin{array}{llll}15.54 & 17.60 \quad 1-2 \quad 5^{1} & (99) \\ & (100)\end{array}$ $5^{1} \mathrm{po}^{\circ}-8^{1} \mathrm{D}$
$(100)$

\section{$\begin{array}{lllllll}8119.73 & \text { A } & 1.5 & 16.40 & 17.93 & -4 & 5^{3} D-9^{3} F^{\circ} \\ 8122.08 & \text { A } & 0.5 & 16.40 & 17.93 & -3 & (110)\end{array}$}

8123.52

6775.97

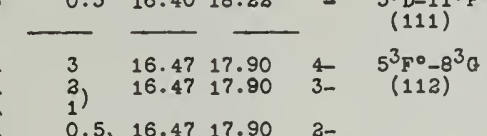

8675.28

8671.28

7709.78

7138.81

8858.39
8858.77

8086.91

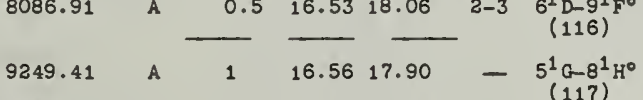

$\begin{array}{lllll}8160.15 & \mathrm{~A} & 3 & 16.5618 .08 & -{ }_{5}(117)_{\mathrm{H}^{\circ}}\end{array}$

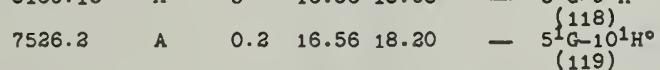

Fine structure

AI III I P 28.33 Anal A L1st A July 1944

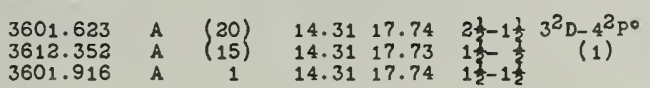

5696.47
5732.65

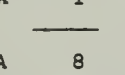

$\begin{array}{llll}15.57 & 17.74 & \frac{1}{3}-1 \frac{1}{\frac{1}{2}} 4^{2} \mathrm{~S}-4^{2} \mathrm{po}_{0} \\ 15.57 & 17.73 & \text { (2) }\end{array}$

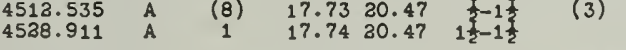
4149.897 A (10) $20.4723 .44 \quad 2 \frac{1}{3}-3 \frac{1}{3} 4^{2} \mathrm{D}-5^{2} \mathrm{FO}^{\circ}$

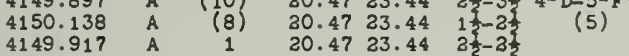

$4701.65 \quad A \quad 6 \quad \overline{20.69} 23.32-4^{2} F^{0}-5^{2} \mathrm{D}$

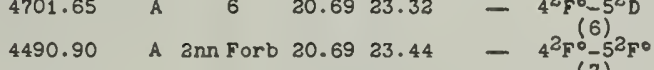
$\begin{array}{lllllll}4479.968 & \text { A } & 4 & 20.69 & 23.45 & 3 \frac{1}{3}- & 4^{2} F^{0}-5^{2} G \\ 4479.891 & \text { A } & 3 & 20.69 & 23.45 & 2 \frac{1}{2}-3 \frac{1}{2} & (8)\end{array}$ $\begin{array}{lllllll}4364.59 & \mathrm{~A} & \text { an } & 22.03 & 24.86 & 1 \frac{1}{3}- & 5^{2} \mathrm{po}^{\circ}-6^{3} \mathrm{D} \\ 4357.24 & \mathrm{P}\end{array} \quad \begin{array}{llll}\frac{1}{2}-1 \frac{1}{2} & (9)\end{array}$ $\begin{array}{lllllll}3387.37 & A & 1 & 22.03 & 25.79 & 1 \frac{1}{2} & 5^{2} \mathrm{P}^{\circ}-7^{2} \mathrm{D}\end{array}$ $\begin{array}{lllllll}3283.11 & A & 0.5 & 22.03 & 25.79 & \frac{1}{2}-1 \frac{1}{2} & (10)\end{array}$ 4903.71 A $4 \quad \overline{23.32} 25.83-5^{2} D-7^{2} F^{\circ}$ $3980.56 \quad \mathrm{~A} \quad$ an $23.3226 .42 \quad-5^{2} \mathrm{D}-8^{2} \mathrm{~F}^{\circ}$ $5260.91 \quad \mathrm{~A}$ On $23.4425 .79-5^{2} \mathrm{~F}^{\circ}-7^{2} \mathrm{D}$ $5150.86 \quad$ A $\quad$ Gn $23.4425 .84 \quad-5^{2} F^{(13)}-7^{2} Q$ $\begin{array}{lllll}4188.88 \quad A \quad 0.5 & 23.4436 .39 & -5^{2} F^{(14)}-8^{2} \mathrm{D}\end{array}$ $4142.15 \quad A \quad$ an $23.4426 .42 \quad-5^{2} F^{\circ}-8^{2} G$ $\begin{aligned} 4141.25 \quad A \quad \text { On Forb } 23.4426 .42 & -5^{2} 5^{\circ}-8^{2} \mathrm{H}^{\circ} \\ (17) & \end{aligned}$ $\begin{array}{lllll}5172.6 \quad 1 & 1 & 23.4525 .83 & 5^{2} G-7^{2} F^{\circ} \\ (18) & \end{array}$
7134.66
7131.29

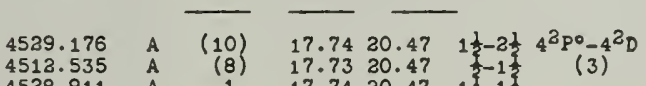

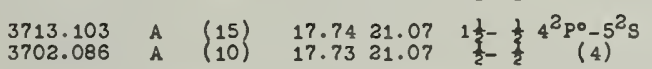

I A Laboratory $_{\text {Ref Int Low }}^{\text {E P }}$ High J Mit1plet Al III continued

$\begin{array}{llllll}5163.90 \quad A \quad & 7 & 33.45 & 35.84 & 5^{2} \mathrm{a}-7^{2} \mathrm{H}^{\circ}\end{array}$ $\begin{array}{lllll}3658.3 & A & (1 n) \quad 23.45 & 26.82 \quad 5^{2} G^{(19)} 9_{H^{\circ}}\end{array}$ (30)

See NSRDS-NBS 3, Section 2, 1967

S1 I P 8.11 Anal B L1st A Aug 1944

$\begin{array}{lllllll}2970.35 & \text { A } & 15 & 0.78 & 4.93 & 2-2 & 3 p^{2}{ }^{1} p-48^{3} p^{\circ} \\ 2987.65 & \text { A } & 25 & 0.78 & 4.91 & 2-1 & (1)\end{array}$

$4102.926 \mathrm{~A} \quad 25 \quad \overline{1.90} \quad \overline{4.91} 0-1 \quad 3 \mathrm{p}^{2} 1 \mathrm{~B}-4 \mathrm{~s}^{3} \mathrm{p}$

$3905.527 \quad$ A $100 \quad 1.90 \quad 5.06 \quad 0-1 \quad 3 p^{2}\left(\mathrm{l}_{\mathrm{S}-4 \mathrm{~s}^{1} \mathrm{po}}\right.$

$\begin{array}{lllllll}12031.49 & \text { A } & 25 & 4.93 & 5.96 & 2-3 & 48^{3} \mathrm{p}^{\circ}-4 \mathrm{p}^{3} \mathrm{D} \\ 11984.20 & \text { A. } & 20 & 4.91 & 5.94 & 1-2 & (4)\end{array}$

$\begin{array}{rrrrrr}11984.20 & \text { A } & 20 & 4.91 & 5.94 & 1-2 \\ 1.991 .57 & \text { A } & 10 & 4.90 & 5.93 & 0-1\end{array}$

$\begin{array}{llllll}12270.50 & \text { A } & 2 & 4.93 & 5.94 & 2-2 \\ 12103.46 & \text { A } & 5 & 4.91 & 5.93 & 1-1 \\ 12395.97 & \text { P } & & 4.93 & 5.93 & 2-1\end{array}$

10837.09 A $100 \quad 4.93 \quad 6.07 \quad 2-2 \quad 48^{3} p^{\circ}-4 p^{3} p$

10749.40 A $60 \quad 4.916 .06 \quad 1-1$

10786.86 A $50 \quad 4.916 .05$

$\begin{array}{llllll}10603.38 & \text { A } & 60 & 4.91 & 6.07 & 1-2 \\ 10660.98 & \text { A } & 50 & 4.90 & 6.06 & 0-1\end{array}$

$10585.12 \quad$ A $\quad 100 \quad 4.93 \quad 6.10 \quad 2-1 \quad 48^{3} p^{\circ}-4 p^{3} s$

$\begin{array}{llllll}10371.33 & \text { A } & 50 & 4.91 & 6.10 & 1-1 \\ 10388.83 & \text { A } & 25 & 4.90 & 6.10 & 0-1\end{array}$

$\begin{array}{lllllll}9768.27 & A & 5 \pi & 4.93 & 6.20 & 2-3 & 48^{3} p^{\circ}-4 p^{1} D \\ 9585.72 & A & 4 & 4.91 & 6.20 & 1-2 & \end{array}$

$\begin{array}{llllll}8435.28 & P & 4.91 & 6.37 & 1-0 & 48^{3} \mathrm{P}^{\circ}-4 \mathrm{p}^{1} \mathrm{~s}\end{array}$

$\begin{array}{ccccccc}5797.912 & \text { A } & 40 & 4.93 & 7.06 & 2-3 & 48^{3} \mathrm{P}^{\circ}-5 p^{3} \mathrm{D} \\ 5793.128 & \text { A } & 30 & 4.91 & 7.04 & 1-2 & (9)\end{array}$

$\begin{array}{llllll}5793.128 & \text { A } & 30 & 4.91 & 7.04 & 1-2 \\ 5780.452 & \text { A } & 25 & 4.90 & 7.03 & 0-1\end{array}$

$\begin{array}{llllll}5859.23 & P & & 4.90 & 7.03 & 0-1 \\ & P & 4.93 & 7.04 & 2-2\end{array}$

$\begin{array}{lllllll}5708.437 & \text { A } & 75 & 4.93 & 7.09 & 2-2 & 48^{3} p \circ-5 p^{3} p\end{array}$

$\begin{array}{lllllll}5690.470 & \text { A } & 40 & 4.91 & 7.08 & 1-1 & \text { (10) }\end{array}$

$\begin{array}{llcccc}5754.258 & \text { A } & 81 & 4.93 & 7.08 & 2-1 \\ 5701.138 & \text { A } & 25 & 4.91 & 7.07 & 1-0\end{array}$

$\begin{array}{llllll}5645.665 & \text { A } & 25 & 4.91 & 7.09 & 1-2 \\ 5665.601 & \text { A } & 25 & 4.90 & 7.08 & 0-1\end{array}$

$5684.523 \quad A \quad 50 \quad 4.93 \quad 7.10 \quad 2-1 \quad 48^{3} p^{0}-5 p^{3} s$

$-\frac{4.91}{5.06}-10^{1-1}$ (11)

$\begin{array}{lllllll}10869.54 & A & 125 & 5.06 & 6.20 & 1-2 & 48\end{array}$

$9413.59 \quad A \quad 200 \quad 5.06 \quad 6.37 \quad 1-0 \quad{ }_{48}\left(\begin{array}{l}130 \\ \mathrm{p}^{\circ}-4 \mathrm{p}^{1} \mathrm{~s}\end{array}\right.$

$6067.62 \quad P \quad 5.06 \quad 7.09 \quad 1-2 \quad 48^{\left\{p_{0}^{0}-5 p^{3} p\right.}$

$5948.584 \quad A \quad 100 \quad 5.06 \quad 7.14 \quad 1-2 \quad 4 B^{1} P_{0}^{15}-5 p^{1} D$

$\begin{array}{llrrrrr}5772.258 & A & 50 & 5.06 & 7.20 & 1-0 & 48(16)^{1-5 p^{1} \mathrm{~S}}\end{array}$

$\begin{array}{llllll}8417.89 & P & 5.59 & 7.06 & 3-3 & 3 \mathrm{~d}^{3} \mathrm{D}^{0}-5 \mathrm{p}^{3} \mathrm{D}\end{array}$

8527.32
8397.96

8514.64

8230.67

8306.80
8317.45

8211.48

8150.57

7995.00

7416.00

7433.54

7409.11
7405.85

7405.85
7424.63

7415.37

7289.25

7275.28
7290.21

7250.69

7250.69
$? 193.56$

7184.89

7193.89

7235.86
7184.54

7235.32

7226.20

6244.56

6254.25

6243.86

6237.34
6254.96

6254.96
6244.13 
16

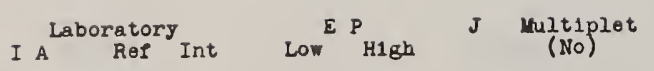

81 I continued

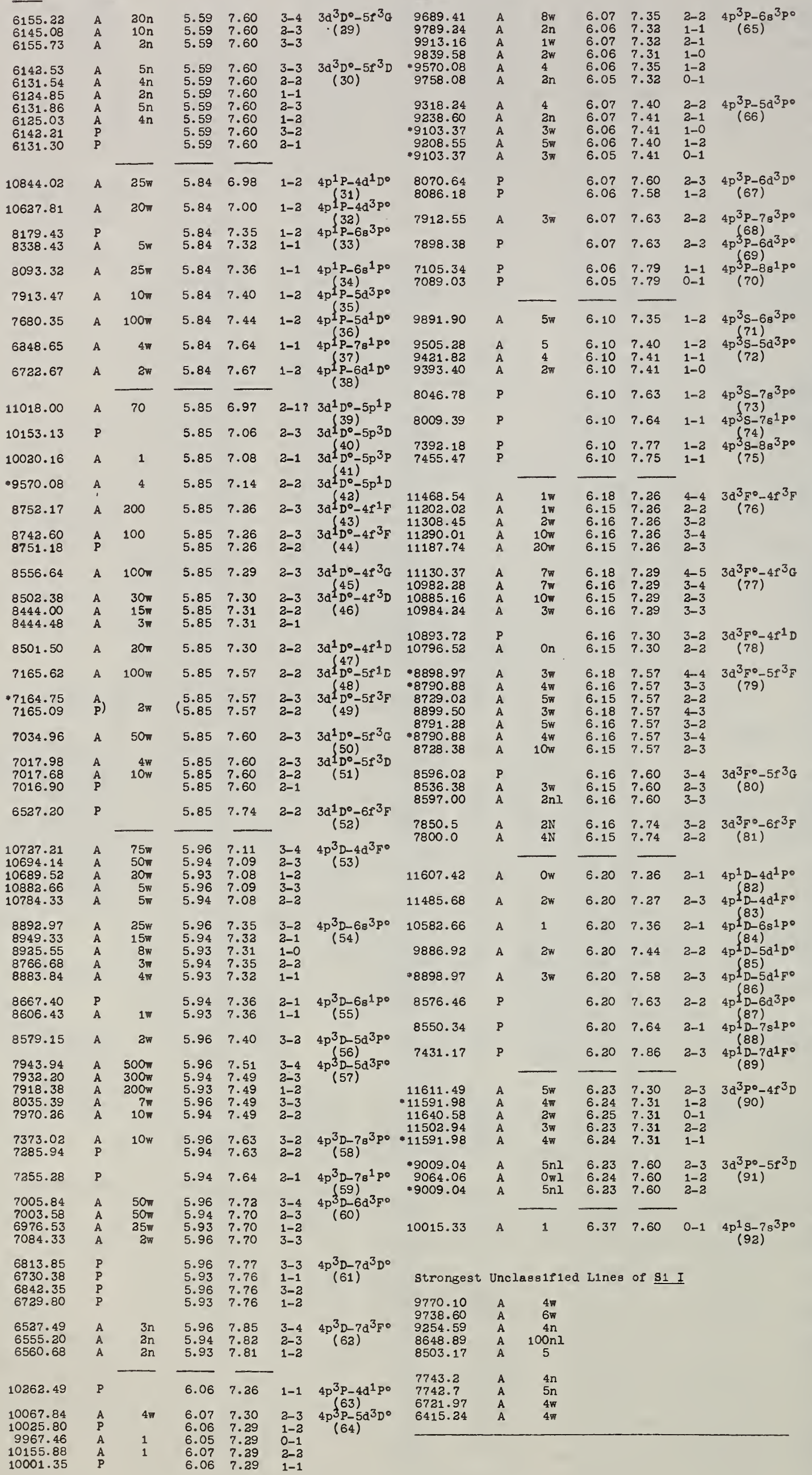

\section{REVIBED MULTIPLET TAB}

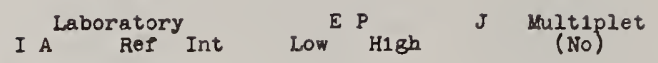

\section{S1 I continued}

REVISED

See NSRDS-NBS 3, Section 1, 196

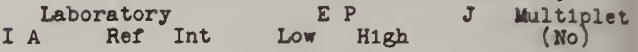

S1 II I P 16.27 Anal B L18t A Aug 1944

$3856.021 \quad \mathrm{~A} \quad 8 \quad 6.83 \quad 10.03 \quad 2 \frac{1}{3}-1 \frac{1}{2} 3 \mathrm{p}^{2} 2 \mathrm{D}-4^{2} \mathrm{p}$ $\begin{array}{lllllll}3862.592 & \mathrm{~A} & 6 & 6.83 & 10.02 & 1 \frac{1}{2} & \text { (1) } \\ 3853.657 & \mathrm{~A} & 3 & 6.83 & 10.03 & 1 \frac{1}{2}-1 \frac{1}{2} & \end{array}$

$\begin{array}{rrrrrrr}6347.091 & \text { A } & 10 & & 8.09 & 10.03 & \frac{1}{\frac{1}{2}-1 \frac{1}{2}} 4^{2} \mathrm{~S}-4^{2} \mathrm{po} \\ 6371.359 & \mathrm{~A} & 8 & 8.09 & 10.02 & \frac{2}{2}-\frac{1}{2} & (2)\end{array}$

$\begin{array}{rrrrrrrr}4130.884 & \text { A } & 10 & & 9.80 & 12.78 & 2 \frac{1}{3}- & 3^{2} \mathrm{D}-4^{2} F^{\circ} \\ 4128.053 & \mathrm{~A} & 8 & 9.79 & 12.78 & 1 \frac{5}{2}-2 \frac{1}{2} & (3)\end{array}$

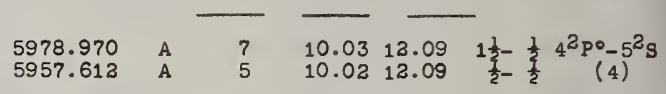

$\begin{array}{rrrrrrr}5056.020 & \text { A } & 10 & 10.03 & 12.47 & 1 \frac{1}{2}-2 \frac{1}{2} & 4^{2} \mathrm{PO}-4^{2} \mathrm{D} \\ 5041.063 & \text { A } & 8 & 10.02 & 12.47 & \frac{1}{3}-1 \frac{1}{2} & (5)\end{array}$

$\begin{array}{rrrrrr}5041.063 & \text { A } & 8 & 10.02 & 12.47 & 1 \frac{1}{3}-1 \frac{1}{3} \\ 5056.353 & \text { A } & \text { Z } & 10.03 & 12.47 & 1 \frac{3}{2}-1 \frac{1}{2}\end{array}$

$\begin{array}{llllll}3339.84 & \mathrm{~A} & 3 & 10.0313 .73 & 1 \frac{1}{3}-\frac{1}{2} & 4^{2} \mathrm{P} 0-6^{2} \mathrm{~S} \\ 3333.16 & \mathrm{~A} & \mathrm{Z} & 10.02 & 13.73 & \end{array}$

$\begin{array}{llllll}3333.16 \quad A & 2 & 10.02 & 13.73 \quad \frac{1}{2}-\frac{1}{2} & (6)\end{array}$

$\begin{array}{lllllll}3210.04 & \text { A } & 3 & 10.03 & 13.87 & 1 \frac{1}{2}- & 4^{2} \mathrm{Po}^{\circ}-5^{2} \mathrm{D} \\ 3203.89 & \mathrm{~A} & \mathrm{Z} & 10.02 & 13.87 & \frac{1}{2}-1 \frac{1}{2} & (7)\end{array}$

5868.404

5846.12
5827.80

5827.80
5915.266

5867.497
5800.48

5800.48
5806.75

5639.493

5576.61

A
A
A
A
A
A
A
A
A
A

$2 \frac{1}{3}-2 \frac{1}{2} 3 p 484 p^{\circ}-3 p 4$

$2 \frac{\frac{1}{2}-1}{\frac{2}{2}}-1 \frac{1}{3}$

$1 \frac{3}{3}-\frac{1}{2}$
$1 \frac{3}{3}-2 \frac{1}{2}$
$\frac{1}{2}-1 \frac{1}{2}$

$2 \frac{1}{2}-1 \frac{1}{2} 304 s^{4} p_{0}-3 p 4 p^{\circ}$

$1 \frac{1}{2}-1 \frac{1}{2}$

Strongest Unclassifled Lines of S1 II

$\begin{array}{lll}5706.375 & \text { A } & 1 \\ 5701.375 & \text { A } & 1\end{array}$

5688.856

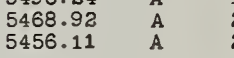

5438.41

294.97

5192.75

5185.09

5181.77

921.69

4906.88
4859.28

$\begin{array}{lll}656.80 & A & 1 \\ & A & 1\end{array}$

4198.174

190.738

$\begin{array}{lll}4190.738 & \text { A } & 3 \\ 4076.78 & \text { A } & 1 \\ 4075.45 & \text { A } & 2 \\ 3998.00 & \text { A } & \text { 1n }\end{array}$

$\begin{array}{lll}3991.77 & \text { A } & \text { 2n } \\ 3199.54 & \text { A } & 1 \\ 3193.10 & \text { A } & 1 \\ 3188.95 & \text { A } & 1\end{array}$

See NSRDS-NBS 3, Section 1, 1965

S1 III I P 33.32 Anal B L1st A Aug 1944

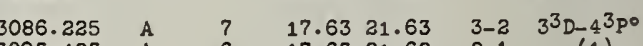

$\begin{array}{lllllll}3093.423 & A & 6 & 17.63 & 21.62 & 3-1 & 35-4 \\ (1)\end{array}$

$\begin{array}{llllll}3096.786 & A & 4 & 17.64 & 21.62 & 1-0 \\ 3086.429 & A & 3 & 17.6321 .63 & 2-2 \\ 3086.620 & A & 1 & 17.64 & 21.63 & 1-2\end{array}$

$\begin{array}{llllll}3093.613 & \text { A } & 3 & 17.64 & 21.62 & 1-1 \\ 3086.620 & \text { A } & 1 & 17.64 & 21.63 & 1-2\end{array}$

$4552.654 \quad \mathrm{~A} \quad 9 \quad \overline{18.92} 21.63 \quad 1-2 \quad 4^{3} \mathrm{~s}-4^{3} \mathrm{po}$

$\begin{array}{lllllll}4567.872 & \text { A } & 7 & 18.92 & 21.62 & 1-1 & \text { (2) } \\ 457.777 & \text { A } & 4 & 18.92 & 21.62 & 1-0 & \end{array}$

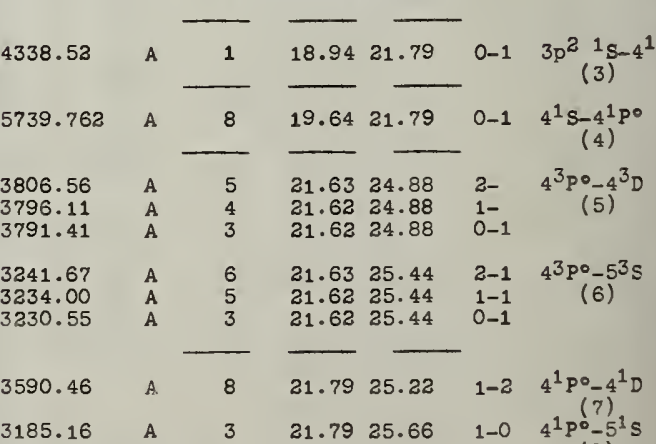




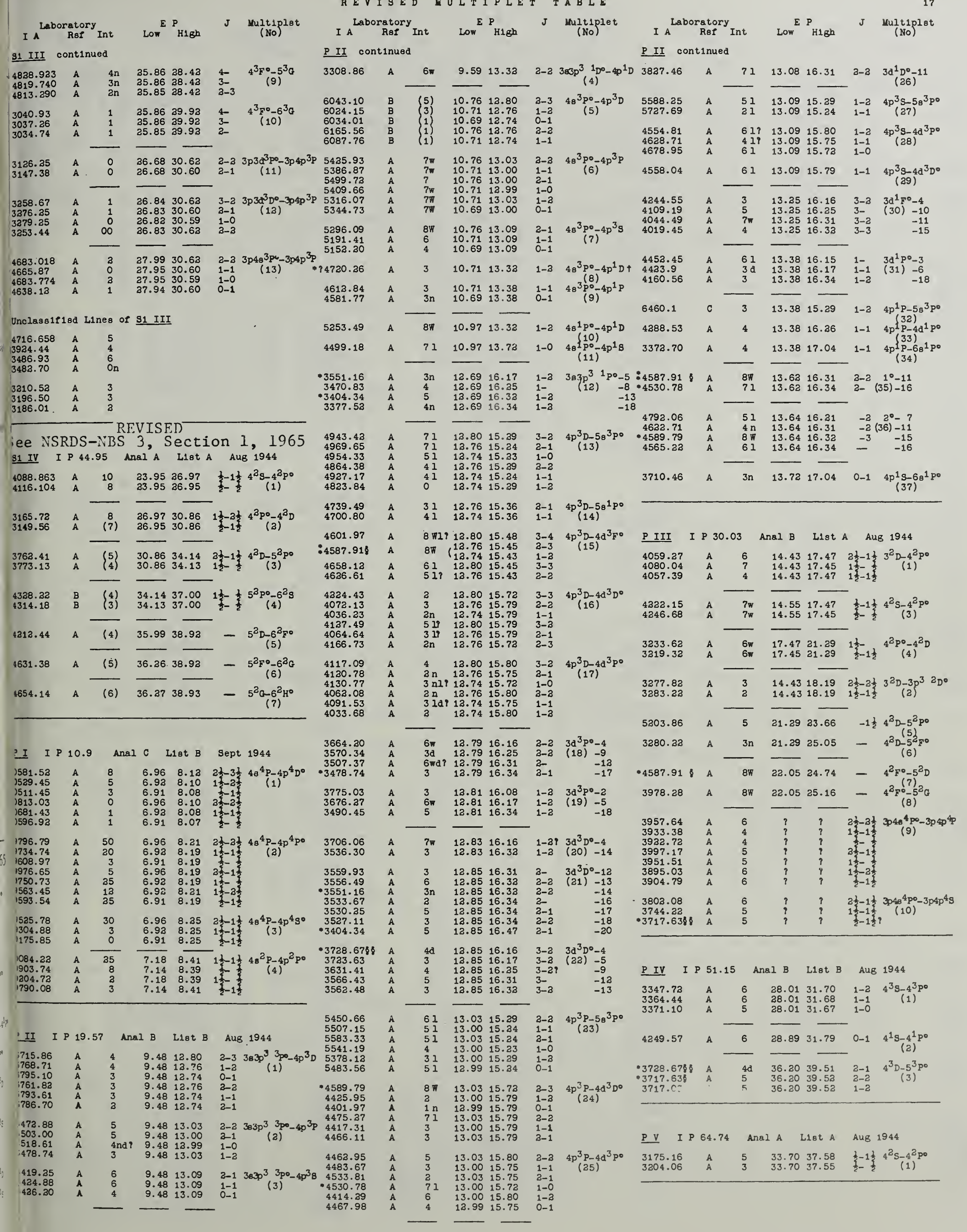


18

Laboratory
Ref Int Low H1gh J thitiplet
(No)

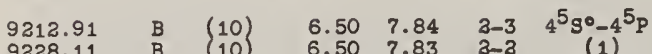
$4694.13 \quad \mathrm{~A} \quad 10 \quad 6.50 \quad 9.13 \quad 3-3 \quad 4^{5} \mathrm{~g}^{0}-5^{5} \mathrm{P}$

$\begin{array}{rrrrrrr}4694.13 & \text { A } & 10 & 6.50 & 9.12 & 3-3 & 45 \\ 4695.45 & \text { A } & 8 & 6.50 & 9.12 & 3-2 & (2) \\ 4696.25 & \text { A } & 6 & 6.50 & 9.12 & 3-1 & \end{array}$

\subsection{7}

10459.46

\subsection{9}

5278.70

5278.10

$\begin{array}{lllc}6.83 & 8.01 & 1-3 & 4^{3} 8^{\circ}-4^{3} p \\ 6.83 & 8.01 & 1-1 & (3) \\ 6.83 & 8.01 & 1-0 & \end{array}$

$\left\{\begin{array}{l}8 \\ 8 \\ 4\end{array}\right)$

$6.83 \quad 9.17 \quad 1-3 \quad 4^{3} \mathrm{~g}^{\circ}-5^{3} \mathrm{P}$

$\begin{array}{lll}6.83 & 9.17 & 1-1 \\ 6.83 & 9.17 & 1-0\end{array}$

(4)

8694.70

8694.70
8680.47

8693.98

8679.70

8670.65
8693.24

8679.00
8670.19

7696.73

7686.13
7679.60

6757.16

6748.79
6743.58

6415.50

6408.13
6403.58

6052.66

6046.04
6041.93

5706.11

5700.24

5507.01

5498.18

9035.92

9036.32
9038.72

9039.27

8452.14

8449.54
8451.55

*7244.77

\section{.9949 .84}

\subsection{6}

-9633. 78

9672.34
9649.94

9649.94
9680.80

9413.46

9413.46
9421.93

.9437 .11

11453
11472
11464

9693.68

9739.74
9741.93

8874.53

8884.23
8882.47

8882.47
8880.70

7923.95

7931.70
7930.33

7928.84

$\begin{array}{llll}6.83 & 9.63 \quad 1-\quad 4^{3} g^{0}-6^{3} \\ (5)\end{array}$

$\begin{array}{llll}7.84 & 9.35 & 3-4 & 4^{5} P-4 \\ 7 & D^{\circ}\end{array}$

$\begin{array}{lll}7.84 & 9.35 & 3-3 \\ 7.83 & 9.36 & 3-2\end{array}$

$\begin{array}{lll}7.83 & 9.26 & 1-1\end{array}$

$\begin{array}{lll}7.84 & 9.36 & 3-2\end{array}$

$\begin{array}{lll}7.83 & 9.36 & 3-1 \\ 7.83 & 9.36 & 1-0\end{array}$

$7.84 \quad 9.44 \quad 3-3 \quad 4^{5} \mathrm{P}-6^{5} \mathrm{~g}^{\circ}$

$\begin{array}{lll}7.83 & 9.44 & 2-2 \\ 7.83 & 9.44 & 1-3\end{array}$

(7)

$\begin{array}{cccc}7.84 & 9.66 & 3- & 4^{5} \mathrm{P}-5^{5} \mathrm{D}^{\circ} \\ 7.83 & 9.66 & 3- & (8)\end{array}$

$\begin{array}{lll}7.83 & 9.66\end{array}$

P-7 5 量。

$\begin{array}{cccc}7.84 & 9.76 & 3-2 & 4^{5} \mathrm{P}_{-7} 5 \mathrm{~S}^{\circ} \\ 7.83 & 9.76 & 3-3 & (9)\end{array}$

$\begin{array}{lll}7.83 & 9.76 & 1-2\end{array}$

$5 p-6^{5} D^{\circ}$

$\begin{array}{cccc}7.84 & 9.87 & 3- & 4^{5} \mathrm{P}-6^{5} \mathrm{D} \\ 7.83 & 9.87 & 2- & (10) \\ 7.83 & 9.87 & 1- & \end{array}$

$7.84 \quad 10.00 \quad 3-\quad 4^{5} \mathrm{P}-7 \mathrm{P}^{5}$

7.8310 .00 32 (11)

$7.84 \quad 10.08 \quad 3-\quad 4^{5} \mathrm{P}-8^{5} \mathrm{D}^{\circ}$

$\begin{array}{lll}7.83 & 10.08 & 3- \\ 7.83 & 10.08 & 1-\end{array}$

(13)

$8.01 \quad 9.38 \quad 2-3 \quad 4^{3} P-4^{3} D^{\circ}$

$\begin{array}{lll}8.01 & 9.38 & 1- \\ 8.01 & 9.38 & 0-1\end{array}$

$8.01 \quad 9.47 \quad 2-1 \quad 4^{3} \mathrm{P}-6^{3} \mathrm{~g}^{\circ}$

(10) $8.37 \quad 9.65$

$\begin{array}{llll}10 & 8.37 & 9.65 & 3-3 \\ (8) & 8.37 & 9.65 & 3-2\end{array}$

$\begin{array}{lllll}(8) & 8.37 & 9.69 & 3-3 & 3^{3} \mathrm{D}^{\circ}-4 \mathrm{p}^{\prime} \\ 8 & 8.37 & 9.68 & 3-3 & (18)\end{array}$

(8) \begin{tabular}{rrr}
8.37 & 9.68 & $1-1$ \\
8.37 & 9.68 & $3-2$ \\
\hline
\end{tabular}

$\begin{array}{lllll}8.38 & 9.46 & 4- & 3^{5} D^{\circ}-4^{5} \\ 8.38 & 9.46 & 3- & (19)\end{array}$

$\begin{array}{llll}8.38 & 9.77 & 1-\end{array}$

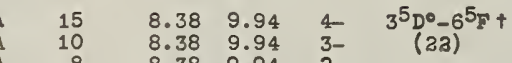
SI I P 10.31 Anal B L1st B Sept 1944

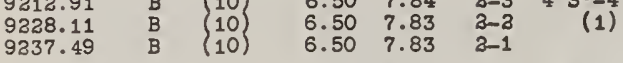

REVISED KULTIPLET TABLE

I A Laboratory ${\text { Ref Int Low }{ }_{\text {High }} \text { J Hultiplet }}_{\text {(NO) }}$

I A Laboratory Rer

LOT $^{\text {E }}{ }_{\text {H1gh }}$

J (xo)

S II I P 33.3 Anal B L1st A Sept $1944 \quad \underline{8 \text { II }}$ cont1nued

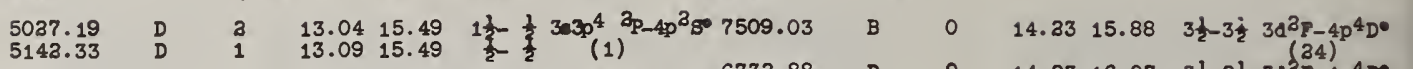

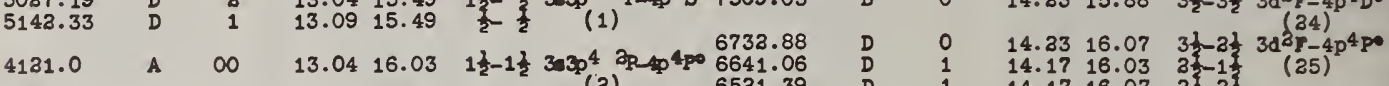

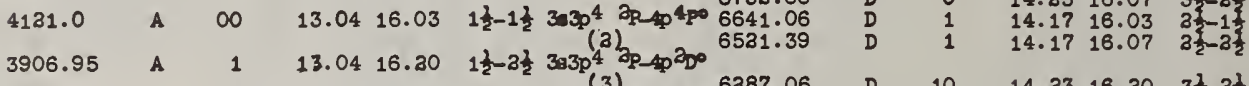

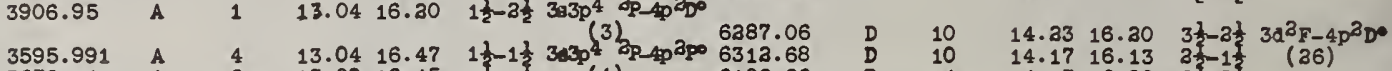

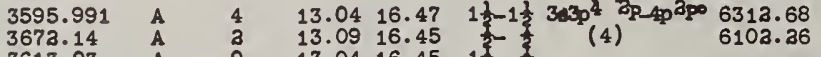

$\begin{array}{llll}14.17 & 16.13 & 2 & 3 \\ 14.17 & 16.30 & 3 & (36)\end{array}$

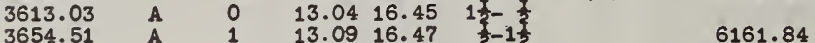

\section{p}

$6386.48 \quad D \quad 3 \quad \overline{13.56} 15.49 \quad 1 \frac{1}{2}-\frac{1}{2} 48^{4} \mathrm{P}-4 \mathrm{p}^{2} \mathrm{~g}^{\circ}$

$14.1716 .18 \quad 3 \frac{1}{2}-1 \frac{1}{2} 3 \mathrm{~d}^{2} \mathrm{~F}-4 \mathrm{p}^{4} \mathrm{~g}$

$\begin{array}{cccccccc}5453.81 & C & 15 & 13.61 & 15.88 & 31-3 \frac{1}{4} & 4 \mathrm{~B}^{4} \mathrm{PP}_{4} \mathrm{P}^{4} \mathrm{D}^{\circ} & 3993.536\end{array}$

5438.64

5564.94

5473.59

$\begin{array}{lll}7.83 & 9.26 & 1-2\end{array}$

5645.62
5556.01

5033.41

4991.94
4943.47

494.47
5103.30

5009.54

4924.08
4925.32

4779.11

4804.13
4681.33

4681.32
4742.4

4815.515

4716.326
4656.74

4193.51

5606.11

5640.32

5664.73

5536.23

5616.63

5466.55
5536.77

$\begin{array}{lll}8.01 & 9.38 & 2-2 \\ 8.01 & 9.38 & 1-1\end{array}$

$\begin{array}{llll}8.01 & 9.47 & 1-1 & (14)\end{array}$

$\begin{array}{llll}8.01 & 9.71 \quad 2-3 & 4^{3} P-5^{3} D^{0}+\end{array}$

8314.73

5996.16
5908.35

6097.12
5932.95

6133.41

5639.96

5646.98
5819.32

$\begin{array}{lllll}8.01 & 9.71 & 2-2 & \text { (15) } & 5819.20 \\ & & & 5014.03\end{array}$

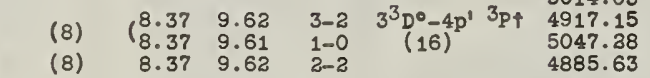

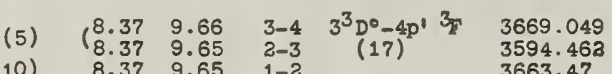

3594.462

3314.50

3314.50

3329.3
3257.83

Strongest Unclase1p1ed L1nea of $S I$

6981.40

7139.79

7317.03

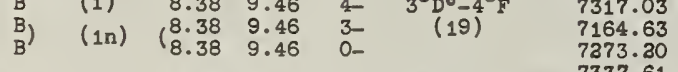

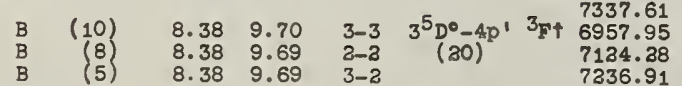

$\begin{array}{llllll}B & (9) & 8.38 & 9.77 & 4 & 3^{5} \mathrm{D}^{0}-5^{5} \mathrm{~F}+ \\ \mathrm{B} & 7 & 8.38 & 9.77 & 3- & (31)\end{array}$

6305.51

6397.30
6413.71

6286.35

6384.89

6274.34

6369.34

6093.13
5895.89

5895.89
6080.85

$9958.90 \quad$ B $\quad(8)$

7629.82

7578.96

4993.51
3867.56

5951.30
5940.69

5927.15

3845.21

3788.6
13.5315 .80 th

$\begin{array}{llll}13.61 & 15.83 & 35 \\ 13.56 & 15.80 & 1\end{array}$

13.5315 .78 曹

$\begin{array}{lll}13.61 & 15.80 & 35 \\ 13.56 & 15.78 & 1 \frac{3}{2} \\ 1 \frac{3}{2}\end{array}$

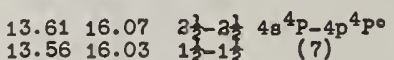

$\begin{array}{lll}13.56 & 16.03 & 1 \\ 13.53 & 16.03 & 1 \\ 13.61 & 16.03 & 3\end{array}$

$\begin{array}{lll}13.61 & 16.03 & 3 \\ 13.56 & 16.02 & 13 \\ 13.56 & 16.07 & 1\end{array}$

$\begin{array}{lll}13.56 & 16.07 & 1 \frac{2}{2}-2 \frac{3}{2} \\ 13.53 & 16.03 & \frac{1}{2}-1 \frac{1}{2}\end{array}$

$\begin{array}{ll} & 8051.91 \\ \frac{1}{2}-1 \frac{3}{2} & 8358.27 \\ & 8377.79\end{array}$

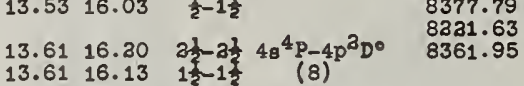

3853.09
3859.26

$\begin{array}{lll}13.56 & 16.30 & 1 \frac{3}{2}-\frac{3}{2} \\ 13.53 & 16.13 & \frac{3}{2}-1 \frac{2}{2}\end{array}$

$13.61 \quad 16.18$ 3द $1 \frac{1}{3} \quad 4 \mathrm{~g}^{4} \mathrm{P}-4 \mathrm{p}^{4} \mathrm{~g}$ 。

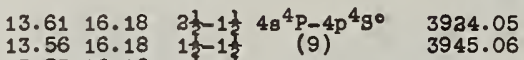

$\begin{array}{llll}13.53 & 16.18 & \frac{1}{2}-1 \frac{1}{2} \\ 13.56 & 16.45 & \frac{1}{2}-1 \frac{1}{2} & 4 \mathrm{~s}^{4} \mathrm{P}-4 \mathrm{p}^{2} \mathrm{po}\end{array}$

$13.5616 .45{ }^{\frac{1}{2}-1 \frac{1}{2} 4 \mathrm{~s}^{4} \mathrm{P}-4 \mathrm{p}^{2} \mathrm{P}}$

8530.23

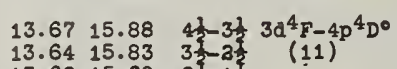

13.6215 .80 a 13 年

13.6415 .88 3.

13.6315 .83 3.3

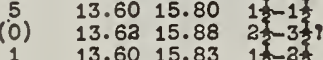

4668.58
4648.17

4755.12
4763.38

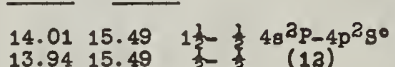

$14.01 \quad 16.07 \quad 1 \frac{1}{3}-2 \frac{1}{2} 4 \mathrm{~s}^{2} \mathrm{p}-4 \mathrm{p}^{4} \mathrm{po}$

13.9416 .03 安 $1 \frac{1}{2}$ (13)

$\begin{array}{lll}14.01 & 16.03 & 1 \frac{1}{3}-1 \frac{1}{2} \\ 13.94 & 16.03 & \\ 14.01 & 16.02 & 1 \frac{2}{2}\end{array}$

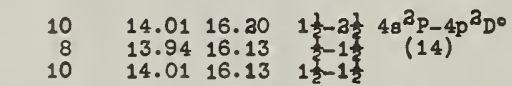

$\frac{1}{7} \quad 14.0116 .47 \quad 1 \frac{1}{2}-1 \frac{1}{2} 48^{3} \mathrm{p}-4 \mathrm{p}^{2} \mathrm{po}$

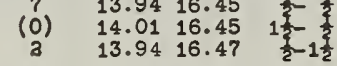

D

4463.582

$\begin{array}{lllllll}14.01 & 17.37 & 1 \frac{1}{2}-2 \frac{1}{2} & 48^{2} \mathrm{P}-4 \mathrm{p}^{\prime} \mathrm{D}^{\circ} & 4483.424 \\ 4486.66\end{array}$

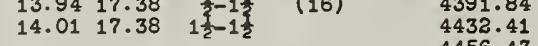

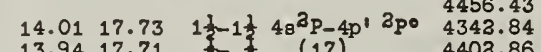

$\begin{array}{llll}13.9417 .71 & 4403.86 \\ 14.01 & 17.71 & 1\end{array}$

$13.9417 .73 \quad .4162 .698$

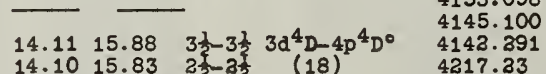

14.1015 .80 1 $14.1 \frac{1}{3}$

$14.11 \quad 15.83 \quad 3 \frac{2}{2}-3 \frac{2}{2}$

14.1015 .78 12.

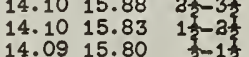

$\begin{array}{llll}14.11 & 16.07 & 3 \frac{1}{2}-2 \frac{1}{3} & 3 a^{4} p-4 p^{4} p o \\ 14.10 & 16.03 & 35-1 & (19)\end{array}$

$\begin{array}{lll}14.10 & 16.02 & 13 \\ 14.10 & 16.07 & 2 \frac{1}{2}\end{array}$

$14.1016 .03 \quad 1 \frac{1}{2} 1 \frac{1}{2}$

$\begin{array}{lll}14.09 & 16.03 & \\ 14.10 & 16.07 & 12 \\ 14.09 & 16.03 & \frac{2}{2}-2 \\ 2 & -1 \frac{3}{2}\end{array}$

14.1010 .13

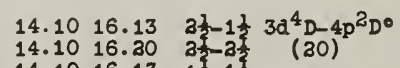

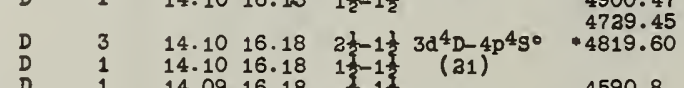

4142.291
4217.23

4189.71
418.70

4168.409
4255.01
4213.5

4038.791

3990.94

3963.13

4050.11

4050.11

3970.69

3950.42
3939.49

4793.02

4835.85

4883.73
4901.30

4900.47
4739.45

$\begin{array}{llll}14.10 & 16.18 & 1 \frac{1}{3}-1 \frac{1}{3} & (21) \\ 14.09 & 16.18 & \frac{3}{2}-1 \frac{3}{2} & 4590.8\end{array}$

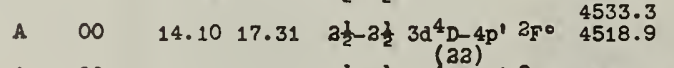

A $\infty$
4007.78
3918.19

3932.30

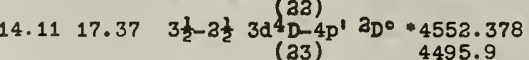

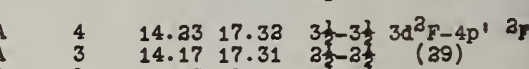

$\begin{array}{rlll}0 & 14.33 & 17.31 & 32 \\ 00 & 14.17 & 17.32 & 3 \frac{3}{2}-3 \frac{3}{2}\end{array}$

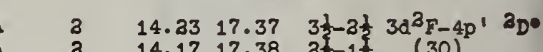

$\begin{array}{llll}14.23 & 17.37 & 3 \frac{1}{2}-2 \frac{1}{2} & 30^{2} F-4 p \\ 14.17 & 17.38 & 3 \frac{1}{2}-1 \frac{1}{2} & (30) \\ 14.17 & 17.37 & 2 \frac{1}{2}-2 \frac{3}{2} & \end{array}$

14.30 $15.88 \quad 3 \frac{1}{3}-3 \frac{1}{2} \quad 3 d^{4} \mathrm{P}-4 \mathrm{p}^{4} \mathrm{D}^{\circ}$

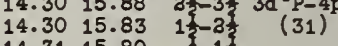

$\begin{array}{lll}14.31 & 15.80 & 15 \\ 14.30 & 15.83 & 3 \frac{2}{3}\end{array}$

14.3015 .80 1 1 - 1 .

$\begin{array}{lll}14.31 & 15.78 & 3- \\ 14.30 & 15.80 & 3 \frac{3}{3}-1 \frac{3}{3}\end{array}$

\begin{tabular}{lll}
14.30 & 15.78 & $1 \frac{3}{2}-\frac{1}{2}$ \\
\hline
\end{tabular}

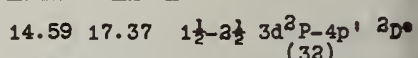

$14.59 \quad 17.73 \quad 1 \frac{1}{2}-1 \frac{1}{3} 3 \mathrm{~d}^{2} \mathrm{P}-4 \mathrm{p}^{\prime} \quad 2 \mathrm{po}$

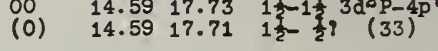

$14.73 \quad 16.18 \quad 2 \frac{1}{2}-1 \frac{1}{2} 3 d^{2} p-4 p^{4} g^{\circ}$

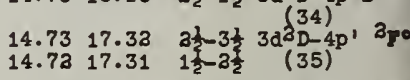

$14.73 \quad 17.37 \quad 2 \frac{1}{2}-2 \frac{1}{3} 3 \mathrm{~d}^{2} \mathrm{D}-4 \mathrm{p}^{\prime} \quad 2 \mathrm{D}^{\circ}$

$\begin{array}{llll}14.73 & 17.38 & 1 \frac{1}{2}-1 \frac{3}{2} & (36)\end{array}$

15.0016 .47 3h-1 $48^{\circ} 3 p-4 p^{3} P^{\circ}$

$15.0017 .33 \quad 3 \frac{1}{2}-3 \frac{1}{2} 4 g^{\prime} 3 p_{-4 p^{\prime}} 2 p$

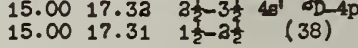




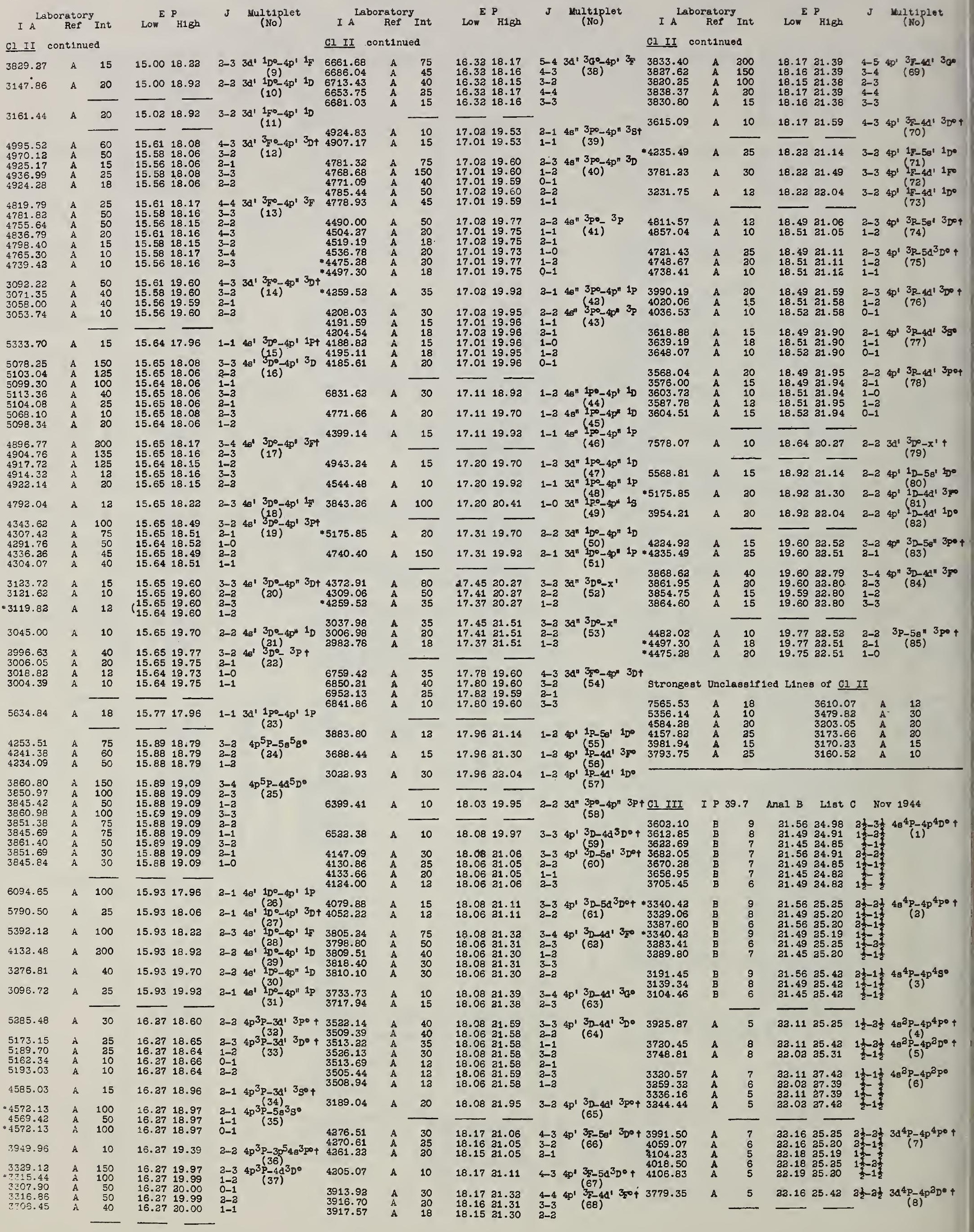




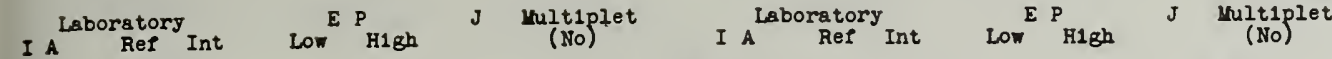
C1 III cont1nued

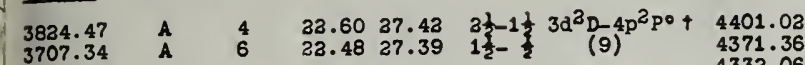
(9) 4371.36

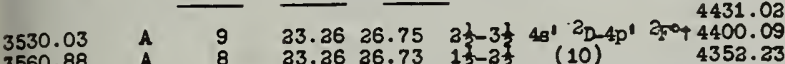
$\begin{array}{lllllll}A & 8 & 23.26 & 36.73 & 1 \frac{1}{2}-2 \frac{1}{2} & \text { (10) } & 4352.23\end{array}$

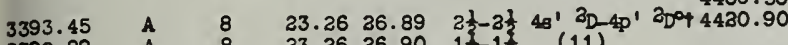
3392.89 3386.23 .

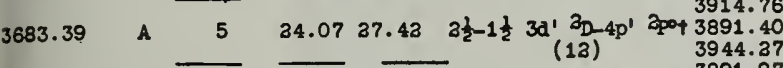
$4608.31 \quad A \quad 5 \quad \overline{24.32} 26.893 \frac{1}{2}-2 \frac{1}{2} 3 a^{\prime} 3-4 p^{\prime} 300+3875.26$ (13)

Ql IV See 1ntroduction
Aral A Llat D $\triangle I$ I P 15.69 AneI A I1日t D 9123.9660
8115.3115
8014.7856
7733.7597
7635.1053
7147.0406
7067.2170
6965.4303
4251.1853
4300.6751
4190.7137
4164.1800
4158.5906
3947.5043
3948.9788

9657.7841 A 1500 $\begin{array}{lll}8424.647 & \text { A } & 2500 \\ 8103.6933 & \text { A } & 3000\end{array}$ 4300.1011 2366. 2867 $\begin{array}{ll}198.3170 & \text { B } \\ 4044.4182 & \text { B }\end{array}\left(\begin{array}{l}100 \\ 100\end{array}\right\}$ 3806.5234

10470.051 A 500 $\begin{array}{lll}7948.1754 & \text { A } & 400\end{array}$ $\begin{array}{rll}7948.1754 & \text { A } & 400 \\ 7734.2064 & \text { A } & 300\end{array}$ 4523.3338 B $(40)$

8784.5010 A 1000

$\begin{array}{llr}9354.218 & \text { A } & 300 \\ 9234.498 & \text { A } & 1000\end{array}$ 8521.4407 A 2000 $\begin{array}{llccc}8408.208 & \text { A } & 3000 & 11.78 & 13.24 \\ 8264.5209 & \text { A } & 1500 & 11.78 & 13.37 \\ 7503.8676 & \text { A } & (15) & 11.78 & 13.42\end{array}$

\subsection{6}

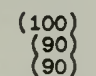

11.7814 .44

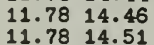

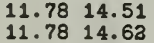

11.7814 .63

$\begin{array}{ll}11.78 & 14.63 \\ 11.78 & 14.87\end{array}$

$\longrightarrow$

\begin{tabular}{|c|c|}
\hline une & 1944 \\
\hline $\begin{array}{l}3-1 \\
3-3 \\
3-3 \\
3-1 \\
2-2 \\
3-1 \\
3-2 \\
3-1\end{array}$ & 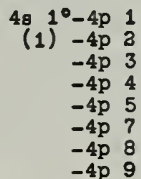 \\
\hline
\end{tabular}

3808.61

3735.49
-3830.43

3830.43
3750.50

\subsection{1}

3631.06

3706.94

$-4 \mathrm{p} 8$
$-4 \mathrm{p} 9$

2-1 48 $1^{\circ}-5 \mathrm{p} 1$

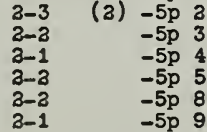

4510.7333

4333.5612

$\begin{array}{llll}4335.3380 & \text { B } & 70 \\ 4259.3818 & \text { B } & (100)\end{array}$

10673.5

6753.833

$12.8514 .01 \quad 1-3 \quad 4 p 10)^{1-58} 1^{\circ}$

(30) $\quad 13.85 \quad 14.88 \quad 1-3 \quad 4 \mathrm{p} \quad 1-4 \mathrm{~d} \quad 3^{\circ}$

(30) $12.85 \quad 15.04 \quad 1-0 \quad 4 p \quad 1-5 \mathrm{~d} 1^{\circ}$

6033.134 A (30) $13.0315 .07 \quad 3-4 \quad 4 p^{3-5 d \quad 4^{\circ}}$ $\begin{array}{lllll}5495.8730 & A & (30) \quad 13.0315 .38 & 3-4 & (13)^{3-5 d} 4^{\circ} \\ (14)^{\circ}\end{array}$

3499.49

3517.90
3531.22

4806.07

4972.16

4735.93
4847.90

5009.35
5063.07

$\begin{array}{rrrr}1-1 & 48 & 3^{\circ}-4 p & 1 \\ 1-2 & (3)^{-4 p} & 3 \\ 1-1 & & -4 p & 4\end{array}$

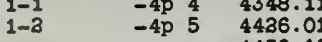

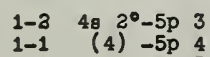

$\begin{array}{ll}1-1 & (4) \\ 1-2 & -5 p 5 \\ 1-0 & -5 p\end{array}$

$48)^{30}-6 p 6$

$\begin{array}{llll}-1 & 4 g & 3^{\circ}-4 p & 1 \\ -1 & (8)^{-4 p} & 4\end{array}$ $-4 p ?$
$-4 p 9$

$\begin{array}{llll}-1 & 48 & 3^{\circ}-5 p & 1 \\ -1 & (7)^{-5 p}-5 & ?\end{array}$

1-3 $484^{\circ}-4 p 3$

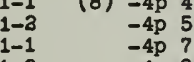
$-4 \mathrm{p}$
$-4 \mathrm{p}$ $-4 \mathrm{p} 1$

$\begin{array}{lcc}-1 & 4 a 4^{0}-5 p & 1 \\ -3 & (9)^{-5 p}-5 & \end{array}$

$\begin{array}{ll}-5 p & 4 \\ -5 p & 6\end{array}$

$\begin{array}{ll}-5 \mathrm{p} & ? \\ -5 \mathrm{p} & 8\end{array}$

$\begin{array}{ll}-5 \mathrm{p} & 9 \\ -5 \mathrm{p} & 10\end{array}$

Por changes in Paschen notation soo text $\$ 37$

4348.11
4436.01
4430.18

4430.18
4266.53

4331.25
4379.74

4379.74
4178.39

4283.90

4083.40
4113.83

3974.76

4338.18

3845.43

3845.43
3974.48

4147.43

3729.29

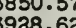

.3923 .54

5950.91

5843.80
6077.43

6077.43
5734.37

5145.36

5388.92
5017.63

4879.90

4965.12
4736.91

4545.08

4889.06

4657.94

4383.79

4375.96
4579.3

4579.39 $\begin{array}{llr}8103.6933 & \text { A } & 3000 \\ 8006.1556 & \text { A } & 600\end{array}$ $\begin{array}{lll}4191.0296 & \text { B } & (100 \\ 4181.8838 & B\end{array}$ 4038.82 3992.06
3931.34 3786.40 3714.74

\section{$P 27.5$ Anal B List A Oct 1944}

(2)

$\begin{array}{llll}18.34 & 19.14 & 3 \frac{1}{2}-2 \frac{1}{2} & 3 d^{4} \mathrm{D}-4 \mathrm{p}^{4} \mathrm{p}^{\circ} \\ 16.35 & 19.18 & 3 & (1)\end{array}$

$16.3719 .32 \quad 1 \frac{1}{2}-$

$16.3719 .18 \quad 1 \frac{1}{16}$

$\begin{array}{lll}16.39 & 19.23 & 1 \\ 16.37 & 19.14 & 12\end{array}$

$\begin{array}{lll}16.37 & 19.14 & 1 \frac{1}{2}-2 \frac{1}{3} \\ 18.39 & 19.18 & \frac{1}{2}-1 \frac{1}{2}\end{array}$

$16.3419 .41 \quad 3 \frac{1}{2}-3 \frac{1}{3} \quad 3 d^{4} D-4 p^{4} D^{\circ}$

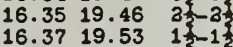

$\begin{array}{ll}16.37 & 19.53 \\ 16.39 & 19.56\end{array}$

16.3519 .53

16.3519 .41

$16.3719 .46 \quad 1 \frac{2}{2}$

$\begin{array}{llll}16.34 & 19.60 & 3 \frac{1}{2}-2 \frac{1}{2} & 3 d^{4} D-4 p^{3} D^{0} \\ 16.35 & 19.68 & 2 \frac{1}{2}-1 \frac{1}{2} & (3)\end{array}$

$\begin{array}{lll}16.35 & 19.68 & 32-1 \\ 16.35 & 19.60 & 32\end{array}$

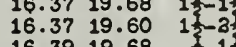

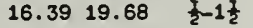

$16.35 \quad 19.78 \quad 3 \frac{1}{2}-1 \frac{1}{2} \quad 3 \mathrm{~d}^{4} \mathrm{p}-4 \mathrm{p}^{2} \mathrm{p}^{\circ}$

16.3719 .78 1

$\begin{array}{cccc}4 & 16.39 & 19.73 & \\ 00 \mathrm{n} & 16.39 & 19.78 & \frac{1}{2}-1 \frac{1}{2}\end{array}$

$5 \quad 16.35 \quad 19.88 \quad 3 \frac{1}{3}-1 \frac{1}{3} \quad 3 d^{4} \mathrm{p}-4 \mathrm{p}^{4} \mathrm{~g}^{\circ}$

3

3

\begin{tabular}{lll}
16.37 & 19.88 & 13 \\
16.39 & 19.88 & 1 \\
\hline
\end{tabular}

\section{0}

16.57 19.14 3t $-3 \frac{1}{3} 48^{4} p-4 p^{4} p^{0}$

$\begin{array}{rll}6 & 16.68 & 19.18 \\ 5 & 16.74 & 19.22\end{array}$

$16.5719 .18 \quad 2 \frac{2}{2}-1$

$\begin{array}{lll}16.68 & 19.22 & 1 \\ 16.68 & 19.14 & 1\end{array}$

$\begin{array}{lll}16.68 & 19.14 & 12 \\ 16.74 & 19.18\end{array}$

3108.82
3979.05

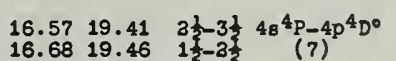

$\begin{array}{lll}16.68 & 19.46 & 1 \frac{1}{2}-3 \\ 18.74 & 19.53 & \end{array}$

16.5719 .46 32.

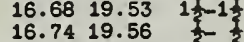

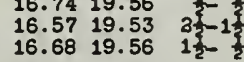

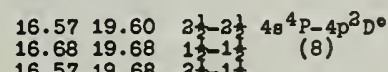

$\begin{array}{lll}16.57 & 19.68 & 33-1 \\ 16.68 & 19.60 & 13\end{array}$

16.7419 .68 (3)

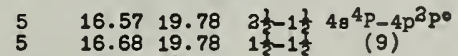

$\begin{array}{llll}\text { in } 16.74 & 19.72 \\ \text { on } 16.74 & 19.78\end{array}$

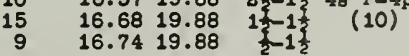

A In 16.7419 .89 t) $\frac{1}{2} 48^{4} \mathrm{P}-4 \mathrm{p}^{2} \mathrm{~S}^{\circ}$

ए $\overline{17.07} 19.14 \quad 1 \frac{1}{3}-2 \frac{1}{2} 48^{2} \mathrm{p}-4 \mathrm{p}^{4} \mathrm{po}$

$\begin{array}{cccc}\infty & 17.07 & 19.18 & 1 \\ 0 & 17.19 & 19.22 & \frac{1}{3} \\ \text { On } & 17.07 & 19.32 & 1 \frac{3}{2} \\ \frac{3}{2}\end{array}$

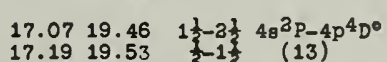

$\begin{array}{lll}17.19 & 19.53 & 1 \\ 17.07 & 19.53 & 1 \frac{3}{2}-1 \frac{3}{2}\end{array}$

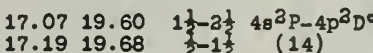

$\begin{array}{lll}17.19 & 19.68 & 1 \\ 17.07 & 19.68 & 1\end{array}$

$17.0719 .78 \quad 1 \frac{1}{2}-1 \frac{1}{2} 4 \mathrm{~s}^{2} \mathrm{P}-4 \mathrm{p}^{2} \mathrm{po}$

17.1919 .78 क्षे

17.0719 .88
17.1919 .88
6
6
6

$16.34 \quad 19.46 \quad 3 \frac{2}{2}-3$

$16.3719 .72 \quad 1 \frac{1}{2} \quad(4)$

4764.89

\begin{tabular}{llllll} 
& 4309.11 \\
\hline & 16.57 & 19.88 & $2 \frac{1}{2}-1 \frac{1}{2}$ & $48^{4} \mathrm{P}-4 \mathrm{p}^{4} \mathrm{~g}^{\circ}$ & 4300.66
\end{tabular}

17.1919 .72 (15) 4127.09

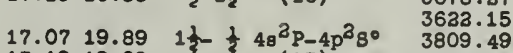

I $A$ Laboratory

Lo P H1gh J Multiplet

A II conținued

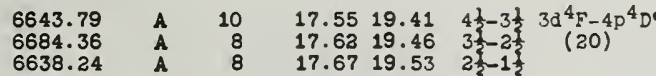

6638.24

40

6863.52

6756.61
7077.03

6990.16

6343.13

6138.67
6399.23

6239.73

(659.16

6130.12

3594.41

6808.55

$\begin{array}{lll}8 & 17.6719 .53 & 23 \\ 7 & 17.7019 .56 & 13\end{array}$

\begin{tabular}{lll}
17.70 & 19.56 \\
17.62 & 19.41 \\
\hline
\end{tabular}

17.6719 .46

17.7019 .53
17.67
19.41

17.6719 .41
17.7019 .46

$17.63 \quad 19.60 \quad 3 \frac{1}{2}-2 \frac{1}{3} \quad 3 d^{4} F-4 p^{2} D^{0}$

17.6719 .68 25 (21)

$\begin{array}{lll}17.67 & 19.60 & 21-2 \\ 17.70 & 19.68 & 13\end{array}$

$\begin{array}{lll}17.70 & 19.68 & 1 \frac{1}{2}-1 \\ 17.70 & 19.60 & 1 \frac{1}{2}-2\end{array}$

$17.7019 .72 \quad 1 \frac{1}{2}-\frac{1}{2} 3 d^{4} F-4 p^{2} p_{0}$

$17.6221 .053 \frac{1}{2}-3 \frac{1}{2} 3 a_{F-4 p^{\prime}}^{(22)}\left(23 F^{\circ}\right.$

$17.8619 .68 \quad 3-1 \frac{1}{3} 3 \mathrm{~d}^{2} \mathrm{P}-4 \mathrm{p}^{2} \mathrm{D}^{0}$

6861.30

6666.3

$17.98 \quad 19.68 \quad 1 \frac{1}{2}-1 \frac{1}{2} \quad(24)$

6437.63

$17.98 \quad 19.78 \quad 1 \frac{1}{2}-1 \frac{1}{3} \quad 3 d^{2} \mathrm{p}-4 \mathrm{p}^{2} \mathrm{po}$

6500.25

6483.10

4043.20

3766.13

3556.91

3682.56
3634.83

3605.89

3490.89
3611.84

5

$\begin{array}{llll}17.86 & 19.72 & 1 \\ 17.86 & 19.78 & \text { (25) }\end{array}$

$17.98 \quad 19.88 \quad 1 \frac{1}{2}-1 \frac{1}{2} \quad 3 a^{2} \mathrm{P}-4 \mathrm{p}^{4} \mathrm{~s}^{\circ}$

$17.9819 .891 \frac{1}{2}-\frac{1}{2} 3 \mathrm{~d}^{2} \mathrm{P}-4 \mathrm{p}^{2} \mathrm{~S}^{\circ}$

$17.9831 .04 \quad 1 \frac{1}{2}-2 \frac{1}{2} 3 \mathrm{~d}^{2} \mathrm{P}-4 \mathrm{p}^{\prime} 2 \mathrm{~F}^{\circ}$

$\begin{array}{llll}17.98 & 21.26 & 1 \frac{1}{2}-1 \frac{1}{2} & 3 \mathrm{~d}^{2}{ }_{\mathrm{P}-4 \mathrm{p}^{\prime}} \quad 3 \mathrm{PO}\end{array}$

17.8631 .33
17.9821 .33
17.8631 .26

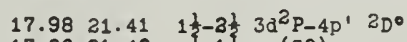

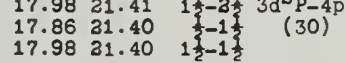

4609.

4589.93
4637.35

$18.3721 .05 \quad 2 \frac{1}{2}-3 \frac{1}{3} 48^{\prime} 2 \mathrm{D}-4 \mathrm{p}^{\prime} 2 \mathrm{p}^{\circ}$ 4277.

4131.73
4237.33

4073.01

4043.91

4079.60
4035.47

4936.13
4904.75

4530.57

4182.98

5141.84
5017.16
5176.38

4732.08

4474.77
4598.77

4481.83

4370.76

4490.99
4362.07

5345.49

- 4073.40

4124.09

3765.27

3720.43
3669.62

3669.62
3678.27

3809.49
3770.54

$\begin{array}{llll}18.35 & 21.04 & 1 \frac{1}{2}-2 \frac{1}{3} & \text { (31) } \\ 18.37 & 21.04 & 2 \frac{1}{2}-2 \frac{1}{2} & \end{array}$

$18.37 \quad 31.26 \quad 2 \frac{1}{2}-1 \frac{1}{2} 4 \mathrm{~g}^{\prime} \cdot{ }^{2} \mathrm{D}-4 \mathrm{p}^{\prime} \mathrm{2} \mathrm{p}$ $\begin{array}{llll}18.35 & 21.33 & 1 \frac{1}{2}-\frac{1}{2} & \text { (32) } \\ 18.35 & 21.36 & 1 \frac{1}{2}-1 \frac{1}{2} & \end{array}$

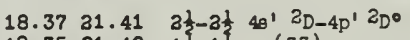
18.3531 .40 13 $-1 \frac{1}{3}$ (33) $\begin{array}{llll}18.37 & 21.40 & 2 \frac{1}{2}-1 \frac{1}{3} \\ 18.35 & 31.41 & 1 \frac{3}{2}-2 \frac{1}{2}\end{array}$

$18.5431 .04 \quad 2 \frac{1}{2}-2 \frac{1}{3} \quad 3 \mathrm{~d}^{2} \mathrm{~F}-4 \mathrm{p}^{1} \quad 2 \mathrm{~F}^{\circ}$ $\begin{array}{llll}18.54 & 31.04 & 21 & 21 \\ 18.54 & 31.05 & 2 \frac{1}{2}-3 \frac{1}{2} & (34)\end{array}$

$18.5421 .26 \quad 2 \frac{1}{2}-1 \frac{1}{2} \quad 3 d^{2} F-4 p^{\prime}{ }^{2} p^{\circ}$

$18.4621 .413 \frac{1}{2}-2 \frac{1}{2} 3 d^{2} F-4 p^{\prime} \quad 2_{D^{\circ}}$

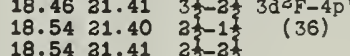

$\overline{18.65} 21.052 \frac{1}{2}-3 \frac{1}{2} 3 \mathrm{~d}^{2} \mathrm{D}-4 \mathrm{p}^{\prime} 2 \mathrm{~F}^{\circ}$ 18.5821 .04 13 3 (37)

$18.6521 .36 \quad 21-1 \frac{1}{3} 3 \mathrm{~d}^{2} \mathrm{D}-4 \mathrm{p}^{\prime}{ }^{2} \mathrm{po}$ $\begin{array}{lll}18.58 & 21.33 & 1 \\ 18.58 & 31.26 & 1\end{array}$

$18.6531 .41 \quad 31-2 \frac{1}{3} 3 \mathrm{a}^{2} \mathrm{D}-4 \mathrm{p}^{\prime} 2 \mathrm{D}^{\circ}$ $\begin{array}{lll}18.58 & 31.40 & 1\end{array}$ $\begin{array}{lll}18.65 & 31.40 & 21 \\ 18.58 & 31.41 & 1 \frac{1}{2}-2 \frac{1}{2}\end{array}$

$19.18 \quad 21.53 \quad 1 \frac{1}{2}-1 \frac{1}{2} 4 p^{4} p^{0}-3 d^{2}: 2 p$ $19.14 \quad 32.17 \quad 3 \frac{1}{2}-1 \frac{1}{2} 4 \mathrm{p}^{4} \mathrm{PO}^{2}-\mathrm{a}^{2} \mathrm{P}$ 
22

Laboratory
I A Per Int Low $_{\text {H1gh }}$

I A Laboratory ${ }_{\text {Ref Int Low }}^{\text {E P1gh }}$ J (Noltiplet $^{\text {(No) }}$

A II continued

3491.54

3491.54

3535.33

3491.24

3454.10

3478.24

3478.24
3521.98

3269.05

3263.60

3221.64
3226.00

3226.00
3194.25

3139.02

3212.54

3181.05

3243.70
3169.68

3249.82

3186.19

$\begin{array}{lll}3146.47 & \text { A } & 3\end{array}$

*6818.39

4561.03

4593.44
4666.28

4649.06

* 4103.91

-10
4033.85

4179.31

4076.6

4267.47
4201.58

3933.19

4011.23

3922.54
4053.56

3780.84

3826.83

3880.34

3763.52

379.39

3844.75

3900.63
3911.58

3856.16

3895.26

3588.44

3576.62

3581.62

3521.27

3548.51

3466.34
3487.33

3370.97

3471.59
3569.94

3569.94

3532.19

3603.46
3480.52

3480.52
3565.02

3438.14

3379.48

3341.77
3430.44

3430.44
.3397 .898

7348.11

7440.54
7090.55

$6376.00 \mathrm{~A}$

4792.12

4867.59
4949.45
$19.1422 .67 \quad 3 \frac{1}{2}-3 \frac{1}{2} \quad 4 p^{4} \mathrm{p}^{0}-4 \mathrm{~d}^{4} \mathrm{D}$ 19.2222 .71 ? 19.1423 .69 aर

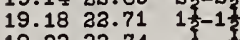
$\begin{array}{lll}19.22 & 22.74 \\ 19.14 & 23.71 & 3 \frac{1}{2}-1 \frac{1}{2}\end{array}$ $\begin{array}{lll}19.14 & 23.71 & 3 \\ 19.18 & 22.74 & 1 \frac{1}{2}\end{array}$

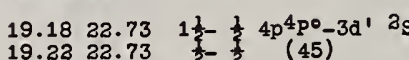

$\begin{array}{lll}19.14 & 22.92 & 2 \frac{1}{2}-3 \frac{1}{2} \\ 19.18 & 22.97 & 4 p^{\circ}-4 d^{4} F \\ 1 \frac{1}{2}-2 \frac{7}{2} ? & (46)\end{array}$

$\begin{array}{lll}19.22 & 23.00 & \frac{1}{2}-1 \frac{1}{2} \\ 19.14 & 22.97 & 2 \frac{1}{3}-2 \frac{1}{2}\end{array}$

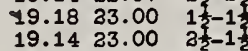

$19.1423 .07 \quad 2 \frac{1}{2}-2 \frac{1}{2} \quad 4 p^{4} P^{0}-4 d^{4} P$

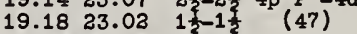

19.2223 .98

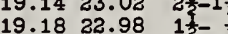

$19.1823 .05 \quad 1 \frac{1}{2}-2 \frac{1}{2} \quad 4 p^{4} P^{0}-4 d^{2} D$

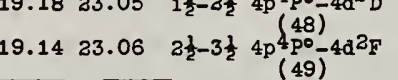

$19.46 \quad 21.27 \quad 2 \frac{1}{3}-2 \frac{1}{2} \quad 4 p^{4} D^{0}-3 d^{\prime} \quad 2 D$

$\begin{array}{llll}19.53 & 21.34 & 1 \frac{1}{2}-1 \frac{1}{2} & (50) \quad 3559.53\end{array}$

$19.46 \quad 22.17 \quad 2 \frac{1}{2}-1 \frac{1}{2} \quad 4 p^{4} D^{0}-a^{2} P$

$\begin{array}{lll}19.53 & 22.21 & 1 \\ 19.53 & 22.17 & 1\end{array}$

$19.4122 .42 \quad 3 \frac{1}{2}-2 \frac{1}{2} 4 \mathrm{p}^{4} \mathrm{D}^{0}-5 \mathrm{~s}^{4} \mathrm{P}$

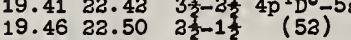

$\begin{array}{lll}19.53 & 22.59 & 1 \frac{1}{3}- \\ 19.46 & 22.42 & 23\end{array}$

19.5322 .50 12-1

$\begin{array}{ll}19.53 & 22.42 \\ 19.56 & 22.50\end{array}$

$19.4622 .60 \quad 2 \frac{1}{3}-1 \frac{1}{2} \quad 4 \mathrm{p}^{4} \mathrm{D}^{0}-5 \mathrm{~s}^{2} \mathrm{P}$

$19.5332 .60 \quad 1 \frac{1}{2}-1 \frac{1}{2} \quad(53)^{4 s}$

$\begin{array}{lll}19.56 & 32.70 & \frac{1}{3}-\frac{1}{2} \\ 19.56 & 32.60\end{array}$

$19.4122 .67 \quad 3 \frac{1}{2}-3 \frac{1}{2} \quad 4 p^{4} D^{\circ}-4 \alpha^{4} D$

$19.46 \quad 22.69 \quad 2 \frac{1}{2}-2 \frac{1}{2} \quad(54)$

$19.5322 .71 \quad 1 \frac{1}{3}-1$

$\begin{array}{lll}19.56 & 22.74 & 3 \\ 19.41 & 22.69 & 3 \frac{3}{2}-2\end{array}$

19.4622 .71 2द 1 1

19.5322 .7

$\begin{array}{lll}19.53 & 22.69 & 1 \frac{1}{2}-2 \frac{1}{2} \\ 19.56 & 22.71 & -1 \frac{1}{2}\end{array}$

$19.5322 .73 \quad 1 \frac{1}{3} 4 p^{4} D^{\circ}-3 d^{\prime} \quad 2 \mathrm{~s} 4243.71$

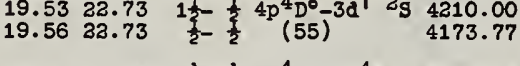

$19.41 \quad 22.85 \quad 3 \frac{1}{2}-4 \frac{1}{2} \quad 4 p^{4} D^{\circ}-4 d^{4} F$

$19.46 \quad 22.92 \quad 2 \frac{1}{2}-3 \frac{1}{2} \quad$ (56)

$19.5322 .97 \quad 1 \frac{1}{3}-2 \frac{2}{3}$

$19.4122 .92 \quad 3 \frac{1}{2}-3$

$19.4622 .97 \quad 2 \frac{2}{2}-2 \frac{1}{2}$

$\begin{array}{lll}19.53 & 23.00 & 1 \frac{2}{2}-1 \frac{2}{2} \\ 19.41 & 22.97 & 3 \frac{3}{3}-2 \frac{1}{2} \\ 19.46 & 23.00 & 2 \frac{1}{2}-1 \frac{1}{2}\end{array}$

$\begin{array}{llll}19.41 & 23.07 & 3 \frac{1}{2}-2 \frac{1}{2} & 4 p^{4} D^{0}-4 d^{4} p \\ 19.46 & 23.02 & 2 \frac{1}{2}-1 \frac{2}{2} & (57)\end{array}$

$\begin{array}{lll}19.53 & 22.98 & 12 \\ 19.46 & 23.07 & 3 \frac{1}{2}-2\end{array}$

$19.5323 .02 \quad 1 \frac{2}{2}-1 \frac{1}{2}$ ?

19.5622 .9

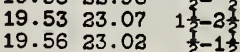

$19.4623 .05 \quad 2 \frac{1}{2}-1 \frac{1}{2} \quad 4 p^{4} D^{0}-4 d^{2} D$

$\begin{array}{llll}19.41 & 23.06 & 3 \frac{1}{2}-3 \frac{1}{2} & 4 p^{4} \\ 19.46 & 53.16 & 2 \frac{5}{2}-2 \frac{1}{2} & (59)\end{array}$

$\begin{array}{lll}19.46 & 23.06 & 3 \frac{1}{2}-3 \frac{1}{2} \\ 19.53 & 23.16 & 1 \frac{1}{2}-2 \frac{1}{2}\end{array}$

$19.60 \quad 31.27 \quad 2 \frac{1}{2}-2 \frac{1}{2} \quad 4 p^{2} D^{0}-3 d^{1} 2_{D}$

$19.68 \quad 21.34 \quad 1 \frac{2}{2}-1 \frac{1}{2} \quad 4 p^{2} D^{\circ}-3 d^{\prime} 2_{D}$

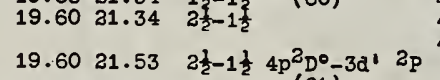

$19.6022 .17 \quad 2 \frac{1}{3}-1 \frac{1}{2} 4 p^{2} D^{\circ}-8^{2} P$

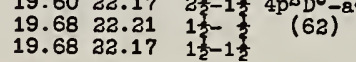

$19.1823 .07 \quad 1 \frac{1}{2}-3$
4373.50

4355.62

4343.71
4502.95

4103.91
4076.96

4076.96
4218.69

4007.66

4096.47
3988.18

4065.14

4031.41

4047.51

3717.17

3656.05
3709.90

3620.82

3550.03

3692.17
3603.91

3733.36
3635.67

3570.77

3545.58
3464.14

3137.66
3373.36

3204.34

3000.45

7617.8

7055.01
6799.32

5165.82
4942.9

4681.53

$\frac{4440.09}{4547.78}$

$+4300.66$

4374.87

4332.67

4275.19

4099.47

3869.61
-3753.53

3751.06

3811.22
3777.55

3855.18

3655.29

3293.66

3307.24
3366.59

3236.82

3093.41
3028.93

4865.96

4721.62
4564.43

4564.43

4535.51
4372.09

4394.65
4358.53

4358.53
4319.69

4338.24

3994.81
3952.74

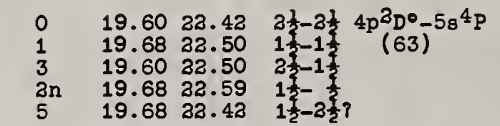 \\ $19.6022 .60 \quad 2 \frac{1}{2}-1 \frac{1}{2} 4 \mathrm{p}^{2} \mathrm{D}^{\circ}-5 \mathrm{~s}^{2} \mathrm{P}$ \\ $\begin{array}{lll}19.68 & 22.70 & 1 \frac{1}{2} \\ 19.68 & 22.60 & 1 \frac{1}{2}-1 \frac{1}{2}\end{array}$ \\ $19.6022 .67 \quad 3 \frac{1}{2}-3 \frac{1}{2} \quad 4 p^{2} D^{\circ}-4 d^{4} D$ \\ $19.6822 .69 \quad 1 \frac{1}{2}-2 \frac{1}{2} \quad(65)$ \\ $\begin{array}{lll}19.60 & 22.69 & 2 \frac{2}{2}-2 \frac{1}{2} \\ 19.68 & 22.71 & 1 \frac{2}{2}-1 \frac{1}{2}\end{array}$ \\ $\begin{array}{lll}19.60 & 22.71 & 3 \frac{1}{3}-1 \frac{1}{2} \\ 19.68 & 22.74 & 1 \frac{1}{2}-\frac{1}{2} 9\end{array}$}

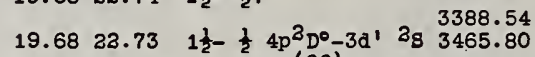

$19.6022 .92 \quad 3 \frac{1}{3}-3 \frac{1}{2} \quad 4 \mathrm{p}^{2} \mathrm{D}^{\circ}-4 \mathrm{~d}^{4} \boldsymbol{F} \quad 3161.38$

$\begin{array}{llll}19.68 & 22.97 & 1 \frac{3}{3}-2 \frac{7}{3} & (67)\end{array}$

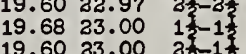

$19.6033 .07 \quad 3 \frac{1}{3}-2 \frac{1}{2} \quad 4 \mathrm{p}^{2} \mathrm{D}^{\circ}-4 \mathrm{~d}^{4} \mathrm{P}$

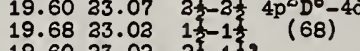

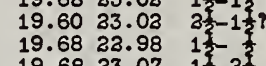

$\begin{array}{lll}19.68 & 33.07 & 12 \\ 2 & -2\end{array}$

$19.6023 .052 \frac{1}{2}-2 \frac{1}{2} \quad 4 \mathrm{p}^{3} \mathrm{D}^{\circ}-4 \mathrm{~d}^{2} \mathrm{D}$

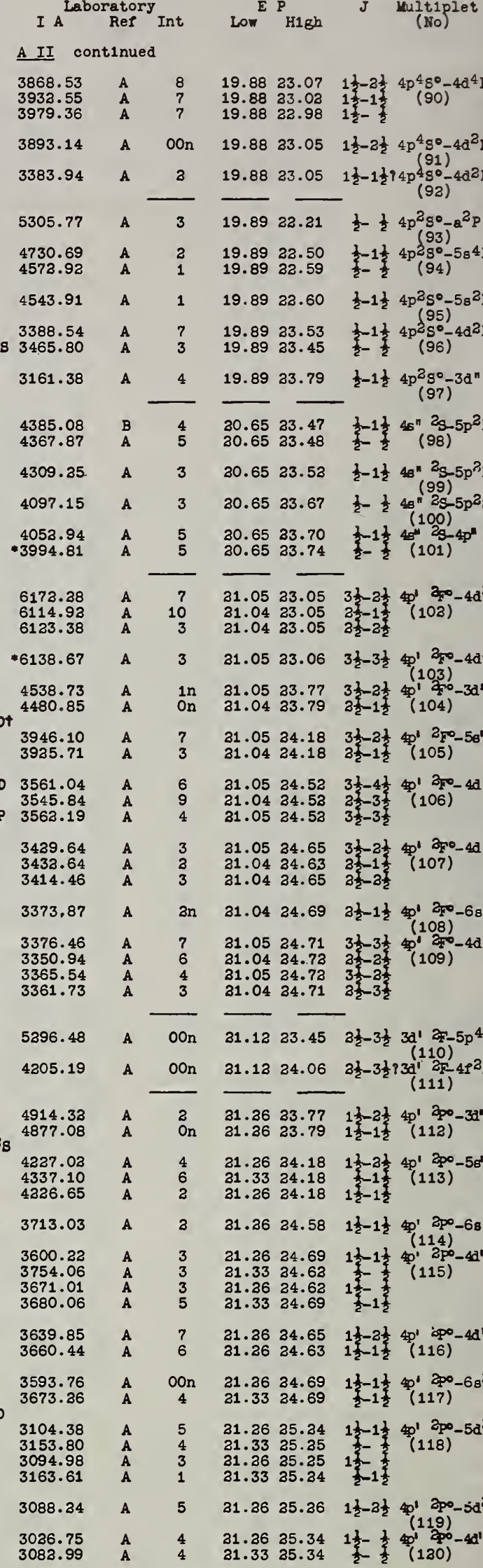

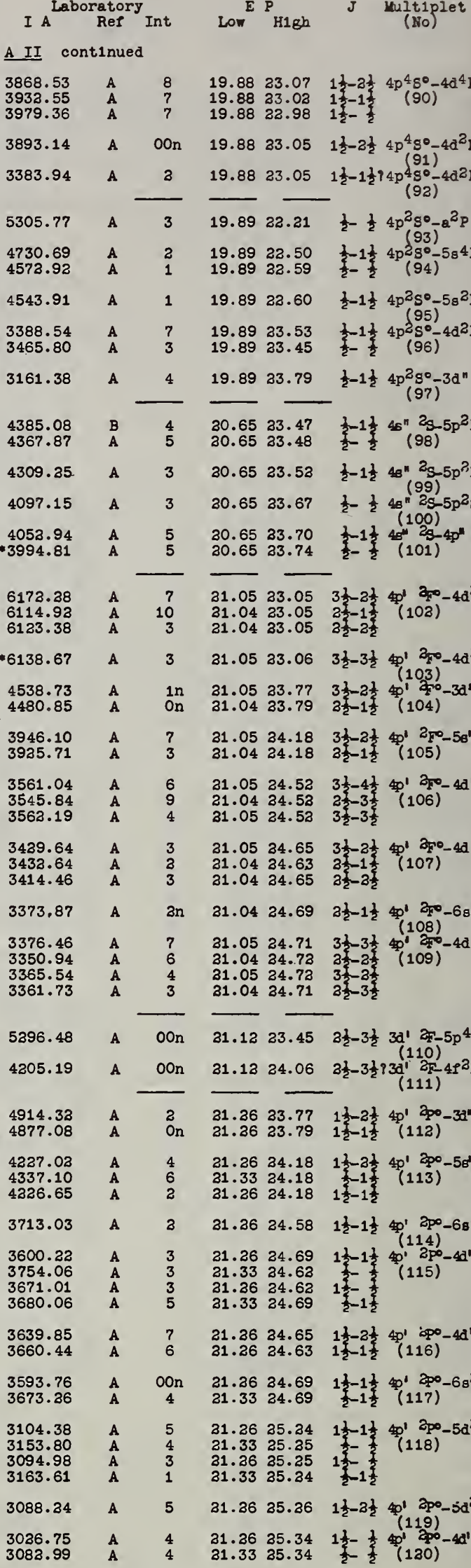

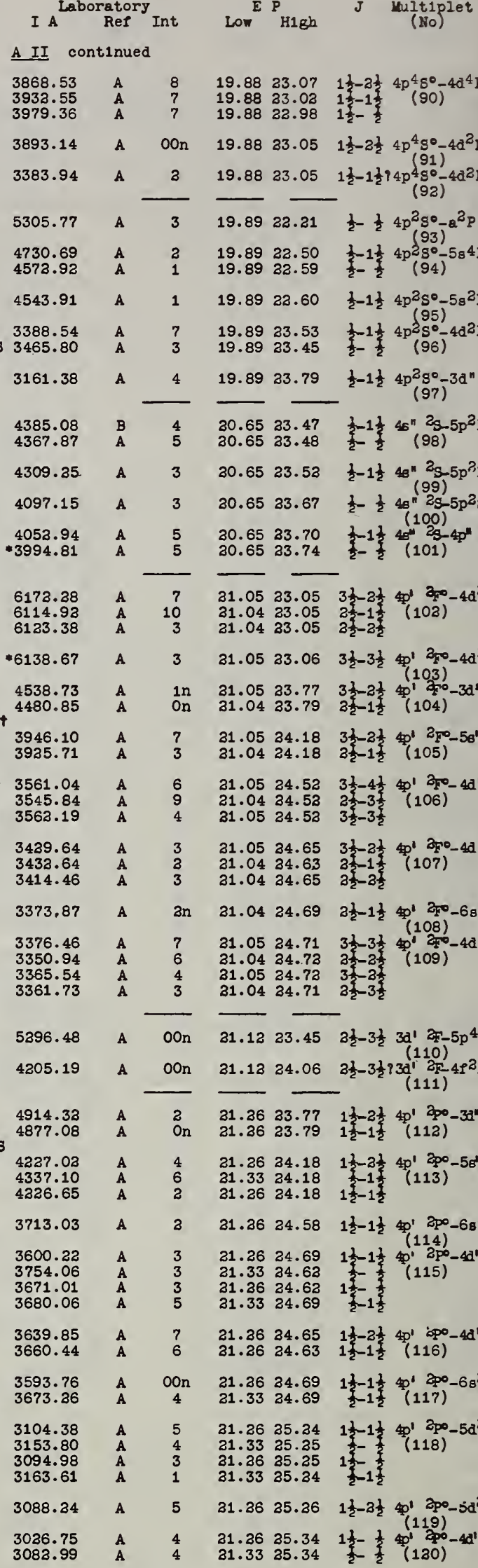

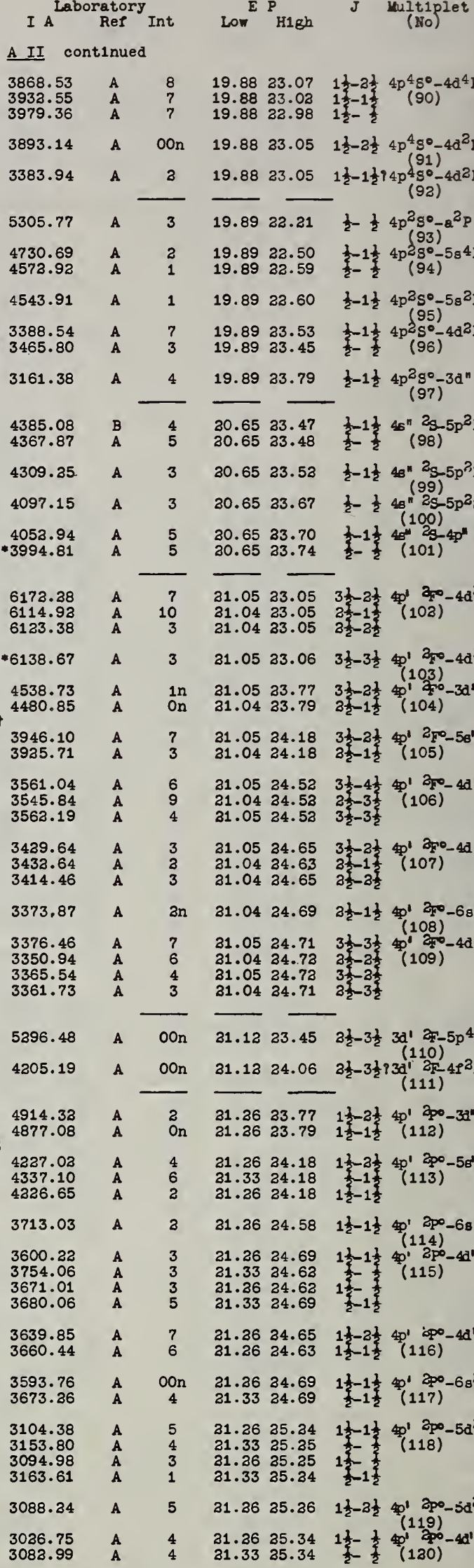

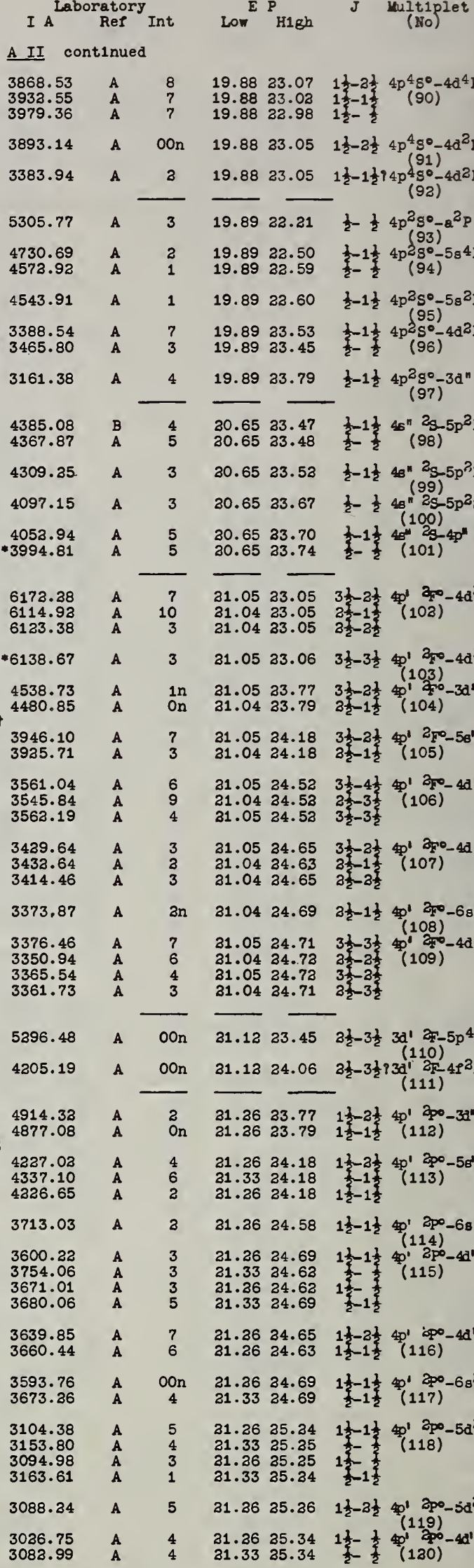

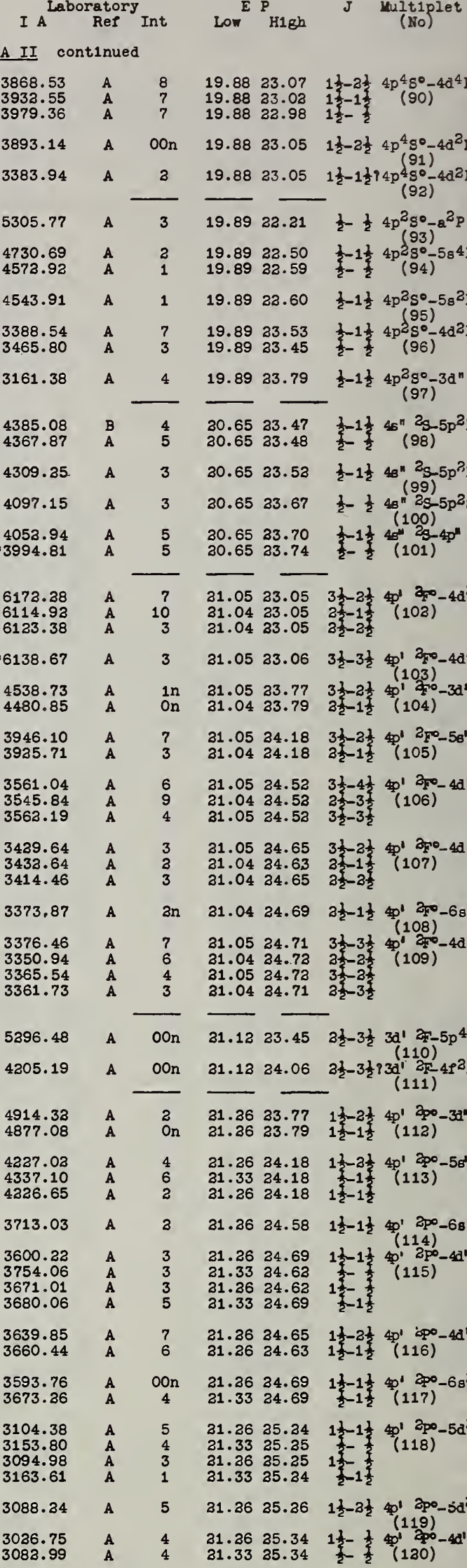

$19.60 \quad 33.06 \quad 3 \frac{1}{2}-3 \frac{1}{2} \quad 4 \mathrm{p}^{2} \mathrm{D}^{\circ}-4 \mathrm{~d}^{2} \mathrm{r}$

$\begin{array}{lll}19.68 & 33.16 & 1 \\ 19.60 & 33.16 & 3\end{array}$

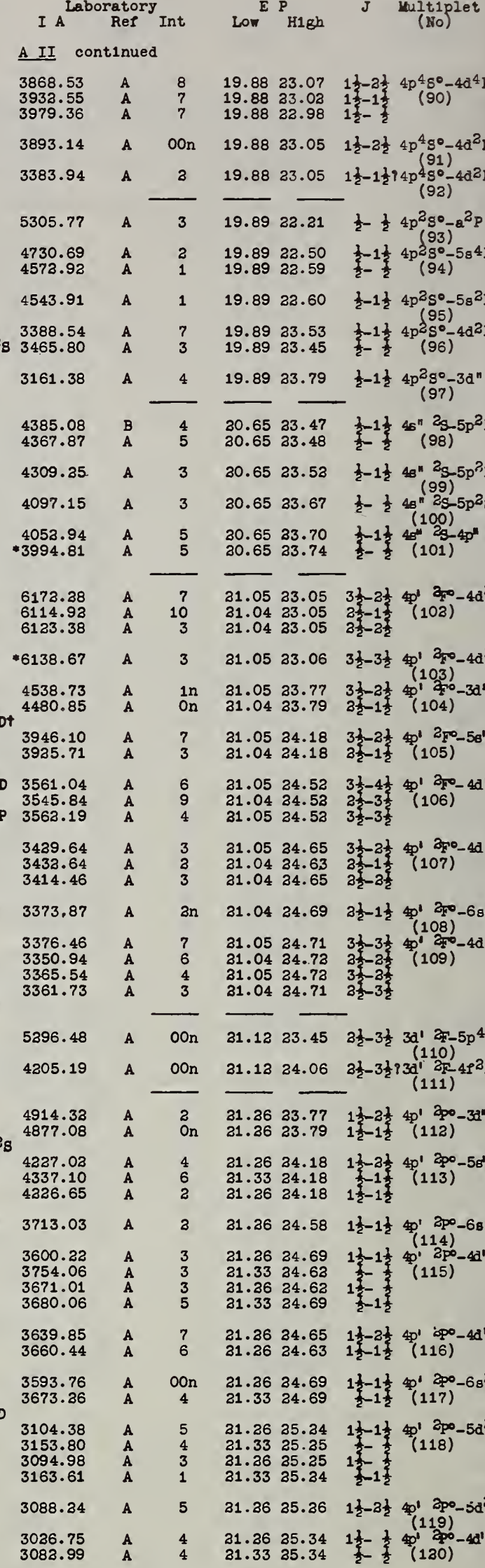

$19.6023 .53 \quad 2 \frac{1}{2}-1 \frac{1}{2} \quad 4 p^{2} D^{\circ}-4 d^{2} p \quad \quad 6138.67$

$\begin{array}{lll}19.68 & 33.45 & 1 \frac{1}{2} \\ 19.68 & 33.53 & 1 \frac{1}{2}-1 \frac{1}{2}\end{array}$

4538.73
4480.85

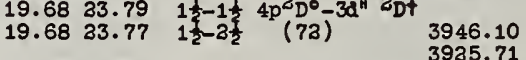

$19.72 \quad 21.34 \quad \frac{1}{2}-1 \frac{1}{2} 4 \mathrm{p}^{2} \mathrm{P}^{\circ}-3 \mathrm{~d}^{1}{ }^{2} \mathrm{D} \quad 3561.04$

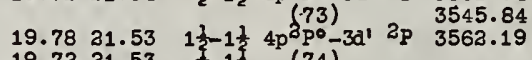

$19.7221 .53 \quad \frac{1}{2}-1 \frac{1}{2}(74)$

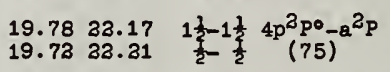

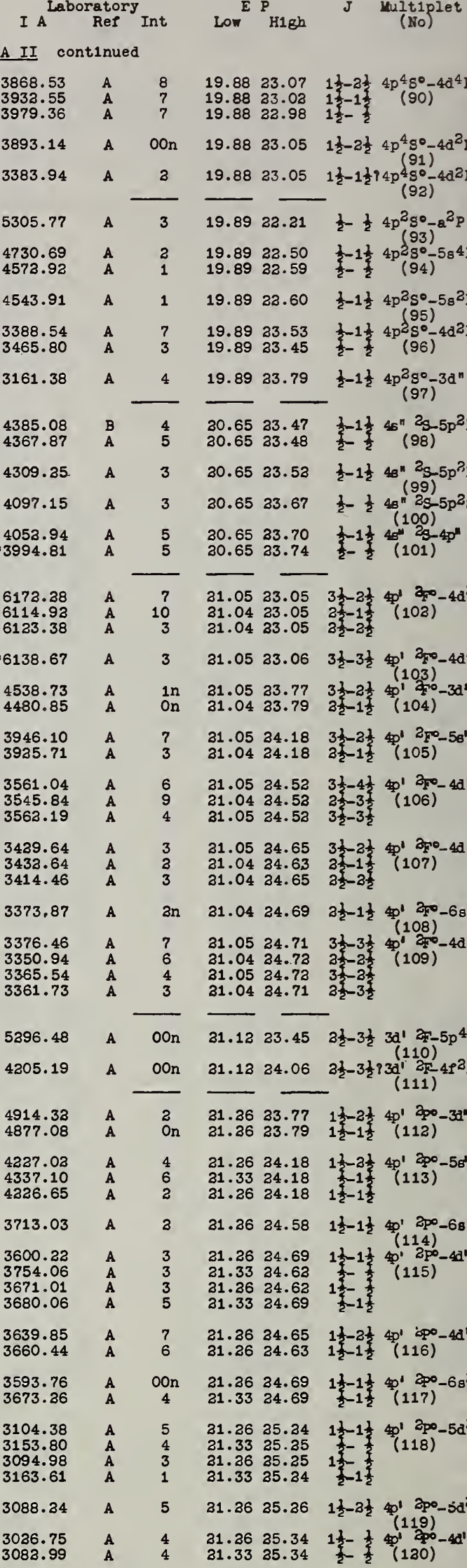

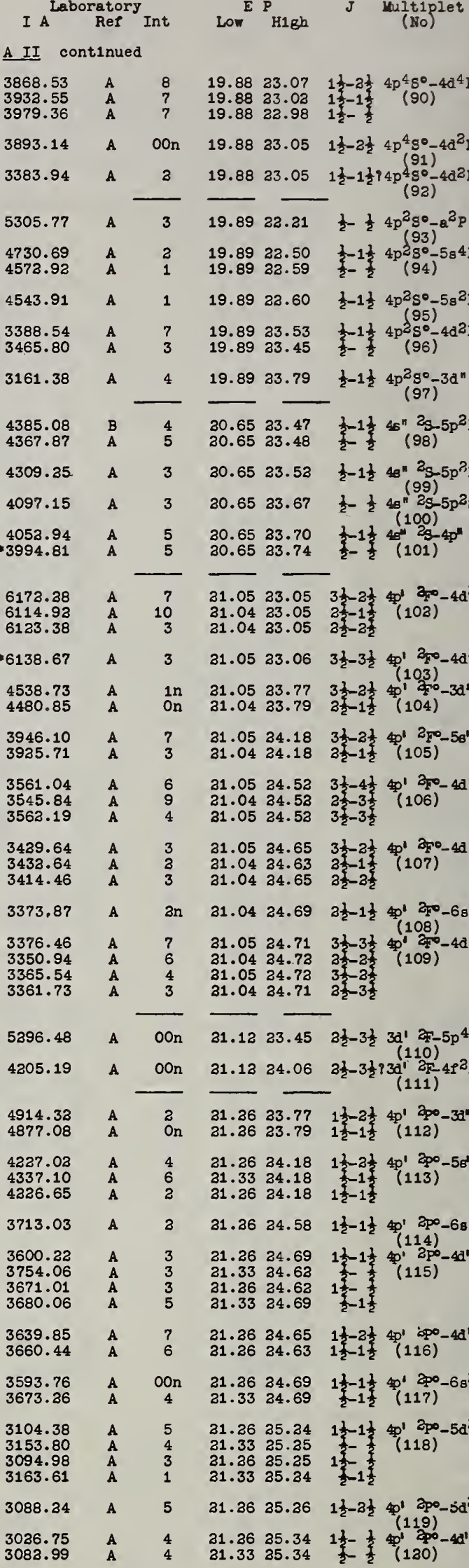

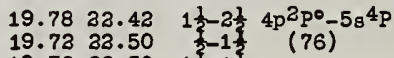

$19.7833 .50 \quad 1 \frac{1}{2}-1$

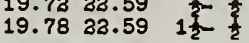

19.78 22.60 $1 \frac{1}{2}-1 \frac{1}{2} 4 \mathrm{p}^{2 \mathrm{p} 0}-5 \mathrm{~s}^{2} \mathrm{P}$

$\begin{array}{lll}19.72 & 22.70 \\ 19.78 & 22.70 & 1 \\ 19.73 & 32.60 & \frac{2}{2}-1\end{array}$

$\begin{array}{llll}19.78 & 22.69 & 1 \frac{1}{2}-2 \frac{1}{2} & 4 p^{3} P^{0}-4 d^{4} D \\ 19.78 & 22.71 & 1 \frac{2}{2}-1 \frac{1}{2} & (78)\end{array}$

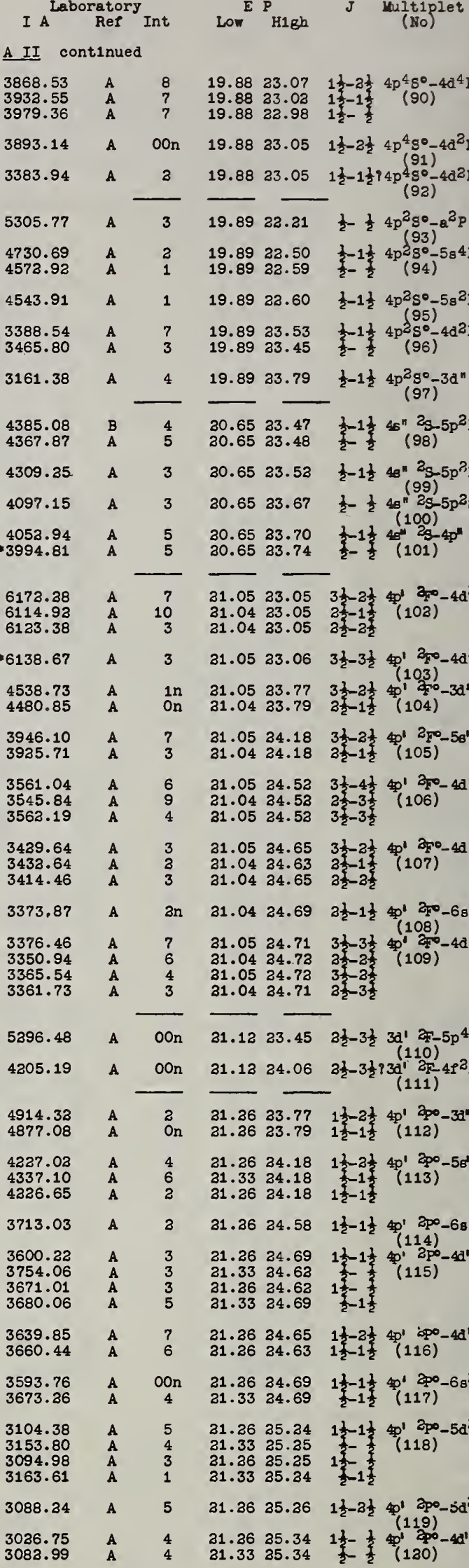

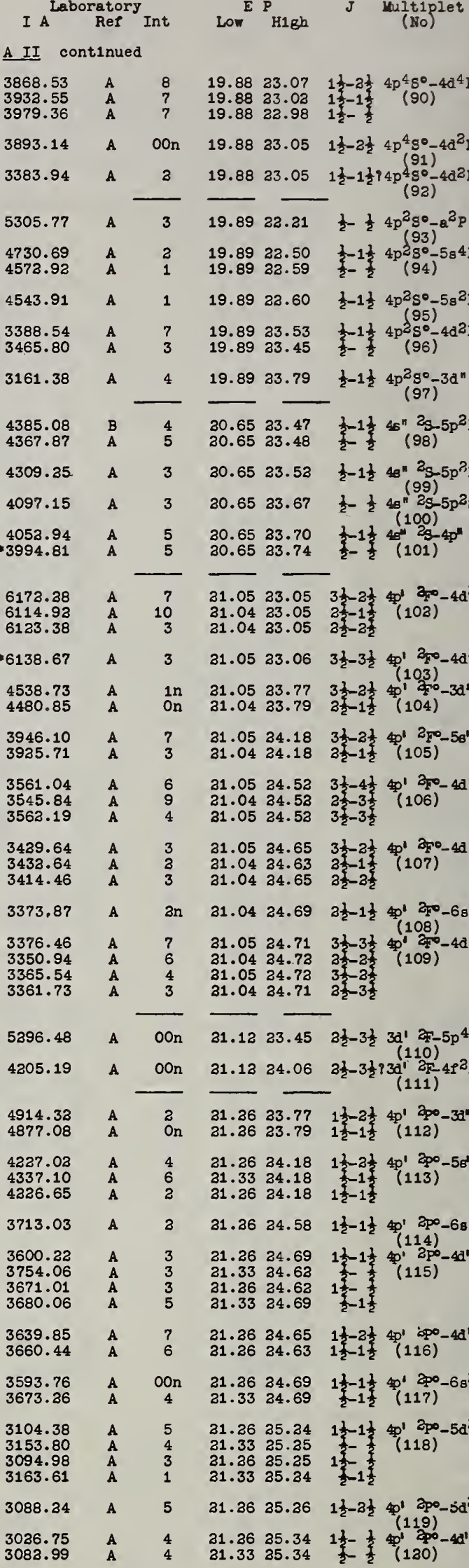

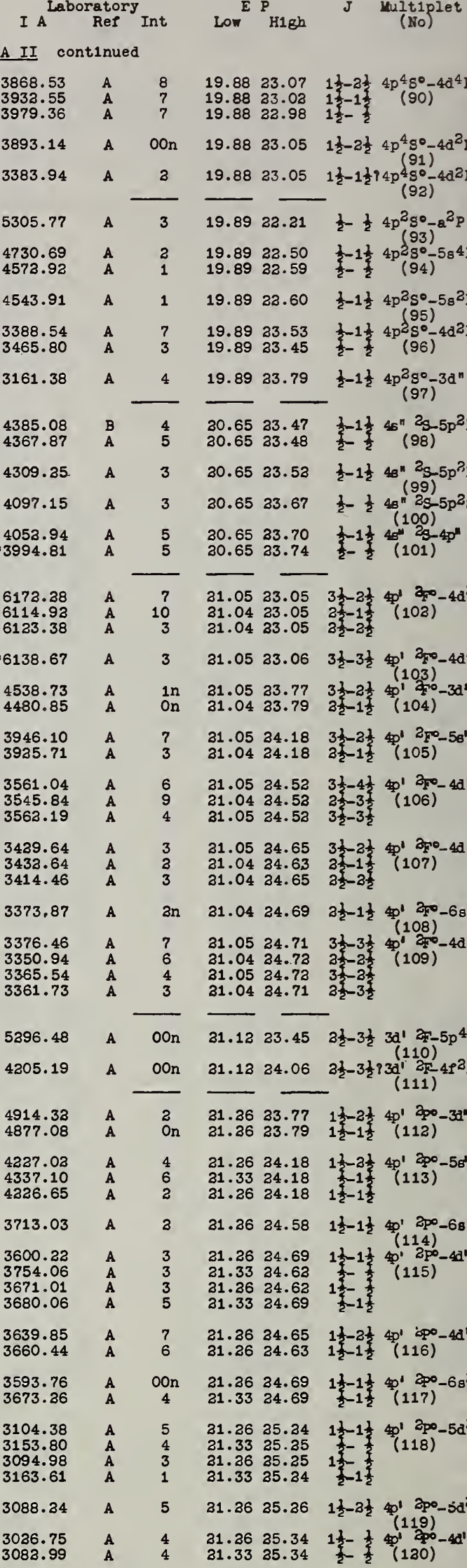

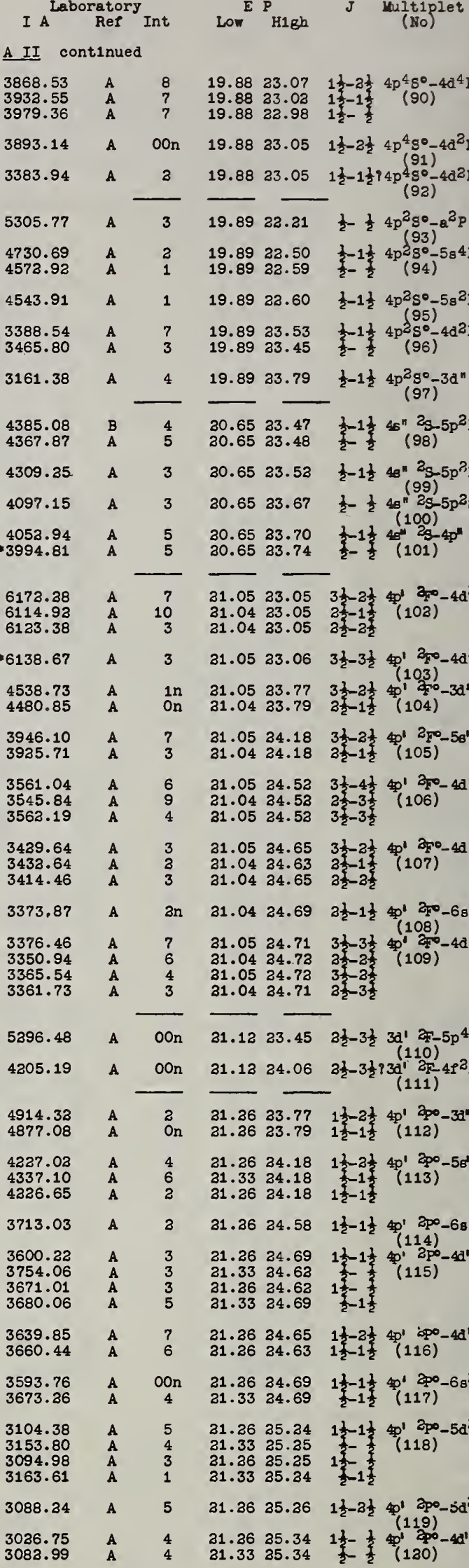

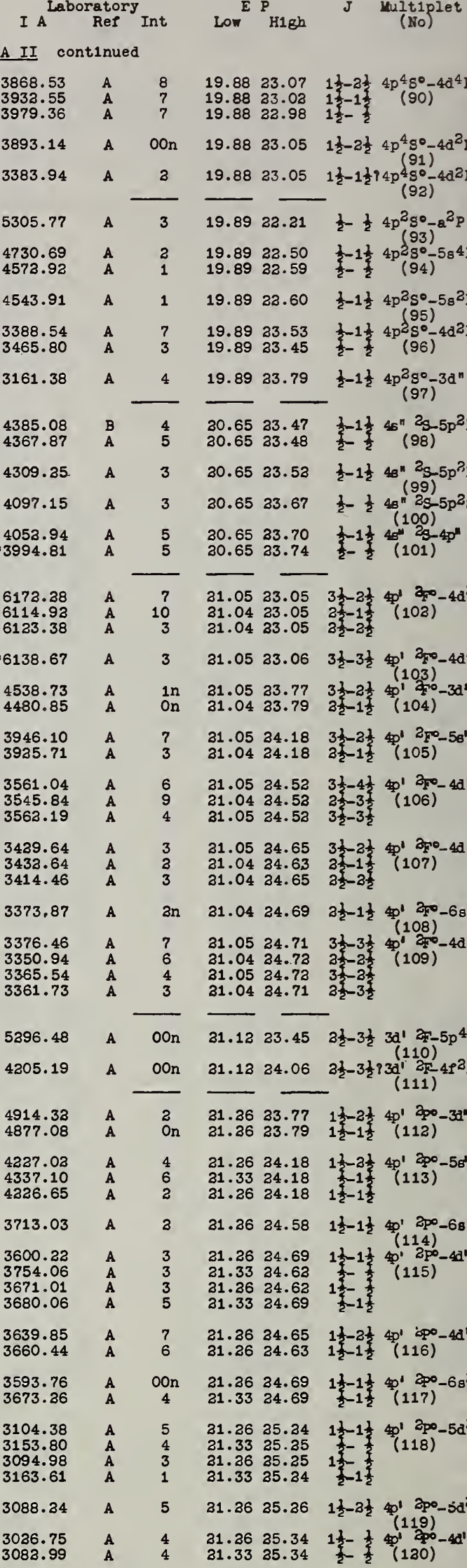

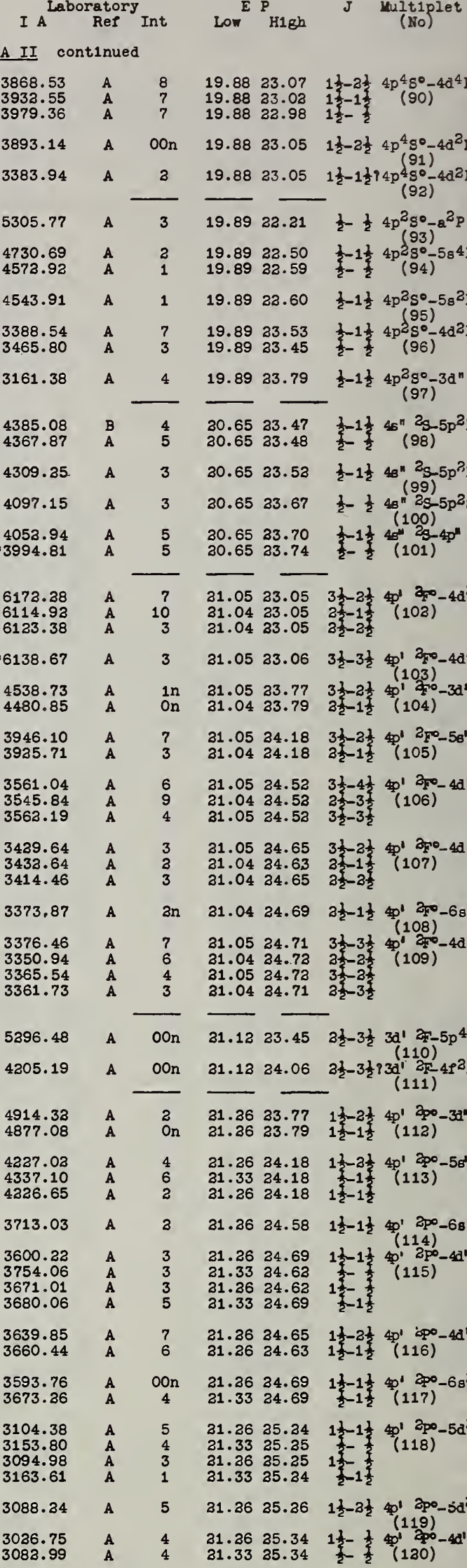

$\begin{array}{lll}19.78 & 22.74 & 1 \frac{1}{2}-\frac{1}{2} \\ 19.72 & 22.73 & \frac{1}{2}-\frac{1}{2} 4 \mathrm{p}^{2} \mathrm{p}^{\circ}-3 \mathrm{~d}^{\prime} 2 \mathrm{~g}\end{array}$

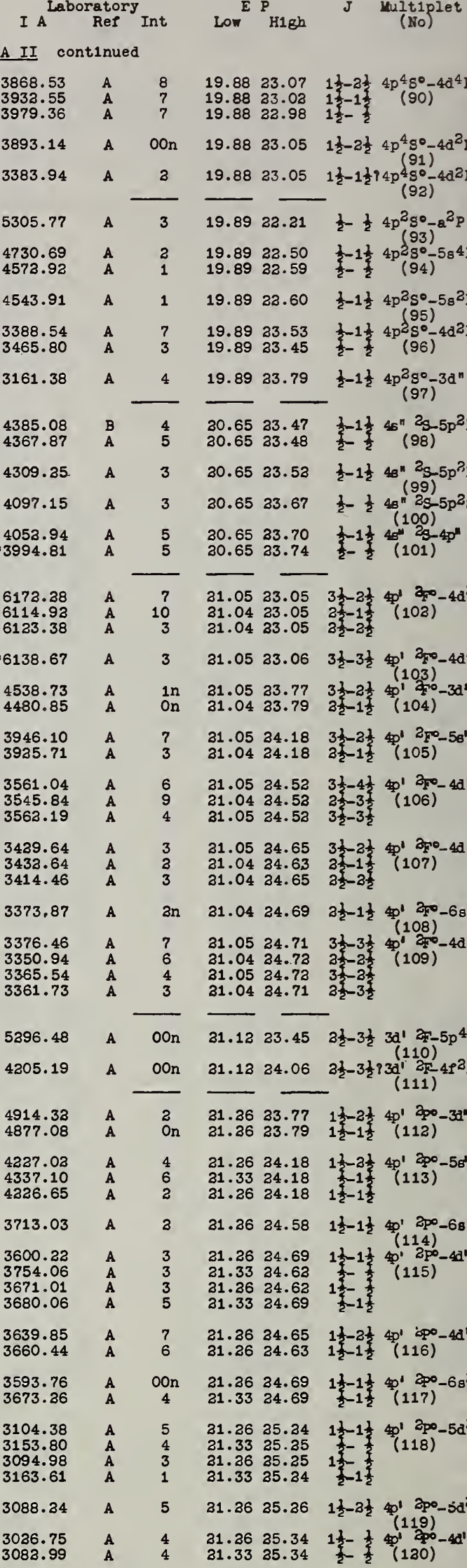

$19.72 \quad 22.73 \quad \frac{1}{2}-\frac{1}{2} 4 \mathrm{p}^{2} \mathrm{p}^{\circ}-3 \mathrm{~d}^{1} \mathrm{2}_{\mathrm{S}}$

$\begin{array}{lll}19.78 & 22.97 & 1 \frac{1}{2}-3 \frac{1}{2}\end{array} 4 \mathrm{p}^{2} \mathrm{PO}^{\circ}-4 \mathrm{~d}^{4} \mathrm{~F} \quad 4337.10$

$19.78 \quad 23.07 \quad 1 \frac{1}{2}-2 \frac{1}{2} \quad 4 \mathrm{p}^{2} \mathrm{p}^{\circ}-4 \mathrm{~d}^{4} \mathrm{P} \quad 3713.03$

$19.7823 .02 \quad 1 \frac{1}{2}(81)^{-4 d^{4}}$

$\begin{array}{lll}19.72 & 22.98 & \frac{1}{2}-\frac{1}{2} \\ 19.78 & 22.98 & 1 \frac{1}{2}-\frac{1}{2}\end{array}$

$19.78 \quad 23.16 \quad 1 \frac{1}{2}-3 \frac{1}{2} ? 4 \mathrm{p}^{2} \mathrm{p}^{0}-4 \mathrm{~d}^{2} \mathrm{~F}$

19.78 a3.53 (83)

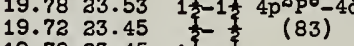

$\begin{array}{lll}19.78 & 23.45 & 1 \frac{1}{2}-\frac{1}{2} \\ 19.72 & 23.53 & \frac{1}{2}-1 \frac{1}{2}\end{array}$

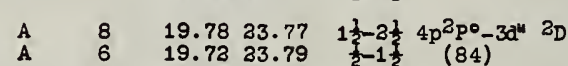

$19.88 \quad 32.42 \quad 1 \frac{1}{2}-2 \frac{1}{2} \quad 4 p^{4} S^{\circ}-5 s^{4} p$

$\begin{array}{llll}19.88 & 22.50 & 1 \frac{1}{2}-1 \frac{1}{2} & (85)\end{array}$

$19.8822 .60 \quad 1 \frac{1}{2}-1 \frac{1}{2} 4 p^{4} g^{0}-5 s^{2} p$

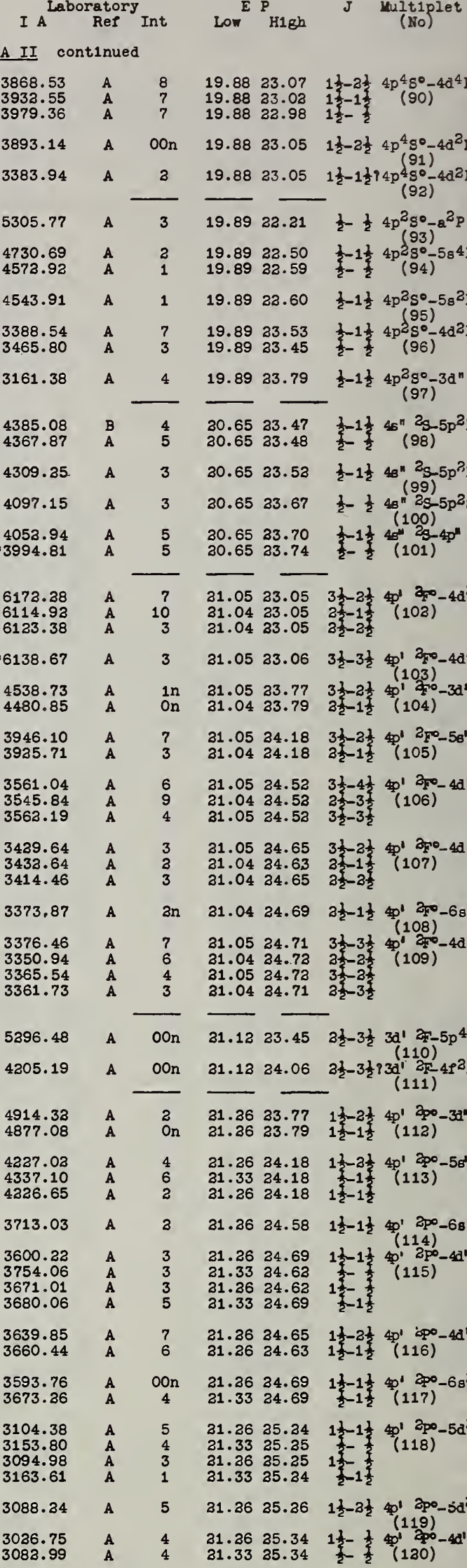

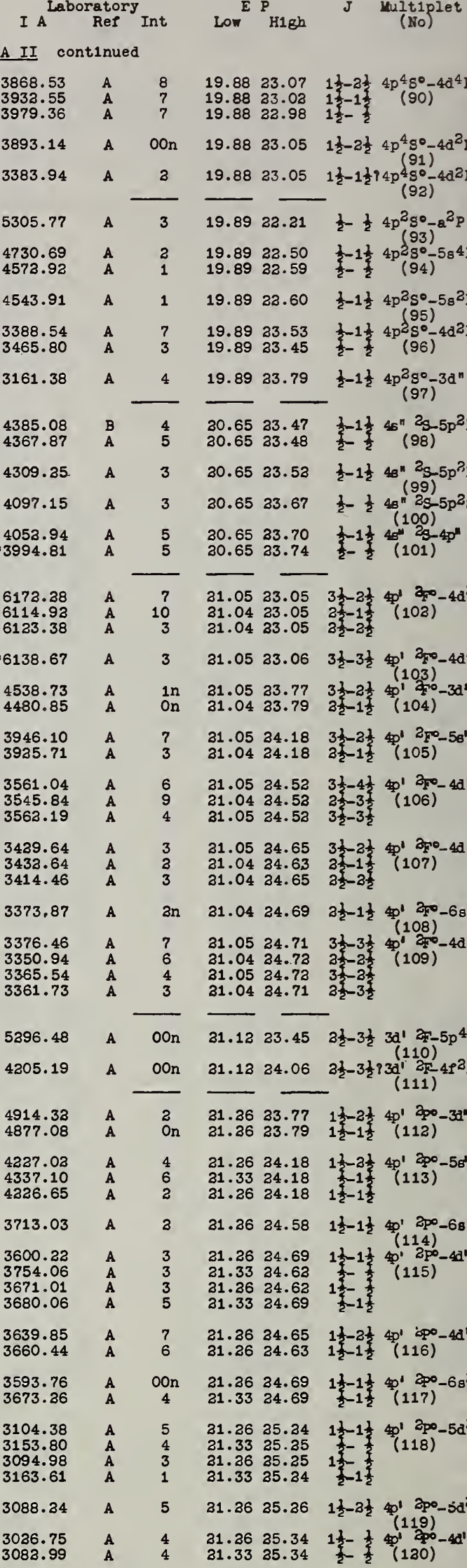

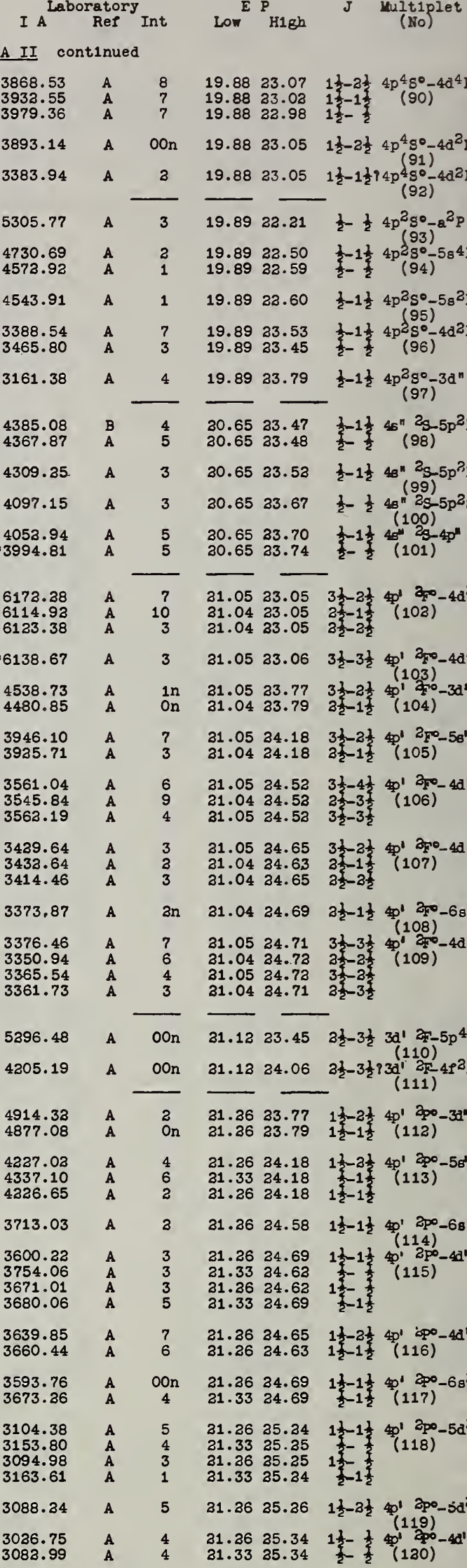

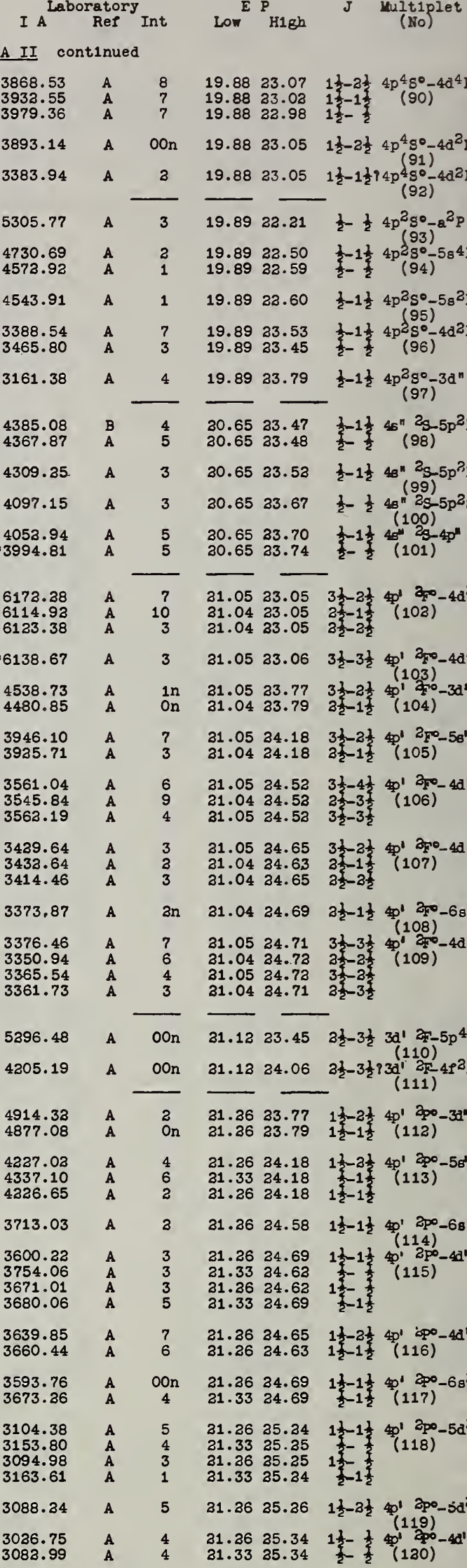

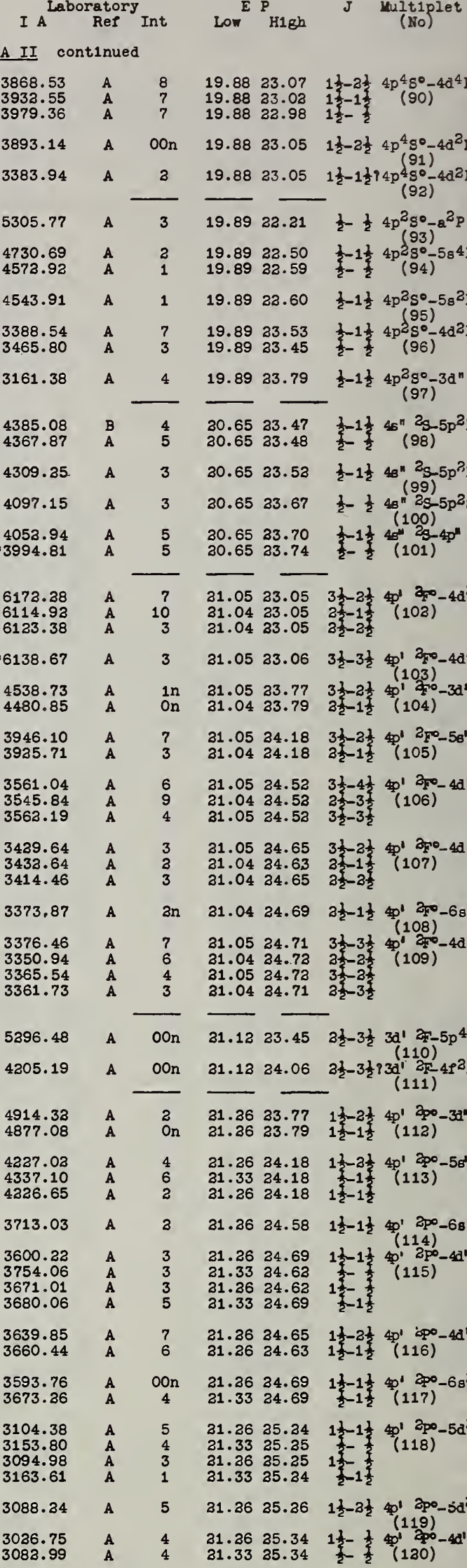

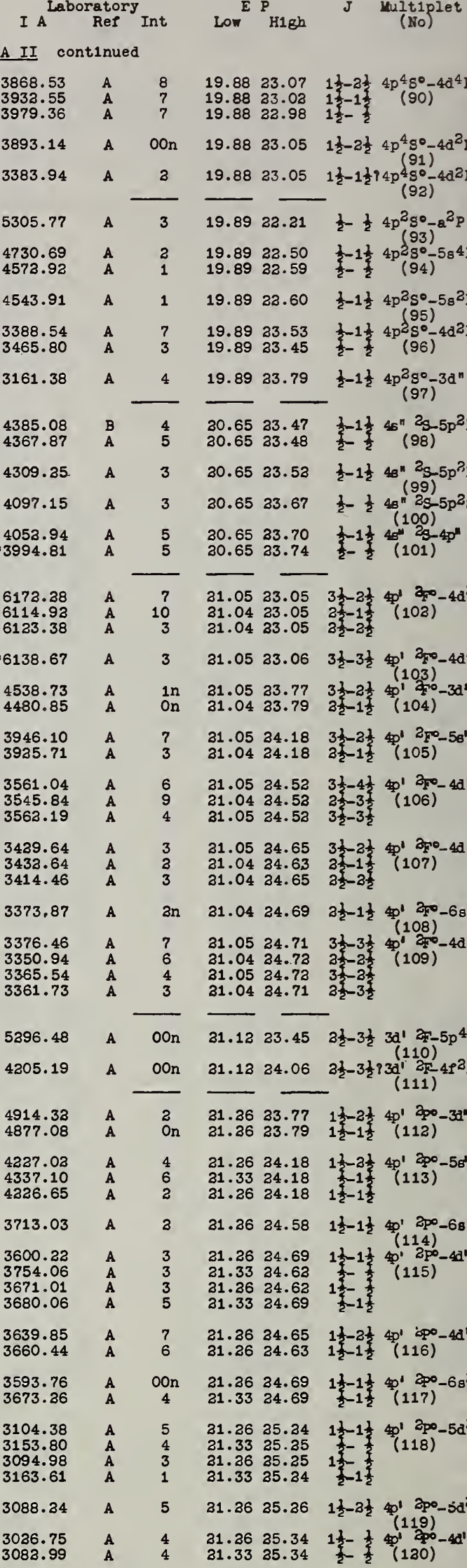

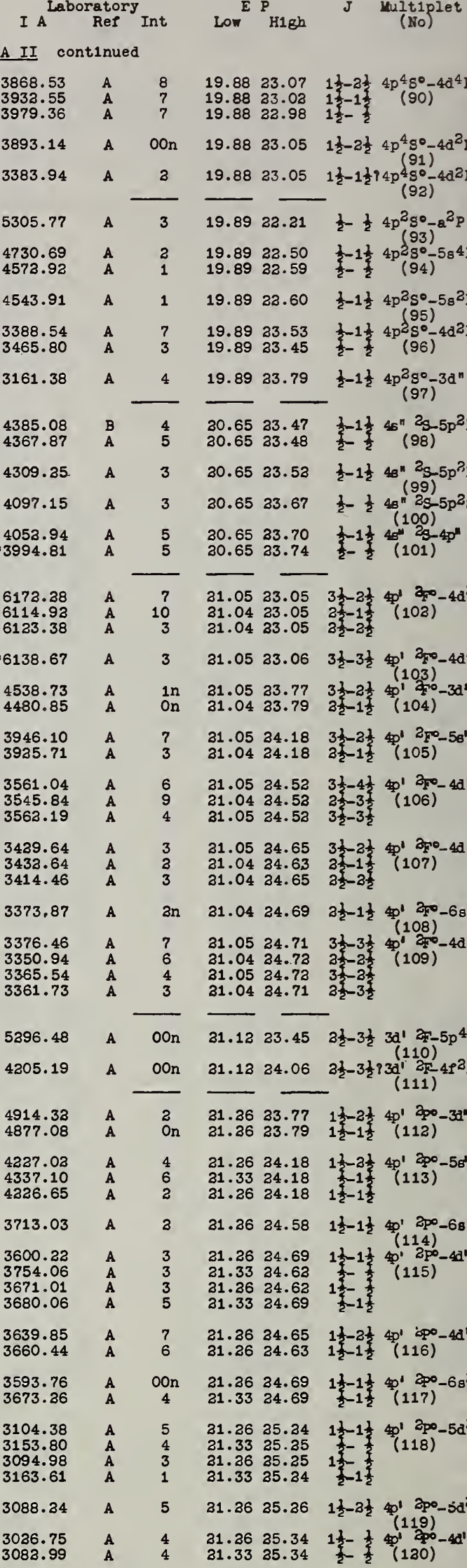

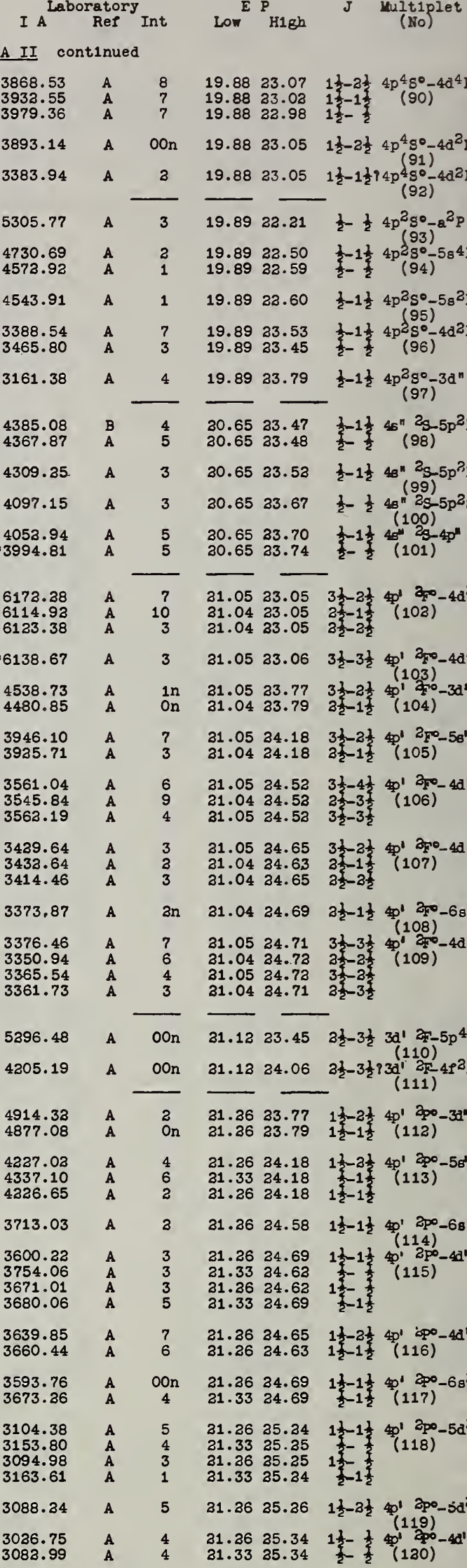

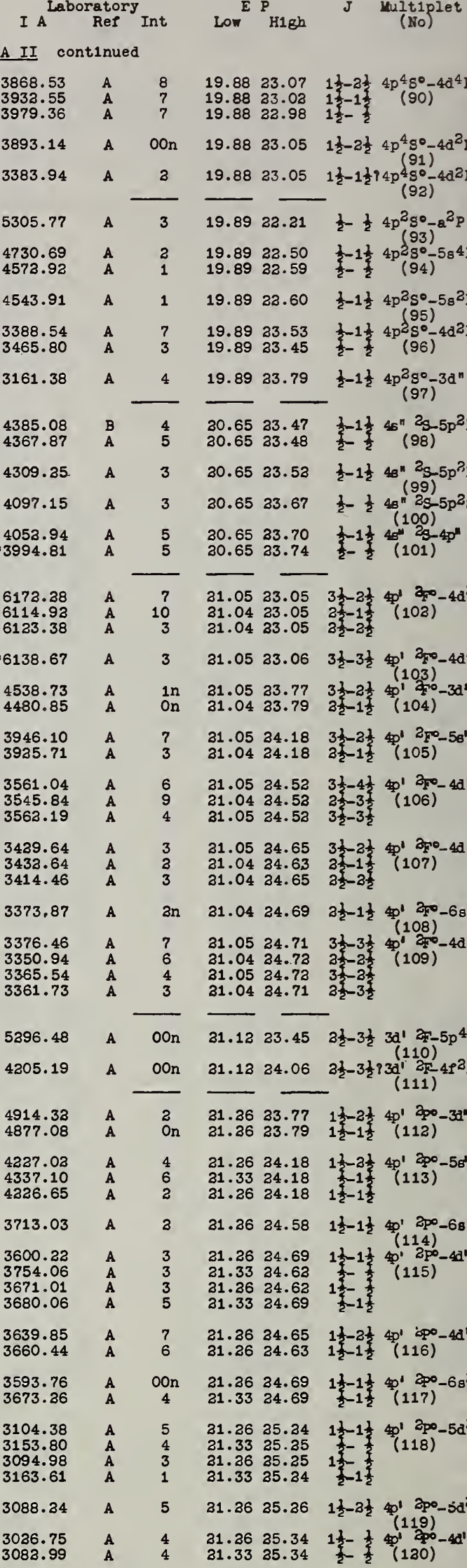

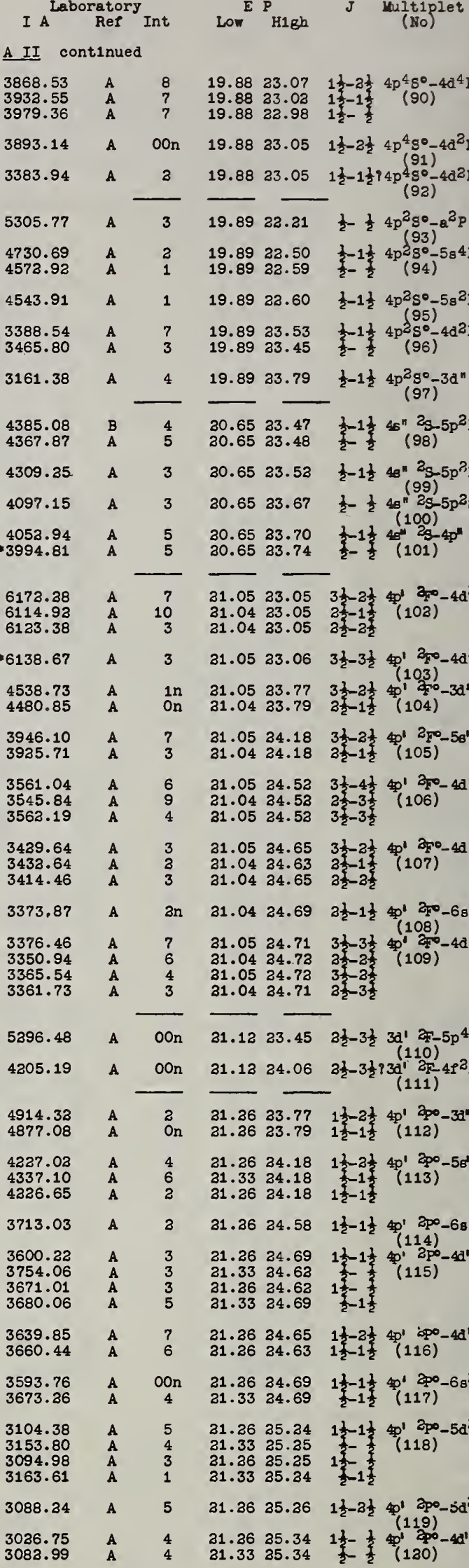

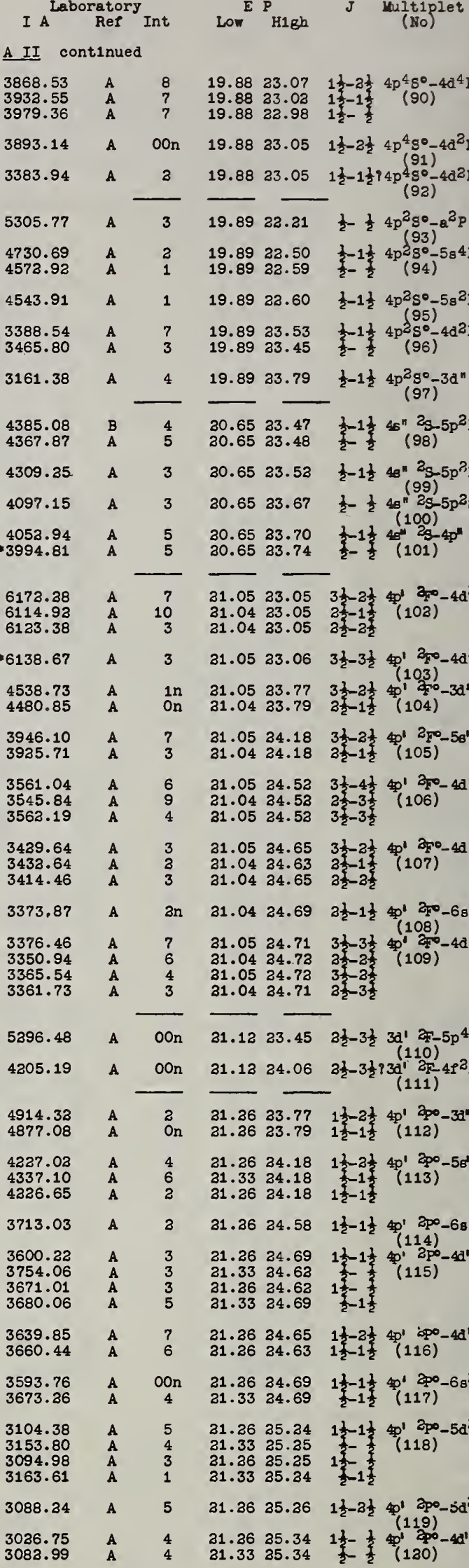

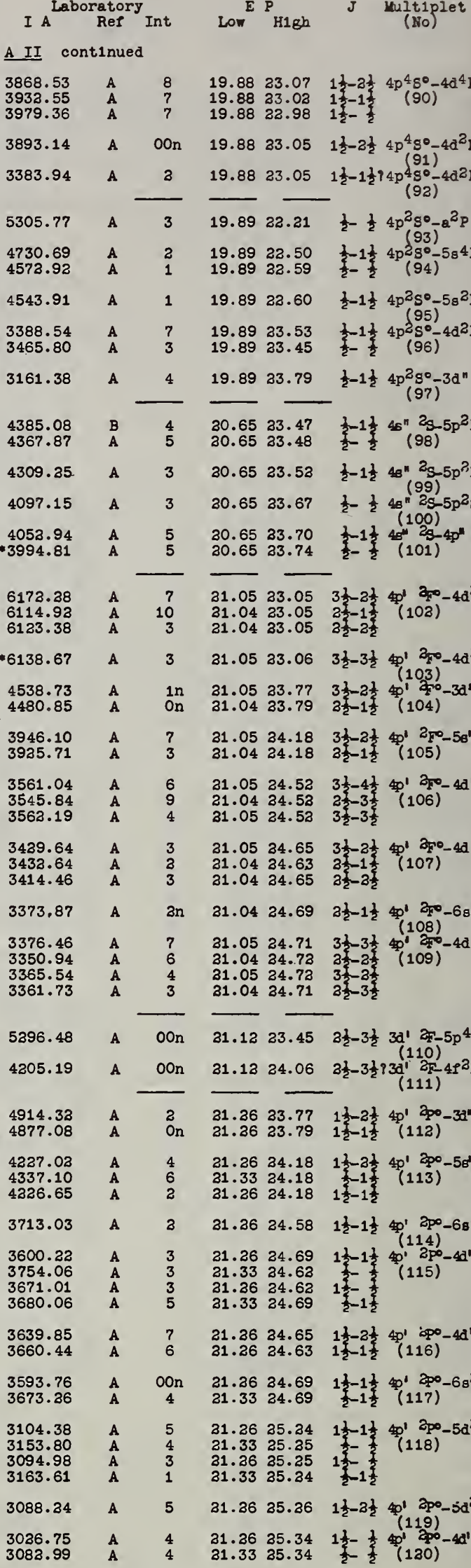

${ }_{A}^{A} \quad$ On

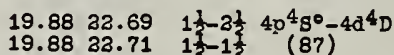

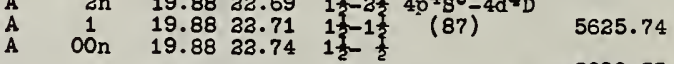

19.88 22.73 $1 \frac{1}{2}-\frac{1}{2} 4 p^{4} \mathrm{~g}^{0}-3 d^{\prime}$ as $\begin{array}{r}5090.55 \\ 5125.84\end{array}$

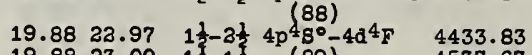

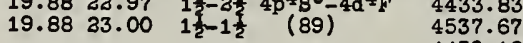
21.27 $33.47 \quad 2 \frac{1}{2}-1 \frac{1}{2} 3 \mathrm{~d}^{\prime} 2 \mathrm{p}-5 \mathrm{p}^{2} \mathrm{po}$

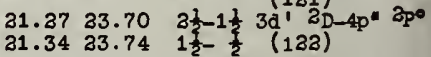
$21.3724 .06 \quad 3 \frac{1}{3}-3 \frac{1}{3} 3 d^{\prime} 2 D-4 I^{2} g^{\circ}$ $\begin{array}{llll}21.34 & 34.06 & 1 \frac{1}{5}-2 \frac{1}{2} & (123) \\ 21.37 & 34.06 & 2 \frac{1}{2}-3 \frac{1}{2}\end{array}$ 


\section{REVISED YULTIPLET TABLE}

23

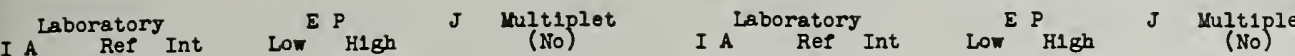
A II continued 4301.98 41169.39

5813.8

\section{8} 5162.8 5304.

4448.88 4439.4 4448.4
-440.09

- 3830.43 - 3753.53

3803.19 3819.0 3796.60 3746.46 3737.89 3718.31
3734.5

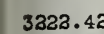
3307.61
3317.70 3205.03

\section{7} 4888.3 4498.5 6985.74 Strongest Unclassified Lines Attributed to A II 7689.3
7380.4
7333.5
6441.9 6441.95
6334.45 5454.41 5407.44 5397.60
4883.25 4703.36
4683.29 tit. 4563.78
4336.51 4297.99 4229.8
4317.4 4303.43
4189.67 4168.98 4138.65
4080.67 3774.54 3637.05

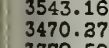
3379.58
3306.50

sas..9. sis.t.

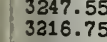

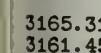
法: 3136.55
3109.75 (31020.63

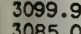
3066.92

\section{A III I P 40.8 Anal C L1st $D$ Nov 1944}

$31.27 \quad 24.27 \quad 3 \frac{1}{2}-3 \frac{1}{3} 3 d^{\prime} \quad 3 \mathrm{p}-49^{2} \mathrm{D}$

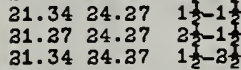

21.41 23.53 3t-1t $200-40^{30}$

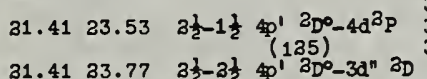
$\begin{array}{llll}31.40 & 33.79 & 1 \frac{1}{3}-1 \frac{1}{2} & (136) \\ 31.40 & 33.77 & 1 \frac{3}{2}-3 \frac{2}{2} & \end{array}$

31.4134 .18 3t $-3 \frac{1}{4} 40^{\prime} 200-58^{\prime}$ 2D 31.4024 .18 13 $1 \frac{1}{2}$ (137) $\begin{array}{lll}31.41 & 34.18 & 3 \frac{1}{2}-1 \frac{1}{2} \\ 31.40 & 34.18 & 1 \frac{2}{2}-3 \frac{3}{2}\end{array}$ $\begin{array}{lllr}31.40 & 34.18 & 1 \frac{1}{2}-3 \frac{1}{2} & 3054.83 \\ 31.40 & 34.63 & 1 \frac{1}{2}-\frac{1}{2} & 4 \mathrm{p}^{\prime} 2 \mathrm{po}^{\circ}-4 \mathrm{~d}^{\prime} \\ 3 \mathrm{p} & 3078.15 \\ 3064.77\end{array}$

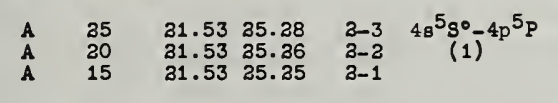
3301.88

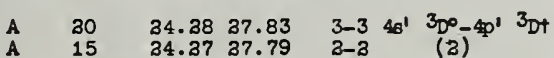
3503.58
3499.67

3336.13 3344.73
3358.49 A 12

\begin{tabular}{ll} 
A & 35 \\
A & 30 \\
A & 15 \\
\hline
\end{tabular} 34.3727 .80

$34.38 \quad 37.98$ \begin{tabular}{lll}
34.38 & 37.98 & $3-4$ \\
34.37 & 37.96 & $2-3$ \\
34.37 & 37.94 & $1-$ \\
\hline
\end{tabular}

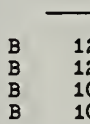

58.58

$35.6 a^{2}$

35.6439 .65

\begin{tabular}{ll}
35.64 & 39.65 \\
35.63 & 39.65 \\
\hline
\end{tabular}

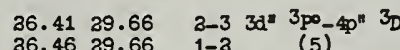

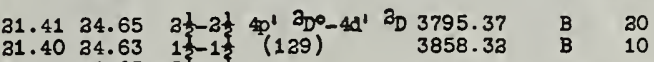

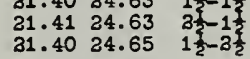

$31.4034 .69 \quad 1 \frac{1}{2}-1 \frac{1}{2} 94 p^{1} 3 D^{\circ}-6 \mathrm{~s}^{3} \mathrm{P}$

3391.85

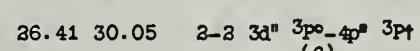
$31.4133 .77 \quad 3 \frac{1}{3}-2 \frac{1}{3} 4 \mathrm{p}^{\prime} 3 \mathrm{DO}^{\circ}-3 \mathrm{~A}^{\mathrm{N}} \mathrm{3 \textrm {D }}$

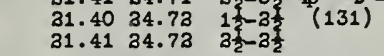

A IV I P 61 Anal B L1st A Nov 1944

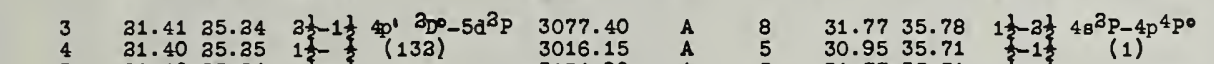

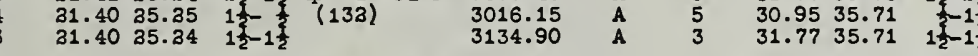
$31.4135 .26 \quad 3 \frac{1}{2}-2 \frac{1}{2} \frac{p^{1}}{200}(133)-5 \mathrm{~d}^{3} \mathrm{D} \quad 3037.98$ $31.7735 .831 \frac{1}{2}-1 \frac{1}{2} 4 \mathrm{~s}^{3} \mathrm{P}-4 \mathrm{p}^{2} \mathrm{D}^{0}+$

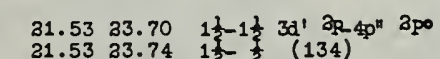
A $0 \quad 31.5334 .06 \quad 1 \frac{1}{2}-2 \frac{1}{2} 3 \mathrm{~d}^{1} 3 \mathrm{P}-4 \mathrm{r}^{2} \mathrm{~F}^{\circ} \quad \underline{\mathrm{X} I}$ I P 4.33 Anal A L1st C Nov 1944

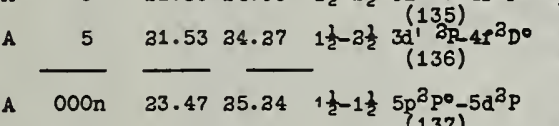
$7664.907 / /$ $\begin{array}{lll}10 R & 0.00 & 1.61\end{array}$ $\frac{1}{3}-1 \frac{1}{2} 4^{2} \mathrm{~S}-4^{3} \mathrm{P}^{\circ}$ 4643.37 H $\quad$ (3)Forb $0.00 \quad 3.66 \quad \frac{1}{3}-3 \frac{1}{2} 4^{2} 8-3^{3} \mathrm{D}$

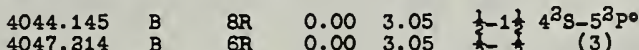

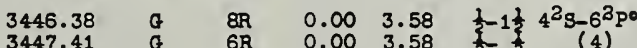

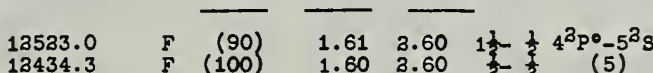
$\begin{array}{lllllll}11773.66 & C & 15 \mathrm{r} & 1.61 & 3.66 & 1 \frac{1}{3}-2 \frac{1}{3} & 4^{3} \mathrm{P}^{0}-3^{2} \mathrm{D}\end{array}$ $\begin{array}{ccccccc}11689.76 & C & 10 & 1.60 & 3.66 & \frac{1}{2}-1 \frac{1}{2} & (6) \\ 11769.41 & D & 3 & 1.61 & 3.66 & 1 \frac{1}{3}-1 & \end{array}$ $6964.69 \quad \mathrm{E} \quad(1) \quad \begin{array}{llll}1.61 & 3.38 & 1 \frac{1}{3}-21 & 4^{3} \mathrm{P}^{\circ}-4^{2} \mathrm{D}\end{array}$ 6964.18 \begin{tabular}{lll}
1.60 & 3.38 & $1 \frac{2}{2}-1 \frac{1}{3}$ \\
1.61 & 3.38 & $1 \frac{1}{2}-1 \frac{3}{2}$ \\
\hline
\end{tabular}

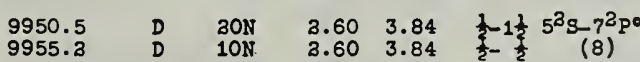

$11033.3 \quad D \overline{10 N T} \overline{3.66} \overline{3.78}-3^{3} \mathrm{D}-5^{3} F^{\circ}$

K II I P 31.7 Anal A L1st D June 1944

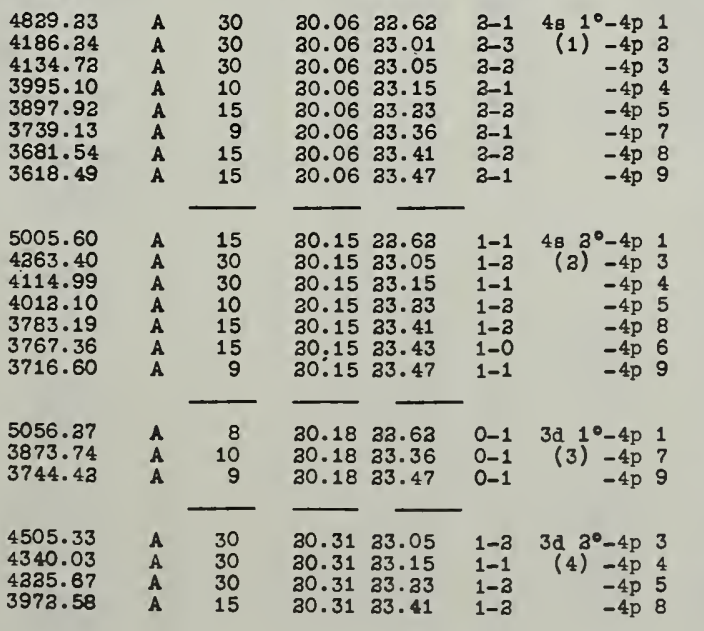

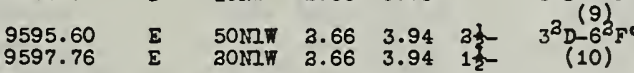

I A Laboratory $_{\text {Fef }}$ Int Low ${ }^{\text {E P1gh }}$

MuItiplet
(No)

K II continued

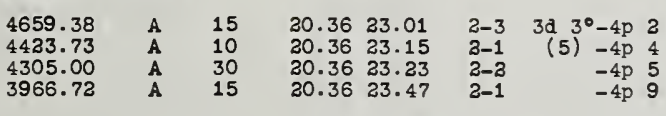

5536.0

4466.65

$3-44 s^{\prime} 3 p^{\circ}-4 p^{\prime} 3 F^{4} 4001.34$

A (3)

$30.3922 .62 \quad 0-1 \quad 48 \quad 3^{\circ}-4 p 1$

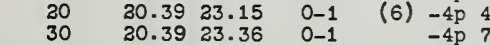
$\begin{array}{lllll}15 & 20.39 & 23.47 & 0-1 & -4 \mathrm{p} 9\end{array}$

(2) $20.5522 .62 \quad 1-1 \quad 4 \mathrm{~g} \quad 4^{\circ}-4 \mathrm{p} 1$ 5969.64 4943.24
4608.45 4388.16 4309.10
4308 4233.97
3530.75

3
30
30
30
30
30

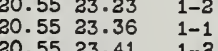

$20.5523 .41 \quad 1-3$

$\begin{array}{lll}30.55 & 33.47 & 1-1 \\ 30.55 & 34.05 & 1-0\end{array}$

$\begin{array}{rl}-4 \mathrm{p} & 5 \\ -4 \mathrm{p} & 7\end{array}$

For changes in Paschen notation see text 37

I III I P 46 Anal D L18t D Nov 1944

$\begin{array}{lllllll}3323.40 & A & 6 & 35.61 & 29.32 & 21-21 & 48^{4} \mathrm{p}-4 \mathrm{p}^{4} \mathrm{po}\end{array}$ $\begin{array}{llllll}3430.83 & \text { A } & 6 & 35.76 & 39.37 & 13-1 \\ 3378.79 & \text { A } & 6 & 25.61 & 29.37 & 21 \\ 3468.32 & \text { A } & 6 & 25.76 & 29.32 & 1 \\ 3 & & \end{array}$ $\begin{array}{llllll}3468.33 & \mathbf{A} & 6 & 25.76 & 29.32 & 1 \frac{2}{2}-2 \frac{2}{2} \\ 3513.88 & \mathbf{A} & 5 & 35.86 & 29.37 & \frac{1}{2}-1 \frac{2}{2}\end{array}$ $\begin{array}{lllllll}2992.34 \quad A & 6 & 35.61 & 29.73 & 21 & -3 \frac{1}{2} & 48^{4} \mathrm{P}-4 \mathrm{p}^{4} \mathrm{D}^{\circ}+\end{array}$ \begin{tabular}{llllll}
3052.07 & $A$ & 6 & 25.76 & 39.81 & $1 \frac{1}{3}-2 \frac{1}{2}$ \\
3056.84 & $A$ & 5 & 35.86 & 39.90 & $\frac{1}{2}-1 \frac{3}{2}$ \\
\hline
\end{tabular}

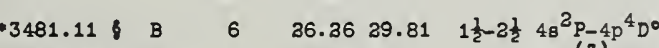

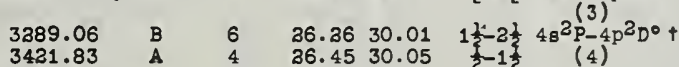
$3301.95 \quad A \quad 6 \quad 36.26 \quad 30.13 \quad 1 \frac{1}{2}-1 \frac{1}{2} \quad 48^{2} \mathrm{P}-4 \mathrm{p}^{2} \mathrm{PO}+$

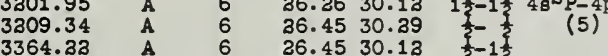

Ca I I P 6.09 Anal A L1st B Mar 1944

$\begin{array}{lllllll}6572.781 & A & 50 & 0.00 & 1.88 & 0-1 & 4^{1} \mathrm{~S}-4^{3} \mathrm{po}^{0}\end{array}$ $4336.738 / /$ A $500 R \quad 0.00 \quad 2.92 \quad 0-1 \quad 4^{1} \mathrm{~s}_{-4}^{(1)} \mathrm{p}$

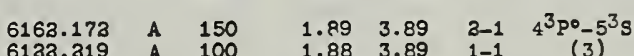
$\begin{array}{rrrrrr}6133.319 & \text { A } & 100 & 1.88 & 3.89 & 1-1 \\ 6103.733 & \text { A } & 80 & 1.87 & 3.89 & 0-1\end{array}$

$\begin{array}{lllllll}4454.781 & \text { A } & 80 & 1.89 & 4.66 & 2-3 & 4^{3} \mathrm{Po}^{2}-4^{3} \mathrm{D} \\ 4434.960 & \text { A } & 60 \mathrm{r} & 1.88 & 4.66 & 1-2 & (4)\end{array}$

$\begin{array}{lllllll}4434.960 & \text { A } & 60 \mathrm{r} & 1.88 & 4.66 & 1-2 \\ 4435.441 & \mathrm{~A} & 50 & 1.87 & 4.66 & 0-1\end{array}$

$\begin{array}{lllllll}4455.887 & A & 40 & 1.89 & 4.66 & 3-\end{array}$

$\begin{array}{llllll}4435.688 & \mathrm{~A} & 40 & 1.88 & 4.66 & 1-1 \\ 4456.613 & \mathrm{~B} & 10 & 1.89 & 4.66 & 3-1\end{array}$

4302.537

4398.986

4318.653
4307.741

4383.010
4389.364

3973.707

3973.707
3957.053
3948.901

3923.50

3933.50

3761.73

3644.410

3634.111

3644.765

3630.974

3630.974
3644.990

3487.598

3474.763
3468.476

3361.918

3350.309

3344.513

3350.361

3362.38

3386.067

3374.661
3369.090

3335.896

3315.145
3309.930

3309.930
3336.129

3326.129
3315.334
$60 x$
30

30
45
45
40

45
40
40

13

(0)

$(0)$
$(0)$

$1.89 \quad 4.76$

$\begin{array}{ll}1.88 & 4.75 \\ 1.89 & 4.75\end{array}$

$\begin{array}{ll}1.89 & 4.75 \\ 1.88 & 4.74\end{array}$

$\begin{array}{ll}1.88 & 4.76 \\ 1.87 & 4.75\end{array}$

$1.89 \quad 5.00$

$\begin{array}{ll}1.89 & 5.00 \\ 1.88 & 5.00\end{array}$

$1.87 \quad 5.00$

$2-2$
$1-1$

$\frac{4^{3} p^{0}-4 p^{2} \quad 3 p}{(5)}$

$2-1$
$1-0$

$1-3$

$5.00 \quad 0-1$

$-1 \quad 4^{3} p^{0}-6^{3} s$

$\begin{array}{ll}1.88 & 5.02\end{array}$

$$
0-1
$$

$(6)$

$5.16 \quad 1-0 \quad 4^{3} \mathrm{po}_{-6}^{1} \mathrm{~s}$

$\begin{array}{llll}1.89 & 5.38 & 2-3 & 4^{3} \mathrm{PO}^{\circ}-5^{3} \mathrm{D}\end{array}$

$\begin{array}{lll}1.88 & 5.28 & 1-2 \\ 1.87 & 5.38 & 0-1\end{array}$

$\begin{array}{lll}1.87 & 5.38 & 0-1 \\ 1.89 & 5.38 & 3-2\end{array}$

$\begin{array}{lll}1.89 & 5.38 & 3-2 \\ 1.88 & 5.38 & 1-1 \\ 1.89 & 5.38 & 3-1\end{array}$

$\begin{array}{ll}1.89 & 5.43 \\ 1.88 & 5.43 \\ 1.87 & 5.43\end{array}$

$\begin{array}{lll}1.87 & 5.43 & 1-1 \\ 1.87 & -1.43\end{array}$

$-14^{3} p^{\circ}-7^{3} s$

$\begin{array}{lll}35 \mathrm{n}^{*} & 1.89 & 5.56 \\ 35 n^{*} & 1.88 & 5.56\end{array}$

$\begin{array}{ccc}35 \mathrm{n}^{\circ} & 1.88 & 5.56 \\ 8 \mathrm{n} & 1.87 & 5.56\end{array}$

$35 n^{*}, \quad 1.89 \quad 5.56$

$\begin{array}{lll}35 n^{*} & 1.88 & 5.56 \\ (0) & 1.89 & 5.56\end{array}$

$4 \quad 1.89 \quad 5.65 \quad 3-1 \quad 4^{3} p^{0}-8^{3} s$

$\begin{array}{llll}3 & 1.88 & 5.65 & 1-1 \\ \text { 1n } & 1.87 & 5.65 & 0-1\end{array}$

$8 n^{*} \quad 1.89 \quad 5.73 \quad 3-3 \quad 4^{3} \mathrm{P}^{0}-7^{3} \mathrm{D}$

$\begin{array}{lllll}8 \mathrm{n}^{*} & 1.89 & 5.72 & 2-3 & 4^{3} \mathrm{p}^{0}-7^{3} \mathrm{D} \\ 5 \mathrm{n}^{*} & 1.88 & 5.73 & 1-2 & (13)^{-2}\end{array}$

$\begin{array}{llll}2 \mathrm{n} & 1.87 & 5.73 & 0-1 \\ 8 \mathrm{n}^{*} & 1.89 & 5.73 & 3-3\end{array}$

$\begin{array}{llll}8 n^{*} & 1.89 & 5.73 & 3-2 \\ 5 n^{\circ} & 1.88 & 5.73 & 1-1\end{array}$
$-3 \quad 4^{3} p^{\circ}-6^{3} D$

$-1$

1 $2-1$
$3-2$
$1-1$
88 - 5.16 1-0 $4^{3} \mathrm{po}^{\mathrm{C}}-4 \mathrm{p}^{2}$ 
24 I A $\begin{gathered}\text { Laboratory } \\ \text { Ref Int } P_{\text {Ligh }}\end{gathered}$ J Multiplet

\begin{tabular}{|c|c|c|c|c|c|c|c|c|c|c|c|c|c|}
\hline $\begin{array}{l}3180.521 \\
3169.854 \\
3164.618\end{array}$ & $\begin{array}{l}\text { B } \\
\text { B } \\
\text { B }\end{array}$ & $\begin{array}{l}1 \mathrm{~N} \\
1 \mathrm{~N} \\
1 \mathrm{~N}\end{array}$ & $\begin{array}{l}1.89 \\
1.88 \\
1.87\end{array}$ & $\begin{array}{l}5.77 \\
5.77 \\
5.77\end{array}$ & $\begin{array}{l}3-1 \\
1-1 \\
0-1\end{array}$ & $\begin{array}{c}4^{3} p^{0}-9^{3} s \\
(14)\end{array}$ & $\begin{array}{l}6798.51 \\
6717.685\end{array}$ & A & $\begin{array}{r}6 n \\
500 n\end{array}$ & $\begin{array}{l}2.70 \\
3.70\end{array}$ & $\begin{array}{l}4.51 \\
4.53\end{array}$ & $\begin{array}{l}2-1 \\
2-1\end{array}$ & $\begin{array}{l}3^{1} p-5^{3} p^{0}+ \\
(31) \\
3^{1} D-3 d 4 p^{1} p^{0}\end{array}$ \\
\hline $\begin{array}{l}3150.738 \\
3140.782 \\
3136.003 \\
3151.280 \\
3141.164\end{array}$ & $\begin{array}{l}A \\
B \\
B \\
B \\
B\end{array}$ & $\begin{array}{l}4 \mathrm{~N}^{*} \\
3 \mathrm{~N}^{*} \\
1 \mathrm{~N} \\
4 \mathrm{~N}^{*} \\
3 \mathrm{~N}^{*}\end{array}$ & $\begin{array}{l}1.89 \\
1.88 \\
1.87 \\
1.89 \\
1.88\end{array}$ & $\begin{array}{l}5.81 \\
5.81 \\
5.81 \\
5.81 \\
5.81\end{array}$ & $\begin{array}{l}2-3 \\
1-2 \\
0-1 \\
2-2 \\
1-1\end{array}$ & $\begin{array}{c}4^{3} p^{0}-8^{3} D \\
(15)\end{array}$ & $\begin{array}{l}5349.473 \\
5041.620 \\
4878.133\end{array}$ & $\begin{array}{l}\text { A } \\
\text { A } \\
\text { A }\end{array}$ & $\begin{array}{l}35 \\
40 \\
50\end{array}$ & $\begin{array}{l}2.70 \\
3.70 \\
3.70\end{array}$ & $\begin{array}{l}5.00 \\
5.15 \\
5.33\end{array}$ & $\begin{array}{l}2-3 \\
3-1 \\
2-3\end{array}$ & $\begin{array}{l}{ }^{1}(33) \\
\left(3-3 d 4 p^{1} F^{\circ}\right. \\
(33) \\
3^{1} \mathrm{D}-5^{1} \mathrm{P}^{\circ} \\
(34)^{\circ} \\
3^{1} \mathrm{D}-4 \mathrm{~F}^{\circ} \\
(35)\end{array}$ \\
\hline $\begin{array}{l}3117.656 \\
3107.388 \\
3102.36\end{array}$ & $\begin{array}{l}B \\
B \\
B\end{array}$ & $\begin{array}{l}1 \mathrm{~N} \\
1 \mathrm{~N} \\
(0)\end{array}$ & $\begin{array}{l}1.89 \\
1.88 \\
1.87\end{array}$ & $\begin{array}{l}5.85 \\
5.85 \\
5.85\end{array}$ & $\begin{array}{l}3-1 \\
1-1 \\
0-1\end{array}$ & $\begin{array}{c}4^{3} p^{\circ}-10^{3} s \\
(16)\end{array}$ & $\begin{array}{l}4526.935 \\
4355.096\end{array}$ & $\begin{array}{l}\text { A } \\
\text { A }\end{array}$ & $\begin{array}{l}30 \\
25\end{array}$ & $\begin{array}{l}2.70 \\
2.70\end{array}$ & $\begin{array}{l}5.43 \\
5.53\end{array}$ & $\begin{array}{l}2-1 \\
2-3\end{array}$ & $\begin{array}{c}3^{1} \mathrm{D}-6_{\mathrm{po}} \\
(36)^{1} \\
3^{1} \mathrm{D}-5^{1} \mathrm{~F}^{\circ} \\
(37)\end{array}$ \\
\hline $\begin{array}{l}3006.858 \\
2999.641 \\
3009.205 \\
3000.863 \\
2997.309 \\
2994.958\end{array}$ & $\begin{array}{l}A \\
A \\
A \\
A \\
B \\
A\end{array}$ & $\begin{array}{l}6 \\
4 \\
5 \\
5 \\
5 \\
5\end{array}$ & $\begin{array}{l}1.89 \\
1.88 \\
1.89 \\
1.88 \\
1.88 \\
1.87\end{array}$ & $\begin{array}{l}6.00 \\
5.99 \\
5.99 \\
5.99 \\
6.00 \\
5.99\end{array}$ & $\begin{array}{l}2-2 \\
1-1 \\
3-1 \\
1-0 \\
1-2 \\
0-1\end{array}$ & $\begin{array}{c}4^{3} \mathrm{P}^{0}-3 \mathrm{~d}^{2}{ }^{3} \mathrm{P} \\
(17)\end{array}$ & $\begin{array}{l}4240.456 \\
4108.554 \\
4058.912 \\
3972.570\end{array}$ & $\begin{array}{l}\text { A } \\
\text { B } \\
\text { B } \\
\text { A }\end{array}$ & $\begin{array}{c}6 \\
10 \mathrm{~N} \\
1 \mathrm{n} \\
(1)\end{array}$ & $\begin{array}{l}2.70 \\
3.70 \\
2.70 \\
2.70\end{array}$ & $\begin{array}{l}5.61 \\
5.70 \\
5.74 \\
5.80\end{array}$ & $\begin{array}{l}2-1 \\
2-3 \\
2-1 \\
2-3\end{array}$ & $\begin{array}{l}{ }^{1}{ }^{1} \mathrm{D}-7^{1} \mathrm{P}^{\circ} \\
(38) \\
{ }^{1} \mathrm{D}-6^{1} \mathrm{~F}^{\circ} \\
(39) \\
(39) \\
{ }^{1} \mathrm{D}-8^{1} \mathrm{P}^{\circ} \\
(40) \\
{ }^{1} \mathrm{D}-\mathrm{F}^{1} \mathrm{~F}^{\circ}\end{array}$ \\
\hline $\begin{array}{l}6439.073 \\
6462.566 \\
6493.780 \\
6471.660 \\
6499.649 \\
6508.742\end{array}$ & $\begin{array}{l}A \\
A \\
A \\
A \\
A \\
B\end{array}$ & $\begin{array}{r}150 \\
125 \\
80 \\
40 \\
30 \\
(1)\end{array}$ & $\begin{array}{l}2.51 \\
2.51 \\
2.51 \\
2.51 \\
2.51 \\
2.51\end{array}$ & $\begin{array}{l}4.43 \\
4.42 \\
4.41 \\
4.42 \\
4.41 \\
4.41\end{array}$ & $\begin{array}{l}3-4 \\
2-3 \\
1-2 \\
3-3 \\
2-2 \\
3-2\end{array}$ & $\begin{array}{l}3^{3} D-3 d 4 p^{3} F^{0} \\
(18)\end{array}$ & $\begin{array}{r}10343.85 \\
7326.146\end{array}$ & $\begin{array}{l}C \\
A\end{array}$ & $\frac{(1)}{500}$ & $\begin{array}{r}\frac{3.70}{2.92} \\
2.92\end{array}$ & $\begin{array}{r}5.87 \\
4.11 \\
4.60\end{array}$ & $\begin{array}{l}1-0 \\
1-2\end{array}$ & $\begin{array}{c}3^{1} \mathrm{p}^{(41)} \\
(42) \\
4^{1} \mathrm{p}^{\circ}-5^{1} \mathrm{~s} \\
(43)^{1} \\
4^{1} \mathrm{p}^{0}-4^{1} \mathrm{D}\end{array}$ \\
\hline $\begin{array}{l}6464.70 \\
6455.500 \\
6449.810\end{array}$ & $\begin{array}{l}D \\
A \\
A\end{array}$ & $\begin{array}{l}(1) \\
10 \\
50\end{array}$ & $\begin{array}{l}2.51 \\
2.51 \\
2.51\end{array}$ & $\begin{array}{l}4.42 \\
4.42 \\
4.42\end{array}$ & $\begin{array}{l}3-2 \\
2-2 \\
1-2\end{array}$ & $\begin{array}{c}3^{3} D-3 d 4 p^{1} D^{\circ} \\
(19)\end{array}$ & $\begin{array}{l}6709.88 \\
5867.573\end{array}$ & $\begin{array}{l}D \\
A\end{array}$ & $\begin{array}{c}(1) \\
1\end{array}$ & $\begin{array}{l}2.92 \\
2.92\end{array}$ & $\begin{array}{l}4.76 \\
5.02\end{array}$ & $\begin{array}{l}1-2 \\
1-0\end{array}$ & 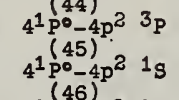 \\
\hline $\begin{array}{l}6169.559 \\
6169.055 \\
6166.443 \\
6161.289 \\
6163.758 \\
6156.10\end{array}$ & $\begin{array}{l}A \\
A \\
A \\
A \\
A \\
\text { E }\end{array}$ & $\begin{array}{l}40 \\
25 \\
15 \\
10 \\
10 \\
(1)\end{array}$ & $\begin{array}{l}2.51 \\
2.51 \\
2.51 \\
2.51 \\
2.51 \\
2.51\end{array}$ & $\begin{array}{l}4.52 \\
4.51 \\
4.51 \\
4.52 \\
4.51 \\
4.52\end{array}$ & $\begin{array}{l}3-2 \\
2-1 \\
1-0 \\
2-2 \\
1-1 \\
1-2\end{array}$ & $\begin{array}{c}3^{3} D-5^{3} p^{0} \\
(20)\end{array}$ & $\begin{array}{l}5857.454 \\
5512.979 \\
5188.848 \\
4847.296\end{array}$ & $\begin{array}{l}A \\
A \\
A\end{array}$ & $\begin{array}{c}100 \\
20 n \\
50\end{array}$ & $\begin{array}{l}2.92 \\
3.92 \\
2.92\end{array}$ & $\begin{array}{l}5.03 \\
5.16 \\
5.30\end{array}$ & $\begin{array}{l}1-2 \\
1-0 \\
1-2 \\
1-0\end{array}$ & $\begin{array}{l}4^{1} \mathrm{p}^{0}-4 \mathrm{p}^{3}{ }^{1} \mathrm{D} \\
(47) \\
4^{1} \mathrm{P}^{0}-6^{1} \mathrm{~s} \\
(48) \\
4^{1} \mathrm{p}^{0}-5^{1} \mathrm{D} \\
(49) \\
4^{1} \mathrm{p}^{0}-7^{1} \mathrm{~s}\end{array}$ \\
\hline $\begin{array}{l}5588.757 \\
5594.468 \\
5598.487 \\
5601.285 \\
5602.846 \\
5581.971 \\
5590.120\end{array}$ & $\begin{array}{l}\hat{A} \\
\hat{A} \\
A \\
\dot{A} \\
A \\
A \\
A\end{array}$ & $\begin{array}{l}80 \\
60 \\
50 \\
30 \\
25 \\
25 \\
20\end{array}$ & $\begin{array}{l}2.51 \\
2.51 \\
2.51 \\
2.51 \\
2.51 \\
2.51 \\
2.51\end{array}$ & $\begin{array}{l}4.72 \\
4.72 \\
4.71 \\
4.72 \\
4.71 \\
4.72 \\
4.72\end{array}$ & $\begin{array}{l}3-3 \\
2-2 \\
1-1 \\
3-2 \\
2-1 \\
2-3 \\
1-2\end{array}$ & $\underset{(21)}{3^{3} D-3 d 4 p^{3} D^{9}}$ & $\begin{array}{l}12816.06 \\
12823.89 \\
12827.09\end{array}$ & $\begin{array}{l}P \\
P \\
P\end{array}$ & $\begin{array}{r}2 \\
13\end{array}$ & $\begin{array}{r}2.93 \\
2.92 \\
\begin{array}{r}3.89 \\
3.89 \\
3.89\end{array}\end{array}$ & $\begin{array}{r}5.47 \\
5.55 \\
4.86 \\
4.86 \\
4.86\end{array}$ & $\begin{array}{l}1-0 \\
1-2 \\
1-2 \\
1-1 \\
1-0\end{array}$ & $\begin{array}{l}4^{1} \mathrm{p}^{0}-7^{1} \mathrm{~S} \\
(50) \\
4^{1} \mathrm{P}^{0}-6^{1} \mathrm{D} \\
(51) \\
5^{3} \mathrm{~S}-3 \mathrm{~d} 4 \mathrm{p}^{3} \mathrm{P}^{0} \\
(52)\end{array}$ \\
\hline $\begin{array}{l}5270.270 \\
5265.557 \\
5262.244 \\
5264.239 \\
5261.706 \\
5260.375\end{array}$ & $\begin{array}{l}A \\
A \\
A \\
A \\
A \\
A\end{array}$ & $\begin{array}{r}60 \\
40 \\
25 \\
20 \\
20 \\
2\end{array}$ & $\begin{array}{l}2.51 \\
2.51 \\
2.51 \\
2.51 \\
2.51 \\
2.51\end{array}$ & $\begin{array}{l}4.86 \\
4.86 \\
4.86 \\
4.86 \\
4.86 \\
4.86\end{array}$ & $\begin{array}{l}3-2 \\
2-1 \\
1-0 \\
2-2 \\
1-1 \\
1-2\end{array}$ & $\begin{array}{l}3^{3} D-3 d 4 p^{3} p^{0} \\
(22)\end{array}$ & $\begin{array}{l}6361.79 \\
6343.29 \\
6318.11 \\
5757.69\end{array}$ & $\begin{array}{l}F \\
F \\
F \\
F\end{array}$ & $\left\{\begin{array}{l}5 n \\
4 n \\
3 n\end{array}\right)$ & $\begin{array}{l}4.43 \\
4.42 \\
4.41 \\
4.43\end{array}$ & $\begin{array}{l}6.37 \\
6.37 \\
6.36 \\
6.57\end{array}$ & $\begin{array}{l}4-5 \\
3-4 \\
2-3 \\
4-4\end{array}$ & $\begin{array}{l}3 d 4 p^{3} p o-3 d 4 d^{3} a \\
(53) \\
3 d 4 p^{3} p^{3}-3 d 4 d^{3} r\end{array}$ \\
\hline $\begin{array}{l}4585.871 \\
4581.402 \\
4578.558 \\
4585.923\end{array}$ & $\begin{array}{l}A \\
A \\
A \\
B\end{array}$ & $\begin{array}{l}50 \\
40 \\
30 \\
(2)\end{array}$ & $\begin{array}{l}2.51 \\
2.51 \\
2.51 \\
2.51\end{array}$ & $\begin{array}{l}5.21 \\
5.21 \\
5.21 \\
5.21\end{array}$ & $\begin{array}{l}3-4 \\
2-3 \\
1-2 \\
3-3\end{array}$ & $\begin{array}{c}3^{3} D-4^{3} F^{\circ} \\
(23)\end{array}$ & $\begin{array}{l}5735.74 \\
5717.99 \\
5761.88 \\
5746.81 \\
5731.70 \\
5707.03\end{array}$ & $\begin{array}{l}F \\
F \\
F \\
F \\
F \\
F\end{array}$ & $\left\{\begin{array}{l}3 n \\
4 n \\
1 n \\
2 N \\
1 n \\
1 n\end{array}\right\}$ & $\begin{array}{l}4.48 \\
4.41 \\
4.43 \\
4.42 \\
4.42 \\
4.41\end{array}$ & $\begin{array}{l}6.57 \\
6.57 \\
6.57 \\
6.57 \\
6.57 \\
6.57\end{array}$ & $\begin{array}{l}3-3 \\
2-2 \\
4-3 \\
3-2 \\
3-4 \\
2-3\end{array}$ & \\
\hline $\begin{array}{l}4509.446 \\
4507.417 \\
4507.854 \\
4506.624 \\
4505.00\end{array}$ & $\begin{array}{l}\mathrm{B} \\
\mathrm{B} \\
\mathrm{B} \\
\mathrm{B} \\
\mathrm{E}\end{array}$ & $\left.\begin{array}{l}3 \\
(1) \\
1 \\
(1) \\
0\end{array}\right)$ & $\begin{array}{l}2.51 \\
2.51 \\
2.51 \\
2.51 \\
2.51\end{array}$ & $\begin{array}{l}5.25 \\
5.25 \\
5.25 \\
5.25 \\
5.25\end{array}$ & $\begin{array}{l}2-1 \\
1-0 \\
2-2 \\
1-1 \\
1-2\end{array}$ & & $\begin{array}{l}9701.81 \\
9688.60 \\
9676.25 \\
9664.29 \\
9663.58\end{array}$ & $\begin{array}{l}P \\
P \\
P \\
P \\
P \\
P\end{array}$ & $\begin{array}{l}20 \\
15 \\
5 \\
5 p ?\end{array}$ & $\begin{array}{r}4.72 \\
4.72 \\
4.71 \\
4.72 \\
4.71\end{array}$ & $\begin{array}{l}6.00 \\
5.99 \\
5.99 \\
6.00 \\
5.99\end{array}$ & $\begin{array}{l}3-2 \\
2-1 \\
1-0 \\
3-2\end{array}$ & $\begin{array}{c}3 d 4 p^{3} p^{0}-3 d^{2} \quad 3 p \\
(55)^{3}\end{array}$ \\
\hline $\begin{array}{l}4098.533 \\
4094.930 \\
4092.633\end{array}$ & $\begin{array}{l}A \\
A \\
A\end{array}$ & $\begin{array}{r}15 \\
12 \\
8\end{array}$ & $\begin{array}{l}2.51 \\
2.51 \\
2.51\end{array}$ & $\begin{array}{l}5.53 \\
5.53 \\
5.53\end{array}$ & $\begin{array}{l}3-4 \\
2-3 \\
1-2\end{array}$ & $\begin{array}{c}3^{3} D-5^{3} F^{\circ} \\
(25)\end{array}$ & 9639.40 & $\mathrm{P}$ & & 4.71 & 6.00 & $\begin{array}{l}1-1 \\
1-2\end{array}$ & \\
\hline $\begin{array}{l}3875.807 \\
3872.552 \\
3870.506\end{array}$ & $\begin{array}{l}\mathrm{B} \\
\mathrm{B} \\
\mathrm{B}\end{array}$ & $\left(\begin{array}{l}4 \\
3 \\
2\end{array}\right)$ & $\begin{array}{l}2.51 \\
2.51 \\
2.51\end{array}$ & $\begin{array}{l}5.70 \\
5.70 \\
5.70\end{array}$ & $\begin{array}{l}3- \\
2- \\
1-\end{array}$ & $\begin{array}{c}3^{3} D-6^{3} F^{\circ} \\
(26)\end{array}$ & $\begin{array}{l}10838.77 \\
10863.72 \\
10869.37 \\
10879.78\end{array}$ & $\begin{array}{l}c \\
c \\
c \\
c\end{array}$ & $\begin{array}{r}10 \\
2 \\
3 \\
4\end{array}$ & $\begin{array}{l}4.86 \\
4.86 \\
4.86 \\
4.86\end{array}$ & $\begin{array}{l}6.00 \\
5.99 \\
5.99 \\
5.99\end{array}$ & $\begin{array}{l}3-2 \\
1-1 \\
2-1 \\
1-0\end{array}$ & $\begin{array}{c}3 d 4 p^{3} p c-3 d^{2} 3 p \\
(56)\end{array}$ \\
\hline $\begin{array}{l}3753.367 \\
3750.349 \\
3748.374\end{array}$ & $\begin{array}{l}\mathrm{B} \\
\mathrm{B} \\
\mathrm{B}\end{array}$ & $\left.\begin{array}{l}1 \\
1 \\
1\end{array}\right\}$ & $\begin{array}{l}2.51 \\
2.51 \\
2.51\end{array}$ & $\begin{array}{l}5.80 \\
5.80 \\
5.80\end{array}$ & $\begin{array}{l}3- \\
2- \\
1-\end{array}$ & $\begin{array}{c}3^{3} D-7^{3} F^{0} \\
(27)\end{array}$ & 10861.51 & $\begin{array}{l}c \\
c\end{array}$ & $\begin{array}{l}4 \\
3\end{array}$ & $\begin{array}{l}4.86 \\
4.86\end{array}$ & $\begin{array}{l}6.00 \\
5.99\end{array}$ & $\begin{array}{l}1-2 \\
0-1\end{array}$ & \\
\hline $\begin{array}{l}3678.240 \\
3675.307 \\
3673.448\end{array}$ & $\begin{array}{l}\text { B } \\
\text { B } \\
\text { B }\end{array}$ & $\begin{array}{l}3 \\
2 \\
1\end{array}$ & $\begin{array}{l}2.51 \\
2.51 \\
2.51\end{array}$ & $\begin{array}{l}5.87 \\
5.87 \\
5.87\end{array}$ & $\begin{array}{l}3- \\
2- \\
1-\end{array}$ & $\begin{array}{c}3^{3} D-8^{3} F^{0} \\
(28)\end{array}$ & $\begin{array}{l}7468.41 \\
6405.89 \\
6395.16\end{array}$ & $\begin{array}{l}F \\
F \\
F\end{array}$ & $\left.\begin{array}{l}311 \mathrm{ec} \\
3 \mathrm{n} \\
3 \mathrm{n}\end{array}\right\}$ & Lines & of $\mathrm{Ca} I$ & & \\
\hline 7202.194 & $A$ & 200 & 2.70 & 4.41 & $2-2$ & $3^{1} D-3 d 4 p^{3} F^{0}$ & $\begin{array}{l}5764.32 \\
5743.38\end{array}$ & $\boldsymbol{F}$ & $\left\{\begin{array}{l}3 n \\
3 n\end{array}\right\}$ & & & & \\
\hline 7148.147 & $A$ & 500 & 2.70 & 4.43 & $2-2$ & $\begin{array}{c}3^{1} D \rightarrow 3 d 4 p^{1} D^{0} \\
(30)\end{array}$ & $\begin{array}{l}5688.47 \\
5682.88\end{array}$ & $\frac{F}{F}$ & $\left\{\begin{array}{l}4 N \\
3 n\end{array}\right\}$ & & & & \\
\hline
\end{tabular}

Ca I continued 3164.61

150.738

3102.36

3006.858

3009.205

999.958

6439.073

6493.780

6508.742

404.70

$449.810 \quad \vec{A}$

5588.757

5581.971

5270.270

$5265.55 ?$
5262.244

5264.239

5260.375

4512.282

4098.533

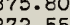

3870.506

3750.367

3675.307

202.194 is 200

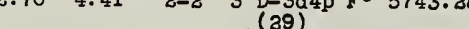

I A Ref Int Low ${ }_{\text {H1gh }}$ J Multiplet

Ca I cont1nued

E P
A Rel Int Jow High Ca II I P 11.82 Anal A L1st B Apr 1944

$3933.664 / /$ A $400 R(K) \quad \begin{array}{llll}K & 0.00 & 3.14 & 1\end{array}$ 8543.089 B $\overline{1500} \quad \overline{1.69} 3.14 \quad 3 \frac{3}{3}-1 \frac{3}{3} \mathrm{D}-4^{2} \mathrm{po}$ $\begin{array}{rrrrrrr}8662.140 & \text { B } & 1000 & 1.69 & 3.11 & 1 \frac{1}{3}-\frac{1}{3} & \text { (2) } \\ 8498.018 & \text { B } & 300 & 1.69 & 3.14 & 13-1 & \end{array}$

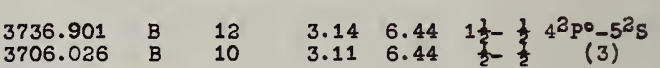

$\begin{array}{lllllll}3179.333 & B & 15 & 3.14 & 7.02 & 1 \frac{1}{2}-2 \frac{1}{2} & 4^{2} \mathrm{P}^{0}-4^{3} \mathrm{D}\end{array}$

$\begin{array}{rrrrrrr}3158.869 & \mathrm{~B} & 10 & 3.11 & 7.02 & 1 \frac{1}{\frac{1}{2}-1 \frac{1}{2}} & (4) \\ 3181.275 & \mathrm{~B} & 4 & 3.14 & 7.02 & 1 \frac{2}{2}-1 \frac{2}{2} & \end{array}$

$11836.4-\longrightarrow$

$6.44 \quad 7.48 \quad \frac{1}{3}-1 \frac{1}{2} 5^{2} 8-5^{3} \mathrm{po}^{\circ}$

$4473.09 \quad D \quad(0) \quad 6.44 \quad 9.30 \quad \frac{1}{2}-1 \frac{1}{2} 5^{2} 8-6^{2} P_{0}$

$4479.39 \mathrm{D} \quad(1) \quad 6.44 \quad 9.20 \quad \frac{1}{2}-\frac{1}{2} \quad(6)$

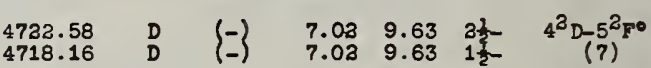

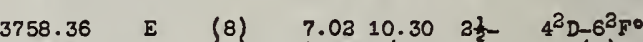

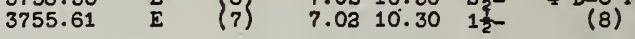

$3346.99 \quad E \quad(10) \quad 7.0210 .71 \quad 2 \frac{1}{2} \quad 4^{3} \mathrm{D}-7^{2} F^{\circ}$

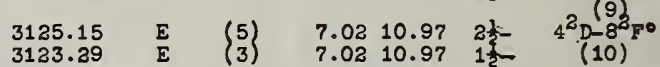

$3989.42 \quad E \quad$ (1) $7.0311 .15 \quad 2 \frac{1}{1} \quad 4^{3} \mathrm{D}-9^{3} \mathrm{~F}^{\circ}$

9933.3

9856.7

8303.2

8256.1

5307.30

5019.979

5001.489

5021.14

4206.21

4109.83

4097.13
4110.33

4110.33

3694.11

3683.71
3694.31

6456.907

5339.29

7.0211 .151

$7.48 \quad 8.73 \quad 1 \frac{1}{2}-\frac{1}{2} 5^{3} \mathrm{P}^{0}-6^{2} \mathrm{~S}$

$7.48 \quad 8.98 \quad 1 \frac{1}{2}-2 \frac{1}{2} 5^{3} \mathrm{P}^{0}-5^{2} \mathrm{D}$

$\begin{array}{llll}7.48 & 8.98 & 1 \frac{1}{2}-2 \frac{1}{2} & 5^{2} \mathrm{P}^{0}-5 \\ 7.47 & 8.98 & \frac{1}{2}-1 \frac{1}{2} & (13)\end{array}$

$\begin{array}{llr}7.47 & 8.98 & \frac{1}{2} \\ 7.48 & 8.98 & 1 \frac{1}{2}-1\end{array}$

$-3 \quad 7.48 \quad 9.81 \quad 1 \frac{1}{2} 5^{3} \mathrm{p}^{0}-7^{2} \mathrm{~S}$

(2) $7.48 \quad 9.94 \quad 1 \frac{1}{3}-2 \frac{1}{2} \quad 5^{2} \mathrm{P}^{0}-6^{2} \mathrm{D}$

(1) $\begin{array}{llll}7.47 & 9.94 & \frac{1}{3}-1 \frac{2}{2} \\ 0 & 7.48 & 9.94 & 1 \frac{1}{2}-1 \frac{1}{2}\end{array}$

-) $\begin{array}{llll}7.48 & 10.41 & 1 \frac{1}{2} & \frac{1}{2} 5^{3} \mathrm{p}^{0}-8^{3} \mathrm{~s}\end{array}$

(1) $\begin{array}{lllll}7.48 & 10.49 & 1 \frac{1}{3}-2 \frac{1}{2} & 5^{2} \mathrm{P}^{0}-7^{2} \mathrm{D} \\ 1 & 7.47 & 10.49 & & (17)\end{array}$

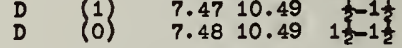

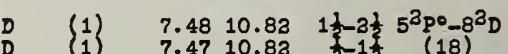

(0) $\begin{array}{llll}7.47 & 10.82 & 10.82 & 1 \frac{1}{2}-1 \frac{1}{2}\end{array}$

(-) $8.4010 .31-4^{2} 5^{\circ}-6^{2} G$

D (-) $8.4010 .71-4^{2} \mathrm{~F}^{\circ}-7^{2}$

Ca III I P 51.00 Anal D L1st A Apr 1944 $3372.68 \quad A \quad 8 \quad 29.94 \quad 33.60 \quad 2-1 \quad 48 \quad 1^{\circ}-4 p$ $\begin{array}{llllllll}3537.75 \quad A & 7 & 30.11 & 33.60 & 1-1 & 4 \mathrm{~g} 3^{\circ}-4 \mathrm{p} & 1\end{array}$ $\begin{array}{llll}30.11 & 34.24 & 1-2 & \text { (2) }-4 \mathrm{p}\end{array}$

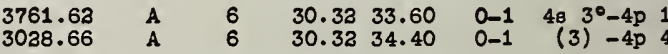

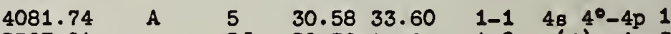

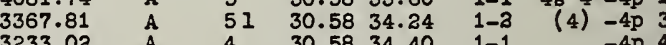
$\begin{array}{lllllll}3233.02 & \text { A } & 4 & 30.58 & 34.40 & 1-1 & -4 \mathrm{p} \\ 3119.66 & \mathrm{~A} & 8 & 30.58 & 34.53 & 1-2 & -4 \mathrm{p} 5\end{array}$ 
I A Laboratory ReI Int Low Pigh

J Hitiplet

T1 I I P 6.81 Anal A L1st B Nov 1940

6295.251

6273.38

6359.896

$\begin{array}{lll}6273.389 & E & \\ 6257.72 & H & \\ 6359.896 & E & \\ 6325.22 & H & \\ 6296.646 & E & (1 \\ 6413.13 & P & \end{array}$

$\left(\begin{array}{l}3 \\ 6 \\ 2 \\ 8 \\ 10 \\ 13\end{array}\right)$

$\begin{array}{ll}0.05 & 3.01 \\ 0.02 & 1.99 \\ 0.00 & 1.97 \\ 0.05 & 1.99 \\ 0.02 & 1.97 \\ 0.00 & 1.96 \\ 0.05 & 1.97 \\ 0.02 & 1.96\end{array}$

$\begin{array}{ll}4-5 & \mathrm{a}^{3} \mathrm{r}-\mathbf{z}^{5} \\ 3-4 & (1) \\ 2-3 & (1) \\ 4-4 & \\ 3-3 & \\ 2-3 & \\ 4-3 & \\ 3-2 & \end{array}$

5940.68

5913.730
6031.68

(2)

$\begin{array}{ll}0.05 & 3.13 \\ 0.02 & 3.11 \\ 0.05 & 3.09 \\ 0.02 & 3.08 \\ 0.00 & 3.08\end{array}$

$\begin{array}{ll}4-5 & a^{3} F-2^{5} F^{\circ} \\ 4-3 & (2)\end{array}$

5984.586
5944.65

5460.503

5426.256
-5396.600
5490.840

$\begin{array}{r}5490.840 \\ -5446.593 \\ \hline\end{array}$

5408.940

5396.600
5376.59

5210.386
5193.971

5173.742

5252.105
5319.697

5153.185
$\mathbf{5 1 4 7 . 4 8 3}$

$\begin{array}{lll}5064.654 & \text { A } & 35 \\ 5039.959 & \text { A } & 32 \\ 5014.185 & \text { A } & (35)\end{array}$

5014.185
5009.652
4997.099

4997.099
4967.30

4681.908

$\begin{array}{lll}4681.908 & \text { A } & 30 \\ 4667.585 & \text { A } & 35 \\ 4656.468 & \text { A } & 35\end{array}$

$\begin{array}{rrr}4715.295 & A & 4 \\ 4693.670 & B & 5\end{array}$

$\begin{array}{lll}4562.637 & B & 6 \\ 4527.455 & \text { E } & (4) \\ 4540 & 0\end{array}$

$\begin{array}{ll}0.05 & 3.31\end{array}$

$\begin{array}{ccc}3 & 0.02 & 3.30 \\ 1 & 0.00 & 3.39 \\ \text { (0) } & 0.05 & 2.30 \\ (1) & 0.02 & 3.39\end{array}$

(1)

$\begin{array}{ll}0.05 & 2.30 \\ 0.03 & 3.39 \\ 0.02 & 2.38 \\ 0.02 & 3.31\end{array}$

$\begin{array}{lll}0.02 & 3.31 & 3 \\ 0.00 & 3.30 & 2-3\end{array}$

4540.483

4496.245
4462.099

(3)

$0.05 \quad 3.43$

$\begin{array}{ll}0.05 & 3.42 \\ 0.02 & 3.40 \\ 0.00 & 3.39\end{array}$

$\begin{array}{lll}0.00 & 3.39 & 2-2 \\ 0.05 & 3.40 & 4-3 \\ 0.02 & 3.39 & 3-3\end{array}$

$\begin{array}{lll}0.03 & 3.43 & 3-4 \\ 0.00 & 3.40 & 2-3\end{array}$

$\begin{array}{cccc}0.05 & 3.48 & 4-3 & a^{3} F-z^{3} D^{\circ} \\ 0.03 & 3.47 & 3-2 & (5)\end{array}$

$\begin{array}{lll}0.03 & 3.47 & 3 \\ 0.00 & 2.46 & 2\end{array}$

$\begin{array}{lll}0.00 & 2.46 & 2-1 \\ 0.02 & 3.48 & 3-3 \\ 0.00 & 3.47 & 2-2\end{array}$

$\begin{array}{lll}0.00 & 3.47 & 2-2 \\ 0.00 & 3.48 & 2-3\end{array}$

$\begin{array}{llll}0.05 & 2.68 & 4-5 & a^{3} \mathrm{~F}^{3} \mathbf{z}^{3} \mathrm{G}^{\circ} \\ 0.03 & 3.67 & 3-4 & (6) \\ 0.00 & 2.65 & 2-3 & (6)\end{array}$

$\begin{array}{lll}0.00 & 2.65 & 2-3 \\ 0.05 & 3.67 & 4-4 \\ 0.02 & 2.65 & 3-3\end{array}$

$\begin{array}{llll}0.03 & 3.73 & 3-2 & a^{3} \mathrm{~F}^{-2^{1} D^{0}} \\ 0.00 & 3.73 & 2-2 & (7)\end{array}$

$\begin{array}{llll}0.05 & 3.77 & 4-3 & 2^{3} \mathrm{~F}_{-2} \mathrm{~F}^{\circ} \\ 0.02 & 3.77 & 3-3 & (8)^{\circ}\end{array}$

$\begin{array}{lll}4112.708 & \text { A } & 20 \\ 4076.370 & B\end{array}$

$0.05 \quad 3.05 \quad 4-4 \quad 2^{3} \mathrm{~F}_{-2} z^{1} \mathrm{G}^{\circ}$

4011.534 E (3)

$\begin{array}{llll}4009.653 & B & 15 \\ 3982.478 & \text { B } & 30\end{array}$

3998.635 A $100 \mathrm{R}$

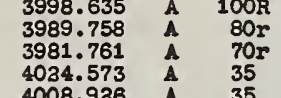

4008.926

$\begin{array}{lll}3964.369 & \text { A } & 35 \\ 3962.851 & \text { A } & 35\end{array}$

$\begin{array}{rrr}3958.306 & \text { A } & 80 \\ 3956.336 & \text { A } & 60 \\ 3948.670 & \text { A } & 60 \\ 3924.537 & \text { A } & 50 \\ 3929.875 & \text { A } & 40 \\ 3898.487 & \text { B } & 8\end{array}$

$\begin{array}{llr}3947.770 & \text { A } & 40 \\ 3914.751 & \text { B } & 5 \\ 3921.423 & \text { A } & 30\end{array}$

$\begin{array}{lll}3914.334 & \text { A } & 35 \\ 3900.958 & \text { B } & 12\end{array}$

3900.958
3889.948

3934.228
3915.879

.3899 .668
3881.399

3881.399
-3875.362

3788.804
3774.331

3753.860 A $80 \mathrm{r}$

$\begin{array}{lll}3741.059 & \text { A } & 60 \\ 3729.806 & \text { A } & 50 \\ 3771.653 & \text { A } & 25 \\ 3753.623 & \text { B } & 35\end{array}$

$\begin{array}{lll}3732.568 & \text { A } & 15 \text { ? } \\ 3717.393 & \text { A } & 30\end{array}$

3689.916

3668.965
3654.592
3660.631

3660.631
3646.198

$\begin{array}{lll}3646.198 & A & 12 \\ 3637.966 & \mathrm{~B} & 12\end{array}$

3653.497

3643.675
3635.463
3671.672

3671.672
3658.097

3687.354

3635.202

3626.085
3606.786

3606.786
3603.845

3804.284 $\begin{array}{llll}0.03 & 3.05 & 3-4 & \\ 0.00 & 3.08 & 2-1 & a^{3} F-2^{3} g^{\circ}\end{array}$

$\begin{array}{llll}0.00 & 3.08 & 2-1 & a^{3} \mathrm{~F}-2^{3} \mathrm{~g}^{\circ} \\ 0.02 & 3.10 & 3-2 & a^{3}(10) \mathrm{F}^{5} \\ 0.00 & 3.10 & 2-2 & (11)\end{array}$

$0.05 \quad 3.13 \quad 4-4 \quad a^{3} \mathrm{~F}-\mathrm{J}^{3} \mathrm{~F}^{\circ}$

$\begin{array}{llll}0.03 & 3.11 & 4-4 & a^{3} \mathrm{~F}-\mathrm{J}^{3} \mathrm{~F} \\ 0.00 & 3.11 & 3-3 & (12)\end{array}$

$0.00 \quad 3.10$

$0.05 \quad 3.11 \quad 4-3$

$\begin{array}{lll}0.02 & 3.10 & 3-2 \\ 0.03 & 3.13 & 3-4 \\ 0.00 & 3.11 & 2-3\end{array}$

$0.05 \quad 3.17 \quad 4-3 \quad a^{3} F-y^{3} D^{\circ}$

$\begin{array}{cccc}0.05 & 3.17 & 4-3 & a^{3} \mathrm{~F}-\mathrm{J}^{3} D^{\circ} \\ 0.08 & 3.14 & 3-3 & (13)\end{array}$

$\begin{array}{lll}0 . \infty & 3.13 \mid & 2-1 \\ 0.02 & 3.17 & 3-3\end{array}$

$\begin{array}{lll}0.00 & 3.14 & 2-2 \\ 0.00 & 3.17 & 2-3\end{array}$

$0.03 \quad 3.15 \quad 3-3, a^{3} p-z^{3} p^{30}$

$\begin{array}{llll}0.03 & 3.15 & 3-2 & a^{3}-2 \\ 0.00 & 3.15 & 2-1 \\ 0.00 & 3.15 & 2-2 & (14)\end{array}$

$\begin{array}{llll}0.05 & 3.30 & 4-4 & a^{3} F-y^{5} D^{\circ} \\ 0.03 & 3.18 & 3-3 & (15) \\ 0.00 & 3.17 & 2-2 & \end{array}$

$\begin{array}{lll}0.00 & 3.17 & 2-3 \\ 0.05 & 3.18 & 43\end{array}$

(3) $\quad \begin{array}{llll}3 & 0.03 & 3.17 & 3-3 \\ \text { (3) } & 0.00 & 3.16 & 2-1\end{array}$

$\begin{array}{cccc}\text { (3) } & 0.00 & 3.16 & 2-1 \\ 4 & 0.03 & 3.30 & 3-4 \\ \text { an } & 0.00 & 3.18 & 2-3\end{array}$

$0.05 \quad 3.31 \quad 4-5 \quad a^{3} 5-y^{5} a^{\circ}$

$\begin{array}{llll}0.02 & 3.39 & 3-4 & (16) \\ 0.05 & 3.34 & 4-4 & 2^{3} 5-x^{3} F^{0}\end{array}$

$\begin{array}{llll}0.05 & 3.34 & 4-4 & a^{3} F-x^{3} p^{\circ} \\ 0.03 & 3.32 & 3-3 & (17)\end{array}$

$0.00 \quad 3.31$

$0.05 \quad 3.33$

$\begin{array}{lll}0.02 & 3.34 \\ 0.00 & 3.33\end{array}$

$\begin{array}{llll}0.05 & 3.39 & 4-3 & a^{3} F-x^{3} D^{0} \\ 0.02 & 3.38 & 3-3 & (18)\end{array}$

$0.00 \quad 3.38$

$\begin{array}{lll}0.00 & 3.38 & 2-3 \\ 0.00 & 3.39 & 2-3\end{array}$

$0.05 \quad 3.43 \quad 4-5 \quad a^{3} \mathrm{~F}-\mathrm{J}^{3} \mathrm{G}^{0}$

0.003 .41

$\begin{array}{ll}0.05 & 3.41 \\ 0.02 & 3.39\end{array}$

$\begin{array}{lll}0.02 & 3.39, & 3-3 \\ 0.05 & 3.39 ! & 4-3\end{array}$

$0.05 \quad 3.44 \quad 4-3 \quad a^{3} \mathrm{p}-z^{5} \mathrm{po}$

$0.02 \quad 3.45 \mid \quad 3-3 \quad 2^{3} \mathrm{~F}-y^{1} \mathrm{D}^{0}$
$0.02 \quad 3.39 \quad 3-3$

$\begin{array}{llll}0.05 & 3.44 & 4-3 & a^{3} p_{-2} z_{p} \\ 0.02 & 3.42 & 3-2 & (30) \\ 0.02 & 3.44 & 3-3 & (30)\end{array}$

$\begin{array}{lll}0.02 & 3.44 & 3-3 \\ 0.00 & 3.42 & 2-2\end{array}$
I A Laboratory $_{\text {Rnt }}^{\text {Low }}{ }_{\text {High }}^{\text {Ligh }}$

I1 I continued

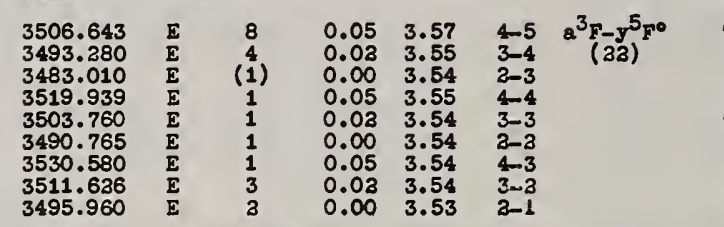

I A Ref Int Lo Plgh

J Multiplot

I1 I continued

$\begin{array}{lllllll}-5238.560 \quad \text { B } & 6 & 0.84 & 3.20 & 5-4 & a^{5} \mathrm{~F}-\mathrm{y}^{5} \mathrm{D}^{\circ}\end{array}$

$$
\text { 列 }
$$

5346.574

5350.95

5251.49
.5348 .402

5211.22
5234.14

5233.14

$\begin{array}{lll}3385.944 & \text { A } & 40 \\ 3377.577 & \text { A } & 301 \\ 3370.436 & \text { A } & 401 \\ 3361.263 & \text { E } & 40 . \\ 3358.271 & \text { A } & 10 \\ 3343.151 & \text { E } & \text { G }\end{array}$

$0.05 \quad 3.69 \quad 4-3 \quad a^{3} 5-m^{3} D^{0}$

$4981.732 / /$

0.023 .67

$\begin{array}{lll}0.00 & 3.66 & 2-1 \\ 0.02 & 3.69 & 3-3 \\ 0.00 & 3.67 & 2-3\end{array}$

$\begin{array}{lll}3371.447 & \text { A } & 80 R \\ 3354.634 & \text { A } & 60 \\ 3341.8758 & \text { A } & 50 \\ 3379.316 & \text { E } & 15\end{array}$

$\begin{array}{lll}0.60 & 3.69 & 2-3\end{array}$

$\begin{array}{lll}3360.990 & \mathrm{E} & 10 \\ 3385.664 & \mathrm{E} & 12\end{array}$

$0.05 \quad 3.71$

$\begin{array}{ll}0.00 & 3.70 \\ 0.00 & 3.69 \\ 0.05 & 3.70\end{array}$

$\begin{array}{ll}0.05 & 3.70 \\ 0.02 & 3.69 \\ 0.05 & 3.69\end{array}$

3369.054

$\begin{array}{llllll}3369.054 & \mathrm{E} & 1 & 0.05 & 3.71 \\ 3352.937 & \mathrm{E} & 6 & 0.02 & 3.70\end{array}$

3342.707
3377.485

3361.835

$\begin{array}{lllll}3348.535 & E & 10 & 0.02 & 3.69\end{array}$

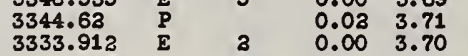

$\begin{array}{ll}0.02 & 3.71 \\ 0.00 & 3.70\end{array}$

$\begin{array}{lllll}3243.803 & E & 4 & 0.05 & 3.85 \\ 3222.741 & E & 3 & 0.02 & 3.85 \\ 3205.848 & E & 5 & 0.00 & 3.85\end{array}$

3205.168
3203.58

$\begin{array}{lll}3 & 0.03 & 3.85 \\ 3 & 0.00 & 3.85\end{array}$

3199.915

3191.994
3186.451
3314.340

3303.82

3160.09

3160.09
3151.11
3143.16

$\begin{array}{lll}3000.868 & \mathrm{E} & 30 \\ 3983.306 & \mathrm{E} & 30\end{array}$

3970.384

3003.738
3985.477

3981.448

3956.133

3967.355
2956.797

10396.85
10496.14

10498.14
10584.66

10681.61

10607.78

10677.04

-10774.9

10792.59

10828.04
10847.73

9638.38

9675.55
9705.64
9738.36

9738.36

9743.60
9770.38

9783.30

9787.6

9783.59
9546.07

9599.53
9647.40

9688.86

8434.98

8435.68

8413.36

8396.93
8364.24

8377.90

8382.82

8307.41
8334.37

8353.15

7858.74
7885.00

7895.50

5361.724

5384.634

5401.33

5366.651
5389.180

5389.38

5323.958

5340.68
5366.49
(2)

$\begin{array}{lll}100 \mathrm{R} & 0.05 \quad 3.90\end{array}$

$\begin{array}{lll}80 \mathrm{R} & 0.02 & 3.89 \\ \text { 60r } & 0.00 & 3.87\end{array}$

$60 r$
12
15

$\begin{array}{ll}0.02 & 3.87 \\ 0.05 & 3.87\end{array}$

tr

$\begin{array}{ll}0.03 & 3.93 \\ 0.00 & 3.92\end{array}$

$\begin{array}{ll}0.00 & 3.92 \\ 0.00 & 3.93\end{array}$

30
30
10
3

$0.05 \quad 4.16$

$0.02 \quad 4.16$

0.004 .15

(3)

$\begin{array}{ll}0.05 & 4.16 \\ 0.02 & 4.15\end{array}$

$\begin{array}{ll}0.03 & 4.16 \\ 0.00 & 4.16\end{array}$

$0.05 \quad 4.33$

\begin{tabular}{ll}
0.05 & 4.31 \\
0.03 & 4.19 \\
\hline
\end{tabular}

-

$\begin{array}{ll}0.84 & 3.03 \\ 0.83 & 3.01 \\ 0.83 & 1.09\end{array}$

$\begin{array}{ll}0.84 & 3.03 \\ 0.83 & 1.99 \\ 0.81 & 1.99\end{array}$

0.811 .97

0.811 .96

$\begin{array}{ll}0.84 & \mathbf{3 . 0 1} \\ 0.83 & 1.99\end{array}$

0.831 .97

$\begin{array}{ll}0.81 & 1.96 \\ 0.84 & 1.99\end{array}$

$\begin{array}{ll}0.84 & 1.99 \\ 0.83 & 1.97\end{array}$

0.821 .96

$\begin{array}{ll}0.84 & 3.13 \\ 0.83 & 3.11\end{array}$

$0.83 \quad 3.09$

0.813 .08

0.84
0.83
3.11

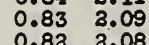

$0.83 \quad 3.08$

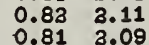

$\begin{array}{ll}0.81 & 3.09 \\ 0.81 & 3.08\end{array}$

$0.84 \quad 3.31$

$\begin{array}{ll}0.83 & 3.30 \\ 0.83 & 3.30\end{array}$

$\begin{array}{ll}0.83 & 3.29 \\ 0.81 & 3.28\end{array}$

$0.81 \quad 3.38$

$\begin{array}{ll}0.83 & 3.31 \\ 0.82 & 3.30\end{array}$

$\begin{array}{ll}0.83 & 3.30 \\ 0.81 & 3.39\end{array}$

$\begin{array}{ll}0.81 & 3.38 \\ 0.83 & 3.31 \\ 0.81 & 3.30\end{array}$

$\begin{array}{ll}0.81 & 3.30 \\ 0.81 & 3.29\end{array}$

$\begin{array}{ll}0.84 & 3.43 \\ 0.83 & 3.40\end{array}$

$\begin{array}{ll}0.83 & 3.40 \\ 0.83 & 3.39\end{array}$

$\left(\begin{array}{l}1 \\ 1 \\ 1\end{array}\right)$

$\begin{array}{ll}0.83 & 3.13 \\ 0.83 & 3.11\end{array}$

$\begin{array}{ll}0.82 & 3.11 \\ 0.81 & 3.10 \\ 0.810\end{array}$

$\begin{array}{ll}0.83 & 3.13 \\ 0.81 & 3.11 \\ 0.81 & 3.10\end{array}$

$\begin{array}{ll}0.81 & 3.11 \\ 0.81 & 3.10\end{array}$

(1) $0.83 \quad 3.17 \quad 4-3 \quad a^{5} F-J^{3} D^{0}$

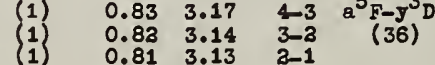

$\begin{array}{lll}0.81 & 3.13 & 2-1 \\ 0.82 & 3.17 & 3-3\end{array}$

$\begin{array}{ll}4-4 & a^{3} F-w^{3} \mathrm{ro}^{\circ} \\ 3-3 & (29) \\ 2-2 & \\ 4-3 & \\ 3-3 & \\ 3-4 & \\ 2-3 & \end{array}$

$\begin{array}{ll}0.00 & 3.87 \\ 0.05 & 3.89 \\ 0.02 & 3.87\end{array}$

$0.83 \quad 3.13$

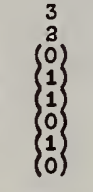

$\begin{array}{llll}0.84 & 3.20 & 5-4 & a^{5} F-y^{5} D^{0} \\ 0.83 & 3.18 & 4-3 & (37) \\ 0.82 & 3.17 & 3-2 & \\ 0.81 & 3.16 & 2-1 & \\ 0.81 & 3.16 & 1-0 & \\ 0.83 & 3.20 & 4-4 & \\ 0.82 & 3.18 & 3-3 & \\ 0.81 & 3.17 & 2-2 & \\ 0.81 & 3.16 & 1-1 & \\ 0.84 & & & \end{array}$

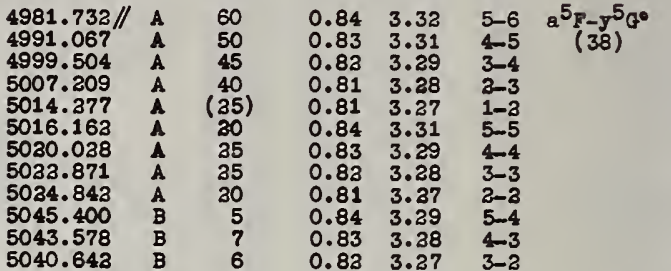

4953.37

4938.895
4941.332

4947.994
4909.105

4936.148
4937.719

4801.93

4801.90
4806.75

$\begin{array}{ll}-3 & a^{3} F-\nabla^{3} D^{0} \\ (36) & \end{array}$

4787.64
4793.34

4793.34
4816.47

4781.718

4781.718
4789.80

$\begin{array}{lc}4-5 & a^{3} p-m^{3} g^{\circ} \\ 3-4 & (37)\end{array}$

4758.913
4771.103

4771.103
4783.306

4533.238

4533.238
4534.782
4535.574

$\begin{array}{ll}-3 & a^{3} p-y^{3} p 0 \\ (38)^{0} & \end{array}$

4535.574
4535.920

4536.051
4555.486

4553.453

4548.764
4544.688

4513.734

4518.023
4532.798 


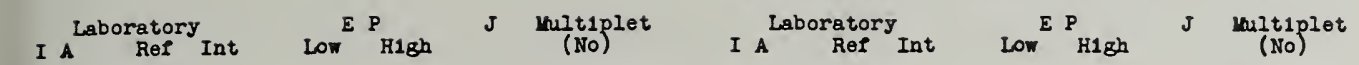
11 I oont1nued

\begin{tabular}{|c|c|c|c|c|c|c|}
\hline .4174 .0888 & B & (1) & 0.90 & 3.85 & $2-3$ & $a^{1} D-\nabla^{3} D^{0}$ \\
\hline 3904.785 & A & $40 n$ & 0.90 & 4.06 & $2-3$ & $a^{1} D-y^{1} F^{\circ}$ \\
\hline 3786.043 & $A$ & 30 & 0.90 & 4.16 & $3-1$ & \\
\hline 3610.154 & A & 12 & 0.90 & 4.31 & $2-1$ & a) \\
\hline 3598.714 & A & 15 & 0.90 & 4.33 & $2-3$ & $a^{1} D-x^{1} D^{\circ}$ \\
\hline $\begin{array}{c}3324.61 \\
.3341 .554\end{array}$ & $\frac{a}{E}$ & $\begin{array}{l}1 \\
1\end{array}$ & $\begin{array}{l}0.90 \\
0.90\end{array}$ & $\begin{array}{l}4.61 \\
4.59\end{array}$ & $\begin{array}{l}2-3 \\
2-1\end{array}$ & $\begin{array}{c}a^{1} D-w^{3} p^{0} \\
(60)\end{array}$ \\
\hline 3299.413 & E & 10 & 0.90 & 4.64 & $2-3$ & $a^{1} p-v^{3} G^{0}$ \\
\hline 3393.078 & $A$ & 30 & 0.90 & 4.64 & $2-3$ & \\
\hline $\begin{array}{l}3278.9238 \\
=3288.59\end{array}$ & $\frac{\mathrm{E}}{\mathrm{P}}$ & $\begin{array}{l}(12) \\
\left.T_{1}\right)\end{array}$ & $\begin{array}{l}0.90 \\
0.90\end{array}$ & $\begin{array}{l}4.66 \\
4.65\end{array}$ & $\begin{array}{l}3-3 \\
3-3\end{array}$ & $\begin{array}{c}a^{1} \frac{u^{3}}{(63)} F^{0} \\
(63)\end{array}$ \\
\hline
\end{tabular}

T1 I continued

$3267.41 \quad E \quad$ tr $\quad 0.90 \quad 4.67 \quad 2-1 \quad Q^{1} \mathrm{D}-\mathrm{u}^{3} \mathrm{D}^{\mathrm{C}}$

$\begin{array}{llllllll}3172.731 & \mathrm{E} & 4 & 0.90 & 4.79 & 2-3 & \mathrm{a}^{1} \mathrm{DD} \mathrm{t}^{3} \mathrm{D} \\ 3179.291 & \mathrm{E} & 3 & 0.90 & 4.78 & 3-3 & (65)\end{array}$

$3141.537 \quad E \quad 15 \quad 0.90 \quad 4.82 \quad 2-1 \quad Q^{1} D-x^{1} P^{0}$

$\begin{array}{llll}0.90 & 4.85 \quad 2-1 & a^{1} D_{-1}{ }^{1} p^{\circ} \\ (67)\end{array}$

$\begin{array}{llll}1.06 & 3.48 & 2-3 & a^{3} P-z^{3} D^{\circ} \\ 1.05 & 3.47 & 1-2 & (68)\end{array}$

$\begin{array}{ll}1.05 & 3.47 \\ 1.04 & 3.46\end{array}$

$\begin{array}{ll}1.04 & 3.46 \\ 1.06 & 3.4 ? \\ 1.05 & 3.46\end{array}$

$\begin{array}{ll}1.05 & 3.46 \\ 1.06 & 3.46\end{array}$

$1.06 \quad 3.08$

$\begin{array}{ll}1.05 & 3.08 \\ 1.04 & 3.08\end{array}$

(0) $\begin{array}{lllll}1.06 & 3.10 & \mathrm{a}-3 & \mathrm{a}^{3} \mathrm{p}_{-2} \mathrm{~s}^{\circ} \\ 1.05 & 3.10 & 1-\mathrm{a} & (70)\end{array}$

2-1 $a^{3} P-z^{3} g^{0}$

6126.31
6085.32
6064.63

6058.7

5918.54

5903.317
5880.306

5866.453

5899.295
5833.113

5937.806

5941.755
5980.89

5814.0
3809.7

5395.781
5382.378

5284.380

5313.239
5300.012

4691.336

.4710 .18

4733.171

4722.60
4747.25

$4690.82 ?$

4675.118

-668.35
-6667.53

4692.45
4679.73

4433.823

4404.397
4394.855

4435.840

405.69
4427.13
4508.51

4308.514
4302.979

4323.440
4310.373

.4288 .16

4293.676

4078.47

4083.456

$\$ 060.263$

3989.581

3972.130
3963.354

3782.139
3786.445

3735.155

3703.291

3480.535

3485.689
3499.099

3495.754

3478.260
3478.918

3476.452
3463.305
$1.06 \quad 3.15 \quad 2-a \quad a^{3} P_{-2} z^{3} p^{0}$

$\begin{array}{llll}1.06 & 3.15 & 3-1 & (71) \\ 1.05 & 3.15 & 1-3 & \end{array}$

$\begin{array}{cccc}1.06 & 3.17 & 3-3 & a^{3} p-y^{3} D^{0} \\ 1.05 & 3.14 & 1-3 & (73)\end{array}$

$\begin{array}{ll}1.05 & 3.14 \\ 1.04 & 3.13\end{array}$

$1.06 \quad 3.14$

$\begin{array}{ll}1.05 & 3.13 \\ 1.06 & 3.13\end{array}$

$\begin{array}{ll}1.06 & 3.18 \\ 1.05 & 3.17\end{array}$

$0-1$
$3-3$

$1.05 \quad 3.17$

$\begin{array}{ll}3-3 & a^{3} P-y^{5} D^{\circ} \\ 1-3 & (73)\end{array}$

$\begin{array}{llll}1.06 & 3.39 & 3-3 & a^{3} P-x^{3} D^{\circ} \\ 1.05 & 3.38 & 1-3 & (74)\end{array}$

(1) $\begin{array}{lll}3 & 1.04 & 3.38 \\ \text { (1) } & 1.06 & 3.38\end{array}$

$\begin{array}{lll}1.06 & 3.38 \\ 1.05 & 3.38 & 1-1\end{array}$

$\begin{array}{ll}1.06 & 3.69 \\ 1.05 & 3.67\end{array}$

1.043 .66

$1.05 \quad 3.66 \quad 1-1$

$\begin{array}{lll}1.06 & 3.66 & 1-1 \\ 1.06 & 3.1\end{array}$

$\begin{array}{llll}1.06 & 3.69 & 2-3 & a^{3} P-x^{3} G^{\circ}\end{array}$

$\begin{array}{cccc}1.06 & 3.70 & 2-3 & a^{3} P-x^{5} D^{\circ} \\ 1.05 & 3.69 & 1-3 & (77)\end{array}$

$\begin{array}{lll}1.04 & 3.69 & 0-1 \\ 1.06 & 3.69 & 2-3\end{array}$

$\begin{array}{lll}1.06 & 3.69 & 2-1 \\ 1.05 & 3.69 & 1-1\end{array}$

$\begin{array}{ccccc}10 & 1.06 & 3.85 & 2-3 & a^{3} P-r^{3} D^{0} \\ 5 & 1.05 & 3.85 & 1-3 & (78)\end{array}$

$\begin{array}{ccc}5 & 1.05 & 3.85 \\ (3) & 1.04 & 3.85 \\ 3 & 1.06 & 3.85 \\ 3 & 1.05 & 3.85\end{array}$

$\begin{array}{ll}1.05 & 3.85 \\ 1.06 & 3.85\end{array}$

$\begin{array}{ll}1.06 & 3.93 \\ 1.05 & 3.93\end{array}$

$\begin{array}{ll}1.05 & 3.93 \\ 1.06 & 3.92 \\ 1.05 & 3.91\end{array}$

$\begin{array}{ll}1.05 & 3.91 \\ 1.05 & 3.93 \\ 1.04 & 3.93\end{array}$

$\begin{array}{ll}1.06 & 4.09 \\ 1.05 & 4.09\end{array}$

$\begin{array}{ll}1.05 & 4.09 \\ 1.06 & 4.09 \\ 1.05 & 4.08\end{array}$

$\begin{array}{ll}1.05 & 4.08 \\ 1.05 & 4.09 \\ 1.04 & 4.09\end{array}$

$\begin{array}{lllll}0 & 0 \\ 0 & 1.06 & 4.16 & 2-1 & a^{3} p-z^{1} p^{p} \\ 0 & 1.05 & 4.16 & 1-1 & (81) \\ 1.04 & 4.16 & 0-1 & \end{array}$

$\begin{array}{llll}1.06 & 4.33 & 2-3 & a^{3} P-x^{1} D^{\bullet} \\ 1.05 & 4.33 & 1-3 & (83)\end{array}$

$\begin{array}{lllc}1.06 & 4.37 & 2-1 & \mathrm{~s}^{3} \mathrm{p}-\mathrm{y}^{3} \mathrm{~g}^{*} \\ 1.05 & 4.37 & 1-1 & (83) \\ 1.04 & 4.37 & 0-1 & \end{array}$

$\begin{array}{llll}1.06 & 4.61 & 2-3 & a^{3} p-\pi^{3} p^{\circ} \\ 1.05 & 4.59 & 1-1 & (84)\end{array}$

$\begin{array}{lll}1.05 & 4.59 & 1-1 \\ 1.06 & 4.59 & 3-1\end{array}$

$\begin{array}{lll}1.05 & 4.58 & 1-0 \\ 1.05 & 4.61 & 1-2\end{array}$

$1.04 \quad 4.59$

$\begin{array}{llll}1.06 & 4.61 & 2-2 & a^{3} P-y^{5} 8^{\circ} \\ 1.05 & 4.61 & 1-3 & \end{array}$
3398.634
3403.369

3405.094
03417.88

$\begin{array}{lll}1.04 & 4.67 & 0-1 \\ 1.06 & 4.69 & a-3\end{array}$

$\begin{array}{lll}1.06 & 4.69 & 2-3 \\ 1.05 & 4.67 & 1-1\end{array}$

(n)

$\begin{array}{llllllll}3314.432 & A & 10 & 1.06 & 4.79 & 2-3 & a^{3} \mathrm{P}-\mathrm{t}^{3} \mathrm{D}^{\circ} \\ 3309.501 & \mathrm{E} & 15 & 1.05 & 4.78 & 1-2 & (87)\end{array}$

1.04 4.77 $0-1$

3331.588

$\begin{array}{llllll}3314.533 & E & 8 & 1.06 & 4.78 & 2-1 \\ 3336.639 & E & 3 & 1.05 & 4.77 & 1-1 \\ 3 & & 1.06 & 4.77 & 3-1\end{array}$

3280.391

$\begin{array}{llll}1.06 & 4.83 & 2-1 & a^{3} P-x^{1} p^{\circ}\end{array}$

$\begin{array}{llll}1.05 & 4.82 & 2-1 & 8^{3}-x^{1} \\ 1.05 & 4.82 & 1-1 & (88) \\ 1.04 & 4.83 & 0-1 & \end{array}$

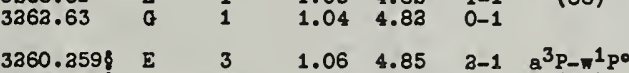

3260.2598
-3248.6028

$\begin{array}{llll}1.06 & 4.85 & 2-1 & a^{3} P-m^{1} P^{\circ} \\ 1.05 & 4.85 & 1-1 & (89)\end{array}$

- 3213.1458

$\begin{array}{lllc}1.06 & 4.90 & 2-3 & \mathrm{a}^{3} \mathrm{P}-\mathrm{s}^{3} \mathrm{D}^{\circ} \\ 1.05 & 4.90 & 1-2 & (90) \\ 1.04 & 4.90 & 0-1 & \end{array}$

3201.594

$\begin{array}{ll}1.04 & 4.90 \\ 1.06 & 4.90 \\ 1.05 & 4.90\end{array}$

3318.683

3137.353

$\begin{array}{ll}1.05 & 4.90 \\ 1.06 & 4.90\end{array}$

$\begin{array}{ccccccc}3137.353 & E & (1) & 1.06 & 5.00 & 2-2 & a^{3} P_{-} r^{3} p 0 \\ 3134.654 & E & 1 & 1.05 & 4.99 & 1-1 & (91)\end{array}$

3145.515

$\begin{array}{ll}1.06 & 4.99 \\ 1.05 & 4.98\end{array}$

1.065 .04

.3100 .666

3113.483

$\begin{array}{ll}1.06 & 5.02 \\ 1.05 & 5.01 \\ 1.06 & 5.01\end{array}$

$1-2$
$0-1$
$2-3$
$1-1$

$3-3 \quad a^{3} P-r^{3} D^{0}$

.3100 .666

$\begin{array}{rrrrrrr}3090.137 & E & 13 & 1.06 & 5.04 & 2-1 & a^{3} P-x^{3} \mathrm{~s} \\ 3084.819 & E & 8 & 1.05 & 5.04 & 1-1 & (93)\end{array}$

2965.707

2965. 231

3965.68

3970.556
2980.296

$\begin{array}{lll}15 & 1.06 \quad 5.32\end{array}$

$\begin{array}{cc}2-3 & a^{3} P-q^{3} D^{\circ} \\ 1-2 & (94) \\ 0-1 & \end{array}$

$\begin{array}{ll}1.06 & 5.31 \\ 1.05 & 5.30\end{array}$

$2-1$
$2-3$
$1-1$
$2-1$

10034.45

10048.78

10189.36

\begin{tabular}{lll}
4 & 1.05 & 5.30 \\
tr & 1.06 & 5.30 \\
\hline
\end{tabular}

$\begin{array}{ll}1.45 & 3.68 \\ 1.44 & 2.67 \\ 1.48 & 3.65\end{array}$

$\begin{array}{llll}1.44 & 2.67 & 4-5 & b^{3} F_{-2} z^{3} \\ 1.40 & 3-4 & (95)\end{array}$

$\begin{array}{ll}1.43 & 3.65 \\ 1.45 & 3.67\end{array}$

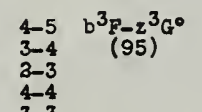

(1) $1.43 \quad 3.10$

$\begin{array}{ll}2-3 & b^{3} F-z^{5} s^{\circ} \\ 4-4 & b^{3}(96) 3^{3} \\ 3-3 & (97)\end{array}$

7344.72

7364.11

7371.41
7399.67

$3 \quad \begin{array}{lll}1.45 & 3.13 \\ 3 & 1.44 & 3.11\end{array}$

(2) $\begin{array}{lll}1.43 & 3.10 \\ 0 & 1.44 & 3.10\end{array}$

$\begin{array}{ll}1.44 & 3.13 \\ 1.42 & 3.11\end{array}$

$3-3$
$3-3$
$3-4$
$3-3$

7316.20

7160.33
7138.05

(3) $\begin{array}{lll}1.44 & 3.15 \\ \text { (3) } & 1.43 & 3.15\end{array}$

3-2 $\mathrm{b}^{3} \mathrm{~F}-\mathrm{z}^{3} \mathrm{p}$

7309.44

7344.86

731.74
7138.91
7188.55

7084.25

$\begin{array}{lllll}30 & 1.45 & 3.17 & 4-3 & b^{3} F-y^{3} D^{0} \\ 10 & 1.44 & 3.14 & 3-3 & (99)\end{array}$

$\begin{array}{llll}8 & 1.43 & 3.13 & 2-1 \\ (1) T 11 & 1.44 & 3.17 & 3-3 \\ 0 & 1.43 & 3.14 & 2-3\end{array}$

$7065.15 ?$

7130.34
.7007 .81

(1) $\begin{array}{ll}1.42 & 3.14 \\ 1.43 & 3.17\end{array}$

(1) $\begin{array}{llll}1.45 & 3.30 & 4-4 & b^{3} 5-y^{5} p^{\circ} \\ 1.45 & 3.18 & 4-3 & (100)\end{array}$

(1) $\begin{array}{lll}1.45 & 3.18 \\ & 1.43\end{array}$

(an) $1.45 \quad 3.31$

$\begin{array}{ll}1.45 & 3.34 \\ 1.44 & 3.32\end{array}$

$\begin{array}{ll}1.44 & 3.32 \\ 1.42 & 3.31\end{array}$

$\begin{array}{ll}1.42 & 3.31 \\ 1.44 & 3.31 \\ 1.44 & 3.34\end{array}$

$\begin{array}{ll}1.44 & 3.34 \\ 1.43 & 3.32\end{array}$

$1.45 \quad 3.39 \quad 4-3 \quad b^{3} F-x^{3} D^{\circ}$

$$
\begin{array}{ll}
4-3 & (100) \\
2-3 &
\end{array}
$$

6556.066

6546.376

6497.689
6508.135

6366.354

6366.354
6336.104

6318.037
6311.289

6393.00

6268.50

6358.706
6358.103

6361.101

6313.240
6303.754

5839.78

5513.539

5514.536

5514.350
5471.198

5481.863

5449.155

5440.53

5490.151
5473.686

8
8
5
$(1)$

$\begin{array}{llll}1.3-3 & (103)\end{array}$

(1) $1.44 \quad 3.39 \quad 3-1$

(a) $\begin{array}{llll}1.42 & 3.38 & 3-2 \\ 1.43 & 3.39 & 2-3\end{array}$

$50 \quad 1.45 \quad 3.43 \quad 4-5 \quad b^{3} 7-y^{3} G^{0}$

$\begin{array}{llll}1.45 & 3.43 & 4-5 & b^{3} 7_{-5} y^{3} a^{0} \\ 1.44 & 3.41 & 3-4 & (104)\end{array}$

$\begin{array}{lll}1.44 & 3.41 & 3-4 \\ 1.43 & 3.39 & 2-3 \\ 1.45 & 3.41 & 4-4 \\ 1.44 & 3.39 & 3-3\end{array}$

$\begin{array}{llll}1.45 & 3.57 & 4-5 & b^{3} F-y^{5} F^{\circ} \\ 1.45 & 3.54 & 4-3 & (105)\end{array}$

$\begin{array}{llll}1.45 & 3.69 & 4-3 & b^{3} F-m^{3} D^{\circ} \\ 1.44 & 3.67 & 3-2 & (106)\end{array}$

$\begin{array}{ll}1.42 & 3.86 \\ 1.44 & 3.69\end{array}$

$\begin{array}{ll}1.44 & 3.69 \\ 1.42 & 3.67\end{array}$

$\begin{array}{llll}1.44 & 3.70 & 3-3 & b^{3} F-x^{5} D^{\circ} \\ 1.43 & 3.69 & 2-2 & (107)\end{array}$

$3-1$
$3-3$
$2-3$

$\begin{array}{lll}1.45 & 3.70 & 4-3 \\ 1.44 & 3.69 & 3-2\end{array}$

(107)

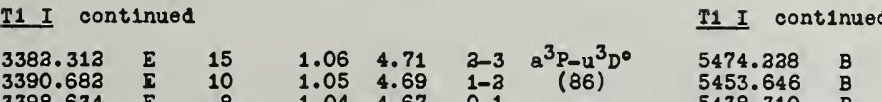

0

5453.646
5438.310

5494.726
5470.50

5470.50
.5511 .795

5145.465

\subsection{8}

5109.437

5081.39

5035.908

5036.468
5038.400

5071.475
5065.985

4742.32

4742.32
4711.68

4687.83

4559.920
4535.87

4518.700
4564.316

4540.873
$\mathbf{m 4 5 3 1 . 6 0}$

$\mathrm{m} 4531.60$
$\mathbf{4 5 1 3 . 7 1 5}$

4457.438
4455.321

4455.321
4453.312

4453.313
.4482 .688
-4474.853

4430.366
.4434 .003

4137.09

3789.293

3795.903
3798.276

3717.259

3715.795
3713.734
3738.676

3704.295

3694.445
3685.964

3651.90

3638.49
3656.73

3487.

3487.80

3443.644
3444.403
3423.173
3430.874

3434.69

3297.68
3309.32

3274.047

3270.563
3325.480

3259.42
3359.04

\section{$\left.\begin{array}{c}6 \\ 3 \\ 1 \\ 1 \\ 2 \\ 3\end{array}\right)$}

$\begin{array}{llll}1.45 & 3.71 & 4-5 & b^{3} F-x^{3} G^{0} \\ 1.44 & 3.70 & 3-4 & (108) \\ 1.43 & 3.69 & 2-3 & \\ 1.45 & 3.70 & 4-4 & \\ 1.44 & 3.69 & 3-3 & \end{array}$

$1.45 \quad 3.69 \quad 4-3$

$1.45 \quad 3.85 \quad 4-3 \quad b^{3} F-\nabla^{3} D^{\circ}$

$\begin{array}{lll}1.44 & 3.85 & 3-3 \\ 1.42 & 3.85 & 2-1\end{array}$

$\begin{array}{lll}1.44 & 3.85 & 3-3 \\ 1.42 & 3.85 & 2-2\end{array}$

$\begin{array}{lll}1.43 & 3.85 & 2-3\end{array}$

$\begin{array}{llll}1.45 & 3.90 & 4-5 & b^{3} F-m^{3} a^{\circ} \\ 1.44 & 3.89 & 3-4 & (110)\end{array}$

$\begin{array}{lll}1.44 & 3.87 & 2-4 \\ 1.45 & 3.89 & 2-3\end{array}$

$\begin{array}{lll}1.45 & 3.89 & 4-4 \\ 1.44 & 3.87 & 3-3\end{array}$

$1.45 \quad 4.06 \quad 4-3 \quad b^{3} \mathrm{~F}-\mathrm{y}^{1} F^{0}$

$\begin{array}{llll}1.44 & 4.06 & 3-3 & \text { (111) } \\ 1.42 & 4.06 & 2-3 & \end{array}$

$1.45 \quad 4.16 \quad 4-4 \quad b^{3} F-\pi^{3} F^{\circ}$

$1.44 \quad 4.16$ 3-3 (112)

$\begin{array}{lll}1.42 & 4.15 & 2-2 \\ 1.45 & 4.16 & 4-3\end{array}$

$\begin{array}{lll}1.44 & 4.15 & 3-2 \\ 1.44 & 4.16 & 3-4\end{array}$

$1.45 \quad 4.33 \quad 4-4 \quad b^{3} F-\nabla^{3} F^{\circ}$

$1.44 \quad 4.31 \quad 3-3 \quad(113)$

$1.42 \quad 4.19 \quad 2-2$

1.44 4.19 3-a

$\begin{array}{llll}1.42 & 4.23 & 3-4\end{array}$

$1.45 \quad 4.44 \quad 4-4 \quad b^{3} F-y^{1} a^{\circ}$

$\begin{array}{llll}1.45 & 4.71 & 4-3 & b^{3} F-u^{3} D^{\circ} \\ 1.44 & 4.69 & 3-2 & (115)\end{array}$ 
I A ${ }_{\text {Ref }}^{\text {Laboratory }}$ Int

\begin{tabular}{|c|c|c|c|c|c|c|c|c|c|c|c|c|c|c|c|c|c|c|c|c|}
\hline 1 I oon & nus & & & & & & $\underline{T 1 I}$ oon & ux & & & & & & T1 I con & tinue & & & & & \\
\hline $\begin{array}{l}8457.10 \\
8494.42 \\
8531.36 \\
8550.54 \\
8565.45\end{array}$ & $\begin{array}{l}D \\
D \\
D \\
D \\
D\end{array}$ & $\begin{array}{l}40 \\
30 \\
15 \\
35 \\
25 \\
\end{array}$ & $\begin{array}{l}1.74 \\
1.73 \\
1.73 \\
1.74 \\
1.73\end{array}$ & $\begin{array}{l}3.20 \\
3.18 \\
3.17 \\
3.18 \\
3.17\end{array}$ & $\begin{array}{l}3-4 \\
3-3 \\
1-2 \\
3-3 \\
2-3 \\
1-9\end{array}$ & $\begin{array}{l}a^{5} P-y^{5} D^{0} \\
(14.1)\end{array}$ & $\begin{array}{r}4363.134 \\
+4274.584 \\
4382.702 \\
4251.618 \\
4265.723\end{array}$ & $\begin{array}{l}\mathrm{A} \\
\mathrm{A} \\
\mathrm{A} \\
\mathrm{B} \\
\mathrm{B}\end{array}$ & $\begin{array}{r}15 \\
15 \\
12 \\
3 \\
4\end{array}$ & $\begin{array}{l}1.88 \\
1.87 \\
1.87 \\
1.87 \\
1.87\end{array}$ & $\begin{array}{l}4.77 \\
4.76 \\
4.75 \\
4.77 \\
4.76\end{array}$ & $\begin{array}{l}5-4 \\
4-3 \\
3-2 \\
4-4 \\
3-3\end{array}$ & $\begin{array}{c}a^{3} G-t^{3} F^{0} \\
(162)\end{array}$ & $\begin{array}{l}3143.350 \\
3139.87 \\
3135.069 \\
3130.175 \\
3127.684\end{array}$ & $\begin{array}{l}\mathrm{E} \\
\mathrm{E} \\
\mathbf{E} \\
\mathrm{E} \\
\mathrm{E}\end{array}$ & $\begin{array}{c}12 \mathrm{~N} \\
10 \mathrm{~N} \\
8 \mathrm{~N} \\
8 \mathrm{~N} \\
8 \mathrm{~N}\end{array}$ & $\begin{array}{l}2.03 \\
3.01 \\
1.99 \\
1.97 \\
1.96\end{array}$ & $\begin{array}{l}5.96 \\
5.94 \\
5.92 \\
5.91 \\
5.91\end{array}$ & $\begin{array}{l}6-7 \\
5-6 \\
4-5 \\
3-4 \\
2-3\end{array}$ & $\begin{array}{c}2^{5} a^{0}-8^{5} k \\
(180)\end{array}$ \\
\hline $\begin{array}{r}8578.40 \\
8612.91 \\
+8600.98\end{array}$ & $\begin{array}{l}D \\
D \\
D\end{array}$ & $\begin{array}{r}15 \\
7 \\
35\end{array}$ & $\begin{array}{l}1.73 \\
1.73 \\
1.73\end{array}$ & $\begin{array}{l}3.16 \\
3.16 \\
3.16\end{array}$ & $\begin{array}{l}1-1 \\
2=1 \\
1-0\end{array}$ & & $\begin{array}{l}4169.330 \\
4166.311\end{array}$ & $\begin{array}{l}B \\
B\end{array}$ & $\begin{array}{l}7 \\
6\end{array}$ & $\begin{array}{l}1.88 \\
1.87\end{array}$ & $\begin{array}{l}4.84 \\
4.83\end{array}$ & $\begin{array}{l}5-6 \\
4-5\end{array}$ & $\begin{array}{l}a^{3} G-x^{3} H^{\circ} \\
(163)\end{array}$ & $\begin{array}{l}3133.769 \\
3118.130\end{array}$ & $\frac{E}{E}$ & $\begin{array}{l}30 \mathrm{n} \\
15\end{array}$ & $\begin{array}{l}3.03 \\
3.01\end{array}$ & $\begin{array}{l}5.98 \\
5.97\end{array}$ & $\begin{array}{l}6-5 \\
5-4\end{array}$ & $\begin{array}{c}2^{5} G^{0}-j^{5} p \\
(181)^{2}\end{array}$ \\
\hline $\begin{array}{l}7474.94 \\
7466.44 \\
7431.98\end{array}$ & $\begin{array}{l}E \\
P \\
P\end{array}$ & (1pq) & $\begin{array}{l}1.74 \\
1.73 \\
1.73\end{array}$ & $\begin{array}{l}3.39 \\
3.38 \\
3.39\end{array}$ & $\begin{array}{l}3-3 \\
2-2 \\
2-3\end{array}$ & $\begin{array}{l}a^{5} P-x^{3} D^{\circ} \\
(14 a)\end{array}$ & $\begin{array}{l}4164.134 \\
4177.357 \\
4173.609\end{array}$ & $\begin{array}{l}\mathrm{B} \\
\mathrm{E} \\
\mathbf{E}\end{array}$ & $\left(\begin{array}{l}4 \\
3 \\
3\end{array}\right)$ & $\begin{array}{l}1.87 \\
1.88 \\
1.87\end{array}$ & $\begin{array}{l}4.83 \\
4.83 \\
4.83\end{array}$ & $\begin{array}{l}3-4 \\
5-5 \\
4-4\end{array}$ & & $\begin{array}{l}3114.092 \\
3111.383 \\
3107.468 \\
3105.320\end{array}$ & $\begin{array}{l}E \\
E \\
E \\
E\end{array}$ & $\begin{array}{l}30 n \\
10 n \\
12 n \\
2 n\end{array}$ & $\begin{array}{l}1.99 \\
1.97 \\
1.96 \\
3.01\end{array}$ & $\begin{array}{l}5.95 \\
5.94 \\
5.93 \\
5.98\end{array}$ & $\begin{array}{l}4-3 \\
3-3 \\
2-1 \\
5-5\end{array}$ & \\
\hline $\begin{array}{r}7253.76 \\
7291.03 \\
7305.87\end{array}$ & $\frac{T}{P}$ & (1pq) & $\begin{array}{l}1.74 \\
1.73 \\
1.73\end{array}$ & $\begin{array}{l}3.44 \\
3.42 \\
3.42\end{array}$ & $\begin{array}{l}3-3 \\
2-2 \\
1-1\end{array}$ & $\begin{array}{c}a^{5} p_{-2} 5 p^{0} \\
(143)\end{array}$ & $\begin{array}{l}3895.59 \\
3885.95 \\
3878.61\end{array}$ & $\begin{array}{l}P \\
P \\
P\end{array}$ & & $\begin{array}{l}1.88 \\
1.87 \\
1.87\end{array}$ & $\begin{array}{l}5.05 \\
5.05 \\
5.05\end{array}$ & $\begin{array}{l}5-4 \\
4-4 \\
3-4\end{array}$ & $\begin{array}{c}a^{3} G-r^{1} G^{\circ} \\
(164)\end{array}$ & $\begin{array}{l}3102.517 \\
3101.526 \\
3101.77\end{array}$ & $\begin{array}{l}\bar{E} \\
\bar{E} \\
G\end{array}$ & $\begin{array}{l}3 n \\
4 n \\
1 n\end{array}$ & $\begin{array}{l}1.99 \\
1.97 \\
1.96\end{array}$ & $\begin{array}{l}5.97 \\
5.95 \\
5.94\end{array}$ & $\begin{array}{l}4-4 \\
3-3 \\
2-2\end{array}$ & \\
\hline $\begin{array}{l}7305.87 \\
7332.26 \\
7330.97 \\
7213.35\end{array}$ & $\begin{array}{l}\text { R } \\
I \\
I \\
\text { E }\end{array}$ & $\left\{\begin{array}{l}19 \\
1 p q \\
1 p^{\prime} \\
1\}\end{array}\right\}$ & $\begin{array}{l}1.73 \\
1.74 \\
1.73 \\
1.73\end{array}$ & $\begin{array}{l}3.46 \\
3.43 \\
3.43 \\
3.44\end{array}$ & $\begin{array}{l}1-1 \\
3-2 \\
2-1 \\
2-3\end{array}$ & & $\begin{array}{l}3786.253 \\
3801.093 \\
3811.385\end{array}$ & $\begin{array}{l}\mathrm{B} \\
\mathrm{B} \\
\mathrm{B}\end{array}$ & $\begin{array}{l}3 \\
3 \\
4 .\end{array}$ & $\begin{array}{l}1.88 \\
1.87 \\
1.87\end{array}$ & $\begin{array}{l}5.14 \\
5.12 \\
5.10\end{array}$ & $\begin{array}{l}5-4 \\
4-3 \\
3-2\end{array}$ & $\begin{array}{l}a^{3} G-8^{3} F^{0} \\
(165)\end{array}$ & $\begin{array}{l}8518.05 \\
8467.15\end{array}$ & $\begin{array}{l}D \\
D\end{array}$ & $\begin{array}{l}60 \\
75\end{array}$ & $\begin{array}{l}3.13 \\
3.11\end{array}$ & $\begin{array}{l}3.57 \\
3.57\end{array}$ & $\begin{array}{l}5-4 \\
4-3\end{array}$ & $\begin{array}{c}2^{5} F^{0}-a^{5} D \\
(182)\end{array}$ \\
\hline $\begin{array}{l}7366.29 \\
6266.021 \\
6364.825 \\
6277.525\end{array}$ & $\begin{array}{l}\bar{E} \\
\bar{E} \\
E \\
E\end{array}$ & $\begin{array}{r}(0) \\
\left(\begin{array}{l}1 \\
0 \\
(00\end{array}\right)\end{array}$ & $\begin{array}{l}1.73 \\
1.74 \\
1.73 \\
1.73\end{array}$ & $\begin{array}{l}3.42 \\
3.71 \\
3.70 \\
3.69\end{array}$ & $\begin{array}{l}1-2 \\
3-4 \\
2-3 \\
1-2\end{array}$ & $\begin{array}{l}a^{5} p-x^{5} D^{0} \\
(144)\end{array}$ & $\begin{array}{l}3733.767 \\
3738.901 \\
3748.101\end{array}$ & $\begin{array}{l}\mathrm{B} \\
\mathrm{E} \\
\mathrm{B}\end{array}$ & $\begin{array}{l}4 n \\
5 n \\
6 n\end{array}$ & $\begin{array}{l}1.88 \\
1.87 \\
1.87\end{array}$ & $\begin{array}{l}5.18 \\
5.17 \\
5.16\end{array}$ & $\begin{array}{l}5-6 \\
4-5 \\
3-4\end{array}$ & $\begin{array}{l}a^{3} G-\pi^{3} H^{0}+ \\
(166)\end{array}$ & $\begin{array}{l}8424.41 \\
8389.48 \\
8417.54 \\
8386.24 \\
8363.58\end{array}$ & $\begin{array}{l}D \\
D \\
D \\
D \\
P \\
P\end{array}$ & $\begin{array}{l}50 \\
25 \\
25\end{array}$ & $\begin{array}{l}2.09 \\
3.08 \\
2.11 \\
3.09 \\
3.08\end{array}$ & $\begin{array}{l}3.56 \\
3.55 \\
3.57 \\
3.57 \\
3.56\end{array}$ & $\begin{array}{l}3-0 \\
3-2 \\
2-1 \\
4-4 \\
3-3 \\
2-2\end{array}$ & \\
\hline $\begin{array}{l}6295.251 \\
6295.949 \\
6298.075\end{array}$ & $\begin{array}{l}\frac{E}{E} \\
\frac{E}{E}\end{array}$ & $\left.\begin{array}{r}\left(\begin{array}{l}3 \\
0 \\
0\end{array}\right) \\
(000\end{array}\right)$ & $\begin{array}{l}1.74 \\
1.73 \\
1.73\end{array}$ & $\begin{array}{l}3.70 \\
3.69 \\
3.69\end{array}$ & $\begin{array}{l}3-3 \\
2-2 \\
1-1\end{array}$ & & $\begin{array}{l}3504.773 \\
3516.838 \\
3525.161\end{array}$ & $\begin{array}{l}\mathrm{E} \\
\mathrm{E} \\
\mathrm{E}\end{array}$ & $\begin{array}{l}2 \\
3 \\
3\end{array}$ & $\begin{array}{l}1.88 \\
1.87 \\
1.87\end{array}$ & $\begin{array}{l}5.40 \\
5.38 \\
5.37\end{array}$ & $\begin{array}{l}5-4 \\
4-3 \\
3-2\end{array}$ & $\begin{array}{c}a^{3} a-r^{3} p^{0} \\
(167)\end{array}$ & $\begin{array}{l}5234.301 \\
5224.938\end{array}$ & A & $\begin{array}{r}15 \\
8\end{array}$ & $\begin{array}{l}3.13 \\
2.11\end{array}$ & $\begin{array}{l}4.49 \\
4.47\end{array}$ & $\begin{array}{l}5-5 \\
4-4\end{array}$ & $\mathbf{2}^{2^{5} \mathbf{F}^{0}-e^{5} \mathbf{F}}$ \\
\hline $\begin{array}{l}4617.369 \\
4623.098 \\
4629.336 \\
4639.669 \\
4639.369 \\
4639.944 \\
4656.048 \\
4650.016 \\
4645.193 \\
4481.261\end{array}$ & $\begin{array}{l}A \\
A \\
A \\
B \\
B \\
B \\
B \\
B \\
A \\
A\end{array}$ & $\begin{array}{r}30 \\
25 \\
15 \\
15 \\
18 \\
15 \\
6 \\
10 \\
13 \\
30\end{array}$ & $\begin{array}{l}1.74 \\
1.73 \\
1.73 \\
1.74 \\
1.73 \\
1.73 \\
1.74 \\
1.73 \\
1.73 \\
1.74\end{array}$ & $\begin{array}{l}4.41 \\
4.40 \\
4.39 \\
4.40 \\
4.39 \\
4.39 \\
4.39 \\
4.39 \\
4.38 \\
4.50\end{array}$ & $\begin{array}{l}3-4 \\
2-3 \\
1-2 \\
3-3 \\
2-3 \\
1-1 \\
3-2 \\
2-1 \\
1-0 \\
3-3\end{array}$ & $\begin{array}{l}a^{5 p-\pi^{5} p^{0}} \\
(145)\end{array}$ & $\begin{array}{r}3428.955 \\
3446.603 \\
-3454.165 \\
3352.43 \\
3358.56 \\
3364.10 \\
3010.42\end{array}$ & $\begin{array}{l}E \\
E \\
E \\
E \\
P \\
P \\
P\end{array}$ & $\begin{array}{l}4 \\
3 \\
1\end{array}$ & $\begin{array}{l}1.88 \\
1.87 \\
1.88 \\
1.88 \\
1.87 \\
1.87 \\
1.87\end{array}$ & $\begin{array}{l}5.48 \\
5.45 \\
5.45 \\
5.56 \\
5.55 \\
5.53 \\
5.97\end{array}$ & $\begin{array}{l}5-5 \\
4-4 \\
5-4 \\
5-4 \\
4-3 \\
3-2 \\
4-3\end{array}$ & $\begin{array}{l}a^{3} G-q^{3} F^{\circ} \\
(169) \\
a^{3} G-u^{1} F^{\circ 0} \\
(170)\end{array}$ & $\begin{array}{r}5324.558 \\
5223.623 \\
5222.685 \\
5263.483 \\
5255.811 \\
5247.293 \\
5238.560 \\
5186.329 \\
5194.043 \\
5201.096 \\
5307.852\end{array}$ & $\begin{array}{l}\text { B } \\
\text { B } \\
\text { B } \\
\text { B } \\
\text { B } \\
\text { B } \\
\text { B } \\
\text { B } \\
\text { E } \\
\text { E } \\
\text { B }\end{array}$ & $\begin{array}{l}6 \\
6 \\
6 \\
3 \\
5 \\
5 \\
6 \\
3 \\
4 \\
4 \\
3\end{array}$ & $\begin{array}{l}2.09 \\
2.08 \\
3.08 \\
2.13 \\
2.11 \\
2.09 \\
2.08 \\
3.11 \\
3.09 \\
2.08 \\
2.08\end{array}$ & $\begin{array}{l}4.46 \\
4.45 \\
4.44 \\
4.47 \\
4.46 \\
4.45 \\
4.44 \\
4.49 \\
4.47 \\
4.46 \\
4.45\end{array}$ & $\begin{array}{l}3-3 \\
2-3 \\
1-1 \\
5-4 \\
4-3 \\
3-2 \\
2-1 \\
4-5 \\
3-4 \\
2-3 \\
1-2\end{array}$ & \\
\hline $\begin{array}{l}4481.261 \\
4480.600 \\
4479.724 \\
4496.146 \\
4489.089 \\
4465.807 \\
4471.238\end{array}$ & $\begin{array}{l}\text { A } \\
\text { B } \\
B \\
\text { A } \\
\text { A } \\
\text { A } \\
\text { A }\end{array}$ & $\begin{array}{r}30 \\
5 \\
9 \\
20 \\
20 \\
20 \\
20\end{array}$ & $\begin{array}{l}1.74 \\
1.73 \\
1.73 \\
1.74 \\
1.73 \\
1.73 \\
1.73\end{array}$ & $\begin{array}{l}4.50 \\
4.49 \\
4.48 \\
4.49 \\
4.48 \\
4.50 \\
4.49\end{array}$ & $\begin{array}{l}3-3 \\
2-2 \\
1-1 \\
3-2 \\
2-1 \\
2-3 \\
1-3\end{array}$ & 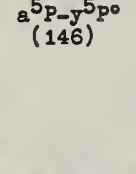 & $\begin{array}{c}5054.070 \\
3601.16\end{array}$ & J & $\begin{array}{l}3 \\
1\end{array}$ & $\begin{array}{l}1.87 \\
1.87\end{array}$ & $\begin{array}{l}4.31 \\
5.30\end{array}$ & $\begin{array}{l}0-1 \\
0-1\end{array}$ & $\begin{array}{l}a^{1} s-z^{1} p^{\circ} \\
(171) \\
a^{1} s-p^{1} p o \\
(173) \\
z^{5} g^{\circ}-e^{5} F\end{array}$ & $\begin{array}{r}4503.762 \\
4497.709 \\
4492.540 \\
m 4488.37 \\
4485.013 \\
+4526.374\end{array}$ & $\begin{array}{l}\text { A } \\
\text { B } \\
B \\
\text { P } \\
B \\
\text { E }\end{array}$ & $\begin{array}{c}4 \mathrm{n} \\
3 \\
3 \\
\mathrm{~T}^{+} \\
1 \\
1\end{array}$ & $\begin{array}{l}2.13 \\
3.11 \\
3.09 \\
2.08 \\
3.08 \\
2.13 \\
2.13\end{array}$ & $\begin{array}{l}4.87 \\
4.85 \\
4.84 \\
4.83 \\
4.83 \\
4.85\end{array}$ & $\begin{array}{l}5-5 \\
4-4 \\
3-3 \\
2-3 \\
1-1 \\
5-4\end{array}$ & $\begin{array}{c}2^{5} F^{0}-P^{5} r \\
(184)\end{array}$ \\
\hline $\begin{array}{r}4305.474 \\
-4291.214\end{array}$ & $\begin{array}{l}\text { B } \\
\text { B }\end{array}$ & $\stackrel{2}{5 n}$ & $\begin{array}{l}1.74 \\
1.73\end{array}$ & $\begin{array}{l}4.61 \\
4.61\end{array}$ & $\begin{array}{l}3-2 \\
2-2\end{array}$ & $a^{5} p-w^{3} p^{\circ}$ & $\begin{array}{l}5013.084 \\
5000.991 \\
4989.140\end{array}$ & $\begin{array}{l}B \\
B \\
B\end{array}$ & & $\begin{array}{l}3.01 \\
1.99 \\
1.97\end{array}$ & $\begin{array}{l}4.47 \\
4.46 \\
4.45\end{array}$ & $\begin{array}{l}5-4 \\
4-3 \\
3-2 \\
2-1\end{array}$ & & $\begin{array}{l}4515.610 \\
4505.715 \\
4496.75 \\
4475.518\end{array}$ & $\begin{array}{l}B \\
E \\
P \\
P\end{array}$ & $\begin{array}{l}1 \\
1\end{array}$ & $\begin{array}{l}2.11 \\
2.09 \\
2.08\end{array}$ & $\begin{array}{l}4.84 \\
4.83 \\
4.83\end{array}$ & $\begin{array}{l}4-3 \\
3-3 \\
2-1\end{array}$ & \\
\hline $\begin{array}{l}4299.229 \\
4284.988 \\
4276.441\end{array}$ & $\begin{array}{l}E \\
B \\
B\end{array}$ & $\begin{array}{r}15 \\
8 \\
8\end{array}$ & $\begin{array}{l}1.74 \\
1.73 \\
1.73\end{array}$ & $\begin{array}{l}4.61 \\
4.61 \\
4.61\end{array}$ & $\begin{array}{l}3-2 \\
2-2 \\
1-2\end{array}$ & $\begin{array}{c}a^{5} p-y^{5} s^{0} \\
(148)\end{array}$ & $\begin{array}{l}4977.731 \\
4973.051 \\
4968.566 \\
4964.713\end{array}$ & $\begin{array}{l}\text { A } \\
B \\
B \\
B \\
B \\
B\end{array}$ & $\begin{array}{r}10 \\
5 \\
6 \\
6 \\
5\end{array}$ & $\begin{array}{l}1.96 \\
2.01 \\
1.99 \\
1.97 \\
1.96\end{array}$ & $\begin{array}{l}4.44 \\
4.49 \\
4.47 \\
4.46 \\
4.45\end{array}$ & $\begin{array}{l}3-1 \\
5-5 \\
4-4 \\
3-3 \\
2-2\end{array}$ & & $\begin{array}{c}4475.518 \\
-4474.852 \\
4475.19 \\
4476.61\end{array}$ & $\begin{array}{l}\mathbf{E} \\
\mathbf{A} \\
\mathbf{P} \\
\mathrm{P}\end{array}$ & $\begin{array}{l}1 \\
8\end{array}$ & $\begin{array}{l}2.11 \\
3.09 \\
2.08 \\
2.08\end{array}$ & $\begin{array}{l}4.87 \\
4.85 \\
4.84 \\
4.83\end{array}$ & $\begin{array}{l}4-5 \\
3-4 \\
2-3 \\
1-2\end{array}$ & \\
\hline $\begin{array}{l}9832.15 \\
9927.35 \\
9997.94\end{array}$ & $\begin{array}{l}D \\
D \\
D \\
D\end{array}$ & $\begin{array}{l}25 \\
20 \\
15 \\
3\end{array}$ & $\begin{array}{l}1.88 \\
1.87 \\
1.87\end{array}$ & $\begin{array}{l}3.13 \\
3.11 \\
3.10\end{array}$ & $\begin{array}{l}5-4 \\
4-3 \\
3-2\end{array}$ & $\begin{array}{c}a^{3} a-y^{3} F^{\circ} \\
(149)\end{array}$ & $\begin{array}{l}4938.04 \\
4941.015 \\
4944.388\end{array}$ & $\begin{array}{l}\mathrm{H} \\
\mathrm{E} \\
\mathbf{E}\end{array}$ & $\left(\begin{array}{l}\left(\begin{array}{l}n \\
1\end{array}\right) \\
0\end{array}\right)$ & $\begin{array}{l}1.99 \\
1.97 \\
1.96\end{array}$ & $\begin{array}{l}4.49 \\
4.47 \\
4.46\end{array}$ & $\begin{array}{l}4-5 \\
3-4 \\
2-3\end{array}$ & & $\begin{array}{l}4030.512 \\
4026.539 \\
4021.812 \\
4017.771\end{array}$ & $\begin{array}{l}\mathrm{A} \\
\mathrm{A} \\
\mathrm{B} \\
\mathrm{A}\end{array}$ & $\begin{array}{l}25 n \\
25 n \\
25 n \\
15 n\end{array}$ & $\begin{array}{l}3.13 \\
2.11 \\
3.09 \\
2.08\end{array}$ & $\begin{array}{l}5.19 \\
5.17 \\
5.16 \\
5.15\end{array}$ & $\begin{array}{l}5-6 \\
4-5 \\
3-4 \\
2-3\end{array}$ & ${ }^{2^{5} F^{\circ}-e^{5} G}(185)$ \\
\hline $\begin{array}{l}9879.41 \\
8468.46 \\
8518.37\end{array}$ & $\begin{array}{l}D \\
D\end{array}$ & $\begin{array}{r}3 \\
100 \\
100\end{array}$ & $\begin{array}{l}1.87 \\
1.88 \\
1.87\end{array}$ & $\begin{array}{l}3.11 \\
3.34 \\
3.32\end{array}$ & $\begin{array}{l}3-3 \\
5-4 \\
4-3\end{array}$ & $\begin{array}{l}a^{3} a-x^{3} F^{0} \\
(150)\end{array}$ & $\begin{array}{l}4355.308 \\
4340.018\end{array}$ & $\frac{E}{E}$ & $\begin{array}{l}1 \\
1\end{array}$ & $\begin{array}{l}3.03 \\
3.01\end{array}$ & $\begin{array}{l}4.87 \\
4.85\end{array}$ & $\begin{array}{l}6-5 \\
5-4 \\
6-6\end{array}$ & $\begin{array}{l}2^{5} G^{0}-p^{5} F \\
(174) \\
2^{5} G^{0}-e^{5} G\end{array}$ & $\begin{array}{l}4015.377 \\
4049.399 \\
4040.310 \\
4031.753\end{array}$ & $\begin{array}{l}A \\
E \\
B \\
B\end{array}$ & $\begin{array}{r}12 n \\
2 n \\
4 n \\
3 n\end{array}$ & $\begin{array}{l}3.08 \\
2.13 \\
3.11 \\
3.09\end{array}$ & $\begin{array}{l}5.15 \\
5.17 \\
5.16 \\
5.15\end{array}$ & $\begin{array}{l}1-2 \\
5-5 \\
4-4 \\
3-3\end{array}$ & \\
\hline $\begin{array}{l}8548.07 \\
8423.10 \\
8483.16\end{array}$ & $\begin{array}{l}D \\
D \\
D\end{array}$ & $\begin{array}{r}100 \\
20 \\
35\end{array}$ & $\begin{array}{l}1.87 \\
1.87 \\
1.87\end{array}$ & $\begin{array}{l}3.31 \\
3.34 \\
3.32\end{array}$ & $\begin{array}{l}3-2 \\
4-4 \\
3-3\end{array}$ & & $\begin{array}{r}3899.668 \\
3888.030 \\
3877.591\end{array}$ & $\begin{array}{l}B \\
B \\
B\end{array}$ & $\begin{array}{r}(3) \\
4 n \\
2 n\end{array}$ & & & $\begin{array}{l}5-5 \\
4-4 \\
3-3\end{array}$ & & $\begin{array}{l}4016.264 \\
4012.786\end{array}$ & B & $\begin{array}{l}6 \\
3\end{array}$ & $\begin{array}{l}3.13 \\
2.11\end{array}$ & $\begin{array}{l}5.30 \\
5.18\end{array}$ & $\begin{array}{l}5-4 \\
4-3\end{array}$ & $\begin{array}{c}z^{5} F^{0}-f^{3} F \\
(186)^{3}\end{array}$ \\
\hline $\begin{array}{r}7978.88 \\
8024.84 \\
8068.34 \\
8066.05 \\
7938.53\end{array}$ & $\begin{array}{l}E \\
E \\
E \\
P \\
P\end{array}$ & $\left\{\begin{array}{l}4 \\
3 \\
3\end{array}\right\}$ & $\begin{array}{l}1.88 \\
1.87 \\
1.87 \\
1.88 \\
1.87\end{array}$ & $\begin{array}{l}3.43 \\
3.41 \\
3.39 \\
3.41 \\
3.43\end{array}$ & $\begin{array}{l}5-5 \\
4-4 \\
3-3 \\
5-4 \\
4-5\end{array}$ & $\begin{array}{l}a^{3} a-y^{3} a^{\circ} \\
(151)\end{array}$ & $\begin{array}{l}3869.275 \\
3928.97 \\
3912.589 \\
3897.390 \\
3884.090 \\
3882.147\end{array}$ & $\begin{array}{l}\text { B } \\
\text { P } \\
B \\
B \\
B \\
B \\
B\end{array}$ & $\begin{array}{l}3 \\
1 \\
(0) \\
15 \mathrm{n}\end{array}$ & $\begin{array}{l}1.96 \\
3.03 \\
3.01 \\
1.99 \\
1.97 \\
3.01\end{array}$ & $\begin{array}{l}5.15 \\
5.17 \\
5.16 \\
5.15 \\
5.15 \\
5.19\end{array}$ & $\begin{array}{l}2-3 \\
6-5 \\
5-4 \\
4-3 \\
3-2 \\
5-6\end{array}$ & & $\begin{array}{l}4013.24 \\
3993.796 \\
3994.56 \\
3975.69 \\
3980.821\end{array}$ & $\begin{array}{l}P \\
B \\
P \\
P \\
P \\
B\end{array}$ & (0) & $\begin{array}{l}2.09 \\
3.11 \\
3.09 \\
2.09 \\
3.08\end{array}$ & $\begin{array}{l}5.17 \\
5.20 \\
5.18 \\
5.20 \\
5.18\end{array}$ & $\begin{array}{l}3-3 \\
4-4 \\
3-3 \\
3-4 \\
3-3\end{array}$ & \\
\hline $\begin{array}{l}6746.433 \\
6748.43 \\
6751.94\end{array}$ & $\begin{array}{l}\mathrm{E} \\
\mathrm{H} \\
\mathrm{H}\end{array}$ & $\left\{\begin{array}{l}1 \\
1 \\
0\end{array}\right\}$ & $\begin{array}{l}1.88 \\
1.87 \\
1.87\end{array}$ & $\begin{array}{l}3.71 \\
3.70 \\
3.69\end{array}$ & $\begin{array}{l}5-5 \\
4-4 \\
3-3\end{array}$ & $\begin{array}{l}a^{3} a-x^{3} a^{\circ} \\
(153)\end{array}$ & $\begin{array}{r}3875.363 \\
3868.397 \\
3863.823\end{array}$ & $\begin{array}{l}\mathrm{A} \\
\mathrm{B}\end{array}$ & $\begin{array}{l}30 n \\
10 n \\
10 n\end{array}$ & $\begin{array}{l}1.99 \\
1.97 \\
1.96\end{array}$ & $\begin{array}{l}5.17 \\
5.16 \\
5.15\end{array}$ & $\begin{array}{l}4-5 \\
3-4 \\
2-3\end{array}$ & & $\begin{array}{l}4013.587 \\
4008.046 \\
4005.953 \\
4007.195\end{array}$ & $\begin{array}{l}\text { A } \\
\text { B } \\
\text { B } \\
\text { B }\end{array}$ & $\begin{array}{r}12 n \\
9 n \\
6 n \\
3 n\end{array}$ & $\begin{array}{l}3.13 \\
3.11 \\
3.09 \\
3.08\end{array}$ & $\begin{array}{l}5.30 \\
5.19 \\
5.17 \\
5.16\end{array}$ & $\begin{array}{l}5-6 \\
4-5 \\
3-4 \\
3-3\end{array}$ & $\begin{array}{c}z^{5} F^{0}-e^{5} H \\
(187)\end{array}$ \\
\hline $\begin{array}{l}6092.814 \\
6121.008 \\
6146.225\end{array}$ & $\frac{E}{E}$ & $\begin{array}{l}4 \\
3 \\
3\end{array}$ & $\begin{array}{l}1.88 \\
1.87 \\
1.87\end{array}$ & $\begin{array}{l}3.90 \\
3.89 \\
3.87\end{array}$ & $\begin{array}{l}5-5 \\
4-4 \\
3-3\end{array}$ & $\begin{array}{c}a^{3} G-\pi^{3} G^{\circ} \\
(153)\end{array}$ & $\begin{array}{l}3882.892 \\
3866.446 \\
3858.133 \\
3853.719\end{array}$ & $\begin{array}{l}\text { B } \\
\text { A } \\
\text { B } \\
\text { B }\end{array}$ & $\begin{array}{l}20 n \\
15 n \\
15 n \\
10 n\end{array}$ & $\begin{array}{l}2.03 \\
2.01 \\
1.99 \\
1.97\end{array}$ & $\begin{array}{l}5.21 \\
5.20 \\
5.19 \\
5.17\end{array}$ & $\begin{array}{l}6-7 \\
5-6 \\
4-5 \\
3-4\end{array}$ & & $\begin{array}{l}4003.789 \\
4002.466 \\
3999.336\end{array}$ & $\begin{array}{l}\text { B } \\
\text { B } \\
\text { B }\end{array}$ & $\begin{array}{r}10 \mathrm{n} \\
9 \mathrm{n} \\
7 \mathrm{n}\end{array}$ & $\begin{array}{l}3.13 \\
3.11 \\
3.09\end{array}$ & $\begin{array}{l}5.31 \\
5.19 \\
5.18\end{array}$ & $\begin{array}{l}5-4 \\
4-3 \\
3-3\end{array}$ & $\begin{array}{c}2^{5} F^{\circ}-0^{5} D \\
(188)^{5}\end{array}$ \\
\hline $\begin{array}{l}5953.162 \\
5965.828 \\
5978.543 \\
5988.560 \\
5996.007\end{array}$ & $\begin{array}{l}A \\
A \\
A \\
E \\
E\end{array}$ & $\begin{array}{r}30 \\
30 \\
25 \\
2 \\
2\end{array}$ & $\begin{array}{l}1.88 \\
1.87 \\
1.87 \\
1.88 \\
1.87\end{array}$ & $\begin{array}{l}3.95 \\
3.94 \\
3.93 \\
3.94 \\
3.93\end{array}$ & $\begin{array}{l}5-6 \\
4-5 \\
3-4 \\
5-5 \\
4-4\end{array}$ & $\begin{array}{c}a^{3} G-z^{3} \mathrm{H}^{\circ} \\
(154)\end{array}$ & $\begin{array}{l}3895.243 \\
3882.313 \\
3873.203 \\
3867.739 \\
3911.362\end{array}$ & $\begin{array}{l}\text { B } \\
\text { A } \\
\text { B } \\
\text { B } \\
\text { B } \\
\text { E }\end{array}$ & $\begin{array}{l}10 \mathrm{n} \\
30 \mathrm{n} \\
10 \mathrm{n} \\
10 \mathrm{n} \\
3 \\
(3)\end{array}$ & $\begin{array}{l}1.96 \\
3.03 \\
3.01 \\
1.99 \\
1.97 \\
3.03\end{array}$ & $\begin{array}{l}5.16 \\
5.20 \\
5.19 \\
5.17 \\
5.16 \\
5.19\end{array}$ & $\begin{array}{l}2-3 \\
6-6 \\
5-5 \\
4-4 \\
3-3 \\
6-5\end{array}$ & & $\begin{array}{l}3994.683 \\
3990.184 \\
3981.466 \\
3984.313 \\
3985.580\end{array}$ & $\begin{array}{l}\text { B } \\
E \\
B \\
B \\
B\end{array}$ & $\begin{array}{l}\left(\begin{array}{l}4 n \\
1 n\end{array}\right) \\
0 \\
3 \\
(1)\end{array}$ & $\begin{array}{l}2.08 \\
3.08 \\
2.11 \\
2.09 \\
2.08\end{array}$ & $\begin{array}{l}5.17 \\
5.17 \\
5.21 \\
5.19 \\
5.18\end{array}$ & $\begin{array}{l}2-1 \\
1-0 \\
4-4 \\
3-3 \\
2-3\end{array}$ & \\
\hline $\begin{array}{l}5409.609 \\
5397.093 \\
5389.996 \\
5391.06 \\
5382.96\end{array}$ & $\begin{array}{l}\mathrm{A} \\
\mathrm{A} \\
\mathrm{A} \\
\mathrm{P} \\
\mathrm{P}\end{array}$ & $\begin{array}{l}6 \\
4 \\
3\end{array}$ & $\begin{array}{l}1.88 \\
1.87 \\
1.87 \\
1.87 \\
1.87\end{array}$ & $\begin{array}{l}4.16 \\
4.16 \\
4.15 \\
4.16 \\
4.16\end{array}$ & $\begin{array}{l}5-4 \\
4-3 \\
3-2 \\
4-4 \\
3-3\end{array}$ & $\begin{array}{l}a^{3} a-\pi^{3} F^{0} \\
(155)\end{array}$ & $\begin{array}{l}3897.581 \\
3887.365 \\
3720.384 \\
3707.549 \\
3696.885\end{array}$ & $\begin{array}{l}\mathrm{E} \\
\mathrm{B} \\
\mathrm{E}\end{array}$ & $\begin{array}{c}1 \\
(1 n) \\
3 \\
10 n \\
3\end{array}$ & $\begin{array}{l}3.01 \\
1.99 \\
3.03 \\
2.01 \\
1.99\end{array}$ & $\begin{array}{l}5.17 \\
5.16 \\
5.35 \\
5.34 \\
5.33\end{array}$ & $\begin{array}{l}5-4 \\
4-3 \\
6-5 \\
5-4 \\
4-3\end{array}$ & $\begin{array}{l}2^{5} g^{0}-8^{5} F \\
(177)^{-1}\end{array}$ & $\begin{array}{l}3838.180 \\
3832.026 \\
3817.639 \\
3814.855 \\
3813.261\end{array}$ & $\begin{array}{l}\text { B } \\
B \\
B \\
B \\
B\end{array}$ & $\begin{array}{c}\left(\begin{array}{l}3 \\
2\end{array}\right) \\
5 \\
4 \\
(0)\end{array}$ & $\begin{array}{l}3.13 \\
2.11 \\
2.09 \\
2.08 \\
3.08\end{array}$ & $\begin{array}{l}5.35 \\
5.34 \\
5.33 \\
5.32 \\
5.31\end{array}$ & $\begin{array}{l}5-5 \\
4-4 \\
3-3 \\
2-3 \\
1-1\end{array}$ & $\begin{array}{l}\left.2^{5} F^{0}-8\right)^{5} r^{4} \\
(189)^{-1}\end{array}$ \\
\hline $\begin{array}{c}5265.967 \\
5283.441 \\
5297.236 \\
5248.402 \\
5269.93\end{array}$ & $\begin{array}{l}A \\
A \\
A \\
E \\
B \\
H\end{array}$ & $\left.\begin{array}{c}10 \\
8 \\
6 \\
1 \\
1 \\
1\end{array}\right)$ & $\begin{array}{l}1.88 \\
1.87 \\
1.87 \\
1.87 \\
1.87\end{array}$ & $\begin{array}{l}4.22 \\
4.21 \\
4.19 \\
4.32 \\
4.21\end{array}$ & $\begin{array}{l}5-4 \\
4-3 \\
3-2 \\
4-4 \\
3-3\end{array}$ & $\begin{array}{l}a^{3} a-v^{3} F^{\circ} \\
(156)\end{array}$ & $\begin{array}{l}3688.27 \\
3681.273 \\
3694.10 \\
3685.47 \\
3679.14 \\
3674.93\end{array}$ & $\begin{array}{l}\bar{P} \\
E \\
P \\
P \\
P \\
P \\
P\end{array}$ & 1 & $\begin{array}{l}1.97 \\
1.96 \\
2.01 \\
1.99 \\
1.97 \\
1.96\end{array}$ & $\begin{array}{l}5.32 \\
5.31 \\
5.35 \\
5.34 \\
5.33 \\
5.32\end{array}$ & $\begin{array}{l}3-2 \\
2-1 \\
5-5 \\
4-4 \\
3-3 \\
2-3\end{array}$ & & $\begin{array}{l}3306.879 \\
3309.730 \\
3312.690 \\
3315.237 \\
3318.362 \\
3325.155\end{array}$ & $\begin{array}{l}\mathbf{E} \\
\mathbf{E} \\
\mathbf{E} \\
\mathbf{E} \\
\mathbf{E} \\
\mathbf{E}\end{array}$ & $\begin{array}{r}10 \\
6 \\
5 \\
3 \\
4 \\
3 \\
3\end{array}$ & $\begin{array}{l}2.13 \\
2.11 \\
3.09 \\
3.08 \\
2.08 \\
3.13\end{array}$ & $\begin{array}{l}5.86 \\
5.84 \\
5.82 \\
5.81 \\
5.80 \\
5.84 \\
5.85\end{array}$ & $\begin{array}{l}5-6 \\
4-5 \\
3-4 \\
2-3 \\
1-3 \\
5-5\end{array}$ & $\begin{array}{c}2^{5} F^{0}-8^{5} G \\
(190)^{5}\end{array}$ \\
\hline $\begin{array}{l}4885.083 \\
4899.910 \\
4913.616 \\
4915.236 \\
4925.396\end{array}$ & $\begin{array}{l}\mathbf{A} \\
\mathbf{A} \\
\mathbf{A} \\
\mathrm{B} \\
\mathrm{B}\end{array}$ & $\begin{array}{r}20 \\
20 \\
30 \\
5 \\
5 .\end{array}$ & $\begin{array}{l}1.88 \\
1.87 \\
1.87 \\
1.88 \\
1.87\end{array}$ & $\begin{array}{l}4.41 \\
4.39 \\
4.38 \\
4.39 \\
4.38\end{array}$ & $\begin{array}{l}5-6 \\
4-5 \\
3-4 \\
5-5 \\
4-4\end{array}$ & $\begin{array}{c}a^{3} \mathrm{G}-\mathrm{y}^{3} \mathrm{H}^{\circ} \\
(157)\end{array}$ & $\begin{array}{c}3366.1768 \\
3361.50 \\
3356.196 \\
+3350.5488 \\
3344.931 \\
3344.630\end{array}$ & $\begin{array}{l}E \\
Q \\
Q \\
E \\
E \\
E \\
E\end{array}$ & $\begin{array}{l}5 \\
1 \\
3 \\
3 \\
1 \\
1\end{array}$ & $\begin{array}{l}3.03 \\
3.01 \\
1.99 \\
1.97 \\
1.96 \\
2.01\end{array}$ & $\begin{array}{l}5.70 \\
5.68 \\
5.67 \\
5.66 \\
5.65 \\
5.70\end{array}$ & $\begin{array}{l}6-5 \\
5-4 \\
4-3 \\
3-2 \\
2-1 \\
5-5\end{array}$ & $\begin{array}{c}2^{5} G^{\circ}-h^{5} F \\
(178)\end{array}$ & $\begin{array}{r}3325.229 \\
\cdot 3324.754 \\
3340.77 \\
3337.40 \\
3334.35\end{array}$ & $\begin{array}{l}\mathbf{E} \\
\mathbf{E} \\
\mathrm{H} \\
G \\
\mathrm{H}\end{array}$ & $\begin{array}{c}3 \\
4 \\
(1) \\
1 \mathrm{n} \\
(1 \mathrm{n})\end{array}$ & $\begin{array}{l}3.11 \\
3.09 \\
2.13 \\
3.11 \\
3.09\end{array}$ & $\begin{array}{l}5.82 \\
5.80 \\
5.83 \\
5.81 \\
5.80\end{array}$ & 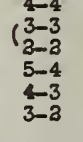 & \\
\hline 4811.074 & B & 4 & 1.88 & 4.44 & $5-4$ & $a^{3}$ & $\begin{array}{l}3344.630 \\
3343.379\end{array}$ & $\frac{\mathbf{E}}{\mathbf{E}}$ & $\begin{array}{l}\operatorname{tr} \\
\operatorname{tr}\end{array}$ & $\begin{array}{l}2.01 \\
1.99\end{array}$ & $\begin{array}{l}5.70 \\
5.68\end{array}$ & 4 & & & $G$ & 18 & 2.13 & 5.98 & $5-5$ & $5^{5}$ \\
\hline $\begin{array}{l}4449.985 \\
4440.345\end{array}$ & $\begin{array}{l}\mathbf{B} \\
\mathbf{A}\end{array}$ & 10 & $\begin{array}{l}1.87 \\
1.87\end{array}$ & $\begin{array}{l}4.64 \\
4.64\end{array}$ & $\begin{array}{l}4-3 \\
3-3\end{array}$ & $\begin{array}{c}a^{3} \bar{a}-x^{1} F^{0} \\
(159)\end{array}$ & & & 1n & & & & & $\begin{array}{l}3198.726 \\
3199.34 \\
.3213 .1458\end{array}$ & $\begin{array}{l}\frac{E}{H} \\
\frac{H}{E}\end{array}$ & $\frac{1 n}{(1 n)}$ & $\begin{array}{l}3.11 \\
3.09 \\
3.13\end{array}$ & $\begin{array}{l}5.97 \\
5.95 \\
5.97\end{array}$ & $\begin{array}{l}4-4 \\
3-3 \\
5-4\end{array}$ & \\
\hline $\begin{array}{l}4449.143 \\
4450.896 \\
4453.708 \\
4463.539 \\
4463.391 \\
4436.586 \\
4441.272\end{array}$ & $\begin{array}{l}\text { A } \\
A \\
A \\
\text { B } \\
\text { B } \\
\text { A } \\
B\end{array}$ & $\begin{array}{r}30 \\
35 \\
30 \\
8 \\
8 \\
4 \\
4 \\
4\end{array}$ & $\begin{array}{l}1.88 \\
1.87 \\
1.87 \\
1.88 \\
1.87 \\
1.87 \\
1.87\end{array}$ & $\begin{array}{l}4.65 \\
4.64 \\
4.64 \\
4.64 \\
4.64 \\
4.65 \\
4.64\end{array}$ & $\begin{array}{l}5-5 \\
4-4 \\
3-3 \\
5-4 \\
4-3 \\
4-5 \\
3-4\end{array}$ & $\begin{array}{c}a^{3} a-\nabla^{3} G^{\circ} \\
(160)\end{array}$ & $\begin{array}{l}3226.138 \\
3223.519 \\
3231.381 \\
3219.312 \\
3217.942 \\
3243.513 \\
3238.324 \\
3232.791\end{array}$ & $\begin{array}{l}E \\
E \\
E \\
E \\
E \\
E \\
E \\
E \\
E\end{array}$ & $\begin{array}{r}12 \\
10 \\
10 \\
8 \\
8 \\
3 \\
4 \\
3\end{array}$ & $\begin{array}{l}2.03 \\
2.01 \\
1.99 \\
1.97 \\
1.96 \\
2.03 \\
2.01 \\
1.99\end{array}$ & $\begin{array}{l}5.86 \\
5.84 \\
5.82 \\
5.81 \\
5.80 \\
5.84 \\
5.82 \\
5.81\end{array}$ & $\begin{array}{l}6-6 \\
5-5 \\
4-4 \\
3-3 \\
2-3 \\
6-5 \\
5-4 \\
4-3\end{array}$ & $\begin{array}{c}2^{5} G^{\circ}-g^{5} G \\
(179)^{5}\end{array}$ & $\begin{array}{c}3311.07 \\
3141.670 \\
3129.075 \\
3128.6408 \\
3125.656 \\
3125.553\end{array}$ & $\begin{array}{l}\mathbf{E} \\
\mathbf{E} \\
\mathbf{E} \\
\mathbf{E} \\
\mathbf{E}\end{array}$ & $\begin{array}{c}10 \\
7 \\
8 \\
(3) \\
3\end{array}$ & $\begin{array}{l}2.11 \\
2.13 \\
2.11 \\
3.09 \\
3.08 \\
3.08\end{array}$ & $\begin{array}{l}5.95 \\
6.05 \\
6.05 \\
6.04 \\
6.03 \\
6.03\end{array}$ & $\begin{array}{l}5-4 \\
4-3 \\
3-2 \\
3-1 \\
1-0\end{array}$ & $\begin{array}{c}2^{5} F^{\circ}-h^{5}{ }^{5} \uparrow \\
(193)\end{array}$ \\
\hline $\begin{array}{r}4417.274 \\
4426.054 \\
4424.003 \\
4404.911 \\
4416.535\end{array}$ & $\begin{array}{l}A \\
A \\
A \\
B \\
B\end{array}$ & $\begin{array}{r}15 \\
10 \\
15 \\
5 \\
4\end{array}$ & $\begin{array}{l}1.88 \\
1.87 \\
1.87 \\
1.87 \\
1.87\end{array}$ & $\begin{array}{l}4.67 \\
4.66 \\
4.65 \\
4.67 \\
4.66\end{array}$ & $\begin{array}{l}5-4 \\
4-3 \\
3-2 \\
4-4 \\
3-3\end{array}$ & $\begin{array}{c}a^{3} G-u^{3} F^{0} \\
(161)\end{array}$ & $\begin{array}{l}3206.344 \\
3206.825 \\
3207.897 \\
3209.030\end{array}$ & $\begin{array}{l}\mathrm{E} \\
\mathrm{E} \\
\mathrm{E} \\
\mathrm{E} \\
\mathrm{E}\end{array}$ & $\begin{array}{l}3 \\
5 \\
5 \\
5 \\
4\end{array}$ & $\begin{array}{l}1.97 \\
2.01 \\
1.99 \\
1.97 \\
1.96\end{array}$ & $\begin{array}{l}5.80 \\
5.86 \\
5.84 \\
5.82 \\
5.81\end{array}$ & $\begin{array}{l}3-2 \\
5-6 \\
4-5 \\
3-4 \\
2-3\end{array}$ & & & & & & & & \\
\hline
\end{tabular}

REVISED UULTIPLET TABLE

Laboratory ${ }_{\text {Ref }}^{\text {Lnt LOW }} P_{\text {H1gh }}$ J Multiplet

I A Laboratory Int EP JuItiplet T1 I continued

3143.350
3139.87
3135.069
3130.175
3137.684
3123.769
3118.130
3114.092
3111.383
3107.468
3105.320
3102.517
3101.536

Low H1gh

No)

11.

8578.40

8612.91
.860 .98

7474.94
7466.44
7431.98

$+7253.76$

7330.9

7266.29

6266.021

6277.525

6295.949

4617.369

4629.336

4481.361

4480.600
449.72

4465.80

4305.474

4299.229

4276.441

9832.15

9879.41

8468.4

8548.07

8483.16

$-7978.88$

8068.3
8066.0

7938.53

6748.43
6751.94

6092.814

6121.008
6146.225

5953.162

59

5409.609

5389.996

4416.535

$1.87 \quad 4.66$ $\begin{array}{llll}1.96 & 5.81 \quad 2-3\end{array}$ 
I A Roboratory

\section{I cont1nued}

10057.69

10011.72
10120.90

10066.47

9941.33
9948.98

$\begin{array}{rrr}9661.42 & D & 10 \\ 9690.63 & \text { I } & 2\end{array}$

8080.55

8098.50
8133.36

8419.15

6381.416
6381.41

8186.14

6138.3

3999.003

6002.640
6018.433

3052.879

5069.351

5023.39

5048.208

4921.768

4928.343

4941.562

4848.487

4864.187
4880.922

4891.828

4893.90

4731.173
4733.426

4733.436
4742.129

4759.74

- 4710.186

4698.86

4724.679

4684.484

4684.484
4686.931

4380.487

4346.810

438.478
4343.798

4389.919

+4300.53
4311.654

4150.963

4159.634
4171.018

4179.860

4099.166

4077.148
4065.595

4079.708

4035.828

4033.883

4034.884
$\$ 553.930$
1043.775

4043.75
4016.943

- 8496.03

8569.72
-8600.98

8618.44

842.98
8495.5

7654.4

7814.50
7580.55

7318.39

7361.56
7337.78

7373.77

6678.60

5780.778

5212.371

5189.61

5312.997
Low ${ }^{\text {E }}$ High

(No)
(Not

L Laboratory

LW E P ${ }_{\text {H1gh }}$

Multiplet I1 I continued

$\begin{array}{lllll}25 & 3.17 & 3.39 & 3-3 & 8^{3} D-x^{3} D^{0}\end{array}$

4995.062 (B)

$\begin{array}{lll}3.14 & 3.38 & 1-1 \\ 3.17 & 3.38 & 3-2 \\ 3.15 & 3.38 & 2-1 \\ 3.15 & 3.39 & 2-3\end{array}$

$\begin{array}{lll}3.15 & 3.39 & 2-3 \\ 3.14 & 3.38 & 1-2\end{array}$

$\begin{array}{llll}2.17 & 3.44 & 3-3 & a^{3} p-z^{5} p o \\ 3.15 & 3.42 & 2-2 & \end{array}$

$\begin{array}{llll}2.17 & 3.69 & 3-3 & 8^{3} D-\pi^{3} D^{0}\end{array}$

$\begin{array}{ll}2.15 & 3.6 .7 \\ 3.14 & 3.66\end{array}$

(2) $\begin{array}{llll}3.17 & 4.09 & 3-3 & 8^{3} p-x^{3} p 0+ \\ 3.15 & 4.09 & 2-1 & \end{array}$

$\begin{array}{lll}1) & 3.15 & 4.09 \\ 1.14 & 4.08\end{array}$

$\begin{array}{llll}2.17 & 4.16 & 3-4 & a^{3} p-m^{3} F^{0}+ \\ 3.15 & 4.16 & 2-3 & (197)\end{array}$

$\begin{array}{lllll}4 \pi & 2.17 & 4.32 & 3-4 & a^{3} D-v^{3} F^{0} \\ (0) & 2.15 & 4.31 & 2-3 & (198) \\ 1 & 3.14 & 4.19 & 1-3 & \end{array}$

$\begin{array}{lllll}8 & 2.17 & 4.61 & 3-2 & 8^{3} p-m^{3} p^{\circ} \\ 7 & 3.15 & 4.59 & 3-1 & (199)\end{array}$

$\begin{array}{clll}7 & 3.15 & 4.59 & 2-1 \\ 5 & 3.14 & 4.58 & 1-0 \\ (\bar{a} & 3.15 & 4.61 & 2-2 \\ 1 & 3.14 & 4.59 & 1-1\end{array}$

(1) 2.14 4.49

$\begin{array}{llllll}3 & 3.17 & 4.67 & 3-4 & 8^{3} D-u^{3} F^{0} \\ 0 & 3.15 & 4.66 & 2-3 & (200)\end{array}$

$123.14 \quad 4.65$

$\begin{array}{lll}2.14 & 4.65 & 1-3 \\ 2.17 & 4.66 & 3-3 \\ 2.15 & 4.65 & 2-3\end{array}$

$\begin{array}{lllll}8 & 3.17 & 4.71 & 3-3 & a^{3} D-u^{3} D^{\circ} \\ 4 & 3.15 & 4.69 & a-2 & (201)\end{array}$

$3 \quad 3.14 \quad 4.67$

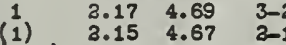

(1pi) $\begin{array}{llll}2.15 & 4.71 & 2-1\end{array}$

$\begin{array}{lll}9 & 2.17 & 4.77 \\ 6 & 3.15 & 4.76 \\ 3 & 3.14 & 4.75\end{array}$

$\begin{array}{lll}3 & 3.14 & 4.75 \\ \text { (1p?) } & 3.17 & 4.76\end{array}$

$\begin{array}{lll}\text { (1p?) } & 3.15 & 4.75 \\ 18 & 3.17 & 4.79\end{array}$

$\begin{array}{lll}18 & 3.17 & 4.79 \\ (69) & 3.15 & 4.78\end{array}$

$\begin{array}{lll}\text { (a) } & 3.14 & 4.77 \\ 3.17 & 4.78\end{array}$

$\begin{array}{ll}3.17 & 4.78 \\ 3.15 & 4.77 \\ 3.15 & 4.79\end{array}$

$\begin{array}{ll}3.15 & 4.79 \\ 3.14 & 4.78\end{array}$

$3.17 \quad 5.00$

$\begin{array}{ll}3.17 & 5.00 \\ 3.15 & 4.99 \\ 3.14 & 4.98 \\ 3.15 & 5.00\end{array}$

$\begin{array}{ll}3.14 & 4.98 \\ 2.15 & 5.00\end{array}$

$\begin{array}{llll}2.14 & 4.99 \quad 1-1\end{array}$

$\begin{array}{lllll}3 & 3.17 & 5.04 & 3-3 & a^{3} D-r^{3} D^{0}\end{array}$

$\begin{array}{lll}3 & 2.15 & 5.03 \\ 2 & 3.14 & 5.01\end{array}$

$\begin{array}{llll}3.17 & 5.14 & 3-4 & a^{3} D-8^{3} p^{0} \\ 3.15 & 5.12 & 2-3 & (306)\end{array}$

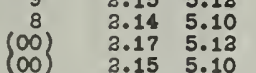

$\begin{array}{llllll}8 & 3.17 & 5.18 & 3-2 & a^{3} p-u^{3} p \circ \\ 4 & 3.15 & 5.18 & a_{-1} & (307)\end{array}$

$\begin{array}{lll}(0) & 2.15 & 5.18 \\ 6 & 3.14 & 5.18 \\ 3 & 3.15 & 5.18\end{array}$

$\begin{array}{ll}3.15 & 5.18 \\ 3.14 & 5.18\end{array}$

$\begin{array}{rrrrr}10 & 3.17 & 5.22 & 3-3 & 8^{3} D-a^{3} D^{\circ} \\ 6 & 3.15 & 5.21 & 2-2 & (308) \\ 5 & 3.14 & 5.30 & 1-1 & \\ 3 & 3.17 & 5.31 & 3-3 & \\ 3 & 3.17 & 5.31 & \end{array}$

$3.17 \quad 5.31$

$\begin{array}{lll}3.15 & 5.20 & 2-1 \\ 3.15 & 5.32 & 2\end{array}$

\begin{tabular}{rrr}
3 & 3.15 & 5.23 \\
$\mathrm{Ti}^{+}$ & 3.14 & 5.31 \\
\hline
\end{tabular}

2.34 $3.69 \quad 2-3 \quad b^{3} P-\pi^{3} D^{\circ}$

$\begin{array}{lllll}60 & 3.24 & 3.69 & 2-3 & b^{3} P-4300 \\ 60 & 2.23 & 3.67 & 1-3 & (209)\end{array}$

$\begin{array}{llll}50 & 2.23 & 3.66 & 0-1 \\ 35 & 2.24 & 3.67 & 3-2\end{array}$

$\begin{array}{lllll}20 & 3.24 & 3.67 & 2-2 \\ 20 & 3.33 & 3.66 & 1-1\end{array}$

$\begin{array}{lllll}20 & 2.24 & 3.70 & 2-3 & b^{3} p-x^{5} p^{\circ} \\ 15 & 2.23 & 3.68 & 1-0, & (310)\end{array}$

(a) $2.34 \quad 3.85 \quad 2-3 \quad b^{3} p-r^{3} D^{0} \uparrow$

$\begin{array}{lllll}\text { ip?) } & 3.33 & 3.85 & 3-3 & \\ 1 & 3.32 & (311) \\ 2.32 & 3.85 & 0-1 & \end{array}$

(2) $\begin{array}{llll}2.24 & 3.93 & 2-3 & b^{3} p-y^{3} p^{p 0} \\ 2.34 & 3.92 & 3-1 & (213)\end{array}$

(0) $\begin{array}{lll}2.34 & 3.92 & 2-1 \\ 2.23 & 3.91 & 1-0 \\ 2.23 & 3.93 & 1-2\end{array}$

$\begin{array}{llll}2.24 & 4.09 \quad 2-2 & b^{3}\left(3 p_{1}-x^{3}\right)^{30}\end{array}$

$\begin{array}{lllll}3 & 2.24 & 4.37 & 2-1 & b^{3} \mathrm{P}-y^{3} \mathrm{~s}^{\circ} \\ 1 & 2.23 & 4.37 & 1-1 & (214)\end{array}$

$\begin{array}{llllll}3 & 2.24 & 4.61 & 2-3 & b^{3} p-m^{3} p_{0} \\ \text { (0) } & 2.23 & 4.59 & 1-1 & (215)\end{array}$

$\begin{array}{llll}\text { (o) } & 2.23 & 4.61 & 1-1 \\ 3.23 & 4.59 & 0-1\end{array}$

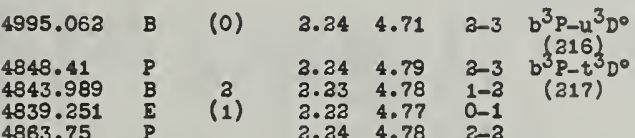

$\begin{array}{llllll}4883.75 & P & (1) & 2.24 & 4.78 & 2-2 \\ 4854.737 & B & (00) & 2.23 & 4.77 & 1-1\end{array}$

$\begin{array}{rrrrrrr}.4404 .276 & B & 10 & 2.24 & 5.04 & 2-3 & b^{3} \mathrm{P}-\mathrm{r}^{3} \mathrm{D}^{\circ} \\ 4441.754 & \mathrm{~A} & 6 & 3.23 & 5.02 & 1-2 & (218) \\ 4431.284 & \mathrm{~B} & 4 & 2.32 & 5.01 & 0-1 & \end{array}$

$\begin{array}{llllll}4438.233 & B & \text { B } & 2.23 & 5.01 & 0-1 \\ 4444.367 & B & \text { (1) } & 3.24 & 5.02 & 3-2 \\ 4.23 & 5.01 & 1-1\end{array}$

$\begin{array}{lllllll}.4404 .276 & B & 10 & 2.24 & 5.04 & 3-1 & b^{3} \mathrm{p}-\mathrm{x}^{3} \mathrm{~g}^{\circ}\end{array}$

$\begin{array}{llrllll}4388.077 & B & 3 & 2.24 & 5.04 & 2-1 & 0 \\ 4375.425 & E & 1 & 2.23 & 5.04 & 1-1 & (219) \\ & & 3.04 & 0.1 & \end{array}$

$\begin{array}{lllllll}4303.465 & B & 8 & 2.24 & 5.18 & 2-2 & b^{3} \mathrm{p}-\mathrm{u}^{3} \mathrm{po}\end{array}$

4186.01

$\begin{array}{lllllll}4200.753 & \mathrm{P} & 6 & 2.23 & 5.18 & 1-1 & \text { (220) } \\ 4.24 & 5.18 & \mathrm{a}-1 & \end{array}$

$\begin{array}{lllllll}4183.294 & \mathrm{~B} & 4 & 3.23 & 5.18 & 1-0 \\ 4188.694 & \mathrm{~B} & 5 & 3.23 & 5.18 & 1-2\end{array}$

4174.473

4136.894

4140.48
4139.48

4139.48
4154.865
4150.809

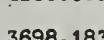

3710.186
3705.53

3705.53
3686.71
3689.671

360.62

10460.07
10553.02

10553.02
10565.97

8438.93

8450.89
8416.97

8416.97
8403.54

7440.60

7489.61
7496.12

6745.56

(1) $\quad 3.24 \quad 5.23 \quad 2-3 \quad b^{3} P-q^{3} p^{\circ}$

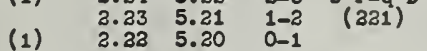

$\begin{array}{llll}3 & 2.24 & 5.21 & 2-2 \\ \text { (0) } & 2.33 & 5.30 & 1-1\end{array}$

$3 \quad \begin{array}{llll}3.34 & 5.58 & 2-3 & b^{3} \mathrm{p}-t^{3} \mathrm{po}^{\circ}\end{array}$

$\begin{array}{lllll}3 & 3.34 & 5.58 & 2-3 & { }^{3} P-t^{3} \\ (0) & 3.34 & 5.57 & 2-1 & (223) \\ 1 & 3.23 & 5.56 & 1-0 & \end{array}$

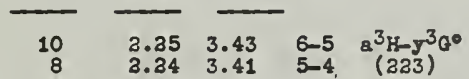

$\begin{array}{llll}2.34 & 3.43 & 6-4 & 838-436 \\ 3.24 & 3.41 & 5-4 & (223) \\ 3.33 & 3.39 & 4-3 & \end{array}$

$\begin{array}{lllll}75 & 3.25 & 3.71 & 6-5 & a^{3} \mathrm{H}-\mathrm{x}^{3} \mathrm{G} \cdot 0 \\ 75 & 3.24 & 3.70 & 5-4 & (324)\end{array}$

$\begin{array}{rrrr}75 & 3.24 & 3.70 & 5 \\ 60 & 3.23 & 3.69 & 4 \\ 5 & 3.24 & 3.71 & 5-5\end{array}$

$\begin{array}{llllll}3 & 3.25 & 3.90 & 6-5 & 8^{3} \mathrm{H}_{-1} \mathrm{~m}^{3} \mathrm{G}^{\circ} \\ 3 & 3.24 & 3.89 & 5-4 & (225) \\ 3 & 3.24 & 3.25 & 3.07 & 4-3 & \end{array}$

$\begin{array}{lllll}P & 3.23 & 4.06 \quad 4-3 & 8^{3} \mathrm{H}-\mathrm{y}^{2} \mathrm{~F}^{\circ}\end{array}$

$\begin{array}{llllllll}5715.123 & \mathbf{A} & 9 & 2.25 & 4.41 & 6-6 & \mathrm{a}^{3} \mathrm{H} / \mathrm{H}^{3} \mathrm{H}^{\circ}\end{array}$ $\begin{array}{lllllll}5715.123 & \mathrm{~A} & 9 & 3.25 & 4.41 & 6-6 & \mathrm{~B}^{3} \mathrm{H}-\mathrm{-}^{3} \mathrm{~K} \\ 5739.164 & \mathrm{~A} & 9 & 3.24 & 4.39 & 5-5 & (228) \\ 5739.975 & \mathrm{~B} & 4 & 3.23 & 4.38 & 4-4 & \\ 5756.45 & \mathrm{P} & & 2.35 & 4.39 & 6-5 & \end{array}$

5597.93

5127.367

$\left.\begin{array}{lll}5132.931 & \text { B } & (1 \\ 5132.082 & B & (00\end{array}\right)$

4856.01

4870.129
4868.264

4882.364
4893.065

4778.259

4759.372
4758.130

4758.120
4743.791
4769.775

4769.775
4766.330

474.680

4734.682

4346.104

4318.631

4325.134

4309.071

$\begin{array}{lll}3.23 & 4.38 & 4-4 \\ 2.35 & 4.39 & 6-5\end{array}$

$\begin{array}{lllll}(2 n) & 2.34 & 4.44 & 5-1 & 8^{3} H-y^{1} a^{\circ} \\ 9 & 3.23 & 4.44 & 4-4 & (229)\end{array}$

$\begin{array}{lllll}(1) & 3.25 & 4.65 & 6-5 & a^{3} \mathrm{~g}-\sigma^{3} \mathrm{G}^{\circ} \\ (0) & 3.24 & 4.64 & 5-4 & (230) \\ 3 & 3.23 & 4.84 & 4-3 & \end{array}$

30
30
18
3

$\begin{array}{llll}3.25 & 4.79 & 6-7 & 8^{3} \mathrm{H}_{-2} \mathrm{z}^{3} \mathrm{I}^{\circ} \\ 3.24 & 4.77 & 5-6 & (331)\end{array}$

$\begin{array}{lll}3.24 & 4.77 & 5-6 \\ 3.23 & 4.76 & 4 \\ 3.25 & 4.77 & 6\end{array}$

$\begin{array}{lll}3.25 & 4.77 & 6-6 \\ 3.34 & 4.78 & 5-5\end{array}$

$2.23 \quad 4.81 \quad 4-4 \quad 8^{3} H-x^{1} G^{\circ}$

$\begin{array}{llll}3.25 & 4.84 & 6-6 & 8^{3} \mathrm{H}-x^{3} \mathrm{H}^{\circ} \\ 2.24 & 4.83 & 5-5 & (233)\end{array}$

$2.23 \quad 4.83 \quad 4-4$

$\begin{array}{ll}3.25 & 4.83 \\ 2.34 & 4.83\end{array}$

$\begin{array}{ll}3.24 & 4.83 \\ 3.24 & 4.84\end{array}$

$\begin{array}{lll}3.24 & 4.84 & 5-6 \\ 3.23 & 4.83 & 4-5\end{array}$

$\begin{array}{llll}2.23 & 5.07 & 4-5 & 8^{3} \mathrm{H}-5^{1} \mathrm{H}^{\circ} \\ 2.25 & 5.10 & 6-5 & 8^{3} \mathrm{H}-\mathrm{u}^{3} \mathrm{G}^{\circ}\end{array}$

$\begin{array}{llll}3.25 & 5.10 & 6-5 & 8^{3} \mathrm{H}-u^{3} \\ 2.24 & 5.09 & 5-4 & (235)\end{array}$

8598.18 D $\overline{60} \quad \overline{3.26} \overline{3.69}+3 b^{1} a-x^{3} \xi^{0}$

$6861.47 \quad E \quad 6 \quad 3.26 \quad 4.06 \quad 4-3 \quad b\left(\frac{a-1}{23}\right)^{F^{\circ}}$

$6091.175 \quad$ A $30 \quad 2.26 \quad 4.38 \quad 45 \quad b^{1}\left(G-2^{1} \mathrm{H}^{\circ}\right.$

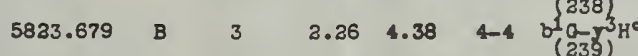

5644.137 A $\left.\quad 18 \quad 2.26 \quad 4.44 \quad 4-4 \quad b 10.8^{1}\right)^{\circ}$

$4836.135 \quad B \quad B \quad 2.28 \quad 4.81 \quad 4-4 \quad b\left(a-x^{1} G\right.$ e

$4799.797 \quad A \quad 13 \quad 3.38 \quad 4.83 \quad 4-4 \quad b \frac{a-x^{3} H^{\circ}}{243}$

$\begin{array}{llllllll}4424.401 & B & 3 & 3.26 & 5.05 & 4 & b^{1}\left(a-\pi_{1}^{1} G^{\circ}\right.\end{array}$

$\begin{array}{llllllll}4393.925 \quad A \quad & 8 & 2.26 & 5.07 & 4-5 & b^{1} \mathrm{a}-y^{1} & \mathrm{H}^{\circ}\end{array}$

$\begin{array}{lllllll}4368.941 \quad B \quad & 2 & 2.26 & 5.08 & 4-3 & b^{3}\left(\begin{array}{l}24)^{3} \\ (245)\end{array}\right.\end{array}$

$\begin{array}{lllllll}3938.005 \quad B & \text { 2n } & 2.26 & 5.39 & 4-4 & b 1 G-y^{1} & 0\end{array}$

$\begin{array}{llllllll}3574.245 & \mathrm{E} & 8 & 2.26 & 5.71 & 4-4 & b^{1}\left(a-u^{1} a^{\circ}\right.\end{array}$

$\begin{array}{lllllllllllllll}9746.86 & D & 15 & 2.31 & 3.57 & 4=4 & z^{5} D^{\circ}-8^{5} D+\end{array}$

$\begin{array}{llrrrrr}9717.00 & D & 10 & 2.30 & 3.57 & 3-3 & (248) \\ 9703.86 & D & 3 & 3.39 & 3.58 & 2-2 & \end{array}$ \begin{tabular}{llll}
1 & \\
0 & 2.23 & 5.58 & $1-2$ \\
0 & 3.22 & 5.57 & $0-1$ \\
\hline
\end{tabular}
I A Laborstory

LOW $^{E} P_{\text {H1Eb }}$

uultiplet

\section{T1 I continued}

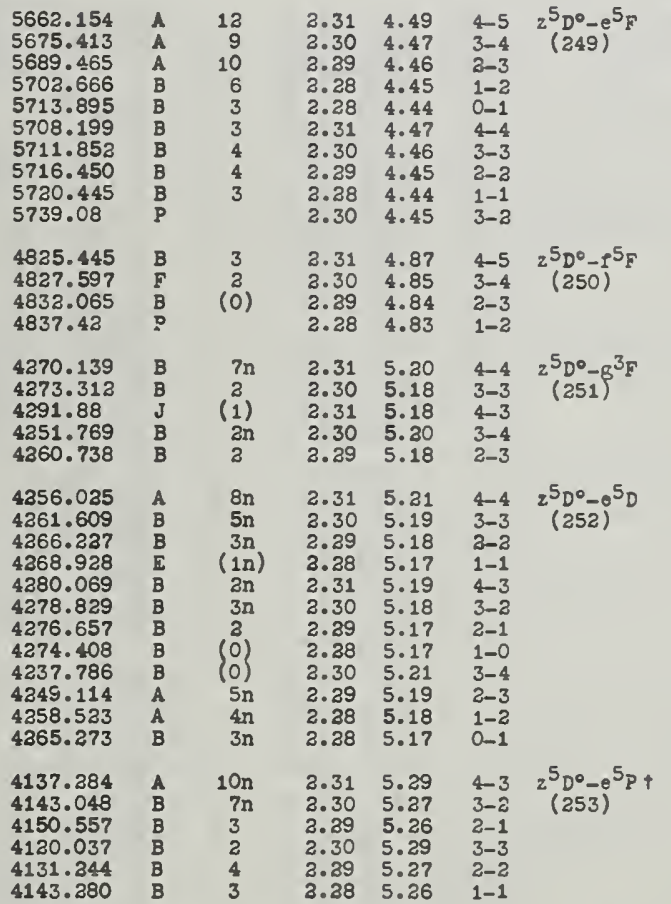

4058.139
4057.613

4060.09

4064.22
4068.66

4074.356

4071.469
4071.211

3323.896

3323.660

3325.365
3328.326

7038.80

7038.80

7050.65

7010.94
6996.63

6017.52

5419.189

5429.139

5448.882

5473.517

5404.023
.5446 .593

4805.418

4792.482
4796.210

4812.240

4637.887

4637.209

4655.712
4640.431

4619.525
4635.539

.4558 .093

4576.551

4598.99

4558.092

6012.53
5982.52
5971.07

5477.695

5481.426

5438.210
5537.606

5518.11

5432.318
5451.965

4563.427

4555.069
4570.906

4570.906
4586.95

4436.64

4430.033
4433.578

3708.625

$\begin{array}{llll}2.31 & 5.35 & 4-5 & 2^{5} D^{0}-8^{5} \\ 2.30 & 5.34 & 3-4 & (254)^{5}\end{array}$

$\begin{array}{lll}2.30 & 5.34 & 3-4 \\ 2.29 & 5.33 & 2-3\end{array}$

2.38 5.32 $1-2$

$\begin{array}{lll}2.28 & 5.31 & 0-1 \\ 2.31 & 5.34 & 4-4\end{array}$

$\begin{array}{lll}2.30 & 5.33 & 3-3 \\ 2.29 & 5.32 & 2-2\end{array}$

$2.31 \quad 6.02 \quad 4-5 \quad z^{5} D^{0}-k^{5} F+$

$\begin{array}{llll}2.30 & 6.01 & 3-4 & 2 \\ 2.0 & (255)\end{array}$

$\begin{array}{lll}2.28 & 5.99 & 1-2\end{array}$

$2.33 \quad 4.09 \quad 2-2 \quad c^{3} \mathrm{P}-\mathrm{x}^{3} \mathrm{p}^{0}$

$\begin{array}{llll}6 & 2.33 & 4.09 & 2-2 \\ 1 & 3.32 & 4.09 & 1-1 \\ 1 & 2.33 & 4.09 & 2-1 \\ 1 & 2.32 & 4.08 & 1-0 \\ 1 & 2.32 & 4.08 & 1-0 \\ 1 & 2.32 & 4.09 & 1-2\end{array}$

$\begin{array}{lll}2.32 & 4.09 & 0-1\end{array}$

$2.32 \quad 4.37 \quad 1-1 \quad c^{3} \mathrm{p}-y^{3} \mathrm{~s}^{0}$

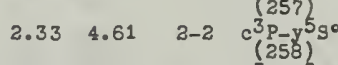

$\begin{array}{llllll}6 & 2.33 & 4.61 & 2-2 & \mathrm{c}^{3} \mathrm{p}-\pi^{3} \mathrm{po} \\ (0) & 2.32 & 4.59 & 1-1 & (259)\end{array}$

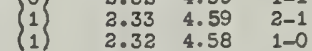

$\begin{array}{lll}2.32 & 4.58 & 1-0 \\ 2.32 & 4.61 & 1-2 \\ 2.32 & 4.59 & 0-1\end{array}$

$\begin{array}{llll}2.33 & 4.90 & 2-3 & c^{3} \mathrm{P}-5^{3} \mathrm{p}^{\circ} \\ 2.32 & 4.90 & 1-2 & (260)\end{array}$

$\begin{array}{lll}2.32 & 4.90 & 1-3 \\ 2.32 & 4.90 & 0\end{array}$

$\begin{array}{lll}2.33 & 4.90 & 2-2 \\ 2.32 & 4.90 & 1-1\end{array}$

$2.33 \quad 5.00 \quad 2-2 \quad c^{3} p-v^{3} p^{0}$

$3.32 \quad 4.99 \quad 1-1 \quad$ (261)

$2.33 \quad 4.99 \quad 2-1$

$\begin{array}{lll}2.32 & 5.00 & 1-2\end{array}$

$\begin{array}{lllll}2 & 2.33 & 5.04 & 2-3 & c^{3} \mathrm{P}-\mathrm{r}^{3} \mathrm{v}^{\circ}\end{array}$ 
I A Laboratory Ref Int Low $_{\text {High }}$ J mitiplet

T1 I continued

$5648.570 \quad A \quad 5 \quad 3.48 \quad 4.67 \quad 3-4 \quad z^{3} D^{0}-0^{3} F$ $\begin{array}{lllllll}5662.891 & \text { B } & 4 & 3.47 & 4.65 & 2-3 & (269) \\ 5679.908 & \text { B } & 3 & 2.46 & 4.63 & 1-2 & \\ 4548.094 & \text { B } & 3 & 2.48 & 5.20 & 3-4 & 2^{3} D^{\circ}-3^{3} \text { I }\end{array}$ $\begin{array}{lllllll}4548.094 & \mathrm{~B} & 3 & 2.48 & 5.30 & 3-4 & z^{3} \mathrm{D}^{\circ}-\mathrm{g}^{3} \mathrm{~J} \\ 4547.850 & \mathrm{E} & 2 & 2.47 & 5.18 & 2-3 & (270)\end{array}$ $\begin{array}{lllllll}4557.857 & \text { B } & 2 & 2.46 & 5.17 & 1-2 & \\ 3481.675 & \text { E } & 3 & 2.48 & 6.03 & 3-3 & 2^{3} D^{\circ}-0^{3} D\end{array}$ $\begin{array}{lllllll}3481.126 & E & 3 & 2.48 & 6.03 & 3-3 & z^{3} D^{\circ}-0^{3} \\ 3472.793 & E & 3 & 2.47 & 6.02 & 3-3 & (271) \\ \end{array}$ 7353.16 ह (1) $2.48 \quad \overline{4.16} \quad 1-1 \quad a^{1} P-z^{1} p^{0}$

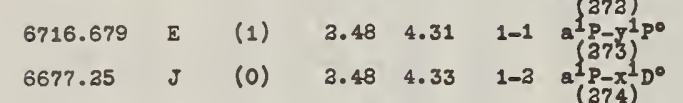
$\begin{array}{lllllll}.5511 .795 \quad B & 2 & 2.48 & 4.73 \quad 1-0 & \begin{array}{l}(374) \\ (375)\end{array}\end{array}$

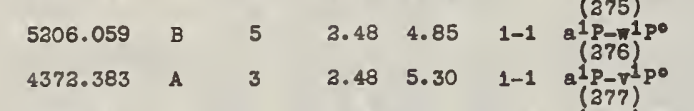

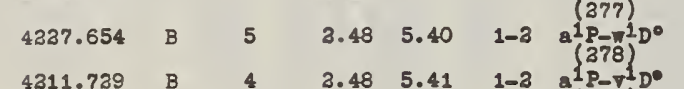

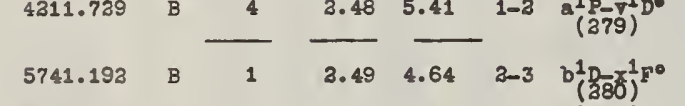
$5398.429 \quad A \quad 4 \quad 3.494 .82 \quad a-1 \quad b^{1}\left(28 x^{1} p^{0}\right.$

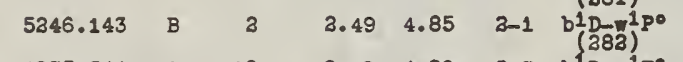
$\begin{array}{rrrrrrr}4975.344 & A & 10 & 2.49 & 4.98 & 3-3 & b \frac{1}{(283)} F^{\circ} \\ 4337.889 & A & 7 & 2.49 & 5.41 & 3-2 & b \\ (38)^{1} D^{\circ}\end{array}$

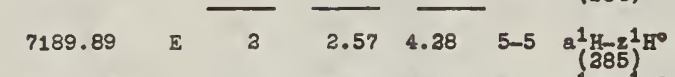

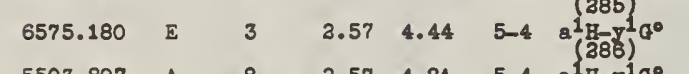
$\begin{array}{rrrrrrr}5503.897 & \text { A } & 8 & 2.57 & 4.81 & 5-4 & a_{R-x^{1}}^{10} \\ 5130.420 & \text { A } & 12 & 3.57 & 4.98 & 5-6 & a^{1}\left(3-z^{1} I^{\circ}\right.\end{array}$ $\begin{array}{lllllll}4938.383 & \text { A } & 8 & 3.57 & 5.07 & 5-5 & \mathrm{a}^{(} \mathrm{B}-\mathrm{H}^{1} \mathrm{H}^{\circ}\end{array}$ $\begin{array}{llllllll}4369.683 & \mathrm{~A} & 5 \mathrm{n} & 2.57 & 5.39 & 5-4 & \mathrm{a}^{1} \mathrm{H}-\mathrm{r}^{1} 0^{\circ} \\ 4278.231 & \mathrm{~B} & 7 & 3.57 & 5.45 & 5-5 & \mathrm{a}^{1}\left(\mathrm{H}-\mathrm{x}^{1} \mathrm{H}^{\circ}\right.\end{array}$

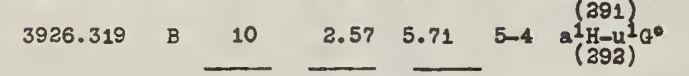
$\begin{array}{lrrrrrr}6215.212 & \mathrm{~B} & 20 & 3.68 & 4.67 & 5-4 & 2^{3} \mathrm{G}^{\circ}-\mathrm{C}^{3} \mathrm{p} \\ 6220.460 & \mathrm{~B} & 13 & 3.67 & 4.65 & 4-3 & (393) \\ 6221.41 & \mathrm{E} & 8 & 3.65 & 4.63 & 3-2 & \end{array}$

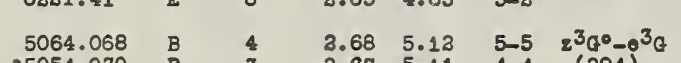
$\begin{array}{ccccccc}5054.070 & \text { B } & 3 & 3.67 & 5.11 & 4-4 & (394) \\ 5068.332 & \text { B } & 3 & 3.65 & 5.09 & 3-3 & \end{array}$

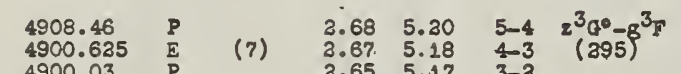
$\begin{array}{lllllll}4127.531 & \mathrm{~A} & 15 & 2.68 & 5.67 & 5-6 & 2^{3} \mathrm{~g}^{\circ}-\mathrm{F}^{3} \mathrm{H}\end{array}$ $\begin{array}{lllllll}4123.559 & \text { B } & 10 & 2.67 & 5.66 & 4-5 & (296) \\ 4122.143 & \text { A } & 10 & 2.65 & 5.64 & 3-4 & \end{array}$ $\begin{array}{llcllll}4149.445 & \text { B } & (0) & 2.68 & 5.66 & 5-5 \\ 4142.480 & \text { B } & 3 & 2.67 & 5.64 & 4-4 & \\ 4032.628 & \text { B } & \text { 3n } & 3.68 & 5.74 & 5-4 & z^{3} G^{\circ}-h^{3} \mathrm{y}\end{array}$ 5259.976 B $\frac{3}{3} \quad \frac{3.68}{3.73} \overline{5.07}^{2-3.2^{1} D^{\circ}-0^{1} \mathrm{~F}}$

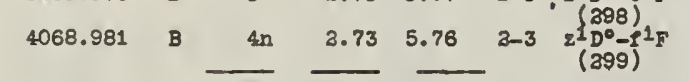
$\begin{array}{lllllllllll}5351.073 & \text { B } & 4 & 2.77 & 5.07 & 3-3 & 2^{1} \frac{F^{0}}{300)^{1} F}\end{array}$ $\begin{array}{lllllll}4224.795 & \text { B } & 5 & 3.77 & 5.69 & 3-4 & 2^{1} F^{0}-\theta^{1 Q}\end{array}$ $\begin{array}{lllllll}4133.287 & B & 5 n & 3.77 & 5.76 & 3-3 & 2 \frac{1}{(301} F^{\circ}-f^{1} F\end{array}$

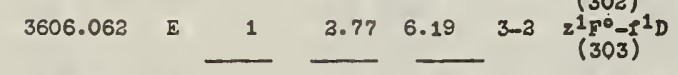
6098.655 B $7 \quad \overline{3.05} 5.074-3 \quad 2^{1} G^{0}-0^{1} \mathrm{~F}$

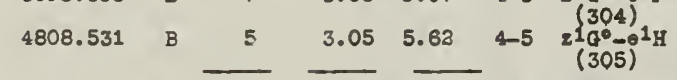

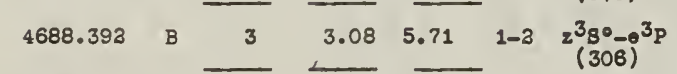
$\begin{array}{lllllll}7069.11 & I & 3 & 3.17 & 4.91 & 3-4 & \mathrm{~J}^{3} \mathrm{D}^{0}-\mathrm{I}^{3} F \\ 7039.36 & \mathrm{E} & \left(\begin{array}{l}1 \\ 1\end{array}\right) & 3.14 & 4.89 & 3-3 & (307) \\ 7035.86 & \mathrm{E} & (2) & 3.13 & 4.88 & 1-3 & \end{array}$ $\begin{array}{llllll}7035.86 & E & \text { (3) } 3.13 \quad 4.88 & 1-2\end{array}$

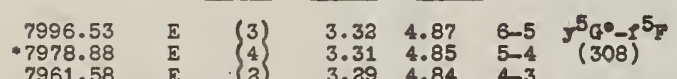

I A Laboratory ${\text { Rof Int Low }{ }^{E} \text { High } J \text { Multiplot }}_{\text {(Ho) }}$ II I cont1nued

$\begin{array}{lllllll}5832.47 & \text { A } & \text { (1) } & 3.32 & 5.44 & 6-6 & J^{5} \mathrm{G}^{\circ}-\mathrm{r}^{5} \mathrm{H} \\ 5812.837 & \mathrm{~B} & 3.31 & 5.43 & 5-5 & \text { oont }\end{array}$

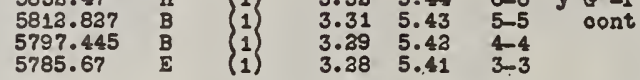

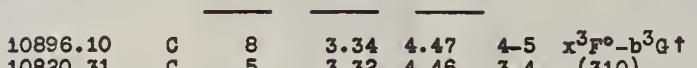
10820.31
10793.65

$$
\begin{aligned}
& 5995.685 \mathrm{2} \quad \overline{3.45} 5.50 \quad 2-3 \frac{J^{1} D^{0}-0^{1} D}{(311)}
\end{aligned}
$$

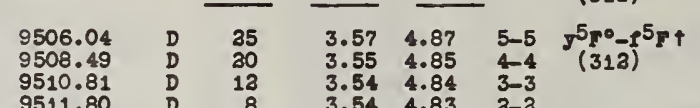

$$
\begin{aligned}
& \begin{array}{rrrrrr}
9510.81 & D & 12 & 3.54 & 4.84 & 3-3 \\
9511.80 & D & 8 & 3.54 & 4.83 & 3-2 \\
9511.55 & D & 10 & 3.53 & 4.83 & 1-1
\end{array} \\
& .8496 .03 \quad D \quad \frac{10}{60} \frac{3.53}{3.68} \frac{4.83}{5.13} 3-3 \quad \mathrm{a}^{1} \bar{F}-\nabla^{2} F^{\circ}
\end{aligned}
$$

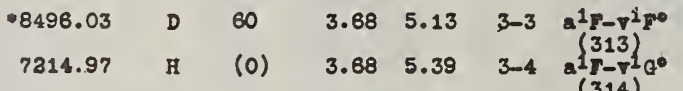

$$
\begin{aligned}
& \begin{array}{llllllll}
10147.09 & c & 4 & 3.90 & 5.12 & 5-5 & \pi^{3} c^{\circ}-0^{3} \theta \\
10119.30 & c & 3 & 3.89 & 5.11 & 4-4 & (315)
\end{array} \\
& \begin{array}{lllllllll}
10119.30 & C & 3 & 3.89 & 5.11 & 4-4 & (315) \\
10179.93 & C & 3 & 3.87 & 5.09 & 3-3 & \\
& & & & & & & &
\end{array} \\
& \begin{array}{llllll}
5341.492 & \mathrm{~B} & 1 & 4.31 & 6.68 & 1-1 \quad \mathrm{~J}_{(316)}^{1} \mathrm{P}^{0}-0^{1 P}
\end{array}
\end{aligned}
$$

Strongest Unolaseleled Lines of T1 I

11609.41
11539.50

11403.89

11381.53
11330.91

10145.48 C 8

$\begin{array}{rrr}9981.16 & D & 5 \\ 8641.47 & D & 40 \\ 8418.70 & D & 10\end{array}$

$\begin{array}{rrrr}8565.62 & \text { I } & 10 & \\ & 4 & \end{array}$

$\begin{array}{llll}5369.635 & A & 4 & \text { III } \\ 4599.236 & A & 5 & \text { IV } \\ 4539.096 & \text { B } & 3 & \text { IV }\end{array}$

$\begin{array}{llll}4511.176 & \mathrm{~B} & 3 & \text { IV } \\ 4495.006 & \mathrm{~B} & 4 & \text { III }\end{array}$

$\begin{array}{llll}4129.166 & \mathrm{~B} & 4 & \text { III } \\ 4121.637 & \mathrm{~B} & 4 & \text { III } \\ 4027.436 & \mathrm{~B} & 4 & \text { IV } \\ 3985 & \end{array}$

$\begin{array}{llll}3985.246 & \mathrm{~B} & 3 & \text { III } \\ 3861.079 & \mathrm{~B} & 3 \mathrm{n} & \text { IV? }\end{array}$

$\begin{array}{llll}3846.438 & \text { B } & 6 n & \text { IV } \\ 3836.763 & \text { B } & 5 & \text { IV } \\ 3833.874 & \text { B } & 4 & \text { III } \\ 3833 & \end{array}$

$\begin{array}{llll}3833.186 & B & 4 & \text { IV } \\ 3735.880 & \text { B } & 4 \mathrm{n} & \text { IV }\end{array}$

3715.371

3700.055
3644.699

3633.458

3585.853

3556.184

3507.436
3459.431

3435.433

.3130 .8048
3007.487

(3)

$\begin{array}{ll}3 \mathrm{n} & \mathrm{IV} \\ \text { 4n } & \mathrm{IV} \\ 4 & \mathrm{IV} \\ 5 & \mathrm{IV} \\ 3 & \mathrm{IV}\end{array}$

$\begin{array}{cc}4 & \text { IIIP } \\ 3 & \text { IV } \\ 3 & \text { IV } \\ 3 & \text { III } \\ 3 & \text { III }\end{array}$

T1 II I P 13.6 Anal A L1st A oot 1940

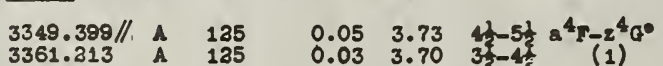

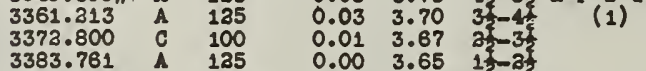

$\begin{array}{rrrrrr}3383.781 & A & 125 & 0.00 & 3.65 & 1 \\ 3330.278 & A & 30 & 0.05 & 3.70 & 4\end{array}$

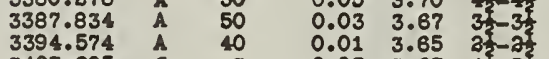

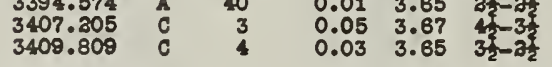

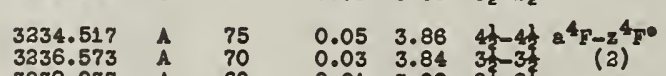

3239.037
3241.984
3254.250

3253.914

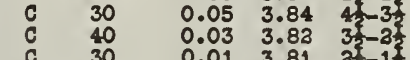

0.013 .81 a

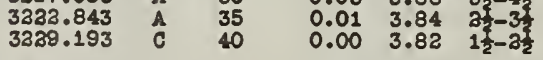

3314.750

3236.771

3197.518
3313.1458

3184.09

3203.435

3143.756
3157.397
3130.8048

3130.8048
3148.033
3121.599

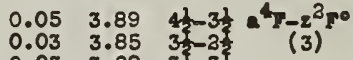

$\begin{array}{lll}0.03 & 3.85 & 3.31 \\ 0.03 & 3.89 & 31 \\ 0.01 & 3.85 & 3\end{array}$

$\begin{array}{lll}0.01 & 3.89 & 2 \\ 0.00 & 3.85 & 1 \frac{1}{2}-2 \frac{1}{2}\end{array}$
I A Laboratory

Low ${ }^{\text {Plgh }}$

s (xo)

I1 II continued

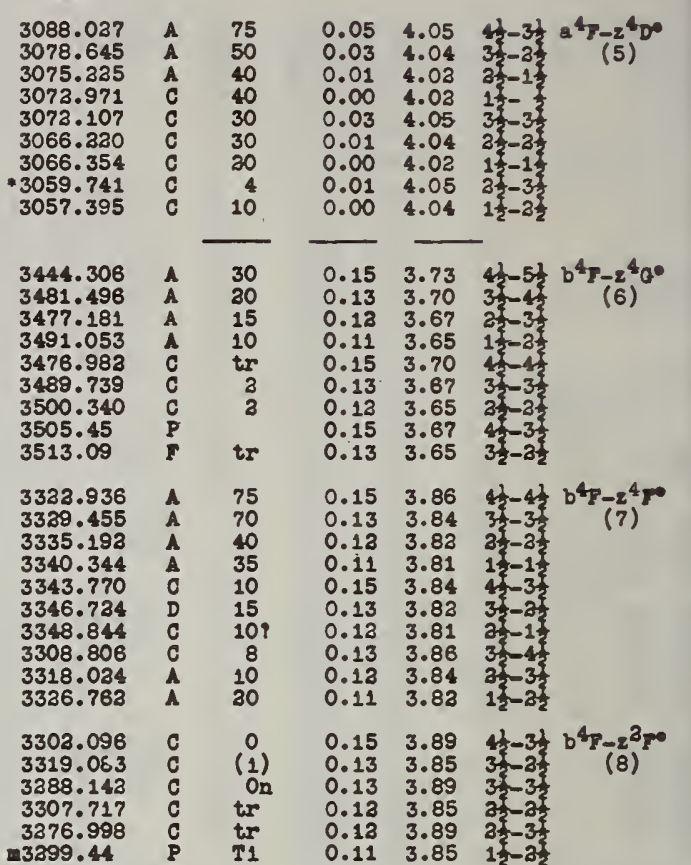

$0.13 \quad 3.95 \quad 3 \frac{1}{3}-2 \frac{1}{2} b^{4}-2^{2} D^{0}$

$\begin{array}{llllll}3248.70 & P & 0.13 & 3.92 & 31 & -1 \\ 3230 & (9)\end{array}$

$\begin{array}{llllll}3230.467 & C & 1 & 0.12 & 3.95 & 2 \\ 3240.71 & 0 & 1 & 0.11 & 3.92 & 1 \\ 3312.7 & P & & 0.11 & 3.95 & 1\end{array}$

$3168.519 \quad A \quad 40 \quad 0.15 \quad 4.05 \quad 4-3 \frac{1}{b^{4} T-2^{4} D^{\circ}}$

$0.13 \quad 4.04 \quad 3-2 \frac{1}{2}(10)$

$\begin{array}{llllll}3161.755 & \text { A } & 30 & 0.12 & 4.02 & 2 \frac{1}{2}-1 \frac{1}{1} \\ 3161.305 & \text { C } & 25 & 0.11 & 4.02 & 1 \frac{1}{2} \\ 3155.670 & \text { C } & 12 & 0.13 & 4.05 & 31-3\end{array}$

$0.13 \quad 4.04$ 3द -2

$\begin{array}{lll}0.11 & 4.02 & 12-1 \frac{1}{2} \\ 0.12 & 4.05 & 32\end{array}$

3154.195

-3145.402
$m 3144.74$

12
0
$1^{+}$

$0.11 \quad 4.04 \quad 1 \frac{1}{2}-3 \frac{3}{2}$

3987.63

3981.998

4025.136

4056.31

3786.33

3786.33
3774.650

3813.390

3796.899
3836.085

3814.580

3759.291

3761.320 A 300

$\begin{array}{lll}3799.81 & \text { p } & \text { tr } \\ 3731.632 & \text { B } & 15\end{array}$

-3685.192 A 250

3649.01

3587.130

3561.575 C

$\begin{array}{lll}3596.048 & \text { A } & 60 \\ 3573.737 & \text { C } & 20\end{array}$

$0.60 \quad 3.70 \quad 3 \frac{1}{2}-4 \frac{1}{2} a^{2} \overline{7}-2^{4} 0$

a

$0.57 \quad 3.67 \quad 21-3 \frac{5}{2} \quad(11)$

(1) $\quad 0.57 \quad 3.65$ a 3.65 और

$0.603 .86 \quad 3 \frac{1}{2}-48^{2} 5-8^{4}$

$3 n$

$\begin{array}{llll}3 & 0.60 & 3.84 & 31 \\ 3 n & 0.57 & 3.82 & 3\end{array}$

$0.603 .82 \quad$ और 3

$0.603 .893 \frac{1}{2}-3 \frac{1}{2} a^{2} F-2^{2} F$

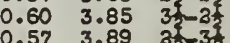

$0.60 \quad 3.95 \quad 3 \frac{1}{2}-3 \frac{1}{2} a^{3} \bar{Y}-2^{2} D$ $\begin{array}{llll}0.57 & 3.98 & 3 & 31 \\ 0.57 & 3.95 & \text { (14) }\end{array}$

0.604 .05 और $-3 \frac{1}{2} \mathrm{a}^{2} \mathrm{~F}-2^{4} \mathrm{D}$

$\begin{array}{llll}0.57 & 4.04 & 3 & 3 \\ 0 & \text { (15) }\end{array}$

$\begin{array}{lll}0.57 & 4.02 & 3 \frac{2}{2}-1 \frac{1}{2} \\ 0.57 & 4.05 & 3 \frac{1}{2}-3 \frac{1}{2}\end{array}$

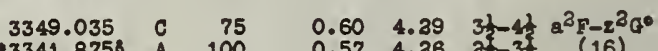

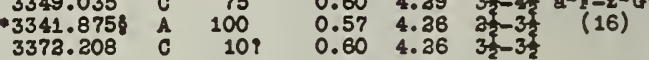

$\begin{array}{lllllll}4762.77 & f & (1) & 1.08 & 3.67 & 2 \frac{1}{2}-3 \frac{1}{2} & a^{2} \mathrm{D}-2^{4} a 0 \\ 4798.535 & \mathrm{C} & (2) & 1.08 & 3.65 & 1 \frac{1}{2}-2 \frac{1}{2} & (17) \\ 4806.33 & \mathrm{P} & & 1.08 & 3.65 & 3 \frac{1}{2}-2 \frac{1}{2} & \end{array}$

4469.160

4493.53

4500.32

45155.21

4395.031

4443.806
4450.487

4294.101
4337.916

437.916
434.291

4287.893

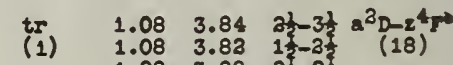

$1.08 \quad 3.82$ 3.

$\begin{array}{lll}-1.08 & 3.81 & 1 \frac{1}{2}-1 \frac{1}{2} \\ 1.08 & 3.81 & 32\end{array}$

4161.52
4167.67

4173.537

184.329
4190.29

.

$1.08 \quad 3.89 \quad 2 \frac{1}{2}-3 \frac{1}{2} a^{2} \mathrm{D}-2^{2} \mathrm{~F}^{\circ}$

$\begin{array}{lll}1.08 & 3.85 & 1 \frac{3}{2} \\ 1.08 & 3.85 & 21\end{array}$

$1.08 \quad 3.95 \quad 2 \frac{1}{2}-2 \frac{1}{2} a^{2} D-z^{2} D^{\circ}$

$\begin{array}{llll}1.08 & 3.92 & 1 \frac{1}{2}-1 \frac{1}{2} & (30)\end{array}$

$\begin{array}{lll}1.08 & 3.92 & 2 \\ 1.08 & 3.95 & 1 \frac{1}{2}-3 \frac{1}{2}\end{array}$

$1.08 \quad 4.05$ और $-3 \frac{1}{2} \mathrm{a}^{3} \mathrm{D}-2^{4} \mathrm{D}$

$\begin{array}{llll}1.08 & 4.04 & 1 \frac{1}{2}-2 a^{2} & (21)\end{array}$

$\begin{array}{llll}1 & 1.08 & 4.04 & 2 \\ 0 & 1.08 & 4.02 & 12\end{array}$

$\begin{array}{llll}0 & 1.08 & 4.02 & 1 \\ \text { (1) } & 1.08 & 4.02 & 3 \\ & 1.08 & 4.03 & 1\end{array}$

$3480.897 \quad C \quad 0 \quad 1.384 .62 \quad 1 \frac{1}{2}-\frac{1}{2} a^{2} D_{-2}^{2} g^{\circ}$ 


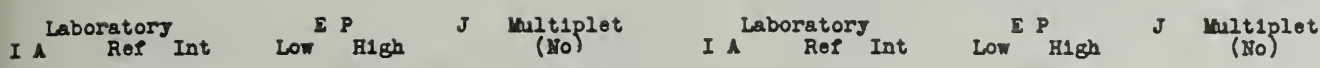
II II oont1nued

\begin{tabular}{|c|c|c|c|c|c|}
\hline $\begin{array}{r}3352.94 \\
3375.293 \\
.3378 .9238 \\
3349.370\end{array}$ & $\begin{array}{l}P \\
c \\
c \\
c\end{array}$ & $\begin{array}{r}\mathrm{T1}^{+} \\
3 \\
35 \\
3\end{array}$ & $\begin{array}{l}1.08 \\
1.08 \\
1.08 \\
1.08\end{array}$ & $\begin{array}{l}4.87 \\
4.84 \\
4.84 \\
4.87\end{array}$ & $\begin{array}{l}3 \frac{1}{2}-2 \frac{1}{2} a^{3} D-r^{3} D^{\circ} \\
1 \frac{x}{2}-1 \frac{1}{2} \quad(23) \\
3 \frac{1}{2}-1 \frac{1}{2} \\
1 \frac{2}{2}-3 \frac{1}{2}\end{array}$ \\
\hline $\begin{array}{l}3339.664 \\
3328.605 \\
3336.123\end{array}$ & $\begin{array}{l}c \\
c \\
c\end{array}$ & $\begin{array}{l}30 \\
30 \\
30\end{array}$ & $\begin{array}{l}1.08 \\
1.08 \\
1.08\end{array}$ & $\begin{array}{l}4.89 \\
4.90 \\
4.89\end{array}$ & $\begin{array}{l}3 \frac{1}{2}-1 \frac{1}{2} a^{3} D_{-2} \mathrm{P}^{0} \\
1 \frac{2}{3}-\frac{1}{2} \\
1 \frac{1}{2}-1 \frac{2}{2}\end{array}$ \\
\hline $\begin{array}{l}3195.717 \\
3192.36\end{array}$ & $\begin{array}{l}c \\
c\end{array}$ & $\begin{array}{l}3 \\
2\end{array}$ & $\begin{array}{l}1.08 \\
1.08\end{array}$ & $\begin{array}{l}4.94 \\
4.94\end{array}$ & $\begin{array}{cc}3 \frac{1}{2}-1 \frac{1}{2} \\
1 \frac{1}{2}-1 \frac{1}{2}\end{array} a^{2} \mathrm{D}-z^{4} \mathrm{~g}^{0}$ \\
\hline $\begin{array}{l}3190.874 \\
3203.535 \\
3205.990\end{array}$ & $\begin{array}{l}\text { C } \\
\text { A } \\
\text { C }\end{array}$ & $\begin{array}{r}30 \\
40 \\
1\end{array}$ & $\begin{array}{l}1.08 \\
1.08 \\
1.08\end{array}$ & $\begin{array}{l}4.95 \\
4.93 \\
4.93\end{array}$ & $\begin{array}{l}2 \frac{1}{2}-3 \frac{1}{2} a^{3} D-y^{3} F^{\circ} \\
1 \frac{1}{2}-3 \frac{2}{2}(36) \\
2 \frac{1}{2}-2 \frac{1}{2}\end{array}$ \\
\hline $\begin{array}{l}3118.824 \\
3136.77 \\
3140.04 \\
3153.14 \\
3155.80 \\
3161.66\end{array}$ & $\begin{array}{l}C \\
F \\
P \\
P \\
P \\
P\end{array}$ & tr & $\begin{array}{l}1.08 \\
1.08 \\
1.08 \\
1.08 \\
1.08 \\
1.08\end{array}$ & $\begin{array}{l}5.04 \\
5.01 \\
5.01 \\
4.99 \\
4.99 \\
4.98\end{array}$ & 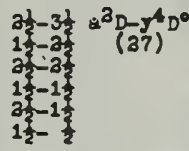 \\
\hline $\begin{array}{l}2987.40 \\
3996.88 \\
2999.92 \\
3003.37 \\
2884.35\end{array}$ & $\begin{array}{l}F \\
P \\
P \\
P \\
P\end{array}$ & 1 & $\begin{array}{l}1.08 \\
1.08 \\
1.08 \\
1.08 \\
1.08\end{array}$ & $\begin{array}{l}5.31 \\
5.19 \\
5.19 \\
5.18 \\
5.31\end{array}$ & 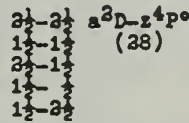 \\
\hline
\end{tabular}

I1 II continued

\begin{tabular}{|c|c|c|c|c|c|}
\hline $\begin{array}{r}m 3318.36 \\
3331.76 \\
3338.36 \\
\mathrm{~m} 3234.50 \\
3331.71\end{array}$ & $\begin{array}{l}P \\
P \\
P \\
P \\
P\end{array}$ & $\mathrm{TI}^{+}$ & $\begin{array}{l}1.18 \\
1.16 \\
1.16 \\
1.18 \\
1.16\end{array}$ & $\begin{array}{l}5.01 \\
4.99 \\
4.98 \\
4.99 \\
4.98\end{array}$ & 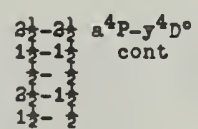 \\
\hline $\begin{array}{r}3058.090 \\
.3059 .741 \\
3063.502 \\
3071.342 \\
3066.514 \\
3046.685 \\
3056.740\end{array}$ & $\begin{array}{l}\mathrm{c} \\
\mathrm{c} \\
\mathrm{c} \\
\mathrm{c} \\
\mathrm{c} \\
\mathrm{c}\end{array}$ & $\begin{array}{r}50 \\
6 \\
4 \\
15 \\
3 \\
30 \\
15\end{array}$ & $\begin{array}{l}1.18 \\
1.16 \\
1.16 \\
1.18 \\
1.16 \\
1.16 \\
1.16\end{array}$ & $\begin{array}{l}5.31 \\
5.19 \\
5.18 \\
5.19 \\
5.18 \\
5.31 \\
5.19\end{array}$ & 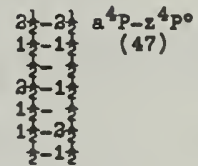 \\
\hline
\end{tabular}

1.13 3.73 $41-5+a^{2} a-2^{4} a^{\circ}$ $\begin{array}{llll}1.11 & 3.70 & 3 .-4 \\ 1.13 & 3.70 & \frac{4}{3}-4 \\ 1.11 & 3.67 & 3\end{array}$

$\begin{array}{lll}1.11 & 3.67 & 3 \\ 1.13 & 3.67 & 4 \\ 1.11 & 3.65 & 35\end{array}$

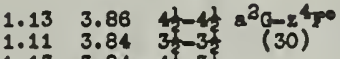

$\begin{array}{lll}1.11 & 3.84 & 3 .-31 \\ 1.13 & 3.84 & 4 \\ 1.11 & 3.83 & 3\end{array}$

1.113 .88 उ -4 ह

$\begin{array}{llll}1.11 & 3.89 & 3 & 3 \\ 1.11 & 3.89 & 35 & 31 \\ 1 & & \end{array}$

$1.11 \quad 3.95 \quad 3 \frac{1}{2}-2 \frac{1}{2} \mathrm{a}^{2} a-\Omega^{2} \mathrm{D}^{\circ}$

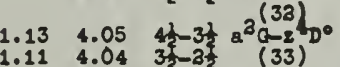

$1.11 \quad 4.05 \quad 3 \frac{1}{2}-3 \frac{1}{2}(33)$

$\begin{array}{lllll}70 & 1.13 & 4.29 & 4 \frac{1}{4}-4 \frac{1}{2} a^{2} a-2^{2} a^{\circ} \\ 80 & 1.11 & 4.26 & 35-3 \frac{1}{2} & (34)\end{array}$

$\begin{array}{lll}1.13 & 4.36 & 41 \\ 1.11 & 4.29 & 3 \frac{1}{3}-4 \frac{1}{2}\end{array}$

$1.114 .87 \quad 3 \frac{1}{2}-2 \frac{1}{2} \mathrm{a}^{2} a-v^{2} \mathrm{D}^{\circ}$

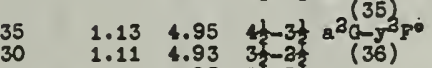

$1.11 \quad 4.95 \quad 3 \frac{1}{2}-3 \frac{3}{2}$

$\begin{array}{llll}1.13 & 5.04 & 41-3 \frac{1}{3} & a^{2} a-y^{4} p^{0} \\ 1.11 & 5.01 & 3 & (37)\end{array}$ \begin{tabular}{lll}
1.11 & 5.01 & 3 \\
1.11 & 5.04 & $3 \frac{1}{2}-3 \frac{1}{2}$ \\
\hline
\end{tabular}

T1 1.18 3.84 $31-3 \frac{1}{2} s^{4}-2^{4} \mathrm{~F}^{\circ}$

T1

$\begin{array}{llll}1.16 & 3.81 & \\ 1.18 & 3.82 & 2\end{array}$

$\begin{array}{lll}1.16 & 3.81 & 12 \\ 1.18 & 3.81 & 21\end{array}$

(1)

$1.18 \quad 3.89 \quad 21-3 \frac{1}{2} a^{4} \mathrm{p}_{-2} \mathrm{z}^{3} \mathrm{pe}$

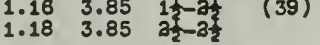

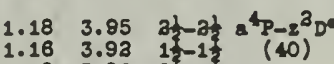

$\begin{array}{lll}1.18 & 3.93 & 31 \\ 1.16 & 3.95 & 1 \frac{2}{3}\end{array}$

$1.16 \quad 3.92$ र-12

$\begin{array}{lll}1.18 & 4.05 & 21-31 \\ 1.16 & 4.04 & 1\end{array}$

$\begin{array}{lll}1.16 & 4.03 & 1 \\ 1.18 & 4.04 & 3\end{array}$

$1.16 \quad 4.03 \quad 15$

$\begin{array}{lll}1.18 & 4.03 & 3 \\ 1.16 & 4.03 & 1\end{array}$

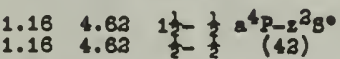

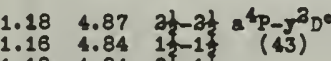

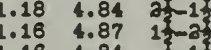

1.164 .84 रू-1द

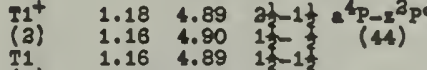

$\begin{array}{lll}1.16 & 4.89 & 15 \\ 1.16 & 4.90 & 1\end{array}$

1.184 .94 at $-1 \frac{1}{2}+{ }^{4} p-24$.

$\begin{array}{llll}1.16 & 4.94 & 1 & 1 \\ 1.16 & 1.94 & (15)\end{array}$

$1.18 \quad 5.04$ की $-3 \frac{1}{2} a^{4} P-C^{4}$

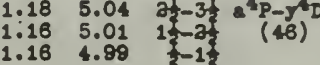

4763.84
4793.39

4708.663

4533.986
4563.761
4589.961

4399.767

4394.05
4418.340

407.678
433.089

3641.330

3624.330
3628

3388.75

3402.433
3416.957

3374.353

3352.071
.3338 .1788

3386.178
3380.16

3337.85

3326.68

3266.43

3283.14

3329.97
3393.48

$\mathbf{2} 101.53$

3103.975

3109.93

4657.310

4698.67

4719.51

4544.009
4580.458

4580.458

4524.732

4568.313
4395.848

4395.818
4390.977

4398.314

4409.23
4409.519

4411.936
4437.90

4427.90
4423.23

3635.36

3394.37

$\mathbf{3 4 1 1 . 6 8}$

3383.57

3379.930

$-3361.07$

3369.312

3362.653

3333.111

3321.700
3315.334

.3348 .6038

- 3261.596

3271.653

-1
0

3388.438

328.575

3108.234
3110.620

3113.050

3119.800
3117.869

3097.186

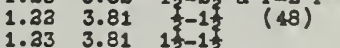

$1.23 \quad 3.85 \quad 1 \frac{1}{2}-2 \frac{1}{2} \mathrm{a}^{3} \mathrm{P}_{-2} \mathrm{z}^{2} \mathrm{~F}^{\circ}$

$1.23 \quad 3.95 \quad 1 \frac{1}{3}-3 a^{2} \mathrm{P}_{-2}^{(49)} \mathrm{a}^{\circ}$

$\begin{array}{llll}1.23 & 3.93 & 1 & 1 \\ 1.33 & 3.93 & 1 \frac{1}{2}-1 \frac{3}{2} & (50)\end{array}$

$1.23 \quad 4.04 \quad 1 \frac{1}{3}-2 \frac{1}{3} \mathrm{a}^{3} \mathrm{P}-2^{4} D^{\circ}$

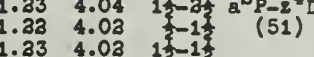

$\begin{array}{lll}1.23 & 4.03 & 1 \\ 1.23 & 4.03 & 1 \frac{3}{2}-\frac{2}{2}\end{array}$

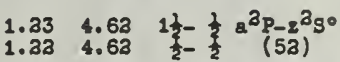

$1.33 \quad 4.87 \quad 2 \frac{1}{3}-2 \frac{1}{2} a^{2} P-y^{3} D^{\circ}$

$\begin{array}{llll}1.32 & 4.84 & \frac{5}{3}-1 \frac{2}{2} \\ 1.23 & 4.84 & 1 \frac{1}{2}-1 \frac{2}{2}\end{array}$

$1.33 \quad 4.89 \quad 1 \frac{1}{2}-1 \frac{1}{2} \mathrm{a}^{3} \mathrm{P}_{-2} \mathrm{p}^{2} \mathrm{O}$

$\begin{array}{lll}1.32 & 4.90 & \frac{1}{2}-\frac{4}{2} \\ 1.33 & 4.90 & 1 \frac{1}{2} \\ 1.33 & 4.89 & \frac{1}{2}-1 \frac{2}{2}\end{array}$

$\begin{array}{lll}1.33 & 4.89 & \frac{1}{2}-1 \frac{1}{2} \\ 1.33 & 4.93 & 1 \frac{1}{2}-2 \frac{1}{2} \mathrm{a}^{2} \mathrm{p}-\mathrm{y}^{3} \mathrm{~F}^{0}\end{array}$

$1.33 \quad 4.94 \quad 1 \frac{1}{3}-1 \frac{1}{2} a^{2}(55) \mathrm{g}^{\circ}$

(1) $1.33 \quad 5.01 \quad 1 \frac{1}{3}-3 a^{2} P-y^{4} D^{\circ}$

1.33 4.99 1 ?

$1.23 \quad 4.98 \quad 1 \frac{2}{2}-\frac{2}{2}$

$1.33 \quad 5.31 \quad 1 \frac{1}{2}-2 \frac{1}{2} \mathrm{a}^{3} \mathrm{P}-z^{4} \mathrm{P}^{\circ}$

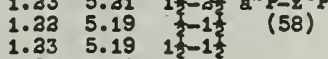

$\begin{array}{lll}1.23 & 5.18 & \frac{1}{2} \\ 1.23 & 5.18 & 1 \frac{1}{2}\end{array}$

tr $1.34 \quad \overline{3.89} 31-31 b^{4} p_{-2} z^{2 t}$

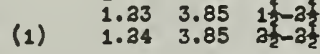

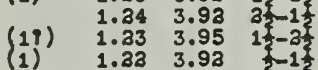

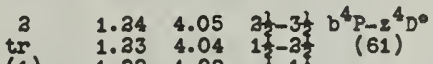

$\begin{array}{llll}\text { (1) } & 1.32 & 4.03 & 1 \\ t r & 1.34 & 4.04 & 31\end{array}$

(1) 1.234 .02

$\begin{array}{lll}1.23 & 4.03 & 1 \\ 1.34 & 4.03 & 3 \\ 1.23 & 4.03 & 1 \frac{1}{2}-1\end{array}$

(1) $\begin{array}{llll}1.33 & 4.63 & 1 & 1 \\ 1.32 & 4.63 & \frac{1}{2} b^{4} P_{-2} z^{2} & (63)\end{array}$

T1 $\quad \begin{array}{llll}1.34 & 4.87 & \text { at-a } & b^{4} \mathrm{P}-y^{3} \mathrm{D}^{\circ}\end{array}$

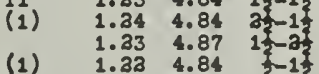

1n $1.34 \quad 4.89$ at $1 \frac{1}{2} \mathrm{~b}^{4} \mathrm{P}-\mathrm{z}^{3} \mathrm{P}$.

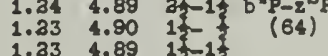

$\begin{array}{lll}1.32 & 4.90 & 1 \\ 1.32 & 4.89 & \frac{1}{2}-1 \frac{3}{2}\end{array}$

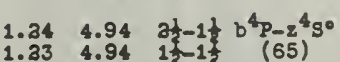

$\begin{array}{llll}1.34 & 5.04 & 21-31 & b^{4} P-J^{4} D^{\circ} \\ 1.33 & 5.01 & 13-25 & (66)\end{array}$

$\begin{array}{lll}1.33 & 5.01 & 11-3 \\ 1.32 & 4.99 & 1\end{array}$

$\begin{array}{lll}1.34 & 5.01 & 32 \\ 1.33 & 4.99 & 13\end{array}$

1.334 .99

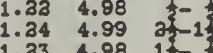

1.345 .31 at $-2 \frac{1}{2} b^{4} P-2^{4} t^{4}$ 。

$\begin{array}{llll}1.24 & 5.31 & 21-21 & b^{4} p-2 \\ 1.33 & 5.19 & 1.13 & (67)\end{array}$

1.235 .18

$1.33 \quad 5.18 \quad 13$

$\begin{array}{lll}1.23 & 5.21 & 1 \\ 1.32 & 5.19 & 2\end{array}$

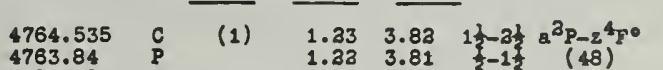

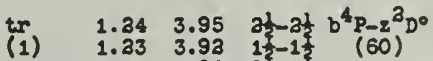

$\begin{array}{lll}1.33 & 4.94 & 1 \frac{1}{1} \\ 1.32 & 4.94 & \frac{1}{2}\end{array}$

I $A_{\text {ReI }}^{\text {Laboratory }}$

LOT $^{\text {E } P}{ }_{\text {H1B }}$

Multiplet

T1 II oontinued

5446.46

5454.05

5490.65

5539.94

5336.809

5381.030
5418.802

5188.700

5336.534

5363.104
5154.061

4995.89

4981.38

5005.18

5037.81

3741.633

3757.684
3776.083
3733.631

3734.10

3696.39
3706.319

3666.11

3648.80

3659.765

3663.237
3679.673

3565.336

3576.38

3596.55

3613.30

3608.89

3110.095

3108.927

3048.766

3043.851

3036.784

5723.87
5781.73

5814.63

5691.99

5396.3

5432.47

5367.95

5313.76

4549.633

4571.971
4539.465

3648.86

3234.241
3318.370

3318.370

3017.187

3029.730

3038.706
3008.333

\subsection{3}

5185.90

5183.72
5131.28

4028.333

4053.814
4039.64

3504.890

3510.840

3509.844
3505.901

- 3361.596

3387.657

3387.657
3386.756

3103.804

3089.40

6491.61

8559.580

1805.105

4779.986

4374.825

4400.63
4331.949

4
1
0
6
5
0
0

tr

11
50
30
6
tr
1
$(1)$
20

60
40
$(3)$
3
$(0 n)$
3
tr

8
3
0
1
6
5
1

$1.57 \quad 3.842 \frac{1}{3}-3 \frac{1}{2} b^{2} \mathrm{D}-2^{4} \mathrm{~F}^{0}$

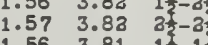

$\begin{array}{lll}1.56 & 3.81 & 1 \frac{1}{2}-1 \frac{1}{2} \\ 1.57 & 3.81 & 2 \frac{1}{2}-1 \frac{1}{2}\end{array}$

$1.57 \quad 3.89 \quad 3 \frac{1}{2}-3 \frac{1}{2} b^{2} \mathrm{D}-8^{3} F^{\circ}$

$\begin{array}{lll}1.56 & 3.85 & 1 \frac{1}{3}-2 \frac{1}{2} \\ 1.57 & 3.85 & 3 \frac{1}{2}-2 \frac{2}{2}\end{array}$

$1.57 \quad 3.95 \quad 3 \frac{1}{2}-3 \frac{1}{2} b^{2} \mathrm{D}-z^{2} \mathrm{D}^{\circ}$

$\begin{array}{lll}1.56 & 3.92 & 1 \frac{1}{2}-1 \frac{1}{2} \\ 1.57 & 3.92 & 3-1 \frac{1}{2}\end{array}$

$1.57 \quad 4.05 \quad 3 \frac{1}{2}-3 \frac{1}{2} b^{2} D-2^{4} D^{0}$

$\begin{array}{lll}1.56 & 4.04 & 12 \\ 1.57 & 4.04 & 2 \frac{1}{3}-2\end{array}$

1.564 .02

$1.56 \quad 4.03 \quad 1$

$1.57 \quad 4.87 \quad 2 \frac{1}{2}-2 \frac{1}{2} b^{2} D-5^{3} b^{\circ}$

$\begin{array}{lll}1.56 & 4.84 & 1 \frac{1}{2}-1 \frac{1}{2} \\ 1.57 & 4.84 & 2 \frac{1}{2}-1 \frac{2}{2} \\ 1.56 & 4.87 & 1 \frac{1}{2}-2 \frac{1}{2}\end{array}$

$1.57 \quad 4.892 \frac{1}{2}-1 \frac{1}{2} b^{2} D_{-2} 2^{2} p_{0}$

$\begin{array}{llll}1.56 & 4.90 & 1 \frac{1}{2}-\frac{1}{3} & (73) \\ 1.56 & 4.89 & 1 \frac{1}{2}-1 \frac{1}{2} & \end{array}$

$\begin{array}{llll}1.57 & 4.94 & 3 \frac{1}{2}-1 \frac{1}{2} & b^{2} \mathrm{D}-z^{4} g^{\circ} \\ 1.56 & 4.94 & 1 \frac{1}{2}-1 \frac{1}{2} & (74)\end{array}$

$1.57 \quad 4.95 \quad 2 \frac{1}{3}-3 \frac{1}{2} b^{2} D-y^{3} F^{\circ}$

$\begin{array}{lll}1.56 & 4.93 & 1 \frac{1}{2}-3 \frac{1}{3} \\ 1.57 & 4.93 & 3 \frac{1}{2}-2 \frac{1}{2}\end{array}$

$1.57 \quad 5.04 \quad 2 \frac{1}{2}-3 \frac{1}{2} b^{2} D-y^{4} D^{0}$

$\begin{array}{lll}1.56 & 5.01 & 11-3 \\ 1.57 & 5.01 & 25 \\ 1 & -2\end{array}$

$1.57 \quad 4.99 \quad 3 \frac{1}{2}-1$

$1.56 \quad 4.98 \quad 1 \frac{2}{2}-\frac{2}{2}$

$1.57 \quad 5.54 \quad 3 \frac{1}{2}-2 \frac{1}{2} b^{2} D-x^{3} D^{\circ}$

$\begin{array}{lll}1.56 & 5.54 & \frac{1}{32}-1 \frac{1}{2} \\ 1.57 & 5.54 & 32\end{array}$

$1.56 \quad 5.54 \quad 1 \frac{2}{2}-2 \frac{1}{2}$

$1.57 \quad 5.62 \quad 2 \frac{1}{2}-1 \frac{1}{2} b^{2} \mathrm{D}-\mathrm{g}^{2} \mathrm{po}^{\mathrm{O}}$ $\begin{array}{lll}1.56 & 5.61 & 1 \frac{1}{2}-\frac{1}{2} \\ 1.56 & 5.62 & 1 \frac{1}{2}-1 \frac{1}{2}\end{array}$

$1.58 \quad 3.73 \quad 5 \frac{1}{2}-5 \frac{1}{2} \mathrm{a}^{2} \mathrm{H-2} \mathrm{2}^{4} \mathrm{CO}^{\circ}$

$\begin{array}{lll}1.56 & 3.70 & 41-4 \\ 1.58 & 3.70 & 53\end{array}$

$\begin{array}{llll}1.56 & 3.67 & 3 \frac{3}{2} \\ 1.56 & 3.73 & -5 \frac{7}{2}\end{array}$

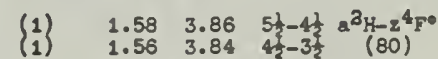

$\begin{array}{lll}1.56 & 3.84 & 4 \frac{1}{2}-3 \frac{1}{3} \\ 1.56 & 3.86 & 4 \frac{1}{2}-4 \frac{1}{2}\end{array}$

$1.56 \quad 3.89 \quad \frac{1}{2}-3 \frac{1}{2} \mathrm{a}^{3} \mathrm{H}-\mathrm{z}^{3} \mathrm{~F}$

$1.58 \quad 4.39 \quad 5 \frac{1}{3}-4 \frac{1}{2} \mathrm{a}^{3} \mathrm{a}_{\mathrm{H}-\mathrm{z}^{3} \mathrm{G}^{\circ}}$

$\begin{array}{lll}1.56 & 4.36 & 4 \frac{1}{2}-3 \frac{1}{1} \\ 1.56 & 4.29 & 4 \frac{1}{2}-4 \frac{1}{2}\end{array}$

$1.56 \quad 4.954 \frac{1}{2}-3 \frac{1}{2} \mathrm{a}^{3} \mathrm{H}-\mathrm{y}^{3} \mathrm{~F}^{\circ}$

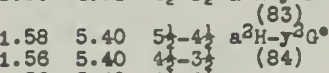

$\begin{array}{lll}1.56 & 5.40 & 4 \\ 1.56 & 5.40 & -3 \frac{1}{2}\end{array}$

$1.58 \quad 5.67 \quad 5 \frac{1}{2}-5 \frac{1}{2} \mathrm{a}^{2} \mathrm{H-z^{2 }} \mathrm{H}^{\circ}$

\begin{tabular}{lll}
1.58 & 5.64 & $5 \frac{1}{4}$ \\
1.58 & 5.67 & $4 \frac{1}{3}-5$ \\
\hline
\end{tabular}

$1.88 \quad 4.29 \quad 4 \frac{1}{3}-4 \frac{1}{2} b^{2} a-z^{2} a^{\circ}$

$\begin{array}{lll}1.88 & 4.36 & 3 \frac{2}{3}-3 \frac{1}{2} \\ 1.88 & 4.26 & 4 \frac{1}{3}-3 \frac{1}{4} \\ 1.88 & 4.39 & 3 \frac{1}{2}-4\end{array}$

$1.88 \quad 4.95 \quad 4 \frac{1}{3}-3 \frac{1}{2} b^{2} a-5^{2} F^{\circ}$

$\begin{array}{lllll}1.88 & 4.93 & 3 \frac{3}{2}-2 \frac{3}{2} & (87) \\ 1.88 & 4.95 & 3 \frac{1}{2}-3 \frac{1}{2} & \end{array}$

$1.88 \quad 5.40 \quad 4 \frac{1}{3}-4 \frac{1}{3} b^{2} a-y^{3} C^{0}$

$\begin{array}{lll}1.88 & 5.40 & 3 \frac{1}{2}-3 \\ 1.88 & 5.40 & 4 \frac{1}{3}-3\end{array}$

$1.88 \quad 5.67 \quad 4 \frac{1}{3}-5 \frac{1}{2} b^{2} a-z^{2} H^{\circ}$

$\begin{array}{llll}1.88 & 5.67 & 41-5 \frac{1}{2} & b^{2} a-z^{2} \\ 1.88 & 5.64 & 3.4 & (89)\end{array}$

$1.88 \quad 5.86 \quad 4 \frac{1}{3}-3 \frac{1}{2} b^{2} a-x^{2} F^{\circ}$

$\begin{array}{llll}1.88 & 5.88 & 33-5 & (90)\end{array}$

$3.053 .95 \quad 1 \frac{1}{3}-2 \frac{1}{2} b^{2} p-z^{2} p^{\circ}$

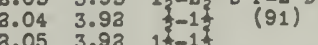

$3.054 .631 \frac{1}{2}+b^{2} P_{-2}{ }^{2} S^{\circ}$

$3.04 \quad 4.62$ 古-

$3.05 \quad 4.87 \quad 1 \frac{1}{2}-2 b^{2} P-y^{3} D^{\circ}$

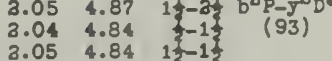




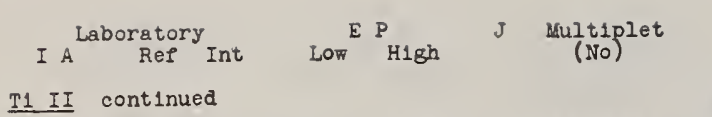

\begin{tabular}{|c|c|c|c|c|c|}
\hline $\begin{array}{l}4350.834 \\
4316.807 \\
4337.33 \\
4330.264\end{array}$ & $\begin{array}{l}\text { C } \\
B \\
C \\
B\end{array}$ & $\begin{array}{c}1 \\
1 \\
(1) \\
0\end{array}$ & $\begin{array}{l}2.05 \\
2.04 \\
2.05 \\
3.04\end{array}$ & $\begin{array}{l}4.89 \\
4.90 \\
4.90 \\
4.89\end{array}$ & $\begin{array}{l}1 \frac{1}{2}-1 \frac{1}{2} b^{2} \mathrm{P}-z^{2} \mathrm{P}^{\circ} \\
\frac{1}{2}-\frac{1}{2} \quad(94) \\
1 \frac{\frac{1}{2}}{2}-\frac{2}{2} \\
\frac{1}{2}-1 \frac{1}{2}\end{array}$ \\
\hline $\begin{array}{l}4271.94 \\
4252.05\end{array}$ & $\begin{array}{l}P \\
P\end{array}$ & & $\begin{array}{l}2.05 \\
2.04\end{array}$ & $\begin{array}{l}4.94 \\
4.94\end{array}$ & $\begin{array}{c}-1 \frac{1}{2} b^{2} P-z^{4} S^{0} \\
-1 \frac{1}{2}\end{array}$ \\
\hline $\begin{array}{l}4173.05 \\
4181.17 \\
4200.40 \\
4197.95 \\
4217.34\end{array}$ & $\begin{array}{l}P \\
P \\
P \\
P \\
P\end{array}$ & & $\begin{array}{l}2.05 \\
2.04 \\
2.05 \\
3.04 \\
2.05\end{array}$ & $\begin{array}{l}5.01 \\
4.99 \\
4.99 \\
4.98 \\
4.98\end{array}$ & $\begin{array}{l}1 \frac{1}{2}-2 \frac{1}{2} b^{3} \mathrm{P}-\mathrm{y}^{4} \mathrm{D}^{\circ} \\
\frac{1}{2}-1 \frac{1}{2}(96) \\
1 \frac{1}{2}=1 \frac{2}{2} \\
\frac{1}{2}-\frac{1}{2} \\
1 \frac{2}{2}=\frac{2}{2}\end{array}$ \\
\hline $\begin{array}{l}3907.65 \\
3912.32 \\
3929.15 \\
3923.39 \\
3940.32\end{array}$ & $\begin{array}{l}\mathrm{P} \\
\mathrm{P} \\
\mathrm{P} \\
\mathrm{P} \\
\mathrm{P}\end{array}$ & & $\begin{array}{l}3.05 \\
2.04 \\
2.05 \\
2.04 \\
2.05\end{array}$ & $\begin{array}{l}5.31 \\
5.19 \\
5.19 \\
5.18 \\
5.18\end{array}$ & 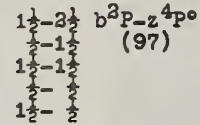 \\
\hline
\end{tabular}

$\begin{array}{rrrrrrr}3535.408 & \mathrm{~A} & 40 & 2.05 & 5.54 & 1 \frac{1}{2}-2 \frac{1}{3} & \mathrm{~b}^{2} \mathrm{P}-\mathrm{x}^{2} \mathrm{D}^{\circ} \\ 3520.253 & \mathrm{C} & 20 & 3.04 & 5.54 & \frac{2}{2}-1 \frac{2}{2} & (98) \\ 3533.868 & \mathrm{C} & 2 & 3.05 & 5.54 & 1 \frac{2}{2}-1 \frac{2}{2} & \end{array}$

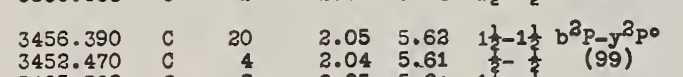

$\begin{array}{llllll}3465.562 & 0 & 3 & 3.05 & 5.61 & 1 \frac{2}{3}-\frac{2}{2} \\ 3443.387 & 0 & 1 & 2.04 & 5.62 & \frac{2}{2}-1 \frac{2}{2}\end{array}$

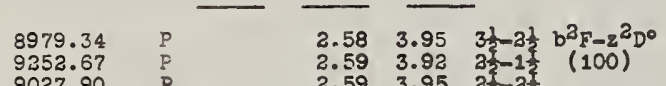

$\begin{array}{lllll}9027.90 & \mathrm{P} & 2.59 & 3.95 & 21 \\ 7214.78 & \mathrm{P} & 2.58 & 4.29 & 31-4 \frac{1}{2} \mathrm{~b}^{3} \mathrm{~F}-\mathrm{z}^{3} \mathrm{G} \text { 。 }\end{array}$

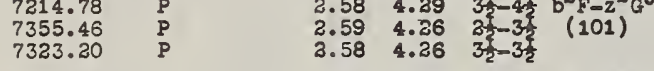

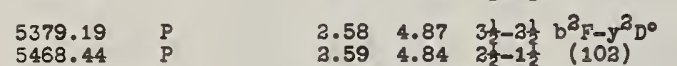

5468.4

5211.544

5258.62
5227.08

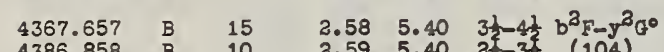

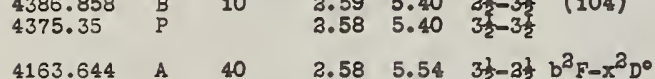

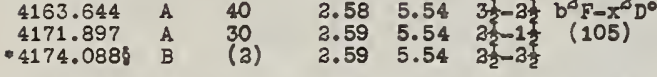

$4064.350 \quad$ B $\quad$ (1) $3.59 \quad 5.62 \quad 3 \frac{1}{2}-1 \frac{1}{2} b^{2} F-y^{3} p_{0}$

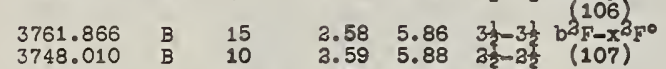

$\begin{array}{llllll}3739.6 & F & \text { tr } & 2.58 & 5.88 & 3 \frac{3}{2} \\ 3770.412 & \mathrm{C} & (1) & \mathbf{3 . 5 9} & 5.86 & 2 \frac{2}{2}-3 \frac{1}{2}\end{array}$

$6212.30 \quad F \quad \overline{(1)} \quad \overline{2.63} \quad \overline{4.63} \quad \frac{1}{2}-\frac{1}{2} \mathrm{a}^{2} \mathrm{~s}-2^{2} \mathrm{~s}^{\circ}$

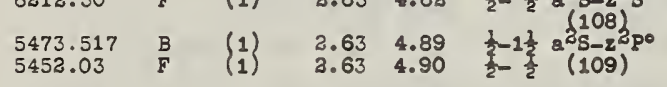

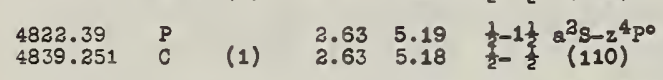

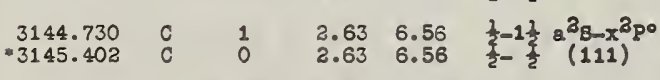

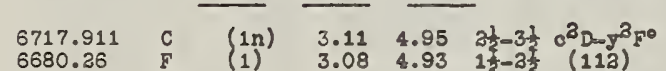

$\begin{array}{lllllll}6680.26 & F & (1) & 3.08 & 4.93 & 1 \frac{2}{3}-25 & (112) \\ 6785.25 & P & & 3.11 & 4.93 & 2 \frac{1}{2}-2 \frac{1}{2} & \end{array}$

$\begin{array}{llrllll}5072.30 & E & 3 & 3.11 & 5.54 & 2 \frac{1}{2}-2 \frac{1}{2} & c^{2} D-x^{3} D^{0} \\ 5010.202 & C & t r & 3.08 & 5.54 & 1 \frac{1}{2}-1 \frac{1}{2} & (113)\end{array}$

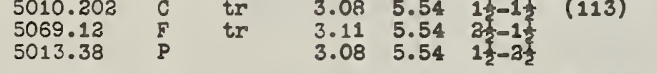

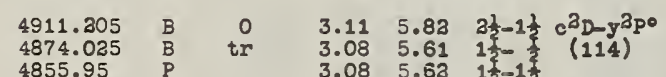

$4488.319 \quad B \quad 15 \quad 3.11 \quad 5.86 \quad 2 \frac{1}{3}-3 \frac{1}{2} c^{2} D-x^{3} F^{\circ}$

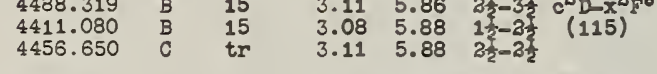

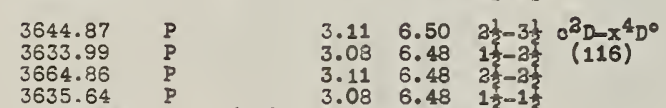

3666.592
3652.81

3578.687

3550.19
3549.27

3524.87

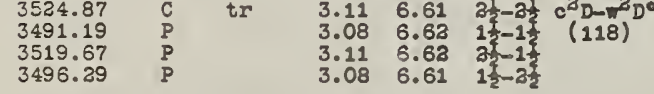

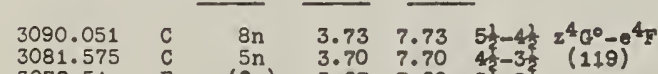

3081.575
3072.54

(On) $\begin{array}{llll}3.11 & 6.48 & 32 \\ 3.08 & 6.46 & 1 \frac{2}{2}\end{array}$

(0) $3.11 \quad 6.56 \quad 3 \frac{1}{2}-1 \frac{1}{3} \quad c^{3} p-x^{3} p_{0}^{0}$ $\begin{array}{llll}3.08 & 6.56 & 1 \frac{1}{2} & (117) \\ 3.08 & 6.56 & 19 & 1\end{array}$

3063.380

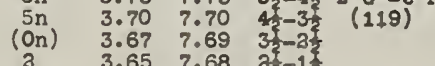

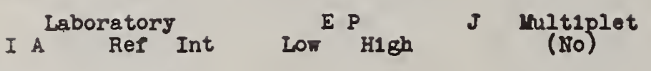
T1 II continued

$\begin{array}{lllllll}3208.607 & c & \text { in } & 3.84 & 7.69 & 31-31 & 2^{4} \mathrm{~F}^{0}-e^{4} \mathrm{~F}\end{array}$

$\begin{array}{lllllll}3175.66 & \mathrm{C} & 2 \mathrm{n} & 3.84 & 7.73 & \frac{3}{3}-4 \frac{3}{2} & \text { cont } \\ 3178.630 & \mathrm{C} & 3 \mathrm{n} & 3.82 & 7.70 & 3 \frac{3}{3} & \\ 3180.325 & \mathrm{C} & 2 \mathrm{n} & 3.81 & 7.69 & 1 \frac{3}{2}-3 \frac{1}{2} & \end{array}$

$\begin{array}{cccc} & & \\ 3128.6408 & c & 10 n \\ 3127.883 & c & 10 n \\ 3155.91 & P & \end{array}$

$\begin{array}{llll}3181.84 & c & 8 \mathrm{n} \\ 3183.57 & \mathrm{C} & 6 \mathrm{n}\end{array}$

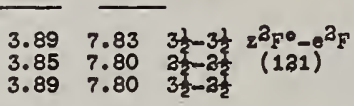

$\begin{array}{llllllll}3990.16 & C & 10 & 3.95 & 8.08 & 32-31 & z^{2} D^{0}-F^{3} F \\ 3979.199 & C & 10 & 3.92 & 8.06 & 1 \frac{1}{2}-3 \frac{1}{2} & (133)\end{array}$

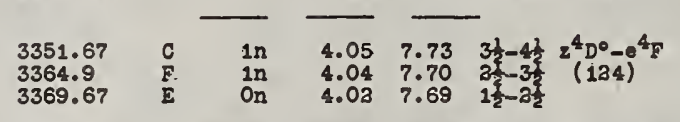

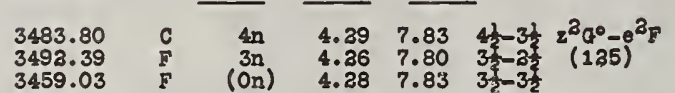

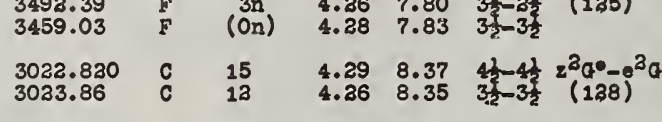

$3414.03 \quad F \quad \overline{(0)} \quad \overline{5.04} 8.53 \quad 3 \frac{1}{2}-4 \frac{1}{2} 8_{(127)}^{4} p^{0}-r^{4} F$

Strongest Unclase1fied Lines of II II

$\begin{array}{lll}3194.76 & C & 6 \mathrm{nP} \\ 3174.80 & \mathrm{C} & 5 \\ 3164.91 & \mathrm{~F} & 8 \mathrm{\nabla}+ \\ 3045.085 & \mathrm{C} & 5 \mathrm{n}\end{array}$

I1 IV I P 43.06 Anal A L1Bt A Dec 1940

$\begin{array}{llllll}5398.82 & A & 8 & 36.3238 .51 & \frac{1}{1}-1 \frac{1}{3} & 5^{3} \mathrm{~S}-5^{2} \mathrm{PO}\end{array}$

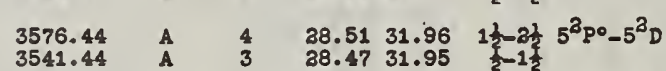

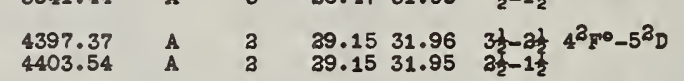

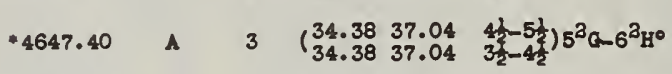

VI I P 6.71 Anal A L1st C Nor 1940

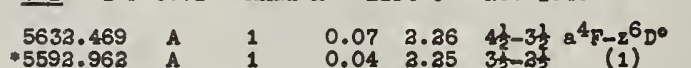

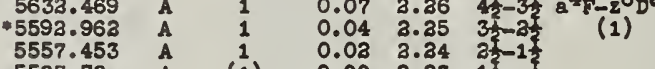

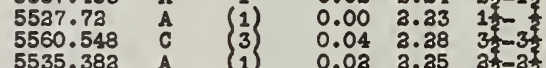

$\left.\begin{array}{llllll}5535.383 & \text { A } \\ 5515.371 & \text { B } & 1 \\ 4\end{array}\right\} \begin{array}{llll}0.02 & 3.25 & 3 \frac{3}{2}-3 \frac{1}{3} \\ 0.00 & 3.34 & 1 \frac{1}{2}-1 \frac{1}{2}\end{array}$

$\begin{array}{lllllll}5515.083 & A & 1 & 0.07 & 2.31 & 4 \\ 5498.020 & B & (3) & 0.04 & 3.29 & a^{4} \mathrm{~F}_{-2} \mathrm{~F}_{\mathrm{F}} \uparrow\end{array}$

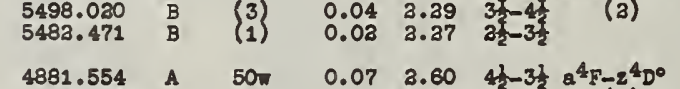

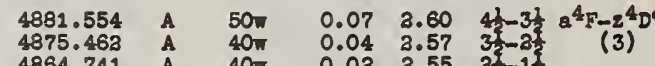

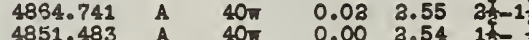

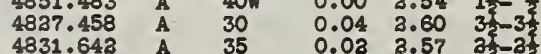

$\begin{array}{llllll}4831.642 & \text { A } & 35 & 0.02 & 3.57 & 3 \\ 4833.437 & \text { A } & 30 & 0.00 & 2.55 & 13-1\end{array}$

$\begin{array}{rrrrrr}4832.437 & \mathrm{~A} & 30 & 0.00 & 2.55 & 1 \\ 4784.480 & \mathrm{~A} & 5 & 0.02 & 2.60 & 2 \\ 4799.786 & \mathrm{~A} & \mathbf{5} & 0.00 & 3.57 & 1 \frac{1}{2}-2 \frac{1}{2}\end{array}$

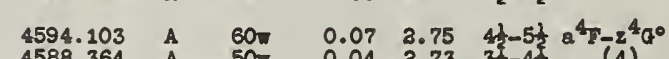

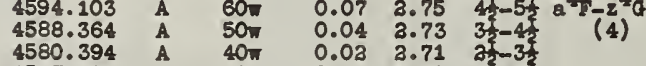

$\begin{array}{r}4330.034 \\ -4384.732 \\ \hline\end{array}$

$\begin{array}{llll}40 \pi & 0.02 & 2.71 & 25 \\ 40 \pi & 0.00 & 3.70 & 13\end{array}$

$\begin{array}{llll}8 & 0.02 & 3.91 & 32 \\ 1 & 0.03 & 2.95 & 25 \\ 8 & 0.00 & 3.91\end{array}$
$3.116 .612 \frac{1}{3}-2 \frac{1}{2} c^{2} D-\pi^{2} D^{\circ}$ \begin{tabular}{lll}
3.11 & 6.62 & 2 \\
3.1 \\
3.08 & 6.61 & $1 \frac{1}{2}-3$ \\
\hline
\end{tabular}

4577.173

4635.176

4606.146
4689.273
4645.971

4353.872

4341.013
4333.823

4368.043

4309.795

4306.314
4307.184

.4234 .000

4259.312

.4234.5348

4176.793

3902.250
3875.075

3864.882

3855.370
+3809.894

3892.859

$\begin{array}{llll}45 & 0.07 & 3.73 & 43\end{array}$

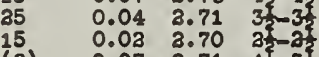

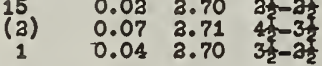

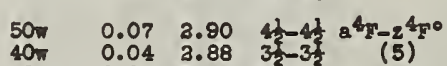

$\begin{array}{llll}40 \pi & 0.04 & 3.88 & 35 \\ 30 \pi & 0.02 & 3.87 & 37 \\ 30 \pi & 0.00 & 2.85 & 13-1\end{array}$

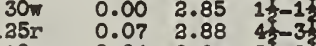

$\begin{array}{llll}10 & 0.04 & 3.87 & 33-23 \\ 10 & 0.03 & 3.85 & 31\end{array}$

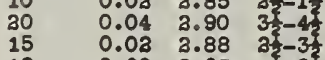

$\begin{array}{llll}12 & 0.02 & 3.88 & 3 \\ 12 & 0.00 & 3.87 & 1 \frac{1}{2}-3 \frac{2}{2}\end{array}$

$13 \quad 0.04 \quad 3.95 \quad 34-21 \quad a^{4} \sqrt{5-2} a^{2} p^{0}$

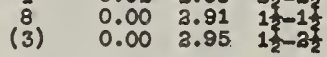

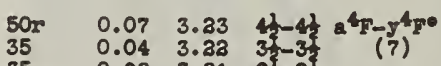

$0.03 \quad 3.31$ 21-3.

$\begin{array}{lll}0.00 & 3.20 & 1 \frac{1}{3} \\ 0.07 & 3.23 & 42\end{array}$

0.04 3. 31 31

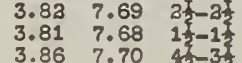

I A Laboratory Ref Int

$\checkmark$ I continued

$3867.602 \quad c \quad 15 \quad 0.04 \quad 3.23 \quad 34-4 \frac{1}{4} a^{4}-y^{4}=0$

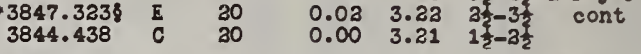

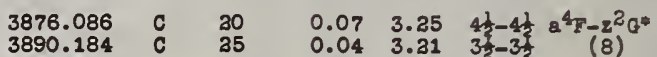

3890.184
.3925 .340

3841.890
3863.323

3855.841

3840.752

3818.344

3813.009

3808.521
-3794.964

3793.814

3817.844

3803.902
3791.326
3781.393

* 3781.393

3713.957
3721.358

3298.139

3383.311
3271.637

3271.637

3308.348
3291.676

3291.676
3277.939

3249.566

3249.566
3230.646
3215.375

3204.198
-3254.7738

3185.398

3183.96

3183.406

3207.410

3202.381

3286.0106
3317.131

3091.437

3091.552

3093.24
3090.40

3069.845

3080.146

3052.194
3060.93

3063.734

3054.89
3050.890

3068.375

3060.460
3056.334

3056.334
3053.65

3082.109

3073.823
3066.51

3066.51

3043.124
304.355

3977.539

3977.539
3962.773
3954.333

3954.332
3957.33

8343.11
8251.83

8251.83

6358.595

6296.518

8385.185

6274.670

6216.368

8330.736
6342.80

8150.133
6170.340

6189.350

6207.251

6213.874
6234.507

8324.507
6233.187

6233.187
6240.137

6245.214

*6268.841

6266.32

6281.236

4460.293

4459.760
4457.479

4437.837

4441.683
4444.207

419.935
4438.515

4438.515
4436.138

$0.07 \quad 3.21 \quad 4 \frac{11}{3}-3$

$\begin{array}{lll}0.04 & 3.25 & 32-4 \frac{1}{3} \\ 0.02 & 3.21 & 2 \frac{2}{2}-3 \frac{1}{2}\end{array}$

$0.07 \quad 3.27 \quad 4 \frac{1}{2}-3 \frac{1}{2} a^{4} F-y^{4} D^{\circ}$

$0.03 \quad 3.24$ as -1

$0.04 \quad 3.37 \quad 35-3$

$\begin{array}{lll}0.02 & 3.25 & 23-3 . \\ 0.00 & 3.24 & 10\end{array}$

$\begin{array}{llll}0.02 & 3.27 & 25 & 31 \\ 0.00 & 3.25 & 1\end{array}$

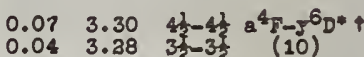

$\begin{array}{lll}0.04 & 3.28 & 31 \\ 0.02 & 3.27 & \frac{31}{2}-2 \frac{1}{2} \\ 0.00 & 3.26 & 1 \frac{1}{2}-1 \frac{2}{2}\end{array}$

$\begin{array}{llll}0.07 & 3.39 & 4 \frac{1}{3}-3 \frac{1}{2} & a^{4} \mathrm{~F}^{2} \mathrm{z}^{2} \mathrm{~F}^{0}+ \\ 0.04 & 3.36 & \frac{\mathrm{T}}{2}-2 \frac{7}{2} & (11)\end{array}$

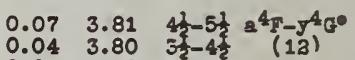

$\begin{array}{lll}0.04 & 3.80 & 35 \\ 0.03 & 3.79 & 33 \\ 0.00 & 3.73\end{array}$

$0.07 \quad 3.80 \quad 43-4$

$\begin{array}{lll}0.04 & 3.79 & 31-3 \frac{1}{2} \\ 0.02 & 3.78 & 2 \frac{1}{2}-\frac{21}{2}\end{array}$

$0.07 \quad 3.87 \quad 4 \frac{1}{3} a^{2} y-x^{4} F^{0}$

$0.04 \quad 3.86 \quad 35$ (13)

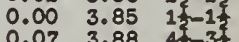

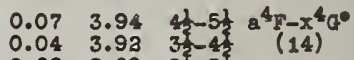

$200 \mathrm{R}$

(125R)

(150R)

$0.00 \quad 3.89 \quad 35-35$

$0.07 \quad 3.92$ 45 -4

$\begin{array}{lll}0.02 & 3.88 & 2 \frac{2}{2}-2 \frac{1}{2} \\ 0.07 & 3.89 & \frac{43}{2}-3 \frac{1}{2} \\ 0.04 & 3.88 & 35-2\end{array}$

10

$30 \quad 0.07 \quad 4.08 \quad 4 \frac{1}{3}-3 \frac{1}{2} a^{4}-x^{4}-0$

8i $\quad 0.04$ 4. 4.01 3 3 -15

(1) $\quad 0.0003 .99$ (1)

$60 \mathrm{r}$

$0.03 \quad 4.03$

$0.00 \quad 4.01 \quad 1 \frac{1}{3}-1$.

$\begin{array}{llll}0.02 & 4.06 & 31 & -3 \\ 0.00 & 4.03 & 1 \frac{2}{2}-2 \frac{1}{2}\end{array}$

$12 \quad 0.03 \quad 4.05 \quad 2 \frac{21}{2}-1 \frac{1}{4} a^{4} F-z^{3} p_{0}$

$\begin{array}{llll}0.00 & 4.04 & 1 \frac{1}{2}-\frac{1}{2} & 2 \\ 0.00 & 4.05 & 1 \frac{1}{2}-1 \frac{1}{2}\end{array}$

$\begin{array}{lllll}125 R & 0.07 & 4.09 & 4 \frac{1}{2}-4 \frac{1}{2} & a^{4}-m^{4} \mathrm{~F}^{\circ} \\ 125 R & 0.04 & 4.07 & 3 \frac{1}{2}-3 \frac{1}{2} & (17)\end{array}$

$\begin{array}{llll}125 R & 0.04 & 4.07 & 35 \\ 100 R & 0.03 & 4.06 & 35 \\ 100 & 3\end{array}$

$\begin{array}{llll}80 \mathrm{R} & 0.00 & 4.04 & 1 \\ 50 \mathrm{r} & 0.07 & 4.07 & 43\end{array}$

$\begin{array}{llll}605 & 0.04 & 4.06 & 35\end{array}$

$\begin{array}{llll}50 \mathrm{r} & 0.04 & 4.09 & 32 \\ 50 \mathrm{r} & 0.04 & 4 & 4\end{array}$

$50 x$

$\begin{array}{llll}0.00 & 4.06 & 1 \frac{2}{2}-3 \frac{1}{2} .\end{array}$

$0.07 \quad 4.31-4 \frac{1}{3}-3 \frac{1}{2} a^{4}-7^{4} 0^{0}$ ?

$0.04 \quad 4.21 \quad 3 \frac{1}{5}-2 \frac{1}{2}$ (18)

\begin{tabular}{lll}
0.02 & 4.19 & $36-1 \frac{1}{2}$ \\
0.04 & 4.21 & $3 \frac{1}{2}-3 \frac{1}{2}$ \\
\hline
\end{tabular}

$0.30 \quad 2.38 \quad 4 \frac{1}{3}-4 \frac{1}{2} a^{6} D-z^{6} D^{0}$

$\begin{array}{llll}0.29 & 3.26 & 35 & -3\end{array}$

$0.37 \quad 3.25 \quad 2.25$

$0.36 \quad 3.23$ s-

0.29 a. 35 31-3.

$0.37 \quad 3.24$ 3. -1

$0.39 \quad 3.38 \quad 3=-1$

$\begin{array}{lll}0.27 & 2.36 & 32-3 \\ 0.37 & 2.25 & 12-37\end{array}$

0.28 3.24 र्के

$0.30 \quad 3.31$ th $-5 \frac{1}{2} a^{8} z^{6} z^{6}$

$\begin{array}{lll}0.39 & 3.29 & 3-4 \\ 0.37 & 3.27 & 37\end{array}$

$0.37 \quad 3.25 \quad 13-3$

$0.30 \quad 3.39$ \& 1

$\begin{array}{lll}0.39 & 3.37 & 3-3 \\ 0.37 & 3.25 & 3\end{array}$

$0.37 \quad 3.24 \quad 11-1$

$\begin{array}{lll}0.36 & 3.24 & 3 \\ 0.30 & 3.27 & 35\end{array}$

$\begin{array}{lll}0.30 & 3.27 \\ 0.39 & 3.25 & 3-3\end{array}$

$\begin{array}{lll}0.37 & 3.24 & 25 \\ 0.37 & 3.24 & 12\end{array}$

$0.30 \quad 3.07 \quad 4 \frac{1}{-3} a^{8} 8 \mathrm{D}_{2} 6 \mathrm{po}$

$0.39 \quad 3.05$ (31)

0.39 3.07 3

$0.37 \quad 3.05$ 3र

$0.37 \quad 3.04 \quad 13$

$\begin{array}{lll}0.37 & 3.07 & 3 \\ 0.27 & 3.05 & 1\end{array}$ 


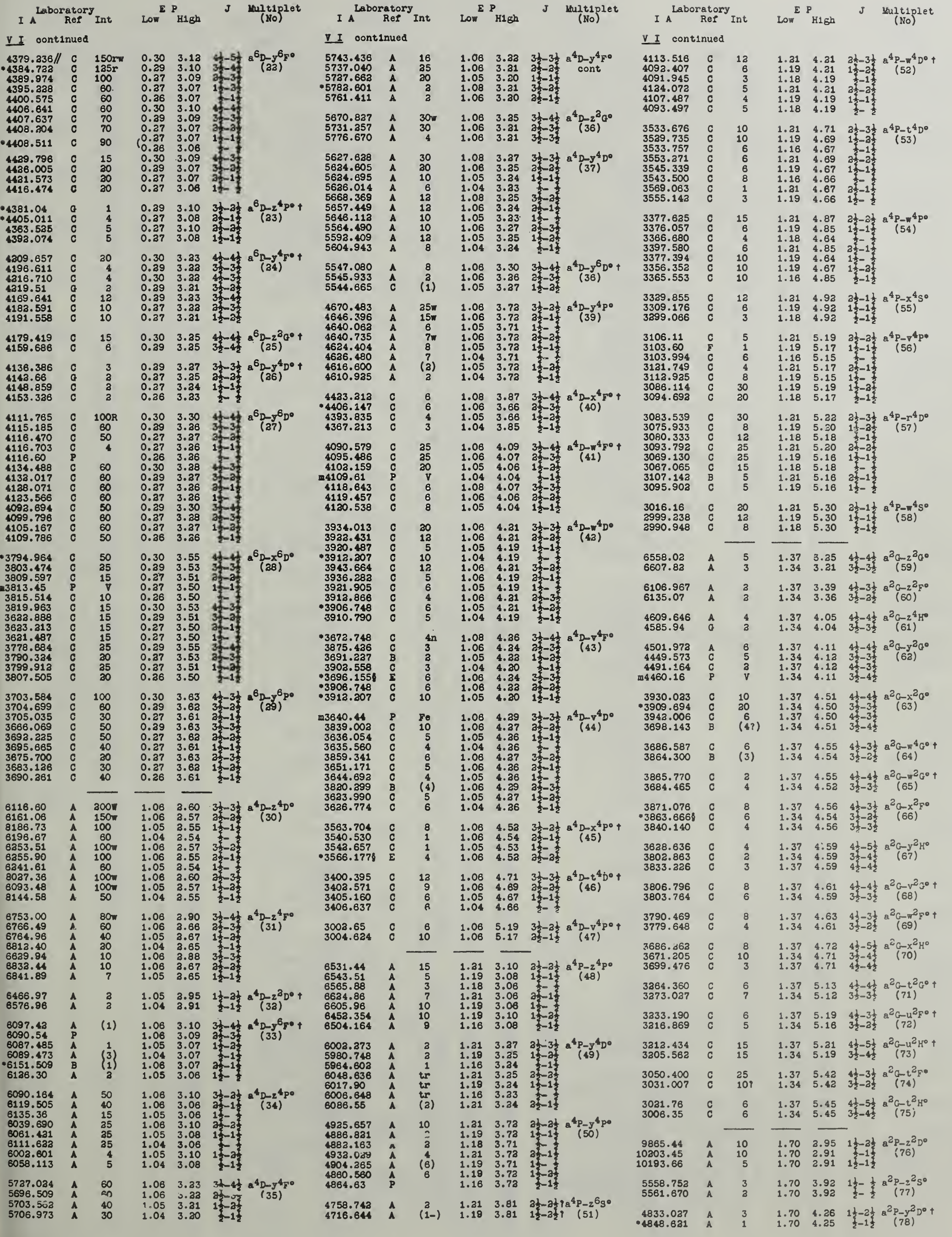




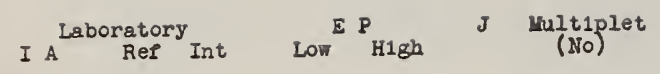 \\ VI continued}

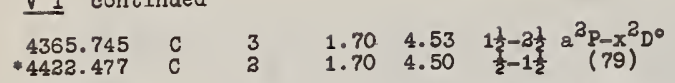

$\begin{array}{rrrrrrr}.4422 .477 & \mathrm{C} & \mathrm{B} & 1.70 & 4.50 & \frac{1}{2}-1 \frac{1}{2} & (79) \\ -3798.661 & \mathrm{C} & 3 & 1.70 & 4.95 & 1 \frac{1}{2}-2 \frac{1}{2} & \mathrm{a}^{2} \mathrm{P}-\mathrm{v}^{2} \mathrm{D}^{\circ} \\ -33834.22 & \mathrm{P} & \mathrm{Fe} & 1.70 & 4.92 & \frac{1}{2}-1 \frac{2}{2} & (80)\end{array}$

$\begin{array}{rrrrrrr}\mathrm{m} 3834.22 & \mathrm{P} & \mathrm{Fe} & 1.70 & 4.92 & \frac{1}{2}-1 \frac{2}{2} & (80) \\ 3832.835 & \mathrm{C} & 4 & 1.70 & 4.92 & 1 \frac{1}{2}-1 \frac{1}{2} & \end{array}$

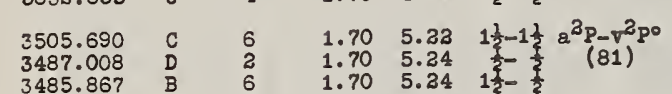

3506.843

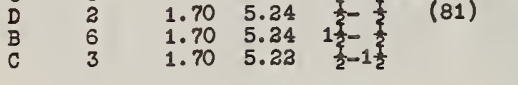

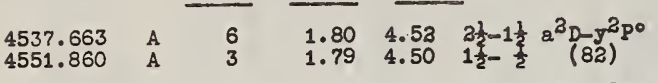

$\begin{array}{lllllll}3639.024 & C & 6 & 1.80 & 5.19 & \frac{31}{3}-3 \frac{1}{2} & \mathrm{a}^{2} \mathrm{D}-\mathrm{u}^{2} F^{\circ}+ \\ 3643.864 & \mathrm{C} & 5 & 1.79 & 5.18 & 1 \frac{2}{2}-\mathrm{a}^{\frac{2}{2}} & (83)\end{array}$

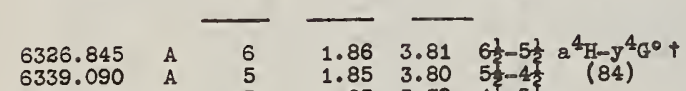

$\begin{array}{llllll}6339.090 & \mathrm{~A} & 5 & 1.85 & 3.80 & 5 \frac{5}{-1}-\frac{4}{2} \\ 6349.477 & \mathrm{~A} & 5 & 1.85 & 3.79 & 4 \frac{1}{2}-\frac{3}{2} \\ 6357.297 & \mathrm{~A} & 4 & 1.84 & 3.78 & 3 \frac{2}{2}-2 \frac{2}{2}\end{array}$

$\begin{array}{lcccccc}5584.738 & \text { A } & \text { (3) } & 1.86 & 4.07 & 6 \frac{1}{2}-6 \frac{1}{2} & 2^{4} \mathrm{H}-z^{4} \mathrm{H}^{\circ}+ \\ 5586.007 & \mathrm{~A} & \mathrm{~B} & 1.85 & 4.06 & 5 \frac{7}{2}-5 \frac{2}{2} & (85)\end{array}$

$\begin{array}{rrcccc}5586.007 & \mathrm{~A} & \mathrm{~B} & 1.85 & 4.06 & 5 \\ .5604 .205 & \mathrm{~A} & 1 & 1.85 & 4.05 & 4 \frac{4}{4} \\ 5622.075 & \mathrm{~A} & \text { (2) } & 1.84 & 4.04 & 3 \frac{3}{2}-3 \frac{3}{2}\end{array}$

4501.256

4496.864
4490.815
+4488.898

4452.008 C 20

$\begin{array}{rrr}4462.363 & C & 20 \\ 4469.710 & C & 15 \\ 4468.010 & C & 8\end{array}$

$\begin{array}{lll}4268.643 & c & 20 \\ 4271.554 & c & 1\end{array}$

$\begin{array}{lll}4271.554 & \mathrm{C} & 12 \\ 4276.958 & \mathrm{C} & 12 \\ 4284.055 & \mathrm{C} & 15\end{array}$

$3998.730 \quad c \quad 15$

$\begin{array}{rrr}3992.801 & c & 12 \\ -3990.566 & \mathrm{C} & 20\end{array}$

$\begin{array}{lll}3988.833 & \mathrm{C} & 5 \\ 3984.000 & \mathrm{C} & 6 \\ 3984.335 & \mathrm{C} & 6\end{array}$

$\begin{array}{llll}1.85 & 4.59 & 5 \frac{1}{2}-5 \frac{1}{2} & a^{4} \mathrm{H}-\mathrm{y}^{3} \mathrm{H}^{\circ} \\ 1.85 & 4.59 & 4 \frac{1}{3}-4 \frac{1}{2} & (86)\end{array}$

$\begin{array}{lll}1.85 & 4.59 & 4 \frac{1}{3}-5 \frac{2}{2} \\ 1.84 & 4.59 & 3 \frac{1}{2}-4 \frac{1}{2}\end{array}$

$1.86 \quad 4.63 \quad 6 \frac{1}{5}-7 \frac{1}{2} a^{4} \mathrm{H}^{4}-2^{4} \mathrm{I}^{\circ}+$

$\begin{array}{llll}1.85 & 4.61 & 4 \frac{1}{2}-5 \frac{1}{2} \\ 1.84 & 4.60 & 3 \frac{1}{2}-4 \frac{1}{2}\end{array}$

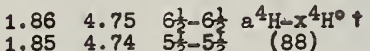

$\begin{array}{lll}1.85 & 4.74 & 5 \frac{1}{2}-5 \frac{3}{3} \\ 1.85 & 4.73 & \frac{43}{3}-4 \frac{1}{2} \\ 1.84 & 4.72 & 3 \frac{1}{2}-3 \frac{3}{2}\end{array}$

$1.86 \quad 4.95 \quad 6 \frac{1}{2}-5 \frac{1}{2} a^{4} H-u^{4} G^{\circ}+$

$\begin{array}{llll}1.86 & 4.95 & 6 \frac{1}{2}-5 \frac{1}{3} & 4 \\ 1.85 & 4.94 & 5 \frac{1}{2}-4 & (89)\end{array}$

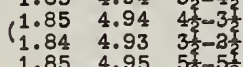

$\begin{array}{llll}1.85 & 4.95 & 5 \frac{1}{3}-5 \frac{1}{2} \\ 1.85 & 4.94 & 4 \frac{3}{3}-4 \frac{2}{2} \\ 1.84 & 4.94 & 3 \frac{2}{2}-3 \frac{1}{2}\end{array}$

$3924.658 \quad c \quad 10 \quad 1.86 \quad 5.00 \quad 6 \frac{1}{2}-6 \frac{1}{2} a^{4} \mathrm{H}^{4}-\pi^{4} \mathrm{H}^{\circ}+$

$\begin{array}{lllllll}3927.926 & C & (3) & 1.85 & 4.99 & 5 \frac{1}{3}-5 \frac{2}{2} & (90)\end{array}$

$\begin{array}{llllll}3931.340 & C & 5 & 1.85 & 4.98 & 4 \frac{4}{3}-4 \frac{3}{3} \\ 3935.141 & C & 6 & 1.84 & 4.98 & 3 \frac{1}{2}-3 \frac{3}{2}\end{array}$

$\begin{array}{ccccccc}-3722.601 \quad C & (3) & 1.86 & 5.17 & 61-5 \frac{1}{2} & a^{4} \mathrm{H}-t^{4} G^{\circ}+\end{array}$

\begin{tabular}{llllll}
3721.998 & $\mathrm{C}$ & 4 & 1.85 & 5.17 & $5 \frac{1}{4}-4 \frac{4}{2}$ \\
3729.035 & $\mathrm{C}$ & 4 & 1.85 & 5.16 & $4 \frac{4}{3}$ \\
3737.992 & $\mathrm{C}$ & 5 & 1.84 & 5.14 & $3 \frac{3}{2}-3 \frac{1}{2}$ \\
\hline
\end{tabular}

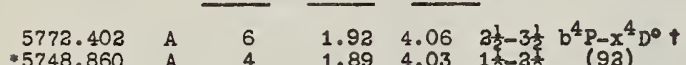

-5748.860
5752.711

5850.286

5788.549

4797.973

4729.544
4686.926

4751.574

4624.657

4706.17

4684.45 ?

4591.991

$\begin{array}{llll}1.89 & 4.03 & 1 & 2 \\ 1.86 & 4.01 & \frac{2}{2} & (93) \\ 1.92 & \end{array}$

$\begin{array}{lll}1.92 & 4.03 & 2 \frac{1}{3}-2 \\ 1.89 & 4.01 & 12\end{array}$

$1.86 \quad 3.99 \quad \frac{1}{2}-\frac{1}{2}$

$1.93 \quad 4.49 \quad 2 \frac{1}{3}-1 \frac{1}{3} b^{4} P-y^{4} s^{\circ}$

$\begin{array}{lll}1.86 & 4.49 & 1 \\ 1.86 & 4.4 \frac{3}{2} & \end{array}$

$1.92 \quad 4.52 \quad 2 \frac{1}{2}-2 \frac{1}{2} b^{4} P-x^{4} p^{\circ}$

$\begin{array}{llll}1.89 & 4.54 & 1 \frac{2}{3}=1 \frac{1}{3} & (94)\end{array}$

$\begin{array}{lll}1.92 & 4.54 & 2 \frac{2}{1}-1 \frac{3}{3} \\ 1.89 & 4.53 & 1 \frac{1}{2}-\end{array}$

(3) $\quad 1.89 \quad 4.52 \quad 1 \frac{1}{2}-2 \frac{1}{2}$

$\begin{array}{ccccc}\text { (0) } & 1.92 & 4.61 & 2 \frac{1}{2}-2 \frac{1}{2} & b^{4} P-m^{2} F^{\circ} \\ 4 & 1.89 & 4.61 & 1 \frac{1}{2}-2 \frac{1}{2} & (95)\end{array}$

$1.924 .952 \frac{1}{2}-3 \frac{1}{2} b^{4} P_{-8}^{4} D^{\circ}+$

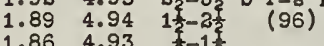

$1.92 \quad 5.19 \quad 2 \frac{1}{2}-2 \frac{1}{2} \quad b^{4} P-\nabla^{4} p \circ$

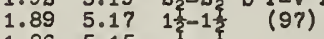

$\begin{array}{lll}1.86 & 5.15 & \frac{1}{2}-1 \frac{1}{2} \\ 1.92 & 5.17 & 2 \frac{3}{2}-1\end{array}$

$\begin{array}{lll}1.89 & 5.15 & 1 \frac{1}{3} \\ 1.89 & 5.19 & 1 \frac{1}{3}-2 \frac{1}{2} \\ 1.86 & 5.17 & \frac{1}{2}-1 \frac{1}{2}\end{array}$

$1.92 \quad 5.22 \quad 2 \frac{1}{2}-3 \frac{1}{2} b^{4} P-r^{4} D^{0}+$

$\begin{array}{llll}1.89 & 5.20 & 1 \frac{1}{2}-2 \frac{3}{2} & (98)\end{array}$

$\begin{array}{lll}1.86 & 5.18 & \\ 1.92 & 5.20 & 2 \frac{3}{2}-2 \\ 1.89 & 5.13 & \end{array}$

$\begin{array}{lll}1.89 & 5.18 & 1 \frac{1}{2}-1 \frac{1}{2} \\ 1.86 & 5.18 & \frac{1}{2}-\frac{1}{2}\end{array}$

$\begin{array}{llll}1.88 & 4.61 & 5 \frac{1}{2}-4 \frac{3}{2} & a^{2} \mathrm{H}-\nabla^{2} G^{0}+ \\ 1.86 & 4.59 & 4 \frac{2}{2}-3 \frac{3}{2} & (99)\end{array}$

$\begin{array}{llll}1.88 & 4.62 & 5 \frac{1}{3}-6 \frac{1}{2} & a^{2} \mathrm{H}-z^{4} I^{\circ} \\ 1.88 & 4.60 & 5 \frac{1}{2}-4 \frac{1}{2} & (100)\end{array}$

$1.88 \quad 4.64 \quad 5 \frac{1}{4}-6 \frac{1}{2} \Omega^{2} \mathrm{H}-z^{3} \mathrm{I}^{\circ}$

$1.86 \quad 4.63 \quad 4 \frac{1}{2}-5 \frac{5}{2}(101)$

$1.86 \quad 4.63 \quad 4 \frac{1}{2}-3 \frac{1}{2} a^{2} \mathrm{H}-w^{2} \xi^{\circ}$

REVISID MULTIPLEE IABLE

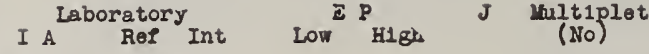

I A Laboratory Rer Int

E P

wiltiplet

VI continued

$\begin{array}{lllllll}4354.979 & \mathrm{C} & 5 & 1.88 & 4.73 & 5 \frac{1}{4}-5 \frac{1}{2} & \mathrm{a}^{2} \mathrm{H}-\mathrm{x}^{2} \mathrm{H}^{\circ} \\ 4342.833 & \mathrm{C} & 6 & 1.86 & 4.71 & 4 \frac{2}{2}-\frac{4}{2} & (103)\end{array}$

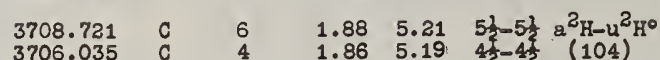

$\begin{array}{rrrrrrr}3082.010 & \mathrm{~B} & 6 & 1.88 & 5.89 & 5 \frac{1}{2}-5 \frac{1}{2} & \mathrm{a}^{3} \mathrm{H}-8^{3} \mathrm{H}^{\circ} \\ 3075.269 & \mathrm{C} & 10 & 1.86 & 5.88 & 4 \frac{4}{2}-4 \frac{2}{2} & (105)\end{array}$

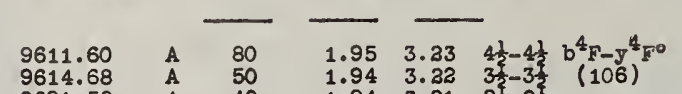

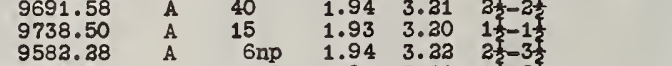

9668.9

6430.471
6431.620

6431.620

6433.17
6435.148

4732.877

4721.524
4730.394

4545.394

4560.710
4571.783

(5)

4570.42

4579.198
4583.783

4474.045

4496.062
4514.191

4525.168
4464.747

4488.898
4509.387

4232.460

4234.000
-4235.756

4104.778

4118.182
4123.188
4138.858

4138.85

4807.53

4796.930
4786.515

4776.364

4766.53
4757.50

4757.37

4753.957
4750.990

4748.525
4746.638

3695.335

3687.473
3680.113

3673.404

3667.741

3717.55

3705.83

3684.332

3675.497

3676.684
3672.403

3665.142

3656.706
-3648.966

3641.096

$3014.3 ?$

3006.90

3006.24

3997.87
2997.08

7338.9

7356.51
7363.16
7361.39

4904.350

4904.44

4900.624
4894.218

4706.574

4710.56

4714.113
4717.692

4291.816

4296.107
4297.681

4297.681
4298.039

$-4051.3528$ 4050.9638
4057.074

$\begin{array}{llll}1.95 & 3.87 & 4 \frac{1}{2}-4 \frac{1}{2} & b^{4} F-x^{4} \\ 1.94 & 3.86 & 3 \frac{1}{2}-3 \frac{1}{2} & (107)\end{array}$

$\begin{array}{lll}1.94 & 3.86 & 2 \frac{1}{2}-3 \frac{3}{2} \\ 1.93 & 3.85 & 1 \frac{3}{2}-1 \frac{1}{2}\end{array}$

$1.95 \quad 4.56 \quad \frac{4}{3}-5 \frac{2}{3} b^{4} F-w^{4} G^{0}+$

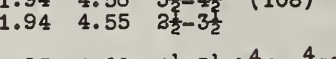

$\begin{array}{llll}1.95 & 4.66 & 4 \frac{1}{3}-5 \frac{1}{2} & b^{4} F-\nabla^{4} G^{\circ} \\ 1.94 & 4.65 & 3 \frac{1}{2}-4 \frac{1}{2} & (109)\end{array}$

$\begin{array}{llll}1.94 & 4.65 & 3 \frac{1}{2}-4 \frac{1}{3} & (109) \\ 1.94 & 4.64 & 35 & 3.3\end{array}$

\begin{tabular}{lll}
1.94 & 4.64 & 35 \\
1.93 & 4.63 & $1 \frac{1}{2}-3$ \\
\hline
\end{tabular}

$\begin{array}{lll}1.95 & 4.65 & 4 \\ 1.94 & 4.64 & 3 \frac{1}{3}-4 \\ 1 & -3\end{array}$

$1.94 \quad 4.63 \quad 3 \frac{1}{2}-6 \frac{4}{2}$

$1.954 .71 \quad b^{4}-3 \frac{1}{2}-t^{4} D^{0}$

$1.94 \quad 4.69 \quad 3 \frac{1}{2}-3 \frac{2}{2}$ (110)

$1.94 \quad 4.67 \quad 33-1$

1.944 .71 3र

$\begin{array}{lll}1.94 & 4.69 & 2 \frac{1}{2}-2 \\ 1.93 & 4.67 & 1 \frac{1}{2}-1 \frac{2}{2}\end{array}$

$1.95 \quad 4.86 \quad \frac{1}{3}-4 \frac{1}{2} b^{4} F-u^{4} F^{0}+$

$1.94 \quad 4.86$ 3?

$\begin{array}{lll}1.94 & 4.85 & 2 \frac{1}{3}-2 \frac{1}{2} \\ 1.93 & 4.85 & 1 \frac{1}{2}-1 \frac{1}{2}\end{array}$

$1.95 \quad 4.95 \quad 4 \frac{1}{3}-3 \frac{1}{2} b^{4} F-8^{4} D^{0}+$

$1.94 \quad 4.94 \quad 3 \frac{1}{3}-2 \frac{1}{2}$ (113)

$\begin{array}{lll}1.94 & 4.93 & 2 \frac{2}{-1} \\ 1.93 & 4.93 & 1 \frac{2}{2}-\frac{1}{2}\end{array}$

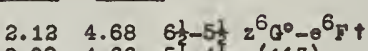

$\begin{array}{llll}2.09 & 4.66 & 5 \frac{1}{2}-4 \frac{1}{3} & 2^{6}(113)\end{array}$

$2.07 \quad 4.64$ 45 -3

$3.03 \quad 4.63$ as -1

$\begin{array}{lll}3.03 & 4.61 & 15- \\ 3.09 & 4.68 & 5\end{array}$

$3.07 \quad 4.66$ 4t

$\begin{array}{lll}3.05 & 4.64 & 3 x-3 \\ 3.03 & 4.63 & 35\end{array}$

$3.03 \quad 4.62 \quad 1 \frac{1}{2}-1 \frac{1}{2}$

$\begin{array}{llll}3.13 & 5.46 & 6 \frac{1}{2}-7 \frac{1}{2} & z^{6} \mathrm{G}^{\circ}-e^{6} \mathrm{H} \\ 3.09 & 5.44 & 5 & -6\end{array}$

$\begin{array}{lll}3.07 & 5.42 & 43 \\ 2.05 & 5.41 & 35\end{array}$

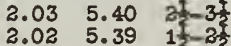

$\begin{array}{lll}2.02 & 5.39 & 11-2 \\ 2.12 & 5.44 & 61-6\end{array}$

$\begin{array}{lll}2.09 & 5.42 & 51-5 \\ 2.07 & 5.41 & 41\end{array}$

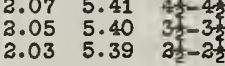

$3.125 .47 \quad 6 \frac{1}{3}-6 \frac{1}{3} z^{6} G^{0}-p^{6} G t$

$\begin{array}{llll}3.12 & 5.47 & 6 \frac{1}{3}-6 \frac{1}{3} & 2^{6} \mathrm{G}^{0}-P^{6} \\ 2.09 & 5.45 & 5 & \end{array}$

$2.05 \quad 5.43 \quad 35-3$

$\begin{array}{lll}2.03 & 5.41 & 2 \frac{1}{2}-2 \frac{9}{2} \\ 3.02 & 5.41 & 1 \frac{1}{2}-1 \frac{1}{2}\end{array}$

$2.126 .21 \quad 6 \frac{1}{2}-7 \frac{1}{2} z^{6} G^{0}-p^{6}$ H

$2.09 \quad 6.19 \quad 5 \frac{1}{2}-6 \frac{1}{2}(116)$

$2.07 \quad 6.17 \quad 43-5$

$3.03 \quad 6.15 \quad 215$

$2.13 \quad 3.81 \quad 5 \frac{1}{2}-5 \frac{1}{2} a^{4} G-y^{4} G^{\circ}+$

$\begin{array}{lll}3.11 & 3.70 \\ 3.11 & 3.78\end{array}$

$\begin{array}{lll}2.11 & 4.63 & 3 \frac{1}{2}-4 \\ 2.11 & 4.63 & 3 \frac{1}{2}-3 \frac{1}{2}\end{array}$

$\begin{array}{lll}2.11 & 4.73 & 32 \\ 3.72 & 2 \frac{2}{2}-3 \frac{1}{2}\end{array}$

$3.13 \quad 5.00 \quad 5 \frac{1}{-1}-\frac{1}{2} 2^{4} G-w^{4} H^{\circ}+$

2.124 .99 4.

$\begin{array}{lll}2.11 & 4.98 & 3 \\ 3.11 & 4.98 & 2 \frac{4}{2}-3 \frac{1}{2}\end{array}$

$3.135 .175-5 \frac{1}{3} a^{4} G-t^{4} G^{0}+$

$2.12 \quad 5.17 \quad 4 \frac{4}{3}-4$ (121)
VI continued

$\begin{array}{lllllll}3571.037 & c & 4 & 3.13 & 5.58 & 5 t-4 \frac{1}{3} & a^{4} a-a^{4} p+t\end{array}$ \begin{tabular}{llllll}
3571.653 & $\mathrm{C}$ & 5 & 2.11 & 5.57 & $3 \frac{1}{2}-23$ \\
3568.940 & $\mathrm{C}$ & 3 & 2.11 & 5.56 & $3 \frac{1}{2}-1 \frac{1}{2}$ \\
\hline
\end{tabular}

$\begin{array}{lllllll}5138.530 & \mathrm{~A} & 7 & 2.28 & 4.68 & 4 \frac{1}{4}-5 \frac{1}{2} & z^{6} D^{0}-e^{6} C_{F}+ \\ 5138.431 & \mathrm{~A} & 5 & 2.26 & 4.66 & 3 \frac{1}{2}-4 \frac{3}{3} & (123)\end{array}$

$\begin{array}{llllll}5148.724 & \mathrm{~A} & 4 & 2.25 & 4.64 & 2 \frac{1}{2}-3 \frac{1}{2} \\ 5159.350 & \mathrm{~A} & 3 & 2.24 & 4.63 & 1 \frac{1}{2}-2 \frac{3}{2}\end{array}$

$3741.504 \quad C \quad 6 \quad 2.28 \quad 5.57 \quad 4 \frac{1}{3}-4 \frac{1}{7} z^{6} D^{0}-8^{6} D+$

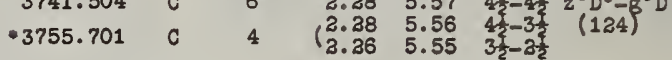

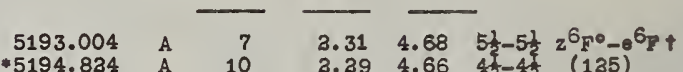

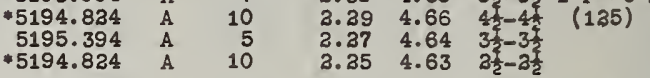

3898.019

3901.152
3900.175

3897.075

5748.412

- 5782.601

5624.223

4776.519

4743.631

5487.915
5507.753

5415.277
-5401.945

5340.878

5234.088

5014.620
5003.330

4591.220

4553.056

3337.409

$\left.3.123 .80 \quad \frac{1}{3}-5 \frac{1}{2} a^{4} G-G^{4} G^{0}+117\right)$

5725.633
5734.004

4705.099

4715.900

3645.596

3265.8998
3256.779

$.315 .47 \quad 5 \frac{1}{2}-6 \frac{1}{2} z^{6} F^{0}-1^{6} G t$

3.39 5.45 $4 \frac{1}{3}-5 \frac{2}{2} \quad(126)$

\begin{tabular}{lll} 
2. 27 & 5.43 & $3 \frac{1}{2}-4 \frac{1}{2}$ \\
3.25 & 5.43 & $2 \frac{1}{2}-3 \frac{1}{2}$ \\
\hline
\end{tabular}

(1) $\quad 3.37 \quad 4.53 \quad 1 \frac{1}{2}-1 \frac{1}{2} b^{2} p-y^{3} p^{\circ}$

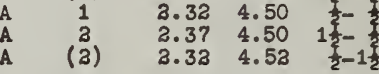

$5 \quad 2.37 \quad 4.95 \quad 1 \frac{1}{2}-2 \frac{1}{2} b^{2} P_{-} v^{3} p^{0}+$ $5 \quad 3.32 \quad 4.92 \quad \frac{2}{2}-1 \frac{2}{2}$ (138)

$\begin{array}{lllllll}5366.118 & \mathrm{~A} & (4) & 2.67 & 5.01 & 4 \frac{1}{2}-3 \frac{1}{2} & b^{2} a-r^{3} F^{\circ} \\ .5401 .945 & \mathrm{~A} & 8 & 2.67 & 4.96 & \frac{3}{2}-2 \frac{2}{2} & (139)\end{array}$

$\begin{array}{lllllll}4373.230 & C & 4 & 2.67 & 5.49 & \frac{41}{2}-4 \frac{1}{2} & b^{2} G-8 G^{\circ} \\ 4375.304 & C & 4 & 3.67 & 5.49 & 3 \frac{2}{2}-3 \frac{3}{2} & (140)\end{array}$

5784.360 A $5 \quad 2.75 \quad 4.89 \quad 5 \frac{1}{2}-4 \frac{1}{2} z^{4} G^{\circ}-P^{4} F$

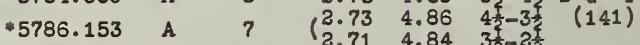

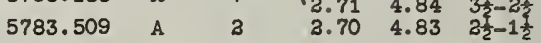

$5846.306 \quad \mathrm{~A} \quad 8 \quad 3.12 \quad 5.23 \quad 5 \frac{1}{3}-6 \frac{1}{2} 5^{6} \mathrm{~F}^{\circ}-\theta^{6} \mathrm{G}$

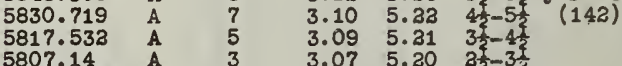

5798.905

$\begin{array}{lll}3.09 & 5.31 & 31 \\ 3.07 & 5.20 & 31\end{array}$

$3.07 \quad 5.19$ 1 $32-3 \frac{1}{3}$

Strongest Unclass1p1ed Lines of $\mathrm{V} I$

4619.648

4549.644

4527.990

4265.170
-3963.6268

$\begin{array}{ll}\text { A } & \\ \text { A } & 10 \\ \text { A } & \\ \text { C } & \end{array}$

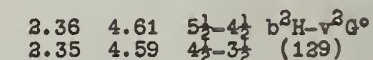

$36 \quad 4.64 \quad 5 \frac{1}{2}-6 \frac{1}{2} b^{2} \mathrm{H}_{-2} \mathrm{z}^{2} \mathrm{I}^{\circ}$

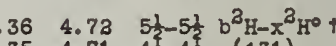

$.36 \quad 4.835 \frac{1}{3}-6 \frac{1}{2} b^{2} \mathrm{H}-\mathrm{y}^{3} \mathrm{I}^{\circ}$

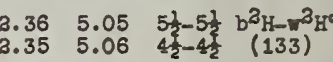

$\begin{array}{llll}3.36 & 6.19 & 5 \frac{1}{2}-6 \frac{1}{2} & b^{2} \mathrm{H}-x^{3} \mathrm{I} \\ 3.35 & 6.17 & 4 \frac{1}{2}-5 \frac{1}{2} & (134)\end{array}$

3898.278

3891.119

3845.974

3425.070

3361.081

3156.222

3153.549

3093.72

3041.86
3003.442

$\begin{array}{lll}2.11 & 5.16 & 3 \frac{2}{2}-3 \frac{1}{2} \\ 2.11 & 5.14 & 3 \frac{2}{2}-2 \frac{3}{2}\end{array}$ 
REVIAED MULIIPLET TABLE

35

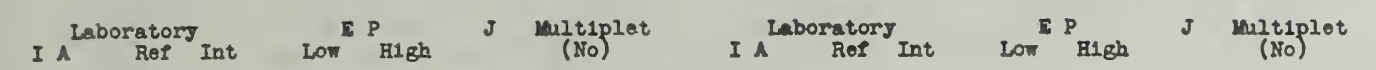

VII I P 14.1 Anal A Liat A Jan 1941 VII continued

$3093.108 / /$ A 3500 R

3103.395 A 3500 R

3118.378

3131.138

3138.315

3130.362

3133.329
-3145.337

$\begin{array}{lll}3145.971 & \text { A } & 30 \\ 3 & 30\end{array}$

3145.337 A 30

$\begin{array}{lll}3953.07 & \text { A } & 150 \\ 3957.530 & \text { A } & 100\end{array}$

$\begin{array}{llll}0.39 & 4.38 & 5-6 & a^{5} F-z^{5} G \\ 0.37 & 4.34 & 4-5 & (1)\end{array}$

$1500 \mathrm{R}$
$1000 \mathrm{R}$
$800 \mathrm{R}$
80
$150 \mathrm{R}$
$100 \mathrm{R}$
$150 \mathrm{x}$
30
30
30
150
100

$0.33 \quad 4.29$

$\begin{array}{lll}0.32 & 4.37 & 1-2\end{array}$

$\begin{array}{lll}0.39 & 4.34 & 5 \\ 0.37 & 4.31 & 4 \\ 0.35 & 4.39 & 3-3\end{array}$

$\begin{array}{lll}0.35 & 4.39 & 3-3 \\ 0.33 & 4.37 & 2-2 \\ 0.39 & 4.31 & 5-4\end{array}$

$\begin{array}{lll}0.39 & 4.31 & 5-4 \\ 0.37 & 4.39 & 4-3 \\ 0.35 & 4.37 & 3-2\end{array}$

$0.35 \quad 4.53 \quad 3-3 \quad a^{5} F-25 F^{0}+$

$(3)$

$0.33 \quad 4.50$

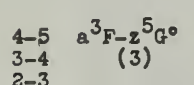

3831.017

3839.53
3853.10

3538.238

3531.48
3535.18

3563.71

3560.594

3593.323 A 600

$\begin{array}{rrr}3592.012 & \text { A } & 800 \\ 3589.745 & \text { A } & 1000\end{array}$

3556.800 A 1500

$\begin{array}{rrr}3545.190 & \text { A } & 1000 \\ 3530.765 & \text { A } & 500\end{array}$

3520.032

3499.833

3516.00
3485.916

3479.837

3504.432

$\begin{array}{llr}3493.163 & \text { A } & 150 \\ 3484.65 & \text { A } & 3 \\ 3461.580 & \text { A } & 5\end{array}$

$\begin{array}{llll}1.12 & 4.34 & 4-5 & a^{3} F-z^{5} G^{0} \\ 1.09 & 4.31 & 3-4 & (3)\end{array}$

$\begin{array}{lll}1.09 & 4.31 & 3-4 \\ 1.07 & 4.39 & 2-3 \\ 1.07 & 4.27 & 2-3\end{array}$

$1.12 \quad 4.61 \quad 4-5 \quad a^{3} F-z^{5} F^{0}$

$\begin{array}{lll}1.09 & 4.59 & 3-4 \\ 1.07 & 4.56 & 3\end{array}$

$\begin{array}{lll}1.13 & 4.59 & 4 \\ 1.09 & 4.56 & 3-3\end{array}$

$\begin{array}{lll}1.09 & 4.56 & 3-3 \\ 1.07 & 4.53 & 3-3\end{array}$

$\begin{array}{lll}1.13 & 4.56 & 4-3\end{array}$

$\begin{array}{lll}1.09 & 4.53 & 3-3 \\ 1.07 & 4.50 & 3-1\end{array}$

$1.13 \quad 4.59 \quad 4-3 \quad a^{3} F-2^{3} D^{0}$

$1.09 \quad 4.5$

$\begin{array}{ll}1.07 & 4.56 \\ 1.09 & 4.59 \\ 1.07 & 4.57\end{array}$

$\begin{array}{ll}1.07 & 4.57 \\ 1.07 & 4.59\end{array}$

3378.13 A $1500 \mathrm{R}$

$\begin{array}{llll}1.13 & 4.63 & 4-4 & a^{3} F-z^{5} D^{0} \\ 1.09 & 4.63 & 3-3 & (6)\end{array}$

$\begin{array}{lll}1.09 & 4.63 & 3 \\ 1.07 & 4.81 & 2\end{array}$

$\begin{array}{lll}1.12 & 4.63 & 4-3 \\ 1.09 & 4.81 & 3-3\end{array}$

$\begin{array}{lll}1.09 & 4.81 & 3-2 \\ 1.07 & 4.60 & 3-1\end{array}$

$\begin{array}{lll}1.07 & 4.60 & 3-1 \\ 1.09 & 4.63 & 3-4\end{array}$

$1.07 \quad 4.83$

$1.13 \quad 4.89 \quad 4-5 \quad a^{3} F-z^{3} G^{\circ}$

$\begin{array}{ll}1.09 & 4.88 \\ 1.07 & 4.84\end{array}$

$\begin{array}{ll}1.13 & 4.86 \\ 1.09 & 4.84\end{array}$

$\begin{array}{lc}4-5 & 25-z^{3} G^{\circ} \\ 3-4 & (7) \\ 2-3 & \end{array}$

3387.709
3298.738

3389.391
3317.395

3190.686

3188.532
3187.717

3214.750
3208.345

3184.83
3168.127

3997.126

3973.642

3968.11

4003.940

4067.03

3951.988
3916.418

3896.155

3929.73

3991.47

3903.27

3888.744
3850.409

3938.497

3883.20
3943.48

3943.48
3891.98

3534.14

$\begin{array}{ccccc}500 R & 1.13 & 4.99 & 4-4 & a^{3} F-z^{3} F^{\circ} \\ 300 R & 1.09 & 4.96 & 3-3 & (8)\end{array}$

$1.07 \quad 4.94 \quad 3-$

$\begin{array}{lll}1.012 & 4.96 & 4-3 \\ 1.09 & 4.94 & 3-3\end{array}$

$\begin{array}{lll}1.09 & 4.99 & 3-4 \\ 1.07 & 4.98 & 3-3\end{array}$

$\begin{array}{llll}1.47 & 4.56 & 2-3 & a^{3} P-z^{5} \\ 1.5\end{array}$

$\begin{array}{lll}1.43 & 4.53 & 1-2 \\ 1.39 & 4.50 & 0\end{array}$

$\begin{array}{lll}1.47 & 4.53 & 3-3\end{array}$

$\begin{array}{lll}1.43 & 4.50 & 1-1 \\ 1.47 & 4.50 & 3-1\end{array}$

$\begin{array}{llll}1.47 & 4.59 & 2-3 & a^{3} P-2^{3} D^{\circ} \\ 1.43 & 4.57 & 1-3 & (10)\end{array}$

$\begin{array}{lll}1.39 & 4.58 & 0 \\ 1.47 & 4.57 & 2\end{array}$

$\begin{array}{lll}1.47 & 4.57 & 2-2 \\ 1.42 & 4.58 & 1-1\end{array}$

$\begin{array}{lll}1.48 & 4.58 & 1-1 \\ 1.47 & 4.58 & 3\end{array}$

$1.47 \quad 4.63 \quad 3-3 \quad a^{3} P-z^{5} D^{\circ}$

$1.43 \quad 4.61 \quad 1-3$ (11)

$\begin{array}{lll}1.39 & 4.60 & 0-1 \\ 1.47 & 4.81 & 3-3\end{array}$

$\begin{array}{lll}1.47 & 4.81 & 2-3 \\ 1.42 & 4.60 & 1-1\end{array}$

$\begin{array}{lll}1.47 & 4.60 & 3-1\end{array}$

$1.47 \quad 4.98 \quad 2-3 \quad a^{3} P-z^{3} F^{\circ}$

$\begin{array}{lllllll}4389.12 & P & P & & & & \\ 4439.11 & P & & 1.57 & 4.38 & 6-6 & a^{3} \mathrm{H}-z^{5} \mathrm{G}^{0} \\ 4.58 & 4.34 & 5-5 & (13)\end{array}$

4463.76

445.77
478.03

478.03
4503.13

437.88
4414.17

4058.370

4075.68

3715.478
3732.760
3745.808

3745.808
3703.832

.3732 .168

$4737.59 \quad A \quad 1 \quad 1.87 \quad 4.37 \quad 2-3 \quad b^{3} \mathrm{~F}-z^{5} \mathrm{G}^{\circ}$

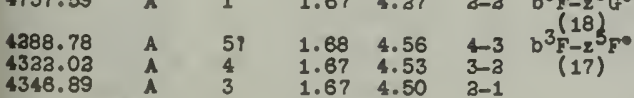

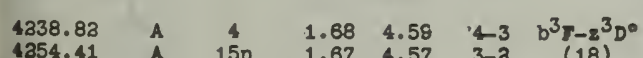
4254.41
.4860 .75

4179.083

4100.86 m3844. 48

3883.43
3875.67
3891.35

3901.33

$\begin{array}{llclllc}3727.351 & \text { A } & 10001 & 1.68 & 4.99 & 4-4 & b^{3} F-z^{3} F^{\circ} \\ 3750.88 & \text { A } 600 & 1.67 & 4.96 & 3-3 & (21)\end{array}$

$\begin{array}{lll}3760.34 & \text { A } & 400 \\ 3778.35 & \text { A } & 140 \\ 3718.159 & \text { A } & 100\end{array}$

$\begin{array}{rrr}3718.159 & \text { A } & 100 \\ 3743.610 & \text { A } & 60\end{array}$

- 3983.009

4370.64

4388.13
4316.358
4313.30
4331.79

. 4260.75

4363.838
4364.50

4302.350

4178.390
4190.40

4304.20
$\mathrm{~m} 4305.05$

4309.74
431.165

4324.51
4320.047

3029.56

\begin{tabular}{l}
3020.6 \\
3022.5 \\
\hline
\end{tabular}

3016.14

3008.610

3001.303
3003.461

3007.296

3016.775
3014.823

3988.027
3995.999

3968.373

2978.517

2976.197

3983.558
3975.650

2983.009
2982.75

4799.94

4844.31

4887.79

4903.89
4938.62

4936.94
4951.86

4951.86
4966.08

4404.68

4434.63

4451.61
4444.30

470.39
4500.88

4385.45

4370.27

4005.713

4033.388
4035.631

4039.574

4051.068
3989.803

4008.17

3878.715

3899.140

3914.333
-3883.818

3884.847
3849.758

3033.821
3053.39
3067.104

3067.104

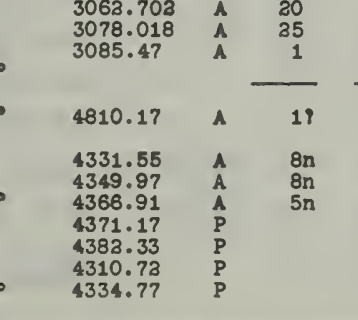

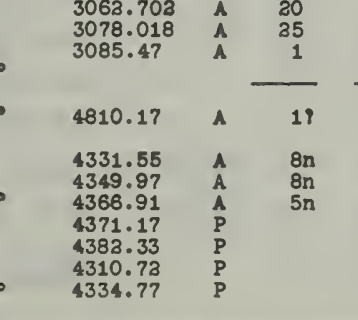

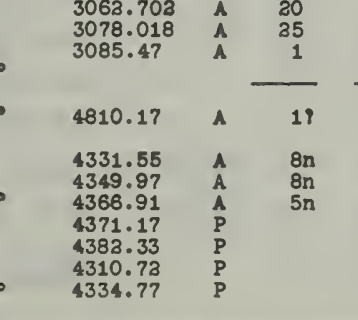

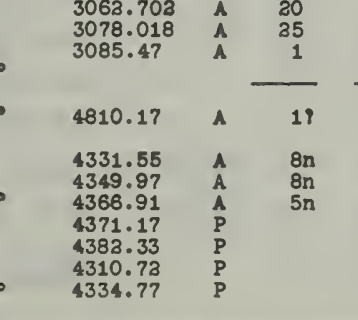

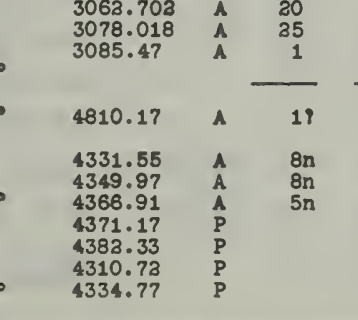

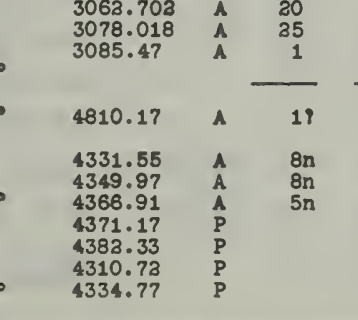

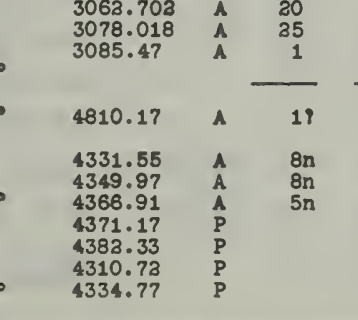

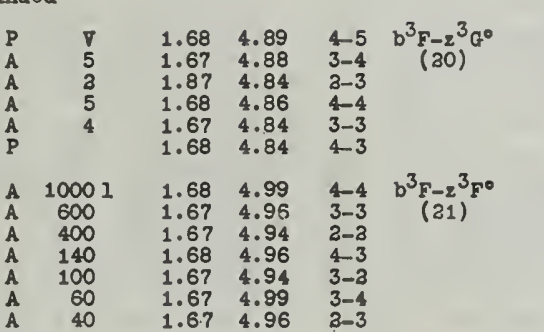

$1.67 \quad 5.81 \quad 3-4 \quad b^{3} \mathrm{~F}-z^{3} \mathrm{H}^{\circ}+$

(33)

$\begin{array}{cccc}1.70 & 4.59 & 3-4 & a^{5} \mathrm{P}-25 F^{5} \\ 1.88 & 4.56 & 2-3 & (33)\end{array}$

$\begin{array}{lll}1.87 & 4.53 & 1-3 \\ 1.70 & 4.56 & 3-3 \\ 1.88 & 1.53 & 3-3\end{array}$

$\begin{array}{llll}1.70 & 4.59 & 3-3 & a^{5} P_{-2} D^{0}\end{array}$

$\begin{array}{lll}1.68 & 4.57 & 2- \\ 1.67 & 4.56 & 1\end{array}$

$\begin{array}{lll}1.68 & 4.59 & 3-3 \\ 1.67 & 4.57 & 1-2\end{array}$

$\begin{array}{llll}1.70 & 4.83 & 3-4 & a^{5} P_{-2} 5 D^{\circ}\end{array}$

$\begin{array}{lll}1.68 & 4.63 & 2-3 \\ 1.67 & 4.81 & 1-2\end{array}$

$1.70 \quad 4.83 \quad 3-3$

$\begin{array}{lll}1.68 & 4.61 & 2-3 \\ 1.67 & 4.60 & 1-1\end{array}$

$\begin{array}{lll}1.70 & 4.61 & 3-2 \\ 1.68 & 4.60 & 3-1\end{array}$

$1.70 \quad 5.77 \quad 3-3 \quad a^{5} P_{-2} z^{3} P^{0}$

$\begin{array}{lll}1.68 & 5.76 & 2-1 \\ 1.67 & 5.75 & 1-0\end{array}$

$\begin{array}{lll}1.68 & 5.77 & 3-3 \\ 1.67 & 5.76 & 1-1 \\ 1.67 & 5.77 & 1-3\end{array}$

$\begin{array}{cccc}1.70 & 5.81 & 3-3 & a^{5} P-z^{5} P^{0} \\ 1.68 & 5.79 & 3-3 & (37)\end{array}$

$\begin{array}{lll}1.68 & 5.79 & 3-2 \\ 1.67 & 5.77 & 1-1\end{array}$

$\begin{array}{lll}1.67 & 5.77 & 1-1 \\ 1.70 & 5.79 & 3-2\end{array}$

$\begin{array}{lll}1.68 & 5.77 & 3-1 \\ 1.68 & 5.81 & 3-3\end{array}$

$\begin{array}{lll}1.68 & 5.81 & 2-3 \\ 1.67 & 5.79 & 1-3\end{array}$

$\begin{array}{cccc}1.70 & 5.85 & 3-4 & a^{5} \mathrm{P}-\mathrm{y}^{5} \mathrm{D}^{\circ} \\ 1.68 & 5.83 & 2-3 & (38)\end{array}$

$\begin{array}{ll}1.68 & 5.82 \\ 1.67 & 5.81\end{array}$

$1.70 \quad 5.83$

1.68 5.81 $3-3$

$\begin{array}{lll}1.67 & 5.82 & 1-1 \\ 1.70 & 5.81 & 3-2\end{array}$

$\begin{array}{llll}1.81 & 4.38 & 5-6 & a^{3} a_{2}{ }^{5} G^{\circ} \\ 1.80 & 4.34 & 4-5 & (39)\end{array}$

$1.79 \quad 4.31$

$\begin{array}{ll}1.81 & 4.34 \\ 1.80 & 4.31\end{array}$

$1.794 .29 \quad 3=$

$\begin{array}{lll}1.81 & 4.31 & 5-4 \\ 1.80 & 4.29 & 4-3\end{array}$

$1.81 \quad 4.61 \quad 5-5 \quad 8^{3}-5^{5}$

$\begin{array}{llll}1.80 & 4.59 & 4-4 & a^{3}-2 \\ (30)\end{array}$

$1.81 \quad 4.59 \quad 5-4$

$1.79 \quad 4.58 \quad 4-3$

$\begin{array}{lll}1.79 & 4.53 & 3-3 \\ 1.80 & 4.61 & 4-5\end{array}$

$\begin{array}{lll}1.79 & 4.59 \quad 3-4\end{array}$

$1.814 .63 \quad 5-4 \quad 8^{3}\left(-z^{5} D^{\circ}\right.$

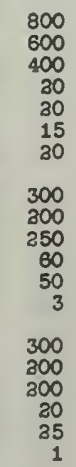

$\begin{array}{cccc}1.81 & 4.89 & 5-5 & a^{3} a-z^{3} G^{\circ} \\ 1.80 & 4.86 & 4-4 & (33)\end{array}$

$\begin{array}{ll}1.80 & 4.86 \\ 1.79 & 4.84\end{array}$

$\begin{array}{ll}1.79 & 4.84 \\ 1.81 & 4.86\end{array}$

$\begin{array}{ll}1.80 & 4.84\end{array}$

$\begin{array}{lll}1.80 & 4.89 & 4-5 \\ 1.79 & 4.88 & 3-4\end{array}$

$\begin{array}{llll}1.81 & 4.99 & 5-4 & a^{3} a-z^{3} F^{\circ} \\ 1.80 & 4.98 & 4-3 & (33)\end{array}$

$\begin{array}{lll}1.80 & 4.98 & 4-3 \\ 1.79 & 4.94 & 3\end{array}$

$\begin{array}{lll}1.79 & 4.98 & 3-3 \\ 1.79 & 4.99 & 3-4\end{array}$

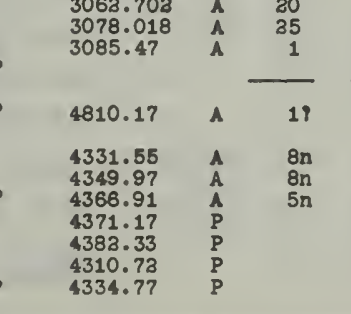

4334.251
4348.820

$\begin{array}{lll}1.67 & 4.99 & 3-4 \\ 1.67 & 4.96 & 3-3\end{array}$

$\begin{array}{lll}1.68 & 5.83 & 2-1 \\ 1.87 & 5.81 & 1-0\end{array}$

$\begin{array}{ll}1.80 & 4.29 \\ 1.79 & 4.37\end{array}$

$\begin{array}{lll}1.80 & 4.99 & 4-4 \\ 1.79 & 4.98 & 3-3\end{array}$

I A Laboratory Ref Int Low $^{\text {E P }}$ H1gh

J Miltiplet

Y II continued

$\begin{array}{llr}4183.435 & \text { A } & 250 \\ 4205.080 & \text { A } & 350 \\ 4335.328 & \text { A } & 120 \\ 4164.015 & \text { A } & 15 \\ 4190.89 & \text { A } & 10 \\ 4150.08 & \text { P } & \end{array}$

$\begin{array}{llll}2.04 & 4.99 & 5-4 & b^{3} G-z^{3} F^{\circ} \\ 2.03 & 4.96 & 4-3 & (37) \\ 3.02 & 4.94 & 3-3 & \\ 2.03 & 4.99 & 4-4 & \\ 2.03 & 4.96 & 3-3 & \end{array}$

-3217.1218 A 400

$\begin{array}{lll}3237.876 & \text { A } & 350 \\ 3354.7738 & \text { A } & 300\end{array}$

3249.617 A 40

m3093.16 P $\mathrm{r}^{+}$

$\begin{array}{lll}3094.196 & \text { A } & 100 \\ 3100.938 & \text { A } & 100\end{array}$

$\begin{array}{lll}3104.906 & \text { A } & 35 \\ 3108.704 & \text { A } & 30 \\ 3082.534 & \text { A } & 40 \\ 3086.507 & \text { A } & 30\end{array}$

$2.04 \quad 5.88 \quad-5 \quad b^{3} \mathrm{G}_{-2} \mathrm{z}^{3}$

$\begin{array}{llll}2.03 & 5.84 & 4-5 & (38)\end{array}$

$\begin{array}{lll}2.03 & 5.81 & 3-4 \\ 3.04 & 5.84 & 5-5\end{array}$

$\begin{array}{llll}2.04 & 6.03 & 5-5 & b^{3} G-y^{3} G^{0}\end{array}$

$\begin{array}{llll}2.03 & 6.03 & 4-4 & (39)\end{array}$

$\begin{array}{lll}2.03 & 6.00 & 3-3 \\ 3.04 & 6.03 & 5-4\end{array}$

$\begin{array}{lll}3.03 & 6.00 & 4-3 \\ 3.03 & 6.03 & 4-5\end{array}$

$\begin{array}{lll}2.03 & 6.03 & 4-5 \\ 3.03 & 6.02 & 3-4\end{array}$

$\begin{array}{lllllll}3053.894 \quad \text { A } & 80 & 3.04 & 6.08 & 5-4 & b^{3} a-y^{3} F^{\circ}\end{array}$

$\begin{array}{llllll}3048.891 & \text { A } & 70 & 3.03 & 6.08 & 4-3 \\ 3042.37 & \text { A } & 80 & 2.03 & 6.07 & 3-2\end{array}$

$\begin{array}{llllll}3043.548 & \text { A } & 40 & 3.03 & 6.08 & 4-4\end{array}$

$\begin{array}{lll}3.03 & 6.08 & 3-3 \\ 3.03 & 6.08 & 3-4\end{array}$

$3.04 \quad 6.13 \quad 5-5 \quad b^{3} a-z^{1} H^{\circ}$

3033.883 A

$3015.98 \quad$ A 10

$\begin{array}{llr}3012.020 & \text { A } & 30 \\ 3001.93 & \text { A } & 2\end{array}$

$\begin{array}{llll}3.03 & 6.13 \quad 4-3 \quad b^{3}(41) \\ (42) & =0\end{array}$

$\begin{array}{cccc}3.04 & 6.14 & 5-4 & b^{3} G_{-2} G^{0} \\ 3.03 & 6.14 & 4-4 & (43)\end{array}$

$2979.103 \quad A \quad 5 \quad 2.03 \quad 6.16 \quad 3-3 \quad b^{3} \mathrm{G}-\mathrm{z}^{1} \mathrm{D}^{0}$

$\begin{array}{llllll}4606.59 & P & 3.21 & 4.89 & 4-5 & a^{1} G-z^{3} G^{\circ}\end{array}$

$\begin{array}{llllll}4651.42 & P & 2.21 & 4.86 & 4-4 & (45) \\ 4688.45 & P & 3.21 & 4.84 & 4-3 & \end{array}$

$\begin{array}{lllllll}4439.43 \quad A & 1 & 2.21 & 4.99 & 4-4 & a^{1}\left(-z^{3} F^{\circ}\right.\end{array}$

$\begin{array}{lllllll}3401.997 & A & a & 2.31 & 5.84 & 4-5 & a^{1} G-z^{3} \mathrm{H}^{0}\end{array}$

$\begin{array}{lllllll}3230.919 & \text { A } & 4 & 3.31 & 6.03 & 4-5 & a^{1} G-y^{3} G \\ 3343.74 & P & & 3.21 & 6.03 & 4-4 & (48)\end{array}$

$\begin{array}{lllllll}3188.10 & \text { A } & 30 & 3.31 & 6.08 & 4-4 & a^{1} a-y^{3} F^{\circ} \\ -3193.978 & \text { A } & 109 & 3.31 & 6.08 & 4-3 & (49)\end{array}$

3157.900 A $40 \quad 3.31 \quad 6.12 \quad 4-3 \quad a^{1}\left(502^{1} F^{0}\right.$

$3155.409 \quad$ A $\quad 60 \quad 3.31 \quad 6.12 \quad 4-5 \quad a^{1}\left(5^{2}\right)^{1} H^{0}$

$\begin{array}{lllllll}3143.484 & A & 150 & 3.21 & 6.14 & 4-4 & a^{1} G-2^{1} G^{0} \\ (53)\end{array}$

3.27 $4.59 \quad 3-4 \quad a^{3} D-2^{5} F^{\circ}$

$\begin{array}{lll}2.26 & 4.56 & 2-3 \\ 3.26 & 4.53 & 1-3\end{array}$

$2.27 \quad 4.56 \quad 3-3$

$\begin{array}{lll}2.26 & 4.53 & 2-2 \\ 2.36 & 4.50 & 1-1\end{array}$

$\begin{array}{lll}2.36 & 4.50 & 1-1 \\ 3.27 & 4.53 & 3-2 \\ 3.26 & 4.50 & 2-1\end{array}$

$\begin{array}{llll}2.37 & 4.59 \quad 3-3 & a^{3} D-2^{3} D^{\circ}\end{array}$

$2.26 \quad 4.56$

$\begin{array}{lll}2.26 & 4.56 & 1-1 \\ 3.37 & 4.57 & 3-2\end{array}$

$\begin{array}{lll}2.36 & 4.59 & 2-3\end{array}$

$\begin{array}{llll}3.36 & 4.57 & 1-2\end{array}$ 


\section{6}

$$
\text { I Laboratory }
$$

3186.86 A 10

3186.10 A

$\begin{array}{cc}3169.21 & \text { A } \\ -3160.781 & \text { A }\end{array}$

$\begin{array}{lll}3050.735 & \text { A } & 15 \\ 3081.254 & \text { A } & 25\end{array}$

$\begin{array}{lll}3081.254 & \text { A } & 25 \\ 3086.210 & \text { A } & 10 \\ 3078.948 & \text { A } & \end{array}$

3054.24

$\begin{array}{lllllll}3054.24 & \text { A } & 7 n & 2.27 & 6.31 & 3-3 & a^{3} D-y^{3} D^{0} \\ 3048.65 & \text { A } & 4 & 2.26 & 6.31 & 2-3 & (67) \\ 3075.474 & \text { A } & & & & \end{array}$

4968.50 A 1

$\begin{array}{ll}3547.07 & \text { A } \\ 3577.644 & \text { A }\end{array}$

$\begin{array}{lll}3361.506 & \text { A } & 60 \\ 3392.659 & \text { A } & 50\end{array}$

$\begin{array}{rrr}3315.176 & \text { A } & 50 \\ 3321.539 & \text { A } & 150\end{array}$

3282.534 A 150

3279.844 A 300

. 3265.8938 A 100

$\begin{array}{ll}3025.68 & \text { A } \\ 3032.187 & \text { A }\end{array}$

\begin{tabular}{llr} 
& & \\
\cline { 3 - 3 } 3621.203 & A & 150 \\
3632.126 & A & 15 \\
3627.713 & A & 60 \\
3645.905 & A & 30 \\
3625.608 & A & 50 \\
3631.482 & A & 10
\end{tabular}

3623.0

$\begin{array}{ccc}35777.8578 & \text { A } \\ 3573.557 & \text { A } & \\ 3588.13 & \text { A } & 1\end{array}$

$\begin{array}{lll}3588.13 & \text { A } & 15 \\ 3578.636 & \text { A } & 1 \\ 3577.220 & \text { A } & 10\end{array}$

3436.393 A

$\begin{array}{lll}3394.92 & \text { A } \\ 3323.731 & \text { A } & \\ 3249.464 & \text { A } & \\ 3128.686 & \text { A } & 20 \\ 3162.714 & \text { A } & 3 \\ 3192.699 & \text { A } & 1 \\ 3159.365 & \text { A } & 2 \\ 3193.200 & \text { A } & 20 \\ 3189.76 & \text { A } & \end{array}$

3125.01

3125.01
3166.39
3163.02

3128.288
3165.89

3024.981 A 50

$\begin{array}{lll}3024.981 & \text { A } & 50 \\ 3028.042 & \text { A } & 50 \\ 3027.600 & \text { A } & 15\end{array}$

$\begin{array}{llrrrrr}3005.813 & \text { A } & 30 & 3.36 & 6.47 & 2-3 & b^{3} P-x^{3} y^{*}\end{array}$

$\begin{array}{lllllll}3022.146 & \text { A } & 4 & 2.37 & 6.45 & 1-2 & (86) \\ 3019.09 & \text { A } & 3 & 2.36 & 6.45 & 2-2 & \end{array}$

$\begin{array}{rrrrrrc}3019.09 & \text { A } & 3 & 2.36 & 6.45 & 2-2 & \\ 2972.263 & \text { A } & 80 & 3.36 & 6.51 & 2-3 & b^{3} P-x^{3} D^{\circ} \\ 2981.200 & \text { A } & 70 & 3.37 & 6.51 & 1-2 & (87)\end{array}$

2989.3
2978.226

2989.7

$\begin{array}{lllc}2.36 & 4.84 & 4-3 & b^{1} G-2^{3} G^{\circ} \\ 2.36 & 5.84 & 4-5 & b^{1} G-2^{3} H^{\circ} \\ 2.36 & 5.81 & 4-4 & (69)\end{array}$

$\begin{array}{llll}2.36 & 6.03 & 4-5 & b^{1} a-y^{3} a^{0} \\ 2.36 & 6.00 & 4-3 & (70)\end{array}$

$\begin{array}{llll}2.36 & 6.08 & 4-4 & b^{1}\left(a-y^{3} 5^{\circ}\right. \\ 2.36 & 6.08 & 4-3 & (71)\end{array}$

$2.36 \quad 6.12 \quad 4-3 \quad b^{1}\left(72^{1}\right)^{\circ}$

$\begin{array}{llll}2.36 & 6.44 & 4-5 & b^{1}(74) \mathrm{J}^{3} \mathrm{H}^{\circ} \\ 2.36 & 6.43 & 4-4 & (75)\end{array}$

$\begin{array}{llll}2.36 & 5.77 & 2-2 & b^{3} p-2^{3} p^{\circ} \\ 3.37 & 5.76 & 1-1 & (76)\end{array}$

$\begin{array}{lll}2.36 & 5.76 & 2-1 \\ 2.37 & 5.75 & 1-0\end{array}$

$\begin{array}{lll}2.37 & 5.77 & 1-2 \\ 2.37 & 5.76 & 0-1\end{array}$

$\begin{array}{cccc}3.37 & 5.79 & 1-3 & b^{3} p-25 p o \\ 2.37 & 5.77 & 0-1 & (77)\end{array}$

$\begin{array}{lllc}2.36 & 5.81 & 2-3 & b^{3} P-y^{5} D^{\circ} \\ 2.37 & 5.82 & 1-1 & (78)\end{array}$

$\begin{array}{lll}2.36 & 5.82 & 2-1 \\ 2.37 & -1\end{array}$

$\begin{array}{lll}2.37 & 5.81 & 1-0 \\ 2.37 & 5.81 & 1-2\end{array}$

$\begin{array}{lll}2.37 & 5.81 & 1-2 \\ 2.37 & 5.83 & 0-1\end{array}$

$2.37 \quad 5.96 \quad 1-0 \quad b^{3} P_{-2} z^{1} S^{0}$

$\begin{array}{llll}2.36 & 6.00 & 8-3 & b^{3} 8-y^{3}\end{array}$

$\begin{array}{llll}2.36 & 6.08 & 2-3 & b^{3} 8-y^{3} F^{\circ}\end{array}$

$\begin{array}{lllc}2.36 & 6.31 & 2-3 & b^{3} 8-7^{3} D^{\circ} \\ 2.37 & 6.27 & 1=3 & (83) \\ 3.37 & 6.23 & 0-1 & \end{array}$

$\begin{array}{lll}3.37 & 6.23 & 0-1 \\ 2.36 & 6.37 & 2-8\end{array}$

$\begin{array}{lll}3.37 & 6.33 & 1-1 \\ 2.36 & 6.23 & 3-1\end{array}$

$\begin{array}{llll}3.36 & 6.31 & 2-a & b^{3} p-y^{3} p^{0} \\ 3.37 & 6.26 & 1-1 & (84)\end{array}$

$\begin{array}{lll}3.37 & 6.26 & 1-1 \\ 2.36 & 6.26 & 2-1\end{array}$

$\begin{array}{lll}3.37 & 6.35 & 1=0 \\ 2.37 & 6.31 & 1-2\end{array}$

$2.36 \quad 6.44 \quad 3-1 \quad b^{3} p_{-2} 38$ e

$3.37 \quad 6.44 \quad 3-1 \quad b^{3} \mathrm{P}-2^{3}$

a.37, $6.44 \quad 0$

$\begin{array}{lll}3.37 & 6.49 & 0-1 \\ 3.36 & 6.51 & 2-2\end{array}$

$\begin{array}{lll}3.36 & 6.51 & 2-2 \\ 3.37 & 6.49 & 1-1\end{array}$

\section{$\nabla$ II continue}

$\begin{array}{llr}3302.711 & \text { A } & \text { Z } \\ 3196.574 & \text { A } & 20\end{array}$

3607.30

3574.340 A 60

3163.024
3174.077
3128

AEV I SED 4 ULIIPLET TABLE

I A Laboratory Int Low ${ }_{\text {Righ }}^{\text {Pef In Nultiplet }}$ VII continued

$5928.86 \quad$ A $\quad 100 \quad 2.51 \quad 4.59 \quad 2-3 \quad 0^{3} \mathrm{P}_{-2} \mathrm{z}^{3}$ $\begin{array}{lrrrrrc}5928.86 & \text { A } & 100 & 2.51 & 4.59 & 2-3 & 0^{3} P-2^{3} D^{\circ} \\ 5897.54 & \text { A } & 50 & 2.48 & 4.57 & 1-2 & (98) \\ 5951.45 & \text { A } & 4 & 3.49 & 4.56 & 0-1 & \end{array}$

$\begin{array}{llll}2.36 & 6.13 & 4-5 & b^{1}(72)^{1} H^{\circ} \\ 3.36 & 6.14 & 4-4 & b^{1}\left(7 z^{1} G^{\circ}\right.\end{array}$

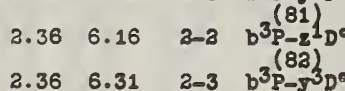

3370.40 A $2 \quad \overline{3.37} 6.03 \quad 6-5 \quad \mathrm{Q}^{1} \mathrm{I}-\mathrm{r}^{3} \mathrm{G}^{\circ}$

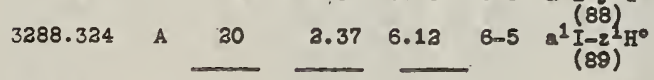

6027.23 A $8 \quad 2.46 \quad 4.50 \quad 0-1 \quad \mathrm{2}^{1} 8=2^{5} 7^{\circ}$

$\begin{array}{lllllll}5862.80 & A & 15+p ? & 3.46 & 4.56 & 0-2 & a^{1}(90)^{3} D^{\circ}\end{array}$

$\begin{array}{lllllll}3731.983 & A & 20 & 0.46 & 5.76 & 0-1 & 191 p^{30}\end{array}$

$\begin{array}{lllllll}3674.691 & A & 30 & 3.46 & 5.83 & 0.1 & 183 \mathrm{D}^{\circ}\end{array}$

$\begin{array}{lrrrrrr}3270.115 & \text { A } & 10 & 0.46 & 6.23 & 0-1 & a^{1} \mathrm{~S}^{3} \mathrm{D}^{\circ} \\ 3057.08 & \mathrm{~A} & 2 & 2.46 & 6.49 & 0-1 & \mathrm{~g}^{1} \mathrm{~g}-\mathrm{x}^{3} \mathrm{D}^{\circ}\end{array}$

$\begin{array}{lrrrrrr}3057.08 & \mathrm{~A} & 2 & 2.46 & 6.49 & 0-1 & \mathrm{a}^{1} \mathrm{~g}-\mathrm{x}^{3} \mathrm{D}^{\circ} \\ 3038.520 & \mathrm{~A} & 30 & 2.46 & 6.53 & 0-1 & \mathrm{a}^{1}(95)^{1} \mathrm{z}^{1} \\ & & & & \end{array}$

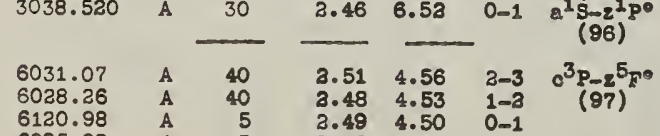

5819.93 A 80

$\begin{array}{rr}3787.235 & \text { A } 150 \\ -3758.238 & \text { A }\end{array}$

$\begin{array}{rrr}3758.238 & \text { A } & 150 \\ 3794.366 & \text { A } & 50 \\ 3772.962 & \text { A } & 80 \\ 3751.222 & \text { A } & 150\end{array}$

$\begin{array}{rrr}3772.962 & \text { A } & 80 \\ 3751.322 & \text { A } & 150 \\ 3767.720 & \text { A } & 40\end{array}$

$2.51 \quad 4.63 \quad 2-3 \quad c^{3} \mathrm{P}-z^{5} \mathrm{D}^{\circ}$

$\begin{array}{llll}2.51 & 5.77 & 2-3 & 0^{3} P_{-2}^{(99} P^{\circ} \\ 2.48 & 5.76 & 1-1 & (100)\end{array}$

$\begin{array}{lll}3.48 & 5.77 & 1-2 \\ 2.49 & 5.76 & 0.1\end{array}$

$3.48 \quad 5.79 \quad 1-3 \quad c^{3} P_{-2} p^{5}$

$\begin{array}{llll}2.51 & 5.82 & 2-3 & \mathrm{c}^{3} \mathrm{P}-\mathrm{J}^{5} \mathrm{D}^{\circ} \\ 3.48 & 5.81 & 1-3 & (102)\end{array}$

$\begin{array}{lll}3.51 & 5.81 & 3-2 \\ 3.48 & 5.83 & 1-1\end{array}$

$\begin{array}{lll}2.51 & 5.88 & 2-1 \\ 2.48 & 5.81 & 1-0\end{array}$

$2.48 \quad 5.96 \quad 1-0 \quad 0^{3} \mathrm{P}-\mathrm{x}^{1} \mathrm{~g}^{\circ}$

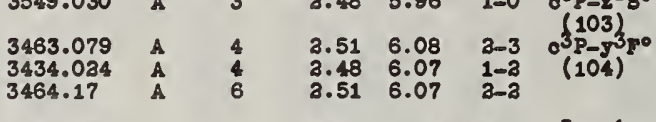

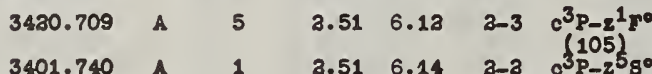

$\begin{array}{lllllll}3401.740 & A & 1 & 3.51 & 6.14 & 2-2 & 0^{3} \mathrm{P}_{2} 5_{\mathrm{g}} \\ 3373.666 & \mathrm{~A} & 3 & 3.48 & 6.14 & 1-2 & (106)\end{array}$

$\begin{array}{lllllll}3382.589 & \text { A } & 30 & 3.51 & 6.16 & \mathrm{a}-3 & \mathrm{o}^{3} \mathrm{~F}-\mathrm{z}^{1} \mathrm{D}^{\circ} \\ 3353.776 & \mathrm{~A} & 30 & 3.48 & 6.16 & 1-2 & (107)\end{array}$

3251.869 A 200

$\begin{array}{rrr}3257.893 & \text { A } & 100 \\ 3397.528 & \text { A } & 30 \\ 3285.023 & \text { A } & 50\end{array}$

$\begin{array}{lll}3285.022 & \text { A } & 50 \\ 3290.240 & \text { A } & 50 \\ 3317.913 & \text { A } & 30\end{array}$

3247.90

3361.80
3388.98

3288.985
3221.380

3119.3

3115.16

3083.208

$\begin{array}{llr}3065.61 & \text { A } & 50 \\ 3081.01 & \text { A } & 30 \\ 3089.633 & \text { A } & 4\end{array}$

$\begin{array}{llr}3074.66 & \text { A } & 4 \\ & & \end{array}$

$\begin{array}{llll}3079.75 & \text { A } & 1 \\ 3062.178 & \text { A } & 3\end{array}$

\begin{tabular}{llr}
3981.924 & $A$ & 15 \\
2992.378 & $A$ & $a$ \\
\hline
\end{tabular}

$\begin{array}{lll}5193.43 & P & \\ 5327.70 & \text { A } & 30 \\ 5263.99 & \text { A } & 15 \\ 5171.13 & \text { P } & \end{array}$

5317.36

3669.410 A 300

3700.33

3711.751
3733.607

3658.266
.3695 .158

3509.024

3513.877

3527.867
3498.83

3498.83
3509.20

3448.69

12

3420.15

3408.06

3408.95

3391.01

3134.92

3139.733

3144.700
3143.477

3126.79
3138.793

3033.445

3048.214 A 200

3063.247
3055.948

3066.80

6236.29

6028.98

6086.93
6083.82
6036.81

$\begin{array}{llll}2.51 & 6.31 & 2-3 & c^{3} P-r^{3} D^{\circ}\end{array}$

$\begin{array}{llll}3.51 & 6.31 & 2-3 & c^{3} P-y^{3} D \\ 3.48 & 6.27 & 1-2 & (108)\end{array}$

$\begin{array}{lll}3.49 & 6.23 & 1-2 \\ 3.49 & 6.27 & 0-1 \\ 3.51 & 6.27 & 2-2\end{array}$

$\begin{array}{lll}3.48 & 6.23 & 1-1 \\ 3.51 & 6.23 & 3-1\end{array}$

$\begin{array}{llll}3.51 & 6.31 & 2-2 & 0^{3} \mathrm{P}-\mathrm{F}^{3} \mathrm{P}^{\circ} \\ 3.48 & 6.26 & 1-1 & (109)^{2}\end{array}$

$\begin{array}{lll}3.51 & 6.26 & 2-1 \\ 3.48 & 6.31 & 1-2\end{array}$

$2.51 \quad 6.47 \quad 2-3 \quad 0^{3} P-x^{3} y^{\circ}$

$3.48 \quad 6.44 \quad 1-1 \quad 0^{3} \mathrm{P}_{-2} 8^{\circ}$

$\begin{array}{llll}3.51 & 6.51 & 2-3 & 0^{3} \mathrm{P}-\mathrm{x}^{3} \mathrm{D}^{\circ} \\ 3.48 & 6.51 & 1-2 & (113)\end{array}$

$3.49 \quad 6.49$

$\begin{array}{lll}3.51 & 6.51 & 2-2 \\ 3.48 & 6.49 & 1-1\end{array}$

$\begin{array}{llll}3.51 & 6.53 & 2-1 & 0^{3} P-z^{1} P^{\circ} \\ 2.49 & 6.53 & 0-1 & (113)\end{array}$

$\begin{array}{llll}2.51 & 6.64 & 2-1 & (114)\end{array}$

$\begin{array}{llll}3.51 & 4.89 & 6-5 & b^{3} 1-z^{3} a^{\circ} \\ 3.50 & 4.86 & 5-4 & (115) \\ 3.50 & 4.84 & 4-3 & \end{array}$

$\begin{array}{ll}3.50 & 4.89 \\ 3.50 & 4.86\end{array}$

3.515 .88

$3.50 \quad 5.84$

$\begin{array}{ll}3.50 & 5.81 \\ 3.51 & 5.84\end{array}$

3.60 5.81

$\begin{array}{ll}3.50 & 5.88 \\ 3.50 & 5.84\end{array}$

$\begin{array}{llll}3.51 & 6.03 & 6-5 & b^{3} H^{3}-5^{3} 0^{\circ} \\ 3.50 & 6.03 & 5-4 & (117)\end{array}$

$\begin{array}{lll}3.50 & 6.00 & 4-3 \\ 3.50 & 6.03 & 5-5\end{array}$

$\begin{array}{lll}3.50 & 6.02 \quad 4-4\end{array}$

3. $50 \quad 6.43$

$3.51 \quad 6.44$

$\begin{array}{ll}3.50 & 6.43 \\ 3.50 & 6.45\end{array}$

$\begin{array}{ll}3.50 & 6.45 \\ 3.50 & 6.44\end{array}$

$\begin{array}{llll}2.51 & 6.58 & 6-7 & b^{3} \mathrm{HL}^{3} \mathrm{z}^{3} \\ 3.50 & 6.55 & 5-6 & (123)\end{array}$

$\begin{array}{lll}3.50 & 6.53 & 4-5 \\ 3.51 & 6.55 & 0-6\end{array}$

$\begin{array}{ll}3.51 & 6.55 \\ 3.50 & 6.53\end{array}$

$3.55 \quad 4.53 \quad a-a \quad b^{3} D-z^{5} y^{0}$ $\begin{array}{lll}3.51 & 5.76 & 2-1 \\ 3.48 & 5.75 & 100\end{array}$

$\begin{array}{lll}2.49 & 5.82 & 0-1\end{array}$

$2.51 \quad 6.65 \quad 2-3 \quad 0^{3} P-\pi^{3} D^{0}+$

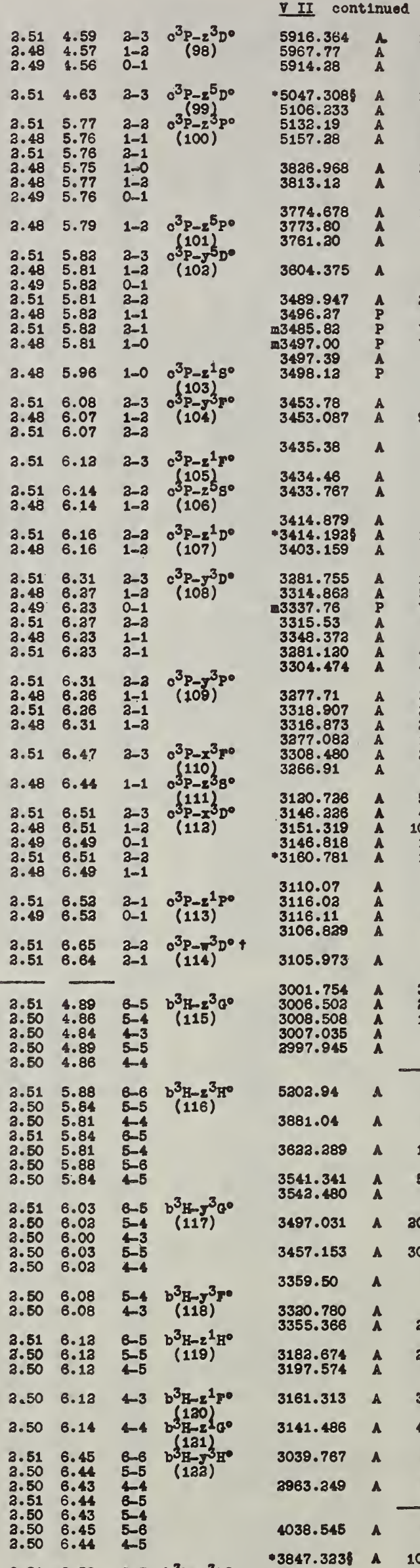

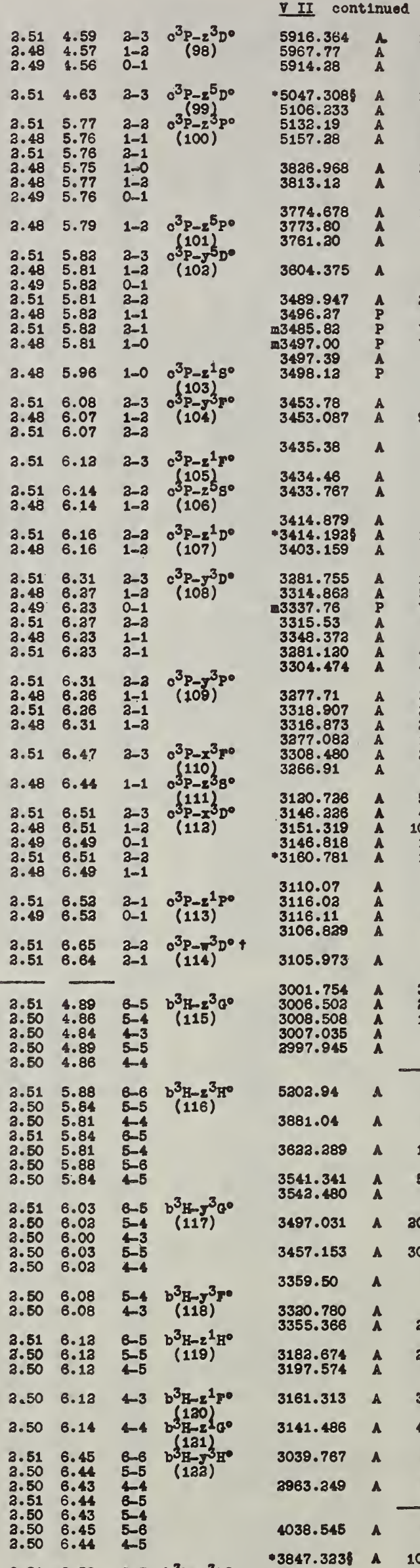

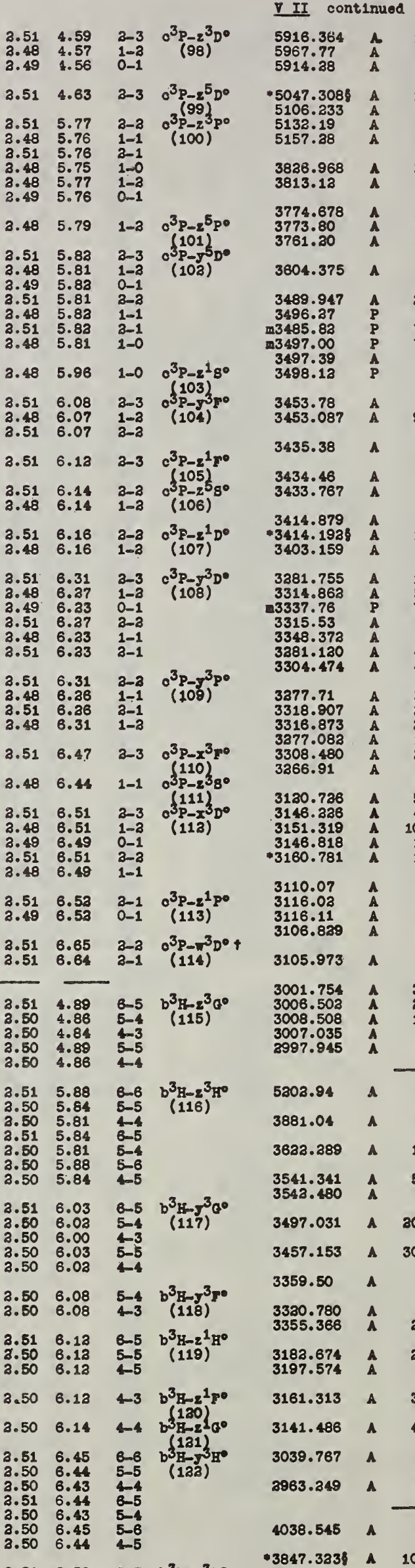

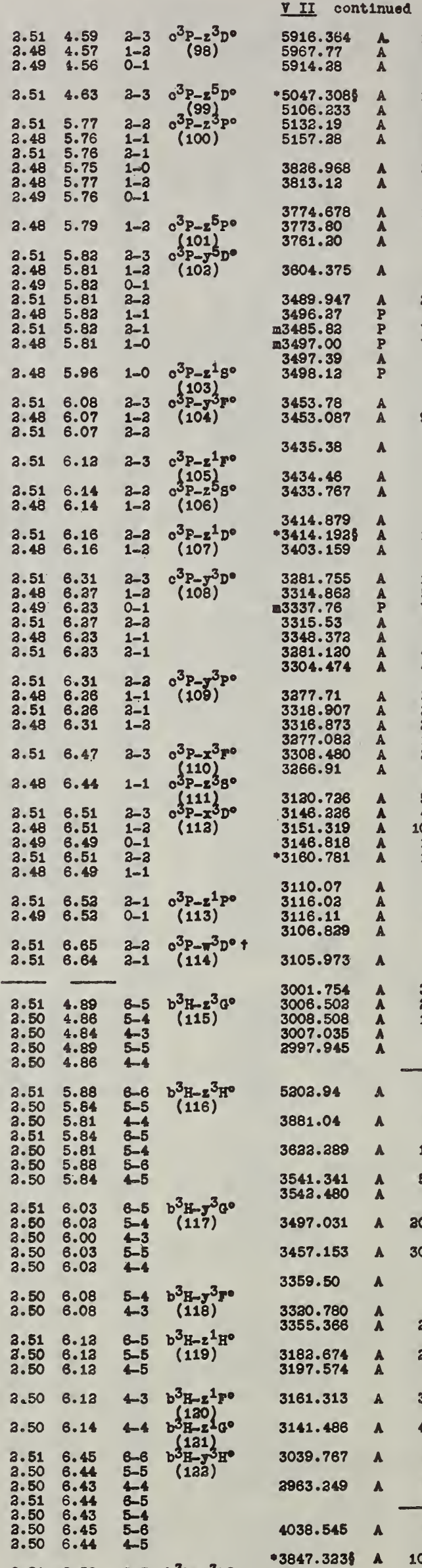

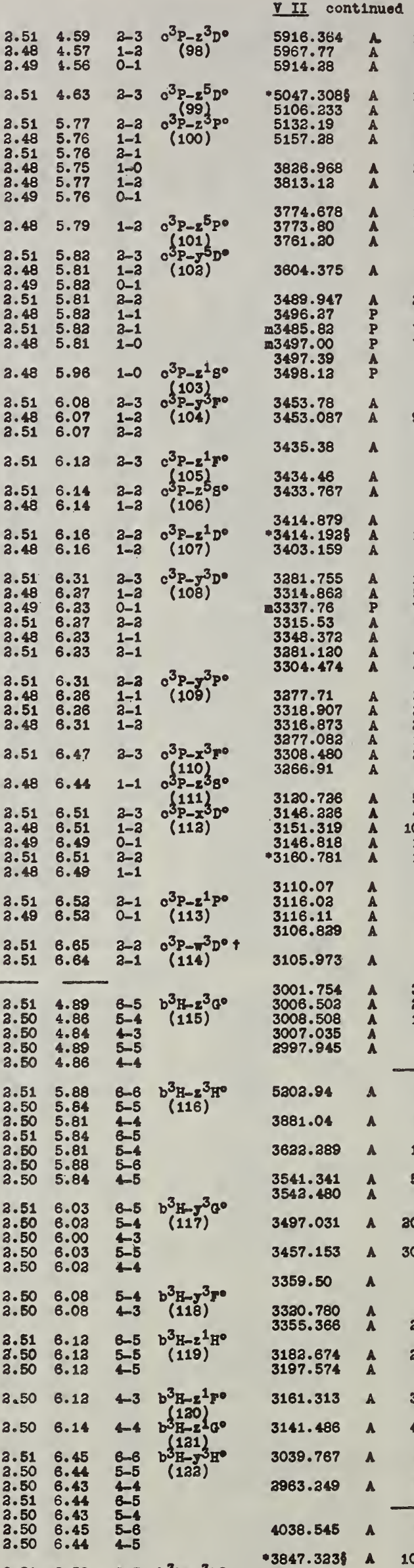

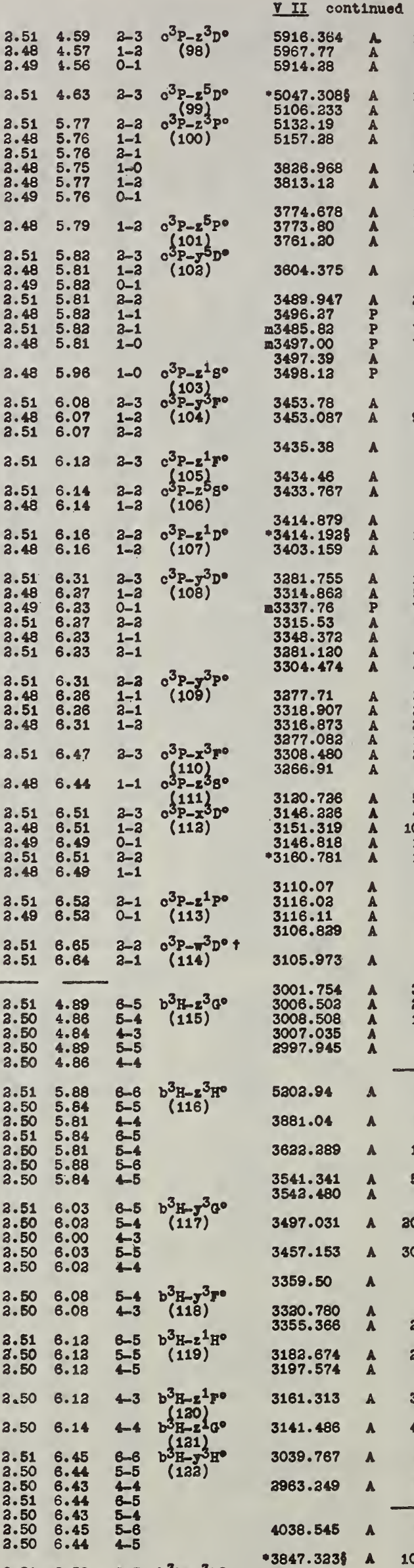

I $A_{\text {Laboratory }}^{\text {Lnt }}$

I I P ${ }^{\mathrm{P}}$

3 tiplot

II continued

$\begin{array}{lrllll}A & 15 & 3.55 & 4.63 & 3-3 & b^{3} D-2^{5} D\end{array}$

-5047.3088

${ }_{5157.28}^{5132.19}$

10
5
3

$\begin{array}{llll}3.55 & 4.99 & 3-4 & b^{3} D-z^{3} \\ 3.50 & 4.96 & 2-3 & (127)\end{array}$

$\begin{array}{lll}2.53 & 4.94 & 1-2 \\ 3.55 & 4.94 & 2-2\end{array}$

$\begin{array}{llll}2.55 & 5.77 & 2-2 & b^{3} D_{-2} p^{0} \\ 3.53 & 5.77 & t-a & (128)\end{array}$

$\begin{array}{llll}2.55 & 5.81 & 2-2 & b^{3} D-y^{5} D^{\circ}\end{array}$

$\begin{array}{llll}2.55 & 5.82 & 3-1 & (129) \\ 2.53 & 5.81 & 1-2 & \end{array}$

$2.53 \quad 5.96 \quad 1-0 \quad b^{3} \mathrm{D}^{2} 2^{1} \mathrm{~g}^{\circ}$

$\begin{array}{llll}2.55 & 6.08 & 3-4 & b^{3} D-y^{3} F^{\circ} \\ 3.55 & 6.08 & 2-3 & (131)\end{array}$

$\begin{array}{lll}3.55 & 6.08 & 2-3 \\ 3.53 & 6.07 & 1-3\end{array}$

$\begin{array}{lll}3.55 & 6.08 & 3-3 \\ 3.55 & 6.07 & 2-2\end{array}$

$3.556 .07 \quad 3-3$

$\begin{array}{llll}3.55 & 6.12 \quad 3-3 & b^{3} D-2^{1} F^{\circ}\end{array}$

$3.55 \quad 6.14 \quad 3-4 \quad b^{3} D-z^{1} a^{\circ}$

$3.556 .14 . \quad 3-a \quad b^{3} D_{-2} 5_{8}$

$\begin{array}{llll}3.55 & 6.14 & 2-2 & (134)\end{array}$

$\begin{array}{llll}3.55 & 6.16 & 3-2 & b^{3} D-z^{1} D^{0} \\ 3.55 & 6.16 & 2-2 & (135)\end{array}$

$\begin{array}{llll}3.53 & 6.16 & 1-3 & (135)\end{array}$

$\begin{array}{llll}2.55 & 6.31 & 3-3 & b^{3} D-J^{3} \\ 0\end{array}$

3.55 6.27 2-2 (136)

$\begin{array}{lll}3.53 & 6.23 & 1-1 \\ 3.55 & 6.37 & 3-3\end{array}$

$\begin{array}{lll}3.55 & 6.37 & 3-2 \\ 3.55 & 6.33 & 2-1\end{array}$

$\begin{array}{lll}3.55 & 6.31 & 2-3 \\ 3.53 & 6.37 & 1-3\end{array}$

$\begin{array}{llll}2.55 & 6.31 & 3-2 & b^{3} D-r^{3} p \\ 3.55 & 6.36 & 2-1 & (137)\end{array}$

$\begin{array}{ll}2.55 & 6.26 \\ 2.53 & 6.25\end{array}$

$\begin{array}{lll}3.55 & 6.31 & 2-2\end{array}$

$\begin{array}{lll}3.53 & 6.36 & 1-1 \\ 3.53 & 6.31 & 1-3\end{array}$

$3.55 \quad 6.50 \quad 3-4 \quad b^{3} D-x^{3}-0$

$\begin{array}{llll}2.55 & 6.47 & 2-3 & (138)\end{array}$

$\begin{array}{lll}3.53 & 6.45 & 1-3 \\ 3.55 & 6.47 & 3-3\end{array}$

$65 \quad 6.45 \quad 2-2$

$\begin{array}{llll}3.55 & 6.51 & 3-3 & b^{3} D-x^{3} D\end{array}$

$\begin{array}{llll}2.55 & 6.51 & 2-3 & \left(D-x^{3}\right)^{2} \\ 3.53 & 6.49 & 139)\end{array}$

$\begin{array}{lll}2.53 & 6.49 & 1-1 \\ 3.53 & 6.51 & 1-2\end{array}$

$3.55 \quad 6.52 \quad a-1 \quad b^{3} D-z^{1} p$

$\begin{array}{llll}3.55 & 6.66 & 3-3 & b^{3}(10) \\ D-1 & 0\end{array}$

$\begin{array}{llll}3.55 & 6.65 & 2-2 & \text { (141) }\end{array}$

$\begin{array}{lll}2.53 & 6.64 & 1-1 \\ 3.55 & 6.65 & 3-2\end{array}$

$\begin{array}{lll}3.53 & 6.65 & 1-2\end{array}$

$2.59 \quad 4.96 \quad 2-3 \quad a^{1} D-2^{3} F^{0}$

$2.595 .77 \quad 2-2 \quad a_{D-z^{3}}$

2.596 .00

2.596 .08

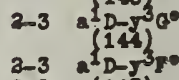

$3.596 .13 \quad 2-3 \quad a^{1} D-2^{1} F^{\circ}$

$\begin{array}{llll}2.59 & 6.16 \quad 2-2 \quad a^{1} \\ (147) & D^{\circ}\end{array}$

$\begin{array}{llll}3.59 & 6.26 & 2-1 & \left(\begin{array}{l}147 \\ D-7\end{array}\right)\end{array}$

$\begin{array}{llll}3.59 & 6.31 & 2-3 & a^{1}-y^{3} D^{0} \\ 3.59 & 6.37 & 2-2 & (149)\end{array}$

$\begin{array}{llll}2.59 & 6.47 & 2-3 & a^{1} D x^{3}-0 \\ 3.59 & 6.45 & a-3 & (150)\end{array}$

$2.59 \quad 6.49 \quad 2-1 \quad a^{1} D-x^{3} D^{2}$

$.596 .52 \quad 2-1 \quad a^{1}\left(\frac{151}{152}\right)$

$\left.2.596 .65 \quad 2-2 \quad \frac{1}{D-5}\right)^{\circ}$

$2.596 .75 \quad 2-1 \quad a_{D-x^{3}}^{3}$

$4038.545 \quad A \quad 2 \quad 2.75 \quad 5.81 \quad 1-0 \quad a^{1} P-J^{5} D^{\circ}$

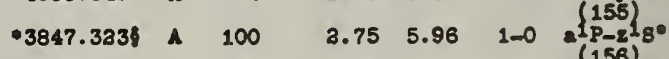

$\begin{array}{llll}3.55 & 4.59 & 3-3 & b^{3} D-s^{3} D^{\circ}\end{array}$
$3713.533 \quad A \quad 8$

3618.924 A 200

3507.534 A 20

3465.35

$3335.488 \triangle 10$

3285.67

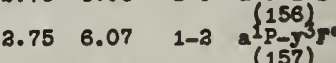

$\left.2.75 \quad 6.16 \quad 1-2 \quad a_{P-8}^{157}\right)_{D}$

$2.75 \quad 6.37 \quad 1-3 \quad l_{\mathrm{P}-\mathrm{D}^{3} \mathrm{D}}$

$2.75 \quad 6.31 \quad 1-2 \quad(160)$

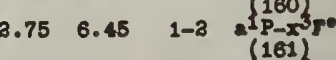

$\begin{array}{llll}3.75 & 6.51 & 1-2 & a_{P-X^{3}}^{10} \\ 0.75 & 6.49 & 1-1 & (163)\end{array}$ 
REVISED KULTIPLET TABLE

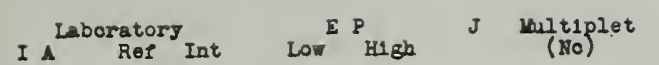
VII oontinued

$\begin{array}{lllllll}3274.50 \quad A \quad & 10 & 3.75 & 6.53 & 1-1 & \alpha^{1} p-z^{1} p^{0}\end{array}$

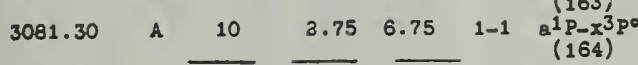

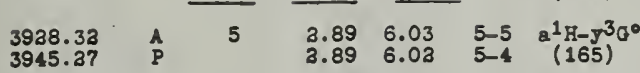

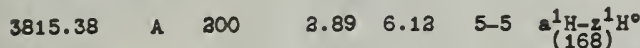
$.3798 .48 \quad$ A $\quad 10 \quad 3.89 \quad 6.14 \quad 5-4 \quad \mathrm{a}^{1 \mathrm{H}-2^{1} \mathrm{G}^{\circ}}$ $\begin{array}{llllllll}3463.831 & A & 4 & 3.89 & 6.45 & 5-6 & \mathbf{a}^{1} \mathrm{H}-\mathrm{y}^{3} \mathrm{H}^{\circ} \\ 3484.32 & \mathrm{~A} & \mathbf{a} & 3.89 & 6.43 & 5-4 & (168)^{2}\end{array}$ $\begin{array}{lllllll}3415.91 \quad A \quad & 3 & 3.89 & 6.50 & 5-4 & 8^{1} \mathrm{H}-x^{3} \xi^{\circ}\end{array}$ $3367.666 \quad A \quad 3 \quad 3.89 \quad 6.55 \quad 5-6 \quad a_{(170)}^{(169)}$ $\begin{array}{llllllll}3250.775 \quad A \quad 300 & 3.89 & 6.68 & 5-4 & 8^{1} H_{1}^{1} 1^{10}\end{array}$ $\begin{array}{lllllllll}3143.183 \quad A \quad 30 & 3.89 & 6.83 & 5-5 & \mathrm{a}^{1 / R-3^{3}}\left(17 \mathrm{G}^{\circ}\right.\end{array}$ $\begin{array}{lllllllll}3133.887 & A & 100 & 3.89 & 6.84 & 5-6 & a^{1}\left(17 z^{1} I^{0}\right.\end{array}$

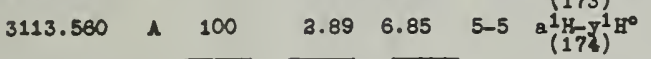
$\begin{array}{lllllll}4162.073 & A & 3 & 3.11 & 6.08 & 2-3 & b^{1} D-y^{3} F^{\circ} \\ 4163.855 & A & 3 & 3.11 & 8.07 & 3-3 & (195)\end{array}$ $\begin{array}{lllllll}.4108 .00 \quad \text { a } & 8 & 3.11 \quad 8.13 & 2-3 & b^{1} D^{2} z^{1} r^{\circ}\end{array}$

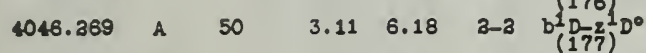
$\begin{array}{lllllll}3907.53 & A & 3 & 3.11 & 6.37 & 2-3 & \left.b \frac{1}{178}\right)^{3} D^{\circ}\end{array}$ $\begin{array}{rllllll}-3695.158 \quad A & 8 & 3.11 & 6.45 & 2-a & b^{1} p-x^{3} F^{\circ} \\ (179)^{\circ}\end{array}$ $\begin{array}{llllllll}3834.13 & A & 1 & 3.11 & 6.51 & 3-3 & b^{1} D-x^{3} D^{0} \\ 3648.848 & \text { A } & 7 & 3.11 & 6.49 & 3-1 & (180)\end{array}$ $3630.496 \quad A \quad 30 \quad 3.11 \quad 8.53 \quad 3-1 \quad b^{1} D-z^{1} P^{0}$ $\begin{array}{llllllll}3478.961 \quad A & 6 & 3.11 & 6.66 & 3-3 & b^{1} D-\pi^{3} D^{\circ}\end{array}$

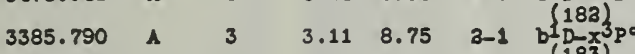
$\begin{array}{lllllll}3337.845 \quad A \quad 200 & 3.11 & 6.81 & 3-3 & b^{1}(1-1)^{1} F^{\circ}\end{array}$

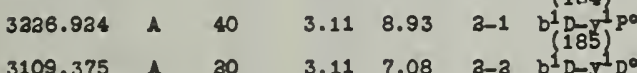
$3109.375 \wedge \quad 30 \quad 3.11 \quad 7.08 \quad 3-3 \quad b^{1} \quad\left(1-y^{1} D\right.$

$4398.53 \quad A \quad$ in $3.31 \quad 6.13 \quad 3-3 \quad \mathrm{a}^{1} F^{1}-2^{1} F^{\circ}$ $4388.67 \quad P \quad 3.31 \quad 6.14 \quad 3-4 \quad 8 \frac{18-21}{18 a^{\circ}}$ $\left.3980.37 \quad A \quad 1 \quad 3.31 \quad 6.43 \quad 3-4 \quad a^{1} F_{18}\right)^{3} \mathrm{H}^{\circ}$

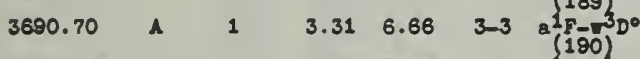
$3681.383 \quad \AA \quad 200 \quad 3.31 \quad 6.68 \quad 3-4 \quad 2 \frac{F}{191} 0^{\circ}$

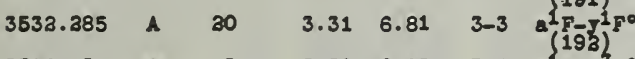
$\begin{array}{llllllll}3512.13 & A & 3 & 3.31 & 6.83 & 3-4 & a_{F-x^{3}}^{100} \\ 3506.57 & A & 7 & 3.31 & 8.83 & 3-3 & (193)\end{array}$ $\begin{array}{llllllll}3377.448 & A & 15 & 3.31 & 7.08 & 3-3 & 8^{1} P_{-1} y^{1} D^{0} \\ (194)\end{array}$

$\begin{array}{ccccccc}.5275 .65 & A & 10 & 3.74 & 6.08 & 4-4 & 0^{3}-y^{3} \\ 5288.31 & A & 5 & 3.74 & 6.08 & 3-3 & (195)\end{array}$ $\begin{array}{llllllll}5380.00 & A & 38 & 3.74 & 6.07 & 2-3 & \\ 5151.87 & A & 3 & 3.74 & 8.14 & 4-4 & 0^{3} F_{-2}^{1} 0^{\circ}\end{array}$ $\begin{array}{lllllllll}4813.952 & A & 50 & 3.74 & 8.31 & 4 & (198) & 0^{3} F-5^{\circ} D^{\circ}\end{array}$

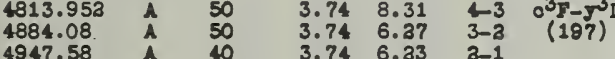
$\begin{array}{llllll}4811.14 & \text { A } & 6 & 3.74 & 6.31 & 3-3 \\ 4874.805 & A & 3.74 & 6.37 & 3-3\end{array}$

$\begin{array}{llllll}475.34 \quad A \quad 1 & 3.74 & 8.50 & 4-4 & 0^{3} r-x^{3} & 7^{0}\end{array}$ $\begin{array}{lllllll}4556.785 & A & 4 & 3.74 & 8.45 & 3-2 & \end{array}$ $\begin{array}{llllllll}4453.35 & A & 30 \mathrm{n} & 3.74 & 6.51 & 4-3 & { }^{3}{ }^{P}-x^{3} D^{0} \\ 464.32 & A & 40 n & 3.74 & 6.51 & 3-3 & (199)\end{array}$ $\begin{array}{rrrrrr}475.70 & A & 30 n & 3.74 & 6.49 & 2-1 \\ 458.53 & A & 3 n & 3.74 & 8.51 & 3-3\end{array}$

$\begin{array}{ccccccc}.4234 .55 & A & 10 \mathrm{n} & 3.74 & 8.66 & 4-3 & 0^{3} \mathrm{~F}-\mathrm{m}^{3} \mathrm{D}^{\circ} \\ 4343.894 & A & 30 \mathrm{n} & 3.74 & 8.65 & 3-3 & (300)\end{array}$ $4257.03 \quad i \quad 15 n$

$\begin{array}{lllllll}4027.30 & A & 1 & 3.74 & 6.81 & 4-3 & 0^{3} y_{-1}^{1} y^{50} \\ 4019.05 & A & 7 n & 3.74 & 8.81 & 3-3 & (301)\end{array}$ $\begin{array}{llllll}4018.83 & \text { an } & 3.74 & 8.83 & 45 & 0^{3} T-x^{3} a^{0}\end{array}$ $\begin{array}{llllllll}3999.195 & \hat{A} & 30 \mathrm{~A} & 3.74 & 8.83 & 3-4 & \text { (303) }\end{array}$ 1001.17 $3970.15 \quad 5$ $\begin{array}{lll}3.74 & 8.83 & 4-4 \\ 3.74 & 8.83 & 3-3\end{array}$ $3.74 \quad 6.85 \quad 45 \quad 0^{3} \mathrm{~T}-\mathrm{y}^{1} \mathrm{H}^{\circ}$

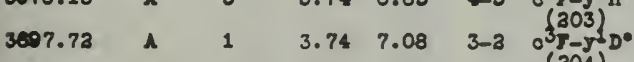

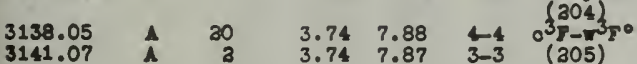

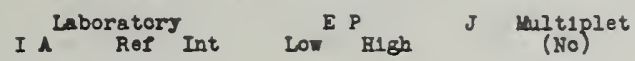
$\checkmark$ II continued

$\begin{array}{lllllll}6080.11 & A & 6 & 3.78 & 5.81 & 4-4 & d^{3} F-z^{3}\end{array}$ $\begin{array}{llllllll}5390.748 \text { A } & 6 & 3.79 & 6.13 & 2-3 & \mathrm{~d}^{3} \mathrm{~F}^{2} 2^{1} \mathrm{~F}^{\circ}\end{array}$ $\begin{array}{llllllll}5191.59 \quad A \quad & 3 & 3.78 & 6.16 & 3-3 & d^{3} F-21 D^{\circ} \\ (308)\end{array}$ $\begin{array}{lrrrrrrr}4883.415 & \mathrm{~A} & 100 & 3.78 & 6.31 & 4-3 & \mathrm{~d}^{3} F-y^{3} \mathrm{D}^{0} \\ 4965.40 & \mathrm{~A} & 40 & 3.78 & 6.37 & 3-3 & (209)\end{array}$ 5048.91
4973.18

$\begin{array}{lllllll}4535.315 \quad A \quad 3 n & 3.78 & 6.50 & 4-4 & d^{3} F-x^{3} F^{0}\end{array}$ $\begin{array}{llllllll}4596.37 & A & 5 \mathrm{n} & 3.78 & 6.47 & 3-3 & (310)\end{array}$ 4590.505 4637.48

4517.35

4512.72

4533.188
4558.46

4518.38

4538.64
4524.81

4304.15

4080.44
4085.87

4065.070
4053.59

.4051 .34
4049.03

4017.29

3167.480
3174.531

3174.531
3182.59

3171.739

3179.416
3170.308

3177.696

3973.975

3985.184
3994.540

$\begin{array}{lll}3.79 & 6.33 & 2-1 \\ 3.79 & 6.27 & 2-2\end{array}$

$\begin{array}{lll}3.78 & 6.47 & 3-3 \\ 3.79 & 6.45 & 2 \\ 3.78 & 8.47 & 4-3\end{array}$

$\begin{array}{lll}3.78 & 8.47 & 4-3 \\ 3.78 & 6.45 & 3-3\end{array}$

$3.79 \quad 6.53 \quad 2-1 \quad d^{3} F-2^{1} p 0$

$\begin{array}{llll}3.78 & 6.51 & 4-3 & d^{3} F-x^{3} D^{\circ}\end{array}$

$\begin{array}{lll}3.79 & 6.49 & 2-1 \\ 3.78 & 6.51 & 3-3 \\ 3.79 & 6.51 & 2-3\end{array}$

$\begin{array}{lll}3.79 & 6.51 & 2-3 \\ 3.79 & 6.51 & 3-3\end{array}$

$\begin{array}{llll}3.78 & 6.65 \quad 3-3 & d^{3} F-x^{3} D^{0}\end{array}$

$\begin{array}{llll}3.78 & 6.81 & 3-3 & d^{3} F-y^{1} F^{\circ} \\ 3.79 & 6.81 & 2-3 & (314)\end{array}$ 10

100
601

$\begin{array}{llll}3.78 & 6.88 & 4-5 & d^{3} F-x^{3} G^{\circ}\end{array}$

$\begin{array}{lll}3.78 & 6.83 & 3-4 \\ 3.79 & 6.83 & 2-3\end{array}$

$\begin{array}{llll}3.78 & 6.83 & 4-4\end{array}$

$\begin{array}{lll}3.78 & 6.85\end{array}$

$\begin{array}{llll}3.78 & 7.68 & 4-4 & d^{3} F-w^{3} F^{\circ} \\ 3.78 & 7.67 & 3-3 & (317)\end{array}$

3.797 .66

$\begin{array}{ll}3.78 & 7.67 \\ 3.78 & 7.66\end{array}$

$\begin{array}{lll}3.78 & 7.68 & 3-4\end{array}$

$\begin{array}{llll}3.79 & 7.67 \quad 3-3\end{array}$

6801.16 \&

$\begin{array}{llll}3.78 & 7.93 & 4-5 & d^{3} F-\pi^{3} a^{0} \\ 3.78 & 7.93 & 3-4 & (318)\end{array}$ $3.79 \quad 7.81 \quad 2-3$

$\begin{array}{lllllll}5801.16 \quad A & 5 & 3.96 & 5.77 & 3-3 & d^{3} P-z^{3} P^{0}\end{array}$ $\begin{array}{llllllllll}4933.75 \quad A \quad & 3 & 3.96 & 6.44 & 2-1 & d^{3} P-z^{3} & 3\end{array}$ $\begin{array}{llllllllll}4913.38 \quad A & a & 3.96 & 6.47 & 2-3 & \mathrm{~d}^{3} \mathrm{P}-x^{3} \mathrm{~F}^{\circ}\end{array}$ $\begin{array}{llllllll}4833.396 & A & 8 & 3.98 & 8.51 & 2-3 & d^{3} P-x^{3} D^{\circ} \\ 4839.08 & A & 3 & 3.96 & 6.51 & 3-3 & (233)\end{array}$

$\begin{array}{llllll}4408.93 \quad A \quad 40197 & 3.96 & 6.75 & 2-3 & d^{3} P-x^{3} P^{0}\end{array}$ $\begin{array}{lllllll}4440.41 & \hat{A} & 5 n & 3.99 & 6.77 & 1-0 & (334) \\ 4483.50 & \hat{A} & 32 & 4.00 & 6.75 & 0-1 & \end{array}$

$\begin{array}{lllllll}4233.085 & A & 80 \mathrm{n} & 3.96 & 6.87 & 2-1 & \mathrm{~d}^{3} \mathrm{P}-\mathrm{y}^{3} \mathrm{~s}^{\circ} \\ 4378.893 & \mathrm{~A} & 60 \mathrm{n} & 3.99 & 6.87 & 1-1 & (325)\end{array}$ $\begin{array}{lllllll}4278.893 & \hat{A} & 60 \mathrm{n} & 3.99 & 6.87 & 1-1 & (225) \\ 4301.130 & \hat{A} & 40 \mathrm{n} & 4.00 & 8.87 & 0-1 & \end{array}$

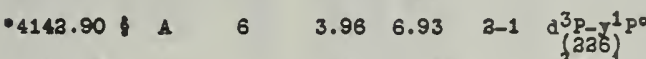

$\begin{array}{cccccccc}-3991.965 & A & 3 & 3.99 & 7.08 & 1-2 & d^{3} P-7^{3} \\ 3070.13 & A & 251 & 3.98 & 7.98 & 2-3 & d^{3} P-\nabla^{3} D^{\circ}\end{array}$

$\begin{array}{llccccc}3070.13 & \mathbf{A} & 251 & 3.98 & 7.98 & 2-3 & \mathrm{~d}^{3} \mathrm{P}-\nabla^{3} \mathrm{D} \\ 3075.58 & \mathrm{~A} & 5 & 3.99 & 8.00 & 1-3 & (328) \\ 3075.043 & \mathrm{~A} & 3 & 4.00 & 8.03 & 0-1 & \end{array}$

\begin{tabular}{llllll}
3075.043 & $A$ & 3 & 4.00 & 8.03 & $0-1$ \\
3051.308 & $\AA$ & 3 & 3.96 & 8.00 & $2-3$ \\
\hline
\end{tabular}

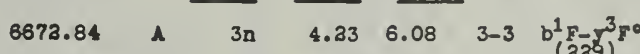

$6517.37 \quad A \quad 15 \mathrm{n} \quad 4.23 \quad 6.13 \quad 3-3 \quad b^{1} F_{-2}-F^{0}$

$8380.11 \quad A \quad 40 \mathrm{n} \quad 4.33 \quad 6.18 \quad 3-3 \quad b^{1} F-21 D^{\circ}$

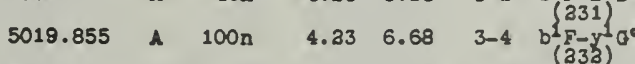

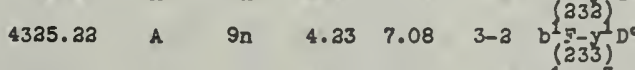

$\begin{array}{lllllll}3343.313 & \hat{A} & 3 & 4.23 & 7.92 & 3-4 & b^{1} F-\pi^{3} \\ 3351.53 & \hat{A} & 1 & 4.23 & 7.91 & 3-3 & (334)\end{array}$

3393.146 A 50

$3167.49 \quad A \quad 30$

$4.33 \quad 7.97 \quad 3-a \quad b^{1} F-x^{2} D^{\circ}$

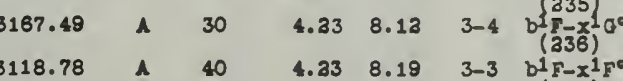

$5843.01 \wedge \overline{60 \mathrm{n}}=4.50 \quad 6.684 \mathrm{c}^{1} \mathrm{G}-\mathrm{y}^{1} \mathrm{G}^{\circ}$

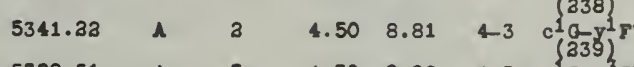

$5333.61 \quad A \quad 5 \quad 4.50 \quad 8.83 \quad 4-5 \quad c\left(a-x^{3} \sigma\right.$

$5341.19 \quad A \quad 100 \mathrm{n} \quad 4.50 \quad 6.85 \quad 45 \quad 0(a-1)^{\circ}$

$\begin{array}{lllllll}3608.33 \quad A & 1 & 4.50 & 7.92 & 4 & 0 & 0 \\ 3 & (243) & 300\end{array}$

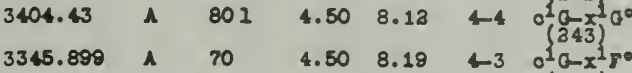

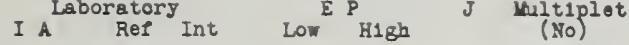
V II continued

$\begin{array}{lllllll}3035.14 & A & 3 N & 4.89 & 8.96 & 5-4 & 2^{3} G^{0}-e^{5} \mathrm{H}\end{array}$

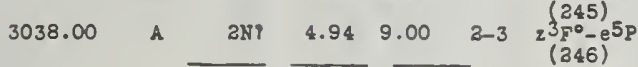

$\begin{array}{lllllll}5530.10 & \text { A } & 4 & 5.44 & 7.68 & 3-4 & c^{3} D-m^{3} F^{\circ} \\ 5562.03 & A & 4 n p ? & 5.45 & 7.67 & 3-3 & (204)^{3}\end{array}$ $\begin{array}{lllllll}5563.03 & A & 4 \mathrm{np} & 5.45 & 7.67 & 3-3 & (247)\end{array}$

$\begin{array}{llllll}44875.49 \quad P & V & 5.44 & 7.98 & 3-3 & c^{3} D-r^{3} D^{\circ}\end{array}$ $\begin{array}{llllll}4842.50 & \text { A } & 3 \mathrm{n} & 5.45 & 8.00 & 3-2 \\ 4813.00 & \text { A } & 19 & 5.45 & 8.02 & 3-1\end{array}$

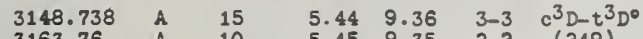
$\begin{array}{llrrrrr}3163.76 & \text { A } & 10 & 5.45 & 9.35 & 2-3 & (349) \\ 3172.330 & \text { A } & 7 & 5.46 & 9.35 & 1-1 & \end{array}$

$\begin{array}{llllll}3154.80 & A & 1 & 5.46 & 9.35 & 1-1 \\ & & 5.45 & 9.36 & 2-3\end{array}$

$3071.77 \quad A \quad$ an $\quad 5.44 \quad 9.46 \quad 3-4 \quad c^{3} D-u^{3} F^{0}$

$5016.60 \quad A \quad 4 \quad 5.51 \quad 7.97 \quad 2-3 \quad c^{1} D-x^{1} D^{\circ}$

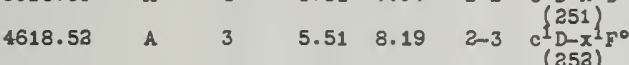

Stronge日t Unclase1 191ed Lines of VII

$\begin{array}{lll}5791.47 & \mathbf{A} & 15 \\ 3611.58 & \text { A } & 10 \mathrm{D} \\ 3301.66 & \mathrm{~A} & 10 \\ 3306.16 & \mathrm{~A} & 15 \mathrm{~N} \\ 3201.58 & \mathrm{~A} & 15 \mathrm{~N} \\ 3195.50 & \mathrm{~A} & 15 \mathrm{~N}\end{array}$

Cr I I P 6.74 Anel A Ligt B Karch 1941

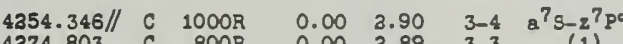
$\begin{array}{lllllll}4374.803 & C & 800 R & 0.00 & 2.89 & 3-3 & \text { (1) } \\ 4389.721 & C & 700 R & 0.00 & 3.88 & 3-3 & \end{array}$

$\begin{array}{llllllll}3733.033 \quad C & 50 & 0.00 & 3.31 & 3-3 & 8^{7} S_{-2} 5 \mathrm{po}\end{array}$ $\begin{array}{lllllll}3732.033 & C & 50 & 0.00 & 3.31 & 3-3 & { }^{7} \mathrm{~S}-z^{2} \mathrm{~S} \\ 3730.807 & \mathrm{C} & 40 & 0.00 & 3.31 & 3-3 & (3)\end{array}$

$\begin{array}{lllllll}3615.645 & C & 30 & 0.00 & 3.41 & 3-4 & a^{7} S-z^{7} D^{\circ}\end{array}$ $\begin{array}{llllllll}3635.381 & C & 10 & 0.00 & 3.39 & 3-3 & \text { (3) }\end{array}$

3578.687 C $1000 \mathrm{R} \quad 0.00 \quad 3.45$ 3-4 $\mathrm{a}^{7} \mathrm{~s}-\mathrm{y}^{7} \mathrm{po}$ $\begin{array}{llllll}3593.488 & C & 900 R & 0.00 & 3.43 & 3-3 \\ 3605.333 & C & 750 R & 0.00 & 3.42 & 3-2\end{array}$

$\begin{array}{lllllll}3351.966 & \mathrm{C} & 13 & 0.00 & 3.68 & 3-3 & \mathrm{a}^{7} \mathrm{~s}-\mathrm{y}^{5} \mathrm{po} \\ 3379.171 & \mathrm{C} & 15 & 0.00 & 3.65 & 3-2 & (5)\end{array}$

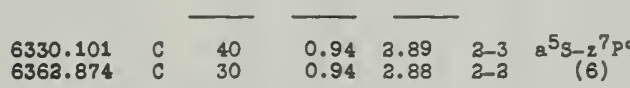

$\begin{array}{lllllll}5308.438 & C & 500 R & 0.94 & 3.31 & 2-3 & { }^{5} S_{-2} 5^{50}\end{array}$ $\begin{array}{lllllll}5208.039 & \mathrm{C} & 300 \mathrm{R} & 0.94 & 3.31 & 2-2 & (7) \\ 5304.518 & \mathrm{C} & 300 R & 0.94 & 3.31 & 3-1 & \end{array}$

$\begin{array}{llllllll}5031.903 & C & 25 & 0.94 & 3.39 & 2-3 & 8^{5} S_{-2} 7^{70}\end{array}$ $\begin{array}{lllllll}5051.900 & c & 40 & 0.94 & 3.38 & 2-3 & (8) \\ 5073.920 & c & 60 & 0.94 & 3.37 & 2-1 & \end{array}$

$\begin{array}{lllllllll}4943.495 & C & 200 & 0.94 & 3.43 & 2-3 & \mathrm{~B}^{5} S-y^{7} \mathrm{P} & \end{array}$ $\begin{array}{lllllll}4964.928 & C & 100 & 0.94 & 3.42 & 2-3 & \text { (9) }\end{array}$

$\begin{array}{llllllll}4496.863 & C & 100 & 0.94 & 3.68 & 2-3 & 8^{5} s_{-y} y_{p o}\end{array}$ $\begin{array}{lllllll}4545.956 & C & 50 & 0.94 & 3.65 & 2-3 & (10) \\ 4580.056 & C & 40 & 0.94 & 3.63 & 2-1 & \end{array}$

$\begin{array}{llllllll}3833.49 & B & 4 & 0.94 & 4.16 & 3-3 & { }_{8}^{5} S_{-2} 5 D^{\circ}\end{array}$ $\begin{array}{llcllll}3852.58 & \text { B } & 15 & 0.94 & 4.16 & 2-3 & 85 \\ 3870.367 & C & 25 n & 0.94 & 4.14 & 3-29 & \text { (11) }\end{array}$

$\begin{array}{lllllll}3758.72 \quad B \quad & 4 & 0.94 & 4.23 & 2-3 & 8^{5} 8-z^{3} p o\end{array}$ $\begin{array}{llllllll}319 a .13 & \text { B } & 5 & 0.94 & 4.80 & 3-3 & 8^{5} & (12) z^{3} D^{\circ}\end{array}$ $\begin{array}{lllllll}3310.62 & \text { B } & \text { a } & 0.94 & 4.78 & 2-3 & \text { (13) }\end{array}$

$\begin{array}{lllllll}2988.649 & C & 35 r & 0.94 & 5.07 & 2-3 & a^{5} 8-x^{5} p o\end{array}$ $\begin{array}{lllllll}3994.069 & C & 18 & 0.94 & 5.06 & 2-3 & (14)\end{array}$

$\begin{array}{lllllll}3984.83 & B & 8 & 0.94 & 5.07 & 3-3 & a^{5} s-y^{5} F^{0}\end{array}$ $\begin{array}{llllllll}2995.10 & \text { B } & 35 & 0.94 & 5.06 & 3-3 & \text { (15) }\end{array}$

$\begin{array}{llrllll}8580.96 & B & 8 & 1.03 & 3.90 & 4-4 & a^{5} D_{-2} p^{0}\end{array}$ $6501.213 \quad D \quad 15 \quad 0.98 \quad 2.88 \quad 3-2$ $\begin{array}{llllll}6830.015 & \mathrm{C} & 25 & 1.03 & 2.89 & 4-3 \\ 6573.900 & \mathrm{C} & 15 & 1.00 & 3.88 & 3-2\end{array}$

$\begin{array}{llllllll}5798.46 & \mathrm{~B} & 25 & 1.03 & 3.15 & 4-5 & \mathrm{a}^{5} \mathrm{D}-z^{7} F^{\circ} \\ 5790.59 & \mathrm{P} & & 1.00 & 3.13 & 3-4 & (17)\end{array}$

.5785 .88

5409.791
5345.807

5298.688
5348.319

5398.389
5364.152
5300.749

5364.152
5300.749

5385.732
5347.584
15
500
500
100
350
100
300
75
100
150 $\begin{array}{lll}0.98 & 3.11 & 2-3 \\ 0.10 & 1-3\end{array}$

$1.03 \quad 3.31 \quad 4-3 \quad a^{5} \mathrm{D}_{2} 5 \mathrm{po}$

$\begin{array}{lll}1.00 & 3.31 & 3-2 \\ 0.98 & 3.31 & 3-1\end{array}$

$\begin{array}{lll}0.98 & 3.31 & 3-1 \\ 1.00 & 3.31 & 3-3\end{array}$

0.98 3. 3112

0.98 3.31 $1-1$

$\begin{array}{lll}0.98 & 3.31 & 2-3 \\ 0.98 & 3.31 & 1-2\end{array}$ $\frac{a^{5}-z^{5}}{(18)}$

1

$-1$ 


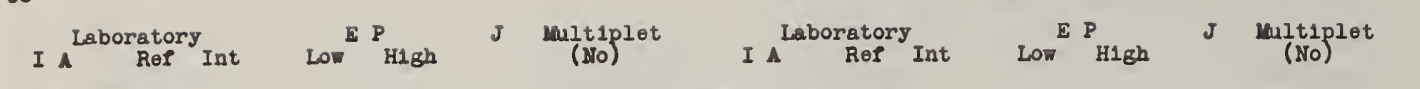

Cr I continued $\begin{array}{lll}5123.121 & \mathrm{C} & 30 \\ 5112.490 & \mathrm{C} & 25\end{array}$

5168.63

5151.83

5138.71
5183.41

5093.41

5068.290
5048.752

5123.465
51205
5

5038.87

5025.54
5019.20

$4646.174 \quad c \quad 100$

4651.28

4616.137

4626.188

4591.394

4613.373

4351.770
4344.507

4344.507
4339.450

4337.566
4339.718

4384.977

4351.051

4391.753

3919.159

3908.755

3903.164

3941.490

3931.023

3916.243

3883.293

3885.218
3894.035

3831.032

3853.218

3806.55

3789.723
3833.533

3366.634

3363.35
3359.60

3244.115
3245.485

3347.274

3333.234

3340.951

3053.880
3039.780

3029.164

(3034.190

18.496

3013.713

3031.558

3017.569
3014.915

3015.194

3037.044

3030.245

3020.673
3025

3049.883

3031.353

2986.473

3985.849

3986.137

3900.890

3991.886

2971.112

2980.791

9290.44
9447.00

9574.25

974.53
$1.03 \quad 3.44$

$1.00 \quad 3.41$

$1.03 \quad 3.41$

$1.00 \quad 3.39$

$0.98 \quad 3.38$

$1.03 \quad 3.45$

$1.00 \quad 3.43$

$1.03 \quad 3.43$

$1.00 \quad 3.45$

$\begin{array}{ll}0.98 & 3.43 \\ 0.96 & 3.42\end{array}$

$1.03 \quad 3.68$

$0.98 \quad 3.63$

$\begin{array}{ll}1.00 & 3.68 \\ 0.98 & 3.65\end{array}$

$0.98 \quad 3.68$

$\begin{array}{ll}0.96 & 3.65 \\ 0.96 & 3.63\end{array}$

$\begin{array}{ll}1.03 & 3.86 \\ 1.00 & 3.84\end{array}$

$0.98 \quad 3.82$

0.963 .80

$\begin{array}{ll}1.03 & 3.84 \\ 1.00 & 3.82\end{array}$

$0.98 \quad 3.81$

$\begin{array}{ll}0.96 & 3.80 \\ 1.03 & 3.82\end{array}$

$\begin{array}{ll}1.00 & 3.81 \\ 0.98 & 3.80\end{array}$

$1.03 \quad 4.17$

1.004 .16

$0.96 \quad 4.13$

$1.03 \quad 4.16$

$\begin{array}{lll}1.00 & 4.14 \\ 0.98 & 4.13\end{array}$

$0.96 \quad 4.12$

$\begin{array}{ll}1.00 & 4.17\end{array}$

$0.96 \quad 4.14$

$1.00 \quad 4.23 \quad 3-3 \quad a^{5} D-z^{3} p^{0}$

$\begin{array}{ll}0.98 & 4.18 \\ 0.96 & 4.17\end{array}$

$0.96 \quad 4.18 \quad 1-1$

$\begin{array}{lll}0.96 & 4.22 & 1-2 \\ 0.96 & 4.18 & 0-1\end{array}$

$1.03 \quad 4.80 \quad 4-3 \quad a^{5} D-z^{3} D^{\circ}$

$\begin{array}{ll}1.00 & 4.78 \\ 0.98 & 4.76\end{array}$

$\begin{array}{ll}1.90 & 4.70\end{array}$

$\begin{array}{ll}0.98 & 4.78 \\ 0.96 & 4.76\end{array}$

$\begin{array}{ll}0.96 & 4.76 \\ 0.98 & 4.80\end{array}$

$1-1$

$\begin{array}{lll}0.96 & 4.78 & 1-3 \\ 0.96 & 4.76 & 0-1\end{array}$

$\begin{array}{ll}1.03 & 5.07 \\ 1.00 & 5.06\end{array}$

$\begin{array}{ll}0.98 & 5.05 \\ 1.00 & 5.07\end{array}$

$0.98 \quad 5.06$

$\begin{array}{ll}0.96 & 5.05 \\ 0.98 & 5.07\end{array}$

$\begin{array}{ll}0.96 & 5.06 \\ 0.96 & 5.05\end{array}$

$\begin{array}{ll}1.03 & 5.11 \\ 1.00 & 5.09\end{array}$

$\begin{array}{lll}1.00 & 5.09 & 3 \\ 0.98 & 5.07 & 2\end{array}$

$\begin{array}{lll}0.96 & 5.06 & 1-2 \\ 0.96 & 5.05 & 0-1\end{array}$

$\begin{array}{lll}1.03 & 5.09 & 4\end{array}$

$\begin{array}{lll}1.00 & 5.07 & 3-3 \\ 0.98 & 5.06 & 2-2\end{array}$

$\begin{array}{lll}0.96 & 5.05 & 1-1 \\ 1.03 & 5.07 & 4-3\end{array}$

$\begin{array}{lll}1.00 & 5.06 & 3-2 \\ 0.98 & 5.05 & 2-1\end{array}$

$1.03 \quad 5.16$

$0.98 \quad 5.11$

0.965 .10

$1.00 \quad 5.11$

$0.98 \quad 5.10$

$\begin{array}{ll}0.96 & 5.09 \\ 1.00 & 5.16\end{array}$

$\begin{array}{lll}0.96 & 5.11 & 1-2 \\ 0.96 & 5.10 & 0-1\end{array}$

$+0.965 .10$

$2.53 \quad 3.86 \quad 6-5 \quad a^{5} a-z^{5} w^{0}$

$\begin{array}{ll}3.53 & 3.81 \\ 3.53 & 3.80\end{array}$

$\frac{a^{5}-y^{7} p^{p o}}{(20)}$

$a^{5} D_{-2}^{5} F^{\circ}$

$\frac{5}{(37)} \frac{5}{50}$
$0.96 \quad 3.63$

$0.98 \quad 4.28 \quad 2-2$

$\begin{array}{lll}1.00 & 5.16 & 3-4 \\ 0.98 & 5.13 & 2-3\end{array}$

$\begin{array}{llll}3.53 & 3.84 & 5-4 & (29) \\ 3.53 & 3.82 & 4-3 & \end{array}$
Cr I continued

$3.53 \quad$ Cr I continued

$\begin{array}{lc}4-5 & a^{5} p-z^{7} D^{0}+ \\ 3-4 & (19) \\ 2-3 & (19) \\ 4-4 & \\ 3-3 & \\ 2-3 & \\ 3-3 & \\ 3-3 & \end{array}$

9394.17 a $\quad 35$

$\begin{array}{lll}3.53 & 3.84 \\ 3.53 & 3.82 \\ 3.53 & 3.81\end{array}$

3.53
3.53
3.81

$\begin{array}{ll}2.53 & 3.86 \\ 2.53 & 3.84\end{array}$

$\begin{array}{lll}4872.03 & \text { B } & 18 \\ 4885.776 & \text { C } & 75\end{array}$

$3.53 \quad 5.07 \quad 4-3 \quad a^{5}\left(-x^{5} p_{0}\right.$

$+$

3768.08

4789.354

4866. 1000 i

$\begin{array}{llll}2.53 & 5.11 & 6-5 & a^{5}(-1)^{5} F^{\circ} \\ 2(31) & 0\end{array}$

$\begin{array}{r}3768.08 \\ \hline 778.08\end{array}$

4888.530

4903.239
-4790.337
+4929.376

4839.376
4861.305

4887.73
-4790.337

4828.66
4860.37

4571.676

4601.021

4621.893
4637.182

4648.126

.4631 .963

4637.773
4648.888

4631.00
-4637.773

4637.772
4649.461

4536.466

4530.755
-4535.731

4535.731
4540.502

4544.619

4535.721
4541.071

454.071
4545.335
4537.339

4527.339
4530.688

4535.146

m4466. 13

4466.13
+4518.63
4561.30

4126.521

4153.816
-4163.625
-491.671

4191.371
4303.590

4153.067

4163.625
4191.750

- 4153.816

4163.16
4190.66

4037.294
4043.346

4046.760

.4033 .95

-4037.294

4046.19

4033.95
4036.80

4037.103

4026.166
4025.012
.4036 .166

- 4035.44

3963.690
3969.748
.3976 .665

3976.665
3983.907

3991.123

$\begin{array}{r}3969.061 \\ -3976.665 \\ \hline\end{array}$

- 3984.338

3976.01
-3984.338

- 3860.13

3817.844

.3816 .173

3832.10

3818.481
-3816.173

3821.583
-3818.481

3818.481
3819.97

$$
\begin{array}{cccc}
100 d 9 & 3.53 & 5.09 & 5-4 \\
75 & 2.53 & 5.07 & 4-3 \\
40 & 3.53 & 5.06 & 3-3 \\
70 & 3.53 & 5.05 & 2-1 \\
30 & 3.53 & 5.11 & 5-5 \\
100 d 9 & 3.53 & 5.09 & 4-4 \\
35 & 3.53 & 5.07 & 3-3 \\
25 & 3.53 & 5.06 & 2-3 \\
20 & 2.53 & 5.11 & 4-5 \\
8 & 3.53 & 5.09 & 3-4 \\
8 & 3.53
\end{array}
$$

$\begin{array}{lll}2.53 & 5.11 & 4-5 \\ 3.53 & 5.09 & 3-4 \\ 3.53 & 5.07 & 2-3\end{array}$

$\begin{array}{lllc}3.53 & 5.23 & 6-7 & a^{5} a_{2} 5_{H^{\circ}} \\ 3.53 & 5.23 & 5-6 & (33) \\ 3.53 & 5.20 & 4-5 & (32\end{array}$

$3.53 \quad 5.19 \quad 3-4$

$\begin{array}{ll}3.53 & 5.19 \\ 2.53 & 5.32\end{array}$

$\begin{array}{ll}3.53 & 5.32 \\ 3.53 & 5.20\end{array}$

$\begin{array}{ll}2.53 & 5.19 \\ 3.53 & 5.19\end{array}$

$\begin{array}{ll}3.53 & 5.30 \\ 3.53 & 5.30 \\ 3.53 & 5.19\end{array}$

$\begin{array}{llll}3.53 & 5.26 & 6-6 & a^{5} a-z^{5} a^{\circ} \\ 3.53 & 5.26 & 5-5 & (33)\end{array}$

$\begin{array}{lll}2.53 & 5.25 & 4-4 \\ 2.53 & 5.25 & 3-3\end{array}$

$3.53 \quad 5.35 \quad 2-2$

$\begin{array}{lll}3.53 & 5.36 & 6-5 \\ 3.53 & 5.35 & 5-4\end{array}$

$\begin{array}{lll}3.53 & 5.25 & 3-2 \\ 3.53 & 5.26 & 5-6 \\ 3\end{array}$

$\begin{array}{lll}3.53 & 5.36 & 4-5 \\ 3.53 & 5.25 & 3-4 \\ 3.53 & 5.35 & 3-3\end{array}$

3.53 $5.30 \quad 5-4 \quad a^{5}\left(-x^{5} D^{0} \uparrow\right.$

$\begin{array}{lll}2.53 & 5.30 & 5-4 \\ 3.53 & 5.36 & 4-3 \\ 3.53 & 5.34\end{array}$

$\begin{array}{llll}3.53 & 5.52 & 6-6 & 2^{5} \mathrm{a}-y^{5} G^{\bullet} \\ 3.53 & 5.50 & 5-5 & (35)\end{array}$

$3.53 \quad 5.50$

$\begin{array}{lll}3.53 & 5.48 & 3-3\end{array}$

$3.53 \quad 5.47 \quad 2-3$

$\begin{array}{lll}3.53 & 5.50 & 6-5 \\ 3.53 & 5.50 & 5-1\end{array}$

$3.53 \quad 5.48 \quad 4-3$

$\begin{array}{lll}3.53 & 5.47 & 3-3 \\ 3 & -5\end{array}$

$\begin{array}{lll}3.53 & 5.53 & 5-6 \\ 3.53 & 5.50 & 4-5 \\ 3.53 & 5.50 & 3-4\end{array}$

$\begin{array}{lll}3.53 & 5.50 & 3-4 \\ 3.53 & 5.48 & 3-3\end{array}$

$\begin{array}{llll}3.53 & 5.59 & 6-5 & a^{5} a-x_{50}^{5} \\ 3.53 & 5.59 & 5-4 & (36)\end{array}$

$3.53 \quad 5.59 \quad 4-3$

$\begin{array}{lll}3.53 & 5.58 & 3-2 \\ 3 & 53 & 5.58\end{array}$

$\begin{array}{lll}3.53 & 5.58 & 3-1 \\ 3.53 & 5.59 & 5-5\end{array}$

$\begin{array}{lll}3.53 & 5.59 & 5-5 \\ 3.53 & 5.59 & 4-4\end{array}$

$3.53 \quad 5.59 \quad 3-3$

$\begin{array}{lll}3.53 & 5.58 & 2-2 \\ 3.53 & 5.59 & 4\end{array}$

$\begin{array}{lll}3.53 & 5.59 & 4 \\ 3.53 & 5.59 & 3\end{array}$

$3.53 \quad 5.60$

$\begin{array}{ll}3.53 & 5.60 \\ 3.53 & 5.60\end{array}$

$\begin{array}{ll}3.53 & 5.60 \\ 3.53 & 5.60 \\ 3.53 & 5.60\end{array}$

$5-6$
$4-5$
$3-4$
$5-5$
$4-4$
$5-4$

$3.53 \quad 5.65 \quad 6-7 \quad a^{5} a-y^{5} H^{\circ}$

3768.63
.3768 .08

3743.884

3743.578
-3748.998
3757.663

3757.662
3768.340

$\begin{array}{r}3743.968 \\ -3748.998 \\ \hline\end{array}$

3758.044
3768.734

3768.734
3744.490

3743.578
3748.614

3748.614
3757.174

3685.548
-3686.803

3686.803
3687.258

3686.18
-3686.803

- 3686.18

3679.070

3688.11
-3694.12

3694.12
3687.545
.3694 .12

3693.56

- 3656.361

3663.206

3666.642
3668.039

3656.361
3663.840

3666.19

3655.92
3662.39

3639.803

3641.830

3653.912 $\begin{array}{llr}9444.36 & \text { A } & 5 \\ 9568.58 & A & 4\end{array}$

$\begin{array}{lll}3.53 & 3.82 & 3-4 \\ 3.3 & \end{array}$

4033.363

- 3984.338

$2-3$
$6-6$
$5-5$
$4-4$
$3-3$
$6-5$
$5-4$
$4-3$

$\begin{array}{lll}2.53 & 5.35 & 4-3 \\ 3.53 & 5.35 & 3-3\end{array}$

I I Laboratory Ref Int Low ${ }_{\text {High }}$

J
$($ Bo) 
I A Reboratory Int

Cr I continu

$\begin{array}{ll}3435.819 & C \\ 3433.31 & B \\ 3431.69 & C \\ 3435.819 & C \\ 3431.995 & D \\ 3431.384 & C \\ 3435.488 & C \\ 3431.59 & C \\ 3363.313 & C \\ 3367.53 & C \\ 3379.8358 & C \\ 3384.65 & B \\ 3388.71 & B \\ 3363.70 & B \\ 3387.53 & C \\ 3379.564 & C \\ 3384.34 & B \\ 3363.70 & B \\ 3061.653 & C \\ 3067.33 & \text { P } \\ 3071.69 & P \\ 3074.47 & B \\ 3076.58 & B\end{array}$

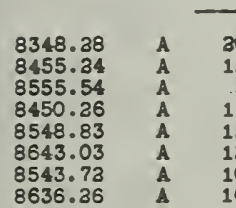

8636.36
8707.43

8396.90

8397.04

8303.19

5335.831 C 50

5337.75
5330.238

5306.15

5334.541
5341.458

5333.676

5308.07

5337.10

5013.316

5067.714

5113.130
5065.910

5110.751

5144.673
5108.93

5143.363
5161.765

4745.308

4806.35
485.34

4804.6

4855.146
4891.97

483.52
489.73

4888.73
4009.87

1697.063
.4898 .615

4898.615
4700.608

459.34

475.30
473.783

487.46
460.769

477.03

4295.757

4341.48

4383.853
4340.130

4381.112
4297.050

4343.163

4108.400

4120.613

4137.643

$\$ 136.099$
4109.584

4133.163

1066.838
1077.088

1077.089
4081.737

1080.331

3093.845

3979.798

3973.688

3871.355

3993.968
3981.333

3980.763
LoT $_{\text {HIgh }}$ J MuItiplet

I A Laboratory Ref Int

Lo: ${ }^{E P}$ High

J untiplet

$\begin{array}{cccc}6 \mathrm{~d} & 3.53 & 6.13 & 5-4 \\ 8 & 3.53 & 6.13 & 4-3 \\ 4 & 3.53 & 6.13 & 3- \\ 6 \mathrm{~d} & 2.53 & 6.13 & 4- \\ 7 & 2.53 & 6.13 & 3- \\ 10 & 3.53 & 6.13 & 2- \\ 3 & 3.53 & 6.13 & 3- \\ 3 & 3.53 & 6.13 & 2-3\end{array}$

$\begin{array}{llllc}30 & 3.53 & 6.20 & 6-5 & a^{5} a-t^{5} F^{\circ} \\ 15 . & 3.53 & 6.30 & 5-4 & (54)\end{array}$

$\begin{array}{lll}3.53 & 6.19 & 4-3\end{array}$

$\begin{array}{ll}3.53 & 6.18 \\ 3.53 & 6.17\end{array}$

$\begin{array}{ll}3.53 & 6.30 \\ 3.53 & 6.30\end{array}$

$\begin{array}{ll}3.53 & 6.20 \\ 3.53 & 6.19 \\ 3.53 & 6.18\end{array}$

$2-1$
$5-5$
$4-4$
$3-3$

$\begin{array}{lll}3.53 & 6.20 & 4-2\end{array}$

$\begin{array}{llll}3.53 & 6.56 & 6-5 & a^{5}\left(-8^{5} F^{\circ}\right. \\ 3.53 & 6.56 & 5-4 & (55) .\end{array}$

$\begin{array}{lll}3.53 & 6.56 & 5-4 \\ 3.53 & 6.55 & 4-3\end{array}$

\begin{tabular}{lll}
3.53 & 6.55 & $3-3$ \\
3.53 & 6.54 & $3-1$ \\
\hline
\end{tabular}

3.70 $4.17 \quad 3-4 \quad a^{5} P_{-2}^{5} D^{0}$

3.70 4.16

$\begin{array}{ll}3.70 & 4.14 \\ 3.70 & 4.16\end{array}$

3.70 4.14

$\begin{array}{ll}3.70 & 4.13 \\ 3.70 & 4.14 \\ 2.70 & 4.13 \\ 3.70 & 4.13\end{array}$

$3.70 \quad 4.18 \quad 3-1 \quad a^{5} p-z^{3} p^{0}$

3. $70 \quad 4.17$

$3.70 \quad 4.18 \quad 1-1$

$(57)$

$\begin{array}{llll}3.70 & 5.06 & 3-3 & a^{5} p-y^{5} F^{\circ} \\ 3.70 & 5.06 & 2-3 & (58)\end{array}$

$3.70 \quad 5.06$

$\begin{array}{cc}1-3 & \\ 3-3 & a^{5} p-x^{5} p \\ 3-3 & (59)\end{array}$

$\begin{array}{ll}3.70 & 5.07 \\ 3.70 & 5.06\end{array}$

$\begin{array}{ll}3.70 & 5.06 \\ 3.70 & 5.05\end{array}$

3.705 .06

$\begin{array}{ll}3.70 & 5.05 \\ 3.70 & 5.07\end{array}$

$2.70 \quad 5.06$

3.70 5.16

$\begin{array}{cc}3-4 & 2^{5} p-5^{5} D^{0} \\ 2-3 & (60)\end{array}$

$\begin{array}{lll}3.70 & 5.13 & 3-3 \\ 3.70 & 5.11 & 1-3 \\ 3.70 & 5.13 & 3-3\end{array}$

3.70 5.13

$\begin{array}{lll}3.70 & 5.11 & 2-2 \\ 3.70 & 5.10 & 1-1\end{array}$

$\begin{array}{lll}3.70 & 5.11 & 3-3 \\ 3.70 & 5.10 & 3-1\end{array}$

$3.70 \quad 5.30 \quad 3-4 \quad a^{5} P-x^{5} D^{\circ}$

$\begin{array}{ll}3.70 & 5.30 \\ 3.70 & 5.36 \\ 3.70 & 5.34\end{array}$

$3.70 \quad 5.36$

$3.70 \quad 5.3$

3.705 .34

$\begin{array}{ll}3.70 & 5.33 \\ 3.70 & 5.31\end{array}$

$\begin{array}{llll}3.70 & 5.33 & 3-3 & a^{5} P-2^{5} 8^{\circ}\end{array}$

$\begin{array}{ll}3.70 & 5.33 \\ 3.70 & 5.33\end{array}$

$$
\begin{aligned}
& 3-3 \\
& 1-3 \\
& 3-3
\end{aligned}
$$

$(61)$

$\begin{array}{ll}3.70 & 5.46 \\ 3.70 & 5.45\end{array}$

$3.70 \quad 5.45$

$\begin{array}{ll}3.70 & 5.45 \\ 3.70 & 5.46\end{array}$

$3.70 \quad 5.45$

$\begin{array}{ll}3.70 & 5.57 \\ 3.70 & 5.54\end{array}$

$\begin{array}{ll}3.70 & 5.54 \\ 3.70 & 5.51\end{array}$

$3.70 \quad 5.54 \quad 3-$

$3.7005 .51 \quad 2-1$

$\begin{array}{lll}3.70 & 5.54 & 1-3\end{array}$

3. $70 \quad 5.70$

$3.70 \quad 5.69$

$3-3$

$\begin{array}{ll}3.70 & 5.69 \\ 3.70 & 5.68 \\ 3.70 & 5.70\end{array}$

$\begin{array}{lll}3.70 & 5.70 & 3-3 \\ 2.70 & 5.68 & 1-3\end{array}$

$\begin{array}{cccc}3.70 & 5.73 & 3-4 & a^{5} P-W^{5} D^{\circ} \\ 3.70 & 5.73 & 3-3 & (88)\end{array}$

$\begin{array}{lll}3.70 & 5.73 & 3-3 \\ 3.70 & 5.73 & 1-3 \\ 3.70 & 5.73 & 3-3\end{array}$

$\begin{array}{lll}3.70 & 5.73 & 2-2 \\ 3.70 & 5.73 & 1-1\end{array}$

$\begin{array}{cccc}3.70 & 5.79 & 3-3 & a^{5} p-u^{5} p^{0} \\ 3.70 & 5.80 & 3-3 & (67)\end{array}$

$\begin{array}{lll}3.70 & 5.80 & 3-2 \\ 3.70 & 5.60 & 1-1 \\ 3.70 & 5.80 & 3-1\end{array}$

$3.70 \quad 5.80$

$\begin{array}{ll}3.70 & 5.80 \\ 3.70 & 5.79\end{array}$

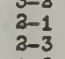

$\begin{array}{cccc}3.70 & 5.81 & 2-1 & { }^{5} 8-z^{3} g^{\circ} \\ 3.70 & 5.81 & 1-1 & (68)\end{array}$

3815.433 C 30

$\begin{array}{cccc}3.70 & 5.93 & 3-4 & a^{5} p-u^{5} F^{\circ} \\ 3.70 & 5.93 & 2-3 & (70)\end{array}$

$3.70 \quad 5.91 \quad 3-1$

3548.731

1
1
4
12
10
12

$\begin{array}{lll}2.70 & 6.15 & 2-3 \\ 3.70 & 6.13 & 1-3\end{array}$

$\begin{array}{lll}3.70 & 6.15 & 3-2 \\ 3.70 & 6.15 & 1-\end{array}$

$3.97 \quad 6.33 \quad 2-1$

$\begin{array}{lll}3.90 & 6.33 & 1-0 \\ 3.90 & 6.33 & 1-3\end{array}$

$3.97 \quad 6.61 \quad 3-3$
Cr I continued

$\begin{array}{lll}3841.377 & C & 50 \\ 3850.043 & C & 50\end{array}$ 3855.571
3848.983

3854.330

3857.631
3853.176

3856.381
3855.386

3819.564

3836.435

3834.735

3833.71

3840.70

$\begin{array}{lll}3786.33 & 3 & 8 \\ 3793.43 & B & 5\end{array}$

$\begin{array}{lll}3755.81 & \text { B } & ? \\ 3756.83 & \text { B } & 3\end{array}$

CrI cont1nued

Cr I continued

$\begin{array}{llll}3.70 & 5.98 & 3-3 & a^{5} p-x^{3} p^{\circ} 4 \\ 3.70 & 5.98 & 2-3 & (73)\end{array}$

$\begin{array}{llll}2.70 & 5.91 & 3-4 & 8^{5} P-r^{5} \\ 3.70 & 5.90 & 0\end{array}$

7462.37

$\begin{array}{lll}3.70 & 5.90 & 1-3 \\ 3.70 & 5.90 & 3-3\end{array}$

$\begin{array}{lll}3.70 & 5.90 & 3-2\end{array}$

$\begin{array}{lll}3.70 & 5.90 & 1-1 \\ 3.70 & 5.90 & 3-3\end{array}$

$\begin{array}{lll}3.70 & 5.90 & 2-1 \\ 3.70 & 5.90 & 1-0\end{array}$

$\begin{array}{lll}3.70 & 5.93 & 1-2\end{array}$

$\begin{array}{lll}3.70 & 5.93 & 3-3 \\ 2.70 & 5.93 & 3-2\end{array}$

$\begin{array}{lll}3.70 & 5.91 & 1-1 \\ 3.70 & 5.93 & 3-3\end{array}$

$3.70 \quad 5.93 \quad 3-4 \quad a^{5} p-u^{\prime} 5_{-4} \cdot 4$

$\begin{array}{llll}3.70 & 5.96 & 3-3 & \text { (71) } \\ 3.70 & 5.95 & 1-3 & \end{array}$

3736.85
-3574.039

3. 706.01

$\begin{array}{ll}1-1 & a^{5} p-x^{3} D^{\circ} \\ 3-3 & a^{5} p-t^{5} p^{\circ}\end{array}$

3603.574

3601.666
3603.745

3574.935
-3603.745

3573.748

3573.643
3574.805

3481.303

3473.613
3471.49

3471.49
3473.764

3470.401
3470.529

3470.73

3307.755

3312.06
-3315.19

3196.37

3301.97
$\mathbf{m} 301.34$

$\mathrm{m} 3301.34$
3304.55

9900.87

9636.30
10197.05

9753.84

9363.06
9313.55

4619.551

4501.788

4501.113
4498.730
4433.175

4537.339
4434.075

4424.075
.4363 .95

4491.858
4377.519

4377.519
4331.238

4387.380

\begin{tabular}{ccc}
.436 .383 & $C$ & 10 \\
4190.16 & B & 13 \\
\hline
\end{tabular}

4118.45

3886.94

3843.64

3819.57
3748.18
3710.60

3710.60
-3676.33

$-3604.54$

3681.691

3599.395
3559.781

3450.00

3453.84

3198.113

3188.011

3159.59

3316.70
3179.283

3339.14
12
18
12

$\begin{array}{lllc}3.70 & 6.15 & 3-3 & a^{5} p_{-t} p^{\circ} \\ 3.70 & 6.12 & 3-3 & (74)\end{array}$

$\begin{array}{lll}3.70 & 6.13 & 1-1 \\ 3.70 & 6.13 & 3-2\end{array}$

$\begin{array}{lll}3.70 & 6.13 & 3-3 \\ 3.70 & 6.13 & 3-1 \\ 2.70 & 6.15 & 2-3\end{array}$

$\begin{array}{lllc}3.70 & 6.15 & 3-3 & a^{5} \mathrm{p}-\mathrm{y}^{5} \mathrm{~S}^{\circ} \\ 3.70 & 6.15 & 3-3 & (75)\end{array}$

$\begin{array}{llll}3.70 & 6.17 \quad 3-1 \quad a^{5} p_{-t} 5 \xi\end{array}$

$\begin{array}{llll}3.70 & 6.34 & 3-4 & a^{5}(76){ }^{5} D^{\circ} \\ 2.70 & 6.35 & 2-3 & (77)\end{array}$

3.70 6.35

$\begin{array}{lll}3.70 & 6.35 & 3-3\end{array}$

$\begin{array}{lll}3.70 & 6.35 & 1-1\end{array}$

$3.70 \quad 6.43 \quad 3-4 \quad a^{5} P-t^{5} D^{0}+$

$\begin{array}{llll}3.70 & 6.56 & 3-4 & a^{5} \mathrm{P}-8^{5} \mathrm{p}^{\circ}\end{array}$

$\begin{array}{lll}3.70 & 6.55 & 3-3 \\ 3.70 & 6.55 & 3-3\end{array}$

$\begin{array}{lllc}3.97 & 4.33 & 2-3 & a^{3} P-z^{3} P^{\circ} \\ 3.90 & 4.18 & 1-1 & (80)\end{array}$

$3.974 .18 \quad 3-1$

$\begin{array}{lll}3.90 & 4.17 & 1-0 \\ 3.90 & 4.32 & 1-3\end{array}$

$3.97 \quad 5.65 \quad 2-3 \quad a^{3} \mathrm{P}-\mathrm{r}^{3} \mathrm{p}^{\circ}$

$\begin{array}{llll}3.97 & 5.65 & 2-3 & a^{3}-\left(-Y^{3} P\right. \\ 3.90 & 5.64 & 1-1 & (81) \\ 3.97 & 5.64 & 3-1 & \end{array}$

$\begin{array}{lll}3.97 & 5.64 & 3-1 \\ 3.90 & 5.64 & 1-0\end{array}$

$\begin{array}{lll}3.90 & 5.65 & 1=3 \\ 3.86 & 5.64 & 0-1\end{array}$

$\begin{array}{lllc}3.97 & 5.70 & 3-3 & a^{3} p-y^{3} D^{\circ} \\ 3.90 & 5.69 & 1-3 & (83)\end{array}$

$\begin{array}{lll}3.90 & 5.69 & 1-2 \\ 3.88 & 5.69 & 0\end{array}$

$\begin{array}{cccc}3.97 & 5.73 & 2-3 & a^{3} P-\pi^{5} D^{\circ} \\ 3.90 & 5.73 & 1-3 & (83)\end{array}$

$\begin{array}{lll}3.90 & 5.73 & 1- \\ 3.86 & 5.73 & 0\end{array}$

$3.97 \quad 5.79 \quad 3-3 \quad a^{3} p-u^{5} p^{0}$

$\begin{array}{lll}3.90 & 5.80 & 1- \\ 3.86 & 5.80 & 0\end{array}$

$3.90 \quad 5.90 \quad 1-3 \quad a^{3} P-r^{5} D^{0}$

$3.97 \quad 6.15 \quad 2-3 \quad a^{3} P-t^{5} P$

$\begin{array}{llll}3.97 & 6.31 & 2-3 & a^{3} \mathrm{P}^{(87)} \mathrm{m}^{\circ}+ \\ 3.90 & 6.30 & 1-3 & (88)\end{array}$

$\begin{array}{lll}3.90 & 6.30 & 1- \\ 3.68 & 6.19 & 0\end{array}$

$3.97 \quad 6.33 \quad 3-3 \quad a^{3} p-\pi^{3} p^{0}$

$\begin{array}{llll}3.90 & 6.33 & 1-1 & (89)\end{array}$

$\begin{array}{lll}3.86 & 6.33 & 0-1\end{array}$

$\begin{array}{llll}3.97 & 6.55 & 2-3 & a^{3} P-a^{5} \\ 3\end{array}$

$\begin{array}{llll}2.97 & 6.55 & 2-3 & a^{3} P-151 \\ 3.97 & 6.55 & 2-2 & (90) \\ 3.90 & 6.54 & 1-1 & \end{array}$

$3.97 \quad 6.83 \quad 3-3 \quad \Omega^{3} p-r^{3} y^{0}$

$\begin{array}{cccc}3.97 & 6.85 & 2-3 & { }^{3} P-7^{3} \\ 3.90 & 6.61 & 1-3 & (92)\end{array}$

$3.66 \quad 6.78$

$\begin{array}{lll}3.90 & 6.78 & 1-1 \\ 3.97 & 6.76 & 3-1\end{array}$ $\begin{array}{ll}3.70 & 6.43 \\ 3.70 & 6.43 \\ 3.70 & 6.43\end{array}$

$3.87 \quad 6.19 \quad 2-3 \quad 3^{3}(86)$

\subsection{4 \\ 5338.339
5397.360
5275.171 \\ 5275.171
5339.12
5397.976 \\ 5275.689
5339.719 \\ 5298.44
5276.03 \\ 4361.354
4373.910 \\ 4373.910
4384.735
4393.565 \\ 4299.718
4305.453 \\ .4320 .592 \\ 4129.21 \\ 41097.65
4128.96
4 \\ 4111.36
309.96 \\ 4130.47}

$\begin{array}{lll}4514.531 & C & 40 \\ 4491.678 & \text { C } & 30 \\ 4475.345 & \text { C } & 50\end{array}$

4111.67
4098.18

8234.09
8361.95

4737.153

4693.949
4666.315

4666.31
4735.95

4692.97
4695.153

4695.153
4667.181

4543.74
.4518 .63

4495.04

4442.268

4410.967

4387.496

4375.333
4363.134

4374.15

4346.833
4325.075

4255.503

$-4240.705$ 
40

I A Laboratory Int Low High J Multiplet

\section{Cr I continued}

3163.756 C 15 $\begin{array}{llr}3155.149 & \text { C } & 13 \\ 3148.445 & \text { C } & 10 \\ 3169.58 & \text { B } & 3\end{array}$ $\begin{array}{ll}3152.881 & D \\ 3141.891 & D\end{array}$ $\square 3039.74 \quad P \quad C r$ \begin{tabular}{rrr}
$\$ 3039.74$ & P & $C r$ \\
3031.486 & $C$ & 4 \\
3024.681 & $C$ & 3 \\
\hline
\end{tabular} $\begin{array}{lll}10486.24 & \text { A } & 2 \\ 10672.17 & \text { A } & 1 \\ 10816.91 & \text { A } & \\ 10647.66 & \text { A } & 1 \\ 10831.63 & \text { A } & 1 \\ 10957.19 & \text { A } & 1 \\ 11044.64 & \text { A } & \\ 10509.96 & \text { A } & 10 \\ 10667.53 & \text { A } & 1 \\ 10801.37 & \text { A } & 1 \\ 10939.90 & \text { A } & 1 \\ 5713.778 & \text { C } & 100 \\ 5788.389 & \text { C } & 2 \\ 5843.24 & \text { B } & 25 \\ 5781.195 & \text { C } & 40 \\ 5844.606 & \text { C } & 40 \\ 5884.453 & \text { C } & 25 \\ 5902.183 & \text { C } & 25 \\ 5719.821 & \text { C } & 40 \\ 5787.036 & \text { C } & 20 \\ 5838.66 & \text { B } & 25 \\ 5876.55 & \text { B } & 25 \\ & & \end{array}$

$\begin{array}{rrr}-5556.19 & \text { B } & 10 \\ 5574.41 & \text { B } & 13\end{array}$

$\begin{array}{rrr}5512.69 & \text { B } & 10 \\ .5556 .19 & \text { B } & 10\end{array}$

$\begin{array}{lll}5004.38 & B & 35 \pi \\ 5028.00 & B & 15 \pi\end{array}$

4981.30

4998.5
4980.30

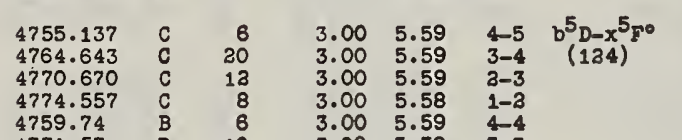

$\begin{array}{llrrrr}477.57 & \mathrm{~B} & 10 & 3.00 & 5.59 & 3-3 \\ 4777.57 & \mathrm{~B} & 7 & 3.00 & 5.56 & 33 \\ 475.6 & & 3 & 3.00 & 5.59 & 1-1\end{array}$

$3.00 \quad 5.56 \quad 3-2$

$\begin{array}{lll}3.00 & 5.58 & 1-1 \\ 3.00 & 5.59 & 4-3 \\ 3.00 & 5.58 & 3-3\end{array}$

$3.00 \quad 5.70$

$3.00 \quad 5.69$

3.00
3.00 5.69

$3.00 \quad 5.69$

$\begin{array}{ll}3.00 & 5.69 \\ 3.00 & 5.70\end{array}$

$\begin{array}{lllll}3.00 & 5.73 & 4-4 & b^{5}-w^{5} D^{0}+\end{array}$

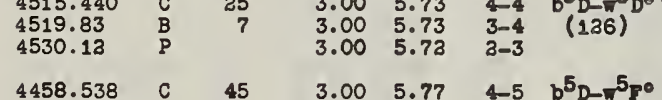

$\begin{array}{lllllll}4458.538 & c & 45 & 3.00 & 5.77 & 4-5 & b^{5} D-w^{5} \mathbf{r}^{0} \\ 4459.738 & c & 25 & 3.00 & 5.77 & 3-4 & (127) \\ 4465.357 & c & 35 & 3.00 & 5.76 & 3-3 & (127\end{array}$

4464.907

4462.774
4455.45

$\begin{array}{ll}3.00 & 5.77 \\ 3.00 & 5.76 \\ 3 & \end{array}$

3.000
3.00
3.76

$\begin{array}{lll}3.00 & 5.76 \\ 3.00 & 5.76\end{array}$

$\begin{array}{ll}3.00 & 5.60 \\ 3.00 & 5.79\end{array}$

$\begin{array}{lll}3.00 & 5.79 \\ 3.00 & 5.78 \\ 3.00 & 5.78 & -1\end{array}$

$\begin{array}{lll}3.00 & 5.78 \\ 3.00 & 5.79 \\ 3.00 & 5.78 \\ 3 & 5.78\end{array}$

$3.00 \quad 5.79$

3.00
3.00
3

$3.00 \quad 5.80$

3.005 .80

$\begin{array}{lll}3.00 & 5.60 \\ 3.00 & 5.60 & 1\end{array}$

$3.00 \quad 5.83$

3.00
3.00
3.63

$\begin{array}{lll}3.00 & 5.81 \\ 3.00 & 5.83 & 1 \\ 3 & & \end{array}$

$\begin{array}{lll}3.00 & \mathrm{E} .83 \\ 3.00 & 5.81\end{array}$

$\begin{array}{llll}3.00 & 5.91 & 4-4 & 0 \\ 3.00 & 5.90 & 3-3 & b^{5}-1 p^{\circ 0} \\ (131)\end{array}$

4252.243
4256.620

4248.344
4257.368

4259.15

4242.83

$b^{5} p-z^{3} a^{\circ}$

$4-3 \quad b^{5} p-u^{5} p_{0}$

$(130)$

$\begin{array}{lll}3.00 & 5.90 & 3-2 \\ 3.00 & 5.90 & 4-3\end{array}$

$\begin{array}{lll}3.00 & 5.90 & 3-2 \\ 3.00 & 5.90 & 3-1\end{array}$

$\begin{array}{lll}3.00 & 5.90 & 2-1 \\ 3.00 & 5.91 & 3-4\end{array}$

REVISED MULTIPLET TABLE

I A Laboratory Int Low ${ }^{E} P_{\text {High }}$ J Multiplet

I A Ref Int

$\operatorname{Low}^{E P}{ }_{\text {HIgh }}$

I Kultiplet

3.00
3.00
3.79
CrI oontinued

$\begin{array}{lllllll}4317.626 & C & 30 & 3.00 & 5.92 & 4-5 & b^{5} D-u^{5} F^{0} \\ 4316.365 & C & 15 & 3.00 & 5.93 & 3-4 & (133)\end{array}$

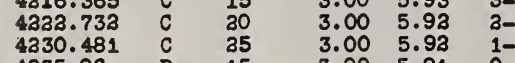

4335.96

\begin{tabular}{lllll}
4323.47 & $B$ & 15 & 3.00 & 5.91 \\
\hline & 3 & 3.00 & 5
\end{tabular}

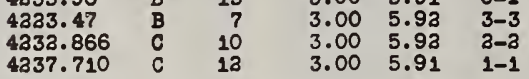

$\begin{array}{llrllll}4311.349 & C & 15 & 3.00 & 5.93 & 3-4 & b^{5} p_{-1} 5_{F 0} \\ 4177.17 & B & 3 & 3.00 & 5.95 & 0-1 & (23)^{2}\end{array}$

$\begin{array}{lrrrrrr}4177.17 & \mathrm{~B} & 3 & 3.00 & 5.95 & 0-1 & (133) \\ 4207.51 & \mathrm{~B} & 3 & 3.00 & 5.93 & 4-4 & \\ 3945.966 & C & 10 & 3.00 & 6.13 & 4-5 & b^{5} p_{-15} \pi^{\circ}\end{array}$

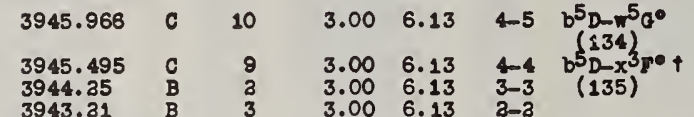

$\begin{array}{lrrrrrrl}3944.25 & \mathrm{~B} & 2 & 3 & 3.00 & 6.13 & 3-3 & (135) \\ 394.21 & \mathrm{~B} & 3 & 3.00 & 6.13 & 3-3 & \\ 3915.843 & \mathrm{C} & 40 & 3.00 & 6.15 & 4-3 & b^{5} \mathrm{p}-t^{5} \mathrm{po}\end{array}$

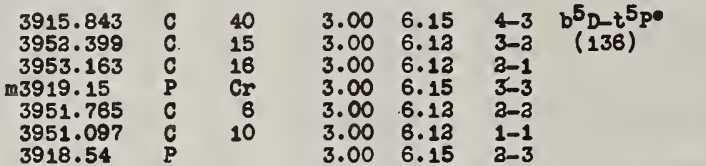

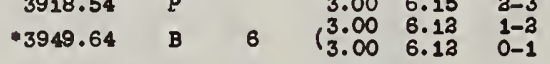

$\begin{array}{llrrrrrr}3917.598 & C & 15 & 3.00 & 6.15 & 3-3 & b^{5} D-y^{5} g^{\circ} \\ 3916.980 & C & 10 & 3.00 & 6.15 & 2-3 & (137) \\ 3914.96 & B & 4 & 3.00 & 6.15 & 1-3 & (137)\end{array}$

3649.365

3658.90

$\begin{array}{llllllll}570 & B & 157 & 3.00 & 6.20 & 4-5 & b^{5} p-t^{5} \mathrm{pr}^{0} \\ (136)\end{array}$

3679.323

3633.680

$\mathrm{m}_{33755.65}$

$\begin{array}{ll}3.00 & 6.16 \\ 3.00 & 6.17 \\ 3.00 & 6.17\end{array}$

$\begin{array}{lll}3.00 & 6.20 \\ 3.00 & 6.19\end{array}$

$\begin{array}{lll}3.00 & 6.18 \\ 3.00 & 6.17 \\ 3 & 1.00 & 6\end{array}$

3.006 .16

$\begin{array}{lll}3.00 & 6.24 \\ 3 & 000 & 6.25 \\ 3.00 & 6.25 & \end{array}$

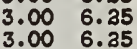

$\begin{array}{ll}3.00 & 6.35 \\ 3 & 600 \\ 3.00 & 6.25 \\ 3\end{array}$

$\begin{array}{ll}3.00 & 6.35 \\ 3.00 & 6.25 \\ 3.00 & \end{array}$

3.006 .35

$\begin{array}{lll}3.00 & 6.34 \\ 3.00 & 6.25 \\ 3 & & \end{array}$

3.006 .35

$3.00 \quad 6.35$

$\begin{array}{ll}3.00 & 6.43 \\ 3.00 & 8.42 \\ 3.00 & 6.42 \\ 3.00 & 6.43\end{array}$

$\begin{array}{lll}3.00 & 8.43 \\ 3.00 & 8.42\end{array}$

3.008 .56

$\begin{array}{lll}3.00 & 6.56 \\ 3.00 & 6.55 \\ 3 & & \\ 3.00 & 6.55 & 1\end{array}$

3.006 .55

3.006 .58

3.006 .55

\begin{tabular}{lll}
3.00 & 6.55 \\
3.00 & 6.54 \\
\hline
\end{tabular}

3475.36
3476.77
3480.28

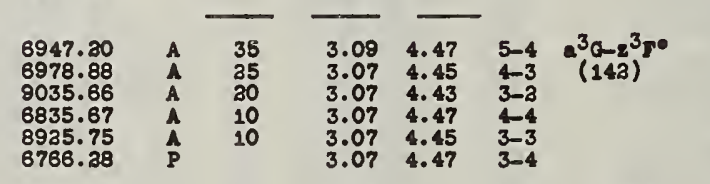

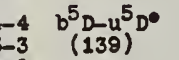

$3-3$
$1-1$
$4-3$

$3-1$
$3=0$
$3=4$

$3-4$
$2-3$
$1-2$
$0-1$

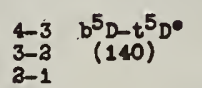

3460.430

347. 87

3477.161

3479.14

4933. 287

4887.013

4920.945

4885.957

4838.857

4814.285

4847.177

4825.51
$482 a .08$

4756.11

4737.350
4730.711

4734.416

4733.03
4710.34

$-4696.615$

4668.847
4684.805

(4856.189

4858.169
4646.495

4614.15

4581.063
4574.45
4576.78

.4555 .09

-4543.681
4541.513

L-5 $b^{5} p-5^{5} F^{0}+$

$1+3$

$\begin{array}{llll}3.09 & 5.60 & 5-8 & a^{3}\left(a-23^{3} H^{\circ}\right. \\ 3.07 & 5.60 & 45 & (143)\end{array}$

$\begin{array}{lll}3.09 & 5.60 \\ 3 & 5.60\end{array}$

$3.07 \quad 5.60$

$3.09 \quad 5.64$

$\begin{array}{ll}3.07 & 5.63 \\ 3.09 & 5.64 \\ 3.07 & 5.63\end{array}$

$5-5$

$5-6$
$4-5$$a^{3}\left(14-5^{5} \mathrm{H}^{\circ}\right.$

$3.07 \quad 5.63$

$3.09 \quad 5.89$

$5-5$
$4-4$
$3-3$

$\begin{array}{ll}3.07 & 5.68 \\ 3.07 & 5.87\end{array}$

$\begin{array}{ll}3.07 & 5.87 \\ 3.07 & 5.69 \\ 3.07 & 5.68\end{array}$

$\begin{array}{cc}5-4 \\ 4-3 & a^{3} a-y^{3} p^{0} \\ (145) & \end{array}$

$\begin{array}{llll}3.07 & 5.89 & 3-4\end{array}$

$3.07 \quad 5.70 \quad 4-3 \quad a^{3} a-y^{3} p^{\circ}$

$\begin{array}{llll}3.07 & 5.89 & 3-3 & (146) \\ 3.07 & 5.70 & 3-3 & \end{array}$

$3.07 \quad 5.73 \quad 4-3 \quad 2^{3} a-w^{5} p^{\circ}+$

$3.09 \quad 5.77 \quad 5-5 \quad a^{3} a-\pi^{5}, 0$

$\begin{array}{lll}3.07 & 5.77 & 4-4 \\ 3.07 & 5.76 & 3-3 \\ 3.07 & 5.78 & 3-2\end{array}$
Cr I continued

\begin{tabular}{|c|c|c|c|c|c|}
\hline $\begin{array}{l}4540.719 \\
4511.903 \\
4500.395 \\
4513.31\end{array}$ & $\begin{array}{l}c \\
C \\
C \\
B\end{array}$ & $\begin{array}{l}50 \\
50 \\
40 \\
8+B\end{array}$ & $\begin{array}{l}3.09 \\
3.07 \\
3.07 \\
3.07\end{array}$ & $\begin{array}{l}5.81 \\
5.81 \\
5.81 \\
5.81\end{array}$ & $\begin{array}{l}5-5 \\
4-4 \\
3-3 \\
4-3\end{array}$ \\
\hline $\begin{array}{l}5.32 \\
4.68\end{array}$ & ${ }_{B}^{B}$ & $\frac{1}{6}$ & $\begin{array}{l}3.09 \\
3.07\end{array}$ & $\begin{array}{l}5.63 \\
5.82\end{array}$ & $\begin{array}{l}5-5 \\
3-3\end{array}$ \\
\hline $\begin{array}{l}5.129 \\
5.26\end{array}$ & ${ }_{B}^{C}$ & $\begin{array}{l}13 \\
18\end{array}$ & $\begin{array}{l}3.09 \\
3.07\end{array}$ & $\begin{array}{l}5.88 \\
5.87\end{array}$ & $\begin{array}{l}5-6 \\
4-5\end{array}$ \\
\hline $\begin{array}{l}4.87 \\
.833\end{array}$ & $\stackrel{\text { B }}{B}$ & $\begin{array}{r}10 \\
4\end{array}$ & $\begin{array}{l}3.09 \\
3.07\end{array}$ & $\begin{array}{l}5.93 \\
5.90\end{array}$ & $\begin{array}{l}5-6 \\
4-5\end{array}$ \\
\hline $\begin{array}{l}1.061 \\
9.951 \\
3.38\end{array}$ & $\begin{array}{l}C \\
C \\
B\end{array}$ & $\begin{array}{r}15 \\
12 \\
8\end{array}$ & $\begin{array}{l}3.09 \\
3.07 \\
3.07\end{array}$ & $\begin{array}{l}5.96 \\
5.96 \\
5.96\end{array}$ & $\begin{array}{l}5-8 \\
4-5 \\
3-4\end{array}$ \\
\hline $\begin{array}{l}9.756 \\
4.514 \\
1.573 \\
9.81 \\
4.895 \\
3.179\end{array}$ & $\begin{array}{l}c \\
c \\
c \\
p \\
c \\
c\end{array}$ & $\begin{array}{l}15 \\
18 \\
25 \\
12 \\
10\end{array}$ & $\begin{array}{l}3.09 \\
3.07 \\
3.07 \\
3.09 \\
3.07 \\
3.07\end{array}$ & $\begin{array}{l}6.03 \\
5.99 \\
5.99 \\
5.99 \\
6.02 \\
5.99\end{array}$ & $\begin{array}{l}5-5 \\
4-4 \\
3-3 \\
5-4 \\
4-5 \\
3-4\end{array}$ \\
\hline & & & 3.09 & & \\
\hline
\end{tabular}

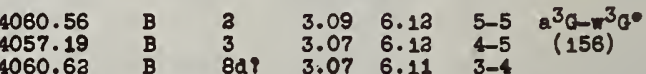

$\begin{array}{lllllll}3566.23 & \mathrm{~B} & 4 & 3.09 & 6.53 & 5-6 & \mathrm{a}^{3} \mathrm{G}-\mathrm{x}^{3} \mathrm{H}^{\circ} \\ 3571.97 & \mathrm{P} & & 3.07 & 6.53 & 4-5 & (157)\end{array}$

$\begin{array}{llllllllll}3553.968 & \mathrm{C} & 5 & 3.07 & 8.54 & 3-4 & & & & 3.07\end{array}$

$\begin{array}{llllllll}3443.56 & \text { B } & 1 & 3.09 & 6.68 & 5-5 & a^{3}\left(6-r^{3} C^{\circ}\right. \\ 3425.96 & \text { B } & 4 & 3.07 & 6.68 & 4-5 & (158)\end{array}$

$\begin{array}{lllllll}\cdot 3349.3238 & 0 & 6 & 3.09 & 6.78 & 5-6 & 2^{3}\left(\mathrm{G}-\mathrm{m}^{3} \mathrm{H}^{\circ}+\right.\end{array}$

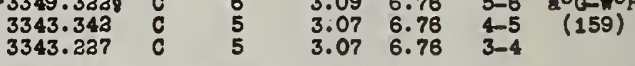

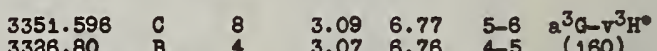

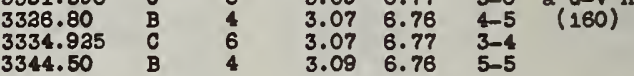

$\begin{array}{lllllll}3313.721 & \mathrm{C} & 3 & 3.09 & 6.63 & 5-4 & \mathrm{a}^{3} \mathrm{a}-\mathrm{m}^{3} \mathrm{ro}^{\circ}\end{array}$

$\begin{array}{lllllll}3298.316 & \mathrm{~B} & 4 & 3.07 & 6.80 & 4-3 & (161) \\ 3303.86 & \mathrm{~B} & 6 & 3.07 & 6.62 & 4-4 & \\ 3 & & 3.07 & 6.60 & 3 & \\ 3 & & & \end{array}$

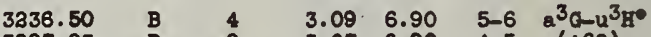

$\begin{array}{llllllll}3237.23 & B & 3 & 3.09 & 6.90 & 5-6 & a^{3}\left(\alpha-4 u^{3}\right) \\ 3 & 3.07 & 6.90 & 4-5 & (162)\end{array}$

$\begin{array}{llllllllll}3119.246 & C & 5 & 3.09 & 7.05 & 5-5 & \mathrm{a}^{3} \mathrm{G}^{3} \mathrm{a}^{\circ}\end{array}$

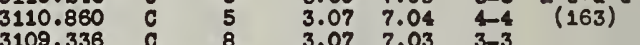

3115.51
3105.57

3104.70

- 3080.83

3058.17
3047.455
305.200

9059.7

9148.45

9363.97

4954.811 C 60

\begin{tabular}{lll}
4936.334 & $C$ & 150 \\
4953.714 & $D$ & 35 \\
\hline
\end{tabular}

$3.07 \quad 7.03$

$3.07 \quad 7.05$

$3.07 \quad 7.04 \quad 3-5$

$3.09 \quad 7.12 \quad 5-5 \quad 2^{3} a-t^{3} a^{0}+$

$\begin{array}{llll}3.07 & 7.11 & 4-4 & 8 \\ & (164)\end{array}$

$\begin{array}{lll}3.07 & 7.12 & 4-5 \\ 3.07 & 7.11 & 3-4\end{array}$

$\begin{array}{lll}4880.08 & B & 35 \\ 4874.651 & 0 & 20\end{array}$

4787.74

4784.70

4754.743
4801.030

4792.513
474.00

$\begin{array}{ll}3.07 & 5.84 \\ 3 & .07 \\ 5.63\end{array}$

4761.242

4759.907
4729.723

4717.88

4706.10
4680.870

4701.93

4640.55

4593.25

4634.59

4599.00
4635.30

4584.095
4588.138
4563.657

4601.15
4596.441

4569.530

4554.830 


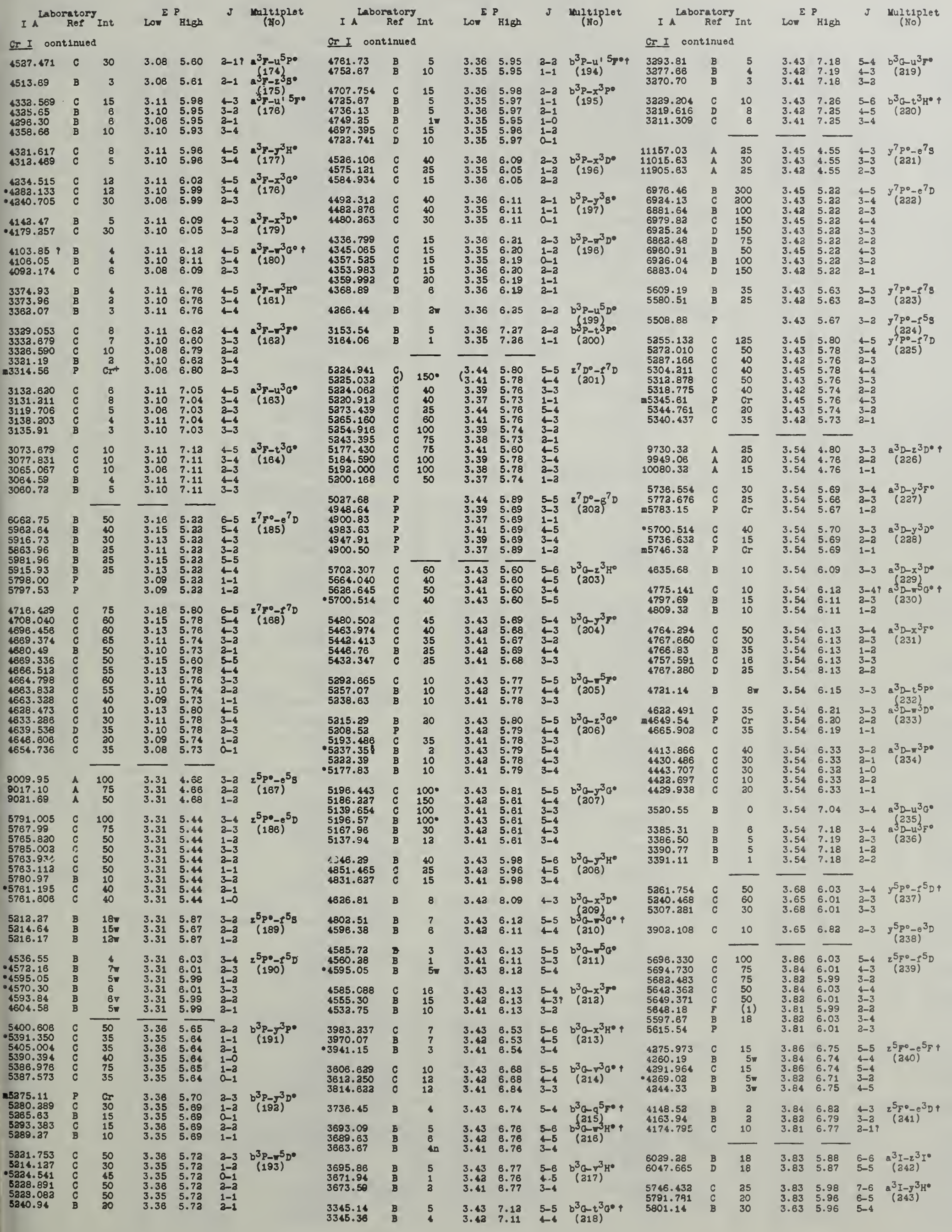


43

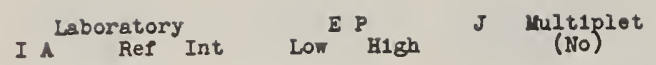

Cr I continued

\begin{tabular}{|c|c|c|c|c|c|c|}
\hline $\begin{array}{c}-4621.963 \\
4625.925 \\
4642.011 \\
4625.65 \\
4640.67\end{array}$ & $\begin{array}{l}\text { C } \\
\text { C } \\
\text { C } \\
\text { B } \\
B\end{array}$ & $\begin{array}{l}45^{\circ} \\
20 \\
10 \\
1 \\
1\end{array}$ & $\begin{array}{l}3.83 \\
3.83 \\
3.83 \\
3.83 \\
3.83\end{array}$ & $\begin{array}{l}6.50 \\
6.50 \\
6.49 \\
6.50 \\
6.49\end{array}$ & $\begin{array}{l}7-7 \\
6-6 \\
5-5 \\
7-6 \\
6-5\end{array}$ & $\begin{array}{l}a^{3} I-y^{3} I^{0} \\
(244)^{\circ}\end{array}$ \\
\hline 4614.523 & C & 12 & 3.83 & 6.51 & $5-4$ & $a^{3} I-1$ \\
\hline $\begin{array}{c}4571.83 \\
4578.334 \\
4563.245 \\
+4572.16 \\
4579.59 \\
4573.38\end{array}$ & $\begin{array}{l}\text { B } \\
\text { C } \\
\text { C } \\
B \\
B \\
B\end{array}$ & $\begin{array}{l}12 \\
12 \\
15 \\
7 \bar{w} \\
3 \\
1 \operatorname{gn} ?\end{array}$ & $\begin{array}{l}3.83 \\
3.83 \\
3.83 \\
3.83 \\
3.83 \\
3.83\end{array}$ & $\begin{array}{l}6.53 \\
6.53 \\
6.54 \\
6.53 \\
6.53 \\
6.53\end{array}$ & $\begin{array}{l}7-6 \\
6-5 \\
5-4 \\
6-6 \\
5-5 \\
5-6\end{array}$ & $\begin{array}{c}a^{3} \frac{I-x^{3} H^{0}}{(246)} \\
(246)\end{array}$ \\
\hline $\begin{array}{l}4263.141 \\
4280.405 \\
4297.738\end{array}$ & $\begin{array}{l}c \\
c \\
c\end{array}$ & $\begin{array}{l}35 \\
25 \\
30\end{array}$ & $\begin{array}{l}3.83 \\
3.83 \\
3.83\end{array}$ & $\begin{array}{l}6.73 \\
6.73 \\
6.71\end{array}$ & $\begin{array}{l}7-8 \\
6-7 \\
5-6\end{array}$ & $\begin{array}{l}a^{3} I-z^{3} K^{0}+ \\
(247)\end{array}$ \\
\hline $\begin{array}{c}4193.662 \\
4209.368 \\
4221.572 \\
4193.89 \\
4194.951\end{array}$ & $\begin{array}{l}\mathrm{C} \\
\mathrm{C} \\
\mathrm{C} \\
\mathrm{B}\end{array}$ & $\begin{array}{r}40 \\
20 \\
25 \\
3 \\
20\end{array}$ & $\begin{array}{l}3.83 \\
3.83 \\
3.83 \\
3.83 \\
3.83\end{array}$ & $\begin{array}{l}6.78 \\
6.76 \\
6.76 \\
6.78 \\
6.78\end{array}$ & $\begin{array}{l}7-6 \\
6-5 \\
5-4 \\
6-6 \\
5-6\end{array}$ & $\begin{array}{c}a^{3} I-m^{3} H^{\circ} \\
(248)\end{array}$ \\
\hline $\begin{array}{r}4197.234 \\
-4186.359 \\
4208.357 \\
4197.47 \\
4198.525\end{array}$ & $\begin{array}{l}\text { C } \\
C \\
C \\
B\end{array}$ & $\begin{array}{r}20 \\
15 \\
15 \\
2 \\
35\end{array}$ & $\begin{array}{l}3.83 \\
3.83 \\
3.83 \\
3.83 \\
3.83\end{array}$ & $\begin{array}{l}6.77 \\
6.78 \\
6.77 \\
6.77 \\
6.77\end{array}$ & $\begin{array}{l}7-6 \\
6-5 \\
5-4 \\
6-6 \\
5-6\end{array}$ & $\frac{a^{3} I-v^{3} H^{\circ}}{(249)}$ \\
\hline $\begin{array}{l}4179.05 \\
.4179 .257\end{array}$ & $\begin{array}{l}\mathrm{B} \\
\mathrm{C}\end{array}$ & 30 & $\begin{array}{l}3.83 \\
3.83\end{array}$ & $\begin{array}{l}6.79 \\
6.79\end{array}$ & $\begin{array}{l}7-7 \\
6-7\end{array}$ & $\begin{array}{c}a^{3} I-z^{1} I^{0} \\
(250)\end{array}$ \\
\hline $\begin{array}{l}4039.100 \\
4048.780 \\
4058.772 \\
4048.56 \\
4057.81 \\
4039.30 \\
4049.783\end{array}$ & $\begin{array}{l}C \\
C \\
C \\
P \\
B\end{array}$ & $\begin{array}{r}30 \\
20 \\
30 \\
\\
8 \\
5 \\
5\end{array}$ & $\begin{array}{l}3.83 \\
3.83 \\
3.83 \\
3.83 \\
3.83 \\
3.83 \\
3.83\end{array}$ & $\begin{array}{l}6.89 \\
6.88 \\
6.87 \\
6.88 \\
6.87 \\
6.89 \\
6.88\end{array}$ & $\begin{array}{l}7-7 \\
6-6 \\
5-5 \\
7-6 \\
6-5 \\
6-7 \\
5-6\end{array}$ & $\begin{array}{c}a^{3} I-x^{3} I^{0} \\
(251)^{\circ}\end{array}$ \\
\hline
\end{tabular}

$\begin{array}{lllllll}\mathrm{m} 3605.52 & \text { P } & \text { Cr } & 3.83 & 7.26 & 7-6 & \mathrm{a}^{3} I-t^{3} \mathrm{H}^{\circ} \\ 3608.401 & \mathrm{C} & 10 & 3.83 & 7.25 & 6-5 & (252)\end{array}$

$\begin{array}{rrrrrrr}3608.401 & C & 10 & 3.83 & 7.25 & 6-5 & \text { (252) } \\ 3612.609 & \mathrm{C} & 4 & 3.83 & 7.25 & 5-4 & \end{array}$

$\begin{array}{llllllll}3458.090 & C & 10 & 3.83 & 7.40 & 7-7 & 8^{3} I-\nabla^{3} I^{0}\end{array}$

$\begin{array}{lllllll}3467.023 & \text { C } & 12 & 3.83 & 7.39 & 6-6 & (253) \\ 3453.23 & \text { B } & 10 & 3.83 & 7.41 & 5-5 & \end{array}$

$\begin{array}{lllllll}3376.397 & \mathrm{C} & 10 & 3.83 & 7.49 & 7-7 & \mathrm{Q}^{3} I-\nabla^{3} I^{\circ}\end{array}$

$\begin{array}{lrrrrrr}3391.372 & \text { C } & 10 & 3.83 & 7.47 & 6-6 & (254) \\ 3403.59 & \text { B } & 8 & 3.83 & 7.46 & 5-5 & \end{array}$

$\begin{array}{lllllll}3316.503 & \mathrm{C} & 5 & 3.83 & 7.55 & 7-6 & \mathrm{a}^{3} \mathrm{I}-8^{3} \mathrm{H}^{\circ}\end{array}$

\begin{tabular}{lllllll}
3336.97 & $B$ & 4 & 3.83 & 7.53 & $6-5$ & $(255)$ \\
\hline
\end{tabular}

$\begin{array}{lllllll}6643.023 & \mathrm{C} & 15 & 3.83 & 5.69 & 3-4 & b^{3} p-g^{3} F^{\circ} \\ 6677.24 & \mathrm{~B} & 10 & 3.83 & 5.68 & 2-3 & (256)\end{array}$

$\begin{array}{llll}3.83 & 5.68 & 2-3 & (256) \\ 3.83 & 5.67 & 1-3 & \end{array}$

$\begin{array}{lllllll}5729.203 & c & 20 & 3.83 & 5.98 & 3-3 & b^{3} D-x^{3} p 0\end{array}$

$\begin{array}{lllllll}5371.48 & \mathrm{~B} & 50 & 3.83 & 6.13 & 3-4 & b^{3} D-x^{3} F^{\circ}\end{array}$

5362.98

4930.183

4944.59

4934.89

4146.20

4109.98

4093.06

4089.63

4131.36

4152.775
4175.227

4149.45

4171.675
4168.31

3907.778

3890.82

3510.40

3521.53
3531.44

7185.50

7188.06

7196.83
7207.85

7218.57

6529.197

6516.026

4840.2

841.5

4841.73
4833.54

4465.15

4490.56
4507.95

$\begin{array}{lll}3.83 & 6.13 & 3-3 \\ 3.83 & 6.13 & 2-0\end{array}$

$3.83 \quad 6.33 \quad 3-3 \quad b^{3} D-\pi^{3} p^{0}$

$\begin{array}{llll}3.83 & 6.33 & 3-1 & \text { (259) }\end{array}$

$\begin{array}{lll}3.83 & 6.32 & 1-0\end{array}$

$\begin{array}{lll}3.83 & 6.33 & 3-2 \\ 3.83 & 6.33 & 1-1\end{array}$

$3.83 \quad 6.80 \quad 3-4 \quad b^{3} D-v^{3} F^{0}$

$\begin{array}{llll}3.83 & 6.80 & 3-4 & b^{3} D-\nabla^{3} \\ 3.83 & 6.83 & 3-3 & (360)\end{array}$

$\begin{array}{lll}3.83 & 6.85 & 1-2 \\ 3.83 & 6.83 & 3-3\end{array}$

$\begin{array}{lll}3.83 & 6.85 & 3-2\end{array}$

$\begin{array}{llll}3.83 & 6.82 & 3-4 & b^{3} D-\pi^{3} F^{\circ} \\ 3.83 & 6.80 & 2-3 & (261)\end{array}$

$\begin{array}{lll}3.83 & 6.79 & 1-2 \\ 3.83 & 6.80 & 3-3\end{array}$

$\begin{array}{lll}3.83 & 6.79 & 3-2 \\ 3.83 & 6.79 & 3-2\end{array}$

$3.83 \quad 6.99 \quad 3-3 \quad b^{3} p-r^{3} p^{0}$

$\begin{array}{llll}3.83 & 7.00 & 3-1 & (262)\end{array}$

$3.83 \quad 7.34 \quad 3-4 \quad b^{3} D-t^{3} F^{\circ}$

$\begin{array}{llll}3.83 & 7.34 & 2-3 & (263) \\ 3.83 & 7.33 & 1-3 & \end{array}$

-

$3.88 \quad 5.59 \quad 5-5 \quad a^{5} F-x^{5} F^{\circ}$

$\begin{array}{llll}3.87 & 5.59 & 5-5 & a \\ 3.87 & 5.59 & 4-4 & (264) \\ 3.87 & 5.59 & 3-3 & \\ 3.87 & 5.58 & 2-3 & \end{array}$

$\begin{array}{lll}3.87 & 5.58 & 1-1\end{array}$

$\begin{array}{llll}3.88 & 5.77 & 5-5 & 85-\pi^{5} F \\ 3.87 & 5.77 & 4-4 & (265)\end{array}$

(1) $\quad 3.88 \quad 6.43 \quad 5-4 \quad a^{5 F-t 5} D^{\circ}$

$\begin{array}{llll}3.87 & 6.42 & 4-3 & (266)\end{array}$

$\begin{array}{lll}3.87 & 6.42 & 3-2 \\ 3.87 & 6.43 & 1-0\end{array}$

$\begin{array}{lll}3.87 & 6.43 & 4-4\end{array}$

$\begin{array}{llll}3.88 & 6.64 & 5-4 & a^{5} \\ F-8 & 5 & D^{0}+\end{array}$

REVISE D U ULTIPLET IABLE

I Aaboratory ReP Int Low $_{\text {H1gh }} \mathrm{J}$ Multiplet $_{(\text {Ho) }}$

I L Laboratory Ref Int

I P ${ }_{\text {High }}$

J (\$o)

Cr. I cont1nued

$\begin{array}{lllllll}3989.986 & C & 15 & 3.88 & 6.97 & 5-6 & a^{5} F-r^{5} G^{\circ}+\end{array}$

$\begin{array}{lllllll}4001.444 & \text { C } & 25 & 3.87 & 6.96 & 4-5 & (268) \\ .4012 .49 \text { B } & \text { B } & 30 & 3.87 & 6.95 & 3-4 & \end{array}$

4022.263

$\begin{array}{rrr}4003.921 & D & 7 \\ 4 & 7\end{array}$

$\begin{array}{rrrrrr}4014.668 & C & 10 & 3.87 & 6.95 & 4-4 \\ 4023.739 & \text { C } & 8 & 3.87 & 6.94 & 3-3\end{array}$

$\begin{array}{lrrrrrr}3716.531 & C & 10 \pi & 3.88 & 7.20 & 5-4 & 0_{T-r^{5}} D^{\circ}+ \\ 3714.39 & \mathrm{~B} & 4 & 3.87 & 7.30 & 4-4 & (369)\end{array}$

$\begin{array}{lllllll}3714.39 & \text { B } & 4 \pi & 3.87 & 7.20 & 4-4 & (269) \\ 3712.50 & \text { B } & 1 \pi & 3.87 & 7.20 & 3-4 & \end{array}$

$4481.44 \quad B \quad{ }_{18} \quad-3.96 \quad \overline{6.73} 6-7 \quad a^{1} I-z^{3} X^{0}+$

$4268.788 \quad C \quad 10 \quad 3.96 \quad 6.85 \quad 6-6 \quad a^{1}\left(\frac{1}{37} z^{1} I^{\circ}\right.$

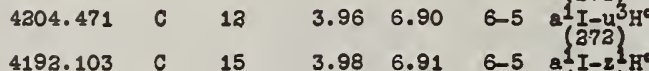

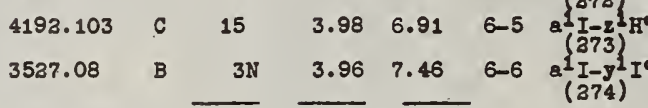

$\begin{array}{llrrrrc}-4542.621 & \mathrm{C} & 35 & 4.09 & 6.80 & 4-4 & \mathrm{~b}^{3} \mathrm{~T}-\mathrm{V}^{3} \mathrm{~F}^{\circ} \\ 4495.275 & \mathrm{C} & 12 & 4.09 & 6.83 & 4-3 & (275) \\ 4531.83 & \mathrm{~B} & 3 & 4.08 & 6.80 & 3-4 & \end{array}$

$\begin{array}{lllllll}4524.841 & C & 15 & 4.09 & 6.82 & 4-4 & b^{3} \mathrm{r}-w^{3} F^{0}+\end{array}$

$\begin{array}{lllllll}4535.731 & C & 60 & 4.08 & 6.80 & 3-3 & (376) \\ 4553.949 & C & 18 & 4.08 & 8.79 & 3-3 & \end{array}$

$\begin{array}{ccccccc}-4521.141 & C & 25 & 4.08 & 6.81 & 2-3 & b^{3} F-v^{3} D^{\circ} \\ 4561.54 & B & 10 . & 4.08 & 6.78 & 3-1 & (377)\end{array}$

$\begin{array}{lllllll}4169.838 & C & 1 & 4.09 & 7.05 & 4-5 & b^{3} F-u^{3} G^{\bullet}+\end{array}$

$\begin{array}{rrrrrrr}4170.202 & C & 15 & 4.08 & 7.04 & 3-4 & (278) \\ 4174.941 & C & 8 & 4.08 & 7.03 & 3-3 & \end{array}$

$\begin{array}{lllllll}4065.716 & C & 13 & 4.09 & 7.12 & 4-5 & b^{3} \vec{F}-t^{3} a^{\circ}\end{array}$

$\begin{array}{lllllll}4076.061 & C & 10 & 4.08 & 7.11 & 3-4 & (279) \\ 4077.677 & \mathrm{C} & 10 & 4.08 & 7.11 & 2-3 & \end{array}$

$\begin{array}{lllllll}3990.16 & \mathrm{~B} & 8 & 4.09 & 7.18 & 4-4 & b^{3} F_{-3}^{3} F^{0}+\end{array}$

$\begin{array}{lllllll}3976.30 & \text { B } & 5 & 4.09 & 7.18 & 4-4 & b^{3}-u^{3} \\ 3979.324 & \text { C } & 7 & 4.08 & 7.19 & 3-3 & (280)\end{array}$

$\begin{array}{ccccccc}3564.30 & \text { B } & 7 & 4.09 & 7.55 & 4-5 & b^{3} F-8^{3} G^{\circ} \\ 3562.48 & \text { B } & 4 & 4.08 & 7.55 & 3-4 & (281)\end{array}$

3565.55
3569.14

6
6
6
6
6
6
6

6661.076 c $50 \quad 4.17 \quad 5.03 \quad 4-4 \quad z^{5} D^{0}-F^{5} D$

$\begin{array}{lllllll}6669.257 & \mathrm{C} & 40 & 4.16 & 6.01 & 3-3 & (382)\end{array}$

$\begin{array}{llllll}6734.16 & \mathrm{~B} & 20 \mathrm{w} & 4.14 & 5.99 & 3-2 \\ & \mathrm{~B} & 30 & 4.17 & 6.01 & 4-3\end{array}$

$\begin{array}{llllll}6880.19 & B & 35 & 4.16 & 5.99 & 3-2 \\ & B & 4.14 & 5.99 & 3-1\end{array}$

$\begin{array}{llllll}6597.556 & \text { C } & 40 & 4.16 & 6.03 & 3-4 \\ 6613.17 & \text { B } & 40 & 4.14 & 6.01 & 3-3\end{array}$

$\begin{array}{lllllll}4796.169 & C & -40 w & 4.17 & 6.75 & 4-5 & 2^{5} D^{\bullet}-0^{5} \mathrm{~F}+\end{array}$

$\begin{array}{lllllll}4783.06 & B & 15 \pi & 4.16 & 6.74 & 3-4 & \text { (383) } \\ 4775.53 & \text { B } & 10 \pi & 4.14 & 6.73 & 2-3 & \end{array}$

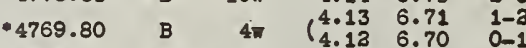

$\begin{array}{llllll}4816.41 & B & 10 \mathrm{w} & 4.17 & 6.74 & 4-4\end{array}$

$\begin{array}{llllll}4805.24 & \text { B } & 15 \% & 4.16 & 6.73 & 3-3 \\ 4796.84 & \text { B } & 12 w & 4.14 & 6.71 & 3-2\end{array}$

3566.10

5385.38

5309.47

4595.590 C 45

$\begin{array}{lll}4514.373 & \text { C } & 30 \\ 4531.141 & \text { C } & 35\end{array}$

$4.17 \quad 7.64 \quad 4-3 \quad 2^{5} D^{0}-P^{5} P$

4506.853 C 30

3747.264

4757.336 C 15

$\begin{array}{lllll}15 & 4.22 & 6.82 & 3-3 & z^{3} \mathrm{p}^{\circ}-e^{3} \mathrm{D}+\end{array}$

\begin{tabular}{llrrrr}
4743.113 & $\mathrm{C}$ & $12 \pi$ & 4.18 & 6.79 & $1-2$ \\
-4751.04 & $\mathrm{~B}$ & $5 \mathrm{~W}$ & 4.17 & 6.77 & $0-1$ \\
\hline
\end{tabular}

$8167.94 \quad \mathrm{4} \quad \overline{4.19} 5.70 \quad 3-3 \quad c^{3} D-J^{3} D^{\circ}$

$\begin{array}{lrrrrrc}4699.589 & \mathrm{C} & 25 & 4.19 & 6.82 & 3-4 & \mathrm{c}^{3} \mathrm{D}-\pi^{3} \mathrm{~F}^{0}+ \\ 4723.18 & \mathrm{~B} & 8 & 4.19 & 6.80 & 2-3 & (293) \\ 4741.089 & \mathrm{D} & 12 & 4.19 & 6.79 & 1-2 & \end{array}$

4488.051 C 30

4232.232 C 15

4000.59

8707.95

8718.70
8732.17

8788.96
8773.56

8397.58
$4.19 \quad 6.94 \quad 3-3 \quad c^{3} D-v^{5} c^{\circ}$

$4.19 \quad 7.11 \quad 2-3 \quad c^{3} p-t^{3} a^{\circ}$

\section{Cr I oontinued}

$\begin{array}{lllllll}8183.22 & \mathbf{A} & 35 & 4.37 & 5.88 & 4-5 & 0^{5} D-T^{5} r \\ 8335.89 & \mathbf{A} & 30 & 4.38 & 5.88 & 3-4 & (398)\end{array}$

8287.38

8338.83

8166.66

8390.62

8333.44

8018.04
8119.13

8185.69

8084.9

8166.68
8218.28

7948.02

7989.36
8061.37

8061.27
8128.28

8169.80

6739.78

.5373 .715
.5391 .350

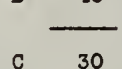

4592.54

4606.375
4609.894

4376.798

$\mathbf{4} 4371.28$
4373.656

4161.415

4165.1919
4142.193

4174.15

4043.696

4056.793
4071.000

3958.08

3979.22
3998.85

3562.29

3554.953
-3574.039

5283.750

5378.262
5387.62

4503.05

35
15
15
8

.395 .88

$\begin{array}{ll}4.40 & 5.88 \\ 4 & 40\end{array}$

4.40
4.37
4.88
4.39

$\begin{array}{lll}4.37 & 5.88 & 4-1 \\ 4.38 & 5.88 & 3-3\end{array}$

$\begin{array}{lll}4.38 & 5.88 & 3-3 \\ 4.39 & 5.88 & 3-2\end{array}$

$\begin{array}{lll}4.39 & 5.88 & 2-3 \\ 4.40 & 5.88 & 1-1\end{array}$

$4.37 \quad 5.91 \quad 4-4 \quad e^{50-1500}$

4.38
4.39

$\begin{array}{lll}4.39 & 5.90 & 2-2 \\ 4.40 & 5.90 & 1-1\end{array}$

$\begin{array}{lll}4.40 & 5.90 & 1-1 \\ 4.38 & 5.91 & 3-4\end{array}$

$\begin{array}{lll}4.39 & 5.90 & 2-3 \\ 4.40 & 5.90 & 1-2\end{array}$

$\begin{array}{llll}4.37 & 5.92 & 4-5 & c^{5} D-u^{5} F^{*}\end{array}$

$\begin{array}{llll}4.38 & 5.93 & 3-4 & (300) \\ 4.39 & 5.92 & 3-3 & \end{array}$

$\begin{array}{lll}4.39 & 5.92 & 2-3 \\ 4.40 & 5.92 & 1-2\end{array}$

$4.37 \quad 6.20 \quad 4-5 \quad c^{5} D-t^{5} y^{0}$

$4.44 \quad 6.73 \quad 6-5 \quad b^{3} H-r^{5} r^{\circ}$

$4.44 \quad 7.12 \quad 6-5 \quad b^{3} \mathrm{H}-t^{3} \mathrm{G}^{\circ}$

$\begin{array}{llll}4.43 & 7.11 & 5-4 & (303) \\ 4.43 & 7.11 & 4-3 & \end{array}$

$4.44 \quad 7.26 \quad 6-6 \quad b^{3} \mathrm{H}-t^{3} \mathrm{H}^{\bullet}+$

$\begin{array}{llll}4.43 & 7.25 & 5-5 & (304) \\ 4.43 & 7.25 & 1-4 & \end{array}$

$\begin{array}{llll}4.44 & 7.40 & 6-7 & b^{3} \mathrm{H}_{-3}^{3} \mathrm{I}^{\circ} \\ 4.43 & 7.39 & 5-6 & (305)\end{array}$

$\begin{array}{lll}4.43 & 7.41 & 4-5 \\ 4.44 & 7.39 & 6-6\end{array}$

$4.44 \quad 7.49 \quad 6-7 \quad b^{3} \mathrm{H}-\mathrm{v}^{3} \mathrm{I} \bullet$

$\begin{array}{llll}4.43 & 7.47 & 5-6 & (306) \\ 4.43 & 7.48 & 4-5 & \end{array}$

$4.44 \quad 7.55 \quad 6-6 \quad b^{3} \mathrm{H}_{-} \mathrm{s}^{3} \mathrm{H}^{\bullet}$

$\begin{array}{llll}4.43 & 7.53 & 5-51 & (307) \\ 4.43 & 7.51 & 4-4 & \end{array}$

$\begin{array}{llll}4.44 & 7.90 & 6-6 & b^{3} \mathrm{H}_{-} \mathrm{a}^{3} \mathrm{~B}^{\circ} \\ 4.43 & 7.89 & 5-5 & (308)\end{array}$

\begin{tabular}{lll}
4.43 & 7.89 & $5-5$ \\
4.43 & 7.88 & $4-4$ \\
\hline
\end{tabular}

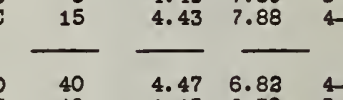

$\begin{array}{lllll}40 & 4.47 & 6.83 & 4-3 & 2^{3} y^{0}-0^{3}-\end{array}$

B 12 4.68 7.48 2-1 $0^{5} 8-x^{3} 80$

$4656.837 \quad D \quad 10 \quad 4.76 \quad 7.41 \quad 5-5 \quad 8^{1 / \mathrm{H}-\mathrm{w}^{3} \mathrm{I}^{\circ}}$

$4564.168 \quad C \quad 40 \quad 4.76 \quad 7.46 \quad 5-6 \quad \begin{aligned} & 311) \\ & (313)\end{aligned}$

$\begin{array}{lllllll}3926.649 & C & 10 & 4.76 & 7.90 & 5-6 & \begin{array}{l}1 \\ a^{1} \mathrm{~B}-q^{3} \mathrm{E} \\ (313)\end{array}\end{array}$

6135.759 C $25 \quad-4.80 \quad \overline{6.82} 3-3 \quad 2^{3} D^{\circ}-e^{3} \mathrm{D}$

$\begin{array}{lllllll}6762.41 & C & 40 & 5.26 & 7.08 & 6-6 & z^{5} G^{\circ}-0^{5} \uparrow \\ 6757.78 & \text { B } & 25 & 5.26 & 7.08 & 5-5 & (315)\end{array}$

\begin{tabular}{lllll} 
B & 15 & 5.35 & 7.08 & $5-4$ \\
B & 18 & 5.25 & 7.08 & $2-3$ \\
\hline
\end{tabular}

$\begin{array}{llllllll}7908.30 & \mathrm{~A} & 20 & & 5.60 & 7.16 & 6-5 & 8^{3} \mathrm{H}^{\circ}-0^{3} \mathrm{G} \\ 79 & \mathrm{~A} & 18 & & 5.60 & 7.16 & 5-4 & (316)\end{array}$

$\begin{array}{llllll}7910.50 & \mathrm{~A} & 18 & 5.60 & 7.16 & 5-4 \\ 7917.85 & \mathrm{~A} & 18 & 5.60 & 7.16 & 4-3\end{array}$

Strongest Unclassified Lines or Cr I

6771.74

5854.37
5796.757

5796.757
5753.693

5713.635

5681.198
5385.28

5370.356

894.949

4753.084

4614.73

4611.968
4594.403

4586.99

$4.37 \quad-79 \quad 4-3 \quad c^{5} D-u^{5} p_{0}+$

4489.471
4403.498

4323.523
4301.178

$\begin{array}{lll}4.39 & 5.80 & 2-1 \\ 4.38 & 5.79 & 3-3\end{array}$

4261.615

$4.39 \quad 5.88 \quad 2-3 \quad c^{5} \mathrm{D}-x^{5} \mathrm{H}^{\circ}$

306.899

4300.103
4138.925
3990.679

15

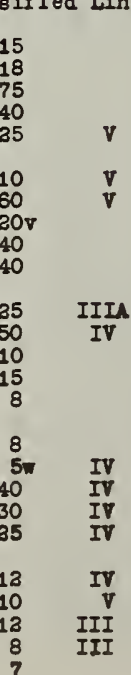




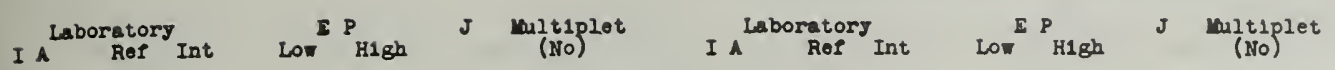
G. I continued

\begin{tabular}{|c|c|c|c|}
\hline 3911.95 & B & $(10 n)$ & (IIII \\
\hline $\begin{array}{l}3830.033 \\
3584.366 \\
3574.38\end{array}$ & $\begin{array}{l}\text { C } \\
\text { D } \\
\text { B }\end{array}$ & $\begin{array}{l}50 \mathrm{~W} \\
30 \mathrm{~W} \\
3 \mathrm{~N}\end{array}$ & $\begin{array}{r}\bar{v} \\
\bar{v} \\
\text { III }\end{array}$ \\
\hline $\begin{array}{l}3559.61 \\
3558.60 \\
3558.953 \\
3550.635 \\
3547.98\end{array}$ & $\begin{array}{l}\text { C } \\
\text { B } \\
\text { C } \\
\text { C } \\
\text { B }\end{array}$ & $\begin{array}{r}3 N \\
7 \pi \\
4 \pi \\
10 n \\
3 n\end{array}$ & $\begin{array}{c}\text { IVY } \\
\text { III } \\
\text { IV } \\
\text { II } \\
\text { III }\end{array}$ \\
\hline $\begin{array}{l}3532.888 \\
3525.44 \\
3508.81 \\
3508.09 \\
3474.379\end{array}$ & $\begin{array}{l}\text { C } \\
\text { B } \\
\text { B } \\
\text { B } \\
C\end{array}$ & $\begin{array}{c}5 \\
4 \mathrm{~N} \\
5 \mathrm{n} \\
5 \\
13\end{array}$ & $\begin{array}{l}\text { V } \\
\text { IV } \\
\text { III } \\
\text { III } \\
\text { III }\end{array}$ \\
\hline $\begin{array}{l}3411.01 \\
3409.38 \\
3408.01 \\
3407.33 \\
3376.18\end{array}$ & $\begin{array}{l}\text { B } \\
\text { B } \\
\text { B } \\
\text { B } \\
\text { E }\end{array}$ & $\begin{array}{l}8 n \\
7 n \\
4 n \\
5 n \\
3 n\end{array}$ & $\begin{array}{l}\text { IV } \\
\text { IV } \\
\text { IV } \\
\text { V }\end{array}$ \\
\hline $\begin{array}{l}3349.073 \\
3346.09 \\
3334.690 \\
3333.605 \\
3387.70\end{array}$ & $\begin{array}{l}\text { C } \\
E \\
C \\
C \\
B\end{array}$ & $\begin{array}{r}30 . \\
5 \pi \\
30 \\
15 \pi \\
315\end{array}$ & $\begin{array}{l}\text { III } \\
\text { III } \\
\text { III } \\
\text { III } \\
\text { V }\end{array}$ \\
\hline $\begin{array}{l}3354.95 \\
3180.7018 \\
3098.531 \\
3095.859 \\
3983.73 \\
3957.38\end{array}$ & $\begin{array}{l}\text { B } \\
\text { C } \\
\text { C } \\
C \\
\text { B } \\
B\end{array}$ & $\begin{array}{l}38 \\
8 \\
5 \pi \\
7 \pi \\
3 \pi \\
3 \pi\end{array}$ & $\begin{array}{r}V \\
\text { III } \\
\text { III } \\
\text { III } \\
\text { III } \\
\text { V }\end{array}$ \\
\hline
\end{tabular}

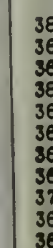

Cr II I P 18.8 Anal B Lat A April 1941

$\begin{array}{cc}-3180.73 & \text { A } \\ 3197.12 & A \\ 3309.31 & A \\ 3317.44 & A \\ 311.438 & \text { B } \\ 3196.96 & A \\ 3308.63 & A \\ .3196 .40 & A\end{array}$

3935.18
3964.64
3985.96
3964.35
3986.03
3999.00
3985.74
3999.07

3748.68 3761.90
3761.69
-3761.90

3631.49

3677.93

3877.69

3713.04

3877.88

3593.02

3585.54
3603.80

3585.31

3613.21

3813.36

3336.1
3349.68 4

851.68

675.00

$(1000.6$

3688.01

$3.69 \quad 5.83 \quad 3 \frac{1}{3}-3 \frac{1}{2} a^{4} P-26 F^{\circ}$

$\begin{array}{lll}3.69 & 5.81 & 1 \frac{3}{3}-3 \frac{1}{2} \\ 3.69 & 5.79 & \end{array}$

$\begin{array}{lll}2.69 & 5.81 & 2 \frac{1}{2}-3 \frac{3}{2} \\ 3.69 & 5.79 & 1 \frac{1}{2}-1 \frac{1}{2}\end{array}$

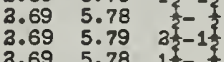

$3.695 .99 \quad 2 \frac{1}{3}-2 a^{4} P_{-2} 6 P^{0}$

$\begin{array}{llll}3.69 & 5.97 & 12-1 & (11) \\ 3.69 & 5.97 & 3 \frac{1}{3}-1 & \\ 3.89 & 5.97 & \frac{1}{2}-1 \frac{3}{2} & \end{array}$

$\begin{array}{llll}3.69 & 6.09 & 31 & -21 \\ 3.69 & 6.05 & z_{-2} p^{\circ}\end{array}$

$\begin{array}{lll}3.89 & 6.03 & \\ 3.69 & 8.05 & 3\end{array}$

$\begin{array}{lll}3.69 & 6.03 & 12 \\ 3.69 & 6.09 & 1\end{array}$

3.896 .13 3h $3 \frac{31}{4} a^{4} P-2^{6} D^{\circ}$

$\begin{array}{lll}3.69 & 6.14 & 2 \\ 3.69 & 6.13 & 1\end{array}$

$\begin{array}{lll}3.69 & 6.11 & \\ 3.89 & 6.13 & 3\end{array}$

$\begin{array}{lll}3.89 & 6.13 & 3 \\ 3.69 & 8.11 & 12\end{array}$

$\begin{array}{llll}3.69 & 6.39 & 31-3 \frac{1}{2} & a^{4} P-z^{4}+{ }^{4} \\ 3.69 & 8.38 & 1 \frac{1}{2}-2 \frac{1}{2} & (14)\end{array}$

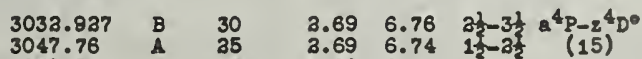

$\begin{array}{ccc}3047.76 & \mathrm{~A} & 35 \\ .3059 .531 & \mathrm{~B} & 35 \\ 3047.63 & \mathrm{~A} & 20 \\ .3059 .531 & \mathrm{~B} & 35 \\ 3067.18 & \mathrm{~A} & 30 \\ 3065.18 & \mathrm{~A} & 30\end{array}$

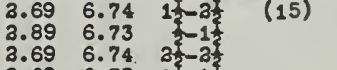

$\begin{array}{llll}3.69 & 6.74 & 25 \\ 3.69 & 6.73 & 12-1 \frac{3}{2}\end{array}$

$\begin{array}{lll}3.69 & 6.73 & 3- \\ 3.69 & 8.73 & 23-1 \frac{3}{3}\end{array}$

3059.41
$\mathbf{m} 3067.33$
Cr II continued

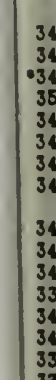

3

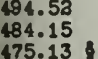
-15
511.84 667.09 462.73

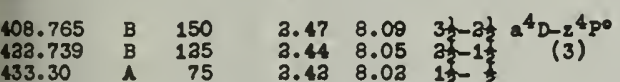
433.30 (382.883 131.20 3381.434

3353.12
.3349 .34 3334.060 3374.95 3342.51 3338.330 3358.50 3347.837

3132.058 3124.978 3118.65 3136.680 3158.10 3145.10

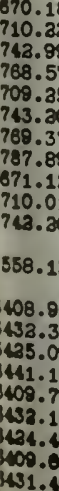

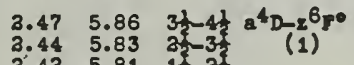
$\begin{array}{lll}3.41 & 5.79 & 13 \\ 3.13 & \end{array}$ $3.44 \quad 5.81$ a 3

$3.43 \quad 5.79 \quad 13-1$

$\begin{array}{lll}3.44 & 5.79 & 35 \\ 3.43 & 5.78 & 1 \frac{1}{2}-\frac{2}{2}\end{array}$

$3.47 \quad 6.00 \quad 3 \frac{1}{3}-3 \frac{1}{4} a^{4} 0-z^{6} p^{0}$

3.445 .99 और

$\begin{array}{lll}3.43 & 5.97 & 13-1 \\ 3.47 & 5.99 & 3\end{array}$

$3.44 \quad 5.97$ 3. -1

8.448 .00 3. 3

$\begin{array}{llll}3.43 & 5.99 & 1 & 1 \\ 3.41 & 5.97 & \frac{2}{2}-1 \frac{2}{2}\end{array}$

3.418 .03 t

$\begin{array}{lll}3.43 & 8.09 & 192 \\ 3.41 & 6.05 & \frac{1}{2}-1 \frac{1}{2}\end{array}$

$3.47 \quad 8.15$ 31 $-4 \frac{1}{3}=D_{-2}^{6} D^{\circ}$

$\begin{array}{lll}3.44 & 8.13 & 3 \\ 3.43 & 8.14 & 1 \\ 3.41 & 6.13 & \end{array}$

$\begin{array}{lll}3.41 & 6.13 & 13 \\ 3.47 & 6.13 & 3\end{array}$

$\begin{array}{lll}3.44 & 6.14 & 3 \\ 3.43 & 8.13 & 13\end{array}$

$\begin{array}{lll}3.42 & 8.13 & 13 \\ 3.41 & 8.11\end{array}$

$\begin{array}{lll}3.47 & 8.14 & 3 \\ 3.44 & 8.13 & 32 \\ 3.43 & 6.11 & 15\end{array}$

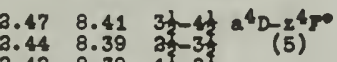

$\begin{array}{lll}3.43 & 8.38 & 12 \\ 3.41 & 8.37 & 3 \\ 3 & 31\end{array}$

$\begin{array}{llll}.47 & 6.39 & 32 & 3 \\ 3.44 & 8.38 & 35\end{array}$

$\begin{array}{lll}3.44 & 8.38 & 35-21 \\ 3.43 & 6.37 & 15\end{array}$

$\begin{array}{lll}3.47 & 8.38 & 31-2 \\ 3.44 & 8.37 & 3 \frac{1}{2}-1 \frac{2}{2}\end{array}$

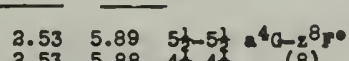

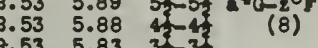

$\begin{array}{lll}3.53 & 5.83 & 3.3 \\ 3.53 & 5.81 & 3.25\end{array}$

$\begin{array}{lll}3.53 & 5.88 & 5\end{array}$

3.535 .81 3.

3.535 .79 a 13

$\begin{array}{lll}3.53 & 5.89 & 12 \\ 3.53 & 5.86 & 3 \\ 3.53 & 5.83 & 3\end{array}$

(1) $\quad 3.53 \quad 8.00 \quad \frac{1}{2} a^{4} 0-z^{6} p^{0}$

$\begin{array}{llll}3.53 & 8.15 & 51-4 & a^{4}-2^{6} D^{\circ} \\ 3.53 & 6.13 & (8) & (8)\end{array}$

$\begin{array}{lll}3.53 & 6.14 & 3 \\ 3.53 & 6.12 & 2\end{array}$

$\begin{array}{lll}3.53 & 6.12 & 2 \\ 3.53 & 6.15 & 1\end{array}$

$\begin{array}{lll}3.53 & 6.15 & -4 \\ 3.53 & 8.13 & 3\end{array}$

$3.53 \quad 8.14$ 25

$\begin{array}{lll}3.53 & 8.15 & 3-4 \\ 3.53 & 6.13 & \text { से }\end{array}$ $\begin{array}{lll}3.41 & 5.78 \\ 3.47 & 5.81 & 3 \\ 3 & -2\end{array}$

3.44 6.09 ato

3379.835
3393.86

\begin{tabular}{|c|c|c|c|}
\hline $\begin{array}{r}75 \\
75 \\
50 \\
50 \\
30 \\
30 \\
30 \\
3\end{array}$ & $\begin{array}{l}3.53 \\
3.53 \\
3.53 \\
3.53 \\
3.53 \\
3.53 \\
3.53 \\
3.53\end{array}$ & $\begin{array}{l}6.41 \\
6.39 \\
6.38 \\
6.37 \\
6.41 \\
6.39 \\
6.38 \\
6.39\end{array}$ & 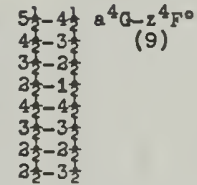 \\
\hline
\end{tabular}

$3.698 .05 \quad \frac{1}{2}-1 \frac{2}{2}$

$\begin{array}{lll}3.69 & 6.14 & 13 \\ 3.69 & 6.13 & \end{array}$

456.84
4507.19
454.70
4571.24
4504.53
4545.49
4573.83
4588.40
4543.77
4573.63
4590.00

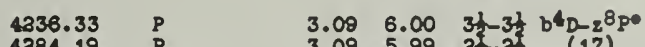

3.095 .99 3 5 (17)

$\begin{array}{lll}3.09 & 5.97 & 1 \%-1 \\ 3.09 & 5.99 & 3\end{array}$

3.095 .97 2.

$\begin{array}{lll}3.09 & 5.99 & 1 \\ 3.09 & 5.97 & -12\end{array}$

438.69
4378.49
4378.94

4111.01

4173.80
4317.07

4113.34
4171.93

4315.77
112.59

112.59
4170.58

4030.38

4083.94
4053.45

4075.63
4081.77

4054.11

408.8

4051.97

4077.50
4088.90

3715.19

3738.38
3754.59

3765.83
3736.56

3755.13

3768.65

3753.38
3787.18

3360.295

3403.43

3378.337
3394.32

$\mathbf{m} 3403.29$
3381.770

3379.371
3393.00

(a)

$3.095 .86 \quad 31-4 \frac{1}{2} b^{4} D_{-2} 6 T^{0}$

$\begin{array}{lll}3.09 & 5.83 \text { 3 } & 3\end{array}$

3.095 .79 s

$3.09 \quad 5.81 \quad 35-3$

. $5.79 \quad 1$ -

3

$3.098 .09 \quad 32-2 b^{4} b_{-2}{ }^{4} p$

3.098 .05 3. 1 (18)

$\begin{array}{lll}3.09 & 6.02 & 1 \\ 3.09 & 6.00 & 2\end{array}$

$\begin{array}{lll}3.09 & 8.02 & \\ 3.09 & 6.09 & 12 \\ 3.09 & 6.05 & \frac{2}{2}\end{array}$

3.096 .05 र $1 \frac{1}{2}$

$3.09 \quad 6.13 \quad 3 \quad-3 \frac{1}{3}(19)$

$3.09 \quad 6.12$ 1.

$3.09 \quad 8.13 \quad 3$ ?

3.096 .14 aर

$3.096 .13 \quad 1$ का

3.096 .14 3र 6 कर

$\begin{array}{lll}3.09 & 8.12 & 2 \\ 3.09 & 6.11 & 1 \frac{1}{2}\end{array}$

3.098 .41 3र $b^{4} D-2^{4} T^{*}$

$\begin{array}{llll}3.09 & 8.38 & 1 & 23 \\ 3.09 & 5.37 & 13\end{array}$

$\begin{array}{llll}3.09 & 8.39 & 35 & 3\end{array}$

$\begin{array}{lll}3.09 & 6.38 & 2 \\ 3.09 & 6.37 & 1\end{array}$

$\begin{array}{lll}3.09 & 6.38 & 32 \\ 3.09 & 8.37 & 32\end{array}$

$\begin{array}{llll}3.09 & 6.78 & 31-3 t & b^{4} D-2^{4} D^{\circ} \\ 3.09 & 8.74 & (31)\end{array}$

$3.098 .73 \quad 13 \frac{1}{3}$

$\begin{array}{lll}3.09 & 6.73 & -2 \\ 3.09 & 6.74 & 3\end{array}$

$3.09 \quad 6.73$ aर 1

$\begin{array}{lll}3.09 & 6.73 & 13 \\ 3.09 & 8.78 & 3\end{array}$

$\begin{array}{lll}3.09 & 8.74 & 1 \\ 3.09 & 6.73 & -1 \frac{1}{2}\end{array}$
I A Laboratory Ref

Low ${ }^{\text {E }}$ H1gh

Multiplot
(No)

Cr II continued

5636.60

5497.86

5419.36
5671.62

5525.90

5407.62

5346.54
5318.41

5510.68

5430.90
5249.40

5249.40
5346.75

5323.78

5097.29

5305.8

5116.08

5346.13
5310.87

477.78
4679.87

4679.87

4805.18

4698.64
4834.97

4179.43
.411 .01

.4111 .01
4073.56

4207.35

4086.14
4339.81

3978.718

3961.733
3953.358

2953.35
3011.43

$3.09 \quad 6.05 \quad 1$ - $1 \frac{2}{2}$

3984.69

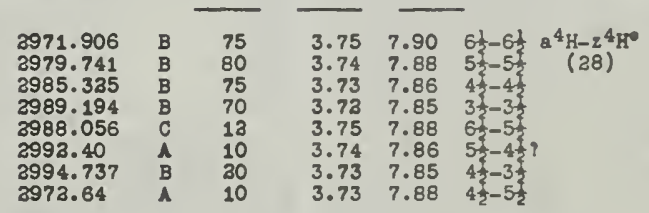

$2973.64 \quad \Lambda \quad 10$

$73 \quad 7.88 \quad 4 \frac{2}{2}-5 \frac{2}{2}$

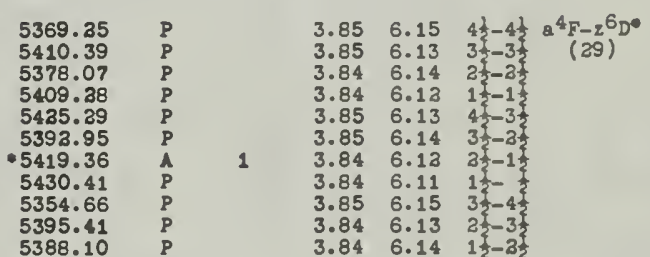

4834.

4848.24

4864.33

4860.20

4876.48

4813.35

4836.33
4856.19

4343.38

4261.92
4275.57

438.21

435.8

4369.38
4324.09

4346.41

3083.84

3968.051

3003.92
3034.54

3055.44
2999.30

5553.8

5731.02

5806.31

5610.01

5464.36
5500.61

5500.61
5446.57

5543.86
5488.97

5488.97
5568.08

4891.55

4984.34
4920.38

4985.46

4941.03

$\begin{array}{llll}3.81 & 6.00 & 2 \frac{1}{2}-3 \frac{1}{2} & b^{4} P-26 p o \\ 3.74 & 5.99 & 1 \frac{1}{3}-3 \frac{1}{2} & (23)\end{array}$

$\begin{array}{lll}3.70 & 5.97 & 1 \\ 3.81 & 5.99 & 2 \\ 3 & -1\end{array}$

$\begin{array}{lll}3.74 & 5.97 & 1 \frac{1}{2}-1 \frac{1}{3} \\ 3.81 & 5.97 & 2 \frac{1}{2}-1 \frac{1}{2}\end{array}$

3.81 $6.09 \quad 2 \frac{1}{3}-2 \frac{1}{2} b^{4} P_{-2} p^{40}$

$\begin{array}{ll}3.70 & 6.02 \\ 3.81 & 6.05 \\ 3.74 & 6.02\end{array}$

$3.74 \quad 6.02$

$3.70 \quad 6.05$ t

$3.81 \quad 6.13 \quad 3 \frac{1}{2}-3 \frac{1}{2} b^{4} P_{-2} 6 D^{0}$

$\begin{array}{lll}3.70 & 6.12 & 1 \\ 3 & -1\end{array}$

3.74 6.13 $13-1 \frac{3}{3}$

$\begin{array}{lll}3.81 & 6.13 & 3 \frac{2}{3}-1 \\ 3.74 & 6.11 & 1 \frac{3}{3}\end{array}$

$3.81 \quad 6.39 \quad 3 \frac{1}{2}-3 \frac{1}{2} b^{4} P-z^{4} F^{0}$

$\begin{array}{lll}3.74 & 6.38 & 1 \frac{1}{3}-3 \\ 3.70 & 6.37 & \end{array}$

$\begin{array}{lll}3.81 & 6.38 & 25 \\ 3.74 & 6.37 & 12 \\ 3.81 & 6.37 & 2 \frac{1}{2} \\ 3.12\end{array}$

$3.81 \quad 6.76 \quad 3 \frac{1}{2}-3 \frac{1}{2} \quad b^{4} P-z^{4} D^{0}$

$\begin{array}{lll}3.74 & 6.74 & 11 \\ 3.70 & 6.73 & 3-1\end{array}$

$\begin{array}{lll}3.81 & 6.74 & 35-2 \frac{1}{2} \\ 3.74 & 6.73 & 15-1\end{array}$

$\begin{array}{lll}3.70 & 6.72 & \frac{1}{2}- \\ 3.81 & 6.73 & 2 \frac{1}{2}-1 \frac{1}{2}\end{array}$

$3.81 \quad 7.96 \quad 2 \frac{1}{2}-3 \frac{1}{2} \quad b^{4} P-y^{4} D^{0}+$

$\begin{array}{lll}3.74 & 7.91 & 1 \frac{1}{2}-1 \\ 3.70 & 7.88 & 3\end{array}$

\begin{tabular}{lll}
3.81 & 7.91 & 31 \\
3.74 & 7.88 & $1 \frac{1}{2}-\frac{1}{2}$ \\
\hline
\end{tabular}

$3.75 \quad 7.90 \quad 6 \frac{1}{3}-6 \frac{1}{4} a^{4} \mathrm{H}-2^{4} \mathrm{HO}^{\circ}$

$\begin{array}{lll}7.86 & 4 \frac{1}{3}-4\end{array}$

$\begin{array}{lll}3.75 & 7.88 & 6\end{array}$

$85 \quad 6.15 \quad 4 \frac{1}{2}-4 \frac{1}{2} a^{4} F-z^{6} D^{0}$

$84 \quad 6.12$ at

$3.846 .13 \quad 23-3$

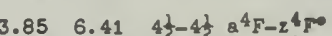

$\begin{array}{llll}3.85 & 6.39 & 35 & 3 \\ 3 & (30)\end{array}$

3.846 .38 31

$\begin{array}{lll}3.85 & 6.39 & 43\end{array}$

$\begin{array}{lll}3.85 & 6.38 & 3 \frac{1}{2}-2 \frac{1}{2}\end{array}$ 


\section{4}

int Cr II continued

$\begin{array}{llllll}4366.33 & P & 3.87 & 6.76 & 3 \frac{1}{2}-3 \frac{1}{2} a^{2} D-z^{4} D^{\circ} \\ 4338.91 & P & 3.89 & 6.74 & 1 \frac{1}{2}-2 & (37) \\ 4295.37 & P & 3.87 & 6.74 & 3 \frac{1}{2}-3 & \\ 4353.68 & P & 3.89 & 6.73 & 1 \frac{1}{2}-1 \frac{1}{2} & \\ 4318.77 & P & 3.87 & 6.73 & 2 \frac{1}{3}-1 \frac{1}{3} & \\ 4368.30 & P & 3.89 & 6.73 & 1 \frac{1}{2}-\frac{1}{2} & \end{array}$

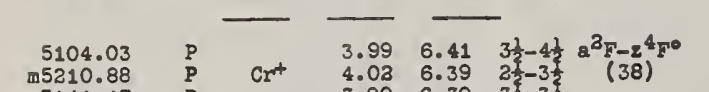

5243.5

5176.26
5267.10

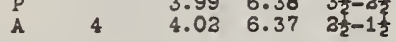

$\begin{array}{rrrrrrr}4539.62 & \text { A } & 3 & 4.02 & 6.74 & 3 \frac{1}{2}-3 \frac{1}{2} a^{2} 8-z^{4} D^{\circ} \\ 4565.78 & \text { A } & 10 & 4.02 & 6.73 & 3 \frac{1}{2}-1 \frac{1}{2} & (39)\end{array}$

$\begin{array}{rrrrrrr}3177.90 & \text { A } & 1 & 4.03 & 7.91 & 3 \frac{1}{2}-1 \frac{1}{2} a^{3}\left\{-D^{4} D^{\circ}\right. \\ 3036.85 & \text { A } & 30 & 3.99 & 8.07 & 3 \frac{1}{2}-4 \frac{1}{2} a^{3} \frac{8}{8}-2^{4} C^{\circ}\end{array}$

3061.59

3038.53
3071.03

2999.96

3034.99
3013.34

5237.34

5274.99

5334.88

5308.44

5337.79

$\$ 3310.70$

4558.659

4688.217
4618.83

4634.11

4555.02

4616.64

4558.8

3225.44

3117.28

3162.46
3203.53

3229.38

3164.28

3042.79 3071.58

3093.97

3073.25

-3094.94

3045.53

3043.90

3058.38

2958.20

5478.35

5502.05

5503.18

5472.63
5477.45

3295.427

3307.044
3311.929

3312.18

3315.29

3322.69
3333.53

3193.41

$-3131.54$

3143.68
3154.04

3123.596
$\mathrm{~m} 3147.19$

${ }_{3150.11}$

3149.83
3154.66

3159.86

3160.11
3115.38

3137.55
3139.91

3125.46

3131.54

3118.14
3121.97

3108.66

$\begin{array}{lll}4.05 & 6.39 & 3 \frac{1}{2}-3 \\ 4.06 & 6.38 & 2 \frac{2}{2}-3\end{array}$

I A Laboratory ${\text { ReP Int Low }{ }_{\text {H1gh }} J \text { Multiplet }}_{\text {(No) }}$

I $A$ Laboratory Int

Low ${ }^{\text {E P1gh }}$

J $(\text { (Ho) })^{208}$

Cr II continued

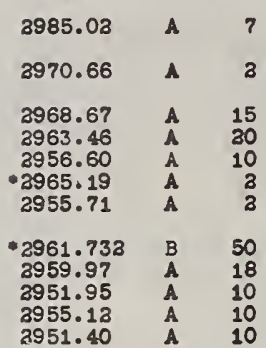

.3431 .63

3460.03
3450.84

$\begin{array}{llll}3.99 & 8.06 & 3 \frac{1}{3}-\frac{3}{3} \\ 4.02 & 8.04 & 3 \frac{1}{2}-3 \frac{7}{2}\end{array}$

$\begin{array}{llll}3.99 & 8.11 & 3 \frac{1}{2}-4 \frac{1}{2} & a^{2} y^{2}-2^{2} a^{\circ} \\ 4.02 & 8.09 & 3 \frac{1}{3}-3 \frac{1}{2} & (42)\end{array}$

\begin{tabular}{lll}
4.02 & 8.09 & $2 \frac{1}{3}-3 \frac{1}{2}$ \\
3.99 & 8.09 & $3 \frac{1}{2}-3 \frac{2}{2}$ \\
\hline
\end{tabular}

$4.06 \quad 6.41 \quad 4 \frac{1}{3}-4 \frac{1}{1} b^{4} y-z^{4} g^{\circ}$

$\begin{array}{lll}4.05 & 6.37 & 1 \frac{1}{3} \\ 4.06 & 6.39 & 4\end{array}$

$4.05 \quad 6.38$ 3र

$\begin{array}{lll}4.06 & 6.37 & 3 \frac{1}{2}-1 \\ 4.05 & 6.41 & 3 \frac{1}{2}-4\end{array}$

$\begin{array}{lll}4.05 & 6.41 & 3 \frac{1}{2}-4 \frac{1}{2} \\ 4.06 & 6.39 & 32-3 \frac{2}{2} \\ 4.05 & 6.38 & 1 \frac{2}{2}-2 \frac{1}{2}\end{array}$

$4.06 \quad 6.78 \quad 4 \frac{1}{2}-3 \frac{1}{2} b^{4} 7-8^{4} D^{\circ}$

4.05 6.74 $35-2 \frac{1}{2} \quad(44)$

$\begin{array}{lll}4.06 & 6.73 & 32 \\ 4.05 & 6.73 & 1\end{array}$

$4.05 \quad 6.76 \quad 35$

$\begin{array}{lll}4.06 & 6.74 & 32 \\ 4.05 & 6.73 & 13\end{array}$

$4.05 \quad 6.74 \quad 1 \frac{1}{2}-3 \frac{2}{2}$

$4.067 .88 \quad 4 \frac{1}{2}-5 \frac{1}{2} b^{4}\left\{\frac{1}{45}\right\}^{4} H^{\circ}$

$\begin{array}{llll}4.06 & 8.01 & 4 \frac{1}{3}-3 \frac{1}{2} & b^{4} \frac{(45)}{8}-y^{4} D^{\circ} \\ 4.05 & 7.96 & 3 \frac{3}{2}-3 \frac{2}{2} & (46)\end{array}$

$4.06 \quad 7.91$ 3.

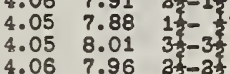

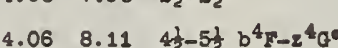

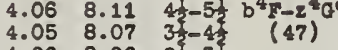

$\begin{array}{lll}4.06 & 8.06 & 2 \frac{2}{2}-3 \\ 4.05 & 8.04 & 12-3\end{array}$

4.068 .07

$\begin{array}{lll}4.05 & 8.06 & 3 \frac{1}{3}-3 \\ 4.06 & 8.04 & 3 \frac{5}{2}-3\end{array}$

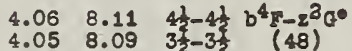

$\begin{array}{lll}4.05 & 8.11 & 3 \\ 4.4 \frac{2}{2}\end{array}$

$4.06 \quad 8.23 \quad 2 \frac{1}{2}-1 \frac{1}{2} b^{4} F-z^{2} D^{0}$

4
$(49)$

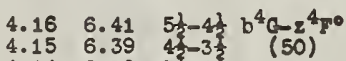

$\begin{array}{lll}4.14 & 6.38 & 3 \%=2 \\ 4.13 & 6.37 & 25\end{array}$

$\begin{array}{lll}4.15 & 6.41 & 4 \frac{1}{3}-4 \frac{1}{2} \\ 4.14 & 6.39 & 3 \frac{3}{3}-3 \frac{1}{2} \\ 4.13 & 6.38 & 25\end{array}$

$4.16 \quad 7.90 \quad 5 \frac{1}{-6}-6 \frac{1}{3} b^{4} a-z^{4} H^{0}$

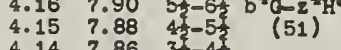

$\begin{array}{ll}4.14 & 7.86 \\ 4.13 & 7.85\end{array}$

$4.16 \quad 7.88 \quad 5 \quad-5$

$\begin{array}{lll}4.15 & 7.86 & 4 \frac{2}{3}-\frac{4}{2} \\ 4.14 & 7.85 & 3 \frac{1}{2}-3\end{array}$

$4.158 .01 \quad 4 \frac{1}{2}-3 \frac{1}{2} b^{4} a-y^{4} D^{\circ}$

$\begin{array}{llll}4.16 & 8.10 & 5 \frac{1}{2}-6 \frac{1}{2} b^{4} a_{2} z^{4} I^{\circ} \\ 4.15 & 8.08 & 4 \frac{1}{2}-5 & (53)\end{array}$

$\begin{array}{lll}4.15 & 8.08 & 41 \\ 4.14 & 8.05 & 3 \frac{2}{2}-4 \frac{1}{2}\end{array}$

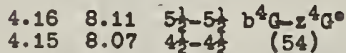

$\begin{array}{lll}4.15 & 8.07 & 42-4 \\ 4.14 & 8.08 & 3 \frac{1}{2}-3 \\ 4.13 & 8.04 & 25\end{array}$

$\begin{array}{lll}4.13 & 8.04 & 2 \\ 4.16 & 8.07 & 5\end{array}$

$\begin{array}{lll}4.16 & 8.07 & 51-4 \\ 4.15 & 8.06 & 4\end{array}$

$\begin{array}{lll}4.14 & 8.04 & 3 \frac{3}{2}-2 \\ 4.15 & 8.11 & 4 \\ 4.14 & 8.07 & 3 \frac{1}{3}-4\end{array}$

$\begin{array}{lll}4.14 & 8.07 & 3 \frac{1}{5}-4 \frac{4}{5} \\ 4.13 & 8.06 & 3 \frac{5}{2}-3 \frac{1}{2}\end{array}$

$\begin{array}{llll}4.18 & 8.11 & 5 \frac{1}{2}-4 \frac{1}{3} b^{4} a_{2}^{2} g^{\circ} \\ 4.15 & 8.09 & 4 \frac{1}{2}-3 & (55)\end{array}$

$\begin{array}{lll}4.15 & 8.09 & 4 \frac{1}{2}-3 \frac{2}{2} \\ 4.15 & 8.11 & 4 \frac{1}{3}-4 \frac{1}{2} \\ 4.14 & 8.09 & 3 \frac{2}{2}-3 \frac{3}{2}\end{array}$

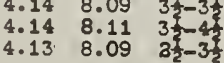

3008.67

$\begin{array}{lll}3145.77 & \hat{A} & 15 \\ 3143.74 & \mathrm{~A} & 10\end{array}$
3370.14 A 40

$\begin{array}{lll}3284.28 & \text { A } & 35 \\ 3250.79 & \text { A } & 10\end{array}$

$4.148 .37 \quad 3 \frac{1}{2}-3 \frac{1}{2} b^{4}(56)^{2} D^{\circ}$

$4.138 .38 \quad 2 \frac{1}{2}-1 \frac{1}{2} b^{4}\left(5 z^{3}\right)$

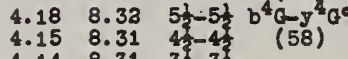

$\begin{array}{lll}4.14 & 8.31 & 3 \\ 4.15 & 8.31 & 4 \\ 4 & -3\end{array}$

$4.14 \quad 8.31$ 3र

$\begin{array}{lll}4.18 & 8.33 & 5 \frac{1}{2}-4 \frac{4}{4} b^{4}\left(-g^{4} r^{0}+\right. \\ 4.15 & 8.33 & 43-3 \frac{5}{3}-(59)\end{array}$

$\begin{array}{llll}4.14 & 8.32 & 31 & -3 \\ 4.15 & 8.33 & 4 \frac{2}{3} \\ 4.14 & 8.32 & 3 \frac{3}{2}-3 \frac{1}{2} \\ 4.14 & \end{array}$

$\begin{array}{lll}4.38 & 7.88 & 4 \\ 4.30 & 7.86 & 5 \\ 4.38 & 7.85 & 4 \frac{1}{2}-3 \\ 4\end{array}$

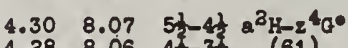

$\begin{array}{llll}4.38 & 8.06 & 4 & -3 \\ 4.38 & 8.07 & \text { (61) }\end{array}$

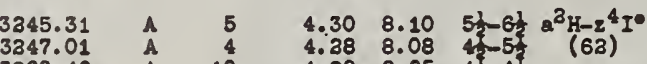

$\begin{array}{lllllll}3268.46 & \hat{A} & 10 & 4.38 & 8.05 & 4 \\ 3388.04 & \mathrm{~A} & 15 & 4.30 & 8.05 & 5 \frac{4}{2}-\frac{4}{2}\end{array}$

3238.77 A 50

$\begin{array}{lll}3334.06 & \text { A } & 50 \\ 3219.79 & \text { A } & 10\end{array}$

3053.65 A 10

3050.137 B 100

$\begin{array}{lll}3040.93 & \text { A } & 70 \\ 3057.88 & \text { A } & 13\end{array}$

2953.706 B 45

2989.67

3400.08

3488.58

3430.43

3389.05
3291.75

3188.75

3154.10

3158.93

3125.02

3153.83

3103.48

3173.08

3131.05

3121.84

3074.67

$\begin{array}{llll}4.30 & 8.11 & 5 t-41 & a^{2} H-z^{2} G^{\circ} \\ 4.38 & 8.09 & 43-3 \frac{1}{3} & (63)\end{array}$

$\begin{array}{lll}4.38 & 8.09 & 4 \\ 4.38 & 8.11 & 42\end{array}$

$4.38 \quad 8.33 \quad 41-5 \frac{1}{2} a^{3} \mathrm{H}-\mathrm{y}^{4} \mathrm{G}^{\circ}$

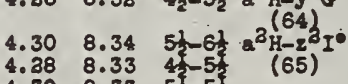

$\begin{array}{llll}4.38 & 8.33 & 4 \\ 4.30 & 8.33 & 5 \frac{5}{2}-5 \frac{1}{2}\end{array}$

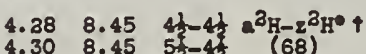

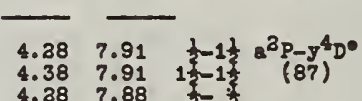

$4.36 \quad 8.03 \quad 1 \frac{1}{2}-\frac{1}{2} \mathrm{a}^{2} \mathrm{p}-z^{2} \mathrm{~g}$ 。

$\begin{array}{llll}4.28 & 8.03 & \frac{1}{2}-\frac{1}{2} & (68)\end{array}$

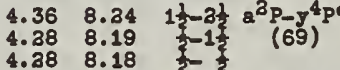

$4.36 \quad 8.37 \quad 1 \frac{1}{2}-2 \frac{1}{2} \mathrm{a}^{2} \mathrm{P}-\mathrm{z}^{2} \mathrm{D}^{\circ}$

$4.36 \quad 8.38 \quad 1 \frac{1}{3}-1 \frac{1}{1} a^{2} p_{-2} z^{2} p_{0}$

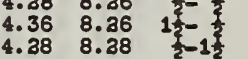

$4.36 \quad 8.33 \quad 1 \frac{1}{2-3 \frac{1}{2}} \mathrm{a}^{2} \mathrm{P}-\mathrm{J}^{4} \mathrm{~m}$

$4.38 \quad 8.38 \quad 12-21 a^{2} P-x^{4} D^{0}$

$4.38 \quad 8.43 \quad 12-1 \frac{1}{3} p_{-2} 40$

3557.85
3566.37

3466.35

3376.37

3357.40
3387.43

3324.346
3335.38

3213.53

$\begin{array}{rrr}3213.53 & \mathbf{A} & 30 \\ 3347.33 & \mathbf{A} & 8\end{array}$

3183.335 B 40

$\begin{array}{llr}3218.55 & \text { A } & 30 \\ 3179.45 & \text { A } & 8\end{array}$

3173.58 A 15

3149.13 A 4

3098.16

3095.32

3098.88
-3094.94

3015.510

3038.125

3031.83

3004.47

$4.40 \quad 7.88$ 3t $-4 \frac{1}{3} b^{2} 5-2^{4}$

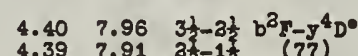

$4.40 \quad 8.05 \quad 3 \frac{1}{2}-4 \frac{1}{2} b^{2} 5-2^{4} I^{\circ}$

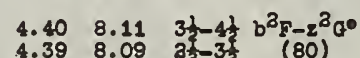

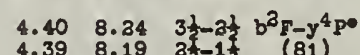

$4.40 \quad 8.37 \quad 3 \frac{1}{2}-2 \frac{1}{2} b^{2} 5-z^{2} D^{\circ}$

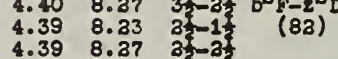

$4.398 .38 \quad 2 \frac{1}{2}-1 \frac{1}{2} P b^{2} T-z^{3} p^{0}$

$4.40 \quad 8.31 \quad 3 \frac{1}{2}-4 \frac{1}{2} b^{3}(83)$

$\begin{array}{llll}4.40 & 8.33 & 3 \frac{1}{2}-2 \frac{1}{2} & b^{2} \frac{(84)}{5}-y^{4} \\ 4.39 & 8.32 & 35 & (85)\end{array}$

$\begin{array}{llll}4.40 & 8.38 & 3 \frac{1}{2}-3 \frac{1}{2} b^{3}-x^{4} D^{\circ} \\ 4.39 & 8.38 & 3 & (88)\end{array}$

$\begin{array}{lll}4.39 & 8.38 & 35 \\ 4.40 & 8.38 & 3 \\ 4.39 & 3.38 & 3 \\ 4 & 25\end{array}$

$\begin{array}{llll}4.40 & 8.49 & 3 \frac{1}{3}-3 \frac{1}{2} & b^{2} \mathrm{~F}-z^{2} \mathrm{~F} 0 \\ 4.39 & 8.47 & 3 \frac{5}{5}-3 & (87)\end{array}$

$\begin{array}{lll}4.40 & 8.47 & 35 \\ 4.39 & 8.49 & 32 \\ 4 & 3 \frac{3}{2}\end{array}$

Cr II continued

(4.30 $7.90 \quad 5 \mathrm{~b}-6 \frac{1}{4} \mathrm{a}^{2} \mathrm{H}-2^{4} \mathrm{H}^{\circ}$

$\begin{array}{llll}4.36 & 8.37 & 1 \frac{1}{1}-1 & { }^{2} \mathrm{P}-2^{2} \mathrm{D} \\ 4.38 & 8.33 & 1 & (70) \\ 4.36 & 8.33 & 1 \frac{1}{2}-1 \frac{1}{2} & (70)\end{array}$

\section{35
35
35
35
357
33
33
3357
337
33
3335}

539
3550
3558
3558
3571
3387
3357
3368
372
3335
3339
333

${ }_{3147}^{3157}$

3135.7

3134.

3038.647

3041.647 B 80

$\begin{array}{rrr}3050.75 & \text { D } & 50 \\ 3017.80 & A & 5\end{array}$

*3968.31 A 3

$\begin{array}{ccc}3958.54 & 1 & 3\end{array}$

3635.30

3681.51

3844.13

3438.94

3438.9

$\begin{array}{lll}3399.54 & \hat{A} & 18 \\ 3395.63 & \mathbf{A} & 30\end{array}$

(1)

30

3415.

3199.87

3079.34

3087.90
3104.29

3077.24

3077.79

2954.67

$\cdot 3957.58$

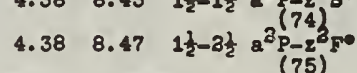

6053.48
6129.23

6129.23

6112.36

6176.95

60.70 .08

6147.15
6308.18

3895.16

3513.03

3513.03

3584.01

3518.63

3588.30

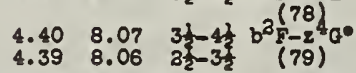

.3506 .61

3478.17

3538.33
.3489 .45

3438.46

3436.1

3437.93
3444.34

3449.28

- 3376.72

3382.79

3278.79

$4.398 .50 \quad 3 \frac{1}{2}-3 \frac{1}{2} b^{3} \mathrm{~F}-y^{4} \mathrm{H}^{\circ}$

\section{(1)}

3201.

3212.91

3208.02

3164.48

3164.48
-3198.40

3073.47
3103.58

$4.41 \quad 7.90 \quad 5 \frac{1}{3}-6 \frac{1}{4} b^{2} \mathrm{H}-8^{4}+1 \mathrm{to}$

$\begin{array}{llll}4.40 & 7.88 & 5 & 5 \\ 4.41 & 7.88 & 53 & \text { (89) }\end{array}$ 
Laboratory
Rer Int Low ${ }_{\text {H1gh }}{ }^{\text {I Multiplot }}$ c. II cont1nued $\begin{array}{lllllll}3727.37 & \text { A } & 40 & 4.76 & 8.07 & 4 \frac{1}{3}-4 \frac{1}{3} b^{2} a_{-2}^{4} a^{\circ} \\ 3737.55 & \text { A } & 10 & 4.75 & 8.06 & 3 \frac{3}{2} & (117) \\ 3751.60 & \text { A } & 3 \mathrm{Zr} & 4.75 & 8.04 & 3 \frac{5}{2}-2 \frac{2}{2} & \end{array}$ $\begin{array}{lllllll}3686.67 & \text { A } & 20 & 4.76 & 8.11 & 4 \frac{1}{2}-4 \frac{1}{2} b^{3} a_{-2} G^{\circ} \\ 3698.00 & \text { A } & 35 & 4.75 & 8.09 & 3 \frac{3}{2}-3 \frac{3}{2} & (118)\end{array}$ 3705.40
3879.3

$3313.08 \quad \& \quad 20 \quad 4.78 \quad 8.49 \quad 4 \frac{1}{2}-5 \frac{1}{2} b^{2} a_{-2}{ }^{3} K^{\circ}$ $\begin{array}{lllllll}33.35 .93 & \mathrm{~A} & 4 & 4.75 & 8.45 & 3 \frac{3}{2}-4 & (119) \\ 3341.98 & \mathrm{~A} & 5 & 4.78 & 8.45 & 4 \frac{1}{2}-4 & \end{array}$

$\cdot 3310.65$ 3324.10
3304.73

3366.25 3279.54

3231.84

3169.20

3189.85
$\mathrm{~m} 3163.77$

3140.21

3140.67

3093.48
3107.58

3112.81

3132.12
3142.9 ?

3096.11

3118.78
3090.94

4161.37

4161.56
4186.08

4179.92
4804.68

3905.88

3915.30

3862.17

3911.33

3911.32

3924.65

3866.54

3868.01

3814.26

3608.68

3583.9

3547.10
3546.15

(3)

3473.07
3489.07

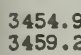

3301.2

3308.1

3289.7

3255.3

3235.26

3219.13

3225.39
3240.07

-3957.56

3945.11

$$
\begin{array}{lll}
4.76 & 8.49 & 42-3 \\
4.75 & 8.47 & b^{3} a-z^{2} F^{\circ} \\
4.75 & 8.49 & (120)
\end{array}
$$

$\begin{array}{llll}4.75 & 8.47 & 35-2 \frac{1}{2} & (120) \\ 4.75 & 8.49 & 3\end{array}$

$4.76 \quad 8.54$ 4h $-5 \frac{1}{2} b^{2} a-y^{4} y^{\circ}$

$4.78 \quad 8.58 \quad 4 \frac{1}{2}-4 \frac{1}{2} b^{2} a-x^{4} F^{0}$

$4.768 .65 \quad 4 \frac{1}{2}-4 \frac{1}{2} b^{3} a-y^{2} G$

$\begin{array}{llll}4.76 & 8.65 & 43-4 \frac{1}{2} & \\ 4.75 & 8.63 & 3 \frac{1}{2}-3 \frac{1}{2} & (123)\end{array}$

$\begin{array}{lll}4.76 & 8.63 & 4 \frac{4}{2}-3 \frac{1}{2} \\ 4.75 & 8.65 & 3 \frac{1}{2}-4 \frac{1}{2}\end{array}$

$4.76 \quad 8.69 \quad 4 \frac{1}{2}-5 \frac{1}{2} b^{2} a-y^{2} H^{\circ}$

$\begin{array}{llll}4.75 & 8.69 & 3 \frac{1}{2}-4 \frac{2}{2} & (124) \\ 4.76 & 8.69 & 4 & \end{array}$

$4.76 \quad 8.75 \quad 4 \frac{1}{2}-5 \frac{1}{2} b^{2} a-x^{4} \sigma^{\circ}$

$\begin{array}{ll}4.75 & 8.75 \\ 4.76 & 8.73\end{array}$

$\begin{array}{lll}4.75 & 8.69 & 3 \frac{1}{2}-3 \\ 4.75 & 8.68 & 3 \frac{1}{2}-2\end{array}$

$4.76 \quad 8.75 \quad 4 \frac{1}{2}-3 \frac{1}{2} b^{2} a-y^{2} F^{\circ}$

$\begin{array}{llll}4.75 & 8.71 & 3 \frac{1}{3}-\frac{2}{2} & (126) \\ 4.75 & 8.75 & 3 \frac{1}{2}-3 \frac{1}{2} & \end{array}$

$\begin{array}{llll}4.92 & 7.88 & 4 \frac{1}{2}-5 \frac{1}{2} & 0^{2} a-z^{4} H^{\circ} \\ 4.90 & 7.88 & 3.5 & (127)\end{array}$

$\begin{array}{lll}4.90 & 7.88 & 3 \\ 4.93 & 7.86 & 4 \\ 4 & 4\end{array}$

$\begin{array}{lll}4.90 & 7.85 & 3 \frac{1}{2}-3 \frac{1}{2} \\ 4.93 & 7.85 & 4 \frac{1}{2}-3 \frac{7}{6}\end{array}$

$4.93 \quad 8.03 \quad 4 \frac{1}{4}-5 \frac{1}{2} c^{3} a-z^{4} I^{0}$

$\begin{array}{llll}4.90 & 8.05 & 2 \frac{1}{2}-4 \frac{1}{2} & (128) \\ 4.93 & 8.05 & 4 \frac{1}{2}-4 \frac{1}{2}\end{array}$

$\begin{array}{llll}4.92 & 8.11 & 4 \frac{1}{3}-5 \frac{1}{2} & c^{2} G-z^{4} \sigma^{0} \\ 4.90 & 8.07 & 3 \frac{1}{2}-4 \frac{1}{2} & (139)\end{array}$

$\begin{array}{lll}4.90 & 8.07 & 3 \frac{1}{2}-\frac{1}{2} \\ 4.92 & 8.07 & 4\end{array}$

$4.90 \quad 8.08 \quad 3 \frac{1}{2}-3$

$4.90 \quad 8.04$ और $-2 \frac{2}{2}$

$4.92 \quad 8.11 \quad 4 \frac{1}{2}-4 \frac{1}{2} 0^{2} a-2^{2} a^{0}$

$4.908 .34 \quad 3 \frac{1}{2}-2 \frac{1}{2} c^{2} G-y^{4} \sum^{4}$

$4.908 .31 \quad 3 \frac{1}{2}-2 \frac{1}{2} 0^{2}(131)$

$\left.4.908 .33 \quad 3 \frac{1}{2}-3 \frac{1}{2} c^{3}\left(\frac{1}{13}\right)^{4}\right)^{\circ}$

$\begin{array}{llll}4.93 & 8.38 & 4 \frac{2}{2}-3 \frac{1}{2} & c^{2} G-x^{4} \\ 4.90 & 8.38 & 3 \frac{2}{2} & (134) \\ 4.90 & 8.38 & 3 \frac{1}{2}-3 \frac{1}{2} & \end{array}$

$4.938 .49 \quad 4 \frac{1}{2}-5 \frac{1}{2} c^{2} a_{-2}^{2} \mathrm{H}^{\circ}$

$\begin{array}{llll}4.90 & 8.45 & 3 \frac{1}{2}-4 \frac{1}{3} & (135) \\ 4.93 & 8.45 & 4 \frac{1}{2}-4 \frac{1}{2} & \end{array}$

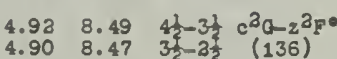

$4.928 .65 \quad 4 \frac{1}{2}-4 \frac{1}{2} c^{2} a-y^{2} a^{\circ}$

$\begin{array}{llll}4.90 & 8.63 & 3 \frac{1}{2}-3 \frac{2}{2} & (137)\end{array}$

$\begin{array}{llll}4.92 & 8.69 & 4 \frac{1}{2}-5 \frac{1}{3} & c^{2} \mathrm{G}^{2} \mathrm{r}^{3} \mathrm{H}^{\circ} \\ 4.90 & 8.69 & 3 \frac{1}{2}-\frac{1}{2} & (138)\end{array}$

$4.90 \quad 8.71 \quad 3 \frac{1}{2}-2 \frac{1}{2} 0^{3} a-y^{2} y^{\circ}$

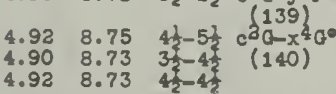

$4.90 \quad 9.07 \quad 3 \frac{1}{2}-3 \frac{1}{2} c^{2} G-\pi^{4} D^{\circ}$

(1) $4.928 .05 \quad 3 \frac{1}{2}-4 \frac{1}{2} c^{2} \mathrm{P}-2^{4} \mathrm{I}$

$\begin{array}{llll}4.92 & 8.05 & 3 \frac{1}{2}-4 \frac{1}{2} & c^{2} P-z^{4} I \\ 4.92 & 8.11 & 3 \frac{1}{2}-4 \frac{1}{2} 0^{2} F-z^{2} G \\ 4.91 & 8.09 & 3 \frac{3}{3}-3 & (143) \\ 4.92 & 8.09 & 3 \frac{1}{2}-3 \frac{1}{2} & \end{array}$

3895.12

$\begin{array}{llrllll}3723.40 & \mathbf{A} & 15 & 4.92 & 8.24 & 3 \frac{1}{2}-2 \frac{1}{2} & c^{2} 8-y^{4} \mathrm{p} \bullet \\ 3758.55 & \mathbf{A} & 3 & 4.91 & 8.19 & 3 \frac{1}{2}-1 & (144)\end{array}$

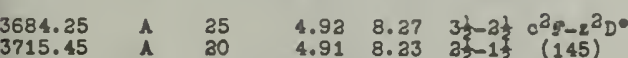

$\begin{array}{llll}4.91 & 8.23 & 2 & 1 \\ 4.91 & 8.37 & 2 & (145)\end{array}$

4.918 .38 2k-1 $1 \frac{1}{2} \mathrm{c}^{3} \mathrm{~F}-2^{3} \mathrm{p}$

$\begin{array}{llll}4.93 & 8.33 & 3 \frac{1}{2}-4 \frac{1}{3} & 0^{6} \mathrm{~F}-\mathrm{y}^{4} \mathrm{~F}^{\bullet} \\ 4.91 & 8.33 & 32 & (147)\end{array}$

$\begin{array}{lll}4.91 & 8.32 & 32 \\ 4.92 & 8.32 & 32 \\ 4.91 & 8.32 & 2.3\end{array}$

$\begin{array}{lll}4.91 & 8.32 & 2 \\ 4.92 & 8.32 & 31 \\ 4.91 & 8.33 & 21 \\ 42-1 \frac{1}{2}\end{array}$

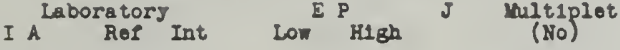
Cr II continued

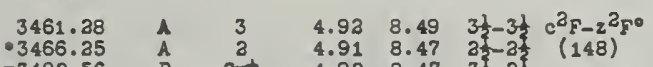

3445.20

$\begin{array}{lll}4.92 & 8.47 & 3 \frac{1}{2}-3 \frac{1}{3} \\ 4.91 & 8.49 & 3 \frac{1}{2}-3 \frac{1}{2}\end{array}$

$\begin{array}{llllll}3374.99 \quad A & 8 & 4.93 & 8.58 & 3 \frac{1}{3}-4 \frac{1}{2} & c^{2} F-x^{4} F\end{array}$

$\begin{array}{rllllll}3377.36 & \mathrm{~A} & 5 & 4.92 & 8.58 & 3 \frac{1}{2}-3 \frac{1}{3} & (149) \\ .3376 .73 & \mathrm{~A} & 5 & 4.91 & 8.56 & 3 \frac{5}{2}-1 \frac{1}{2} & \end{array}$

$3306.95 \quad \hat{A} \quad 50 \quad 4.92 \quad 8.65 \quad 3 \frac{1}{2}-4 \frac{1}{3} c^{2} F-y^{2} G^{\circ}$

$\begin{array}{rrr}3314.57 & \hat{A} & 35 \\ 3329.45 & \mathbf{A} & 4\end{array}$

$\begin{array}{llll}4.91 & 8.63 & 2 \frac{2}{3}-3 & (150) \\ 4.92 & 8.63 & 3 \frac{1}{2}-3 \frac{1}{2}\end{array}$

$3375.92 \quad A \quad 10$

$4.93 \quad 8.69 \quad 3 \frac{1}{2}-4 \frac{1}{2} c^{3} P-y^{3} H^{0}$

$3258.01 \quad A \quad 3$

$\begin{array}{llll}4.91 & 8.69 & 2 \frac{1}{2}-3 \frac{1}{2} & c^{3} 5-x^{4} c^{\circ} \\ 4.91 & 8.68 & 3 \frac{2}{2}-3 \frac{1}{2} & (152)\end{array}$

3237.48

3341.38

325.62

3044.24
3038.04

4237.7

3650.3

3684.9

.3503 .368

3539.00
-3508.61

3310.65
3314.06

$3358.77 \quad A \quad 30$

$\begin{array}{rrr}3383.04 & \hat{A} & 30 \\ 3281.58 & \hat{A} & 4\end{array}$

- 3965.19

4195.41

4278.10

4145.77

4324.85
4309.02

4181.05

4135.77

4185.50
4151.00

4089.49

4081.2

4083.30

4003.48
4017.98

3865.59

3905.6

(8) 14

3701.9

3694.

$\begin{array}{llll}3631.48 & P & \mathrm{Cr}^{+}\end{array}$

$\begin{array}{llll}4.92 & 8.75 & 3 \frac{1}{2}-3 \frac{1}{2} & c^{2} F-y^{3} F \\ 4.91 & 8.71 & 3 \frac{2}{2}-a^{2} & (153)\end{array}$

$4.92 \quad 8.71 \quad 3 \frac{3}{2}-3 \frac{2}{3}$

$4.92 \quad 8.98 \quad 3 \frac{1}{2}-4 \frac{1}{2} c^{2} 5-x^{2} G^{0}$

$4.918 .97 \quad 2 \frac{1}{2}-3 \frac{1}{2}$ (154)

$4.967 .88 \quad 6 \frac{1}{2}-5 \frac{1}{2} b^{2} I-z^{4} \mathrm{H}^{\circ}$

$4.968 .34 \quad 6 \frac{1}{2}-6 \frac{1}{2} b^{2} I-2^{2} I^{\circ}$

$\begin{array}{lll}4.96 & 8.33 & 6 \frac{2}{2}-5 \frac{1}{2} \\ 4.97 & 8.34 & 5 \frac{1}{2}-6 \frac{1}{2}\end{array}$

$4.96 \quad 8.49 \quad 6 \frac{1}{2}-5 \frac{1}{2} b^{2} I-z^{2} H^{\circ}$ $\begin{array}{llll}4.97 & 8.45 & 5 \frac{1}{2}-4 \frac{1}{2} & \text { (157) } \\ 4.97 & 8.49 & 5 \frac{2}{2}-5 \frac{1}{2} & \end{array}$

$\begin{array}{llll}4.96 & 8.69 & 6 \frac{1}{2}-5 \frac{1}{2} & b^{2} I-y^{2} H^{\circ} \\ 4.97 & 8.69 & 5 \frac{2}{2}-4 \frac{1}{2} & (158)\end{array}$

$4.96 \quad 8.75 \quad 6 \frac{1}{2}-5 \frac{1}{2} b^{2} I-x^{4} a^{0}$

$\begin{array}{llll}4.97 & 8.73 & 5 & 4 \\ 4.97 & 8.75 & 5 \frac{1}{2}-5 \frac{1}{2} & \text { (159) }\end{array}$

$4.96 \quad 9.13 \quad 6 \frac{1}{2}-6 \frac{1}{2} b^{2} I-10$

$5.30 \quad 0.34 \quad 3 \frac{1}{5}-2 \frac{1}{2} b^{2} D-y^{4} p$

$5.318 .19 \quad 1 \frac{1}{2}-1 \frac{1}{2}$ (161)

$5.30 \quad 8.37 \quad 2 \frac{1}{2}-2 \frac{1}{2} b^{2} D_{-2} D^{0}$

$\begin{array}{lll}5.30 & 8.23 & 2 \frac{1}{2}-1 \frac{1}{5} \\ 5.31 & 8.27 & 1 \frac{1}{2}-2 \frac{1}{2}\end{array}$

$5.308 .28 \quad 2 \frac{1}{3}-1 \frac{1}{2} b^{2} D-z^{2} p^{0}$

$\begin{array}{llll}5.31 & 8.26 & 1 \frac{1}{2} & (163) \\ 5.31 & 8.28 & 1 & \end{array}$

$5.30 \quad 8.31 \quad 2 \frac{1}{2}-2 \frac{1}{2} b^{3} D-y^{4} c^{\circ}$

5.308 .32 2र $-3 \frac{1}{2} b^{2}$ D- 184 .

$\begin{array}{llll}5.30 & 8.32 & 3 & 3 \\ 5.31 & 8.32 & 1 \frac{1}{2}-1 \frac{2}{2} & (165)\end{array}$

$\begin{array}{lllll}5.31 & 8.47 & 1 \frac{1}{2}-2 \frac{1}{2} & \text { (167) } \\ 5.30 & 8.47 & 2 \frac{1}{2}-2 \frac{1}{2} & \end{array}$

$5.30 \quad 8.63 \quad 2 \frac{1}{2}-3 \frac{1}{2} b^{2} D-y^{3} G^{\circ}$

$5.30 \quad 8.64 \quad 3 \frac{2}{2}-a \frac{1}{2} b^{6} \mathrm{D}-y^{3} \mathrm{D}$

$\begin{array}{llll}5.30 & 8.69 & 2 \frac{1}{2}-3 \frac{1}{2} & b^{2} D-x^{4} G \cdot \\ 5.31 & 8.68 & 1 \frac{1}{2}-2 \frac{2}{2} & (170)\end{array}$

$5.30 \quad 8.75 \quad 2 \frac{1}{2}-3 \frac{1}{2} b^{2} D-y^{3} F^{\circ}$

$\begin{array}{llll}5.31 & 8.71 & 12 & 2 \\ 5.30 & 8.71 & 21 & \text { (171) }\end{array}$

$5.30 \quad 9.06 \quad 3 \frac{1}{2}-1 \frac{1}{2} b^{2} D-m^{4} D^{0}$

$5.319 .06 \quad 1 \frac{1}{2} \frac{1}{2} \quad(173)$

$5.30 \quad 9.15 \quad 2 \frac{1}{2}-3 \frac{1}{2} b^{2} D-x^{2} F^{\bullet}$

$5.30 \quad 9.19 \quad 2 \%$

$5.319 .17 \quad 1 \frac{1}{2}-2 \frac{1}{2} b^{2} D-\pi^{4} y^{\circ}$

$5.30 \quad 9.23 \quad 3 \frac{2}{2}-1 \frac{1}{2} b^{2} D-x^{2} P^{0}$

$\begin{array}{llll}5.65 & 8.24 & 21-2 \frac{1}{2} & c^{3} D-y^{4} p * \\ 5.84 & 8.19 & 1 \frac{1}{2}-1 \frac{1}{2} & (178)\end{array}$

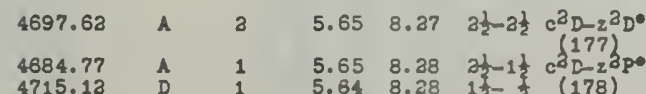

$\begin{array}{lllllll}4715.12 & D & 1 & 5.84 & 8.28 & 1 \frac{1}{2}-1 & (178) \\ 4671.38 & P & & 5.84 & 8.28 & 1 \frac{1}{2}-1 \frac{1}{2} & \end{array}$

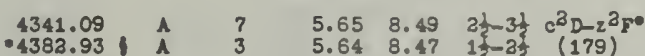

$\begin{array}{rlllllll}4382.93 & \mathrm{~A} & 3 & 5.64 & 8.47 & 1 \frac{1}{2}-23 & (179) \\ 4374.81 & \mathrm{P} & & 5.65 & 8.47 & 3 & -2 \frac{1}{2} & \end{array}$

4304.83
4199.02

4209.84
4232.00

4323.00
4332.88

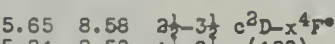

5.848 .58 1.2 $(180)$

$5.85 \quad 8.58 \quad 3 .-3$

$\begin{array}{lll}5.64 & 8.56 & 1 \\ 5.65 & 8.56 & 31\end{array}$
$5.30 \quad 8.38 \quad 2 \frac{21}{2}-3 \frac{1}{2} b^{2} D-x^{4} D^{0}$

$5.30 \quad 8.49 \quad 2 \frac{1}{3}-3 \frac{1}{2} b^{2} D-z^{2} F^{0}$

$\begin{array}{llll}5.31 & 8.64 & 1 \frac{5}{2}-2 \frac{1}{2} & \text { (169) }\end{array}$

I $A$ Laboratory Int

Low H1gh

Hultiplet
(No)

Cr II continued

\begin{tabular}{|c|c|c|c|c|c|c|}
\hline $\begin{array}{l}4127.08 \\
4170.86 \\
4181.50 \\
4116.66\end{array}$ & $\begin{array}{l}A \\
A \\
A \\
A\end{array}$ & $\begin{array}{l}3 \\
1 \\
1 \\
3\end{array}$ & $\begin{array}{l}5.65 \\
5.64 \\
5.65 \\
5.64\end{array}$ & $\begin{array}{l}8.64 \\
8.60 \\
8.60 \\
8.64\end{array}$ & $\begin{array}{l}2 \frac{1}{2}-2 \frac{1}{2} \\
1 \frac{1}{\frac{1}{2}}-1 \frac{1}{2} \\
2 \frac{1}{2}-1 \frac{1}{2} \\
1 \frac{1}{2}-2 \frac{1}{2}\end{array}$ & $\begin{array}{c}c^{2} D-y^{2} D^{0} \\
(181)\end{array}$ \\
\hline $\begin{array}{l}4048.02 \\
4056.07 \\
4066.16\end{array}$ & $\begin{array}{l}\mathrm{P} \\
\mathrm{A} \\
\mathrm{P}\end{array}$ & 4 & $\begin{array}{l}5.65 \\
5.64 \\
5.65\end{array}$ & $\begin{array}{l}8.69 \\
8.68 \\
8.68\end{array}$ & $\begin{array}{l}2 \frac{1}{2}-3 \frac{1}{2} \\
\frac{12}{2}-2 \frac{2}{2} \\
2 \frac{1}{2}-2 \frac{2}{2}\end{array}$ & $\begin{array}{c}c^{2} D-x^{4} G^{0} \\
(182)\end{array}$ \\
\hline $\begin{array}{c}3979.51 \\
.4012 .50 \\
4022.36\end{array}$ & $\begin{array}{l}\hat{A} \\
\hat{A}\end{array}$ & $\begin{array}{r}20 \\
30 \\
3\end{array}$ & $\begin{array}{l}5.65 \\
5.64 \\
5.65\end{array}$ & $\begin{array}{l}8.75 \\
8.71 \\
8.71\end{array}$ & $\begin{array}{l}2 \frac{1}{2}-3 \frac{1}{2} \\
1 \frac{1}{2}-2 \frac{1}{2} \\
2 \frac{1}{2}-2 \frac{2}{2}\end{array}$ & $\begin{array}{c}c^{2} D-y^{2} F^{\circ} \\
(183)\end{array}$ \\
\hline 3522.13 & $\boldsymbol{\Lambda}$ & 7 & 5.65 & 9.15 & $2 \frac{1}{2}-3 \frac{1}{2}$ & $c^{2} D-x^{2} F^{\circ}$ \\
\hline $\begin{array}{r}\text { ‥3484.16 } \\
\cdot 3489.45\end{array}$ & $\begin{array}{l}\text { P } \\
\text { f }\end{array}$ & $\begin{array}{c}3 r^{+} \\
2\end{array}$ & $\begin{array}{l}5.65 \\
5.64\end{array}$ & $\begin{array}{l}9.19 \\
9.17\end{array}$ & $\begin{array}{l}2 \frac{1}{2}-3 \frac{1}{2} \\
1 \frac{1}{2}-2 \frac{1}{2}\end{array}$ & $\begin{array}{c}c^{2} D-\pi^{4} F \\
(185)\end{array}$ \\
\hline $\begin{array}{l}3125.79 \\
3113.59\end{array}$ & $\hat{A}$ & $\begin{array}{l}5 \\
5\end{array}$ & $\begin{array}{l}5.65 \\
5.64\end{array}$ & $\begin{array}{l}9.59 \\
9.60\end{array}$ & $\begin{array}{l}2 \frac{1}{2}-1 \frac{1}{2} \\
1 \frac{1}{2}-\frac{1}{2}\end{array}$ & $\begin{array}{c}c^{2} D-y^{3} p o \\
(186)\end{array}$ \\
\hline $\begin{array}{l}6089.69 \\
6179.17 \\
6188.00\end{array}$ & $\begin{array}{l}\mathbf{A} \\
\mathbf{A}\end{array}$ & $\begin{array}{l}15 \\
10\end{array}$ & $\begin{array}{l}6.46 \\
6.46 \\
6.46\end{array}$ & $\begin{array}{l}8.49 \\
8.45 \\
8.45\end{array}$ & $\begin{array}{l}4 \frac{1}{2}-5 \frac{1}{2} \\
3 \frac{1}{2}-4 \frac{1}{2} \\
4 \frac{1}{2}-4 \frac{1}{2}\end{array}$ & $\begin{array}{c}d^{2} G-z^{2} H^{\circ} \\
(187)\end{array}$ \\
\hline $\begin{array}{l}6081.51 \\
6138.77\end{array}$ & $\hat{A}$ & $\begin{array}{l}3 \\
2\end{array}$ & $\begin{array}{l}6.46 \\
6.46\end{array}$ & $\begin{array}{l}8.49 \\
8.47\end{array}$ & $\begin{array}{l}4 \frac{1}{3}-3 \frac{1}{2} \\
3 \frac{1}{2}-2 \frac{1}{2}\end{array}$ & $\begin{array}{c}d^{2} G-z^{2} F^{\circ} \\
(188)\end{array}$ \\
\hline $\begin{array}{l}5820.63 \\
5678.42\end{array}$ & $\stackrel{A}{A}$ & $\begin{array}{l}12 \\
10\end{array}$ & $\begin{array}{l}6.46 \\
6.46\end{array}$ & $\begin{array}{l}8.65 \\
8.63\end{array}$ & $\begin{array}{l}4 \frac{1}{2}-4 \frac{1}{2} \\
3 \frac{1}{2}-3 \frac{1}{2}\end{array}$ & $d^{2} a-y^{3} \sigma^{\circ}$ \\
\hline $\begin{array}{l}4901.65 \\
4913.49\end{array}$ & $\hat{A}$ & $\begin{array}{l}15 \\
12\end{array}$ & $\begin{array}{l}6.46 \\
6.46\end{array}$ & $\begin{array}{l}8.98 \\
8.97\end{array}$ & $\begin{array}{l}4 \frac{1}{2}-4 \frac{1}{2} \\
3 \frac{1}{2}-3 \frac{1}{2}\end{array}$ & $\begin{array}{c}d^{2} G-x^{2} G^{\circ} \\
(190)\end{array}$ \\
\hline $\begin{array}{l}4465.78 \\
4511.82 \\
4516.56\end{array}$ & $\begin{array}{l}\mathrm{A} \\
\mathrm{P} \\
\mathrm{P}\end{array}$ & 4 & $\begin{array}{l}6.46 \\
6.46 \\
6.46\end{array}$ & $\begin{array}{l}9.22 \\
9.19 \\
9.19\end{array}$ & $\begin{array}{l}4 \frac{1}{2}-5 \frac{1}{2} \\
3 \frac{1}{2}-4 \frac{1}{2} \\
4 \frac{1}{2}-4 \frac{1}{2}\end{array}$ & $\begin{array}{c}d^{2} G-x^{2} H^{\bullet} \\
(191)\end{array}$ \\
\hline $\begin{array}{l}4256.16 \\
4268.93\end{array}$ & $\hat{A}$ & $\begin{array}{l}5 \\
1\end{array}$ & $\begin{array}{l}6.46 \\
6.46\end{array}$ & $\begin{array}{l}9.36 \\
9.35\end{array}$ & $\begin{array}{l}4 \frac{1}{2}-4 \frac{1}{2} \\
3 \frac{1}{2}-3 \frac{1}{2}\end{array}$ & $\begin{array}{c}d^{2} G-n^{3} G^{\circ} \\
(193)\end{array}$ \\
\hline $\begin{array}{l}4070.90 \\
4049.14 \\
4067.05\end{array}$ & $\begin{array}{l}\hat{A} \\
\hat{A}\end{array}$ & $\begin{array}{l}10 \\
18\end{array}$ & $\begin{array}{l}6.46 \\
6.46 \\
6.46\end{array}$ & $\begin{array}{l}9.49 \\
9.50 \\
9.49\end{array}$ & $\begin{array}{l}4 \frac{1}{2}-3 \frac{1}{3} \\
3 \frac{1}{2}-2 \frac{1}{2} \\
3 \frac{1}{2}-3 \frac{1}{2}\end{array}$ & $\begin{array}{c}d^{2} G-w^{2} F^{\bullet} \\
(193)\end{array}$ \\
\hline $\begin{array}{l}4038.03 \\
4 C 03.33 \\
4007.04\end{array}$ & $\begin{array}{l}\hat{A} \\
\hat{A} \\
\mathbf{P}\end{array}$ & $\begin{array}{l}25 \\
35\end{array}$ & $\begin{array}{l}6.46 \\
6.46 \\
6.46\end{array}$ & $\begin{array}{l}9.52 \\
9.54 \\
9.54\end{array}$ & $\begin{array}{l}4 \frac{1}{2}-5 \frac{1}{2} \\
3 \frac{1}{2}-4 \frac{1}{2} \\
4 \frac{1}{2}-4 \frac{1}{2}\end{array}$ & $\begin{array}{c}a^{2} G-w^{3} H^{\bullet} \\
(194)\end{array}$ \\
\hline 3089.75 & A & 1 & 6.46 & 10.45 & $4 \frac{1}{2}-3 \frac{1}{2}$ & $\begin{array}{c}d^{2} a-u^{2} p^{\circ} \\
(195)\end{array}$ \\
\hline $\begin{array}{l}6418.87 \\
6271.83 \\
6168.46 \\
6415.59 \\
6382.92 \\
6274.94\end{array}$ & $\begin{array}{l}\mathbf{A} \\
\hat{A} \\
\hat{A} \\
\mathbf{A} \\
\hat{A} \\
\hat{A}\end{array}$ & $\begin{array}{l}? \\
5 \\
2 \\
1 \\
2 \\
1\end{array}$ & $\begin{array}{l}6.66 \\
6.61 \\
6.58 \\
6.66 \\
6.61 \\
6.61\end{array}$ & $\begin{array}{l}8.58 \\
8.58 \\
8.58 \\
8.58 \\
8.58 \\
8.58\end{array}$ & $\begin{array}{l}4 \frac{1}{2}-4 \frac{1}{2} \\
3 \frac{1}{2}-3 \frac{1}{2} \\
2 \frac{1}{2}-2 \frac{1}{2} \\
4 \frac{1}{2}-3 \frac{1}{2} \\
3 \frac{1}{2}-2 \frac{1}{2} \\
3 \frac{1}{2}-4 \frac{1}{2}\end{array}$ & $\begin{array}{c}c^{4} F-x^{4} p^{\bullet} \\
(196)\end{array}$ \\
\hline $\begin{array}{l}6088.00 \\
6069.69\end{array}$ & $\hat{A}$ & $\begin{array}{l}7 \\
1\end{array}$ & $\begin{array}{l}6.66 \\
6.66\end{array}$ & $\begin{array}{l}8.69 \\
8.69\end{array}$ & $\begin{array}{l}4 \frac{1}{2}-5 \frac{1}{2} \\
4 \frac{1}{2}-4 \frac{1}{2}\end{array}$ & $\begin{array}{c}c^{4} F-y^{2} H^{\circ} \\
(197)\end{array}$ \\
\hline $\begin{array}{l}5895.90 \\
5841.86 \\
5827.24\end{array}$ & $\begin{array}{l}\hat{A} \\
\hat{A}\end{array}$ & $\begin{array}{l}4 \\
5 \\
5\end{array}$ & $\begin{array}{l}6.66 \\
6.61 \\
6.58\end{array}$ & $\begin{array}{l}8.75 \\
8.73 \\
8.69\end{array}$ & $\begin{array}{l}4 \frac{1}{2}-5 \frac{1}{2} \\
3 \frac{2}{2}-4 \frac{2}{2} \\
2 \frac{1}{2}-3 \frac{1}{2}\end{array}$ & $\begin{array}{c}c^{4} F-x^{4} G^{6} \\
(198)\end{array}$ \\
\hline 5110.43 & A & 3 & 6.68 & 9.07 & $4 \frac{1}{2}-3 \frac{1}{2}$ & $c^{4} F-w^{4} D^{0}$ \\
\hline 4857.60 & A & 3 & 6.66 & 9.20 & $4 \frac{1}{2}-4 \frac{1}{2}$ & $\begin{array}{c}c_{F}-T_{F} \\
(200)\end{array}$ \\
\hline $\begin{array}{l}5137.09 \\
5091.14 \\
5076.15\end{array}$ & $\hat{\hat{A}}$ & $\begin{array}{l}7 \\
3 \\
4\end{array}$ & $\begin{array}{l}6.79 \\
6.80 \\
6.79\end{array}$ & $\begin{array}{l}9.20 \\
9.22 \\
9.22\end{array}$ & $\begin{array}{l}2 \frac{1}{2}-2 \frac{1}{2} \\
1 \frac{1}{2}-1 \frac{1}{2} \\
2 \frac{1}{2}-1 \frac{1}{2}\end{array}$ & $\begin{array}{c}c^{4} p-x^{4} p^{0} \\
(201)\end{array}$ \\
\hline
\end{tabular}

Strongest Unclass1fled Lines of $\mathrm{Cr}$. II

6305.60

5913.87

4952.78

3814.00
3801.21

3778.69

3750.56
3711.29

3495.5
Kn I I P 7.40 Anal B L1gt B Hay 1941

$\begin{array}{ccccccc}5394.674 & \text { B } & 10 & 0.00 & 2.39 & 2 \frac{1}{2}-3 \frac{1}{2} & a^{6} \mathrm{~S}-z^{8} \mathrm{p} \\ 5433.548 & \text { B } & 4 & 0.00 & 2.27 & 2 \frac{1}{2}-2 \frac{1}{2} & (1)\end{array}$

$4030.755 / /$ B $200 R \quad 0.00 \quad 3.06 \quad 2 \frac{1}{2}-3 \frac{1}{2} a^{6} s-z^{6} P^{0}$

$\begin{array}{lllllll}4033.073 & \text { B } & 150 R & 0.00 & 3.06 & 2 \frac{2}{2} & \text { (2) } \\ 4034.490 & \text { B } & 100 R & 0.00 & 3.06 & 2 \frac{1}{2}-1 & \end{array}$

$\begin{array}{lllllll}3224.761 & \mathrm{~B} & 10 & 0.00 & 3.83 & 2 \frac{1}{2}-2 \frac{1}{2} & \mathrm{a}^{6} \mathrm{~S}-2^{4} \mathrm{P}\end{array}$

$\begin{array}{lllllllllll}3216.946 & B & 8 & 0.00 & 3.84 & 2 \frac{1}{2}-1 \frac{1}{2} & \text { (3) }\end{array}$

$\begin{array}{lllllll}5341.065 & \mathrm{~B} & 20 & 2.11 & 4.42 & 4 \frac{1}{3}-3 \frac{3}{2} & \mathrm{a}^{6} \mathrm{D}_{-\mathrm{y}^{6} \mathrm{P}^{0}} \\ 5420.362 & \mathrm{~B} & 10 & 2.13 & 4.41 & 3 \frac{1}{2}-2 \frac{1}{2} & \end{array}$

5481.396
5407.424

5470.638

5516.771
5457.471

5505.889

5537.756
$2.15 \quad 4.41 \quad 25-1$

$2.13 \quad 4.42 \quad 3 \frac{1}{2}-3$

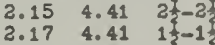

$2.15 \quad 4.42 \quad 2 \frac{1}{2}-3$

$\begin{array}{lll}2.17 & 4.41 & 1 \frac{1}{2}-2 \frac{2}{3} \\ 2.18 & 4.41 & \frac{1}{2}-1 \frac{1}{2}\end{array}$ 
${ }_{1} x^{2}$

In oontinued

\begin{tabular}{|c|c|c|c|c|c|}
\hline $\begin{array}{l}4041.361 \\
4055.543 \\
4063.528 \\
4068.003 \\
4070.279 \\
4018.102 \\
4035.728 \\
4048.755 \\
4058.930 \\
4079.241 \\
4083.628 \\
4082.944 \\
4079.422\end{array}$ & $\begin{array}{l}\text { B } \\
\text { B } \\
\text { B } \\
\text { B } \\
\text { B } \\
\text { B } \\
\text { B } \\
\text { B } \\
\text { B } \\
\text { B } \\
\text { B } \\
\text { B } \\
\text { B }\end{array}$ & $\begin{array}{l}50 r \\
30 \\
8 \\
(3) \\
5 \\
20 \\
15 \\
15 \\
10 \\
12 \\
12 \\
12 \\
10\end{array}$ & $\begin{array}{l}2.11 \\
2.13 \\
3.15 \\
3.17 \\
3.18 \\
2.11 \\
2.13 \\
2.15 \\
2.17 \\
2.13 \\
2.15 \\
2.17 \\
2.18\end{array}$ & $\begin{array}{l}5.16 \\
5.18 \\
5.19 \\
5.20 \\
5.21 \\
5.18 \\
5.19 \\
5.20 \\
5.21 \\
5.16 \\
5.18 \\
5.19 \\
5.20\end{array}$ & 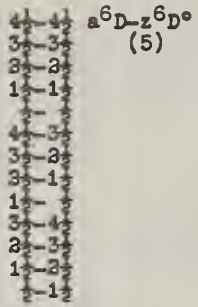 \\
\hline $\begin{array}{l}3806.719 \\
3823.513 \\
3834.364 \\
3841.082 \\
3843.983 \\
3790.215 \\
3809.592 \\
3823.893 \\
3833.862 \\
3839.777 \\
377.6 .527 \\
3799.259 \\
3816.753 \\
3829.680\end{array}$ & $\begin{array}{l}\text { B } \\
\text { B } \\
\text { B } \\
\text { B } \\
\text { B } \\
\text { B } \\
\text { B } \\
\text { B } \\
\text { B } \\
\text { B } \\
\text { B } \\
\text { B } \\
\text { B } \\
\text { B }\end{array}$ & $\begin{array}{l}20 r \\
20 r \\
12 \\
10 \\
7 \\
10 \\
10 \\
10 \\
8 \\
8 \\
(1) \\
4 \\
5 \\
5\end{array}$ & $\begin{array}{l}2.11 \\
2.13 \\
3.15 \\
2.17 \\
2.18 \\
2.11 \\
2.13 \\
2.15 \\
2.17 \\
2.18 \\
2.11 \\
2.13 \\
2.15 \\
2.17\end{array}$ & $\begin{array}{l}5.35 \\
5.36 \\
5.37 \\
5.38 \\
5.39 \\
5.36 \\
5.37 \\
5.38 \\
5.39 \\
5.39 \\
5.37 \\
5.38 \\
5.39 \\
5.39\end{array}$ & 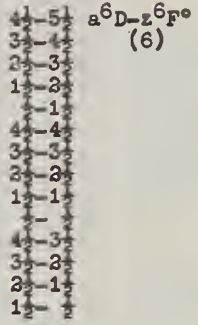 \\
\hline
\end{tabular}

$\begin{array}{lllllll}3670.517 & \mathrm{~B} & 5 & 2.11 & 5.47 & 4 \frac{1}{2}-4 \frac{1}{2} & a^{6} \mathrm{D}-2^{4} \mathrm{~F}^{\circ}\end{array}$

3669.838
3669.399

3639.14
3701.730

3692.121
3685.212

3678.46

3577.880
3586.543

3586.543
3595.119
3607.537

3608.494

3610.299

3623.792
3619.284
3350.68

3330.668

3343.731
3351.424

3280.756
3311.905

3296.027

3308.785
3316.334

3254.039

3278.553
3296.883

3240.399

3228.090

3236.778

3248.516
3256.137

3260.231

3230.719
3212

3252.948

3558.413

3226.034

3240.616
3251.135

3044.567

3054.362
3062.119
3065.019

3070.266

3073.126

3001.330
3079.627

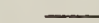

4783.420
4754.042

4370.875

3569.493
3547.802

3547.802
3531.848

3569.804

3548.029
3531.998

3570.041

3548.202
3532.121

3178.495

3161.039
3148.179

4965.881
5004.907

5029.812

4942.418

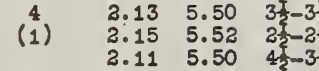

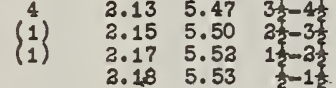

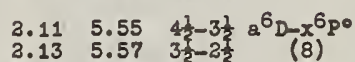

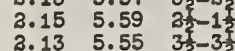

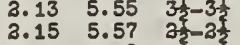

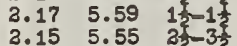

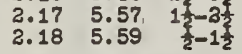

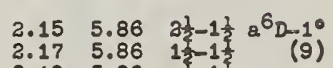

3.185 .86 3

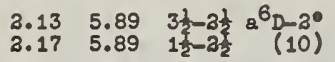

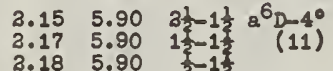

3.11 5.90 4t-31 $\mathrm{a}^{6} \mathrm{D}-30^{\circ}$

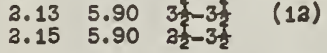

$\begin{array}{llll}2.11 & 5.91 & 41 & 0 \\ 2.13 & 5.91 & 0-50 & (13)\end{array}$

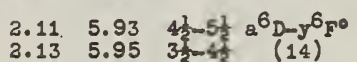

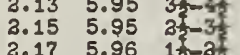

$2.18 \quad 5.96 \quad$ s.

$\begin{array}{lll}2.11 & 5.95 & 4 \\ 2.13 & 5.95 & 3=-3\end{array}$

$3.15 \quad 5.96 \quad 32$

$\begin{array}{lll}2.17 & 5.96 & 17-1 \\ 2.18 & 5.96 & \end{array}$

3.11 5.95 4 4 - 3 3

$\begin{array}{lll}2.13 & 5.96 & 36-25 \\ 2.15 & 5.96 & 37 \\ 2.17 & 5.96 & 1 \\ 1 \frac{1}{2}-\frac{1}{2}\end{array}$

$2.116 .16 \quad 4 \frac{1}{3}-3 \frac{1}{2} a^{6} D_{-1} 6 p^{6}$

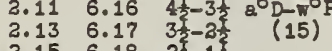

$2.15 \quad 6.18$ 3.1

$\begin{array}{llll}2.15 & 6.17 & 3 \\ 2.15 & 25\end{array}$

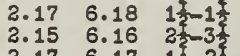

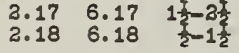

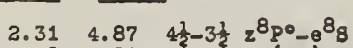

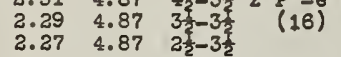

(1) $2.29 \quad 5.11 \quad 3 \frac{1}{2}-3 \frac{1}{2} 2^{8} \mathrm{po}^{0}-8^{6} \mathrm{~s} 4$

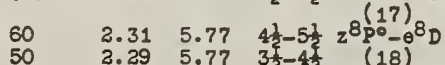

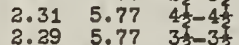

$\begin{array}{lll}2.39 & 5.77 & 35-3 \\ 2.27 & 5.77 & 35 \\ 2 & 2.35\end{array}$

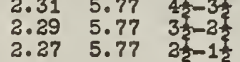

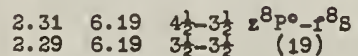

$\begin{array}{lll}3.29 & 6.19 & 3 \\ 3.27 & 6.19 & 3 \frac{3}{2}-3 \frac{3}{2} \\ 3 & 3 \frac{2}{2}\end{array}$

$2.885 .36 \quad 3 \frac{1}{2}-4 \frac{1}{3} a^{4} D_{-2} z^{6}{ }^{\circ}$

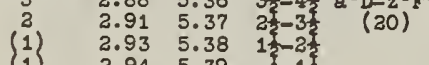

$\begin{array}{lll}2.93 & 5.38 & 1 \\ 2.94 & 5.39 & 3 \\ 2.88 & 5.37 & 3 \frac{1}{2}-3 \frac{2}{2}\end{array}$

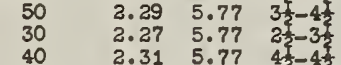

\section{Laboratory $E P$ J multiplet}

\section{$\underline{\underline{n} I}$ continued}

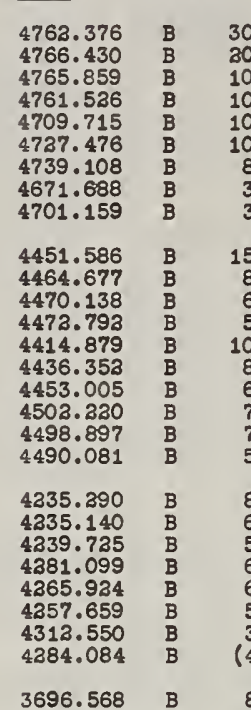

3696.568

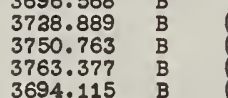

3726.931

3601.783

3601.782
3583.691
3589.676

3407.960

6021.803

6016.637
6013.498

4462.023
4458.262
4455.821

4455.821
4461.085

445.549
4455.318
4460.377

4460.377
4457.045

4455.013

4059.393
4057.950

3317.305

3314.876
-3313.534

3316.440
3314.393

5504.2

5480.6

5444.096
5510.174

5255.325

5150.890

5260.77

5197.216

3986.836

3987.098
3958.241
398.5

398.583

398.464

3986.395

3047.035

3045.593
3043.356

3040.603
3043.770
303.743

3043.143
3041.22

3048.86

3045.808
3042.733

3023.749
3016.454

301.454

3011.376
3007.655

3014.668
3011.162
30162

3008.265

5388.521

5348.069
5317.095

5334.804

5307.53

$\left(\begin{array}{l}8 \\ 1 \\ 2 \\ 3 \\ 1 \\ 1\end{array}\right\}$

(3) $3.36 \quad 5.65 \quad 2 \frac{1}{2}-3 \frac{1}{3} \mathrm{a}^{4} \mathrm{P}-2^{4} \mathrm{D}$

$\left(\begin{array}{l}1 \\ 2 \\ 1\end{array}\right)$

$\begin{array}{llll}3.36 & 5.67 & 13-3 \frac{1}{2} & 8^{4} \mathrm{P}-2^{4}=\mathrm{D} \\ 3.37 & 5.69 & (36)\end{array}$

$\begin{array}{lll}3.37 & 5.69 & 1 \\ 3.36 & 5.67 & 2\end{array}$

$\begin{array}{lll}3.36 & 5.69 & 1 \frac{1}{1}-1 \frac{2}{2} \\ 3.37 & 5.70 & \end{array}$

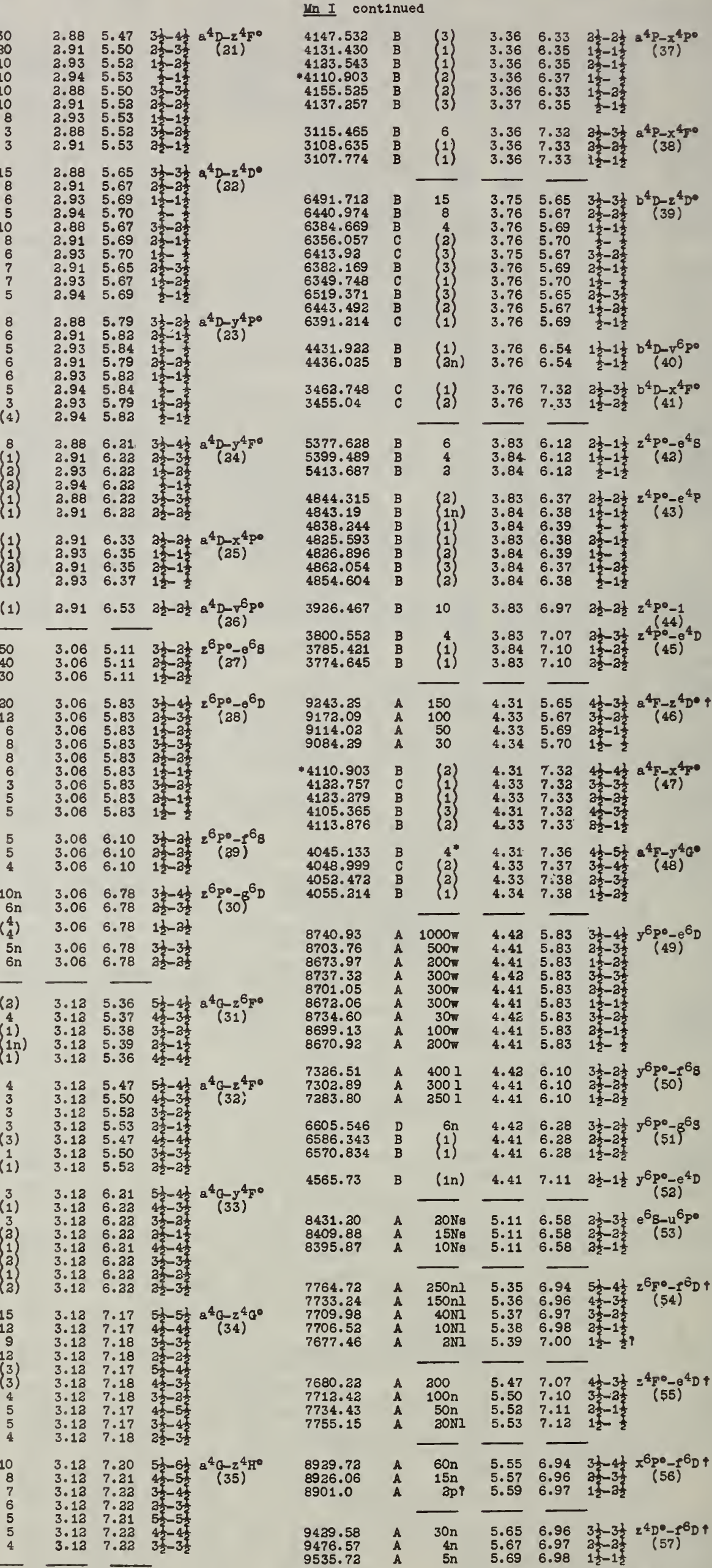

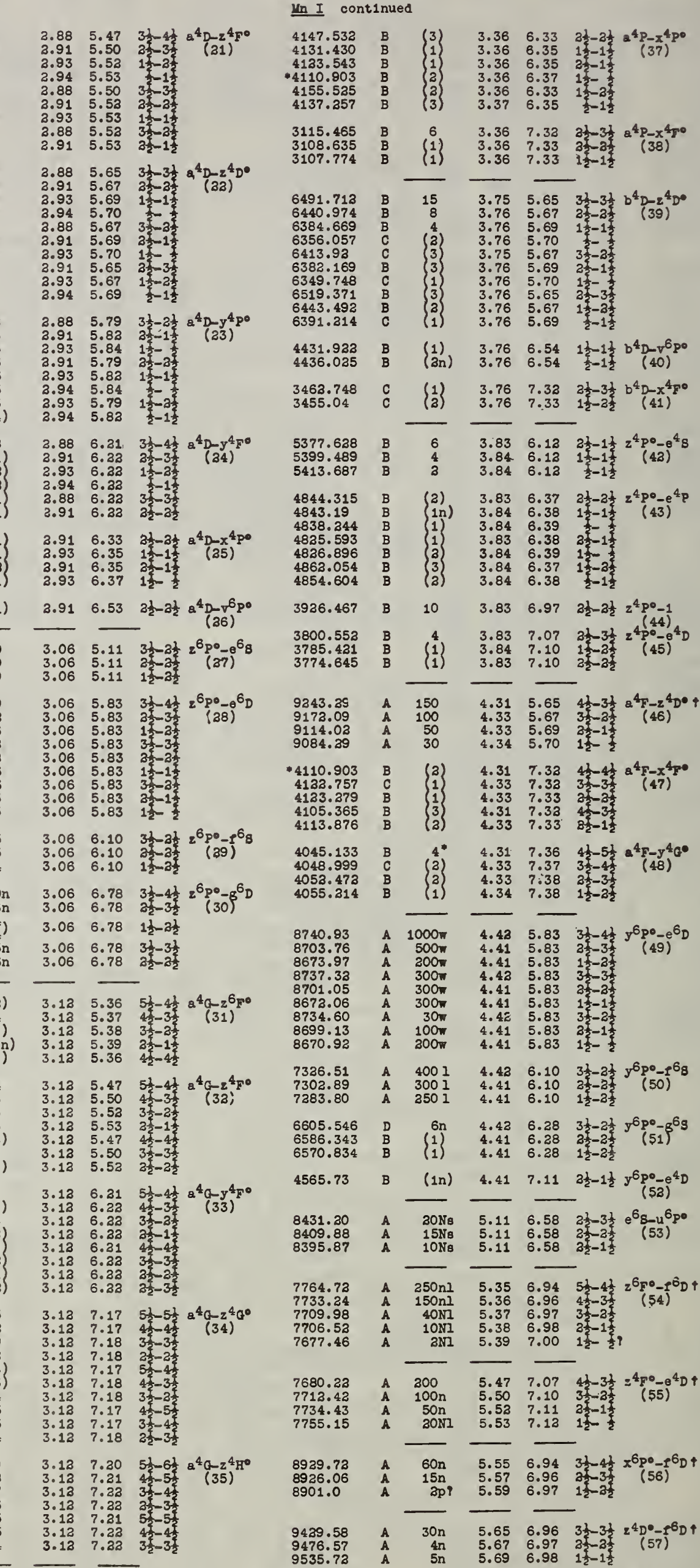

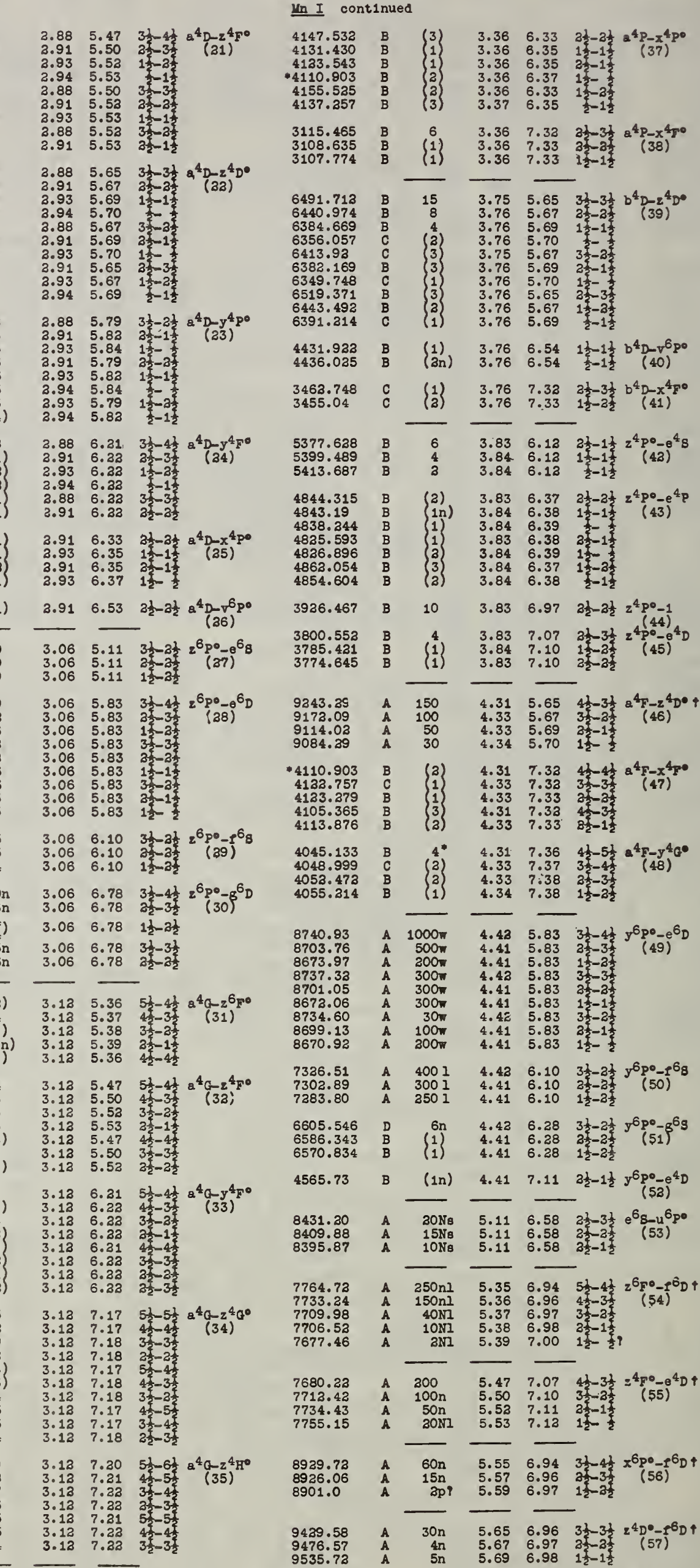

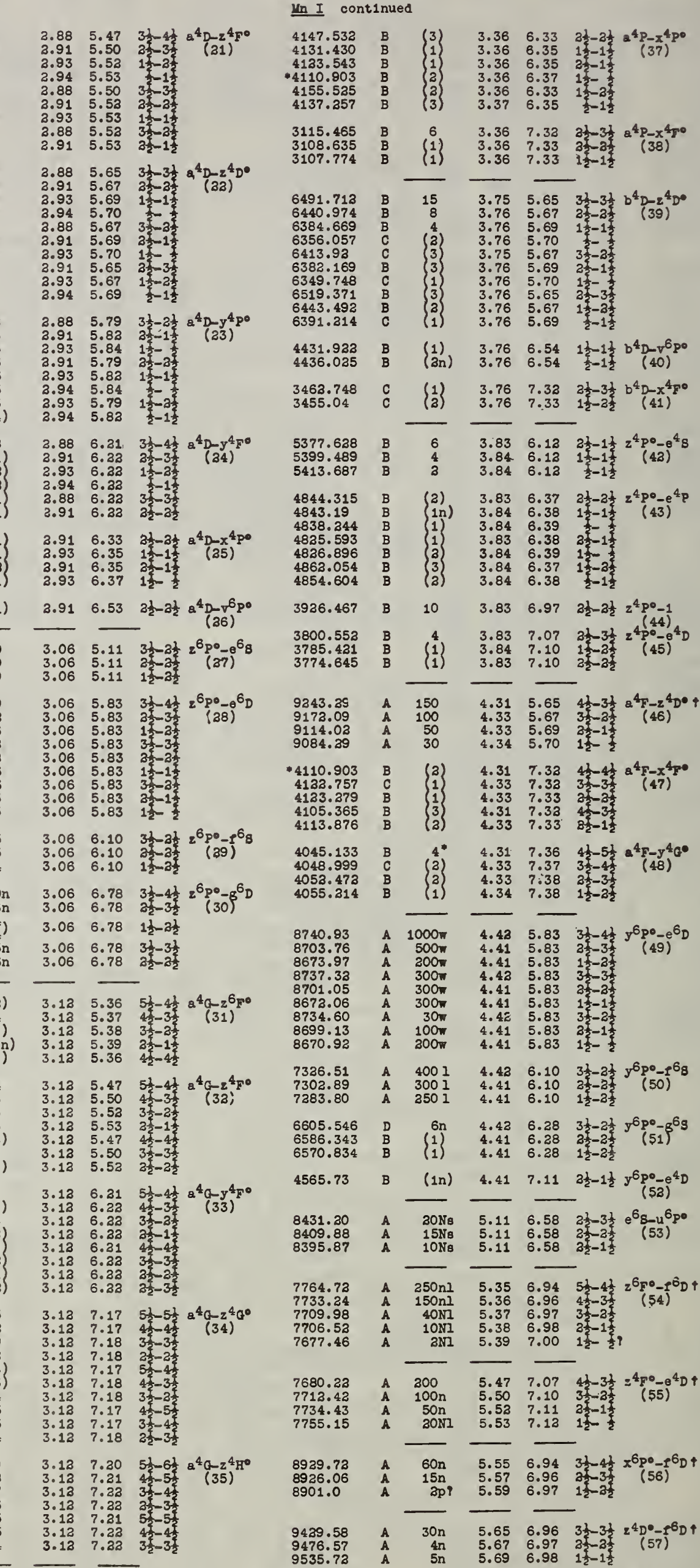

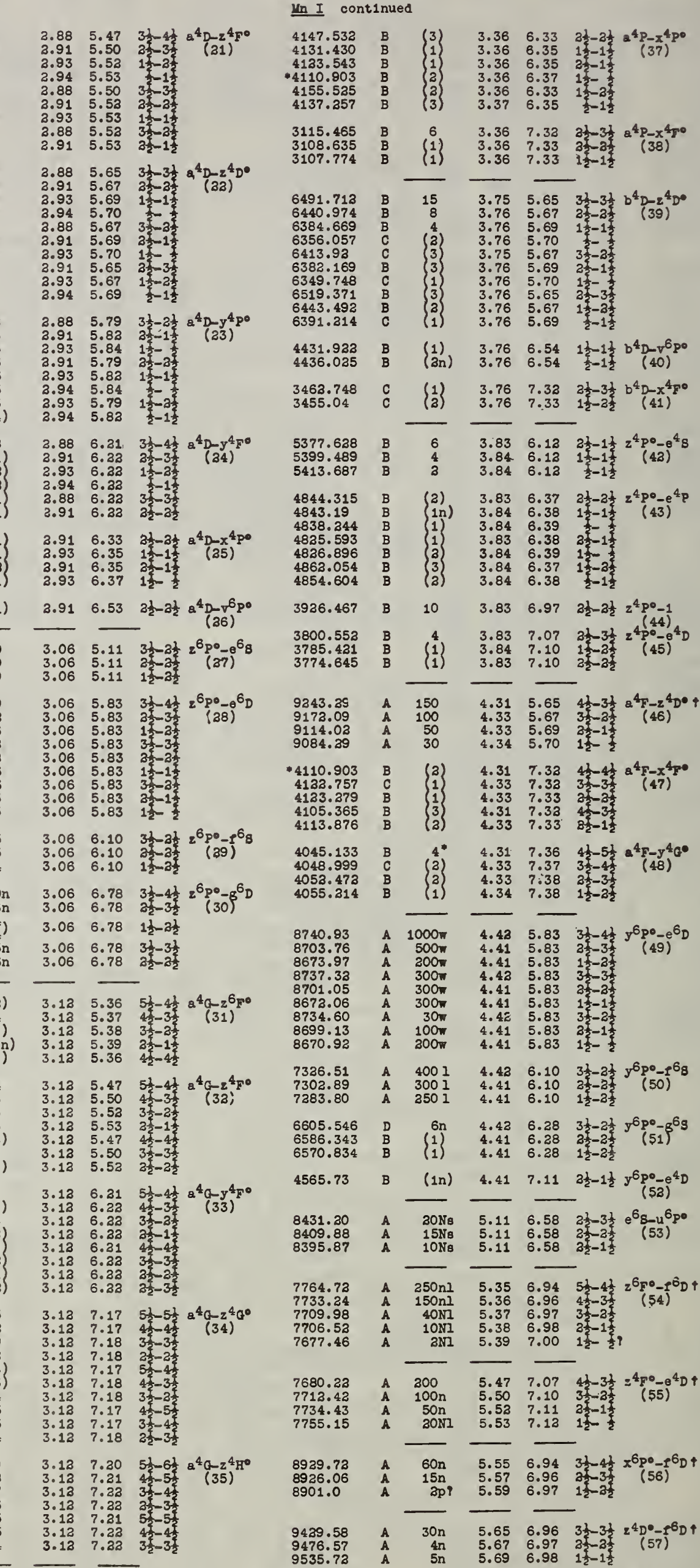

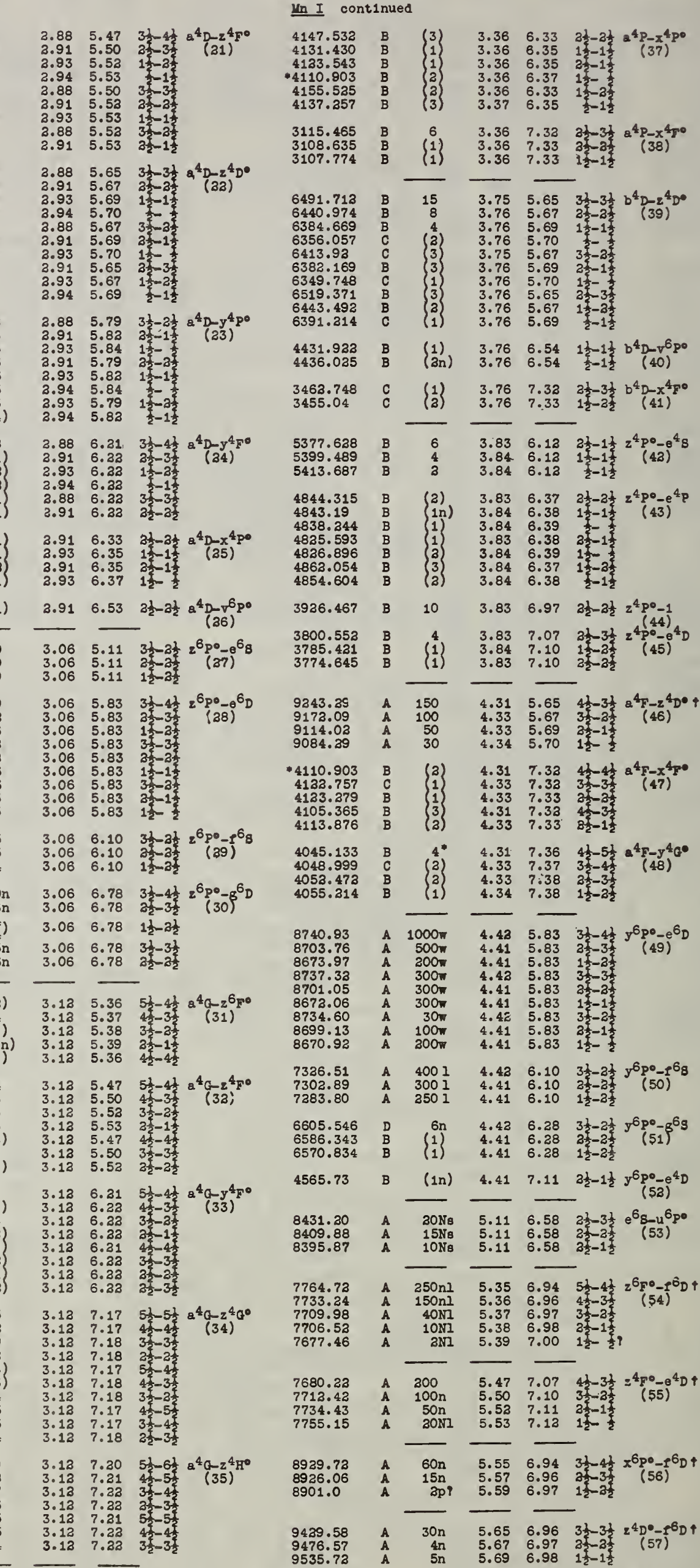

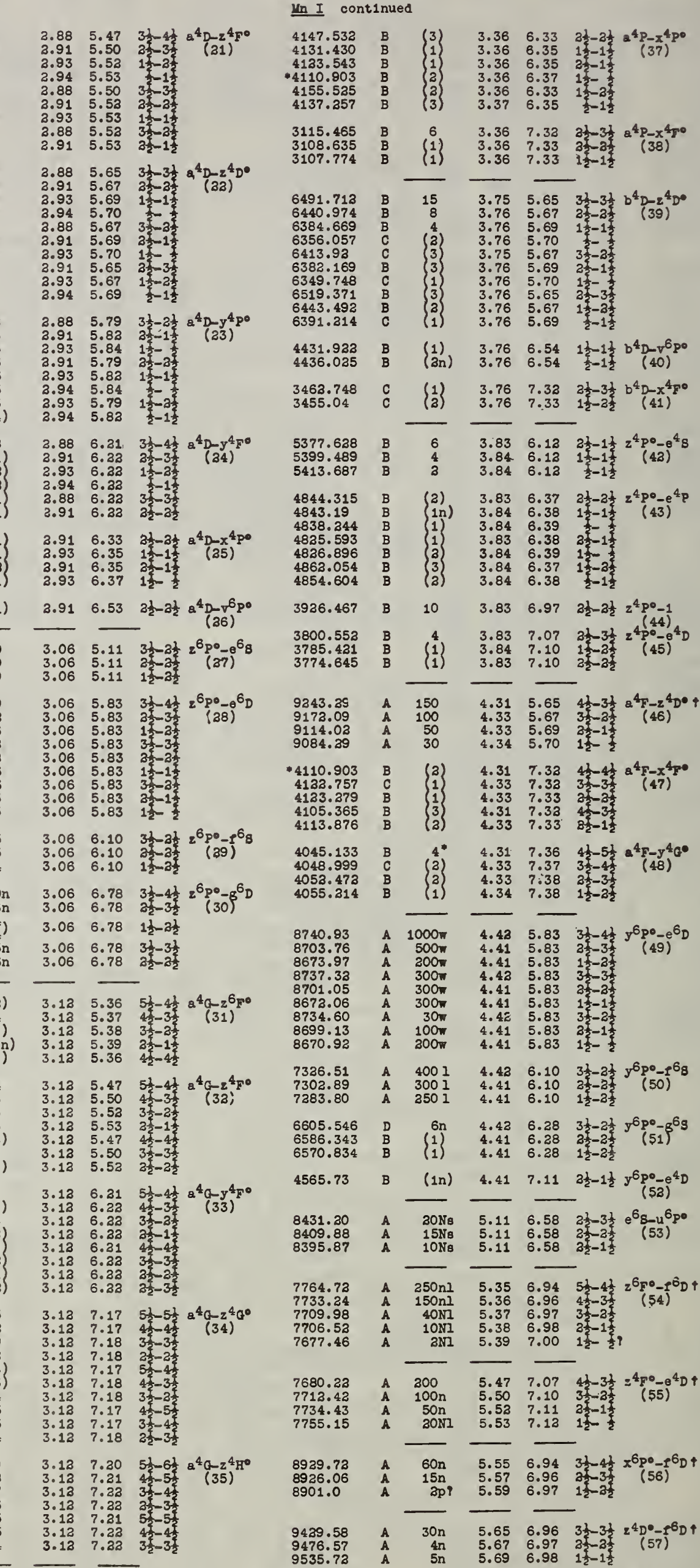

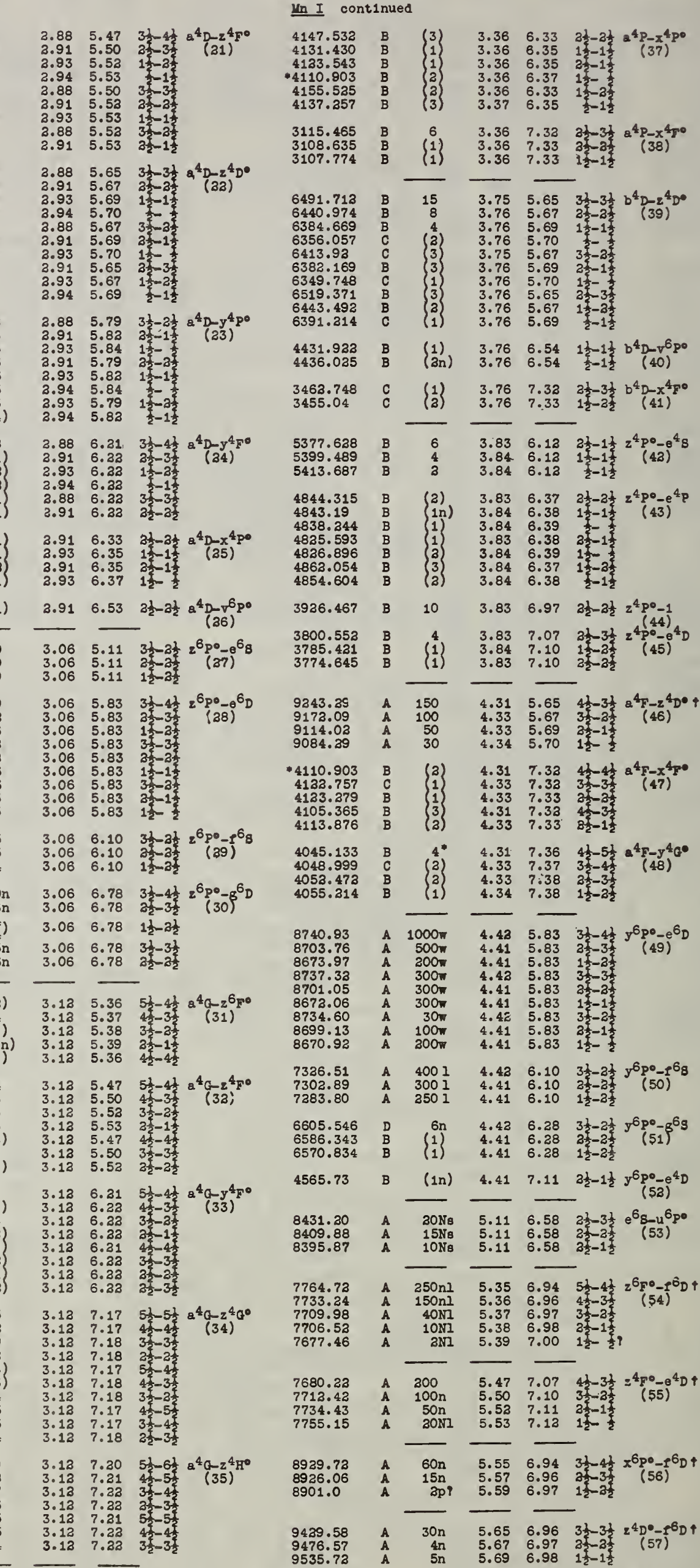

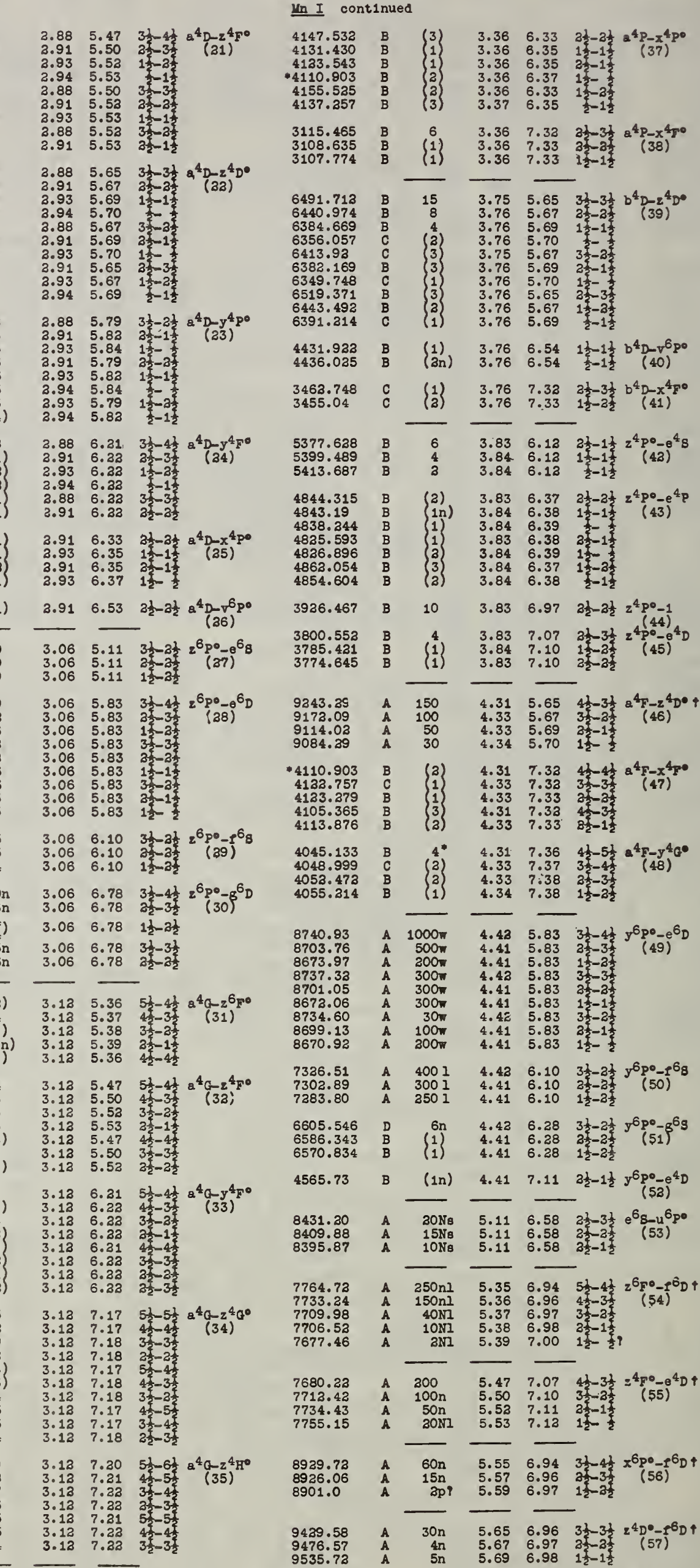

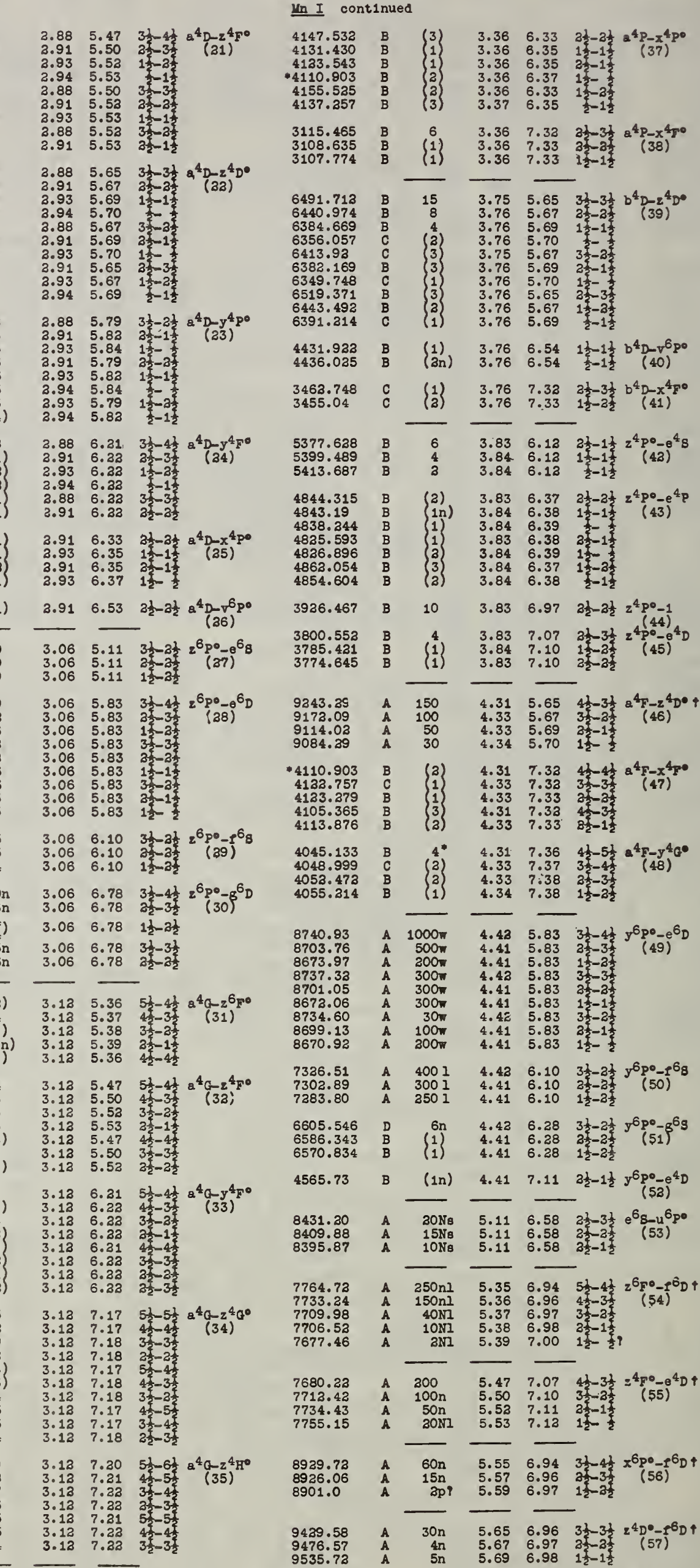

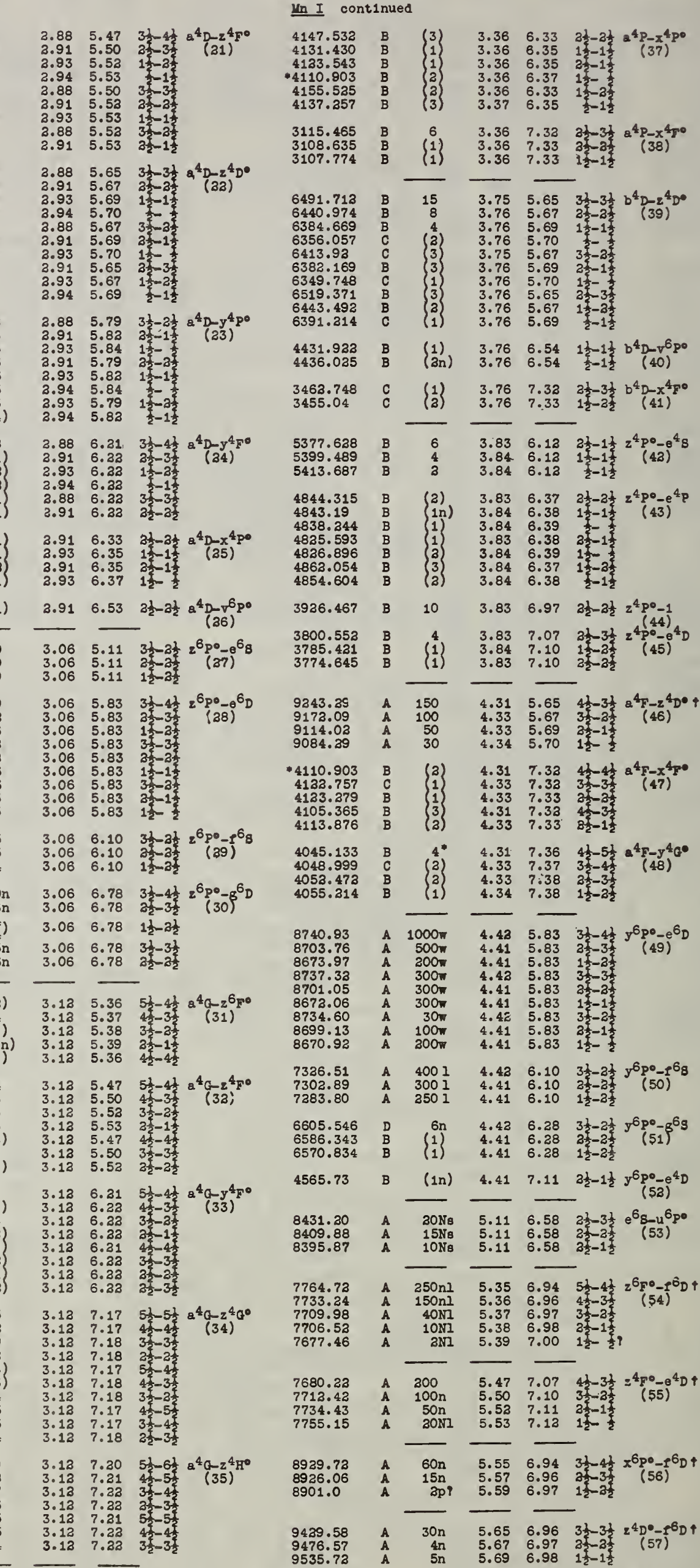

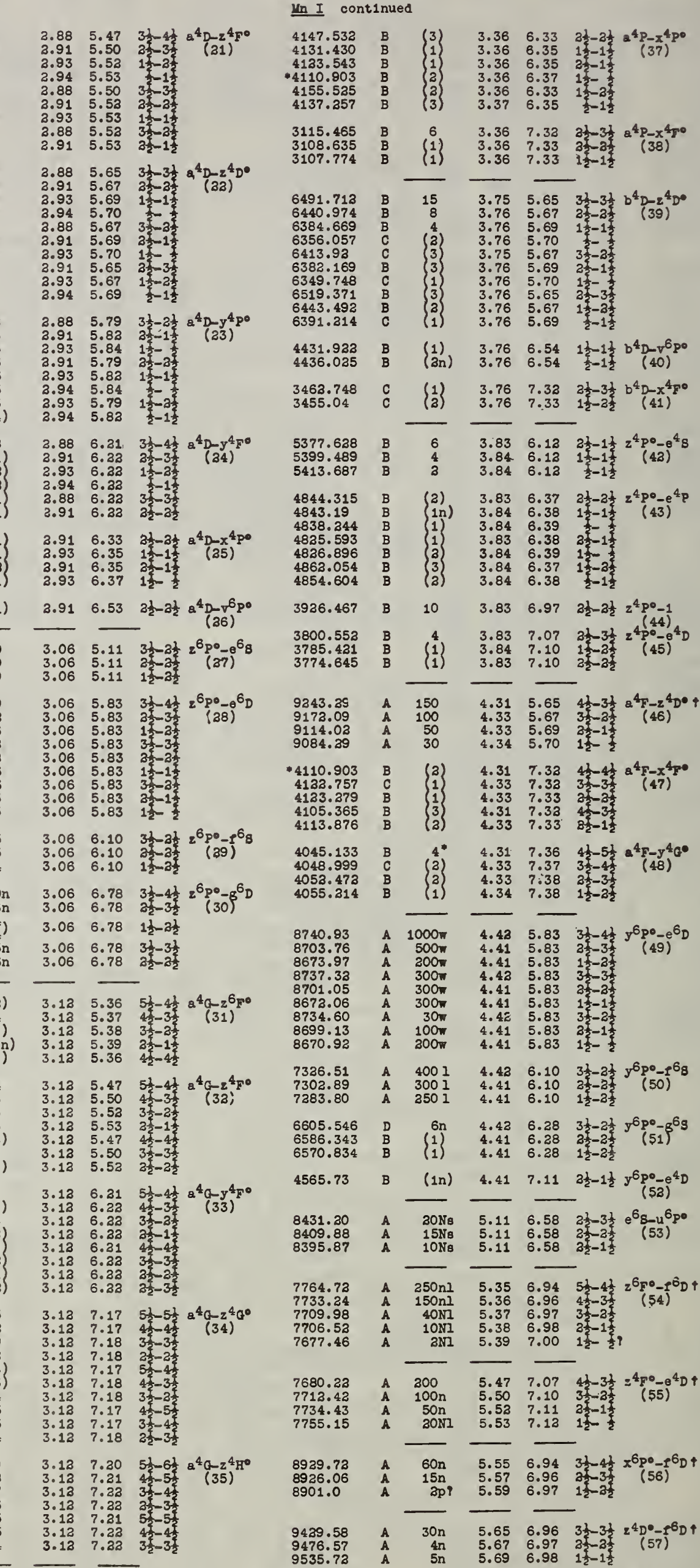

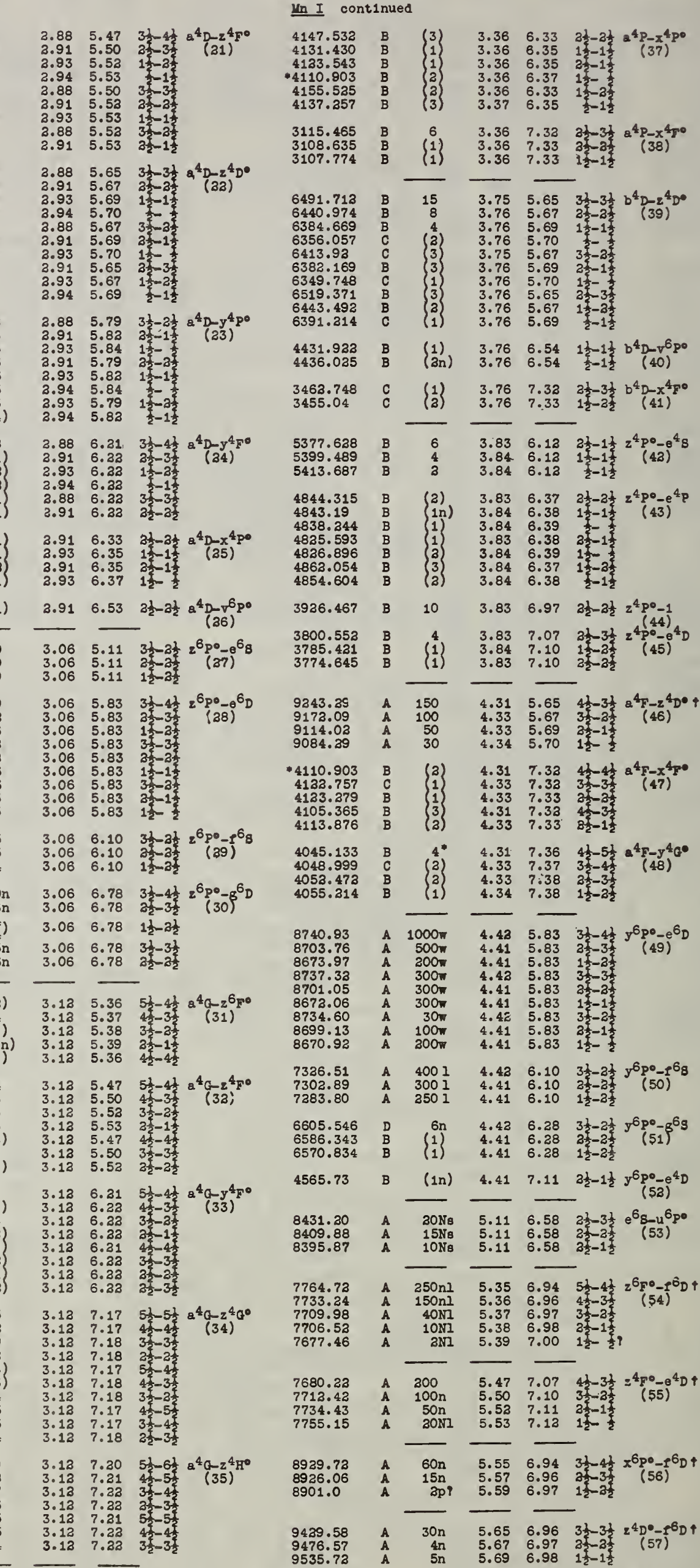

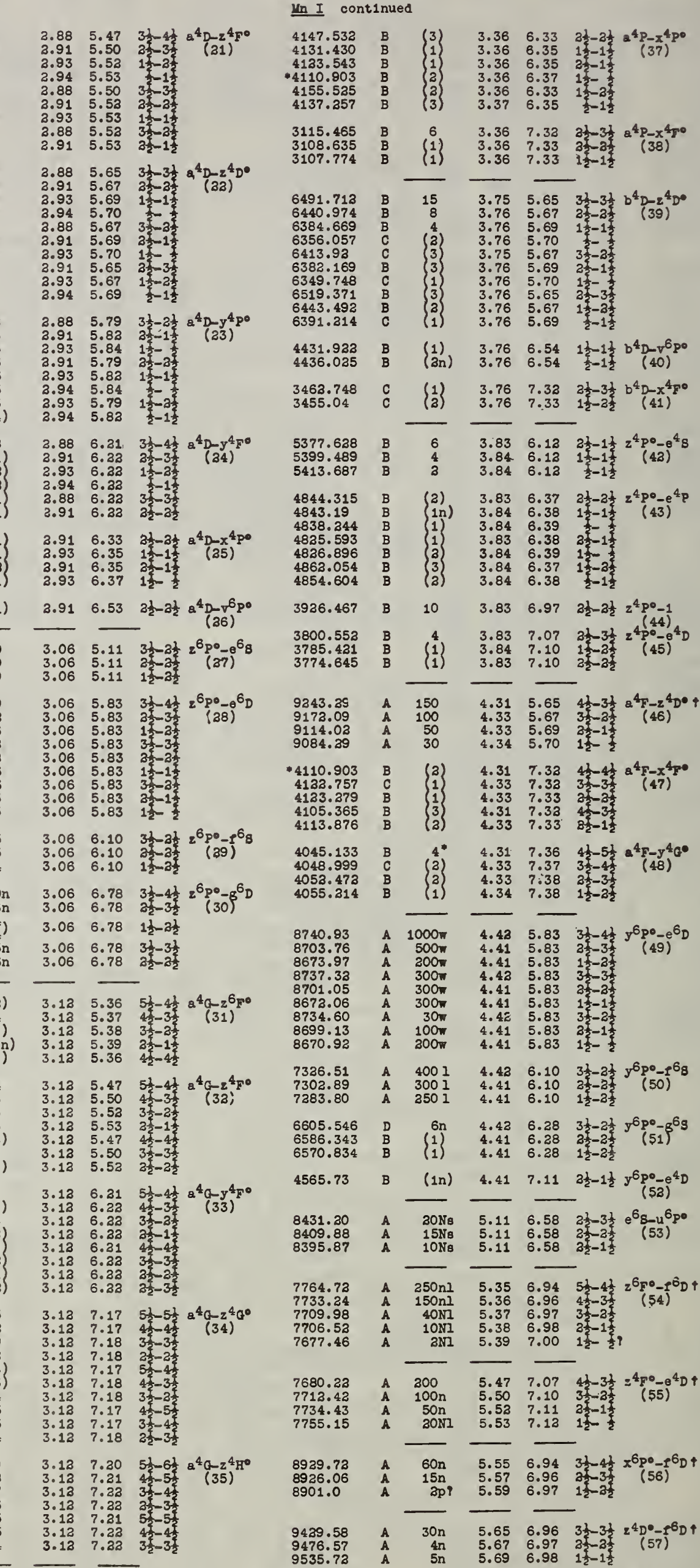

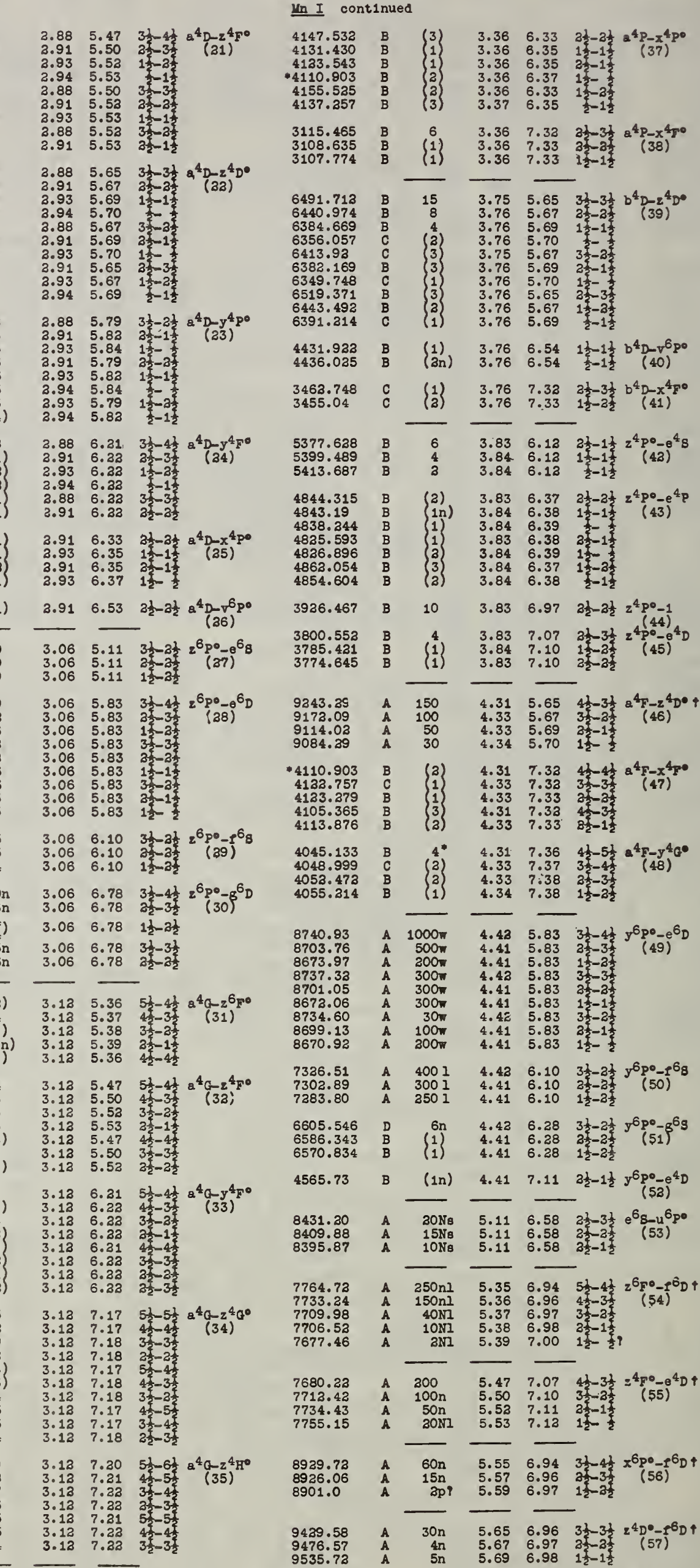

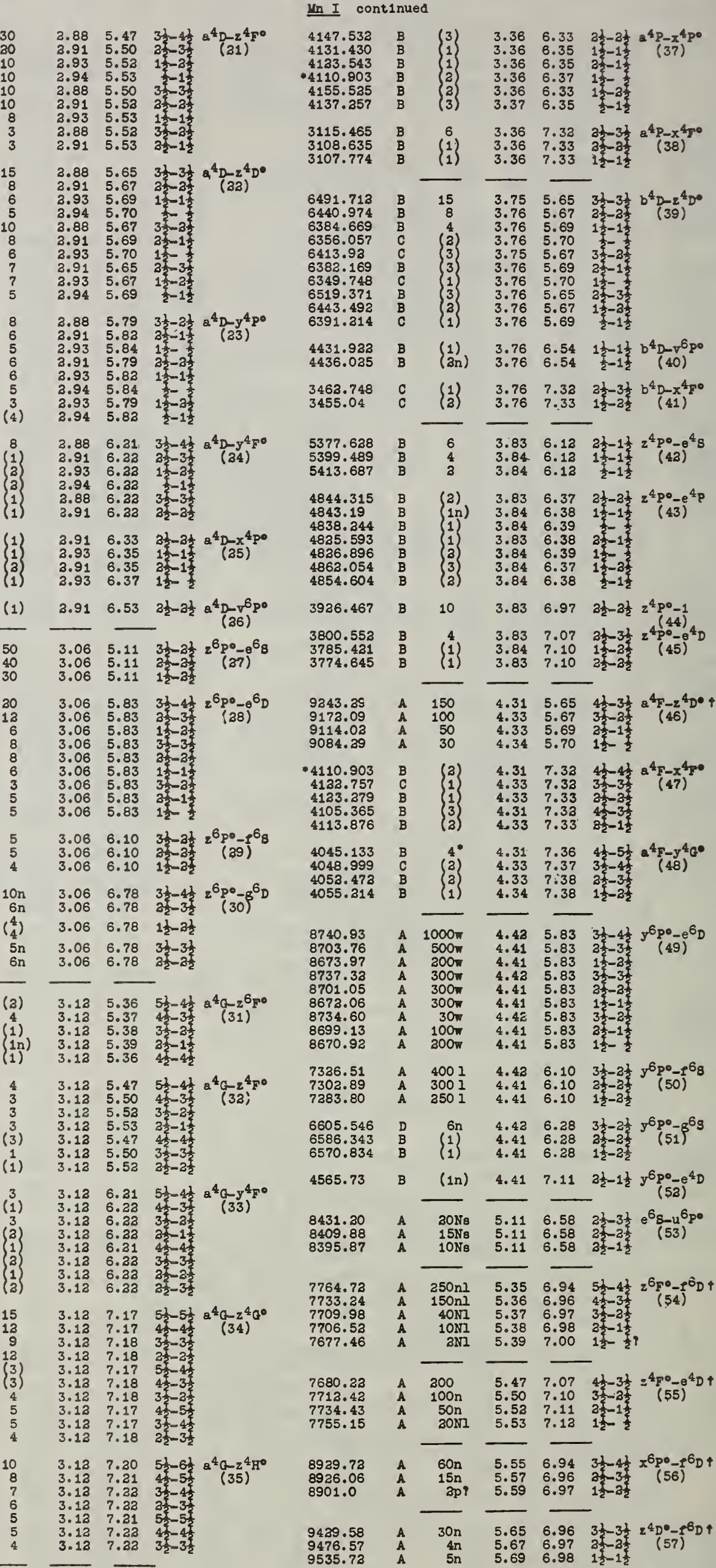

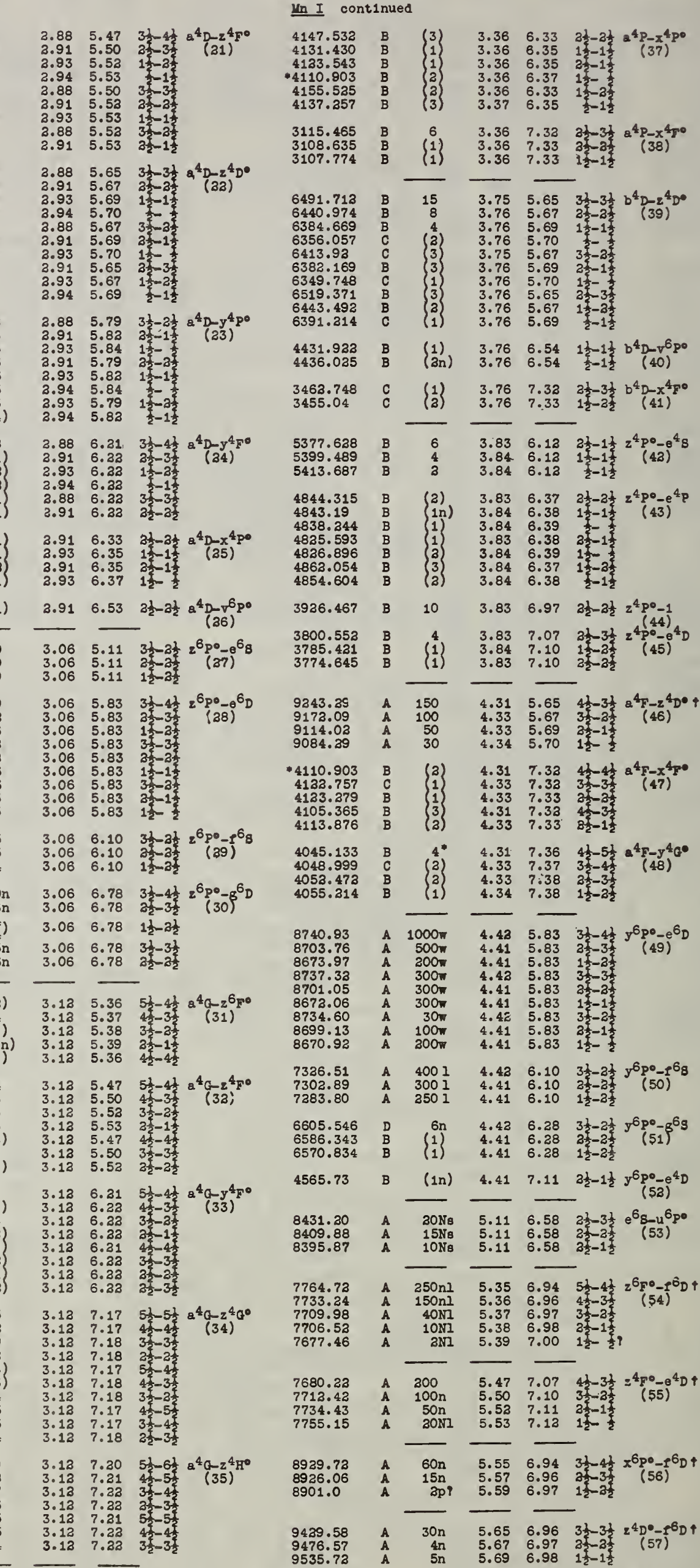

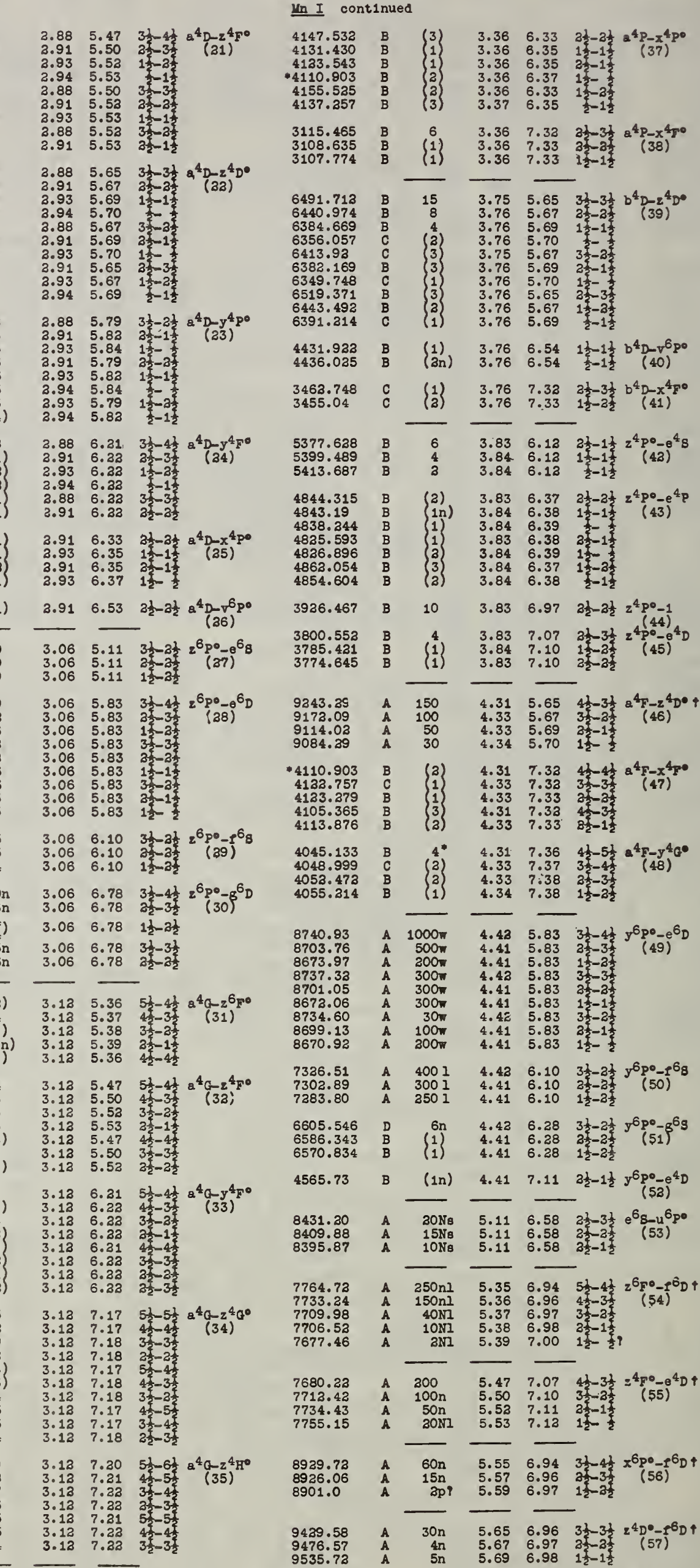

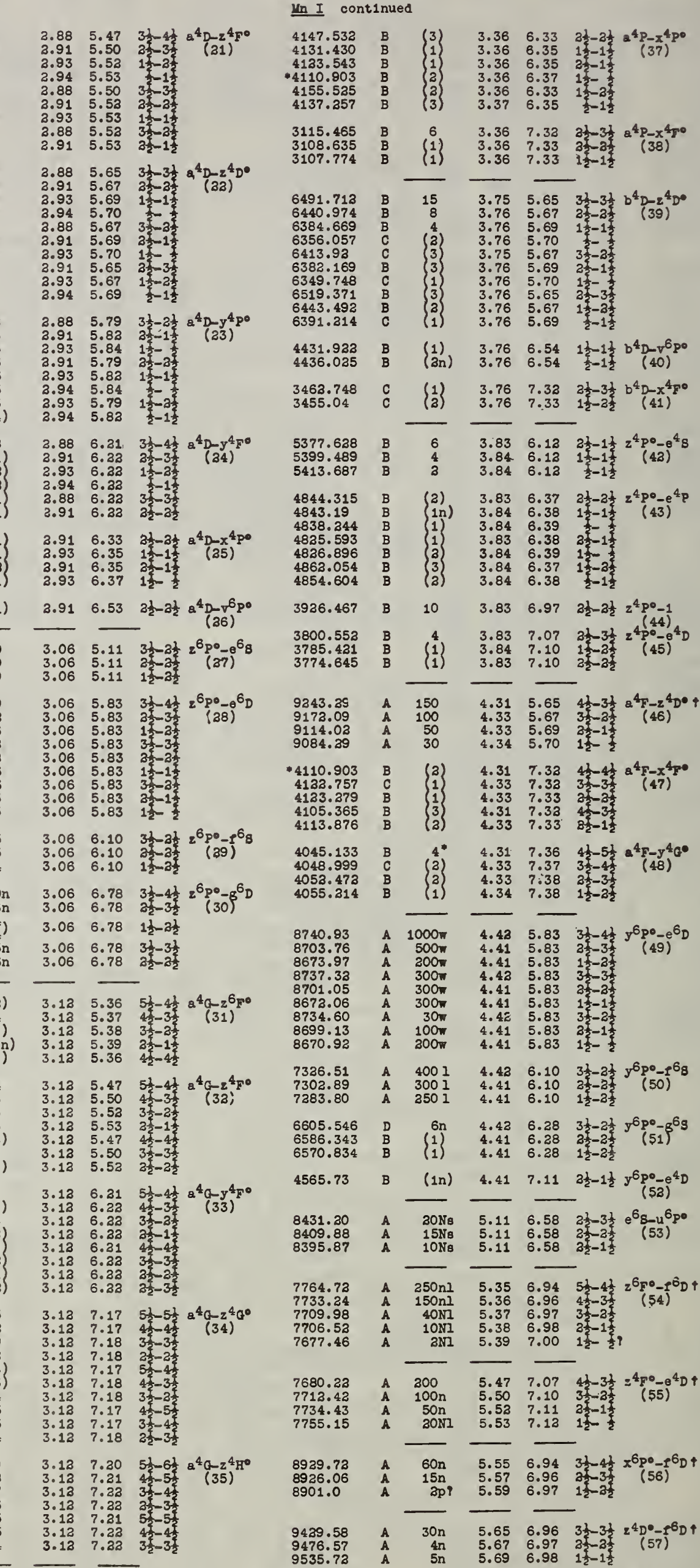

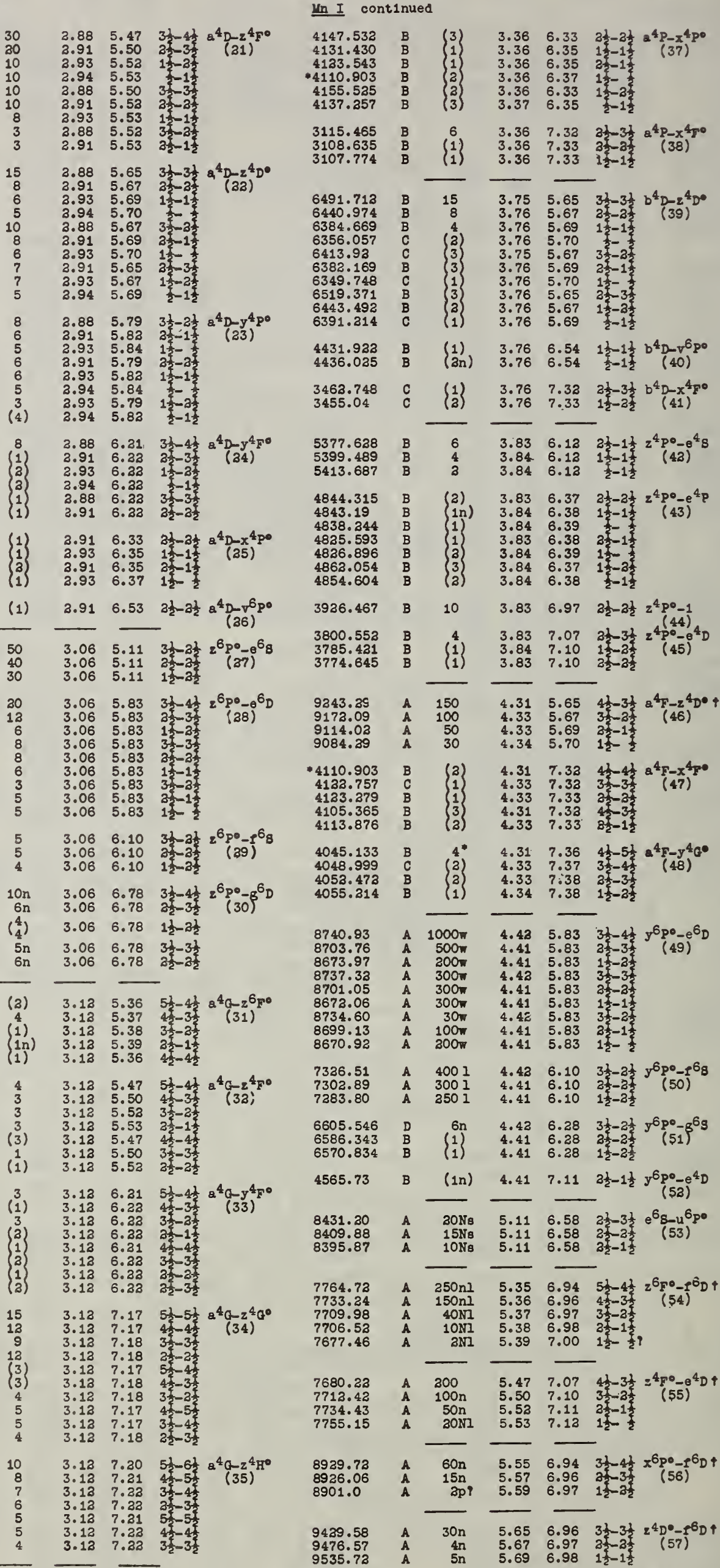

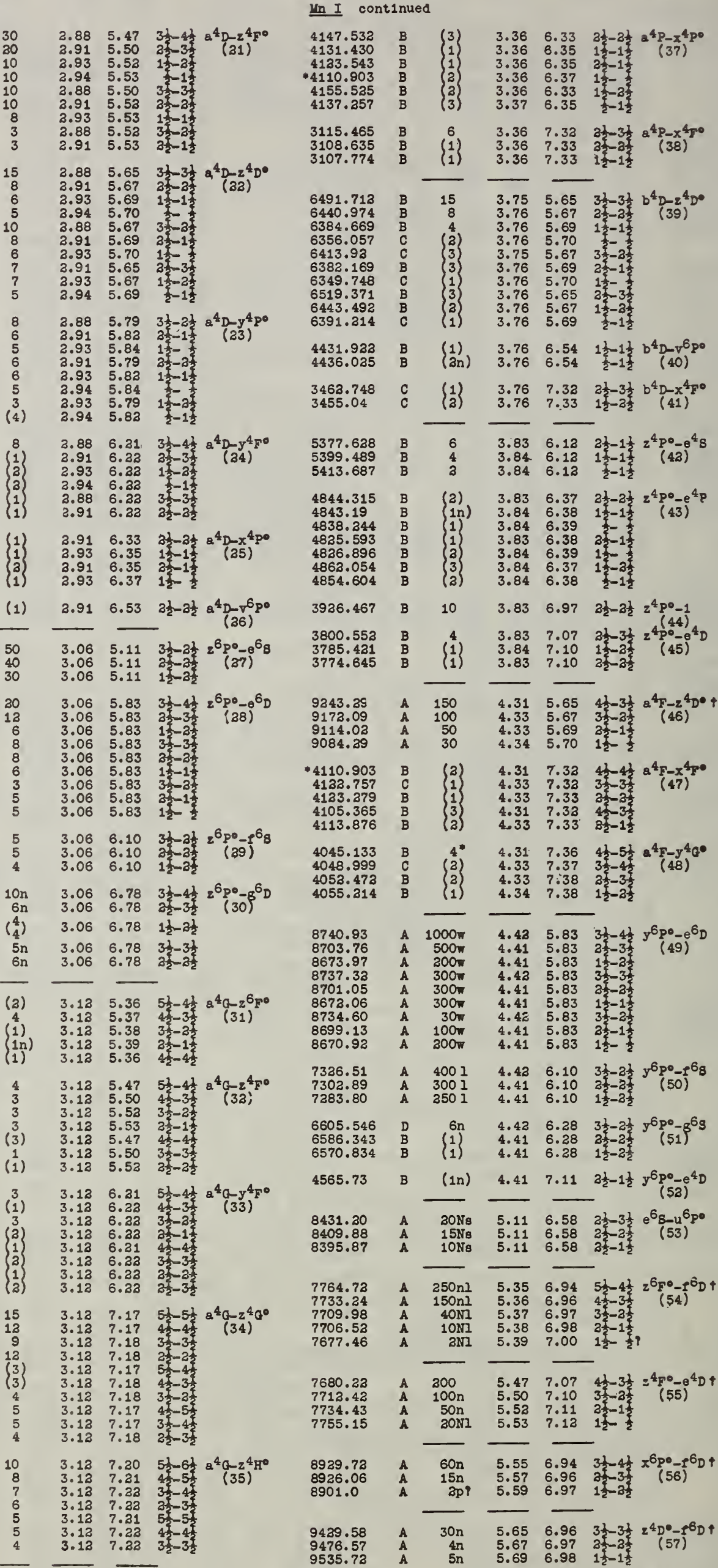

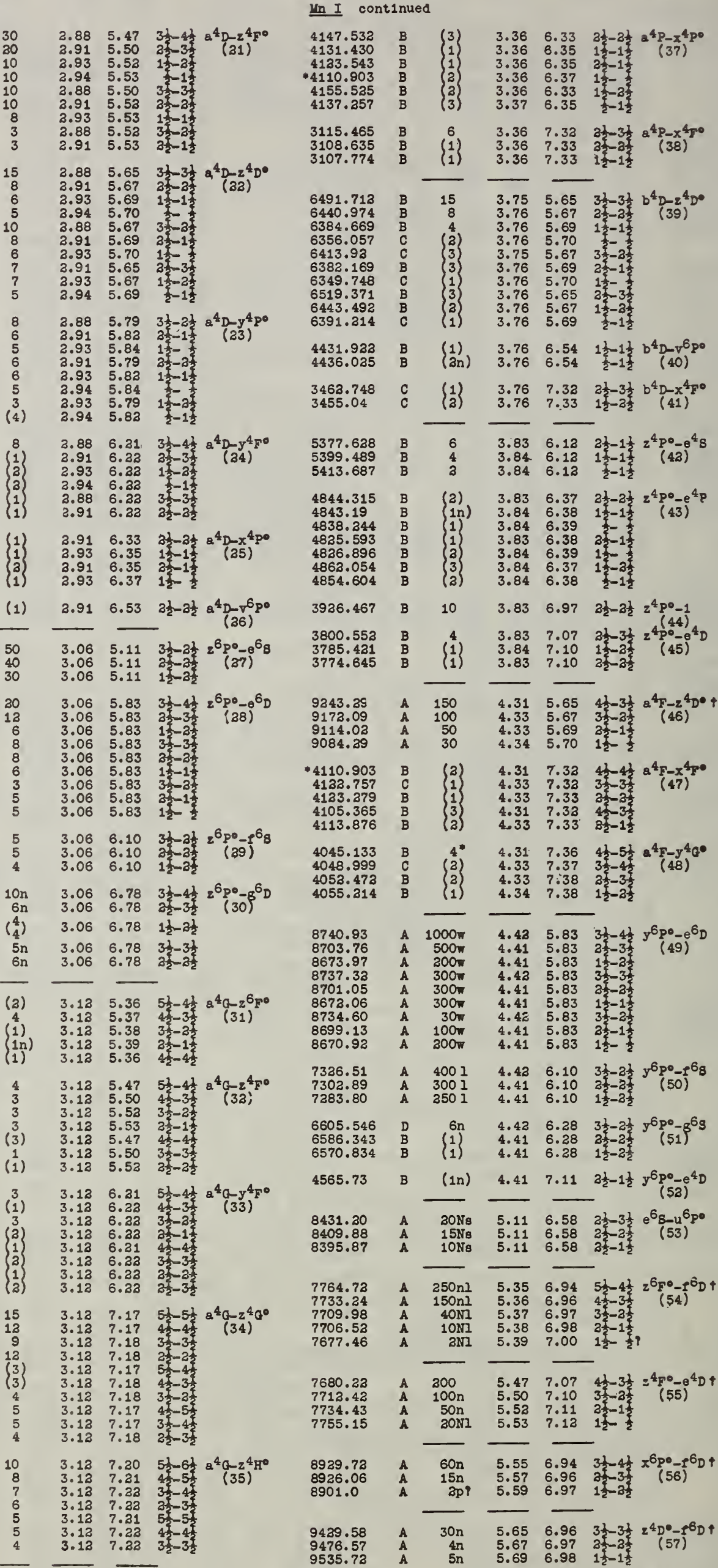

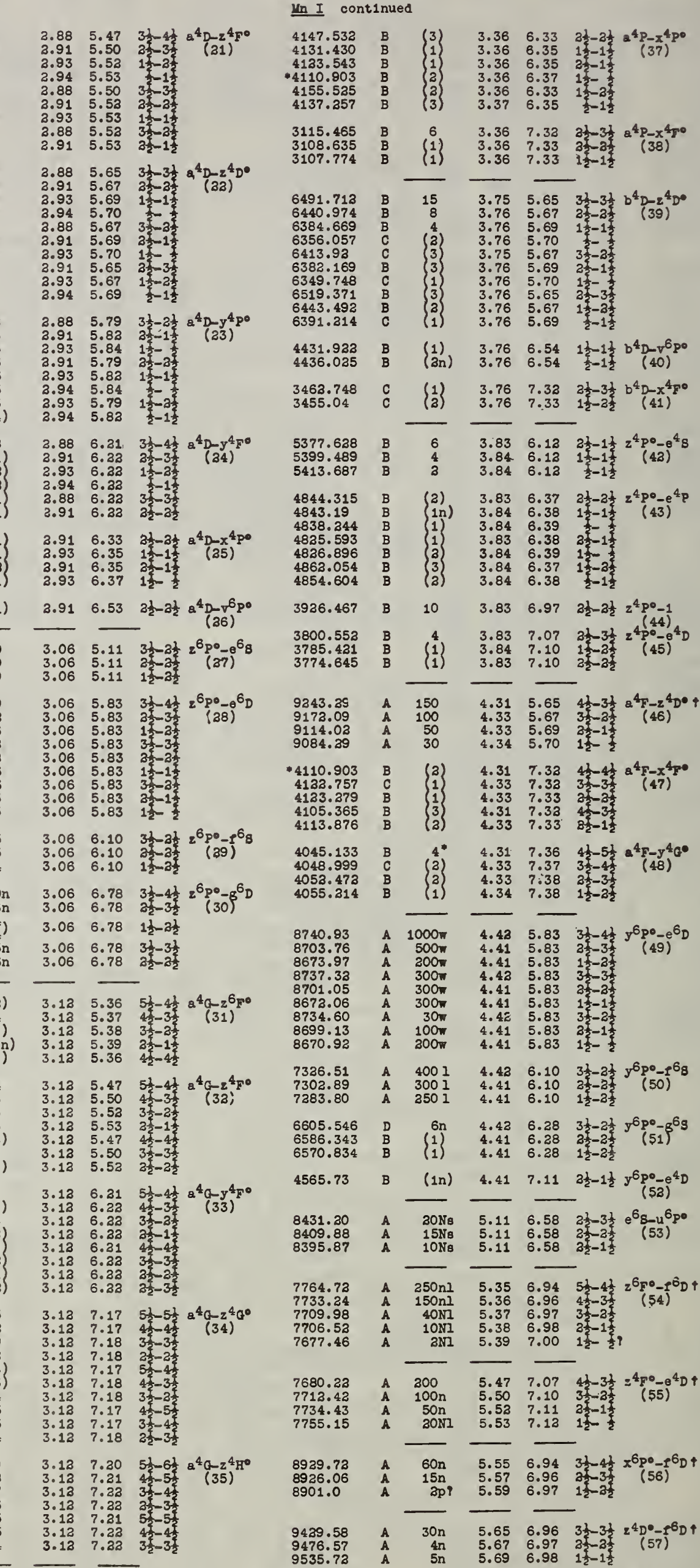

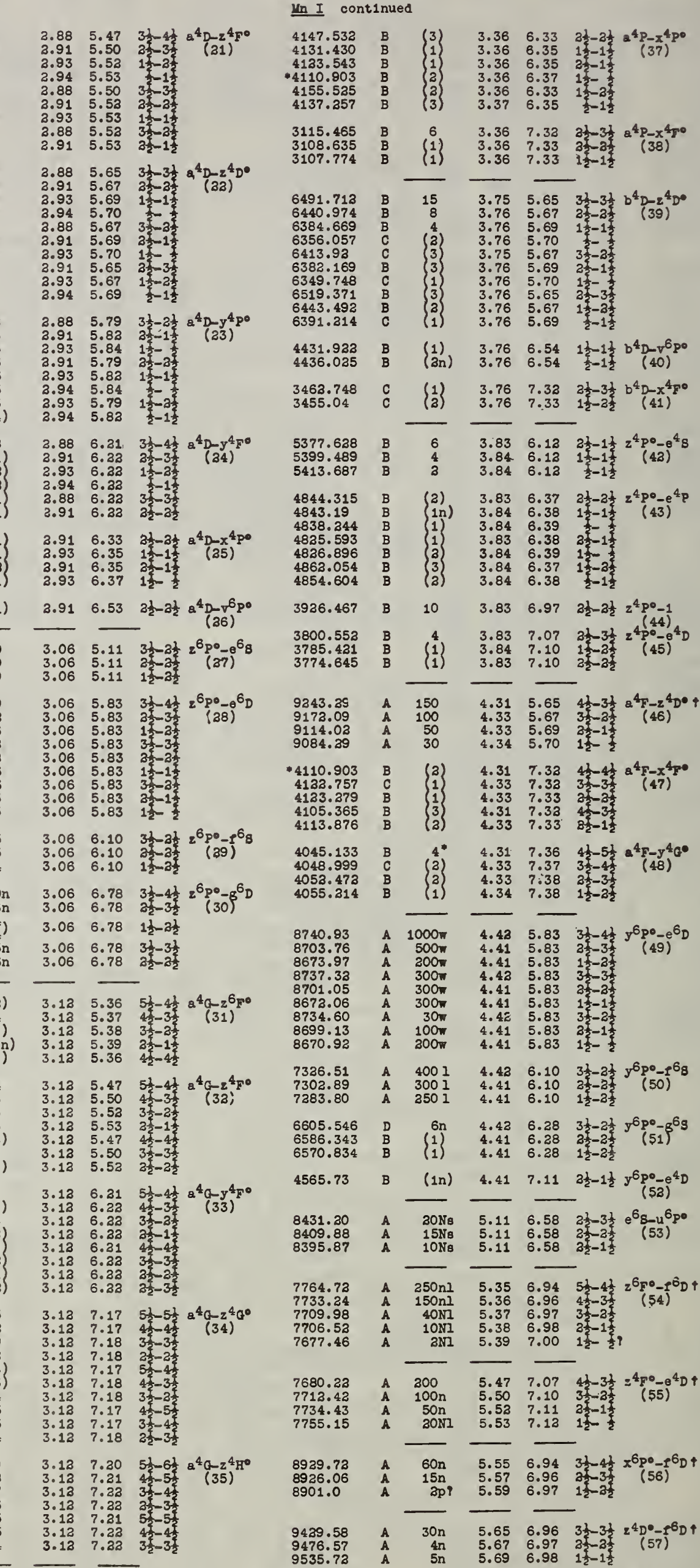

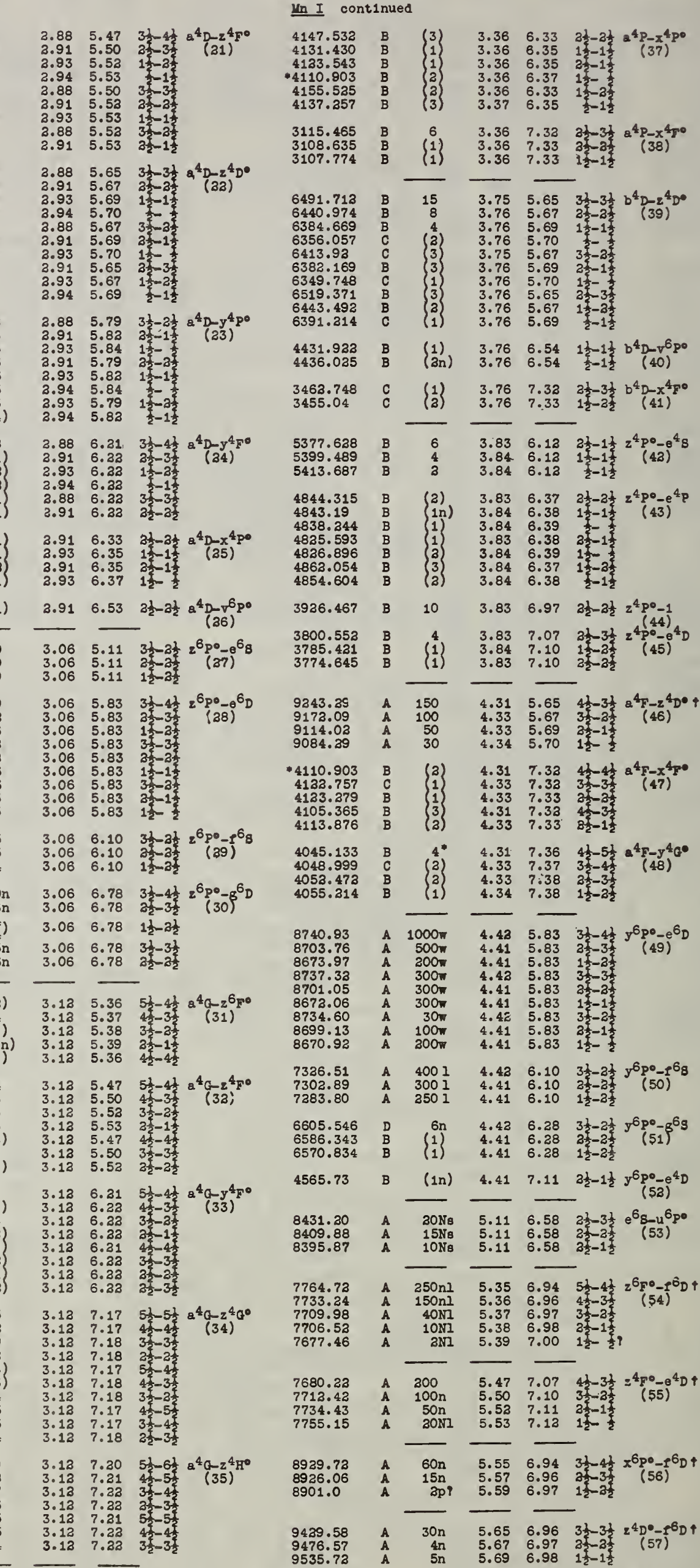

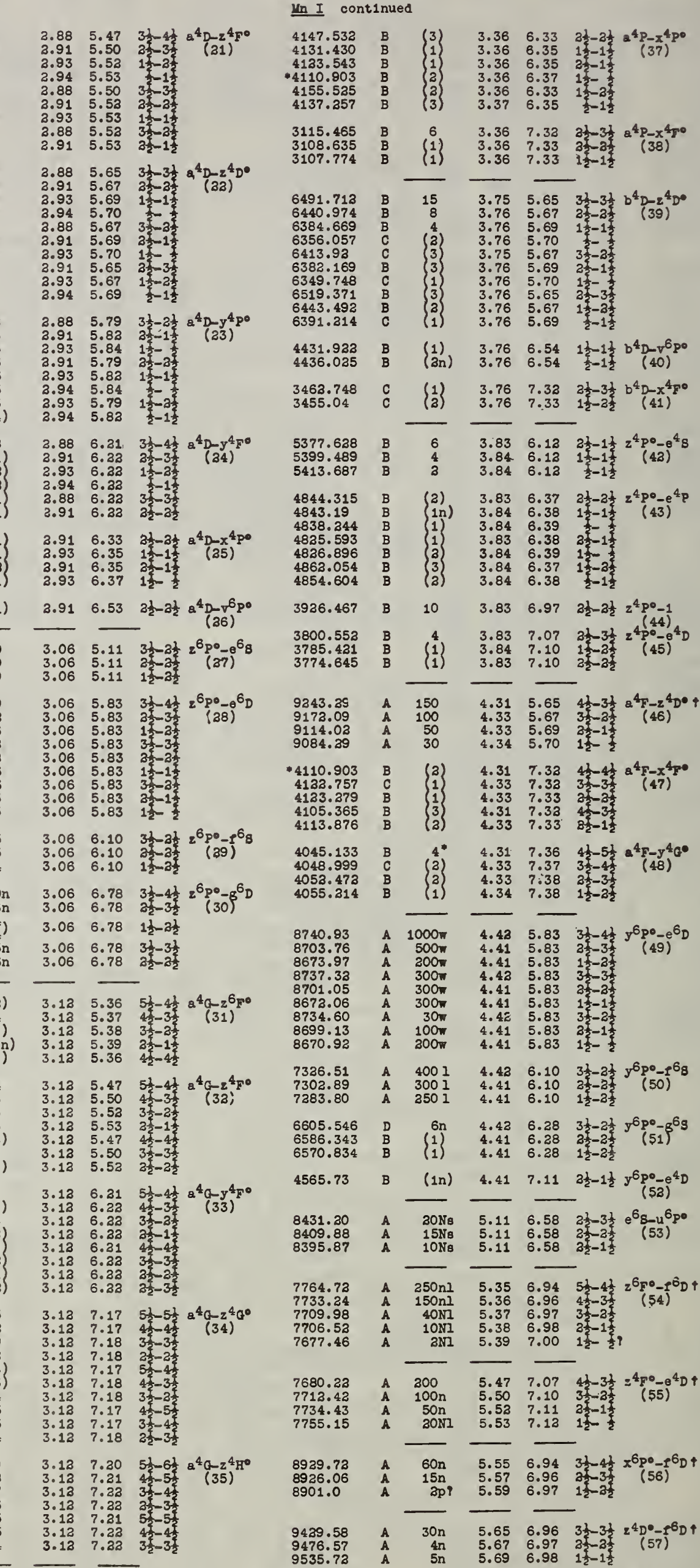

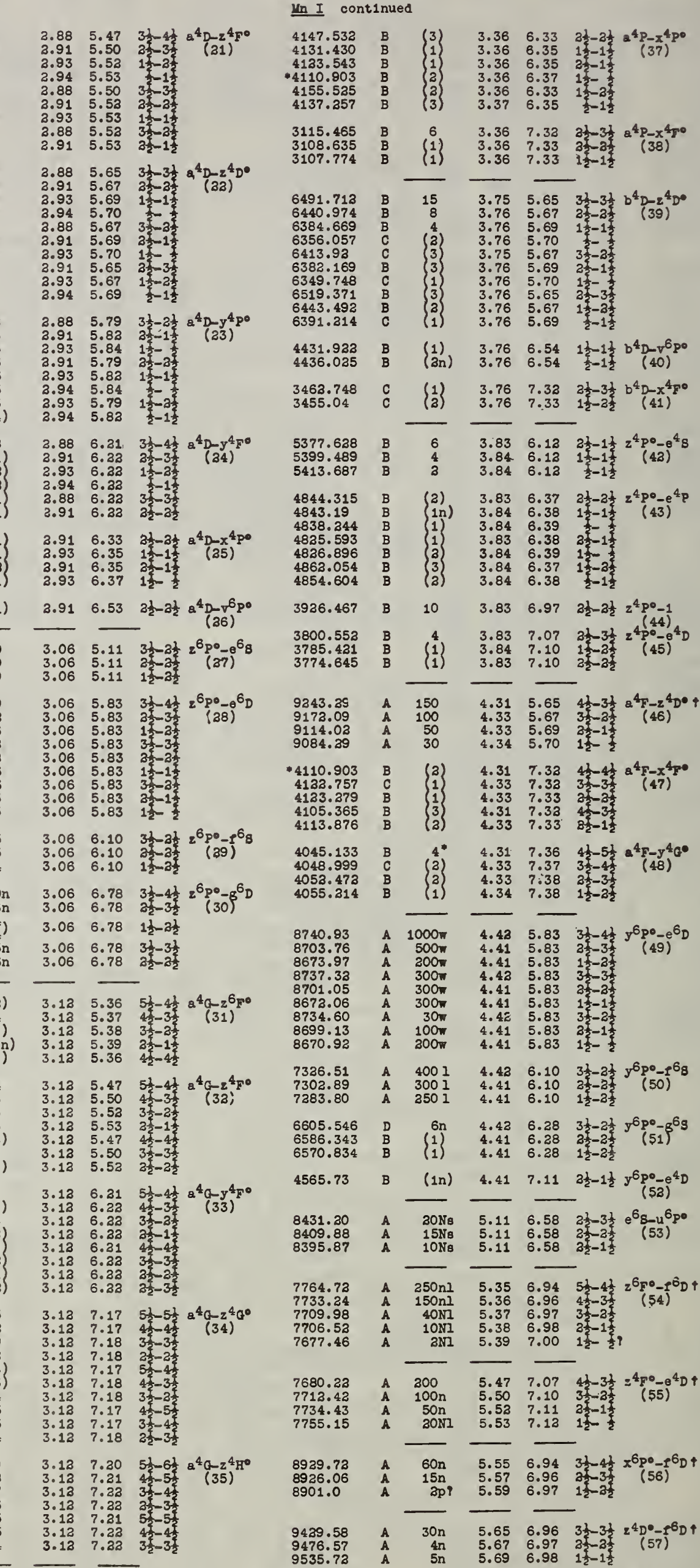

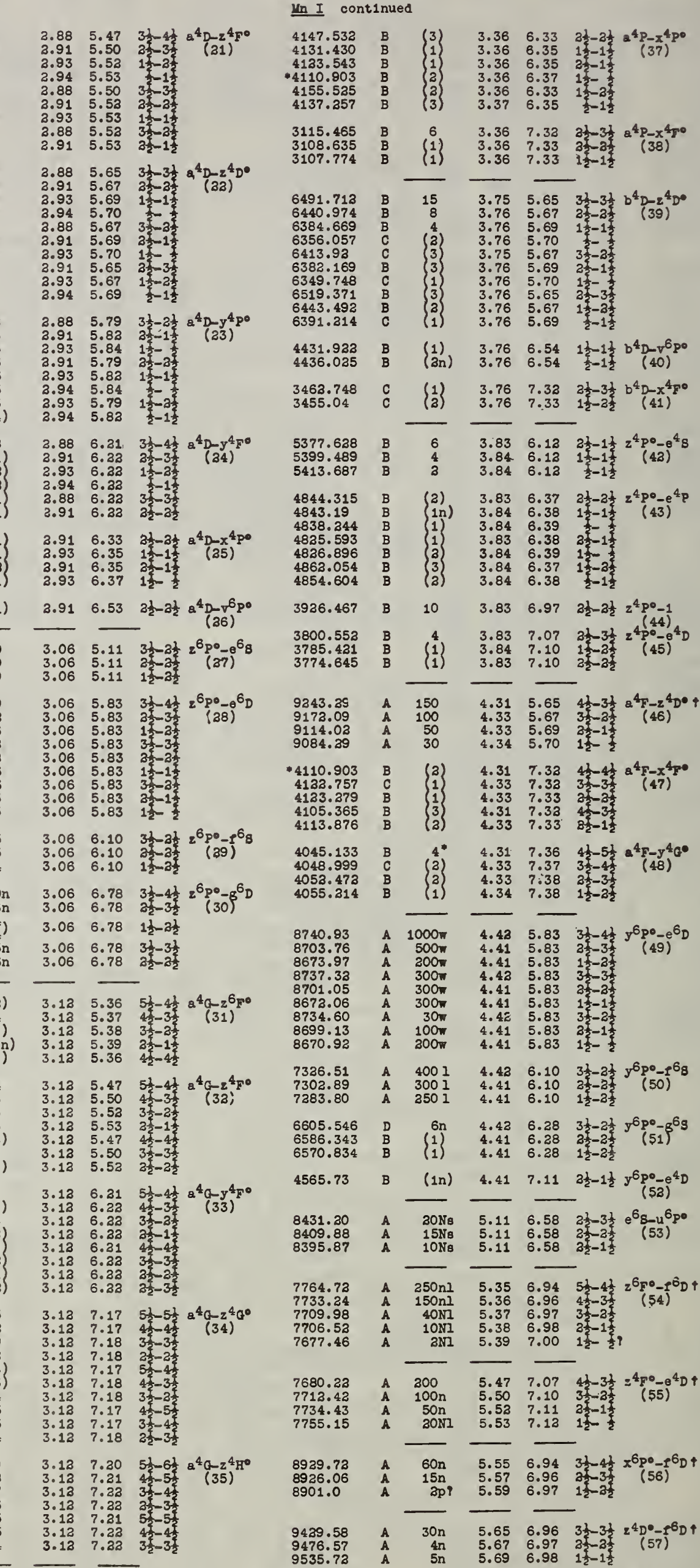

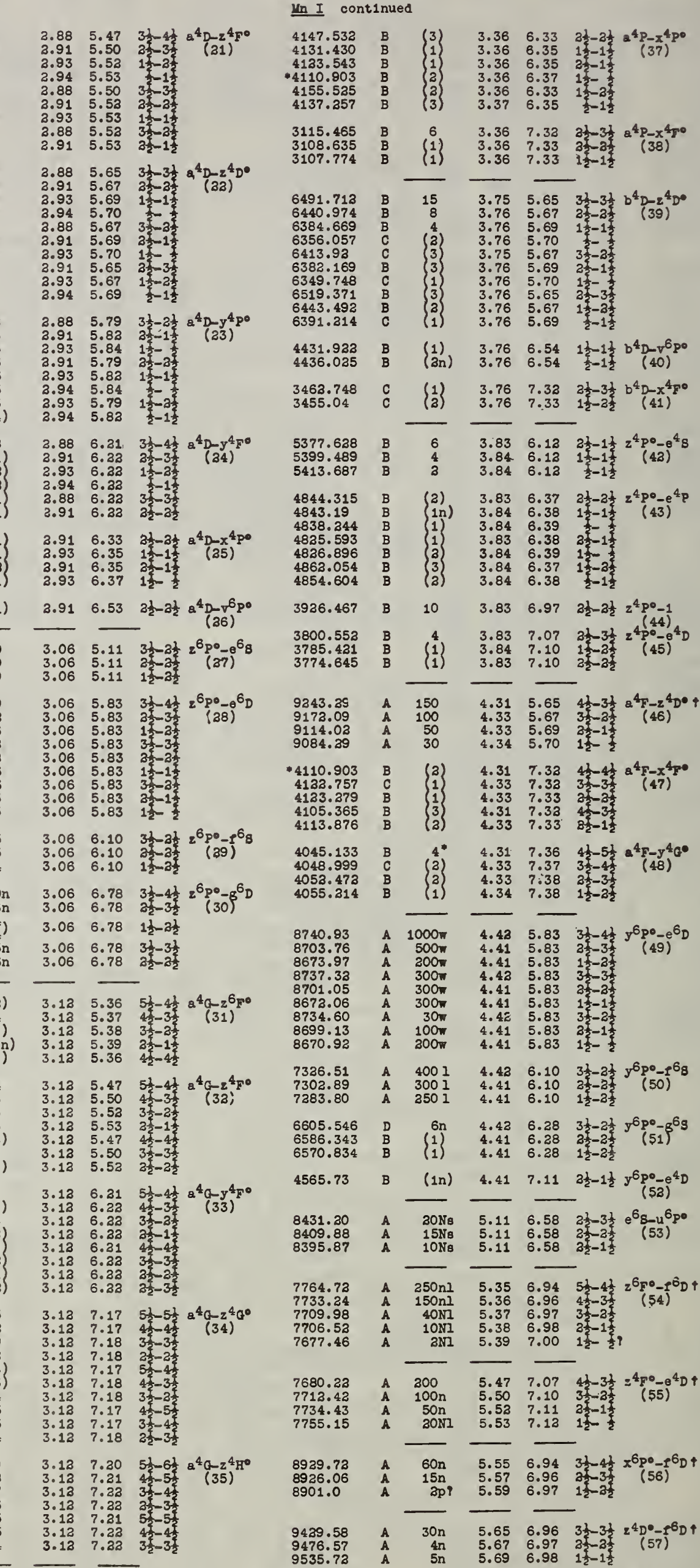

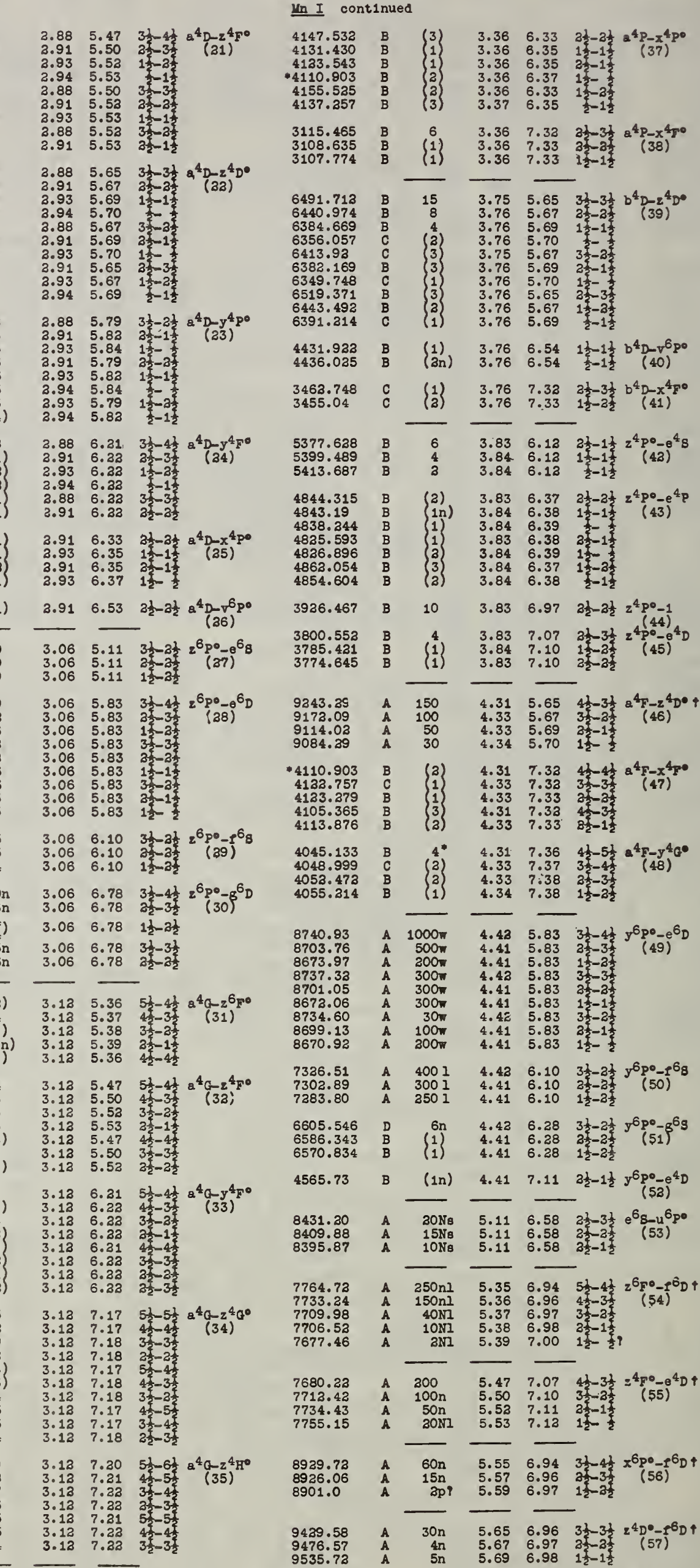

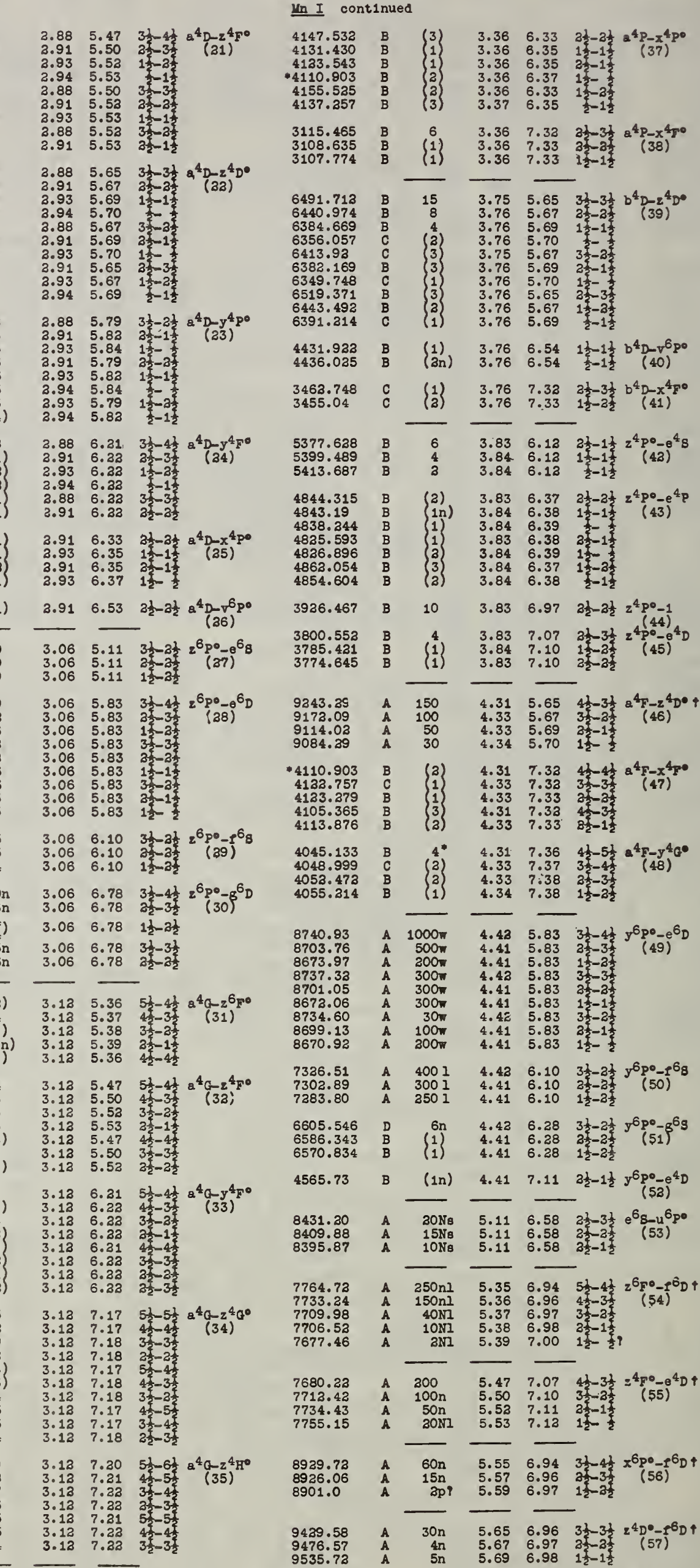

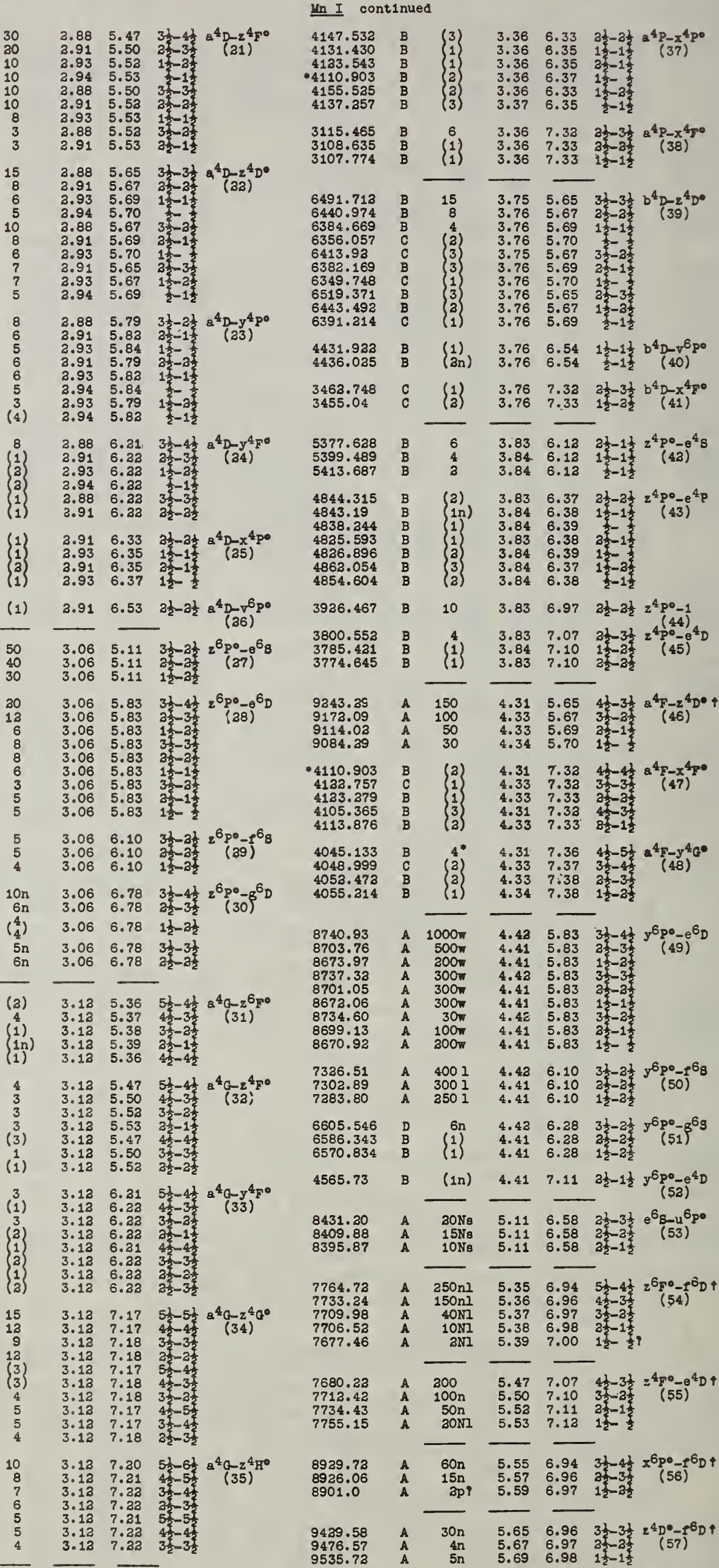

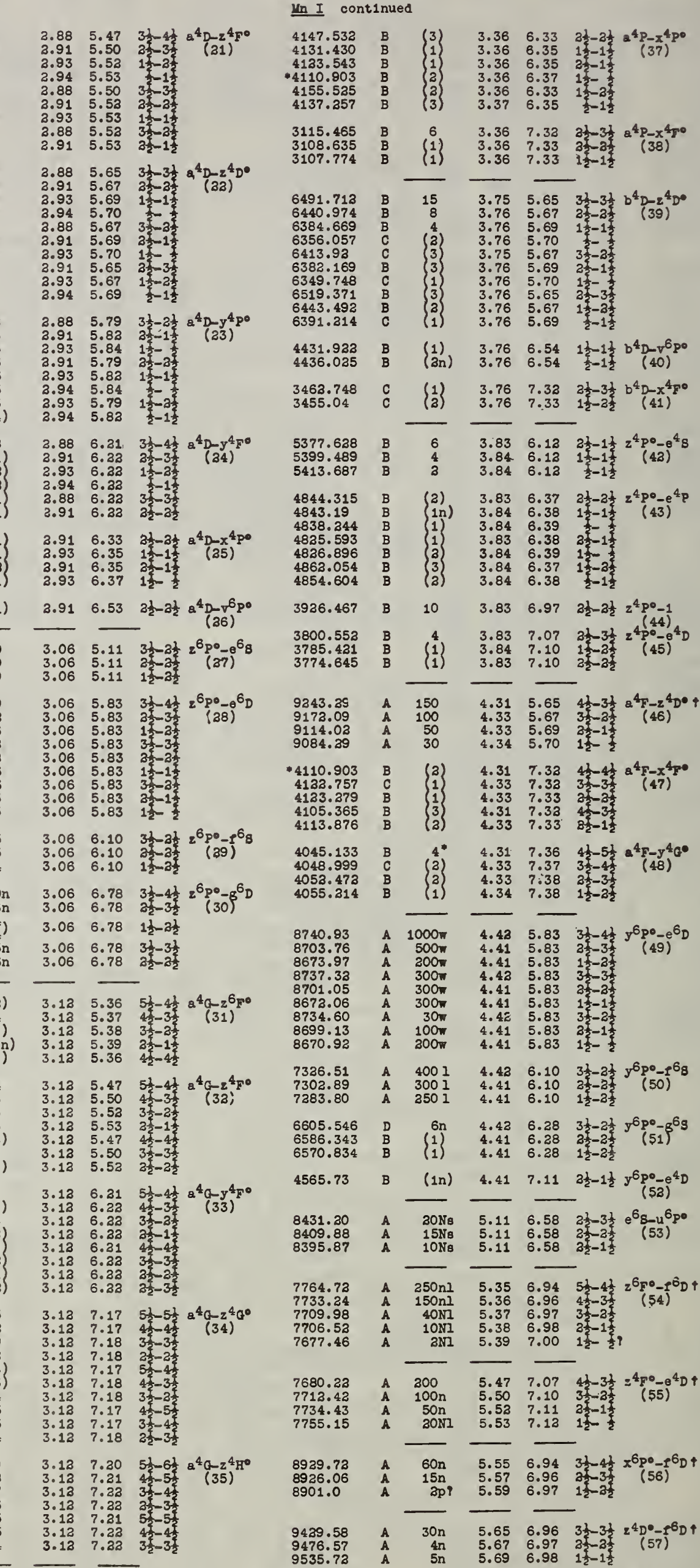

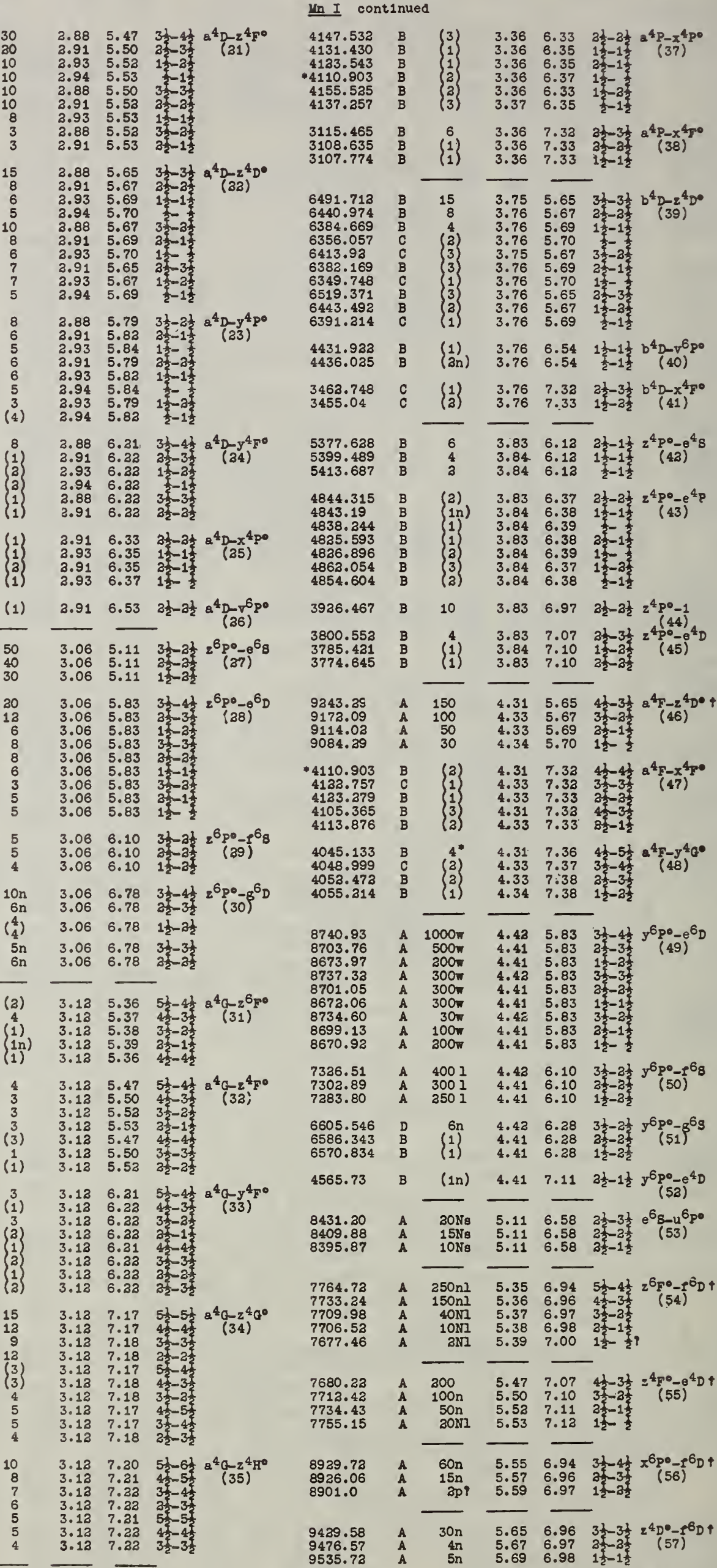

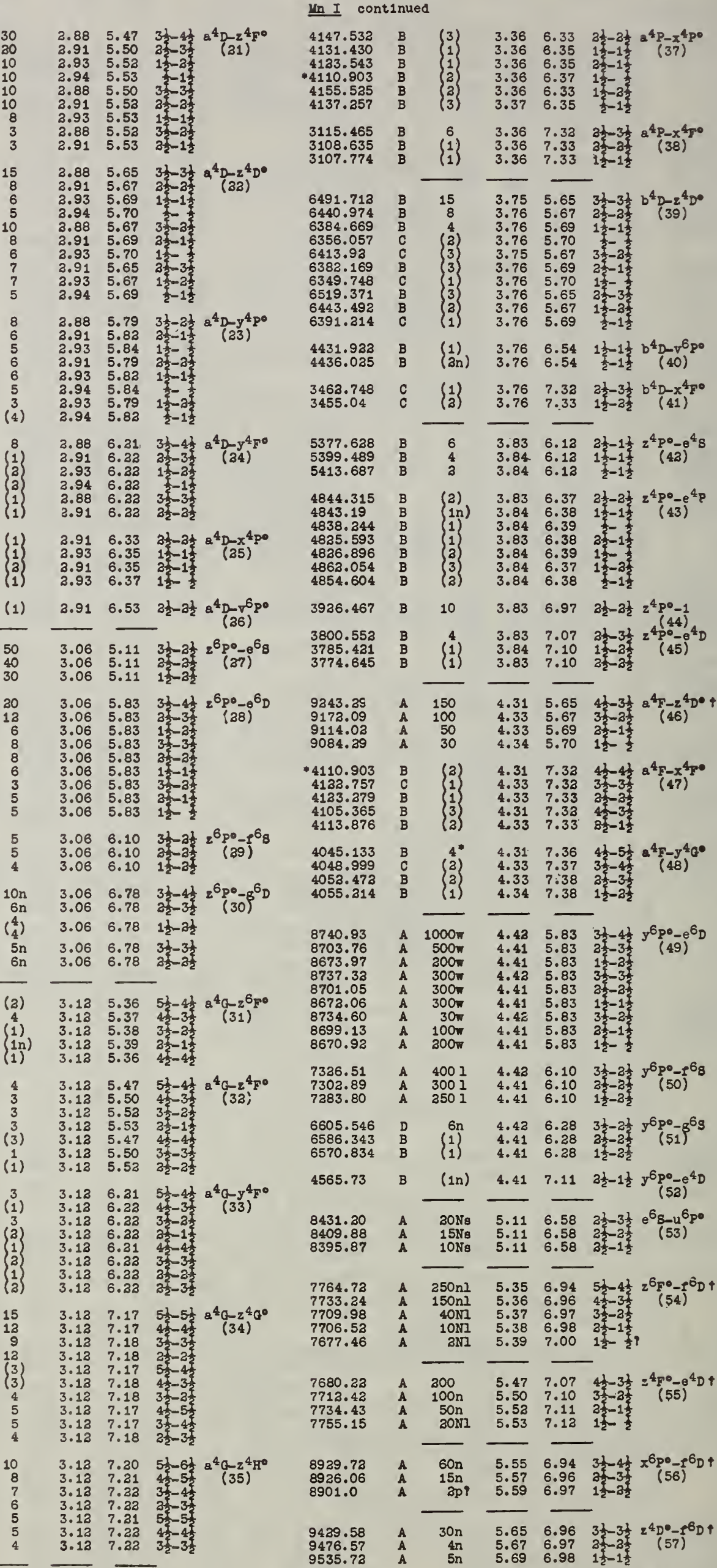

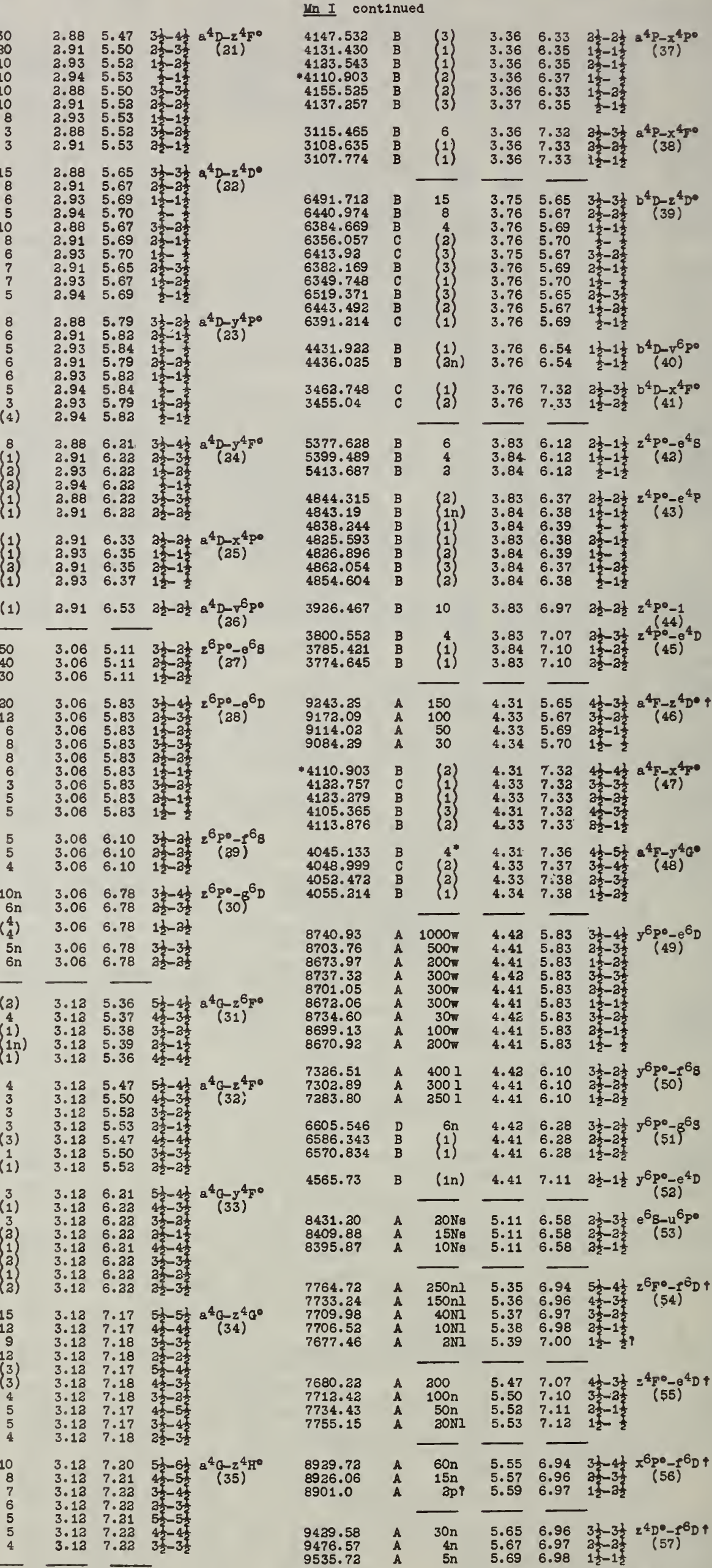

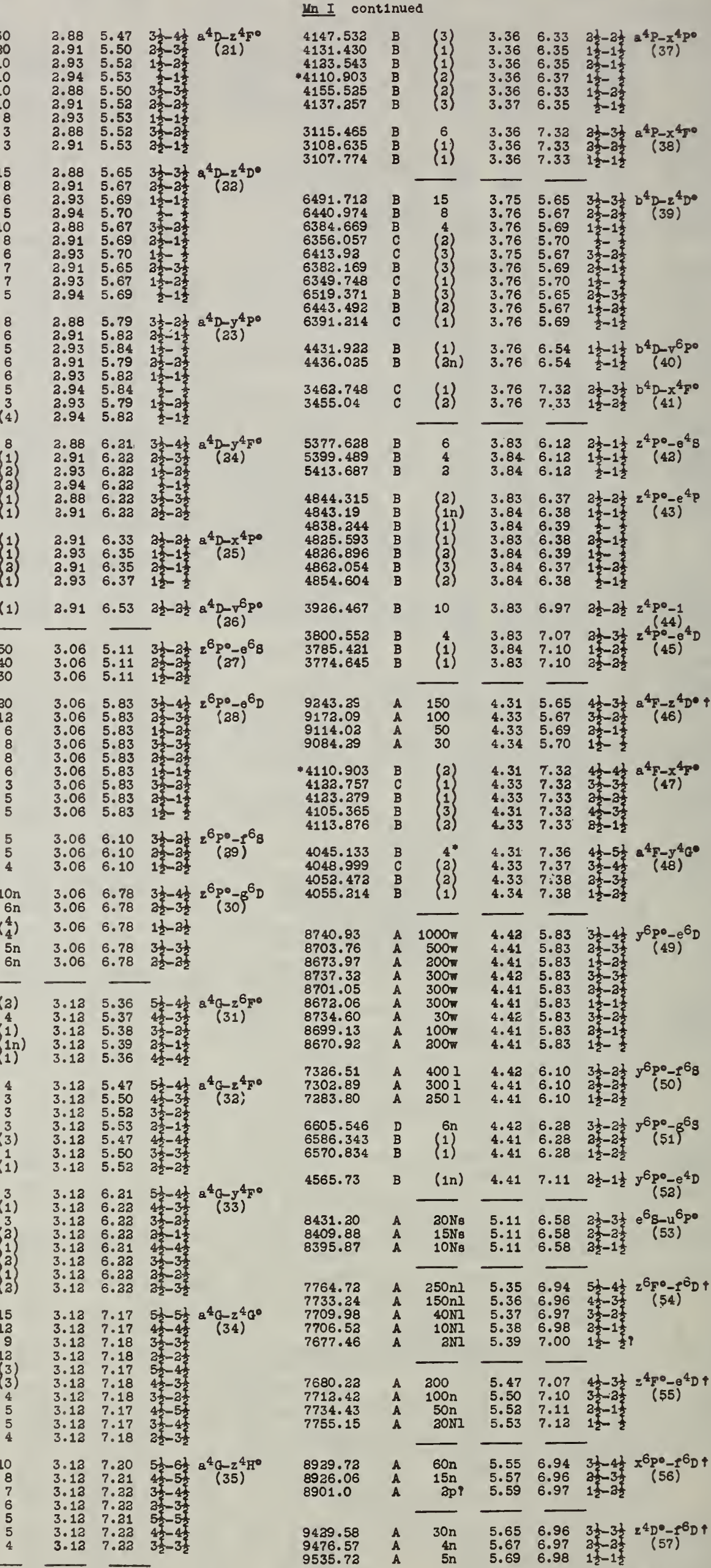

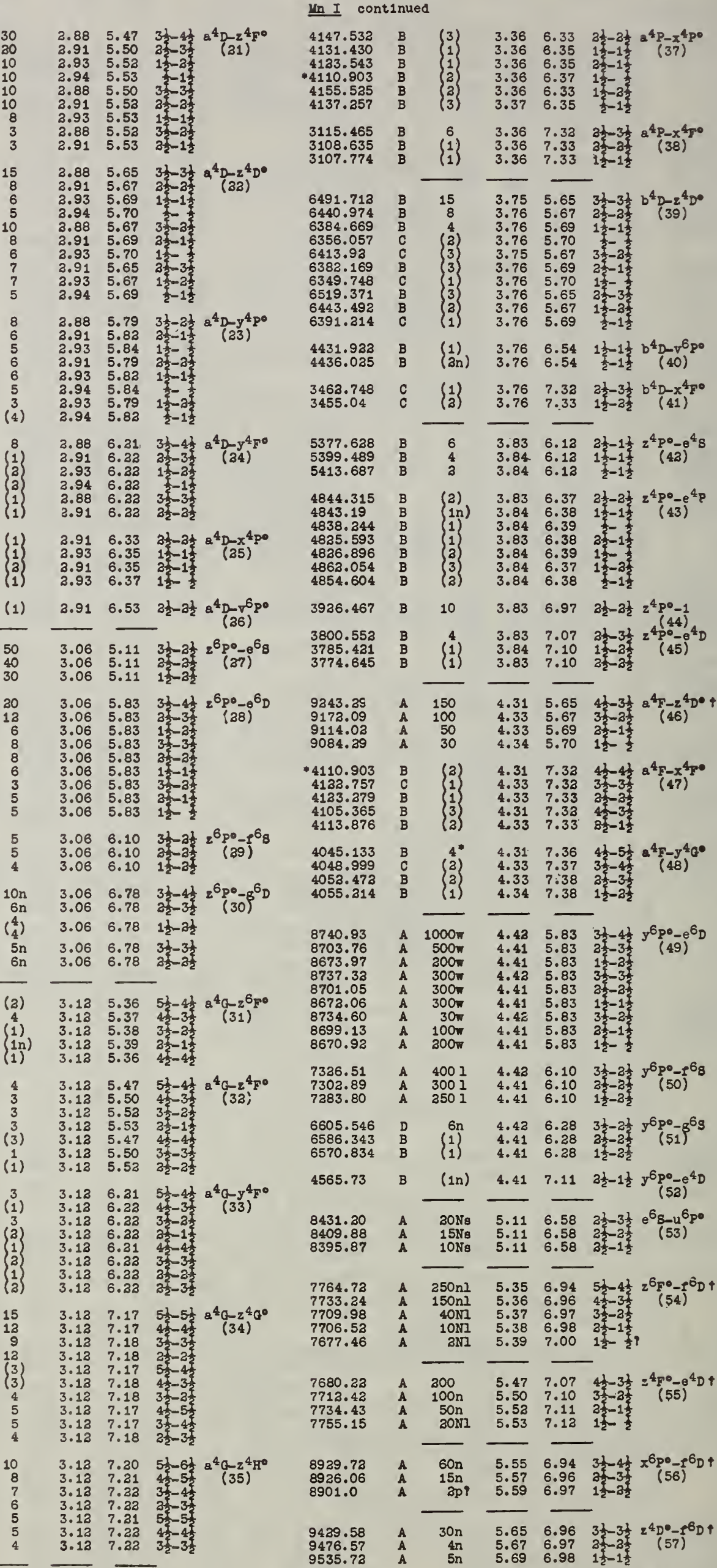

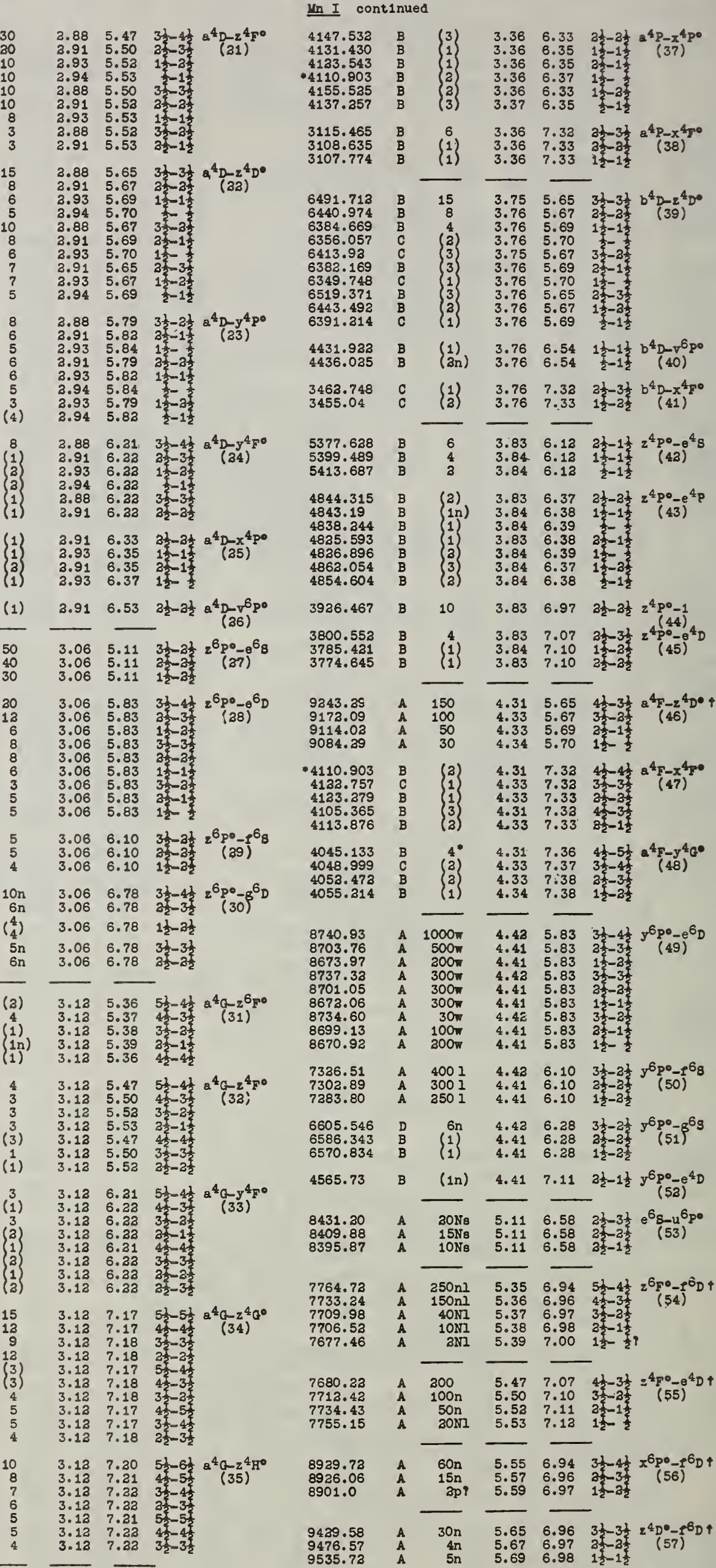

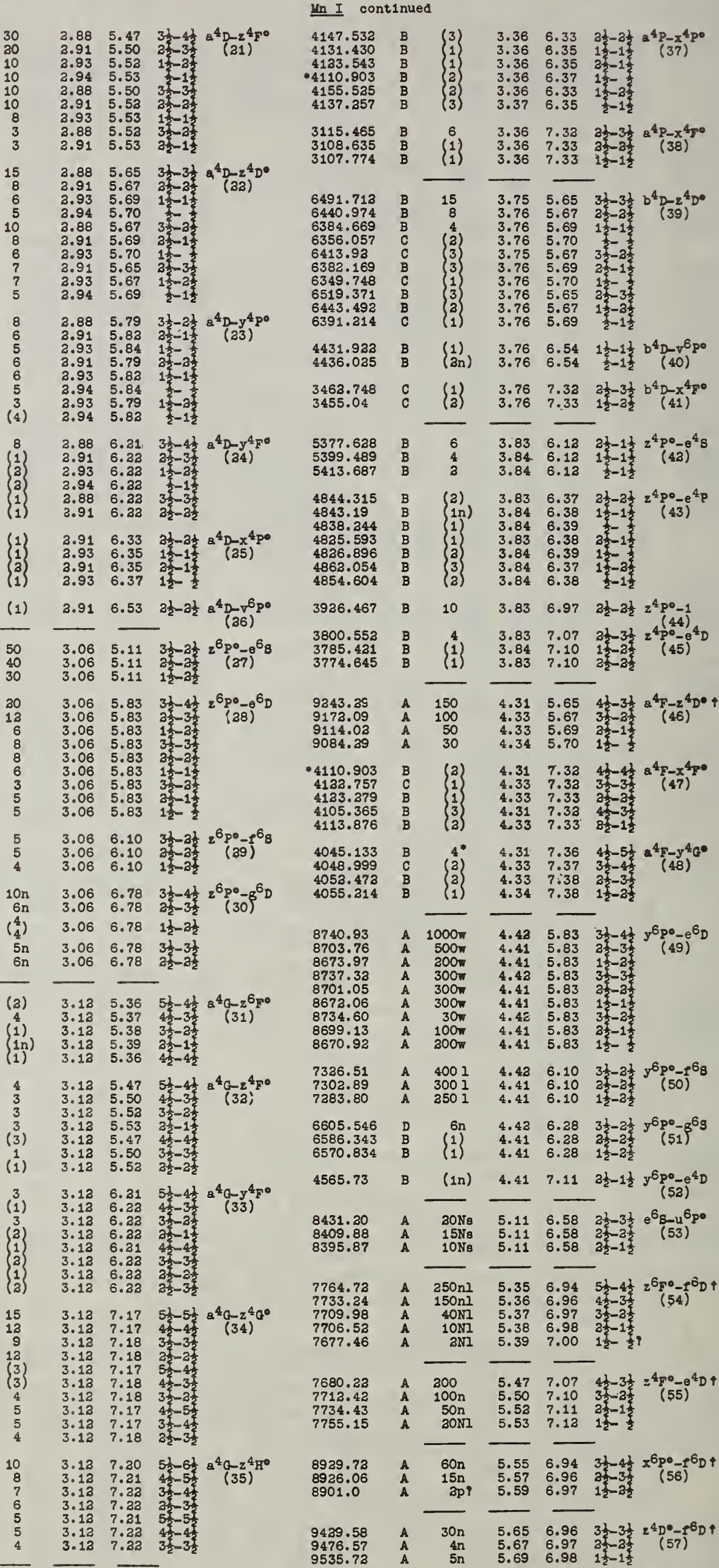

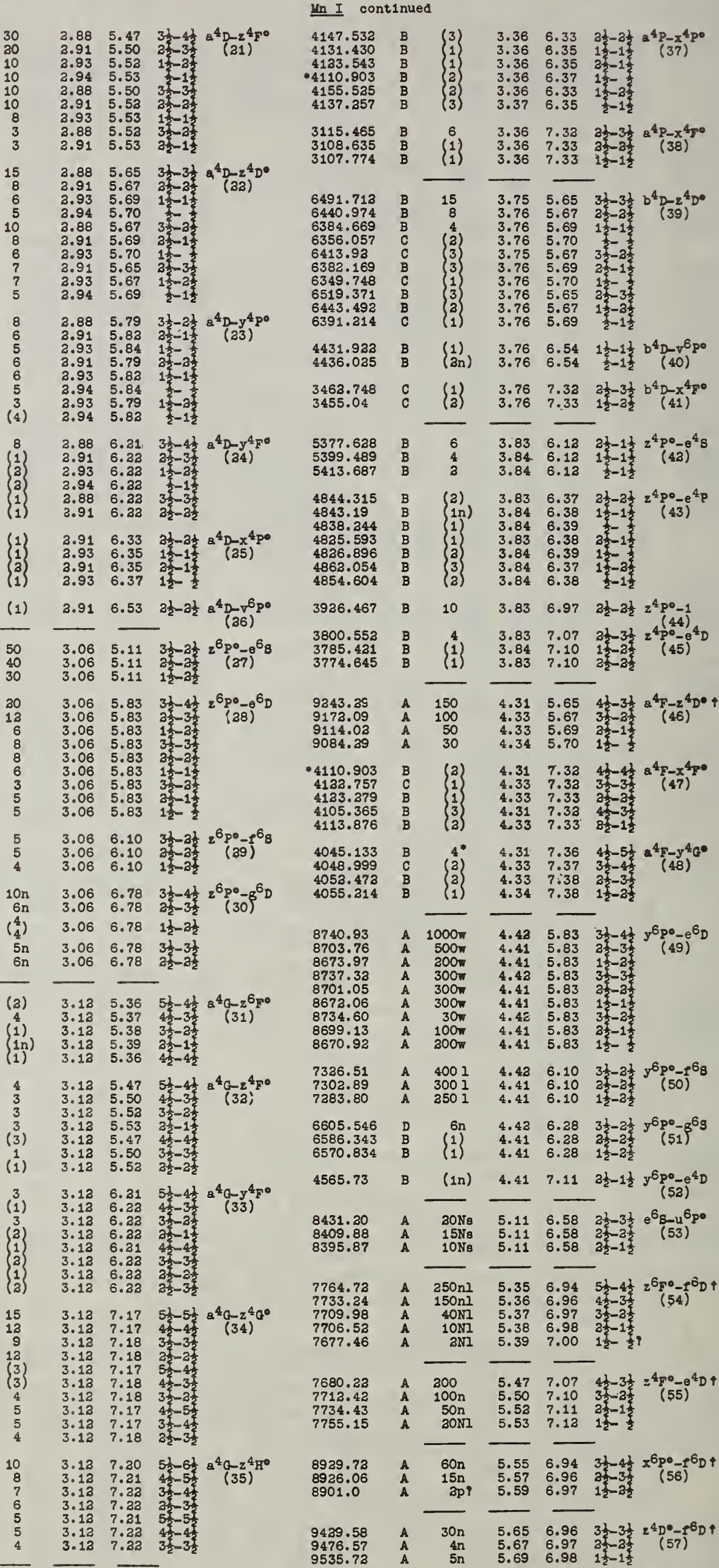

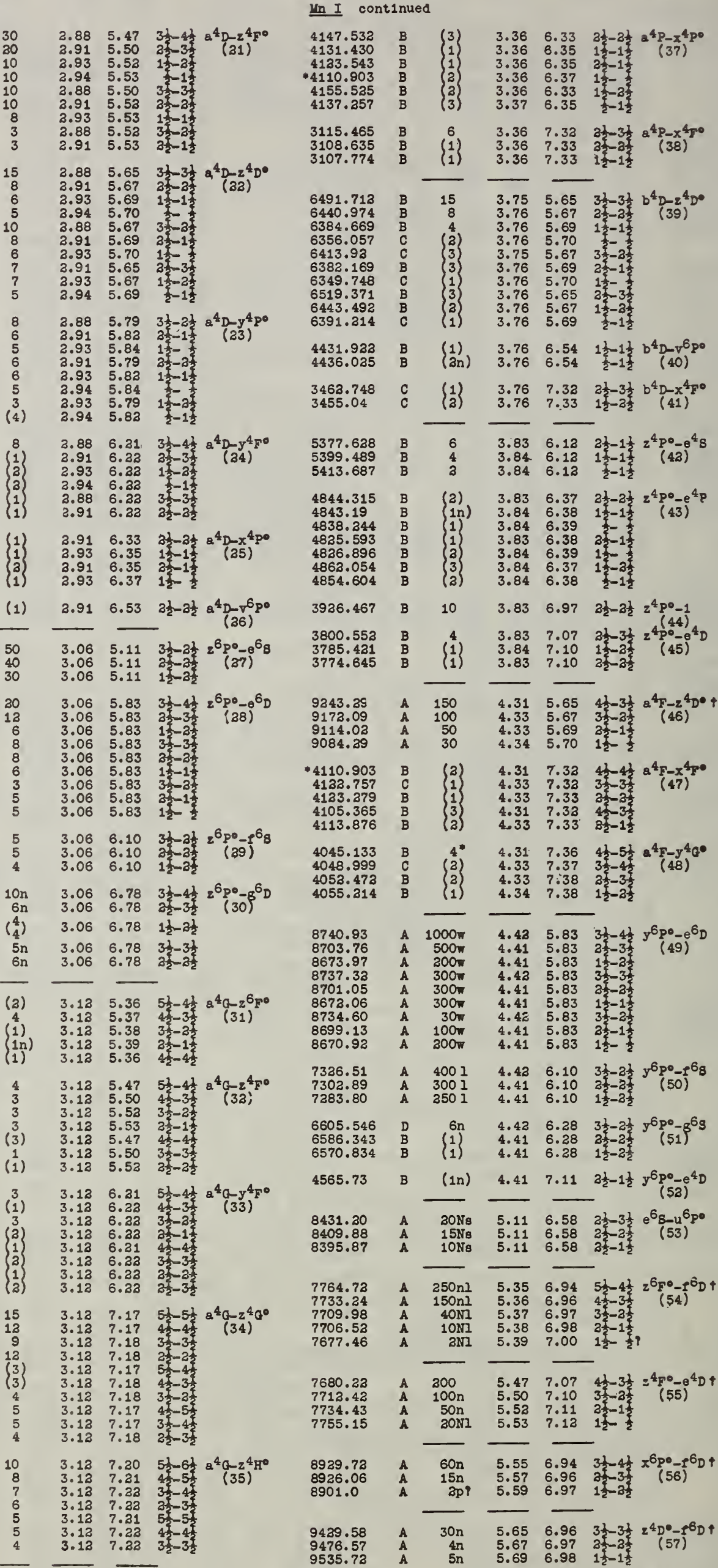

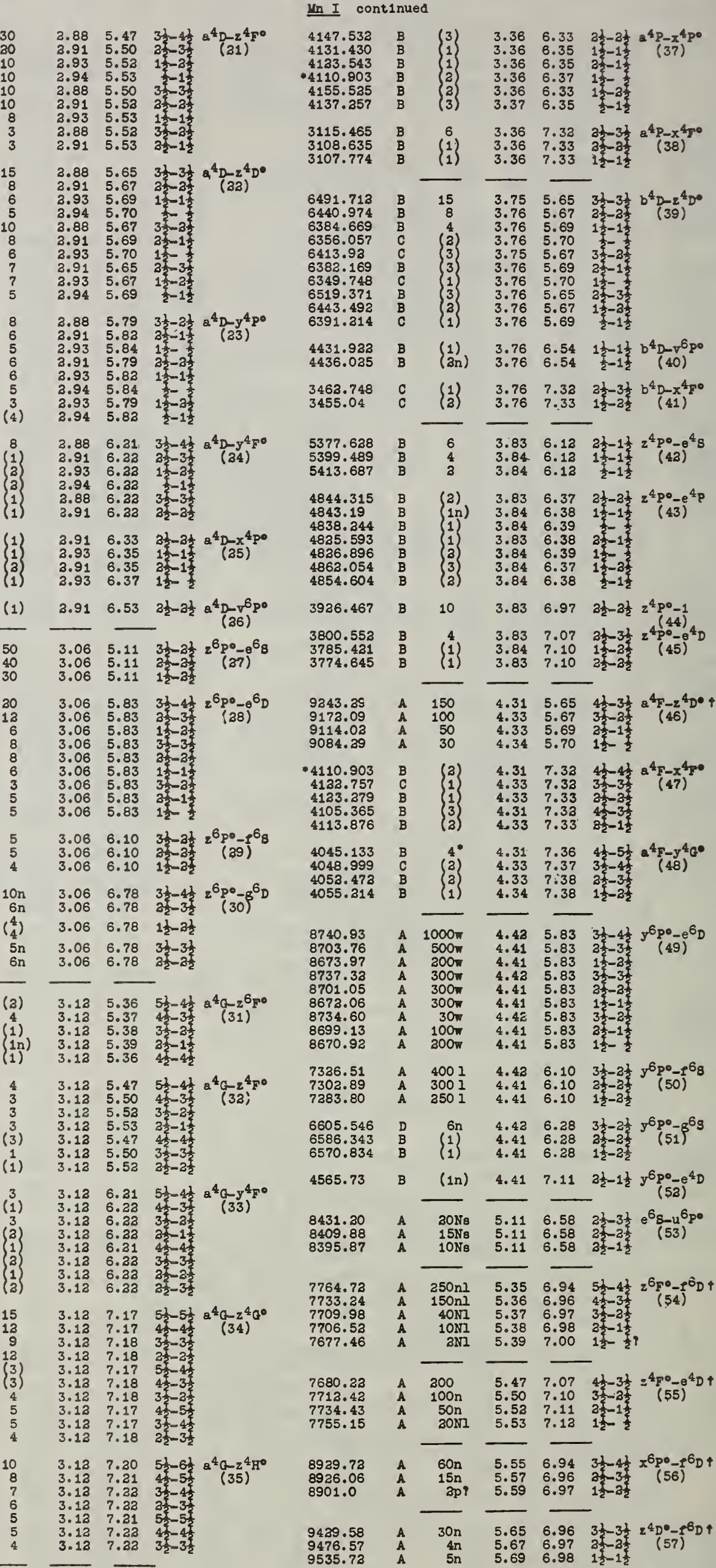

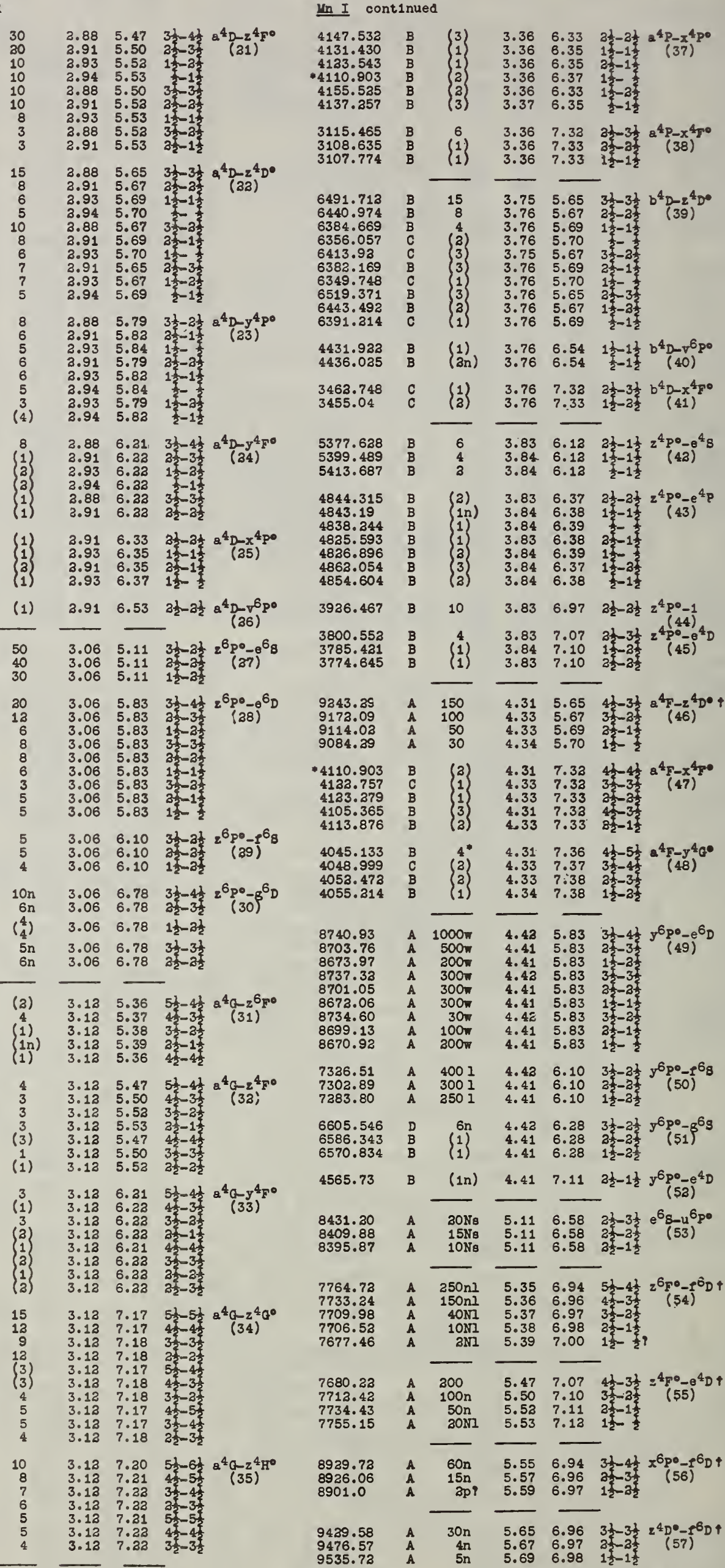

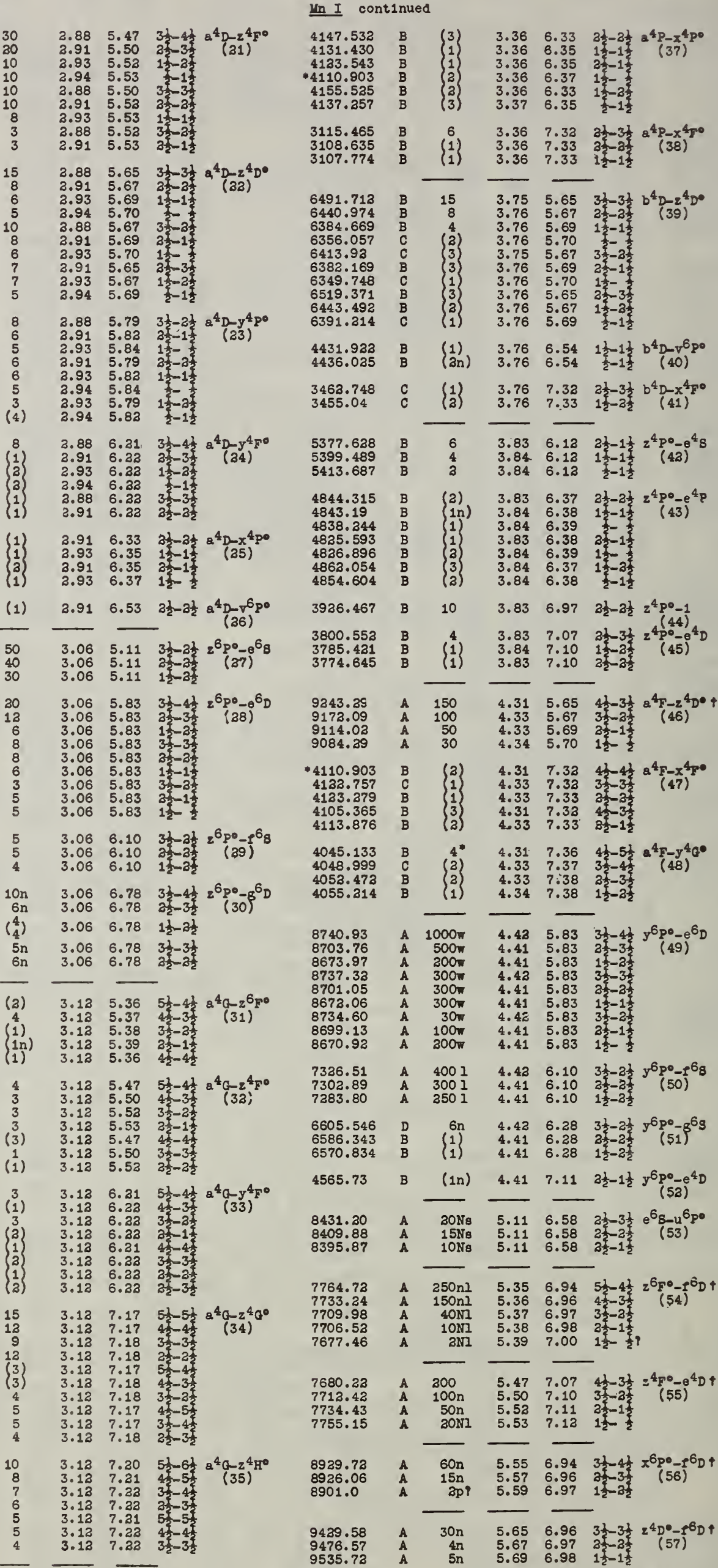

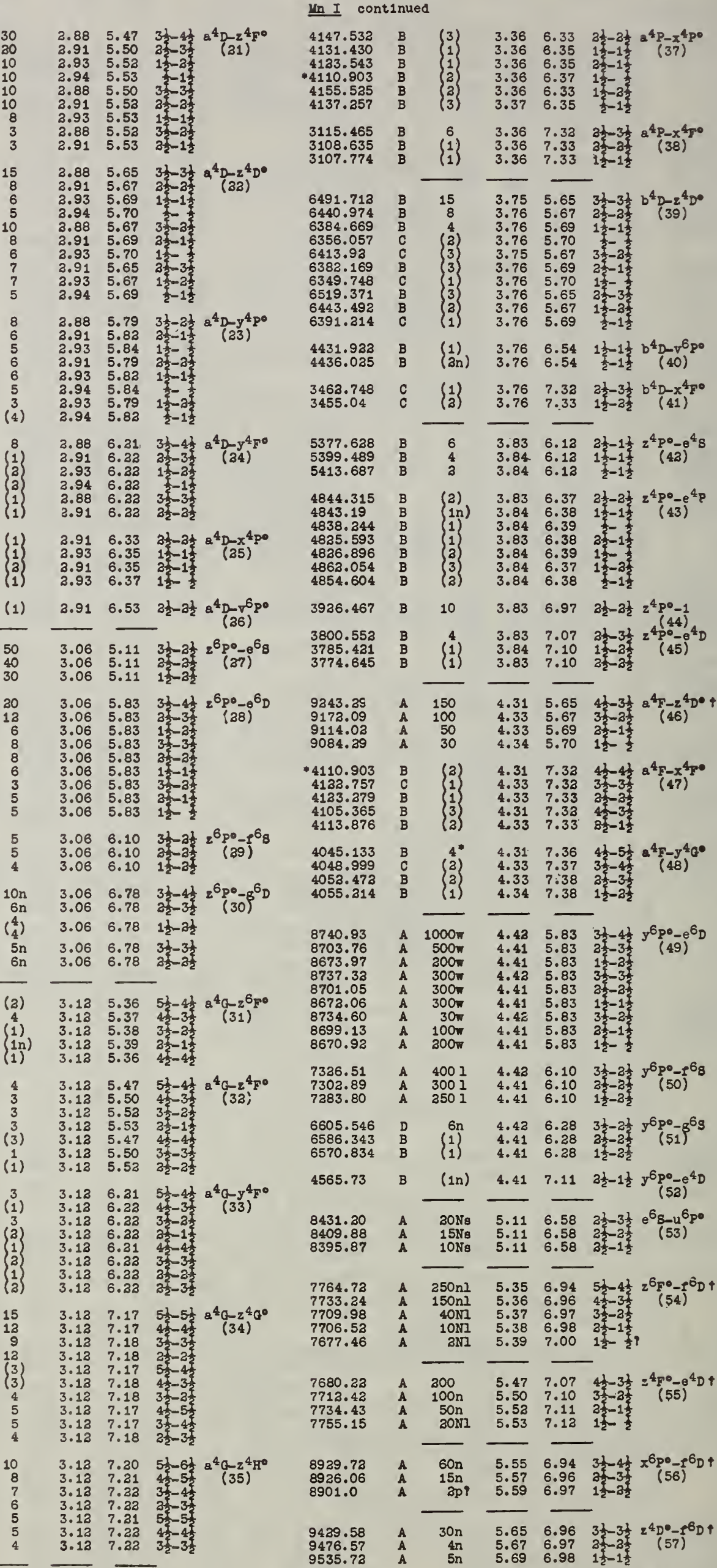

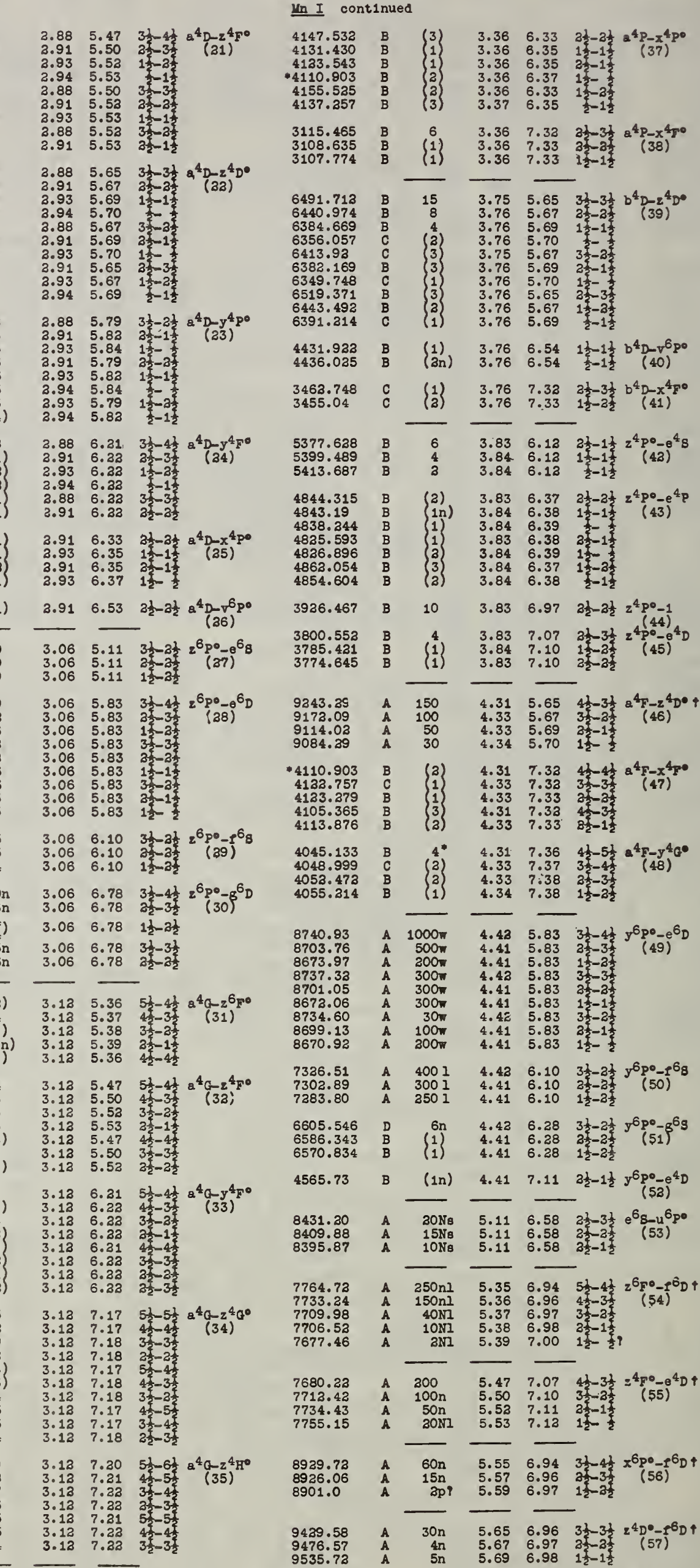

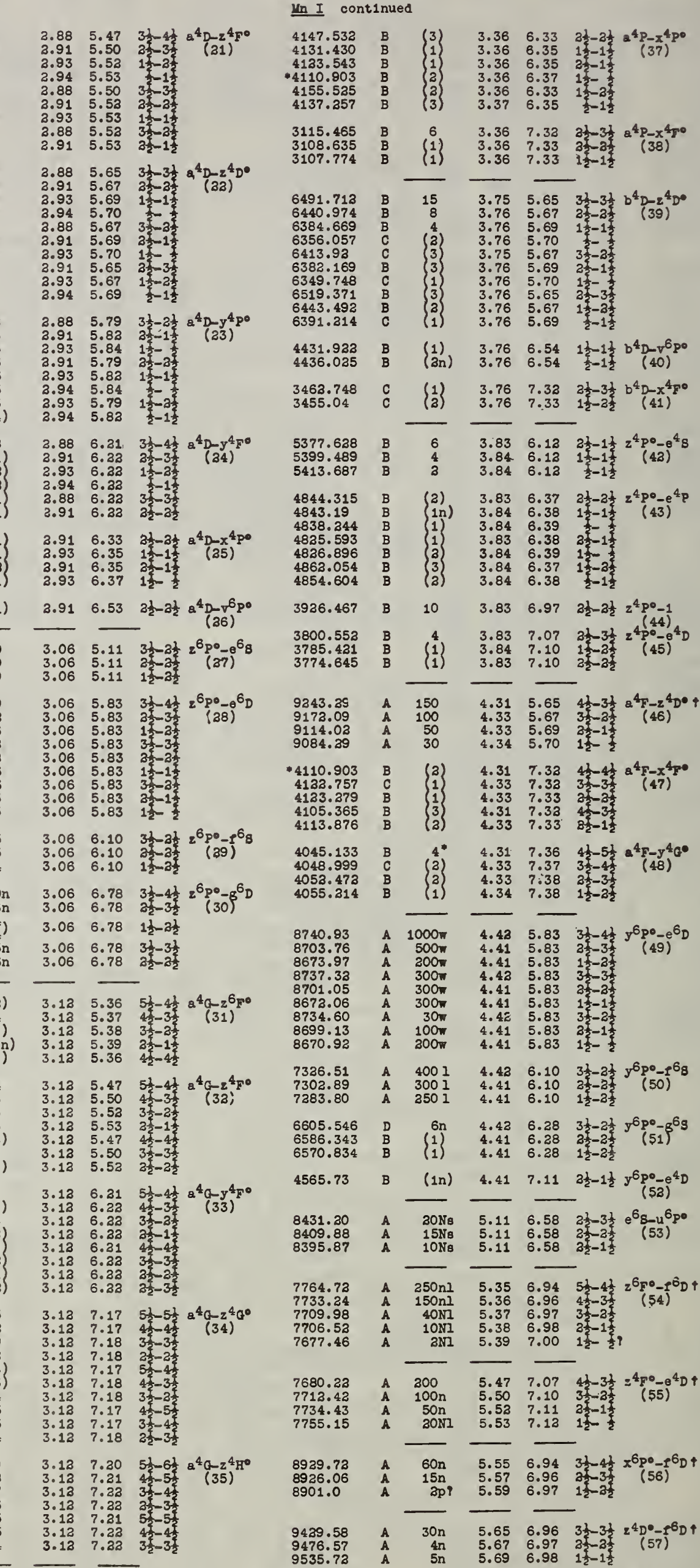

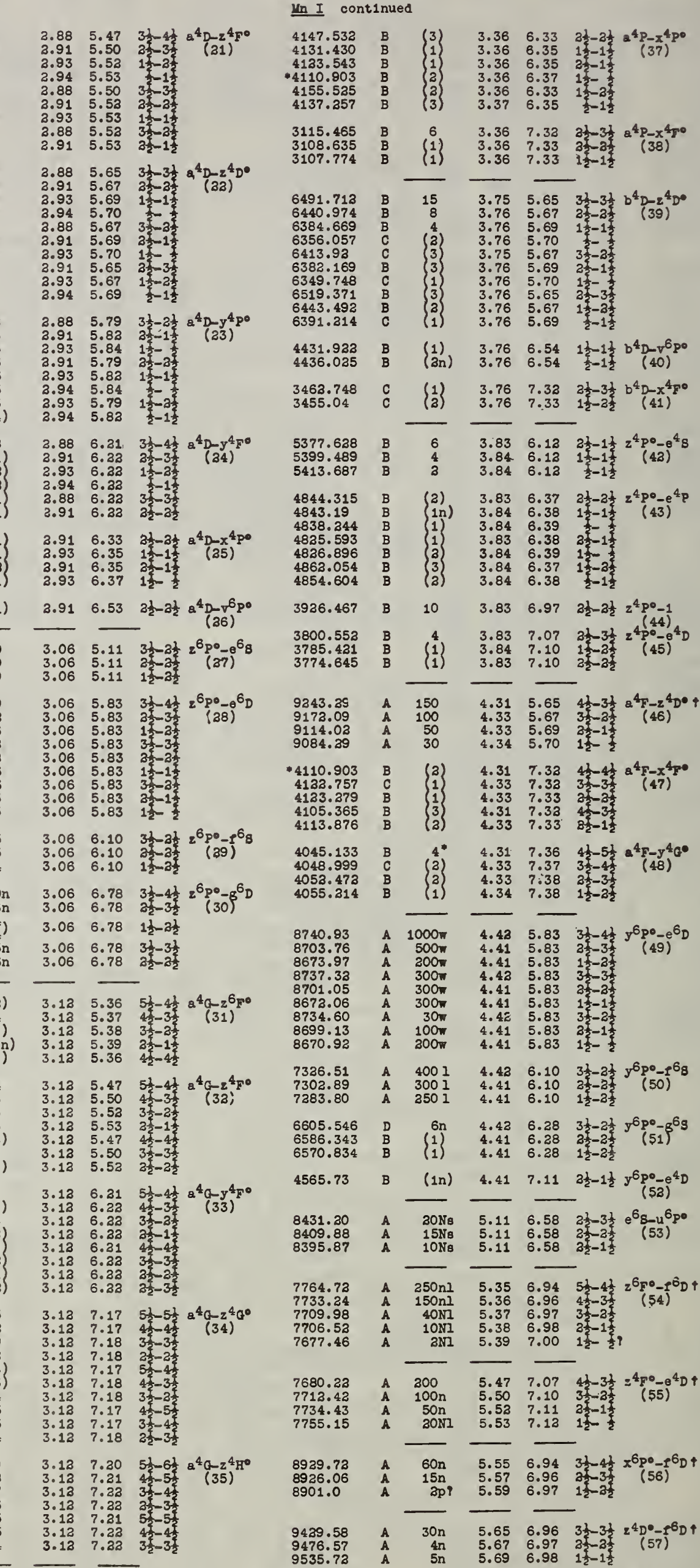

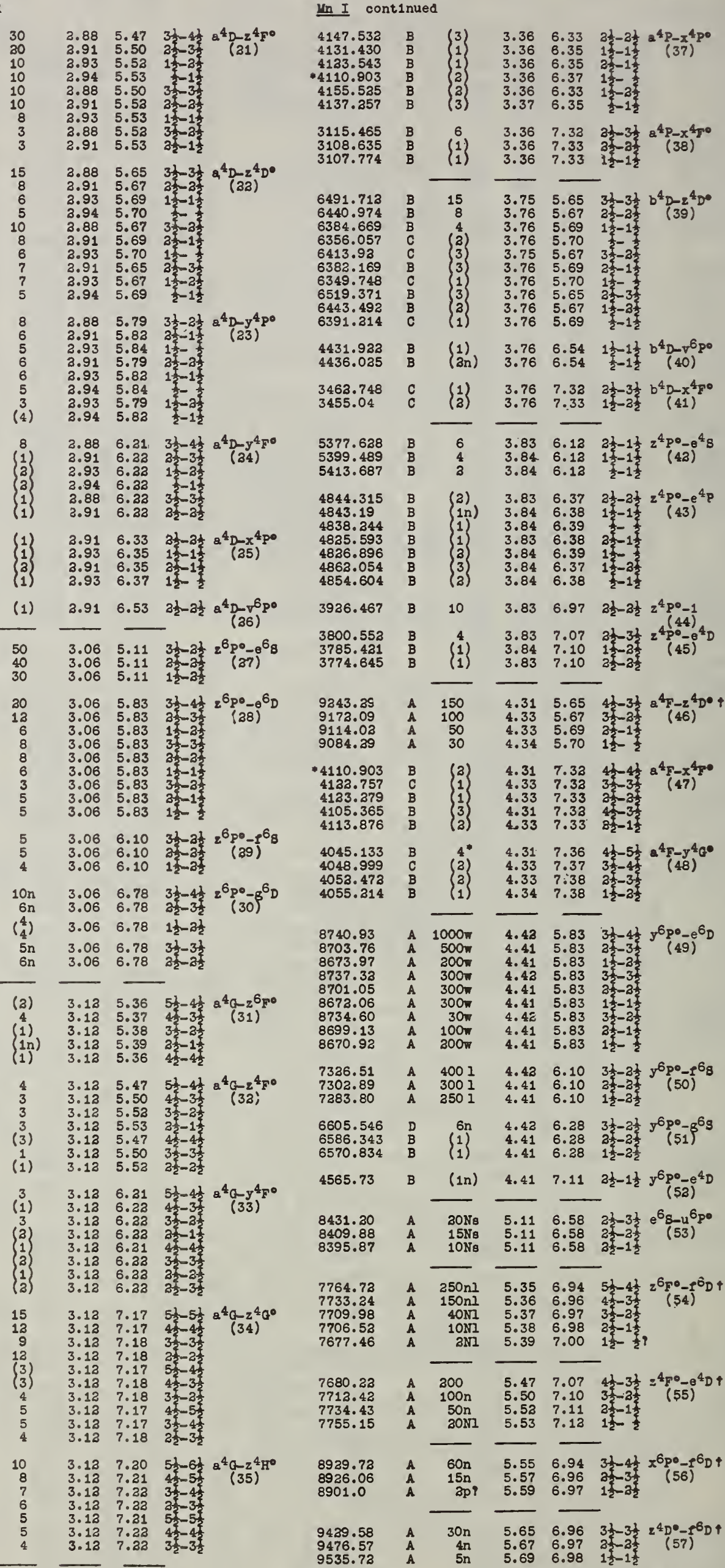

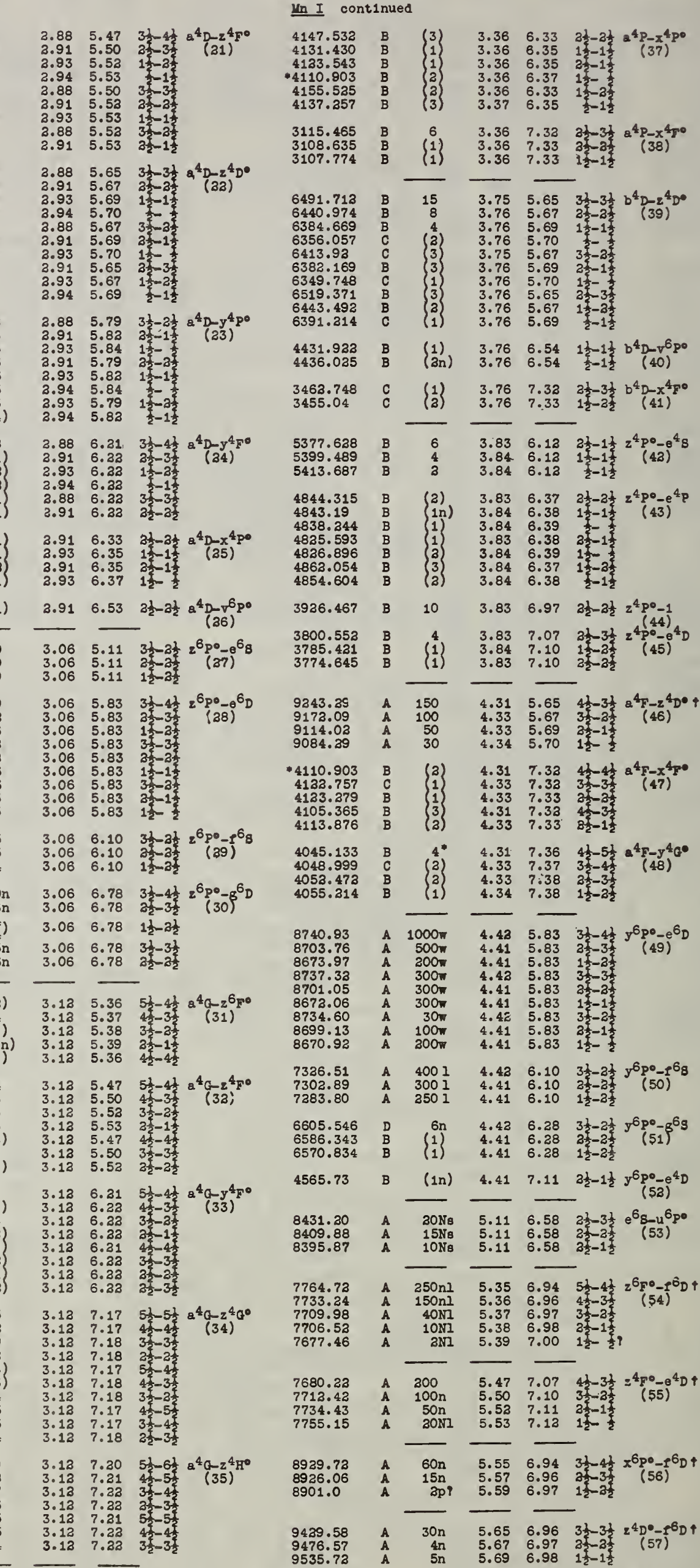

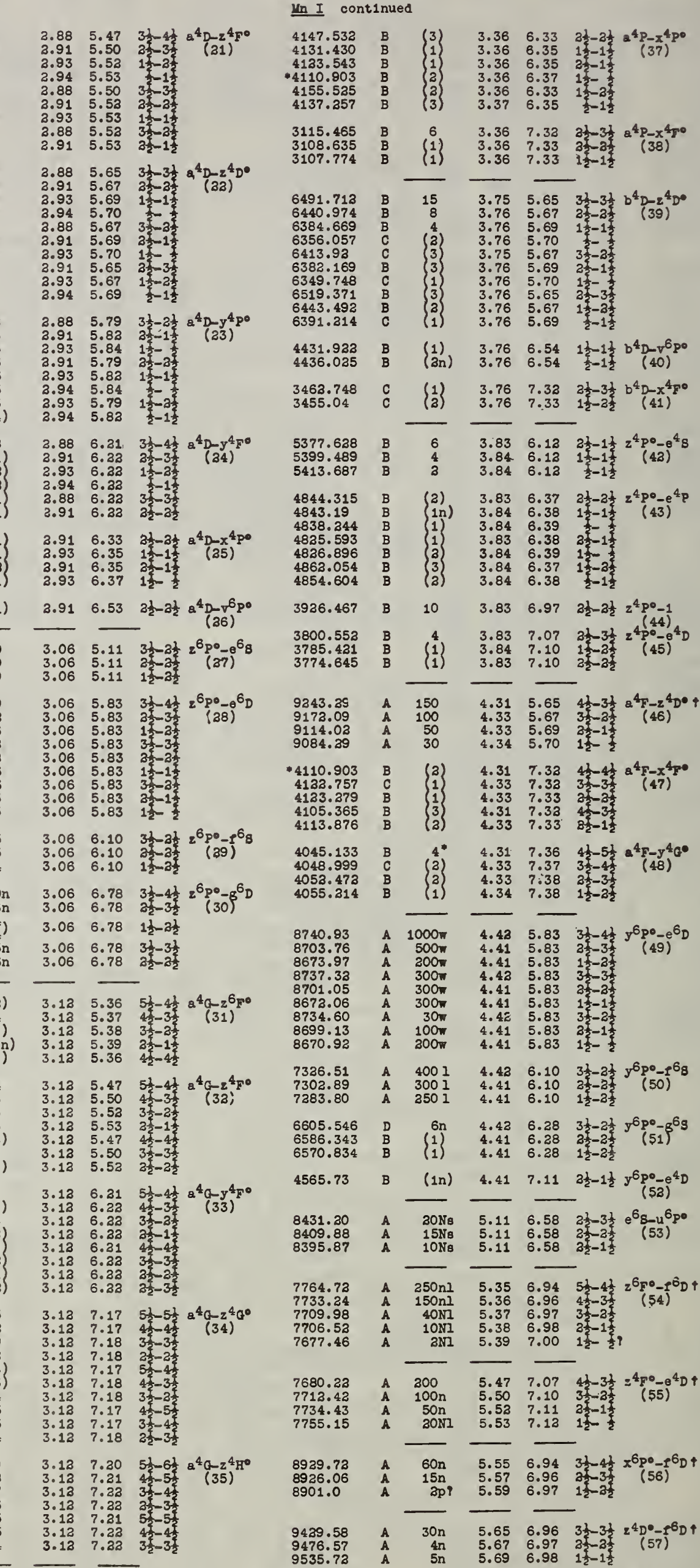

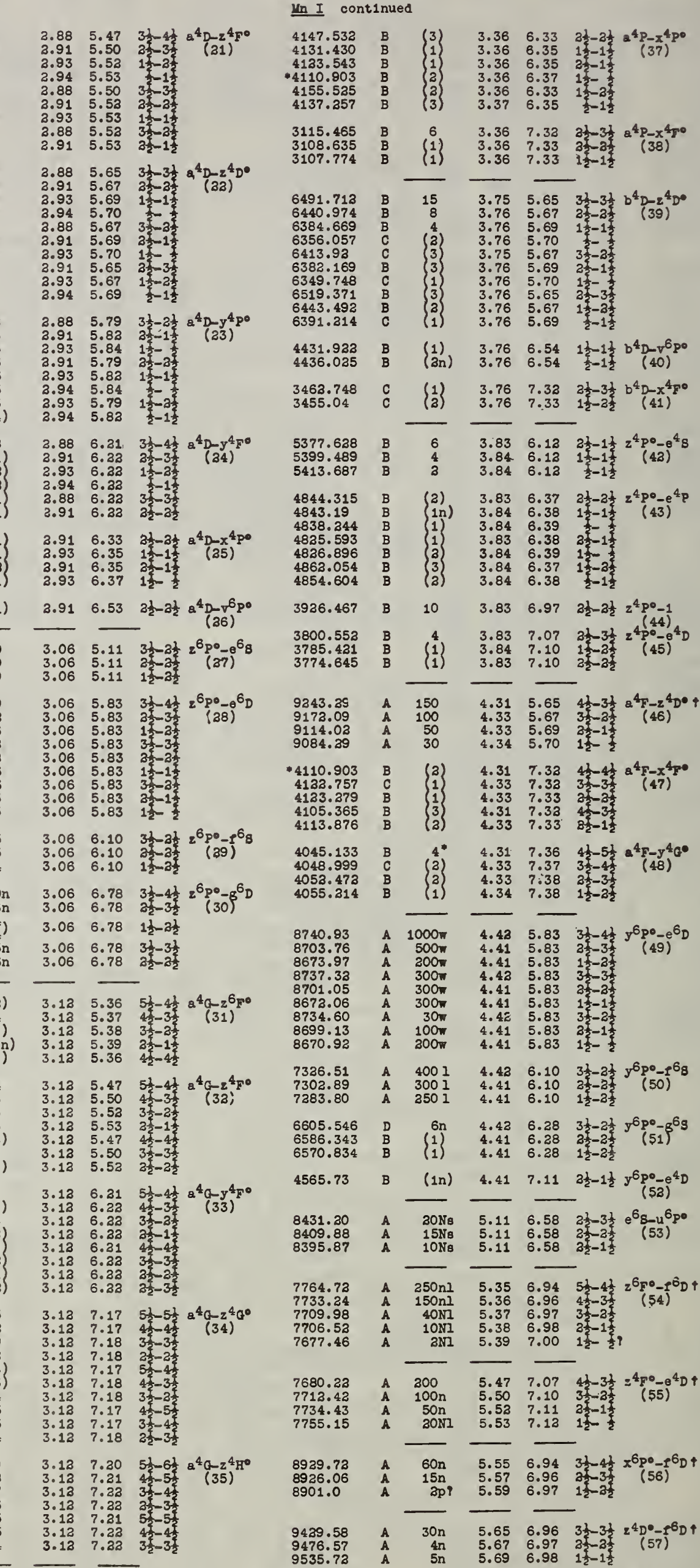

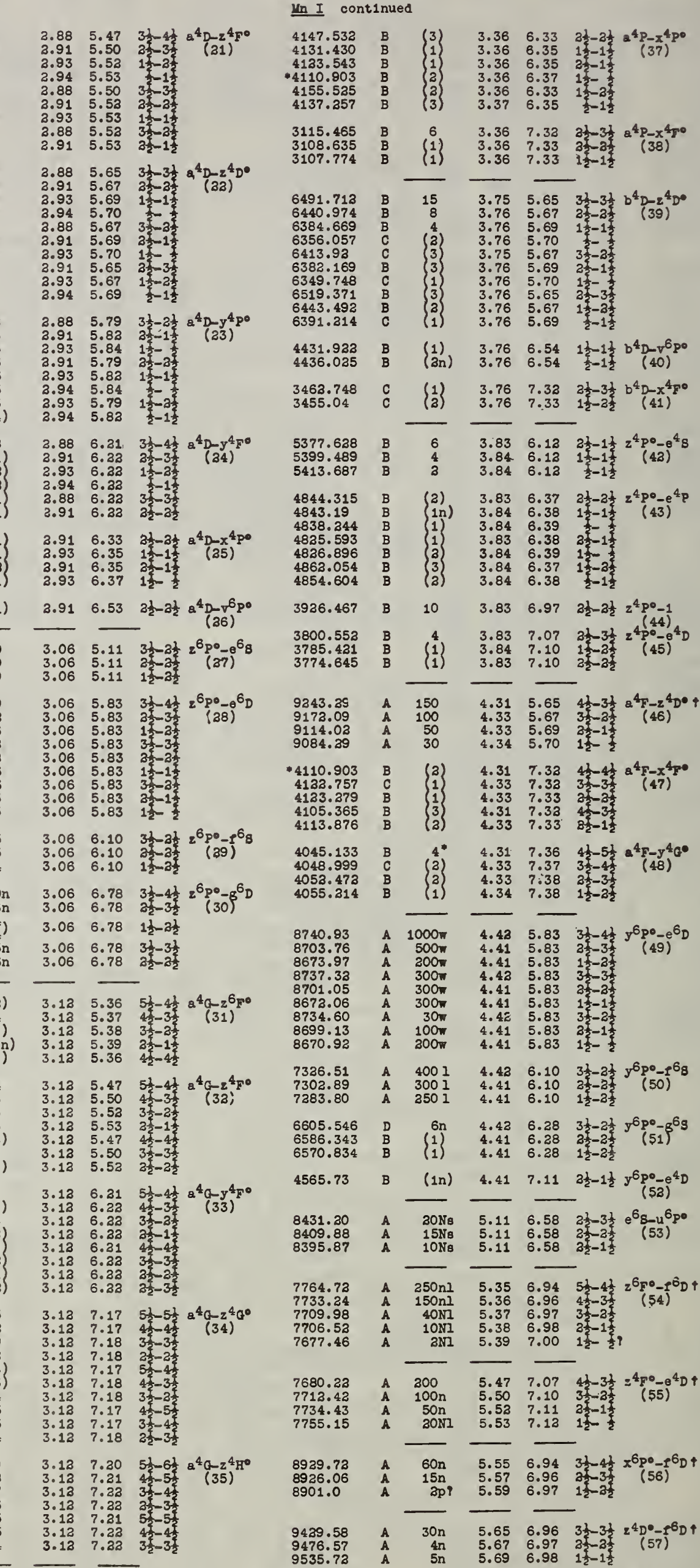

I \& Ref Int

E P

(No)

in I continued

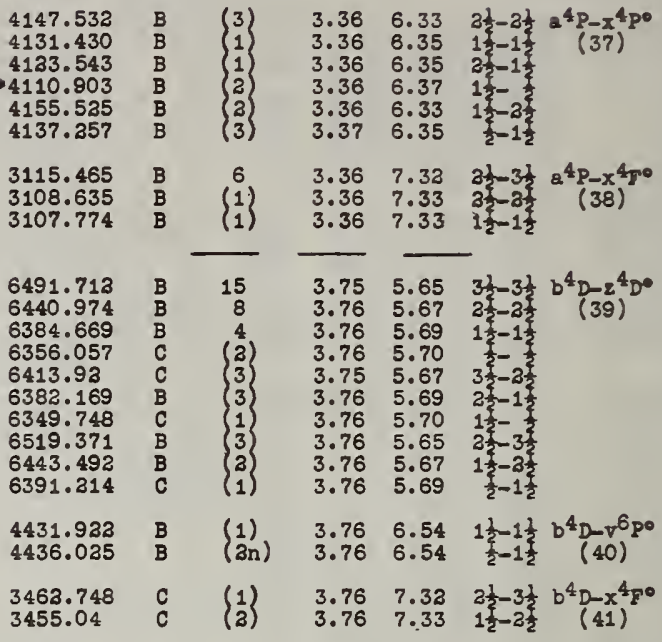

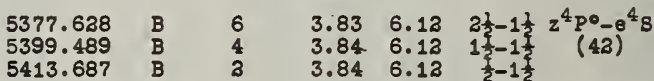

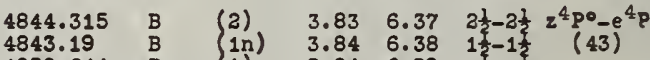

4825.593
4826.896

4854.604

3926.467

3800.552
3785.421
3774.645

9343.35

9114.02

1

4110.903
412.757
4133.279
4105.365

$\left(\begin{array}{l}3 \\ 1 \\ 3 \\ 3 \\ 3\end{array}\right.$

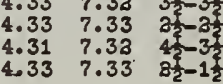

4045.133
4048.999

4055.214

$\left(\begin{array}{l}4 \\ 2 \\ 2 \\ 1\end{array}\right)$

31.7 .36

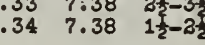

$\begin{array}{llll}.42 & 5.83 & 3 \frac{1}{2}-4 \frac{1}{2} & y^{6} \mathrm{P}^{\circ}-\mathrm{e}^{6 \mathrm{D}} \\ 41 & 5.83 & 3 \frac{1}{2}-3 \frac{3}{2} & (49)\end{array}$

$415.83 \quad 13-3$

415.83 at-a

$41 \quad 5.83$ at

$\begin{array}{llll}.42 & 6.10 & 3 \frac{1}{3}-2 \frac{1}{3} & y^{6} \mathrm{p}^{\circ}-\mathrm{r}^{6} \mathrm{~B} \\ .41 & 6.10 & 3 \frac{1}{3}-2 \frac{3}{2} & (50)\end{array}$

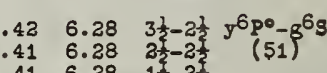

4565.73

$(25)$

$\begin{array}{lllllll}8431.20 & \text { A } & 20 \mathrm{Ns} & 5.11 & 6.58 & 2 \frac{1}{2}-3 \frac{1}{2} & e^{6} \mathrm{~s}-\mathrm{u}^{6} \mathrm{po} \\ 8409.88 & \text { A } & 15 \mathrm{Ng} & 5.11 & 6.58 & 25-2 \frac{2}{2} & (53) \\ 8395.87 & \text { A } & 10 \mathrm{Ng} & 5.11 & 6.58 & 3 \frac{2}{2}-1 \frac{2}{2} & \end{array}$

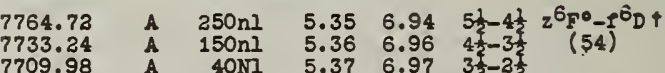

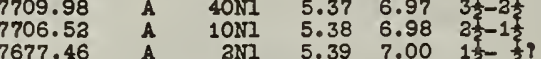

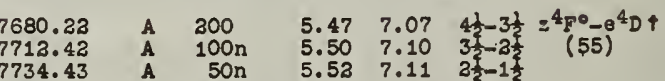

8926.06
8901.0

9535.72

$\begin{array}{rrrrrrrr}9336.47 & \mathrm{~A} & 40 \mathrm{n} & 5.65 & 6.97 & 3 \frac{1}{2}-2 \frac{1}{2} & 2^{4} \mathrm{D}^{0}-1 \\ 9502.12 & \mathrm{~A} & 8 \mathrm{n} & 5.67 & 6.97 & 3 \frac{1}{2}-2 \frac{1}{2} & (58) \\ 9633.02 & \mathrm{~A} & 4 \mathrm{p} ? & 5.69 & 6.97 & 1 \frac{1}{2}-2 \frac{1}{2} & \end{array}$

$\begin{array}{lllllll}8654.63 & \text { A } & 40 \mathrm{n} & 5.65 & 7.07 & 3 \frac{1}{3}-3 \frac{1}{3} & z^{4} D^{0}-0^{4} D \\ 8659.38 & \text { A } & 10 \mathrm{n} & 5.67 & 7.10 & 2 \frac{1}{2}-2 \frac{2}{2} & (59)\end{array}$ 


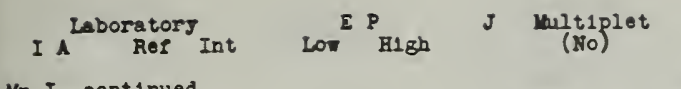

I I continued

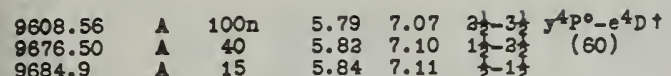

Dtrongest Uncles81f18d Lins of MnI

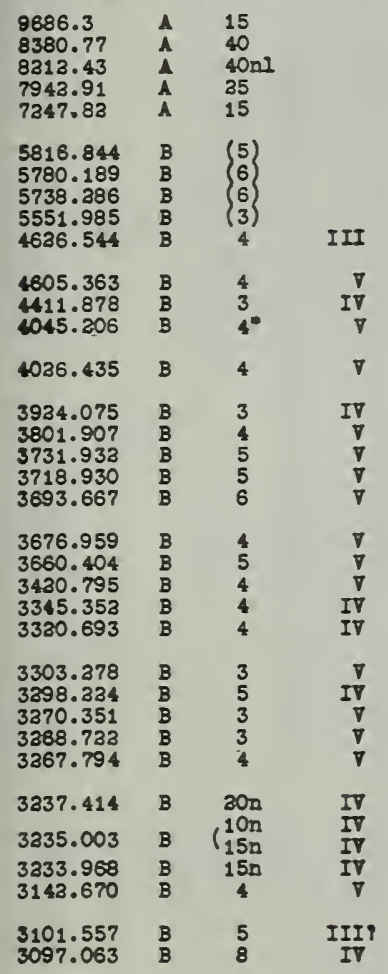

VII I P 15.8 inal $C$ List $\Lambda$ Aug 1941 $\begin{array}{rrrrrrr}3438.978 & A & 30 & 1.17 & 4.76 & 2-3 & a^{5} \mathrm{~g} \\ 3460.039 & \text { A } & 8 & 1.17 & 4.74 & 2-3 & (1)\end{array}$

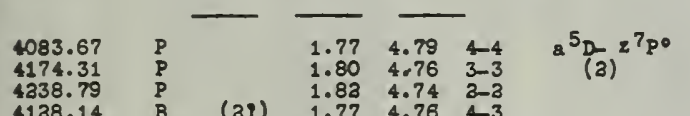
4138.14 4205.37 4138.87 4360.47

(3i) $1.77 \quad 4.76$ $\begin{array}{lll}1.80 & 4.79 & 3-4 \\ 1.83 & 4.76 & 3-3\end{array}$

341.983

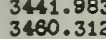
3480.313
3474.124

${ }^{5} z^{5} z^{50}$
RIVIBID YULTIPLEI TABLE

I L Laboratory Int Lor Figh J Hultiplet m II continued

3474.037 3482.905
3488.676 3498.814 3497.531

7415.78
7369.73
7347.72
7353.52
7330.54
7432.37
7387.10

4755.728

4755.728
$4764 . ?$ 4738.39
4730.361
4737.9

4343.987
4336.756 4336.756
4292.246 4383.77 4384.485 4335.1 4 .197

4306.375 359.303 4353.02 4244.36

3685.049

3708.06

3709.88

3717.53

3706.91

3729.49
3735.29

3688 . 20

- 3509.971

3482.06
3457.809

3457.809

3446.0

3089.041 3039.551
3046.266

5302.320

5299.278

5396.968
5395.393

5394.316

3466.336

3465.03

3464.043

3463.330

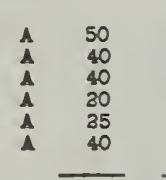

$\begin{array}{llll}1.80 & 5.35 & 3-3 & 8^{5} D-2^{5} p^{\circ}\end{array}$

$\begin{array}{lll}1.83 & 5.37 & 2-3 \\ 1.84 & 5.38 & 1-1\end{array}$

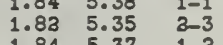

$\begin{array}{lll}1.83 & 5.35 & 2-3 \\ 1.84 & 5.37 & 1-3 \\ 1.85 & 5.38 & 0-1\end{array}$

$1.85 \quad 5.38$

$\begin{array}{llllll}P & & & & & \\ P & & 3.89 & 5.35 & 3-3 & 8^{5} P_{-} z^{5} P_{0} \\ P & & 3.69 & 5.37 & 2-3 & (4) \\ P & & 3.70 & 5.38 & 1-1 & \\ P & & 3.69 & 5.37 & 3-2 & \\ P & & 3.69 & 5.38 & 2-1 & \\ P & & 3.69 & 5.35 & 2-3 & \\ P & & 3.70 & 5.37 & 1-2 & \end{array}$

$-3.70 \quad 5.37$

\begin{tabular}{cccccc} 
A On & 5.37 & 7.97 & $5-6$ & $\mathrm{a}^{5} \mathrm{~F}_{-2} \mathrm{z}^{\circ}$ \\
$\mathrm{P}$ & - & 5.37 & 7.96 & $4-5$ & $(5)$ \\
\hline & 5.36 & 7.96 & $3-4$ &
\end{tabular}

$\begin{array}{lll}5.35 & 7.96 & 2-3 \\ 5.35 & 7.96 & 1-3\end{array}$

$\begin{array}{lll}2 N & 5.37 & 8.21\end{array}$

(3) $5.37 \quad 8.23$

5.358 .33

$\begin{array}{ll}5.35 & 8.23 \\ 5.37 & 8.33\end{array}$

5.378 .31

(1)

$5.36 \quad 8.33$

ON $5.37 \quad 8.31$

(3)

$\begin{array}{ll}5.37 & 8.37 \\ 5.36 & 8.36\end{array}$

$\begin{array}{ll}5.36 & 8.36 \\ 5.35 & 8.26\end{array}$

$\begin{array}{lll}-11 & 5.37 & 8.73 \\ (1) & 5.37 & 8.70\end{array}$

$\begin{array}{ll}5.37 & 8.70 \\ 5.36 & 8.68\end{array}$

$\begin{array}{ll}5.36 & 8.68 \\ 5.35 & 8.67\end{array}$

$5.35 \quad 8.66$

$\begin{array}{ll}5.37 & 8.70 \\ 5.37 & 8.58\end{array}$

$\begin{array}{ll}5.37 & 8.68 \\ 5.36 & 8.67\end{array}$

8.72

$5.37 \quad 8.89$

$\begin{array}{ll}5.37 & 8.92 \\ 5.36 & 8.93\end{array}$

$\begin{array}{ll}5.36 & 8.93 \\ 5.35 & 8.93\end{array}$

$5.35 \quad 8.93 \quad 1-$

$5-5 \quad 8^{5} T-2^{5} F^{0}$

$4-4$
$3-3$
$2-2$
$1-1$
$5-$
3

$\begin{array}{cc}5-4 & 8^{5} T-2^{5} D^{\circ} \\ 4-3 & (7)\end{array}$

$3-3$

$2-5 \quad a_{2} 2-z^{2} g^{2}$

$4-4$
$3-3$
$2-3$

$3-3$
$1-1$
$5-4$

$4-3$
$3-3$
$4-5$

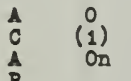

5.358

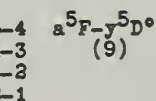

$\begin{array}{llll}5.35 & 9.43 & 3-2 & 2^{5} p^{\circ}-e^{5} 8 \\ 5.37 & 9.43 & 2-3 & \end{array}$

$\begin{array}{lll}5.37 & 9.43 & 2- \\ 5.38 & 9.43 & 1-\end{array}$

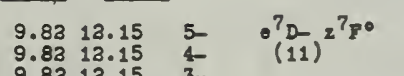

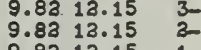

$9.8313 .151-$

$9.83 \quad 13.38$

9.8313 .38

9.82
9.83
9.83 .38

$\begin{array}{lll}9.83 & 13.38 & 2-\end{array}$

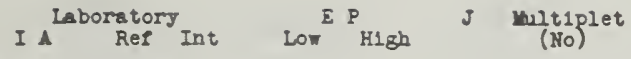

Kn II continued

$6133.438 \quad A \quad 40 \quad 10.14 \quad 13.16 \quad 4-5 \quad{ }^{5} \mathrm{D} \times \mathrm{x}^{5}$ 6135.855 6130.794 6131.917 6132.799 6126.210

6131.005

6133.164

6136.516
6129.355

3800.240

3801.633

3803.881

3804.476

3134.819

3135.507
3136.315

6105.381

6050.446

6107.393

6051.860

(20)

000

4530.03

4510.210

4496.989

4639.150

4652.816

6446.381

$\begin{array}{lll}25 & 10.14 & 13.16 \\ 30 & 10.14 & 13.16\end{array}$

10.1412 .16

10.1413 .16

10.1412 .16

10.1412 .16

$10.14 \quad 13.16$

$10.1413 .16 \quad 4-$

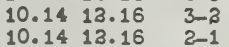

$10.1413 .39 \quad 4-5 \quad 0^{5} \mathrm{D} \mathrm{V}^{5} \mathrm{~F}^{\circ}$

10.1413 .39

10.1413 .39

$\begin{array}{ll}10.14 & 13.39 \\ 10.14 & 13.39\end{array}$

$2-4$

$10.1414 .08 \quad 4-5 \quad 0^{5} \mathrm{D}^{5} \mathrm{~F}^{\circ}$ $\begin{array}{llll}10.14 & 14.08 & 3-4 & (15) \\ 10.14 & 14.08 & 2-3 & \end{array}$

$\longrightarrow$ $\begin{array}{lllc}10.31 & 12.33 & 4-5 & 5^{7} P^{0}-I^{7} \mathrm{D} \\ 10.29 & 12.33 & 3-4 & (16)\end{array}$ $\begin{array}{ll}10.28 & 12.33 \\ 10.31 & 13.33\end{array}$ 10.2912 .33

10.3812 .33

10.3113

$\begin{array}{lll}10.31 & 13.33 & 4-3\end{array}$

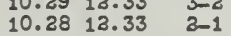

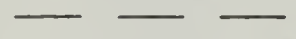

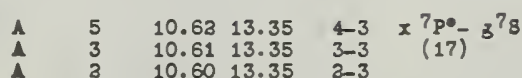

- 3 -

$10.7313 .39 \quad 3-2=5 p^{\circ}-B^{5}$

6.43.6.

6463.195
6463.799

6462.454
6463.210

A $\quad \begin{aligned} & 2 \\ & \mathbf{A}\end{aligned}$

10.7313 .39

$2-$

A 501

$\overline{13.15} 14.06 \quad 6 \quad 2^{7} F^{0}-e^{7}$

\section{3}

$\overline{13.16} 14.06 \quad 5-6 \quad x^{5} \mathrm{po}^{0}-\mathrm{P}^{5} \mathrm{G}$ 12.1614 .06 $\begin{array}{lll}12.16 & 14.06 & 2-3 \\ 12.16 & 14.06 & 1-3\end{array}$

3050.661

3043.132

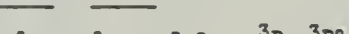

25

(sagurss 1nadequate for preparat10n of $118 \mathrm{t}$ of Masurs 8 Inadequa te for preparation of 
48 REVIBED UULTIPLEI TABLE

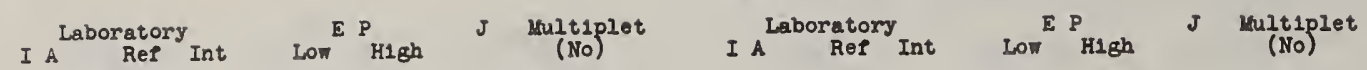
I A Laboratory ${ }_{\text {Ref Int Lom }}^{\text {E } P_{\text {H1gh }} \text { J Multiplet }}$ Fe I I P 7.858 Anel A List A Feb 1943 Fe I continued

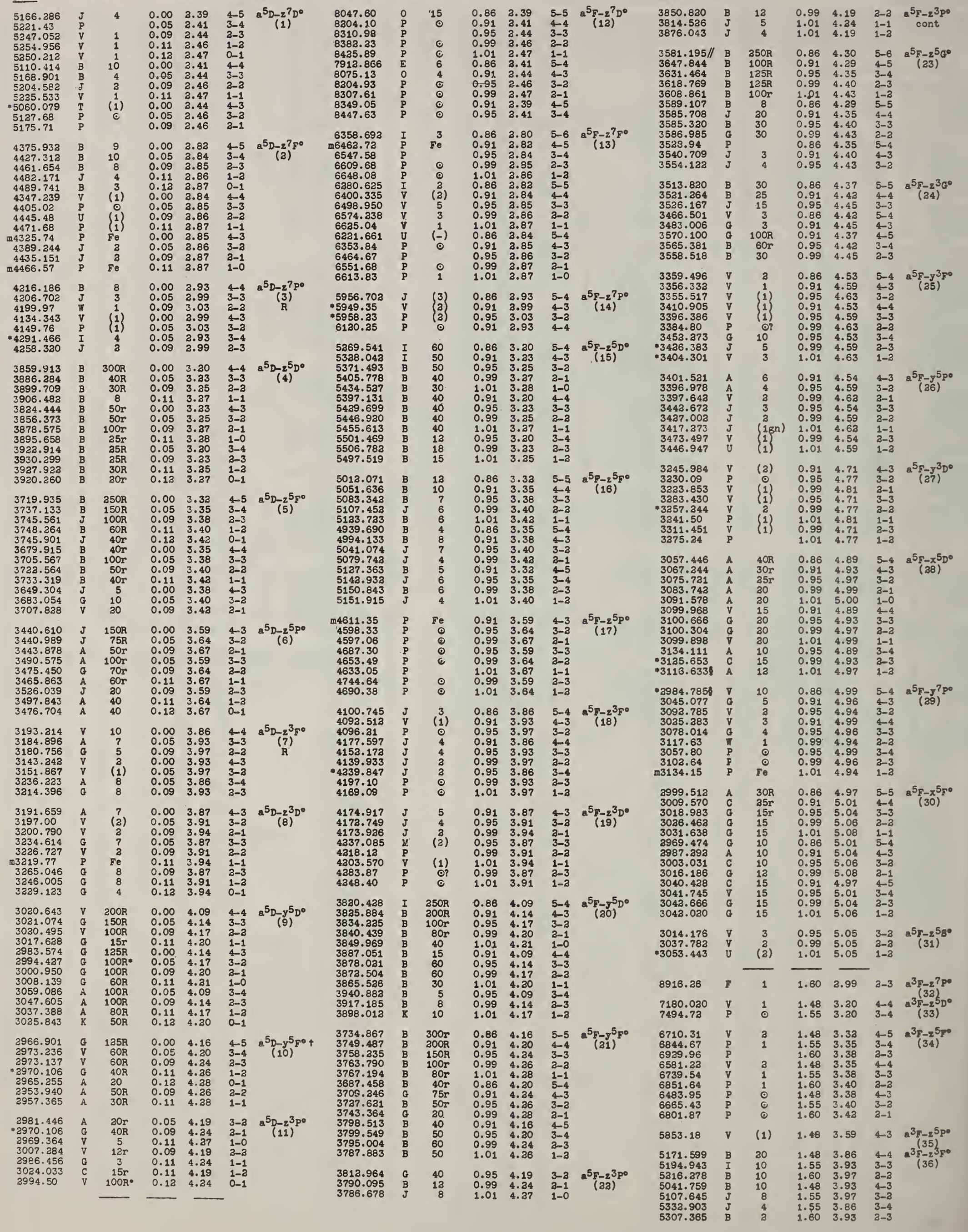




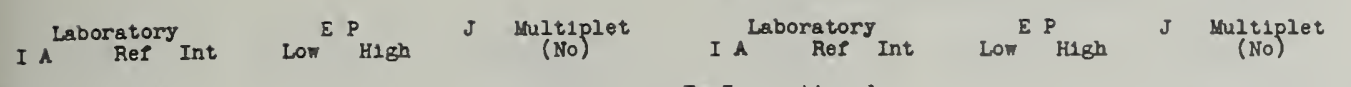

\section{Fe I continued}

\begin{tabular}{|c|c|}
\hline $\begin{array}{r}5167.491 \\
5227.192 \\
5270.360 \\
5338.534 \\
5341.026 \\
\text { m5446.87 }\end{array}$ & $\begin{array}{l}B \\
B \\
B \\
P\end{array}$ \\
\hline $\begin{array}{l}4733.596 \\
.4772 .817 \\
4798.736 \\
4643.20 \\
4868.38 \\
4867.53\end{array}$ & $\begin{array}{l}\text { B } \\
\text { B } \\
\text { V }\end{array}$ \\
\hline $\begin{array}{l}4602.944 \\
4654.501 \\
4680.297 \\
4531.153 \\
4592.655 \\
4632.915 \\
4472.52 \\
4547.022 \\
4602.005\end{array}$ & $\begin{array}{l}\text { B } \\
\text { J } \\
\text { J } \\
\text { B } \\
\text { B } \\
\text { J } \\
\text { P } \\
\text { J } \\
\end{array}$ \\
\hline $\begin{array}{l}4674.65 \\
4672.83 \\
4765.485\end{array}$ & $\begin{array}{l}P \\
v\end{array}$ \\
\hline $\begin{array}{r}4383.547 \\
4404.753 \\
4415.125 \\
4394.128 \\
4337.049 \\
4367.906 \\
4329.760 \\
-4391.466\end{array}$ & $\begin{array}{l}\text { B } \\
B \\
B \\
\text { J } \\
\text { J } \\
\text { I }\end{array}$ \\
\hline
\end{tabular}

$\begin{array}{lllll}40 & 1.48 & 3.87 & 4-3 & \mathrm{a}^{3} \mathrm{~F}_{-2} \mathrm{Z}^{3} \mathrm{D}^{\circ} \\ 40 & 1.55 & 3.91 & 3-2 & (3700 \\ 30 & 1.60 & 3.94 & 2-1 & (37) \\ 15 & 1.55 & 3.87 & 3-3 & \\ 20 & 1.60 & 3.91 & 2-2 & \\ \mathrm{Fe} & 1.60 & 3.87 & 2-3 & \\ & & & & \end{array}$

$\left(\begin{array}{ll}6 \\ a \\ a\end{array}\right)$

$\begin{array}{llll}1.48 & 4.09 & 4-4 & a^{3} F-y^{5} D^{\circ} \\ 1.55 & 4.14 & 3-3 & (38) \\ 1.60 & 4.17 & 3-2 & \\ 1.48 & 4.14 & 4-3 & \\ 1.55 & 4.09 & 3-4 & \end{array}$

$1.60 \quad 4.14$

$\begin{array}{llll}1.48 & 4.16 & 4-5 & 8^{3} \mathrm{~F}-y^{5} F^{\circ} \\ 1.55 & 4.30 & 3-4 & (39)^{3}\end{array}$ $\begin{array}{lll}1.60 & 4.24 & 2-3 \\ 1.48 & 4.20 & 4 \\ 1.55 & 4.34 & 3-3\end{array}$ $\begin{array}{lll}1.55 & 4.24 & 3-3 \\ 1.60 & 4.36 & 2-3\end{array}$

$\begin{array}{lll}1.60 & 4.36 & 2-2 \\ 1.48 & 4.24 & 43\end{array}$

$\begin{array}{lll}1.48 & 4.24 & 4-3 \\ 1.55 & 4.26 & 3-2\end{array}$

(- $\quad 1.55 \quad 4.19 \quad 3-2 \quad a^{3} F-z^{3} p^{0}$

(1) $1.60 \quad 4.24 \quad 2-1$

$\begin{array}{llll}45 r & 1.48 & 4.29 & \\ 30 & 1.55 & 4.35 & 3-4 \\ 20 & 1.60 & 4.40 & 2-3 \\ 15 & 1.48 & 4.35 & 4 \\ 10 & 1.55 & 4.40 & 3-3 \\ 3 & 1.60 & 4.43 & 2-3 \\ (1) & 1.48 & 4.40 & 4-3 \\ 4 & 1.55 & 4.43 & 3-2\end{array}$

4271.764

4307.906
4325.765
4

4302.031
4250.790

4147.673

4045.815
4063.597

4063.597
4071.740

3969.261
4005.246

4143.871
4132.060

4032.636 4064.46
4090.34
.4130 .035
-4132.94 4132.94
4200.78

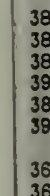

38
38
38
38
38
38
36

15.842

\section{5}

$1.48 \quad 4.37 \quad 4-5 \quad 8^{3} F-z^{3} G^{\circ}$

$\begin{array}{lll}1.55 & 4.42 & 3-4 \\ 1.60 & 4.45 & 2 \\ 1.48 & 4.43 & 4-4\end{array}$

$\begin{array}{lll}1.48 & 4.42 & 4-4 \\ 1.55 & 4.45 & 3-3\end{array}$

$\begin{array}{lll}1.58 & 4.45 & 4\end{array}$

$\begin{array}{lllll}60 \mathrm{r} & 1.48 & 4.53 & 4-4 & a^{3} F-y^{3} F \\ 45 & 1.55 & 4.59 & 3-3 & (43)\end{array}$

$\begin{array}{ll}1.55 & 4.59 \\ 1.60 & 4.63\end{array}$

$1.48 \quad 4.59$

$\begin{array}{ll}1.55 & 4.63 \\ 1.55 & 4.53\end{array}$

$1.60 \quad 4.59 \quad 2-3$

$\begin{array}{lll}\text { (2) } & 1.48 & 4.54 \\ \text { (2) } & 1.55 & 4.59 \\ 0 & 1.60 & 4.62\end{array}$

(i)

$\begin{array}{lll}1.60 & 4.62 & 2-1 \\ 1.55 & 4.54 & 3-3\end{array}$

$\begin{array}{lll}1.60 & 4.59 & 2-2 \\ 1.60 & 4.54 & 2-3\end{array}$

$\begin{array}{ll}1.48 & 4.71 \\ 1.55 & 4.77\end{array}$

$\begin{array}{lll}1.60 & 4.81 & 2-1 \\ 1.55 & 4.71 & 3-3\end{array}$

$\begin{array}{lll}1.55 & 4.71 & 3-3 \\ 1.60 & 4.77 & 2-3\end{array}$

(1) $\quad 1.48 \quad 4.89 \quad 4-4 \quad a^{3} F-x^{5} D$

$\begin{array}{lll}3 & 1.55 & 4.93 \\ (1) & 1.60 & 4.97\end{array}$

$\begin{array}{lll}3 & 1.48 & 4.93 \\ 1 & 1.55 & 4.97\end{array}$

$\begin{array}{ll}1.55 & 4.97 \\ 1.60 & 4.99\end{array}$

$\begin{array}{ll}1.55 & 4.89 \\ 1.60 & 4.93\end{array}$

$\begin{array}{ll}1.48 & 4.99 \\ 1.48 & 4.96\end{array}$

$\begin{array}{ll}1.48 & 4.96 \\ 1.55 & 4.94 \\ 1.55 & 4.99 \\ 1.60 & 4.96\end{array}$

(1) $\begin{array}{llll}1.48 & 4.97 & 45 & a^{3}-x^{5} F^{\circ} \\ 1.48 & 5.01 & 4-4 & (48)\end{array}$

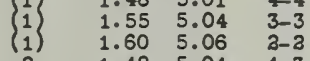

$\begin{array}{llll}3 & 1.48 & 5.04 & 4-3 \\ \text { (1) } & 1.55 & 5.06 & 3-2\end{array}$

$4 \quad 1.60 \quad 5.08$

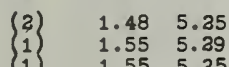

(1) $1.48 \quad 5.30$

$3-3$

(1)

$1.48 \quad 5.30$

(1) $1.60 \quad 5.33$

$\begin{array}{cc}4-5 & a^{3} F-y^{5} G^{\circ} \\ 3-4 & (50) \\ 2-3 & (50)\end{array}$

(1) $\quad 1.48 \quad 5.31 \quad 4-5 \quad 8^{3} \mathrm{~F}-2^{5} \mathrm{H}^{\circ}$

(1) $1.55 \quad 5.32$

$\begin{array}{llll}1.48 & 5.37 & 4-4 & a^{3} F-m^{5} D^{\circ}\end{array}$

$1.55 \quad 5.42 \quad 3-3$

$\begin{array}{clll}\odot & 1.60 & 5.45 & 2-2 \\ 5 & 1.48 & 5.42 & 4-3 \\ 4 & 1.55 & 5.45 & 3-2 \\ \text { (1) } & 1.60 & 5.48 & 2-1\end{array}$

$\begin{array}{llll}1.48 & 5.50 & 4-3 & 2^{3} F-v^{5} p^{\circ} \\ 1.55 & 5.48 & 3-4 & (53) \\ 1.48 & 5.48 & 4-4 & \end{array}$

$\begin{array}{llllll}138.40 & \mathrm{P} & \odot & 1.55 & 5.48 & 3-4 \\ 1081.83 & \mathrm{P} & \odot & 1.48 & 5.48 & 4-4 \\ 1125.03 & \mathrm{P} & \odot & 1.55 & 5.50 & 3-3 \\ 1154.11 & \mathrm{P} & \odot & 1.60 & 5.51 & 2-2\end{array}$

\$138.901
Fe I oontinued

$\begin{array}{rrrrrrr}3007.146 & \text { V } & 8 & 1.48 & 5.58 & 4-3 & 8^{3} F-x^{3} D^{0} \\ 3055.263 & C & 12 & 1.55 & 5.59 & 3-2 & (55)\end{array}$ $\begin{array}{rrrrrrr}3068.175 & G & 8 & 1.60 & 5.69 & 3-2 & (55) \\ 3060.984 & G & 4 & 1.55 & 5.58 & 3-1 & \\ 3093.806 & \mathrm{~V} & 3 & 1.60 & 5.59 & 2-2 & \end{array}$ 3000.452 3041.639 3029.237
2976.50

3962.11

3004.62
3034.51

11973.01 11882.80 11884.13 11638.25 11689.98

11374.02

11422.30
11593.55

10395.75

10340.77

10155.18

10167.4
10265.33

9987.8

4058.38

$8688.633 \quad E \quad 1500$

8514.075

8468.413
8387.781

8327.063
8824.337

8824.237
8661.908

7367.00
7101.38

7101.28
7037.04

6430.851
6335.335

6335.335
6297.800
6265.140

6219.290

6151.624

6173.343

6062.89

6031.83

5958.34

5943.58
5963.25

5881.76
5893.80

6097.08

$\mathrm{m} 6012.31$

6082.718

523.30

5143.73
5102.24

5202.339

5145.105
5131.475

5098.703

5079.226
5250.650

5198.714

4847.09

4771.702
4745.129

4745.129
4731.77

4700.42
4889.009

4817.773

4528.619
4494.568

4494.568
4482.357

4459.121
4442.343

4447.723
4407

4407.714
4408.419

4430.618

4371.00
4447.134

4518.58

4412.43
4478.040
4443.835

4443.835

438.260
4324.961

4324.961
4329.54

4329.54
4292.13

4292.293

4308.54

$\mathbf{4} 4259.95$
4271.65

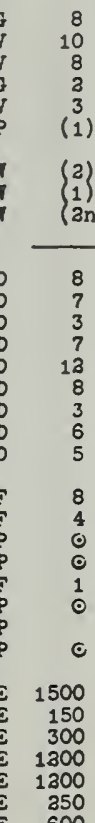

10

$\left(\begin{array}{l}5 \\ 2 \\ 2\end{array}\right)$

$\begin{array}{llllll}18 & 2.17 & 4.89 & 3-4 & a^{5} P-x^{5} D^{\circ} \\ 12 & 2.19 & 4.93 & 2-3 & (68)\end{array}$

$\begin{array}{rrrr}6 & 2.21 & 4.97 & 1-2 \\ 10 & 2.17 & 4.93 & 3-3\end{array}$

$\begin{array}{llll}10 & 2.17 & 4.93 & 3-3 \\ 12 & 2.19 & 4.97 & 2-2\end{array}$

$\begin{array}{lll}2.19 & 4.97 & 2-2 \\ 2.31 & 4.99 & 1-1\end{array}$

$\begin{array}{lll}3.17 & 4.97 & 3-3 \\ 3.19 & 4.99 & 2-1\end{array}$

(2) un : $\begin{array}{llll}2.17 & 4.99 & 3-4 & 2^{5} \mathrm{p}-y^{7} \mathrm{po}^{2} \\ 2.19 & 4.96 & 2-3 & (69)^{2}\end{array}$

$\begin{array}{llll}0 & 3.21 & 4.94 & 1-2 \\ (1) & 3.17 & 4.96 & 3-3 \\ (1) & 3.19 & 4.94 & 3-3\end{array}$

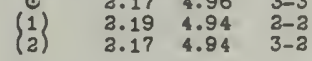

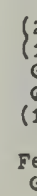

$\begin{array}{llll}2.17 & 5.01 & 3-4 & 2^{5} p-x^{5} F^{\circ}\end{array}$ 3988.468

6009.45

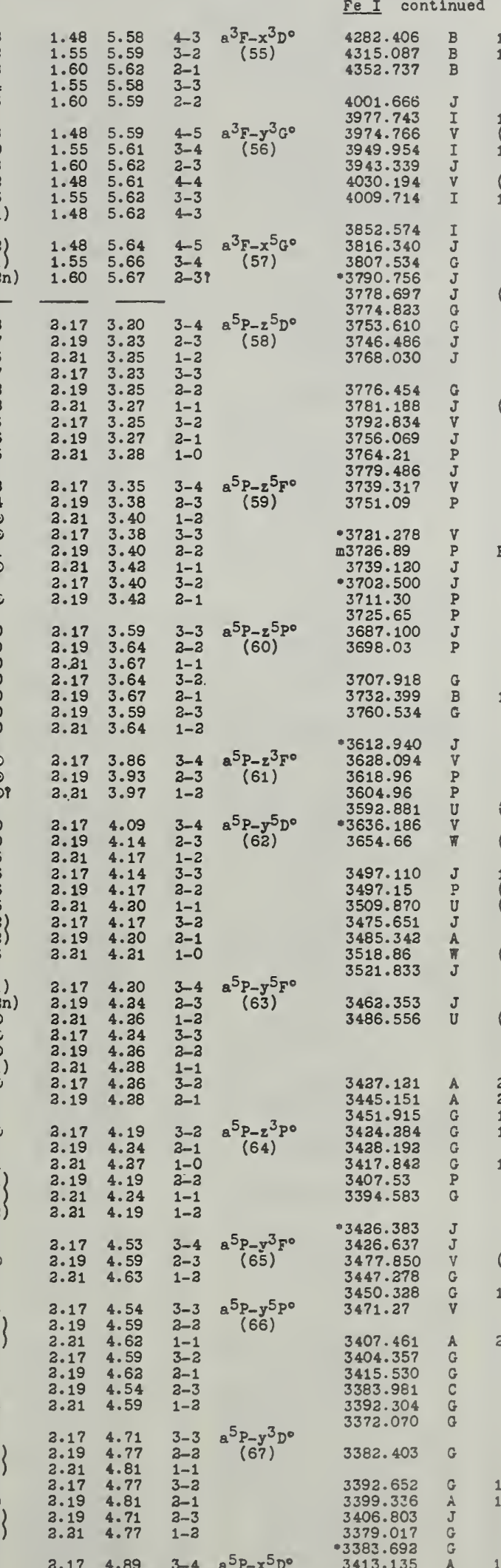

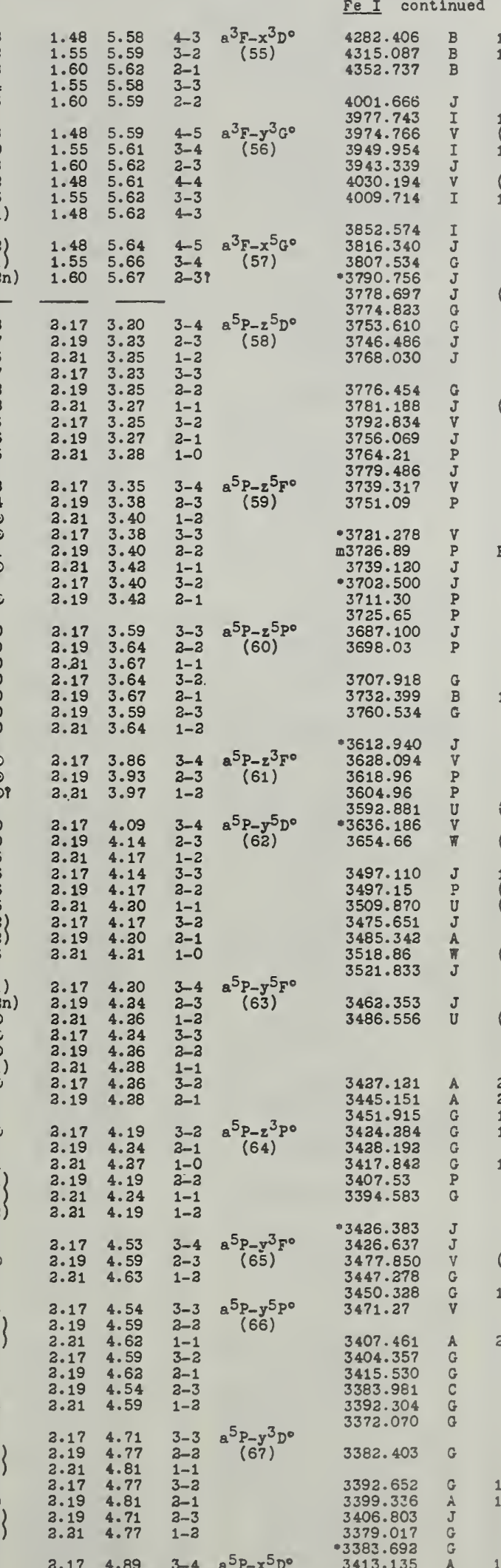

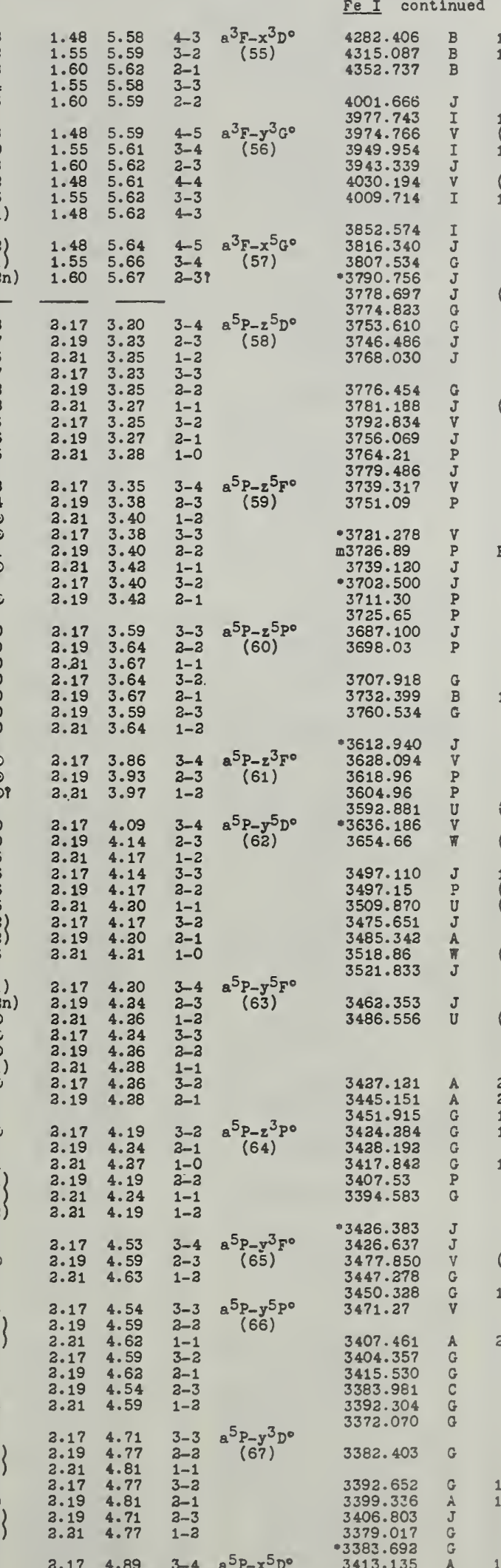

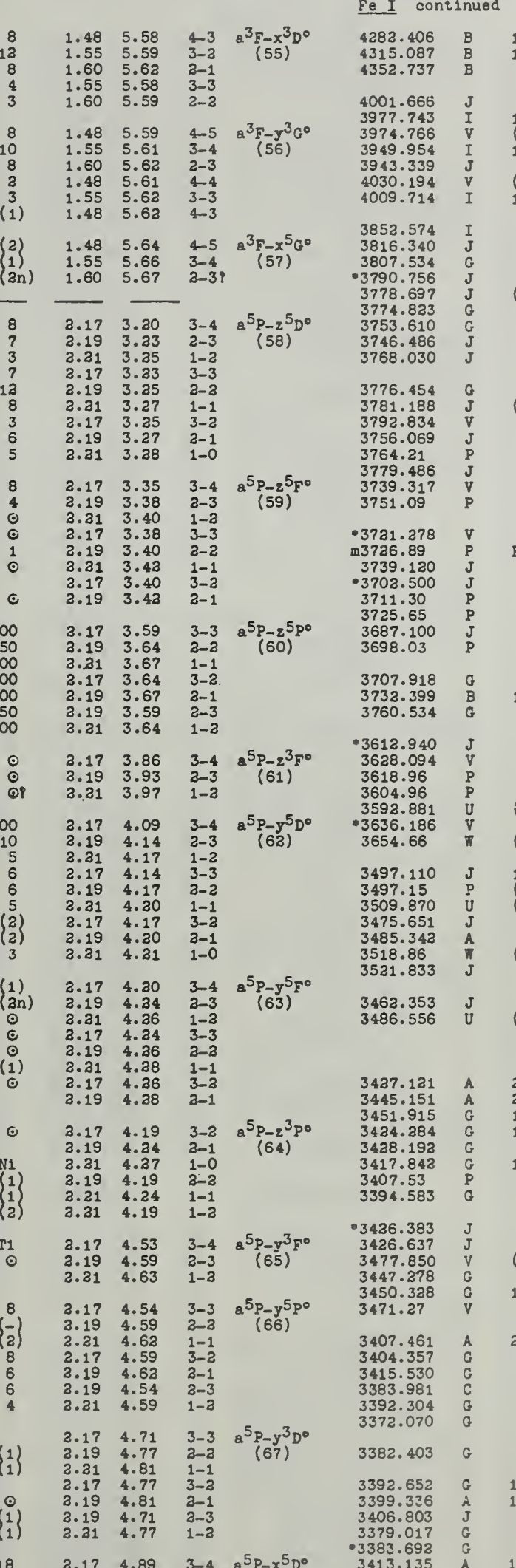

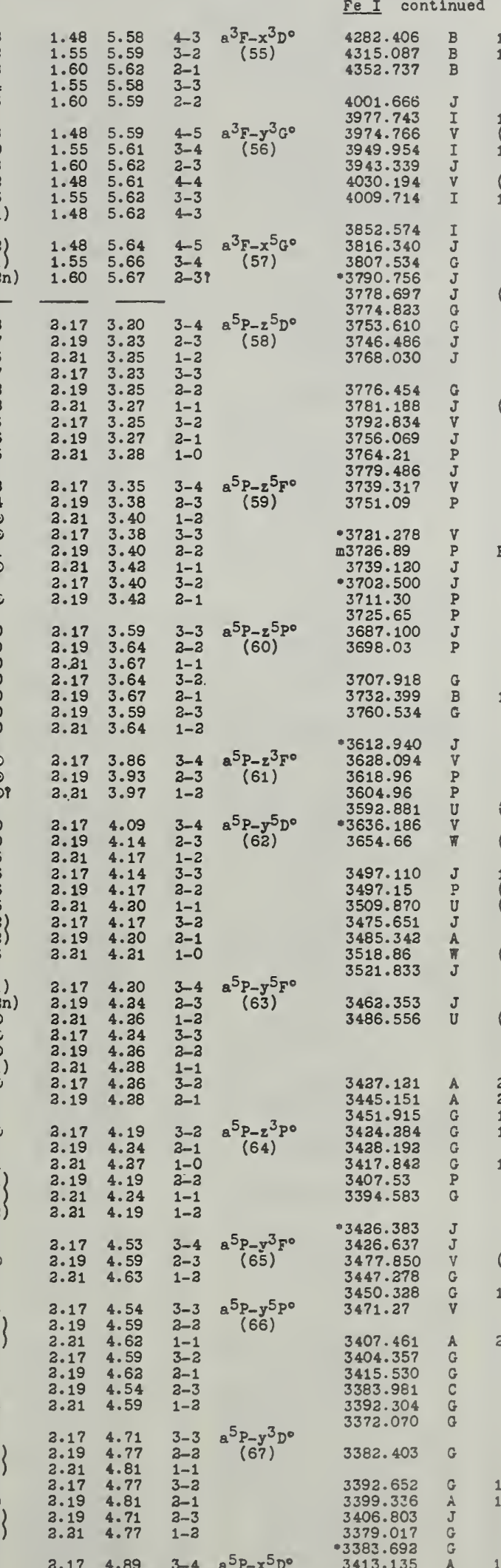

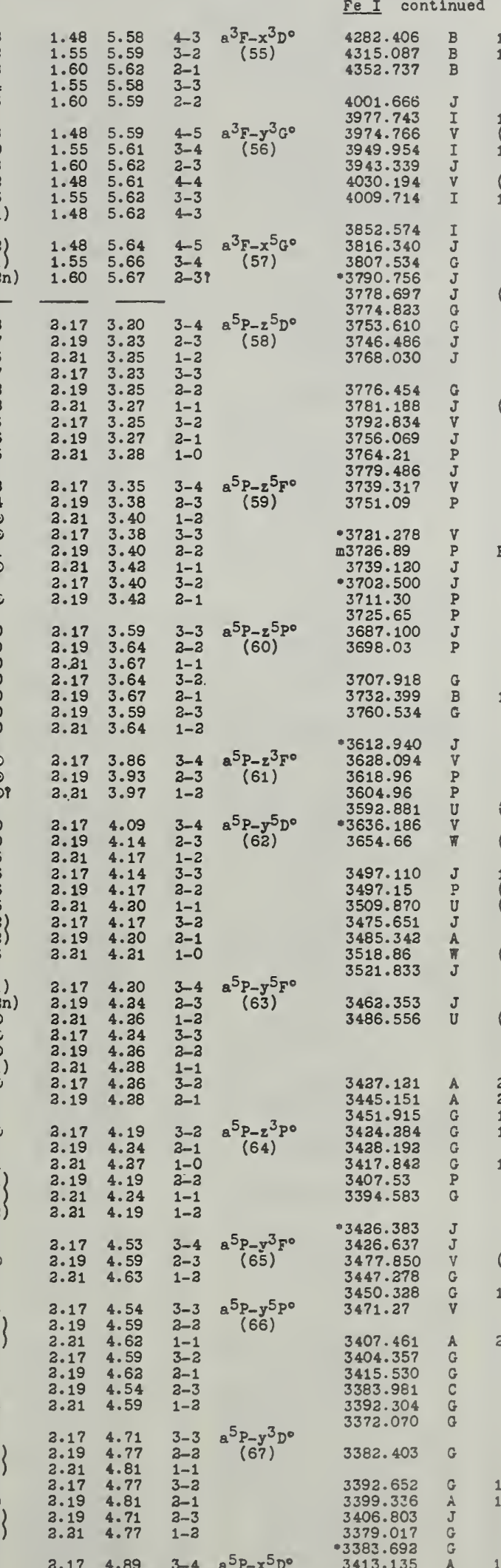

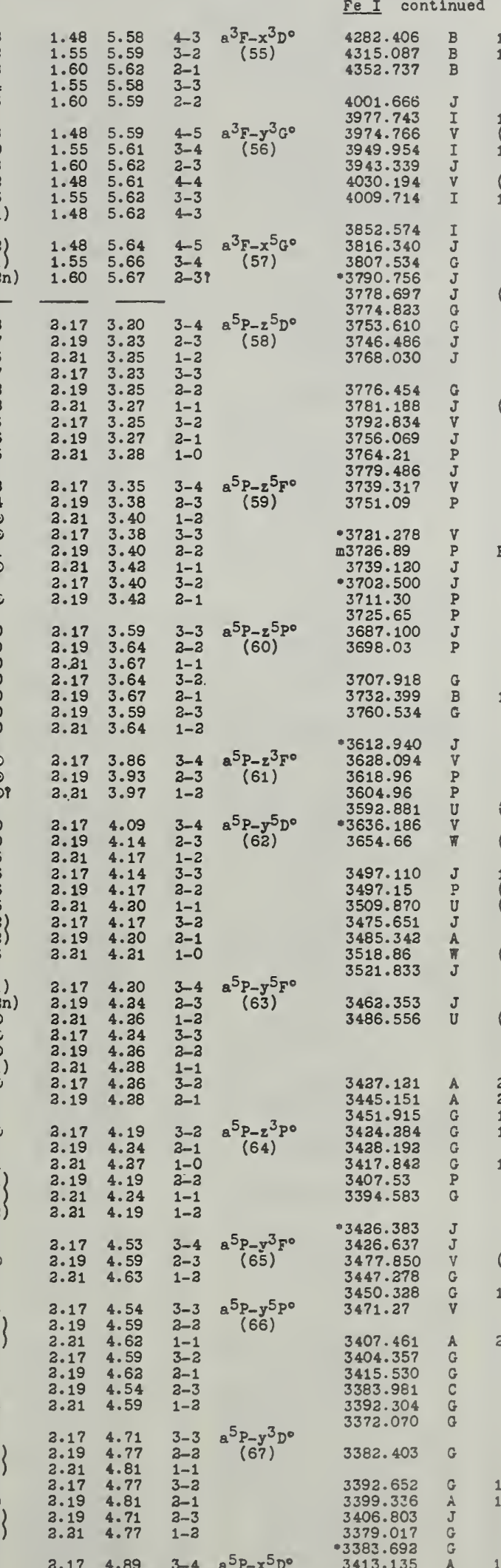

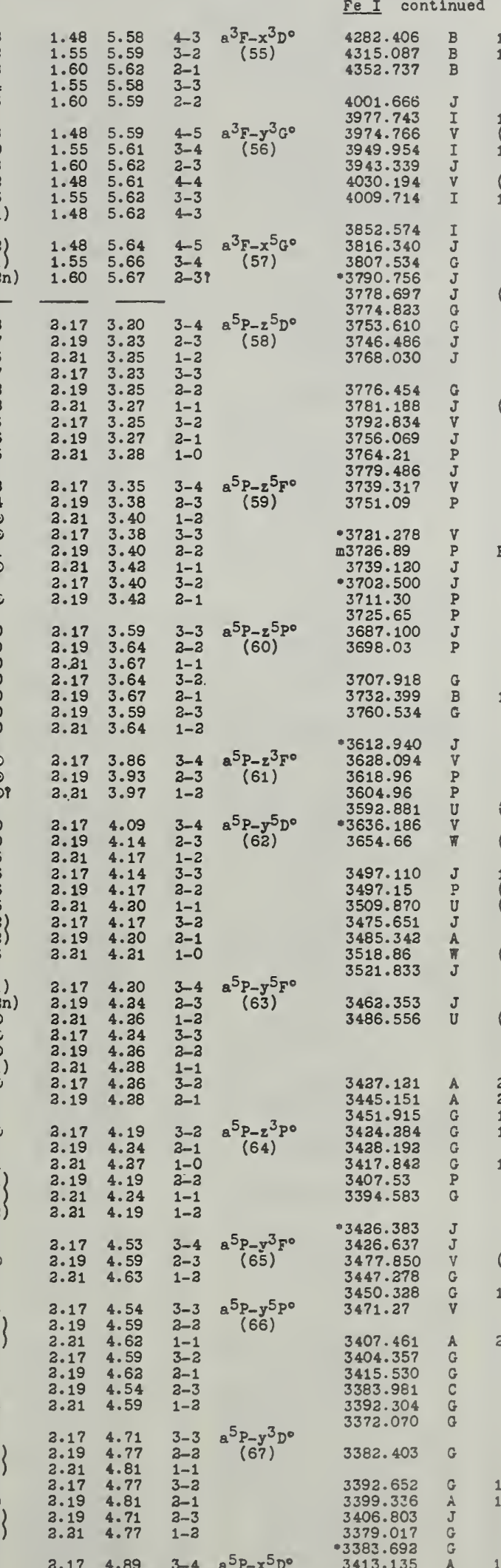

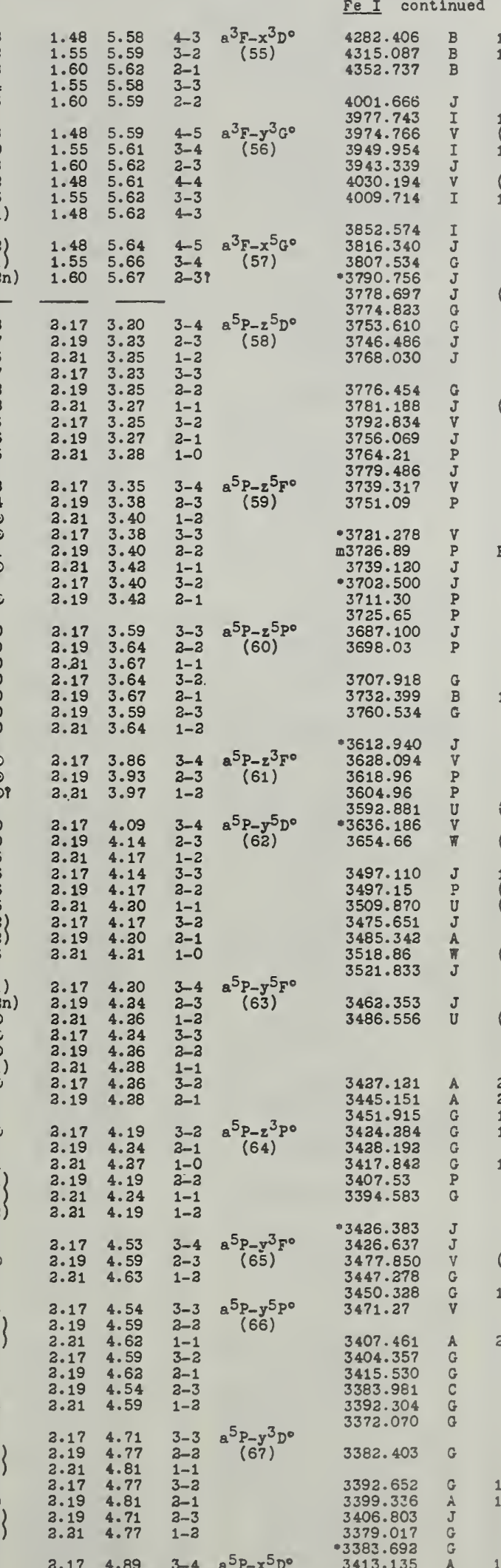

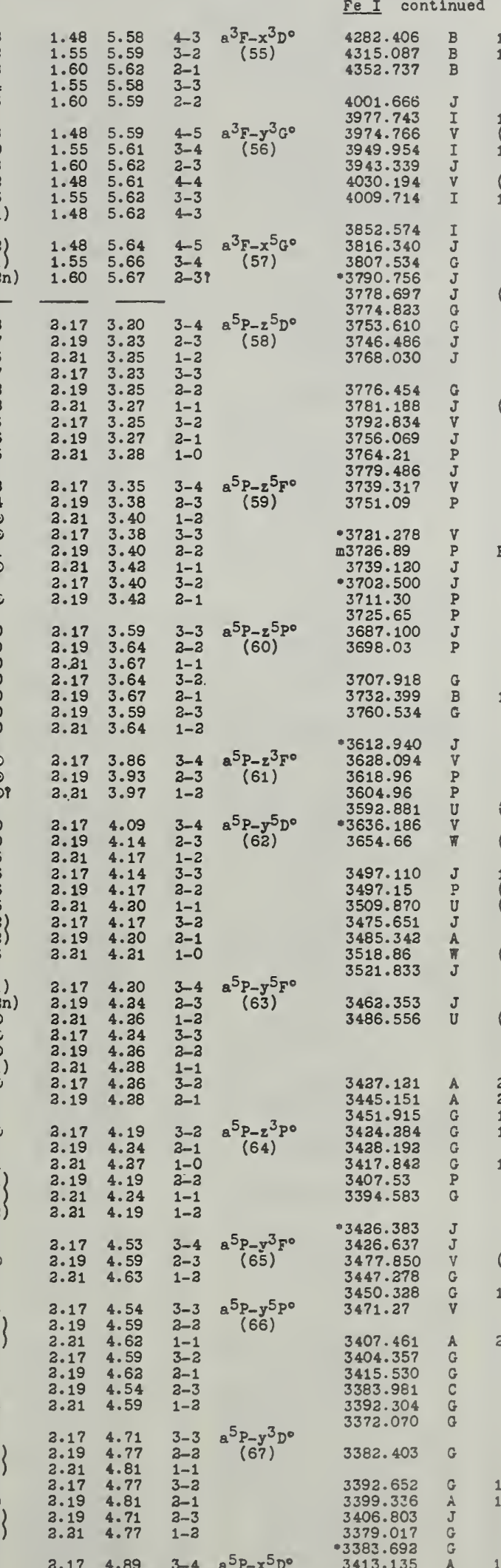

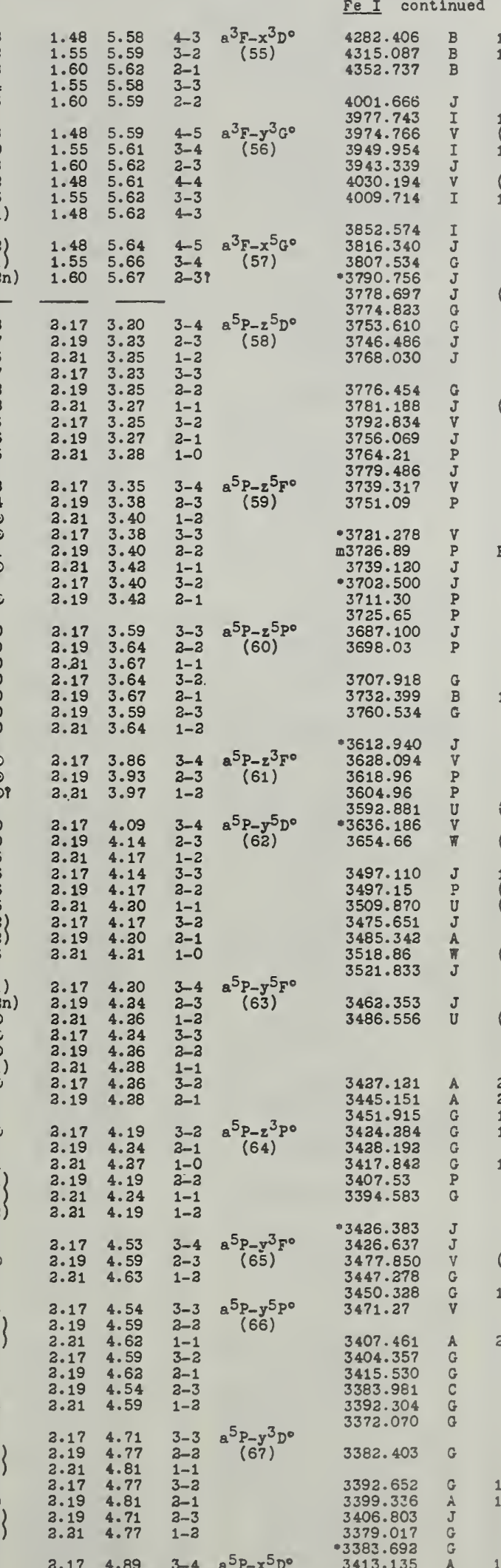

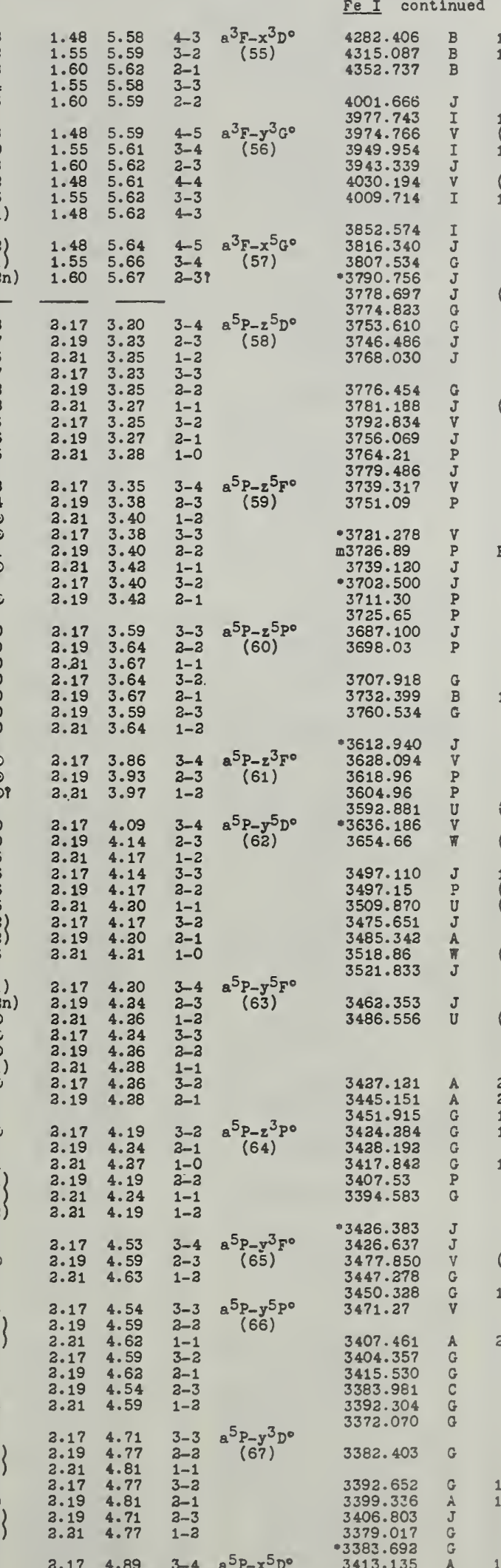

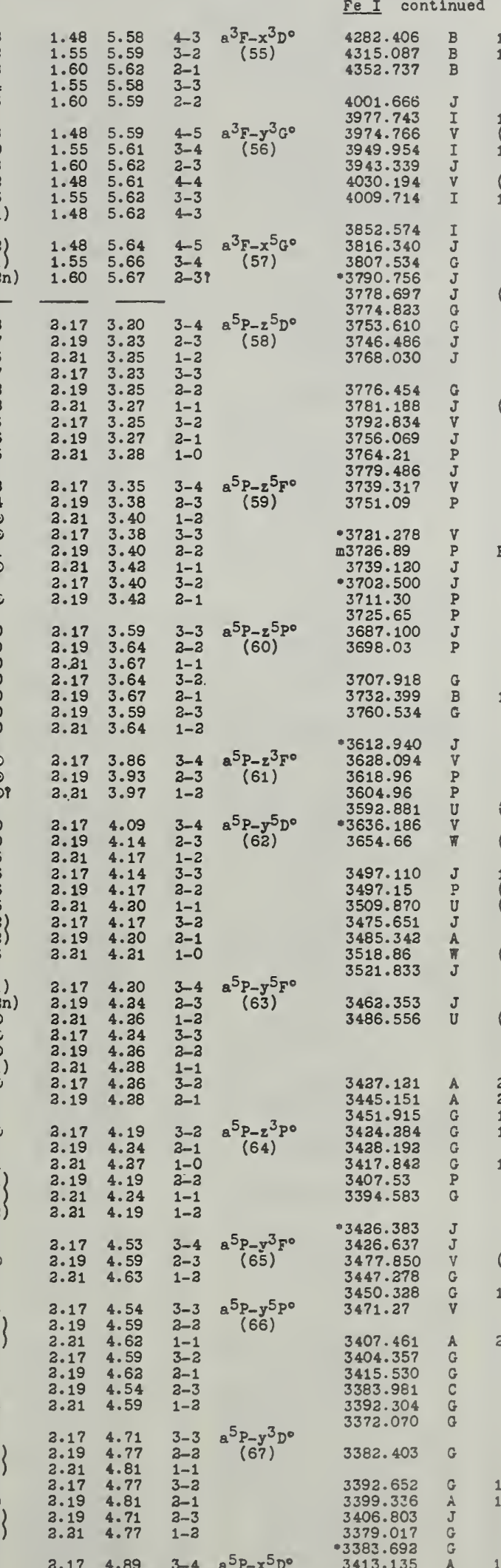

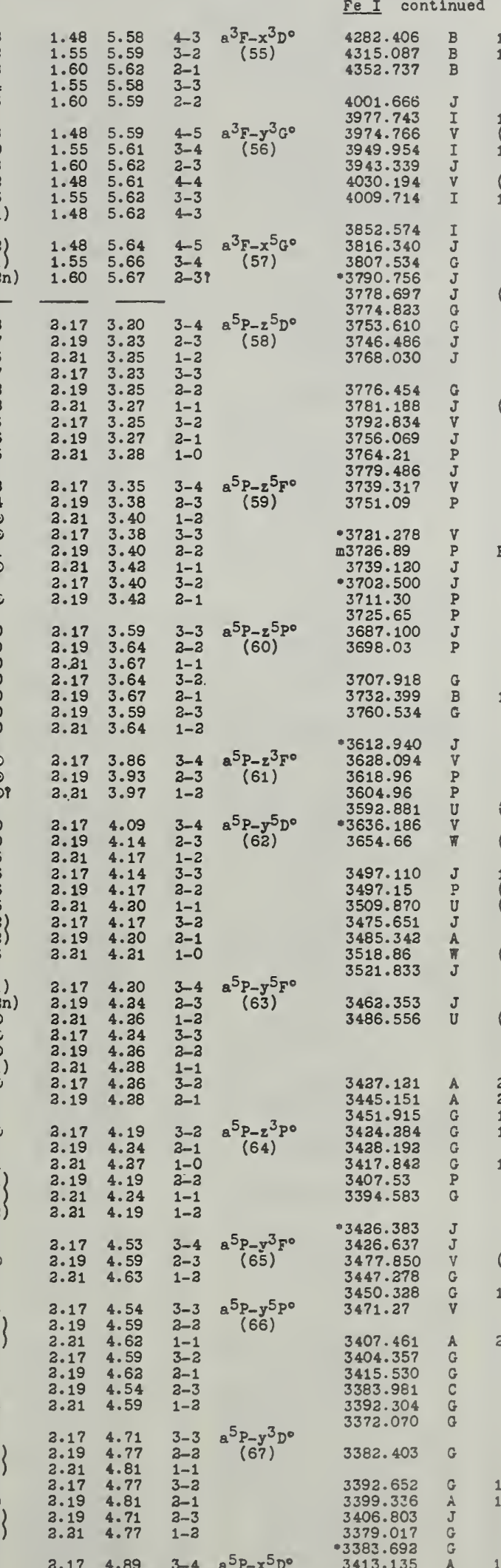

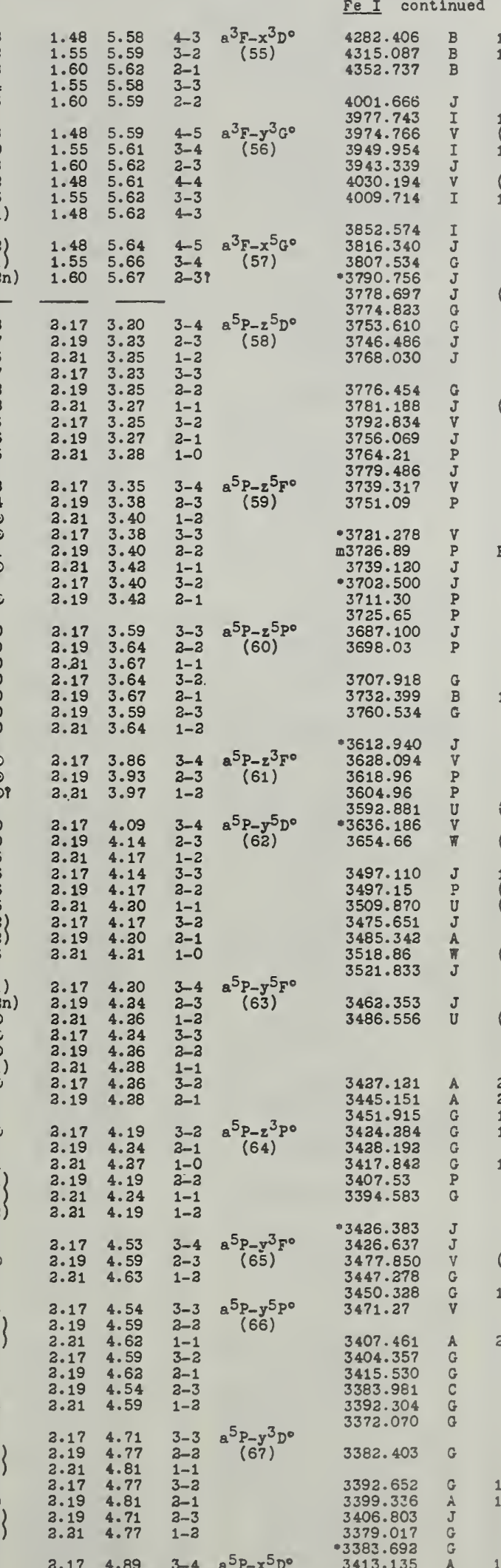

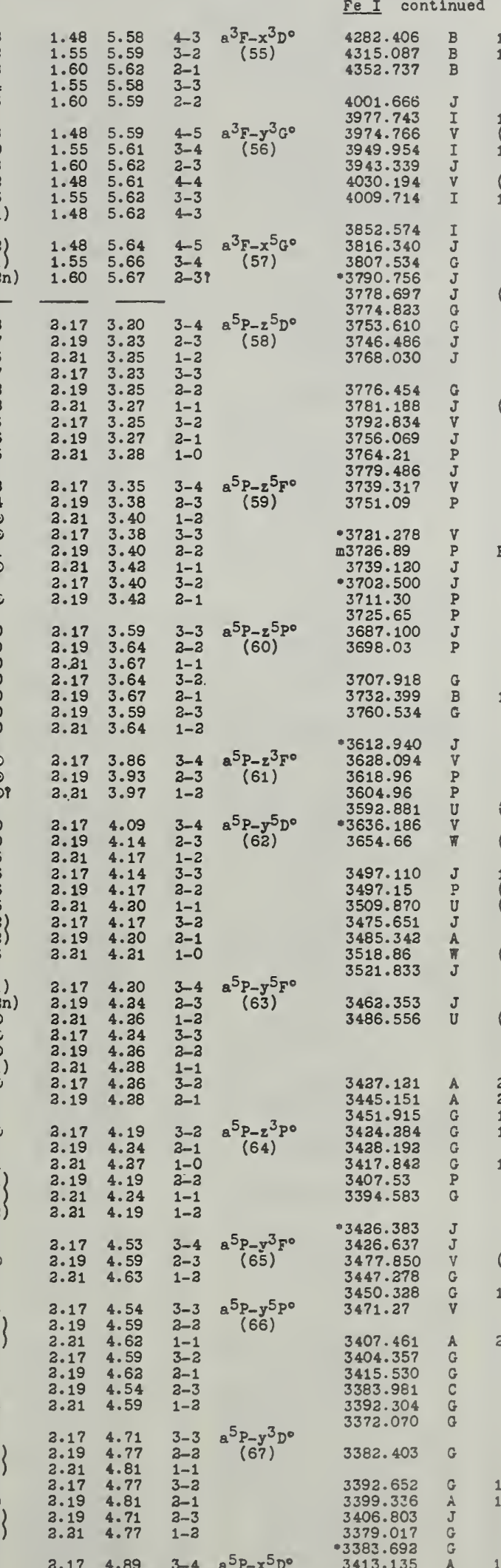

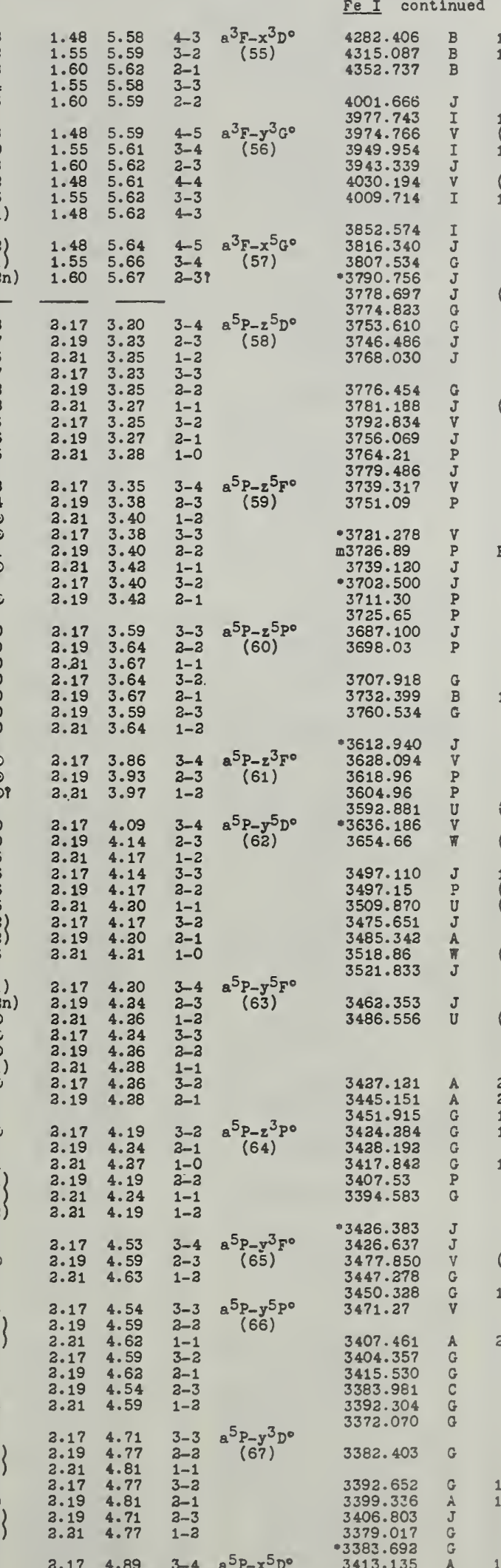

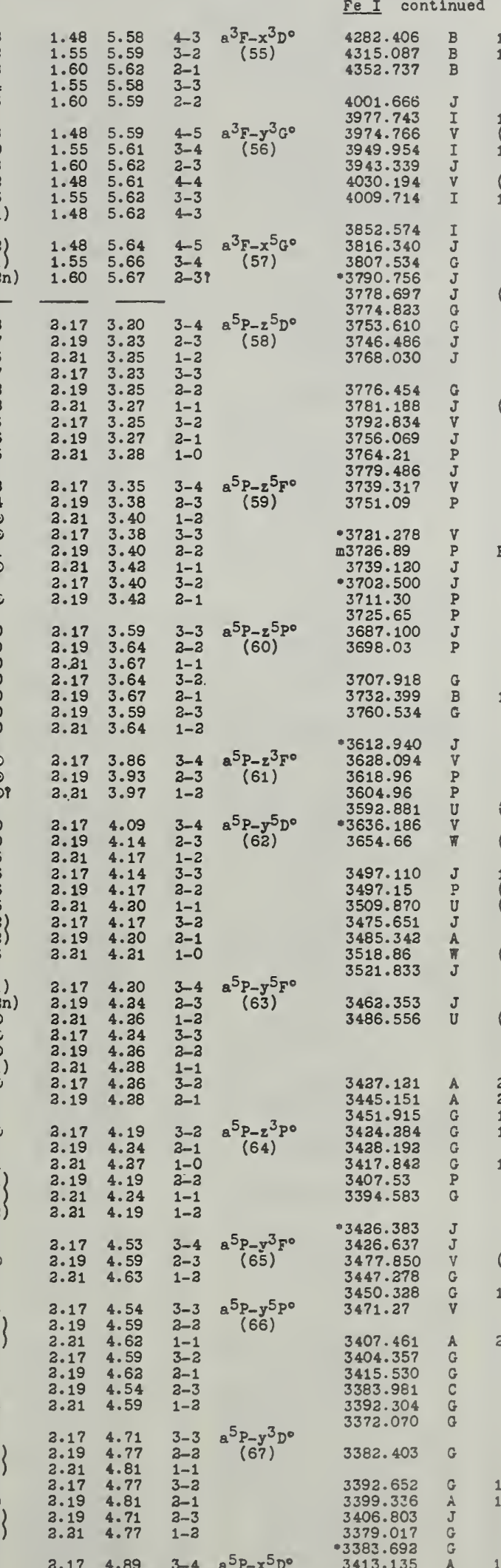

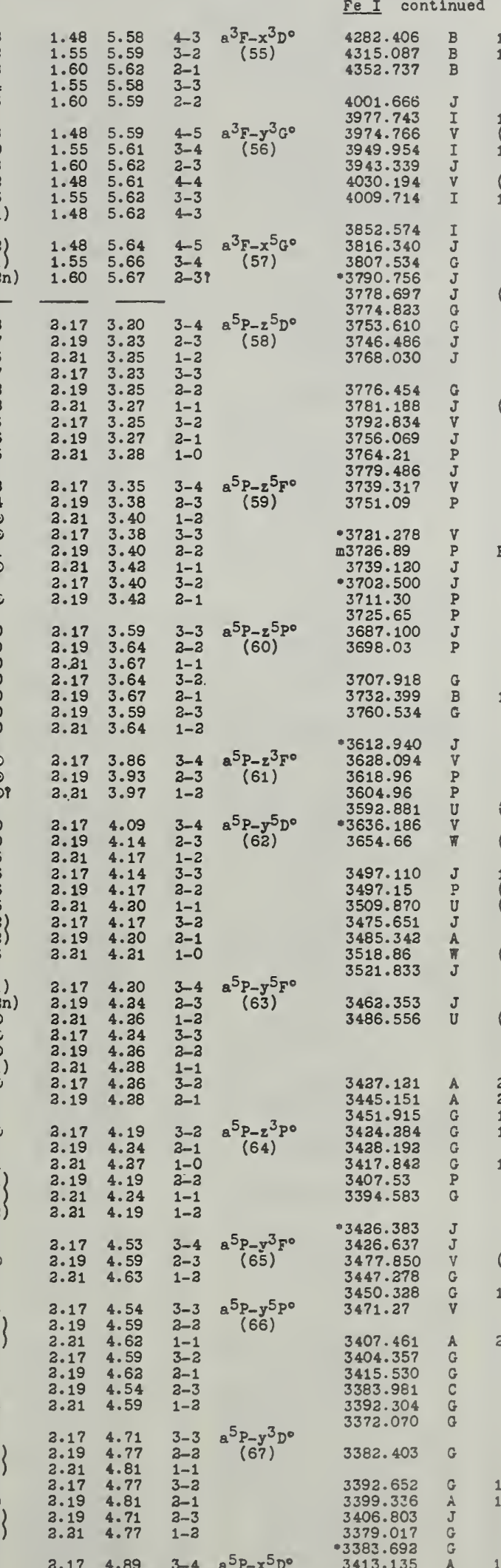

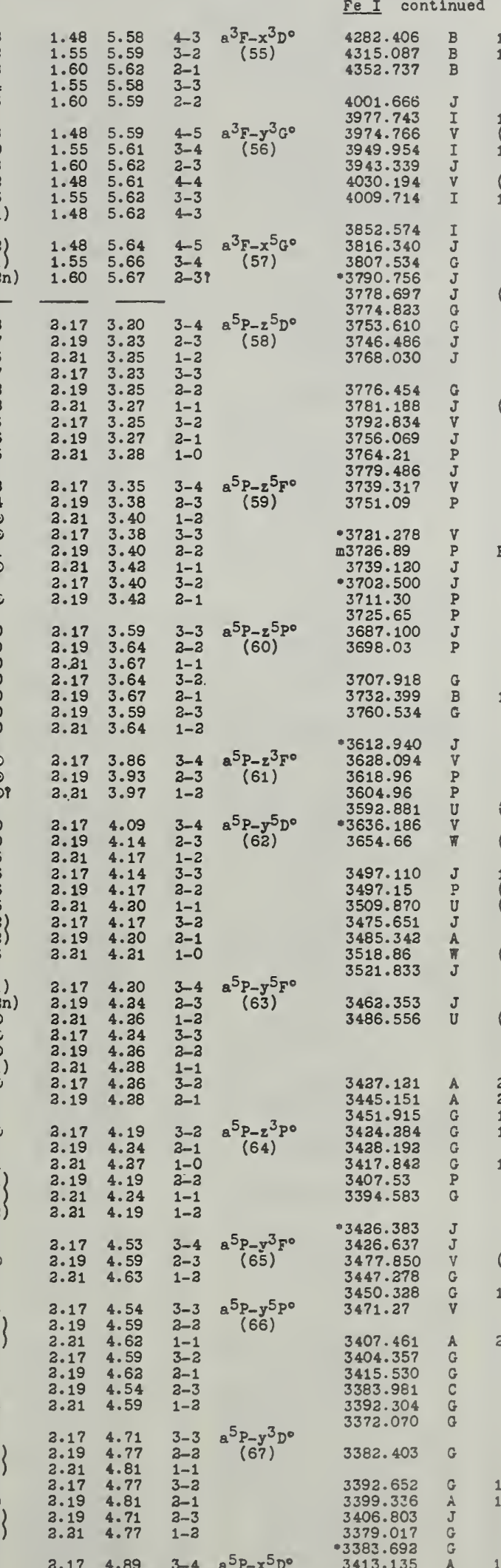

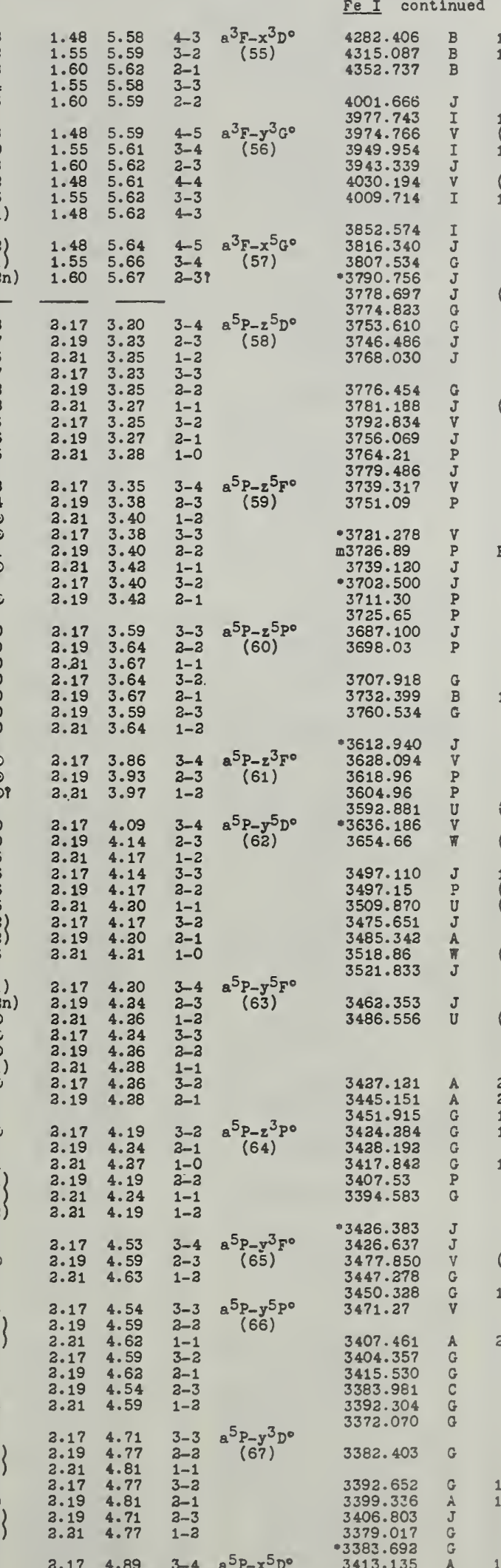

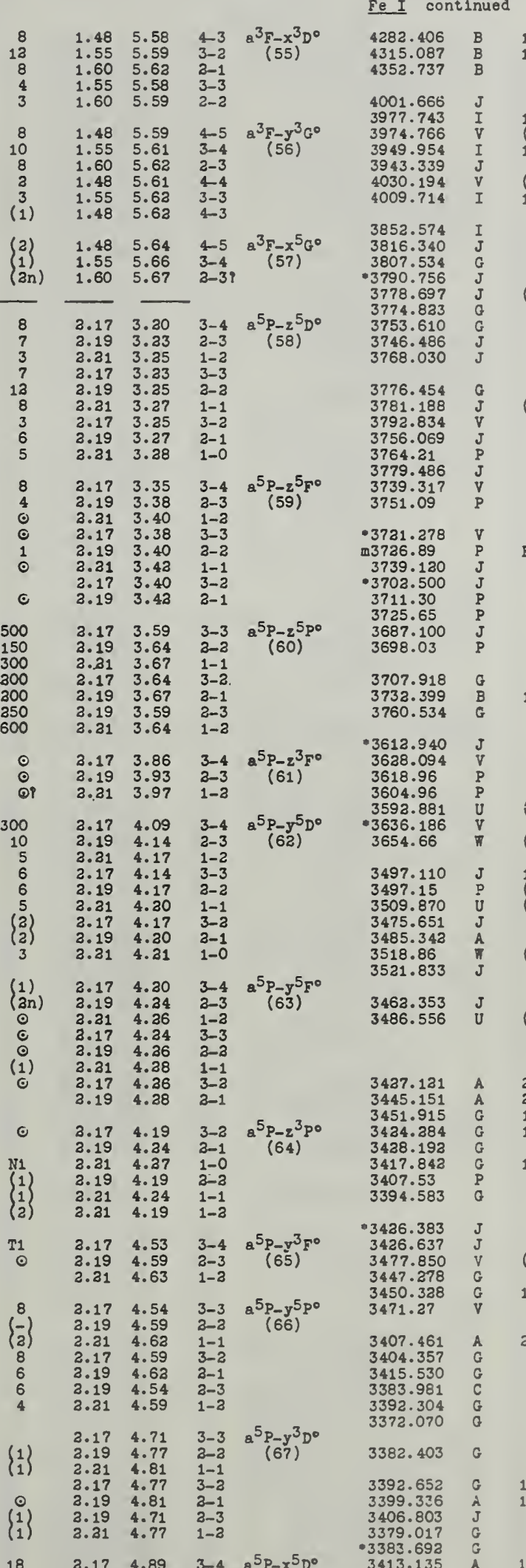

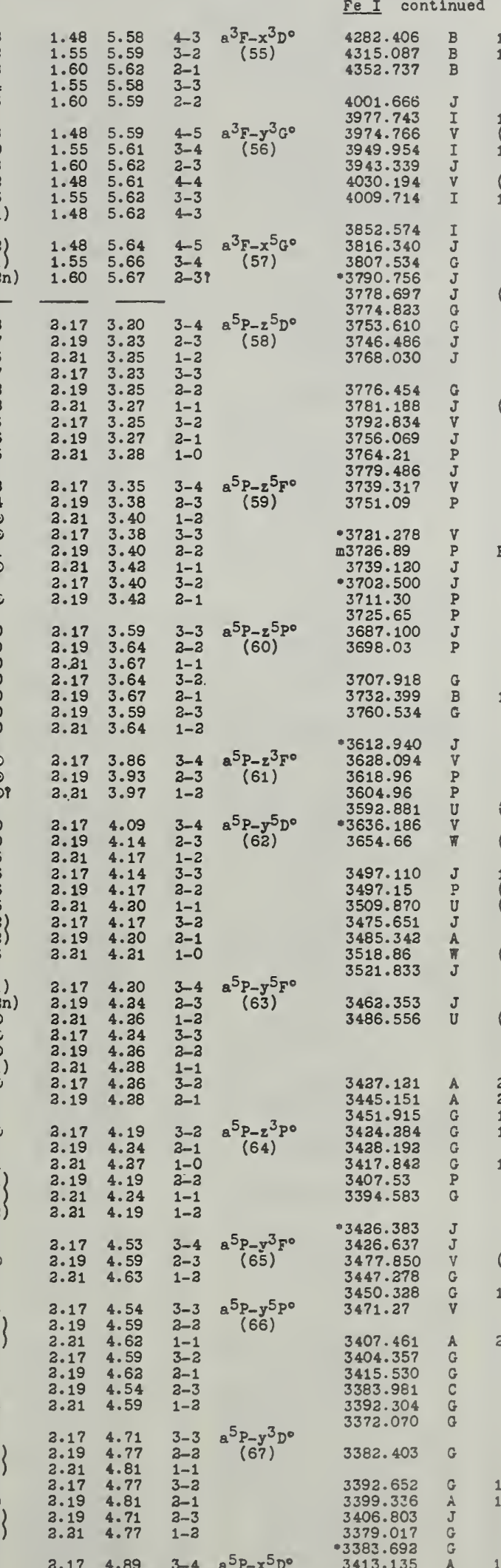

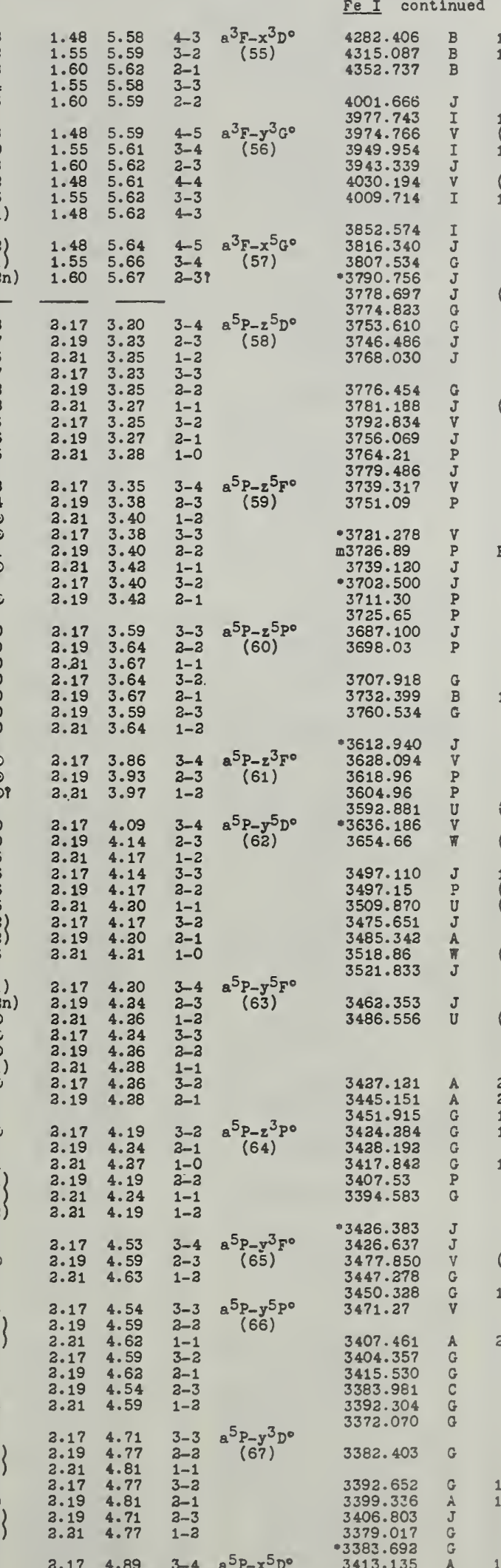

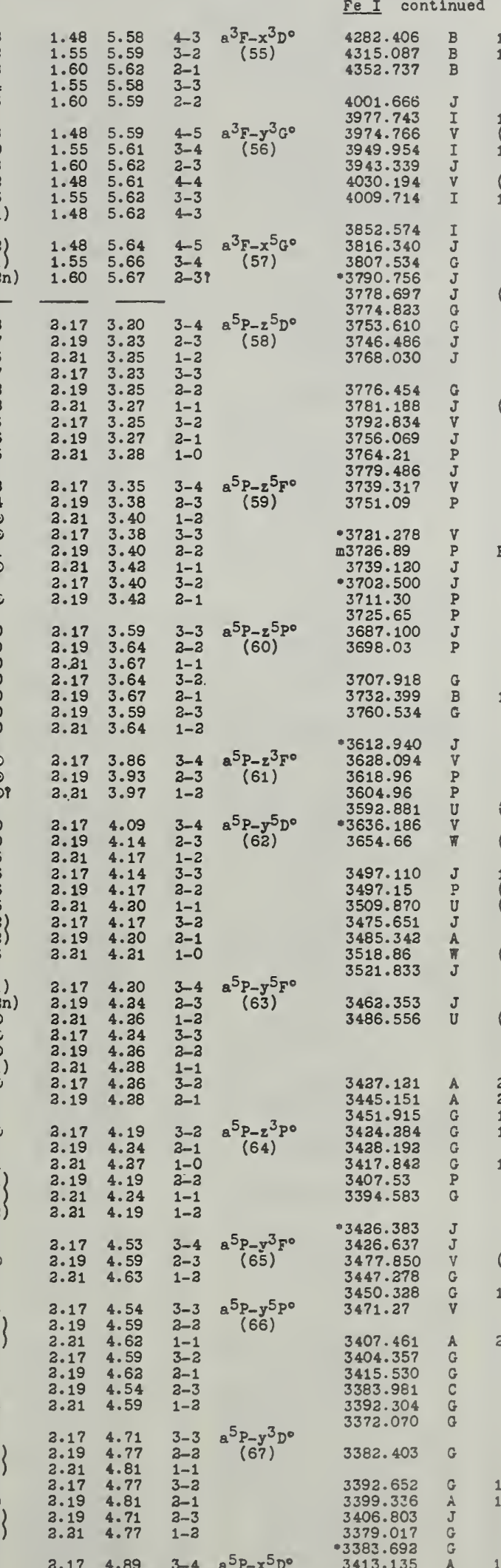

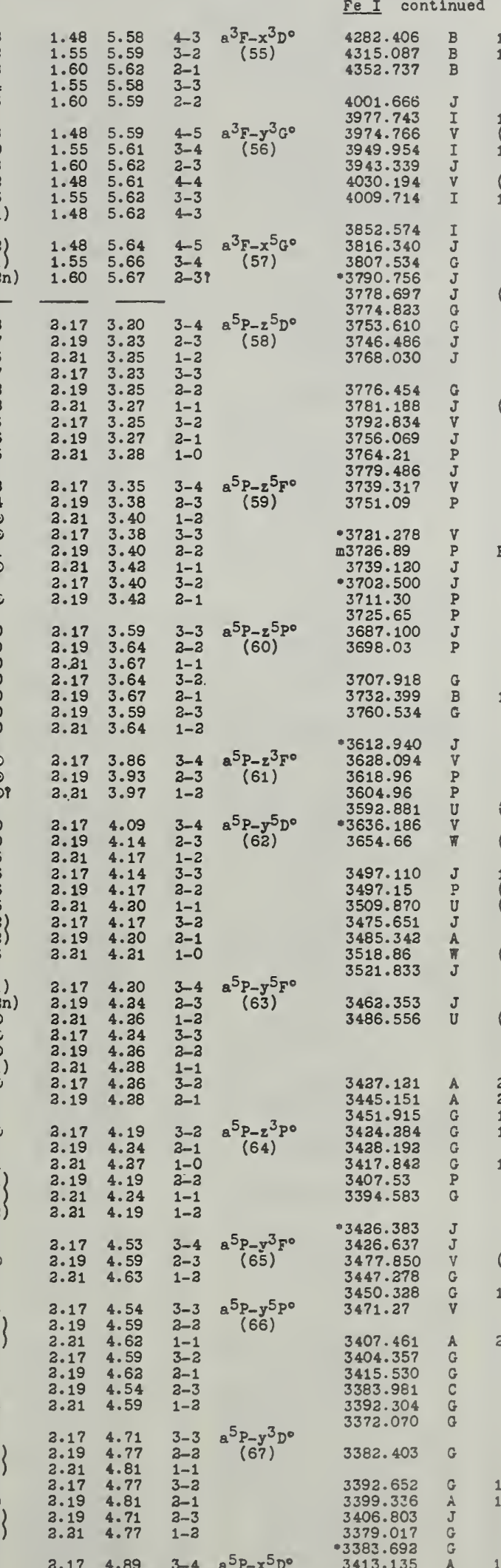

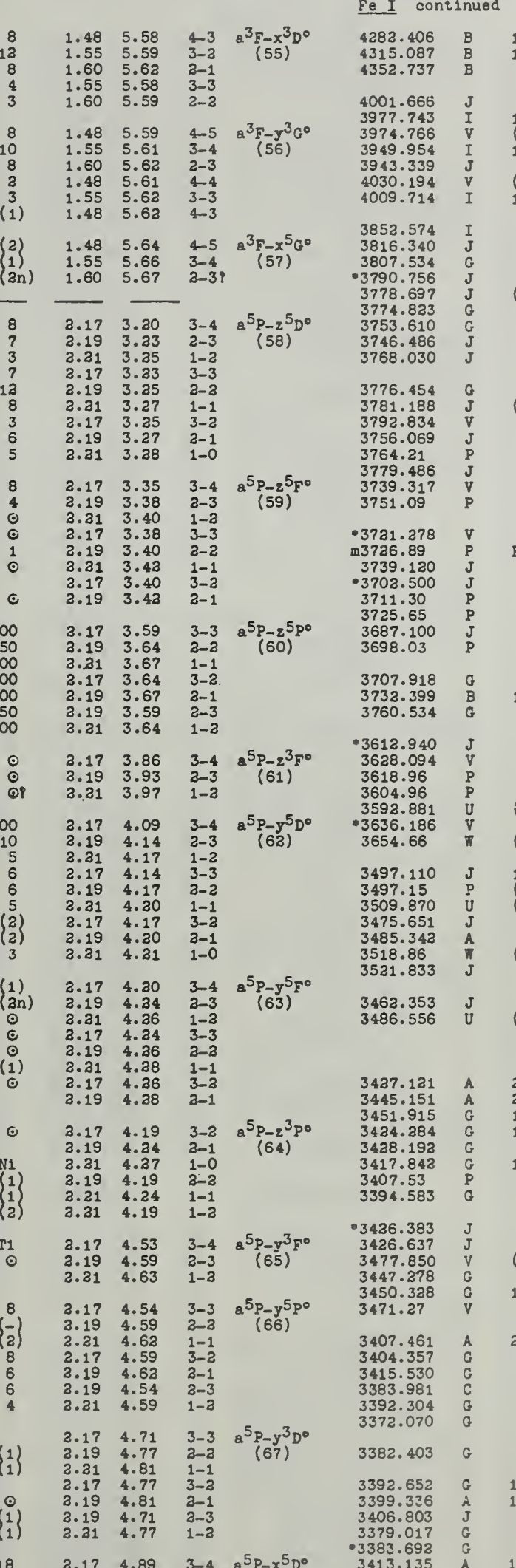

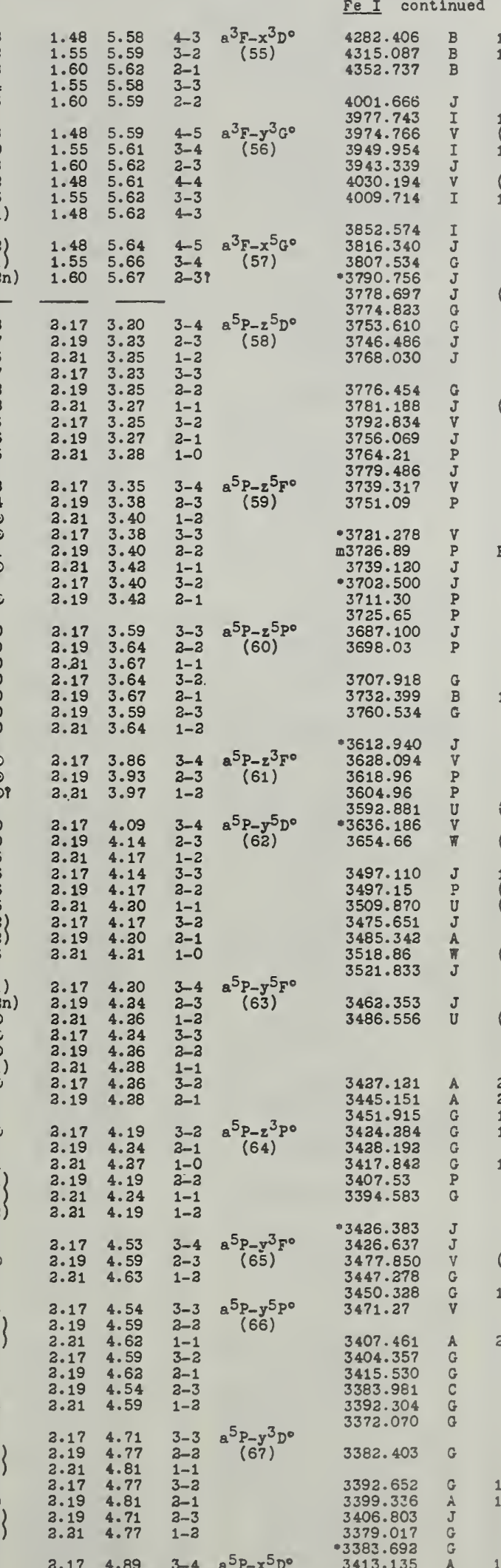

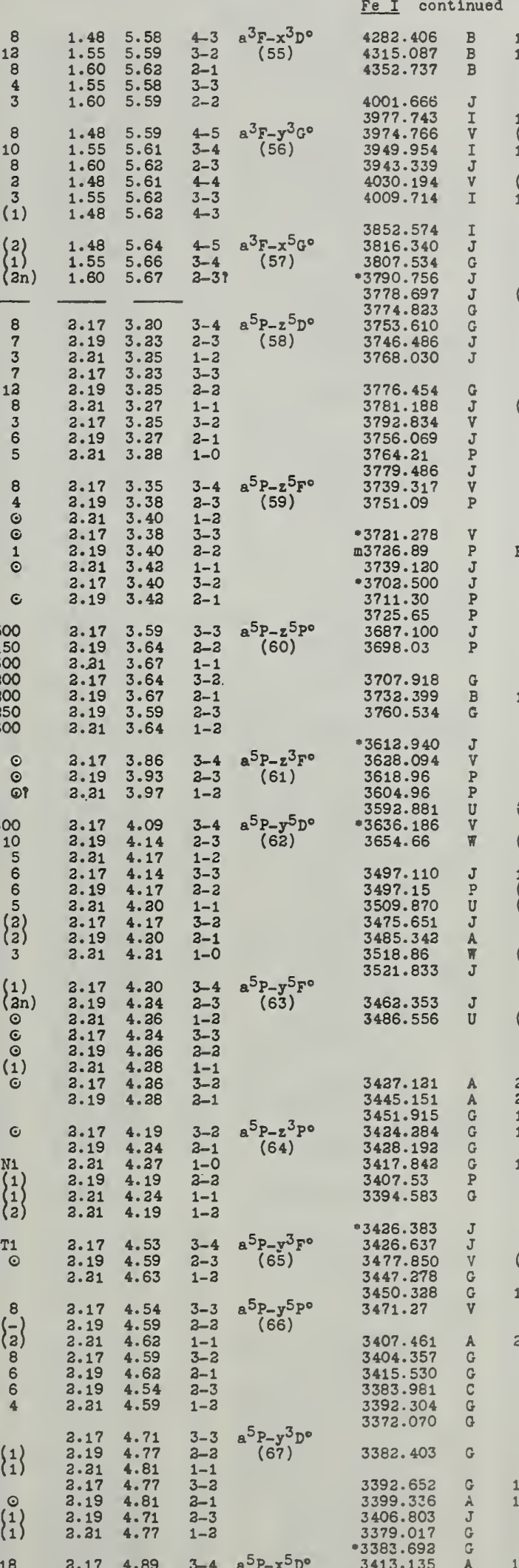

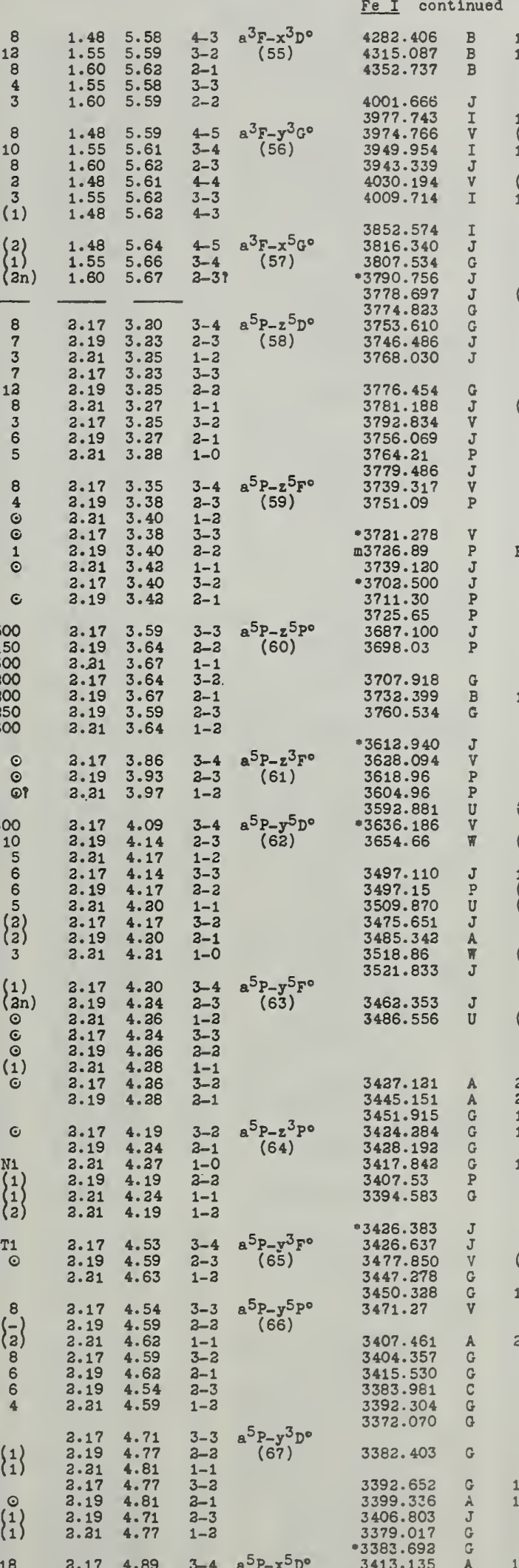

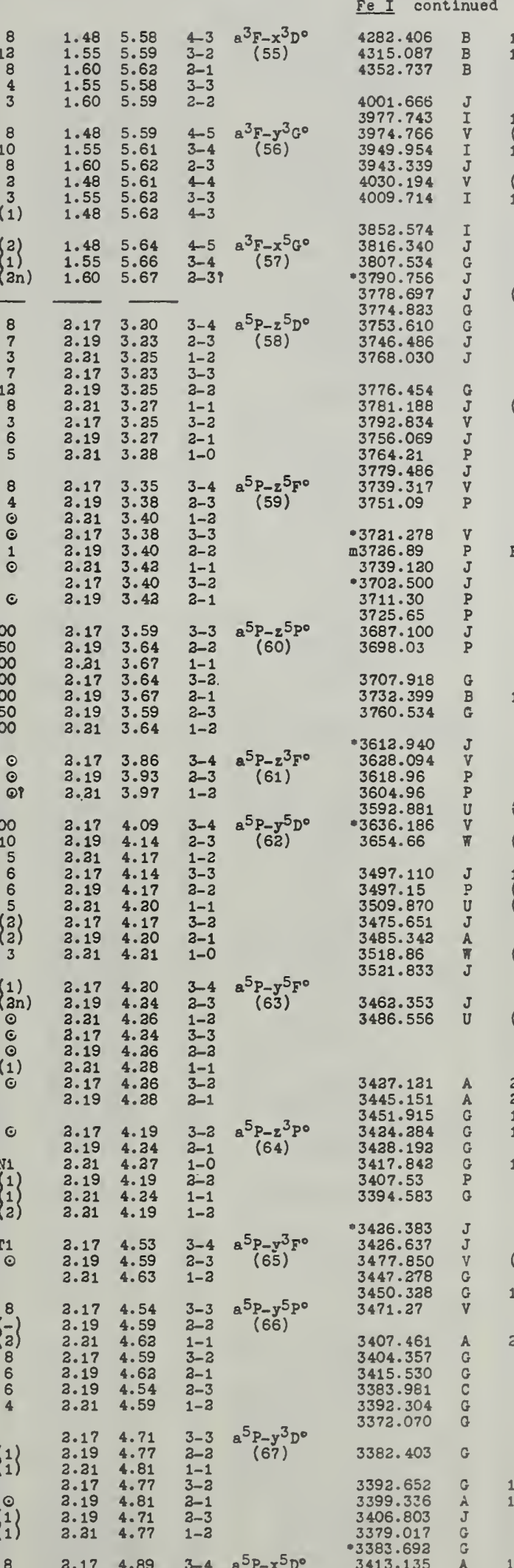

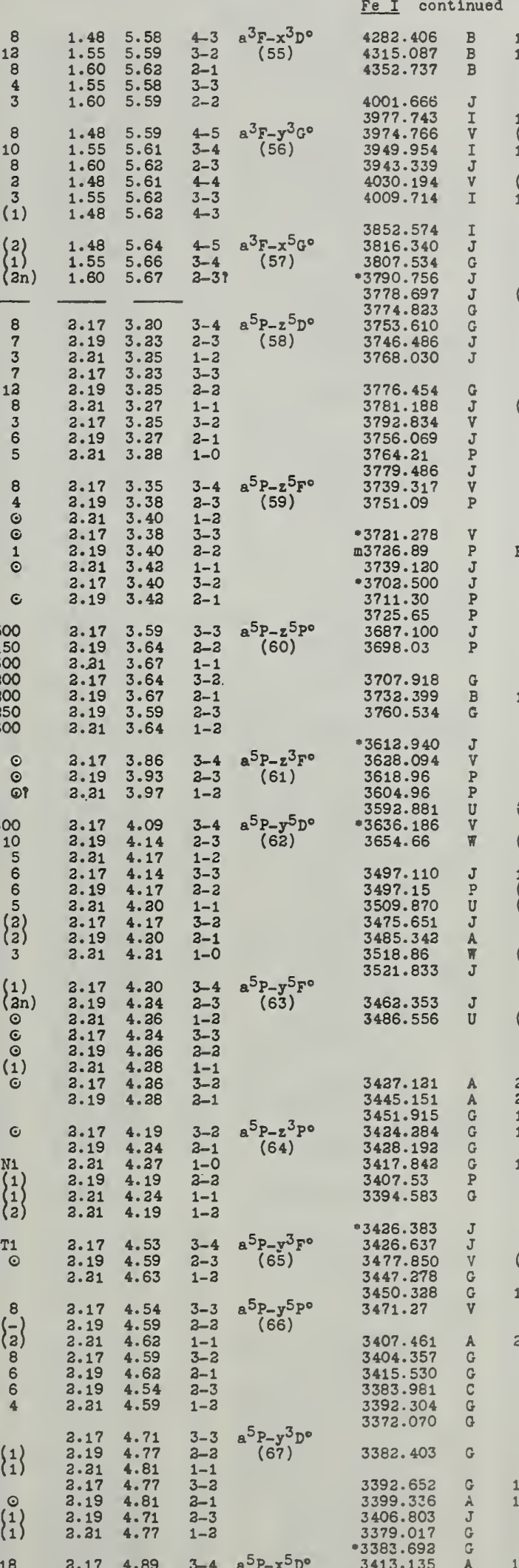

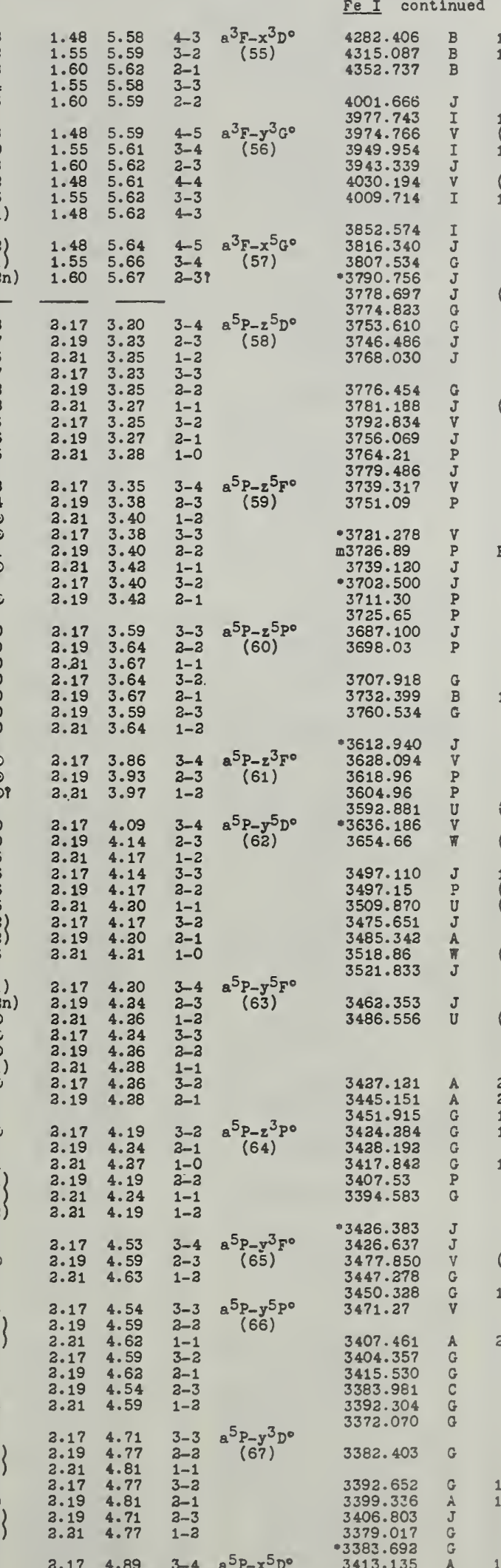

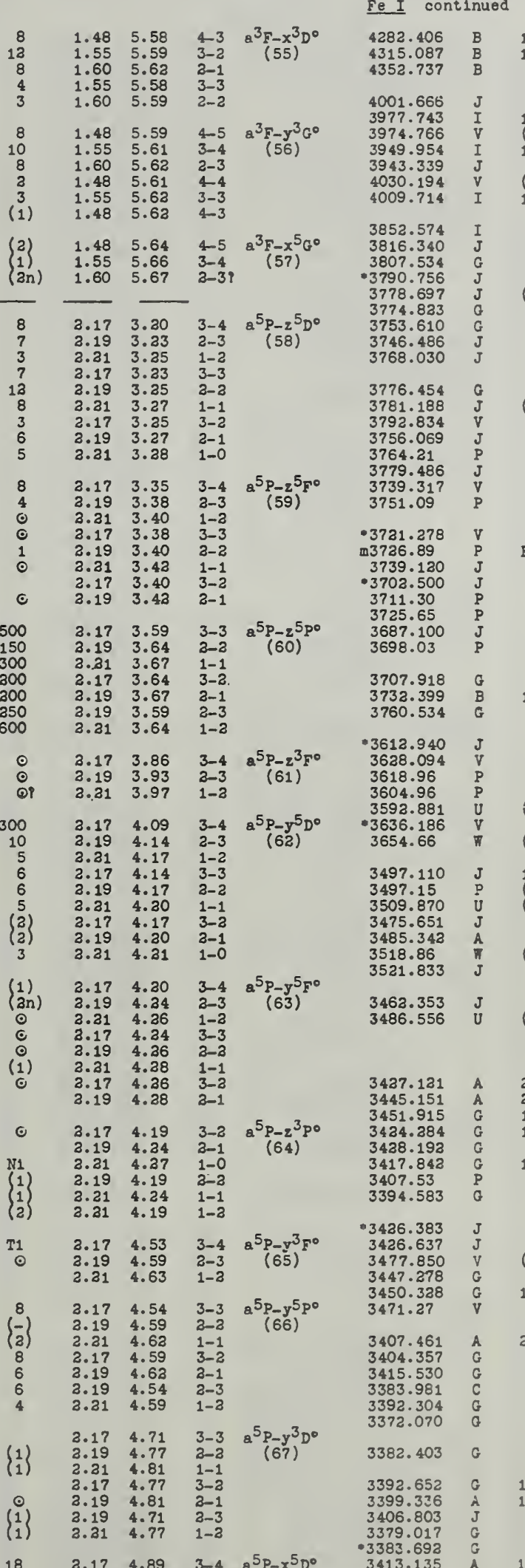

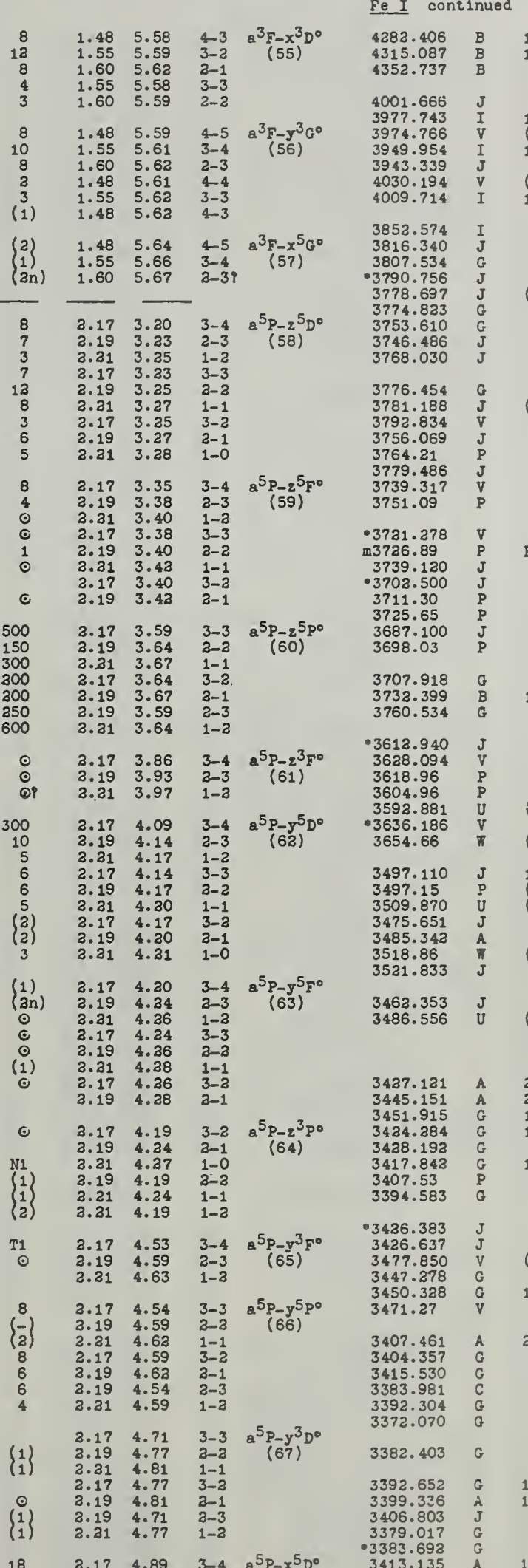

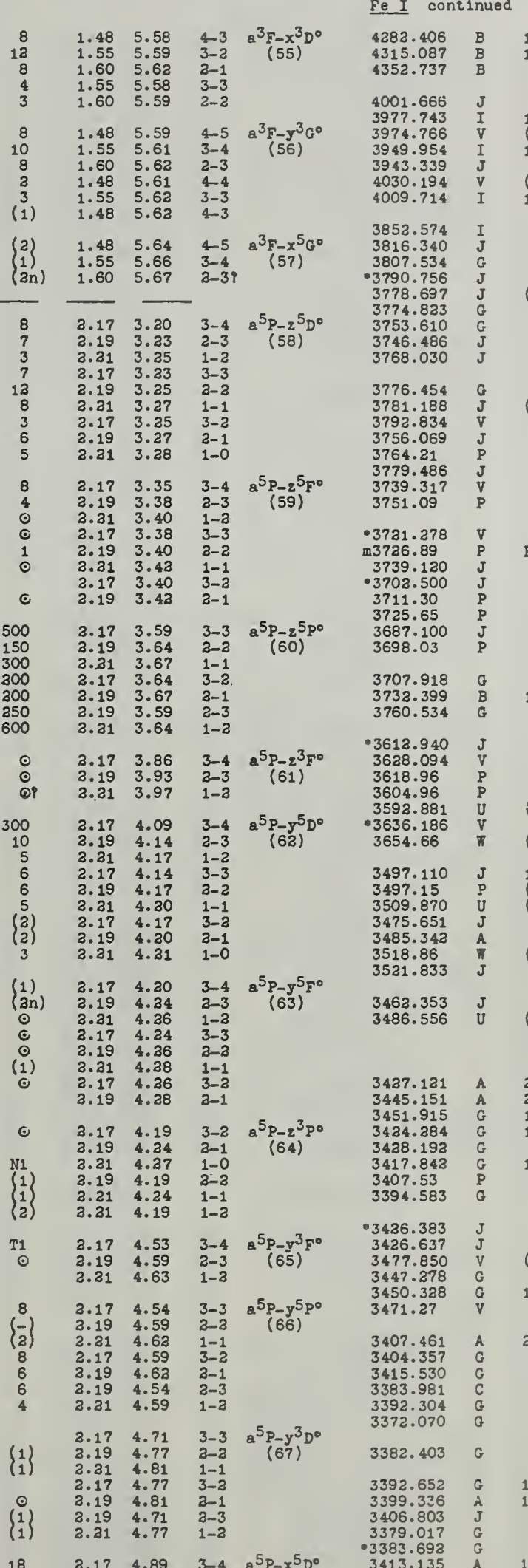

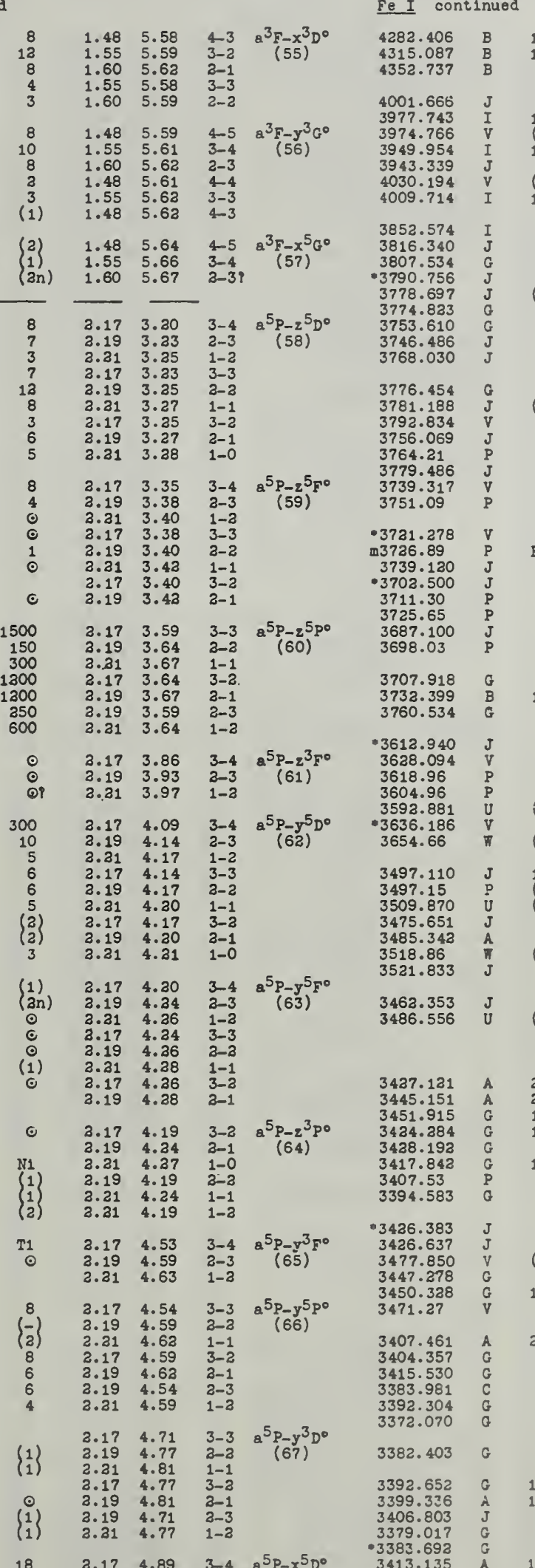

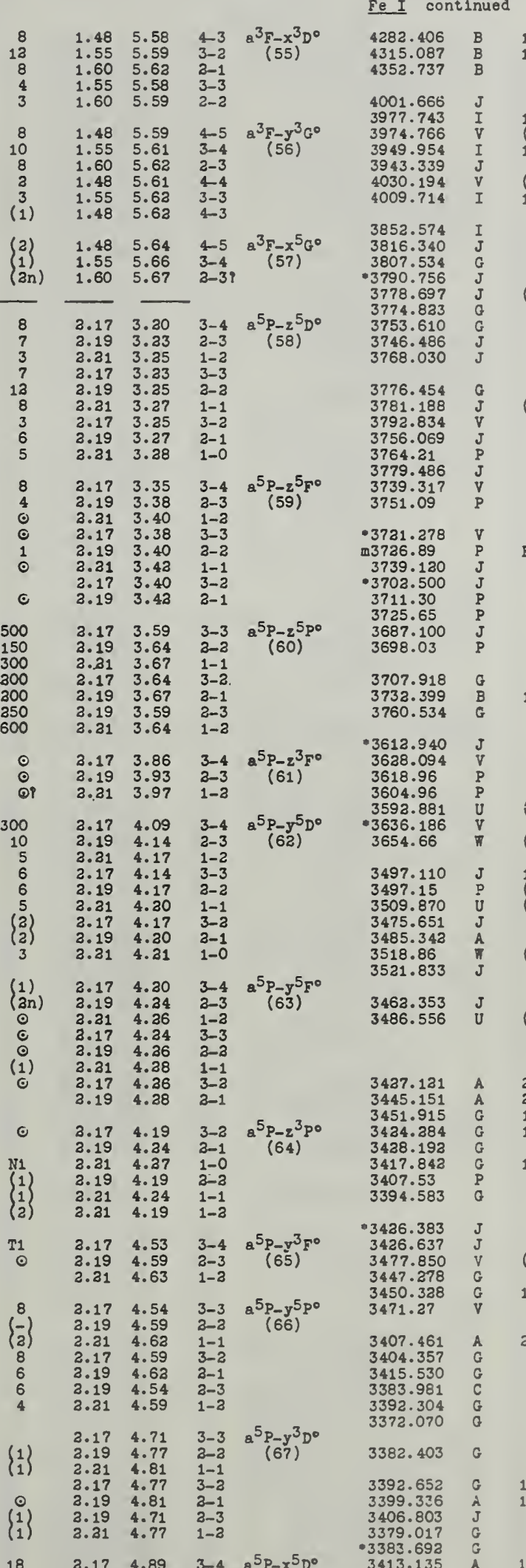

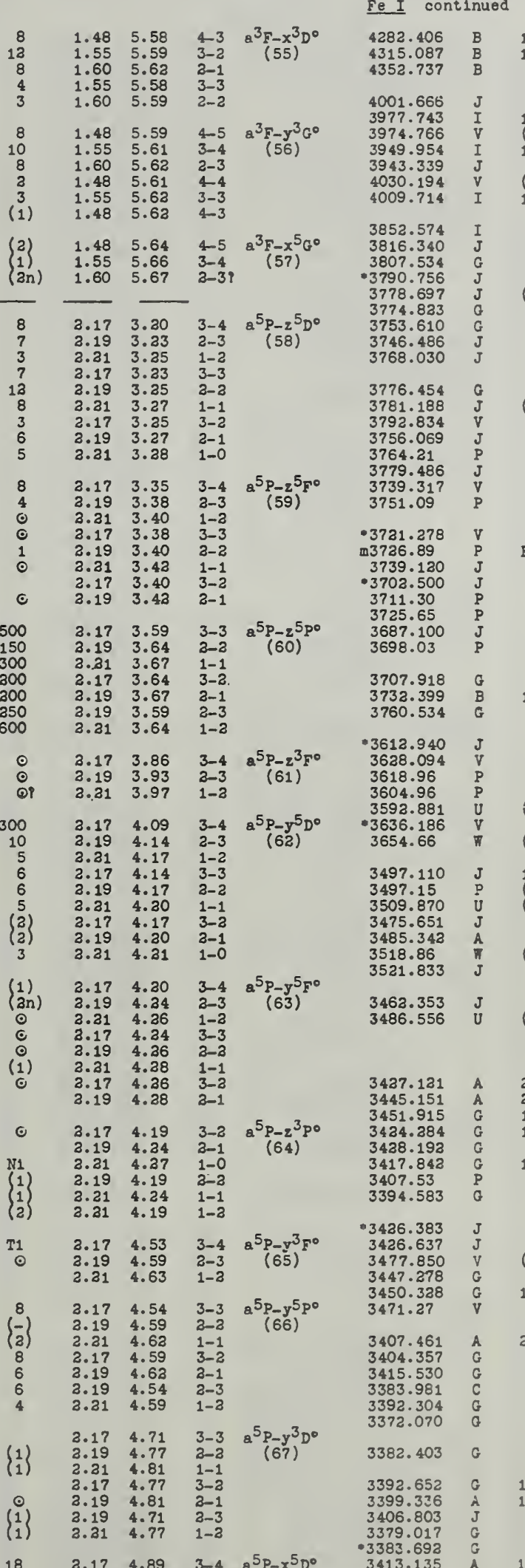

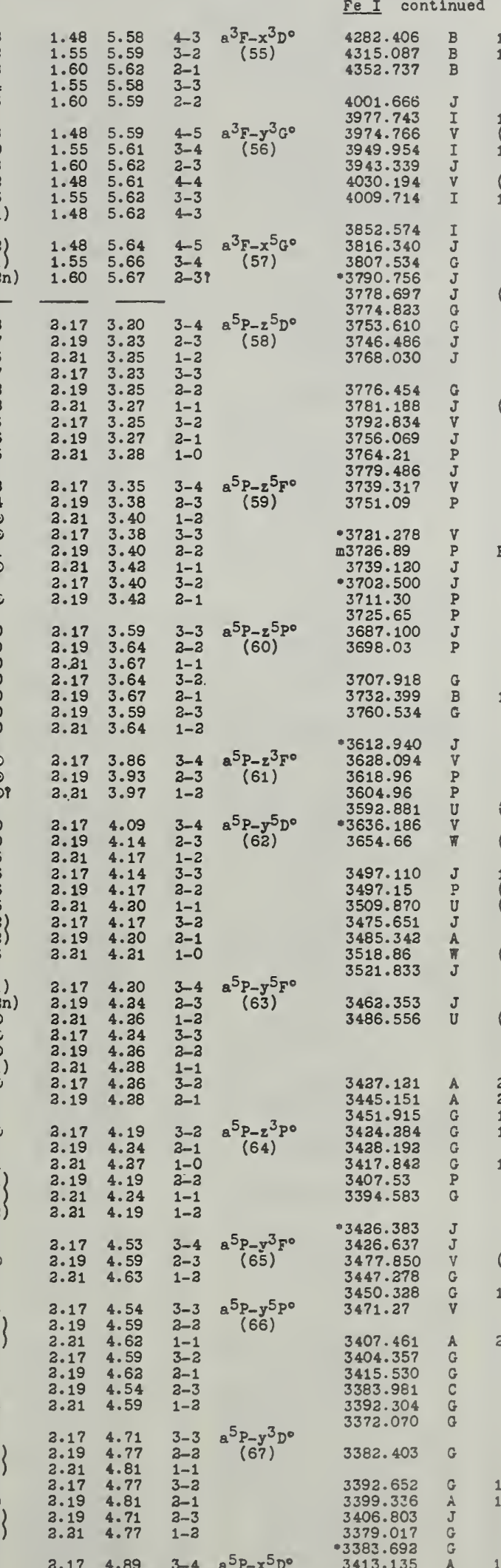

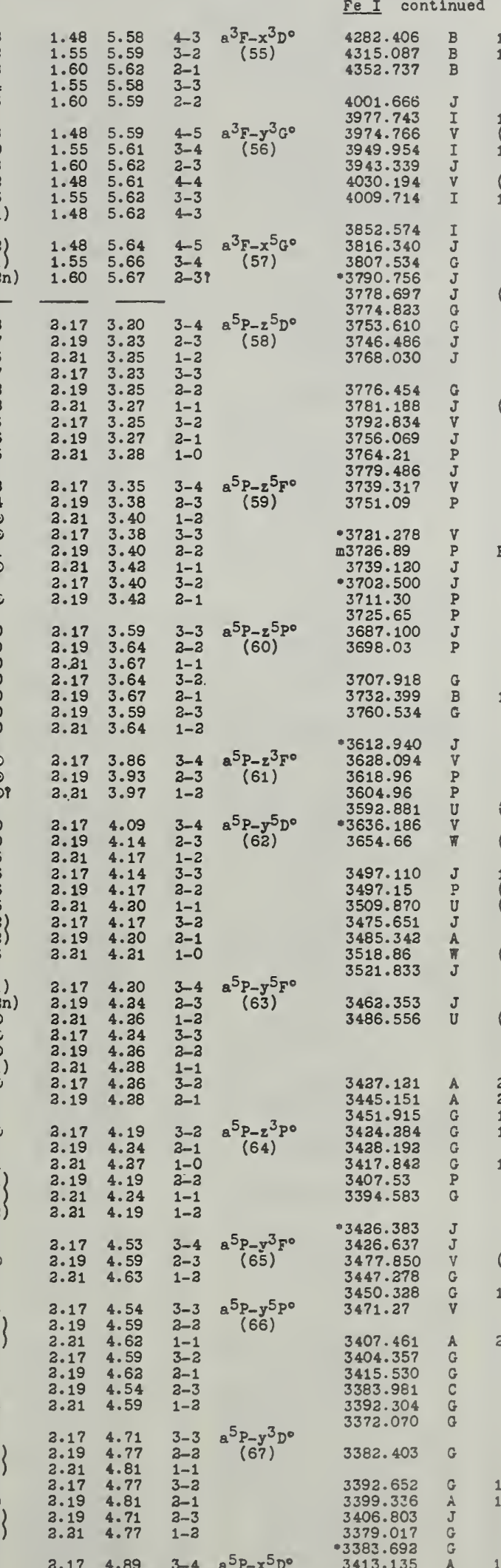

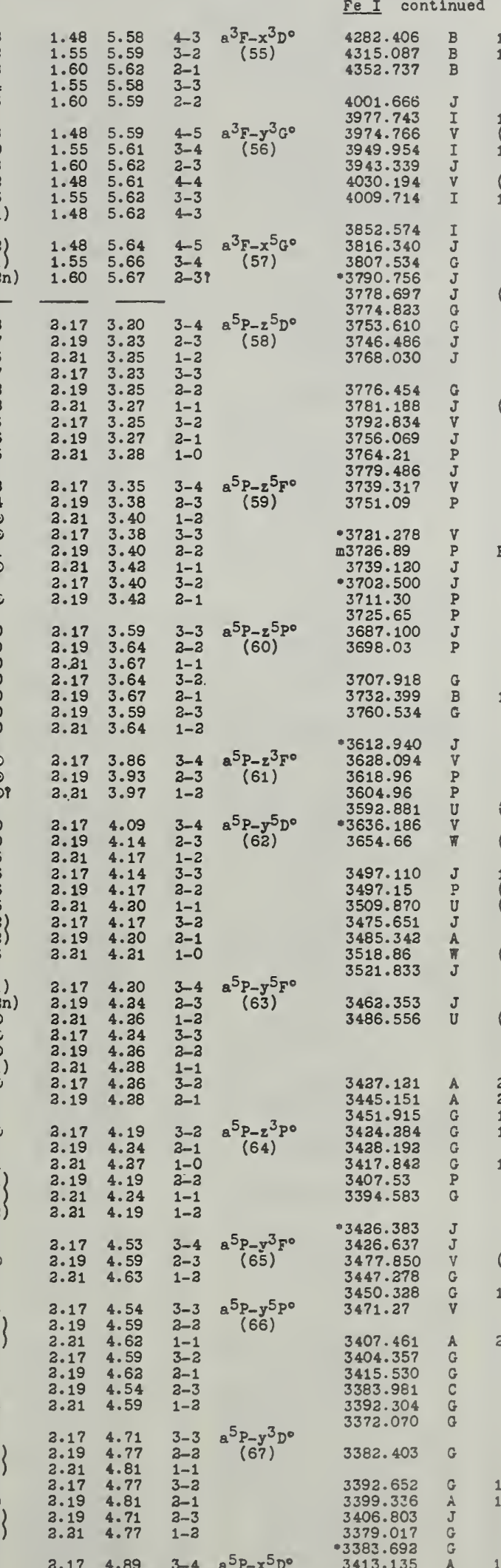

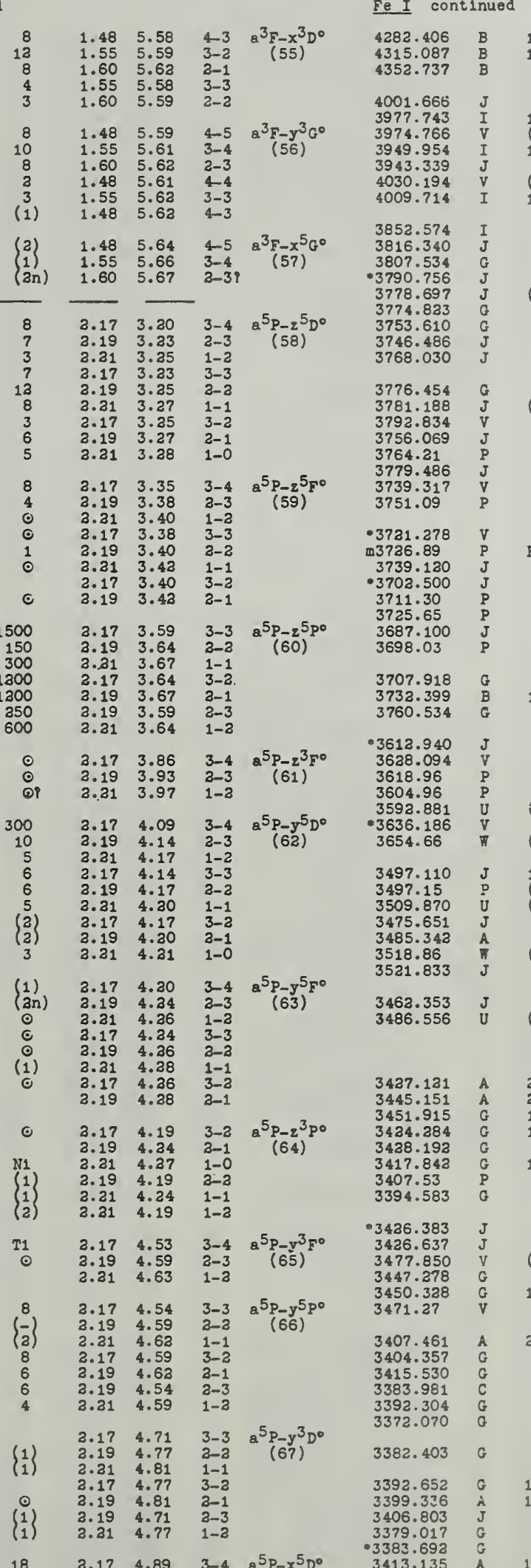

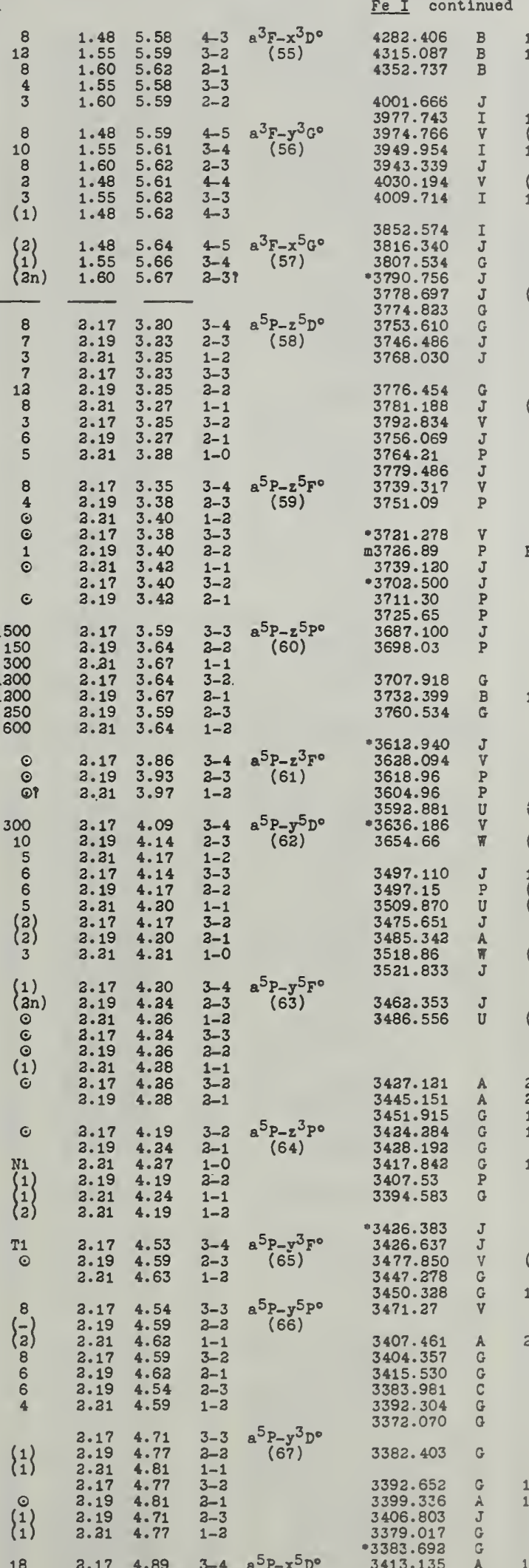

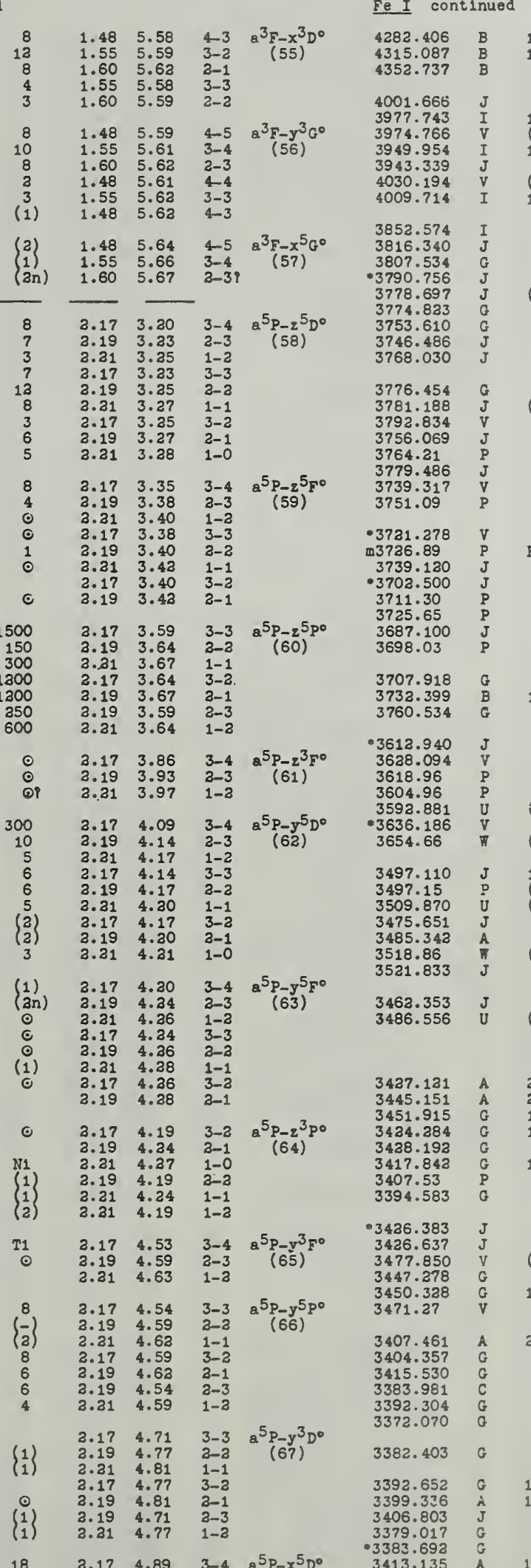

I A ${ }_{\text {Ref }}^{\text {Laboratory }}$ Int Low ${ }^{\sum P}$ High

J Multiplet Fe I continued

$\begin{array}{lllllll}4282.406 & B & 13 & 2.17 & 5.05 & 3-2 & a^{5} \mathrm{P}-z^{5} \mathrm{~S}^{0}\end{array}$ $\begin{array}{rrrrrr}4352.737 & \text { B } & 10 & 2.19 & 5.05 & 2-2 \\ & 9 & 3.21 & 5.05 & 1-2\end{array}$

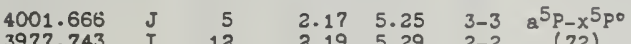

3974.

$\begin{array}{lll}12 & 3.19 & 5.29 \\ (1) & 2.21 & 5.32\end{array}$

$\begin{array}{llll}2 & 2.17 & 5.29 & 3-2 \\ 1 & 2.19 & 5.32 & 2-1 \\ 13 & 2.19 & 5.25 & 2-3\end{array}$

$\begin{array}{llll}\text { (3) } & 2.19 & 5.25 & 2-3 \\ 10 & 2.21 & 5.29 & 1-2\end{array}$

$\begin{array}{llllll} & 6 & 2.17 & 5.37 & 3-4 & 8^{5} \mathrm{P}_{-} 5 \mathrm{D}\end{array}$

$\begin{array}{llll}2.1 .7 & 5.37 & 3-4 & a^{5} P-\pi^{5} D \\ 2.19 & 5.42 & 2-3 & (73)\end{array}$

$\begin{array}{lll}2.19 & 5.42 & 2-3 \\ 2.21 & 5.45 & 1-2\end{array}$

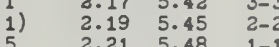

$\begin{array}{lll}2.17 & 5.45 & 3-1 \\ 2.19 & 5.48 & 2-1\end{array}$

$\begin{array}{lll}2.21 & 5.49 & 1-0\end{array}$

$\begin{array}{lllll}6 & 2.17 & 5.43 & 3-4 & \mathrm{a}^{5} \mathrm{P}-\pi^{5} \mathrm{~F}^{0}\end{array}$

$\begin{array}{lll}2.19 & 5.45 & 2-3 \\ 2.21 & 5.47 & 1-2\end{array}$

$\begin{array}{lll}2.17 & 5.45 & 3-3 \\ 2.19 & 5.47 & 2-2\end{array}$

$2.21 \quad 5.48 \quad 1-1$

$\begin{array}{llll}2.19 & 5.48 & 2-1\end{array}$

$\begin{array}{lllll}2.17 & 5.48 & 3-4 & Q^{5} P_{-1} v^{5} & \end{array}$

$\begin{array}{lll}2.19 & 5.50 & 2-3 \\ 2.21 & 5.51 & 1-2\end{array}$

$2.17 \quad 5.50 \quad 3-3$

$\begin{array}{lll}2.21 & 5.53 & 1-1\end{array}$

$\begin{array}{lll}2.17 & 5.51 & 3-2 \\ 2.19 & 5.53 & 2-1\end{array}$

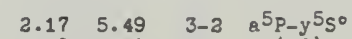

$\begin{array}{llll}2.19 & 5.49 & 2-2 & (76) \\ 2.21 & 5.49 & 1-2 & \end{array}$

$\begin{array}{llll}3.17 & 5.5 \varepsilon & 3-3 & a^{5} \mathrm{P}-\mathrm{x}^{3} \mathrm{D}\end{array}$

$\begin{array}{lll}2.19 & 5.59 & 2-2 \\ 2.21 & 5.52 & 1-1\end{array}$

$\begin{array}{lll}2.21 & 5.52 & 1-1 \\ 2.17 & 5.59 & 3-2\end{array}$

$\begin{array}{llll}\text { (1) } & 2.19 & 5.62 & 2-1 \\ 2 & 2.19 & 5.58 & 2-3\end{array}$

(1) $\quad \begin{array}{lll}2.21 & 5.59 & 1-2\end{array}$

$\begin{array}{lllll}10 & 2.17 & 5.70 & 3-3 & Q^{5} P-\pi^{5} P^{C} \\ \text { (1) } & 2.19 & 5.72 & 2-2 & (78)\end{array}$

1) $2.21 \quad 5.73 \quad 1-1$

(2) $\quad \begin{array}{lll}2.19 & 5.73 & 2-1\end{array}$

$\begin{array}{llll}\text { (2) } & 2.19 & 5.70 & 2-3 \\ 2 & 2.21 & 5.72 & 1-2\end{array}$

$\begin{array}{lllll}2 & 2.19 & 5.75 & 2-1 & 8^{5} p_{-z^{3}} S^{\circ}\end{array}$

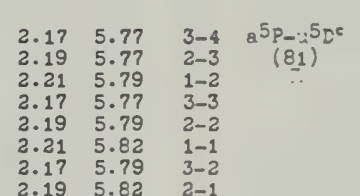

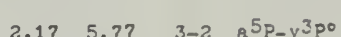

$\begin{array}{lllll}5 \mathrm{~d} & 2.17 & 5.77 & 3-2 & \mathrm{a} \\ 5 & 2.19 & 5.79 & 2-1 & (82)\end{array}$

$\begin{array}{clll}\text { (2) } & 2.21 & 5.76 & 1-0 \\ 8 & 2.19 & 5.77 & 2-2\end{array}$

$\begin{array}{lll}2.21 & 5.79 & 1-1 \\ 2.21 & 5.77 & 1-2\end{array}$

$\begin{array}{cccc}2.17 & 5.79 & 3-4 & a^{5} P-x^{3} F^{0} \\ 2.19 & 5.81 & 2-3 & (83)\end{array}$

$\begin{array}{lll}2.21 & 5.63 & 1-2 \\ 2.17 & 5.81 & 3-3\end{array}$

$\begin{array}{lll}2.19 & 5.83 & 2-2 \\ 2.17 & 5.83 & 3-2\end{array}$

$2.17 \quad 5 . \& 2 \quad 3-4 \quad 8^{5} \mathrm{P}_{-2} 2^{3} \mathrm{H}^{\circ}$

$\begin{array}{cccc}2.17 & 5.80 & 3-3 & { }_{8} 5 \mathrm{P}-\mathrm{F}^{3} \mathrm{DO}^{\circ} \\ 2.19 & 5.82 & 2-2 & (85)\end{array}$

(3383.692

3413.135
3422.656

3327.961

3346.942

3366.870
3389.748

3343.243

$\begin{array}{ll}2.19 & 5.82 \\ 2.21 & 5.8\end{array}$

$2.17 \quad 5.82$

$\begin{array}{ll}2.19 & 5.8 \\ 2.19 & 5.8\end{array}$

(1) $\quad 2.17 \quad 5.87 \quad 3-4 \quad 8^{5} P-.^{5} G^{\circ}$

3351.529
3374.221

$2.17 \quad 5.85$

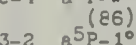

$\begin{array}{llll}2.19 & 5.85 & 2-2 & (87)\end{array}$

(1) $\quad 2.17 \quad 5.86 \quad 3-4 \quad a^{5 P-z^{1} G^{c}}$

$\begin{array}{llll}2.19 & 5.04 & 2-3 & (70)\end{array}$

3386.755

3284.588
3292.590

3265.616

3271.002
3305.971

3305.971
3305.256

$\begin{array}{cccc}2.17 & 5.86 & 3-4 & a^{5} P-z^{1} G^{c} \\ 2.19 & 5.87 & 2-1 & 588) \\ 2.2-y^{3} S^{\circ} \\ 2.21 & 5.87 & 1-1 & (89)\end{array}$

$2.17 \quad 5.92 \quad 3-3 \quad a^{5} P-v^{5} p o$

$\begin{array}{lll}2.19 & 5.95 & 2-2 \\ 2.21 & 5.96 & 1-1\end{array}$

$2.17 \quad 5.95 \quad 3-$ 
50

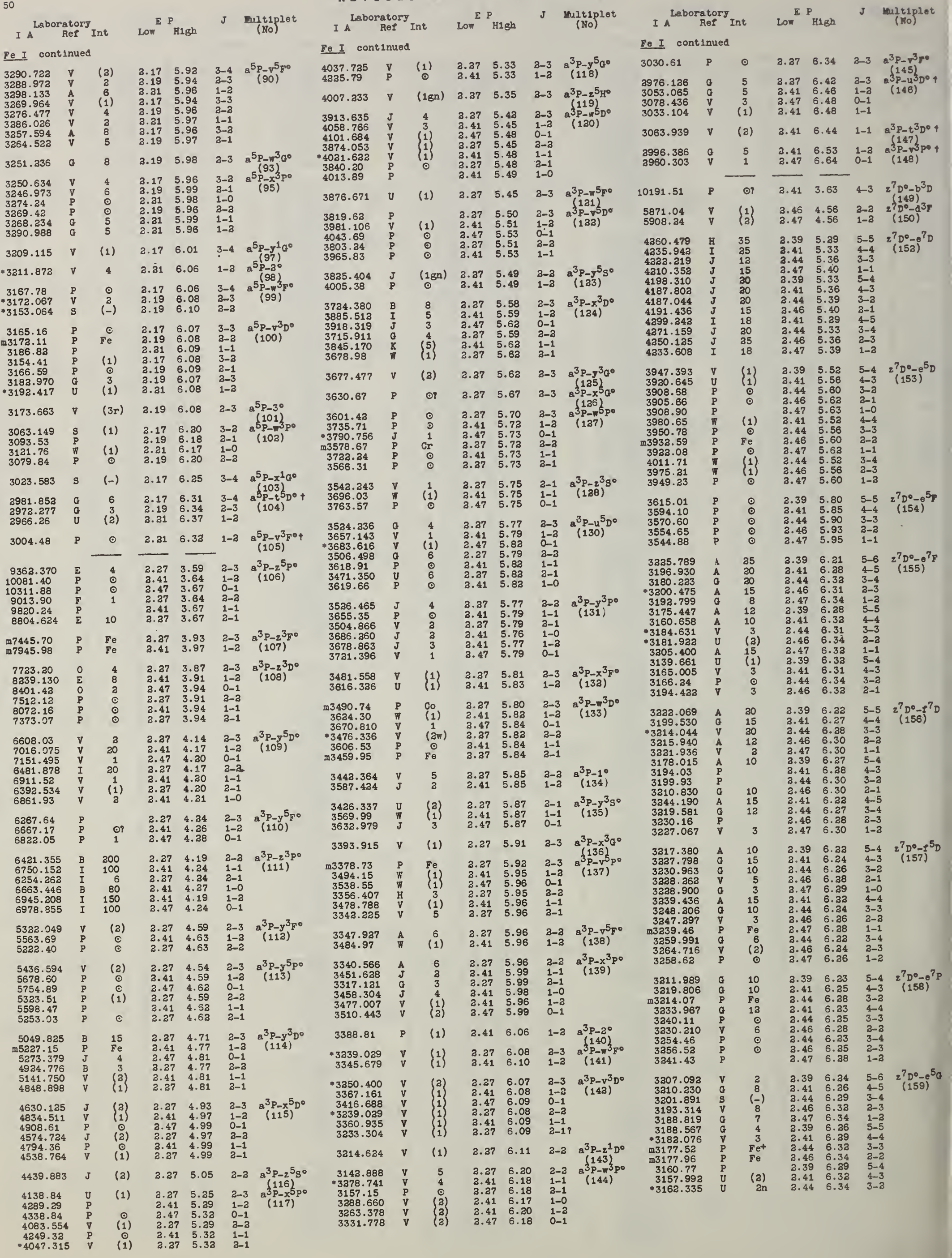




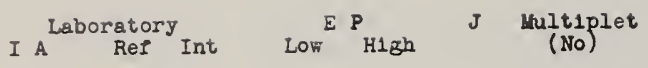

Fe I continued

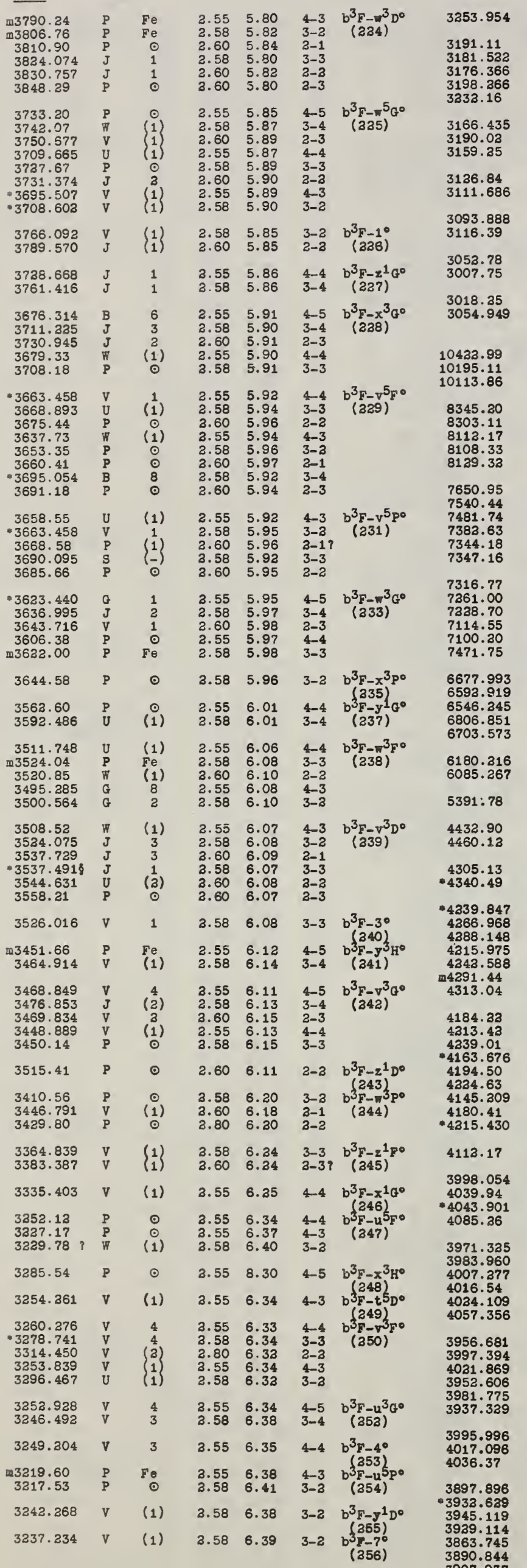

3890.844
3907.937

\section{REVIBED YULIIPLET TABLE}

I A Laboratory ${\text { Ref Int Low }{ }_{\text {High }} \text { J miltiplet }}_{(N O)}$ I A Laboratory ReI Int Low ${ }_{\text {H1gh }}$ (xo)
$($ Mortor Fe I continued

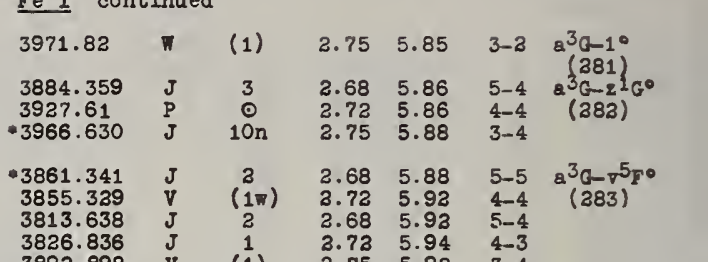

$\begin{array}{llllll}3826.836 & \mathrm{~J} & 1 & 2.68 & 5.92 & 5-4 \\ 3893.898 & \mathrm{~V} & (1) & 3.73 & 5.94 & 4-3 \\ 3.75 & 5.92 & 3-4\end{array}$

$\begin{array}{lllllll}3837.573 & J & 1 & 2.68 & 5.91 & 5-5 & a^{3}\left(a-x^{3} G^{*}\right.\end{array}$

$\begin{array}{lllllll}3873.923 & \mathrm{~V} & 1 & 2.68 & 5.91 & 5-5 & 2 \\ 3907.464 & \mathrm{~J} & (1) & 3.73 & 5.90 & 4-4 & (284) \\ 3 & 2.75 & 5.91 & 3-3 & \end{array}$

$\begin{array}{llcccc}3907.464 & \mathrm{~J} & (1) & 2.75 & 5.91 & 3-3 \\ 3830.850 & \mathrm{~J} & 1 & 3.68 & 5.90 & 5-3\end{array}$

$\begin{array}{llllll}3869.590 & \mathrm{~V} & 1 & 3.68 & 5.90 & 5-4 \\ 3 & 3.73 & 5.91 & 4-3 \\ 3869.562 & \mathrm{~V} & 3 . & 3.72 & 5.91 & 4-5\end{array}$

$\begin{array}{llllll}3869.562 & \mathrm{~K} & 3 * & 2.73 & 5.91 & 4-5 \\ 3910.845 & \mathrm{~J} & (3) & 2.75 & 5.90 & 3-4\end{array}$

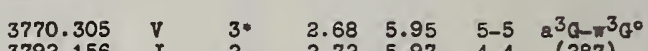

$\begin{array}{lllllll}3770.305 & v & 3 . & 2.68 & 5.95 & 5-5 & 2^{3}\left(a-\pi^{3} G\right. \\ 3792.156 & J & 2 & 2.72 & 5.97 & 4-4 & (287) \\ 3811.893 & J & 2 & 2.75 & 5.98 & 3-3 & \end{array}$

3751.830

$\begin{array}{lll}2 & 2.75 & 5.98 \\ (1) & 3.68 & 5.97 \\ 1 & 3.72 & 5.98\end{array}$

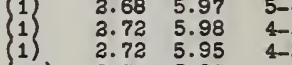

$\begin{array}{cccccc}-3811.05 & U \\ 3838.510 & V & (1) & 2.72 & 5.95 & 4-5 \\ \text { 1n) } & 3.75 & 5.97 & 3-4\end{array}$

$\begin{array}{llll}3.68 & 3.86 & 5-4 & 8^{3} a-z^{3} F^{\circ} \\ 3.78 & 3.93 & 4-3 & (264)\end{array}$

3748.91

$\begin{array}{rrr}\circ & \begin{array}{l}3.68 \\ 3\end{array} & 5.97 \\ 3.73 & 5.97\end{array}$

$\begin{array}{llll}2.68 & 4.16 & 5-5 & a^{3} G-y^{5} F^{\circ} \\ 2.72 & 4.30 & 4-4 & (365) \\ 2.68 & 4.20 & 5-4 & \\ 2.72 & 4.34 & 4-3 & \\ 2.75 & 4.3 & 3-2 & \end{array}$

3704.463

3704.463
3743.78
3779.213

$\begin{array}{llll}2.68 & 4.39 & 5-5 & a^{3} a-z^{5} G^{\circ} \\ 3.73 & 4.35 & 4-4 & (266)\end{array}$

$\begin{array}{lll}10 & 2.68 & 6.01 \\ \text { (0) } & 2.72 & 6.01\end{array}$

3649.508

3669.523
3677.630

$\begin{array}{lll}2.75 & 4.40 & 3-3 \\ 3.68 & 4.35 & 5-4\end{array}$

$\begin{array}{lll}3.68 & 4.35 & 5-4 \\ 2.73 & 4.40 & 4-3 \\ 2.75 & 4.43 & 3-2\end{array}$

.3703 .556

$\begin{array}{llll}2.75 & 4.43 & 3-3\end{array}$

3722.028

$\begin{array}{llll}3.68 & 4.37 & 5-5 & 2^{3}\left(a-z^{3} G 0\right. \\ 3.73 & 4.43 & 4-4 & (387)\end{array}$

$2.68 \quad 4.43 \quad 5-4$

$\begin{array}{lll}2.68 & 4.43 & 5-4 \\ 3.72 & 4.45 & 4-3\end{array}$

3684.108

3703.556
3718.407

3705.71

3606.679

$\begin{array}{llll}3.68 & 4.53 & 5-4 & a^{3} a-y^{3} \\ 7\end{array}$

3.75 4.63

$\begin{array}{lll}2.75 & 4.63 & 3-2 \\ 2.72 & 4.53 & 4\end{array}$

$\begin{array}{lll}2.73 & 4.53 & 4-4 \\ 2.75 & 4.59 & 3-3\end{array}$

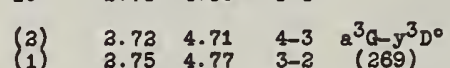

3638.296
3584.663

3584.663
3605.450
3568.977

3603.305

3603.305
3618.392
361.01

3633.001
3581.645
3589

$\begin{array}{llll}3.68 & 4.97 \quad 5-5 & 2^{3} a-x^{5} F^{\circ}\end{array}$

3640.38

$\begin{array}{llll}3.72 & 5.50 & 4-3 & 2^{3}\left(a-v^{5} D^{\circ}\right. \\ 3.75 & 5.51 & 3-2 & (271)\end{array}$

3651.469

\section{(1)}

$\begin{array}{ll}2.72 & 6.01 \\ 2.75 & 6.01\end{array}$

$\begin{array}{ccc}12 & 2.68 & 6.06 \\ 10 & 2.72 & 6.08 \\ 12 & 2.75 & 6.10 \\ 4 & 2.72 & 6.06 \\ 5 & 2.75 & 6.08 \\ (1) & 3.75 & 6.06\end{array}$

(1)

$\begin{array}{lll}2.72 & 6.07\end{array}$

$\begin{array}{ll}3.75 & 6.08 \\ 3.75 & 6.07\end{array}$

$\circ$

$\begin{array}{lll}3.75 & 6.08\end{array}$

$\begin{array}{llll}3.72 & 5.58 & 4-3 & a^{3} a-x^{3} D \\ 3.75 & 5.59 & 3-3 & (373)\end{array}$

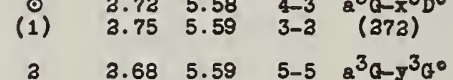

$\begin{array}{lllll}3 & 3.68 & 5.59 & 5-5 & 8^{3}-y^{3} \\ 3 & 3.72 & 5.61 & 4-4 & (273) \\ 3 & 3.75 & 5.62 & 3-3 & (273) \\ 1 & 3.68 & 5.61 & 5-4 & \\ 1 & 3.73 & 5.63 & 4-3 & \end{array}$

$\left(\begin{array}{l}3 \\ 1 \\ 1 \\ \mathrm{Fe}\end{array}\right.$
$\mathrm{g}$

$\begin{array}{ccccc}\text { (1) } & 3.68 & 5.63 & 5-6 & 8^{3}\left(-x^{5} G^{\circ}\right. \\ 0 & 3.73 & 5.64 & 4-5 & (.274) \\ 0 & 3.75 & 5.66 & 3-4 & \end{array}$

$\begin{array}{cccc}\text { (1) } & 3.75 & 5.66 & 3-4 \\ \text { (1) } & 3.68 & 5.64 & 5-5\end{array}$

$\begin{array}{lll}\text { (1) } & 3.78 & 5.66 \\ 0 & 3.75 & 5.67\end{array}$

$\begin{array}{ccc}(1) & 3.75 & 5.67 \\ (1) & 3.68 & 5.66 \\ \circ & 3.73 & 5.67 \\ & 3.75 & 5.67\end{array}$

$$
\circ
$$

20
15
13
8
15
4
10
2
2
13
1
3
15
30
0

$\begin{array}{ll}3.68 & 6.10 \\ 3.73 & 6.12 \\ 2.75 & 6.14 \\ 2.68 & 6.13 \\ 3.73 & 6.14\end{array}$

$\begin{array}{ll}2.68 & 6.13 \\ 2.73 & 6.14\end{array}$

$\begin{array}{ll}3.73 & 6.14 \\ 3.68 & 6.14\end{array}$

$\begin{array}{lll}2.68 & 6.11 & 5\end{array}$

$\begin{array}{ll}2.72 & 6.13 \\ 3.75 & 6.15 \\ 2.68 & 6.13\end{array}$

$\begin{array}{lll}2.68 & 6.13 \\ 2.72 & 6.15\end{array}$

$\begin{array}{ll}3.72 & 6.15 \\ 3.75 & 6.11\end{array}$

$\begin{array}{ll}3.73 & 6.11 \\ 3.75 & 6.13\end{array}$

$5-5 a^{3} a-2^{1} \mathrm{H}^{\bullet}$

$\begin{array}{cc}5-4 & a^{3} a-y^{1} G^{\circ} \\ 4-4 & (290)\end{array}$

$a^{3} a-x^{3} F^{\circ}$

$-2$

$a^{3}\left(-r^{3} p^{\circ}\right.$

(293)

$-3 \quad 2^{3} a-3^{\circ}$

$303\}$
$30-5 \mathrm{H}^{\circ}$
$(394)$

$-4$

$5 a^{3} a-r^{3} G$

(295)

$\begin{array}{cccc}(1) & 3.73 & 5.77 & 4 \\ 5 n & 3.72 & 5.77 & 4 \\ 0 & 3.75 & 5.77 & 3\end{array}$

$\begin{array}{rllll}9 & 3.68 & 5.79 & 5-4 & a^{3}\left(a-x^{3} F 0\right. \\ 10 & 3.72 & 5.81 & 4-3 & (277)\end{array}$

$\begin{array}{lll}3.75 & 5.83 & 3-4 \\ 3.68 & 5.80 & 5-5 \\ 3.68 & 5.83 & 5.4\end{array}$

$\begin{array}{lll}2.75 & 6.24\end{array}$

$5-4$
$4-3$
$4-5$

$\begin{array}{llll}3.68 & 5.83 & 5-4 & \\ 3.73 & 5.80 & 4-3 & 8^{3}\left(a-w^{3} \mathrm{D}^{\circ}\right.\end{array}$

$\begin{array}{lllll}4 & 3.73 & 5.80 & 4-3 & 8^{3}\left(-w^{3} D^{2}\right. \\ \text { (1) } & 3.75 & 5.82 & 3-3 & (379) \\ 0 & 3.75 & 5.80 & 3-3 & \end{array}$

3354.734

3335.592
3537.90

$\begin{array}{lll}3.72 & 5.59 & 4-5 \\ 3.75 & 5.61 & 3-4\end{array}$

$4-4$
$3-3$
$5-4$
$4-3$
$3-2$

$3.68 \quad 5.68$

$\begin{array}{cc}5-6 & a^{3}\left(a-2^{3} I^{\circ}\right. \\ 5-4 & (375) b^{\circ} \\ -4 & a^{3} a-u^{5}\end{array}$

3411.88

3411.134

3405.83

3404.923

3404.75
3438.10
3434.029

3438.10
3434.039

3378.676
-3404.301

3404.301
3453.022
3411.353

3411.353
3440.74

3366.790
3399.230

3399.230
3438.41

3341.906

$\begin{array}{llll}3.73 & 5.77 & 4-3 & (376) \\ 3.72 & 5.77 & 4-4 & \end{array}$

3370.786

$\begin{array}{llll}3.75 & 5.83 & 3-2 & 3369.549 \\ 3.72 & 5.79 & 4-4 & 3380.111\end{array}$

$\begin{array}{llll}3.73 & 5.79 & 4-4 & 3380.111 \\ 3.75 & 5.81 & 3-3 & 3337.666 \\ 3.75 & 5.79 & 3-4 & 3351.750\end{array}$

$3.68 \quad 5.80 \quad 5-6 \quad 8^{3} \mathrm{a}-8^{3} \mathrm{H}^{\circ}$

3351.750
3403.299

3403.299
3398.236

$\begin{array}{llll}3.73 & 5.80 & 4-5 & (378)\end{array}$

$\begin{array}{lll}3.68 & 5.80 & 5-5 \\ 3.72 & 5.83 & 4-4 \\ 3.88 & 5.83 & 5-4\end{array}$

.3393 .609

$\left(\begin{array}{l}3 \\ 2\end{array}\right)$

3387.410

3335.72
3363.81

3365.55

$\begin{array}{llll}3.68 & 5.85 & 5-6 & a^{3} a-\pi^{5} G^{\circ}\end{array}$

3349.037
3319.37

$\left.\begin{array}{c}\odot \\ 1 \\ 1 \\ 2\end{array}\right)$

$\begin{array}{ll}3.68 & 6.25 \\ 2.72 & 6.35\end{array}$

$3.68 \quad 6.30$

$\begin{array}{ll}3.68 & 6.30 \\ 3.73 & 6.30 \\ 3.68 & 6.30\end{array}$

(1) $3.68 \quad 6.31$

$\begin{array}{lll}(1) & 3.73 & 6.34 \\ \circ & 3.73 & 6.31 \\ \text { (1i) } & 3.75 & 6.34\end{array}$

6
3
$(3)$
3
0

$\begin{array}{lll}3.68 & 6.33\end{array}$

$\begin{array}{ll}3.73 & 6.34 \\ 3.75 & 6.32 \\ 3.73 & 6.33\end{array}$

$\begin{array}{ll}3.75 & 6.33 \\ 3.73 & 6.33 \\ 3.75 & 6.33\end{array}$

(1)

$3.68 \quad 6.3$

5

$\begin{array}{ll}3.75 & 6.35\end{array}$

$\begin{array}{lll}3.68 & 6.37\end{array}$

(1)

10
8
8
6
3
3
3

$\begin{array}{lll}3.68 & 6.34\end{array}$

$3.73 \quad 6.38$

$\begin{array}{ll}3.75 & 6.40 \\ 3.68 & 6.38\end{array}$

$\left.\begin{array}{lll}3 & 3.73 & 6.40 \\ (3) & 3.73 & 6.34 \\ 1\end{array}\right)$

3-3 $a^{3}\left(a-2^{1}+5\right.$

$5-4 a^{3}(a-x)$
$4-4$
$(297)$

$3.75 \quad 5.87 \quad 3-4$

$\begin{array}{lll}3.75 & 5.89 & 3-3 \\ 3.68 & 5.87 & 5-4\end{array}$

$\begin{array}{lll}3.68 & 5.87 & 5-4 \\ 3.72 & 5.89 & 4-3 \\ 3.75 & 5.90 & 3-2\end{array}$
5-5 $a^{3} a-u^{5} \mathrm{~F}$

$\begin{array}{cc}5-8 & (398) \\ 4-5 & \left(39 x^{3}\right. \\ 5-5 & (309)\end{array}$

$\begin{array}{ll}5-4 & a^{3} a-t^{5} D \\ 4-3 & (300)\end{array}$

3-4

$\begin{array}{ll}5-4 & a^{3} a-r^{3} p \\ 4-3 & (301)\end{array}$

-3
-2
-4

$5-4 \quad a^{3}\left(a-4^{0}\right.$

$\begin{array}{ll}5-5 & a^{2}\left(a-6^{\circ}\right. \\ 4-5 & (303)\end{array}$

$\begin{array}{ll}5-5 & 2^{3}\left(4-u^{3} G^{\circ}\right. \\ 4-4 & (304) \\ 3-3 & \end{array}$

$4-3$ 


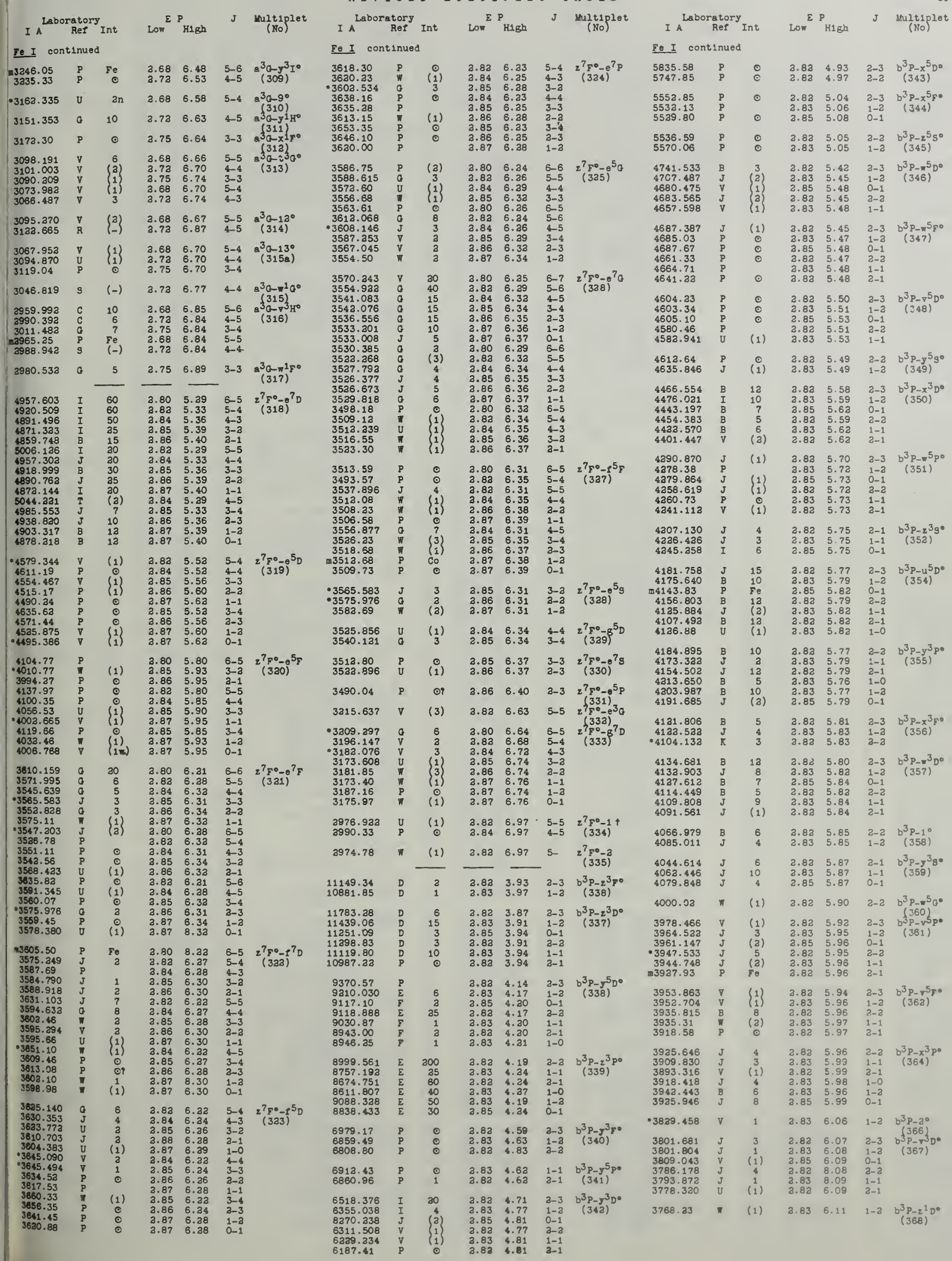




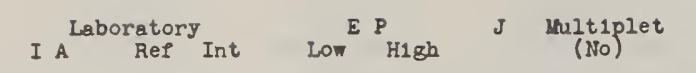

Fe I continued

$\begin{array}{rrrrrrr}3655.465 & \mathrm{~J} & 4 & 3.82 & 6.20 & 3-2 & \mathrm{~b}^{3} \mathrm{P}-\mathrm{r}^{3} \mathrm{Po}^{\circ} \\ .3689 .457 & \mathrm{6} & 12 & 2.83 & 6.18 & 1-1 & (369)\end{array}$

$\begin{array}{llllll}3674.766 & \mathrm{~J} & 2 & 2.82 & 6.18 & 2-1 \\ 3702.033 & \mathrm{~J} & 3 & 3.83 & 6.17 & 1-0\end{array}$

$\begin{array}{llllll}3670.035 & \mathrm{~V} & 3 & 2.83 & 6.20 & 1-2 \\ 3703.824 & \mathrm{~J} & 3 & 2.85 & 6.18 & 0-1\end{array}$

$\begin{array}{lllllll}3602.77 \quad P & P & 3.82 & 6.34 & 3-3 & b^{3} P-2^{1} F^{\circ}\end{array}$

$3504.455 \quad U \quad(1) \quad 3.82 \quad 6.34 \quad 2-3 \quad b^{3} P-v^{3} F^{\circ}$

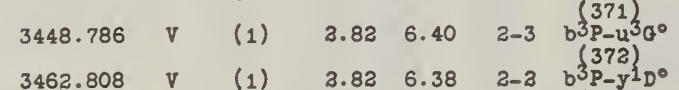

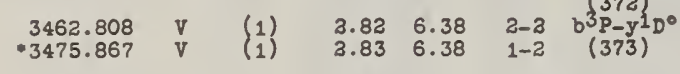

$\begin{array}{lllllll}-3457.090 & v & (3 \pi) \quad 3.82 & 6.39 & 2-2 & b^{3} P-70\end{array}$

$\begin{array}{ccccccc}3469.390 & \mathrm{~V} & (1) & 3.83 & 6.39 & 1-2 & \mathrm{~b}^{3} \mathrm{P}-\mathrm{x}^{1} \mathrm{D}^{\circ} \\ 3431.815 & \mathrm{~J} & 3 & 2.82 & 6.42 & 2-3 & \mathrm{~b}^{3} \mathrm{3}-\mathrm{u}^{3} \mathrm{D}^{\circ} \\ 3406.17 & \mathrm{P} & \odot & 2.83 & 6.46 & 1-2 & (376) \\ 3393.382 & \mathrm{~V} & (1) & 2.85 & 6.48 & 0-1 & \\ 3393.609 & \mathrm{~V} & (1) & 2.82 & 6.46 & 2-2 & \end{array}$

17) $\quad 2.82 \quad 6.46 \quad 2-2$

$\left.\begin{array}{lllll}3368.983 & V & 2 \\ 1\end{array}\right) \quad \begin{array}{lll}2.83 & 6.48 & 1-1 \\ 2.82 & 6.48 & 2-1\end{array}$

$\begin{array}{lllllll}3403.29 & P \\ 3361.959 & V \\ 3432.023 & V \\ 3349.739 & V \\ 3419.706 & V\end{array} \quad\left\{\begin{array}{l}1 \\ 3407.06\end{array}\right.$

$\begin{array}{lllllll}3342.298 & V & 4 & 3.83 & 6.53 & 1-1 & b^{3} P-80 \\ 3354.068 & V & 3 & 2.85 & 6.53 & 0-1 & (378)\end{array}$

$\begin{array}{llcllll}3323.737 & \mathrm{C} & 7 & 2.82 & 6.53 & 2-a & \mathrm{~b}^{3} P-v^{3} p_{0} \\ 3239.35 & \mathrm{P} & & 2.83 & 6.64 & 1-1 & (379)\end{array}$

$\begin{array}{llcllll}3239.35 & P & & 2.83 & 6.64 & 1-1 & (379) \\ 3228.003 & V & (3) & 3.82 & 6.64 & 2-1 & \\ 3335.776 & V & 4 & 2.83 & 6.53 & 1-2 & \\ 3250.400 & V & (2) & 2.85 & 6.64 & 0-1 & \\ 3389.442 & V & (2) & 2.83 & 6.57 & 3-1 & b^{3} P-z^{1} P^{\circ} \\ 3301.227 & V & (2) & 2.83 & 6.57 & 1-1 & (380)\end{array}$

$\begin{array}{ccccccc}3301.227 & V & (2) & 2.83 & 6.57 & 1-1 & (380) \\ 3343.406 & V & 3 & 2.82 & 6.62 & 3-3 & b^{3} P-y^{1} F^{\circ} \\ 3 & & & & & & \end{array}$

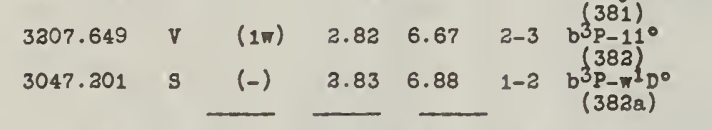

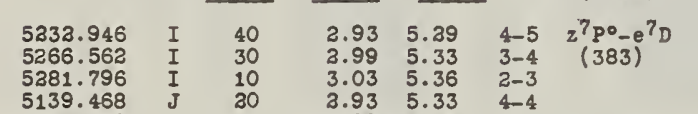

$\begin{array}{llllll}5192.350 & I & 30 & 2.99 & 5.36 & 3-3\end{array}$

$\begin{array}{llllll}5068.774 & \mathrm{~J} & 10 & 3.93 & 5.36 & 4-2 \\ 5139.260 & \mathrm{~J} & 10 & 3.99 & 5.39 & 4-3 \\ 5191.460 & \mathrm{~J} & 30 & 3.03 & 5.40 & 3-2\end{array}$

$\begin{array}{lllllll}4768.397 & v & 3 n & 2.93 & 5.52 & 4-4 & z^{7} P^{\circ}-e^{5} \mathrm{D}\end{array}$

4787.84
4800.14

4682.58

$\begin{array}{lllll}1 & \\ 1 & & 2.93 & 5.56 & 4-3 \\ 1 & 3.99 & 5.80 & 3-2\end{array}$

$\begin{array}{llllll}4760.07 & P & \odot & 3.03 & 5.62 & 2-1 \\ 4877.61 & P & \odot & 3.99 & 5.52 & 3-4 \\ 4863.78 & P & \odot & 3.03 & 5.56 & 2-3\end{array}$

$\begin{array}{ccccccc}3685.998 & G & 15 n & 3.93 & 6.28 & 4-5 & 2^{7} P^{0}-e^{7} \mathrm{~F} \\ 3701.086 & G & 20 & 3.99 & 6.32 & 3-4 & (385) \\ 3752.420 & \mathrm{~J} & 1 \mathrm{w} & 3.03 & 6.31 & 2-3 & \end{array}$

3637.862
-3707.048

3736.927
3643.627

3682.17
3744.105

3748.969
3754.506

3754.506
3793.28

3689.457
3746.931

3773.69

3682.15
3727.809
3766.665

3742.621

3793.478
3816.92

$\begin{array}{lllll}3727.096 & \mathrm{~J} & 4 & 3.93 & 6.24 \\ 3769.995 & \mathrm{~V} & 4 & 2.99 & 6.26 \\ 3790.656 & \mathrm{~J} & (1) & 3.03 & 6.28\end{array}$

$\begin{array}{lllllll}3735.325 & \mathrm{~J} & 6 & 3.93 & 6.23 & 4-4 & \mathrm{z}^{7} \mathrm{P}^{\circ}-\theta^{7} \mathrm{P}\end{array}$

$\begin{array}{lllll}3782.450 & \mathrm{~J} & 1 & 3.99 & 6.35 \\ 3793.354 & \mathrm{~V} & 1 & 3.03 & 6.38\end{array}$

3716.442
3747.00

3703.697

$\mathrm{m} 3730.46$
3742.56

3666.34

3687.426

3721.512
3634.326

3634.326
3876.879

3633.07

3681.84
3709.03

3709.03
3664.69

3664.69
3602.70

3.936 .31

$\begin{array}{ll}2.99 & 6.34 \\ 3.03 & 8.32\end{array}$

$\begin{array}{ll}3.93 & 6.22 \\ 3.99 & 6.27\end{array}$

$\begin{array}{ll}3.03 & 6.28 \\ 3.93 & 6.37\end{array}$

$\begin{array}{ll}3.99 & 6.28 \\ 3.03 & 6.30\end{array}$

$\begin{array}{ll}3.93 & 6.38 \\ 2.99 & 6.30\end{array}$

$3.03 \quad 6.30$

2.936 .23

3.996 .34

$3.03 \quad 6.26$

$\begin{array}{ll}3.03 & 6.28 \\ 3.93 & 6.25 \\ 3.99 & 6.28\end{array}$

$2.93 \quad 6.36$

$\begin{array}{rrr}3 & 2.93 & 6.36 \\ \text { Co } & 3.99 & 6.29 \\ 0 & 3.03 & 6.32 \\ 1 & 3.93 & 6.39\end{array}$

$\begin{array}{lll}1 & 3.83 & 6.39 \\ 8 n & 3.89 & 6.33\end{array}$

$\begin{array}{lll}8 \mathrm{n} & 3.89 & 6.32 \\ 3 & 3.03 & 6.34\end{array}$

$\begin{array}{lll}6 n & 3.93 & 6.32 \\ \text { (1) } & 2.99 & 6.34\end{array}$

(1)

$\begin{array}{ll}3.93 & 6.32\end{array}$

$\begin{array}{ll}3.99 & 6.34 \\ 3.03 & 6.35\end{array}$

$\begin{array}{ll}3.99 & 6.35 \\ 3.93 & 6.35\end{array}$

$3-3$

$3-3$
$4-3$

$3 \div 2$
$3-1$

$\begin{array}{cc}4-5 & 2^{7} p^{\circ}-f^{7} \mathrm{D} \\ 3-4 & (388)\end{array}$

$4-4 \quad 2^{7} P^{0}-r^{5} \mathrm{D}$

4-5 $2^{7} p^{\circ}-e^{50}$

$4-4$
$3-3$
$3-2$

$4-5$
$3-4$
$2^{7} p^{0}-e^{7} \mathrm{G}$
$(390)$

$2-3$

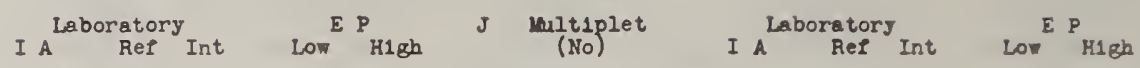

J cultiplot

\section{Fe I continued} $\begin{array}{lllllll}3649.70 & p & \odot & 3.93 & 6.31 & 4-5 & z^{7} p^{\circ}-p^{5} \\ 3664.537 & q & 2 & 2.99 & 6.35 & 3-4 & (391) \\ 3689.37 & p & \odot & 3.03 & 6.37 & 3-3 & \end{array}$

$+3645.494$

3707.048
-3752.420

3752.420

3679.53
3666.85

3588.5

3650.03

3554.44
3584.960
3633.64

3633.64
3614.77

3627.35
3657.89

3322.474
3338.643

3338.643
3342.76
3287.117

3287.117
3308.703
3320.800

3320.800
3285.20

3256.52

3238.535

$-3053.443$

10086.27

9038.84

9375.14
9156.23

9089.413
8975.408

8868.43

8638.71

8621.613
8582.26

8515.08

8343.21
8358.53

8878.36
8747.33

7748.381

7884.302

7583.798
7954.94

7904.13

7112.176
6971.95

6310.543

6539.72

5261.49

5326.42
5288.38

5318.04
5196.24

.5336 .154

5385.35
5304.95

$\begin{array}{lllll}\text { (i) } & 2.99 & 6.39 & 3-3 & z^{7} \mathrm{P} 0 \\ \text { (1) } & 2.99 & 6.34 & 3-4 & \left(393{ }^{5} \mathrm{D}\right.\end{array}$

$\vdots 0.0 .040$

$\begin{array}{ll}4-3 & 2^{7} p^{0}-e^{5} p \\ 3-3 & (395) \\ 3-1 & \end{array}$

$\begin{array}{lll}3.03 & 6.42 & 3-1 \\ 2.99 & 6.40 & 3-3\end{array}$

$\begin{array}{llll}- & 3.03 & 6.43 & 3-2 \\ 1 & 3.03 & 6.40 & 2-3\end{array}$

$\begin{array}{lll}5 n & 2.93 & 6.64\end{array}$

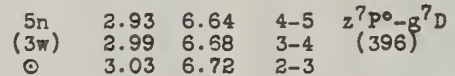

(1iv) $2.93 \quad 6.68$

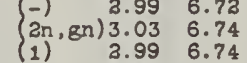

$4-4$
$3-3$
$2-2$

$\begin{array}{lllll}\circ & 2.99 & 6.77 \quad 3-2 & z^{7} \mathrm{po}^{3}-\mathrm{e}^{3 \mathrm{P}}\end{array}$

(a) $\quad \begin{array}{llll}2.93 & 6.97 & 4 & z^{7} p^{\mathrm{po}}-3\end{array}$

๑) $3.94 \quad 4.16 \quad 5-5 \quad b^{3} \mathrm{a}-\mathrm{y}^{5} \mathrm{~F}^{\circ}$

$\begin{array}{ccc}10 & 2.98 & 4.35 \\ 3 & 3.00 & 4.40 \\ (10) & 3.94 & 4.35\end{array}$

$\begin{array}{ccc}(10) & 3.94 & 4.35 \\ \odot & 3.98 & 4.40\end{array}$

$\begin{array}{cc}5-6 & (400) \\ 4-5 & (4-5)\end{array}$

$3-4$
$5-5$

$4-4$
$3-3$
$5-4$

$\begin{array}{lllll}10 & 3.94 & 4.37 & 5-5 & b^{3} a-z^{3} g^{\circ} \\ 15 & 2.98 & 4.42 & 4-4 & (401)\end{array}$

$\begin{array}{lll}15 & 2.98 & 4.42 \\ 30 & 3.00 & 4.45\end{array}$

$\odot$ ค $3.94 \quad 4.42$

$\begin{array}{lll}\circ & 3.94 & 4.42 \\ \circ & 2.98 & 4.45 \\ \circ & 3.98 & 4.37\end{array}$

$5-4$
$4-3$
$4-5$
$3-4$

$3.00 \quad 4.43$

$\begin{array}{llll}2.94 & 4.53 & 5-4 & b^{3} a-y^{3} F 0 \\ 2.98 & 4.59 & 4-3 & (403)\end{array}$

$\begin{array}{lll}3.00 & 4.83 & 3-3 \\ 3.98 & 4.53 & 4-4\end{array}$

$\begin{array}{llll}3.98 & 4.54 & 4-3 & b^{3} a-y 5 p o \\ 3.00 & 4.59 & 3-3 & (403)\end{array}$

$\begin{array}{llll}3.98 & 4.71 & 4-3 & b^{3} a-y^{3} D^{\circ} \\ 3.00 & 4.77 & 3-3 & (404)\end{array}$

$\begin{array}{lllll}(1) & 3.94 & 4.89 & 5-4 & b^{3} a-x^{5} D^{\circ} \\ 2 & 3.00 & 4.89 & 3-4 . & (405)\end{array}$

$\begin{array}{lllll}\odot & 3.94 & 5.38 & 5-6 & b^{3} a-y^{5} g^{\circ} \\ \odot & 3.94 & 5.30 & 5-5 & (406)\end{array}$

$\begin{array}{llll}\odot & 2.98 & 5.31 & 4-4 \\ \odot & 3.00 & 5.33 & 3-3 \\ \odot & 3.94 & 5.31 & 5-4\end{array}$

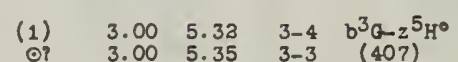

4773.52
4787.50

4647.437

4691.414
4710.386

4618.76

4881.975
-4730.997

4720.997
4740.343

4826. 758

4836.758
+4556.129
4603.956

4603.956
4633.764

4494.47

4358.505
4418.432

4433.39

4423.145
4461.80

4336.762

4351.549
-4373.563
4390.460

4309.388

4367.581

4390.954
4304.552

4348.939
4386.440

4386.440

$\left(\begin{array}{l}a \\ 3 \\ 1 \\ 1 \\ 1\end{array}\right)$

$\begin{array}{llll}3.94 & 5.59 & 5-5 & b^{3}\left(a-y^{3} a^{\circ}\right. \\ 3.98 & 5.61 & 4-4 & (409)\end{array}$

$3.00 \quad 5.62$

$\begin{array}{ll}3.98 & 5.62 \\ 3.98 & 5.5\end{array}$

3.005 .6

$5-4$
$4-3$
$4-5$

$\begin{array}{lllll}(-) & 3.98 & 5.64 & 4-5 & b^{3} G-x^{5} c^{\circ} \\ \text { in } & 3.94 & 5.64 & 5-5 & (410) \\ \text { (1) } & 3.98 & 5.66 & 4-4 & \end{array}$

$\left(\begin{array}{l}1 \\ 1\end{array}\right)$

$\begin{array}{ll}3.98 & 5.64 \\ 3.00 & 5.67\end{array}$

3.94 5.68
Fe I continued

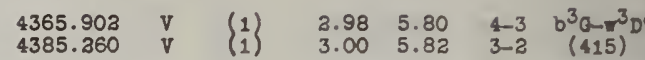

4239.735 4290.382
4299.65

$\begin{array}{lllll}3 & 2.94 & 5.85 & 5-6 & b^{3} a-\pi^{5} \\ \text { (3) } & 2.98 & 5.85 & 4-5 & (416)\end{array}$

4229.516

4259.34
4280.63

$\begin{array}{llll}1 \mathrm{gn}) & 2.94 & 5.85 & 5-5\end{array}$

$\begin{array}{llll}\circ & 3.98 & 5.87 & 4-4 \\ 0 & 3.00 & 5.89 & 3-3\end{array}$

4199.37

(1)

$\begin{array}{ll}2.94 & 5.87 \\ 3.00 & 5.90\end{array}$

(1)

$2.94 \quad 5.86$

4223.73
4284.415

(1)

$\begin{array}{ll}3.94 & 5.88 \\ 3.00 & 5.94\end{array}$

$5-4 \quad b^{3} a-z^{1} a$

4196.533

4203.30

(1)

$2.94 \quad 5.9$

4156.670

(1)

$\begin{array}{ll}2.98 & 5.94 \\ 3.00 & 5.92\end{array}$

4219.41
.4254 .938

4160.561

$\left.\begin{array}{l}(1) \\ 0 \\ 1 \\ 1\end{array}\right\}$

$2.94 \quad 5.91$

4215.430
4258.956

(i)

$\begin{array}{ll}2.98 & 5.9 \\ 3.00 & 5.91 \\ 2.94 & 5.90\end{array}$

$\begin{array}{ll}2.94 & 5.90 \\ 3.98 & 5.91\end{array}$

4089.225

- 4123.748

$\left\{\begin{array}{l}1 \\ 1 \\ 1 \\ 0\end{array}\right\}$

$2.94 \quad 5.95$

4067.49
4104.46
4146.071

(3)

(2)

3.98

$\begin{array}{ll}2.98 & 5.97 \\ 3.00 & 5.98\end{array}$

$2.94 \quad 5.97$

$\begin{array}{ll}2.98 & 5.95 \\ 3.00 & 5.97\end{array}$

4064.07

4130.211

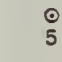

$\begin{array}{ll}3.94 & 5.97 \\ 3.98 & 5.97\end{array}$

4011.89
4066.597

\begin{tabular}{lll}
- & 3.94 & 4.30 \\
& 2.98 & 4.29 \\
\hline & 3.00 & 4.35
\end{tabular}

$\begin{array}{rrr}\circ & 3.00 & 4.35 \\ 30 & 3.94 & 4.29 \\ 10 & 2.98 & 4.35\end{array}$

4045.13

(1)

$3.94 \quad 6.01$

.3947 .533

3979.12

4000.466

4014.38
4035.98

4035.98

4014.28
4031.73

125

3981.62

3897.449

3922.68

3871.750

3853.462

(1)

(1)

5
$\odot$
$\odot$
$\vdots$
3
$(1)$
$\odot$

$3.00 \quad 6.0$

3893.39

3919.069

3968.34

3885.15

3944.890
3953.15

3978.885

3777.061

3726.0

3716.7
3696.81

3696.81

3670.071

3709.535
3663.95

3662.90

3669.58
3708.45

3708.45
3699.55 


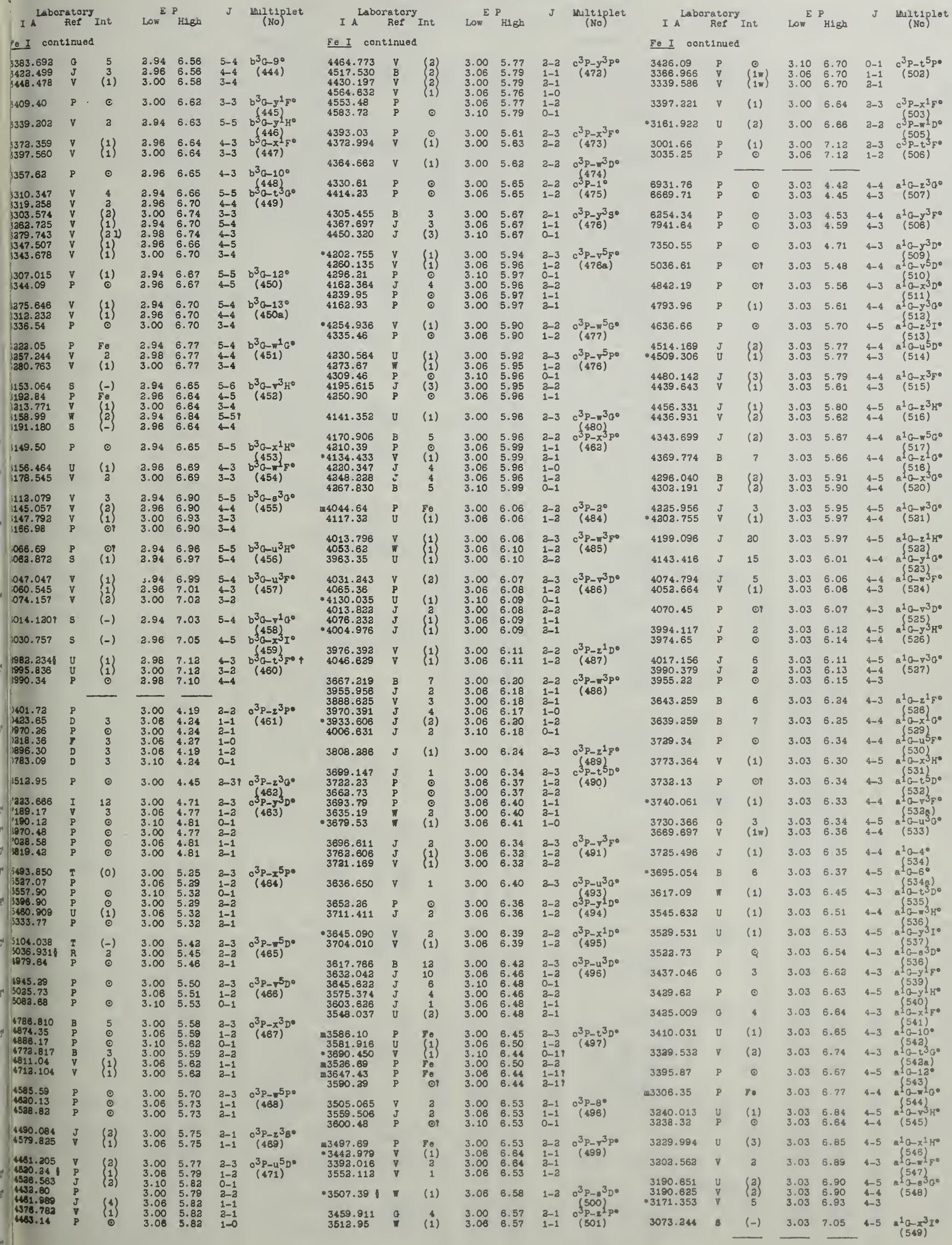




\section{6}

I A Rel Int Low ${ }^{\text {Laboratory }}$ High

\begin{tabular}{|c|c|c|c|c|c|c|}
\hline 6831.44 & $P$ & $\odot ?$ & 3.30 & 5.00 & $4-3$ & $z^{5} D^{0}-\Omega^{1} F$ \\
\hline $\begin{array}{l}6677.54 \\
6647.90 \\
6786.41 \\
6737.39 \\
6879.59 \\
6801.31\end{array}$ & $\begin{array}{l}\mathrm{P} \\
\mathrm{P} \\
\mathrm{P} \\
\mathrm{P} \\
\mathrm{P} \\
\mathrm{P}\end{array}$ & $\begin{array}{l}\circ \\
\stackrel{\circ}{\circ} \\
\stackrel{\circ}{\circ} \\
\stackrel{\circ}{\circ}\end{array}$ & $\begin{array}{l}3.20 \\
3.23 \\
3.33 \\
3.25 \\
3.25 \\
3.27\end{array}$ & $\begin{array}{l}5.05 \\
5.08 \\
5.05 \\
5.08 \\
5.05 \\
5.08\end{array}$ & $\begin{array}{l}4-3 \\
3-3 \\
3-3 \\
3-3 \\
2-3 \\
1-3\end{array}$ & $\begin{array}{l}2^{5} D^{\circ}-x \\
(551)\end{array}$ \\
\hline $\begin{array}{c}5909.99 \\
5872.73 \\
.5848 .09 \\
5837.89 \\
5807.79 \\
5791.044 \\
5780.621 \\
.5780 .83 \\
5714.88\end{array}$ & $\begin{array}{l}\text { W } \\
\text { P } \\
\text { W } \\
\text { P } \\
\text { P } \\
\text { V } \\
\text { V } \\
\text { V } \\
P\end{array}$ & $\begin{array}{l}(3) \\
(2 n) \\
0 \\
0 \\
0 \\
2 \\
3 \\
1 \\
0 \\
0\end{array}$ & $\begin{array}{l}3.20 \\
3.23 \\
3.25 \\
3.27 \\
3.28 \\
3.20 \\
3.23 \\
3.25 \\
3.23\end{array}$ & $\begin{array}{l}5.39 \\
5.33 \\
5.36 \\
5.39 \\
5.40 \\
5.33 \\
5.36 \\
5.39 \\
5.39\end{array}$ & $\begin{array}{l}4-5 \\
3-4 \\
2-3 \\
1-3 \\
0-1 \\
4-4 \\
3-3 \\
2-3 \\
3-3\end{array}$ & $\begin{array}{c}z^{5} D^{0}-e^{7 D} \\
(552)\end{array}$ \\
\hline $\begin{array}{r}5324.185 \\
5283.628 \\
5263.314 \\
5253.479 \\
5217.395 \\
5208.601 \\
5215.185 \\
.5229 .857 \\
5393.174 \\
5339.935 \\
5302.307 \\
5273.176\end{array}$ & $\begin{array}{l}I \\
I \\
\text { J } \\
\text { V } \\
\text { J } \\
\text { J } \\
\text { J } \\
\text { J } \\
\text { I } \\
\text { I } \\
\text { I } \\
\text { K }\end{array}$ & $\begin{array}{l}30 \\
18 \\
8 \\
(2) \\
5 \\
7 \\
6 \\
5 n \\
10 \\
13 \\
10 \\
(5)\end{array}$ & $\begin{array}{l}3.20 \\
3.23 \\
3.35 \\
3.27 \\
3.20 \\
3.23 \\
3.25 \\
3.27 \\
3.23 \\
3.25 \\
3.27 \\
3.28\end{array}$ & $\begin{array}{l}5.52 \\
5.56 \\
5.60 \\
5.62 \\
5.56 \\
5.60 \\
5.62 \\
5.63 \\
5.52 \\
5.56 \\
5.60 \\
5.62\end{array}$ & $\begin{array}{l}4-4 \\
3-3 \\
2-3 \\
1-1 \\
4-3 \\
3-3 \\
3-1 \\
1-0 \\
3-4 \\
2-3 \\
1-2 \\
0-1\end{array}$ & $\begin{array}{l}2^{5} D^{0}-e^{5} D \\
(553)\end{array}$ \\
\hline $\begin{array}{r}4736.780 \\
4707.381 \\
4668.142 \\
4637.512 \\
4613.210 \\
.4654 .628 \\
4625.052 \\
-4607.655 \\
4598.122 \\
4574.240 \\
4565.684 \\
4568.789\end{array}$ & $\begin{array}{l}I \\
\text { I } \\
\text { J } \\
\text { J } \\
\text { J } \\
\text { J } \\
\text { I } \\
\text { J } \\
\text { J } \\
\text { V } \\
\text { V } \\
\text { V }\end{array}$ & $\left.\begin{array}{l}12 \\
8 \\
6 \\
3 \\
2 n \\
5 \\
3 \\
3 n \\
3 n \\
2 n \\
1 \\
2 \\
1 \\
1\end{array}\right)$ & $\begin{array}{l}3.20 \\
3.23 \\
3.25 \\
3.27 \\
3.28 \\
3.20 \\
3.23 \\
3.25 \\
3.27 \\
3.20 \\
3.23 \\
3.25\end{array}$ & $\begin{array}{l}5.80 \\
5.85 \\
5.90 \\
5.93 \\
5.95 \\
5.85 \\
5.90 \\
5.93 \\
5.95 \\
5.90 \\
5.93 \\
5.95\end{array}$ & $\begin{array}{l}4-5 \\
3-4 \\
2-3 \\
1-3 \\
0-1 \\
4-4 \\
3-3 \\
2-3 \\
1-1 \\
4-3 \\
3-3 \\
3-1\end{array}$ & $\begin{array}{c}z^{5} D^{0}-\theta^{5} F \\
(554)^{-1}\end{array}$ \\
\hline $\begin{array}{c}4531.633 \\
4464.69 \\
4425.75 \\
4581.517 \\
4504.838\end{array}$ & $\begin{array}{l}\mathrm{J} \\
\mathrm{P} \\
\mathrm{P} \\
\mathrm{J} \\
\mathrm{J}\end{array}$ & $\begin{array}{l}(3) \\
\left(\begin{array}{c}2 \\
2 \\
3\end{array}\right)\end{array}$ & $\begin{array}{l}3.20 \\
3.23 \\
3.25 \\
3.23 \\
3.25\end{array}$ & $\begin{array}{l}5.93 \\
5.99 \\
6.04 \\
5.93 \\
5.99\end{array}$ & $\begin{array}{l}4-4 \\
3-3 \\
2-2 \\
3-4 \\
3-3\end{array}$ & $\begin{array}{l}z^{5} D^{0}-e^{3} F \\
(555)\end{array}$ \\
\hline $\begin{array}{l}4009.54 \\
3990.55 \\
1029.640 \\
4022.744 \\
3997.49 \\
4000.266 \\
4042.75 \\
3959.46 \\
4020.05\end{array}$ & $\begin{array}{l}P \\
P \\
P \\
V \\
U \\
P \\
\text { P } \\
\text { P } \\
P \\
P\end{array}$ & 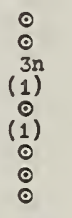 & $\begin{array}{l}3.20 \\
3.23 \\
3.25 \\
3.27 \\
3.23 \\
3.25 \\
3.27 \\
3.20 \\
3.25\end{array}$ & $\begin{array}{l}6.38 \\
6.32 \\
6.31 \\
6.34 \\
6.31 \\
6.34 \\
6.33 \\
6.31 \\
6.33\end{array}$ & $\begin{array}{l}4-5 \\
3-4 \\
2-3 \\
1-3 \\
3-3 \\
3-3 \\
1-1 \\
4-3 \\
2-1\end{array}$ & $\begin{array}{c}2^{5} D^{0}-e^{7} F \\
(556)\end{array}$ \\
\hline $\begin{array}{c}4084.17 \\
4052.73 \\
4076.810 \\
4080.886 \\
4013.641 \\
4043.901 \\
4054.18 \\
4069.08 \\
4004.976 \\
4021.622 \\
4046.07\end{array}$ & $\begin{array}{l}P \\
P \\
J \\
V \\
J \\
V \\
V \\
U \\
U \\
J \\
V \\
P\end{array}$ & 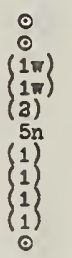 & $\begin{array}{l}3.20 \\
3.23 \\
3.25 \\
3.28 \\
3.20 \\
3.23 \\
3.25 \\
3.37 \\
3.20 \\
3.23 \\
3.25\end{array}$ & $\begin{array}{l}6.32 \\
6.27 \\
6.28 \\
6.30 \\
6.27 \\
6.28 \\
6.30 \\
6.30 \\
6.28 \\
6.30 \\
6.30\end{array}$ & $\begin{array}{l}4-5 \\
3-4 \\
3-3 \\
0-1 \\
4-4 \\
3-3 \\
2-3 \\
1-1 \\
4-3 \\
3-3 \\
3-1\end{array}$ & $\begin{array}{c}2^{5} D^{0}-R^{7} D \\
(557)\end{array}$ \\
\hline
\end{tabular}

$\begin{array}{lllll}4076.636 \quad J & 8 n & 3.20 & 6.23\end{array}$

4098.183

4104.133
4097.099

4058.237

4070.788
4073.760

4080.326

4116.97

4131.97
-4127.807
4109.070

\begin{tabular}{lll}
$4 n$ & 3.23 & 6.24 \\
3 & 3.25 & 6.26 \\
\hline & 3.37 & 0.28
\end{tabular}

(1) $3.27 \quad 6.28$

$4 n \quad 3.20 \quad 6.24$

$4 n \quad 3.25 \quad 6.28$

$\begin{array}{lll}3 \mathrm{n} & 3.27 & 6.39 \\ \text { (1) } & 3.33 & 6.32\end{array}$

$\begin{array}{lll}0 & 3.25 & 6.24 \\ 3 n & 3.27 & 6.36\end{array}$

$\begin{array}{lll}3 \mathrm{n} & 3.27 & 6.36 \\ \text { (1) } & 3.38 & 6.28\end{array}$

4067.984

4085.312
4076.89

4045.59

4043.98

4108.13
4118.904

4030.499
4024.735

4024.735
4018.283

4018.282
4016.432

43986.18
3986.30

3986.30
3994.00

3948.48
3962.42

3947.002

3967.964
3979.65

3979.65
3989.34

3986.26

3948.28
3984.93

.3966 .630

3948.105
3957.027
3963.108

3963.108

8n $\quad 3.30 \quad 6.23$

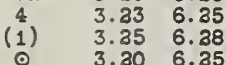

$\begin{array}{lll}\circ & 3.30 & 6.35 \\ \circ & 3.33 & 6.38\end{array}$

(1) $\quad 3.33$ 6. $\quad 3.23$

(8) $3.30 \quad 6.36$

$\begin{array}{lll}6 n & 3.23 & 6.39 \\ (4) & 3.35 & 6.32 \\ 3 & 3.37 & 6.34\end{array}$

$\begin{array}{lll}\text { (2) } & 3.37 & 6.34 \\ \text { Fe } & 3.30 & 6.39\end{array}$

$\begin{array}{ll}3.30 & 6.39 \\ 3.33 & 6.32\end{array}$

$\begin{array}{ll}3.25 & 6.34 \\ 3.20 & 6.32\end{array}$

$\stackrel{3}{\odot} \quad 3.23 \quad 6.32$

$\begin{array}{lll}4 \mathrm{n} & 3.30 & 6.33 \\ 4 \mathrm{n} & 3.23 & 6.34\end{array}$

(1) $\begin{array}{lll}4.23 & 6.34 \\ & 3.35 & 6.35\end{array}$

$\begin{array}{ll}3.27 & 6.36 \\ 3.38 & 6.37\end{array}$

$\begin{array}{ll}3.28 & 6.37 \\ 3.23 & 6.35 \\ 3.37 & 6.37\end{array}$

$\frac{4}{3} z^{5} D^{0}-p^{5} D$

$2-2$
$1-1$
$4-3$

$2-1$
$3-3$
$2-1$

$2-1$
$1-0$
$3-4$

$3-4$
$2-3$
$1-2$
$0-1$

$\begin{array}{ll}4-4 & 2^{5} D^{0}-e^{7 p} \\ 3-3 & (559)^{7 p}\end{array}$

$3-3$
$4-3$
$3-3$

$3-3$
$3-4$
$3-3$

$\begin{array}{ll}4-5 & 2^{5} D^{0}-0^{5 a} \\ 3-4 & (560)\end{array}$

$3-4$
$3-3$

$\begin{array}{lll}3-1 & \end{array}$

$\begin{array}{rrrrr}10 \mathrm{n} & 3.30 & 6.31 & 4-5 & \mathbf{2}^{5} \mathrm{D}^{0}-\mathbf{F}^{5} \mathrm{~T} \\ 6 \mathrm{n} & 3.23 & 6.35 & 3-4 & (563)\end{array}$

$\begin{array}{llll}4 n & 3.25 & 6.37\end{array}$

$\begin{array}{lll}6 \text { n } & 3.37 & 6.38 \\ \text { (1n) } & 3.38 & 6.39\end{array}$
I A Laboratory Int Low ${ }^{E} P_{\text {High }}$ J Lultiplet Fe I continued

$\begin{array}{ll}3911.00 & P \\ 3926.001 & \mathrm{~V} \\ 3941.283 & \mathrm{~J} \\ 3955.353 & \mathrm{~J} \\ 3889.33 & \mathrm{P} \\ 3910.52 & \mathrm{P} \\ 3933.606 & \mathrm{~J} \\ 3997.48 & \mathrm{P} \\ .4039 .640 & \mathrm{~V}\end{array}$

(1) $\begin{array}{llll}3.30 & 6.35 & 4 \\ 3.23 & 6.37 & 3-3\end{array}$

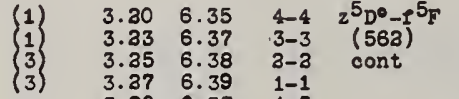

$\begin{array}{lll}3.37 & 6.39 \\ 3.30 & 6.37\end{array}$

$\begin{array}{lll}3.30 & 6.37 & 4-1 \\ 3.33 & 6.38 & 3-3\end{array}$

(a) $\begin{array}{llll}3.23 & 6.38 & 3-3 \\ \text { (a) } & 3.25 & 8.39 & 3-1\end{array}$

.4039 .640
4052.466

3936.79
3905.18
3889.92
3974.397

3974.397
-3935.86

3935.86
3911.18

4006.16
3957.62

3938.085

3900.519

3888.43

3864.30

3858.48
3863.70

3879.19

3931.123

3909.664
3895.44

3894.49
3962.353

3854.375

3855.846
3892.98

3890.39

3885.76
3914.273

3920.839
3906.97

3906.97
3925.201

3680.675
3668.214

3636.49
3598.93

3591.485

3636.186
3582.34

.3667 .999

3647.84

3615.19

3616.15
359.68
359.65

3597.05
3700.61

3667.253

3644.798

3624.08
3671.51

3651.03

3651.03
.3618 .392
3593.33

(1)

$\begin{array}{llll}3.23 & 6.31 & 3-3 & 2^{5} D^{0}-e^{5} s \\ 3.35 & 6.31 & 2-3 & (563)\end{array}$

$\begin{array}{lllll}\circ & 3.30 & 6.33 & 4-3 & 2^{5} \mathrm{D}^{\circ}-\mathrm{e}^{3} \mathrm{D} \\ \odot & 3.33 & 6.39 & 3-2 & (564)\end{array}$

(1) $\begin{array}{ll}3.25 & 6.42 \\ 3.23 & 6.33\end{array}$

$\begin{array}{lll}3.25 & 6.39 \\ 3.27 & 6.42\end{array}$

$\begin{array}{lll}3.27 & 6.42 & 1-1 \\ 3.25 & 6.33 & 2-3\end{array}$

$\begin{array}{llll}(1) & 3.25 & 6.33 & 3-3 \\ (1) & 3.27 & 6.39 & 1-2 \\ \circ & 3.38 & 6.43 & 0-1\end{array}$

$\begin{array}{lllll}\text { (1) } & 3.30 & 6.34 & 4-4 & z^{5} D^{0}-8^{5} \mathrm{D} \\ 3 & 3.23 & 6.39 & 3-3 & (565)\end{array}$

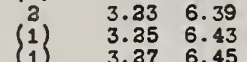

$\begin{array}{ccc}(1) & 3.37 & 6.45 \\ \circ & 3.20 & 6.39\end{array}$

$\begin{array}{lll}\circ & 3.23 & 6.43 \\ \circ & 3.35 & 6.45 \\ \circ & 3.27 & 6.45\end{array}$

$\left(\begin{array}{lll}\circ & 3.27 & 6.45 \\ 3 & 3.23 & 6.34 \\ 3 & 3.25 & 6.39 \\ 1 & 3 & 37 \\ 1\end{array}\right.$

$3-3$
$2-2$
$1-1$
$4-3$
$3-2$
$2-1$
$1-0$
$3-4$
$2-3$
$1-2$
$0-1$

$\begin{array}{lll}(1) & 3.20 & 6.37 \\ \text { (2) } & 3.25 & 6.37\end{array}$

$\begin{array}{ll}4-3 & 2^{5} D^{0}-e^{7} S \\ 2-3 & (566)^{7}\end{array}$

(1) $\begin{array}{lllll}\text { (1) } & 3.30 & 6.40 & 4-3 & 2^{5} D^{\circ}-e^{5} p \\ \text { 1iv } & 3.33 & 6.43 & 3-2 & (567)\end{array}$

(1)

$\begin{array}{ll}3.35 & 6.42 \\ 3.23 & 6.40\end{array}$

$\begin{array}{ll}3.35 & 6.43 \\ 3.37 & 6.42\end{array}$

$\begin{array}{llll}\text { (1) } & 3.37 & 6.42 & 1-1 \\ 1 & 3.25 & 6.40 & 3-3 \\ 0 & 3.27 & 6.43 & 1-3 \\ \text { (1) } & 3.28 & 6.42 & 0-1\end{array}$

$\begin{array}{lllll}3 \mathrm{n} & 3.20 & 6.55 & 4-5 & 2^{5} D^{\circ}-8^{5} \mathrm{~F} \\ (1) & 3.33 & 6.59 & 3-4 & (568)^{2}\end{array}$

$\begin{array}{lll}(1) & 3.23 & 6.59 \\ \circ & 3.25 & 6.65 \\ 1 & 3.37 & 6.70 \\ 1 & 3.38 & 6.71\end{array}$

$\begin{array}{cccc}(1) & 3.37 & 6.70 & 1-3 \\ 1 & 3.28 & 6.71 & 0-1 \\ 0 & 3.20 & 6.59 & 4-4\end{array}$

$\begin{array}{llll}1 & 3.20 & 6.59 & 4-4 \\ \circ & 3.27 & 6.71 & 1-1\end{array}$

$\begin{array}{ccccc}1 & 3.30 & 6.56 & 4-4 & 2^{5} D^{0}-h^{5} D \\ r e & 3.33 & 6.61 & 3-3 & (569)\end{array}$

(1) $\begin{array}{lll}3.25 & 6.66 \\ 1 & 3.37 & 6.68 \\ 3.20 & 6.61\end{array}$

$\begin{array}{llll}1 & 3.20 & 6.61 & 4-3 \\ 1 & 3.32 & 6.66 & 3-3 \\ 3 n & 3.25 & 6.68 & 2-1\end{array}$

$\begin{array}{lllll}3 \mathrm{n} & 3.20 & 6.56 & 4-3 & 2^{5} \mathrm{p}^{0}-\mathrm{p}^{5} \mathrm{p} \\ 3 \mathrm{i} & 3.33 & 6.61 & 3-2 & (570)\end{array}$

$\begin{array}{lllll}\text { (1) } & 3.33 & 6.61 & 3-2 & (570) \\ 0 & 3.35 & 6.66 & 3-1 & \\ 1 & 3.25 & 6.61 & 3-3 & \\ 1 & 3.37 & 6.61 & 1-3 & \end{array}$

$\begin{array}{ccccc}\circ & 3.30 & 6.58 & 4-5 & 2^{5} D^{\circ}-15^{5} \\ 3 & 3.23 & 6.64 & 3-4 & (571) \\ (1) & 3.35 & 6.69 & 3-3 & \end{array}$

$\begin{array}{cccc}\text { (1) } & 3.23 & 6.64 & 3-4 \\ 0 & 3.25 & 6.69 & 3-3 \\ 0 & 3.35 & 6.71 & 3-3\end{array}$

3558.08
3496.60

3590.99

3590.99
3579.83

3621.19
3645
353.353
353

3518.23

$\begin{array}{llllllll}3473.23 & P & \odot & 3.30 & 6.75 & 4-4 & (575) \\ 3419.157 & \mathrm{~V} & (1) & 3.23 & 6.84 & 3-2 & (576)\end{array}$

3503.46

3473.01
3459.29

3418.176 U (2w) $3.37 \quad 6.88 \quad 1-0 \quad z^{5} D^{\circ}-e^{3} \mathrm{P}$

$\begin{array}{lllllll}3503.85 & P & 0 & 3.35 & 6.88 & 1-0 & 2^{5} p^{\circ}-e^{3} p \\ 3459.81 & P & 0 & 3.27 & 6.77 & 3-2 & (577)\end{array}$

3459.61

$3.30 \quad 7.14$

6007.75
6085.81
3705.70

3586.114
-3584.960

3573.896

3583.301

3582.201
3613.95

3576.760
3613.51
$1-3$
$4-4$

$3-3$
$2-3$
$4-3$

$\underbrace{4-3}_{3-4} \quad 2^{5} D^{0}-0^{7} a$

$3-4$
$3-3$
$1-3$

$1-3$
$0-1$
$3-3$

(561)

3143.990
3156.275
3160.200

3132.514
3140.385

3167.807
3174.28

3150.301

$\begin{array}{lllll}8 & 3.20 & 7.12 & 4-4 & 2^{5} D^{0}-1^{5} D \\ 5 n & 3.23 & 7.14 & 3-3 & (578) \\ (3 n) & 3.35 & 7.16 & 3-2 & \end{array}$

(an) $3.25 \quad 7.16$

$\begin{array}{ll}3.23 & 7.16 \\ 3.33 & 7.12 \\ 3.37 & 7.18\end{array}$

$3-2$
$4-3$
$3-2$
$3-4$
$1-3$

3765.543
3821.181

3821.181
3805.345
3785.706

3785.706
3765.70

3704.336
3738.308

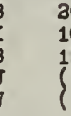
(1)

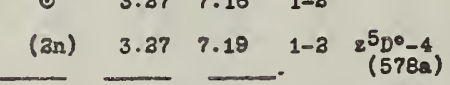

$\begin{array}{lllllll}10780.71 & p & \odot & 3.32 & 4.37 & 6.5 & b^{3} \mathrm{H}-z^{3} G^{\circ} \\ 10816.75 & P & \odot & 3.25 & 4.43 & 5-4 & (579) \\ 10577.14 & P & \odot & 3.39 & 4.45 & 4-3 & \end{array}$

9673.16

5807.23
6007.75

$\begin{array}{llll}3.23 & 6.50 & 6-7 & b^{3} h_{-3}^{3} I^{\bullet} \\ 3.25 & 6.48 & 5-6 & (608)\end{array}$

$\begin{array}{lll}3.29 & 6.53 & 4-5 \\ 3.23 & 6.48 & 6-6\end{array}$

$\begin{array}{lll}3.23 & 6.48 & 6-6 \\ 3.25 & 6.53 & 5-5\end{array}$

$3.22 \quad 6.55 \quad 6-6 \quad b^{3} \mathrm{H}-z^{1} \mathrm{I}^{\bullet}$

$3.35 \quad 6.58 \quad 5-4 \quad b^{3} \mathrm{H}-9 \cdot$

$3-3$
$1-3$
$0-1$

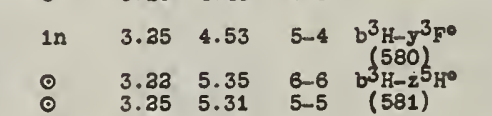


I A Laboratory Int

E. I oontinu

$\begin{array}{llllll}3513.74 & P & \circ & 3.25 & 6.77\end{array}$

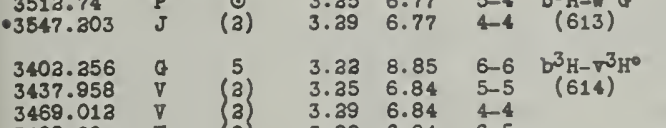
3409.20 3436.045 3430.88 3398.1
3436.67 3438.0 3307.234 3338.867
3355.338 3301.93 3384.372 3359.814

$\begin{array}{lllllll}3305.75 & \mathrm{P} & \odot & 3.35 & 6.99 & 5-4 & \mathrm{~b}^{3} \mathrm{H}-\mathrm{u}^{3} \mathrm{~F}^{\circ} \\ 3315.17 & \mathrm{P} & \odot & 3.39 & 7.01 & 4-3 & (618)\end{array}$ $\begin{array}{rllllll}3336.363 & \nabla & (3) & 3.29 & 6.99 & 4-4 & \\ .3396 .8068 & \nabla & (1) & 3.39 & 7.03 & 4-4 & b^{3} \mathrm{H}-\nabla^{1} G^{\circ}\end{array}$ $\begin{array}{ccccccc}3396.8068 & \nabla & (1) & 3.39 & 7.03 & 4-4 & b^{3} \mathrm{H}-\nabla^{1} G^{\circ} \\ 3233.053 & G & 8 & 3.33 & 7.04 & 6-7 & (619) \\ 33-x^{3} I^{\circ}\end{array}$ 3254.363
3280.26

$10145.00 \quad P \quad$ op $\quad \overline{3.34} \quad \overline{4.45} 3-3 \quad \mathrm{a}^{3} \mathrm{D}-z^{3} \mathrm{G}^{\circ}$ $\begin{array}{lllllll}9556.56 & F & 1 & 3.24 & 4.53 & 3-4 & (631) \\ 9485.93 & \mathrm{P} & & 3.39 & 4.59 & 3-3 & (632) \\ 8994.63 & \mathrm{P} & \odot & 3.26 & 4.63 & 1-3 & \end{array}$ $\begin{array}{llllll}9140.15 & P & 0 & 3.24 & 4.59 & 3-3 \\ 9173.83 & U & \text { (1) } & 3.39 & 4.63 & 3-3\end{array}$ $\begin{array}{lllllll}8365.643 & \mathrm{E} & 35 & 3.34 & 4.71 & 3-3 & \mathbf{a}^{3} \mathrm{D}-\mathrm{v}^{3} \mathrm{D}^{0} \\ 8393.537 & \mathrm{E} & 30 & 3.39 & 4.77 & 3-3 & (633)\end{array}$ 7941.09
9037.96 8080.66 8654.40
8146.67 6130.37 8157.4
5996. 8009.83

6384.

5787.2 5648 .

5617.32 .5563 .71

5455.09

5383.8

5358.1

5346.00

5030.67
5031.89 4871.9 4813.1

4876.19 4930.0 4973.9
4919.7 4790. 4808 . 491.2
4780.8 480.8
481. 416.85
4807 4716.85
-4807.34
-4757.58 : $\begin{array}{lllllll}4838.36 \quad P & \circ & 3.34 & 5.91 & 3-3 & a^{3} \mathrm{D}-x^{3} \sigma^{\circ}\end{array}$

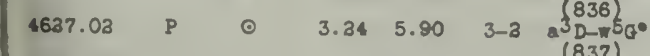

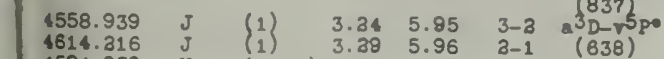
4614.216
4594.959 4516.08
493.98 $(3.8$ ?) $3.35 \quad 5.95$ $\begin{array}{ll}3.34 & 5.87 \\ 3.34 & 5.98\end{array}$ ${ }_{3-3}^{3-4} \stackrel{30-\pi^{3} g \cdot 0}{(639)}$ 4579.0
4555.7 455.75 4587.796
4533.530
453 $\begin{array}{ll}1-3 & a^{3} p-r^{5} \mathbf{r}^{-0} \\ 1-1 & (840)\end{array}$

(1) $3.34 \quad 5.96 \quad 3-3 \quad a^{3} D-x^{3} p_{0}$

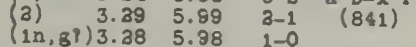

I L Laboratory Ref Int Low ${ }_{\text {H1gh }}$ J mitiplet

I A $A_{\text {Ref Int }}^{\text {Laboratory }}$ Low ${ }_{\text {High }}$

thitiplot
(No)

\section{Fe I continued}

$\begin{array}{lllllll}4611.05 & \mathrm{P} & \odot & 3.29 & 5.96 & \mathrm{a}-3 & \mathrm{a}^{3} \mathrm{D}-\mathrm{x}^{3} \mathrm{po}\end{array}$

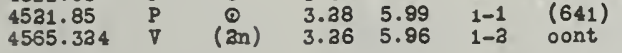

$\begin{array}{lllll}4414.47 \quad P & \text { (1) } 3.36 \quad 6.06\end{array}$

4368.66 4419.78
4341.57

4343.33

$\begin{array}{lllllll}4343.357 & J & (a) & 3.24 & 6.08 & 3-3 & a^{3} D-v^{3} D^{0} \\ 4409.133 & J & \{1) & 3.29 & 6.09 & 3-1 & (645) \\ 4440.973 & \nabla & 3.29 & 6.07 & 3-3 & \end{array}$

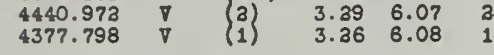

$\begin{array}{ccccccc}4432.883 & \nabla & (1 n) & 3.39 & 8.08 & 3-3 & a^{3} D-3^{\circ} \\ 4304.15 & P & \odot & 3.39 & 6.15 & 3-3 & 8^{3} D-\nabla^{3} a^{\circ}\end{array}$

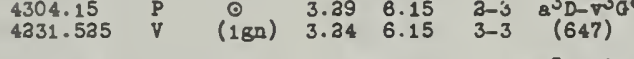

$\begin{array}{lllllll}4399.49 & P & \odot & 3.34 & 6.11 & 3-3 & 8^{3} D-z^{1} D^{0} \\ 4374.495 & \nabla & (1) & 3.39 & 6.11 & 3-3 & (848)\end{array}$

$\begin{array}{lllllll}4173.126 & J & 5 & 3.34 & 6.30 & 3-3 & a^{3} \mathrm{D}-\mathrm{m}^{3} \mathrm{po}\end{array}$

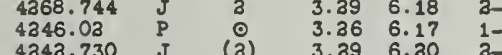

$\begin{array}{llllll}4343.730 & \mathrm{~J} & (3) & 3.29 & 6.30 & 3-2 \\ .4339 .516 & \mathrm{~J} & (\mathrm{gn}) & 3.26 & 6.18 & 1-1\end{array}$

4103.63
4171.804

(a) $\quad 3.24 \quad 8.34 \quad 3-3 \quad 3^{3} \mathrm{D}-z^{1} \mathrm{y}^{\circ}$

.4099 .08

(1) $\quad 3.34 \quad 8.35 \quad 3-4 \quad \mathrm{a}^{3} \mathrm{D}-\mathrm{x}^{1} \mathrm{~g}^{\circ}$

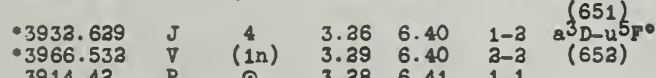

$\begin{array}{llllll}3914.43 & P & \odot & 3.38 & 6.41 & 1-1 \\ 3948.00 & P & \odot & 3.39 & 6.41 & 3-1\end{array}$

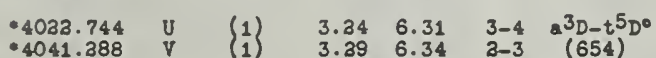
$\begin{array}{ccccccc}.4041 .388 & \mathrm{~V} & (1) & 3.29 & 6.34 & 3-3 & (654) \\ 3963.43 & \mathrm{P} & 0 & 3.36 & 6.37 & 1-3 & \end{array}$

$\begin{array}{lllllll}3986.176 & J & 5 & 3.24 & 6.33 & 3-4 & a^{3} D-v^{3} \% 0 \\ 4040.650 & J & 4 & 3.29 & 6.34 & 3-3 & (655)\end{array}$ $\begin{array}{lllllll}4031.968 & \mathrm{~V} & 4 & 3.26 & 6.33 & 1-3 \\ 3976.564 & \mathrm{~V} & \text { (1) } & 3.34 & 6.34 & 3-3\end{array}$

$\begin{array}{llllll}4067.60 & P & 0 & 3.39 & 6.33 & 2-3 \\ .4003 .665 & \mathrm{~V} & \text { (1) } & 3.34 & 8.33 & 3-2\end{array}$

$3969.638 \quad J \quad$ (1) $3.34 \quad 6.35 \quad 3-4 \quad \mathrm{a}^{3} \mathrm{D}-4^{\circ}$

$\begin{array}{lllll}3965.446 \quad \nabla & (1) \quad 3.34 & 6.35\end{array}$

$\begin{array}{lll}3939.308 & J & (1 \\ 3966.834 & J & 1\end{array}$

3935.55

3925.55

3889.38
3914.50

3923.03

3985.393
3951.184

3914.73

3914.73
.3978 .885

3883.383

3894.005
-3839.458

3834.46

3861.60

3846.803

3836.332
3878.726

3778.509

3911.899
3906.748

3810.759
3779.444

3779.444

3740.347

3751.058

3796.80

$\begin{array}{llllllll}3757.459 & \mathrm{~J} & 1 & 3.39 & 8.57 & 3-1 & \mathrm{a}^{3} \mathrm{D}-z^{1} \mathrm{P} \\ 3737.03 & \mathrm{P} & \odot & 3.26 & 8.57 & 1-1 & (868)\end{array}$

$\begin{array}{llllll}3688.478 \quad V & \text { (1i) } \quad 3.34 & 8.58 & 3-4 & 0^{3} D_{-9}\end{array}$

3843.80

3897.510

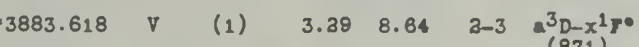

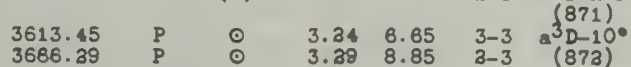

$\begin{array}{lllllll}3568.838 & v & \text { (a) } & 3.24 & 8.70 & 3-4 & 30-t^{3} \sigma^{\circ}\end{array}$

$\begin{array}{lllllll}3573.103 & \nabla & 3 & 3.29 & 8.74 & 2-3 & (873) \\ 3533.18 & \mathrm{P} & \odot & 3.34 & 8.74 & 3-3 & \end{array}$

$\begin{array}{llllll}3598.71 & 1 \\ .3851 .10 & 3.34 & 6.87 & 3-3 & a^{3} D-111^{\circ}\end{array}$

$3580.705 \quad \nabla \quad 5 \quad 3.34 \quad 8.70 \quad 3-4 \quad \mathrm{a}^{3} \mathrm{D}-130$

3431.815

3408.443

$\cdot 3381.340$

$\begin{array}{llll}(1) & 3.34 & 6.50 & 3-3 \\ (1) & 3.39 & 6.44 & 3-19 \\ 3 & 3.39 & 6.45 & 3-3\end{array}$

(1) $\quad 3.39 \quad 6.53 \quad 3-3 \quad a^{3} p-\nabla^{3} p_{0}$
Fe I oontinued

$\begin{array}{lllllll}3388.35 & \mathrm{P} & \odot & 3.34 & 6.90 & 3-4 & \mathrm{a}^{3} \mathrm{D}-\mathrm{s}^{3} \mathrm{G} \cdot \\ 3391.84 & \mathrm{P} & \odot & 3.39 & 6.93 & 3-3 & (678)\end{array}$

$3310.498 \quad \nabla \quad(3) \quad 3.34 \quad 6.97 \quad 3-4 \quad 8^{3} \mathrm{D}-u^{3} \mathrm{H}^{*}$

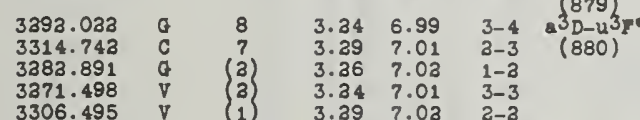

$\begin{array}{llllll}3363.45 & P & \odot & 3.34 & 7.03 & 3-3\end{array}$

$\begin{array}{llllllll}3353.610 & \nabla & 4 & 3.34 & 7.03 & 3-4 & \mathrm{a}^{3} \mathrm{D}-\nabla^{1} \sigma_{0}\end{array}$

$\begin{array}{lllllll}3191.41 & \mathrm{P} & \odot & 3.34 & 7.10 & 3-4 & \mathrm{a}^{3} \mathrm{(b-t3)} \\ 3333.08 & \mathrm{P} & \odot & 3.39 & 7.13 & 2-3 & (682)\end{array}$

\begin{tabular}{llllll}
3193.74 & $\mathrm{P}$ & $\odot$ & 3.36 & 7.13 & $1-3$ \\
3316.08 & $\mathrm{P}$ & $\odot$ & 3.39 & 7.13 & $3-3$ \\
\hline
\end{tabular}

\begin{tabular}{|c|c|c|c|c|c|c|}
\hline 7478.87 & P & ○ & 3.35 & 5.00 & $4-3$ & $z^{5} F^{0}-a^{1}$ \\
\hline .78 & $\stackrel{P}{P}$ & $\stackrel{\circ}{\circ}$ & $\begin{array}{l}3.40 \\
3.42\end{array}$ & $\begin{array}{l}5.08 \\
5.08\end{array}$ & $\begin{array}{l}3-3 \\
1-3\end{array}$ & $\begin{array}{r}25 F^{\circ}-x \\
(684)\end{array}$ \\
\hline $\begin{array}{l}6371.389 \\
6349.65 \\
6333.735 \\
6319.54 \\
6137.51 \\
6145.43 \\
6388.41 \\
6339.96\end{array}$ & $\begin{array}{l}D \\
P \\
Q \\
P \\
P \\
P \\
P\end{array}$ & $\begin{array}{c}(1) \\
0 \\
(-) \\
\circ \\
\circ \\
\circ \\
\bullet \\
\odot\end{array}$ & $\begin{array}{l}3.32 \\
3.35 \\
3.38 \\
3.40 \\
3.33 \\
3.35 \\
3.35\end{array}$ & $\begin{array}{l}5.39 \\
5.33 \\
5.36 \\
5.39 \\
5.33 \\
5.36 \\
5.39\end{array}$ & $\begin{array}{l}5-5 \\
4-4 \\
3-3 \\
3-3 \\
5-4 \\
4-3 \\
4-5\end{array}$ & $\begin{array}{l}2^{5} \mathrm{~F}^{\circ}-\mathrm{e}^{7} \mathrm{D} \\
(685)^{-3}\end{array}$ \\
\hline
\end{tabular}

5615.653

50

$\begin{array}{llll}3.33 & 5.53 & 5-4 & 2^{5} F^{0}-e^{5 \eta} \\ 3.35 & 5.56 & 4-3 & (686) \\ 3.38 & 5.60 & 3-3 & \\ 3.40 & 5.63 & 3-1 & \\ 3.43 & 5.63 & 1-0 & \\ 3.35 & 5.53 & 4-4 & \\ 3.38 & 5.56 & 3-3 & \\ 3.40 & 5.60 & 3-3 & \\ 3.43 & 5.63 & 1-1 & \\ 3.38 & 5.53 & 3-4 & \\ 3.40 & 5.56 & 2-3 & \\ 3.43 & 5.60 & 1-2 & \\ 3.33 & 5.80 & 5-5 & 2^{5} F^{\circ}-e^{5} \\ 3.35 & 5.85 & 4-4 & (687) \\ 3.38 & 5.90 & 3-3 & \\ 3.40 & 5.93 & 3-3 & \\ 3.43 & 5.95 & 1-1 & \\ 3.33 & 5.85 & 5-4 & \\ 3.35 & 5.90 & 4-3 & \\ 3.38 & 5.93 & 3-3 & \\ 3.40 & 5.95 & 3-1 & \\ 3.35 & 5.80 & 4-5 & \\ 3.38 & 5.85 & 3-4 & \\ 3.40 & 5.90 & 2-3 & \\ 3.43 & 5.93 & 1-3 & \end{array}$

5573.849

5576.097

5709.378
5658.836

5634.549
5603.955

5784.69
5713.150

5713.150
5658.543

4966.096

4946.394

4910.037
4883.151

4883.653
4875.89

4855.883
4843.155

4838.519
5039.368

4950.113

4907.743

4741.081
4679.228

4643.58

4739.699

4739.69
4678.41

4860.98
4786.87

4766.87
4701.90

4359.988

4224.176

-4338.037

4334.509
4173.641

$\begin{array}{lllll}4 & 3.34 & 5.45 & 3-3 & 8^{3} D-t^{3} \\ \text { (a) } & 3.39 & 8.50 & 3-3 & (664)\end{array}$

4308.610
4305.548

4346.59

4111.06

4168.635

$\mathrm{m} 4176.5 ?$
$\mathrm{~m} 4327.43$

4353.53

4328.71

4256.313

4307.08
4369.87

4345.358

4378.33
4330.36

4320.36
4330.53

4306.58
431.23

4351.37
-4340.51

m4235.96

4384.309
4336.86

4337.434

437.432

4338.816
4235.460
4317.551

4317.551

4195.337

4198.318

4198.645
4147.34

4156.480
4169.777
$3.43 \quad 5.93$

(1) $3.33 \quad 5.93$

() $\quad 3.38$ 6.04

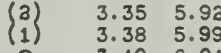

$\begin{array}{ll}3.38 & 5.92 \\ 3.40 & 5.99\end{array}$

3.426 .0

(3)

$3 \mathrm{n} \quad 3.38 \quad 6.32$

$\begin{array}{ll}3.40 & 6.31 \\ 3 & \end{array}$

$\left(\begin{array}{l}3 n \\ 1 \\ 1\end{array}\right)$

$\left(\begin{array}{l}3 n \\ a \\ 1 \\ 1\end{array}\right\}$

$\begin{array}{ll}3.32 & 6.34 \\ 3 & .35\end{array}$

$3.35 \quad 6.32$

$\begin{array}{lll}3.40 & 6.34\end{array}$

3.43 6.32

3. 32 6. 63

re

$\begin{array}{ll}3.38 & 6.34 \\ 3.40 & 6.35\end{array}$

$\stackrel{\odot}{\odot}$

$\begin{array}{ll}3.33 & 6.23 \\ 3.35 & 8.37\end{array}$

$\begin{array}{lll}3.35 & 8.37 \\ 3.32 & 6.37\end{array}$

$\begin{array}{ll}3.40 & 6.30 \\ 3.35 & 6.32\end{array}$

$\begin{array}{ll}3.35 & 6.32 \\ 3.38 & 6.37\end{array}$

tr? $\quad 3.33 \quad 6.33 \quad 5-4 \quad 2^{5} F^{0}-850$

$\left\{\begin{array}{l}1 \\ 1 \\ 1\end{array} \begin{array}{lll}3.35 & 6.34 \\ 3 & 3.38 & 6.34 \\ 3 & 3.40 & 6.36\end{array}\right.$

$\begin{array}{lll}3.40 & 6.36 & 2-3\end{array}$

$\begin{array}{lll}3.43 & 6.38 & 1-1 \\ 3.38 & 6.32 & 3-4\end{array}$

(i) $\begin{array}{llll}\odot .40 & 6.34 & 3-3 \\ & 3.43 & 6.38 & 1-3\end{array}$

$\begin{array}{llllll}\mathrm{Pe} & 3.33 & 6.33 & 5-4 & x^{5} \mathrm{~F}^{\bullet}-0^{7 p} \\ \text { (3) } & 3.35 & 6.35 & 4-3 & (693)^{7}\end{array}$

$\begin{array}{lllll}30 & 3.40 & 6.35 & 3-3 & \\ 30 & 3.33 & 6.34 & 5-6 & 2^{5} F^{\circ}-050\end{array}$

$\begin{array}{rrrr}10 n & 3.38 & 6.29 & 3-4 \\ 6 n & 3.40 & 6.33 & 3-3\end{array}$

$\begin{array}{llll}7 \mathrm{n} & 3.43 & 6.34 & 1-2 \\ 5 & 3.32 & 6.36 & 5-5\end{array}$

(1) $\begin{array}{llll}3.35 & 8.39 & 4-4 \\ 3 & 3.38 & 6.33 & 3-3\end{array}$

in $3.40 \quad 8.34 \quad 3-2$

$\begin{array}{llll}(1) & 3.33 & 8.39 & 5-4 \\ (1) & 3.35 & 6.33 & 4-3 \\ 1 & 3.38 & 6.34 & 3-3\end{array}$
(691) (i) $\quad 3.40 \quad 6.04$

(3) 3.326 .31

$\begin{array}{lllll}30 & 3.33 & 6.34 & 5-6 & 25 \\ 13 & 3.35 & 6.38 & 4-5 & (693)\end{array}$ 
I A Raboratory Ref Int Low High ${ }^{E}$ Multiplet $_{\text {(NO) }}$ Fe I continued

Fe I continued

\begin{tabular}{|c|c|c|c|c|c|c|}
\hline $\begin{array}{l}4149.373 \\
4154.812 \\
4175.89 \\
4182.790 \\
4187.59 \\
4104.97 \\
4136.513 \\
4154.109 \\
4168.942 \\
4087.099 \\
4140.441 \\
4164.34\end{array}$ & $\begin{array}{l}\text { V } \\
\text { b } \\
J \\
\end{array}$ & $\begin{array}{l}5 n \\
9 n \\
0 \\
\left(\begin{array}{ll}3 b \\
1 \\
1 \\
1 \\
1 \\
1 \\
1 \\
1 \\
1 \\
0\end{array}\right) \\
0\end{array}$ & $\begin{array}{r}3.32 \\
3.35 \\
3.38 \\
3.40 \\
3.42 \\
3.32 \\
3.35 \\
3.38 \\
3.40 \\
3.32 \\
3.38 \\
3.40\end{array}$ & $\begin{array}{l}6.39 \\
6.32 \\
6.34 \\
6.35 \\
6.36 \\
6.32 \\
6.34 \\
6.35 \\
6.36 \\
6.34 \\
6.36 \\
6.37\end{array}$ & $\begin{array}{l}5-6 \\
4-5 \\
3-4 \\
2-3 \\
1-3 \\
5-5 \\
4-4 \\
3-3 \\
2-3 \\
5-4 \\
3-3 \\
3-1\end{array}$ & $\begin{array}{l}2^{5} F^{\circ}-e^{7 G} \\
(694)\end{array}$ \\
\hline $\begin{array}{c}4136.193 \\
4114.957 \\
4139.46 \\
.4140 .441 \\
4150.358 \\
4066.02 \\
4090.984 \\
4113.35 \\
4131.94 \\
4176.571 \\
4153.906 \\
4157.788 \\
4158.798\end{array}$ & $\begin{array}{l}\mathrm{J} \\
\mathrm{P} \\
\mathrm{V} \\
\mathrm{J} \\
\mathrm{P} \\
\mathrm{V} \\
\mathrm{V} \\
\mathrm{P} \\
\mathrm{J} \\
\mathrm{J} \\
\mathrm{J}\end{array}$ & $\begin{array}{c}3 n \\
(1 w) \\
0 \\
(1) \\
\left(\begin{array}{l}1 \\
4\end{array}\right) \\
0 \\
\left(\begin{array}{l}1 \\
1\end{array}\right) \\
0 \\
7 n \\
10 n \\
8 n \\
5 n\end{array}$ & $\begin{array}{l}3.32 \\
3.35 \\
3.38 \\
3.40 \\
3.42 \\
3.32 \\
3.35 \\
3.38 \\
3.40 \\
3.35 \\
3.38 \\
3.40 \\
3.42\end{array}$ & $\begin{array}{l}6.31 \\
6.35 \\
6.37 \\
6.38 \\
6.39 \\
6.35 \\
6.37 \\
6.38 \\
6.39 \\
6.31 \\
6.35 \\
6.37 \\
6.38\end{array}$ & $\begin{array}{l}5-5 \\
4-4 \\
3-3 \\
2-3 \\
1-1 \\
5-4 \\
4-3 \\
3-3 \\
2-1 \\
4-5 \\
3-4 \\
2-3 \\
1-3\end{array}$ & $\begin{array}{c}2^{5} r 0^{5} p^{5} \\
(695)\end{array}$ \\
\hline $\begin{array}{r}4208.610 \\
-4338.0 \% 7\end{array}$ & $\begin{array}{l}\mathrm{J} \\
\mathrm{J}\end{array}$ & $\begin{array}{l}3 n \\
4\end{array}$ & $\begin{array}{l}3.38 \\
3.40\end{array}$ & $\begin{array}{l}6.31 \\
6.31\end{array}$ & $\begin{array}{l}3-3 \\
2-3\end{array}$ & $\begin{array}{c}2^{5} F^{\bullet}-e^{5} 8 \\
(696)\end{array}$ \\
\hline $\begin{array}{c}4143.50 \\
4106.437 \\
4083.780 \\
4183.025 \\
.4134 .433 \\
4213.06\end{array}$ & $\begin{array}{l}P \\
\text { V } \\
\text { J } \\
\text { V } \\
\text { V } \\
\text { P }\end{array}$ & $\left\{\begin{array}{l}0 \\
1 \\
1 \\
1 \\
1 \\
0\end{array}\right\}$ & $\begin{array}{l}3.35 \\
3.38 \\
3.40 \\
3.38 \\
3.40 \\
3.40\end{array}$ & $\begin{array}{l}6.33 \\
6.39 \\
6.43 \\
6.33 \\
6.39 \\
6.33\end{array}$ & $\begin{array}{l}4-3 \\
3-3 \\
3-1 \\
3-3 \\
2-3 \\
2-3\end{array}$ & $\begin{array}{c}\varepsilon^{5} F^{0}-e^{3} D \\
(697)\end{array}$ \\
\hline $\begin{array}{l}4084.498 \\
4063.286 \\
4054.833 \\
4054.883 \\
4065.402 \\
4133.869 \\
4101.273 \\
4083.125 \\
4072.518 \\
4173.18 \\
4139.23 \\
409.99\end{array}$ & $\begin{array}{l}\mathrm{J} \\
\mathrm{J} \\
\mathrm{V} \\
\mathrm{V} \\
\mathrm{V} \\
\mathrm{J} \\
\mathrm{J} \\
\mathrm{J} \\
\mathrm{V} \\
\mathrm{P} \\
\mathrm{U} \\
\mathrm{P}\end{array}$ & $\begin{array}{l}6 \\
3 \\
3 \\
1 \\
3 \\
3 \\
3 \\
3 \\
1 \\
1 \\
3 \\
0 \\
0 \\
(1) \\
0\end{array}$ & $\begin{array}{l}3.32 \\
3.35 \\
3.38 \\
3.40 \\
3.42 \\
3.35 \\
3.38 \\
3.40 \\
3.42 \\
3.38 \\
3.40 \\
3.42\end{array}$ & $\begin{array}{l}6.32 \\
6.34 \\
6.26 \\
6.38 \\
6.39 \\
6.32 \\
6.24 \\
6.36 \\
6.38 \\
6.32 \\
6.34 \\
6.36\end{array}$ & $\begin{array}{l}5-4 \\
4-3 \\
3-3 \\
3-1 \\
1-0 \\
4-4 \\
3-3 \\
3-3 \\
1-1 \\
3-4 \\
2-3 \\
1-2\end{array}$ & $\begin{array}{l}2^{5} g^{\circ}-8^{5} \mathrm{D} \\
(698)^{-3}\end{array}$ \\
\hline$\bullet 4163.676$ & $\nabla$ & (1) & 3.40 & 6.37 & $2-3$ & $2^{5} F^{\circ}-e^{7} s$ \\
\hline $\begin{array}{c}4052.313 \\
4051.923 \\
4090.085 \\
4079.18 \\
4105.06 \\
4117.873 \\
4097.08\end{array}$ & 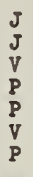 & $\begin{array}{c}\left(\begin{array}{l}1 \\
3 \\
1 \\
1\end{array}\right. \\
0 \\
0 \\
(1) \\
0\end{array}$ & $\begin{array}{l}3.35 \\
3.38 \\
3.38 \\
3.40 \\
3.42 \\
3.40 \\
3.42\end{array}$ & $\begin{array}{l}6.40 \\
8.43 \\
6.40 \\
6.43 \\
6.43 \\
6.40 \\
6.43\end{array}$ & $\begin{array}{l}4-3 \\
3-3 \\
3-3 \\
2-3 \\
1-1 \\
2-3 \\
1-2\end{array}$ & $\begin{array}{l}2^{5} \mathrm{~F}^{0}-e^{5 p} \\
(700)\end{array}$ \\
\hline $\begin{array}{l}3817.64 \\
3811.80 \\
3860.74 \\
3845.31\end{array}$ & $\begin{array}{l}\text { Th } \\
\text { P } \\
\text { P } \\
P\end{array}$ & $\begin{array}{l}3 n \\
\stackrel{\circ}{\circ} \\
\stackrel{\circ}{ }\end{array}$ & $\begin{array}{l}3.33 \\
3.35 \\
3.35 \\
3.38\end{array}$ & $\begin{array}{l}6.55 \\
6.59 \\
6.55 \\
6.59\end{array}$ & $\begin{array}{l}5-5 \\
4-4 \\
4-5 \\
3-4\end{array}$ & $\begin{array}{l}2^{5} F^{0}-g^{5} F \\
(701)^{5}\end{array}$ \\
\hline $\begin{array}{l}3804.013 \\
3789.82\end{array}$ & $\mathfrak{p}^{\mathrm{s}}$ & $\stackrel{(2)}{0}$ & $\begin{array}{l}3.32 \\
3.35\end{array}$ & $\begin{array}{l}6.56 \\
6.61\end{array}$ & $\begin{array}{l}5-4 \\
4-3\end{array}$ & $\begin{array}{l}2^{5} p^{\circ}-h^{5} D \\
(70 a)\end{array}$ \\
\hline $\begin{array}{l}3846.001 \\
3819.50 \\
3791.73 \\
3843.72 \\
3905.01\end{array}$ & $\begin{array}{l}V \\
P \\
U \\
P\end{array}$ & $\begin{array}{l}(1 \pi) \\
\stackrel{0}{(1)} \\
\stackrel{0}{\circ} \\
\stackrel{0}{0}\end{array}$ & $\begin{array}{l}3.35 \\
3.38 \\
3.40 \\
3.40 \\
3.40\end{array}$ & $\begin{array}{l}6.56 \\
6.61 \\
6.66 \\
6.61 \\
6.56\end{array}$ & $\begin{array}{l}4-3 \\
3-2 \\
2-1 \\
2-3 \\
3-3\end{array}$ & $\begin{array}{c}2^{5} F^{\circ}-P^{5} P \\
(703)\end{array}$ \\
\hline $\begin{array}{l}3801.975 \\
3758.11 \\
3743.937 \\
3785.78 \\
3717.19 \\
3703.43 \\
3705.26\end{array}$ & 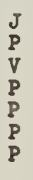 & $\begin{array}{l}(2 \pi) \\
\circ \\
\circ \\
(0) \\
\circ \\
\circ \\
\circ \\
\circ\end{array}$ & $\begin{array}{l}3.32 \\
3.40 \\
3.43 \\
3.32 \\
3.33 \\
3.35 \\
3.38\end{array}$ & $\begin{array}{l}6.56 \\
6.69 \\
8.71 \\
6.58 \\
6.64 \\
6.69 \\
6.71\end{array}$ & $\begin{array}{l}5-6 \\
3-3 \\
1-3 \\
5-5 \\
5-4 \\
4-3 \\
3-2\end{array}$ & $\begin{array}{l}2^{5} p^{\circ}-f^{5} b \\
(704)^{-1}\end{array}$ \\
\hline $\begin{array}{c}-3731.278 \\
-3716.442 \\
3762.205 \\
3737.53\end{array}$ & $\begin{array}{l}\mathrm{V} \\
\mathrm{g} \\
\mathrm{y}\end{array}$ & $\begin{array}{c}3 \\
12 \\
(1) \\
0\end{array}$ & $\begin{array}{l}3.33 \\
3.35 \\
3.35 \\
3.40\end{array}$ & $\begin{array}{l}6.63 \\
6.67 \\
6.63 \\
6.71\end{array}$ & $\begin{array}{l}5-5 \\
4-4 \\
4-5 \\
2-3\end{array}$ & $\begin{array}{c}2^{5} F^{\bullet}-e^{3} G \\
(705)\end{array}$ \\
\hline $\begin{array}{l}3761.06 \\
3717.84\end{array}$ & $\begin{array}{l}P \\
P\end{array}$ & $\stackrel{\circ}{\circ}$ & $\begin{array}{l}3.35 \\
3.40\end{array}$ & $\begin{array}{l}6.64 \\
6.73\end{array}$ & $\begin{array}{l}4-3 \\
3-1\end{array}$ & $\begin{array}{c}2^{5} F^{0}-f^{3} D \\
(706)\end{array}$ \\
\hline $\begin{array}{c}3695.507 \\
3691.53 \\
.3740 .061 \\
3732.77\end{array}$ & $\begin{array}{l}\mathrm{V} \\
\mathrm{P}\end{array}$ & $\begin{array}{l}(1) \\
0 \\
(1) \\
0\end{array}$ & $\begin{array}{l}3.40 \\
3.42 \\
3.38 \\
3.40\end{array}$ & $\begin{array}{l}6.74 \\
6.76 \\
6.88 \\
6.72\end{array}$ & $\begin{array}{l}3-8 \\
1-1 \\
3-4 \\
3-3\end{array}$ & $\begin{array}{c}2^{5} F^{0}-8^{7} \mathrm{D} \\
(707)^{-1}\end{array}$ \\
\hline 3416.58 & $P$ & $\odot$ & 3.35 & 6.97 & $4-5$ & $2^{5}$ \\
\hline 3380.004 & v & (1) & 3.33 & 6.97 & 5 & \\
\hline 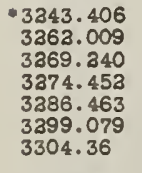 & $\begin{array}{l}\mathrm{V} \\
\mathrm{v} \\
\mathrm{v}\end{array}$ & $\left(\begin{array}{l}3 \\
3 \\
1 w \\
a w \\
2 w \\
1 w \\
0\end{array}\right)$ & $\begin{array}{l}3.32 \\
3.35 \\
3.38 \\
3.35 \\
3.38 \\
3.38 \\
3.40\end{array}$ & $\begin{array}{l}7.13 \\
7.14 \\
7.16 \\
7.12 \\
7.14 \\
7.12 \\
7.14\end{array}$ & $\begin{array}{l}5-4 \\
4-3 \\
3-2 \\
4-4 \\
3-3 \\
3-4 \\
3-3\end{array}$ & $\begin{array}{c}2^{5} F^{\circ}-1^{5} D \\
(710)\end{array}$ \\
\hline $\begin{array}{r}3311.693 \\
-3314.044 \\
-3311.872 \\
-3309.397 \\
3308.470 \\
-3192.417 \\
3197.53\end{array}$ & 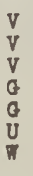 & $\left.\begin{array}{r}8 \\
30 \\
4 \\
6 \\
4 \\
1 \\
1 \\
1\end{array}\right)$ & $\begin{array}{l}3.32 \\
3.35 \\
3.38 \\
3.40 \\
3.43 \\
3.38 \\
3.40\end{array}$ & $\begin{array}{l}7.16 \\
7.19 \\
7.38 \\
7.25 \\
7.36 \\
7.35 \\
7.36\end{array}$ & $\begin{array}{l}5-6 \\
4-5 \\
3-4 \\
2-3 \\
1-3 \\
3-3 \\
3-3\end{array}$ & 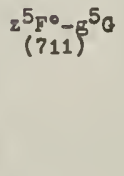 \\
\hline $\begin{array}{l}3361.333 \\
3372.71\end{array}$ & $\begin{array}{l}\mathrm{v} \\
\mathrm{u}\end{array}$ & $\begin{array}{l}\text { (a) } \\
\text { (1) }\end{array}$ & $\begin{array}{l}3.40 \\
3.42\end{array}$ & $\begin{array}{l}7.19 \\
7.19\end{array}$ & $\begin{array}{l}3-3 \\
1-3\end{array}$ & $\begin{array}{l}z^{5} F^{0}-4 \\
(712)\end{array}$ \\
\hline
\end{tabular}
$5551.39 \quad P \quad \odot \quad 3.40 \quad 5.63 \quad 1-1 \quad a^{1} P-x^{3} D^{\circ}$

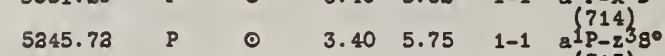

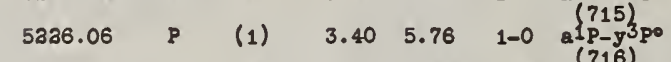
$\begin{array}{llllllll}5167.70 & P & \odot & 3.40 & 5.79 & 1-3 & \begin{array}{l}716) \\ 715 D^{\circ}\end{array} \\ 5091.73 & P & \odot & 3.40 & 5.82 & 1-1 & (717)\end{array}$ $5039.633 \quad V \quad$ (1) $3.40 \quad 5.85 \quad 1-3 \quad a^{1} P-10$ $\begin{array}{lllllll}4818.66 & P & \odot & 3.40 & 5.96 & 1-1 & \mathrm{a}_{\mathrm{P}-75 \mathrm{p}}\end{array}$ $\begin{array}{lllllll}4815.32 & P & 0 & 3.40 & 5.96 & 1-3 & \begin{array}{l}\text { a } \\ 477-x^{3 p 0}\end{array} \\ 4779.444 & J & (1) & 3.40 & 5.98 & 1-0 & (730)\end{array}$ $\begin{array}{lllllll}4804.59 \quad P & \text { (1) } \quad 3.40 & 5.97 & 1-1 & a^{1} p-v^{5} F_{0}\end{array}$ $4566.990 \quad V \quad$ (1) $\quad 3.40 \quad 6.10 \quad 1-3 \quad a_{P-1350}$

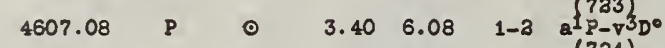

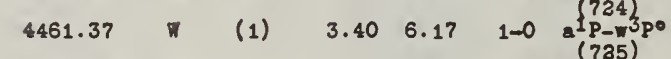

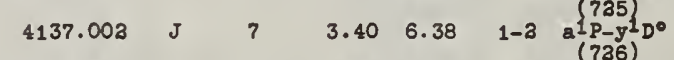
$\begin{array}{lllllll}4137.807 & J & 3 n & 3.40 & 6.39 & 1-3 & { }_{8}^{1} P_{P-x^{1}} D^{\circ}\end{array}$

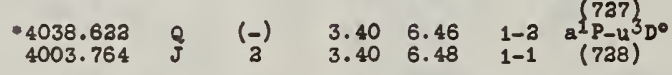
$3976.615 \quad \mathrm{~J} \quad 4 \quad 3.40 \quad 6.50 \quad 1-2 \quad \mathrm{a}^{1} \mathrm{P}-\mathrm{t}^{3} \mathrm{p}^{0}$ $\begin{array}{lllllll}3977.66 & \mathrm{P} & \odot & 3.40 & 6.44 & 1-19 & (739)\end{array}$ $3949.14 \quad(1) \quad 3.40 \quad 6.53 \quad 1-1 \quad a^{1} p-8^{\circ} j$ $\begin{array}{llclllll}3940.044 & V & (1) & 3.40 & 6.53 & 1-3 & a^{(730)} \\ 3806.303 & J & 3 & 3.40 & 6.64 & 1-1 & (731)\end{array}$ $\begin{array}{lllllll}3885.07 & P & \circ & 3.40 & 6.58 & 1-3 & 8^{1} P-8^{3} D^{\circ}\end{array}$

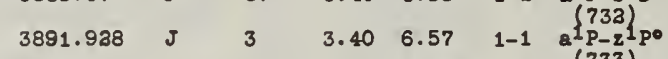

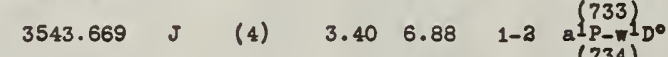
$3410.171 \quad 0 \quad 3 \quad 3.40 \quad 7.03 \quad 1-3 \quad a_{P-43 F}$

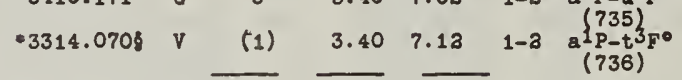
$\begin{array}{llllllll}9924.35 & P & P & \odot & 3.53 & 4.77 & 2-3 & a^{1} D-y^{3} D^{\circ} \\ 9620.93 & P & \odot & 3.53 & 4.81 & 3-1 & (737)\end{array}$ $6016.66 \quad$ (3) $3.53 \quad 5.58 \quad 2-3 \quad 2^{1} D-x^{3} D^{0}$

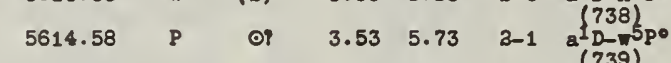
$\begin{array}{llllllll}5555.17 \quad P & \circ & 3.53 & 5.75 & 3-1 & a_{D-2} 38^{\circ}\end{array}$

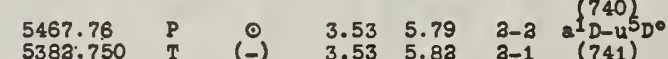
$5375.30 \quad P \quad \odot \quad 3.53 \quad 5.87 \quad 2-1 \quad a^{1} D_{-1}^{3} \mathrm{~g}^{\circ}$

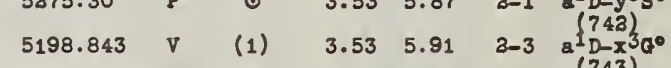

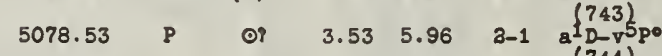

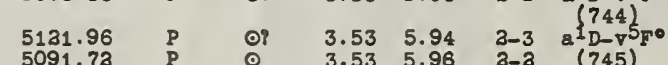
$\begin{array}{lllllll}-5031.030 \quad R \quad & \text { a } & 3.53 & 5.98 & 3-3 & a^{1} D_{-1}^{3} G^{\circ}\end{array}$

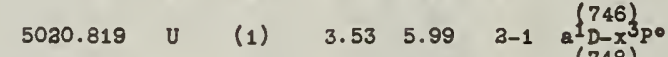

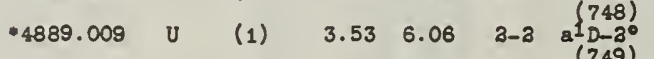

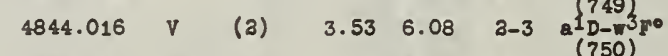
$\begin{array}{llllllll}4869.45 & P & \odot & 3.53 & 6.07 & 3-3 & a_{0-F^{3} D}^{750}\end{array}$

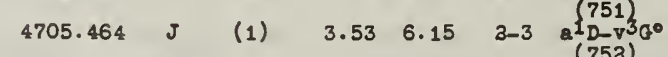

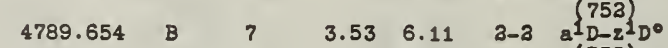
$\begin{array}{llllllll}4633.14 & P & \odot & 3.53 & 6.30 & 3-3 & 0 & 753 \\ 4663.183 & J & \text { (1) } & 3.53 & 6.18 & 3-1 & (754)\end{array}$ $\begin{array}{lllllll}4547.851 \quad B & 4 & 3.53 & 6.34 & 3-3 & a^{1} D-2^{1} F^{0}\end{array}$ $\begin{array}{llllllll}4343.86 & P & \odot & 3.53 & 6.37 & 3-3 & a^{1}-u_{50} \\ 4304.87 & P & \odot & 3.53 & 8.40 & 3-3 & (756)\end{array}$ $\begin{array}{lllllll}4392.31 & P & \odot & 3.53 & 6.34 & 3-3 & a^{1} D-v^{3} F^{0} \\ 4434.194 & V & (1) & 3.53 & 6.32 & 3-3 & (757)\end{array}$

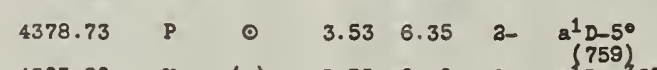

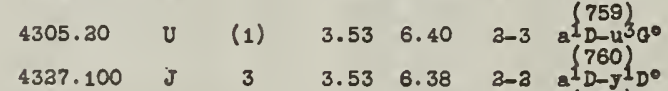

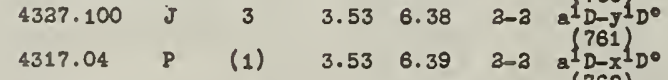
$4151.857 \quad V \quad$ (1) $3.53 \quad 6.50 \quad 3-3 \quad a^{1} D-t^{3} D^{0}$ $\begin{array}{lllllll}4340.373 & \mathrm{~J} & \left(\begin{array}{l}1 \\ a\end{array}\right) & 3.53 & 6.44 & 3-19 & (764)\end{array}$ $\begin{array}{lllllll}4133.00 & P & \odot & 3.53 & 6.53 & 2-1 & 8^{1} D-8^{\circ}\end{array}$ $\begin{array}{lllllll}8978.17 & \mathrm{P} & \odot & 3.40 & 4.77 & 1-3 & \mathrm{a}^{1} \mathrm{P}-\mathrm{y}^{3} \mathrm{D}^{\circ} \\ 8739.13 & \mathrm{P} & 3 & 3.40 & 4.81 & 1-1 & (713)\end{array}$ $\begin{array}{llllllll}4647.73 \quad P & \circ & 3.40 & 6.06 & 1-3 & a^{(731)} \\ P-3^{\circ}\end{array}$ $\begin{array}{lllllll}4319.59 \quad P & P & 3.53 & 6.46 & 3-3 & (763) & \left(70-D^{\circ}\right.\end{array}$

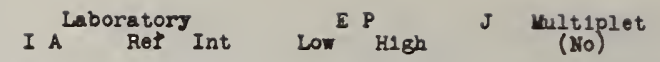
Fe. I continued

$\begin{array}{lllllll}4113.09 & P & \circ & 3.53 & 6.53 & 2-3 & a^{1} p-n^{3} p o \\ .3966 .533 & V & \text { (in) } & 3.53 & 6.64 & 2-1 & (78 B)\end{array}$ $\begin{array}{llllllll}4059.736 \quad v & 3 & 3.53 & 6.57 & 3-1 & 8^{1} D-z^{1} p_{0}\end{array}$

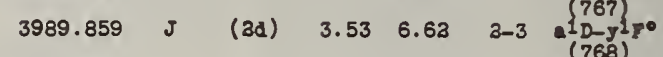

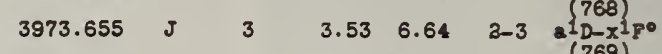

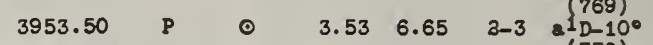

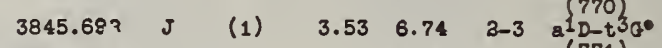
$3683.236 \quad J \quad 20 \quad 3.53 \quad 6.88$ a-3 $\quad$ a $\left\{\begin{array}{llll}771 & D^{\circ}\end{array}\right.$ $\begin{array}{llllllll}3677.309 & J & 3 & 3.53 & 6.89 & 2-3 & a_{0-15} & (773)\end{array}$ $\begin{array}{lllllll}3636.23 & \text { (1) } \quad 3.53 & 6.93 & 2-3 & \mathbf{a}_{D-8360}^{773}\end{array}$ 3538.31 (1) $3.53 \quad 7.03 \quad 3-3 \quad \begin{array}{llll}(774) \\ 075 *\end{array}$

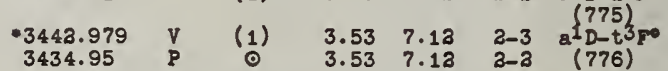

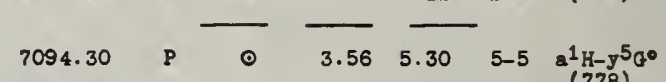

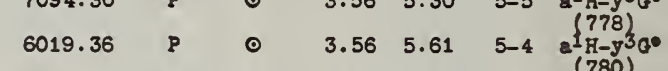

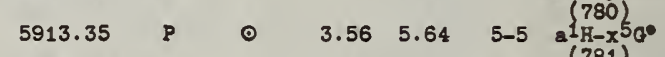

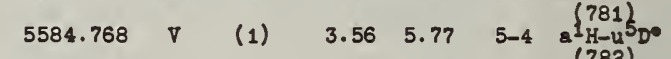

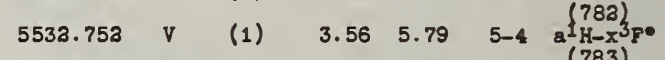

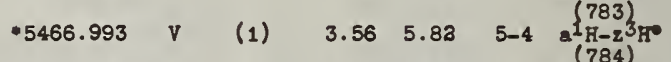

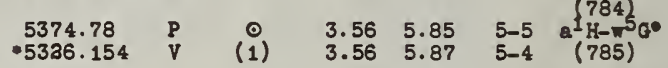
$\begin{array}{lllllll}5365.403 & \mathrm{~J} & 3 & 3.56 & 5.86 & 5-4 & \mathrm{a}^{1} \mathrm{H}_{-2} \mathrm{z}^{1} \mathrm{G}^{\circ}\end{array}$ $\begin{array}{lllllll}5331.41 & U & \text { (1) } 3.56 \quad 5.93 & 5-4 & (786) \\ \left(1 \mathrm{H}-755^{\circ}\right. & (787)\end{array}$

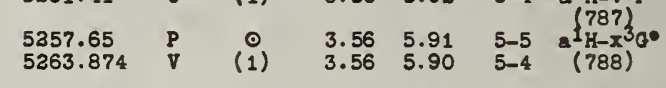
$\begin{array}{lllllll}5150.19 & P & \odot & 3.56 & 5.95 & 5-5 & a^{1} 1 /-n^{3} \\ 5115.788 & T & (1) & 3.56 & 5.97 & 5-4 & (789)\end{array}$

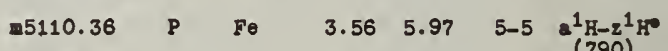
$5038.139 \quad \mathrm{~J} \quad 4 \quad 3.56 \quad 6.01 \quad 5-4 \quad \begin{array}{lllll}(790) \\ (791)^{\circ}\end{array}$

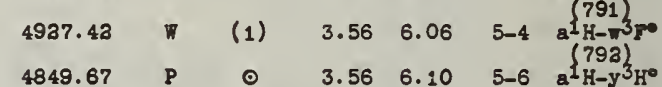
$\begin{array}{lllllll}4849.67 & P & \odot & 3.56 & 6.10 & 5-6 & a^{1} H^{3} \mathrm{H}^{3} \\ 4809.94 & V & (1) & 3.56 & 6.13 & 5-5 & (793)\end{array}$ $\begin{array}{lllllll}4843.39 & P & \odot & 3.56 & 6.11 & 5-5 & a^{1} \mathrm{~K}-\nabla^{3} G^{\circ} \\ 4804.539 & \mathrm{~V} & \text { (1) } & 3.56 & 6.13 & 5-4 & (794)\end{array}$ $\begin{array}{llllll}4587.133 & J & \text { (a) } 3.56 & 6.35 & 5-4 & \mathrm{a}^{1} \mathrm{H}-\mathrm{x}^{1} \mathrm{G}^{\circ}\end{array}$

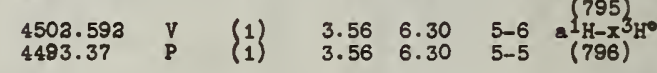

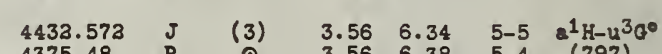
$\begin{array}{llcllll}4375.48 & P & \odot & 3.56 & 6.38 & 5-4 & (797) \\ 4435.663 & V & (1) & 3.56 & 6.35 & 5-4 & 11^{1}-4^{\circ}\end{array}$ $\begin{array}{lllllll}4435.663 & \vee & \text { (1) } & 3.56 & 6.35 & 5-4 & \mathrm{a}^{1} \mathrm{H}-4^{\circ} \\ 4382.777 & \mathrm{~V} & \text { (a) } & 3.56 & 6.37 & 5-5 & \mathrm{a}_{\mathrm{H}-6^{\circ}}\end{array}$

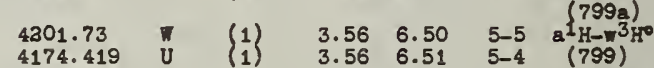
$\begin{array}{llllllll}4319.364 \quad B \quad & 12 & 3.56 & 6.48 & 5-6 & \mathrm{a}^{1} \mathrm{H}-\mathrm{y}^{3} \mathrm{I} & & \end{array}$ $\begin{array}{llllllll}4118.549 & B & 15 & 3.56 & 6.55 & 5-6 & \mathrm{a}_{\mathrm{H}-\mathrm{z}}^{(8.1} \text {. }\end{array}$

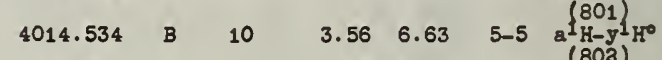

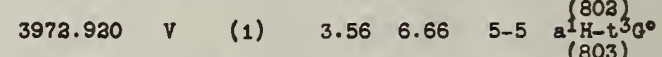

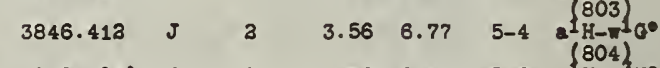

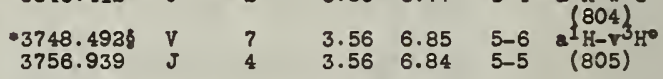

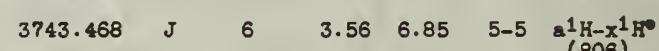

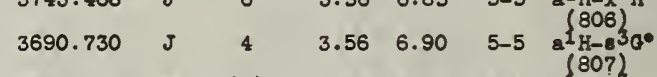

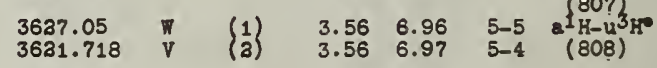

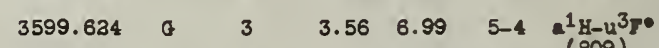
$3553.741 \quad 6 \quad 6 \quad 3.56 \quad 7.03 \quad 5-4 \quad \begin{array}{lllll}(809) \\ (810)\end{array}$

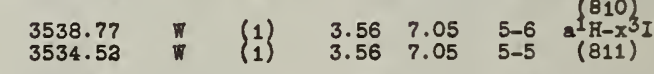
$\begin{array}{rllllll}-3479.683 & \mathrm{~V} & \text { (1) } \quad 3.56 \quad 7.10 & 5-4 & \mathrm{a}^{1} \mathrm{H}-\mathrm{t}^{3} & \mathrm{r0}\end{array}$

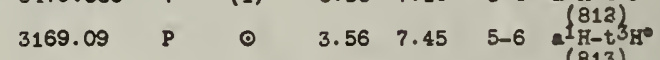

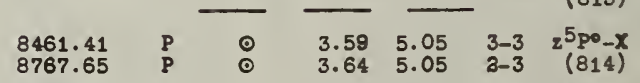
$\begin{array}{lllllll}\cdot 7086.76 & \mathrm{~V} & 3 & 3.59 & 5.33 & 3-4 & 2^{5} \mathrm{P} \\ 7 & -0^{7} \mathrm{D}\end{array}$ 7158.502
6953.01 6953.01
7057.96 $\begin{array}{lll}3.64 & 5.36 & 3-3 \\ 3.59 & 5.36 & 3-3 \\ 3.64 & 5.39 & 3-2 \\ 3.67 & 5.10 & 1-1\end{array}$ 
I A Laboratory Rer Int

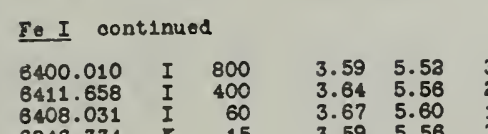

6346.334
6301.515

6336.835

6141.734
6333.661

5456.48

5461.80

5346.34

5384.23
5331.30

5388.34
5133.32

4516.37

4611.39
4638.6

4525.15
-4573.86

4488.140

4598.74

4596.059

1873.169

4584.733

4643.468

4678.853

4745.80

4768.33
-4854.638

4709.092

4619.394

4869.174
4704.958

4887.459

4738.555
4638.016

4673.38
4584.82

4560.096

4586.433
4510.82

4564.715
4480.27

487.38
4468.30

- 461.989

4516.45
4433.793

4433.793
4495.986

4414.03

4535.142

4611.285
4668.0 ?

4495.568

488.91
4581

4580.800
4542.730

4484.387

4482.75

4401.39

4437.30

447.854
4395.388

4438.353

4440.479
4533.403

4388.412

4423.858
4485.679

4485.678
4433.323

469.381
476.082

4107.75

4103.61

4147.49

414.37
4087.7

1087.79
4818.74

4127.7
4108.3

3001.03
3896.83

$\begin{array}{ll}3.64 & 5.56 \\ 3.67 & 5.60 \\ 3.59 & 5.56\end{array}$

$\begin{array}{ll}3.59 & 5.56 \\ 3.64 & 5.60\end{array}$

$\begin{array}{ll}3.59 & 5.60 \\ 3.64 & 5.63 \\ 3.67 & 5.63\end{array}$

(1) $3.59 \quad 5.85$

$\begin{array}{ll}3.64 & 5.90 \\ 3.67 & 5.93 \\ 3.59 & 5.90 \\ 3.64 & 5.93\end{array}$

$\begin{array}{lll}\circ & 3.59 & 5.93 \\ \circ & 3.59 & 5.99\end{array}$

3.67
$0 \quad 3.34$

$\begin{array}{lll}\text { (1) } & 3.59 & 6.31 \\ (3 n) & 3.54 & 6.34\end{array}$

- $3.64 \quad 6.38$

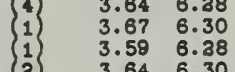

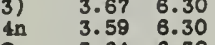

$\begin{array}{ll}3.59 & 6.30 \\ 3.64 & 6.30\end{array}$

$\begin{array}{lll}7 & 3.59 & 6.32\end{array}$

$\begin{array}{lll}3 n & 3.64 & 6.34 \\ \text { (1) } & 3.67 & 6.36\end{array}$

$\begin{array}{lll}\text { (1) } & 3.67 & 6.36 \\ 5 & 3.59 & 6.34 \\ \text { 3) } & 3.64 & 6.38\end{array}$

$\begin{array}{lll}3 n & 3.59 & 6.36 \\ (4) & 3.64 & 6.38 \\ 5 & 3.67 & 6.39\end{array}$

$\begin{array}{lll}3 \mathrm{n} & 3.64 & 6.35 \\ 3 & 3.59 & 6.35\end{array}$

$\begin{array}{lll}(\circ & 3.64 & 6.38 \\ \text { (3) } & 3.59 & 6.38\end{array}$

(a) $3.59 \quad 6.39$

$\begin{array}{ccc}0 & 3.58 & 6.33 \\ \text { (1) } & 3.64 & 6.34\end{array}$

$\begin{array}{ll}3.64 & 6.34 \\ 3.59 & 6.34\end{array}$

(3n) 3.596 .37

$\begin{array}{ll}3.64 & 6.38 \\ 3.59 & 6.38\end{array}$

$3.59 \quad 6.31$

- 3.676 .31

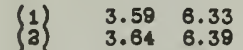

$\begin{array}{ll}3.64 & 6.39 \\ 3.67 & 6.48\end{array}$

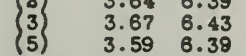

$\begin{array}{lll}\text { (a) } & 3.64 & 6.43 \\ \text { (a) } & 3.67 & 6.45\end{array}$

3
1
1
3
3 $\quad \begin{array}{ll}3.67 & 6.45 \\ 3.59 & 6.43 \\ 3.64 & 6.45 \\ 3.67 & 6.45\end{array}$

(a) $3.67 \quad 6.43$

$\begin{array}{ll}3.59 & 6.59 \\ 3.64 & 6.65\end{array}$

$\begin{array}{ll}3.64 & 6.61 \\ 3.64 & 6.68\end{array}$

RIVISED YULIIPLIT TABLE

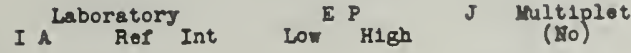

I A Reboratory Int

Lo: ${ }^{I} \mathrm{P}$ Heh

(yo)

\section{Fe I continued}

$3.64 \quad 5.60 \quad 3-3$

$\begin{array}{lll}3.67 & 5.63 & 1-1 \\ 3.59 & 5.60 & 3-2\end{array}$

$\begin{array}{lll}3.59 & 5.90 & 3-3 \\ 3.64 & 5.93 & 2-3\end{array}$

$3.64 \quad 5.95 \quad 3-1$

$\begin{array}{ccccc}\circ & 3.59 & 6.33 & 3-4 & 2^{5} P^{\bullet}-0^{7} \mathrm{~F} \\ r & 3.64 & 6.31 & 2-3 & (819)\end{array}$

(3n) $3.59 \quad 6.37$

$\begin{array}{lll}3 & 3.64 & 6.30 \\ 3) & 3.67 & 6.30\end{array}$

$\begin{array}{lll}3 n & 3.64 & 6.36 \\ 3 n & 3.67 & 6.38\end{array}$

$\begin{array}{lllll}6 & 3.59 & 6.33 & 3-4 & z^{5}{ }^{50}-0^{7 p} p \\ 3 n & 3.64 & 6.35 & 2-3 & (833)\end{array}$

$\begin{array}{lll}-4 & 2^{5} p^{0}-0^{5} D & 3490.47 \\ -3 & (816) & 3526.96 \\ -3 & & 3476.336 \\ -3 & & 3507.14 \\ -2 & & 3457.090 \\ -1 & & 3438.746 \\ -2 & & 3477.98 \\ -1 & & 3510.18\end{array}$

$\begin{array}{lllll}\circ & 3.59 & 7.13 & 3-4 & 2^{5}-1^{5} D \\ (3 w) & 3.64 & 7.14 & 3-3 & (835) \\ (3.59 & 3.14 & 3-3 & \end{array}$

$\begin{array}{cccc}0 & 3.64 & 7.16 & 3-3 \\ \text { (3i) } & 3.59 & 7.16 & 3-3\end{array}$

$z^{5} p^{\circ}-e^{5} \mathrm{~F}$
$(817)$

\begin{tabular}{ccccc}
$(3)$ & 3.59 & 7.19 & $3-3$ & $2^{5} p^{\circ}-4$ \\
$\circ$ & 3.64 & 7.19 & $2-3$ & $(836)$ \\
$\odot$ & 3.67 & 7.19 & $1-3$ & \\
\hline
\end{tabular}

$5981.38 \quad P \quad 0 \quad \overline{3.63} 5.68 \quad 6-6 \quad a^{1} I-z^{3} I^{\circ}$

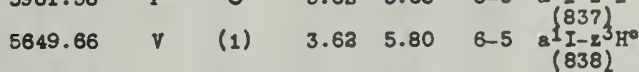

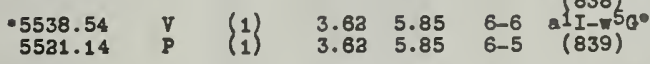

$5465.04 \quad P \quad(1) \quad 3.63 \quad 5.88 \quad 6-5 \quad \mathrm{a}^{1} I-\nabla^{5}$

$5397.60 \quad(1) \quad 3.63 \quad 5.91 \quad 6-5 \quad\left(\frac{1}{I-x^{3} 0^{\circ}}\right.$

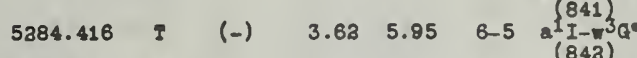

$5343.495 \quad B \quad 4 \quad 3.635 .87$ Q 5 a ( 1842$)_{H^{\circ}}$

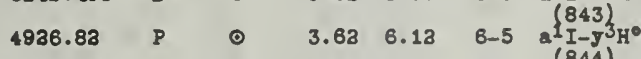

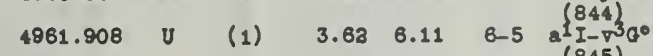

$\begin{array}{lllllll}4604.85 & P & 0 & 3.63 & 6.30 & 6-6 & a^{(845)} I-x^{3} \\ 4 & P & 0 & 3.63 & 6.30 & 6-5 & (846)\end{array}$

$.4531 .633 \mathrm{~J} \quad$ (3) $3.63 \quad 6.34 \quad 6-5 \quad \mathrm{a}^{1} I-u^{3} \mathrm{G}^{\circ}$

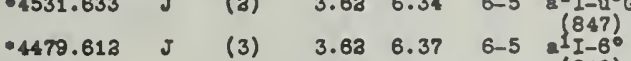

$\begin{array}{llcllll}4309.036 & J & (3) & 3.63 & 6.48 & 6-6 & (848) \\ 4338.61 & \mathrm{P} & 0 & 3.63 & 6.53 & 6-5 & (849)\end{array}$

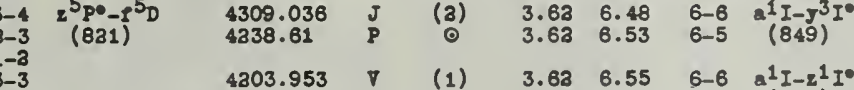

4303.953

. 4053.313

.4047 .315

3813,891

3759.155

3597.34

$\begin{array}{ll}3-4 & =5 \\ 3-3 & (833) \\ 3-3 & (830\end{array}$

$3-3$
$2-2$
$3-2$

11355.97
10735.19 10338.33

7333.38
7368.46

6749.58
6603.67$$
\text { (1) }
$$

3.636.

6-5 $\left(\begin{array}{l}850 \\ I-J^{1}\end{array}\right.$

(1) $\quad 3.68 \quad 6.68 \quad 6-5 \quad \begin{array}{lll}(851) \\ I-t 30^{\circ}\end{array}$

(1) $3.63 \quad 6.67 \quad 6-5 \quad a^{(853)}$

$\odot \quad 3.59 \quad 6.35 \quad 3-3 \quad(834)$

$\begin{array}{llllll}\text { (4) } & 3.59 & 6.35 & 3-4 & 2^{5} p^{5} p^{5} \\ 0 & 3.64 & 6.37 & a-3 & (835)\end{array}$

$3-3$
$3-3$

$3-3$
$3-2$
$3-1$

6474.6

$\begin{array}{ll}-3 & (836) \\ -3 & 2^{2}+e^{5} 8\end{array}$

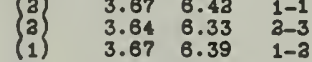

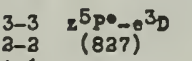

$\begin{array}{lllll}4 & 3.59 & 6.34 & 3-4 & z^{5}-z^{5} \mathrm{D} \\ \text { (3) } & 3.64 & 6.39 & 3-3 & (838)^{5}\end{array}$

$\begin{array}{ll}3-3 & (838) \\ 1-3 & \\ 3-3 & \\ 3-3 & \end{array}$

6603.80

6307.85

$\begin{array}{lllllll}6307.85 & P & \circ & 3.63 & 5.58 & 3-3 & b^{3} D-x^{3} D \\ 6301.86 & P & \odot & 3.63 & 5.58 & a-3 & (863) \\ 6043.738 & 0 & (1) & 3.63 & 5.67 & 3-3 & b^{3} D-x^{5}\end{array}$

5763.434

5754.41

5707.35

5609.97
-5600.348

5760.351

5761.37

5707.068

(1) $\begin{array}{llll}3.59 & 6.37 & 3-3 & 2^{5} \mathrm{p}^{\circ}-0^{7} \mathrm{~g}\end{array}$

$\begin{array}{lllll}\text { an } & 3.59 & 6.40 & 3-3 & 2^{5} p_{0.07}^{5} p \\ \text { (31) } & 3.64 & 6.43 & 2-3 & (830)\end{array}$

$\begin{array}{lll}3 n & 3.64 & 6.43 \\ 5 n & 3.64 & 6.40\end{array}$

$\begin{array}{llll}\text { (4) } & 3.64 & 6.40 & 2-3 \\ & 3.67 & 6.43 & 1-2\end{array}$

$\begin{array}{ll}3-4 & 2^{5} p \bullet-\beta^{5} \\ 3-3 & (831)\end{array}$

3.596 .65

$\begin{array}{llll}3.59 & 6.58 & 3-3 & z^{5} p^{6-p^{5}} p \\ 3.64 & 6.61 & 2-3 & (83 a)\end{array}$

$2-1$
$2-3$

$\begin{array}{llll}3.64 & 6.64 & 2-3 & z^{5} p-f^{3} D \\ 3.67 & 6.67 & 1-3 & (833)\end{array}$

$\begin{array}{lllll}0 & 3.59 & 6.75 & 3-4 & 250-p^{3}- \\ 0 & 3.64 & 6.81 & 2-3 & (834)\end{array}$ seas

$\begin{array}{llcllll}5568.81 & 0 & (1) & 3.63 & 5.84 & 1-1 & b^{3} D-\pi^{3} D \\ 5660.79 & 7 & (1) & 3.62 & 5.80 & 2-3 & (869) \\ 5611.35 & 8 & 0 & 3.63 & 5.82 & 1-2 & \end{array}$

$\begin{array}{llllllll}5487.49 \quad P & P & 3.63 & 5.87 & 3-4 & b^{3} D-T_{0}^{5}\end{array}$

$\begin{array}{lllllll}5458.119 & U & (1) & 3.63 & 5.89 & 2-3 & (870) \\ 5411.39 & P & 0 & 3.63 & 5.90 & 2-3 & \end{array}$

$5529.15 \quad$ (3) $3.63 \quad 5.86 \quad 3-4 \quad b^{3} D-2^{1} a^{\circ}$

$\begin{array}{lllllll}5493.33 & P & 0 & 3.63 & 5.87 & 2-1 & b^{3} D-J^{3} \\ 5483.26 & P & 0 & 3.62 & 5.87 & 1-1 & (873)\end{array}$

$5386.958 \quad J \quad$ (1) $\quad 3.63 \quad 5.98 \quad 3-4 \quad b^{3} D-r^{5}$

$\begin{array}{lllllll}5337.35 & P & 0 & 3.63 & 5.94 & 2-3 & (875) \\ 5384.37 & P & 0 & 3.62 & 5.96 & 1-3 & \end{array}$

$\begin{array}{llllll}5294.555 & \text { T } & (-) & 3.62 & 5.96 & 1-2 \\ 5353.35 & 8 & 0 & 3.62 & 5.96 & 2-2\end{array}$

$\begin{array}{llllll}5353.35 & p & 0 & 3.68 & 5.97 & 1-1 \\ 5398.789 & \vee & (1) & 3.63 & 5.98 & 3-2\end{array}$

$\begin{array}{lllllll}5315.78 & P & \odot & 3.68 & 5.95 & a-a & b^{3} p-r^{5} p\end{array}$

$\begin{array}{lllllll}5370.08 & P & 0 & 3.68 & 5.96 & 1-1 & (877)\end{array}$ $\begin{array}{llllll}5539.28 & \\ .5534 .68 & (1) & 3.63 & 5.85 & 3-3 & b^{3} D-1 \\ 1 & 3.63 & 5.85 & 3-3 & (871)\end{array}$

$\begin{array}{lllllll}5431.40 & P & \circ & 3.63 & 5.90 & 3-4 & b^{3} D-x^{3} Q^{\circ}\end{array}$
Po I oontinued

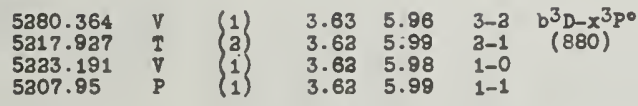

$\begin{array}{lllllll}5066.38 & P & 0 & 3.63 & 6.06 & 1-3 & b^{3} D-3\end{array}$

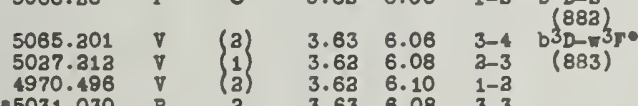

$\begin{array}{cccccc}-5031.030 & R & 3 & 3.63 & 6.08 & 3-3 \\ 4979.58 & \text { (1) } & 3.68 & 6.10 & 3-2\end{array}$

5058.50 (1) $3.63 \quad 6.07 \quad 3-3 \quad b^{3} D-r^{3} D^{\circ}$

$\begin{array}{llcllll}5054.647 & \mathrm{~T} & 1 & 3.63 & 6.07 & 2-3 & (884) \\ 5018.03 & \mathrm{P} & 0 & 3.63 & 6.08 & 1-2 & \end{array}$

$\begin{array}{lllllll}5035.085 & R & 3 & 3.63 & 6.08 & 3-3 & b^{3} \mathrm{D}-3^{\circ}\end{array}$

$\begin{array}{lllllll}4935.43 \quad P & \circ & 3.63 & 6.13 & 3-4 & b^{3} D-\nabla^{3} & 885\end{array}$

$4868.709 \quad V \quad$ (1) $3.63 \quad 6.11 \quad 3-3 \quad b^{3} D_{-2} I_{D}$

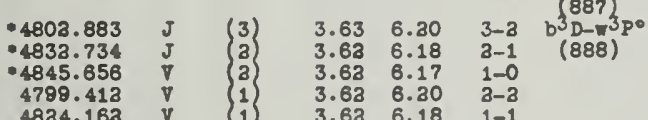

4824.163 v l $13.63 \quad 6.18 \quad 1-1$

$4708.873 \quad \vee \quad(1) \quad 3.63 \quad 6.34 \quad 3-3 \quad b^{3} D-z^{1} F^{0}$

$4706.31 \quad P \quad \circ \quad 3.63 \quad 6.35 \quad 3-4 b^{3} D-x^{100}$

$\begin{array}{lllllll}4490.63 & P & \circ & 3.63 & 6.37 & 3-3 & b^{3} D-u^{5}, 0\end{array}$

$\begin{array}{llllllll}4605.99 & P & \circ & 3.63 & 6.31 & 3-4 & b^{3} D-t^{5} D^{\circ}\end{array}$

$\begin{array}{llllll}4543.32 & P & \odot & 3.63 & 6.34 & 2-3 \\ 4481.04 & \mathrm{P} & \odot & 3.63 & 6.37 & 1-2\end{array}$

$\begin{array}{lllllll}-4558.108 & \mathrm{~J} & \text { (1) } 3.63 & 6.33 & 3-4 & \mathrm{~b}^{3} \mathrm{D}-\mathrm{r}^{3} \mathrm{~F}\end{array}$

$\begin{array}{lllllll}4543.432 & \mathrm{v}\end{array} \quad\left\{\begin{array}{l}1 \\ \mathrm{a} \\ 1\end{array} \quad \begin{array}{llll}3.63 & 6.33 & 3-4 & \mathrm{~b}^{3} \mathrm{D}-\mathrm{r}^{3} \mathrm{r} \\ 3.63 & 6.34 & 3-3 & (894)\end{array}\right.$

$\begin{array}{llllll}4545.54 & P & \odot & 3.63 & 6.34 & 3-3 \\ 4579.68 & P & \odot & 3.63 & 6.33 & 3-3\end{array}$

$\begin{array}{llllll}4536.509 & U & (1) \quad 3.63 & 6.35 & 3-4 & b^{3} D-4^{\circ}\end{array}$

4587.90

(1) $\quad 3.63 \quad 6.35 \quad 3-\quad b^{3} D-5$ ( 696$)$

$\begin{array}{lllllll}4483.78 & P & \circ & 3.63 & 6.38 & 3-4 & b^{3}{ }^{3}-u^{3} a^{\circ} \\ 4453.33 & P & \circ & 3.63 & 6.40 & 3-3 & (898)\end{array}$

$\begin{array}{lllllll}4479.01 \quad P & \circ & 3.63 & 6.38 & 3-3 & b^{3} D-u^{5}\end{array}$

4435.79
4386.6

4438.74

4393.70

$\mathbf{4} 475.99$
$\mathbf{4 4 1 8 . 6 0}$

$\begin{array}{llll}\circ & 3.62 & 6.41 & 3-2 \\ \text { (1.i) } & 3.62 & 6.43 & 1-1 \\ 0 & 3.63 & 6.41 & 3-2\end{array}$

$\begin{array}{llll}\circ & 3.63 & 6.41 & 3-2 \\ \circ & 3.63 & 6.43 & 3-1\end{array}$

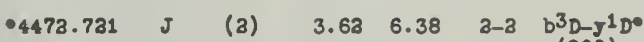

$\begin{array}{llcllll}4466.183 & \nabla & (1) & 3.63 & 6.39 & 3-3 & b^{3} D-70 \\ 4463.16 & P & 0 & 3.62 & 6.39 & 2-3 & (901)\end{array}$

$\begin{array}{lllllll}4461.989 & \mathrm{~J} & (4) & 3.68 & 6.39 & 2-3 & b^{3} D-x^{1} D\end{array}$

$\begin{array}{lllllll}4454.655 & J & (1) & 3.68 & 6.39 & 1-2 & (903)\end{array}$

$\begin{array}{lllllll}4360.813 \quad \vee \quad(1) & 3.63 & 6.46 \quad 3-3 & b^{3} D-u^{3} D^{\circ}\end{array}$

$\begin{array}{lllllll}4376.783 & v & \text { (1) } & 3.63 & 6.45 & 3-3 & b^{3} D-t 3 D^{\circ}\end{array}$

$\begin{array}{lllllll}4385.833 & \mathrm{~V} & (1) & 3.63 & 6.50 & \mathrm{a}-\mathrm{z} & (904) \\ 4373.90 & \mathrm{P} & 0 & 3.63 & 6.45 & 3-3 & \end{array}$

$\begin{array}{lllllll}4353.93 & P & 0 & 3.62 & 6.53 & 2-1 & b^{3} D-8\end{array}$

$\begin{array}{lllllll}4347.29 & P & \circ & 3.63 & 6.53 & 1-1 & (905)\end{array}$

4346.090

4088.567

4843.368

4083.44
4336.78

$\begin{array}{lllll}3 & 3.63 & 6.53 & 3-3 & b^{3} p-r^{3} p \\ 1 & 3.63 & 6.64 & 3-1 & (808)\end{array}$

$\left\{\begin{array}{lll}3 \\ 3\end{array} \quad \begin{array}{lll}3.62 & 6.53 & 3-3 \\ 3.62 & 6.64 & 1-1\end{array}\right.$

$\begin{array}{lll}3.63 & 6.64 & 1-1 \\ 3.62 & 6.53 & 1-2\end{array}$

4339.36

$\begin{array}{lllll}\circ & 3.63 & 6.54 \quad 3-3 \quad b^{3} D-a^{3} D\end{array}$

$\begin{array}{lllll}-3.63 & 6.54 & 3-3 & (907)\end{array}$

$\begin{array}{llllll}4181.30 \quad P & \circ & 3.63 & 6.57 & 1-1 & b^{3} D-z^{1} p e\end{array}$

$\left.4173.97 \quad P \quad 0 \quad 3.63 \quad 6.58 \quad 3-4 \quad b^{3} D_{-9}\right)$

$4115.69 \quad P \quad \odot \quad 3.63 \quad 6.68 \quad 3-3 \quad b^{3} D_{-J} 150$

$4096.118 \nabla(1) \quad 3.63 \quad 6.64 \quad 2-3 \quad b^{3} \mathrm{D}-x^{1} \mathrm{~F}$

$4074.70 \quad P \quad 0 \quad 3.63 \quad 6.65 \quad 2-3 \quad b^{3} D-10$.

$\begin{array}{llcllll}4030.490 & \mathrm{v} & (1) & 3.63 & 6.70 & 3-4 & b^{3} \mathrm{D}-\mathrm{t}^{3} \mathrm{a}^{\circ} \\ 3980.284 & \mathrm{~J} & (1) & 3.62 & 6.74 & 3-3 & (913) \\ 3962.85 & P & 0 & 3.63 & 6.74 & 3-3 & \end{array}$

$\begin{array}{lllllll}4058.46 & P & 0 & 3.63 & 6.67 & 3-3 & b^{3} \mathrm{D}-11 \\ 4055.98 & \mathbb{1} & (1) & 3.68 & 6.67 & 2-3 & (914)\end{array}$

4010.18 (1) $3.63 \quad 6.70 \quad 3-4 \quad b^{3} D-13$

$3787.164 \quad J \quad$ (1) $3.63 \quad 6.88 \quad a-3 \quad b^{3} D-11$

$\left.3781.938 \mathrm{~J} \quad(1) \quad 3.63 \quad 6.89 \quad 3-3 \quad b^{3}(916)\right)^{3}$

$\begin{array}{lllllll}3787.73 & P & \odot & 3.63 & 6.90 & 3-4 & b^{3} D-3_{0} \\ 3738.51 & P & 0 & 3.63 & 6.93 & 2-3 & (918)\end{array}$ 
60

I A Laboratory Ref Int Low High J Multiplet
Se I continued

3635.08 continued

$\begin{array}{lllllll}3635.08 & P & \odot ? & 3.63 & 7.03 & 2-3 & b^{3} \mathrm{D}-\mathrm{u}^{3} \mathrm{~F}^{\circ} \\ 3345.80 & \mathrm{P} & \odot & 3.63 & 7.43 & 3-4 & \mathrm{~b}^{3} \mathrm{P}-\mathrm{r}^{3} \mathrm{G}^{\circ}\end{array}$ $\begin{array}{lllllll}3224.05 & P & \odot & 3.62 & 7.45 & 2-3 & (930) \\ 3325.607 & U & (1) & 3.63 & 7.45 & 3-3 & \end{array}$

\begin{tabular}{|c|c|c|c|c|c|c|}
\hline $\begin{array}{l}6451.58 \\
6396.39\end{array}$ & $\begin{array}{l}P^{2} \\
\end{array}$ & $\stackrel{(2)}{(2)}$ & $\begin{array}{l}3.68 \\
3.68\end{array}$ & $\begin{array}{l}5.59 \\
5.61\end{array}$ & $\begin{array}{l}4-5 \\
4-4\end{array}$ & $\begin{array}{l}b^{1} G-y^{3} G^{0} \\
(931)\end{array}$ \\
\hline $\begin{array}{r}5849.67 \\
.5780 .83\end{array}$ & $\mathrm{v}$ & (i) & $\begin{array}{l}3.68 \\
3.68\end{array}$ & $\begin{array}{l}5.79 \\
5.81\end{array}$ & $\begin{array}{l}4-4 \\
4-3\end{array}$ & $\begin{array}{l}b^{1} G-x^{3} F^{\circ} \\
(92 a) a^{2}\end{array}$ \\
\hline 5619.23 & $P$ & ๑ & 3.68 & 5.87 & $4-4$ & $b^{1} a-m^{5} a^{\circ}$ \\
\hline 5662.94 & v & (1) & 3.68 & 5.86 & $4-4$ & \\
\hline 5513.86 & $P$ & $\odot$ & 3.68 & 5.93 & $4-4$ & \\
\hline $\begin{array}{l}5543.04 \\
559.94 \\
5543.184\end{array}$ & & $\left(\begin{array}{c}0 \\
\vdots \\
3 \\
3\end{array}\right)$ & $\begin{array}{l}3.68 \\
3.68 \\
3.68\end{array}$ & $\begin{array}{l}5.91 \\
5.90 \\
5.91\end{array}$ & $\begin{array}{l}4-5 \\
4-4 \\
4-3\end{array}$ & $\begin{array}{c}b 1 G-x^{3} G^{\circ} \\
(926))^{\circ}\end{array}$ \\
\hline
\end{tabular}

$\begin{array}{llllllll}5423.73 & \mathrm{P} & \odot & 3.68 & 5.95 & 4-5 & \mathrm{~b}^{1} \mathrm{G}-\mathrm{m}^{3} \mathrm{G}^{\circ} \\ 5385.58 & \mathrm{P} & \odot & 3.68 & 5.97 & 4-4 & (937)\end{array}$

$\begin{array}{llllll}5379.580 & \mathrm{~J} & \text { (2) } 3.68 & 5.97 & 4-5 & \mathrm{~b}^{1} \mathrm{G-2} 2^{1} \mathrm{H}^{\circ} \\ (928)\end{array}$

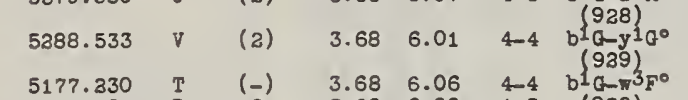

$\begin{array}{llcllll}5177.230 & \mathrm{~T} & (-) & 3.68 & 6.06 & 4-4 & \mathrm{~b} 1\left(\mathrm{G}-\mathrm{m}^{3} \mathrm{~F}^{\circ}\right. \\ 5141.55 & \mathrm{P} & \odot & 3.68 & 6.08 & 4-3 & (930)\end{array}$

$\begin{array}{lllllll}5145.73 & P & \odot & 3.68 & 6.08 & 4-3 & b^{1} G-3^{\circ} \\ 5084.55 & P & \odot & 3.68 & 6.11 & 4-5 & b^{1}(931)^{3} G^{\circ}\end{array}$

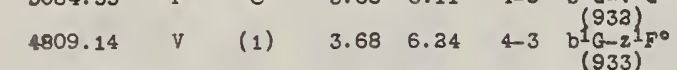

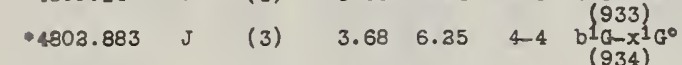

$\begin{array}{ccccccc}4700.171 & J & (2 n) & 3.68 & 6.30 & 4-5 & b(934) \\ .4579 .344 & \text { V } & (1) & 3.68 & 6.37 & 4-5 & (935)\end{array}$

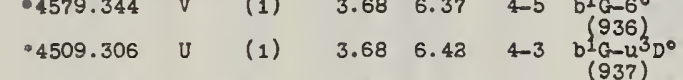

$\begin{array}{lllllll}4382.02 & P & \odot & 3.68 & 6.50 & 4-5 & b(G-7) \\ 4248.72 & P & \odot & 3.68 & 6.58 & 4-4 & (938) \\ b\left(G-9^{\circ}\right.\end{array}$

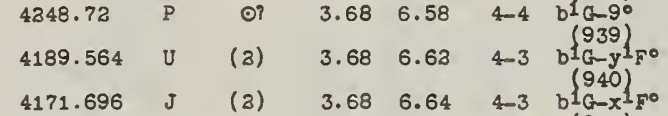

$\begin{array}{lllllll}4149.49 & P & \odot & 3.68 & 6.65 & 4-3 & b 1\left(a 10^{\circ}\right.\end{array}$

$\begin{array}{lllllll}4090.75 & P & \odot & 3.68 & 6.70 & 4-4 & (943) \\ 4030.90 & P & \odot & 3.68 & 6.74 & 4-3 & (943)\end{array}$

$\begin{array}{llllllll}4080.08 & P & \odot & 3.68 & 6.70 & 4-4 & b^{1}\left(a-13^{\circ}\right.\end{array}$

$\begin{array}{llllllll}3996.968 & \mathrm{~J} & 2 & 3.68 & 6.77 & 4-4 & \mathrm{~b}(944) \\ \left(9-\pi_{1} G^{\circ}\right.\end{array}$

$\begin{array}{lllllll}3885.93 & P & \odot & 3.68 & 6.85 & 4-5 & \begin{array}{l}(945) \\ b(9-x) \\ (946)\end{array}\end{array}$

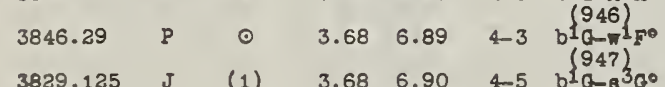

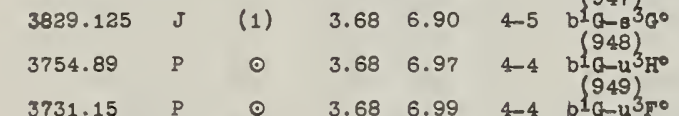

$\begin{array}{llllllll}3731.15 & \mathrm{P} & \odot & 3.68 & 6.99 & 4-4 & 01 \mathrm{G}-\mathrm{u}^{3} \mathrm{~F}^{\circ} \\ 3704.80 & \mathrm{P} & \odot & 3.68 & 7.01 & 4-3 & (950)\end{array}$

$\begin{array}{lllllll}3681.87 & \text { (1) } 3.68 & 7.03 & 4-4 & b^{1} G-\nabla^{1} G^{\circ}\end{array}$

$\begin{array}{llllllll}3661.25 & P & \odot & 3.68 & 7.05 & 4-5 & b(951) & \left(9-x^{3} I^{\circ}\right.\end{array}$

$\begin{array}{lllllll}3590.66 \quad T & (1) & 3.68 & 7.13 & 4-3 & b\left(G-t 3 F^{\circ}\right. \\ 3391.44 & P & 0 & 3.68 & 7.43 & 4-4 & (953)\end{array}$

\begin{tabular}{llllllll}
3391.44 & $\mathrm{P}$ & $\odot$ & 3.68 & 7.43 & $4-4$ & $b_{10-r_{3} 0^{\circ}}$ \\
3370.69 & $\mathrm{P}$ & $\odot$ & 3.68 & 7.45 & $4-3$ & $(954)$ \\
\hline
\end{tabular}

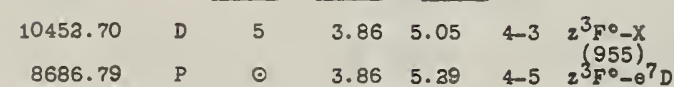

$\begin{array}{lllllll}8686.79 & P & \circ & 3.86 & 5.29 & 4-5 & 2^{3} F^{\circ}-\theta^{7} \mathrm{D} \\ 8801.78 & \mathrm{P} & & 3.93 & 5.33 & 3-4 & (956)\end{array}$

$\begin{array}{lcccccc}7477.53 & \mathrm{P} & \odot & 3.86 & 5.53 & 4-4 & 2^{3} F^{\circ}-e^{5} \mathrm{D} \\ 7541.61 & \mathrm{U} & (1) & 3.93 & 5.56 & 3-3 & (957) \\ 753.76 & \mathrm{P} & \odot & 3.97 & 5.60 & 3-2 & \end{array}$

7368.58

7474.50
7766.7

6330.7
6419.65

$\begin{array}{lllllll}6003.033 & K & 8 & 3.88 & 5.93 & 4-4 & 2^{3} F^{\circ}-0^{3} \\ 5976.799 & K & 5 & 3.93 & 5.99 & 3-3 & (959)\end{array}$

58504.06

5838.418
6188.037

(1) $\begin{array}{lll}3.97 & 6.04 \\ 1 & 3.86 & 5.99 \\ 1 & 3.93 & 0.04\end{array}$

(1) 3.93 6. 6.04

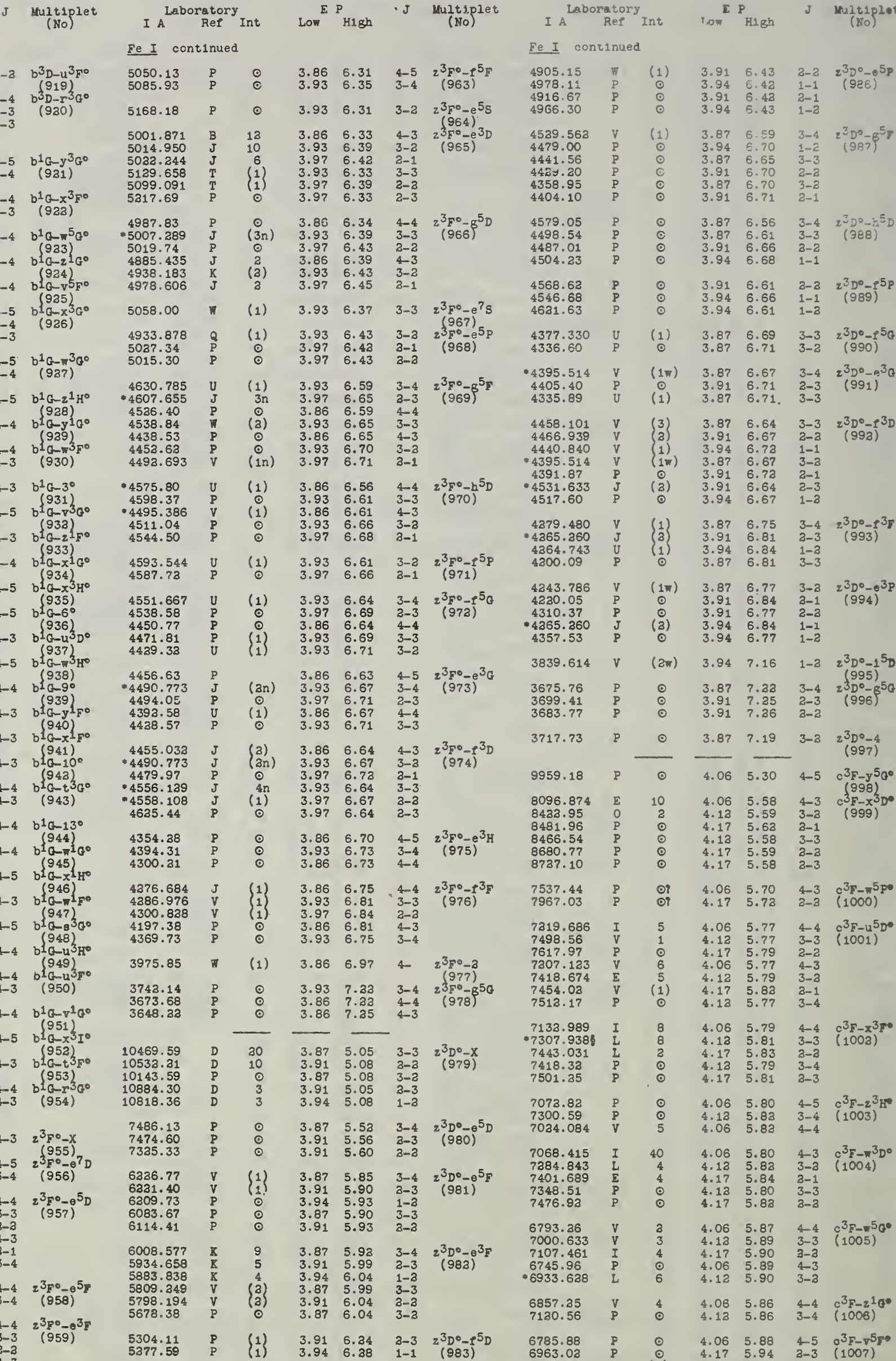

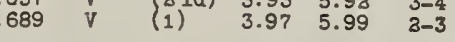

$\begin{array}{lllllll}5119.90 & P & \odot & 3.86 & 6.38 & 4-5 & z^{3} F^{\circ}-e^{7} F \\ 5027.51 & P & \odot & 3.86 & 6.33 & 4-4 & (960)\end{array}$

$\begin{array}{lllllll}5027.51 & \mathrm{P} & \odot & 3.86 & 6.33 & 4-4 & (960) \\ 5168.19 & \mathrm{P} & \odot & 3.93 & 6.31 & 3-3 & \end{array}$

$\begin{array}{lllllll}5126.598 & U & (1) & 3.86 & 6.27 & 4-4 & z^{3} F^{0}-P^{7} D \\ 5385.60 & P & (1) & 3.97 & 6.30 & 2-1 & (961)\end{array}$

$\begin{array}{lllllll}5813.80 \quad P & \odot & 3.93 & 6.29 & 3-4 & z^{3} F^{\circ}-0_{0}\end{array}$

$\begin{array}{lllllll}5213.80 & \mathrm{P} & \odot & 3.93 & 6.29 & 3-4 & \mathrm{z}^{3} F^{0}-0^{50} \\ 5238.25 & \mathrm{P} & \odot & 3.97 & 6.33 & 3-3 & (963) \\ 5081.86 & \mathrm{P} & \odot & 3.86 & 6.39 & 4-4 & \end{array}$

\section{REVISED NULTIPLET TABLE}

$$
\frac{4}{4300.8}
$$

\begin{tabular}{|c|c|c|c|c|c|c|}
\hline $\begin{array}{l}4376.684 \\
4286.976 \\
4300.838 \\
4197.38 \\
4369.73\end{array}$ & $\begin{array}{l}V \\
v \\
P \\
P\end{array}$ & $\begin{array}{c}1 \\
1 \\
1 \\
\circ \\
\bullet\end{array}$ & $\begin{array}{l}3.86 \\
3.93 \\
3.97 \\
3.86 \\
3.93\end{array}$ & $\begin{array}{l}6.75 \\
6.81 \\
6.84 \\
6.81 \\
6.75\end{array}$ & $\begin{array}{l}4-4 \\
3-3 \\
2-2 \\
4-3 \\
3-4\end{array}$ & $\begin{array}{c}2^{3} 5^{0}-f^{3} \\
(976)\end{array}$ \\
\hline 3975.85 & $\pi$ & (1) & 3.86 & 6.97 & 4 & $2^{3} F^{\circ}-3$ \\
\hline $\begin{array}{l}3742.14 \\
3673.68 \\
3648.32\end{array}$ & $\begin{array}{l}P \\
P \\
P\end{array}$ & $\stackrel{\circ}{\circ}$ & $\begin{array}{l}3.93 \\
3.86 \\
3.86\end{array}$ & $\begin{array}{l}7.33 \\
7.33 \\
7.35\end{array}$ & $\begin{array}{l}3-4 \\
4-4 \\
4-3\end{array}$ & $\begin{array}{c}23 F^{\circ}-8^{50} \\
(978)^{56}\end{array}$ \\
\hline $\begin{array}{l}10469.59 \\
10533.31 \\
10143.59 \\
10884.30 \\
10818.36\end{array}$ & $\begin{array}{l}D \\
D \\
P \\
D \\
D\end{array}$ & $\begin{array}{r}30 \\
10 \\
0 \\
3 \\
3\end{array}$ & $\begin{array}{l}3.87 \\
3.91 \\
3.87 \\
3.91 \\
3.94\end{array}$ & $\begin{array}{l}5.05 \\
5.08 \\
5.08 \\
5.05 \\
5.08\end{array}$ & $\begin{array}{l}3-3 \\
2-2 \\
3-2 \\
2-3 \\
1-3\end{array}$ & $\begin{array}{l}2^{3} D^{0}-X \\
(979)\end{array}$ \\
\hline $\begin{array}{l}7486.13 \\
7474.60 \\
7335.33\end{array}$ & $\begin{array}{l}P \\
P \\
P\end{array}$ & $\stackrel{\circ}{\circ}$ & $\begin{array}{l}3.87 \\
3.91 \\
3.91\end{array}$ & $\begin{array}{l}5.53 \\
5.56 \\
5.60\end{array}$ & $\begin{array}{l}3-4 \\
3-3 \\
3-3\end{array}$ & $\begin{array}{c}2^{3} D^{0}-e^{5} D \\
(980)\end{array}$ \\
\hline $\begin{array}{l}6326.77 \\
6321.40 \\
6309.73 \\
6083.67 \\
6114.41\end{array}$ & $\mathrm{v}$ & $\begin{array}{c}1 \\
1 \\
\vdots \\
\vdots \\
0\end{array}$ & $\begin{array}{l}3.87 \\
3.91 \\
3.94 \\
3.87 \\
3.91\end{array}$ & $\begin{array}{l}5.85 \\
5.90 \\
5.93 \\
5.90 \\
5.93\end{array}$ & $\begin{array}{l}3-4 \\
3-3 \\
1-3 \\
3-3 \\
3-3\end{array}$ & $\begin{array}{l}z^{3} D^{\circ}-e^{5} r \\
(981)\end{array}$ \\
\hline $\begin{array}{l}6008.577 \\
5934.658 \\
5883.838 \\
5809.349 \\
5798.194 \\
5678.38\end{array}$ & $\begin{array}{l}\mathbb{Z} \\
\mathbb{R} \\
\mathbb{K} \\
\mathbf{V}\end{array}$ & $\begin{array}{r}9 \\
5 \\
4 \\
3 \\
3 \\
3 \\
0\end{array}$ & $\begin{array}{l}3.87 \\
3.91 \\
3.94 \\
3.87 \\
3.91 \\
3.87\end{array}$ & $\begin{array}{l}5.92 \\
5.99 \\
6.04 \\
5.99 \\
6.04 \\
6.04\end{array}$ & $\begin{array}{l}3-4 \\
2-3 \\
1-3 \\
3-3 \\
3-3 \\
3-3\end{array}$ & $\begin{array}{c}2^{3} D^{0}-e^{3} F \\
(983)\end{array}$ \\
\hline
\end{tabular}

5304.11
5377.59

5005.730
4985.361
4973.108

4985.361

4896.437

4911.786
5098.594

5048.454

$\begin{array}{rrrrr}10 & 3.87 & 6.33 & 3-3 & 2^{3} D^{\circ}-0^{3} D \\ 7 & 3.91 & 6.39 & 2-3 & (984)\end{array}$

$3.94 \quad 6.42$

$\begin{array}{ll}3.87 & 6.39 \\ 3.91 & 6.42\end{array}$

4977.653

4970.66
4889.113

4909.387
4930.331
(1) $3.91 \quad 6.39$

$\begin{array}{lll}1 & & \\ 0 & 3.91 & 6.39 \\ 3 & 3.94 & 6.43 \\ 1 & 3.87 & 6.39 \\ 1 & 3.91 & 6.43\end{array}$ $\begin{array}{ll}8-3 & 2^{3} D^{\circ}-8^{5} D \\ 1-3 & (985)^{5}\end{array}$
6963.03

6639.90

6796.11

6683.33
6943.83

6943.83
7105.90

6633.78
.6777 .44

6509.56 $\circ$
$\circ$
$(3)$
3
3

$\begin{array}{llll}4.17 & 5.94 & 3-3 & (1007) \\ 4.06 & 5.93 & 4-4 & \end{array}$

$\begin{array}{lll}4.06 & 5.93 & 4-4 \\ 4.13 & 5.94 & 3-3\end{array}$

$\begin{array}{lll}4.12 & 5.94 & 3-3 \\ 4.06 & 5.94 & 4-3\end{array}$

$4.06 \quad 5.91 \quad 4-5 \quad 8^{3} F-x^{3} \cdot$

$4.06 \quad 5.92 \quad 4-3 \quad c^{3} \bar{r}-r^{5} p_{0}$ $\begin{array}{llll}4.13 & 5.90 & 3-4 & (1008) \\ 4.17 & 5.91 & 3-3 & \end{array}$

(a) 


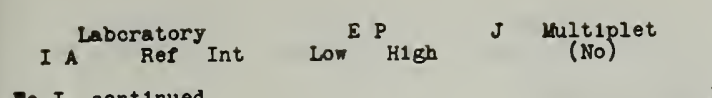

Pe I continued

\begin{tabular}{|c|c|c|c|c|c|c|}
\hline $\begin{array}{r}8713.14 \\
-6777.44 \\
6875.98\end{array}$ & 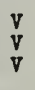 & $\begin{array}{l}6 \mathrm{~d} \\
1 \\
1\end{array}$ & $\begin{array}{l}4.13 \\
4.17 \\
4.17\end{array}$ & $\begin{array}{l}5.96 \\
5.99 \\
5.96\end{array}$ & $\begin{array}{l}3-3 \\
2-1 \\
3-3\end{array}$ & $\begin{array}{c}\mathrm{c}^{3} \mathrm{~F}-\mathrm{x}^{3} \mathrm{po} \\
(1013)\end{array}$ \\
\hline 6315.814 & v & (a) & 4.06 & 6.01 & $4-4$ & $c^{3} F-y^{1} g^{\circ}$ \\
\hline $\begin{array}{l}6157.734 \\
8315.316 \\
6380.748 \\
6107.33 \\
6340.366\end{array}$ & $\begin{array}{l}U \\
\\
I \\
J \\
J \\
\end{array}$ & $\begin{array}{c}(4 \\
(3) \\
3 \\
\odot \\
(1)\end{array}$ & $\begin{array}{l}4.06 \\
4.13 \\
4.17 \\
4.06 \\
4.13\end{array}$ & $\begin{array}{l}6.06 \\
6.08 \\
6.10 \\
6.08 \\
6.10\end{array}$ & $\begin{array}{l}4-4 \\
3-3 \\
2-3 \\
4-3 \\
3-3\end{array}$ & $\begin{array}{c}c \\
0 \\
(1015)\end{array}$ \\
\hline $\begin{array}{l}6147.85 \\
6315.43 \\
6436.43\end{array}$ & $\begin{array}{l}\mathrm{V} \\
\mathrm{P} \\
\mathrm{V}\end{array}$ & $\begin{array}{l}(-) \\
\circ \\
(1)\end{array}$ & $\begin{array}{l}4.06 \\
4.13 \\
4.17\end{array}$ & $\begin{array}{l}6.07 \\
6.08 \\
6.09\end{array}$ & $\begin{array}{l}4-3 \\
3-3 \\
3-1\end{array}$ & $\begin{array}{c}0^{3} F-v^{3} D^{\circ} \\
(1016)\end{array}$ \\
\hline $\begin{array}{c}5975.355 \\
6127.913 \\
5931.89\end{array}$ & $\begin{array}{l}\mathrm{J} \\
\mathrm{J} \\
\mathrm{P}\end{array}$ & $\stackrel{4}{(3)}$ & $\begin{array}{l}4.06 \\
4.13 \\
4.06\end{array}$ & $\begin{array}{l}6.13 \\
6.14 \\
6.14\end{array}$ & $\begin{array}{l}4-5 \\
3-4 \\
4-4\end{array}$ & $\begin{array}{c}c^{3} F-y^{3} H^{\circ} \\
(1017)\end{array}$ \\
\hline $\begin{array}{l}6037.057 \\
8165.366 \\
6315.153 \\
6081.85\end{array}$ & $\begin{array}{l}B \\
\text { J } \\
\text { J } \\
P\end{array}$ & $\left.\begin{array}{c}4 \\
3 \\
3 \\
0\end{array}\right)$ & $\begin{array}{l}4.06 \\
4.13 \\
4.17 \\
4.13\end{array}$ & $\begin{array}{l}6.11 \\
6.13 \\
6.15 \\
6.15\end{array}$ & $\begin{array}{l}4-5 \\
3-4 \\
3-3 \\
3-3\end{array}$ & $\begin{array}{c}0^{3} r-v^{3} g^{\circ} \\
(1018)\end{array}$ \\
\hline
\end{tabular}

$6363.889 \quad V \quad$ (3) $4.17 \quad 6.11 \quad 3-3 \quad c^{3} F-2^{1} p^{\circ}$

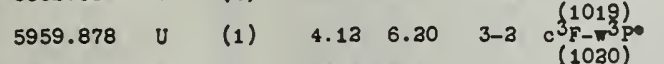

$\begin{array}{llllllll}5643.94 & \mathrm{P} & \odot & 4.06 & 6.34 & 4-3 & (1030) \\ 5943.11 & \mathrm{P} & \odot & 4.17 & 6.34 & 3-3 & (1031)\end{array}$

$\begin{array}{lllllll}5811.93 & V & (1) \quad 4.13 & 6.35 & 3-4 & c^{3} F-x^{1} G^{\circ}\end{array}$

$\begin{array}{llcllll}5811.93 & \mathrm{~V} & (1) & 4.12 & 6.35 & 3-4 & \mathrm{c}^{3} \mathrm{~F}^{1} \mathrm{C}^{\circ} \\ 5510.23 & \mathrm{P} & \odot & 4.06 & 6.30 & 4-5 & (1023) \\ 5563.69 & \mathrm{P} & \odot & 4.12 & 6.34 & 3-4 & (1023) \\ 5333.15 & \mathrm{P} & \odot & 4.06 & 6.37 & 4-3 & \end{array}$

$5494.468 \quad \mathrm{~V} \quad(1) \quad 4.06 \quad 6.30 \quad 4-5 \quad c^{3} \mathrm{~F}-x^{3} \mathrm{H}^{\circ}$

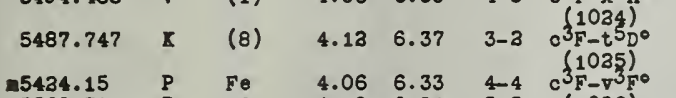

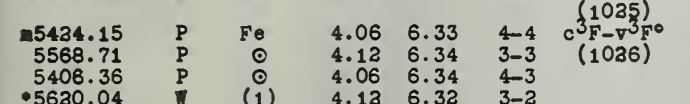

.5630 .04

\begin{tabular}{|c|c|c|c|c|c|c|}
\hline 5329.994 & $\mathrm{~s}$ & (2) & 4.06 & 6.37 & $4-5$ & $c^{3} F-6^{0}$ \\
\hline $\begin{array}{r}5403.823 \\
5476.298 \\
.5535 .419 \\
5319.33 \\
5439.43 \\
5375.00\end{array}$ & $\begin{array}{l}V \\
\mathrm{~J} \\
\mathrm{~J} \\
\mathrm{P} \\
\mathrm{P} \\
\mathbf{1}\end{array}$ & $\begin{array}{c}\left(\begin{array}{l}1 \\
3 \\
2 \\
2\end{array}\right) \\
\circ \\
\odot \\
(1 \mathrm{n})\end{array}$ & $\begin{array}{l}4.06 \\
4.13 \\
4.17 \\
4.06 \\
4.13 \\
4.06\end{array}$ & $\begin{array}{l}6.34 \\
6.38 \\
6.40 \\
6.38 \\
6.40 \\
6.40\end{array}$ & $\begin{array}{l}4-5 \\
3-4 \\
2-3 \\
4-4 \\
3-3 \\
4-3\end{array}$ & $\begin{array}{c}c^{3} F-u^{3} G^{\circ} \\
(1029)\end{array}$ \\
\hline
\end{tabular}

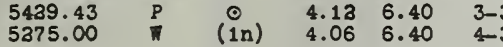

$\begin{array}{lllllll}5464.386 & V & (1) & 4.13 & 6.38 & 3-3 & c^{3} F-y^{1} D^{\circ}\end{array}$

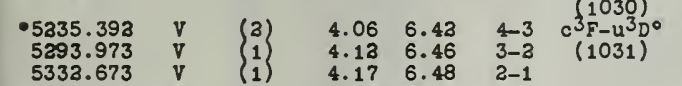

$\begin{array}{llllll}5387.51 & R & 3 & 4.12 & 6.43 & 3-3 \\ 5394.683 & 1 & (3) & 4.17 & 6.46 & 2-3\end{array}$

$\begin{array}{llllll}5394.683 & T & \text { T } \\ 5491.84 & \text { I } & 4.17 & 6.46 & 2-2 \\ 4.17 & 6.42 & 3-3\end{array}$

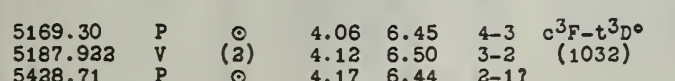

$\begin{array}{llllll}5438.71 & \mathrm{P} & \odot & 4.17 & 6.44 & 2-1 \\ 5317.53 & \mathrm{P} & \odot & 4.13 & 6.45 & 3-3 \\ 5384.62 & \mathrm{P} & \odot & 4.17 & 6.50 & 3-2\end{array}$

$\begin{array}{llllllll}5164.932 & 8 & (-) & 4.13 & 6.51 & 3-4 & \mathrm{c}^{3} \mathrm{~F}-\pi^{3} \mathrm{H}^{\circ}\end{array}$

$\begin{array}{lllllll}5236.189 & V & (1) & 4.17 & 6.53 & 3-1 & c^{3}(1033)\end{array}$

$\begin{array}{lllllll}4978.70 & P & \odot & 4.06 & 6.54 & 4-3 & (1034) \\ 5134.17 & P & (1) & 4.17 & 6.58 & 2-2 & (1035)\end{array}$

$5136.09 \quad(1) \quad 4.17 \quad 6.57 \quad 3-1 \quad c^{3} F_{-2} p^{0}$

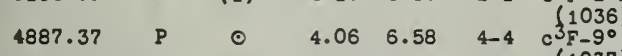

$\begin{array}{lllllll}4839.68 & P & \odot & 4.13 & 6.68 & 3-3 & (1037) \\ 4875.33 & P & \odot & 4.17 & 6.70 & 3-1 & (1038)\end{array}$

$\begin{array}{lllllll}4809.36 & P & (1) & 4.06 & 6.63 & 4-3 & c^{3} F-y^{1} F^{\circ}\end{array}$

$\begin{array}{lllllll}4913.53 & P & \odot ? & 4.13 & 6.64 & 3-3 & c^{3}(1039) \\ 4999.114 & T & \text { (1) } & 4.17 & 6.64 & 2-3 & (1040)\end{array}$

$\begin{array}{llllll}-4881.736 & J & \text { (a) } 4.13 & 6.65 & 3-3 & c^{3} F-10^{\circ}\end{array}$

$\begin{array}{lllllll}4735.846 & J & (3) & 4.08 & 6.66 & 4-5 & \mathrm{c}^{3}(1041) \\ 2 & -t^{3} G^{\circ}\end{array}$

$\left.\begin{array}{lllllll}4800.653 & \mathrm{~J} \\ 4798.369 & \mathrm{~V} & \{ & 3 \\ 1\end{array}\right) \begin{array}{llll}4.12 & 6.70 & 3-4 & (1042) \\ 4.17 & 6.74 & 3-3 & \end{array}$

$\begin{array}{ccccccc}4854.89 & V & (1 n) & 4.13 & 6.67 & 3-3 & c^{3} F-11 \circ \\ 4939.46 & P & \odot & 4.17 & 6.67 & 2-3 & (1043)\end{array}$

$\begin{array}{lllllll}4739.038 & \mathrm{~J} & \text { (1) } \quad 4.06 & 6.67 & 4-5 & \mathrm{c}^{3} F-12^{\circ}\end{array}$

$\begin{array}{lllllll}4785.963 & \mathrm{~V} & (1) & 4.13 & 6.70 & 3-4 & (1043 \mathrm{a}) \\ 4665.56 & \mathrm{P} & \odot & 4.06 & 6.70 & 4-4 & (1044)\end{array}$

$\begin{array}{lllllll}4673.03 \quad P & \odot & 4.13 & 6.77 & 3-4 & c^{3} F-\pi^{1} G^{\circ}\end{array}$

$\begin{array}{llllllll}4413.40 \quad P & P & 4.06 & 6.85 & 4-5 & c^{3} F-x^{1} \mathrm{~K}^{\circ}\end{array}$

$\begin{array}{llllllll}4546.47 \quad P & \odot & 4.17 & 6.88 & 3-3 & c^{3}(1046) \\ & (10.7)^{\circ}\end{array}$

$\begin{array}{lllllll}4467.446 & U & (1) & 4.13 & 6.89 & 3-3 & (1047) \\ 4538.95 & P & \odot & 4.17 & 6.89 & 3-3 & \left(10 . F^{3}\right.\end{array}$

$\begin{array}{llllllll}4153.07 \quad P & \odot & 4.06 & 7.03 & 4-4 & c^{3} \mathrm{~F}-v^{1} G^{\circ}\end{array}$
REVISED UULTIPLET TABLE

I A Laboratory ${ }_{\text {Ref }}$ Int Low ${ }_{\text {H1gh }}^{\text {Le Multiplet }}$ Fe I continued

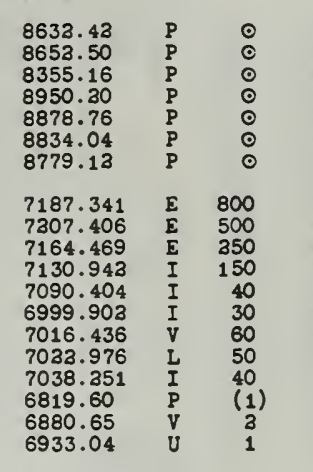

$\begin{array}{llll}4.09 & 5.52 & 4-4 & y^{5} D^{\circ}-e^{5} D \\ 4.14 & 5.56 & 3-3 & (1050) \\ 4.09 & 5.56 & 4-3 & \\ 4.14 & 5.53 & 3-4 & \\ 4.17 & 5.56 & 2-3 & \\ 4.30 & 5.60 & 1-2 & \\ 4.3 & 5.62 & 0-1 & \end{array}$

$\begin{array}{lll}4.30 & 5.60 & 1-3 \\ 4.31 & 5.63 & 0-1\end{array}$

$4.09 \quad 5.80$

$\begin{array}{ll}4.14 & 5.85 \\ 4.17 & 5.90 \\ 4.20 & 5\end{array}$

$\begin{array}{ll}4.30 & 5.93 \\ 4.31 & 5.95\end{array}$

$\begin{array}{ll}4.09 & 5.85 \\ 4.14 & 5.90\end{array}$

$\begin{array}{ll}4.14 & 5.90 \\ 4.17 & 5.93 \\ 4.30 & 5.95 \\ 4.09 & 5.90\end{array}$

$\begin{array}{ll}4.09 & 5.90 \\ 4.14 & 5.93 \\ 4.17 & 5.95\end{array}$

$\begin{array}{cc}4-5 & y^{5} D^{0}-e^{5} F \\ 3-4 & (1051)\end{array}$

$3-3$
$1-3$
$0-1$
$4-4$
$3-3$
$3-3$
$1-1$
$4-3$
$3-3$
$3-1$

6735.39
6653.88
6916.703
6786.88
6704.48

$\begin{array}{ccccc}3 & 4.09 & 5.93 & 4-4 & y^{5} D^{0}-e^{3} F \\ (1) & 4.14 & 5.99 & 3-3 & (1053)\end{array}$

$\begin{array}{lll}60 & 4.14 & 5.93 \\ 5 & 4.17 & 5.99\end{array}$

5668.837

5703.09

(1)

$4.30 \quad 6.04$

$3-4$
$3-3$
$1-3$

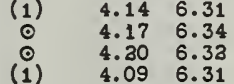

5737.71 ,

5859.96

5760.53

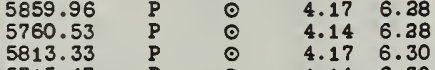

5715.47
5796.67

$\begin{array}{llll}4.17 & 6.38 & 3-3 & y^{5} D^{\circ}-r^{7} \mathrm{D} \\ 4.14 & 6.38 & 3-3 & (1054)\end{array}$

$\begin{array}{ll}4.17 & 6.30 \\ 4.14 & 6.30\end{array}$

5871.389

5928.50

5733.86
5815.16

5893.34
5974.63

(1) $4.14 \quad 6.24$

$\begin{array}{lll}\circ & 4.30 & 6.38 \\ \odot & 4.09 & 6.34\end{array}$

$\begin{array}{lll}\text { (1) } & 4.14 & 6.36 \\ \odot & 4.30 & 6.39\end{array}$

5844.879

5707.70
5760.71

(1) $4.14 \quad 6.35$

5947.30

5677.68

5731.70
5739.78

5761.08
5644.35

5644.35
5516.39

5607.66

5705.66
5481.253
556.44

5481.25
5568.44
5636.00

5551.77

5653.01

5443.41
5534.35

5534.35
5583.97

.5666 .837

$\begin{array}{lll}\odot & 4.09 & 6.35 \\ \odot & 4.14 & 6.38 \\ \odot & 4.17 & 6.35\end{array}$

$\begin{array}{lll}\odot & 4.09 & 6.36 \\ \odot & 4.14 & 6.39\end{array}$

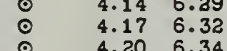

$\begin{array}{lll}\odot & 4.20 & 6.34 \\ \odot & 4.14 & 6.33 \\ \circ & 4.09 & 6.32\end{array}$

\begin{tabular}{cll}
$\odot$ & 4.14 & 6.34 \\
$\odot$ & 4.20 & 6.36 \\
$(2)$ & 4.09 & 6.34 \\
\hline & 4.14 & 0.35
\end{tabular}

$\begin{array}{ccc}(2) & 4.09 & 6.34 \\ \odot & 4.14 & 6.35 \\ \odot & 4.17 & 6.36\end{array}$

- 4.096 .31

$\begin{array}{lll}\odot & 4.14 & 6.35 \\ \odot & 4.30 & 6.38\end{array}$

$\begin{array}{lll}(0 & 4.09 & 6.35 \\ \text { (i) } & 4.09 & 6.35\end{array}$

(1)

$\begin{array}{lll}4.14 & 6.37 \\ 4.17 & 6.38\end{array}$

(1) $4.14 \quad 6.31 \quad 3-2 \quad y^{5} D^{\circ}-e^{5} s$

5493.508

5481.451
5630.537

5573.10

5547.00
.5715 .107
5579.34

$\begin{array}{lllll}1 & 1 \\ 1 & 4.09 & 6.33 & 4-3 & (1060) \\ 4 & y^{5} D^{\circ}-e^{3} \\ 4.14 & 6.39 & 3-2 & (1061)\end{array}$

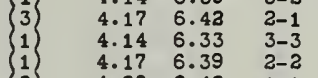

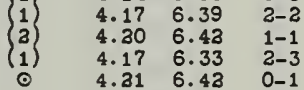

5476.571
5473.908

5473.908
5478.48

-5493.850
5353.386

5391.493
5429.53

5429.53
5480.873

5603.788
5563.604

5563.604
5543.930
5535.553

$\begin{array}{lll}10 & 4.09 & 6.34 \\ 3 & 4.14 & 6.39 \\ 1) & 4.17 & 6.43 \\ 0 & 4.30 & 6.45\end{array}$

$\begin{array}{lll}\text { a) } & 4.20 & 6.45 \\ \text { 2) } & 4.09 & 6.39\end{array}$

\begin{tabular}{lll} 
1) & 4.14 & 6.43 \\
0 & 4.17 & 6.45 \\
\hline &
\end{tabular}

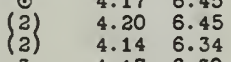

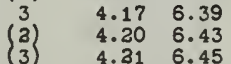

.5534 .68

(1)

$4.31 \quad 6.45$

$\begin{array}{ll}4-4 & y^{5} D^{\circ}-8^{5} \mathrm{D} \\ 3-3 & (1063) \\ 3-2 & \end{array}$

$3-2$
$1-1$
$4-3$
$3-2$

$3-2$
$2-1$
$1-0$

$1-0$
$3-4$

$3-4$
$3-3$
$1-2$

5334.32

5487.52

5453.98
5473.18

5553.22
5543.03
-5538.54

5543.03
.5538 .54

.5007 .389

5037.136
4991.377

4939.344
4933.348

4935.38
4887.189

4983.855
4988.963

4988.963
4957.68

4957.68
4969.927

4969.927
4888.651
4886.335

Fe I continued

$\begin{array}{lllllll} & & & & & & \\ 5 & 1 & 4.14 & 6.56 & 3-4 & y^{5} D^{\circ}-h^{5} D\end{array}$

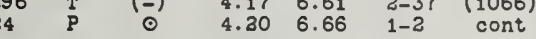

$\begin{array}{lllllll}J & 8 n & 4.09 & 6.56 & 4-3 & y^{5} p^{\circ}-15 p\end{array}$

4983.25

5086.77

.4953 .646

4934.023

4790.56

(3)

$\begin{array}{ll}4.14 & 6.61 \\ 4.17 & 6.66 \\ 4.14 & 6.56\end{array}$

(1) $4.14 \quad 6.56$

$3-1$
$3-3$
$3-2$

(1n) $\quad \begin{array}{llll}4.09 & 6.58 & 4-5 & y^{5} D^{0}-r^{5} G\end{array}$

(ii) 4.17 6.69 $3-3$

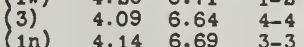

$\begin{array}{llll}\text { 1) } & 4.17 & 6.71 & 2-2 \\ 3 \mathrm{n} & 4.09 & 6.69 & 4-3\end{array}$

(1) $\quad 4.09 \quad 6.63 \quad 4-5 \quad y^{5} D^{0}-e^{3 c} c$

$\begin{array}{lllll}\odot & 4.14 & 6.67 & 3-4 & (1069) \\ \odot & 4.17 & 6.71 & 3-3 & \end{array}$

$\begin{array}{lllll}\text { T1 } & 4.09 & 6.64 & 4-3 & y^{5} D^{0}-p^{3} \mathrm{D}\end{array}$

$\begin{array}{llll}(1) & 4.14 & 6.67 & 3-2 \\ \text { (1) } & 4.17 & 6.72 & 3-1\end{array}$

$\begin{array}{llll}\text { (3) } & 4.14 & 6.64 & 3-3 \\ \odot & 4.17 & 6.67 & 3-3\end{array}$

\begin{tabular}{llll} 
(1) & 4.17 & 6.67 & $3-2$ \\
$\circ$ & 4.30 & 6.72 & $1-1$ \\
\hline & 4.17 & 6.64 & $2-3$
\end{tabular}

$\begin{array}{llll}\circ & 4.17 & 6.64 & 2-3 \\ \text { (1) } & 4.30 & 6.67 & 1-2 \\ 1 & 4.21 & 6.72 & 0-1\end{array}$

$\begin{array}{llllll}\odot & 4.09 & 6.75 & 4-4 & \mathrm{y}^{5} \mathrm{D}^{0}-P^{3} \mathrm{~F} \\ \stackrel{\circ}{ } & 4.09 & 6.81 & 4-3 & (1071)\end{array}$

(1) $4.14 \quad 6.75$

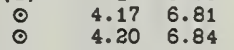

$4.14 \quad 6.77 \quad 3-3 \quad y^{5} p^{0}-e^{3} p$

$\odot \quad 4.17 \quad 6.77 \quad 3-3 \quad(1072)$

(1) $4.17 \quad 7.16 \quad 2-2 \quad y^{5} D^{0}-15 D$

$\begin{array}{llll}(1) & 4.09 & 7.14 & 4-3 \\ \text { (1) } & 4.14 & 7.16 & 3-2\end{array}$

$\begin{array}{lll}4.17 & 7.14 & 2-3 \\ 4.20 & 7.16 & 1-2\end{array}$

$\begin{array}{llll}4.09 & 7.19 & 4-5 & y^{5} D^{\circ}-z^{5} c\end{array}$

$\begin{array}{llll}4.14 & 7.19 & 3-2 & y^{5} D^{0}-4\end{array}$

\begin{tabular}{llll}
4.20 & 7.19 & $1-2$ & $(1075)$ \\
\hline
\end{tabular}

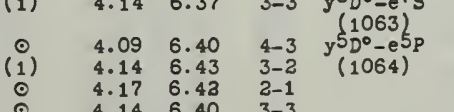

$\begin{array}{llll}\odot & 4.14 & 6.40 & 3-3 \\ \odot & 4.17 & 6.43 & 3-2\end{array}$

$\circ \quad 4.2006 .42 \quad 1-1$

$\begin{array}{llll}(1) & 4.17 & 6.40 & 2-3 \\ \text { (1) } & 4.20 & 6.43 & 1-2\end{array}$

4917.35

$\begin{array}{llllcl}(3 n) & 4.09 & 6.55 & 4-5 & y^{5} D^{\circ}-8^{5} F & 5742.95 \\ 5 n & 4.14 & 6.59 & 3-4 & (1065) & 5738.23 \\ 3) & 4.17 & 6.65 & 2-3 & & 5786.99\end{array}$

$\begin{array}{lllll}2) & 4.30 & 6.70 & 1-2 & 5826.64 \\ \mathrm{an}) & 4.31 & 6.71 & 0-1 & 5859.20 \\ 1 & 4.09 & 6.59 & -1 & 5691.69\end{array}$

$\begin{array}{lllll}\text { in) } & 4.21 & 6.79 & 0-1 & \\ 1-3 & 4.09 & 6.59 & 4-4 & 5637.08 \\ -3 & 4.17 & 6.70 & 2-2 & 5691.69\end{array}$

$\begin{array}{cccccc}6 n & 4.09 & 6.56 & 4-4 & y^{5} D^{\circ}-h^{5} D & 5753.38 \\ (6) & 4.14 & 6.61 & 3-3 & (1066) & 5858.88 \\ 0 & 4.17 & 6.66 & 2-3 & & 5835.10\end{array}$

5835.10
5861.11
5876.27

$\begin{array}{ll}4.09 & 6.61 \\ 4.14 & 6.61\end{array}$

$\begin{array}{ll}4.14 & 6.66 \\ 4.17 & 6.68\end{array}$ $\begin{array}{llll}4.16 & 5.53 & 5-4 & y^{5} F^{0}-e^{5 D} \\ 4.20 & 5.56 & 4-3 & (1076)\end{array}$

$4.16 \quad 5.80 \quad 5-5 \quad y^{5} p^{\circ}-05 F$

$\begin{array}{llll}4.20 & 5.85 & 4-4 & (1077)\end{array}$

$4.34 \quad 5.90 \quad 3-3$

$4.28 \quad 5.95 \quad 1-1$

$4.16 \quad 5.85 \quad 5-4$

$\begin{array}{lll}4.20 & 5.90 & 4-3 \\ 4.24 & 5.93 & 3-2\end{array}$

$\begin{array}{lll}4.26 & 5.95 & 2-1 \\ 4.20 & 5.80 & 4-5\end{array}$

$\begin{array}{lll}4.24 & 5.85 & 3-4 \\ 4.26 & 5.90 & 2-3\end{array}$

$\begin{array}{lll}4.26 & 5.90 & 2-3 \\ 4.38 & 5.93 & 1-2\end{array}$

$\begin{array}{lllll}5 & 4.16 & 5.92 & 5-4 & \mathrm{y}^{5} \mathrm{~F}^{\circ}-\mathrm{e}^{3} \mathrm{~F} \\ 3 & 4.20 & 5.99 & 4-3 & (1078)\end{array}$

$\begin{array}{lll}4.20 & 5.93 & 4-4 \\ 4.34 & 5.99 & 3-3\end{array}$

(1) $4.26 \quad 6.04 \quad 2-2$

$\begin{array}{llll}1 \mathrm{n} & 4.24 & 5.93 & 3-4 \\ (-) & 4.26 & 5.99 & 2-3\end{array}$

(1) $4.16 \quad 6.21 \quad 5-6 \quad y^{5} F^{\circ}-e^{7} F$

$\begin{array}{ccccc}(3 n) & 4.26 & 6.31 & 2-3 & (1079) \\ \odot & 4.38 & 6.34 & 1-2 & \end{array}$

$\begin{array}{lllll}\circ & 4.16 & 6.22 & 5-5 & y^{5} F^{\circ}-r^{7} D\end{array}$

(i) $\quad 4.30 \quad 6.24 \quad 4-3 \quad y^{5} 5^{\circ}-p^{5} \mathrm{D}$

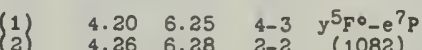

$\begin{array}{lllll}\text { (2) } & 4.16 & 6.24 & 5-6 & \mathrm{y}^{5} \mathrm{~F}^{\circ}-\mathrm{e}^{5 \mathrm{G}} \\ 0 & 4.36 & 6.32 & 2-3 & (1083)\end{array}$

$\begin{array}{llll}\text { (1) } & 4.16 & 6.26 & 5-5 \\ 0 & 4.20 & 6.29 & 4-4\end{array}$

$\begin{array}{llll}\text { (1) } & 4.24 & 6.32 & 3-3\end{array}$

(1) $\quad 4.16 \quad 6.31 \quad 5-5 \quad y^{5} F^{0}-P^{5} F$

$\begin{array}{lllll}\odot & 4.20 & 6.35 & 4-4 & (1084)\end{array}$

$\begin{array}{lll}(1) & 4.26 & 6.38 \\ \text { (i) } & 4.28 & 6.39\end{array}$

$\begin{array}{lll}4.16 & 6.35 \\ 4 & 6.30 & 6.37 \\ 4 & .24 & 6.38\end{array}$

4.206 .37

$\begin{array}{ll}4.26 & 6.39 \\ 4.20 & 6.31\end{array}$

4.206 .3

$\begin{array}{lll}4.24 & 6.35 & 3-4 \\ 4.26 & 6.37 & 2-3 \\ 4.28 & 6.38 & 1-2\end{array}$

$\begin{array}{lll}4.26 & 6.37 & 2-3 \\ 4.28 & 6.38 & 1-2\end{array}$
(1) $4.34 \quad 6.04 \quad 3-2$ 
62

$$
\text { I A }{ }_{\text {Fe I continued }}^{\text {Laboratory }}
$$

$\begin{array}{lllllll}5943.62 & P & \circ & 4.24 & 6.31 & 3-2 & y^{5} F^{\circ}-e^{5} \mathrm{~s} \\ 6021.82 & \mathrm{~W} & (2 \mathrm{n}) & 4.36 & 6.31 & 3-2 & (1085)\end{array}$

5793.933

5741.861
5715.107

5892.71

5814.80
5762.84
5969.554

5969.554
5864.24

5662.525

5638.266

5641.464
5658.67

5691.509
5775.090

5775.090
5731.771

5711.867

5705.48
5873.211

5804.478
.5759 .56

5617.14
5635.85

5635.85
5721.71

5709.93
5706.11

5162.288

5126.218
5072.077

5076.388

5076.288
5075.17

5051.39
5016.48

5016.48
5255.68
5343.798

5343.798
5184.292

5137.388

5125.130

5104.47

5229.857
5202.27

5148.061

5228.408

5196.100
5159.066

5308.71

5255.76
5197.93

5133.693

5195.471
-5142.541
5096.998

5079.002

5104.21
5067.162
5040.902

5040.902
4986.90

5085.68

5040.25
5021.60

5021.60
5012.68

4954.30
4866.77

4987.62

4991.86
4985.98

4985.98
5074.757

5065.020
+5040.902

5072.690

5064.95
5023.226

5148.234

5121.646

$\begin{array}{lll}5206.80 & P & \odot \\ 5159.95 & P & \odot\end{array}$

$\left(\begin{array}{l}2 \\ 3 \\ 1 \\ 3 \\ 1 \\ 0 \\ 0 \\ 1\end{array}\right)$

$\begin{array}{llll}4.20 & 6.33 & 4-3 & y^{5} F^{0}-e^{3} \mathrm{D} \\ 4.24 & 6.39 & 3-2 & (1086)\end{array}$

$\begin{array}{lll}\text { (3) } & 4.24 & 6.33 \\ 1 & 4.26 & 6.39 \\ 0 & 4.28 & 6.42 \\ 0 & 4.28 & \end{array}$

$\begin{array}{lll}4.26 & 6.33 & 1-1 \\ 4.38 & 6.39 & 1-2\end{array}$

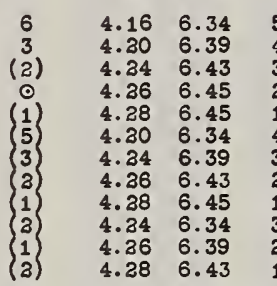

$\begin{array}{llllll}9 & 4.20 & 6.40 & 4-3 & y^{5} F^{0}-e^{5 p} \\ (1) & 4.24 & 6.43 & 3-2 & (1088)\end{array}$

$\begin{array}{lll}\odot & 4.26 & 6.42 \\ \odot & 4.24 & 6.40 \\ \odot & 4.26 & 6.43\end{array}$

$\begin{array}{lllll}10 n & 4.16 & 6.55 & 5-5 & y^{5} F^{\circ}-8^{5} \\ 4 & 4.20 & 6.59 & 4-4 & (1089) \\ 1 & 4.24 & 6.65 & 3-3 & \end{array}$

$\begin{array}{ll}5-4 & 5^{5} \mathrm{~F}^{\circ}-8^{5} \mathrm{D} \\ 4-3 & (1087) \\ 3-2 & \\ 2-1 & \\ 1-0 & \\ 4-4 & \end{array}$

REVISED Y ULT I P.LET T A L E

I A Reboratory Int Low ${ }_{\text {Righ }}^{\text {Lab }}$ Lult1plet $_{(\text {No) }}$

I A Laboratory Rer Int Low $_{\text {H1gh }}^{\text {L }}$

J $\underset{(\text { No })}{\operatorname{lng} \text { (Not }}$

\section{Fe I continued}

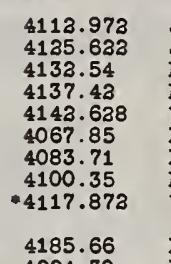

$$
\begin{array}{cccc}
\text { 3n } & 4.16 & 7.16 & 5-6 \\
(1) & 4.20 & 7.19 & 4-5 \\
0 & 4.24 & 7.23 & 3-4 \\
0 & 4.26 & 7.25 & 2-3 \\
\text { (1N) } & 4.28 & 7.26 & 1-3 \\
0 & 4.16 & 7.19 & 5-5 \\
& 4.20 & 7.23 & 4-4 \\
0 & 4.24 & 7.25 & 3-3 \\
\text { (1) } & 4.26 & 7.26 & 2-3
\end{array}
$$

$\begin{array}{lllllll}4185.66 & P & \odot & 4.24 & 7.19 & 3-3 & y^{5} F^{\circ}-4 \\ 4224.30 & P & \odot & 4.26 & 7.19 & 3-2 & (1104)\end{array}$

$\begin{array}{llllllll}7339.885 & I & 6 & & 4.19 & 5.90 & 2-3 & z^{3} p^{\circ}-e^{5} F \\ 7311.26 & \mathrm{p} & \odot & 4.24 & 5.93 & 1-2 & (1105)\end{array}$

$\begin{array}{llllll}7311.26 & \text { P } & \odot & 4.24 & 5.93 & 1-2 \\ 7095.425 & I & 3 & 4.19 & 5.93 & 2-2 \\ 7213.84 & \text { P } & \odot & 4.24 & 5.95 & 1-1\end{array}$

$\begin{array}{llcllll}5955.682 & U & (1) & 4.24 & 6.31 & 1-2 & z^{3} P^{\circ}-e^{5} \mathrm{~g} \\ 5762.992 & \mathrm{~K} & 10 & 4.19 & 6.33 & 2-3 & z^{3} P^{\circ}-\theta^{3} \mathrm{D} \\ 5753.136 & \mathrm{~J} & 5 & 4.24 & 6.39 & 1-2 & (1107) \\ 5717.845 & \mathrm{~L} & (3) & 4.27 & 6.42 & 0-1 & \end{array}$

$\begin{array}{llllll}5753.136 & J & 5 & 4.24 & 6.39 & 1- \\ 5717.845 & \text { L } & (3) & 4.27 & 6.42 & 0-1 \\ 5618.646 & \text { V } & \text { (1) } & 4.19 & 6.39 & 2-\end{array}$

$\begin{array}{llrlll}+5655.506 & V & 4 & 4.34 & 6.42 & 1-1 \\ \mathrm{~m} 5525.48 & P & 58 & 4.19 & 6.42 & 2-1\end{array}$

5608.98

5652.3

5661.36
5522.46
+5600.242

$\left(\begin{array}{c}0 \\ 1 \\ 1 \\ 2 \\ 1 \\ 1 \\ 1\end{array}\right)$

$\begin{array}{llll}4.19 & 6.39 & 2-3 & \varepsilon^{3} p^{\circ}-g^{5} D\end{array}$

5473.720

$$
\text { (1) }
$$$$
4.276 .45
$$

4.19 6.43 $6-2$

$\begin{array}{lll}4.24 & 6.45 & 1-1 \\ 4.19 & 6.45 & 2-1\end{array}$

5588.07

5646.70
5734.445
5517.08

5517.08
5661.97

5027.785

5025.08

$\begin{array}{llll}(1) & 4.24 & 6.59 & 3-4 \\ 3 n) & 4.26 & 6.65 & 2-3 \\ 3) & 4.28 & 6.70 & 1-2\end{array}$

$\begin{array}{lllll}6 n & 4.16 & 6.56 & 5-4 & \mathrm{y}^{5} F^{\circ}-\mathrm{h}^{5} \mathrm{D}\end{array}$

$\begin{array}{cll}6 n & 4.30 & 6.61 \\ (6 n) & 4.24 & 6.66\end{array}$

(1) $\quad 4.26 \quad 6.68$

$\begin{array}{lll}5 \mathrm{n} & 4.20 & 6.56 \\ \text { (1) } & 4.24 & 6.61 \\ 3 & 4.26 & 6.66\end{array}$

$\begin{array}{llll}3 & 4.36 & 6.66 & 3-2 \\ 3 \pi) & 4.38 & 6.68 & 1-1\end{array}$

(1n) $\begin{array}{lllll}4.20 & 6.56 & 4-3 & \mathrm{y}^{5} \mathrm{~F}^{\circ}-\mathrm{F}^{5 \mathrm{P}} \\ 4.34 & 6.61 & 3-2 & (1091)\end{array}$

$\left\{\begin{array}{l}2 \pi \\ \text { ant }\end{array}\right\}$

$\begin{array}{ll}4.24 & 6.61 \\ 4.26 & 6.66\end{array}$

$\begin{array}{lll}4.24 & 6.56 & 3-3 \\ 4.26 & 6.61 & 2-2 \\ 4.28 & 6.66 & 1-1\end{array}$

$\begin{array}{lllll}30 n & 4.16 & 6.56 & 5-6 & y^{5} F^{\circ}-P^{5} 5_{G}\end{array}$

$\begin{array}{lllll}30 n & 4.16 & 6.56 & 5-6 & y^{5} F^{\circ}-P^{5} \\ 8 \text { (8) } & 4.20 & 6.58 & 4-5 & (1093) \\ 3 \text { (i) } & 4.24 & 6.64 & 3-4 & \end{array}$

(6)

$\begin{array}{lll}4.24 & 6.64 & 3-4 \\ 4.26 & 6.69 & 2-3\end{array}$

$\begin{array}{lll}4.28 & 6.71 & 1-2 \\ 4.16 & 6.58 & 5-5\end{array}$

$\begin{array}{lll}4.16 & 6.58 & 5-5 \\ 4.30 & 6.64 & 4-4 \\ 4.24 & 6.69 & 3-3\end{array}$

$\begin{array}{lll}4.24 & 6.69 & 3-3 \\ 4.24 & 6.71 & 3-2\end{array}$

$\begin{array}{llll}4.16 & 6.59 & 5-6 & y^{5} F^{\circ}-e^{5} \mathrm{H} \\ 4.20 & 6.65 & 4-5 & (1093)\end{array}$

4.24 6.70 $3-4$

$\begin{array}{lll}4.16 & 6.73 & 2-3 \\ 4.16 & 6.65 & 5-5 \\ 4.16 & 6.70 & 5-4\end{array}$

$\begin{array}{lllll}\odot & 4.16 & 6.63 & 5-5 & y^{5} F^{0}-e^{3} G \\ \odot & 4.20 & 6.67 & 4-4 & (1094)\end{array}$

$\begin{array}{cccc}\odot & 4.20 & 6.67 & 4-4 \\ \circ & 4.24 & 6.71 & 3-3 \\ 10 \mathrm{n} & 4.20 & 6.63 & 4-5\end{array}$

$\begin{array}{llll}6 \mathrm{n} & 4.24 & 6.67 & 3-4 \\ (2) & 4.26 & 6.71 & 2-3\end{array}$

$\begin{array}{lllll}\text { (1) } & 4.20 & 6.64 & 4-3 & \mathrm{y}^{5} \mathrm{~F}^{\circ}-\mathrm{P}^{3} \mathrm{D} \\ (0 & 4.24 & 6.67 & 3-3 & (1095)\end{array}$

$\left(\begin{array}{llll}(-) & 4.24 & 6.67 & 3-2 \\ (-) & 4.26 & 6.72 & 2-1 \\ (3) & 4.24 & 6.64 & 3-3\end{array}\right.$

$\begin{array}{llll}3 \text { 3n) } & 4.24 & 6.64 & 3-3 \\ \text { an } & 4.26 & 6.67 & 2-2\end{array}$

\begin{tabular}{clll}
1 & 4.38 & 6.72 & $1-1$ \\
$\odot$ & 4.26 & 6.64 & $2-3$ \\
\hline & 4.28 & 6.67 & $1-3$
\end{tabular}

$\begin{array}{llll}\text { (1) } 4.16 & 6.64 & 5-5 & y^{5} F^{0}-g^{7} \mathrm{D}\end{array}$

$\begin{array}{llll}4.16 & 6.68 & 5-4 & (1096) \\ 4.20 & 6.72 & 4-3 & \end{array}$

$\begin{array}{lll}4973.39 & P & \\ 4893.59 & P & \circ \\ 4906.80 & P & \odot\end{array}$

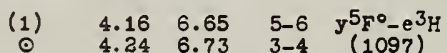

4962.564
4942.59

4942.59
4872.91

4749.25
4842.71

4807.343
-4799.06

4911.52

4858.37
+4832.734

4465.33

4460.55

4384.13

4325.71

4256.79
4278.01 $\begin{array}{lllll}\odot & 4.24 & 6.84 & 3-2 & y^{5} F^{\circ}-F^{3} F \\ \stackrel{\odot}{\circ} & 4.20 & 6.75 & 4-4 & (1098) \\ (-) & 4.24 & 6.81 & 3-3 & \end{array}$

$\begin{array}{clll}\circ & 4.24 & 6.75 & 3-4 \\ \text { (3) } & 4.26 & 6.81 & 2-3 \\ \text { (3) } & 4.28 & 6.84 & 1-2\end{array}$

(1) $\quad 4.30 \quad 6.97 \quad 4-5 \quad y^{5} F^{\circ}-1$

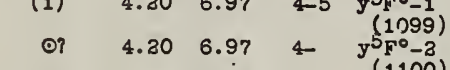

๑?

$\begin{array}{llll}4.20 & 7.03 & 4-4 & y^{5} F^{\circ}-3\end{array}$

$\begin{array}{ccccc}\odot & 4.20 & 7.12 & 4-4 & y^{5} F^{\circ}-15_{D} \\ (1) & 4.24 & 7.14 & 3-3 & (1102) \\ \odot & 4.34 & 7.12 & 3-4 & \end{array}$ \begin{tabular}{cccc}
$(-)$ & 4.24 & 6.81 & $3-3$ \\
$\circ$ & 4.26 & 6.84 & $3-2$ \\
\hline & 4.24 & 6.75 & $3-4$
\end{tabular}
5041.33
4932.18

4992.80

$4993.687 \quad U \quad$ (1) $4.19 \quad 6.66 \quad 3-2 \quad 2^{3 p^{0}-h^{5} \mathrm{D}}$

$\begin{array}{lllllll}5056.856 & \mathrm{U} & \text { 1) } & 4.24 & 6.68 & 1-1 & \text { (1111) } \\ .4953 .646 & \mathrm{~V} & \text { (n) } & 4.19 & 6.68 & 2-1 & \end{array}$

$\begin{array}{ccccccc}4945.65 & \text { (1) } & 4.19 & 6.69 & 2-3 & 2^{3} p^{0}-p^{5} G \\ 4995.41 & P & \odot & 4.24 & 6.71 & 1-2 & (1113)\end{array}$

$\begin{array}{lllllll}4893.70 & P & \odot & 4.19 & 6.71 & 3-2 & \\ 4730.56 & P & \odot ? & 4.19 & 6.81 & 3-3 & z^{3} p^{0}-I^{3} F\end{array}$

$\begin{array}{lllllll}4775.87 & P & & 4.19 & 6.77 & 2-2 & 2^{3}(114)-e^{3} \\ .4757 .582 & J & \text { (a) } & 4.24 & 6.84 & 1-1 & (1115)\end{array}$

$\begin{array}{lcccccc}4665.84 & J & (2) & 4.24 & 6.84 & 1-1 & (1115) \\ 465.24 & P & \odot & 4.19 & 6.84 & 2-1 & \\ 465.69 & P & \odot & 4.34 & 6.77 & 1-2 & \end{array}$

$\begin{array}{llllll}4873.69 & \mathrm{P} & \odot & 4.34 & 6.77 & 1-2 \\ 4801.63 & \mathrm{P} & \odot & 4.27 & 6.84 & 0-1\end{array}$

$\begin{array}{lllllll}4160.78 \quad P & \odot & 4.19 & 7.16 & 2-2 & 2^{3} P^{\circ}-1^{5} \mathrm{D} \\ (1116)\end{array}$

$8356.07 \quad P \quad \odot \quad \overline{4.38} 5.75 \quad 2-1 \quad b^{1} D-z^{3} g^{\circ}$

$\begin{array}{lllllll}7820.80 & P & \odot & 4.28 & 5.85 & 2-3 & \begin{array}{l}(1117) \\ b\left(1-10^{\circ}\right. \\ (1118)\end{array}\end{array}$

$\begin{array}{lllllll}6756.56 & P & \odot & 4.38 & 6.10 & 3-3 & b^{1} D-m^{3} F^{\circ}\end{array}$

$6571.32 \quad U \quad$ (1) $4.38 \quad 6.15 \quad 2-3 \quad b^{(1120)}\left(\frac{11 v^{3} G^{\circ}}{6}\right.$

$\begin{array}{llllllll}6736.56 & P & \odot & 4.28 & 6.11 & 2-2 & b^{1}\left(11212^{1} D^{\circ}\right.\end{array}$

$6267.845 \quad U \quad$ (1) $\quad 4.28 \quad 6.34 \quad 2-3 \quad b(1122)$

$\begin{array}{lllllll}5883.06 & P & \odot & 4.38 & 6.37 & 2-2 & b^{(1123)}\left(1-t^{\circ}\right.\end{array}$

$\begin{array}{lllllll}6035.34 & P & \odot & 4.38 & 6.32 & 2-2 & (1124) \\ 5976.18 & P & \odot & 4.28 & 6.34 & 2-3 & (1125)\end{array}$

$\begin{array}{lllllll}5816.07 & P & \quad & 4.38 & 6.40 & 2-3 & b^{1} \mathrm{D}-\mathrm{u}^{3} \mathrm{G}^{\circ}\end{array}$

$\begin{array}{lrrrrrr}5816.07 & P & \odot ? & 4.38 & 6.40 & 2-3 & b^{1} D-u^{3} G^{\circ} \\ 5856.084 & V & (2) & 4.38 & 6.38 & 2-3 & b^{1}(127)^{1} D^{\circ}\end{array}$

$5837.709 \quad V \quad(1) \quad 4.28 \quad 6.39 \quad 2-3 \quad b^{(1128)}$

$\begin{array}{lllllll}5539.831 & U & (1) & 4.28 & 6.50 & 2-2 & b(1129) \\ (1)-t^{3} D^{\circ}\end{array}$

$\begin{array}{lllllll}5469.09 & P & \odot & 4.38 & 6.53 & 2-2 & b^{1} \mathrm{D}-v^{3} \mathrm{p} \text { 。 }\end{array}$

$5376.849 \quad U \quad(2) \quad 4.28 \quad 6.57 \quad 2-1 \quad b^{1}{ }_{D-2}^{1131}$ )

$\begin{array}{lllllll}4734.100 & J & (1) & 4.38 & 6.88 & 2-2 & b(1133)^{\circ}\end{array}$

$\begin{array}{lllllll}4735.94 & V & (1 \mathrm{n}) & 4.28 & 6.89 & 2-3 & b^{(1133)}\left(1-F^{1} F^{\circ}\right.\end{array}$

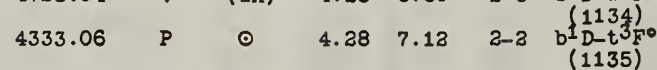

$\begin{array}{rrrrrrr}8220.406 & E & 1500 & 4.30 & 5.80 & 6-5 & z^{5} G^{\circ}-e^{5} F \\ 7937.166 & E & 700 & 4.39 & 5.85 & 5-4 & (1136)\end{array}$

$\begin{array}{llllll}7937.166 & E & 700 & 4.29 & 5.85 & 5-4 \\ 7998.973 & E & 700 & 4.35 & 5.90 & 4-3 \\ 8046.073 & E & 600 & 4.40 & 5.93 & 3-2\end{array}$

$\begin{array}{llllll}8046.073 & \mathrm{E} & 600 & 4.40 & 5.93 & 3-2 \\ 8085.300 & \mathrm{E} & 500 & 4.43 & 5.95 & 2-1\end{array}$

$\begin{array}{llclll}8085.300 & \mathrm{E} & 500 & 4.43 & 5.95 & 2-1 \\ 8179.03 & 0 & (1) & 4.29 & 5.80 & 5-5\end{array}$

$\begin{array}{llllll}8179.03 & 0 & (1) & 4.29 & 5.80 & 5-5 \\ 8248.151 & \mathrm{E} & 30 & 4.35 & 5.85 & 4-4\end{array}$

$\begin{array}{llllll}8232.347 & \mathrm{E} & 50 & 4.40 & 5.90 & 3-3 \\ 8207.767 & \mathrm{E} & 40 & 4.43 & 5.93 & 2-2\end{array}$ $\begin{array}{llrllll}5205.31 & P & \odot & 4.24 & 6.61 & 1-3 & z^{3} P^{\circ}-P^{5} P \\ 5004.034 & T & (1) & 4.19 & 6.66 & 2-1 & (1112)\end{array}$

Fo I cont1nued

$8509.63 \quad P \quad \circ \quad 4.35 \quad 5.80 \quad 4-5 \quad z^{5} \mathrm{G}^{0}-0^{5} \mathrm{Y}$

$\begin{array}{llllllc}8496.51 & P & \odot & 4.40 & 5.85 & 3-4 & (1136) \\ 8401.68 & P & \odot & 4.43 & 5.90 & 2-3 & \text { cont }\end{array}$

$\begin{array}{rrrrrrr}7586.044 & \mathrm{E} & 150 & 4.29 & 5.92 & 5-4 & \varepsilon^{5} G^{0}-e^{3} F \\ 7531.171 & \mathrm{E} & 60 & 4.35 & 5.99 & 4-3 & (1137) \\ 7507.300 & \mathrm{~L} & 8 & 4.40 & 6.04 & 3-2 & \end{array}$

7869.65
7737.67

7647.83

6488.80

$\stackrel{\circ}{\circ}$

$\begin{array}{lll}4.40 & 6.04 & 3-3 \\ 4.35 & 5.92 & 4-4 \\ 4.40 & 5.99 & 3-3\end{array}$

$\begin{array}{llllll} & \text { (1) } \quad 4.35 \quad 6.27 \quad 4-4 & 2^{5} G^{0}-r^{7} \mathrm{D}\end{array}$

$\begin{array}{lllllll}6543.98 \quad U & (1) \quad 4.35 & 6.24 & 4-3 & 25 G^{\circ}-1^{5} \mathrm{D}\end{array}$

$\begin{array}{lllllll}6376.23 & P & \odot & 4.30 & 6.34 & 6-6 & { }^{5} 5^{\circ}-e^{5} \\ 6303.46 & \mathrm{~V} & (1 \mathrm{n}) & 4.30 & 6.26 & 6-5 & (1140)\end{array}$

6351.29

6148.65

(1)

$\begin{array}{lll}4.30 & 6.26 & 6-5 \\ 4.29 & 6.24 & 5-6 \\ 4.35 & 6.26 & 4-5\end{array}$

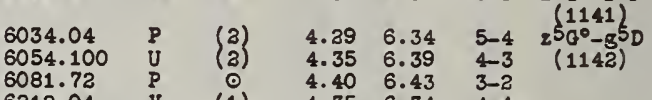

(i)

$\begin{array}{lll}4.40 & 6.43 \\ 4.35 & 6.34 & 4\end{array}$

5361.637

(1)

4.40 . 6.70

5395.25
5469.29

5512.277

5487.16

$\left(\begin{array}{l}0 \\ 1 \\ 1 \\ 2 n\end{array}\right)$

4.436 .71

5615.18

$\left(\begin{array}{c}1 \\ 2 n)\end{array}\right.$

$\begin{array}{ll}4.35 & 6.59 \\ 4.40 & 6.65\end{array}$

4.43 6.70

(1)

$4.29 \quad 6.5$

5441.331
5466.404

5446.58
5470.17

5470.17
5520.19

(1)

$\begin{array}{ll}4.43 & 6.68 \\ 4.43 & 6.66\end{array}$

(5)

4.436 .66

$\left(\begin{array}{l}5 \\ 5 \\ 1 \\ 1\end{array}\right)$$$
\begin{aligned}
& 4.30 \\
& 4.29 \\
& 4.35
\end{aligned}
$$

4.296 .5

m5404.12

5389.461

5398.285

5265.42

5327.86
5437.19

5546.512

5505.893

4.40
4.43 6.69

$4.43 \quad 6.71$

$\begin{array}{ll}4.39 & 6.64\end{array}$

$\begin{array}{lll}\odot ? & 4.40 & 6.71 \\ \circ & 4.29 & 6.56\end{array}$

(1) 4.356 .58

(in) $\begin{array}{lll}4.40 & 6.64 \\ 4.43 & 6.69\end{array}$

5424.072

5383.374

4.306.

5367.470

5364.874

5336.38

5267.28
5395.316

5290.79

5290.79
5184.17

5273.62
5315.07

5326.793
5409.125

$35 n$

4.356 .65

4.406 .70

4.436 .73

० $\quad 4.396 .65$

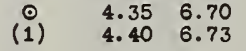

$\stackrel{\odot}{\circ}$

4. 306.

$\begin{array}{ll}4.30 & 6.63 \\ 4.29 & 6.67 \\ 4.29 & 6.63\end{array}$

$\begin{array}{ll}4.29 & 6.63 \\ 4.35 & 6.67\end{array}$

$\begin{array}{ll}4.40 & 6.71\end{array}$

$\begin{array}{lll}4.35 & 6.63\end{array}$

5406.77

5417.03
5512.40

(i)

$4.35 \quad 6.6$

$\begin{array}{ll}4.40 & 6.67 \\ 4.40 & 6.64\end{array}$

$\begin{array}{ll}4.40 & 6.64 \\ 4.43 & 6.67\end{array}$

5262.61

5262.61

5130.91

5245.62
5259.09

$\stackrel{\circ}{\stackrel{0}{1}}$

$\begin{array}{ll}4.30 & 6.65 \\ 4.29 & 6.70\end{array}$

$4.29 \quad 6.73$

$\begin{array}{ll}4.29 & 6.65 \\ 4.35 & 6.70\end{array}$ 


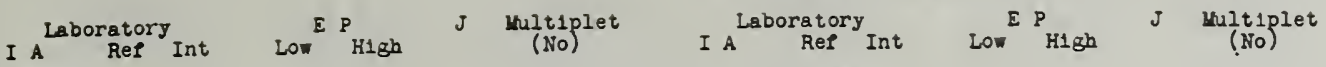 \\ Fe I continued \\ Fe I continued} $\begin{array}{llllllll}6623.41 & P & \odot & 4.37 & 6.23 & 5-4 & z^{3} G^{\circ}-e^{7} p & 5816.36 . \\ 6735.00 & P & \odot & 4.42 & 6.25 & 4-3 & (1157) & 5855.136 \\ 6879.51 & P & \odot & 4.45 & 6.35 & 3-3 & & 5891.16\end{array}$

6438.775

5653.889

5631.72
5549.55

5449.55

- 5780.83

5624.056
5589.00 5749.65 5749.65
5733.66

5619.60 5708.109

5553.586 5528.89 5436.399
5435.17

5562.12 5521.28
5505.75 5405.35 5412.80
5429.83 5301.33
5339.40

5445.045 5463.282
5462.970 5349.742 m5371. 43 5557.954 5560.230

\subsection{1}

5415.301
5404.144 5410.913 5331.106

5178.798 5164.56 5180.065 5249.099
5373.704 4838.81 4744.13 4534.63
4566.03 4367.07 4320.13
4357.50

9786.62 9350.46
9437.91

8866.961 8793.376
8764.000 8439.603 8497.00
9258.30 9258.30
9079.599 6843.671 6858.164
6885.773 7074.45
7031.03

6881.07 6727.558
6715.410
6804.020 6105.15 6159.409
5983.704 5997.808 5848.09 5927.798 6079.02 5929.700
.5949 .35 6018.34 6351.36 6093.66
6094.419 6024.066 6020.173 5853.19 5981.28 5720.89
5807.97
(1) $4.42 \quad 6.33 \quad 4-3 \quad z^{3} G^{0}-e^{3} D$ $\left(\begin{array}{c}1 \\ 1 \\ 3\end{array}\right)$

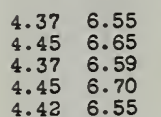

$\begin{array}{llll}4.37 & 6.56 & 5-4 & z^{3} G^{0}-h^{5} D \\ 4.45 & 6.66 & 3-2 & (1160) \\ 4.42 & 6.56 & 4-4 & \\ 4.45 & 6.61 & 3-3 & \end{array}$

(1)

$\begin{array}{ll}4.42 & 6.58 \\ 4.45 & 6.64 \\ 4.42 & 6.64\end{array}$ $\begin{array}{ll}4.42 & 6.64 \\ 4.45 & 6.69 \\ 4.37 & 6.64\end{array}$ $\begin{array}{ll}4.37 & 6.64 \\ 4.42 & 6.69\end{array}$

$\begin{array}{llll}4.37 & 6.59 & 5-6 & 2^{3} G^{0}-e^{5} H \\ 4.42 & 6.65 & 4-5 & (1162) \\ 4.45 & 6.70 & 3-4 & \\ 4.37 & 6.65 & 5-5 & \end{array}$

$\begin{array}{lll}4.42 & 6.70 & 4 \\ 4.45 & 6.73 & 3-3 \\ 4.37 & 6.70 & 554 \\ 4.42 & 6.73 & 4-3\end{array}$

$$
\begin{array}{cc}
5-6 & 2^{3} G^{\circ}-P^{5} G \\
4-5 & (1161) \\
3-4 &
\end{array}
$$

$\begin{array}{lllll}15 n & 4.37 & 6.63 & 5-5 & 2^{3} G^{\circ}-e^{3} G \\ 10 n & 4.42 & 6.67 & 4-4 & (1163) \\ (2) & 4.45 & 6.71 & 3-3 & \\ 3 & 4.37 & 6.67 & 5-4 & \end{array}$

$\left(\begin{array}{l}10 n \\ 2 \\ 3\end{array}\right)$

( $\left.\begin{array}{l}\mathrm{Fe} \\ 2 \\ 1\end{array}\right)$

$\begin{array}{ll}4.43 & 6.71 \\ 4.42 & 6.63\end{array}$

$\begin{array}{ll}4.42 & 6.63 \\ 4.45 & 6.67\end{array}$

$4-3$
$4-5$
$3-4$

(1)

$\begin{array}{ll}4.43 & 6.64 \\ 4.45 & 6.67\end{array}$

$4.37 \quad 6.65$
4.45

$\begin{array}{lll}35 \mathrm{n} & 4.37 & 6.65 \\ 30 \mathrm{n} & 4.42 & 6.70 \\ 15 \mathrm{n} & 4.45 & 6.73\end{array}$

$\begin{array}{ccc}15 \mathrm{n} & 4.45 & 6.73 \\ 0 & 4.37 & 6.70 \\ \text { (1) } & 4.43 & 6.73\end{array}$

$\begin{array}{lll}6 & 4.37 & 6.73 \\ \text { (1n) } & 4.37 & 6.75\end{array}$

$\begin{array}{lll}1 n) & 4.37 & 6.75 \\ 1) & 4.42 & 6.81 \\ -3 & 4.45 & 6.84 \\ 6 & 4.42 & 6.75\end{array}$

$\begin{array}{lll}\text { (in) } & 4.42 & 6.75 \\ \text { 1) } & 4.45 & 6.81 \\ & 4.45 & 6.75\end{array}$

$\begin{array}{cc}4-3 & 2^{3} G^{0}-P^{3} D \\ 3-2 & (1164) \\ 5-6 & 2^{3} G^{0}-e^{3} H\end{array}$

$\begin{array}{cc}5-6 & z^{3} \sigma^{0}-\theta^{3} H \\ 4-5 & (1165) \\ 3-4 & \end{array}$

$3-4$
$5-5$
$4-4$
$5-4$

$\begin{array}{ll}5-4 & 2^{3} G^{0}-f^{3} F \\ 4-3 & (1166)\end{array}$

$4-4$
$3-2$
$4-4$
$3-3$
$3-4$

$4.42 \quad 6.97 \quad 4-5 \quad z^{3} \mathrm{G}^{0}-1$

$\begin{array}{lllll}\text { C8 } & 4.37 & 6.97 & 5- & z^{3} G^{0}-3\end{array}$

$\begin{array}{lllll}\odot & 4.42 & 7.14 & 4-3 & z^{3} G^{\circ}-15 D \\ \odot & 4.45 & 7.16 & 3-2 & (1169)\end{array}$

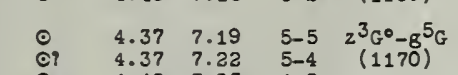

$-\frac{C}{2} \frac{4.42}{4.59} \frac{7.25}{5.85} 3-4 \quad \mathrm{y}^{3} \mathrm{~F}^{\circ}-\mathrm{e}^{5 \mathrm{~F}}$

$\begin{array}{rllll}10 & 4.53 & 5.85 & 4-4 & (1171) \\ 2 & 4.59 & 5.90 & 3-3 & \end{array}$

$\begin{array}{llllll}150 & 4.53 & 5.93 & 4-4 & \mathrm{y}^{3} F^{\circ}-\mathrm{e}^{3} F \\ 120 & 4.59 & 5.99 & 3-3 & (1172)\end{array}$

$\begin{array}{rrrrr}120 & 4.59 & 5.99 & 3-3 & (1172) \\ 100 & 4.63 & 6.04 & 2-2 & \\ 20 & 4.53 & 5.99 & 4-3 & \\ 8 & 4.59 & 6.04 & 3-2 & \\ 2 & 4.59 & 5.93 & 3-4 & \end{array}$

$\begin{array}{rrrr}8 & 4.59 & 6.04 & 3-2 \\ 20 & 4.59 & 5.93 & 3-4 \\ 8 & 4.63 & 5.99 & 2-3\end{array}$

$\begin{array}{lllll}40 & 4.59 & 6.39 & 3-2 & \text { (1173) } \\ 30 & 4.63 & 6.42 & -21 & \end{array}$

$\begin{array}{lll}4.63 & 6.42 & 3-1 \\ 4.59 & 6.33 & 3-3 \\ 4.63 & 6.39 & 2-3\end{array}$

$\begin{array}{lllll}c & 4.63 & 6.43 & 2-2 & \mathrm{y}^{3} \mathrm{~F}^{\circ}-8^{5} \mathrm{D} \\ 5 & 4.53 & 6.39 & 4-3 & (1174)\end{array}$

$\begin{array}{lll}4.59 & 6.43 & 3-2 \\ 4.63 & 6.45 & 2-1\end{array}$

$\begin{array}{cll}\odot & 4.53 & 6.55 \\ (1 \mathrm{n}) & 4.59 & 6.59 \\ 6 & 4.53 & 6.59\end{array}$

(1) 4.53 6.59

$\begin{array}{lll}(0 & 4.63 & 6.70 \\ (2 \mathrm{n}) & 4.59 & 6.70 \\ \text { aw,d) } & 4.63 & 6.71\end{array}$

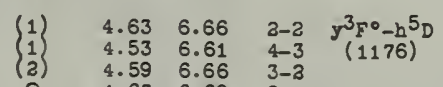

$\begin{array}{clll}(2) & 4.59 & 6.66 & 3-3 \\ \odot & 4.63 & 6.68 & 2-1 \\ \odot & 4.59 & 6.56 & 3-4\end{array}$

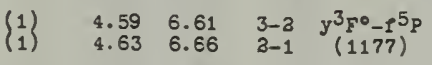

$\begin{array}{lllll}15 & 4.53 & 6.58 & 4-5 & \mathrm{y}^{3} F^{\circ}-\mathbf{r}^{5} \mathrm{G} \\ 10 \mathrm{n} & 4.59 & 6.64 & 3-4 & (1178)\end{array}$

$\begin{array}{lll}(3 n) & 4.59 & 6.64 \\ 3 n) & 4.63 & 6.69 \\ 2 n) & 4.53 & 6.64\end{array}$

$\begin{array}{llll}\circ & 4.53 & 6.64 & 4-4 \\ 0 & 4.59 & 6.69 & 3-3\end{array}$

$\begin{array}{lll}\odot & 4.53 & 6.69 \\ \odot & 4.59 & 6.71\end{array}$

5855.126
5891.16

5891.16
5696.10

5554.895

5565.708
5598.303

5598.303
5421.85

5488.14
5705.988

5705.988
$\mathbf{5 6 7 9 . 0 2 3}$

5642.75
5759.270

5759.270
5057.83

7053.48

6864.31
7115.25

7120.01

7330.16

7473.56

7421.60
7175.937

7385.386
7366.37

7292.856

7430.90

7431.94
7221.22
7295.27
7093.10

7109.67
7161.04

6917.53

7034.08
6845.93

6989.64
6803.84

6803.30

6920.16
6838.08

6692.47
6848.86

.6951 .261

6881.74

6881.74
6855.74

6833.24

6676.86
6717.556

7071.88
6976.306

$\begin{array}{cc}4-5 & y^{3} F^{0}-8^{5} F \\ 3-4 & (1175)\end{array}$

6855.176

6841.349

6663.26

6713.14
6753.724

6541.49
6639.71

6753.45
6936.48

6633.764

6705.117
6842.668

6842.668
6533.97

6726.668
6810.28

6810.38
6820.43

6012.75

5995.93

5933.80
5715.80
(1) $\quad 4.37 \quad 6.56 \quad 5-6 \quad z^{3} G^{0}-r^{5} G$

$\begin{array}{lllll}60 & 4.53 & 6.33 & 4-3 & y^{3} F^{\circ}-e^{3} D\end{array}$ (3d) $4.53 \quad 6.65 \quad 4-5 \quad y^{3} 5^{0}-0^{5}-\frac{1}{2}$ cont1nued

5862.357

5914.16
5930.173
5752.043
580.727
5650.31

5859.608

-5914.16
5905.673

5686.533
5747.95

5747.95
5594.661

$\begin{array}{clll}(1) & 4.59 & 6.70 \\ \odot & 4.63 & 6.73 \\ \odot & 4.53 & 6.70 \\ (1) & 4.59 & 6.73\end{array}$

$\begin{array}{lc}4-5 & \mathrm{y}^{3} \mathrm{~F}^{0}-e^{5} \mathrm{H} \\ 3-4 & (1179) \\ 3-3 & (119) \\ 4-4 & \\ 3-3 & \end{array}$

$\begin{array}{lllll}8 & 4.53 & 6.63 & 1-5 & y^{3} F^{\circ}-e^{3} G \\ 8 & 4.59 & 6.67 & 3-4 & (1180) \\ 8 & 4.63 & 6.71 & 3-3 & (110)\end{array}$

$\begin{array}{cccc}8 & 4.63 & 6.71 & 3-3 \\ (3) & 4.53 & 6.67 & 4-4 \\ 3 & 4.59 & 6.71 & 3-3 \\ 0 & 4.53 & 6.71 & 4-3\end{array}$

$\begin{array}{lllll}5 & 4.53 & 6.64 & 4-3 & y^{3} F^{\circ}-f^{3} \mathrm{D} \\ 8 & 4.59 & 6.67 & 3-2 & (1181) \\ 3 \mathrm{n} & 4.63 & 6.73 & 3-1 & (12\end{array}$

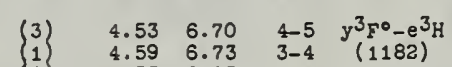

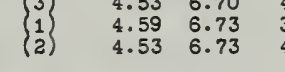

$\begin{array}{lllll}4 & 4.53 & 6.75 & 4-4 & \mathrm{y}^{3} F^{\circ}-P^{3} \\ 4 & 4.59 & 6.81 & 3-3 & (1183)\end{array}$

$\begin{array}{llll}4 & 4.63 & 6.84 & 2-2 \\ \odot & 4.53 & 6.81 & 4-3 \\ \odot & 4.59 & 6.84 & 3-3\end{array}$

(1)

$\begin{array}{llll}4.59 & 6.77 & 3-2 & \mathrm{y}^{3} F^{\circ}-e^{3} \mathrm{p} \\ 4.63 & 6.77 & 3-3 & (1184)\end{array}$

$\begin{array}{lllllll}5057.83 \quad P & \odot & 4.53 & 6.97 \quad 4-\quad y^{3} F^{\circ}-3 \\ (1185)\end{array}$

$\begin{array}{lrrrrrr}6930.35 & \mathrm{P} & \odot & 4.54 & 6.33 & 3-4 & \mathrm{y}^{5} \mathrm{P}^{\circ}-\mathrm{-}^{7} \mathrm{~F} \\ .7145 .317 & \mathrm{~V} & 5 & 4.59 & 6.31 & 2-3 & (1186) \\ .6951 .361 & \mathrm{I} & 25 & 4.54 & 6.31 & 3-3 & \end{array}$

7395.00
.7356 .81
.7232 .88

7320.6948

7034.06

6862.481

6639.71

$\begin{array}{lll}4.54 & 6.31 & 3-3 \\ 4.59 & 6.34 & 3-2\end{array}$

$\begin{array}{lll}4.59 & 6.34 & 3-2 \\ 4.54 & 6.34 & 3-2\end{array}$

$\begin{array}{lllll}\text { O? } & 4.59 & 6.32 & 3-1 & \\ \circ & 4.54 & 6.37 & 3-4 & \text { y } 5 \text { po-7 } 7 \mathrm{D}\end{array}$

9881.5

9747.24
9950.70

10084.42

$\begin{array}{lllll}1 & 4.59 & 6.28 & 3-3 & (1187) \\ 0 & 4.62 & 6.30 & 1-2 & \\ 0 & 4 & \end{array}$

$\begin{array}{cccc}\odot & 4.63 & 6.30 & 1-1 \\ 10 n & 4.54 & 6.30 & 3-2\end{array}$

$\begin{array}{rrrrr}\text { Sn } & 4.54 & 6.32 & 3-4 & \mathrm{y}^{5} \mathrm{Po}-\mathrm{P} 5 \mathrm{D} \\ (1) & 4.59 & 6.24 & 3-3 & (1188) \\ 3 \mathrm{n} & 4.54 & 6.34 & 3-3 & \end{array}$

$4.596 .36 \quad 3-3$

$\begin{array}{lll}4.62 & 6.38 & 1-1 \\ 4.54 & 6.26 & 3-2\end{array}$

$\begin{array}{lll}4.59 & 6.38 & 3-1 \\ 4.62 & 6.39 & 1-0\end{array}$

$\begin{array}{lllll}3 \mathrm{n} & 4.54 & 6.23 & 3-4 & \mathrm{y}^{5} \mathrm{po}^{-}-\mathrm{e}^{7 \mathrm{p}} \\ 1 & 4.59 & 6.25 & 2-3 & (1189)\end{array}$

$\begin{array}{ll}4.59 & 6.25 \\ 4.62 & 6.28\end{array}$

$\begin{array}{lll}4.54 & 6.25 & 3-3 \\ 4.59 & 6.28 & 3-2\end{array}$

$\begin{array}{lll}4.59 & 6.28 & 2-2 \\ 4.54 & 6.38 & 3-2\end{array}$

$\begin{array}{llll}4.54 & 6.29 & 3-4 & y^{5 \mathrm{p}^{\circ}}-\mathrm{e}^{5} \mathrm{G} \\ 4.59 & 6.32 & 2-3 & (1190)\end{array}$

$\begin{array}{ll}4.63 & 6.34\end{array}$

$\begin{array}{lll}4.59 & 6.34 & 2-2 \\ 4.54 & 6.34 & 3-2\end{array}$

9937.10

10036.10

9839.38

9955.85

9636.69

9235.55

8848.46

8576.50

8525.04

8253.78

8149.59
8002.55

8002.55
8196.53

8269.66

.8149 .59

7129.30

7125.28
6949.37

$\begin{array}{lllll}4 \mathrm{n} & 4.54 & 6.34 & 3-4 & \mathrm{y}^{5 \mathrm{p}^{\circ}}-\mathrm{e}^{70} \mathrm{G}\end{array}$

$\begin{array}{lllll}\odot & 4.59 & 6.35 & 2-3 & \text { (1191) } \\ \odot & 4.54 & 6.35 & 3-3 & \end{array}$

$\begin{array}{ccccc}\odot & 4.54 & 6.35 & 3-4 & y^{5 p^{\circ}}-p^{5} F \\ \odot & 4.59 & 6.37 & 2-3 & (1192)\end{array}$

$\begin{array}{llll}4 \mathrm{nI} & 4.54 & 6.37 & 3-3 \\ 1 & 4.54 & 6.38 & 3-2\end{array}$

$\begin{array}{llll}4.54 & 6.31 & 3-2 & \mathrm{y}^{5} \mathrm{p}^{\circ}-\mathrm{e}^{5} \mathrm{~s} \\ 4.59 & 6.31 & 3-2 & (1193)\end{array}$

$\begin{array}{llll}4.54 & 6.33 & 3-3 & \mathrm{r}^{5} \mathrm{P}^{\circ}-\mathrm{e}^{3} \mathrm{D}\end{array}$

$\begin{array}{llll}4.59 & 6.39 & 2-3 & (1194)\end{array}$

$\begin{array}{lll}4.54 & 6.39 & 3-2 \\ 4.59 & 6.42 & 3-1\end{array}$

$\begin{array}{lll}4.59 & 6.42 & 3-1 \\ 4.59 & 6.33 & 2-3\end{array}$

$\begin{array}{lll}4.63 & 6.39 & 1-2\end{array}$

6805.72

6983.53
6822.00

6822.00

7089.73
6932.49

7011.364

6947.501
7010.362

7010.363
7027.60

6976.93
6930.64

6960.334

6926.40

.6947 .50

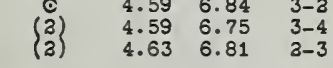

7381.54

(1) $4.596 .30 \quad 2-3$

$4.54 \quad 6.33 \quad \frac{1-2}{3-3}$

$\begin{array}{lll}4.63 & 6.42 & 1-1 \\ 4.54 & 6.39 & 3.2\end{array}$
I A Laboratory Ref Int Low ${ }_{\text {High }}^{\text {L }}$

Yultiplet

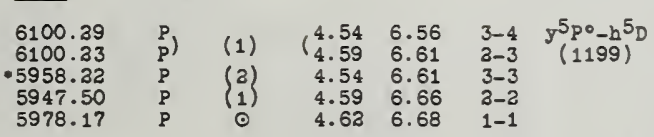

$\begin{array}{lllllll}6098.28 & P & \odot & 4.54 & 6.56 & 3-3 & y^{5} P^{\circ}-P^{5} P\end{array}$

$\begin{array}{lllllll}6091.74 & P & \odot & 4.59 & 6.61 & 2-2 & (1200) \\ 5950.13 & P & \odot & 4.54 & 6.61 & 3-2 & \end{array}$

$\begin{array}{llcllll}5880.00 & \mathrm{~V} & (2 \mathrm{md}) & 4.54 & 6.64 & 3-4 & \mathrm{y}^{5} \mathrm{P} \circ-\mathrm{P}^{5} \mathrm{G} \\ 5879.49 & \mathrm{P} & \odot & 4.59 & 6.69 & 2-3 & (1201) \\ 5893.46 & \mathrm{P} & \odot & 4.62 & 6.71 & 1-2 & \end{array}$

$5640.46 \quad$ (1n) $4.54 \quad 6.73 \quad 3-3 \quad y^{5} \mathrm{P}^{\circ}-e^{5 \mathrm{H}}$

$\begin{array}{lllllll}5887.46 & P & \odot & 4.54 & 6.64 & 3-3 & (1203) \\ 5867.01 & P & \odot & 4.62 & 6.72 & 1-1 & (1203) \\ 5778.81 & P & \odot & 4.54 & 6.67 & 3-2 & \end{array}$

$\begin{array}{lllllll}.5759 .57 & P & (2) & 4.54 & 6.68 & 3-4 & y^{5} P_{0}-g^{7} D \\ 5727.75 & U & (1) & 4.59 & 6.74 & 2-2 & (1204)\end{array}$

$\begin{array}{lllllll}-5620.04 \quad \text { (1) } & 4.54 & 6.73 & 3-4 & y^{5} \mathrm{po}^{\circ}-\mathrm{e}^{3 \mathrm{H}}\end{array}$

$\begin{array}{lllllll}4776.34 & \mathrm{~V} & (1 \mathrm{n}) & 4.54 & 7.12 & 3-4 & (1205) \\ 4839.77 & \mathrm{P} & \circ & 4.59 & 7.14 & 2-3 & (1206) \\ 479.93 & \mathrm{~V} & (1) & 4.54 & 7.14 & 3-3 & \\ 48.53 & \end{array}$

$\begin{array}{llllll}4802.53 & \mathrm{P} & (1) & 4.54 & 7.14 & 3-3 \\ 4714.074 & \mathrm{~V} & (1 \mathrm{n}) & 4.59 & 7.16 & 2-2 \\ 4.54 & 7.16 & 3-2\end{array}$

$\begin{array}{llllllll}4661.538 & J & (2 n) & 4.54 & 7.19 & 3-2 & y^{5} p^{\circ}-4\end{array}$

$\begin{array}{lllllll}10333.24 & P & \odot & 4.57 & 5.77 & 4-4 & d^{3} F-u^{5} D^{\circ} \\ 10307.48 & P & \odot & 4.57 & 5.77 & 4-3 & (1208)\end{array}$

$\begin{array}{lllllll}10156.50 & P & \odot & 4.57 & 5.79 & 4-4 & d^{3} F-x^{3} F^{\circ}\end{array}$ 
64 I A A Reoratory Int Low ${ }_{\text {H1gh }}$ J Multiplet
Po I continued

$\begin{array}{lllllll}5955.13 & \mathrm{P} & \odot & 4.56 & 6.64 & 3-3 & \mathrm{~d}^{3} F-\mathrm{x}^{1} F^{\circ} \\ 5942.71 & \mathrm{P} & \odot & 4.56 & 6.64 & 3-3 & (1233)\end{array}$ $\begin{array}{llllllll}5903.53 \quad V & \text { (1) } 4.57 \quad 6.66 & 4-5 & d^{3} F-t^{3} G^{\circ}\end{array}$ $\begin{array}{lllllll}5791.53 & \mathrm{P} & \odot & 4.56 & 6.70 & 3-4 & (1234) \\ 5661.03 & \mathrm{P} & \odot & 4.56 & 6.74 & 3-3 & \end{array}$ $\begin{array}{llllll}5661.03 & P & C & 4.56 & 6.74 & 3-3 \\ 5815.33 & F & (1) & 4.57 & 6.70 & 4-4 \\ 5672.38 & P & (1) & 4.56 & 6.74 & 3-3\end{array}$

$\begin{array}{lllllll}5895.007 & U & (1) & 4.57 & 6.67 & 4-3 & d^{3} F-11^{\circ} \\ 5870.65 & P & \odot & 4.56 & 6.67 & 3-3 & (1235)\end{array}$ $\begin{array}{lllllll}5891.89 & P & \text { (1) } & 4.57 & 6.67 & 4-5 & \mathrm{~d}^{3} F-12^{\circ}\end{array}$ $\begin{array}{llllllll}5793.70 & P & \odot & 4.57 & 6.70 & 4-4 & d^{3}(1336) \\ 5770.17 & P & \odot & 4.56 & 6.70 & 3-4 & (1236 \mathrm{a})\end{array}$ $\begin{array}{llllllll}5438.04 & P & \odot & 4.57 & 6.84 & 4-5 & d^{3} F-v^{3} H^{\circ} \\ 5412.56 & P & \odot & 4.56 & 6.84 & 3-4 & (1237)\end{array}$ $\begin{array}{llllllll}5313.839 & T & (-) & 4.56 & 6.88 & 2-2 & d^{3} F-\pi^{1} D^{\circ}\end{array}$ $\begin{array}{lllllll}5313.41 & P & \odot & 4.56 & 6.89 & 3-3 & \mathrm{~d}^{3} F-w^{1} F^{\circ}\end{array}$ $\begin{array}{llllllll}5300.41 & P & \odot & 4.57 & 6.90 & 45 & d^{3} F-8^{3} G^{\circ} \\ 5381.18 & P & \odot & 4.56 & 6.90 & 3-4 & (1240)\end{array}$

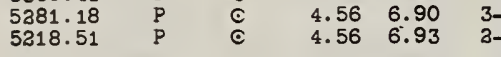

$\begin{array}{lllllll}5170.08 & P & \odot & 4.57 & 6.96 & 4-5 & \mathrm{~d}^{3} \mathrm{~F}-\mathrm{u}^{3} \mathrm{H}^{\circ}\end{array}$ $\begin{array}{lllllll}5114.52 & P & \odot & 4.57 & 6.99 & 4-4 & \mathrm{~d}^{3}(1241) \\ 5047.14 & P & \odot & 4.56 & 7.01 & 3-3 & (1242)\end{array}$ $\begin{array}{llllll}504.14 & \mathrm{P} & \odot & 4.56 & 7.02 & 2-2 \\ 5019.18 & \mathrm{P} & \odot & 4.56 & 6.99 & 3-4\end{array}$

4875.72

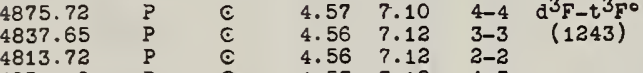

$\begin{array}{llllll}485.72 & P & \odot & 4.56 & 7.12 & 2-2 \\ 4854.18 & P & \odot & 4.57 & 7.12 & 4-3\end{array}$

$\begin{array}{lllllllllll}4369.29 & P & \odot & 4.57 & 7.40 & 4-5 & \mathrm{~d}^{3} F-r^{3} G^{\circ}\end{array}$

$\begin{array}{llllllll}4253.55 & P & \odot & 4.57 & 7.47 & 4-5 & \mathrm{~d}^{3} \mathrm{~s}-\mathrm{t}^{3} \mathrm{H}^{\circ} \\ 4203.67 & P & \odot & 4.56 & 7.50 & 3-4 & (1245)\end{array}$

$\begin{array}{lllllll}10863.60 & D & 5 & 4.71 & 5.85 & 3-4 & y^{3} D^{\circ}-e^{5 F} \\ 11013.27 & D & 1 & 4.77 & 5.90 & 2-3 & (1246)\end{array}$

$\begin{array}{llllll}10435.38 & P & 4.71 & 5.90 & 3-3 & \end{array}$

$\begin{array}{rrrrrrr}10316.351 & E & 100 & 4.71 & 5.92 & 3-4 & y^{3} D^{\circ}-e^{3} F \\ 10145.601 & E & 80 & 4.77 & 5.99 & 2-3 & (1247)\end{array}$

10065.080

9653.143

9753.129

$8016.51 \quad P \quad \odot$

7620.538
7653.783

7664.15

7370.16

7924.14
7844.55

7353.528

7476.40

7563.03

7205.51
7385.54

7196.37

6569.231

6597.607

๓6416.94

6495.779
6364.384

6673.84

6604.67

6468.86

6671.43

6713.76
$6 \in 96.30$

6494.52

6860.13

6411.10

6456.87

6253.82
6171.01

6224.23

6419.982

$\varepsilon 496.456$

6390.968

6338.896

6634.10
6633.44

6055.987

6078.496 6102.178
5898.212

$\begin{array}{lll}4.81 & 6.04 & 1-3 \\ 4.71 & 5.99 & 3-3\end{array}$

$\begin{array}{lll}4.71 & 5.99 & 3-3 \\ 4.77 & 6.04 & 2-3 \\ 4.71 & 6.04 & 3-2\end{array}$

$\begin{array}{llll}4.77 & 6.31 & 2-3 & \mathrm{y}^{3} \mathrm{D}^{0}-\mathrm{e}^{5} \mathrm{~S}\end{array}$

$\begin{array}{llll}4.71 & 6.33 & 3-3 & \mathrm{y}^{3} \mathrm{D}^{\circ}-\mathrm{e}^{3} \mathrm{D} \\ 4.77 & 6.39 & 2-2 & (1350)\end{array}$

$\begin{array}{lll}4.81 & 6.42 & 1-1 \\ 4.71 & 6.39 & 3-2\end{array}$

$\begin{array}{llll}1 & 4.71 & 6.39 & 3-2 \\ \text { (1) } & 4.77 & 6.42 & 2-1 \\ 0 & 4.77 & 6.33 & 2-3\end{array}$

$\begin{array}{lll}4.77 & 6.33 & 2-3 \\ 4.81 & 6.39 & 1-2\end{array}$

$\begin{array}{lllll}1 & 4.71 & 6.39 & 3-3 & \mathrm{y}^{3} \mathrm{D}^{0}-\mathrm{g}^{5} \mathrm{D} \\ \text { (1) } & 4.77 & 6.43 & 2-2 & (1251)\end{array}$

$\begin{array}{lll}4.81 & 6.45 & 1-1 \\ 4.71 & 6.43 & 3-2\end{array}$

$\begin{array}{lll}4.77 & 6.45 & 2-1\end{array}$

$$
\odot
$$

$4.71 \quad 6.43 \quad 3-2 \quad y^{3} p^{\circ}-e^{5 P}$

$\begin{array}{llllll}50 \mathrm{n} & 4.71 & 6.59 & 3-4 & \mathrm{y}^{3} \mathrm{D}^{\circ}-8^{5} \mathrm{~F} \\ 15 n & 4.77 & 6.65 & 2-3 & (1253)\end{array}$

$\begin{array}{cccc}15 \mathrm{n} & 4.77 & 6.65 & 2-3 \\ \mathrm{C} & 4.71 & 6.65 & 3-3 \\ \mathrm{Fe}^{+} & 4.77 & 6.70 & 2-2\end{array}$

$\begin{array}{cccc}\mathrm{Fe}^{+} & 4.77 & 6.70 & 2-2 \\ 3 & 4.81 & 6.71 & 1-1\end{array}$

$\begin{array}{lllll}(0 & 4.71 & 6.56 & 3-4 & \mathrm{y}^{3} \mathrm{D}^{\circ}-\mathrm{h}^{5} \mathrm{D} \\ \text { (i) } & 4.81 & 6.68 & 1-1 & (1254)\end{array}$

$\begin{array}{llll}\text { (1) } & 4.81 & 6.68 & 1-1 \\ \text { in) } & 4.71 & 6.66 & 3-2 \\ \text { C } & 4.77 & 6.68 & 2-1\end{array}$

$\begin{array}{llllll}P & & 4.71 & 6.56 & 3-3 & y^{3} D^{0}-P^{5 p} \\ V & 3 n & 4.77 & 6.61 & 2-2 & (1255) \\ P & & 4.81 & 6.66 & 1-1 & \end{array}$

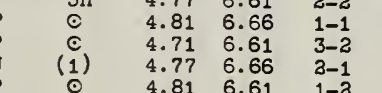

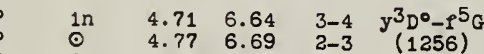

$\begin{array}{llllll}\mathrm{P} & \odot & 4.77 & 6.69 & 2-3 & (1256) \\ \mathrm{P} & \odot & 4.71 & 6.69 & 3-3 & \\ \mathrm{P} & \odot & 4.71 & 6.71 & 3-2 & \end{array}$

$\begin{array}{lllll}30 n & 4.71 & 6.64 & 3-3 & y^{3} D^{\circ}-f^{3} \mathrm{D} \\ 20 n & 4.77 & 6.67 & 2-2 & (1258)\end{array}$

$\begin{array}{llll}15 \mathrm{n} & 4.81 & 6.72 & 1-1 \\ 3 \mathrm{n} & 4.71 & 6.67 & 3-2\end{array}$

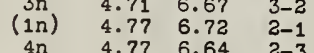

$\begin{array}{llll}4 \mathrm{n} & 4.77 & 6.64 & 2-3 \\ 4 \mathrm{n} & 4.81 & 6.67 & 1-2\end{array}$

$\begin{array}{lllll}4 & 4.71 & 6.75 & 3-4 & y^{3} p^{\circ}-p^{3 F} \\ 4 n & 4.77 & 6.81 & 2-3 & (1259)\end{array}$

$\begin{array}{llll}5 & 4.81 & 6.84 & 1-2 \\ \text { (1) } & 4.71 & 6.81 & 3-3\end{array}$ $\mathrm{m} 8930.02$
.9157 .08

$\begin{array}{lllll}4 & 4.71 & 6.70 & 3-4 & \mathrm{y}^{3} \mathrm{D}^{\circ}-\mathrm{e}^{5} \mathrm{H}\end{array}$

\author{
I A Raboratory ReP Int Low ${ }^{E} P_{\text {H1gh }}$
}

\section{Fe I continued}

$\begin{array}{lllllll}5984.805 & \mathrm{~K} & 8 & 4.71 & 6.77 & 3-2 & \mathrm{y}^{3} \mathrm{D}^{\circ}-\mathrm{e}^{3 \mathrm{P}} \\ 5987.057 & \mathrm{~K} & 6 & 4.77 & 6.84 & 3-1 & (1260) \\ .5975 .355 & \mathrm{~J} & 4 & 4.81 & 6.88 & 1-0 & \\ 6170.492 & \mathrm{~K} & 4 \mathrm{n} & 4.77 & 6.77 & 2-2 & \\ 6103.190 & \mathrm{X} & 3 & 4.81 & 6.84 & 1-1 & \\ 6293.93 & \mathrm{P} & \odot & 4.81 & 6.77 & 1-3 & \end{array}$

9328.64

9006.72
9253.73

9298.05

9178.57
9392.77

9342.32

9259.05

$9462.9 ?$
9550.90

9164.51
9318.13

9388.28
-9452.45

9214.45
9394.71

9394.71
9404.80
9100.50

9024.47
.9080 .48

9080.48
9116.89
8805.21

8805.21
8887.10

8616.27
8796.42

8796.43
8902.94
8978.04

8978.04
8538.02

8700.34
8956.26

8956.26
8447.41

8710.29

8699.43
8790.62

Multiplet

$\begin{array}{cccccc}\mathrm{Fe} & & 4.89 & 6.38 & 4-5 & \mathrm{x}^{5} \mathrm{D}^{0}-\mathrm{e}^{7} \mathrm{~F} \\ (3) & 4.97 & 6.31 & 2-3 & (1261) \\ \odot & 5.00 & 6.33 & 0-1 & \\ \odot & 4.89 & 6.33 & 4-4 & \\ 1 & 4.97 & 6.34 & 2-2 & \\ 0 & 4.99 & 6.32 & 1-1 & \end{array}$

$\begin{array}{llll}1 & 4.97 & 6.34 & 2-2 \\ \odot & 4.99 & 6.32 & 1-1\end{array}$

$\begin{array}{llllll}\circ & 4.89 & 6.22 & 4-5 & x^{5} D^{0}-P^{7} \mathrm{D} \\ \text { in } & 4.93 & 6.28 & 3-3 & (1263)\end{array}$

$\begin{array}{llll}0 & 4.99 & 6.30 & 1-1 \\ 2 & 4.97 & 6.30 & 2-1\end{array}$

$\begin{array}{rllll}15 & 4.89 & 6.22 & 4-4 & x^{5} p^{\circ}-p^{5} D \\ 2 & 4.93 & 6.34 & 3-3 & (1263)\end{array}$

(1) $4.97 \quad 6.26$

$\begin{array}{llll}3 & 4.93 & 6.24 & 4-3 \\ 3 \mathrm{n} & 4.97 & 6.26 & 3-2\end{array}$

$\begin{array}{llll}2 & 4.99 & 6.29 & 1-1 \\ 2 & 4.9 & \end{array}$

$\begin{array}{llllll}6 & 4.89 & 6.23 & 4-4 & x^{5} D^{\circ}-e^{7} P \\ 3 n & 4.93 & 6.25 & 3-3 & (1264)\end{array}$

$\begin{array}{llll}\circ \mathrm{n} & 4.97 & 6.38 \\ 5.89 & 6.35\end{array}$

\begin{tabular}{ccc}
15 & 4.89 & 6.26 \\
$3 \mathrm{n}$ & 4.93 & 6.29 \\
\hline & 4.99 & 6.34
\end{tabular}

$\begin{array}{lll}\odot & 4.99 & 6.34 \\ \circ & 4.89 & 6.29 \\ \odot & 4.93 & 6.32\end{array}$

c 4.896 .32

$\begin{array}{ll}4.89 & 6.32 \\ 4.93 & 6.34 \\ 4.97 & 6.35 \\ 4.99 & 6.36\end{array}$

$\begin{array}{ll}4.97 & 6.35 \\ 4.99 & 6.36 \\ 4 & .99 \\ 4.93 & 6.35\end{array}$

$\begin{array}{ll}4.89 & 6.34 \\ 4.93 & 6.35\end{array}$

$\begin{array}{ll}4.93 & 6.35 \\ 4.99 & 6.37\end{array}$

$\begin{array}{ll}4.99 & 6.37 \\ 4.89 & 6.35 \\ 4 & 6.37\end{array}$

$\begin{array}{lll}\odot & 4.89 & 6.35 \\ \odot & 4.97 & 6.37\end{array}$

$\begin{array}{lll}20 \mathrm{n} & 4.89 & 6.31 \\ (4 \mathrm{n}) & 4.93 & 6.35\end{array}$

$\begin{array}{ccc}(4 n) & 4.93 & 6.35 \\ 10 n & 4.97 & 6.37 \\ 5 & 4.99 & 6.38 \\ 3 & 5.00 & 6.39\end{array}$

(an) $\begin{array}{ll}4.89 & 6.35 \\ 4.93 & 6.37\end{array}$

$\begin{array}{ccc} & \\ 10 & 4.97 & 6.38 \\ 4 \mathrm{n} & 4.99 & 6.39\end{array}$

852.97

8713.19
8808.17
8519.05

-9157.07

8567.78

8493.79

8686.77
8592.10

9036.74
8819.48
8656.67

8819.48
8656.67

8526.685

8471.75
8459.01
8465.23

8465.23
8275.91
8342.95

8342.95
8434.51

8784.44
8663.73

8584.82
8527.88

8369.87
8816.86

8816.86
8186.80

8186.80
8263.86

8480.63
8424.14

8424.14
8446.42

8607.08
8613.93
8571.84

8571.84
8671.86

7440.98

7440.98
7447.43
7351.160

7351.160
7216.68

7216.68
7194.92

7261.29
7212.47

7212.47
7127.58

6997.13
7062.80

7389.34

7363.96
7278.48
7382.39

7181.93
7142.522

7191.66

7582.15
7508.53

$\begin{array}{cc}-5 & x^{5} D^{\circ}-e^{5} a \\ -4 & (1265)\end{array}$

$\begin{array}{cc}-5 & x^{5} D^{0}-e^{7} G \\ -4 & (1266)\end{array}$

$4-4$
$3-3$

$3-3$
$1-1$
$1-3$

$-5 \quad x^{5} D^{0}-t^{5} F$

3-4 (1267)

$1-2$

-4
-3

$\begin{array}{lll}4.93 & 6.38 \quad 3-2\end{array}$

$\begin{array}{llll}\text { (a) } \quad 4.97 & 6.31 & 2-2 & x^{5} D^{0}-e^{5} s\end{array}$

$\begin{array}{lllll}\odot & 4.89 & 6.33 & 4-3 & x^{5} D^{0}-e^{3} \mathrm{D} \\ \odot & 4.93 & 6.39 & 3-2 & (1269)\end{array}$

$\begin{array}{llll}\odot & 4.97 & 6.42 & 2-1 \\ \odot & 4.93 & 6.33 & 3-3\end{array}$

$\therefore \quad 4.976 .39 \quad 3-2$

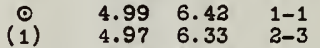

$\begin{array}{clll}\odot & 4.99 & 6.39 & 1-2 \\ \odot & 5.00 & 6.42 & 0-1\end{array}$

$\begin{array}{lllll}8 & 4.89 & 6.34 & 4-4 & x^{5} \mathrm{D}^{0}-8^{5} \mathrm{D} \\ 2 & 4.93 & 6.39 & 3-3 & (1270) \\ 0 & 4.97 & 6.43 & 2-2 & (\end{array}$

$\begin{array}{lll}4 \mathrm{n} & 4.99 & 6.45 \\ & 4.93 & 6.43\end{array}$

$\begin{array}{ccc}(-) & 4.97 & 6.45 \\ 0 & 4.99 & 6.45\end{array}$

4.996 .45

\begin{tabular}{ll}
4.93 & 6.34 \\
4.97 & 6.39 \\
\hline & .99 \\
5.00 & 6.45
\end{tabular}

$\begin{array}{ll}4.99 & 6.43 \\ 5.00 & 6.45\end{array}$

$\begin{array}{ll}4.89 & 6.37 \\ 4.97 & 6.37\end{array}$

$2-2$
$1-1$
$3-2$

2-1

$1-0$
$3-4$
$2-3$

$2-3$
$1-2$
$0-1$

10nd? $4.896 .40-4-3 x^{5} 0^{\circ}-5^{5}$

$\begin{array}{ll}4.93 & 6.40 \\ 4.97 & 6.43\end{array}$

$4.97 \quad 6.43 \quad 3-2$

$\begin{array}{llll}\odot & 4.97 & 6.40 & 3-3 \\ \odot & 4.99 & 6.43 & 1-2 \\ \odot & 5.00 & 6.42 & 0-1\end{array}$

$\begin{array}{llllll}\text { 2n } & 4.89 & 6.55 & 4-5 & x^{5} D^{\circ}-8^{5} F \\ 1 & 4.93 & 6.59 & 3-4 & (1273) \\ \text { 2n } & 4.97 & 6.65 & 2-3 & \end{array}$

$\begin{array}{ll}4.97 & 6.65 \\ 4.99 & 6.70\end{array}$
8828.08

$\begin{array}{llll}\odot & 4.99 & 6.43 & 1-1 \\ \odot & 4.97 & 6.40 & 3-3\end{array}$

I A Laboratory $_{\text {Reft Lo }}^{\text {E P }}$

J Multiplet Fe I continued

$\begin{array}{lllllll}7386.394 & \mathrm{~L} & 8 \mathrm{n} & 4.89 & 6.56 & 4-3 & \mathrm{x}^{5} \mathrm{D}^{0}-\mathrm{P}^{5} \mathrm{P}\end{array}$ $\begin{array}{llllll}7300.47 & 0 & 4 & 4.93 & 6.61 & 3-2 \\ 7495.67 & \mathrm{P} & 0 & 4.97 & 6.66 & 2-1 \\ & 0 & 4.97 & 6.61 & 2-2\end{array}$

$\begin{array}{lllllll}7320.6948 & \mathrm{~L} & 5 \mathrm{n} & 4.89 & 6.58 & 4-5 & \mathrm{x}^{5} \mathrm{D}^{\circ}-\mathrm{r}^{5} \mathrm{G}\end{array}$

$\begin{array}{lllllll}7344.86 & \mathrm{~V} & \mathrm{Sn} & 4.89 & 6.58 & 4-5 & \mathrm{x}^{5} \mathrm{D}^{\circ}-\mathrm{F}^{5} \mathrm{C} \\ 7176.886 & \mathrm{~V} & 2 \mathrm{n} & 4.93 & 6.64 & 3-4 & (1276)\end{array}$

$\begin{array}{llllll}7176.886 & \mathrm{~V} & 2 \mathrm{n} & 4.97 & 6.69 & 2-3 \\ 7155.64 & \mathrm{~V} & 3 \mathrm{n} & 4.99 & 6.71 & 1-2\end{array}$

$\begin{array}{llllll}7068.60 & P & \odot & 4.89 & 6.64 & 4-4 \\ 7044.60 & 0 & \text { (1) } & 4.93 & 6.69 & 3-3\end{array}$

$\begin{array}{llllll}7068.02 & P & \odot & 4.97 & 6.71 & 3-3\end{array}$

$\begin{array}{lllllll}7083.396 & Y & \text { 1n } & 4.89 & 8.63 & 4-5 & x^{5} D^{0}-e^{3} G \\ 7091.91 & P & (1) & 4.93 & 6.67 & 3-4 & (1277)\end{array}$

$\begin{array}{llclll}7091.91 & P & (1) & 4.93 & 6.67 & 3-4 \\ 7066.15 & \mathrm{P} & \odot & 4.97 & 6.71 & 2-3\end{array}$

$\begin{array}{lllllll}7079.32 & P & 0 & 4.89 & 6.64 & 4-3 & x^{5} D^{0}-P^{3} \mathrm{D} \\ 7091.83 & 0 & (1) & 4.93 & 6.67 & 3-2 & (1278)\end{array}$

$\begin{array}{llllll}7031.42 & P & 0 & 4.97 & 6.72 & 3-1 \\ 7256.13 & P & (1) & 4.93 & 6.64 & 3-3\end{array}$

$\begin{array}{llllll}7225.82 & P & 0 & 4.97 & 6.67 & 2-2 \\ 72128 & P & 0 & 4.99 & 6.62 & 1-1\end{array}$

$\begin{array}{llllll}7118.12 & P & \odot & 4.99 & 6.72 & 1-1 \\ 7396.50 & P & \odot & 4.97 & 6.64 & 2-3\end{array}$

$\begin{array}{llllll}7317.40 & P & \odot & 4.99 & 6.67 & 1-2 \\ 7162.37 & P & \odot & 5.00 & 6.72 & 0-1\end{array}$

$\begin{array}{lllllll}6639.35 & P & \odot & 4.89 & 6.75 & 4-4 & x^{5} D^{0}-P^{3} F \\ 6794.60 & P & \odot & 4.93 & 6.75 & 3-4 & (1279) \\ 6713.44 & P & \odot & 4.97 & 6.81 & 2-3 & \end{array}$

6601.13

Fe

$4.93 \quad 6.77 \quad 3-2 \quad x^{5} p^{0}-e^{3} p$

$\begin{array}{lllll}\odot & 4.97 & 6.84 & 3-1 & (1280) \\ \odot & 4.99 & 6.88 & 1-0 & \end{array}$

$\therefore \quad 4.97 \quad 6.77 \quad 2-2$

$\begin{array}{lllllll}5531.949 \quad U & (1) \quad 4.89 & 7.12 & 4-4 & x^{5} D^{0}-1^{5} \mathrm{D}\end{array}$

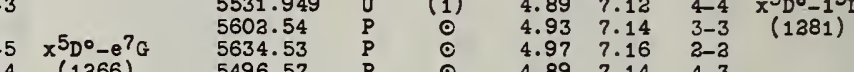

5552.70

5685.86

5479.95

5559.64
5613.70

9603.07

8863.64

9944.13

9608.89

9248.80

9811.36
9383.40

8967.53

8798.05

7909.60

6813.55

6345.84

5678.04
5748.15

(2)

$\circ$
$\circ$
$\circ$

$4.897 .14 \quad 4-3$

$\begin{array}{lll}4.93 & 7.16 & 3-2 \\ 4.97 & 7.14 & 2-3 \\ 4-9 & 7.16 & 1-3\end{array}$

$\begin{array}{lll}4.97 & 7.14 & 2-3 \\ 4.99 & 7.16 & 1-2\end{array}$

$\begin{array}{lllll}\circ & 4.93 & 7.19 & 3-2 & x^{5} D^{0}-4 \\ \circ & 4.97 & 7.19 & 2-3 & (1383)\end{array}$

$\begin{array}{llllll}2 & 4.99 & 6.28 & 4-5 & y^{7} p^{0}-e^{7} \bar{F} \\ 1 \mathrm{p}, & 4.94 & 6.34 & 3-2 & (1283)\end{array}$

$\begin{array}{lllll}3 n & 4.96 & 6.28 & 3-3 & y^{7} P^{\circ}-p^{7} D\end{array}$

$\begin{array}{llllll}3 n & 4.99 & 6.23 & 4-4 & \left.y^{3 p 0} \mathrm{p}^{3}\right) \\ \text { of } & 4.96 & 6.25 & 3-3 & (1285)\end{array}$

$\begin{array}{llll}\circ & 4.96 & 6.25 & 3-3 \\ 2 & 4.94 & 6.28 & 2-2 \\ 2 & 4.99 & 6.35 & 4-3 \\ 0 & 4.96 & 6.28 & 3-2\end{array}$

$\begin{array}{lllll}\circ & 4.99 & 6.37 & 4-3 & y^{7} \mathrm{p}^{0}-\mathrm{e}^{7} \mathrm{~g}\end{array}$

$\begin{array}{lllll}\circ & 4.96 & 6.37 & 3-3 & (1286) \\ \odot & 4.94 & 6.37 & 2-3 & \end{array}$

$\begin{array}{lllll}\text { of } & 4.99 & 6.55 & 4-5 & y^{7} P^{0}-g^{5}\end{array}$

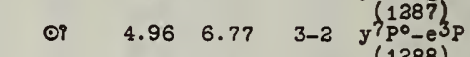

$\begin{array}{lllll}\text { (1) } & 4.99 & 6.97 & 4-5 & y^{7}(1288) \\ P^{2}-1\end{array}$

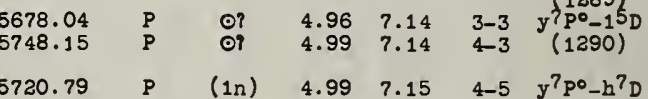

$\begin{array}{lrrrrrr}9913.19 & \mathrm{P} & \odot & 4.97 & 6.31 & 5-6 & x^{5} F^{0}-\mathrm{e}^{7} \boldsymbol{F} \\ 9763.913 & \mathrm{E} & 15 & 5.01 & 6.28 & 4-5 & (1293)\end{array}$

$\begin{array}{llrrrr}9763.913 & \mathrm{E} & 15 & 5.01 & 6.28 & 4-5 \\ 9658.94 & \mathrm{~F} & 3 & 5.04 & 6.32 & 3-4 \\ .9868 .09 & \mathrm{~F} & 3 & 5.06 & 6.31 & 2-3\end{array}$

$\begin{array}{llllll}.9868 .09 & F & 3 & 5.06 & 6.31 & 2-3 \\ .9800 .79 & P & 5 & 5.08 & 6.34 & 1-2\end{array}$

$\begin{array}{rrrrrr}9800.79 & \mathrm{P} & \odot & 5.08 & 6.34 & 1-2 \\ .9452 .45 & \mathrm{~F} & 2 & 4.97 & 6.38 & 5-5\end{array}$

9433.29

.9699.70

9693.69

9531.23

9878.18

9977.52
10016.67

10080.44
9967.32

9834.04
10057.64

10057.64
10142.82
10137.06

10137.06
10149.09 
Laboratory
PeI continued

9333.94 9401.09
9537.73 9537.73
9573.65

9113.35

9307.94

9415.04

9217.5
9199.5

9389.39

9410.15

8923.6

9203.10

9414.1

9454.34

-9699. 70

9868.09

9343.40

9173.63

9334.07

9433.07

9013.098

8945.304

8929.0

8984.87

9294.66

9147.800

0019.84

9155.67

8892.13

8905.99

7807.97

7551.10

7553.79

7453.08

7964.93
7803.49

7751.18

7719.05

7959.21

7730.68

7689.10
7980.04

7955.81

7855.41

7745.48
7965.52

7813.62

7743.71

7879.75

(7)

7508.30

7505.98

7484.38

7538.15

7463.38

7420.30

7341.78
7194.03

7415.19

7384.96

7559.68

7483.30

7443.36
7746.56

7746.56
7661.46

7359.95

7312.05
7160.85

- 7323.8

-7086. 7

6027.76
6153.82

$4-4$
$3-3$
$5-4$

$\begin{array}{ll}5.04 & 6.35 \\ 4.97 & 6.34\end{array}$

$\begin{array}{ll}4.97 & 6.31 \\ 5.01 & 6.35\end{array}$

5.046 .37

4.976 .35

5.016 .37

5.016 .31

$5.04 \quad 6.35$

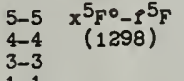

$4-5$
$3-4$
$2-3$
$1-3$

$\begin{array}{lll}6 n & 5.04 & 6.31 \\ 3 & 5.06 & 6.31\end{array}$

$\begin{array}{lll}3 & 5.01 & 6.33 \\ \text { 4nd } & 5.04 & 6.39\end{array}$

$\begin{array}{lll}3 & 5.06 & 6.42 \\ \text { (1) } & 5.06 & 6.39\end{array}$

$\begin{array}{ll}3-2 & x^{5} F^{0}-\theta^{5} s \\ 2-2 & (1299)\end{array}$

$\begin{array}{cc}4-3 & x^{5} F^{\circ}-\theta^{3} D \\ 3-3 & (1300)\end{array}$

$3-1$

$\begin{array}{llll}4.97 & 6.34 & 5-4 & x^{5} F^{\circ}-g^{5} D \\ 5.01 & 6.39 & 4-3 & (1301)\end{array}$

$\begin{array}{ll}5.04 & 6.43 \\ 5.06 & 6.45\end{array}$

$5.08 \quad 6.45$

5.016 .34

$5.04 \quad 6.39$

$\begin{array}{ll}5.06 & 6.43 \\ 5.08 & 6.45\end{array}$

$\begin{array}{ll}5.08 & 6.45 \\ 5.08 & 6.43\end{array}$

$5.01 \quad 6.40$

$4.97 \quad 6.55$

5.016 .59

$\begin{array}{ll}5.06 & 6.70 \\ 5.01 & 6.65\end{array}$

$5.04 \quad 6.70$

$\begin{array}{ll}5.04 & 6.59 \\ 5.06 & 6.65\end{array}$

4.976 .56

5.016 .61

$5.04 \quad 6.66$

$\begin{array}{ll}5.01 & 6.56 \\ 5.06 & 6.66\end{array}$

$\begin{array}{ll}5.06 & 6.66 \\ 5.08 & 6.68\end{array}$

$\begin{array}{ll}5.08 & 6.68 \\ 5.06 & 6.61\end{array}$

$\begin{array}{ll}5.01 & 6.56 \\ 5.04 & 6.61\end{array}$

$\begin{array}{ll}5.04 & 6.61 \\ 5.06 & 6.66\end{array}$

$\begin{array}{ll}5.06 & 6.66 \\ 5.06 & 6.61\end{array}$

$5.08 \quad 6.66$

$\begin{array}{ll}4.97 & 6.56 \\ 5.01 & 6.58\end{array}$

$\begin{array}{ll}5.01 & 6.58 \\ 5.04 & 6.64\end{array}$

$\begin{array}{ll}5.04 & 6.64 \\ 5.08 & 6.71\end{array}$

5.016 .64

$5.04 \quad 6.69$

$\begin{array}{ll}5.06 & 6.71 \\ 4.97 & 6.64\end{array}$

$5.01 \quad 6.65 \quad 4-5 \quad x^{5} F^{\circ}-e^{5} H$

$\begin{array}{llll}5.04 & 6.70 & 3-4 & (1307)\end{array}$

$\begin{array}{lll}5.06 & 6.73 & 2-3 \\ 4.97 & 6.65 & 5-5\end{array}$

$\begin{array}{lll}4.97 & 6.65 & 5-5 \\ 5.01 & 6.73 & 4-3\end{array}$

$4.97 \quad 6.63 \quad 5-5 \quad x^{5} F^{0}-e^{3} a$

$5.04 \quad 6.71 \quad 3-3 \quad(1308)$

5.016 .63

$\begin{array}{ll}5.04 & 6.67 \\ 5.06 & 6.71\end{array}$

$4-5$
$3-4$
$2-3$

$5.06 \quad 6.73 \quad 2-1 \quad x^{5} F^{\circ}-p^{3}$

$4.97 \quad 6.65 \quad 5-6 \quad x^{0} F^{\circ}-e^{3} H$

$\begin{array}{llll}5.01 & 6.70 & 4-5 & (1310) \\ 5.01 & 6.73 & 4-4\end{array}$

$\begin{array}{lllll}\text { (1) } & 5.04 & 6.75 & 3-4 & x^{5} F^{\circ}-r^{3} F \\ 2 & 5.06 & 6.81 & 2-3 & (1311)\end{array}$

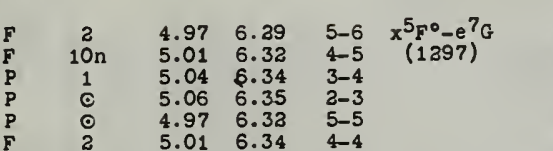

Laboratory
Aef Int Low ${ }_{\text {H1gh }}$ J Multiplet
(No) Fe I continued

$\begin{array}{lllllll}5733.39 & \mathrm{P} & \stackrel{\odot}{*} & 4.97 & 7.13 & 5-4 & \mathrm{x}^{5} \mathrm{~F}^{\circ}-1{ }^{5} \mathrm{D} \\ 5805.76 & \mathrm{P} & (1) & 5.01 & 7.14 & 4-3 & (1313)\end{array}$ 5835.41 5845.27 5890.48

$\begin{array}{ll}5.06 & 6.37 \\ 5.08 & 6.38\end{array}$

5633.970

5655.506

5650.71

5650.01

5549.66
5577.03

5595.06

5614.2

5474.09
5518.57

$\begin{array}{llll}5.04 & 6.64 & 3-3 & (1309) \\ 5.06 & 6.57 & 2-3\end{array}$

11479.87

$\begin{array}{llllll}P & 5.00 & 6.08 & 3-3 & a^{1} F-v^{3} D^{0}\end{array}$

$\begin{array}{lllll}\circ & 5.00 & 6.14 & 3-4 & a^{1}-y^{3} H^{\circ}\end{array}$

$9917.93 \quad F \quad z \quad 5.00 \quad 6.35 \quad 3-4 \quad a^{1} \frac{1316}{F-x^{1} g^{0}}$

$8853.30 \quad P \quad$ or $5.00 \quad 6.40 \quad 3-3 \quad a_{F-45 \%}^{(1317)}$

$9483.82 \quad P \quad \circ \quad 5.00 \quad 6.31 \quad 3-4 \quad a^{(1318)}$

$8959.88 \quad P \quad$ or $\quad 5.00 \quad 6.38 \quad 3-3 \quad a^{(1319)}\left(\begin{array}{llll}(19) & \end{array}\right.$

$8559.98 \quad$ (1) $5.00 \quad 6.45 \quad 3-3 \quad a^{1}\left(132 t^{3} D^{\circ}\right.$

$8171.30 \quad P \quad 0 \quad 5.00 \quad 6.51 \quad 3-4 \quad a^{(1321)^{3}}$

$\begin{array}{llllllll}7846.47 & P & 0 & 5.00 & 6.58 & 3-3 & a^{1} F-8^{3} D^{\circ}\end{array}$

$\begin{array}{lllllllll}7107.30 & P & \odot & 5.00 & 6.74 & 3-3 & a^{(1333)}(132)\end{array}$

$6552.77 \quad \pi \quad$ (2) $5.00 \quad 6.89 \quad 3-3 \quad a^{(1324)}$

$6134.08 \quad P \quad \circ \quad 5.00 \quad 7.02 \quad 3-3 \quad a^{(1325)}$

$6089.566 \quad L \quad$ (1) $5.00 \quad 7.03 \quad 3-4 \quad a^{(1326) V^{1}}$

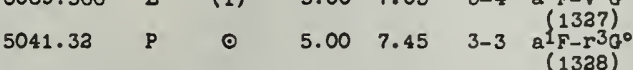

$9008.37 \quad F \quad 2 \quad 5.05 \quad 6.42 \quad 3-3 \quad x-u^{3} D^{\circ}$

$\begin{array}{lllllll}8814.50 \quad P & 2 & 5.05 & 6.45 & 3-3 & x-t^{3} D^{\circ}\end{array}$

$\begin{array}{lllllll}8689.83 & \mathrm{P} & \odot & 5.08 & 6.50 & 2-2 & (1330) \\ 8464.02 & \mathrm{P} & \odot & 5.05 & 6.50 & 3-2 & \end{array}$

$8300.01 \quad P \quad 0 \quad 5.05 \quad 6.53 \quad 3-2 \quad X-v^{3} P^{\circ}$

$\begin{array}{lllllll}8374.28 & 0 & 6 & 5.05 & 6.54 & 3-3 & x-83 D^{\circ}\end{array}$

$\begin{array}{llllllr}8264.27 \quad M & 3 & 5.08 & 6.58 & 2-2 & (1332)\end{array}$

$\begin{array}{lllllll}6700.90 & \mathrm{P} & \odot & 5.05 & 6.89 & 3-3 & \mathrm{X}-\nabla^{1} \mathrm{~F}^{\circ} \\ 6841.65 & \mathrm{P} & \odot & 5.08 & 6.89 & 2-3 & (1333)\end{array}$

$6406.42 \quad(1) \quad 5.08 \quad 7.01 \quad 2-3 \quad x-u^{3} F^{\circ}$

6217.388

(1) $\quad 5.05 \quad 7.03 \quad 3-4 \begin{gathered}x-v^{1} 6^{\circ} \\ (1335)\end{gathered}$

$10012.15 \quad P \quad \odot \quad 5.05 \quad \overline{5} 6.28 \quad 3-1 \quad z^{5} S^{\circ}-P^{5} D$

$9683.57 \quad F \quad 1 \quad 5.05 \quad 6.33 \quad 2-3 \quad 2^{5} S^{\circ}-e^{50}$

$\begin{array}{lllllll}9335.27 & P & c & 5.05 & 6.37 & 2-3 & 2^{5} \mathrm{~S}^{\circ}-\mathrm{P}^{5}\end{array}$

$\begin{array}{lllllll}-7148.69 & R & (-) & 5.05 & 6.77 & 3-2 & z^{5} \mathrm{~S}^{\circ}-e^{3} \mathrm{P}\end{array}$

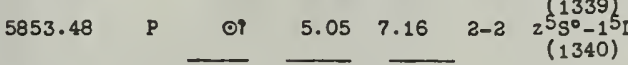

$\begin{array}{lllllll}10890.13 & P & C ? & 5.29 & 6.42 & 2-1 & x^{5} p \circ-e^{3} D \\ 1542.96 & P & c ? & 5.32 & 6.39 & 1-3 & (1341)\end{array}$

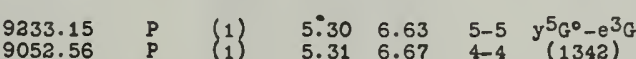

6671.36

6450.99

6402 .

(3)

$5.30 \quad 7.15 \quad 5-5 \quad y^{5} c^{\circ}-h^{7}$

(1) $\begin{array}{lllll}5.31 & 7.32 & 4-4 & 5^{5} G^{\circ}-8^{5 G} \\ 5.33 & 7.36 & 2-37 & (1344)\end{array}$

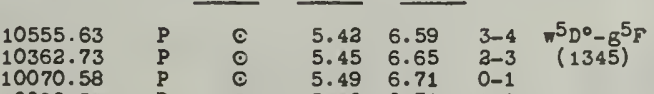

10022.34
9676.42

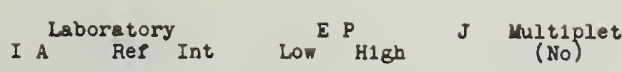

Fe I cont1nued

$\begin{array}{ccccccc}10353.85 & P & (3 n) & 5.37 & 6.56 & 4-4 & w^{5} D^{\circ}-h^{5} D \\ 10388.73 & P & \odot & 5.42 & 6.61 & 3-3 & (1346)\end{array}$ $\begin{array}{lllllll}10383.87 & P & \odot & 5.42 & 6.61 & 3-3 & (1346) \\ 1028 & \odot & 5.48 & 6.68 & 1-1 & \end{array}$ $\begin{array}{llllll}9951.15 & P & \odot & 5.37 & 6.61 & 4-3 \\ 9953.45 & P & \odot & 5.42 & 6.66 & 3-2\end{array}$

$\begin{array}{lllllll}10348.16 & F & 4 n & 5.37 & 6.56 & 4-3 & { }^{5} D^{0}-p^{5} p \\ 10364.13 & P & \odot & 5.42 & 6.61 & 3-3 & (1347)\end{array}$

$\begin{array}{ccccccc}10153.30 & \mathrm{P} & & 5.42 & 6.64 & 3-4 & { }^{5} D^{0}-\mathrm{P}^{5} \mathrm{G} \\ 10019.77 & \mathrm{P} & \odot & 5.45 & 6.69 & 2-3 & (1348)\end{array}$

$\begin{array}{rlllll}10032.84 & \mathrm{P} & \odot & 5.48 & 6.71 & 1-2 \\ 9764.40 & \mathrm{P} & \odot & 5.42 & 6.69 & 3-3\end{array}$

$\begin{array}{lllllll}6943.67 \quad P & \text { of } & 5.37 & 7.15 & 4-5 & -5 D^{\circ}-h^{7} D \\ (1349)\end{array}$

$10925.80 \quad D \quad \begin{array}{lllll}5.46 & 6.59 & 5-4 & -5 F^{\circ}-8^{5} F\end{array}$

$7430.73 \quad 0 \quad$ (1) $\quad 5.46 \quad 7.12 \quad 5-4 \quad 75 F^{\circ}-15 D$

$\begin{array}{lllllll}7526.72 & \mathrm{P} & \odot & 5.48 & 7.13 & 4-4 & \mathrm{v} 5 D^{\circ}-15 \mathrm{D} \\ 7537.97 & \mathrm{P} & \odot & 5.50 & 7.14 & 3-3 & (1353)\end{array}$

$\begin{array}{llllll}7461.28 & \mathrm{P} & \odot & 5.48 & 7.14 & 4-3 \\ 7448.00 & \mathrm{P} & \odot & 5.50 & 7.16 & 3-2\end{array}$

Additions to Kultiplets of Fe I

$\begin{array}{lllllll}4232.724 & V & 1 & 0.11 & 2.99 & 1-2 & a^{5} \mathrm{D}-2^{7} \mathrm{P} \\ 0\end{array}$

$\begin{array}{lllllll}m 3199.50 & P & F e & 0.11 & 3.97 & 1-2 & a^{5} D-z^{3} F^{\circ}\end{array}$

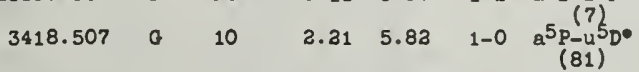

Strongest Unclass1fled Lines of Fe I

9666.59

9637.55

9529.31

8145.47

8024.50

$\begin{array}{llcc}7994.473 & \mathrm{E} & 30 & \text { IV } \\ 7808.04 & 0 & 6 \mathrm{n} & \mathrm{V}\end{array}$

$\begin{array}{llll}7573.53 & 0 & 2 n & V \\ 7546.177 & \text { L } & 4 & \text { IV. }\end{array}$

-7376.4348

7254.649
6975.46

6975.46
6902.80

6881.46

6838.86

$\begin{array}{llll}6838.86 & V & 3 n & V \\ 6793.62 & \text { U } & 1 & \text { V } \\ 6755.609 & V & 3 & \text { IV* }\end{array}$

$\begin{array}{llcl}6726.78 & D & (3) & \\ 6609.56 & V & 1 & V\end{array}$

6538.53

6501.681

6043.093

4552.544

4237.162

4100.17

3851.58
3739.52

3681.774 V

3656.227

3634.698

3616.572

3614.550

3587.752

$\begin{array}{llcl}3506.40 & \text { V } & (3) & \\ 3438.306 & V & (3 \pi) \\ 3262.384 & V & 4 & \text { IV }\end{array}$

$\begin{array}{llll}3179.538 & V & 3 & \text { IV }\end{array}$

$\begin{array}{lll}3139.908 & V & 4 n \\ 3136.17 & \text { W }\end{array}$

$\begin{array}{llll}3126.175 & G & 8 \mathrm{n} & \text { IV } \\ 3103.71 & & \text { (4) }\end{array}$

3991.632 \& $5 \mathrm{n}$ IV 


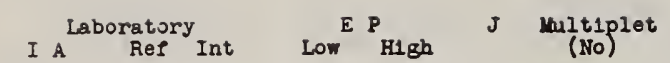
Fe II I P 16.16 Anal A List A July 1941

3277.347 A $\quad 9 \quad 0.98 \quad 4.75 \quad 3 \frac{1}{2}-4 a^{4} D-z^{6} D^{\circ}$ 3302.861
3313.707 3313.996 3281.293 3295.814 3303.466 3234.923 3264.76
3285.425

2953.774

2970.510
2979.349

2975.938
2961.372

3969.40

3969.38

3981.61

3945.21

3966.43
3914.480

3930.31

3475.74
3487.990

3508.213

3463.974

3503.474

3456.00
3475.25

3435.582

3381.36

3358.78

3316.18

-3227.7328 A

3213.311

3192.917

3186.740

3166.670

3170.337

3196.070

3183.115

3163.091

3142.220

2984.831

2965.036
2964.639

2985.545

4420.75

4484.93

4445.26

4525.75

3521.64

3458.13

3587.95

$4818.26 \quad P=\overline{3.27} \overline{4.83} 1 \frac{1}{2}-\frac{1}{2} \mathrm{a}^{2} \mathrm{P}_{-2} \mathrm{z}^{0}$

$4169.98 \quad P \quad 2.27 \quad 5.33 \quad 1 \frac{1}{3}-3 \frac{1}{2} \mathrm{a}^{2} \mathrm{P}_{2}^{(11} \mathrm{C}_{\mathrm{F}}$

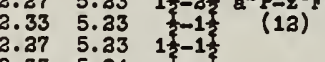

$\begin{array}{llll}2.33 & 5.24 & \frac{1}{2} \\ 2.37 & 5.24 & 1 \frac{1}{2}-\frac{1}{2}\end{array}$

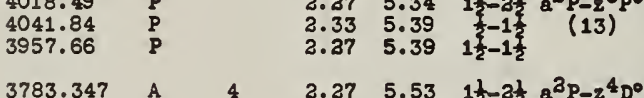

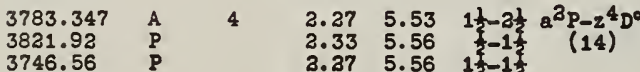

3798.36
3723.92

3741.56

3786.37
3712.39

3494.672
3507.387

3543.83

3478.55
3416.021

4622.40

4591.56

4664.79
4724.07

5696.11

$1.66 \quad 5.37 \quad 3 \frac{2}{3}-\frac{1}{2} Q^{4} \mathrm{P}_{-2}{ }^{6} \mathrm{PO}$

$\begin{array}{lll}1.66 & 5.34 & \frac{21}{2}-\frac{21}{2} \\ 1.69 & 5.39 & 1 \frac{1}{2}-1 \frac{3}{2} \\ 1.66 & 5.39 & 25-1 \frac{1}{2}\end{array}$

$1.66 \quad 5.49 \quad 2 \frac{1}{3}-3 \frac{1}{3} Q^{4} P-2^{4} D^{\circ}$

$\begin{array}{lll}1.72 & 5.56 & 1 \\ 1.66 & 5.53 & 3 \frac{1}{2}-3\end{array}$

1.735 .58 s

$1.695 .58 \quad 1 \frac{1}{2}-\frac{1}{2}$

$1.66 \quad 5.52 \quad 3 \frac{1}{2}-3 \frac{1}{2} Q^{4} \mathrm{P}_{-2} \mathrm{~F}_{5} \mathrm{C}$

$\begin{array}{lll}1.72 & 5.59 & 1 \\ 1.66 & 5.57 & 3 \\ 3 & -3\end{array}$

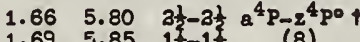

$\begin{array}{lll}1.73 & 5.88 & 12 \\ 1.69 & 5.80 & 1 \frac{2}{2}-2 \frac{1}{2}\end{array}$

$1.96 \quad 4.75 \quad 4 \frac{4}{2}-4 \frac{1}{2} a^{3} a-z^{6} D^{\circ}$

$\begin{array}{lll}2.03 & 4.80 & \frac{3}{2}-2 \\ 2.02 & 4.75 & 3 \frac{2}{2}-4\end{array}$

$1.96 \quad 5.46 \quad 4 \frac{1}{3}-4 \frac{1}{2} a^{2}\left(c-2^{4} F 0\right.$

$\begin{array}{lll}1.96 & 5.52 & 4 \frac{1}{3}-3 \frac{5}{2} \\ 2.02 & 5.57 & 3 \frac{1}{3}-3\end{array}$

$\begin{array}{lll}3.37 & 5.56 & 1 \frac{1}{6}-1 \\ 2.33 & 5.58 & \end{array}$

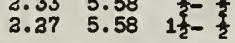

3. $37 \quad 5.57 \quad 1 \frac{1}{2}-3 \frac{1}{2} a^{3} \mathrm{P}-2^{4} \mathrm{~F}^{\circ}$

$\begin{array}{lllll}2.33 & 5.59 & \frac{2}{2}-1 \frac{2}{2} & \text { (15) } \\ 3.27 & 5.59 & 1 \frac{5}{2}-1 \frac{3}{2} & \end{array}$

$\begin{array}{llll}2.27 & 5.80 & 1 \frac{1}{2}-2 \frac{1}{2} & a^{3} \mathrm{P}-2^{4} \mathrm{po}^{\circ} \\ 2.33 & 5.85 & & (16)\end{array}$

$\begin{array}{llll}2.33 & 5.85 & \frac{2}{3}-1 \frac{1}{2} & \text { (16) } \\ 2.27 & 5.85 & 1\end{array}$

$\begin{array}{lll}2.27 & 5.85 & 1 \frac{1}{2}-1 \\ 2.33 & 5.88 & \end{array}$

$2.27 \quad 5.88 \quad 1 \frac{1}{2}-$

$2.515 .18 \quad 5 \frac{1}{2}-5 \frac{1}{2} a^{2} \mathrm{H}_{2} 8^{6} \mathrm{~F}^{\circ}$

2.575 .20 43

$\begin{array}{lll}2.51 & 5.20 & 53-4 \\ 2.57 & 5.21 & 4 \frac{1}{3}-3 \\ 2.57 & 5.18 & 4\end{array}$

$2.57 \quad 5.18 \quad 42-5$
2. $27 \quad 5.34 \quad 1 \frac{1}{3}-2 \frac{1}{2} a^{3} \mathrm{P}-2^{6} \mathrm{PO}$

AEVISED UULTIPLET TABLE
Laboratory

I A ${ }_{\text {Ref }}^{\text {Laboratory }}$ Int Low ${ }_{\text {H1gh }}^{E P}$ Jultiplet

Fe II continued

$\begin{array}{llllll}4603.75 & P & 3.53 & 5.31 & 3 \frac{1}{2}-3 \frac{1}{2} & a^{2} D_{-2} F^{6} \\ 4583.13 & P & 3.53 & 5.33 & 3 \frac{2}{2}-6 \frac{2}{2} & (19)\end{array}$

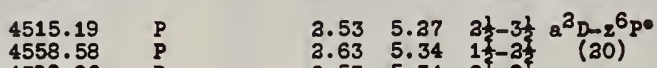

$\begin{array}{lll}2.63 & 5.39 & 1 \frac{2}{2}-1 \frac{2}{2} \\ 2.53 & 5.39 & 2 \frac{2}{2}-1 \frac{2}{2}\end{array}$

$3.53 \quad 5.49 \quad 3 \frac{1}{2}-3 \frac{1}{2} a^{2} D-2^{4} D^{\circ}$

$\begin{array}{lll}2.63 & 5.53 & 1 \\ 3.53 & 5.53 & 3 \\ 2.63 & 5.56 & 1\end{array}$

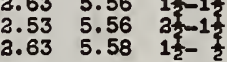

$2.53 \quad 5.53 \quad 3 \frac{1}{3}-3 \frac{1}{2} a^{3} D-2^{4} g^{\circ}$

$\begin{array}{llll}3.63 & 5.57 & 15 & 13 \\ 3.53 & 5.57 & 3-3 & (32)\end{array}$

$\begin{array}{llll}3.63 & 5.59 & 1 \frac{1}{2}-1 \frac{1}{2} \\ 2.53 & 5.59 & 3 \frac{1}{2}-1 \frac{1}{2}\end{array}$

$3.53 \quad 5.80 \quad 3 \frac{1}{2}-2 \frac{1}{2} a^{2} p_{-2} p^{4}$

$2.635 .85 \quad 13$ (33)

$\begin{array}{lll}2.53 & 5.85 & 3 \\ 3.63 & 5.88 & 1 \frac{1}{3} \\ 3.63 & 5.80 & 1 \frac{3}{2}-2 \frac{1}{2}\end{array}$

$3.57 \quad 4.77$ at $-3 \frac{1}{2} b^{4} P_{-2}{ }^{6} D^{\circ}$

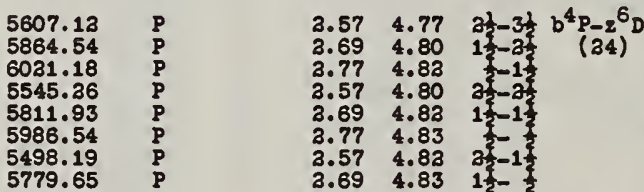

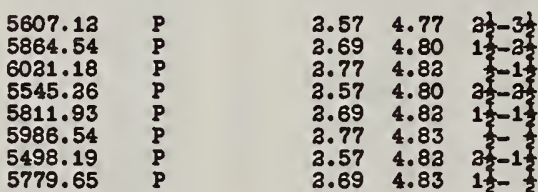

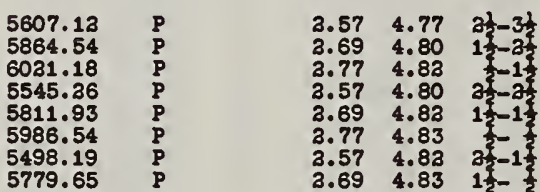

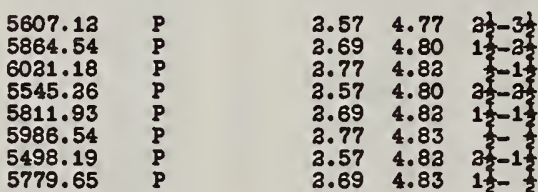

$2.634 .80 \quad 1 \frac{1}{2}-2 \frac{1}{2} a^{3} D-2^{6} D^{\circ}$

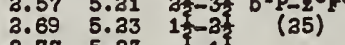

$\begin{array}{lll}3.77 & 5.23 & \\ 3.57 & 5.83 & 21\end{array}$

3.69 $5.33 \quad 1 \frac{1}{3}-1$

$\begin{array}{lll}3.57 & 5.34 & 25 \\ 3.69 & 5.34 & 1\end{array}$

3.57 $5.27 \quad 3 \frac{1}{2}-3 \frac{1}{2} b^{4} \mathrm{P}_{-2} \mathrm{PO}$

$\begin{array}{lll}3.69 & 5.34 & 1 \frac{2}{2}-3 \\ 3.77 & 5.39 & \\ 3.57 & 5.34 & 3\end{array}$

$\begin{array}{lll}3.57 & 5.34 & 3 \\ 3.69 & 5.39 & 1\end{array}$

$\begin{array}{lll}3.69 & 5.39 & 1 \frac{1}{2}-1 \frac{1}{2} \\ 3.57 & 5.39 & 3.2\end{array}$

$3.57 \quad 5.49 \quad 2 \frac{1}{2}-3 \frac{1}{2} b^{4} P-2^{4} D^{\circ}$

$\begin{array}{lll}3.69 & 5.53 & 1 \\ 3.77 & 5.56 & =1\end{array}$

$\begin{array}{lll}3.57 & 5.53 & 32 \\ 3.69 & 5.56 & 1 \frac{1}{2}-1\end{array}$

$\begin{array}{lll}3.69 & 5.56 & 1 \frac{1}{2}-1 \\ 3.77 & 5.58 & 3 \frac{1}{2} \\ 3.57 & 5.56 & 3 \frac{3}{5}-1\end{array}$

$\begin{array}{lll}3.57 & 5.56 & 3 \frac{1}{1} \\ 3.69 & 5.58 & 1 \frac{1}{2}\end{array}$

$3.57 \quad 5.53 \quad 31-3 \frac{1}{2} b^{4} P-2^{4} F^{\circ}$

$\begin{array}{lll}2.69 & 5.57 & 1 \\ 3.77 & 5.59 & \\ 2.57 & 5.57 & 3\end{array}$

$\begin{array}{lll}2.69 & 5.59 & 1 \frac{1}{2}-1 \frac{7}{2} \\ 3.57 & 5.59 & 3 \frac{1}{2}-1 \frac{7}{2}\end{array}$

$3.57 \quad 5.80 \quad 31-3 \frac{1}{2} b^{4} \mathrm{P}-2^{4} \mathrm{po}$

$\begin{array}{llll}3.57 & 5.80 & 21-21 & b^{4} p_{-2} \\ 3.69 & 5.85 & 1 & 13 \\ 3 & (29)\end{array}$

$\begin{array}{lll}3.77 & 5.88 \\ 3.57 & 5.85 & 31\end{array}$

3.695 .88 1

\begin{tabular}{lll}
3.69 & 5.80 & $1 \frac{3}{3}-2 \frac{1}{2}$ \\
3.77 & 5.85 & $\frac{1}{2}-1 \frac{1}{2}$ \\
\hline
\end{tabular}

3.635 .18 6t $-5 \frac{1}{2} \mathrm{a}^{4} \mathrm{H}_{-2} \mathrm{z}^{6} \mathrm{~F}$

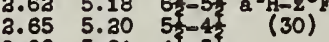

$2.66 \quad 5.21 \quad 43-3$

$3.65 \quad 5.18 \quad 5 \div 5$

$3.66 \quad 5.30$

$\begin{array}{llll}3.68 & 5.21 & 3 & 3 \\ 3.66 & 5.18 & 4 & 5\end{array}$

$3.66 \quad 5.37 \quad 4 \frac{1}{3}-3 \frac{1}{2} \mathrm{a}^{4} \mathrm{H}-\mathrm{z}^{6} \mathrm{po}$

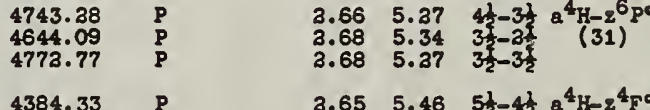

4384.33
.4314 .2898

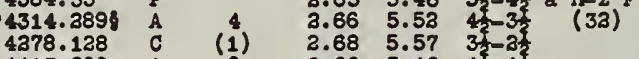

4413.600

43388.70
4439.13

4373.28
4332.88

4332.88
4397.27

6239.36

6239.34

$\begin{array}{lll}2.68 & 5.57 & 3 \\ 3.66 & 5.46 & 4\end{array}$

$\begin{array}{llll}3.68 & 5.53 & 3 \\ 3.68 & 5.46 & 35 & 35\end{array}$

$3.665 .49 \quad 4 \frac{1}{3}-3 \frac{1}{2} Q^{4} H-2^{4} D$

$\begin{array}{lll}3.68 & 5.53 & 3 \\ 3.68 & 5.49 & 3\end{array}$

$3.79 \quad 4.77$ at $-31 b^{4} 7-8^{6} D^{0}+$

$\begin{array}{lll}2.83 & 4.83 & 25 \\ 3.84 & 4.83 & 1\end{array}$

$\begin{array}{llll}3.79 & 5.18 & 42-51 & b^{4} F-z^{6} F^{0} \\ 3.82 & 5.20 & 35 & (35)\end{array}$

5171.68

180.53

5178.95

5146.12 $\begin{array}{llll}2.63 & 5.34 & 1 \frac{1}{3} \\ 2.53 & 5.34 & 25 \\ 3.63 & 5.39 & 13-1\end{array}$

I A Laboratory Ref Int Low ${ }_{\text {H1gh }}^{\text {P }}$

$\operatorname{mintiplet}_{(\text {No }}$

Fe II continued

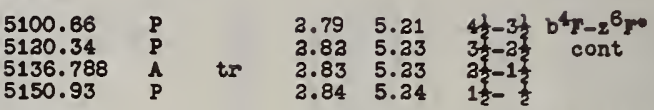

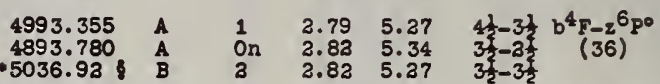

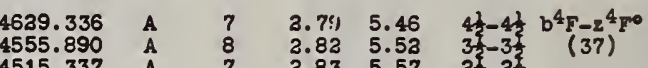

$\begin{array}{llllll}4515.337 & A & 7 & 2.83 & 5.57 & 31-3 \\ 4491.401 & A & 5 & 3.84 & 5.59 & 17-1\end{array}$

4530.325

4473.921

4666.750
4582.835

4534.166

4583.829

4549.467

4508.383

4620.513

4571.523

4648.33

$4670.170 \quad A \quad 0 \quad 3.57 \quad 5.31 \quad 31-3 \frac{1}{3} b^{4} P_{-2} 6_{50}$

4138.40

4088.75

4160.62

4104.18

$3.79 \quad 5.52$

$3.82 \quad 5.57$

$3.83 \quad 5.46$

$\begin{array}{ll}2.83 & 5.52 \\ 3.84 & 5.57\end{array}$

3.79 5.49

\begin{tabular}{ll}
3.89 & 5.49 \\
2.83 & 5.53 \\
\hline
\end{tabular}

$3.84 \quad 5.58$

$\begin{array}{ll}3.82 & 5.49 \\ 3.83 & 5.53\end{array}$

$\begin{array}{ll}3.83 & 5.53 \\ 3.84 & 5.56\end{array}$

$\begin{array}{ll}3.84 & 5.56 \\ 3.83 & 5.49\end{array}$

$3.84 \quad 5.53$

$3.83 \quad 5.80$

$\begin{array}{ll}3.83 & 5.85 \\ 3.84 & 5.88\end{array}$

\begin{tabular}{ll}
3.83 & 5.80 \\
3.83 & 5.85 \\
\hline
\end{tabular}

$1 \frac{1}{3}-1 \frac{1}{2}$
$4 \frac{3}{2}-3 \frac{1}{2}$

$\frac{3}{3}-4$

$\begin{array}{lrrrrrr}6516.053 & \text { B } & 30 & 3.88 & 4.77 & 21-3 \frac{1}{2} & a^{6} 82^{6} D^{\circ} \\ 6433.654 & \text { B } & 8 & 3.88 & 4.80 & 3 & 3 \\ 6369.45 & B & 4 & 3.88 & 4.83 & 3 \frac{1}{2}-1 \frac{1}{2} & (40)\end{array}$

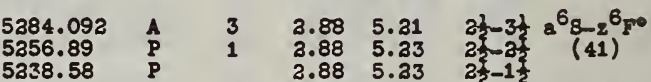

$5169.030 \quad A \quad 13 \quad 3.88 \quad 5.37 \quad 2 \frac{1}{3}-3 \frac{1}{2} a^{6} s_{-2}{ }^{6} \mathrm{po}$

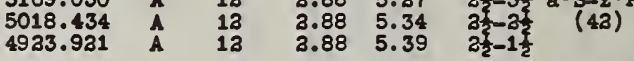

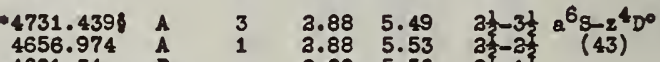

$\begin{array}{lllllll}4656.974 & A & 1 & 3.88 & 5.53 & 3 \frac{1}{3}-2 & (43) \\ 4601.34 & P & & 3.88 & 5.56 & 3 \frac{1}{2}-1 \frac{1}{2} & \end{array}$

$4663.700 \quad \mathrm{~A} \quad 0 \quad 3.88 \quad 5.53 \quad 2 \frac{1}{2}-3 \frac{1}{2} \mathrm{a}^{6} 8-2^{4} F^{\circ}$

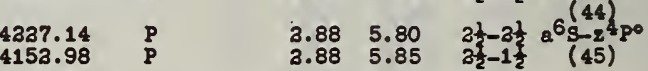

5146.12
5154.10
5161.18 a.

3.79 5.20 1

3.825 .31 3

$\begin{array}{lll}3.83 & 5.23 & 2 \\ 3.84 & 5.33 & 12\end{array}$
6044.53

6150.1

6141.01

6084.11

6113.33

6185.34

6196.71

5932.05

5793.16
5691.38

2091.38

5362.864
5316.777

5264.801

5414.089
5337.713

(5316.609

5316.609

5334.620

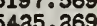

5325.559

5254.92

5346.56

4763.79
4780.60

4780.60
4685.95

5738.74

5605.91

5990.59

5362.48
5518.83

4

5470.81

5148.1

5181.97

$3.14 \quad 5.18$ $3.19 \quad 5.20$

3. $31 \quad 5.21$

$\begin{array}{ll}3.14 & 5.30\end{array}$

$\begin{array}{ll}3.14 & 5.30 \\ 3.19 & 5.21\end{array}$

$\begin{array}{ll}3.21 & 5.33 \\ 3.32 & 5.33\end{array}$

$\begin{array}{ll}3.32 & 5.33 \\ 3.19 & 5.18\end{array}$

$3.31 \quad 5.30$

$3.19 \quad 5.37$

$3.31 \quad 5.34$

$3.22 \quad 5.39$

$3.19 \quad 5.49$

3.325 .56

$3.32 \quad 5.53$

3.145 .46

$3.31 \quad 5.57$

$3.19 \quad 5.46$

$3.31 \quad 5.52$

$\begin{array}{ll}3.32 & 5.57 \\ 3.31 & 5.46\end{array}$

$3.23 \quad 5.52 \quad 2 \frac{2}{2}-3 \frac{1}{2}$

$\begin{array}{llll}3.21 & 5.80 & 31-21 & a^{4} a-z^{4} p 0 \\ 3.22 & 5.80 & 25-35 & (50)\end{array}$

$\begin{array}{lll}3.32 & 5.85 & 25 \\ 3 & -1\end{array}$

5136.19

4730.15

4886.92

4831.11

4577.78

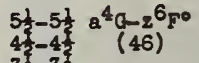

促

4t.

$\frac{3}{32}-4$

$4 \frac{1}{3}-3 \frac{1}{2} a^{4} a-2^{4} D^{\circ}$
$4-3 \frac{1}{2} a^{4} a-2^{6} p 0+$ 


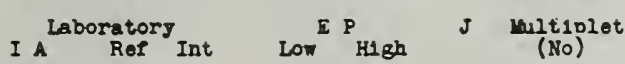

I A Laboratory Ref Int Low ${ }_{\text {H1gh }}^{\text {L }}$

Hultiplet

ro II continued

\begin{tabular}{|c|c|c|c|c|c|}
\hline $\begin{array}{l}5534.860 \\
5433.96 \\
5591.36\end{array}$ & $\begin{array}{l}A \\
\stackrel{A}{P} \\
P\end{array}$ & 4 & $\begin{array}{l}3.23 \\
3.25 \\
3.25\end{array}$ & $\begin{array}{l}5.46 \\
5.53 \\
5.46\end{array}$ & 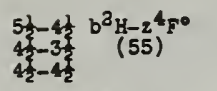 \\
\hline 5525.14 & $P$ & & 3.25 & 5.49 & $-4 \frac{1}{2}-3 \frac{1}{2} b^{2} \frac{H-2}{(56)}{ }^{4} D^{\circ}$ \\
\hline $\begin{array}{l}5909.38 \\
5834.93 \\
5732.72 \\
5735.95 \\
5627.49 \\
5657.93\end{array}$ & $\begin{array}{l}P \\
P \\
P \\
P \\
P \\
P\end{array}$ & & $\begin{array}{l}3.37 \\
3.41 \\
3.37 \\
3.41 \\
3.37 \\
3.41\end{array}$ & $\begin{array}{l}5.46 \\
5.52 \\
5.52 \\
5.57 \\
5.57 \\
5.59\end{array}$ & 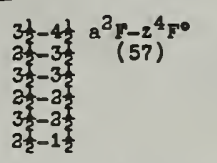 \\
\hline $\begin{array}{l}5835.43 \\
5834.40 \\
5733.56 \\
5737.68 \\
5941.36\end{array}$ & $\begin{array}{l}P \\
P \\
P \\
P \\
P\end{array}$ & & $\begin{array}{l}3.37 \\
3.41 \\
3.37 \\
3.41 \\
3.41\end{array}$ & $\begin{array}{l}5.49 \\
5.53 \\
5.53 \\
5.56 \\
5.49\end{array}$ & 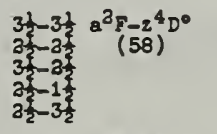 \\
\hline $\begin{array}{l}3031.40 ? \\
3965.395\end{array}$ & $\hat{A}$ & $\frac{1}{3}$ & $\begin{array}{l}3.37 \\
3.41\end{array}$ & $\begin{array}{l}7.46 \\
7.57\end{array}$ & 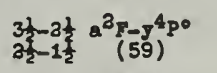 \\
\hline $\begin{array}{l}2984.89 \\
3998.655 \\
2971.616 \\
2991.244 \\
3964.131\end{array}$ & $\begin{array}{l}\mathbf{P} \\
\mathbf{A} \\
\mathbf{A} \\
\mathbf{A} \\
\mathbf{A}\end{array}$ & $\begin{array}{l}3 \\
1 \\
0 \\
7\end{array}$ & $\begin{array}{l}3.37 \\
3.41 \\
3.37 \\
3.41 \\
3.37\end{array}$ & $\begin{array}{l}7.51 \\
7.53 \\
7.53 \\
7.54 \\
7.54\end{array}$ & 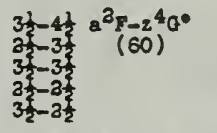 \\
\hline $\begin{array}{r}3988.738 \\
.2980 .963 \\
2954.050\end{array}$ & $\stackrel{\hat{A}}{\hat{A}}$ & $\begin{array}{l}3 \\
4 \\
4\end{array}$ & $\begin{array}{l}3.37 \\
3.41 \\
3.37\end{array}$ & $\begin{array}{l}7.53 \\
7.55 \\
7.55\end{array}$ & 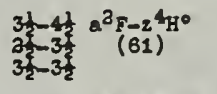 \\
\hline $\begin{array}{l}2959.601 \\
.2986 .6178\end{array}$ & $\hat{A}$ & $\begin{array}{l}? \\
4\end{array}$ & $\begin{array}{l}3.37 \\
3.41\end{array}$ & $\begin{array}{l}7.54 \\
7.54\end{array}$ & 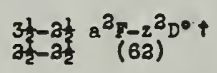 \\
\hline 6983.54 & $P$ & & 3.80 & 5.57 & $\left.-2 \frac{1}{2} b^{2} q-2^{4}\right\}^{0}$ \\
\hline 3327.63 & $P$ & & 3.60 & 7.51 & 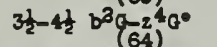 \\
\hline $\begin{array}{l}3377.853 \\
3307.57 \\
3368.938 \\
3389.347 \\
3249.16\end{array}$ & $\begin{array}{l}\mathbf{A} \\
\mathbf{P} \\
\mathbf{A} \\
\hat{A} \\
\vec{P}\end{array}$ & $\begin{array}{l}0 \\
4 \\
7\end{array}$ & $\begin{array}{l}3.75 \\
3.80 \\
3.75 \\
3.80 \\
3.75\end{array}$ & $\begin{array}{l}7.53 \\
7.53 \\
7.53 \\
7.55 \\
7.55\end{array}$ & 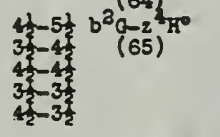 \\
\hline $\begin{array}{l}3154.201 \\
3167.853 \\
3130.561 \\
3193.059\end{array}$ & $\begin{array}{l}\hat{A} \\
\hat{A} \\
\hat{A}\end{array}$ & $\begin{array}{r}12 \\
11 \\
2 \\
3\end{array}$ & $\begin{array}{l}3.75 \\
3.80 \\
3.75 \\
3.80\end{array}$ & $\begin{array}{l}7.66 \\
7.69 \\
7.69 \\
7.66\end{array}$ & 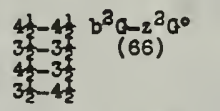 \\
\hline $\begin{array}{l}3146.748 \\
=3193.65 \\
3155.950 \\
3165.095 \\
3164.43\end{array}$ & $\begin{array}{l}\hat{A} \\
\mathbf{P} \\
\mathbf{A} \\
\hat{\mathbf{P}}\end{array}$ & $\begin{array}{c}3 \\
r e^{+} \\
2 \\
1\end{array}$ & $\begin{array}{l}3.75 \\
3.79 \\
3.75 \\
3.80 \\
3.80\end{array}$ & $\begin{array}{l}7.67 \\
7.66 \\
7.66 \\
7.67 \\
7.67\end{array}$ & 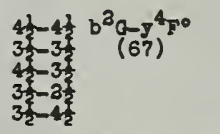 \\
\hline $\begin{array}{l}3070.693 \\
3075.838 \\
3106.559\end{array}$ & $\stackrel{\hat{A}}{\hat{A}}$ & $\begin{array}{l}4 \\
3 \\
4\end{array}$ & $\begin{array}{l}3.75 \\
3.60 \\
3.80\end{array}$ & $\begin{array}{l}7.77 \\
7.61 \\
7.77\end{array}$ & 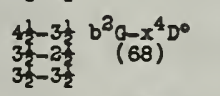 \\
\hline $\begin{array}{l}2985.39 \\
3012.59 \\
3978.850 \\
3004.849 \\
3970.688 \\
3000.059\end{array}$ & $\begin{array}{l}\mathbf{P} \\
p \\
\hat{A} \\
\hat{A} \\
\hat{A} \\
\hat{A}\end{array}$ & $\begin{array}{l}3 \\
3 \\
5 \\
5\end{array}$ & $\begin{array}{l}3.75 \\
3.80 \\
3.75 \\
3.80 \\
3.75 \\
3.80\end{array}$ & $\begin{array}{l}7.89 \\
7.89 \\
7.89 \\
7.91 \\
7.91 \\
7.91\end{array}$ & 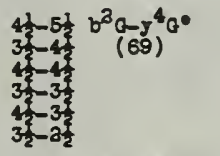 \\
\hline $\begin{array}{l}2969.934 \\
2982.339\end{array}$ & $\hat{A}$ & $\begin{array}{l}8 \\
3\end{array}$ & $\begin{array}{l}3.80 \\
3.80\end{array}$ & $\begin{array}{l}7.95 \\
7.94\end{array}$ & $\begin{array}{l}\frac{31}{3}-2 \frac{1}{2} b^{2} a-z^{2} g^{0}+4 \\
(70)\end{array}$ \\
\hline $\begin{array}{l}9403.36 \\
9314.65 \\
9112.95 \\
9081.33\end{array}$ & $\begin{array}{l}P \\
P \\
P \\
P\end{array}$ & & $\begin{array}{l}3.89 \\
3.87 \\
3.67 \\
3.67\end{array}$ & $\begin{array}{l}5.30 \\
5.21 \\
5.33 \\
5.23\end{array}$ & 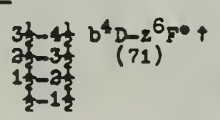 \\
\hline $\begin{array}{l}7841.40 \\
7479.70 \\
7389.05 \\
7181.31 \\
7533.42 \\
7301.57 \\
7179.16\end{array}$ & $\begin{array}{l}p \\
P \\
P \\
P \\
B \\
P \\
P \\
P\end{array}$ & $\begin{array}{l}3 \\
3\end{array}$ & $\begin{array}{l}3.89 \\
3.87 \\
3.67 \\
3.87 \\
3.89 \\
3.87 \\
3.67\end{array}$ & $\begin{array}{l}5.46 \\
5.52 \\
5.57 \\
5.59 \\
5.52 \\
5.57 \\
5.59\end{array}$ & 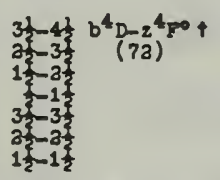 \\
\hline $\begin{array}{c}7711.71 \\
7163.38 \\
.7307 .87 \\
7324.51 \\
7515.88 \\
.7330 .70 \\
7332.39 \\
7655.47 \\
7419.34 \\
7310.34\end{array}$ & $\begin{array}{l}\text { B } \\
\text { B } \\
\text { B } \\
\text { B } \\
\text { B } \\
\text { B } \\
\text { B } \\
\text { B } \\
\text { B } \\
\text { B }\end{array}$ & $\begin{array}{r}15 \\
20 \\
50 \\
12 \\
6 \\
60 \\
6 \\
1 \\
8 \\
6\end{array}$ & $\begin{array}{l}3.89 \\
3.67 \\
3.87 \\
3.87 \\
3.89 \\
3.67 \\
3.87 \\
3.67 \\
3.87 \\
3.87\end{array}$ & $\begin{array}{l}5.49 \\
5.53 \\
5.56 \\
5.58 \\
5.53 \\
5.56 \\
5.58 \\
5.49 \\
5.53 \\
5.56\end{array}$ & 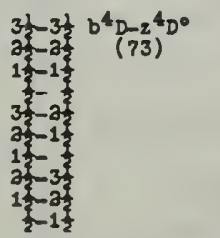 \\
\hline
\end{tabular}

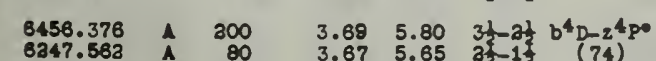

8247.582
.6147 .7358
$A$

8416.905

6338.375
3149.238

8407.30

6339.95

3535.638

353.19

3456.938

3369.349

3445.58

3389.80

3443.79
3336.34

3.87 7.46 3.

$\begin{array}{lll}3.87 & 7.57 & 1 \\ 3.53 & \end{array}$

$\begin{array}{llll}3.67 & 7.46 & 1 \frac{1}{3}-2 \frac{1}{2} \\ 3.87 & 7.57 & \end{array}$ re II continued

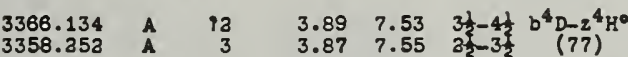

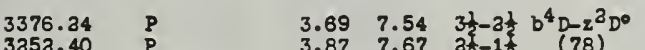

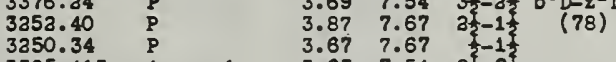

$\begin{array}{llllll}3365.413 & A & 1 & 3.67 & 7.54 & 31-3 \\ 3249.911 & A & 1 & 3.67 & 7.67 & 11-1 \\ 3 & & \end{array}$

$\begin{array}{llllll}3362.764 & A & 1 & 3.67 & 7.67 & 13-1 \\ & A & 3.67 & 7.54 & 1 \frac{1}{2}-2 \frac{1}{2}\end{array}$

3305.634

3193.76

3163.66
3177.65

3303.509
3166.32

3177.260
3295.340

3191.374

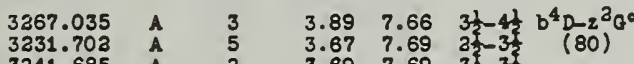

$\begin{array}{rrrrrll}3341.685 & \mathrm{~A} & 3 & 3.69 & 7.69 & 3 \frac{1}{2}-\frac{31}{2} \\ 3259.048 & \mathrm{~A} & 10 & 3.89 & 7.67 & 3 \frac{1}{2}-4 \frac{1}{2} b^{4} \mathrm{D}-\mathrm{y}^{4} \mathrm{~F}^{\circ}\end{array}$

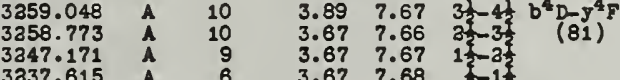

3268.92

3237.403

3259.75
3239.87

3177.53

3135.360
3114.295
3105.548

3105.548
3144.751

3105.166
3167.94

3133.048

3114.680

3070.591

3047.60
3025.99

3034.713
3038.777

3033.859

2997.749

3989.01

2969.367

2966.91
2989.731
3967.37

2967.

7638.09
7534.63

3519.72

3386.452
3496.67

3470.242

3430.15
3452.33

3420.164
3443.339

3448.433

3408.76
3438.64

3436.113

3414.144

3323.086

3376.606
.3286 .6364

3314.80
3304.433

3325.012

3395.06

3384.996

3257.35

3330.496
3177.61

3196.63
3158.32

3158.32
3311.073

3139.013

3120.023

3097.415
3115.493

3096.296

3085.315

3083.034
$\mathbf{3} 3078.44$

3044.643
3002.330

3027.38

$\begin{array}{llll}3.69 & 7.62 & 3 \frac{1}{3}-3 \frac{1}{2} & b^{4} D-j^{4} D^{\circ} \\ 3.87 & 7.74 & 2 \frac{1}{2}-3 \frac{1}{2} & (79)\end{array}$

$3.87 \quad 7.77 \quad 1 \frac{1}{3}-1$

$3.89 \quad 7.74 \quad 3 \frac{1}{3}-2$

$3.87 \quad 7.76 \quad 1 \frac{1}{3}$

$3.67 \quad 7.74 \quad 1 \frac{1}{3}-2$

$3.87 \quad 7.77$ क्ष

3.677 .68 द

$\begin{array}{lll}3.67 & 7.67 & 35 \\ 31 & 2\end{array}$

$\begin{array}{lll}3.67 & 7.68 & 11-1 \\ 3.69 & 7.67 & 3 \\ 3.67 & 7.68 & 3\end{array}$

$3.89 \quad 7.77 \quad 3 \frac{1}{3}-\frac{11}{2} b^{4} D-x^{4} D^{0}$

$\begin{array}{llll}3.87 & 7.61 & 3 & 3 \\ 3 & (83)\end{array}$

$\begin{array}{lll}3.67 & 7.63 & 1 \\ 3.87 & 7.65\end{array}$

$\begin{array}{lll}3.89 & 7.61 & 3 \frac{2}{3}-2 \frac{1}{3}\end{array}$

$\begin{array}{lll}3.67 & 7.63 & 35 \\ 3.87 & 7.65 & 1\end{array}$

$\begin{array}{lll}3.87 & 7.61 & 1 \\ 3.87 & 7.83 & 2\end{array}$

$3.697 .91 \quad 3 \frac{1}{2}-3 \frac{1}{2} b^{4}\left(-y^{4}\right)^{\circ} 0^{\circ}$

$\begin{array}{llll}3.69 & 7.94 & 3 \frac{1}{2}-3 \frac{1}{2} & b^{4} \mathrm{p}_{2}^{2} \mathrm{~F}^{\circ} \\ 3.67 & 7.95 & 35-2 \frac{1}{2} & (84)\end{array}$

$\begin{array}{lll}3.67 & 7.94 & 3 \\ 3.67 & 7.95 & 1 \frac{3}{2}-3 \frac{3}{2}\end{array}$

$3.67 \quad 6.002 \frac{1}{3}-1 \frac{1}{1} b^{4} p_{-2}^{(65)} 2^{30}$

$\begin{array}{lllll}3.67 & 8.00 & 1 \frac{2}{2}-\frac{1}{3} & (66) \\ 3.87 & 6.00 & 1 & \end{array}$

$\begin{array}{lll}3.67 & 6.00 & \frac{1}{2}-\frac{1}{2} \\ 3.67 & 6.00 & \frac{1}{2}-1 \frac{1}{2}\end{array}$

$\begin{array}{llll}3.95 & 5.52 & 31 & -3 \\ 3.93 & 5.57 & b^{3} 5-2^{4} 5^{\circ}+\end{array}$

$3.95 \quad 7.48 \quad 3 \frac{1}{2}-2 \frac{1}{3} b^{3} 5-y^{4} p^{0}$

$\begin{array}{llll}3.93 & 7.57 & 3125 \\ 3.93 & 7.46 & 3 \frac{1}{2}-13 & (66)\end{array}$

$\begin{array}{llll}3.95 & 7.51 & 3-4 \frac{1}{2} & \mathrm{~b}^{2} \mathrm{~F}_{-2} \mathrm{G}^{\circ} \\ 3.93 & 7.53 & 32 & (69)\end{array}$

$\begin{array}{lll}3.95 & 7.53 & 3 \\ 3.93 & 7.54 & 3\end{array}$

$3.95 \quad 7.54 \quad 3 \frac{1}{2}-2 \frac{1}{2}$

$\begin{array}{llll}3.95 & 7.53 & 3 \frac{1}{2}-4 \frac{1}{3} b^{2} 5-2^{4} \mathrm{~K}^{\circ} \\ 3.93 & 7.55 & { }^{2}-3 & (90)\end{array}$

$\begin{array}{llll}3.93 & 7.55 & \frac{2}{3} & \\ 3.95 & 7.55 & \text { 3र } & (90)\end{array}$

$\begin{array}{llll}3.95 & 7.54 & 3 \frac{1}{2}-3 \frac{1}{2} b^{2} 7-2^{2} D^{\circ} \\ 3.93 & 7.67 & 35-1 & (91)\end{array}$

$\begin{array}{lll}3.93 & 7.67 & 3 \\ 3.93 & 7.54 & 3 \frac{1}{2}-2 \frac{1}{2}\end{array}$

$3.95 \quad 7.66 \quad 31-4 \frac{1}{2} b^{2} \mathrm{r}_{-2} \mathrm{z}_{6}$.

$\begin{array}{llll}3.93 & 7.69 & 3 & 3 \\ 3.95 & 7.69 & \text { (9) }\end{array}$

$3.95 \quad 7.67 \quad 3 \frac{1}{2}-\frac{1}{2} b^{2} F-y^{4} F^{\circ}$

$3.95 \quad 7.66$ औ

$\begin{array}{lll}3.93 & 7.67 & 31 \\ 3.95 & 7.67 & 3\end{array}$

$3.93 \quad 7.68$ aर

$3.95 \quad 7.74 \quad 3 \frac{1}{2}-2 \frac{1}{2} b^{2} 5-y^{4} D^{\circ}$

$\begin{array}{lll}3.93 & 7.63 & 2 \frac{1}{3}-1 \frac{1}{2} \\ 3.93 & 7.77 & 3 \frac{1}{2}-3 \frac{1}{2}\end{array}$

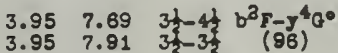

$\begin{array}{lll}3.95 & 7.91 & 3 \\ 3.93 & 7.91 & 3 \\ 3.95 & 7.91 & 3\end{array}$

$3.95 \quad 7.94$ 3र-3t $b^{3} \mathrm{~F}_{-2} \mathrm{z}^{2}$

$\begin{array}{lll}3.93 & 7.95 & 31-25 \\ 3.95 & 7.95 & 3 \\ 3.93 & 7.94 & 32\end{array}$

3.956 .00 और $b^{2} T-5^{3} G^{\circ}$

$\begin{array}{llll}3.93 & 6.04 & \text { 2द } \\ 3 & -3 \frac{1}{2} & (98)\end{array}$
-3116.5908

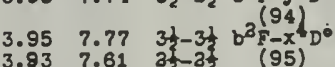

3.95 7.81 और

$3.936 .00 \quad 2 \frac{1}{2}-1 \frac{1}{2} b^{3} \mathrm{p}^{2}-z^{2} \mathrm{P}^{0}$
$(99)$ $\begin{array}{lll}3.67 & 7.77 & 32-3 \\ 3.87 & 7.61 & 15-2\end{array}$

$3.898 .00 \quad 3 \frac{1}{2}-4 \frac{1}{2} b^{4} D-5^{3} a^{\circ}$

Fe II continued

\begin{tabular}{|c|c|c|c|c|}
\hline $\begin{array}{l}3602.60 \\
3583.54 \\
3607.05\end{array}$ & $\begin{array}{l}P \\
P \\
P\end{array}$ & $\begin{array}{l}4.06 \\
4.06 \\
4.06\end{array}$ & $\begin{array}{l}7.48 \\
7.51 \\
7.48\end{array}$ & $\begin{array}{l}6 \frac{1}{2}-5 \frac{1}{2} a^{2} I-z^{4} a^{\circ} \\
\frac{51}{2}-\frac{4}{2} \\
\frac{2}{2}-5 \frac{2}{2}\end{array}$ \\
\hline $\begin{array}{l}3511.25 \\
3493.34 \\
3489.17 \\
3486.08 \\
3481.92\end{array}$ & $\begin{array}{l}P \\
p \\
p \\
p\end{array}$ & $\begin{array}{l}4.06 \\
4.06 \\
4.06 \\
4.06 \\
4.06\end{array}$ & $\begin{array}{l}7.57 \\
7.60 \\
7.60 \\
7.60 \\
7.60 \\
7.50\end{array}$ & $\begin{array}{l}6 \frac{1}{2}-7 \frac{1}{2} a^{2} I-2^{4} I^{\circ} \\
5 \frac{1}{2}-6 \frac{1}{2} \\
6 \frac{1}{2}-6 \frac{1}{2} \\
5 \frac{1}{2}-5 \frac{1}{2} \\
6 \frac{1}{2}-5 \frac{1}{2}\end{array}$ \\
\hline
\end{tabular}

3481.92
3495.16

3426.81

3418.02

3396.355

3360.103
$\mathrm{~m} 3356.24$

3320.635

3131.719

3077.168
3062.234
3060.405

3056.602

3049.16
3060.023

3020.001

$\begin{array}{ll}4.06 & 7.60 \\ 4.06 \quad 7.59\end{array}$

$4.067 .66 \quad 5 \frac{1}{2}-4 \frac{1}{2} a^{2} I-2^{2} g^{\circ}$

$4.06 \quad 7.67$

$4.06 \quad 7.69$

$\begin{array}{ccc}4 & 4.06 & 7.69 \\ 3 & 4.06 & 7.74 \\ \mathrm{Fe}^{+} \quad 4.06 & 7.74 \\ & 4.06 & 7.69\end{array}$

$5 \frac{1}{2}-4 \frac{1}{2} a^{2} I-y^{4}$

3680.98

3661.17
3656.50

3641.23

3636.61
3629.99

3645.78

3636.90
3632.292

3614.673
3610.33

3555.06

3566.97
3564.54

3493.468

3468.680

3464.497

-3484.3488

3499.677
3495.616

3424.17

3391.303

3357.965
3395.336

3387.468

3363.40

3379.649
3373.499

3369.772
3368.512

3243.723

3232.791
3247.392

3167.294

40

3159.32

3134.17

3134.17
3116.74

3079.356

3068.757

3071.65

3071.653
3040.629

3029.681

(1)

4270.39
4247.43

4046.61

4013.467
4033.946

4024.552

$\mathrm{m} 3845.18$
.3863 .953

4004.15

3872.98

3841.35

3827.67

3846.31
$4.06 \quad 7.69$

4.068 .00

$\begin{array}{ll}4.06 & 8.07 \\ 4.06 & 8.09 \\ 4.06 & 8.07\end{array}$

$4.06 \quad 8.10$

$\begin{array}{ll}4.06 & 8.11 \\ 4.06 & 6.10\end{array}$

$4.06 \quad 8.15$

$\begin{array}{ll}4.13 & 7.48\end{array}$

4.147 .5

$4.14 \quad 7.53$

$4.13 \quad 7.53$

$4.13 \quad 7.53$

$\begin{array}{ll}4.14 & 7.53 \\ 4.13 & 7.53 \\ 4.14 & 7.55\end{array}$

.137 .55

$4.13 \quad 7.60$

4.147 .59

$4.13 \quad 7.66$

$4.14 \quad 7.69$

4.137 .69

$4.13 \quad 7.67$

4.147 .66

$4.13 \quad 7.7$

$4.13 \quad 7.77$

$\begin{array}{ll}4.14 & 7.61 \\ 4.14 & 7.77\end{array}$

$4.13 \quad 7.69$

$4.14 \quad 7.69$

4.147 .91

$\begin{array}{ll}4.13 & 7.91 \\ 4.14 & 7.91\end{array}$

$4.13 \quad 7.94$

$\begin{array}{ll}4.14 & 7.95 \\ 4.14 & 7.94\end{array}$

$4.13 \quad 8.00$

$\begin{array}{ll}4.13 & 8.00 \\ 4.14 & 8.04\end{array}$

$\begin{array}{ll}4.13 & 8.04 \\ 4.14 & 6.00\end{array}$

$4.13 \quad 6.07$

4.146 .09

$4.13 \quad 8.14$

$\begin{array}{ll}4.14 & 8.16 \\ 4.14 & 8.11\end{array}$

$\begin{array}{ll}4.13 & 8.15 \\ 4.14 & 6.19\end{array}$

\begin{tabular}{ll}
4.13 & 6.20 \\
4.14 & 8.23 \\
\hline
\end{tabular}

4.48 7.36

$4.46 \quad 7.3$

$\begin{array}{ll}4.48 & 7.53 \\ 4.46 & 7.5\end{array}$

$\begin{array}{ll}4.46 & 7.54 \\ 4.48 & 7.54\end{array}$

$4.48 \quad 7.54$

$\begin{array}{ll}4.46 & 7.67 \\ 4.48 & 7.67\end{array}$

$\begin{array}{ll}4.48 & 7.67 \\ 4.46 & 7.5\end{array}$

$\begin{array}{ll}4.48 & 7.66\end{array}$

$4.46 \quad 7.67$

$\begin{array}{lll}4.46 & 7.68 & 13 \\ 4.48 & 7.68 & \frac{1}{2}-1\end{array}$
$-5 \frac{1}{2}$

$5 \frac{1}{2}-4 \frac{1}{2} a^{2} I-y^{4} G^{\circ}$

$5 \frac{1}{2}-4 \frac{1}{2} a^{3} I-y^{2} G^{\circ}$

$6 \frac{1}{2}-5 \frac{1}{3} a^{2} I-z^{2} H^{\circ}$
$(108)$

$6 \frac{1}{2}-5 \frac{1}{2} a^{2} I-x^{4} G^{\circ}$

$5 \frac{1}{2}-4 \frac{1}{2} a^{2} I-x^{4} F^{0}$ (110)

$\frac{4}{3}-5 \frac{1}{2} c^{2} a-2^{4} c^{\circ}$
$(111)$

$3 \frac{1}{3}-3$
$4 \frac{1}{3}-3$
$3 \frac{1}{2}-2$

$4-5 \frac{1}{4} c^{2}\left(-24^{4}\right.$

$4 \frac{2}{3}-4$
$3 \frac{1}{3}$
$4 \frac{1}{3}-3$

$4 \frac{1}{3}-5 \frac{1}{2} c^{2} a-2^{4} I 0$

4) $-4 \frac{1}{2}(113)$

$4 \frac{1}{3}-4 \frac{1}{3} c^{2} a-z^{2} a$

$3 \frac{2}{2}-4 \frac{1}{2}$

$4 \frac{1}{3}-4 \frac{1}{2} c^{2} a-g^{4} F$
$3 \frac{3}{4}-3 \frac{3}{2}$
$(115)$

$4 \frac{1}{2}-5 \frac{1}{2} c^{2} a-z^{2} I^{0}$

$4 \frac{1}{3}-3 \frac{1}{2} c^{2} G-x^{4} D^{\circ}$

$3 \frac{3}{2}-3 \frac{3}{2}$ (117)

4) $-5 \frac{1}{2} c^{2} G-y^{4} G^{0}$

(118)

$4 \frac{1}{2}-3 \frac{1}{2}$
$3 \frac{1}{2}-2 \frac{1}{2}$

$4 \frac{1}{3}-3 \frac{1}{2} c^{2} G-2^{2} F^{\circ}$

4t-4t $c^{2} G-y^{2} G^{0}$

$3 \frac{1}{2}-3 \frac{2}{2}(120)$

$4 \frac{1}{2}-5 \frac{1}{2} c^{2} G-z^{2} H^{\circ}+$

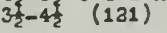

$4 \frac{1}{3}-3 \frac{1}{2} c^{2} a-x^{4} a^{\circ}$

$4 \frac{1}{3}-4 \frac{1}{3} c^{2} a-x^{4} F^{0}$

3द $-3 \frac{1}{2}$ (123)

$4 \frac{1}{3}-5 \frac{1}{2} c^{2} a-g^{4} H^{\circ}$
$3 \frac{2}{2}-3 \frac{3}{2}(124)$

$2 \frac{1}{3}-1 \frac{1}{2} b^{2} D-z^{4} g^{\circ}$
$1 \frac{1}{2}-1 \frac{3}{2}$
$(125)$

$2 \frac{1}{2}-3 \frac{1}{2} b^{2} D-z^{4} a^{\circ}$

$1 \frac{1}{2}-2 \frac{1}{2}$
$2 \frac{1}{2}-2 \frac{1}{2}$ 


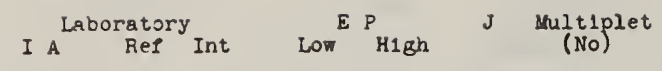

Fe II continued

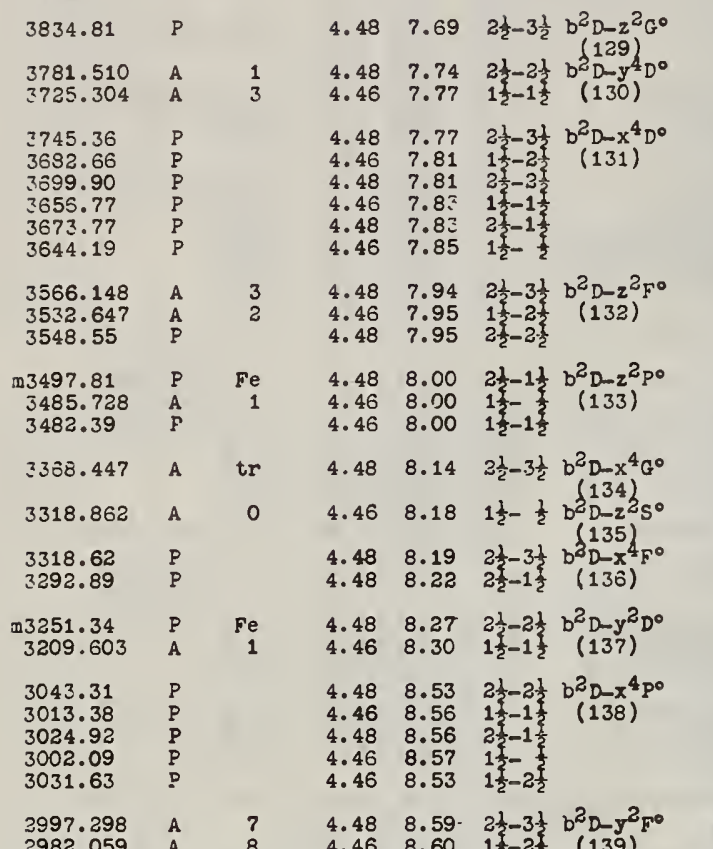

\begin{tabular}{lllllll}
2982.059 & A & $?$ & 4.48 & 8.59 & $2 \frac{1}{2}-3 \frac{1}{3}$ & $b^{2} D-y^{2} F^{\circ}$ \\
2993.366 & A & 1n & 4.46 & 8.60 & $1 \frac{1}{2}-2 \frac{1}{2}$ & $(139)$ \\
\hline
\end{tabular}

$4455.85 \mathrm{P}-\overline{4.60} \overline{7.37} \frac{1}{\frac{1}{2}-1 \frac{1}{2}} \mathrm{a}^{2} \mathrm{~S}-z^{4} \mathrm{~S}^{0}$

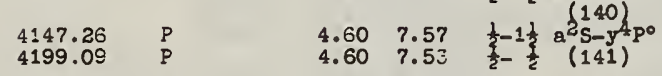

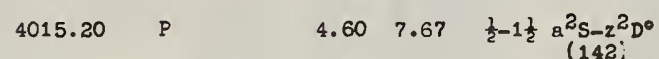

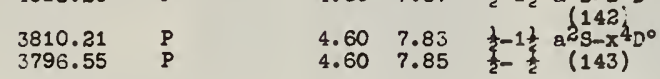

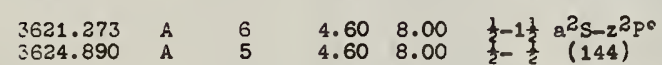

$3444.76 \quad P \quad 4.608 .18 \frac{1}{2}-\frac{1}{2} \mathrm{a}_{(145)}^{2} \mathrm{~S}_{-2}^{2} \mathrm{~S}^{\circ}$

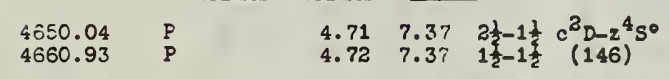

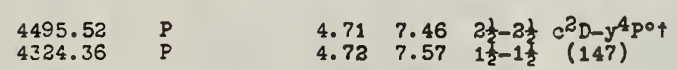

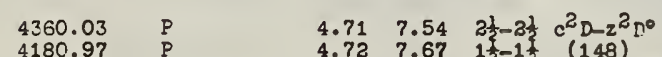

$\begin{array}{llllll}4180.97 & \mathrm{P} & 4.72 & 7.67 & 1 \frac{1}{2}-1 \frac{1}{2} & (148) \\ 4172.20 & \mathrm{P} & 4.71 & 7.67 & 2 \\ 4269.61 & \mathrm{P} & 4.72 & 7.54 & 1 \frac{1}{2}-1 \frac{3}{2} & \end{array}$

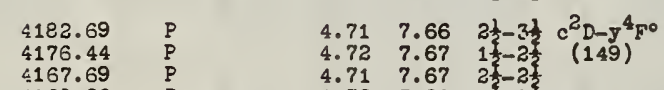

4160.28
4151.60

$4138.21 \quad P \quad 4.71 \quad 7.69 \quad 2 \frac{1}{2}-3 \frac{1}{2} c^{2} D-z^{2} G^{\circ}$

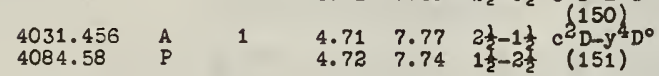

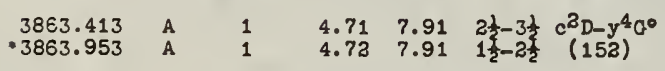

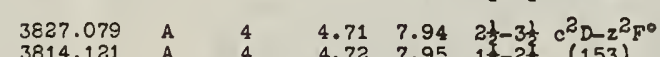

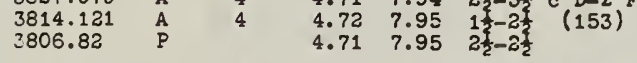

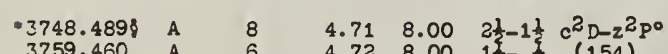

$\begin{array}{lllllll}3759.460 & \mathrm{~A} & 6 & 4.72 & 8.00 & 1 & 1 \\ 3755.563 & \mathrm{~A} & 4 & 4.72 & 8.00 & 1 \\ 1 \frac{1}{2}-1 \frac{1}{2} & (154)\end{array}$

$\begin{array}{lllllll}3566.052 \quad \mathrm{~A} & 2 & 4.72 & 8.18 & 1 \frac{1}{2}-\frac{1}{2} & \mathrm{c}^{2} \mathrm{D}-z^{2} \mathrm{~S}^{\circ}\end{array}$

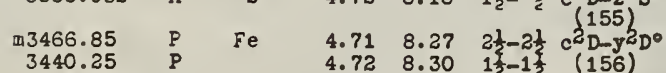

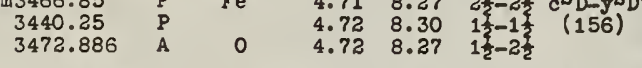

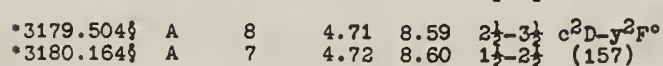

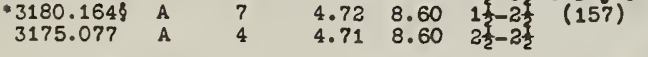

$\begin{array}{lllllll}3089.388 & A & 4 & 4.71 & 8.71 & 2 \frac{1}{2}-3 \frac{1}{2} & c_{(158)}^{2} D_{-1}{ }^{2} G^{\circ}\end{array}$

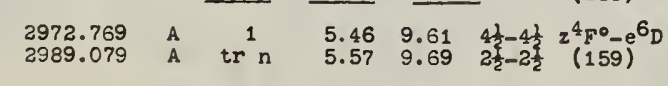

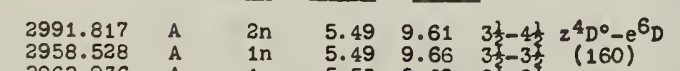

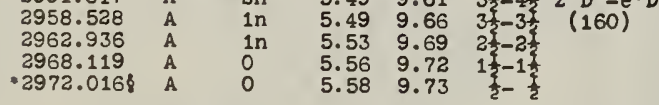

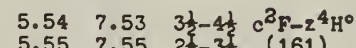

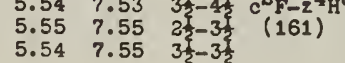

REVISED MULTIPLET TABLE

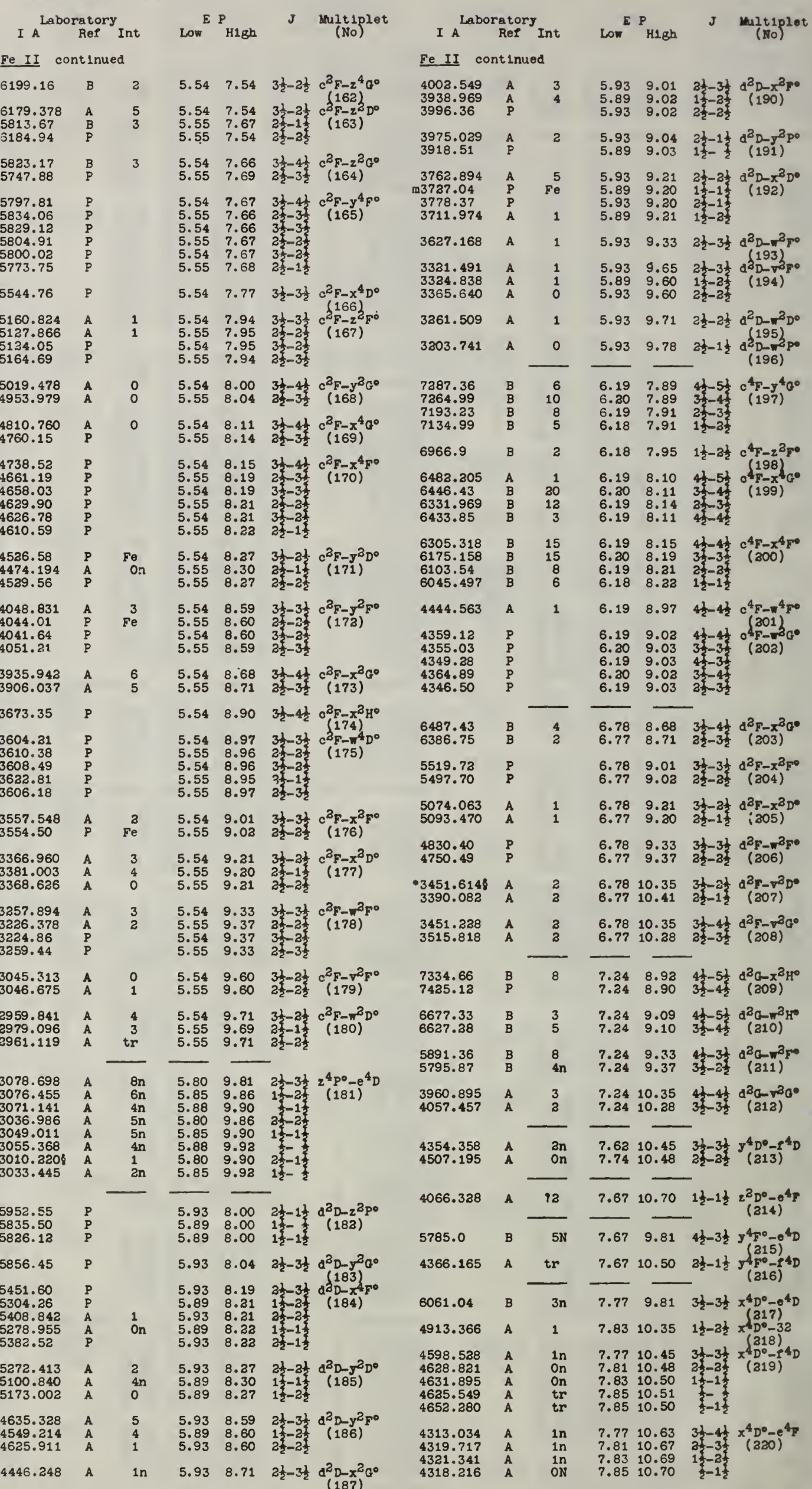

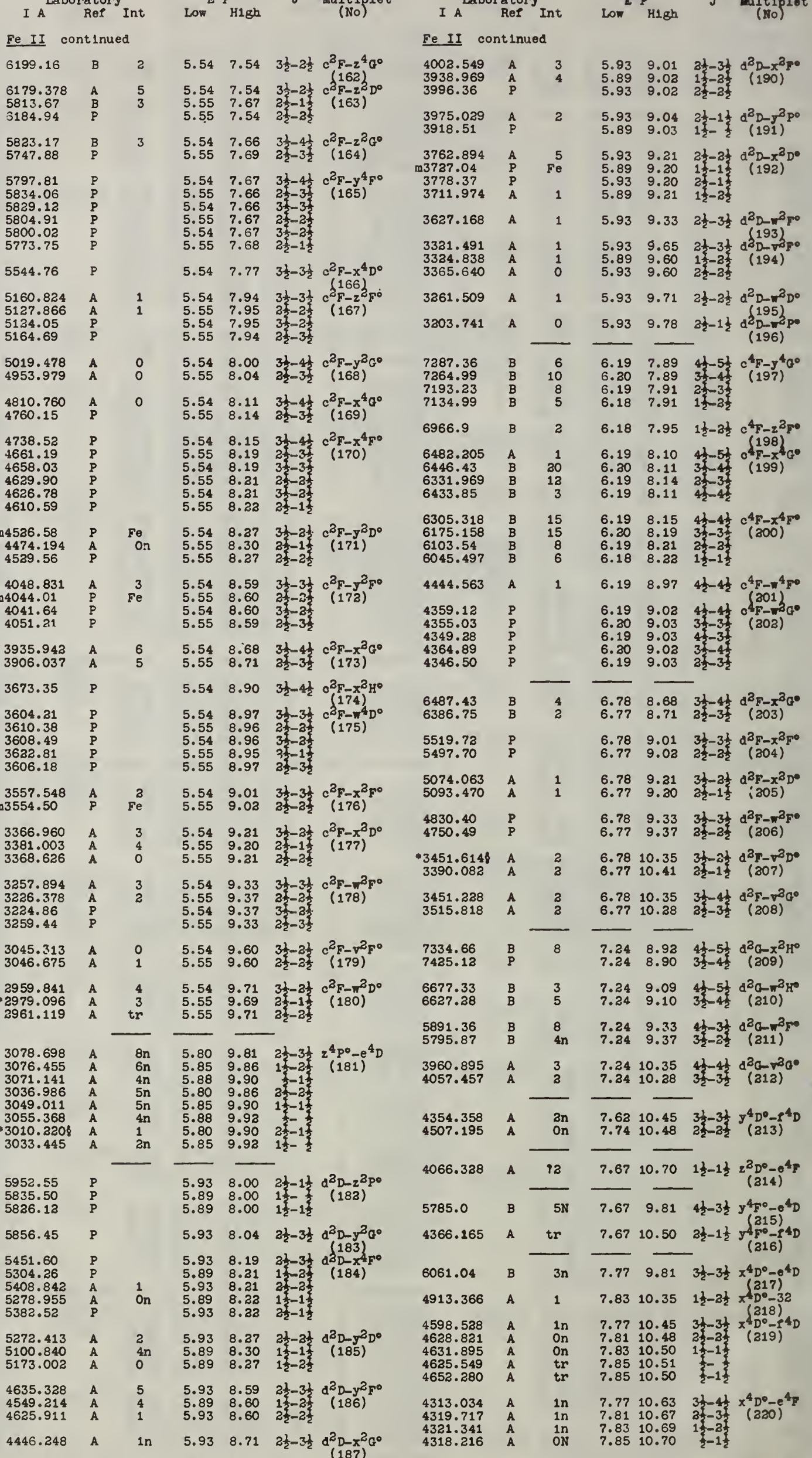

\begin{tabular}{|c|c|c|c|c|c|}
\hline 6199.16 & B & 2 & 5.54 & 7.54 & $3 \frac{1}{2}-2 \frac{1}{2} c^{2} F-2^{4} G^{\circ}$ \\
\hline $\begin{array}{l}6179.378 \\
5813.67 \\
3184.94\end{array}$ & $\begin{array}{l}\mathrm{A} \\
\mathrm{B} \\
\mathrm{P}\end{array}$ & $\begin{array}{l}5 \\
3\end{array}$ & $\begin{array}{l}5.54 \\
5.55 \\
5.5+5\end{array}$ & $\begin{array}{l}7.54 \\
7.67 \\
7.54\end{array}$ & 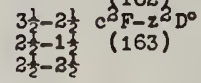 \\
\hline $\begin{array}{l}5823.17 \\
5747.88\end{array}$ & $\stackrel{B}{P}$ & 3 & $\begin{array}{l}5.54 \\
5.55\end{array}$ & $\begin{array}{l}7.66 \\
7.69\end{array}$ & $\begin{array}{l}3 \frac{1}{2}-4 \frac{1}{3} c^{2} F-z^{2} G^{\circ} \\
2 \frac{2}{2}-3 \frac{1}{2} \\
(164)\end{array}$ \\
\hline $\begin{array}{l}5797.81 \\
5834.06 \\
5829.12 \\
5804.91 \\
5800.02 \\
5773.75\end{array}$ & $\begin{array}{l}P \\
P \\
P \\
P \\
P \\
P \\
P\end{array}$ & & $\begin{array}{l}5.54 \\
5.55 \\
5.54 \\
5.55 \\
5.54 \\
5.55\end{array}$ & $\begin{array}{l}7.67 \\
7.66 \\
7.66 \\
7.67 \\
7.67 \\
7.68\end{array}$ & $\begin{array}{l}3 \frac{1}{3}-4 \frac{1}{3} c^{2} F-y^{4} F^{\circ} \\
2 \frac{1}{2}-3 \frac{1}{2} \\
3 \frac{1}{2}-3 \frac{1}{2} \\
2 \frac{1}{2}-2 \frac{1}{2} \\
3 \frac{1}{3}-2 \frac{1}{2} \\
2 \frac{1}{2}-1 \frac{1}{2}\end{array}$ \\
\hline
\end{tabular}

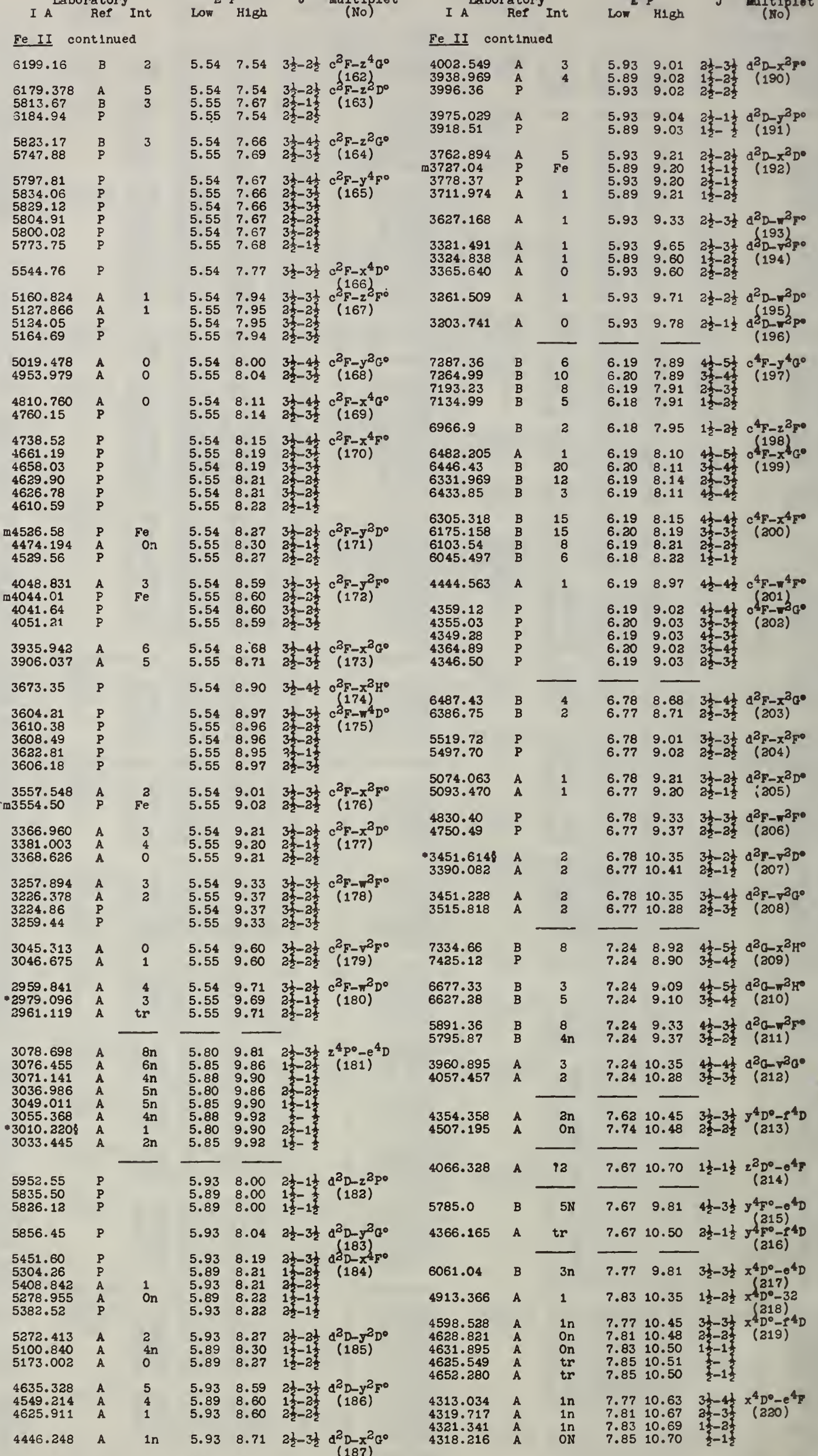

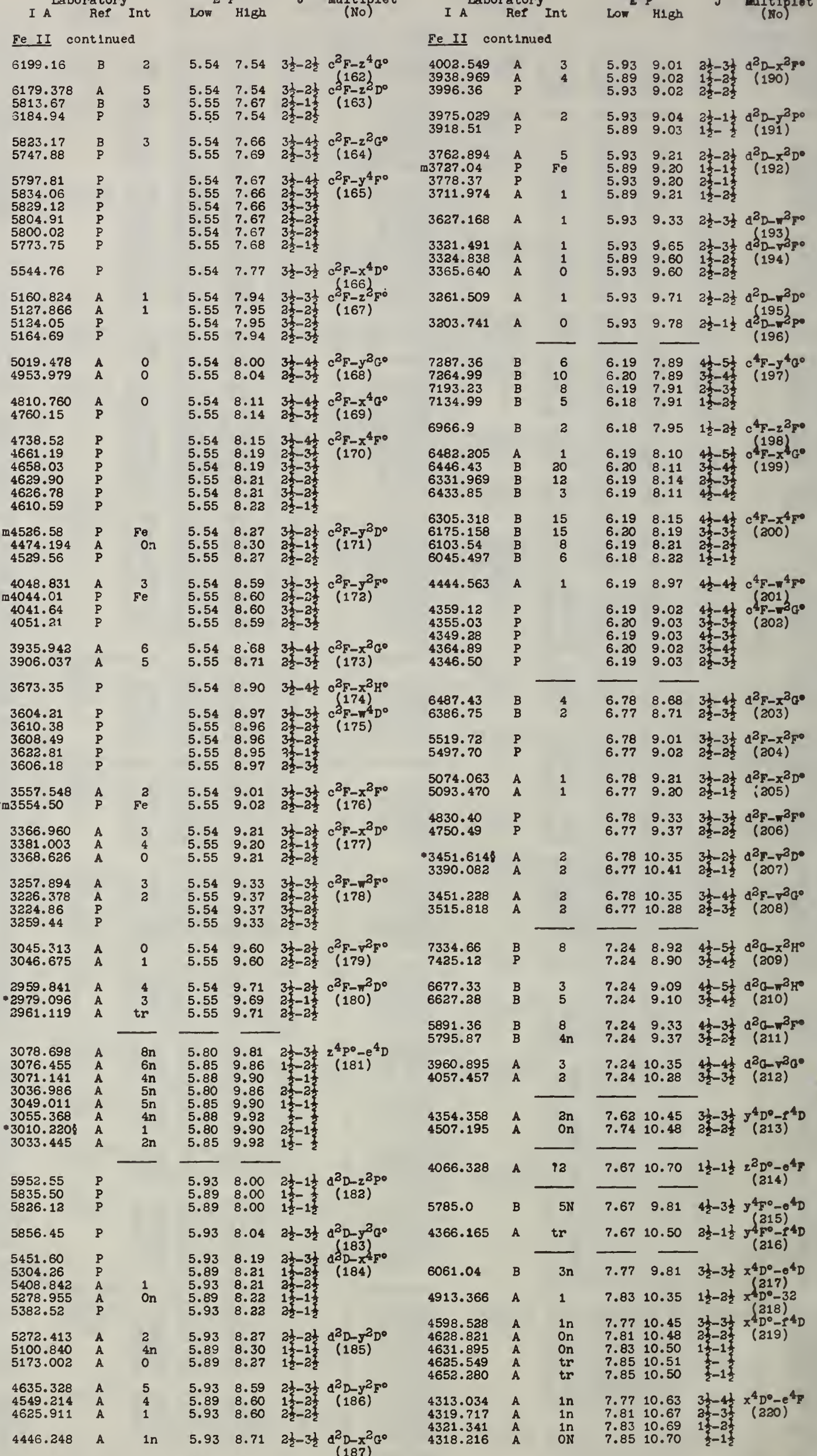

5124.05
5164.69

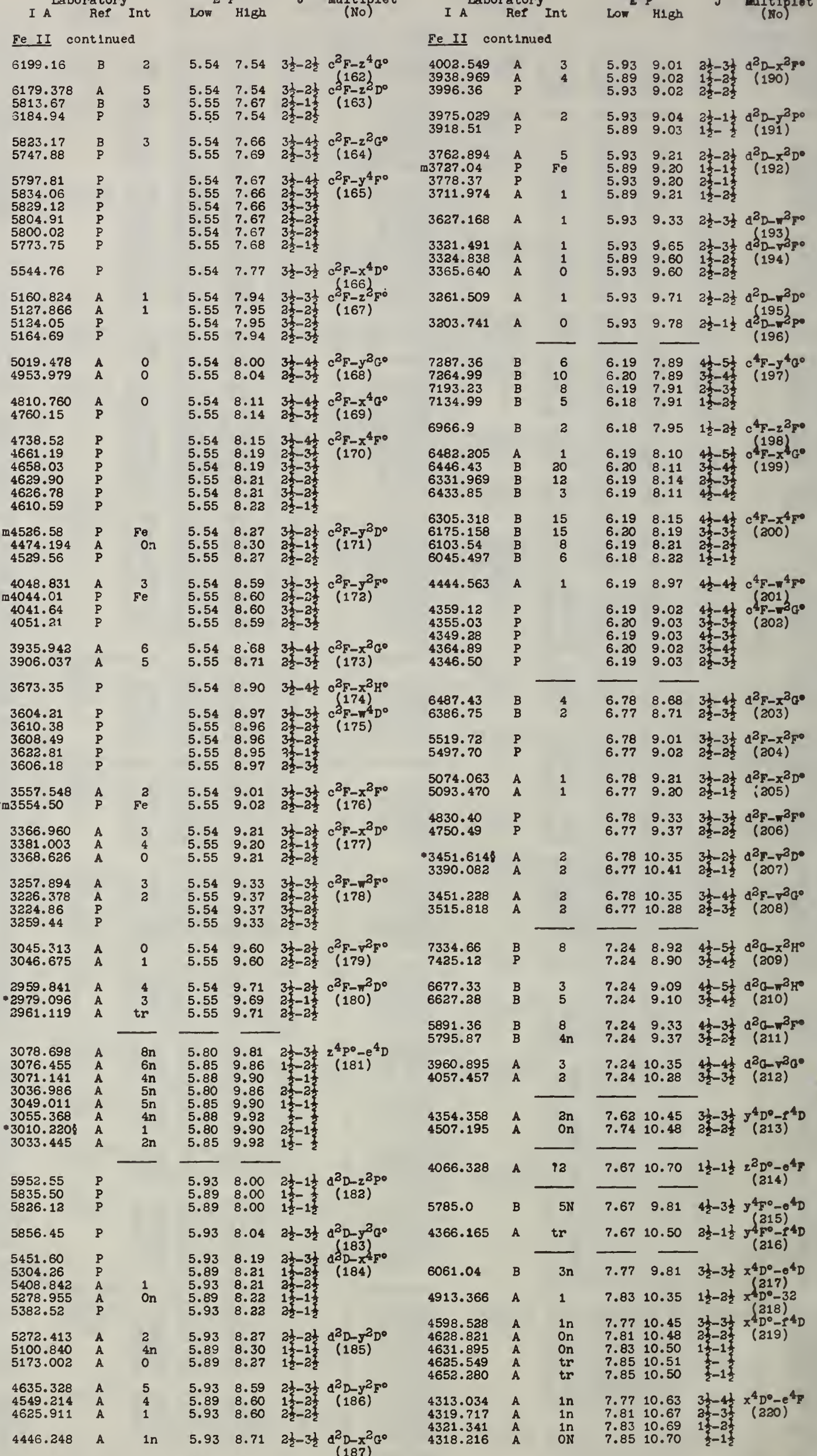

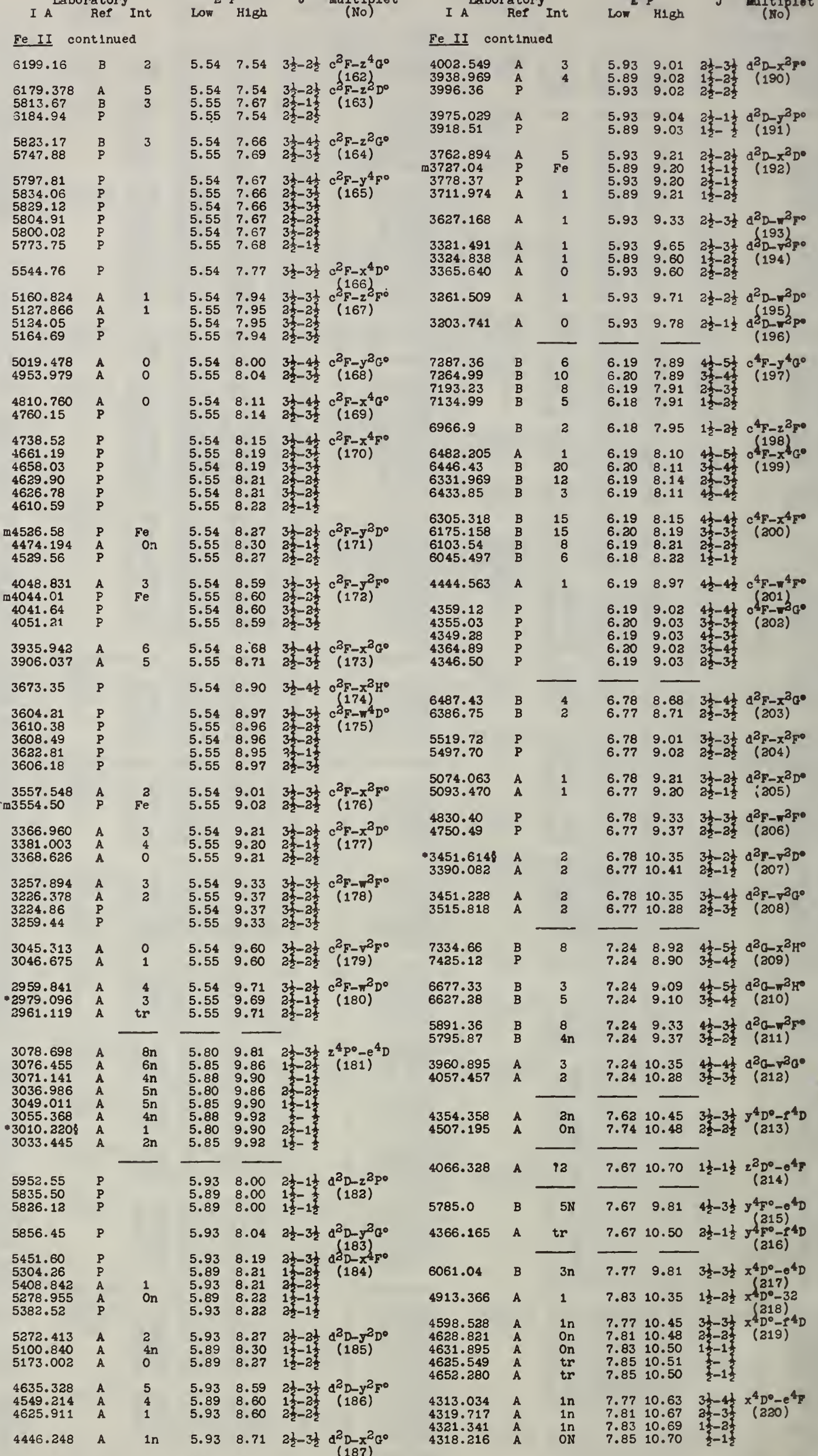

4760.15
4738.52

4661.19
4658.03

4629.90
4626.78

4626.78
4610.59

$\mathrm{m} 4526.58$
4474.194

4474.194
4529.56

4048.831

\begin{tabular}{c}
$\begin{array}{c}4048.831 \\
\mathrm{~m} 4044.01 \\
4041.64 \\
4051.21\end{array}$ \\
\hline
\end{tabular}

3935.942

3935.942
3906.037

3673.35

3604.21
3610.38
3608.49

3622.81

3557.548
$\mathrm{~m} 3554.50$

3366.960
3381.003

3381.003
3368.626

3257.894
3226.378
3224.86

3224.86
3259.44

3045.313
3046.675

2959.841
.2979 .096

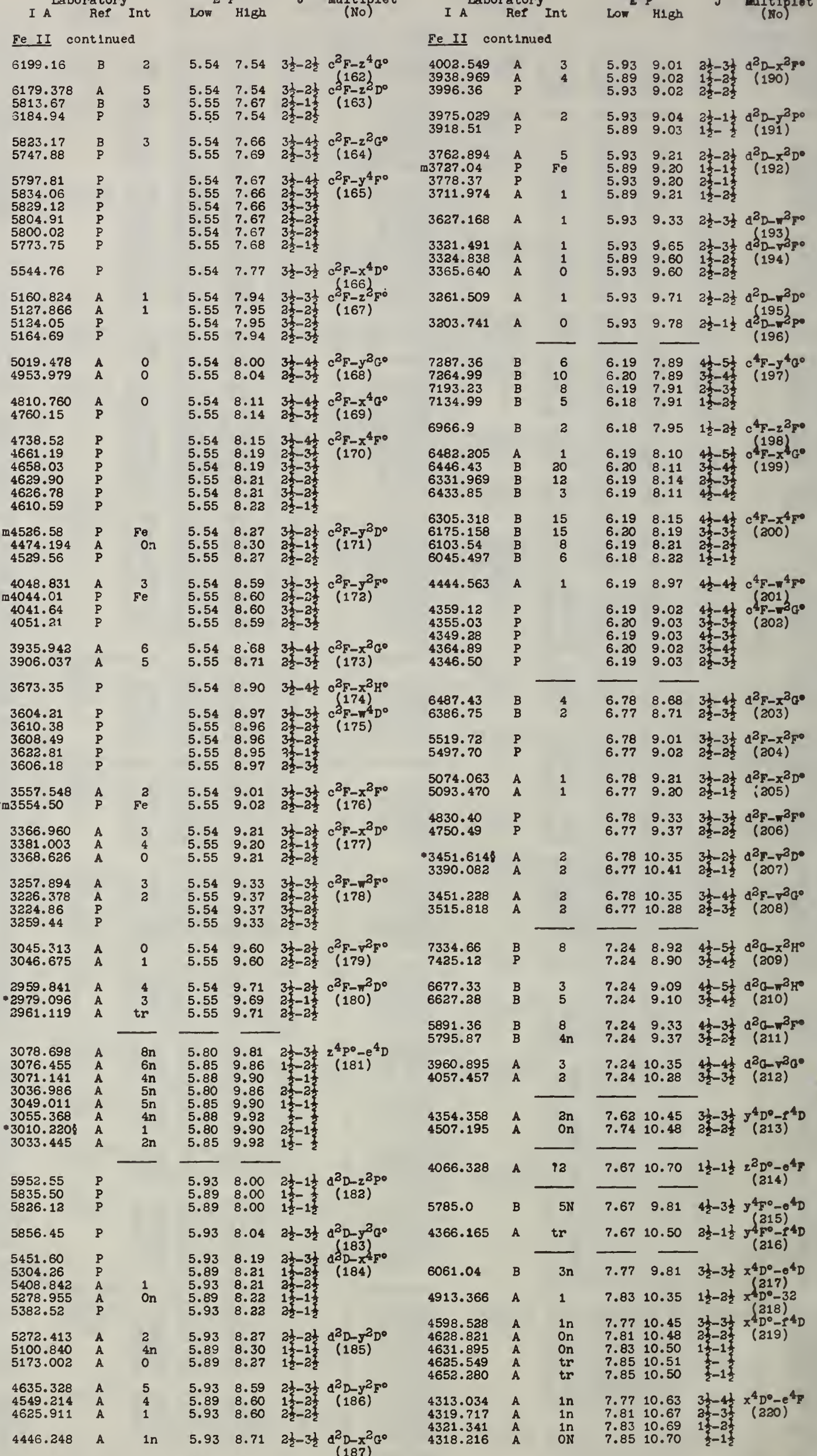

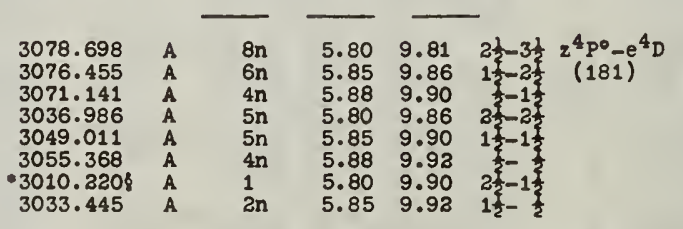

5952.55

5835.50

5856.45

5451.60

5304.26

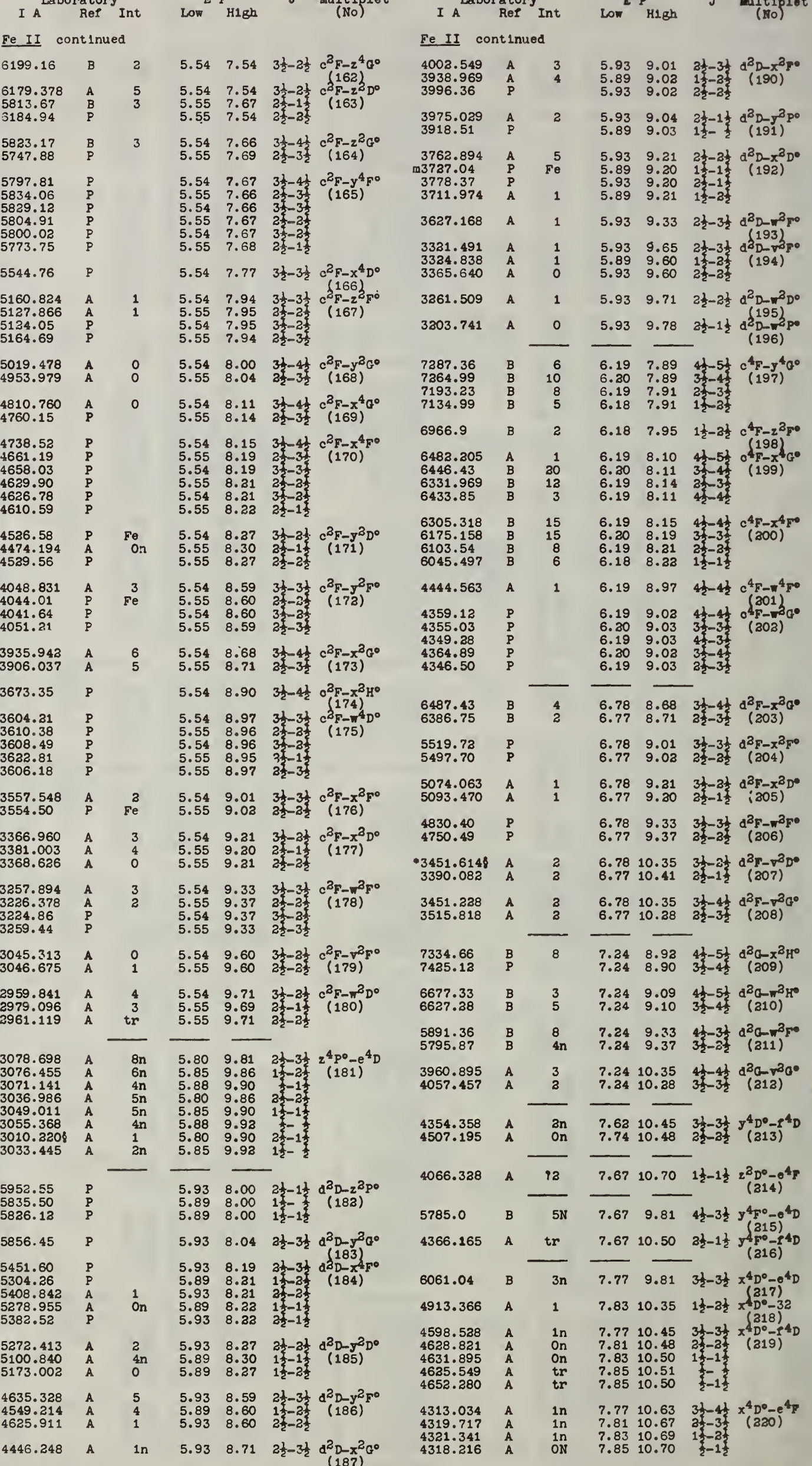

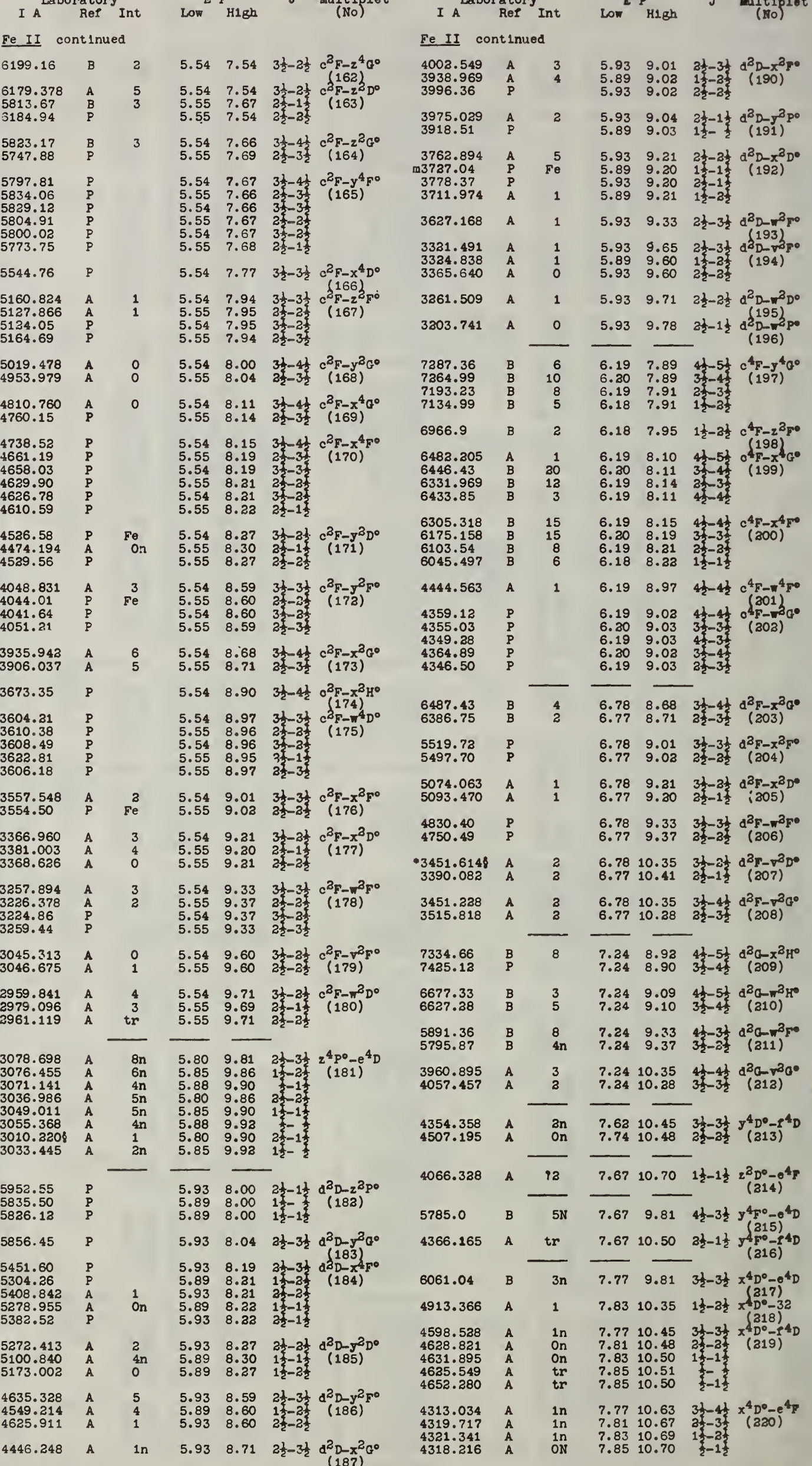

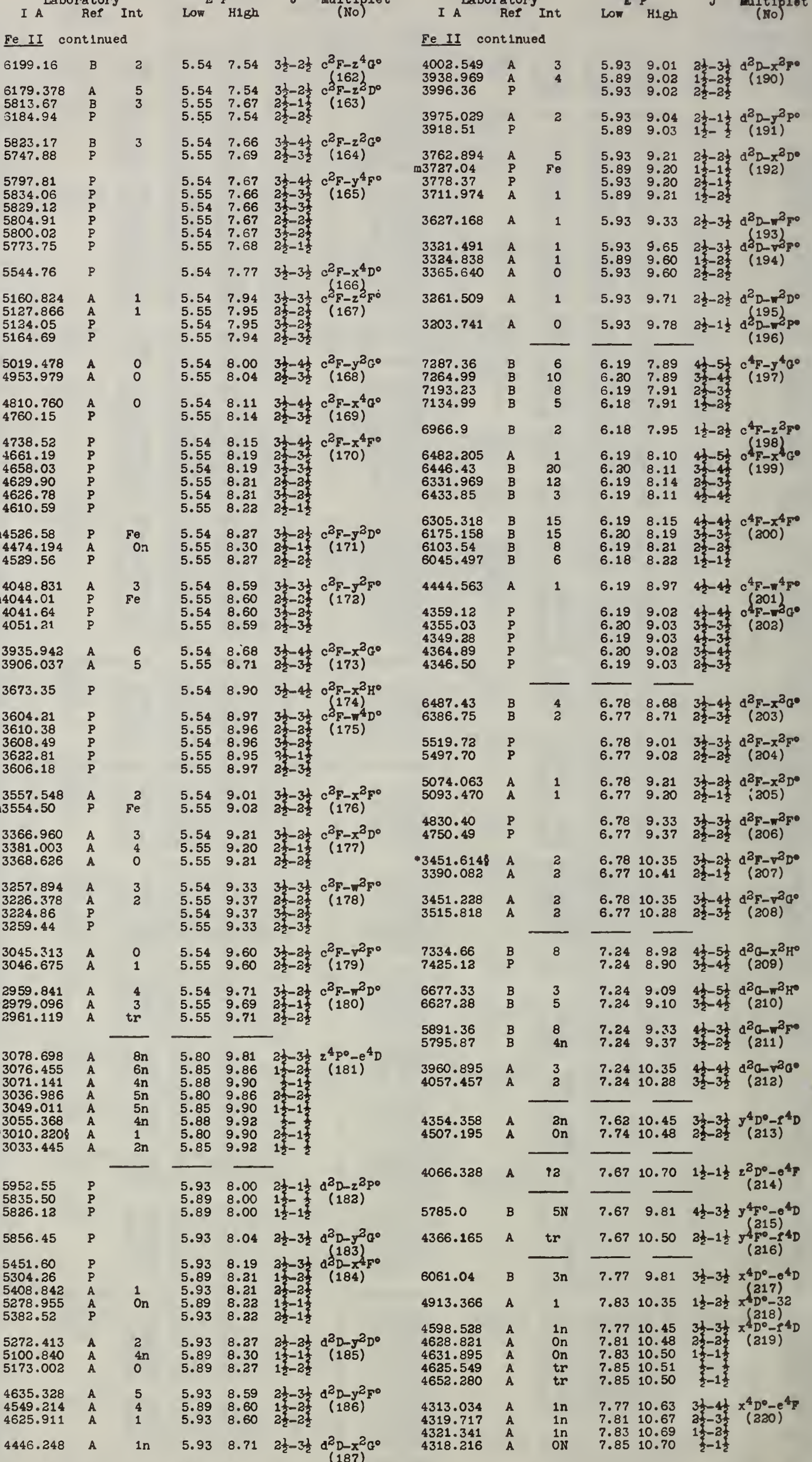

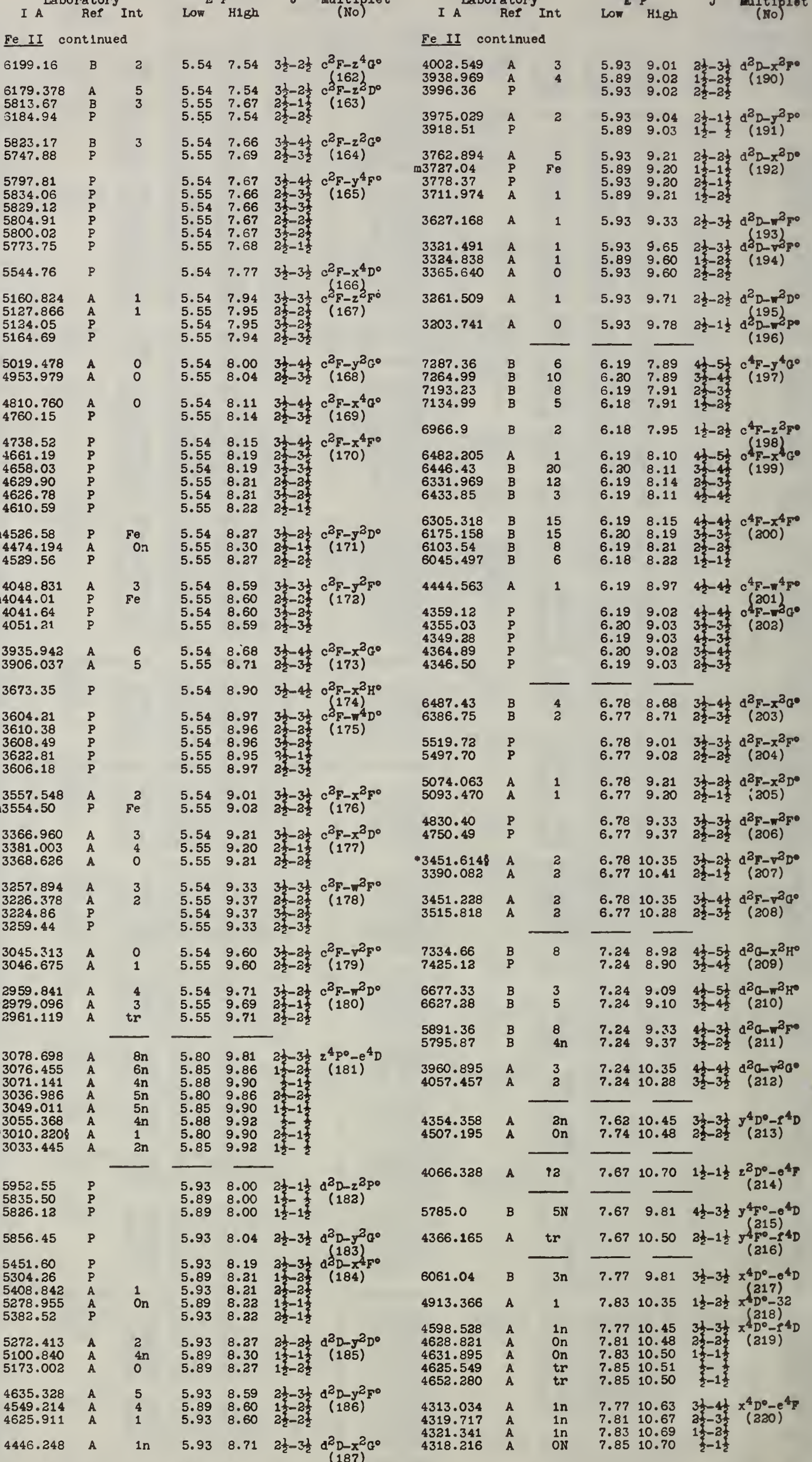

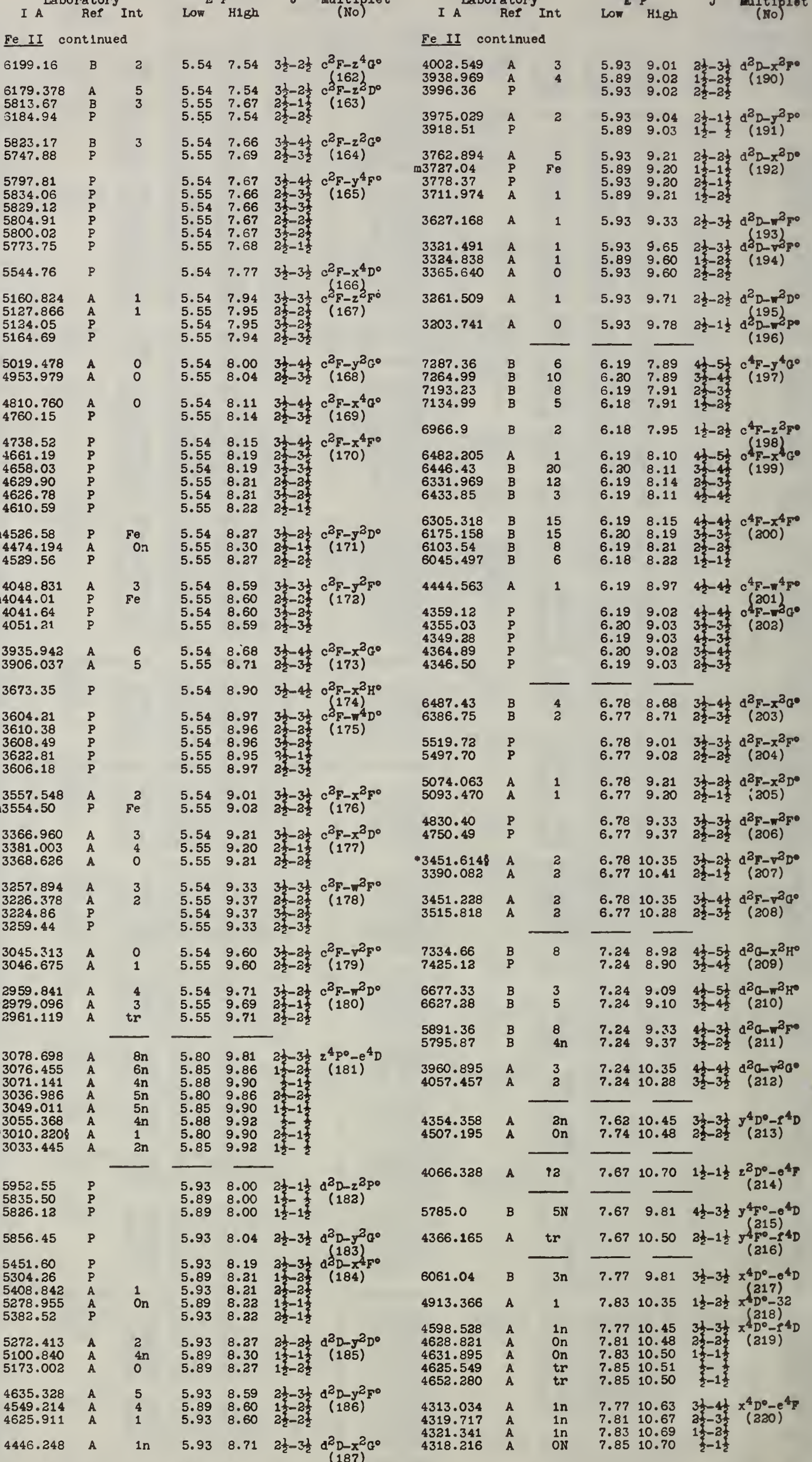

4111.902

4069.883
4131.17

4131.17
4081.42
4143.07

4143.07

4061.787
4007.73

I A ${ }_{\text {ReP }}^{\text {Laboratory }}$ Int Low ${ }^{E}{ }_{\text {HIgh }}$

${ }_{(\mathrm{NO})}^{41 \mathrm{p})^{20 t}}$ e II continued

3711.97

261.509

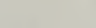

7264.

7134.9

6966.9

6305.318

6103.54

045.497

4444.

4364.89

4346.50

6386.7

5093.470

4750.49

3515.81

7425.1

6627.28

5891.36

3960.895
4057.457

4066.32

5785.0

$5.93 \quad 8.04$ at

$5.93 \quad 8.19 \quad 2 \frac{1}{3}-3 \frac{1}{2} d^{3} D-x^{4} F^{\circ}$

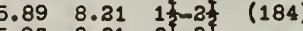

$\begin{array}{lll}5.89 & 8.22 & 1 \frac{1}{2}-1 \\ 5.93 & 8.22 & 2 \frac{2}{2}-1\end{array}$

$5.93 \quad 8.27 \quad 2 \frac{1}{2}-2 \frac{1}{3} d^{2} D-r^{2} D$

(5.93 8.59 (2)

$\begin{array}{llll}5.89 & 8.60 & 1 \frac{1}{3}-2 \frac{1}{2} & \text { (186) } \\ 5.93 & 8.60 & 2 & 2\end{array}$

$5.93 \quad 8.712 \frac{2}{2}-3 \frac{1}{2} d^{2} D-x^{2} G^{\circ}$

$\begin{array}{llll}5.93 & 8.93 & 2 \frac{1}{2}-3 \frac{1}{2} & \mathrm{~d}^{2} D-\pi^{4} \mathrm{~F}^{\circ} \\ 5.89 & 8.92 & 1 \frac{1}{3}-25 & (188)\end{array}$

$\begin{array}{llll}5.89 & 8.92 & 1 \frac{1}{3}-2 \frac{2}{3} & (188) \\ 5.93 & 8.92 & 232 & -3\end{array}$

$\begin{array}{lll}5.89 & 8.91 & \frac{1}{2}-1 \frac{1}{2} \\ 5.93 & 8.91 & 3 \frac{1}{2}-1 \frac{1}{2}\end{array}$

$\begin{array}{llll}5.93 & 8.97 & 2 \frac{1}{2}-3 \frac{1}{2} & d^{2} D-\pi^{4} D^{\circ} \\ 5.89 & 8.96 & 1 \frac{1}{2}-2 \frac{2}{2} & (189)\end{array}$
5081.920 A $\frac{\text { tr }}{4318.216}$

4493.579

4449.663
4431.626

4431.626
$\mathbf{m} 4508.26$
$7.91 \quad 10.33 \quad 3 \frac{1}{2}-3 \frac{1}{2}, \mathrm{r}^{4} \mathrm{a}^{\circ}-30$

7.8910 .63 5t-4t $\mathrm{r}^{4} \mathrm{0}^{\circ}-\mathrm{C}^{\circ} \mathrm{r}$

$7.89 \quad 10.67$ 43 3 (232)

\begin{tabular}{lll}
7.91 & 10.67 & 42 \\
7.91 & 10.69 & $3 \frac{7}{2}$ \\
7.89 & 10.63 & $4 \frac{1}{2}-4 \frac{1}{2}$ \\
\hline
\end{tabular} 


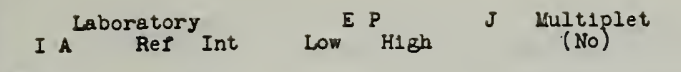

Fe II continued

$3987.543 \quad \mathrm{~A} \quad$ in $\quad 8.0012 .13 \quad 4 \frac{1}{2}-5 \frac{1}{2} \mathrm{y}^{2} \mathrm{C}^{0} \mathrm{C}-\mathrm{e}^{4} \mathrm{H}$

$5529.940 \mathrm{~A}=\frac{3}{8.18} 10.41 \quad \frac{1}{2}-1 \frac{1}{2} z^{2} \mathrm{~S}^{\circ}-e^{6 \mathrm{P}}$ $\begin{array}{llllllll}5303.419 & \text { A } & \text { On } & 8.15 & 10.48 & 4 \frac{1}{3}-5 \frac{1}{4} & x^{4} F^{0}-e^{4} G \\ 5315.618 & A & \text { On } & 8.19 & 10.52 & 31 & 4 \frac{1}{3} & (225) \\ 5278.265 & A & \text { On } & 8.21 & 10.55 & 2 \frac{1}{2}-3 \frac{1}{2} & \end{array}$

$3117.505 \quad \mathrm{~A} \quad 0 \quad 8.1912 .15 \quad 3 \frac{1}{2}-4 \frac{1}{2} \mathrm{x}^{4} \mathrm{~F}^{0}-\mathrm{e}^{4} \mathrm{H}$

$\begin{array}{lllllll}.3140 .6938 & \mathrm{~A} & 1 & 8.20 & 12.13 & 5 \frac{1}{4}-5 \frac{1}{3} & \mathrm{y}^{4} \mathrm{H}^{\circ}-\mathrm{e}^{4} \mathrm{H} \\ 3138.207 & \mathrm{~A} & \text { in } & 8.22 & 12.15 & 4 \frac{1}{2}-4 \frac{1}{2} & (227)\end{array}$

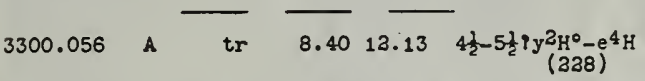

Strongest Unclassifled Lines of Fe II

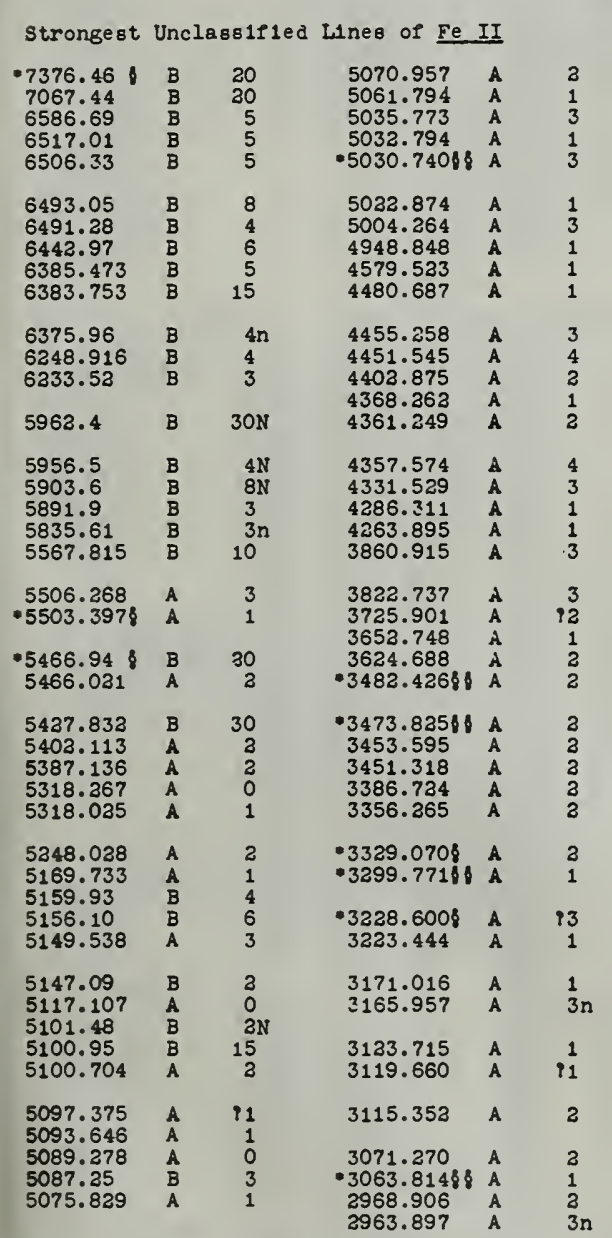

3109.32

3129.048

$\begin{array}{ll}3013.125 & A \\ 3001.589 & A \\ 3002.99 & A \\ 3015.230 & A \\ 3008.506 & A \\ 3000.836 & \\ 3012.847 & B \\ 3027.46 & A \\ 3055.55 & A \\ 3007.803 & A \\ 3023.85 & A \\ 3054.134 & A \\ 3018.744 & A \\ 3050.463 & A\end{array}$

3419.49
3421.97

3108.85

3143.36

$\begin{array}{llllllll}3283.30 & \mathrm{~A} & \boldsymbol{2} & & 10.85 & 14.61 & 3-4 & \mathrm{~b}^{1} \mathrm{~F}-2^{3} \mathrm{~F}^{\circ} \\ 3294.50 & \mathrm{~A} & 4 & 10.85 & 14.60 & 3-3 & (14)\end{array}$

$4003.41 \quad A \quad \frac{4}{10.95} \quad \overline{14.03} 4-4 \quad b^{3} H_{-2} 5_{G^{\circ}}$

$\begin{array}{llllllll}3464.27 \quad B & 1 & 10.95 & 14.51 & 4-4 & b^{3} \mathrm{H}_{-2} \mathrm{z}^{\circ}\end{array}$

$\begin{array}{llllllll}3367.54 \quad \text { A } & 3 & 10.95 & 14.61 & 4-4 & b^{3} \mathrm{H}_{-2}^{3} \mathrm{~F}^{\circ}\end{array}$

$\begin{array}{ccccccc}3396.71 & \text { A } & 7 & 10.98 & 14.61 & 6-6 & b^{3} \mathrm{H}_{-2} 3 \mathrm{H}^{\circ} \\ 3347.70 & \text { A } & 8 & 10.95 & 14.64 & 5-5 & (18) \\ 3329.89 & \text { A } & 7 & 10.95 & 14.65 & 4-4 & \end{array}$

3373.51

.3333 .27

$\begin{array}{lllllll}3357.07 & B & \text { tr } & 10.95 & 14.62 & 4-3 & b^{3} \mathrm{H}-y^{5} p^{\circ}\end{array}$

$\begin{array}{llllllll}3090.772 \quad B & 1 & 10.95 & 14.94 & 4-3 & b^{3} H_{-1} y^{\circ}\end{array}$

$\begin{array}{llcllll}3026.985 & \text { A } & \cdot 6 & 10.98 & 15.05 & 6-5 & \mathrm{~b}^{3} \mathrm{H}^{3} \mathbf{2}^{3} \mathrm{G}^{\circ}\end{array}$

m3006.95

.3004 .109

$\begin{array}{lll}\mathrm{P} & \mathrm{Fe} & \mathrm{III} \\ \mathrm{B} & 4 & 10 \\ & & \end{array}$

10.9515 .05

$4205.92 \quad A \quad 2 \quad 11.10 \quad 14.03 \quad 5-5 \quad c^{3} G_{2} 5_{G}$ o

4184.09
4196.69

$\begin{array}{rrrrrrr}-3947.10 & \text { A } & 4 & 11.08 & 14.21 & 4-4 & c^{3} G-25 H^{\circ} \\ 3663.98 & \text { B } & \operatorname{tr} & 11.08 & 14.45 & 4-3 & (23) \\ 3664.98 & \text { B } & 1 & 11.07 & 14.44 & 3-2 & (24)\end{array}$

$\begin{array}{llllllllll}3620.37 \quad A & 3 & 11.10 & 14.51 & 5-4 & c^{3} G-z^{5} D^{\circ}\end{array}$

$\begin{array}{lllllll}3514.87 & A & 2 & 11.10 & 14.61 & 5-4 & c^{3} G-2^{3} F^{\circ} \\ 3512.34 & \text { P } & & 11.08 & 14.60 & 4-3 & (26)\end{array}$

$\begin{array}{lrrrrrr}3512.34 & P & & 11.0814 .60 & 4-3 & (26) \\ 3511.93 & \text { B } & \operatorname{tr} & 11.0714 .59 & 3-27 & \\ 3499.57 & \text { A } & 7 & 11.0814 .61 & 4-4 & \end{array}$

3501.75
3489.07

3514.39

3514.39
3474.41
3448.63

3448.63
3489.48

3458.91
.3473 .82

3167.54

.3120 .03

3108.78

3102.5

3110.85

3107.950
3100.48

3070.072

3035.802

3011.060

$11.0814 .64 \quad 4-5$ $\begin{array}{lll}10.95 & 15.05 & 4-3 \\ 10.95 & 15.05 & 4-4\end{array}$

I A Laboratory ${ }_{\text {Ref Int Low }{ }_{\text {H1gh }} \text { I Multiplet }}$ Fe III cont1nued

$\begin{array}{lllllll}4323.81 & B & 2 & 11.17 & 14.02 & 3-3 & \mathrm{~d}^{3} \mathrm{~F}-2^{5} \mathrm{G}^{\circ}\end{array}$ $\begin{array}{llllllll}4057.51 & B & 4 & 11.17 & 14.21 & 4-4 & \mathrm{~d}^{3} \mathrm{~F}-2^{5} \mathrm{H}^{\circ}\end{array}$

$\begin{array}{lllllllll}3773.80 & B & \text { tr } & 11.17 & 14.44 & 3-2 & \mathrm{~d}^{3} \mathrm{~F}-2^{5} \mathrm{~F}^{\circ}\end{array}$

$\begin{array}{lllllll}-3697.4586 & \mathrm{~B} & 3 & 11.17 & 14.51 & 3-4 & (34) \\ 3845.68 & \mathrm{~B} & 1 & 11.16 & 14.37 & 2-2 & (35)\end{array}$

$\begin{array}{rrrrrrr}3586.12 & A & 9 & 11.17 & 14.61 & 4-4 & \mathrm{~d}^{3} \mathrm{~F}-2^{3} \mathrm{~F}^{\circ} \\ 3600.93 & \mathrm{~A} & 10 & 11.17 & 14.60 & 3-3 & (36)\end{array}$

$\begin{array}{rrrrrr}3600.93 & \text { A } & 10 & 11.17 & 14.60 & 3-3 \\ 3603.88 & \text { A } & 9 & 11.16 & 14.59 & 2-2\end{array}$

$\begin{array}{llllll}3599.49 & \text { A } & 3 & 11.17 & 14.60 & 4-3 \\ 3611.72 & \text { A } & 3 & 11.17 & 14.59 & 3-2\end{array}$

$\begin{array}{llllll}3587.53 & \text { A } & 3 & 11.17 & 14.61 & 3-4 \\ 3593.15 & \text { A } & 4 & 11.16 & 14.60 & 2-3\end{array}$

$\begin{array}{lllllll}3250.27 & \text { B } & 1 & 11.17 & 14.97 & 3-4 & \mathrm{~d}^{3} F-y^{5} F^{\circ} \\ 3294.85 & \text { B } & 1 & 11.17 & 14.92 & 3-2 & (37) \\ 3302.19 & B & 1 & 11.16 & 14.90 & 2-1 & \end{array}$

3302.19

3176.00

3178.03
3174.09

3176.86

3180.17
3179.08

$\begin{array}{lllll}10 & 11.17 & 15.05 & 4-5 & d^{2} F-z^{3} G^{0} \\ 10 & 11.17 & 15.05 & 3-4 & (38)\end{array}$

$\begin{array}{rrrr}10 & 11.17 & 15.05 & 3-4 \\ 10 & 11.16 & 15.05 & 2-3 \\ 2 & 11.17 & 15.05 & 4-4 \\ & 11.17 & 15.05 & 3-3\end{array}$

$\begin{array}{lll}11.17 & 15.05 & 3-3 \\ 11.17 & 15.05 & 4-3\end{array}$

$\begin{array}{ccccccc}3136.43 & A & 10 & 11.17 & 15.10 & 4-3 & \mathrm{~d}^{3} \mathrm{~F}-2^{3} \mathrm{p}^{\circ} \\ 3110.052 & \mathrm{~A} & 10 & 11.17 & 15.14 & 3-2 & (39) \\ 3083.68 & \mathrm{P} & \mathrm{Fe} & 11.16 & 15.16 & 2-1 & \end{array}$

$\begin{array}{lllllll}3089.649 & \text { B } & 1 & 11.17 & 15.16 & 4-3 & d^{3} F-y^{5} D^{\circ} \\ 3084.09 & \text { A } & 6 & 11.17 & 15.17 & 3-2 & \end{array}$

$\begin{array}{lllllllll}-3004.109 & B & 3 & 11.17 & 15.28 & 4-3 & d^{3} F-x^{5} \text { po }\end{array}$

$\begin{array}{lllllll}-3004.109 & \text { B } & 3 & 11.17 & 15.28 & 4-3 & \mathrm{~d}^{3} \text { F-x } \\ 3009.998 & \mathrm{~B} & 1 & 11.17 & 15.27 & 3-2 & (41) \\ 3004.490 & \text { B } & 1 & 11.16 & 15.27 & 2-2 & \end{array}$

$4391.26 \quad$ B $\overline{1} \quad \overline{11.42} 14.23 \quad 5-5 \quad \mathrm{a}^{1} \mathrm{H}_{\left(-z^{5} \mathrm{H}^{\circ}\right.}$

$\begin{array}{lllllll}4927.56 \quad A \quad 2 & 11.53 & 14.03 & 4-5 & e^{3} F-z^{5} G^{\circ}\end{array}$

$\begin{array}{lllllll}4226.14 & B & 2 & 11.53 & 14.45 & 2-3 & e^{3} F_{-2} 5 F^{\circ}\end{array}$

$\begin{array}{lllllll}m 4005.04 & P & F e & 11.53 & 14.61 & 4-4 & e^{3} F^{(44)} z^{3} \text { o }\end{array}$

$\begin{array}{lcccccc}4022.36 & \mathrm{~A} & 4 & 11.53 & 14.60 & 3-3 & \mathrm{e} \\ 4039.12 & \mathrm{~A} & 3 & 11.53 & 14.59 & 2-2 & \end{array}$

$\begin{array}{llll}4021.75 & P & 11.5314 .60 & 4-3 \\ 4035.82 & P & 11.53 & 14.59\end{array}$

$\begin{array}{lllll}4035.82 & \mathrm{P} & 11.53 & 14.59 & 3-2 \\ 4005.64 & \mathrm{P} & 11.53 & 14.61 & 3-4\end{array}$

$\begin{array}{lllll}4005.64 & P & 11.53 & 14.61 & 3-4 \\ 4025.67 & P & 11.53 & 14.60 & 2-3\end{array}$

$3990.81 \quad B \quad$ tr $\quad 11.53 \quad 14.62 \quad 3-3 \quad e^{3} F-y 5 p c$

$3800.43 \quad B \quad 1 \quad 11.53 \quad 14.78 \quad 3-29 e^{3 \frac{F-z}{3} p_{0}}$

$\begin{array}{rllllll}3500.29 & \mathrm{~A} & 7 & 11.53 & 15.05 & 4-5 & \mathrm{e}^{3 \mathrm{~F}-23} \mathrm{z}_{\mathrm{G}} \\ .3501 .75 & \mathrm{~A} & 8 & 11.53 & 15.05 & 3-4 & (48)\end{array}$

$\begin{array}{llllll}3506.93 & A & 5 & 11.53 & 15.05 & 2-3\end{array}$

$\begin{array}{llllll}3501.32 & \mathrm{P} & & 11.53 & 15.05 & 4-4 \\ 3504.40 & \mathrm{~A} & 2 & 11.53 & 15.05 & 3-3\end{array}$

$3503.96 \quad P \quad 11.5315 .05 \quad 4-3$

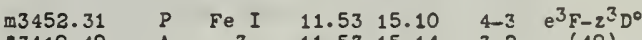

$\begin{array}{lllllll}3419.49 & \text { A } & 3 & 11.53 & 15.14 & 3-2 & \text { (49) }\end{array}$

$3301.09 \quad B \quad$ tr $11.53 \quad 15.27 \quad 2-2 \quad e^{2} F-x^{5} p^{\circ}$

$\begin{array}{rllllll}-3118.75 & A & 5 & 11.53 & 15.49 & 4-4 & e^{3} F^{2}-y^{3} F^{\circ}\end{array}$

3098.93

$\begin{array}{llll}11.53 & 15.51 & 3-3 & (51) \\ 11.53 & 15.51 & 2-2 & \end{array}$

$\begin{array}{llrllll}4590.68 & \text { B } & \operatorname{tr} & 11.54 & 14.23 & 4-5 & \mathrm{c}^{1} \mathrm{G}-z^{5} \mathrm{H}^{\circ} \\ 4663.78 & \mathrm{~B} & 1 & 11.54 & 14.19 & 4-3 & (52)\end{array}$

$\begin{array}{llllllll}4025.07 \quad A & 3 & 11.54 & 14.61 & 4-4 & c^{1} G_{2} F^{\circ}\end{array}$

$\begin{array}{lllllll}3515.57 & A & 5 & 11.54 & 15.05 & 4-5 & c^{1} G-z^{3} G^{\circ}\end{array}$

$\begin{array}{lllllll}3516.58 & \mathrm{P} & & 11.54 & 15.05 & 4-4 & (54) \\ 3519.25 & \mathrm{~B} & 1 & 11.54 & 15.05 & 4-3 & \end{array}$

$\begin{array}{lllllll}3139.74 \quad A \quad & 3 & 11.54 & 15.41 & 4-3 & C^{1} G-y^{3} D^{\circ} \\ (55)\end{array}$

$5532.65 \quad B \quad \overline{1} \quad \overline{11.98} 14.21 \quad 3-4 \quad c^{1} F-2^{5} H^{\circ}$

$\begin{array}{lllllll}4714.53 & B & 1 & 11.98 & 14.60 & 3-3 & c^{1} F-2^{3} F^{\circ}\end{array}$

$\begin{array}{llllllll}4671.25 & B & \text { tr } & 11.98 & 14.62 & 3-3 & c^{1} F-y^{5 p o}\end{array}$

$11.0714 .61 \quad 3-4$

$\begin{array}{llll}11.10 & 14.61 & 5-6 & c^{3} G-z^{3} H^{\circ} \\ 11.08 & 14.64 & 4-5 & (27)\end{array}$

$11.1014 .64 \quad 5-5$

$\begin{array}{lll}11.08 & 14.65 & 4-4 \\ 11.10 & 14.65 & 5-4\end{array}$

$11.10 \quad 15.00 \quad 5-5 \quad c^{3} G-y^{5} F^{\circ}$

3519.8

$\begin{array}{llll}11.98 & 15.49 & 3-4 & (58) \\ c^{1} F-y & (59) & \end{array}$

3525.17

3403.5

$13.07 \quad 16.58 \quad 2-2 \quad d^{3} p-x^{3} p^{0}$

3403.5

.3410 .74

$13.07 \quad 16.61$

$13.07 \quad 16.70 \quad 2-2 \quad d^{3} D-x^{3} D^{0}$

$11.10 \quad 15.05 \quad 5-5 \quad c^{3} G_{-2}^{3} G^{\circ}$

.3410 .7

$\begin{array}{lll}11.07 & 15.05 \\ 11.10 & 15.05\end{array}$

11.0815 .05

$4-3$
$3-3$
$5-4$
$4-3$
$4-5$
$3-4$

$\begin{array}{lll}11.08 & 15.05 & 4-5 \\ 11.07 & 15.05 & 3-\end{array}$

-3357.40
-3370.23
-3329.0486

$11.08 \quad 15.10 \quad 4-3 \quad c^{3} a-z^{3} p^{\circ}$

3263.04

3238.74
3264.22

3096.86

3096.86
3099.05 $\begin{array}{lll}13.08 & 16.70 & 3-2 \\ 13.07 & 16.69 & 2-1\end{array}$

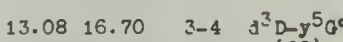

$\begin{array}{llll}13.08 & 16.75 & 3-3 & d^{3} 2 \pi^{3} 3 D^{\circ}\end{array}$

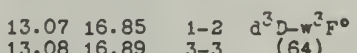

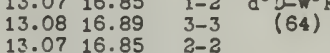

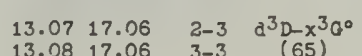

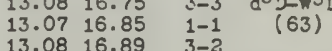




\section{0}

\section{A Laboratory ReP Int Low High $_{\text {H }}$ Multiplet $_{\text {(No) }}$}

Fe III continued

$\begin{array}{lllllll}4570.34 & \mathrm{~A} & 4 & 13.53 & 16.33 & 2-2 & \mathrm{c}^{1} \mathrm{D}-z^{1} \mathrm{D}^{\circ} \\ 3432.97 & \mathrm{~A} & 3 & 13.53 & 17.12 & 3-1 & \mathrm{c}^{1} \mathrm{D}-z^{1} \mathrm{P}^{\circ} \\ (67)\end{array}$

$\begin{array}{llllllc}5573.3 & \mathrm{~A} & 4 & 14.11 & 16.33 & 5-6 & \mathrm{~d}^{3} \mathrm{G}-\mathrm{-}^{3} \mathrm{H}^{\circ} \\ 5485.6 & \mathrm{~A} & 3 & 14.12 & 16.37 & 4.5 & (68) \\ 5460.8 & \mathrm{~A} & 3 & 14.12 & 16.38 & 3-4 & (68)\end{array}$

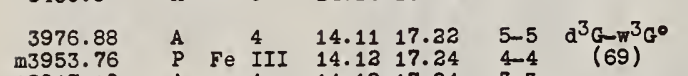

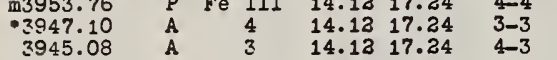

$\begin{array}{rlllllll}-3828.44 \quad \text { A } & 2 & 14.13 & 17.34 & 4-3 & d^{3} G-y^{1} F^{0}\end{array}$

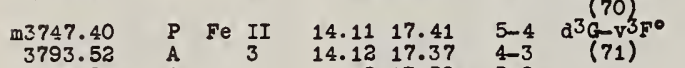

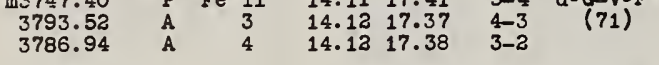

$\begin{array}{lllllll}3382.19 & A & 6 & 14.11 & 17.76 & 5-5 & d^{3}\left(-w^{3} G^{\circ}\right. \\ 3360.84 & A & 6 & 14.12 & 17.79 & 4-4 & (72)\end{array}$

3358.

3359.18
.3357.

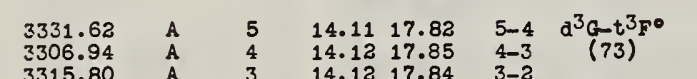

$\begin{array}{rlllll}3315.80 & \mathrm{~A} & 3 & 14.12 & 17.84 & 3-2 \\ .3333 .27 & \mathrm{~A} & 3 & 14.12 & 17.82 & 4-4\end{array}$

$\begin{array}{llllllll}3362.44 & A & 6 & 14.11 & 17.90 & 5-4 & d^{3} G-x^{1} a^{\circ}\end{array}$

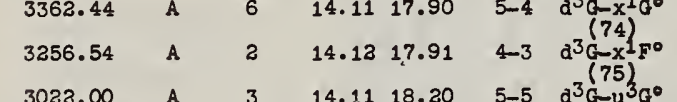

$\begin{array}{llllllll}3022.00 & A & 3 & 14.11 & 18.20 & 5-5 & d^{3} G-G_{G}^{3} G^{\circ} \\ 3045.877 & A & 3 & 14.12 & 18.17 & 4-4 & (76)\end{array}$

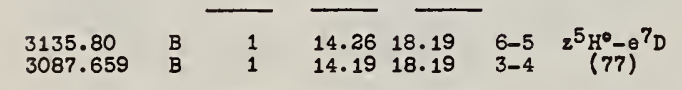

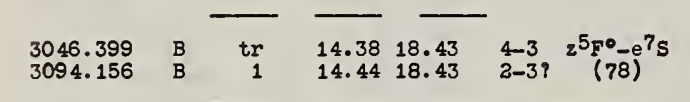

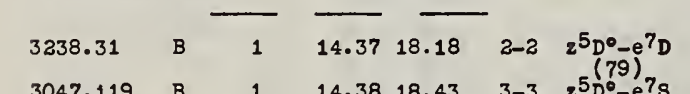

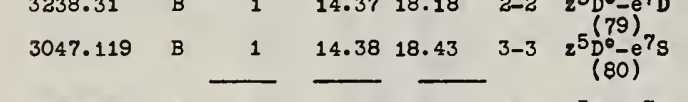

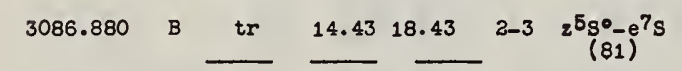

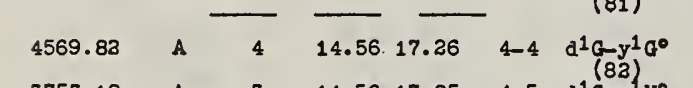

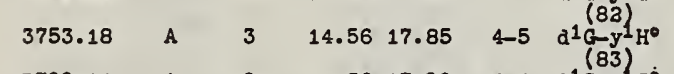

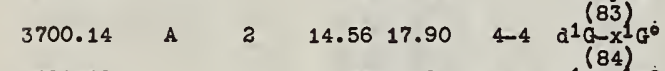

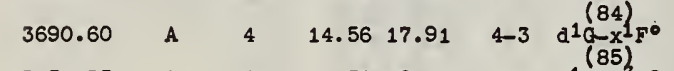

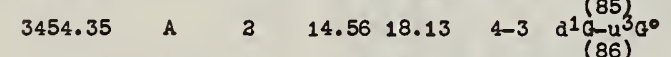

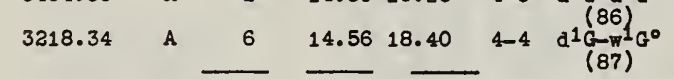

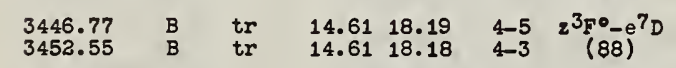

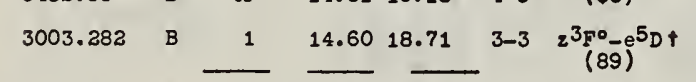

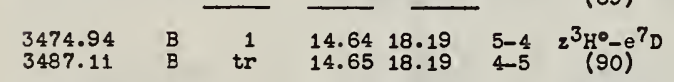

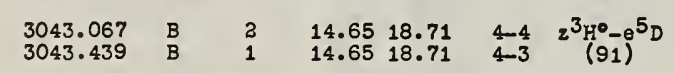

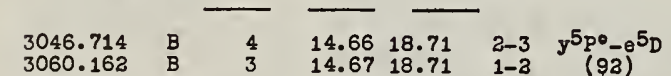

$3624.25 \quad B \quad \frac{1}{14.78} \frac{18.18}{2-1} z^{3} P^{0}-e^{7} D$

$\begin{array}{rrrrrrr}3624.25 & \mathrm{~B} & 1 & 14.78 & 18.18 & 2-1 & z^{3} \mathrm{P}^{0}-\mathrm{e}^{7} \mathrm{D} \\ 3660.85 & \mathrm{~B} & 3 & 14.81 & 18.18 & 1-2 & (93)^{2} \\ 3688.71 & \mathrm{~B} & \text { tr } & 14.84 & 18.18 & 0-1 & \end{array}$

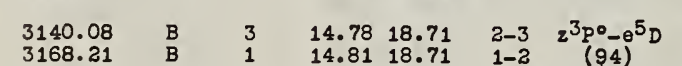

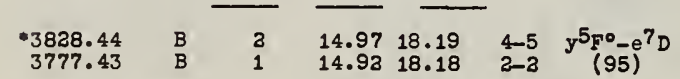

$\begin{array}{lllllll}3324.72 & \mathrm{~A} & 3 & 15.00 & 18.71 & 5-4 & \mathrm{y}^{5} \mathrm{FO}^{\circ}-0^{5} \mathrm{D} \\ 3300.20 & \mathrm{~A} & 4 & 14.97 & 18.71 & 4.3 & (96) \\ 3274.95 & \mathrm{~A} & 3 & 14.94 & 18.71 & 3-3 & (96)\end{array}$

3274.95
-3299.77
3255.49

3274.65

3377.77

4051.08

$\begin{array}{lllllll}4051.08 & \text { B } & 2 & 15.14 & 18.18 & 2-3 & 2^{3} \mathrm{D}^{\circ}-\mathrm{e}^{7 \mathrm{D}} \\ 4053.59 & \mathrm{~B} & 1 & 15.14 & 18.18 & 2-2 & (98) \\ 3711.32 & \mathrm{~B} & 3 & 15.10 & 18.43 & 3-3 & 3^{3} \mathrm{D}^{\circ}-9^{7 \mathrm{~g}}\end{array}$

$\begin{array}{lll}14.94 & 18.71 & 3-2 \\ 14.97 & 18.71 & 4-4 \\ 14.92 & 18.71 & 3-4\end{array}$

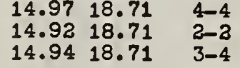

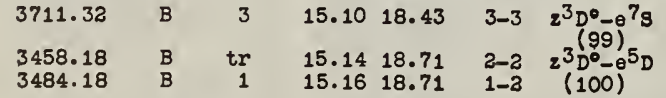

REVISED UULTIPLET TABLE

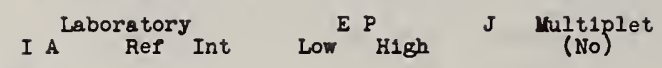

I A Laboratory Ref Int Low ${ }^{\text {E } P}$ High

J viltiplet

\section{Fe III continued}

$\begin{array}{llllllll}4098.54 & B & 1 & 15.17 & 18.18 & 2-2 & y^{5} D^{0}-e^{7} D\end{array}$

$3788.91 \quad B \quad$ tr $\quad 15.1718 .43 \quad 2-3 \quad \begin{gathered}(101) \\ \mathrm{y}^{5} \mathrm{D}^{\circ}-\mathrm{e}^{7 \mathrm{~S}} \\ (102)\end{gathered}$

$\begin{array}{llllllll}3496.29 & \mathrm{~A} & 4 & 15.18 & 18.71 & 4-4 & \mathrm{y}^{5} \mathrm{D}^{\circ}-\theta^{5} \mathrm{D} \\ 3482.36 & \mathrm{~B} & 4 \mathrm{~d} & 15.1618 .71 & 3-4 & (103)\end{array}$

\begin{tabular}{ccc}
3482.36 & B & $4 \mathrm{~d}$ \\
3491.16 & $\mathrm{~B}$ & $\mathrm{Z}$ \\
\hline
\end{tabular}

$\begin{array}{lllllll}4237.21 & B & 2 & 15.27 & 18.18 & 2-2 & x^{5} P^{\circ}-e^{7} D\end{array}$

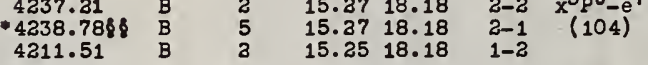

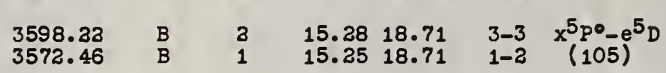

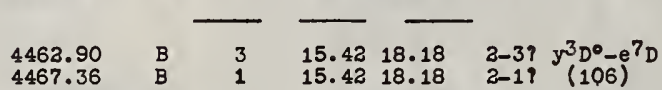

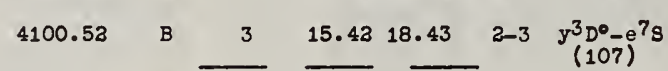

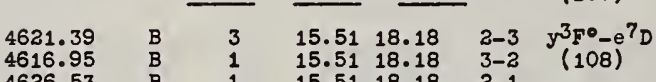

$\begin{array}{rrrrrr}4626.53 & B & 1 & 15.51 & 18.18 & 2-1 \\ 4624.42 & B & \text { tr } & 15.51 & 18.18 & 2-2\end{array}$

$\begin{array}{llrllll}3831.75 & \text { B } & \text { tr } & 15.49 & 18.71 & 4-3 & y^{3} F^{\circ}-e^{5} \mathrm{D} \\ 3860.46 & \text { B } & 1 & 15.51 & 18.71 & 3-3 & (109)\end{array}$

$\begin{array}{lllllll}5436.80 & \text { B } & 1 & 15.91 & 18.18 & 2-3 & y^{3} \mathrm{p}^{0}-\mathrm{e}^{7} \mathrm{D}\end{array}$

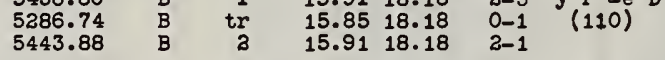

$\begin{array}{lllllll}4908.74 & \text { B } & 1 & 15.91 & 18.43 & 2-3 & \mathrm{y}^{3} \mathrm{P}^{0}-\mathrm{e}^{7} \mathrm{~S}\end{array}$

5269.15 B $4 \quad \frac{1}{16.37} 18.71 \quad 5-4 \mathrm{y}^{3} \mathrm{H}^{\circ}-e^{5} \mathrm{D}$

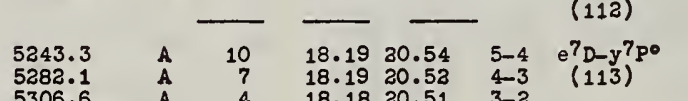

$\begin{array}{llllll}5282.1 & \text { A } & 7 & 18.19 & 20.52 & 4-3 \\ 5306.6 & \text { A } & 4 & 18.18 & 20.51 & 3- \\ 5235.3 & \text { A } & 5 & 18.19 & 20.54 & 4\end{array}$

$\begin{array}{llllll}5302.5 & \text { A } & 6 & 18.18 & 20.51 & 2-2 \\ 5329.57 & \text { B } & \text { B } & 18.18 & 20.54 & 3-4 \\ 5072.0 & \text { A } & 3 & 18.18 & 20.52 & 2-3\end{array}$

5272.0
5299.9

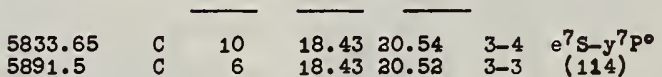

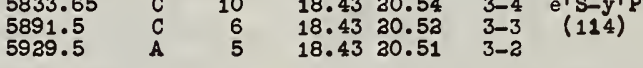

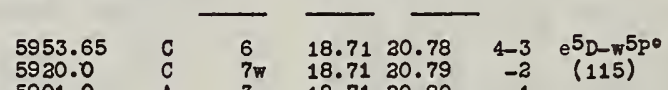

3007.8

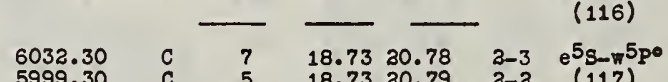

$\begin{array}{lllllll}5999.30 & \mathrm{C} & ? & 18.73 & 30.78 & 2-3 & \mathrm{e}^{5} \mathrm{~S}-\mathrm{F}^{5} \\ 5978.90 & \mathrm{C} & 5 & 18.73 & 30.79 & 2-2 & (117) \\ & \mathrm{C} & 5 \mathrm{n} & 18.73 & 20.80 & 3-1 & \end{array}$

$\begin{array}{llllllll}4164.79 & \text { A } & 30 & & 20.54 & 23.51 & 4-5 & y^{7 P^{0}-F^{7} D} \\ 4137.93 & \text { A } & 10 & 20.52 & 23.51 & 3-4 & (118)\end{array}$

4137.93
4120.97

4166.86

4132.37

4168.41
4140.51

4123.98

4081.19

4053.28
4035.54

.3954 .38

3968.78

3978.43
3954.38

m3969. 43

3979.42
3980.14

$\begin{array}{ll}20.51 & 23.50 \\ 20.54 & 23.51\end{array}$

$\begin{array}{ll}20.54 & 23.51 \\ 20.52 & 23.51\end{array}$

20.5123 .50

$\begin{array}{ll}20.54 & 23.51 \\ 20.52 & 23.50\end{array}$

$\begin{array}{ll}20.52 & 23.50 \\ 20.51 & 23.50\end{array}$

$\begin{array}{llll}20.54 & 23.57 & 4-3 & y^{7} p^{\circ}-f^{7} s \\ 20.53 & 23.57 & 3-3 & (119)\end{array}$

$\begin{array}{lll}20.52 & 23.57 & 3-3 \\ 30.51 & 23.57 & 2-3\end{array}$

\begin{tabular}{cc}
$A$ & 12 \\
\cline { 2 - 2 } & 8 \\
$A$ & 4 \\
$A$ & 12 \\
$P$ & $F \ominus I$ \\
$A$ & 5 \\
$A$ & 3
\end{tabular}

$\begin{array}{llll}30.78 & 33.90 & 3-4 & w^{5} P^{\circ}-P^{5} D \\ 20.79 & 23.90 & 2-3 & (120) \\ 20.80 & 23.90 & 1-2 & \end{array}$

$\begin{array}{ll}20.80 & 23.90 \\ 30.78 & 23.90\end{array}$

$30.7823 .90 \quad 3-3$

$\begin{array}{lll}20.79 & 23.90 & 2-2 \\ 30.80 & 23.90 & 1-1\end{array}$

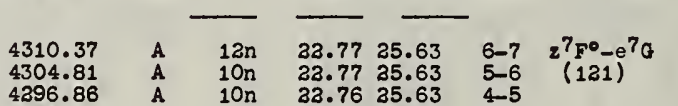

4286.13
4273.42

ss2.4.

$\begin{array}{rrrrr}\mathrm{A} & 10 \mathrm{n} & 22.77 & 35.63 & 5-6 \\ \mathrm{~A} & 10 \mathrm{n} & 22.76 & 35.63 & 4-5 \\ \mathrm{~A} & 10 \mathrm{n} & 22.75 & 35.63 & 3-4 \\ \mathrm{~A} & 7 \mathrm{n} & 32.75 & 35.63 & 2-3\end{array}$

20wn 23.8125 .63

Strongest Unclassified Lines of Fe III
(Some poss1bly Fe II)

$w^{5} \mathrm{~F}^{\circ}-\theta^{5} \mathrm{G}$

6185.1
5875.6

5875.6

5587.9
5466.46

(1)

5291.78

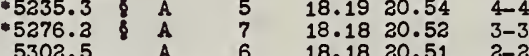

$\begin{array}{lllllll}5920.0 & \mathrm{C} & 7 \mathrm{w} & 18.71 & 30.79 & -2 & \text { (115) } \\ 5901.0 & \mathrm{~A} & 3 & 18.71 & 20.80 & -1 & \end{array}$
Fe III continued

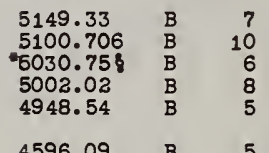

$\begin{array}{lll}4143.87 & \text { B } & 7 \\ 4121.31 & \text { B } & 6 \\ 4113.45 & \text { B } & ? \\ 4113.23 & \text { B } & 7 \\ 4109.95 & \text { B } & 5\end{array}$

4596.09

4535.50
4271.47

4366.88

4255.20
4249.95

4343.85
4235.54

4222.39

4220.32
4210.87

4210.87
4200.38

4200.06

4189.10
4179.25

4179.25
4174.27

4154.98
4145.74
4008.81
3964.11
3743.40

3652.65
3589.77

3367.02

3338.72
3309.40

3304.31
-3295.248

3190.81
3151.86

3151.86
3133.18

3121.08
3086.31

3044.438
4573.14
4559.09

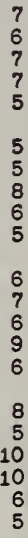

\section{Co I I P.7.84 Anal A L1st B Feb 1942}

4233.996

4339.13
4361.913

4361.913
4361.031

4190.712

4252.302
4285.782

4303.235
-4109.706

4179.90

4229.955
4268.032

4059.331

4088.291

4108.488
3956.270

4011.089

4054.618
4198.425

4189.50

4177.59

3909.933

3979.518

4037.032

4057.195
3993.014

3526.847

3575.361

3594.870
3602.079

3602.079
+3474.018

3520.075

3550.592
3631.390

3652.541

3647.658

3465.792

3513.478

353.35

3533.356
3415.519

3456.924

3412.633

3412.633
3431.582

3442.918

3455.237
3510.426

3502.63

3491.316

3584.801
3552.720

3153.692

3132.218

3237.028
3191.297

3136.726

3219.150

3186.350

3281.585
3237.753

3121.415
3139.947

3139.947
3149.310

3149.310
-3159.662

3303.026
3199.323

3189.753

$\mathrm{m} 3264.83$
3241.05

(3) $\left(\begin{array}{l}1 \mathrm{~N} \\ 1 \mathrm{n}\end{array}\right.$
20
12
6
3
$(10)$
$\left(\begin{array}{l}2 \mathrm{n} \\ 1 \mathrm{n}\end{array}\right.$
$\left(\begin{array}{l}1 \\ 1\end{array}\right.$
$\left(\begin{array}{l}1 \\ 2 \\ 2 \\ 3 \\ 3 \\ 2 \\ 1 \\ 1\end{array}\right)$

15
10
10
5

10
5
(1)

$100 \mathrm{R}$

$60 \mathrm{r}$
$50 \mathrm{R}$

$40 \mathrm{R}$
$10 \mathrm{R}$
15
$20 \mathrm{r}$

20r

15

$100 \mathrm{R}$

100R

$30 r$

5
(6)
(6)

(6)

$80 \mathrm{R}$
$50 \mathrm{r}$

$40 r$

30r

$20 r$

15
8

(1)

4
8
4

$0.00 \quad 3.16 \quad 4 \frac{1}{2}-5 \frac{1}{3} a^{4} 5-2^{6} 0^{\circ}+$

$\begin{array}{llll}0.00 & 3.16 & 4 \frac{1}{2}-5 \frac{1}{2} & a^{4} 5-2 \\ 0.10 & 3.20 & 3 \frac{1}{3}-4 \frac{1}{2} & (3)\end{array}$

$\begin{array}{lll}0.22 & 3.27 & 1 \frac{1}{3}-2 \frac{1}{2} \\ 0.17 & 3.26 & 2 \frac{1}{2}-2 \frac{1}{2}\end{array}$

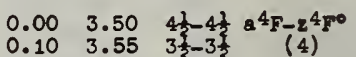

$\begin{array}{lll}0.10 & 3.55 & 3 \frac{1}{3}-3 \\ 0.17 & 3.61 & 2 \frac{1}{3}-2\end{array}$

$0.22 \quad 3.65 \quad 1 \frac{1}{3}-1$

$0.10 \quad 3.61 \quad 3 \frac{1}{2}-2$

$\begin{array}{lll}0.17 & 3.65 & 2 \frac{1}{3}-1 \frac{1}{3} \\ 0.10 & 3.50 & 3 \frac{1}{3}-4\end{array}$

$\begin{array}{lll}0.17 & 3.55 & 3 \frac{1}{2}-3 \\ 0.22 & 3.61 & 1 \frac{1}{2}-2 \frac{1}{2}\end{array}$

$0.00 \quad 3.56 \quad 4 \frac{1}{3}-5 \frac{1}{3} \mathrm{a}^{4} \mathrm{~F}-2^{4} \mathrm{G}^{0}$

$0.1003 .61 \quad 3 \frac{1}{3}-4$

$\begin{array}{lll}0.17 & 3.67 & 2 \frac{1}{3}-3 \\ 0.22 & 3.72 & 1 \frac{1}{2}-2\end{array}$

$0.10 \quad 3.67 \quad 3 \frac{1}{2}-3$

$0.17 \quad 3.72 \quad 2 \frac{2}{2}-3 \frac{1}{2}$

$0.00 \quad 3.62 \quad 4 \frac{1}{3}-3 \frac{1}{3} a^{4} \mathrm{r}-2^{4} D^{\circ}$

$0.10 \quad 3.70 \quad 3 \frac{1}{2}-2 \frac{1}{2} \quad(6)$

$0.23 \quad 3.80 \quad 12$

$0.10 \quad 3.62 \quad 3 \frac{1}{2}-3$

$0.22 \quad 3.76 \quad 13$

$\begin{array}{lll}0.22 & 3.76 & 1 \frac{1}{2}-1 \\ 0.17 & 3.62 & 2 \frac{1}{2}-3 \\ 0.32 & 3.70 & 1 \frac{1}{2}-2.2\end{array}$

$0.00 \quad 3.91 \quad 4 \frac{1}{2}-4 \frac{1}{2} 8^{4} \mathrm{~F}-z^{2} \mathrm{G}^{\circ}$

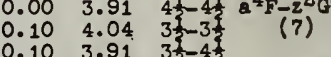

$\begin{array}{lll}0.10 & 3.91 & 3 \frac{1}{3}-4 \frac{1}{3} \\ 0.17 & 4.04 & 3 \frac{1}{2}-3 \frac{1}{2}\end{array}$

$0.00 \quad 3.93 \quad 4 \frac{1}{2}-3 \frac{1}{2} \mathrm{a}^{4} \mathrm{~F}-2^{3} \mathrm{~F}$

$\begin{array}{llll}0.00 & 3.93 & 4 \frac{1}{3}-3 \frac{1}{2} & a^{4}-2 \\ 0.10 & 3.93 & 3 \frac{1}{2}-3 \frac{1}{2} & (8)\end{array}$

0.177 .05 2 3.93

$\begin{array}{lllll}0.00 & 3.95 & 4 \frac{1}{3}-3 \frac{1}{3} & a^{4}-y^{4} D^{*} \\ 0.10 & 4.03 & 3 \frac{1}{2} & (9)\end{array}$

$\begin{array}{lll}0.10 & 4.03 & 3 \frac{1}{3}-3 \\ 0.17 & 4.09 & 3 \frac{1}{2}-1\end{array}$

$0.22 \quad 4.13$ 1

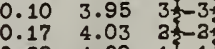

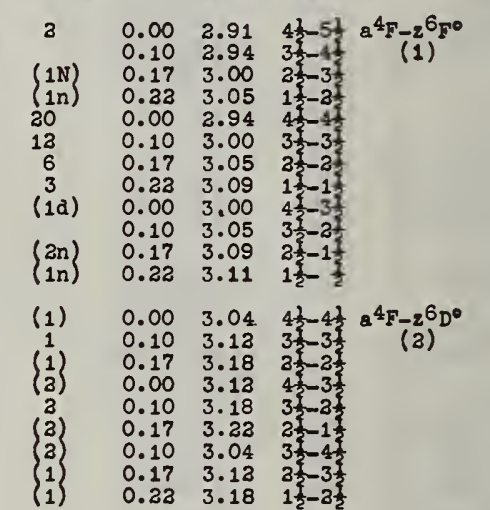

$0.23 \quad 4.05 \quad 1 \frac{1}{2}-2 \frac{1}{2}$

$\begin{array}{lll}0.22 & 4.09 & 12-1 \\ 0.17 & 3.95 & 3\end{array}$

5430.14

5402.27

5375.68

5373.86

5272.86
5260.25

5227.53
5216.99

.




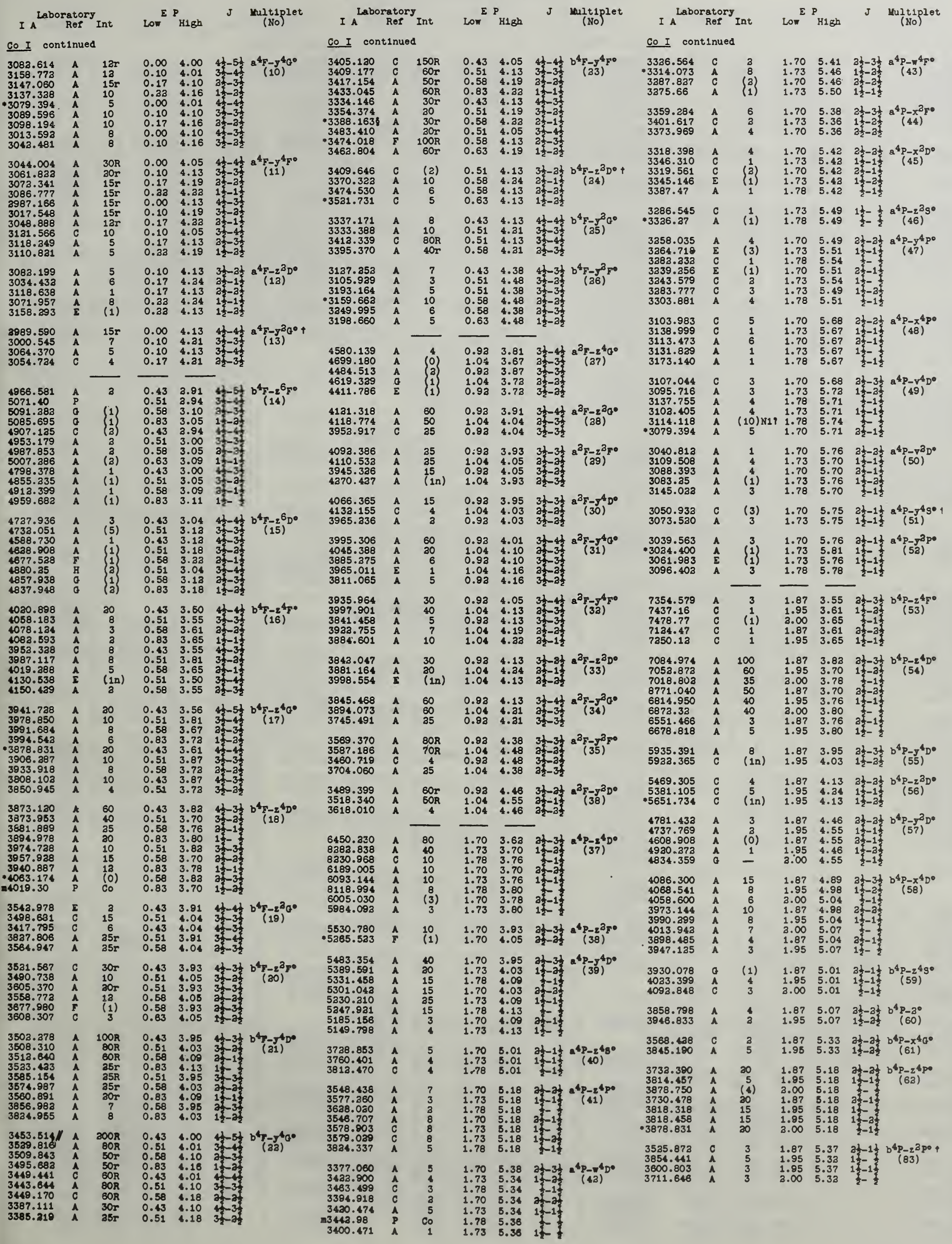


Laboratory
ReI Int Low ${ }_{\text {H1gh }}$ J Multiplet
(No)

I CoI continued

Fe III

4570.3

$\begin{array}{llr}3543.256 & \text { A } & 15 \\ 3639.443 & \text { A } & 10 \\ 3693.364 & \text { C } & 2 \\ 3562.912 & \text { A } & 7 \\ 3636.713 & \text { A } & 6 \\ 3670.041 & \text { A } & 3 \\ 3560.306 & \text { C } & 5 \\ 3614.10 & \text { A } & (0)\end{array}$

$5573 .:$

5485.8

$3976 .:$
$\mathrm{m} 3953$.

m3953.

3945.
-3828 .

m374.

3786 .

3382 .

3360.

3359.
.3357.

3331
3306
3315

- 3333

3362

3256

3023
3045

3135
$308 i$

$304 t$
309.

32

304

308

$45 \epsilon$

375

$37 c$
365

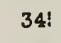

32:

34
34

30

34
34

${ }_{36}^{3 c}$

31

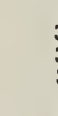$$
\begin{aligned}
& 3 \\
& 3 \\
& 3 \\
& 3
\end{aligned}
$$

(1)

\section{$\begin{array}{lllllll}3487.712 & A & 8 & 1.87 & 5.41 & 2 \frac{1}{2}-3 \frac{1}{2} & b^{4} P-\pi^{4} F^{\circ}+ \\ 3516.675 & \text { C } & 1 & 1.95 & 5.46 & 1 \frac{2}{2}-6 \frac{1}{2} & (65)\end{array}$ \\ $\begin{array}{lllllll}3523.701 & \mathrm{C} & 7 & 1.87 & 5.38 & 2 \frac{1}{3}-3 \frac{1}{2} \mathrm{~b}^{4} \mathrm{P}-\mathrm{x}^{3} \mathrm{~F} \\ 3615.387 & \mathrm{~A} & 6 & 1.95 & 5.36 & 1 \frac{5}{2}-3 \frac{7}{2} & (66)\end{array}$}

$\begin{array}{lllllll}3478.744 & \mathrm{C} & 7 & 1.87 & 5.42 & 2 \frac{1}{2}-2 \frac{1}{4} & \mathrm{~b}^{4} \mathrm{P}-\mathrm{x}^{2} \mathrm{D}^{\circ} \\ 3553.989 & \AA & 8 & 1.95 & 5.42 & 1 \frac{1}{2}-1 \frac{3}{3} & (67)\end{array}$

3480.012

$\begin{array}{lll}3551.666 & \mathrm{C} & 2 \\ 3607.04 & \mathrm{~A} & (0)\end{array}$

$\begin{array}{lllllll}3485.700 & \mathrm{~A} & 4 & 1.95 & 5.49 & 1 \frac{1}{3}-\frac{1}{1} \mathrm{~b}^{4} \mathrm{P}_{-\mathrm{z}^{2} \mathrm{~S}^{\circ}} \\ 3537.707 & \mathrm{~A} & 1 & 2.00 & 5.49 & \frac{1}{2}-\frac{2}{2} & (68)\end{array}$

$\begin{array}{ccccccc}3243.840 & \mathrm{~A} & 8 & 1.87 & 5.68 & 3 \frac{1}{3}-2 \frac{1}{2} & \mathrm{~b}^{4} \mathrm{P}-\mathrm{C}^{4} \mathrm{po} \\ 3317.93 & \mathrm{~A} & (\mathrm{O}) & 1.95 & 5.67 & 1 \frac{2}{3}-1 \frac{1}{2} & (69)\end{array}$

3359.06

3254.202
3312.148

3307.156
3365.014

3247.170

3325.24

3209.80

3279.254
3298.680

3216.996

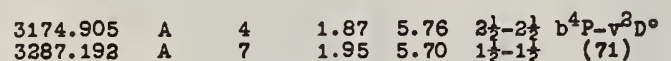

3224.632

m3333.41

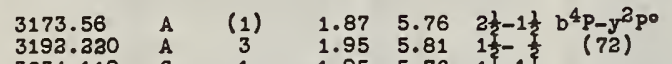

3234.11

3235.783
3278.842

3154.794

3161.652
3182.118

3103.73
3139.98

3152.707

3082.844
3111.339

$3120.10 \quad A \quad$ (3) $1.87 \quad 5.83 \quad 21-3 \frac{1}{2} b^{4} p_{-1} \pi^{2} F^{0}$

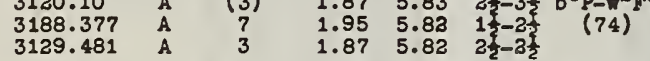

$\begin{array}{lllllll}3099.667 & \mathrm{~A} & 2 & 1.95 & 5.93 & 1 \frac{1}{2}-\frac{1}{2} \mathrm{~b}^{4} \mathrm{P}-\mathrm{x}^{3} \mathrm{~S}^{\circ} \\ 3140.715 & \mathrm{~A} & 2 & 3.00 & 5.93 & \frac{2}{2}-\frac{2}{2} & (75)\end{array}$

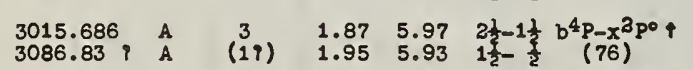

$\begin{array}{lllllll}3026.373 & \mathrm{~A} & 6 & 1.87 & 5.95 & 2 \frac{1}{3}-3 \frac{1}{2} & \mathrm{~b}^{4} \mathrm{P}-\mathrm{t}^{4} \mathrm{D}^{\circ} \\ 3060.048 & \mathrm{~A} & 5 & 1.95 & 5.98 & 1 \frac{2}{3}-2 \frac{2}{3} & (77)\end{array}$

3090.251

3005.766

3087.806

3996.549
3048.108

3017.25
$\mathrm{~m} 3044.04$

7809.24
.7996 .80

6563.403
-6450.09

m6450.09

6146.38
6910.84

6490.344

6429.913
6829.93

5890.487

5890.487 A 12

$\begin{array}{lll}5659.131 & \text { A } & 10 \\ 6168.86 & \text { D } & \text { (1) }\end{array}$

$\begin{array}{lll}5266.506 & \text { A } & 25 \\ 5335.188 & \text { A } & 15\end{array}$

$\begin{array}{llllllll}3712.177 & \mathrm{~A} & 6 & 2.03 & 5.36 & 4 \frac{1}{3}-3 \frac{1}{2} & \mathrm{a}^{2} a-\pi^{4} \mathrm{p}^{\circ} \\ 3843.693 & \mathrm{~A} & 4 & 3.13 & 5.34 & 3 \frac{1}{2}-3 \frac{7}{2} & (84)\end{array}$

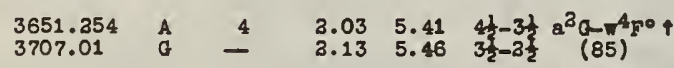

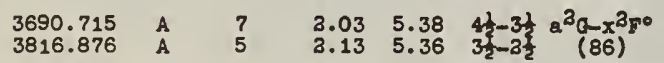

$\begin{array}{lllllll}3438.713 & C & 4 & 3.03 & 5.63 & 4 \frac{1}{3}-5 \frac{1}{2} a^{3} a-z^{2} H^{\circ} \\ 3586.082 & E & 3 & 3.13 & 5.57 & 3 \frac{1}{2}-4 \frac{1}{2} & (87)\end{array}$

$\begin{array}{lllllll}3381.498 & C & 4 & 3.03 & 5.68 & 41-4 \frac{1}{3} a^{2} a-x^{3} a^{\circ} \\ 3503.717 & C & 3 & 3.13 & 5.65 & 3 \frac{1}{2}-3 \frac{1}{2} & (88)\end{array}$
I A ${ }_{\text {Ref Int Low }}^{\text {Laboratory }}{ }_{\text {High }}$ J Multiplet Co I continued

7987.36 7417.38
7590.57 7590.57
7154.688 7315.73
7004.81

5991.890

5590.744

5883.421

m5354. 01

5034.06
$\mathbf{5 1 1 3 . 3 3 2}$

5176.085

4974.47

499.47
5094.955

4371.130
4187.346

4192.856
4139.452

3735.928
3749.930

3749.930
3693.476

3755.447

3734.139
3777.543
3731.268

3731.268
3774.599
3707.465

3693.106

3605.015

3645.440
3559.597

3733.483

3708.823
3751.625

3683.047

3643.181
3684.479

3684.479
3641.784

3585.808

3521.731
3458.028

33334.12
3368.67

3368.67
3431.628

3420.790

3432.418

3390.396
3304

3424.500

3348.112
3355.940

3356.464

-3322. 198

3343.734

3264.842
3308.688

3331.913

3233.874
3365.352

3265.352
3310.319

3180.290

3383.466

3260.814

3168.060

3154.678
3137.454

3169.766

3169.766
3110.031

6946.31

6632.438
6417.324

5647.334

5523.310
5408.119

3915.503

3977.184
3917.115

3835.497
3893.067

3662.158
3611.701

3611.701
3562.097

3620.423

3684.960
3633.340

3633.340
3677.835

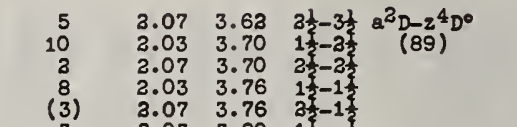 $\begin{array}{llll}3 & 3.07 & 3.76 & 31 \\ 3 & 3.03 & 3.80 & 1 \frac{1}{2}-\frac{1}{2} \\ 3 & 3.07 & 4.13 & 02-3 t\end{array}$}

20
10

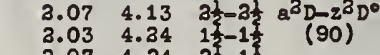

$\begin{array}{llll}3 & 3.07 & 4.24 & 3 \frac{1}{2}-1 \\ \text { (3) } & 3.03 & 4.13 & 1 \frac{1}{2}-2 \frac{3}{2}\end{array}$

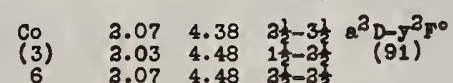

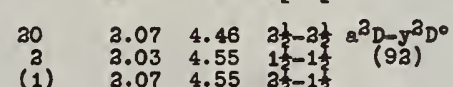

$\begin{array}{clll}\text { (1) } & 3.07 & 4.55 & 2 \frac{1}{2}-1 \frac{2}{3} \\ 8 & 2.03 & 4.46 & 1 \frac{1}{2}-2 \frac{1}{2}\end{array}$

5
4

$\begin{array}{llll}3.07 & 4.89 & 31-3 \frac{1}{2} & a^{2} p-x^{4} D^{0}+ \\ 3.03 & 4.98 & 1 \frac{1}{2}-2 & (93)\end{array}$

$\begin{array}{lllll}(2 \mathrm{~N}) & 3.07 & 5.01 & 2 \frac{1}{3}-1 \frac{1}{3} & \mathrm{a}^{2} \mathrm{D}_{2} 2^{4} \mathrm{~S}^{\circ} \\ 3 & 2.03 & 5.01 & 1 \frac{1}{2}-1 \frac{2}{2} & (94)\end{array}$

3.07 $5.37 \quad 2 \frac{1}{2}-1 \frac{1}{2} \mathrm{a}^{2} \mathrm{D}-z^{2} \mathrm{po}^{\circ}$

$\begin{array}{lll}2.03 & 5.33 & 1 \frac{1}{3}-\frac{1}{3} \\ 3.03 & 5.37 & 1 \frac{9}{2}-1 \frac{1}{2}\end{array}$

$2.07 \quad 5.36 \quad 3 \frac{1}{2}-3 \frac{1}{2} a^{3} D-\pi^{4} D^{0}$

$\begin{array}{lll}2.03 & 5.34 & 1 \frac{1}{2}-3 \\ 2.07 & 5.34 & 2 \frac{3}{2}-2 \\ 2.03 & 5.34 & 1\end{array}$

$\begin{array}{lll}2.03 & 5.34 & 1 \frac{1}{1}-1 \\ 2.07 & 5.34 & 2 \frac{1}{3}-1 \\ 2.03 & 5.36 & 1 \frac{1}{2}-\end{array}$

3.07 $5.41 \quad 3 \frac{3}{3}-3 \frac{1}{2} \mathrm{a}^{2} \mathrm{D}-\pi^{4} \mathrm{r}^{\circ}$

$\begin{array}{lll}3.03 & 5.46 & 1 \frac{1}{2}-\frac{2}{2} \\ 3.07 & 5.46 & 2 \frac{2}{2}-2 \frac{2}{3} \\ 3.03 & 5.50 & 1 \frac{1}{2}-1 \frac{7}{2}\end{array}$

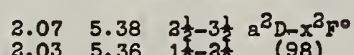

$\begin{array}{lll}2.03 & 5.36 & 1 \frac{1}{2}-3 \frac{2}{2} \\ 2.07 & 5.36 & 2 \frac{1}{2}-3 \frac{2}{2}\end{array}$

$\begin{array}{llll}2.07 & 5.42 & 2 \frac{1}{3}-3 \frac{1}{2} & a^{2} D-x^{2} D^{\circ} \\ 2.03 & 5.43 & 1 \frac{1}{2}-1 \frac{1}{2} & (99)\end{array}$

$\begin{array}{lll}2.03 & 5.42 & 1 \frac{1}{3}-1 \frac{1}{3} \\ 3.07 & 5.42 & 3 \frac{1}{3}-1 \frac{1}{2} \\ 2.03 & 5.43 & 1 \frac{1}{2}-2 \frac{3}{2}\end{array}$

$\begin{array}{llll}3.07 & 5.51 & 2 \frac{1}{3}-1 \frac{1}{3} & a^{2} D-y^{4} \mathrm{po}^{\circ} \\ 3.03 & 5.54 & 1 \frac{3}{2}-\frac{1}{2} & (100)\end{array}$

$3.07 \quad 5.64 \quad 21-2 \frac{1}{2} a^{2} D-m^{2} D^{\circ}$

$\begin{array}{cccc}\text { Co } & 3.03 & 5.73 & 1 \frac{1}{3}-1 \frac{1}{2} \\ \text { (1) } & 3.07 & 5.73 & 3 \frac{1}{3}-1 \frac{1}{2} \\ 3 & 3.03 & 5.64 & 1 \frac{2}{2}-6 \frac{2}{2}\end{array}$

$\begin{array}{llll}3.07 & 5.68 & 2 \frac{1}{3}-3 \frac{1}{3} & a^{3} p-x^{4} p^{\circ} \\ 2.03 & 5.67 & 1 \frac{1}{2}-1 \frac{2}{2} & (103)\end{array}$

$\begin{array}{lll}3.07 & 5.67 & 2 \frac{1}{2}-1 \frac{1}{2} \\ 3.03 & 5.67 & 1 \frac{2}{2}-\frac{1}{2}\end{array}$

$2.07 \quad 5.68 \quad 3 \frac{1}{2}-3 \frac{1}{2} a^{3} p-v^{4} D^{0}+$

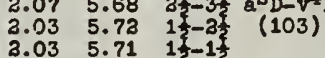

$\begin{array}{llll}3.07 & 5.75 & 2 \frac{1}{2}-1 \frac{1}{2} & \mathrm{a}^{2} \mathrm{D}-\mathrm{y}^{4} \mathrm{~s}^{\circ} \\ 3.03 & 5.75 & 1 \frac{1}{2}-1 \frac{1}{2} & (104)\end{array}$

$2.07 \quad 5.76 \quad 2 \frac{1}{3}-1 \frac{1}{2} a^{2} \mathrm{D}-\mathrm{y}^{2} \mathrm{po}$

$\begin{array}{llll}3.03 & 5.81 & 1 & 1 \\ 3.03 & 5.76 & 1 & 1\end{array}$

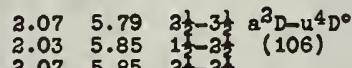

$\begin{array}{llll}3.07 & 5.85 & 35 \\ 3.03 & 5.88 & 1 \frac{2}{2} \\ 3.03 & 5.91 & 1 \frac{2}{2}-\frac{1}{2}\end{array}$

$3.07 \quad 5.83 \quad 3 \frac{1}{2}-3 \frac{1}{2} a^{2} p-w^{2} F^{\circ}$

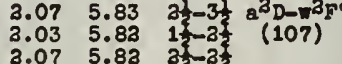

3.07 5.97. $3 \frac{1}{5}-1 \frac{1}{2} a^{3} p-x^{3} p 0$

$\begin{array}{llll}2.07 & 5.95 & 1 \\ 3.03 & 5.97 & 1 & 1 \\ 3.03 & (108)\end{array}$

$\begin{array}{llll}3.07 & 5.96 & 3 \frac{1}{2}-3 \frac{1}{2} & a^{3} \mathrm{D}-v^{2} \mathrm{~F}^{\circ} \\ 3.03 & 6.00 & 1 \frac{2}{2}-3 \frac{2}{2} & (109)\end{array}$

(a) $\overline{3.37} \overline{0.05}$

$\begin{array}{llll}3.37 & 4.05 & 1 \frac{1}{2}-2 \frac{1}{2} & \mathrm{a}^{2} \mathrm{P}-z^{2} \mathrm{~F}^{0} \\ 3.37 & 4.13 & 11\end{array}$

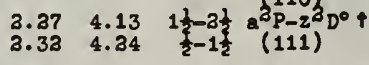

$\begin{array}{llll}3.37 & 4.46 & 1 \frac{1}{2}-2 \frac{1}{3} & \mathrm{a}^{3} \mathrm{P}-\mathrm{y}^{2} \mathrm{D}^{\circ} \\ 3.32 & 4.55 & \frac{1}{3}-1 \frac{1}{2} & (113) \\ 2.27 & 4.55 & 1 \frac{1}{2}-1 \frac{3}{2} & \end{array}$

(a) $\quad 3.37 \quad 5.42 \quad 1 \frac{1}{2}-2 \frac{1}{2} a^{2} P-x^{2} D^{\circ}$

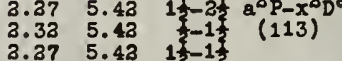

$\begin{array}{llll}3.27 & 5.49 & 1 \frac{1}{3}-\frac{1}{3} a^{2} P_{-2}{ }^{2} \mathrm{~S}^{\circ} \\ 3.32 & 5.49 & \frac{1}{2}-\frac{1}{2} & (114)\end{array}$

3. $37 \quad 5.64 \quad 1 \frac{1}{2}-3 \frac{1}{2} a^{3} P_{-} \nabla^{3} D^{\circ}$

$\begin{array}{llll}3.32 & 5.73 & 15 \\ 3.27 & 5.73 & 1 \frac{1}{2}-1 \frac{1}{2} & (115)\end{array}$

3.37 $5.68 \quad 1 \frac{1}{2}-3 \frac{1}{2} a^{2} p_{-} x^{4} p^{\circ}$

3.33 5.67 (116)

$\begin{array}{lll}3.37 & 5.67 & 1 \\ 3.32 & 5.67 & -1\end{array}$

I A ${ }_{\text {Ref }}^{\text {Laboratory }}$ Int Low ${ }_{\text {H1gb }}^{E P}$

$\operatorname{(xo)~}_{(x)}$

Co I continued

$3578.076 \quad A \quad 6 \quad 2.27 \quad 5.73 \quad 1 \frac{1}{3}-31+a^{2} P-V^{4} D^{\circ}$

$\begin{array}{lllllll}3637.319 & A & 4 & 2.32 & 5.71 & \frac{1}{3}-1 \frac{3}{2} & (117) \\ 3556.120 & \text { G } & \text { (1) } & 3.27 & 5.74 & 1 \frac{5}{2}-\frac{1}{2} & \end{array}$

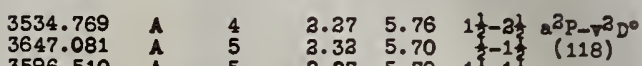

3596.510

$\begin{array}{lllll}3431.039 \quad & 3.37 \quad 5.70 \quad 1 \frac{1}{2}-1 \frac{3}{2}\end{array}$

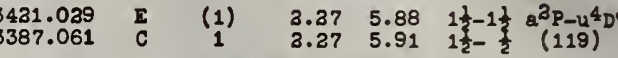

$\begin{array}{lllllll}3478.555 \quad C & 8 & 3.27 & 5.83 & 1 \frac{1}{2}-2 \frac{1}{2} & a^{2} \mathrm{P}-w^{2} \mathrm{~F}^{\circ}\end{array}$

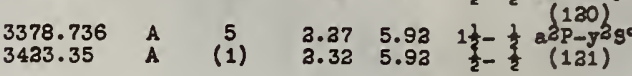

$3373.326 \quad A \quad 7 \quad 3.27 \quad 5.93 \quad 1 \frac{1}{3} a^{2} p-x^{2} 8$

$37.97 \quad 1+-1 \frac{2}{2}-x^{3} p_{0}$

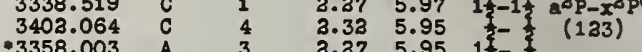

$\begin{array}{llllll}3382.071 & \mathrm{~A} & 3 & \mathbf{3} .37 & 5.95 & 1 \frac{1}{2}-\frac{1}{2} \\ 3 & \mathbf{3} 32 & \mathbf{5 . 9 7} & \frac{1}{2}-1 \frac{1}{2}\end{array}$

3233.213
3236.986

3.773 .664
$3 \times 07.540$

4

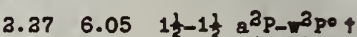

$\begin{array}{llll}3.32 & 6.29 & 1 \frac{1}{1}-1 \frac{1}{2} a^{2} \mathrm{P}-v^{2} \\ 3.32 & 6.29 & \frac{1}{2}-\frac{1}{2} & (135)\end{array}$

$7712.661 \quad A \quad 6 \quad 3.53 \quad 4.13 \quad 1 \frac{1}{3}-2 \frac{1}{2} b^{2} \mathrm{P}-2^{2} \mathrm{D}^{\circ}$

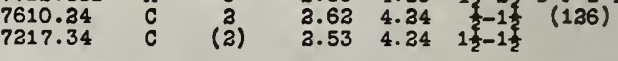

$4268.446 \quad \mathrm{~A} \quad 3 \quad 3.53 \quad 5.42 \quad 1 \frac{1}{3}-2 \frac{1}{2} b^{2} P-x^{2} D^{\circ}$

4404.932

3969.116
3960.997
3851.848

3870.534
3991.831

3819.908

4003.59
3892.11

3817.940

3863.600

3759.68

3754.346

3631.94

3591.746

3591.746

3614.34
3686.477

3504.738

3502.998

3417.353
$\mathbf{m} 3594.87$

3496.070
3604.469

3604.46

3469.683

3553.161

3174.140
-3235.53

3235.532
3345.750

7388.689

7586.72

6937.81

7054.042
7385.38

4634.56

5004.187

8

$2.53 \quad 5.64 \quad 1 \frac{1}{2}-2 \frac{1}{2} b^{2} P_{-}-m^{2} D^{0}$ $\begin{array}{llll}3.63 & 5.73 & 1 \frac{3}{3}-1 & \\ 3.53 & 5.73 & 1 \frac{1}{2}-1 & (128)\end{array}$

$4 \quad 2.53 \quad 5.72 \quad 1 \frac{1}{3}-21 b^{2} P-V^{4} D^{0} \uparrow$

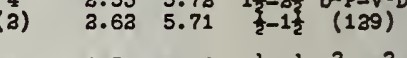

4543.810

4815.900

4545.985
$\mathrm{~m} 4813: 45$

4375.540
4431.608

4431.608

4158.420

3676.553

3702.237
3699.017

3649.339
3634.713

3632.839
3609.752 
-3945.

-3828.

m3747.

3786 .

3382.
3360
3358

3358

3359
.3357

${ }_{3306}^{3331}$

.3333

3362

3256

3022
3045

3135
$308 i$

3044
309.

323

304

308

$45 €$

375

37

36 ?

32

34
34

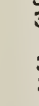

\section{(1)}

$\underset{(N O)}{\operatorname{Milt1plet}}$

N1 I I P 7.61 Angl A L1st $B$
I A Laboratory ${ }_{\text {Ref Int Low }{ }_{\text {H1gh }} \text { J Uultiplet }}$ N1 I continued

$\begin{array}{lllllll}3392.992 & \text { B } & 100 R & 0.03 & 3.66 & 3-3 & a^{3} \mathrm{D}-z^{3} \mathrm{D}^{\circ}\end{array}$ $\begin{array}{lcccccc}3446.263 & \text { B } & 100 R & 0.11 & 3.69 & 2-2 & \text { (20) } \\ 3423.711 & \text { B } & 50 R & 0.21 & 3.82 & 1-1 & \\ 3367.892 & \text { B } & 8 & 0.03 & 3.69 & 3-2 & \end{array}$ $\begin{array}{llclll}3367.892 & \mathrm{~B} & 8 & 0.03 & 3.69 & 3-2 \\ 3328.714 & \mathrm{~B} & 5 & 0.11 & 3.82 & 2-1 \\ 3472.545 & \mathrm{~B} & 7 \mathrm{OR} & 0.11 & 3.66 & 2-3\end{array}$ $\begin{array}{llllll}3472.545 & \mathrm{~B} & 7 \mathrm{OR} & 0.11 & 3.66 & 2-3 \\ .3548 .185 & \mathrm{~B} & 20 \mathrm{r} & 0.21 & 3.69 & 1-2\end{array}$

$\begin{array}{lllllll}3749.045 & \mathrm{P} & & 0.16 & 3.39 & 3-3 & \mathrm{~A}^{3} \mathrm{~F}_{-2} \mathrm{z}^{5} \\ 3832.873 & \mathrm{~B} & 5 & 0.00 & 3.39 & 4-3 & (16 \\ 3 & 3.38 & 3-2 & (1)\end{array}$ 3885.87
4093.62

3624.733

3739.22

3561.751

3730.751

3498.19

3502.595

3602.281
3437.280

3507.694
3577.240

3351.06
3467.502
-3548.185

3670.427
3664.095

3664.095
3793.608

3391.050

3571.869

3519.766
3409.578

3413.478

3551.534
3688.415

3369.573

3500.852
3483.774

3483.774
3537.982

3612.741
3641.641

3232.963

3371.993
3380.885

3226.984

3282.696
3145.121

3221.653

3366.168
3469.486

3320.257
3420.741

3349.440

3031.870
3145.719

3145.719
3184.367

3019.143
3097.118

3159.521
3335.753

2984.131

3045.006
3105.469

3105.469
3107.714
3129.314
3195.573

3099.115

. 2991.09

3913.979
3889.671

3889.67
3778.063
3811.32

3811.33
.3674 .06

3693.933
3772.530

3587.931

3609.314
3661.951

3523.444
3553.483

3461.652
3453.890

3452.890
-3513.9338
-3374.331

3374.231
3413.939
3485

3413.939
3337.888
3387.014

3534.541

3492.956
3510.338

3610.462
3597.705

3597.705
3722.484

$3414.765 / /$

3515.054
3458.474

3433.558
3361.556
3286.948

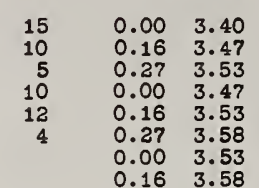

$\begin{array}{rrrr}8 & 0.00 & 3.52 \\ 15 & 0.16 & 3.59\end{array}$

$\begin{array}{ccc}30 R & 0.00 & 3.59 \\ 8 & 0.16 & 3.68\end{array}$

$0.27 \quad 3.72$

$\begin{array}{lll}12 & 0.00 & 3.68 \\ & 0.16 & 3.72\end{array}$

20

20

$\begin{array}{ll}0.16 & 3.53 \\ 0.27 & 3.64\end{array}$

$\begin{array}{llll}5^{\prime} \mathrm{R} & 0.00 & 3.64\end{array}$

$\begin{array}{lll}5 \mathrm{LR} & 0.16 & 3.62 \\ 20 \mathrm{R} & 0.27 & 3.78\end{array}$

2OR

$25 R$

$\begin{array}{ll}0.00 & 3.62 \\ 0.16 & 3.78\end{array}$

$\begin{array}{ll}0.16 & 3.64 \\ 0.27 & 3.62\end{array}$

$\begin{array}{lll}80 \mathrm{R} & 0.00 & 3.66\end{array}$

$\begin{array}{lll}25 \mathrm{R} & 0.16 & 3.69 \\ 25 \mathrm{R} & 0.27 & 3.82\end{array}$

15
$30 \mathrm{R}$

$\begin{array}{lll}25 \mathrm{R} & 0.00 & 3.82\end{array}$

$15 r$

$\begin{array}{ll}0.00 & 3.82 \\ 0.16 & 3.82\end{array}$

$\begin{array}{ll}0.27 & 3.92 \\ 0.00 & 3.82 \\ 0.16 & 3.82\end{array}$

$\begin{array}{ll}0.00 & 3.82 \\ 0.16 & 3.92 \\ 0.00 & 3.92\end{array}$

$\begin{array}{llllc}10 \mathrm{r} & 0.00 & 3.83 & 4-3 & \mathrm{a}^{3} F_{-1} z^{\circ} F^{\circ} \\ 3 \mathrm{OR} & 0.16 & 3.83 & 3-3 & (8) \\ 15 & 0.27 & 3.83 & 2-3 & (8)\end{array}$

$0.16 \quad 3.88 \quad 3-3 \quad a^{3} F-2^{1} D^{\circ}$

20R

6

$10 r$
8

$0.27 \quad 4.07$

$0.00 \quad 4.07$

$\begin{array}{ll}0.00 & 4.07 \\ 0.16 & 4.08 \\ 0.27 & 4.15\end{array}$

$\begin{array}{cccc}8 & 0.27 & 4.15 & 3-3 \\ 8 & 3-2 \\ 20 \mathrm{R} & 0.00 & 4.09 & 4-3 \\ 15 \mathrm{r} & 0.16 & 4.15 & 3-2 \\ 3 & 0.16 & 4.07 & 3-4\end{array}$

$\begin{array}{cccc}15 \mathrm{r} & 0.16 & 4.15 & 3-2 \\ 3 & 0.16 & 4.07 & 3-4 \\ 4 & 0.27 & 4.09 & 3-3\end{array}$

$\begin{array}{lllll}12 R & 0.00 & 4.14 & 4-3 & a^{3} F-y^{3} D^{0} \\ 10 r & 0.16 & 4.22 & 3-2 & (12)\end{array}$

$15 r$

$0.27 \quad 4.25$

$\begin{array}{lll}0.16 & 4.14 & 3-3 \\ 0.27 & 4.22 & 2-2\end{array}$

$13 \mathrm{r} \quad 0.16 \quad 4.15 \quad 3-4 \quad a^{3} F_{-2} \varepsilon^{10}$

\begin{tabular}{ccccc}
$12 r$ & 0.16 & 4.15 & $3-4$ & $a^{3} F-z^{1} G^{\circ}$ \\
4 & 0.37 & 4.40 & $2-3$ & $a^{3}(13) y^{1} F^{\circ}+$ \\
\hline
\end{tabular}

$\begin{array}{lllll}5 & 0.03 & 3.18 & 3-4 & 2^{3} \mathrm{D}_{-2} 5 \mathrm{D}^{\circ} \\ 15 & 0.31 & 3.38 & 1-2 & (15)\end{array}$

$\begin{array}{llll}5 & 0.03 & 3.39 & 3-3 \\ \text { (3) } & 0.31 & 3.45 & 1-1 \\ 10 & 0.03 & 3.38 & 3-0\end{array}$

$\begin{array}{rrrr}10 & 0.03 & 3.38 & 3-3 \\ 8 & 0.11 & 3.45 & 3-1 \\ 6 & 0.21 & 3.48 & 1-0\end{array}$

$\begin{array}{lllll}12 & 0.03 & 3.47 & 3-4 & \mathrm{a}^{3} \mathrm{D}-2^{5} \mathrm{G}^{\circ} \\ 15 & 0.11 & 3.53 & 3-3 & (16)\end{array}$

$\begin{array}{rlll}1 & 0.21 & 3.58 & 1-2 \\ 10 & 0.03 & 3.53 & 3-3 \\ 7 & 0.11 & 3.58 & 3-2\end{array}$

$\begin{array}{ccccc}7 & 0.11 & 3.58 & 2-2 & \\ 125 R & 0.03 & 3.59 & 3-4 & 2^{3} \mathrm{D}-25_{F^{\circ}} \\ 40 R & 0.11 & 3.68 & 2-3 & (17) \\ 15 & 0.31 & 3.73 & 1-3 & \\ 15 r & 0.03 & 3.68 & 3-3 & \end{array}$

$\begin{array}{lll}15 r & 0.03 & 3.68 \\ 12 r & 0.11 & 3.72 \\ 10 & 0.31 & 3.75\end{array}$

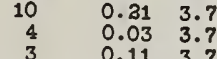

$\begin{array}{ccccc}300 R & 0.03 & 3.53 & 3-2 & \mathrm{a}^{3} \mathrm{p}_{-2} \mathbf{z}^{3} \mathrm{P} \\ 150 \mathrm{R} & 0.11 & 3.64 & 2-1 & (18) \\ 80 R & 0.21 & 3.73 & 1-0 & (1-2)\end{array}$

$6 O R$

$0.11 \quad 3.53 \quad 2-3$

$\begin{array}{lll}0.21 & 3.64 & 1-1 \\ 0.31 & 3.53 & 1-2\end{array}$

$150 R$

$\begin{array}{llll}0.03 & 3.64 & 3-4 & a^{3} \mathrm{D}^{3} \mathrm{~F}^{\circ} \\ 0.11 & 3.62 & 3-3 & (19)\end{array}$

$\begin{array}{lll}0.11 & 3.62 \\ 0.21 & 3.78 \\ 0.03 & 3.62\end{array}$

$\begin{array}{lll}0.03 & 3.62 & 3-3 \\ 0.11 & 3.78 & 2-2 \\ 0.03 & 3.78 & 3-2\end{array}$ $4-\quad .37 \quad 3.69$
3248.457

$\begin{array}{ccccccc}3234.649 & \text { B } & 8 & 0.03 & 3.82 & 3-4 & a^{3} \mathrm{D}-2 \mathrm{z}^{3} \mathrm{G} \\ 3165.508 & \text { B } & 3 & 0.11 & 3.92 & 2-3 & (21)\end{array}$

$\begin{array}{lllllll}3243.058 & \mathrm{~B} & 25 \mathrm{R} & 0.05 & 3.83 & 3-3 & \mathrm{a}^{3} \mathrm{p}-\mathrm{z}^{1} \mathrm{~F}^{\circ}\end{array}$

$\begin{array}{rrrrrrr}3200.423 & \mathrm{~B} & 5 & 0.03 & 3.88 & 3-2 & \mathrm{~B}^{3} \mathrm{D}-\mathrm{z}^{1} \mathrm{D}^{0} \\ 3271.118 & \mathrm{~B} & 10 & 0.11 & 3.88 & 2-2 & (23) \\ 3362.806 & \mathrm{~B} & 6 & 0.31 & 3.88 & 1-2 & (2)\end{array}$

3114.124
3197.113

3050.819
3101.554

3101.554
3134.108
3037.935

3054.316

2992.595

3002.491
3003.629

3057.63

3981.651

3064.623
3080.755

3994.460

4298.76

4164.636
4074.897

3972.171
3904.64

3783.530

3736.813
3705.12

3973.56
3831.6

3858.3

3858.301
-3674.15

$\begin{array}{lllllll}3775.572 & \text { B } & 35 r & 0.42 & 3.66 & 2-3 & a^{1} D_{-2} z^{0} \\ 30 r & 0.42 & 3.69 & 2-2 & (33)\end{array}$

$\begin{array}{rrrrrrrr}3634.941 & \text { B } & 13 & 0.42 & 3.82 & 2-1 & \\ 3523.074 & \text { B } & 4 & 0.42 & 3.92 & 2-3 & a^{1} D_{-2}^{3} a^{\circ}\end{array}$

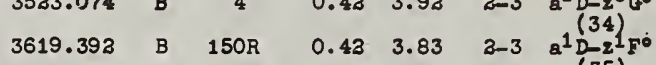

3566.373 B $100 \mathrm{R}$

3380.574 B $80 \mathrm{R}$

$0.42 \quad 3.88$

$0.42 \quad 4.07$

$\begin{array}{cccccccc}3365.766 & \mathrm{~B} & 15 \mathrm{r} & 0.42 & 4.09 & 2-3 & \mathrm{a}^{1} \mathrm{D}-\mathrm{y}^{3} \mathrm{~F}^{\circ} \\ 3310.202 & \mathrm{~B} & 5 & 0.42 & 4.15 & \mathrm{a}-2 & (\mathrm{~B})\end{array}$

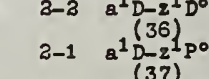

$\begin{array}{lllllll}3322.310 & B & 15 r & 0.42 & 4.14 & 2-3 & a^{1} \mathrm{D}-\mathrm{y}^{3} \mathrm{D}^{\circ}\end{array}$

$\begin{array}{ccccccc}3350.743 & \mathrm{~B} & 9 & 0.42 & 4.23 & 2-2 & \text { (39) } \\ 3235.020 & \mathrm{~B} & 10 \mathrm{r} & 0.42 & 4.25 & 2-1 & \end{array}$

3101.879 B $40 \mathrm{P}$

$0.43 \quad 4.40 \quad 2-3 \quad a^{1} D-y^{1} F^{\circ}$

3012.004 B $75 \mathrm{R}$

6128.990

6007.313

5925.81

(3)

$0.43 \quad 4.52$

$\begin{array}{ll}2^{1} D-y^{1} F^{\circ} \\ -2 & (40) \\ -2 & a^{1}-y^{1} D^{\circ} \\ (41)\end{array}$

$\begin{array}{lllllll}6643.641 & \mathrm{C} & 30 & 1.67 & 3.53 & 2-3 & \mathrm{~b}^{1} \mathrm{D}-2^{3} \mathrm{po} \\ 6256.365 & \mathrm{C} & 15 & 1.67 & 3.64 & 3-1 & (43)\end{array}$

$\begin{array}{lllllll}6327.603 & B & 5 & 1.67 & 3.62 & 3-3 & b^{1} D-z^{3} F^{0} \\ 5847.010 & B & (3) & 1.67 & 3.78 & 3-2 & (44)\end{array}$

$6191.186 \quad B \quad 12 \quad 1.67 \quad 3.66 \quad 3-3 \quad b^{1} D-z^{3} D^{\circ}$

$\begin{array}{rrrrrrr}6108.121 & B & 12 & 1.67 & 3.66 & 2-3 & b^{1} D_{-2} D^{D} \\ 5748 & C & 8 & 1.67 & 3.69 & 3-3 & (45)\end{array}$

$5709.559 \quad B \quad 12 \quad 1.67 \quad 3.83 \quad 3-3 \quad b^{1} D_{-2} F^{\circ}$

$\begin{array}{llllllll}5578.734 & B & 5 & 1.67 & 3.88 & 3-3 & b^{1} D_{-2} 1_{D^{\circ}}\end{array}$

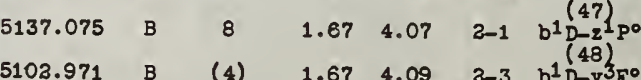

$\begin{array}{lllllll}5003.751 & \mathrm{~B} & (2) & 1.67 & 4.14 & 3-3 & \mathrm{~b}^{1} \mathrm{p}-\mathrm{y}^{3} \mathrm{p}^{\circ} \\ 4843.165 & \mathrm{~B} & (3) & 1.67 & 4.32 & 2-3 & (50) \\ 4786.393 & \mathrm{~B} & (3) & 1.67 & 4.35 & 3-1 & (5)\end{array}$

4519.986

4331.645

3435.489

3338.758

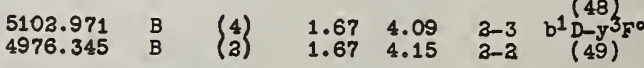

I A Laboratory Ref Int Low $_{\text {High }}^{\text {E }}$

J (No)

N1 I cont1nued

$\begin{array}{lllllll}3287.321 & \text { B } & 2 & 1.67 & 5.43 & 2-2 & b^{1} D-x^{1} D^{\circ}\end{array}$

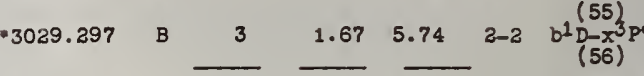

$6767.778 \quad C \quad \overline{20} \quad \overline{1.83} \quad \overline{3.64} \quad 0-1 \quad a^{1} 5-z^{3} p^{\circ}$

$\begin{array}{llllllll}6177.258 & B & (3) & 1.82 & 3.82 & 0-1 & \mathrm{a}^{1}\left(57 z^{3} \mathrm{D}\right. & (58)\end{array}$

$\begin{array}{llllllll}5476.906 & B & 50 & 1.82 & 4.07 & 0-1 & 8^{1} 82^{1} P_{0} \\ (59)\end{array}$

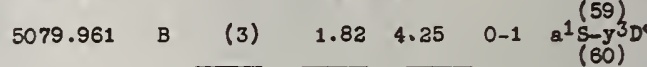

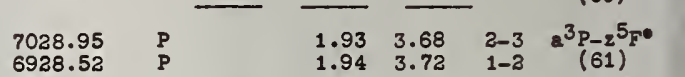

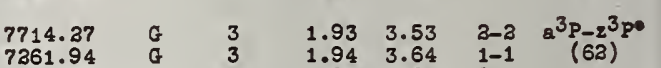

$\begin{array}{llrrrr}7261.94 & G & 3 & 1.94 & 3.64 & 1-1 \\ 7197.07 & G & 5 & 1.93 & 3.64 & 2-1 \\ 6914.563 & D & 50 & 1.94 & 3.73 & 1-0 \\ 778.65 & G & 2 & 1.94 & 3.53 & 1-2\end{array}$

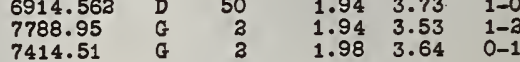

$\begin{array}{llllllll}7291.48 & \text { B } & \text { (8) } & 1.93 & 3.62 & 2-3 & \mathrm{a}^{3} \mathrm{P}_{-2} \mathrm{z}^{30}\end{array}$

$\begin{array}{lllllll}7110.91 & \mathrm{~B} & (6) & 1.93 & 3.66 & \mathrm{a}-3 & \mathrm{a}^{3 \mathrm{P}-z^{3} \mathrm{D}^{0}}\end{array}$

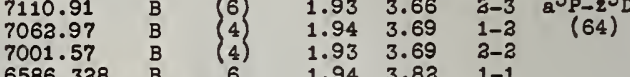

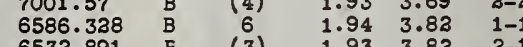

$\begin{array}{llllll}6532.891 & B & \text { (3) } & 1.93 & 3.82 & 2-1\end{array}$

.6180 .093

(1n) $\quad \begin{array}{lllll}.93 & 3.93 & 2-3 & a^{3} \mathrm{P}_{-2} \mathrm{z}^{30}\end{array}$

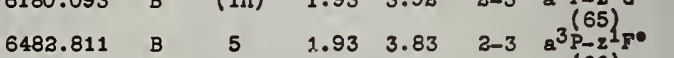

$\begin{array}{llllllll}6314.666 & C & 15 & 1.93 & 3.88 & 2-3 & a^{3} P_{-2}(66) \\ 6364.597 & D & (1) & 1.94 & 3.88 & 1-3 & (67)\end{array}$

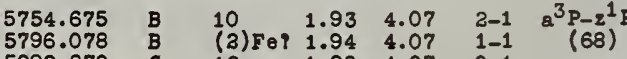

$12 \quad 1.98 \quad 4.07 \quad 0-1$

5593.283
5553.693

5587.865

5424.654

5435.871

5353.415

4762.637
4791.00

4019.055

3564.67

3696.65

3713.336

3713.696

3642.387

3529.625
3545.16

3176.293

3181.740

3183.25

3170.715
3154.585

3164.166

.2991 .095

11196.70

8770.68

8702.49
7385.24

\section{(1)}

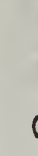

\section{(a)}

$\begin{array}{ccccc}5 & 1.93 & 4.14 & 2-3 & \mathrm{a}^{3} \mathrm{p}-\mathrm{y}^{3} \mathrm{po}^{\circ} \\ 4 & 1.94 & 4.32 & 1-2 & (70) \\ 5 & 1.98 & 4.25 & 0-1 & \end{array}$

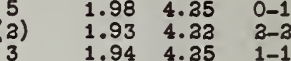

4837.65

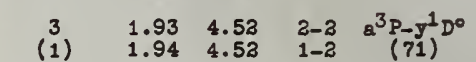

(3) $\quad 1.93 \quad 5.00 \quad 3-2 \quad \mathrm{a}^{3 \mathrm{P}-3^{\circ}}$

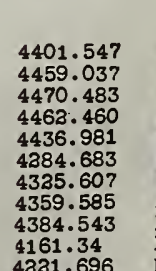

$\frac{4321.696}{4285.19}$

4389.870

4389.870
4574.03
4443.441 
REVISED NULTIPLET TABLE

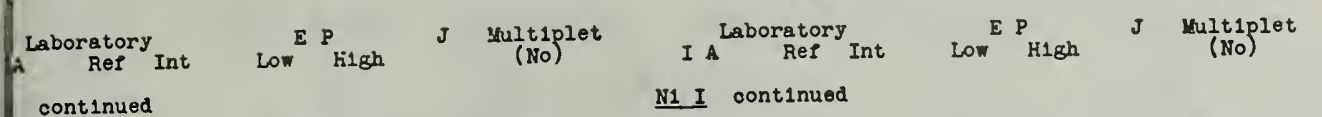

\begin{tabular}{|c|c|c|c|c|}
\hline $\begin{array}{l}(2) \\
5 \\
(4)\end{array}$ & $\begin{array}{l}3.29 \\
3.39 \\
3.38 \\
3.15\end{array}$ & $\begin{array}{l}6.33 \\
6.33 \\
6.33 \\
6.42\end{array}$ & $\begin{array}{l}3-3 \\
3-4 \\
2-3 \\
1-2\end{array}$ & $\begin{array}{c}z^{5} D^{\circ}-r^{3} F \\
(89)\end{array}$ \\
\hline$\left\{\begin{array}{l}4 \\
1 \\
1\end{array}\right)$ & $\begin{array}{l}3.39 \\
3.38 \\
3.45 \\
3.38\end{array}$ & $\begin{array}{l}7.00 \\
7.13 \\
7.22 \\
7.00\end{array}$ & $\begin{array}{l}3-2 \\
3-1 \\
1-0 \\
3-2\end{array}$ & $\begin{array}{c}z^{5} D^{0}-f^{3} P \\
(90)\end{array}$ \\
\hline
\end{tabular}

N1 I continued

6928.25

$\begin{array}{llll}3.18 & 7.01 & 4-3 & 2^{5} p^{0}-e^{5 p} \\ 3.29 & 7.11 & 3-3 & (91)\end{array}$

$\begin{array}{ll}3.29 & 7.11 \\ 3.38 & 7.32 \\ 3.45 & 7.22\end{array}$

$\begin{array}{lll}3.45 & 7.22 & 1-1 \\ 3.48 & 7.32 & 0\end{array}$

$\begin{array}{llll}3.18 & 7.01 & 4-4 & 2^{5} D^{0}-13_{F} \\ 3-29 & 73)^{3}\end{array}$

3.29 7.01

$\begin{array}{llll}3.18 & 7.02 & 4-4 & 2^{5} D^{0}-e^{5} D+ \\ 3.29 & 7.13 & 3-3 & (93)\end{array}$

$\begin{array}{cccc}3.18 & 7.03 & 4-5 & z^{5} p^{\circ}-r^{5} F \\ 3.29 & 7.14 & 3-4 & (94)\end{array}$

$\begin{array}{lll}3.38 & 7.23 & 2-3 \\ 3.45 & 7.28 & 1-2\end{array}$

$\begin{array}{lll}3.48 & 7.31 & 0-1 \\ 3.18 & 7.14 & 4-4\end{array}$

$\begin{array}{llll}3.18 & 7.14 \quad 4-5 \quad 2^{5} \mathrm{p}^{0}-0^{5} \mathrm{G}\end{array}$

$\begin{array}{cccc}3.29 & 7.05 & 3-3 & 2^{5} \mathrm{D}^{\circ}-13^{3} \mathrm{D} \\ 3.38 & 7.05 & 2-3 & (96)\end{array}$

- -

$\begin{array}{ll}3.53 & 5.38 \\ 3.58 & 5.45 \\ 3.53 & 5.36\end{array}$

$\begin{array}{lll}3.53 & 5.36 \\ 3.58 & 5.38\end{array}$

$\begin{array}{ll}3.37 & 5.98 \\ 3.40 & 6.06 \\ 3.53 & 6.32\end{array}$

$\begin{array}{lll}3.40 & 6.06 \\ 3.47 & 6.15\end{array}$

$\begin{array}{ll}3.47 & 6.15 \\ 3.53 & 6.22\end{array}$

$\begin{array}{ll}3.59 & 6.36 \\ 3.40 & 5.98\end{array}$

$\begin{array}{ll}3.40 & 5.98 \\ 3.47 & 6.06\end{array}$

$\begin{array}{ll}3.47 & 6.06 \\ 3.53 & 6.15 \\ 3 & 3\end{array}$

$\begin{array}{ll}3.58 & 6.32 \\ 3.47 & 5.98\end{array}$

$\begin{array}{ll}3.47 & 5.98 \\ 3.53 & 6.06 \\ 3.58 & 6.15\end{array}$

$\begin{array}{lll}3.37 & 6.07\end{array}$

$\begin{array}{ll}3.37 & 6.07 \\ 3.47 & 6.07 \\ 3.53 & 8.26\end{array}$

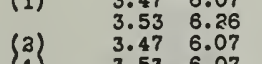

$\left(\begin{array}{l}3 \\ 1 \\ 1\end{array}\right) \begin{array}{ll}3.47 & 6.07 \\ 3.58 & 6.07\end{array}$

$\begin{array}{llll}3.58 & 6.26 & 3-1 & 2^{5} a^{\circ}-P^{3} \mathrm{D} \\ 3.53 & 6.08 & 3-3 & (100)\end{array}$

$\begin{array}{ll}3.53 & 6.08 \\ 3.58 & 6.09\end{array}$

$\left.\begin{array}{lll}(4 n) & 3.40 & 6.09 \\ 1 \\ 3\end{array}\right) \quad \begin{array}{ll}3.47 & 8.09 \\ 3.47 & 6.09\end{array}$

$\begin{array}{ll}3.40 & 6.33 \\ 3.47 & 6.33\end{array}$

$\begin{array}{ll}3.47 & 6.33 \\ 3.47 & 6.33 \\ 3 & 0.33\end{array}$

$\begin{array}{ll}3.53 & 6.33 \\ 3.53 & 6.23\end{array}$

$\begin{array}{lll}3.58 & 6.33\end{array}$

$3.53 \quad 6.36$

$\begin{array}{ll}3.40 & 6.99 \\ 3.47 & 7.13\end{array}$

$\begin{array}{ll}3.47 & 7.13 \\ 3.53 & 7.23\end{array}$

$\begin{array}{lll}3.40 & 7.13\end{array}$

$\begin{array}{ll}3.40 & 7.01 \\ 3.47 & 7.13\end{array}$

$\begin{array}{ll}3.47 & 7.01 \\ 3.53 & 7.13\end{array}$

$3.37 \quad 7.03$

$\begin{array}{ll}3.40 & 7.13 \\ 3.47 & 7.32\end{array}$

$\begin{array}{ll}3.53 & 7.29 \\ 3.58 & 7.31\end{array}$

$\begin{array}{ll}3.58 & 7.31 \\ 3.40 & 7.22\end{array}$

$\begin{array}{ll}3.37 & 7.03 \\ 3.40 & 7.14\end{array}$

$\begin{array}{ll}3.40 & 7.14 \\ 3.47 & 7.33 \\ 3.53 & 7.38 \\ 3 & 58\end{array}$

$\begin{array}{ll}3.53 & 7.28 \\ 3.58 & 7.31 \\ 3.41 & 7.14\end{array}$

$\begin{array}{ll}3.40 & 7.03 \\ 3.47 & 7.14\end{array}$

$\begin{array}{ll}3.47 & 7.14 \\ 3.53 & 7.23\end{array}$

$\begin{array}{ll}3.37 & 7.03 \\ 3.40 & 7.14\end{array}$

$\begin{array}{ll}3.40 & 7.14 \\ 3.47 & 7.37 \\ 3.53 & 7.34\end{array}$

$\begin{array}{ll}3.47 & 7.37 \\ 3.53 & 7.24 \\ 3.58 & 7.30\end{array}$

$\begin{array}{ll}3.58 & 7.30 \\ 3.40 & 7.37\end{array}$

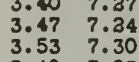

$\begin{array}{ll}3.53 & 7.30 \\ 3.40 & 7.03 \\ 3.47 & 7.14\end{array}$

\begin{tabular}{lll}
3.47 & 7.14 \\
3.58 & 7.34 \\
\hline
\end{tabular} 7167.01
7836.81
7917.48
7386.56
8034.56

$\begin{array}{ll}4-3 & 2^{5} G^{\circ}-e^{3} \mathrm{D} \\ 3-2 & (97)^{-2}\end{array}$

(3) $3.68 \quad 5.46 \quad 3-2 \quad z^{5} F^{0}-e^{1 D}$

$\begin{array}{llrlllll}5017.591 & \mathrm{~B} & 10 & 3.52 & 5.98 & 5-5 & 2^{5110} \mathrm{~F}^{\circ}-\mathrm{e}^{5} \mathrm{~F} \\ 4998.233 & \mathrm{~B} & \mathrm{Z} & 3.59 & 6.06 & 4-4 & (111)\end{array}$

5012.464

4913.030

4866.367
4831.183
4873.437

4873.437
4857.382
5157.993

5157.993
5192.524
5096.874

5096.874
5010.045

4849.12

4976.155
4980.161
5168.660

5168.660
4873.27

4953.334
5128.03

4863.931
$\mathrm{~m} 5143.98$

5216.513

4808.52

4808.52
4941.920
4760.23

4760.23
4937.337

$\begin{array}{lll} & 4937.337 \\ 5-5 & 2^{5} a^{\circ}-e^{5} & 5131.770 \\ -4 & (98) & 4836.37 \\ -3 & & 5220.307 \\ 3-3 & & 4890.45\end{array}$

4559.945

4501.692

4675.639
4655.661

4845.17

4738.42
4617.94

4325.361

$2^{5} a^{\circ}-0^{3} \mathrm{G}$

4531.93

3908.931
4025.44

3559.930

3496.350

3485.110
3396.50

3543.00
3611.418

$5-4 \quad 2^{5} a^{0}-a^{3}$
$4-3 \quad(101)$

$\begin{array}{ll}4-4 & (101) \\ 4-3 & z^{5} a^{0}-r^{3} F \\ (103)\end{array}$

3537.634

3482.73

3483.63
-3606.853

$4-4$
$3-3$

$3-4$
$2-3$

3-4 $2^{5} a^{0}-0^{1} a$

3575.952

3530.595
3488.293

3599.530

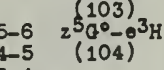

m3624.73

3431.343

3326.184

3444.251

$\begin{array}{ll}5-4 & 2^{5} G^{0}-8^{3} G \\ 5-5 & (105)\end{array}$

$4-4$
$4-5$
$3-4$

$6-7 \quad 2^{5} a^{0}-e^{5} \mathrm{H}$

$4-5$
$3-4$
$2-3$
$5-5$

$\begin{array}{cc}6-5 & z^{5} a^{\circ}-r^{5} F \\ 5-4 & (107)\end{array}$

$4-3$
$3-2$

5-1

$5-5$
$3-3$

$2^{6-6} \quad z^{5} a^{\circ}-0^{5} a$

3337.36
$\mathbf{3} 405.50$

3516.234

- 3480.183

3476.63
3467.732

3467.12

3415.67

3438.42
3573.27

3517.03

3518.634
3477.864

3477.864
$344 \dot{4} .559$

3511.94
-3480.183

3480.183
$\mathbf{m} 3413.46$

3471.63

3191.875

7122.24

7183.00

7182.00
7030.06

8843.07
8433.00

6370.383

6773.36

$\begin{array}{lll}3 & 3.75 & 6.36 \\ 3 & 3.52 & 6.36\end{array}$

(1) $3.53 \quad 6.09$

$\begin{array}{llll}3.68 & 7.24 & 3-3\end{array}$

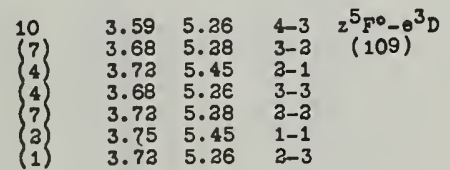

\begin{tabular}{lll}
3 & 3.68 & 6.15 \\
3 & 3.72 & 5.32 \\
\hline & 3.75 & 6.36
\end{tabular}

\begin{tabular}{lll}
4 & 3.68 & 6.23 \\
3 & 3.73 & 6.36 \\
\hline & 3.59 & 5.98
\end{tabular}

(a) $3.59 \quad 5.98$

\begin{tabular}{lll}
3 & 3.68 & 6.06 \\
3 & 3.73 & 6.15 \\
\hline & 3.75 & 6.32
\end{tabular}

(1n) $\begin{array}{llll}3.52 & 6.07 & 5-5 & 2^{5} 5^{\circ}-e^{3 G} \\ 3.59 & 6.07 & 4-4 & (113)\end{array}$

$\begin{array}{ccc}12 & 3.59 & 6.07 \\ 6 & 3.68 & 6.07 \\ & 3.70 & 6.36\end{array}$

(1n) $3.59 \quad 6.08$

$\begin{array}{lll}\text { (1) } & 3.68 & 6.09 \\ \text { an) } & 3.73 & 6.26\end{array}$

$\begin{array}{lll}\text { N1 } & 3.68 & 6.08 \\ 3 & 3.73 & 6.09\end{array}$

$\begin{array}{lll}3 & 3.73 & 6.09 \\ \text { (3) } & 3.75 & 6.26\end{array}$

$\begin{array}{ll}3.59 & 6.09 \\ 3.68 & 6.28\end{array}$

$\begin{array}{ll}3.68 & 6.28 \\ 3.59 & 6.09\end{array}$

$\begin{array}{ccc}4 & 3.58 & 6.09 \\ 3 & 2.68 & 6.09 \\ (1) & 3.73 & 6.28 \\ 2 & 3.72 & 0.09\end{array}$

$\begin{array}{ll}3.72 & 6.28 \\ 3.72 & 6.09 \\ 3.75 & 6.28\end{array}$

(3) $3.53 \quad 6.23$

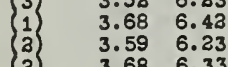

$\left(\begin{array}{l}a \\ 1 \\ 1 \\ 1\end{array}\right)$

$\begin{array}{ll}3.68 & 6.33 \\ 3.68 & 6.23 \\ 3.73 & 6.33\end{array}$

$\begin{array}{ll}3.73 & 6.33 \\ 3.75 & 6.42\end{array}$

(1)

$\begin{array}{ll}3.59 & 6.44 \\ 3.72 & 6.45\end{array}$

$\begin{array}{cccc}8 n & 3.59 & 6.75 \\ \text { (1N) } & 3.68 & 6.75\end{array}$

$\begin{array}{lll}3 & 3.53 & 6.99 \\ 5 & 3.59 & 7.13\end{array}$

$\begin{array}{lll}5 & 3.59 & 7.13 \\ \text { an } & 3.68 & 7.32 \\ & 3.59 & 7.23\end{array}$

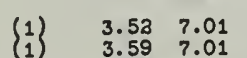

(1) $\begin{array}{ll}3.52 & 7.01 \\ 3.59 & 7.13\end{array}$

$\begin{array}{ll}3.59 & 7.13 \\ 3.68 & 7.23\end{array}$

$\begin{array}{ll}3.68 & 7.23 \\ 3.59 & 7.01\end{array}$

3 $3.68 \quad 7.13 \quad 4-4$

$\begin{array}{lllll}4 & 3.52 & 7.02 & 5-4 & 2^{5} F^{0}-e^{5} \mathrm{D} \\ 3 & 3.59 & 7.13 & 4-3 & (131)^{2} \\ \text { (1) } & 3.59 & 7.02 & 4-4 & \end{array}$

$\begin{array}{llll}\text { (1) } & 3.59 & 7.03 & 4-4 \\ \text { N1 } & 3.73 & 7.13 & 3-3\end{array}$

$7 \quad 3.537 .13$

$\begin{array}{lll}7 & 3.52 & 7.13 \\ 6 & 3.59 & 7.32 \\ 4 & 3.68 & 7.39\end{array}$

$\begin{array}{lll}4 & 3.68 & 7.39 \\ 5 & 3.72 & 7.31 \\ & 3.59 & 7.39\end{array}$

$\begin{array}{ll}5-6 & 2^{5} 5^{\circ}-0^{5} H \\ 4-5 & (133)^{5}\end{array}$

$\begin{array}{ll}3.59 & 7.39 \\ 3.68 & 7.31\end{array}$

$3-4$
$3-3$
$4-4$
$3-3$

$\begin{array}{llllll}8 & 3.53 & 7.03 & 5-5 & z^{5} F^{\circ}-r^{5} F \\ 4 & 3.59 & 7.14 & 4-4 & (133)\end{array}$

$\begin{array}{llll}\text { an } & 3.68 & 7.23 & 3-3 \\ 4 & 3.72 & 7.28 & 2-3\end{array}$

(1) $3.75 \quad 7.31 \quad 1-1$

$\begin{array}{lll}3.52 & 7.14 & 5-4 \\ 3.68 & 7.38 & 3-2\end{array}$

$\begin{array}{lll}3.68 & 7.28 & 3-2 \\ 3.68 & 7.14 & 3-4 \\ 3.73 & 7.33 & 2-3\end{array}$

$\begin{array}{lllll}8 & 3.53 & 7.03 & 5-6 & 2^{5} 5^{0}-0^{5} a \\ 3 & 3.59 & 7.14 & 4-5 & (124) \\ 4 n & 3.68 & 7.37 & 3-4 & \end{array}$

$\begin{array}{lll}4 n & 3.68 & 7.37 \\ \text { (1) } & 3.73 & 7.34\end{array}$

$\begin{array}{lll}4 & 3.75 & 7.30 \\ \mathrm{~N} 1 & 3.53 & 7.14\end{array}$

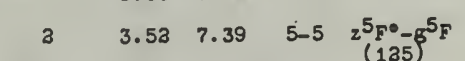

$\begin{array}{lllll}100 & 3.53 & 5.26 & 2-3 & 2^{3} P^{\circ}-0^{3} \mathrm{D} \\ 3 & 3.64 & 5.28 & 1-2 & (128)\end{array}$

$\begin{array}{llll}3 & 3.64 & 5.28 & 1-3 \\ 8 & 3.73 & 5.45 & 0-1 \\ 3 & 3.53 & 5.38 & 3-3\end{array}$

$\begin{array}{llll}3 & 3.53 & 5.38 & 3-2 \\ 8 & 3.84 & 5.45 & 1-1 \\ \text { (1) } & 3.53 & 5.45 & 3-1\end{array}$

$\begin{array}{ccccc}(4) & 3.53 & 5.46 & 2-3 & 2^{3} P^{\circ}-0^{1} D \\ 5 & 3.64 & 5.46 & 1-3 & (127)\end{array}$ \begin{tabular}{lll}
10 & 3.52 & 6.06 \\
10 & 3.59 & 6.15 \\
\hline & 3.68 & 6.33
\end{tabular}

(2) $\begin{array}{llll}3.75 & 6.22 & 1-2\end{array}$
I A ${ }_{\text {Lef }}^{\text {Laboratory }}$ Int Low ${ }^{\text {E P1gh }}$

J $\underset{(\mathrm{NO})}{\operatorname{mult})^{2}}$

N1 I continued

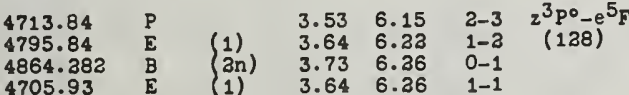

$3.64 \quad 6.26 \quad 1-1$

$\begin{array}{lllll}10 & 3.53 & 6.04 & 2-1 & z^{3} \mathrm{p}^{\circ}-\mathrm{e}^{3} \mathrm{~s} \\ 3 & 3.64 & 6.04 & 1-1 & (129)\end{array}$

$\begin{array}{llllll}38.70 & P & & 3.64 & 6.04 & 1-1 \\ & & 3.73 & 6.04 & 0-1\end{array}$

$\begin{array}{lllllll}4855.414 & \mathrm{~B} & 15 & 3.53 & 6.07 & 2-3 & \mathrm{z}^{3} \mathrm{po}^{\circ}-\mathrm{e}^{3} \mathrm{P} \\ & \mathrm{B}^{2} & 3.64 & 6.07 & 1-1 & (10)\end{array}$

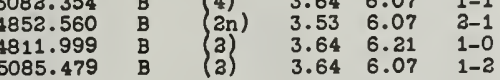

$\begin{array}{lllllll}3.53 & 6.08 & 2-3 & 2^{3} \mathrm{p}^{\circ}-f^{3} \mathrm{D}\end{array}$

$\begin{array}{lllll}4 & 3.64 & 6.09 & 1-2 & (131) \\ 3 & 3.73 & 6.26 & 0-1 & \end{array}$

(1) $\begin{array}{llll}3.53 & 6.09 & 2-2\end{array}$

$\begin{array}{llll}3.53 & 6.26 & 2-1\end{array}$

$4 \quad 3.64 \quad 6.24 \quad 1-1 \quad z^{3} p^{0}-e^{1 p}$

4506.303 $D \quad(1) \quad 3.53 \quad 6.27 \quad 3-2 \quad z^{3} \mathrm{P}^{\circ}-\mathrm{f}^{1} \mathrm{D}$

$\begin{array}{rlllll}4490.541 \quad B & (3) \quad 3.53 & 6.28 & 2-3 & 2^{3} P^{0}-e^{1 F}\end{array}$

$\begin{array}{lllllll}4553.175 \quad \mathrm{~B} & \text { (3) } \quad 3.64 & 6.35 & 1-0 & 2^{3} \mathrm{PO}^{30} \mathrm{e}^{1} \mathrm{~S}\end{array}$

$\begin{array}{llllll}5 & 3.53 & 6.44 & 2-3 & 2^{3}(135) \\ 3 P^{\circ}-8^{3} D\end{array}$

$\begin{array}{lll}3.73 & 6.63 & 0-1\end{array}$

$\begin{array}{llllll}(3 \mathrm{~N}) & 3.53 & 6.74 & 2-1 & z^{3} \mathrm{po}^{\circ}-\mathrm{f}^{3} \mathrm{~S} \\ \text { a) } & 3.64 & 6.74 & 1-1 & (137)\end{array}$

$\begin{array}{llll}3.53 & 6.86 & 2-3 & 2^{3} P^{\circ}-f^{1} F \\ (138)\end{array}$

$\begin{array}{llll}3.64 & 5.26 & 4-3 & z^{3} F^{\circ}-e^{3} D \\ 3.62 & 5.28 & 3-2 & (139)\end{array}$

$\begin{array}{lll}3.78 & 5.45 & 2-1 \\ 3.62 & 5.26 & 3-3\end{array}$

$\begin{array}{lllll}2 & 3.63 & 5.46 & 3-2 & z^{3} F^{\circ}-e^{1} D \\ 4 & 3.78 & 5.46 & 3-2 & (140)\end{array}$

$\begin{array}{llllll}2 & 3.64 & 5.98 & 4-5 & z^{3} F^{0}-e^{5} F \\ 2 & 3.62 & 6.06 & 3-4 & (141)\end{array}$

$\begin{array}{llll}5 & 3.64 & 6.06 & 4-4 \\ \text { (3) } & 3.62 & 6.15 & 3-3\end{array}$

$\begin{array}{llll}1 & 3.78 & 6.22 & 2-2 \\ 2 & 3.64 & 6.15 & 4-3\end{array}$

(1) $\begin{array}{llll}3.62 & 6.22 & 3-2 \\ & 3.78 & 6.26 & 2-1\end{array}$

(ar) $\quad 3.62 \quad 6.07 \quad 3-2 \quad 2^{3} F^{0}-e^{3 P}$

$\begin{array}{lllll}30 & 3.64 & 6.07 & 4-5 & 2^{3}(142) \\ 30 & 2^{0}-0^{3} a\end{array}$

$\begin{array}{llll}10 & 3.62 & 6.07 & 3-4 \\ 13 & 3.78 & 6.26 & 3-3 \\ \text { (3) } & 3.64 & 6.07 & 4-4\end{array}$

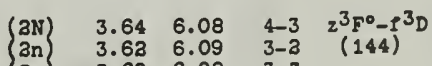

(3N) $\quad 3.64 \quad 6.09 \quad 4-4 \quad z^{3} F^{0}-e^{3} F$

$\begin{array}{lllll}4 & 3.63 & 6.09 & 3-3 & (145)\end{array}$

$\begin{array}{lll}3.78 & 6.38 & 2-2\end{array}$

$\begin{array}{lll}3.62 & 6.28 & 3-2 \\ 3.62 & 6.09 & 3-4 \\ 3.78 & 6.09 & 3-3\end{array}$

$\begin{array}{lllll}4 & 3.64 & 6.23 & 4-4 & 2^{3} F^{0}-r^{3} \\ 3 & 3.62 & 6.33 & 3-3 & (146)\end{array}$

(3) $\begin{array}{llll}3.78 & 6.42 & 3-3 \\ \text { (3) } & 3.64 & 6.33 & 4-3\end{array}$

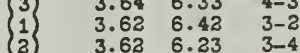

$\begin{array}{lll}3.78 & 6.33 & 3-3\end{array}$

(1) $\begin{array}{lllll}3.62 & 6.27 & 3-2 & 2^{3} F^{\circ}-f^{1} D \\ 3.78 & 6.27 & 2-2 & (147)\end{array}$

$\left.\begin{array}{lllll}1 & 1 \\ 3\end{array}\right) \quad \begin{array}{llll}3.62 & 6.28 & 3-3 & z^{3} F^{0}-e^{1} F \\ 3.78 & 6.28 & 2-3 & (148)\end{array}$

$\begin{array}{lllll}3 & 3.64 & 6.44 & 4-3 & 2^{3} F^{0}-\beta^{3} D \\ 3 & 3.62 & 6.45 & 3-2 & (149)^{3}\end{array}$

(3) $\begin{array}{llll}3.78 & 6.63 & 2-1 \\ & 3.62 & 6.44 & 3-3\end{array}$

(3) $\quad \begin{array}{lllll}3.64 & 6.70 & 4-4 & 2^{3} F^{\circ}-6^{3} F \\ 3.62 & 6.76 & 3-3 & (150)^{3}\end{array}$

$\begin{array}{lll}3.78 & 6.76 & 3-3 \\ 3.78 & 6.90 & 2-2\end{array}$

$\begin{array}{lll}3.64 & 6.70 & 4-3 \\ 3.78 & 6.70 & 3-3\end{array}$

$\begin{array}{lllll}10 n & 3.64 & 6.75 & 4-5 & z^{3} F^{0}-r^{30}\end{array}$

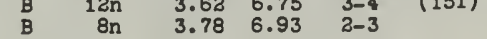

$\begin{array}{lllllll}3511.613 \quad D & 2 & 3.62 & 7.13 & 3-49 & z^{3} F^{\circ}-\beta^{3 a} a\end{array}$

$\begin{array}{lllllll}3651.67 & P & & 3.64 & 7.03 & 4-4 & 2^{3}(153) \\ 3537.243 & D & \text { (1) } & 3.64 & 7.13 & 4-3 & (153)\end{array}$

$\begin{array}{lllllll}3538.891 & B & (3) & 3.64 & 7.14 & 4-4 & z^{3} F^{0}-F^{5} F\end{array}$

$\mathbf{m} 3523.47$
3494.703

3536.540

(1) $3.78 \quad 7.31 \quad 3-1$

$3 \quad 3.64 \quad 7.14 \quad 4-5 \quad \frac{2^{3} F^{\circ}-0^{50}}{(155)^{5}}$ 
FINDING LIST

Type Element

I A Type Elewent Multiplet Mo.

6560.68

6561.032

$\mathrm{Zr} \mathrm{I} \quad 65$

Fe II

$\begin{array}{ll}\text { N1 } & \text { I } \\ \text { II }\end{array}$

Mn I

N II

Fe II

21
91
39

45

18

Gd II 123

Fe I 1253

Fe I

Al II

1255
168

Fe I 1253

Fe I 1258

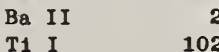

La II 104

$\mathrm{Ba} I$

13

Fe I

N I

Cr I

Fe I

Sr I

$\begin{array}{ll}\mathrm{V} & I \\ \mathrm{~N} & I\end{array}$

Fe II

6562.817

6563.403

6563.86

6565.62

6567.22

6567.39

6568.00

6569.281

6569.31

6527.10

6570.96

6571.22

6572.781

6572.900

6574.238

$\begin{array}{lr}\text { He II } & 2 \\ \text { S1 I } & 62 \\ \text { D } & 1 \\ \text { H } & 1 \\ \text { Co I } & 80 \\ \text { HF II } & 81 \\ \text { TI I } & \\ \text { V I } & 48 \\ \text { Fe I } & 168 \\ \text { HI II } & 90\end{array}$

2
62
1
1
80
81
48
168
90
121

Fe I $\quad 1253$

$\begin{array}{lr}\text { Sm II } & 62 \\ \text { He II } & 7\end{array}$

$\begin{array}{lr}\text { He II } & 7 \\ \text { Mn I } & 51 \\ \text { IR II } & 47\end{array}$

La II 1121

Fe

$\begin{array}{ll}\mathrm{Ca} & \mathrm{I} \\ \mathrm{Cr} & \mathrm{I}\end{array}$

Fe I

1121
1
16

$6575.022 \quad F e ~ I-206$

$\begin{array}{lll}6575.022 & \text { Fe I } & 206 \\ 6575.180 & \text { T1 I } & 286\end{array}$

$\begin{array}{llll}6576.96 & P & \text { N1 I } & 283\end{array}$

$\begin{array}{lll}6578.03 & \text { La I } & 1\end{array}$

6578.51

6578.96

6580.22

6580.96

6581.22

6582.85

V I

N1 I

Cr I

Fe I

6584.53

6584.89

Ne I

T1 I

$\mathrm{Ca} I$

A II

Fe I

Hf II

Cr I

Fe II

Fe II

$\mathrm{Fe} I$

Fe I

S II

N II

Cl II

Le II

S1 I

21
3
102
18
21
1012
69
49
265
40

6586.328

6586.69

6587.75

6588.91

6591.32

6591.834

6592

230

6592.472

6592.91

6592.919

6593.878

6595.326

6585.868

6697.556

6597.607

6598.59

6598.9528

\section{He II}

Y I
N1

Mn I

Fe II

C I

Sm I

Co I
C IV

1228

6599.112

6601.13

$6604.60 \quad$ SC II

6604.67 Fe I 1254

$6605.546 \quad$ Mn I 51

$\begin{array}{llll}6605.98 & & \text { V I } & 48 \\ 6607.02 & \text { P } & \text { T1 II } & 91\end{array}$

$\begin{array}{lll}6607.82 & \text { V I } & 59\end{array}$

6608.03

6609.116

6609.20

6609.56

6609.64

6609.68

6610.04

6612.17

6613.74

$6613.83 \quad P \quad$ Fe $I \quad 13$

6615.03 P $\quad$ Fe I 115

6617.126 Co I

$\begin{array}{llll}6617.14 & P & \text { N1 I } & 248\end{array}$

$\begin{array}{lll}6617.266 & \text { N1 I } & 97\end{array}$

$\begin{array}{rllr}6622.28 & & \text { Gd II } & 110\end{array}$

$\begin{array}{rllr}6622.41 & P & \text { Fe I } & 1157 \\ 6622.53 & & \text { N I } & 20\end{array}$

$\begin{array}{llll}6623.78 & P & \text { Fe } I & 1010\end{array}$

6624.86

6625.04

6627.558

6627.62

6630.015

6630.5

6632.438

6633.44

6633.764

$\begin{array}{lllr}6634.10 & \text { P } & \text { Fe I } & 1258 \\ 6634.36 & & \text { Gd II } & 94 \\ 6635.15 & & \text { N1 I } & 264 \\ 6635.68 & \text { P } & \text { Fe I } & 1155 \\ 6636.53 & & \text { L I I } & 61 \\ 6637.01 & & \text { N I } & 20 \\ 6638.24 & & \text { A II } & 20 \\ 6639.35 & \text { P } & \text { Fe I } & 1279 \\ 6639.71 & \text { P } & \text { Fe I } & 1195 \\ 6639.72 & & \text { A II } & 20\end{array}$

$6639.90 \quad P \quad$ Fe I 1007

$6640.90 \quad$ S II

6643.536 Sr I

6643.641 N II $\quad 20$

6644.60 HP II 34

6644.96

N I

- 8

$\begin{array}{rrrrr}6646.52 & & \text { N I } & 1156\end{array}$

6646.98 Fe I $\quad 200$

$\begin{array}{llll}6647.06 & \text { HP II } & \\ 6547.00 & P & \text { Fe I } & \end{array}$

$6647.90 \quad$ P $\quad$ Fe I

6653.41 N I 20

$\begin{array}{lll}6653.75 & \text { C1 II } & 38\end{array}$

6653.88 Fe I 1052

6656.61

6657.54

6660.49

6661.078

6661.68

6663.26

6663.446

Fe I $\quad 20$

Cr I 282

Cr'I 282

N1 I 246

$6665.43 \quad P \quad$ Fe I

T1 I 101

$\begin{array}{rllr}6667.42 & \text { P } & \text { Fe I } & 168 \\ 8667.73 & & \text { Fe I } & 1228\end{array}$

6669.257 Cr I 282

$6671.36 \quad$ Fe I 1343

$6671.43 \quad P \quad$ Fe I $\quad 125$

6671.51 Sm I

$\begin{array}{lll}6671.88 & \text { S1 II } & \\ 6672.84 & \text { V II } & 228\end{array}$

$\begin{array}{llll}6672.88 & P & F e & 206\end{array}$

P $\quad$ Fe I 125

6676.86 P $\quad$ Fe I 118

$6677.24 \quad$ Cr I 256

6677.33 Fe II 210

$\begin{array}{rllr}6677.49 & P & F e ~ I ~ & 1280\end{array}$

551

$6677.993 \quad \mathrm{Fe} \mathrm{I} \quad 268$

$6678.03 \quad$ Zr II 128

6678.149 He I 46

$\begin{array}{lll}6678.19 & 0 & \text { II } \\ 6678.2764 & \text { Ne I } & 85\end{array}$

$\begin{array}{lllll}6678.60 & P & \text { T1 } & \text { I } & 213\end{array}$

6678.818 Co I

$6680.19 \quad$ Cr I

$6681.03 \quad$ Cl II 38

$\begin{array}{llll}6682.23 & P & F e & 1\end{array}$

6683.2

6684.36

6686.04

He II

A II

20

6687.57

6690.80

6692.47

6693.842

6695.97

6696.30

6698.63

6699.14

6699. 46

6
6699.14
6699.46

Y

N1 I

Al

Fe I

AI I

Al I

Al II
6641.06 La II 103

6642.78 Cr I 256

Cl II 38

$\mathrm{Fe} I \quad 1185$

6666.36 A II 10

6667.17 P $\quad$ Fe I 110

$6680.26 \quad$ T1 II 112

$\begin{array}{llll}6681.23 & & \text { Gd II } & 94 \\ 6681.34 & P & \text { Fe I } & 1155\end{array}$ 


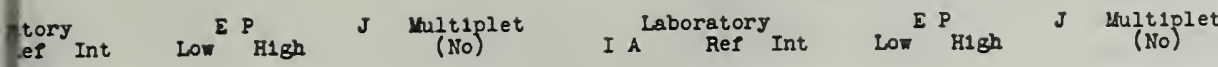
nued

(1) $\quad 4.23 \quad 6.37 \quad 3-2 \quad y^{3} D^{0}-1^{10}$ an $\quad 4.22 \quad 6.28 \quad 2-3 \quad \mathrm{y}^{3} \mathrm{D}^{0}-\mathrm{e}^{1} \mathrm{r}$ (2) $\quad 4.25 \quad 6.35 \quad 1-0 \quad \mathrm{y}^{3} \mathrm{D}^{\circ}-\mathrm{e}^{15}$

(2) $\quad 4.14 \quad 6.70 \quad 3-4 \quad \mathrm{y}^{3} \mathrm{D}^{\circ}-\beta^{3 F}$

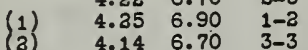

(3) $\quad \begin{array}{llll}4.14 & 7.13 & 3-4 & y^{3} D^{\circ}-g^{3} G \\ 4.22 & 7.23 & 2-3 & (255)\end{array}$

$\begin{array}{lllll} & 4.14 & 7.05 & 3-3 & \mathrm{y}^{3} \mathrm{D}^{0}-1^{3} \mathrm{D} \\ \text { (1) } \quad 4.32 & 7.05 & 3-3 & (256)\end{array}$

(2) Fe? $4.15 \quad 6.06 \quad 4-4 \quad \mathrm{z}^{1} G^{0}-\theta^{5} \mathrm{~F}$

(5n) $\quad \begin{array}{llll}4.15 & 6.07 \quad 4-5 & 2^{1} 0^{\circ}-0^{30}\end{array}$

(1) $\quad \begin{array}{lllll}4.15 & 6.23 & 4-4 & \mathrm{z}(258) \\ \left(G^{0}-F^{3} F\right. & \end{array}$

$\begin{array}{lllll}\text { (1) } & 4.15 & 6.70 & 4-4 & z^{1} 0^{\circ}-\beta^{3} F \\ 2 & 4.15 & 6.70 & 4-3 & (260)^{2}\end{array}$

$4.15 \quad 6.86 \quad 4-3 \quad 2^{1} G^{0}-P^{1} \mathrm{~F}$

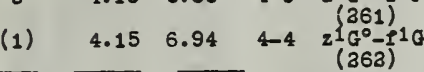

(2) Fei $4.40 \quad 6.08 \quad 3-3 \quad y^{1} F^{\circ}-F^{3} D$

$\begin{array}{llllll}3 & 4.40 & 6.36 & 3-4 & \mathrm{y}^{1} F^{\circ}-\mathrm{e}^{16}\end{array}$

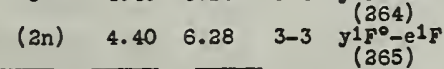

(1) $4.52 \quad \overline{6.07} 3-1 \quad \mathrm{~J}^{1} \mathrm{D}^{\circ}-\mathrm{e}^{3 P}$

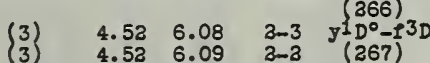

(3) $\left.\begin{array}{lll}4.52 & 6.09 & 2-2 \\ 1\end{array}\right) \quad \begin{array}{lll}4.52 & 6.26 & 2-1\end{array}$

(5) $4.52 \quad 6.09 \quad 2-3 \quad \mathbf{J}^{1} D^{\circ}-\mathrm{e}^{3} F$

(a) $\quad 4.52 \quad 6.34 \quad 3-1 \quad \mathrm{y}^{1} D^{\circ}-\theta^{1 P}$

(2) $\quad \begin{array}{llll}4.52 & 6.27 \quad 2-2 \quad y^{1} D^{\circ}-I^{1} D\end{array}$

$4.526 .28 \quad 2-3 \quad \mathrm{y}^{1}\left(\mathrm{D}^{\circ}-0^{1}\right.$

$4.52 \quad 6.70 \quad 2-3 \quad \mathrm{y}^{1} \mathrm{D}^{\circ}-\mathrm{s}^{35}$

4.526 .60 2-3 $\mathrm{y}^{1} \mathrm{D}^{\circ}-1^{1 F}$

$4.52 \quad 7.00 \quad 2-29 \mathrm{~J}^{1} \frac{(273)}{(274)}(2 \mathrm{P}$

$5.26 \quad 6.70 \quad 3-4 \quad x^{3} D^{0}-5^{3} F$

(4) Fe? $5.26 \quad 7.00 \quad 3-2 \quad x^{3} D^{\circ}-P^{3} P$

$\begin{array}{lllll}\text { (2) } 5.26 & 7.01 \quad 3-4 \quad x^{3} D^{\circ}-135 \\ (277) & \end{array}$

(3) $5.39 \quad 7.00 \quad 3-2 \quad x^{3} F^{\circ}-p^{3} P$

(1) $5.26 \quad 7.01 \quad 45 x^{3} F^{0}-5^{3} G$

(3) $\quad \begin{array}{llll}5.39 & 7.05 \quad 3-3 \quad \begin{array}{l}x^{3} F^{\circ}-13 \mathrm{D} \\ (280)\end{array}\end{array}$

(1) $5.27 \quad 6.86 \quad 3-3 \quad y^{3} p^{0}-p^{1} F$

(2) $5.28 \quad 7.00 \quad 3-3 \quad w^{3} D^{0}-p^{3} P$

(3) $\left.\begin{array}{llll}5.49 & 7.13 & 2-1 & (282) \\ 1\end{array}\right) \quad \begin{array}{llll}5.57 & 7.22 & 1-0 & \end{array}$

(1) $5.28 \quad 7.01 \quad 3-4 \quad \omega^{3} D^{0}-1^{3} \xi$

$\begin{array}{llll}5.49 & 7.16 & 2-3 & (283)\end{array}$

\begin{tabular}{lll}
5.57 & 7.24 & $1-2$ \\
5.28 & 7.16 & $3-3$ \\
\hline
\end{tabular}

$5.32 \quad 6.70 \quad 5-4 \quad y^{3} G^{\circ}-g^{3}$

$\begin{array}{llll}5.32 & 6.70 & 5-4 & y^{3} G^{\circ}-g^{3} \\ 5.70 & 4-3 & (284)^{2}\end{array}$

$\begin{array}{llll}5.47 & 6.86 & 4-3 & y^{3} G^{0}-f^{1} \\ 5.59 & 6.86 & 3-3 & (285)\end{array}$

$\left\{\begin{array}{lllll}7 & 5.32 & 6.99 & 5-6 & \mathrm{y}^{3} \mathrm{G}^{\circ}-\mathrm{e}^{3 \mathrm{H}} \\ 5 & 5.47 & 7.12 & 4-5 & (286)\end{array}\right.$

$\begin{array}{llll}5.47 & 7.12 & 4-5 & (286) \\ 5.59 & 7.22 & 3-4 & \end{array}$

$\left\{\begin{array}{llll}1 \\ 2 \\ 2\end{array}\right\} \begin{array}{llll}5.32 & 7.01 & 5-5 & r^{3} \mathrm{G}^{\circ}-\beta^{3} \mathrm{c} \\ 5.47 & 7.13 & 4-4 & (287)^{2} \\ 5.59 & 7.23 & 3-3 & \end{array}$

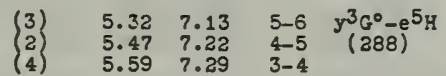

$\begin{array}{rllll}30 & 5.34 & 6.70 & 4-4 & x^{3} g^{\circ}-g^{3} F \\ 5 & 5.50 & 6.70 & 3-3 & (389)^{3}\end{array}$

(1) $5.61 \quad 7.13 \quad 2-1 \quad 3^{3} F^{0}-P^{3 P}$

(4) $\begin{array}{llllc}5.34 & 7.01 & 4-4 & (390) \\ 1 & 5.50 & 7.16 & 3-3 & (291) \\ 1 & 5.61 & 7.24 & 2-2 & \end{array}$
N1 I cont1nued $\begin{array}{lllllll}7381.94 & B & (5) & 5.34 & 7.01 & 4-5 & w^{3} F^{\circ}-g^{3} G \\ 7559.62 & B & & \end{array}$

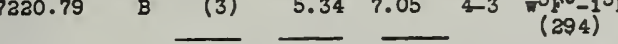
$9689.35 \quad A \quad 3 \quad 5.43 \quad 6.70 \quad 3-3 \quad x^{1} D^{\circ}-5^{3} F$

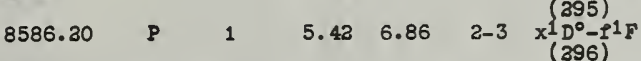

Strongest Unclass1f1ed Lines of N1. I

10395.05

\begin{tabular}{llll}
10295.05 & $A$ & 5 & \\
9396.57 & $A$ & 20 & \\
6362.414 & $D$ & $\{5\}$ & \\
6012.251 & $B$ & 5 & \\
4594.908 & $B$ & $(5 n)$ & \\
4142.320 & $B$ & $(4)$ & \\
4006.136 & $B$ & 3 & $V$ \\
3762.618 & $B$ & $(3)$ & III \\
3665.924 & $B$ & $Z$ & II \\
3647.71 & E & Z & II \\
3335.59 & E & $2 n$ & $V$ \\
3332.180 & $B$ & $6 n$ & $V$ \\
3309.428 & $B$ & $2 n$ & $V$ \\
3368.064 & $B$ & $4 n$ & $V$ \\
3264.44 & E & $2 n$ & $V$ \\
3333.88 & E & 2 & $V$ \\
3199.342 & $B$ & $3 n$ & $V$ \\
3151.259 & $B$ & $4 n$ & $V$ \\
\hline
\end{tabular}

N1 II I P 18.4 Anal C L1st A Apr 1942

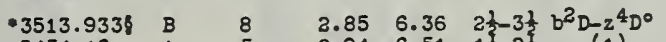
$\begin{array}{lllllll}3454.16 & \mathrm{~A} & 5 & 2.94 & 6.51 & 1 \frac{1}{2}-2 \frac{1}{2} & \text { (1) }\end{array}$

$\begin{array}{llllll}3373.98 & \mathrm{~A} & 4 & 2.85 & 6.51 & 2 \frac{1}{2}-2 \frac{1}{2} \\ 3350.42 & \mathrm{~A} & 5 & 2.94 & 6.62 & 1 \frac{1}{2}-1 \frac{1}{2}\end{array}$

$\begin{array}{llllll}3274.90 & \text { A } & 3 & 2.85 & 6.62 & 2 \frac{1}{2} \\ 3390.69 & \text { A } & 1 & 2.94 & 6.69 & 1 \frac{1}{2}-\frac{1}{2}\end{array}$

$3208.91 \quad A \quad 1 \quad 2.85 \quad 6.70 \quad 2 \frac{1}{2}-3 \frac{1}{2} b^{2} D-2^{4} G^{\circ}$

$\begin{array}{lllllll}3032.44 & \mathrm{~A} & 2 & 2.85 & 6.92 & 2 \frac{1}{3}-2 \frac{1}{2} & b^{2} \mathrm{D}-\mathrm{z}^{4} \mathrm{~F}^{\circ} \\ 3063.93 & \mathrm{~A} & \mathrm{a} & 2.94 & 6.97 & 1 \frac{1}{2}-1 \frac{1}{2} & (3)\end{array}$

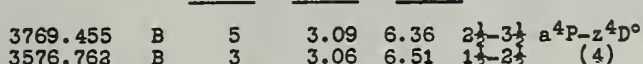

3576.763 B $33 \quad 3.06 \quad 6.51 \quad 1 \frac{2}{3}-2 \frac{2}{3} \quad$ (4)

3471.35

$3608 . ?$

3465.62

3407.30

$3.096 .62 \quad 2 \frac{2}{3-1}$

$3390.54 \quad$ A $\quad 19 \quad 3.09 \quad 6.84 \quad 2 \frac{1}{2}-3 \frac{1}{2} 8^{4} P_{-} z^{4} 7^{\circ}$

$\begin{array}{lllllll}2988.05 & A & 5 & 3.09 & 7.22 & 2 \frac{1}{2}-2 \frac{1}{2} a^{4} \mathrm{P}-z^{2} \mathrm{~F}^{\circ}\end{array}$

$3087.07 \quad$ A $\quad 20 \quad 3.09 \quad 7.09 \quad 2 \frac{1}{2}-2 \frac{1}{2} a^{4} P-z^{2} D^{0}+$

$3397.83 \mathrm{~A} \quad 1 \quad 3.597 .22 \quad 1 \frac{1}{2}-2 \frac{1}{2} \mathrm{a}^{2} \mathrm{P}-2^{2} \mathrm{~F}^{\circ}$

$4362.10 \quad C \quad 1 \quad 4.01 \quad 6.84 \quad 4 \frac{1}{3}-3 \frac{1}{\frac{1}{2}} \mathrm{a}^{2} \mathrm{G}-\mathrm{z}^{4} \mathrm{~F}^{\circ}$

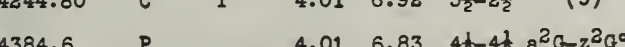

$\begin{array}{lllllll}4384.6 & P & & 4.01 & 6.83 & 4 \frac{1}{2}-4 \frac{1}{2} & a^{2} a-z^{2} \\ 4192.07 & C & 1 & 4.01 & 6.96 & 3 \frac{1}{2}-3 \frac{1}{2}\end{array}$

$4067.051 \quad B \quad 3 \quad 4.01 \quad 7.05 \quad 4 \frac{1}{2}-3 \frac{1}{2} a^{2} G-z^{2} F^{0}$

$\begin{array}{lllllll}3849.58 & \mathrm{~B} & \mathrm{C} & 4.01 & 7.22 & 3 \frac{2}{2}-2 \frac{2}{2} & \text { (11) } \\ 4 & \mathrm{P} & & 4.01 & 7.05 & 3 \frac{1}{2}-3 & \end{array}$

$4015.50 \quad C \quad 1 \quad 4.01 \quad 7.09 \quad 3 \frac{1}{2}-2 \frac{1}{2} a^{2} G-z^{2} D^{\circ}$

$3881.93 \mathrm{C} \quad 1 \quad \overline{8.22} 11.40 \quad 2 \frac{1}{2}-3 \frac{1}{2} \mathrm{z}^{4} \mathrm{p}_{(13)}^{0}-\mathrm{e}^{4} \mathrm{~F}$

stronger unclassifled lines of $\mathrm{N1}$ I

Cu I I P 7.69 Anal A List $D$ Apr 1942

$\begin{array}{llrllll}3247.540 / / & \text { A } & 1000 & 0.00 & 3.80 & \frac{1}{2}-1 \frac{1}{2} 4^{2} s-4^{2} P_{0} \\ 3273.957 & \text { A } & 600 & 0.00 & 3.77 & \frac{1}{2}-\frac{1}{2} & \text { (1) }\end{array}$

5105.541 A $300 \quad-1.38 \quad 3.80 \quad 2 \frac{1}{3}-1 \frac{1}{3} 48^{2} \quad 2_{D-4} P^{\circ}$

3093.989

3208.231

3010.838

3194.099

2997.364

3997.364
3036.101 $\begin{array}{lll}1.38 & 5.48 & 2 \frac{1}{3}-2 \frac{1}{2} \\ 1.64 & 5.50 & 1 \frac{1}{2}-1 \frac{1}{2}\end{array}$ $6861.34 \quad \mathrm{~B} \quad\left(\begin{array}{l}3 \\ 3\end{array}\right) \quad 5.34 \quad 7.14 \quad 4-5 \quad \pi^{3} F^{\circ} e^{5} \mathrm{C}$ $\begin{array}{llllll}7220.79 & B & \text { (3) } 5.34 & 7.05 & 4-3 & -350-13 \mathrm{D}\end{array}$

Neasures Inadequate for preparation of 11st of

$\begin{array}{lllllll}5782.132 & \text { A } & 300 & 1.38 & 3.80 & 23-1 & 48 \\ 5700.240 & \text { A } & 30 & 1.64 & 3.77 & 13 & \text { (2) }\end{array}$
I A Raboratory Int

\section{Cu I continued}

$\begin{array}{lllllll}8092.634 & \text { A } & 300 & 3.80 & 5.33 & 1 \frac{1}{2}-\frac{1}{3} & 4^{2} \mathrm{P} 0-5^{2} \mathrm{~S} \\ 7933.130 & \text { A } & 160 & 3.77 & 5.33 & \frac{1}{2}-\frac{1}{2} & (6)\end{array}$

$\begin{array}{rrrrrrr}5218.202 & \text { A } & 80 & 3.80 & 6.17 & 1 \frac{1}{2}-2 \frac{1}{3} & 4^{2} \mathrm{P} 0 \\ 5 & -4^{2} \mathrm{D}\end{array}$

$\begin{array}{lll}5153.235 & \text { A } & 100 \\ 5220.070 & \text { A }\end{array}$

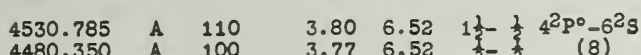

Qu II I P 30.18 Anal A L1st D May 1942

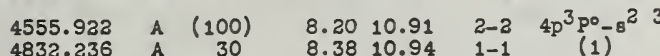

$\begin{array}{rrrrrr}4832.236 & \mathrm{~A} & 30 & 8.38 & 10.94 & 1-1\end{array}$

$\begin{array}{cccccc}4758.421 & \text { A } & (75) & 8.20 & 10.94 & 2-1 \\ 40 & 8.38 & 10.98 & 1-0\end{array}$

$\begin{array}{llllll}4889.690 & \text { A } & 30 & 8.38 & 10.91 & 1-2 \\ 5060.635 & \text { A } & 30 & 8.50 & 10.94 & 0-1\end{array}$

3686.555 A $\overline{(100)} \quad \overline{8.45} 11.80 \quad 3-4 \quad 4 p^{3} F_{(2)}^{\circ}-8^{2} 1 G$

$4043.502 A \quad 75 \quad \frac{8.75}{11.80} 3-4 \quad 4 p^{3} \mathrm{p}^{\circ}-8^{2} 1 \mathrm{G}$

$\begin{array}{lllllll}4671.686 & \text { A } & 40 & 14.14 & 16.78 & 1-2 & 4 \mathrm{~d}^{3} \mathrm{~S}-4 \mathrm{P}^{3} \mathrm{P}^{\circ}\end{array}$

$\begin{array}{llllll}4681.990 & \text { A } & 50 & 14.14 & 16.7 ? & 1-1 \\ 4673.555 & \text { A } & 30 & 14.14 & 16.78 & 1-\end{array}$

4909.736 A $100 \quad 514.27 \quad 16.78$ 5-6 $4 \mathrm{~d}^{3} \mathrm{G}-4 \mathrm{P}^{3} \mathrm{H}^{\circ}$

$\begin{array}{rrrrrr}4931.653 & \text { A } & 100 & 14.28 & 16.78 & 4-5 \\ 4918.373 & \text { A } & 30 & 14.54 & 17.05 & 3-4\end{array}$

$\begin{array}{llllll}4918.373 \quad A \quad 30 & 14.54 & 17.05 & 3-4\end{array}$

$4985.503 \quad A \quad 40 \quad 14.33 \quad 16.81 \quad 3-4 \quad 4 d^{3} D-4 r^{3} F^{\circ}$

$\begin{array}{llllll}5088.260 & \text { A } & 30 & 14.37 & 16.79 & 2-3 \\ 4937.196 & \text { A } & 20 & 14.56 & 17.06 & 1-2\end{array}$

5051.778 A $\quad 60 \quad 14.3716 .81 \quad 4-5 \quad 4 d^{3} F-4 r^{3} G^{\circ}$

$\begin{array}{lllllll}5012.611 & \text { A } & 20 & 14.36 & 16.82 & 3-4 & \text { (7) } \\ 5067.082 & \text { A } & 30 & 14.63 & 17.07 & 2-3 & \end{array}$

$4812.940 \quad A \quad 40 \quad \overline{14.47} 17.04 \quad 1-2 \quad 4 \mathrm{~d}^{1} \mathrm{P}-41^{1} \mathrm{D}^{\circ}$

$4953.733 \quad$ A $50 \quad 14.55 \quad 17.04 \quad 4-5 \quad 4 \mathrm{~d}^{1} \mathrm{G}-4 \mathrm{I}^{1} \mathrm{H}^{\circ}$

5006.787 A $30 \quad \overline{14.59} \quad 17.05 \quad 2-3 \quad 4 \mathrm{~d}^{1} \mathrm{D}-4 \mathrm{I}^{1} \mathrm{~F}^{\circ}$

$5065.448 \quad A \quad 40 \quad 14.6317 .06 \quad 3-4 \quad 4 \mathrm{~d}^{1} \mathrm{~F}-4 \mathrm{I}^{1} \mathrm{G}^{\circ}$

Zn I I P 9.35 Anal A L1st B Vay 1942

$3075.901 \quad$ A $\quad 90 \quad 0.00 \quad 4.01 \quad 0-1 \quad 4^{1} S-4^{3} P^{0}$

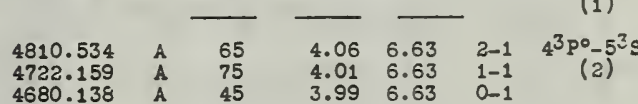

$4292.885 \quad A \quad 8 \quad 4.01 \quad 6.89 \quad 1-0 \quad 4^{3} p^{0}-5^{1} s$

$\begin{array}{lllllll}3345.020 & A & 150 & 4.06 & 7.75 & 2-3 & \left.4^{3} \mathrm{P}^{\circ}-4\right)^{3}\end{array}$

$\begin{array}{llllllc}3302.588 & \text { A } & 150 & 4.06 & 7.75 & 2-3 & 438 \\ 3.4 & 7.75 & 1-2 & (4)\end{array}$

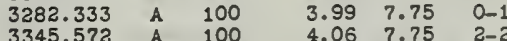

3302.941 A $125 \quad 4.01 \quad 7.75 \quad 1-1$

$\begin{array}{llllll}3345.934 & A & 30 & 4.06 & 7.75 & 2-1\end{array}$

$\begin{array}{lllllll}3072.062 & A & 70 & 4.06 & 8.08 & 2-1 & 4^{3} P^{0}-6^{3} S\end{array}$

$\begin{array}{llllll}3035.781 & \text { A } & 35 & 4.01 & 8.08 & 1-1 \\ 3018.352 & \text { A } & 30 & 3.99 & 8.08 & 0-1\end{array}$

6362.347 A $100 \quad-5.77 \quad 7.71 \quad 1-2 \quad 4^{1} \mathrm{P}^{\circ}-4^{1} \mathrm{D}$

$\begin{array}{lllllll}5181.995 \quad A \quad 30 & 5.77 & 8.15 & 1-0 & 4^{1} p^{0}-6^{1 S}\end{array}$

$4629.814 \quad A \quad 12 \quad 5.77 \quad 8.44 \quad 1-2 \quad 4^{1 \mathrm{Po}^{(7)}}$

$\begin{array}{lllllll}4113.210 \quad A \quad 12 & 5.77 & 8.77 \quad 1-2 & 4^{1} P^{\circ}-6^{1} \mathrm{D}\end{array}$

6928.319 A $10 \quad 6.63 \quad 8.41 \quad 1-2 \quad 5^{3} \mathrm{~g}-6^{3} \mathrm{p}^{\circ}$

\begin{tabular}{llllll}
6938.472 & A & 6 & 6.63 & 8.40 & $1-1$ \\
6943.202 & A & 2 & 6.63 & 8.40 & $1-0$ \\
\hline
\end{tabular}

2n II I P 17.89 Anal A L18t $C$ Vay 1942

$\begin{array}{lllllll}7478.79 \quad B \quad 20 & 3.09 & 7.74 & 1 \frac{1}{2}-2 \frac{1}{2} & 4^{2} P^{\circ}-d^{9}{ }_{8} 2 D\end{array}$

$\begin{array}{llllll}5894.351 & \text { A } & 20 & 5.98 & 8.08 & \frac{1}{3}-1 \frac{1}{2} \\ 6214.58 & \mathrm{~B} & 12 & 6.09 & 8.08 & 1 \frac{1}{2}-1 \frac{1}{2}\end{array}$

\section{$-15-$}

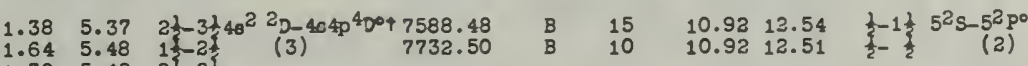

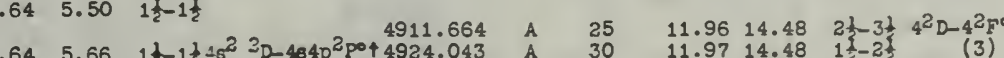




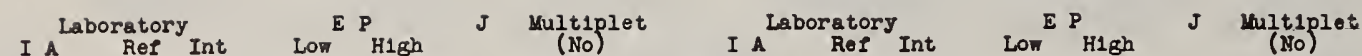
Ge I I P 5.97 Anal A L1st B May 1943

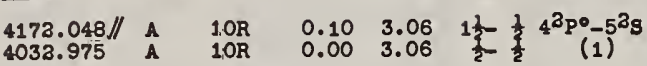
Qe II See introduction

Qe I I P 8.09 Anal A L1st B June 1942 $\begin{array}{lllllll}3124.817 & B & (30) & 0.88 & 4.83 & 2-3 & 4 \mathrm{p} .1 \mathrm{D}-5 \mathrm{~B}^{3} \mathrm{p} \bullet\end{array}$ $\begin{array}{llllllc}3269.494 & B & (40) & 0.88 & 4.65 & 3-1 & (1) \\ 3039.064 & B & (60) & 0.88 & 4.94 & 3-1 & 4 F^{1} D-581 P^{2}\end{array}$

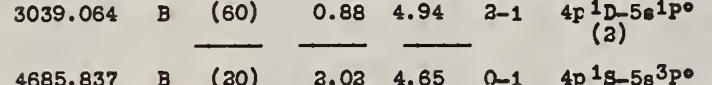

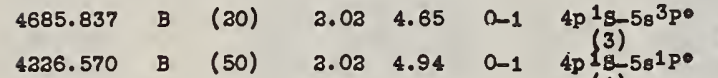

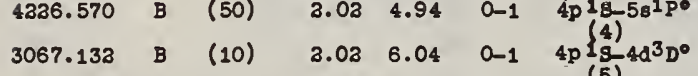

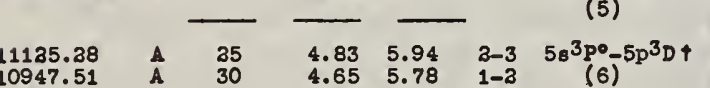
$\begin{array}{lll}11125.28 & \text { A } & 25 \\ 10947.51 & \text { A } & 30 \\ 10734.14 & \text { A } & \end{array}$ $\begin{array}{lll}4.65 & 5.78 & 1-3\end{array}$ $4.83 \quad 6.03 \quad 2-3 \quad 5 s^{3} p^{0}-5 p^{3} p+$ 10405.05
0625.73 $\begin{array}{cccc}4.83 & 6.02 & 2-2 & 5 s^{3} p^{0}-5 p \\ 4.65 & 5.94 & 1-1 & (7)\end{array}$

\section{-}

De II I P 15.9 Anal A List B Lay 1943

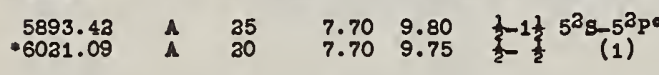

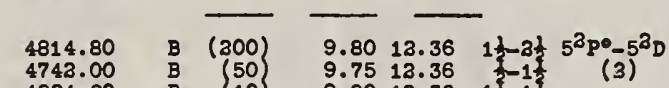

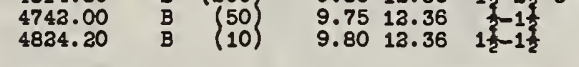

\section{AB I I P 10 Anal B L18t B Hay 1943}

3032.8 3119.6 9923.03 10023.9
9833.76 8654.1 8564.7
8541.6
9836.69 9826.69 9367.39
8831.76 9597.9 $\begin{array}{lll}8869.69 & \text { A } & 100 \\ 8428.94 & \text { A } & 100\end{array}$ 9300.63 8305.62

Br II See introduotion

Kr I See Introduction

Kr II See introduction

Rb I I P 4.16 Anal A L1st C lay 1943

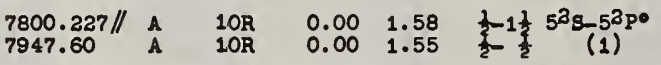

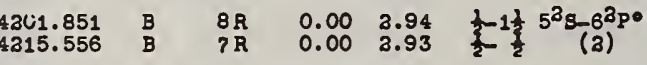

Rb II See introduction

SF I I P 5.67 Anal A L1st C Lay 1943 $6892.585 \quad A \quad 300 \quad 0.00 \quad 1.79 \quad 0-1 \quad 5^{1} \mathrm{~s}-5^{3} \mathrm{po}$ $4607.331 / / A \quad 600 \mathrm{R} \quad 0.00 \quad 3.68 \quad 0-1 \quad 518-51 \mathrm{po}$ $\begin{array}{lllllll}7070.071 & \text { A } & 2000 & 1.84 & 3.58 & 2-1 & 5^{3} \mathrm{P}^{\circ}-6^{3} \mathrm{~g} \\ 6878.313 & \mathrm{~A} & 1000 & 1.79 & 3.58 & 1-1 & (3) \\ 6791.038 & \mathbf{A} & 500 & 1.77 & 3.58 & 0-1 & \end{array}$ $\begin{array}{lllllll}4962.263 \quad A & 40 & 1.84 & 4.33 & 2-3 & 5^{3} \mathrm{P} & -5^{3} \mathrm{D}\end{array}$ .4873 .493 4967.944 4876.06
4971.668

4811.881 4784.320
4876.325 -4832.075
4722.278
4741.923 4438.044
4361.710
4326.445 $\begin{array}{lll}4361.710 & A & 35 \\ 4336.445 & A & \end{array}$ 3351.346 3323.231
3366.333
3339.988 3329.988
3307.534 3307.534
3301.734 A 100

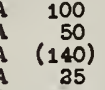
$2.30 \quad 6.37 \quad 1 \frac{1}{3}-1 \frac{1}{3} 4 \mathrm{p}^{2} \mathrm{P} 0-5 \mathrm{~s}^{4} \mathrm{P}$ $\begin{array}{lll}3.30 & 6.26 & \\ 3.36 & 1 \frac{2}{2}\end{array}$

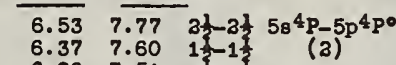
6.367 .51 ?

4
$(3)$ $\begin{array}{lll}6.53 & 7.96 & 31-3 \\ 6.37 & 7.81 & 13 \\ 6.26 & 7.70 & 3\end{array}$ $\begin{array}{lll}6.26 & 7.70 & 1 \\ 6.53 & 7.81 & 2 \frac{3}{3}\end{array}$ $6.37 \quad 7.70 \quad 13-1$ A (100) $6.37 \quad 7.66 \quad 1 \frac{1}{2}-\frac{1}{2}$ $6.53 \quad 7.93 \quad 2 \frac{1}{3}-3 \frac{1}{2} 58^{4} \mathrm{P}-5 \mathrm{p}^{2} \mathrm{D}^{0}+$ $\begin{array}{llll}6.53 & 7.86 & 2 \frac{1}{2}-1 \frac{1}{2} & 58^{4} \mathrm{P}-5 \mathrm{p}^{4} \mathrm{~g}^{\circ} \\ 6.37 & 7.86 & 1 \frac{1}{2}-1 \frac{2}{2} & (5)^{2}\end{array}$ 6408.463 A 100 6503.989 6546.791 6643.536

5480.865
5504.184 5504.184
5531.785

$\begin{array}{lll}1.84 & 4.37 & 2-1 \\ 1.79 & 4.34 & 1-0\end{array}$

$\begin{array}{lll}1.79 & 4.40 & 1-3 \\ 1.77 & 4.37 & 0-1\end{array}$

$\begin{array}{llll}1.84 & 4.63 & 3-1 & 5^{3} \mathrm{po}^{\circ}-7^{3} \mathrm{~S} \\ 1.79 & 4.63 & 1-1 & (8)\end{array}$

$\begin{array}{lll}1.84 & 5.51 & 2-1 \\ 1.79 & 5.50 & 1-0\end{array}$

\begin{tabular}{lll}
1.79 & 5.52 & $1-2$ \\
1.77 & 5.51 & $0-1$ \\
\hline
\end{tabular}

$\begin{array}{lll}3.25 & 4.15 & 2-3 \\ 3.24 & 4.11 & 1-3\end{array}$

(8)

As II I P 19.78 Anal B L1st C June 1943 $\begin{array}{lllllll}6170.47 & \text { A } & 10 & 9.77 & 11.77 & 1-1 & 5 \mathrm{~s}^{3} \mathrm{po}^{\circ}-5 \mathrm{p}^{1} \mathrm{P} \\ 6023.81 & \mathrm{~A} & 10 & 9.73 & 11.77 & 0_{-1} & (1)\end{array}$ 534.794
5540.051 5450.836 5486 4891.980
4868.700 $\begin{array}{lll}5651.53 & \text { A } & 10 \\ 5558.31 & \text { A } & 10\end{array}$

5331.54 4985.60 5105.8
4552.37 \begin{tabular}{rr}
$A$ & 10 \\
$A$ & 8 \\
\hline
\end{tabular} $10.0612 .25 \quad 2-3 \quad .58^{3} p^{0}-5 p^{3} D+5156.040$ $10.06 \quad 12.38 \quad 2-2 \quad 58^{3} \mathrm{po}^{\circ}-5 \mathrm{p}^{3} \mathrm{p}+6550.344$

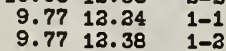
$(3)$

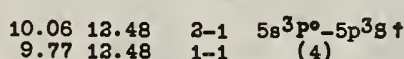

$\begin{array}{lllllll}5110.30 & \mathrm{~A} & 10 & 10.23 & 12.34 & 1-1 & 58^{1} \mathrm{PO}^{\circ}-5 \mathrm{p}^{3} \mathrm{P} \uparrow \\ 5107.80 & \mathrm{~A} & 10 & 10.22 & 12.64 & 1-2 & 5 \mathrm{~s}^{100} \mathrm{PO}^{\circ}-5 \mathrm{p}^{1} \mathrm{D}\end{array}$

$\begin{array}{rllllll}452.35 \quad A & 10 & 10.32 & 13.06 & 1-0 & 581 \mathrm{po}^{\circ}-5 p^{1 \mathrm{~s}} \\ & & & \end{array}$

Se I I P 9.71 Anal B L1st C June 1943

89

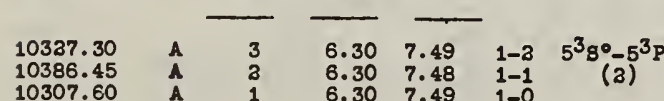

Sr II I P 10.98 Anal \& L1st A Kay 1943

$\begin{array}{lllllll}4077.714 / / & A & 400 \mathrm{r} & 0.00 & 3.03 & \frac{1}{2}-1 \frac{1}{5} 5^{2} \mathrm{~g}-5^{2} \mathrm{p} 0 \\ 4315.534 & A & 300 \mathrm{r} & 0.00 & 3.93 & \frac{3}{2}-\frac{2}{2} & (1)\end{array}$

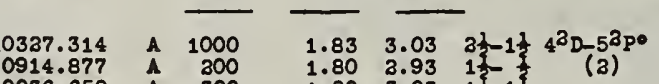
$\begin{array}{lllllll}10914.877 & \mathrm{~A} & 200 & 1.80 & 3.93 & 1 \frac{3}{3} & \text { (a) } \\ 10036.658 & \mathrm{~A} & 300 & 1.80 & 3.03 & 1 \frac{1}{2}-1 \frac{3}{2} & \end{array}$ $\begin{array}{lllllll}4305.447 & A & 40 & 3.03 & 5.89 & 1 \frac{1}{3} & 5^{2} \mathrm{PO}^{\circ}-6^{2} \mathrm{~g} \\ 4161.796 & A & 30 & 3.93 & 5.89 & \frac{1}{2} & (3)\end{array}$ $3464.157 \quad$ A $\quad 50 \quad 3.03 \quad 6.59 \quad 1 \frac{1}{2} \quad 5^{2} \mathrm{PO}^{0}-5^{2} \mathrm{D}$

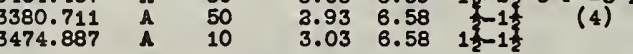

I I P 6.5 Anal A L1st C June 1943
6584.89
6557.40 657.40
6793.71 $6687.5 \%$ 8933.55

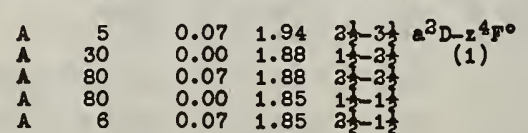

I A Reboratory Int

Lor ${ }^{\text {E }}{ }_{\text {H1gh }}$

J (Noltiplot

\section{$\underline{\text { Y }}$ continued}

$\begin{array}{lllllll}6435.03 & \text { A } & 500 & 0.07 & 1.98 & 2 \frac{1}{3}-2 \frac{1}{3} & a^{2} D-z^{2} D^{\circ} \\ 6191.73 & \text { A } & 1009 & 0.00 & 1.99 & 1 \frac{1}{3}-1 \frac{1}{3} & (3)\end{array}$ $\begin{array}{llcllll}6402.005 & \text { B } & 50 & 0.07 & 1.99 & 33 \\ 6232.59 & \text { A } & 508 & 0.00 & 1.98 & 1 \frac{1}{2}-2\end{array}$

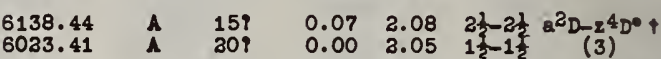

$4674.84 \quad A \quad 135 \quad 0.07 \quad 3.71 \quad 21-3 \frac{1}{3} a^{2} D_{-2}{ }^{2} F^{\circ}$

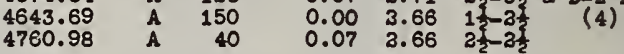

4138.31 143.86 4335.94
4039.83

4174.14 4047.64
4083.71

4103.38 4077.38

3620.95

3592.93
3553.70

3021.74

3996.94

3045.36
3005.36

A 300

$\begin{array}{llll}0.07 & 3.05 & 2 \frac{1}{2}-2 \frac{1}{2} & a^{2} \mathrm{D}-\mathrm{y}^{2} \mathrm{p}^{0} \\ 0.00 & 3.98 & 1 \frac{1}{2}-1 \frac{1}{2} & (5)\end{array}$

$\begin{array}{lll}0.07 & 3.98 & 21 \\ 0.00 & 3.05 & 1 \frac{1}{2}-2 \frac{1}{2}\end{array}$

$0.07 \quad 3.02 \quad 2 \frac{1}{3}-1 \frac{1}{3} a^{2} \mathrm{D}-\mathrm{y}^{3} \mathrm{po}$

$\begin{array}{lll}0.00 & 3.05 & 1 \\ 0.00 & 3.02 & 1\end{array}$

$0.07 \quad 3.07 \quad 3 \frac{1}{3}-3 \frac{1}{2} a^{2} \mathrm{D}-\mathrm{y}^{2} \mathrm{~F}^{0}$

$0.00 \quad 3.03 \quad 13 \frac{13}{0.07}$

$0.07 \quad 3.47 \quad 21-1 \frac{1}{3} a^{3} D-x^{300}$

$\begin{array}{llll}0.07 & 3.47 & 21-1 & \\ 0.00 & 3.43 & 13 & (8)\end{array}$

$0.07 \quad 4.15 \quad 2 \frac{1}{3}-3 \frac{1}{2} a^{2} p-y^{4} D^{0}$

$0.00 \quad 4.13 \quad 130.37$

$\begin{array}{lll}0.07 & 4.13 & 3 \\ 0.00 & 4.11 & 1\end{array}$

$0.07 \quad 4.30 \quad 2 \frac{1}{3}-3 \frac{2}{2} a^{2} \mathrm{p}-x^{3}$

$0.00 \quad 4.15 \quad \frac{1}{3}-2 \frac{3}{2}(10)$

3974.59

$\hat{A} \quad 13$

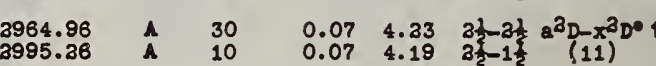

$\begin{array}{lllcl}1.84 & 4.40 & 2-2 & 5^{3} \mathrm{P}^{0}-5 \mathrm{p}^{3} & 3 \mathrm{p} \\ 1.79 & 4.37 & 1-1 & (5) & \end{array}$

$\begin{array}{lll}1.79 & 4.62 & 1-1 \\ 1.77 & 4.62 & 0-1\end{array}$

$.5466 .46 / /$

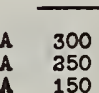

$1.43 \quad 3.68 \quad 4 \frac{1}{3}-5 \frac{1}{2} 2^{4} \mathrm{~F}-2^{4} \sigma^{\circ}+$

5581.87

5630.14

4839.87

4845.67
4852.69

4859.84

4906.11
4893.44

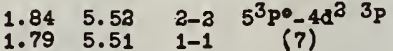

4781.04

4799.30
4819.64

4537.35

4527.80

$\begin{array}{llllll}3.26 & 4.19 & 3-4 & 4^{3} p-4 a 5 p^{3} r & 4505.95 \\ 4487.47\end{array}$

4475.72
4487.28

4477.45

$\begin{array}{llllll}3.26 & 4.51 & 3-3 & 4^{3} p-4 d 5 p^{3} p^{\circ} \quad 4513.58\end{array}$

$\begin{array}{lllll}3.25 & 4.49 & 3-3 & (9) & 4581.32\end{array}$

$\begin{array}{lll}3.26 & 4.49 & 3-2\end{array}$

$\begin{array}{lll}3.35 & 4.51 & 2-3 \\ 3.34 & 4.49 & 1-3\end{array}$

6845.24

6845.24
6950.32

$\begin{array}{ll}\mathbf{A} & 150 \\ \mathbf{A} & 100\end{array}$

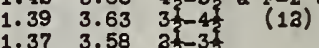

$1.35 \quad 3.54 \quad 1 \frac{1}{2}-2 \frac{1}{2}$

$1.42 \quad 3.97 \quad 4 \frac{1}{3}-4 \frac{1}{3} a^{4} 7-5^{4} 7^{\circ}$

1.39 3.94 $31-3 \frac{1}{2} \quad(13)$

1.35 3.89 11.1

$1.43 \quad 3.94$ 43 3 .

$1.37 \quad 3.89$

$\begin{array}{lll}1.39 & 3.97 \quad 31 & -1\end{array}$

$\begin{array}{lll}1.37 & 3.94 & 2 \frac{1}{2}-3 \frac{1}{2} \\ 1.35 & 3.91 & 1 \frac{1}{2}-2 \frac{1}{2}\end{array}$

$1.43 \quad 4.15 \quad 4 \frac{1}{3}-3 \frac{1}{2} a^{4} T-y^{400}+$

$1.39 \quad 4.12 \quad 353 \frac{1}{3} \quad(14)$

$1.37 \quad 4.11 \quad 33-1 \frac{1}{3}$

1.39 4.15 13

$\begin{array}{lll}1.37 & 4.12 & 3 \\ 1.35 & 4.11 & 1\end{array}$

1.35

$\begin{array}{lll}1.89 & 4.63 & 32-2 \frac{1}{2} \mathrm{a}^{2} \mathrm{~F}-\mathrm{y}^{4} \mathrm{p}^{\circ} \\ 1.89 & 4.59 & 31\end{array}$

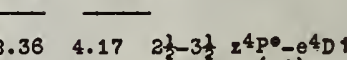

$3.26 \quad 4.78 \quad 3-4 \quad 4^{3} p-43 r^{\circ} \quad$ Strongest Unclassifled L1nes of $\underline{y} I$

$\begin{array}{lllll}3.24 & 4.78 & 1-3 & & 3587.75 \\ 3.2 & \end{array}$

$\begin{array}{rrrr}3434.16 & \text { A } & \text { AO } & \text { III } \\ 3278.43 & \text { I } & \text { IIIA }\end{array}$

\begin{tabular}{rrrr}
3278.43 & $A$ & 5 & IIIA \\
3091.70 & A & 15 & III \\
\hline
\end{tabular}

$Y$ II I P 12.3 Anal A L1st A June 1943

$\begin{array}{lllllll}4204.69 \quad A & 10 & 0.00 & 2.94 & 0-1 & a^{1} s_{-2} 3 p^{2}\end{array}$ $3633.13 \quad$ A $200 \quad 0.00 \quad 3.40 \quad 0-1 \quad a^{1} s_{-2}(1)$

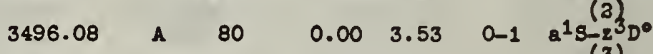
$\begin{array}{llllllll}3113.05 & A & 4 & 0.00 & 3.97 & 0-1 & 2^{1} 8 \begin{array}{l}(3) \\ (4)\end{array}\end{array}$

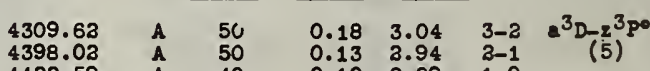
$\begin{array}{llllll}4398.03 & \mathbf{A} & 50 & 0.13 & 3.94 & 3-1 \\ 4423.59 & \mathbf{A} & 40 & 0.10 & 2.89 & 1-0 \\ 4335.73 & \mathbf{A} & 30 & 0.13 & 3.04 & 3-2\end{array}$ $\begin{array}{llrrrr}4235.73 & \text { A } & 30 & 0.13 & 3.04 & 3-2 \\ 4358.73 & \text { A } & 30 & 0.10 & 3.94 & 1-1 \\ 4199.27 & \text { A } & 5 & 0.10 & 3.04 & 1-2\end{array}$

$\begin{array}{llllll}4047.88 \quad P \quad & 0.18 & 3.23 & 3-3 & a^{3} D_{-2} 1 D^{\circ}\end{array}$

$\begin{array}{llllll}3982.59 & \text { A } & 150 & 0.13 & 3.23 & 3- \\ 3950.35 & \text { A } & 200 & 0.10 & 3.23 & 1-\end{array}$

$3710.30 / /$ A 500 3774.33

3788.70

3832.89
3818.34

3818.34
3878.28

3776.56

$0.18 \quad 3.51$

$\begin{array}{ll}0.13 & 3.40 \\ 0.10 & 3.36 \\ 0.18 & 3.40\end{array}$

$\begin{array}{lll}0.10 & 3.36 & 1-2 \\ 0.18 & 3.40 & 3\end{array}$

$3-4 a^{3} D-z^{3} r^{0}$

3747.55 $\begin{array}{lll}0.13 & 3.36 & 3-2 \\ 0.18 & 3.36 & 3-2\end{array}$

$0.13 \quad 3.40 \quad 3-1 \quad a^{3} \mathrm{D}-\mathrm{z}^{1} \mathrm{Pe}^{0}$ 


\section{:} II continued

$\begin{array}{ll}3600.74 & \text { A } \\ 3611.06 & \text { A } \\ 3601.93 & \text { A } \\ 3664.62 & \text { A } \\ 3628.71 & \text { A } \\ 3549.02 & \text { A } \\ 3584.53 & \text { A } \\ 3242.30 & \text { A } \\ 3216.70 & \text { A } \\ 3303.33 & \text { A } \\ 3300.28 & \text { A } \\ 3195.63 & \text { A } \\ 3179.43 & \text { A } \\ 3135.17 & \text { A }\end{array}$

$\begin{array}{cc}\mathbf{A} & 300 \\ \mathbf{A} & 200 \\ \mathbf{A} & 100 \\ \mathbf{A} & 150 \\ \mathbf{A} & 100 \\ \mathbf{A} & 100 \\ \mathbf{A} & 100 \\ \mathbf{A} & 150 \\ \mathbf{A} & 100 \\ \mathbf{A} & 60 \\ \mathbf{A} & 5 \\ \mathbf{A} & 5\end{array}$

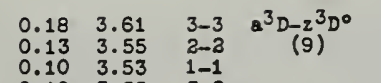

$\begin{array}{lll}0.10 & 3.53 & 1-1 \\ 0.18 & 3.55 & 3-2 \\ 0.13 & 3.53 & 2-1\end{array}$

$\begin{array}{llll}0.13 & 3.61 & 2-3\end{array}$

$0.18 \quad 3.99 \quad 3-3 \quad a^{3} \mathrm{D}-\mathrm{y}^{3} \mathrm{po}$

0.13 3.97 $2-1$ (10)

$0.13 \quad 3.99 \quad 2-3$

$\begin{array}{lll}0.13 & 3.99 & 20 \\ 0.10 & 3.97 & 1-1\end{array}$

$0.10 \quad 3.99$

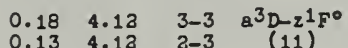

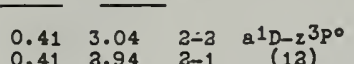

882.33
4881.44
4374.94

4374.94

$0.41 \quad 3.23 \quad 2-3 \quad a^{1} D_{-2} D^{1} D^{0}$

$\begin{array}{llll}0.41 & 3.40 & a-3 & a^{1} D_{-2} F^{\circ} \\ 0.41 & 3.36 & a-2 & (14)\end{array}$

$0.41 \quad 3.40 \quad 3-1 \quad \mathrm{Q}^{1} \mathrm{D-z} \mathbf{2}^{1 \mathrm{PO}}$

$\begin{array}{llll}0.41 & 3.61 & 2-3 & \mathbf{a}^{1} \mathrm{D}-23 \mathrm{D}^{\circ} \\ 0.41 & 3.55 & 3-2 & (16)\end{array}$

$\begin{array}{lll}0.41 & 3.55 & 3-2 \\ 0.41 & 3.53 & a-1\end{array}$

4127.5

3857.36

3951.59

$\begin{array}{ll}3448.82 & \text { A } \\ 3467.88 & \text { A }\end{array}$

$\begin{array}{llll}0.41 & 3.99 & 2-3 & 1 p-y^{3} p o \\ 0.41 & 3.97 & a-1 & (17)\end{array}$

$0.41 \quad 4.13 \quad 2-3 \quad Q^{1} \mathrm{D}_{-1} \mathrm{~F}^{\circ}$

100

$\frac{0.41}{1.03} \frac{4.13}{3.23}$

${ }_{(18)}^{1}$

5610.36

5087.42

5205.73

5300.42
5330.78

5389.8

4982.13
5119.12

5123.2

4883.6

4854.87
4786.58

4823.31
4713.26

4173.78

4064.9

3997.4

7450.3

7406.33

6613.7

-6795.4

6833.45

6896.0

5497.42

5521.56
5546.02

5544.61
$5473.4 C$

5480.75

5196.

3380 .

8835.85

8066.20

7881.90

sa.t.

7193.74
7364.19

5728.91

5781.69

3402.7

7388.4

7388.46
5668.95

3225.

$\begin{array}{llll}1.03 & 3.99 & 3-3 & a^{3} F-y^{3} p \\ 1.08 & 4.13 & 4-3 & a^{3} F-z^{2} F^{\circ}\end{array}$

$\begin{array}{llll}1.08 & 4.12 & 4-3 & a^{3} F-2 \\ 1.03 & 4.18 & 3-3 & (34)\end{array}$

$0.99 \quad 4.12 \quad 2-3$

$\begin{array}{lllc}1.74 & 3.40 & 2-1 & a^{3} P-2^{1} P^{\circ} \\ 1.73 & 3.40 & 1-1 & (35)\end{array}$

$1.74 \quad 3.61 \quad 2-3 \quad Q^{3} P-2^{3} D^{\circ}$

$\begin{array}{rrrc}1.74 & 3.61 & 2-3 & a^{3} P-2^{3} D \\ 1.73 & 3.55 & 1-2 & (26)\end{array}$

$\begin{array}{lll}1.71 & 3.53 & 0-1 \\ 1.74 & 3.55 & 2-2\end{array}$

$\begin{array}{lll}1.74 & 3.55 & 2-3 \\ 1.73 & 3.53 & 1-1\end{array}$

1.743 .97

$1.73 \quad 3.96 \quad 1-0$

$\begin{array}{lll}1.73 & 3.99 & 1-3 \\ 1.71 & 3.97 & 0-1\end{array}$

$1.74 \quad 4.13 \quad 3-3 \quad a^{3} P-z^{1} F^{\circ}$

$1.74 \quad 5.50 \quad 2-1 \quad \begin{gathered}3 P^{3}-y^{1} \\ (29)\end{gathered}$

$1.83 \quad 3.33 \quad 3-3 \quad b^{1} D_{-2}^{1} D^{\circ}$

$1.83 \quad 3.36 \quad 2-2 \quad b^{1} D_{-2}^{(30} F^{\circ}$

$1.83 \quad 3.40 \quad 2-1 \quad b^{1} D_{2}^{(31)}$

$\begin{array}{lllc}1.83 & 3.61 & 2-3 & b^{1} D_{2} 3 D^{3} \\ 1.83 & 3.55 & 2-2 & (33)\end{array}$

$\begin{array}{llll}1.83 & 3.55 & 2-2 & (33) \\ 1.83 & 3.53 & 2-1 & \end{array}$

$1.83 \quad 3.99 \quad 3-3 \quad b^{1} D-y^{3} P^{\circ}$

$\begin{array}{llllll}A & 51 & 1.83 & 3.97 & 3-1 & (34)\end{array}$

$A$ 30nl $1.83 \quad 5.50 \quad 2-1 \quad b^{1} \frac{D-y 1 p^{\prime}}{(36)}$

A 1 1.94 $3.61 \quad 4-3 a^{1} a_{-2}{ }^{3} D^{\circ}$

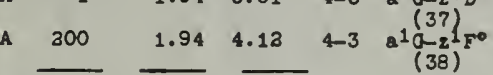

$5 n 1 \quad 3.04 \quad 6.87 \quad 2-3 \quad 2^{3} P^{0}-0^{3} \mathrm{D}$
REVISED MULTIP LET TA B L E

I A Reforatory Int Low ${ }_{\text {High }}^{\text {Lab }}$ Jultiplet $_{\text {(No) }}$ Y II continued

$\begin{array}{llrrrrr}3461.0 & \text { A } & \text { 20nl } & 3.23 & 6.79 & 2-2 & z^{1} D^{0}-e^{3} \mathrm{D}\end{array}$ $3380.114 \quad C \quad 5 n I \quad 3.23 \quad 6.88 \quad 2-2 \quad z^{1} D^{0}-e^{1} D$ $\begin{array}{lllllll}3086.858 & C & 30 \mathrm{nl} & 3.23 & 7.23 & 2-3 & \mathrm{z}^{1} \mathrm{D}^{0}-\mathrm{e}^{1 \mathrm{~F}}\end{array}$ $3069.36 \quad A \quad 5 n \quad 3.23 \quad 7.25 \quad 2-1 \quad 2^{1} D^{0}-f^{3} D$ $\begin{array}{llllllll}3026.47 & C & 10 \mathrm{nl} & 3.23 & 7.31 & 2-3 & \mathrm{z}^{1} \mathrm{D}^{\circ}-\mathrm{e}^{3 \mathrm{O}}\end{array}$ $2978.18 \quad A \quad 3 n \quad 3.23 \quad 7.37 \quad 3-1 \quad z^{1} D^{\circ}-e^{1 P}$ $\begin{array}{lllllll}3668.489 & \text { C } & 50 \mathrm{nl} & 3.51 & 6.87 & 4-3 & \mathrm{z}^{3} \mathrm{~F}^{\circ}-\mathrm{e}^{3} \mathrm{D}\end{array}$ $\begin{array}{llrrrr}3605.46 & \mathrm{C} & \text { 10nl } & 3.36 & 6.78 & 3-1 \\ 3556.083 & \mathrm{C} & \text { 5nl } & 3.40 & 6.87 & 3-3\end{array}$

$3507.964 \quad C \quad 8 n l \quad 3.36 \quad 6.88 \quad 2-3 \quad z^{3} F^{\circ}-e^{1} D$

$3193.48 \quad A \quad 2 n l \quad 3.36 \quad 7.23 \quad 2-3 \quad 2^{3} F^{\circ}-e^{1 F}$

$\begin{array}{llllll}3332.00 \quad A \quad 3 n & 3.51 & 7.32 & 4-3 & 2^{3} \frac{F}{0}^{\circ}-f^{3} \mathrm{D}\end{array}$

$\begin{array}{llllll}3182.42 & \text { A } & 3 \mathrm{nl} & 3.40 & 7.28 & 3-2 \\ 3144.37 & \text { A } & 3 \mathrm{n} & 3.40 & 7.32 & 3-3\end{array}$

$\begin{array}{lllll}3114.45 \text { A } 10 \mathrm{n} & 3.36 & 7.32 & 2-3 \text { ? }\end{array}$

$\begin{array}{ccccccc}-3110.65 & \text { A } & \text { an } & 3.40 & 7.37 & 3-21 & z^{3} F^{\circ}-e^{3 p} \\ 3081.600 & \text { C } & \text { an } & 3.36 & 7.37 & 2-3 & (50)\end{array}$

3173.07 A $100 \mathrm{nl} \quad 3.51 \quad 7.39 \quad 4-5 \quad z^{3} \mathrm{~F}^{\circ}-\mathrm{e}^{3} \mathrm{C}$

$\begin{array}{rrrrrrr}3129.933 & \text { C } & 40 \mathrm{nl} & 3.40 & 7.34 & 3-4 & (51) \\ 3138.789 & \text { C } & 20 \mathrm{nl} & 3.36 & 7.31 & 2-3 & \end{array}$

$3077.14 \quad A \quad 4 n \quad 3.36 \quad 7.37 \quad 2-1 \quad 2^{3} F^{\circ}-e^{1 P}$

$\begin{array}{lllllll}3001.43 & \mathrm{~A} & 3 & 3.36 & 7.47 & 2-2 & z^{3} \mathrm{~F}^{\circ}-\mathrm{P}^{1} \mathrm{D}\end{array}$

$\begin{array}{llrlllll}2980.69 & \text { C } & 30 n I & 3.51 & 7.65 & 4-4 & 2^{3} F^{0}-e^{3 F} \\ 3006.0 & \text { A } & \text { anl } & 3.51 & 7.61 & 4-3 & (54)\end{array}$

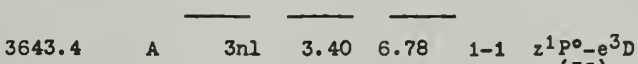

$\begin{array}{lllllll}3643.4 & A & 3 n I & 3.40 & 6.78 & 1-1 & z^{1} P^{0}-e^{3} D \\ 3544.001 & \text { C } & 3 n I & 3.40 & 6.88 & 1-3 & z^{1} P^{0}-e^{1 D} D\end{array}$

$\begin{array}{lllllll}3109.3 & \text { A } & \text { 1 } & 3.40 & 7.37 & 1-2 & z^{1} \mathrm{Po}_{-} \mathrm{e}^{3 \mathrm{P}} \\ 3160.60 & \text { A } & \text { In } & 3.40 & 7.30 & 1-1 & (57)\end{array}$

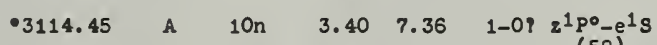

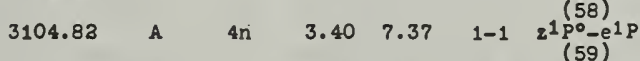

3037.75

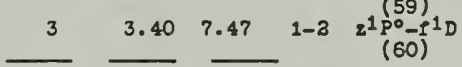

$\begin{array}{lllllll}3782.302 & C & 50 \mathrm{nl} & 3.61 & 6.87 & 3-3 & 2^{3} \mathrm{D}^{\circ}-\mathrm{e}^{3} \mathrm{D}\end{array}$

3800.883
3793.56

3873.308

$\begin{array}{llll}1.74 & 3.99 & 2-3 & a^{3} P-y^{3} P^{0} \\ 1.73 & 3.97 & 1-1 & (37)\end{array}$

\begin{tabular}{llllll}
\hline$n 1$ & 3.04 & 6.87 & $2-3$ & $2^{3} \mathrm{p}^{\circ}-0^{3} \mathrm{D}$ \\
$\mathrm{C}$ & $\mathrm{3n}$ & $\mathbf{3 . 9 4}$ & 6.79 & $1-3$ & $(39)$ \\
\hline
\end{tabular}
3714.3

3703.333

3409.87

3319.78

3308.4

3318.6
3333.606

3393.9

3282.51

3386.71
3312.39

3331.20

3304.01
3336.25

3313.40

3055.3

3036.59
3053.27

3082.16
3066.06

3050.5

$\begin{array}{llllllc}4279.3 & \text { A } & 5 n I & 3.99 & 6.87 & 2-3 & y^{3} \mathrm{P}^{\circ}-e^{3} \mathrm{D} \\ 4364.01 & \text { A } & \mathbf{l} & 3.97 & 6.79 & 1-2 & (70)\end{array}$

$4264.88 \quad A \quad$ in $\quad 3.99 \quad 6.88 \quad 2-2 \quad y^{3} \mathrm{p}^{0}-\mathrm{e}^{1 \mathrm{D}}$

$\begin{array}{lllllll}3848.194 & C & 8 \mathrm{nl} & 3.99 & 7.19 & 2-1 & \mathrm{y}^{3} \mathrm{po}^{\circ}-\mathrm{e}^{3 \mathrm{~S}} \\ 3834.78 & \mathrm{C} & 5 \mathrm{nl} & 3.97 & 7.19 & 1-1 & (72)\end{array}$ $\begin{array}{llllll}3834.78 & \mathrm{C} & 5 \mathrm{nl} & 3.97 & 7.19 & 1-1 \\ 3813.8 & \mathbf{A} & 2 \mathrm{nl} & 3.96 & 7.19 & 0-1\end{array}$

3808 .

$\begin{array}{llllllll}3696.6 & \text { A } & 35 n l & 3.99 & 7.33 & 2-3 & y^{3} \mathrm{p}^{0}-\mathrm{p}^{3} \mathrm{D}\end{array}$

$\begin{array}{llllll}3737.09 \quad C & \text { and } & 3.97 & 7.28 & 1-2 & (74)\end{array}$

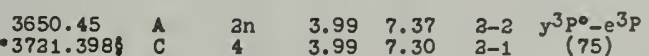

3731.3988
3689.2

3716.91

$5 n 1 \quad 3.55 \quad 6.87 \quad 3-3$

$5 n] \quad 3.53 \quad 6.88 \quad 1-3 \quad(63)$

4nl $3.61 \quad 7.23 \quad 3-3 \quad z^{3} D^{\circ}-e^{1}$
I A Ref Int

Low ${ }^{\text {P }}$ High

$\underset{(N O)}{\text { Multiplet }}$

$\underline{Y \text { II continued }}$

$\begin{array}{lllllll}3457.088 & C & 4 \pi l & 3.99 & 7.56 & 2-1 & y^{3} \mathrm{p}^{\circ}-\mathrm{f}^{3} \mathrm{~S} \\ 3429.42 & \mathrm{C} & 3 \mathrm{n} & 3.96 & 7.56 & 0-1 & (77)\end{array}$

$\begin{array}{rrrrrrc}3093.76 & \text { A } & 10 \mathrm{n} & 3.99 & 7.97 & 2-2 & \mathrm{y}^{3} \mathrm{p}^{\circ}-\mathrm{f}^{3} \mathrm{P} \\ -3110.65 & \mathrm{~A} & \mathrm{Zn} & 3.97 & 7.93 & 1-1 & (78)\end{array}$

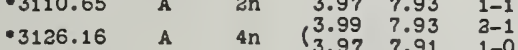

$\begin{array}{lllll}3078.64 \quad A & 4 n & 3.97 & 7.97 & 1-2\end{array}$

$\begin{array}{lllllll}3030.214 & C & 4 n & 3.99 & 8.06 & 2-3 & y^{3} P^{\circ}-F^{3} D\end{array}$

$4607.94 \quad A-5.126 .79 \quad 3-29 z^{1} F^{0}-e^{3} D$

$\begin{array}{lllllll}4465.4 \quad A & 10 \mathrm{nl} & 4.12 & 6.88 & 3-2 & z^{1} \mathrm{~F}^{\circ}-\mathrm{e}^{1} \mathrm{D}\end{array}$

$3967.69 \quad \mathrm{C} \quad 15 \mathrm{nl} \quad 4.12 \quad 7.23 \quad 3-3 \quad 2^{1} \mathrm{~F}^{\circ}-\mathrm{e}^{1 \mathrm{~F}}$

$\begin{array}{llllllll}3846.516 \quad C & 3 n & 4.12 & 7.32 & 3-3 & 21 \frac{18}{80}-5^{3} D\end{array}$

$\begin{array}{lllllll}3675.64 & C & 5 n 1 & 4.13 & 7.47 & 3-2 & 2^{1} 7^{0}-1^{1} \mathrm{D}\end{array}$

$\begin{array}{lllllll}3330.880 \quad \mathrm{C} & \mathrm{an} \mathrm{O} 1 & 4.12 & 7.83 & 3-4 & \begin{array}{c}(34) \\ 2^{1} \mathrm{~F}^{0}-e^{10} \\ (85)\end{array}\end{array}$

3896.804 C $\quad{ }_{10 n l} \quad 5.50 \quad 8.67 \quad 1-2 y_{(86)}^{1 P^{0}-h^{1} D}$

Strongest Unclassified Lines of $\underline{Y}$ II

$\begin{array}{llr}8429.36 & \text { A } & 10 n \\ 4734.52 & \text { A } & 5 n \\ 3407.7 & \text { A } & 3 n\end{array}$

Zr I I P 6.93 Anal A L1st C June 1943

$\begin{array}{lllllll}6832.93 & \text { A } & 12 & 0.07 & 1.88 & 3-3 & 8^{3} F-z^{5} G^{\circ} \\ 6762.38 & \text { A } & 30 & 0.00 & 1.83 & 2-2 & \end{array}$

6127.49 A $200 \quad 0.15 \quad 2.17 \quad 4-4 \quad a^{3} F-z^{3} F^{0}$

$\begin{array}{llllllc}6137.23 & \text { A } & 150 & 0.07 & 2.08 & 3-3 & a^{3}-2{ }^{3} \\ 6134.58 & \text { A } & 125 & 0.00 & 3.01 & 2-3 & (3)\end{array}$

6134.58
6407.03

6357.10

5885.61
5935.23

6062.88

5955.37

5879.79

5797.76
5735.70

4688.45

4633.99
4575.52

3916.64

3879.0

3849.26
3900.51

3989.29

$\begin{array}{lll}0.00 & 3.01 & 2-2 \\ 0.15 & 2.08 & 4-3\end{array}$

$\begin{array}{lll}0.07 & 2.01 & 3-2 \\ 0.07 & 2.17 & 3-4\end{array}$

$\begin{array}{rlll}8 & 0.07 & 2.17 & 3-4 \\ 10 & 0.00 & 2.08 & 2-3\end{array}$

$\begin{array}{rrrr}10 n] & 3.53 & 6.78 & 1-1 \\ 5 n 1 & 3.61 & 6.79 & 3-2\end{array}$

3968.25

-3929.538

3890.32

3890.33
3863.88

3835.96

3966.65

3921.80
3791.39

3780.53

12
13

$0.07 \quad 2.11$

3-2 $a^{3} F-z^{5} F$

$\begin{array}{llll}0.00 & 2.07 & 3-1 & \text { (3) }\end{array}$

20

$\begin{array}{llll}0.15 & 2.25 & 4-3 & a^{3} F-z^{3} D^{0}+\end{array}$

$\begin{array}{lll}0.07 & 3.20 & 3- \\ 0.00 & 2.15 & 2-\end{array}$

$0.15 \quad 2.79 \quad 4-5 \quad a^{3} F-z^{3} G^{0}$ A 50

$0.07 \quad 2.73 \quad 3$

$0.00 \quad 2.70 \quad 2-3$

(5)

$(10)$

$0.15 \quad 3.30$

$0.07 \quad 3.25$

$4-5 \quad a^{3} F-y^{5} a^{\circ}$

$0.00 \quad 3.16 \quad 2-2$

$0.15 \quad 3.36 \quad 4-5 \quad a^{3} F-y^{3} G^{0}$

$\begin{array}{lll}0.07 & 3.31 & 3-4\end{array}$

$\begin{array}{cccc}0.15 & 3.33 & 4-4 & a^{3} F-x^{3} F^{0} \\ 0.07 & 3.26 & 3-3 & (8)\end{array}$

$\begin{array}{lll}0.07 & 3.26 & 3-3 \\ 0.00 & 3.23 & 2-2\end{array}$

$0.15 \quad 3.26 \quad 4-3$

$\begin{array}{lll}0.07 & 3.23 & 3-2 \\ 0.07 & 3.33 & 3-4\end{array}$

3856.53

0.003 .26

$0.07 \quad 3.34 \quad 3-3 \quad a^{3} F-x^{1} F^{0}$

3864.33

3822.41

3764.38

3891.39

3663.64

3623.87

3586.28
3714.13

3661.20

3575.79

3550.46

3601.18

3547.69
3519.60

3533.23

3501.33

3566.10 A 100

$\begin{array}{cc}3509.32 & \text { A } 100 \\ -3471.4 \text { A A } 100\end{array}$

$\begin{array}{cccc}0.15 & 3.35 & 4-3 & \left(95-x^{3} D^{\circ}\right. \\ 0.07 & 3.38 & 3-2 & (10)\end{array}$

$0.00 \quad 3.23 \quad 2-1$

$0.00 \quad 3.38 \quad 2-2$

$0.15 \quad 3.32 \quad 4-4 \quad{ }^{3} F-2^{1} G^{0}$

$\begin{array}{llll}0.15 & 3.53 & 4-4 & (11) \\ 0.5 & 3 & \end{array}$

$0.07 \quad 3.48 \quad 3$

$\begin{array}{ll}0.00 & 3.44 \\ 0.15 & 3.48\end{array}$

$0.07 \quad 3.44$

$\begin{array}{ll}0.07 & 3.53 \\ 0.00 & 3.48\end{array}$

$0.15 \quad 3.58$

$\begin{array}{ll}0.15 & 3.58 \\ 0.07 & 3.55\end{array}$

$\begin{array}{ll}0.07 & 3.55 \\ 0.00 & 3.51\end{array}$

$0.15 \quad 3.65$

$\begin{array}{ll}0.15 & 3.65 \\ 0.07 & 3.60\end{array}$

$0.15 \quad 3.61 \quad 3-3 \quad 35-\pi^{3}$

$0.07 \quad 3.59 \quad 3-2$ (15)

$3447.36 \quad$ A 100

$0.00 \quad 3.58 \quad 2-1 \quad a^{3} F-z^{1} p$

3430.29

3465.63
-3414.66
3368.63 $\begin{array}{llll}0.15 & 3.75 & 4-4 & a^{3} F-x^{5} D^{\circ}+\end{array}$

$\begin{array}{cccc}0.15 & 3.71 & 4-3 & (17) \\ 0.07 & 3.68 & 3 & \end{array}$ $\begin{array}{lll}0.07 & 3.68 & 3-2 \\ 0.00 & 3.66 & 2-1\end{array}$ 


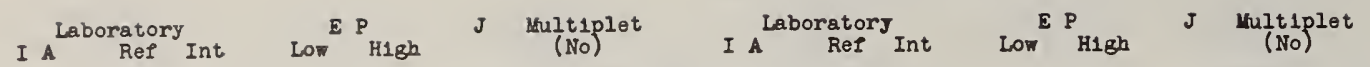
ZrI continued

$3353.65 \quad$ A 20 3234.13 A 100 $\begin{array}{lll}3213.02 & \text { A } & 100 \\ 3191.23 & \text { A } & 100\end{array}$ $\begin{array}{rrr}3282.73 & \text { A } & 15 \\ -3250.428 & \text { A } & 35\end{array}$ $0.15 \quad 3.83$ $\begin{array}{llll}0.15 & 3.97 & 4-5 & a^{3} F-\pi^{3} G^{\circ} \\ 0.07 & 3.91 & 3-4 & (19)\end{array}$ $\underline{\text { zrI }}$ continued

3085.34 A 12

3014.44 A 15

3029.52 A 300

\begin{tabular}{lll}
3011.73 & A & 250 \\
2985.36 & A & 200 \\
\hline
\end{tabular}

7111.71 A 20

7439.81

7336.03

6140.50 6192.96 6304.35 6134.8

5630.16

A 15

5385.14 A 40

$\begin{array}{lllllll}5133.43 & \mathbf{A} & 6 & 0.52 & 2.92 & 2-3 & \mathrm{a}^{3} \mathrm{P}^{3} \mathrm{y}^{3} \mathrm{D}^{\circ}+ \\ 5277.40 & \mathbf{A} & 8 & 0.54 & 2.88 & 1-3 & (27)\end{array}$

$\begin{array}{llllll}5311.42 & \text { A } & 6 & 0.52 & 2.84 & 0-1 \\ 5234.94 & \text { A } & 6 & 0.52 & 2.88 & 2-2 \\ 5362.56 & \text { A } & 6 & 0.54 & 2.84 & 1-1\end{array}$

$0.53 \quad 3.01 \quad 3-3 \quad a^{3} \mathrm{P}-\mathrm{y}^{1} \mathrm{~F}^{\circ}$

$\begin{array}{lllll}0.52 & 3.20 & 2-3 & a^{3} \mathrm{P}_{2} \mathrm{z}^{50}+\end{array}$

$\begin{array}{llll}0.53 & 3.24 & 2-3 & 2^{3} \mathrm{P}_{-1} \mathrm{x}_{\mathrm{F}}\end{array}$

$0.52 \quad 3.35 \quad 2-3 \quad a^{3} \mathrm{P}-x^{3} D^{\circ}+$

$\begin{array}{lll}0.54 & 3.28 & 1-2 \\ 0.52 & 3.23 & 0-1\end{array}$

$\begin{array}{llll}0.52 & 3.57 & 2-3 & a^{3} p-x^{3} p 0 \\ 0.54 & 3 & \end{array}$

$\begin{array}{ll}0.54 & 3.54 \\ 0.54 & 3.53\end{array}$

$0.52 \quad 3.93$

$0.52 \quad 4.29$

$0.53 \quad 4.30$

$0.53 \quad 4.5 ?$

$\begin{array}{ll}0.52 & 4.50 \\ 0.54 & 4.45\end{array}$

$\begin{array}{ll}0.54 & 4.57 \\ 0.52 & 4.50\end{array}$

$\begin{array}{ll}0.52 & 4.47 \\ 0.54 & 4.48\end{array}$

$\begin{array}{ll}0.52 & 4.46 \\ 0.52 & 4.48\end{array}$

$\begin{array}{ll}0.52 & 4.48 \\ 0.54 & 4.46\end{array}$

$\begin{array}{lll}0.54 & 4.46 & 1-1 \\ 0.52 & 4.46 & 2-1\end{array}$

$0.53 \quad 4.62 \quad 2-3 \quad 2^{3} \mathrm{P}-\mathrm{u}^{3} \mathrm{PO}+$

$\begin{array}{llll}0.54 & 4.51 & 1-0 & (38)\end{array}$

$0.73 \quad 2.10 \quad 5-6 \quad a^{5} \mathrm{~F}-2^{5} \mathrm{G}^{0}+$

$\begin{array}{llll}0.68 & 2.01 & 4-5 & (39)\end{array}$

$\begin{array}{lll}0.65 & 1.94 & 3-4 \\ 0.63 & 1.88 & 2-3 \\ 0.60 & 1.83 & 1-2\end{array}$

$\begin{array}{lllll}25 & 25 \\ 20 & 0.73 & 2.26 & 5-5 & 2^{5} \mathrm{~F}_{-2} \mathrm{z}^{\circ} \\ 18 & 0.68 & 2.20 & 4-4 & (40)\end{array}$

$\begin{array}{ll}0.65 & 3.15 \\ 0.62 & 3.11\end{array}$

$0.60 \quad 2.07$

$\begin{array}{ll}0.73 & 2.20 \\ 0.68 & 2.15\end{array}$

$\begin{array}{ll}0.68 & 2.15 \\ 0.65 & 2.11\end{array}$

$0.62 \quad 3.07$

$\begin{array}{ll}0.68 & 3.26 \\ 0.65 & 2.20\end{array}$

$\begin{array}{ll}0.62 & 3.15 \\ 0.60 & 2.11\end{array}$

$0.68 \quad 3.25$

$\begin{array}{ll}0.68 & 3.25 \\ 0.65 & 3.30\end{array}$

0.73 .

$\begin{array}{cc}4-3 & a^{5} F-z^{3} D^{0}+ \\ (41) & \end{array}$

7870.00

8058.14 A $\quad$ ?

$\begin{array}{llll}0.73 & 2.45 & 5-4 & a^{5} \mathrm{~F}-z^{5} D^{0}+ \\ 0.68 & 2.42 & 4-3 & (43)\end{array}$

7169.14

7103.95 A 150

$\begin{array}{lll}7103.77 & \text { A } & 40 \\ 7087.35 & \text { A } & \end{array}$

4687.80 // A 150

4710.08

4739.48

4815.62

4805.88

4824.29
4851.36

4887.72

4893.12
4905.09

$0.65 \quad 2.39$

$\begin{array}{lll}0.62 & 3.36 & 2-1 \\ 0.60 & 2.34 & 1-0\end{array}$

$0.73 \quad 3.36 \quad 5-6 \quad a^{5}-y^{5} 6^{\circ}$

$0.65 \quad 3.25$

$0.62 \quad 3.21$

$0.60 \quad 3.16 \quad 1-2$

$0.73 \quad 3.30 \quad 5-5$

$0.65 \quad 3.31$

$0.62 \quad 3.16$

$0.73 \quad 3.25$

$\begin{array}{llll}3 & 0.68 & 3.21 & 4-3 \\ \text { (4) } & 0.65 & 3.16 & 3-3\end{array}$

$3-3$
$2-2$
$5-4$

$\begin{array}{llrllll}4866.07 & \text { A } & 5 & 0.73 & 3.26 & 5-5 & { }^{5} 5-y^{3} G^{0}+ \\ 4883.61 & \text { A } & 5 & 0.68 & 3.21 & 4-4 & (44) \\ 4881.25 & \text { A } & 4 & 0.65 & 3.18 & 3-3 & \\ 4784.94 & \text { A } & 13 & 0.68 & 3.26 & 4-5 & \\ 4815.05 & \text { A } & 12 & 0.65 & 3.21 & 3-4 & \end{array}$

3340.55
3356.08
3333.13
3214.19
3231.69
3272.31

$\begin{array}{lllll}0.73 & 3.65 & 5-5 & a^{5} F-y^{5} F^{\circ} & 3288.81 \\ 0.68 & 3.60 & 4-4 & (45) & 3319.03\end{array}$

4327.76 A 200

$\begin{array}{rrr}4239.31 & \text { A } & 150 \\ 4241.68 & \text { A } & 80 \\ 4241.30 & \text { A } & 50\end{array}$

$\begin{array}{lll}4368.01 & \text { A } & 20 \\ 4166.37 & \text { A } & 20 \\ 4187.56 & \text { A } & 20\end{array}$

$\begin{array}{lll}4187.56 & \text { A } & 20 \\ 4201.45 & \text { A } & 20 \\ 4313.86 & \text { A } & 15\end{array}$

4081.32 A 100

$\begin{array}{lllllll}4081.32 & \text { A } & 100 & 0.73 & 3.75 & 5-4 & a^{5} F-x^{5} D^{0} \\ 4073.71 & \text { A } & 100 & 0.68 & 3.71 & 4-3 & (46)\end{array}$

$\begin{array}{lll}0.62 & 3.53 & 2-2 \\ 0.60 & 3.51 & 1-1\end{array}$

$\begin{array}{lll}0.60 & 3.51 & 1-1 \\ 0.73 & 3.60 & 5-4\end{array}$

$\begin{array}{lll}0.68 & 3.56 & 4-3 \\ 0.65 & 3.53 & 3-2\end{array}$

$\begin{array}{lll}0.65 & 3.53 & 3-2 \\ 0.62 & 3.51 & 2-1\end{array}$

$\begin{array}{lll}0.68 & 3.65 & 4-5 \\ 0.65 & 3.60 & 3-4\end{array}$

$\begin{array}{lll}0.65 & 3.60 & 3-4 \\ 0.63 & 3.56 & 3-3\end{array}$

$\begin{array}{llll}0.60 & 3.53 & 1-2\end{array}$

4064.16

4044.57

4023.99

4027.30

3977.32

3988.68
4002.55

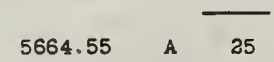

$4732.34 \quad A \quad 15$

4542.32 A 20

4135.68 A 10

$4183.31 \quad A \quad 10$

$3530.23 \quad$ A $\quad 15$

$3360.45 \quad A \quad 25$

$\begin{array}{llr}3090.44 & \text { A } & 6 \\ 3136.95 & \text { A } & 30\end{array}$

$\begin{array}{lll}0.65 & 3.68 & 3-2 \\ 0.62 & 3.66 & 2\end{array}$

$0.60 \quad 3.65 \quad 1-0$

$\begin{array}{lll}0.68 & 3.75 & 4-4 \\ 0.65 & 3.71 & 3-3\end{array}$

$0.63 \quad 3.68 \quad 2-2$

$\begin{array}{lll}0.60 & 3.66 & 1-1 \\ 0.65 & 3.75 & 3-4\end{array}$

\begin{tabular}{lll}
0.62 & 3.71 & $2-3$ \\
0.60 & 3.68 & $1-2$ \\
\hline
\end{tabular}

$\overline{0.63} \quad 3.81 \quad 2=3 \quad 2^{1} D-y^{1} D^{\circ}$

$0.63 \quad 3.34 \quad 2-3 \quad \begin{array}{lll}1 & 47 x_{F}\end{array}$

$\begin{array}{llll}0.63 & 3.35 & 3-3 & 2^{1} \mathrm{D}^{3} \mathrm{x}^{3} \mathrm{D}^{0}+\end{array}$

$0.63 \quad 3.61 \quad 3-3 \quad 21 D_{-13}^{10} D^{\circ}+$

$0.63 \quad 3.58 \quad 3-1 \quad a^{1} D_{z-1} p^{p}$

$0.63 \quad 4.13 \quad 2-3 \quad a^{1} D-u^{3} F^{0}+$

$0.63 \quad 4.30 \quad 2-3 \quad a^{1} D-\pi^{1} D^{\circ}$

$\begin{array}{llll}0.63 & 4.63 & 2-2 & a^{1} D u^{3} \\ 0.63 & 4.56 & 3-1 & (54)\end{array}$

$\begin{array}{llll}0.63 & 4.54 \quad 2-3 & Q^{1} D_{-1}^{1} F^{\circ}\end{array}$

$\begin{array}{llll}0.63 \quad 4.56 \quad 2-3 \quad a^{1} D-v^{3} G^{\circ} & (56)^{2}\end{array}$

$0.99 \quad 3.91 \quad 4-3 \quad a^{1} a-y^{3} F^{0}$

$\begin{array}{lll}6445.76 & \text { A } & 10 \\ 3877.60 & \text { A } & 40\end{array}$

$\begin{array}{llll}0.99 & 4.18 & 4-5 & a^{1} G-z H^{\circ} \\ 0.99 & 4.49 & 4-4 & a^{1}(58) K^{\circ}\end{array}$

3005.50

60

$\begin{array}{rrr}4341.13 & \mathrm{~A} & 30 \\ 4366.45 & \mathrm{~A} & 15 \\ 4394.94 & \mathrm{~A} & \mathbf{8} \\ 4413.04 & \mathrm{~A} & 12 \\ 4430.45 & \mathrm{~A} & 12 \\ 4431.48 & \mathrm{~A} & 10\end{array}$

$\begin{array}{llll}0.99 \quad 5.10 \quad 4-4 & 21 \mathrm{G}-\pi 160 \\ (60)\end{array}$

$\begin{array}{llll}1.39 & 4.23 & 3-4 & a^{5} P-\pi^{5} D^{0}+ \\ 1.36 & 4.19 & 3-3 & (61)\end{array}$

$\begin{array}{lll}1.36 & 4.19 & 3-3 \\ 1.34 & 4.15 & 1-3 \\ 1.39 & 4.19 & 3-3\end{array}$

$\begin{array}{lll}1.36 & 4.19 & 3-3 \\ 1.3-3\end{array}$

4431.48 A 10

$\begin{array}{lll}5046.61 & \text { A } & 10 \\ 5064.93 & \text { A } & 15 \\ 5078.28 & \text { A } & 30\end{array}$

$\begin{array}{llll}1.34 & 4.13 & 1-1\end{array}$

$1.53 \quad 3.97 \quad 4-5 \quad b^{3} F-m^{3} G^{0} \uparrow$

$\begin{array}{llll}1.48 & 3.91 & 3-4 & (63) \\ 1.44 & 3.87 & 3-3 & \end{array}$

$\begin{array}{rrr}4683.43 & \text { A } & 15 \\ 4707.78 & \text { A } & 4\end{array}$

4711.91

4657.64
.4644 .83

A

$1.53 \quad 4.16 \quad 4-4 \quad b^{3} F-v^{3} F^{0}+$

$\begin{array}{llll}1.58 & 4.16 & 4-4 & b^{3} \\ 1.4-163) & 3-3 & (63)\end{array}$

$\begin{array}{llll}1.52 & 4.14 & 4-4 & b^{3} \mathrm{~F}^{3}-\mathrm{u}^{3} \mathrm{~F}^{\circ}+ \\ 1.48 & 4.13 & 3-3 & (64)\end{array}$

$\begin{array}{lllllll}6313.05 & A & 50 & 1.58 & 3.53 & 5-6 & a^{3} G-z^{3} H^{\circ}+ \\ 6470.35 & A & 15 & 1.58 & 3.48 & 4-5 & (65)\end{array}$

\begin{tabular}{lll}
6470.25 & A & 1 \\
6489.68 & A & a \\
\hline
\end{tabular}

$\begin{array}{rrrrrrrr}4753.06 & \mathrm{~A} & \mathbf{3} & & 1.87 & 4.46 & 6-7 & \mathrm{a}^{3} \mathrm{H}-\mathrm{z}^{3} \mathrm{I}^{\circ} \\ 4719.13 & \mathrm{~A} & 10 & & 1.85 & 4.47 & 5-6 & (66) \\ 4763.78 & \mathrm{~A} & 8 & & 1.83 & 4.42 & 4-5 & \end{array}$

Zr II I P 13.97 Anal A L1st A July 1942

3391.96 // A 100

3496.18
3573.47

3505.67

3551.94
3613.08

3673.65

3419.10
3478.29
3424.82

3424.82
3305.15

3373.04

323.04

3306.37
3357.26

3241.01
3284.72

3208.32

3165.98
3138.66

3138.66
3125.76

3125.92
3110.87

3110.87
3095.0 ?

3099.22
3068.03

3061.33

3060.11
3019.84

3030.91
3991.40

3697.49

3766.83

3934.14

3832.94

3903.77
3984.76

3729.74

3814.97
3667.06

3756.96

3613.43
3711.95

3556.61

3576.88
3614.79

3674.74
3636.46

3668.46

3718.86
3499.58

3525.81
3573.09

3588.33

3630.03

3536.94
3587.98

3497.00

3430.53
3410.26

3410.26
3404.84

3399.36

3377.45
3363.81

3367.81

3331.90
3327.67

3375.15

3287.31

2998.34

2968.95

3978.07
3979.18

3738.13

3800.73

3838.28

3915.94
3955.82

3750.65

3817.59
3855.43

3855.43
3714.77

$\begin{array}{llll}0.16 & 3.80 & \frac{4}{3}-5 \frac{1}{2} & \mathrm{a}^{4} \mathrm{~F}-z^{4} \mathrm{G}^{\circ} \\ 0.09 & 3.68 & 3 \frac{1}{3}-4 \frac{1}{2} & (1)\end{array}$

$\begin{array}{llll}0.04 & 3.57 & 3 \frac{1}{3}-3 \frac{1}{2} \\ 0.00 & 3.45 & 1\end{array}$

$\begin{array}{lll}0.00 & 3.45 & 15 \\ 0.16 & 3.68 & 4 \\ 0.09 & 3.57\end{array}$

$\begin{array}{llll}0.04 & 3.54 & 3 & 3 \\ 0.04 & 3.45 & 3 \\ 0.09 & 3.45 & 3 \frac{1}{2}-3 \frac{3}{2}\end{array}$ $\begin{array}{lll}4241.30 & \text { A } & 50 \\ 4240.35 & \text { A } & 50 \\ 4302.88 & \text { A } & 15 \\ 4394.78 & \text { A } & 30\end{array}$

$\begin{array}{rrr}4394.78 & \text { A } & 30 \\ .4282 .20 & \text { B } & 30 \\ 4368.01 & \text { A } & 20 \\ 436 . .37 & \text { A } & 20\end{array}$

$0.65 \quad 3.56 \quad 3-3$

$3535.16 \quad A \quad 30$

I A Laboratory ${ }_{\text {Ref }}$ Int Low ${ }_{\text {H1gh }}$

J $\underset{(\text { No })}{\text { Multiplet }}$

Zr II continued

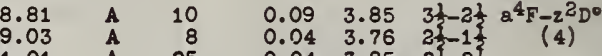

$\begin{array}{rr}\mathbf{A} & 15 \\ \mathbf{A} & 18 \\ \mathbf{A} & 10 \\ \mathbf{A} & 40 \\ \mathbf{A} & 30 \\ \mathbf{A} & 8 \\ \mathbf{A} & 10 \\ \mathbf{A} & 8 \\ \mathbf{A} & 25 \\ \mathbf{A} & 20 \\ \mathbf{A} & 4 \\ \mathbf{A} & 10 \\ \mathbf{A} & 25 \\ \mathbf{A} & 13 \\ \mathbf{A} & 12 \\ \mathbf{A} & 8 \\ \mathbf{A} & 13 \\ \mathbf{A} & 10 \\ \mathbf{A} & 2 \\ \mathbf{A} & 2\end{array}$

$0.16 \quad 3.86 \quad 4 \frac{1}{3}-3 \frac{1}{2} Q^{4} F-2^{4} F^{\circ}$

0.043 .68 2t $-1 \frac{1}{3}$

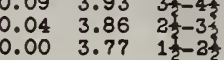

$0.04 \quad 3.85 \quad 31-23$

$0.00 \quad 3.85 \quad 1 \frac{1}{2}-2 \frac{1}{2}$

$0.16 \quad 4.06 \quad 41-3 \frac{1}{2} \mathrm{a}^{4} \mathrm{~F}-\mathrm{z}^{4} \mathrm{D}^{\circ}$

$\begin{array}{lll}0.09 & 4.03 & 3 \\ 0.04 & 3.98 & 2 \frac{1}{3}\end{array}$

$0.00 \quad 3.95 \quad 1 \frac{1}{3}$

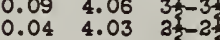

$0.00 \quad 3.98$ 1 $31 \frac{1}{2}$

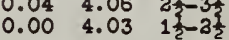

$0.09 \quad 4.13 \quad 3 \frac{1}{2}-2 \frac{1}{2} a^{4} F-y^{2} D^{\circ}$

$0.04 \quad 4.07 \quad 3 \frac{1}{3} a^{2}-y^{2}(6)$

$\begin{array}{lll}0.04 & 4.13 & 21 \\ 0.00 & 4.07 & 1\end{array}$

$0.00 \quad 4.13 \quad 1 \frac{2}{2}-2 \frac{2}{2}$

30
25
30
30
1
1

$\begin{array}{llll}0.46 & 3.80 & 4 \frac{1}{3}-5 \frac{1}{4} b^{4} \mathrm{~F}-z^{4} \mathrm{G}^{\circ} \\ 0.41 & 3.68 & 3 & (7)\end{array}$

$\begin{array}{lll}0.41 & 3.68 & 3 \\ 0.36 & 3.57 & 25\end{array}$

$0.32 \quad 3.45$ 13-23

$\begin{array}{lll}0.41 & 3.57 & 3 \frac{1}{2}-3 \frac{2}{2} \\ 0.36 & 3.45 & 2 \frac{1}{2}-3 \frac{2}{2}\end{array}$

$0.46 \quad 3.77 \quad 4 \frac{1}{3}-3 \frac{1}{2} b^{4} 7-z^{2} \mathrm{pro}^{0}$

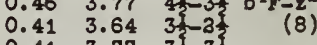

$\begin{array}{lll}0.41 & 3.77 & 31 \\ 0.36 & 3.64 & 21\end{array}$

$\begin{array}{lll}0.36 & 3.77 & 2 \frac{1}{3}-\frac{1}{3} \\ 0.32 & 3.64 & 1 \frac{1}{2}-3 \frac{1}{2}\end{array}$

$0.46 \quad 3.93 \quad 41-41 b^{4} \mathrm{r}-z^{4} \mathrm{~F}^{\circ}$

$\begin{array}{lll}0.41 & 3.86 & 31 \\ 0.36 & 3.77 & 21\end{array}$

$\begin{array}{lll}0.36 & 3.77 & 21.25 \\ 0.32 & 3.68 & 13\end{array}$

$0.46 \quad 3.86 \quad 43$

$0.41 \quad 3.77 \quad 3 \frac{1}{3}-2$

$\begin{array}{lll}0.36 & 3.68 & 2 \frac{1}{3}-1 \frac{1}{3} \\ 0.41 & 3.93 & 3 \frac{1}{3}\end{array}$

$\begin{array}{lll}0.36 & 3.86 & 21 \\ 0.32 & 3.77 & 1 \frac{3}{2}-3 \frac{3}{2}\end{array}$

$0.41 \quad 3.85 \quad 31-3 \frac{1}{3} b^{4} F-z^{3} D^{0}$

$\begin{array}{llll}0.41 & 3.85 & 31-2 \frac{1}{3} & b^{4} F-z^{2} \\ 0.36 & 3.76 & 3 & \end{array}$

$\begin{array}{lll}0.36 & 3.85 & 3 \\ 0.32 & 3.76 & 13 \\ 0.32 & 3.85 & 1\end{array}$

$0.32 \quad 3.85 \quad 1 \frac{1}{2}-3 \frac{1}{2}$

$0.46 \quad 4.06 \quad 41-3 \frac{1}{3} \quad b^{4} F-2^{4} D^{0}$

$0.41 \quad 4.03 \quad 3=2 \frac{3}{3} \quad(11)$

$0.32 \quad 3.08$ 3.1

$0.41 \quad 4.06 \quad 33-3$

$0.36 \quad 4.03 \quad 2 \frac{1}{2}-3 \frac{3}{3}$

$\begin{array}{lll}0.32 & 3.98 & 1 \frac{1}{3}-1 \frac{1}{3} \\ 0.36 & 4.06 & 2 \frac{3}{2}-3 \frac{1}{2}\end{array}$

$0.32 \quad 4.03 \quad 1 \frac{1}{2}-2 \frac{1}{2}$

$0.36 \quad 4.13 \quad 2 \frac{1}{2}-2 \frac{1}{2} b^{4} 5-y^{3} D^{\circ}$

$\begin{array}{llll}0.33 & 4.07 & 13-13 & (13) \\ 0.32 & 4.13 & 13 & 13\end{array}$

$0.33 \quad 4.43 \quad 1 \frac{1}{2}-1 \frac{1}{2} b^{4} \mathrm{~F}-\mathrm{z}^{3} \mathrm{p} 0$

$0.464 .63 \quad 4 \frac{1}{3}-3 \frac{1}{3} \mathrm{~b}^{4} \mathrm{~F}^{(13)} \mathrm{F}+$ \begin{tabular}{lll}
0.41 & 4.55 & $3 \frac{1}{2}-3 \frac{1}{2}$ \\
0.36 & 4.50 & $3 \frac{1}{2}-1 \frac{1}{2}$ \\
\hline
\end{tabular}

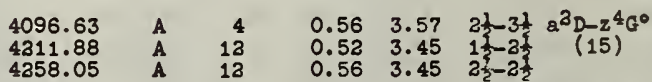

$\begin{array}{lll}3836.76 & \text { A } & 60 \\ 3958.24 & \text { A } & 50 \\ 3998.98 & \text { A } & 30\end{array}$

$0.56 \quad 3.77 \quad 21-3 \frac{1}{3} \mathrm{a}^{2} \mathrm{D}-\mathrm{z}^{2} \mathrm{~F}^{\circ}$

$\begin{array}{rrr}3530.87 & \text { A } & 5 \\ 3556.54 & \text { P } & \mathrm{zr}^{+}\end{array}$

$\begin{array}{llll}0.56 & 3.64 & 1 & 3 \\ 0.64 & (16)\end{array}$

$0.56 \quad 3.86 \quad 2 \frac{1}{3}-3 \frac{1}{2} \mathrm{a}^{2} \mathrm{D}-2^{4} \mathrm{~F}^{\circ}$

(17) 


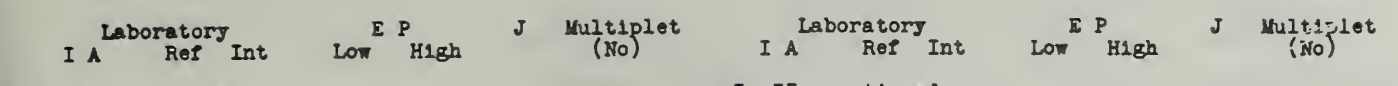
2r II continued

\begin{tabular}{|c|c|c|c|c|c|}
\hline $\begin{array}{l}3981.02 \\
3036.50 \\
3086.44 \\
3111.15 \\
3125.21\end{array}$ & $\begin{array}{l}\hat{A} \\
\hat{A} \\
\hat{A} \\
\mathbf{A}\end{array}$ & $\begin{array}{r}12 \\
7 \\
3 \\
3 \\
4 \\
4\end{array}$ & $\begin{array}{l}0.56 \\
0.52 \\
0.52 \\
0.56 \\
0.52\end{array}$ & $\begin{array}{l}4.70 \\
4.59 \\
4.52 \\
4.52 \\
4.47\end{array}$ & $\begin{array}{l}3 \frac{1}{2}-3 \frac{1}{3} a^{2} D-y^{4} D^{0} \\
1 \frac{1}{2}-2 \frac{1}{2} \quad(24) \\
1 \frac{1}{2}-1 \frac{2}{2} \\
3 \frac{1}{2}-1 \frac{1}{2} \\
1 \frac{1}{2}-\frac{1}{2}\end{array}$ \\
\hline $\begin{array}{l}3036.39 \\
3064.64 \\
3089.00\end{array}$ & $\hat{\hat{A}}$ & $\begin{array}{r}15 \\
3 \\
1\end{array}$ & $\begin{array}{l}0.56 \\
0.53 \\
0.56\end{array}$ & $\begin{array}{l}4.62 \\
4.55 \\
4.55\end{array}$ & $\begin{array}{l}3 \frac{2}{3}-3 \frac{1}{2} a^{2} D-5^{4} F^{\circ} \\
1 \frac{1}{3}-3 \frac{1}{2} \\
3 \frac{1}{2}-3 \frac{1}{2}\end{array}$ \\
\hline $\begin{array}{l}3003.73 \\
3030.45 \\
3044.12\end{array}$ & $\begin{array}{l}\hat{A} \\
\hat{A}\end{array}$ & $\begin{array}{r}15 \\
5 \\
4\end{array}$ & $\begin{array}{l}0.56 \\
0.52 \\
0.56\end{array}$ & $\begin{array}{l}4.66 \\
4.61 \\
4.61\end{array}$ & $\begin{array}{l}3 \frac{1}{2}-3 \frac{1}{2} a^{2} D-y^{2} F^{\circ} \\
\frac{1}{2}-2 \frac{1}{2} \\
\frac{1}{2}-2 \frac{1}{2}\end{array}$ \\
\hline $\begin{array}{l}3013.32 \\
2990.10\end{array}$ & $\hat{A}$ & $\begin{array}{l}8 \\
3\end{array}$ & $\begin{array}{l}0.56 \\
0.52\end{array}$ & $\begin{array}{l}4.65 \\
4.65\end{array}$ & $\begin{array}{l}2 \frac{1}{2}-1 \frac{1}{2} a^{2} D-2^{4} S^{\circ} \\
1 \frac{1}{2}-1 \frac{1}{2} \quad(27)\end{array}$ \\
\hline 4373.52 & A & 4 & 0.75 & 3.64 & $-3 \frac{1}{2} a^{2} P-z^{2} F^{\circ}$ \\
\hline $\begin{array}{l}4090.52 \\
4156.24 \\
4234.27\end{array}$ & $\begin{array}{l}\hat{A} \\
\mathbf{A} \\
\mathbf{A}\end{array}$ & $\begin{array}{r}10 \\
15 \\
3\end{array}$ & $\begin{array}{l}0.75 \\
0.71 \\
0.75\end{array}$ & $\begin{array}{l}3.77 \\
3.68 \\
3.68\end{array}$ & $\begin{array}{c}1 \frac{1}{2}-2 \frac{1}{2} a^{2} P-2^{4} F^{\circ} \\
\frac{1}{3}-1 \frac{1}{2} \quad(39) \\
1 \frac{1}{2}-1 \frac{1}{2}\end{array}$ \\
\hline $\begin{array}{l}3991.14 \\
4045.63 \\
4110.05\end{array}$ & $\hat{A}$ & $\begin{array}{r}40 \\
15 \\
3\end{array}$ & $\begin{array}{l}0.75 \\
0.71 \\
0.75\end{array}$ & $\begin{array}{l}3.85 \\
3.76 \\
3.76\end{array}$ & $\begin{array}{l}1 \frac{1}{2}-3 \frac{1}{2} a^{2} P-z^{2} D^{\circ} \\
\frac{1}{2}-1 \frac{1}{2} \quad(30) \\
1 \frac{1}{2}-1 \frac{1}{c}\end{array}$ \\
\hline $\begin{array}{l}3773.06 \\
3767.89 \\
3833.73 \\
3807.41\end{array}$ & $\begin{array}{l}\hat{A} \\
\hat{A} \\
\hat{A} \\
\mathbf{A}\end{array}$ & $\begin{array}{l}4 \\
5 \\
1 \\
3\end{array}$ & $\begin{array}{l}0.75 \\
0.71 \\
0.75 \\
0.71\end{array}$ & $\begin{array}{l}4.03 \\
3.98 \\
3.98 \\
3.95\end{array}$ & $\begin{array}{c}2 \frac{1}{2} a^{2} P-z^{4} D^{0} \\
(31)\end{array}$ \\
\hline $\begin{array}{l}3660.93 \\
3667.40 \\
3720.29\end{array}$ & $\stackrel{A}{A}$ & $\begin{array}{l}3 \\
1\end{array}$ & $\begin{array}{l}0.75 \\
0.71 \\
0.75\end{array}$ & $\begin{array}{l}4.13 \\
4.07 \\
4.07\end{array}$ & $\begin{array}{c}1 \frac{1}{2}-2 \frac{1}{2} a^{2} P-y^{2} D^{\circ} \\
\frac{1}{3}-1 \frac{1}{3} \quad(32) \\
1 \frac{1}{2}-1 \frac{1}{2}\end{array}$ \\
\hline $\begin{array}{l}3483.54 \\
3437.16\end{array}$ & $\hat{A}$ & $\begin{array}{l}13 \\
10\end{array}$ & $\begin{array}{l}0.75 \\
0.71\end{array}$ & $\begin{array}{l}4.30 \\
4.30\end{array}$ & $\begin{array}{c}1 \frac{1}{2}-\frac{1}{2} a^{2} P-z^{2} S^{\circ} \\
\left.\frac{1}{2}-\frac{33}{2}\right)\end{array}$ \\
\hline $\begin{array}{l}3354.39 \\
3280.75 \\
3333.99 \\
3311.34\end{array}$ & $\begin{array}{l}\mathbf{A} \\
\mathbf{A} \\
\mathbf{A} \\
\mathbf{A}\end{array}$ & $\begin{array}{r}7 \\
3 \\
10 \\
4\end{array}$ & $\begin{array}{l}0.75 \\
0.71 \\
0.75 \\
0.71\end{array}$ & $\begin{array}{l}4.43 \\
4.47 \\
4.47 \\
4.43\end{array}$ & $\begin{array}{l}1 \frac{1}{2}-1 \frac{1}{2} a^{2} P-z^{2} p^{0} \\
1 \frac{2}{2}-\frac{1}{2}(34) \\
\frac{1}{2}-1 \frac{1}{2}\end{array}$ \\
\hline 3218.68 & $\mathbf{A}$ & 1 & 0.75 & 4.59 & $1 \frac{1}{2}-2 \frac{1}{3} a^{2} P-J^{4} D^{\circ}$ \\
\hline
\end{tabular}

$3395.03 \quad A \quad 1 \quad 0.75 \quad 4.50 \quad 1 \frac{1}{2}-1 \frac{1}{2} a^{3} P-y^{4} F^{0}$

$3300.67 \quad A \quad 3 \quad 0.75 \quad 4.61 \quad 1 \frac{1}{2}-2 \frac{1}{2} \mathrm{a}^{3} \mathrm{P}-\mathrm{y}^{3} \mathrm{~F}^{\circ}$

$\begin{array}{ccccccc}3088.28 & A & 1 & 0.75 & 4.75 & 1 \frac{1}{3}-2 \frac{1}{2} a^{2} P_{-2}(37) \\ 3138.79 & 4 & 1 & 0.75 & 4.70 & 1 & (38)\end{array}$

$\begin{array}{llllll}3138.79 & \text { A } & 1 & 0.75 & 4.70 & 1 \frac{1}{2} \\ 3091.30 & \text { A } & 1 & 0.71 & 4.70 & \frac{1}{2}\end{array}$

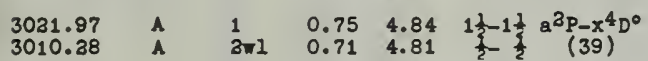

$4377.37 \quad \hat{A} \quad \begin{aligned} & 4.80 \\ & 0.71\end{aligned}$

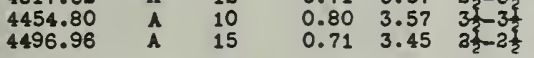

$4149.33 \quad A \quad 75 \quad 0.803 .77 \quad 3 \frac{1}{-3}-3 \frac{1}{2} \mathrm{a}^{2} \mathrm{~F}_{-2} 2_{F 0}$

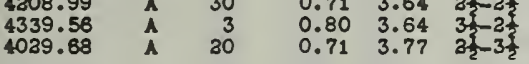

3936.07

3921.02

4034.10

4150.97

$4048.68 \quad A \quad 25 \quad 0.80 \quad 3.85 \quad 3 \frac{1}{2}-2 \frac{1}{2} a^{2} F-2^{2} D^{\circ}$

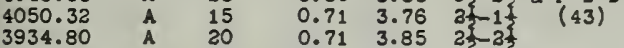

3783.34

3823.41

3771.98
3683.67

3709.37

3671.28
-3613.43

3481.14

$3479.39 \quad A \quad 35 \quad 0.80 \quad 4.34 \quad \frac{3}{2}-4 \frac{4}{3} a^{3} F-2^{3} G^{\circ}$

10.804 .36 3र

$\begin{array}{lllllll}3168.39 & A & 8 & 0.80 & 4.70 & 3-3 \frac{1}{3} & a^{3} F-y^{4} D^{0}\end{array}$

$\begin{array}{lllllll}3181.94 \quad A & 7 & 0.71 & 4.59 & 31 & 3 \frac{1}{2} & \end{array}$

$\begin{array}{rrrrrrr}3338.81 & \text { A } & 15 & 0.80 & 4.63 & 3 \frac{1}{3}-3 \frac{1}{3} & a^{3} F-y^{4} F \\ 3313.85 & \text { A } & 6 & 0.71 & 4.55 & 31-3 \frac{3}{3} & (49)\end{array}$

3256.53

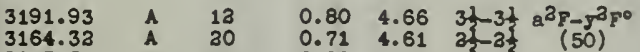

3237.54
3130.72

3123.61

3013.66

Zr II continued

$4442.50 \quad A \quad 3 \quad 0.99 \quad 3.77 \quad 2 \frac{1}{2}-3 \frac{1}{2} a^{4} P_{-2} F_{F} 0$

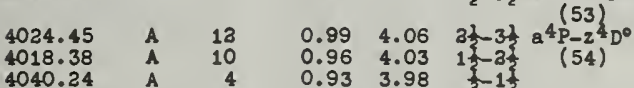

3414.658

(a)

$\begin{array}{lll}0.80 & 4.75 & 3 \\ 0.71 & 4.75 & 2\end{array}$

$0.80 \quad 4.89 \quad 3 \frac{1}{2}-3 \frac{1}{2} a^{2} F-x^{4} D^{0}$
4040.2

4071.09
4077.05

4085.68

4131.31
4123.38

3941.92

3693.60

3588.80

3512.6
3485.3

3334.25
3396.34

3432.41
3433.90

3458.93

3480.40

3497.90
3507.66

3403.69

3431.57
3454.5 ?

3454.57
3469.94

3481.44
$\mathrm{~m} 3520.91$

3362.70

3376.25
3413.39

3374.71

3338.41
3313.70

3285.89

3388.81
3272.30

3324.03

3396.41

3264.81

3106.58

3133.49
3155.68

3165.45
3178.10

3178.10
3181.58

3210.98
3204.36

3975.16
3009.8

3009.85
3048.43

4816.4

4461.3

4613.9
4399.4

$4315 . ?$

4386.5

4034.84

3698.17

3751.60

3796.47
3655.56

3344.80

3408.09
3309.90

3443.5
3378.3

3373.48

3387.8
3337.9

3115. 73

3054.84

3038.05
3057.33

3057.32
3025.70

2976.61

$0.994 .03 \quad 2 \frac{2}{2}-2 \frac{1}{2}$

$0.93 \quad 3.95$ L

$\begin{array}{lll}0.99 & 3.98 & 3 \frac{1}{2}-1 \frac{1}{4} \\ 0.96 & 3.95 & 1 \frac{1}{2}\end{array}$

$0.99 \quad 4.13 \quad 2 \frac{1}{2}-2 \frac{1}{2} a^{4} p-y^{3} D^{\circ}$

$0.96 \quad 4.30 \quad 1 \frac{1}{2} \frac{1}{2} a^{4} \mathrm{P}_{-2} \mathrm{z}^{2}$

$\begin{array}{cccc}0.99 & 4.43 & 3 \frac{1}{2}-1 \frac{1}{2} & a^{4} \mathrm{P}-z^{3} \mathrm{P}^{\circ} \\ 0.96 & 4.47 & 1 & (57)\end{array}$

$\begin{array}{lll}0.96 & 4.47 & 1 \frac{1}{2} \\ 0.93 & 4.47 & \frac{1}{2}\end{array}$

$\begin{array}{llll}0.99 & 4.70 & 3 \frac{1}{3}-3 \frac{1}{2} & a^{4} P-y^{4} D^{\circ} \\ 0.96 & 4.59 & 1 \frac{1}{3}-3 \frac{1}{4} & (58)\end{array}$

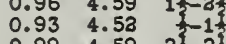

0.96 4.52 $1 \frac{3}{2}-1 \frac{1}{2}$

$\begin{array}{lll}0.93 & 4.47 & \\ 0.99 & 4.52 & 2 \frac{1}{3}-1\end{array}$

$0.964 .47 \quad 1 \frac{1}{2}-\frac{1}{2}$

$\begin{array}{llll}0.99 & 4.62 & 2 \frac{1}{3}-3 \frac{1}{3} & a^{4} P-y^{4} F^{\circ} \\ 0.96 & 4.55 & 1 \frac{1}{2}-2 \frac{1}{5} & (59)\end{array}$

$0.93 \quad 4.50$ ?

$\begin{array}{lll}0.99 & 4.55 & 2 \frac{1}{2}-2 \frac{1}{3} \\ 0.96 & 4.50 & 1 \frac{1}{2}-1 \frac{1}{3}\end{array}$

$0.99 \quad 4.66 \quad 2 \frac{1}{2}-3 \frac{1}{4} a^{4} p-y^{3} F^{\circ}$

$\begin{array}{lll}0.96 & 4.61 & 12-2 \frac{1}{2} \\ 0.99 & 4.61 & 2 \frac{2}{2}-2 \frac{1}{2}\end{array}$

$0.99 \quad 4.65 \quad 2 \frac{1}{2}-1 \frac{1}{2} a^{4} p-z^{4} g^{\circ}$

$\begin{array}{llll}0.96 & 4.65 & 1 \frac{1}{3} & (61) \\ 0.93 & 4.65 & & \end{array}$

$0.99 \quad 4.75 \quad 2 \frac{1}{2}-2 \frac{1}{2} \quad a^{4} P-z^{4} P^{\circ}$

$\begin{array}{llll}0.99 & 4.75 & 2 \frac{1}{2}-2 \frac{1}{3} & a^{4} P-z^{4} \\ 0.96 & 4.71 & 1 \frac{1}{2}-1 \frac{1}{2} & (62)\end{array}$

$0.99 \begin{array}{lll}4.71 & 2 \frac{3}{3}-1\end{array}$

$\begin{array}{lll}0.96 & 4.70 & 1 \frac{1}{3} \\ 0.96 & 4.75 & 1 \frac{1}{2}-2 \frac{1}{2} \\ 0.93 & 4.71 & \end{array}$

$\begin{array}{cccc}0.99 & 4.97 & 3 \frac{1}{3}-3 \frac{1}{3} & a^{4} P-x^{4} D^{\circ} \\ 0.96 & 4.89 & 1 \frac{1}{3}-2 \frac{1}{3} & (63)\end{array}$

$\begin{array}{lll}0.93 & 4.84 & \\ 0.99 & 4.89 & 3 \frac{1}{3}-2 \frac{1}{3}\end{array}$

$0.96 \quad 4.84 \quad 1 \frac{1}{3}-1 \frac{1}{4}$

$\begin{array}{lll}0.93 & 4.81 & \frac{1}{2}-\frac{1}{2} \\ 0.99 & 4.84 & 2 \frac{1}{3}-1 \frac{1}{2} \\ 0.96 & 4.81 & 1 \frac{2}{2}-\frac{1}{2}\end{array}$

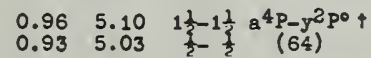

$\begin{array}{lll}0.99 & 5.04 \quad 2 \frac{1}{2}-3 \frac{1}{2} a^{4} P-y^{2} G^{0} \\ (65) & \end{array}$

$1.013 .57 \quad 4 \frac{1}{2}-3 \frac{1}{2} a^{2} a-2^{4} \sigma^{0}$

$\begin{array}{llll}1.01 & 3.77 & 4 \frac{1}{3}-3 \frac{1}{2} & a^{2} G-2350 \\ 0.97 & 3.64 & 3 \frac{1}{2}-2 \frac{1}{2} & (67)\end{array}$

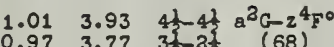

$0.97 \quad 3.853 \frac{1}{2}-2 \frac{1}{2} a^{2} G-2^{2} D^{\circ}$

$0.97 \quad 4.03 \quad 3 \frac{1}{2}-2 \frac{1}{2} a^{2} G-2^{4} D^{\circ}$

$\begin{array}{llll}1.01 & 4.34 & 4 \frac{1}{3}-4 \frac{1}{2} a^{3} G-z^{2} G^{\circ}\end{array}$

$\begin{array}{lll}1.01 & 4.34 & 4 \\ 0.97 & 4.26 & 3 \frac{1}{3}-3 \frac{1}{3} \\ 1.01 & 4.26 & 4 \frac{1}{3}-3 \frac{1}{3}\end{array}$

$1.01 \quad 4.70 \quad 4 \frac{1}{2}-3 \frac{1}{2} a^{2} c-y^{4} D^{0}$

$\begin{array}{lllll}0.97 & 4.59 & 3 \frac{1}{3} & -2 \frac{1}{3} & \text { (72) } \\ 0.97 & 4.70 & 3 \frac{1}{2}-3 \frac{1}{2} & \end{array}$

$1.01 \quad 4.63 \quad 4 \frac{1}{3}-3 \frac{1}{3} a^{2} a-y^{4} F^{\circ}$

$\begin{array}{llll}0.97 & 4.55 & 3 \frac{1}{3}-2 \frac{1}{3} & (73) \\ 0.97 & 4.62 & 3 \frac{1}{2}-3 \frac{1}{3}\end{array}$

$\begin{array}{lll}1.01 & 4.66 & 4 \\ 0.97 & 4.61 & a^{3} a-y^{2} F^{\circ}\end{array}$

$\begin{array}{lll}0.97 & 4.61 & 3 \frac{1}{2}-2 \frac{1}{3} \\ 0.97 & 4.66 & 3 \frac{1}{2}-3 \frac{1}{2}\end{array}$

$1.01 \quad 4.97 \quad 4 \frac{1}{2}-3 \frac{1}{2} \mathrm{a}^{3} a-x^{4} D^{\circ}$

$1.01 \quad 5.05 \quad \frac{1}{3}-4 \frac{1}{3} a^{2} a-5^{3} a^{\circ}$

$\begin{array}{lll}1.01 & 5.04 & 4 \frac{1}{2}-3 \frac{1}{2} \\ 0.97 & 5.05 & 3 \frac{2}{2}-4 \frac{1}{2}\end{array}$

$1.015 .15 \quad 4-4 \frac{1}{2} a^{2} a-z^{2} H^{0}+$

$4854.65 \quad A \quad 0 \quad 1.33 \quad 3.77 \quad 3 \frac{1}{2}-3 \frac{1}{2} b^{4} P_{-2}^{2} F^{\circ}$

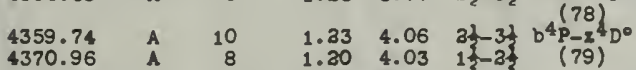

4370.96
4403.35

4414.54

4457.45
.445

4485.44

$\begin{array}{lll}1.18 & 3.98 & 1-1 \\ 1.33 & 4.03 & 35-2\end{array}$

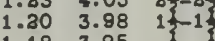

1.23 3.98 $3 \frac{1}{1}$
$0.964 .81 \quad 1 \frac{2}{2}$

I A Laboratory

Lo P

Multiple

Zr II continued

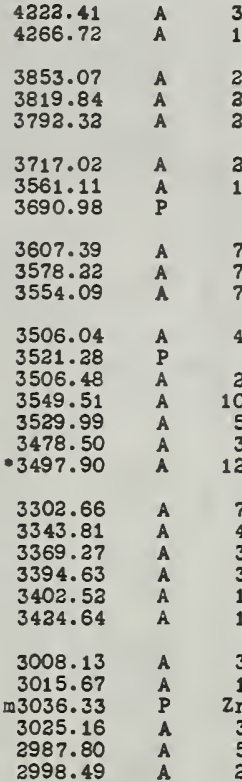

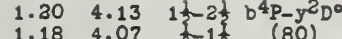

$1.23 \quad 4.43 \quad 2 \frac{1}{2}-1 \frac{1}{3} b^{4} \mathrm{P}-z^{2} \mathrm{po}^{\circ}$

$\begin{array}{lll}1.20 & 4.43 & 1 \frac{1}{3}-1 \frac{1}{3} \\ 1.18 & 4.43 & \end{array}$

$\begin{array}{llll}1.20 & 4.52 & 1 \frac{1}{2}-1 \frac{1}{3} & b^{4} P-y^{4} D^{0} \\ 1.23 & 4.70 & 2 \frac{1}{2}-3 \frac{1}{2} & (82) \\ 1.18 & 4.52 & \frac{1}{2}-1 \frac{1}{2} & \end{array}$

$1.23 \quad 4.65 \quad 2 \frac{1}{3}-1 \frac{1}{2} b^{4} \mathrm{P}-z^{4} \mathrm{~S}^{\circ}$

$\begin{array}{lll}1.20 & 4.65 & 1 \frac{1}{2}-1 \frac{1}{2} \\ 1.18 & 4.65 & \frac{1}{3}-1 \frac{1}{2}\end{array}$

$1.23 \quad 4.75 \quad 3 \frac{1}{3}-2 t \quad b^{4} P_{-2} P^{\circ}$

$1.20 \quad 4.71 \quad 1 \frac{1}{3}-1 \frac{1}{4} \quad(84)$

1.234 .7

$1.204 .701 \frac{1}{3}$

1.18 4.71 $12-1 \frac{1}{2}$

$1.23 \quad 4.97 \quad 2+3 \frac{1}{2} b^{4} P-x^{4} D^{0}$

$\begin{array}{ll}.23 & 4.89 \\ 1.30 & 4.8\end{array}$

$\begin{array}{ll}1.20 & 4.8 \\ 1.18 & 4.81\end{array}$

$1.20 \quad 4.81,3$

$1.23 \quad 5.33 \quad 2 \frac{1}{3}-2 \frac{1}{5} b^{4} \mathrm{P}-\mathrm{y}^{4} \mathrm{p}$

$1.20 \quad 5.30 \quad 1230$

$1.23 \quad 5.30 \quad 23-1$

$1.20 \quad 5.33 \quad 13-2 \frac{1}{3}$

$1.53 \quad 3.93 \quad 5 \frac{1}{3}-4 \frac{1}{3} \mathrm{a}^{2} \mathrm{H}-2^{4} \mathrm{~F}^{\circ}$

5124.98

4379.78

4442.99
4308.9

3874.37

3505.47

3463.02
3459.95

$332 \epsilon .81$

3402.

3285.77

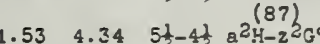

$\begin{array}{cccc}1.53 & 4.34 & 5 \frac{1}{3}-4 \frac{1}{3} & 0^{2} 4-22 \\ 1.48 & 4.26 & 4 \frac{1}{3}-3 \frac{1}{2} & (88) \\ 1.48 & 4.34 & 4 & \end{array}$

$1.48 \quad 4.66 \quad 4 \frac{1}{2}-3 \frac{1}{3} \mathrm{~g}^{2} \mathrm{H}-\mathrm{y}^{2} \mathrm{~F}^{\circ}$

$\begin{array}{llll}1.53 & 5.05 & 5 t-4 \frac{1}{3} & (89) \\ 1.48 & 5.04 & 41-31 & (90)\end{array}$

$\begin{array}{lll}1.48 & 5.04 & 4+3 \\ 1.48 & 5.05 & 4 \frac{9}{2}-4 \frac{1}{3}\end{array}$

$1.53 \quad 5.24 \quad 5 \frac{1}{2}-5 \frac{1}{3} a^{2} H_{-2}{ }^{2} H^{\circ}$

$1.48 \quad 5.15 \quad 45-4 \frac{1}{3} \quad$ (91)

$\begin{array}{lll}1.53 & 5.15 & 51-4 \\ 1.48 & 5.24 & 4 \frac{1}{2}-5 \frac{1}{2}\end{array}$

$1.48 \quad 5.25 \quad 4 \frac{1}{2}-3 \frac{1}{3} a^{2} \mu-x^{2} F^{\circ}$

$\begin{array}{lllllll}6100.04 & \text { A } & 2 & 1.75 & 3.77 & 2 \frac{1}{3}-3 \frac{1}{2} & 0^{2} 2-2^{4} 50 \\ 6114.78 & \text { A } & 2 & 1.66 & 3.68 & 1 \frac{1}{3}-1 \frac{1}{3} & (93)\end{array}$

5418.0

$.75 \quad 4.03 \quad 2 \frac{1}{3}-2 \frac{1}{2} b^{2} \mathrm{C}-2^{4}=0$

$\begin{array}{llll}1.75 & 4.13 & 2 \frac{1}{2}-2 \frac{1}{2} & b^{2}(94) \\ 1.66 & 4.07 & 1 \frac{1}{2}-1 & (95)\end{array}$

$1.75 \quad 4.07$ ? $31-1 \frac{1}{3}$

$1.66 \quad 4.43 \quad 1 \frac{1}{2}-1 \frac{1}{2} b^{2} D-z^{2} \geq 0$

$1.75 \quad 4.70 \quad 2 \frac{1}{2}-3 \frac{1}{2} b^{2} 2-y^{4} D^{\circ}$

$\begin{array}{lll}1.66 & 4.59 & 13-2 \\ 1.66 & 4.47 & 1\end{array}$

$1.75 \quad 4.62 \quad 2 \frac{1}{3}-3 \frac{1}{2} b^{2} D-y^{4} p^{\circ}$ $\begin{array}{llll}1.66 & 4.55 & 11-21 & (98)\end{array}$

$1.75 \quad 4.66 \quad 22-3 \frac{1}{3} b^{2} 0-y^{2} 5^{\circ}$ $\begin{array}{lll}1.66 & 4.61 & 1 .-21 \\ 1.75 & 4.61 & 2 \frac{1}{3}-2\end{array}$

$1.75 \quad 4.97 \quad 2 \frac{1}{3}-3 \frac{1}{3} b^{2} D-x^{4} D^{\circ}$ $\begin{array}{llll}1.66 & 4.89 & 1 & -21 \\ 1.75 & 4.89 & 21 & (100)\end{array}$

$1.75 \quad 5.10 \quad 2 \frac{1}{2}-1 \frac{1}{2} b^{2} D-y^{2} p_{0}$ $\begin{array}{lll}1.66 & 5.03 & 1 \frac{1}{2} \\ 1.66 & 5.10 & 1 \frac{1}{2}-1 \frac{1}{2}\end{array}$

$1.75 \quad 5.15 \quad 2 \frac{1}{2}-2 \frac{1}{2} b^{2} D-x^{2} \partial^{\circ}$

$\begin{array}{llll}1.66 & 5.12 & 1 \frac{1}{3}-1 & \text { (103) }\end{array}$

$\begin{array}{lll}1.75 & 5.12 & 2 \frac{1}{3} \\ 1.66 & 5.15 & 1 \frac{1}{2}-2 \frac{1}{2}\end{array}$

$1.75 \quad 5.35 \quad 2 \frac{1}{2}-3 \frac{1}{2} b^{2} D-x^{2} \xi^{\circ}$

$\begin{array}{llll}1.66 & 5.29 & 1 & 1 \\ 1.75 & 5.29 & 2 & (103)\end{array}$

$1.75 \quad 5.58 \quad 2+3+b^{2} 2-2^{2} 2^{\circ}$

$1.66 \quad 5.56 \quad 1 \frac{1}{3}-1 \frac{1}{3} \quad(104)$

$1.75 \quad 5.63 \quad 3 \frac{1}{12} b^{2} D-x^{2} p^{0}$

$\left.1.665 .67 \quad 1 \frac{1}{105}\right)$

3074.5

-6106. 47

$1.75 \quad 3.77 \quad 4 \frac{1}{2}-3 \frac{1}{3} b^{2} G-2^{2} 5$

1.754 .34 4t $\left.45 b^{2} a-2^{2}\right)^{\circ}$

$\begin{array}{llll}1.75 & 4.34 & 41-4 \frac{1}{3} b^{2} a-z^{2} \\ 1.74 & 4.36 & 37-3 \frac{1}{3} & (107)\end{array}$

4894.43
4925.90

$1.75 \quad 4.70 \quad 4 \frac{1}{2}-3 \frac{1}{2} b^{2} a-y^{4} D^{\circ}$

4191.50
4335.64 
82 I A Ref Int Low ${ }_{\text {High }}^{\text {Laboratory }}$ J $\begin{gathered}\text { Lultiplet } \\ \text { (No) }\end{gathered}$ 2r II continued

$\begin{array}{rrr}4301.81 & \text { A } & 5 \\ 4382.95 & \text { A } & 1 \\ 4236.56 & \text { A } & 5 \\ 4293.14 & \text { A } & 7 \\ 3818.78 & \text { A } & 1 \\ 3745.97 & \text { A } & 40 \\ 3731.26 & \text { A } & 35 \\ 3749.55 & \text { P } & \\ 3727.72 & \text { A } & 10 \\ 3542.65 & \text { A } & 25 \\ 3611.90 & \text { A } & 15 \\ 3629.12 & \text { A } & 1 \\ 3530.85 & \text { A } & 6 \\ 3471.14 & \text { A } & 8 \\ 3514.64 & \text { A } & 4 \\ & & \end{array}$

$\begin{array}{llll}1.75 & 4.62 & 4 \frac{1}{3}-3 \frac{1}{2} & b^{2} G-y^{4} F^{b} \\ 1.74 & 4.55 & 3 \frac{1}{2}-2 \frac{1}{2} & (109)\end{array}$ $\begin{array}{llll}1.75 & 4.66 & 4 \frac{1}{3}-3 \frac{1}{2} & b^{2} G-y^{2} F^{\circ} \\ 1.74 & 4.61 & 3 \frac{1}{2}-2 \frac{3}{2} & (110)\end{array}$ $1.74 \quad 4.97 \quad 3 \frac{1}{2}-3 \frac{1}{2} b^{2} a-x^{4} D^{0}$ $1.75 \quad 5.05 \quad 4 \frac{1}{2}-4 \frac{1}{3} b^{2}\left(111 y^{2} G^{\circ}\right.$ $\begin{array}{llll}1.75 & 5.05 & 4 \frac{1}{3}-4 \frac{1}{3} & b^{2}\left(a-y^{2}\right. \\ 1.74 & 5.04 & 3 \frac{1}{3}-3 \frac{1}{3} & (113)\end{array}$ $\begin{array}{lll}1.75 & 5.04 & 4 \frac{1}{3}-3 \frac{1}{2} \\ 1.74 & 5.05 & 3 \frac{1}{2}-4 \frac{1}{3}\end{array}$

$1.75 \quad 5.24 \quad 4 \frac{1}{3}-5 \frac{1}{3} b^{3} G-z^{2} H^{\circ}$ $\begin{array}{llll}1.74 & 5.15 & 3 \frac{3}{3}-4 \frac{1}{3} & (113) \\ 1.75 & 5.15 & 4 \frac{1}{2}-4 \frac{1}{3} & \end{array}$ $1.75 \quad 5.25 \quad 43-3 \frac{1}{3} b^{2} G-x^{2} 5^{0}$ $\begin{array}{llll}1.74 & 5.29 & 3 \frac{1}{2}-2 \frac{1}{2} & (114) \\ 1.74 & 5.25 & 3 \frac{1}{2}-3 \frac{1}{2} & \end{array}$

$\begin{array}{lllllll}5350.10 & \mathrm{~A} & 5 & 1.82 & 4.13 & 2 \frac{1}{3}-2 \frac{1}{3} & \mathrm{c}^{2} \mathrm{D}-\mathrm{y}^{3} \mathrm{D}^{\circ} \\ 5350.36 & \mathrm{~A} & 5 & 1.77 & 4.07 & 1 \frac{1}{3}-1 \frac{1}{2} & (115)\end{array}$ $\begin{array}{lllllll}5477.82 & \mathrm{~A} & 2 & 1.82 & 4.07 & 2 \frac{2}{2}-1 \frac{1}{2} & \\ & & & 1 & & & \end{array}$ $\begin{array}{lllllll}4719.80 & \text { A } & 1 & 1.83 & 4.43 & 2 \frac{1}{2}-1 \frac{1}{3} c^{2} D-z^{2} \mathrm{P} \\ 4565.43 & \text { A } & 3 & 1.77 & 4.47 & 1 \frac{1}{3} & (116) \\ 4624.86 & \text { A } & 1 & 1.77 & 4.43 & 1 \frac{3}{2}-1 \frac{1}{2} & \end{array}$ $4289.18 \quad A \quad 2 \quad 1.82 \quad 4.70 \quad 2 \frac{1}{2}-3 \frac{1}{2} c^{2} D-y^{4} D^{\circ}$ $\begin{array}{lllllll}4404.81 & \text { A } & 2 & 1.82 & 4.62 & 2 \frac{1}{3}-3 \frac{1}{2} & c^{2} D-y^{4} F^{\circ} \\ 4429.34 & \text { A } & 2 & 1.77 & 4.55 & 1 \frac{1}{2}-3 \frac{2}{2} & (118)\end{array}$ $\begin{array}{lllllll}4336.36 & \text { A } & 2 & 1.82 & 4.66 & 2 \frac{1}{2}-3 \frac{1}{2} & c^{3} D-y^{3} g^{\circ} \\ 4337.63 & \text { A } & 5 & 1.77 & 4.61 & 1 \frac{3}{2}-3 \frac{1}{2} & (119)\end{array}$ $\begin{array}{lllllll}3757.80 & \text { A } & 8 & 1.83 & 5.10 & 2 \frac{1}{2}-1 \frac{1}{1} & \mathrm{c}^{2} \mathrm{D}-\mathrm{y}^{2} \mathrm{P}^{\circ} \\ 3783.72 & \mathrm{~A} & 5 & 1.77 & 5.03 & 1 \frac{1}{2}-\frac{1}{2} & (130)\end{array}$ $3827.51 \quad A \quad 1 \quad 1.82 \quad 5.04 \quad 3 \frac{1}{2}-3 \frac{1}{2} c^{2} D-y^{3} G^{\circ}$ $\begin{array}{lllllll}3710.47 & A & 1 & 1.83 & 5.15 & 2 \frac{1}{2}-2 \frac{1}{2} & c^{2} D-x^{2} D^{\circ}\end{array}$ $\begin{array}{lllllll}3679.64 & \text { A } & 1 & 1.77 & 5.12 & 1 \frac{1}{3}-1 \frac{1}{2} & \text { (122) } \\ 3651.50 & \text { A } & 2 & 1.77 & 5.15 & 1 \frac{1}{2}-2 \frac{1}{2} & \end{array}$ $\begin{array}{lllllll}3599.91 & \text { A } & 7 & 1.82 & 5.25 & 2 \frac{1}{2}-3 \frac{1}{2} & c^{3} D-x^{2} F^{\circ} \\ 3500.15 & \text { A } & 4 & 1.77 & 5.39 & 12-2 & (123)\end{array}$ $\begin{array}{lllllll}3511.55 \quad A \quad 2 & 1.82 & 5.33 & 2 \frac{1}{2}-2 \frac{1}{2} & c^{2} D-y^{4} \mathrm{Po}\end{array}$ $\begin{array}{llllll}3550.11 & P & 1.82 & 5.30 & 2 \frac{1}{2}-1 \frac{1}{2} & (124)\end{array}$ $\begin{array}{rrrrrrr}3282.84 & \mathrm{~A} & 13 & 1.82 & 5.58 & 2 \frac{1}{3}-2 \frac{1}{2} & \mathrm{c}^{2} \mathrm{D}-\pi^{2} \mathrm{D} \\ 3250.44 & \mathrm{~A} & 20 & 1.77 & 5.56 & 1 \frac{1}{2}-1 \frac{1}{2} & (125) \\ 3236.61 & \mathrm{~A} & 4 & 1.77 & 5.58 & 1 \frac{1}{2}-2 \frac{1}{2} & \end{array}$ $\begin{array}{llllll}3242.18 \quad A \quad 3 & 1.82 & 5.63 & 3 \frac{1}{2}-1 \frac{1}{3} \mathrm{c}^{2} \mathrm{D}-\mathrm{x}^{2} \mathrm{P}^{0}\end{array}$ $\begin{array}{lllllll}3159.12 & \text { A } & 5 & 1.77 & 5.67 & 1 \frac{1}{2} & (126) \\ 3197.08 & \text { A } & 3 & 1.77 & 5.63 & 1 \frac{1}{2}-1 \frac{1}{2} & \end{array}$ $3015.86 \quad A \quad 3 \quad 1.825 .91 \quad 3 \frac{1}{2}-2 \frac{1}{2} c^{3} D-m^{2} F^{\circ}$ $\begin{array}{lllllll}6346.54 & \mathrm{~A} & 1 & 2.40 & 4.34 & 3 \frac{1}{3}-4 \frac{1}{3} & \mathrm{~b}^{2} \mathrm{~F}-\mathrm{z}^{2} \mathrm{G}^{\circ} \\ 6678.03 & \mathrm{~A} & 3 & 3.41 & 4.36 & 3 \frac{1}{2}-3 \frac{3}{2} & (138)\end{array}$ $\begin{array}{lllllll}4661.78 & \text { A } & 5 & 2.40 & 5.05 & 3 \frac{1}{2}-4 \frac{1}{2} & b^{2} F-y^{2} G^{\circ} \\ 4685.19 & \text { A } & 4 & 2.41 & 5.04 & 3 \frac{1}{2}-3 \frac{1}{2} & (139)\end{array}$ $\begin{array}{rrrrrrr}4494.41 & \text { A } & 8 & 2.40 & 5.15 & 3 \frac{1}{2}-2 \frac{1}{2} & b^{2} F-x^{2} D^{\circ} \\ 4553.96 & \text { A } & 12 & 2.41 & 5.12 & 2 \frac{1}{2}-1 \frac{1}{2} & (130)\end{array}$ $4482.04 \quad A \quad 3 \quad 2.40 \quad 5.15 \quad 3 \frac{1}{2}-4 \frac{1}{2} b^{3} \mathrm{~F}-z^{3} \mathrm{H}^{\circ}$

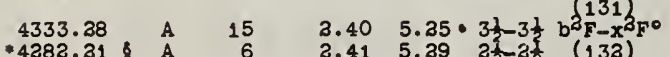
$\begin{array}{lllllll}4282.218 & A & 6 & 2.41 & 5.29 & 2 \frac{1}{3}-2 \frac{2}{2} & \text { (132) } \\ 4267.30 & \mathrm{P} & & 2.40 & 5.29 & 31 & \end{array}$ $\begin{array}{lllll}4348.64 & P & 2.40 & 5.29 & 3 \frac{1}{3}-2 \frac{2}{2} \\ 4 & 2.41 & 5.25 & 2 \frac{1}{2}-3 \frac{1}{2}\end{array}$

$4305.91 \quad \mathrm{~A} \quad 2 \quad 2.40 \quad 5.33 \quad 3 \frac{1}{2}-2 \frac{2}{2} \mathrm{~b}^{2} \mathrm{~F}-\mathrm{y}^{4} \mathrm{PO}^{\circ}$ $\begin{array}{lllllll}3881.97 & \text { A } & 7 & 2.40 & 5.58 & 3 \frac{1}{2}-2 \frac{1}{2} & \text { b } 25-T^{2} D^{\circ} \\ 3914.36 & \text { A } & 7 & 3.41 & 5.56 & 3 \frac{1}{2}-1 \frac{1}{2} & (134)\end{array}$

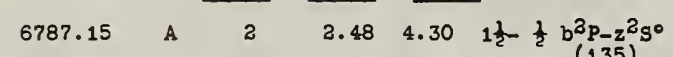

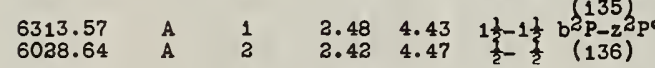
$\begin{array}{llllll}6106.47 \quad A \quad 2 & 2.48 & 4.50 \quad 1 \frac{1}{2}-1 \frac{1}{2} b^{3} \mathrm{P}-y^{4} \mathrm{~F}^{\circ}\end{array}$

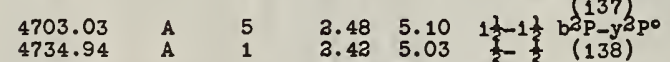

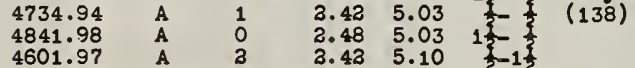
$4629.07 \quad A \quad 5 \quad 3.48 \quad 5.15 \quad 1 \frac{1}{2}-2 \frac{1}{3} b^{2} P-x^{3} D^{\circ}$

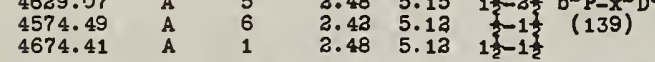
$4388.50 \quad A \quad 3 \quad 3.48 \quad 5.39 \quad 1 \frac{1}{2}-2 \frac{1}{2} b^{2} \mathrm{P}-x^{3} \mathrm{~F}^{\circ}$ $4323.63 \quad A \quad 2 \quad 2.48 \quad 5.33 \quad 1 \frac{1}{2}-2 \frac{1}{2} \quad$ b $2 \frac{140)}{P-y^{4} \circ}$

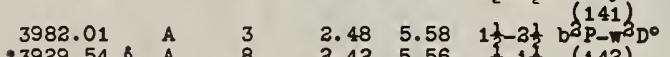

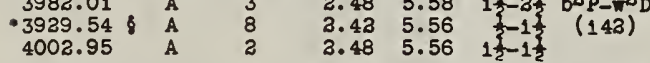
$3922.36 \quad A \quad 1 \quad 2.48 \quad 5.63 \quad 1 \frac{1}{2}-1 \frac{1}{2} b^{2} \mathrm{P}-x^{2} \mathrm{po}$

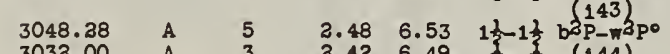
$\begin{array}{lllllll}3032.00 & \text { A } & 3 & 3.42 & 6.49 & \frac{1}{3}-\frac{1}{3} & (144) \\ 3075.55 & \text { A } & 1 & 3.48 & 6.49 & 1 \frac{1}{2} & \end{array}$
I A Reboratory Int Low H1gh J Multiplet I A Raboratory Ref Int Low ${ }_{\text {H1gh }}^{\text {L }}$ J vultiplet 2r II continued

$4908.67 \quad \mathrm{~A} \quad 1 \quad 3.11 \quad 5.63 \quad \frac{1}{2}-1 \frac{1}{2} \mathrm{a}^{2} \mathrm{~S}-\mathrm{x}^{3} \mathrm{po}$

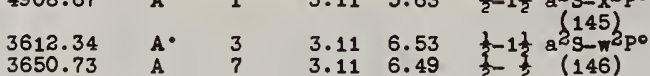
Cb II continued

$\begin{array}{llllllc}3781.379 & \text { A } & 200 & 1.69 & 4.95 & 4-5 & b^{3} F-z^{3} \sigma^{\circ}+ \\ 3898.293 & \text { A } & 200 & 1.69 & 4.86 & 3-4 & (9)\end{array}$ $\begin{array}{llllll}3898.292 & \text { A } & 200 & 1.69 & 4.86 & 3-4 \\ 3863.056 & \text { A } & 150 & 1.58 & 4.78 & 2-3\end{array}$ $\begin{array}{llccccc}3763.13 & \text { A } & 8 \mathrm{n} & 1.69 & 4.97 & 4-4 & \mathrm{~b}^{3} F-z^{3} F^{\bullet}+ \\ 3831.840 & \text { A } & 200 & 1.69 & 4.91 & 3-3 & (10)\end{array}$ $\begin{array}{lllllllllllll}3026.18 & \text { A } & 3 w 1 & 3.93 & 8.01 & 4 \frac{1}{3}-4 & z^{4} F^{\circ}-e^{4} \mathrm{~F}+ & 3818.862 & \text { A } & 200 & 1.58 & 4.81 & 2-2 \\ 3018.53 & \text { A } & 3 w & 3.86 & 7.95 & 3 \frac{3}{2}-3 \frac{1}{2} & (147) & 3952.367 & \text { A } & 100 \text { n } & 1.69 & 4.81 & 3-2\end{array}$

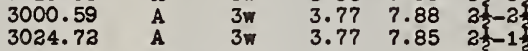

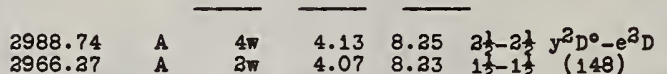
Strongest Unclassified Lines of Cb II

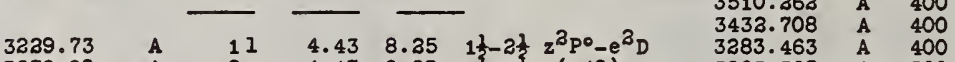

$\begin{array}{llllllllll}3229.73 & \mathrm{~A} & 11 & 4.43 & 8.25 & 1 \frac{1}{2}-2 \frac{1}{2} & \mathrm{z}^{2} \mathrm{P}^{\circ}-\mathrm{e}^{2} \mathrm{D} & 3283.463 & \mathrm{~A} & 400 \\ 3278.89 & \mathrm{~A} & \mathbf{2} & 4.47 & 8.23 & \frac{1}{2}-1 \frac{1}{2} & (149) & 3263.365 & \mathrm{~A} & 300\end{array}$

Strongest Unclassified Lines of $\underline{\mathrm{Zr}}$ II

$\begin{array}{lll}3827.27 & \text { A } & 1 \\ 3423.82 & \text { A } & 31 \\ 3068.32 & \text { A } & \text { 3 } \\ 3063.63 & \text { A } & 3 \text { WI } \\ 3038.59 & \text { A } & \text { 3 }\end{array}$

$\begin{array}{lll}3018.08 & \text { A } & 2 \text { w } \\ 2994.05 & \text { A } & 4 \%\end{array}$

Cb I I P P Anal C L18t D July 1942

$\begin{array}{llllll}4058.933 / / \text { A } & 3000 & 0.13 & 3.17 & 4 \frac{1}{3}-5 \frac{1}{3} & a^{6} \mathrm{D}-\mathrm{y}^{6} \mathrm{~F}^{\circ}+\end{array}$

4100.918 A $600 \mathrm{w} \quad 0.05 \quad 3.06 \quad 3 \frac{3}{3} \quad$ (1)

4123.812 A $400 \quad 0.02 \quad 3.01 \quad 1 \frac{2}{2}-3 \frac{1}{2}$

$\begin{array}{llllll}4137.090 & \text { A } & 200 & 0.00 & 2.98 & 13 \\ 4139.703 & \text { A } & 400 \mathrm{w} & 0.13 & 3.11 & 4 \frac{1}{3}\end{array}$

$\begin{array}{llllll}4152.575 & \text { A } & 500 & 0.09 & 3.06 & 3\end{array}$

$\begin{array}{llllll}4163.658 & \text { A } & 250 & 0.03 & 2.98 & 1 \frac{1}{2}-1 \frac{1}{2} \\ 4168.133 & \text { A } & 250 w & 0.00 & 3.96 & \text { 至 }\end{array}$

3791.209 A $300 \mathrm{r} \quad 0.13 \quad 3.38 \quad 4 \frac{1}{3} a^{6} \mathrm{D}-\mathrm{y}^{6} \mathrm{D}^{0}+$

$\begin{array}{llllllll}3824.883 & \text { A } & 100 & 0.09 & 3.31 \quad 3 \frac{1}{2}-3 \frac{1}{2} \quad \text { (3) }\end{array}$

$\begin{array}{lllllll}3713.018 & \text { A } & 300 r & 0.13 & 3.45 & 4 \frac{1}{2}-4 \frac{1}{2} & a^{6} D-x^{6} D^{\circ} \\ 3739.80 & \text { A } & 300 r & 0.09 & 3.39 & 3 \frac{1}{2}-3 \frac{1}{3} & (3)\end{array}$

3759.556 A $200 \mathrm{r} \quad 0.05 \quad 3.33$ 3.

$\begin{array}{llllll}3790.138 & \text { A } & 300 \mathrm{r} & 0.13 & 3.39 & 4 \\ 3803.938 & \text { A } & 400 \mathrm{r} & 0.09 & 3.33 & 3=-3\end{array}$

3798.127 A $300 \mathrm{r} \quad 0.05 \quad 3.30$ at $1 \frac{1}{3}$

$\begin{array}{llllll}3787.064 & \text { A } & 150 & 0.02 & 3.38 & 12 \\ 3697.850 & \text { A } & 200 & 0.05 & 3.39 & 2 \frac{1}{3}\end{array}$

$\begin{array}{llllll}3726.335 & \text { A } & 250 & 0.02 & 3.33 & 1 \frac{1}{2}-2 \frac{1}{2} \\ 3742.393 & \text { A } & 300 \mathrm{r} & 0.00 & 3.30 & \frac{1}{2}-1 \frac{1}{2}\end{array}$

3580.377 A $400 \mathrm{r} \quad 0.13 \quad 3.58 \quad 4 \frac{1}{3}-3 \frac{1}{3} a^{6} \mathrm{D}-\mathrm{y}^{6} \mathrm{p}^{0}+$

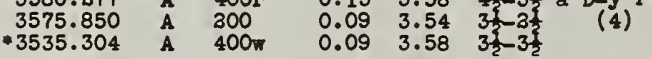

Cb II I P P Anal C L18t C July 1943

$3094.173 / /$ A $3000 \mathrm{wR} \quad 0.51 \quad 4.50 \quad 5-6 \quad \mathrm{a}^{5} \mathrm{~F}-\mathrm{z}^{5} \mathrm{G}^{\circ}+$ 3130.780 A $1500 \mathrm{wR}$ O.44 4.38 4-5 3163.403 A $1000 \mathrm{R} \quad 0.37$ 4.38 $3-4$ $\begin{array}{llllll}3194.983 & \text { A } & 700 R & 0.32 & 4.19 & 2-3 \\ 3225.478 & \text { A } & 500 w R & 0.29 & 4.12 & 1-2\end{array}$ $\begin{array}{llllll}3225.478 & \text { A } & 500 w R & 0.29 & 4.12 & 1-2 \\ 3191.096 & \text { A } & 300 \text { w } & 0.51 & 4.38 & 5-5\end{array}$ $\begin{array}{llllll}3215.595 & \text { A } & 300 \mathrm{wr} & 0.44 & 4.28 & 4-4 \\ 3236.403 & \mathrm{~A} & 300 \mathrm{r} & 0.37 & 4.19 & 3-3\end{array}$ $\begin{array}{llllll}3236.403 & \text { A } & 300 \mathrm{r} & 0.37 & 4.19 & 3-3 \\ 3254.070 & \text { A } & 200 \mathrm{r} & 0.32 & 4.12 & 2-2\end{array}$

3038.436 A $300 \mathrm{w}$

3076.864 A 200

3099.180

$\begin{array}{lll}0.44 & 4.51 & 4-3 \\ 0.37 & 4.39 & 3-2\end{array}$

$0.32 \quad 4.31 \quad 2-1$

$\begin{array}{llllll}3412.934 & \text { A } & 150 & 0.90 & 4.51 & 2-3 \\ 3408.678 & \text { A } & 100 & 0.76 & 4.39 & 1-2\end{array}$

\begin{tabular}{llllll}
3409.191 & $A$ & 100 & 0.76 & 4.39 & $1-2$ \\
& & & 0.69 & 4.31 & $0-1$ \\
\hline
\end{tabular}

3619.514 A 200

$\begin{array}{lll}3619.514 & \text { A } & 200 \\ 3651.182 & \text { A } & 300\end{array}$

$\begin{array}{lll}1.03 & 4.51 & 4-3\end{array}$

$a^{5} \mathrm{~F}-\mathrm{z}^{3} \mathrm{D}^{\circ}+$

3180.390 A $500 \mathrm{rs} \quad 1.03 \quad 4.95 \quad 4-5$

$\begin{array}{llllll}3180.390 & \text { A } & 400 & 0.98 & 4.86 & 3-4 \\ 3206.350 & \text { A } & 300 \mathrm{rs} & 0.93 & 4.78 & 2-3\end{array}$

\begin{tabular}{llllll}
3223.332 & A & 100 & 1.03 & 4.86 & $4-4$ \\
3347.478 & A & 150 & 0.98 & 4.78 & $3-3$ \\
\hline
\end{tabular}

3440.589 A $300 \quad 1.36 \quad 4.95 \quad 5-5 \quad a^{3} a-z^{3} c^{0}+$

$\begin{array}{llllll}3479.567 & \text { A } & 150 & 1.31 & 4.86 & 4-4 \\ 3515.421 & \text { A } & 300 \text { w } & 1.37 & 4.78 & 3-3\end{array}$

(6)

3425.432 A 300 - $1.36 \quad 4.97 \quad 5-4 \quad a^{3} G-z^{3} 5^{\circ}+$

$\begin{array}{llllll}3426.562 & \text { A } & 350 \mathrm{~W} & 1.31 & 4.97 & 5-4 \\ 3478.79 & \mathrm{~A} & 100 & 1.37 & 4.81 & 3-3 \\ \end{array}$

4367.966 A $100 \mathrm{n} \quad \overline{1.69} \quad \overline{4.51} 4-3 \quad \mathrm{~b}^{3} \mathrm{~F}-\mathrm{z}^{3} \mathrm{D}^{0}+$

$\begin{array}{rrrrrr}4579.446 & \text { A } & 150 \mathrm{n} & 1.69 & 4.39 & 3-2 \\ 4527.648 & \text { A } & 50 \mathrm{n} & 1.58 & 4.31 & 2-1\end{array}$
3360.564 A 350 w

$\begin{array}{lll}3127.526 & \text { A } & 500 \\ 3064.530 & \text { A } & 250 \mathrm{r}\end{array}$

$\begin{array}{lll}3034.95 & \text { A } & 200 \mathrm{wR} \\ 3032.767 & \text { A } & 400 \mathrm{rs}\end{array}$

3994.725 A $300 \mathrm{rs}$
Mo I I P 7.06 Anal C L18t D July 1943

$3798.259 / /$ A $\quad 50 R \quad 0.00 \quad 3.25 \quad 3-4 \quad a^{7} S-z^{7} p$

$\begin{array}{lllllll}3864.115 & \text { A } & \text { 5OR } & 0.00 & 3.19 & 3-3 & \text { (1) } \\ 3902.968 & \text { A } & \text { 5OR } & 0.00 & 3.16 & 3-3 & \end{array}$

3113.125

3158.156

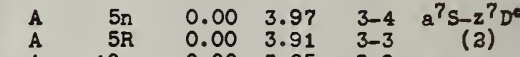

(a)

3133.591

3170.333
3193.969

5506.51

5533.01

6030.

5791.86

5751.41

4686.467

4663.767

4661.933
4534.344

4576.500

4277.246

4288.65
4293.228

3833.757

3838.883

3826.701
3822.987

901.775

3886.325
3869.085

3847.252

3763.356
3770.517

3781.597

3405.934

3384.617

3358.130
3344.750

3337.308

3361.371

(3)

1OR

$\begin{array}{cccc}0.00 & 3.94 & 3-4 & a^{7} s-y^{7} p^{0} \\ 0.00 & 3.89 & 3-3 & (3)\end{array}$

IOR

$0.00 \quad 3.86 \quad 3-3$

$1.33 \quad 3.57 \quad 3-3 \quad a^{5} s_{-2} 50$

$1.33 \quad 3.56 \quad 2-2$

$(4)$

$\begin{array}{lll}1.52 & 3.57 & 4.3\end{array}$

$\begin{array}{lll}1.46 & 3.56 & 3-2 \\ 1.41 & 3.54 & 2-1 \\ 1.46 & 3.57 & 3-3\end{array}$

$\begin{array}{lll}1.41 & 3.56 & 2-3 \\ 1.38 & 3.54 & 1-1\end{array}$

$1.53 \quad 4.19 \quad 4-3 \quad a^{5} \mathrm{D}-\mathrm{y}^{5} \mathrm{po}$

$\begin{array}{lll}1.46 & 4.11 & 3-2\end{array}$

$\begin{array}{lll}1.41 & 4.06 & 3-1 \\ 1.46 & 4.19 & 3-3\end{array}$

$\begin{array}{lll}1.41 & 4.11 & 2-2 \\ 1.38 & 4.06 & 1-1\end{array}$

$1.53 \quad 4.41 \quad 4-5 \quad a^{5} \mathrm{D}-z^{5} F^{0} \uparrow$

$\begin{array}{lll}1.52 & 4.41 & 4-5 \\ 1.46 & 4.34 & 3-4\end{array}$

(?)

$1.52 \quad 4.74 \quad 4-4 \quad a^{5} \mathrm{D}-z^{5} \mathrm{D}^{\circ}$

$\begin{array}{lllll}10 \mathrm{n} & 1.52 & 4.74 & 4- \\ 10 & 1.46 & 4.69 & 3-3\end{array}$

$(8)$

$\begin{array}{lll}1.41 & 4.64 & 2-2\end{array}$

$\begin{array}{lll}1.38 & 4.60 & 1-1 \\ 1.52 & 4.69 & 4-3\end{array}$

$\begin{array}{lll}1.52 & 4.69 & 4-3 \\ 1.46 & 4.64 & 3-2\end{array}$

$1.41 \quad 4.60 \quad 2-1$

$\begin{array}{lll}1.38 & 4.58 & 1-0\end{array}$

$\begin{array}{lll}1.46 & 4.74 & 3-4 \\ 1.41 & 4.69 & 2-3\end{array}$

$\begin{array}{lll}1.41 & 4.69 & 2-3 \\ 1.38 & 4.64 & 1-2\end{array}$

$\begin{array}{lllc}1.52 & 5.15 & 4-5 & a^{5} D-y^{5} F^{0}+ \\ 1.46 & 5.11 & 3-4 & (9)\end{array}$

$\begin{array}{ll}1.41 & 5.09\end{array}$

$\begin{array}{lll}1.38 & 5.07 & 1-2 \\ 1.35 & 5.06 & 0-1\end{array}$

$1.53 \quad 5.30 \quad 4-4 \quad a^{5} \mathrm{D}-8^{\circ}+$

$89.016 \quad A \quad 10 r \quad 1.41 \quad 5.17 \quad 2-3 \quad a^{5} \mathrm{D}^{(10)}+$

$\begin{array}{lllllll}4012.51 & \mathrm{C} & (1) & 3.07 & 5.15 & 6-5 & \mathrm{a}^{5} \mathrm{G}-\mathrm{y}^{5} \mathrm{~F}^{\circ}+ \\ 4062.09 & \mathrm{~A} & 5 \mathrm{Nr} & 3.07 & 5.11 & 5-4 & (13)\end{array}$

$\begin{array}{lllllll}063.09 & \text { A } & 5 \mathrm{Nr} & 3.07 & 5.11 & 5-4 & \text { (12) }\end{array}$

\begin{tabular}{llllll}
4102.158 & A & 10 & 2.05 & 5.06 & $2-1$ \\
4056.027 & A & 10 & 3.07 & 5.11 & $4-4$ \\
\hline
\end{tabular}
5888.32

$\begin{array}{lll}1.41 & 3.54 & 2-1 \\ 1.46 & 3.57 & 3-3 \\ 1.41 & 3.56 & 2-3\end{array}$

$\begin{array}{llllll}107.477 & A & 8 \mathrm{r} & 2.06 & 5.07 & 3-2\end{array}$
Yo II I P ? Anal D L18t $D$ Aug 1943

$\begin{array}{lllllll}3446.085 & A & 6 & 2.94 & 6.53 & 42-4 \frac{1}{2} & 4 \mathrm{~F}-{ }^{4} \mathrm{~F}^{\circ} \\ 3524 & 8 & 3.94 & 6.45 & 3 \frac{1}{3} & (1)\end{array}$ 3596.351

3523.063

3585.91

3643.47

3448.542
3534.688

3534.688
3623.850

3136.465

3187.592
3350.747 $\begin{array}{lll}3.94 & 6.45 & 3 \frac{3}{2}-3 \\ 3.95 & 6.39 & 3 \frac{1}{2}-2 \frac{1}{2}\end{array}$

$2.98 \quad 6.34 \quad 121$

$\begin{array}{lll}2.94 & 6.45 & 4 \frac{1}{2}-3 \\ 2.94 & 6.39 & 3 \frac{1}{3}-2\end{array}$

2.95 6.34 $25-1$

$\begin{array}{lll}2.94 & 6.52 & 3-4 \\ 3.95 & 6.45 & 2\end{array}$

$\begin{array}{lll}3.95 & 6.45 & 2 \\ 3.98 & 6.39 & 1 \frac{1}{2}-3 \frac{1}{2}\end{array}$

$2.94 \quad 6.88 \quad 4 \frac{1}{3}-3 \frac{1}{2} \quad 4 \mathrm{~T}-\mathrm{C}^{4}+$

$\begin{array}{lll}2.94 & 6.82 & 3 \frac{1}{2}-2 \frac{1}{2} \\ 3.95 & 6.75 & 2 \frac{1}{2}-1 \frac{1}{5}\end{array}$ 
REVISED Y ULTIPLET TABLE

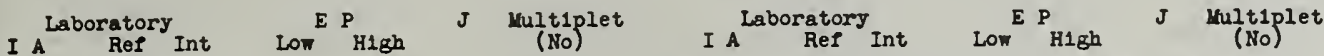
עo II continued

\begin{tabular}{|c|c|c|c|c|c|c|}
\hline $\begin{array}{l}4850.689 \\
4363.644 \\
4433.501 \\
4309.649 \\
4879.023 \\
4377.765\end{array}$ & $\begin{array}{l}\hat{A} \\
\hat{A} \\
\mathbf{A} \\
\hat{A} \\
\hat{A} \\
\mathbf{A}\end{array}$ & $\begin{array}{r}10 \\
10 \\
8 \\
5 \\
10 \\
10\end{array}$ & $\begin{array}{l}3.13 \\
3.10 \\
3.04 \\
3.10 \\
3.04 \\
3.01\end{array}$ & $\begin{array}{l}6.03 \\
5.93 \\
5.83 \\
6.03 \\
5.93 \\
5.83\end{array}$ & $\begin{array}{l}3 \frac{1}{3}-2 \frac{1}{2} \\
3 \frac{1}{2}-1 \frac{1}{2} \\
1 \frac{1}{2}-\frac{1}{2} \\
2 \frac{1}{3}-3 \frac{1}{2} \\
1 \frac{1}{2}-1 \frac{1}{2} \\
\frac{1}{2}-\frac{1}{2}\end{array}$ & ${ }^{4} D_{-3)^{4}} \mathrm{P}^{\circ}+$ \\
\hline $\begin{array}{l}3941.478 \\
3961.503 \\
3986.301\end{array}$ & $\begin{array}{l}\hat{A} \\
\hat{A}\end{array}$ & $\begin{array}{r}10 \\
15 \\
6\end{array}$ & $\begin{array}{l}3.13 \\
3.13 \\
3.10\end{array}$ & $\begin{array}{l}6.36 \\
6.34 \\
6.30\end{array}$ & $\begin{array}{l}\frac{31}{3}-4 \frac{1}{2} \\
\frac{3}{2}-3 \frac{1}{2} \\
3 \frac{1}{2}-1 \frac{1}{2}\end{array}$ & ${ }_{(4)}^{4}{ }^{6} D^{0} \uparrow$ \\
\hline $\begin{array}{l}3835.144 \\
3888.307 \\
3893.645 \\
3702.553 \\
3719.74 \\
3755.54 \\
3748.34\end{array}$ & $\begin{array}{l}\hat{A} \\
\mathbf{A} \\
\hat{A} \\
\hat{A} \\
\hat{A} \\
\hat{A} \\
\hat{A}\end{array}$ & $\begin{array}{r}30 \\
15 \\
10 \\
8 \\
3 \\
5 \\
6\end{array}$ & $\begin{array}{l}3.13 \\
3.10 \\
3.04 \\
3.01 \\
3.13 \\
3.10 \\
3.04\end{array}$ & $\begin{array}{l}6.53 \\
6.45 \\
6.39 \\
6.34 \\
6.45 \\
6.39 \\
6.34\end{array}$ & $\begin{array}{l}3 \frac{1}{2}-4 \frac{1}{2} \\
3 \frac{1}{2}-3 \frac{1}{2} \\
1 \frac{1}{2}-3 \frac{1}{2} \\
\frac{1}{3}-1 \frac{1}{2} \\
3 \frac{2}{2}-3 \frac{1}{2} \\
3 \frac{1}{2}-2 \frac{1}{2} \\
1 \frac{1}{2}-1 \frac{2}{2}\end{array}$ & ${ }^{4} D-{ }_{(5)}^{4} F^{0}+$ \\
\hline $\begin{array}{l}3393.313 \\
3330.903 \\
3339.315 \\
3347.369 \\
3346.403 \\
3380.315 \\
3379.783 \\
3367.639 \\
3671.866 \\
3397.684\end{array}$ & $\begin{array}{l}\mathbf{A} \\
\hat{A} \\
\mathbf{A} \\
\hat{A} \\
\mathbf{A} \\
\hat{A} \\
\mathbf{A} \\
\mathbf{A} \\
\hat{A} \\
\mathbf{A}\end{array}$ & $\begin{array}{r}13 \\
5 \\
5 \\
3 \\
4 \\
3 \\
3 \\
3 \\
4 \\
3\end{array}$ & $\begin{array}{l}3.13 \\
3.10 \\
3.04 \\
3.01 \\
3.13 \\
3.10 \\
3.04 \\
3.10 \\
3.04 \\
3.01\end{array}$ & $\begin{array}{l}6.88 \\
6.83 \\
6.75 \\
8.70 \\
6.83 \\
6.75 \\
8.70 \\
6.88 \\
6.82 \\
6.75\end{array}$ & $\begin{array}{l}3 \frac{1}{2} \\
3 \frac{1}{2}-1 \frac{1}{1} \\
1 \frac{1}{2} \\
3 \frac{1}{2} \\
1 \frac{1}{2}-3 \frac{1}{2} \\
\frac{1}{2}-1 \frac{1}{2}\end{array}$ & ${ }_{(8)}^{{ }^{4} D-D^{\circ}}$ \\
\hline
\end{tabular}

Fu I I 7.5 Ansl C L18t $D$ Aug 1948

\begin{tabular}{|c|c|c|c|c|c|c|c|c|c|c|c|c|}
\hline 3799 & $A$ & $(8)$ & 0.00 & 3.25 & $5-4$ & $a^{5} F-z^{5} D^{0} \uparrow$ & Rh I & 7.7 & Anal & \multicolumn{2}{|c|}{ List $D$} & Aug 1943 \\
\hline $\begin{array}{l}3738.030 \\
3736.926 \\
3730.433 \\
3743.280\end{array}$ & $\hat{A}$ & $\begin{array}{l}\left(\begin{array}{l}10 R \\
10 R\end{array}\right) \\
(4) \\
(10)\end{array}$ & $\begin{array}{l}0.00 \\
0.15 \\
0.26 \\
0.33\end{array}$ & $\begin{array}{l}3.31 \\
3.46 \\
3.57 \\
3.63\end{array}$ & $\begin{array}{l}5-5 \\
4-4 \\
3-3 \\
3-3\end{array}$ & $\frac{a^{5} F-z^{5} F^{\circ} \uparrow}{(z)}$ & $\begin{array}{l}3693.357 \\
3657.987 \\
3596.194 \\
3613.470\end{array}$ & $\begin{array}{l}\mathbf{A} \\
\mathbf{A} \\
\mathbf{A} \\
\mathbf{A}\end{array}$ & $\begin{array}{l}50 \\
50 \\
20 \\
15\end{array}$ & $\begin{array}{l}0.00 \\
0.19 \\
0.33 \\
0.43\end{array}$ & $\begin{array}{l}3.34 \\
3.56 \\
3.75 \\
3.85\end{array}$ & $\begin{array}{l}4 \frac{1}{3}-3 \frac{1}{2} a^{4} F-2^{4} D^{0}+ \\
3 \frac{2}{2}-(1) \\
2 \frac{2}{2}-1 \frac{1}{2} \\
1 \frac{1}{2}-\frac{1}{2}\end{array}$ \\
\hline 3760.031 & A & (4) & 0.38 & 3.67 & $1-1$ & & $\begin{array}{l}3434.893 / / \\
3700.909\end{array}$ & $\stackrel{A}{A}$ & $\begin{array}{c}300 R \\
30\end{array}$ & $\begin{array}{l}0.00 \\
0.19\end{array}$ & $\begin{array}{l}3.59 \\
3.53\end{array}$ & $\frac{4 \frac{1}{3}-5 \frac{1}{3} a^{4} F-z^{4} \sigma^{0}+}{(a)}$ \\
\hline $\begin{array}{l}3661.353 \\
3417.353 \\
3430.773\end{array}$ & $\begin{array}{l}\hat{A} \\
\hat{A}\end{array}$ & $\begin{array}{r}16 \\
(20 \\
(7)\end{array}$ & $\begin{array}{l}0.15 \\
0.36 \\
0.33\end{array}$ & $\begin{array}{l}3.58 \\
3.87 \\
3.93\end{array}$ & $\begin{array}{l}4-5 \\
3-4 \\
3-3\end{array}$ & $\frac{a^{5} F-z^{3} G^{0} \uparrow}{(3)}$ & $\begin{array}{l}3507.316 \\
3474.780 \\
3502.524\end{array}$ & A & $\begin{array}{l}30 \\
30 \\
50\end{array}$ & $\begin{array}{l}0.32 \\
0.43 \\
0.00\end{array}$ & $\begin{array}{l}3.84 \\
3.98 \\
3.53\end{array}$ & $\begin{array}{l}3 \frac{2}{2}-3 \frac{1}{2} \\
1 \frac{2}{2}-2 \frac{1}{2} \\
4 \frac{1}{2}-4 \frac{1}{2}\end{array}$ \\
\hline $\begin{array}{l}3498.948 / / \\
3436.737 \\
3596.179 \\
3593.032 \\
3589.315 \\
3301.587\end{array}$ & $\begin{array}{l}\hat{A} \\
\hat{A} \\
\hat{A} \\
\hat{A}\end{array}$ & $\left(\begin{array}{l}50 R \\
30 R \\
30 \\
20 \\
5 \\
8\end{array}\right)$ & $\begin{array}{l}0.00 \\
0.15 \\
0.36 \\
0.33 \\
0.38 \\
0.00\end{array}$ & $\begin{array}{l}3.53 \\
3.74 \\
3.89 \\
3.77 \\
3.83 \\
3.74\end{array}$ & $\begin{array}{l}5-6 \\
4-5 \\
3-4 \\
3-3 \\
1-3 \\
5-5\end{array}$ & $\begin{array}{c}a^{5} F-z^{5} G^{0} \uparrow \\
(4)\end{array}$ & $\begin{array}{l}3396.85 \\
3538.034 \\
3462.040 \\
3470.657 \\
3583.098 \\
3668.315\end{array}$ & $\begin{array}{l}\mathbf{A} \\
\mathbf{A} \\
\mathbf{A} \\
\mathbf{A} \\
\mathbf{A} \\
\mathbf{A}\end{array}$ & $\begin{array}{c}100 \mathrm{R} \\
30 \\
30 \\
30 \\
10 \\
15\end{array}$ & $\begin{array}{l}0.00 \\
0.19 \\
0.33 \\
0.43 \\
0.19 \\
0.32\end{array}$ & $\begin{array}{l}3.83 \\
3.69 \\
3.89 \\
3.98 \\
3.63 \\
3.69\end{array}$ & $\begin{array}{l}4 \frac{1}{2}-4 \frac{1}{3} a^{4} F-z^{4} F^{0} \uparrow \\
3 \frac{2}{2}-\frac{1}{2}(3) \\
3 \frac{2}{2}-2 \frac{2}{2} \\
1 \frac{2}{2}-1 \frac{2}{2} \\
3 \frac{2}{2}-\frac{4}{2} \\
3 \frac{2}{2}-3 \frac{2}{2}\end{array}$ \\
\hline $\begin{array}{l}4554.509 \\
4397.711\end{array}$ & $\hat{A}$ & $\left(\begin{array}{l}10 R) \\
10 \\
(8\end{array}\right)$ & $\begin{array}{l}0.81 \\
1.00\end{array}$ & $\begin{array}{l}3.52 \\
3.87\end{array}$ & $\begin{array}{l}4-5 \\
3-4\end{array}$ & $\begin{array}{l}F-z^{3} G^{0}+ \\
(5)\end{array}$ & $\begin{array}{l}3333.093 \\
3383.573\end{array}$ & $\hat{A}$ & $\begin{array}{l}\text { 5OR } \\
\text { 30R }\end{array}$ & $\begin{array}{l}0.19 \\
0.32\end{array}$ & $\begin{array}{l}3.90 \\
4.08\end{array}$ & $\frac{3 \frac{1}{2}-4 \frac{1}{2}}{3 \frac{1}{2}-\frac{1}{2}} a^{4} F-z^{2} a^{0}+$ \\
\hline 4313.063 & $\boldsymbol{A}$ & (10) & 0.81 & 3. & $4-5$ & -200 & 3597.147 & A & 30 & 0.41 & 3.84 & $\begin{array}{c}3 \frac{1}{2}-3 \frac{1}{2} a^{3} D-z^{4} c^{0} \uparrow \\
(5)\end{array}$ \\
\hline $\begin{array}{l}4584.445 \\
4681.786\end{array}$ & $\hat{A}$ & $\begin{array}{l}30 \\
10\end{array}$ & $\begin{array}{l}1.00 \\
1.13\end{array}$ & $\begin{array}{l}3.69 \\
3.77\end{array}$ & $\begin{array}{l}3-4 \\
3-3\end{array}$ & & $\begin{array}{l}3478.908 \\
3543.948\end{array}$ & $\mathbf{A}$ & $\begin{array}{l}15 \\
10\end{array}$ & $\begin{array}{l}0.41 \\
0.70\end{array}$ & $\begin{array}{l}3.96 \\
4.18\end{array}$ & $\begin{array}{c}3 \frac{1}{2}-3 \frac{1}{2} \\
1 \frac{1}{2}-1 \frac{1}{2}\end{array}$ \\
\hline $\begin{array}{l}4080.600 \\
4144.164\end{array}$ & $\hat{A}$ & $\left\{\begin{array}{l}30 \\
10\end{array}\right)$ & $\begin{array}{l}0.81 \\
1.00\end{array}$ & $\begin{array}{l}3.83 \\
3.98\end{array}$ & $\begin{array}{l}4-3 \\
3-3\end{array}$ & $\frac{a^{3} F-y^{5} F^{\circ}+}{(7)}$ & $\begin{array}{l}3371.812 \\
3788.474\end{array}$ & $\stackrel{A}{A}$ & $\begin{array}{l}10 \\
15\end{array}$ & $\begin{array}{l}0.41 \\
0.70\end{array}$ & $\begin{array}{l}4.18 \\
3.96\end{array}$ & \\
\hline 4199.903 & A & (10) & 0.81 & 3.75 & $4-4$ & $\begin{array}{l}F-z^{3} F^{\circ}+ \\
(8)\end{array}$ & $\begin{array}{l}3856.515 \\
3958.865\end{array}$ & $\hat{A}$ & $\begin{array}{l}10 \\
30\end{array}$ & $\begin{array}{l}0.70 \\
0.96\end{array}$ & $\begin{array}{l}3.90 \\
4.08\end{array}$ & $\begin{array}{c}3 \frac{2}{2}-4 \frac{1}{2} \\
3 \frac{1}{2}-3 \frac{1}{2}\end{array} a^{3} \vec{F}-2^{2} \sigma^{0}+$ \\
\hline $\begin{array}{l}3984.858 \\
4097.791\end{array}$ & $\hat{\Lambda}$ & $\left\{\begin{array}{l}10 \\
10\end{array}\right\}$ & $\begin{array}{l}.81 \\
1.00 \\
1.13\end{array}$ & $\begin{array}{l}4.00 \\
4.10 \\
4.15\end{array}$ & $\begin{array}{l}4-3 \\
3-3 \\
3-1\end{array}$ & & $\begin{array}{l}3799.311 \\
3833.363 \\
4128.870\end{array}$ & $\begin{array}{l}\mathbf{A} \\
\mathbf{A}\end{array}$ & $\begin{array}{l}30 \\
15 \\
30\end{array}$ & $\begin{array}{l}0.70 \\
0.96 \\
0.96\end{array}$ & $\begin{array}{l}3.95 \\
4.19 \\
3.95\end{array}$ & $\begin{array}{l}3 \frac{1}{2}-3 \frac{1}{2} a^{3} \mathrm{~F}-2^{3} \mathrm{~F}^{0} \\
3 \frac{2}{2} \\
3(8)\end{array}$ \\
\hline 5309.367 & $A$ & 30 & 0.93 & 3.35 & $4-4$ & $a^{5} D-z^{5} D^{0} \uparrow$ & & A & 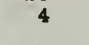 & & & \\
\hline $\begin{array}{l}5636.235 \\
5155.138\end{array}$ & $\hat{A}$ & $\begin{array}{l}35 \\
12\end{array}$ & $\begin{array}{l}1.08 \\
1.13\end{array}$ & $\begin{array}{l}3.25 \\
3.51\end{array}$ & $\begin{array}{l}3-4 \\
1-3\end{array}$ & & $\begin{array}{l}3793.217 \\
3833.889\end{array}$ & $\stackrel{A}{A}$ & $\begin{array}{l}15 \\
10\end{array}$ & $\begin{array}{l}0.70 \\
0.98\end{array}$ & $\begin{array}{l}3.96 \\
4.18\end{array}$ & $\begin{array}{c}3 \frac{1}{2}-3 \frac{1}{3} a^{2} F-z^{3} D^{0} \\
(9)\end{array}$ \\
\hline 517 & $\boldsymbol{A}$ & 40 & & & $4-$ & $a^{5} p$ & & $\mathbf{A}$ & 15 & 0.96 & & \\
\hline
\end{tabular}

\section{Ru II I P Aral D L1at C Sept 1943}

$3690.033 \quad$ A $\quad 80 \quad 3.39 \quad 5.74 \quad 3 \frac{1}{4}-4{ }^{4} D_{-} 6_{D^{\circ}+}$

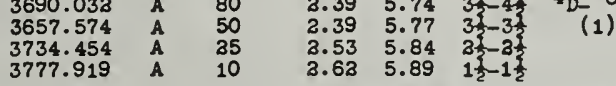
$\begin{array}{rrrrrrr}3177.060 & \text { A } & 100 & 3.39 & 6.38 & 3 \frac{1}{3}-4 \frac{1}{3} & { }^{4} D_{-} 6 F^{\circ}+ \\ 3294.320 & \text { A } & 80 & 2.53 & 6.38 & 2 \frac{1}{3}-3 & (3)\end{array}$

$\begin{array}{llllll}3175.317 & \text { A } & 10 & 2.39 & 6.38 & 3 \frac{1}{3}-3 \\ 3143.657 & \text { A } & 15 & 3.39 & 6.33 & 3 \frac{1}{2}-2 \frac{1}{2}\end{array}$

$3107.586 \quad A \quad 10 \quad 3.39 \quad 8.36 \quad 3 \frac{1}{2} \quad 4 D-6 P^{\circ}+$ $\begin{array}{rrr}3094.555 & \text { A } & 8 \\ 3331.378 & \text { A } & 15\end{array}$

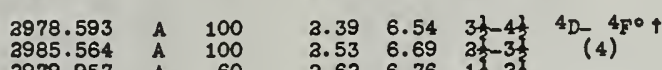
$\begin{array}{rrrrrr}3979.957 & \text { A } & 60 & 3.63 & 6.76 & 1 \frac{1}{2}-3 \\ 3977.326 & \text { A } & 30 & 3.67 & 6.82 & \frac{1}{2}-1 \frac{1}{2}\end{array}$

$3979.736 \quad A \quad 40 \quad 3.53 \quad 6.67 \quad 3 \frac{1}{2}-2 \frac{1}{3} \quad{ }^{4} D_{-}{ }^{4} D^{\circ}+$

$\begin{array}{lllllll}3991.626 & \text { A } & 40 & 3.63 & 6.75 & 1 \frac{2}{3} & \text { (5) } \\ 3998.896 & \text { A } & 30 & 3.67 & 6.79 & & \end{array}$

3359.0079 A $30 \quad 3.75 \quad 6.54 \quad 3 \frac{1}{30} 3_{F-} 4_{F 0}+$

$\begin{array}{lllllll}3060.353 & \text { A } & 8 & 3.66 & 6.69 & 3 \frac{1}{2}-3 \frac{1}{2} & (8)\end{array}$

$\begin{array}{rrrrrrr}3331.978 & \text { A } & 30 & 3.75 & 6.58 & 3 \frac{1}{1}-3 \frac{1}{2} & { }^{3} 7-D^{4}+1 \\ 3075.336 & \text { A } & 6 & 3.66 & 6.67 & 3 \frac{1}{2}-3 \frac{1}{2} & (7)\end{array}$

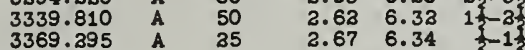

I A Ref Int

Low ${ }^{E} P_{\text {High }}$

J Multiplet

\section{Rh II continued}

\begin{tabular}{|c|c|c|c|c|c|c|}
\hline $\begin{array}{l}3477.838 \\
3093.481 \\
3096.740 \\
3062.301 \\
3008.996 \\
3047.160 \\
2979.382 \\
3962.167 \\
3035.013\end{array}$ & $\begin{array}{l}\mathbf{A} \\
\mathbf{A} \\
\mathbf{A} \\
\mathbf{A} \\
\mathbf{A} \\
\mathbf{A} \\
\mathbf{A} \\
\mathbf{A} \\
\mathbf{A}\end{array}$ & $\begin{array}{r}300 \\
300 \\
150 \\
100 \\
300 \\
300 \\
3 \\
3 \\
75 \\
300\end{array}$ & $\begin{array}{l}3.43 \\
3.47 \\
3.59 \\
3.43 \\
3.47 \\
3.59 \\
3.43 \\
3.47 \\
3.59\end{array}$ & $\begin{array}{l}6.98 \\
7.46 \\
7.57 \\
7.46 \\
7.57 \\
7.64 \\
7.57 \\
7.64 \\
7.66\end{array}$ & $\begin{array}{l}3-4 \\
2-3 \\
1-2 \\
3-3 \\
3-2 \\
1-1 \\
3-2 \\
2-1 \\
1-0\end{array}$ & ${ }^{5} P_{-}{ }^{5} D^{\circ}$ \\
\hline $\begin{array}{l}3187.889 \\
3307.362 \\
3364.391 \\
3166.948 \\
3173.678 \\
3081.585\end{array}$ & $\begin{array}{l}\mathbf{A} \\
\mathbf{A} \\
\mathbf{A} \\
\mathbf{A} \\
\mathbf{A} \\
\mathbf{A}\end{array}$ & $\begin{array}{r}200 \\
300 \\
75 \\
200 \\
100 \\
100\end{array}$ & $\begin{array}{l}3.43 \\
3.47 \\
3.59 \\
3.47 \\
3.59 \\
3.47\end{array}$ & $\begin{array}{l}7.30 \\
7.30 \\
7.37 \\
7.37 \\
7.48 \\
7.48\end{array}$ & $\begin{array}{l}3-4 \\
3-3 \\
1-2 \\
2-2 \\
1-1 \\
3-1\end{array}$ & $\frac{5 P_{-}}{(5)} 5_{F^{\circ}}$ \\
\hline $\begin{array}{l}3367.480 \\
3340.516 \\
3311.947\end{array}$ & $\begin{array}{l}\mathbf{A} \\
\hat{A} \\
\mathbf{A}\end{array}$ & $\begin{array}{l}350 \\
150 \\
150\end{array}$ & $\begin{array}{l}3.93 \\
4.03 \\
4.18\end{array}$ & $\begin{array}{l}7.69 \\
7.83 \\
8.03\end{array}$ & $\begin{array}{l}5-4 \\
4-3 \\
3-2\end{array}$ & ${ }^{3_{G}}{ }_{(6)}^{3_{F}}{ }^{\circ}$ \\
\hline $\begin{array}{l}3815.013 \\
3754.13 \\
3730.69\end{array}$ & $\begin{array}{l}\mathrm{A} \\
\mathrm{P} \\
\mathrm{P}\end{array}$ & 100 & $\begin{array}{l}4.33 \\
4.39 \\
4.32\end{array}$ & $\begin{array}{l}7.46 \\
7.57 \\
7.64\end{array}$ & $\begin{array}{l}2-3 \\
1-2 \\
0-1\end{array}$ & ${ }^{3} P_{-}{ }_{(7)}^{5} D^{0}$ \\
\hline $\begin{array}{l}3269.60 \\
3188.603 \\
3147.931 \\
3140.373 \\
3119.837\end{array}$ & $\begin{array}{l}\mathbf{P} \\
\mathbf{A} \\
\mathbf{A} \\
\mathbf{A} \\
\mathbf{A}\end{array}$ & $\begin{array}{r}150 \\
8 \\
50 \\
5\end{array}$ & $\begin{array}{l}4.33 \\
4.39 \\
4.32 \\
4.33 \\
4.39\end{array}$ & $\begin{array}{l}8.00 \\
8.16 \\
8.34 \\
8.16 \\
8.34\end{array}$ & $\begin{array}{l}2-3 \\
1-3 \\
0-1 \\
2-2 \\
1-1\end{array}$ & ${ }^{3} P_{-}{ }^{3} D^{\circ}$ \\
\hline
\end{tabular}

Pd I I P 8.30 Anal A L1st D Aug 1943

$\begin{array}{lllllll}3634.71 & B & 700 R & 0.81 & 4.31 & 3-2 & 5 s^{3} \mathrm{D}-5 \mathrm{p}^{3} \mathrm{po}\end{array}$ $\begin{array}{llllllc}3518.95 & \text { A } & 500 \mathrm{r} & 0.96 & 4.47 & 2-1 & 5 \theta^{3} \mathrm{D}-5 \mathrm{p}^{3} \\ & & & \end{array}$ $\begin{array}{llllll}3571.16 & \text { A } & 200 & 0.96 & 4.47 & 2-1 \\ 3799 & 1.25 & 4.70 & 1-0\end{array}$ $\begin{array}{rrrrrr}3799.17 & \text { A } & 75 & 1.25 & 4.70 & 1-0 \\ 3832.31 & \text { B } & 75 & 1.35 & 4.47 & 1-2 \\ & & & & \end{array}$

$3404.60 / /$ A $1000 \mathrm{R} \quad 0.81 \quad 4.44 \quad 3-4 \quad 58^{3} \mathrm{D}-5 \mathrm{p}^{3} \mathrm{~F}^{\circ}$ $\begin{array}{lllllll}3609.56 & \text { A } & 1000 \mathrm{R} & 0.81 & 4.44 & 3-4 & 58^{3} \mathrm{D}-5 \mathrm{p}^{3} \mathrm{~F} \\ 3600 \mathrm{R} & 0.96 & 4.38 & 2-3 & (3)\end{array}$ $\begin{array}{lllll}3481.17 & \text { A } 400 \mathrm{r} & 1.35 & 4.79 & 1-2\end{array}$ $\begin{array}{llclll}3460.76 & \text { A } & 300 r & 0.81 & 4.38 & 3-3 \\ 3318.98 & \text { A } & 30 & 0.96 & 4.79 & 3-2\end{array}$

$3342.73 \quad$ A $1000 R \quad 0.81 \quad 4.63 \quad 3-3 \quad 58^{3} D-5 p^{3} D^{\circ}$ $\begin{array}{llcllll}3431.34 & \text { A } & 500 & 0.96 & 4.56 & 2-3 & 58 \\ 3303.15 & \text { A } & 400 & 1.25 & 4.98 & 1-1 & (3)\end{array}$ $\begin{array}{rrrrrr}3302.15 & \text { A } & 400 & 1.25 & 4.98 & 1-1 \\ 3287.36 & \text { A } & 50 & 0.81 & 4.56 & 3-2\end{array}$ $\begin{array}{llllll}3065.30 & A & 100 & 0.96 & 4.98 & 3-1\end{array}$ $\begin{array}{llllll}3372.02 & A & 300 & 0.96 & 4.62 & 3-3 \\ 3718.93 & \text { B } & 100 & 1.25 & 4.56 & 1-3\end{array}$ $3003.66 \quad A \quad 50 \quad 0.81 \quad 4.93 \quad 3-3 \quad 58^{3} \mathrm{D}-5 \mathrm{p}^{1} \mathrm{~F}^{\circ}$ $\begin{array}{rrrrrrc}3002.66 & A & 50 & 0.81 & 4.93 & 3-3 & 58^{3} \mathrm{D}-5 \mathrm{p}^{1} \\ 3114.05 & \mathrm{~A} & 300 & 0.96 & 4.92 & 3-3 & (4)\end{array}$ $\begin{array}{llllllll}3027.93 & \text { A } & 100 & 0.96 & 5.03 & 2-3 & 58^{3} \mathrm{D}-5 \mathrm{p}^{1} \mathrm{D}^{\circ}+ \\ 3358.80 & \text { A } & 300 & 1.25 & .5 .03 & 1-2 & (5)\end{array}$ $\begin{array}{rrrrrrr}3021.74 & \text { A } & 10 & 0.96 & 5.04 & 2-1 & 58^{3} \mathrm{D}-5 \mathrm{p}^{1} \mathrm{P}^{\circ} \\ 3351.68 & \text { A } & 300 & 1.25 & 5.04 & 1-1 & \end{array}$ $\begin{array}{lllllll}3251.68 & \text { A } & 300 & 1.25 & 5.04 & 1-1 & (6) \\ \end{array}$

$\begin{array}{ccccccc}4313.95 & \text { B } & 300 & 1.45 & 4.38 & 3-3 & 5 B^{1} D-5 p^{3} F^{\circ} \\ 3690.35 & \text { B } & 200 & 1.45 & 4.79 & 3-3 & (7)\end{array}$ $\begin{array}{ccccccc}3894.19 & \text { B } & 300 & 1.45 & 4.62 & 2-3 & 58^{1} \mathrm{D}-5 \mathrm{p}^{3} \mathrm{D}^{\circ}\end{array}$ $\begin{array}{llllll}3958.66 & \text { B } & 300 & 1.45 & 4.56 & 3-3 \\ 3489.79 & \text { A } & 300 \mathrm{r} & 1.45 & 4.98 & 3-1\end{array}$

$3553.10 \quad A \quad 500 \mathrm{r} \quad 1.45 \quad 4.93 \quad 3-3 \quad 58^{1} \mathrm{D}-5 \mathrm{p}^{1} \mathrm{~F}^{\circ}$ $\begin{array}{lllllll}3441.40 \quad A \quad 300 & 1.45 & 5.03 & 3-3 & 58^{1} D_{-5 p^{1} D^{\circ}}\end{array}$ $\begin{array}{lllllll}3433.44 \quad A \quad 350 & 1.45 & 5.04 & 3-1 & 58 & 10-5 p^{1} P^{\circ}\end{array}$

Rh II I P Anal C L1st D Nov 1943

$3307.297 \quad A \quad 350 \quad 3.13 \quad 6.98 \quad 4-4 \quad 3 F_{-} 5^{50} \uparrow$ $\begin{array}{rrrrrrr}3038.808 & A & 75 & 3.39 & 7.98 & 4-4 & 5-5 \\ 3 & 75 & & 3.3 & (1)\end{array}$ $\begin{array}{llllll}3074.061 & \text { A } & 50 & 3.56 & 7.57 & 3-3 \\ 3434.57 & \text { P } & & 3.39 & 8.98 & 3-4\end{array}$ $\begin{array}{llllll}3183.384 \quad \text { A } & 100 \quad 3.58 & 7.46 & 3-3\end{array}$

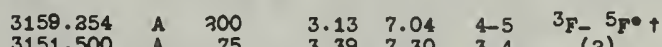
$\begin{array}{rrrrrrc}3151.500 & \text { A } & 75 & 3.13 & 7.04 & 4-5 & 35-5 \\ 3388.139 & \text { A } & 3 & 3.39 & 7.30 & 3-4 & \text { (3) }\end{array}$

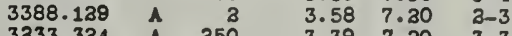
$\begin{array}{rrrrrr}3333.334 & \text { A } & 250 & 3.39 & 7.30 & 3-3 \\ 3339.101 & \mathbf{A} & 30 & 3.56 & 7.37 & 3-3\end{array}$ $\begin{array}{llrlllc}3019.819 & \text { A } & 150 & 3.56 & 7.85 & 3-3 & 3 r_{-} 5_{G \bullet} \uparrow \\ 3988.387 & \Lambda & 50 & 3.56 & 7.89 & 3-3 & (3)\end{array}$

Pd II 8ee introduction
As I I P 7.54 Anal A List C May 1943

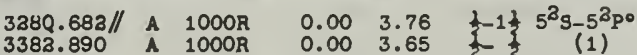

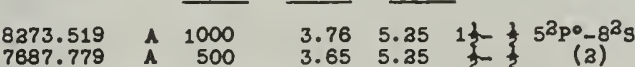


94

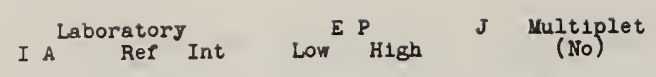
Cd I P 8.96 Anal A List D Aug 1943 $3261.050 \quad \mathrm{~A} \quad 10 \mathrm{R} \quad 0.00 \quad 3.78 \quad 0-1 \quad 5^{1} \mathrm{~s}-5^{3} \mathrm{po}^{0}$ $\begin{array}{lllllll}5085.824 & \text { A }{ }_{100 R} & 3.93 & 6.36 & 3-1 & 5^{3} \mathrm{P}^{0}-6^{3} \mathrm{~S}\end{array}$ $\begin{array}{llcllll}4799.918 & \text { A } & 100 R & 3.78 & 6.36 & 1-1 & \text { (3) } \\ 4678.160 & \text { A } & 50 & 3.72 & 6.36 & 0-1 & \end{array}$

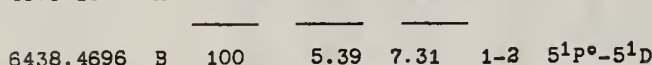
(3)

\section{Cd II See introduction}

In I I P 5.76 Anal A L18t $D$ Aug 1943

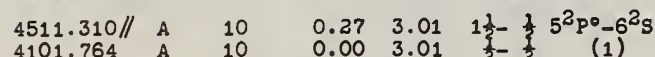
In II see introduction

SnI I P 7.30 Anal A L1Bt D Aug 1943

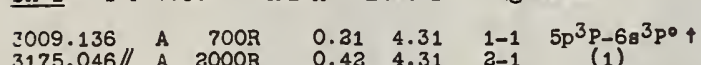
$3175.046 / /$ A 2000 R

3330.620 A $500 \mathrm{r} \quad \frac{1.06}{4.77} 2-2 \quad 5 \mathrm{p}^{1} \mathrm{D}-6 \mathrm{~B}^{3} \mathrm{p}^{\circ}$ $\begin{array}{rrrrrrc}3330.620 & \text { A } & 500 \mathrm{r} & 1.06 & 4.77 & 2-2 & 5 \mathrm{p}^{1} \mathrm{D}-6 \mathrm{~B}^{3} \mathrm{p} \\ 3801.022 & \text { A } & 2000 \mathrm{R} & 1.06 & 4.31 & 2-1 & \text { (2) }\end{array}$ 3262.340 A $2500 R \quad 1.06 \quad 4.85 \quad 2-1 \quad \frac{5 p^{1} D-68^{1} p^{\circ}}{(3)}$ 5631.707 A $500 \quad \overline{2.12} 4.31 \quad 0-1 \quad 5 \mathrm{p}^{1} \mathrm{~S}-6 \mathrm{~B}^{3} \mathrm{po}$

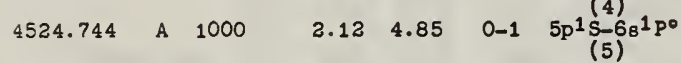

Sn II I P 14.57 Anal A List $D$ Aug 1942 $\begin{array}{lllllll}6453.50 & A & 70 & 7.02 & 8.93 & \frac{1}{2}-1 \frac{1}{2} & 6^{2} \text { S }-6^{2} P_{0} \\ 6844.05 & A & 25 & 7.02 & 8.83 & \frac{1}{2} & (1)\end{array}$ 3351.97 A $60 \quad 7.3411 .03 \quad 2 \frac{1}{3}-3 \frac{1}{3} p^{2}{ }^{3} D_{-4} F^{\circ}$ $\begin{array}{llllll}3283.21 \quad A \quad 50 \quad 7.26 & 11.02 \quad 1 \frac{1}{\geqslant}-2 \frac{1}{2} & \text { (2) }\end{array}$

Sb I I P 8.64 Anal A L1st D Sept 1943

\begin{tabular}{|c|c|c|c|c|c|}
\hline $\begin{array}{l}33.47 .10 \\
3383.15 \\
3637.83 \\
3722.79 \\
4033.55\end{array}$ & $\begin{array}{l}A \\
A \\
A \\
A \\
A\end{array}$ & $\begin{array}{l}5 \\
100 \\
250 \\
200 r \\
200\end{array}$ & $\begin{array}{l}2.28 \\
2.02 \\
2.28 \\
2.02 \\
2.28\end{array}$ & $\begin{array}{l}5.97 \\
5.67 \\
5.67 \\
5.34 \\
5.34\end{array}$ & 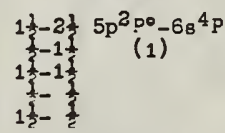 \\
\hline $\begin{array}{l}3232.52 \\
3267.51 \\
3504.48 \\
3029.83\end{array}$ & $\begin{array}{l}\text { A } \\
\text { A } \\
\text { A } \\
\text { A }\end{array}$ & $\begin{array}{l}600 r \\
700 r \\
50 \\
500 r\end{array}$ & $\begin{array}{l}2.28 \\
2.02 \\
2.28 \\
2.02\end{array}$ & $\begin{array}{l}6.10 \\
5.80 \\
5.80 \\
6.10\end{array}$ & 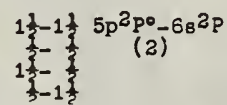 \\
\hline
\end{tabular}

Sb II See 1ntroduction

Te I I P 8.96 Anal B List D June 1943

$\begin{array}{rrrrrrc}9722.88 & \text { A } & 100 & 5.46 & 6.73 & 2-3 & 6^{5} \mathrm{~S}^{0}-6^{5} \mathrm{P} \\ 10051.55 & \text { A } & 50 & 5.46 & 6.69 & 2-2 & \text { (1) }\end{array}$ \begin{tabular}{llllll}
10051.55 & A & 50 & 5.46 & 6.69 & $2-2$ \\
10091.13 & A & 25 & 5.46 & 6.69 & $2-1$ \\
\hline
\end{tabular}

I I See introduction

I II See introduction

Xe I See introduction

Xe II see introduction
REVISED MULTIPLET TABLE

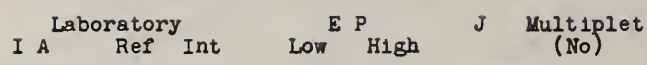
I A Laboratory

Low ${ }^{\text {H P gh }}$ J $\underset{\text { (No) }}{\text { (Notet }}$ CBI I P 3.88 Anal A L1st $D$ Nov 1942

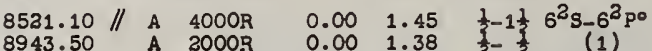
455.50 A $2000 \mathrm{R} \quad 0.001 .38$ 年

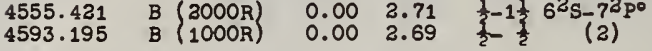

CB II See introduction

Ba II cont1nued

$\begin{array}{ccccccc}5013.00 & D & (10) & 5.99 & 8.45 & 3 \frac{1}{3} & 4^{2} F^{\circ}-6^{2} G \\ 4957.15 & D & (10) & 5.96 & 8.45 & 3 \frac{1}{2}- & (10)\end{array}$

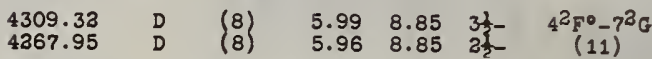
$6378.91 \quad D \quad\{5\} \quad-6.178 .11 \quad 1 \frac{1}{3}-\frac{1}{2} 7^{2} \mathrm{p}^{0}-9^{3} 8$ $6135.83 \quad D \quad(4) \quad 6.10 \quad 8.11 \quad \frac{1}{2}-\frac{1}{2} \quad(12)$ $\begin{array}{lllllll}5981.25 & D & (8) & 6.17 & 8.24 & 1 \frac{1}{3}-2 \frac{1}{2} 7^{2} \mathrm{P}^{0}-8^{2} \mathrm{D} \\ 8 & 6.10 & 8.23 & & \mathrm{D}\end{array}$

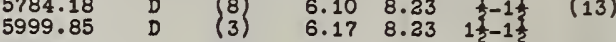

Ba I I P 5.19 Anal A List C Nov 1943 7911.338 A $(200) \quad 0.00 \quad 1.56 \quad 0-1 \quad 6^{1} \mathrm{~S}-6^{3} \mathrm{p}_{0}$ $5535.484 / /$ A $\left.1000 \mathrm{R} \quad 0.00 \quad 3.33 \quad 0-1 \quad 6^{1} \mathrm{~s}-6^{(1)}\right)_{\mathrm{po}}$ 3501.107 A $200 \mathrm{R} \quad 0.00 \quad 3.53 \quad 0-1 \quad 6^{1} \mathrm{~S}_{-5 \mathrm{~d} 6 \mathrm{p}^{1} \mathrm{p}}$ 3071.583 A $100 \mathrm{R} \quad 0.00 \quad 4.03 \quad 0-1 \quad 6^{1} \mathrm{~S}_{-7}^{(3)} \mathrm{p}^{\circ}$

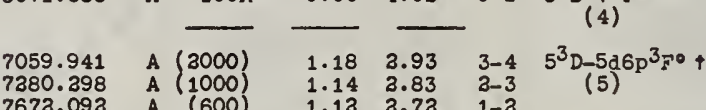

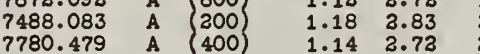

$\begin{array}{lllllll}6498.759 & \text { A } & 300 \mathrm{r} & 1.18 & 3.08 & 3-3 & 5^{3} \mathrm{D}-5 \mathrm{a} 6 \mathrm{p}^{3} \mathrm{D}^{\circ} \\ 6537.312 & \text { A } & 250 & 1.14 & 3.03 & 2-3 & (6)\end{array}$ $\begin{array}{rrrrrrr}6595.326 & \text { A } & 250 & 1.14 & 3.03 & 2-2 & (6) \\ & 1.12 & 2.99 & 1-1 & \end{array}$ $\begin{array}{llllll}6693.842 & A & 70 & 1.18 & 3.03 & 3-3\end{array}$ 6675.271 A $80 \quad 1.14 \quad 2.99 \quad 2-1$ $\begin{array}{llllll}6341.682 & \text { A } & 150 & 1.14 & 3.08 & 2-3 \\ 6450.854 & \text { A } & 125 & 1.13 & 3.03 & 1-\end{array}$

$\begin{array}{llllll}4997.81 \quad D \quad & \text { (3) } & 6.17 & 8.64 & 1 \frac{1}{5}-\frac{1}{2} 7^{2} \mathrm{P}^{0}-10^{2} \mathrm{~g}\end{array}$ $4847.14 \quad D \quad(3) \quad 6.10 \quad 8.64 \quad \frac{1}{2}-\frac{1}{2} \quad(14)$ $\begin{array}{lllllll}4843.46 & \mathrm{D} & (8) & 6.17 & 8.73 & 1 \frac{1}{2}-2 \frac{1}{7} & 7^{2} \mathrm{P}^{\circ}-9^{2} \mathrm{D}\end{array}$

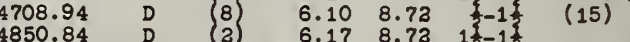
$4405.33 \quad D \quad(4) \quad 6.17 \quad 8.98 \quad 1 \frac{1}{3}-\frac{1}{7} 7^{2} \mathrm{p}^{0}-11^{2} \mathrm{~S}$ 887.80

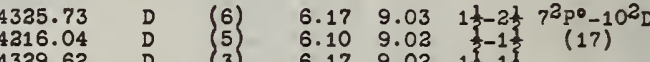
4329.62 $\begin{array}{lllc}1.18 & 3.20 & 3-3 & 5^{3} \mathrm{D}-5 \mathrm{a} 6 \mathrm{p}^{3} \mathrm{p}^{\circ}+ \\ 1.14 & 3.17 & 2-1 & (7)\end{array}$

$\begin{array}{ll}1.12 & 3.17 \\ 1.14 & 3.20\end{array}$

$\begin{array}{lll}1.14 & 3.20 & 2-2 \\ 1.12 & 3.17 & 1-1\end{array}$

$\begin{array}{llll}1.18 & 4.28 & 3-4 & 5^{3} D-4^{3} F^{\circ} \\ 1.14 & 4.27 & 3-3 & (8)\end{array}$

$\begin{array}{ll}1.14 & 4.27 \\ 1.12 & 4.37\end{array}$

$\begin{array}{ll}1.12 & 4.27 \\ 1.18 & 4.37\end{array}$

\begin{tabular}{ll}
1.18 & 4.37 \\
1.14 & 4.27 \\
\hline
\end{tabular}

-

1.67 $3.80 \quad 2-3 \quad 6^{3} \mathrm{P}^{0}-6^{3} \mathrm{D}+$

5777.622

519.047 A 400 r

5800.229

7905.751

7392.411
7195.235 200
100
100

$\begin{array}{lll}1.51 & 3.79 & 0-1\end{array}$

(9)

$\begin{array}{lll}1.67 & 3.80 & 2-2\end{array}$

$\begin{array}{llll}1.67 & 3.23 & 3-1 & 6^{3} \mathrm{p}^{0}-7^{3} \mathrm{~s}\end{array}$
5424.551 $\begin{array}{lll}1.51 & 3.23 & 0-1\end{array}$

$5930.61 \quad A \quad(200) \quad 0.13 \quad 3.21 \quad 2 \frac{1}{2}-3 \frac{1}{2} \mathrm{a}^{2} \mathrm{D}-\mathrm{y}^{2} \mathrm{~F}^{\circ}$
Ba II I P 9.96 Anal A List B Nov 1943

$\begin{array}{llrllll}4554.033 / / & A & 1000 R & 0.00 & 2.71 & \frac{1}{2}-1 \frac{1}{3} & 6^{2} \mathrm{~S}-6^{2} \mathrm{P}^{\circ} \\ 4934.086 & \mathrm{~B} & 700 \mathrm{R} & 0.00 & 3.50 & \frac{1}{2}-\frac{1}{2} & (1)\end{array}$

6141.718 A $600 \mathrm{r} \quad 0.70 \quad 3.71 \quad 2 \frac{1}{-1 \frac{1}{1}} 5^{2} \mathrm{D}-6^{3} \mathrm{po}$

$\begin{array}{lllllll}6496.896 & \text { A } & 600 \mathrm{r} & 0.60 & 2.50 & 1 \frac{1}{3} & \text { (3) } \\ 5853.675 & \text { A } & 200 & 0.60 & 2.71 & 1 \frac{1}{2} & \end{array}$

$\begin{array}{llllllll}4899.934 & A & 35 & & 2.71 & 5.23 & 1 \frac{1}{1}-\frac{1}{2} & 6^{2} \mathrm{P}^{0}-7^{3} \mathrm{~S} \\ 4524.928 & \mathrm{~A} & 35 & 2.50 & 5.23 & \frac{1}{2}-\frac{1}{2} & (3)\end{array}$

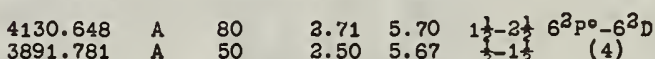

$\begin{array}{lllllll}3891.781 & \text { A } & 50 & 2.50 & 5.67 & \frac{1}{3}-1 \frac{1}{3} & \text { (4) } \\ 4166.003 & \text { A } & 20 & 2.71 & 5.67 & 1 \frac{1}{2}-1 \frac{1}{2} & \end{array}$

$\begin{array}{lllllll}8710.83 & C & (3 n) & 5.70 & 7.11 & 2 \frac{1}{3}-3 \frac{1}{3} & 6^{2} \mathrm{D}-5^{2} F^{\circ} \\ 8737.74 & C & (3 n) & 5.67 & 7.08 & 1 \frac{1}{2}-2 \frac{2}{2} & (5)\end{array}$

$5391.60 \quad \mathrm{D} \quad(10) \quad 5.70 \quad 7.99 \quad 3 \frac{1}{2}-3 \frac{1}{2} 6^{2} \mathrm{D}-6^{2} F^{\circ}$

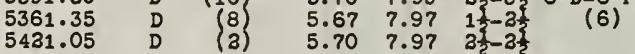

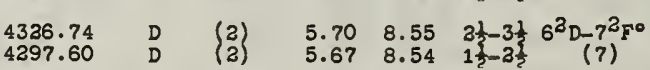

$\begin{array}{lllllll}6874.09 & D & (10) & 5.99 & 7.78 & 3 \frac{1}{3}- & 4^{2} F^{0}-5^{2} G \\ 6769.63 & D & \left(\begin{array}{lllll}10 \\ 10\end{array}\right. & 5.96 & 7.78 & 2 \frac{3}{5} & (8)\end{array}$

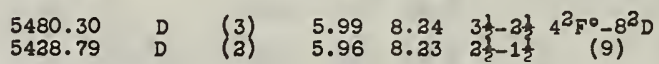

La I I P 5.59 Anal A L18t C Nor 1948

6455.99 A $300 \quad 0.13 \quad 2.04 \quad 2 \frac{1}{3}-3 \frac{1}{3} a^{2} D-2^{3} F^{\circ}$ $\begin{array}{lllllll}6578.51 & \text { A } & 400 & 0.00 & 1.88 & 1 \frac{1}{2}-2 \frac{1}{3} & \text { (1) } \\ 7068.37 & \text { A } & 100 & 0.13 & 1.88 & 2 \frac{1}{2}-3 \frac{1}{2} & \end{array}$

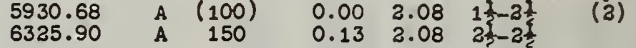

$5455.14 \quad$ A $400 \quad 0.13 \quad 2.39 \quad 2 \frac{1}{2}-2 \frac{1}{2} a^{2} D-y^{2} D^{0}+$ 5501.34 A $300 \quad 0.00 \quad 2.24 \quad 1 \frac{1}{2}-1 \frac{1}{2} \quad(3)$ $\begin{array}{lllllll}5271.18 & \text { A } & 150 & 0.13 & 2.47 & 2 \frac{1}{1}-1 \frac{1}{2} & a^{2} D-y^{3} p+ \\ 4949.76 & \text { A } & 200 & 0.00 & 3.49 & 1 \frac{1}{2}-\frac{1}{2} & (4)\end{array}$ 4280.37 A $100 \quad 0.13 \quad 3.01 \quad 2 \frac{1}{3}-3 \frac{1}{2} a^{3} \mathrm{D}-w^{2} F^{\circ}$ $\begin{array}{lllllll}4187.31 & \text { A } & 125 & 0.00 & 3.95 & 1 \frac{1}{2}-2 \frac{1}{2} & \text { (5) }\end{array}$

$6709.49 \quad A \quad 200 \quad 0.37 \quad 2.31 \quad 21-3 \frac{1}{3} a^{4} F-y^{2} F^{\circ}+$ $\begin{array}{lllllll}7045.96 & \text { A } & 200 & 0.37 & 2.21 & 2 \frac{1}{3}-3 \frac{1}{3} & a^{4} F-y^{2} \\ 70 & & 0.33 & 2.08 & 1 \frac{1}{2}-2 \frac{1}{2} & (6)\end{array}$

6249.92 /I A $500 \quad 0.512 .48 \quad 4 \frac{1}{-51} a^{4} F-2^{4} G^{0}+$ $\begin{array}{llllll}6394.23 & \text { A } & 600 & 0.43 & 2.36 & 3 \frac{1}{3}-4 \\ 6410.98 & \text { A } & 300 & 0.37 & 2.30 & 2 \frac{1}{3}-3\end{array}$ $\begin{array}{llllll}6410.98 & \text { A } & 300 & 0.37 & 2.30 & 2 \frac{1}{2}-3 \frac{1}{1} \\ 6543.17 & \text { A } & 500 \mathrm{~d} & 0.33 & 2.22 & 1 \frac{1}{2}-2 \frac{1}{2}\end{array}$

5791.32 789.32 769.32

5740.6

5211.85 5177.30 5145.42
5106.33

5334.3 4570.02 4567.90 $\begin{array}{r}4549.50 \\ 4494.71 \\ \hline\end{array}$

$\begin{array}{cccc}0.51 & 3.64 & 4 \frac{1}{2}-4 \frac{1}{3} & a^{4} F-y^{4} F^{0}+ \\ 0.43 & 3.56 & 3 \frac{1}{2}-3 \frac{1}{3} & (8)\end{array}$

$\begin{array}{lll}0.43 & 3.56 & 3 \frac{1}{3}-3 \\ 0.37 & 2.51 & 2 \frac{1}{3}-2 \frac{1}{2} \\ 0.33 & 3.48 & 1 \frac{1}{2}-1 \frac{1}{2}\end{array}$

$0.51 \quad 2.88 \quad 4 \frac{1}{3}-3 \frac{1}{2} a^{4} F-J^{4} D^{0} 4$

$\begin{array}{lll}0.43 & 2.82 & 3 \frac{2}{2}-2 \frac{1}{2} \\ 0.37 & 2.77 & 2 \frac{1}{3}-1 \frac{2}{2}\end{array}$

$\begin{array}{lll}0.37 & 2.77 & 2 \frac{2}{3}-1 \frac{1}{3} \\ 0.33 & 3.75 & 1 \frac{1}{2}\end{array}$

$0.512 .87 \quad 4 \frac{1}{2}-3 \frac{1}{2} a^{4} \mathrm{~F}-1^{\circ}+$

$0.513 .21 \quad 4 \frac{1}{3}-4 \frac{1}{4} a^{4}-x^{4} F^{0}$ $0.43 \quad 3.13 \quad 3 \frac{1}{3}-3 \frac{1}{3}$ (11) $\begin{array}{lll}0.37 & 3.08 & 2 \frac{1}{3}-2 \frac{1}{3} \\ 0.33 & 3.08 & 12-1\end{array}$

La II I P 11.38 Anal A List $B$ Nov 1943

\begin{tabular}{|c|c|c|c|c|c|c|}
\hline $\begin{array}{l}7283.36 \\
7483.48 \\
7066.34 \\
6808.88 \\
6954.54\end{array}$ & $\begin{array}{l}\mathbf{A} \\
\mathbf{A} \\
\mathbf{A} \\
\mathbf{A} \\
\mathbf{A}\end{array}$ & $\begin{array}{r}150 \\
30 \\
300 \\
30 \\
30\end{array}$ & $\begin{array}{l}0.24 \\
0.13 \\
0.00 \\
0.13 \\
0.00\end{array}$ & $\begin{array}{l}1.94 \\
1.77 \\
1.75 \\
1.94 \\
1.77\end{array}$ & $\begin{array}{l}4-4 \\
3-3 \\
2-2 \\
3-4 \\
2-3\end{array}$ & $\begin{array}{c}a^{3} F-z^{3} F^{0}+ \\
\text { (1) }\end{array}$ \\
\hline 6774.38 & $A$ & 100 & 0.13 & 1.95 & $3-3$ & $\begin{array}{c}a^{3} F-z^{1} F^{0} \uparrow \\
(\text { a) }\end{array}$ \\
\hline 6834.07 & A & 30 & 0.34 & 3.05 & $4-4$ & $a^{3} F-2 G^{0}+$ \\
\hline $\begin{array}{l}5797.57 \\
5805.77 \\
5808.31 \\
6146.53 \\
6172.72 \\
5493.45 \\
5482.27\end{array}$ & $\begin{array}{l}A \\
A \\
A \\
A \\
A \\
A \\
A\end{array}$ & $\begin{array}{r}150 \\
130 \\
60 \\
15 \\
10 \\
30 \\
40\end{array}$ & $\begin{array}{l}0.24 \\
0.13 \\
0.00 \\
0.24 \\
0.13 \\
0.13 \\
0.00\end{array}$ & $\begin{array}{l}3.37 \\
3.35 \\
3.13 \\
3.35 \\
3.12 \\
3.37 \\
3.25\end{array}$ & $\begin{array}{l}4-4 \\
3-3 \\
3-3 \\
4-3 \\
3-2 \\
3-4 \\
3-3\end{array}$ & $\begin{array}{c}a^{3} F-y^{3} F^{6} \\
(4)\end{array}$ \\
\hline
\end{tabular}


I Laboratory $A$ Res Int Low ${ }_{\text {High }} J$ multiplet Laboratory $E P$ J multiplet La II continued

$6305.46 \quad$ A $\quad 10$

$5390.83 \quad$ A 50

4931.80 A 300

$\begin{array}{rrr}4930.98 & \text { A } & 300 \\ 4899.92 & \text { A } & 300 \\ 5163.61 & \text { A } & 40\end{array}$

$\begin{array}{lll}5156.74 & \text { A } & 40 \\ 5433.82 & \text { A } & 4\end{array}$

4860.90 A 80

$\begin{array}{lll}4740.27 & \text { A } & 130 \\ 4663.51 & \text { A } & 300 \\ 4645.28 & \text { A } & 100 \\ 4523.37 & \text { A } & 400\end{array}$

$\begin{array}{rrr}-4522.37 & \text { A } & 400 \\ 4435.84 & A & 10\end{array}$

$4300.44 \quad \triangle \quad 60$

4088.72 A 300

$\begin{array}{lll}4432.95 & \text { A } & 30 \text { ? } \\ 4076.71 & \text { A } & 40\end{array}$

$\begin{array}{lll}3794.78 & \text { A } 400 \\ 3790.83 & \text { A } 300\end{array}$

3849.03

3650.19

3530.67

3759.08

3871.64
3784.81

3936.22

3638.83
3725.05

3645.43 A 300

$\begin{array}{rrr}3510.00 & \text { A } & 15 \\ 3550.82 & \text { A } & 6\end{array}$

3108.46 A 8

\begin{tabular}{rrr}
3306.98 & A & 8 \\
3104.58 & A & 50 \\
\hline
\end{tabular}

6952.53 A 10

$\begin{array}{rrr}5936.33 & \text { A } & 30 \\ 8320.39 & \text { A } & 300\end{array}$

5713.39 A 30

$5259.38 \quad$ A 50

$\begin{array}{rrr}4738.41 \quad A \quad 100 \\ 4838.87 & \text { A }\end{array}$

$4988.82 \quad \hat{A} \quad 100$

$\begin{array}{rrr}4574.87 & \text { A } & 300 \\ 4691.17 & \text { A } & 50\end{array}$

4333.76 A 500

4333.51 A 100

$\begin{array}{lll}3713.54 & \text { A } \quad 100 \\ 3846.00 & \text { A } 20\end{array}$

$\begin{array}{rrr}3846.00 & \text { A } & 20 \\ 4067.39 & \text { A } 100\end{array}$

$\begin{array}{lll}3929.22 & \text { A } 300 \\ 3995.74 & \text { A } & 400\end{array}$

3840.73 A 60

$\begin{array}{rrr}3557.26 & \text { A } & 8 \\ 3735.85 & \text { A } & 10\end{array}$

3453.18 A 40

$\begin{array}{lll}3142.76 & \text { A } & 40 \\ 3348.35 & \text { A } & 80\end{array}$

3345.13

A 150

363.30

8390.48
8526.99

6871.41

6859.03

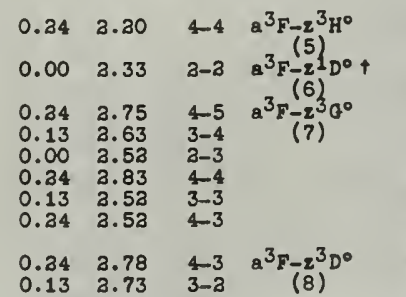

$\begin{array}{ll}0.00 & 3.85 \\ 0.13 & 3.78 \\ 0.00 & 3.73\end{array}$

$\begin{array}{lll}0.00 & 3.78 & 3-3\end{array}$

$0.00 \quad 3.87 \quad 3-a \quad a^{3} F-z^{3} p^{0}+$

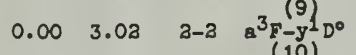

$\begin{array}{llll}0.24 & 3.03 & 4-3 & a^{3} F_{-y} F^{10}+ \\ 0.00 & 3.03 & 3-3 & (11)\end{array}$

$\begin{array}{llll}0.24 & 3.50 & 4-3 & a^{3} F-y^{3} D^{0} \\ 0.3 & 3.38 & 3-2 & (13)\end{array}$

$\begin{array}{ll}0.13 & 3.38 \\ 0.00 & 3.21 \\ 0.13 & 3.50\end{array}$

$\begin{array}{lll}.13 & 3.51 & 3-1 \\ 0.13 & 3-3\end{array}$

$\begin{array}{lll}0.00 & 3.38 & 2-3 \\ 0.00 & 3.50 & 2-3\end{array}$

$0.34 \quad 3.53 \quad 4-4 \quad a^{3} F-x^{3} F^{0}$

$0.13 \quad 3.31 \quad 3-3$ (13)

$\begin{array}{lll}0.00 & 3.26 & 2-3 \\ 0.13 & 3.26 & 3-2\end{array}$

$\begin{array}{lll}0.13 & 3.53 & 3-4 \\ 0.00 & 3.31 & 2-3\end{array}$

$0.00 \quad 3.39 \quad 3-1 \quad 8^{3} F-2^{1} p_{0}$

$\begin{array}{llll}0.13 & 3.64 & 3-3 & a^{3} F-y^{3} p 0 \\ 0.00 & 3.48 & 2-1 & (15)\end{array}$

$0.00 \quad 3.97 \quad 2-1 \quad a^{3} F-x^{3} p_{0}$

$0.24 \quad 3.98 \quad 4-3 \quad a^{3} F-x^{3} F^{0}+$

0.17 $1.95 \quad 2-3 \quad a^{1} D_{-2} F^{\circ}$

$\begin{array}{llll}0.17 & 1.95 & 2-3 & a^{1}-z^{1} F^{\circ} \\ 0.17 & 3.35 & 3-3 & a^{1} D-y^{3} F^{\circ} \\ 0.17 & 3.13 & 3-3 & (19)\end{array}$

$0.17 \quad 3.33 \quad 3-3 \quad a^{1} D-2^{1} D^{\circ}$

$\begin{array}{lllll}0.17 & 2.53 & 3-3 & a^{1 D-2} 0_{0} & (30)\end{array}$

$\begin{array}{cccc}0.17 & 3.78 & 2-3 & a^{1} D-23 D^{\circ} \\ 0.17 & 3.73 & 2-3 & (23) \\ 0.17 & 3.85 & 2-1 & \end{array}$

$\begin{array}{llll}0.17 & 2.87 & 2-a & a^{1} p-z^{3} p^{0} \\ 0.17 & 3.80 & 3-1 & (23)\end{array}$

$0.17 \quad 3.03 \quad 3-3 \quad a^{1} D-y^{1} D^{0}$

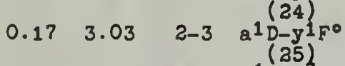

$\begin{array}{cccc}0.17 & 3.50 & 2-3 & a^{1} D-y^{3} D^{\circ} \\ 0.17 & 3.38 & 2-3 & (26)\end{array}$

$\begin{array}{cccc}0.17 & 3.31 & 3-3 & a^{1} D-x^{3} F^{\circ} \\ 0.17 & 3.26 & 3-3 & (27)\end{array}$

$0.17 \quad 3.39 \quad 2-1 \quad a^{1} D-2^{1} p \circ$

$\begin{array}{cccc}0.17 & 3.64 & 2-3 & a^{1} p-y^{3} p 0 \\ 0.17 & 3.48 & 2-1 & (29)\end{array}$

$0.17 \quad 3.75 \quad 3-1 \quad a^{1} D-y^{1} p_{0}$

$\begin{array}{llll}0.17 & 4.10 & 2-3 & a^{1} D-x^{3} p 0\end{array}$

$0.17 \quad 3.98 \quad 2-3 \quad a^{1} D-x^{1} F^{0}$

$\overline{0.40} \quad-3.37 \quad 3-4 \quad a^{3} \mathrm{p}^{3} \mathrm{y}^{\circ}$

$\begin{array}{lll}0.32 & 2.25 & 3-3 \\ 0.33 & 2.12 & 1-3\end{array}$

$\begin{array}{lll}0.23 & 2.12 & 1-2 \\ 0.40 & 2.25 & 3-3\end{array}$

$\begin{array}{lll}0.32 & 2.12 & 3-3\end{array}$

$0.40 \quad 3.20 \quad 3-4 \quad a^{3} \mathrm{D}-2^{3} \mathrm{~K}^{\circ}$
Le II continued $\begin{array}{lll}0.17 & 3.73 & 2-3 \\ 0.17 & 3.85 & 3-1\end{array}$

$\begin{array}{lll}0.17 & 3.21 & 3-1\end{array}$

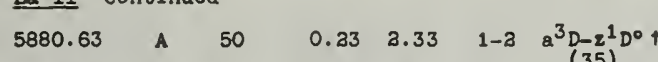

5183.43 A 400

$\begin{array}{lll}5114.95 & \hat{A} & 300 \\ 5301.97 & \hat{A} & 300 \\ 5300\end{array}$

$\begin{array}{rrr}5301.97 & \text { A } & 300 \\ 4946.47 & \text { A } & 100 \\ 40\end{array}$

4998.46 A 300

4809.00

4840.03

4804.04
4683.13

4713.93
4570.87

$\begin{array}{rrr}4570.87 & \text { A } & 40 \\ 4439.90 & \text { A } & 10\end{array}$

$\begin{array}{rrr}4699.62 & \text { A } & 50 \\ 4558.46 & \text { A } & 300\end{array}$

3988.51 A 500
4031.68

$\begin{array}{lll}4031.68 & \text { A } & 300 \\ 4151.98 & \text { A } & 350\end{array}$

4151.98

$\begin{array}{lll}4141.73 & \text { A } & 200 \\ 4275.64 & \text { A } & 100\end{array}$

$\begin{array}{lll}3886.37 & \hat{A} & 150 \\ 3921.54 & \mathbf{A} & 300\end{array}$

$\begin{array}{ll}3949.10 & \text { II A } 600 \\ 4123.33 & \text { A } 400\end{array}$

$\begin{array}{lll}4123.23 & \text { A } & 400 \\ 4077.35 & \text { A } & 300 \\ 4338.38 & \text { A } & 400\end{array}$

4196.55

4315.90

4025.87

3916.05 A 300

3808.79

3910.81

3601.07

3601.07
3512.93

3337.49
3380.91

3344.56

3303.11

3453.17

6129.57

6100.37

6174.15
.8396 .08

6358.12
6570.96

5874.00

6067.13
5892.66

5703.32

$\begin{array}{lll}5737.29 & \mathbf{A} & 20 \\ & & \end{array}$

5464.37 A 25

4526.13 A 200

4613.38

4824.05

4935.61

.4850 .58

-4850.58
4830.51

4716.44

4605.78

4296.05

4455.79

4559.28
4580.05

4204.03
4364.66

4143.77

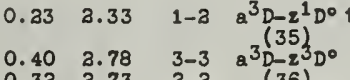

$\begin{array}{lll}0.23 & 2.65 & 1-1 \\ 0.40 & 2.73 & 3-2\end{array}$

$\begin{array}{lll}0.40 & 2.73 & 3-2 \\ 0.32 & 3.65 & 3-1 \\ 0.33 & 3.73 & 1-3\end{array}$

$0.40 \quad 3.87 \quad 3-3 \quad a^{3} p-2^{3} p^{0}$

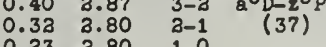

$\begin{array}{lll}0.33 & 3.80 & 1-0 \\ 0.32 & 3.87 & 3-2\end{array}$

$\begin{array}{lll}0.33 & 3.80 & 1-1 \\ 0.33 & 3.87 & 1-2\end{array}$

$0.40 \quad 3.02 \quad 3-2 \quad a^{3} D-y^{1} D^{\circ}$

$\begin{array}{llll}0.32 & 3.03 & 2-2 & (38) \\ 0.33 & 3.02 & 1-2 & \end{array}$

$\begin{array}{llll}0.40 & 3.03 & 3-3 & a^{3}-y^{1} F^{\circ} \\ 0.32 & 3.03 & 2-3 & (39)\end{array}$

$\begin{array}{llll}0.40 & 3.50 & 3-3 & a^{3} D-y^{3} D^{\circ} \\ 0.32 & 3.38 & 3-2 & (40)\end{array}$

$0.33 \quad 3.31$

$\begin{array}{lll}0.40 & 3.38 \\ 0.32 & 3.31 \\ 0.32 & 3.50\end{array}$

$0.32 \quad 3.21 \quad 3-1$

$\begin{array}{lll}0.32 & 3.50 & 2-3 \\ 0.33 & 3.38 & 1-2\end{array}$

$0.23 \quad 3.38$

$\begin{array}{llll}0.40 & 3.53 & 3-4 & a^{3} p-x^{3} F^{\circ} \\ 0.32 & 3.31 & 3-3 & (41)\end{array}$

$0.23 \quad 3.36 \quad 1-3$

$\begin{array}{lll}0.40 & 3.31 & 3-3 \\ 0.32 & 3.26 & 2-2\end{array}$

$\begin{array}{lll}0.32 & 3.26 & 2-2 \\ 0.40 & 3.26 & 3-2\end{array}$

$0.33 \quad 3.39 \quad 2-1 \quad a^{3} D-2^{1} p^{0}$

$0.23 \quad 3.3$

1-1 (4a)

$\begin{array}{lcccc}15 & 0.40 & 3.64 & 3-2 & a^{3} \mathrm{p}-\mathrm{y}^{3} \mathrm{po} \\ 101 & 0.32 & 3.48 & 3-1 & (43) \\ 50 & 0.32 & 3.64 & 2-2 & \end{array}$

$20 \mathrm{nl}$
10

$0.32 \quad 3.75 \quad 3-1 \quad a^{3} \mathrm{D}-\mathrm{y}^{1} \mathrm{po}^{\circ}$

$0.40 \quad 4.10 \quad 3-3 \quad a^{3} p-x^{3}$ po

$\begin{array}{cccc}0.40 & 4.10 & 3-2 & a^{3} D-x^{3} \\ 0.32 & 3.97 & 3-1 & (45)\end{array}$

$0.23 \quad 3.93 \quad 1-0$

$\begin{array}{lll}0.32 & 4.10 & 3-2 \\ 0.33 & 3.97 & 1-1\end{array}$

$\begin{array}{lll}0.23 & 4.10 & 1-2\end{array}$

$\begin{array}{cccc}0.40 & 3.98 & 3-3 & a^{3} \mathrm{D}-\mathrm{x}^{1} \mathrm{~F}^{\circ} \\ 0.32 & 3.98 & 2-3 & (46)\end{array}$

$-1$

$0.77 \quad 3.78$

$0.71 \quad 3.73$

$\begin{array}{ll}0.65 & 2.65 \\ 0.77 & 3.73\end{array}$

$\begin{array}{ll}0.71 & 3.75 \\ 0.77 & 3.65\end{array}$

$0.77 \quad 3.87$

$\begin{array}{lll}0.77 & 3.87 \\ 0.71 & 3.80\end{array}$

$\begin{array}{lll}0.71 & 3.80 & 1-0 \\ 0.71 & 2.87 & 1-2 \\ 0.65 & 3.80 & 0-1\end{array}$

$0.77 \quad 3.03 \quad 2-3 \quad a^{3} \mathrm{P}-y^{1} F^{0}$

$\begin{array}{cccc}0.77 & 3.50 & 2-3 & a^{3} P-y^{3} D^{\circ} \\ 0.71 & 3.38 & 1-2 & (50)\end{array}$

$\begin{array}{lll}0.65 & 3.21 & 0-1 \\ 0.77 & 3.38 & 2-2\end{array}$

$0.77 \quad 3.38 \quad 2-2$

$\begin{array}{lll}0.71 & 3.21 & 1-1 \\ 0.77 & 3.21 & 2-1\end{array}$

$\begin{array}{cccc}0.77 & 3.31 & 2-3 & 8^{3} p-x^{3} F^{\circ} \\ 0.71 & 3.26 & 1-2 & (51)\end{array}$

$0.77 \quad 3.39 \quad 3-1 \quad a^{3} p_{-2} p^{1} 0$

$\begin{array}{lllc}0.77 & 3.64 & 2-2 & a^{3} p-y^{3} p^{\circ} \\ 0.71 & 3.48 & 1-1 & (53)\end{array}$

$\begin{array}{lll}0.71 & 3.64 & 1-2 \\ 0.65 & 3.48 & 0-1\end{array}$

$\begin{array}{cccc}0.77 & 3.75 & 3-1 & a^{3} p-y^{1} p 0 \\ 0.71 & 3.75 & 1-1 & (54)\end{array}$ $\begin{array}{llll}0.40 & 3.78 & 3-3 & a^{3} D-z^{3} D \\ 0.32 & 3.73 & 3-3 & (36) \\ 0.33 & 3.65 & 1-1 & \end{array}$

$\begin{array}{llll}0.71 & 3.39 & 1-1 & (5 a)\end{array}$

$\begin{array}{lll}0.77 & 3.48 & 3-1 \\ 0.71 & 3.40 & 1-0\end{array}$

$0.71 \quad 3.40 \quad 1-0$

I A ${ }_{\text {Ref }}^{\text {Laboratory }}$ Int Low ${ }_{\text {High }}^{\text {S P Hult1plet }}$

La II continued

$\begin{array}{lllllll}3705.81 & \text { A } & 80 & 0.77 & 4.10 & 2-2 & a^{3} \text { P-X } \\ 3780.67 & \text { A } & 509 & 0.71 & 3.97 & 1-1 & (55) \\ 3854.91 & \text { A } & 30 & 0.77 & 3.97 & 3-1 & \\ 3835.09 & \text { A } & 50 & 0.71 & 3.92 & 1-0 & \end{array}$

3714.87

\begin{tabular}{ll} 
A & 50 \\
A & 40 \\
\hline
\end{tabular}

5380.87

100

$4991.37 \quad$ A 80

$4354.40 \quad$ A 200

$4036.59 \quad A \quad 15 d$

9657.00 A 30

6636.53 A 5

5863.70 A 80

4796.67 A 25

4739.80 A 15

4748.73 A 150

4043.91 A 300

6958.11

5486.86
.6296 .08

100

6296.08

A 300

$\begin{array}{rrr}5971.09 & \text { A } & 8 \\ 6136.09 & \text { A } & 50\end{array}$

5769.06 A 60

5535.66 A 80

4934.83 A 100

4530.54 A 15

-453a.37 A 400

$\begin{array}{lll}.71 & 3.92 & 1-0 \\ 0.71 & 4.10 & 1-2\end{array}$

\begin{tabular}{lll}
0.71 & 4.10 & $1-2$ \\
0.65 & 3.97 & $0-1$ \\
\hline
\end{tabular}

$0.91 \quad 3.21 \quad 0-1 \quad a^{1} s-y^{3} D^{0}$

$\begin{array}{lllll}0.91 & 3.39 & 0-1 & a^{15-2} & (56)\end{array}$

$0.91 \quad 3.75 \quad 0-1 \quad a^{1} \mathrm{~g}^{5} \mathrm{y}^{1} \mathrm{po}$

$\begin{array}{lllll}0.91 & 3.97 & 0-1 & a^{1} 8 x^{3} p_{0} \\ (59) & & & \end{array}$

$0.92 \quad 3.30 \quad 4-4 \quad a^{1} a-z^{3} H^{0}$

$\begin{array}{llll}0.92 & 3.78 & 4-3 & a^{1} G-z^{3} D^{\circ}\end{array}$

$0.92 \quad 3.03 \quad 4-3 \quad a^{1} G-y^{1} F^{\circ}$

$0.92 \quad 3.50 \quad 4-3 \quad a^{1} G-y^{3} D^{0}$

$0.92 \quad 3.53 \quad 4-4 \quad a^{1} G-x^{3} F^{\circ}+$

$0.92 \quad 3.52 \quad 4-5 \quad a^{1} G-z^{1} H^{\circ}$

$\begin{array}{llll}0.92 & 3.98 & 4-3 & a^{1} G x^{1} F^{\circ} \\ (66)\end{array}$

$\begin{array}{llll}1.25 & 3.02 \quad 2-2 & b^{1} D-y^{1} D^{\circ}\end{array}$

$\begin{array}{llll}1.25 & 3.50 & \bar{a}-3 & \mathrm{~b}^{1} \mathrm{D}-\mathrm{y}^{3} \mathrm{D}^{\circ}+ \\ 1.25 & 3.21 & 2-1 & (68)\end{array}$

$1.25 \quad 3.31 \quad 2-3 \quad b^{1} D-x^{3} F^{\circ}$

$\begin{array}{llll}1.25 & 3.39 & 2-1 & b^{1} D-z^{1} p o\end{array}$

$1.35 \quad 3.48$ a-1 b1 (70)

$\left.1.35 \quad 3.75 \quad 2-1 \quad b^{1}\right)_{-y} p^{2}$

$\begin{array}{llll}1.25 & 3.97 & 2-1 & b^{1} D-x^{3} p^{\circ}\end{array}$

$\begin{array}{llll}1.25 & 3.98 & 2-3 & b^{1} D-x^{1} \\ & & & (73) \\ \end{array}$

4286.97 A 300

4385.30

4655.49
4743.08

$\begin{array}{ll}\text { A } & 300 \\ \text { A } & 400 \\ \text { A } & 250\end{array}$

4525.31
4427.52

4619.87

4668.91

4383.44

4647.50

4378.10
4334.96

4217.56

4193.35

4099.54

3994.50
4152.78

4249.99
4033.58

$\begin{array}{lllc}1.94 & 4.82 & 4-5 & z^{3} F^{0}-e^{3} G t \\ 1.77 & 4.59 & 3-4 & (75) \\ 1.75 & 4.38 & 2-3 & \end{array}$

$\begin{array}{lll}1.94 & 4.59 & 4-4 \\ 1.77 & 4.38 & 3-3\end{array}$

$1.94 \quad 4.67 \quad 4-4 \quad z^{3} \mathrm{~F}^{0}-\mathrm{e}^{3} \mathrm{~F}$

$1.75 \quad 4.42$

$\begin{array}{lll}1.94 & 4.56 & 4-3 \\ 1.77 & 4.43 & 3-2\end{array}$

$\begin{array}{lll}1.77 & 4.42 & 3-2 \\ 1.77 & 4.67 & 3-4 \\ 1.75 & 4.56 & 3-3\end{array}$

$\begin{array}{llll}1.94 & 4.59 & 4-3 & z^{3} F^{0}-e^{1 F} \\ 1.77 & 4.59 & 3-3 & (77)\end{array}$

$\begin{array}{lll}1.77 & 4.59 & 3-3 \\ 1.75 & 4.59 & 2-3\end{array}$

$1.94 \quad 4.86 \quad 4-3 \quad z^{3} F^{\circ}-e^{3} D+$

$\begin{array}{lll}1.77 & 4.72 & 3-2 \\ 1.75 & 4.76 & 2-1\end{array}$

$1.77 \quad 4.86 \quad 3-3$

$\begin{array}{llll}1.75 & 4.72 & 2-2\end{array}$

$\begin{array}{llll}1.94 & 4.84 & 4-4 & 2^{3} F^{0}-e^{1} G \\ 1.77 & 4.84 & 3-4 & (79)\end{array}$

$4671.82 \quad A \quad 300$

$1.95 \quad 4.59 \quad 3-4 \quad z^{1} \mathrm{~F}^{0}-\mathrm{e}^{3} \mathrm{O}$

$\begin{array}{lllllll}4540.71 \quad A \quad 10 & 1.95 & 4.67 & 3-4 & z^{1} F^{\circ}-e^{3} F+\end{array}$

4663.76

$1.95 \quad 4.59 \quad 3-3 \quad 2^{1} \mathrm{~F}^{\circ}-\mathrm{e}^{1} \mathrm{~F}$

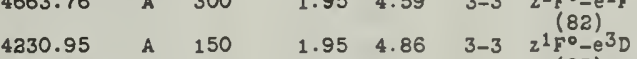


86

\begin{tabular}{|c|c|c|c|c|c|c|c|c|c|c|c|c|c|c|c|c|c|c|c|c|}
\hline \multicolumn{3}{|c|}{ I A $\stackrel{\text { Laboratory }}{\text { Ref }}$ Int } & \multirow{2}{*}{\multicolumn{2}{|c|}{ Low ${ }_{\text {High }}^{\text {P }}$}} & \multirow[t]{2}{*}{$\mathrm{J}$} & \multirow[t]{2}{*}{$\begin{array}{l}\text { Mult1plet } \\
\text { (No) }\end{array}$} & \multicolumn{3}{|c|}{ I A ${ }_{\text {Ref }}^{\text {Laboratory }}$ Int } & \multirow{2}{*}{\multicolumn{2}{|c|}{${ }_{w}^{E} P_{\text {H1gh }}$}} & \multirow[t]{2}{*}{$\mathrm{J}$} & \multirow[t]{2}{*}{$\begin{array}{l}\text { Multiplet } \\
(\text { No) }\end{array}$} & \multicolumn{3}{|c|}{ I A $\stackrel{\text { Ret }}{\text { Laboratory }}$ Int $^{\text {Lab }}$} & \multirow[t]{2}{*}{$\operatorname{Low}^{E}$} & \multirow[t]{2}{*}{${ }_{\mathrm{H} 1 \mathrm{gh}}$} & \multirow[t]{2}{*}{$\mathrm{J}$} & \multirow[t]{2}{*}{$\underset{\text { (No) }}{\text { Mult1plet }}$} \\
\hline & & & & & & & La II $c$ & tin & & & & & & Le II $c 0$ & ontin & & & & & \\
\hline $\begin{array}{l}4717.58 \\
4911.34\end{array}$ & A & $\begin{array}{l}50 \\
10\end{array}$ & $\begin{array}{l}3.05 \\
2.05\end{array}$ & $\begin{array}{l}4.67 \\
4.56\end{array}$ & $\begin{array}{l}4-4 \\
4-3\end{array}$ & $\begin{array}{c}z^{1} G^{0}-e^{3} F \\
(87)\end{array}$ & $\begin{array}{l}3049.39 \\
3054.02 \\
3081.42\end{array}$ & $\begin{array}{l}\mathbf{A} \\
{ }_{A}^{A}\end{array}$ & $\begin{array}{l}5 \\
6 \\
6 n\end{array}$ & $\begin{array}{l}3.78 \\
2.73 \\
2.65\end{array}$ & $\begin{array}{l}6.83 \\
6.77 \\
6.65\end{array}$ & $\begin{array}{l}3-4 \\
3-3 \\
1-2\end{array}$ & $\begin{array}{l}2^{3} D^{0}-f^{3} F \uparrow \\
(115)\end{array}$ & 4600.59 & A & $5 n$ & $\begin{array}{l}3.64 \\
3.48\end{array}$ & $\begin{array}{l}6.33 \\
6.16\end{array}$ & $\begin{array}{l}2-3 \\
1-2\end{array}$ & $\begin{array}{l}y^{3} P^{0}-t^{3} D+ \\
(148)\end{array}$ \\
\hline 4850.58 & A & 30 & 2.05 & 4.59 & $4-3$ & $\mathrm{z}^{1} G^{0}-\mathrm{e}^{1} F$ & 3022.36 & A & $5 \mathrm{n} 1$ & & & & & 4538.87 & A & $8 \mathrm{nl}$ & 3.64 & 6.36 & $2-2$ & $y^{3} P^{\circ}-P^{1} D$ \\
\hline 4419.16 & A & 30 & 2.05 & 4.84 & $4-4$ & $\begin{array}{c}\mathrm{z}^{1} \mathrm{G}^{\circ}-\mathrm{e}^{1} \mathrm{G} \\
(89)\end{array}$ & C & $\infty$ & . & W.10 & 0.01 & $\infty-\infty$ & 20 & 4132.50 & A & $10 \mathrm{nl}$ & 3.64 & 6.63 & $3-3$ & $\beta^{3 p}$ \\
\hline $\begin{array}{l}5048.04 \\
5279.11 \\
5480.73\end{array}$ & $\begin{array}{l}A \\
A\end{array}$ & $\begin{array}{l}301 \\
40 \\
35\end{array}$ & $\begin{array}{l}2.3 ? \\
2.25 \\
2.13\end{array}$ & $\begin{array}{l}4.82 \\
4.59 \\
4.38\end{array}$ & $\begin{array}{l}4-5 \\
3-4 \\
2-3\end{array}$ & $\begin{array}{c}y^{3} F^{\circ}-e^{3} G \\
(90)\end{array}$ & $\begin{array}{l}6188.09 \\
6443.05 \\
6307.25 \\
6315.79\end{array}$ & $\begin{array}{l}\mathbf{A} \\
\mathbf{A} \\
\mathbf{A} \\
\mathbf{A}\end{array}$ & $\begin{array}{l}1001 \\
50 \mathrm{n} \\
20 \mathrm{n} \\
50\end{array}$ & $\begin{array}{l}3.87 \\
3.80 \\
3.80 \\
3.80\end{array}$ & $\begin{array}{l}4.86 \\
4.73 \\
4.76 \\
4.76\end{array}$ & $\begin{array}{l}2-3 \\
1-2 \\
0-1 \\
1-1\end{array}$ & $\begin{array}{l}z^{3} p^{\circ}-e^{3} D \dagger \\
(117)\end{array}$ & $\begin{array}{l}3767.05 \\
3885.09\end{array}$ & A & $\begin{array}{l}5 n \\
4\end{array}$ & $\begin{array}{l}3.64 \\
3.64\end{array}$ & $\begin{array}{l}6.92 \\
6.82\end{array}$ & $\begin{array}{l}2-2 \\
3-1\end{array}$ & $\begin{array}{l}\mathrm{y}^{3} \mathrm{P}^{0}-\mathrm{e}^{3 P} \uparrow \\
(151)\end{array}$ \\
\hline $\begin{array}{l}5566.92 \\
5806.56\end{array}$ & $\stackrel{A}{A}$ & $\begin{array}{r}40 \\
8\end{array}$ & $\begin{array}{l}2.37 \\
2.25\end{array}$ & $\begin{array}{l}4.59 \\
4.38\end{array}$ & $\begin{array}{l}4-4 \\
3-3\end{array}$ & & 5808.63 & $A$ & 8 & 2.87 & 4.99 & $3-3$ & $z^{3} P^{0}-e^{1} D$ & 9346.69 & A & 15 & 3.53 & 4.84 & $5-4$ & $\begin{array}{l}2^{1} \mathrm{~K}^{0}-e^{1} \mathrm{G} \\
(153)\end{array}$ \\
\hline $\begin{array}{l}5381.77 \\
5340.66\end{array}$ & A & $\begin{array}{r}50 \\
100\end{array}$ & $\begin{array}{l}2.37 \\
3.35\end{array}$ & $\begin{array}{l}4.67 \\
4.56\end{array}$ & $\begin{array}{l}4-4 \\
3-3\end{array}$ & $\begin{array}{c}y^{3} F^{0}-e^{3} F \dagger \\
(91)\end{array}$ & 3460.31 & $A$ & 51 & 2.87 & 6.44 & $2-3$ & $2^{3} \mathrm{PO}^{3}-\mathrm{F}^{1} F$ & 4880.30 & A & $10 \mathrm{n}$ & 4.10 & 6.63 & $3-3$ & $x^{3} P^{0}-\beta^{3} D^{3}$ \\
\hline 5381.91 & A & 100 & 2.13 & 4.43 & $2-3$ & & $\begin{array}{l}3283.95 \\
3329.07\end{array}$ & $\stackrel{A}{A}$ & $\begin{array}{l}8 \mathrm{n} \\
8\end{array}$ & $\begin{array}{l}3.87 \\
3.80\end{array}$ & $\begin{array}{l}6.63 \\
6.51\end{array}$ & $\begin{array}{l}3-3 \\
1-3\end{array}$ & $\begin{array}{l}{ }^{2} P^{\circ}-g^{3} D \dagger \\
(130)^{-1}\end{array}$ & 4502.16 & A & $10 \mathrm{n}]$ & 3.97 & 6.71 & $1-1$ & $x^{3} p^{0}-e^{3} 8$ \\
\hline $\begin{array}{l}4952.06 \\
5002.13 \\
4688.65\end{array}$ & $\begin{array}{l}A \\
A \\
A\end{array}$ & $\begin{array}{l}40 \\
40 \\
40\end{array}$ & $\begin{array}{l}3.37 \\
3.35 \\
2.12\end{array}$ & $\begin{array}{l}4.86 \\
4.73 \\
4.76\end{array}$ & $\begin{array}{l}4-3 \\
3-2 \\
2-1\end{array}$ & ${ }^{y^{3} F^{0}}$ & 3336.31 & A & 5 & 3.87 & 6.58 & $3-1$ & $\begin{array}{l}z^{3} p^{0}-e^{1} p+ \\
(121)\end{array}$ & $\begin{array}{l}3411.76 \\
3580.10 \\
3578.89\end{array}$ & $\begin{array}{l}\mathbf{A} \\
\mathrm{A} \\
\mathrm{A}\end{array}$ & $\begin{array}{l}20 \mathrm{nl} \\
8 \mathrm{n} \\
5 \mathrm{n}\end{array}$ & $\begin{array}{l}4.10 \\
4.10 \\
3.97\end{array}$ & $\begin{array}{l}7.73 \\
7.55 \\
7.43\end{array}$ & $\begin{array}{l}3-3 \\
3-1 \\
1-0\end{array}$ & $\begin{array}{l}x^{3 P 0}-150 P \\
(155)\end{array}$ \\
\hline 4996.82 & A & 50 & 2.37 & 4.84 & $4-4$ & $\begin{array}{c}y^{3} F^{0}-e^{1} G \\
(93)^{1}\end{array}$ & 3618.56 & $\mathrm{~A}$ & ${ }^{5}$ & 8.87 & 6.71 & $8-1$ & $(12$ & 3407.00 & $\stackrel{A}{A}$ & 8 In & $\begin{array}{l}3.92 \\
3.92\end{array}$ & 7.55 & $\begin{array}{l}1-z \\
0-1\end{array}$ & \\
\hline 4498.76 & A & 10 & 3.25 & 4.99 & $3-2$ & $\begin{array}{c}\mathrm{y}^{3} \mathrm{~F}^{\circ}-\mathrm{e}^{1} \mathrm{D} \\
(94)\end{array}$ & $\begin{array}{l}3932.53 \\
3694.37\end{array}$ & $\begin{array}{l}\mathbf{A} \\
\mathbf{A}\end{array}$ & $\begin{array}{r}101 \\
7 \mathrm{n}\end{array}$ & 3.03 & $\begin{array}{l}6.16 \\
6.36\end{array}$ & $\begin{array}{l}3-2 \\
2-3\end{array}$ & $\begin{array}{l}y^{1} D^{0}-f^{3} D \uparrow \\
(123) \\
y^{1} D^{0}-P^{1} D\end{array}$ & $\begin{array}{l}3217.12 \\
3112.63\end{array}$ & $\stackrel{A}{A}$ & $\begin{array}{l}8 \mathrm{n} \\
8 \mathrm{n}\end{array}$ & $\begin{array}{l}4.10 \\
3.97\end{array}$ & $\begin{array}{l}7.94 \\
7.94\end{array}$ & $\begin{array}{l}2-3 \\
1-a\end{array}$ & $x^{3} p^{0}-g^{3} p \uparrow$ \\
\hline $\begin{array}{l}5188.21 \\
5377.08 \\
5671.54\end{array}$ & $\begin{array}{l}A \\
A \\
A\end{array}$ & $\begin{array}{l}500 \\
200 \\
100\end{array}$ & $\begin{array}{l}2.44 \\
3.29 \\
2.20 \\
2.20\end{array}$ & $\begin{array}{l}4.82 \\
4.59 \\
4.38\end{array}$ & $\begin{array}{l}6-5 \\
5-4 \\
4-3 \\
5-5\end{array}$ & $\begin{array}{c}z^{3} H^{\circ}-e^{3} G \\
(95)\end{array}$ & 3612.34 & A & 50 & 3.02 & 6.44 & $3-3$ & $\begin{array}{l}(124) \\
J^{1}\left(D^{0}-P^{1 F}\right. \\
(125)\end{array}$ & $\begin{array}{l}3174.88 \\
3191.39\end{array}$ & $\stackrel{A}{A}$ & $\begin{array}{l}10 \mathrm{nl} \\
10 \mathrm{n}\end{array}$ & $\begin{array}{l}4.10 \\
4.10\end{array}$ & $\begin{array}{l}7.99 \\
7.97\end{array}$ & $\begin{array}{l}2-3 \\
2-2\end{array}$ & $\begin{array}{l}x^{3} p^{0}-h^{3} p+ \\
(157)\end{array}$ \\
\hline $\begin{array}{l}4891.43 \\
5167.28\end{array}$ & $\begin{array}{l}A \\
A\end{array}$ & $\begin{array}{l}10 \\
10\end{array}$ & $\begin{array}{l}2.29 \\
2.20\end{array}$ & $\begin{array}{l}4.83 \\
4.59\end{array}$ & $\begin{array}{l}5-5 \\
4-4\end{array}$ & & $\begin{array}{l}3420.54 \\
3520.72\end{array}$ & A & $\begin{array}{c}5 n \\
10 n 1\end{array}$ & $\begin{array}{l}3.03 \\
3.03\end{array}$ & $\begin{array}{l}6.63 \\
6.53\end{array}$ & $3-3$ & $\begin{array}{l}\mathrm{J}^{1} \mathrm{D} \\
{ }_{1}^{1}\end{array}$ & 5173.83 & A & 251 & 3.98 & 6.36 & $3-3$ & $x^{1} F$ \\
\hline $\begin{array}{l}5204.14 \\
5226.20\end{array}$ & $\begin{array}{l}A \\
A\end{array}$ & $\begin{array}{l}300 \\
401\end{array}$ & $\begin{array}{l}2.29 \\
2.20\end{array}$ & $\begin{array}{l}4.67 \\
4.56\end{array}$ & $\begin{array}{l}5-4 \\
4-3\end{array}$ & $\begin{array}{c}z^{3} H^{\circ}-e^{3} F \\
(96)\end{array}$ & 3397.77 & A & $40 \mathrm{nl}$ & 3.02 & 6.00 & $3-2$ & $\begin{array}{c}\left.1 D^{07}\right) \\
\mathrm{D}^{0}-f^{3} F \\
(128)\end{array}$ & 5014.45 & A & $30 \mathrm{nl}$ & 3.98 & 6.44 & $3-3$ & \\
\hline 5157.43 & A & 150 & 3.30 & 4.59 & $4-3$ & $\mathrm{z}^{3} \mathrm{H}^{0}-\mathrm{e}^{1} \mathrm{~F}$ & 6718.68 & $\mathbf{A}$ & 60 & 3.03 & 4.86 & $3-3$ & $\mathrm{~J}^{1} \mathrm{~F}^{0}-\mathrm{e}^{3} \mathrm{D}+$ & 4194.36 & A & 30n & 3.98 & 6.92 & $3-4$ & $(160)$ \\
\hline 4843.29 & A & 5 & 2.29 & 4.84 & $5-4$ & $\begin{array}{c}z^{3} H^{0}-e^{1 G} \\
(98)\end{array}$ & 6801.38 & A & 5 & 3.03 & 4.84 & $3-4$ & $\mathrm{y}^{1}$ & 4563.5 & A & $5 n$ & 4.38 & 7.08 & $3-3$ & $e^{3} c$ \\
\hline 5458.68 & A & 50 & 3.33 & 4.59 & $3-3$ & $z^{1} D^{0}-e^{1} F$ & 6373.76 & A & 100 & 3.03 & 4.99 & $3-3$ & $y^{1}=$ & 5066.99 & A & $20 \mathrm{n}$ & 4.83 & 7.25 & $5-4$ & \\
\hline $\begin{array}{l}5172.89 \\
5090.56\end{array}$ & $\stackrel{A}{A}$ & $\begin{array}{l}301 \\
301\end{array}$ & $\begin{array}{l}2.33 \\
3.33\end{array}$ & $\begin{array}{l}4.73 \\
4.76\end{array}$ & $\begin{array}{l}2-2 \\
2-1\end{array}$ & $\begin{array}{l}z^{1} D^{0}-e^{3} D+ \\
(100)\end{array}$ & 3437.57 & $\mathbf{A}$ & 8 & 3.03 & 6.63 & $3-3$ & $\begin{array}{l}\mathrm{y}^{1} F^{\circ}-8^{3} \mathrm{D} \\
(132)^{3}\end{array}$ & 4341.30 & A & $15 n ?$ & 4.59 & 7.50 & $4-4$ & \\
\hline $\begin{array}{l}4636.43 \\
3007.33\end{array}$ & $\begin{array}{l}\text { A } \\
\text { A }\end{array}$ & $\begin{array}{r}80 \\
5\end{array}$ & $\begin{array}{l}3.33 \\
2.33\end{array}$ & $\begin{array}{l}4.99 \\
6.44\end{array}$ & $\begin{array}{l}3-3 \\
2-3\end{array}$ & $\begin{array}{l}z^{1} D^{0}-e^{1} D \\
(101) \\
z^{1} D^{0}-f^{1} F \\
(103)\end{array}$ & $\begin{array}{l}4363.05 \\
4443.94 \\
4307.61 \\
4634.95\end{array}$ & $\begin{array}{l}\mathbf{A} \\
\mathbf{A} \\
\mathbf{A} \\
\mathbf{A}\end{array}$ & $\begin{array}{l}501 \\
20 \mathrm{nl} \\
101 \\
351\end{array}$ & $\begin{array}{l}3.50 \\
3.38 \\
3.21 \\
3.50\end{array}$ & $\begin{array}{l}6.33 \\
6.16 \\
6.14 \\
6.16\end{array}$ & $\begin{array}{l}3-3 \\
3-3 \\
1-1 \\
3-3\end{array}$ & $\begin{array}{l}y^{3} D^{0}-P^{3} D \\
(133)\end{array}$ & 4304.11 & A & $\begin{array}{c}6 \mathrm{n} \\
10 \mathrm{n} 1\end{array}$ & $\begin{array}{l}4.67 \\
4.67\end{array}$ & $\begin{array}{l}7.08 \\
7.53\end{array}$ & $4-3$ & $\begin{array}{c}e^{0} F-10 \\
(164) \\
e^{3 F-50}+ \\
(165)\end{array}$ \\
\hline $\begin{array}{l}5973.53 \\
6310.91 \\
6642.79\end{array}$ & A & $\begin{array}{l}1201 \\
200 \\
100\end{array}$ & $\begin{array}{l}2.75 \\
2.63 \\
2.52\end{array}$ & $\begin{array}{l}4.83 \\
4.59 \\
4.38\end{array}$ & $\begin{array}{l}5-5 \\
4-4 \\
3-3\end{array}$ & $\begin{array}{l}2^{3} G^{0}-e^{3} G+ \\
(103)\end{array}$ & $\begin{array}{r}4474.03 \\
\mathrm{~m} 4193.37 \\
4180.97\end{array}$ & $\begin{array}{l}\ddot{A} \\
\mathbf{P} \\
\mathbf{A}\end{array}$ & $\begin{array}{l}10 \\
\mathrm{La}^{+} \\
121\end{array}$ & $\begin{array}{l}3.38 \\
3.38 \\
3.31\end{array}$ & $\begin{array}{l}6.14 \\
6.32 \\
6.16\end{array}$ & $\begin{array}{l}3-1 \\
3-3 \\
1-2\end{array}$ & & 4113.38 & A & 401 & 4.59 & 7.59 & 3 & $\begin{array}{l}e^{1 F-6^{0}} \\
(166)\end{array}$ \\
\hline $\begin{array}{l}6642.79 \\
6714.08 \\
5652.3\end{array}$ & $\begin{array}{l}A \\
A \\
A\end{array}$ & $\begin{array}{l}100 \\
80 \\
10 \mathrm{n}\end{array}$ & $\begin{array}{l}2.53 \\
2.75 \\
2.63\end{array}$ & $\begin{array}{l}4.38 \\
4.59 \\
4.83\end{array}$ & $\begin{array}{l}3-3 \\
5-4 \\
4-5\end{array}$ & & $\begin{array}{l}3939.85 \\
3816.25\end{array}$ & $\stackrel{A}{A}$ & $\begin{array}{l}301 \\
10 \mathrm{n}\end{array}$ & $\begin{array}{l}3.50 \\
3.21\end{array}$ & $\begin{array}{l}6.63 \\
6.44\end{array}$ & $\begin{array}{l}3-3 \\
1-1\end{array}$ & $\begin{array}{l}y^{3} D^{0}-8^{3} D \uparrow \\
(134)^{3}\end{array}$ & $\begin{array}{l}4131.74 \\
3817.34\end{array}$ & $\begin{array}{l}\mathbf{A} \\
\mathbf{A}\end{array}$ & $\begin{array}{l}5 n \\
8 n\end{array}$ & $\begin{array}{l}4.86 \\
4.73\end{array}$ & $\begin{array}{l}7.85 \\
7.95\end{array}$ & $\begin{array}{l}3- \\
3-3\end{array}$ & $\begin{array}{r}(167) \\
e^{3} D-80 \\
(168)\end{array}$ \\
\hline $\begin{array}{l}6446.62 \\
6399.04 \\
6498.19\end{array}$ & $\begin{array}{l}A \\
A \\
A\end{array}$ & $\begin{array}{l}200 \\
400 \\
250\end{array}$ & $\begin{array}{l}2.75 \\
2.63 \\
3.53 \\
2.52\end{array}$ & $\begin{array}{l}4.67 \\
4.56 \\
4.43\end{array}$ & $\begin{array}{l}5-4 \\
4-3 \\
3-2\end{array}$ & $\begin{array}{c}z^{3} G^{0}-e^{3} F \dagger \\
(104)\end{array}$ & $\begin{array}{l}3925.09 \\
3701.81 \\
3641.66\end{array}$ & $\begin{array}{l}\mathbf{A} \\
\mathbf{A} \\
\mathbf{A}\end{array}$ & $\begin{array}{c}5 \\
401 \\
501\end{array}$ & $\begin{array}{l}3.38 \\
3.50 \\
3.38\end{array}$ & $\begin{array}{l}6.53 \\
6.83 \\
6.77\end{array}$ & $\begin{array}{l}2-3 \\
3-4 \\
2-3\end{array}$ & $\begin{array}{l}y^{3} D^{\circ}-f^{3} G \\
(135) \\
y^{3} D^{D^{0}} f^{3} F+ \\
(136) \\
(15)\end{array}$ & $\begin{array}{l}\text { Strongest } \\
\text { ( Some }\end{array}$ & 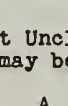 & $\begin{array}{l}881 \\
\mathbf{L}\end{array}$ & & & & \\
\hline 5948.30 & A & 30 & 2.53 & 4.59 & $3-3$ & $z^{3} G^{0}-e^{1} F$ & 3581.68 & $\ddot{A}$ & $20 \mathrm{nl}$ & 3.31 & & $1-$ & & $\begin{array}{l}5317.83 \\
4516.38\end{array}$ & $\stackrel{A}{A}$ & $\begin{array}{c}10 \mathrm{n} \\
5 \mathrm{nI}\end{array}$ & & & & \\
\hline $\begin{array}{l}5532.17 \\
5610.53\end{array}$ & ${ }_{A}^{A}$ & $\begin{array}{l}10 \\
20\end{array}$ & $\begin{array}{l}3.63 \\
2.53\end{array}$ & $\begin{array}{l}4.86 \\
4.78\end{array}$ & $\begin{array}{l}4-3 \\
3-3\end{array}$ & $\begin{array}{c}\mathbf{z}^{3} G^{\circ}-e^{3} D \\
(106)\end{array}$ & 3731.42 & A & $8 n$ & 3.50 & 6.80 & $3-4$ & $\begin{array}{c}\mathrm{y}^{3} \mathrm{D}^{0}-\mathrm{e}^{3} \mathrm{H} \\
(137)\end{array}$ & $\begin{array}{l}4210.23 \\
4301.50 \\
4193.34\end{array}$ & $\begin{array}{l}A \\
A\end{array}$ & $\begin{array}{l}50 \mathrm{nl} \\
6 \mathrm{n} \\
5\end{array}$ & & & & \\
\hline 5901.95 & A & 401 & 3.75 & 4.84 & $5-4$ & $\begin{array}{c}z^{3} G^{0}-e^{1} G \\
(107)\end{array}$ & $\begin{array}{l}4411.21 \\
4337.78 \\
4098.73\end{array}$ & $\begin{array}{l}\mathbf{A} \\
\mathbf{A} \\
\mathbf{A}\end{array}$ & $\begin{array}{c}25 \mathrm{nl} \\
101 \\
5\end{array}$ & $\begin{array}{l}3.53 \\
3.31 \\
3.31\end{array}$ & $\begin{array}{l}6.32 \\
6.16 \\
6.32\end{array}$ & $\begin{array}{l}4-3 \\
3-3 \\
3-3\end{array}$ & $\begin{array}{c}x^{3} F^{0}-P^{3} D t \\
(138)\end{array}$ & $\begin{array}{l}4161.94 \\
4133.33\end{array}$ & $\stackrel{A}{A}$ & $\begin{array}{l}8 \mathrm{n} \\
6 \mathrm{nn}\end{array}$ & & & & \\
\hline 6830.83 & A & 6 & 2.78 & 4.59 & $3-4$ & $z^{3} D^{\circ}-$ & 3981.36 & A & 101 & 3.36 & 6.36 & $2-3$ & $x^{3} F^{0}$ & & A & & & & & \\
\hline $\begin{array}{l}6554.18 \\
6732.80 \\
6968.78\end{array}$ & $\begin{array}{l}A \\
A \\
A\end{array}$ & $\begin{array}{l}? \\
40 \\
25\end{array}$ & $\begin{array}{l}2.78 \\
2.73 \\
3.65\end{array}$ & $\begin{array}{l}4.67 \\
4.56 \\
4.42\end{array}$ & $\begin{array}{l}3-4 \\
3-3 \\
1-2\end{array}$ & $\begin{array}{l}{ }^{2} \mathrm{D}^{\circ}- \\
(109\end{array}$ & 3979.08 & $A$ & 81 & 3.53 & 6.63 & $4-3$ & $x^{3} F^{\circ}-g^{3} D \dagger$ & & A & & & & & \\
\hline $\begin{array}{l}6813.68 \\
5927.71\end{array}$ & A & 50 & 2.78 & 4.59 & $3-3$ & $\begin{array}{l}z^{3} D^{\circ}-e^{1} F \dagger \\
(110) \\
z^{3} D^{0}-e^{3} D \dagger\end{array}$ & $\begin{array}{l}3864.49 \\
3773.13 \\
3780.53\end{array}$ & $\begin{array}{l}\mathbf{A} \\
\mathbf{A} \\
\mathbf{A}\end{array}$ & $\begin{array}{r}1001 \\
1501 \\
509\end{array}$ & $\begin{array}{l}3.53 \\
3.31 \\
3.36\end{array}$ & $\begin{array}{l}6.73 \\
6.58 \\
6.53\end{array}$ & $\begin{array}{l}4-5 \\
3-4 \\
2-3\end{array}$ & ${ }^{x} F^{6}$ & $\begin{array}{l}366 \\
361 \\
325 \\
320\end{array}$ & $\begin{array}{l}\mathrm{A} \\
\mathrm{A}\end{array}$ & $\begin{array}{c}101 \\
301 \\
5 \mathrm{n} \\
6\end{array}$ & & & & \\
\hline $\begin{array}{l}6203.51 \\
5848.95 \\
6374.08 \\
6085.43\end{array}$ & $\begin{array}{l}\text { A } \\
\text { A } \\
\text { A } \\
\text { A } \\
\text { A }\end{array}$ & $\begin{array}{l}30 \\
501 \\
30 \\
30 \\
10\end{array}$ & $\begin{array}{l}2.78 \\
2.73 \\
3.65 \\
3.78 \\
3.73\end{array}$ & $\begin{array}{l}4.86 \\
4.73 \\
4.76 \\
4.73 \\
4.76\end{array}$ & $\begin{array}{l}3-3 \\
3-3 \\
1-1 \\
3-2 \\
3-1\end{array}$ & 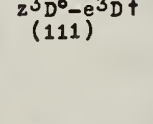 & $\begin{array}{l}3736.41 \\
3570.10 \\
3474.84\end{array}$ & $\begin{array}{l}\mathbf{A} \\
\mathbf{A} \\
\mathbf{A}\end{array}$ & $\begin{array}{l}151 \\
30 \mathrm{nl} \\
81\end{array}$ & $\begin{array}{l}3.53 \\
3.31 \\
3.36\end{array}$ & $\begin{array}{l}6.83 \\
6.77 \\
6.81\end{array}$ & $\begin{array}{l}4-4 \\
3-3 \\
2-3\end{array}$ & $\begin{array}{l}x^{3} F^{0}-f^{3} F \dagger \\
(14 z) \\
x^{3} F^{0}-g^{1} D \dagger\end{array}$ & $\begin{array}{l}3018.95 \\
3004.68\end{array}$ & $\stackrel{A}{A}$ & $\begin{array}{l}6 \mathrm{nl} \\
5 \mathrm{n}\end{array}$ & & & & \\
\hline 5447.59 & A & 10 & 2.73 & 4.99 & $2-2$ & $\begin{array}{l}z^{3} D^{0}-e^{1} D \uparrow \\
(112) \\
(112)\end{array}$ & 3423.9 & A & 5 & 3.31 & 6.93 & $3-4$ & $\frac{1}{35}$ & Le III & I $P$ & .1 & Ans1 C & L1st A & Nor & 194 \\
\hline $\begin{array}{l}3484.39 \\
3433.81\end{array}$ & $\stackrel{A}{A}$ & 0 & $\begin{array}{l}3.78 \\
2.73\end{array}$ & $\begin{array}{l}6.32 \\
6.32\end{array}$ & $\begin{array}{l}3-3 \\
3-3\end{array}$ & $(113)$ & 2985.43 & A & 5 & 3.36 & 7.39 & $3-3$ & & $\begin{array}{l}3171.68 \\
3517.14\end{array}$ & $\stackrel{A}{A}$ & $\begin{array}{l}300 \\
300\end{array}$ & $\begin{array}{l}1.68 \\
1.68\end{array}$ & $\begin{array}{l}5.57 \\
5.19\end{array}$ & $\frac{1}{\frac{1}{2}-1 \frac{1}{3}}$ & $\frac{6^{2} s-6^{2} p^{0}}{(1)}$ \\
\hline $\begin{array}{l}3209.13 \\
3263.98\end{array}$ & $\stackrel{A}{A}$ & $\begin{array}{l}6 \\
5\end{array}$ & $\begin{array}{l}2.78 \\
3.73\end{array}$ & $\begin{array}{l}6.63 \\
6.51\end{array}$ & $\begin{array}{l}3-3 \\
2-3\end{array}$ & $\begin{array}{l}z^{3} D^{0}-g^{3} D+ \\
(114)^{3}\end{array}$ & 4481.21 & A & $25 \mathrm{nl}$ & 3.39 & 6.14 & $1-1$ & $\mathbf{z}_{1}^{1} P$ & & & & & & & \\
\hline & A & $10 \mathrm{n}$ & & & & & 3059.91 & A & 8 & 3.39 & 7.43 & $1-0$ & $\begin{array}{l}{ }^{2} p^{0}-f^{3} P \uparrow \\
(147)\end{array}$ & Ce I No & ans. & & 1943 & $(2)-3)$ & atur & \\
\hline
\end{tabular}




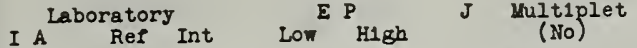
Ce II I P ? Anal B L18t B Dec 1941

Group I

$\begin{array}{cc}4186.599 & \mathrm{C} \\ 4248.676 & \mathrm{C} \\ 4306.734 & \mathrm{C} \\ 4562.360 & \mathrm{~A} \\ 4528.477 & \mathrm{~A} \\ 4572.377 & \mathrm{~A} \\ 4638.160 & \mathrm{~A} \\ 4418.784 & \mathrm{~A} \\ 4382.167 & \mathrm{~A} \\ 4296.680 & \mathrm{~A} \\ 4460.213 & \mathrm{~A} \\ 4680.458 & \mathrm{C} \\ 4560.959 & \mathrm{C} \\ 4533.077 & \mathrm{~A} \\ 4151.970 & \mathrm{C} \\ 4137.646 & \mathrm{C} \\ 4239.912 & \mathrm{~A} \\ 4483.900 & \mathrm{~A} \\ 4450.733 & \mathrm{~A} \\ .4309 .4098 & \mathrm{~A} \\ 4198.734 & \mathrm{~A} \\ 3934.46 & \mathrm{P} \\ 4144.493 & \mathrm{~A} \\ .4133 .800 & \mathrm{C} \\ 4127.367 & \mathrm{C} \\ .4133 .800 & \mathrm{C} \\ 4073.477 & \mathrm{C} \\ 4081.223 & \mathrm{~A} \\ 4101.773 & \mathrm{~A} \\ 4388.007 & \mathrm{~A}\end{array}$

$\begin{array}{lllll}300 & 0.38 & 3.18 & 6 \frac{1}{6}-6 \frac{1}{2} & a^{4} H_{-} z^{4} \mathrm{H}^{\circ} \\ 300 & 0.20 & 3.02 & 5 \frac{1}{5}-5 \frac{1}{2} & (2)\end{array}$

$\begin{array}{llll}300 & 0.20 & 3.02 & 5 \frac{1}{3}-5 \frac{1}{3} \\ 300 & 0.04 & 3.91 & 41\end{array}$

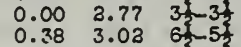

$\begin{array}{lll}0.38 & 3.02 & 63 \\ 0.20 & 3.91 & 5 \\ 0.43\end{array}$

$\begin{array}{lll}0.04 & 3.77 & 43 \\ 0.20 & 3.18 & 5 \frac{1}{3}-6 \frac{1}{3}\end{array}$

$\begin{array}{llll}0.04 & 3.02 & 43 & 3 \\ 0.00 & 2.91 & 3 \frac{1}{2}-4 \frac{1}{2}\end{array}$

$0.38 \quad 3.14 \quad 6 \frac{1}{2}-5 \frac{1}{2} a^{4} \mathrm{H}-2^{2} \mathrm{H}^{\circ}$

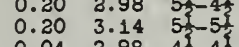

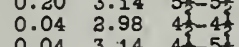

$\begin{array}{llll}0.04 & 3.14 & 43 \\ 0.00 & 3.98 & 3 \frac{1}{2}-4 \frac{13}{3}\end{array}$

$500 \quad 0.38 \quad 3.37 \quad 6 \frac{1}{3}-5 \frac{1}{2} \quad a^{4} \mathrm{H}_{-2} z^{4} \mathrm{G}^{\circ}$

$\begin{array}{lll}0.00 & 3.02 & 3 \frac{1}{3}-2 \frac{1}{3} \\ 0.00 & 3.03 & 3-3 \frac{1}{2}\end{array}$

$\begin{array}{llll}0.38 & 3.39 & 6 \frac{1}{-}-6 \frac{1}{4} & a^{4} H-z^{2} I^{\circ} \\ 0.38 & 3.20 & 6 \frac{1}{3}-5 \frac{1}{2} & (5)\end{array}$

$\begin{array}{lllllllll}4606.402 & A & \text { A } & 50 & 0.43 & 3.11 & 5 \frac{1}{3}-\frac{1}{4} & \mathrm{a}^{2} \mathrm{H}-2^{4} \mathrm{I}^{\circ} \\ 4593.932 & \mathrm{~A} & 200 & 0.22 & 3.90 & 4 \frac{1}{2}-5 \frac{1}{2} & (6)\end{array}$

$\begin{array}{ccccccc}4198.669 & A & 75 & 0.43 & 3.02 & 5 \frac{1}{2}-5 \frac{1}{1} & \mathrm{a}^{2} \mathrm{H}-2^{4} \mathrm{H}^{\circ} \\ 4582.502 & \mathrm{~A} & (10) & 0.22 & 2.91 & 4 \frac{10}{2} & (7)\end{array}$

4560.380
4471.240

4471.240
4844.87

4227.746

4144.995

4165.606

4142.398
4461.138

4285.366

3993.832

.3918 .276

5330.583

5075.304

5079.681

5187.452
5374.244

53.53 .534

5044.008
5022.871

4467.537

4714.83
4846.574

4846.574
4773.942

4680.127

4429.270

4442.73
4078.321

444.393

4317.591

4361.164

4463.410

4565.843

4433.678

4119.784
4067.279

4123.488

$\mathbf{4 1 1 5 . 3 7 4}$
$\mathbf{4 1 6 1 . 1 7 5}$

5117.175
5365.710

5409.324

$-5468.37$

5473.297

5513.085

3380.541

3334.455
3312.315

3325.339 $\begin{array}{lll}0.43 & 2.98 & 5 \frac{1}{3}-4 \frac{1}{2} \\ 0.33 & 3.14 & 43-5 \frac{1}{2}\end{array}$

$0.32 \quad 3.30 \quad 4 \frac{1}{2}-4 \frac{1}{2} \mathrm{a}^{2} \mathrm{H}-2^{4} \mathrm{G}^{\circ}$

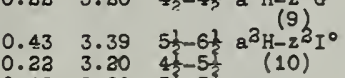
$\begin{array}{lll}0.23 & 3.20 & 41 \\ 0.43 & 3.30 & 5 \frac{1}{2}-5\end{array}$

$0.43 \quad 3.31 \quad 5 \frac{1}{3}-4 \frac{1}{2} \mathrm{a}^{2} \mathrm{H-z^{2 }} \mathrm{G}^{\circ}$

$0.43 \quad 3.53 \quad 5 \frac{1}{3}-4 \frac{1}{3} a^{2} \mathrm{H}-\mathrm{y}^{2} \mathrm{G}^{\circ}$ $\begin{array}{llll}0.22 & 3.37 & 4 \frac{1}{3}-3 \frac{3}{3} & (12)\end{array}$

$25 \quad 0.39 \quad 3.71 \quad 4 \frac{1}{2}-4 \frac{3}{3} b^{3} H-z^{4} I^{\circ}$ $0.55 \quad 3.98 \quad 5 \frac{1}{2}-4 \frac{1}{3} b^{2} \mathrm{H}_{-2}^{(13)} \mathrm{H}^{\circ}$

$0.90 \quad 3.33 \quad 8 \frac{1}{3}-7 \frac{1}{3} a^{4} K-2^{4} I^{\circ}$

$0.73 \quad 3.11$ 71-6 (15)

$\begin{array}{llll}0.56 & 3.90 & 6 \frac{1}{2} & 5 \frac{1}{2} \\ 0.40 & 3.71 & 5 \frac{3}{2}-4 \frac{1}{2}\end{array}$

$\begin{array}{llll}0.73 & 3.18 & 7 \frac{1}{1}-6 \frac{1}{3} & a^{4} \mathrm{~K}-2^{4} \mathrm{H}^{\circ} \\ 0.56 & 3.02 & 6 \frac{1}{4} 5 \frac{1}{3} & (16)\end{array}$

$0.61 \quad 3.37 \quad 43 a^{4} F-z^{4} G^{\circ}$

$0.58 \quad 3.30$ 33 $4 \frac{1}{3} \quad$ (17)

$\begin{array}{lll}0.48 & 3.02 & 21-3 \frac{1}{3} \\ 0.44 & 3.03 & 13-2 \frac{1}{3}\end{array}$

$0.58 \quad 3.213 \frac{1}{2}-3 \frac{1}{2} a^{4} F-2^{2} G^{\circ}$

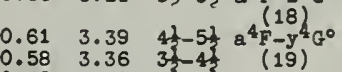

$\begin{array}{lll}0.58 & 3.36 & 31-4 \\ 0.48 & 3.50 & 2 \frac{1}{3}\end{array}$

$\begin{array}{lll}0.44 & 3.22 & 12 \\ 0.61 & 3.36 & 43\end{array}$

$0.58 \quad 3.50 \quad 3 \frac{3}{3}-\frac{31}{3}$

$\begin{array}{lll}0.48 & 3.22 & 3 \\ 0.61 & 3.50 & 45\end{array}$

$0.48 \quad 3.24 \quad 2 \frac{2}{2}-1 \frac{1}{2} \mathrm{a}^{4} F-2^{4} D^{\circ}$

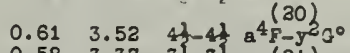

$\begin{array}{llll}0.58 & 3.37 & 31 & 3 \\ 0.48 & 3.37 & 3 & 3\end{array}$

$0.61 \quad 3.60 \quad 4 \frac{1}{3} \mathrm{a}^{4} F-2^{4} F^{\circ}$

$0.58 \quad 3.61 \quad 33-31 a^{4} F-z^{4}$

$\begin{array}{llll}0.48 & 3.47 & 21-2 \frac{1}{1} \\ 0.44 & 3.44 & 13-1 \frac{1}{1} \\ 0.48 & 3.44 & 21-1\end{array}$

$0.93 \quad 3.33 \quad 7 \frac{1}{3}-7 \frac{1}{3} a^{4} I-2^{4} I^{0}$

$0.77 \quad 3.11$ G

$\begin{array}{llll}0.63 & 3.90 & 5 & -5 \\ 0.53 & 3.71 & 43\end{array}$

$0.93 \quad 3.18 \quad 7 \frac{1}{3}-6 \frac{1}{2} a^{4} I-84 H^{\circ}$

$\begin{array}{lll}0.77 & 3.02 & 6 \frac{1}{3}-5 \frac{1}{3} \\ 0.82 & 3.91 & 5 \frac{1}{3}-\frac{13}{3} \\ 0.53 & 3.77 & 41-3 \frac{1}{2}\end{array}$

$0.93 \quad 4.59 \quad 7 \frac{1}{2}-7 \frac{1}{3} a^{4} I-y^{4} I^{\circ}$

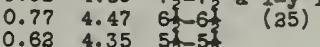

REVISED UULTIPLET TABLE

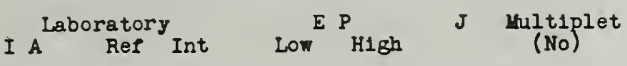

I A Laboratory

Low ${ }^{\text {H1gh }}$

$\underset{\text { (No) }}{\text { Multiplet }}$

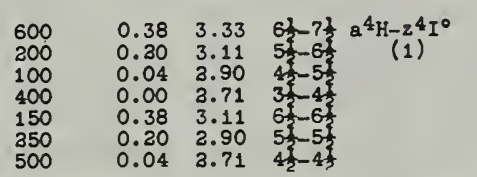

$\begin{array}{lllll}150 & 0.30 & 3.20 & 53 & 51 \\ 500 & 0.04 & 3.02 & (4) & (4)\end{array}$

Ce II continued

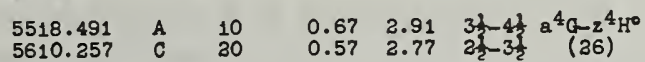

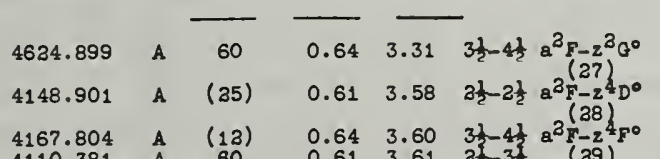
$\begin{array}{ccccccc}4167.804 & \text { A } & (12) & 0.64 & 3.60 & 3 \frac{1}{2}-4 \frac{1}{3} & a^{2} F-2 \\ 4110.381 & \text { A } & 80 & 0.61 & 3.61 & 3 \frac{1}{3} \\ 4155.533 & \text { A } & \text { (6) } & 0.64 & 3.61 & 3 \frac{3}{2}-3 \frac{1}{2} & (39)\end{array}$

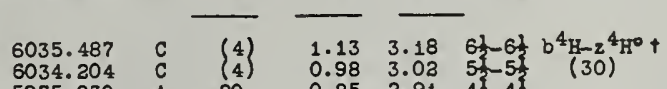

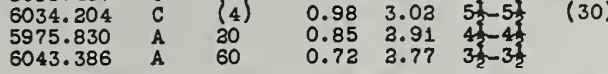

$4893.968 \quad C \quad 15 \quad 0.85 \quad 3.37 \quad 4 \frac{1}{2}-3 \frac{1}{2} b^{4} H-5^{2} G^{\circ}$

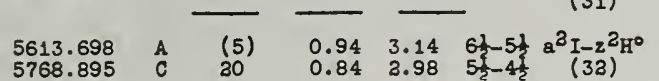

4410.641 c $\quad \frac{30}{0.87} \quad \overline{3.66} 4 \frac{1}{3}-3 \frac{1}{3} \mathrm{a}^{2} \mathrm{G}_{-} \mathrm{y}^{2} \mathrm{~F}^{\circ}$

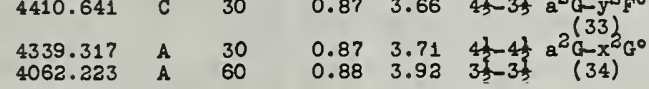

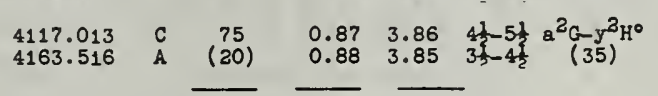

Ce II Group II See Introduction

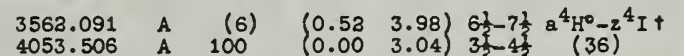

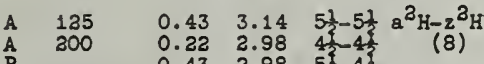

$\begin{array}{lll}0.62 & 4.35 & 5 \\ 0.53 & 4.34 & -513\end{array}$

\section{$\begin{array}{lllllll}3848.597 & \mathrm{C} & 150 & 0.52 & 3.73 & 6 \frac{1}{3}-6 \frac{1}{2} \\ 4080.435 & \mathrm{~B} & (5) & 0.36 & 3.38 & 5 \frac{1}{2} \\ 4223.599 & \mathrm{~A} & 300 & 0.12 & 3.04 & 4 \frac{1}{2}-4 \frac{1}{2}\end{array}$}

3718.380

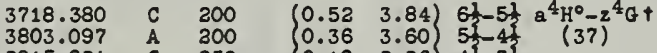

$\begin{array}{llll}3815.831 & \mathrm{C} & 350 \\ 3943.151 & \mathrm{~B} & 125\end{array} \quad\left\{\begin{array}{ll}0.36 \\ 0.12 & 3.36 \\ 0.00 & 3.13\end{array}\right) \frac{43}{3}$

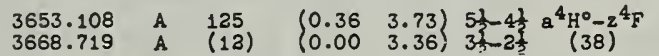

3853.164 A $125 \quad(0.00 \quad 3.20) \quad 3 \frac{1}{3}-3 \frac{1}{2} \mathrm{a}^{4} \mathrm{H}^{\circ}-2^{2} \mathrm{G}$

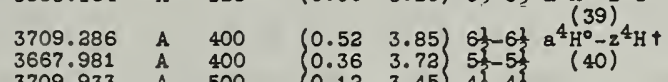

$\begin{array}{lllll}3709.933 & \text { A } & 500 \\ 3716.365 & \text { A } & 600 & (0.12 & 3.45 \\ 0.00 & 3.32 & 3 \frac{1}{3}-4 \frac{1}{3}\end{array}$

3764.117 A $150 \quad(0.36 \quad 3.63) 5 \frac{1}{3}-5 \frac{1}{3} \mathrm{a}^{4} \mathrm{H}^{\circ}-\mathrm{y}^{2} \mathrm{H}$

$3660.641 \quad C \quad 250 \quad(0.13 \quad 3.49)$ 4

3927.383 B (4) $\quad(0.36 \quad 3.50) 51-4) \quad \mathrm{a}^{4} \mathrm{H}^{\circ}-\mathrm{x}^{2} \mathrm{H}$

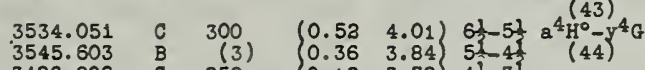

$\begin{array}{llllll}3426.208 & \mathrm{~B} & 250 \\ 3485.054 & \mathrm{C} & 400 & (0.00 & 3.54 & 3 \frac{13}{4}-2\end{array}$

$3441.210 \quad C \quad 150 \quad(0.36 \quad 3.94) \quad 5 \frac{1}{3}-4 \frac{1}{3} \mathrm{a}^{4} \mathrm{H}^{0}-159$

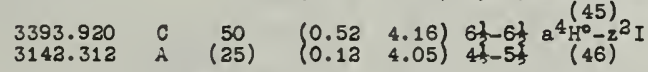

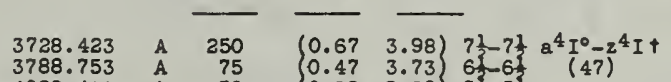

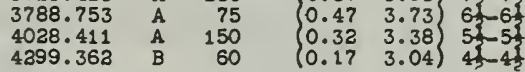

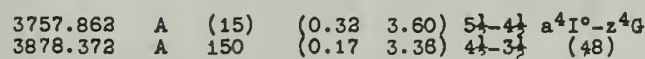

$4034.491 \quad C \quad 60 \quad(0.47 \quad 3.53) 6 \frac{1}{51} \quad a^{4} I^{0}-z^{2} H$

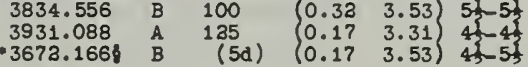

$3889.980 \quad C \quad 300 \quad(0.67 \quad 3.85) \quad 71-61 a^{4} 1^{0}-z^{4} \mathrm{H}$

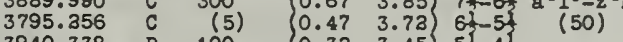

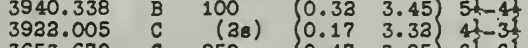

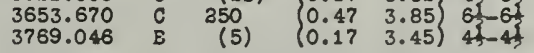

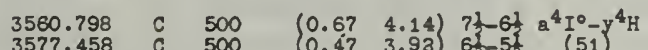

3577.458

3786.632

3436.583

3655.85

3898.373

3719.78

3718.290

3659.327

3530.53

3448.731

(5) $\quad \begin{array}{lll}0.32 & 3.69 \\ 0.17 & 3.63 & 51 \\ 0.17 & 3.43\end{array}$

150

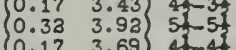

$\left\{\begin{array}{lll}0.17 & 3.69 & \frac{3}{3} \\ 0.32 & 3.69 & 5 \frac{1}{2}-4 \frac{1}{2}\end{array}\right.$

(15)

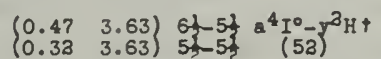

150

$(0.173 .49)$ 4h-31 $2^{4} I^{0}-116$

$(0.17 \quad 3.55) \quad 4+3 \frac{1}{2} e^{4} \frac{(53)}{10}-123$

150

$(0.173 .68)$ 4t-3) $a^{4} I^{0}-133$

(15)
Ce II continued

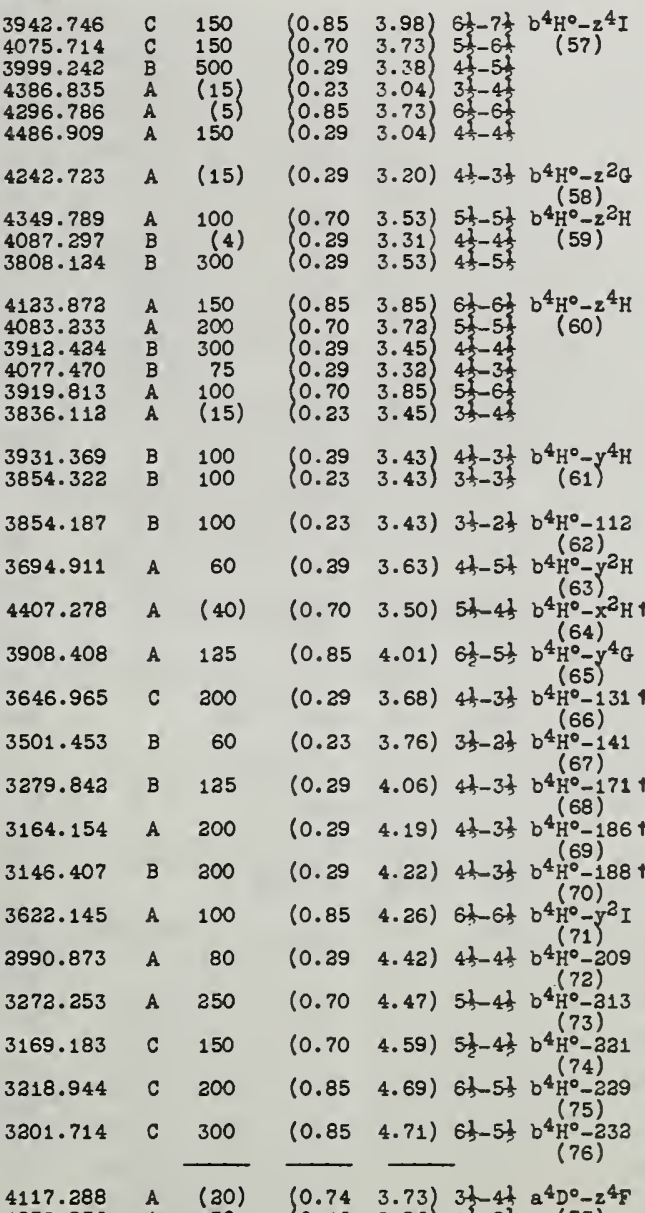

$\begin{array}{lll}4117.288 & \text { A } & (20) \\ 4253.356 & \text { A } & 50 \\ 4 & & \end{array}$

$\left\{\begin{array}{llll}0.74 & 3.73 & 31-41 & a^{4} 0^{0}-2^{4-5} \\ 0.46 & 3.36 & 1 \frac{1}{3}-2 \frac{1}{3} & (77)\end{array}\right.$

3914.949 A $\quad(18)$

4193.094 C 50

$(0.463 .61) \quad 1 \frac{1}{3}-2 \frac{1}{3} \mathrm{a}^{4} \mathrm{D}^{0}-126$

3234.274 C 300

3933.731 C $(60)$

$4046.341 \quad B \quad 100$

4071.81

4391.661
4255.784

4398.787
4399.203

4068.83

4330.445
4337.777

3876.974

4119.877 A (30)

$\begin{array}{lll}3967.048 & \text { A } & 100 \\ 3960.914 & \text { A } & 125\end{array}$

4193.874 A (35)

4187.323 A (35)

3882.446 A 75

$3631.194 \quad B \quad 135$

4338.255 A 50

3722.759 A (12)

4098.981

2904.340

$(12)$

3760.694

3376.35

(25)

\subsection{A (15)}

-3337.1148 A 300

3405.977 C 100

3379.172 C 100

$\begin{array}{lll}3366.554 & \text { B } & 150 \\ 3171.615 & \text { B } & 300\end{array}$
3189.638 A (30)
$(0.743 .68) \quad 3 \frac{1}{3}-3 \frac{1}{3} a^{4} D^{\circ}-132$

$(0.26 \quad 4.08) \quad+1 \frac{1}{4} a^{4} D^{\circ}-173$
$(80)$

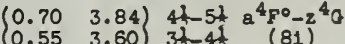

0.33 3. 36 21 31

$\left(\begin{array}{lll}0.32 & 3.13 \\ 0.70 & 3.60 & 13-2 \\ 0.33 & 3.13 & 3.4\end{array}\right.$

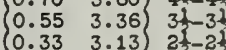

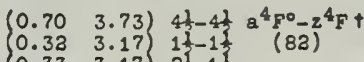

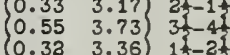

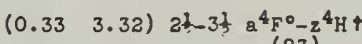
$\left(\begin{array}{ll}0.33 & 3.44 \\ 0.32 & 3.44\end{array}\right) \begin{array}{ll}2 \frac{1}{3}-1 \frac{1}{3} & a^{4} \mathrm{~F}^{\circ}-2^{2} \mathrm{D} \\ 0.1 \frac{1}{3} & (84)\end{array}$

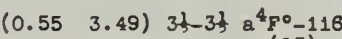
$(0.553 .50) \quad 3+4 \frac{1}{3} a^{4} F^{0}-x^{2} H$ $(0.333 .50) 1 \frac{1}{3}-\frac{1}{3} a^{4} F^{\circ}-z^{4} D$ $(0.33 \quad 3.72) 2 \frac{1}{-3}-3 \frac{1}{3} 8^{4} F^{\circ}-y^{4}$

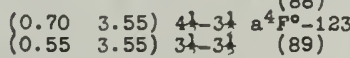
$(0.333 .64)$ 1t-1t $\mathrm{a}^{4} \mathrm{~F}^{\circ}-139$ $\left(\begin{array}{ll}0.70 & 3.71 \\ 0.55 & 3.71\end{array}\right)$ 4h-31 $a^{4} \mathrm{~F}^{0}-136$ $(0.553 .83) 31-2 \frac{1}{4} 8^{4} F^{0}-148$ (0.33 4.09) $2 k-2 t a^{4} F^{0}-175$

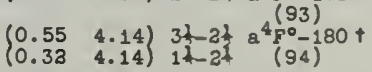
(0.33 4.14) 1t-13 $a^{4} F^{0}-181$ $\left(0.55\right.$ 4.17) $3 \frac{1+4 t}{4} a^{4} F^{\circ}-184$ $(0.334 .19)$ 3b-3! $a^{4} F^{\circ}-186$

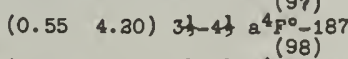

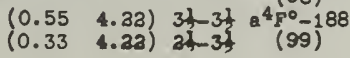

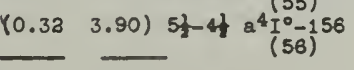




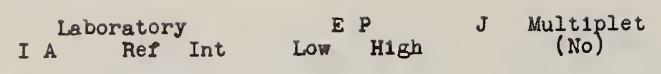
Ce II continued

\begin{tabular}{|c|c|c|c|c|c|}
\hline 3417.450 & A & 125 & 10.70 & $4.31)$ & $\begin{array}{c}4 \frac{1}{2}-4 \frac{1}{2} \quad a^{4} F^{0}-199 \\
(100)\end{array}$ \\
\hline 3236.735 & A & 150 & 10.55 & $4.36)$ & $3 \frac{1}{2}-3 \frac{1}{2} a^{4} F^{0}-205$ \\
\hline 3317.797 & A & 30 & 10.70 & $4.42)$ & $\begin{array}{c}4 \frac{1}{2}-4 \frac{1}{2} a^{4} F^{c}-209 \\
(102)\end{array}$ \\
\hline $\begin{array}{l}3304.836 \\
3177.137\end{array}$ & $\hat{A}$ & $\begin{array}{c}60 \\
(20)\end{array}$ & $\left\{\begin{array}{l}0.70 \\
0.55\end{array}\right.$ & $\begin{array}{l}4.44) \\
4.44)\end{array}$ & $\begin{array}{l}4 \frac{1}{2}-3 \frac{1}{3} \\
3 \frac{1}{2}-3 \frac{1}{2} \quad(103)\end{array}$ \\
\hline 3274.864 & C & 150 & 10.70 & $4.47)$ & $\begin{array}{c}4 \frac{1}{2}-4 \frac{1}{2} a^{4} F^{\circ}-213 \\
(104)\end{array}$ \\
\hline 3082.304 & A & (20) & 10.55 & $4.55)$ & $\begin{array}{c}3 \frac{1}{2}-3 \frac{1}{2} a^{4} F^{0}-217 \\
(105)\end{array}$ \\
\hline 3199.279 & A & (25) & 10.70 & $4.56)$ & $4 \frac{1}{2}-4 \frac{1}{2} a^{4} F^{0}-218$ \\
\hline 3017.195 & $A$ & 80 & 10.70 & $4.79)$ & $\begin{array}{c}4 \frac{1}{2}-3 \frac{1}{2} a^{4} F^{0}-237 \\
(107)\end{array}$ \\
\hline $\begin{array}{l}4031.339 \\
4539.755 \\
4527.348\end{array}$ & $\begin{array}{l}\text { B } \\
\text { B } \\
\text { A }\end{array}$ & $\begin{array}{l}150 \\
200 \\
200\end{array}$ & $\left\{\begin{array}{l}0.32 \\
0.33 \\
0.32\end{array}\right.$ & $\left.\begin{array}{l}3.38 \\
3.04 \\
3.04\end{array}\right\}$ & $\begin{array}{l}4 \frac{1}{2}-5 \frac{1}{2} a^{2} \sigma^{0}-2^{4} I \\
3 \frac{1}{2}-4 \quad(108) \\
4 \frac{1}{2}-4 \frac{1}{2}\end{array}$ \\
\hline
\end{tabular}

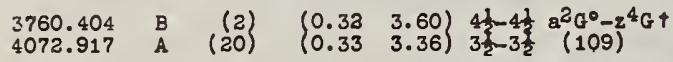

$3613.701 \quad A \quad 150 \quad(0.32 \quad 3.73) 4 \frac{1}{2}-4 \frac{1}{2} \mathrm{a}^{2} \mathrm{G}^{\circ}-2^{4} \mathrm{~F}$

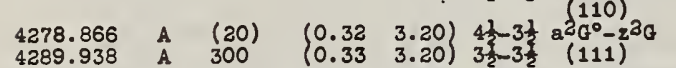

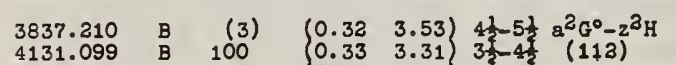

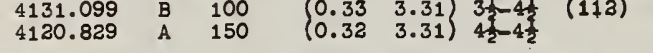

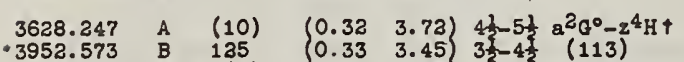

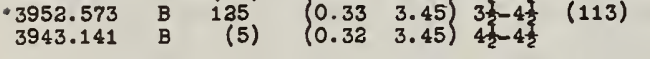

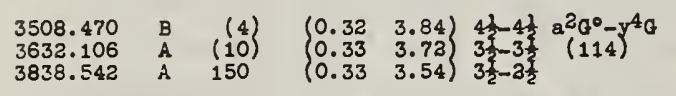

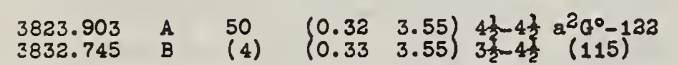

$\begin{array}{llllllll}3598.196 & \text { B } & 50 & (0.33 & 3.76) & 3 \frac{1}{2}-2 \frac{1}{2} & \mathrm{a}^{2} \mathrm{G}^{\circ}-141\end{array}$

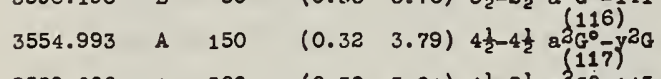

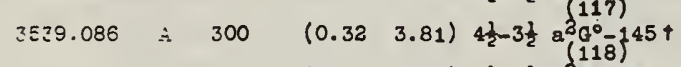

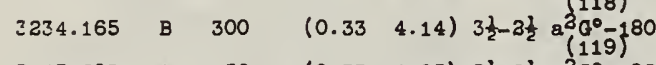

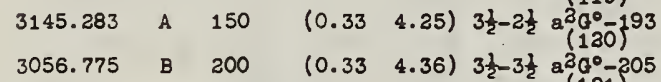

3008.789 A $125 \quad(0.32 \quad 4.42) \frac{1}{2}-4 \frac{1}{2} a^{2} a^{\circ}-2009$

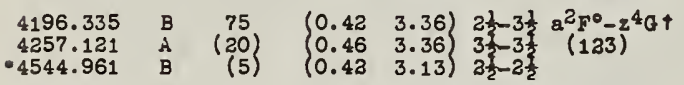

3766.514 B $(4 n)(0.46 \quad 3.73) \quad 3 \frac{1}{3}-4 \frac{1}{2} a^{2} F^{0}-2^{4} F_{1}$

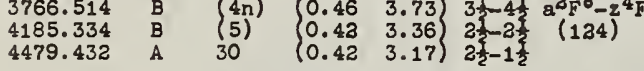

$4320.723 \quad A \quad 60 \quad(0.46 \quad 3.31) \quad 3 \frac{1}{2}-4 \frac{1}{2} a^{230}-z^{2} \mathrm{H}$

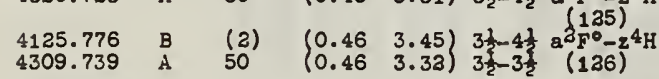

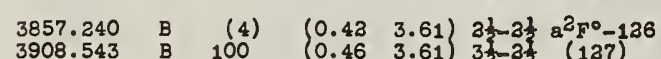

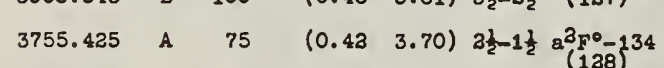

3792.326 A $50 \quad\left(\begin{array}{llll}0.46 & 3.71) & 3 \frac{1}{2}-3 \frac{1}{2} \mathrm{a}^{2} \mathrm{~F}^{\circ}-136 t \\ (39) & \end{array}\right.$

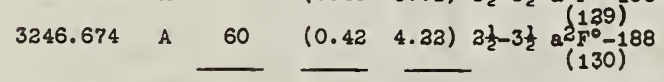

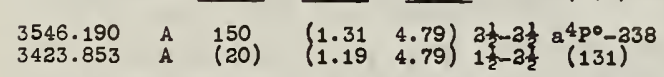

$3476.842 \quad A \quad 150 \quad\left(\begin{array}{ll}1.31 & 4.86) 3 \frac{1}{2}-3 \frac{1}{2} a^{4 p 0}-344 \\ (132)\end{array}\right.$

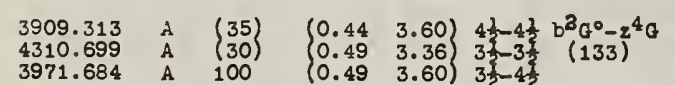

$\begin{array}{llllll}3971.684 & \mathrm{~A} & 100 & (0.49 & 3.60) & 3 \frac{1}{2}-4 \frac{1}{2} \\ 3992.386 & \mathrm{~A} & 125 & (0.44 & 3.53) & 4 \frac{1}{3}-5 \frac{1}{3} b^{2} G^{\circ}-z^{2} \mathrm{H}\end{array}$

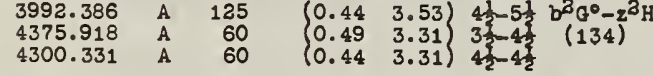

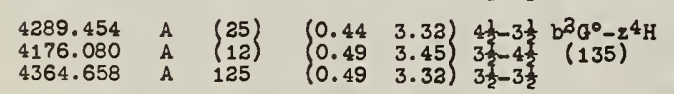

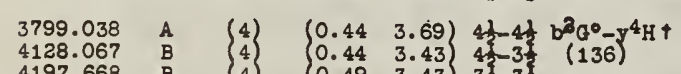

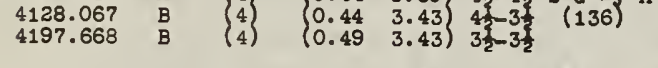

$\begin{array}{lllll}4113.726 \quad A & (30) & (0.49 & 3.49) & 3 \frac{1}{2}-3 \frac{1}{2} b^{2} a^{\circ}-116\end{array}$

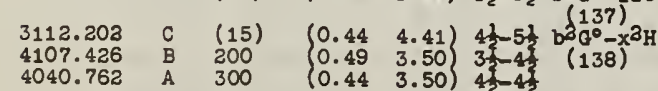

$\begin{array}{lllllll}4040.762 & \text { A } & 300 & (0.44 & 3.50 & 41 & 4 \\ 4106.881 & \text { B } & (5 \mathrm{~d}) & (0.49 & 3.50) & 3 \frac{1}{2}-3 \frac{1}{2} \\ b^{2} \mathrm{G}^{\circ}-118\end{array}$

$\begin{array}{ccccccc}4106.881 & \text { B } & (5 \mathrm{~d}) & (0.49 & 3.50) & 3 \frac{1}{2}-2 \frac{1}{2} & b^{2} G^{\circ}-118 \\ 4042.584 & \text { A } & 200 & (0.49 & 3.55) & 3 \frac{1}{2}-4 \frac{1}{2} & \left.b_{6}^{209}\right) \\ 432 & & (0.132\end{array}$

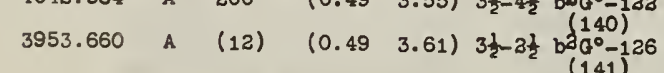

I A Laboratory ${\text { Ref Int Low }{ }^{E} \text { High } J \text { Multiplet }}_{\text {(No) }}$

Ce II continued

3782.524 B $75 \quad(0.49 \quad 3.76) \quad 3 \frac{1}{2}-2 \frac{1}{2} b^{2} G^{0}-141$

3687.802 A $30 \quad(0.44 \quad 3.79) 4 \frac{1}{2}-4 \frac{1}{2} b^{2} 0^{\circ}-y^{3} \mathrm{C}$

3423.708 C $300 \quad(0.44 \quad 4.05) 4 \frac{1}{2}-5 \frac{1}{2} b^{2} G^{0}-z^{2} I$

3390.515 A (20) $(0.44 \quad 4.08) 4 \frac{1}{2}-3 \frac{1}{2} b_{(145)}^{(144)}$

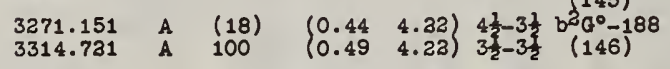

3295.289 A $80 \quad(0.49 \quad 4.24) 3 \frac{1}{2}-2 \frac{1}{2} b^{2} \mathrm{G}^{\circ}-193$

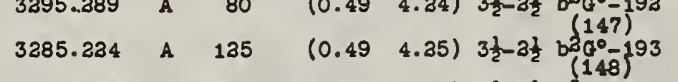

3231.236 A $200 \quad(0.494 .31) 3 \frac{1}{2}-2 \frac{1}{2} b 3 a^{\circ}-300$

3137.530 A $80 \quad(0.44 \quad 4.39) \quad 4 \frac{1}{2}-3 \frac{1}{2} b 00^{\circ}-307 t$

3103.377 A $125 \quad(0.44 \quad 4.48) 4 \frac{1}{2}-4 \frac{1}{2}$ b $(150)$

$\begin{array}{lllll}3110.278 \quad A \quad 100 \quad(0.49 & 4.46) \quad 3 \frac{1}{2}-2 \frac{1}{2} b^{2} 0^{\circ}-313 \\ (153)\end{array}$

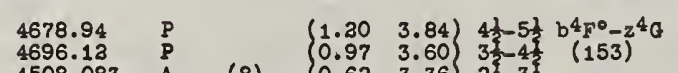

$\begin{array}{lll}4508.083 & \text { A } & (8) \\ 4725.090 & \text { C } & 20\end{array} \quad\left\{\begin{array}{lll}10.62 & 3.36 \\ 0.53 & 3.13\end{array}\right\} \begin{aligned} & 3 \frac{1}{2}-2 \frac{3}{2} \\ & 1 \frac{1}{2}-2\end{aligned}$

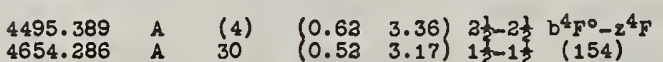

4380.060 A (30) $(0.63 \quad 3.44) 3 \frac{1}{2}-1 \frac{1}{2} b^{4} F^{0}-z^{2} D$

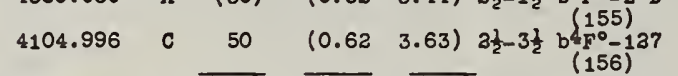

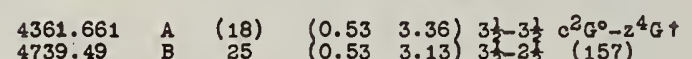

$\begin{array}{lll}4739.49 & \mathrm{~B} & 25 \\ 4014.899 & \mathrm{~A} & 125\end{array} \quad\left\{\begin{array}{lll}0.53 & 3.13 \\ 0.53 & 3.60\end{array}\right) \begin{aligned} & 3 \frac{1}{2}-2 \frac{1}{2} \\ & 3 \frac{1}{2}\end{aligned}$

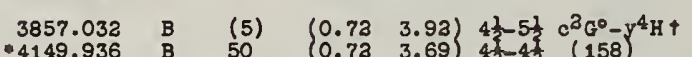

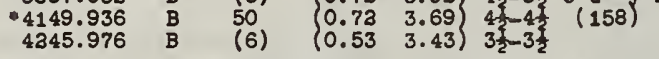

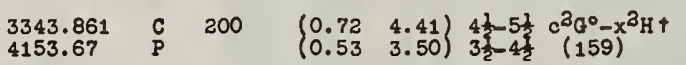

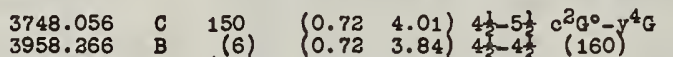

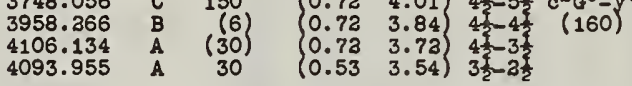

$4169.773 \quad \mathrm{~A} \quad(12) \quad(0.73 \quad 3.68) 4 \frac{1}{2}-3 \frac{1}{2}, c^{2} G^{0}-131$

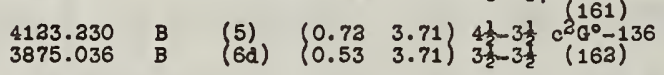

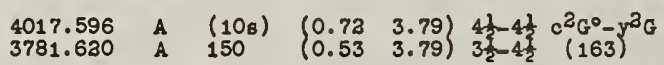

$3357.315 \quad A \quad 135 \quad(0.53 \quad 4.30) 3 \frac{1}{2}-4 \frac{1}{2} c^{2} G^{\circ}-187$

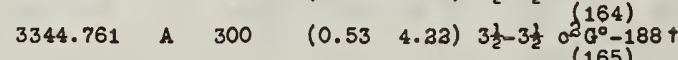

$3300.153 \quad c \quad 60 \quad(0.72 \quad 4.46) 4 \frac{1}{2}-3 \frac{1}{2} c^{3} 0^{\circ}-20^{\circ}-211$

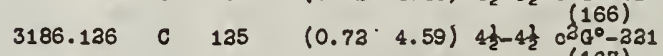

$3976.905 \quad \mathrm{~A} \quad 100 \quad(0.53 \quad 4.67) \quad 3 \frac{1}{2}-2 \frac{1}{2} \mathrm{c}^{\left(066^{\circ}-23\right)}(168)$

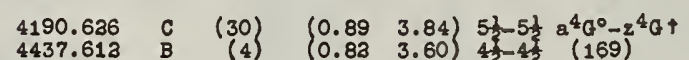

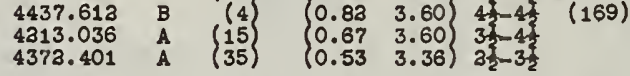

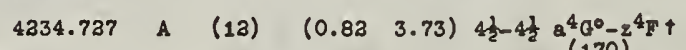

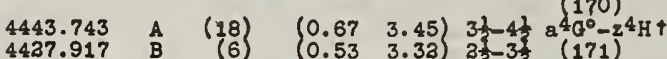

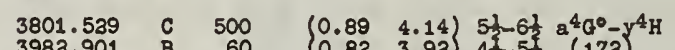

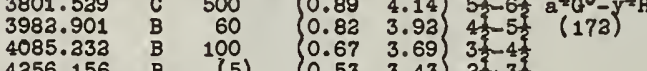

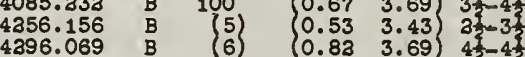

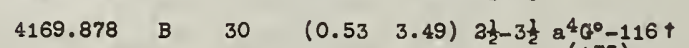

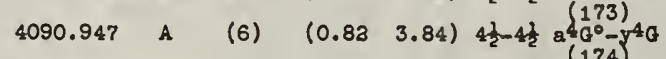

$3978.650 \quad C \quad 135 \quad(0.53 \quad 3.64) \quad 3 \frac{1}{2}-1 \frac{1}{2} \mathrm{a}^{\left(\frac{100}{40}-129\right.}$

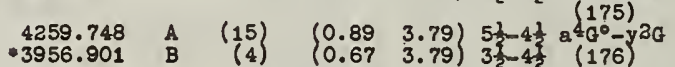

$\begin{array}{lllllll}-3953.573 \quad B \quad 125 & (0.83 & 3.94) & 4 \frac{1}{2}-4 \frac{1}{2} 8^{4} G^{\circ}-159\end{array}$

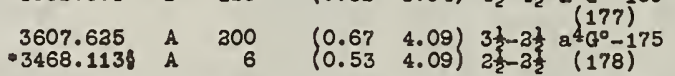

$3609.887 \quad C \quad 350 \quad(0.89 \quad 4.31) \quad 5 \frac{1}{2}-4 \frac{1}{2} a^{4} G^{\circ}-199$

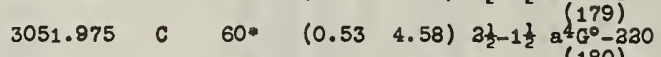

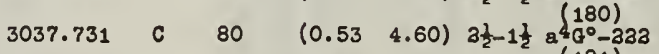

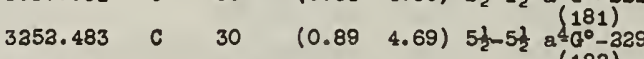

$\begin{array}{llllllll}3995.644 & A & 80 & (0.67 & 4.79) & 3 \frac{1}{2}-3 \frac{1}{2} & 80(183) & (183)\end{array}$

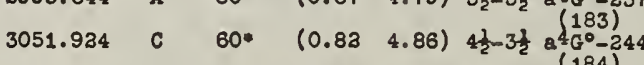

3063.010 C 400
I A Raboratory Int

Low ${ }^{\text {P }}$ High

VuIt1plet
$($ (ID)

Ce II continued

4303.944 A 150

$3488.553 \wedge \quad 75$

4003.772 C 100

$\begin{array}{lll}4135.443 & \hat{A} & (30 \\ 3896.804 & \text { A } & 100\end{array}$

.4149 .936 B 60

3924.644 B 60

3923.109 C 125

3913.191 B (5)

4001.049 B (4)

3980.895 В 100

3921.731 B 100

3442.380 A 75

3439.831 C 60

3341.868 A 100

-3286.0398 A (18)

3349.967 A (30)

3055.243 C 150

$\begin{array}{lllr}3956.284 & \text { C } & 150 \\ 4373.818 & \text { A } & 50\end{array}$

$\begin{array}{llr}4373.818 & \text { A } & 50 \\ 4449.336 & \text { A } & 200\end{array}$

$\begin{array}{lll}4214.041 & \text { B } & 50 \\ 4479.359 & \text { A } & 50\end{array}$

$\begin{array}{lll}4479.359 & \text { A } & 50 \\ 4146.234 & \text { A } & 75\end{array}$

$\begin{array}{ccc}3809.324 & \text { A } & (25) \\ 4270.189 & \text { A } & 60\end{array}$

$\begin{array}{lll}3938.086 & \text { B } & \left(\begin{array}{l}7 \\ 4\end{array}\right)\end{array}$

$\begin{array}{lll}4075.853 & \text { B } & 125 \\ 4275.561 & \text { C } & (25)\end{array}$

4275.561
4012.389

4198.431

$\begin{array}{lll}3830.871 & \text { B } & (5 \\ 3765.044 & \text { A } & (12)\end{array}$

$\begin{array}{ccc}4197.9988 & \text { B } & (5) \\ 4130.706 & \text { A } & 100\end{array}$

$\begin{array}{lll}4130.706 & \text { A } & 100 \\ 3895.114 & \text { B } & 125\end{array}$

$\begin{array}{lll}3895.114 & \text { B } & 125 \\ 3521.880 & \text { C } & 200\end{array}$

3373.739 B 125

3377.127 C 300

3243.370 A 200

3331.171 A 250

3183.523 A 250

3194.825 C 300

$\begin{array}{lll}3155.704 & \text { A } & 300 \\ & & \end{array}$

4037.665 A (25)

3599.974 A (10)

4352.733

4007.589

3817.455

3699.930

4336.82

4880.14

3993.913

4368.23

4139.176
.5347 .806

4684.605
4789.68

4551.297

3517.38

4469.850

3725.675

$(0.934 .01) \quad 5 \frac{1}{3}-5 \frac{1}{3} b^{4} 0^{\circ}-5^{4} a$

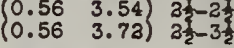

$(0.713 .68) \quad 3 \frac{1}{2}-3 \frac{1}{2} b^{4} a^{0}-132$

$-1 \frac{1}{2} b^{4} g^{0}-13$

(191)

$(0.713 .79) \quad 3 \frac{1}{2}-4 \frac{1}{2} b 40^{\circ}-7^{2 c}$

3.81) $3 \frac{1}{2}-3 \frac{1}{2} b^{4} G^{\circ}-145$

56 (195)

$\left(0.56\right.$ 4.14) $2 \frac{1}{2}-1 \frac{1}{2} b \frac{196}{0}-181$

(0.56 4.25) $2 \frac{1}{2}-2 \frac{1}{2} \quad b \frac{197}{60}-193$

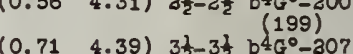

$\begin{array}{ll}(0.564 .60) & 2 \frac{1}{2}-1 \frac{1}{2} b 4 \\ \left(G^{\circ}-201\right. & (200) \\ (201)\end{array}$

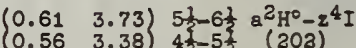

$\left(\begin{array}{lll}0.61 & 3.38\end{array}\right) 5 \frac{4}{2}-5$

$(0.613 .53) 5 \frac{1}{3}-5 \frac{1}{2} a^{2} H^{\circ}-2^{2} H$ $\left(\begin{array}{lll}0.56 & 3.53 & 4 \frac{2}{2}-5 \frac{1}{2}\end{array}\right.$

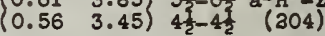

$(0.56 \quad 3.43) \quad 4 \frac{1}{2}-3 \frac{3}{2} \quad(205)$

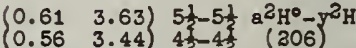

$\left(\begin{array}{lll}0.56 & 3.63\end{array}\right) \quad 4 \frac{2}{2}-5 \frac{1}{2}$

$(0.56 \quad 3.84) \quad 4 \frac{1}{2}-4 \frac{1}{2} \quad(308)$

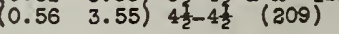

(0.61 3.77) $5 \frac{1}{2}-4 \frac{1}{2} a^{3} H^{\circ}-143$

(0.61 4.11) $5 \frac{1}{2}-4 \frac{1}{2} a^{3} H^{\circ}-177$

(0.56 4.23) $4 \frac{1}{2}-3 \frac{1}{2} \mathrm{a}^{2} \mathrm{H}^{\circ}-188$

$(0.614 .26) \quad 5 \frac{1}{2}-6 \frac{1}{2} a^{2} H^{\circ}-7^{3}$

$\begin{array}{ll}(0.564 .36) & 4 \frac{1}{2}-3 \frac{1}{2} 8^{2} H^{\circ}-205 \\ (314)\end{array}$

$\left.(0.56 \quad 4.39) 4 \frac{1}{2}-3 \frac{1}{2} a^{2} H^{\circ}-307\right)$
$(315)$

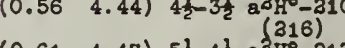

$\begin{array}{llll}0.61 & 4.47) & 5 \frac{1}{5}-4 \frac{1}{2} & a^{3} \mathrm{H}^{\circ}-21 \\ .56 & 4.47) & 4 \frac{1}{2}-4 \frac{1}{2} & (317)\end{array}$

3590.598 A 125

3672.789 


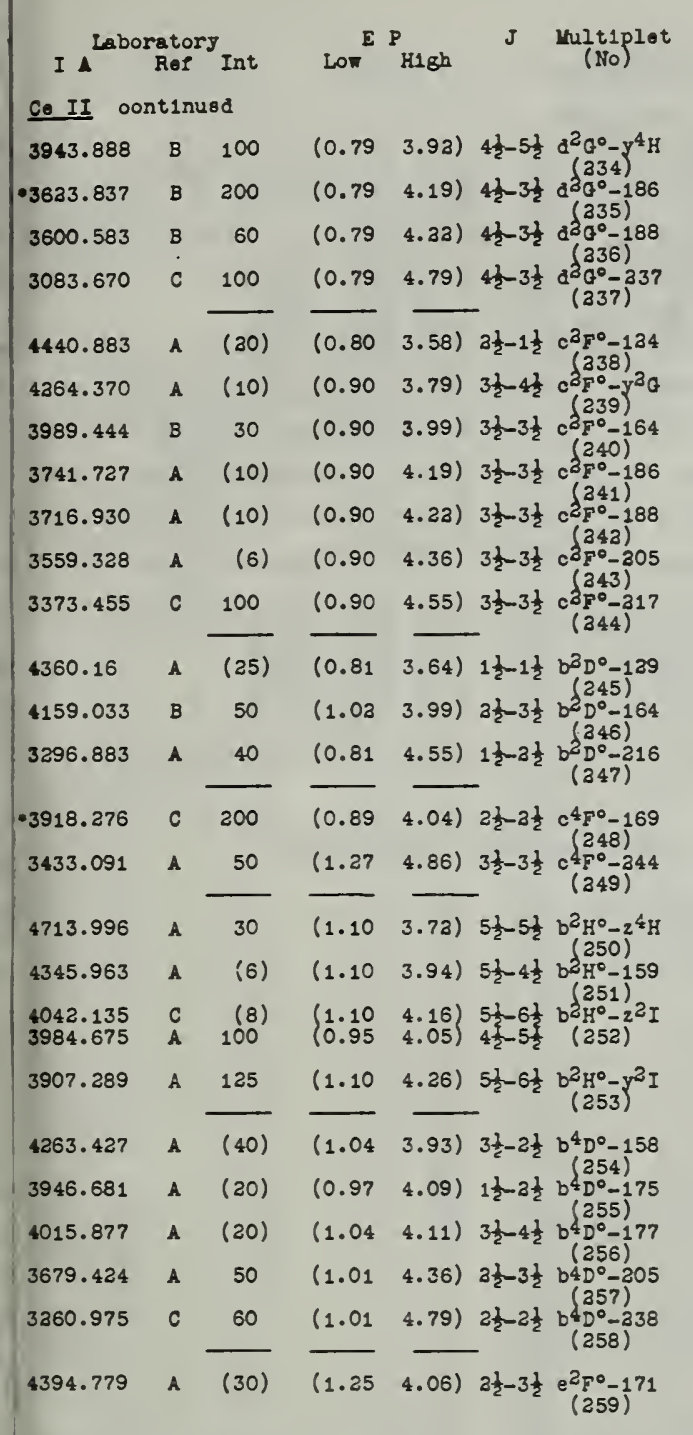

strongest Unclass1f18d Lines of Ce II

\begin{tabular}{llll}
6343.963 & $\mathrm{C}$ & 20 & $\mathrm{~V}$ \\
4971.475 & $\mathrm{C}$ & 20 & $\mathrm{~V}$ \\
4883.463 & $\mathrm{C}$ & 40 & $\mathrm{~V}$ \\
4757.842 & $\mathrm{C}$ & 15 & $\mathrm{~V}$ \\
4747.143 & $\mathrm{C}$ & 30 & $\mathrm{~V}$ \\
4737.383 & $\mathrm{C}$ & 60 & $\mathrm{~V}$ \\
4669.503 & $\mathrm{C}$ & 20 & $\mathrm{~V}$ \\
4444.704 & $\mathrm{C}$ & 75 & $\mathrm{~V}$ \\
4231.745 & $\mathrm{C}$ & 30 & $\mathrm{~V}$ \\
4111.394 & $\mathrm{C}$ & 60 & $\mathrm{~V}$ \\
4070.094 & $\mathrm{C}$ & 15 & $\mathrm{~V}$ \\
3633.837 & $\mathrm{~B}$ & 60 & $\mathrm{~V}$ \\
\hline
\end{tabular}

$\cong$

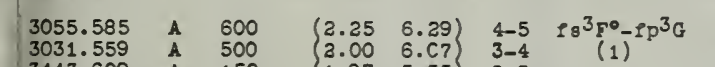
3443.60 3228.564

3353.262
3143.95 3427.33
3356.35 3454.368 3141.247
3131.548 3459.374 3110.516
3106.974 3106.974
3057.575
3085.089 $\begin{array}{lll}3085.089 & \text { A } & 100 \\ 308 & & \end{array}$

3033.736

3504.596 A 100 3147.05 A 300 3057.314 A 300 $3056.556 \quad$ A $\quad 125$

Pr I No analy818 Yay 1942 (Temperature CLa88)

\begin{tabular}{ll|l}
2.00 & 5.93 & $3-4$ \\
1.97 & 5.93 & $2-3$
\end{tabular}

(3.25 5.81) 4-3 $\rho^{3} \mathrm{~g}^{3} F^{0}-1 \mathrm{P}^{1} F \uparrow$ $\left(\begin{array}{llll}2.25 & 6.31 \\ 2.00 & 5.97 & 4-3 & 18^{3} F^{\circ}-1 \mathrm{p}^{3} \mathrm{D} \uparrow \\ 3-2 & (4)^{4}\end{array}\right.$ $\begin{array}{lll}1.97 & 6.01 & 2-1 \\ 1.97 & 5.97 & 3-2\end{array}$

(a.25 6.33) $4-4 \quad 8^{3} \mathrm{~F}^{\circ}-\mathrm{P}^{1} \mathrm{G}$

(a.29 5.81) $3-3 \quad \mathrm{Sg}^{1} \mathrm{~F}^{\circ}-\mathrm{Pp}^{1} \mathrm{~F}$

(3.29 6.31) 3-3 $18^{1} \mathrm{~F}^{\circ}-1 \mathrm{p}^{3} \mathrm{D}$ $\begin{array}{llll}(3.29 & 6.33) & 3-4 & 8^{1} \mathrm{~F}^{\circ}-\mathrm{Pp}^{1} \mathrm{G}\end{array}$

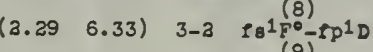

\begin{tabular}{|c|c|c|c|c|c|c|}
\hline $\begin{array}{l}3353.262 \\
3143.956 \\
3427.333 \\
2356.35 \\
3454.368 \\
3141.247 \\
3121.548\end{array}$ & $\begin{array}{l}\mathbf{A} \\
P \\
\hat{A} \\
\hat{A} \\
A\end{array}$ & $\begin{array}{l}150 \\
200 \\
125 \\
C 8^{+} \\
150 \\
250 \\
400\end{array}$ & $\left\{\begin{array}{l}2.35 \\
3.00 \\
1.97 \\
2.25 \\
2.00 \\
2.00 \\
1.97\end{array}\right.$ & $\left.\begin{array}{l}5.93 \\
5.92 \\
5.57 \\
5.92 \\
5.57 \\
5.93 \\
5.92\end{array}\right)$ & $\begin{array}{l}4-4 \\
3-3 \\
2-2 \\
4-3 \\
3-2 \\
3-4 \\
2-3\end{array}$ & $\begin{array}{c}\rho \theta^{3} F^{\circ}-\rho p^{3} F \\
(2)^{2}\end{array}$ \\
\hline 3459.374 & A & 300 & (3.25 & 5.81) & $4-3$ & $P g^{2} F^{0}-P p^{1} F \dagger$ \\
\hline $\begin{array}{l}3110.516 \\
3106.974 \\
3057.575 \\
3085.089\end{array}$ & $\begin{array}{l}\mathbf{A} \\
{ }_{A}^{A} \\
A\end{array}$ & $\begin{array}{l}200 \\
200 \\
100 \\
200\end{array}$ & $\left\{\begin{array}{l}2.25 \\
2.00 \\
1.97 \\
1.97\end{array}\right.$ & $\left.\begin{array}{l}6.31 \\
5.97 \\
6.01 \\
5.97\end{array}\right)$ & $\begin{array}{l}4-3 \\
3-2 \\
2-1 \\
3-2\end{array}$ & $\left.8^{3} F^{3}-1\right)^{3} D+$ \\
\hline 3033.736 & $A$ & 200 & (3.35 & $6.33)$ & $4-4$ & ${ }^{P B^{3}} F^{\circ}(5)^{-1 p^{1} G}$ \\
\hline 3504.596 & $A$ & 100 & (a.29) & $5.81)$ & $3-3$ & $\rho q^{1} F^{0}-\rho p^{1} F$ \\
\hline 3147.05 & A & 300 & (3.29) & $6.31)$ & $3-3$ & $-p^{3} D$ \\
\hline 3057.314 & A & 300 & (3.29 & 6.33) & $3-4$ & $6 a_{1} p_{0}-t p_{1} a$ \\
\hline 3056.556 & $A$ & 135 & (3.29 & $6.33)$ & $3-3$ & 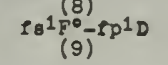 \\
\hline
\end{tabular}

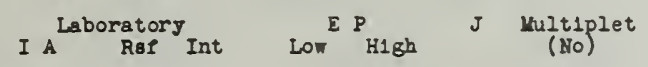

I L Laboratory

Lo: ${ }^{\text {HIgh }}$ Yult1plet
(No)
PrII I P, Anal C L1st B Dec 1941

$4628.751 \quad A \quad 100 \quad 0.05 \quad 3.73 \quad 5-5 \quad 8^{5} I^{0}$

$\begin{array}{lllllll}4517.595 \quad A \quad 40 & 0.05 & 2.79 & 5-5 & \mathrm{a}^{5} \mathrm{I}^{\circ}-3+\end{array}$

$\begin{array}{rllllll}44239.338 & \text { A } & 60 & 0.00 & 3.79 & 4-5 & \text { (3) }\end{array}$

$\begin{array}{lll}4744.935 & \text { A } & 40 \\ 4487.831 & \text { A } & 30\end{array}$

$4100.746 / /$ A 150

4143.136 A 150

4179.422 A 150
4323.98

$\begin{array}{lll}4223.98 & \text { A } & 150 \\ 4408.844 & \text { A } & 200\end{array}$

4408.844
4405.849

.4429 .238

4449.867

$\begin{array}{llll}4734.177 & \text { A } & 25 \\ 4754.635 & \text { A } & (15)\end{array}$

$\begin{array}{rrr}4707.541 & \text { A } & 20 \\ 4454.383 & \text { A } & 30 \\ 4368.327 & \text { A } & 150\end{array}$

4651.517 A 75

4297.764 A 80

4206.739 A 100

4189.518

4118.481

4225.337

4458.336
4413.155

4413.155
4333.913

4305.763

3966.573

3965.263

4044.818

3953.516

4241.019 A $\quad 60$

$\begin{array}{lll}4141.257 & \text { A } & 80 \\ 4578.139 & \text { A } & 25\end{array}$

3908.033

3918.856

3947.633
3994.834

3908.431

3699.952
3925.456

4191.615 A 20

3989.718 A 100

4421.231 A 40

$\begin{array}{llr}4173.273 & \text { A } & 50 \\ 3972.164 & \text { A } & 100\end{array}$

4081.018 A 50

3823.571 A (10)

$\begin{array}{lll}4273.271 & \text { A } & 80 \\ 4039.357 & \text { A } & 30\end{array}$

4171.834 A 40

$\begin{array}{rlr}3949.438 & \text { A } & 125 \\ 3769.695 & \text { A } & \end{array}$

3912.898 A 135

3885.190
.3711 .099
A A (35)

4283.440 A 60

4033.857 A $\quad 75$

$\begin{array}{rlr}4534.154 & \text { A } & 60 \\ 4510.160 & \text { A } & 100 \\ 4488.712 & \text { A } & 150 \\ 4879.121 & \text { A } & 30 \\ 4825.649 & \text { A } & \end{array}$

$\begin{array}{lll}4879.121 & \mathbf{A} & 30 \\ 4826.649 & \mathbf{A} & 40 \\ 5251.738 & \mathbf{A} & 12\end{array}$

4672.081 A 40

$4646.059 \quad$ A 30

4323.551
4261.796

4180.68
4589.76

4589.76
4492.427

$\begin{array}{lll}4351.849 & \hat{A} & 50 \\ 4561.461 & \mathbf{A} & (6)\end{array}$

5292.10 A 60.

$\begin{array}{rrr}4859.038 & \text { A } & 12 \\ .4496 .429 & \text { A } & 350\end{array}$

4063.817
4496.429
$4734.177 \quad$ A
450

4056.543 A 80

4413.765
4359.795

4359.795
4763.737 $\begin{array}{llll}0.00 & 3.79 & 4-5 & \text { (3) } \\ 0.30 & 3.80 & 6-6 & 5 I^{\circ}\end{array}$

$\begin{array}{llll}0.30 & 3.80 & 6-6 & 8^{5} \mathrm{I}^{0}-4 \\ 0.05 & 2.80 & 5-6 & (3)\end{array}$

$\begin{array}{llll}0.55 & 3.56 & 8-9 & a^{5} I^{0}-z^{5} K \\ 0.37 & 3.35 & 7-8 & (4)\end{array}$

$\begin{array}{lll}0.37 & 3.35 & 7-8 \\ 0.30 & 3.16 & 6-7\end{array}$

0.052 .98

$0.00 \quad 3.80$

$0.55 \quad 3.35$

$0.20 \quad 2.98$

$0.05 \quad 3.80$

$0.20 \quad 2.80$

$\begin{array}{llll}0.30 & 2.83 & 6-5 & \mathrm{a}^{5} \mathrm{I}^{0}-5 \\ 0.05 & 2.83 & 5-5 & (5) \\ 0.00 & 2.83 & 4-5 & \end{array}$

$0.00 \quad 3.83$

$0.20 \quad 2.86$

$0.00 \quad 2.87$

6-6 $\quad a^{5} I^{0}-6$

$\begin{array}{ll}0.55 & 3.48 \\ 0.37 & 3.32 \\ 0.20 & 3.17\end{array}$

$\begin{array}{ll}0.37 & 3.32 \\ 0.20 & 3.17 \\ 0.05 & 3.05\end{array}$

$\begin{array}{ll}0.20 & 3.17 \\ 0.05 & 3.05\end{array}$

$\begin{array}{ll}0.00 & 2.92 \\ 0.55 & 3.32\end{array}$

$\begin{array}{ll}0.55 & 3.32 \\ 0.37 & 3.17\end{array}$

$\begin{array}{ll}0.20 & 3.05 \\ 0.05 & 2.92\end{array}$

$\begin{array}{ll}0.05 & 2.92 \\ 0.37 & 3.48\end{array}$

$0.20 \quad 3.32$

$\begin{array}{ll}0.05 & 3.17 \\ 0.00 & 3.05\end{array}$

$0.55 \quad 3.67$

$\begin{array}{ll}0.37 & 3.46 \\ 0.55 & 3.46\end{array}$

$0.55 \quad 3.53$

$\begin{array}{ll}0.55 & 3.53 \\ 0.37 & 3.07\end{array}$

$0.55 \quad 3.71$

$0.37 \quad 3.52$

$0.05 \quad 3.14$

$\begin{array}{ll}0.05 & 3.14 \\ 0.00 & 3.16\end{array}$

$0.37 \quad 3.71$

$0.30 \quad 3.15$

$0.05 \quad 3.15$

$0.37 \quad 3.16$

$0.20 \quad 3.16$

$0.30 \quad 3.23$

$0.05 \quad 3.23$

$\begin{array}{lll}0.37 \quad 3.26 \\ 0.30 & 3.26\end{array}$

$0.37 \quad 3.33$

$\begin{array}{ll}0.37 & 3.33 \\ 0.05 & 3.33 \\ 0.33\end{array}$

$0.20 \quad 3.36$

$\begin{array}{ll}0.30 & 3.38 \\ 0.05 & 3.38\end{array}$

$\begin{array}{cc}4-5 & \left.a^{5} I^{0}-7\right) \\ 8-8 & a^{5} I^{0}-2^{5} I \\ 7-7 & (8)\end{array}$

$6-6$
$5-5$

$5-5$
$4-4$

$8-7$
$7-6$
$6-5$

$6-5$
$5-4$

$7-8$
$6-7$

$5-6$
$4-5$

$\begin{array}{cc}8-8 & 8^{5} I^{0}-z^{3} K+ \\ 7-7 & (9)\end{array}$

$\begin{array}{cc}8-7 & 8^{5} I^{0}-z^{3} I \\ 7-6 & (10)\end{array}$

$\begin{array}{lc}8-7 & 8^{5} I^{\circ}-2^{5} \mathrm{H} \\ 7-6 & (11)\end{array}$

$7-6$
$6-5$
$5-4$

$5-4$
$4-3$

$4-4$

$\begin{array}{cc}6-5 & a^{5} I^{\circ}-16 \\ 5-5 & (12)\end{array}$

7-6 $\quad a^{5} I^{0}-17$

$5-6 \quad$ (13)

$\begin{array}{cc}6-5 & a^{5} I^{0}-19 \\ 5-5 & (14)\end{array}$

$5-5$
$4-5$

7-6 $a^{5} I^{\circ}-23$

$-6 \quad a^{5} I^{0}-2^{3} \mathrm{H}$

$6-5 \quad a^{5} I^{\circ}-36$

$\begin{array}{ll}0.55 & 3.43 \\ 0.37 & 3.43\end{array}$

$0.63 \quad 3.35$

$\begin{array}{ll}0.42 & 3.16 \\ 0.32 & 2.98\end{array}$

$\begin{array}{lll}0.32 & 2.98 & 5-6 \\ 0.63 & 3.16 & 7-7\end{array}$

$\begin{array}{lll}0.63 & 3.16 & 7-7 \\ 0.42 & 3.98 & 6-6\end{array}$

$0.63 \quad 3.98 \quad 7-6$

$0.23 \quad 3.86$

5-6 $a^{3} I^{0}-64$

$\begin{array}{llll}0.33 & 2.87 & 5-5 & 8^{3} I^{\circ}-7\end{array}$

$0.63 \quad 3.48 \quad 7-8 \quad a^{3} I^{(23)}-2^{5} I$

$0.43 \quad 3.33 \quad 6-7 \quad(23)$

$0.32 \quad 3.17 \quad 5-6$

$0.43 \quad 3.17 \quad 6-7$

$\begin{array}{lll}0.23 & 3.05 & 5-6 \\ 0.32 & 3.92 & 5-4\end{array}$

$\begin{array}{llll}0.63 & 2.96 & 7-8 & 2^{3} I^{0}-10\end{array}$

$\begin{array}{llll}0.43 & 2.96 & 6-6 & a^{3} \frac{}{0}^{0}-11+ \\ 0.32 & 2.96 & 5-6 & (25)^{-1}\end{array}$

$\begin{array}{llll}0.63 & 3.67 & 7-8 & a^{3} I^{0}-2^{3} \mathbf{r} \\ 0.43 & 3.46 & 6-7 & (26)^{2}\end{array}$

$\begin{array}{lll}0.43 & 3.46 & 6-7 \\ 0.23 & 3.01 & 5-6\end{array}$

$\begin{array}{lll}0.23 & 3.01 & 5-6 \\ 0.63 & 3.46 & 7-7\end{array}$

$\begin{array}{lll}0.43 & 3.01 & 6-6\end{array}$
Pr II continued

\begin{tabular}{|c|c|c|c|c|c|c|}
\hline $\begin{array}{l}4354.420 \\
4664.647 \\
3971.164 \\
4329.415\end{array}$ & ${ }_{A}^{A}$ & $\begin{array}{l}30 \\
30 \\
40 \\
35\end{array}$ & $\begin{array}{l}0.63 \\
0.42 \\
0.43 \\
0.32\end{array}$ & $\begin{array}{l}3.53 \\
3.07 \\
3.53 \\
3.07\end{array}$ & $\begin{array}{l}7-7 \\
6-6 \\
6-7 \\
5-6\end{array}$ & $\begin{array}{c}a^{3} I^{0}-z^{3} I^{4} \\
(27)\end{array}$ \\
\hline $\begin{array}{l}4008.714 \\
3982.063 \\
3962.445\end{array}$ & $\begin{array}{l}A \\
A \\
A\end{array}$ & $\begin{array}{r}75 \\
150 \\
40\end{array}$ & $\begin{array}{l}0.63 \\
0.42 \\
0.32\end{array}$ & $\begin{array}{l}3.71 \\
3.53 \\
3.33\end{array}$ & $\begin{array}{l}7-7 \\
6-6 \\
5-5\end{array}$ & $\left.{ }^{25}\right)^{5 \mathrm{H}}$ \\
\hline $\begin{array}{l}4395.788 \\
4096.823\end{array}$ & $\hat{A}$ & $\begin{array}{l}30 \\
35\end{array}$ & $\begin{array}{l}0.42 \\
0.32\end{array}$ & $\begin{array}{l}3.23 \\
3.23\end{array}$ & $\begin{array}{l}6-5 \\
5-5\end{array}$ & $\begin{array}{c}a^{3} I^{0}-19 \\
(29)\end{array}$ \\
\hline $\begin{array}{l}4347.490 \\
4054.845\end{array}$ & $\hat{\mathbf{A}}$ & $\begin{array}{l}30 \\
80\end{array}$ & $\begin{array}{l}0.43 \\
0.23\end{array}$ & $\begin{array}{l}3.26 \\
3.26\end{array}$ & $\begin{array}{l}6-6 \\
5-6\end{array}$ & $a^{3} I^{\circ}-23+$ \\
\hline 4338.694 & A & 25 & 0.42 & 3.26 & $6-7$ & \\
\hline $\begin{array}{l}4303.100 \\
4015.389\end{array}$ & $\stackrel{A}{A}$ & $\begin{array}{c}(60) \\
40\end{array}$ & $\begin{array}{l}0.42 \\
0.22\end{array}$ & $\begin{array}{l}3.29 \\
3.29\end{array}$ & $\begin{array}{l}6-5 \\
5-5\end{array}$ & \\
\hline $\begin{array}{l}4568.545 \\
4243.528 \\
3964.261\end{array}$ & $\stackrel{\hat{A}}{A}$ & $\begin{array}{l}(30) \\
20 \\
40\end{array}$ & $\begin{array}{l}0.63 \\
0.42 \\
0.23\end{array}$ & $\begin{array}{l}3.33 \\
3.33 \\
3.33\end{array}$ & $\begin{array}{l}7-6 \\
6-6 \\
5-6\end{array}$ & $\begin{array}{c}a^{3} I^{0}-z^{3} \mathrm{H} \\
(33)\end{array}$ \\
\hline 4403.605 & A & 25 & 0.63 & 3.43 & $7-8$ & $\frac{a^{3} I^{0}-30}{(34)}$ \\
\hline $\begin{array}{l}5110.768 \\
5173.898 \\
5220.113 \\
5259.743 \\
5322.778\end{array}$ & $\begin{array}{l}\mathbf{A} \\
\hat{A} \\
\hat{A} \\
\mathbf{A} \\
\mathbf{A}\end{array}$ & $\begin{array}{l}60 \\
60 \\
50 \\
80 \\
60\end{array}$ & $\begin{array}{l}1.14 \\
0.96 \\
0.79 \\
0.63 \\
0.48\end{array}$ & $\begin{array}{l}3.56 \\
3.35 \\
3.16 \\
2.98 \\
3.80\end{array}$ & $\begin{array}{r}10-9 \\
9-8 \\
8-7 \\
7-6 \\
6-5\end{array}$ & $\frac{a^{5} I^{0}-2^{5} X+}{(35)}$ \\
\hline 4801.150 & A & 15 & 0.48 & 3.05 & $6-5$ & $\frac{a^{5} \frac{L^{0}-2^{5} I}{(36)}}{(3)}$ \\
\hline $\begin{array}{l}5034.415 \\
5135.125 \\
5219.053 \\
5292.630 \\
5381.262\end{array}$ & $\begin{array}{l}\mathbf{A} \\
\hat{A} \\
\hat{A} \\
\hat{A} \\
\mathbf{A}\end{array}$ & $\begin{array}{l}20 \\
20 \\
20 \\
30 \\
60\end{array}$ & $\begin{array}{l}1.11 \\
0.95 \\
0.79 \\
0.65 \\
0.51\end{array}$ & $\begin{array}{l}3.56 \\
3.35 \\
3.16 \\
2.98 \\
2.80\end{array}$ & $\begin{array}{l}9-9 \\
8-8 \\
7-7 \\
6-6 \\
5-5\end{array}$ & $\begin{array}{c}a^{5} K^{0}-2^{5} K+ \\
(37 !\end{array}$ \\
\hline $\begin{array}{l}5195.110 \\
5206.562 \\
5195.307 \\
5129.520 \\
5110.382\end{array}$ & $\begin{array}{l}\mathbf{A} \\
\mathbf{A} \\
\mathbf{A} \\
\mathbf{A} \\
\mathbf{A}\end{array}$ & $\begin{array}{l}20 \\
20 \\
30 \\
40 \\
60\end{array}$ & $\begin{array}{l}1.11 \\
0.95 \\
0.79 \\
0.65 \\
0.51\end{array}$ & $\begin{array}{l}3.48 \\
3.32 \\
3.17 \\
3.05 \\
3.92\end{array}$ & $\begin{array}{l}9-8 \\
8-7 \\
7-6 \\
6-5 \\
5-4\end{array}$ & $\begin{array}{c}8^{5} K^{0}-2^{5} I \\
(38)^{2}\end{array}$ \\
\hline $\begin{array}{l}6025.733 \\
6305.262 \\
6244.344 \\
6161.194 \\
6165.945\end{array}$ & $\begin{array}{l}A \\
A \\
A \\
A \\
A\end{array}$ & $\begin{array}{r}20 \\
4 \\
5 \\
50 \\
60\end{array}$ & $\begin{array}{l}1.43 \\
1.36 \\
1.19 \\
1.05 \\
0.92\end{array}$ & $\begin{array}{l}3.48 \\
3.32 \\
3.17 \\
3.05 \\
2.92\end{array}$ & $\begin{array}{l}8-8 \\
7-7 \\
6-6 \\
5-5 \\
4-4\end{array}$ & $\frac{b^{5} I^{0}-2^{5} I \uparrow}{(39)}$ \\
\hline
\end{tabular}

Stronzest Unclassified Lines of pr II

3880.466 B 100

5877.225 B $\quad 100$

3865.458 B 100

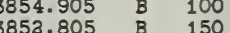

$3851.617 \quad$ B 200

$3850.825 \quad$ B 150

3830.719 B 125

3818.281

$\begin{array}{lll}3816.166 & B & 125 \\ 38 & 125\end{array}$

$\begin{array}{lll}3773.854 & B & 100 \\ & B & 100\end{array}$

$\begin{array}{lll}3764.811 & \mathrm{~B} & 125 \\ 3761.867 & \mathrm{~B} & 250\end{array}$

$\begin{array}{lll}3761.867 & \text { B } & 250 \\ 3739.193 & \text { B } & 100 \\ 3787.039 & \text { B } & 125\end{array}$

\begin{tabular}{lll}
3687.039 & B & 125 \\
3668.830 & B & 150 \\
\hline
\end{tabular}

Nd I No anslys1s May 1942 (Temperature Class)

Nd II I P A Anal C L1st B Apr 1942

$\begin{array}{llllll}-4959.1308 \text { A } & 60 & 0.06 & 2.55 & 4 \frac{1}{3}-4 \frac{1}{2} & \mathrm{a}^{6} I-10\end{array}$

4930.692

4799.433

4859.030

4825.488

4811.343
4706.5438

4609.148

4414.432

4505.75

4680.734

4569.849

4763.865

4556.136
4451.978

4709.714
4506.583

$$
\begin{gathered}
V \\
V \\
V \\
V \\
V \\
V \\
V \\
V \\
V \\
V \\
V \\
V \\
V \\
V \\
V \\
V \\
V \\
I V \\
V \\
I V \\
I V \\
I V
\end{gathered}
$$

$\begin{array}{lll}3800.303 & B & 300\end{array}$ 
REVISED UULTIPLET TABLE

I A Laboratory ReP Int $^{\text {E P }}$ LOw ${ }^{\text {H1gh }}$ J Mult1plet

Nd II continued

\begin{tabular}{|c|c|c|c|c|c|}
\hline $\begin{array}{l}4411.052 \\
4342.071 \\
4375.039 \\
4232.378 \\
4247.367\end{array}$ & $\begin{array}{l}A \\
A \\
A \\
A \\
A\end{array}$ & $\begin{array}{r}150 \\
20 \\
30 \\
150 \\
200\end{array}$ & $\begin{array}{l}0.18 \\
0.06 \\
0.00 \\
0.06 \\
0.00\end{array}$ & $\begin{array}{l}2.98 \\
2.91 \\
2.82 \\
2.98 \\
2.91\end{array}$ & 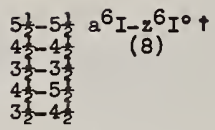 \\
\hline $\begin{array}{l}4412.265 \\
4314.511\end{array}$ & $\stackrel{A}{A}$ & $\begin{array}{l}20 \\
50\end{array}$ & $\begin{array}{l}0.06 \\
0.00\end{array}$ & $\begin{array}{l}3.86 \\
2.86\end{array}$ & 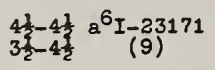 \\
\hline $\begin{array}{l}4012.250 \\
4061.085 \\
4109.455 \\
4156.083 \\
4177.321 \\
4303.573 \\
4284.518 \\
4325.766 \\
4358.169 \\
4351.295 \\
4400.828\end{array}$ & $\begin{array}{l}A \\
A \\
A \\
A \\
A \\
A \\
A \\
A \\
A \\
A \\
A\end{array}$ & $\begin{array}{r}300 \\
200 \\
200 \\
250 \\
200 \\
400 \\
100 \\
150 \\
200 \\
40 \\
100\end{array}$ & $\begin{array}{l}0.63 \\
0.47 \\
0.32 \\
0.18 \\
0.06 \\
0.00 \\
0.63 \\
0.47 \\
0.32 \\
0.18 \\
0.06\end{array}$ & $\begin{array}{l}3.70 \\
3.51 \\
3.32 \\
3.15 \\
3.02 \\
2.87 \\
3.51 \\
3.32 \\
3.15 \\
3.02 \\
2.87\end{array}$ & 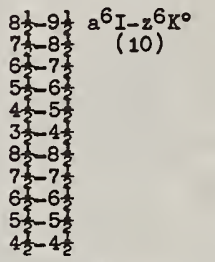 \\
\hline $\begin{array}{l}4368.632 \\
4272.789\end{array}$ & $\hat{A}$ & $\begin{array}{l}60 \\
30\end{array}$ & $\begin{array}{l}0.06 \\
0.00\end{array}$ & $\begin{array}{l}2.89 \\
2.89\end{array}$ & $\frac{3 \frac{1}{2}}{3^{\frac{1}{2}}} a^{6} \frac{I-6^{0}}{(11)}$ \\
\hline $\begin{array}{l}4556.735 \\
4366.315 \\
4270.565\end{array}$ & $\stackrel{A}{A}$ & $\begin{array}{l}12 \\
12 \\
25\end{array}$ & $\begin{array}{l}0.18 \\
0.06 \\
0.00\end{array}$ & $\begin{array}{l}2.89 \\
2.89 \\
2.89\end{array}$ & $\begin{array}{l}-7^{\circ} \\
13)\end{array}$ \\
\hline $\begin{array}{l}4465.601 \\
4282.570\end{array}$ & A & $\begin{array}{l}10 \\
15\end{array}$ & $\begin{array}{l}0.18 \\
0.06\end{array}$ & $\begin{array}{l}2.95 \\
2.95\end{array}$ & $-5 \frac{1}{2} a^{6} \frac{I-8^{0}+}{(13)}$ \\
\hline $\begin{array}{l}4246.879 \\
4156.265\end{array}$ & $\stackrel{\mathbf{A}}{\mathbf{A}}$ & $\begin{array}{c}(10) \\
30\end{array}$ & $\begin{array}{l}0.06 \\
0.00\end{array}$ & $\begin{array}{l}2.97 \\
2.97\end{array}$ & $\frac{4 \frac{1}{2}}{4 \frac{1}{2}} a^{6} \frac{I-9^{0} \uparrow}{(14)}$ \\
\hline $\begin{array}{l}4374.923 \\
4199.099 \\
4110.472\end{array}$ & $\begin{array}{l}\mathbf{A} \\
\mathrm{A} \\
\mathrm{A}\end{array}$ & $\begin{array}{l}20 \\
10 \\
40\end{array}$ & $\begin{array}{l}0.18 \\
0.06 \\
0.00\end{array}$ & $\begin{array}{l}3.00 \\
3.00 \\
3.00\end{array}$ & $\begin{array}{l}5 \frac{1}{3}-4 \frac{1}{2} a^{6} I-10^{0} \\
\frac{4}{3}-4 \frac{1}{2} \\
3 \frac{1}{2}-4 \frac{1}{2}\end{array}$ \\
\hline $\begin{array}{l}4173.379 \\
4085.815\end{array}$ & $\stackrel{A}{A}$ & $\begin{array}{r}8 \\
30\end{array}$ & $\begin{array}{l}0.06 \\
0.00\end{array}$ & $\begin{array}{l}3.02 \\
3.02\end{array}$ & $\begin{array}{l}4 \frac{1}{2}-3 \frac{1}{2} \\
3 \frac{1}{2}-3 \frac{1}{2}\end{array} a^{6} \frac{I-111^{\circ}}{(16)}$ \\
\hline $\begin{array}{l}4277.279 \\
4109.073\end{array}$ & A & $\begin{array}{r}6 \\
100\end{array}$ & $\begin{array}{l}0.18 \\
0.06\end{array}$ & $\begin{array}{l}3.07 \\
3.07\end{array}$ & $\frac{5 \frac{1}{3}-5 \frac{1}{2}}{4 \frac{1}{2}-5 \frac{1}{2}} a^{6} \frac{I}{(17)}$ \\
\hline $\begin{array}{l}4457.179 \\
4080.227\end{array}$ & $\stackrel{A}{A}$ & $\begin{array}{l}(5) \\
50\end{array}$ & $\begin{array}{l}0.32 \\
0.06\end{array}$ & $\begin{array}{l}3.09 \\
3.09\end{array}$ & $\begin{array}{l}\frac{1}{2}-5 \frac{1}{2} a^{6} I-25014 \\
\frac{4 \frac{1}{2}-5 \frac{1}{2}}{(18)}\end{array}$ \\
\hline $\begin{array}{l}3973.269 \\
3990.103 \\
4020.872 \\
3951.154 \\
4018.826 \\
3991.743 \\
4205.595 \\
4227.719 \\
4133.361 \\
4179.585 \\
4075.272 \\
3780.391 \\
3805.359 \\
3848.233 \\
3807.227 \\
3937.5758\end{array}$ & $\begin{array}{l}A \\
A \\
A \\
A \\
A \\
A \\
A \\
A \\
A \\
A \\
A \\
A \\
A \\
A \\
A \\
A \\
A\end{array}$ & $\begin{array}{r}80 \\
60 \\
60 \\
150 \\
30 \\
80 \\
40 \\
30 \\
50 \\
30 \\
50 \\
20 \\
100 \\
50 \\
(15) \\
5\end{array}$ & $\begin{array}{l}0.63 \\
0.47 \\
0.32 \\
0.18 \\
0.06 \\
0.00 \\
0.63 \\
0.47 \\
0.32 \\
0.18 \\
0.06 \\
0.47 \\
0.32 \\
0.18 \\
0.06 \\
0.00\end{array}$ & $\begin{array}{l}3.73 \\
3.56 \\
3.39 \\
3.31 \\
3.13 \\
3.09 \\
3.56 \\
3.39 \\
3.31 \\
3.13 \\
3.09 \\
3.73 \\
3.56 \\
3.39 \\
3.31 \\
3.13\end{array}$ & $\frac{1}{a^{6} I-y^{6} I^{\circ}}(19)^{\circ}$ \\
\hline
\end{tabular}

$\begin{array}{lll}4234.196 & \text { A } & 6 \\ 4069.267 & \text { A } & 80\end{array}$

.3976 .8368 A 60

$\begin{array}{lll}4961.396 & \text { A } & 10 \\ 4413.784 & \text { A }\end{array}$

$\begin{array}{rrr}4034.012 & \text { A } & (4) \\ 3952.195 & \text { A } & 100\end{array}$

$\begin{array}{lll}4391.110 & \text { A } & 10 \\ 4186.033 & \text { A } & 8 \\ 4024.785 & \text { A } & 30\end{array}$

$\begin{array}{lll}4113.826 & \text { A } & 30 \\ 3958.001 & \text { A } & 40\end{array}$

3863.409 A 60

$\begin{array}{rrr}3941.513 & \text { A } & 150 \\ 3863.327 & \text { A } & 20\end{array}$

$3838.981 \quad$ A $\quad 80$

3894.627 A 40

4040.796 A 100

4038.124 A 30

$\begin{array}{lll}3887.8668 & \text { A } & 30 \\ 3811.774 & \text { A } & 20\end{array}$

4220.258 A 20

$\begin{array}{lll}4030.470 & \text { A } & 25 \\ 3880.779 & \text { A } & 40\end{array}$

3826.416 A 60
3752.679 A 30

4043.596 A 15

3851.748 A 60

4328.800 A 8

$\begin{array}{lll}4228.200 & \text { A } & 8 \\ 4021.330 & \text { A } & 80\end{array}$

3973.650 A 60

3614.673 A (8)

$\begin{array}{lll}\mathbf{4} 75.606 & \text { A } & 50 \\ 3963.114 & \text { A } & 60\end{array}$

I A Laboratory Ref

Low ${ }_{\text {H1gh }}$

J Nult1plet
(No)

Nd II continued

3328.270 A 80

$\begin{array}{rrr}3339.063 & \text { A } & 60 \\ 3282.777 & \text { A } & 8\end{array}$

$\begin{array}{lll}3334.471 & \text { A } & 50 \\ .3231 .3498 & \text { A } & \text { (8) } \\ & & \end{array}$

5255.510 A 50

5213.365 A 30

$\begin{array}{llr}5603.651 & \text { A } & 5 \\ .5191 .4488 & \text { A } & 100\end{array}$

5361.174

5328.427
5089.837

4867.839

4647.759 A

4820.336 A 30

5093.797 A 30

4446.387 A 200

4715.589 A 12

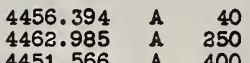

$\begin{array}{lll}4451.566 & \text { A } & 400 \\ 4385.663 & \text { A } & 150\end{array}$

4597.013 A 20

$\begin{array}{rlr}4914.385 & \text { A } & 15 \\ 4594.447 & \text { A } & 6\end{array}$

4501.808 A 50

$\begin{array}{rrr}4763.624 & \text { A } & 5 \\ 4463.407 & \text { A } & 30\end{array}$

4703.578 A 15

4381.290 A (10)

4120.654

4106.582
4100.240

3979.479

4358.699

4217.282

$\begin{array}{lll}4541.269 & \text { A } & 50 \\ 4266.716 & \text { A } & 30\end{array}$

4256.239 A 8

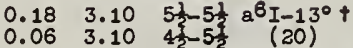

$0.00 \quad 3.10 \quad 3 \frac{1}{2}-3 \frac{1}{2} a^{6} I-25138$

$\begin{array}{llll}0.63 & 3.12 & 8 \frac{1}{2}-7 \frac{1}{3} & a^{6} \frac{(21)}{1}-25235 \\ 0.32 & 3.12 & 6 \frac{3}{2}-7 \frac{1}{2} & (22)\end{array}$

$\begin{array}{llll}0.06 & 3.12 & 4 \frac{1}{3}-3 \frac{1}{3} & a^{6} I-14^{\circ} \\ 0.00 & 3.12 & 3 \frac{3}{2}-3 \frac{2}{2} & (23)\end{array}$

$\begin{array}{llll}0.32 & 3.13 & 6 \frac{1}{4}-5 \frac{1}{2} & { }^{6} I-15^{\circ} \\ 0.18 & 3.13 & 5 & 5\end{array}$

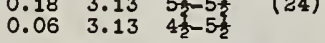

$\begin{array}{llll}0.18 & 3.18 & 5 \frac{1}{3}-4 \frac{1}{3} & a^{6} I_{-16^{\circ}} \\ 0.06 & 3.18 & 4 \frac{3}{2}-4 \frac{3}{2} & (35)^{2}\end{array}$

$0.003 .193 \frac{1}{2}-2 \frac{1}{2} a^{6} I-y^{6} H^{\circ}$ $\begin{array}{llll}0.06 & 3.19 & 4 \frac{1}{2}-4 \frac{1}{2} & a^{6} \frac{(26)}{I-17^{\circ}}+ \\ 0.00 & 3.19 & 3 \frac{1}{2}-4 \frac{1}{2} & (27)\end{array}$ $0.00 \quad 3.21 \quad 3 \frac{1}{2}-2 \frac{1}{2} a^{6} I-26041$ $0.06 \quad 3.23 \quad 4 \frac{1}{2}-5 \frac{1}{2} a^{6} \frac{(28)}{I-26182}$ $0.18 \quad 3.24 \quad 5 \frac{1}{2}-6 \frac{1}{2} a^{6} \mathrm{I}^{(29)}$ $0.18 \quad 3.24 \quad 5 \frac{1}{2}-4 \frac{1}{3} a^{6} I^{(30)}$ $\begin{array}{lll}0.06 & 3.24 & 4 \frac{1}{3}-4 \frac{1}{2} \\ 0.00 & 3.24 & 3 \frac{1}{2}-4 \frac{1}{2}\end{array}$

$0.32 \quad 3.24 \quad 6 \frac{1}{2}-5 \frac{1}{2} a^{6} I-21^{\circ}$

$\begin{array}{lllll}0.18 & 3.24 & 5 \frac{3}{3}-5 \frac{1}{2} & (32) \\ 0.06 & 3.24 & 4 \frac{1}{2}-5 \frac{1}{2} & \end{array}$

$\begin{array}{llll}0.06 & 3.29 & \frac{41}{3}-3 \frac{1}{2} & a^{6} I-20^{\circ} \\ 0.00 & 3.29 & 3 \frac{1}{2}-3 \frac{1}{2} & (33)\end{array}$

$\begin{array}{llll}0.32 & 3.37 & 6 \frac{1}{2}-6 \frac{1}{2} & a^{6} \mathrm{I}-22^{\circ} \\ 0.18 & 3.37 & 5 \frac{3}{2}-6 \frac{3}{2} & (34)\end{array}$

$\begin{array}{llll}0.18 & 3.39 & 5 \frac{1}{2}-4 \frac{1}{3} & a^{6} \mathrm{I}-23^{\circ} \\ 0.06 & 3.39 & 4 \frac{1}{2} & (35)\end{array}$

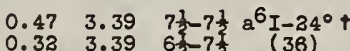

$0.33 \quad 3.43 \quad 6 \frac{1}{2}-6 \frac{1}{2} a^{6} I-37744$

$0.06 \quad 3.48 \quad 4 \frac{1}{2}-4 \frac{1}{2} a^{6} \frac{(37)}{(-28170}$

$\begin{array}{lllll}0.63 & 3.58 & 8 \frac{1}{2}-8 \frac{1}{2} & 6 I-29027 \\ 0.47 & 3.58 & 7 \frac{1}{2}-8 \frac{3}{2} & (39)\end{array}$
4797.157 A 20

4144.553 A 30

4075.116 A 60

$\begin{array}{lll}4307.778 & \text { A } & 15 \\ 4059.961 & \text { A } & 50\end{array}$

4000.493 A 30

4123:881 A 40

4051.145 A 60

$\begin{array}{lll}3982.355 & \text { A } & 30 \\ 3769.644 & \text { A } & 40\end{array}$

4338.697 A 80

$\begin{array}{lll}3811.073 & \text { A } & 20 \\ 3615.817 & \text { A } & 30\end{array}$

3470.866 A 20

$\begin{array}{lll}3522.044 & \text { A } & 25 \\ 3354.621 & \text { A } & 10\end{array}$

6257.834 A (25)

5548.474 A 8

$\begin{array}{lll}5361.474 & A & 60 \\ 5234.195 & A & 50\end{array}$

5130.596

5192.621
5249.585

$\begin{array}{rrr}5273.431 & A & 50 \\ 5310 & & \end{array}$

5442.274 A 40

5165.140 A 10

5934.747 A (10) $\begin{array}{lll}5811.572 & \text { A } & 13 \\ 5703.244 & \text { A } & 20\end{array}$

5371.935 A 20

$\begin{array}{rrr}5485.699 & \text { A } & 80 \\ 5594.425 & \text { A } & 150\end{array}$ $\begin{array}{lll}5594.425 & \text { A } & 150 \\ 5688.525 & \text { A } & 150\end{array}$
5293.168 $\begin{array}{llll}0.00 & 3.71 & 3 \frac{1}{2}-2 \frac{1}{2} & a^{6} I-30037 \\ 0.06 & 3.76 & 43-3 \frac{1}{4} & 0^{6} I^{-30)}\end{array}$

$\begin{array}{llll}0.06 & 3.76 & 43-3 \frac{1}{3} & 26 \frac{(4-30453}{(41)} \\ 0.00 & 3.76 & 3 \frac{3}{2}-\frac{3}{2} & (41)\end{array}$

$\begin{array}{llll}0.18 & 3.88 & 5 \frac{1}{4}-4 \frac{1}{2} & a^{6} \mathrm{I}-25^{\circ} \\ 0.06 & 3.88 & 4 \frac{1}{2}-4 \frac{1}{2} & (42)\end{array}$

$0.20 \quad 2.57 \quad 4 \frac{1}{2}-3 \frac{1}{2} 8^{4} \frac{4}{I}-20830$

$\begin{array}{llll}0.38 & 2.58 & 5 \frac{1}{3}-5 \frac{1}{2} & a^{4} \frac{(44)}{1}-2 \\ 0.20 & 3.58 & 4 \frac{1}{2}-5 \frac{2}{2} & (45)\end{array}$

$0.56 \quad 3.86 \quad 6 \frac{1}{2}-5 \frac{1}{3} a^{4} I-z^{6} H^{\circ}$

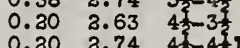

$\begin{array}{lll}0.30 & 3.74 & 42 \\ 0.20 & 2.86 & 4 \frac{14}{2}\end{array}$

$0.20 \quad 3.76 \quad 4 \frac{1}{2}-3 \frac{1}{2} a^{4} I-3^{\circ}$

$0.38 \quad 3.80 \quad 5 \frac{1}{2}-5 \frac{1}{2} a^{4}\left(4-5^{\circ}+\right.$

$0.20 \quad 3.98 \quad 4 \frac{1}{3}-5 \frac{1}{2} a^{4} I-26 I$.

$\begin{array}{llll}0.20 & 3.91 & 4 \\ 0.20 & 3.82 & 43 & 4\end{array}$

$0.74 \quad 3.51 \quad 7 \frac{1}{2}-8 \frac{1}{2} a^{4} I-z^{6} E^{\circ}+$

$\begin{array}{lll}0.38 & 3.15 & 51-6 \\ 0.30 & 3.03 & 4\end{array}$

$0.20 \quad 2.89 \quad 4 \frac{1}{2}-3 \frac{1}{2} \mathrm{a}^{4} \mathrm{I}-6^{\circ}$

$\begin{array}{lllll}0.38 & 2.89 & 51-4 \frac{1}{3} & a^{4} I-70 \\ 0.30 & 3.89 & 4 \frac{1}{2}-4 \frac{1}{2} & (52)\end{array}$

$0.20 \quad 2.95 \quad 4 \frac{1}{2}-5 \frac{1}{2} a^{4} I-8^{\circ}+$

$\begin{array}{llll}0.38 & 3.97 & 5 \frac{1}{4}-4 \frac{1}{3} & a^{4} I-90 \\ 0.20 & 3.97 & \frac{1}{2}-4 \frac{1}{2} & (54)\end{array}$

$0.38 \quad 3.00 \quad 5 \frac{1}{2}-4 \frac{1}{2} a^{4} I-10^{\circ}$

$0.20 \quad 3.03 \quad 4 \frac{1}{2}-3 \frac{1}{2} \mathrm{a}^{4} I_{-11}(56)$

$\begin{array}{lllll}0.74 & 3.73 & 7 \frac{1}{3}-8 \frac{1}{2} & a^{4} I-y^{6} I^{\circ} \\ 0.56 & 3.56 & 6 \frac{3}{3}-7 \frac{1}{3} & (57)\end{array}$

$0.38 \quad 3.39 \quad 5 \frac{1}{3}-6 \frac{1}{3}$

$\begin{array}{lll}0.20 & 3.31 & 43 \\ 0.74 & 3.56 & 73-7\end{array}$

$0.38 \quad 3.31 \quad 5 \frac{1}{3}-5$

$\begin{array}{llll}0.38 & 3.10 & 5 \frac{1}{2}-5 \frac{1}{2} & a^{4} I-13^{\circ} \\ 0.20 & 3.10 & 4 \frac{3}{2}-5 \frac{2}{2} & (58)\end{array}$

$0.20 \quad 3.10 \quad 4 \frac{1}{2}-3 \frac{2}{2} a^{4} I-25138$

$0.563 .136 \frac{1}{2}-5 \frac{1}{2} 8^{4} \frac{(59)}{\left(-15^{\circ}+\right.}$

$0.20 \quad 3.18 \quad 4 \frac{1}{2}-4 \frac{1}{2} a^{4} I_{-16^{\circ}}$

$0.20 \quad 3.23 \quad 4 \frac{1}{2}-5 \frac{1}{2} a^{4} I-26183$ t

$\begin{array}{llll}0.38 & 3.24 & 5 \frac{1}{3}-5 \frac{1}{2} & a^{4} \mathrm{I}-21^{\circ} \\ 0.20 & 3.24 & 4 & (63)\end{array}$

$0.20 \quad 3.29 \quad 4 \frac{1}{2}-3 \frac{1}{2} a^{4} I-20^{\circ}$

$0.38 \quad 3.37 \quad 5 \frac{1}{2}-6 \frac{1}{2} a^{4} \frac{(64)}{(-25)}$

$0.38 \quad 3.43 \quad 5 \frac{1}{2}-6 \frac{1}{2} a^{4} I-27744+$

$0.74 \quad 3.58 \quad 7 \frac{1}{2}-8 \frac{1}{2} a^{4} I-29037$

$\begin{array}{lllll}0.38 & 3.62 & 51-4 \frac{1}{2} & a^{4} I-29298 \\ 0.30 & 3.62 & 4 \frac{2}{2}-4 \frac{2}{2} & (69)\end{array}$

$0.20 \quad 3.76 \quad 4 \frac{1}{2}-3 \frac{1}{2} a^{4} I-30453$

$\begin{array}{llll}0.80 & 3.88 & 4 \frac{1}{2}-4 \frac{3}{2} & (71)\end{array}$

$0.55 \quad 3.77 \quad 5 \frac{1}{2}-4 \frac{1}{2} a^{6} 6 \frac{73}{4}$

$0.68 \quad 2.98 \quad 6 \frac{1}{2}-5 \frac{1}{2} a^{6} \mathrm{~L}^{6} \mathrm{z}^{6} \mathrm{I}^{\circ}$

$1.30 \quad 3.70 \quad 10 \frac{1}{2}-9 \frac{1}{2} a^{6} \mathrm{~L}-z^{6} \mathrm{~K}^{0}+$

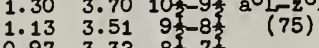

$0.82 \quad 3.15 \quad 73-6$

$\begin{array}{lll}0.82 & 3.15 & 71-6 \\ 0.68 & 3.02 & 61 \\ 0.55 & 3.87 & 5\end{array}$

$0.68 \quad 3.95 \quad 6 \frac{1}{2}-5 \frac{1}{2} a^{6} \mathrm{~L}-8^{\circ}$

$\begin{array}{lll}0.68 & 3.07 \quad 6 \frac{1}{2}-5 \frac{1}{2} & 0 \\ 0 & 6 & (76) \\ (77) & \end{array}$

$0.74 \quad 2.82 \quad 4 \frac{1}{3}-3 \frac{1}{3} a^{6} \mathrm{~K}-2^{6} I^{\circ}$

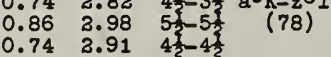

$1.41 \quad 3.70 \quad 9 \frac{1}{2}-9 \frac{1}{\frac{1}{3}} a^{6} \mathrm{~K}-2^{6} \mathrm{~K}^{\circ}+$

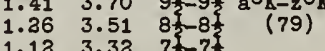

$\begin{array}{lll}1.12 & 3.32 & 7 \\ 0.98 & 3.15 & 6 \frac{1}{3}\end{array}$
$0.20 \quad 2.55 \quad 4 \frac{1}{2}-4 \frac{1}{2} a^{4} I-1^{\circ}$

$\begin{array}{lllll}0.38 & 3.48 & 5 \frac{1}{2}-4 \frac{1}{3} & 24 & 4-28170 \\ 0.20 & 3.48 & 4 & (66) & (67)\end{array}$

$0.38 \quad 3.88 \quad 5 \frac{1}{3}-4 \frac{1}{3} a^{4} \frac{(70)}{2}(71)^{\circ}$

$0.55 \quad 2.55 \quad 5 \frac{1}{2}-4 \frac{1}{2} a^{6} \mathrm{I}-10$
I A $\stackrel{\text { Ref }}{\text { Laboratory }}$ Int $^{2}$

E P

MuIt1plet

Na II continued

$\begin{array}{lll}5708.280 & \text { A } & 40 \\ 5804.020 & \text { A } & 60\end{array}$

$0.86 \quad 3.02 \quad 5 \frac{1}{3}-5 \frac{1}{1} a^{6} \mathrm{~K}-2^{6} \mathrm{~K}^{\circ}+$

5421.559 A $\quad 20$

$\begin{array}{llll}0.74 & 2.87 & 43-4 \frac{13}{2} \\ 0.74 & 3.02 & 4 \frac{3}{2}-5 \frac{2}{2}\end{array}$

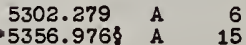

$\begin{array}{lll}5356.9768 & \text { A } & 15 \\ 5431.526 & \text { A } & 40\end{array}$

$1.41 \quad 3.73 \quad 9 \frac{1}{2}-8 \frac{1}{3} a^{6} \mathrm{~K}-\mathrm{y}^{6} \mathrm{I}^{\circ}+$

$\begin{array}{lll}1.26 & 3.56 & 83-7 \frac{1}{3} \\ 1.12 & 3.39 & 71-6 \frac{1}{3} \\ 0.98 & 3.31 & 61-5 \frac{1}{3}\end{array}$

$\begin{array}{lll}0.98 & 3.31 & 61-54 \\ 0.86 & 3.13 & 51-43\end{array}$

$\begin{array}{lll}0.86 & 3.13 & 5 \frac{1}{3}-4 \frac{1}{3} \\ 0.74 & 3.09 & 4 \frac{1}{2}-3 \frac{1}{2}\end{array}$

5250.816 A 8

$0.86 \quad 3.19 \quad 5 \frac{1}{2}-4 \frac{1}{2} a^{6} \mathrm{~K}-17^{\circ}$

5474.734 A 10

$0.98 \quad 3.24 \quad 6 \frac{1}{2}-6 \frac{1}{2} a^{6} \frac{(81)}{\left(-188^{\circ}\right.}$

5455.815 A 20

$0.98 \quad 3.24 \quad 6 \frac{1}{2}-5 \frac{1}{2} a^{6} 6-21^{0}$

5668.868 A 15

.6385 .1968 A $\overline{150}$

.5620 .628 P 500

$\begin{array}{rrr}5718.120 & \text { A } & 12 \\ 5843.391 & \text { A } & 8\end{array}$

$\begin{array}{rrr}5740.862 \text { A } & 15 \\ 5891.528 & \text { A } & 15\end{array}$

$\begin{array}{lll}5891.528 & \text { A } & 15 \\ 5706.206 & \text { A } & 15\end{array}$

$\left.1.41 \quad 3.58 \quad 9 \frac{1}{2}-8 \frac{1}{2} a^{6} \mathrm{~K}-293\right)$

$\overline{1.16} \quad \widetilde{3.09} 5 \frac{1}{2}-5 \frac{1}{2} 7 b^{6} \frac{(84)}{(-25014}$

$\begin{array}{rlllll}5614.303 \quad A \quad(10) & 1.04 & 3.24 & 4 \frac{1}{2}-4 \frac{1}{2} & b^{6} \frac{\mathrm{I}-19^{\circ}}{(87)}\end{array}$

$1.54 \quad 3.73 \quad 8 \frac{1}{3}-8 \frac{1}{1} b^{6} I_{-5}^{(85)} I^{\circ}+$

1.28 3.39 6 6 $6 \frac{1}{3}$

$\begin{array}{lll}1.16 & 3.31 & 51 \\ 1.04 & 3.13 & 4 \frac{3}{3} \\ 0.93 & 3.09 & 3\end{array}$

$\begin{array}{lll}1.04 & 3.13 & 43 \\ 0.93 & 3.09 & 3 \frac{3}{2}-3 \frac{1}{2}\end{array}$

Strongest Unclass1f1ed Lines of Na II

$\begin{array}{rrrr}5451.115 & \text { B } & 100 & \text { IV } \\ 4632.276 & \text { B } & 20 & \text { III } \\ 4542.603 & \text { B } & 60 & \text { IV } \\ 4282.443 & \text { B } & 50 & \text { IV } \\ 4135.325 & \text { B } & 50 & \text { IV }\end{array}$

4031.807 B 100

$\begin{array}{lll}4023.002 & B & \\ 4012.704 & B & 50 \\ 4007.435 & \text { B }\end{array}$

4007.435
4004.010

3994.684

3953.535

3930.965

3911.169

3905.886

3901.850

3900.226

3890.580

3889.929

3878.582

3848.524

3814.725

3808.772

3803.474
3784.250

3763.475

3758.944

3728.130

3723.506

3685.804
3673.542

3672.363

3665.180
3609.788

3592.595

3587.504

3543.352

3393.641

3364.950
3300.148

3300.148
3285.093

3275.218

3134.897

3133.603
3116.141

3115.172

3098.476

3092.915

3075.380

3014.165
3007.975

100
80
50
50
60

IV

80
60
50

60
50
100

III

100
50
60
60
50

III

50

50
80
60

60

30
40
80
60
40

III

IV
IV
IV
IV
III
III
III
III
III
III

$\begin{array}{ll}50 & \text { III } \\ 50 & \text { III } \\ 50 & \text { III } \\ 60 & \text { IV } \\ 50 & \end{array}$

$\begin{array}{ll}50 & V \\ 50 & \text { IV } \\ 40 & \text { III } \\ 60 & \text { IV } \\ 50 & \text { IV }\end{array}$

Sm I I P 5.69 Anal C L1st D Apr 1942

$\begin{array}{lllllll}6671.51 & \text { A } & 800 & 0.50 & 2.35 & 6-7 & a^{7} F^{-2} 9^{\circ}+ \\ 6588.91 & \text { A } & 500 & 0.39 & 2.26 & 5-6 & (1)\end{array}$

$\begin{array}{lllllll}5659.86 & \text { B } & 400 & 0.10 & 2.38 & 2-1 & a^{7} F-38^{\circ}\end{array}$

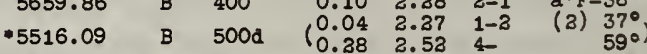

$\begin{array}{rllllll}4841.701 & \mathrm{~B} & 400 & 0.50 & 3.05 & 6-5 & 102^{\circ}\end{array}$

$\begin{array}{lllllll}3925.216 & \mathrm{~B} & 400 & 0.10 & 3.24 & 2-1 & 118^{\circ} \\ .3756 .4118 & \mathrm{~B} & 600 & 0.10 & 3.39 & 2-3 & 127^{\circ}\end{array}$

$4296.743 / /$ B 300

$0.50 \quad 3.37 \quad 6-7$

$a^{7} F^{-2^{7} G^{0}+}$ 


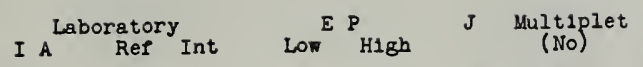

Sm II I P 11.4 Anal B L18t C $\operatorname{Mar} 1942$

\begin{tabular}{|c|c|c|c|c|c|}
\hline $\begin{array}{l}4704.397 \\
4648.160 \\
4606.514 \\
4092.266 \\
3970.528 \\
3941.874\end{array}$ & $\begin{array}{l}\text { A } \\
\text { A } \\
\text { A } \\
\text { A } \\
\text { A } \\
\text { A }\end{array}$ & $\begin{array}{l}500 \\
100 \\
100 \\
400 \\
200 \\
300\end{array}$ & $\begin{array}{l}0.00 \\
0.00 \\
0.00 \\
0.00 \\
0.00 \\
0.00\end{array}$ & $\begin{array}{l}2.62 \\
3.66 \\
2.68 \\
3.02 \\
3.11 \\
3.13\end{array}$ & 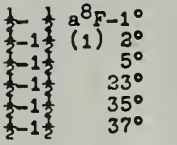 \\
\hline $\begin{array}{c}3745.605 \\
-3693.9898 \\
3304.523 \\
3250.372 \\
3207.185\end{array}$ & $\begin{array}{l}\hat{A} \\
\mathbf{A} \\
\mathbf{A} \\
\mathbf{A} \\
\mathbf{A}\end{array}$ & $\begin{array}{c}200 \mathrm{~d} \\
1200 \\
200 \\
200 \mathrm{~d} \\
400\end{array}$ & $\begin{array}{l}0.00 \\
0.00 \\
0.00 \\
0.00 \\
0.00\end{array}$ & $\begin{array}{l}3.29 \\
3.34 \\
3.73 \\
3.80 \\
3.85\end{array}$ & $\begin{array}{r}a^{8} F-2^{8} G^{\circ} \\
(2)^{8} G^{\circ} \\
124^{\circ} \\
129^{\circ} \\
134^{\circ}\end{array}$ \\
\hline
\end{tabular}

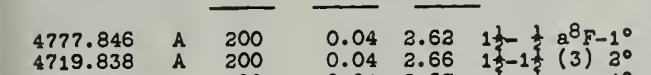

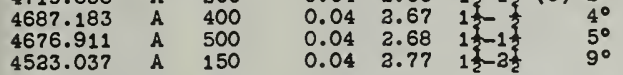

$\begin{array}{lllllll}4329.704 & \text { A } & 300 & 0.04 & 3.96 & 1 \frac{1}{2}-1 \frac{1}{3} 8^{8} F-18^{\circ}\end{array}$

$\begin{array}{lllllll}4183.764 & \text { A } & 150 & 0.04 & 3.99 & 1 & 1 \\ 4043.723 & \text { A } & 200 & 0.04 & 3.09 & 1 \frac{1}{2}-1 & 30^{\circ} \\ 33^{\circ}\end{array}$

$\begin{array}{lllllll}4023.231 & \text { A } & 300 & 0.04 & 3.11 & 1 \frac{1}{2}-\frac{1}{3} & 34^{\circ} \\ 3993.308 & \text { A } & 200 & 0.04 & 3.13 & 1 \frac{1}{2}-1 \frac{1}{2} & 37^{\circ}\end{array}$

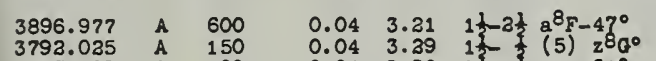

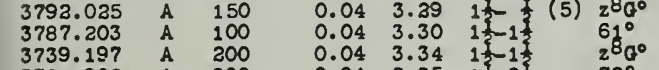

$\begin{array}{lllllll}3739.197 & \text { A } & 200 & 0.04 & 3.34 & 1 \frac{1}{2} & z^{8} 0^{\circ} \\ 3724.902 & \text { A } & 200 & 0.04 & 3.35 & 1 \frac{1}{2}-2 \frac{1}{2} & 72^{\circ} \\ 3708.410 & \text { A } & 200 & 0.04 & 3.3 ? & 1 \frac{1}{2}-2 \frac{1}{2} & 76^{\circ}\end{array}$

3661.365

3634.928
3622.504

3340.579

3254.377
3241.161

4791.584

4745.680
4669.396

4458.517
4345.858

4323.284

4237.663
4310.352

4155.217
4149.831

4042.905

3976.270
3948.113

3943.239
3917.442

3880.766

3862.054
3830.293

3812.067
3805.626

3797.283

3793.971
3731.258

3688.418
3670.840

3627.971

3623.316
3621.229

3584.259
3511.227

3295.813

3216.850
-3187.316

4815.808

4693.638
4674.599

4591.818 A 100

4390.858

4329.016
4309.012

4265.075
-4220.659

- 4178.019

4152.309
4113.902

4047.160
4019.982

3986.682

3946.511
3928.279

3890.080
3875.545

3871.778

3835.725
3824.175

-3760.694
-3743.868

3736.805
3710.869

3708.654
3645.290

3645.290
3634.290 $\begin{array}{llll}0.04 & 3.41 & 1 \frac{1}{2}-2 \frac{1}{2} & 8^{8} F-z^{8} \sigma^{\circ} \\ 0.04 & 3.44 & 1 \frac{1}{2}-1 \frac{1}{2} & (6) 85^{\circ}\end{array}$ $0.043 .45 \quad 1 \frac{1}{3} \quad 2 \frac{1}{3} \quad 86^{\circ}$ $\begin{array}{llll}0.04 & 3.73 & 1 \frac{2}{3} & 134^{\circ} \\ 0.04 & 3.83 & 13-2 \frac{1}{2} & 133^{\circ} \\ 0.04 & 3.85 & 1 \frac{1}{2}-1 \frac{2}{2} & 134^{\circ}\end{array}$ $0.043 .851 \frac{1}{2-1 \frac{1}{2}} \quad 134^{\circ}$

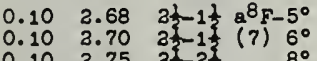

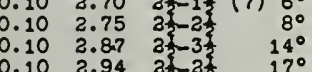
$\begin{array}{llll}0.10 & 3.96 & 3 \frac{1}{3}-1 \frac{1}{3} & 8^{8} F-18^{\circ} \\ 0.10 & 3.02 & 3 & \end{array}$ $\begin{array}{llll}0.10 & 3.03 & 2 \frac{2}{3} & 24^{\circ} \\ 0.10 & 3.07 & 2 \frac{3}{3} & 30^{\circ} \\ 0.10 & 3.08 & 2 & 32^{\circ}\end{array}$

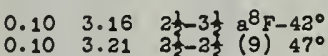

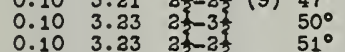

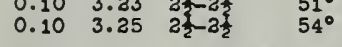
$\begin{array}{lll}0.10 & 3.28 & 2 \frac{1}{2}-1 \frac{1}{2} 8^{8} F-59^{\circ} \\ 0.10 & 3.30 & 2 \frac{1}{3}(10) \\ 0.10\end{array}$

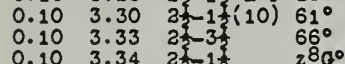
$\begin{array}{llll}0.10 & 3.34 & 2 \frac{1}{2} & 280^{\circ} \\ 0.10 & 3.35 & 2 \frac{1}{2}-3 \frac{1}{2} & 71^{\circ}\end{array}$

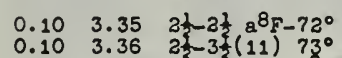

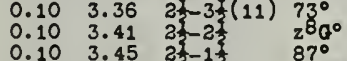
$\begin{array}{llll}0.10 & 3.45 & 2 \frac{1}{2}-1 \frac{1}{3} & 87^{\circ} \\ 0.10 & 3.47 & 3 \frac{1}{2}-3 \frac{1}{2} & 2^{8} 0^{\circ}\end{array}$ $0.10 \quad 3.51 \quad 31-2 \frac{1}{3} 8^{8} F-95^{\circ}$

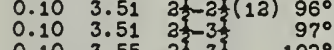
$\begin{array}{rrrr}0.10 & 3.51 & 3 \frac{3}{2} & 97^{\circ} \\ 0.10 & 3.55 & 3 \frac{3}{2} & 103^{\circ} \\ 0.10 & 3.62 & 2 \frac{1}{2} & 108^{\circ}\end{array}$ $0.10 \quad 3.85 \quad 3 \frac{1}{2}-1 \frac{1}{2} 8^{8} F-134^{\circ}$ $0.18 \quad 2.75 \quad 3 \frac{1}{2}-2 \frac{1}{2} 8^{8}-8^{\circ}$ $0.18 \quad 2.81 \quad 3 \frac{1}{3}-3 \frac{3}{3}(14) 11^{\circ}$ $\begin{array}{llll}0.18 & 2.82 & 3 \frac{1}{2}-3 \frac{1}{2} & 12^{\circ} \\ 0.18 & 2.87 & 3 \frac{1}{2}-3 & 14^{\circ} \\ 0.18 & 2.92 & 3 \frac{1}{2}-4 \frac{1}{2} & 15^{\circ}\end{array}$

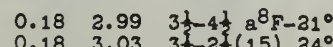
$\begin{array}{lll}0.18 & 3.03 & 3 \frac{1}{3}-2 \frac{2}{3}(15) 34^{\circ} \\ 0.18 & 3.05 & 3 \frac{1}{3}-3\end{array}$ $\begin{array}{llll}0.18 & 3.05 & 3 \frac{1}{2}-3 \frac{1}{2} & 37^{\circ} \\ 0.18 & 3.08 & 3 \frac{1}{2}-2 \frac{1}{2} & 32^{\circ} \\ 0.18 & 3.11 & 3 \frac{1}{2}-3 \frac{1}{2} & 34^{\circ}\end{array}$ $0.18 \quad 3.14 \quad 3 \frac{1}{2}-2 \frac{1}{3} a^{8} F-39^{\circ}$

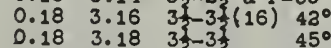
$\begin{array}{llll}0.18 & 3.18 & 3 \frac{1}{2}-3 \frac{1}{2} & 45^{\circ} \\ 0.18 & 3.23 & 3 \frac{1}{2}-2 \frac{1}{2} & 51^{\circ} \\ 0.18 & 3.25 & 3 \frac{1}{2}-2 \frac{1}{2} & 54^{\circ}\end{array}$ $0.18 \quad 3.38 \quad 3 \frac{1}{3}-4 \frac{1}{3} 8^{8} \mathrm{~F}-58^{\circ}$

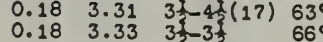
$\begin{array}{llll}0.18 & 3.33 & 3 \frac{3}{3} & 66^{\circ} \\ 0.18 & 3.36 & 3 \frac{1}{2}-3 \frac{1}{2} & 73^{\circ} \\ 0.18 & 3.37 & 3 \frac{3}{2}-2 \frac{1}{2} & 76^{\circ}\end{array}$ $0.18 \quad 3.37 \quad 3 \frac{1}{3}-4 \frac{1}{2} a^{8} 5-77^{\circ}$

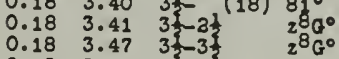
$\begin{array}{llll}0.18 & 3.48 & 3 \frac{1}{2}-4 \frac{1}{2} & 2^{2} \mathrm{G}^{\circ}\end{array}$ $\begin{array}{lllllr}\text { A } & 100 & 0.18 & 3.50 & 3 \frac{1}{2}-4 \frac{1}{2} 8^{8} F-94^{\circ} \\ \text { A } & 100 & 0.18 & 3.51 & 3 \frac{1}{3}-2(18) & 96^{\circ} \\ \text { A } & 300 & 0.18 & 3.51 & 3 \frac{3}{3} & 97^{\circ}\end{array}$

R EVISED $\triangle$ UL T I P LET TABLE

I A Laboratory Ref Int LoF $_{\text {H1 gh }}^{\text {L }}$

(No)

Sm II continued

$\begin{array}{lllllll}3601.692 & \text { A } & 200 & 0.18 & 3.61 & 3 \frac{1}{3}-3 \frac{1}{2} 8^{8} F-107^{\circ} \\ 3583.394 & \text { A } & 150 & 0.18 & 3.63 & 3 \frac{3}{3}(20) & 110^{\circ} \\ 3530.600 & \text { A } & 150 & 0.18 & 3.68 & 3 \frac{3}{3}-4 \frac{1}{3} & 118^{\circ}\end{array}$ $\begin{array}{lllllll}3530.600 & \text { A } & 150 & 0.18 & 3.68 & 3 \frac{1}{3}-4 \frac{1}{2} & 118^{\circ} \\ 3382.399 & \text { A } & 600 & 0.18 & 3.83 & 3 \frac{1}{2}-2 \frac{1}{2} & 133^{\circ} \\ 3320.155 & \text { A } & 600 \text { d } & 0.18 & 3.90 & 3 \frac{3}{2}-2 \frac{3}{2} & 136^{\circ}\end{array}$

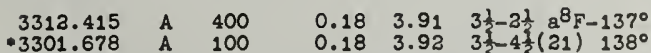

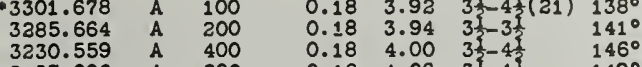
$\begin{array}{lllllll}3187.006 & \text { A } & 200 & 0.18 & 4.06 & 3 \frac{1}{2}-4 \frac{1}{2} & 149^{\circ} \\ 3178.125 & \text { A } & 200 & 0.18 & 4.07 & 3 \frac{1}{2}-4 \frac{1}{2} & 151^{\circ}\end{array}$

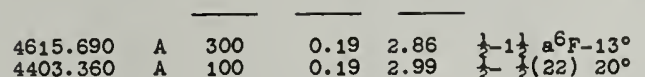
$\begin{array}{llllllll}4403.360 & \text { A } & 100 & 0.19 & 2.99 & \frac{1}{3}(22) & 20^{\circ} \\ 4225.328 & \text { A } & 400 & 0.19 & 3.11 & \frac{1}{2}-1 \frac{1}{3} & 35^{\circ} \\ 4041.675 & \text { A } & 200 & 0.19 & 3.24 & \frac{3}{2} & 53^{\circ} \\ 3891.210 & \text { A } & 100 & 0.19 & 3.36 & \frac{1}{3}-\frac{3}{3} & 74^{\circ}\end{array}$ $\begin{array}{rlllllr}3891.210 & \text { A } & 100 & 0.19 & 3.36 & \frac{1}{3} & 74^{\circ} \\ 3799.542 & \text { A } & 300 & 0.19 & 3.44 & \frac{2}{3}-1 \frac{1}{2} & 85^{\circ} \\ 3241.586 & \text { A } & 100 & 0.19 & 3.99 & \frac{3}{2}-1 \frac{1}{2} & 145^{\circ}\end{array}$

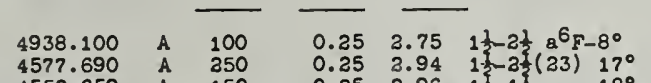

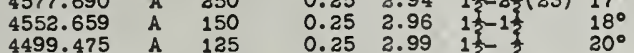
$\begin{array}{lllllll}4499.475 & \text { A } & 125 & 0.25 & 2.99 & 1 \frac{2}{3} & 20^{\circ} \\ 4360.730 & \text { A } & 150 & 0.25 & 3.08 & 1 \frac{1}{2}-2 \frac{2}{2} & 32^{\circ}\end{array}$

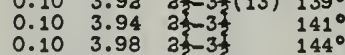

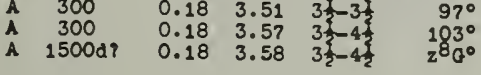

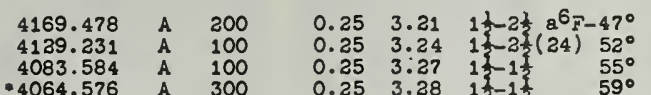
$\begin{array}{rrrrrrr}.4064 .576 & \text { A } & 300 & 0.25 & 3.28 & 1 \frac{1}{2}-1 \frac{1}{2} & 59^{\circ} \\ 3966.045 & \text { A } & 150 & 0.25 & 3.36 & 1 \frac{1}{2}-\frac{1}{2} & 74^{\circ}\end{array}$

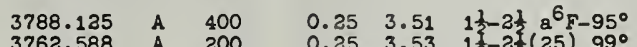
$\begin{array}{rllllll}3762.588 & \text { A } & 200 & 0.25 & 3.53 & 1 \frac{1}{3}-2 \frac{1}{3}(25) & 99^{\circ} \\ 3712.764 & \text { A } & 200 & 0.25 & 3.57 & 1 \frac{1}{2}-2 \frac{1}{2} & 104^{\circ} \\ 3711.543 & \text { A } & 200 & 0.25 & 3.57 & 1 & 100\end{array}$ $\begin{array}{lllllll}3711.543 & \text { A } & 200 & 0.25 & 3.57 & 1 \frac{1}{2}-1 \frac{1}{3} & 105^{\circ} \\ 3650.188 & \text { A } & 200 & 0.25 & 3.63 & 1 \frac{1}{2}-2 \frac{1}{2} & 111^{\circ} \\ 3214.125 & \text { A } & 150 & 0.25 & 4.09 & 1 \frac{1}{2}-2 \frac{1}{2} & 152^{\circ}\end{array}$ 4844.308

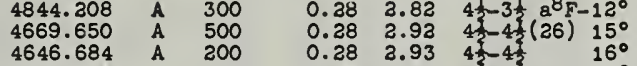
$\begin{array}{lllllll}4646.684 & \text { A } & 200 & 0.28 & 3.93 & 4 \frac{4}{3} & 15^{\circ} \\ 4473.015 & \text { A } & 150 & 0.28 & 3.04 & 4 \frac{5}{2} & 25^{\circ} \\ 4452.727 & \text { A } & 250 & 0.28 & 3.05 & 4 \frac{1}{2}-3 \frac{2}{2} & 37^{\circ}\end{array}$ $\begin{array}{lllllll}4334.153 & \text { A } & 400 & 0.28 & 3.12 & 4 \frac{1}{2}-3 \frac{1}{2} a^{8} F-36^{\circ} \\ 4318.936 & \text { A } & 500 & 0.28 & 3.13 & 4 \frac{1}{3}(27) & 38^{\circ}\end{array}$

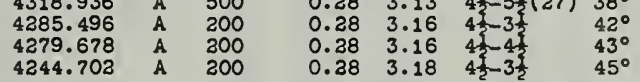
$\begin{array}{lllllll}4109.405 & \text { A } & 150 & 0.28 & 3.28 & 4 \frac{1}{2}-4 \frac{1}{2} 8^{8} F-58^{\circ} \\ 4066.737 & \text { A } & 200 & 0.28 & 3.31 & 4 & -4\end{array}$ $\begin{array}{rrrrrrr}4066.737 & \text { A } & 200 & 0.28 & 3.31 & 4 \frac{1}{2}-4 \frac{1}{3}(28) & 63^{\circ} \\ 3987.438 & \text { A } & 80 & 0.38 & 3.37 & 4 \frac{1}{2} & 77^{\circ}\end{array}$ $\begin{array}{lllllll}3935.764 & \text { A } & 150 & 0.38 & 3.41 & 4 \frac{1}{2}-5 \frac{1}{2} & 83^{\circ} \\ 3857.913 & \text { A } & 100 & 0.28 & 3.48 & 4 \frac{1}{2}-5 \frac{1}{2} & 91^{\circ}\end{array}$ $\begin{array}{lllllll}3851.880 & \text { A } & 150 & 0.28 & 3.48 & 4 \frac{1}{2}-4 \frac{1}{2} a^{8} \mathrm{~F}-93^{\circ} \\ 3833.838 & \text { A } & 300 & 0.38 & 3.50 & 4 \frac{1}{3}(29) & 94^{\circ}\end{array}$

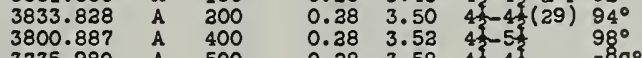
$\begin{array}{lllllll}3735.980 & \text { A } & 500 & 0.28 & 3.58 & \frac{4}{3} & z^{8} 6^{\circ} \\ 3692.221 & \text { A } & 150 & 0.28 & 3.62 & \frac{1}{2}-5 \frac{1}{2} & 109^{\circ}\end{array}$

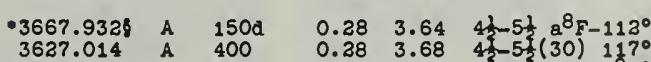

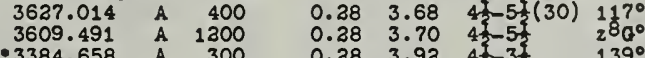

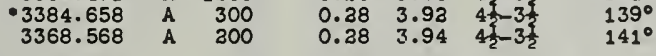
$3336.124 \quad$ A $200 \quad 0.38 \quad 3.98 \quad 41-3 \frac{1}{3} a^{8} 5-144^{\circ}$

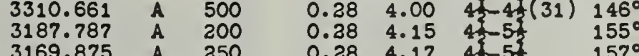

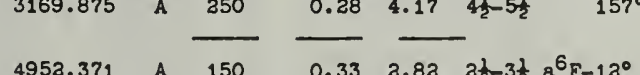

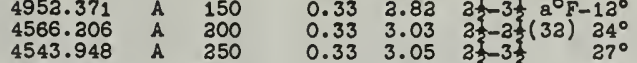

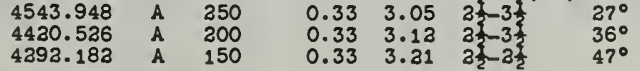

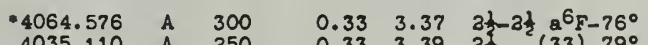

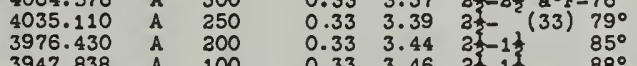
$\begin{array}{lllllll}3947.838 & \text { A } & 100 & 0.33 & 3.46 & 3 \frac{2}{2}-1 \frac{1}{3} & 88^{\circ} \\ 3881.383 & \text { A } & 100 & 0.33 & 3.51 & 3 \frac{1}{2}-3 \frac{1}{2} & 97^{\circ}\end{array}$ 3847.511 A $150 \quad 0.33 \quad 3.54 \quad 2 \frac{1}{3} a^{6} \mathrm{~F}-100^{\circ}$

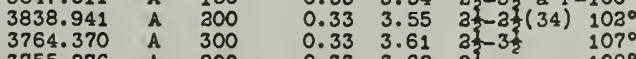

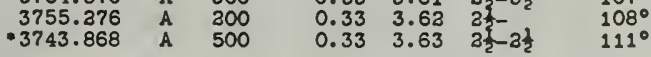
$\begin{array}{ccccccc}3707.1678 & \text { A } & 100 \mathrm{~d} & 0.33 & 3.66 & 2 \frac{1}{2}-1 \frac{1}{2} \mathrm{a}^{6} \mathrm{~F}-116^{\circ} \\ 3645.387 & \text { A } & 300 & 0.33 & 3.73 & 2 \frac{3}{2}-3 \frac{3}{3}(35) & 123^{\circ}\end{array}$ $\begin{array}{lllllll}3369.455 & \text { A } & 200 & 0.33 & 3.99 & 2 \frac{1}{3} & 145^{\circ} \\ 3305.185 & \text { A } & 200 & 0.33 & 4.07 & 2 \frac{1}{2}-2 \frac{1}{2} & 150^{\circ}\end{array}$

$\begin{array}{lllllll}4854.365 & \mathrm{~A} & 150 & & 0.38 & 3.92 & 5 \frac{1}{2}-4 \frac{1}{a^{8} F-15^{\circ}} \\ 4839.568 & \mathrm{~A} & 250 & 0.38 & 2.93 & 5 \frac{1}{3}(36) & 16^{\circ}\end{array}$

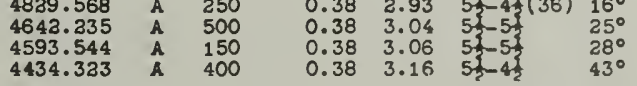
4421.138 A $300 \quad 0.38 \quad 3.17 \quad 5 \frac{1}{3}-6 \frac{1}{2} \mathrm{a}^{8} \mathrm{~F}-44^{\circ}$ $\begin{array}{lllllll}4421.138 & \text { A } & 200 & 0.38 & 3.17 & 53-67 & 8^{8} F-44 \\ 4368.031 & \wedge & 150 d & 0.38 & 3.20 & 5 \frac{1}{3}-6 \frac{2}{3}(37) & 46^{\circ}\end{array}$

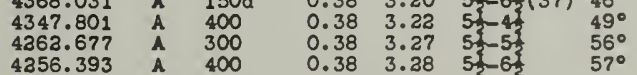

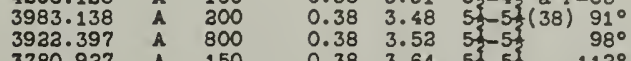

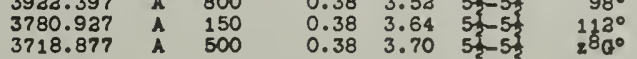
4306.138 A $100 \quad 0.38 \quad 3.31 \quad 5 \frac{1}{3}-4 \frac{1}{3} 8^{8} F-63^{\circ}$

I A Laboratory $_{\text {Ref }}$ Int

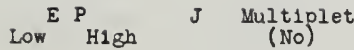

Sm II continued

3662.905 A 200

$\begin{array}{rrr}3592.603 & \text { A } & 1500 \\ 3402.464 & \text { A } & 500\end{array}$

$\begin{array}{rrr}3384.658 & \text { A } & 500 \\ 3354.185 & \text { A } & 150\end{array}$

$\begin{array}{lll}3354.185 & \text { A } & 150 \\ 3344.353 & \text { A } & 200\end{array}$

3321.179 A 800

3253.943 A 200

\begin{tabular}{rrr}
3196.182 & A & 150 \\
.3187 .216 & A & 300 \\
\hline
\end{tabular}

4961.936 A 250

$\begin{array}{lll}4816.012 & \text { A } & 100 \\ 4717.718 & \text { A } & 150\end{array}$

$\begin{array}{lll}4523.912 & \text { A } & 250 \\ 4433.885 & \text { A } & 300\end{array}$

4373.463 A 100

$\begin{array}{llll}42866.640 & \text { A } & 100 \\ 4234.573 & \text { A } & 200 \\ 4 & \end{array}$

$\begin{array}{lll}4203.051 & A & 125 \\ 4068.334 & A & 100\end{array}$

3971.397 A 300

$\begin{array}{lll}3843.500 & \text { A } & 200 \\ 3831.501 & \text { A } & 400 \\ 3 & & \end{array}$

$\begin{array}{rrr}3800.370 & A & 100 \\ .3774 .294 & A & 150\end{array}$

- 3756.4118 a 600

$\begin{array}{llll}3355.653 & A & 150 \\ 3396.187 & \text { A } & & \\ 250 & & \end{array}$

4834.618 A 100
4559.291 A

$\begin{array}{llll}4537.952 & \mathrm{~A} & 250 \\ 4424.339 & \mathrm{~A} & 200 \\ 4 & & \end{array}$

4424.339
4362.040

4350.465 A 300 d

4123.956

3767.35

3706.752

3649.52
3604.28

3568.27

3418.51

3347.298

$-3306.38$

3286.229
3276.747

3276.747
3239.657

4948.62

4713.057

4519.63

$-4230.659$

4188.12

4155.217

4075.845

3979.200

3826.202
.3760 .694

3650.998

3389.325

3371.209

3238.78

4913.248

4847.760
4718.339

4467.34

4378.236
4236.745

4153.33

4118.551
4083.600

3728.469

3467.874
3440.502

7082.77
6862.82

6790.00

5116.700

.7039 .22

7042.24 $\begin{array}{llll}0.38 & 3.75 & 5 \frac{1}{2}-4 \frac{1}{2} 8^{8} F-125^{\circ} \\ 0.38 & 3.81 & 5 \frac{1}{3}-6 \frac{1}{3}(39) & z^{8} 6^{\circ}\end{array}$

$\begin{array}{llll}0.38 & 4.00 & 5 \frac{1}{4}-4 \frac{1}{3} & 146^{\circ} \\ 0.38 & 4.02 & 51 & 5 \frac{1}{3}\end{array}$

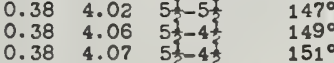

$0.38 \quad 4.09 \quad 5 \frac{1}{3}-5 \frac{1}{2} 8^{8} F-153^{\circ}$

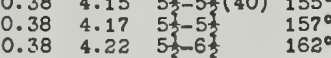

$\begin{array}{llll}0.38 & 4.24 & 5 \frac{1}{3}-5 \frac{1}{3} & 164^{\circ} \\ 0.38 & 4.25 & 5 \frac{1}{2}-5 \frac{1}{2} & 166^{\circ}\end{array}$

$\begin{array}{lll}0.43 & 2.92 & 31 \\ 0.43 & 2.91 & a^{6} F-15^{\circ}\end{array}$

$\begin{array}{lllll}0.43 & 3.05 & 313 & 31 & 27^{\circ} \\ 0.43 & 3.16 & 31 & 41\end{array}$

$\begin{array}{lll}0.43 & 3.16 & 3 \frac{1}{3}-4 \\ 0.43 & 3.22 & 3 \frac{1}{2}-4\end{array}$

$0.43 \quad 3.25 \quad 3 \frac{1}{2}-2 \frac{1}{6} a^{6} F-54^{\circ}$

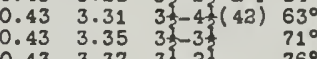

$\begin{array}{llll}0.43 & 3.37 & 3 \frac{1}{3}-2 \frac{1}{3} & 76^{\circ} \\ 0.43 & 3.47 & 3 \frac{1}{2}-3 \frac{1}{2} & z^{8} G^{\circ}\end{array}$

$0.43 \quad 3.54 \quad 3 \frac{1}{3}-2 \frac{1}{3} a^{6} F-100^{\circ}$

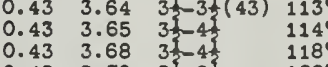

$0.43 \quad 3.72 \quad 3 \frac{1}{3}-3 \frac{1}{2} a^{6} F-123^{\circ}$

$\begin{array}{llll}0.43 & 3.92 & 3 \frac{1}{2}-3 \frac{1}{3}(44) & 1399^{\circ} \\ 0.43 & 4.07 & 3 \frac{1}{2}-2 \frac{1}{2} & 150^{\circ}\end{array}$

$0.48 \quad 3.04 \quad 6 \frac{1}{3}-5 \frac{1}{2} 8^{8} \mathrm{~F}-25^{\circ}$

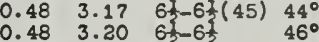

$\begin{array}{llll}0.48 & 3.20 & 65 & 46 \\ 0.48 & 3.27 & 6 \frac{1}{3} & 56^{\circ} \\ 0.48 & 3.31 & 6 \frac{3}{2}-5 \frac{1}{2} & 64^{\circ}\end{array}$

$0.48 \quad 3.33 \quad 6 \frac{1}{2}-7 \frac{1}{8} a^{8} F-65^{\circ}$

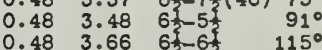

$0.48 \quad 3.76 \quad 6 \frac{1}{2}-6 \frac{1}{2} \quad 126^{\circ}$

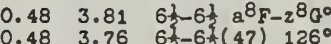

$\begin{array}{llll}0.48 & 3.91 & 6 \frac{1}{2} & 128^{\circ} \\ 0.48 & 3.94 & 6 \frac{1}{3} & 2^{8} 6^{\circ} \\ 0.48 & 4.09 & 6 & \end{array}$

$0.48 \quad 4.09 \quad 6 \frac{1}{2}-5 \frac{1}{2} \quad 153^{\circ}$

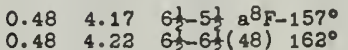

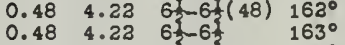

$\begin{array}{llll}0.48 & 4.24 & 61-5 \frac{1}{3} & 164^{\circ} \\ 0.48 & 4.25 & 6 \frac{1}{3}-5 \frac{1}{3} & 166^{\circ} \\ 0.48 & 4.29 & 6 & \end{array}$

$0.48 \quad 4.29 \quad 6 \frac{1}{2}-6 \frac{1}{2} \quad 168^{\circ}$

$0.54 \quad 3.04 \quad 4 \frac{1}{4}-5 \frac{1}{4} \mathrm{a}^{6} \mathrm{~F}-25^{\circ}$

$0.543 .16 \quad 434 \frac{1}{3}(49) \quad 43^{\circ}$

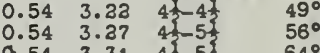

$0.54 \quad 3.47 \quad 4 \frac{1}{3}-3 \frac{1}{2} a^{6} F-z^{8} 0^{\circ}$

$0.54 \quad 3.49$ 43 $3 \frac{3}{3}(50) 93^{\circ}$

$\begin{array}{llll}0.54 & 3.50 & 41-41 & 94^{\circ} \\ 0.54 & 3.51 & 43-3 \frac{3}{3} & 97^{\circ} \\ 0.54 & 3.55 & 43 & -45\end{array}$

$0.54 \quad 3.57 \quad 4 \frac{1}{3}-4 \frac{1}{2} a^{6} F-103^{\circ}$

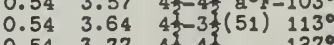

$\begin{array}{llll}0.54 & 3.77 & 42-4 \frac{1}{3} & 127^{\circ} \\ 0.54 & 3.82 & 43-43 & 173^{\circ} \\ 0.54 & 3.92 & 4 & \end{array}$

$0.54 \quad 4.184-3 \frac{1}{2} a^{6} \mathrm{~F}-159^{\circ}$

$0.54 \quad 4.20 \quad 4 \frac{1}{3}(52) 161^{\circ}$

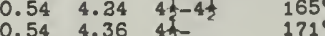

$0.66 \quad 3.17 \quad 5+61 a^{6}-44^{\circ}$

$0.663 .205 \frac{5}{3}-6 \frac{1}{3}(53) \quad 46^{\circ}$

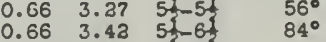

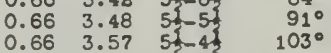

$0.66 \quad 3.63 \quad 5+4 \frac{1}{3} 8^{6} F-110^{\circ}$

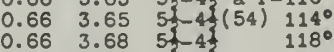

$0.66 \quad 3.97 \quad 53-6 \frac{1}{143^{\circ}}$

$\begin{array}{llll}0.66 & 4.22 & 5 \frac{1}{2}-6 \frac{1}{3} & 163^{\circ} \\ 0.66 & 4.24 & 5 \frac{1}{2}-4 \frac{1}{2} & 165^{\circ}\end{array}$

$\begin{array}{lll}0.88 & 2.62 & 1 \frac{1}{2}-\frac{1}{3} 8^{8} \mathrm{H}-1^{\circ} \\ 0.88 & 2.68 & 1 \frac{1}{2}-1 \frac{1}{2}(55) 5^{\circ}\end{array}$

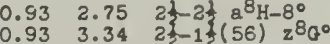

$0.99 \quad 2.25 \quad 3 \frac{1}{2}-2 \frac{1}{2} 8^{8} \mathrm{H}-8$

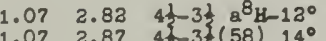




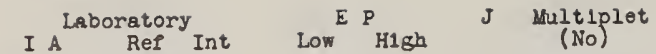
Sil II continued

$7030.44 \quad A \quad 800 d$

\begin{tabular}{lllll}
$800 \mathrm{~d}$ & 1.16 & 3.92 & $5 \frac{1}{2}-4 \frac{1}{2}$ & $a^{8} \mathrm{H}-15^{\circ}$ \\
\hline
\end{tabular}

6473.34 A $300 \mathrm{~d} \quad 1.373 .387 \frac{1}{2}-6 \frac{1}{2} a^{8} \mathrm{H}-57^{\circ}$

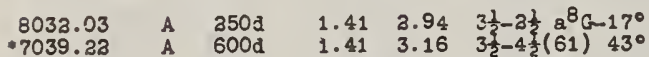

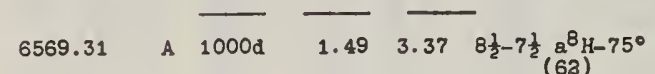

$8035.12 \mathrm{~A}+\frac{100}{1.51} 3.05{ }^{3 \frac{1}{2}-3 \frac{1}{2}}(63)^{8} \mathrm{~F}-27^{\circ}$

8510.90 A $200 \mathrm{~d} \quad 1.59 \quad 3.04 \quad 4 \frac{1}{3}-5 \frac{1}{2} \mathrm{~b}^{5}-25^{\circ}$ $\begin{array}{lllllll}8348.68 & \text { A } & 150 \mathrm{~d} & 1.59 & 3.06 & 4 \frac{2}{2}-5 \frac{3}{2}(64) & 38^{\circ} \\ 7837.27 & \text { A } & 400 & 1.59 & 3.16 & 4 \frac{1}{2}-4 \frac{1}{2} & 43^{\circ}\end{array}$

7928.14 A $800 \quad \overline{1.60} 3.163 \frac{1}{2}-4 \frac{1}{2} a_{(65)}^{8}{ }^{8}-43^{\circ}$

$8485.99 \mathrm{~A} \quad 400 \mathrm{~d} \quad 1.683 .136 \frac{1}{2}-5 \frac{1}{2} a^{8}(66)-38^{\circ}$

$\begin{array}{lllllll}8048.70 & \text { A } & 400 \mathrm{~d} & 1.74 & 3.37 & 6 \frac{1}{2}-5 \frac{1}{2} & b^{8} \mathrm{~F}-56^{\circ} \\ 8026.32 & \text { A } & 500 \mathrm{~d} & 1.74 & 3.28 & 6 \frac{1}{2}-6 \frac{1}{2}(67) & 57^{\circ}\end{array}$

8068.46 A $800 \quad 1.743 .274 \frac{1}{2}-5 \frac{1}{2} a^{8 P}(68)^{\circ}$

$8305.79 \quad$ A $500 d \quad 1.79 \quad 3.38 \quad 7 \frac{1}{3}-6 \frac{1}{2} 8^{8} \alpha-57^{\circ}$

Strongest Unclassifled Lines of Sm II

\begin{tabular}{|c|c|c|c|}
\hline $\begin{array}{l}4515.094 \\
4478.557 \\
4444.259 \\
4352.101 \\
4381.009\end{array}$ & $\begin{array}{l}A \\
A \\
A \\
A \\
A\end{array}$ & $\begin{array}{l}150 \\
125 \\
150 \\
200 \\
100\end{array}$ & $\begin{array}{l}\text { III } \\
\text { IV } \\
\text { IV } \\
\text { IV } \\
\text { III }\end{array}$ \\
\hline $\begin{array}{l}3962.995 \\
3959.527 \\
3903.417 \\
3875.193 \\
3854.209\end{array}$ & $\begin{array}{l}\mathrm{A} \\
\mathrm{A} \\
\mathrm{A} \\
\mathrm{A} \\
\mathrm{A}\end{array}$ & $\begin{array}{l}200 \mathrm{~d} \\
100 \\
500 \\
100 \\
300\end{array}$ & $\begin{array}{l}I I \\
I I \\
I I \\
I I \\
I I\end{array}$ \\
\hline $\begin{array}{l}3848.779 \\
3797.730 \\
3780.763 \\
3778.136 \\
3767.755\end{array}$ & $\begin{array}{l}A \\
A \\
A \\
A \\
A\end{array}$ & $\begin{array}{l}200 \mathrm{~d} \\
600 \\
200 \\
400 \\
150\end{array}$ & II \\
\hline $\begin{array}{l}3758.968 \\
3757.529 \\
3741.288 \\
3739.117 \\
3737.141\end{array}$ & $\begin{array}{l}A \\
A \\
A \\
A \\
A\end{array}$ & $\begin{array}{l}200 \\
300 \\
300 \\
300 \\
300\end{array}$ & II \\
\hline $\begin{array}{l}3721.847 \\
3712.109 \\
3706.979 \\
3700.922 \\
3677.793\end{array}$ & $\begin{array}{l}A \\
A \\
A \\
A \\
A\end{array}$ & $\begin{array}{l}400 \\
100 \\
200 \\
150 \\
200\end{array}$ & II \\
\hline $\begin{array}{l}3670.677 \\
3662.693 \\
3656.221 \\
3638.767 \\
3631.126\end{array}$ & $\begin{array}{l}A \\
A \\
A \\
A \\
A\end{array}$ & $\begin{array}{l}150 \\
300 \\
300 \\
400 \\
400\end{array}$ & II \\
\hline $\begin{array}{l}3580.941 \\
3566.836 \\
3559.101 \\
3418.151 \\
3408.676\end{array}$ & $\begin{array}{l}\mathrm{A} \\
\mathrm{A} \\
\mathrm{A} \\
\mathrm{A} \\
\mathrm{A}\end{array}$ & $\begin{array}{l}300 \\
150 \\
300 \mathrm{~d} \\
300 \\
400\end{array}$ & $\begin{array}{l}\text { II } \\
\text { II } \\
\text { II } \\
\text { II }\end{array}$ \\
\hline $\begin{array}{l}3365.863 \\
3350.875 \\
3348.683 \\
3343.494 \\
3325.258\end{array}$ & $\begin{array}{l}\mathbf{A} \\
\mathbf{A} \\
\mathbf{A} \\
\mathbf{A} \\
\mathbf{A}\end{array}$ & $\begin{array}{l}400 \\
200 \\
200 \\
200 \\
300\end{array}$ & \\
\hline $\begin{array}{l}3316.579 \\
3307.017 \\
3298.104 \\
3273.483 \\
3253.401\end{array}$ & $\begin{array}{l}\mathrm{A} \\
\mathrm{A} \\
\mathrm{A} \\
\mathrm{A} \\
\mathrm{A}\end{array}$ & $\begin{array}{l}300 \\
500 \\
500 \\
500 \\
300\end{array}$ & I \\
\hline $\begin{array}{l}3236.638 \\
3218.614 \\
3211.734 \\
3193.014 \\
3183.916 \\
3158.535\end{array}$ & $\begin{array}{l}\text { A } \\
\text { A } \\
\text { A } \\
\text { A } \\
\text { A } \\
\text { A }\end{array}$ & $\begin{array}{l}500 \\
300 \\
400 \\
300 \\
400 \\
300\end{array}$ & \\
\hline
\end{tabular}

DEISED UULTIPLET TABLE

I A Laboratory Ref Int $^{\text {I P }}$ Low $J$ gultiplet Eu I I P 5.64 Ang? A L1st D Apr 1948 $4594.03 / /$ A $10000 R \quad 0.00$ a. $69 \quad 3 \frac{1}{3}-4 \frac{1}{3} a^{8} \mathrm{~S}^{0}-y^{8} \mathrm{P}$ $\begin{array}{llllll}4627.22 & \text { A } 8000 \mathrm{R} & 0.00 & 3.67 & 3 \frac{1}{3}-3 \frac{2}{2} & \text { (1) } \\ 4661.88 & \text { A } 7000 \mathrm{R} & 0.00 & 2.65 & 3 \frac{2}{2}-2 \frac{1}{2} & \end{array}$

Eu II I P 11.21 Anal $B$ List $C$ May 1942

$3819.67 / /$ A $6000 \mathrm{R} \quad 0.00 \quad 3.33$ 4-5 $\mathrm{a}^{9} \mathrm{~S}^{0}-2^{9} \mathrm{P}$

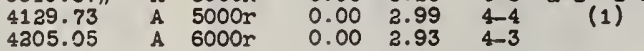

$\begin{array}{llllllc}3734.94 & \text { A } & 4000 & 0.00 & 3.31 & 4-4 & a^{9} \mathrm{~S}^{0}-z^{7 P} \\ 3688.42 & \text { A } & 1500 & 0.00 & 3.35 & 4-3 & (2)\end{array}$

$3991.33 \quad$ A $300 \quad 0.00 \quad 4.13 \quad 4-5 \quad a^{9} S^{0}-2^{9} D$

$\begin{array}{lllllll}3077.358 & \text { A } & 300 & 0.00 & 4.01 & 4-4 & (3) \\ 3173.607 & \text { A } & 100 & 0.00 & 3.89 & 4-3 & \end{array}$

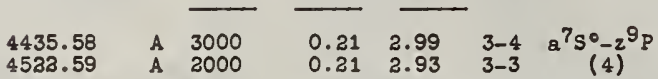

$\begin{array}{ccccccc}3971.98 & \text { A } & 4000 \mathrm{r} & 0.21 & 3.31 & 3-4 & \mathrm{a}^{7} \mathrm{~S}^{0}-2^{7} \mathrm{P} \\ 3930.50 & \text { A } 4000 \mathrm{r} & 0.21 & 3.35 & 3-3 & (5)\end{array}$

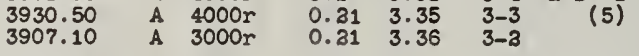

3097.45 A $100 \quad 0.21 \quad 4.19 \quad 3-2 \quad a^{7} S^{0}-103$

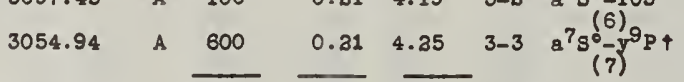

$\begin{array}{llllll}6645.11 & \text { A } 8000 & 1.37 & 3.33 & 6-5 & a^{9} D^{0}-2^{9} \mathrm{P}\end{array}$

$\begin{array}{lllllll}7370.23 & \text { A } & 2500 & 1.31 & 3.99 & 5-4 & (8) \\ 7426.57 & \text { A } & 1500 & 1.37 & 3.93 & 4-3 & \end{array}$

$\begin{array}{llllll}6437.64 & \text { A } & 4000 & 1.31 & 3.33 & 5-5\end{array}$

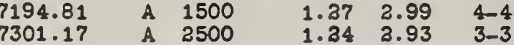

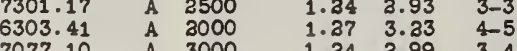

$\begin{array}{llllll}7077.10 & \text { A } & 3000 & 1.24 & 3.99 & 3-4 \\ 7317.55 & \text { A } & 1500 & 1.33 & 3.93 & 2-3\end{array}$

6173.05 A 2000

$\begin{array}{lll}6173.05 & \text { A } 2000 \\ 6049.51 & \text { A } 2000\end{array}$

$\begin{array}{rrr}5873.98 & \text { A } & 500 \\ 5966.07 & \text { A } & 1200\end{array}$

$\begin{array}{lll}5966.07 & \text { A } & 1200 \\ 5818.74 & \text { A } & 1000\end{array}$

3917.39 \& A 60

$\begin{array}{rrr}4017.58 & \text { A } & 100 \\ 4151.52 & \text { A } & 30\end{array}$

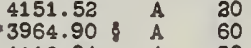

$\begin{array}{lll}4112.04 & \text { A } & 30 \\ 3938.87 & \text { A } & 15\end{array}$

$\begin{array}{lll}3938.87 & \text { A } & 15 \\ 4085.38 & \text { A } & 40 .\end{array}$

$\begin{array}{lll}3741.31 & \text { A } & 400 \\ 3761.13 & \text { A } & 300\end{array}$

$\begin{array}{lll}3761.13 & \text { A } & 300 \\ 3799.009 & \text { A } & 100\end{array}$

3674.634

3714.904

$\begin{array}{llr}3743.556 & \text { A } & 40\end{array}$

3713.45 A 135

3508.853 A 20

$\begin{array}{lll}3508.731 & \text { A } & 10 \\ 3646.75 & \text { A } & 35\end{array}$

$\begin{array}{lll}3440.999 & \text { A } & 80 \\ 3461.38 & \text { A } & 80\end{array}$

3710.870 A 80

3611.57 A 100

3603.20 A 200

$\begin{array}{rrr}3391.989 & \text { A } & 100 \\ 3542.152 & \text { A } & 80\end{array}$

3623.54 A 150

3553.516 A 100

$1.31 \quad 3.31$

$1.37 \quad 3.31$

$\begin{array}{ll}1.34 & 3.35 \\ 1.34 & 3.31\end{array}$

$1.22 \quad 3.35$

$1.37 \quad 4.53$

$\begin{array}{ll}.31 & 4.39\end{array}$

$\begin{array}{ll}1.37 & 4.35 \\ 1.27 & 4.39\end{array}$

$\begin{array}{ll}1.34 & 4.39 \\ 1.34 & 4.35\end{array}$

$\begin{array}{ll}1.24 & 4.39 \\ 1.23 & 4.25\end{array}$

$5-4$
$4-4$
$3-3$
$3-4$

$\begin{array}{cc}5-4 & a^{9} D^{0}-2^{7} P+ \\ 4-4 & (9)\end{array}$

$1.37 \quad 4.67$

$1.31 \quad 4.60$

$\begin{array}{ll}1.37 & 4.52 \\ 1.31 & 4.67\end{array}$

$\begin{array}{ll}1.37 & 4.60\end{array}$

$\begin{array}{lll}1.34 & 4.53 & 3-3\end{array}$

$\begin{array}{lll}1.32 & 4.53 & 3-3\end{array}$

$1.34 \quad 4.57 \quad 3-\hat{a} \quad a^{9} D^{\circ}-115$

$\begin{array}{llll}1.31 & 4.83 & 5-4 & a^{9} D^{\circ}-Y^{7 P} \\ 1.27 & 4.79 & 4-3 & (13)\end{array}$

$1.22 \quad 4.61$

$\begin{array}{lll}1.24 & 4.83 & 3-4 \\ 1.22 & 4.79 & 2-3\end{array}$

$1.31 \quad 4.64 \quad 5-6 \quad a^{9} D^{0}-116 \uparrow$

$1.37 \quad 4.69 \quad 4-4 \quad a^{9} D^{(14)}-131+$

$1.37 \quad 4.70 \quad 4-4 \quad a^{9} D^{\circ}-123 t$

$\begin{array}{cccc}1.34 & 4.88 & 3-4 & 2^{9} D^{\circ}-x^{7} \mathrm{P} \uparrow \\ 1.32 & 4.71 & 2-3 & (17)\end{array}$

$1.37 \quad 4.78 \quad 6-7 \quad a^{9} D^{0}-125$

$\begin{array}{rrr}3369.055 & \text { A } & 200 \\ 3425.022 & \text { A } & 80\end{array}$

1.314 .79

5-5 a $a^{9}(18)-126+$

$\begin{array}{llll}1.31 & 4.98 & 5-6 & 8^{9} D^{\circ}-131 \\ 1.37 & 4.98 & 6-6 & (20)\end{array}$

3321.857

$\begin{array}{rrrrrrc}4355.09 & \text { A } & 300 & 3.33 & 6.06 & 5-4 & 2^{9} P_{-} e^{9} S^{\circ} \\ 4011.69 & \text { A } & 100 & 3.99 & 6.06 & 4-4 & (23) \\ 3943.08 & \text { A } & 40 & 3.93 & 6.06 & 3-4 & \end{array}$

100

$1.37 \quad 5.09$

6

$9 D^{\circ}-13$
$(21)$

$3380.25 \quad$ A 100

3521.09 A 100

$\begin{array}{lll}3301.95 & \text { A } & 150 \\ 3366.39 & \text { A } & 300\end{array}$
I A ${ }^{\text {Laboratory }}$ Ref Int

E P

tultiplet

Eu II continued

$\begin{array}{lllllll}3531.151 \quad \text { A } & 60 & 3.23 & 5.73 & 5-5 & 2^{9} \mathrm{P}-\mathrm{e}^{9} \mathrm{D}^{0}+\end{array}$ $\begin{array}{lllllll}3313.33 & \text { A } & 400 & 2.99 & 6.71 & 4-4 & (24) \\ 3372.77 & \text { A } & 400 & 2.93 & 6.71 & 3-3 & \text { cont }\end{array}$

$\begin{array}{rlrrrr}3319.89 & \text { A } & 80 & 2.99 & 6.71 & 4-3 \\ 3277.78 & \text { A } & 600 & 2.93 & 6.70 & 3-3\end{array}$

$\begin{array}{lllllll}3308.02 & \text { A } & 200 & 3.99 & 6.73 & 4-5 & 2^{9} \mathrm{P}-e^{7} \mathrm{D}^{\circ}+\end{array}$

4485.15 A $\overline{100} \quad \overline{3.31} \quad \overline{6.06} 4-4 \quad 2^{7} \mathrm{P}_{-\mathrm{e}^{9} \mathrm{~S}^{0} \mathrm{t}}$

$\begin{array}{llllllll}4383.17 & \text { A } & 200 & 3.31 & 6.13 & 4-3 & 2^{7} P-e^{7} S^{\circ}+ \\ 4464.97 & \text { A } & 200 & 3.36 & 6.13 & 2-3 & (27)\end{array}$

$\begin{array}{lrrrrrr}3616.152 & \text { A } & 100 & 3.31 & 6.73 & 4-5 & z^{7} \mathrm{P}-\mathrm{e}^{9} \mathrm{D}^{\circ}+ \\ 3673.19 & \text { A } & 80 & 3.35 & 6.71 & 3-3 & (38)\end{array}$

3678.359 A $100 \quad 3.36 \quad 6.72 \quad 2-1 \quad 2^{7} P_{-9} D^{0}+$

$\begin{array}{llllll}3396.58 \quad A \quad 300 & 3.31 & 6.95 \quad 4-5 & z^{7} P_{-P^{7}}^{(29)} D^{\circ}+\end{array}$

Strongest Unclassifled Lines of Eu II

$\begin{array}{llll}3861.18 & \text { A } & 80 & \text { V } \\ 3815.495 & \text { A } & 80 & \text { V } \\ 3738.08 & \text { A } & 80 & \text { V }\end{array}$

$\begin{array}{llll}3738.08 & \text { A } & 80 & \text { V } \\ 3717.69 & \text { A } & 80 & \text { V }\end{array}$

$\begin{array}{llll}3687.78 & \text { A } & 80 & \text { V } \\ 3679.500 & \text { A } & 80 & \text { V } \\ 3390.783 & \text { A } & 80 & \text { V } \\ 3130.73 & \text { A } & 80 & \text { V }\end{array}$

Gd I I P 6.16 Anal C L1st D June 1943

$\begin{array}{llllll}7168.37 & \text { A } 3000 & 0.31 & 1.93 & 6-5 & a^{9} D^{0}-2^{9} P 4\end{array}$

$6730.73 \quad$ A $1500 \quad 0.13 \quad 1.96 \quad 5-6 \quad 8^{9} 0^{\circ}-8^{11}+$

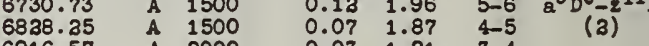

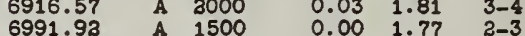

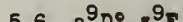

$\begin{array}{lllllll}5696.23 & \text { A } 8000 & 0.07 & 2.23 & 4-5 & \text { (3) }\end{array}$

5643.24 A $6000 \quad 0.03 \quad 2.21 \quad 3-4$

$\begin{array}{llllll}6114.07 & \text { A } & 4000 & 0.00 & 2.30 & 2-3 \\ 5 & \text { A } & 0.200 & 0.31 & 2.33 & 6-6\end{array}$

$\begin{array}{llllll}5851.63 & \text { A } & 5000 & 0.12 & 3.23 & 5-5 \\ 5632.35 & \text { A } 3500 & 0.00 & 3.19 & 2-2\end{array}$

$\begin{array}{lllll}5791.38 \text { A } & 3500 & 0.00 & 3.19 & 2-3 \\ 5 & 0.07 & 2.30 & 4-3\end{array}$

$\begin{array}{llllll}5701.35 & \text { A } & 4000 & 0.07 & 2.30 & 4-3 \\ & & & 0.03 & 2.19 & 3-3\end{array}$

$\begin{array}{lllllll}4235.850 / / & \text { A } & 3000 & 0.21 & 3.13 & 6-7 & a^{9} D^{0}-y^{9} F+ \\ 4346.458 & \text { A } & 1500 & 0.12 & 2.96 & 5-5 & (4)\end{array}$

4346.458 A 1500

4313.845 A 3000

$5-5$
$3-3$

$\begin{array}{lll}0.03 & 3.89 & 3-3 \\ 0.00 & 3.87 & 3-3\end{array}$

$\begin{array}{lllllll}4175.538 & \text { A } & 3000 & 0.31 & 3.17 & 6-6 & a^{9} D^{0}-y^{9} D+ \\ 4053.643 & \text { A } & 3500 & 0.13 & 3.17 & 5-6 & (5)\end{array}$

$\begin{array}{lll}4078.700 & \text { A } 3000 \\ 4058.219 & \text { A } 2500\end{array}$

\begin{tabular}{lll}
0.07 & 3.09 & $4-5$ \\
0.03 & 3.07 & $3-4$ \\
\hline
\end{tabular}

5015.04 A $1500 \quad 1.05 \quad 3.51 \quad 8-9 \quad a^{11} \mathrm{~F}^{\circ}-z^{1104}$

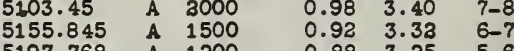

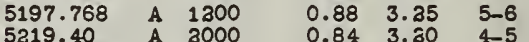

5255.805 A $1500 \quad 0.81 \quad 3.16 \quad 3-4$

$\begin{array}{llllll}5251.180 & \text { A } & 2000 & 1.05 & 3.40 & 8-8 \\ 5383.076 & \text { A } 3000 & 0.98 & 3.32 & 7-7\end{array}$

5301.67 A $4000 \quad 0.93 \quad 3.35 \quad 6-6$

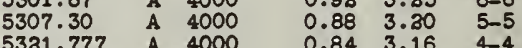

$\begin{array}{llllll}5331.777 & \text { A } & 4000 & 0.84 & 3.16 & 4-4 \\ 5303.76 & \text { A } & 3000 & 0.81 & 3.14 & 3-3\end{array}$

$\begin{array}{llllll}5321.496 & \text { A } & 3000 & 0.79 & 3.11 & 3-3 \\ 5348.67 & \text { A } & 3000 & 0.79 & 3.09 & 3-1\end{array}$

$\begin{array}{llllllll}5350.38 & \text { A } & 4000 & & 1.54 & 3.85 & 7-8 & 8^{9} F^{\circ}-2^{9}+\uparrow \\ 5353.26 & \text { A } 3000 & 1.46 & 3.77 & 6-7 & (7)\end{array}$

$\begin{array}{llllll}5353.26 & \text { A } & 3000 & 1.46 & 3.77 & 6-7 \\ 5343.00 & \text { A } & 3000 & 1.39 & 3.70 & 5-6 \\ 5333.30 & \text { A } & 3000 & 1.34 & 3.66 & 4-5\end{array}$

Qd II I P ? Anal C L1st B Sept 1943

$3763.00 \quad A \quad 50 \quad 0.24 \quad 3.52 \quad 6 \frac{1}{2}-5 \frac{1}{2} a^{10} D^{\circ}-2^{10}$ P

$\begin{array}{lllllll}3952.00 & \text { A } 300 & 0.14 & 3.27 & 5 & 54 & \text { (1) }\end{array}$

3993.313

3871.54

3871.54

3587.186

3816.64
3894.696

A $1500 \quad 0.14 \quad 3.52 \quad 5-5$

A $300 \% \quad 0.08$ 3.27 $4 \frac{4}{2}-4$

$\begin{array}{ccccc}\text { A } & 3009 & 0.03 & 3.17 & 3 \frac{1}{3}-3 \\ \text { A } & 40 & 0.08 & 3.52 & 42\end{array}$

$\begin{array}{lll}0.08 & 3.52 & 45 \\ 0.03 & 3.37 & 3\end{array}$ 


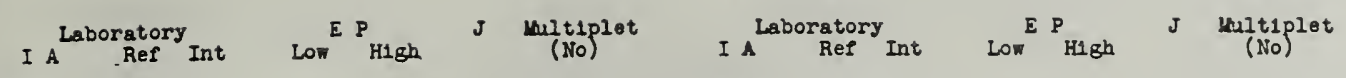

Gd II continued

$\begin{array}{lll}3432.466 & \text { A } & 1000 \\ 3545.797 & \text { A } & 3000 \\ 3671.20 & \text { A } & 1500 \\ 3716.36 & \text { A } & 100 \\ 3759.00 & \text { A } & 30 \\ 3646.19 & \text { A } & 300 \\ 3743.47 & \text { A } & 2000 \\ 3768.39 & \text { A } & 300 \\ 3796.37 & \text { A } & 350 \\ 3813.97 & \text { A } & 200 \\ 3855.56 & \text { A } & 20 \\ 3844.579 & \text { A } & 50 \\ 3850.69 & \text { A } & 80 \\ 3852.45 & \text { A } & 100 \\ 3850.97 & \text { A } & 130\end{array}$

3850.97 A 1300

$\begin{array}{llr}3988.261 & \text { A } & 80 \\ 3887.157 & \text { A } & 40 \\ 3831.80 & \text { A } & 100\end{array}$

3367.093 A 100

$\begin{array}{lll}3654.62 & \text { A } & 2000 \\ 3697.73 & \text { A } 1000\end{array}$

3308.51 ?

$\begin{array}{lll}360.665 & \text { A } & 100 \\ 3362.26 & \text { A } & \\ 3368.335 & \text { A } & 400\end{array}$

3571.933 A 300

3733.45 A 100

$\begin{array}{lll}3524.196 & \text { A } 1000 \\ 3491.954 & \text { A } 2000\end{array}$

3439.990 A 6000

$\begin{array}{rrr}3454.145 & A & 1500 \\ 3518.633 & \text { A } & 30\end{array}$

3449.616 A 800

3423.92 A 1500

359.365

3494.404

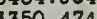

3392.530

3418.733

3462.997

3432.751

3401.067

3360.711

3382.233

A 8000

3196.533 A 150

$\begin{array}{llr}3052.511 & \text { A } & 50 \\ 3133.094 & \text { A } & 150\end{array}$

3161.369 A 2500

3145.00 A 3500

3098. 899

3134.350

3119.336

3093.846

3083.350

3160.69 A 300

$\begin{array}{rrr}3135.034 & \text { A } & 300 \\ 3156.533 & \text { A } 2000\end{array}$

(3123.

3119.941

3098.644 A 800

3100.504 A 10000

3081.993 A 8000

3032.845

3034.051

3980.154
3999.045

3010.129

4508.931

4325.148

4446.487
4494.853

4341.382

\section{$0.24 \quad 3.84 \quad 6 \frac{1}{2}-7 \frac{1}{2} a^{10} D^{0}-z^{1 O_{F}} \quad 4078.444$ A 1200

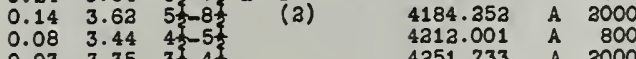

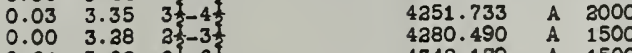 \\ 0.343 .62 61 $-6 \frac{2}{2} \quad 4342.179$ A 1500

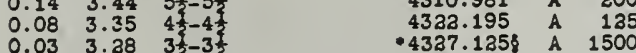

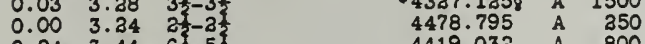

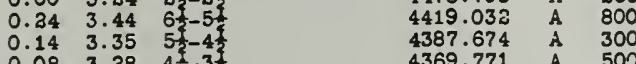 \\ $\begin{array}{llll}0.08 & 3.38 & 4 \frac{1}{2}-3 \frac{1}{3} \\ 0.03 & 3.34 & 3 \frac{1}{2}-2 \frac{1}{3}\end{array}$ \\ $\begin{array}{llll}0.03 & 3.24 & 3 \frac{1}{2}-2 \frac{1}{2} \\ 0.00 & 3.20 & 3 \frac{1}{2}-1 \frac{2}{2}\end{array}$ \\ 4360.917 A 250}

$\begin{array}{llll}0.14 & 3.25 & 51-4 \frac{1}{3} \mathrm{a}^{10} \mathrm{D}^{0}-2 \\ 0.08 & 3.25 & 4 \frac{3}{3} & (3)^{2} \\ 0.03 & 3.25 & 3 \frac{1}{2}-4 \frac{3}{2} & \end{array}$

$\begin{array}{llr}3843.80 & \text { A } & 35 \\ 4162.733 & \text { A } & 500\end{array}$

4188.099 A 60

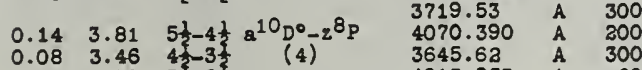

$\begin{array}{lll}0.03 & 3.37 & 3 \frac{1}{2}-2 \frac{2}{2} \\ 0.08 & 3.81 & 47-4 \frac{1}{2}\end{array}$

$0.03 \quad 3.46 \quad 3 \frac{1}{0}-3 \frac{1}{2}$

$\begin{array}{llll}0.03 & 3.81 & 3 \frac{1}{3}-4 \frac{2}{2} \\ 0.00 & 3.46 & 3 \frac{1}{2}-3 \frac{1}{2}\end{array}$

$0.08 \quad 3.38 \quad 4 \frac{1}{2}-3 \frac{1}{2} 2^{10} D^{0}-3$

$\begin{array}{llll}0.03 & 3.53 & 3 \frac{1}{3}-3 \frac{1}{3} & \mathrm{a}^{10} 0^{(5)}-4 \\ 0.00 & 3.53 & 3 \frac{1}{2}-2 \frac{1}{2} & (6)\end{array}$

$\begin{array}{rrr}4013.953 & \text { A } & 60\end{array}$

4167.159 A 40

3822.17 A 80

$\begin{array}{llr}3826.05 & \text { À } & 200 \\ 3902.398 & \text { A } & 1000\end{array}$

$\begin{array}{rrr}3957.872 & \text { A } & 1000 \\ 3987.314 & \text { A } & 600\end{array}$

$0.24 \quad 3.83 \quad 6 \frac{1}{0}-8 \frac{1}{10} \mathrm{a}^{10}-2^{10 D}$

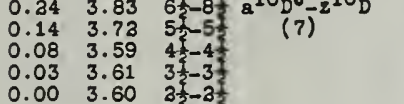

$0.24 \quad 3.72$ 6

$\begin{array}{lll}0.14 & 3.59 & 51-4 \frac{1}{3} \\ 0.08 & 3.51 & 43\end{array}$

$\begin{array}{lll}0.03 & 3.60 & 3 \\ 0.14 & 3.83 & 5\end{array}$

$\begin{array}{lll}0.08 & 3.72 & 4 \frac{1}{2}-5 \frac{1}{2} \\ 0.03 & 3.59 & 3 \frac{2}{2}-4 \frac{2}{2} \\ 0.00 & 3.61 & 3 \frac{2}{2}-3 \frac{2}{2}\end{array}$

$\begin{array}{rrr}3875.62 & \text { A } & 60 \\ -4130.372 & \text { A } 3000\end{array}$

$\begin{array}{llr}3916.508 & \text { A } 3000 \\ 3838.91 & \text { A } 300\end{array}$

3838.91 A 300

$\begin{array}{lll}3760.93 & \text { A } & 100 \\ 3699.73 & \text { A } & 800\end{array}$

3969.293 A 300

$\begin{array}{lll}3839.64 & \text { A } & 300 \\ 3767.04 & \text { A } & 500\end{array}$

4

3787.56

3758.31
3712.70

$0.14 \quad 3.71 \quad 5 \frac{1}{1}-4 \frac{1}{3} \mathrm{a}^{10} \mathrm{D}^{0}-\mathrm{z}^{8} \mathrm{Dt}$

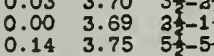

$0.08 \quad 3.71 \quad 4 \frac{4}{3}$

$0.03 \quad 3.70 \quad 3 \frac{3}{3}-3$

3687.74

3578.598

3409.397
3331.348

$\begin{array}{rrr}3369.818 & \text { A } & 400\end{array}$

$\begin{array}{lll}0.00 & 3.70 & 35-2 \frac{1}{2} \\ 0.08 & 3.75 & 43-5 \frac{1}{5}\end{array}$

$\begin{array}{lll}0.03 & 3.71 & 3 \frac{1}{2}-4 \frac{1}{2} \\ 0.00 & 3.70 & 3 \frac{1}{2}-3 \frac{1}{2}\end{array}$

3481.275 A 5000

3450.376 A 4000

$0.08 \quad 3.94 \quad 4 \frac{1}{2}-3 \frac{1}{3} \mathrm{a}^{10 \mathrm{D}^{0}-2^{6} \mathrm{P}+}$

$\begin{array}{llll}0.00 & 4.04 & 31 & 3 \\ 0.00 & 3.94 & 3 & 3\end{array}$

$0.24 \quad 4.14$ 6t $6 \frac{1}{2} \mathrm{a}^{10} \mathrm{D}^{0}-2^{8} \mathrm{~F}_{F}$

$0.14 \quad 4.07 \quad 57-5 \frac{1}{2}(10)$

$\begin{array}{lll}0.08 & 4.03 & 4-4 \frac{1}{3} \\ 0.03 & 4.01 & 3-3\end{array}$

$\begin{array}{llll}0.00 & 3.98 & 3 & 2 \\ 0.24 & 4.07 & 5 & -5\end{array}$

$0.14 \quad 4.03 \quad 5=-4$

0.08 4.01 4.013

$0.00 \quad 3.98$ 3: 13

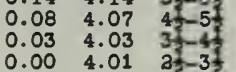

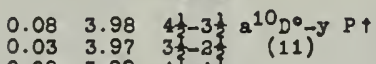

$\begin{array}{lll}0.08 & 3.99 & 4 \frac{1}{3}-4 \frac{2}{2} \\ 0.03 & 3.98 & 3 \frac{3}{2}-3\end{array}$

$\begin{array}{lll}0.03 & 3.99 & 3 \frac{2}{2}-4 \frac{1}{2} \\ 0.00 & 3.98 & 3 \frac{1}{2}-3\end{array}$

$0.24 \quad 4.22 \quad 6 \frac{1}{3}-5 \frac{1}{2} \mathrm{a}^{10} \mathrm{D}^{0}-\mathrm{y}^{10 \mathrm{P}}$

$0.14 \quad 4.15 \quad 5 \frac{2}{2} \quad(12)^{-y}$

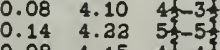

$\begin{array}{lll}0.08 & 4.15 & 43-4 \\ 0.03 & 4.10 & 3\end{array}$

$\begin{array}{lll}0.08 & 4.32 & 42-5 \\ 0.03 & 4.15 & 3 \frac{1}{2}-4 \frac{1}{3} \\ 0.00 & 4.10 & 2 \frac{1}{2}-3 \frac{1}{2}\end{array}$

$0.43 \quad 3.163 \frac{1}{2}-4 \frac{1}{2} \mathrm{a}^{8} \mathrm{D}^{0}-1+$

3399.406 A 500

$\begin{array}{lll}3399.991 & \text { A } & 1200 \\ 3557.053 & \text { A } & 1000\end{array}$

3481.797 A 3000

3439.784 A 1500

3590.468 A 100

3505.512 A 2000

$\begin{array}{lll}3467.267 & \text { A } & 3500 \\ 3451.333 & \text { A } & 2000 \\ 3432.994 & \text { A } & \end{array}$

$\begin{array}{llll}0.60 & 3.99 & 5 \frac{1}{2}-4 \frac{1}{2} & 8^{8} D^{0}-r^{8} P \uparrow \\ 0.43 & 3.97 & 3 \frac{3}{3}-2 \frac{3}{2} & (23)\end{array}$

$\begin{array}{lll}0.49 & 3.99 & 4 \frac{1}{3}-4 \frac{2}{3} \\ 0.42 & 3.98 & 3.3\end{array}$

0.38 3.97 $2 \frac{1}{3}-2 \frac{1}{2}$

$\begin{array}{lll}0.42 & 3.99 & 3 \frac{1}{2}-4 \frac{1}{3} \\ 0.38 & 3.98 & 2 \frac{1}{3}-3 \frac{1}{3} \\ 0.35 & 3.97 & 1 \frac{1}{2}-2\end{array}$

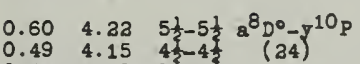

$\begin{array}{lll}0.42 & 4.10 & 3 \frac{3}{3}-3 \frac{2}{2} \\ 0.49 & 4.10 & 4 \frac{1}{3}-3 \frac{2}{2}\end{array}$

$\begin{array}{llll}0.49 & 4.22 & 4 \frac{2}{2}-5 \frac{1}{1} \\ 0.42 & 4.15 & 3 \frac{1}{4}-4\end{array}$

$\begin{array}{lll}0.42 & 4.15 & 3 \frac{1}{3}-4 \frac{1}{3} \\ 0.38 & 4.10 & 3 \frac{1}{2}-3 \frac{1}{2}\end{array}$

$\begin{array}{llll}0.60 & 4.50 & 51-5 \frac{1}{2} & 8^{8} D^{0}-y^{8} D \uparrow \\ 0.42 & 4.53 & 3 \frac{1}{2}-3 \frac{3}{2} & (25)^{-1}\end{array}$

$\begin{array}{lll}0.42 & 4.53 & 3 \frac{1}{3}-3 \frac{1}{2} \\ 0.38 & 4.55 & 2 \frac{1}{3}-2 \frac{3}{3}\end{array}$

$0.80 \quad 4.52 \quad 52-4 \frac{1}{2}$

$\begin{array}{lll}0.49 & 4.53 & 41-3 \frac{1}{2} \\ 0.42 & 4.55 & 3 \frac{1}{2}-2 \frac{1}{2}\end{array}$

$0.43 \quad 4.52 \quad 3 \frac{3}{3}-4$

$0.60 \quad 4.87 \quad 5 \frac{1}{2}-4 \frac{1}{2} a^{8} D^{0}-7$ t

3028.981 A 200
I A Laboratory

Low ${ }_{\text {H1gh }}$

thitiplet

Gd II continued

2969.267 A 50

$\begin{array}{lll}2965.428 & \text { A } & 400 \\ 3012.190 & \text { A } 600\end{array}$

4510.380 A 30 d?

$\begin{array}{llr}4344.487 & \text { A } & 40 \\ 4498.276 & \text { A } & 300\end{array}$

$\begin{array}{lll}4215.023 & \text { A } & 600 \\ 4390.953 & \text { A } & 300\end{array}$

4364.140 A 25

$\begin{array}{lll}4073.195 & \text { A } & 400 \\ 4191.067 & \text { A } & 800\end{array}$

-4170.1088 A 150

3881.84 A 50

3760.71 A 200

$\begin{array}{rrr}3763.33 & \text { A } & 60 \\ 3769.45 & \text { A } & 100\end{array}$

3512.219 A 800

$\begin{array}{lll}3441.790 & \text { A } & 400 \\ 3469.307 & \text { A } & 100\end{array}$

3463.984 A 5000

$\begin{array}{rrr}3468.989 & \text { A } & 3000 \\ 3482.602 & \text { A } & 800\end{array}$

$\begin{array}{lll}3315.590 & \text { A } & 400 \\ 3358.434 & \text { A } & 300\end{array}$

$\begin{array}{lll}3010.899 & \text { A } & 250 \\ 3993.038 & \text { A } & 500\end{array}$

4734.427 A 100

4802.575 A 80

$\begin{array}{llr}4627.66 & \text { A } & 40 \\ 4719.040 & \text { A } & 60\end{array}$

4719.040
4237.140 A
A 200

4073.759 A 1500

- 4262.0928 A 2500

4438.266 A 150

4521.296 A 100

4558.080 A 250

4394.719 A 25

4581.086 A 200

-4597.91 A A 500

4601.05 A 500

4596.978 A 400

. 4163.0928 A 250

4344.300 A 100

.4466 .5478 A 500

4757.791 A 80

4331.110 A 200

4382.061 A 60

4253.368 A 800

3791.72 A 30

$\begin{array}{rrr}4204.857 & \text { A } & 300 \\ 4296.076 & \text { A } & 1000\end{array}$

$\begin{array}{rrr}-4359.152 & \text { A } & 40 \\ 4308.233 & \text { A } & 40\end{array}$

4140.450 A 100

$\begin{array}{lll}4094.478 & \text { A } & 300 \\ 4063.59 & \text { A } & 200\end{array}$

4098.606 A 3000

$\begin{array}{rrr}-4130.372 & \text { A } 3000 \\ 4217.195 & \text { A } & 500\end{array}$

$\begin{array}{lll}4217.195 & \text { A } & 500 \\ 4098.900 & \text { A } & 400\end{array}$

$\begin{array}{rrr}4045.148 & \text { A } & 100 \\ 3983.008 & \text { A } & 80\end{array}$

4037.332 A 1500

4132.275 A 2000

4001.257 A 1200

3959.436 A 300 ?

$\begin{array}{lll}4070.288 & \text { A } & 600 \\ 3994.165 & \text { A } & 800 \\ 3971.754 & \text { A } & 300\end{array}$
$0.60 \quad 4.75 \quad 5 \frac{1}{2}-5 \frac{1}{2} \mathrm{a}^{8} \mathrm{D}^{\circ}-9$

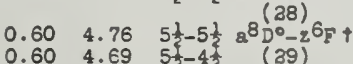

$0.43 \quad 3.16 \quad 3 \frac{1}{2}-4 \frac{1}{2} \mathrm{x}^{8} \mathrm{~s}^{\circ}-1$

$\begin{array}{llll}0.43 & 3.27 & 3 \frac{1}{3}-4 \frac{1}{2} & 8^{8} \mathrm{~S}^{\circ}-210_{\mathrm{P}} \\ 0.43 & 3.17 & 3 \frac{1}{2}-3 \frac{1}{2} & (31)\end{array}$

$0.43 \quad 3.35 \quad 3 \frac{1}{2}-4 \frac{1}{3} 8^{8} \mathrm{~S}^{0}-2^{10} \mathrm{~F}$

$0.43 \quad 3.25 \quad 3 \frac{1}{2}-4 \frac{1}{2} 8^{8} 5^{0}-3$

$\begin{array}{llll}0.43 & 3.46 & 3 \frac{1}{3}-3 \frac{1}{2} & 8^{8} \mathrm{~S}^{\circ}-2^{8} \mathrm{P} \uparrow \\ 0.43 & 3.37 & 3 \frac{3}{2}-3 \frac{3}{2} & (34)\end{array}$

$0.43 \quad 3.38 \quad 3 \frac{1}{2}-3 \frac{1}{2} \mathrm{a}^{8} \mathrm{~S}^{0}-3$

$0.43 \quad 3.60 \quad 3 \frac{1}{2}-2 \frac{1}{2} \mathrm{a}^{8} \mathrm{~S}^{\circ}-2^{(35)} 10_{\mathrm{D}} \uparrow$

$0.43 \quad 3.71 \quad 31-4 \frac{1}{3} \mathrm{a}^{8} \mathrm{~S}^{(36)}-2^{8} \mathrm{D}$

$\begin{array}{lll}0.43 & 3.70 & 3 \frac{1}{2}-3 \frac{1}{2} \\ 0.43 & 3.70 & 3 \frac{3}{2}-2 \frac{1}{2}\end{array}$

$0.43 \quad 3.943 \frac{1}{2}-3 \frac{1}{2} 8^{8} 8^{\circ}-26 p \uparrow$

$\begin{array}{llll}0.43 & 4.01 & 3 \frac{1}{2}-3 \frac{1}{2} & \left.a^{8} \mathrm{~S}^{0}-28\right) \\ 0.43 & 3.98 & 3 \frac{3}{2}-2 \frac{1}{2} & (39)\end{array}$

$\begin{array}{llll}0.43 & 3.99 & 3 \frac{1}{2}-4 \frac{1}{2} & a^{8} S^{0}-y^{8} p \\ 0.43 & 3.98 & 3 \frac{1}{3}-3 & (40)\end{array}$

$\begin{array}{lll}0.43 & 3.98 & 3 \frac{1}{2}-3 \\ 0.43 & 3.97 & 3 \frac{1}{2}-2\end{array}$

$\begin{array}{llll}0.43 & 4.15 & 3 \frac{1}{3}-4 \frac{1}{3} & 8 \\ 0.45 & 4.10 & 3 \frac{1}{2}-3 \frac{3}{2} & (41)^{10 P}\end{array}$

$\begin{array}{llll}0.43 & 4.52 & 3 \frac{1}{2}-4 \frac{1}{2} & 8^{8} \mathrm{~S}^{\circ}-y^{8} \mathrm{D} \\ 0.43 & 4.55 & 3 \frac{2}{2}-2 \frac{1}{2} & (42)\end{array}$

$0.66 \quad 3.27 \quad 5 \frac{1}{3}-4 \frac{1}{3} \mathrm{a}^{10_{F^{0}}-2^{10} P_{\uparrow}}$

$0.60 \quad 3.17 \quad 4 \frac{3}{2}-3 \frac{3}{3} \quad(43)$

$\begin{array}{ll}0.66 & 3.5 \\ 0.60 & 3.27\end{array}$

$0.55 \quad 3.17 \quad 3 \frac{1}{2}-3$

$0.82 \quad 3.84 \quad 7 \frac{2}{3}-7 \frac{1}{3} \mathrm{a}^{1 \mathrm{O}^{0}}-\mathrm{z}^{1 \mathrm{O}_{\mathrm{F}}}$

$0.73 \quad 3.52 \quad 65-6 \frac{2}{2} \quad(44)$

$0.60 \quad 3.35 \quad 43-4$

$0.55 \quad 3.28 \quad 31-3 \frac{1}{2}$

$0.82 \quad 3.6272-6$

$0.60 \quad 3.28 \quad 4 \frac{1}{3}-3 \frac{1}{2}$

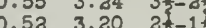

$0.73 \quad 3.84 \quad 6 \frac{1}{2}$

$0.86 \quad 3.62 \quad 5$ - 6

$0.55 \quad 3.35 \quad 3 \frac{1}{3}-4 \frac{3}{3}$

$0.50 \quad 3.24 \quad 1 \frac{1}{2}-2 \frac{1}{2}$

$0.66 \quad 3.25 \quad 5 \frac{1}{2}-4 \frac{1}{2} 8^{10} F^{\circ}-3+$

$0.60 \quad 3.46 \quad 4 \frac{1}{2}-3 \frac{1}{2} \mathrm{a}^{1} \mathrm{C}_{\mathrm{F}}^{(45)}-\mathrm{z}^{\mathrm{P}} \mathrm{P} \uparrow$

$\begin{array}{lll}0.55 & 3.37 & 3=2 \\ 0.55 & 3.46 & 31-3\end{array}$

$\begin{array}{lll}0.52 & 3.37 & 2 \\ 0.55 & 3.81 & 3\end{array}$

$\begin{array}{lll}0.52 & 3.46 & 21-3 \frac{1}{3} \\ 0.50 & 3.37 & 1 \frac{1}{2}-2 \frac{1}{3}\end{array}$

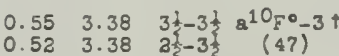

$0.55 \quad 3.53 \quad 3 \frac{1}{2}-2 \frac{1}{2} \mathrm{a}^{10} F^{\circ}-4$

$\begin{array}{llll}0.52 & 3.53 & 2 \frac{1}{3}-2 \frac{1}{3} & 2\end{array}$

$0.82 \quad 3.83 \quad 7 \frac{1}{3}-6 \frac{1}{3} \mathrm{a}^{10} \mathrm{~F}^{\circ}-2^{10}{ }^{0}+$

$0.73 \quad 3.72 \quad 6 \frac{1}{2}-5 \frac{1}{3} \quad(49)$

$0.60 \quad 3.61 \quad 43-3$

$0.55 \quad 3.60 \quad 3 \%-2$

$3.72 \quad 55-5$

$\begin{array}{lll}0.60 & 3.59 & 45 \\ 0.55 & 3.61 & 3\end{array}$

$0.52 \quad 3.60 \quad 31-23$

$0.55 \quad 3.59 \quad 35$

$\begin{array}{lll}0.52 & 3.61 & 21 \\ 0.50 & 3.60 & 1 \frac{1}{2}-2 ?\end{array}$
$0.66 \quad 3.35 \quad 5$. 
94

I Aaboratory Int

4085.564 A 2000 4049.429 A 1200 $\begin{array}{llr}3973.981 & \text { A } & 500 \\ 3923.246 & \text { A } 300\end{array}$ 3895.230 3971.062 3916.61
3881.94

3867.26

3918.236
3875.46

A 100

3709.13 A 50 3576.772 A 25

3591.912 A 30

$\begin{array}{lll}3569.566 & \text { A } & 40 \\ 3567.654 & \text { A } & 40\end{array}$

$\begin{array}{lll}3542.768 & \text { A } & 500 \\ 3558.468 & \text { A } & 250\end{array}$

$\begin{array}{llr}3558.468 & \text { A } & 250 \\ 3544.985 & \text { A } & 60\end{array}$

3593.445 A 60

$\begin{array}{lll}3564.046 & \text { A } & 60 \\ 3554.802 & \text { A }\end{array}$

3466.498 A 150

3193.174 A 200

3137.940

3212.274

3162.764
3108.230

3075.422 A 30

$\begin{array}{lll}3058.119 & \text { A } & 80 \\ 2987.074 & \text { A } & 80\end{array}$

5860.73 A 1000

5010.821 A 400

$\begin{array}{rrr}6049.50 & \text { A } & 80 \\ 5583.68 & \text { A } & 800\end{array}$

$\begin{array}{lll}5956.48 & \text { A } & 200 \\ 5096.063 & \text { A } & 200\end{array}$

4805.817 A 100

$\begin{array}{rlr}5267.322 & \text { A } & 40 \\ 5176.285 & \text { A } & 800\end{array}$

-5469.72 A A 800

$\begin{array}{lll}5728.32 & \text { A } & 60 \\ 5371.621 & \text { A } & 40\end{array}$

$\begin{array}{lll}5644.84 & \text { A } & 300 \\ 5856.96 & \text { A } & 150\end{array}$

$\begin{array}{rrr}5856.96 & \text { A } & 150 \\ 6011.12 & \text { A } & 30\end{array}$

5616.31 A 300

4483.328 A 300

$\begin{array}{lll}4551.455 & \text { A } & 30 \\ 5304.923 & \text { A } & 25\end{array}$

$\begin{array}{lrr}5394.321 & \text { A } & 125 \\ 5470.53 & \text { A } & 50\end{array}$

4453.931 A 60
4711.975 A

$\begin{array}{rrr}5023.133 & \text { A } 200 \\ -5020.3688 \text { A } 80\end{array}$

5062.862 A 150

-4958.788 A 800

$\begin{array}{lll}4573.896 & \text { A } & 30 \\ 5031.562 & \text { A } & 80\end{array}$

$\begin{array}{lll}4878.049 & \text { A } & 30 \\ 4910.838 & \text { A } & 50\end{array}$

$\begin{array}{lll}4910.838 & \text { A } & 50 \\ 4984.905 & \text { A } & 60\end{array}$

4582.53 A 400

$\begin{array}{rrr}4728.468 & \text { A } & 300 \\ 4791.150 & \text { A } & 40\end{array}$

$\begin{array}{lll}4894.30 & \text { A } & 600 \\ 4654.986 & \text { A } & 100\end{array}$

$\begin{array}{lll}4654.986 & \text { A } & 100 \\ 4732.60 & \text { A } & 600\end{array}$

4801.05

4865.02
4786.908

4834.232
4873.339
Low $P_{\text {High }} \quad J \quad \frac{\text { LuIt1plet }}{(\text { No) }}$

I A Laboratory ${ }_{\text {ReP Int Low }}{ }_{\text {High }}$

Gd II continued

$\begin{array}{llll}0.73 & 3.75 & 6 \frac{1}{2}-5 \frac{1}{3} & a^{10} F^{0}-2^{8} D+ \\ 0.66 & 3.71 & 5 \frac{2}{2}-4 \frac{1}{3} & (50)\end{array}$

$\begin{array}{lll}0.66 & 3.71 & 5 \frac{5}{2}-4 \frac{1}{3} \\ 0.60 & 3.70 & 4 \frac{3}{3}-3 \frac{1}{3}\end{array}$

$\begin{array}{lll}0.55 & 3.70 & 3 \frac{1}{3}-2 \frac{1}{2} \\ 0.52 & 3.69 & 2 \frac{3}{2}-1 \frac{1}{2}\end{array}$

$\begin{array}{lll}0.60 & 3.71 & 4 \frac{2}{2}-4 \frac{1}{2} \\ 0.55 & 3.70 & 3 \frac{1}{2}-3 \frac{1}{2}\end{array}$

$\begin{array}{lll}0.52 & 3.70 & 2 \frac{1}{2}-2 \frac{1}{2} \\ 0.50 & 3.69 & 1 \frac{1}{2}-1 \frac{1}{2}\end{array}$

$\begin{array}{lll}0.60 & 3.75 & 4 \frac{1}{2}-5 \frac{1}{3} \\ 0.52 & 3.70 & 3 \frac{3}{2}-3 \frac{1}{2}\end{array}$

$\begin{array}{lll}0.52 & 3.70 & 3 \frac{1}{2}-3 \frac{1}{2} \\ 0.50 & 3.70 & 1 \frac{1}{2}-20 \frac{4}{2}\end{array}$

$0.82 \quad 4.14 \quad 7 \frac{1}{2}-6 \frac{1}{2} a^{10} F^{0}-z^{8} F+$

$\begin{array}{lll}0.50 & 3.95 & 1 \frac{1}{3}- \\ 0.73 & 4.14 & 6 \frac{1}{3}-6 \frac{1}{2}\end{array}$

$\begin{array}{lll}0.73 & 4.14 & 6 \frac{1}{3}-6 \frac{1}{3} \\ 0.60 & 4.03 & 4 \frac{1}{2}-4 \frac{1}{4}\end{array}$

$\begin{array}{lll}0.55 & 4.01 & 3 \frac{1}{2}-3 \frac{1}{2} \\ 0.50 & 3.96 & 1 \frac{1}{2}-1 \frac{1}{2}\end{array}$

$\begin{array}{lll}0.50 & 3.96 & 1 \frac{1}{2}-1 \frac{1}{2} \\ 0.66 & 4.14 & 5 \frac{1}{3}-6 \frac{1}{2}\end{array}$

$\begin{array}{lll}0.60 & 4.07 & 4 \frac{1}{2}-5 \frac{1}{4} \\ 0.55 & 4.03 & 3 \frac{1}{2}-4 \frac{1}{2}\end{array}$

$0.553 .99 \quad 3 \frac{1}{2}-4 \frac{1}{2} a^{10} F^{0}-y^{8} P+$

$\begin{array}{lllll}0.55 & 3.99 & 3 \frac{1}{2}-4 \frac{1}{2} \mathrm{a}^{10} \mathrm{~F}^{0}-\mathrm{y}^{8} \mathrm{P}+ & 3558.189 \\ 0.52 & 3.98 & 2 \frac{1}{2}-3 & 352.709 \\ 0.50 & 3.97 & 1 \frac{1}{2}-2 \frac{2}{2} & (52) & 3608.753 \\ & & 3613.392\end{array}$

$0.664 .225 \frac{1}{2}-5 \frac{1}{2} a^{10} F^{\circ}-y^{10} \mathrm{P} \uparrow$

3625.26
3634.75

$0.66 \quad 4.56 \quad 5 \frac{1}{5}-4 \frac{1}{2} a^{1 \delta_{F}^{0}-y^{8} D+}$

$\begin{array}{lll}0.60 & 4.53 & 4 \frac{1}{2}-3 \\ 0.55 & 4.55 & 3 \frac{1}{2}-2 \\ 0.66 & 4.50 & 5 \frac{1}{2}-5\end{array}$

$\begin{array}{lll}0.66 & 4.50 & 5 \frac{1}{3}-5 \frac{1}{2} \\ 0.60 & 4.50 & 4 \frac{1}{3}-5 \frac{1}{2} \\ 0.55 & 4.52 & 3 \frac{1}{2}-4 \frac{1}{2}\end{array}$

$0.60 \quad 4.66 \quad 4 \frac{1}{2}-3 \frac{1}{2} a^{10} F^{0}-6$

$0.66 \quad 4.67 \quad 5 \frac{1}{2}-4 \frac{1}{2} a^{1}\left(5 F^{\circ}-7 \uparrow\right.$

$\begin{array}{rrr}3650.95 & \text { A } & 100 \\ 3649.44 & \text { A } & 100\end{array}$

$\begin{array}{ccr}3412.583 & \text { A } & 60 \\ -3451.9148 & \text { A } & 35\end{array}$

3388.065 A 40

$\begin{array}{rrr}3336.984 & \text { A } & 200 \\ 3374.584 & \text { A } & 50\end{array}$

$0.66 \quad 4.69 \quad 5 \frac{1}{3}-4 \frac{1}{2} a^{1}(56)-265 \uparrow$

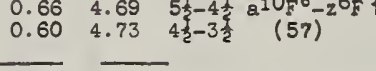

$1.06 \quad 3.16 \quad 5 \frac{1}{2}-4 \frac{1}{2} b^{8} D^{0}-1$

$\begin{array}{lll}1.06 & 3.52 & 5 \frac{1}{2}-5 \frac{1}{2} b^{8} D^{0}-z^{10} P \uparrow\end{array}$

$\begin{array}{lll}1.13 & 3.17 & 3 \frac{1}{3}-3 \\ 1.06 & 3.27 & 5 \frac{1}{3}-4\end{array}$

$\begin{array}{lll}1.10 & 3.17 & 4 \frac{1}{3}-3 \frac{1}{2} \\ 1.10 & 3.52 & 4 \frac{1}{2}-5 \frac{1}{2}\end{array}$

$1.06 \quad 3.62 \quad 5 \frac{1}{2}-6 \frac{1}{3} \mathrm{~b}^{8} \mathrm{D}^{0}-\mathrm{z}^{10} \mathrm{~F}+$

$\begin{array}{llll}1.10 & 3.44 & 4 \frac{1}{2}-5 \frac{1}{3} & (60)\end{array}$

$1.10 \quad 3.35 \quad 43-4$

$\begin{array}{lll}1.06 & 3.35 & 5 \frac{1}{2}-4 \frac{1}{3}\end{array}$

$\begin{array}{lll}1.10 & 3.28 & 4 \frac{1}{3}-3 \frac{1}{2} \\ 1.13 & 3.24 & 3 \frac{1}{5}-2 \frac{1}{3}\end{array}$

$\begin{array}{lll}1.13 & 3.24 & 3 \frac{1}{3}-2 \frac{1}{3} \\ 1.15 & 3.20 & 3 \frac{1}{2}-1 \frac{1}{2}\end{array}$

$1.06 \quad 3.25 \quad 5 \frac{1}{2}-4 \frac{1}{2} b^{8} D^{0}-2$

$\begin{array}{lll}1.06 & 3.81 & 5 \frac{1}{2}-4 \frac{1}{3} b^{8} D^{0}-28 P+\end{array}$

$\begin{array}{lll}1.10 & 3.81 & 4 \frac{1}{2}-4 \frac{1}{3} \\ 1.13 & 3.46 & 3 \frac{1}{2}-3 \frac{1}{2}\end{array}$

$\begin{array}{llll}1.10 & 3.38 & 4 \frac{1}{3}-3 \frac{1}{3} & b^{8} D^{0}-34 \\ 1.13 & 3.38 & 3 & (63)^{4}\end{array}$

$\begin{array}{llll}1.06 & 3.83 & 5 \frac{1}{2}-6 \frac{1}{2} & b^{8} D^{\circ}-2^{10} \mathrm{D} \\ 1.10 & 3.73 & 4 \frac{1}{3}-5 \frac{13}{2} & (64)\end{array}$

$\begin{array}{lll}1.13 & 3.59 & 3 \\ 1.15 & 3.61 & 2 \frac{1}{3}-3 \frac{1}{2}\end{array}$

$1.17 \quad 3.60$ 12 $2 \frac{2}{3}$

$\begin{array}{lll}1.10 & 3.53 & 4 \frac{1}{2}-4 \frac{1}{2}\end{array}$

$\begin{array}{lll}1.13 & 3.61 & 3 \frac{1}{2}-3 \frac{1}{3} \\ 1.15 & 3.60 & 2 \frac{5}{5}-3 \frac{2}{3}\end{array}$

$\begin{array}{lll}1.06 & 3.59 & 5 \frac{1}{2}-4 \frac{1}{2} \\ 1.10 & 3.61 & \frac{4}{2}-3 \frac{1}{3} \\ 1.13 & 3.60 & 3 \frac{1}{2}-2 \frac{1}{2}\end{array}$

$1.06 \quad 3.75 \quad 5 \frac{1}{3}-5 \frac{1}{2} b^{8} D^{0}-z^{8} D+$

$\begin{array}{lll}1.10 & 3.71 & 4 \frac{1}{3}-4 \\ 1.13 & 3.70 & 335\end{array}$

$1.17 \quad 3.69 \quad 1 \frac{1}{2}-1 \frac{1}{2}$

$\begin{array}{lll}1.06 & 3.71 & 5 \frac{1}{3}-4 \frac{1}{2} \\ 1.10 & 3.70 & 4 \frac{1}{3}-3 \frac{1}{2}\end{array}$

$\begin{array}{lll}1.13 & 3.70 & 3 \\ 1.15 & 3.69 & 3 \frac{1}{2}\end{array}$

$\begin{array}{lll}1.15 & 3.69 & 2 \frac{1}{2}-1 \frac{1}{2} \\ 1.13 & 3.71 & 3 \frac{1}{2}-4 \frac{1}{2}\end{array}$

$\begin{array}{lll}1.15 & 3.70 & 2 \frac{2}{2}-3 \frac{1}{2} \\ 1.17 & 3.70 & 1 \frac{2}{2}-2 \frac{2}{2}\end{array}$
3333.133 A 1000

$\begin{array}{rrr}3332.133 & \text { A } & \\ 3426.342 & \text { A } & 50 \\ 3413.030 & \text { A } & 300\end{array}$

$\begin{array}{lll}3413.030 & \text { A } & 300 \\ 3403.081 & \text { A } 150\end{array}$

3391.294 A 150
3390.498 A

3388.912

3460.776

3300.976

$\begin{array}{rrr}3300.976 & \text { A } & 60 \\ 3292.310 & \text { A } & 800 \\ 3329.345 & \text { A } & 400\end{array}$

3320.438 A 50

3350.097 A 400

3226.318 A 1000

3236.106 A 150

3249.747

3263.373

.3263 .515

3389.150

3102.551 A 1000

$\begin{array}{lll}3145.516 & \text { A } & 800 \\ 3146.878 & \text { A } & 250 \\ 3142.900 & \text { A } & 300\end{array}$

3142.900
3101.407

3120.181

3128.560 A 200

$\begin{array}{lll}3130.812 & \text { A } & 300 \\ 3129.955 & \text { A } & 100\end{array}$

$\begin{array}{llr}3129.955 & \text { A } & 100 \\ 3117.974 & \text { A } & 40 \\ 3131.760 & \text { A } & 80\end{array}$

3975.110 A 30

$\begin{array}{llr}2973.17 & \text { A } & 100 \\ 2985.531 & \text { A } & 100\end{array}$

$\begin{array}{llr}2985.531 & \text { A } & 100 \\ 3983.060 & \text { A } & 60 \\ 3002.197 & \text { A } & 50\end{array}$

3002.71

5586.16

\begin{tabular}{ll} 
A & 50 \\
A & 60 \\
\hline
\end{tabular}

5871.81

A 100

$\begin{array}{llr}5303.43 & \text { A } & 30 \\ 5330.297 & \text { A } & 300\end{array}$

$\begin{array}{rrr}5019.361 & \text { A } & 100 \\ 5107.406 & \text { A } & 60\end{array}$ $\begin{array}{rrr}3390.878 & \text { A } & 200 \\ -3369.618 & \text { A } & 400\end{array}$

$\begin{array}{lll}3133.852 & \text { A } 1000 \\ 3145.516 & \text { A } & \end{array}$
$1.15 \quad 3.94 \quad 3 \frac{1}{2}-3 \frac{1}{2} b^{8} D^{0}-2^{6} P+$

$\begin{array}{llll}1.06 & 4.14 & 5 \frac{1}{2}-6 \frac{1}{2} & b^{8} D^{\circ}-2^{8} \\ 1.10 & 4.07 & 4 \frac{1}{3}-5 \frac{1}{4} & (67)\end{array}$

$\begin{array}{lll}1.13 & 4.03 & 3 \frac{1}{5}-4 \frac{1}{5} \\ 1.15 & 4.01 & 3 \frac{1}{3}-3\end{array}$

$\begin{array}{lll}1.15 & 4.01 & 3 \frac{1}{3}-3 \frac{1}{2} \\ 1.17 & 3.98 & 1 \frac{2}{2}-3 \frac{1}{2}\end{array}$

$1.15 \quad 3.98$ 23 -2

$\begin{array}{lll}1.17 & 3.96 & 1 \frac{1}{3}-1 \frac{1}{2} \\ 1.15 & 3.96 & 3 \frac{1}{3}-1 \frac{1}{2}\end{array}$

$\begin{array}{llll}1.10 & 3.99 & 4 \frac{1}{2}-4 \frac{1}{2} & b^{8} D^{0}-r^{8} P+ \\ 1.13 & 3.98 & 3 \frac{1}{2}-3 \frac{1}{2} & (68)\end{array}$

$\begin{array}{lll}1.15 & 3.97 & 3 \frac{1}{3}-2 \frac{1}{2} \\ 1.13 & 3.99 & 3 \frac{1}{3}-4 \frac{1}{2}\end{array}$

1.153 .98 3र $3 \frac{2}{2}$

$1.06 \quad 4.50 \quad 5 \frac{1}{5}-5 \frac{1}{5} b^{8} D^{0}-y^{8} D$

$\begin{array}{llll}1.06 & 4.50 & 5 & 5 \\ 1.10 & 4.52 & 4 \frac{1}{2}-4 \frac{1}{3} & (69)\end{array}$

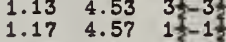

$1.064 .52 \quad 52-4$

$1.10 \quad 4.53 \quad 42-3$

$\begin{array}{lll}1.13 & 4.55 & 32-3 \\ 1.15 & 4.57 & 25-1\end{array}$

$1.104 .50 \quad 4 \frac{2}{2}$

$\begin{array}{lll}1.13 & 4.52 & 3 \\ 1.15 & 4.53 & 3 \%-3 \frac{1}{2} \\ 1.17 & 4.55 & 1 \frac{1}{2}-3 \frac{1}{2}\end{array}$

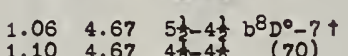

$1.06 \quad 4.70 \quad 5 \frac{1}{2}-4 \frac{1}{2} \quad b^{8} D^{0}-8+$

$\begin{array}{rrrr}1.06 & 4.75 & 5 \frac{1}{2}-5 \frac{1}{1} & 8000 \\ 1.10 & 4.75 & 4 \frac{1}{2}-5 \frac{1}{2} & (73)\end{array}$

$1.06 \quad 4.76 \quad 5 \frac{1}{2}-5 \frac{1}{3} b^{8} D^{0}-z^{6} F+$

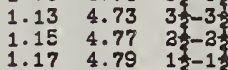

$\begin{array}{lll}1.17 & 4.79 & 1 \frac{1}{2}-1 \\ 1.06 & 4.69 & 5\end{array}$

$\begin{array}{lll}1.13 & 4.77 & 3 \frac{1}{2}-3 \\ 1.15 & 4.79 & 3 \frac{1}{2}-1\end{array}$

1.174 .8

$\begin{array}{lll}1.10 & 4.76 & 4 \frac{1}{2}-5 \frac{1}{2} \\ 1.13 & 4.69 & 3 \frac{3}{2}-4 \frac{1}{2}\end{array}$

$\begin{array}{cccc}1.10 & 4.84 & 4 \frac{1}{3}-3 \frac{1}{\frac{1}{2}} & b^{8} D^{0}-x^{8} P+ \\ 1.10 & 4.85 & 4 \frac{1}{2}-4 \frac{1}{2} & (74)\end{array}$

$\begin{array}{lll}1.10 & 4.85 & 45-4 \\ 1.13 & 4.84 & 35-3 \frac{1}{2}\end{array}$

$\begin{array}{lll}1.15 & 4.82 & 32 \\ 1.13 & 4.85 & 3 \frac{1}{2}-4\end{array}$

$\begin{array}{lll}1.15 & 4.84 & 3 \frac{2}{2}-3 \frac{1}{2} \\ 1.17 & 4.82 & 1 \frac{1}{2}-3 \frac{1}{2}\end{array}$

$\begin{array}{llll}1.06 & 4.88 & 5 \frac{1}{3}-4 \frac{1}{2} & b^{8} D^{0}-z^{6} D+ \\ 1.10 & 4.91 & 4 \frac{1}{3}-3 \frac{1}{2} & (75)\end{array}$

$\begin{array}{lll}1.10 & 4.91 & 4 \frac{1}{2}-3 \\ 1.13 & 4.93 & 3 \frac{1}{5}-3 \\ 1.15 & 4.95 & 3 \frac{1}{2}-1\end{array}$

$\begin{array}{ll}1.15 & 4.95 \\ 1.10 & 4.88\end{array}$

1.104 .88 4t-4

$\begin{array}{lll}1.15 & 4.93 & 2 \frac{1}{2}-2 \frac{1}{2} \\ 1.13 & 4.88 & 3 \frac{1}{2}-4\end{array}$

$1.06 \quad 5.03 \quad 5 \frac{1}{2}-6 \frac{1}{3} b^{8} D^{0}-y^{8} F+$

$\begin{array}{lll}1.10 & 5.03 & 4 \frac{1}{2}-5 \\ 1.13 & 5.05 & 33-4\end{array}$

$1.15 \quad 5.07 \quad 3 \%$

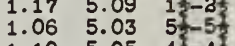

$\begin{array}{lll}1.10 & 5.05 & 4 \\ 1.13 & 5.07 & 3\end{array}$

$1.15 \quad 5.09$ 32-35

$\begin{array}{lll}1.17 & 5.11 & 1 \\ 1.15 & 5.11 & 3 \\ 1.17 & 5.12 & 1\end{array}$

$1.13 \quad 5.38 \quad 3 \frac{1}{2}-3 \frac{1}{3} b^{8} D^{0}-y^{6} P$

$\begin{array}{llll}1.13 & 5.38 & 3 \frac{1}{2}-3 \frac{3}{3} & b^{8} D^{0}-y^{6} \\ 1.15 & 5.30 & 3 \frac{1}{3}-1 \frac{1}{5} & (77)\end{array}$

$\begin{array}{lll}1.13 & 5.36 & 3 \\ 1.17 & 5.30 & 1\end{array}$

$\begin{array}{lll}1.15 & 5.36 & 3 \frac{1}{2}-3 \frac{1}{2} \\ 1.17 & 5.38 & 1 \frac{1}{2}-3 \frac{1}{2}\end{array}$

1.175.

$1.25 \quad 3.46 \quad 4 \frac{1}{2}-3 \frac{1}{2} a^{6} D^{0}-z^{8} p$

$1.38 \quad 3.38 \quad 3 \frac{1}{2}-3 \frac{1}{2} a^{6} D^{\circ}-3+$

$\begin{array}{llll}1.38 & 3.61 & 3 \frac{1}{3}-3 \frac{1}{2} & a^{6} \mathrm{D}^{\circ}-\mathrm{z}^{10} \mathrm{D}+ \\ 1.31 & 3.61 & 3 \frac{2}{2}-3 \frac{1}{2} & (80)\end{array}$

$\begin{array}{llll}1.25 & 3.70 & 4 \frac{1}{2}-3 \frac{1}{2} & a^{6} D^{\circ}-z^{8} D \dagger \\ 1.38 & 3.70 & 3 \frac{1}{2}-3 \frac{1}{2} & (81)\end{array}$
I A Laboratory Int

Low ${ }^{\text {P }}$ High

J Inutiplet

Gd II continued

4582.38
4471.29

4433.635
4646.336

4520.070
4467.327

4467.327
4554.989

4488.401

4374.343
4463.247

4463.247
4721.273

A
A
200
A

$\begin{array}{llll}1.35 & 3.94 & 4 \frac{1}{3}-3 \frac{1}{2} & a^{6} D^{0}-z^{6} P \uparrow \\ 1.28 & 4.04 & 3 \frac{1}{2}-2 \frac{1}{2} & (82)\end{array}$

4570.977

4509.083

3791.17
3807.65

3767.65
3754.60

3641.39

3613.490

3517.890

3512.496

3617.164

3610.76
3592.709

3580.618
3579.549

3567.116

3553.716

3428.467

3464.133
3503.206

3395.130

3402.072
3407.56

3413.373
3417.330

3367.661

3379.756
3393.630

3405.038

3430.238

3437.362
3435.624

3357.073

3274.183

3282.305

3279.529

3242.304

3264.137

3270.515

32.

3250.187

3259.250

3073.565

3089.954

3093.058

3101.185
3113.173

3108.360

3108.360
3129.696

3118.600

5877.36

6634.36

6681.33
6846.60

5733.86

5597.31

5951.60
6106.19

$\begin{array}{lll}6727.83 & \text { A } & 125 \\ 6346.65 & \text { A } & 400\end{array}$

5164.543 A 150

$\begin{array}{lll}5987.11 & \text { A } & 150 \\ 5749.41 & \text { A } & 500\end{array}$

5545.01 A 250

$1.314 .10 \quad 3 \frac{5}{3}-1$

$\begin{array}{lll}1.28 & 3.94 & 3 \frac{5}{2}-3 \\ 1.31 & 4.04 & 3 \frac{1}{3}-2 \frac{1}{4}\end{array}$

$\begin{array}{lll}1.33 & 4.10 & 1 \\ 1.33 & 4.04 & 1 \frac{1}{2}-3\end{array}$

$1.35 \quad 4.10 \quad \frac{1}{2}-1 \frac{1}{2}$

$1.25 \quad 4.07 \quad 4 \frac{1}{2}-5 \frac{1}{2} a^{6} D^{0}-2^{8} F+$

$\begin{array}{lll}1.35 & 4.01 & 4 \frac{1}{2}-3 \frac{1}{2} \\ 1.33 & 3.95 & 1 \frac{1}{2}-\frac{1}{2}\end{array}$

$1.38 \quad 3.98 \quad 3 \frac{1}{2}-3 \frac{1}{2} a^{6} D^{0}-p^{8} p+$

$1.35 \quad 4.50 \quad 4 \frac{1}{3}-5 \frac{1}{2} a^{6} D^{0}-Y^{8} D+$

$\begin{array}{lll}1.38 & 4.52 & 3 \frac{1}{2}-4 \frac{1}{2} \\ 1.35 & 4.53 & 4 \frac{1}{2}-4 \frac{1}{5}\end{array}$

$\begin{array}{lll}1.35 & 4.53 & 42 \\ 1.25 & 4.53 & 4 \frac{1}{2}-3 \frac{2}{2}\end{array}$

$1.38 \quad 4.67 \quad 3 \frac{1}{2}-4 \frac{1}{2} a^{6} D^{0}-7 \dagger$

$1.38 \quad 4.70 \quad 3 \frac{1}{2}-4 \frac{1}{2} a^{6} D^{\circ}-8$

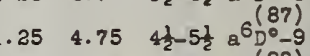




\section{REVISED 4 ULTI PLET TA BLE}

I A ${ }_{\text {Gaboratory }}^{\text {La II continued }}$

5135.56 A 400

$\begin{array}{ccc}5253.144^{8} & \text { A } & 500 \\ 5419.876^{-} & \text {A } & 150\end{array}$

5372.316 A 300

$\begin{array}{lll}5560.69 & \text { A } & 600 \\ 5500.43 & \text { A } & 600 \\ 5375.393 & \text { A } & 100\end{array}$

$\begin{array}{lll}5393.659 & \text { A } & 100 \\ 5179.919 & \text { A } & 125\end{array}$

.4668 .4488 A 40

4803.536 A 80

$\begin{array}{rrr}4716.576 & \text { A } & 30 \\ -4639.001 & \text { A } & 200\end{array}$

4406.67 A 400

$\begin{array}{lll}4421.24 & \text { A } & 200 \\ -4522.82 \text { B } & \text { A } & 250\end{array}$

$\begin{array}{ccc}4522.82{ }^{8} & \text { A } & 250 \\ 4514.505^{4} & \text { A } & 200 \\ 4325.560^{2} & \text { A } & 300\end{array}$

$\begin{array}{lll}4325.566 & \text { A } & 200 \\ 4347.310 & \text { A } & 400\end{array}$

4003.850 A 30

3748.88 A 50

3489.381 A 40

3414.307 A 60

8610.04 A 80

6480.11 A 300

$\begin{array}{lll}6480.11 & \text { A } & 300 \\ 7173.26 & \text { A } 600\end{array}$

$\begin{array}{rrr}7252.70 & \text { A } & 400 \\ 7394.90 & \text { A } & 150 \\ 7505.35 & \text { A } & 80\end{array}$

$\begin{array}{rrr}5731.99 & \text { A } & 200 \\ 8704.18 & \text { A } & 60\end{array}$

$\begin{array}{lll}8704.18 & \text { A } & 60 \\ 6623.38 & \text { A } & 50\end{array}$

$\begin{array}{rrr}6260.31 & \text { A } \\ 6180.42 & \text { B } \\ 6 & 300\end{array}$

$\begin{array}{ccc}6180.42 & \text { A } & 300 \\ 6380.95 & \text { A } & 600\end{array}$

$\begin{array}{lll}6080.65 & \text { A } 300\end{array}$

6004.5

5855.24

5845.71
5884.59

5897.62

5840.47

5815.85

5801.30

5807.05

-4881.935

5093.351

5108.91

5098.38

5175.839

5256.030
5178.104

5178.104
5187.337

5186.915

4954.035

5050.878

5071.023

5111.930
5156.76

5310.488

5191.081

5199.211
5160.896

5180.105

5149.841

5130.38
5140.839

4936.155

4808.165
4893.11

4436. 335

4296.30
4329.803

4339.803
4173.558

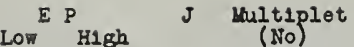

I A Laboratory Ref Int

Low ${ }^{\text {P }}$ High

Sultiplet

Gd II continued

$1.43 \quad 3.83 \quad 5 k-6 \frac{1}{2} a^{10} \mathrm{PO}^{2}-\mathrm{z}^{10} \mathrm{D}+4341.376$ A 80

$\begin{array}{rrrrrrr}1.37 & 3.72 & 4 \frac{1}{2}-5 \frac{1}{2} & (99) & 4197.069 & \text { A } & 150 \\ 1.31 & 3.59 & 3 \frac{1}{3}-4 \frac{1}{2} & & 4153.510 & \text { A } & 135 \\ 1.42 & 3.72 & 5 \frac{1}{2}-5 \frac{1}{3} & & 4115.376 & \text { A } & 80\end{array}$

$\begin{array}{lll}1.37 & 3.59 & 4 \frac{1}{2}-4 \frac{1}{2} \\ 1.37 & 3.61 & 4 \frac{1}{3}-3 \frac{1}{3}\end{array}$

$\begin{array}{lll}1.37 & 3.61 & 4 \frac{1}{2}-3 \frac{1}{3} \\ 1.31 & 3.60 & 3 \frac{1}{2}-3 \frac{1}{2}\end{array}$

$\begin{array}{llll}1.42 & 3.71 & 5 \frac{1}{3}-4 \frac{1}{3} & \mathrm{a}^{10} \mathrm{P}^{\circ}-\mathrm{z}^{8} \mathrm{D} \uparrow \\ 1.37 & 3.75 & 4 \frac{1}{2}-5 \frac{1}{2}\end{array}$

$1.374 .01 \quad 4 \frac{1}{2}-3 \frac{1}{2} a^{10} p^{0}-2^{8} F+$

$1.43 \quad 3.99 \quad 5 \frac{1}{3}-4 \frac{1}{3}$ a $\left(10 \mathrm{P}^{\circ}-\mathrm{y}^{8} \mathrm{P} 4\right.$

$\begin{array}{llll}1.37 & 3.98 & 4 \frac{1}{3}-3 \frac{1}{2} & (103) \\ 1.31 & 3.97 & 3 \frac{1}{2}-3 \frac{3}{2}\end{array}$

$1.42 \quad 4.325 \frac{1}{2}-5 \frac{1}{2} \mathrm{a}^{10} \mathrm{po}^{\circ}-\mathrm{y}^{10} \mathrm{p}+$ $1.31 \quad 4.10 \quad 3 \frac{1}{3}-3 \frac{1}{2} \quad(103)$

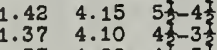

$\begin{array}{lll}1.37 & 4.22 & 4 \frac{1}{5}-5 \frac{1}{2} \\ 1.31 & 4.15 & 3 \frac{2}{2}-4 \frac{1}{2}\end{array}$

$1.424 .50 \quad 5 \frac{1}{2}-5 \frac{1}{2} \mathrm{a}^{10} \mathrm{P}^{\circ}-\mathrm{y}^{8} \mathrm{D}+$

$1.374 .88 \quad 4 \frac{1}{2}-3 \frac{1}{2} \mathrm{a}^{(10 \mathrm{Po}-6}$

$1.314 .853 \frac{1}{2}-4 \frac{1}{2} \mathrm{a}^{1}(105) \mathrm{P}-\mathrm{x}^{8 \mathrm{P}}$

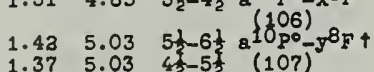

$1.853 .535 \frac{1}{2}-5 \frac{1}{2} \mathrm{a}^{8} \mathrm{~F}^{0}-\mathrm{z}^{10 \mathrm{P} \uparrow}$

$1.73 \quad 3.83 \quad 6 \frac{1}{2}-6 \frac{1}{2} a^{8}(108)-210 F+$

$1.73 \quad 3.44 \quad 6 \frac{1}{3}-5 \frac{1}{3} \quad(109)$

$\begin{array}{lll}1.65 & 3.35 & 5 \frac{1}{2}-4 \frac{1}{2} \\ 1.61 & 3.38 & 4 \frac{1}{2}-3 \frac{1}{2} \\ 1.59 & 3.34 & 3 \frac{3}{2}-3 \frac{2}{2}\end{array}$

$1.65 \quad 3.81 \quad 5 \frac{1}{3}-4 \frac{1}{3} a^{8} F^{0}-z^{8} p+$

$\begin{array}{llll}1.81 & 3.46 & 4 & 3 \\ 1.59 & 3.48 & 3 & (110)\end{array}$

$1.61 \quad 3.59 \quad 4 \frac{1}{2}-4 \frac{1}{3} 8^{8} F^{0}-z^{10 D}$

$\begin{array}{llll}1.61 & 3.59 & 4 & 4 \\ 1.72 & 3.72 & 6 \frac{1}{2}-5 \frac{2}{2} & (111) \\ 1.65 & 3.59 & 5 \frac{2}{2}-4 \frac{1}{2} & \end{array}$

$\begin{array}{llll}1.73 & 3.75 & 6 \frac{1}{2}-5 \frac{1}{2} & a^{8} F^{0}-z^{8} \mathrm{D} \\ 1.65 & 3.71 & 5 \frac{2}{2}-4 \frac{2}{2} & (113)\end{array}$

$\begin{array}{ll}1.65 & 3.71 \\ 1.61 & 3.70\end{array}$

$1.59 \quad 3.70 \quad 3 \frac{1}{2}-2 \frac{1}{2}$

$\begin{array}{lll}1.58 & 3.59 & 3 \frac{1}{2}-1 \frac{1}{2} \\ 1.65 & 3.75 & 5 \frac{5}{3}-5 \frac{1}{3}\end{array}$

$1.61 \quad 3.71 \quad 5 \frac{5}{2}-\frac{4}{3}$

1.58 3.70

$1.57 \quad 3.69$

$1.58 \quad 3.69$

$1.57 \quad 4.10 \quad 1 \frac{1}{2}-1 \frac{1}{2} 98^{8} F^{\circ}-z^{6}{ }^{6}+$

$1.734 .14 \quad 8 \frac{1}{5}-6 \frac{1}{2}$ a $87^{\circ}-285+$

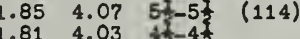

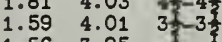

$\begin{array}{lll}1.56 & 3.95 & 3- \\ 1.73 & 4.07 & 8\end{array}$

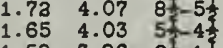

$1.58 \quad 3.96$ at $-1 \frac{1}{2}$

$\begin{array}{lll}1.57 & 3.95 & 1 \frac{1}{2}-6 \frac{1}{2} \\ 1.65 & 4.14 & 5\end{array}$

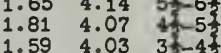

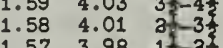

$\begin{array}{lll}1.57 & 3.98 & 1 \frac{2}{2} \\ 1.56 & 3.96 & -1 \frac{2}{2}\end{array}$

$1.61 \quad 3.98 \quad 41-3 \frac{1}{3} a^{8} 5^{\circ}-5^{8}+$

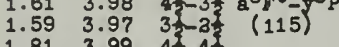

$\begin{array}{lll}1.81 & 3.99 & 4 \frac{1}{2}-4 \frac{1}{2} \\ 1.59 & 3.98 & 3 \frac{1}{2}-3 \frac{1}{3}\end{array}$

$1.58 \quad 3.97 \quad 3 \frac{2}{2}-2 \frac{7}{2}$

$\begin{array}{lll}1.59 & 3.99 & 3-4 \\ 1.58 & 3.98 & 3\end{array}$

$\begin{array}{lll}1.58 & 3.98 & 3 \\ 1.57 & 3.97 & 1 \frac{5}{2}-3 \frac{1}{2}\end{array}$

$1.73 \quad 4.33 \quad 8 \frac{1}{2}-5 \frac{1}{8} a^{8} F^{\circ}-7^{10} P+$

$\begin{array}{llll}1.65 & 4.23 & 5 & 5 \frac{1}{2} \\ 1.58 & 4.10 & 31 & (118)\end{array}$

$\begin{array}{lll}1.73 & 4.50 & 61-5 \frac{1}{2} a^{8} F^{0}-Y^{8} D t \\ 1.85 & 4.53 & \left.5-4 \frac{7}{117}\right)\end{array}$

$\begin{array}{lll}1.85 & 4.53 & 5 \\ 1.81 & 4.53 & 4\end{array}$

$\begin{array}{lll}1.59 & 4.55 & 3 \\ 1.58 & 4.57 & 3\end{array}$ $\begin{array}{llll}1.61 & 4.53 & 4 \frac{1}{2}-4 \frac{1}{3} & a^{8} F^{\circ}-y^{8} D+\end{array}$

$\begin{array}{lll}1.57 & 4.57 & 1 \frac{2}{2}-1 \frac{1}{2} \\ 1.57 & 4.55 & 1 \frac{2}{2}-2 \frac{1}{2}\end{array}$

$\begin{array}{lll}1.57 & 4.55 & 1 \frac{1}{2}-2 \frac{1}{2} \\ 1.56 & 4.57 & \frac{1}{2}-1 \frac{1}{2}\end{array}$

$\begin{array}{lll}4141.017 & \text { A } & 35 \\ 4108.401 & \text { A } & 50\end{array}$

4059.370 A 80

3732.068 A 100

- -

$1.73 \quad 4.76 \quad 6 \frac{1}{2}-5 \frac{1}{2} 8^{8} F^{\circ}-2^{6} F+$

$1.735 .036 \frac{1}{2}-5 \frac{1}{2} a(118)-y^{8}+1$
$(119)$

$2.19 \quad 3.75 \quad 5 \frac{1}{2}-5 \frac{1}{2} \quad c^{8} D^{0}-2^{8} D+$

$\begin{array}{llll}3.19 & 4.14 & 5 \frac{1}{3}-6 \frac{1}{2} & \mathrm{c}^{8 D^{\circ}-Z^{8}}+\uparrow \\ 2.37 & 3.98 & 1 \frac{1}{2}-3 \frac{13}{3} & (131)\end{array}$

$\begin{array}{lll}2.37 & 3.98 & 1 \frac{1}{2}-2 \frac{1}{2} \\ 3.19 & 4.07 & 5 \frac{1}{2}-5 \frac{2}{2} \\ 3.23 & 3.98 & 2 \frac{1}{2}-3 \frac{1}{2}\end{array}$

$3.19 \quad 3.99 \quad 5 \frac{1}{2}-4 \frac{1}{2} 0^{8} D^{0}-Y^{8} P$

$\begin{array}{llll}2.30 & 3.98 & 4 \frac{1}{2}-3 \frac{1}{2} & (133) \\ 3.31 & 3.97 & 3 \frac{1}{3}-3 \frac{2}{3} & \end{array}$

$\begin{array}{lll}3.21 & 3.97 & 3 t-2 \frac{1}{2} \\ 3.20 & 3.99 & 4.3-4 \frac{1}{3}\end{array}$

$\begin{array}{lll}3.31 & 3.98 & 3 \frac{1}{2}-3 \frac{1}{2} \\ 3.32 & 3.97 & 3 \frac{2}{2}-3 \frac{3}{2}\end{array}$

$\begin{array}{llll}3.19 & 4.15 & 5 \frac{1}{2}-4 \frac{1}{2} & c^{8} D^{0}-y^{10} \mathrm{P} \\ 3.20 & 4.10 & 4 \frac{2}{2}-3 \frac{1}{2} & (123)\end{array}$

$2.19 \quad 4.67 \quad 5 \frac{1}{2}-4 \frac{1}{2} \quad c^{8} D^{\circ}-7$

$3.19 \quad 4.70 \quad 5 \frac{1}{2}-4 \frac{1}{2} c^{8}(124)$

$3.19 \quad 4.76 \quad 52-5 \frac{1}{3} c^{(125)} D^{0}-26 F$

$3.31 \quad 4.73 \quad 3 \frac{1}{2}-3 \frac{1}{2} \quad(126)$

$\begin{array}{lll}3.22 & 4.77 & 3 \frac{1}{3}-2 \frac{1}{3} \\ 3.19 & 4.69 & 5 \frac{1}{2}-4 \frac{1}{3} \\ 3.20 & 4.73 & 4 \frac{1}{2}-3 \frac{1}{2}\end{array}$

$2.30 \quad 4.85 \quad 4 \frac{1}{2}-4 \frac{1}{2} c^{8} D^{0}-x^{8} P+$

3.19 $5.035 \frac{1}{2}-6 \frac{1}{2} c^{8 D^{\circ}-y^{8}}+$

$\begin{array}{llll}3.19 & 5.03 & 5 \frac{1}{2}-6 \frac{1}{3} & \mathrm{CD}^{\circ}-\mathrm{y}^{8} \\ 3.19 & 5.03 & 5 \frac{2}{2} & (128)\end{array}$

$\begin{array}{lll}3.21 & 5.07 & 3 \frac{1}{2}-3 \frac{1}{2} \\ 3.22 & 5.09 & 2 \frac{2}{2}-2 \frac{1}{2}\end{array}$

$3.19 \quad 6.08 \quad 5 \frac{1}{5}-4 \frac{1}{3} c^{8} D^{0}-w^{8} P+$

$\begin{array}{lllll}2.20 & 6.09 & 4 & -3 \frac{1}{2} & (129)\end{array}$

$\begin{array}{lll}2.20 & 6.06 & 4 \frac{1}{3}-4 \frac{1}{5} \\ 3.31 & 6.09 & 3 \frac{1}{3}-3 \frac{1}{2}\end{array}$

$2.37 \quad 6.13 \quad 1 \frac{1}{2}-3 \frac{1}{2}$

$\begin{array}{llll}2.32 & 4.14 & 7 \frac{1}{2}-6 \frac{1}{2} & a^{8} G^{0}-z^{8} F+ \\ 3.31 & 4.07 & 6 \frac{1}{2}-5 \frac{1}{2} & (130)\end{array}$

$\begin{array}{lll}2.30 & 4.03 & 5 \frac{1}{2}-4 \frac{1}{2} \\ 3.36 & 4.01 & 4 \frac{1}{2}-3 \frac{1}{3}\end{array}$

$\begin{array}{lll}3.26 & 4.01 & 4 \frac{3}{3}-3 \\ 2.34 & 3.98 & 3 \frac{2}{2}-2\end{array}$

$\begin{array}{lll}2.23 & 3.96 & 2 \frac{1}{3}-1 \\ 3.31 & 4.14 & 8 \frac{1}{2}-8 \\ 3.30 & 4.07 & 5\end{array}$

$3.36 \quad 4.03 \quad 42-4$

$3.24 \quad 4.01 \quad 3 \frac{1}{2}-3$

3.3358

$\begin{array}{rrrrrr}7116.77 & \text { A } & 150 & 2.33 & 3.96 & 1 \frac{1}{2}-1 \frac{2}{2} \\ 7146.13 & \text { A } & 40 & 3.32 & 3.95 & \frac{1}{3}- \\ 8703.13 & \text { A } & 40 & 3.30 & 4.14 & 5 \frac{1}{2}-6 \frac{1}{2}\end{array}$

3703.12

7141.17

$3.34 \quad 3.97 \quad 3 \frac{1}{2}-3 \frac{1}{2} a^{8} G^{0}-Y^{8} P+$

$3.314 .50 \quad 6 \frac{1}{2}-5 \frac{1}{3} 8^{8} G^{\circ}-48 D+$

$\begin{array}{rrr}5621.43 & \text { A } & 100 \\ 5510.58 & \text { A } & 80\end{array}$

4998.373

$\begin{array}{rrr}4881.925 & \text { A } & 300 \\ 4831.955 & \text { A } & 60\end{array}$

$\begin{array}{lll}4800.100 & \text { A } & 60 \\ 4772.728 & \text { A } & 30\end{array}$

4755.347 A 30

4540.016 A 300

$\begin{array}{lll}4531.94 & \text { A } & 150 \\ 4486.352 & \text { A } & 100\end{array}$

$\begin{array}{llr}4486.353 & \text { A } & 100 \\ 4517.10 & \text { A } & 30\end{array}$

$\begin{array}{lll}3287.193 & \text { A } & 40 \\ 3252.743 & \text { A } & 30\end{array}$

$3.26 \quad 4.73 \quad 4 \frac{1}{2}-3 \frac{1}{2} a^{8} G^{0}-2^{6}+$

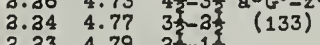

$\begin{array}{lll}2.23 & 4.79 & 2 \frac{1}{2}-1 \frac{1}{2} \\ 3.22 & 4.79 & 1 \frac{2}{2}-1 \frac{2}{2}\end{array}$

4.81

3.32 $4.83 \quad 1 \frac{1}{2}-3 \frac{1}{2} a^{8} G^{0}-x^{8} P+$

$3.325 .03 \quad 7 \frac{1}{2}-6 \frac{1}{2} 8^{8} 6^{\circ}-y^{8} 8+$

$\begin{array}{lll}3.30 & 5.05 & 5 \frac{1}{2}-4 \frac{1}{2} \\ 3.30 & 5.03 & 5 \frac{1}{2}\end{array}$

$\begin{array}{llll}3.30 & 8.08 & 5 \frac{1}{2}-4 \frac{1}{2} & a^{8} G^{0}-n^{8} P+ \\ 3.36 & 6.06 & 4 \frac{1}{2}-4 \frac{1}{2} & (136)\end{array}$

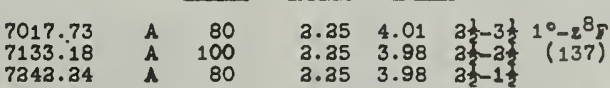

7189.57 A 800

$3.253 .97 \quad 3 \frac{1}{2}-3 \frac{1}{2} 1^{0}-y^{8} \mathrm{p}$

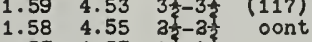

$\begin{array}{llll}3.31 & 5.03 & 8 \frac{2}{2}-5 \frac{1}{2} & a^{8} \sigma^{\circ}-y^{8} \\ 3.30 & 5.05 & 5 & (135)\end{array}$
I A Raboratory Int

Low $^{E \text { PIgh }}$

Nult1plet

od II continued

7385.97 A 80

$5162.47 \quad A \quad 50 d \quad 3.34 \quad 4.73 \quad 2 \frac{1}{2}-3 \frac{1}{2} 3^{0}-26 F+$

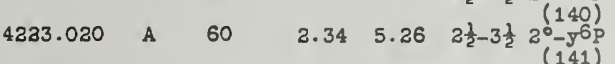

7748.37 A $40 \quad 3.40 \quad 3.99 \quad 4 \frac{1}{2}-4 \frac{1}{2} \quad 3^{\circ}-y^{8 p}+$

$4965.047 \quad$ A $\quad 60 \quad 2.40 \quad 4.88 \quad 4 \frac{1}{2}-4 \frac{1}{2} \quad 3^{\circ}-2^{6}$ D

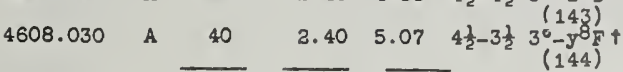

$8089.96 \quad$ A $\quad 60 \quad 3.46 \quad 3.99 \quad 3 \frac{1}{2}-4 \frac{1}{2} 4^{4}-y^{8} p \uparrow$

$5441.17 \quad A \quad 40 \quad 2.46 \quad 4.73 \quad 3 \frac{1}{2}-3 \frac{1}{2} \quad 4^{\circ}-26 \frac{(145)}{8}$

$\begin{array}{lllllll}5178.8438 & \text { A } \quad 100 & 3.46 & 4.85 & 3 \frac{1}{3}-4 \frac{1}{3} & 4^{\circ}-2^{8} \mathrm{p}\end{array}$

4736.735 A 40

$\begin{array}{llllll}3402.072 \quad \text { A } 1000 & 2.46 & 6.09 & 3 \frac{1}{2}-3 \frac{1}{2} & 4^{0}-\pi^{8} P \uparrow\end{array}$

Strongest Unclass1f1ed Lines of Gd II

8443.58

$\begin{array}{rrr}8443.58 & \text { A } & 300 \\ 8316.38 & \text { A } & 500 \\ 7963.35 & \text { A } & 500 \\ 7930.35 & \text { A } & 2000\end{array}$

$\begin{array}{lll}7930.35 & \text { A } 2000 \\ 7846.35 & \text { A } & 3000\end{array}$

7844.87 \& 300

7324.89 A 400

$\begin{array}{lll}7147.31 & \text { A } & 500 \\ 7135.73 & \text { A } & 250 \\ 7037.26 & \text { A } & 600\end{array}$

6985.89 A 1500

6980.86 \& A 250

$\begin{array}{lll}6887.63 & \text { A } & 300 \\ 5913.55 & \text { A } & 800 \\ 5 & & \end{array}$

5911.45 A 500

5754.178 A 250
5538.32

$\begin{array}{lll}5538.32 & \text { A. } & 300 \\ 4397.51 & \text { A } & 300 \\ 4304.895 & \text { A } & 400\end{array}$

$\begin{array}{lll}4304.895 & \text { A } & 400 \\ 4297.173 & \text { A } & 400\end{array}$

4253.612 A 800

$\begin{array}{lll}4238.782 & \text { A } 500 \\ 4197.681 & \text { A } 800\end{array}$

$\begin{array}{lll}4197.681 & \text { A } & 800 \\ 4137.104 & \text { A } & 500\end{array}$

4111.438 A 500

4063.390 A 1500

4062.590 A 500

$\begin{array}{lll}4053.294 & \text { A } 1000 \\ 4049.858 \text { A } & 3000\end{array}$

4023.333 A 300

4013.798 A 350

4008.913 A 400

3895.791 A 400

3842.20 A 400

3801.29

3733.08 A 300

3664.60 A 3000

3457.0478 A 300

3364.241 A 500

$\begin{array}{lll}3330.340 & \text { A } & 800 \\ 3235.460 & \text { A } & 600\end{array}$

3084.007 A 250

3005.09 A 300

$\begin{array}{rrr}963.605 & \text { A } & 1000 \\ & \text { A } & 400\end{array}$

Tb I No analyo1s Kay 1942 (Temperature Class)

To II No analyo1s May 1942 (Temperature Claso) 


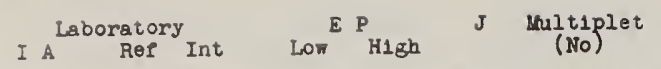

Dy I No analys18 May 1943 (Temperature Class)

Dy II No analy818 May $194 \mathrm{a}$ (Temperature Clas8)

Ho I No analysis May 1943 (Temperature Class)

Ho II No analys18 May 1942 (Temperature Clas8)

Er Not separated May 1943

Tm I P ? Anal D L18t D Jan 1943

\begin{tabular}{|c|c|c|c|c|c|c|}
\hline $\begin{array}{l}4386.434 \\
4359.929 \\
3887.347\end{array}$ & $\begin{array}{l}\mathrm{A} \\
\mathrm{A} \\
\mathrm{A}\end{array}$ & $\begin{array}{l}200 \\
300 \\
200\end{array}$ & $\begin{array}{l}0.00 \\
0.00 \\
0.00\end{array}$ & $\begin{array}{l}2.81 \\
2.83 \\
3.17\end{array}$ & & $\begin{array}{r}8^{2} F^{0}-2 \\
(1) \begin{array}{r}3 \\
5\end{array}\end{array}$ \\
\hline $\begin{array}{l}3949.275 \\
3916.476\end{array}$ & A & $\begin{array}{l}100 \\
200\end{array}$ & $\begin{array}{l}1.08 \\
1.08\end{array}$ & $\begin{array}{l}4.21 \\
4.23\end{array}$ & & $\begin{array}{l}a^{3} F^{0}-15 \\
(2)^{0} 16\end{array}$ \\
\hline Strongest & Unc & $28 s 11$ & Lines & of $\underline{\underline{m} m}$ & & \\
\hline $\begin{array}{l}5971.28 \\
5895.646 \\
5764.300 \\
5675.853 \\
5631.404 \\
5307.121\end{array}$ & $\begin{array}{l}\mathrm{A} \\
\mathrm{A} \\
\mathrm{A} \\
\mathrm{A} \\
\mathrm{A} \\
\mathrm{A}\end{array}$ & $\begin{array}{l}200 \\
300 \\
200 \\
400 \\
150 \\
200\end{array}$ & $\begin{array}{l}I \\
I \\
I \\
I \\
I \\
I\end{array}$ & & & \\
\hline $\begin{array}{l}4203.730 \\
4187.616 \\
4105.843 \\
4094.188\end{array}$ & $\begin{array}{l}\mathrm{A} \\
\mathrm{A} \\
\mathrm{A} \\
\mathrm{A}\end{array}$ & $\begin{array}{l}300 \\
500 \\
600 \\
700\end{array}$ & $\begin{array}{l}\text { I } \\
\text { I } \\
\text { I } \\
\text { I }\end{array}$ & & & \\
\hline $\begin{array}{l}3883.132 \\
3751.812 \\
3744.066 \\
3717.915\end{array}$ & $\begin{array}{l}\mathrm{A} \\
\mathrm{A} \\
\mathrm{A} \\
\mathrm{A}\end{array}$ & $\begin{array}{l}400 \\
100 \\
300 \\
500\end{array}$ & $\begin{array}{l}I \\
I \\
I \\
I\end{array}$ & & & \\
\hline
\end{tabular}

Tri II I P A Anal B L18t $B$ Jan 1942

\begin{tabular}{|c|c|c|c|c|c|c|}
\hline $\begin{array}{l}4526.565 \\
4481.273 \\
4199.918 \\
3958.101 \\
3890.528\end{array}$ & $\begin{array}{l}\text { A } \\
\text { A } \\
\text { A } \\
\text { A } \\
\text { A }\end{array}$ & $\begin{array}{r}80 \\
200 \\
100 \\
200 \\
60\end{array}$ & $\begin{array}{l}0.00 \\
0.00 \\
0.00 \\
0.00 \\
0.00\end{array}$ & $\begin{array}{l}2.67 \\
2.75 \\
2.94 \\
3.12 \\
3.17\end{array}$ & $\begin{array}{l}4- \\
4- \\
4-4 \\
4-4 ? \\
4-\end{array}$ & $\begin{array}{r}a^{3} F^{0}-1 \\
(1) \\
2 \\
3 \\
4 \\
5\end{array}$ \\
\hline $\begin{array}{l}3848.023 \\
3761.913 \\
3761.331 \\
3701.364 \\
3668.088\end{array}$ & $\begin{array}{l}\mathrm{A} \\
\mathrm{A} \\
\mathrm{A} \\
\mathrm{A} \\
\mathrm{A}\end{array}$ & $\begin{array}{r}1000 \\
600 \\
800 \\
250 \\
120\end{array}$ & $\begin{array}{l}0.00 \\
0.00 \\
0.00 \\
0.00 \\
0.00\end{array}$ & $\begin{array}{l}3.21 \\
3.28 \\
3.28 \\
3.33 \\
3.36\end{array}$ & $\begin{array}{l}4- \\
4-4 \\
4-3 \\
4-4 \\
4-4\end{array}$ & $\begin{array}{r}a^{3} F^{0}-6 \\
(2) \frac{7}{7} \\
8 \\
9 \\
10\end{array}$ \\
\hline $\begin{array}{l}3608.766 \\
3536.576 \\
3425.630 \\
3397.499 \\
3291.001\end{array}$ & $\begin{array}{l}\text { A } \\
\text { A } \\
\text { A } \\
\text { A }\end{array}$ & $\begin{array}{r}200 \\
80 \\
150 \\
100 \\
120\end{array}$ & $\begin{array}{l}0.00 \\
0.00 \\
0.00 \\
0.00 \\
0.00\end{array}$ & $\begin{array}{l}3.42 \\
3.49 \\
3.60 \\
3.63 \\
3.75\end{array}$ & $\begin{array}{l}4-3 \\
4-3 \\
4-4 \\
4-3 \\
4-4\end{array}$ & $\begin{array}{r}2^{3} \mathrm{~F}^{0}-11 \\
(3)-12 \\
13 \\
14 \\
15\end{array}$ \\
\hline $\begin{array}{l}3276.811 \\
3258.048 \\
3241.530 \\
3210.825 \\
3133.886\end{array}$ & $\begin{array}{l}\mathrm{A} \\
\mathrm{A} \\
\mathrm{A} \\
\mathrm{A} \\
\mathrm{A}\end{array}$ & $\begin{array}{r}50 \\
150 \\
200 \\
50 \\
250\end{array}$ & $\begin{array}{l}0.00 \\
0.00 \\
0.00 \\
0.00 \\
0.00\end{array}$ & $\begin{array}{l}3.77 \\
3.79 \\
3.81 \\
3.84 \\
3.94\end{array}$ & $\begin{array}{l}4-4 \\
4-3 \\
4-4 \\
4-4 \\
4-4\end{array}$ & $\begin{array}{r}a^{3} F^{0}-16 \\
(4) \\
17 \\
18 \\
20 \\
22\end{array}$ \\
\hline $\begin{array}{l}4677.858 \\
4529.376 \\
4242.153 \\
3995.586 \\
3883.437\end{array}$ & $\begin{array}{l}A \\
A \\
A \\
A \\
A\end{array}$ & $\begin{array}{r}40 \\
80 \\
300 \\
80 \\
200\end{array}$ & $\begin{array}{l}0.03 \\
0.03 \\
0.03 \\
0.03 \\
0.03\end{array}$ & $\begin{array}{l}3.67 \\
3.75 \\
3.94 \\
3.12 \\
3.21\end{array}$ & $\begin{array}{l}3- \\
3- \\
3-4 \\
3-49 \\
3-\end{array}$ & $\begin{array}{r}a^{3} F^{\circ}-1 \\
(5)-\frac{1}{3} \\
3 \\
4 \\
6\end{array}$ \\
\hline $\begin{array}{l}3795.759 \\
3795.169 \\
3734.124 \\
3700.256 \\
3566.472\end{array}$ & $\begin{array}{l}\mathrm{A} \\
\mathrm{A} \\
\mathrm{A} \\
\mathrm{A} \\
\mathrm{A}\end{array}$ & $\begin{array}{r}600 \\
80 \\
300 \\
300 \\
100\end{array}$ & $\begin{array}{l}0.03 \\
0.03 \\
0.03 \\
0.03 \\
0.03\end{array}$ & $\begin{array}{l}3.28 \\
3.28 \\
3.33 \\
3.36 \\
3.49\end{array}$ & $\begin{array}{l}3-4 \\
3-3 \\
3-4 \\
3-4 \\
3-3\end{array}$ & $\begin{array}{r}a^{3} F^{0}-7 \\
(6) 8 \\
9 \\
10 \\
13\end{array}$ \\
\hline $\begin{array}{l}3453.665 \\
3425.082 \\
3316.875 \\
3302.454 \\
3283.400\end{array}$ & $\begin{array}{l}\mathrm{A} \\
\mathrm{A} \\
\mathrm{A} \\
\mathrm{A} \\
\mathrm{A}\end{array}$ & $\begin{array}{r}200 \\
300 \\
60 \\
150 \\
50\end{array}$ & $\begin{array}{l}0.03 \\
0.03 \\
0.03 \\
0.03 \\
0.03\end{array}$ & $\begin{array}{l}3.60 \\
3.63 \\
3.75 \\
3.77 \\
3.79\end{array}$ & $\begin{array}{l}3-4 \\
3-3 \\
3-4 \\
3-4 \\
3-3\end{array}$ & 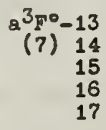 \\
\hline $\begin{array}{l}3266.633 \\
3235.448 \\
3172.828 \\
3157.344 \\
3098.597 \\
3015.296\end{array}$ & $\begin{array}{l}\mathrm{A} \\
\mathrm{A} \\
\mathrm{A} \\
\mathrm{A} \\
\mathrm{A} \\
\mathrm{A}\end{array}$ & $\begin{array}{r}80 \\
90 \\
200 \\
180 \\
100 \\
100\end{array}$ & $\begin{array}{l}0.03 \\
0.03 \\
0.03 \\
0.03 \\
0.03 \\
0.03\end{array}$ & $\begin{array}{l}3.81 \\
3.84 \\
3.92 \\
3.94 \\
4.01 \\
4.12\end{array}$ & $\begin{array}{l}3-4 \\
3-4 \\
3-3 \\
3-4 \\
3-39 \\
3-4\end{array}$ & 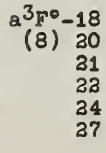 \\
\hline $\begin{array}{l}3900.790 \\
3810.734 \\
3756.860 \\
3704.848\end{array}$ & $\begin{array}{l}\mathrm{A} \\
\mathrm{A} \\
\mathrm{A} \\
\mathrm{A}\end{array}$ & $\begin{array}{r}90 \\
50 \\
100 \\
50\end{array}$ & $\begin{array}{l}1.08 \\
1.08 \\
1.08 \\
1.08\end{array}$ & $\begin{array}{l}4.35 \\
4.32 \\
4.37 \\
4.41\end{array}$ & $\begin{array}{l}2-29 \\
2-3 \\
2- \\
2-3\end{array}$ & $\begin{array}{r}a^{3} \mathrm{~F}^{0}-33 \\
(9) \quad 37 \\
40 \\
41\end{array}$ \\
\hline $\begin{array}{l}3665.812 \\
3653.614 \\
3557.796 \\
3481.750 \\
3285.609\end{array}$ & $\begin{array}{l}\mathrm{A} \\
\mathrm{A} \\
\mathrm{A} \\
\mathrm{A} \\
\mathbf{A}\end{array}$ & $\begin{array}{l}60 \\
80 \\
80 \\
30 \\
60\end{array}$ & $\begin{array}{l}1.08 \\
1.08 \\
1.08 \\
1.08 \\
1.08\end{array}$ & $\begin{array}{l}4.45 \\
4.46 \\
4.55 \\
4.63 \\
4.84\end{array}$ & $\begin{array}{l}2-3 \\
2-3 \\
3-3 \\
2-2 \\
2-2\end{array}$ & $\begin{array}{r}a^{3} \mathrm{~F}^{0}-44 \\
(10) \quad 45 \\
47 \\
48 \\
56\end{array}$ \\
\hline $\begin{array}{l}3929.583 \\
3838.198 \\
3798.753 \\
3783.561 \\
3730.810\end{array}$ & $\begin{array}{l}\mathrm{A} \\
\mathrm{A} \\
\mathrm{A} \\
\mathrm{A} \\
\mathrm{A}\end{array}$ & $\begin{array}{r}100 \\
300 \\
80 \\
60 \\
40\end{array}$ & $\begin{array}{l}1.11 \\
1.11 \\
1.11 \\
1.11 \\
1.11\end{array}$ & $\begin{array}{l}4.25 \\
4.32 \\
4.35 \\
4.37 \\
4.41\end{array}$ & $\begin{array}{l}3-3 ? \\
3-2 \\
3-2 \\
3- \\
3-3\end{array}$ & $\begin{array}{r}a^{1} F^{0}-33 \\
(11) \quad 37 \\
39 \\
40 \\
41\end{array}$ \\
\hline
\end{tabular}

REVISED UULTIPLET TABLE

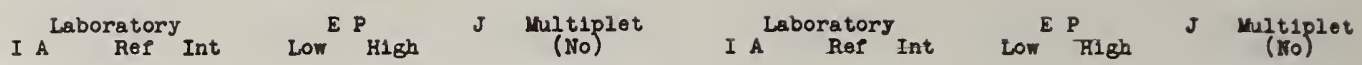
Tm II continued

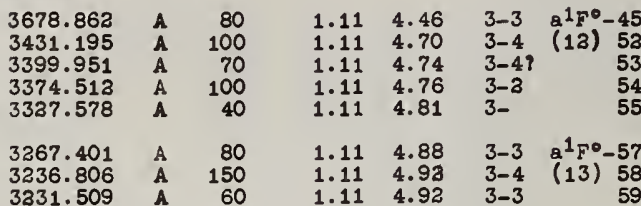

Strongest Unclassiried Lines or Tm II

$\begin{array}{rrrr}5782.356 & \text { B } & 100 & \text { V } \\ 5709.976 & \text { B } & 100 & \text { IV } \\ 4626.565 & \text { B } & 80 & \text { IV } \\ 3996.518 & \text { B } & 200 & \text { III } \\ 3817.395 & \text { B } & 100 & \text { III } \\ 3725.061 & \text { B } & 200 & \text { III } \\ 3535.523 & \text { B } & 100 & \text { III } \\ 3463.198 & \text { B } & 300 & \text { III } \\ 3441.505 & \text { B } & 300 & \text { III } \\ 3363.619 & \text { B } & 300 & \text { III } \\ 3309.804 & \text { B } & 100 & \text { IV } \\ 3240.230 & \text { B } & 125 & \text { IV } \\ 3151.036 & \text { B } & 200 & \text { IV } \\ 3131.257 & \text { B } & 400 & \text { IV }\end{array}$

He I continued

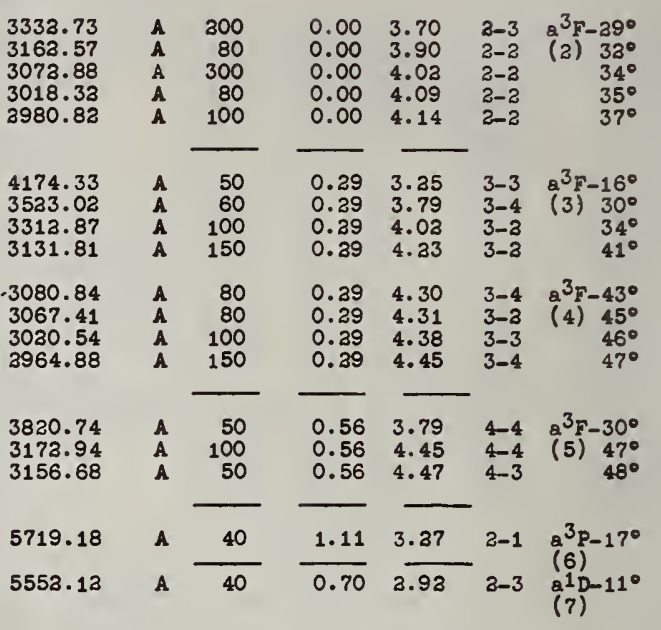

Yb I I P 6.33 Anal B L1st D May 1943

$5556.48 \quad$ A $1500 \quad 0.00 \quad 2.38 \quad 0-1 \quad 6^{1} \mathrm{~S}-6^{3} \mathrm{po}$

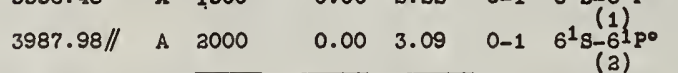
$\begin{array}{rrrrrrrrr}7699.49 & \text { A } & 1500 & & 3.43 & 4.04 & 3-1 & 6^{3} \mathrm{P}^{0}-7^{3} \mathrm{~S} \\ 6799.61 & \mathrm{~A} & 1000 & & 2.32 & 4.04 & 1-1 & (3)^{2} \\ 6489.10 & \mathrm{~A} & 800 & & 3.13 & 4.04 & 0-1 & \end{array}$

Yb II I P 13.05 Anal D L18t D May 1943 $\begin{array}{llrllll}3289.36 & \text { A } & 800 & 0.00 & 3.75 & \frac{1}{1}-1 \frac{1}{3} & 6^{2} \mathrm{~s}-6^{2} \mathrm{po} \\ 3694.19 / / & \text { A } & 1000 & 0.00 & 3.34 & \frac{1}{2}-\frac{2}{2} & (1)\end{array}$

Lu I I P 5? Anal B L1Bt D Kay 1943

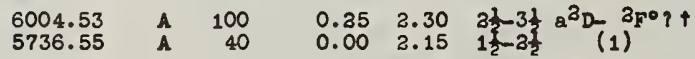

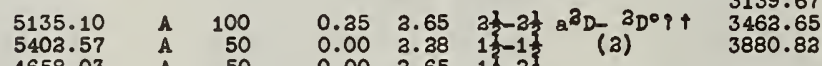
$\begin{array}{lllllll}4658.03 & A & 50 & 0.00 & 3.65 & 1 \frac{1}{2}-2 \frac{1}{2} & 3505.32\end{array}$

$3841.17 \quad$ A $100 \quad 0.25 \quad 3.46 \quad 2 \frac{1}{1}-1 \frac{1}{3} a^{2} \mathrm{D}-3 \mathrm{por}$ $\begin{array}{rrrrrrr}4124.73 & \text { A } & 100 & 0.00 & 3.99 & 1 & 1 \\ 3567.84 & \text { A } & 80 & 0.00 & 3.46 & 1 \frac{1}{2}-1 \frac{1}{2} & (3)\end{array}$

Strongest Unolassified Lines of Lu I

$\begin{array}{rrrr}5001.15 & \text { A } & 100 & \text { III } \\ 4518.58 & \text { A } & 300 & \text { II } \\ 3647.77 & \text { A } & 50 & \text { III }\end{array}$

Lu II I P I Anal A L1st B May 1943

\begin{tabular}{|c|c|c|c|c|c|c|}
\hline 3507.39 & A & 100 & 0.00 & 3.52 & $0-1$ & $a^{1} s-z^{3} p^{0}$ \\
\hline $\begin{array}{l}5476.69 \\
6231.88 \\
6463.11 \\
4994.14 \\
5983.90 \\
4839.62\end{array}$ & $\begin{array}{l}\mathbf{A} \\
\mathbf{A} \\
\mathbf{A} \\
\mathbf{A} \\
\mathbf{A} \\
\mathbf{A}\end{array}$ & $\begin{array}{l}300 \\
300 \\
300 \\
120 \\
100 d \\
30 d\end{array}$ & $\begin{array}{l}1.75 \\
1.54 \\
1.46 \\
1.54 \\
1.46 \\
1.46\end{array}$ & $\begin{array}{l}4.01 \\
3.52 \\
3.37 \\
4.01 \\
3.52 \\
4.01\end{array}$ & $\begin{array}{l}3-3 \\
3-1 \\
1-0 \\
2-3 \\
1-1 \\
1-3\end{array}$ & $\begin{array}{c}a^{3} p-z^{3} p o \\
(a)\end{array}$ \\
\hline 3876.65 & A & 100 & 1.54 & 4.73 & $3-1$ & $a^{3} D-z^{1} p^{0}$ \\
\hline $\begin{array}{l}3077.59 \\
3397.07 \\
3254.32 \\
3473.48\end{array}$ & $\begin{array}{l}\mathbf{A} \\
\mathbf{A} \\
\mathbf{A} \\
\mathbf{A}\end{array}$ & $\begin{array}{r}150 \\
150 \\
90 \\
130\end{array}$ & $\begin{array}{l}1.54 \\
1.46 \\
1.75 \\
1.54\end{array}$ & $\begin{array}{l}5.55 \\
5.09 \\
5.55 \\
5.09\end{array}$ & $\begin{array}{l}2-3 \\
1-2 \\
3-3 \\
2-2\end{array}$ & $\begin{array}{c}a^{3} D_{-2}^{3} F^{\circ}+ \\
(4)^{2}+\end{array}$ \\
\hline 4785.43 & A & 60 & 3.14 & 4.73 & $a-1$ & $a^{1} D-2^{1} p_{0}$ \\
\hline $\begin{array}{l}3623.98 \\
4184.36\end{array}$ & A & $\begin{array}{r}40 \\
130\end{array}$ & $\begin{array}{l}3.14 \\
3.14\end{array}$ & $\begin{array}{l}5.55 \\
5.09\end{array}$ & $\begin{array}{l}2-3 \\
2-2\end{array}$ & $a^{1} a_{(6)}^{3} F^{0}$ \\
\hline 3554.43 & A & 200 & 3.14 & 5.61 & $3-3$ & $\begin{array}{c}a^{1} D-2^{1} D^{\circ} \\
(7)\end{array}$ \\
\hline
\end{tabular}

He I I P A Anal D L1st D Dec 1943

5550.60 5181.86
3777.64 3682.25 3497.49
3472.38

50
40
50
300
150
100

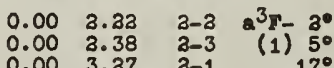
$\begin{array}{lll}0.00 & 3.37 & 2-1 \\ 0.00 & 3.35 & 2-3\end{array}$ $\begin{array}{lll}0.00 & 3.35 & 2-3 \\ 0.00 & 3.53 & 2-3\end{array}$ $\begin{array}{llll}0.00 & 3.53 & 2-3 & 24^{\circ} \\ 0.00 & 3.55 & 2-1 & 35^{\circ}\end{array}$
He II I P 14.8 Anal B List B Nor 1943

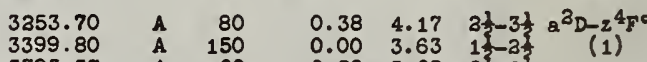

$\begin{array}{rrrrrr}3793.37 & \text { A } & 60 & 0.38 & 3.63 & 3 \frac{3}{2}-2 \frac{1}{2} \\ 3561.65 & \text { A } & 80 & 0.00 & 3.47 & 1 \frac{1}{2}-1 \frac{1}{2}\end{array}$

$3193.53 \quad \mathrm{~A} \quad 40 \quad 0.38 \quad 4.34 \quad 3 \frac{1}{2}-3 \frac{1}{3} \mathrm{a}^{3} \mathrm{D}-\mathrm{z}^{4} \mathrm{D}^{\circ} 4$

$\begin{array}{lllllll}3145.33 & \text { A } & 45 & 0.00 & 3.93 & 1 \frac{2}{3}-1 \frac{1}{3} & (3) \\ 3479.39 & \text { A } & 40 & 0.38 & 3.93 & 3 & \text { (a) }\end{array}$

3438.37 A $30 \quad 0.00 \quad 3.60 \quad 1 \frac{1}{2}-\frac{1}{2}$

$3000.09 \quad$ A $40 \quad 0.38 \quad 4.49 \quad 31-1 \frac{1}{2} \mathrm{a}^{2} \mathrm{D}-\mathrm{z}^{2} \mathrm{po}+$

$\begin{array}{rrrrrrr}3000.09 & \mathrm{~A} & 40 & 0.38 & 4.49 & 3 \frac{1}{1}-1 \frac{1}{2} \mathrm{a}^{2} \mathrm{D}-z^{2} \mathrm{P} \\ 3016.94 & \mathrm{~A} & 6 & 0.00 & 4.09 & 1 \frac{1}{2}-\frac{1}{2} & (3)\end{array}$

$3317.99 \quad A \quad 20 \quad 0.38 \quad 4.10 \quad 3 \frac{1}{2}-3 \frac{1}{2} a^{3} \mathrm{D}-z^{3} \mathrm{D}^{0}+$

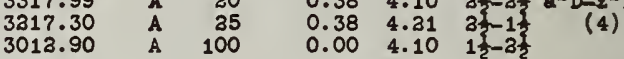

$3134.73 \quad \mathrm{~A} \quad 150 \quad 0.38 \quad 4.31 \quad 3 \frac{1}{2}-3 \frac{1}{2} a^{3} D_{-2} \mathrm{C}^{0}+$

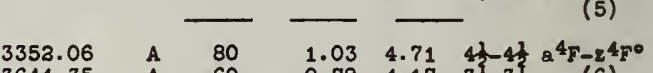

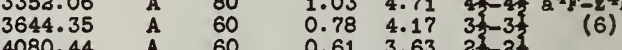

$\begin{array}{rrrrrr}4080.44 & \text { A } & 60 & 0.61 & 3.63 & 23\end{array}$

$\begin{array}{rrrrrr}3933.65 & \text { A } & 150 & 0.45 & 3.47 & 12-1 \\ 4 & 1.03 & 4.17 & 4 & -3\end{array}$

4335.15 A $\quad 5 \quad 0.78 \quad 3.63 \quad 3-3 \frac{1}{3}$

$0.78 \quad 4.71 \quad 3 \frac{1}{3}-4 \frac{1}{3}$

$\begin{array}{lll}0.61 & 4.17 & 2 \frac{1}{3}-3 \frac{1}{3} \\ 0.45 & 3.63 & 1 \frac{1}{2}-2 \frac{1}{2}\end{array}$

3505.32
3569.03

3569.03
3719.27

3719.27
3918.10

3273.66
3394.58

3394.58
3553.70

3126.37

3176.85

3389.83

3054.58

3279.98

3384.70

3975.89

3194.19
3109.11

3338.31

3031.16

3025.29

3101.39

3968. 82

4936.99

6379.84
6835.39

4373.85

4664.14
5040.82

5040.82
5399.85

5809.50

5463.38

4367.90

4945.38
4362.73

4362.72
4719.10

5075.93

4844.00

4934.46

4097.31 $\begin{array}{llll}1.03 & 4.55 & 4 \frac{1}{3}-3 \frac{1}{3} & a^{4} 7^{2}-2^{4} D^{0} \\ 0.78 & 4.34 & 3 \frac{1}{2}-3 \frac{1}{2} & (7)\end{array}$

$\begin{array}{lll}0.78 & 4.34 & 35 \\ 0.61 & 3.93 & 3 \frac{1}{3}-1 \frac{1}{3}\end{array}$

$\begin{array}{llll}.78 & 4.55 & 3 & 3\end{array}-3 \frac{1}{2}$

0.61 4.24 31 2

$0.61 \quad 4.55$ 35 $3 \frac{13}{3}$

$0.45 \quad 4.84 \quad 1 \frac{1}{2}-31$

$0.61 \quad 4.49 \quad 2 \frac{1}{2}-1 \frac{1}{2} a^{4}-z^{2} p^{30}$

$\begin{array}{llll}0.45 & 4.09 & 1 \frac{1}{2} & (8) \\ 0.45 & 4.49 & 13 & 1 \frac{1}{2}\end{array}$

$0.61 \quad 4.10 \quad 3 \frac{1}{3}-3 \frac{1}{3} \mathrm{a}^{4} \mathrm{~F}-2^{2} \mathrm{D}^{0}+$

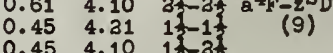

$0.61 \quad 4.75 \quad 3 \frac{1}{3}-3 \frac{1}{2} a^{4} \bar{\gamma}-2^{4} G^{0}+$

$0.45 \quad 4.31 \quad 13 \frac{1}{3}$ (10)

$0.78 \quad 4.75 \quad 31-3 \frac{1}{3}$

$\begin{array}{lll}0.61 & 4.31 & 32 \\ 0.78 & 4.31 & 3 \frac{1}{2}-2 \frac{3}{2}\end{array}$

$0.614 .68 \quad 3 \frac{1}{2}-1 \frac{1}{2} a^{4} \mathrm{~F}^{\mathrm{F}}-\mathrm{y}^{2} \mathrm{D}^{\circ} \uparrow$

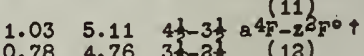

\begin{tabular}{lll}
0.78 & 4.76 & $3 \frac{1}{3}-2 \frac{1}{2}$ \\
0.61 & 4.76 & $3 \frac{1}{2}-2 \frac{1}{2}$ \\
\hline
\end{tabular}

$1.66 \quad 4.17 \quad 3 \frac{1}{3}-3 \frac{1}{4} a^{4} \mathrm{p}-2^{4} \mathrm{p}^{\circ}+$

$\begin{array}{llll}1.66 & 3.63 & 21 & 2 \frac{1}{3} \\ 1.66 & 3.47 & 3 \frac{1}{2}-1 \frac{1}{2} & \text { (13) }\end{array}$

$1.66 \quad 4.55 \quad 2 \frac{1}{2}-3 \frac{1}{3} 2^{4} P-2^{4} D^{\circ}+$

$\begin{array}{lll}1.48 & 3.92 & 1 \frac{1}{3} \\ 1.60 & 3.93 & 1 \frac{1}{2}\end{array}$

$\begin{array}{lll}1.48 & 3.60 & \\ 1.66 & 3.92 & 2 \frac{1}{2}-1 \frac{1}{2}\end{array}$

$1.66 \quad 4.49$ 3र $1 \frac{1}{3} a^{4} p_{-2}^{3} p_{0}$

$\begin{array}{lll}1.60 & 4.09 & 13\end{array}$

$\begin{array}{lll}1.60 & 4.49 & 1 \\ 1.48 & 4.09 & 1\end{array}$

$1.66 \quad 4.10 \quad 31-2 \frac{1}{3} a^{4} P-z^{3} D^{0} \uparrow$

$\begin{array}{llll}1.66 & 4.10 & 3 & 3 \\ 1.66 & 4.31 & 3 & 1 \\ 1.60 & 4.10 & 1 \frac{1}{2}-2 \frac{1}{2} & (16)\end{array}$

$1.66 \quad 4.68 \quad 3 \frac{1}{2}-1 \frac{1}{2} a^{4} \mathrm{P}-\mathrm{y}^{3} \mathrm{p}^{\circ}$
$1.60 \quad 4.34$ 13 $(14)$ 
REVISED UULTIPLET TABLE

I A Laboratory Int

Rf II continued

3699.73 3800.39

3883.77

3823.91

3665.35

$\begin{array}{ll}3413.74 & \text { A } \\ 3349.17 & \text { A }\end{array}$

3303.67 A 10

\begin{tabular}{|c|c|c|c|c|c|}
\hline $\begin{array}{l}4605.79 \\
5348.40 \\
5767.18 \\
6980.91 \\
6248.95\end{array}$ & $\begin{array}{l}\mathbf{A} \\
\mathbf{A} \\
\mathbf{A} \\
\mathrm{A} \\
\mathrm{A}\end{array}$ & $\begin{array}{r}30 \\
15 \\
30 \\
300 \\
100\end{array}$ & $\begin{array}{l}1.49 \\
1.86 \\
1.49 \\
1.86 \\
1.49\end{array}$ & $\begin{array}{l}4.17 \\
4.17 \\
3.63 \\
3.63 \\
3.47\end{array}$ & $\begin{array}{l}3 \frac{1}{2}-3 \frac{1}{2} a^{2} F-2^{4} F^{0} \uparrow \\
3 \frac{1}{3}-3 \frac{1}{2} \quad(3 a) \\
3 \frac{1}{2}-3 \frac{1}{2} \\
3 \frac{1}{3}-3 \frac{1}{2} \\
3 \frac{1}{2}-1 \frac{1}{2}\end{array}$ \\
\hline
\end{tabular}

4586.85

4486.14

5071.23

4029.1

4113.

5524.

4753.

3861.

3782.

4369.67

$\begin{array}{lll}3747.48 & \text { A } & \text { ? } \\ 3872.55 & \text { A } & 30\end{array}$

3933.40 A 10

$\begin{array}{llr}3797.95 & \text { A } & 10 \\ 3771.36 & \text { A } & 8 \\ 3407.76 & \text { A } & 15\end{array}$

3230.66

3092.26
3410.18

3163.6

$\begin{array}{lll}3495.94 & \text { A } & 10 \\ 3383.39 & \text { A } & \end{array}$

3376.68

3140.77 A $\quad 15$

3046.03 A 30

3116.95

6644.

$\begin{array}{llr}4999.69 & \text { A } & 40 \\ 8935.16 & \text { A } & 50 \\ 6754.61 & & \text { A }\end{array}$

$5360.44 \quad \hat{A} \quad 40$

$\begin{array}{lll}5324.36 & \text { A } \\ 451.31 & \text { A }\end{array}$

$\begin{array}{llr}5058.18 & \text { A } & 10 \\ 5311.60 & \text { A } & 150\end{array}$

4731.36 A 40

$\begin{array}{ll}4249.33 & \text { A } \\ 3648.35 & \text { A }\end{array}$

$\begin{array}{rrr}4330.69 & \text { A } & 40 \\ 4030.25 & \text { A } & 5\end{array}$

4573.81 A 30

$\begin{array}{lll}4158.90 & \text { A } & 30 \\ 4127.80 & \text { A } & 40\end{array}$

3698.39 A 1

3935.64 A 30

$\begin{array}{rrr}3485.16 & \text { A } & 3 \\ 3518.75 & \text { A } & 15\end{array}$

$\begin{array}{llr}3659.02 & \text { A } & 4 \\ 3384.14 & \text { A } & 10\end{array}$

3195.63 A 8

3110.87 A 40

$3034.78 \quad$ A 15

5391.36

5391.36

6531.66 A 30

$\begin{array}{llr}6513.61 & \text { A } & 10 \\ 5298.06 & \text { A } & 100\end{array}$

$5842.33 \quad$ A 80

$\begin{array}{rr}4177.50 & \text { A } \\ 4417.37 & 30\end{array}$
Lor ${ }^{\mathrm{P}}$ Hsgh $J \quad$ Multiplet $_{(\text {No })}$

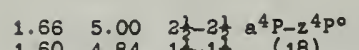

$\begin{array}{llll}1.60 & 5.00 & 2 \frac{1}{2}-2 \frac{1}{2} & a^{4} P-z \\ 1.60 & 4.84 & 1 \frac{1}{3}-1 \frac{1}{3} & (18)\end{array}$

$\begin{array}{lll}1.48 & 4.74 \\ 1.66 & 4.84 & 3\end{array}$

$\begin{array}{lll}1.60 & 4.74 & 1 \frac{1}{3} \\ 1.60 & 5.00 & 1\end{array}$

$1.48 \quad 4.84 \quad \frac{1}{2}-1 \frac{1}{2}$

$1.66 \quad 4.76 \quad 3 \frac{1}{2}-3 \frac{1}{2} a^{4} \mathrm{P}-\mathrm{z}^{2} \mathrm{~F}^{\circ}+$

$\begin{array}{llll}1.66 & 5.38 & 2 \frac{1}{3}-1 \frac{1}{2} a^{4} \mathrm{P}_{-\mathrm{y}^{3} \mathrm{P}^{\circ}+}+ \\ 1.60 & 5.38 & 1 \frac{1}{2}-1 \frac{1}{2} & (30)\end{array}$

$1.665 .53 \quad 3 \frac{1}{2}-3 \frac{1}{2} a^{4} \mathrm{P}-\mathrm{y}^{3} \mathrm{~F}^{0}+$

$\begin{array}{lll}1.49 & 4.17 & 3 \frac{1}{3}-3 \frac{1}{2} a^{2} F-2^{4} F^{\circ} \\ (3 a) & \end{array}$

$\begin{array}{lll}1.86 & 3.63 & 3 \frac{1}{2}-2 \frac{1}{3} \\ 1.49 & 3.47 & 3 \frac{1}{2}-1 \frac{1}{2}\end{array}$

$\begin{array}{llll}1.86 & 4.55 & 3 \frac{1}{2}-3 \frac{1}{2} & a^{2} F-2^{4} D^{0} \\ 1.49 & 4.34 & 3 \frac{1}{2}-3 \frac{1}{2} & (23)\end{array}$

$\begin{array}{lll}1.86 & 4.34 & 3 \frac{2}{2}-3 \frac{1}{2} \\ 1.49 & 3.93 & 3 \frac{1}{2}-1 \frac{1}{2}\end{array}$

$1.49 \quad 4.55$ at

$1.49 \quad 4.49 \quad 3 \frac{1}{2}-1 \frac{1}{2} a^{2} F-\varepsilon^{2} p_{0}$

$1.86 \quad 4.10 \quad 3 \frac{1}{2}-2 \frac{1}{2} a^{3} \frac{(34)}{2}-2^{\circ}$

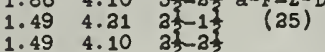

$1.86 \quad 5.23 \quad 3 \frac{1}{2}-4 \frac{1}{3} a^{2} F-2^{4} a^{0}$

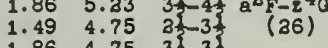

$\begin{array}{lll}1.86 & 4.75 & 3 \frac{2}{2}-3 \frac{1}{2} \\ 1.49 & 4.31 & 21-2 \frac{1}{2}\end{array}$

$1.86 \quad 4.31 \quad 3 \frac{1}{2}-3 \frac{1}{2}$

$1.86 \quad 5.16 \quad 3 \frac{1}{2}-2 \frac{1}{3} a^{2} F-y^{3} D^{0}+$

$1.865 .00 \quad 3 \frac{1}{2}-3 \frac{1}{2} a^{2} \bar{F}-2^{4} p 0+$

$1.865 .11 \quad 3 \frac{1}{3}-3 \frac{1}{3} a^{2} \frac{(28)}{F-2} F^{\circ}$

$\begin{array}{llll}1.49 & 4.76 & 3 \frac{1}{2}-3 \frac{1}{3} & (29) \\ 1.49 & 5.11 & 3 \frac{1}{2}-3 \frac{1}{2} & \end{array}$

$1.86 \quad 5.69 \quad 3 \frac{1}{3}-4 \frac{1}{3} a^{2} 5-y^{4} 5^{\circ}$

$\begin{array}{lll}1.49 & 5.48 & 3 \\ 1.3 & \end{array}$

$\begin{array}{lll}1.49 & 5.39 & 21-2 \\ 1.86 & 5.39 & 3\end{array}$

$1.49 \quad 5.25 \quad 2 \frac{1}{2}-1 \frac{1}{2}$

$1.86 \quad 5.52 \quad 3 \frac{1}{2}-3 \frac{1}{2} a^{2} F-y^{3} F^{0}+$

$\begin{array}{llll}1.49 & 5.42 & 3 \frac{1}{2}-2 \frac{1}{2} & (31) \\ 1.49 & 5.52 & 2 \frac{1}{2}-3 \frac{3}{2} & \end{array}$

$1.86 \quad 5.91 \quad 3 \frac{1}{2}-2 \frac{1}{2} a^{2} F-y^{4} D^{0}+$

$\begin{array}{lll}1.865 .82 \quad 3 \frac{1}{2}-3 \frac{1}{2} a^{3} F-z^{2} G^{\circ}+ & (33)\end{array}$

$1.77 \quad 3.63 \quad 1 \frac{1}{2}-2 \frac{1}{2} \mathrm{~b}^{2} \mathrm{D}-2^{4} \mathrm{~F}^{\circ}+$

$\begin{array}{llll}1.77 & 4.24 & 1 \frac{1}{3}-2 \frac{1}{3} & b^{2} D_{-2}^{(34)} D^{\circ} 4 \\ 3.14 & 3.92 & 3 \frac{1}{3}-1 \frac{1}{3} & (35)\end{array}$

$\begin{array}{lll}3.14 & 3.92 & 21-1 \\ 1.77 & 3.60 & 1\end{array}$

$3.14 \quad 4.49 \quad 2 \frac{1}{2}-1 \frac{1}{2} b^{3} \mathrm{D}-\mathrm{z}^{2} \mathrm{P}$ 。

$\begin{array}{llll}1.77 & 4.09 & 1 \frac{1}{3}-\frac{1}{3} & \text { (36) } \\ 1.77 & 4.49 & 1 \frac{1}{2}-1 \frac{1}{2} & \end{array}$

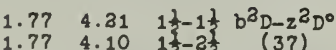

$2.14 \quad 4.75 \quad 2 \frac{1}{2}-3 \frac{1}{2} b^{2} \mathrm{D}-z^{4} G^{\circ}$

$\begin{array}{llll}1.77 & 4.68 & 1 \frac{1}{2}-1 \frac{1}{2} & b^{2} \mathrm{D}-\mathrm{y}^{2} \mathrm{D}^{\circ} \\ 1.77 & 5.16 & 1 \frac{1}{3}-3 \frac{1}{2} & (39)\end{array}$

$2.14 \quad 5.00 \quad 2 \frac{1}{2}-2 \frac{1}{2} b^{2} \mathrm{D}-2^{4} \mathrm{po}$

$\begin{array}{llll}1.77 & 4.84 & 1 \frac{1}{3}-1 \frac{1}{3} & (40) \\ 3.14 & 4.84 & 3 \frac{1}{3}-1 & \end{array}$

$\begin{array}{llll}3.14 & 5.11 & 3 \frac{1}{3}-3 \frac{1}{2} & b^{2} \mathrm{D}-z^{3} F^{\circ} \uparrow \\ 1.77 & 4.76 & 1\end{array}$

$3.14 \quad 5.48 \quad 2 \frac{1}{2}-3 \frac{1}{2} b^{3} \mathrm{D}-\mathrm{y}^{4} \mathrm{~F}^{0}+$

$3.14 \quad 5.38 \quad 3 \frac{1}{2}-1 \frac{1}{2} b^{2} \mathrm{D}-\mathrm{y}^{2} \mathrm{po}$

$\begin{array}{lll}1.77 & 5.31 & 1 \frac{1}{3}-\frac{1}{3} \\ 1.77 & 5.38 & 1 \frac{1}{2}-1 \frac{1}{2}\end{array}$

$\begin{array}{llll}3.14 & 5.53 & 3 \frac{1}{3}-3 \frac{1}{2} & b^{2} \mathrm{D}-\mathrm{y}^{3} \mathrm{~F}^{\circ} \\ 1.77 & 5.43 & 1 \frac{2}{2}-2 \frac{1}{2} & (44)\end{array}$

$1.77 \quad 5.63 \quad 1 \frac{1}{2}-\frac{1}{2} b^{2} \mathrm{D}-\mathrm{y}^{4} \mathrm{D}^{\circ}+$

$1.77 \quad 5.74 \quad 1 \frac{1}{2}-\frac{1}{2} b^{3} D_{-2}^{2} z^{\circ}$

$3.14 \quad 6.33 \quad 3 \frac{1}{2}-1 \frac{1}{2} b^{2}{\mathrm{D}-\mathrm{y}^{4}}^{40+}(47)$

$2.20 \quad 4.49 \quad 1 \frac{1}{2}-1 \frac{1}{2} \mathrm{a}^{3} \mathrm{P}-\mathrm{2}^{3} \mathrm{PO}+$

$\begin{array}{llll}1.88 & 4.09 & \frac{1}{3} & (48) \\ 3.20 & 4.09 & 1 \frac{1}{2}\end{array}$

$3.20 \quad 4.10 \quad 1 \frac{1}{2}-2 \frac{1}{3} a^{2} P-z^{2} D^{0}+$

$2.30 \quad 4.31 \quad 1 \frac{1}{2}-2 \frac{1}{2} a^{3} \mathrm{P}-2^{4} \mathrm{G}^{\circ}$

$\begin{array}{lll}2.30 & 4.31 & 1 \frac{1}{2}-2 \frac{1}{2} \mathrm{a}^{3} \mathrm{P}-z^{4} \mathrm{G}^{\circ} \\ 3.20 & 5.16 & 11-3 \frac{1}{3} \mathrm{a}^{2} \mathrm{P}-\mathrm{y}^{2} \mathrm{D}^{\circ}\end{array}$

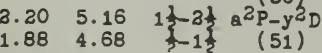

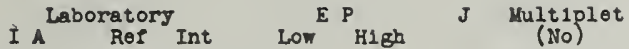

I A Laboratory

E Pigh

Multiplet

HF II continued

$4319.51 \quad A \quad 8 \quad 1.88 \quad 4.74 \quad \frac{1}{2}-\frac{1}{2} \mathrm{a}^{2} \mathrm{P}-\mathrm{z}^{4} \mathrm{PO}+$

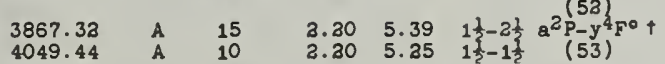

4008.46 3597.42
3964.96

3487.57

3199.99

3306.77
3055.43

4807.1

5138.5

$4050.6 ?$

3998.51

4735.75

5801.71

4162.40
4790.72

3478.98

3701.15
3849.53

3661.73

3817.20
3705.40

3080.64

3394.99
3358.30

3011.24

6647.0
8336.13

6041.44
7338.6

7377.67

7861.32

8581.88

9743.28
6557.91

7030.33

7757.89
5969.38

- 6156.35

7021.33

7663.09

4334.65
4817.32

$5444.0 ?$
6330.84

5194.5 ?
5939.35

6511.62

4623.71

5264.95

5057.03

4699.72

4350.53

4245.84

4232.43
4703.63

4535.38
4466.41

4187.68

4640.14

4490.60
4336.66

4306.59
4071.22

3877.11

3806.07
3766.92

3737.88

3744.98
3917.47

$3438.24 \quad A \quad 15$

3318.30

3323.35

7561.08

6306.17

6455.85

$\begin{array}{rrrcc}8 & 3.20 & 5.38 & 1 \frac{1}{3}-1 \frac{1}{3} a^{3} \mathrm{P}-\mathrm{y}^{2} \mathrm{p}^{\circ} \uparrow \\ 10 & 1.88 & 5.31 & (54)\end{array}$

$\begin{array}{lll}1.88 & 5.31 & \frac{1}{2} \\ 3.30 & 5.31 & 1 \frac{1}{2}\end{array}$

$\begin{array}{lll}3.30 & 5.74 & 1 \frac{1}{2}-\frac{1}{2} e^{3} \mathrm{P}-z^{2} \mathrm{~g}^{\circ} \\ 1.88 & 5.74 & (55)\end{array}$

3. $30 \quad 6.05 \quad 1 \frac{1}{3}-2 \frac{1}{3} a^{2} P-x^{3} D^{\circ}$

$3.15 \quad 4.71 \quad 4 \frac{1}{3}-4 \frac{1}{3} \cdot a^{2} a-2^{4} 5^{\circ}$

$3.154 .554 \frac{1}{2}-3 \frac{1}{2} a^{3} G-2^{4} D^{0}+$

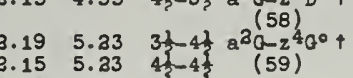

$\begin{array}{lll}2.15 & 5.23 & 41-4 \frac{1}{3} \\ 3.19 & 4.75 & 3 \frac{3}{3}-3 \frac{1}{3} \\ 3.15 & 4.75 & 4 \frac{1}{3}-3 \frac{1}{3}\end{array}$

$3.194 .31 \quad 3 \frac{1}{2}-3 \frac{1}{2}$

$\begin{array}{llll}3.15 & 5.11 & 4 \frac{1}{3}-3 \frac{1}{3} & a^{2} a-z^{2} F^{0} \uparrow\end{array}$

$3.15 \quad 5.59 \quad 4 \frac{1}{2}-4 \frac{1}{3} \mathrm{a}^{3} \mathrm{G}-\mathrm{y}^{4} F^{\circ} \uparrow$

$\begin{array}{llll}3.15 & 5.48 & 4 \frac{1}{2}-3 \frac{1}{2} & \text { (61) } \\ 2.19 & 5.39 & 3 \frac{1}{5}-2 \frac{1}{2} & \end{array}$

$2.15 \quad 5.53 \quad 4 \frac{1}{3}-3 \frac{1}{3} a^{2} G-y^{3} F^{\circ}$

$\begin{array}{lll}2.19 & 5.42 & 3 \frac{1}{3}-2 \frac{1}{2} \\ 3.19 & 5.53 & 3 \frac{1}{3}\end{array}$

$3.15 \quad 6.15 \quad 4 \frac{1}{2}-4 \frac{1}{3} a^{2} G-2^{2} G^{\circ}+$

$\begin{array}{lll}3.19 & 5.82 & 3 \frac{1}{3}-3 \frac{1}{2} \\ 3.15 & 5.82 & 4\end{array}$

$2.19 \quad 6.28 \quad 3 \frac{1}{2}-2 \frac{1}{2} a^{2} a-x^{2} F^{\circ}+$

$\begin{array}{llll}3.86 & 4.71 & 4 k-4 \frac{1}{3} & b^{4} F-z^{4} F^{\circ} \\ 3.67 & 4.17 & 3 & 3\end{array}$

$\begin{array}{lll}3.67 & 4.71 & 3 \frac{3}{2}-4 \frac{1}{3} \\ 3.49 & 4.17 & 3 \frac{2}{2}-3 \frac{1}{3}\end{array}$

$\begin{array}{llll}3.86 & 4.55 & 4 \frac{1}{3}-3 \frac{1}{3} & b^{4} \\ 3 & -z^{4} D^{0}+\end{array}$

$\begin{array}{lll}3.67 & 4.24 & 3 \\ 3.49 & 3.92 & 21\end{array}$

$2.33 \quad 3.60 \quad 1 \frac{2}{3}$

$\begin{array}{lll}3.67 & 4.55 & 3 \frac{1}{2}-3 \frac{1}{3} \\ 2.49 & 4.34 & 3 \frac{1}{2}-3 \frac{1}{2}\end{array}$

$\begin{array}{lll}2.49 & 4.24 & 3 \frac{1}{3}-3 \frac{1}{2} \\ 3.33 & 3.92 & 1 \frac{1}{3}-1 \frac{1}{3} \\ 3.49 & 4.55 & 2 \frac{1}{5}-3 \frac{1}{3}\end{array}$

3. $49 \quad 4.49 \quad 2 \frac{1}{5}-1 \frac{1}{2} b^{4} F-\varepsilon^{2} p 0$

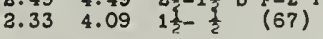

$2.49 \quad 4.10 \quad 2 \frac{1}{2}-2 \frac{1}{2} b^{4} \mathrm{~F}-z^{2} D^{0}+$

$\begin{array}{llll}3.86 & 5.70 & 4 \frac{1}{3}-5 \frac{1}{3} & b^{4} \mathrm{~F}-\mathrm{z}^{4} \mathrm{G}^{\circ} \\ 2.67 & 5.23 & 3 \frac{1}{2}-4 \frac{1}{3} & (69)\end{array}$

$\begin{array}{lll}2.67 & 5.23 & 3 \frac{1}{3}-4 \\ 3.49 & 4.75 & 2 \frac{1}{4}-3\end{array}$

$2.33 \quad 4.31 \quad 1 \frac{1}{6}-2 \frac{1}{3}$

$\begin{array}{lll}3.67 & 4.75 & 3 \frac{1}{2}-3 \frac{1}{3}\end{array}$

$\begin{array}{cccc}2.49 & 5.16 & 2 \lambda-2 \frac{1}{2} & b^{4} F-y^{2} D^{0} \\ 2.33 & 4.68 & 1 \frac{1}{2}-1 \frac{1}{2} & (70)\end{array}$

$2.67 \quad 5.11 \quad 3:-3 \frac{i}{3} b^{4} F-z^{2} F^{\circ}+$

2. $49 \quad 5.11 \quad-3 \frac{1}{2} \quad$ (71)

$2.86 \quad 5.69 \quad 4 \frac{1}{3}-4 b^{4} \mathrm{~F}-\mathrm{y}^{4} \mathrm{~F}^{\circ}+$

$2.49 \quad 5.39 \quad 2 \frac{1}{3}-2 \frac{1}{2} \quad$ (72)

$3.33 \quad 5.25 \quad 1 \frac{1}{3}-1 \frac{1}{3}$

$\begin{array}{lll}3.86 & 5.48 & 4 \frac{3}{3}-3 \frac{1}{3} \\ 2.67 & 5.39 & 3 \frac{1}{3}-2 \frac{1}{3}\end{array}$

$3.33 \quad 5.28 \quad 1 \frac{1}{2}-1 \frac{1}{2} b^{4} F-y^{3} p^{0} \uparrow$

$2.86 \quad 5.53 \quad 4 \frac{1}{3}-3 \frac{1}{3} b^{4} F-y^{3} F^{\circ}$

$\begin{array}{llll}3.67 & 5.42 & 3 & 3 \\ 3 & & (74)\end{array}$

$\begin{array}{lll}3.49 & 5.42 & 2 \\ 3.49 & 5.52 & 3\end{array}$

$3.86 \quad 6.04 \quad 43-3 \frac{1}{3} b^{4} F-y^{4} D^{0} \uparrow$

$\begin{array}{lll}3.67 & 5.91 & 3 \frac{1}{3}-2 \frac{1}{3} \\ 3.49 & 5.76 & 2 \frac{1}{3}-1 \frac{1}{2} \\ 2.33 & 5.63 & 1 \frac{1}{2}-\frac{1}{2}\end{array}$

$3.86 \quad 6.15 \quad 4 \frac{1}{3}-\frac{1}{3} b^{4} 8-2^{3} G^{\circ}$

$2.335 .93 \quad 1 \frac{1}{2}-1 \frac{1}{2} b^{4} \frac{7}{7}-x^{2} D^{0}+$

$3.676 .51 \quad 3 \frac{1}{2}-2 \frac{1}{4} b^{4} \mathrm{~F}-\mathrm{T}^{2} \mathrm{D}^{\circ}$

$\begin{array}{lll}3.86 & 6.57 \quad 4 \frac{1}{2}-3 \frac{1}{2} b^{4} & (78) \\ (79) & \end{array}$

$3.37 \quad 5.00 \quad 1 \frac{1}{2}-2 \frac{1}{2} b^{4} \mathrm{P}-z^{4} \mathrm{P}^{0}$

$3.53 \quad 5.48 \quad 2 \frac{1}{3}-3 \frac{1}{3} b^{4} \mathrm{P}^{3} \mathrm{y}^{4} \mathrm{~F}$

$3.37 \quad 5.38 \quad 1 \frac{1}{2}-1 \frac{1}{2} b^{4} P-y^{2} P^{0}+$

HS II continued

4904.51

4848.46
5080.44

5164.56

5156.06

4765.7

4760.59
4570.70

4321.36
4368.10

4426.18

4007.36

6997.83

6135.10

6027.57

6473.89

4599.46

5847.10

4675.45

4125.10

4452.70
442.10

4123.5

3979.40

3864.75

6584.53

7016.99

5389.98

6542.80

5565.56

3762.51

4683.68

3900.64

4613 . ?

4047.96
$4524 . ?$

6609.30

5110.6

4486.6

4486.65
4241.93

3945.3

6719.4
7398.9

6550.0
7278.7

5673.58

5493.32

4179.55

3946.00

Strongest Unclassirled Lines of Hr II

7061.90

6850.07
6548.72

6548.72

4519.02
443.07

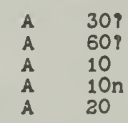

Ta I I P, Anal C L18t $D$ Dec 1942

5402.51

5312.75
4574.32

4574.32
3970.10

3077.24 
II I P ? I Anal D L1st D Dec 1943 $\begin{array}{lllllll}3641.43 & \text { A } & 150 & 1.08 & 4.46 & 1 \frac{1}{2} & 4 T_{-1} \\ 3286.57 & \AA & 100 & 1.08 & 4.83 & 1 \frac{2}{2}-1 \frac{1}{2} & (1)^{\circ}\end{array}$
98

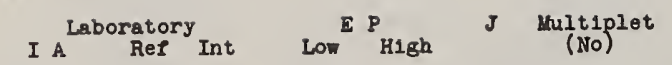
Ta I continued

$5811.10 \quad$ A $20 d 9 \quad 0.49 \quad 3.61 \quad 3 \frac{1}{2}-2 \frac{1}{2} 2^{4} 8-6^{\circ}$ 5461.31 A $35 \quad \overline{0.69} 3.95 \quad 4 \frac{1}{3}-3 \frac{1}{3} a^{4} F-12^{\circ}$ $3063.56 \quad$ A $\quad 18 x \quad 0.69 \quad 4.73 \quad \frac{4}{2}-3 \frac{3}{2} \quad(4) 78^{\circ}$

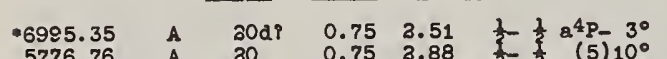
5776.7 5413.47
5349.08 5136.47 4921.39

\section{$5419 \cdot 19$} 4936.02

5939.75 A 20 \% $\begin{array}{ll}0.75 & 3.88 \\ 0.75 & 3.03\end{array}$ 0.753 .03 का $14^{\circ}$ $0.753 .05 \quad-1 \frac{1}{3} \quad 15^{\circ}$ $\begin{array}{llll}0.75 & 3.15 & \frac{1}{2} & 18^{\circ} \\ 0.75 & 3.25 & \frac{1}{2}-1 \frac{1}{2} & 31^{\circ}\end{array}$

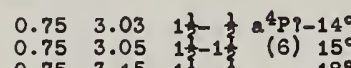

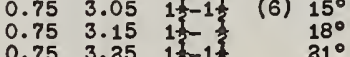
$0.75 \quad 3.25 \quad 1 \frac{1}{2}-1 \frac{1}{2} \quad 21^{\circ}$

$1.30 \quad 3.28 \quad \frac{1}{2}-1 \frac{1}{2} 8^{6} \mathrm{D}-23^{\circ}$ $1.33 \quad 3.31 \quad 1 \frac{1}{2}-2 \frac{1}{2} 3-24^{\circ}$

5944.01 A 30d? $1.353 .621 \frac{1}{2}-3 \frac{1}{2} a^{6} D ?-35^{\circ}$

$5435.27 \quad$ A $\quad 30$ $1.35{ }^{3.62} 1 \frac{2}{2}-6 \frac{21}{2} a^{6} D i-350$

\begin{tabular}{llll}
$6045.38 \quad \mathrm{~A} \quad 30 \quad 1.39 \quad 3.43 \quad 3 \frac{1}{2}-3 \frac{1}{2} \mathrm{a}^{6} \mathrm{D}-38^{\circ}$ \\
\hline
\end{tabular}

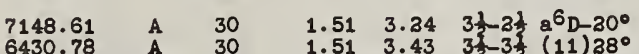
$\begin{array}{rrrrrrr}6430.78 & \text { A } & 30 & 1.51 & 3.43 & 3 \frac{3}{3}-3 \frac{1}{2} & (11) 28^{\circ} \\ 4936.41 & \text { A } & 30 & 1.51 & 4.01 & 3 \frac{2}{2}-2 \frac{2}{2} & 58^{\circ}\end{array}$

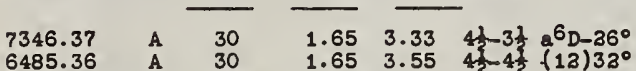
$\begin{array}{rrrrrrr}6485.36 & \text { A } & 30 & 1.65 & 3.55 & 4 \frac{1}{2} 4 \frac{1}{3} & (13) 33^{\circ} \\ 5997.34 & \text { A } & 35 & 1.65 & 3.71 & 4 \frac{1}{3}-4 & 39^{\circ}\end{array}$

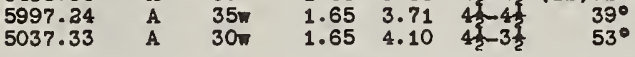

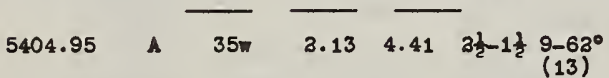

Ta II see introduction

\section{I P 7.94 Anal A List D June 1941} 3631.

. 3682.101

$:_{3872.835}^{3757}$

4047.946
3877.501

.3570 .663 3326.194 3315.578 3191.577 3046.453 3041.876 $\begin{array}{rrr}4008.769 & \text { A } 1000 \\ 4074.374 & \text { A } 500\end{array}$ 4294.623 A 1000 $\begin{array}{rrr}4302.123 & \text { À } & 500 \\ 4757.565 & \text { A } & 300\end{array}$

3617.532
3780.770 A 800
3.2007 .218

3307.248 60
3867.986 A 300

REVIBED MULTIPLET TABLE I A Laiooratory ${\text { Ref Int Low }{ }_{\text {High }} \text { I Multiplet }}_{\text {(No) }}$

II continued

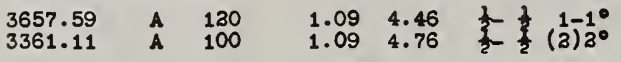

3573.48 A $\left.300 \quad \overline{1.31} 4.761 \frac{1}{2}-\frac{1}{2}(3)^{2}\right)^{\circ}$

$3024.51 \quad$ A $300 \quad 1.40 \quad 5.48 \quad 3 \frac{1}{2}-2 \frac{1}{2} \quad 3-7^{\circ}$

3149.87 A $500 \quad 1.63 \quad 5.54 \quad \frac{1}{2}-1 \frac{1}{2} \quad 4-11^{\circ}$

$\begin{array}{lllllll}-3189.34 \quad \text { A } 1001 & 1.66 & 5.53 & 31 & 41 & 5-9^{\circ}\end{array}$

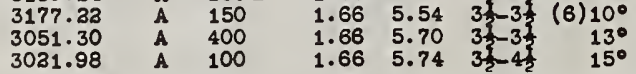

3463.53 A $300 \quad \frac{1.66}{5.23} 3 \frac{1}{13}-\frac{1}{3} 6-5^{\circ}$

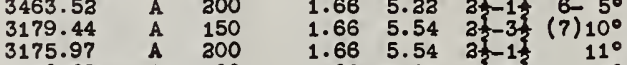
$\begin{array}{lllllll}3036.68 & \text { A } & 100 & 1.66 & 5.72 & 3 \frac{1}{2}-1 \frac{1}{2} & 114^{\circ} \\ 30 & & & \end{array}$

$\begin{array}{lllllll}3343.40 & \text { A } & 120 & 1.83 & 5.53 & 41-4 & 8-9^{\circ} \\ 3160.03 & \text { A } & 300 & 1.83 & 5.74 & 4 \frac{1}{2}-4 & (8) 15^{\circ}\end{array}$

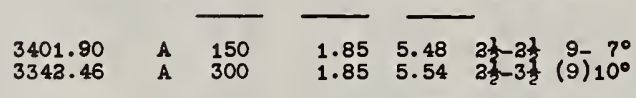

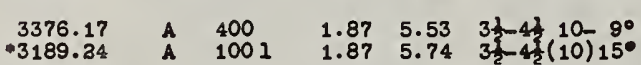
3555.18 A $130 \quad 3.00 \quad 5.48 \quad 3 \frac{1}{3}-2111-7^{\circ}$ 3486.14 A $100 \quad 3.00 \quad 5.54 \quad 3 \frac{3}{2}-1 \frac{1}{2}(11)_{11}$ 3529.57 A $100 \quad 3.04 \quad 5.54 \quad 4 \frac{1}{2}-3 \frac{1}{2} 13-10^{\circ}$ 3549.08 A $150 \quad 3.05 \quad 5.53 \quad 3 \frac{1}{3}-413-9^{\circ}$ 3358.63 A $300 \quad 3.05 \quad 5.73 \quad 33-3 \frac{3}{3}(13) 14^{\circ}$

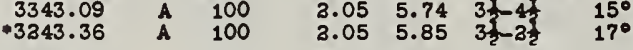

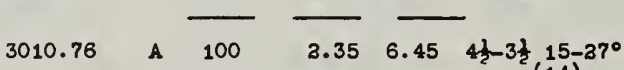
.3243 .36 A $100 \quad \overline{3.47} 6.283 \frac{1}{2}-4 \frac{1}{2}(15)-23^{\circ}$

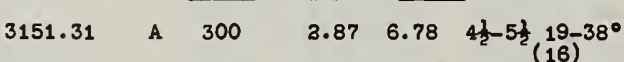

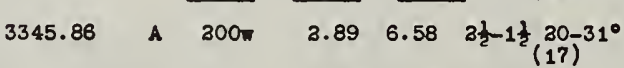
$\begin{array}{lll}0.77 & 3.63 & 1-1 \\ 0.60 & 2.33 & 4-3\end{array}$ $\begin{array}{lll}0.60 & 2.96 & 3-2 \\ 0.41 & 3.65 & 3-1\end{array}$ $0.77 \quad 3.78 \quad 4-3 \quad d^{4} 8^{2} 5 D_{-} d^{4} 855$ por $\begin{array}{lll}0.60 & 3.78 & 3-3 \\ 0.41 & 3.63 & 3-3\end{array}$

\section{(3) $0.77 \quad 4.05$} $\begin{array}{lll}0.77 & 4.05 \\ 0.41 & 3.60 \\ 0.31 & 3.43 & 3.8\end{array}$ $\begin{array}{lll}0.21 & 3.43 & 1-1 \\ 0.77 & 3.69 & 4-3\end{array}$ $\begin{array}{lll}0.60 & 4.05 & 3-4 \\ 0.41 & 3.69 & 3-3\end{array}$ $\begin{array}{lll}0.41 & 3.69 & 2-3 \\ 0.31 & 3.60 & 1-2\end{array}$

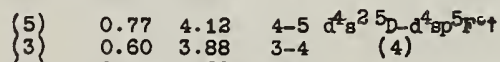
4
3 $\begin{array}{lll}0.41 & 3.60 & 3-3 \\ 0.21 & 3.26 & 1-3\end{array}$ $0.00 \quad 3.31 \quad 0-1$ $\begin{array}{rrrr}0.41 & 3.87 & 3-1 & d^{4} \sigma^{2} \\ 0.77-313^{\circ} & 4.48 & 4-5 & (5) 362^{\circ}\end{array}$ $\begin{array}{rrrr}0.60 & 4.34 & 3-4 & 351^{\circ} \\ 0.77 & 4.61 & 4-5 & 373^{\circ}\end{array}$ $0.00 \quad 3.87 \quad 0-1 \quad 313^{\circ}$ $\begin{array}{llll}0.21 & 4.09 & 1-2 & 331^{\circ} \\ 0.31 & 4.26 & 1-3 & 344^{\circ} \\ 0.41 & 4.47 & 0-1 & 361^{\circ}\end{array}$ $\begin{array}{lll}0.41 & 4.47 & 3-1\end{array}$

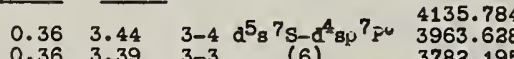
$\begin{array}{lllll}0.36 & 3.39 & 3-3 & (6) & 3782.195 \\ 0.36 & 3.24 & 3-2 & & 3336.150\end{array}$ $\begin{array}{llllll}0.36 & 3.56 & 3-4 & a^{5} 8^{7} 8-a^{4} 8 p^{7} D^{\circ} & 3232.290 \\ 0.36 & 3.23 & 3-3 & \end{array}$ $\begin{array}{llll}0.36 & 3.23 & 3-3 & (7) \\ 0.36 & 3.96 & 3-3 & \end{array}$

$\begin{array}{lllll}0.36 & 3.78 & 3-3 & a^{5} 878-d^{4} 8 p^{5} p_{0} & 4173.234 \\ 3977.331\end{array}$ $\begin{array}{lllll}0.36 & 3.63 & 3-2 & (8) & 3370.588\end{array}$ $\begin{array}{llll}0.36 & 4.21 & 3-4 & d^{5} B^{7} 8-341^{\circ} \\ 0.36 & 4.41 & 3-2 & (9) 357^{\circ}\end{array}$
Os I I P 8.7 Ansi C L18t $D$ Dec 1943 $\begin{array}{lllllll}4420.468 & \text { A } & 400 R & 0.00 & 3.79 & 4-4 & 1-36^{\circ} \\ 4260.854 & \text { A } & 300 & 0.00 & 3.90 & 4-5(1) 37^{\circ}\end{array}$ $\begin{array}{lllllll}4260.854 & \text { A } & 300 & 0.00 & 3.90 & 4-5 & (1) 27^{\circ} \\ 3528.603 & \text { A } & 400 \mathrm{R} & 0.00 & 3.50 & 4-4 & 33^{\circ}\end{array}$ $\begin{array}{lllllll}3301.559 & \text { A } & 500 R & 0.00 & 3.74 & 4-4 & 32 \\ 3267 & 37^{\circ}\end{array}$ $\begin{array}{llllllll}3058.66 & \text { A } & 400 \mathrm{R} & 0.00 & 3.78 & 4-4 & 39^{\circ} \\ & \text {. } & 000 \mathrm{R} & 0.00 & 4.03 & 4-4 & 41^{\circ}\end{array}$ $\begin{array}{r}3049.694 \\ 3017.447 \\ \hline\end{array}$ $\begin{array}{rrrr}0.36 & 4.41 & 3-2 & (9) 357^{\circ} \\ 0.36 & 4.45 & 3-4 & 360^{\circ}\end{array}$

\begin{tabular}{rllllll}
3049.694 & $\mathrm{~A}$ & 60 & 0.36 & 4.41 & $3-2$ & (9) $357^{\circ}$ \\
3017.447 & $\mathrm{~A}$ & 60 & 0.36 & 4.45 & $3-4$ & $360^{\circ}$ \\
\hline
\end{tabular}

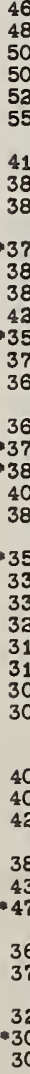

I A ${ }_{\text {Ref Int }}^{\text {Laboratory }}$ Low $_{\text {High }}^{\text {P }}$ J Kuitiplet

Ir I I P 9.3 Anal B L1et D Deo 1948

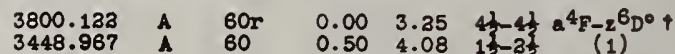

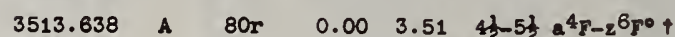

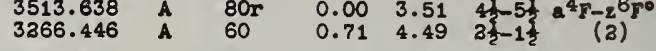
$3437.006 \quad$ A $\quad 60 \quad 0.78 \quad 4.37 \quad 3 \frac{1}{2}-3 \frac{1}{2} a^{4} F-2^{6} 0^{0}+$ $4268.096 \quad A \quad \overline{80} \quad \overline{0.88} \quad \overline{3.77} 3 \frac{1}{2}-3 \frac{1}{2} b^{4} 7_{-2} 6 D^{0}+$ 3368.473 A $60 \quad 0.35 \quad 4.01 \quad 4 \frac{1}{3}-4 \frac{1}{3} b^{4} F_{-2}(4)+4=+$ $\begin{array}{cccccc}3992.114 & \mathrm{~A} & 80 & 1.32 & 4.31 & 21-3 \frac{1}{3} \\ 3320.773 & \mathrm{~A} & 100 \mathrm{r} & 0.35 & 4.18 & 4 \frac{1}{2}-3 \frac{1}{2}\end{array}$

$3915.384 \quad A \quad 60 \quad 1.22 \quad 4.37 \quad 3 \frac{1}{2}-3 \frac{1}{2} b^{4} F_{-2} 60 \%+$ $3068.897 \quad A \quad 60 \quad 0.35 \quad 4.37 \quad 4 \frac{1}{2}-3 \frac{1}{2} \quad(6)$ 3198.917 A $\quad 60 \quad 0.88 \quad 4.74 \quad 3 \frac{1}{2}-2 \frac{1}{2} \quad b^{4} F_{-2}{ }^{4} D^{\circ}+$

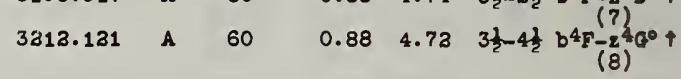

Pt I I P 9.8 Anal B L18t D Dec 1948

\begin{tabular}{|c|c|c|c|c|c|c|}
\hline $\begin{array}{l}3315.05 \\
3290.23\end{array}$ & & $\begin{array}{l}8 \\
6\end{array}$ & $\begin{array}{l}0.00 \\
1.25\end{array}$ & $\begin{array}{l}3.72 \\
5.00\end{array}$ & $\begin{array}{l}3-4 \\
1-2\end{array}$ & $\begin{array}{c}a^{3} D-z^{5} D^{0} \uparrow \\
\text { (1) }\end{array}$ \\
\hline $\begin{array}{r}3064.71 \\
3139.39 \\
3156.59\end{array}$ & & & $\begin{array}{l}0.00 \\
0.10 \\
1.25\end{array}$ & $\begin{array}{l}4.03 \\
4.03 \\
5.16\end{array}$ & $\begin{array}{l}3-2 \\
3-2 \\
1-1\end{array}$ & $\begin{array}{c}a^{3} D_{-z^{3}} \mathrm{P}^{\circ}+ \\
(\mathrm{a})\end{array}$ \\
\hline 97.97 & $\mathbf{A}$ & 30 & 0.10 & 4.31 & $2-3$ & $\begin{array}{c}a^{3} D-z^{3} z^{6} \\
(3)\end{array}$ \\
\hline $\begin{array}{l}3408.14 \\
3966.37\end{array}$ & & $\begin{array}{r}15 \\
6\end{array}$ & $\begin{array}{l}.10 \\
.25\end{array}$ & $\begin{array}{l}3.72 \\
4.36\end{array}$ & $\begin{array}{l}4-4 \\
3-3\end{array}$ & $\frac{a^{3} F-z^{5} D^{0}+}{(4)}$ \\
\hline 3042.65 & A & 30 & 0.10 & 4.16 & $4-5$ & $a^{3}$ \\
\hline $\begin{array}{l}4164.54 \\
3638.80 \\
5368.97\end{array}$ & & $\begin{array}{l}5 \\
8 \\
3\end{array}$ & $\begin{array}{l}1.25 \\
1.25 \\
1.91\end{array}$ & $\begin{array}{l}4.31 \\
4.64 \\
4.21\end{array}$ & $\begin{array}{l}3-3 \\
3-4 \\
2-3\end{array}$ & $(6)^{\circ}$ \\
\hline 3301.8 & A & 10 & 0.81 & 4.55 & $2-2$ & $a^{3} p_{i}$ \\
\hline
\end{tabular}

Pt II see introduction

Au I I 9 9.3 Anal A L1st D Deo 1943

$3132.783 \quad B \quad(150) \quad 1.13 \quad 5.08 \quad 3 \frac{1}{2}-1 \frac{1}{2} a^{2} D_{-} 6^{2} p^{\circ}$ $\begin{array}{lllllll}6278.30 & \text { A } & 35 \mathrm{n} & 3.65 & 4.61 & 1 & 1 \\ .5064 .69 & \text { A } & 15 & 3.65 & 5.08 & 1 & \text { (1) }\end{array}$

7510.74 A $\overline{300} \quad \frac{5.08}{6.73} 1 \frac{1}{1} 6^{2} \mathrm{po}^{0}-7^{3} 8$ $\begin{array}{rrrrrrrr}7510.74 & \text { A } & 300 & 5.08 & 6.73 & 1 \frac{1}{3} & 6^{2} P^{0}-7^{3} 8 \\ 5837.29 & \text { A } & 40 & 4.61 & 6.73 & \frac{1}{2} & (a)\end{array}$ $4792.63 \quad$ A $100 \quad 5.08 \quad 7.66 \quad 1 \frac{1}{3}-3 \frac{1}{3} \quad 6^{2} \mathrm{P}^{0}-6^{2} \mathrm{D}$

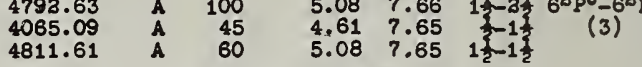

Au II See introduction

HB I I P 10.39 Anal A L1st $D$ Deo 1943

$\begin{array}{lllllll}5460.743 & \text { A } 500 \mathrm{R} & 5.44 & 7.70 & 3-1 & 6^{3} \mathrm{po}^{0}-7^{3} \mathrm{~s}\end{array}$ $\begin{array}{lllllll}4358.343 & \text { A } & 300 & 4.87 & 7.70 & 1-1 & \text { (1) } \\ 4046.557 & \text { A } & 100 & 4.65 & 7.70 & 0-1 & \end{array}$ $\begin{array}{lllllll}3663.274 & A & 50 \mathrm{R} & 5.44 & 8.81 & 3-3 & 6^{3} \mathrm{P}^{\circ}-6^{1} \mathrm{D}\end{array}$ $\begin{array}{lllllll}3131.845 & A & 100 & 4.87 & 8.81 & 1-2 & \text { (3) }\end{array}$

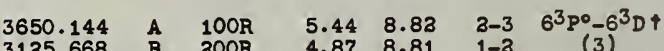
3135.668 B $300 R \quad 4.87 \quad 8.81 \quad 1-3 \quad$ (3) 5790.659 A $\overline{300} \quad \overline{6.67} 8.81 \quad 1-3 \quad 6^{1} P^{\circ}-6^{1} \mathrm{D}$ $5769.598 \quad$ A $300 \quad 6.67 \quad 8.81 \quad 1-3 \quad 6^{1} \mathrm{P}^{\circ}-6^{3} \mathrm{D}+$ Wany lines show fine struoture Hg II See introduction

TI I I P 6.08 Anal A L18t D Deo 1948

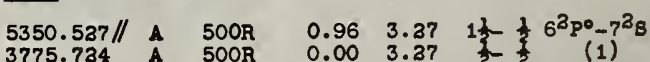
$3519.24 \quad$ B $500 \mathrm{R} \quad 0.96 \quad 4.47 \quad 1 \frac{1}{3}-2 \frac{1}{6} 6^{3} \mathrm{po}-6^{2} \mathrm{D} \uparrow$ $\begin{array}{lllllll}3519.34 & \mathrm{~B} & 500 \mathrm{R} & 0.96 & 4.47 & 1 & -37 \\ 3529.38 & \mathrm{~B} & 100 \mathrm{R} & 0.96 & 4.46 & 1 \frac{1}{2}-1 \frac{1}{2} & \text { (3) }\end{array}$ 
REVIBID 1 ULTIPLET TABLE Anal A Llot D Dec 1943

Po I I P 7.38 Anal $A$ Liot D

3639.568 A $500 R \quad 0.97 \quad 4.36 \quad 1-1 \quad 6 p^{3} p_{-7} a^{3} p^{0} \uparrow$

$4057.813 / /$

$1000 \mathrm{R}$

$\begin{array}{ll}1.31 & 4.36\end{array}$

$2-1$
$1-0$

(1)

An I So0 1ntroduotion

$3.65 \quad 5.95 \quad 3-3 \quad 6 p^{1} \mathrm{D}-7 \mathrm{~s}^{3} \mathrm{p}$

$\begin{array}{lll}4.36 & 2-1 & \text { (3) }\end{array}$

$\begin{array}{ll}3739.940 & \text { A }(3000)\end{array}$

$3.65 \quad 6.10 \quad 2-1 \quad 6 p^{1} \frac{D-7 .}{(3)}$

Ra I I 5.35 Anal A List D KaJ 1943

3573.734

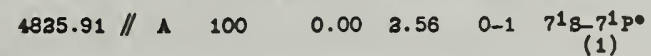

Po II Soo Introduotion

B1 I I P Anal B Llet D Doo 1943

$3087.713 / / \quad 9 \mathrm{R} \quad 0.004 .031 \frac{1}{2}-\frac{1}{2} 6 \mathrm{p}^{4} \mathrm{~g}^{\circ}{ }^{-1}$

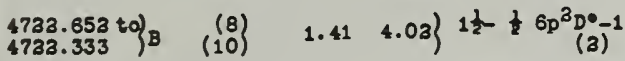

T1de s1ne structure
I A Laborators Rer Int Low ${ }^{E}$ High $J$ Multiplet B1 II See introduction

I A Laboratory ${ }_{\text {Ref Int Low }}^{\text {E P }}$ Figh J Multiplet Th II I P P Ansl C Llat D JuIy 1944 3539.589 A $400 \quad 0.00 \quad 3.49 \quad 1 \frac{1}{2}-2 \frac{1}{2} \mathrm{a}^{2} \mathrm{D}-2^{4} \mathrm{~F}+\uparrow$

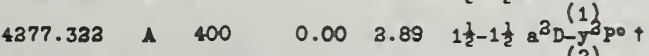
$\begin{array}{lllllll}3610.794 & \mathbf{A} & 30 & 0.51 & 3.93 & 3 \frac{1}{3}-3 \frac{1}{3} & \mathrm{a}^{3} \mathrm{D}-\mathrm{J}^{3} \mathrm{FO}+\end{array}$ $3180.199 \quad \overline{400} \overline{0.19} 4.07 \quad 3 \frac{1}{2}-3 \frac{1}{2} \mathrm{a}^{4} \mathrm{~F}-2^{4} F_{0}$ \%

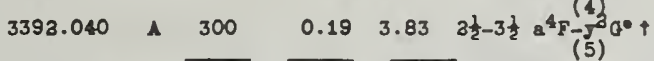

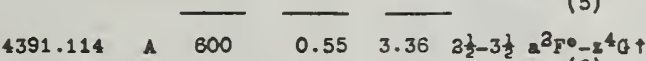
$4919.814 \quad 500 \quad 0.76 \quad 3.37 \quad 3 \frac{1}{2}-3 \frac{1}{2} 8^{4} H^{\circ}-2^{4}+0+$

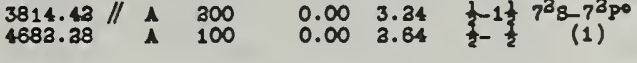

Th I Mo analy:10 Doo 1943

E Iot eoparated Doo 1943 


\section{CORBIDDEN LINES}

I A Low ${ }_{\text {High }}$ J Multiplet

Be I I P 9.28

$4548.3 \quad 0.00(2.71) \quad 0-1 \quad 38_{(1 F)^{2}}^{1 / 28 p^{3} p o}$

C I I P 11.20

\begin{tabular}{|c|c|c|c|c|}
\hline $\begin{array}{l}9849.5 \\
9823.4 \\
9808.9\end{array}$ & $\begin{array}{l}0.01 \\
0.00 \\
0.00\end{array}$ & $\begin{array}{l}1.26 \\
1.26 \\
1.26\end{array}$ & $\begin{array}{l}2-2 \\
1-2 \\
0-3\end{array}$ & $\begin{array}{l}2 p^{2}{ }^{3} p-2 p^{21} D \\
(1 F)\end{array}$ \\
\hline $\begin{array}{l}4627.3 \\
4621.5\end{array}$ & $\begin{array}{l}0.01 \\
0.00\end{array}$ & $\begin{array}{l}3.67 \\
2.67\end{array}$ & $\begin{array}{l}2-0 \\
1-0\end{array}$ & $\begin{array}{c}2 p^{2} 3^{3 p-2 p^{2}}(2 F)^{2}\end{array}$ \\
\hline 737.4 & 1.26 & 2.67 & $2-0$ & $\begin{array}{c}2 p^{2}{ }^{1} p-2 p^{2} \\
(3 F)\end{array}$ \\
\hline
\end{tabular}

\section{NI I P 14.49}

$5200.7 \quad 0.00 \quad 2.37 \quad \frac{1 \frac{1}{2}-2 \frac{1}{2}}{2 p^{3}} \quad{ }^{4} \mathrm{~s}^{0}-2 \mathrm{p}^{3} \quad 2_{\mathrm{D}} 0$ $\begin{array}{llllll}5198.5 & 0.00 & 2.37 & 1 \frac{1}{2}-1 \frac{1}{2} & (1 F) \\ 3466.4 & 0.00 & 3.56 & 1 \frac{1}{2}- & 2 p^{3} \mathrm{p}^{\circ}-2 \mathrm{p}^{3} & 2 \mathrm{po}\end{array}$

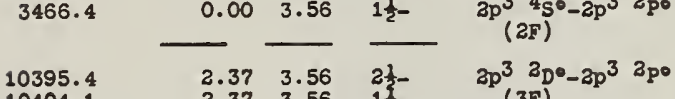
\begin{tabular}{lllll}
10404.1 & 3.37 & 3.56 & $1 \frac{1}{2}$ & (3F) \\
\hline
\end{tabular}

\section{II I P 29.49}

\begin{tabular}{|c|c|c|c|c|c|}
\hline $\begin{array}{l}6583.6 \\
6548.1 \\
6537.4\end{array}$ & $\underset{N}{N}$ & $\begin{array}{l}0.02 \\
0.01 \\
0.00\end{array}$ & $\begin{array}{l}1.89 \\
1.89 \\
1.89\end{array}$ & $\begin{array}{l}3-2 \\
1-2 \\
0-2\end{array}$ & $\begin{array}{l}2 p^{2} 3 p-2 p^{2} 1_{D} \\
(1 F)\end{array}$ \\
\hline $\begin{array}{l}3070.8 \\
3063.0\end{array}$ & & $\begin{array}{l}0.02 \\
0.01\end{array}$ & $\begin{array}{l}4.04 \\
4.04\end{array}$ & $\begin{array}{l}2-0 \\
1-0\end{array}$ & $\begin{array}{l}2 \mathrm{p}^{2}{ }^{3} \mathrm{p}-2 \mathrm{p}^{2}{ }^{1} \mathrm{~s} \\
(2 \mathrm{~F})\end{array}$ \\
\hline 3754.8 & 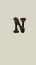 & 1.89 & 4.04 & $2-0$ & $\begin{array}{l}2 p^{2}{ }^{1} p-2 p \\
(3 F)\end{array}$ \\
\hline
\end{tabular}

\section{OI I P 13.56}

6300.23 L $\quad 0.00 \quad 1.96 \quad 2-3 \quad 2 p^{4} \quad{ }^{3} \mathrm{P}-2 \mathrm{p}^{4} \quad 1_{\mathrm{D}}$ $\begin{array}{lllll}5363.88 \mathrm{~L} & 0.02 & 1.96 & 1-2 & 2 \mathrm{p} \\ (1 F)\end{array}$ $\begin{array}{llllll}2972.3 & 0.02 & 4.17 & 1-0 & 2 p^{4} 3 \mathrm{p}-2 \mathrm{p}^{4} & 1 \mathrm{~s} \\ (2 \mathrm{~F})\end{array}$

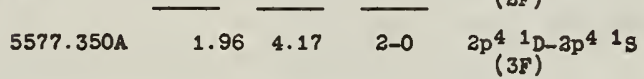

O II I P 35.00

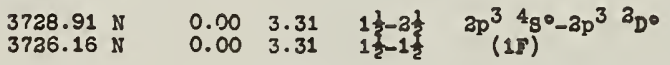

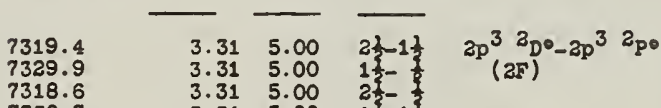
\begin{tabular}{llll}
7318.6 & 3.31 & 5.00 & $2 \frac{1}{3}$ \\
7330.7 & 3.31 & 5.00 & $1 \frac{3}{2}-1 \frac{3}{2}$ \\
\hline
\end{tabular}

O III I P 54.71

\begin{tabular}{|c|c|c|c|c|}
\hline $\begin{array}{l}5006.84 \mathrm{~N} \\
4958.91 \mathrm{~N} \\
4931.8\end{array}$ & $\begin{array}{l}0.04 \\
0.01 \\
0.00\end{array}$ & $\begin{array}{l}2.50 \\
2.50 \\
2.50\end{array}$ & $\begin{array}{l}2-2 \\
1-2 \\
0-2\end{array}$ & $\begin{array}{l}2 p^{2} p^{3} p-2 p^{3} 1^{1} D \\
(1 F)\end{array}$ \\
\hline 4363.21 & 2.50 & 5.33 & $2-0$ & $\begin{array}{c}2 p^{2} 1_{\left(p-2 p^{2}\right.}^{1}{ }_{(2 F)} \\
\end{array}$ \\
\hline
\end{tabular}

F II I P 34.84

\begin{tabular}{|c|c|c|c|c|}
\hline $\begin{array}{l}4789.5 \\
4869.3\end{array}$ & $\begin{array}{l}0.00 \\
0.04\end{array}$ & $\begin{array}{l}3.58 \\
2.58\end{array}$ & $\begin{array}{l}2-2 \\
1-2\end{array}$ & $\begin{array}{l}2 p^{4}{ }^{3} P-2 p^{4} \\
(1 F)\end{array}$ \\
\hline 4157.5 & 2.58 & 5.55 & $3-0$ & $\begin{array}{l}2 p^{4}{ }^{1} p-2 p^{4} \\
(2 F)\end{array}$ \\
\hline
\end{tabular}

III I P 62.39

$\begin{array}{llllll}5721.2 & 4.21 & 6.36 & 2 \frac{1}{-} & 2 p^{3} 2 p^{\circ}-2 p^{3} & 2 p \\ 5733.0 & 4.21 & 6.36 & 1 \frac{1}{2} & (1 F) & (1 F)\end{array}$
I A Low ${ }^{\text {HIgh }}$ J Multiplet

F IV I P 86.88

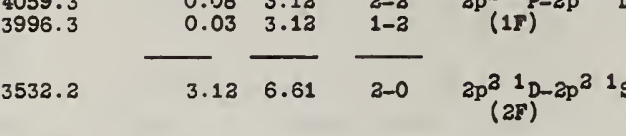

Ne III I P 63.3

$3868.74 \mathrm{~N} \quad 0.00 \quad 3.19 \quad 2-2 \quad 2 \mathrm{p}^{4} 3 \mathrm{p}-2 \mathrm{p}^{4} \quad 1_{\mathrm{D}}$

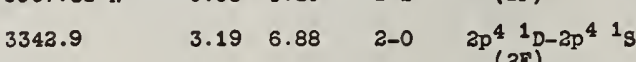

Ne IV I P 96

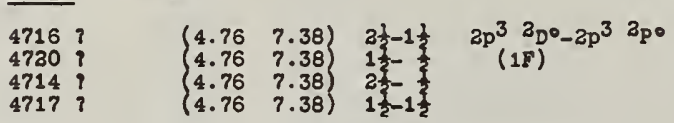

Ne V I P 136

$3425.8 N \quad 0.10(3.74) \quad 2-2 \quad 2 p^{2} 3 p-2 p^{2} 1 D$ 3973 , $\quad\left(3.74 \overline{7.89)} \frac{2-0}{2 p^{3} 11_{-2} p^{2} 1_{s}}\right.$

Na IV I P 98.5

$\begin{array}{lllll}3319.3 & 0.00 & 3.72 & 2-2 & 2 p^{4} 3 p-2 p^{4} .1 D \\ 3445.9 & 0.14 & 3.72 & 1-2 & (1 F)\end{array}$ \begin{tabular}{l}
$3445.9 \quad 0.14 \quad 3.72 \quad 1-2 \quad(15)$ \\
\hline
\end{tabular}

Na V I P 138.0

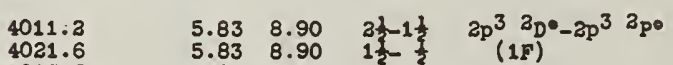
$\begin{array}{lllll}4021.6 & 5.83 & 8.90 & 1 \frac{1}{2} & \text { (1F) } \\ 4017.5 & 5.83 & 8.90 & 2 \frac{1}{2} & \\ 4015.3 & 5.83 & 8.90 & 1 \frac{3}{2}-1 \frac{3}{2} & \end{array}$

Yg VI I P 186.1

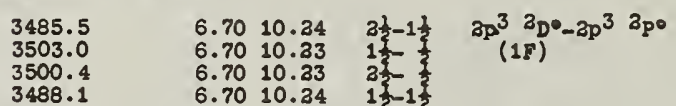

$\begin{array}{lll}6.70 & 10.23 & 3 \frac{3}{2} \\ 6.70 & 10.24 & 1 \frac{1}{2}-1 \frac{3}{2}\end{array}$

AI VII I P 241.1

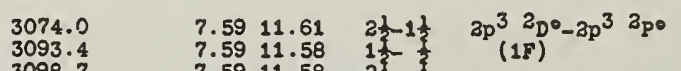
3098.7

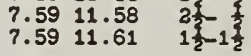

S1 I I P 8.12

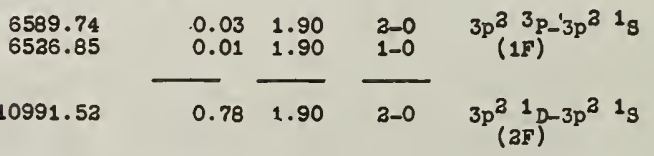

P I I P 10.9

$\begin{array}{llllll}8787.6 & 0.00 & 1.40 & 1 \frac{1}{2}-2 \frac{1}{2} & 3 \mathrm{p}^{3} 4 \mathrm{~g}^{\circ}-3 \mathrm{p}^{3} & 2 \mathrm{D}^{\circ} \\ 8799.1 & 0.00 & 1.40 & 1 \frac{1}{2}-1 \frac{2}{2} & (1 F) & \end{array}$ $\begin{array}{llllll}5332.4 & 0.00 & 2.31 & 1 \frac{1}{2}-1 \frac{1}{2} & 3 \mathrm{p}^{3} \mathrm{4}^{4}-3 \mathrm{p}^{3} & 2 \mathrm{p} 0 \\ 5339.7 & 0.00 & 2.31 & 1 \frac{2}{2}-\frac{1}{2} & (\mathrm{aF})\end{array}$

\section{P II I P 19.57}

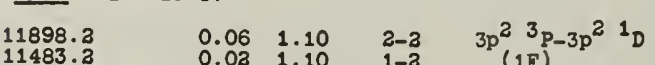
$\begin{array}{llllll}4736.6 & 0.06 & 2.66 & 3-0 & 3 p^{2} 3 p^{2}-3 p^{2} & 1 s\end{array}$

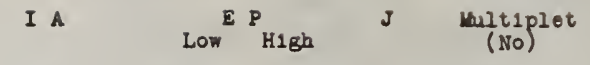

P II continued

$\begin{array}{lllll}7869.5 & 1.10 & 2.66 & 2-0 & 3 p^{2}{ }^{1} p-3 p^{2} 1_{8} \\ (3 F)\end{array}$

S I I P 10.31

\begin{tabular}{|c|c|c|c|c|}
\hline $\begin{array}{l}10819.8 \\
11305.8\end{array}$ & $\begin{array}{l}0.00 \\
0.05\end{array}$ & $\begin{array}{l}1.14 \\
1.14\end{array}$ & $\begin{array}{l}2-2 \\
1-2\end{array}$ & $\begin{array}{c}3 p^{4} 3^{3} p-3 p^{4}-1 D \\
(1 F)\end{array}$ \\
\hline $\begin{array}{l}4506.9 \\
4589.0\end{array}$ & $\begin{array}{l}0.00 \\
0.05\end{array}$ & $\begin{array}{l}2.74 \\
2.74\end{array}$ & $\begin{array}{l}2-0 \\
1-0\end{array}$ & $\begin{array}{c}3 p^{4}{ }^{3} p-3 p^{4} \\
(2 p)\end{array}$ \\
\hline 7724.7 & 1.14 & 2.74 & $2-0$ & $\begin{array}{c}3 p^{4}{ }^{1} p-3 p^{4} \\
(3 F)\end{array}$ \\
\hline
\end{tabular}

S II I P 33.3

$4068.63 \mathrm{~N} \quad 0.00 \quad 3.03 \quad 1 \frac{1}{2}-1 \frac{1}{2} \quad 3 \mathrm{p}^{3} 4 \mathrm{~g}^{0}-3 \mathrm{p}^{3} 2_{\mathrm{p}}$ $4076.32 \mathrm{~N} \quad 0.00 \quad 3.03 \quad 1 \frac{1}{2}-\frac{1}{2} \quad$ (1F)

$6717.0 \quad 0.00 \quad 1.84 \quad 1 \frac{1}{2}-2 \frac{1}{2} \quad 3 p^{3} 4 g^{0}-3 p^{3} \quad 30^{0}$ $\begin{array}{lllll}6731.3 & 0.00 & 1.83 & 1 \frac{1}{2}-1 \frac{1}{2} & (2 F)\end{array}$

$\begin{array}{cccccc}10317.7 & 1.84 & 3.03 & 2 \frac{1}{1}-1 \frac{1}{2} & 3 p^{3} \quad D^{\circ}-3 p^{3} & 3 p 0 \\ 10336.0 & 1.83 & 3.03 & 1 \frac{2}{2}-\frac{1}{2} & (3 F)\end{array}$ $\begin{array}{lllll}10336.0 & 1.83 & 3.03 & 1 \frac{1}{2}-\frac{1}{2} & \text { (35) } \\ 10369.7 & 1.84 & 3.03 & 3 & \end{array}$

\begin{tabular}{llll}
10369.7 & 1.84 & 3.03 & 2 \\
10284.3 & 1.83 & 3.03 & $1 \frac{1}{2}-1 \frac{1}{2}$ \\
\hline
\end{tabular}

SIII I P 34.9

\begin{tabular}{|c|c|c|c|c|}
\hline $\begin{array}{l}9532.1 \\
9069.4\end{array}$ & $\begin{array}{l}0.10 \\
0.04\end{array}$ & $\begin{array}{l}1.40 \\
1.40\end{array}$ & $\begin{array}{l}3-2 \\
1-2\end{array}$ & $\begin{array}{c}3 p^{2}{ }^{3} p-3 p^{2}{ }^{1} D \\
(1 F)\end{array}$ \\
\hline $\begin{array}{l}3796.7 \\
3721.1\end{array}$ & $\begin{array}{l}0.10 \\
0.04\end{array}$ & $\begin{array}{l}3.35 \\
3.35\end{array}$ & $\begin{array}{l}2-0 \\
1-0\end{array}$ & $\begin{array}{l}3 p^{2}{ }^{3} p-3 p^{2} \\
(2 F)\end{array}$ \\
\hline 6310.2 & 1.40 & 3.35 & $2-0$ & $\begin{array}{c}3 p^{2}{ }^{1} p-3 p^{3} \\
(3 F)\end{array}$ \\
\hline
\end{tabular}

S VIII I P ? $\begin{array}{rlllll}9917.9 & 0.00 & 1.34 & 1 \frac{1}{2}-\frac{1}{2} & 2 p^{5} 2 p^{\circ}-2 p^{5} & 3 p_{0} \\ (1 F)\end{array}$

S XII I P 1

$\begin{array}{lllll}7536 & 0.00 & 1.64 & \frac{1}{2}-1 \frac{1}{2} & 2 p^{2} p-3 p^{2} p \\ (1 F)\end{array}$

C1 II I P 23.70

\begin{tabular}{|c|c|c|c|c|}
\hline $\begin{array}{l}8579.5 \\
9125.8\end{array}$ & $\begin{array}{l}0.00 \\
0.09\end{array}$ & $\begin{array}{l}1.44 \\
1.44\end{array}$ & $\begin{array}{l}2-3 \\
1-2\end{array}$ & $\begin{array}{c}3 p^{4}{ }^{3} p-3 p^{4}{ }^{1} D \\
(1 F)\end{array}$ \\
\hline $\begin{array}{l}3583.2 \\
3675.0\end{array}$ & $\begin{array}{l}0.00 \\
0.09\end{array}$ & $\left\{\begin{array}{l}3.44 \\
3.44\end{array}\right)$ & $\begin{array}{l}2-0 \\
1-0\end{array}$ & $\begin{array}{c}3 p^{4} 3^{3} p-3 p^{4} 1^{1} s \\
(2 F)\end{array}$ \\
\hline 6152.9 & 1.44 & $(3.44)$ & $2-0$ & $\begin{array}{c}3 p^{4}{ }^{1} D-3 p^{4} \\
(3 F)\end{array}$ \\
\hline
\end{tabular}

CI III I P 39.7

\begin{tabular}{|c|c|c|c|c|}
\hline $\begin{array}{l}3342.7 \\
3353.4\end{array}$ & $\begin{array}{l}0.00 \\
0.00\end{array}$ & $\begin{array}{l}3.69 \\
3.68\end{array}$ & $\begin{array}{l}1 \frac{1}{2}-1 \frac{1}{2} \\
1 \frac{2}{2}\end{array}$ & $\begin{array}{c}3 p^{3}{ }^{4} g^{0}-3 p^{3} \\
(2 F)\end{array}$ \\
\hline $\begin{array}{l}5517.2 \\
5537.7\end{array}$ & $\begin{array}{l}0.00 \\
0.00\end{array}$ & $\begin{array}{l}2.24 \\
2.23\end{array}$ & $\begin{array}{l}1 \frac{1}{2}-2 \frac{1}{2} \\
1 \frac{1}{2}-1 \frac{2}{2}\end{array}$ & $\begin{array}{c}3 p^{3}{ }^{4} s^{0}-3 p^{3} \\
(1 F)\end{array}$ \\
\hline $\begin{array}{l}8481.6 \\
8501.8 \\
8550.5 \\
8433.7\end{array}$ & $\begin{array}{l}3.24 \\
2.23 \\
2.24 \\
3.23\end{array}$ & $\begin{array}{l}3.69 \\
3.68 \\
3.68 \\
3.69 .\end{array}$ & $\begin{array}{l}3 \frac{1}{2}-1 \frac{1}{2} \\
1 \frac{1}{2}-\frac{1}{2} \\
3 \frac{1}{3}-\frac{1}{3} \\
1 \frac{1}{2}-1 \frac{1}{2}\end{array}$ & $\begin{array}{c}3 p^{3}{ }^{2} p^{0}-3 p^{3} \\
(3 F)^{(3)}\end{array}$ \\
\hline
\end{tabular}

CI IV I P 53.2

\begin{tabular}{|c|c|c|c|c|}
\hline $\begin{array}{l}8046.1 \\
7530.9\end{array}$ & $\begin{array}{l}0.17 \\
0.06\end{array}$ & $\begin{array}{l}1.70 \\
1.70\end{array}$ & $\begin{array}{l}2-2 \\
1-2\end{array}$ & $\begin{array}{l}3 p^{2}{ }^{3} p-3 p^{2}{ }^{1} D \\
(1 F)\end{array}$ \\
\hline $\begin{array}{l}3203.3 \\
3118.3\end{array}$ & $\begin{array}{l}0.17 \\
0.06\end{array}$ & $\begin{array}{l}4.02 \\
4.02\end{array}$ & $\begin{array}{l}2-0 \\
1-0\end{array}$ & $\begin{array}{l}3 p^{2} 3 p-3 p^{2} 1_{s} \\
(2 F)\end{array}$ \\
\hline 5322.2 & 1.70 & 4.02 & $2-0$ & $\begin{array}{c}3 p^{2}{ }^{1} p-3 p^{2}{ }^{1} s \\
(3 F)\end{array}$ \\
\hline
\end{tabular}


I A Low ${ }_{\text {H1gh }}$ J Multiplet

A III I P 40.8

\begin{tabular}{|c|c|c|c|c|}
\hline $\begin{array}{l}7135.8 \\
7751.0\end{array}$ & $\begin{array}{l}0.00 \\
0.14\end{array}$ & $\begin{array}{l}1.73 \\
1.73\end{array}$ & $\begin{array}{l}2-2 \\
1-3\end{array}$ & $\begin{array}{c}3 p^{4} 3 p-3 p^{4} \\
(1 F)\end{array}$ \\
\hline $\begin{array}{l}3005.1 \\
109.0\end{array}$ & $\begin{array}{l}0.00 \\
0.14\end{array}$ & $\left\{\begin{array}{l}4.11 \\
4.11\end{array}\right\}$ & $\begin{array}{l}2-0 \\
1-0\end{array}$ & $\begin{array}{c}3 p^{4} 3^{3} p-3 p^{4} \\
(2 F)\end{array}$ \\
\hline 91.4 & 1.73 & $.11)$ & $2-0$ & $\begin{array}{c}3 p^{4}{ }^{1} D- \\
(3 F)\end{array}$ \\
\hline
\end{tabular}

A IV I P 61

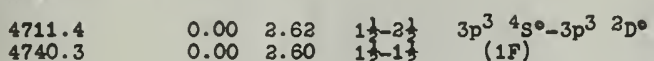

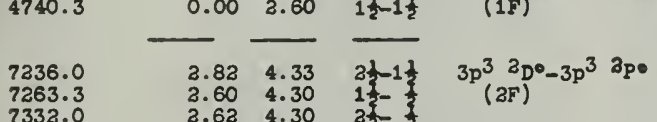
\begin{tabular}{llll}
7263.3 & 3.62 & 4.30 & 25 \\
7332.0 & 3.62 & 4.30 & 2 \\
7169.0 & 3.60 & 4.33 & $1 \frac{3}{2}-1 \frac{3}{2}$ \\
\hline
\end{tabular}

A Y I P 78

$\begin{array}{ccccc}7006.3 & 0.25 & 2.01 & 2-2 & 3 \mathrm{p}^{2} 3 \mathrm{P}-3 \mathrm{p}^{2}{ }^{1} \mathrm{D} \\ 6434.9 & 0.09 & 2.01 & 1-2 & (1 \mathrm{~F})\end{array}$

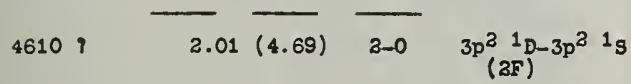

A $X$ I P ?

$\begin{array}{lllll}5534.6 & 0.00 & 2.23 & 1 \frac{1}{2}-\frac{1}{2} & 2 p^{5} 2 p^{0}-2 p^{5} \\ (1 F) & 2 p 0\end{array}$

$\begin{array}{llllll}6918 & 0.00 & 1.78 & 2-1 & 2 p^{4} 3 p-2 p^{4} & 3 p \\ (1 F)\end{array}$

A XIV I P ?

$4359, \quad 0.00 \quad 2.83 \quad \frac{1}{2-1 \frac{2}{2}} \quad \frac{2 \mathrm{p}^{2} \mathrm{p} 0-2 \mathrm{p}^{2} \mathrm{po}}{(1 \mathrm{~F})}$

K IV I P 62.5

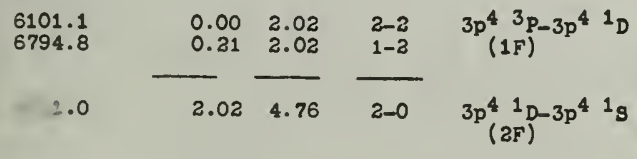

$\underline{X V}$ I P ?

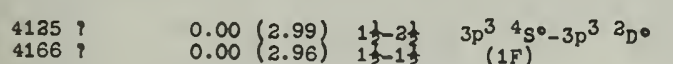

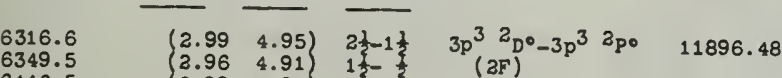
6446.5

6223.4

$\left.\begin{array}{lll}2.99 & 4.91 \\ 2.96 & 4.95\end{array}\right) \begin{aligned} & 2 \frac{1}{2}-1 \frac{2}{2} \\ & 1 \frac{1}{2}\end{aligned}$

$\underline{X I} I P$ ?

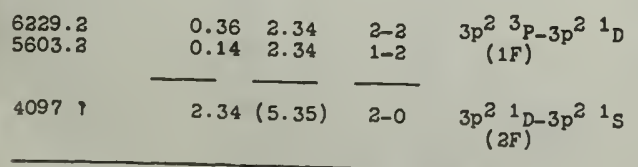

Ca I I P 6.09

$\begin{array}{ccccc}4912.82 & 0.00 & 2.51 & 0-2 & 4^{1} S-3^{3} D \\ 4916.18 & 0.00 & 2.51 & 0-1 & (1 F) \\ 4575.46 & 0.00 & 2.70 & 0-2 & 4^{1} S-3^{1} D\end{array}$

$\begin{array}{lllll}4575.46 & 0.00 & 2.70 & 0-2 & 4^{1} \mathrm{~S}-3^{1} \mathrm{D} \\ (25)\end{array}$

Ca II I P 11.82

$\begin{array}{ccccc}7291.46 & 0.00 & 1.69 & \frac{1}{2}-2 \frac{1}{2} & 4^{2} S-3^{2} D \\ 7383.88 & 0.00 & 1.69 & \frac{1}{2}-1 \frac{1}{2} & (15)\end{array}$

8347.24

8307.67
8279.99

8384.28

8326.66

8261.32

8403.62

1896.48

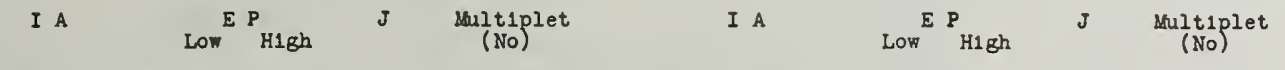

Ca V I P 84

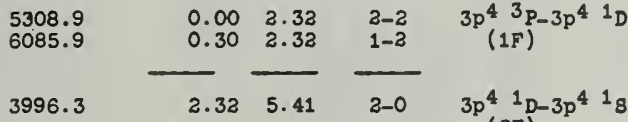

Sc VII I P ?

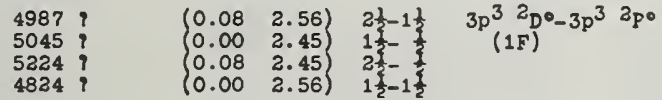

T1 I I P 6.81

Ca VI I P ?

$3646.3 \quad 0.00 \quad 3.38 \quad 1 \frac{1}{2} \quad 3 \frac{1}{3} \quad 3 p^{3}{ }^{4} g^{0}-3 p^{3} \quad D^{0}$

\begin{tabular}{|c|c|c|c|c|}
\hline $\begin{array}{l}5587.2 \\
5631.0 \\
5766.4 \\
5460.0\end{array}$ & $\begin{array}{l}3.38 \\
3.33 \\
3.38 \\
3.33\end{array}$ & $\begin{array}{l}5.59 \\
5.52 \\
5.52 \\
5.59\end{array}$ & $\begin{array}{l}2 \frac{1}{2}-1 \frac{1}{2} \\
1 \frac{1}{2}-\frac{1}{2} \\
2 \frac{1}{2}-\frac{1}{2} \\
1 \frac{1}{2}-1 \frac{1}{2}\end{array}$ & $\begin{array}{c}3 p^{3}{ }^{2} p^{0}-3 p^{3} \\
(2 F)\end{array}$ \\
\hline
\end{tabular}

Ca VII I P ?

$\begin{array}{ccccc}5615.8 & 0.50 & 2.70 & 2-2 & 3 p^{2} 3 p-3 p^{2}{ }^{1} D \\ 4938.6 & 0.20 & 2.70 & 1-2 & (1 F)\end{array}$

$3688, \quad 2.70 \overline{(6.05)} \quad \frac{2-0}{3 \mathrm{p}^{2}{ }_{(2 \mathrm{D})}-3 \mathrm{p}^{2}{ }^{1} \mathrm{~s}}$

Ca XII I P 655

$3329.3 \quad 0.00 \quad 3.71 \quad 1 \frac{1}{2}-\frac{1}{2} \quad 2 p^{5}{ }^{2} p^{\circ}-3 p^{5} \quad 2 p 0$

Ca XIII I P ?

$\begin{array}{lllll}4086.5 & 0.00 & 3.02 & 2-1 & 2 p^{4} 2 p-2 p^{4} 2_{p} \\ (1 F)\end{array}$

Ca XV I P ?

$5648 ? \quad 0.00(2.19) \quad 0-1 \quad 2 p^{2} 3 p-2 p^{2} 3 p$

Sc II I P 12.8

$\begin{array}{ccccc}9285.20 & 0.02 & 1.35 & 3-2 & a^{3} D-b^{1} D \\ 9191.34 & 0.01 & 1.35 & 2-2 & (1 F)\end{array}$

$9134.50 \quad 0.001 .35 \quad 1-2 \quad 5509.51$

$8649.11 \quad 0.02 \quad 1.45 \quad 3-0 \quad 0^{3} 0^{1} \mathrm{~s}$

$\begin{array}{llllc}8649.11 & 0.02 & 1.45 & 3-0 & a^{3} D-a^{1} S \\ 8567.60 & 0.01 & 1.45 & 2-0 & (2 F) \\ 8518.20 & 0.00 & 1.45 & 1-0 & \end{array}$

8225.35

$\begin{array}{cccc}0.02 & 1.50 & 3-2 & a^{3} D-a^{3} P \\ 0.01 & 1.49 & 2-1 & (3 F)\end{array}$

0.001 .49

$\begin{array}{ll}0.02 & 1.49 \\ 0.01 & 1.49\end{array}$

$\begin{array}{lll}0.01 & 1.49 & 2-0 \\ 0.01 & 1.50 & 2\end{array}$

$\begin{array}{lll}0.01 & 1.50 & 2-2 \\ 0.00 & 1.49 & 1-1 \\ 0.02 & 1.49 & 3-0\end{array}$

$0.02 \quad 1.49$

$0.00 \quad 1.50 \quad 1-2$

-

$0.31 \quad 1.35 \quad 2-2 \quad a^{1} D-b^{1} D$

$\begin{array}{lllll}0872.05 & 0.31 & 1.45 & 2-0 & a^{1}(4 F)\end{array}$

$10399.33 \quad 0.31 \quad 1.50 \quad 2-2 \quad a^{1}(5 F)^{(5 F}$

$\begin{array}{lllll}10456.86 & 0.31 & 1.49 & 2-1 & (6 F) \\ 10486.97 & 0.31 & 1.49 & 2-0 & \end{array}$

$\begin{array}{llllll} & & & & & \\ 10780.17 & 0.62 & 1.76 & 4-4 & a^{3} F-a^{1} G \\ 10660.35 & 0.60 & 1.76 & 3-4 & (7 F) \\ 10569.44 & 0.59 & 1.76 & 3-4 & \end{array}$

SC III I P 24.65

$\begin{array}{lllll}3945.34 & 0.02 & 3.15 & 2 \frac{1}{1}-\frac{1}{2} & 3^{2} D-4^{2} S \\ 3914.83 & 0.00 & 3.15 & 1 \frac{1}{2}-\frac{1}{2} & (1 F)\end{array}$

Sc VI I P 111 ?

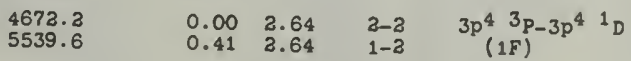

$3590.8 \quad-\quad-646.08 \quad 2-0 \quad 3 p^{4}{ }_{(2 F)}^{1} D-3 p^{4}{ }^{1} S$
12168.80

12012.60
11849.83

11856.02

11771.95

8777.26

8716.24

8669.38
8884.13

8799.09

8613.35
8588.84

8970.23

8488.93

8521.66
8367.07

8367.07
8249.61

7287.35

7213.88

7150.21

7238.29

7173.92

7126.40
7087.39

6739.63

6670.76

768.65

6692.48

6791.02
6642.57

6595.88

5828.12

5794.16
5755.60

5867.87

5812.53

5755.39
5737.59

5737.59
5699.57

5629.54

5887.73

555.33

535.09
5509.51

5614.62
5562.94

5542.54
5630.85

5630.85
5595.31

5595.31
5664.02

5584.81

5518.00
5466.67

5396.71

5358.79

5312.52

5334.30

5310.36
5286.31

5025.53

4982.92

4988.75

4946.76

4898.49

4847.01

11933.60

11881.68

11835.06

12024.89

$11950.7 ?$

11792.55

11767.30

11748.60

11679.85

11681.81
11690.94

9258.83

9288.45

9281.86

9189.22

9235.10

9245.82

9137.01
9199.44
$0.05 \quad 1.06$

$0.02 \quad 1.05$

$0.00 \quad 1.04$

$\begin{array}{ll}0.02 & 1.06 \\ 0.00 & 1.05\end{array}$

$0.00 \quad 1.06$

$0.05 \quad 1.45$

$0.02 \quad 1.44$

$0.00 \quad 1.42$

$\begin{array}{ll}0.05 & 1.44 \\ 0.02 & 1.42\end{array}$

$\begin{array}{ll}0.02 & 1.42 \\ 0.02 & 1.45\end{array}$

$0.00 \quad 1.44$

$0.05 \quad 1.42$

$0.05 \quad 1.50$

$0.02 \quad 1.50$

0.051 .74

$0.05 \quad 1.74$

0.001 .73

0.051 .73

0.021 .74

$\begin{array}{ll}0.00 & 1.73 \\ 0.00 & 1.74\end{array}$

$0.05 \quad 1.88$

$0.02 \quad 1.87$

$0.00 \quad 1.87$

$\begin{array}{ll}0.05 & 1.87 \\ 0.02 & 1.87\end{array}$

0.051 .87

$\begin{array}{ll}0.00 & 1.88\end{array}$

$0.05 \quad 2.17$

$0.02 \quad 2.15$

$0.00 \quad 2.14$

$\begin{array}{ll}0.05 & 2.15 \\ 0.02 & 2.14\end{array}$

$0.02 \quad 2.17$

$\begin{array}{ll}0.00 & 2.15 \\ 0.00 & 2.17\end{array}$

$0.05 \quad 2.24$

$0.02 \quad 2.23$

$0.00 \quad 2.22$

$\begin{array}{ll}0.02 & 2.24 \\ 0.00 & 2.23\end{array}$

$\begin{array}{ll}0.00 & 2.23 \\ 0.00 & 2.24\end{array}$

$0.05 \quad 2.25$

$\begin{array}{ll}0.02 & 2.24 \\ 0.00 & 2.23\end{array}$

$0.05 \quad 2.24$

$0.02 \quad 2.23$

$0.05 \quad 2.23$

$0.05 \quad 2.26$

$\begin{array}{ll}0.02 & 3.26 \\ 0.00 & 2.26\end{array}$

$0.05 \quad 2.33$

$0.02 \quad 2.32$

$\begin{array}{ll}0.00 & 2.32 \\ 0.02 & 2.33\end{array}$

$0.00 \quad 2.32$

$0.00 \quad 2.33$

$0.02 \quad 2.48$

$0.00 \quad 2.48$

$0.05 \quad 2.49$

$0.02 \quad 2.49$

$0.05 \quad 2.57$

$0.02 \quad 2.57$

$0.84 \quad 1.88$

$0.84 \quad 1.88$

$\begin{array}{ll}0.83 & 1.87 \\ 0.82 & 1.87\end{array}$

$0.84 \quad 1.87$

$\begin{array}{ll}0.84 & 1.87 \\ 0.83 & 1.87\end{array}$

$0.83 \quad 1.88$

$\begin{array}{ll}0.83 & 1.88 \\ 0.82 & 1.87\end{array}$

$0.81 \quad 1.87$

0.841 .8 ?

$0.82 \quad 1.88$

0.811 .87

$0.83 \quad 2.17$

$0.82 \quad 2.15$

$0.81 \quad 2.14$

0.812 .15

$0.81 \quad 2.14$

$0.81 \quad 2.17$ $\begin{array}{cc}4-2 & a^{3} F-a^{3} P \\ 3-1 & (1 F) \\ 2-0 & \\ 3-2 & \\ 2-1 & \\ 2-2 & \\ 4-4 & a^{3} F-b^{3} F \\ 4-3 & (2 F) \\ 2-2 & \\ 4-3 & \\ 3-2 & \\ 3-4 & \\ 3-3 & \\ 4-2 & \\ 2-4 & \\ 4-4 & a^{3} F-a^{1} Q \\ 3-4 & (3 F) \\ 2-4 & \\ 4-3 & a^{3} F-a^{5} P \\ 3-2 & (4 F) \\ 2-1 & \\ 4-2 & \\ 3-1 & \\ 3-3 & \\ 2-2 & \\ 3-3 & \\ 4-5 & a^{3} F-a^{3} G \\ 3-4 & (5 F) \\ 2-3 & \end{array}$

$a^{3} F-a^{3} D$

3-a $\quad(6 F)$

3-1

$2-2$
$2-3$

4-2 $\quad a^{3} F-b^{3} P$

3-2

2-2

4-6 $\quad a^{3} F-a^{3} H$

$2-4$
$4-5$

$3-4$
$4-4$

$\begin{array}{cc}4-4 & a^{3} F-b^{1} c \\ 3-4 & (9 F)\end{array}$

4-2 $\quad a^{3} F-c^{3} p$

3-1 (10F)

3-2

$2-2$

3-1 $a^{3} F-a^{1} P$

4-2 $\quad a^{3} F-b^{1} D$

(12F)

$\begin{array}{cc}4-5 & a^{3} F-a^{1} H \\ 3-5 & (13 F)\end{array}$

$\begin{array}{cc}5-5 & a^{5} F-a^{3} G \\ 4-4 & (14 F)\end{array}$

$5-4$

$4-3$

$3-4$
$2-3$ 
10 I A LOW ${ }_{\text {H1gh }}$ J Lurtiplet T1 I continued \begin{tabular}{ll} 
& 8705. \\
& 8721 \\
Be $\quad 8739$ \\
\hline $45 \quad 8658$ \\
\hline & 8689 \\
\hline & 8626 \\
\hline
\end{tabular} $705.08 \quad 0.82 \quad 0.81$ 739.71 8626.85

8808.47 8787.81 8848.50 8851.45 5708.23

8160.66 8176.33 8153.46 8119.46 8143.37
8091.87

4521.76

4515.52

4504.71

4504.71

4536.05

4526.55

4508.52

4501.56

4498.90

4497.23

4484.84

4488.76

4472.37

9720.20

9831.29
9884.29

9180.13

9308.03

8576.73

8645.95

7805.66

7717.29

4430.79

11185.14

11193.04
11191.43

11332.50

11261.79

11402.97

11049.28
11123.53

10475.96

10447.44

10568.84

10356.68

10386.86

10642.86
10297.14

9697.42

9674.66

9778.67

9681.84

(622.68

9786.00
9544.00

8723.13

8640.27
8598.79

8612.91

8532.12

11665.66

11520.46

11084.87

11521.31

$\begin{array}{ll}0.82 & 2.24 \\ 0.81 & 2.23 \\ 0.81 & 2.22 \\ 0.81 & 2.24 \\ 0.81 & 2.23 \\ 0.81 & 2.24 \\ 0.84 & 2.25 \\ 0.83 & 2.24 \\ 0.82 & 2.23 \\ 0.84 & 2.24 \\ 0.83 & 2.23 \\ 0.84 & 2.23 \\ 0.83 & 2.25 \\ 0.82 & 2.24 \\ 0.81 & 2.23\end{array}$

$0.82 \quad 2.33$

$0.81 \quad 2.32$

$\begin{array}{ll}0.81 & 2.32 \\ 0.81 & 2.33\end{array}$

$\begin{array}{ll}0.81 & 2.33 \\ 0.81 & 2.32\end{array}$

$0.81 \quad 2.33$

$0.84 \quad 3.57$

$0.83 \quad 3.57$

0.813 .55

$0.81 \quad 3.55$

$0.84 \quad 3.57$

$0.82 \quad 3.55$

0.81 3.55

$0.82 \quad 3.57$

0.81 3.56

$\begin{array}{ll}0.81 & 3.55 \\ 0.82 & 3.57\end{array}$

$0.81 \quad 3.57$

.81 3.56

$0.81 \quad 3.5$ ?

$0 . 9 0 \longdiv { 2 . 1 7 }$

$\begin{array}{ll}0.90 & 2.17 \\ 0.90 & 2.15\end{array}$

$0.90 \quad 2.24$

$\begin{array}{ll}0.90 & 2.24 \\ 0.90 & 2.23 \\ 0.90 & 2.22\end{array}$

$0.90 \quad 2.33$

$\begin{array}{ll}0.90 & 2.33 \\ 0.90 & 2.32 \\ 0.90 & 2.32\end{array}$

$0.90 \quad 2.49$

-

$\begin{array}{ll}1.06 & 2.17 \\ 1.05 & 2.15\end{array}$

$1.04 \quad 2.14$

$1.06 \quad 2.15$

$1.05 \quad 2.14$

$\begin{array}{ll}1.06 & 2.14 \\ 1.05 & 2.17 \\ 1.04 & 2.15\end{array}$

$1.06 \quad 2.24$
1.05

$\begin{array}{ll}1.06 & 2.24 \\ 1.06 & 2.23 \\ 1.23\end{array}$

$\begin{array}{ll}1.06 & 2.23 \\ 1.05 & 2.22\end{array}$

$\begin{array}{ll}1.05 & 2.22 \\ 1.05 & 2.24\end{array}$

$1.04 \quad 2.23$

$\begin{array}{ll}1.06 & 2.22 \\ 1.04 & 2.24\end{array}$

\begin{tabular}{ll}
$1.06 \quad 2.33$ \\
\hline
\end{tabular}

$\begin{array}{ll}1.05 & 2.32 \\ 1.06 & 2.32\end{array}$

$\begin{array}{ll}1.06 & 2.32 \\ 1.05 & 2.32\end{array}$

$\begin{array}{ll}1.05 & 2.33 \\ 1.04 & 2.32\end{array}$

$\begin{array}{ll}1.04 & 2.32 \\ 1.06 & 2.32\end{array}$

$1.04 \quad 2.33$

$1.06 \quad 2.48$

$\begin{array}{ll}1.05 & 2.48 \\ 1.04 & 2.48\end{array}$

$1.06 \quad 2.49$

$1.05 \frac{2.49}{2.49}$

$\begin{array}{ll}1.44 & 2.49 \\ 1.42 & 2.49\end{array}$

$1.45 \quad 2.57$

$1 . 5 0 \longdiv { 2 . 5 7 }$
$0.90 \quad 2.48$ $\begin{array}{lc}3-2 & a^{5} F-b^{3} P \\ 2-1 & (16 F)\end{array}$

$2-2$

$1-1$
$1-2$

$\begin{array}{ll}5-6 & a^{5} F-a^{3} H \\ 4-5 & (17 F)\end{array}$

$5-5$
$4-4$

$-6$

$\begin{array}{ll}3-2 & a^{5} F-c^{3} P \\ 2-1 & (18 F) \\ 1-0 & \end{array}$

$1-2$
$2-2$

$1-1$
$1-2$

5-4 $\quad a^{5} F-a^{5} D$

$4-3$
$3-2$
$2-1$

$2-1$
$1-0$
$5-3$
$4-2$
$3-1$

$4-2$
$3-1$

$2-0$
$4-4$

$3-3$
$2-2$
$1-1$

$\frac{1-1}{3-4}$

2-3

$1-2$
$2-4$
$1-3$

$1-3$

$2-3$
$2-2$
$2-1$

$a^{1} D-a^{3} D$

$a^{1} D-b^{3} p$

$\begin{array}{ll}2-2 & a \\ 2-1 & (21 F)\end{array}$

$\begin{array}{cc}2-\hat{2} & a^{1} D-c^{3} P \\ 2-1 & (22 F)\end{array}$

2-1 $\quad a^{1} D-a^{1} p$

$2-2 \quad a_{D-b}\left(\frac{23 F}{D}\right)$

2-3 $\quad \begin{aligned} & 24 F \\ & (2-a F)\end{aligned}$

$a^{3} p-a^{3} p$
$(26 F)$

$\begin{array}{ll}2-3 & a^{3} P-a^{3} D \\ 1-0 & (26 F)\end{array}$

$0-1$
$2-2$

$\frac{1-1}{2-1}$

$2-1$
$1-3$
$0-2$

$\begin{array}{ll}2-2 & a^{3} P-b^{3} p \\ 1-1 & (27 F)\end{array}$

$1-1$
$2-1$
$1-0$

$2-1$
$1-0$
$1-2$

$0-1$
$2-0$

$0-3$

$\begin{array}{ll}2-2 & a^{3} P-c^{3} P \\ 1-1 & (28 F)\end{array}$

$2-1$

$1-0$
$1-3$
$0-1$

O-1

$0-2$

$\begin{array}{cc}2-1 & a^{3} p-a^{1} p \\ 1-1 & (29 F)\end{array}$

$2-2 \quad a^{3} P-b^{1} D$

$\overline{3-2} \quad b^{3} F-b^{1} D$

$(31 \mathrm{~F})$

$(32 F)$
${ }^{3}-a^{1}$
$(G-a$

$a^{1} G-a^{1} H$

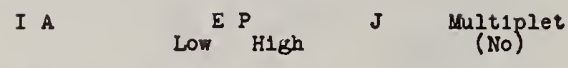

T1 II I P 13.6

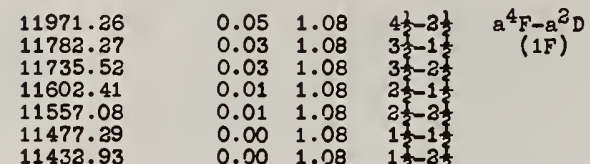

11458.27

11396.50

11618.68

11342.12

11228.14
11078.26

11110.92

10956.10
10901.79

10784.80
10758.32

10758.32

10747.64
10676.61

10608.18

10640.19
10503.47

10116.66

$10148.5 ?$

10379.73

10300.86

10203.05

10163.13

10125.99
10066.92

10066.98

8085.17

8060.16

7975.58

7894.10

7916.25
7835.98

8074.29

8028.94

7966.36
7945.03

6725.67

6647.05
6732.03

6650.61

6589.42

6548.87

6095.96

6087.77

6047.46

6053.14
6013.28

4877.01

4823.44
4862.80

4837.43

4793.03
4806.83

4771.54
4785.21

4031.15

4041.57

4004.07
4020.20

3983.08

4005.07
3968.23

11857.96

11884.57

11714.28

11778.39
11611.10

11117.80

11178.94
11024.82

11185.70

11173.94

11151.54

11080.02

0965.77

8661.20
8661.96

8565.9

8585.04

8490.71
8529.50

8436.37

\subsection{1 .08}

$0.05 \quad 1.13$

$\begin{array}{ll}0.03 & 1.11 \\ 0.05 & 1.11\end{array}$

$0.03 \quad 1.13$

$0.01 \quad 1.13$

0.001 .11

$0.03 \quad 1.16$

0.031 .18

0.011 .16

$0.01 \quad 1.18$

$\begin{array}{ll}0.00 & 1.16 \\ 0.00 & 1.18\end{array}$

$0.01 \quad 1.23$

$\begin{array}{ll}0.00 & 1.22 \\ 0.00 & 1.23\end{array}$

$0.05 \quad 1.24$

$0.01 \quad 1.22$

$0.03 \quad 1.24$

$0.00 \quad 1.22$

$\begin{array}{ll}0.01 & 1.24 \\ 0.00 & 1.23\end{array}$

0.001 .24

$0.05 \quad 1.57$

$\begin{array}{ll}0.03 & 1.56 \\ 0.03 & 1.57\end{array}$

$0.01 \quad 1.56$

0.011 .57

0.001 .57

$0.05 \quad 1.58$

$0.05 \quad 1.56$

$\begin{array}{ll}0.03 & 1.58 \\ 0.01 & 1.56\end{array}$

$0.05 \quad 1.88$

$0.03 \quad 1.88$

$0.03 \quad 1.88$

0.011 .88

0.001 .88

$0.03 \quad 2.05$

$0.01 \quad 2.04$

$0.00 \quad 2.04$

$0.00 \quad 2.05$

$\begin{array}{ll}0.05 & 2.58 \\ 0.03 & 3.59\end{array}$

$\begin{array}{ll}0.03 & 3.59 \\ 0.05 & 3.59\end{array}$

$0.03 \quad 3.58$

$\begin{array}{ll}0.01 & 3.59 \\ 0.01 & 2.58\end{array}$

$\begin{array}{ll}0.01 & 2.59 \\ 0.00 & 2.58\end{array}$

$0.05 \quad 3.11$

$\begin{array}{ll}0.03 & 3.08 \\ 0.03 & 3.11 \\ 0.01 & 3\end{array}$

$\begin{array}{ll}0.03 & 3.11 \\ 0.01 & 3.08\end{array}$

0.013 .11

\begin{tabular}{ll}
0.00 & 3.08 \\
0.00 & 3.11 \\
\hline
\end{tabular}

$0.13 \quad 1.18$

$\begin{array}{ll}0.12 & 1.16 \\ 0.11 & 1.16\end{array}$ 


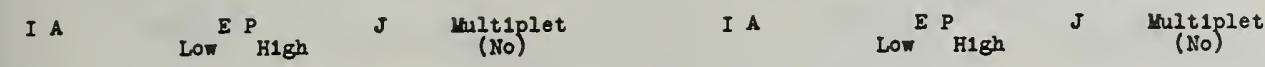

I1 II continued

\begin{tabular}{|c|c|c|c|c|}
\hline 11478.93 & 1.56 & 3.63 & $1 \frac{1}{2}-\frac{1}{2}$ & $b^{2} p-a^{3} s$ \\
\hline $\begin{array}{l}8039.68 \\
8106.38 \\
8193.33 \\
7956.90\end{array}$ & $\begin{array}{l}1.57 \\
1.56 \\
1.57 \\
1.56\end{array}$ & $\begin{array}{l}3.11 \\
3.08 \\
3.08 \\
3.11\end{array}$ & $\begin{array}{l}2 \frac{1}{2}-3 \frac{1}{2} \\
1 \frac{1}{2} \\
2 \frac{1}{2} \\
1 \frac{1}{2} \\
1 \frac{1}{2}-3 \frac{1}{2}\end{array}$ & $\begin{array}{l}b^{2} \mathrm{D}-\mathrm{C}^{3 D} \mathrm{D} \\
(39 \mathrm{~F})\end{array}$ \\
\hline
\end{tabular}

T1 III I P 37.6

13417.8 13061.0

9706.8

9594.5

488.

9438.3

9334.8

7153.8

7033.0

$3 m \cdot \frac{3}{30}$

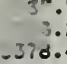

3008.4

3608.5

3682.9

3638.4

3640.6

3593.3

3328.

3307.6

$\begin{array}{lllll}4140.4 ? & 1.73 & 4.73 & 0-39 & a^{1} S-a^{3} \mathrm{D} \\ \$ 183.8 & 1.73 & 4.70 & 0-11 & (8 F)\end{array}$

3815.5

$1.73 \quad 5.15 \quad 0-3 \quad a^{1} s-b^{1} D$

4160.9
4300.6

$\begin{array}{llll}1.78 & 4.74 & 4-3 & a^{1} G-a^{3} D \\ 1.78 & 4.72 & 4-3 & (10 F)\end{array}$

3681.3

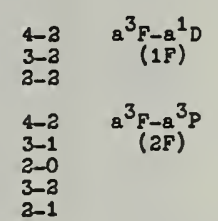

0.001 .33

$\begin{array}{cccc}0.05 & 1.78 & 4-4 & a^{3} F-8^{1} G \\ 0.02 & 1.78 & 3-4 & (3 F) \\ 0.00 & 1.78 & 2-4 & \end{array}$

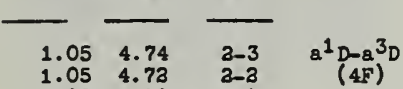

$1.05 \quad 5.15 \quad 2-2 \quad a^{1} D-b^{1} D$

$\begin{array}{llll}1.32 & 4.74 & -2-3 & a^{3} P-a^{3} D \\ 1.31 & 4.73 & -1-2 & (6 F)\end{array}$

$\begin{array}{lll}1.30 & 4.70 & 0-1\end{array}$

1.314 .70

$.31 \quad 4.74 \quad 1-3$

$\begin{array}{llll}1.32 & 5.15 & 2-3 & a^{3} P-b^{1} D \\ 1.31 & 5.15 & 1-3 & (7 F)\end{array}$ $\begin{array}{lll}1.33 & 4.70 \quad 3-1\end{array}$

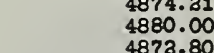

4873.80

4871.43

9570.24

9454.15

9358.90

9395.23

9256.51

9209.25

9379.59

9368.77

9144.25

9165.30

9166.00

9043.53
9096.76

8698.69

8627.35

8579.15

8698.18

8553.8

8510.34
8490.44

7477.36

7431.08

7387.74

7540.14
7475.84

7475.84
7370.00

7344.03
7321.87

VII I P 14.1

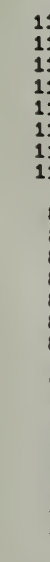

$\begin{array}{lllc}0.04 & 1.12 & 4-4 & a^{5} D-a^{3} F \\ 0.03 & 1.09 & 3-3 & (1 F) \\ 0.01 & 1.07 & 2-2 & \\ 0.04 & 1.09 & 4-3 & \\ 0.03 & 1.07 & 3-3 & \\ 0.03 & 1.12 & 3-4 & \\ 0.01 & 1.09 & 2-3 & \\ 0.00 & 1.07 & 1-2 & \\ 0.03 & 1.47 & 3-3 & a^{5} D-a^{3} P \\ 0.01 & 1.42 & 2-1 & (3 F) \\ 0.00 & 1.39 & 1-0 & \\ 0.01 & 1.47 & 2-3 & \\ 0.00 & 1.43 & 1-1 & \\ 0.00 & 1.47 & 1-3 & \\ 0.00 & 1.43 & 0-1 & \\ 0.04 & 1.68 & 4-4 & { }^{5} D-b^{3} F \\ 0.03 & 1.87 & 3-3 & (3 F) \\ 0.01 & 1.67 & 3-2 & \\ 0.04 & 1.87 & 4-3 & \\ 0.03 & 1.67 & 3-3 & \\ 0.03 & 1.68 & 3-4 & \\ 0.01 & 1.87 & 2-3 & \\ 0.00 & 1.67 & 1-3 & \end{array}$

6497.76

6497.76
6456.04

6415.69

6431.11

6405.6

8381.13

6373.11

5662.63

5605.36

5611.94

5575.69

5573.84
5550.35

7533.84

7497.68
7469.44

7571.69

7526.94

7440.63
7430.36
$0.39 \overline{1.68}$

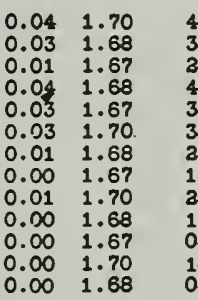

$0.04 \quad 3.37$ $0.03 \quad 3.26$

0.013 .36

0.013 .36

$\begin{array}{ll}0.00 & 3.36 \\ 0.01 & 3.37\end{array}$

$\begin{array}{lll}0.01 & 2.37 \\ 0.00 & 3.26 \\ 0.00 & 3.36\end{array}$

$0.03 \quad 2.36$

$0.01 \quad 3.37$

$0.01 \quad 3.36$

$0.00 \quad 3.37$

$\begin{array}{ll}0.00 & 3.36 \\ 0.00 & 3.37\end{array}$

$0.03 \quad 3.51$

$\begin{array}{ll}0.01 & 3.48 \\ 0.00 & 3.48\end{array}$

$\begin{array}{ll}0.01 & 3.51 \\ 0.00 & 3.51\end{array}$

$0.00 \quad 3.48$

$0.00 \quad 3.48$

$0.04 \quad 3.55$

$\begin{array}{ll}0.03 & 3.55 \\ 0.01 & 3.53\end{array}$

$0.03 \quad 3.55$

0.01 3.55

$0.01 \quad 3.55$

$\begin{array}{lll}0.00 & 3.55 \\ 0.00 & 3.53\end{array}$

$0.39 \begin{array}{ll}0.68 \\ 0.37 & 1.67\end{array}$

0.351 .67

$0.35 \quad 1.67$

$\begin{array}{lll}0.33 & 1.67 & 2-3 \\ 0.35 & 1.68 & 3-4 \\ 0.33 & 1.67 & 2-3\end{array}$

$\begin{array}{lll}0.33 & 1.67 & 2-3 \\ 0.32 & 1.67 & 1-3\end{array}$

$\begin{array}{ll}0.37 & 1.70 \\ 0.35 & 1.68\end{array}$

$\begin{array}{ll}0.35 & 1.68 \\ 0.33 & 1.67\end{array}$

$0.35 \quad 1.70$

0.331 .68

0.331 .67

$\begin{array}{ll}0.33 & 1.70 \\ 0.33 & 1.68\end{array}$

$0.39 \quad 1.81$

$\begin{array}{ll}0.37 & 1.81 \\ 0.35 & 1.70 \\ 0.39 & 1.80\end{array}$

$0.39 \quad 1.80$

$0.37 \quad 1.79$

$\begin{array}{ll}0.37 & 1.81 \\ 0.35 & 1.80\end{array}$

$0.33 \quad 1.79$

$\begin{array}{ll}0.39 & 3.04 \\ 0.37 & 3.03\end{array}$

$\begin{array}{ll}0.37 & 3.03 \\ 0.35 & 2.03\end{array}$

$0.39 \quad 2.03$

$0.37 \quad 2.03$

$\begin{array}{ll}0.37 & 3.04 \\ 0.35 & 3.03\end{array}$
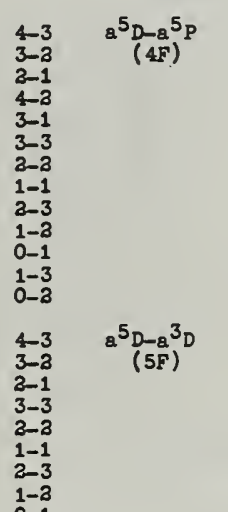

$3-3$
$2-1$
$1-0$
$2-2$
$1-1$
$1-2$
$0-1$

$\frac{a^{5} p-b^{3} p}{(6 F)}$

$\begin{array}{lc}3-3 & a^{5} p-c^{3} p \\ 1-0 & (75)\end{array}$

$1-1$
$1-2$
$0-1$

$\begin{array}{cc}4-3 & a^{5} \mathrm{D}-\mathrm{b}^{3} \mathrm{D} \\ 3-2 & (8 \mathrm{~F}) \\ 2-1 & \end{array}$

$0.37 \quad 3.37$

$0.35 \quad 2.36$

$0.33 \quad 3.26$

$0.35 \quad 3.37$

$0.33 \quad 3.36$

$0.33 \quad 2.36$

$0.32 \quad 3.36$

$0.37 \quad 3.55$

$0.35 \quad 3.55$

0.35 3.55

$0.33 \quad 3.55$

0.323 .53

\begin{tabular}{ll}
0.33 & 3.55 \\
0.32 & 3.55 \\
\hline
\end{tabular}

$1.13 \overline{2.27}$

$\begin{array}{ll}1.12 & 2.27 \\ 1.09 & 2.36\end{array}$

$1.07 \quad 3.26$

$\begin{array}{ll}1.09 & 2.27\end{array}$

$\begin{array}{ll}1.07 & 3.26 \\ 1.07 & 2.27\end{array}$
I A ${ }_{\text {LOI }}{ }^{\text {H1gh }}$

J Multiplet

V II cont1nued

\begin{tabular}{|c|c|c|c|c|}
\hline $\begin{array}{l}9982.17 \\
9733.53\end{array}$ & $\begin{array}{l}1.13 \\
1.09\end{array}$ & $\begin{array}{l}3.36 \\
2.36\end{array}$ & $\begin{array}{l}4-4 \\
3-4\end{array}$ & $\begin{array}{c}a^{3} F-b^{1} G G \\
(16 F)\end{array}$ \\
\hline $\begin{array}{l}8674.27 \\
8490.18 \\
8413.83 \\
8485.90 \\
8347.16 \\
8343.03\end{array}$ & $\begin{array}{l}1.13 \\
1.09 \\
1.07 \\
1.09 \\
1.07 \\
1.07\end{array}$ & $\begin{array}{l}3.55 \\
2.55 \\
3.53 \\
2.55 \\
2.55 \\
3.55\end{array}$ & $\begin{array}{l}4-3 \\
3-3 \\
2-1 \\
3-3 \\
2-3 \\
2-3\end{array}$ & $\begin{array}{l}a^{3} F-b^{3} D \\
(17 F)\end{array}$ \\
\hline $\begin{array}{l}8335.69 \\
8101.03\end{array}$ & $\begin{array}{l}1.09 \\
1.07\end{array}$ & $\begin{array}{l}3.59 \\
3.59\end{array}$ & $\begin{array}{l}3-3 \\
3-3\end{array}$ & $\begin{array}{l}a^{3} F-a^{1} D \\
(18 F)\end{array}$ \\
\hline $\begin{array}{l}6114.85 \\
6040.31\end{array}$ & $\begin{array}{l}1.09 \\
1.07\end{array}$ & $\begin{array}{l}3.11 \\
3.11\end{array}$ & $\begin{array}{l}3-3 \\
2-2\end{array}$ & $\begin{array}{l}a^{3} F-b^{1} D \\
(19 F)\end{array}$ \\
\hline $\begin{array}{l}5634.78 \\
5554.68 \\
5493.10\end{array}$ & $\begin{array}{l}1.13 \\
1.09 \\
1.07\end{array}$ & $\begin{array}{l}3.31 \\
3.31 \\
3.31\end{array}$ & $\begin{array}{l}4-3 \\
3-3 \\
3-3\end{array}$ & $\begin{array}{c}a^{3} F-a^{1} F \\
(a O F)\end{array}$ \\
\hline 11918.75 & 1.43 & 3.46 & $1-0$ & $a^{3} P-a^{1} s$ \\
\hline $\begin{array}{l}11852.49 \\
11658.88 \\
12219.66 \\
11568.38 \\
11324.18 \\
11368.31\end{array}$ & $\begin{array}{l}1.47 \\
1.48 \\
1.47 \\
1.48 \\
1.43 \\
1.39\end{array}$ & $\begin{array}{l}3.51 \\
3.48 \\
2.48 \\
3.49 \\
3.51 \\
2.48\end{array}$ & $\begin{array}{l}2-3 \\
1-1 \\
2-1 \\
1-0 \\
1-3 \\
0-1\end{array}$ & 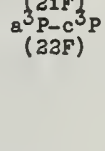 \\
\hline $\begin{array}{l}11471.69 \\
10983.33 \\
10835.23 \\
11479.51 \\
11098.96 \\
11606.00\end{array}$ & $\begin{array}{l}1.47 \\
1.42 \\
1.39 \\
1.47 \\
1.48 \\
1.47\end{array}$ & $\begin{array}{l}2.55 \\
3.55 \\
3.53 \\
3.55 \\
3.53 \\
2.53\end{array}$ & $\begin{array}{l}2-3 \\
1-2 \\
0-1 \\
2-3 \\
1-1 \\
2-1\end{array}$ & $\begin{array}{l}a^{3}{ }^{P}-b^{3} D \\
(a 3 F)\end{array}$ \\
\hline $\begin{array}{l}11019.11 \\
10561.05\end{array}$ & $\begin{array}{l}1.47 \\
1.48\end{array}$ & $\begin{array}{l}3.59 \\
3.59\end{array}$ & $\begin{array}{l}2-3 \\
1-2\end{array}$ & $\begin{array}{c}a^{3} P-a^{1} D \\
(34 F)\end{array}$ \\
\hline $\begin{array}{l}9644.96 \\
9392.19 \\
9106.60\end{array}$ & $\begin{array}{l}1.47 \\
1.48 \\
1.39\end{array}$ & $\begin{array}{l}2.75 \\
3.75 \\
3.75\end{array}$ & $\begin{array}{l}2-1 \\
1-1 \\
0-1\end{array}$ & $\begin{array}{c}\mathrm{a}^{3} \mathrm{P}_{\mathrm{P}} \mathrm{g}^{1} \mathrm{P} \\
(35 \mathrm{~F})\end{array}$ \\
\hline $\begin{array}{l}7536.46 \\
7309.90\end{array}$ & $\begin{array}{l}1.47 \\
1.42\end{array}$ & $\begin{array}{l}3.11 \\
3.11\end{array}$ & $\begin{array}{l}2-3 \\
1-3\end{array}$ & $\begin{array}{l}a^{3} P-b^{1} D \\
(36 F)\end{array}$ \\
\hline $\begin{array}{l}9356.40 \\
9382.92 \\
9317.51\end{array}$ & $\begin{array}{l}1.57 \\
1.56 \\
1.55\end{array}$ & $\begin{array}{l}3.89 \\
3.89 \\
3.89\end{array}$ & $\begin{array}{l}6-5 \\
5-5 \\
4-5\end{array}$ & $\begin{array}{l}a^{3} \mathrm{~K}-\mathrm{a}^{1} \mathrm{H} \\
(\mathrm{a} 7 \mathrm{~F})\end{array}$ \\
\hline $\begin{array}{l}8582.53 \\
8544.49\end{array}$ & $\begin{array}{l}1.67 \\
1.67\end{array}$ & $\begin{array}{l}3.11 \\
3.11\end{array}$ & $\begin{array}{l}3-3 \\
3-3\end{array}$ & $\begin{array}{c}b^{3} F-b^{1} D \\
(38 F)\end{array}$ \\
\hline $\begin{array}{l}7556.03 \\
7518.35 \\
7489.15\end{array}$ & $\begin{array}{l}1.68 \\
1.67 \\
1.67\end{array}$ & $\begin{array}{l}3.31 \\
3.31 \\
3.31\end{array}$ & $\begin{array}{l}4-3 \\
3-3 \\
2-3\end{array}$ & $\begin{array}{c}b^{3} F-a^{1} F \\
(a 9 F)\end{array}$ \\
\hline $\begin{array}{l}11444.66 \\
11315.58\end{array}$ & $\begin{array}{l}1.81 \\
1.80\end{array}$ & $\begin{array}{l}3.89 \\
3.89\end{array}$ & $\begin{array}{l}5-5 \\
4-5\end{array}$ & $\begin{array}{c}a^{3} G-a^{1} H \\
(30 F)\end{array}$ \\
\hline $\begin{array}{l}8138.62 \\
8076.58\end{array}$ & $\begin{array}{l}1.80 \\
1.79 \\
\end{array}$ & $\begin{array}{l}3.31 \\
3.31\end{array}$ & $\begin{array}{l}4-3 \\
3-3\end{array}$ & $\begin{array}{c}a^{3} a-a^{1} F \\
(31 F)\end{array}$ \\
\hline $\begin{array}{l}9595.85 \\
9538.24\end{array}$ & $\begin{array}{l}3.03 \\
3.03\end{array}$ & $\begin{array}{l}3.31 \\
3.31\end{array}$ & $\begin{array}{l}4-3 \\
3-3\end{array}$ & $\begin{array}{l}b^{3} a-a^{1} F \\
(3 a F)\end{array}$ \\
\hline
\end{tabular}

VIII I P 39.6

\begin{tabular}{|c|c|c|c|c|}
\hline $\begin{array}{l}8745.0 \\
8735.0 \\
8683.4 \\
8599.1 \\
8625.8 \\
8493.1\end{array}$ & $\begin{array}{l}0.04 \\
0.03 \\
0.00 \\
0.03 \\
0.00 \\
0.00\end{array}$ & $\begin{array}{l}1.45 \\
1.43 \\
1.43 \\
1.45 \\
1.43 \\
1.45\end{array}$ & 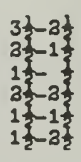 & $\begin{array}{l}3 d^{3}{ }^{4} p-3 d^{3}{ }^{4} p \\
(1 F)^{-1}\end{array}$ \\
\hline $\begin{array}{l}8615.4 \\
8598.3 \\
8782.6 \\
8437.9 \\
8457.3\end{array}$ & $\begin{array}{l}0.07 \\
0.04 \\
0.07 \\
0.04 \\
0.03\end{array}$ & $\begin{array}{l}1.50 \\
1.48 \\
1.48 \\
1.50 \\
1.48\end{array}$ & $\begin{array}{l}4 \frac{1}{3}-4 \frac{1}{3} \\
3 \frac{1}{2}-3 \frac{3}{3} \\
4 \frac{1}{3}-3 \frac{1}{2} \\
3 \frac{1}{2}-4 \frac{1}{2} \\
3 \frac{1}{2}-3 \frac{2}{2}\end{array}$ & $\begin{array}{c}3 d^{3}{ }^{4} F-3 d^{3}{ }^{3} o \\
(a F)\end{array}$ \\
\hline $\begin{array}{l}6233.9 \\
6215.6 \\
6159.3 \\
6160.1 \\
6104.8\end{array}$ & $\begin{array}{l}0.04 \\
0.02 \\
0.02 \\
0.00 \\
0.00\end{array}$ & $\begin{array}{l}2.02 \\
3.00 \\
3.02 \\
3.00 \\
3.02\end{array}$ & $\begin{array}{l}3 \frac{1}{3}-3 \frac{1}{2} \\
2 \frac{1}{2}-1 \frac{1}{3} \\
3 \frac{2}{2}-2 \frac{1}{2} \\
13 \frac{1}{2} \\
1 \frac{3}{2}-2 \frac{1}{2}\end{array}$ & $\begin{array}{c}3 d^{3} 4 F-3 d^{3} a_{D} \\
(3 F)\end{array}$ \\
\hline $\begin{array}{l}6098.1 \\
6065.3\end{array}$ & $\begin{array}{l}0.07 \\
0.04\end{array}$ & $\begin{array}{l}3.10 \\
3.08\end{array}$ & 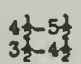 & ${ }^{3 d^{3}}(4 F)-3 d^{3}$ \\
\hline
\end{tabular}

IIV I P 48.3

\begin{tabular}{|c|c|c|c|c|}
\hline $\begin{array}{l}8815.9 \\
8575.4\end{array}$ & $\begin{array}{l}0.04 \\
0.00\end{array}$ & $\begin{array}{l}1.44 \\
1.44\end{array}$ & $\begin{array}{l}3-3 \\
2-3\end{array}$ & $\begin{array}{l}3 d^{2} 3 p-3 d^{3}{ }^{1} D \\
(1 F)\end{array}$ \\
\hline $\begin{array}{l}7811.3 \\
7551.9 \\
7431.2\end{array}$ & $\begin{array}{l}0.04 \\
0.00 \\
0.00\end{array}$ & $\begin{array}{l}1.66 \\
1.63 \\
1.66\end{array}$ & $\begin{array}{l}3-3 \\
2-1 \\
2-3\end{array}$ & $\begin{array}{l}3 d^{2} 3 F-3 d^{2} 3 p \\
(3 F)\end{array}$ \\
\hline $\begin{array}{l}5448.0 \\
5326.5 \\
5337.7\end{array}$ & $\begin{array}{l}0.09 \\
0.04 \\
0.00\end{array}$ & $\begin{array}{l}3.36 \\
2.36 \\
3.36\end{array}$ & $\begin{array}{l}4-4 \\
3-4 \\
2-4\end{array}$ & $\begin{array}{c}3 d^{2} 3_{F}-3 d^{2} 1_{a} \\
(3 F)\end{array}$ \\
\hline V VIII & I P 173\% & & & \\
\hline $\begin{array}{l}3686 \\
4734 \\
\end{array}$ & $\begin{array}{l}0.00 \\
0.74\end{array}$ & $\begin{array}{l}3.35 \\
3.35\end{array}$ & $\begin{array}{l}3-2 \\
1-3\end{array}$ & $\begin{array}{l}3 p^{4} 3 p-3 p^{4} 11_{D} \\
(1 F)\end{array}$ \\
\hline
\end{tabular}


FORBIDDEAN LINES

I A Low ${ }^{\text {High }}$ J Muitiplet Cr I I P 6.74

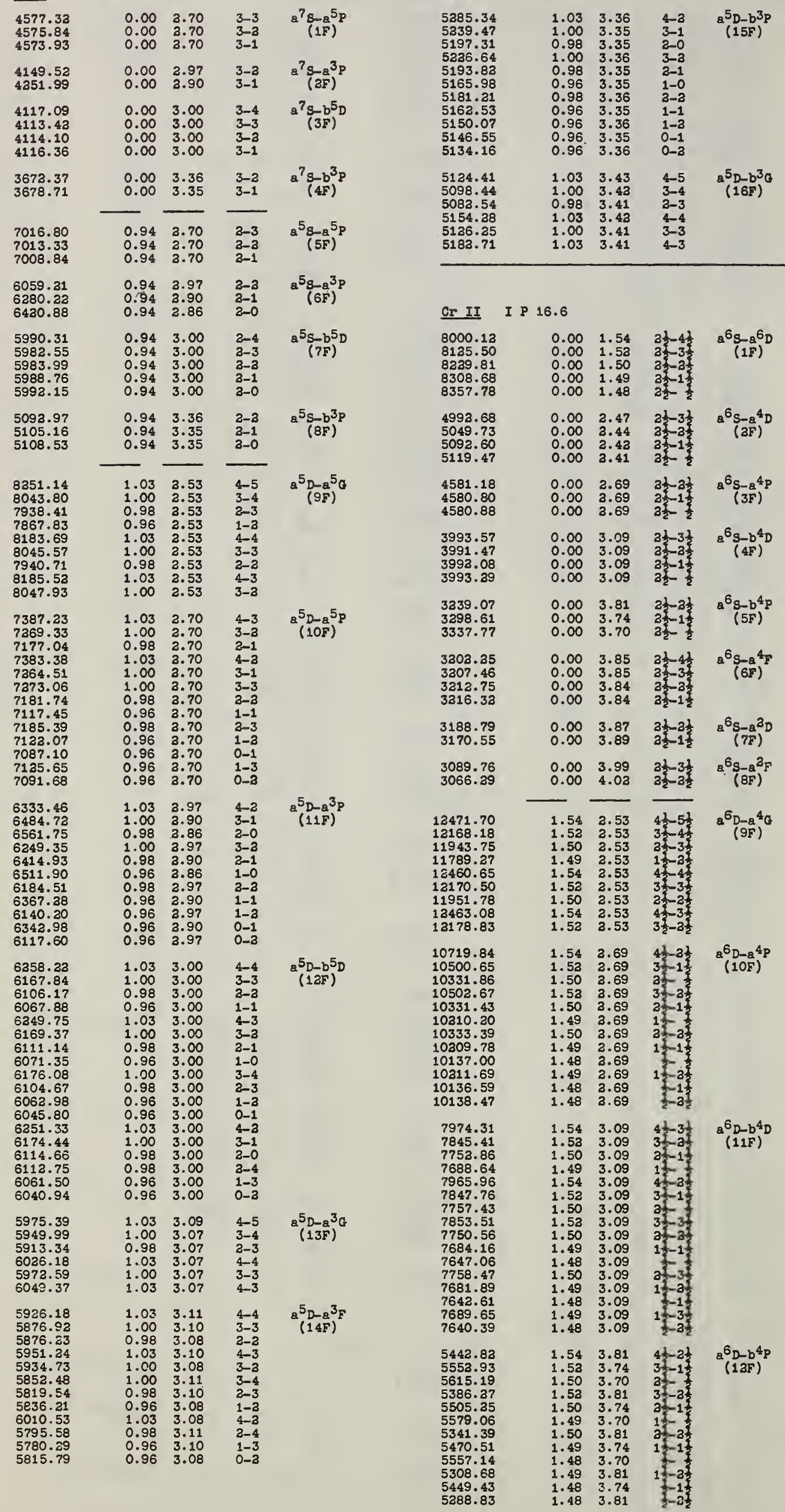

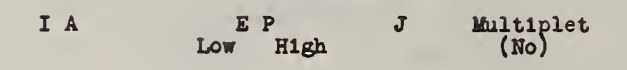

Cr I continued

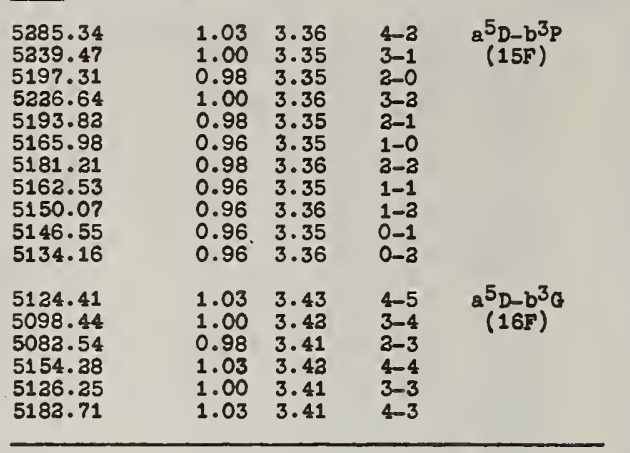

Cr II I P 16.6

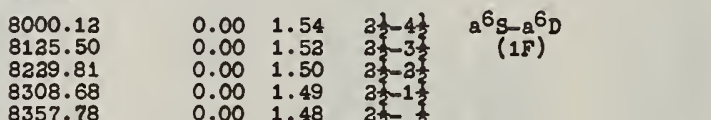

4993.68

5092.

4581.

4580.88

3993.

3993.

3339.07

3337.

3307.

3212.75

3188.79

3089.7

12471.70

11943.75

(2460.65

11951.78

3178.83

(0500.65

10331.86

10331.43

0333.39
I A

Low ${ }^{E}$ High

cr II continued

53
52
52
53
535
53
52
528
5
5
5
53
5
52
53
53
5
515

5299.42
5270.19

5347.8

5354.15
5313.88

5379.80

5385.21
5355.97

5338.3

5328.44
5368.91

5323.64
5242.00

5324.30
5319.03

5348.64

5157.59

5127.09
5174.95

5174.95
5108.57

5034.05

4924.81
4985.64

4887.37

4947.17
4859.87

9223.25

9512.5

9033.73

9033.73
9364.08

9590.9

8899.71
937.58

9806.20

9651.03

9866.49

8939.91

8793.09
8703.79

8703.79
8653.17

8970.56

8831.9

9013.04

8858.94

8826.03

8520.22

85

8400.89
8530.15

8530.15
8328.78

8106.88

7806.88

7947.28

7706.58

10373.30

10388.07
10380.40

10119.57

10233.37

10305.67

10366.26

10215.85

10373.30

10373.98

9337.40

9388.13

9433.18
9457.95

9343.61

9386.74

9427.18

9343.24
9381.78

9228.60

9072.86

8446.39

8272.21

8445.38

8268.36
$8441.3 ?$

11056.70

$\div 785.1$

13300.16

11783.63
13300.77

13300.77
11058.94

11058.94
11784.63 $\begin{array}{ll}1.49 & 3.84 \\ 1.54 & 3.85\end{array}$

$1.53 \quad 3.84$

$1.50 \quad 3.85$

$\begin{array}{ll}1.49 & 3.84 \\ 1.48 & 3.84\end{array}$

$1.48 \quad 3.84$

$1.53 \quad 3.87$

$\begin{array}{ll}1.50 & 3.99 \\ 1.49 & 4.03\end{array}$

-

$2.47 \quad 3.81$

$\begin{array}{ll}3.44 & 3.74 \\ 2.42 & 3.70\end{array}$

$\begin{array}{ll}2.42 & 3.70 \\ 2.44 & 3.81\end{array}$

$2.43 \quad 3.74$

$3.42 \quad 3.81$

$\begin{array}{ll}3.42 & 3.81 \\ 3.41 & 3.74\end{array}$

$3.47 \quad 3.73$

$3.44 \quad 3.72$

$3.47 \quad 3.85$

$\begin{array}{ll}3.47 & 3.85 \\ 3.44 & 3.85\end{array}$

$\begin{array}{ll}2.42 & 3.84 \\ 2.41 & 3.84\end{array}$

$\begin{array}{ll}2.41 & 3.84 \\ 3.47 & 3.85\end{array}$

$3.44 \quad 3.84$

$\begin{array}{ll}3.42 & 3.84 \\ 3.47 & 3.84\end{array}$

$3.44 \quad 3.84$

$3.47 \quad 3.87$

$\begin{array}{ll}3.47 & 3.87 \\ 2.44 & 3.89\end{array}$

2.443 .87

$\begin{array}{ll}2.42 & 3.89 \\ 2.42 & 3.87\end{array}$

$\begin{array}{ll}2.42 & 3.87 \\ 2.41 & 3.89\end{array}$

$2.47 \quad 3.99$

$2.44 \quad 4.03$

$\begin{array}{ll}2.47 & 4.02 \\ 2.44 & 3.99\end{array}$

\begin{tabular}{ll}
2.44 & 3.99 \\
2.43 & 4.03 \\
\hline
\end{tabular}

-

$2.53 \quad 3.72$

$7.53 \quad 3.72$

$2.53 \quad 3.74$

$2.53 \quad 3.73$

2.53 3.72

$\begin{array}{ll}3.53 & 3.74 \\ 3.53 & 3.73\end{array}$

$\begin{array}{ll}2.53 & 3.73 \\ 3.53 & 3.72\end{array}$

$\begin{array}{ll}2.53 & 3.73 \\ 2.53 & 3.72\end{array}$

$2.53 \quad 3.85$

$2.53 \quad 3.85$

$2.53 \quad 3.84$

$2.53 \quad 3.85$

$\begin{array}{ll}2.53 & 3.85 \\ 3.53 & 3.85\end{array}$

$2.53 \quad 3.84$

$\begin{array}{ll}2.53 & 3.85 \\ 2.53 & 3.85\end{array}$

$2.53 \quad 3.8$ ?

$\begin{array}{ll}2.53 & 3.87 \\ 2.53 & 3.89 \\ 3.53 & 3.87\end{array}$

$2.53 \quad 3.99$

$\begin{array}{ll}2.53 & 3.99 \\ 3.53 & 4.02 \\ 3.53 & 3.99\end{array}$

\begin{tabular}{ll}
2.53 & 4.08 \\
2.53 & 3.99 \\
\hline
\end{tabular}

-
$1.54 \quad 3.85$ $\begin{array}{ll}1.52 & 3.85 \\ 1.50 & 3.84 \\ 1.49 & 3.84\end{array}$

$\begin{array}{ll}1.50 & 3.84 \\ 1.53 & 3.85\end{array}$

$1.54 \quad 3.84$

$1.53 \quad 3.84$

$\begin{array}{ll}1.50 & 3.85 \\ 1.49 & 3.85\end{array}$

$\begin{array}{ll}1.50 & 3.89 \\ 1.50 & 3.87 \\ 1.40 & 3.89\end{array}$

1.493 .89

$\begin{array}{ll}1.49 & 3.87 \\ 1.48 & 3.89\end{array}$

$1.54 \quad 3.99$

$\begin{array}{ll}1.52 & 4.02 \\ 1.52 & 3.99\end{array}$

$\begin{array}{ll}1.52 & 3.99 \\ 1.50 & 4.03\end{array}$

$2.53 \quad 3.99$

$2.69 \quad 3.81 \quad 3 \frac{1}{2}-2 \frac{1}{5} \quad a^{4} \mathrm{P}-\mathrm{b}^{4} \mathrm{P}$

$\begin{array}{llll}2.69 & 3.81 & 37-2 \frac{1}{2} & a^{4} P-b 4 P \\ 3.69 & 3.74 & 15-1 & (36 F)\end{array}$

$\begin{array}{lll}2.69 & 3.70 \\ 2.69 & 3.74 & 2 \frac{2}{3}-1\end{array}$

$2.69 \quad 3.70 \quad 1$ -

$\begin{array}{lll}2.69 & 3.81 & 1 \\ 3.69 & 3.74 & -3 \frac{1}{2}\end{array}$

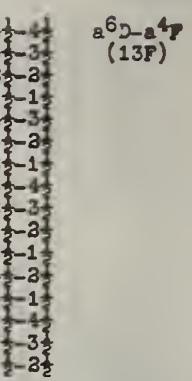

$\begin{array}{ll}3 \frac{1}{2}-2 \frac{1}{3} & a^{6} D-8^{2} D \\ 3 \frac{2}{2}-1 \frac{2}{2} & (14 F)\end{array}$

$1 \frac{1}{3}$

$\begin{array}{ll}4 \frac{1}{3}-3 \frac{1}{2} & a^{6} \mathrm{D}-a^{2} 5 \\ 3 \frac{3}{3}-3 \frac{1}{2} & (15 F)\end{array}$

$3 \frac{1}{2}-3 \frac{1}{3}$
$3 \frac{1}{2}-3 \frac{1}{2}$
$1 \frac{1}{2}-2 \frac{1}{2}$

$\begin{array}{ll}3 \frac{2}{2}-2 \frac{1}{3} & a^{4} D-b^{4} p \\ 23-1 \frac{1}{2} & (16 F)\end{array}$

3t-2

$\frac{1}{2}-1 \frac{1}{2}$

$\begin{array}{cc}3 \frac{1}{2}-4 \frac{1}{3} & a^{4} D-a^{4} H \\ 3 & (17 F) \\ 3 \frac{3}{2}-3 \frac{1}{2} & (17)\end{array}$

$\begin{array}{ll}3 \frac{1}{2}-4 \frac{1}{3} & a^{4} D-a^{4} r \\ (18 F) & \end{array}$

$3 \frac{2}{3}-3 \frac{1}{2}$

$\begin{aligned} & 1 \\ & 3 \\ & 3\end{aligned}-1 \frac{1}{2}$

$\begin{array}{cc}3 \frac{1}{2}-a \frac{1}{2} & a^{4} D-a^{2} \mathrm{D} \\ 2 & (19 F)\end{array}$

$\begin{array}{ll}2 \\ 1 \\ 1 & =1 \frac{1}{2} \\ 2 & =1 \frac{1}{2}\end{array}$

$\begin{array}{cc}\frac{31}{2}-3 \frac{1}{2} & a^{4} D-a^{3} F \\ 2-3 \frac{2}{2} & (20 F)\end{array}$

$3 \frac{1}{2}-3 \frac{2}{2}$
$1 \frac{1}{2}-2 \frac{7}{2}$

$5+-6 \frac{1}{2} \quad a^{4} G-a^{2} I$

$5 \frac{1}{2}-6 \frac{1}{2} \quad a^{4} G-a^{4} H$

$2 \frac{1}{3}-3 \frac{1}{2}$

$4 \frac{1}{3}-4 \frac{1}{2}$

$5 \frac{1}{2}-4 \frac{1}{2}$

$5 \frac{1}{43}-4$
$a^{4} G-a^{4} F$
$(23 F)$

$3 \frac{1}{3}-1 \frac{2}{3}$

$3 \frac{1}{3}$

$\begin{array}{ll}3 & 5 \\ 3 \frac{1}{2}- & -3 \frac{1}{2}\end{array}$

$\begin{array}{ll}3 \frac{1}{3}-2 \frac{1}{2} & a^{4} G-8^{2} D \\ 2 & (34 F)\end{array}$

$\begin{array}{ll}4 \frac{1}{3}-3 \frac{1}{2} & a^{4}\left(a-a^{2} F\right. \\ (25 F) & \end{array}$

3
3
3 


\begin{tabular}{ccccc} 
I A & \multicolumn{2}{c}{ E P } & L & Mult1plet \\
(NO)
\end{tabular}

Cr III I P 31

\begin{tabular}{|c|c|c|c|c|}
\hline $\begin{array}{l}5785.4 \\
5945.1 \\
5712.7 \\
5884.9 \\
5689.3 \\
5843.6 \\
5618.9 \\
5833.2 \\
5600.1\end{array}$ & $\begin{array}{l}0.07 \\
0.04 \\
0.04 \\
0.02 \\
0.02 \\
0.01 \\
0.01 \\
0.00 \\
0.00\end{array}$ & $\begin{array}{l}2.20 \\
2.12 \\
2.20 \\
2.12 \\
2.20 \\
2.12 \\
2.20 \\
3.12 \\
3.20\end{array}$ & $\begin{array}{l}4-2 \\
3-1 \\
3-2 \\
2-1 \\
3-2 \\
1-1 \\
1-2 \\
0-1 \\
0-2\end{array}$ & $\begin{array}{l}3 d^{4} 5 p-3 d^{4} 3 p \\
(1 F)^{3}\end{array}$ \\
\hline $\begin{array}{l}5550.3 \\
5505.1 \\
5471.3 \\
5572.6 \\
5523.3 \\
5483.3 \\
5453.4 \\
5435.6 \\
5591.3 \\
5432.1 \\
.5418 .0\end{array}$ & $\begin{array}{l}0.07 \\
0.04 \\
0.02 \\
0.07 \\
0.04 \\
0.04 \\
0.02 \\
0.01 \\
0.07 \\
0.02 \\
10.01 \\
0.00\end{array}$ & $\begin{array}{l}2.29 \\
2.29 \\
2.28 \\
2.29 \\
2.28 \\
2.29 \\
2.29 \\
2.28 \\
2.28 \\
2.29 \\
2.29 \\
3.28\end{array}$ & $\begin{array}{l}4-4 \\
3-3 \\
2-2 \\
4-3 \\
3-2 \\
3-4 \\
2-3 \\
1-2 \\
4-2 \\
2-4 \\
1-3 \\
0-2\end{array}$ & $\begin{array}{l}3 d^{4}{ }^{5} p-3 d^{4}{ }^{3} F \\
(a F)\end{array}$ \\
\hline $\begin{array}{r}4894.1 \\
4876.0 \\
4870.8 \\
4928.9 \\
4911.9 \\
.4842 .4 \\
4835.4 \\
.4842 .4 \\
4965.6\end{array}$ & $\begin{array}{l}0.07 \\
0.04 \\
0.02 \\
0.07 \\
0.04 \\
0.04 \\
0.02 \\
0.01 \\
0.07\end{array}$ & $\begin{array}{l}2.59 \\
2.57 \\
2.56 \\
2.57 \\
2.56 \\
3.59 \\
2.57 \\
2.56 \\
3.56\end{array}$ & $\begin{array}{l}4-5 \\
3-4 \\
2-3 \\
4-4 \\
3-3 \\
3-5 \\
2-4 \\
1-3 \\
4-3\end{array}$ & $\begin{array}{c}3 d^{4} 5 p-3 d^{4} 3 a \\
(3 F)^{3}\end{array}$ \\
\hline
\end{tabular}

C. IV I P 50.4 7338.

7233.

7180.

?171.

7021.

8906.1

6915.

6893.

7086.

6731.2

6591.0

5396.

5145.5

5071.

4976.5

4899

1894.

4843.1
4838.7

4907.

4873.4
4969.

4814.0 $0.07 \quad 1.75$ $0.03 \quad 1.75$ $\begin{array}{ll}0.00 & 1.74 \\ 0.03 & 1.79\end{array}$ $0.00 \quad 1.75$

$0.12 \quad 1.90$ $0.37 \quad 1.86$ 0.121 .88 $0.03 \quad 1.86$ 0.031 .90 0.001 .86 $0.07 \quad 2.40$ $0.00 \quad 2.40$ $\begin{array}{ll}0.12 & 2.55 \\ 0.07 & 2.55\end{array}$ $0.07 \quad 3.55$ $0.03 \quad 2.55$ 0.032 .55 $\begin{array}{ll}0.00 & 2.55 \\ 0.00 & 2.55\end{array}$ $0.12 \quad 2.63$ $\begin{array}{ll}0.07 & 2.60 \\ 0.12 & 2.60\end{array}$ $\begin{array}{ll}0.07 & 2.63 \\ 0.03 & 2.60\end{array}$ $\begin{array}{ll}0.03 & 1.74 \\ 0.07 & 1.79\end{array}$
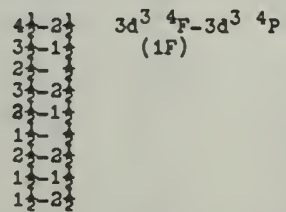

$\begin{array}{cc}4 \frac{1}{2}-4 \frac{1}{3} & 3 d^{3}-\frac{4}{5}-3 d^{3}{ }^{2} \sigma\end{array}$

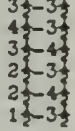

$3 \frac{1}{2}-1 \frac{1}{2} \quad 3 d^{3} 4 p-3 d^{3} a_{p}$

$\begin{array}{lll}4 \frac{1}{2}-2 \frac{1}{3} & 3 d^{3} 4 F-3 d^{3} \quad 2 D\end{array}$

2
$1 \frac{1}{3}-1 \frac{1}{3}$
$1 \frac{1}{2}-3 \frac{1}{2}$

$3 \frac{1}{-5} \quad 3 d^{3} 4 F-3 d^{3}{ }^{2} H$

CrV I P 72.8

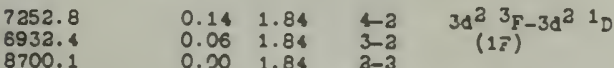
8700.1

6705.5

6588.

8430.

6376.

6230.

4523.6 4396.9
4302.3
$0.14 \quad 1.98 \quad 4-2 \quad 3 d^{2} 3 p-3 d^{2} 3 p$

$0.06 \quad 1.94$

$0.06 \quad 1.98$

0.001 .98

$\begin{array}{lllc}0.14 & 2.87 & 4-4 & 34^{2} 3 F-3 d^{2} 10 \\ 0.06 & 2.87 & 3-4 & (3 F) \\ 0.00 & 2.87 & 3-4 & \end{array}$
I A Low ${ }_{\text {High }}$ I Multiplet

Cr V continued

$\begin{array}{ccccc}9635.9 & 1.84 & 3.12 & 3-0 & 3 d^{2} 1 D^{2}-3 d^{2}{ }^{1} \mathrm{~g} \\ (4 F)\end{array}$

$\begin{array}{llllll}10807.8 & 1.98 & 3.12 & 2-0 & 3 d^{2} 3 p-3 d^{2} 1 g \\ 10394.3 & 1.94 & 3.12 & 1-0 & (5 F) & \end{array}$

Cr VIII I P $184 \%$

$10098.2 \quad 0.00 \quad 1.22 \quad 1 \frac{1}{2}-\frac{1}{2} \quad 3 p^{5} 2 p^{0}-3 p^{5} \quad 3 p^{0}$

Cr IX I P 2099

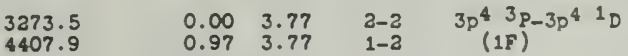

(n II I P 15.57

6978.57

6850.42
6763.56

6709.08

3344.72
3341.38

3049.05

3043.61

3044.52

4896.65
4889.49
4881.87

sas..25.

4274.87
4278.97

7547.77
7696.30

7805.96

7540.74

7693.38
7805.47

7537.93
7692.91

6423.45

6523.23
6590.10

6590.10

6603.99

6642.66
6617.06

6656.77

5415.04
5473.94

5530.11

5574.04
5394.78

5394.78
5473.37

5536.98
5579.73

5494.80
5530.69

5530.69
5567.08

5561.21

\begin{tabular}{|c|c|c|c|}
\hline $0 . \infty 0$ & 1.17 & $3-2$ & $a^{7} s-a^{5} s$ \\
\hline $\begin{array}{l}0.00 \\
0.00 \\
0.00 \\
0.00\end{array}$ & $\begin{array}{l}1.77 \\
1.80 \\
1.82 \\
1.84\end{array}$ & $\begin{array}{l}3-4 \\
3-3 \\
3-2 \\
3-1\end{array}$ & $\begin{array}{c}a^{7}{ }_{3-a^{5} D} D \\
(2 F)\end{array}$ \\
\hline $\begin{array}{l}0.00 \\
0.00 \\
0.00\end{array}$ & $\begin{array}{l}3.69 \\
3.69 \\
3.70\end{array}$ & $\begin{array}{l}3-3 \\
3-2 \\
3-1\end{array}$ & $\begin{array}{c}a^{7} g-a^{5} P \\
(3 F)\end{array}$ \\
\hline $\begin{array}{l}0.00 \\
0.00 \\
0.00 \\
0.00\end{array}$ & $\begin{array}{l}4.05 \\
4.06 \\
4.06 \\
4.05\end{array}$ & $\begin{array}{l}3-4 \\
3-3 \\
3-2 \\
3-1\end{array}$ & $\begin{array}{c}a^{7} S-b^{5} D \\
(4 F)\end{array}$ \\
\hline $\begin{array}{l}1.17 \\
1.17 \\
1.17\end{array}$ & $\begin{array}{l}3.69 \\
3.69 \\
3.70\end{array}$ & $\begin{array}{l}2-3 \\
2-2 \\
2-1\end{array}$ & $\begin{array}{c}a^{5} g-a^{5} P \\
(5 F)\end{array}$ \\
\hline $\begin{array}{l}1.17 \\
1.17 \\
1.17\end{array}$ & $\begin{array}{l}4.06 \\
4.06 \\
4.05\end{array}$ & $\begin{array}{l}2-3 \\
2-2 \\
2-1\end{array}$ & $\begin{array}{c}a^{5} S-b^{5} D \\
(6 F)\end{array}$ \\
\hline $\begin{array}{l}1.77 \\
1.80 \\
1.82 \\
1.84 \\
1.77 \\
1.80 \\
1.82 \\
1.77 \\
1.80\end{array}$ & $\begin{array}{l}3.40 \\
3.41 \\
3.41 \\
3.41 \\
3.41 \\
3.41 \\
3.41 \\
3.41 \\
3.41\end{array}$ & $\begin{array}{l}4-5 \\
3-4 \\
2-3 \\
1-2 \\
4-4 \\
3-3 \\
2-2 \\
4-3 \\
3-2\end{array}$ & $\begin{array}{c}a^{5} D-a^{5} a \\
(7 F)\end{array}$ \\
\hline $\begin{array}{l}1.77 \\
1.80 \\
1.82 \\
1.80 \\
1.82 \\
1.84 \\
1.82 \\
1.84 \\
1.85\end{array}$ & $\begin{array}{l}3.69 \\
3.69 \\
3.70 \\
3.69 \\
3.69 \\
3.70 \\
3.69 \\
3.69 \\
3.70\end{array}$ & $\begin{array}{l}4-3 \\
3-2 \\
2-1 \\
3-3 \\
2-2 \\
1-1 \\
2-3 \\
1-2 \\
0-1\end{array}$ & $\begin{array}{c}a^{5} D-a^{5} p \\
(8 F)\end{array}$ \\
\hline $\begin{array}{l}1.77 \\
1.80 \\
1.82 \\
1.84 \\
1.77 \\
1.80 \\
1.82 \\
1.84 \\
1.80 \\
1.82 \\
1.84 \\
1.85\end{array}$ & $\begin{array}{l}4.05 \\
4.06 \\
4.06 \\
4.05 \\
4.06 \\
4.06 \\
4.05 \\
4.05 \\
4.05 \\
4.06 \\
4.06 \\
4.05\end{array}$ & $\begin{array}{l}4-4 \\
3-3 \\
2-2 \\
1-1 \\
4-3 \\
3-2 \\
2-1 \\
1-0 \\
3-4 \\
2-3 \\
1-2 \\
0-1\end{array}$ & $\begin{array}{c}a^{5} D-b^{5} D \\
(9 F)\end{array}$ \\
\hline
\end{tabular}

VI IV I P 52

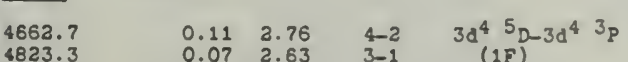

4823.3
4908.8

4591.4

4781.9

4863.9
$4535 . ?$

$4535 . ?$
$4719 . ?$

497.4
4699.3

4699.3
4478.8

4528.3

4480.8

442.0
4548.5

4495.3
$4 \$ 61.0$

461.0
4427.7

4427.7
4405.2

4563.

4408.5
4391.1

$\$ 387.4$
IA Low ${ }^{\text {H1gh }}$

J Lultiplet

Kn V I P 75.7

\begin{tabular}{|c|c|c|c|c|}
\hline $\begin{array}{l}6396.2 \\
6346.2 \\
6220.7 \\
6167.7 \\
6159.3 \\
6088.5 \\
5991.0 \\
6029.7 \\
5868.3\end{array}$ & $\begin{array}{l}0.17 \\
0.10 \\
0.04 \\
0.10 \\
0.04 \\
0.00 \\
0.04 \\
0.00 \\
0.00\end{array}$ & $\begin{array}{l}2.10 \\
2.05 \\
2.03 \\
2.10 \\
2.05 \\
2.03 \\
2.10 \\
2.05 \\
2.10\end{array}$ & 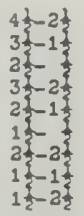 & $\begin{array}{c}3 d^{3}{ }^{4} F-3 d^{3}{ }^{4} P \\
(1 F)^{-1}\end{array}$ \\
\hline $\begin{array}{l}5889.0 \\
5863.1 \\
6069.2 \\
5694.8 \\
5703.3 \\
5543.9 \\
5591.9\end{array}$ & $\begin{array}{l}0.17 \\
0.10 \\
0.17 \\
0.10 \\
0.04 \\
0.04 \\
0.00\end{array}$ & $\begin{array}{l}2.27 \\
2.21 \\
2.21 \\
2.27 \\
2.21 \\
2.27 \\
2.21\end{array}$ & $\begin{array}{l}4 \frac{1}{2}-4 \frac{1}{2} \\
3 \frac{2}{2}-3 \frac{2}{2} \\
4 \frac{2}{2}-3 \frac{2}{2} \\
3 \frac{1}{2}-4 \frac{1}{2} \\
2 \frac{1}{2}-3 \frac{2}{2} \\
2 \frac{2}{2}-4 \frac{2}{2} \\
1 \frac{1}{2}-3 \frac{1}{2}\end{array}$ & $\begin{array}{c}3 d^{3}{ }^{4} F-3 d^{3}{ }^{2} G \\
(2 F)\end{array}$ \\
\hline $\begin{array}{l}4528.7 \\
4398.4 \\
4432.8 \\
4331.9 \\
4365.2\end{array}$ & $\begin{array}{l}0.10 \\
0.04 \\
0.04 \\
0.00 \\
0.00\end{array}$ & $\begin{array}{l}2.83 \\
2.85 \\
2.83 \\
2.85 \\
2.83\end{array}$ & $\begin{array}{l}3 \frac{1}{2}-1 \frac{1}{2} \\
2 \frac{1}{2}-\frac{1}{2} \\
2 \frac{1}{2}-1 \\
1 \frac{1}{2}-\frac{1}{2} \\
1 \frac{1}{2}-1 \frac{1}{2}\end{array}$ & $\begin{array}{c}3 d^{3}{ }^{4} F-3 d^{3} 2 p \\
(3 F)\end{array}$ \\
\hline $\begin{array}{l}4308.4 \\
4196.3 \\
4203.5 \\
4113.7 \\
4120.7 \\
4055.5 \\
4062.2\end{array}$ & $\begin{array}{l}0.17 \\
0.10 \\
0.10 \\
0.04 \\
0.04 \\
0.00 \\
0.00\end{array}$ & $\begin{array}{l}3.04 \\
3.04 \\
3.04 \\
3.04 \\
3.04 \\
3.04 \\
3.04\end{array}$ & $\begin{array}{l}4 \frac{1}{2}-2 \frac{1}{2} \\
3 \frac{1}{2}-1 \frac{1}{2} \\
3 \frac{1}{3}-2 \frac{1}{3} \\
2 \frac{1}{2}-1 \frac{1}{2} \\
2 \frac{1}{2}-2 \frac{1}{3} \\
1-1 \frac{1}{3} \\
1 \frac{1}{2}-2 \frac{1}{2}\end{array}$ & $\begin{array}{c}3 d^{3}{ }^{4} F-3 d^{3}{ }^{2} D \\
(4 F)\end{array}$ \\
\hline
\end{tabular}

In VI I P ?

\begin{tabular}{|c|c|c|c|c|}
\hline $\begin{array}{l}6277.3 \\
5933.4 \\
5679.3\end{array}$ & $\begin{array}{l}0.21 \\
0.09 \\
0.00\end{array}$ & $\begin{array}{l}2.17 \\
2.17 \\
2.17\end{array}$ & $\begin{array}{l}4-2 \\
3-2 \\
2-2\end{array}$ & $\begin{array}{c}3 d^{2} 3 F-3 d^{2} \\
(1 F)^{2}\end{array}$ \\
\hline $\begin{array}{l}5907.1 \\
5783.4 \\
5625.0 \\
5601.6 \\
5541.7 \\
5374.6\end{array}$ & $\begin{array}{l}0.21 \\
0.09 \\
0.00 \\
0.09 \\
0.00 \\
0.00\end{array}$ & $\begin{array}{l}2.30 \\
2.23 \\
2.19 \\
2.30 \\
2.23 \\
2.30\end{array}$ & $\begin{array}{l}4-2 \\
3-1 \\
2-0 \\
3-2 \\
2-1 \\
2-2\end{array}$ & $\begin{array}{c}3 d^{2}{ }^{3} F-3 d^{2} \\
(2 F)^{2}\end{array}$ \\
\hline $\begin{array}{l}3866.9 \\
3733.6 \\
3631.4\end{array}$ & $\begin{array}{l}0.21 \\
0.09 \\
0.00\end{array}$ & $\begin{array}{l}3.40 \\
3.40 \\
3.40\end{array}$ & $\begin{array}{l}4-4 ? \\
3-4 ? \\
2-4 ?\end{array}$ & $\begin{array}{c}3 d^{2}{ }^{3} F-3 d^{2} \\
(3 F)\end{array}$ \\
\hline
\end{tabular}

Yn IX I P 221 ?

$7978.7 \quad 0.00 \quad 1.55 \quad 1 \frac{1}{2}-\frac{1}{2} \quad 3 p^{5} \quad 3 p^{0}-3 p^{5} \quad 3 p^{\circ}$ 15

$\underline{\underline{\ln } X \quad I} \quad \mathrm{P} 2479$

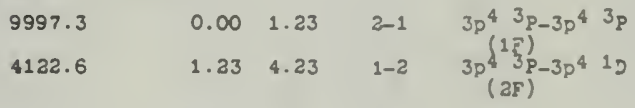

Fe I I P 7.858

\begin{tabular}{|c|c|c|c|c|}
\hline $\begin{array}{l}8347.55 \\
8231.57 \\
8151.33 \\
7959.00 \\
7964.27 \\
8647.89 \\
8431.56 \\
8275.57 \\
7708.83 \\
8868.91 \\
8564.56 \\
8337.65\end{array}$ & $\begin{array}{l}0.00 \\
0.05 \\
0.09 \\
0.00 \\
0.05 \\
0.05 \\
0.09 \\
0.11 \\
0.00 \\
0.09 \\
0.11 \\
0.12\end{array}$ & $\begin{array}{l}1.48 \\
1.55 \\
1.60 \\
1.55 \\
1.60 \\
1.48 \\
1.55 \\
1.60 \\
1.60 \\
1.48 \\
1.55 \\
1.60\end{array}$ & $\begin{array}{l}4-4 \\
3-3 \\
2-2 \\
4-3 \\
3-2 \\
3-4 \\
2-3 \\
1-2 \\
4-2 \\
2-4 \\
1-3 \\
0-2\end{array}$ & $a^{5}-a^{3} F$ \\
\hline $\begin{array}{l}5696.36 \\
5775.05 \\
5804.45 \\
5639.55 \\
5708.96 \\
5834.64 \\
5872.77 \\
5867.17 \\
5934.41 \\
5936.99 \\
5898.30 \\
5999.99 \\
5968.87\end{array}$ & $\begin{array}{l}0.00 \\
0.05 \\
0.09 \\
0.00 \\
0.05 \\
0.05 \\
0.09 \\
0.11 \\
0.09 \\
0.11 \\
0.12 \\
0.11 \\
0.12\end{array}$ & $\begin{array}{l}2.17 \\
2.19 \\
2.21 \\
2.19 \\
2.21 \\
2.17 \\
2.19 \\
2.21 \\
2.17 \\
2.19 \\
2.21 \\
2.17 \\
2.19\end{array}$ & $\begin{array}{l}4-3 \\
3-2 \\
2-1 \\
4-2 \\
3-1 \\
3-3 \\
2-2 \\
1-1 \\
2-3 \\
1-2 \\
0-1 \\
1-3 \\
0-2\end{array}$ & $\begin{array}{c}a^{5} D-a^{5} p \\
(2 F)\end{array}$ \\
\hline $\begin{array}{l}5439.72 \\
5224.15 \\
5170.84 \\
5565.68 \\
5303.99 \\
5220.56 \\
5656.39 \\
5356.32 \\
5715.94 \\
5382.26 \\
5745.49\end{array}$ & $\begin{array}{l}0.00 \\
0.05 \\
0.09 \\
0.05 \\
0.09 \\
0.11 \\
0.09 \\
0.11 \\
0.11 \\
0.12 \\
0.12\end{array}$ & $\begin{array}{l}2.37 \\
2.41 \\
2.47 \\
2.27 \\
2.41 \\
2.47 \\
2.27 \\
2.41 \\
2.27 \\
2.41 \\
2.27\end{array}$ & $\begin{array}{l}4-2 \\
3-1 \\
2-0 \\
3-2 \\
2-1 \\
1-0 \\
2-2 \\
1-1 \\
1-2 \\
0-1 \\
0-2\end{array}$ & $\begin{array}{c}a^{5} D-a^{3} p \\
(3 \bar{r})\end{array}$ \\
\hline
\end{tabular}




\section{FORBIDDEN LINES}

I A Low P High J turitiplet

Fe I continued

4843.34

4886.56

4916.26
4789.19

4847.58

4942.95
4956.35

4961.18

4751.75
5014.37

5014.37

4983.42

4603.66

4631.93
4640.05

4640.05
4544.36

4578.83

4693.56
4694.59

4680.05

4493.23

4377.37

4437.10
4473.46

4458.57

4494.57

4510.63
4516.60

4532.09

4554.49
4550.64

4573.23

4203.39

4217.71
4239.86

4144.97

4178.93

4278.21
4269.60

4263.07

4103.02

4104.59

4179.45
4153.72

4153.72
4130.47

4230.40

4185.74

4263.6

4301.56
4380.04

3812.07

3814.58

388.58

3754.98
3846.46

3873.51

3856.98

3917.64
3817.33

3884.57

3931.50

3945.70
3898.19

3403.65

3454.34

3493.55

3405.39
3458.73

3452.54

3489.07

3516.17
3487.23

3511.64
3537.33

3509.78

3522.76

9886.83

9998.31

10178.39

10262.84

10339.79

10443.95

9386.96

8643.14
8413.97

8412.97

8771.3

9778.70
$0.00 \quad 3.55$

$\begin{array}{ll}0.05 & 2.58 \\ 0.09 & 2.60\end{array}$

$\begin{array}{ll}0.00 & 2.58 \\ 0.05 & 2.60\end{array}$

$\begin{array}{ll}0.05 & 2.55 \\ 0.09 & 2.58\end{array}$

0.112 .60

$\begin{array}{ll}0.00 & 2.60 \\ 0.09 & 2.55\end{array}$

$0.11 \quad 3.58$

$0.00 \quad 3.68$

$0.05 \quad 2.72$

$\begin{array}{ll}0.09 & 2.75 \\ 0.00 & 3.72\end{array}$

$0.05 \quad 3.75$

$0.09 \quad 2.72$

$\begin{array}{ll}0.11 & 2.75 \\ 0.00 & 2.75\end{array}$

$0.00 \quad 2.82$

$\begin{array}{ll}0.05 & 2.83 \\ 0.09 & 2.85\end{array}$

$\begin{array}{ll}0.05 & 2.82 \\ 0.09 & 2.83\end{array}$

$0.11 \quad 2.85$

0.112 .83

$\begin{array}{ll}0.11 & 2.83 \\ 0.13 & 2.83\end{array}$

$0.12 \quad 2.82$

$0.00 \quad 2.94$

$\begin{array}{ll}0.05 & 3.98 \\ 0.09 & 3.00\end{array}$

$0.00 \quad 2.98$

$\begin{array}{ll}0.05 & 3.00 \\ 0.05 & 2.94\end{array}$

$0.09 \quad 2.98$

$\begin{array}{ll}0.00 & 3.00\end{array}$

$0.00 \quad 3.00$

0.053 .00

0.093 .06

$0.11 \quad 3.10$

$\begin{array}{ll}0.09 & 3.00 \\ 0.11 & 3.06\end{array}$

$0.11 \quad 3.00$

$\begin{array}{ll}0.12 & 3.06 \\ 0.12 & 3.00\end{array}$

$0.00 \quad 3.34$

$\begin{array}{ll}0.05 & 3.29 \\ 0.09 & 3.26\end{array}$

$0.00 \quad 3.29$

$\begin{array}{ll}0.05 & 3.26 \\ 0.05 & 3.34\end{array}$

$\begin{array}{ll}0.05 & 3.34 \\ 0.09 & 3.29\end{array}$

0.113 .36

$\begin{array}{ll}0.09 & 3.34 \\ 0.11 & 3.29\end{array}$

0.123 .36

$\begin{array}{ll}0.11 & 3.24 \\ 0.12 & 3.29\end{array}$

$0.00 \quad 3.63$

$\begin{array}{ll}0.05 & 3.62 \\ 0.09 & 3.68\end{array}$

$0.00 \quad 3.62$

$0.05 \quad 3.68$

0.093 .62

0.113 .62

$0.11 \quad 3.62$

$0.12 \quad 3.62$

$\begin{array}{ll}0.11 & 3.63 \\ 0.13 & 3.62\end{array}$

0.912 .17

$0.95 \quad 2.19$

$0.99 \quad 2.21$

0.95 2.17

$1.01 \quad 2.31$

$\begin{array}{ll}0.99 & 2.17 \\ 1.01 & 6.19\end{array}$

$0.95 \quad 3.37$

$0.99 \quad 2.41$

$1.01 \quad 3.47$

$\begin{array}{ll}1.99 & 2.87 \\ 1.01 & 2.41\end{array}$

$\begin{array}{ll}1.01 & 3.41 \\ 1.01 & 3.27\end{array}$
$0.05 \quad 3.06$

$\frac{a^{5} D-a^{3} G}{(5 F)}$

$\begin{array}{ll}3-4 & (5 \xi) \\ 3-3 & \end{array}$

$3-3$

$3-5$
$2-4$
$1-3$

4-3

$\begin{array}{cc}4-3 & a^{5} D-b^{3} p \\ 3-1 & (6 F)\end{array}$

$3-2$

$3-1$
$1-0$

$2-2$
$1-1$

$1-2$
$0-1$
$0-2$

$\begin{array}{cc}4-5 & a^{5} p-b^{3} a \\ 3-4 & (7 F) \\ 2-3 & \end{array}$

$4-4$
$3-3$

$3-5$
$2-4$

$4-3$

$\begin{array}{cc}4-2 & a^{5} D-c^{3} p \\ 3-1 & (B F) \\ 3-0 & \end{array}$

$3-2$
$2-1$

$1-0$

$3-3$
$1-1$
$1-3$

$1-3$
$0-1$
$0-3$

$\begin{array}{cc}4-3 & a^{5} D-a^{3} D \\ 3-3 & (9 F)\end{array}$

$2-1$
$4-3$

3-1

$2-2$
$1-1$

$2-3$
$1-2$

$1-2$
$0-1$
$1-3$

$\begin{array}{cc}4-3 & a^{5} D-b^{3} D \\ 3-3 & (10 F) \\ 2-1 & \end{array}$

$2-1$
$4-3$
$3-1$

$3-1$

$2-2$
$1-1$
$2-3$

$2-3$
$1-2$
$0-1$

$1-1$
$1-3$
$0-3$

$4-3$
$3-3$
$2-1$
$3-3$
$2-2$
$1-1$
$2-3$
$1-2$
$3-2$
$2-1$
$1-0$
$2-2$
$1-1$
$1-2$

$a^{5} p-a^{5} p$

$\begin{array}{lc}3-a & a^{5} p-a^{3} p \\ 2-1 & (12 F)\end{array}$
I A

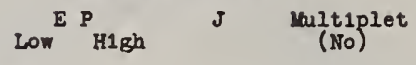

Fe I continued

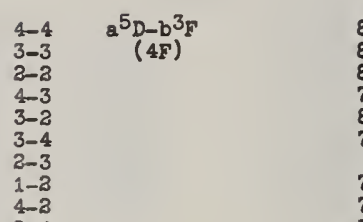

8164.85
8289.45

7876.34
8054.83

8054.83
7773.91

7290.42

7406.61

7510.54
7168.48

7168.48
7317.43

$\begin{array}{ll}0.86 & 2.39 \\ 0.91 & 2.43 \\ 0.95 & 2.44 \\ 0.86 & 2.42 \\ 0.91 & 2.44 \\ 0.86 、 & 3.44\end{array}$

$\begin{array}{ll}0.86 & 3.55 \\ 0.91 & 2.58\end{array}$

$\begin{array}{ll}0.91 & 2.58 \\ 0.95 & 2.60\end{array}$

0.862 .58

$\begin{array}{ll}0.91 & 2.60 \\ 0.91 & 3.55\end{array}$

$\begin{array}{ll}0.91 & 2.55 \\ 0.95 & 2.58\end{array}$

$0.99 \quad 2.60$

$0.95 \quad 2.55$

$0.99 \quad 2.58$

758.8

7756.59

7759.25

7899.63
7859.60

$0.99 \quad 3.55$

6760.61

6836.94

6884.50
6633.48

6633.48
6731.89

6972.07

7005.23

6525.11

7134.08

7092.89

6616.18

668.18

6730.99

6808.48

5931.19

6018.54

5815.53

5893.89

6093.32
6099.31

6099.31

6113.40
5743.07

6336.6

6196.75

6019.63

(1)

6016.15

5218.95

5213.95
5268.82

5368.82
5389.66

5147.16

5193.13

5180.78

5194.19

5352.39

5290.75

5487.17
5404.80

5404.80

5363.91

5481.17

5413.97

0.862 .68

$\begin{array}{ll}0.86 & 2.68 \\ 0.91 & 2.72\end{array}$

0.95 2.75

$\begin{array}{ll}0.86 & 2.72 \\ 0.91 & 3.75\end{array}$

0.912 .68

$0.95 \quad 2.73$

$\begin{array}{ll}0.99 & 2.75 \\ 0.86 & 2.75\end{array}$

$0.95 \quad 3.68 \quad 5-3$

$0.99 \quad 3.73 \quad 2-4$

$\begin{array}{lll}0.95 & 3.82 & 3-2 \\ 0.99 & 3.83 & 2-1\end{array}$

$\begin{array}{lll}0.99 & 3.83 & 2-1 \\ 1.01 & 3.85 & 1-0\end{array}$

$\begin{array}{lll}0.99 & 2.83 & 2-2 \\ 1.01 & 3.83 & 1-1\end{array}$

$\begin{array}{ll}0.86 & 2.94 \\ 0.91 & 2.98\end{array}$

$\begin{array}{ll}0.91 & 2.98 \\ 0.95 & 3.00\end{array}$

$0.86 \quad 3.98$

0.913 .00

$\begin{array}{ll}0.91 & 3.94 \\ 0.95 & 2.98\end{array}$

0.993 .06

0.863 .0

$0.95 \quad 3.94$

$\begin{array}{ll}0.99 & 3.98 \\ 1.01 & 3.00\end{array}$

$0.95 \quad 3.00$

$\begin{array}{ll}0.95 & 3.00 \\ 0.99 & 3.06\end{array}$

1.013 .10

$\begin{array}{ll}0.99 & 3.00 \\ 1.01 & 3.06\end{array}$

1.013 .00

0.863 .32

$0.91 \quad 3.25$

0.953 .29

$\begin{array}{ll}0.86 & 3.25 \\ 0.91 & 3.29\end{array}$

0.863 .29

$0.86 \quad 3.34$

0.913 .29

0.913 .34

$\begin{array}{ll}0.91 & 3.24 \\ 0.95 & 3.29\end{array}$

0.993 .26

$0.95 \quad 3.24$

0.993 .29

$\begin{array}{ll}1.01 & 3.26\end{array}$

$\begin{array}{ll}1.99 & 3.24 \\ 1.01 & 3.29\end{array}$

1.013 .24

$0.86 \quad 3.63$

0.913 .62

0.913 .63 


\section{REVISED YULTIPLET IABLE TORBIDDEN LINES}

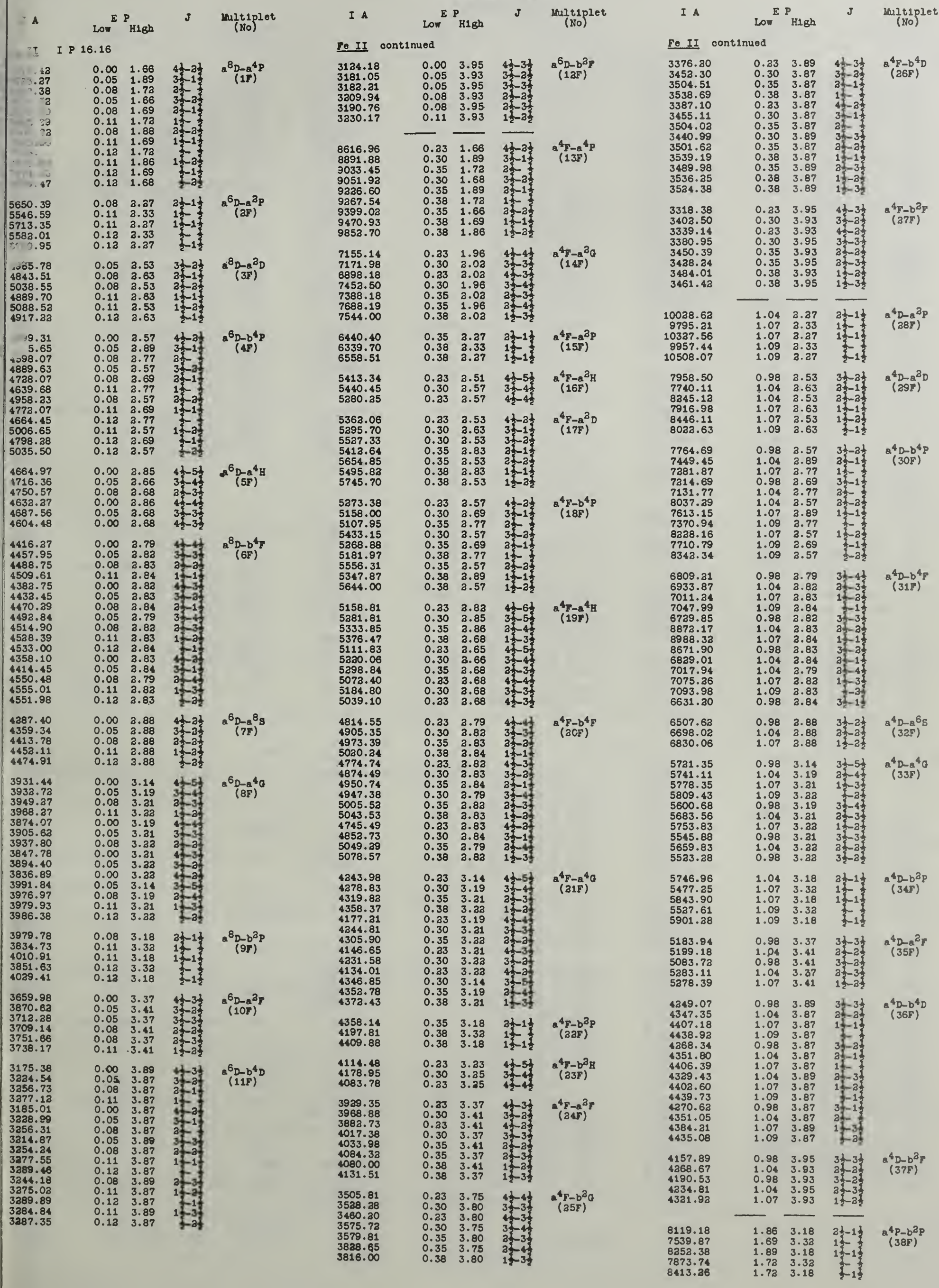


REVISED U ULTIPLET TABLE FORBIDDEN LINES

\begin{tabular}{|c|c|c|c|c|}
\hline & \multicolumn{2}{|c|}{ Low ${ }^{E P}{ }_{\text {H1gh }}$} & & $\underset{\text { (No) }}{\text { Multiple }}$ \\
\hline Fe II $c 0$ & nued & & & \\
\hline $\begin{array}{l}5551.31 \\
5643.44 \\
5725.92 \\
5580.82 \\
5650.94 \\
5724.62 \\
5588.15 \\
5649.67\end{array}$ & $\begin{array}{l}1.66 \\
1.69 \\
1.72 \\
1.66 \\
1.69 \\
1.72 \\
1.66 \\
1.69\end{array}$ & $\begin{array}{l}3.89 \\
3.87 \\
3.87 \\
3.87 \\
3.87 \\
3.87 \\
3.87 \\
3.87\end{array}$ & 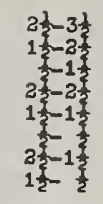 & $\begin{array}{c}a^{4} P-b^{4} D \\
(39 F)\end{array}$ \\
\hline $\begin{array}{r}10431.10 \\
10594.89 \\
10036.79 \\
10400.53 \\
9862.21 \\
10321.34\end{array}$ & $\begin{array}{l}1.96 \\
2.02 \\
1.96 \\
2.02 \\
1.96 \\
2.02\end{array}$ & $\begin{array}{l}3.14 \\
3.19 \\
3.19 \\
3.21 \\
3.21 \\
3.22\end{array}$ & $\begin{array}{l}4 \frac{1}{2}-5 \frac{1}{2} \\
3 \frac{1}{2}-4 \frac{2}{2} \\
4 \frac{1}{2}-4 \frac{1}{2} \\
3 \frac{1}{3}-3 \frac{1}{3} \\
4 \frac{1}{2}-3 \frac{1}{2} \\
3 \frac{2}{2}-2 \frac{2}{2}\end{array}$ & $\begin{array}{c}a^{2} a-a^{4} a \\
(40 F)\end{array}$ \\
\hline $\begin{array}{r}9682.13 \\
10013.88 \\
9513.87\end{array}$ & $\begin{array}{l}1.96 \\
2.02 \\
1.98\end{array}$ & $\begin{array}{l}3.23 \\
3.25 \\
3.25\end{array}$ & $\begin{array}{l}4 \frac{1}{3}-5 \frac{1}{2} \\
3 \frac{2}{2}-4 \frac{1}{2} \\
4 \frac{3}{2}-4 \frac{1}{2}\end{array}$ & $\begin{array}{l}a^{2} a-b^{2} H \\
(41 F)\end{array}$ \\
\hline $\begin{array}{l}8715.84 \\
8885.66 \\
9133.63\end{array}$ & $\begin{array}{l}1.96 \\
2.02 \\
2.02\end{array}$ & $\begin{array}{l}3.37 \\
3.41 \\
3.37\end{array}$ & $\begin{array}{l}4 \frac{1}{2}-3 \frac{1}{2} \\
3 \frac{2}{2}-3 \frac{2}{2} \\
3 \frac{1}{2}-3 \frac{1}{2}\end{array}$ & $\begin{array}{c}a^{2} G-a^{2} F \\
(42 F)\end{array}$ \\
\hline $\begin{array}{l}6873.87 \\
6944.91 \\
6700.68 \\
7131.13\end{array}$ & $\begin{array}{l}1.96 \\
2.02 \\
1.96 \\
2.02\end{array}$ & $\begin{array}{l}3.75 \\
3.80 \\
3.80 \\
3.75\end{array}$ & $\begin{array}{l}4 \frac{1}{3}-4 \frac{1}{2} \\
3 \frac{1}{2}-3 \frac{1}{2} \\
4 \frac{2}{3}-3 \frac{1}{2} \\
3 \frac{2}{2}-4 \frac{2}{2}\end{array}$ & $\begin{array}{c}a^{2} a-b^{2} a \\
(43 F)\end{array}$ \\
\hline $\begin{array}{l}6188.55 \\
6473.86 \\
6396.30\end{array}$ & $\begin{array}{l}1.96 \\
2.02 \\
2.02\end{array}$ & $\begin{array}{l}3.95 \\
3.93 \\
3.95\end{array}$ & $\begin{array}{l}4 \frac{1}{2}-3 \frac{1}{2} \\
3 \frac{1}{2}-2 \frac{2}{2} \\
3 \frac{1}{2}-3 \frac{1}{2}\end{array}$ & $\begin{array}{l}a^{2} a-b^{2} F \\
(44 F)\end{array}$ \\
\hline 10796.48 & 2.27 & 3.41 & $1 \frac{1}{2}-2 \frac{1}{2}$ & $a^{2}{ }_{P-a^{2}}^{a_{F}}$ \\
\hline $\begin{array}{l}7674.06 \\
8012.08 \\
7687.94 \\
8009.53 \\
7685.58\end{array}$ & $\begin{array}{l}2.27 \\
2.33 \\
2.27 \\
3.33 \\
2.27\end{array}$ & $\begin{array}{l}3.87 \\
3.87 \\
3.87 \\
3.87 \\
3.87\end{array}$ & $\begin{array}{l}1 \frac{1}{2}-2 \frac{1}{2} \\
\frac{1}{2}-1 \frac{2}{2} \\
1 \frac{2}{2}-1 \frac{2}{2} \\
1 \frac{5}{2}-\frac{2}{2}\end{array}$ & $\begin{array}{c}\left.a^{25 F}\right) \\
a_{P-b} d D \\
(46 F)\end{array}$ \\
\hline 7432.23 & 2.37 & 3.93 & $1 \frac{1}{2}-3 \frac{1}{2}$ & $\begin{array}{c}a^{2} P-b^{2} F \\
(47 F)\end{array}$ \\
\hline $\begin{array}{r}9949.32 \\
10038.79 \\
10432.60\end{array}$ & $\begin{array}{l}2.51 \\
2.57 \\
2.57\end{array}$ & $\begin{array}{l}3.75 \\
3.80 \\
3.75\end{array}$ & $\begin{array}{l}5 \frac{1}{2}-4 \frac{1}{2} \\
4 \frac{1}{3}-3 \frac{1}{2} \\
4 \frac{1}{2}-4 \frac{1}{2}\end{array}$ & $\begin{array}{c}a^{2} H-b^{2} G \\
(48 F)\end{array}$ \\
\hline 8931.47 & 2.57 & 3.95 & $4 \frac{1}{2}-3 \frac{1}{2}$ & $\begin{array}{c}a^{2} \mathrm{H}-b^{2}{ }_{F} \\
(49 F)\end{array}$ \\
\hline 9755.81 & 2.53 & 3.80 & $2 \frac{1}{2}-3 \frac{1}{2}$ & $a^{2} D-b^{2} a$ \\
\hline $\begin{array}{l}9116.41 \\
9918.01 \\
9196.26 \\
9941.20 \\
9216.20 \\
9937.27\end{array}$ & $\begin{array}{l}2.53 \\
2.63 \\
2.53 \\
2.63 \\
2.53 \\
2.63\end{array}$ & $\begin{array}{l}3.89 \\
3.87 \\
3.87 \\
3.87 \\
3.87 \\
3.87\end{array}$ & $\begin{array}{l}2 \frac{1}{2}-3 \frac{1}{2} \\
1 \frac{2}{2}-2 \frac{1}{2} \\
2 \frac{1}{2}-2 \frac{1}{2} \\
1 \frac{1}{2}-1 \\
2 \frac{1}{2}-1 \frac{3}{2} \\
1 \frac{2}{2}-\frac{1}{2}\end{array}$ & $\begin{array}{c}a^{2} D-b^{4} D \\
(51 F)\end{array}$ \\
\hline $\begin{array}{l}8706.79 \\
9517.76 \\
8851.13\end{array}$ & $\begin{array}{l}2.53 \\
2.63 \\
2.53\end{array}$ & $\begin{array}{l}3.95 \\
3.93 \\
3.93\end{array}$ & $\begin{array}{l}2 \frac{1}{2}-3 \frac{1}{2} \\
\frac{13}{3}-2 \frac{1}{2} \\
2 \frac{1}{2}-2 \frac{1}{2}\end{array}$ & $\begin{array}{c}a^{2} D-b^{3} F \\
(58 F)\end{array}$ \\
\hline
\end{tabular}

Fe III I P 30.48

\begin{tabular}{|c|c|c|c|c|}
\hline $\begin{array}{l}5151.9 \\
4936.4 \\
4883.9 \\
5270.4 \\
5011.3 \\
4930.5 \\
5355.9 \\
5060.3 \\
5412.0 \\
5084.8 \\
5439.9\end{array}$ & $\begin{array}{l}0.00 \\
0.05 \\
0.09 \\
0.05 \\
0.09 \\
0.12 \\
0.09 \\
0.13 \\
0.13 \\
0.13 \\
0.13\end{array}$ & $\begin{array}{l}2.40 \\
3.55 \\
3.62 \\
2.40 \\
2.55 \\
2.62 \\
2.40 \\
2.55 \\
2.40 \\
2.55 \\
2.40\end{array}$ & $\begin{array}{l}4-3 \\
3-1 \\
2-0 \\
3-3 \\
2-1 \\
1-0 \\
3-3 \\
1-1 \\
1-2 \\
0-1 \\
0-2\end{array}$ & $\begin{array}{c}a^{5} p-a^{3} p \\
(1 F)\end{array}$ \\
\hline $\begin{array}{l}4985.9 \\
5032.7 \\
5063.7 \\
4924.5 \\
4987.2 \\
4881.0\end{array}$ & $\begin{array}{l}0.00 \\
0.05 \\
0.09 \\
0.00 \\
0.05 \\
0.00\end{array}$ & $\begin{array}{l}2.48 \\
2.51 \\
2.53 \\
2.51 \\
2.53 \\
2.53\end{array}$ & $\begin{array}{l}4-6 \\
3-5 \\
2-4 \\
4-5 \\
3-4 \\
4-4\end{array}$ & $\begin{array}{c}a^{5} D_{-} a^{3} H \\
(2 F)^{3}\end{array}$ \\
\hline $\begin{array}{l}4658.1 \\
4701.5 \\
4733.9 \\
4607.0 \\
4667.0 \\
4754.7 \\
4769.4 \\
4777.7 \\
4573.9 \\
4824.1 \\
4813.9 \\
4799.5\end{array}$ & $\begin{array}{l}0.00 \\
0.05 \\
0.09 \\
0.00 \\
0.05 \\
0.05 \\
0.09 \\
0.12 \\
0.00 \\
0.09 \\
0.12 \\
0.13\end{array}$ & $\begin{array}{l}2.65 \\
2.68 \\
2.70 \\
2.68 \\
2.70 \\
2.65 \\
2.68 \\
2.70 \\
2.70 \\
2.65 \\
2.68 \\
2.70\end{array}$ & $\begin{array}{l}4-4 \\
3-3 \\
2-2 \\
4-3 \\
3-2 \\
3-4 \\
2-3 \\
1-2 \\
4-2 \\
2-4 \\
1-3 \\
0-2\end{array}$ & $\frac{a^{5} \frac{p-a^{3}}{(3 F)}}{(3 F)}$ \\
\hline $\begin{array}{l}4070.7 \\
4079.7 \\
4096.6 \\
4008.3 \\
4046.4\end{array}$ & $\begin{array}{l}0.00 \\
0.05 \\
0.09 \\
0.00 \\
0.05\end{array}$ & $\begin{array}{l}3.03 \\
3.08 \\
3.10 \\
3.08 \\
3.10\end{array}$ & $\begin{array}{l}4-5 \\
3-4 \\
2-3 \\
4-4 \\
3-3\end{array}$ & $\frac{a^{5} D-a^{3} a}{(4 F)}$ \\
\hline
\end{tabular}

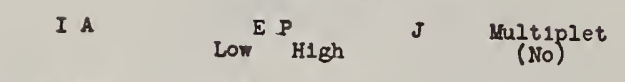

\section{Fe III continued}

\begin{tabular}{|c|c|c|c|c|}
\hline $\begin{array}{l}3976.2 \\
4144.3 \\
4130.7 \\
4129.4\end{array}$ & $\begin{array}{l}0.00 \\
0.05 \\
0.09 \\
0.12\end{array}$ & $\begin{array}{l}3.10 \\
3.03 \\
3.08 \\
3.10\end{array}$ & $\begin{array}{l}4-3 \\
3-5 \\
2-4 \\
1-3\end{array}$ & $\begin{array}{c}a^{5} p-a^{3} a \\
(4 r) \\
\text { cont }\end{array}$ \\
\hline $\begin{array}{l}3322.54 \\
3371.4 \\
3406.2 \\
3428.8\end{array}$ & $\begin{array}{l}0.00 \\
0.05 \\
0.09 \\
0.13\end{array}$ & $\begin{array}{l}3.71 \\
3.71 \\
3.71 \\
3.71\end{array}$ & $\begin{array}{l}4-3 \\
3-3 \\
2-3 \\
1-3\end{array}$ & $\begin{array}{c}a^{5} p-a^{7} s \\
(5 r)\end{array}$ \\
\hline $\begin{array}{l}3239.7 \\
3301.6 \\
3333.8 \\
3254.7 \\
3300.5 \\
3286.2 \\
3334.9 \\
3355.5 \\
3319.2 \\
3356.6 \\
3366.3 \\
3340.7 \\
3367.3\end{array}$ & $\begin{array}{l}0.00 \\
0.05 \\
0.09 \\
0.00 \\
0.05 \\
0.05 \\
0.09 \\
0.12 \\
0.09 \\
0.12 \\
0.13 \\
0.13 \\
0.13\end{array}$ & $\begin{array}{l}3.81 \\
3.79 \\
3.79 \\
3.79 \\
3.79 \\
3.81 \\
3.79 \\
3.79 \\
3.81 \\
3.79 \\
3.79 \\
3.81 \\
3.79\end{array}$ & $\begin{array}{l}4-3 \\
3-2 \\
3-1 \\
4-2 \\
3-1 \\
3-3 \\
2-2 \\
1-1 \\
2-3 \\
1-3 \\
0-1 \\
1-3 \\
0-2\end{array}$ & $\begin{array}{c}a^{5} D-a^{3} D \\
(6 F)\end{array}$ \\
\hline $\begin{array}{l}3236.7 \\
3283.1 \\
3316.1\end{array}$ & $\begin{array}{l}0.00 \\
0.05 \\
0.09\end{array}$ & $\begin{array}{l}3.81 \\
3.81 \\
3.81\end{array}$ & $\begin{array}{l}4-4 \\
3-4 \\
2-4\end{array}$ & $\frac{a^{5} p-a^{1} a}{(75)}$ \\
\hline $\begin{array}{r}8728.9 \\
9969.6 \\
10504.3 \\
8838.2 \\
9960.0 \\
8830.7\end{array}$ & $\begin{array}{l}2.40 \\
2.55 \\
2.62 \\
2.40 \\
2.55 \\
3.40\end{array}$ & $\begin{array}{l}3.81 \\
3.79 \\
3.79 \\
3.79 \\
3.79 \\
3.79\end{array}$ & $\begin{array}{l}2-3 \\
1-3 \\
0-1 \\
2-3 \\
1-1 \\
2-1\end{array}$ & $\begin{array}{c}a^{3} P-8^{3} D \\
(8 F)\end{array}$ \\
\hline 7078.2 & 2.55 & 4.30 & $1-0$ & $a^{3} p-a^{1} s$ \\
\hline $\begin{array}{l}6096.3 \\
6614.0\end{array}$ & $\begin{array}{l}2.40 \\
2.55 \\
\end{array}$ & $\begin{array}{l}4.42 \\
4.42\end{array}$ & $\begin{array}{l}2-3 \\
1-2 \\
\end{array}$ & $\begin{array}{c}a^{3} P-a^{1} D \\
(10 F)\end{array}$ \\
\hline $\begin{array}{l}9701.3 \\
9943.2\end{array}$ & $\begin{array}{l}2.48 \\
2.51\end{array}$ & $\begin{array}{l}3.75 \\
3.75\end{array}$ & $\begin{array}{l}6-6 \\
5-6\end{array}$ & $\begin{array}{c}a^{3} H-a^{1} I \\
(11 F)\end{array}$ \\
\hline $\begin{array}{l}9444.2 \\
9608.6\end{array}$ & $\begin{array}{l}2.51 \\
3.53\end{array}$ & $\begin{array}{l}3.81 \\
3.81\end{array}$ & $\begin{array}{l}5-4 \\
4-4\end{array}$ & 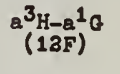 \\
\hline $\begin{array}{l}0840.4 \\
1088.0 \\
1373.6 \\
0916.5 \\
1384.9 \\
1107.3\end{array}$ & $\begin{array}{l}3.65 \\
2.68 \\
3.70 \\
2.68 \\
2.70 \\
3.70\end{array}$ & $\begin{array}{l}3.81 \\
3.79 \\
3.79 \\
3.81 \\
3.79 \\
3.81\end{array}$ & $\begin{array}{l}4-3 \\
3-3 \\
2-1 \\
3-3 \\
2-2 \\
2-3\end{array}$ & $a^{3} F-a^{3} D$ \\
\hline $\begin{array}{l}0608.1 \\
0882.6\end{array}$ & $\begin{array}{r}2.65 \\
3.68\end{array}$ & $\begin{array}{l}3.81 \\
3.81\end{array}$ & $\begin{array}{l}4-4 \\
3-4\end{array}$ & $\begin{array}{c}a^{3} \sqrt{F}-a^{1} g \\
(14 F)\end{array}$ \\
\hline $\begin{array}{l}7088.3 \\
7220.0\end{array}$ & $\begin{array}{l}3.68 \\
2.70\end{array}$ & $\begin{array}{l}4.43 \\
4.42\end{array}$ & $\begin{array}{l}3-2 \\
2-3\end{array}$ & $a^{3} F-a^{1} D$ \\
\hline
\end{tabular}

Fe $\mathrm{V}$ I P ?

$3970.1 \quad 0.16 \quad 3.27 \quad 4-2 \quad 3 d^{4} \quad 50-3 d^{4} \quad 3 p$

$\begin{array}{lllll}4136.4 & 0.10 & 3.08 & 3-1 & 3 \mathrm{a} \\ 4329.8 & 0.05 & 8.97 & 2-0 & (1 F)\end{array}$ $\begin{array}{lll}3895.7 & 0.05 & 3.97 \\ & 0.10 & 3.27\end{array}$ $4071.5 \quad 0.053 .08$ $4026.6 \quad 0.02 \quad 3.08$ $\begin{array}{lll}3798.3 & 0.02 & 3.27 \\ 3003 & 0.3 & 0.00\end{array}$ $\begin{array}{lll}3777.4 & 0.00 & 3.08 \\ 37.00 & 3.27\end{array}$

4123.9 4093.0

4077.5

4175.2
4142.5

4226.8

3891.8

3838.9

3911.1

3850.8

3820.3

3782.9

3933.5

3764.8

3744.1

3430.3

3406.6

3400.3

3463.4

3374.6

3368.9
3503.5 $0.03 \quad 3.97$

$0.16 \quad 3.15$ $0.16 \quad 3.11$ 0.16 3.08 $0.10 \quad 3.31$ $0.05 \quad 3.30$ $0.16 \quad 3.31$ $0.10 \quad 3.33$ $0.05 \quad 3.31$ $\begin{array}{ll}0.08 & 3.30 \\ 0.16 & 3.30\end{array}$ 0.05 3.33 $\begin{array}{ll}0.02 & 3.31 \\ 0.00 & 3.30\end{array}$ $\begin{array}{lllc}0.16 & 3.76 & 4-5 & 3 d^{4} 5 p-3 d^{4} 3 G \\ 0.10 & 3.72 & 3-4 & (45) \\ 0.05 & 3.68 & 2-3 & \end{array}$ $0.05 \quad 3.68$ 0.163 .73 $0.10 \quad 3.76$ 0.053 .73 \begin{tabular}{ll}
0.16 & 3.68 \\
\hline
\end{tabular} 0.1053 .11 $0.10 \quad 3.08$ a-1

$3-2$
$3-1$
$1-0$

$1-0$
$2-3$

$1-1$
$1-2$

$0-2$

$\begin{array}{ll}4-6 & 3 d^{4} 5 p-3 d^{4} \quad 3 H \\ 3-5 & (3 F)\end{array}$

$\begin{array}{lcl}4-4 & 3 q^{4} 5 p-3 d^{4} \quad 3 F \\ 3-3 & (3 F)\end{array}$

$3-3$
$2-2$

$4-3$
$3-3$
$3-4$

$3-3$
$3-4$
$2-3$

$2-3$
$1-3$
$4-3$

$4-3$
$3-4$

$3-4$
$2-4$
$4-4$

$3-3$

$2-4$
$1-3$
$4-3$
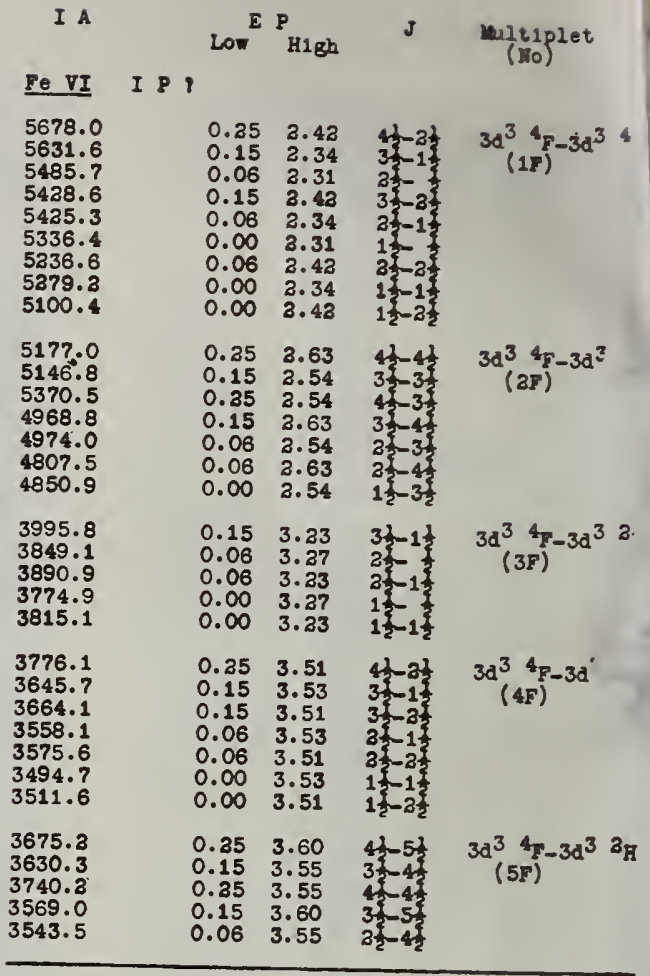

Fe VII I P ?

\begin{tabular}{|c|c|c|c|c|}
\hline $\begin{array}{l}6599.7 \\
6085.5 \\
5730.9\end{array}$ & $\begin{array}{l}0.29 \\
0.13 \\
0.00\end{array}$ & $\begin{array}{l}3.16 \\
3.16 \\
3.16\end{array}$ & $\begin{array}{l}1-2 \\
3-2 \\
2-2\end{array}$ & $\begin{array}{l}3 d^{3}{ }_{(1 F)}^{3}-3 d^{2}{ }^{1} D \\
\end{array}$ \\
\hline $\begin{array}{l}5276.1 \\
5158.3 \\
4989.4 \\
4943.3 \\
4893.9 \\
4699.0\end{array}$ & $\begin{array}{l}0.29 \\
0.13 \\
0 . \infty \\
0.13 \\
0.00 \\
0.00\end{array}$ & $\begin{array}{l}2.63 \\
3.53 \\
3.47 \\
3.63 \\
3.58 \\
3.63\end{array}$ & $\begin{array}{l}4-3 \\
3-1 \\
2-0 \\
3-2 \\
2-1 \\
2-3\end{array}$ & $\begin{array}{l}3 d^{2}{ }^{3} p-3 d^{3} 3 p \\
(a F)^{3} p\end{array}$ \\
\hline $\begin{array}{l}3759.9 \\
3587.2 \\
3457.3\end{array}$ & $\begin{array}{l}0.29 \\
0.13 \\
0.00\end{array}$ & $\begin{array}{l}3.57 \\
3.57 \\
3.57\end{array}$ & $\begin{array}{l}4-4 \\
3-4 \\
3-4\end{array}$ & ${ }^{3 d^{2} 3}(3)^{-3 d^{2}}$ \\
\hline 8738.1 & 3.16 & 3.57 & $2-4$ & $\begin{array}{c}3 d^{2}{ }^{1} p-3 d^{3} \\
(4 r !\end{array}$ \\
\hline
\end{tabular}

FeX I P 261?

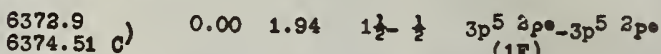
Te XI I P 389 ?

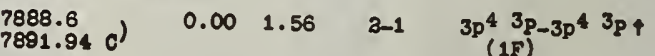
$3986.1 \quad 1.56 \quad 4.66 \quad 1-2 \quad 3 p^{4}(3 p)-3 p^{4}{ }^{4} D+$ (3F)

Fe XIII I P 355 ? $\begin{array}{llllll}10797.95 & c \\ 10749.7 & 0.00 & 1.15 & 0-1 & (1 F)^{-3 p^{2} 3 p}\end{array}$ $3387 . ?$ $\begin{array}{llll}2.39 & 5.93 \quad 2-2 & 3 p^{2} 3 p-3 p^{3} & 10 \\ (25) & \end{array}$

Fo XIV I P 3909

$\begin{array}{lllll}5303.6 & 0.00 & 2.33 & \frac{1}{2}-1 \frac{1}{2} \quad 3 p^{2} p^{0}-3 p^{2} p^{0}\end{array}$

Ye XV I P 454?

$\left.\begin{array}{l}7080.3 \\ 7059.63 \mathrm{c}\end{array}\right) \quad\left(\begin{array}{llll}29.8 & 31.6) & 1-3 \quad 3 \mathrm{p}^{3} \mathrm{p}^{-}-3 \mathrm{p}^{3} \mathrm{p} \bullet\end{array}\right.$ 
REVISED UULTIPLET TABLE FORBIDDEN LINES

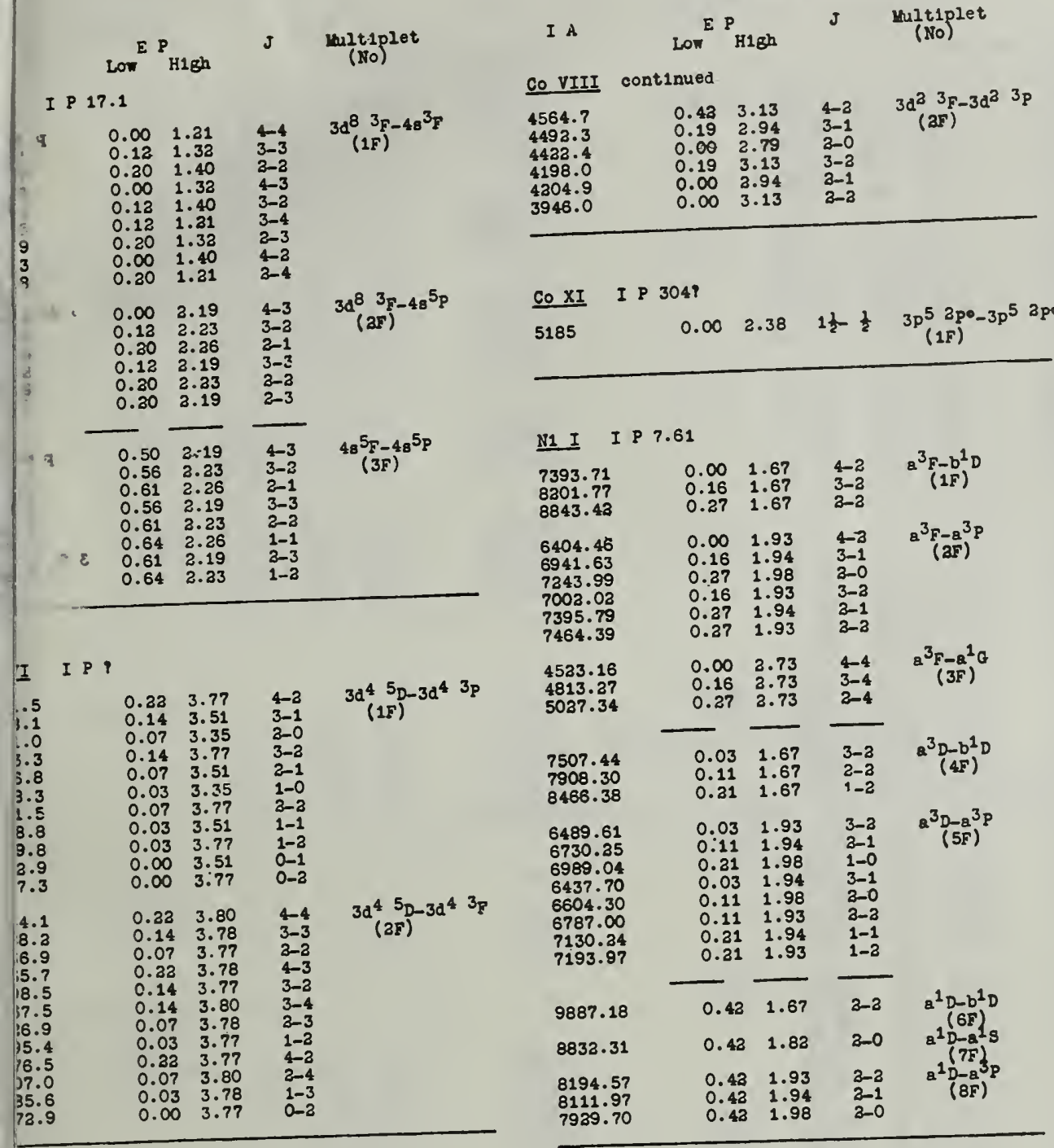

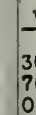

I P ?

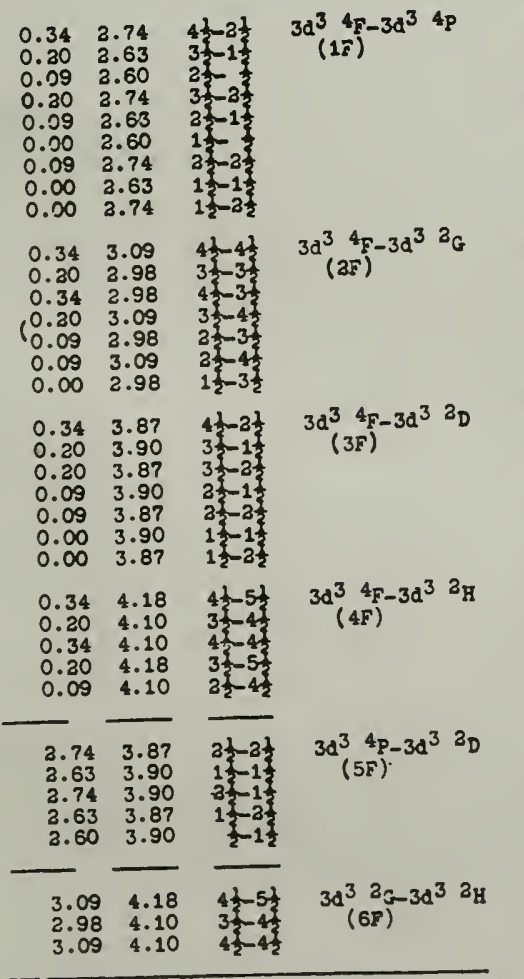

N1 II I P 18.4

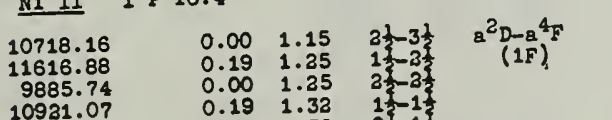
9377.33

7379.57

7413.35

8303.23

4326.85

4485.87

4385.87
4301.74

3993.65

3993.65
4294.70

4033.56

4249.48

3439.29

3439.29
3559.86

3378.55

3627.35

3074.11

6794.37

6911.05

7307.82

7694.82
7612.96

8033.86

6467.52

6791.61

6365.52

7054.37

6700.61

7078.25
6958.25

$0.00 \quad 1.32$

$\begin{array}{ll}0.00 & 1.67 \\ 0.19 & 1.85 \\ 0.00 & 1.85\end{array}$

0.00
0.19
0.19

$0.00 \quad 3.85$

$0.19 \quad 2.94$

$0.19 \quad 3.85$

$0.00 \quad 3.09$

$0.19 \quad 3.06$

$\begin{array}{ll}0.00 & 3.06 \\ 0.19 & 3.07\end{array}$

$0.19 \quad 3.09$

$0.00 \quad 3.59$

$0.00 \quad 3.65$

$0 . \infty \quad 4.01$

$-1.04 \frac{4.01}{2.85}$

$\begin{array}{ll}1.04 & 2.85 \\ 1.15 & 2.94\end{array}$

$\begin{array}{ll}1.15 & 3.85 \\ 1.35 & 3.94\end{array}$

$1.25 \quad 3.85$

$\begin{array}{ll}1.32 & 3.94 \\ 1.33 & 3.85\end{array}$

$\begin{array}{ll}1.04 & 3.09 \\ 1.15 & 3.06\end{array}$

$1.35 \quad 3.07$

$\begin{array}{ll}1.15 & 3.09 \\ 1.25 & 3.06\end{array}$

$1.32 \quad 3.07$

$\begin{array}{ll}1.25 & 3.09 \\ 1.32 & 3.05\end{array}$

$\begin{array}{ll}1.32 & 3.09\end{array}$

$\begin{array}{lllc}0.42 & 2.77 & 4-2 & 3 d^{2} 3 F-3 d^{2}{ }^{1} D \\ 0.19 & 2.77 & 3-2 & (15)\end{array}$

$3-3$
$2-2$

(15)
I A Low ${ }_{\text {H1gh }}$ J Multiplet

N1 II continued

\begin{tabular}{|c|c|c|c|c|}
\hline $\begin{array}{l}4147.30 \\
4310.46 \\
4143.17 \\
4314.92 \\
4461.54 \\
4466.33 \\
4573.45\end{array}$ & $\begin{array}{l}1.04 \\
1.15 \\
1.04 \\
1.15 \\
1.25 \\
1.25 \\
1.32\end{array}$ & $\begin{array}{l}4.01 \\
4.01 \\
4.01 \\
4.01 \\
4.01 \\
4.01 \\
4.01\end{array}$ & $\begin{array}{l}4 \frac{1}{3}-4 \frac{1}{2} \\
3 \frac{1}{2}-3 \frac{1}{2} \\
4 \frac{1}{2}-3 \frac{2}{3} \\
3 \frac{1}{3}-4 \frac{1}{2} \\
2 \frac{1}{3}-3 \frac{1}{\frac{1}{2}} \\
2 \frac{1}{2}-4 \frac{1}{3} \\
1 \frac{1}{2}-3 \frac{1}{2}\end{array}$ & $\begin{array}{c}a^{4} F-a^{2} G \\
(10 F)\end{array}$ \\
\hline $\begin{array}{l}10459.79 \\
11359.87 \\
12323.37\end{array}$ & $\begin{array}{l}1.67 \\
1.85 \\
1.85\end{array}$ & $\begin{array}{l}2.85 \\
3.94 \\
3.85\end{array}$ & $\begin{array}{l}3 \frac{1}{2}-2 \frac{1}{2} \\
3 \frac{1}{2}-1 \frac{1}{2} \\
2 \frac{1}{2}-2 \frac{1}{2}\end{array}$ & $\begin{array}{c}\mathrm{a}^{2} F-b^{2} \mathrm{D} \\
(11 F)\end{array}$ \\
\hline $\begin{array}{r}8704.24 \\
10309.10 \\
9957.33\end{array}$ & $\begin{array}{l}1.67 \\
1.85 \\
1.85\end{array}$ & $\begin{array}{l}3.09 \\
3.06 \\
3.09\end{array}$ & $\begin{array}{l}3 \frac{1}{2}-2 \frac{1}{2} \\
2 \frac{3}{2}-1 \frac{1}{3} \\
2 \frac{1}{2}-2 \frac{1}{2}\end{array}$ & $\begin{array}{c}a^{3} F-8^{4} P \\
(12 F)\end{array}$ \\
\hline 7102.84 & 1.85 & 3.59 & $3 \frac{1}{2}-1 \frac{1}{2}$ & $\begin{array}{l}a^{2} F-a^{2} p \\
(13 F)\end{array}$ \\
\hline $\begin{array}{l}5275.83 \\
5703.64 \\
5269.16 \\
5711.46\end{array}$ & $\begin{array}{l}1.67 \\
1.85 \\
1.67 \\
1.85\end{array}$ & $\begin{array}{l}4.01 \\
4.01 \\
4.01 \\
4.01\end{array}$ & $\begin{array}{l}3 \frac{1}{2}-4 \frac{1}{2} \\
2 \frac{1}{3}-3 \frac{1}{3} \\
3 \frac{3}{2}-3 \frac{2}{2} \\
2 \frac{1}{2}-4 \frac{2}{2}\end{array}$ & $\begin{array}{c}a^{2} F-a^{2} G \\
(14 F)\end{array}$ \\
\hline
\end{tabular}

N1 VII I P P

\begin{tabular}{|c|c|c|c|c|}
\hline $\begin{array}{l}3191.2 \\
3379.7 \\
3503.8 \\
3106.0 \\
3299.6 \\
3440.3 \\
3038.3 \\
3243.2 \\
3990.4 \\
3214.5\end{array}$ & $\begin{array}{l}0.29 \\
0.19 \\
0.10 \\
0.19 \\
0.10 \\
0.03 \\
0.10 \\
0.03 \\
0.03 \\
0.00\end{array}$ & $\begin{array}{l}4.16 \\
3.84 \\
3.62 \\
4.16 \\
3.84 \\
3.62 \\
4.16 \\
3.84 \\
4.16 \\
3.84\end{array}$ & $\begin{array}{l}4-2 \\
3-1 \\
2-0 \\
3-2 \\
2-1 \\
1-0 \\
2-2 \\
1-1 \\
1-2 \\
0-1\end{array}$ & $\begin{array}{l}3 d^{4} 5 p-3 d^{4} 3 p \\
(1 F)\end{array}$ \\
\hline $\begin{array}{l}3413.3 \\
3396.7 \\
3486.6\end{array}$ & $\begin{array}{l}0.29 \\
0.19 \\
0.29\end{array}$ & $\begin{array}{l}3.91 \\
3.83 \\
3.83\end{array}$ & $\begin{array}{l}4-5 \\
3-4 \\
4-4\end{array}$ & $\begin{array}{l}3 d^{4} 5 D-3 d^{4} 3 H \\
(3 F)\end{array}$ \\
\hline $\begin{array}{l}3165.4 \\
3106.1 \\
3048.8 \\
319: .3 \\
3117.1 \\
3081.6 \\
3038.4 \\
3000.6\end{array}$ & $\begin{array}{l}0.29 \\
0.19 \\
0.10 \\
0.29 \\
0.19 \\
0.19 \\
0.10 \\
0.03\end{array}$ & $\begin{array}{l}4.19 \\
4.16 \\
4.15 \\
4.16 \\
4.15 \\
4.19 \\
4.16 \\
4.15\end{array}$ & $\begin{array}{l}4-4 \\
3-3 \\
2-2 \\
4-3 \\
3-2 \\
3-4 \\
2-3 \\
1-2\end{array}$ & $\begin{array}{l}3 d^{4} 5 D-3 d^{4} 3_{F} \\
(3 F)\end{array}$ \\
\hline
\end{tabular}

111 VIII I P ?

\begin{tabular}{|c|c|c|c|c|}
\hline $\begin{array}{l}4772.4 \\
4644.2 \\
4493.3 \\
4446.2 \\
4404.4 \\
4297.8 \\
4225.9 \\
4216.4 \\
4053.5\end{array}$ & $\begin{array}{l}0.46 \\
0.27 \\
0.13 \\
0.27 \\
0.13 \\
0.00 \\
0.13 \\
0.00 \\
0.00\end{array}$ & $\begin{array}{l}3.05 \\
2.93 \\
2.87 \\
3.05 \\
2.93 \\
2.87 \\
3.05 \\
2.93 \\
3.05\end{array}$ & 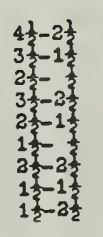 & $\begin{array}{l}3 d^{3}{ }^{4} F-3 d^{3} d^{4} p \\
(1 F)^{2}\end{array}$ \\
\hline $\begin{array}{l}4106.1 \\
4032.3 \\
4298.8 \\
3862.3 \\
3850.3 \\
3695.0 \\
3705.8\end{array}$ & $\begin{array}{l}0.46 \\
0.27 \\
0.46 \\
0.37 \\
0.13 \\
0.13 \\
0.00\end{array}$ & $\begin{array}{l}3.46 \\
3.33 \\
3.33 \\
3.46 \\
3.33 \\
3.46 \\
3.33\end{array}$ & $\begin{array}{l}4 \frac{1}{3}-4 \frac{1}{2} \\
3 \frac{1}{2}-3 \frac{1}{3} \\
4 \frac{1}{3}-3 \frac{1}{3} \\
3 \frac{1}{2}-4 \frac{1}{2} \\
2 \frac{3}{2}-3 \frac{2}{2} \\
2 \frac{1}{2}-4 \frac{1}{2} \\
1 \frac{1}{2}-3 \frac{1}{2}\end{array}$ & $\begin{array}{l}3 d^{3} d^{4} F-3 d^{3} 2_{G} \\
(2 F)\end{array}$ \\
\hline $\begin{array}{l}3228.2 \\
3035.3 \\
3075.6\end{array}$ & $\begin{array}{l}0.46 \\
0.27 \\
0.37\end{array}$ & $\begin{array}{l}4.38 \\
4.34 \\
4.28\end{array}$ & $\begin{array}{l}4 \frac{1}{3}-2 \frac{1}{2} \\
3 \frac{1}{3}-1 \frac{1}{2} \\
3 \frac{1}{2}-2 \frac{1}{2}\end{array}$ & $\begin{array}{l}3 d^{3}{ }^{4} F-3 d^{3} 2 g \\
(3 F)\end{array}$ \\
\hline 3036.4 & 0.46 & 4.54 & $4 \frac{1}{2}-4 \frac{1}{2}$ & $\begin{array}{c}3 d^{3}{ }^{4} F-3 d^{3} 2_{H} \\
(4 F)\end{array}$ \\
\hline $\begin{array}{l}9977.1 \\
8761.8 \\
9565.8 \\
9105.8 \\
8430.1\end{array}$ & $\begin{array}{l}3.05 \\
2.93 \\
3.05 \\
3.93 \\
2.87\end{array}$ & $\begin{array}{l}4.38 \\
4.34 \\
4.34 \\
4.28 \\
4.34\end{array}$ & 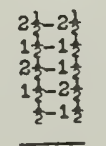 & $\begin{array}{l}3 d^{3} 4 p-3 d^{3} 2 g \\
(5 F)\end{array}$ \\
\hline $\begin{array}{l}10627.5 \\
10225.3 \\
11509.6\end{array}$ & $\begin{array}{l}3.46 \\
3.33 \\
3.46\end{array}$ & $\begin{array}{l}4.63 \\
4.54 \\
4.54\end{array}$ & $\begin{array}{l}4 \frac{1}{2}-5 \frac{1}{2} \\
3 \frac{1}{2}-4 \frac{1}{3} \\
4 \frac{1}{2}-4 \frac{1}{2}\end{array}$ & $\begin{array}{c}3 d^{3}{ }^{2} G-3 d^{3}{ }^{2} H \\
(6 F)\end{array}$ \\
\hline III IX & I P P & & & \\
\hline $\begin{array}{l}5056.5 \\
4331.7 \\
4043.4\end{array}$ & $\begin{array}{l}0.61 \\
0.20 \\
0.00\end{array}$ & $\begin{array}{l}3.05 \\
3.05 \\
3.05\end{array}$ & $\begin{array}{l}4-3 \\
3-2 \\
2-2\end{array}$ & $\begin{array}{l}3 d^{2} 3 F-3 d^{2} 1 p \\
(1 F)^{2}\end{array}$ \\
\hline $\begin{array}{l}4190.6 \\
4065.7 \\
4112.7 \\
3680.3 \\
3810.6 \\
3470.0\end{array}$ & $\begin{array}{l}0.61 \\
0.20 \\
0.00 \\
0.20 \\
0.00 \\
0.00\end{array}$ & $\begin{array}{l}3.56 \\
3.24 \\
3.00 \\
3.56 \\
3.24 \\
3.56\end{array}$ & $\begin{array}{l}4-2 \\
3-1 \\
2-0 \\
3-2 \\
2-1 \\
2-2\end{array}$ & $\begin{array}{l}3 d^{2} 3 \bar{F}-3 d^{2} 3 p \\
(a F)\end{array}$ \\
\hline N1XII & I P ? & & & \\
\hline 4231.4 & 0.00 & $(3.93)$ & $1 \frac{1}{2}-\frac{1}{2}$ & $\begin{array}{l}3 p^{5} 2 p^{0}-3 p^{5} 2 p^{0} \\
(1 F)\end{array}$ \\
\hline
\end{tabular}




\section{A Low H1gh J Multiplet}

N1 XIII I P 350?

5116.3
$5116.03 c) \quad 0.00(2.41) \quad \hat{b}-1 \quad 3 p^{4} 3 p-3 p^{4} 3 p$
$(1 F)$

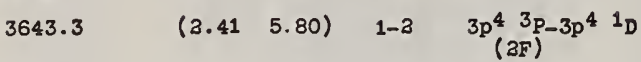

N1 XV I P 455 ?

8024 c) $\quad\left(\begin{array}{llll}1.84 & 3.38) & 1-2 \quad 3 p^{2} 3 p-3 p^{2} 3 p\end{array}\right.$ 6700.6 ( )

Y1 XVI I $P$ ?

$\begin{array}{lllll}3601.3 & 0.00 & 3.43 \quad \frac{1}{2}-1 \frac{1}{2} & 3 p^{2} p^{\circ}-3 p^{2} p^{\circ} \\ (1 F)\end{array}$

Cu II I P 20.18

$\begin{array}{ccccc}4375.71 & 0.00 & 2.82 & 0-2 & 3 \mathrm{~d}^{10}{ }^{1} \mathrm{~S}-4 \mathrm{~B}^{3} \mathrm{D} \\ 4165.79 & 0.00 & 2.96 & 0-1 & (1 F) \\ 3806.34 & 0.00 & 3.24 & 0-3 & 3 \mathrm{~d}^{101}{ }^{1} \mathrm{~S}-4 \mathrm{~B}^{1} \mathrm{D}\end{array}$

Ir. III I P 36.8

$\begin{array}{ccccc}6826.9 & 0.00 & 1.81 & 2-2 & 4 p^{4} 3 p-4 p^{4} 1_{D} \\ 9902.2 & 0.56 & 1.81 & 1-2 & (1 F)\end{array}$

Sr. II I P 10.98

$\begin{array}{ccccc}6738.40 & 0.00 & 1.83 & \frac{1}{2}-2 \frac{1}{2} & 5^{2} \mathrm{~S}-4^{3} \mathrm{D} \\ 6868.18 & 0.00 & 1.80 & \frac{1}{2}-1 \frac{1}{2} & (1 F)\end{array}$

III I P 12.3

\begin{tabular}{|c|c|c|c|c|}
\hline $\begin{array}{l}7091.17 \\
7131.55\end{array}$ & $\begin{array}{l}0.00 \\
0.00\end{array}$ & $\begin{array}{l}1.74 \\
1.73\end{array}$ & $\begin{array}{l}0-2 \\
0-1\end{array}$ & $\frac{a^{1} 8-a^{3} p}{(1 F)^{2}}$ \\
\hline 6739.91 & 0.00 & 1.83 & $0-3$ & $D$ \\
\hline $\begin{array}{l}7904.04 \\
7706.06 \\
7664.67 \\
7954.24 \\
7787.00 \\
7658.92 \\
7586.23 \\
7540.54\end{array}$ & $\begin{array}{l}0.18 \\
0.13 \\
0.10 \\
0.18 \\
0.13 \\
0.13 \\
0.10 \\
0.10\end{array}$ & $\begin{array}{l}1.74 \\
1.73 \\
1.71 \\
1.73 \\
1.71 \\
1.74 \\
1.73 \\
1.74\end{array}$ & $\begin{array}{l}3-2 \\
2-1 \\
1-0 \\
3-1 \\
3-0 \\
2-2 \\
1-1 \\
1-2\end{array}$ & $\begin{array}{c}a^{3} p-a^{3} p \\
(3 F)\end{array}$ \\
\hline $\begin{array}{l}7470.10 \\
7350.78 \\
7144.60\end{array}$ & $\begin{array}{l}0.18 \\
0.13 \\
0.10\end{array}$ & $\begin{array}{l}1.83 \\
1.83 \\
1.83\end{array}$ & $\begin{array}{l}3-2 \\
2-2 \\
1-2\end{array}$ & $\frac{a^{3} D-b^{1} D}{(4 F)}$ \\
\hline $\begin{array}{l}9255.10 \\
9334.01 \\
9442.77\end{array}$ & $\begin{array}{l}0.41 \\
0.41 \\
0.41\end{array}$ & $\begin{array}{l}1.74 \\
1.73 \\
1.71\end{array}$ & $\begin{array}{l}2-2 \\
2-1 \\
2-0\end{array}$ & $\begin{array}{c}a^{1} D-g^{3} P \\
(5 F)\end{array}$ \\
\hline 665.66 & 0.41 & 1.83 & $2-2$ & $\begin{array}{c}a^{1} D-b^{1} D \\
(6 F)\end{array}$ \\
\hline
\end{tabular}

$\underline{Y}$ V I P 77

$8284.1 \quad 0.00 \quad 1.49 \quad 1 \frac{1}{2} \frac{1}{2} \quad 4 p^{5} 2 p^{\circ}-4 p^{5} 3_{p 0}$

2r II I P 13.97

10860.44 10603.65
10464.94 10464.94
10355.58 10261.18
10028.71

10028.71

9058.16

8909.40
9376.93

7786.03

7893.57

7460.93

7623.44
7219.15

7444.80
7058.76

7769.35 7518.81 7849.08 7445.63
7273.33 (2) 7204.83
7110.54
I A Low $P_{\text {H1gh }}$ Jultiplet

Zr II continued

77

7454.82
7386.11
7156.26
7149.08
6933.5
6991.75
6785.4
5855.3
5932.8
5669.5
5778.9
5528.8
5675.73
5434.3
5520.1
5331.4
5495.4
5354.7
5206.8
5229.0
5122.8
5144.3

9607.90

9870.08

9490.96

8873.37

8625.25

9582.55

9291.03
9704.10

9179.54

8954.34

8702.70

9108.53

9089.24

8743.65
8766.76

8444.83

8525.41
8230.64

6829.24

694.07

6622.05
6793.01

6793.01
6449.31

6646.31

6317.64

6377.59

6165.35
6344.56

6344.56
6196.53

6015.36

6044.9

5900.64
5939.20

10351.92

10890.02

10083.37

9774.53

10208.43

7196.91

7479.79

7626.54

6697.09

6548.47
6660.68

6583.66

6418.86

6506.40
6617.17

6617.17
6314.58

11595.50

1659.62

12211.22
11096.98

8137.88

$8408: 39$
8691.53

8691.53
7889.15

7156.94

7197.88
7404.36

7404.36
6963.85

12094.78

11698.63
11132.34

8380.68

8438.62

8969.06
7906.95

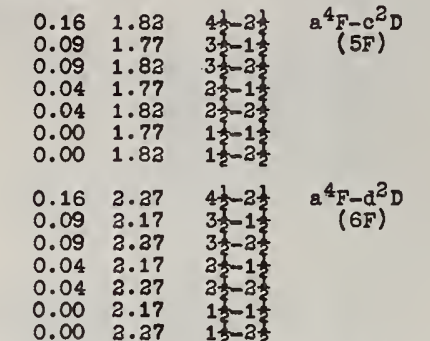

$0.16 \quad 2.40$

0.162 .41

$0.04 \quad 2.41$

$0.04 \quad 2.40$

$\begin{array}{ll}0.00 & 2.41 \\ 0.00 & 3.40\end{array}$

- $0.46 \overline{1.75}$

$0.41 \quad 1.66$

$0.36 \quad 1.66$

$0.36 \quad 1.75$

$\begin{array}{ll}0.32 & 1.76\end{array}$

$0.46 \quad 1.75$

$\begin{array}{ll}0.41 & 1.74 \\ 0.46 & 1.74\end{array}$

$0.41 \quad 1.75$

$\begin{array}{ll}0.36 & 1.74 \\ 0.36 & 1.75\end{array}$

$\begin{array}{ll}0.36 & 1.75 \\ 0.32 & 1.74\end{array}$

$\begin{array}{ll}0.46 & 1.82\end{array}$

$\begin{array}{ll}0.41 & 1.77 \\ 0.41 & 1.82\end{array}$

$\begin{array}{ll}0.41 & 1.88 \\ 0.36 & 1.77\end{array}$

$\begin{array}{ll}0.36 & 1.82 \\ 0.32 & 1.77\end{array}$

$\begin{array}{ll}0.32 & 1.77 \\ 0.32 & 1.82\end{array}$

$0.46 \quad 3.37$

$\begin{array}{ll}0.46 & 2.37 \\ 0.41 & 3.17\end{array}$

0.413 .37

$0.36 \quad 2.37$

$0.32 \quad 2.17$

$0.32 \quad 2.27$

$0.46 \quad 3.40$

$0.41 \quad 3.41$

$\begin{array}{ll}0.46 & 3.41 \\ 0.41 & 2.40\end{array}$

$0.36 \quad 3.41$

$\begin{array}{ll}0.32 & 3.41 \\ 0.32 & 2.40\end{array}$

$0.56 \quad 1.75$

$0.52 \quad 1.66$

$\begin{array}{ll}0.56 & 1.66 \\ 0.52 & 1.75\end{array}$

$0.56 \quad 1.83$

0.521 .77

$\begin{array}{ll}0.56 & 1.77 \\ 0.52 & 1.82\end{array}$

0.562 .27

$0.52 \quad 3.17$

$\begin{array}{ll}0.56 & 2.17 \\ 0.52 & 2.27\end{array}$

$0.56 \quad 2.40$

$\begin{array}{ll}0.52 & 2.41 \\ 0.56 & 2.41 \\ 0.52 & 2.40\end{array}$

$0.56 \quad 3.48$

$\begin{array}{ll}0.52 & 3.42 \\ 0.56 & 3.42\end{array}$

$\begin{array}{ll}0.52 & 2.48\end{array}$

$0.75 \quad 1.83$

$\begin{array}{ll}0.71 & 1.77 \\ 0.75 & 1.77\end{array}$

$\begin{array}{ll}0.75 & 1.77 \\ 0.71 & 1.82\end{array}$

$0.75 \quad 2.87$

$\begin{array}{ll}0.71 & 3.17 \\ 0.75 & 2.17\end{array}$

$\begin{array}{ll}0.75 & 2.17 \\ 0.71 & 2.27\end{array}$

$0.75 \quad 2.48$

$\begin{array}{ll}0.75 & 2.48 \\ 0.71 & 2.42\end{array}$

$\begin{array}{ll}0.75 & 2.42 \\ 0.71 & 2.48\end{array}$

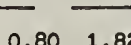

$\begin{array}{ll}0.80 & 1.82 \\ 0.71 & 1.77\end{array}$

0.711 .82

$0.80 \quad 2.27$

$0.80 \quad 2.17$

$\begin{array}{ll}0.80 & 2.17 \\ 0.71 & 2.27\end{array}$
I A Low ${ }^{E P}$ H1gh J

Multiplet
(Wo $)$

Zr II continued

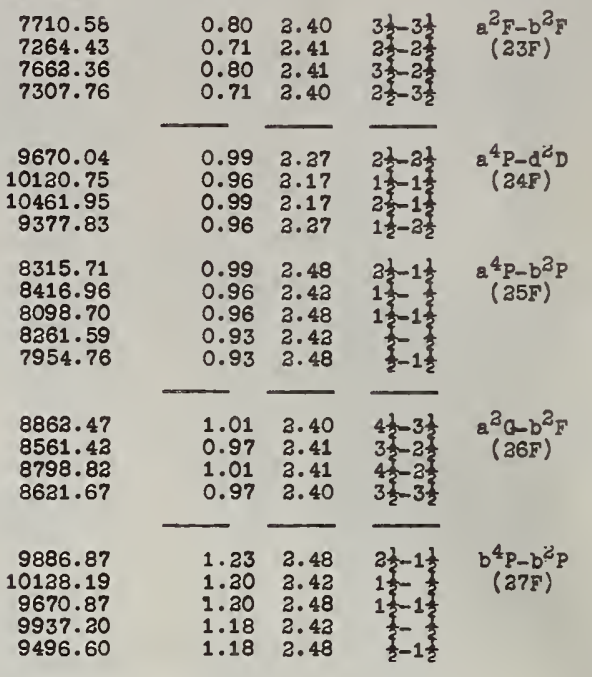

Zr III I P 24.0

\begin{tabular}{|c|c|c|c|c|}
\hline $\begin{array}{l}5539.74 \\
5517.24 \\
5433.69 \\
5773.51 \\
5643.08 \\
5303.37 \\
5316.97 \\
5118.07\end{array}$ & $\begin{array}{l}0.18 \\
0.08 \\
0.00 \\
0.18 \\
0.08 \\
0.08 \\
0.00 \\
0.00\end{array}$ & $\begin{array}{l}2.41 \\
2.32 \\
2.37 \\
2.32 \\
2.37 \\
2.41 \\
2.32 \\
2.41\end{array}$ & $\begin{array}{l}4-3 \\
3-2 \\
2-1 \\
4-2 \\
3-1 \\
3-3 \\
2-2 \\
2-3\end{array}$ & $\begin{array}{l}4 d^{2}{ }^{3} F-5 s^{3} D \\
(1 F)^{-1}\end{array}$ \\
\hline 7853.3 & 0.43 & 1.99 & $2-2$ & $4 a^{2}{ }^{1} D-5 s^{1} D$ \\
\hline $\begin{array}{l}6193.7 \\
6487.5 \\
6661.7\end{array}$ & $\begin{array}{l}0.42 \\
0.42 \\
0.42\end{array}$ & $\begin{array}{l}2.41 \\
2.32 \\
2.27\end{array}$ & $\begin{array}{l}2-3 \\
2-2 \\
2-1\end{array}$ & $\begin{array}{l}4 d^{2} P_{D-5 s^{3} D} \\
(3 F)\end{array}$ \\
\hline 6864.4 & 0.47 & 2.27 & $0-1$ & $\begin{array}{l}4 d^{3}{ }^{1} S-5 s^{3} D \\
(4 F)^{-1}\end{array}$ \\
\hline $\begin{array}{r}9349.2 \\
9543.3 \\
9671.3 \\
10034.9 \\
9926.0 \\
8931.0 \\
9307.5 \\
10458.9\end{array}$ & $\begin{array}{l}1.09 \\
1.03 \\
1.00 \\
1.09 \\
1.03 \\
1.03 \\
1.00 \\
1.09\end{array}$ & $\begin{array}{l}2.41 \\
2.32 \\
2.27 \\
2.32 \\
2.27 \\
2.41 \\
2.32 \\
2.27\end{array}$ & $\begin{array}{l}2-3 \\
1-2 \\
0-1 \\
2-2 \\
1-1 \\
1-3 \\
0-2 \\
2-1\end{array}$ & $\begin{array}{l}4 d^{2} 3 p-5 s^{3} D \\
(5 F)\end{array}$ \\
\hline
\end{tabular}

Zr VI I P 99

$6408.5 \quad 0.00 \quad 1.93 \quad 1 \frac{1}{2}-\frac{1}{2} \quad 4 p^{5}{ }^{2} p^{0}-4 p^{5} \quad 2 p^{0}$

Xe II I P 31.1

$9487.5 \quad 0.00 \quad 1.30 \quad 1 \frac{1}{2}-\frac{1}{2} \quad 5 p^{5} \quad 2_{p 0}-5 p^{5} \quad 2_{p 0}$ (1r)

Xe III I P 32.0

$10206.5 \quad 0.00 \quad 1.31 \quad 2-1 \quad 5 p^{4} 3 p-5 p^{4} 3 p$

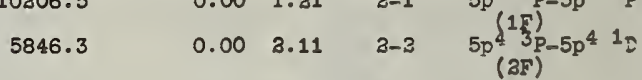

La II I P 11.38

$\begin{array}{rrrrc}11011.70 & 0.13 & 1.25 & 3-2 & { }^{3} F-D^{1} D \\ 9903.31 & 0.00 & 1.25 & 2-2 & (1 F)\end{array}$

$\begin{array}{lllll}11490.57 & 0.17 & 1.25 & 3-2 & a^{1} D-b^{1} D \\ (a F)\end{array}$

La III I P 19.1

$\begin{array}{ccccc}8339.72 & 0.20 & 1.68 & 2 \frac{1}{2}-\frac{1}{2} & 5^{2} \mathrm{D}-6^{2} \mathrm{~S} \\ 7355.92 & 0.00 & 1.68 & 1 \frac{1}{2} & (12)\end{array}$

Eu II I. P 11.21

$8983.71 \quad 0.00 \quad 1.37 \quad 4-6 \quad a^{9} \mathrm{~S}-\mathrm{a}^{9} \mathrm{D}^{\circ}$

$\begin{array}{lllll}9392.85 & 0.00 & 1.31 & 4-5 & (1 F)\end{array}$

$\begin{array}{rrrr}9916.30 & 0.00 & 1.34 & 4-3 \\ 10074.84 & 0.00 & 1.32 & 4-3\end{array}$

$\begin{array}{lllll}5929.31 & 0.00 & 2.08 & 4-5 & a^{9} \mathrm{~S}-a^{7} D^{\circ}\end{array}$

$\begin{array}{rllll}5879.32 & 0.00 & 3.10 & 4-4 & 8^{9} S-8700 \\ 5832.40 & 0.00 & 2.12 & 4-3 & (35)\end{array}$

$5796.28 \quad 0.00 \quad 2.13 \quad 4-3$ 
Part II -Finding List

of All Lines in the Table of Multiplets 

TABLE OF CONTENTS

Part iI. Finding List

PAGE

1. Introduction.

2. Part A-Observed and Permitted Predicted Lines

3. Blends.

4. Scope.

5. Part B-Forbidden Lines

6. Contents of Section on Forbidden Lines

7. Index Card

8. Errata

Finding List

Body of Multiples Table

Forbidden Lines.

$1-86$ 



\title{
A MULTIPLET TABLE OF ASTROPHYSICAL INTEREST
}

PART II

\author{
Finding List of All Lines in the Table of Multiplets
}

\section{Introduction}

Any arrangement of the wave-lengths in a given spectrum, by multiplets makes it inconvenient to locate a given line. The difficulty is greatly increased when many spectra are involved. Consequently a "Finding List" containing all lines in the Revised Multiplet Table has been included as Part II of this Contribution. As in the R M T the Finding List is in two parts. The first contains permitted lines observed in the laboratory, permitted predicted lines, and a few forbidden lines observed in the laboratory when a strong electric field is present. The second contains only forbidden lines of the nebular, auroral and coronal type. See $\$ 5$.

2. Part $\mathrm{A}-$ Observed and Permitted Predicted Lines

The lines are listed in order of increasing wave-length and cover the range $\lambda \lambda 2951-13164$. At the violet end of this long range, the proportion of known lines included is smaller than in the main body of the list, due to the masking by the ozone in our atmosphere of all but the strongest lines. The number of lines in the same wave-length interval decreases from the violet to the red. The incompleteness of laboratory material accentuates this in the infra-red. The total number of lines in this section is approximately 23,200.

Three entries are given for each line and a fourth if the line is predicted or forbidden. All entries are copied directly from Part I of this Contribution. The first is the laboratory wavelength. The source from which the wave-length is taken can be found from the references $A, B, C$ etc. in the R M T and Table 7.

The second entry headed "Type" is blank for all lines observed in the laboratory, except the selected forbidden ones that appear under special conditions, (due to Stark effect). These are marked "Forb" and include 11 lines of $H e$ I, 6 of $N a$ I, 11 of $A l$ II, 2 of $A l$ III and 2 of $K$ I.

Predicted Lines. These fall into three classes. (a) For some faint lines observed in the laboratory but not well-measured, a predicted wave-length obtained from the spectroscopic term values is preferable to the observed value. (b) It is well-known that many predicted lines not yet observed in the laboratory are important astrophysically, and an attempt has been made to include these in the R M T. (c) If a line that would otherwise be included is masked by a strong line in the laboratory, the predicted position of the masked line is entered. Such cases are carefully noted and explained in the $\mathrm{R} \mathrm{MT}$. In every case where a predicted wave-length is used, the entry "P" occurs in the column headed "Type" in the Finding List. This column contains only the two entries "Forb" and "P". All other lines are observed laboratory wavelengths in the usual sense of the word "observed".

The third entry for each line is the spectrum to which the line belongs. Here the chemical symbols of the elements are used and Roman numerals denote arc spectra (I) and spark spectra in successive stages of ionization, i.e. first spark spectrum (II), second spark spectrum (III) etc.

Finally the number of the multiplet to which the line of a given spectrum belongs, is given under the heading "Multiplet No." This number appears under the "Multiplet Designation" of each multiplet in the R M T and the numbers start with 1 for each spectrum. All lines of a given multiplet have the same multiplet number. A blank in this column indicates that the line is unclassified. In the R M T, under a given spectrum, unclassified lines follow the multiplets.

When two or more numbers appear in this column, the line is a blend and occurs in each of the multiplets indicated.

Examples: $\lambda 2957.56$ is due to $C r$ II and appears in Multiplets 104 and 141 of $C r$ II (See pp. 44 and 45 of the $\mathrm{R} \mathrm{M} \mathrm{T).}$

$\lambda 2984.89$ is a predicted wave-length. The line is in Multiplet No. 60 of Fe II (See p. 67 of the $\mathrm{R} M \mathrm{M}$ ).

$\lambda 2991.632$ is an unclassified line of $F_{e}$ I (See p. 65 of the R M T). 
3. Blends

Reference has been made above to a line appearing in two multiplets of a given spectrum, for example $\lambda$ 2957.56. Such blends can be readily detected in the Finding List by the presence of more than one multiplet number. In the $\mathrm{R} \mathrm{M} \mathrm{T} \mathrm{they} \mathrm{are} \mathrm{noted} \mathrm{by} \mathrm{an} \mathrm{asterisk.} \mathrm{This} \mathrm{applies}$ to blended lines in the same spectrum.

If, however, an arc and spark line of an element are blended the wave-length is repeated in the Finding List; or nearly identical wave-lengths are entered, if different measures were used in the two instances. For example $\lambda 2988.952$ appears in Multiplet No. 11 of Sc I and in Multiplet No. 34 of $S c$ II. In the R M T such lines have an asterisk preceding the wave-length and the symbol " $\$$ " following it.

A careful examination of close pairs of lines of a given element in the Finding List will doubtless reveal more blends than have been noted in the R M T. Similarly, it is probable that erroneous identifications of lines due to impurities that have not heretofore been suspected, can be detected.

The predicted wave-lengths of masked lines ( $\$ 2$ ) fall close to observed lines. For example $\lambda 2965.25$ is the predicted position of the line of $F e$ I in Multiplet No. 316, masked by the strong $F e$ I line at $\lambda 2965.255$, which occurs in Multiplet No. 10.

All predicted lines have separate entries in the Finding List, regardless of how close the pairs in a given spectrum may be-for example, $\lambda \lambda 2990.33$ and 2990.34 are both predicted lines of $\mathrm{Fe}$ I. If observed in the laboratory these lines would undoubtedly be blended.

When identical wave-lengths appear in spectra of different elements, the lines are arranged in the alphabetical order of the chemical symbol. When similar wave-lengths occur in spectra of different stages of ionization of a given element, the arc spectrum comes first, then the spark spectra, in order of increasing ionization.

4. Scope of the Finding List

The users of this Finding List are emphatically warned that the list is not complete. The range is that useful to the astrophysicist, having the violet limit $\lambda 2951$. Within the range covered, the elements to be included have been selected according to their astrophysical importance. For a given element, the spectra for different degrees of ionization and the lines of each have been similarly selected. It is fairly complete for the first spark specira through the first long period. It lists only the leading arc lines for many elements, but includes all observed classified lines of $F e$ I. For any element, the List grading in the R M T can be used as a guide to the completeness of selection. On account of these restrictions this book is not a list of "Hauptlinien" or a compendium of wave-lengths of elements in general. On the other hand it does contain a large number of predicted lines which invite the attention of the laboratory worker in spectroscopy.

5. Part B. Forbidden Lines-Nebular, Auroral, Coronal etc.

The second part of the Finding List contains only forbidden lines. Here the word "forbidden" applies in the general sense-i.e. lines due to downward transitions from metastable states in the atoms. The number of lines listed is roughly 2550.

The arrangement is similar to Part A of the Finding List, with the exception that in Part B the great majority of lines are predicted. Consequently no column headed "Type" is given. The wave-lengths that are not predicted are noted by the following letters:

$\mathrm{N}$ Nebular Wave-length

L Laboratory Wave-length

\section{A Auroral Wave-length \\ C Coronal Wave-length}

Column two contains the chemical symbol and stage of ionization of the spectrum as in Part A, and column three the Multiplet Number. In order to avoid confusion with Multiplet Numbers in Part A, all Multiplets of forbidden lines have the letter " $F$ " accompanying the Multiplet Number. 


\section{Contents}

A complete list of all possible forbidden lines in the region useful to the astrophysicist would be prohibitively long. For simple spectra the lines are few, but for the complex spectra, particularly in the first long period, fairly rigid selection has been made. Anyone desiring to construct complete lists is advised to consult the references to the analysis of the various spectra.

7. Index of The Finding Lists

In order to facilitate the work of transferring from the Multiplet Number of the Finding List to the Multiplet in the R M T, a separate card is enclosed in the Finding List, containing an index of the $\mathrm{R} \mathrm{M} \mathrm{T.} \mathrm{The} \mathrm{elements} \mathrm{are} \mathrm{in} \mathrm{order} \mathrm{of} \mathrm{increasing} \mathrm{atomic} \mathrm{number.} \mathrm{This} \mathrm{index}$ gives the multiplets of each element contained on each page of the R M T.

For example, $\lambda 2980.296$ is in Multiplet No. 94 of $T i{ }_{I}$. On the index card hunt $T i$ I and then this Multiplet Number. It is to be found on page 27 of the R M T, which contains Multiplets of $T i$ I from No. 55 through No. 140.

8. Errata

After the tabular material in the Finding List had been completed for publication, four errors were detected, as follows:

I A Element Multiplet No. $3497.137 \mathrm{Fe}$

$4618.568 \mathrm{Fe} \mathrm{I}$

1151

78

\section{$4061.3 \quad S c$ III}

4068.7 Sc III
I A Type Element Multiplet No. $\begin{array}{lllll}\text { should read } & 3497.15 & \mathrm{P} & \mathrm{Fe}_{\mathrm{I}} & 78\end{array}$

Reject-Wave-length erroneous

should read 4061.3

should read 4068.7
Sc III

Sc III
1

The writer will be grateful to those who use this Table if they will call to her attention any errors they detect, so that a list of errata may be published. In-the compilation of a list containing about 25,750 lines, doubtless there are a number of mistakes in spite of the care that has been taken to avoid errors. 

I A

$\begin{array}{llr}2972.277 & \text { Fe I } & 104 \\ 2972.64 & \text { Cr II } & 28 \\ 2972.742 & \text { Gd II } & 25 \\ 2972.768 & \text { Fe II } & 159 \\ 2973.137 & \text { Fe I } & 10 \\ 2973.236 & \text { Fe I } & 10 \\ 2973.975 & \text { V II } & 218 \\ 2974.006 & \text { Sc I } & 11 \\ 2974.59 & \text { I I } & 10 \\ 2974.78 & \text { Fe I } & 335\end{array}$

2985.849

2985.98

2985.895

2986.137

2986.456

2986.473

2986.617

2986.655

2986.81

2887.074

T1 I

2974. 934

2975.110

84

Cr II 58

$\begin{array}{ll}\text { T1 I } & 30 \\ \text { Cr I I } & 58\end{array}$

T1 I 30

Cr

V I 18

$\begin{array}{llr}\text { Fe I } & 10 \\ V & \text { II } & 2\end{array}$

Cr II 104,141

Cr II

Cr II

Fe II

Fo II

Cr II

$\mathrm{Fe} I$

Fe I

Gd II. 2

Fo II 180

$\mathrm{Fe}$ II

Cr II

Fe I

Rh II

$\checkmark$ I

Fe II

Cr II

Gd II

Cr I

2975.16

2975.483

2975.650

2875.89

4.838

2976.197
2976.50

Zr II

Cr I

$\begin{array}{ll}\mathrm{H} & \text { II } \\ \text { HF II } & \end{array}$

$\mathrm{Fe}$ II

$\mathrm{Fe} I$

V I I
Fe I

2887.166

2987.27

2987.292

2987.40

2987.542

2987.65

2987.72

2987.80

2988.027

2988.05

V I I

2976.517

2976.61

2976.718

2976.805

2976.922

2977.226

2977.539

2978.07

2978.18

Ru II

Cr II

Ce II

Fe I

Ru II

$\checkmark$ I

Zr II

2978.226

2978.850

2978.05

2979.102

2979.102
2979.18

2979.199

2979.348

2979.382

2979.683

$Y$ II

2988.058

2988.367

2888.468

2988.61

2988.648

2988.74

2988.942

2988.952

2988.952

2889.01

V II
Fe II

$\triangle$ II

Fe II

$\checkmark$ II

Zr II

T1 II

Fe II

Rh II

$8 \mathrm{C}$ II

2989.078

2989.194

2989.30

2989.306

2989.387

2989.42

2889.590

2989.594

2989.731

2989.74

$2990 \cdot 10$

2978.741

Pus II

Fe II

Fe II

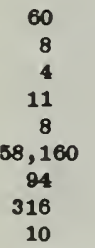

2979.957

2980.154

2980.296

2980.532

2980.60

2980.68

2980.752

2980.791

Cr II

Ru II

Gd II

T1 I

Fe I

Fe I

Sc I

100,180

14
123

4

5

28

12

84

Cr II 58,160

T1 I

Fo I

201
54

54
11
28

2980.82

2980.963

2981.02

2981.200

2981.446

2981.448

2981.651

2881.852

2981.824

2982.058

Bf I

Fe II

Zr II

$\checkmark$ II

Fe I

T1 I

N1 I

V II

990.16

2990.34

2990.392

2990.873

2990.948

2891.095

2991.244

2991. 33

2981.40

2991.520

2891.626

2991.632

2991.817

2991.886

2992.11

2982. 24

2892. 378

2992.40

2982.585

2892.63

2993.038

2993.366

2994.05

2984.069

2984.258

2984.427

2984.460

2894.50

2994.540

2994.725

2984.737

2994.858

2985.10

2995.26

2985.530

2995.644
2995.838

2995.989

2908.386

2996.51

2896.548

2996.580

2996.63

2998.70

2996.88

2996. 94

2987.08

Cr I

S II

Cr I

Cr I

Fe I
Cr I

Fe II

Fe I

Gd II $\quad 56$

Co I 11

Fe II 86

Fe I 30

$\begin{array}{llr}\text { T1 I I } & 28 \\ \text { Fe II } & 223\end{array}$

S1 I

Ca II

Zr II

$\checkmark$ II

N1 II

Cr II 28

Fe I

Ca III

Cr I

Zr II

Fe I

Sc I

Sc II

Fe II

Fe II 158

Cr II

Ce III

$\checkmark$ II

Fe II

Ca II

Co I

$\checkmark$ II

Fe II

$\checkmark$ II

Zr II

T1 II

$\mathrm{Fe} I$

Fe I

Ce II

$\checkmark I$

N1 I

Fe II

Br II

Gd II

Gd II

Fe I

Fe $\Pi 160$

Cr I

o III

I III

$\checkmark$ II

Cr II

N1 I

C I

Gd II

Fe II

Zr II

Cr I

Al II

N1 
I A Type Element Multiplet No.

I A Type Element Multiplet No.

I A

$\begin{array}{ll}0 & \text { I I } \\ \mathrm{Ca} & \text { I } \\ \mathrm{N} & \mathrm{II} \\ \mathrm{V} & \mathrm{I} \\ \mathrm{Fe} & \text { II I } \\ \mathrm{CI} & \text { I I } \\ \mathrm{V} & \text { II } \\ \mathrm{Na} & \text { I I } \\ 0 & \text { II }\end{array}$

17
18
116
21
53
141
12
74
55

Fe III 116

Fe I

$\begin{array}{ll}\text { Fe } & \text { I } \\ V & \text { II }\end{array}$

La II

Na II

T1 I

Mn I

3007.487

3007.655

3007.74

3007.75

2999.512

2999.641

Cr II

$\begin{array}{ll}\mathrm{Fe} & \mathrm{I} \\ \mathrm{Ca} & \mathrm{I}\end{array}$

30

2999.92 P $\quad$ T1 II

2999. 96

3000.059

3000.09

3000.14

3000.45

3000.452

$\mathbf{3 0 0 0 . 5 9}$

3000.836

3000.863

3000.868

3000.890

3000,950

3001.203

3001.42

3001.589

3001.65

3001.66
3001.754

T1 II

Fr II

Hf II

A II

Fe I

Fe I

Zr II

Fe III

28

42
69
3

3007.975

3008.13

3008.139

008.265

3008.28

3008.322

3008.506

3008.508

3008.610

3008.67

3008.789

3008.79

3008.996

3009.098

3009.136

3009.138

3009.205

3009.366

3009.570

3009.650

3009.85

3009.998

3010.129

3010.220

3010.28

3010.42

3010.76

3010.838

3010.899

3011.060

3011.162

3011 . 24

3011. 376

3011.42

3011.482

3011.73

3012 . 004

3012. 01

3012.020

3012.190

$$
\begin{array}{ll}
0 & \text { I I } \\
\text { Fe } & \text { I }
\end{array}
$$

Fe III

116
11
27
102
1
35
74
262
10

Nd II

$\begin{array}{ll}\text { Zr II } & \text { I }\end{array}$

Mn I

0 II

T1 II

Fe III
$V$ II

$\begin{array}{ll}V & \text { II } \\ \text { Cr } & \text { II }\end{array}$

Ce II

0 II I

Rh II

Fe I

Sn I

$\mathrm{Na}$ II

Ca I

Gd II

Fe I
Gd II

7r II

Fe III

Gd II

Fe II

Zr II

T1 I

$\begin{array}{ll}\text { W I I } \\ \text { Cu } & \text { I }\end{array}$

Gd II

Fe III

86
9

9
35
74

74
85
9

9
141

141
26
75

122

122
10

4
198

198
13

13
17

25
30

30
27

64

41
12
181

181

39
170

170
14
3

3
42
31

Mn I

Mn I

Cr II

$\mathrm{Fe} I$

Zr I

Cr II

$\checkmark$ II

Gd II

35

35

64
35
27

27
316

22

41

87
43
29

$\begin{array}{lll}3012.34 & \text { Cr II } & 42\end{array}$

3012.59 P $\quad$ Fe II

3012.847 Fe II I

3012.90

3013.030

3013. 102

3013. 125

3013. 32

3013.37

3013.38

3013.592

3013.66

3013. 713

3013.802

3014.120

3014.165

3014. 176

3014.37

3014.44

3014.49

Hf II

Cr I

V II

Fe III

Zr II

0 II

3014.668

3014.760

3014.822

3014.915

3015.19

3015.230

3015.296

3015 . 364

3015.400

3015.510
3015.67

3015.68
$\mathbf{3 0 1 5} .86$

3015. 913

3015.98

3016. 14

3016.15

3016.16

3016.186

3016.454

3016. 775

3016.94

3017.187

3017.195

3017.254

3017. 34

3017.447

3017.548

3017.569

3017.63

3017.80

3018.08

3018. 134

3018.25

3018.32

3018.352

3018.496

3018.53

3018.744

3018.82

3018.821

3018.95

3018.983

3019. 09

3019.143

3019.291

3019.350

3019.819

3019.84

3020.001

3020.45

3020.495

3020.54

3020.643

3020.65

3020.673

3021.074

3021.407

3021.558

3021.74

3021.74

3021.78

3021.97

3021.98

3022.00

3022.146

3022.26

3022 . 28

3022.57

3022.736

3022.749

3022.804

3022.820

3022.93

3023.45

3023.50

3023.583

3023.80

3023.85

3023.859

3023.86

3023.882

3024.033

3024.05

3024.098

3024.114

3024 . 350

3024. 36

3024.400

3024.51

3024.57

3024.681

3024.72

3024.78

3024.92

3024.981

3025.16

3025 . 283

3025.29

Type Element Multiplet No.

$\begin{array}{llr}\text { Zr II } & 86 \\ \text { Co I } & 76 \\ \text { Zr II } & 127 \\ \text { Fe I r } & 198 \\ \text { V } & \text { II } & 42 \\ \text { V } & \text { II } & 26 \\ \text { A } & \text { IV } & 1 \\ \text { V I } & 58 \\ \text { Fe I } & 30 \\ \text { Mn I } & 35 \\ & & \\ \text { V II } & 27 \\ \text { HE II } & 3 \\ \text { TI II. } & 85 \\ \text { Ce II } & 107 \\ \text { Co I } & 78 \\ \text { Ne II } & 8 \\ \text { W I } & 9 \\ \text { Co I } & 11 \\ \text { Cr I } & 27 \\ \text { Fe I } & 9\end{array}$

0 III

Cr II 95

Zr II

Fe I 199

$\mathrm{Fe} I \quad 263$

Hf I 1

Cr I

Zr II

Fe III 147

C1 II 22

Cr I 26

La II 
I A Type Element Multiplet No.

\begin{tabular}{|c|c|c|c|}
\hline 3025.40 & $\mathbf{P}$ & $\mathrm{Cr}$ & \\
\hline 3025.638 & & $\mathrm{Fe}$ & \\
\hline 3025.68 & & v & I I \\
\hline 3025.70 & & $\mathrm{Zr}$ & I. I \\
\hline 3025.75 & & 0 & II \\
\hline 3025.843 & & $\mathrm{Fe}$ & I \\
\hline 3025.99 & $\mathbf{P}$ & $\mathrm{Fe}$ & II \\
\hline 3026.18 & & $\mathrm{Zr}$ & II \\
\hline 3026.373 & & Co & I \\
\hline 3026.462 & & $\mathrm{Fe}$ & I \\
\hline
\end{tabular}

$3028.47 \quad$ Y II

$\begin{array}{llr}3026.75 & \text { Cr II } & 95 \\ 3020.776 & \text { A II } & 120\end{array}$

$\begin{array}{llll}3026.776 & \text { P } & \text { Al II } & 13 \\ 3026.781 & \text { P } & \text { Al II } & 13\end{array}$

3026.781

$\begin{array}{ll}3026.85 & \text { Cr II } \\ 3026.985 & \text { Fe III }\end{array}$

3027.04 Ne II

3027.38 P $\quad$ Fe II

$3027.46 \quad$ Fe III

3027.600

3027.602

3027.75

3027.92

3028.04

3038.05

3028.125

3028,436

3028.608

V II

G I I I

Pd I

0 IV

I II

Zr II

Cr II

Rh II

Ca III

Ne II

A II

Gd II

Mn II

Cr I

Fe I

Ni I

Zr I

V II

Fe II

T1 II

$\mathrm{Sb} I$

Fe I

Y II

Cr I

$\mathrm{Fe} \mathrm{I}$

Fe I

Ne II

Zr II

$\mathrm{V} I$

Hf II

$\mathrm{Fe} I$

$\begin{array}{ll}\text { Cr } & \text { I } \\ \text { Cr } & \text { I }\end{array}$

Cr I
Ce III

Cr II

Fe II

27

\begin{tabular}{r}
75 \\
76 \\
84 \\
9 \\
84 \\
147 \\
77 \\
30 \\
\hline 44 \\
45 \\
120 \\
13 \\
13 \\
41 \\
21 \\
8
\end{tabular}

8
99
10

85

12
60

5

5

85
76
87

87
2

3

$\begin{array}{r}3 \\ \hline\end{array}$

4

84
26

10

26

56
56

26

124

85
2

198

79

79
27
145

145
459

10

17

74

11

198
27

117

1
87

138

Fe I

$\begin{array}{ll}\text { N1 } & \text { I } \\ \text { Zr } & \text { II }\end{array}$

$\begin{array}{ll}\mathrm{Zr} & \text { II } \\ 0 & \text { II }\end{array}$

V II

N1 II

0 II

Cb II

Gd II

As $I$

Cr II

Fe I

Fe II
$\mathrm{V}$ II

A II

Nn II

$\checkmark$ II

Cr II

Gd I I

Cr I

0 III

Co I

Ne II

Fe I

Cr II

F III

Fe II

S1 III
I A Type Element Hultiplet No.

$\begin{array}{ll}\mathrm{Cb} & \text { II } \\ \mathrm{Cr} & \text { I I } \\ \mathrm{Rh} & \text { II } \\ \mathrm{V} & \text { II } \\ \mathrm{Fe} & \text { I } \\ 0 & \text { II I } \\ \mathrm{Zn} & \text { I } \\ \mathrm{Fe} & \text { II I } \\ \mathrm{Ne} & \text { II } \\ \mathrm{V} & \text { I I }\end{array}$

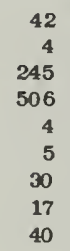

Cu I

3036.101

3036. 33

3036. 39

3036.50

3036.68

3036.784

3036.986

3037.044

3037.388

3037.73

3037.731

3037.782

3037.935

3037.98

3037.98

3038.04

3038.52

3038.520

3038.59

3038.706

3038.777

3039.064

3039.254

3039.32

3039.551

3039.563

3039.65

3039.67

3039.74

3039.746

3039.767

3039.780

3039.92

3040 . 34

3040.428

3040.603

3040.812

3040.829

3040.846

3040.92

3040.93

3041.224

3041.42

3041.639

3041.74

3041.745

3041.86

3041.876

3042.020

3042.27

3042.481

3042.65

3042.666

3042. 733

Zr II

Zr II

Y II

W II

T1 II

Fe II

S1 III

Fe I

$\mathrm{Ne}$ II

Fe I

N1 I

A IV

Cl II

V II

Cr II

V II

T1 II

Fe II

Ge I

F III

$\mathrm{Fe} \mathrm{I}$

0 II

Mn II

Ne II

C II

Cr I
F II

O II

II

Cr I

Sc II

Gd I I

Mn I

Co I

$\mathrm{Fe}$ II

Cr I

Cr II

S1 III

$\begin{array}{ll}\text { S1 } & \text { II } \\ \text { Mn } & \text { I } \\ \text { Al } & \text { II }\end{array}$

Fe I

Cr II

Fe I

I

Fe I

$\checkmark$ II

Co I

Pt

Fe I

Mn I

3042. 79

3043.02

3043.06?

3043. 124

3043. 132

3043. 143

3043. 31

3043. 356

3043. 439

3043.54

Cr II

0 III

Fe III

In II

un I

Mn I

Fe III

3043. 555

3043. 770

3043. 851

3043.90

3044.004

3044.04

3044.12

3044.16

3044.24

3044.438

I A Type Elerent Multiplet No.

3044.567

3044.843

3044.936

3045.00

3045.006

3045.077

3045.085

3045. 313

3045. 36

3045.53

Mn I

Fe II

V I

C1 II

$\mathrm{Fe} I$

T1 II

Fe II

$\begin{array}{ll}\text { Y } & \text { I } \\ \text { II }\end{array}$

15

17

12

179

179
9

3045. 58

3045.593

3045. 593

3045.594

3045. 714

3045.808

3045.82

3045.877

3046.03

3046. 266

3046. 399

3046.452

3046.675

3046. 685

3046.714 


\section{FINDING LIST}

I A Type Element Multiplet No. I A Type Element Multiplet No.

\begin{tabular}{|c|c|c|c|}
\hline 3063.25 & & Co & I \\
\hline 3063.280 & & T1 & II \\
\hline 3063.411 & & Cu & I \\
\hline 3063.46 & & 0 & IV \\
\hline 3063.502 & & T1 & II \\
\hline 3063.56 & & $\mathrm{Ta}$ & I \\
\hline 063.58 & & $\mathrm{Zr}$ & I \\
\hline 063.63 & & $\mathrm{zr}_{\mathbf{r}}$ & II \\
\hline 63.734 & & $\mathbf{v}$ & I \\
\hline 3063.814 & & Fe & II \\
\hline 1063.84 & & $\mathrm{Cr}$ & II \\
\hline 3063.93 & & N1 & II \\
\hline 3063.939 & & $\mathrm{Fe}$ & I \\
\hline 064.302 & & $\mathrm{Al}$ & I \\
\hline 064.370 & & Co & I \\
\hline 64.372 & & $\mathrm{Na}$ & II \\
\hline 064.530 & & $\mathrm{Cb}$ & II \\
\hline 3064.623 & & N1 & I \\
\hline 3064.64 & & $\mathrm{Zr}$ & II \\
\hline 3064.68 & & Hf & II \\
\hline 3064.71 & & Pt & I \\
\hline 3064.77 & & $A$ & II I \\
\hline 3065.01 & & 0 & III \\
\hline 3065.067 & & $\mathrm{Cr}$ & I \\
\hline 3065.106 & & Sc & II \\
\hline 065.20 & & $\mathbf{Z r}$ & II \\
\hline 65.30 & & $\mathbf{P d}$ & I \\
\hline 65.315 & & $\mathrm{Fe}$ & II \\
\hline 3065.61 & & $\mathbf{v}$ & II \\
\hline 3066.019 & & 站 & I \\
\hline 3066.02 & & $\mathbf{Y}$ & II \\
\hline 3066.158 & & Al & I \\
\hline 3066.220 & & T1 & I I \\
\hline 3066.354 & & T1 & II \\
\hline 3066.375 & & $\mathbf{v}$ & I \\
\hline 3066.487 & & $\mathrm{Fe}$ & I \\
\hline 3066.51 & & $\mathbf{v}$ & I. \\
\hline 066.514 & & T1 & II \\
\hline 66.536 & & $\mathrm{Na}$ & II \\
\hline 66.69 & $\mathbf{P}$ & $\mathrm{Fe}$ & I \\
\hline 3066.80 & & $\mathbf{v}$ & II \\
\hline 066.92 & & $\mathbf{A}$ & II \\
\hline $3067 \cdot 104$ & & v & II \\
\hline 3067.123 & & $\mathrm{Fe}$ & I \\
\hline $3067 \cdot 132$ & & Ge & I \\
\hline 3067.18 & & $\mathrm{Cr}$ & II \\
\hline 3067.22 & $\mathbf{P}$ & $\mathrm{Cr}$ & I \\
\hline 3067.23 & $\mathbf{P}$ & $\mathrm{Cr}$ & II \\
\hline 7.244 & & $\mathrm{Fe}$ & I \\
\hline 3067.41 & & Hf & I \\
\hline
\end{tabular}

97.664 3072.68

3072.88

3072.971

3073. 126

3073. 244

3073. 25

$3073 . \overline{5} 20$

3073.679

3073.823

3073.982

3074.061

3074. 15

3074.334

3074.47

3074.55

3074.665

3074.67

3074.68

3074.81

3075.043

3075. 19

3075. 225

3075.228

3075. 289

3075.32

3075. 336

3075. 38

3075.380

3075.422

3075.474

3075.55

3075. 721

3075. 901

3075.933

3075.95

3076.016

3076.455

3076. 58

3076.864

3076. 925

3077.077

3077.14

3077.168

3077.24

3077.24

3077. 358

3077.40

3077.59

3077.79

3077.831

3078.014

3078. 315

3078.316
3078.436

3078.44

3078.64

3078.646

3078. 698

3078.948

3079.34

3079. 356

3079.394

3079.627

3079.75

3079.84

3080.146

3080 . 333

3080,405

3080.64

3080.72

3080.755

3080.84

3081.01

3081. 254

3081.30

3081.330

3081.42

3081.46

3081.575

3081.585

3081.600

3081.83

3081.993

3082.010

3082.052

3082. 1109
Type El ement Multiplet No.

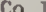

Co I

125

Ne II 17,48

Hf I

$T i$

Fe I

Cr II

Co I

Cr I

47
51

284

Fe I 313

0 III 1

Fe I 457

Na. II 9

Cr

Zr II $\quad 105$

V II 112

Al II 27

Cr II 73

0 III 26

Cr II 73

V II 228

T1 II $\quad 5$

$\mathrm{Fe}$ II

V I

As I

Ru II

Se II

105

105
1
7

Nd II

Gd II

Zr II

V II

Fe I

Zn I

V I

0 III

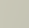

56

67
144

228

28

1
67

II

Fe II 181

Cr I 55

2

Gd II

I II 52

Fe II 108

II 103

$\begin{array}{ll}\text { Ta I } & 1 \\ \text { EU II } & 3\end{array}$

A IV 1

Lu II 208

Cr I 18

Fe 1

III

Na II $\quad 2,8$

Fe II $\quad 97$

T1 II 5

Fe II 181

V II $\quad 66$

Fe II 12

Co I 10,49

$\begin{array}{lr}\text { Mn I } & 15 \\ V & \text { II }\end{array}$

Fe I 102

V I 5?

Fe II 108

HF II 63

Cr I 184

$\begin{array}{ll}\text { N1 I } & 26 \\ \text { HP I } & 4\end{array}$

V II 112

II

Mn I 15

La II 115

$\begin{array}{lll}0 & \text { II } & 119\end{array}$

Rh II

I II

Fe I

Gd II

V I

Mn I 


\begin{tabular}{|c|c|c|c|}
\hline 3082.159 & & Al & I \\
\hline 3082.16 & & $\mathbf{Y}$ & II \\
\hline 3082.304 & & $\mathrm{Ce}$ & II \\
\hline 3082.524 & & $\mathbf{v}$ & II \\
\hline 3082.56 & & Sc & II \\
\hline 3082.614 & & Co & I \\
\hline 3082.844 & & Co & I \\
\hline 3082.99 & & A & II \\
\hline 3083.024 & & $\mathrm{Fe}$ & II \\
\hline 3083.07 & P & Sc & II \\
\hline 3083.152 & & $\mathrm{Fe}$ & I \\
\hline 083.208 & & $\mathbf{v}$ & II \\
\hline 083.350 & & lid & I I \\
\hline 083.539 & & $v$ & I \\
\hline 5083.62 & & $C_{r}$ & II \\
\hline 3083.65 & & 0 & III \\
\hline 3083.670 & & C. & II \\
\hline 3083.68 & $P$ & Fe & III \\
\hline 3083.742 & & $\mathrm{Fe}$ & I \\
\hline 3084.007 & & Gd & II \\
\hline 3084.09 & & Fo & III \\
\hline 84.46 & & $\mathrm{Cr}$ & II \\
\hline 84.59 & & $\mathrm{Cr}$ & I \\
\hline 184.63 & & 0 & II I \\
\hline 84.819 & & T1 & I \\
\hline 35.05 & & A & I I \\
\hline 3085.089 & & $\mathrm{Ce}$ & III \\
\hline 3085.34 & & $\mathrm{Zr}$ & I \\
\hline 3085.36 & & $C_{r}$ & I I \\
\hline 3085.47 & & $\mathrm{v}$ & II \\
\hline
\end{tabular}

\begin{tabular}{|c|c|c|c|c|}
\hline 3092.915 & & Nd & II & \\
\hline 3092.997 & & $\mathrm{Mg}$ & I & 5 \\
\hline 3093.108 & & v & II & 1 \\
\hline 3093.16 & $\mathbf{P}$ & $\mathrm{v}$ & II & 39 \\
\hline 3093.24 & & $v$ & I & 15 \\
\hline
\end{tabular}

3101.557

3201.878

3101.911

3102.295

3102.36

3102.406

3102.517

3093.423

3093.48

S1 III

481 .

Cr II 47,86

Co I

2r II

S1 III

3086.507
3086.620

3086. 777

Co I

F. III

Al I 19

$\checkmark \mathrm{I}$

N1 II ?

Co I 77

$\begin{array}{lr}\text { Cr II } & 102 \\ \text { TI II } & 8\end{array}$

3087.806

3087.90

3088.027

- III

3088.04

3088.114

3088.23

3088.24

208.28

2088.

3089.130

089.204

3089.388

3089.401

3089.596

3089.633

3089.648

3089.75

3089.854

3090.081

3090.137

3080.209

3090.251

3090.40

3090.44

3090.772

3090.84

3091.078

3091.30

5091.437

091.552

3091.578

3091.70

5082.058

3092.22

3092. 26

3092.519

3092.716

3092.72

1082. 729

5092.785

3092.843

3082.81
3093.481

3093.613 SI III

3083.76

3093.806

3093.846

3093.888

3093.97

3093.989

3094,08

3094.08

Y II

F. I

Gd II

Fe I

Cr II

Cu I

F. I

3094. 156

3094. 172

3094. 196

3094.555

3094.682

3094.79

3094.870

3094.94

3094.98

3095.07

Ne II

Cb II

$\checkmark$ II

Ru I

$\checkmark I$

$\mathrm{Zr}$

Fe I

$\begin{array}{llr}\text { A II } & 118 \\ \text { ZF II } & 5\end{array}$

3095. 22

3095. 270

3095. 716

3095.81

3095.82

3095.859

3095.88

3095.902

3096. 11

3096. 296

Cr II

Fo I

0 III

2r I

Cr I

$\begin{array}{ll}\text { I } & \text { II } \\ \text { C } & \text { II }\end{array}$

Cr II

Fe II

Co I

TI II

3096.424

3096.531

3096. 72

3098.740

3096. 77

3096. 786

3096.86

3096,802

3097.063

Cl II

Rh II

Sc II

S1 III

$\mathrm{Mg} I$

3097.118

3097.15

3097.186

3097.415

3097 , 45

3097.46

3097.40

3097.626

3088. 16

3098. 191

$$
\text { Mn I }
$$

86

314
49

28

38

11
57

126

52

77

31

4
6
1
85

85
5

NI I

Ne II

TI II

Fe II

$S$ IV

Fo I

TI II

Cr II

3102. 55

3102.58

3102.63

3102. 64

3102.71

3102.975

3103.3

3103.377

3103.48

3103.60

3103. ? 35

3103.804

3103.983

3103.994

3104 . 29

3104.38

3104.396

3104.46

3104.58

3104.593

$3104.7 \mathrm{C}$

3104.713

3104.805

3104.82

3104.906

3105.084

3105. 166

3105.220

3105.469

3105.348

3105.57

3105.973

3106.11

3106. 234

3106.542

3106.559

3106,58

3106.806

3106.829

3106.974

3107.044

3107.142

3107.387

3107.388

3107.468

3107.528

3107.540

3207.88

3107.686

3107.714

3107.774

3107.960

3208.230

3108.36

3108.360

3108.46

3108.511

3108.635

3108.66

3108.704

N1 I

3099.115

CD II

3108.78

3108.82

3108.85

3108.927

3109.05

3109.11

3209.3

3109.32

3109. 336

3208.378

3109.806

3109.60

3109.76

3108.92

3110.021

3110.052

3110.07

3110.096

3110.278

3110.510
3105.928

un I

$\begin{array}{ll}\text { I1 } & \text { I } \\ \text { N1 } & I\end{array}$

Gd II

V II

Ca I

Co

T1 I

Fe III

Gd II 20

Cr II 116

A II

20

$\mathrm{Fe} I$

T1 II 58

$\begin{array}{llr}\text { I II } & 78 \\ \text { C. II } & 151\end{array}$

Cr II 71

$\begin{array}{lll}\text { V } & I & 56 \\ \text { Co } & I & 73\end{array}$

I1 II $\quad 90$

Co I 48

$\checkmark$ I 58

Cr II 102

$\mathrm{Ne}$ II 118

Cl II

II

$\begin{array}{lr}\text { Cr I } & 90 \\ & 163\end{array}$

Mg II

$\mathrm{Ng}$ II

Y II

TI II 
I A Type Element Nultiplet No.

I A Type El ement MuItiplet No.

I A

As I $\quad 1 \quad 3128.640$

$\begin{array}{lll}\mathrm{Ca} \text { III } & 4 & 3128.686 \\ \mathrm{Fe} & & 3128.699\end{array}$

Cr I $183 \quad 3128.789$

$\begin{array}{lll}\text { T1 I } & 137 & 3128.79\end{array}$

$\begin{array}{lll}\text { TI II } & 67 & 3128.901\end{array}$

$\begin{array}{lll}\text { C1 II } & 20 & 3129.013\end{array}$

Rh II $\quad 8 \quad 3129.04$

Gd II $11 \quad 3129.075$

3129.16

3119.94

3120.023

Fe II 96

T1 I 181

3111.283

3111.339

311.609
3111.686

3111.95

3112.05

3112.050

3112.079
3112.125

3112.202

Co I

Fe III

$\mathrm{Fe} I$

Cr II

$\begin{array}{ll}\text { Y } & \text { II } \\ \text { T1 } & \text { II }\end{array}$

$\mathrm{Fe} I$

Mo I

Ce II

$3120.03 \quad P \quad F e$

$\begin{array}{lllr}3120.03 & \text { P } & \text { Fe I } & 161 \\ 3120.03 & & \text { Fe III } & 29 \\ 3120.10 & & \text { Co I } & 74\end{array}$

3120.181

Co I

Gd II

3120.24

3120.371

3120.435

3120.72

3120.726

$\mathrm{Fe}$ III

$\begin{array}{ll}\text { Cr } & \text { II } \\ \text { Fe } & \text { I }\end{array}$

Fe I

3120.74

V II

3129. 18

3129.314

3129. 334

3129.368

3129.44

3129.481

3129.696

3129.76

3129.933

3129.955

Type

El ement

YuI tiplet No.

3112.482

\section{T1 I}

La II

3112.81

3112.925

3113. 172

3113. 31

3113.473

3113. 560

3113.579

92

3120.84

3121.05

3121. 138

3121.415

3121.515

3121.548

3121. 566

3121. 599

3121.62

Fe III

Cr II

Fe III

Co I

F III

Ce III

Co I

TI II

3121.71

3121. 749

3121. 76

3121. 760

3121.84

3121.97

3122.065

3122. 542

3122. 596

3122.61

o III

V I

Fe I

Gd II

Cr II

r II

T1 II

Cr II

49,58

Y II

2r II

3130.05

3130.175

3130.262

3130.416

3130.561

3130.73

3130.780

3130.804

3130.804

3130.812

3131.064

3131. 11

3131.211

3131.257

3131.54

3131. 719

3131.81

3131.829

3131.845

3132.058

II I
II

$\begin{array}{ll}V & \text { II } \\ \text { Cr } & \text { II }\end{array}$

Y II

r II

$\mathrm{Fe}$ I

Fe III

T1 I

2r II

121

3122.62

3122.665

3122.887

3122.954

3123.074

3123. 18

3123. 29

3123. 353

3123. 715

$\begin{array}{ll}0 & \text { II } \\ \text { Fe } & \text { I }\end{array}$

Fe I

v II

Sc II

T1 I

Fe III

C\& II

Fe I

Fe II

3132. 06

3 132. 12

3132. 218

3132.22

3132.514

3132.591

3132.793

3132.820

3132.86

3133.048

$\mathrm{Fe}$

$\mathrm{N1} I$

$\mathrm{Na} I I$

0 II

Co I

2r II

I II

Gd II

2r I

V II

Be II

Fe II

Cb II

T1 I

T1 II

Gd II

Be II

$\begin{array}{rrr}\text { Zr I } & 37 \\ \text { Cr } & \text { I } & 183\end{array}$

Tw II

Cr II 53,55

Fe II

Hf I

Co I

Hg I

107
3

3123.72

CI II

T1 I

Gd II

Fe II

Fe I

F III

Ge I

Cr II

3124. 76

3124.817

3124.978

3125.01

V II

3133.094

3133.096

3133. 329

3133. 49

3133. 603

3133.852

3133.886

3133.96

3134.08

3134. 108

Co I

$\mathrm{Ne} I$

$\mathrm{Fe} I$

Yo I

V II

0 III

Fe II

Gd II

Sc II

V II

Zr II

Nd II

Gd II

Tा II

$\mathrm{Fe} I$

Fe I

3134.111

Fe I

3125.02

Cr II

$\begin{array}{ll}\mathrm{Fe} & \mathrm{I} \\ \mathrm{Ca} & \mathrm{II}\end{array}$

Zr II

V II

Cr II

Ti I

Fe I

Ti I

Hg I

$\mathrm{Fe} I$

Cr II

Zr II

III

$\begin{array}{ll}\text { Fe } & \text { I } \\ \text { V } & \text { II }\end{array}$

S1 III

HP II

70

3134.17

3134.208

3134.32

3134.33

3134.654

3134.72

3134.819

3134.82

Fe II

F III

0 II

Cr II

T1 I

HS II

Mn II

3134.897

3134.90

3134.928

3135.034

3135.069

3135.17

3135.35

3135. 360

3135.483

3135. 507

Nd II

$A$ IV

V II 122

Gd II

T1 I

Y II

Cr II

Fe II

Na II

Mn II 
I A Type Element Multiplet No.

\begin{tabular}{|c|c|c|c|}
\hline 3136.17 & & $\mathrm{Fe}$ & \\
\hline 3136.315 & & Mn & II \\
\hline 3136.43 & & $\mathrm{Fe}$ & III \\
\hline 3198.465 & & Mo & II \\
\hline$\$ 136.503$ & & $\mathbf{v}$ & II \\
\hline 3136.55 & & $\Delta$ & II \\
\hline 3136.680 & & Cr & II \\
\hline 3136.726 & & Co & \\
\hline 3136.77 & & T1 & II \\
\hline 3136.95 & & $\mathbf{Z r}$ & I \\
\hline 3136.999 & & Co & I \\
\hline 3137.328 & & co & I \\
\hline 3137.352 & & Ti & I \\
\hline 3137.454 & & Co & \\
\hline 5137.55 & & $\mathrm{Cr}_{\mathrm{r}}$ & II \\
\hline 3137.66 & & $\mathbf{A}$ & II \\
\hline 3137.755 & & Co & I \\
\hline 3187.940 & & Gd & II \\
\hline 3138.05 & & $\mathbf{v}$ & II \\
\hline 3138.094 & & Gd & II \\
\hline 3138.203 & & Cr & I \\
\hline 3138.207 & & $\mathrm{Fe}$ & II \\
\hline 3138.40 & $\mathbf{P}$ & $\mathrm{Fe}$ & I \\
\hline 3138.44 & & 0 & II \\
\hline 3138.46 & & $8 c$ & II \\
\hline 3138.66 & & $\mathrm{zr}$ & II \\
\hline 3139.02 & & $\mathbf{A}$ & II \\
\hline 3139.10 & $\mathbf{P}$ & Fe & I \\
\hline 3139.34 & & C1 & III \\
\hline 3139.39 & & Pt & I \\
\hline 3139.60 & $\mathbf{P}$ & $F_{e}$ & I \\
\hline 3139.661 & & $F_{\theta}$ & I \\
\hline 3139.67 & & Hf & II \\
\hline 3139.729 & & Sc & II \\
\hline 3139.733 & & $\mathbf{v}$ & II \\
\hline 3139.77 & & 0 & II \\
\hline 3139.78 & & $\mathrm{Zr}_{\mathbf{r}}$ & I \\
\hline 3139.87 & & T1 & I \\
\hline 3139.908 & & Fe & I \\
\hline 3139.91 & & Cr & II \\
\hline
\end{tabular}

$\begin{array}{lllr}3139.847 & & \text { Co } I & 9 \\ 3139.98 & P & \text { Co } I & 73\end{array}$

$\begin{array}{llll}3140.04 & P & \text { TI IF } & 27\end{array}$

Fe III

Cr II

Rh II

Fe I

Cr II $\quad 124$

Fo II $\quad 227$

3140.87

s140.692

3140.715

3140.77

3140.782

3141.07

3141.164

3141.247

3141.35

3141.486

3141.537

3141.670

3141.80

3141.891

3142.183

3142.22

3142.220

3142.312

3142.445

3142.484

$\$ 142.670$

3142.74

3142.76

J142.777

3142.888

3142.900

3142.97

3143.131

3143.16

143.242

3143. 36

3143.477

Co I

15

2

5

8

27

48
10

10

54

71

49

10

.

.

53

14
39
5

5
47
61

2

61

6

39

22

I A Type Element Multiplet No.

I A Type Eloment Multiplet No.

\begin{tabular}{|c|c|c|c|c|}
\hline 3144.488 & & Fe & I & 161 \\
\hline 3144.68 & & 0 & v & 2 \\
\hline 3144.700 & & v & II & 122 \\
\hline 3144.730 & & T1 & II & 111 \\
\hline 3144.74 & $\mathbf{P}$ & T1 & II & 10 \\
\hline 3144.751 & & $\mathrm{Fe}$ & II & 82 \\
\hline 3144.92 & $\mathbf{P}$ & $F_{\theta}$ & I & 195 \\
\hline 3145.00 & & Gd & II & 10 \\
\hline 3145.022 & & Co & I & 50 \\
\hline 3145.057 & & $\mathrm{Fe}$ & I & 455 \\
\hline
\end{tabular}

$\begin{array}{lllr}3154.195 & & \text { T1 II } & 10 \\ 3154.201 & & \text { Fe II } & 66 \\ 3154.387 & & \text { F III } & 4 \\ 3154.41 & \text { P } & \text { Fe I } & 100 \\ 3154.510 & & \text { Fe I } & 161 \\ 3154.585 & & \text { N1 I } & 78 \\ 3154.66 & \text { P } & \text { Cr II } & 64 \\ 3154.678 & & \text { Co I } & 108 \\ 3154.794 & & \text { Co I } & 73 \\ 3154.80 & & \text { V II } & 249\end{array}$

\begin{tabular}{|c|c|c|c|c|}
\hline 3145.10 & & $\mathbf{C r}$ & II & 5 \\
\hline 3145.121 & & N1 & I & 7 \\
\hline 3145.283 & & Ce & I I & 120 \\
\hline 3145.32 & & Hf & II & 2 \\
\hline 3145.337 & & v & II & 1 \\
\hline 3145.402 & & T1 & II & 10,111 \\
\hline 3145.405 & & $\mathrm{Cb}$ & II & 5 \\
\hline 3145.46 & $\mathbf{P}$ & $\mathrm{Fe}$ & I & 160 \\
\hline 3145.515 & & T1 & I & 91 \\
\hline 3145.516 & & Gd & II & 76 \\
\hline
\end{tabular}

$\begin{array}{lllr}3154.82 & & \text { Ne II } & 14 \\ 3155.12 & P & \text { Fe I } & 161 \\ 3155.149 & & \text { Cr I } & 115 \\ 3155.293 & & \text { Fe I } & 193 \\ 3155.409 & & \text { V II } & 51 \\ 3155.50 & \text { P } & \text { TI II } & 27 \\ 3155.63 & \text { P } & \text { TI II } & 37 \\ 3155.670 & & \text { TI II } & 10 \\ 3155.68 & & \text { Zr II } & 63 \\ 3155.704 & & \text { Ce II } & 217\end{array}$

\begin{tabular}{|c|c|c|c|c|}
\hline 3145.536 & & $F$ & III & 1 \\
\hline 3145.719 & & N1 & I & 11 \\
\hline 3146.77 & & $\mathrm{Cr}_{\mathbf{r}}$ & II & 85 \\
\hline 3145.971 & & $\mathbf{v}$ & II & 1 \\
\hline 3146.226 & & v & II & 138 \\
\hline 3146.407 & & $\mathrm{Ce}$ & II & 70 \\
\hline 3146.47 & & $\mathbf{A}$ & II & 49 \\
\hline 3146.475 & & $\mathrm{Fe}$ & I & 160 \\
\hline 3146.748 & & $\mathrm{Fe}$ & II & 67 \\
\hline 3146.818 & & $\mathbf{V}$ & II & 138 \\
\hline 3146.878 & & Gd & II & 76 \\
\hline 3146.91 & & Sc & II & 39 \\
\hline 3146.962 & & $\mathbf{F}$ & III & 1 \\
\hline 3147.05 & & $\mathrm{Ce}$ & III & 7 \\
\hline 3147.060 & & Co & I & 10 \\
\hline $3147 \cdot 19$ & $\mathbf{P}$ & $\mathrm{Cr}_{\mathbf{r}}$ & II & 54 \\
\hline 3147.227 & & $\mathrm{Cr}$ & II & 5 \\
\hline 3147.38 & & S1 & III & 11 \\
\hline 3147.792 & & $\mathrm{Fe}$ & I & 455 \\
\hline 3147.84 & & $\mathrm{Cr}_{\mathbf{r}}$ & I I & 93 \\
\hline
\end{tabular}

$$
31
$$

\begin{tabular}{|c|c|c|c|c|}
\hline 3155.80 & $\mathbf{P}$ & $F_{\theta}$ & I & 1920 \\
\hline 3155.91 & $\mathbf{P}$ & T1 & II & 121 \\
\hline 3155.95 & & $\mathbf{Z r}$ & I I & 49 \\
\hline 3155.950 & & $F_{\theta}$ & II & 67 \\
\hline 3156.11 & & $\mathbf{F}$ & III & 4 \\
\hline 3156.222 & & v & I & \\
\hline 3156.248 & & 08 & I & 4 \\
\hline 3156.275 & & $F_{\theta}$ & I & 578 \\
\hline 3156.464 & & Fe & I & 454 \\
\hline 3156.532 & & Gd & I I & 11 \\
\hline 3156.59 & & Pt & I & 2 \\
\hline 3156.68 & & HP & I & 5 \\
\hline 3157.00 & & $\mathbf{z r}$ & I I & 23 \\
\hline 3157.040 & & $F_{\theta}$ & I & 160 \\
\hline 3157.15 & $\mathbf{P}$ & Fe & I & 144 \\
\hline 3157.344 & & Tm & II & 8 \\
\hline 3157.397 & & $T_{1}$ & II & 4 \\
\hline 3157.44 & P & Sc & II & 32 \\
\hline 3157.52 & & $\mathrm{Cr}_{\boldsymbol{r}}$ & II & 93 \\
\hline 3157.82 & & $\mathbf{Z r}$ & I & 36,65 \\
\hline 3157.88 & & $\mathrm{Fe}$ & I & 164 \\
\hline 3157.900 & & v & II & 50 \\
\hline 3157.992 & & Fe & I & 159 \\
\hline 3158.03 & & $\mathrm{Cr}_{\mathbf{r}}$ & II & 70 \\
\hline 3158.156 & & Mo & I & 2 \\
\hline 3158.21 & $\mathbf{P}$ & $F_{\theta}$ & I & 160 \\
\hline 3158.293 & & Co & I & 12 \\
\hline 3158.32 & $\mathbf{P}$ & $\mathrm{Fe}$ & II & 95 \\
\hline 3158.772 & & Co & I & 10 \\
\hline 3158.869 & & Ca & I I & 4 \\
\hline
\end{tabular}

3158.99 Fe I 452

$\begin{array}{lll}3149.12 & \text { Cr II } & 84\end{array}$

3149.267 Na II 4

$\begin{array}{lllr}3149.310 & & \text { Co I } & 9 \\ 3149.50 & \text { P } & \text { Fe I } & 453\end{array}$

$\begin{array}{lll}3148.50 & P & \text { Se IV }\end{array}$

$3148.56 \quad 81$ IV 2

$\begin{array}{llr}3149.83 & \text { Cr II } & 54 \\ 3149.87 & \text { W II } & 5\end{array}$

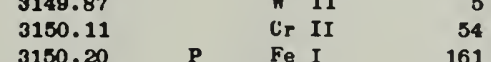

$\begin{array}{llll}3150.301 & P & \text { Fe I } & 161 \\ 3150.301 & & \text { Fe I } & 578 \text { a }\end{array}$

\begin{tabular}{|c|c|}
\hline 3150.568 & $\begin{array}{ll}v & \text { I } \\
\text { Ca } & \text { I }\end{array}$ \\
\hline
\end{tabular}

3151.036

3151.11

3151.16

3151.258

3151.31

3151.319

3151.353

3151.500

3151.86

3151. 867

3152.14

3152.21

3152.251
3152.525

3152.707

3152. 881

3153.064

Ca I

T1 I 28

Ne II 16

N1 I

Ca I 15

$\begin{array}{rrr}\text { W } & \text { II } & 16 \\ V & \text { II } & 138\end{array}$

$\begin{array}{ll}\text { Fe I } & 138 \\ & 311\end{array}$

Rh II 2

Fe I

$\begin{array}{lll}\text { T1 II } & 27 \\ \text { Cr } & \text { II } & 71\end{array}$

$\begin{array}{lll}\text { Cr II } & 71 \\ \text { T1 II } & 10\end{array}$

8n II

Co $I \quad 73$

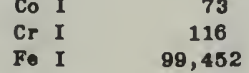

$\begin{array}{lll}3153.200 & \text { Fo I } & 161\end{array}$

3153.322

3153.54

3153.549

3153.692

3153.80

3154.04
3154.10

3154.10
3154.11

$\begin{array}{ll}\text { Pe I } & 160 \\ \text { Cr I } & 200\end{array}$

$\begin{array}{llr}\text { V } & I & 200 \\ \text { Co } & I & 7\end{array}$

$\begin{array}{llr}\text { Co I } & 7 \\ \text { A II } & 118\end{array}$

A II II

3143.990

3144.37

Ce III

Fo I

Cr I

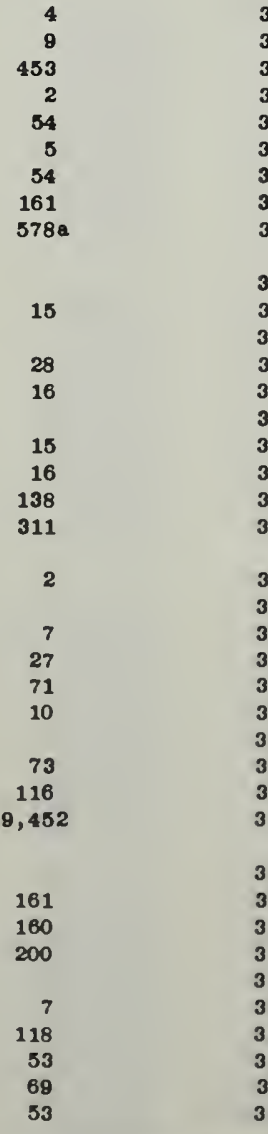

3159.10

$\begin{array}{llll}3158.25 & P & \text { Fe I } & 259\end{array}$

$\begin{array}{lllr}159.254 & & \text { Rh II } & 2 \\ 3159.32 & P & \text { Fe II } & 120\end{array}$

$\begin{array}{llr}3159.365 & \text { V II } & 83 \\ 3159.521 & \text { N1 I } & 11\end{array}$

$\begin{array}{lll}3159.59 & \text { Cr I } & 92\end{array}$

159.602 Co I 9,26

3159.86

160.03

3160.11

3160.200

160.342

3160.52

$\begin{array}{llr}3160.60 & \text { Y II } & 57 \\ 3160.61 & \text { Cr I } & 115 \\ 3160.658 & \text { Fe I } & 155\end{array}$

3160.69 Gd II 11

$\begin{array}{lllr}3160.77 & P & \text { Fo I } & 11 \\ & & \text { V II } & 159\end{array}$

$\begin{array}{llll}3160.781 & & \text { V II } & 65,136 \\ 3160.92 & \text { P } & \text { Fe I } & 160\end{array}$

3161.01

$\begin{array}{lllr}3161 & \text { P } & \text { N V } & 2 \\ 3161.01 & & \text { Zr II } & 104 \\ 3161.039 & & \text { Mn I } & 19 \\ 3161.205 & & \text { T1 II } & 10\end{array}$

$\begin{array}{rlr}3161.313 & \text { V II } & 151 \\ 3161.369 & \text { Od II } & 10\end{array}$

$\begin{array}{lll}3161.370 & \text { Fe I } & 52\end{array}$

$\begin{array}{lll}3161.38 & \text { A II } & 97 \\ 3161.44 & \text { CI II } & 11\end{array}$

$\begin{array}{llll}3161.45 & & \text { A II } & 11 \\ 3161.55 & \text { P } & \text { Fo I } & 195\end{array}$

$\begin{array}{rrrr}3161.638 & \text { P } & \text { Fo I } & 195 \\ 3161.652 & & \text { Gd II } & 25 \\ 3161.68 \text { Co I } & 73\end{array}$

$\begin{array}{lllll}3161.652 & & \text { Co I } & 73 \\ 3161.66 & P & \text { T1 II } & 27\end{array}$

$\begin{array}{rrrr}3161.755 & & \text { T1 II } & 27 \\ 3161.945 & & \text { TI II } & 10 \\ & \text { Fe II } & 7\end{array}$ 
I \& Type Element Multiplet No.

3161.949

3162. 284

3162.46

3162.57

3.162.570

\$162. 61

3162. 714

3162.764

3162. 799

3163.024

3163.403

3163.61

3163. 731

3163.756

3163.76

3163.77

3163.86

3163.93

3164.06

3164. 154

3164. 166

3164. 26

3164.28

3164. 308

3164.32

3164.46

3164.48
3164.618

3164.67

3164.82

3165.005

3165.08

3165.16

3165. 24

3168. 31

3165.45

3165.508

3165.51

3165.70

3165.72

3165.86

3165. 860

3165.89

3165. 94

3165.857

3166.98

3165. 99

3166.22 P $\quad$ Fe II

3166.24 P Fe I

3166.29 Zr II

3166.39

3166.495 Fe $I$

$\begin{array}{lll}3166.59 & P & \mathrm{Fe} I\end{array}$

$\$ 166.670$ Fe II

3166.948 Ph II

3166.98 $P$ Fe $I$

$\mathbf{9 1 6 7 . 4 2 0}$

V II

160
1
159,310
46
2
10
30
83
54
120

84

84
7
1
118
7
115
249
123
79
69

200

200
69
79
79
46
163
50
13
115
14

\section{8}

165

194

100
37

63

21

9
13

13
2
1

160
84

14

s

79

155

48
84

269

100

6

456

3167.49 V II 236

3167.54 Fe III

$\begin{array}{lll}\mathbf{3 1 6 7 . 7 8} P & \mathrm{Fe} I\end{array}$

3167.853 Fe II

3167.907 Fe I

3167.94 P Fe II

3167.95 C II

3168.060 Co I

3168.127 V II

3168. 21

Fe III

3168.519 T1 II

$\begin{array}{lll}3168.86 & & \text { Fe I } \\ 3168.94 & P & \text { Fe I }\end{array}$

$\begin{array}{llll}3168.94 & P & \text { Fe I } \\ 3168.98 & & \text { Yg II }\end{array}$

$3169.09 \quad P \quad$ Fe $I$

3169.183 Ce II

3169.20 Cr II

\$169.21 V II

3160

3169.58

Ne II

$\begin{array}{lll}3169.68 & P & \text { Fe I } \\ 3169.68 & & \text { A II }\end{array}$

$\begin{array}{ll}3169.68 & \text { A II } \\ 3169.766 & \text { Co I }\end{array}$

3169.85 Cr II

3169.864

3169.875

3170.16

$\mathbf{3 1 7 0 . 2 0 8}$

Ca I

8w II

2170.28

$\begin{array}{ll}\mathrm{C} & \text { III }\end{array}$
I A Type Element Multiplet No.

3170.337

3170.40

3170.715

3171.016

3171.09

3171.14
3171.353

3171.615

3171.659

3171.739

3172.067

3172.08

3172.11
3172.168

3172. 169

3172.30

3172. 731

3172.828

9172.94

3173.07

3173. 140

3173.40

3173.56

3173.58

3173. 58

3173.607

3173.608

3173.66

3173. 663

3173.678

3174.077

3174.09

3174.125

3174.140

3174.22

3174.531

3174. 725

9174.80

3174. 905

3175.046

3176.077

3175. 16

3175.317

3175.447

3175. 66

3175.84

$\$ 175.97$

$\$ 175.97$

3176.00

$\$ 176.16$

3176. 292

3176. 366

3176.602

3176.70

3176.85

3176.86

3177.060

3177. 137

3177.22

3177.260

3177.266

3177.490

3177.52

3177.531

$\$ 177.61$

\$177. 65

$\$ 177.696$

3177.80

3177.90

3177.96

3178.015

3178.03

3178. 10

3178.120

3178.495

3178.645

$\$ 178.650$

3178.79

$\mathbf{3 1 7 8 . 9 7 0}$

3179.055

3179.08

3179. 283

3179.291

3179. 332

3179.416

Fe I

Sc II

N1 I

Fe II

N III

Fe I

Ce II
Fe

La III

$\begin{array}{ll}\text { V } & \text { II } \\ \text { Fe } & \text { I } \\ \text { Cr } & \text { II } \\ \text { Fe } & \text { I } \\ \text { Gd } & \text { II } \\ \text { V } & \text { II } \\ \text { Fe } & \text { I } \\ \text { TI } & \text { I } \\ \text { Mg } & \text { II } \\ \text { Tn } & \text { II }\end{array}$

Hf I

N III

Y II

Co I

Co I

Cr II

Ne II

Bu II

Fe I

CI II

Fe I
Rh II

Rh II
V II

Fe III

F III

Co I

V II

F III

6
32
78
10
52,548
99
160
1

217
99,193

71
100

129

249

312

65
13

8

5
51
48
333
72
83
13
3
333

TI II

La II

Co I

Sn I

Fe II

Ru II

Fe I

T1 II
Mg II

Fe I

W II

Fe III

N1 I

Fe I

Fe I

Sc II

HP II

101
5
84
38
2
138
578
217
2

157

71

1
157

1

155

13

393

7

16
77

258

5

32
8
38

Ru II

Ce II

Fe II

Co I

Gd II

Fe I

Fe II

Fe II

V II

o IV

Cr II

$\mathrm{Fe} I$

$\mathrm{Fe}$ I

Ir II

8n II

Mn I

Fe I

TI II

Cr II

Fe I

Na II

Fe III

Cr 1

T1 1

Ca II
$V$ II 
I A Type Element Multiplet No.

3188.95

3189.04

3189.24

3188.52

3189.638

3188.74

3189.752

3189.76

3189.783

3189.85

3190.02

3190.403

3190.651

3190.686

3190.69

3190.81

3190.825

3190.84

3190.86

3190.874

3191.005

3191.044

3191.096

3191.11

3191.180

3191.23

3191. 297

3191.374

3191.39

3191.41

3191.45

3191.577

3191.659

3191.875

3191.83

3181.994

3192.058

3192.12

3182.220

3192. 26

3182.417

3192.68

3182.689

3192.798

3182.84

3192.817

3193.02

$\$ 193.10$

3193.164

3193. 174

3193.200

3183.21

3193.314

3183.41

\$193.48

3193.53

3193.74

3193.75

3193. 76

S1 II

CI II

T1 II

Fe II

Co I

$\mathrm{V}$ II

$\mathrm{Na}$ II

Cr II

Fe I
Sc II

Fe I

V II

Cr II

Fe III

Fe I

Fe II

Ne II

T1 II

65
6,10
120
97
55
9
83
4
123

259
42
548
8
174

548
120
13
26

I A Type

Element Multiplet No.

I A

Type Element

Multiplet No.

$\begin{array}{llrl}3196.532 & \text { Gd II } & 9 & 3206.16\end{array}$

$\begin{array}{llll}3196.574 & V & 62 & \end{array}$

$\begin{array}{lllll}3196.63 & P & \text { Fe II } & 95 & 3206.350\end{array}$

$\begin{array}{llll}3196.930 & \text { Fe I } & 155 & 3206.466\end{array}$

3196.96 Cr II

3197.00

3197.08

3197.113

3197.12

$\begin{array}{lll}\mathrm{Zr} & \text { II } \\ \mathrm{N} 1 & \mathrm{I}\end{array}$

Cr II

8
126

3197.518

3197.53

3197.574

3198.012

3198.112

3198.266

3198.42

3198.62

3188.660

3198.726

Fe I

$\begin{array}{ll}V & \text { II } \\ \text { Cr } & \text { II }\end{array}$

$\mathrm{V} I$

Cr I

$\begin{array}{ll}\mathrm{Fe} & \mathrm{I} \\ \mathrm{Y} & \mathrm{II}\end{array}$

I I
Ne II

Co I

T1 I

42

Gd II

$\mathrm{Cb}$ II

Fe I

Fe I

Zr I

Co I

Fe II

La II

129
1
258

3198.81

3198.88 P Fe III

3198.917 Ir I

3199. 279 Ce II

3199.322

3199.34

3198.342

3199. 37

3198.43

3199. 50

3199.53

CI III

Fe I

N1 I

Zr II

T1 I

Fe II

Cr I

Co I

T1 II

Fe I
I1 II

$\checkmark$ II

Fe I

$\mathrm{Fe}$ I

La II

S1 II

Co I

26

Gd II

$\begin{array}{ll}V & I I \\ \text { Pe I }\end{array}$

$\mathrm{Fe} I$

Cr II

HP II

Fe I

N1 I

Fe II

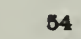

84
83
7

169

48

682

3193. 809 Fo II

$3193.86 \quad P \quad$ Fe II

3193.969 Mo I

3193.97 V I

$3194.03 \quad P \quad F e I$

$\begin{array}{ll}3184.099 & \text { Cu I } \\ 3194.19 & \text { HF II }\end{array}$

3194. 25

3194.28

3194.422

3194.56

3184.61

3194.63

3194.78

3194.76

3194.76

3194.983

3185.30

3195.573

Hf II

A II

$\begin{array}{ll}\mathrm{T} 1 & \mathrm{II} \\ \mathrm{Fe} & \mathrm{I}\end{array}$

3189.530

3199.54

3199.87

3189.915

3199.83

3199.89

3200.28

3200.423

$3200 \cdot 45$

3200.454

3200.475

3200.67

3200.790

3200.95

3201.24

3201.26

3201.584

3201.714

3201.891

3201.90

3201.95

3201.8

3202.142

3202.381

3202.52

3202.536

3202.562

3202.68

3202.711

3202.740

3203.026

3203.05

3203.104

3203.33

3203.39

3203.435

3203.508

3203.53

3203.58

3203.67

3203.741

3203.828

3203.88

3204.06

3204. 196

3204.34

3204 . 36

3204.56

3204.76

3204.870

3205.03

3205. 11

3205. 168

3205.400

3205.382

3205.64

3205.848

3205.800

3206.77

3206.825

3206.908

3206.852

3206.98
3207.092

3207.12

3207.185

3207.248

3207.297

3207.337

3207.410

3207.61

3207.649

3207.897

3208.02

3208.13

3208.231

3208.32

3208.345

3208.470

3208.607

3208.62

3208.838

3208.91

3208.98

3209.030

3200.115

3209.13

3209.21

3208.297

3209.34

3209.38

3208.603

3208.64

3208.80

3209.912

3209.930

3210.04

3210.218

3210.230

3210.448

3210.62

3210.82

3210.825

3210.830 
I A Type Element Multiplet No. I A Type Element Multiplet No.

I A Type Element Multiplet No.

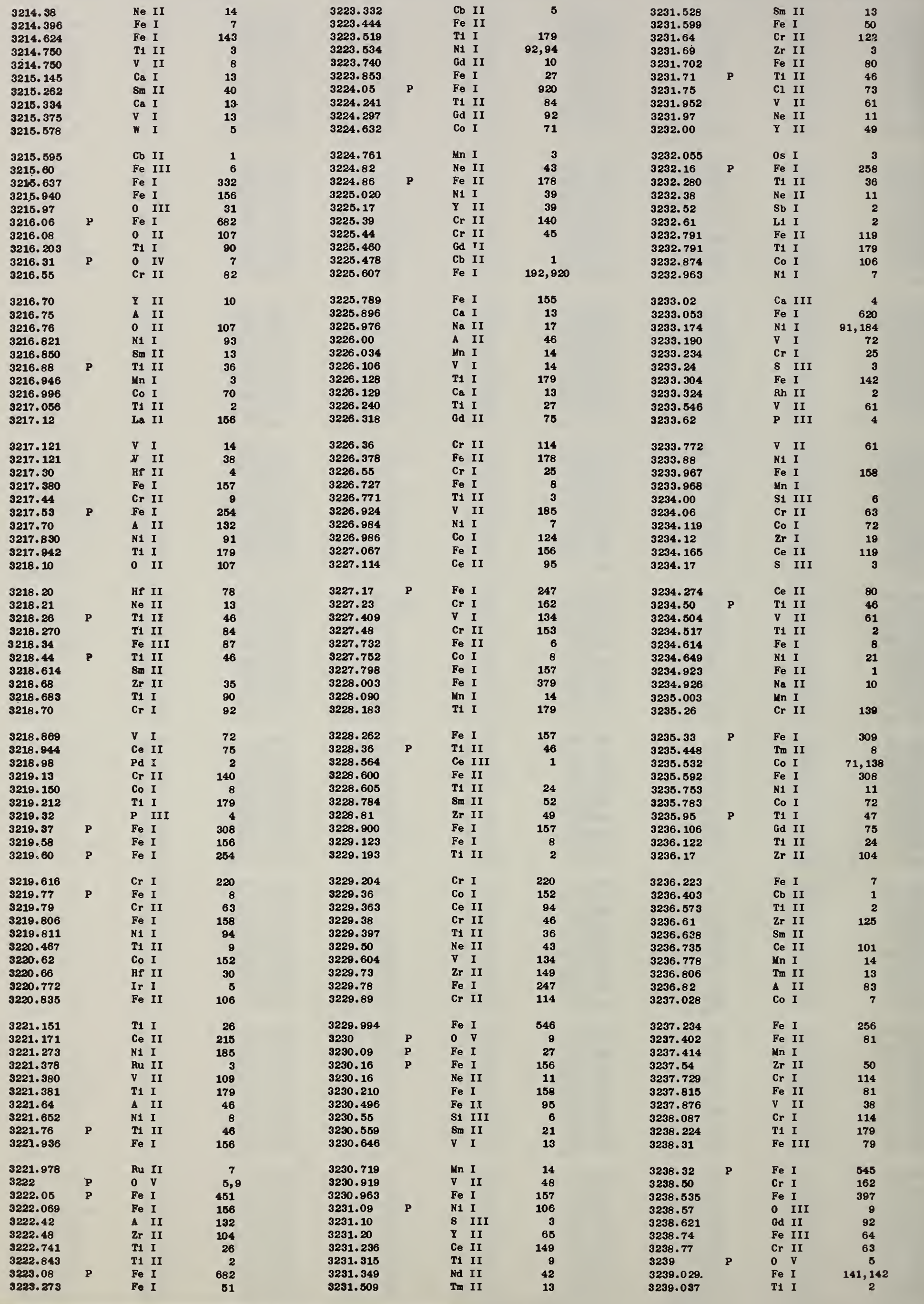


Type Blement

Mul tiplet No.

\begin{tabular}{|c|c|c|c|c|c|c|c|c|}
\hline 3239.04 & & Fe & III & 63 & 3248.516 & & Mn & $I$ \\
\hline 3239.101 & & $\mathrm{Rh}$ & II & 2 & 3248.602 & & T1 & I \\
\hline 3239.14 & & $C_{r}$ & $\mathbf{I}$ & 82 & 3248.602 & & T1 & II \\
\hline 3239.256 & & Co & I & 47 & 3248.70 & & T1 & II \\
\hline 3239.35 & $\mathbf{P}$ & $F e$ & I & 378 & 3248 & $\mathbf{P}$ & 0 & $\mathbf{v}$ \\
\hline 3239.496 & & $\mathbf{F e}$ & I & 157 & 3248.037 & & Fo & I \\
\hline 3239.46 & $\mathbf{P}$ & $F e$ & I & 157 & $\$ 249 \cdot 16$ & $\mathbf{P}$ & Fe & II \\
\hline 3238.657 & & Sem & II & 48 & 3249.204 & & Fe & $I$ \\
\hline 3239.664 & & T1 & II & 24 & 3240.35 & & La & II \\
\hline 3238.833 & & $v$ & II & 61 & 3249.370 & & T1 & II \\
\hline 3238.87 & $\mathbf{P}$ & Fo & II & 81 & 3248.440 & & N1 & I \\
\hline 3240.013 & & $F_{e}$ & I & 545 & 3249.464 & & $\mathbf{v}$ & II \\
\hline 3240.07 & & $C_{r}$ & II & 140 & 3248.566 & & v & I \\
\hline 3240.11 & $\mathbf{P}$ & $F_{\theta}$ & I & 158 & 3248.617 & & v & II \\
\hline 3240.230 & & Tw & II & & 3248.657 & & $\mathrm{Fe}$ & II \\
\hline 3240.389 & & $N_{n}$ & I & 13 & 3249.742 & & Gd & II \\
\hline $\mathbf{3 2 4 0 . 5 1 6}$ & & $\mathbf{R h}$ & II & 6 & 3248.82 & & $\mathbf{A}$ & II \\
\hline 3240.616 & & Mn & I & 14 & 3248.811 & & $\mathrm{Fe}$ & II \\
\hline 3240.71 & & $\mathbf{T 1}$ & I I & 8 & 3248.895 & & Co & I \\
\hline 3240.785 & & $\mathbf{v}$ & II & 61 & 3250.187 & & ad & I.I \\
\hline 3240.84 & $\mathbf{P}$ & $\mathbf{T} \mathbf{1}$ & I & 47 & 3250.27 & & $\mathrm{Fe}$ & III \\
\hline 3240.85 & $\mathbf{P}$ & $\mathbf{z r}$ & II & 12 & 3250.34 & $\mathbf{P}$ & $\mathrm{Fe}$ & II \\
\hline 3240.951 & & $\mathrm{Cr}$ & I & 25 & 3250.372 & & $8 m$ & II \\
\hline 3241.01 & & $\mathbf{z r}$ & II & 4 & 3250.400 & & Fe & I \\
\hline$\$ 241.05$ & & co & I & 8 & 3260.42 & & $\mathbf{z r}$ & I \\
\hline 3241.161 & & So & I I & 6 & 3260.44 & & $\mathbf{z r}$ & II \\
\hline 3241.38 & & $C_{r}$ & II & 153 & 3250.51 & & Co & I \\
\hline 3241.43 & $\mathbf{P}$ & $\mathbf{F e}$ & $I$ & 188 & 3250.58 & & Cr & I \\
\hline 3241.50 & $\mathbf{P}$ & $\mathrm{Fe}$ & I & 27 & 3250.634 & & $\mathrm{Fe}$ & I \\
\hline 3241.530 & & Tw & II & 4 & 3250.743 & & N1 & I \\
\hline 3241.586 & & Sin & II & 22 & 3250.747 & & Mo & II \\
\hline 3241.67 & & 81 & III & 6 & 3280.775 & & $\mathbf{v}$ & II \\
\hline 3241.685 & & $\mathrm{Fe}$ & II & 80 & 3250.79 & & $\mathrm{Cr}_{\mathbf{r}}$ & II \\
\hline 3241.835 & & $\mathrm{Be}_{\theta}$ & II & 6 & 3251.135 & & in & I \\
\hline 3241.984 & & T1 & II & 2 & 3251.236 & & $\mathrm{Fe}$ & I \\
\hline \$242. 18 & & $\mathbf{z r}$ & II & 126 & 3251.32 & & $8 c$ & II \\
\hline 3242.268 & & $\mathrm{Fe}$ & I* & 255 & 3251.34 & $\mathbf{P}$ & $\mathbf{F e}$ & II \\
\hline 3242.30 & & 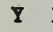 & II & 10 & 3251.46 & $\mathbf{P}$ & $2 \mathbf{r}$ & II \\
\hline 3242.304 & & ad & II & 82 & 3251.656 & & Co & I \\
\hline 3242.72 & & Pd & I & 3 & 3251.68 & & Pd & I \\
\hline 3242.834 & & Gd & II & 75 & 3251.836 & & $\mathrm{Cr}_{\mathrm{r}}$ & I \\
\hline 3243.058 & & N1 & I & 22 & 3251.868 & & $\mathbf{v}$ & II \\
\hline 3243.118 & & $\mathrm{Fe}$ & I & 182 & 3251.811 & & T1 & II \\
\hline 3243.34 & & Ne & II & 13 & $325 \dot{2} \cdot 12$ & $\mathbf{P}$ & $\mathrm{Fe}$ & $I$ \\
\hline 3243.96 & & w & II & 13,15 & 3252.40 & $\mathbf{P}$ & $\mathrm{Fe}$ & II \\
\hline 3243. 370 & & $C_{\theta}$ & II & 214 & 3252.483 & & $\mathrm{Ce}$ & II \\
\hline 3243.406 & & $\mathrm{Fe}$ & I & 381,710 & 3252.743 & & od & II \\
\hline 3243.513 & & $\mathbf{T 1}$ & I & 179 & 3252.814 & & T1 & II \\
\hline 3243.579 & & Co & I & 47 & 3252.828 & & Fe & I \\
\hline 3245. 70 & & $\Delta$ & II & 47 & 3252.84 & & 0 & II I \\
\hline 3243.729 & & $\mathrm{Pe}$ & II & 110 & 3252.84 & $\mathbf{P}$ & $\mathbf{T} 1$ & II \\
\hline 3243.74 & $\mathbf{P}$ & $\mathbf{v}$ & II & 48 & 3252.848 & & Yn & I \\
\hline 3243.780 & & Yn & I & 14 & 3253.26 & & $\mathrm{Cr}$ & I \\
\hline 3243.803 & & T1 & I & 26 & 3253.401 & & $8 \mathrm{sis}$ & II \\
\hline 3243.840 & & Co & I & 68 & 3253.41 & & Le & II \\
\hline 3244.115 & & $C_{r}$ & I & 20 & 3253.416 & & Co & I \\
\hline 3244.15 & & $\mathrm{Ne}$ & II & 14 & 3253.44 & & 81 & III \\
\hline $3244 \cdot 17$ & $\mathbf{P}$ & $8 c$ & II & 5 & 3253.610 & & $F_{e}$ & I \\
\hline 3244.190 & & $\mathrm{Fe}_{\mathrm{e}}$ & I & 156 & 3253.70 & & Be & II \\
\hline 324.44 & & Cl & I II & 6 & 3253.839 & & $\mathrm{Fe}$ & I \\
\hline 3244.53 & $\mathbf{P}$ & T1 & I & 47 & 3263.943 & & $8 m$ & II \\
\hline 3244.69 & & $\mathrm{Cr}_{\mathbf{r}}$ & I & 114 & 3253.854 & & Fe & I \\
\hline 3246 & $\mathbf{P}$ & 0 & $\bar{v}$ & 9 & 3254.03 & & $A$ & II \\
\hline 3245.13 & & Lo & II & 32 & 3254.039 & & Yn & I \\
\hline 3245.31 & & $\mathrm{Cr}_{\mathbf{r}}$ & I I & 62 & 3254.070 & & $\mathrm{Cb}$ & II \\
\hline 3245.370 & & N1 & I & 108 & 3254.202 & & Co & I \\
\hline 3245.485 & & $C_{r}$ & I & 25 & 3254.250 & & $\mathbf{T 1}$ & II \\
\hline 3245.542 & & $C_{r}$ & I & 113 & 3284.261 & & $\mathrm{Fe}_{\mathbf{B}}$ & I \\
\hline 3245.780 & & Co & I & 138 & 3254.32 & & Lu & II \\
\hline 3245.80 & $\mathbf{P}$ & $\mathrm{Fe}$ & I & 820 & 3254.363 & & F• & I \\
\hline 3245.884 & & $\mathbf{F e}$ & I & 27 & 3254.377 & & 8 & II \\
\hline 3246.005 & & Fe & I & 8 & 3254.46 & $\mathbf{P}$ & Fe & I \\
\hline 3246.05 & $\mathbf{P}$ & Fe & I & 308 & 3254.63 & & $\mathrm{Co}_{0}$ & I \\
\hline 3246.482 & & Fe & I & 252 & 3254.734 & & Fe & I \\
\hline 9246.674 & & Ce & II & 130 & 3254.773 & & $\mathbf{v}$ & I \\
\hline 3246.873 & & Fe & I & 95 & 3254.779 & & $\mathbf{v}$ & II \\
\hline 3247.01 & & $\mathrm{Cr}$ & I I & 62 & 3254.85 & & $\mathrm{Cr}$ & I \\
\hline 3247.170 & & co & I & 70 & 3266.28 & & Bf & II \\
\hline 3247.171 & & Fo & II & 81 & 3255.30 & & $\mathrm{Cr}_{\mathbf{r}}$ & II \\
\hline 3247.274 & & $C_{r}$ & I & 25 & 3255.39 & & Ne & II \\
\hline 3247.207 & & Fe & I & 157 & 3255.49 & & Fe & III \\
\hline 3247.33 & & $\mathrm{Cr}_{\mathbf{r}}$ & II & 81 & 3255.62 & & $\mathrm{Cr}_{r}$ & II \\
\hline 3247.392 & & Fo & II & 110 & 3255.678 & & $8 e$ & I \\
\hline 3247.478 & & $\mathrm{Cb}$ & II & 5 & 3255.818 & & Gd & II \\
\hline 3247.840 & & $\mathrm{Cu}$ & I & 1 & 3258.884 & & Fe & II \\
\hline 3247.56 & & $\mathbf{A}$ & II & & 3256.137 & & Mn & I \\
\hline 3247.908 & & $\mathbf{v}$ & II & 108 & 3256.82 & & Fe & I \\
\hline 3248.15 & & Ne & II & 15 & 3266.52 & $\mathbf{P}$ & Pe & I \\
\hline 3248.206 & & Fe & I & 157 & 3256.53 & & $\mathbf{z r}$ & II \\
\hline 3248.467 & & N1 & I & 21 & 3286.84 & & Fe & III \\
\hline
\end{tabular}




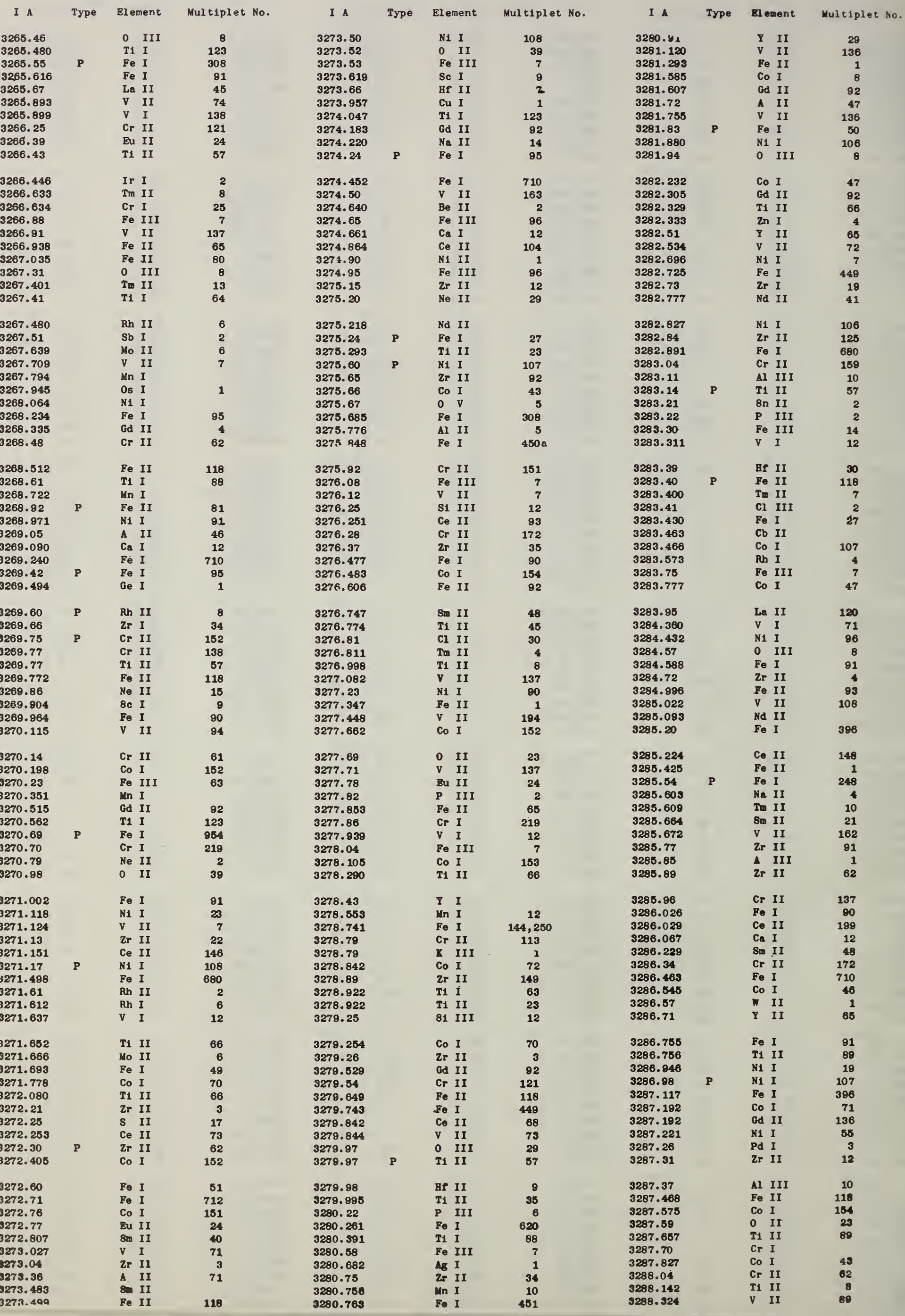


I A Type Element Multiplet No.

I A Type Element Multiplet No.

I A Type Element

$\begin{array}{llr}3307.717 & \text { T1 II } & 8 \\ 3307.755 & \text { Cr I } & 78 \\ 3307.90 & \text { CI II } & 37 \\ 3308.02 & \text { EU II } & 25 \\ 3308.15 & \text { Cr II } & 137 \\ 3308.246 & \text { V I } & 12 \\ 3308.391 & \text { TI I } & 87 \\ 3308.4 & \text { Y II } & 64 \\ 3308.480 & \text { V II } & 137 \\ 3308.482 & \text { Co I } & 155\end{array}$

Ce II 166

3300.152

3300.20

3300.818

3300.905

3900.976

3301.09

3301.21

3301.227

3301.558

3301.56

Co I

0 II I

T1 I

T1 1

Fe III

Fe II

Fe II

228

Fe III

V I I

Gd II

Fe III

Cr II

$\mathrm{Fe} I$

081

151

3
35

61
8

49

8

3301.587

3301.66

3301.678

3301.71

3301.734

3301.87

9301.88

3301.927

3301.95

3302.096

Ru I

V II

St II

T1 II

8r I

Pt I

A III

Fe I

TI II

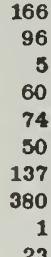

3308.517

3308.688

3308.75

3308.785

3308.814
3308.86

3308. 81

3309.176

3309.32

3309.32

3309.40

3309.428

3309.501

3309.53

3309.582

3509.730

3309.78
3309.804

3309.82

Od II

Co I

$\mathrm{Fe} I$

T1 II

$\begin{array}{ll}\text { Co } & \text { I } \\ P & \text { II }\end{array}$

N1 I

V I

N1 I

T1 I

Fe III

N1 I

T1 I

T1 II

T1 I

Ne II

To II

3509.90

3302 . 16

Pd I

$\begin{array}{ll}\mathrm{Fe} & \text { II } \\ \mathrm{Na} & \mathrm{I}\end{array}$

Tr I I I

Zn I

3302.454

3302.588

3302.66

3302.86

3302.861

3302.94

3302. 941

2r II

Cr I

Fe II

Na I

Co I

154

T1 II

A II

N1 I

Cr I

$\begin{array}{ll}\text { Co } & I \\ Y & \text { I I }\end{array}$

Y I I

Co I

Ru II

Zn I

3303.11

3303.278

3303.466

3303.574

3303.881

3304.01

3304.119

3304 . 31

3304. 36

3304.433

La II
Mn I

Fe II

Fe I

Co I

Y II

Co I

3294.220

La II

2
155

3294.50

Fe III

Co I

Fe III

Zr II

$\mathrm{Fe}$ II

0 II

Fe III

$\mathrm{Fe}$ II

Cr II

Fe II

V II

Sm II

3304.523

3304.73

3304.836

3304.950

3305.15

3305.15

3305. 185

3305.22

3305.75

3305.77

3305.971

3306.053

3306.27

3306.35

3306. 356

3306.388

3306.45

Cr II

Ce II

N1 I

0 II

Zr II

Stm II

Fe III

Pe II

3510 . 202

3910.347

3310.486

3310.65

3310.65

3310.661

3311.25

3311. 30

3311. 34

3311.451

3311. 708

3311.805

3311.928

3912.06

3312. 148

3312.18

3312.215

3312. 232

3312 . 30

3312. 920

3312. 39

3312.415

3312.690

3312. 707

3912. 736

3312. 78

3312.87

3312.80

3312.992

3313.08

3313.116

3313.33

3913. 344

3313.470

3313.524

3313.539

3313.70

3313.721

3313.723

3313. 731

3313. 996

3314

3314. 06

3314.070

3314.073

3314.345

3311.393

3314.422

3314 . 450

Zr II

N1 I

Fe I

Fe I

Ne II

Cr II

Si II

Ne II

2r II

$\mathrm{Fe} I$

Sc II

Un I

Cr I

$\begin{array}{ll}\text { Cr } & \text { I } \\ \text { Co I } & \text { I }\end{array}$

Cr II

Co II

Fo I

0 III

4

105

163

107

6

$10 B$

122

87

44
24

190

161

72

38
449

678

120,158

3

2

3

N1

8. II

T1 I

Fe II

8c. II

C1 I

He I

T1 II
N1 I

41

68

460.

3314.49

3314.50

3314. 523

3314.56

3314.57

3314.60

3314.721

3314.742

Cr II

Cr II 


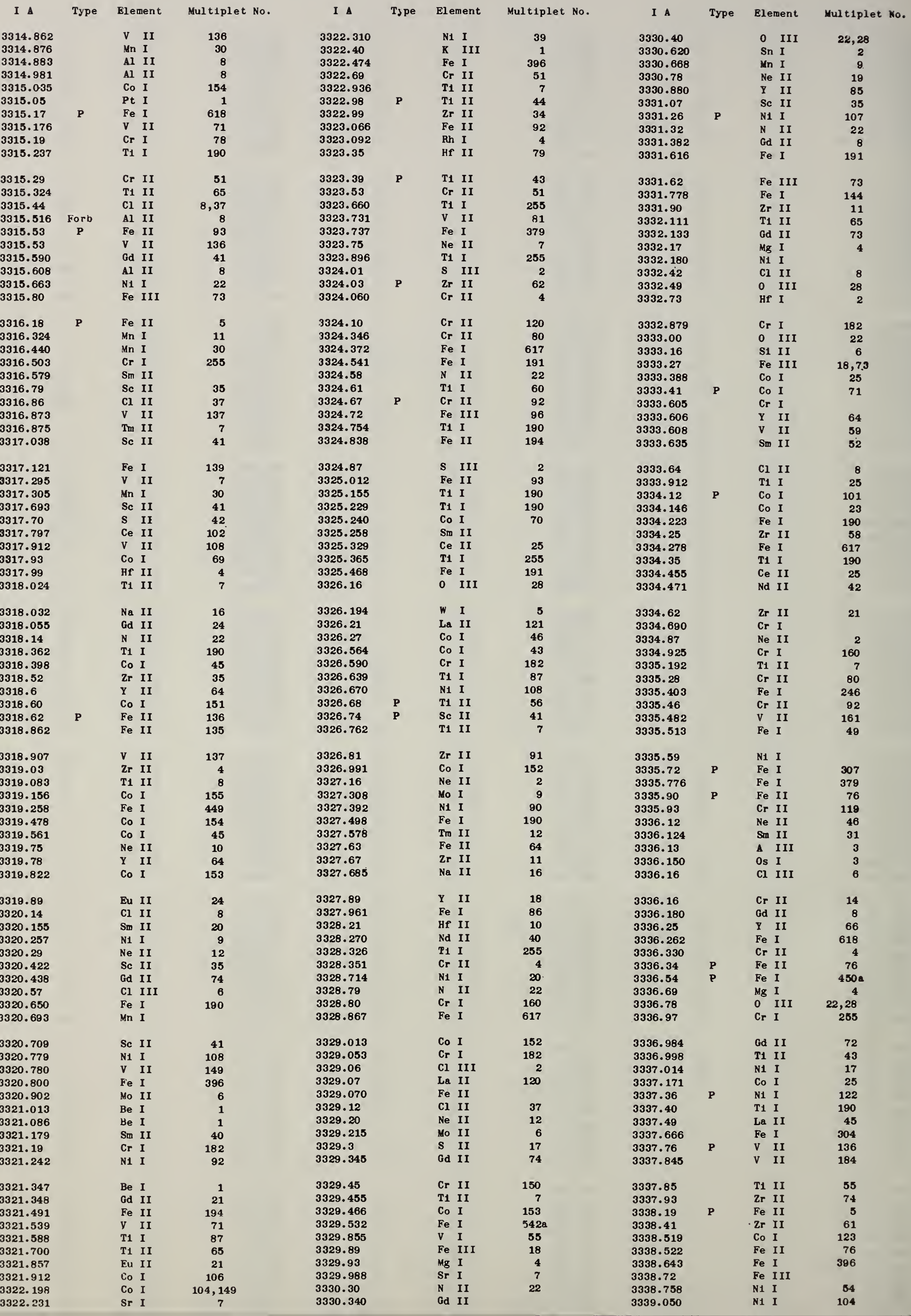


I A Type Element Multiplet No.

\begin{tabular}{|c|c|c|c|c|c|c|c|c|}
\hline 3339.063 & & Nd & I I & 41 & 3347 . 298 & & $\mathrm{Sm}$ & II \\
\hline 3339. 15 & & Co & I & 148 & 3347.507 & & $\mathrm{Fe}$ & I \\
\hline 3339.202 & & $\mathrm{Fe}$ & I & 180,446 & 3347.70 & & $\mathrm{Fe}$ & I I I \\
\hline $\mathbf{3 3 3 9} \cdot \mathbf{3 6}$ & & $\mathrm{Fe}$ & I I I & 7 & $3347 \cdot 72$ & & $\mathbf{P}$ & IV \\
\hline 3339.54 & & T1 & I & 178 & 3347.837 & & $\mathrm{Cr}_{\mathbf{r}}$ & I I \\
\hline 3339.588 & & $\mathrm{Fe}$ & $I$ & 602 & 3347.927 & & $\mathrm{Fe}$ & I \\
\hline 3339.780 & & Co & I & 155 & 3348.05 & & 0 & III \\
\hline 3339.804 & & Cr & I I & 4 & 3348.08 & & 0 & IV \\
\hline 3339.810 & & Ru & II & $?$ & $3348 \cdot 112$ & & Co & I \\
\hline 3339.84 & & S1 & II & 6 & 3348.372 & & v & II \\
\hline 3339.90 & & Cr & II & 92 & 3348.535 & & $\mathrm{~T} 1$ & I \\
\hline 3340.344 & & T1 & II & 7 & 3348.683 & & Sm & I I \\
\hline 3340.42 & & Cl & I I I & 2 & 3348.844 & & $\mathrm{~T} 1$ & I I \\
\hline 3340.55 & & 2 r & II & 3 & 3349.035 & & $\mathrm{~T} 1$ & I I \\
\hline 3340.566 & & $\mathrm{Fe}$ & I & 139 & 3349.072 & & $\mathbf{C r}$ & I \\
\hline 3340.579 & & Sm 1 & II & 6 & 3349.11 & & 0 & IV \\
\hline 3340.74 & & 01 & I I I & 3 & $3349 \cdot 17$ & & Hf & II \\
\hline 3340.77 & & T1 & I & 190 & 3349.322 & & $\mathrm{Cr}$ & I \\
\hline 3341.341 & & Co & I & 148 & 3349.34 & & $\mathrm{Cr}$ & II \\
\hline 3341.554 & & T1 & I & 60,178 & 3349.399 & & T1 & I I \\
\hline 3341.77 & & A & II & 59 & 3349.68 & $\mathbf{P}$ & $\mathrm{Cr}$ & II \\
\hline 3341.868 & & $\mathrm{Ce}$ & II & 198 & 3349.739 & & $\mathrm{Fe}$ & I \\
\hline 3341.875 & & T1 & I & 24 & 3349.967 & & $\mathrm{Ce}$ & I I \\
\hline 3341.875 & & T1 & I I & 16 & 3350.097 & & Gd & I I \\
\hline 3341.806 & & $\mathrm{Fe}$ & I & 303 & 3350.209 & & $\mathrm{Ca}$ & I \\
\hline 3341.98 & & $\mathrm{Cr}$ & I I & 119 & 3350.284 & & $\mathrm{Fe}$ & I \\
\hline 3342.151 & & $\mathrm{~T} 1$ & I & 23 & 3350.361 & & $\mathrm{Ca}$ & I \\
\hline 3342.225 & & $\mathrm{Fe}$ & I & 137 & 3350.42 & & N1 & I I \\
\hline 3942.298 & & $\mathrm{Fe}$ & I & 378 & 3350.474 & & Gd & II \\
\hline 3342.46 & & w & I I & 9 & 3360.548 & & T1 & I \\
\hline 3342.51 & & Cr & I I & 4 & 3350.548 & & $\mathrm{~T} 1$ & I I \\
\hline 3342.707 & & T1 & 1 & 25 & 3350.68 & & 0 & I I I \\
\hline 3342.734 & & Co & I & 105 & 3350.875 & & Sm & I I \\
\hline 3342.76 & $\mathbf{P}$ & $\mathrm{Fe}$ & I & 396 & 3350.94 & & $\mathbf{A}$ & II \\
\hline 9342.77 & & $\mathbf{N}$ & II I & 7 & 3350.99 & & 0 & I I I \\
\hline $\mathbf{3 3 4 3 . 0 9}$ & & w & I I & 13 & 3351.06 & $\mathbf{P}$ & N1 & I \\
\hline 3343.227 & & $\mathrm{Cr}$ & I & 159 & 3351.138 & & Co & I \\
\hline 3343.243 & & $\mathrm{Fe}$ & I & 88 & 3351.246 & & Sr & I \\
\hline 3343.27 & & Sc & II & 35 & 3351.424 & & Mn & I \\
\hline 3343.312 & & v & I I & 234 & 3351.456 & & Al & II \\
\hline 3343.342 & & $\mathrm{Cr}$ & I & 159 & 3351.529 & & $\mathrm{Fe}$ & I \\
\hline 3343.379 & & T1 & I & 178 & 3351.53 & & $\mathbf{v}$ & II \\
\hline 3943.40 & & * & II & 8 & 3351.596 & & $\mathrm{Cr}$ & I \\
\hline 3343.494 & & Sto & I I & & 3351.67 & & $\mathrm{~T} 1$ & I I \\
\hline 3343.530 & & Co & I & 151 & 3351.750 & & $\mathrm{Fe}$ & $\mathbf{I}$ \\
\hline 3343.678 & & $\mathrm{Fe}$ & I & 449 & 3351.966 & & $\mathrm{Cr}$ & $\mathbf{I}$ \\
\hline 3343.731 & & Mn & I & 9 & 3351.97 & & Sn & II \\
\hline 3343.770 & & T1 & I I & 7 & 3352.048 & & Sc & II \\
\hline 3343. 81 & & $\mathrm{zr}$ & II & 85 & 3352.06 & & Hf & I I \\
\hline 3343.861 & & $\mathrm{Ce}$ & II & 159 & 3352.071 & & $\mathrm{~T} 1$ & II \\
\hline 3344.09 & $P$ & $\mathrm{Fe}$ & I & 450 & 3352.43 & $\mathbf{P}$ & $\mathrm{T} 1$ & I \\
\hline 3344,26 & & 0 & I I I & 22,28 & 3352.80 & & Co & I I \\
\hline 3344. 353 & & Sim & II & 39 & 3352.928 & & $\mathrm{Fe}$ & I \\
\hline 3344.43 & & $\mathrm{Ne}$ & II & 2 & 3352.937 & & $\mathrm{~T} 1$ & I \\
\hline 3344.50 & & $\mathbf{C r}$ & I & 160 & 3353.026 & & $\mathrm{Cr}$ & $\mathbf{I}$ \\
\hline 3344.513 & & $\mathrm{Ca}$ & I & 11 & 3353.12 & & $\mathrm{Cr}$ & I I \\
\hline 3344.56 & & $\mathbf{L a}$ & II & 45 & 3353.262 & & Ce & I I I \\
\hline 3344.62 & $\mathbf{P}$ & $\mathrm{T} 1$ & I & 25 & 3353.268 & & Fe & I \\
\hline 3344.630 & & $\mathbf{T} 1$ & I & 178 & 3353.39 & & $\mathrm{Cl}$ & I I \\
\hline 3344.72 & & $\mathbf{A}$ & II I & 3 & 3353.63 & & $\mathrm{Ne}$ & I I \\
\hline 3344.750 & & Mo & I & 9 & 3353.65 & & $\mathrm{Zr}$ & $\mathbf{I}$ \\
\hline 3344.761 & & $\mathrm{Ce}$ & I I & 165 & 3353.734 & & Sc & II \\
\hline 3344.80 & & $\mathbf{z r}$ & II & 72 & 3353.776 & & $v$ & II \\
\hline 3344.931 & & $\mathrm{~T} 1$ & I & 178 & 3353.78 & & $\mathrm{~N}$ & I I I \\
\hline 3345.020 & & $\mathbf{z n}$ & I & 4 & 3354.068 & & $\mathrm{Fe}$ & I \\
\hline 3345.14 & & $\mathrm{Cr}$ & I & 218 & 3354.185 & & Sm & I I \\
\hline 3345.146 & & Co & I & 45 & 3354.213 & & Co & I \\
\hline 3345.352 & & Mn & I & & 3354.29 & & $N$ & II I \\
\hline 3345. 36 & & $\mathrm{Cr}$ & I & 218 & 3354.31 & & 0 & IV \\
\hline 3345.49 & & $\mathrm{Ne}$ & II & 10 & 3354.374 & & Co & I \\
\hline 3345.572 & & $\mathrm{zn}$ & I & 4 & 3354.39 & & $\mathbf{Z r}$ & II \\
\hline 3345.679 & & $\mathrm{Fe}$ & I & 141 & 3364.54 & $\mathbf{P}$ & $\mathbf{T} 1$ & I I \\
\hline 3345.86 & & $w$ & I I & 17 & 3354.550 & & He & I \\
\hline 3345.88 & & Ne & I I & 10,12 & 3354.621 & & Nd & II \\
\hline 3345.899 & & v & I I & 244 & 3354.634 & & T1 & I \\
\hline 3345.934 & & $\mathrm{Zn}$ & $I$ & 4 & 3355.05 & & $\mathrm{Ne}$ & I I \\
\hline 3345.985 & & Gd & II & 8 & 3355.228 & & $\mathrm{Fe}$ & I \\
\hline 3346.018 & & $\mathrm{Cr}$ & I & 112 & 3355.366 & & $\mathbf{v}$ & I I \\
\hline 3346.09 & & $\mathrm{Cr}$ & I & & 3355.47 & & $N$ & I I I \\
\hline 3346.310 & & Co & I & 45 & 3355.817 & & $F_{e}$ & I \\
\hline 3346.403 & & Mo & I I & 6 & 3355.92 & & 0 & I I I \\
\hline 3346.71 & & $\mathrm{Cr}$ & I & 112 & 3355.940 & & Co & I \\
\hline 3346.724 & & $\mathrm{~T} 1$ & I I & 7 & 3356.08 & & $\mathbf{Z r}$ & II \\
\hline 3346.78 & & $\mathrm{Cr}$ & I & 112 & 3356.196 & & $\mathrm{~T} 1$ & I \\
\hline 3346.91 & $\mathbf{P}$ & $\mathrm{T} 1$ & I I & 43 & 3356.24 & $\mathbf{P}$ & $\mathrm{Fe}$ & II \\
\hline 3346.932 & & Co & I & 153 & 3356.263 & & $\mathrm{Fe}$ & II \\
\hline 3546.942 & & $\mathrm{Fe}$ & I & 87 & 3356.332 & & $F_{0}$ & I \\
\hline 3346.99 & & $\mathrm{Ca}$ & II & 9 & 3356.35 & $\mathbf{P}$ & Ce & III \\
\hline 3347.10 & & Sb & $I$ & 1 & 3356.36 & & Ne & II \\
\hline $3547 \cdot 268$ & & Mo & II & 6 & 3366.352 & & v & I \\
\hline
\end{tabular}

I A Type Element

Multiplet No.

$\begin{array}{llr}3356.407 & \text { Fe I } & 137 \\ 3356.464 & \text { Co I } & 104 \\ 3356.513 & \text { Gd I I } & 24 \\ 3356.842 & \text { Co I } & 151 \\ 3357.07 & \text { Fe I I } & 19 \\ 3357.215 & \text { Ce I I } & 164 \\ 3357.26 & \text { Zr II } & 3 \\ 3357.40 & \text { Cr II } & 79 \\ 3357.40 & \text { Fe II I } & 63,72 \\ 3357.72 & \text { Cr II } & 91\end{array}$

$3357.82 \quad P \quad$ Fe I

$\begin{array}{llr}3357.90 & \text { Ne II } & 12\end{array}$

$\begin{array}{lll}3357.965 & \text { Fe II } & 117\end{array}$

$3358.130 \quad$ Mo I $\quad 8$

3358.252 Fe II 77

$\begin{array}{lll}3358.271 & \text { T1 I } & 23\end{array}$

$3358.30 \quad$ HF II $\quad 63$

3358.49 A I I I

$\begin{array}{lll}3358.501 & \text { Cr II } & 4 \\ 3358.56 & \text { TI I }\end{array}$

3358.59 Co II 2

3358.62 W II 13

$\begin{array}{llr}3358.620 & \text { Gd I I } & 8 \\ 3358.72 & \text { N I I I } & 5\end{array}$

3358.74 Fe II I 72

$\begin{array}{llll}3358.78 & P & \text { Fe II } & 5\end{array}$

$\begin{array}{rrr}3359.066 & \text { Co I } & 69 \\ 3359.106 & \text { N1 I } & 108\end{array}$

$\begin{array}{lll}3359.18 & \text { Fe III } & 72\end{array}$

3359.284 Co I 44

$\begin{array}{llr}3359.496 & \text { Fe I } & 25 \\ 3359.50 & \text { V I I } & 148\end{array}$

3359.679 Sc II

$\begin{array}{llr}3359.814 & \text { Fe I } & 617 \\ 3359.96 & \text { Zr I I } & 91\end{array}$

$\begin{array}{llr}3359.96 & \text { Zr I I } & 91 \\ 3360.103 & \text { Fe II } & 105\end{array}$

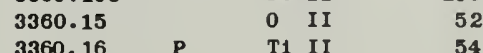

$\begin{array}{lll}3360.295 & \text { Cr II } & 21\end{array}$

$\begin{array}{lll}3360.45 & \mathrm{Zr} \text { I } & 53 \\ 3360.541 & \text { Ce II } & 55\end{array}$

$\begin{array}{llr}3360.541 & \text { Ce II } & 25 \\ \mathbf{3 3 6 0 . 6 3} & \text { Ne II }\end{array}$

3360.711 Gd II

$3360.84 \quad$ Fe III

$3360.835 \quad$ Fe I 142

$\begin{array}{lllll}3360.980 & & \text { T1 I } & 24 \\ 3361.07 & \text { P } & \text { T1 I I } & 64\end{array}$

$\begin{array}{rrrr}3361.09 & \text { C II } & 7\end{array}$

$\begin{array}{lll}3361.11 & \text { W I I } & 2\end{array}$

3361.241 N1 I 107

$\begin{array}{lll}3361.263 & \text { T1 I } & 23\end{array}$

$\begin{array}{rrr}3361.270 & \text { Sc II } & 4\end{array}$

$\begin{array}{lrr}3361.371 & \text { Mo I } & 10 \\ 3361.50 & \text { T1 I } & 178\end{array}$

$\begin{array}{llll}3361.506 & V & \text { II } & 70\end{array}$

$\begin{array}{rrr}3361.553 & \text { Co I } & 157 \\ 3361.556 & \text { N1 I } & 19\end{array}$

$\begin{array}{llll}3361.73 & \text { A II } & 109\end{array}$

$\begin{array}{llll}3361.75 & \text { C } & \text { I I } & 7\end{array}$

$\begin{array}{lll}3361.770 & \text { Cr II } & 21 \\ 3361.835 & \text { T1 I } & 25\end{array}$

3361.835 T1 I 25

$\begin{array}{llr}3361.918 & \text { Ca. I } & 11\end{array}$

$\begin{array}{llr}\mathbf{3 3 6 1 . 9 3 5} & \text { Sc II } & 4 \\ \mathbf{3 3 6 1 . 9 5 9} & \text { Fe I } & \mathbf{3 7 7}\end{array}$

$\begin{array}{lllr}3362.00 & X & \text { II } & 36\end{array}$

3362.191 Ce I 11

3362.213 Cr I 54

$\begin{array}{llr}3362.233 & \text { Gd II } & 8 \\ 3362.28 & \text { Ca I } & 11\end{array}$

$\begin{array}{llll}3362.38 & 0 & \text { III } & 22\end{array}$

$\begin{array}{lll}3362.618 & \text { Tw II } \\ 3362.63 & \text { P IV }\end{array}$

$\begin{array}{llllr}3362.63 & \text { P } & \text { 0 IV } & 8 \\ 3362.653 & & \text { T1 II } & 64\end{array}$

$\begin{array}{lll}3362.70 & \text { Cr I } & 54\end{array}$

$\begin{array}{lll}3362.70 & \text { Zr II } & 60 \\ 3362.764 & \text { Fe II } & 78\end{array}$

$3362.806 \quad$ N1 I 23

3362.89 Ne II 12

$\begin{array}{rrr}3363.601 & \text { Sc I I } & 38 \\ 3363.613 & \text { N1 I } & 105\end{array}$

$\begin{array}{llr}3363.613 & \text { N1 I } & 105 \\ 3363.71 & \text { Cr II } & 3\end{array}$

303.71

$\begin{array}{llr}3363.81 & \text { Zr II } & 11 \\ 3363.816 & \text { Fe I } & 307\end{array}$

$\begin{array}{llll}3363.83 & 0 & \text { III } & 11\end{array}$

3363.974 Od II 107 


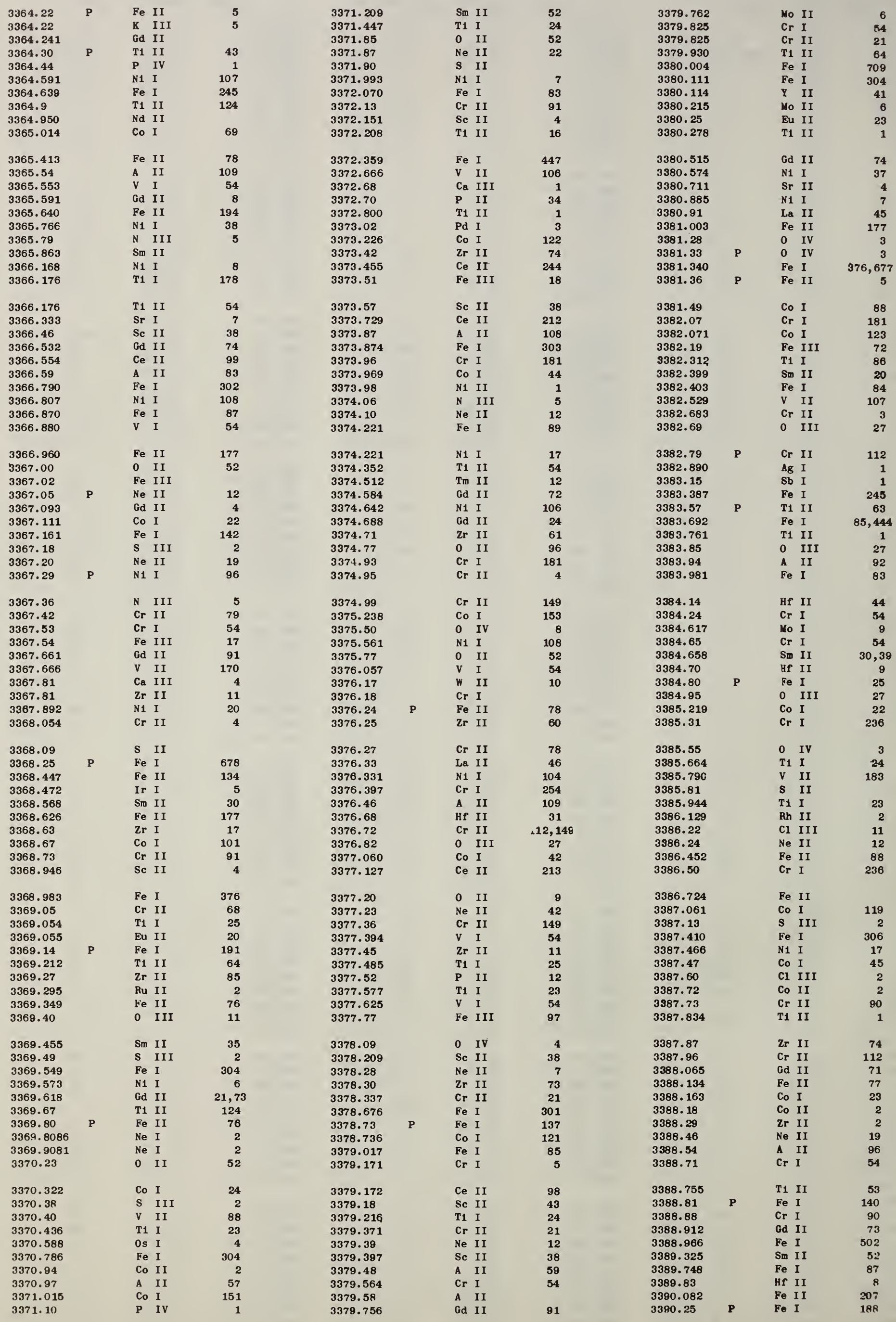




$\begin{array}{lllr}3390.25 & & \text { O II } & \\ 3380.37 & \text { P } & \text { 0 IV } & \\ 3390.396 & & \text { Co I } & 10 \\ 3390.498 & & \text { Gd II } & 7 \\ 3390.315 & & \text { Ce II } & 14 \\ 3380.66 & & \text { Ne II } & 1 \\ 3380.682 & & \text { TI I } & 86 \\ 3380.77 & & \text { Cr I } & 23 \\ 3390.783 & & \text { Bu II } & \\ 3390.878 & & \text { Grd II } & 7 \\ 3391.01 & & \text { V II } & 12 \\ 3391.050 & & \text { N1 I } & \\ 3391.11 & & \text { Cr I } & 23 \\ 3391.294 & & \text { Gd II } & 73 \\ 3391.303 & & \text { Fe II } & 11 \\ 3391.372 & & \text { Cr I } & 25 \\ 3391.434 & & \text { Cr II } & \\ 3391.84 & \text { P } & \text { Fr I } & 67 \\ 3391.85 & & \text { A III } & \\ 3391.96 & & \text { Zr II } & \end{array}$

3391. 989

3392.018

3392.040

3392.304

3392.530

3392.652

3392.659

3992.713

3392.78

3392.89

3392.992

3383.00

3393.12

3383.382

3393.46

3393.609

3393.630

3393. 641

3393.86

3593. 916

3393.920

3394.086

3394.26

3394.29

3394.32

3394. 37

3394.58

3394.583

3394.63

3394.916

3394.92

3304.90

3395. 120

3395.338

$3395.37 \mathrm{C}$

3395.62

3395.87
3395.90

3996. 184

3396. 187

3396.34

3386.386

3396.467

3386.60

3386.58

3396.66

3396.71

3396.83

3396.85

3396. 878

3397.07

3397.221

3397.498

3387.600

3397.680

3387.642

3397.77

3397.82

3387.69

3397.80

3388.12

3398. 226

3398. 358

3398. 634

3388.811

3399.250

3399.336

3399.36

3399.406
Multiplet No.

I

Type Element Multiplet No.

Cr II 100

3399.54

3398.80

3399.991

3400.08

3400.110

3400.395

3400.471

3401.067

3401.166

3401.521

3401.617

3401.740

3401.76

3401.90

3401.913

3402.064

3402.072

3402. 256

3402.32 P $\quad \mathrm{Fe} I I$

3402.422

3402.43

3402.464

3402.52

3402.571

3402.87

3403.081

3403.159

3403.29

3403.29 P

3403.299

3403.322

3403.368

3403.432

3403.61

3403.58

3403.59

3403.69

3404.301

3404.34

3404.43

3404.43

3404. 755

\$404.77

3404.84

3404.923

3404.97

3405.038

3406.084

3405.120

3405. 160

3405.50

3405. 74

3405.83

3405.934

3406.97

3405.977

3406.06

3406.17 P

4406.18 Fe I

3406.442 Fe III

3406.76 P Fe II

3400.803 Fe I

3406.837

3406. 88

3407.00

207.00

$\begin{array}{ll}3407.205 & \text { Fe I } \\ & \text { T1 II }\end{array}$

3407.22

Cr I

3407.30

3407.38

3407.461

3407.63

3407.66

3407.61

3407.7

3407.76

3407.960

N1 II

0 II

Fe I

ad 1

ad 1

I II

He II
Mn I

100
1
12
22
67
4
46
42
8
107
26
44
106
4
9
167
47
123
1,149
614

I $\boldsymbol{A}$

Type Element

Mul tiplet No.

3409.177 Co I

3409.20

3409.297

3409.36

$3409.40 \quad P \quad F e$ I

3409.578 N1 I

$3409.60 \quad P \quad$ Cr II

3409.646 Co I

$3409.75 \quad 0$ IV

3409.78 P $\quad$ Cr II

3409.809 T1 II

$\begin{array}{llll}3409.84 & 0 & \text { II } & 44 \\ 3409.87 & Y & \text { II }\end{array}$

$3410.031 \quad$ Fe I $\$ 342$

$3410.171 \quad$ Fe I 735

3410.18 HF II 30

3410.26 Zr II

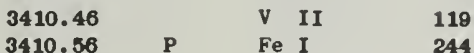

3410.74 Fe III 61,62

3410.805 Fe I

3411.01 Cr

$3411.134 \quad$ Fe I 298

3411.353 Fe I 301

$\begin{array}{llll}3411.68 & P & \text { TI II } & \end{array}$

3411.76 La II 155

$\begin{array}{lll}3411.76 & & 0 \text { IV } \\ 3411.88 & P & \mathrm{Fe} I\end{array}$

$3412.020 \quad$ Gd II

$3412.339 \quad$ Co $I$ I

$\mathbf{3 4 1 2 . 4 7} P$ P 1 I

3412. 683 Gd II $\quad 70$

3418.70

3412.934

3413.13

3413.135

3413.273

Gd II

Cb II

Ne II

Fe I

Gd II

3413.39

2r II

$\begin{array}{llll}3413.46 & P & N 1 & I\end{array}$

$3413.478 \quad N 1$

3413.71

3413.74

3413.939

3414.02

3414.144

3414. 192

3414.207

$\begin{array}{ll}0 & \text { IV } \\ \text { HF II }\end{array}$

N1 I

T1 II

Fe II

$V$ II
Gd II

5414. 46

3414. 65

3414.66

3414. 766

3414.82

3414. 678

3416. 29

3415.47

3416.519

A II

2r II

Zr I

Ne II

V II

0 III

Cr II

Co I

3416. 530

F. I

3415.67

3415. 78

3416.021

1416.

3416.674

3416.688

3416.87

$\$ 416.848$

3416.957

N1 I

Co II

F. II

Fo

Bc I

Fe $\mathbf{l}$

No II

Gd II

3417.154

3417.273

3417.330

3417.353

\$417.353

3417.450

3417.673

3417.71

3417. 795

3417.842

Co

Fe I

Od II

Co $I$

Au I

Co II

Co I

Ne II

Co I
Fe I

\begin{tabular}{|c|c|c|c|}
\hline 3408.13 & 0 & I II & 15 \\
\hline 3408.136 & $\boldsymbol{N}$ & II & 7 \\
\hline 3408.14 & Pt & I & 4 \\
\hline 3408.676 & on & II & \\
\hline 3408.678 & $\mathrm{CO}$ & II & 3 \\
\hline 3408.765 & $\mathrm{Cr}_{\mathbf{r}}$ & II & 3 \\
\hline 3408.956 & $\mathbf{v}$ & II & 120 \\
\hline
\end{tabular}

72
15
4
3
3
8


I A Type Element Multiplet No.

\begin{tabular}{|c|c|c|c|c|}
\hline 3419.10 & & $2 r$ & II & 2 \\
\hline 3419.157 & & $\mathrm{Fe}$ & I & 576 \\
\hline 3419.25 & & $\mathbf{P}$ & II & 3 \\
\hline 3419.358 & & Sc & I & 21 \\
\hline 3419.49 & & $\mathrm{Fe}$ & III & 11,49 \\
\hline 3419.706 & & $\mathbf{F e}$ & I & 377 \\
\hline 3419.87 & & 0 & II & \\
\hline 3420.15 & & v & I I & 119 \\
\hline 3420.184 & & $\mathrm{Fe}$ & II & 89 \\
\hline 3420.474 & & Co & I & 42 \\
\hline 3420.54 & & $\mathbf{L a}$ & I I & 126 \\
\hline 3420.61 & & 0 & II & \\
\hline 3420.709 & & $\mathbf{v}$ & II & 105 \\
\hline 3420.741 & & N1 & I & 9 \\
\hline 3420.790 & & Co & I & 102 \\
\hline 3420.795 & & Mn & I & \\
\hline 3420.82 & & $\mathbf{K}$ & III & 1 \\
\hline 3421.029 & & Co & I & 119 \\
\hline 3421.20 & & $\mathbf{C r}_{\mathbf{r}}$ & II & 3 \\
\hline 3421.22 & $\mathbf{P}$ & $N_{1}$ & I & 105 \\
\hline 3421.24 & & $\mathbf{P d}$ & I & $\mathbf{3}$ \\
\hline 3421.342 & & N1 & I & 122 \\
\hline 3421.62 & & Cr & I I & 60 \\
\hline 3421.628 & & Co & I & 101 \\
\hline 3421.64 & & $\mathbf{A}$ & I I & 57 \\
\hline 3421.83 & & $\mathbf{K}$ & III & 4 \\
\hline 3421.97 & & $\mathrm{Fe}$ & III & 11 \\
\hline 3422. 332 & & N1 & I & 105 \\
\hline 3422.466 & & Gd & I I & 2 \\
\hline 3422.499 & & $\mathrm{Fe}$ & I & 444 \\
\hline
\end{tabular}

\subsection{6}

3422.661
3422.708

3422. 739

3422. 751

3422. 878

3422.900

3423. 172

3423.35

3423.711

3423.82

3423.853

3423.9

3423.92

3424.16

3424. 17

3424.284

3424.43
3424.500

3424. 592

3424.64

3424.82

3424.88

3425.009

3425.022

3425.070

3425.082

3425.09
3425.432

3425.57

3425.582

3425. 624

3425.630

3425.930

3425.96

3426

3426.09

3426.13

Fe I

T1 II

Cr II

Gd II

N1 I

Co I

Ti I

Co I

N1 I

Zr II

Co II

Ce II

La II

Gd II

I I

Fe II

Fe I

Cr II
Co I

Gd II

Zr II

Zr II

P II

Fe I

Eu. II

V I

Tm II

Cr II

o IV

Fe II

ad II

Tn II

Gd II

$\begin{array}{ll}\text { Cr } & \text { I } \\ 0 & \text { VI }\end{array}$

Fe I

Cr II

3426.20

3426. 208

3426. 342

3426.383

3426.562

3426.583

3426.637

3426.67
3426.81

3427.002

3427. 121

3427. 332

3427.362

3427.57

3428.01

3428.192

(420.

3428.41
3428.42

3428.467

122
120

121
I A Type Element Multiplet No.

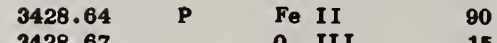

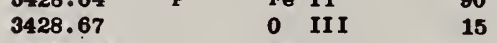

3428.746 $\quad$ Fe I $\quad 836$

3428.76

3428.916

3428.94

3428.955

3429.206

3429.42

Fe I

Al II

Cr II

T1 I

Sc I

3429.64

3429.80

P Fe I

$\begin{array}{lll}3429.82 & \text { P } & \text { Fe I } \\ 3430.15 & \text { P } & \text { Fe II }\end{array}$

3430.238 Gd II

340.20

3430.42

3430.44

3430.53

Zr I

Cr I I

$A$ II

3430.60

$\begin{array}{ll}\text { Zr } & \text { II } \\ 0 & \text { III }\end{array}$

3430.772

$\mathbf{3 4 3 0 . 8 7 4}$

$\begin{array}{llll}3430.88 & P & F e ~ I & 614\end{array}$

3431.195

3431.284

3431.358

$\begin{array}{lll}3431.45 & P & C_{T} \text { II } \\ 3431.57 & & \end{array}$

3431.582

Zr II
Co I

3431.59

3431. 69

3431.895

3432.023

3432.12

3432. 31

3432.318

3432.32

3432.41

Cr I

$\begin{array}{ll}\text { Cr } & \text { I } \\ \text { Fe } & \text { I } \\ \text { Cr } & \text { I }\end{array}$

Fe I

3432.64

3432. 708

3432.81

3432.97
$\mathbf{3 4 3 2 . 9 9 4}$

3433

3433.046

3433. 091

3433.30

3433. 44

Cr II
Cr I

Co I

Cr II

zr II

A I II
Cb II

La II

Fe III

Od II

0 VI

Ce II

Ce II

Pd I

3433. 558

3433.598

3433.767

3433.90

3434

3434.024

3434.020

3434. 112

3434.46

3434. 57

3434. 69

3494.883

3434. 95

3435.38

3435.408

3435.432

3435.488

3435.489

3435.555

3435.679

N1 I

$\begin{array}{ll}\text { Cr } & \text { I } \\ \text { v } & \text { II }\end{array}$

$\begin{array}{ll}\text { V } & \text { II } \\ \text { Zr } & \text { II }\end{array}$

0 VI

V II

Fo I

Cr I

V II

T1 I

Rh I

Fe I

v II

T1 II

T1 I
Cr I

Cr I

Sc I

Cr I

3435.819

Cr I

3436.112

3436.187

3436. 304

3436.393

3436. 737

3437.006

3437.046

$\mathbf{3 4 3 7 . 1 6}$

Fe II

Cr I

Ce II

$\checkmark$ II

Ru I

Ir I

Fe I
Zr II

42
25
99
168
21
77
21

107
244

540

89
91
17

17

59
11
15

3

3
120

12

21

8
59
6

53

376,676

53

377

8
53
102

102
8

58

107

113

67

22
8

23

249

3
11

19
52

134
58

9

104

300
52

134

121

2
776

133

98

53

53

21
52

53

614

91
62

94
79

4
3
539

3457.162

3437.280

3437.631

3437.680

3437.93

3437.958

3438

3438.10

3438. 23

N II

N1 I

Fe I

Co I

Cr II

ve I

I A

Type

Element

Multiplet No.

3438.306

3438.46

3438.713

3438.97

3438. 978 
I A Type Element MuItiplet No.

\begin{tabular}{|c|c|c|c|c|c|c|c|c|}
\hline 3447.430 & & $\mathrm{Cr}$ & I & 52 & 3457.047 & & Gd & II \\
\hline 3447.594 & & $\mathrm{He}$ & I & 7 & $\mathbf{3 4 5 7 . 0 8 8}$ & & $\mathbf{Y}$ & II \\
\hline 3447.760 & & $\mathrm{Cr}$ & I & 52 & $\mathbf{3 4 5 7 . 0 9 0}$ & & $\mathrm{Fe}$ & I \\
\hline 3447.98 & & 0 & I I & 27 & 3457.153 & & $\mathbf{v}$ & II \\
\hline 3448.05 & $P$ & 0 & III & 25 & 3457.16 & $\mathbf{P}$ & $\mathrm{Ne}$ & II \\
\hline 3448.19 & $\mathbf{P}$ & $\mathrm{Fe}$ & I & 186 & 3457.298 & & $\mathbf{T} 1$ & I \\
\hline 3448.255 & & Ti & $\dot{I}$ & 46 & 3457.494 & & $\mathbf{T 1}$ & I \\
\hline 3448.358 & & Co & I & 163 & 3457.512 & & $\mathrm{Fe}$ & I \\
\hline 3448.433 & & $\mathrm{Fe}$ & I I & 90 & 3457.56 & & $\mathrm{Zr}$ & II \\
\hline 3448.478 & & $\mathrm{Fe}$ & I & 444 & 3457.62 & & $\mathrm{Cr}$ & I I \\
\hline 3448.503 & & Sc & I & 21 & $\$ 457.809$ & & Mn & II \\
\hline 3448.542 & & Mo & II & 1 & 3457.99 & & 0 & II \\
\hline 3448.63 & $\mathbf{P}$ & $\mathrm{Fe}$ & I II & 27 & 3458.020 & & $\mathrm{~T} 1$ & I \\
\hline 3448.69 & & v & II & 118 & 3458.028 & & Co & I \\
\hline 3448.786 & & $\mathrm{Fe}$ & I & 372 & 3458.090 & & $\mathrm{Cr}$ & I \\
\hline 3448.82 & & $Y$ & II & 17 & 3458.13 & $\mathbf{P}$ & $\mathrm{Fe}$ & I I \\
\hline 3448.869 & & $\mathrm{Fe}$ & I & 242 & 3458.18 & & $\mathrm{Fe}$ & IIII \\
\hline 3448.967 & & I $\mathbf{r}$ & I & 1 & 3458.230 & & Al & I \\
\hline 3449.06 & $\mathbf{P}$ & Fe & I & 442 & 3458.304 & & $\mathrm{Fe}$ & I \\
\hline 3449.170 & & Co & I & 22 & 3458.474 & & N1 & I \\
\hline 3449.28 & & $\mathrm{Cr}$ & II & 111 & 3458.91 & & $\mathrm{Fe}$ & III \\
\hline 3449.441 & & Co & I & 22 & 3458.93 & & $\mathbf{Z r}$ & II \\
\hline 3448.5 & $\mathbf{P}$ & Mn & II & 9 & 3459.03 & & T1 & II \\
\hline 3449.616 & & $\mathrm{Gd}$ & II & 7 & 3459.07 & & 0 & II \\
\hline 3449.874 & & T1 & I & 46 & 3459.29 & & $\mathrm{Cr}$ & II \\
\hline 3450.00 & $\mathbf{P}$ & $\mathrm{Cr}$ & I & 90 & 3459.29 & $\mathbf{P}$ & $\mathrm{Fe}$ & I \\
\hline 3450.14 & $\mathbf{P}$ & $\mathrm{Fe}$ & I & 242 & 3459.374 & & $\mathrm{Ce}$ & III \\
\hline 3450.328 & & $\mathrm{Fe}$ & I & 82 & 3459.38 & & $\mathrm{Ne}$ & II \\
\hline 3450.376 & & Gd & II & 22 & 3459.429 & & $\mathrm{Fe}$ & I \\
\hline 3450.735 & & $\mathbf{T 1}$ & I & 46 & 3459.431 & & T1 & I \\
\hline 3450.84 & & $\mathrm{Cr}$ & II & 60 & 3459.52 & & 0 & III \\
\hline 3450.94 & & 0 & II I & 25 & 3459.61 & $\mathbf{P}$ & $\mathrm{Fe}$ & I \\
\hline 3451.046 & & v & II & 118 & 3459.911 & & $\mathrm{Fe}$ & I \\
\hline 3451.228 & & $\mathrm{Fe}$ & II & 208 & 3459.95 & $\mathbf{P}$ & $\mathrm{Fe}$ & I \\
\hline 3451.233 & & Gd & I I & 22 & 3459.95 & & $\mathrm{Zr}$ & II \\
\hline 3451.318 & & $\mathrm{Fe}$ & II & & 3459.98 & & 0 & II \\
\hline 3451.33 & & 0 & II I & 25 & 3460.03 & & $\mathrm{Cr}$ & II \\
\hline 3451.41 & & B & II & 1 & 3460.039 & & Mn & II \\
\hline 3451.614 & & $\mathrm{Fe}$ & II & 207 & 3460.31 & & La & II \\
\hline 3451.628 & & $\mathrm{Fe}$ & I & 139 & 3460.312 & & Mn & II \\
\hline 3451.66 & $\mathbf{P}$ & $\mathrm{Fe}$ & I & 241 & 3460.430 & & $\mathrm{Cr}$ & I \\
\hline 3451.88 & & $\operatorname{Re}$ & $I$ & 2 & 3460.47 & & $\mathrm{Re}$ & I \\
\hline 3451.914 & & Gd & II & 70 & 3460.719 & & Co & I \\
\hline 3451.915 & & $\mathrm{Fe}$ & I & 81 & 3460.76 & & $\mathbf{P d}$ & I \\
\hline 3452.18 & & Co & I & 160 & 3460.776 & & Gd & II \\
\hline 3452.18 & & La & II & 30 & $\$ 461.0$ & & $Y$ & II \\
\hline 3452.273 & & $\mathrm{Fe}$ & I & 25 & 3461.173 & & Co & I \\
\hline 3452.31 & $\mathbf{P}$ & $\mathrm{Fe}$ & I II & 49 & 3461.28 & & $\mathrm{Cr}$ & II \\
\hline 3452.33 & $\mathbf{P}$ & $\mathrm{Fe}$ & II & 89 & 3461.34 & & $N$ & IV \\
\hline 3452.470 & & T1 & II & 99 & 3461.38 & & $\mathrm{Eu}$ & II \\
\hline 3452.55 & & $\mathrm{Fe}$ & III & 88 & 3461.496 & & T1 & II \\
\hline 3452.670 & & Al & I & 2 & 3461.580 & & v & II \\
\hline 3452.890 & & N1 & I & 17 & 3461.652 & & N1 & I \\
\hline 3453.022 & & $\mathrm{Fe}$ & I & 301 & 3461.952 & & Gd & II \\
\hline 3453.087 & & v & I I & 132 & 3462.040 & & Rh & I \\
\hline 3453.10 & & $\mathrm{Ne}$ & I I & 21 & $3462.1^{\circ} 8$ & & $\mathrm{Tm}$ & I I \\
\hline 3453.17 & & La & II & 46 & 3462.353 & & $\mathrm{Fe}$ & I \\
\hline 3453.23 & & $\mathrm{Cr}$ & I & 253 & 3462.494 & & $\mathrm{Na}$ & I I \\
\hline 3453.31 & & 0 & II & 71 & 3462.65 & & Hf & II \\
\hline 3453.328 & & $\mathrm{Cr}$ & I & 52 & 3462.73 & & $\mathrm{Cr}$ & II \\
\hline 3453.514 & & Co & I & 22 & 3462.748 & & $M n$ & I \\
\hline 3453.595 & & $\mathrm{Fe}$ & I I & & 3462.804 & & Co & I \\
\hline 3453.654 & & T1 & I & 46 & 3462.808 & & $\mathrm{Fe}$ & I \\
\hline 3453.665 & & $\mathrm{Tm}$ & II & 7 & 3462.878 & & $M n$ & II \\
\hline 3453. 743 & & $\mathrm{Cr}$ & I & 52 & 3462.997 & & Gd & II \\
\hline 3453.78 & & $\mathrm{v}$ & II & 132 & 3463.02 & & $\mathrm{Zr}$ & I I \\
\hline 3453.84 & $\mathbf{P}$ & $\mathrm{Cr}$ & I & 90 & 3463.079 & & $\mathbf{v}$ & I I \\
\hline 3454 & $\mathbf{P}$ & $\mathbf{N}$ & IV & 7 & 3463.205 & & T1 & I \\
\hline $3454 \cdot 10$ & & $\mathbf{A}$ & I I & 44 & 3463.305 & & $\mathrm{Fe}$ & I \\
\hline 3454.145 & & Gd & II & 7 & $\mathbf{3 4 6 3 . 3 3 0}$ & & Mn & II \\
\hline 3454.16 & & $\mathrm{~N} 1$ & II & 1 & 3463.36 & & $\mathbf{N}$ & IV \\
\hline 3454.165 & & T1 & I & 168 & 3463.499 & & Co & I \\
\hline 3454.35 & & $\mathrm{Fe}$ & I I I & 86 & 3463.52 & & $w$ & II \\
\hline 3454.368 & & $\mathrm{Ce}$ & I I I & 2 & 3463.63 & & $\mathbf{A l}$ & II \\
\hline 3454.57 & & $\mathrm{Zr}$ & I I & 59 & 3463.831 & & v & II \\
\hline 3454.90 & & 0 & I II & 25 & 3463.974 & & $\mathrm{Fe}$ & I I \\
\hline 3454.904 & & Gd & II & 7 & 3463.984 & & Gd & II \\
\hline 3454.98 & & $\mathrm{Cr}$ & II & 136 & 3464.02 & & $\mathrm{Cr}$ & II \\
\hline 3455.04 & & $\mathrm{Mn}$ & I & 41 & 3464.043 & & $M n$ & II \\
\hline 3455.12 & & 0 & II I & 25 & 3464.132 & & $G d$ & II \\
\hline 3455.237 & & Co & I & 6 & $3464 \cdot 14$ & & $A$ & I I \\
\hline 3455. 281 & & $\mathrm{Cr}$ & I & 51 & 3464.17 & & V & II \\
\hline 3455.602 & & $\mathrm{Cr}$ & I & 51 & 3464.27 & & $\mathrm{Fe}$ & II I \\
\hline 3455.755 & & T1 & I & 46 & 3464.457 & & $\mathrm{Sr}$ & I I \\
\hline 3456.00 & $\mathbf{P}$ & $\mathrm{Fe}$ & I I & 4 & 3464.497 & & $\mathrm{Fe}$ & I I \\
\hline 3456.390 & & $\mathrm{~T} 1$ & I I & 99 & 3464.72 & & $\mathrm{Re}$ & I \\
\hline 3456.661 & & $\mathbf{T} 1$ & I & 134 & 3464.82 & & $\mathrm{Cr}_{\mathbf{r}}$ & I \\
\hline 3456.68 & & $\mathrm{Ne}$ & II I & 28 & 3464.914 & & $\mathrm{Fe}$ & I \\
\hline 3456.924 & & Co & I & 5 & 3465.037 & & Mn & II \\
\hline 3456.928 & & $\mathrm{Fe}$ & I I & 76 & 3465.245 & & $\mathrm{Cr}$ & \\
\hline
\end{tabular}


I A Type Element Nultiplet No.

I A Type Element Multiplet No.

I A Type Element Multiplet No.

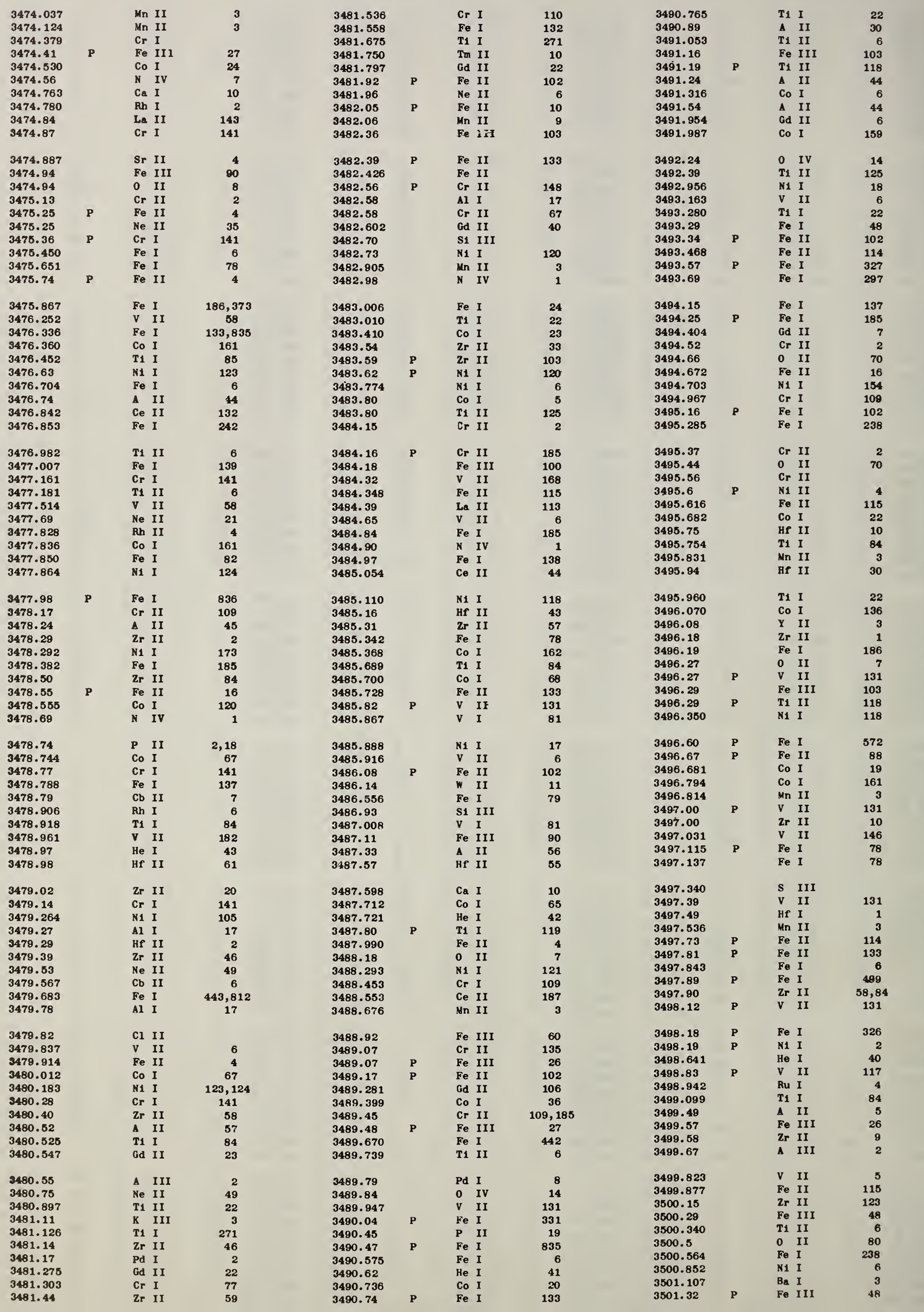


I $A$ 3509.843 Co I

\begin{tabular}{|c|c|c|c|c|}
\hline 3501.33 & & $\mathbf{z r}$ & & 14 \\
\hline 9601.416 & & 8 & II & 3 \\
\hline 9501.453 & & Ce & II & 87 \\
\hline 3601.67 & & 0 & II & 70 \\
\hline 3501.73 & & Co & II & 2 \\
\hline 3501.75 & & $\mathrm{Fe}$ & III & 26,48 \\
\hline 3502.2 & $\mathbf{P}$ & 0 & IV & 15 \\
\hline 3502.278 & & co & I & 21 \\
\hline 3502.381 & & He & I & 39 \\
\hline 3502.46 & $\mathbf{P}$ & $\mathrm{Fe}$ & I & 576 \\
\hline 3502.324 & & $\mathrm{Rh}$ & I & 2 \\
\hline 3502.595 & & N1 & I & 3 \\
\hline 3502.63 & & co & I & 6 \\
\hline 3502.85 & $\mathbf{P}$ & Fe & I & 577 \\
\hline 3502.954 & & $F$ & II & 3 \\
\hline 3502.998 & & co & I & 135 \\
\hline 3303.00 & & $\mathbf{P}$ & II & 2 \\
\hline 3503.095 & & $F$ & II & 3 \\
\hline 3503.206 & & Gd & II & 90 \\
\hline 3603.36 & & $\mathbf{C r}$ & II & $15 i$ \\
\hline 3503.38 & & $C_{r}$ & I & 109 \\
\hline 3503.474 & & $F_{\theta}$ & II & 4 \\
\hline 3603.68 & & $\Delta$ & III & 2 \\
\hline 3803.61 & & $\mathrm{Ne}$ & II & 28 \\
\hline 3503.717 & & co & I & 88 \\
\hline 3503.760 & & Ti & I & 22 \\
\hline 3503.96 & $\boldsymbol{F}$ & $F_{e}$ & III & 48 \\
\hline 3604.40 & & $\mathrm{Fe}$ & III & 48 \\
\hline 3504.432 & & $\mathbf{v}$ & II & 6 \\
\hline 3504.455 & & $F e$ & I & 371 \\
\hline
\end{tabular}

\begin{tabular}{|c|c|c|c|}
\hline 3509.843 & & Co I & 22 \\
\hline 3508.844 & & II II & 88 \\
\hline 3509.870 & & $F e I$ & 78 \\
\hline 3508.971 & & Mn II & 9 \\
\hline 3510.00 & & the II & 15 \\
\hline 3510.133 & & Gd II & 21 \\
\hline 3510.18 & $\mathbf{P}$ & Fe I & 836 \\
\hline 3510.262 & & Cb II & \\
\hline 3510.338 & & N1 I & 18 \\
\hline 3510.40 & & Cr I & 263 \\
\hline
\end{tabular}

3510.426

3510.443

3510.538

3511.227

3511.25

3511.55

3511.613

Co $I$

Fe I

Cr II

TI II

Sm II

Fe II

2r II

6
139
20

20
109

109
88
12

12
102
57

57

N1 I

152

3511.626
3511.748
3511.84
3511.93
3511.94
3512.08
3512.13
3512.219
$3512.239 \quad$
$3512.34 \quad P$

I1 I 22

$\begin{array}{lr}\text { Fe I } & 238 \\ \text { Cr II } & 2\end{array}$

$\begin{array}{lr}\text { Cr II } & 2 \\ \text { Fe IIJ } & 28\end{array}$

N1 I

$\mathrm{Fe} I$

v II

Ge II

Fe I

327

193

$\begin{array}{llll}3512.34 & P & \text { Fe III } & 26\end{array}$

3604.48

3504. 596

3504.728

3504.773

3504.868

3504.890

3505.088

3505. 138

3605.22

sb I

Co III

Co I

T1 I

Fo I

Ti II

Fo I

Co I

Bf II

2
6

138

3512.496

3512.511

9512.640

3512.68

3512.70

3512.74

3512.83

3512.95

Gd II

3505.44

TI II

3513.03

3605.47

3505.812

3608.614

3505.67

3505.690

3505.801

9506.02

3506.04

3506.23

Gr II

F I:

2r II

v I

I1 II

0 II

Fe II

88
498
160

7
64

3506.310

3506.40

3606.48

3506.498

3506.57

3506.68

3506.643

3506.843

5306.93

Co I

Fo I

2r II

Fe I

V. II

Fe I

Cr II 108,157

$\begin{array}{ll}\text { T1 } & I \\ V & I\end{array}$

Fo III

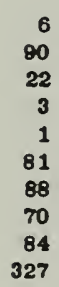

$3507.14 \quad P \quad$ Fo 


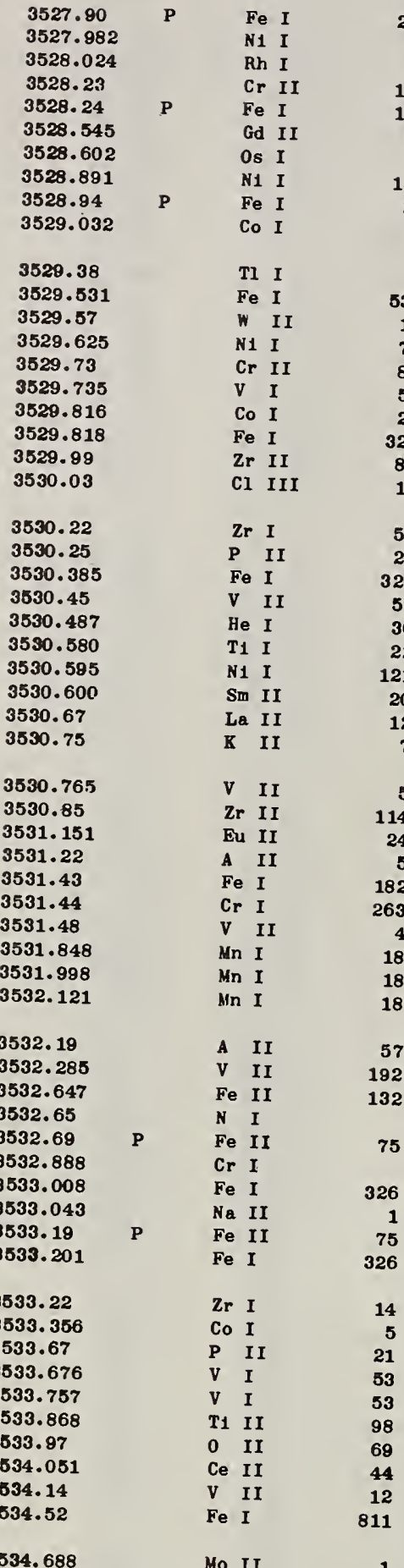

296
6
3
109
182
23
1
154
23
5

I A Type Element

Multiplet No.

2

12

76
89

83
22

22

326
84

52

52
21

326
57

57
36

22

121
20
12

7

5

114
24

182

4
18

18
18

57

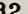

75

326

1
75

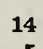

5
21

53

98

69
44

12
811

3534.688

3534.914

3535.04

3535. 16

3535. 18

3535. 33

3535. 408

3535.522

3535.54

3535.628

3535.653

3535.729

3536.30

3536. 556

3536.576

3536.820

3536.838

3536.89

3536.94

3537.243

3537.25

3537.491

3537.634

3537.729

3537.75

3537.896

3537. 99
Mo II

Fe I

$\mathrm{Mg}$ II

Zr I

V II

$\mathrm{Cb} \mathrm{I}$

A II

Trm II

HP II

Fe II

Sm I I

$P$ II

Fe I

Tm II

He I
F II

Cr I

Zr II

$\begin{array}{ll}\text { N1 } & \text { I } \\ \text { Cr } & \text { I }\end{array}$

Fe I

N1 I

Fo I

$\begin{array}{ll}\mathrm{Fe} & \text { I } \\ \mathrm{Ca} & \text { II }\end{array}$

Fe I

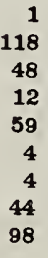

9

75

20

326

3
35

6

10

10
153
50

239

120
68

68
239
2
327

3538.142
3538.238
$\mathbf{3 5 3 8 . 3 1}$
$\mathbf{3 5 3 8 . 5 5}$
$\mathbf{3 5 3 8 . 7 7}$
$\mathbf{3 5 3 8 . 8 6}$
$\mathbf{3 5 3 9 . 0 0}$
$\mathbf{3 5 3 9 . 0 5}$
$\mathbf{3 5 3 9 . 0 8 6}$
$\mathbf{3 5 3 9 . 5 8 \varepsilon}$

Rh I

$\begin{array}{ll}\text { V } & \text { II } \\ \mathrm{Fe} & \text { I } \\ \mathrm{Fe} & \text { I } \\ \mathrm{Fe} & \text { I } \\ \mathrm{Mg} & \text { II } \\ \mathrm{Cr} & \text { I I } \\ \mathrm{Zr} & \text { II } \\ \mathrm{Ce} & \text { II }\end{array}$

8
4
775
137
811
12
157
102
118
1

3540.28

3540.530

3540.709

3540.961

3541.083

3541.22

3541. 341

Ne II

Fe I

Cr I I

Fe I

$\begin{array}{ll}\mathrm{Fe} & \mathrm{I} \\ \mathrm{Cb} & \mathrm{II}\end{array}$

Fe I

3541.44

3541. 765

3542.00

3542.076

3542 - 152

3542 . 243

3542 . 28

3542.480

3542.56
3542.65

3542.657

V I I

$\begin{array}{ll}\text { II IV } & \text { II }\end{array}$

50

329
89
45

45
23
4

4
326

47

3542.768

3542.90

3542.976

3543.09

3543. 16

3543. 256

3543. 352

3543. 39

3543.500

3543. 669

N1

Fe I

Eu II

Fe I
Ne II

$\begin{array}{ll}\mathrm{Ne} & \text { II } \\ \mathrm{V} & \mathrm{II}\end{array}$

$\begin{array}{ll}V & \text { II } \\ \text { Fe I }\end{array}$

$\begin{array}{ll}\text { Zr } & \text { II } \\ \text { V } & \text { I }\end{array}$

145

6

326

17

128

145

321

113
45

Gd II

Ne II

Co I

Fe I

Co I

Nd II

$\mathrm{Fe}$

$\begin{array}{ll}\text { V } & I \\ \text { Fe } & I\end{array}$

183

3543.948

3544.001

3544.631

3544.88

3544.985

3545.03

3545.16

3545.190

Fe

$\begin{array}{ll}\text { Rh } & \text { I } \\ \text { I } & \text { II }\end{array}$

Fe I

Fe I

Gd II

Co II

Co II

V II

v $I$

3545.603

3545. 639

3545. 797

3545.832

3545.84

3546.15

3546.190

3546.21

3546.22

3546. 707

A II

Ce II

Fe I

Fe I

Fe I

$\begin{array}{ll}\text { A } & \text { II } \\ \text { Cr } & \text { II }\end{array}$

Ce II

Fe I

Ne II

Co I

51

34

19
182

3547.029

3547.07

3547.10

3547.203

3547.69

3547.802

3547.98

3548.029

3548.037

3548.185

T1 I

133

Cr II $\quad$\begin{tabular}{rr}
134 \\
\hline
\end{tabular}

Fe I $\quad 321,613$

Zr I 18

Cr I

In I

$\begin{array}{ll}\text { Mn } & \text { I } \\ \text { Fe } & \text { I } \\ \text { N1 } & \text { I }\end{array}$

18
496

3548.202

3548.51

3548.55

3548.731

3549.02

3549.08

3549.27
3549.365

\section{Mn I}

3549.51

3549.61

3549.72

3549.868

3550.03

3550.1

3550.18

3550.46

3550.592

3550.635
Co $I$

A II

Fe II

Cr I

Y II

$\begin{array}{ll}\text { V } & \text { II } \\ \text { W } & \text { II }\end{array}$

T1 II

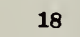

18

41
56
132
76
9
103
13
117
7

Zr II

$\begin{array}{ll}\mathrm{Mg} & \text { II } \\ \mathrm{S} & \text { II }\end{array}$

Fe I

A II

A. II

$\begin{array}{ll}\text { Zr } & \text { II } \\ \text { T1 II } & \text { Zr I }\end{array}$

Zr I

Cr I
6
56

239

154
51

1
76
5

53
70

44
321

2
536

106

131

183

27
41

63
69
613
13
18

18

20
3540.121

3545. 339 
FINDING LIST

Type Element Multiplet No.

I A Type Element Multiplet No.

I

Type

Element Multiplet No.

\begin{tabular}{|c|c|c|c|c|c|c|c|c|c|}
\hline 4 & & $\mathbf{A}$ & II & 106 & 3670.77 & & $\boldsymbol{A}$ & II & 68 \\
\hline-1 & & $\mathbf{2 r}$ & II & 82 & 3571.037 & & V & I & 122 \\
\hline 3 & & $\mathrm{Ne}$ & II & 31 & 3571.16 & & Pd & I & 1 \\
\hline$=.875$ & & Ti & II & 15 & 3571.228 & & $\mathrm{Fe}$ & I & 46 \\
\hline 3061.65 & & Hr & II & 1 & 3571.26 & & Ne & II & 31 \\
\hline 3561.751 & - & N1 & I & 2 & 3571.37 & & $\mathbf{C r}$ & I I & 107 \\
\hline 3561.810 & & $\mathbf{T} \mathbf{1}$ & II & 42 & 3571.64 & $\mathbf{P}$ & $\mathrm{Cr}_{\boldsymbol{r}}$ & II & 89 \\
\hline 3562.091 & & Ce & II & 36 & 3571.653 & & $\mathbf{v}$ & I & 122 \\
\hline 3562.097 & & Co & I & 115 & 3571.869 & & N1 & I & 5 \\
\hline 3562.19 & & $\mathbf{A}$ & II & 106 & 3571.933 & & Gd & II & 4 \\
\hline \$562. 29 & & $\mathrm{Cr}$ & I & 308 & 3571.87 & $\mathbf{P}$ & $\mathrm{Cr}$ & I & 157 \\
\hline 3562.48 & & $\mathrm{Cr}$ & I & 281 & 3571.995 & & $\mathrm{Fe}$ & I & 321 \\
\hline 9562.48 & & $\mathbf{P}$ & II & 22 & 3572.32 & $\mathbf{P}$ & Fe & I & 182 \\
\hline 3562.60 & $\mathbf{P}$ & $\mathrm{Fe}$ & I & 237 & 3572.46 & & $\mathrm{Fe}$ & III & 105 \\
\hline 3562.912 & & Co & I & 64 & 3572.47 & & $\mathbf{Z r}$ & II & 1 \\
\hline 3562.950 & & $\mathrm{He}$ & I & 33 & 3572.48 & & $w$ & II & 3 \\
\hline 3583.36 & & 0 & IV & 12 & 3572.523 & & Sc & II & 3 \\
\hline 3563.61 & $\mathbf{P}$ & $\mathrm{Fe}$ & I & 325 & 3572.60 & & $\mathrm{Fe}$ & I & 325 \\
\hline 3563.71 & & v & II & 4 & 3572.734 & & $\mathbf{P b}$ & I & 3 \\
\hline 3563.82 & & $\mathbf{C r}_{\boldsymbol{r}}$ & II & 134 & 3572.748 & & $\mathrm{Cr}_{\boldsymbol{r}}$ & I & 75 \\
\hline 3564.046 & & Gd & II & 52 & 3573.09 & & $\mathbf{Z r}$ & II & 9 \\
\hline 3564.11 & & $\mathrm{Fe}$ & I & 48 & 3573.27 & $\mathbf{P}$ & Ni & I & 123 \\
\hline 3564.115 & & Co & I & 159 & $35,73,403$ & & $\mathrm{Fe}$ & I & 673 \\
\hline 3664.30 & & $\mathrm{Cr}$ & I & 281 & 3573.516 & & V & I & 122 \\
\hline 3564.34 & & $\mathbf{A}$ & II & 43 & 3573.557 & & v & II & 78 \\
\hline 3564.51 & $\mathbf{P}$ & $\mathrm{Fe}$ & I & 183 & 3573.643 & & $\mathrm{Cr}_{\boldsymbol{r}}$ & I & 75 \\
\hline 3664.54 & $\mathbf{P}$ & $\mathrm{Fe}$ & II & 113 & 3573.737 & & $\mathbf{T 1}$ & II & 15 \\
\hline 3564.56 & $\mathbf{P}$ & $\mathrm{Fe}$ & I & 183 & 3573.842 & & $\mathrm{Fe}$ & I & 181 \\
\hline 3564.67 & $\mathbf{P}$ & N1 & I & 73 & 3573.896 & & $\mathrm{Fe}$ & I & 611 \\
\hline 3564.947 & & Co & I & 19 & 3574.038 & & $\mathbf{C r}_{\boldsymbol{r}}$ & I & 74,308 \\
\hline 3564.953 & & Cr & I & 308 & 3574.23 & & $\mathrm{Ne}$ & II & 9 \\
\hline 9565.02 & & $A$ & II & 57 & 3574.245 & & $\mathbf{T i}$ & $I$ & 247 \\
\hline 3565.15 & & $\mathrm{C}_{\mathrm{r}}$ & I & 50 & 3574.340 & & $\mathbf{v}$ & II & 78 \\
\hline 3565.31 & & $\mathrm{Cr}_{\boldsymbol{r}}$ & II & 107 & 3574.37 & $\mathbf{P}$ & $\mathrm{Fe}$ & I & 181 \\
\hline 3565. 326 & & Ti & II & 76 & 3574.38 & & $C_{r}$ & I & \\
\hline 3565. 381 & & $\mathrm{Fe}$ & I & 24 & 3574.64 & & Ne & II & 9 \\
\hline 3565.41 & & $\mathbf{Z r}_{r}$ & II & 102 & 3574.805 & & $\mathrm{Cr}_{\boldsymbol{r}}$ & I & 7.5 \\
\hline 9565.55 & & $\mathrm{Cr}_{\mathrm{r}}$ & I & 50,281 & 3574.935 & & $\mathbf{C r}$ & I & 74 \\
\hline 3565.583 & & $\mathrm{Fe}$ & I & 321,328 & 3574.867 & & Co & I & 21 \\
\hline 3565.83 & $\mathbf{P}$ & $\mathrm{Fe}$ & I & 571 & 3575.11 & & $\mathrm{Fe}$ & I & 321 \\
\hline 3585.84 & & $\mathrm{Ne}$ & II & 34 & 3575.249 & & $\mathrm{Fe}$ & I & 322 \\
\hline 3566.00 & & T1 & II & 42 & 3575.361 & & Co & I & 4 \\
\hline 3566.052 & & $\mathrm{Fe}$ & II & 155 & 3575.374 & & $\mathrm{Fe}$ & I & 496 \\
\hline 3566.10 & & $\mathrm{Cr}$ & I & 284 & 3575.69 & $\mathbf{P}$ & $C_{r}$ & II & 107 \\
\hline $3566 \cdot 10$ & & $\mathbf{z r}$ & I & 15 & 3575.79 & & $\mathbf{z}_{\boldsymbol{r}}$ & I & 12 \\
\hline 3566.148 & & $\mathrm{Fe}$ & II & 132 & 3575.850 & & $\mathrm{Cb}$ & I & 4 \\
\hline 3566.177 & & $\mathbf{v}$ & I & 45 & 3575.852 & & N1 & I & 120 \\
\hline 3566.177 & & $v$ & II & 4 & 3575.976 & & $\mathrm{Fe}$ & I & 321,328 \\
\hline 3566.31 & $\mathbf{P}$ & $\mathrm{Fe}$ & I & 127 & 3576.00 & & Cl & II & 78 \\
\hline 3566.37 & & $\mathrm{Cr}_{\boldsymbol{r}}$ & II & 76 & 3576.23 & $\mathbf{P}$ & $\mathbf{C r}_{\mathbf{r}}$ & II & 171 \\
\hline 3566. 372 & & N1 & I & 36 & 3576.340 & & Sc & II & 3 \\
\hline 3566.43 & & $\mathbf{P}$ & II & 22 & 3576.38 & & $\mathbf{T 1}$ & II & 76 \\
\hline 3566.472 & & Tm & II & 6 & 3576.44 & & $\mathrm{~T} 1$ & IV & \\
\hline 3566.59 & & $\mathrm{Fe}$ & I & 181 & 3576.62 & & $\mathbf{A}$ & II & 56 \\
\hline 3586.836 & & Sm & II & & 3576.760 & & $\mathrm{Fe}$ & I & $613 a$ \\
\hline 3587.045 & & Fe & I & 325 & 3576.762 & & N1 & II & 4 \\
\hline 3567.116 & & Gd & II & 89 & 3576.772 & & Gd & II & 51 \\
\hline 3567.171 & & $\mathbf{s}$ & II & 56 & 3576.88 & & $\mathbf{Z r}$ & II & 9 \\
\hline 3567.36 & & $\mathrm{Fe}$ & I & 183 & 3577.220 & & $\mathbf{v}$ & II & 78 \\
\hline 3567.654 & & ad & II & 51 & 3577.240 & & N1 & I & 3 \\
\hline 3567.701 & & Sc & II & 3 & 3577.260 & & Co & I & 41 \\
\hline 3567.84 & & Lu & I & 3 & 3577.458 & & $\mathrm{Ce}$ & II & 51 \\
\hline 3568.04 & & Cl & II & 78 & 3577.644 & & v & II & 68 \\
\hline 3568.14 & & $\mathrm{Zr}$ & II & 46 & 3577.857 & & v & II & 78 \\
\hline 3668.271 & & Sm & II & 47 & 3577.880 & & Mn & I & 8 \\
\hline 3568.36 & & $\mathrm{Cr}$ & I & 284 & 3578.03 & & Co & II & 1 \\
\hline 3568.423 & & $\mathbf{F e}$ & I & 321 & 3578.076 & & Co & $\mathbf{I}$ & 117 \\
\hline 3568.426 & & Co & I & 61 & 3578.22 & & $\mathrm{Zr}$ & II & 83 \\
\hline 3568.53 & & Ne & II & 9 & 3578.380 & & $\mathrm{Fe}$ & I & 321 \\
\hline 3668.828 & & $\mathrm{Fe}$ & I & 673 & 3578.588 & & Gd & II & 21 \\
\hline 3568.940 & & $\mathbf{v}$ & I & 122 & 3578.636 & & $\mathbf{v}$ & II & 78 \\
\hline 3588.97 & $\mathbf{P}$ & $\mathrm{Fe}$ & II & 113 & 3578.67 & $\mathbf{P}$ & $F_{e}$ & I & 127 \\
\hline 3568.977 & & $\mathbf{F e}$ & I & 294 & 3578.687 & & $\mathrm{Cr}_{\mathrm{r}}$ & I & 4 \\
\hline 3569.03 & & Hr & II & 7 & 3578.687 & & T1 & II & 117 \\
\hline 3668.083 & & $\mathbf{v}$ & I & 53 & 3578.89 & & La & II & 168 \\
\hline 3569.14 & & $\mathrm{Cr}$ & $\mathbf{I}$ & 281 & 3578.803 & & Co & I & 41 \\
\hline 3569.370 & & Co & I & 35 & 9579.029 & & Co & I & 41 \\
\hline 3569.493 & & Mn & I & 18 & 3579.549 & & od & II & 89 \\
\hline 3569.566 & & od & II & 51 & 3579.83 & $\mathbf{P}$ & Fo & I & 573 \\
\hline 3569.804 & & mn & I & 18 & 3680.10 & & Le & II & 155 \\
\hline 3569.94 & & $\Delta$ & II & 57 & 3580.277 & & $\mathrm{Cb}$ & I & 4 \\
\hline 3568.88 & & $\mathrm{Fe}$ & I & 135 & 3880.618 & & Gd & II & 89 \\
\hline 3570.041 & & Mn & I & 18 & 3580.71 & $\mathbf{P}$ & sc & II & 40 \\
\hline 3570.10 & & La & II & 142 & 3580.927 & & $8 c$ & II & 3 \\
\hline 3570.100 & & $\mathrm{Fe}$ & I & 24 & 3680.041 & & Sor & II & \\
\hline 3570.243 & & $\mathrm{Fe}$ & I & 326 & 3581.195 & & $F_{0}$ & I & 23 \\
\hline 3570.34 & & $\mathbf{P}$ & II & 18 & 3881.62 & & $\Delta$ & II & 36 \\
\hline 3570.57 & $\mathbf{P}$ & $\mathrm{Cr}_{\boldsymbol{r}}$ & II & 89 & 3581.645 & & $F_{e}$ & I & 205 \\
\hline $\begin{array}{l}3570.60 \\
3570.662\end{array}$ & $\mathbf{P}$ & $\begin{array}{l}\mathrm{Fe} \\
w\end{array}$ & $\begin{array}{l}\text { I } \\
\text { I }\end{array}$ & $\begin{array}{l}184 \\
3,5\end{array}$ & 3581.68 & & $\begin{array}{l}\text { La } \\
\text { C }\end{array}$ & II & $\begin{array}{r}136 \\
23\end{array}$ \\
\hline
\end{tabular}




\begin{tabular}{|c|c|c|c|c|c|c|c|c|c|}
\hline 3589.973 & & Mn & & 25 & 3601.666 & & Cr & & 74 \\
\hline 3580.08 & & $\mathrm{Fe}$ & $\mathbf{I}$ & 440 & 3601.692 & & Sm & II & 20 \\
\hline 3590.29 & $\mathbf{P}$ & $\mathrm{Fe}$ & $\mathbf{I}$ & 497 & 3601.782 & & Mn & I & 25 \\
\hline 3590,46 & & si & III & 7 & 3601.916 & & Al. & III & 1 \\
\hline 3580.468 & & Gd & II & 22 & 3601.93 & & $\mathbf{Y}$ & II & 9 \\
\hline 3690.47 & & Ne : & II & 32 & 3602.079 & & Co & I & 4 \\
\hline 3590.475 & & Sc & II & 3 & 3602.10 & & C1 & III & 1 \\
\hline 3580.598 & & $\mathrm{Ce}$ & II & 232 & 3602.10 & & $\mathrm{Fe}$ & I & 322 \\
\hline 3580.66 & & $\mathrm{Fe}$ & I & 953 & 3602.281 & & N1 & I & 3 \\
\hline 3500.87 & & c I & II & 23 & 3602.46 & & $\mathrm{Fe}$ & $\mathbf{I}$ & 322 \\
\hline 3590.98 & & Fe & $\mathbf{I}$ & 573 & 3602.534 & & $\mathrm{Fe}$ & I & 324,391 \\
\hline 3591.345 & & $\mathrm{Fe}$ & I & 321 & 3602.574 & & $\mathrm{Cr}_{\boldsymbol{r}}$ & I & 74 \\
\hline 3591.485 & & Fe & $\mathbf{I}$ & 568 & 3602.60 & $\mathbf{P}$ & $\mathrm{Fe}$ & II & 101 \\
\hline 3591.746 & & Co & I & 134 & 3602.61 & $\mathbf{P}$ & $\mathrm{Cr}_{\mathbf{r}}$ & I & 140 \\
\hline 3591.912 & & Gd & II & 51 & 3602.70 & $\mathbf{P}$ & $\mathrm{Fe}$ & I & $39 c$ \\
\hline 3592.012 & & v & II & 4 & 3602.77 & $\mathbf{P}$ & $\mathrm{Fe}$ & I & 370 \\
\hline 3592.486 & & Fe I & I & 237 & 3603.20 & & Eu & II & 16 \\
\hline 3592.595 & & Nd & II & & 3603.205 & & Fe & I & 295 \\
\hline 3592.603 & & Sm & II & 39 & 3603.46 & & $\mathbf{A}$ & II & 57 \\
\hline 3592.68 & & Fe & I & 669 & 3603.572 & & $\mathrm{Fe}$ & I & 181 \\
\hline 35़92. 709 & & Gd $]$ & II & 69,89 & 3603.61 & & $\mathbf{C r}_{\mathbf{r}}$ & II & 13 \\
\hline 3592.881 & & Fe & I & 77 & 3603.72 & & C1 & II & 78 \\
\hline 3592.92 & & $\mathbf{Y}$ & $\mathbf{I}$ & 8 & 3603.745 & & $\mathbf{C r}$ & $\mathbf{I}$ & 74 \\
\hline 3593.02 & $\mathbf{P}$ & Cr & II & 13 & 3603.80 & & $C_{r}$ & II & 13 \\
\hline 3593.022 & & Ru & I & 4 & 3603.828 & & $\mathrm{Fe}$ & I & 496 \\
\hline 3593.093 & & T1 & II & 76 & 3603.845 & & $\mathbf{T} 1$ & I & 20 \\
\hline 3593.15 & & Fe & III & 36 & 3603.86 & & $\mathrm{Cr}$ & II & 13 \\
\hline 3593.323 & & v $\mathbf{I}$ & II & 4 & 3603.88 & & $\mathrm{Fe}$ & III & 36 \\
\hline 3593.33 & & $\mathrm{Fe}$ & $\mathbf{I}$ & 671 & 3603.91 & & $\Delta$ & II & 43,68 \\
\hline 3593.445 & & Gd & II & 52 & 3604.21 & $\mathbf{P}$ & $\mathrm{Fe}$ & II & 175 \\
\hline 3593.488 & & Cr & I & 4 & 3604.284 & & $\mathbf{T 1}$ & I & 21 \\
\hline 3593.5259 & & Ne I & $\mathbf{I}$ & 7 & 3604.285 & & Stan & II & 47 \\
\hline 3593.60 & & N I & II & 26 & 3604.375 & & $\mathbf{v}$ & II & 130 \\
\hline 3593.76 & & A I & II & 117 & 3604.383 & & $\mathrm{Fe}$ & I & 323 \\
\hline 3593.80 & $\mathbf{P}$ & Fe I & I & 182 & 3604.469 & & Co & I & 136 \\
\hline 3594.10 & $\mathbf{P}$ & $\mathrm{Fe}$ & I & 154 & 3604.51 & & Cl & II & 78 \\
\hline 3594.13 & $\mathbf{P}$ & Sc 1 & II & 40 & 3604.54 & & $\mathrm{Cr}$ & I & 49,89 \\
\hline 3594.18 & & $\mathrm{Ne} I$ & II & 34 & 3604.95 & $\mathbf{P}$ & $\mathbf{C r}$ & I & 74 \\
\hline 3594.41 & & $\Delta \mathrm{I}$ & II & 23 & 3604.96 & $\mathbf{P}$ & $\mathrm{Fe}$ & I & 77 \\
\hline 3594.462 & & s & II & 16 & 3605.010 & & Co & I & 87 \\
\hline 3594.632 & & $\mathrm{Fe}$ & I & 322 & 3605.05 & $\mathbf{P}$ & $\mathbf{C r}$ & I & 49 \\
\hline 3594.87 & $\mathbf{P}$ & Co 1 & I & 136 & 3605.333 & & $\mathrm{Cr}$ & I & 4 \\
\hline 3594.870 & & Co 1 & I & 4 & 3605.370 & & Co & I & 20 \\
\hline 3594.89 & $\mathbf{P}$ & Se I & II & 40 & 3605.41 & $\mathbf{P}$ & $\mathbf{C r}$ & I & 49 \\
\hline 3595.119 & & Mn I & I & 8 & 3608.450 & & $\mathrm{Fe}$ & I & 294 \\
\hline 3595. 294 & & $\mathrm{Fe}$ & I & 322 & 3605.46 & & $Y$ & II & 46 \\
\hline 3595.66 & & $\mathrm{Fe} I$ & I & 322 & 3605.60 & $\mathbf{P}$ & $\mathrm{Fe}$ & I & 322 \\
\hline 3596.87 & & $\mathrm{Fe}$ & I & 181 & 3605.60 & $\mathbf{P}$ & $8 c$ & II & 40 \\
\hline 3595.991 & & $\mathbf{s}$ & II & 4 & 3605.62 & $\mathbf{P}$ & $\mathrm{Cr}_{\mathbf{r}}$ & I & 252 \\
\hline 3596.048 & & T1 & II & 15 & 3605.665 & & Gd & II & 4 \\
\hline 3596.179 & & Ru : & I & 4 & 3605.691 & & Mn & I & 25 \\
\hline 3596.194 & & Rh & I & 1 & 3605.89 & & $\Delta$ & II & 30 \\
\hline 3596.20 & & $\mathrm{Fe}$ & I & 181 & 3606.062 & & $\mathbf{T 1}$ & I & 303 \\
\hline 3596.351 & & Mo & II & 1 & 3606.18 & $\mathbf{P}$ & $\mathrm{Fe}$ & II & 175 \\
\hline 3596.510 & & Co : & I & 118 & 3606.38 & $\mathbf{P}$ & $\mathrm{Fe}$ & I & 233 \\
\hline 3596.55 & & T1 : & II & 76 & 3606.5224 & & $A$ & I & 5 \\
\hline 3587.05 & & Fe & I & 869 & 3606.53 & $\mathbf{P}$ & $\mathrm{Fe}$ & $\mathbf{I}$ & 133 \\
\hline 3597.147 & & Rh & $I$ & 5 & 3606.679 & & Fe & I & 294 \\
\hline 3597.24 & $\mathbf{P}$ & $\mathbf{F e}$ & I & 856 & 3606.786 & & $\mathbf{T 1}$ & I & 20 \\
\hline 3597.39 & $\mathbf{P}$ & Sc & II & 40 & 3606.852 & & N1 & I & 120,173 \\
\hline 3597.42 & & H $\mathbf{f}$ & II & 54 & 3607.04 & & Co & I & 67 \\
\hline 3597.50 & & Al. & II & 52 & 3607.08 & $\mathbf{P}$ & $F_{\theta}$ & II & 101 \\
\hline 3597.705 & & N1 & I & 18 & 3607.25 & $\mathbf{P}$ & $\mathrm{Cr}_{\mathbf{r}}$ & $\mathbf{I}$ & 140 \\
\hline 3598.196 & & $\mathrm{Ce}$ & II & 116 & 3607.30 & & $\mathbf{v}$ & II & 77 \\
\hline 3598.22 & & Fe & III & 105 & 3607.39 & & $\mathbf{Z r}$ & II & 83 \\
\hline 3598.71 & & Fe & I & 674 & 3607.637 & & Mn & I & 8 \\
\hline 3598.714 & & T1 & $\mathbf{I}$ & 69 & 3607.626 & & $\mathrm{Ce}$ & I I & 178 \\
\hline 3598.93 & & $\mathrm{Fe}$ & I & 668 & 3607.92 & $\mathbf{P}$ & $\mathrm{Cr}$ & I & 140 \\
\hline 3598.98 & & $\mathbf{F e}$ & I & 322 & $3608 \cdot 146$ & & $\mathrm{Fe}$ & I & 325,438 \\
\hline 3599.304 & & He & I & 30 & 3608.307 & & Co & I & 20 \\
\hline 3599.395 & & $\mathrm{Cr}$ & I & 89 & 3608.32 & & $\mathbf{v}$ & II & 242 \\
\hline 3599.442 & & He & $\mathbf{I}$ & 30 & 3608.401 & & Cr & I & 252 \\
\hline 3599.49 & & $\mathbf{F e}$ & III & 36 & 3608.49 & $\mathbf{P}$ & Fe & II & 175 \\
\hline 3599.530 & & N1 & I & 121 & 3608.494 & & Mn & I & 8 \\
\hline 3599.624 & & Fe & I & 809 & 3608.68 & $\mathbf{P}$ & $\mathrm{Cr}_{\mathbf{r}}$ & I & 140 \\
\hline 3599.91 & & $\mathbf{z r}$ & II & 123 & 3608.66 & & $\mathrm{Cr}$ & II & 133 \\
\hline 3599.974 & & $\mathrm{Ce}$ & II & 219 & 3608.7 & $\mathbf{P}$ & N1 & II & 4 \\
\hline 3600.22 & & $A$ & II & 115 & 3608.753 & & od & II & 69 \\
\hline 3600.48 & $\mathbf{P}$ & $\mathrm{Fe}$ & I & 498 & 3608.766 & - & Tw & II & 3 \\
\hline 3600.583 & & $\mathrm{Ce}$ & II & 236 & 3608.861 & & Fe & I & 23 \\
\hline 3600.74 & & $Y$ & II & 9 & 3608.89 & $\mathbf{P}$ & T1 & II & 76 \\
\hline 3600.803 & & Co & I & 63 & 3608.96 & & C & III & 10 \\
\hline 3600.83 & & Fe & III & 36 & 3609.04 & & Cr & I & 49 \\
\hline 3600.963 & & Gd & II & 68 & 3609.09 & & N & I I & 26 \\
\hline 3601.07 & & La & II & 44 & 3609.314 & & N1 & I & 16 \\
\hline 3601.16 & & T1 & I & 172 & 3609.46 & $\mathbf{P}$ & $\mathrm{Fe}_{\mathrm{e}}$ & I & 322 \\
\hline 3601.18 & & $\mathrm{Zr}$ & I & 13 & 3609.479 & & $\mathrm{Cr}$ & I & 49 \\
\hline 3601.42 & $\mathbf{P}$ & $\mathrm{Fe}$ & I & 127 & 3609.491 & & $8 \mathrm{~m}$ & II & 30 \\
\hline 3601.51 & & A & II & 4 & 3609.66 & & $\mathbf{P d}$ & I & 2 \\
\hline
\end{tabular}


I A Type Element Multiplet No.

\begin{tabular}{|c|c|c|c|}
\hline 3618.49 & & & II \\
\hline $\begin{array}{l}3618.62 \\
3618.769\end{array}$ & $\mathbf{P}$ & Fe & I \\
\hline $\begin{array}{l}3618.769 \\
3618.88\end{array}$ & & $\begin{array}{l}\text { Fe } \\
\text { C1 }\end{array}$ & I \\
\hline $\begin{array}{l}3618.88 \\
3618.81\end{array}$ & $\mathbf{P}$ & Fe & I \\
\hline 3618.824 & & v & II \\
\hline 3618.96 & $\mathbf{P}$ & $\mathbf{F e}$ & \\
\hline 3619.284 & & Mn & \\
\hline 3619.392 & & & \\
\hline 3618.460 & & Cr & \\
\hline 3619.514 & & cb & II \\
\hline 3619.68 & $\mathbf{P}$ & $\mathrm{Fe}$ & I \\
\hline 3619.76 & & Fe & \\
\hline 3620.00 & $\mathbf{P}$ & Fe & \\
\hline 3620.23 & & Fe & \\
\hline 3620.27 & & $\mathrm{Fe}$ & III \\
\hline 3620.422 & & Co & I \\
\hline 5620.496 & & $\mathbf{v}$ & II \\
\hline 3620.82 & & $\mathbf{A}$ & II \\
\hline 3620.87 & $\mathbf{P}$ & $\mathbf{F e}$ & I \\
\hline 3620.88 & $\mathbf{P}$ & $\mathrm{Fe}$ & I \\
\hline 3620.96 & & $Y$ & I \\
\hline 3621.06 & & $A$ & II \\
\hline 3621.19 & $\mathbf{P}$ & Fe & \\
\hline 3621.203 & & $\mathbf{v}$ & II \\
\hline 3621.22 & & Co & II \\
\hline 3621.229 & & Sm & II \\
\hline 3621.273 & & $\mathbf{F e}$ & II \\
\hline 3621.463 & & $\mathrm{Fe}$ & I \\
\hline 3621.51 & & $C_{r}$ & II \\
\hline
\end{tabular}

$\begin{array}{lllr}3621.718 & & \text { Fe I } & 80 \\ 3622 & \text { P } & \text { O VI } & \\ 3622.00 & \text { F } & \text { Fe I } & 233 \\ 3622.001 & & \text { Fe I } & 29 \\ 3622.145 & & \text { Ce II } & 71 \\ 3622.15 & & \text { A II } & 42 \\ 3622.289 & & \text { V II } & 144 \\ 3622.45 & & \text { Cr II } & 17 \\ \$ 622.504 & & \text { 8 II II } & \\ 302.04 & & \text { B II } & \end{array}$

3022.64 Bu II 18

3022.69 CI III

3622.81 P Fe II

2022.850

3623.03

3623.187

$\begin{array}{ll}3623.187 & \text { Fo I } \\ 3623.316 & \text { Sin II }\end{array}$

Fo I

3623.77

\$623. 792

พ623.897

3623.837

3623.87

5623.98

3624.00

$\$ 624.06$

3624.11

3624.25

3624.30

3624.397

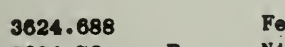

$3624.72 \quad P \quad$ Ni

4624.739

3624.82

3624.880

3624.856

3625.140

3625.20

3625.30

$\mathbf{3 6 2 5 . 6 0 8}$

3028.92

3626.020

3626.085

3826. 32

3626.63

3627.014

3627.06

3027.168

3627.36

3627.63

9627.71

3627.713

3627.971

$\mathbf{3 2 8 . 0 6}$

3628.094

3028.247

3628.71

3628.82

5628.83
I A Type Element Multiplet No.

I A

Type Element

Multiplet No.

$\begin{array}{lllr}3629.10 & \text { P } & \text { Sc II } & 18 \\ 3629.12 & & \text { Zr II } & 113 \\ 3629.51 & & \text { Gd II } & 69 \\ 3629.741 & & \text { Mn I } & 8 \\ 3629.906 & & \text { N1 I } & 182 \\ 3629.99 & \text { P } & \text { Fe II } & 111 \\ 3630.03 & & \text { Zr II } & 10 \\ 3630.26 & \text { P } & \text { N1 I } & 180 \\ 3630.353 & & \text { Fe I } & 323 \\ 3630.67 & \text { P } & \text { Fe I } & 126\end{array}$

$\begin{array}{lllr}3637.15 & & \text { La II } & 35 \\ 3637.251 & & \text { Fe I } & 180 \\ 3637.319 & & \text { Co I } & 117 \\ 3637.73 & & \text { Fe I } & 228 \\ 3637.83 & & \text { 8b I } & \\ 3637.862 & & \text { Fe I } & 385 \\ 3637.89 & & \text { A II } & \\ 3637.866 & & \text { II I } & 18 \\ 3638.15 & & \text { S II I } & \\ 3638.16 & \text { P } & \text { Fe I } & 32 \\ 3638.296 & & \text { Fe I } & 29 \\ 3638.49 & \text { P } & \text { TI I } & 11 \\ 3638.70 & & \text { O II I } & 3 \\ 3638.767 & & \text { Sm II } & \\ 3638.80 & & \text { Pt I } & \\ 3639.024 & & \text { V I } & 83 \\ 3639.14 & \text { P } & \text { Mn I } & \\ 3639.19 & & \text { CI I I } & 77 \\ 3639.443 & & \text { Co I } & 6 \\ 3639.568 & & \text { Pb I } & \end{array}$

Sc II

$3630 \cdot 740$

Ca I

Ca I

Fe I

3630.974

3631.126

3631.184

3631.266

3631.41

3631.464

Ce II

$\mathrm{Na}$ II

$\begin{array}{ll}\text { Co } & \text { I } \\ P & \text { II }\end{array}$

$\mathrm{Fe} \mathrm{I}$

18
13
69
8
82
11
10
80
323
126

2
9
9
322

88

2

22

$\begin{array}{llll}3631.48 & P & \text { Cr II } & 170\end{array}$

$\begin{array}{llll}3631.482 & \text { V II } & 76 \\ 3031.48 & \text { Cr II } & 12\end{array}$

3631.72

3631.948

3631.959

Cr II

Co I

3632.022

3632.042
3632.106

T1 I

8 III

Fe I

1

3632.126

3632.292

$3632: 46$

3632.558

3632.76

3632.839

3632.839

3632.879

3633.07

Ce II

114

III 76

Fe II

Cr I

Fe I

Co I

Cr I

3633.13

Fe I

Po I

$\begin{array}{llll}3633.16 & P & \text { Cr II } & 147\end{array}$

3635.340 Co I 116

3633.468 T1 I

$3633.49 \quad 2$ II $\quad 102$

$\begin{array}{llll}3633.64 & P & F_{0} I & 396\end{array}$

$3633.837 \quad$ Fe I 440

$\begin{array}{llll}3633.89 & P & \text { TI II } & 116\end{array}$

3634.10 P Forb ge I

$\begin{array}{llll}3634.10 & P \text { Forb } & \text { Ye I } & \\ 3634.13 & & V & \end{array}$

3634.235

He I

3634.280

8m II

3634.326

3634.373

$3634.62 \quad P$

$3634.698 \quad$ Fe I

3634.71

3634.713

3634. 757

3634.83

639.76

3639.802

3639.86

3640.388

3640.38

3640.891

3641.01

3641.096

3641.22

3641.330

3641.39

3641.42

3641.45

3641.470

3641.641

3641.66

3641.784

3641.830

3641.986

Sc II

Cr I

A II

Fe I

Cr I

F II

Cr I

V I

180

228

385

18

24

3642.387

3642.675

3642.785

3642.798

3643.181

3643.22

3643.4

3643.47

3643.716

T1 II

Gd II

Fe I

Cr I

N1 I

La II

Co I

Cr I

N1 I

T1 I

Sc II

F II

Co I

C. II

I II

Mo II

Fe I

$\begin{array}{lllr}3643.80 & \text { P } & \text { Fe I } & 67 \\ 3643.82 & \text { P } & \text { Fe I } & 4 \\ 3643.864 & & \text { V I } & 83 \\ 3643.89 & & \text { Ne II } & \\ 3643.841 & & \text { N1 I } & 17 \\ 3644.12 & \text { P } & \text { Cr II } & 98 \\ 3644.19 & \text { P } & \text { Fe II } & 13 \\ 3644.36 & & \text { HF II } & \\ 3644.410 & & \text { Ca I } & \\ 3644.47 & & \text { He II } & \end{array}$

3634.928 8ः II 6

$3634.041 \quad N 1$ I 33

3636.08 P $\quad$ Fe I

3636. 13 A II

3635.144 Mo II

3038.18

Fo I

3636.202

T1 I

$\begin{array}{lll}3636.28 & P & \text { Fe } \\ 3635.281 & & \text { Cr }\end{array}$

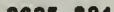

I II

919
4

3844.699

3644.70

3644.766

3644.798

3644.86

3644.87

3644.990

3645.090

3646.190

3645.20

3645.280

3645.311

3645.387

3646.43

3645.440

3645.484

3645.69

3646.596

3645.62

T1 I

Cr II

Ca I

Fe I

Ne II

I1 I

Ca I

Fe I
Co I

9
, 495

3645.78

3645.822

3645.905

3645.981

3646. 10

3646. 161

3646.19

3646. 188

3646.75

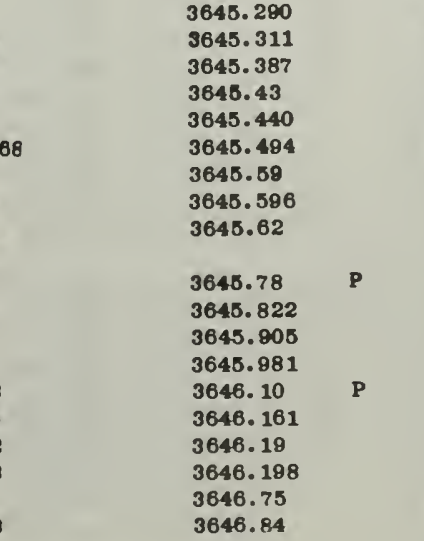

\begin{tabular}{|c|c|}
\hline I I & 18 \\
\hline I & 47 \\
\hline II & 116 \\
\hline II & 23 \\
\hline 1 & 298 \\
\hline I & 47 \\
\hline II & 11 \\
\hline I & 47 \\
\hline I & 115 \\
\hline II & 111 \\
\hline I I & 52 \\
\hline II & 86 \\
\hline II & 1 \\
\hline I & 323 \\
\hline I & 47 \\
\hline I & 6 \\
\hline II & 136 \\
\hline I & 99 \\
\hline I & 47 \\
\hline II & 11 \\
\hline I & 75 \\
\hline I & 18 \\
\hline II & 2 \\
\hline I I & 11 \\
\hline I & 98 \\
\hline II & 1 \\
\hline II & 56 \\
\hline I I & 1 \\
\hline I & 385 \\
\hline I & 233 \\
\hline
\end{tabular}

$\begin{array}{llr}\text { O III } & 35 \\ \text { Sw II } & 19 \\ \text { 8c II } & 2 \\ \text { S. II } & 35 \\ \text { LE II } & 14 \\ \text { Co I } & 97 \\ \text { Fo I } & 323,391,441 \\ \text { Cr I } & 48 \\ \text { V } & \text { I } & 137 \\ \text { Od II } & 17 \\ \text { Fo II } & 112 \\ \text { Fe I } & 496 \\ \text { V } & \text { II } & 76 \\ \text { H } & & 7 \\ \text { Fe I } & 324 \\ \text { Cr I } & 48 \\ \text { Od II } & 2 \\ \text { T1 I } & 18 \\ \text { Bu II } & 13 \\ \text { O } & \text { III } & 36\end{array}$


I A Type Element Multiplet No.

I A Type Element Multiplet No.

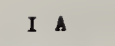

Type Element

Multiplet No.

\begin{tabular}{|c|c|c|c|c|c|c|c|c|c|}
\hline 3646.848 & & $\mathbf{v}$ & II & $\begin{array}{r}180 \\
66\end{array}$ & 3656.261 & & $\mathrm{Cr}$ & I & 46 \\
\hline 3647.081 & & $\begin{array}{l}\mathrm{Ce} \\
\mathrm{Co}\end{array}$ & I I & $\begin{array}{r}66 \\
118\end{array}$ & 3656.319 & & Al & II I & 51 \\
\hline 3647.40 & & $\begin{array}{l}\text { Co } \\
\text { Cr }\end{array}$ & I & $\begin{array}{r}118 \\
1\end{array}$ & $\begin{array}{l}3656.35 \\
3656.50\end{array}$ & $\begin{array}{l}\mathbf{P} \\
\mathbf{P}\end{array}$ & $\begin{array}{l}\mathrm{Fe} \\
\mathrm{Fe}\end{array}$ & I & 323 \\
\hline 3647.427 & & $\mathrm{Fe}$ & I & $\begin{array}{r}1 \\
46\end{array}$ & 3656.61 & & s & III & $\begin{array}{r}111 \\
6\end{array}$ \\
\hline 3647.43 & $\mathbf{P}$ & $\mathrm{Fe}$ & I & 497 & 3656.666 & & $\mathbf{H}$ & & 7 \\
\hline 3647.56 & $\mathbf{P}$ & $\mathrm{Fe}$ & $\mathbf{I}$ & 574 & 3656.706 & & $\mathbf{v}$ & I & 115 \\
\hline 3647.658 & & Co & I & 4 & 3656.73 & $\mathbf{P}$ & T1 & I & 118 \\
\hline 3647.71 & & $N i$ & I & & 3656.77 & $\mathbf{P}$ & $\mathrm{Fe}$ & I I & 131 \\
\hline 3647.77 & & Lu & I & & 3656.95 & & Cl & III & 1 \\
\hline 3647.84 & $\mathbf{P}$ & $\mathrm{Fe}$ & I & 569 & 3656.962 & & Co & $I$ & 21 \\
\hline 3647.844 & & $\mathrm{Fe}$ & I & 23 & 3657.143 & & $\mathrm{Fe}$ & I & 130 \\
\hline 3648.07 & & $\mathrm{Cl}$ & II & 77 & 3657.269 & & $\mathrm{H}$ & & 7 \\
\hline 3648.22 & $\mathbf{P}$ & $\mathrm{Fe}$ & I & 978 & 3657.574 & & Ru & II & 1 \\
\hline 3648.35 & & Hf & II & 39 & 3657.59 & & $w$ & II & 2 \\
\hline 3648. 534 & & $\mathbf{C r}$ & I & 47 & 3657.698 & & N1 & I & 183 \\
\hline 3648.80 & $\mathbf{P}$ & $\mathbf{T} 1$ & II & 74 & 3657.89 & & $\mathrm{Fe}$ & I & 395 \\
\hline 3648.86 & & $\mathbf{T} 1$ & I I & 83 & 3657.926 & & H & & 7 \\
\hline 3648.966 & & $\mathbf{v}$ & I & 115 & 3657.94 & & $\mathrm{Cr}_{\mathbf{r}}$ & II & 170 \\
\hline 3648.997 & & $\mathrm{Cr}$ & I & 47 & 3657.987 & & $\mathrm{Rh}$ & I & 1 \\
\hline 3649.01 & $\mathbf{P}$ & T1 & I I & 14 & 3658.02 & $\mathbf{P}$ & $\mathrm{Fe}$ & I & 438 \\
\hline 3649.184 & Forb & Al & II & 12 & 3658.097 & & $\mathbf{T} 1$ & I & 19 \\
\hline 3649.20 & & 0 & II I & 35 & 3658.19 & & $\mathrm{Cr}$ & II & 98,146 \\
\hline 3649.232 & Forb & Al & I I & 12 & 3658.266 & & v & II & 116 \\
\hline $3649: 304$ & & $\mathrm{Fe}$ & I & 5 & 3658.3 & & Al & III & 20 \\
\hline 3649.329 & & Co & $\mathbf{I}$ & 146 & 3658.38 & & Cl & II & 7 \\
\hline 3649.44 & & Gd & I I & 69 & 3658.55 & & $\mathrm{Fe}$ & $\mathbf{I}$ & 231 \\
\hline 3649.508 & & $\mathrm{Fe}$ & I & 291 & 3658.641 & & H & & 7 \\
\hline 3649.527 & & Sm & II & 47 & 3659.02 & & H f & II & 44 \\
\hline 3649.70 & $\mathbf{P}$ & $\mathrm{Fe}$ & I & 391 & 3659.227 & & $\mathrm{Ce}$ & II & 54 \\
\hline 3660.031 & & $\mathrm{Fe}$ & I & 394 & 3659.423 & & $\mathrm{H}$ & & 6 \\
\hline 3650.13 & & $\mathrm{Cl}$ & II & 7 & 3659.516 & & $\mathrm{Fe}$ & I & 180 \\
\hline 3650.144 & & $\mathrm{Hg}$ & I & 3 & 3659.602 & & $\mathrm{Cb}$ & II & \\
\hline 3650.188 & & Sm & II & 25 & 3659.765 & & T1 & II & 75 \\
\hline 3650.19 & & La & II & 12 & 3659.84 & & Cl & II & 7 \\
\hline 3650.19 & & $\mathbf{N}$ & I & & 3659.93 & & $\mathrm{Ne}$ & II & 33 \\
\hline 3650.280 & & $\mathrm{Fe}$ & I & 180 & 3660.279 & & H & & 6 \\
\hline 3650.37 & & $\mathrm{Cr}$ & II & 156 & 3660.33 & & $\mathrm{Fe}$ & I & 323 \\
\hline 3650.45 & & $\mathbf{Y}$ & I I & 75 & 3660.404 & & Mn & I & \\
\hline 3650.70 & & 0 & III & 35 & 3660.41 & $\mathbf{P}$ & $\mathrm{Fe}$ & I & 229 \\
\hline 3650.73 & & $\mathbf{Z r}$ & II & 146 & 3660.44 & & $\mathbf{A}$ & II & 116 \\
\hline 3650.90 & & $\mathbf{A}$ & II & 43 & 3660.631 & & $T 1$ & I & 18 \\
\hline 3650.95 & & Gd & II & 69 & 3660.641 & & $\mathrm{Ce}$ & II & 42 \\
\hline 3650.988 & & Sm & I I & 51 & 3660.85 & & $\mathrm{Fe}$ & II I & 93 \\
\hline 3651.03 & $\mathbf{P}$ & $\mathrm{Fe}$ & I & 571 & 3660.92 & & $\mathbf{Z r}$ & II & 32 \\
\hline 3651.065 & Forb & Al & II & 12 & 3661.05 & & Hf & II & 26 \\
\hline 3651.096 & & Al & II & 12 & 3661.17 & $\mathbf{P}$ & $\mathrm{Fe}$ & II & 111 \\
\hline 3651.10 & & $\mathrm{Fe}$ & I & 322,674 & 3661. 20 & & $\mathbf{Z r}$ & I & 12 \\
\hline 3651.182 & & $\mathrm{Cb}$ & II & 4 & 3661.221 & & H & & 6 \\
\hline 3651.254 & & Co & I & 85 & 3661.25 & $\mathbf{P}$ & $\mathrm{Fe}$ & I & 952 \\
\hline 3651.469 & & $\mathrm{Fe}$ & I & 295 & 3661.33 & & $\mathbf{Z r}$ & II & 102 \\
\hline 3651.50 & & $\mathbf{Z r}$ & II & 122 & 3661.353 & & Ru & I & 2 \\
\hline 3651.67 & $\mathbf{P}$ & $\mathbf{N} 1$ & I & 153 & 3661.36 & & $\mathrm{Fe}$ & $\mathbf{I}$ & 179 \\
\hline 3651.68 & & $\mathrm{Cr}$ & II & 1 & 3661.365 & & $8 m$ & II & 6 \\
\hline 3651.798 & & Sc & II & 2 & 3661.383 & & $\mathbf{v}$ & II & 191 \\
\hline 3651.90 & $\mathbf{P}$ & $\mathbf{T} 1$ & I & 118 & 3661.44 & & $\mathrm{Cr}$ & II & 156 \\
\hline 3651.971 & & $\mathrm{He}$ & I & 27 & 3661.73 & & Hr & II & 62 \\
\hline 3652.119 & & $\mathrm{He}$ & I & 27 & 3661.951 & & N1 & I & 16 \\
\hline 3652.26 & $\mathbf{P}$ & $\mathrm{Fe}^{\prime}$ & I & 494 & 3662.005 & & $\mathbf{s}$ & I I I & 6 \\
\hline 3652.541 & & Co & I & 4 & 3662.08 & & $\mathbf{L a}$ & II & 12 \\
\hline 3652.65 & & $\mathrm{Fe}$ & I I I & & 3662.14 & & $\mathbf{Z r}$ & II & 101 \\
\hline 3652.748 & & $\mathrm{Fe}$ & II & & 3662.158 & & Co & I & 115 \\
\hline 3652.81 & $\mathbf{P}$ & T1 & II & 116 & 3662.237 & & T1 & II & 75 \\
\hline 3653.00 & & 0 & III & 35 & 3662.258 & & H & & 6 \\
\hline 3653.108 & & $\mathrm{Ce}$ & I I & 38 & 3662.26 & & Gd & II & 4 \\
\hline 3653.35 & $\mathbf{P}$ & $\mathrm{Fe}$ & I & 229,324 & 3662.39 & & $\mathbf{C r}$ & I & 46 \\
\hline 3653.497 & & T1 & I & 19 & 3662.62 & $\mathbf{P}$ & $\mathrm{Cr}$ & II & 1 \\
\hline 3653.614 & & Tr & II & 10 & 3662.693 & & $\mathrm{Sm}$ & II & \\
\hline 3653.62 & $\mathbf{P}$ & Sc & II & 18 & 3662.73 & $\mathbf{P}$ & $\mathrm{Fe}$ & I & 490 \\
\hline 3653.670 & & $\mathrm{Ce}$ & II & 50 & 3662.840 & & $\mathrm{Cr}$ & I & 46 \\
\hline 3653.763 & & $\mathrm{Fe}$ & I & 180 & 3662.90 & $\mathbf{P}$ & $\mathrm{Fe}$ & I & 436 \\
\hline 3653.85 & $\mathbf{P}$ & $\mathrm{Cr}$ & I I & 156 & 3662.905 & & $\mathrm{Sm}$ & I I & 39 \\
\hline 3653.912 & & $\mathrm{Cr}$ & I & 47 & 3663.206 & & $\mathrm{Cr}$ & I & 46 \\
\hline 3654.441 & & Co & I & 63 & 3663.25 & & $\mathrm{Fe}$ & I & 439 \\
\hline 3654.51 & & $\mathrm{~s}$ & I I & 4 & 3663.274 & & $\mathrm{Hg}$ & I & 2 \\
\hline 3654.592 & & $\mathrm{~T} 1$ & I & 18 & 3663.406 & & $\mathbf{H}$ & & 6 \\
\hline 3654.62 & & Gd & II & 4 & 3663.458 & & $\mathrm{Fe}$ & I & 229,231 \\
\hline 3654.66 & & $\mathrm{Fe}$ & I & 77 & 3663.47 & & $\mathbf{s}$ & I I & 16 \\
\hline 3654.995 & & Al. & I I & 12 & 3663.594 & & v & I & 114 \\
\hline 3655.29 & & A & II & 82 & 3663.64 & & $\mathbf{Z r}$ & I & 12 \\
\hline 3655.35 & $\mathbf{P}$ & $\mathrm{Fe}$ & I & 131 & 3663.95 & & $\mathrm{Fe}$ & I & 435 \\
\hline 3655.465 & & $\mathrm{Fe}$ & I & 369 & 3663.98 & & $\mathrm{Fe}$ & II I & 24 \\
\hline 3655.56 & & $\mathbf{Z r}$ & II & 71 & 3664.09 & & $\mathrm{Ne}$ & I I & 1 \\
\hline 3655.851 & & $\mathrm{Ce}$ & I I & 51 & 3664.095 & & N1 & I & 4 \\
\hline 3655.92 & $\mathbf{P}$ & $\mathrm{Cr}$ & I & 46 & 3664.20 & & $\mathbf{P}$ & II & 18 \\
\hline 3656.05 & & $\boldsymbol{\Lambda}$ & II & 67 & 3664.254 & & Sc & II & 10 \\
\hline 2656.135 & & H & & 7 & 3664.537 & & $\mathrm{Fe}$ & I & 391 \\
\hline 3656.152 & & Gd & II & 1 & 3664.60 & & Gd & II & \\
\hline 3656.221 & & Sm & I I & & 3664.62 & & $\mathbf{Y}$ & II & 9 \\
\hline 3656.227 & & $\mathrm{Fe}$ & I & & 3664.679 & & H & & 6 \\
\hline
\end{tabular}


I Iype Element Multiplet No.

$3873.58 \quad \mathrm{Cr}$

$\begin{array}{llll}3673.59 & & \text { Cr I } & 217 \\ 3673.68 & \text { P } & \text { Fe I } & 978 \\ 3673.761 & & \text { H } & \text { 5 }\end{array}$

3673.77 P Fe II

3673.83 Cl I

$3674.06 \quad P \quad N 1$

3874.634 Eu II

$3674.681 \quad V$ II

3674. 7

3674.766 Fe I 36

P T1 I 368

3675.00 P Cr I

3675.265 Sc I

$3675.307 \quad \mathrm{Ca} I$

$\begin{array}{llll}3675.44 & P & \text { Fo I } & 228\end{array}$

$\begin{array}{lllr}3675.487 & \text { V I } & 114 \\ 3675.64 & \text { Y II } & 84\end{array}$

$\begin{array}{llllr}3675.700 & & \text { V } & I & 29 \\ 3675.76 & P & \text { Fe } & I & 896\end{array}$

$\begin{array}{lll}3676.27 & \text { P II } & 18\end{array}$

3676. 365 H

$\begin{array}{lllr}3676.50 & \text { P } & \text { Cr II } & 1 \\ 3676.552 & & \text { Co I } & 145\end{array}$

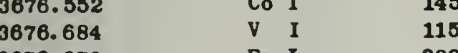

$3676.879 \quad$ Fe I 388

$\begin{array}{lll}3677.309 & \text { Fe I } & 773\end{array}$

$3677.477 \quad$ Fe I 125

3677.68

3677.793

3677.835

3677.86

3678.13

3678.240

3678.256

3678.27

3678.34

$\begin{array}{lll}3678.342 & & \text { Sc I } \\ 3678.46 & P & \text { Mn I } \\ 3678.862 & & \text { Tw I I }\end{array}$

3678.863 Tw

3678.91

3678.98

$\begin{array}{llll}3679.33 & & \text { Fo I } & 228 \\ 3679.34 & P & \text { Cr II } & 118\end{array}$

3678.355

3679.424

3678.500

3678.5

3679.64

3678.80

3678.818

3678.915

3680.06

3680.801

3680.98

3681.272

3881.5

3681.64

Cr II

Co I

Cr II

Co I

$\begin{array}{ll}\text { s } & \text { I I } \\ \text { C. I }\end{array}$

12

12
12

20

28

Bu II 20

II 42

I

I

II

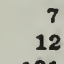

12
131

131

124
48

177

228
118
4

257

Ce II

Eu II

Fe I

2r II

T1 II

Cr I

393,490

122

75

41

48

Fo I

$\begin{array}{lll}\mathrm{A} & 1 & 114\end{array}$

$\begin{array}{ll}\text { Cr I } & 48 \\ \text { Fe I } & \$ 68\end{array}$

Fe $I$

Fe II 111

T1 I

X II

Fe I

1

89

3681.774

3681.87

3682.05

3682.101

3682.15

3682.17

3682.220

3682.66

Cr I

Fe 1

Cl III

Fo I

Fo I

Fo I

He I

A II

3682.66

3682.67

3682.810

3683.047

3683.054

3683.126

3683.287

3683.39

3683.616

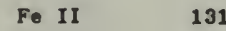

Zr II

Co

Fe I

Eu II

CI III

$\mathrm{Pb} I$

Fe I
I Type Element Multiplet No.

$\begin{array}{lllr}3683.67 & & \text { Cr I } & 216 \\ 3683.71 & & \text { Ca I I } & 18 \\ 3683.77 & \text { P } & \text { Fe I } & 896 \\ 3684.1 & & \text { LI I I } & 2 \\ 3684.108 & & \text { Fe I } & 292 \\ 3684.25 & & \text { Cr II } & 145 \\ 3684.332 & \text { V I } & 114 \\ 3684.478 & & \text { Co I } & 98 \\ 3684.803 & & \text { Y II } & 62 \\ 3684.960 & & \text { Co I } & 116\end{array}$

3885.049

Mn II

3685. 192

3685.212

3685.47

3685.548

3685.68

3686.804
3685.864

3885.988

3686.18

3686.20

3686.260

3686.477

3686.555

3686.67

3686.71

3686.803

686.833

3687.039

3687.100

3687.252

3687.354

3687.458

3687.473

3687.545

3687.74

3687.78

3687.802

3688.01

3688.06

3688.11

3688.27
3688.307

3688.416

3688.418

3688.42

3888.44

3688.457

3688.476

3688.71

3688.877

3688

3688.02

3689.2

3689.302

3689.305

3688.37

688.457

3689.63

3689.671

3689.816

3690.032

3600.095

3680.28

3690.35

3680.450

3690.60

3680.70

3690.715

3680.730

3690.98

3691.18

3691.53

3601.887

3692. 17

3692.221

$\begin{array}{ll}\text { T1 } & 1 \\ \text { Mn } I\end{array}$

T1 I

Cr I

Fe I

Nd II

$\begin{array}{ll}\text { T1 } & \text { I } \\ \text { Fe } & \text { I } \\ \text { Cr } & \text { I }\end{array}$

Mn II

Fe

Co I

Cu II

Cr II

T1 I

H

Pr II

Fe

$\begin{array}{ll}\text { Fe } & \text { I } \\ \text { Cr } & \text { I } \\ \text { T1 } & \text { I }\end{array}$

Fe I

$\begin{array}{ll}\text { Cr } & \text { I }\end{array}$

Fe I

od II

Bu II

Cr II

V I

Cr I

T1 I
Mo II

x) 1

8. II

Eu II

Cl II
Cr I

$\mathrm{Fe} I$

Fe II

IV

Fe I

Y II

Cr I

$\begin{array}{ll}\text { N1 } & \text { I } \\ \text { Pe } & I \\ \text { Fe } & \text { I }\end{array}$

Pe

Cr

Ti I

T1 I

Ru II

Fe I
I

Pd I

He I 497,570

Fe III

85

V II 190

Co I 86

Fe I 807

$\begin{array}{lr}\text { ZF II } & 82 \\ \text { Fo I } & 228\end{array}$

Fo I

$\begin{array}{ll}H & \\ 0 & V \\ \text { I } & \text { II }\end{array}$

A. II

3682.220

3692.33

3682. 357

3692.44

3692.60

3692.645

3692.812

3693.008

3693.08

3693. 106

I A Type

ewent Multiplet No.

$\begin{array}{lllr}3683.364 & & \text { Co I } & 64 \\ 3683.476 & & \text { Co I } & 95 \\ 3693.56 & & \text { Cr I } & 45 \\ 3683.667 & & \text { Mn I } & \\ 3693.78 & \text { P } & \text { Fe I } & 46 \\ 3683.79 & \text { P } & \text { Fe I } & 480 \\ 3683.832 & & \text { N I I } & 15 \\ 3693.889 & & \text { Sm I I } & 2 \\ 3694.005 & & \text { Fe I } & 384 \\ 3694.10 & \text { P } & \text { TI I } & 177\end{array}$

$\begin{array}{llr}3694.11 & \text { C. II } & 18 \\ 3694.115 & \text { Mn I } & 24 \\ 3694.12 & \text { Cr I } & 45 \\ 3694.18 & \text { Yo II } & 1 \\ 3694.22 & \text { Ne II } & 1 \\ 3694.27 & \text { Lo II } & 124 \\ 3694.31 & \text { Ca II } & 18 \\ 3694.445 & \text { TI I } & 117 \\ 3694.622 & \text { V I } & 114 \\ 3694.911 & \text { Ce II } & 63\end{array}$

3604.08

3695.054

3695.158

3695.335

3695. 37

3685.507

3685. 86

3685.865

686

$368 € .29$

3686.38

3696.568

3696.6

3696.65

3696.78

3696.81 
I I Type Element Multiplet No.

I Type Element Multiplet No.

I

Type Elenent

Multiplet No.

\begin{tabular}{|c|c|c|c|c|c|c|c|c|}
\hline 3702.237 & & Co 1 & I & 145 & 3710.46 & & $\mathbf{P}$ & II \\
\hline 3702.201 & & T1 1 & I & 83 & 3710.47 & & $\mathbf{z r}$ & II \\
\hline 3702.500 & & $\mathrm{Fe} 1$ & I & 46,75 & 3710.60 & & $\mathrm{Cr}$ & I \\
\hline 3702.653 & & Mo 1 & II & 5 & 3710.869 & & $\mathrm{Sm}$ & II \\
\hline 3702.75 & & 01 & III & 14 & 3710.870 & & Eu & II \\
\hline 3702.942 & & T1 1 & I & 132 & 3711.074 & & $\mathrm{Na}$ & II \\
\hline 3703 & $\mathbf{P}$ & $0 V$ & $\mathbf{v}$ & 8 & 3711.099 & & Pr & I I \\
\hline 3703.217 & & AI I & II & 18 & 3711.118 & & $\mathbf{v}$ & II \\
\hline 3703.323 & & $\mathbf{Y} I$ & II & 62 & 3711.225 & & $\mathrm{Fe}$ & $\mathbf{I}$ \\
\hline 3703.37 & & 01 & III & 21 & 3711.29 & & $\mathrm{Cr}$ & II \\
\hline 3703,43 & $\mathbf{P}$ & Fe I & I & 704 & 3711.30 & $\mathbf{P}$ & $\mathrm{Fe}$ & I \\
\hline 3703.52 & & C I & III & 12 & 3711.32 & & Fe & I I I \\
\hline 3703.656 & & $\mathrm{Fe} \mathrm{I}$ & I & 291,292 & 3711.411 & & $\mathbf{F e}$ & I \\
\hline 3703.684 & & V I & I & 29 & 3711.543 & & Sm & II \\
\hline 3703.697 & & $\mathrm{Fe} I$ & I & 389 & 3711.646 & & Co & I \\
\hline 3703.824 & & $\mathrm{Fe} \mathrm{I}$ & I & 369 & 3711.751 & & $\mathbf{v}$ & II \\
\hline$\$ 703.832$ & & V I & II & 16 & 3711.02 & $\mathbf{P}$ & $\mathrm{Fe}$ & $I$ \\
\hline 3703.855 & & H & & 3 & 3711.95 & & $\mathbf{z r}$ & II \\
\hline 3704.010 & & $F \in I$ & I & 495 & 3711.973 & & H & \\
\hline 3704.060 & & Co 1 & I & 35 & 3711.974 & & $\mathbf{F e}$ & II \\
\hline 3704.295 & & T1 I & I & 117 & 3712.109 & & Sm & II \\
\hline 3704. 336 & & Fe I & I & 609 & 3712.177 & & Co & I \\
\hline 3704.463 & & $F \in I$ & I & 290 & 3712.38 & $\mathbf{P}$ & $\mathrm{Fe}$ & II \\
\hline 3704.699 & & V I & I & 29 & 3712.48 & & 0 & III \\
\hline 3704.73 & & 01 & III & 21 & 3712.50 & & $\mathrm{Cr}$ & I \\
\hline 3704.79 & P Forb & He & I & 26 & 3712.533 & & $\mathbf{v}$ & II \\
\hline 3704.80 & $\mathbf{P}$ & $\mathrm{Fe}$ & I & 950 & 3712.70 & & Gd & II \\
\hline 3704.848 & & $\mathrm{Tm}$ & II & 9 & 3712.75 & & 0 & II \\
\hline 3705.003 & & He I & I & 25 & 3712.764 & & Sin & II \\
\hline 3705.035 & & $v^{*}$ & I & 29 & 3712.97 & & $\mathbf{C r}$ & II \\
\hline 3705.12 & $\mathbf{P}$ & N1 & I & 30 & 3713.018 & & $\mathrm{Cb}$ & I \\
\hline 3705.140 & & He 1 & I & 25 & 3713.03 & & $A$ & II \\
\hline $3705 \cdot 26$ & $\mathbf{P}$ & $\mathrm{Fe}$ & $\mathbf{I}$ & 704 & 3713.04 & & $\mathrm{Cr}$ & II \\
\hline 3705.40 & $\mathbf{P}$ & $\mathrm{Cr}$ & II & 118 & 3713.09 & & $\mathrm{Ne}$ & II \\
\hline 3705.40 & & Hf & II & 62 & 3713.103 & & $\mathbf{A l}$ & I I I \\
\hline 3705.45 & & Cl & III & 1 & 3713.336 & & N1 & I \\
\hline 3705.63 & & T1 & I & 222 & 3713.46 & & $\mathrm{Bu}$ & II \\
\hline 3705.567 & & $\mathrm{Fe}$ & I & 5 & 3713.64 & & $\mathbf{L a}$ & II \\
\hline 3706.70 & $\mathbf{P}$ & $\mathrm{Fe}$ & I & 610 & 3713.56 & & $\mathbf{v}$ & I \\
\hline 3706.71 & $\mathbf{P}$ & $\mathrm{Fe}$ & I & 293 & 3713.696 & & N1 & I \\
\hline 3705.81 & & La 1 & II & 56 & 9713.734 & & T1 & I \\
\hline 3705.83 & & v & I & 114 & 3713.957 & & $\mathbf{v}$ & I \\
\hline 3706.026 & & $\mathrm{Ca}$ : & II & 3 & 3714 & $\mathbf{P}$ & $\mathbf{N}$ & IV \\
\hline 3706.035 & & V I & I & 104 & 3714.03 & & 0 & III \\
\hline 3706.06 & & P & II & 20 & 3714.13 & & $\mathbf{z r}$ & I \\
\hline 3706.219 & & T1 & II & 73 & 3714.3 & & $\mathbf{Y}$ & II \\
\hline 3706.752 & & $8 \mathrm{~m}$ & II & 47 & 3714.39 & & $\mathrm{Cr}$ & $I$ \\
\hline 3706.91 & & in & II & 8 & 3714.74 & & $\mathbf{A}$ & II \\
\hline 3706.94 & & $\mathbf{A}$ & II & 4 & 3714.77 & & $\mathbf{Z r}$ & II \\
\hline 3706.979 & & $8 \mathrm{~m}$ & II & & 3714.808 & & Nd & II \\
\hline 3707.01 & & Co 1 & I & 85 & 3714.87 & & La & II \\
\hline$\$ 707.048$ & & $\mathrm{Fe}$ & I & 885,392 & 3714.904 & & $\mathrm{Eu}$ & II \\
\hline 3707.13 & & Cr & II & 169 & 3715.08 & & 0 & III \\
\hline 3707.167 & & $\mathrm{Sm}$ & II & 35 & 3716.18 & & $\mathrm{Cr}$ & II \\
\hline 3707.24 & & 01 & III & 14 & 3715.371 & & T1 & I \\
\hline 3707.34 & & Cl & III & 9 & 3716.40 & & Cr & II \\
\hline 3707.466 & & Co & I & 96 & 3715.476 & & $\mathbf{v}$ & II \\
\hline 3707.849 & & T1 & I & 177 & 3715.499 & & $\mathbf{N}_{1}$ & I \\
\hline 3707.828 & & $\mathrm{Fe}$ & I & 6 & 3715.53 & & $\mathbf{L a}$ & II \\
\hline 3707.918 & & $\mathrm{Fe}$ & I & 76 & 3715.795 & & T1 & I \\
\hline 3708.06 & & Mn & II & 8 & 3715.86 & & $\mathbf{P}$ & II \\
\hline 3708.18 & $\mathbf{P}$ & $\mathrm{Fe}$ & I & 228 & 3715.911 & & $\mathrm{Fe}$ & I \\
\hline 9708.410 & & 8in & II & .5 & 3716.36 & & Gd & II \\
\hline 3708.46 & $\mathbf{P}$ & $\mathrm{Fe}$ & I & 436 & 3716.366 & & $\mathrm{Ce}$ & II \\
\hline 3708.602 & & $\mathrm{Fe}$ & I & 178,226 & 3716.442 & & $\mathrm{Fe}$ & I \\
\hline 3708.625 & & T1 & $\mathbf{I}$ & 268 & 3716.531 & 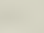 & Cr & I \\
\hline 3708.654 & & $8 \mathrm{~m}$ & II & 18 & 3716.60 & & K & II \\
\hline 3708.721 & & v & I & 104 & 3716.71 & $\mathbf{P}$ & $\mathrm{Fe}$ & I \\
\hline 3708.823 & & Co & $\mathbf{I}$ & 98 & 3716.91 & & $\mathbf{Y}$ & II \\
\hline 3709.03 & $\mathbf{P}$ & $\mathrm{Fe}$ & I & 390 & 3716.030 & & $\mathrm{Ce}$ & II \\
\hline 3709.13 & & $\mathrm{Gd}$ & II & 51 & 3717 & $\mathbf{P}$ & 0 & $\mathbf{v}$ \\
\hline 3709.246 & & $\mathrm{Fe}$ & I & 21 & 3717.02 & & $\mathbf{2 r}$ & II \\
\hline 3709.25 & $\mathbf{P}$ & $\mathrm{Cr}_{\mathbf{r}}$ & II & 6 & 3717.03 & & $\mathbf{P}$ & IV \\
\hline 3709.27 & & $\mathbf{Z r}$ & II & 45 & 9717.06 & & Co & II \\
\hline 3709.286 & & $\mathrm{Ce}$ & II & 40 & 3717.17 & & $\Delta$ & II \\
\hline $3709-335$ & & $\mathbf{v}$ & II & 102 & 3717.19 & $\mathbf{P}$ & $\mathrm{Fe}$ & I \\
\hline 3709.371 & & 8 & III & 1 & 3717.259 & & T1 & I \\
\hline$\$ 709.52$ & & 0 & II I & 21 & 3717.393 & & T1 & I \\
\hline 3709.535 & & Fe & I & 435 & 3717.63 & & Mn & I I \\
\hline 3709.64 & & $\mathrm{Ne}$ & II & 1 & 3717.65 & & $\mathbf{v}$ & I \\
\hline 3709.665 & & $\mathrm{Fe}_{\theta}$ & I & 225 & 3717.63 & & $\mathbf{P}$ & III \\
\hline 3709.88 & & Mn & II & 8 & 3717.68 & & $\mathbf{P}$ & IV \\
\hline 3709.90 & & $\Lambda$ & II & 67 & 3717.69 & & Eu & II \\
\hline 3709.933 & & $\mathrm{Ce}$ & II & 40 & $371 ? .79$ & $\mathbf{P}$ & $\mathrm{Fe}$ & I \\
\hline 3709.963 & & $\mathbf{T 1}$ & I & 83 & 3717.775 & & $\mathbf{8}$ & III \\
\hline 3710.01 & $\mathbf{P}$ & $\mathrm{Cr}$ & II & 6 & 3717.84 & $\mathbf{P}$ & $\mathrm{Fe}$ & I \\
\hline 3710.186 & & $\mathbf{T 1}$ & I & 222 & 3717.915 & & Tn & I \\
\hline 3710.22 & $\mathbf{P}$ & $\mathrm{Cr}$ & II & 6 & 3717.84 & 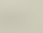 & Cl & II \\
\hline 3710.30 & & $\mathbf{Y}$ & II & 7 & 3718.159 & & $\mathbf{v}$ & II \\
\hline 3710.42 & 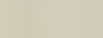 & 8 & III & 1 & 3718.190 & & $\mathrm{Ce}$ & II \\
\hline
\end{tabular}


FINDING LIST

I I Type Element Multiplet No.

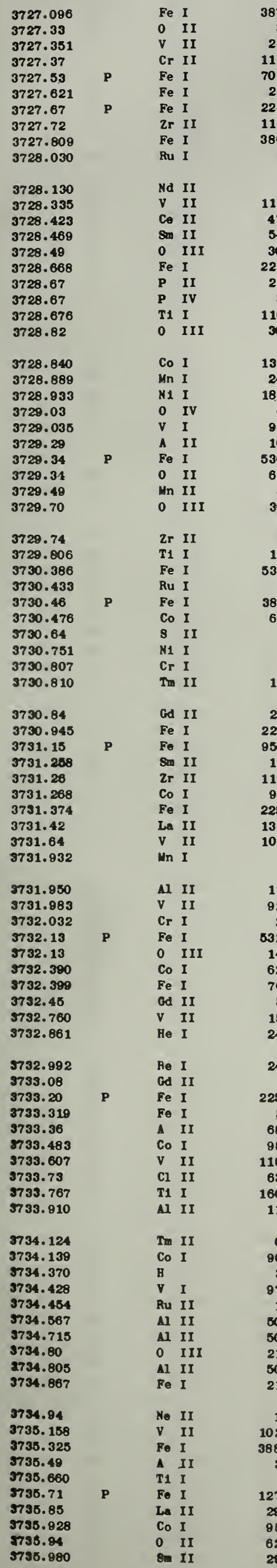

I $\triangle$ Type Element Multiplet No.

$\begin{array}{llr}\text { V } & \text { II } & 102 \\ \text { Be I } & 6 \\ \text { Le II } & 142 \\ \text { Cr I } & 215 \\ \text { Cr II } & 20 \\ \text { O } & \text { IV } & 6 \\ \text { N I I } & 30 \\ \text { Ca II } & 3 \\ \text { Fe I } & 5\end{array}$

3737.133

3737.68

3737.88

3737.89

3737.892

3738.003

3738.08

3738.13

3738.308

3738.38

3738.51

3738.757

3738.801

3739.117

3739.120

3739.13

3739.183

3739.197

3739.229

3739.317

3739.6

3739.782

3739.80

3739.82

3739.940

3740.061

3740.241

3740.247

3741.058

3741.288

Sm II

Cr II

$\triangle$ II

$\begin{array}{ll}\text { AI I I } & \text { I } \\ \text { A } & \end{array}$

Eu II

Zr II

Fe I

Cr II

117
75

131

91
11

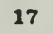

609

609
20

\section{I}

T1 I

Sim II

Fo I

I II

Pr II

Sw II

Fe I

Fe I

T1 II

N1 I

0 II

$\mathrm{Pb} I$

Fe I

$\checkmark$ I

Fe I

TI I

3741.31

3741.42,

3741.504

3741.56

3741.633

$\mathbf{3 7 4 1 . 7 2 7}$

3742.07

$\begin{array}{lll}3742.14 & \text { P } & \text { Fe I } \\ 3742.20 & \text { P } & \text { Cr II }\end{array}$

Sw II

87

166

76

1

5

74

Bu II

Nd II

$\begin{array}{ll}V & \text { I } \\ \text { Fe } & \text { II }\end{array}$

Fe II

0 II

Ce II

Fe I

3742. 280

3742.34

3742.393

Cb I

$P \quad F$

3742.937

3742.968

3742.98 P

778.20 P

3743. 364

Cr I I
Fe I

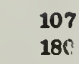

180

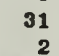

532a, 707

98
667

17

11

124

15
72

38

241

225

878
6

Ru I

743.40

3743.47

3743.856

3743.678

3743.610

3743.78

3743. 868

3743. 884

3744.066

Fe I

ad II

Bu II

Cr I

V II

Fe I

8m II

Cr I

3744.105

3744.22

3744.42

3744.490

3744.662

3744.66

3744.73

3744.98

3745. 36

3745.491

3746.561

3746.606

3745.806

3746.83

3746. 901

3745.97

3746.46

3746.486

3746.56

3746.92

18,34

Fe I $\quad 385$

Cr I

Ne II

$\begin{array}{ll}0 & \text { IV } \\ \text { HP II }\end{array}$

Fe II

Fe I

8a II

N III

Fo $I$

2r II

II

Po I

Fo II
I A Type

Blement Multiplet

$\begin{array}{lllr}3746.931 & & \text { Fe I } & 386 \\ 3747 & \text { P } & \text { O V } & 8 \\ 3747.00 & \text { P } & \text { Fe I } & 388 \\ 3747.264 & & \text { Cr I } & 289 \\ 3747.40 & \text { P } & \text { Fe III } & 71 \\ 3747.48 & & \text { HF II } & 27 \\ 3747.55 & & \text { Y II } & 8 \\ 3747.66 & & \text { N IV } & 8 \\ 3747.90 & & \text { S III } & 1 \\ 3747.96 & & \text { La II } & \end{array}$

3747.982

3748.010

3748.056

3748.101

3748. 18

3748.264

3748.374

3748.46

3748.489

La II

V I 97,98

Ti II 107

Ce II 160

T1 I 166

Cr I

Fe I

Ca I

Cl II

$\mathrm{Fe} I$

6

27
6

3748.492

Cr I

Cr II

8 III

C1 III

3748.73

3748.81

3748.88

3748.989

3749.045

Fe I

Fe I

Cr I

3749.487

N1 I

0 II

$\begin{array}{lll}3749.55 & \text { P } & \text { Zr II } \\ 3749.930 & & \text { Co I }\end{array}$

$\begin{array}{ll}3749.930 & \text { Co I } \\ 3760.00 & \text { C1 II }\end{array}$

3750.154

3750.349

3750.50

3750.56

3750.68

3750.677

B

Ca I

Cr II

Zr II

Fe I

220

3750.74

3750.763

3750.88

3751.059

3751.06

A II 81

$\begin{array}{lllr}3751.222 & P & \text { Fe I } & 7 \\ 37 \text { II } & 100\end{array}$

3751.60

3761.60

Ne II

Zr II

\begin{tabular}{|c|c|c|c|c|}
\hline 3751.628 & & Co & I & 88 \\
\hline 3751.812 & & $\mathrm{Tm}$ & I & \\
\hline 3751.820 & & $\mathrm{Fe}$ & I & 287 \\
\hline 3752.420 & & $\mathrm{Fe}$ & I & 385,392 \\
\hline 3752.524 & & Os & I & 2 \\
\hline 3762.65 & $\mathbf{P}$ & $N$ & I I I & 11 \\
\hline 3752.679 & & Nd & II & 33 \\
\hline 3752.860 & & T1 & I & 17 \\
\hline 3753. 10 & & Al & I I & 39 \\
\hline 3753.154 & & $\mathrm{Fe}$ & I & 177 \\
\hline
\end{tabular}

$\begin{array}{lll}3783.18 & \text { Fe III } & 83\end{array}$

$\begin{array}{llll}3753.26 & P & \text { Cr II } & 20\end{array}$

$\begin{array}{llr}3753.367 & \text { Ca I } & 27 \\ 3753.53 & \text { A II } & 80,128\end{array}$

3753.610

3753.623

3753.83

3754.06

3754.12

3754.346

Fe I

TI I

Ne II

A II

Rh II

73

3754.606

3754.59

3754.62

3754.67

3764.89

3785.13

5758. 276

3755.425

3758.447

3786.54

Co I

38

118

3755.66

3755.583

3755.61

3768.701

3755.81

3755.82

3786.068

5756.10

3756.411

3756.411

Fe I

132

3

95

18

1

21

1

$$
\begin{aligned}
& 81 \\
& 74 \\
& 00
\end{aligned}
$$
7

87 
I $A$

Type

Element

Multiplet No.

\begin{tabular}{|c|c|c|c|c|c|c|c|c|}
\hline 3756.55 & & $\mathbf{C r}$ & II & 144 & 3765.542 & & $\mathrm{Fe}$ & I \\
\hline 3756.83 & & $\mathrm{Cr}$ & I & 72 & 3765.62 & & $\mathrm{Cr}$ & I I \\
\hline 3756.860 & & $\mathrm{Tm}$ & II & 9 & 3765.70 & & $\mathrm{Fe}$ & I \\
\hline 3756.939 & & $\mathrm{Fe}$ & $I$ & 805 & 3765.93 & & Eu & II \\
\hline 3756.96 & & $\mathbf{Z r}$ & II & 8 & 3766.092 & & $\mathrm{Fe}$ & I \\
\hline 3757.093 & & $w$ & I & 3 & 3766.13 & & $\mathbf{A}$ & I I \\
\hline $3757 \cdot 174$ & & $\mathrm{Cr}$ & I & 43 & 3766.29 & & $\mathrm{Ne}$ & II \\
\hline 3757.21 & & 0 & III & 2 & 3766.445 & & $T 1$ & I \\
\hline 3757.459 & & $\mathrm{Fe}$ & I & 668 & 3766.514 & & $\mathrm{Ce}$ & I I \\
\hline 3757.529 & & $\mathrm{Sm}$ & II & & 3766.65 & & $\mathrm{Cr}$ & I I \\
\hline 3757.60 & $\mathbf{P}$ & $\mathbf{N}$ & III & 11 & 3766.665 & & $\mathrm{Fe}$ & I \\
\hline 3757.66 & $\mathbf{P}$ & $N$ & I II & 11 & 3766.71 & & $\mathrm{zr}$ & I \\
\hline 3757.662 & & $\mathrm{Cr}$ & I & 43 & 3766.83 & & $\mathbf{Z r}$ & I I \\
\hline 3757.684 & & $T 1$ & II & 72 & 3766.92 & & $\mathrm{Hf}$ & II \\
\hline 3757.80 & & $\mathbf{Z r}$ & II & 120 & 3767.04 & & Gd & II \\
\hline 3757.862 & & $\mathrm{Ce}$ & II & 48 & 3767.05 & & La & II \\
\hline 3757.929 & & $w$ & I & 4 & 3767.18 & $\mathbf{P}$ & $\mathrm{Cr}$ & I.I \\
\hline 3758.044 & & $\mathrm{Cr}_{\mathbf{r}}$ & I & 43 & 3767.194 & & $\mathrm{Fe}$ & I \\
\hline 3758.11 & $\mathbf{P}$ & $\mathrm{Fe}$ & I & 704 & 3767.358 & & Sm & II \\
\hline 3758.22 & & $\mathrm{v}$ & II & 100 & 3767.36 & & $\mathbf{K}$ & Il \\
\hline 9758.235 & & $\mathrm{Fe}$ & I & 21 & 3767.431 & & $\mathrm{Cr}$ & I \\
\hline 3758.31 & & Gd & II & 20 & 3767.57 & & Cl & I I \\
\hline 3758.36 & & $\mathrm{Ca}$ & II & 8 & 3767.720 & & $\mathbf{v}$ & II \\
\hline 3758.45 & & 0 & IV & 6 & 3767.73 & $\mathbf{P}$ & F'e & I \\
\hline 3758.72 & & $\mathrm{Cr}$ & I & 12 & 3767.755 & & Sm & II \\
\hline 3758.9 & & $\mathbf{Y}$ & II & 74 & 3767.89 & & $\mathrm{Zr}$ & I I \\
\hline 3758.944 & & $\mathrm{Nd}$ & II & & 3768.030 & & $\mathrm{Fe}$ & I \\
\hline 3758.968 & & Sm & I I & & 3768.08 & & $\mathrm{Cr}$ & I \\
\hline 3759.00 & & Gd & II & 2 & 3768.13 & & $\mathrm{Cl}$ & II \\
\hline 3759.08 & & $\mathbf{L a}$ & II & 13 & 3768.23 & & $\mathrm{Fe}$ & I \\
\hline 3759.155 & & $\mathrm{Fe}$ & I & 855 & 3768.240 & & $\mathrm{Cr}$ & I \\
\hline 3759.291 & & Ti & II & 13 & 3768.39 & & Gd & I I \\
\hline 3759.460 & & $\mathrm{Fe}$ & II & 154 & 3768.57 & $\mathbf{P}$ & $\mathrm{Cr}$ & I I \\
\hline 3759.556 & & $\mathrm{Cb}$ & I & 3 & 3768.62 & & $\mathrm{Cr}$ & I \\
\hline 3759.684 & & Co & I & 131 & 3768.71 & & $\mathbf{P}$ & II \\
\hline 3759.87 & & 0 & III & 2 & 3768.734 & & $\mathrm{Cr}_{\mathbf{r}}$ & I \\
\hline 3760.031 & & Pu & I & 2 & 3768.81 & & $\mathrm{He}$ & I \\
\hline 3760.052 & & $\mathrm{Fe}$ & I & 177 & 3769.00 & & $\mathrm{Cr}$ & $\mathbf{I}$ \\
\hline 3760.133 & & $w$ & I & 3 & 3769.046 & & $\mathrm{Ce}$ & II \\
\hline 3760.24 & & v & II & 21 & 3769.13 & & $\mathrm{Cl}$ & II \\
\hline 3760.401 & & Co & I & 40 & 3769.37 & $\mathbf{P}$ & $\mathrm{Cr}$ & II \\
\hline 9760.404 & & $\mathrm{Ce}$ & II & 109 & 3769.45 & & Gd & II \\
\hline 3760.534 & & $\mathrm{Fe}$ & I & 76 & 3769.455 & & N1 & II \\
\hline 3760.694 & & $\mathrm{Ce}$ & II & 92 & 3769.644 & & Nd & II \\
\hline 3760.694 & & Sn & II & 18,51 & 3769.695 & & Pr & II \\
\hline 3760.71 & & Gd & II & 37 & 3769.995 & & $\mathrm{Fe}$ & I \\
\hline 3760.92 & & Gd & II & 20 & 3770.305 & & $\mathrm{Fe}$ & I \\
\hline 3761.06 & $\mathbf{P}$ & $\mathrm{Fe}$ & I & 706 & 3770.37 & $\mathbf{P}$ & $\mathrm{N}$ & III \\
\hline 3761.12 & & Eu & II & 11 & 3770.405 & & $\mathrm{Fe}$ & I \\
\hline 3761.20 & & $\mathbf{v}$ & II & 129 & 3770.412 & & Ti & II \\
\hline 3761.320 & & T1 & II & 13 & 3770.517 & & Mo & I \\
\hline 3761.331 & & $T w$ & II & 2 & 3770.54 & & $\mathbf{A}$ & II \\
\hline 3761.416 & & $\mathrm{Fe}$ & I & 227 & 3770.632 & & $\mathrm{H}$ & \\
\hline 3761.442 & & v & I & 97 & 3770.69 & 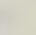 & Gd & II \\
\hline 3761.62 & & $\mathrm{Ca}$ & III & 3 & 3770.974 & & $\mathbf{v}$ & II \\
\hline 3761.69 & & $\mathbf{C r}$ & II & 11 & 3771.08 & & $\mathbf{N}$ & III \\
\hline 3761.72 & . & $\mathrm{Ca}$ & I & 8 & 3771.36 & & Hf & II \\
\hline 3761.82 & & $\mathbf{P}$ & I I & 1 & 3771.45 & $\mathbf{P}$ & $\mathbf{N}$ & I II \\
\hline 3761.866 & & $\mathrm{~T} 1$ & II & 107 & 3771.50 & $\mathbf{P}$ & $\mathrm{Fe}$ & I \\
\hline 3761.867 & & Pr & II & & 3771.652 & & T1 & I \\
\hline 3761.90 & & $\mathbf{C r}$ & II & 11 & 3771.98 & & $\mathrm{Zr}$ & II \\
\hline 9761.913 & & Tm & II & 2 & 3772.06 & & $2 \mathbf{r}$ & II \\
\hline 3762 & $\mathbf{P}$ & 0 & v & 8 & 3772.530 & & N1 & I \\
\hline 3762.205 & & $\mathrm{Fe}$ & I & 705 & 3772.854 & & Pr & I I \\
\hline 3762.41 & & $\mathbf{S i}$ & IV & 3 & 3772.962 & & $\mathbf{v}$ & I I \\
\hline 3762.51 & & Hf & II & 101 & 3773.12 & & La & II \\
\hline 3762.588 & & $\mathrm{Sm}$ & I I & 25 & 3773.13 & 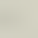 & $\mathbf{S 1}$ & IV \\
\hline 3762.618 & & N1 & I & & 3773.364 & & $\mathrm{Fe}$ & I \\
\hline 3762.62 & $\mathbf{P}$ & $\mathbf{N}$ & I II & 11 & 3773.68 & & CI & II \\
\hline 3762.63 & . & 0 & II & 31 & 3773.699 & & $\mathrm{Fe}$ & I \\
\hline 3762.894 & & $\mathrm{Fe}$ & II & 192 & 3773.80 & & $\mathrm{Fe}$ & I I I \\
\hline 3763.00 & & Gd & I I & 1 & 3773.80 & & v & II \\
\hline 3763.13 & & $\mathrm{Cb}$ & II & 10 & 3774.00 & & 0 & I II \\
\hline 3763.141 & & v & I & 98 & 3774.25 & & $\mathrm{Cl}$ & II \\
\hline 3763.33 & & Gd & II & 37 & 3774.294 & & Stm & II \\
\hline 3763. 356 & & Mo & I & 8 & 3774.3 & & Al & II \\
\hline 3763.377 & & Mn & I & 24 & 3774.33 & & $\mathbf{Y}$ & I I \\
\hline 3763.475 & & Nd & II & & 3774.331 & & $\mathbf{T} \mathbf{1}$ & I \\
\hline 3763.52 & & $\mathbf{A}$ & II & 54 & 3774.38 & $\mathbf{P}$ & 0 & IV \\
\hline 3763.57 & $\mathbf{P}$ & $\mathrm{Fe}$ & I & 128 & 3774.52 & 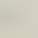 & $\mathbf{s}$ & III \\
\hline 3763. 790 & & $\mathrm{Fe}$ & I & 21 & 3774.54 & & A & II \\
\hline 3764.09 & . & $\mathrm{Fe}$ & II & 29 & 3774.599 & & Co & I \\
\hline 3764.117 & & Ce & II & 41 & 3774.645 & & Mn & I \\
\hline 3764.21 & $\mathbf{P}$ & $\mathrm{Fe}$ & I & 74 & 3774.650 & & $\mathrm{~T} 1$ & II \\
\hline 3764.370 & & $\mathrm{Sm}$ & II & 34 & 3774.678 & & $\mathrm{v}$ & II \\
\hline 3764.38 & & $\mathbf{Z r}$ & I & 10 & 3774.823 & & $\mathrm{Fe}$ & I \\
\hline 3764.60 & & Gd & II & 85 & 3775.03 & & $\mathbf{P}$ & II \\
\hline 3764.811 & & Pr & II & & 3775.187 & & $\mathrm{v}$ & I \\
\hline 3765.044 & & $\mathrm{Ce}$ & II & 208 & 3775.572 & & N1 & I \\
\hline & & & & & & & & \\
\hline
\end{tabular}

3775.860

3776.062

3776.527

3776.56

3776.80

3777.061

3777.16

3777.32

3777.43

3777.448

3777.55

3777.60

3777.64

3777.919

3777.93

3778.063

3778.136

3778.320

e I

Fe I

Mn I

Y II

S II

Fe I

Cr I

Fe III

287

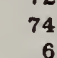

Fe I 223

Co I $\quad 96$

0 II 31

Hf I

Ru II

Cr I

N1 I

Sm II

Fe I $\quad 367$

$\begin{array}{llll}3778.37 & P & \text { Fe II } & 192\end{array}$

$\begin{array}{llr}3778.509 & \text { Fe I } & 664 \\ 3778.684 & \text { V I } & 28\end{array}$

3778.69

3778.697

3778.90

$\begin{array}{llll}3779.23 & \text { P } & \text { Fe I } & 290 \\ 3779.35 & & \text { CI III } & 11\end{array}$

3779.424

$\mathrm{Fe} I \quad 222$

$3779.444 \quad$ Fe I $\quad 665$

$3779.486 \quad$ Fe I $\quad 74$

$3779.58 \quad P \quad$ Fe II $\quad$ P

3779.648

$\begin{array}{lll}3780.09 & \text { HF II } & 18 \\ 3780.391 & \text { Nd II } & 19\end{array}$

3780.53

La. II

$\mathrm{Zr}$ I

La II

3780.763

3780.770

3780.84

3780.927

3781.188

3781.23

3781.379

3781. 393

3781.510

3781.597

Sw II

A I

Sin II

Fe I

Cl II

Cb II

V I

$\mathrm{Fe} I$

Ce II

3781.620

3781.938

3782. 139

3782.195

3782.24

3782.302

3782.34

3782.450

3782.524

3782.6

3782.608

3782.72

3782.78

3783. 16

3783. 19

3783.347

3783. 530

3783.561

3784. 250

$\mathrm{Fe} I$

T1 I

os I

Zr II

Y II

Gd II

$\begin{array}{ll}\mathrm{Fe} & \mathrm{I} \\ \mathrm{Ce} & \text { II }\end{array}$

S II 29

$\mathrm{Fe} \mathrm{I} \quad 491$

Zr II 120

$S$ II

K II

Fe II 14

$\begin{array}{lll}\text { N1 } & \text { I } & 30 \\ \text { Tm II } & \end{array}$

3784.27 P $\quad$ Fe I

$\begin{array}{lllr}3784.81 & P & \text { Fe I } & 607 \\ & & \text { La I I } & 13\end{array}$

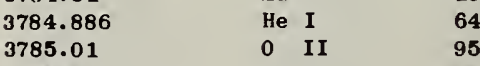

$3785.421 \quad$ Mn I 45

$\begin{array}{lll}3785.706 & \text { Fe I } & 608\end{array}$

$\begin{array}{lllll}3785.78 & P & \text { Fe } & I & 704\end{array}$

$\begin{array}{lll}3785.950 & \text { Fe I } & 177\end{array}$

$\begin{array}{lll}3786.043 & \text { Fe I } & 367\end{array}$

3786.22 Cr I 71

$\begin{array}{lllr}3786.253 & & \text { Ti I } & 165\end{array}$

$\begin{array}{llll}3786.33 & P & \text { TI II } & 12\end{array}$

$3786.632 \quad \mathrm{Ce}$ II

$\begin{array}{llr}3786.678 & \text { Fe I } & 22 \\ 3786.70 & \text { P II } & 1\end{array}$

3786.94

3787.064
3778.357 $\quad$ V II $\quad 21$

5 III 5

3786.40 A II 
I A Type Element Multiplet No.

3787.164

3787.203 3787.235

3787.56

3787.883

$3788.125 \quad$ Sm II

788.474 Rh I

3788.753

Y II
Ce II

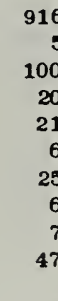

3788.804

3788.864

3788.91

3789.178

3789.293

3789.49

3789.570

3789.723

3789.82

T1 I

Fe III 102

Fe I

T1

Cr I

Fe I

Cr I

$\mathrm{Fe} I$

3790.138

3790.21

3790.228

3790.24

3790.324

3790.454

3790.656

1790.656

3790.83

3790.96

3791. 17

3791. 209

3791.26

3791.326

3791.376

3791.39

3791.41

3791.504

3791.72

3791.73

3792.025

3792.137

3792.156

3792.32

3792. 326

3792.337

3792.42

3792.46

$\mathrm{Cb}$

Mn I

Cr I

Fe I

$\begin{array}{ll}V & I \\ \text { Cr } & I\end{array}$

$\checkmark$ I

$\mathrm{Fe} I \quad 387$

Fe I $\quad 73,127$

La II 12

Ne II 30

Gd II $\quad 85$

0 III

I I
Cr

Cr I

$\mathrm{Fe} I$

Gd II

3
6
139

139

224

28
139
69

3792.524

3792.56

3792.834

3793.217

3793.28

3793. 289

3793.35

3793.478
3793.52

3793. 608

3793.61

3793.614

3793.75

3793.872

3793.879

3793.971

3794. 340

3794.366

3794.48

3794.608

3794.69

3794.78

3794.964

3795.004

3795.10

3795.168

3795.256

3795.37

3795. 759

37.95 .903

3796.00

3796.11

3796.33

3796.37

3796.47

3796.48

3796.55

3796.60

3796. 899

$\mathrm{Fe} \mathrm{I} \quad 703$

$\begin{array}{lr}\text { Sm II } & 5 \\ \text { Cr I } & 139\end{array}$

$\mathrm{Fe} \mathrm{I} \quad 287$

Zr II 81

Ce II 129

N1 I

Cr I

Pr II

71
50

Y I

Fe I

$N$ III

Rh I

Fe I

Cr I

Fe I

Hf II

$\mathrm{Fe} \mathrm{I} \quad 387$

71

$\begin{array}{lll}\text { N1 } & \text { I } & 4 \\ \text { P } & \text { II } & 1 \\ \text { V } & \text { I } & 9\end{array}$

C1 II

Fe I 367

Cr I 139

$\begin{array}{lr}\text { Sm II } & 11 \\ \text { Fe I } & 177\end{array}$

$\begin{array}{ll}\text { V } & \text { II } \\ 0 & \text { II }\end{array}$

177
100
34

Cr I $\quad 139$

$\begin{array}{lll}S & \text { III } & 10 \\ \text { La II } & \text { II }\end{array}$

V I 9,28

Fe I

$P$ II

Tm II

Ce II

A II

Tm II

T1

Fe I

S1 III

He II

Gd II

$\mathrm{Zr} I$

$\mathrm{V}$ II

$\mathrm{Fe}$ II

A II

T1 II
I A Type Element Multiplet No.

$\begin{array}{llr}\text { Fe I } & 667 \\ \text { Cr I } & 41 \\ \text { Cr I } & 139 \\ \text { Sm I I } & 11 \\ \text { Fe I } & 607 \\ \text { Cr I } & 139 \\ \text { Sm II } & \\ \text { H } & 2 \\ \text { Fe I } & 222 \\ \text { H I I } & 29\end{array}$

$\mathrm{Cb} \mathrm{I}$

Mo 1

Fe II

$\mathrm{Fe} I$

Fe II

Tm II

Cl II

Ru I

Eu II

Ce II

Mn I

Ru I

Ru I

Sm II

$\mathrm{Fe} I$

T1 II

$\mathrm{V} I$

Ne II

Ir I

Pn II

Sm II

Hf II

Fe III

Mn I

$\begin{array}{ll}Y & \text { II } \\ \text { Sin } & \text { II }\end{array}$

Sn I

$\begin{array}{ll}\text { T1 } & \text { I } \\ \text { Cr } & \text { II }\end{array}$

Cr II

Gd II

Ce II

Fe I

Mn I

$\begin{array}{ll}\mathrm{Fe} & \mathrm{I} \\ \mathrm{P} & \text { III }\end{array}$

Fe I

$S$ II

V I

Cb I

n II

Ce II

II 129

Fe I 122

Nd II

V I

V I

Mn II

I I

$\mathrm{Fe} I$

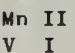

3
1
115

115
14

14
21
23

23
80

11
62

62
1

11

11
136
1

1
6
8

1
54

22

21
13

28

39

1
14

43

18

47
45

17

61

29
2
165

165

172

14
367

367

Cr I 139

Cl II

Fe I $\quad 608$

Stin II 10

He I 63

HF II 75

Fe I $\quad 731$

Cr I 24

S1 III 5

Fe I

$\begin{array}{ll}M n & I \\ \text { Fe I } & \\ \mathrm{V} & \mathrm{I}\end{array}$

V I

Fe II
Cr I

N1 I

Nd II

I A

Type Element

Multiplet No.

$\begin{array}{llr}3807.505 & \text { V I } & 28 \\ 3807.534 & \text { Fe I } & 73 \\ 3807.65 & \text { Gd II } & 85 \\ 3807.926 & \text { Cr I } & 139 \\ 3808.102 & \text { Co I } & 17 \\ 3808.124 & \text { Ce II } & 59 \\ 3808.286 & \text { Fe I } & 489 \\ 3808.521 & \text { V I } & 9 \\ 3808.61 & \text { A II } & 3 \\ 3808.7 & \text { Y II } & 73\end{array}$

3808.731

3808.772

3808.79

3809.043

3809.49

3809.51

3809.592

3809.597

3809.67

3810.10

3810.21

3810.724

(3810.759

48

3810.96

3811.05

3811.065

3811.073

3811.22

3811.32

3811.35

3811.385

3811.774

3811.80
3811.80 
I A Type Element Multiplet No.

\begin{tabular}{|c|c|}
\hline \\
\hline \multicolumn{2}{|l|}{3817.639} \\
\hline \multicolumn{2}{|l|}{3817.64} \\
\hline \multicolumn{2}{|l|}{3817.844} \\
\hline \multicolumn{2}{|l|}{3817.844} \\
\hline \multicolumn{2}{|l|}{3817.940} \\
\hline \multicolumn{2}{|l|}{3818.244} \\
\hline \multicolumn{2}{|l|}{3818.27} \\
\hline \multicolumn{2}{|l|}{3818.281} \\
\hline \multicolumn{2}{|l|}{3818.34} \\
\hline \multicolumn{2}{|l|}{3818.40} \\
\hline \multicolumn{2}{|l|}{3818.44} \\
\hline \multicolumn{2}{|l|}{3818.481} \\
\hline \multicolumn{2}{|l|}{3818.78} \\
\hline \multicolumn{2}{|l|}{3818.862} \\
\hline \multicolumn{2}{|l|}{3819.04} \\
\hline 3819.25 & P Forb \\
\hline 3819.50 & $\mathbf{P}$ \\
\hline \multicolumn{2}{|l|}{3819.564} \\
\hline 3819.57 & P \\
\hline
\end{tabular}

$\begin{array}{ll}\text { Zr } & \text { II } \\ \text { TI } & \text { I } \\ \text { Fe } & \text { I } \\ \text { Cr } & \text { I } \\ \text { V } & \text { I } \\ \text { Co } & \text { I } \\ \text { V } & \text { I } \\ \text { N } & \text { I } \\ \text { Pr } & \text { II } \\ \text { Y } & \text { II }\end{array}$

18
189
701
40
10
131
9
11
7

C1 II

$\mathrm{Ne}$ II

Cr I
Zr II

$\mathrm{Zr}$ II

A II

He I

$\begin{array}{ll}\mathrm{Fe} & \mathrm{I} \\ \mathrm{Cr} & \mathrm{I}\end{array}$

$\begin{array}{ll}\text { Cr } & \text { I } \\ \text { Cr } & \text { I }\end{array}$

62
39
40

40
111

10
129

129

23
703

70
88

3819.606
$3819.62 \quad P$
3819.67

3819.67

3819.761

3819.908

3819.963

3819.97

3820.25

3820.428

3820.74

3820.871

3820.874

3821.181

3821.487

3821.582

3821.68

3821.834
3821.92

3822.009

3822.026

3822.07

3822.10

3822.17

3822.262

3822.41

3822.63

3822.737

3822.888

He I

Fe I
Eu II

He I

Zr II

Co I

$\begin{array}{ll}\text { V' } & \text { I } \\ \text { Cr } & \text { I }\end{array}$

Cl II

$\mathrm{V} I$

$\begin{array}{ll}\mathrm{Fe} & \mathrm{I} \\ \mathrm{Hf} & \mathrm{I}\end{array}$

Ce II

Cr I

$\mathrm{Fe} I$

$\begin{array}{ll}V & I \\ \text { Cr } & \text { I }\end{array}$

0 II

Fe I

Fe II

$\begin{array}{ll}V & I \\ T I & I\end{array}$

TI I

Cr I

Gd II

Rh I

Zr I

$\begin{array}{ll}0 & \text { I } \\ \text { Fe } & \text { II }\end{array}$

V I

Mo I

3822.987
3823.213

3823.41

3823.469

3823.513

3823.522

3823.571

3823.72

3823.893

3823.903

3823.990

3824

3824.074

3824.175

3824.306

3824.425

3824.444

3824.47

3824.73

3824.78

3824.882

3824.913

3825.090

3825.248

3825.380

3825.404

3825.530

3825.70

3825.884

3826.05

V I
Zr I

0 I

Mn I

Cr I

Pr II

Zr II

Mn I

Ce II

V I

N IV

Sm II

$\mathrm{Fe} I$

$\begin{array}{ll}0 & I \\ \mathrm{Fe} & \mathrm{I}\end{array}$

C1 III

$\mathrm{Fe} \mathrm{I}$

$\mathrm{Cb} I$

Fe II

0 I

0 I

$\begin{array}{ll}\mathrm{Cr} & \mathrm{I} \\ \mathrm{Fe} & \mathrm{I}\end{array}$

Fe I

A II

$\mathrm{Fe} I$

Gd II

22

12

22

81

130

28
40

68

20

5
208

208
40

608

34

222
14

9
189

189

11
40

19

10

28

8

28
44

36

6
14
31

31
6

115

44

10
224

18

607

36

9

221

3826.292

3826.416

3826.425

3826.63

3826. 701

3826.774

3826.83

3826.83

3826.968

Sm II

Pr II

Nd II

Cr I

$\mathrm{Fe} \mathrm{I}$

Mo I

1

A II

$\begin{array}{ll}\text { Fe } & \text { I } \\ \text { V } & \text { II }\end{array}$

I A Type Element Multiplet No.

3827.079
3827.27
3827.46
3827.51
3827.572
3827.62
3827.67
3827.825
3828.180
3828.44
3828.510

$\begin{array}{llr}\text { Fe II } & 153 \\ \text { Zr II } & \\ \text { P II } & 26 \\ \text { Zr II } & 121 \\ \text { Fe I } & 284 \\ \text { CI II } & 69 \\ \text { Fe II } & 128 \\ \text { Fe I } & 45 \\ \text { TI I } & 189 \\ \text { Fe II I } & 70,95\end{array}$

Fe I

3828.836

3828.883

3829.125

3829. 133

3829.27

3829.3549

3829.458
$3829.47 \quad P$ Forb

$\begin{array}{ll}V & I \\ V & I\end{array}$

Mo I

Fe I

$\begin{array}{ll}W & I \\ \text { CI } & \text { II }\end{array}$

$\begin{array}{ll}\text { CI } & \text { II } \\ \mathrm{Mg} & \text { I }\end{array}$

$\mathrm{Fe} I$

3829.53

3829.655

3829.680

3829. 77

3829.771

3829.80
3830.032

3830.032
3830.293

3830.39

3830.43

3830.45

3830.719

3830.80

3830.850

3831.017

3831.032

3831.41

3831.501

3831.690

3831.75

3831.80

3831.840

3831.85
3832.12

3832. 2996

3832. 3037

3832.31

3832.32

3832.745

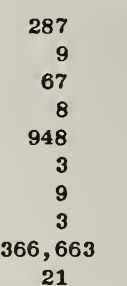

V II

$\begin{array}{ll}V & \text { II } \\ \text { Mn } & I\end{array}$

Ne II

Fe I

N I I

$\begin{array}{ll}\text { Cr } & \text { I } \\ \text { Sm } & \text { II }\end{array}$

Sm II
N I

A II

0 I I

Pr II

C1 II

$\mathrm{Fe} \mathrm{I}$

V II

Cr I

II

Sm II

Fe III

Gd II

$\mathrm{Cb}$ II

S III

C II

$\mathrm{Mg}$

Mg

$\begin{array}{ll}\text { Pd } & \text { I } \\ \text { Cr } & \text { I }\end{array}$

Ce II

3

3
3
6
39
221
30
10
11
3,128

3,128

34

224

69
284

284
3

24

43

31

3832.835

3832.873

3832.89

3832.94

Zr II

3833. 059 Sc II

3833.10

3833.186

3833. 226

3833.311

3833.40

3833.49

3833. 574

3833. 674

3833.71

3833.757

3833.80

3833.828

3833.862

3833. 87

3833.889

3834.22

3834.22

3834.24

3834.24

3834. 364

3834.46
$\mathbf{3 8 3 4} .55$

3834.735

3834.81

3835.058

3835.09

3835. 386

3835.497

3835.560

3835. 725 
I 4 Typo Element Multiplet No.

3846.803 3846.949 3847.01 3847.086 3847.252 3847.323

3847.323

3847.38

3847.501

3847.511

3847.89

3848.023

3848.194

3848.233

3848.24

3848.29

3848.524

3848.597

3848.779

3848.983

3848.02

3848.26

3849.324

3849.52

3849.534

3848.58

3848.758

3848.869

3848.987

3850.042

3850.40

3850.409

3850.67

3850.68

3850.81

3850.820

3850.93

3850.945

3850.97

3850.97

3851.04

3851.171

3851.28

3851.47

3851.58

3851.66

$\$ 881.69$

3851.748

3851.848

3861.880

3852.218

3852.45

$\$ 852.674$

$\$ 852.08$

5852.805

3883.07

3853.09

\$863. 164

3853. 176

3853.462

3853.657

3853.718

3854.187

3864. 208

2884.220

3854.322

3854. 378

3854.76

384.91

3856.08

2855.18

3856.286

3858.328

3855.370

3855.43

3855.06

3835.571

3858.68

3855.841

$\$ 855.846$

5856.021

3856.07

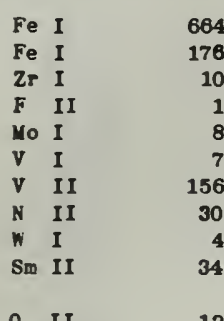

$\begin{array}{lll}0 & \text { II } & 12\end{array}$

TI II II 2

$\begin{array}{llr}\text { I II } & 72 \\ \text { Md II } & 19 \\ \text { Yg II } & 5\end{array}$

Fe I

Nd II

Ce II 38

Sim II

Cr I

69

$\begin{array}{ll}\text { La II } & 12 \\ \text { ZF I } & 6\end{array}$

$\mathrm{V}$ I

Cr I

HP II

Cr I

N1 II

$\checkmark$ II

Fe $I$

Cr I

Mg II

A II

Gd II

0 II

Fe I

Pr II

Co I

cl II

Gd II

$\begin{array}{ll}0 & \text { II } \\ \text { V } & \text { I }\end{array}$

c1 II

0 II

Fe I

Pr II

F II 1

Nd II 35

Co I

Sm II

II

Cr I

II

Fe I

Pr II

Pr II

Zr II

Co II

Cr I

Pe I

81 II

TI T

Gd II

Co II

CF I 68

$\begin{array}{lr}\text { Ce II } & 61 \\ \text { Fe I } & 677\end{array}$

Cl II 84

Pr II

Lo II

II

III

Cr. I

$\begin{array}{ll}V & \text { I } \\ \text { Zr } & \text { II }\end{array}$

od II

Cr I

Cr I

7 I

Fo I

81 II

if II
I A Type Element Multiplet No.

0 II 12

3856.16

3856.281

3856.373

3856.515

3856. 796

3857.032

3857.18

3857.240

3857. 26

20

3858.07

3858.133

3858.301

3858.32

3858.48

3858.90

3859.24

3859.26

Cr I

Fe I

Rh I

Co I

Ce II

Ce II

I II

Cr I

Sm II

T1 I

NI I

A III

Fe I

$\mathrm{Fe} I$

$\mathrm{Mg} \mathrm{I}$

S II

3859.3

V I

P $\quad$ Sc II

$\begin{array}{lll}3858.813 & & \mathrm{Fe} \text { I } \\ 3860.12 & \mathrm{P} & \mathrm{Fe} \text { II }\end{array}$

3860.13 Cr I

3860.15

3860.46

3860.64

$\$ 860.64$

S II

Fe III
S II

$3860.74 \quad P \quad P$

$\begin{array}{llll}3860.74 & \text { P } & \text { Fe I } & 701 \\ \$ 860.80 & & \text { Cl II } & 26\end{array}$

$\mathbf{3 8 6 0 . 8 1 5}$

3861.079

3861.164

3861.18

3861. 341

3861.40

3861.60

C1 II

c1 II

T1 I

Co I

Fe I 285, 683

C1 II 25

$\begin{array}{lll}\$ 861.95 & \text { Cl II } & 84\end{array}$

$3862.054 \quad P \quad$ Sin II 10

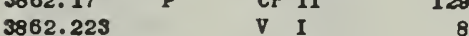

$\begin{array}{rlr}3862.592 & \text { S1 II } & 1 \\ 3862.823 & \text { TI I } & 175\end{array}$

3862.823

3863.056

3863.072

3863.329

3863. 409

T1 I

$\mathrm{Cb}$ II

N1 I

Nd II

Nd II

181

3863.60

3869.607

(283.70

3863.74

3863.81

3863.866

3863. 88

3863.963

3864.115

$\mathrm{Fe}$ II

o II

Co I

Fo $I$

V II

V I

Zr I

Fe II

Mo I

0 II

3864.13

384 13

3864.300

3864.31

3864.35

3864.336

3864.45

3864.49

3864.60

3864.68

3864.75

$\$ 864.862$

3866.458

5865.626

3865.68

3866.72

3866.01

3866.160

3866.446

3866.34

3866.744

3867.219

3867.26

$\$ 867.32$

3867.46

3807.477

3867.56

3867.602

3867.631

3867.730

I A

Type

Blement

Multiplet No.

3867.825

3867.986 


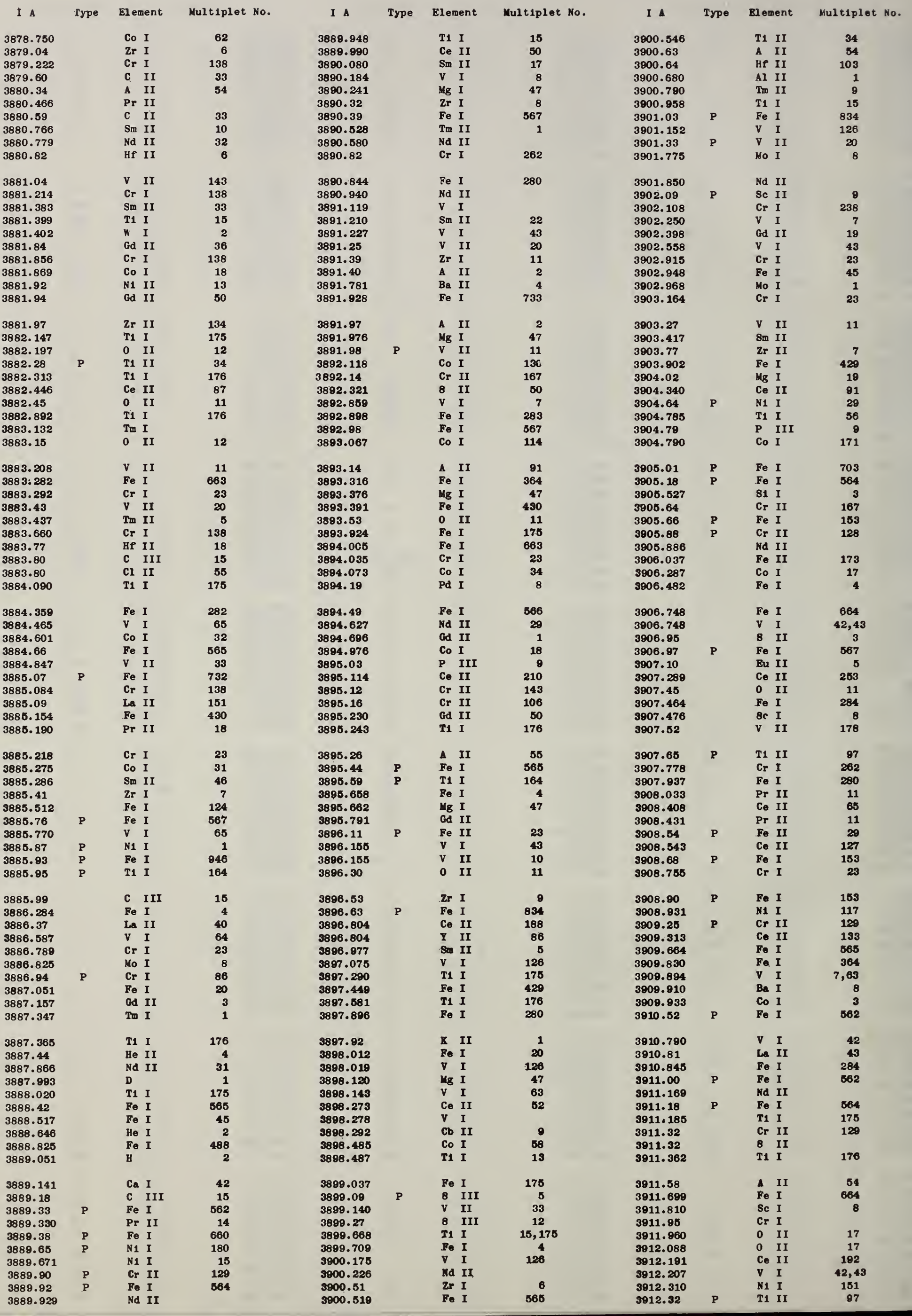




\begin{tabular}{|c|c|c|c|c|c|c|c|c|}
\hline 3912.424 & & $\mathrm{Ce}$ & II & 60 & 3923.03 & $\mathbf{P}$ & $\mathrm{Fe}$ & I \\
\hline 3912.689 & & T1 & I & 175 & 3923.109 & & $\mathrm{Ce}$ & I I \\
\hline 3912.886 & & $\mathbf{v}$ & I & 42 & $3823.24 \mathrm{C}$ & & Gd & II \\
\hline 3812.898 & & Pr & II & 17 & 3923.38 & $\mathbf{P}$ & $\mathbf{T 1}$ & II \\
\hline 3912.978 & & N1 & I & 15 & 3923.48 & & He & II \\
\hline 3913.464 & & T1 & II & 34 & 3923.483 & & 3 & II \\
\hline 9913.635 & & $\mathrm{Fe}$ & I & 120 & 3923. 60 & & $\mathrm{Ca}$ & I \\
\hline 3913.92 & & Cl & II & 68 & 3823.503 & & $8 c$ & II \\
\hline 3914.273 & & $\mathrm{Fe}$ & I & 567 & 3923.91 & & He & II \\
\hline 3914.333 & & $\mathbf{v}$ & II & 33 & 3923.82 & & $2 \mathbf{r}$ & II \\
\hline 3914.334 & & T1 & I & 16 & 3924.05 & & $\mathbf{s}$ & II \\
\hline 3914.36 & & $\mathbf{Z r}$ & II & 134 & 3924.075 & & Mn & $\mathbf{I}$ \\
\hline 3914.42 & $\mathbf{P}$ & Fe & $\mathbf{I}$ & 652 & 3924.18 & $\mathbf{P}$ & N1 & $\mathbf{I}$ \\
\hline 3914.480 & & $\mathrm{Fe}$ & II & 3 & 3924.44 & & 81 & I II \\
\hline 3914.50 & $\mathbf{P}$ & Fe & I & 660 & 3924.627 & & T1 & I \\
\hline 3814.73 & & Fo & I & 682 & 3924.644 & & Ce & II \\
\hline 3914.751 & & T1 & $\mathbf{I}$ & 14 & 3924.66 & $\mathbf{P}$ & $\mathbf{C r}$ & II \\
\hline 3814.76 & & $\boldsymbol{A}$ & I I & 2 & 3924.658 & & $\mathbf{v}$ & I \\
\hline 3914.949 & & $\mathrm{Ce}$ & II & 78 & 3925.08 & & La & III \\
\hline 3814.96 & & $\mathbf{C r}$ & I & 137 & 3925.161 & & Co & I \\
\hline 3815.30 & $\mathbf{P}$ & $\mathrm{Cr}$ & II & 128 & 3925.201 & & $\mathrm{Fe}$ & I \\
\hline 3916.384 & & Ir & I & 6 & 3925.216 & & $\sin$ & I \\
\hline 3916.603 & & Co & I & 113 & 3925.240 & & v & I \\
\hline 9815.843 & & $\mathbf{C r}$ & I & 136 & 3925.456 & & $\mathbf{P r}$ & II \\
\hline 3915.878 & & T1 & I & 16 & 3825.65 & $\mathbf{P}$ & $\mathrm{Fe}$ & I \\
\hline 3915.94 & & $2 r$ & II & 17 & 3925.646 & & $\mathrm{Fe}$ & I \\
\hline 3916.05 & & La & II & 42 & 3925.71 & & $\mathbf{A}$ & II \\
\hline 3916.243 & & Cr & $\mathbf{I}$ & 23 & 3925.87 & & Cl & III \\
\hline 3916.418 & & $\mathbf{v}$ & II & 10 & 3925.846 & & $F \theta$ & I \\
\hline 3916.476 & & $\mathrm{Tm}$ & I & 2 & 3926.001 & & $\mathrm{Fe}$ & I \\
\hline 3916.508 & & od & II & 20 & 3926.319 & & $T 1$ & I \\
\hline 9916.61 & $\mathbf{P}$ & Cd & II & 60 & 3826.32 & & $\mathbf{v}$ & II \\
\hline 3916.64 & & $\mathbf{Z r}$ & I & 6 & 3826.467 & & Mn & $I$ \\
\hline 9916.70 & & Cl & II & 68 & 3926.497 & & $\mathbf{v}$ & II \\
\hline 9916.733 & & Fe & I & 806 & 3926.580 & & He & $\mathbf{I}$ \\
\hline 3916.980 & & $\mathrm{Cr}$ & I & 137 & 3926.68 & $\mathbf{P}$ & 0 & I I \\
\hline 9917.116 & & Co & I & 113 & 3926.648 & & $\mathbf{C r}$ & I \\
\hline 3917.185 & & Fe & I & 20 & 3927.383 & & Ce & II \\
\hline 3917.29 & & $\mathrm{Bu}$ & II & 10 & 3927.61 & $\mathbf{P}$ & Fo & I \\
\hline 9917.442 & & $8 m$ & II & 8 & 3927.822 & & $\mathrm{Fe}$ & I \\
\hline 3917.47 & & HP & II & 76 & 3927.926 & & $\mathbf{v}$ & I \\
\hline $391 \% .67$ & & $\mathrm{Cl}$ & II & 68 & 3927.83 & $\mathbf{P}$ & $\mathrm{Fe}$ & I \\
\hline 3917.686 & & $\mathrm{Cr}$ & I & 137 & 3928.085 & & $\mathrm{Fe}$ & I \\
\hline 9918.10 & & BF & II & 7 & 3928.278 & & $8 m$ & II \\
\hline $3918 \cdot 18$ & & 8 & II & 29 & 3928.615 & & 8 & III \\
\hline 3918.236 & & ad & II & 60 & 3828.62 & & $\boldsymbol{A}$ & II \\
\hline 3918.276 & & Ce & II & 12,248 & 3928.636 & & $\mathbf{C r}_{\mathbf{r}}$ & I \\
\hline 3918.319 & & Fe & $\mathbf{I}$ & 124 & 3828.87 & & $\mathrm{Bu}$ & II \\
\hline 3918.418 & & Fe & I & 364 & 3928.87 & $\mathbf{P}$ & T1 & I \\
\hline 9918.51 & $\mathbf{P}$ & Fe & II & 181 & 3929.114 & & $\mathbf{F e}$ & I \\
\hline 3918.54 & $\mathbf{P}$ & $\mathrm{Cr}$ & I & 136 & 3929.15 & $\mathbf{P}$ & T1 & II \\
\hline 3918.68 & $\mathbf{P}$ & Fe & I & 382 & 3929.208 & & $\mathrm{Fe}$ & $\mathbf{I}$ \\
\hline 3918.641 & & Fe & I & 430 & 3829.22 & & La & II \\
\hline 3918.856 & & Pr & II & 11 & 3929.63 & & $\mathbf{Z r}$ & $\mathbf{I}$ \\
\hline 3918.977 & & C & II & 4 & 3829.64 & & $\mathbf{Z r}$ & II \\
\hline 3918.005 & & $\mathbf{N}$ & II & 17 & 3929.583 & & $\mathrm{Tm}$ & II \\
\hline 3918.068 & & $\mathrm{Fe}$ & I & 430 & 3928.734 & & $\mathbf{v}$ & I I \\
\hline 3918.15 & $\mathbf{P}$ & $\mathbf{C r}$ & I & 136 & 3929.875 & & $\mathbf{T 1}$ & I \\
\hline 3918.169 & & $\mathrm{Cr}$ & $\mathbf{I}$ & 23 & 3030.023 & & $\mathbf{v}$ & $\mathbf{I}$ \\
\hline 3818.287 & & 0 & II & 17 & 3930.076 & . & Co & I \\
\hline 3918.813 & & Ce & II & 60 & 3930.298 & & $\mathrm{Fe}$ & I \\
\hline 3919.822 & & T1 & I & 130 & 3830.31 & $\mathbf{P}$ & $\mathbf{F e}$ & II \\
\hline 3920.260 & & Fe & I & 4 & 3830.60 & & Bu & II \\
\hline 3920.37 & & 8 & I II & 8 & 3930.63 & $\mathbf{P}$ & 0 & IV \\
\hline 3920.487 & & $\mathbf{v}$ & $\mathbf{I}$ & 42 & 3930.66 & & $\mathbf{Y}$ & II \\
\hline 3920.524 & & $\mathbf{P r}$ & II & 12 & 3930.88 & $\mathbf{P}$ & $\mathbf{C r}$ & II \\
\hline 3920.648 & & Fe & I & 163 & 3931.088 & & Ce & II \\
\hline 3920.677 & & C & II & 4 & 3931.122 & & Fe & I \\
\hline 3920.839 & & Fe & I & 367 & 3931.24 & & $\mathbf{A}$ & II \\
\hline 3820.965 & & Nd & II & & 3931.340 & & $\mathbf{v}$ & I \\
\hline 3821.02 & $\mathbf{P}$ & $\mathbf{Z r}$ & II & 42 & 3931.369 & & Ce & II \\
\hline 3821.022 & & $\mathrm{Cr}$ & I & 23 & 3931.838 & & 8 & II \\
\hline 3921.27 & & Po & I & 220 & 3831.87 & & $\mathbf{M}$ & I \\
\hline 3921.423 & & T1 & $\mathbf{I}$ & 14 & 9832.007 & & T1 & II \\
\hline 3921.54 & & La & II & 40 & 3932.30 & & 8 & II \\
\hline 3921.731 & & $\mathrm{Ce}$ & II & 198 & 3832.40 & & Br & II \\
\hline 3921.80 & & $2 \mathbf{r}$ & I & 8 & 3952. 63 & & La & I I \\
\hline 3921.905 & & v & I & 42 & 3932.56 & & $A$ & II \\
\hline 3822.005 & & Ce & II & 50 & 3932.68 & $\mathbf{P}$ & Fe & I \\
\hline 3922.08 & $\mathbf{P}$ & Fe & I & 153 & 3932.628 & & Fo & I \\
\hline 3922.09 & $\mathbf{P}$ & Fe & $I$ & 664 & 3933.18 & & $\Lambda$ & II \\
\hline 3922.36 & & $\mathbf{z r}$ & II & 143 & 3933.294 & & 8 & I I \\
\hline 3922.397 & & $8 a$ & II & 38 & 3933.38 & & $\mathbf{P}$ & III \\
\hline 3922.431 & & $\mathbf{v}$ & I & 42 & 3933.38 & & $8 c$ & I \\
\hline 3922.64 & & A & II & 11,53 & 393ง. 606 & & Fo & I \\
\hline 3922.63 & & $\mathbf{s}$ & II & 60 & 3933.65 & & нг & II \\
\hline 3922. 68 & $\mathbf{P}$ & $\mathrm{Fe}$ & I & 420 & 3933.664 & & Ca & II \\
\hline 3922.72 & & $\mathbf{P}$ & III & 9 & 3933.731 & & $\mathrm{Ce}$ & II \\
\hline 3922.755 & & Co & I & 32 & 3933.918 & & Co & I \\
\hline 3922.814 & & $\mathrm{Fe}$ & & & s934.013 & & $\mathbf{v}$ & \\
\hline
\end{tabular}


I A Type Element Multiplet No.

\begin{tabular}{|c|c|c|c|c|c|c|c|c|}
\hline & $\mathbf{P}$ & N1 & I & 1 & $\begin{array}{l}3956.82 \\
3956.901\end{array}$ & $\mathbf{P}$ & $\begin{array}{l}0 \\
\text { Ce }\end{array}$ & IV \\
\hline $\begin{array}{l}3946.21 \\
3946.35\end{array}$ & & $\begin{array}{l}Y \\
C\end{array}$ & II & $\begin{array}{c}24 \\
31,32\end{array}$ & 3957.027 & & $\mathrm{Fe}$ & II \\
\hline & & & & & & & & \\
\hline 3946.406 & & Al & II & 63 & 3957.053 & & $\mathrm{Ca}$ & I \\
\hline 3946.511 & & Sm & II & 17 & 3957.62 & & $\mathrm{Fe}$ & I \\
\hline 3946.633 & & Co & I & 60 & 3957.64 & & $\mathbf{P}$ & III \\
\hline 3916.681 & & $\mathrm{Ce}$ & II & 255 & 3957.66 & $\mathbf{P}$ & $\mathrm{Fe}$ & II \\
\hline 3946.98 & & $\mathbf{s}$ & II & 46 & 3957.672 & & Gd & II \\
\hline 3947.002 & & $\mathrm{Fe}$ & I & 561 & 3957.928 & & Co & I \\
\hline 3947.10 & & Fe & III & 23,69 & 3958.001 & & Nd & II \\
\hline 3947.125 & & Co & I & 58 & 3958.08 & & $\mathrm{Cr}$ & I \\
\hline 3947.301 & & 0 & I & 3 & 3958.101 & & $\mathrm{Tm}$ & II \\
\hline 3947.393 & & $\mathrm{Fe}$ & I & 153 & 3958.206 & & $\mathbf{T 1}$ & I \\
\hline 3947.489 & & 0 & I & 3 & 3958.24 & & $\mathbf{z r}$ & II \\
\hline 3947.6043 & & $\Delta$ & I & 2 & 3958.266 & & $\mathrm{Ce}$ & II \\
\hline 3947.533 & & Fe & I & 361,426 & 3958.39 & & $\mathbf{A}$ & II \\
\hline 3947.694 & & 0 & I & 3 & 3958.60 & $\mathbf{P}$ & N1 & I \\
\hline 3947.60 & & c & II & 31 & 3958.66 & & $P d$ & I \\
\hline 3947.633 & & Pr & II & 11 & 3958.865 & & $\mathbf{R h}$ & I \\
\hline 3947.770 & & TI & I & 14 & 3959.01 & $\mathbf{P}$ & Sc & II \\
\hline 3947.838 & & So & II & 33 & 3959.436 & & Gd & II \\
\hline 3948.00 & $\mathbf{P}$ & $\mathrm{Fe}$ & I & 652 & 3959.46 & $\mathbf{P}$ & $\mathrm{Fe}$ & I \\
\hline 3948.105 & & Fe & I & 562 & 3959.523 & & Gd & II \\
\hline 3948.113 & & Sm & II & 9 & 3959.527 & & $8 m$ & II \\
\hline 3948.15 & & c & II & 32 & 3960.284 & & $\mathrm{Fe}$ & I \\
\hline $3948 . \overline{28}$ & $\mathbf{P}$ & $\mathrm{Fe}$ & I & 561 & 3860.37 & & $\mathbf{v}$ & II \\
\hline 3948.48 & $\mathbf{P}$ & $\mathrm{Fe}$ & I & 560 & 3960.763 & & $\mathrm{Cr}$ & I \\
\hline 3948.670 & & T1 & I & 13 & 3960.895 & & $\mathrm{Fe}$ & II \\
\hline 3948.779 & & $\mathrm{Fe}$ & I & 604 & 3960.914 & & $\mathrm{Ce}$ & II \\
\hline 3948.801 & & $\mathrm{Ca}$ & I & 6 & 3960.997 & & Co & I \\
\hline 3948.9788 & & $\Delta$ & I & 2 & 3961.147 & & $\mathrm{Fe}$ & I \\
\hline 3949.10 & & Le & II & 41 & 3961.603 & & Mo & II \\
\hline 3949.14 & & $\mathrm{Fe}$ & I & 730 & 3961.523 & & $\mathbf{\Delta 1}$ & I \\
\hline 3949.23 & $\mathbf{P}$ & $\mathrm{Fe}$ & I & 153 & 3961.55 & & $\mathbf{8}$ & III \\
\hline 3949.275 & & To & I & 2 & $\$ 961.59$ & & 0 & III \\
\hline 8949.438 & & Pr & II & 16 & 3962.03 & & La & II \\
\hline 8949.46 & & C & II & 31 & 3962.12 & & N1 & I \\
\hline 3048.64 & & $\mathbf{C r}$ & I & 136 & 3962.19 & & Cr & I \\
\hline 3949.964 & & $\mathrm{Fe}$ & I & 72 & 3962.353 & & $\mathrm{Fe}$ & $\mathbf{I}$ \\
\hline 3949.96 & & Cl & II & 36 & 3962.42 & $\mathbf{P}$ & $\mathrm{Fe}$ & $\mathbf{I}$ \\
\hline 3960.36 & & $\mathbf{Y}$ & II & 6 & 3962.445 & & Pr & II \\
\hline 8960.42 & & 8 & II & 45 & 3962.65 & $\mathbf{P}$ & $\mathrm{Fe}$ & I \\
\hline 3960.78 & $\mathbf{P}$ & Fe & I & 153 & 3962.851 & & $\mathbf{T 1}$ & I \\
\hline 3961.097 & & Cr & I & 136 & 3962.995 & & 80 & II \\
\hline 3951.154 & & Nd & II & 19 & 3963.04 & & La & II \\
\hline 3961.164 & & $F_{\theta}$ & I & 661 & 3963.108 & & $\mathrm{Fe}$ & $\mathbf{I}$ \\
\hline 3961.51 & & $\mathbf{P}$ & III & 9 & 3963.114 & & Nd & II \\
\hline 8951.59 & & $Y$ & II & 16 & $\$ 963.13$ & & 0 & II \\
\hline s951. 717 & & Co & I & 171 & 3963.13 & & 8 & II \\
\hline 3951.765 & & Cr & I & 136 & 3963.354 & & T1 & I \\
\hline 3951.968 & & $\boldsymbol{\nabla}$ & I I & 10 & 3963.43 & $\mathbf{P}$ & $\mathrm{Fe}$ & $\mathbf{I}$ \\
\hline 8961.987 & & 0 & I & 30 & 3963.626 & & $\mathbf{v}$ & I \\
\hline 3952.00 & & Gd & II & 1 & 3963.628 & & Os & $\mathbf{I}$ \\
\hline$\$ 962.08$ & & C & II & 32 & 3963.690 & & Cr & I \\
\hline 3952.195 & & Nd & Ii & 23 & 3964.09 & $\mathbf{P}$ & $\mathrm{Fe}$ & II \\
\hline 3962.326 & & Co & $I$ & 16 & 3964.11 & & $\mathrm{Fe}$ & III \\
\hline 3952. 367 & & $\mathrm{Cb}$ & II & 10 & 3964.261 & & Pr & II \\
\hline 3952.398 & & $\mathrm{Cr}$ & I & 136 & 3964.269 & & T1 & I \\
\hline 3952.673 & & Ce & II & 113,177 & 3964.35 & $\mathbf{P}$ & Cr & II \\
\hline 3952.606 & & Fe & I & 278 & 3964.522 & & Fe & I \\
\hline 3952.704 & & $\mathrm{Fe}$ & I & 362 & 3964.57 & $\mathbf{P}$ & Fe & I: \\
\hline 3952.74 & & $A$ & II I & 89 & 3964.64 & $\mathbf{P}$ & $\mathrm{Cr}$ & II \\
\hline $3952: 917$ & & Co & I & 28 & 3964.727 & & He & I \\
\hline 3952.982 & & 0 & I & 30 & 3964.825 & & Pr & II \\
\hline 3953.056 & & 0 & I & 30 & 3964.80 & & Eu & II \\
\hline 3958.166 & & $\mathrm{Fe}$ & I & 430 & 3964.96 & & Hf & II \\
\hline 3953.163 & & $\mathrm{Cr}_{\mathbf{r}}$ & I & 136 & 3965.011 & & Co & I \\
\hline 3958.50 & $\mathbf{P}$ & $\mathrm{Fe}$ & I & 770 & 3965.236 & & Co & $I$ \\
\hline 3953.616 & & Pr & II & 9 & 3965.263 & & Pr & II \\
\hline 3953.525 & & Nd & II & & 3965.446 & & $\mathrm{Fe}$ & I \\
\hline 3953.660 & & $\mathrm{Ce}$ & II & 141 & 3965.511 & & $\mathrm{Fe}$ & $I$ \\
\hline 3953.76 & $\mathbf{P}$ & $\mathrm{Fe}$ & III & 68 & 3965.83 & $\mathbf{P}$ & Fe & I \\
\hline 3953. 863 & & $\mathrm{Fe}$ & I & $36 \%$ & 3966.045 & & $8 \mathrm{~m}$ & I I \\
\hline 3954.21 & & c1 & II & 82 & 3966.066 & & Fe & I \\
\hline 3954.372 & & 0 & II & 6 & 3966.37 & & Pt & $I$ \\
\hline 3964.38 & & $\mathrm{Fe}$ & III & 120 & 3966.43 & $P$ & Fe & II \\
\hline 3964.596 & & 0 & I & 30 & 3966.532 & & $\mathrm{Fe}$ & I \\
\hline 3954.687 & & 0 & I & 30 & 3966.573 & & Pr & II \\
\hline 3954.715 & & Pe & I & 606 & 3966.630 & & Fe & I \\
\hline 3955.22 & $\mathbf{P}$ & $\mathrm{Fe}$ & I & 527 & 3966.65 & & $\mathbf{z r}$ & $I$ \\
\hline 3955. 352 & & $\mathrm{Fe}$ & I & 562 & 3966.72 & & $\mathbf{E}$ & II \\
\hline 8956.77 & $\mathbf{P}$ & $\mathrm{Fe}$ & I & 219 & 3966.824 & & Fe & $I$ \\
\hline 3965. 82 & $\mathbf{P}$ & $\mathbf{z r}$ & I I & 17 & 3967.048 & & $\mathrm{Ce}$ & II \\
\hline 3955.851 & & $\mathbf{N}$ & II & 6 & 3967.423 & & $\mathrm{Fe}$ & $I$ \\
\hline 3955.956 & & $\mathbf{F e}$ & I & 488 & 3967.441 & & 0 & I I \\
\hline 3966.270 & & Co & I & 2 & 3967.69 & & $I$ & II \\
\hline 3966.284 & & Ce & II & 202 & 3967.964 & & $\mathrm{Fe}$ & I \\
\hline 3956.336 & & T1 & I & 13 & 3968.11 & & v & II \\
\hline 2056.459 & & Fe & I & 604 & 3968.25 & & $\mathbf{z r}$ & I \\
\hline 9956.681 & & Fo & I & 278 & 3968.261 & & Gd & II \\
\hline
\end{tabular}


3978.650

3078.650

5978.677

3978.864

3978.87

3978.08

3979.12

3979.200

3979.22

3979.324

3979.36

3979.40

9979.42

3979.479

3979.51

3979.518

3979.65

3979.788

3879.86

3980.14

3980.35

3980.56

3980.65

3980.821

3980.885

3981.106

3981.233

3981.36

3981.466

3881.61

3981.62

3881.775

3981.94

3981.998

3882.01

3982.063

3982.355

3982.478

3982.583

3982.58

3982.718

3882.901

3983.008

3983.138

3983. 257

9883.35

9883.7

983.77

3983.83

3983.807

983.960

3984.03

3984.140

3884.177

3984.313

984.335

9884.338

3884.46

3984.600

Ce

Co

17

67
173

CO II $\quad 173$

Le II 140

Fe $I \quad 426$

Cr I $\quad 307$

Cr I 280

A II 90

if II $\quad 97$

$\begin{array}{lr}\text { Fe III } & 120 \\ \text { Nd II } & 57\end{array}$

Co I

Fe I

Cr I

$\begin{array}{ll}8 & \text { II } \\ \text { Fe } & \text { III }\end{array}$

3

561

67

$12 n$

C II 37

Al II

Fe I

Ce II

Fe I

Cr I

Lo II

T1 I

Fe II

Fe I

Fe I

C1 II

TI II

Zr II

Pr II

Sd II

Ti I

7 II

$\begin{array}{ll}\text { I } & \text { II } \\ 0 & \text { II }\end{array}$

Ce II

od II

Son II

Cr I

Fe I

AI II

$\mathrm{Fe} I$

37
12
153

153
186

194

22

138

188

428
12

12

11

142
28

67

11
33

6

172

48
38

213

485

32,48
8

426

Cr $1 \quad 38$

$\begin{array}{lr}\text { Pe I } & 277 \\ \text { HF II } & 19\end{array}$

N1 I 171

Un I

TI I

i I

Cr I

Fe I

3884.675

C. II

Zr II

$868 \quad$ Ru I

1884.93

3985.241

3885.246

3985. $32 \quad P$

3985,393

3985.46

3885.680

3985.74

3985.783

3985. 96

2985. 97

2086.03

9886. 170

3886.18

1086.201

3986.29

9806. 682

3986.7533

2086.820

2987.090

3987.098

9987.117

3987.214

9987.428

987.46

5987.63

188

38

219
89

252

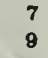

661

19

$\begin{array}{llr}\text { Fe I } & 661 \\ 0 & \text { II } & 22\end{array}$

T1 188

Cr II 10

$\checkmark$ II

$\begin{array}{ll}\text { Cr } & \text { II } \\ 8 & \text { III }\end{array}$

Cr II

Fe I

Fe I

Yo II

F. I

Mn I

202

8
10

656

560

800
33

80 II

48 I

Nn I

Mn I

co I

od II

8. II

Wn I

II II

$\begin{array}{lllr}3987.88 & & \text { Yb I } & 2 \\ 3988.18 & & \text { A II } & 65 \\ 3988.51 & & \text { La II } & 40 \\ 3988.68 & & \text { Zr I } & 46 \\ 3988.833 & & \text { V I } & 89 \\ 3989.06 & & \text { Sc II } & 8 \\ 3989.24 & \text { P } & \text { Fe I } & 561 \\ 3989.29 & & \text { Zr I } & 6 \\ 3989.444 & & \text { Ce II } & 240 \\ 3989.581 & & \text { TI I } & 81 \\ & & & \\ 3989.60 & \text { P } & \text { Fe I } & 605 \\ 3989.718 & & \text { Pr II } & 12 \\ 3989.758 & & \text { TI I } & 12 \\ 3989.803 & & \text { V II } & 32 \\ 3989.859 & & \text { Fe I } & 768 \\ 3989.958 & & \text { Mn I } & 33 \\ 3989.986 & & \text { Cr I } & 268 \\ 3990.103 & & \text { Nd II } & 19 \\ 3990.16 & \text { Cr I } & 280 \\ 3990.184 & & \text { TI I } & 186\end{array}$

$3990.19 \quad$ CI II

$\begin{array}{rlr}3990.298 & \text { Co I } & 58 \\ 3990.379 & \text { Fe I } & 527\end{array}$

\begin{tabular}{|c|c|}
\hline 3990.55 & $\mathbf{P}$ \\
\hline
\end{tabular}

$3990.566 \quad$ V I $\quad 89$

$3990.81 \quad$ Fe III

$3990.94 \quad$ S II 45

$3991.123 \quad$ Cr I $~ 38$

$3991.14 \quad \mathrm{Zr}$ II

$\begin{array}{llr}3991.50 & \text { CI III } & 7 \\ 3991.528 & \text { Co I } & 173\end{array}$

Co I

To I

$3991.743 \quad$ Nd II 19

3991.77

3991.831

3991.865

3992.014

3992.06

Co I $\quad 129$

Co 1 3

3992.11 Cr I 38

3982. 114 IF I

3892.386 Ce II 134

3992.395 $\quad$ Fe I $\quad 604$

3982.64 P $\quad$ Fe I 218

$\begin{array}{llll}3992.801 & \text { V } & & 88 \\ 3992.845 & \text { Cr I } & 67\end{array}$

$\begin{array}{rlr}3992.813 & \text { Ce II } & 226\end{array}$

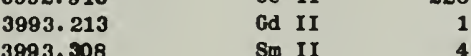

3993.401

3993.526

3993.796

3993.822

3993.952

$\begin{array}{lllr}3993.952 & & \text { N1 I } & 170 \\ 3993.968 & & \text { Cr I } & 67 \\ 3994.00 & \text { P } & \text { Fe I } & 560 \\ 3994.117 & & \text { Fe I } & 526\end{array}$

$\begin{array}{lllr}3993.952 & & \text { N1 I } & 170 \\ 3993.968 & & \text { Cr I } & 67 \\ 3994.00 & \text { P } & \text { Fe I } & 560 \\ 3994.117 & & \text { Fe I } & 526\end{array}$

$\begin{array}{lll}3994.165 & \text { Gd II } & 48\end{array}$

$\begin{array}{llll}3994.27 & P & \text { Fe } I & 320\end{array}$

3994. 50 Lo II 78

3994.542 $\quad$ Co II $\quad 78$

$\begin{array}{lllr}3894.56 & P & T 1 & 1\end{array}$

$\begin{array}{lll}3984.683 & \text { TI I } & 188\end{array}$

3994.81

3995.10

3995. 17

A II 89,101

Pr II

N II 11

Fe 1 604

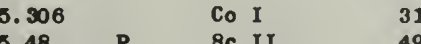

$\begin{array}{llll}3995.48 & \text { P } & 8 \text { C II } & 48 \\ 3995.48 & \text { P } & 8 \text { C II } & 16\end{array}$

3995.586 Th II

$\begin{array}{lll}3995.656 & \text { Ba } I & 8\end{array}$

3995.74 LA II 27

$\begin{array}{llll}3995.83 & \text { P } & \text { N1 I } & 238 \\ 3995.860 & & \text { AI II } & 47\end{array}$

$\begin{array}{rlr}3985.860 & \text { Al II } & 47 \\ 3995.996 & \text { Fe I } & 279\end{array}$

$\begin{array}{lllll}3996.075 & & \text { AI II } & 47 \\ 3996.159 & & \text { AI II } & 47 \\ 3996.182 & \text { Forb } & \text { AI II } & 47\end{array}$

$\begin{array}{lclr}3906.182 & \text { Forb } & \text { Al II } & 47 \\ 3996.28 & \text { P } & \text { Fe I } & 561\end{array}$

$\begin{array}{llll}3996.28 & P & \text { Fe I } & 427 \\ 3996.320 & & \text { Gd II } & \end{array}$

$\begin{array}{llll}3996.320 & & \text { Gd II } & \\ 3996.325 & & \text { AI II } & \$ 7\end{array}$

$\begin{array}{lllr}3996.36 & \text { P } & \text { Fe II } & 180 \\ 3996.381 & & \text { AL II } & 47\end{array}$

T. II

11

3896.518

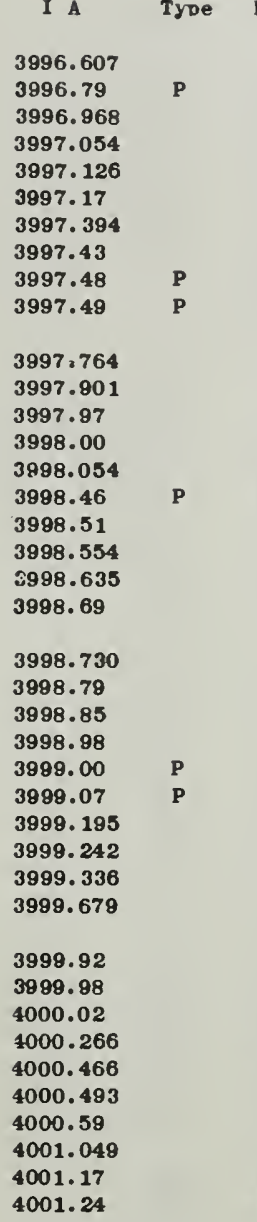

$\begin{array}{llr}\text { Sc } & 1 & 7 \\ \text { Fe } & 1 & 1074\end{array}$

945

Pr II

P III

Fe I

II

Gd II 67

132

8 III

S1 II

Fe $I \quad 276$

606

III 50

$\begin{array}{lll}\text { Co } & I & 33 \\ \text { T1 } & 1 & 12\end{array}$ 
I A Type Element Niultiplet No.

I A Type Element Multiplet No.

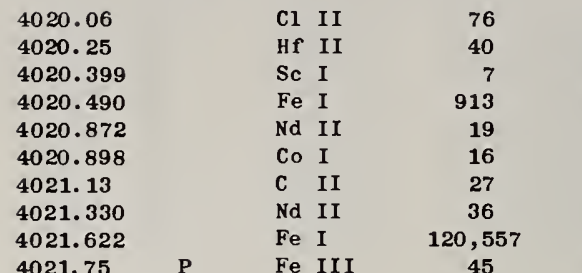

4021.812

4021.869

4021.925

4022.263

4022.333

4022.36

4022.36

4022.45

4022. 744

4023.002

4023. 231

4023.388

4023.399

4023.58

4023.688

4023.739

4023.986

4023.99

4023.99

4024.04

4024. 109

4024.45

4024.491

4024.552

4024.573

4024.727

4024. 735

4024.785

4024.92

4025.010

4025.012

4025.07

4025.07

4025.114

4025.136

4025.44

4025.44

4025.49 P Forb

T1 I

$\begin{array}{ll}\mathrm{Fe} & \text { I } \\ \mathrm{V} & \mathrm{I} \\ \mathrm{N} 1 & \mathrm{I}\end{array}$

Cr

Gd II

Cr II

Fe III

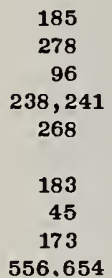

Nd II

Sm II

Co I

La II

Sc I

Cr I

He I

$\begin{array}{ll}\text { N1 } & \text { I } \\ \text { Zr } & \text { I }\end{array}$

0 I I

Fe I

Zr II

Ce II

Fe II

Ti I

F II

Fe I

Nd II

F II

Cr I

Fe III

T1 I

N1

TI II

Cr I

Ni I

4025.495

F II

4025.60

1025.67 He II

4025.87

4026.080

4026.166

4026.189

4026.362

4026.40

4026.435

4026.5

4026. 539

4027.032

4027.103

4027.20

4027.30

4027.426

4028. 332

4028.411

4028.79

4029.16

Fe III

La II

N I I

Cr I

He

He I

o II

Mn I

Al II

556.654

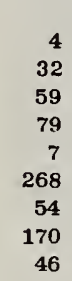

4

32
59

79
7

54

170

99

277

54
49
127

2
560

560
24

46

2

53

208

240

11
37

117

19
2

4029.32 P N1

4029.640

4029.68

4030.03

4030.19

4030.28

4030.470

4030.499

4030.512

T1 I

Co I

Cr I

Z I I
I

T1 I

Ti II

Ce II

HI I I
Hf

$\begin{array}{ll}\text { Ni I } & \text { I } \\ \text { T1 } & \text { I I }\end{array}$

Fe I

Zr II

Zr I

4018.102

4018.282

4018.38

4018.49
4018.50

$\mathrm{Fe} I$

Fe II

4018.826

4019.05

4019.05

4019.055

4019.137

4019.288

4019.30

4019.45

4019.982

4020.05
Nd II

' 19

V II $\quad 201$

Th II

Co I

Co $I$

P II

Sm II

Fe I

I A

Type

Element

Multiplet No.

$4031.456 \quad$ Fe II 151

Al II

$\begin{array}{lll}4031.68 & \text { La II } & 40\end{array}$

$\begin{array}{llll}4031.73 & P & \text { Fe I } & 427 \\ 4031.753 & & \text { TI I } & 185\end{array}$

4031.807

4031.968

4032.46

4032.628

4032.636

Fe I

655

$\begin{array}{lr}\text { T1 I } & 297 \\ \text { Fe I } & 44\end{array}$

4032.946

4032.975

4033.073

4033.18

0 II 50

$\begin{array}{llll}4033.19 & P & \text { Fe } & \\ & & & 218\end{array}$

4033.55

4033.83

Sb I

P II

4033.857

4033.883

4033.95

4034. 012

4034.10

4034.490

4034.84

4034.884

4035.087

4035.09

A II

Pr II 19

Ti I 208

Cr I

Nd II

Zr II

Mn I

Zr II

T1 I

N II

4035.110

0 II

$4035.25 \quad P$

$\mathrm{Fe} \mathrm{I}$

4035.54

4035.542

4035.631

4035.728

4035.82

4035.828

4035.96

4035.98

4036.23

4036.37

4036.53

4036.59

4036.779

4036.80

4037.665

4037.725

4038.12

4038.27

4038.622

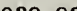

4039.100

$\mathrm{Fe} \mathrm{I}$

Fe II
Fe I

Fe III

Co I

V II

Mn I

Mn I
Fe III

TI I

59

4039.12

4039.30

4039.302

4039.357

4039.397

4039.574

4039.83

4039.94

4040.24

4040.310

Ni I

$\begin{array}{ll}\text { Fe } & \text { I } \\ P & \text { II }\end{array}$

Fe I

Cl II

La II

$\checkmark$ I

Cr I

Gd I I

33

831

33
22

119 
I $\Delta$ Type Blement Multiplet No.

4042.81

4042.91
4043.502

043.002

4043.537

4043.57

4043.596

4043.68

043.696

4043. 775

4043.901

$\begin{array}{ll}4043.88 & P \\ 4044.01 & P\end{array}$

(044.01

4044. 145

4044.4182
4044.49

4044.49

404.67

4044.614

4044.75

4044.818

4044.86

4045. 133

4045. 139

1045. 148

4045. 16 P Forb

045.20

4045.38

4045.58

4045.6

4046.81

$4046.07 \quad P$

4046.15

1046.19

4046.268

4046.341

4046.46

4046.557

1046.620

4046.760

4046.81 P Fe II

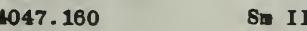

4047.214

047.315

4047.51

1047.64

047.782

$\begin{array}{llll}047.88 & P & \text { Y II }\end{array}$

(1047.8

$\begin{array}{ll}\text { Bf II } & \text { I } \\ & \end{array}$

$\begin{array}{ll}\text { Fe } & \text { I } \\ 0 & \text { II }\end{array}$

Cr I

V II
Ce II

Fe I

$\mathrm{Hg}$

Fe I

S. I

Fe I

Y I

Be II

4048.0

$4048.56 \quad P \quad$ Cr I

$1048.68 \quad$ Zr II

4048.755

4048.780

4048.831

1048.889

049.03

1049.14

4048. 336

1049. 389

4048.428

049.44

049.71
4049.783

049.858

1030.02

050.08

4050.11

4050.32

4060.67

050.863

4051.06

051.08

4051.140

4081.18

081.3

4051.352

4051.923

4051.87

1052. 22

052.312

1052.456

032.472

052.66

1052. 72

4052.83

4052.84

Mn I

Cr I

Pe II

Nn I

$\checkmark$ II

Fe I

Gd II

He II

N1 I

Cr I

Gd II

Cr I

La II

5 II

Zr II

11

V II

Fe II

Nd II

N1 I

Fo II

$\begin{array}{ll}\text { V } & \text { II } \\ \text { V } & \text { I }\end{array}$

33
66
3
39
32
34
122
306
208
276,557

I

Type Blewent

Multiplet No.

$\begin{array}{ll}\text { Fe III } & 11 \\ \text { Gd II } & \\ \text { Cr II } & 1 \\ \text { Ce II } & 3 \\ \text { A II } & 5 \\ \text { Fe III } & 88 \\ \text { V II } & 21 \\ \text { Gd I } & \\ \text { TI II } & 87 \\ \text { Fe I } & 48\end{array}$

119

4053.28
4053.294

4053.45

4053.506

4053.56

4053.59

4053.69

4U53.642

4053. 814

4053.82

4054.10

4054.11

4054.18

4054.55

4054.555

034.618

4054.833

4054.845

4054.883

4054.991

4055.011

4055.03

4055.046

4055.214

4055.543

4055.88

4056.027

4056.06

4056.07

4056.212

4056.270

4056.53

4056.543

4056.793

4056.8

4037.00

4057.074

4057.19

4087.195

4057.347

0 II 50,98

Cr II 19

Fe I 557

$\begin{array}{llr}0 & \text { II } & 98 \\ \text { Sc I } & 6\end{array}$

Co I

Fo I

Pr II

Fe I 698

Ce II 82

T1 I 80

Fe I I

Mn I

Yn I

Fe I

Mo I

C III

Cr II

T1 II

$\begin{array}{lll}V & I I & 14 \\ \text { Fe } & \text { I }\end{array}$

Pr II 26

Cr I 306

$\Delta 1$ II 88

$\begin{array}{llr}\text { N } & \text { II } & 39 \\ \text { V } & \text { I } & 121\end{array}$

Cr I

Co I

N1 I

Fe I

$P$ III

4057.39

4057.467

4057.5052

4087.51

4057.612

4057.68

4057.72

4057.80

4057.81

4057.812

4057.950

4058.08

4058.139

4058.183

4058.219

4058.227

4058.46

4058.600

4058.7

4058.766

4058.77

4058.772

4088.812

4058.930

4058.933

4058.07

4058.27

4059.321

4059.370

4059.392

4059.726

4059.861

1060.08

4080.263

4060.58

4060.62

4060.98

4061.085

4061.3

$\begin{array}{ll}\text { Fe } & \text { II } \\ \text { Mg } & \text { I }\end{array}$

Fe III

T1 I

Fe I

A II

Cr I

19
36
33
98
15
5
87
5
98
9
7
8
6
2
8
0
8
8

5

914
12

24

182

14
88
38
156

166
3
89

277

1

212
16

33

254

729

9
3

251

$\mathrm{Pb} I$

Mn I

La II

T1 I

Co I

Gd I

Fe I

Fe I

Co I

$\mathrm{Fe} I$

$S$ II

Cr I

Ca I

$\begin{array}{ll}\text { Yn } & \text { I } \\ \mathrm{Cb} & \mathrm{I}\end{array}$

Cl III

P III

Co I

Gd II 118

Yn I 29

Fe I $\quad 767$

Nd II

T1 I

$\begin{array}{ll}\text { T1 } & \text { I } \\ 0 & \text { II }\end{array}$

Cr I

0 II

$\begin{array}{ll}\text { Nd } & \text { I I } \\ 8 \mathrm{c} & \text { III }\end{array}$

$\$ 081.742 \quad$ Mn I

1001.787

4062.08

4062.223

4062.446

062.580

4062.817 
I A Type Element Multiplet No. I A Type Element Multiplet No.

I A Type Element Multiplet No.

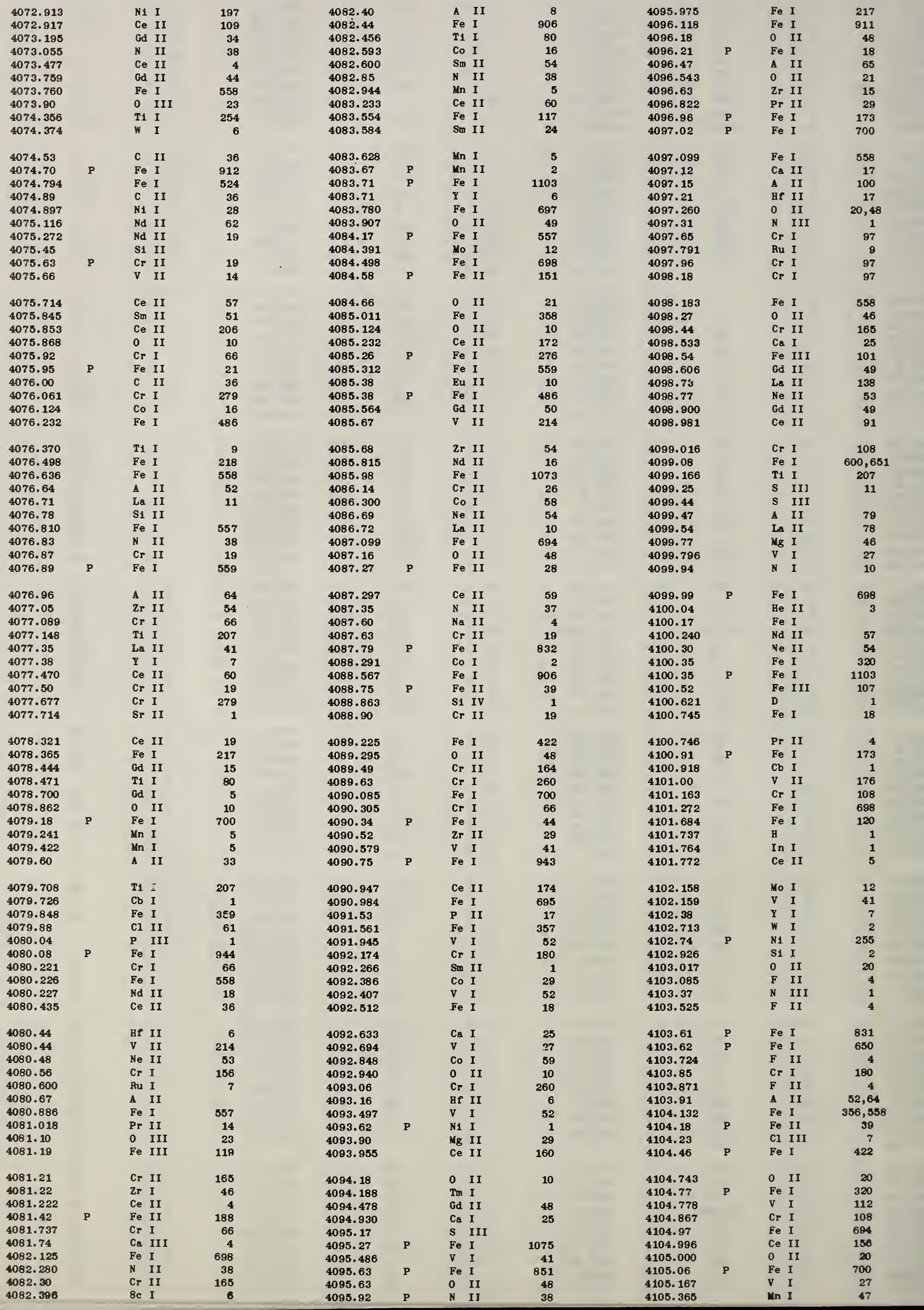


I I Type Blewent Multiplet No.

4105.843

4106.03

4106.05

4106.134

4106.266

4106.437

4106.582

4106.71

4106.83

4106.88

4107.07

107.387
1107.426

4107.477
4

4107.487

4107.492

4107.75

4108.13

4108.31

4108.39

Ton I

0 II 10

Ce II

Fe I

Fo I

Nd I

Cr I

Ce II

10
180
100

160
217

697

260

138

0 II

Sin II

ce II

Yo I

Fo I

Fe I

$\mathrm{Zr} I$

Cr I

Gd II

Co I

$\begin{array}{lll}\text { Ca } & \text { I } \\ 0 & \text { II }\end{array}$

Fe I

Nd II

F II

S I I II

Nd II

Mg II

Cr I

Co $I$

$\mathrm{Fe} I$

$\checkmark$ I

Ca II

Fo III

47

47
50
138

138
12

52

334

831
559

835

4109.54

4109.706

409.786
4109.808

4109.81

4109.85

4109.98

N I

4109.98

$\$ 110.00$

4110.05

4110.20

4110.33
4110.381

4110.472

4110.532

110.795

$\begin{array}{lll}4110.903 & \text { Mn I } & 37,47 \\ 4111.01 & \text { Cr II } & 18,26\end{array}$

$\begin{array}{llll}411.06 & P & \text { Fo I } & 689\end{array}$

$\begin{array}{lll}4111.36 & \text { Cr I } & 97\end{array}$

$\begin{array}{lll}411.438 & \text { Gd II }\end{array}$

4111.56

111.67

1111,785

4111.802

8 III

Cr I 97

V I 27

4112.018 08 I

4112.029

4112.04

4112.09

4112.17

112.3

4112.58

412.708
4112.83

412.83
411.972

4113.210

4113.23

4113.2

1113. 28

4113.45

$\$ 113.518$

4113.68

4113.728

1113.82

1113.826

4113.876

4113.002

1114.00

4114.449

4114.52

4114.85

4114.95 ?

4114.99

4115.185

0 II

Bu II

Fo I

Fe I

Cr II

Cr II

F. II

65

2

48
558

17

30
28

10

21

1
27
357

357
41
17

260

10

4
30
37

37

28

29

20
97

47
, 26
89
97

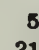

5
21

10
766
275

275

685

18
9
8

1103

Zn I

Fe III

Cr II $\quad 18$

Fo III

HP II

0 II

Nd II

Mn I

8u II

Fe I

A II

Ne II

Fe I

I II

v I

C. II

I A Type Element Kultiplet No.

$\begin{array}{llr}\text { Gd II } & 117 \\ \text { Fe I } & 910 \\ \text { N1 I } & 255 \\ \text { S1 IV } & 1 \\ \text { A I I } & 124 \\ \text { V I I } & 27 \\ \text { F } & \text { II } & 5 \\ \text { V } & \text { I } & 27 \\ \text { Cr II } & 181 \\ \text { V } & \text { I } & 27\end{array}$

Fo I 558

4116.97

4117.013

4117.09

4117.288

117.32

4117.71

4117.872

4118.10

4118.182

4118.45

4118.481

4118.551

4118.643

4118.774

4118.015

4119.219

4119. 221

4119.44

4119.457

$\begin{array}{ll}4119.53 & P \\ 4119.66 & P\end{array}$

4119.784

4119.877

4120

4120.037

4120.21

4120.279

4120.538

4120.584

4120.613

4120.654

4120.78

4120.812

4120.829

4120.97

4120.993

4121.0

4121.31

4121.318

4121.48

4121.48

4121.637

4121.682

4121.7

4121.806

4121.817

4121.95

Ce II

P II

Fe I

Fe I

Fe I

Ne II

v I

Cr I

Fe I

Sa II

$\checkmark$

co

Fe I

Ce II

$\begin{array}{ll}\text { F } & \text { II } \\ \text { O } & \text { II }\end{array}$

35
17
77
484
833
700,1103
54
11
112

Cr I

$\checkmark I$

Fo II

Fe I

Ce II

$0 \mathrm{~V}$

II I

Fe I

$\checkmark$ I

0 II

cr I

Nd II

P II

Be I

Ce II

Fe III

He $I$

8 II

85

801

B

41
28

558

89

20

65

41
21

320

83

263

423
20

$\begin{array}{llll}4122.00 & P & \text { Fo I } & 765\end{array}$

$\begin{array}{llll}4122.05 & C \text { III } & 17\end{array}$

4122.149

4122.162

4122.522

4122. 638

4122.757

4122.98

4123

4123.068

4123.188

4123.23

4123.230

4123.278

4123.287

4123. 38

4123.387

4123.488

1123. 34

Fe III

Co I

$2 r$ I

T1 I

Rh I

$0 \mathrm{v}$

Fe I

Cr I 
I A Type Blement Multiplet No.

$\begin{array}{lllc}4134.19 & \text { P } & \text { Fe I } & 217 \\ 4134.343 & & \text { Fe I } & 3 \\ 4134.433 & & \text { Fe I } & 482.697 \\ 4134.488 & & \text { V I } & 27 \\ 4134.681 & & \text { Fe I } & 357 \\ 4134.72 & & \text { K II } & 1 \\ 4135.325 & & \text { Nd I } & \\ 4135.443 & & \text { Ce II } & 188 \\ 4135.68 & & \text { Zr I } & 50 \\ 4135.77 & \text { P } & \text { Cr II } & 163 \\ 4135.77 & & \text { Fe I } & 1073 \\ 4135.784 & & \text { Os I } & 3 \\ 4135.9 & \text { P } & \text { O V } & 11 \\ 4136.386 & & \text { V I } & 26 \\ 4136.512 & & \text { Fe I } & 694 \\ 4136.894 & & \text { T1 I } & 221 \\ 4137.002 & & \text { Fe I } & 726 \\ 4137.090 & & \text { Cb I } & 1 \\ 4137.104 & & \text { Gd II } & \\ 4137.257 & & \text { Mn I } & 37 \\ & & & \end{array}$

4137.284 T1 I 253

$\begin{array}{lll}4137.42 & P & \mathrm{Fe} \\ \$ 137.63 & & \mathrm{~N}\end{array}$

4137.93

$4137.97 \quad$ Fe III

$\begin{array}{lll}4138.21 & P & \text { Fe I }\end{array}$

4138.40 P Fe II

$4138.52 \quad$ Ni I

$\mathrm{Fe} I$

4139.37

4139.452

4139.48

4139. 702

$4139.933 \quad \mathrm{Fe}$

$4140.24 \quad P \quad$ Fe I

$4140.304 \quad P \quad$ Sc I

$\begin{array}{lll}4140.42 & P & \text { TI I } \\ 4140.441 & & \text { Fe I }\end{array}$

4140.450

Gd II

1103

2

118

320

150
39

237

4140.5

4140.74

4141.017

4141.25 Forb

4141. 257

4141.352

4141.73

1141.84

4141.862

4141.96

Fe III

0 II

Gd II

AI II I
Pr II

Pr I
Fe I

La II

HP II

$\begin{array}{ll}\text { Fe } & \text { I } \\ 0 & \text { II }\end{array}$

0 II

4142.08

4142.15
4142.184

4142.184
4142.193

4142. 24

4142. 291

4142.320

4142. 398

4142.47

4142. 480

4142. 628

4142. 66

4142.86

4142.90

$\mathbf{4 1 4 3 . 0 4 8}$

4143.07

4143. 136

4143.280

4143.418

4143.4

4143.50 P

4143.758

4143.77

4143.77

4143.83

4143.87

4143. 871

4144. 492

4144.553

4144. 995

4145. 100

4145. 209

4145.74
4145.764

4145. 77

4145.90

4146.071

4146.09

O II

NI I

Cr I

0 II

S II

Ni I

Ce II

Cr I
TI I

Fe I

$\begin{array}{ll}V & \text { I } \\ \text { Y } & \text { I }\end{array}$

V. II

Ti I

Fe II

Pr II

TI I

Fe I

$\begin{array}{ll}\text { Fe } & \text { I } \\ 0 & \text { II }\end{array}$

He I

La II

0 II

Fe I
Fe III

$\mathrm{Fe} I$

Ce II

Nd II

Ce II
$S$ II

Fe I

Fe III

$\begin{array}{ll}N & \text { II } \\ \text { Cr } & \text { II }\end{array}$

0 II

Fe I

118

94

221
1
18

18

20

221

48
485

118

19
117

17

10
480

40

87

422
106

106

16
212

305

106

4

10
179
296

109

1103

5
226

226

253
188

4

253
523

6

697

106

53

106

43

43
7

61

91
44
274

65

162
106

422
I A Type Element Multiplet No.

$\begin{array}{lllr}4146.20 & & \text { Cr I } & 260 \\ 4146.234 & & \text { Ce II } & 203 \\ 4146.47 & & \text { Cr I } & 108 \\ 4146.695 & & \text { Cr I } & 107 \\ 4146.94 & & \text { S II } & 65 \\ 4147.09 & & \text { CI II } & 60 \\ 4147.26 & \text { P } & \text { Fe II } & 141 \\ 4147.34 & \text { P } & \text { Fe I } & 693 \\ 4147.43 & & \text { A II } & 9 \\ 4147.49 & \text { P } & \text { Fe I } & 832\end{array}$

4147.532 Mn I 37

$\begin{array}{lllr}4147.673 & & \text { Fe } & \text { I } \\ 4148.27 & P & \text { Fe } I & 832\end{array}$

$\begin{array}{rrrr}4148.52 & & C r & 241 \\ 4148.75 & P & N 1 & \end{array}$

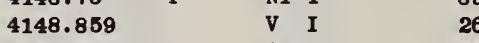

4148.901

4148.91

4149.19

4149.372

4149.445

4149.45

4149.49 $P$ P

4149.76 P Fe

4148.83

4149.897

4149.917

4149.936

4150.08
4150.138

4150.258

4150.366

4150.429

4150.557

4150.67

4150.809

4150.963

4151

4151.00

4151.46

4151.52

4151.60

4131.79

4151.970

4151. 98

4152.07

4152. 172

4152. 209

4152. 355

4152.43

4162.575

4152.775

4152. 78

4152.98

4153.067

4153.302

4133. 328

4153. 332

4153.510

4153.67

4153.816

4153.906

4154. 109

4154.502

4154.812

$\mathbf{4 1 5 4 . 8 6 2}$

4154.865

4154.98

4155.217

4155. 525

4155.532

4156.083

4156.11

4156. 24

4156.3

4156.460

4156.50

4156.54

4156.670

4156.8

4156.803

4157.788

4157.82

4158.420

4168.45
III

158,189

P V II

37

Al III

$\mathrm{Fe} \mathrm{I}$

N1 I

Co I

T1 I

T1 I

T1 I

$\begin{array}{ll}\mathrm{Zr} & \text { II } \\ 0 & \mathrm{~V}\end{array}$

Cr II

N I

Eu II

I A

Type Element

Multiplet No.

\begin{tabular}{|c|c|c|c|c|}
\hline & $\Delta$ & & \\
\hline & & 0 & v & 1 \\
\hline \multicolumn{2}{|l|}{$\begin{array}{l}4158.76 \\
4158.798\end{array}$} & $\mathrm{Fe}$ & I & 60 \\
\hline \multicolumn{2}{|l|}{4158.90} & Hf & II & 4 \\
\hline \multicolumn{2}{|l|}{4159.038} & Ce & II & 24 \\
\hline \multicolumn{2}{|l|}{4159.407} & Al & II & 7 \\
\hline \multicolumn{2}{|l|}{4159.450} & Al & II & \\
\hline \multicolumn{2}{|l|}{ \$159.634 } & T1 & I & 20 \\
\hline \multicolumn{2}{|l|}{$4159 \cdot 686$} & $\mathbf{v}$ & I & 2 \\
\hline \multicolumn{2}{|l|}{4169.725} & Al & II & \\
\hline \multicolumn{2}{|l|}{4159.809} & $\mathbf{A l}$ & II & \\
\hline \multicolumn{2}{|l|}{4160.239} & Al & II & \\
\hline \multicolumn{2}{|l|}{4160.263} & Al & II & \\
\hline 4160.28 & P & Fe & I I & 1 \\
\hline 4160.56 & & $\mathbf{P}$ & II & 3 \\
\hline 4160.561 & & Fe & I & 41 \\
\hline 4160.62 & $\mathbf{P}$ & $\mathrm{Fe}$ & II & \\
\hline 4160.78 & $\mathbf{P}$ & $\mathrm{Fe}$ & I & 111 \\
\hline 4160.8 & & $\mathbf{N}$ & II & 50 , \\
\hline 4161.05 & & $\mathrm{Cr}$ & I I & 16 \\
\hline
\end{tabular}

$\begin{array}{lll}4161.080 & \text { Fe I } & 689\end{array}$

4161.175

4161. 20

4161.27

4161.34 P

4161.415

4161.488

4161.524

4161.56

4161.796

Fe I

Ce II

Cr II

N1 I

Cr I

Fe I

Cr II

4161.94
4162.072

4162.39

162.40

4162. 698

1162.732

4162.80

4162.93

4163.09

4163.16

4163.35

4163.625

4163.644

4163.665

4163.658

4163. 676

4163.94

4164.015

4164. 134

La II

$\begin{array}{ll}\text { V } & \text { II } \\ 8 & \text { II }\end{array}$

He II

8 II

Gd II

C III

Fe I

od II 
I A Type Element Multiplet No.

4169.330

4169.478

4169.773

4169.777
4169.838

4169.878

4169.98

4170.108

P Cr I

$\begin{array}{ll}4170.86 & \text { Cr II } \\ 4170.906 & \text { Fe I }\end{array}$

4171.018

4171.608

4171.675

4171.696

4171.824

4171.897

4171.904
4171.92

$4172.048 \quad G a$ I

4172.126 Fe I

$\begin{array}{lll}4172.20 & P & \text { Fe II } \\ 4172.273 & & \text { Pr II }\end{array}$

4172.60

4172.609

4172.641

4172.749

$\begin{array}{lll}4172.97 & \text { P } & \text { Fe I } \\ 4173.05 & \text { P } & \text { T1 II }\end{array}$

4173.18 P Fe

4173. 234

4173.322

4173.379
4173.450

4173.51

4173.537

4173.556

4173.75

4173.76

4173.77

4173.92

4174

4174.042

4174.088
$\mathbf{4 1 7 4 . 0 8 8}$

4174. 14

4174.15

4174. 27

4174. 300

$\begin{array}{lll}4174.31 & P & \text { Mn II } \\ 4174.33 & & \text { Hf I }\end{array}$

4174.33

4174.419

4174.472

4174.785

4174.917

4174. 941

4175.227

4175.538

4175.606

4175. 640

4175.89

$\mathbf{4 1 7 5 . 9 4 5}$

4176.080

4176.164

4176.44

4176.57

4176.57

4176. 793

4177.07

4177. 17

4177. 321

4177.357

4177.50

4177.5

4177.54

$\mathbf{4 1 7 7 . 5 8}$

4177.587

4177.70
4178.019

4178. 39

4178.390

4178.8

4179

4179.062

4179.226

4179.25

4179. 257

4179.31

$\mathrm{Os} I$

Fe I

Fe II

N II

T1 II

Gd II

$\begin{array}{ll}\mathbf{N} & \text { II } \\ \mathbf{Y} & \text { II }\end{array}$

A II

$\begin{array}{ll}\text { Fe } & \text { I } \\ \text { N } & \text { IV }\end{array}$

S II

T1 I

TI II

Y I

Fe III

S II

Fe I

$\begin{array}{ll}\text { T1 } & \text { I } \\ \text { Cr } & \text { I }\end{array}$

$\mathrm{Fe} I$

Cr I

Cr I$$
\begin{array}{ll}
\text { Gd I } \\
\text { Nd II }
\end{array}
$$

Fe I

$$
\begin{array}{ll}
\mathrm{Fe} & \mathrm{I} \\
\mathrm{Cr} & \mathrm{I}
\end{array}
$$$$
\begin{array}{ll}
\text { Cr } & \text { I } \\
\text { Ce } & \text { II }
\end{array}
$$$$
\text { N II }
$$$$
\text { Fe II }
$$

$\mathrm{Fe} I$

Fe I

P $\quad \mathrm{Fe} I$

$\mathrm{Cr}$

Cr I
Nd

\section{T1 I}

$$
\text { Hf II }
$$$$
\begin{array}{ll}
\mathrm{Fe} & \mathrm{I} \\
\mathrm{Y} & \mathrm{II}
\end{array}
$$

Y II
Co $I$

Fe I

Fe II

8 II

\section{A II}

Fe II

$\begin{array}{ll}\text { Fe } & 11 \\ 0 & V\end{array}$

Cr I

$\begin{array}{ll}V & I \\ \text { Co I }\end{array}$

Fe III

$\begin{array}{ll}\text { Cr } & \text { I } \\ \text { A } & \text { II }\end{array}$
Gd II

163
24
161
693
278
173
12
35
278
18

181
482
206
43
261
941
16
105
650
18

1
649

649
148

13
18
163

163
689
19

19
909,1073

96

698

16,50

179,250
I A Type Element

Multiplet No.

$\checkmark \quad I$

4179.419

4179.422

4179.43

4179.585

4179.667
4179.81

4179.81

4179.860

$\begin{array}{lll}4179.90 & \text { P } & \text { Co I } \\ 4179.92 & \text { P } & \text { Cr II }\end{array}$

25

Pr II

Hf II

Nd II

$N$ II

Zr II

T1 I

$\begin{array}{llll}4180.41 & P & \text { Fe } I & 274\end{array}$

$4180.498 \quad$ TI I $\quad 206$

4180.68

4180.7

4180.86

V II

4180.97 P $\quad$ Fe II

4180.97 La II

4181.17 P $\quad$ TI II

4181.20 P Fe I

$\begin{array}{llll}4181.50 & & \text { Cr II } & 181 \\ 4181.55 & \text { P } & \text { Fe I } & 763\end{array}$

$\begin{array}{lll}4181.758 & \text { Fe } I & 35\end{array}$

4181.8838 A I

$4182.384 \quad$ Fe I

4182.591 P $V I$

4182.69 P $\quad F$ I

$\begin{array}{llll}4182.98 & & \text { Fe I } \\ 4183 & \text { P } & \text { N II } \\ \text { IV }\end{array}$

7
$476 a$

$476 a$
24

24
149

694

36
15

$4183.025 \quad$ Fe I 697

$\begin{array}{lllr}4183.025 & & \text { Fe I } & 697 \\ 4183.20 & \text { P } & \text { Fe II } & 21 \\ 4183.294 & & \text { T1 I } & 220\end{array}$

$\begin{array}{llr}4183.294 & \text { T1 } I & 220 \\ 4183.31 & \text { Zr I } & 51\end{array}$

4183.435

4183.764

4184.08

4184.22

4184.252

4184. 26

$\checkmark$ II

$\begin{array}{ll}\text { Sin } & \text { II } \\ \text { Fe III }\end{array}$

Fe I

Gd II

4184. 329

4184.475

4184.895

4184.895

4185.334

4185. 345

$4185.456 \quad$ Cr I

$4185.50 \quad P \quad$ Cr II

4185.61

$\begin{array}{rllr}4185.66 & P & \text { Fo } I & 43\end{array}$

$\begin{array}{llll}4185.95 & & \text { S II } & \\ 4186.01 & \text { P } & \text { T1 I } & 220\end{array}$

$\begin{array}{llll}4186.033 & & \text { Nd II } & 24 \\ 4186.08 & P & \text { Cr II } & 127\end{array}$

$4186.119 \quad$ T1 I $\quad 129$

$\begin{array}{llr}4186.24 & \text { K } \text { II } & 1 \\ 4186.359 & \text { Cr I } & 248\end{array}$

4186.359

4186. 898

4186.70

Ce II

Ce II

Fe I

4187.05

4187.246

4187.31

4187.323

4187.56

4187.59

4187.616

4187.68

4187.802

4188.099

C III

Co I

Ce II

Zr I

Fe I

Tm I

HP II 73

$\begin{array}{ll}\text { Fe I } & 152 \\ \text { Od II } & 17\end{array}$

4188.128

4188.694

4188.82

4188.88

4189. 10

4189.50

489.518
4189.564

1189.67

4189.71

8in II $\quad 50$

$\begin{array}{llr}\text { T1 I } & 220 \\ \text { C1 II } & 43\end{array}$

$\Delta I$ III 15

$\mathrm{Fe}$ III

Co $I$

Pr II

$\begin{array}{ll}\text { Pe } & \text { I } \\ \text { II }\end{array}$

8 II

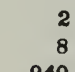

$\$ 189.788$

4189.841

4189.96

4190.16

4190.29

4190.40

4190.626

4190.66

4190.712

4190.7127

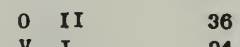

$\begin{array}{lll}0 & I I & 36 \\ V & I & 24\end{array}$

Cr I 106

Cr I

T1 II

Ce II

Cr I

$\begin{array}{ll}\text { Co } & \text { I } \\ \text { A } & I\end{array}$

I A Type Element

Multiplet No.

4190.738 S1

4181.0296

4191.067

4191.271

4191. 436

4191.50 
I A Type Element Multiplet No.

I A Type Element Multiplet No.

I A

Type Element

Multiplet No.

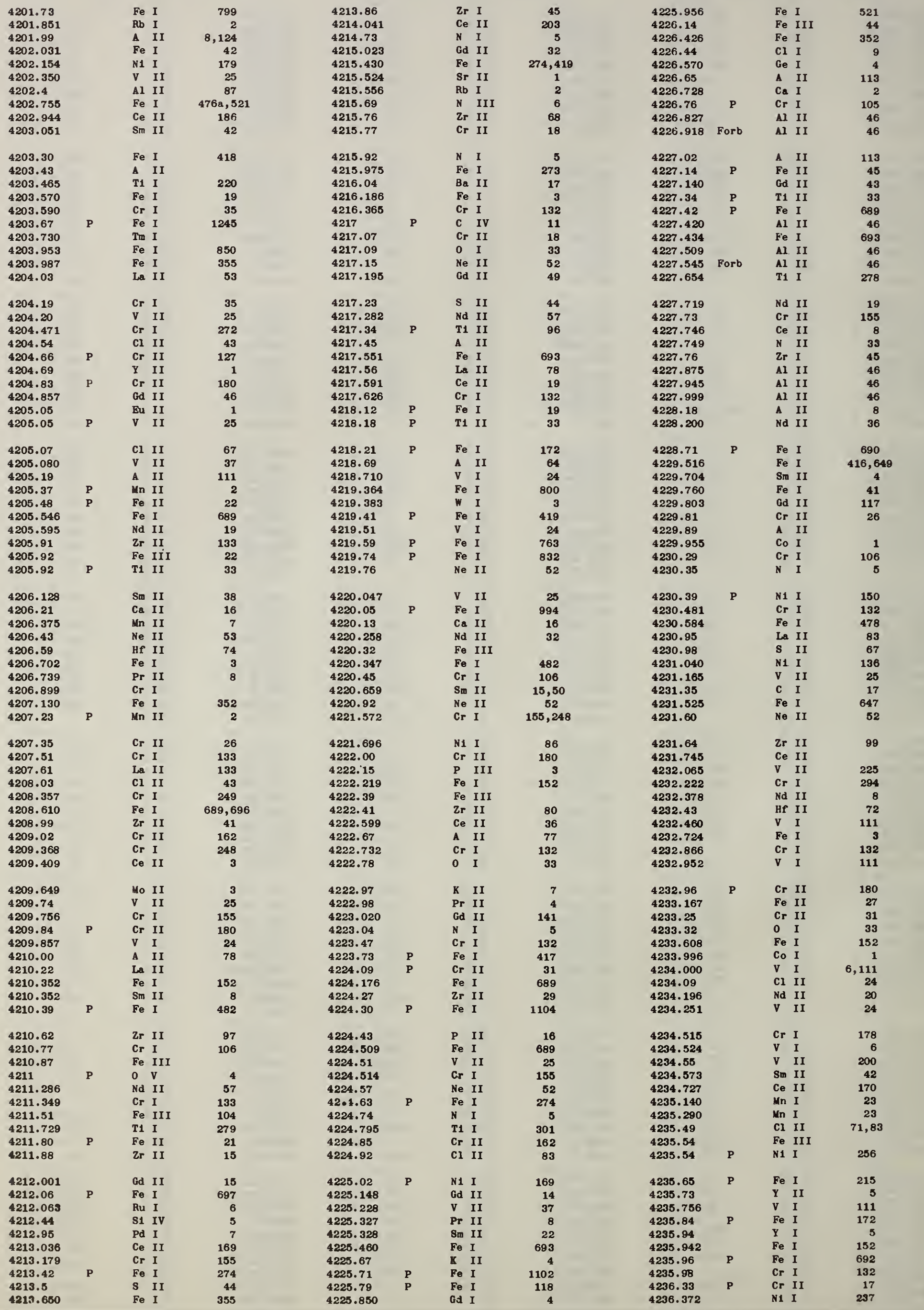


I A Type Nement Kultiplot No.

\begin{tabular}{|c|c|c|c|c|}
\hline 4236.56 & & $2 r$ & II & 110 \\
\hline 4236.66 & $\mathbf{P}$ & Po & I & 907 \\
\hline 4236.745 & & 80 & II & 53 \\
\hline 4236.76 & & $\mathrm{Pe}$ & I & 808 \\
\hline 4236.82 & & $\mathrm{v}$ & II & 18 \\
\hline 4226.930 & & N & II & 48 \\
\hline 4237.048 & & N & II & 48 \\
\hline 4237.085 & & Po & I & 18 \\
\hline 4237.162 & & $\mathrm{Fe}$ & I & \\
\hline 4237.21 & & Fe & III & 104 \\
\hline 4237.23 & & $\boldsymbol{A}$ & II & 32 \\
\hline 4237.27 & & $\mathrm{Cr}$ & I & 106 \\
\hline 4237.57 & $\mathbf{P}$ & $\mathbf{A l}$ & II & 23 \\
\hline 4237.663 & & Sin & II & 8 \\
\hline 4237.67 & $\mathbf{P}$ & $\mathrm{Fe}$ & I & 418 \\
\hline 4237.710 & & $\mathrm{Cr}_{\mathbf{r}}$ & I & 132 \\
\hline 4237.786 & & I1 & I & 252 \\
\hline 4237.889 & & I1 & $I$ & 284 \\
\hline 4238.027 & & $\mathbf{F e}_{\mathbf{0}}$ & I & 689,696 \\
\hline 4238.38 & & La & II & 41 \\
\hline & $\mathbf{P}$ & $\mathrm{Pe}$ & I & 848 \\
\hline
\end{tabular}

$\begin{array}{lllr}4238.61 & \text { P } & \text { Po I } & 849 \\ 4238.69 & \text { P } & \text { Cr II } & 17 \\ 4238.78 & & \text { Fo III } & 104 \\ 4238.782 & & \text { Gd II } & \\ 4238.79 & \text { P } & \text { In II } & 2 \\ 4238.816 & & \text { Pe I } & 683 \\ 4238.957 & & \text { Cr I } & 131 \\ 4239.01 & \text { P } & \text { Fo I } & 274 \\ 4239.31 & & \text { Zr I } & 45 \\ 4239.36 & \text { P } & \text { Fo I } & 907\end{array}$

(1)

4239.5

4239.725

4239.735

4239.912

4239.85

4239.95

4240.35

4240.372

4240.456

4240.705

4240.75

4241.018

4241.112

4241.20

4241.20

4241.276

4241.38

4241.68

4241.787

4241.93

4242.153

4242.20

4242.38

4242.47

4242.588

4242.723

4242.730

4242.82

4242.894

4243.368

4243.528

4243.60

4243.786

4243.85

424.17

424. 26

4244.33

4244.374

$\begin{array}{llr}0 & \text { III } \\ \text { Yn I } & 23 \\ \text { Fo I } & 416\end{array}$

807

$\begin{array}{cc}\text { Pe I } & 416 \\ \text { Pe I } & 18,273\end{array}$

C. II

Pe I

Ne II

$\mathrm{Zr} I$

Po I

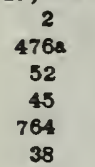

Cr I 105,178

Al II

Fe I

Le II

2r I

C1 II

Zr I

N II

36
9
351
165
45
117
24
45

He II

T. II

Fe II

Cr II

Ys II

$\mathrm{Pe} I$

Ce II

$\mathrm{Pe} I$

Cr I
V II

47,48

108

5
52

52
31
20

20
273

58
649

649
131

200

P. I 900

Pr II

B III

A II

Pe I

Fe III

Re II

Un II

w I

908
35
1
63,78

63,78

62

62
7
240

240
1

12
30

4244.55

Pe II

P II

M1 II

4244.80

4245.258

4243.358

1245. 84

4243.876

4246.02

4246.090

\section{Fo I}

Fe I

C. II

P. I

F. I

30
27
8
352
691
72
158
649
806

4246.41

$\begin{array}{ll}4246.16 & \text { F II } \\ 4246.41 & \text { Cr II } \\ 4246.368 & \text { Od II }\end{array}$

4216.68 Po I

4216.68

4246.711

4246. 78

4246.828

4247.29
I I Type Blement Multiplet No.

$\begin{array}{lllr}4247.31 & \text { P } & \text { Fo I } & 172 \\ 4247.367 & & \text { Nd II } & 8 \\ 4247.43 & \text { P } & \text { Fo II } & 125 \\ 4247.432 & & \text { Fo I } & 693 \\ 4247.36 & & \text { C III } & 11 \\ 4248.228 & & \text { Fo I } & 482 \\ 4248.344 & & \text { Cr I } & 131 \\ 4248.40 & \text { P } & \text { Fo I } & 19 \\ 4248.676 & & \text { C. II } & 1 \\ 4248.72 & \text { P } & \text { Fo I } & 939\end{array}$

$\begin{array}{lll}4248.73 & \text { Cr I } & 105\end{array}$

$\begin{array}{rlr}4248.820 & \text { V II } & 24 \\ 4249.114 & \text { TI I } & 252\end{array}$

$\begin{array}{llll}4249.32 & P & \text { Fo I } & 117\end{array}$

$\begin{array}{llr}4249.35 & \text { HF II } & 39 \\ 4249.57 & \text { P IV } & 2\end{array}$

$\begin{array}{rllr}4249.57 & & \text { P IV } & \\ 4249.81 & P & \text { Cr I } & 155 \\ 8 & & 8 \text { II } & 66\end{array}$

$\begin{array}{ll}4249.82 & 8 \text { II } \\ & \text { Fo III }\end{array}$

4248.98

La II

$\begin{array}{lll}4250.125 & \text { Fe I } & 152\end{array}$

4250.68

4250.790 Fo I

$4250.90 \quad P \quad \mathrm{Fe}$

4251.1852 P A I

4251.618 Ti I

4251.733 Gd II

4251.768

It I

$\begin{array}{llll}4251.88 & P & \text { Fo I } & 216\end{array}$

$\begin{array}{lllr}4252.05 & P & \text { T1 II } & 85 \\ 432.107 & & \text { N1 I } & 136\end{array}$

$\begin{array}{lll}4252.107 & \text { N1 I } & 136 \\ 4252.243 & \text { Cr I } & 131\end{array}$

4252.302

4252.62

4253.02

4253.28

4253.356

Co I

Cr II

Mn II

C. II

Gd II

31

7

4
77
46

4253.51 CI II 24

$\begin{array}{rllr}4253.52 & \text { P } & \text { Fo I } & 690 \\ 4253.55 & \text { P } & \text { Fo I } & 1245\end{array}$

$4253.593 \quad$ S III

4253.612

$\begin{array}{llll}4253.74 & 0 & \text { II } & 101\end{array}$

$\begin{array}{llll}4253.74 & & \text { Pe I } & 101 \\ 4253.83 & P & \text { Pe I } & 905\end{array}$

$4253.86 \quad 0$ II 101

$\begin{array}{llr}4254.346 & \text { Cr I } & 1 \\ 4254.41 & \text { V II } & 18\end{array}$

$\begin{array}{lll}4254.420 & \text { PT II } & 27 \\ 4254.7 & \text { N I }\end{array}$

4254.958

4255.01

4255.20

4255.499

4255.502

4255.62

1255.784

4256.025

4256.156

4256.16

4256.212

4256.239

4256.32

4256.393

4256.620

4256.78

4257.02

4257.121

4257.368

4257.42

4257.658
4257.82

4258.05

4258.155

4258.320

4258.35

4258.525

4258.618

Fe I 419,477

8 II 44

P. III

CrI 110

A II 63

$\begin{array}{llr}\text { Ce II } & 81 \\ \text { I1 I } & 232\end{array}$

Co II 172

Cr II 192

Fo I 680

Nd II 58

Fe I 172

8. II

$\begin{array}{lr}\text { Cr I } & 131 \\ \text { P. I } & 1102\end{array}$

$\begin{array}{ll}V \text { II } & 200 \\ \text { C. II } & 123\end{array}$

Cr I 131

8 II 60

Yn I 25

Zr II 15

Po II

Fo I

P. I

Fe II

80 I

4258.856

4258.15

4258.18

4239.203

4258.312 V I

4259.34 P FoI

4259.2618

4259.52

4259.748

1259.25

I A Type Blewent

Multiplet No.

\begin{tabular}{|c|c|c|c|c|}
\hline 4259.888 & & Fo I & I & 688 \\
\hline 4260.135 & & Fo $I$ & I & $476 a$ \\
\hline 4260.19 & \multirow{3}{*}{$\mathbf{P}$} & CrI & $I$ & 240 \\
\hline 4260.47 & & Kn I & II & 2 \\
\hline 4260.479 & & Fo I & I & 152 \\
\hline 4260.73 & \multirow[t]{10}{*}{$\mathbf{P}$} & Fo : & I & 351 \\
\hline 4260.738 & & I1 & I & 251 \\
\hline 4260.75 & & V I & II & 18,24 \\
\hline 4260.854 & & Os & I & 1 \\
\hline 4261.164 & & Co 1 & II & 18 \\
\hline 4261.22 & & CI I & II & 66 \\
\hline 4261.354 & & $\mathrm{Cr}_{\mathbf{r}}$ & I & 96 \\
\hline 4261.609 & & T1 & I & 252 \\
\hline 4281.615 & & $\mathrm{Cr}_{\mathbf{r}}$ & I & \\
\hline 4281.796 & & Pr & II & 23 \\
\hline 4261.80 & \multirow[t]{11}{*}{$\mathbf{P}$} & $\mathrm{Cr}$ & I I & 17 \\
\hline 4261.92 & & $\mathrm{Cr}_{\mathbf{r}}$ & II & 31 \\
\hline 4262.092 & & Gd & II & 44 \\
\hline 4262.133 & & $\mathrm{Cr}$ & I & 84,178 \\
\hline 4262.38 & & $\mathrm{Cr}$ & I & 154 \\
\hline 4262.677 & & $\mathrm{~S}=$ & II & 37 \\
\hline 4282.72 & & HP & II & 15 \\
\hline 4263.134 & & T1 & $I$ & 162 \\
\hline 4263.141 & & $\mathrm{C}_{\mathbf{r}}$ & I & 247 \\
\hline 4263.40 & & $\mathbf{X}$ & II & 2 \\
\hline 4263.427 & & ce & II & 254 \\
\hline 4263.48 & \multirow[t]{4}{*}{$\mathbf{P}$} & $\mathrm{Cr}_{\mathbf{r}}$ & II & 17 \\
\hline 4263.59 & & La & II & 84 \\
\hline 4263.836 & & $\nabla$ & II & 24 \\
\hline 4263.895 & & Fe & II & \\
\hline 4264.19 & \multirow[t]{9}{*}{$\mathbf{P}$} & $\mathrm{Cr}_{\mathrm{r}}$ & II & 17 \\
\hline 4264.209 & & Fo & I & 692 \\
\hline 4264.370 & & Co & II & 239 \\
\hline 4264.50 & & V & II & 24 \\
\hline 4264.743 & & Fe & I & 893 \\
\hline 4264.88 & & $\mathbf{Y}$ & II & 71 \\
\hline 4264.91 & & $\mathbf{Z r}$ & II & 98 \\
\hline 4265.075 & & Sin & II & 15 \\
\hline 4265.170 & & $v$ & $I$ & \\
\hline
\end{tabular}

4265.260 Po I 993,994

$\begin{array}{lll}4265.273 & \text { Ti I } & 252\end{array}$

$4265.723 \quad$ T1 I 162

4265.824 in I

4266.227 T1 I

4266.23 P Cr II 37

$\begin{array}{lll}4266.2867 & \text { A } & \text { I } \\ 4266.44 & \text { Cr I } & 198\end{array}$

4266.53

4286.716

4266.72

A II

Nd II

Zr II

5

4286.82

4266.88
4266.968

4267.02

42057.27

4267.30

4267.47

4287.802

4287.830

4267.95

Cr I

Fe III

Fe I

C II

Zr II

A II

8 II

Fe $I$

4268.01

4268.032

4268.090

4268.10

4268.446

4268.645

4268.731

4268.744

4268.788

4268.928

ZF I

Co I

Ir I

HI II

Co I

$\checkmark$ I

Ge II

$\begin{array}{ll}\text { Cr } & I \\ \text { I1 } & I\end{array}$

Cr II

1208.83

4268.99

4289.02

4268.28

4269.50

4268.67

4268.76

4289.87

4269.951 
I A Type Element Multiplet No.

\begin{tabular}{|c|c|c|c|c|}
\hline 4271.47 & & $\mathrm{Fe}$ & III & \\
\hline 4271.554 & & $\mathbf{v}$ & I & 88 \\
\hline 4271.65 & $\mathbf{P}$ & $\mathrm{Fe}$ & I & 70 \\
\hline 4271.764 & & $\mathrm{Fe}$ & I & 42 \\
\hline 4271.94 & $\mathbf{P}$ & $\mathbf{T 1}$ & II & 95 \\
\hline 4271.95 & $\mathbf{P}$ & $\mathrm{Fe}$ & I & 171 \\
\hline 4272.1690 & & $\mathbf{A}$ & I & 4 \\
\hline 4272.271 & & Pr & II & 15 \\
\hline 4272.440 & & T1 & I & 44 \\
\hline 4272.789 & & Nd & II & 11 \\
\hline 4272.85 & & Hf & II & 14 \\
\hline 4272.910 & & $\mathrm{Cr}$ & I & 96 \\
\hline 4273.17 & & 0 & II & 68 \\
\hline 4273.312 & & Ti & I & 251 \\
\hline 4273.317 & & $\mathrm{Fe}$ & II & 27 \\
\hline 4273.42 & & $\mathrm{Fe}$ & III & 121 \\
\hline 4273.52 & & $\mathbf{Z r}$ & II & 28 \\
\hline 4273.87 & & $\mathrm{Fe}$ & I & 478 \\
\hline 4274.13 & & 0 & II & 68 \\
\hline 4274.408 & & $\mathbf{T} \mathbf{1}$ & I & 252 \\
\hline 4274.584 & & $\mathbf{T 1}$ & I & 44,162 \\
\hline 4274.803 & & $\mathrm{Cr}$ & I & 1 \\
\hline 4275.19 & & $\mathbf{A}$ & II & 77 \\
\hline 4275.52 & & 0 & II & 67 \\
\hline 4275.561 & & $\mathrm{Ce}$ & II & 206 \\
\hline 4275.57 & & $\mathrm{Cr}$ & II & 31 \\
\hline 4275.64 & & La: & I I & 40 \\
\hline 4275.72 & & $\mathrm{Fe}$ & I & 215 \\
\hline 4275.90 & & 0 & I I & 68 \\
\hline 4275.973 & & $\mathrm{Cr}_{\mathbf{r}}$ & I & 240 \\
\hline 4276.21 & & 0 & II & 68 \\
\hline 4276.441 & & T1 & I & 148 \\
\hline 4276.51 & & $\mathrm{Cl}$ & II & 66 \\
\hline 4276.657 & & T1 & I & 252 \\
\hline 4276.684 & & $\mathrm{Fe}$ & I & 976 \\
\hline 4276.71 & & 0 & I I & 54,67 \\
\hline 4276.958 & & v & I & 88 \\
\hline 4277.246 & & Mo & I & 7 \\
\hline 4277.279 & & Nd & II & 17 \\
\hline 4277.322 & & Th & II & 2 \\
\hline 4277.37 & & $\mathrm{Zr}$ & I I & 40 \\
\hline 4277.40 & & 0 & II & 67,68 \\
\hline 4277.41 & $\mathbf{P}$ & $\mathrm{Fe}$ & I & 214 \\
\hline 4277.55 & & $\mathbf{A}$ & I I & 32 \\
\hline 4277.68 & & $\mathrm{Fe}$ & I & 172 \\
\hline 4277.90 & & 0 & II & 67 \\
\hline 4278.01 & $\mathbf{P}$ & $\mathrm{Fe}$ & I & 1102 \\
\hline 4278.10 & & $\mathrm{Cr}$ & II & 161 \\
\hline 4278.128 & & $\mathrm{Fe}$ & II & 32 \\
\hline 4278.231 & & Ti & I & 291 \\
\hline
\end{tabular}

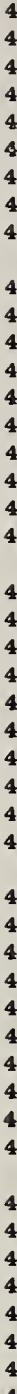

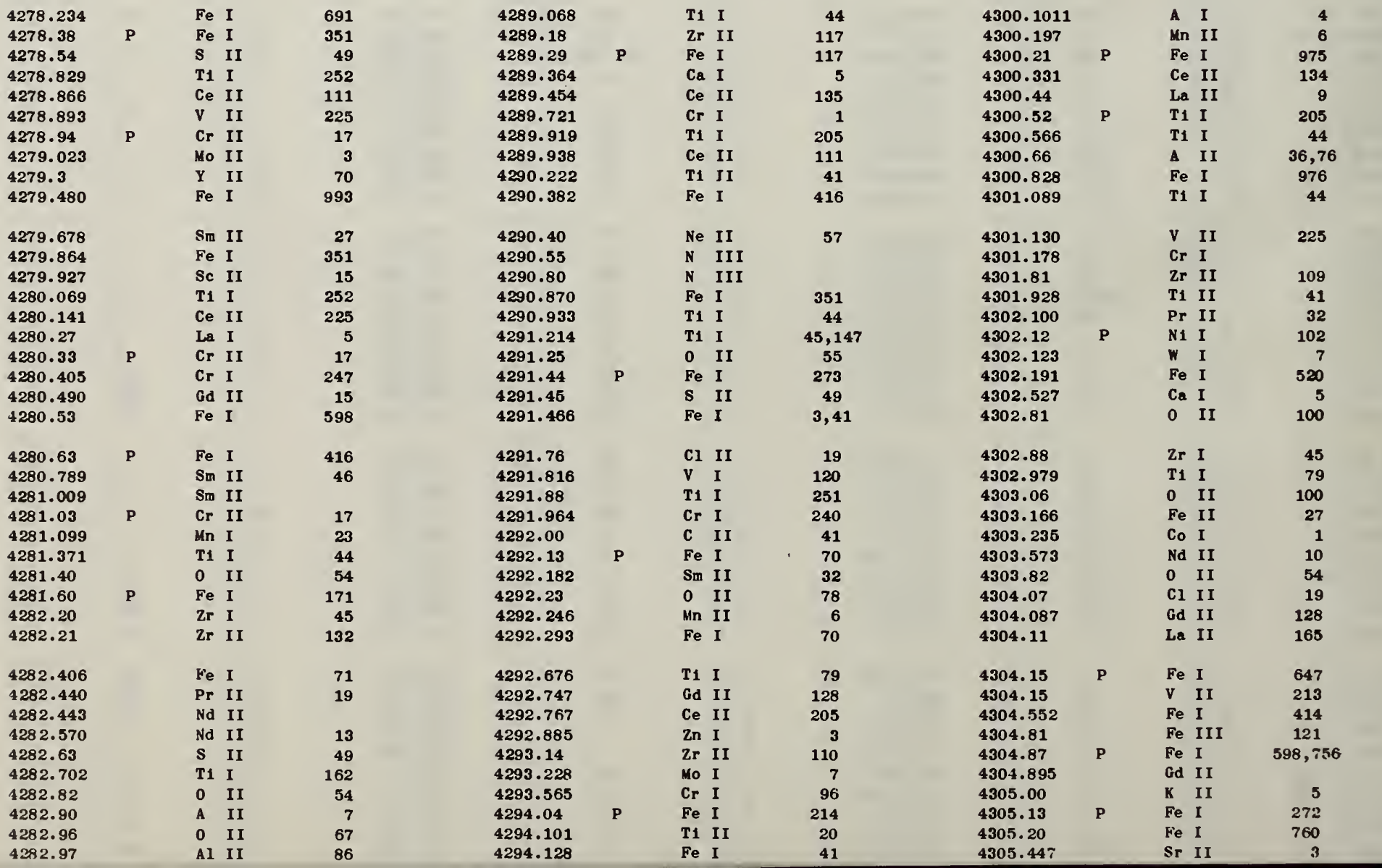

I A Type Element Multiplet No. I A Type Element Multiplet No.

\begin{tabular}{|c|c|c|c|c|c|c|c|c|c|}
\hline 4283.010 & & $\mathrm{Ca}$ & I & 5 & 4294.432 & & s & II & 49 \\
\hline 4283.13 & & 0 & II & 67 & 4294.623 & & w & I & 6 \\
\hline 4283.40 & $\mathbf{P}$ & $\mathrm{Fe}$ & I & 215 & 4294.76 & & N & III & \\
\hline 4283.70 & & $\mathbf{s}$ & III & & 4294.767 & & Sc & II & 15 \\
\hline 4283.75 & & 0 & II & 67 & 4294.78 & & $2 r$ & I & 45 \\
\hline 4283.772 & & Mn & I I & 6 & 4294.82 & & 0 & II & 54 \\
\hline 4283.87 & $\mathbf{P}$ & $\mathrm{Fe}$ & I & 19 & 4294.939 & & $\mathrm{Fe}$ & I & 598 \\
\hline 4284.055 & & v & I & 88 & 4295.37 & $\mathbf{P}$ & $\mathrm{Cr}$ & II & 37 \\
\hline 4284.084 & & Mn & I & 23 & 4295.751 & & T1 & I & 44 \\
\hline 4284.21 & & $\mathrm{Cr}_{\mathrm{r}}$ & II & 31 & 4295.757 & & $\mathrm{Cr}$ & I & 64 \\
\hline
\end{tabular}

\begin{tabular}{|c|c|c|c|c|}
\hline 4284.415 & & $\mathrm{Fe}$ & & 417 \\
\hline 4284.425 & & Mn & I I & 6 \\
\hline 4284.51 & & $\mathbf{N}$ & I I I & \\
\hline 4284.518 & & Nd & II & 10 \\
\hline 4284.683 & & N1 & I & 86 \\
\hline 4284.725 & & $\mathrm{Cr}$ & I & 96 \\
\hline 4284.988 & & T1 & I & 148 \\
\hline 4284.991 & & $\mathbf{s}$ & II I & 4 \\
\hline 4285.19 & $\mathbf{P}$ & N1 & I & 86 \\
\hline 4285.366 & & $\mathrm{Ce}$ & I I & 11 \\
\hline
\end{tabular}

4295.888

4296.05

4296.069

4296.076

4296. 107

4296.11

4296. 30

4296. 30

4296.567

4296.680

N1 I 178

4296. 74

4285.445

4285.496

4285.70

4285.782

Fe I $\quad 597$

Sm II

0 II $\quad 78$

Co I

$\mathrm{Fe} I$

4285.96
4286.006

4286.13

4286.13

4286.311

T1 I

Fe III

Fe II

4286.440

4286.51

4286.640

4286.976

4287.405

4287.71

4287.893

4288.005

Fe I

Zr II

Sin II

La II

Fe I

T1 I

Ba II

TI II

N1 I

4296. 743

4296.86

4297.050

4297.173

4297.60

4297.681

4297.711

4297.738

La II

Ce II

Gd II

$\checkmark$ I

C I

Cr I

Gd II

Ce II

172
46

46
120

42
176
117

4297.764

4297.99

4298.029

4298.040

4298 . 21

4298.515

4298.664

4298.767

4298.986

4299.17

Zr I

Sin I

Fe III

Cr I

Gd II

$\begin{array}{ll}\mathrm{Ba} & \mathrm{I} \\ \mathrm{V} & \mathrm{I}\end{array}$

Ru I

Cr I

4288.148
4288.161

Fe I

N III

273
43,79

4288.21

4288.53

4288.65

4288.72

4288.78

4288.78

4288.78
4288.83

4288.83
4288.962

P II

Mo I

N III

T1 I

V II

Fe I I

45

33
7

4299.177

49

400.25

4299.362

4299.49

4299.636

4299.65

4299.718

4300.052

Pr II

A II

7

I 120

Fe I $\quad 520$

N1 I $\quad 178$

T1 I

N1 I

$\begin{array}{ll}\text { Ca } & \text { I } \\ \text { T1 } & \text { I }\end{array}$

44
28

5
45

4289.068

4289.29

290.40

4290.80

T1

T1 I

Fe I

Ce II

Fe I

T1 I

$\begin{array}{ll}\mathrm{Fe} & \mathrm{I} \\ \mathrm{Cr} & \mathrm{I}\end{array}$

$\begin{array}{ll}\text { Cr I } \\ \text { TI } & \text { I I }\end{array}$

7

148

152

47
648

648

416

98 
FINDING LIST

I Type Blement Multinlet No.

I A Type Blement Multiplet No.

I A Type Element Multiplet No.

\begin{tabular}{|c|c|c|c|c|c|c|c|c|}
\hline 4305.453 & & $\mathrm{Cr}$ & $\mathbf{I}$ & 96 & 4319.45 & $\mathbf{P}$ & $\mathrm{Fe}$ & I \\
\hline 4305.455 & & $\mathrm{Fe}$ & I & 476 & 4318.51 & & He & II \\
\hline $4305.46^{\circ}$ & & N & I & & 4318.631 & & $\mathrm{~J}$ & II \\
\hline 4305.474 & & T1 & $I$ & 147 & 4319.641 & & $\mathrm{C}_{\boldsymbol{r}}$ & I \\
\hline 4305.53 & & 0 & II & 58 & 4318.68 & & $\Delta$ & II \\
\hline 4305.715 & & Sc & II & 15 & 4919.717 & & $\mathrm{Fe}$ & II \\
\hline 4305.763 & & Pr & II & 8 & 4519.93 & & 0 & II \\
\hline 4305.810 & & T1 & I & 44 & 4320.13 & $\mathbf{P}$ & $\mathrm{Fe}$ & I \\
\hline 4306.214 & & $\mathbf{v}$ & I & 5 & 4320.36 & $\mathbf{P}$ & $\mathrm{Fe}$ & I \\
\hline 4306.340 & & od & I & 4 & 4320.52 & & $\mathrm{Fe}$ & I \\
\hline 4306.58 & $\mathbf{P}$ & $\mathrm{Fe}$ & I & 681 & 4320.682 & & $\mathrm{Cr}_{\mathbf{r}}$ & I \\
\hline 1306.724 & & $\mathrm{Ce}$ & II & 1 & 4320.68 & & Hf & II \\
\hline 1306.945 & & T1 & I & 43 & 4320.723 & & $\mathrm{Ce}$ & I I \\
\hline 1507.08 & $\mathbf{P}$ & $\mathrm{Fe}$ & I & 690 & 4320.745 & & $\mathrm{Sc}$ & II \\
\hline 1307.184 & & $\mathbf{v}$ & I & 5 & 4320.965 & & T1 & II \\
\hline 4307.20 & & Al & II & 85 & 4321.110 & & Gd & II \\
\hline 4307.31 & & 0 & II & 53 & 4321.238 & & $\mathrm{Cr}$ & I \\
\hline 4307.42 & & $\mathrm{Cl}$ & II & 19 & 4321.341 & & $\mathrm{Fe}$ & II \\
\hline 4307.741 & & $\mathrm{Ca}$ & I & 5 & 4321.96 & & HP & II \\
\hline 4307.778 & & Nd & II & 63 & 1321.37 & & $\mathbf{N}$ & II I \\
\hline 4307.900 & & T1 & II & 41 & 4321.617 & & Cr & $I$ \\
\hline 4307.906 & & $\mathrm{Fe}$ & $I$ & 42 & 4321.655 & & T1 & I \\
\hline 4308.233 & & Gd & II & 47 & 4321.85 & & C & II \\
\hline 4308.514 & & T1 & I & 78 & 4322.02 & & $\mathbf{v}$ & II \\
\hline 4308.54 & $\mathbf{P}$ & $\mathrm{Fe}$ & I & 70 & 4322.195 & & Gd & II \\
\hline 4308.94 & & $\mathrm{Zr}$ & II & 88 & 4322.51 & & La & II \\
\hline 4308.96 & & 0 & II & 64 & 4322.66 & & Ye & II \\
\hline 4309.012 & & Sm & II & 15 & 4822.70 & $\mathbf{P}$ & $\mathrm{Fe}$ & I \\
\hline 4309.036 & & $\mathrm{Fe}$ & I & 848 & 4323.284 & & Sn & II \\
\hline 4308.06 & & Cl & II & 82 & 4323.35 & & $\mathrm{Cl}$ & I \\
\hline 4309.071 & & T1 & I & 235 & 4323.37 & $\mathbf{P}$ & $\mathrm{Fe}$ & I \\
\hline $4309 \cdot 10$ & & $\mathbf{K}$ & II & 7 & 4323.440 & & $\mathbf{T 1}$ & I \\
\hline 4309.11 & & $\Delta$ & I I & 36 & 4323.520 & & $\mathbf{C}_{\mathbf{r}}$ & I \\
\hline 4309.25 & & 1 & II & 89 & 4325.551 & & Pr & II \\
\hline 4309.32 & & $\mathrm{Ba}$ & I I & 11 & 4325.62 & & $\mathbf{Z r}$ & II \\
\hline 4309.382 & & $\mathrm{Fe}$ & I & 414 & 4323.81 & & $\mathrm{Fe}$ & III \\
\hline 4309.46 & $\mathbf{P}$ & $\mathrm{Fe}$ & I & 478 & 4523.83 & & $\mathbf{M}$ & III \\
\hline 4309.62 & & $Y$ & II & 5 & 4324.064 & & Cd & II \\
\hline 4308.739 & & $\mathrm{Ce}$ & II & 126 & 4324.36 & $\mathbf{P}$ & Fe & II \\
\hline 4309.795 & & $\mathbf{v}$ & I & 5 & 4324.961 & & Fe & I \\
\hline 4310.37 & $P$ & $\mathrm{Fe}$ & I & 894 & 4325.010 & & sc & II \\
\hline 4310.37 & & $\mathrm{Fe}$ & III & 121 & 4325.076 & & $\mathbf{C}_{\mathbf{r}}$ & $I$ \\
\hline 4310.373 & & T1 & $I$ & 79 & 4825.1 & $\mathbf{P}$ & Mn & II \\
\hline 4310.698 & & $\mathrm{Ce}$ & II & 193 & 4325.134 & & $\mathbf{T 1}$ & $I$ \\
\hline 4310.72 & $\mathbf{P}$ & $\mathbf{v}$ & II & 36 & 4325.22 & & $\mathbf{v}$ & II \\
\hline 4310.981 & & ad & II & 15 & 4325.361 & & $\mathbf{N 1}$ & I \\
\hline 4311.654 & & $T_{1}$ & I & 206 & 4925.568 & & Gd & II \\
\hline 4312.10 & & 0 & II & 78 & 4325.607 & & N1 & I \\
\hline 4312.23 & & $\mathbf{Z r}$ & II & 98 & 4320.64 & & $\mathbf{Z r}$ & II \\
\hline 4312.469 & & $\mathrm{Cr}$ & I & 177 & 4325.65 & & $\mathrm{Cr}_{\mathbf{r}}$ & I \\
\hline 4312. 550 & & Mn & I & 23 & 4325.7 & & L1 & II \\
\hline 4312.861 & & T1 & I & 41 & 4325.70 & & C & III \\
\hline 4313.034 & & $\mathrm{Fe}$ & II & 220 & 4325.73 & & Bs & II \\
\hline 4313.04 & $\mathbf{P}$ & $\mathrm{Fe}$ & I & 273 & 4325.74 & $\mathbf{P}$ & $\mathrm{Fe}$ & I \\
\hline 4318.11 & & $\mathbf{N}$ & I & & 4325.765 & & $\mathrm{Fe}$ & I \\
\hline 4313.30 & & $\mathbf{v}$ & II & 23 & 4325.766 & & $\mathbf{R d}$ & II \\
\hline 4313.43 & & 0 & If & 78 & 4325.77 & & 0 & II \\
\hline 4313.50 & & C & I I & 28 & 4325.88 & & C & II \\
\hline 4313.845 & & Gd & I & 4 & 4325.95 & $\mathbf{P}$ & $\mathrm{Fe}$ & I \\
\hline 4314.084 & & $\mathrm{Sc}$ & II & 15 & 4326.359 & & T1 & I \\
\hline 4314.18 & & s1 & IV & 4 & 4326.445 & & Sr & I \\
\hline 4314.288 & & $\mathrm{Fe}$ & II & 32 & 4326.74 & & $\mathrm{Ba}$ & II \\
\hline 4314.356 & & T1 & I & 45 & 4326.756 & & Mn & II \\
\hline 4314.511 & & Nd & II & $\theta$ & 4326.762 & & $\mathrm{Fe}$ & I \\
\hline 4314.74 & $\mathbf{P}$ & T1 & I & 43 & 4326.826 & & $\mathrm{Ce}$ & II \\
\hline 4314.801 & & $\mathbf{T 1}$ & I & 43 & 4326.986 & & $\mathbf{T} \mathbf{1}$ & I \\
\hline 4314.979 & & T1 & II & 41 & 4827.04 & $\mathbf{P}$ & Fe & II \\
\hline 4315.087 & & $\mathrm{Fe}$ & I & 71 & 4327.100 & & $\mathrm{Fe}$ & I \\
\hline 4316.35 & & 0 & II & 64,78 & 4327.125 & & od & II \\
\hline 4315.80 & & 0 & II & 78 & 4327.48 & & 0 & II \\
\hline 4315.80 & & La & II & 41 & 4327.89 & & 0 & II \\
\hline 4315.95 & $\mathbf{P}$ & $\mathrm{Fe}$ & I & 171 & 4827.92 & & Fe & I \\
\hline 4316.052 & & od & II & 43 & 1328.16 & & N & III \\
\hline 4316.258 & & $\mathbf{v}$ & II & 23 & 4328.22 & & 81 & IV \\
\hline 4316.266 & & ad & II & 67,68 & 4328.62 & & 0 & II \\
\hline 4316.807 & & T1 & II & 94 & 4328.91 & $\mathbf{P}$ & $\mathrm{C}_{\mathbf{r}}$ & II \\
\hline 4317.04 & $\mathbf{P}$ & Pe & I & 762 & 4329.016 & & $8 m$ & II \\
\hline 4317.139 & & 0 & II & 2 & 4329.415 & & Pr & II \\
\hline 4317.32 & & $\mathbf{Z r}_{\mathbf{r}}$ & II & 40 & 4329.64 & $\mathbf{P}$ & $\mathrm{Fe}$ & I \\
\hline 4317.42 & & C & II & 28 & 4329.62 & & Ba & II \\
\hline 4317.65 & & 0 & II & 83 & 4330.024 & & $\mathbf{v}$ & I \\
\hline $4317 \cdot 70$ & & N & I & & 4330.14 & & N & III \\
\hline 4318.216 & & Fe & II & 220 & 4330.264 & & T1 & I I \\
\hline 4318.631 & & T1 & I & 205 & 4330.44 & & $\mathbf{N}$ & II I \\
\hline 4318.652 & & Ca & I & 5 & 4330.445 & & $\mathrm{Ce}$ & II \\
\hline 4318.68 & & $\mathbf{s}$ & II & 48 & 4330.606 & & Gd & II \\
\hline 4318.77 & $\mathbf{P}$ & $\mathrm{Cr}_{\mathbf{r}}$ & II & 37 & 4330.708 & & $\mathbf{T} 1$ & II \\
\hline 4318.81 & $\mathbf{P}$ & $\mathrm{Fe}$ & I & 215 & 4330.720 & & N1 & I \\
\hline 4318.92 & & $c$ & II & 28 & 4330.81 & $\mathbf{P}$ & $\mathrm{Fe}$ & I \\
\hline 4318.936 & & $8 m$ & II & 27 & 4530.862 & & $\mathrm{Fe}$ & \\
\hline
\end{tabular}


I A Type

Rlement

Mult1plet No.

\begin{tabular}{|c|c|c|c|c|}
\hline 4342.00 & & 0 & II & 7 \\
\hline 4342.071 & & Nd & II & \\
\hline 4342.179 & & Gd & II & 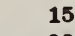 \\
\hline 4342.23 & & $\mathbf{Z r}$ & II & \\
\hline 4342.83 & & 0 & II & 10 \\
\hline 4342.832 & & $\mathbf{v}$ & I & 10 \\
\hline 4342.84 & & $\mathbf{s}$ & II & \\
\hline 4343.163 & & $\mathrm{Cr}$ & $\mathbf{I}$ & \\
\hline 4343.22 & $\mathbf{P}$ & $\mathrm{Fe}$ & $\mathbf{I}$ & 64 \\
\hline 4343.257 & & $\mathrm{Fe}$ & $\mathbf{I}$ & B \\
\hline 4343.36 & & 0 & II & 75 \\
\hline 4343.62 & & C1 & II & 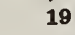 \\
\hline 4343.699 & & $\mathrm{Fe}$ & I & 51 \\
\hline 4343.798 & & T1 & I & 20 \\
\hline 4343.86 & $\mathbf{P}$ & $\mathrm{Fe}$ & I & 75 \\
\hline 4343.987 & & Mn & II & \\
\hline 4344.291 & & T1 & II & 2 \\
\hline 4344.300 & & Gd & II & \\
\hline 4344.42 & & 0 & II & \\
\hline 4344.487 & & Gd & II & \\
\hline 4344.507 & & $\mathbf{C r}$ & I & \\
\hline 4345.085 & & $\mathbf{C r}_{\mathbf{r}}$ & $\mathbf{I}$ & 18 \\
\hline $4345 \cdot 1682$ & & $\Delta$ & I & \\
\hline 4345.562 & & 0 & II & \\
\hline 4345.6 & $\mathbf{P}$ & Mn & II & \\
\hline 4345.858 & & $8 m$ & II & \\
\hline 4345.963 & & $\mathrm{Ce}$ & II & 20 \\
\hline 4346.104 & & T1 & I & 25 \\
\hline 4946.458 & & ed & I & \\
\hline 4346.50 & $\mathbf{P}$ & $\mathrm{Fe}$ & II & 20 \\
\hline 4346.558 & & $\mathrm{Fe}$ & I & 58 \\
\hline 4346.610 & & $\mathbf{T 1}$ & I & 20 \\
\hline 4346.833 & & $\mathrm{Cr}_{\mathbf{r}}$ & I & 10 \\
\hline 4346.866 & & Al & II & 7 \\
\hline 4346.89 & & $\mathbf{v}$ & II & 17 \\
\hline 4346.918 & & $\mathbf{A 1}$ & II & 70 \\
\hline 4347.223 & & $\mathbf{A l}$ & II & 7 \\
\hline 4347.239 & & $\mathrm{Fe}$ & I & \\
\hline 4347.310 & & Gd & II & $10:$ \\
\hline 4947.316 & & $\mathbf{A l}$ & II & \\
\hline 4347.425 & & 0 & II & \\
\hline 4347.490 & & Pr & II & 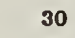 \\
\hline 4347.785 & & $\mathbf{A I}$ & II & 70 \\
\hline 4347.801 & & Sw & II & \\
\hline 4847.802 & & $\mathbf{A 1}$ & II & 7 \\
\hline 4347.854 & & $\mathrm{Fe}$ & $\mathbf{I}$ & 826 \\
\hline 4348.11 & & $\mathbf{A}$ & II & \\
\hline 4848.36 & & $N$ & III & 10 \\
\hline 4348.64 & $\mathbf{P}$ & $\mathbf{Z r}$ & II & 13 \\
\hline 1848.939 & & $\mathrm{Fe}$ & I & 41 \\
\hline 4349.28 & $\mathbf{P}$ & $\mathrm{Fe}$ & II & \\
\hline 4349.426 & & 0 & II & \\
\hline 4349.789 & & $\mathrm{Ce}$ & II & \\
\hline 4349.97 & & $\mathbf{v}$ & II & \\
\hline 4350.465 & & Sm & I I & \\
\hline 4350.52 & & Hf & II & \\
\hline 4350.834 & & T1 & II & \\
\hline 4351.051 & & $\mathrm{Cr}$ & $\mathbf{I}$ & \\
\hline 4351.269 & & 0 & II & \\
\hline 4351.295 & & Nd & II & \\
\hline 4351.37 & $\mathbf{P}$ & $\mathrm{Fe}$ & $\mathbf{I}$ & 69 \\
\hline 4351.549 & & $\mathrm{Fe}$ & I & 41 \\
\hline 4351.764 & & $\mathrm{Fe}$ & I I & \\
\hline 4351.770 & & $\mathrm{Cr}$ & I & \\
\hline 4351.849 & & Pr & I I & \\
\hline 4351.8941 & & $\mathrm{Mg}$ & I & \\
\hline 4351.9056 & & $\mathrm{Mg}$ & I & \\
\hline 4352.1 & & c & I & \\
\hline 4352.101 & & Sm & II & \\
\hline 4352.23 & & $\mathbf{A}$ & II & \\
\hline 4352.25 & & As & II & \\
\hline 4352.68 & $\mathbf{P}$ & $\mathrm{Cr}$ & II & \\
\hline 4352.70 & & $\mathrm{Fe}$ & III & \\
\hline 4352.733 & & $\mathrm{Ce}$ & II & 2 \\
\hline 4352.737 & & $\mathrm{Fe}$ & I & \\
\hline 4352.872 & & $\mathbf{v}$ & I & \\
\hline 4353.60 & & 0 & II & \\
\hline 4353.66 & & $\mathbf{N}$ & III & \\
\hline 4353.983 & & $\mathrm{Cr}$ & I & 10 \\
\hline 4354.064 & & $\mathrm{T1}$ & I & \\
\hline 4354.28 & $\mathbf{P}$ & $\mathrm{Fe}$ & I & 9 \\
\hline 4354.358 & & $\mathrm{Fe}$ & II & \\
\hline 4354.40 & & Ia & II & \\
\hline 4354.540 & & $\mathrm{Mg}$ & I & \\
\hline 4354.56 & & $\mathbf{s}$ & III & \\
\hline 4354.609 & & $\mathrm{Sc}$ & II & \\
\hline 4354.979 & & $\mathbf{v}$ & I & 1 \\
\hline 4355.03 & P & $\mathrm{Fe}$ & II & \\
\hline 4365.09 & & Eu & II & \\
\hline 4255.096 & & $\mathrm{Ca}$ & & \\
\hline
\end{tabular}

\begin{tabular}{|c|c|c|c|c|}
\hline 4355.308 & & T1 & I & 174 \\
\hline 4355.911 & & N1 & I & 149 \\
\hline 4355.943 & & $\mathbf{v}$ & I & 5 \\
\hline 4356.711 & & $\mathbf{A 1}$ & II & 60 \\
\hline 4356.760 & & Er & I & 130 \\
\hline 4356.807 & & $\mathbf{A l}$ & II & 60 \\
\hline 4357.24 & $\mathbf{P}$ & Al & III & 9 \\
\hline $4357 \cdot 25$ & & 0 & IJ & $18,63,64$ \\
\hline 4357.50 & $\mathbf{P}$ & $\mathrm{Fe}$ & I & 1170 \\
\hline 4357.525 & & $\mathrm{Cr}$ & I & 198 \\
\hline 4357.53 & $\mathbf{P}$ & $\mathrm{Fe}$ & I & 994 \\
\hline 4357.574 & & $\mathrm{Fe}$ & II & \\
\hline 4957.85 & $\mathbf{P}$ & N1 & I & 256 \\
\hline 4358.169 & & Nd & II & 10 \\
\hline 4358.27 & & $\mathbf{N}$ & I & \\
\hline 4358.343 & & $\mathrm{Hg}$ & I & 1 \\
\hline 4358.40 & & 0 & II & 64 \\
\hline 4358.505 & & $\mathrm{Fe}$ & $\mathbf{I}$ & 412 \\
\hline $4 J 58.53$ & & $A$ & II & 87 \\
\hline 4358.66 & & $\mathrm{Cr}$ & I & 176 \\
\hline 4358.699 & & Nd & II & 57 \\
\hline 4358.73 & & $\mathbf{Y}$ & II & 5 \\
\hline 4358.95 & $\mathbf{P}$ & $\mathrm{Fe}$ & $\mathbf{I}$ & 987 \\
\hline 4359.12 & $\mathbf{P}$ & $\mathrm{Fe}$ & II & 202 \\
\hline 4359.152 & & Gd & II & 47,68 \\
\hline 4359.38 & & 0 & II & 26 \\
\hline 4359.685 & & N1 & I & 86 \\
\hline 4359.631 & & $\mathrm{Cr}$ & I & 22 \\
\hline 4359.636 & & Inl & II & 67 \\
\hline 4359.74 & & $\mathbf{Z r}$ & II & 79 \\
\hline
\end{tabular}

4368.14 C II 45

$\begin{array}{lllll}4368.14 & & \text { C } & \text { II I } & \\ 4368.20 & \text { P } & \text { Cr II } & 37\end{array}$

$\begin{array}{rrr}4368.234 & \text { Ce II } & 37 \\ 4368.252 & & \text { Cr II }\end{array}$

$4368.252 \quad$ Cr I 130

4368.262 Fe II

$4368.30 \quad 001$ 5

$\begin{array}{llr}4368.312 & \text { N1 I } & 102 \\ 4368.327 & \text { Pr II } & 5\end{array}$

$$
4808
$$

$\begin{array}{lllr}4368.66 & \text { P } & \text { Fe I } & 64 \\ 4368.67 & \text { P } & \text { V II } & 18 \\ 4368.89 & & \text { Cr I } & 19 \\ 4368.941 & & \text { T1 I } & 24 \\ 4369.28 & & \text { O II } & 28 \\ 4369.29 & \text { P } & \text { Fe I } & 124 \\ 4369.404 & & \text { Fe II } & 2 \\ 4369.52 & & \text { CI I } & \\ 4369.61 & \text { P } & \text { Fe I I } & 148 \\ 4369.682 & & \text { T1 I } & 290\end{array}$

$\begin{array}{lll}4369.682 & \text { T1 I } & 290\end{array}$

$\begin{array}{llll}4369.73 & P & \text { Fe } I & 976\end{array}$

$\begin{array}{llr}4369.77 & \text { Pe I } & 976 \\ & \text { Ne II } & 56\end{array}$

4369.774 Fe I 518

$\begin{array}{lll}4370.041 & \text { N1 I } & 148\end{array}$

$\begin{array}{llr}4370.27 & \text { V II } & 31\end{array}$

$4370.76 \quad A$ II

$4370.875 \quad$ Mn I 17

$\begin{array}{lll}4370.95 & \text { HF II } & 26 \\ 4370.96 & \text { Zr II } & 79\end{array}$

43597795

\section{Pr II}

26

4359.929

1950.98

4360.16

4360.487

Th $I$

Cr I

Fe II

T1 I

8 II

4360.48

4360.690

4360.720
4360.80

Be II

Sin II

4371.00 P $\mathrm{Fe}$

$\begin{array}{llll}4371.00 & \text { P } & \text { Fe I } & 69 \\ 4371.069 & & \text { Nd II } & 5 \\ 4371.10 & & \text { Fe II I } & \\ 4371.130 & & \text { Co I } & 9 \\ 4371.17 & \text { P } & \text { V II } & 3 \\ 4371.279 & & \text { Cr I } & 2 \\ 4371.28 & \text { P } & \text { Cr I } & 30 \\ 4371.33 & & \text { C I } & 1 \\ 4371.36 & & \text { A II } & \\ 4371.59 & & \text { C II } & 45\end{array}$

4360.813

4360.917

4361.025

4361.031

4361.249

4361.53

4361.661

4361.710

4861.85

4361.913

Fe I

Be II

Co I

Fe II

8 III

Ce II

8 I

C III

1
198

148

204

4362.040

4362.07

4362.10

4362.93

4362.95

4363.05

4363.134

4363. 30

4363.525

4363.644

Co I

4
23
31

8 II II

A II

Cr II

Cr I
La II

Cr I

$\begin{array}{ll}\text { Cr } & \text { I } \\ \text { C1 } & \text { I } \\ \text { V } & \text { I }\end{array}$

Mo II

903

4364. 01

4364.140

4364. 17

4364.59

4364.658

4364.66

4364.73

4364.87

4364. 89

Y II

Cr I.

Gd II

Y II
AI II

Ce II

La II

8 III

Cr I

16
4
1
4
157
6

4371.65

$\begin{array}{lll}4371.65 & 0 & I \\ 4372.09 & \text { I } & \text { I }\end{array}$

4372.208 Ru I

4372.22 P Fe I

4372.383

4372.4

4372.401

4372.49

$\begin{array}{lllll}4372.50 & & \text { A } & \text { II } & 63 \\ 4972.88 & \text { P } & \text { V } & \text { II } & 13\end{array}$

T1 I

\begin{tabular}{|c|c|c|c|c|}
\hline 4372.91 & & Cl & I I & 52 \\
\hline 4372.994 & & $\mathrm{Fe}$ & I & 473 \\
\hline 4373.230 & & v & $\mathbf{I}$ & 140 \\
\hline 4373.254 & & $\mathrm{Cr}$ & I. & 22 \\
\hline 4373.462 & & Sm & II & 42 \\
\hline 4373.563 & & $\mathrm{Fe}$ & I & 214,413 \\
\hline 4373.656 & & Cr & I & 304 \\
\hline 4373.818 & & Ce & II & 202 \\
\hline 4373.90 & $\mathbf{P}$ & Fe & I & 904 \\
\hline 4374.158 & & $\mathbf{C r}$ & I & 104 \\
\hline 1374.243 & & Gd & II & 83 \\
\hline 1374.28 & & c & II & 46 \\
\hline 1374.455 & & Sc & II & 14 \\
\hline$: 374.495$ & & $\mathrm{Fe}$ & I & 648 \\
\hline$: 374.61$ & $\mathbf{P}$ & $\mathrm{Cr}$ & I I & 179 \\
\hline 4374.825 & & T1 & II & 93 \\
\hline 4374.87 & & $\mathbf{A}$ & II & 77 \\
\hline 4374.918 & & Co & I & 150 \\
\hline 4374.923 & & Nd & I I & 15 \\
\hline 4374.94 & & $\mathbf{Y}$ & II & 13 \\
\hline 4375.00 & & N & II & 16 \\
\hline 4375.039 & & Nd & II & 8 \\
\hline 4375.304 & & v & I & 140 \\
\hline 4875.333 & & Cr & I & 103 \\
\hline 4375.35 & $\mathbf{P}$ & T1 & II & 104 \\
\hline 4375.425 & & T1 & I & 218 \\
\hline 4375.48 & $\mathbf{P}$ & $\mathrm{Fe}$ & I & 797 \\
\hline 4375.540 & & Co & I & 143 \\
\hline 4375.918 & & $\mathrm{Ce}$ & II & 134 \\
\hline 4375.932 & & Fe & I & 2 \\
\hline 4375.96 & & $\mathbf{A}$ & II & 17 \\
\hline 4376.78 & & C & II & 46 \\
\hline 4376.782 & & Fe & I & 471,804 \\
\hline 4976.798 & & $\mathrm{Cr}$ & I & 304 \\
\hline 4377.330 & & Fe & I & 990 \\
\hline 4377.549 & & $\mathbf{C r}$ & I & 83 \\
\hline 4377.765 & & Mo & II & 3 \\
\hline 4377.796 & & Fe & I & 645 \\
\hline 4377.95 & & Ne & II & 66 \\
\hline 1378.01 & & 0 & II & 102 \\
\hline
\end{tabular}

4365.5

4365.72

4365.902

4366.165

4366.315

4366.33

4366.45

4366.896

4366.91

$\begin{array}{r}45 \\ 39 \\ \hline 9\end{array}$

9
179

82
133

103

8
23

70

130

33
70

9
135

53

7
158
202

Fe III 4

Ne II 57

V I

Fe I

Fe II

Nd II

Cr I

Zr I

$\begin{array}{ll}3 & \text { II } \\ 7 & \text { II }\end{array}$

57
79
415
216
12
153
61
2
36

$\begin{array}{llll}4367.07 & P & \text { Fe } I & 1170\end{array}$

$\begin{array}{rlrr}4367.36 & \text { P } & \text { N1 I } & 1170\end{array}$

4367.581

4367.657

4367.87

4367.80

4367.906

4367.966

4368.031

Fe I

T1 II

A II

Hr I

Fe I

Sin II

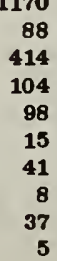

102 
I A Type Element Multiplet No.

\begin{tabular}{|c|c|c|c|c|}
\hline 4978.10 & & La & II & 77 \\
\hline 4978.236 & & Sm & II & 53 \\
\hline 4378.41 & & 0 & II & 102 \\
\hline 4378.73 & $\mathbf{P}$ & $\mathrm{Fe}$ & I & 759 \\
\hline 4379.09 & & N & III & 17 \\
\hline 4379.238 & & v & I & 22 \\
\hline 4379.25 & & $\mathbf{A}$ & II & 63 \\
\hline 4379.50 & & $\mathrm{Ne}$ & II & 56 \\
\hline 4379.74 & & A & II & 7 \\
\hline 4379.78 & & $\mathrm{Zr}$ & II & 88 \\
\hline
\end{tabular}

$\begin{array}{lll}4379.782 & \text { Cr I } & 130\end{array}$

4379.90

4378.97

4380.060

4380.38

4380.55

4380.642

4381.04

4381.112

c III

Ce II

$\mathrm{Mg} I$

Gid II

G I I

Cr I

Nd II

7
14

155
12

130

68

23

$4381.79 \quad P \quad$ Fe II

$\begin{array}{llll}4382.02 & P & \text { Fe } I & 938\end{array}$

4382.061

4882.167

4382.31

4382.33

4382.777

4382.853

4382.95

4383.10

ad II

Ce II

Fe III

V II

Fe I

Cr I

Zr II

Gd II

4383.17

4383.44

4383.547

4383.78

384.08

4384.13

4384.33

4384. 543

4384.6

4384. 643

384.72

4384.813

985.00

4385.08

4385.20

4385.260

Bu II

c III

La II

$\triangle$ II

Ne II

Fe I

Fe II

N1 I

N1 II

Me II

Fe I

Sc II

Cr I

Ne II

A II

Le II

4385.381

4385.45

385.663

4386.434

4386.46

4386.6

4386.835

4386.888

4387.213

4387.380

4387.486

4387.674

4987.887

4387.928

4388.007

4388.077

988.16

4388.24

4388.412

4388.50

4389.12

78

4889.870

4389.97

990.14

1380.322

390.460

4380.585

4380.858

4390.853

$43 \% 0.854$

4380.877

4381.110

391.11

4391.26

4391.568

381.661

4381.763

T1 II

104
40

Cr I

Cr I II

Fe I

Be I

Ce II

T1 I

I II

Pe I
I $\triangle$ Type Element Multiplet No.

$\begin{array}{lllr}4391.84 & & \text { S II } & 43 \\ 4391.87 & \text { P } & \text { Fe I } & 992 \\ 4391.94 & & \text { Ne II } & 57 \\ 4392.074 & & \text { V I } & 23 \\ 4392.26 & & \text { Cr I } & 130 \\ 4392.31 & \text { P } & \text { Fe I } & 757 \\ 4392.58 & & \text { Fe I } & 973 \\ 4393.03 & \text { P } & \text { Fe I } & 473 \\ 4393.45 & & \text { Na I } & 17 \\ 4393.534 & & \text { Cr I } & 102 \\ & & & \\ 4393.70 & \text { P } & \text { Fe I } & 899 \\ 4393.835 & & \text { V I } & 40 \\ 4393.925 & & \text { T1 I } & 244 \\ 4394.057 & & \text { T1 II } & 51 \\ 4394.31 & \text { P } & \text { Fe I } & 975 \\ 4394.65 & & \text { A II } & 87 \\ 4394.719 & & \text { Gd II } & 44 \\ 4394.779 & & \text { Ce II } & 259 \\ 4394.83 & & \text { Cr I } & 130 \\ 4394.855 & & \text { T1 I } & 78\end{array}$

4394.94 Zr I $\quad 61$

4395.031

4395.228

4395.288

4995.417

4395.514

4995.78

4395.788

4395.848

4395.95

$\nabla$ I

Fe I

Cr I

$\mathrm{Fe} I$

Fe III

Pr II

0 II

$\begin{array}{lllr}4397.251 & & \text { Cr I } & 129 \\ 4397.27 & \text { P } & \text { Fe II } & 33\end{array}$

4397.37 T1 IV

4397.51 Gd II

4398.02

4398.314

4388.52

4398.625

4398.787

Ne II

I II

T1 II

N1 I

Ce II

4399.14

(380.

4398.607

4399. 767

4399.823

4398. 86

4400.09

4400.18

4400.26

CI II

Fe II

Zr II

N1 I

T1 II

Cr I

Fe II

A II

G1 I I

61
18
22
828

129

891,992

4
29

61

26

56

61

187

102

46

81

81
67
196

196
51
129

120

1
67

1400.356

4400.575

4400.82

400.870

4401.02

4401.293

4401.35

401.447

401.847

$8 \mathrm{c} \mathrm{II}$

$\begin{array}{ll}\nabla & \text { I } \\ \text { T1 } & \text { II }\end{array}$

Nd II

N1 I

A II

Fe I

Zr. II

Fe I

4401.74

4401.97

4402.86

$\$ 402.875$

4403.03

4403.35

4403.360

4403.372

4403.488

4403.54

N1 I

A II

$\begin{array}{ll}\text { P } & \text { II } \\ \text { S } & \text { II }\end{array}$

Fe II

Cl I

Zr II

8w II

CrI

Cr I

4403.605

4404.10

404.276

404.39

404.68

404.752

1404.81

404.811

4404.932

$\$ 408.011$

Pr II

\section{Pe}

T1 I 218,218

Ti I

$\checkmark$ II

$\mathrm{Pe} I$

Zr II

Co I

14
22
93
10
148
1
828
68
350
86

76

43

6

22

128

405.23

406.40

405.69

4405.848

406.02

4406.147

4406.22

4106.26

$\$ 406.641$

I A Type Element Multiplet No.

4406.67 ad II 103

4407.278 Ce II

$\begin{array}{lll}4407.637 & \text { TI II } & \text { I } \\ 4407.678 & \text { Ve I }\end{array}$

4407.714 Fe I

$4407.72 \quad$ Cr I 129

$4408.204 \quad$ V I

$\begin{array}{lll}4408.248 & \text { Gd II } & 4 \\ 4408.419 & \text { Fe I } & 68\end{array}$

$4408.511 \quad V$ I 22

$\begin{array}{lll}4408.844 & \text { Pr II } & \\ 4408.92 & \text { V II } & 224\end{array}$

4409.123 Fe I 645

$\begin{array}{lll}4409.22 & \text { Ti II } & 61 \\ 4409.30 & \text { Ne II } & 57\end{array}$

$4409.519 \quad$ T1 II

$4409.84 \quad \mathrm{~kg}$

$\$ 410.026$ Ru

4410.06

C II

4410.516

4410.641

4410.967

4411.052 
I A Type Element Multiplet No.

\begin{tabular}{|c|c|c|c|c|c|c|c|c|}
\hline 4421.138 & & Sm I & II & 37 & 4431.922 & & Mn & I \\
\hline 4421.231 & & Pr 1 & II & 13 & 4432.089 & & T1 & II \\
\hline 4421.24 & & Gd I & II & 103 & 4432.175 & & $\mathrm{Cr}$ & I \\
\hline 4421.337 & & Co 1 & I & 150 & 4432.26 & & $\mathrm{Ne}$ & II \\
\hline 4421.38 & & $\mathrm{Ne} \mathrm{I}$ & II & 66 & 4432.41 & & $\mathbf{s}$ & II \\
\hline 4421.573 & & V I & I & 22 & 4432.572 & & $\mathrm{Fe}$ & I \\
\hline 4421.754 & & T1 1 & I & 218 & 4432.739 & & $\mathbf{N}$ & I I \\
\hline 4421.949 & & T1 1 & II & 93 & 4432.80 & $\mathbf{P}$ & $\mathrm{Fe}$ & I \\
\hline 4422.477 & & v I & I & 79 & 4432.82 & & Al & II \\
\hline 4422.570 & & Fe 1 & I & 350 & 4432.90 & $\mathbf{P}$ & $\mathrm{Fe}$ & I \\
\hline 4422.59 & & $\mathbf{Y}$ & II & 5 & 4432.95 & & La & II \\
\hline 4422.697 & & Cr & I & 234 & 4433. 223 & & $\mathrm{Fe}$ & $\mathbf{I}$ \\
\hline 4422.76 & & HF I & II & 103 & 4433.39 & $\mathbf{P}$ & $\mathrm{Fe}$ & $\mathbf{I}$ \\
\hline 4422.823 & & T1 & I & 78 & 4433.48 & & $\mathbf{N}$ & II \\
\hline 4422.882 & & $\mathrm{Fe}$ & I & 646 & 4433.501 & & Mo & II \\
\hline 4423.000 & & N1 1 & I & 168 & 4433.578 & & $\mathbf{T} \mathbf{1}$ & I \\
\hline 4423.145 & & Fe 1 & I & 412 & 4433.635 & & Gd & II \\
\hline 4423.212 & & v & I & 40 & 4433.793 & & $\mathrm{Fe}$ & I \\
\hline 4423.22 & $\mathbf{P}$ & T1 & II & 61 & 4433.83 & & $A$ & II \\
\hline 4423.31 & & $\mathrm{Na}$ & I & 16 & 4433.886 & & $8 m$ & II \\
\hline 4423.318 & & Cr & I & 128 & 4433.968 & & $\mathrm{Cr}$ & I \\
\hline 4423.678 & & Ce 1 & II & 21 & 4433.891 & & $\mathrm{Mg}$ & II \\
\hline 4423.73 & & R I & II & 5 & 4434.003 & & $T 1$ & I \\
\hline 4423.858 & & $\mathrm{Fe} I$ & I & 830 & 4434.323 & & Sm & II \\
\hline 4423.9 & & P & II & 31 & 4434.75 & & $\mathrm{Cr}$ & I \\
\hline 4424.075 & & Cr & I & 82 & 4434.960 & & $\mathrm{Ca}$ & $\mathbf{I}$ \\
\hline 4424.102 & & Ga & II & 67 & 4435.151 & & $\mathrm{Fe}$ & $\mathbf{I}$ \\
\hline 4424.194 & & $\mathrm{Fe}$ & I & 757 & 4435.58 & & Eu & II \\
\hline 4424.281 & & Cr & I & 128 & 4435.688 & & $\mathrm{Ca}_{\mathrm{a}}$ & I \\
\hline 4424.339 & & Sm & II & 45 & 4435.84 & & La & II \\
\hline 4424.401 & & T1 & I & 243 & 4486.025 & & Mn & I \\
\hline 4424.62 & $\mathbf{P}$ & $\mathbf{v}$ & II & 30 & 4436.138 & & $\mathbf{v}$ & $\mathbf{I}$ \\
\hline 4424.84 & & N1 & $\mathbf{I}$ & 262 & 4436.225 & & Gd & II \\
\hline 4425.129 & & $\mathrm{Cr}$ & I & 152 & 4436.362 & & Mn & I \\
\hline 4425.441 & & $\mathrm{Ca}$ & I & 4 & 4436.48 & & $\mathrm{Mg}$ & II \\
\hline 4425.662 & & $\mathrm{Fe}$ & I & 788 & 4436.686 & & T1 & I \\
\hline 4425.75 & $\mathbf{P}$ & $\mathrm{Fe}$ & I & 655 & 4436.64 & & $\mathbf{T 1}$ & I \\
\hline 4425.79 & $\mathbf{P}$ & $\mathrm{Fe}$ & I & 889 & 4436.931 & & $\mathrm{Fe}$ & I \\
\hline 4425.840 & & T1 & I & 78 & 4436.981 & & N1 & I \\
\hline 4425.95 & & $\mathbf{P}$ & II & 24 & 4437.549 & & $\mathrm{He}$ & I \\
\hline 4426.005 & & v & I & 22 & 4437.570 & & N1 & I \\
\hline 4426.01 & & $\mathbf{A}$ & II & 7 & 4437.612 & & $\mathrm{Ce}$ & II \\
\hline 4426.054 & & T1 & $\mathbf{I}$ & 161 & 4437.837 & & $\mathbf{v}$ & I \\
\hline 4426.151 & & Gd & II & 14 & 4438.044 & & Sr & I \\
\hline 4426.18 & & He & II & 87 & 4438.12 & & $\mathbf{A}$ & II \\
\hline 4427.098 & & T1 & $\mathbf{I}$ & 128 & 4438.13 & & Gd & II \\
\hline 4427.12 & $\mathbf{P}$ & T1 & I & 78 & 4438.232 & & T1 & I \\
\hline 4427.21 & & $\mathbf{N}$ & II & 66 & 4438.266 & & Gd & II \\
\hline 4427.30 & $\mathbf{P}$ & $\mathrm{Fe}$ & $\mathbf{I}$ & 828 & 4438.353 & & $\mathrm{Fe}$ & I \\
\hline $442, .312$ & & $\mathrm{Fe}$ & I & 2 & 4438.48 & & Cl & I \\
\hline 4427.62 & & Le & II & 76 & 4438.53 & $\mathbf{P}$ & $\mathrm{Fe}$ & I \\
\hline 4427.606 & & od & II & 66 & 4439.13 & $\mathbf{P}$ & $\mathrm{Fe}$ & II \\
\hline 4427.71 & & Cr & I & 129 & 4439.30 & & Ne & II \\
\hline 4427.90 & $\mathbf{P}$ & T1 & II & 61 & 4439.42 & & $\mathbf{v}$ & II \\
\hline 4427.917 & & $\mathrm{Ce}$ & II & 171 & 4439.45 & & $\mathbf{A}$ & II \\
\hline 4427.97 & & $\mathbf{N}$ & I I & 55 & 4439.643 & & $\mathrm{Fe}$ & I \\
\hline 4427.995 & & $\mathbf{M g}$ & I I & 9 & 4439.87 & & 8 & III \\
\hline 4428.501 & & $\mathrm{Cr}$ & I & 129 & 4439.883 & & $\mathrm{Fe}$ & I \\
\hline 4428.615 & & $\mathbf{v}$ & I & 21 & 4439.95 & & $\mathrm{Ne}$ & II \\
\hline 4428.54 & & $\mathrm{Ne}$ & II & 57,61 & 4440.09 & & $A$ & II \\
\hline 4428.57 & $\mathbf{P}$ & $\mathrm{Fe}$ & I & 973 & 4440.1 & & 0 & III \\
\hline 4428.74 & $\mathbf{P}$ & $\mathrm{Fe}$ & I & 899 & 4440.345 & & $\mathbf{T} \mathbf{1}$ & I \\
\hline 4429.11 & $\mathbf{P}$ & v & II & 13 & 4440.41 & & $\mathbf{v}$ & II \\
\hline 4429.20 & $\mathbf{P}$ & $\mathrm{Fe}$ & I & 987 & 4440.45 & & $\mathrm{Zr}$ & II \\
\hline 4429.238 & & Pr & II & 2,4 & $444 C .479$ & & $\mathrm{Fe}$ & I \\
\hline 4429.270 & & $\mathrm{Ce}$ & I I & 19 & 4440.840 & & $\mathrm{Fe}$ & I \\
\hline 4429.32 & & Fo & I & 972 & 4440.883 & & $\mathrm{Ce}$ & II \\
\hline 4429.34 & & $\mathbf{Z r}$ & II & 118 & 4440.972 & & $\mathrm{Fe}$ & I \\
\hline 4429.60 & & $\mathrm{Ne}$ & II & 74 & 4441.272 & & T1 & I \\
\hline 4429.796 & & $\mathbf{v}$ & I & 22 & 4441.56 & $\mathbf{P}$ & $\mathrm{Fe}$ & I \\
\hline 1429.90 & & Lo & II & 38 & 4441.683 & & $\mathbf{v}$ & I \\
\hline 4429.938 & & $\mathrm{Cr}_{\mathbf{r}}$ & I & 234 & 4441.73 & $\mathbf{P}$ & TI & II \\
\hline 4430.023 & & T1 & I & 267 & 4441.81 & & C & IV \\
\hline 4430.18 & & A & II & 7 & 4441.99 & & $\mathrm{~N}$ & II \\
\hline 4430.197 & & $\mathrm{Fe}$ & $\mathbf{I}$ & 472 & 4442.268 & & $\mathbf{C r}_{\mathbf{r}}$ & I \\
\hline 4430.366 & & T1 & I & 113 & 4442.343 & & $\mathrm{Fe}$ & I \\
\hline 4430.486 & & $\mathrm{Cr}$ & I & 234 & 4442.441 & & N1 & $\bar{I}$ \\
\hline 4430.51 & $\mathbf{P}$ & $\mathrm{Cr}$ & I & 128 & 4442.50 & & $\mathbf{Z r}$ & II \\
\hline 4430.618 & & $\mathrm{Fe}$ & I & 68 & 4442.67 & & $\mathrm{Ne}$ & II \\
\hline 4430.90 & & $\mathrm{Ne}$ & II & 56 & 4442.72 & $\mathbf{P}$ & $\mathrm{Ce}$ & II \\
\hline 4430.95 & & $\mathrm{Fe}$ & III & 4 & 4442.835 & & $\mathrm{Fe}$ & I \\
\hline 4431.02 & & A & II & 1 & 4442.99 & & $\mathrm{Zr}$ & II \\
\hline 4431.02 & & $\mathbf{s}$ & II & 32 & 4443.05 & & 0 & II \\
\hline 4431.284 & & T1 & I & 218 & 4443.07 & & Hf & I I \\
\hline 4431.369 & & $8 c$ & II & 14 & 4443.197 & & $\mathrm{Fe}$ & I \\
\hline 4431.48 & & $\mathrm{Zr}$ & I & B1 & 4443.707 & & $\mathbf{C r}$ & I \\
\hline 4431.608 & & Co & I & 143 & 4443.743 & & $\mathrm{Ce}$ & II \\
\hline 4431.626 & & $\mathrm{Fe}$ & II & 222 & 4443.802 & & T1 & II \\
\hline 4431.67 & & Ne & II & 74 & 4443.94 & & La & II \\
\hline 4431.82 & & $\mathbf{N}$ & II & 55 & 4444.20 & $\mathbf{P}$ & $\mathbf{v}$ & II \\
\hline
\end{tabular}


I 4 Type Element Multiplet No.

4457.758

4458.101

4458.262

4458. 336

4458.517

458.538

4459.037

4459.121

4459.34

4459.798

4459.760

4458.9

4460.12

4460.1

4460.213

4460.377

4460.55

4460.56

$\$ 460.769$

$\mathrm{V}$

Fe I $\quad 101$

Nn I 28

Pr II

Sin I

Cr I

Ni I

$\mathrm{Fe} I$

Cr I

V I

$\begin{array}{ll}V & \text { II }\end{array}$

Fe I

$\begin{array}{ll}V & \text { I } \\ \text { Ce II } & \end{array}$

$\mathrm{V} I$

in I

Fe I

Cr I

Yn I

Fe II

$\begin{array}{ll}\text { Fe } & I \\ \text { Zr II }\end{array}$

Fe I

Fe II

$\begin{array}{ll}0 & \text { II } \\ \mathrm{Fe} & \mathrm{I}\end{array}$

Fe I

Fe I $471,825,902$

4461.989

in I

T1 I

Pe I

Nd II

M1 I

I II

Fe III
$P$ II

28
8
824
87
54
86
13
127
106
25

Nd II

Pe I

$\begin{array}{lll}\text { Pe I } & \text { Od II } \\ \text { T1 I } & \text { Co }\end{array}$

Ce II

N1 I

T1 I

S II

8 II

T1 II
Cr I

Cr I

$\mathrm{Fe} I$

$\mathrm{V} I$

Fe I

Cr I

Bu II

Cr I

Fe I $\quad 1098$

Cr I 127

I II 81

0 II

N II

Nd II

T1 I 146

Cr I

Cr I

Fe I

0 II

N1 I

HP II

Gd I

Fe I

Fo I

Co I

$\mathrm{Fe} I$

od II

Sa II

Fe I

Ce II

Cr I

$\begin{array}{ll}\text { Cr I } \\ \text { S } & \text { III } \\ 0 & \text { III }\end{array}$

50

901

83
160
20

20
102

160
43
189

40
127
22

555

110
472

127
27

27
5

Type Blement Multiplet No.

$\mathbf{4 6 7 . 9 8}$
$\mathbf{4 6 8 . 0 1 0}$
$\mathbf{4 6 8 . 3 8}$
$\mathbf{4 6 8 . 4 9 3}$
$\mathbf{4 6 8 . 7 1 2}$
$\mathbf{4 6 8 . 7 5 9}$
$\mathbf{4 6 8 . 9 1}$
$\mathbf{4 6 9 . 1 6 0}$
$\mathbf{4 4 6 9 . 3 2}$
$\mathbf{4 4 6 9 . 3 7}$

$\begin{array}{llr}\text { P } & \text { II } & 25 \\ \text { V } & \text { I } & 87 \\ \text { CF } & \text { I } & 127 \\ \text { T1 II } & 31 \\ \text { Pr } & \text { II } & 20 \\ \text { V } & \text { I } & 102 \\ \text { Ne } & \text { II } & 61 \\ \text { T1 } & \text { II } & 18 \\ 0 & \text { II } & 59,94 \\ \text { CI } & \text { I } & 15\end{array}$

$\begin{array}{lll}4469.381 & \text { Fe I } & 830\end{array}$

4469.710

4469.850

4469.92 P Forb

4470.138 Yn I

$\begin{array}{lllll}4470.39 & P & V & \text { II } & 30\end{array}$

$\begin{array}{lll}\mathbf{4 4 7 0 . 4 8 3} & \text { N1 I } & 86 \\ \mathbf{4 4 7 0 . 8 6 4} & \text { T1 I I } & 40\end{array}$

4471.238

T1 I

146

4471.240 Ce II 8

4471.29

4471.477

4471.52

He I

$\begin{array}{lll}441.550 & \text { Co } I & 150\end{array}$

$\begin{array}{llll}447.68 & P & F e & \end{array}$

$\begin{array}{lllr}4471.688 & & \text { He I } & 14 \\ 4471.81 & P & \mathrm{Fe} I & 972\end{array}$

$\begin{array}{rrrr}4471.81 & P & \mathrm{Fe} I & 972 \\ 4472.09 & & \mathrm{Ca} \text { II } & 6\end{array}$

$\begin{array}{llll}4472.52 & P & \mathrm{Fe} I & 39\end{array}$

$\begin{array}{lllr}4472.57 & \text { P } & \text { Fe I } & 411 \\ 4472.721 & & \text { Fe I } & 595,90\end{array}$

4472.792

4472.921

4473.015

4473.782

4474.03

4474.045

4474. 194

4474.714

$\mathrm{Fe}$

Fe II

Sw II

Cr I

La II

$\begin{array}{ll}\text { V } & \text { I } \\ \text { Fe } & \text { II }\end{array}$

$\begin{array}{llll}4474.77 & & \text { A II } & 38 \\ 4474.852 & & \text { TI I } & 113,184 \\ 4474.85 & & \text { O III } & 37 \\ 4475.19 & \text { P } & \text { T1 I } & 184 \\ 4475.20 & \text { P } & \text { Cr I } & 63 \\ 4475.22 & & \text { Ne II } & 65 \\ 4475.24 & & \text { V II } & 198 \\ 4475.27 & & \text { P II } & 24 \\ 4475.28 & & \text { CI II } & 41,85 \\ 4475.31 & & \text { CI I } & 7\end{array}$

22
37

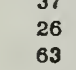

63
133

133

171
101

4475. 345

4475.518

4475.70

$\$ 475.72$

$4475.99 \quad P \quad F e$

$4476.021 \quad \mathrm{Fe}$

446.08

4476.082

4476.61

$\begin{array}{ll}0 & \text { II } \\ \text { Pe I } & \end{array}$

4477.02

T1 I

Cr I

4477.45

1 I

477.74
4477.88

N II

4478.03 0 II

$4478.040 \quad F e I$

448.318

4478.48

4478.657

4478.795 Gd II

$\begin{array}{llll}479.00 & P & \text { Fe I } & 987\end{array}$

478.01

$\mathbf{4 7 9 . 4 3 2}$

4479.612

4479.724

4478.89

479.97

95

198

14
889

350

87
830

184

14

88

13

68
150

480.142

480.263

480.27

4480.250

480.46

4480.570

4480.600

4480.687

4480.85

4481.04
I A Type

Element Multiplet No.

\begin{tabular}{|c|c|c|c|}
\hline 4481.056 & & Gd & II \\
\hline 4481.129 & & $\mathrm{Mg}$ & I I \\
\hline 4481.21 & & La & II \\
\hline 4481.23 & & N1 & I \\
\hline 4481.261 & & Ti & I \\
\hline 4481.273 & & Tm & I I \\
\hline 4481.327 & & $\mathrm{Ng}$ & II \\
\hline 4481.44 & & $\mathrm{C}_{5}$ & I \\
\hline 4481.621 & & $\mathrm{Fe}$ & I \\
\hline 4481.83 & & A & II \\
\hline 4482.02 & & Cl & I I \\
\hline 4482.04 & & $\mathrm{Zr}$ & I I \\
\hline 4482.171 & & $\mathrm{Fe}$ & I \\
\hline 4482.257 & & $\mathrm{Fe}$ & I \\
\hline 4482.40 & $\mathbf{P}$ & T1 & II \\
\hline 4482.688 & & T1 & I \\
\hline 4482.750 & & $\mathrm{Fe}$ & I \\
\hline 4482.878 & & $C_{r}$ & I \\
\hline 4483.328 & & Gd & II \\
\hline 4483.424 & & $\mathbf{s}$ & II \\
\hline
\end{tabular}

$\begin{array}{llll}4483.67 & P & \text { II } & 224\end{array}$

$\begin{array}{llll}4483.78 & P & \mathrm{Fe} I & 898\end{array}$

Ce II

4484.227 Fe I 828

4484.513 Co I

$\begin{array}{lll}4484.54 & \text { N1 I } & 102\end{array}$

$\begin{array}{llll}4484.93 & P & \text { Fe II } & 9\end{array}$

4485.013 TI I 184

$\begin{array}{lll}4485.15 & \text { Bu II } & 26 \\ 4485.44 & \text { Zr II }\end{array}$

$\begin{array}{llll}4485.679 & & \mathrm{Fe} I & 830 \\ 4485.97 & P & \mathrm{Fe} I & 825\end{array}$

$\begin{array}{lll}4486.14 & \text { Fe I } & 825 \\ 4 & \text { HF II } & 23\end{array}$

4486.352 Gd II 135

$\begin{array}{rlr}4486.65 & \text { HF II } & 107 \\ 4486.66 & S \text { II } & 43\end{array}$

$\begin{array}{lll}4486.909 & \text { Ce II } & 57\end{array}$

\begin{tabular}{|c|c|c|c|c|}
\hline 4487.01 & $\mathbf{P}$ & $\mathrm{Fe}$ & & 988 \\
\hline 4487.28 & & $\mathbf{Y}$ & I & 14 \\
\hline $4487 \cdot 36$ & $\mathbf{P}$ & $\mathrm{Fe}$ & I & 824 \\
\hline 4487.46 & & B & I I I & 2 \\
\hline 4487.46 & & $\mathrm{Cr}_{\mathrm{r}}$ & I & 63 \\
\hline 4487.47 & & $\mathrm{Y}$ & I & 14 \\
\hline 4487.72 & & 0 & I I & 104 \\
\hline 4487.74 & $\mathbf{P}$ & $\mathrm{Fe}$ & I & 394 \\
\hline 4487.821 & & Pr & II & \\
\hline 4488.051 & & $\mathrm{Cr}_{\mathrm{r}}$ & I & 29 \\
\hline
\end{tabular}

$\begin{array}{llll}4488.09 & 0 & \text { II } & 104\end{array}$

$\begin{array}{lll}4488.140 & \text { Fe I } & 819\end{array}$

$\begin{array}{lllr}4488.15 & & \text { N II } & 21 \\ 4488.27 & \text { P } & \text { TI I } & 184\end{array}$

$\begin{array}{lll}4488.319 & \text { TI I I } & 115\end{array}$

$\begin{array}{lll}4488.401 & \text { Gd II } & 82\end{array}$

$4488.898 \quad$ V I 86,110

$4488.917 \quad$ Fe I $\quad 213,82$

$\begin{array}{lll}4489.089 & \text { Ti I } & 146 \\ 4489.185 & \text { Fe II } & \end{array}$

4489.471

4489.48

4489.741

4489.87

4490.00

4490.081

4490.084

4490.24

4490.541

4490.56

Cr I

$\begin{array}{lll}0 & \text { II } & 86\end{array}$

Fe I 2

A1 II 107

CI II $\quad 41$

$\begin{array}{lll}\text { C1 II } & 41 \\ \text { Mn I } & 22\end{array}$

Fe I $\quad 469$

15
6

4490.60

N1 I 134,235

$\begin{array}{llll}490.60 & & \text { HP II } & \mathbf{7 4}\end{array}$

$\begin{array}{lr}\text { Fe I } & 891 \\ \text { Fe } & 974,97\end{array}$

$\begin{array}{rrr}4490.815 & V & 974,974\end{array}$

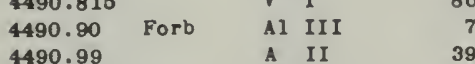

$\begin{array}{llll}4491.10 & P & \text { Ce II } & 19\end{array}$

$\begin{array}{llll}491.164 & V & \text { I }\end{array}$

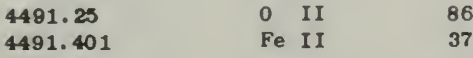

4491.678

4491.858

4492. 312

4482.3

4492.40

4482.427

4.492. 540

$4492: 693$

4492.98

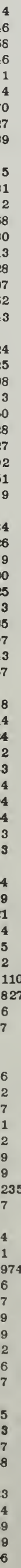


I A Type Element Multiplet No. I A Type Element Multiplet No.

I A Type Element Multiplet No.

\begin{tabular}{|c|c|c|c|c|c|c|c|c|}
\hline 4493.53 & & Ti & I I & 18 & 4507.854 & & $\mathrm{Ca} \mathrm{I}$ & \\
\hline 4493.579 & & $\mathrm{Fe}$ & II & 222 & 4507.95 & & $\mathrm{Cr}$ & I \\
\hline 4494.05 & $\mathbf{P}$ & $\mathrm{Fe}$ & $\mathbf{I}$ & 973 & 4508.083 & & $\mathrm{Ce}$ & II \\
\hline 4494.180 & & $\mathrm{Na}$ & I & 15 & 4508.21 & & Ne 1 & I I \\
\hline 4494.41 & & $\mathrm{Zr}$ & II & 130 & 4508.26 & $\mathbf{P}$ & $\mathrm{Fe}$ & II \\
\hline 4494.47 & $\mathbf{P}$ & $\mathrm{Fe}$ & I & 411 & 4508.283 & & $\mathrm{Fe}$ & II \\
\hline 4494.568 & & $\mathrm{Fe}$ & $\mathbf{I}$ & 68 & 4508.48 & & La 1 & I I \\
\hline 4494.67 & & N & I & & 4509.0 & & s 1 & II \\
\hline 4494.71 & & La & $\mathbf{I}$ & 11 & 4509.082 & & Gd 1 & I I \\
\hline 4494.746 & & Co & $\mathbf{I}$ & 168 & 4509.13 & $\mathbf{P}$ & $\mathrm{Fe}$ & $\mathbf{I}$ \\
\hline 4494.853 & & Gd & II & 14 & 4509.287 & & v I & I \\
\hline 4495 & $\mathbf{P}$ & N & IV & 6 & $\mathbf{4 5 0 9 . 3 0 6}$ & & $\mathrm{Fe}$ & I \\
\hline 4495.006 & & T1 & $\mathbf{I}$ & & 4509.446 & & $\mathrm{Ca}$ & I \\
\hline 4495.04 & & $\mathrm{Cr}$ & $\mathbf{I}$ & 101 & 4510.160 & & Pr & II \\
\hline 4495.275 & & $\mathrm{Cr}_{\mathbf{r}}$ & $\mathbf{I}$ & 275 & 4510.210 & & Mn & I I \\
\hline 4495.386 & & $\mathrm{Fe}$ & $\mathbf{I}$ & 319,970 & 4510.380 & & Gd & I I \\
\hline 4495.389 & & $\mathrm{Ce}$ & I I & 154 & 4510.7333 & & A I & I \\
\hline 4495.44 & & $\mathrm{Zr}$ & II & 79 & 4510.82 & $\mathbf{P}$ & $\mathrm{Fe}$ & $\mathbf{I}$ \\
\hline 4495.46 & $\mathbf{P}$ & T1 & II & 40 & 4510.92 & & $\mathbf{N}$ & III \\
\hline 4495.52 & $\mathbf{P}$ & $\mathrm{Fe}$ & I I & 147 & 4511.04 & $\mathbf{P}$ & $\mathrm{Fe}$ & I \\
\hline 4495.566 & & $\mathrm{Fe}$ & I & 827 & 4511.176 & & TA & I \\
\hline 4495.9 & & $\mathbf{s}$ & II & 48 & 4511.29 & & $\mathrm{Ne}$ & II \\
\hline 4495.986 & & $\mathrm{Fe}$ & I & 825 & 4511.310 & & In 1 & I \\
\hline 4496.062 & & $\mathbf{v}$ & I & 110 & 4511.37 & & $\mathrm{Ne}$ & II \\
\hline 4496.146 & & T1 & I & 146 & 4511.82 & $\mathbf{P}$ & $\mathrm{Cr}$ & II \\
\hline 4496.245 & & T1 & I & 8 & 4511.829 & & Sm I & II \\
\hline 4496.429 & & $\operatorname{Pr}$ & I I & 4,25 & 4511.903 & & $\mathrm{Cr}$ & I \\
\hline 4496.75 & $\mathbf{P}$ & Ti & I & 184 & 4512.282 & & $\mathrm{Ca}$ & I \\
\hline 4496.862 & & $\mathrm{Cr}_{\mathbf{r}}$ & I & 10 & 4512.535 & & Al I & III \\
\hline 4496.864 & & v & I & 86 & 4512.72 & & v I & II \\
\hline 4496.96 & & $\mathbf{Z r}$ & II & 40 & 4512.734 & & T1 & I \\
\hline 4496.989 & & Mn & I 1 & 17 & 4512.995 & & N1 & I \\
\hline 4497.30 & & $\mathrm{Cl}$ & I I & 41,85 & 4513.21 & & $\mathrm{Cr}_{\mathbf{r}}$ & I \\
\hline 4497.58 & & B & I I I & 3 & 4513.58 & & $\mathbf{Y}$ & I \\
\hline 4497.657 & & $\mathrm{Na}$ & I & 15 & 4513.715 & & T1 & I \\
\hline 4497.709 & & Ti & I & 184 & 4513.72 & $\mathbf{P}$ & $\mathrm{Fe}$ & I \\
\hline 4497.849 & & $\mathrm{Ce}$ & I I & 19 & 4513.89 & & $C_{\mathbf{r}}$ & I \\
\hline 4497.88 & & s & I I & 53 & 4513.90 & $\mathbf{P}$ & NI & I \\
\hline 4498.276 & & Gd & I I & 31 & 4514.189 & & $\mathrm{Fe}$ & I \\
\hline 4498.54 & $\mathbf{P}$ & $\mathrm{Fe}$ & I & 988 & 4514.191 & & v & I \\
\hline 4498.55 & & A & II & 136 & 4514.373 & & $\mathrm{Cr}$ & I \\
\hline 4498.730 & & $\mathrm{Cr}$ & I & 81 & 4514.505 & & Gd : & II \\
\hline 4498.76 & & $\mathrm{La}$ & II & 94 & 4514.531 & & $\mathrm{Cr}_{\mathbf{r}}$ & I \\
\hline 4498.897 & & Mn & I & 22 & 4514.80 & & $\mathrm{Ne}$ & II \\
\hline 4498.94 & & $\mathrm{Ne}$ & II & 64 & 4514.89 & & $\mathbf{N}$ & III \\
\hline 4499.18 & & $\mathbf{P}$ & II & 11 & 4515.094 & & Sm & I I \\
\hline 4499.29 & & $\mathbf{s}$ & III & 7 & 4515.17 & $\mathbf{P}$ & $\mathrm{Fe}$ & I \\
\hline 4499.475 & & Sm & II & 23 & 4515.19 & $\mathbf{P}$ & $\mathrm{Fe}$ & I I \\
\hline $4500 \cdot 295$ & & $C=$ & $I$ & 150 & 4515.337 & & $\mathrm{Fe}$ & II \\
\hline 4500.32 & $\mathbf{P}$ & Ti & II & 18 & 4515.440 & & Cr & I \\
\hline 4500.86 & $\mathbf{P}$ & $\mathbf{v}$ & I I & 30 & 4515.558 & & $\mathbf{v}$ & I \\
\hline 4501.112 & & $\mathrm{Cr}_{\mathbf{r}}$ & I & 81 & 4515.610 & & T1 & I \\
\hline 4501.256 & & $\mathbf{v}$ & I & 86 & 4516.02 & & c & I I I \\
\hline 4501.270 & & $\mathbf{T} \mathbf{1}$ & II & $\mathbf{3 1}$ & 4516.08 & $\mathbf{P}$ & $\mathrm{Fe}$ & $I$ \\
\hline 4501.692 & & N1 & $\mathbf{I}$ & 115 & 4516.27 & $\mathbf{P}$ & $\mathrm{Fe}$ & I \\
\hline 4501.788 & & $\mathrm{Cr}_{\mathbf{r}}$ & I & 81 & 4516.38 & & La & II \\
\hline 4501.808 & & $\mathrm{Nd}$ & I I & 53 & 4516.45 & $\mathbf{P}$ & $\mathrm{Fe}$ & $\mathbf{I}$ \\
\hline 4501.972 & & v & I & 62 & 4516.56 & $\mathbf{P}$ & $\mathrm{Cr}$ & II \\
\hline 4502.16 & & La & II & 154 & 4516.93 & & C & III I \\
\hline 4502.220 & & Mn & I & 22 & 4517.094 & & Co & I \\
\hline 4502.52 & & $\mathrm{Ne}$ & I I & 56 & $4517 \cdot 10$ & & Gd & II \\
\hline 4502.592 & & $\mathrm{Fe}$ & I & 796 & 4517.35 & & $\mathbf{v}$ & II \\
\hline 4502.95 & & $\mathbf{A}$ & I I & 63 & 4517.43 & P Forb & $\mathrm{He}$ & I \\
\hline 4503.05 & & $\mathrm{Cr}$ & I & 310 & 4517.530 & & $\mathrm{Fe}$ & I \\
\hline 4503.13 & $\mathbf{P}$ & $\mathbf{v}$ & II & 13 & 4517.595 & & $\mathrm{Pr}$ & II \\
\hline 4503.762 & & $\mathbf{T}_{\mathbf{1}}$ & $\mathbf{I}$ & 184 & 4517.60 & $\mathbf{P}$ & $\mathrm{Fe}$ & I \\
\hline 4504.23 & $\mathbf{P}$ & $\mathrm{Fe}$ & I & 988 & 4517.79 & & $\mathrm{Ne}$ & II \\
\hline 4504.27 & & $\mathrm{Cl}$ & I I & 41 & 4517.81 & & N1 & I \\
\hline 4504.52 & $\mathbf{P}$ & $\mathrm{Cr}$ & I I & 16 & 4518.022 & & $\mathrm{~T} 1$ & I \\
\hline 4504.838 & & $\mathrm{Fe}$ & I & 555 & $4518 \cdot 18$ & & $\mathbf{N}$ & III \\
\hline 4505.00 & & $\mathrm{Ca}$ & I & 24 & 4518.30 & $\mathbf{P}$ & $T_{1}$ & II \\
\hline 4505.22 & & $\mathrm{Cr}$ & I & 151 & 4518.38 & & $\mathbf{v}$ & II \\
\hline 4505.33 & & $\mathbf{K}$ & II & 4 & 4518.45 & & $\mathrm{Fe}$ & I \\
\hline 4505.715 & & $\mathbf{T} \mathbf{1}$ & $\mathbf{I}$ & 184 & 4518.58 & $\mathbf{P}$ & $\mathrm{Fe}$ & $\mathbf{I}$ \\
\hline 4505.75 & & $\mathrm{Nd}$ & II & 3 & 4518.58 & & Lu & I \\
\hline 4505.95 & & $\mathbf{Y}$ & I & 14 & 4518.63 & & $\mathrm{Cr}$ & I \\
\hline 4505.997 & & $\mathrm{Cu}$ & II & 1 & 4518.700 & & $\mathrm{~T} 1$ & $\mathbf{I}$ \\
\hline 4506.302 & & N1 & I & 133 & 4518.9 & & $\mathbf{s}$ & II \\
\hline 4506.333 & & $G d$ & I I & 4 & 4519.02 & & Hf & II \\
\hline 4506.50 & & 0 & I I & & 4519.19 & & $\mathrm{Cl}$ & II \\
\hline 4506.582 & & Nd & II & ? & 4519.633 & & Sm & II \\
\hline 4506.624 & & $\mathrm{Ca}$ & I & 24 & 4519.83 & & $\mathrm{Cr}_{r}$ & I \\
\hline 4506.74 & $\mathbf{P}$ & $\mathrm{T} 1$ & II & 3 & 4519.986 & & N1 & I \\
\hline 4506.853 & & $\mathrm{Cr}$ & $\mathbf{I}$ & 288 & 4520.070 & & Gd & I I \\
\hline 4506.931 & & Gd & II & 13 & 4520.225 & & $\mathrm{Fe}$ & I I \\
\hline 4507.11 & & $\mathrm{Zr}$ & I & 31 & 4520.24 & $\mathbf{P}$ & $\mathrm{Fe}$ & I \\
\hline 4507.19 & $\mathbf{P}$ & $\mathrm{Cr}$ & II & 16 & 4520.37 & $\mathbf{P}$ & T1 & II \\
\hline 4507.195 & & $\mathrm{Fe}$ & II & 213 & 4521.141 & & $\mathrm{Cr}$ & I \\
\hline 4507.417 & & $\mathrm{Ca}$ & I & 24 & 4521.296 & & Gd & I I \\
\hline 4507.559 & & $\mathbf{N}$ & II & 21 & 4521.65 & $\mathbf{P}$ & $\mathrm{Fe}$ & $\mathbf{I}$ \\
\hline
\end{tabular}




\begin{tabular}{|c|c|c|c|c|c|c|c|c|}
\hline 4531.82 & & $\mathrm{Cr}$ & I & 275 & 4545.335 & & $\mathrm{Cr}$ & I \\
\hline 1532.188 & & v & II & 212 & 4545.394 & & $\mathbf{v}$ & I \\
\hline 4632.75 & & $\mathrm{Cr}$ & I & 212 & 4545.49 & $\mathbf{P}$ & $\mathbf{C r}_{\mathbf{r}}$ & II \\
\hline 4533.143 & & $\mathrm{Fe}$ & I & 641 & 4545.54 & $\mathbf{P}$ & $\mathrm{Fe}$ & $\mathbf{I}$ \\
\hline 4533.18 & & Hr & II & 25 & 4545.956 & & $\mathbf{C r}_{\mathbf{r}}$ & I \\
\hline 4533.238 & & $\mathbf{T} \mathbf{1}$ & I & 42 & 4545.985 & & Co & I \\
\hline 4533.3 & & $\mathbf{s}$ & II & 47 & 4546.36 & & $\mathbf{N}$ & III \\
\hline 4533.81 & & $\mathbf{P}$ & II & 25 & 4546.47 & P & $\mathrm{Fe}$ & I \\
\hline 4533.966 & & $\mathbf{T 1}$ & I I & 50 & 4546.68 & $\mathbf{P}$ & $\mathrm{Fe}$ & I \\
\hline 4533.885 & & Co & I & 150 & 4546.930 & & N1 & I \\
\hline 4534.154 & & Pr & II & 20 & 4547.022 & & $\mathrm{Fe}$ & I \\
\hline 4534.166 & & $\mathrm{Fe}$ & II & 37 & 4547.234 & & N1 & I \\
\hline 4534.26 & & $\mathrm{Mg}$ & II & 26 & 4547.34 & & $\mathbf{N}$ & I I I \\
\hline 4534.57 & & $\mathbf{N}$ & I II & 3 & 4547.78 & & A & II \\
\hline 4534.62 & $\mathbf{P}$ & $\mathrm{Fe}$ & I & 1169 & 4547.850 & & $\mathbf{T} \mathbf{1}$ & I \\
\hline 4534.66 & & $\mathrm{Ne}$ & II & 58 & 4547.851 & & $\mathrm{Fe}$ & I \\
\hline 4534.782 & & T1 & I & 42 & 4548.094 & & $\mathbf{T 1}$ & I \\
\hline 4535.11 & & $\mathbf{N}$ & III & 13 & 4548.764 & & T1 & I \\
\hline 4535.146 & & $\mathrm{Cr}$ & I & 33 & 4549.214 & & $\mathrm{Fe}$ & II \\
\hline 4535.215 & & $\mathbf{v}$ & II & 210 & 4549.467 & & $\mathrm{Fe}$ & II \\
\hline 4535.38 & & Hf & II & 72 & 4549.50 & & La & $I$ \\
\hline 4535.47 & & $\mathrm{Ne}$ & II & 55 & 4549.547 & & $\mathbf{s}$ & II \\
\hline 4535.50 & & $\mathrm{Fe}$ & II I & & 4549.622 & & $\mathbf{T 1}$ & I I \\
\hline 4535.51 & & $\Lambda$ & II & 86 & 4549.644 & & $\mathbf{v}$ & I \\
\hline 4535.574 & & $\mathbf{T 1}$ & I & 42 & 4549.658 & & Co & I \\
\hline 4535.721 & & $C_{r}$ & I & 33,276 & 4549.82 & $P$ & $\mathbf{T 1}$ & II \\
\hline 4535.75 & & $\mathbf{Z r}$ & I & 30 & 4550.954 & & Gd & II \\
\hline 4535.87 & $\mathbf{P}$ & $\mathbf{T} 1$ & I & 112 & 4551.236 & & N1 & I \\
\hline 4535.920 & & $\mathbf{T} \mathbf{1}$ & I & 42 & 4551.297 & & $\mathrm{Ce}$ & II \\
\hline 4535.921 & & Pr & II & 1 & 4551.455 & & Gd & II \\
\hline 4536.051 & & T1 & I & 42 & 4551.667 & & $\mathrm{Fe}$ & I \\
\hline 4536.509 & & $\mathrm{Fe}$ & I & 896 & 4551.860 & & $\mathbf{v}$ & $\mathbf{I}$ \\
\hline 4536.55 & & $\mathrm{Cr}$ & I & 180 & 4552.25 & $\mathbf{P}$ & $\mathbf{T} 1$ & II \\
\hline 4536.78 & & Cl & II & 41 & 4552.37 & & As & II \\
\hline 4537.683 & & $\mathbf{v}$ & I & 82 & 4552.378 & & $\mathbf{s}$ & II \\
\hline 4537.67 & & $\mathbf{A}$ & II & 123 & 4552.453 & & $\mathbf{T 1}$ & I \\
\hline 4537.677 & & $\mathrm{Fe}$ & I & 594 & 4552.536 & & $\mathbf{N}$ & II \\
\hline 4537.751 & & $\mathrm{Ne}$ & I & 11 & 4552.544 & & $\mathrm{Fe}$ & $\mathbf{I}$ \\
\hline 1537.952 & & Sin & I I & 45 & 4552.654 & & $\mathbf{s}$ & III \\
\hline 4538.20 & $\mathbf{P}$ & $\mathrm{Fe}$ & I & 1071 & 4552.659 & & $\mathrm{Sm}$ & II \\
\hline 4538.58 & $\mathbf{P}$ & $\mathrm{Fe}$ & I & 972 & 4553.01 & & $\mathbf{Z r}$ & I \\
\hline 4538.64 & & v & II & 212 & 4553.056 & & $\mathbf{v}$ & I \\
\hline 4538.73 & & $\mathbf{A}$ & I I & 104 & 4553.16 & & $\mathrm{Ne}$ & II \\
\hline 4538.764 & & $\mathrm{Fe}$ & I & 115 & 4553.175 & & N1 & I \\
\hline 4638.84 & & $\mathrm{Fe}$ & I & 968 & 4653.48 & $\mathbf{P}$ & $\mathrm{Fe}$ & I \\
\hline 4538.87 & & La & I I & 149 & 4553.949 & & $\mathrm{Cr}$ & I \\
\hline 4538.95 & $\mathbf{P}$ & $\mathrm{Fe}$ & I & 1048 & 4553.96 & & $\mathrm{Zr}$ & II \\
\hline 4539.096 & & $\mathbf{T} 1$ & I & & 4554.033 & & $\mathrm{Ba}$ & II \\
\hline 4538.62 & & $\mathrm{Cr}$ & II & 39 & 4554.28 & & 0 & V \\
\hline 4538.755 & & Ce & I I & 108 & 4554.467 & & $\mathrm{Fe}$ & I \\
\hline 4659.788 & & $\mathrm{Cr}$ & I & 33 & 4554.509 & & $\mathrm{Ru}$ & I \\
\hline 4540.014 & & $\mathbf{V}$ & I & 100 & 4554.81 & & $\mathbf{P}$ & II \\
\hline 4640.016 & & Gd & II & 135 & 4554.830 & & $\mathrm{Cr}$ & I \\
\hline 4540.376 & & $\mathrm{Ne}$ & I & 17 & 4554.889 & & Gd & II \\
\hline 4540.483 & & T1 & I & 8 & 4555.02 & & $\mathrm{Cr}$ & II \\
\hline 4540.502 & & $\mathrm{Cr}$ & I & 33 & 4555.069 & & $\mathbf{T 1}$ & I \\
\hline 4540.71 & & Le & II & 81 & 4555.09 & & $\mathrm{Cr}$ & I \\
\hline 4540.719 & & $\mathrm{Cr}$ & I & 150 & 4555.30 & & $\mathrm{Cr}$ & I \\
\hline 4540.873 & & $\mathbf{T 1}$ & I & 112 & 4555.30 & & 0 & I I I \\
\hline 4641.071 & & $\mathrm{Cr}$ & I & 33 & 4555.421 & & $C_{8}$ & I \\
\hline 4641.269 & & Nd & II & 58 & 4555.486 & & $\mathbf{T 1}$ & I \\
\hline 4541.31 & & Hf & II & 36 & 4555.75 & $\mathbf{P}$ & $\mathrm{Fe}$ & I \\
\hline 4541.513 & & $\mathrm{Cr}$ & I & 148 & 4555.890 & & $\mathrm{Fe}$ & II \\
\hline 4541.523 & & Fe & II & 38 & 4555.822 & & $\mathrm{Cu}$ & II \\
\hline 4641.68 & & He & II & 2 & 4556.129 & & $\mathrm{Fe}$ & I \\
\hline 4641.671 & & Na & I & 14 & 4656.136 & & $N d$ & II \\
\hline 4541.953 & & $\mathrm{Fe}$ & I & 593 & 4556.169 & & $\mathrm{Cr}$ & I \\
\hline 4542.22 & & $\mathbf{Z r}$ & I & 49 & 4556.735 & & Nd & II \\
\hline 4542.422 & & Fe & I & 894 & 4656.765 & & v & II \\
\hline 4642.603 & & Nd & II & & 4556.939 & & $\mathrm{Fe}$ & I \\
\hline 4542.621 & & $\mathrm{Cr}$ & I & 148,275 & 4557.237 & & $8 c$ & I \\
\hline 4542.720 & & Fe & I & 827 & 4557.857 & & T1 & I \\
\hline 4542.77 & $\mathbf{P}$ & $\mathrm{Cr}$ & II & 16 & 4658.04 & & $\mathbf{P}$ & II \\
\hline 4543.22 & $\mathbf{P}$ & Fe & I & 893 & 4558.080 & & Gd & II \\
\hline 4343.74 & & $\mathrm{Cr}$ & I & 100 & 4558.092 & & $\mathbf{T} 1$ & I \\
\hline 4543.810 & & Co & I & 142 & 4558.108 & & $\mathrm{Fe}$ & I \\
\hline 4643.91 & & $\mathbf{A}$ & II & 95 & 4588.46 & & La & II \\
\hline 4643.948 & & 8 & II & 32 & 4538.46 & & $\mathbf{v}$ & I I \\
\hline 4644.000 & & $\mathbf{T} 1$ & II & 60 & 4558.58 & $\mathbf{P}$ & $\mathrm{Fe}$ & II \\
\hline 4644.11 & & No & II & 64 & 4558.659 & & $\mathrm{Cr}$ & II \\
\hline 4644.48 & & C1 & II & 48 & 4558.83 & & $\mathrm{Cr}_{\mathbf{r}}$ & II \\
\hline 1644.50 & $\mathbf{P}$ & Fe & I & 870 & 4559.09 & & $\mathbf{F}$ & III \\
\hline 4644.619 & & $C_{r}$ & I & 33 & 4559.28 & & La & II \\
\hline 4644.688 & & T1 & I & 42 & 4558.920 & & $\mathbf{T 1}$ & I \\
\hline 4544.70 & $\mathbf{P}$ & $\mathrm{Cr}_{\boldsymbol{r}}$ & II & 16 & 4558.945 & & N1 & I \\
\hline 4644.80 & & $N$ & III & 12 & 4660.096 & & $\mathrm{Fe}$ & I \\
\hline 4644. 861 & & Ce & II & 123 & 4560.26 & & $\mathrm{Cr}$ & I \\
\hline 4646.08 & & $A$ & II & 15 & 4660.280 & & $\mathrm{Ce}$ & II \\
\hline $\begin{array}{l}4645.144 \\
4645.218\end{array}$ & & $\begin{array}{l}T_{1} \\
\mathrm{Ne}_{2}\end{array}$ & II & 30 & 4660.710 & & v & I \\
\hline & & Ne & & 14 & 4560.960 & & Ce & II \\
\hline
\end{tabular}




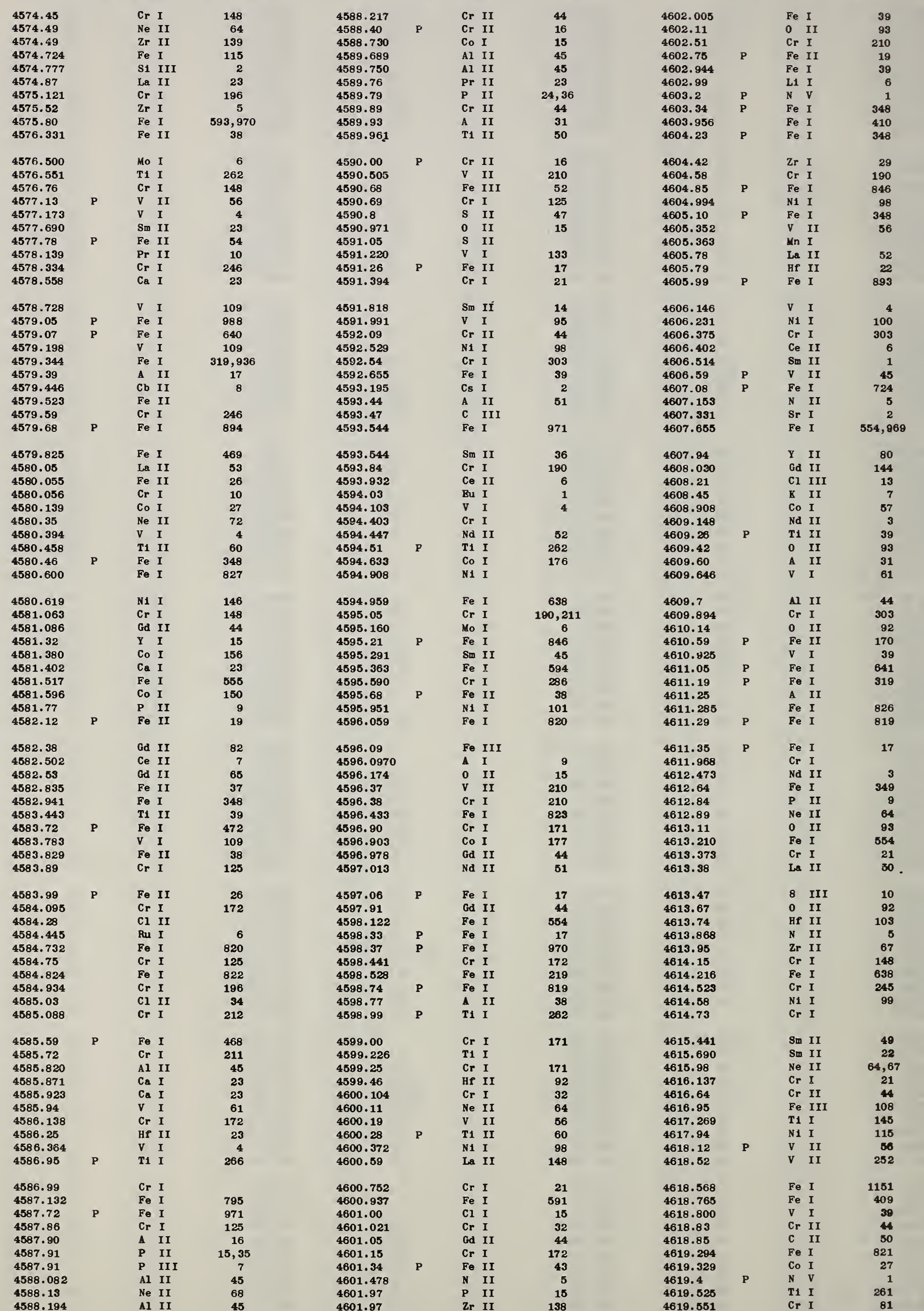




\begin{tabular}{|c|c|c|c|c|c|c|c|c|}
\hline 4619.648 & & v & I & & 4634.16 & & $\mathbf{N}$ & III \\
\hline 4619.771 & & $\mathbf{v}$ & I & 4 & 4634.21 & & v & II \\
\hline 4619.87 & & La & I I & 76 & 4634.59 & & $\mathrm{Cr}$ & $I$ \\
\hline 4620.13 & $\mathbf{P}$ & $\mathrm{Fe}$ & I & 468 & 4634.60 & $\mathbf{P}$ & $\mathrm{Fe}$ & I I \\
\hline 4620.38 & & N1 & I & 163 & 4634.73 & & $\mathrm{Ne}$ & II \\
\hline 4620.513 & & $\mathrm{Fe}$ & I I & 38 & 4634.95 & & La & II \\
\hline 4621.00 & & $\mathrm{Cr}$ & I & 32 & 4635.176 & & V & I \\
\hline 4621.28 & & 0 & II & 92 & 4635.328 & & $\mathrm{Fe}$ & II \\
\hline 4621.39 & & $\mathrm{Fe}$ & III & 108 & 4635.539 & & T1 & I \\
\hline 4621.392 & & N & II & 5 & 4635.62 & $\mathbf{P}$ & $\mathrm{Fe}$ & I \\
\hline 4621.41 & $\mathbf{P}$ & $\mathrm{Cr}$ & I I & 25 & 4635.7 & & Al & II \\
\hline 4621.63 & $\mathbf{P}$ & $\mathrm{Fe}$ & I & 989 & 4635.846 & & $\mathrm{Fe}$ & I \\
\hline 4621.893 & & $\mathrm{Cr}$ & I & 32 & 4636.345 & & T1 & II \\
\hline 4621.963 & & $\mathrm{Cr}$ & I & 32,244 & 4636.42 & & La & I I \\
\hline 4622.40 & $\mathbf{P}$ & $\mathrm{Fe}$ & II & 17 & 4636.66 & $\mathbf{P}$ & $\mathrm{Fe}$ & I \\
\hline 4622.491 & & $\mathrm{Cr}$ & I & 233 & 4637.182 & & $\mathrm{Cr}$ & I \\
\hline 4622.71 & & He & II & 70 & 4637.209 & & T1 & I \\
\hline 4622.71 & & $\mathbf{P}$ & II & 36 & 4637.25 & & A & II \\
\hline 4622.761 & & $\mathrm{Cr}_{r}$ & I & 81 & 4637.512 & & $\mathrm{Fe}$ & I \\
\hline 4623.020 & & Co & $I$ & 156 & 4637.772 & & $\mathrm{Cr}$ & I \\
\hline 4623.098 & & T1 & I & 145 & 4637.887 & & T1 & I \\
\hline 4624.11 & & $\mathbf{s}$ & I I & & 4638.016 & & $\mathrm{Fe}$ & I \\
\hline 4624.404 & & $\mathbf{v}$ & I & 39 & $4638 \cdot 12$ & & S1 & III \\
\hline 4624.42 & & $\mathrm{Fe}$ & III & 108 & 4638.854 & & 0 & II \\
\hline 4624.561 & & Co & I & 141 & 4639.001 & & Gd & II \\
\hline 4624.657 & & $\mathbf{v}$ & I & 94 & 4639.150 & & Mn & II. \\
\hline 4624.86 & & $\mathrm{Zr}$ & II & 116 & 4639.326 & & Al & II \\
\hline 4624.899 & & $\mathrm{Ce}$ & I I & 27 & 4639.369 & & T1 & I \\
\hline 4625.052 & & $\mathrm{Fe}$ & I & 554 & 4639.384 & & Al & II \\
\hline 4625.30 & & $\mathrm{Cr}$ & I & 171 & 4639.538 & & $\mathrm{Cr}$ & I \\
\hline 4625.44 & $\mathbf{P}$ & $\mathrm{Fe}$ & I & 974 & 4639.668 & & T1 & I \\
\hline 4625.549 & & $\mathrm{Fe}$ & II & 219 & 4639.725 & & Al & II \\
\hline 4625.65 & & $\mathrm{Cr}$ & I & 244 & 4638.833 & & Al & II \\
\hline 4625.71 & & C & II & 49 & 4639.944 & & T1 & I \\
\hline 4625.767 & & Co & I & 176 & 4640.062 & & v & I \\
\hline 4625.911 & & $\mathrm{Fe}$ & II & 186 & 4640.14 & & Hf & II \\
\hline 4625.925 & & $\mathrm{Cr}$ & I & 244 & 4640.309 & & $\mathrm{v}$ & I \\
\hline 4626.188 & & $\mathrm{Cr}$ & I & 21 & 4640.362 & & Al & II \\
\hline 4626.36 & $\mathbf{P}$ & $\mathrm{Fe}$ & I & 636 & 4640.384 & & Al & II \\
\hline 4626.467 & & Mo & I & 6 & 4640.431 & & $\mathrm{~T} 1$ & I \\
\hline 4626.480 & & V & I & 39 & 4640.55 & & $\mathrm{Cr}_{\mathbf{r}}$ & I \\
\hline 4626.53 & & $\mathrm{Fe}$ & III & 108 & 4640.64 & & $\mathbf{N}$ & III \\
\hline 4626.544 & & Mn & I & & 4640.67 & & $\mathrm{Cr}$ & I \\
\hline 4626.565 & & Tm & II & & 4640.735 & & $\mathbf{v}$ & I \\
\hline 4626.61 & & $\mathbf{P}$ & II & 15 & 4641.22 & $\mathbf{P}$ & $\mathrm{Fe}$ & I \\
\hline 4626.758 & & $\mathrm{Fe}$ & I & 410 & 4641.77 & Forb & $\mathbf{K}$ & I \\
\hline 4626.78 & $\mathbf{P}$ & $\mathrm{Fe}$ & II & 170 & 4641.811 & & 0 & II \\
\hline 4626.81 & & $\mathrm{Cr}$ & I & 209 & 4641.90 & & $\mathbf{N}$ & III \\
\hline 4627.02 & $\mathbf{P}$ & $\mathrm{Fe}$ & I & 637 & 4642.011 & & $\mathrm{Cr}$ & I \\
\hline 4627.22 & & $\mathrm{Bu}$ & I & 1 & 4642.235 & & Sm & II \\
\hline 4627.48 & & v & II & 210 & 4642.27 & Forb & $\mathbf{K}$ & I \\
\hline 4627.66 & & Gd & II & 43 & 4642.68 & $\mathbf{P}$ & $\mathrm{Fe}$ & I \\
\hline 4627.85 & & $\mathrm{Ne}$ & II & 73 & 4643.086 & & $N$ & I I \\
\hline 4627.86 & $\mathbf{P}$ & $\mathrm{Fe}$ & II & 54 & 4643.20 & $\mathbf{P}$ & $\mathrm{Fe}$ & I \\
\hline $4628 \cdot 160$ & & $\mathrm{Ce}$ & II & 1 & 4643.468 & & $\mathrm{Fe}$ & I \\
\hline 4628.4410 & & $\mathbf{A}$ & I & 9 & 4643.69 & & $Y$ & $\mathbf{I}$ \\
\hline 4628.473 & & $\mathrm{Cr}$ & I & 186 & 4644.09 & $\mathbf{p}$ & $\mathrm{Fe}$ & II \\
\hline 4628.68 & $\mathbf{P}$ & $\mathrm{Fe}$ & I & 819 & 4644.82 & & $\mathbf{Z r}$ & I \\
\hline 4628.71 & & $\mathbf{P}$ & II & 28 & 4645.193 & & T1 & I \\
\hline 4628.751 & & Pr & II & 1 & 4645.28 & & Le & II \\
\hline 4628.821 & & $\mathrm{Fe}$ & II & 219 & 4646.971 & & $\mathbf{v}$ & I \\
\hline 4628.808 & & Co & I & 15 & 4646.059 & & $\mathrm{Pr}$ & II \\
\hline 4629.07 & & $2 r$ & II & 139 & 4646.174 & & $\mathrm{Cr}$ & I \\
\hline $4620 \cdot 28$ & $\mathbf{P}$ & T1 & II & 38 & 4846.326 & & Gd & II \\
\hline 4629.336 & & $\mathrm{Fe}$ & II & 37 & 4646.396 & & $\mathbf{v}$ & I \\
\hline 4629.336 & & T1 & I & 146 & 4646.495 & & $\mathrm{Cr}$ & I \\
\hline 4629.359 & & Co & I & 156 & 4646.684 & & $\mathrm{Sm}$ & II \\
\hline 4629.7 & & Al & II & 35 & 4646.808 & & $\mathrm{Cr}$ & I \\
\hline 4629.814 & & $2 n$ & I & 8 & 4646.94 & $\mathbf{P}$ & N1 & I \\
\hline 4628.90 & $\mathbf{P}$ & $\mathrm{Fe}$ & II & 170 & 4647 & $\mathbf{P}$ & C & IV \\
\hline 4622.98 & $\mathbf{P}$ & N1 & I & 223 & 4647.34 & & $\mathrm{Ne}$ & II \\
\hline 4630.125 & & $\mathrm{Fe}$ & I & 115 & 4647.40 & & c & III \\
\hline 4630.52 & & C & II & 49 & 4647.40 & & T1 & IV \\
\hline 4630.537 & & $\mathbf{N}$ & II & 5 & 4647.42 & $\mathbf{P}$ & N1 & I \\
\hline 4630.785 & & $\mathrm{Fe}$ & I & 969 & $4647^{\circ} .437$ & & $\mathrm{Fe}$ & I \\
\hline 4631.03 & $\mathbf{P}$ & $\mathrm{Fe}$ & I & 1071 & 4647.50 & & La & II \\
\hline $4631 \cdot 38$ & & s1 & IV & 6 & 4647.585 & & Mn & II \\
\hline 4631.49 & & $\mathrm{Fe}$ & I & 1152 & 4647.72 & $\mathbf{P}$ & $\mathrm{Fe}$ & I \\
\hline 4631.5 & & Al & II & 97 & 4647.758 & & Nd & II \\
\hline 4631.895 & & $\mathrm{Fe}$ & II & 219 & 4648.128 & & $\mathrm{Cr}$ & I \\
\hline 4632.14 & $\mathbf{P}$ & $\mathrm{Fe}$ & I & 754 & $4648 \cdot 160$ & & Sm & II \\
\hline 4632.180 & & $\mathrm{Cr}$ & I & 171 & 4648.17 & & $\mathbf{s}$ & II \\
\hline 4632.83 & $P$ & $\mathrm{Fe}$ & I & 820 & 4648.23 & $\mathbf{P}$ & $\mathrm{Fe}$ & II \\
\hline 4632.915 & & $\mathrm{Fe}$ & I & 39 & 4648.62 & & Al & II \\
\hline 4633.05 & $\mathbf{P}$ & $\mathrm{Fe}$ & I & 17 & 4648.659 & & N1 & I \\
\hline 4633.2 & & Al & I I & 97 & 4648.868 & & $\mathrm{Cr}$ & I \\
\hline 4633.236 & & $\mathrm{Cr}$ & I & 186 & 4648.933 & & $\mathrm{Fe}$ & II \\
\hline 4633.764 & & $\mathrm{Fe}$ & I & 410 & 4649.06 & & $A$ & II \\
\hline 4633.98 & & $\mathbf{Z r}$ & I & 5 & 4648.139 & & 0 & II \\
\hline & & $\mathrm{Cr}$ & II & 44 & 4649.461 & & $\mathrm{Cr}$ & \\
\hline
\end{tabular}




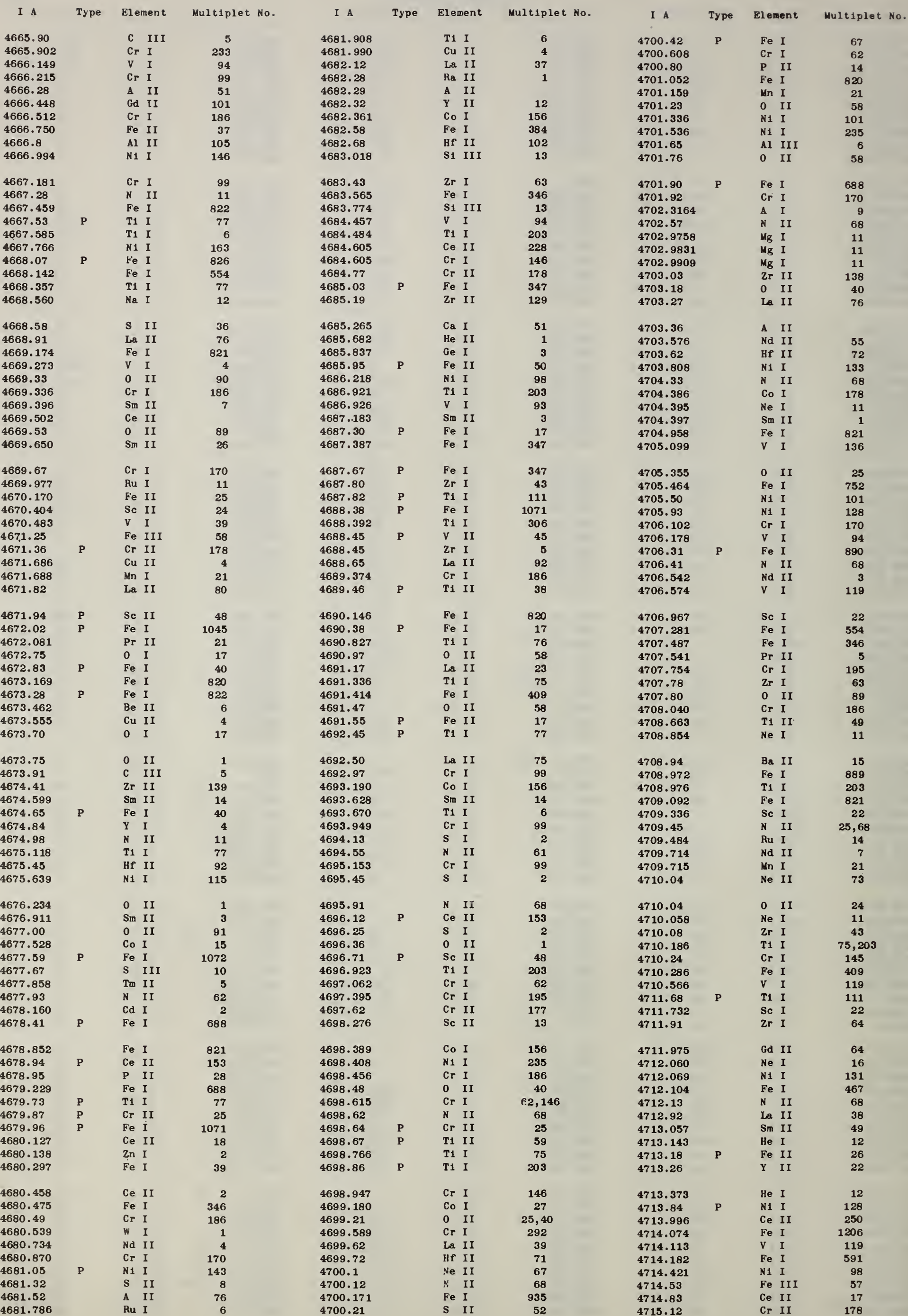


I A Type Element Multiplet No.

I A Type Element Multiplet No.

I A Type Element Multiplet No.

4715.295

$\mathbf{4 7 1 5 . 5 8 9}$

4715.778

4715.900

$4716.13 \quad$ P $\quad$ Sc II

$\begin{array}{lll}4716.226 & \text { S II } \\ 4716.44 & \text { La II }\end{array}$

4716.644

4716.658 S1 III

$\begin{array}{lll}4716.85 & P & \text { Fe I }\end{array}$

$\$ 717.031$ Sc I

4717.58 La II

4717.688

4717.692

4717.718

4718.16

4718.329

4718.429

4718.43

4719.040

4719.10

4719.12
4719

4719.515

4719.80

4719.838

4719.93 La II

$4720.15 \quad P \quad F e$ II

$4720.26 \quad P \quad P$ II

$\begin{array}{lll}4720.56 & P & \mathrm{Fe} \\ 4720.830 & & \mathrm{Sc}\end{array}$

4720.897

4721.14

4721.273

4721.43

4721.524

4721.59

4721.62

4722.159

4722.278

4722.333

4722.58

4722.603

4722.652

4722.741

4722.877

4729

4723.06

4723.18

4723.37

423.88
4724.07

4724.07

4724.416

4724.42

7725.090

4725.67

4725.94
4725.95

4726.165

4726.725

4726.91

4727.01

4727.153

4727.21

1727.405

4727.476

4727.85

4727.8

4727.936

4728.41

4728.42

4728.468

4728.555

4728.768

4729.028

4729.226

4720.291

4720.45

4729.844

4729.699

4729.723

4750.028

4730.24

4730.361

4730.321

4730.69

\begin{tabular}{|c|c|c|c|c|}
\hline 4730.711 & & $\mathrm{Cr}$ & I & 145 \\
\hline 4730.92 & & As & II & 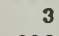 \\
\hline 4731.172 & & T1 & I & 202 \\
\hline 4731.36 & & He & II & 38 \\
\hline 4731.439 & & $\mathrm{Fe}$ & I I & 43 \\
\hline 4731.77 & $\mathbf{P}$ & $\mathrm{Fe}$ & I & 67 \\
\hline 4731.809 & & N1 & I & 168 \\
\hline 4732.051 & & Co & I & 15 \\
\hline 4732.08 & & $A$ & II & 38 \\
\hline 4732.34 & & $\mathrm{Zr}$ & I & 48 \\
\hline 4732.465 & & N1 & I & \\
\hline 4732.53 & & Ne & II & \\
\hline 4732.60 & & Gd & II & \\
\hline 4732.86 & $\mathbf{P}$ & T1 & II & \\
\hline 4733 & $\mathbf{P}$ & N & IV & \\
\hline 4733.426 & & T1 & I & \\
\hline 4733.596 & & Fe & I & \\
\hline 4734.094 & & Sc & I & \\
\hline 4734.100 & & $\mathrm{Fe}$ & I & 11 \\
\hline 4734.177 & & Pr & II & \\
\hline 4734.427 & & Gd & II & \\
\hline 4734.52 & & $Y$ & II & \\
\hline 4734.682 & & T1 & I & \\
\hline 4734.75 & & c & II & \\
\hline 4734.828 & & Co & I & 15 \\
\hline 4734.84 & & $\mathbf{z r}$ & I I & 1 \\
\hline 4735.67 & & He & II & \\
\hline 4735.75 & & Hf & II & \\
\hline 4735.846 & & $\mathrm{Fe}$ & I & 10 \\
\hline 4735.93 & & $\mathbf{A}$ & II & \\
\hline 4736.13 & & $\mathrm{Cr}$ & $I$ & \\
\hline 4796.50 & & Ni & I & \\
\hline 4736.780 & & $\mathrm{Fe}$ & $i$ & \\
\hline 4737 & $\mathbf{P}$ & c & IV & \\
\hline 4737.282 & & $\mathrm{Ce}$ & II & \\
\hline 4737.350 & & $\mathrm{Cr}$ & I & \\
\hline 4737.58 & & v & II & \\
\hline 4737.639 & & $\mathrm{Fe}$ & I & \\
\hline 4737.642 & & Sc & I & \\
\hline 4737.769 & & Co & I & \\
\hline
\end{tabular}

C II

4738.11

4738.28

CI II

$4738.52 \quad P \quad$ Pe II

4739.108 In I

4739.42 CI II

$4739.48 \quad 2 r$ I

$\begin{array}{ll}4739.49 & \text { Ce II } \\ 4739.49 & P \text { II }\end{array}$

4739.59

$\begin{array}{ll}\text { P II } & \text { II }\end{array}$

4739.80 La II

$\begin{array}{lll}4740 & \text { P } & \text { N IV } \\ 4740.165 & & \text { N1 I }\end{array}$

$\begin{array}{ll}4740.165 & \text { N1 I } \\ 4740.27 & \text { Le II }\end{array}$

4740.343

4740.40

$\mathbf{4 7 4 1 . 0 1 8}$

4741.081

$\begin{array}{lll}474.34 & \text { Cr I } & \text { N1 I }\end{array}$

4741.539 $\quad \mathrm{Fe} I$

471.53

4741.922

4742.00

Ge II

4742.4

4742.631

4742.791

4742.93

3
3
38
43
67
63
15
38
48

4748.525

4748.67

4748.73

4749.25

4749.25

4749.93

$\mathbf{4 7 5 0 . 4 9}$
$\mathbf{4 7 5 0 . 9 9 0}$

$\begin{array}{lllll}4751 & & \text { V } & \text { I } & 113 \\ 4 & \text { N } & \text { V } & \end{array}$

475

4751.04

4751.34

4751.574

4751.8

4752
4752.084

4752.124

4732.426

4752.70

$\begin{array}{ll}\text { V } & \text { I } \\ \text { CI } & \text { II } \\ \text { La } & \text { II } \\ \text { Cr } & \text { I } \\ \text { Fe } & \text { I } \\ \text { Co } & \text { I } \\ \text { Fe } & \text { I } \\ \text { Fe } & \text { I I } \\ \text { V } & \text { I } \\ \text { N } & \text { V }\end{array}$

113

0 VI

$\begin{array}{ll}\text { Cr } & \text { I } \\ 0 & \text { II }\end{array}$

V I

$\mathrm{Na} I$

$N$ IV

Cr I

N1 I

N1 I

4752.7313

4752.87

4753.06

4753.152

4753.957

4754.042

4754.358

4754.38

4754.635

4754.743

o II

4754.768

4755.12

4755.137

4755.347

4755.64

4755.728

4756.113

4756.519

4756.722

4757.37

4757.50

4757.582

4757.591

4757.791

4757.841

4757.842

4758.120

4758.42

Ne I

$\mathrm{Cr}$

V I

In I

Co I

T1 I

Pr II

Cr I

10

290

24
94

11

132

4758.421

4758.742

4758.913

4759.272

4759.74

4759.74

4759.907

4760.07

4760.15

4760.23

N1

14

Cr I 124

Gd II 134

Cl II

Cr I

N1 I

Co

Cr I 180

VI

$\begin{array}{ll}V & I \\ W & I\end{array}$

Fe I

Cr I

Gd II

Ru I

Ce II

T1 I

N1 I

Cu II

$\checkmark$ I

T1 I

T1 I

Cr I

Cr I

$\mathrm{Fe}$

Fe II

N1 I

HF II

4760.58

4760.98

4761.242

4761.42

4761.526

4761.67

4761.73

4762

4762.376

4762.41

$\mathrm{X}$
$\mathrm{C}$

Cr I
Cr II

Mn I

Zr II

Cr I

N IV

Mn I

21

66

113

16
156

202

168

4762.627

4762.727

4762.77

4762.78

4763.38

4763.624

4763.78

4763.84

4763.865

4763.950

N1 I

Pr II

II II

Zr I

$S$ II

Nd II

Fo II

TI II

474.925

4745.129

Pr I

4745. 680

4764.294

4764.535

4764.64

4764.7

4764. 89

4765.30

4765.485

4765.78

4765.859

4766.330

4746.638

4747.149

4747.256

4747.680 
I A Type Element Multiplet No.

\begin{tabular}{|c|c|c|c|c|}
\hline 4766.430 & & Mn & I & 21 \\
\hline 4766.62 & & C & I & 6 \\
\hline 4766.63 & & $\mathrm{Cr}$ & I & 231 \\
\hline 4766.635 & & $\mathrm{v}$ & I & 113 \\
\hline 4766.66 & $\mathbf{P}$ & $\mathrm{Cr}$ & I & 124 \\
\hline 4766.87 & $\mathbf{P}$ & $\mathrm{Fe}$ & I & 688 \\
\hline 4767.142 & & Co & I & 182 \\
\hline 4767.280 & & $\mathrm{Cr}$ & I & 231 \\
\hline 4767.30 & $\mathbf{P}$ & T1 & II & 29 \\
\hline 4767.860 & & $\mathbf{C r}$ & I & 231 \\
\hline 4768.072 & & Co & I & 156 \\
\hline 4768.334 & & $\mathrm{Fe}$ & I & 821 \\
\hline 4768.397 & & $\mathrm{Fe}$ & I & 384 \\
\hline 4768.68 & & Cl & II & 40 \\
\hline 4769.775 & & T1 & I & 233 \\
\hline 4769.80 & & $\mathrm{Cr}$ & I & 283 \\
\hline 4770.00 & & C & I & 6 \\
\hline 4770.670 & & $\mathrm{Cr}$ & I & 124 \\
\hline 4771.09 & & Cl & II. & 40 \\
\hline 4771.103 & & T1 & I & 41 \\
\hline 4771.108 & & Co & I & 156 \\
\hline 4771.57 & & $\mathrm{Cr}$ & I & 124 \\
\hline 4771.66 & & Cl & II & 45 \\
\hline 4771.702 & & $\mathrm{Fe}$ & I & 67 \\
\hline 4771.72 & & C & I & 6 \\
\hline 4772.32 & & $\mathrm{Zr}$ & I & 43 \\
\hline 4772.54 & & 0 & I & 16 \\
\hline 4772.57 & & 0 & IV & 9 \\
\hline 4772.728 & & Gd & I I & 133 \\
\hline 4772.77 & $\mathbf{P}$ & $\mathrm{Fe}$ & II & 31 \\
\hline 4772.817 & & $\mathrm{Fe}$ & I & 38,467 \\
\hline 4772.89 & & N1 & I & 162 \\
\hline 4772.89 & & 0 & I & 16 \\
\hline 4773.412 & & N1 & I & 167 \\
\hline 4773.52 & $\mathbf{P}$ & $\mathrm{Fe}$ & I & 408 \\
\hline 4773.76 & & 0 & I & 16 \\
\hline 4773.942 & & $\mathrm{Ce}$ & II & 17 \\
\hline 4774.222 & & $\mathbf{N}$ & II & 20 \\
\hline 4774.557 & & $\mathbf{C r}$ & I & 124 \\
\hline 4775.141 & & $\mathrm{Cr}$ & I & 230 \\
\hline 4775.53 & & $\mathrm{Cr}$ & I & 283 \\
\hline 4775.87 & & c & I & 6 \\
\hline 4775.87 & $\mathbf{P}$ & $\mathrm{Fe}$ & I & 1115 \\
\hline 4776.075 & & $\mathrm{Fe}$ & I & 635 \\
\hline 4776.311 & & Co & I & 158 \\
\hline 4776.34 & & $\mathrm{Fe}$ & I & 1206 \\
\hline 4776.364 & & $\mathbf{v}$ & I & 113 \\
\hline 4776.519 & & $\mathbf{v}$ & I & 128 \\
\hline 4777.57 & & $\mathrm{Cr}$ & I & 124 \\
\hline 4777.78 & $\mathbf{P}$ & $\mathbf{C r}$ & II & 25 \\
\hline 4777.846 & & Sm & II & 3 \\
\hline 4778.233 & & Co & I & 186 \\
\hline 4778.259 & & T1 & I & 232 \\
\hline 4778.50 & & $\mathrm{Cr}$ & I & 124 \\
\hline 4778.93 & & Cl & II & 40 \\
\hline 4779.09 & & 0 & IV & 9 \\
\hline 4779.11 & & s & II & 8 \\
\hline 4779.347 & & Sc & I & 5 \\
\hline 4779.444 & & $\mathrm{Fe}$ & I & 720 \\
\hline 4779.710 & & $\mathbf{N}$ & II & 20 \\
\hline 4779.87 & & Cr & I & 124 \\
\hline 4779.979 & & Co & I & 158 \\
\hline 4779.986 & & $T 1$ & II & 92 \\
\hline 4780.60 & $\mathbf{P}$ & $\mathrm{Fe}$ & II & 50 \\
\hline 4780.81 & $\mathbf{P}$ & $\mathrm{Fe}$ & I & 633 \\
\hline 4781.04 & & $\mathbf{Y}$ & I & 13 \\
\hline 4781.168 & & $\mathbf{N}$ & II & 20 \\
\hline 4781.32 & & Cl & II & 40 \\
\hline 4781.432 & & Co & I & 57 \\
\hline 4781.718 & & T1 & I & 41 \\
\hline 4781.82 & & Cl & II & 13 \\
\hline 4781.95 & & $\mathrm{Ne}$ & II & 71 \\
\hline 4782.79 & $\mathbf{P}$ & $\mathrm{Fe}$ & I & 588 \\
\hline 4783.06 & & $\mathrm{Cr}$ & I & 283 \\
\hline 4783.306 & & T1 & I & 41 \\
\hline 4783.420 & & Mn & I & 16 \\
\hline 4783.43 & & 0 & IV & 9 \\
\hline 4784.320 & & $\mathbf{S r}$ & I & 5 \\
\hline 4784.480 & & $\mathbf{v}$ & I & 3 \\
\hline 4784.70 & $\mathbf{P}$ & $\mathrm{Cr}$ & I & 168 \\
\hline 4784.94 & & $\mathbf{z r}$ & I & 44 \\
\hline 4785.070 & & Co & I & 186 \\
\hline 4785.42 & & Lu & II & 5 \\
\hline 4785.44 & & Cl & II & 40 \\
\hline 478.5 .963 & & $\mathrm{Fe}$ & I & 1044 \\
\hline 4786.293 & & N1 & I & 50 \\
\hline 4786.515 & & $\mathbf{v}$ & I & 113 \\
\hline 4786.541 & & N1 & I & 98 \\
\hline 4786.58 & & $\mathbf{Y}$ & II & 22 \\
\hline
\end{tabular}

I A Type Element Multiplet No.

$\begin{array}{lllr}4786.908 & & \text { Gd II } & 65 \\ 4787.50 & \text { P } & \text { Fe I } & 408 \\ 4787.64 & \text { P } & \text { TI I } & 40 \\ 4787.74 & & \text { Cr I } & 168 \\ 4787.84 & & \text { Fe I } & 384 \\ 4788.126 & & \text { N I I } & 20 \\ 4788.69 & & \text { Zr I } & 43 \\ 4788.757 & & \text { Fe I } & 588 \\ 4788.9258 & & \text { Ne I } & 15 \\ 4789 & \text { P } & \text { C IV } & 5\end{array}$

$\begin{array}{lll}4789.354 & \text { Cr I } & 31\end{array}$

$\begin{array}{rrr}4789.654 & \text { Cr I } & 31 \\ 4 & \text { Fe I } & \mathbf{7 5 3}\end{array}$

$\begin{array}{llll}4789.68 & P & \text { Ce II } & 228\end{array}$

$\begin{array}{rrrr}4789.803 & \text { T1 } I & 41\end{array}$

$\begin{array}{lll}4790.218 & \text { Ne I } & 32 \\ 4790.337 & \text { Cr I } & 31\end{array}$

$\begin{array}{lllr}4790.337 & & \text { Cr I } & 31 \\ 4790.36 & P & \text { Fe I } & 1068\end{array}$

$\begin{array}{rrr}4790.72 & \text { HP II } & 60\end{array}$

$\begin{array}{rllr}4790.75 & \text { P } & \text { Fe I } & 632 \\ 4791.00 & & \text { N1 I } & 71\end{array}$

$4791.150 \quad$ Gd II
45

$4791.250 \quad$ Fe I 633

4791.500 Sc I

$4791.584 \quad$ Sin II

4792.02

4792.04

4792.06

4792.12

$P \quad T 1$

4792.482
41 I

$\begin{array}{lll}4792.513 & \text { Cr I } & 168\end{array}$

$\begin{array}{lll}4792.63 & \text { Au I } & 3\end{array}$

$\begin{array}{lll}4792.855 & \text { Co } I & 168 \\ 4793.47 & \text { N1 I } & 158\end{array}$

$\begin{array}{llr}4793.47 & \text { N1 I } & 158 \\ 4793.656 & \text { N II } & 20\end{array}$

$\begin{array}{llll}4793.96 & P & \mathrm{Fe} I & 512\end{array}$

$\begin{array}{lllr}4794.22 & 0 & \text { IV } & 9\end{array}$

$\begin{array}{rrlr}4794.36 & P & \text { Fe I } & 115 \\ 4794.54 & & \text { Cl II } & 1\end{array}$

$\begin{array}{llll}4794.84 & P & \text { T1 II } & 29\end{array}$

$\begin{array}{lll}4795.62 & \text { Ne II } & 71\end{array}$

$\begin{array}{lll}4795.84 & \text { N1 I } & 128\end{array}$

$4795.853 \quad$ Co I 186

$4796.210 \quad$ T1 I 260

4796.378 $\quad$ Co I 14

$\begin{array}{lll}4796.67 & \text { Lo II } & 63\end{array}$

$\begin{array}{lll}4796.84 & \text { Cr I } & 283 \\ 4796.930 & V & 1\end{array}$

$4797.157 \quad$ Nd II $\quad 60$

$4797.69 \quad$ Cr I 280

$\begin{array}{lll}4797.973 & V & 93\end{array}$

4797.983

4798.25

4788.40

4798.535

4798.736

4799.06

4789.30

4799.412

4799.786

4799.797

4799.83

4799.858

4799.918

4789.94

4800.100

o IV

Fe I

C1 II

T1 II

Fe I

9
1042

1042
13

17
38

$\begin{array}{llr}\text { I } & \text { I } & 13 \\ \text { Fe } & I & 888\end{array}$

Nd II

V I

$\begin{array}{ll}\text { V I } & I \\ \text { T1 }\end{array}$

N1 I

Cd I

V II
Gd II

$A$

4804.12

5 II 8

$\begin{array}{llll}4804.59 & P & \text { Fe } I & 794 \\ 4804.64 & & \text { Cr } I & 721\end{array}$

$4804.64 \quad$ Cr I $\quad 61$

4805.105 T1 II 92

$\begin{array}{rrlr}4805.18 & \text { P } & \text { Cr II } & 25 \\ 4805.24 & & \text { Cr I } & 283\end{array}$

$\begin{array}{lll}4805.24 & \text { Cr I } & 283 \\ 4805.416 & \text { T1 I } & 260\end{array}$

1805.817 Gd II 60

Zr I 4

4806.07

4806.165

4806.33 P T1 II

$4806.75 \quad P \quad T 1$

4806.998

4807.14

4807.243

4807.537

4807.725

4808.155

4808.52

4808.531

4808.864

4809.00

4809.14

4809.18

4809.26

4809.32

4809.94

N1 I

N1 I
HP II

Fe I

$\checkmark$ I

Fe I

6

4810.06

4810.286

4810.534

4810.733

4810.760

4811.04

4811.074

4811.14

4811.343

$\begin{array}{ll}\text { Fe } & \text { I } \\ \text { N1 } & I \\ \text { T1 } & I\end{array}$

N1 I

La II

Fe I

Hf II

Fe I

$\begin{array}{ll}\mathrm{Fe} I & \mathbf{2 3 0} \\ & \end{array}$

4811.57

4811.61

4811.881

4811.999

4812. 240

4812.35

4812.84

4812.906

4812.940

C1 II

$\mathrm{V}$ II

II 20

7n

Cr I

Fe II

Fe I

T1

V II

Nd II

C1 II

Au I

Sr I

N1 I

T1 I

Cr II

C I

T1 1

Cu II

4813.11
4813.290

4813.45

1813. 476

4813.72

4813.952

4813.966

4814.266

4814. 617

0 IV

4800.14 Fe I 38

4800.55. P $\quad$ Fe I $\quad 590$

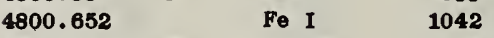

$\begin{array}{llllr}4800.77 & P & 0 & \text { IV } & 8 \\ 4801.030 & & \text { Cr } & \text { I } & 168\end{array}$

$\begin{array}{llr}4801.030 & \text { Cr I } & 168 \\ 4801.05 & \text { Gd II } & 65\end{array}$

$4801.150 \quad$ Pr II 36

$\begin{array}{rrlr}4801.63 & P & \mathrm{Fe} I & 1115 \\ 4801.80 & & 0 & I\end{array}$

$\begin{array}{llll}4801.80 & & 0 & I \\ 4801.90 & P & \text { T1 I } & 40\end{array}$

$\begin{array}{llll}4601.93 & P & T 1 & I\end{array}$

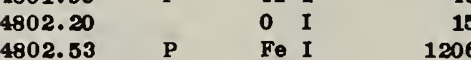

$\begin{array}{lllr}4802.53 & P & \text { Fe I } & 1206 \\ 4802.575 & & \text { Gd II } & 43\end{array}$

4802.81

4802.883

4803.00

4803.272

4803.272

4804.04

\begin{tabular}{|c|c|c|c|c|}
\hline 4814.80 & & $\mathrm{Ge}$ & II & $\cdot 2$ \\
\hline 4815.05 & & $\mathbf{Z r}$ & I & 44 \\
\hline 4815.22 & $\mathbf{P}$ & $\mathrm{Fe}$ & I & 720 \\
\hline 4815.515 & & 8 & II & 9 \\
\hline 4815.62 & & $\mathbf{Z r}$ & I & 43 \\
\hline 4815.808 & & Sm & II & 14 \\
\hline 4815.900 & & Co & I & 142 \\
\hline 4815.92 & & N1 & I & 131 \\
\hline 4816.012 & & $\mathrm{Sm}$ & II & 41 \\
\hline 4816.41 & & $\mathrm{Cr}$ & I & 283 \\
\hline 4816.47 & $\mathbf{P}$ & T1 & I & 40 \\
\hline 4816.47 & & $\mathbf{2 r}$ & II & 66 \\
\hline 4816.67 & $\mathbf{P}$ & $\mathrm{Fe}$ & I & 588 \\
\hline 4817.22 & & He & II & 69 \\
\hline 4817.33 & & c & I & $\mathbf{5}$ \\
\hline 4817.773 & & Fe & I & 67 \\
\hline 4817.847 & & N1 & I & 254 \\
\hline 4818.26 & $\mathbf{P}$ & $\mathbf{F e}$ & II & 11 \\
\hline 4818.66 & $\mathbf{P}$ & Fe & I & 718 \\
\hline 4819.46 & & $\mathrm{Cl}$ & II & 1 \\
\hline 4819.60 & & $\mathbf{s}$ & II & 46,62 \\
\hline 4819.64 & & $\mathbf{Y}$ & I & 13 \\
\hline 4819.740 & & S1 & I II & 9 \\
\hline 4819.79 & & c1 & II & 13 \\
\hline 4820.336 & & Nd & II & 47 \\
\hline 4820.410 & & T1 & I & 126 \\
\hline 4821.01 & $\mathbf{P}$ & T1 & II & 29 \\
\hline 4821.143 & & N1 & I & 254 \\
\hline 4821.29 & & T1 & I & 201 \\
\hline 4821.858 & & Gd & II & 138 \\
\hline
\end{tabular}


Type Blewent Multiplet No.

$\begin{array}{lllr}4840.329 & & \text { Fe I } & 1068 \\ 4840.874 & & \text { TI I } & 53 \\ 4840.89 & \text { P } & \text { Fe I } & 1070 \\ 4841.52 & \text { P } & \text { Cr I } & 266 \\ 4841.65 & \text { P } & \text { Fe I } & 635 \\ 4841.67 & \text { P } & \text { N I I } & 164 \\ 4841.701 & & \text { Sin I } & 2 \\ 4841.73 & & \text { Cr I } & 266 \\ 4841.80 & & \text { Fe I } & 1070 \\ 4841.98 & & \text { Zr II } & 138\end{array}$

4841.98

260

$\begin{array}{lll}4842.01 & \text { N1 I } & 260\end{array}$

\begin{tabular}{|c|c|}
\hline 4842.19 & $\mathbf{P}$ \\
\hline
\end{tabular}

$\begin{array}{rllr}4842.50 & & \text { V II } & 248 \\ 4842.71 & \text { P } & \text { Fe I } & 1098\end{array}$

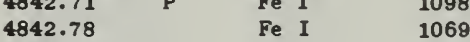

4843.155 $\quad \mathrm{Fe} \mathrm{I}$

4843.165 N1 I 50

4848.19

4843.26

4843. 29

N1 I

Mn I

$\begin{array}{ll}0 & \text { II } \\ \text { Ls } & \text { II }\end{array}$

50
43
105

$\begin{array}{llll}4843.39 & P & \text { Fe } I & 794\end{array}$

$\begin{array}{lll}4843.454 & \text { Co I } & 158\end{array}$

$\begin{array}{lllr}4843.46 & & \text { Ba II } & 15 \\ 4843.53 & P & \text { N1 I } & 235\end{array}$

$4843.829 \quad W \quad I \quad 1$

$\begin{array}{lll}4843.989 & \text { T1 I } & 217\end{array}$

$\begin{array}{llr}4844.00 & \text { HF II } & 16 \\ 4844.016 & \text { Fe I } & 750\end{array}$

$\begin{array}{rrr}4844.208 & \text { Fe I } & 750 \\ \text { Sw II } & 26\end{array}$

$\begin{array}{lllll}4844.31 & P & V & \text { II } & 29\end{array}$

$\begin{array}{llll}4844.315 & & \text { Mn I } & 43\end{array}$

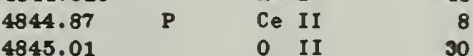

$4845.17 \quad$ N1 I $\quad 115$

$\begin{array}{lll}\mathbf{4 8 4 5 . 6 5 6} & \text { Fe I } & 588,888\end{array}$

4845.67

4846.29

4846.47 $\quad \mathrm{C}$ I

4846.574 Fe II

$\begin{array}{llll}4847.09 & P & \mathrm{Fe} I & 17 \\ & & & \end{array}$

$4847.14 \quad$ Be II $\quad 14$

$\begin{array}{lll}4847.177 & C_{5} I & 144\end{array}$

4847.286 $\quad \mathrm{Ca} \mathrm{I} . \quad 50$

$\begin{array}{llll}4847.61 & \text { P } & \text { Fe II } & 30 \\ 4847.760 & & \text { 8w II } & 53\end{array}$

$\begin{array}{lrr}4847.760 & \text { Sw II } & 53 \\ 4847.90 & & 6\end{array}$

$\begin{array}{rlll}4848.24 & & \text { Cr II } & 30 \\ 4848.41 & \text { P } & \text { I1 I } & 217\end{array}$

$\begin{array}{rrrrr}4848.41 & \text { P } & \text { TI I } & 217 \\ 4848.46 & & \text { HF II } & 83\end{array}$

$\begin{array}{lll}4848.487 & \text { T1 I } & 201\end{array}$

$\begin{array}{llll}4848.821 & \text { V } & \text { I } & 78\end{array}$

$\begin{array}{rrr}4848.898 & \text { Fe } I & 114\end{array}$

$\begin{array}{lllr}4849.12 & \text { P } & \text { N1 I } & 112 \\ 4849.18 & \text { P } & \text { T1 II } & 29\end{array}$

$4849.4 \quad \mathrm{Ne} I I \quad 71$

$\begin{array}{rrrr}4849.67 & \text { P } & \text { Fe I } & 793 \\ 4850.58 & & \text { L II } & 51,88\end{array}$

$\begin{array}{lll}4850.58 & \text { La II } & 51,88 \\ 4850.84 & \text { Ba II } & \text { IJ }\end{array}$

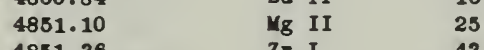

$\begin{array}{lll}4851.36 & \text { Zr I }\end{array}$

$\begin{array}{lll}4851.465 & C r & 208\end{array}$

$\begin{array}{rrrr}4851.483 & \text { V I } & 3 \\ 4852.560 & \text { N1 I } & 130\end{array}$

$\begin{array}{llll}4852.69 & Y & 1 & 13\end{array}$

$\begin{array}{llll}4853.30 & P & \text { N1 } & I\end{array}$

$\begin{array}{llll}4853.52 & P & C r & 1\end{array}$

$\begin{array}{rrrr}4853.74 & & \text { N1 I } & 99\end{array}$

$\begin{array}{llr}4854.365 & \text { St II } & 36\end{array}$

4854.604 Mn I

$\begin{array}{llr}4854.65 & \text { Zr II } & 78\end{array}$

$\begin{array}{llr}4854.727 & \text { T1 I } & 217 \\ 4854.87 & \text { I II } & 22\end{array}$

$\begin{array}{rlrr}4854.87 & \text { I II } & 22 \\ 4854.89 & \text { Fe I } & 1043\end{array}$

4855.045

4855.146

4855.235

4855.414

4855.54

4853.683

ST I

Cr I

Co I

N1 I

$\begin{array}{llll}4855.95 & P & \text { TI II } & 114\end{array}$

$\begin{array}{lll}4856.012 & \text { T1 I } & 231\end{array}$

4856.19

4856.49

4856.76

4857.382

4857.60

4857.938
I A Type Element Multiplet No.

$\begin{array}{lllr}4858.24 & \text { P } & \text { Fe I } & 1068 \\ \mathbf{4 8 5 8 . 2 7} & \text { P } & \text { Fe I } & 1098 \\ 4858.74 & & \text { N III } & 8 \\ \mathbf{4 8 5 8 . 8 8} & & \text { N III } & 9 \\ \mathbf{4 8 5 9 . 0 3 0} & & \text { Nd II } & 3 \\ \mathbf{4 8 5 9 . 0 3 8} & & \text { Pr II } & 25 \\ \mathbf{4 8 5 9 . 1 2} & & \text { Fe I } & 1068 \\ \mathbf{4 8 5 9 . 1 8} & & \text { La II } & 86 \\ \mathbf{4 8 5 9 . 2 8} & & \text { SI II } & \\ \mathbf{4 8 5 9 . 3 1} & \text { P } & \text { Fe I } & 632 \\ & & & \\ 4859.323 & & \text { He II } & 2 \\ \mathbf{4 8 5 9 . 7 4 8} & & \text { Fe I } & 318 \\ \mathbf{4 8 5 9 . 8 4} & & \text { Y I } & 13 \\ \mathbf{4 8 6 0 . 0 2 9} & & \text { D } & 1 \\ \mathbf{4 8 6 0 . 2 0} & & \text { CF II } & 30 \\ \mathbf{4 8 6 0 . 3 5} & & \text { N II } & 67 \\ \mathbf{4 8 6 0 . 3 7} & & \text { Cr I } & 31 \\ 4860.90 & & \text { La II } & 8 \\ \mathbf{4 8 6 0 . 9 8} & \text { P } & \text { Fe I } & 688 \\ 4861.03 & & \text { O II } & 57\end{array}$

4861.205

4861.33 N III $\quad 9$

$4861.842 \quad \mathrm{Cr} I$

4862.054 P Mn I $\quad 43$

$\begin{array}{llll}4862.54 & \text { P } & \text { Fe I } & 1070 \\ 4862.60 & \text { P } & \text { Fe I } & 1069\end{array}$

$4863.653 \quad$ Fe I 687

$\begin{array}{lllll}4863.75 & P & \text { T1 } & I & 217\end{array}$

4863.78 P $\quad$ Fe I

$4863.931 \quad$ N1 I $\quad 113$

$4864.282 \quad N 1$ I 128

4864.32 Cr II

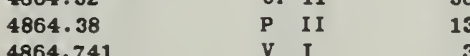

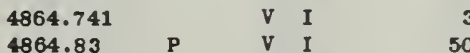

4864.95 O II

$\begin{array}{lll}4865.02 & \text { Gd II } & 65 \\ 4865.43 & \text { HF II } & 93\end{array}$

4865.620 T1 II

$\begin{array}{lll}4865.96 & \text { A II }\end{array}$

$\begin{array}{lll}4866.07 & \text { Zr I } & 44 \\ 4866.267 & \text { N1 I }\end{array}$

$\begin{array}{llll}4866.77 & P & \text { Fe I } & 1093\end{array}$

4867.18 N III

$4867.53 \quad P \quad$ Fe I

$\begin{array}{rllr}4867.59 & & \text { A II } & 62 \\ 4867.64 & P & \text { Fe I } & 587\end{array}$

$\begin{array}{rllr}4867.64 & \text { P } & \text { Fe I } & 587 \\ 4867.73 & \text { P } & \text { Fe II } & 30\end{array}$

$\begin{array}{lllll}4867.79 & P & V & \text { II } & 29\end{array}$

4867.839 Nd II

$4867.870 \quad$ Co I 158

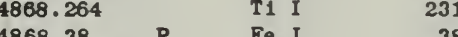

$\begin{array}{llll}4868.38 & \text { P } & \text { Fe I } & 38 \\ 4868.700 & & \text { Sr I } & 10\end{array}$

$4868.82 \quad P \quad$ Fe II

4869.153 P Ru I $\quad 11$

4869.8 Ne II

$\begin{array}{llll}4870.05 & P & \text { Fe } I & 985\end{array}$

$4870.129 \quad$ P $\quad$ TI I $\quad 231$

$4870.796 \quad$ Cr I $\quad 143$

$4870.845 \quad$ NI I 131

$\begin{array}{lllr}4871.27 & P & F e & \\ 4871.323 & & F e & 25 \\ & & & \end{array}$

$\begin{array}{lllr}4871.58 & 0 & \text { II } & 57\end{array}$

$\begin{array}{rrrr}4871.94 & \text { P } & \text { Fe I } & 630 \\ 4872.02 & & \text { Cr I } & 30\end{array}$

$\begin{array}{lllr}4872.144 & & \text { Fe I } & 318 \\ 4872.493 & & \text { Sr I } & 4 \\ 4872.69 & \text { P } & \text { Fe I } & 1115 \\ 4872.91 & \text { P } & \text { Fe I } & 1097 \\ 4873.27 & \text { P } & \text { N I I } & 112 \\ 4873.339 & & \text { Gd II } & 65 \\ 4873.437 & & \text { N1 I } & 111 \\ 4873.58 & & \text { N III } & 9 \\ 4873.74 & \text { P } & \text { Fe I } & 633 \\ 4874.025 & & \text { TI II } & 114 \\ & & & \\ 4874.35 & \text { P } & \text { Fe I } & 467 \\ 4874 . F .51 & & \text { Cr I } & 167 \\ 4874.805 & & \text { V II } & 197 \\ 4874.809 & & \text { NI I } & 98 \\ 4875.32 & \text { P } & \text { Fe I } & 1038 \\ 4875.462 & & \text { V I } & 3 \\ 4875.49 . & \text { P } & \text { V II } & 248 \\ 4875.72 & \text { P } & \text { Fe I } & 1243 \\ 4873.89 & & \text { Fe I } & 687 \\ 4873.966 & & \text { Gd II } & 126\end{array}$


I A Type Blement Multiplet No. I A Type Element Multiplet No.

\begin{tabular}{|c|c|c|c|c|c|c|c|c|}
\hline 4876.06 & & $\mathrm{Sr}$ & I & 4 & & 4896.71 & & N \\
\hline 4876.19 & $\mathbf{P}$ & $\mathrm{Fe}$ & I & 631 & & 4896.77 & & Cl \\
\hline 4876.325 & & $\mathbf{S r}$ & $\mathbf{I}$ & 5 & & 4898.52 & & Al \\
\hline 4876.41 & & $\mathrm{Cr}$ & II & 30 & & 4898.76 & & $\mathbf{A l}$ \\
\hline 4876.48 & $\mathbf{P}$ & $\mathbf{C r}$ & II & 30 & & 4899.520 & & Co \\
\hline 4877.08 & & $\mathbf{A}$ & II & 112 & & 4899.64 & & Al \\
\hline 4877.61 & $\mathbf{P}$ & $\mathrm{Fe}$ & I & 384 & & 4899.90 & $\mathbf{P}$ & $\mathrm{Fe}$ \\
\hline 4878.049 & & Gd & II & 64 & & 4899.910 & & T1 \\
\hline 4878.132 & & $\mathrm{Ca}$ & I & 35 & & 4899.92 & & La \\
\hline 4878.218 & & $\mathrm{Fe}$ & I & 318 & & 4899.934 & & $\mathrm{Ba}$ \\
\hline 4879.121 & & Pr & II & 20 & & 4900.03 & $\mathbf{P}$ & T1 \\
\hline 4879.90 & & $\mathbf{A}$ & II & 14 & & 4900.13 & & $\mathbf{Y}$ \\
\hline 4880.06 & & $\mathrm{Cr}$ & I & 167 & & 4900.47 & & 8 \\
\hline 4880.20 & & $\mathrm{La}$ & II & 153 & & 4900.50 & $\mathbf{P}$ & $\mathrm{Cr}_{\mathbf{r}}$ \\
\hline 4880.25 & & Co & $\mathbf{I}$ & 13 & & 4900.624 & & $\mathrm{v}$ \\
\hline 4880.30 & $\mathbf{P}$ & v & II & 29 & & 4900.625 & & T1 \\
\hline 4880.560 & & $\mathbf{v}$ & I & 50 & & 4900.83 & $\mathbf{P}$ & $\mathrm{Cr}_{\mathrm{r}}$ \\
\hline 4880.922 & & $\mathbf{T} \mathbf{1}$ & $\mathbf{I}$ & 201 & 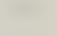 & 4900.97 & & N1 \\
\hline 4881.25 & & $2 r$ & I & 44 & & 4901.30 & & $\mathbf{s}$ \\
\hline 4881.3 & & Li & I I & 4 & 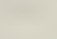 & 4901.65 & & $\mathrm{Cr}_{\boldsymbol{r}}$ \\
\hline 4881.44 & & $\mathbf{Y}$ & II & 12 & & 4902.77 & & Al \\
\hline 4881.564 & & v & I & 3 & & 4902.89 & $\mathbf{P}$ & v \\
\hline 4881.726 & & $\mathrm{Fe}$ & I & 588,1041 & & 4903.10 & $\mathbf{P}$ & $\mathrm{Fe}$ \\
\hline 4881.81 & & N & III & 9 & 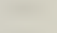 & 4903.238 & & $\mathrm{Cr}$ \\
\hline 4881.925 & & Gd & II & 113,133 & & 4903.317 & & $\mathrm{Fe}$ \\
\hline 4882.151 & & $\mathrm{Fe}$ & $I$ & 687 & & 4903.71 & & Al \\
\hline 4882.183 & & v & I & 30 & & 4903.85 & $\mathbf{P}$ & $\mathrm{Fe}$ \\
\hline 4882.25 & & $\mathbf{A}$ & II & & & 4904.172 & & Co \\
\hline 4882.326 & & $\mathbf{T} \mathbf{1}$ & $I$ & 231 & & 4904.285 & & $\mathrm{~V}$ \\
\hline 4882.462 & & $\mathrm{Ce}$ & II & & & 4904.350 & & V \\
\hline 4882.704 & & Co & $\mathbf{I}$ & 158 & 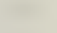 & 4904.413 & & N1 \\
\hline 4883.415 & & $\mathbf{v}$ & II & 209 & & 4904.447 & & $\mathrm{v}$ \\
\hline 4883.61 & & $\mathbf{Z r}_{\mathbf{r}}$ & I & 44 & & 4904.51 & & HP \\
\hline 4883.69 & & $\mathbf{Y}$ & II & 22 & & 4904.75 & & A \\
\hline 4883.73 & & $\mathbf{s}$ & II & 46 & 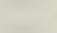 & 4904.76 & & $\mathrm{Cl}$ \\
\hline 4884.06 & & v & II & 197 & & 4905.09 & & $\mathrm{Zr}_{\mathbf{r}}$ \\
\hline 4884.14 & & $\mathbf{N}$ & III & 9 & . & 4905.15 & & $\mathrm{Fe}$ \\
\hline 4884.57 & & $\mathrm{C}_{\boldsymbol{r}}$ & II & 30 & 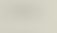 & 4906.11 & & $Y$ \\
\hline 4884.915 & & Ne & $\mathbf{I}$ & 20,35 & 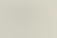 & 4906.80 & $\mathbf{P}$ & $\mathrm{Fe}$ \\
\hline 4884.949 & & $\mathrm{Cr}$ & I & & & 4906.88 & & 0 \\
\hline 4885.082 & & Ti & I & 137 & 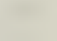 & 4906.88 & & $\mathbf{s 1}$ \\
\hline 4885.435 & & $\mathrm{Fe}$ & I & 966 & 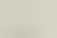 & 4907.125 & & Co \\
\hline 4883.63 & & $\mathbf{s}$ & II & 15 & & 4907.17 & & $\mathrm{Cl}$ \\
\hline 4885.776 & & $\mathrm{Cr}_{\mathbf{r}}$ & I & 30 & 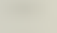 & $4907 \cdot 7.43$ & & $\mathrm{Fe}$ \\
\hline 4885.957 & & $\mathrm{Cr}$ & $\mathbf{I}$ & 143 & - & 4907.888 & & Ru \\
\hline 4886.17 & $\mathbf{P}$ & $\mathrm{Fe}$ & I & 467 & 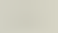 & 4908.46 & & T1 \\
\hline 4886.336 & & $\mathrm{Fe}$ & I & 1066 & 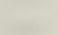 & 4908.61 & $P$ & $\mathrm{Fe}$ \\
\hline 4886.725 & & N1 & $\mathbf{I}$ & 158 & 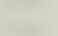 & 4908.67 & & $\mathrm{zr}$ \\
\hline 4886.821 & & v & $\mathbf{I}$ & 50 & 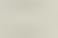 & 4908.74 & & $\mathrm{Fe}$ \\
\hline 4886.92 & $\mathbf{P}$ & $\mathrm{Fe}$ & II & 54 & 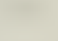 & 4909.105 & & T1 \\
\hline 4886.992 & & N1 & I & 141 & 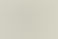 & 4909.387 & & $\mathrm{Fe}$ \\
\hline 4887.013 & & $\mathrm{Cr}$ & I & 143 & & 4909.726 & & $\mathrm{Cu}$ \\
\hline 4887.189 & & $\mathrm{Fe}$ & $\mathbf{I}$ & 1065 & 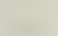 & 4909.87 & & $\mathrm{Cr}$ \\
\hline 4887.37 & $\mathbf{P}$ & $\mathrm{Fe}$ & I & 1037 & 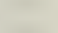 & 4910.027 & & $\mathrm{Fe}$ \\
\hline 4887.72 & & $\mathbf{Z r}$ & I & 43 & 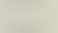 & 4910.328 & & $\mathrm{Fe}$ \\
\hline 4887.73 & & $\mathrm{Cr}$ & I & 31 & 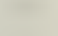 & 4910.570 & & $\mathrm{Fe}$ \\
\hline 4888.29 & & A & II & 135 & & 4910.838 & & Gd \\
\hline 4888.530 & & $\mathrm{Cr}$ & I & 31 & & 4811.205 & & $T 1$ \\
\hline 4888.542 & & Gd & II & 126 & & 4911.34 & & Lo \\
\hline 4888.651 & & $\mathrm{Fe}$ & I & 1066 & 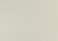 & 4911.52 & $\mathbf{P}$ & $\mathrm{Fe}$ \\
\hline 4889.009 & & $\mathrm{Fe}$ & I & 67,749 & 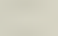 & 4911.593 & & Ru \\
\hline 4889.06 & & $\mathbf{A}$ & II & 15 & 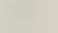 & 4911.664 & & $2 n$ \\
\hline 4889.113 & & $\mathrm{Fe}$ & I & 985 & & 4911.786 & & $\mathrm{Fe}$ \\
\hline 4889.15 & & $\operatorname{Re}$ & I & 1 & & 4912.030 & & Ni \\
\hline 4889.690 & & $\mathrm{Cu}$ & II & 1 & & 4912.38 & & $\mathrm{v}$ \\
\hline 4889.73 & & $\mathrm{Cr}_{\mathrm{r}}$ & I & 61 & & 4912.399 & & Co \\
\hline 4880.45 & $\mathbf{P}$ & N1 & I & 114 & 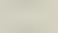 & 4912.49 & & $\mathrm{Cr}_{\mathbf{r}}$ \\
\hline 4890.762 & & $\mathrm{Fe}$ & I & 318 & & 4912.52 & $\mathbf{P}$ & $\mathrm{Fe}$ \\
\hline 4890.93 & & 0 & II & 28 & & 4913.248 & & Sin \\
\hline 4891.43 & & La & II & 95 & 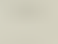 & 4913.366 & & $\mathrm{Fe}$ \\
\hline 4881.486 & & $\mathrm{Fe}$ & I & 318 & 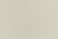 & 4913.616 & & T1 \\
\hline 4891.56 & $\mathbf{P}$ & $\mathrm{Cr}$ & II & 36 & & 4913.970 & & N1 \\
\hline 4881.828 & & T1 & I & 201 & 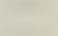 & 4914.32 & & $A$ \\
\hline 4881.97 & & $\mathrm{Cr}$ & I & 61 & & 4914.32 & & $\mathrm{Cl}$ \\
\hline 4891.980 & & $\mathbf{S r}$ & I & 10 & & 4914.385 & & Nd \\
\hline 4892.11 & & $G d$ & II & 116 & & 4914.80 & & $\mathrm{~N}$ \\
\hline 4892.86 & & $\mathrm{Fe}$ & I & 1070 & & 4915.236 & & $\mathbf{T} \mathbf{1}$ \\
\hline 4893.065 & & T1 & I & 231 & 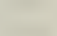 & 4916.67 & $\mathbf{P}$ & $\mathrm{Fe}$ \\
\hline 4893.12 & & $\mathbf{z r}$ & I & 43 & 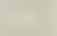 & 4916.78 & & Gd \\
\hline 4893.44 & & $Y$ & I & 13 & & 4917.15 & & s \\
\hline 4893.59 & $\mathbf{P}$ & $\mathrm{Fe}$ & I & 1096 & & 4917.25 & & $\mathrm{Fe}$ \\
\hline 4893.70 & $\mathbf{P}$ & $\mathrm{Fe}$ & I & 1113 & & 4817.72 & & $\mathrm{Cl}$ \\
\hline 4893.780 & & $\mathrm{Fe}$ & II & 36 & & 4918.05 & & $\mathrm{Fe}$ \\
\hline 4893.90 & & T1 & I & 201 & & 4918.363 & & Ni \\
\hline 4893.968 & & $\mathrm{Ce}$ & II & 31 & & 4918.373 & & $\mathrm{Cu}$ \\
\hline 4894.218 & & V & I & 118 & & 4918.712 & & N1 \\
\hline 4894.30 & & Gd & II & 65 & & 4918.98 & & Al \\
\hline 4894.43 & & $\mathrm{Zr}_{\boldsymbol{r}}$ & II & 107 & & 4918.999 & & $\mathrm{Fe}$ \\
\hline 4895.20 & & N & II & 1 & & 4919.73 & $\mathbf{P}$ & $\mathrm{Fe}$ \\
\hline 4896.437 & & $\mathrm{Fe}$ & I & 884 & & 4919.814 & & Th \\
\hline
\end{tabular}




\section{FINDING LIST}

I $\triangle$

Type Blement Multiplet No.

\begin{tabular}{|c|c|c|}
\hline N1 & I & 114 \\
\hline Mn & I & 20 \\
\hline s & II & 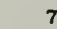 \\
\hline $\mathrm{Cr}$ & I & \\
\hline $\mathrm{Fe}$ & I & 1097 \\
\hline A & I I & 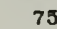 \\
\hline $\mathbf{N}$ & $\mathbf{v}$ & 9 \\
\hline 0 & II & 38 \\
\hline T1 & I & 52 \\
\hline Cl & II & 17 \\
\hline $\mathbf{R}$ & I I & \\
\hline $\mathbf{P}$ & I I & 13 \\
\hline T1 & I & 175 \\
\hline $\mathrm{Cr}_{\mathbf{r}}$ & I & 258 \\
\hline $\boldsymbol{N}$ & $\mathbf{v}$ & 10 \\
\hline $\mathrm{Fe}$ & I & 466 \\
\hline Hf & II & 15 \\
\hline N1 & I & 145 \\
\hline $\mathrm{Fe}$ & I & 1113 \\
\hline N1 & I & 148 \\
\hline $\mathrm{Fe}$ & I & 68 \\
\hline La & II & 36 \\
\hline$v$ & I I & 197 \\
\hline $\mathrm{Cr}$ & I & 202 \\
\hline T1 & I & 38 \\
\hline $\mathrm{T} 1$ & I & 200 \\
\hline Fe & III & \\
\hline Sm & II & 48 \\
\hline $\mathrm{Cr}$ & I & 202 \\
\hline $\mathbf{Z r}$ & I & \\
\hline
\end{tabular}

I A Type Element Multiplet No.

4967.944

4968.50

4968.566

4968.575

4968.709

4968.76

4968.65

4969.927

4970.12

4970.39

4970.496

4970.66

4971.475

4971.668

4971.92

4971.935

4972.16

4972.39

4972.90

4973.051

4973.108

4973.16

4973.4
4973.896

4974.47

4975.344

4975.415

4976.155

4976.345

$4976.71 \quad P \quad N 1 I$

$\begin{array}{llll}4977.6 & \text { P Forb } & \mathrm{Na} & \mathrm{I} \\ 4977.653 & & \mathrm{Fe} & \mathrm{I}\end{array}$

4977.731 T1 I

4978.11 P $\quad$ Fe I

4978.191 T1

$\begin{array}{ll}4978.541 & \mathrm{Na} I \\ 4978.606 & \mathrm{Fe} I\end{array}$

4978.70 P Fe I

$4979.58 \quad \mathrm{Fe} I$

4979.84 P $\quad \mathrm{Fe}$

$\begin{array}{lll}4980.161 & & \text { N1 } \\ 4980.30 & P & C r\end{array}$

$\begin{array}{llll} & \mathrm{CI} & \mathrm{Cr} & 123\end{array}$

$\begin{array}{llll}4981.30 & P & C r ~ I & 123\end{array}$

4981.732 $\quad P \quad$ T1

4982.13 Y I

4982.507

4982.813

4983.258

Fe I

Na I

$\begin{array}{llll}4983.63 & P & \text { Cr I } & 202\end{array}$

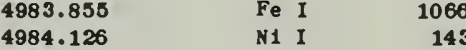

4984.905

4985.261

$\begin{array}{lll}4985.503 & \text { Cr II } \\ & & \text { Cu II }\end{array}$

$4985.553 \quad \mathrm{Fe} I$

4985.60
4985.98

4986.24

4986.82 La II 22

$\begin{array}{lllr}4986.90 & \text { P } & \text { Fe I } & 1092 \\ 4987.377 & & \text { N II } & 24\end{array}$

$\begin{array}{llll}4987.62 & P & \text { Fe } I & 1094\end{array}$

$\begin{array}{llll}4987.83 & P & \text { Fe } I & 966\end{array}$

$4987.853 \quad$ Co I 14

$\begin{array}{rrr}4988.963 & \text { Fe I } & 1066 \\ 4989.140 & \text { TI I } & 173\end{array}$

4991.067

T1 1

$4991.11 \quad P \quad$ Fe II

4991.22

4991.27

4991.277

4991.86

4891.94

4992.80

4993.355

4993.687

4994.133

4994.14

4994.358

4998.062

4995.41

4995.52

4995.65

4995.89

4996.82

4996.850
$N$ II. 25

La II

Fe I

Fe 1

8 II

Fe I

s I

Fe I

Fe I

L II

$\begin{array}{ll}N & \text { II } \\ \text { TI } & \text { I }\end{array}$

T1 I

C1 II

N1 I

T1 II

La II
N1 I
I A Type

lement

Multiplet No.

\begin{tabular}{|c|c|c|c|c|}
\hline \multirow{2}{*}{\multicolumn{2}{|c|}{$\begin{array}{l}4997.099 \\
4987.23\end{array}$}} & T1 & I & 5 \\
\hline & & N & I I & 64 \\
\hline 4997.81 & & $\mathrm{Ba}$ & I I & 14 \\
\hline \multicolumn{2}{|l|}{4998.233} & N1 & I & 111 \\
\hline \multicolumn{2}{|l|}{4998.373} & Gd & II & 133 \\
\hline 4998.43 & \multirow[t]{5}{*}{$\mathbf{P}$} & Al & II & 30 \\
\hline 4998.55 & & $\mathrm{Cr}$ & I & 123 \\
\hline 4999.114 & & $\mathrm{Fe}$ & I & 1040 \\
\hline 4999.46 & & La & I I & 37 \\
\hline 4999.504 & & T1 & I & 38 \\
\hline \multicolumn{2}{|l|}{$\begin{array}{l}4999.69 \\
5000.335\end{array}$} & $\mathrm{HF}$ & II & 35 \\
\hline 5000.335 & \multirow{9}{*}{$\mathbf{P}$} & N1 & 1 & 145 \\
\hline 5000.73 & & Fe & II & 25 \\
\hline 5000.91 & & $\mathrm{zr}$ & II & 95 \\
\hline 5000.97 & & Al & II & 78 \\
\hline 5000.991 & & T1 & I & 173 \\
\hline 5001.128 & & $\mathbf{N}$ & II & 18 \\
\hline 5001.15 & & Lu & I & \\
\hline 5001.469 & & N & II & 19 \\
\hline 5001.489 & & $\mathrm{Ca}$ & I I & 15 \\
\hline \multicolumn{2}{|l|}{5001.871} & $\mathrm{Fe}$ & I & 865 \\
\hline \multicolumn{2}{|l|}{5002.02} & $\mathrm{Fe}$ & I I I & \\
\hline \multicolumn{2}{|l|}{5002.12} & La & I I & 9 \\
\hline \multicolumn{2}{|l|}{5002.320} & V & I & 132 \\
\hline \multirow{2}{*}{\multicolumn{2}{|c|}{$\begin{array}{l}5002.682 \\
5002.800\end{array}$}} & $\mathbf{N}$ & II & \\
\hline & & Fe & I & 687 \\
\hline \multicolumn{2}{|l|}{5003.751} & N1 & I & 50 \\
\hline 5003.85 & \multirow[t]{3}{*}{$\mathbf{P}$} & $\mathrm{Fe}$ & I & 211 \\
\hline 5004.034 & & $\mathrm{Fe}$ & I & 1112 \\
\hline 8004.187 & & Co & I & 141 \\
\hline & Fe & II & \\
\hline \multicolumn{2}{|l|}{5004.38} & $\mathrm{Cr}_{\mathrm{r}}$ & I & 122 \\
\hline \multirow{2}{*}{\multicolumn{2}{|c|}{$\begin{array}{l}5004.907 \\
5005.140\end{array}$}} & mn & I & 2 \\
\hline & & N & II & 19,6 \\
\hline \multicolumn{2}{|l|}{5005.160} & $\mathrm{Ne}$ & I & 2 \\
\hline 5005.18 & $\mathbf{P}$ & T1 & II & 71 \\
\hline \multicolumn{2}{|l|}{5005.60} & $\mathbf{K}$ & II & \\
\hline \multicolumn{2}{|l|}{$\begin{array}{l}5005 \cdot 720 \\
5006 \cdot 126\end{array}$} & $\mathrm{Fe}$ & I & 984 \\
\hline \multirow{2}{*}{\multicolumn{2}{|c|}{$\begin{array}{l}5006.126 \\
5006.169\end{array}$}} & $\mathrm{Fe}$ & I & 318 \\
\hline & & w & I & \\
\hline
\end{tabular}

5006,71

S I

5006.72

5006.787

5007.209

5007.286

5007.289

5007.316

5008.35

5009.54

Cu II

T1 I

Co I

Fe I

N II

A II

T1 I

5010.045

5010.202

5010.30

5010.620

5010.82

5010.961

5011.24

5011.24

5012.026

5012.071

N1 I

TI II

Fe I

$\begin{array}{ll}N & \text { II } \\ \text { Gd II }\end{array}$

N1 I

$\mathrm{Fe}$

18

094

5012.16

5012.461

5012.611

5012.68

5013.00

5013.284

5013.316

5013.38

5013.712

5014.03

$\begin{array}{ll}N & \text { II } \\ \text { N } & \text { II }\end{array}$

Fe I

966,1066

5014.185

5014.277

5014.45

5014.620

5014.950

5015.04

5015.30

5015.675

6016.162

Fe I

N1 I

Cu II

Fe I

Ba II

T1 I

Cr I

T1 II

8 II

5016.387

T1

$5016.48 \quad \mathrm{P}-\mathrm{Fe}$

$\begin{array}{llll}5016.60 & \text { P } & \text { Fe I } & 108 \\ & & \text { V II } & 251\end{array}$

5017.16

6017.591

0017.68

6018.02

5018.294

5018.43

5018.434

6018.78
57

10

14

24

7

111

211

59

144

64

$$
16
$$

1070

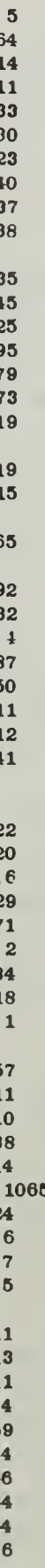

7
1093
10
173
60
113
71
15

5
38
159
132
985
6
968
4
38
19
1089
251
37
111
13
884
162
585
42
13




\begin{tabular}{|c|c|c|c|c|c|c|c|c|c|}
\hline $\begin{array}{l}5019.18 \\
5019.20\end{array}$ & $\mathbf{P}$ & $\begin{array}{l}\mathrm{Fe} \\
\mathrm{Cr}\end{array}$ & I & $\begin{array}{r}1242 \\
20\end{array}$ & $\begin{array}{l}5040.902 \\
5041.063\end{array}$ & & $\begin{array}{l}\mathrm{Fe} \\
\mathrm{S1}\end{array}$ & $\begin{array}{l}\text { I } \\
\text { I I }\end{array}$ & $1092, \underset{5}{1094}$ \\
\hline 5019.34 & & 0 & I & 13 & 5041.074 & & $\mathrm{Fe}$ & I & 16 \\
\hline 5019.361 & & Gd & II & 81 & 5041.077 & & N1 & I & 158 \\
\hline 5019.478 & & $\mathrm{Fe}$ & II & 168 & 5041.32 & $\mathbf{P}$ & $\mathrm{Fe}$ & I & 1328 \\
\hline 5019.74 & $\mathbf{P}$ & $\mathrm{Fe}$ & $I$ & 966 & 5041.33 & $\mathbf{P}$ & $\mathrm{Fe}$ & I & 1110 \\
\hline 5019.855 & & $\mathbf{v}$ & II & 232 & 5041.620 & & $\mathrm{Ca}$ & I & 34 \\
\hline 5019.979 & & $\mathrm{Ca}$ & II & 15 & 5041.66 & & C & I & 4 \\
\hline 5020.028 & & $\mathbf{T} \mathbf{1}$ & I & 38 & 5041.759 & & $\mathrm{Fe}$ & I & 36 \\
\hline 5020.13 & & 0 & I & 13 & 5042.195 & & N1 & I & 131 \\
\hline 5020.368 & & Gd & II & 64 & 5042.589 & & $M n$ & I & 20 \\
\hline 5020.67 & $\mathbf{P}$ & $\mathrm{Fe}$ & I & 629 & 5043.578 & & T1 & I & 38 \\
\hline 5020.819 & & $\mathrm{Fe}$ & I & 748 & 5044.008 & & $\mathrm{Ce}$ & II & 16 \\
\hline 5021 & $\mathbf{P}$ & C & IV & 3 & $5044.22 i$ & & $\mathrm{Fe}$ & I & 318 \\
\hline 5021.141 & & $\mathrm{Ca}$ & II & 15 & 5044.8 & & C & II & 35 \\
\hline 5021.60 & $\mathbf{P}$ & $\mathrm{Fe}$ & I & 1093 & 5045.098 & & N & II & 4 \\
\hline 5021.68 & $\mathbf{P}$ & $\mathrm{Fe}$ & I & 1067 & 5045.400 & & T1 & I & 38 \\
\hline 5021.894 & & $\mathrm{Fe}$ & I & 629 & 5046.61 & & $\mathbf{Z r}$ & I & 62 \\
\hline 5021.903 & & $\mathrm{Cr}$ & I & 8 & 5047.14 & $\mathbf{P}$ & $\mathrm{Fe}$ & I & 1242 \\
\hline 5022.244 & & $\mathrm{Fe}$ & I & 965 & 5047.2 & & c & II & 35 \\
\hline 5022.82 & $\mathbf{P}$ & T1 & I I & 71 & 5047.28 & & $\mathbf{s}$ & II & 15 \\
\hline 5022.871 & & $\mathrm{Ce}$ & II & 16 & 5047.308 & & v & II & 127 \\
\hline 5022.871 & & T1 & I & 38 & 5047.736 & & $\mathrm{He}$ & I & 47 \\
\hline 5022.874 & & $\mathrm{Fe}$ & II & & 5048.04 & & $\mathbf{L a}$ & I I & 90 \\
\hline 5023 & $\mathbf{P}$ & C & IV & 3 & 5048.082 & & N1 & I & 161 \\
\hline 5023.11 & & $\mathbf{N}$ & I I & 64 & 5048.208 & & $\mathbf{T} 1$ & I & 199 \\
\hline 5023.133 & & Gd & II & 64 & 5048.454 & & $\mathrm{Fe}$ & I & 884 \\
\hline 5023.226 & & $\mathrm{Fe}$ & I & 1085 & 5048.752 & & $\mathrm{Cr}$ & I & 20 \\
\hline 5023.39 & & T1 & I & 189 & 5048.851 & & N1 & I & 195 \\
\hline 5023.476 & & $\mathrm{Fe}$ & I & 1150 & 5048.91 & & $\mathbf{v}$ & II & 209 \\
\hline 5024.842 & & $\mathrm{~T} 1$ & I & 38 & 5049.825 & & $\mathrm{Fe}$ & I & 114 \\
\hline 6025.08 & $\mathbf{P}$ & $\mathrm{Fe}$ & I & 1110 & 5050.13 & $\mathbf{P}$ & $\mathrm{Fe}$ & I & 963 \\
\hline 5025.54 & & $\mathrm{Cr}$ & I & 20 & 5050.878 & & Gd & II & 114 \\
\hline 5025.570 & & $\mathrm{~T} 1$ & I & 173 & 5051.29 & $\mathbf{P}$ & $\mathrm{Fe}$ & I & 1089 \\
\hline 5025.665 & & $\mathbf{N}$ & II & 19 & 5051.527 & & $\mathrm{~N} 1$ & I & 144 \\
\hline 5025.73 & $\mathbf{P}$ & $\mathrm{Fe}$ & I & 466 & 5051.636 & & $\mathrm{Fe}$ & I & 16 \\
\hline 5026.50 & & $\mathrm{~N} 1$ & I & 158 & 5051.778 & & $\mathrm{Cu}$ & I I & 7 \\
\hline 5027.136 & & $\mathrm{Fe}$ & I & 1065 & 5051.900 & & $\mathrm{Cr}$ & I & 8 \\
\hline 5027.19 & & $\mathbf{s}$ & II & 1 & 5052.122 & & c & I & 12 \\
\hline 5027.212 & & $\mathrm{Fe}$ & I & 883 & 5052.879 & & T1 & I & 199 \\
\hline 5027.34 & $\mathbf{P}$ & $\mathrm{Fe}$ & I & 968 & 5052.97 & $\mathbf{P}$ & $\mathrm{Fe}$ & $\mathbf{I}$ & 585 \\
\hline 5027.51 & $\mathbf{P}$ & $\mathrm{Fe}$ & I & 960 & 5053.300 & & $w$ & I & 1 \\
\hline 5027.66 & $\mathbf{P}$ & $\mathrm{Cr}$ & I & 202 & 5054.070 & & $T_{1}$ & I & 171,294 \\
\hline 5027.785 & & $\mathrm{Fe}$ & $\mathbf{I}$ & 1110 & 5054.647 & & $\mathrm{Fe}$ & $\mathbf{I}$ & 884 \\
\hline 5028.00 & & $\mathrm{Cr}$ & I & 122 & 5056.00 & & $\mathrm{Fe}$ & I & 1149 \\
\hline 5028.129 & & $\mathrm{Fe}$ & $\mathbf{I}$ & 781 & 5056.020 & & si & II & 5 \\
\hline 5029.623 & & $\mathrm{Fe}$ & I & 718 & 5056.27 & & $\mathbf{K}$ & II & 3 \\
\hline 5029.812 & & Mn & I & 20 & 5056.353 & & $\mathbf{S 1}$ & II & 5 \\
\hline 5030.740 & & $\mathrm{Fe}$ & II & & 5056.856 & & $\mathrm{Fe}$ & I & 1111 \\
\hline 5030.75 & & $\mathrm{Fe}$ & III & & 5057.03 & & HP & II & 71 \\
\hline 5030.784 & & $\mathrm{Fe}$ & I & 585 & 5057.49 & & $\mathrm{Fe}$ & $\mathbf{I}$ & 1067,1160 \\
\hline 5031.019 & & Sc & II & 23 & 5057.83 & $\mathbf{P}$ & $\mathrm{Fe}$ & I & 1185 \\
\hline 5031.030 & & $\mathrm{Fe}$ & I & 746,883 & 5058.00 & & $\mathrm{Fe}$ & I & 967 \\
\hline 5031.290 & & Gd & I I & 114 & 5058.03 & & $\mathbf{N 1}$ & $\mathbf{I}$ & 141 \\
\hline 5031.562 & & Gd & I I & 64 & 5058.18 & & Hf & II & 37 \\
\hline 5031.901 & & $\mathrm{Fe}$ & I & 1150 & 5058.50 & & $\mathrm{Fe}$ & I & 884 \\
\hline 5032.41 & & $\mathbf{s}$ & II & 7 & 5060.079 & & $\mathrm{Fe}$ & I & 1,1095 \\
\hline 5032.748 & & N1 & I & 207 & 5060.635 & & $\mathrm{Cu}$ & II & 1 \\
\hline 5032.794 & & $\mathrm{Fe}$ & II & & 5061.794 & & $\mathrm{Fe}$ & II & \\
\hline 5033.2 & & C & II & 17 & 5062.07 & & A & II & 6 \\
\hline 5034.06 & & Co & I & 81 & 5062.112 & & $T 1$ & I & 189 \\
\hline 5034.33 & & Hf & II & 26 & 5062.862 & & Gd & II & 64 \\
\hline 5034.415 & & Pr & II & 37 & 5062.81 & & La & II & 50 \\
\hline 5035.025 & & $\mathrm{Fe}$ & I & 885 & 3063.296 & & $\mathrm{Fe}$ & I & 1066 \\
\hline 5035.374 & & N1 & I & 143 & 5063.30 & & $\mathrm{Fe}$ & III & 5 \\
\hline 5035.773 & & $\mathrm{Fe}$ & I I & & 5064.068 & & $\mathbf{T 1}$ & I & 294 \\
\hline 5035.908 & & $\mathbf{T} \mathbf{1}$ & I & 110 & 5064.321 & & Sc & I & 13 \\
\hline 5035. 961 & & N1 & I & 145 & 5064.654 & & $\mathbf{T 1}$ & I & 5 \\
\hline 5036.294 & & $\mathrm{Fe}$ & $\mathbf{I}$ & & 5064.69 & & $\mathrm{Au}$ & I & 1 \\
\hline 5036.468 & & Ti & I & 110 & 5064.92 & & $\mathbf{Z r}$ & I & 62 \\
\hline 5036.92 & & $\mathrm{Fe}$ & II & 36 & 5064.95 & $\mathbf{P}$ & $\mathrm{Fe}$ & I & 1095 \\
\hline 5036.931 & & $\mathrm{Fe}$ & $\mathbf{I}$ & 465 & 5065.020 & & $\mathrm{Fe}$ & $\mathbf{I}$ & 1094 \\
\hline 5037.0 & & C & II & 17 & 5065.201 & & $\mathrm{Fe}$ & I & 883 \\
\hline 5037.33 & & $\mathrm{Ta}$ & I & 12 & 5065.448 & & $\mathrm{Cu}$ & II & 11 \\
\hline 5037.65 & & $\mathrm{Ta}$ & I & 2 & 5065.910 & & $\mathrm{Cr}_{\mathrm{r}}$ & I & 60 \\
\hline 5037.7505 & & Ne & I & 14 & 5065.885 & & $\mathbf{T 1}$ & $\mathbf{I}$ & 110 \\
\hline 5037.81 & $\mathbf{P}$ & $\mathbf{T} \mathbf{1}$ & II & 71 & 5066.28 & $\mathbf{P}$ & $\mathrm{Fe}$ & I & 882 \\
\hline 5038.400 & & $\mathbf{T} 1$ & I & 110 & 5066.99 & & La & II & 162 \\
\hline 5038.599 & & N1 & I & 166 & 5067 & $\mathbf{P}$ & $\mathbf{N}$ & v & 6 \\
\hline 5038.81 & $\mathbf{P}$ & $\mathrm{Fe}$ & I & 510 & 5067.082 & & $\mathrm{Cu}$ & II & 7 \\
\hline 5038.87 & $\mathbf{P}$ & $\mathrm{Cr}$ & I & 20 & 5067.162 & & $\mathrm{Fe}$ & I & 1092 \\
\hline 5039.05 & & C & $\mathbf{I}$ & 4 & 5067.714 & & $\mathrm{Cr}$ & I & 60 \\
\hline 5039.259 & & N1 & I & 142 & 5067.82 & & N1 & I & 141 \\
\hline 5039.266 & & $\mathrm{Fe}$ & I & 687 & 5068.10 & & $\mathrm{Cl}$ & II & 16 \\
\hline 5039.959 & & T1 & I & 5 & 5068.290 & & $\mathrm{Cr}_{\mathbf{r}}$ & I & 20 \\
\hline 5040.25 & $\mathbf{P}$ & $\mathrm{Fe}$ & I & 1093 & 5068.332 & & $\mathrm{~T} 1$ & I & 294 \\
\hline 5040.642 & & T1 & I & 38 & 5068.774 & & $\mathrm{Fe}$ & I & 383 \\
\hline 5040.744 & & Ru & I & 11 & 5069.12 & & $\mathbf{T} \mathbf{1}$ & II & 113 \\
\hline 5040.76 & & $\mathbf{N}$ & II & 19 & 5069.351 & & $\mathbf{T} \mathbf{1}$ & I & 189 \\
\hline 5040.82 & & HP & II & 14 & 5069.60 & $\mathbf{P}$ & $\mathrm{Fe}$ & & 211 \\
\hline
\end{tabular}


I $A$

Type

Element Multiplet No.

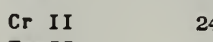

C1 II

Gd II

$\mathrm{Fe}$ I

$\mathrm{Fe}$ I

Sc I

Cl II

N1 I

24
16
114
984
66
965
13
16
141

Type

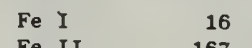

$\begin{array}{lllr}\mathbf{3 1 2 3 . 7 2 3} & & \text { Fe I } & 16 \\ \mathbf{5 1 2 4 . 0 5} & \text { P } & \text { Fe II } & 167 \\ \mathbf{5 1 2 4 . 1 7} & \text { P } & \text { Fe I } & 1035 \\ \mathbf{5 1 2 4 . 6 0} & \text { P } & \text { Fe I } & 585 \\ \mathbf{5 1 2 4 . 7 1 8} & & \text { Co I } & 197 \\ \mathbf{5 1 2 4 . 8 8} & & \text { Zr II } & 87 \\ \mathbf{5 1 2 5 . 1 3 0} & & \text { Fe I } & 1090 \\ \mathbf{5 1 2 5 . 2 1 1} & & \text { NI I } & 160 \\ \mathbf{5 1 2 5 . 5 6} & & \text { Gd II } & 99 \\ \mathbf{5 1 2 5 . 7 1 5} & & \text { Co I } & 181 \\ & & & \\ 5125.84 & & \text { A II } & 122 \\ \mathbf{5 1 2 6 . 1 3} & & \text { S II } & 57 \\ \mathbf{5 1 2 6 . 1 9} & \text { P } & \text { Fe II } & 53 \\ \mathbf{5 1 2 6 . 2 0 1} & & \text { Co I } & 170 \\ \mathbf{5 1 2 6 . 2 1 8} & & \text { Fe I } & 1089 \\ \mathbf{5 1 2 6 . 5 9 8} & & \text { Fe I } & 961 \\ \mathbf{5 1 2 7 . 3 2} & & \text { Fe III } & 5 \\ \mathbf{5 1 2 7 . 3 6 3} & & \text { Fe I } & 16 \\ \mathbf{5 1 2 7 . 3 6 7} & & \text { TI I } & 230 \\ \mathbf{5 1 2 7 . 6 8} & \text { P } & \text { Fe I } & 1\end{array}$

5127.866

5128.03

5128.53

5128.530

5129.143

5129.383

5129.520

5129.658

3130.28

5130.389

Fe II

N1 I

HF II

$\begin{array}{ll}\mathrm{V} & \mathrm{I} \\ \mathrm{T} & \mathrm{II}\end{array}$

T1 II

N1 I

Pr II

$\mathrm{Fe} \mathrm{I}$

Gd II

$\begin{array}{ll}\mathrm{Fe} & \mathrm{I} \\ \mathrm{N} & \mathrm{II}\end{array}$

092
34

Fe I

Cu I

As II

Lo I

Gd II

Ge II

La II

$\mathrm{Fe} I$

1090

5105.80

5107.406

5107.45

5107.645

5107.70

5107.80

5108.903

5108.91

5108.93

5109.427

5109.662

5110.36

5110.382

3110.414

5110.43

5110.61

5110.751

5110.768

5111.930

5112

3112.28

5112.490

5113.130

5113.232

5113.36

5113.448

5114

5114.07

5114.10

5114.52

5114.35

5115.387

5115.78

5116.700

5117.107

5117.175

5117.93 ?

5118.12

5118.55

5119.90

5120.420

5120.89

5121.570

5121.646

5121.68

$\mathbf{3 1 2 1 . 9 6}$

5122.082

5122.121

3122.76

3122.99

5123.

5123.28
5130.53

5130.596

$5131.28 \quad \mathrm{P} \quad \mathrm{Fe} I$

$5131.475 \quad \mathrm{Fe} \mathrm{I}$

5131.475

3132.19

$5132.67 \quad P \quad V$ II

5132.831

5133.22 $P \quad \mathrm{Fe}$

5133.29 C II

5133.42

5133.467
5133.692

5135.10

5135.125

3136.09

5136.47

3136.788

5137.075

5137.09

5137.26

5137.388

3137.94

5138.431

5138.71

5139.21

5139.255

3139.260

5139.468

3139.65

3140.839

5141.55

5141.63

5141.750

5141.84

5142.263

5142.33

5142.541

5142.763

5142.771

5142.932

5142.98

5143.49

5143.73

5144.413

5144.47

5144.672

5144.9376

5144.998

5145.011

5145.105

5145.16

5145.36

5145.42

5145.465

5145.654

II

Zr I

Co I

Fe $I$

Pr II

Fe I

Ta I
Fe II

c II

Fe I

Cr I

C I
C

Cr I

N1 I

Fe I

Fe I

Cr I

Gd II

$\mathrm{Fe} \mathrm{I}$

Fe I

A II

Cr I
3132.96 C II

5144.875

167

113

123

86

159

38
965

115

29,39

75
1148

88

66

114

35

230

818
16

16
27

180

1092
2

37

1036

5
35

N1 I 48

Cr II 201

$\begin{array}{llc}\text { S } & \text { II } & 1 \\ \text { Fe } & \text { I } & 1090,1092\end{array}$

16
1090

109

123

19
16

129

383

383

207

930

6
114

37

60

I A

\begin{tabular}{|c|c|c|c|c|}
\hline 5146.06 & & & & 28,38 \\
\hline 5146.12 & $\mathbf{P}$ & $\mathrm{Fe}$ & II & 35 \\
\hline 5146.30 & $\mathbf{P}$ & $\mathrm{Fe}$ & I & 1150 \\
\hline 5146.478 & & N1 & I & 162 \\
\hline 5146.753 & & Co & I & 170 \\
\hline 5147.09 & & $\mathrm{Fe}$ & II & \\
\hline 5147.483 & & T1 & I & 4 \\
\hline 5148.061 & & $\mathrm{Fe}$ & & 1090 \\
\hline 5148.19 & $\mathbf{P}$ & $\mathrm{Fe}$ & II & 52 \\
\hline 5148.234 & & $\mathrm{Fe}$ & I & 1095 \\
\hline 5118.65 & & N1 & I & 158 \\
\hline 5148.724 & & $\mathrm{v}$ & I & 123 \\
\hline 5148.838 & & $\mathrm{Na}$ & I & \\
\hline $51+9.13$ & & $4 n$ & I & 32 \\
\hline 5149.33 & & $\mathrm{Fe}$ & I I I & \\
\hline 5149.538 & & $\mathrm{Fe}$ & II & \\
\hline 5149.796 & & Co & & 39 \\
\hline 5149.841 & & Gd & II & 115 \\
\hline 5150.19 & $\mathbf{P}$ & $\mathrm{Fe}$ & I & 789 \\
\hline 5150.843 & & $\mathrm{Fe}$ & & 16 \\
\hline
\end{tabular}

5150.86

5150.890

3150.93

5151.08

151.83

5151.87

5151.915

5152.185

5152.20

5153.235

5153.402

5153.48

5154.061

3154.40

5155.136

5153.140

5155.764

5155.845

3156.0

5156.040

3156.08

$\$ 156.10$

5156.366

5156.74

5156.76

\$157.28

5157.43

5157.993

5158.187

3158.854

5159.066

3159.350

5159.93

5159.95

5160.02

5160.105

3160.824

5160.896

5161.18

5161.765

5162. 288

5162.34

5162.38

5162.47

5162.80

5162.93

5163.61

5163.74

5163.90

3164.542

A1 III

Mn I

Fe II

c II

Cr I

$\checkmark$ II

$\mathrm{Fe} I$

T1 I

Cu I

$\begin{array}{ll}\mathrm{Na} & \text { I } \\ \mathrm{Cr} & \text { II }\end{array}$

TI II

Fe II

Ru I

N1

$\begin{array}{ll}\text { N1 } & \text { I } \\ \text { Gd I I }\end{array}$

Fe III

Fe III
Sr I

HF II

$\mathrm{Fe}$ II

Co I

La II 


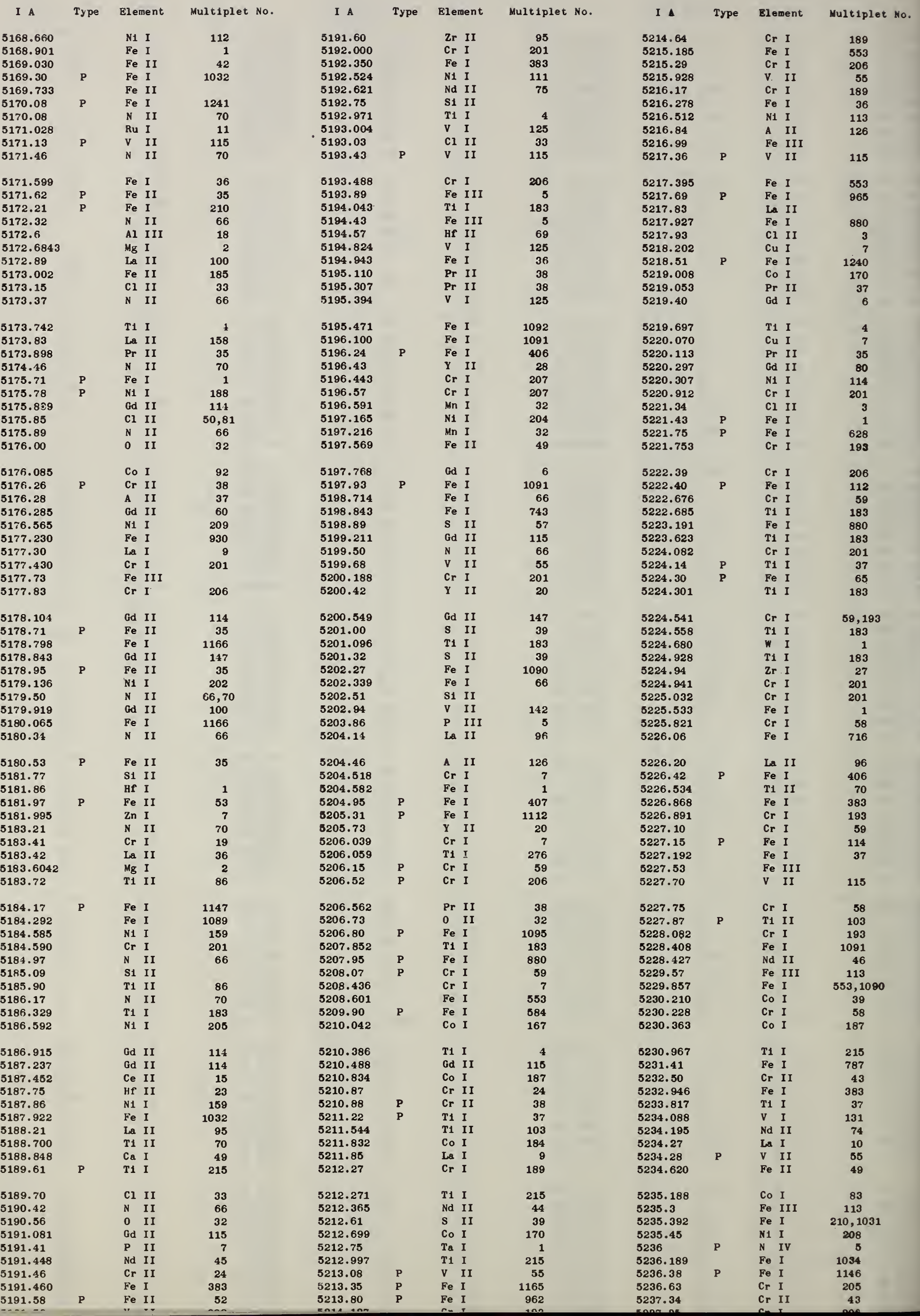


I A Type Biement Multiplet No.

\begin{tabular}{|c|c|c|c|c|c|c|c|c|}
\hline 5238.25 & $\mathbf{P}$ & $\mathrm{Fe}$ & I & 962 & 5261.754 & & $\mathrm{Cr}$ & I \\
\hline 5238.560 & & T1 & I & 37,183 & 5262.104 & & T1 & II \\
\hline 5238.58 & $\mathbf{P}$ & $\mathrm{Fe}$ & II & 41 & 5262.244 & & $\mathrm{Ca}$ & I \\
\hline 5238.971 & & $\mathrm{Cr}_{r}$ & I & 69 & 5262.48 & $\mathbf{P}$ & $\mathrm{Fe}$ & II \\
\hline 5239.823 & & Sc & I I & 26 & 5262.61 & $\mathbf{P}$ & $\mathrm{Fe}$ & I \\
\hline 5239.942 & & T1 & I & 37 & 5262.89 & $\mathbf{P}$ & $\mathrm{Fe}$ & I \\
\hline 5240.36 & $\mathbf{P}$ & $\mathrm{Fe}$ & I & 584 & 3263.314 & & $\mathrm{Fe}$ & I \\
\hline 5240.468 & & $\mathrm{Cr}$ & I & 237 & 5263.483 & & $\mathrm{~T} 1$ & I \\
\hline 5240.878 & & $v$ & I & 131 & 5263.750 & & $\mathrm{Cr}$ & I \\
\hline 5240.94 & & $\mathrm{Cr}_{\mathrm{r}}$ & I & 193 & 5263.874 & & $\mathrm{Fe}$ & I \\
\hline 5240.97 & $\mathbf{P}$ & $\mathbf{v}$ & II & 55 & 5263.99 & & $\mathbf{v}$ & II \\
\hline 5241.19 & & v & II & 241 & 5264.14 & & $\mathrm{Mg}$ & I I \\
\hline 5241.458 & & $\mathrm{Cr}_{r}$ & I & 59 & 3264.152 & & $\mathrm{C}_{\mathbf{r}}$ & I \\
\hline 5241.90 & $\mathbf{P}$ & $\mathrm{Fe}$ & I & 1150 & 5264.239 & & $\mathrm{Ca}$ & I \\
\hline 6242.495 & & $\mathrm{Fe}$ & I & 843 & 5264.49 & $\mathbf{P}$ & v & I I \\
\hline 6243.3 & & $\mathrm{Fe}$ & III & 113 & 5264.801 & & $\mathrm{Fe}$ & II \\
\hline 6243.395 & & $\mathrm{Cr}$ & I & 201 & 5264.95 & & H & II \\
\hline 6243.50 & $\mathbf{P}$ & $\mathrm{Cr}$ & II & 38 & 5265.160 & & $\mathrm{Cr}$ & I \\
\hline 6243.798 & & $\mathrm{Fe}$ & I & 1089 & 5265.25 & $\mathbf{P}$ & $\mathrm{Fe}$ & I \\
\hline 5244.5 & & C & I I I & 4 & 526.5 .42 & $\mathbf{P}$ & $\mathrm{Fe}$ & I \\
\hline 6245 & $\mathbf{P}$ & $N$ & IV & 5 & 5265.523 & & Co & I \\
\hline 6245.49 & & $\Delta$ & II & 40 & 5265.557 & & $\mathrm{Ca}$ & I \\
\hline 6245.62 & $\mathbf{P}$ & $\mathrm{Fe}$ & I & 1149 & 5265.710 & & $\mathrm{Ce}$ & II \\
\hline 5245.72 & $\mathbf{P}$ & $\mathrm{Fe}$ & I & 715 & 5265.722 & & $\mathrm{Cr}$ & I \\
\hline 5246.00 & $\mathbf{P}$ & $\mathrm{Fe}$ & I & 628 & 5265.748 & & N1 & I \\
\hline 5246.143 & & T1 & I & 282 & 5265.786 & & Co & I \\
\hline 5246.574 & & T1 & I & 37 & 5265.94 & $\mathbf{P}$ & $\mathrm{Fe}$ & I \\
\hline 5246.75 & & $\mathrm{Cr}$ & II & 23 & 5265.967 & & $\mathbf{T i}$ & I \\
\hline 6247.052 & & $\mathrm{Fe}$ & I & 1 & 5266.118 & & $\mathbf{v}$ & I \\
\hline 6247.10 & & $\mathrm{Hf}$ & II & 92 & 5266.302 & & Co & I \\
\hline 6241.293 & & $\mathrm{~T}_{1}$ & I & 183 & 5266.49 & $\mathbf{P}$ & T1 & I \\
\hline 6247.564 & & $\mathrm{Cr}$ & I & 18 & 5266.506 & & Co & I \\
\hline 5247.921 & & Co & I & 38 & 3266.562 & & $\mathrm{Fe}$ & I \\
\hline 6248.028 & & $\mathrm{Fe}$ & II & & 5267.10 & & $\mathrm{Cr}_{r}$ & I I \\
\hline 6248.402 & & T1 & I & 37,156 & 5267.28 & $\mathbf{P}$ & $\mathrm{Fe}$ & I \\
\hline 6249.099 & & $\mathrm{Fe}$ & I & 1166 & 5267.322 & & Gd & I I \\
\hline 6248.22 & & V & II & 220 & 5268.06 & & 0 & I II \\
\hline 5249.40 & & $\mathrm{Cr}$ & II & 23 & 5268.348 & & N1 & I \\
\hline 5249.43 & & C & II & 30 & 5268.498 & & Co & $\mathbf{I}$ \\
\hline 5248.585 & & $\mathrm{Nd}$ & I I & 75 & 5268.62 & & $T 1$ & II \\
\hline 5249.6 & & C & I I I & 23 & 5269.15 & & $\mathrm{Fe}$ & III \\
\hline 5250.003 & & Co & I & 190 & 5269.541 & & $\mathrm{Fe}$ & I \\
\hline 5250.212 & & $\mathrm{Fe}$ & I & 1 & 5269.93 & & T1 & I \\
\hline 5250.650 & & $\mathrm{Fe}$ & I & 66 & 5270.06 & $\mathbf{P}$ & $\mathrm{Fe}$ & I \\
\hline 5250.816 & & $\mathrm{Nd}$ & I I & 80 & 5270.270 & & $\mathrm{Ca}$ & $I$ \\
\hline 5250.95 & & T1 & I & 37 & 5270.322 & & $\mathrm{Be}$ & II \\
\hline 5251.180 & & Gd & I & 6 & 5270.360 & & $\mathrm{Fe}$ & I \\
\hline 5251.49 & & T1 & I & 37 & 5270.59 & & $N$ & I I I \\
\hline 3251.738 & & $\operatorname{Pr}$ & II & 20 & 5270.843 & & $\mathrm{Be}$ & II \\
\hline 5252.04 & $\mathbf{P}$ & T1 & II & 103 & 5271.18 & & La & I \\
\hline 3252.105 & & $\mathrm{T}_{1}$ & I & 4 & 5271.26 & $\mathbf{P}$ & $\mathbf{v}$ & I I \\
\hline 5252.14 & & Gd & II & 99 & 5272.0 & & $\mathrm{Fe}$ & I I I \\
\hline 5253.03 & $\mathbf{P}$ & $\mathrm{Fe}$ & I & 113 & 5272.010 & & $\mathrm{Cr}$ & I \\
\hline 5253.25 & $\mathbf{P}$ & $\mathrm{Fe}$ & I & 875 & 5272.413 & & $\mathrm{Fe}$ & I I \\
\hline 5253.479 & & $\mathrm{Fe}$ & I & 553 & 5272.56 & & c & III \\
\hline 5253.49 & & $\mathbf{P}$ & I I & 10 & 5272.60 & & $N$ & I I I \\
\hline 5253.55 & & c & II & 30 & 5272.86 & & $\mathrm{Fe}$ & I I I \\
\hline 5253.55 & & c & I I I & 4 & 5273 & $\mathbf{P}$ & N & $\mathbf{v}$ \\
\hline 5254.652 & & Co & I & 187 & 5273.176 & & $\mathrm{Fe}$ & I \\
\hline 5254.918 & & $\mathrm{Cr}$ & I & 201 & 5273.379 & & Fe & I \\
\hline 5254.92 & $\mathbf{P}$ & $\mathrm{Fe}$ & II & 48 & 5273.431 & & Nd & II \\
\hline 5254.956 & & $\mathrm{Fe}$ & I & 1 & 5273.439 & & $\mathrm{Cr}$ & I \\
\hline 5255.132 & & $\mathrm{Cr}$ & I & 225 & 5273.62 & $\mathbf{P}$ & $\mathrm{Fe}$ & I \\
\hline 5255.325 & & Mn & I & 32 & 5274.244 & & $\mathrm{Ce}$ & II \\
\hline 5255.510 & & Nd & I I & 43 & 5274.99 & & $\mathrm{Cr}$ & II \\
\hline 5255.68 & $\mathbf{P}$ & $\mathrm{Fe}$ & I & 1089 & 5275.00 & & $\mathrm{Fe}$ & I \\
\hline 5256.76 & $\mathbf{P}$ & $\mathrm{Fe}$ & I & 1081 & 5275.08 & & 0 & I \\
\hline 5255.805 & & Gd & I & 6 & 5275.11 & $\mathbf{P}$ & $\mathrm{Cr}$ & I \\
\hline 5255.811 & & $T 1$ & I & $183^{\circ}$ & 5275.171 & & $\mathrm{Cr}$ & I \\
\hline 5256.030 & & $\mathrm{Gd}$ & II & 114 & 5275.30 & $\mathbf{P}$ & $\mathrm{Fe}$ & I \\
\hline 5256.89 & $\mathbf{P}$ & $\mathrm{Fe}$ & I I & 41 & 5275.54 & & $\mathrm{Re}$ & I \\
\hline 5257.07 & & $\mathrm{Cr}$ & I & 205 & 5275.68 & & $\mathbf{v}$ & I I \\
\hline 5257.36 & & C & II & 30 & 5275.689 & & $\mathrm{Cr}$ & I \\
\hline 5257.51 & $\mathbf{P}$ & v & II & 55 & 5275.994 & & $\mathrm{Fe}$ & II \\
\hline 5257.621 & & Co & I & 188 & 5276.03 & & $\mathrm{Cr}$ & I \\
\hline 5257.65 & $\mathbf{P}$ & $\mathrm{Fe}$ & I & 788 & 5276.183 & & Co & I \\
\hline 5258.333 & & $\mathrm{Sc}$ & I & 23 & 5276.2 & & $\mathrm{Fe}$ & I I I \\
\hline 5259.08 & $\mathbf{P}$ & $\mathrm{Fe}$ & I & 1148 & 5276.42 & & $\mathbf{A 1}$ & II \\
\hline 5259.38 & & La & I I & 21 & 5276.81 & & Al & II \\
\hline 5259.62 & & C & II & 30 & 5276.879 & & $\mathrm{Nd}$ & I I \\
\hline 5259.743 & & Pr & II & 35 & 5277.31 & $\mathbf{P}$ & Fe & I \\
\hline 5259.976 & & $\mathbf{T} 1$ & I & 298 & 5277.32 & $\mathbf{P}$ & $\mathrm{Fe}$ & I \\
\hline 5260.25 & & $\mathrm{Fe}$ & II I & & 5277.40 & & $2 r$ & I \\
\hline 5260.375 & & $\mathrm{Ca}$ & I & 22 & 5277.58 & $\mathbf{P}$ & Fo & I \\
\hline 5260.44 & & не & I I & 36 & 5277.68 & & A1 & II \\
\hline 5260.771 & & Mn & I & 32 & $5278 \cdot 10$ & & $s$ & I \\
\hline 5260.81 & & Al & I I I & 13 & 5278.262 & & $\mathrm{Cr}$ & I \\
\hline 6260.81 & & $N$ & I I I & 15 & 5278.265 & & $\mathrm{Fe}$ & II \\
\hline 5261.49 & $\mathbf{P}$ & $\mathrm{Fe}$ & I & 406 & 5278.62 & & A1 & II \\
\hline & & & & & & & 8 & $\mathbf{T}$ \\
\hline
\end{tabular}

I A Type Element Multiplet No.

I A Type Element Multiplet.No.

$5278.955 \quad$ Fe II 18

5 I

$P \quad 0 \mathrm{VI}$

5279.11 La II

$5279.65 \quad P \quad \mathrm{Fe} \mathrm{I}$

Cr II $\quad 584$

$5280.00 \quad$ V II $\quad 195$

$\begin{array}{lll}5280.08 & \text { Cr II } & 43 \\ 5280.21 & \text { AI II } & 95\end{array}$

$\begin{array}{lll}5280.289 & \text { Cr I } & 182\end{array}$

$\begin{array}{lll}5280.364 & \text { Fe I } & 880\end{array}$

5280.62 P $\quad V$ II $\quad 55$

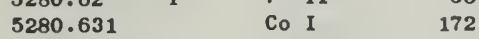

$\begin{array}{lllr}5280.81 & \text { P } & \text { Fe I } & 210 \\ 5281 & \text { P } & \text { N IV } & 5\end{array}$

$\begin{array}{llll}5281.18 & P & \text { Fe } & 1\end{array}$

$\begin{array}{lll}5281.18 & \text { N I } & 14\end{array}$

$\begin{array}{lll}5281.692 & \text { N1 I } & 231 \\ 5281.796 & \text { Fe } & 383\end{array}$

5282.1 Fe III

$\begin{array}{lll}5282.378 & \text { T1 I } & 74\end{array}$

$\begin{array}{llr}5282.52 & \text { N III } & 15 \\ 5283.076 & \text { Gd I } & 6\end{array}$

$\begin{array}{lll}5283.441 & \text { T1 I } & 156\end{array}$

$5283.628 \quad$ Fe I $\quad 553$

5283.77 Al II 95

$\begin{array}{rlr}5284.092 \quad & \text { Fe II } & 41 \\ 5284.27 & \text { Fe I } & 875\end{array}$

$\begin{array}{lll}5284.380 & \text { Ti I } & \mathbf{7 4}\end{array}$

$\begin{array}{lll}5284.416 & \text { Fe I } & 842\end{array}$

$\begin{array}{llll}5284.62 & P & F e & I\end{array}$

$\begin{array}{llll}5284.85 & & \text { Fe III } & \\ 5285.12 & P & \text { Fe I } & 1166\end{array}$

$5285.34 \quad$ Ca II 14

$\begin{array}{lll}5285.38 & \text { Cr I } & 285\end{array}$

$3285.48 \quad$ C1 II 32

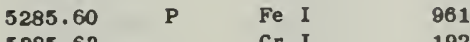

3285.63

$\begin{array}{llr}5285.752 & \text { Sc I } & 23 \\ 5285.85 & \text { A1 I I } & 102\end{array}$

$\begin{array}{lllll}6286.42 & P & V & \text { II } & 54\end{array}$

5286.74 Fe III 110

$\begin{array}{lll}5286.92 & \text { A II } & 13\end{array}$

$5287.188 \quad$ Cr I 225

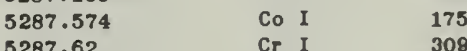

$\begin{array}{llll}5287.785 & \text { Cr I } & 308\end{array}$

$\begin{array}{llll}5288.21 & P & \text { N1 I } & 202\end{array}$

$\begin{array}{llll}5288.24 & \text { P } & \text { Fe I } & 818\end{array}$

$5288.38 \quad P \quad \mathrm{Fe} I$

$\begin{array}{llll}5288.38 & P & \text { Fe I } & 406 \\ 5288.533 & & \text { Fe I } & 928\end{array}$

$\begin{array}{llll}5289 & P & 0 & \text { VI }\end{array}$

$\begin{array}{llr}5289.27 & \text { Cr I } & 192\end{array}$

$\begin{array}{lll}5289.28 & \text { T1 I } & 36 \\ 5289.82 & Y \text { II } & 20\end{array}$

5289.82 HF II $\quad 100$

$\begin{array}{rllr}5290.74 & & \text { V II } & 207 \\ 5290.79 & P & \text { Fe I } & 1147\end{array}$

5290.83 La II

$\begin{array}{lllll}5291 & P & 0 & V I & 18\end{array}$

$\begin{array}{llll}5291.78 & & \text { Fe III } & \\ 5292 & P & 0 & \text { VI }\end{array}$

$5292.10 \quad$ Pr II 24

5292.630 Pr II

5292.861 Mn I

$5292.865 \quad$ Cr I 205

$\begin{array}{lllr}5293.03 & P & \text { Fe I } & 1165\end{array}$

$\begin{array}{lll}5293.168 & \text { Cr I } & 192\end{array}$

5293.973 Fe I 1031

$5294.216 \quad$ Yn II 11

5294.97 S1 II

$\begin{array}{lllll}5295.292 & & \text { Mn II } & \text { 11 } \\ 5295.30 & \text { P } & \text { Sc II } & 22\end{array}$

$5295.316 \quad$ Fo I 1146

$\begin{array}{lll}5295.781 & \text { T1 I } & 74\end{array}$

$\begin{array}{llll}5296.09 & \text { P } & \text { II } & \\ 5296.48 & \text { A II } & 110\end{array}$

$\begin{array}{lll}5290.686 & \text { Cr I } & 18\end{array}$

5296.968 Mn II 11

$\begin{array}{rrr}5297.236 & \text { Ti I } & 156 \\ 5297.360 & \text { Cr I } & 9\end{array}$

$\begin{array}{lll}5297.360 & \text { Cr I } & \text { भI } \\ 5297.86 & \text { N III } & 15\end{array}$

$5297.976 \quad$ CF I $\quad 94$

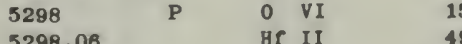

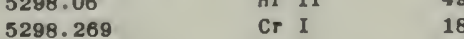

$\begin{array}{rrr}5298.429 & \text { TI I } & 18\end{array}$ 
I A Type Element Multiplet No.

$\begin{array}{ll}\mathbf{5 2 9 8 . 4 4} & \mathbf{P} \\ \mathbf{5 2 9 8 . 7 8 9} & \\ \mathbf{5 2 9 8 . 9 3} & \\ \mathbf{5 2 9 9 . 0 0} & \\ \mathbf{5 2 9 9 . 2 7 8} & \\ \mathbf{5 2 9 9 . 8 5} & \\ \mathbf{5 2 9 9 . 9} & \\ \mathbf{5 3 0 0 . 0 1 2} & \\ \mathbf{5 3 0 0 . 4 1} & \mathbf{P} \\ \mathbf{5 3 0 0 . 7 4 9} & \end{array}$

$\begin{array}{llr}\text { Cr } & \text { I } & 94 \\ \text { Fe } & I & 875\end{array}$

$\begin{array}{llr}\text { Fe I } & 875 \\ \text { III } & \text { II }\end{array}$

$\begin{array}{lll} & 111 & 15 \\ 0 & I & 26\end{array}$

Mn II

Hf II

Fe III $\quad 113$

$\begin{array}{llr}\text { T1 } & \text { I } & 74 \\ \text { Fe } & \text { I } & 1240\end{array}$

$\begin{array}{llr}\text { Fe } & \text { I } & 1240 \\ \text { Cr } & \text { I } & 18\end{array}$

Co $I \quad 39$

Fe I $\quad 1162$

Gd I

Sc I

La I I

Nd II

$\begin{array}{ll}\mathrm{Fe} & \text { I } \\ \mathrm{Mn} & \text { II }\end{array}$

Fe III

Le II

5303.26

5303.419

5303.43

5303.54

5304.11 P

5304.211

5304.26

5305.3

$5305.41 \quad P$

Gd I

Fe II

Gd II

La II

$\mathrm{Fe} I$

$\begin{array}{ll}\mathrm{Cr} & \text { I } \\ \mathrm{Fe} & \text { II }\end{array}$

Gd II

0 IV

Fe I

A II

Fe III

Tm I

Cr I

Gd I

$\mathrm{Fe} I$

5307.30

5307.365

5307.53

Mn I

5308.71

5309.26

5309.47

5310.219

5310.70

5310.76

5311.42

5311.461
5311.60

Cr II

Fe I

Ru I

Cr I

Cr II

Al II

Zr I

Nd II

Zr II

5311.78

5312.32

5312.650

5312.878

313.239

5313.41

5313.43

5313.59

5313.839

5314.45

5315.07

5315.618

5315.78

5316.07
5316.07

5316.609

5316.772

3316.777

5317.095

Al II

Co I

Cr I

T1

Fe I

$N$ II

Cr II

$\mathrm{Fe} I$

N III

Fe I

Fe I

Al II

$\begin{array}{ll}P & \text { II } \\ \mathrm{Fe} & \text { II }\end{array}$

Co I

Fe II

Mn I

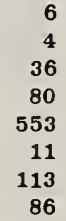

36

11
113
86

6

225
80

36

983

225

184
62

11

877

24

113

237

14
6

36
36

43

1091

10
285

196

43

94
27

80
37

95
94
197

225

74

1239

43
81

1238

15

1147
225

877

94
6
49

$1 \sqcup 2$

48

Fe I $\quad 584$

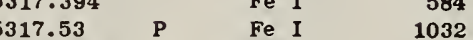

5318.025 Fe II

$\begin{array}{lll}5318.04 & \text { P } & \text { Fe I } \\ 5318.267 & & \text { Fe II }\end{array}$

5318.337 Sc II

$5318.41 \quad$ Cr II

$\begin{array}{llll}5318.61 & P & V & \text { II }\end{array}$

5318775

5319.22

5319.818

5320.048

5320.70

5320.78

5320.96

5321.106

5321.496

5321.777

5322.049

5322.778

I A Type Element Multiplet No.

$\begin{array}{lll}5322.78 & \text { P } & \text { Cr II } \\ 5322.81 & & \text { V I I } \\ 5323.51 & \text { P } & \text { Fe I } \\ 5323.958 & & \text { TI I } \\ 5324.185 & & \text { Fe I } \\ 5324.26 & & \text { Hf II } \\ 5324.61 & & \text { Al I I } \\ 5325.276 & & \text { Co I } \\ 5325.559 & & \text { Fe II } \\ 5325.71 & \text { P } & \text { V } \text { II }\end{array}$

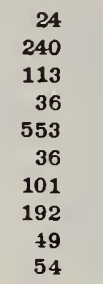

5325.949

5326.154

5326.247

5326.793

5327.25

5327.45

5327.86

5328.338

5328.38

\section{Co I}

$\mathrm{Fe}$

$\mathrm{Fe}$ I

$\mathrm{Fe} I$

N II

$\mathrm{Fe} I$

$\begin{array}{ll}\mathrm{Fe} & \mathrm{I} \\ \mathrm{Cr} & \mathrm{I}\end{array}$

$\begin{array}{ll}\text { Cr } & \text { I } \\ \text { Ta } & \text { I }\end{array}$

194
407,785
175
1147
875
69
1145
15
94
2

5328.534

5328.70

5328.70

5328.98

5329.12

5329.59
5329.719

5329.994

5330.582

5330.66

Fe I

$\begin{array}{ll}\text { N } & \text { I } \\ \text { N1 } & \text { I }\end{array}$

0 I

Cr I

0 I

$\mathrm{Cr}$ I

$\begin{array}{ll}\mathrm{Fe} & \text { I } \\ \mathrm{Ce} & \text { II }\end{array}$

0 I

$\begin{array}{lll}5330.779 & & \text { Ne } \\ 5331.20 & P & \text { Fe } \\ 5331.456 & & \text { Co }\end{array}$

$\begin{array}{lll}5331.456 & & \text { Co } \\ 5331.48 & P & \mathrm{Fe}\end{array}$

$\begin{array}{ll}5331.48 & P\end{array}$ As II

5332.65

5332.652

5332.673

5332.903

5333.15

Co I

Fe I

$\mathrm{Fe} I$

5333.30 Gd

5333.647

5333.70

Co I

$5333.77 \quad P \quad F e I$

5334.228 Sc II

$5334.32 \quad P \quad F e I$

Mn I

5334.88

5336.163

$\begin{array}{ll}\text { Co } & \text { I } \\ \text { Cr } & \text { II }\end{array}$

Co I

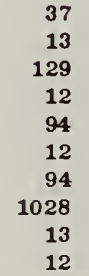

5336.7

5336.809

5337.713

5337.79

5338.326

5338.66

5339.29

5339.40

5339.528

5339.92

5339.935

5340.20

5340.437
5340.66

5340.68

5340.92

5341.026

5341.040

5341.065

5341.096

C II

T1 II

Fe II

Cr II

T1 I

N II

Ca I I

$\mathrm{Fe} I$

Fe III

9
817

39
210

210

3
54
170

170

1031

1023

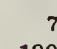

7
190
15

15
464

30

1064

36
191

43
191

11

69
48
43

43

35
69

69
20
1162

199

Fe I

N II

La I

Fe III

Fe I

$\mathrm{Mn} I$
$\mathrm{Ne} I$

553

68
225

91

36 
I A

Type Blement Multiplet No.
I A

Type Element Multiplet No.

5405.778

$\begin{array}{lllr}5406.36 & \text { P } & \text { Fe I } & 1026\end{array}$

$\begin{array}{llll}5406.77 & \text { P } & \text { Fe I } & 1148\end{array}$

5407.14

5407.520

5407.62

5408.119

5408.59

3408.842

$5408 \cdot 9+0$

5409.125

5.109 .224

5409.28

5409.609

5409.66

5409.791

5410

5410.39
5410.76

A II

Co I

Co I

0 I

192
23
112

53

T1 I 3

Fe I

$\mathrm{Ce}$ II

Cr I I

T1 I

$\begin{array}{ll}\text { P } & \text { II } \\ \text { Cr } & \text { I }\end{array}$

0 VI

Cr II

3
1147
23

23
29
155

155
6

18
13

29
51,52

$\begin{array}{rrr}5410.913 & \text { Fe I } & 1165 \\ 5411.227 & \text { N1 I } & 222\end{array}$

$\begin{array}{llll}5411.39 & P & F e & I\end{array}$

$\begin{array}{lllr}5411.524 & & \text { He I I } & 2 \\ 5412.56 & \text { P } & \text { Fe I } & 1237\end{array}$

$\begin{array}{llll}5412.80 & P & \text { Fe } I & 1162\end{array}$

$\begin{array}{llr}\mathbf{5 4 1 3 . 4 7} & \text { Ta I } & 5 \\ \mathbf{5 4 1 3 . 6 8 7} & \text { Mn I } & 42\end{array}$

$\begin{array}{lllr}\mathbf{5 4 1 3 . 6 8 7} & & \text { Mn I } & \mathbf{4 2} \\ \mathbf{5 4 1 4 . 0 8 9} & & \text { Fe II } & 48 \\ \mathbf{5 4 1 4 . 9 1} & \text { P } & \text { Fe I } & \mathbf{8 7 4}\end{array}$

$\begin{array}{lll}5415.201 & \mathrm{Fe} I & 1165\end{array}$

$\begin{array}{lllr}\mathbf{5 4 1 5 . 2 0 1} & & \text { Fe I } & 1165 \\ \mathbf{5 4 1 5 . 2 7 7} & & \text { V I } & 130 \\ \mathbf{5 4 1 6 . 3 8 1} & & \text { Nd I I } & 80 \\ \mathbf{5 4 1 7} & \text { P } & \text { 0 V } & 13 \\ \mathbf{5 4 1 7 . 0 3} & & \text { Fe I } & \mathbf{1 1 4 8} \\ \mathbf{5 4 1 8 . 0 1} & & \text { Zr II } & 94 \\ \mathbf{5 4 1 8 . 8 0 2} & \text { T1 I I } & 69 \\ \mathbf{5 4 1 9 . 1 8 9} & \text { T1 I } & 258 \\ \mathbf{5 4 1 9 . 1 9} & & \text { Ta I } & 6 \\ \mathbf{5 4 1 9 . 3 6} & & \text { Cr II } & 22,29\end{array}$

Cr II 22,29

$\begin{array}{lll}5419.876 & \text { Gd } & \text { II }\end{array}$

5420.362

5420.90

5421.05

Ba II

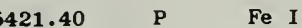

$\begin{array}{lll}5421.559 & 874\end{array}$

$\begin{array}{lllr}5421.85 & P & \text { Fe I } & 79 \\ 5 & & 1183\end{array}$

$\begin{array}{llll}5422.15 & P & F e & \end{array}$

$\begin{array}{rrr}5422.47 & \text { T1 II } & 80 \\ 5423.25 & \text { CI II } & 2\end{array}$

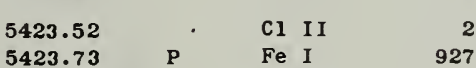

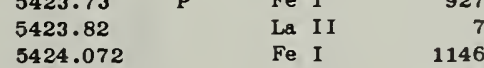

$\begin{array}{llll}5424.072 & & \text { Fe I } & 1146 \\ 5424.15 & \text { P } & \text { Fe I } & 1026\end{array}$

$\begin{array}{lll}5424.36 & \text { Cl II } & 2 \\ 5424.551 & \text { Ba I } & 9\end{array}$

$\begin{array}{llll}5424.551 & & \text { N1 } & \\ 5424.56 & \text { P } & \text { N1 } & 231\end{array}$

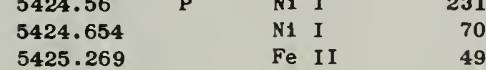

$\begin{array}{llll}5425.29 & P & \text { Cr II } & 29\end{array}$

$\begin{array}{lllr}5425.29 & \text { P } & \text { Cr II } & 29 \\ 5425.621 & & \text { Co I } & 196 \\ 5425.93 & & \text { P II } & 6\end{array}$

$\begin{array}{lll}5425.93 & \text { P II } & 6 \\ 5426.256 & \text { TI I } & 3\end{array}$

$5427.832 \quad$ Fe II

$5428.64 \quad S$ II

$\begin{array}{llll}5428.71 & P & F e I & 1032\end{array}$

$\begin{array}{rlr}5428.79 & \text { Ba II } & 9 \\ 5428.85 & \text { N1 I } & 161\end{array}$

$\begin{array}{lll}5429.139 & \text { T1 I } & 258\end{array}$

$\begin{array}{llll}5429.43 & P & F e & I\end{array}$

$\begin{array}{llll}5429.52 & P & F e & \\ 5429.699 & & F e & 1062 \\ 54 & 15\end{array}$

$\begin{array}{llll}5429.83 & P & F e & 1162\end{array}$

$5430.14 \quad$ Fe III

$\begin{array}{lllll}5430.41 & \text { P } & \text { Cr II } & 39 \\ 5431.526 & & \text { Nd I I } & 80\end{array}$

$\begin{array}{llll}5431.526 & & \text { Nd I I } & 80 \\ 5432 & P & 0 & \text { V }\end{array}$

5432.09 V II

5432.318

T1 I

5432.347

5432.548

5432.77

5432.95

5432.98

5434.527

5435.16

5435.17

5435.27

$\mathbf{5 4 3 5 . 7 6}$
I A

Type Element

Nultiplet No.

$\begin{array}{lllr}\mathbf{5 4 3 5 . 7 9} & \text { P } & \text { Fe II } & 48 \\ \mathbf{5 4 3 5 . 8 7 1} & & \text { N1 I } & 70 \\ \mathbf{5 4 3 6 . 2 9 9} & & \text { Fe I } & 1161 \\ \mathbf{5 4 3 6 . 5 9 4} & & \text { Fe I } & 113 \\ 5436.703 & & \text { T1 I } & 51 \\ 5436.80 & & \text { Fe I I I } & 110 \\ \mathbf{5 4 3 6 . 8 3} & & \text { 0 I } & 11 \\ \mathbf{5 4 3 7 . 1 9} & \text { P } & \text { Fe I } & 1145 \\ \mathbf{5 4 3 8 . 0 4} & \text { P } & \text { Fe I } & 1237 \\ \mathbf{5 4 3 8 . 3 1 0} & & \text { TI I } & 108\end{array}$

$\begin{array}{llll}\mathbf{5 4 3 8 . 4 1} & & \text { S1 I I } & \\ \mathbf{5 4 3 9 . 3 0} & & \text { V } \text { I I } & \\ \mathbf{5 4 4 0 . 5 3} & \text { P } & \text { T1 I } & \mathbf{1 0 7} \\ \mathbf{5 4 4 1 . 1 7} & & \text { Gd I I } & \mathbf{1 4} \\ \mathbf{5 4 4 1 . 3 2 1} & & \text { Fe I } & \mathbf{1 1} \\ \mathbf{5 4 4 2 . 2 7 4} & & \text { Nd I I } & \\ \mathbf{5 4 4 2 . 4 1 3} & & \text { Cr I } & 204 \\ \mathbf{5 4 4 3 . 4 1} & \text { P } & \text { Fe I } & 1059 \\ \mathbf{5 4 4 3 . 4 2} & & \text { CI I I } & \\ \mathbf{5 4 4 3 . 8 8} & & \text { Fe II } & 1\end{array}$

$5443.88 \quad$ Fe III

5444.07 HF II 68

5414.096 Mn I

$\begin{array}{lllr}5444.25 & \text { Cl } & \text { II } & 2 \\ 5444.585 & \text { Co } & \text { I } & 196\end{array}$

5444.99 $\quad$ Cl II

$5445.97 \quad \mathrm{Fe} I \quad 1163$

$\begin{array}{llll}5446.46 & \text { P } & \text { T1 II } & 68\end{array}$

$\begin{array}{rllr}5446.57 & \text { P } & \text { Cr I I } & 35 \\ 5446.58 & \text { P } & \text { Fe I } & 1144\end{array}$

$\begin{array}{lll}5146.593 & \text { T1 I } & 3,259\end{array}$

5446.76 Cr I 204

5446.87 P $\quad$ Fe I $\quad 37$

$5446.920 \quad$ Fe I 15

$5147.59 \quad$ La II 112

$\begin{array}{lll}5448.882 & \text { T1 I } & 259 \\ 5449.155 & \text { T1 I } & 107\end{array}$

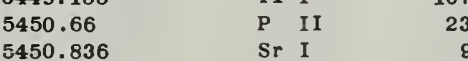

5451.115 Nd II

$\begin{array}{llll}5451.60 & \text { P } & \text { Fe II } & 184\end{array}$

$\begin{array}{lll}5451.965 & \text { T1 I } & 265 \\ 5452.03 & \text { T1 II } & 109\end{array}$

$\begin{array}{lll}5452.119 & \text { Fe I } & 870\end{array}$

$\begin{array}{lll}5452.12 & \text { N II }\end{array}$

$5452.305 \quad$ Co I 175

5453.255 N1 I

5453.338 Co I 19

$\begin{array}{lll}5453.616 & \text { T1 I } & 108 \\ 5453.81 & \text { S II } & \end{array}$

$\begin{array}{llll}5453.98 & \text { P } & \text { Fe I } & 1064\end{array}$

5454.26 N II 29

$\begin{array}{lll}5454.41 & \text { A I I } & \\ 5454.573 & \text { Co I } & 195\end{array}$

$\begin{array}{llll}\mathbf{5 4 5 4 . 5 7 3} & & \text { Co I } & 195 \\ \mathbf{5 4 5 5 . 0 9} & \text { P } & \text { Fe I } & 627\end{array}$

5455.11 LA I

$5455.433 \quad$ Fe I $\quad 1145$

$\begin{array}{lll}\mathbf{5 4 5 5 . 6 1 3} & \text { Fe I } & 15 \\ \mathbf{5 4 5 5 . 8 0} & \text { Cr II } & 50\end{array}$

$\begin{array}{lll}5455.815 & \text { Nd I I } & 83\end{array}$

$\begin{array}{ll}5456.11 & \text { S1 II } \\ \mathbf{5 4 5 6 . 2 7} & \text { CI II }\end{array}$

$\begin{array}{lll}\mathbf{5 4 5 6 . 2 7} & \text { Cl II } & \\ \mathbf{5 4 5 6 . 4 8} & \text { Fe I } & 817\end{array}$

$5457.02 \quad$ Cl II

5457.47 CI I I

$\begin{array}{ll}\mathbf{5 4 5 7 . 4 7 1} & \text { Mn I } \\ \mathbf{5 4 5 8 . 6 8} & \text { La II }\end{array}$

$\begin{array}{llr}5458.68 & \text { La II } & 99 \\ 541 & \text { T1 } & 3\end{array}$

$5460.644 \quad$ Mn I 31

$5460.742 \quad \mathrm{Hg} \mathrm{I}$

$5460.8 \quad$ Fe III

5460.909 Fe I 164

$\begin{array}{llr}\mathbf{5 4 6 1 . 3 1} & \text { Ta I } & 4 \\ \mathbf{5 4 6 1 . 5 4} & \text { Fe I } & \mathbf{1 1 4 5}\end{array}$

$\begin{array}{rlrr}5461.54 & & \mathrm{Fe} I & 1145 \\ 5461 \text { R? } & P & \mathrm{Fe} \mathrm{I} & 817\end{array}$

$5462.487 \quad$ N1 J 192

$\begin{array}{lll}5462.62 & \text { N } & \text { II } \\ 5462.970 & \text { Fe I } & 1163\end{array}$

$\begin{array}{lll}5463.282 & \text { Fe I } & 1163\end{array}$

$\begin{array}{llr}5463.38 & \text { HF II } & 14\end{array}$

$5464.286 \quad$ Fe I 1030

$\begin{array}{llll}5464.36 & P & C r & \text { II }\end{array}$

$\begin{array}{rllr}5464.37 & & \text { LQ II } & 49 \\ 5465.01 & \text { P } & \text { Fe I } & 840\end{array}$

$5466.021 \quad$ Fe II

$5466.46 \quad$ Fe III 114 
I A Type Element Multiplet No. I A Type Element Multiplet No.

I A Type Element Multiplet No.

\begin{tabular}{|c|c|c|c|c|c|c|c|c|}
\hline 5466.46 & & $\mathbf{Y}$ & I & 12 & 5490.65 & $\mathbf{P}$ & T1 & II \\
\hline 5466.55 & & $\mathbf{s}$ & II & 11 & 5490.840 & & T1 & I \\
\hline 5466.94 & & $\mathrm{Fe}$ & I I & & 5491.84 & & $\mathrm{Fe}$ & I \\
\hline 6466.993 & & $\mathrm{Fe}$ & I & 784,817 & 5492.43 & & T1 & IV \\
\hline 5467.76 & $\mathbf{P}$ & $\mathrm{Fe}$ & I & 741 & 5492.8 & & 0 & I \\
\hline 5468.101 & & N1 & I & 192 & 5492.82 & $\mathbf{P}$ & T1 & II \\
\hline 5468.37 & $\mathbf{P}$ & $\mathrm{Ce}$ & I I & 24 & 5493.22 & & $\mathrm{HP}$ & I I \\
\hline 5468.44 & $\mathbf{P}$ & $T_{1}$ & II & 102 & 5493.33 & $\mathbf{P}$ & $\mathrm{Fe}$ & I \\
\hline 5468.92 & & S1 & II & & 6493.45 & & $\mathrm{La}$ & II \\
\hline 5469.09 & $\mathbf{P}$ & $\mathrm{Fe}$ & I & 1131 & 5493.508 & & $\mathrm{Fe}$ & I \\
\hline 5469.29 & $\mathbf{P}$ & $\mathrm{Fe}$ & I & 1143 & 5493.850 & & $\mathrm{Fe}$ & I \\
\hline 5469.305 & & Co & I & 56 & 5494.35 & $\mathbf{P}$ & $\mathbf{v}$ & I I \\
\hline 5469.72 & & Gd & II & 60 & 5494.468 & & $\mathrm{Fe}$ & I \\
\hline 5470.17 & & $\mathrm{Fe}$ & I & 1144 & 5494.726 & & T1 & I \\
\hline 5470.460 & & Co & I & 175 & 5494.890 & & $\mathbf{N 1}$ & I \\
\hline 5470.50 & & $\mathrm{~T} 1$ & I & 108 & 5495.682 & & Co & I \\
\hline 5470.53 & & Gd & I I & 63 & 5495.70 & & $\mathbf{N}$ & II \\
\hline 5470.638 & & Mn & I & 4 & 5495.8720 & & $\mathbf{A}$ & I \\
\hline 6470.81 & $\mathbf{P}$ & $\mathrm{Fe}$ & II & 52 & 5496.020 & & v & I \\
\hline 5471.198 & & T1 & I & 106 & 5496.24 & & si & II \\
\hline 5472.297 & & $\mathrm{Ce}$ & II & 24 & 5496.57 & $\mathbf{P}$ & $\mathrm{Fe}$ & I \\
\hline 5472.63 & & $\mathrm{Cr}_{\mathbf{r}}$ & II & 50 & 5497.42 & & $\mathbf{Y}$ & I I \\
\hline 5472.696 & & $\mathrm{~T} 1$ & I & 107 & 5497.519 & & $\mathrm{Fe}$ & I \\
\hline 5472.720 & & $\mathrm{Fe}$ & I & 1108 & 5497.70 & $\mathbf{P}$ & $\mathrm{Fe}$ & II \\
\hline 5473 & $\mathbf{P}$ & 0 & v & 13 & 5497.86 & $\mathbf{P}$ & $\mathrm{Cr}$ & II \\
\hline 5473.18 & $\mathbf{P}$ & $\mathrm{Fe}$ & I & 1064 & 3497.92 & $\mathbf{P}$ & $\mathbf{T} 1$ & I \\
\hline 5473.40 & & $\mathbf{Y}$ & II & 27 & 5498.18 & & $\mathbf{s}$ & I \\
\hline 5473.517 & & T1 & I & 259 & 5488.19 & $\mathbf{P}$ & $\mathrm{Fe}$ & I I \\
\hline 5473.517 & & T1 & I I & 109 & 5499.39 & $\mathbf{P}$ & N1 & $\mathbf{I}$ \\
\hline 5473.59 & & $\mathbf{s}$ & II & 6 & 5499.60 & $\mathbf{P}$ & $\mathrm{Fe}$ & I \\
\hline 6473.908 & & $\mathrm{Fe}$ & I & 1062 & 5499.72 & & $\mathbf{P}$ & II \\
\hline 5474.09 & $\mathbf{P}$ & $\mathrm{Fe}$ & I & 1314 & 5500.43 & & ad & II \\
\hline 5474.228 & & $\mathrm{~T} 1$ & I & 108 & 5500.61 & $\mathbf{P}$ & $\mathrm{Cr}_{\mathbf{r}}$ & II \\
\hline 5474.449 & & $\mathrm{T1}$ & I & 258 & 5501.34 & & La & I \\
\hline 5474.734 & & Nd & II & 82 & 5501.469 & & $\mathrm{Fe}$ & I \\
\hline 5475.57 & & N1 & I & 159 & 5501.54 & & $\mathbf{s}$ & I \\
\hline 6476.298 & & $\mathrm{Fe}$ & I & 1029 & 5502.05 & & $\mathbf{C r}$ & II \\
\hline 5476.571 & & $\mathrm{Fe}$ & I & 1062 & 5502.88 & & Al & II \\
\hline 5476.69 & & Lu & II & 2 & 5503.18 & & $\mathrm{Cr}$ & II \\
\hline 5476.906 & & N1 & I & 59 & 5503.387 & & $\mathrm{Fe}$ & II \\
\hline 5477.089 & & Co & I & 175 & 5503.897 & & $\mathrm{~T} 1$ & I \\
\hline 6477.45 & $\mathbf{P}$ & $\mathrm{Cr}$ & I I & 50 & 5504.120 & & N1 & I \\
\hline 5477.67 & $\mathbf{P}$ & $\mathrm{Fe}$ & II & 49 & 5504.184 & & $\mathrm{sr}$ & I \\
\hline 5477.685 & & $T \mathbf{I}$ & I & 265 & 5504.21 & & Mn & I \\
\hline 5477.82 & & $\mathrm{Zr}$ & I I & 115 & 5505.75 & $\mathbf{P}$ & $\mathrm{Fe}$ & I \\
\hline 5478.13 & & N & I I & 29 & 5505.869 & & Mn & I \\
\hline 5478.35 & & $\mathrm{Cr}$ & II & 50 & 5505.893 & & $\mathrm{Fe}$ & I \\
\hline 5478.48 & & $\mathrm{Fe}$ & I & 1062 & 5506.268 & & $\mathrm{Fe}$ & I I \\
\hline 5478.6 & & C & II & 34 & 5506.51 & & Mo & I \\
\hline 5479.85 & $\mathbf{P}$ & $\mathrm{Fe}$ & I & 1282 & 5506.782 & & $\mathrm{Fe}$ & I \\
\hline 5480.10 & & $\mathrm{~N}$ & I I & 29 & 5507.01 & & $\mathbf{s}$ & I \\
\hline 5480.30 & & $8 a$ & II & 9 & 5507.15 & & $\mathbf{P}$ & II I \\
\hline 5480.502 & & $C_{r}$ & I & 204 & 5507.753 & & $\mathbf{v}$ & I \\
\hline 6480.72 & & $\mathbf{L a}$ & II & 90 & 5508.11 & & 0 & III \\
\hline 5480.75 & & $\mathbf{Y}$ & I I & 27 & 5508.60 & & $\mathrm{Cr}$ & II \\
\hline 5480.865 & & $\mathrm{Sr}$ & I & 9 & 5508.88 & $\mathbf{P}$ & $\mathbf{c}_{\boldsymbol{T}}$ & I \\
\hline 5480.872 & & $\mathrm{Fe}$ & I & 1062 & 5509.67 & & $\mathbf{s}$ & II \\
\hline$\$ 480.893$ & & $\mathrm{Ni}$ & I & 191 & 5509.91 & & $\mathbf{Y}$ & II \\
\hline 5481.252 & & $\mathrm{Fe}$ & I & 1058 & 5510.001 & & N1 & I \\
\hline 5481.396 & & $M n$ & I & 4,31 & 5510.174 & & $M n$ & I \\
\hline 5481.426 & & $\mathrm{Ti}$ & I & 265 & 5510.23 & $\mathbf{P}$ & $\mathrm{Fe}$ & I \\
\hline 5481.451 & & $\mathrm{Fe}$ & I & 1061 & 5510.58 & & Gd & I I \\
\hline 5481.862 & & $\mathbf{T} \mathbf{1}$ & I & 106 & 5510.68 & & $\mathrm{Cr}$ & I I \\
\hline 5481.989 & & $\mathrm{Sc}$ & I & 16 & 5511.795 & & $\mathbf{T 1}$ & I \\
\hline 5482.26 & $\mathbf{P}$ & $\mathrm{Fe}$ & I & 873 & 5512.085 & & $\mathrm{Ce}$ & I I \\
\hline 5482.27 & & La & II & 4 & 5512.277 & & $\mathrm{Fe}$ & I \\
\hline 5482.471 & & $\mathbf{v}$ & I & 2 & 5512.40 & $\mathbf{P}$ & $\mathrm{Fe}$ & I \\
\hline 5483.111 & & $\mathrm{Fe}$ & I & 1061 & 5512.529 & & T1 & I \\
\hline 5483.354 & & Co & I & 39 & 5512.69 & & $\mathrm{Cr}$ & I \\
\hline 5483.55 & & L1 & I I & 1 & 5512.71 & & 0 & I \\
\hline 5483.56 & & $\mathbf{P}$ & I I & 23 & 5512.979 & & $\mathrm{Ca}$ & I \\
\hline 5483.962 & & Co & I & 175 & 5513.86 & $\mathbf{P}$ & $\mathrm{Fe}$ & I \\
\hline 5484.618 & & Sc & I & 16 & 5514.215 & & Sc & I \\
\hline 5485.6 & & $\mathrm{Fe}$ & I I I & 68 & 5514.350 & & $T 1$ & I \\
\hline 5485.65 & & L1 & I I & 1 & 5514.536 & & T1 & I \\
\hline 5485.699 & & $\mathrm{Nd}$ & I I & 79 & 5514.712 & & w & I \\
\hline 5486.136 & & $\mathrm{Sr}$ & I & 9 & 5514.80 & & N1 & I \\
\hline 5486.6 & & 0 & I & 63 & 5515.083 & & $\mathbf{v}$ & I \\
\hline 5486.86 & & $\mathrm{La}$ & I I & 68 & 5515.371 & & v & I \\
\hline 5487.00 & & v & I I & 53 & 5515.990 & & Co & I \\
\hline 5487.16 & & $\mathrm{Fe}$ & I & 1143 & 5516.09 & & $\mathrm{Sm}$ & I \\
\hline 5487.49 & $\mathbf{P}$ & $\mathrm{Fe}$ & I & 870 & 5516.29 & $\mathbf{P}$ & $\mathrm{Fe}$ & I \\
\hline 5487.52 & $\mathbf{P}$ & $\mathrm{Fe}$ & I & 1064 & 5516.771 & & Mn & I \\
\hline 5487.747 & & $\mathrm{Fe}$ & I & 1025 & 5517.08 & & $\mathrm{Fe}$ & I \\
\hline 5487.915 & & $\mathbf{v}$ & I & 129 & 5518.11 & $\mathbf{P}$ & Ti & I \\
\hline 5488.14 & $\mathbf{P}$ & $\mathrm{Fe}$ & I & 1183 & 5518.491 & & $\mathrm{Ce}$ & II \\
\hline 5488.210 & & $\mathrm{~T} \mathbf{1}$ & I & 265 & 5518.57 & $\mathbf{P}$ & $\mathrm{Fe}$ & I \\
\hline 5488.97 & $\mathbf{P}$ & $\mathrm{Cr}$ & I I & 35 & 5518.74 & & $\mathbf{s}$ & II \\
\hline 5489.85 & $\mathbf{P}$ & $\mathrm{Fe}$ & I & 1148 & 5519.047 & & $\mathrm{Ba}$ & $\mathbf{I}$ \\
\hline 5490.151 & & $\mathbf{T} \mathbf{1}$ & I & 107 & 5519.72 & $\mathbf{P}$ & $\mathrm{Fe}$ & I I \\
\hline
\end{tabular}


I A Type Blement Multiplet No.

I A Type Blement Multiplet No.

$\begin{array}{lllr}5587.865 & & \text { N1 I } & 70 \\ 5587.9 & & \text { Fe III } & \\ 5588.07 & \text { P } & \text { Fe I } & 110 \\ 5588.25 & & \text { P II } & 27 \\ 5588.757 & & \text { Cs I } & 2 \\ 5589.00 & \text { P } & \text { Fe I } & 1160 \\ 5589.384 & & \text { N1 I } & 20 \\ 5590.120 & & \text { Ca I } & 21 \\ 5590.73 & & \text { HF II } & 48 \\ 5580.744 & & \text { Co I } & 80 \\ & & & \\ 5591.322 & & \text { Sc I } & 18 \\ 5591.38 & \text { P } & \text { Fe II } & 55 \\ 5592.146 & & \text { N1 I } & 250 \\ 5592.283 & & \text { N1 I } & 6 \\ 5592.37 & & \text { O III } & 5 \\ 5592.409 & & \text { V I } & 37 \\ 5582.962 & & \text { V I } & \\ 5593.23 & & \text { AI II } & 16 \\ 5593.735 & & \text { N1 I } & 200 \\ 5594.425 & & \text { Nd II } & 79\end{array}$

$\begin{array}{llll}5557.453 & & \text { V I } & 1 \\ 5557.80 & \text { P } & \text { Fe I } & 164,1164 \\ 5557.95 & & \text { AI I } & 6 \\ 5557.854 & & \text { Fe I } & 1163 \\ 5568.31 & & \text { As II } & 2 \\ 5658.752 & & \text { V I } & 77 \\ 5558.825 & & \text { Co I } & 168 \\ 5558.06 & & 8 \text { II } & 61 \\ 5559.64 & \text { P } & \text { Fe I } & 282 \\ 5560.250 & & \text { Fe I } & 1164\end{array}$

$\$ 560.37$ N I

5660.548

8560.68

$8561.670 \quad$ V I

$\mathbf{5 5 6 2 . 0 2 \quad V \text { II }}$

$8562.12 \quad P \quad$ Fe I

6062.712

$B 663.604$

\section{Ne I}

714
1059
63

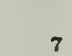

Bc II

Fe I

Fe I

1281

344
1064

1161
69

34
1183

24
740

6

1
6

5594.425

$5594.468 \quad \mathrm{Ca} I \quad 21$

$\begin{array}{llll}5594.681 & & \text { Pe I } & 1182 \\ 5598.06 & P & \text { Fe I } & 1314\end{array}$

5697.21 Gd II 95

$6587.87 \quad$ Cr I 239

$\begin{array}{llr}5597.92 & \text { T1 I } & 229 \\ 5588.303 & \text { Fo I } & 1183\end{array}$

$\begin{array}{lllr}5588.303 & & \text { Fo I } & 1183 \\ 6588.47 & \text { P } & \text { Fo I } & 113\end{array}$

$\begin{array}{llll}5688.487 & & C a & \\ 5600 & P & 0 & V\end{array}$

$\begin{array}{lll}5600.038 & \text { N1 I } & 218\end{array}$

$5600.242 \quad$ Fe I $\quad 866,1108$

5601.285

5602

$5602.54-P$ P

$5602.788 \quad$ P. $\quad$ Fe I $\quad 1062$

$\begin{array}{llr}5602.846 & \mathrm{Ca} I & 21 \\ 5602.855 & \mathrm{Fe} \mathrm{I} & 686\end{array}$

3603.651

26,116

3604.205

Nd I
V I

45
85

112,1023

$\mathrm{Fe} \mathrm{I}$

6863.68

8564.881

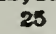

0564.94

606.30

8585.476

6865.86

6565.708

6666.08

6866.82

(5) I

3 II

III

20

18
6
63

63
229

0

af II

Fe $I$

5566.82

6667.401

5687.815

6868.07

6668.14

6668.71

6608.8

6568.81

6.669 .625

6570.06

5870.46

6571

6572.848

5673

6573. 10

8673.3

0574.41

6576.097

6.876 .61

5577.03

1183

625

Po I

90

If II

Fo II

209

Fo I

Fo I

1068

Fo I

Cl I:

Fo I

Fo

1026
80

869
686

345

6677.70

6578.734

6679.85

6879.3

6680.6

6881.87

681.971

5682

5683.33

Mo I

$\begin{array}{ll}\text { N } & \text { IV } \\ \text { Fo } & \text { I } \\ 0 & \text { V }\end{array}$

Fo I

Fo III

Cr I

Fo I

81 II

4

13
686

1061

120
686

686
8
1314

1314

1 II

8 II

Fo I

I I

Ca I

$0 \mathrm{~V}$

6583.68

P II

5883.97 F Fo

3684.490

6884.720

3684.768

5886.007

8686.16

0886.709

5887.38

$68 \times 87.082$

I

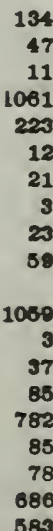

Po I

$3604.943 \quad$ V I 37

$\begin{array}{llll}5605.91 & \text { P } & \text { Pe II } & 51 \\ 5606 & \text { P } & 0 & \text { V }\end{array}$

5608.11

5807.00

P Fo II 2

P $\quad \mathrm{FeI}$

5608.98

6608.19

$0 \mathrm{~V}$

Fo I

Cr I

70

5625.326

5625.43

$5625: 74$
5626.014

5626.60

5627.08

5627.49

5627.628

5628.347

5628.645

5630.14

5631.404

5631.707

5631.72

5632.25

5632.468

5633.970

$\mathbf{5 6 3 4 . 5 3}$

Type Blement

Multiplet No.

$\begin{array}{llll}8609.97 & P & \text { Fo I } & 868\end{array}$

5610.01 P Cr II

$\$ 610.257$

5610.36 I II

8610.83 La II

6011.38 P Fo I

$3013.19 \quad 41$ II 77

$\begin{array}{llll}0613.688 & & \text { Co II } & 32 \\ 5613.70 & P & \text { Fo I } & 1282\end{array}$

$\begin{array}{llll}6614.29 & P & \text { Fo I } & 1314\end{array}$

$6614.303 \quad$ NQ II $\quad 87$

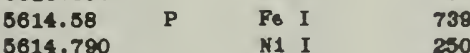

$\begin{array}{llll}5615.18 & P & \mathrm{Pe} I & 1143\end{array}$

5615.308 Fe I 208

$\begin{array}{llll}5615.64 & P & \text { Cr I } & 239 \\ 5615.652 & & \text { Fo I } & 680\end{array}$

5616.21 od II 61

5616.84

3616.63

I I

$6617.14 \quad P \quad$ I 1088

5617.22 t 1 (

$\begin{array}{llr}5617.91 & \text { Od I } & 3 \\ 5618.646 & \text { Fo I } & 1107\end{array}$

$\begin{array}{lllr}6618.646 & & \text { Fo I } & 1107 \\ 6618.23 & P & \text { Fo I } & 923\end{array}$

$5619.60 \quad$ Fe I 1161

5620.04

8620.10

5620.527

Po. I 1020,1200

20

5634.84

8635.85

5636.00

5636.235

5636.708

5637.121

5637.734

5638.266

5638.82

5639.492

$\begin{array}{ll}V & I \\ V & I\end{array}$

N1 I

N I

$\begin{array}{ll}\text { A } & \text { II } \\ \text { V } & \text { I }\end{array}$

Cr II

Fe I

Fe II

N1 I

Cr I

I I

Tw I

Sn

Gd I

$\checkmark$ I

$\begin{array}{ll}\mathrm{Fe} & I \\ \mathrm{Fe} & \mathrm{I}\end{array}$

37

39

24

121
37

22

1084

57
37

215

203
12

1158

5639.96

5640.32

5640.46

5640.971

5641.112

3641.464

5641.880

5642.01

5642.362

CI II

Fe I

Fe I

Ru I

N1 I

Co I

$\mathrm{Fe}$

N1 I

31 II

S II

Fe I

Fe 1

Sc II

N1 I

Fe I

N1 I

III

Cr

N1

$\mathrm{Fe} \mathrm{I}$

5643.099 N1

584.089

$\begin{array}{lll}5643.94 & P & F e \\ 5644.137 & & \text { T1 }\end{array}$

$5644.35 \quad P \quad \mathrm{Fe}$

5644.84 Gd II

8645.665

$S$ II

811

115

1314

1281

5646.112 
I A Type Element Multiplet No.

\begin{tabular}{|c|c|c|c|c|c|c|c|c|}
\hline 5661.97 & $\mathbf{P}$ & $\mathrm{Fe}$ & I & 1109 & 5701.138 & & S1 & I \\
\hline 5662.154 & & $\mathbf{T} \mathbf{1}$ & I & 249 & 5701.35 & & Gd & I \\
\hline 5662.51 & & C & II & 15 & 5701.375 & & S1 & II \\
\hline 5662.525 & & $\mathrm{Fe}$ & I & 1087 & 5701.46 & $\mathbf{P}$ & $\mathbf{C r}$ & II \\
\hline 5662.58 & $\mathbf{P}$ & $\mathrm{Cr}$ & I I & 34 & 5701.553 & & $\mathrm{Fe}$ & I \\
\hline 5662.891 & & T1 & I & 269 & 5702.244 & & Nd & I I \\
\hline 5662.94 & & $\mathrm{Fe}$ & I & 924 & 5702.307 & & $\mathrm{Cr}$ & I \\
\hline 5662.95 & & $\mathrm{Y}$ & II & 38 & 5702.434 & & $\mathrm{Fe}$ & I \\
\hline 5664.017 & & $\mathrm{N1}$ & I & 272 & 5702.666 & & T1 & I \\
\hline 5664.040 & & $\mathrm{Cr}$ & I & 203 & 5703.09 & $\mathbf{P}$ & $\mathrm{Fe}$ & I \\
\hline 5664.55 & & $\mathrm{zr}$ & I & 47 & 5703.32 & & La & II \\
\hline 5664.73 & & $\mathrm{~s}$ & II & 11 & 5703.562 & & $\mathrm{v}$ & I \\
\hline 5665.601 & & S1 & I & 10 & 5705.32 & $\mathbf{P}$ & $\mathrm{Fe}$ & I \\
\hline 5666.64 & & $N$ & II & 3 & 5705.48 & & $\mathrm{Fe}$ & I \\
\hline 5666.78 & $\mathbf{P}$ & $\mathrm{N} 1$ & I & 233 & 5705.988 & & $\mathrm{Fe}$ & I \\
\hline 5666.837 & & $\mathrm{Fe}$ & I & 1053,1060 & 5706.11 & $\mathbf{P}$ & $\mathrm{Fe}$ & I \\
\hline 5667.164 & & $\mathrm{Sc}$ & II & 29 & 5706.11 & & $\mathbf{s}$ & I \\
\hline 5667.67 & $\mathbf{P}$ & $\mathrm{Fe}$ & I & 209 & 5706.206 & & $\mathrm{Nd}$ & II \\
\hline 5668.369 & & $\mathbf{v}$ & I & 37 & 5706.375 & & S1 & II \\
\hline 5668.868 & & Nd & II & 84 & 5706.973 & & $\mathbf{v}$ & I \\
\hline 5668.901 & & $\mathrm{Ce}$ & II & 23 & 5707.03 & & $\mathrm{Ca}$ & I \\
\hline 5669.030 & & Sc & II & 29 & 5707.068 & & $\mathrm{Fe}$ & I \\
\hline 5669.590 & & S1 & II & & 5707.25 & $\mathbf{P}$ & $\mathrm{Fe}$ & I \\
\hline 5669.8 & P Forb & $\mathrm{Na}$ & I & 7 & 5707.70 & $\mathbf{P}$ & $\mathrm{Fe}$ & I \\
\hline 5669.945 & & $\mathbf{N 1}$ & I & 250 & 5708.109 & & $\mathrm{Fe}$ & I \\
\hline 5670.827 & & $\mathrm{~V}$ & I & 36 & 5708.199 & & T1 & I \\
\hline 5671.54 & & La & I I & 95 & 5708.280 & & Nd & II \\
\hline 5671.62 & $\mathbf{P}$ & $\mathrm{Cr}$ & I I & 22 & 5708.437 & & S1 & I \\
\hline 5671.805 & & Sc & I & 12 & 5708.600 & & Sc & I \\
\hline 5672.28 & $\mathbf{P}$ & $\mathrm{Fe}$ & I & 1234 & 5709.378 & & $\mathrm{Fe}$ & I \\
\hline 5673.58 & & $\mathrm{Hf}$ & II & 112 & 5709.559 & & N1 & I \\
\hline 5675.08 & $\mathbf{P}$ & $\mathrm{Fe}$ & I & 583 & 5709.93 & $\mathbf{P}$ & $\mathrm{Fe}$ & I \\
\hline 5675.3 & P Forb & $\mathrm{Na}$ & I & 7 & 5709.976 & & $\mathrm{Tm}$ & II \\
\hline 5675.413 & & $\mathbf{T 1}$ & I & 249 & 5710.76 & & $\mathbf{N}$ & II \\
\hline 5675.853 & & $\mathrm{Tm}$ & I & & 5711.0735 & & $\mathrm{Mg}$ & I \\
\hline 5676.02 & & $\mathbf{N}$ & II & 3 & 5711.0831 & & $\mathrm{Mg}$ & I \\
\hline 5677.68 & $\mathbf{P}$ & $\mathrm{Fe}$ & I & 1057 & 5711.0912 & & $\mathrm{Mg}$ & I \\
\hline 5678.04 & $\mathbf{P}$ & $\mathrm{Fe}$ & I & 1290 & 5711.754 & & $\mathrm{Sc}$ & I \\
\hline 5678.38 & $\mathbf{P}$ & $\mathrm{Fe}$ & I & 982 & 5711.852 & & T1 & I \\
\hline 5678.42 & & $\mathrm{Cr}$ & II & 189 & 5711.867 & & $\mathrm{Fe}$ & I \\
\hline 5678.60 & $\mathbf{P}$ & $\mathrm{Fe}$ & I & 113 & 5711.905 & & N1 & I \\
\hline 5679.023 & & $\mathrm{Fe}$ & I & 1183 & 5712.150 & & $\mathrm{Fe}$ & I \\
\hline 5679.56 & & $\mathrm{~N}$ & II & 3 & 5712.39 & & $\mathbf{L a}$ & II \\
\hline 5679.908 & & T1 & I & 269 & 5712.635 & & $\mathrm{Cr}$ & I \\
\hline 5680.26 & & $\mathrm{Fe}$ & I & 1026 & 5712.778 & & $\mathrm{Cr}$ & I \\
\hline 5680.93 & & $\mathrm{zr}$ & I & 25 & 5713.895 & & T1 & I \\
\hline 5681.198 & & $\mathrm{Cr}$ & I & & 5714.88 & $\mathbf{P}$ & $\mathrm{Fe}$ & I \\
\hline 5682.204 & & $\mathbf{N i}$ & I & 232 & 5715.086 & & $\mathrm{~N} 1$ & I \\
\hline 5682.483 & & $\mathrm{Cr}$ & I & 239 & 5715.107 & & $\mathrm{Fe}$ & I \\
\hline 5682.633 & & $\mathrm{Na}$ & I & 6 & 5715.123 & & T1 & I \\
\hline 5682.88 & & $\mathrm{Ca}$ & I & & 5715.47 & $\mathbf{P}$ & $\mathrm{Fe}$ & I \\
\hline 5684.190 & & Sc & II & 29 & 5715.80 & $\mathbf{P}$ & $\mathrm{Fe}$ & I \\
\hline 5684.523 & & S1 & I & 11 & 5716.450 & & T1 & I \\
\hline 5685.86 & $\mathbf{P}$ & $\mathrm{Fe}$ & I & 1281 & 5717.30 & & Sc & I \\
\hline 5686.21 & & $\mathbf{N}$ & II & 3 & 5717.845 & & $\mathrm{Fe}$ & I \\
\hline 5686.532 & & $\mathrm{Fe}$ & I & 1182 & 5717.99 & & $\mathrm{Ca}$ & I \\
\hline 5686.826 & & Sc & I & 12 & 5718.120 & & Nd & II \\
\hline 5688.193 & & $\mathrm{Na}$ & I & 6 & 5719.18 & & Hf & I \\
\hline 5688.205 & & $\mathrm{Na}$ & I & 6 & 5719.2254 & & $\mathrm{Ne}$ & I \\
\hline 5688.47 & & $\mathrm{Ca}$ & I & & 5719.821 & & $\mathrm{Cr}$ & I \\
\hline 5688.525 & & Nd & II & 79 & 5720.445 & & $\mathrm{~T} 1$ & I \\
\hline 5688.593 & & Co & I & 90 & 5720.613 & & 0 & I \\
\hline 5688.856 & & S1 & II & & 5720.79 & $\mathbf{P}$ & $\mathrm{Fe}$ & I \\
\hline 5689.22 & & Mo & I & 5 & 5720.89 & $\mathbf{P}$ & $\mathrm{Fe}$ & I \\
\hline 5689.465 & & T1 & I & 249 & 5721.02 & $\mathbf{P}$ & $\mathrm{Cr}$ & II \\
\hline 5690.07 & $\mathbf{P}$ & $\mathrm{Fe}$ & I & 1281 & 5721.70 & $\mathbf{P}$ & $\mathrm{Fe}$ & I \\
\hline 5690.470 & & S1 & I & 10 & 5721.71 & $\mathbf{P}$ & $\mathrm{Fe}$ & I \\
\hline 5691.38 & $\mathbf{P}$ & $\mathrm{Fe}$ & II & 47 & 5721.99 & & Gd & II \\
\hline 5691.509 & & $\mathrm{Fe}$ & I & 1087 & 5722.56 & $\mathbf{P}$ & $\mathrm{Fe}$ & II \\
\hline 5691.52 & & N1 & I & 228 & 5722.65 & & Al & III \\
\hline 5691.69 & $\mathbf{P}$ & $\mathrm{Fe}$ & I & 1084 & 5723.66 & $\mathbf{P}$ & $\mathrm{Fe}$ & I \\
\hline 5691.71 & & $\mathbf{A}$ & II & 134 & 5723.87 & $\mathbf{P}$ & $\mathbf{T} 1$ & II \\
\hline 5691.99 & $\mathbf{P}$ & T1 & II & 79 & 5724.073 & & Sc & I \\
\hline 5694.46 & & He & II & 8 & 5724.37 & & $\mathbf{A}$ & II \\
\hline 5694.730 & & $\mathrm{Cr}$ & I & 239 & 5724.445 & & $\mathrm{Fe}$ & I \\
\hline 5694.998 & & N1 & $\mathbf{I}$ & 220 & 5725.633 & & $\mathbf{v}$ & I \\
\hline 5696.0 & & C & I I I & 2 & 5725.95 & $\mathbf{P}$ & $\mathrm{Fe}$ & II \\
\hline 5696.10 & $P$ & $\mathrm{Fe}$ & I & 1179 & 5727.024 & & $\mathrm{v}$ & I \\
\hline 5696.11 & $\mathbf{P}$ & $\mathrm{Fe}$ & II & 18 & 5727.29 & & $\mathbf{L a}$ & II \\
\hline 5696.22 & & Gd & $\mathbf{I}$ & 3 & 5727.662 & & $\mathbf{v}$ & I \\
\hline 5696.47 & & Al & III & 2 & 5727.69 & & $\mathbf{P}$ & I I \\
\hline 5696.63 & & $\mathrm{~s}$ & I & 11 & 5727.75 & & $\mathrm{Fe}$ & I \\
\hline 5698.05 & & $\mathrm{Fe}$ & I & 867 & 5728.32 & & Gd & II \\
\hline 5698.330 & & $\mathrm{Cr}$ & I & 239 & 5728.74 & $\mathbf{P}$ & $\mathrm{Fe}$ & II \\
\hline 5698.37 & & $\mathrm{Fe}$ & I & 1130 & 5728.91 & & $\mathrm{X}$ & II \\
\hline 5698.509 & & V & I & 35 & 5729.203 & & $\mathrm{Cr}$ & I \\
\hline $5700 \cdot 14$ & & Sc & I & 12 & 5730.67 & & $\mathbf{N}$ & II \\
\hline 5700.24 & & $s$ & I & 11 & 5731.103 & & 0 & I \\
\hline 5700.240 & & $\mathrm{Cu}$ & I & 2 & 5731.257 & & v & I \\
\hline 5700.514 & & $\mathrm{Cr}$ & I & 203,228 & 5731.70 & & $\mathrm{Ca}$ & I \\
\hline
\end{tabular}




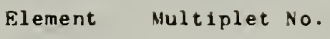

I A Tjpe Element Multiplet No.

$\begin{array}{lllr}5770.17 & \text { P } & \text { Fe I } & 12368 \\ 5772.258 & & \text { SI I } & 17 \\ 5772.402 & & \text { V I } & 92 \\ 5772.676 & & \text { Cr I } & 227 \\ 5773.75 & \text { P } & \text { Fe I I } & 165 \\ 5774.037 & & \text { TI I } & 309 \\ 5775.090 & & \text { Fe I } & 1087 \\ 5776.670 & & \text { V I } & 36 \\ 5776.76 & & \text { Ta I } & 5 \\ 5777.622 & & \text { Ba I } & 9 \\ & & & \\ 5777.77 & & \text { Cr I } & 257 \\ 5778.47 & & \text { Fe I } & 209\end{array}$

$\begin{array}{lllr}8801.71 & & \text { HF II } & 59 \\ 5804.020 & & \text { Nd II } & 79 \\ 5804.06 & & \text { Fe I } & 959 \\ 5804.265 & & \text { TI I } & 309 \\ 5804.4488 & & \text { Ne I } & 19 \\ 5804.478 & & \text { Fe I } & 1087 \\ 5804.91 & \text { P } & \text { Fe II } & 165 \\ 5805.233 & & \text { NI I } & 234 \\ 5805.76 & & \text { C I } & 18 \\ 5805.76 & \text { P } & \text { Fe I } & 1313\end{array}$

5805.77 La II 1

209

5806.31 La II

$5806.56 \quad$ La II

5806.727 Fe I

$5806.75 \quad$ Si II

Mn I

S1 I

Fe I 552

9
552
217
214

$\begin{array}{llc}\text { T I } & \text { I } & 214 \\ \text { Fe } & \text { I } & 552,922,1159\end{array}$

$5807.05 \quad$ Gd II

5807.

5780.77

5780.778

$52,922,11$

$\begin{array}{llll}5807.22 & \text { P } & \text { Fe I } \\ 5807.79 & \text { P } & \text { Fe I }\end{array}$

31
90
1180
8
21
112
142
581
552

Cr I 188

5780.97

Cr I

119,188
34

Y II
TI

Cr I

Cu I

Tm I I

$\begin{array}{llc}V & I & 35,12 \tau\end{array}$

Cr I $\quad 227$

$\begin{array}{lll}5807.97 & P & \text { Fe I }\end{array}$

5808.31 La II

5808.63 La II

5809.249

3809.75

HS II

188

5782.132
5782.356

5782.601

5783.112
5783.15

$\mathrm{VI}$

227

$5809.88 \quad \mathrm{P} \quad \mathrm{Fe}$

$5811.10 \quad$ Ta I

5811.93

$\mathrm{Fe} I$

5783.509

5783.934

5784.18

5784.69

5785.0

$\mathbf{5 7 8 5 . 0 0 2}$

5785.08

5785.64

5785.67

$\mathrm{Cr} I$

Ba II

Fe I

Fe II 215

$\begin{array}{lll}C r & I & 188 \\ \text { Cg } & \text { I } & 24\end{array}$

SI II

T1 I 309

$\begin{array}{ll}\text { Cr } & \text { I } \\ \text { Cr } & \text { I } \\ \text { Ti } & \text { I }\end{array}$

Ti I

$\mathrm{V}$ I

$\mathrm{Fe} I$

Fe I

625

$\begin{array}{lll}\text { Cr } & \text { I } & 188 \\ \text { Cr } & I & 119\end{array}$

1

$5811.93 \quad P \quad$ Fe II

$\begin{array}{llll}5812 & \text { P } & \text { N } & \text { IV } \\ 5812.14 & & \text { C } & \text { IV }\end{array}$

$5812.81 \quad$ A II

5812.827

$\begin{array}{lll}5813.67 & \text { P } & \text { Fe I } \\ 5 & & \text { Fe II }\end{array}$

5814.00

$5814.62 \quad P \quad$ T1 II

5814.80

$\begin{array}{ll}\text { TI } & \text { II } \\ \text { Fe } & \text { I }\end{array}$

4
118
982

14

73
1084

1084
3

1022

5815.16 Fe I 1055

$\begin{array}{llll}5815.23 & P & \text { Fe } & \\ 5815.42 & 1234\end{array}$

$\begin{array}{rllr}5815.42 & P & \text { Fe I } & 1053 \\ 5815.85 & & \text { Gd II } & 112\end{array}$

$\begin{array}{llll}5816.07 & P & \mathrm{Fe} \mathrm{I} & 1127\end{array}$

$\begin{array}{llr}5816.36 & \text { Fe I } & 1179 \\ 5816.48 & \text { N I } & 32\end{array}$

5816.48

5817.063

$\begin{array}{lr}\text { Mn. I } \\ V & I\end{array}$

5817.532

$\begin{array}{lrr}\text { V I } & 92 \\ \end{array}$

5817.87

5818.74

5819.22

5819.93

5820.155

5820.99

5823.13

5823.17

5823.67

C II

Eu II

$\begin{array}{ll}\mathrm{S} & \text { II } \\ \mathrm{V} & \text { II }\end{array}$

Gd II

C II

Fe II

Gd I

Fe I

1234

$\begin{array}{ll}\text { Cr } & \text { I } \\ \text { Mo } & \text { I } \\ \text { S1 } & \text { I }\end{array}$

Si I

e II

$\begin{array}{ll}\mathrm{C} & \text { I } \\ \mathrm{Fe} & \text { I } \\ \mathrm{N} & \mathrm{IV}\end{array}$

N IV

Fe II

Ni I

243

$\begin{array}{rrrrr}5826.61 & P & \mathrm{Fe} & \mathrm{I} & 169 \\ & & \mathrm{C} & \mathrm{III} & \end{array}$

5827.1

5827.24

5827.80

$\begin{array}{llll}5827.80 & & \text { S1 II } & \\ 5827.89 & \text { P } & \text { Fe I } & 55\end{array}$

$\begin{array}{lllll}5828 & P & \text { N } & \text { IV } & 15\end{array}$

$\begin{array}{llll}5829.12 & P & \text { Fe II } & 165\end{array}$

$\begin{array}{llll}5829.53 & \text { N } & \text { I } & 32\end{array}$

$5830.719 \quad$ V I $~ 142$

5832.47
5833.65

5833.93

5834.06

5834.93

5835.10

5835.41

33,250

$\begin{array}{llr}\text { N1 } & 1 & 233,250 \\ \text { T1 } & \text { I } & 309\end{array}$

$\begin{array}{lll}\text { TI I } & 309 \\ \text { Fe III } & 114\end{array}$

Fe I 209

Fe II

Fo II

Fe I

165

57
1084

$\begin{array}{lllll}5835.43 & P & \text { Fe } & \text { II } & 58\end{array}$

$\begin{array}{llll}5835.50 & P & \text { Fe II } & 182\end{array}$

$\begin{array}{llll}5835.58 & P & F e ~ & 343\end{array}$

5835.61

5836.31

5837.29

5837.709

5838.418

5838.66

$\mathbf{5 8 3 9 . 7 8}$

22
2
1129
959
119
105

$5840.17 \quad$ Gd II $\quad 112$

$5841.01 \quad N$ I 32

$\begin{array}{lll}5841.86 & \text { Cr II } & 198\end{array}$

$\begin{array}{lll}5842.23 & \text { HF II } & 50 \\ 5842.391 & \text { Nd II } & 86\end{array}$

$5843.21 \quad$ Cr I $\quad 119$

$\begin{array}{llll}5843.77 & \text { C II } & 22\end{array}$

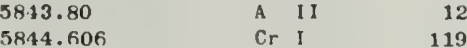

$\begin{array}{rrr}5844.879 & \text { re } I & 113\end{array}$

$5815.27 \quad P \quad$ Fe I 1313

$5845.71 \quad$ Gd II $\quad 112$

5846.12

5846.306

3846.575

$5 \times 47.010$

5848.09

5848.95

5849.67

5850.286

5851.63

5852.19

5852.4878

5853.18

5853.62 AI II

5853.675 Ba II

$5854.1 \quad$ Fe III

$5854.16 \quad$ N I

$5854.27 \quad \mathrm{Cr}$

$5854.31 \quad P \quad$ Sc II

$\begin{array}{lll}5855.126 & \text { Fe I } & 1179\end{array}$

$5855.24 \quad$ Gd II 112

$\begin{array}{llr}5855.24 & \text { Gd } & 11 \\ 5856.084 & \text { Fe I } & 1128\end{array}$

$5856.09 \quad$ C II $\quad 22$

$5856.22 \quad$ Gd I $\quad 3$

$5856.96 \quad$ Gd $\mathrm{II} \quad 60$

$\begin{array}{lll}5857.454 & \mathrm{Ca} I & 47\end{array}$

$\begin{array}{llll}5857.755 & & \text { Ni I } & 228\end{array}$

C III 20

$\begin{array}{rrrr}5858.27 & \text { P } & \text { Fe I } & 170 \\ 5858.28 & & \text { Mo I } & 5\end{array}$

$\begin{array}{llll}5858.28 & & \text { Mo I } & \\ 5858.77 & P & \text { Fe I } & 1084\end{array}$

5859.20 Fe I 1084

$5859.23 \quad P \quad$ Si I

$\begin{array}{llll}5859.608 & & \text { Fe I } & 1181 \\ 5859.96 & P & \text { Fe I } & 1054\end{array}$

$\begin{array}{lll}\mathbf{5 8 6 0 . 7 3} \text { Gd II } & 58\end{array}$

$\begin{array}{lllll}5860.92 & \text { P } & \text { Ti II } & 79\end{array}$

$\begin{array}{llll}5861.11 & P & \text { Fe I } & 1084\end{array}$

$\begin{array}{llr}5861.53 & \text { Al II } & 11 \\ 5862.357 & \text { Fe I } & 1180\end{array}$

$\begin{array}{lll}5862.80 & \text { V II } & 91\end{array}$

5863.70 LA II 62

$\begin{array}{lll}5863.96 & \mathrm{Cr} \text { I } & 185\end{array}$

$5863.97 \quad$ NI I 253

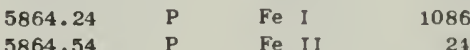

$\begin{array}{llll}5866.453 & \text { T1 I } & 72\end{array}$

$5867.01 \quad$ P $\quad$ Fe I $\quad 1203$

$5867.497 \quad$ SI II $\quad 8$

5867.81 Al II 41

5868.404 S1 II

$\begin{array}{llll}5870.65 & P & \mathrm{Fe} & 1235\end{array}$

$\begin{array}{llr}5871.04 & \text { Fe I } & 150 \\ 5871.289 & \text { Fe I } & 1055\end{array}$

$\begin{array}{lllll}5871.6 & P & C & \text { III } & 20\end{array}$

$5871.81 \quad$ Gd II $\quad 79$

$5872.73 \quad P \quad$ Fe I $\quad 552$

5872.98 Eu II

$\begin{array}{lll}587 \mathrm{~J} .211 & \text { Fe I } & 1087\end{array}$

5874.00

5875.6

5875.618

$5875.65 n$

5875.999

La II

Fe III

$\begin{array}{llll}5876.27 & P & \text { Fe } I & 1084\end{array}$

5876.55

5877.26 Gd II

$5877.770 \quad$ Fe I 1083

$\begin{array}{llll}5879.49 & P & \text { Fe } I & 1201\end{array}$

$\begin{array}{lll}5879.79 & \text { Zr I } & \\ 58800 & \text { Fe I } & 1201\end{array}$

$5880.306 \quad$ T1 I $\quad 71$

$\begin{array}{lllr}5880.63 & & \text { La II } & 35 \\ 5881.28 & \text { P } & \text { Fe I } & 1178\end{array}$ 


\section{A Type Element Mult1plet No.}

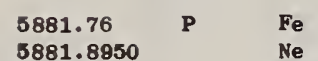

Ne I

5883.06 P $\quad$ Fe

5883.421 Co I

5883.838

$\mathbf{6 8 8 4 . 4 5}$

8884.59

5885.61

6887.46

5889. $95:$

3889.97

8800.02

P Sc

5890.487 Co

5891.18

5891.36

5891.528

5891.5

5891.65

581.65

5891.

5892.4

589.71

5882.80

5892.878

5893.24

5893.42

5894.1

5894.351

5895.007

5895.616

5895.88

5895.90

5895. 923

5897.62

5898. 212

6899.295

5001.0

5801.53

5801.85

5802.182

5802.52

5903.317

5803.6

5804.07

5805.673

5907.36

5908.24

5908.25

5909.38

5809.98

6911.45

5813.35

5813.730

5813.87

5814.16

5914.28

5914.92

5915.123

5915.266

5915.551

5815.83

5916.250

5916. 364

5916.73

5918.548

5918.93

5818.60

5820.0

5820.520

5922.112

5822.365

5923.930

$6925.81 \quad P \quad N 1$

$\begin{array}{lll}5926.83 & P & \text { Fe } \\ 5927.15 & & S\end{array}$

$\begin{array}{ll}5927.15 & S \text { II } \\ 5827.71 & \text { La II }\end{array}$

$\mathbf{6 8 2 7 . 7 9 8}$

5827.82

6828.50

6928.86

5828.35

5829.5

63
1
1124
90
982
119
112
2
1203
5

1

21
1313

82

Fe I 581

Fe I 1178

Nd II $\quad 86$

Fe III

114

C II 5

Fe II

Fe I

La II

$\mathrm{Fe} I$

Fe I

N1 I

Ge II

C III

Fe I

$\begin{array}{ll}\text { Tw } & \text { I } \\ S & \text { II }\end{array}$

$\begin{array}{ll}S & \text { II } \\ \text { Cr } & \text { II }\end{array}$

$\mathrm{Na} \mathrm{I}$

od II

1201

48
1086

250

63
68

1055

1

20
1
1235

20

198
1

1
88

Fe I $\quad 1259$

T1 I 72

Fe III 115

$\begin{array}{llr}\text { Fe I } & 1083 \\ \text { L } & \text { II } & 107\end{array}$

Cr I

T1 I

Fe II

Gd II

119
1234

71

112

Fe I 1181

T1 I

$\mathrm{Fe} I$

S II

Fe II

Fo I

Gd II

Fe I

T1 I

Cr II

V II

C II

T1 I

Co I

is $I$

Fe I

V II

T1 I

Fe I

C II
III

$\mathrm{Fe} I$

T1 I

Co I

105

44
150

13

652

781

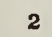

1180,1181

126
44

228
8

8
82

185

170

126
185

185
71

1083

44
115

581

72

55
258

I 42

$\begin{array}{ll}\text { Fe } & \text { I } \\ \text { N } & \text { II }\end{array}$

Fe I

V II

He II

Fe III

1231

21
111

1175

28
1055

9E

$9 E$
68
114
I A Type Blement Multiplet No.

$\mathbf{5 9 2 8 . 7 0 0}$
5930.173
5930.61
5930.68
5931.79
5931.89
5932.05
5932.85
5933.80
$\mathbf{5 9 3 4 . 6 5 8}$

$\begin{array}{llr}\text { Fe I } & 1176 \\ \text { Fe I } & 1180 \\ \text { La I } & 2 \\ \text { La I } & 2 \\ \text { N } & \text { I I } & 28 \\ \text { Fe I } & 1017 \\ \text { Fe I I } & 47 \\ \text { 8 } & \text { II } & 13 \\ \text { Fe I } & 1188 \\ \text { Fe I } & 982\end{array}$

5934.747

5935.23

5935.381

5836.22

5937.806

5939.75

5940.25

5840.68

5940.972

Nd II

Zr I

$\begin{array}{ll}\text { Co } & \text { I } \\ \text { La } & \text { II }\end{array}$

T1 I

Ta I

N II

$\begin{array}{ll}\text { T1 } & \text { I } \\ \text { S II }\end{array}$

Fe I

78

2
55

$N$ I I

T1 I

Fe I

5942.71

5943.11

$5943.58 \quad P \quad$ Fe

5943.62 P Fe

$\begin{array}{lll}5944.01 & & \text { Ta I } \\ 5044.65 & P & T 1\end{array}$

5944.8342 Ne

5946.484

5947.50
5948.30

5948.584

5949.35

5850.13

5850.81

5861.30

5851.45

Co I

Fe I

In II

S1 [

Fe I

Fe I

8 II

V II

\section{Cd II}

$\begin{array}{llll}5951.60 & & \text { Fe I } \\ 5952.19 & P & \text { N II } \\ 5952.39 & & & \text { Fe I I I }\end{array}$

$\begin{array}{llll}5852.39 & & \text { N II } \\ 5852.55 & P & \text { Fe II }\end{array}$

5852.749

5953.162

5953.65

$\begin{array}{lll}5855.37 & \text { Fe I } \\ & \text { Zr I }\end{array}$

5855.682

6956.48

5956.5

5956.702

5957.612

5958.22

5858.23

5958.34

5858.46

5958.63

5959.878

5960.23

5961.91

5963.25

5965.040

5965.474

5965.828

5966.07

5967.77

5968.38

5968.564

5969.64

5971.07

5971.09

5871.28

5971.698

5971.94

5973.37

5973.52

5873.66 $\begin{array}{llll}5974.628 & P & \text { Fe I } & 1055\end{array}$

5875.355

$\mathbf{5 8 7 5 . 5 3 4 0}$

5975.830

5976.18

5976.799

5978.17

5978.543

5978.80 


\begin{tabular}{|c|c|c|c|c|c|c|c|}
\hline I A & Type & Elen & ment & Multiplet No. & I A & Type & Blem \\
\hline 6021.08 & & $\mathrm{Ge}$ & II & 1 & 6080.68 & & Od \\
\hline 6021.18 & $\mathbf{P}$ & Fe & II & 24 & 6080.85 & & $\mathbf{s}$ \\
\hline $6021: 802$ & & Mn & I & 27 & 6081.421 & & $\mathbf{v}$ \\
\hline 6021.82 & & Fe & $\mathbf{I}$ & $63,1079,1085$ & 6081.51 & & $\mathrm{Cr}$ \\
\hline 6022.81 & & As & II & 1 & 6081.72 & $\mathbf{P}$ & $\mathrm{Fe}$ \\
\hline 6023.41 & & $\mathbf{Y}$ & I & 3 & 6.081 .85 & $\mathbf{P}$ & $\mathrm{Fe}$ \\
\hline 6024.066 & & $\mathrm{Fe}$ & I & 1178 & 6082.431 & & Co \\
\hline 6024.15 & & $\mathbf{P}$ & II & 5 & 6082.718 & & Fe \\
\hline 6025.723 & & Pr & II & 39 & 6083.67 & $\mathbf{P}$ & $\mathrm{Fe}$ \\
\hline 6025.73 & & M1 & I & 251 & 6083.82 & & $\mathbf{v}$ \\
\hline 6028.81 & & $\mathbf{v}$ & II & 120 & 6084.11 & & Fe \\
\hline 6027.057 & & $\mathrm{Fe}$ & I & 1018 & 6085.228 & & T1 \\
\hline 6027.23 & & $\mathbf{v}$ & II & 80 & 6085.267 & & $\mathbf{F e}$ \\
\hline 6027.67 & & He & II & 91 & 6085.43 & & La \\
\hline 6027.76 & $\mathbf{P}$ & Fe & I & 1312 & 6086.290 & & N1 \\
\hline 6028.26 & & $\mathbf{v}$ & II & 97 & 6086.55 & & v \\
\hline 6028.64 & & $\mathbf{Z r}$ & II & 136 & 6086.663 & & Co \\
\hline 0028.88 & & $\mathbf{v}$ & II & 128 & 6086.93 & & $\mathbf{v}$ \\
\hline 6029.28 & & $\mathrm{Cr}$ & $\mathbf{I}$ & 242 & 6087.485 & & $\mathbf{v}$ \\
\hline 6029.9971 & & Ne & I & 3 & 6087.76 & & $\mathbf{P}$ \\
\hline 6030.66 & & Ko & I & 5 & 6089.473 & & $\mathbf{v}$ \\
\hline 0031.07 & & $\mathbf{v}$ & II & 97 & 6089.566 & & $\mathbf{F e}$ \\
\hline 6031.68 & & T1 & I & 2 & 6089.69 & & $\mathrm{Cr}$ \\
\hline 6032.124 & & $\mathbf{\Lambda}$ & $I$ & 13 & 6090.184 & & $\mathbf{v}$ \\
\hline 6032.30 & & Fe & III & 117 & 6090.54 & $\mathbf{P}$ & $v$ \\
\hline 6032.67 & & Fe & I & 1082 & 6091.175 & & $\mathbf{T 1}$ \\
\hline 6034.01 & & $\mathbf{P}$ & II & 5 & 6091.74 & $\mathbf{P}$ & $\mathrm{Fe}$ \\
\hline 6034.04 & $\mathbf{P}$ & Fe & I & 1142 & 6092.13 & & 8 \\
\hline 6034.204 & & ce & II & 30 & 6092.814 & & $\mathrm{~T} 1$ \\
\hline 6035.34 & $\mathbf{P}$ & Fe & $I$ & 1125 & 6093.144 & & Co \\
\hline 6035.487 & & Ce & II & 30 & 8093. 66 & & Fe \\
\hline 6036.17 & & $8 c$ & I & & 6094.419 & & Fe \\
\hline 6036.7 & & He & II & 8 & 6094.66 & & $\mathrm{Cl}$ \\
\hline 6039.312 & & N1 & $I$ & 248 & 6086.37 & & C \\
\hline 6039.690 & & $\mathbf{v}$ & I & 34 & 6085.93 & & $\mathbf{v}$ \\
\hline 6041.44 & & не & II & 65 & 6096.689 & & Fe \\
\hline 6041.93 & & 8 & $I$ & 10 & 6097.08 & $\mathbf{P}$ & Fe \\
\hline 6042.092 & & $\mathbf{F e}$ & I & & 6087.12 & & $\mathbf{s}$ \\
\hline 6043.10 & & $\mathbf{P}$ & II & b & 6097.42 & & $\mathbf{v}$ \\
\hline 6043.386 & & $\mathrm{Ce}$ & II & 30 & 6098.28 & $\mathbf{P}$ & Pe \\
\hline 6043.738 & & Fe & I & 864 & 6098.62 & & C \\
\hline 0044.63 & $\mathbf{P}$ & Fe & II & 46 & 6098.665 & & T1 \\
\hline 6046.38 & & $\mathrm{Ta}$ & $I$ & 10 & 6100.04 & & $\mathbf{Z r}$ \\
\hline 6046.497 & & $\mathbf{F e}$ & II & 200 & 6100.23 & $\mathbf{P}$ & Fe \\
\hline 6048.04 & & 8 & I & 10 & 6100.29 & $\mathbf{P}$ & Pe \\
\hline 0046.26 & & 0 & I & 22 & 6100.37 & & Is \\
\hline 6046,46 & & 0 & $I$ & 22 & 6102.178 & & $\mathbf{P e}$ \\
\hline 6047.606 & & $\mathrm{Cr}_{\mathbf{r}}$ & I & 242 & 6102.26 & & 8 \\
\hline 6048.636 & & $\mathbf{v}$ & I & 40 & 6102.58 & & c \\
\hline 6048.110 & & Co & I & 201 & 6102.69 & $\mathbf{P}$ & F• \\
\hline 6049.60 & & Gd & II & 60 & 6102.722 & & $\mathrm{Ca}$ \\
\hline 6049.51 & & Bu & II & 8 & 6103.190 & & Fe \\
\hline 6060.446 & & in & II & 16 & 6103.54 & & Fe \\
\hline 6081.00 & $\mathbf{P}$ & Fe & I & 207 & 8103.68 & & $\Lambda$ \\
\hline 6061.860 & & yn & II & 16 & 6103.642 & & $\mathbf{L}$ \\
\hline 6052.66 & & $\mathrm{~s}$ & $I$ & 10 & 6100.16 & $\mathbf{P}$ & Fe \\
\hline 6002.892 & & in & II & 16 & 6106.381 & & $m$ \\
\hline 6053.48 & & $\mathrm{Cr}_{\mathbf{r}}$ & II & 108 & 6106.19 & & od \\
\hline 6063.680 & & N1 & I & 247 & 6106.25 & & 0 \\
\hline 6004.100 & & $\mathbf{F e}$ & I & 1142 & $\dot{6100.47}$ & & $\mathbf{Z r}$ \\
\hline 6065.087 & & Fe & $\Sigma$ & 1260 & 6106.84 & $\mathbf{P}$ & Fo \\
\hline 0058.118 & & $\mathbf{v}$ & I & 34 & 8106.867 & & $\mathbf{v}$ \\
\hline 6038.76 & $\mathbf{P}$ & $\mathbf{T 1}$ & 1 & 70 & 6107.08 & $\mathbf{P}$ & Fe \\
\hline 6060.20 & $\mathbf{P}$ & sc & I & 20 & 6107.293 & & m \\
\hline 6000.81 & $\mathbf{P}$ & Fe & I & 1081 & 6107.32 & $\mathbf{P}$ & Fe \\
\hline 6001.04 & & Fe & I & 217 & 6108.121 & & M1 \\
\hline 6081.11 & & 11 & 1 & 98 & 6108.8 & $\mathbf{P}$ & in \\
\hline 6069.75 & & Cr & I & 185 & 6109.318 & & Fe \\
\hline 6002.88 & & $\mathbf{z r}$ & I & 3 & 6110.50 & & is \\
\hline 6062.89 & & Fe & $I$ & 63 & 6110.784 & & Ba \\
\hline 6068.117 & & Be & I & 7 & 6111.06 & & N1 \\
\hline 6064.631 & & $\mathbf{T 1}$ & I & 69 & 6111.622 & & $\mathbf{y}$ \\
\hline 6065.487 & & Fe & I & 207 & 6112.26 & & Cr \\
\hline 6066.5 & & $N$ & II & 27 & 6113.33 & & Pe \\
\hline 6068.81 & $\mathbf{P}$ & Fe & I & 581 & 6114.07 & & od \\
\hline 6066.32 & & $\Delta 1$ & II & 82 & 6114.41 & $\mathbf{P}$ & Te \\
\hline 6060.44 & & $\Lambda$ & II & 92 & 6114.6 & & N \\
\hline 6007.13 & & La. & II & 48 & $6114.7 \%$ & & $\mathbf{z r}$ \\
\hline 6007.62 & P & 31 & $I$ & 15 & 6114.02 & & 1 \\
\hline 6068.00 & & Cr & II & 197 & 6116.21 & & $\vec{c}$ \\
\hline 0008.46 & & AI & II & 92 & 6116.04 & $\mathbf{P}$ & Fe \\
\hline 6060.60 & & $\bar{c}_{r}$ & II & 197 & 6116.181 & & N1 \\
\hline 6070.08 & & Cr & II & 105 & 6116.094 & & Co \\
\hline 6078.29 & & 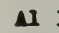 & II & 92 & 6118.00 & & N1 \\
\hline 6074.1 & & म० : & II & 8 & 6118.2 & & He \\
\hline 6074.5377 & & Ne & I & 3 & 6119.008 & & $\mathbf{v}$ \\
\hline 6077.43 & & $\Delta$ & II & 12 & 6119.780 & & M1 \\
\hline 6078.496 & & Fe & I & 1250 & 6120.12 & & $\Lambda$ \\
\hline 9070.02 & & Fe & 1 & 1176 & 6120.26 & $\mathbf{P}$ & Fo \\
\hline 6000.11 & & & & & 6120.80 & & \\
\hline
\end{tabular}


I A

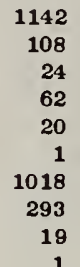

$\begin{array}{llr}6212.04 & \text { Fe I } & 1142 \\ 6212.30 & \text { TI I } & 108 \\ 6213.06 & \text { Zr I } & 24 \\ 6213.438 & \text { Fe I } & 62 \\ 6213.874 & \text { V I } & 20 \\ 6214.58 & \text { Zn I I } & 1 \\ 6215.152 & \text { Fe I } & 1018 \\ 6215.212 & \text { TI I } & 293 \\ 6216.368 & \text { V I } & 19 \\ 6217.2813 & \text { Ne I } & 1\end{array}$

$6217.288 \quad$ Fe I $\quad 1335$

$\begin{array}{lllr}6217.95 & P & \text { Fe I I } & 1335 \\ 6219.290 & & \text { Fe I } & 62\end{array}$

$\begin{array}{rrrr}6219.35 & P & \text { Fe II } & 161\end{array}$

$\begin{array}{llll}6219.54 & P & \text { Fe I } & 685\end{array}$

$6219.54 \quad P$

$6220.460 \quad \mathrm{~T} 1 \mathrm{I}$

6220.78

6221.40

6221.41

$\mathrm{Fe}$

$\mathrm{Fe} I$

6165.56

$\begin{array}{ll}\mathrm{Fe} & \text { I } \\ \mathrm{P} & \text { II }\end{array}$

1018
5

6165.945

6167.82

6168.46

6168.86

6169.055

6169.559

6170.16

6170.340

6170.47

$\begin{array}{llc}\text { Pr } & \text { I I } & 39 \\ \text { Ca } & \text { I } & 20 \\ \text { N } & \text { I I } & 36,60 \\ \text { Cr } & \text { I I } & 196 \\ \text { Co } & \text { I } & 82 \\ \text { Ca } & \text { I } & 20 \\ \text { Ca } & \text { I } & 20 \\ \text { N } & \text { I I } & 36 \\ \text { V } & \text { I } & 20 \\ \text { As } & \text { I I } & 1\end{array}$

6170.492

6170.568

6170.6

$6171.01 \quad P$

6172.28

6172.72

6173.05

6173.343

6173.40

6174.15

Fe I $\quad 1260$

N1 I 228,230

$\begin{array}{llr}\text { He } & \text { I I } & 8 \\ \text { Fe } & \text { I } & 1256\end{array}$

A I I

La II

Eu II

Fe I

$\begin{array}{ll}\text { N } & \text { II } \\ \text { La } & \text { II }\end{array}$

102

9
62

36
47

6175.158

6175.424

6176.813

6177.258

6177.49

6178.13

6179.17

6179.378

6180.093

6180.216

6180.42

6181.57

6181.68

6182.28

6182.45

6183.42

6183.892

6184.94

6185.1

6185.34

6186.14

6186.740

6187.41

6188.00

6188.03

6188.09
6189.005

6189.350

Fe II

N1

Cr II

N1 I

N1 I

Fe II

Cr II

Fe II

Ne

Gd II

Al II

Al II

Al II

Al II

Al II

N1 I

Fe III

Fe II

T1 I

N1

Fe I

Cr II

$\mathrm{Fe} I$

La II

$\begin{array}{ll}\text { Co } & \text { I } \\ \text { V } & \text { I }\end{array}$

200

217

105

58
244

46

187

65,217

269
111
66

66

66
66

66
66
66

66

226
163

6191.186

6191.562

6191.73

6192.96

6193.672

6195. 18

6196.71

6199. 16

6199.202

N1

Fe I

Y I
Zr

Cr II

Cr II

Fe II

$\checkmark$ I

6200.323

6201.52

6201.70

6202. 31

6203.51

6204.640

6207.251

6208.18

6209.73

Fe I

46
197

229

342

187

959

117
37

20

45

169
2

24

3
105

46
162

19

Fe I

Al II

207

$\mathrm{Fe} I$

57
57

208

$\begin{array}{lll}\text { La I I } & 111 \\ \text { N1 } & \text { I } & 226\end{array}$

$\begin{array}{llr}V & \text { I } & 20 \\ C & \text { II }\end{array}$

Cr II

105

Sc I

\begin{tabular}{|c|c|c|c|}
\hline 6221.661 & & $\mathrm{Fe}$ & I \\
\hline 6221.88 & & Lu & I I \\
\hline 6222.59 & & $Y$ & I \\
\hline 6222.81 & & IIf & I I \\
\hline 6223.994 & & N1 & I \\
\hline 6224.23 & $\mathbf{P}$ & $\mathrm{Fe}$ & I \\
\hline 6224.26 & $\mathbf{P}$ & v & I \\
\hline 6224.507 & & v & I \\
\hline 6226.18 & & Al & I I \\
\hline 6226.29 & & v & I I \\
\hline 6226.66 & & $\mathrm{Cr}$ & I I \\
\hline 6226.77 & & $\mathrm{Fe}$ & I \\
\hline 6229.234 & & $\mathrm{Fe}$ & I \\
\hline 6229.34 & $\mathbf{P}$ & $\mathrm{Fe}$ & I I \\
\hline 6230.115 & & N1 & I \\
\hline 6230.728 & & $\mathrm{Fe}$ & I \\
\hline 6230.736 & & v & I \\
\hline 6230.84 & & $\mathrm{Hf}$ & I I \\
\hline 6230.968 & & Co & I \\
\hline 6231.76 & & Sc & I \\
\hline
\end{tabular}

A1 I I

6231.78

6232.661

6232.735

6233.187

6233.8

6237.34

6237.62

6238.375

6239.36

6239.410

6239.64

6239.73

6239.77

6239.778

6239.95

6240.137

6240.266

6240.656

6242.52

6242.80

6243.11

6243.13

6243.36

6243.86

6244.344

6244.51

6244.56

6245.214

6245.629

6245.84

6246.334

6247.562

6248.916

6248.95

6249.65

6249.92

$6,251.83$

6252.561

6253.82

6254.25

6254.262

6254.96

6256.365

6256.370

6256.84

6256.906

6257.72
6258.103

6260.31

6261.55

6262.30

6264.55

6266.021

6266.32

6266.89

6267.64

6268.50

6268.841

6270.238

6271.289

6271.52

6271.83

$627+.34$

6274.94

278.30

6279.84

6280.625

6282.9

6284.

6286.35

6287.06

6290.55

6257.834

6258.591

6258.595

6258.706

6258.962

6259.615

6261.101

Type Element

Multiplet No.

6264.825

6265.140

6266.4950

6267.845

6272.650

6273.389

6273.76

T1

N1 I

T1 I

Sc I

$\begin{array}{ll}\text { N1 } & \text { I } \\ \text { Gd II }\end{array}$

T1 I

20

6274.670

6276.310

6277.525

6279.757

6282.636

0 I

Lo. II

T1 I

$\mathrm{Fe}$

$\begin{array}{ll}\text { T1 } & \text { I } \\ \text { V I }\end{array}$

$\mathrm{Ne} I$

$\mathrm{Ne}$

$\begin{array}{ll}0 & \text { I } \\ \text { Fe } & \text { I }\end{array}$

50

33

19

62
144

144
20

48
110

6284.30

6285.185

6290.968

6292.858 
I 1 Type Element Multiplet No.
6314.22

6314.2

$6314.668 \quad S$ S II

$6314.67 \quad P \quad$ N1 $I$

$6315.316 \quad P \quad F e$

$\begin{array}{lll}6315.42 & P & \text { Fe } I \\ 6315.79 & & \text { La II }\end{array}$

6316.61

6318.022

6318.027

6318.11

6318.23

6318.75

6320.38

6320.854

6322.165

6322.693

6322.98

6323.3

6324.46

6325.22

6325.90

6326.43

6326.845

6327.603

6328.6

6329

6330.101

6330.856

6331.968

6395.335

6335.74

6336.104

6336.835

6338.886

6338.080

6339.148

6338.96

6340.67

6341.682

6342.682

6343.29

6343.863

6344.154

6344.831

6346.54

6346.65

6346.67

6347.081

6347.1

6347.843

6348.50

6349.477

6348.748

6351.17

6351.28

6351.448 Cn

$6353.84 \quad P \quad \mathrm{Pe}$

$6356.038 \quad F$

6356.057

6356.283

6357.0

6357.297

6358.12

6358.692

6359.896

6360.798

6361.41

6361.78

6362.286

6362.34

6362.874

6362.888

6364.384

6364.597

6364.717

6364.92

6366.7

6366.33

6366.354

6366.483

6369.34

6369.46

6370.383

136
121
28
67
249
1015
1016
117
1014
248

Fo I 168

$\begin{array}{ll}\text { T1 } & \text { I } \\ \text { Ca } & I\end{array}$

Mg I

$\mathrm{Mg} I$

La II

N1 I

Fe I

0 I

$\begin{array}{ll}A & \text { II } \\ 0 & \text { I }\end{array}$

T1 I

Le I

$\begin{array}{ll}\text { S } & \text { II } \\ \text { V } & \text { I }\end{array}$

N1 I

N II

$\begin{array}{ll}\text { Cr } & \text { I } \\ \text { Fe } & \text { I }\end{array}$

P.e I 1250

Ne I

Fo I

AI II

Fo I

$\begin{array}{lll}\text { Fe I } & \text { I } \\ \text { V I }\end{array}$

N1 I 248

Fo I

Ba II

Sc II

Ca I

Ce II

Fe I

Zr II

Gd II

$\begin{array}{ll}M 1 & \text { II } \\ 81 & \text { II }\end{array}$

N II

Co I

$\begin{array}{ll}\text { F } & \text { I } \\ \text { V } & \text { I }\end{array}$

n I

0 I

Cn I 200

13

In I 39

Fe I

Zr I

La II

Fo I

T1 I

N1 I

T1 I

Ca I

8c I

Zn I

Cr

Fe I

Fe I

N1 I

Fe I

$\begin{array}{ll}\text { T1 } & \text { I } \\ \text { N } & \text { II }\end{array}$

0 I

T1 I

N1 I

S II

Fo II

N1 I

I A Type Blement Nultiplet No.

Type Blewent

Multiplet No.

6371.359

6374.08

6374.31

6375.86

6376.00

6376.22

6378.824

6378.91

6379.63

S1 II 2

$\begin{array}{llr}\text { Le } & \text { II } & 111 \\ 0 & I & 68\end{array}$

Fo II

A II 61

N1 I 247

Sc I

Ba II

$6380 \cdot 11$

6380.748

6380.95

6381.416

6382.169

6382.991

6383

6383.753

6384.669
6384.687

\section{II}

231

$\begin{array}{llr}\text { Fe I } & 1015 \\ \text { Od } & 111\end{array}$

$\begin{array}{llr}\text { T1 } & \text { I } & 196 \\ \text { Mn I } & 39\end{array}$

$\begin{array}{ll}\text { Ne I } \\ N & \text { IV }\end{array}$

Fe II

N1 I

3

3384.89

6385.186

6385.473

6385.74

6386.48

6386. 75

$\begin{array}{lll}6390.48 & \text { La II } & 33 \\ 6391.214 & \text { Mn I } & 39\end{array}$

6392.534 Fe I 109

6393.605

6394.23

6395.158

6395.16

6385.27

6386. 39

6387.30

6398.05

8399.04

6398.23

8 II

Fe II

Fe I

8 II

109

$\begin{array}{llr}\text { Fe I } & 168 \\ \text { L } & I & 7\end{array}$

Co 1

Ca I

S II

$\begin{array}{ll}\text { Fe } & \text { I } \\ \text { S } & \text { III }\end{array}$

s II

Lo II

10
18

104

6398.41

6400.010

6400.335

6402.005

6402.2455

6402.43

6403.58

B405. 88

6406.3

6406.42

C1 II

Fe I

$\mathrm{Fe} I$

$\begin{array}{ll}\mathrm{N} & \mathrm{I} \\ \mathrm{N} & \mathrm{I}\end{array}$

Ne I

8 I

C8 I

Fe I

58
816

13

1344

6407.03

6407.30

6408.031

6408.13

6408.463

6410.98

6411.10

6411.658

6412.20

\section{Zr I}

Fo II

Fe I

$\begin{array}{ll}8 & I \\ 8 r & I\end{array}$

Le. I

Lo I

Fo I

$\mathrm{Fe} \mathrm{I}$

Fe I
T1 I

6418. 353

6413.66

6413.71

8413.92

6414.603

6415.50

6415.59

6416.905

Se I

$\begin{array}{ll}\text { F } & \text { I } \\ 8 & \text { II }\end{array}$

Mn I

N1 I

81 I

8 I
Cr II

Fe II

6416.94

Fe I

7
1334

2 
I A Type Element Multiplet No. I A Type Elewent Multiplot No. I I Type Element Multiplet Xo.

\begin{tabular}{|c|c|c|c|c|c|c|c|c|}
\hline 6488.10 & & Yb & I & 3 & 6560.098 & & He & I1 \\
\hline 6489.68 & & Zr: & I & 65 & 6560.68 & & 81 & I \\
\hline 6480.344 & & Co & I & 81 & 6561.032 & & D & \\
\hline 6491.28 & & Fe : & II & & 6562.817 & & H & \\
\hline 6481.28 & & N & I & 21 & 6563.403 & & Co & I \\
\hline 6481.61 & $\mathbf{P}$ & Ti & II & 81 & 6563.86 & & Вr & II \\
\hline 6491.712 & & Mn & I & 39 & 6565.62 & & T1 & I \\
\hline 6492.0 & & N & II & 45 & 6565.88 & & $v$ & I \\
\hline 6483.05 & & $\mathrm{Fe}$ & II & & 6567.22 & $\mathbf{P}$ & Fe & I \\
\hline 6493.780 & & $\mathrm{Ca}$ & I & 18 & 6667.39 & & Hr & II \\
\hline 6494.11 & & Gd: & I & 123 & 6568.00 & & Gd & II \\
\hline 6484.52 & $\mathbf{P}$ & Fe & I & 1255 & 6569.281 & & Fe & I \\
\hline 6484.985 & & Fe & I & 168 & 6568.31 & & Sm & II \\
\hline 6495.45 & & 41 & II & 65 & 6570.0 & & He & II \\
\hline 6495.779 & & Fe & I & 1253 & 6570.834 & & Mn & I \\
\hline 6496.456 & & $\mathrm{Fe}$ & I & 1258 & 6570.96 & & Ia & II \\
\hline 6496.896 & & $\mathrm{Ba}$ & I I & 2 & 6571.22 & & $\mathrm{Fe}$ & I \\
\hline 6497.689 & & T1 & I & 102 & 6572.781 & & Ce & I \\
\hline 6488.19 & & La & I I & 104 & 6572.900 & & $\mathrm{Cr}$ & I \\
\hline 6498.759 & & $\mathrm{Ba}$ & I & 6 & 6574.238 & & $\mathrm{Fe}$ & I \\
\hline 6498.950 & & Fe & $I$ & 13 & 6575.022 & & Fo & I \\
\hline 6498.52 & & N & I & 21 & 6575.180 & & T1 & I \\
\hline 6489.649 & & $\mathrm{Ca}$ & I & 18 & 6576.95 & $\mathbf{P}$ & N1 & I \\
\hline 6500.26 & & $\Delta$ & II & 26 & 6578.03 & & C & II \\
\hline 6501.212 & & Cr & I & 16 & 6578.51 & & La & I \\
\hline 6601.681 & & $\mathrm{Fe}$ & I & & 6578.96 & & $\mathbf{v}$ & I \\
\hline 6603.989 & & $\mathbf{8 r}$ & I & 8 & 6580.22 & & N1 & I \\
\hline 6504.164 & & $v$ & $I$ & 48 & 6580.96 & & $\mathrm{Cr}$ & I \\
\hline 6504.9 & & $\mathbf{N}$ & II & 45 & 6581.22 & & Fe & I \\
\hline 6506.33 & & $\mathrm{Fe}$ & II & & 6582.85 & & C & II \\
\hline 6506.45 & & $\mathbf{N}$ & I & 21 & 6584.53 & & HP & II \\
\hline 6506.5279 & & $\mathrm{Ne}$ & $\mathbf{I}$ & 3 & 6584.89 & & $\mathbf{Y}$ & I \\
\hline 6508.135 & & T1 & I & 102 & 6586.328 & & N1 & 1 \\
\hline 6808.742 & & $\mathrm{Ca}$ & I & 18 & 6886.343 & & Mn & I \\
\hline 6509.16 & & $\Delta$ & II & 21 & 6586.69 & & Fe & II \\
\hline 8609.56 & & $\mathrm{Fe}$ & I & 1012 & 6587.76 & & C & I \\
\hline 0011.62 & & нг & II & 69 & 6588.81 & & 8ri & I \\
\hline 6512.61 & & НP & II & 49 & 6591.32 & & Fe & I \\
\hline 6516.026 & & Cr & I & 265 & 6591.834 & & Co & I \\
\hline 6516.053 & & $\mathbf{F e}$ & II & 40 & 6592 & $\mathbf{P}$ & C & IV \\
\hline 6617.01 & & $\mathrm{Fe}$ & II & & 6592.472 & & N1 & I \\
\hline 6517.27 & & $\mathbf{v}$ & II & 230 & 6592.91 & $\mathbf{P}$ & T1 & I \\
\hline 6518.376 & & $\mathrm{Fe}$ & I & 342 & 6592.919 & & $\mathrm{Fe}$ & $I$ \\
\hline 6619.371 & & un & I & 39 & 6593.878 & & Fe & I \\
\hline 6521.39 & & 8 & II & 25 & 6595.326 & & Ba & I \\
\hline 6822.3 & & $N$ & II & 46 & 6585.869 & & Co & $I$ \\
\hline 6522.38 & & Cl & II & 59 & 6597.556 & & $\mathrm{Cr}$ & I \\
\hline $6524 \cdot 76$ & $\mathbf{P}$ & $\mathrm{Fe}$ & $I$ & 1280 & 6597.607 & & Fe & I \\
\hline 6526.99 & & La & II & 33 & 6598.594 & & N1 & $I$ \\
\hline 6527.20 & $\mathbf{P}$ & $\mathbf{s 1}$ & I & 52 & 6598.9529 & & Ne & I \\
\hline 6527.312 & & Ba & I & 6 & 6599.112 & & $\mathbf{T 1}$ & I \\
\hline 6527.49 & & 81 & I & 62 & 6601.13 & $\mathbf{P}$ & Fe & I \\
\hline 6528.53 & & Fe & I & & 6603.20 & $\mathbf{P}$ & Fe & I \\
\hline 6529.197 & & $\mathrm{Cr}$ & I & 265 & 6803.67 & $\mathbf{P}$ & Fe & I \\
\hline 6531.44 & & v & I & 48 & 6604.60 & & $8 c$ & II \\
\hline 6531.66 & & HP & II & 48 & 6604.67 & & Fe & I \\
\hline 6532.891 & & N1 & I & 64 & 6605.546 & & Mn & $\mathbf{I}$ \\
\hline 6533.0 & & $\mathbf{N}$ & II & 45 & 6605.98 & & $\mathbf{v}$ & $\mathbf{I}$ \\
\hline 6533.97 & & Fe & I & 1187 & 6607.02 & $\mathbf{P}$ & T1 & II \\
\hline $6537: 921$ & & $\mathbf{C r}$ & I & 16 & 6607.82 & & $\mathbf{v}$ & I \\
\hline 6539.72 & & Fe & I & 405 & 6608.05 & & F• & $I$ \\
\hline 6541.49 & $\mathbf{P}$ & Fe & I & 1196 & 6609.116 & & Fe & I \\
\hline 6542.80 & & Hr & II & 100 & 6609.20 & & HF & II \\
\hline 6643.17 & & La & I & 7 & 6609.56 & & $\mathrm{Fe}$ & $I$ \\
\hline 6543.61 & & $\mathbf{v}$ & I & 48 & 6609.64 & & A1 & II \\
\hline 6643.98 & & $\mathrm{Fe}$ & I & 1139 & 6609.68 & $\mathbf{P}$ & $\mathrm{Fe}$ & I \\
\hline 6545.2 & & $\mathbf{N}$ & II & 45 & 6610.04 & & Gd & II \\
\hline 6545.80 & & $\mathrm{Mg}$ & II & 23 & 6610.58 & & N & II \\
\hline 6546.245 & & Fe & I & 268 & 6612.17 & & $\mathrm{Cr}$ & I \\
\hline 6546.276 & & $\mathbf{T 1}$ & I & 102 & 6613.74 & & $\mathbf{Y}$ & II \\
\hline 6546.791 & & $8 r$ & I & 8 & 6613.83 & $\mathbf{P}$ & $\mathrm{Fe}$ & I \\
\hline 6547.58 & $\mathbf{P}$ & $\mathrm{Fe}$ & $\mathbf{I}$ & 13 & 6616.03 & $\mathbf{P}$ & $\mathrm{Fe}$ & $I$ \\
\hline 6548.72 & & HP & II & & 6617.126 & & Co & I \\
\hline 6550.01 & & HY & II & 111 & 6617.14 & $\mathbf{P}$ & N1 & I \\
\hline 6550.244 & & Sr & I & 12 & 6617.266 & & Sr & I \\
\hline 6651.466 & & Co & I & B4 & 6621.24 & & N1 & I \\
\hline 6551.68 & $\mathbf{P}$ & $\mathrm{Fe}$ & I & 13 & 6622.28 & & od & II \\
\hline 6552.77 & & $\mathrm{Fe}$ & I & 1325 & 6622.41 & $\mathbf{P}$ & $\mathrm{Fe}$ & $I$ \\
\hline 6554.18 & & La & I I & 109 & 6622.53 & & $N$ & I \\
\hline 6554.226 & & $\mathbf{T 1}$ & I & 102 & 6623.78 & $\mathbf{P}$ & Fe & I \\
\hline 6555.20 & & 81 & I & 62 & 6624.86 & & $\mathbf{v}$ & I \\
\hline 6555.87 & $\mathbf{P}$ & $\mathbf{F e}$ & $I$ & 1007 & 6625.04 & & Fo & I \\
\hline 6556.066 & & $\mathbf{T 1}$ & $\mathbf{I}$ & 102 & 6627.28 & & $\mathrm{Fe}$ & II \\
\hline 6556.79 & & $\mathbf{F e}$ & I & 1255 & 6627.568 & & Fe & I \\
\hline 6557.40 & & $\mathbf{Y}$ & I & 1 & 6627.62 & & 0 & II \\
\hline 6567.87 & & Sc & I & 24 & 6630.016 & & $\mathbf{C r}$ & I \\
\hline 6557.91 & & HP & II & 66 & 6630.5 & & $N$ & II \\
\hline 6558.02 & & v & I & 59 & 6632.438 & & Co & I \\
\hline 6558.05 & & $8 c$ & I & 24 & 6633.44 & & Fe & I \\
\hline 6558.580 & & T1 & II & 91 & & & $\mathrm{Fe}$ & \\
\hline
\end{tabular}




\begin{tabular}{|c|c|c|c|c|c|c|c|}
\hline I $A$ & Type & Blede & sent & Multiplet No. & I $\mathrm{A}$ & Ts pe & Blow \\
\hline 6700.88 & $\mathbf{P}$ & $\mathrm{Fe} I$ & I & 1156 & 6752.832 & & A I \\
\hline 6700.80 & $\mathbf{P}$ & Fe $I$ & I & 1333 & 6753.00 & & 81 \\
\hline 6700.80 & $\mathbf{P}$ & N1 I & I & 248 & 6753.45 & $\mathbf{P}$ & $\mathbf{F e}$ \\
\hline 6701.64 & & Cr 1 & I & 256 & 6734.61 & & He I \\
\hline $6702 \cdot 12$ & & Gd I & I I & 130 & 6754.75 & & C I \\
\hline 6703.573 & & Fe $I$ & I & 268 & 6785.609 & & $\mathrm{Fe} \mathrm{I}$ \\
\hline 6704.18 & & Gd I & II & 110 & 6756.86 & $\mathbf{P}$ & Fe 1 \\
\hline 6704.48 & $\mathbf{P}$ & $\mathrm{Fe} I$ & I & 1062 & 6756.61 & & A 1 \\
\hline 6705.117 & & $\mathrm{Fe} I$ & I & 1197 & $6757 \cdot 16$ & & s I \\
\hline 6705.13 & $\mathbf{P}$ & Fe I & I & 1280 & 6757.78 & & Cr \\
\hline 6706.20 & & N I & I & 31 & 6758.60 & & N I \\
\hline 6707.74 & & L1 I & I & 1 & 6759.41 & & N1 \\
\hline 6707.89 & & L1 I & I & 1 & 6789.42 & & C1 \\
\hline 6708.27 & & $F I$ & I & 2 & 6761.07 & $\mathbf{P}$ & Fe I \\
\hline 6708.81 & & N I & I & & 6762.38 & & $2 \times 1$ \\
\hline 6709.48 & & La 1 & I & 6 & 6762.41 & & Cr \\
\hline 6709.88 & & Ca I & I & 46 & 6764.13 & $\mathbf{P}$ & Fe \\
\hline 6710.31 & & Pe I & I & 34 & 8768.48 & & v I \\
\hline 6711.24 & $\mathbf{P}$ & Fe I & I & 1220 & 6767 & $\mathbf{P}$ & $0 V$ \\
\hline 6712.44 & $\mathbf{P}$ & Fe I & I & 1278 & 6767.778 & & N1 \\
\hline 6712.68 & $\mathbf{P}$ & Fe I & I & 206 & 6769.62 & & Bo 1 \\
\hline 6713.14 & & Fe I & I & 1018,1186 & 6769.66 & $\mathbf{P}$ & Fe 1 \\
\hline 6713.43 & & C1 I & II & 38 & 6771.040 & & Co : \\
\hline 6713.76 & & Fe I & $I^{-2}$ & 1255 & 6772.36 & & N1 \\
\hline 6714.08 & & Ls I & II & 103 & 6773.97 & & F \\
\hline 6715.38 & & Cr I & I & 282 & 6774.28 & & La \\
\hline 6718.410 & & Fe I & I & 1174 & 6778.97 & & Al I \\
\hline 6716.24 & & Fe I & I & 1225 & 6777.44 & & Fe 1 \\
\hline 6716.679 & & Ti I & I & 273 & 6779.74 & & c \\
\hline 6717.656 & & Pe 1 & I & 1194 & 6780.27 & & C I \\
\hline 6717.685 & & Ca I & I & 32 & 6783.27 & $\mathbf{P}$ & $\mathrm{Fe}$ \\
\hline 6717.911 & & Ti I & II & 112 & 6783.71 & & Fo 1 \\
\hline 6718.14 & & ad 1 & II & 130 & 6783.78 & & C I \\
\hline 6718.68 & & In I & II & 128 & 6784.98 & & v \\
\hline 6710 & $\mathbf{P}$ & $x V$ & $\mathbf{v}$ & 11 & 6785.25 & $\mathbf{P}$ & T1 1 \\
\hline 6710.40 & & Bf I & II & 110 & 6786.76 & $\mathbf{P}$ & Fo 1 \\
\hline 6721.35 & & 01 & II & 4 & 6785.88 & $\mathbf{P}$ & I. 1 \\
\hline 6721.97 & & 811 & I & & 6786.41 & $\mathbf{P}$ & Fe 1 \\
\hline 6722.67 & & 811 & I & 38 & 6786.88 & & Pe : \\
\hline 6723.12 & & * 1 & I & 31 & 6787.09 & & C I \\
\hline 6725.39 & & Fo 1 & I & 1052 & 6787.16 & & $2 \times 1$ \\
\hline 6726.25 & & 01 & I & 2 & 6787.61 & $\mathbf{P}$ & Fe : \\
\hline 8728.50 & & 01 & I & 2 & 6789.17 & & Cr \\
\hline 6726.668 & & Fe I & I & 1187 & 6780 & $\mathbf{P}$ & $0 \mathrm{~V}$ \\
\hline 6726.78 & & Fo I & I & & 6780.00 & & 8. 1 \\
\hline 6726.84 & & C I & II & 21 & 6791.022 & & $8 r$ \\
\hline 6727.1 & $\mathbf{P}$ & C I & III & 3 & 6791.30 & & C I \\
\hline 6727.83 & & ad I & II & 96 & 6798.20 & & Fo I \\
\hline 6729.72 & & C. I & I & 301 & 6793.62 & & Fe I \\
\hline 6729.80 & $\mathbf{P}$ & 81 I & I & 61 & 6783.71 & & 11 \\
\hline 8720.38 & $\mathbf{P}$ & $81 \mathrm{I}$ & I & 61 & 6794.60 & $\mathbf{P}$ & Fo 1 \\
\hline 6730.7 & $\mathbf{P}$ & C I & III & 3 & 6795.41 & & 11 \\
\hline 6750.73 & & CA I & I & 2 & 6795.82 & & F I \\
\hline 6750.79 & & C I & II & 21 & 6796.11 & & F• I \\
\hline 6731.84 & & 8. I & II & 89 & 6788.04 & & C I \\
\hline 6752.06 & & Fe I & I & 1225 & 6788.61 & & Ca $I$ \\
\hline 6732.80 & & Is I & II & 109 & 6789.32 & & 1 \\
\hline 6732.88 & & 81 & II & 20 & 6799.61 & & To 1 \\
\hline 6725.164 & & Fo I & I & 1198 & 6800.80 & & C I \\
\hline 6723.48 & & N I & I & 31 & 6801.16 & & $\nabla I$ \\
\hline 0735.86 & & c 1 & II & 21 & 6801.91 & $\mathbf{P}$ & Fe I \\
\hline 6734.16 & & Cr $I$ & I & 282 & 6801.38 & & Le I \\
\hline 6735.00 & $P$ & Fe 1 & I & 1167 & 6801.87 & $\mathbf{P}$ & Fe 1 \\
\hline 6736.68 & $\mathbf{P}$ & Fe 1 & I & 1122 & 6803.30 & $\mathbf{P}$ & Fe I \\
\hline 6737.20 & $\mathbf{P}$ & Fe 1 & I & 881 & 6803.84 & $\mathbf{P}$ & Fe I \\
\hline 6737.87 & & $8 c 1$ & I & & 6804.020 & & Fo 1 \\
\hline 6738.36 & & C I & II & 21 & 6804.27 & & Fe I \\
\hline 6738.81 & & Cr 1 & I & 318 & 6808.72 & $\mathbf{P}$ & Fe I \\
\hline 6739.84 & & Fo 1 & I & 34 & 6806.861 & & Fe I \\
\hline 6741.20 & & N 1 & I & 31 & 6808.66 & & A I \\
\hline 6742.08 & & c I & II & 21 & 6808.80 & $\mathbf{P}$ & Fe I \\
\hline 6743.124 & & T1 & I & 48 & 6808.88 & & Le: \\
\hline 6745.88 & & 8 & I & 8 & 6810.28 & & Fe I \\
\hline 6744.2 & $\mathbf{P}$ & C I & III & 3 & 6812.10 & & C \\
\hline 6744.06 & & Cr 1 & I & 218 & 6812.26 & & N I \\
\hline 6746.11 & & 70 : & I & 1287 & 6812.40 & & v \\
\hline 6745.66 & $P$ & T1 & I & 220 & 6813.80 & $\mathbf{P}$ & Fo : \\
\hline 6745.86 & $\mathbf{P}$ & Fe & $I$ & 1008 & 6813.898 & & K1 \\
\hline 6746.438 & & Ti: & $I$ & 182 & 6813.68 & & I. \\
\hline 6746.86 & $\mathbf{P}$ & Fo & I & 206 & 6813.86 & $\mathbf{P}$ & 81 \\
\hline 6748.43 & & T1 & I & 162 & 3814.960 & & Co: \\
\hline 6748.79 & & 3 & I & 8 & 1816.90 & & 11 \\
\hline 0749.83 & $\mathbf{P}$ & Fo & I & 860 & 6817.08 & & $8 c$ \\
\hline 6760.262 & & F• & I & 111 & 6818.30 & & $1:$ \\
\hline $6700: 82$ & & C & II & 21 & 6810 & $\mathbf{P}$ & 0 \\
\hline 6761.28 & & Cr & I & ik & 6819.42 & $\mathbf{P}$ & Fe : \\
\hline 6751.94 & & T1 & $I$ & 182 & 6819.00 & $\boldsymbol{P}$ & Fo : \\
\hline 6762.40 & & $x$ & I & 30 & 6820.43 & & Fo \\
\hline 6782.67 & & ad & II & 130 & 6822.00 & $\mathbf{P}$ & Fo \\
\hline 6782.724 & & F• & I & 1108 & 6822.06 & $\mathbf{P}$ & Fo : \\
\hline
\end{tabular}


I A Type Element Multiplet No.

6882.48
6883.04
6885.07
6885.772
6886.57
6887.63
6888.7
6890.88
6892.585
6894.92

6896

6898.31

6900.73

6901.52

6902.46

6902.80

6906.54

6908.08

6908.11

Cr I 222

Cr I 222

Fe I $\quad 1173$

A II

Gd II

II

He II

Mg I

7

1
34

0 II 45

$\mathrm{Y}$ II

Fe I

Gd II

Co I

$\mathrm{Fe} I$

$\begin{array}{ll}0 & \text { II } \\ \text { Co I }\end{array}$

$\begin{array}{ll}\text { Co } & \text { I } \\ 0 & \text { II }\end{array}$

45
26
1078

1078
122

164

45

164
45

6909

6909.82

6910.75

6910.84

6911.52

6912.43

6914.562

6916.57

6916.702

6917.52

o $\mathrm{V}$

$\begin{array}{ll}\text { F } & \text { I } \\ \text { O } & \text { II }\end{array}$

Co I

Fe I

$\mathrm{Fe} I$

N1 I

Gd I

$\mathrm{Fe} \mathrm{I}$

12

2
45

80

109

341

62
2

$\begin{array}{lll}617.93 & \text { Al II } & 75\end{array}$

6919.96 Al II

6920.62

6924.13

6925.24

6926.04

6926.40

6926.90

6928.25

dd II

Cr I

Cr I

$\mathrm{Fe} I$

N I

N1 I

122
222
222

222

$\begin{array}{lll}6928.319 & \text { Zn I } \\ 6928.52 & P & \text { N1 }\end{array}$

$6929.4678 \quad \mathrm{Ne}$

$6929.96 \quad P \quad$ Fe I

$6930.35 \quad \mathrm{P} \quad \mathrm{Fe} I$

6930.64 P $\quad \mathrm{Fe} I$

$\begin{array}{lll}6932.49 & \text { P } & \text { Fe } \\ 6933.04 & & \text { Fe I } \\ 6933.55 & & \text { Y I } \\ 6933.628 & & \text { Fe I }\end{array}$

6933.55
6933.628

HP II

6935.16

6936.2

6936.48

6937.8

Zn I

6943.202

6943.67

6945.208

Zn I

Fe I

Fe I

$\begin{array}{ll}N & \text { I } \\ \text { Gd } & \text { II }\end{array}$

6945.88

6946.31

6947.501

6949.37

6950.3

$6951.261 \quad P \quad F \quad F$

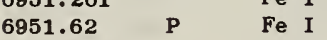

$6951.68 \quad Y$ II

C1 II

6952.52 La II

$6953.01 \quad P \quad$ Fe I

6954.54 La II

6957.95

6858.11

6959.24

Gd II

$\mathrm{Fe} \mathrm{I}$

$6964.18 \quad K$

$6964.69 \quad \mathrm{~K}$

$6965.42 \quad P \quad M g$

6965.4302

6966.35

6966.8

6968.78

6970.48

6970.48
6971.66

\begin{abstract}
1 A Type Elemert Multiplet No.
\end{abstract}
6971.95

6975.46

6976.53

6976.8

6976.934

6978.46

6978.855

6979.10

Fe I $\quad 404$

Fe I 1194

S1 I 60

$\begin{array}{llr}N & \text { I I } & 53 \\ \mathrm{Fe} & I & 1221\end{array}$

Fe I 1225

Cr I 222

$\begin{array}{llr}\mathrm{Fe} & \mathrm{I} & 111 \\ \mathrm{~N} & \mathrm{I} & 29\end{array}$

$\begin{array}{llll}6979.17 & P & \text { Fe I } & 340\end{array}$

$\begin{array}{lll}6979.82 & \text { Cr I } & 222\end{array}$

6980.86

6980.91

6980.91

6981.40

$\begin{array}{lllr}6983.53 & P & \text { Fe I } & 1220\end{array}$

$\begin{array}{rllr}6983.54 & \text { P } & \text { Fe II } & 63 \\ 6985.74 & & \text { A II } & 137\end{array}$

6985.89

Gd II

$\begin{array}{lll}6988.530 & \text { Fe I } & 167\end{array}$

6988.75 Gd II 130

$\begin{array}{lllr}6989.64 & P & \mathrm{Fe} I & 1191\end{array}$

6990.16 A II 20

6991.92

6995.35

6996.63

6996.76

$\begin{array}{rllr}6996.76 & & \text { Gd II } & 121 \\ 6997.13 & \text { P } & \text { Fe I } & 1273 \\ 6997.83 & & \text { HF II } & 89\end{array}$

Gd I

Ta I

Ti I 256

6899.902

7000.633

7000.75

7001.57

7001.83

7002.22

7003.0

7003.58

7004.60

7004.81

7005.84

7006.16

7007.81

7008.014

7008.35

7010.362

7010.94

7011.364

7014.99

7015. 3

7016. 075

7016.436

7016.602

7016.80

7016. 99

7017.68

7017.73

7017.98

7020.44

7021.23

$\begin{array}{llll}7022.39 & P & F e & I\end{array}$

$\begin{array}{llll}7022.976 & & \text { Fe I } & 1078 \\ 7024.0508 & & \text { Fe I } & 1051\end{array}$

$\begin{array}{lll}7024.0508 & \text { Ne I } & 103 \\ 7024.084 & \text { Fe I } & 1003\end{array}$

$\begin{array}{lll}7024.649 & \text { Fe I } & 1187\end{array}$

$7024.86 \quad$ N1 I

7025.52

7027.60

$7027.797 \quad$ Co I $\quad 1221$

$\begin{array}{llll}7028.58 & P & \text { Fe } & \end{array}$

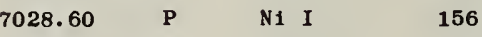

$\begin{array}{rrrr}7028.95 & P & N 1 & 61\end{array}$

$\begin{array}{lll}7030.06 & \text { Ni I } & 126\end{array}$

$\begin{array}{rlrr}7030.33 & & \text { Hf II } & 66 \\ 7031.02 & \mathrm{Fe} I & 1173\end{array}$

$\begin{array}{rlrr}7031.02 & \text { P } & \text { Fe I } & 1173 \\ 7031.42 & P & \text { Fe } I & 1278\end{array}$

$7032.16 \quad N 1$ I $\quad 279$

$\begin{array}{llll}7034.06 & P & \text { Fe } & \\ 7034.08 & P & \text { Fe } & 1190\end{array}$

$\begin{array}{llll}7034.06 & \text { P } & \text { Fe } I & 1190 \\ 7034.08 & \text { P } & \text { Fe I } & 1190\end{array}$

$\begin{array}{lll}7034.42 & N 1 & 97\end{array}$

S1 $1 \quad 50$

$\begin{array}{rllr}7037.04 & P & \text { Fe } I & 61\end{array}$

7037.26

7037.37

7037.45

7038.251

7038.80

7038.818

I A Type

Element Multiplet No.

$\begin{array}{rlr}7039.22 & \text { Sm II } & 57,6 \\ 7039.36 & \text { T1 I } & 307\end{array}$

7042.06 Al II

$\begin{array}{rlr}7042.24 & \text { Str II } & 58 \\ 7044.60 & \text { Fe I } & 1276\end{array}$

$7015.8 \quad$ C II 26

7045.96

$\begin{array}{lll}7050.65 & \text { TI I } & 256\end{array}$

$\begin{array}{llr}7051.00 & \text { Gd II } & 122 \\ 7052.872 & \text { Co } & \text { I }\end{array}$

$\begin{array}{llll}7052.9 & \text { C II } & 26\end{array}$

$\begin{array}{llll}7053.48 & P & F e & \\ 7054.042 & & 1186\end{array}$

$\begin{array}{rrr}7054.042 & \text { Co I } & 140\end{array}$

$\begin{array}{llr}7054.62 & \text { Gd II } & 130 \\ 7055.01 & \text { A II } & 74\end{array}$

7056.60 Al II 3

$\begin{array}{rlll}7057.9 f_{i} & P & \text { Fe I } & 815\end{array}$

$\begin{array}{llr}7058.02 & \text { Gd II } & 130 \\ 7059.941 & \text { Ba I } & 5\end{array}$

$\begin{array}{llll}7060.43 & P & \text { Mg I } & 32\end{array}$

7061.90 HF II

$\begin{array}{rllr}7062.80 & \text { P } & \text { Fe I } & 1273 \\ 7062.97 & & \text { N1 I } & 64\end{array}$

$7063.4 \quad$ C II

$\begin{array}{rlr}7063.57 & \text { N1 I } & 270 \\ 7063.64 & \text { Al II } & 3\end{array}$

$\begin{array}{llr}7063.64 & \text { Al II } & 3 \\ 7065.15 & \text { T1 I } & 100\end{array}$

$\begin{array}{lll}7065.188 & \text { He I } & 10\end{array}$

$\begin{array}{llll}7066.15 & P & \mathrm{Fe} I & 1277\end{array}$

$\begin{array}{lll}7066.24 & \text { La II } & 1 \\ 7067.2170 & \text { A I } & 1\end{array}$

$7067.44 \quad$ Fe II

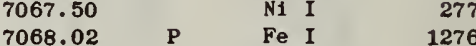

$\begin{array}{rrrr}7068.02 & P & \text { Fe I } & 1276 \\ 7068.37 & & \text { La I } & 1\end{array}$

$7068.415 \quad \mathrm{Fe} I \quad 1004$

$\begin{array}{llll}7068.60 & P & \text { Fe I } & 1276\end{array}$

$\begin{array}{llll}7069.11 & & \text { T1 I } & 307 \\ 7069.54 & P & \text { Fe I } & 205\end{array}$

$7070.071 \quad$ Sr I

$7071.88 \quad 1194$

$\begin{array}{llll}7074.45 & P & \text { Fe I } & 1173\end{array}$

$\begin{array}{rlr}7077.03 & \text { A II } & 20 \\ 7077.10 & \text { Eu II } & 8\end{array}$

$\begin{array}{llll}7079.32 & P & F e & 1278\end{array}$

$\begin{array}{rlr}7082.22 & \text { N1 I } & 267 \\ 7082.37 & \text { Sm II } & 55\end{array}$

$\begin{array}{llr}7083.396 & \text { Sm II } & 55 \\ & \text { Fe I } & 1277\end{array}$

7084.25 P $\quad$ Ti I

$\begin{array}{lll}7084.33 & \text { Si } & \\ 7084.974 & \text { Co } I & 60\end{array}$

7085.52 Gd II 130

7086.76 Fe I 815,131

$\begin{array}{lll}7087.35 & \text { Zr I } & 42\end{array}$

$\begin{array}{llll}7089.03 & P & \text { Si I } & 70\end{array}$

$\begin{array}{llll}7089.73 & \text { P } & \text { Fe I } & 1220 \\ 7090.404 & & \text { Fe I } & 1051\end{array}$

$\begin{array}{rrr}7090.55 & \text { A II } & 60\end{array}$

7091.83

$\begin{array}{llll}7091.91 & \text { P } & \text { Fe I } & 1278 \\ 7093.10 & \text { P } & \text { Fe I } & 1277\end{array}$

$\begin{array}{rrrr}7094.30 & \mathrm{P} & \mathrm{Fe} \mathrm{I} & \mathbf{7 1 8 9}\end{array}$

7095.40 N1 I 276

$\begin{array}{llr}7095.425 & \text { Fe I } & 1105\end{array}$

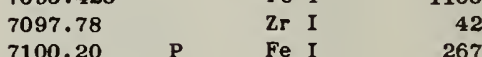

$\begin{array}{llll}7101.28 & P & \text { Fe. I } & 61\end{array}$

$\begin{array}{lll}7102.95 & \text { Zr I }\end{array}$

$\begin{array}{llll}7103.15 & P & \text { Fe } I & 167\end{array}$

$\begin{array}{llll}7103.77 & & \text { Zr I } & 42 \\ 7105.34 & P & \text { S1 I } & 70\end{array}$

$\begin{array}{rlrr}7105.34 & \text { P } & \text { S1 I } & 70 \\ 7105.90 & \text { P } & \text { Fe I } & 1008\end{array}$

$\begin{array}{llll}7107.30 & P & F e ~ I ~ & 1324 \\ 7107.461 & & \text { Fe I } & 1005\end{array}$

$\begin{array}{lll}7109.48 & N \text { IV } & 4\end{array}$

$\begin{array}{llll}7109.67 & P & \text { Fe I } & 1190\end{array}$

$\begin{array}{lll}7110.91 & \text { N1 I }\end{array}$

7111.28

7111.71

7112.176 
I A Type

\begin{tabular}{|c|c|c|c|c|c|c|c|c|}
\hline 7118.86 & & Gd & II & 130 & 7193.74 & & $\mathbf{Y}$ & II \\
\hline 7119.45 & & C & II & 20 & 7193.89 & & S1 & I \\
\hline 7120.01 & $\mathbf{P}$ & $\mathrm{Fe}$ & I & 1187 & 7184.02 & $\mathbf{P}$ & Fe & I \\
\hline 7120.56 & $\mathbf{P}$ & $\mathrm{Fe}$ & I & 1006 & 7184.81 & & Bu & II \\
\hline 7122.24 & & N1 & I & 126 & 7194.92 & & $\mathrm{Fe}$ & $I$ \\
\hline 7123.10 & & $\mathbf{N}$ & IV & 4 & 7195.235 & & Ba & $I$ \\
\hline 7124.28 & & $\mathbf{s}$ & II & 18 & 7196.37 & $\mathbf{P}$ & $\mathrm{Fe}$ & $I$ \\
\hline 7124.47 & & Co & I & 53 & 7196.83 & & $\mathrm{Cr}_{\mathbf{r}}$ & - I \\
\hline 7125.00 & $\mathbf{P}$ & $\mathrm{Fe}$ & I & 815 & 7197.07 & & $\mathbf{N 1}$ & I \\
\hline 7125.28 & $\mathbf{P}$ & Fe & I & 1220 & 7197.08 & & Gd & II \\
\hline 7125.49 & & C & II & 20 & 7202.194 & & Ca & $I$ \\
\hline 7126.71 & & N1 & I & 97 & 7202.37 & & $\mathbf{F}$ & I \\
\hline 7127.21 & & N & IV & 4 & 7205.51 & $\mathbf{P}$ & Fe & I \\
\hline 7127.58 & $\mathbf{P}$ & $\mathrm{Fe}$ & I & 1273 & 7207.123 & & $\mathrm{Fe}$ & I \\
\hline 7127.88 & & F & I & 6 & 7207.406 & & $\mathrm{Fe}$ & I \\
\hline 7129 & $\mathbf{P}$ & $\mathbf{N}$ & IV & 4 & 7207.85 & & $\mathrm{Cr}_{\mathrm{r}}$ & I \\
\hline 7129.30 & $\mathbf{P}$ & $\mathrm{Fe}$ & I & 1219 & 7208.20 & & S1 & I \\
\hline 7130.34 & $\mathbf{P}$ & $\mathrm{T} 1$ & I & 100 & 7209.44 & & T1 & I \\
\hline 7130.942 & & $\mathrm{Fe}$ & I & 1051 & 7212.47 & & $\mathrm{Fe}$ & I \\
\hline 7131.29 & & Al & II & 114 & 7213.35 & & T1 & I \\
\hline 7132.989 & & $\mathrm{Fe}$ & $\mathbf{I}$ & 1002 & 7213.84 & $\mathbf{P}$ & $\mathrm{Fe}$ & I \\
\hline 7133.16 & & Gd & II & 137 & 7214.78 & $\mathbf{P}$ & T1 & II \\
\hline 7133.52 & & C & II & 20 & 7214.97 & & T 1 & I \\
\hline 7134.290 & & Co & I & 179 & 7216.20 & & T1 & I \\
\hline 7134.66 & & $\mathbf{A 1}$ & II & 114 & 7216.68 & $\mathbf{P}$ & $\mathrm{Fe}$ & $I$ \\
\hline 7134.99 & & $\mathrm{Fe}$ & I I & 197 & 7217.0 & & $\mathrm{~N}$ & II \\
\hline 7135.73 & & Gd & I I & & 7217.34 & & Co & I \\
\hline 7138.05 & $\mathbf{P}$ & T1 & I & 98 & 7217.55 & & Eu & II \\
\hline 7138.81 & & A] & II & 114 & 7218.57 & & $\mathrm{Cr}$ & I \\
\hline 7138.91 & & T1 & I & 99 & 7219.686 & & $\mathrm{Fe}$ & I \\
\hline 7139.79 & & $\mathbf{s}$ & II & 18 & 7220.79 & & N1 & I \\
\hline 7139.8 & & $\mathbf{N}$ & II & 52 & 7221.22 & & $\mathrm{Fe}$ & I \\
\hline 7141.17 & & Gd & II & 131 & 7222.39 & & $\mathrm{Fe}$ & II \\
\hline 7141.62 & & N1 & I & 283 & 7222.88 & & $\mathrm{Fe}$ & I \\
\hline 7142.522 & & $\mathrm{Fe}$ & I & 1274 & 7223.668 & & $\mathrm{Fe}$ & I \\
\hline 7145.317 & & $\mathrm{Fe}$ & $\mathbf{I}$ & 1186,1193 & 7224.51 & & $\mathrm{Fe}$ & II \\
\hline 7146.13 & & Gd & II & 130 & 7225.82 & $\mathbf{P}$ & $\mathrm{Fe}$ & $\mathbf{I}$ \\
\hline 7147.0406 & & $\Delta$ & I & 1 & 7226.20 & & S1 & I \\
\hline 7147.31 & & Gd & II & & 7228.70 & & $\mathrm{Fe}$ & I \\
\hline 7148.147 & & $\mathrm{Ca}$ & I & 30 & 7228.974 & & $\mathrm{~Pb}$ & I \\
\hline 7148.61 & & Ta & I & 11 & 7231.12 & & c & II \\
\hline 7148.69 & & $\mathrm{Fe}$ & I & 1078,1339 & 7233.58 & & $\mathbf{A}$ & II \\
\hline 7151.18 & $\mathbf{P}$ & Sc & II & 27 & 7235.32 & & S1 & I \\
\hline 7151.495 & & $\mathrm{Fe}$ & I & 109 & 7235.86 & & S1 & I \\
\hline 7154.688 & & Co & I & 89 & 7236.19 & & c & II \\
\hline 7155.64 & & $\mathrm{Fe}$ & I & 1276 & 7236.81 & & $\mathbf{s}$ & II \\
\hline 7156.80 & & 0 & I & 38 & 7229.885 & & $\mathrm{Fe}$ & I \\
\hline 7158.502 & & $\mathrm{Fe}$ & I & 815 & 7242.24 & & ad & I I \\
\hline 7160.33 & & T1 & I & 98 & 7244.77 & & $\mathbf{s}$ & I \\
\hline 7160.85 & $\mathbf{P}$ & $\mathrm{Fe}$ & I & 1310 & 7244.86 & & $\mathrm{Fe}$ & I \\
\hline 7161.04 & $\mathbf{P}$ & $\mathrm{Fe}$ & I & 1190 & 7244,86 & & $\mathbf{T 1}$ & I \\
\hline 7162.37 & $\mathbf{P}$ & $\mathrm{Fe}$ & I & 1278 & 7245.1668 & & Ne & I \\
\hline 7164.469 & & $\mathrm{Fe}$ & I & 1051 & 7247.82 & & Mn & I \\
\hline 7164.63 & & $\mathbf{s}$ & II & 18 & 7250.12 & & Co & I \\
\hline 7164.75 & & S1 & I & 49 & 7250.69 & & S1 & $\mathbf{I}$ \\
\hline 7164.90 & & Gd & II & 130 & 7251.74 & & T1 & I \\
\hline 7165.09 & $\mathbf{P}$ & S1 & I & 49 & 7252.70 & & Gd & II \\
\hline 7165.62 & & S1 & I & 48 & 7253.76 & & $\mathbf{T} 1$ & I \\
\hline 7167.01 & & N1 & I & 109 & 7254.19 & & 0 & I \\
\hline 7168.37 & & Gd & I & 1 & 7254.47 & & 0 & I \\
\hline 7169.14 & & $\mathbf{Z}_{\mathbf{r}}$ & I & 42 & 7254.649 & & $\mathrm{Fe}$ & I \\
\hline 7170.14 & & N1 & I & 282 & 7255.28 & $P$ & S1 & I \\
\hline 7172.26 & & Gd & II & 108 & 7256.13 & $\mathbf{P}$ & $\mathrm{Fe}$ & I \\
\hline 7173.73 & & N1 & I & 269 & 7256.63 & & Cl & I \\
\hline 7173.9389 & & $\mathrm{Ne}$ & I & 6 & 7256.72 & & N1 & I \\
\hline 7175.937 & & $\mathrm{Fe}$ & I & 1188 & 7256.96 & & $\mathbf{s}$ & II \\
\hline 7176.886 & & $\mathrm{Fe}$ & I & 1276 & 7259.3 & & $\mathbf{N}$ & II \\
\hline 7177.50 & & He & II & 6 & 7261.00 & $\mathbf{P}$ & $\mathrm{Fe}$ & I \\
\hline 7178.33 & $\mathbf{P}$ & Sc & II & 27 & 7261.29 & $\mathbf{P}$ & $\mathrm{Fe}$ & I \\
\hline 7179.16 & $\mathbf{P}$ & $\mathrm{Fe}$ & II & 72 & 7261.54 & & $\mathrm{Fe}$ & I \\
\hline 7180.020 & & $\mathrm{Fe}$ & I & 33 & 7261.84 & & N1 & I \\
\hline 7181.21 & $\mathbf{P}$ & $\mathrm{Fe}$ & II & 72 & 7262.46 & $\mathbf{P}$ & $\mathrm{Fe}$ & I \\
\hline 7181.222 & & $\mathrm{Fe}$ & I & 1078 & 7264.19 & & $Y$ & II \\
\hline 7181.93 & & $\mathrm{Fe}$ & I & 1274 & 7264.90 & & $\mathrm{Fe}$ & II \\
\hline 7182.00 & & N1 & I & 126 & 7266.22 & & N1 & I \\
\hline 7184.64 & & S1 & I & 25 & 7266.29 & & $\mathrm{~T} 1$ & I \\
\hline 7184.89 & & $S_{1}$ & I & 25 & 7267.00 & $\mathbf{P}$ & $\mathrm{Fe}$ & I \\
\hline 7185.50 & & $\mathrm{Cr}$ & I & 264 & 7268.58 & $\mathbf{P}$ & Fe & I \\
\hline 7187.341 & & $\mathrm{Fe}$ & I & 1051 & 7271.41 & & T1 & I \\
\hline 7188.06 & & $\mathrm{Cr}$ & I & 264 & 7273.20 & & $\mathbf{s}$ & II \\
\hline 7188.56 & & $\mathbf{T 1}$ & I & 98 & 7273.77 & & $T_{1}$ & I \\
\hline 7188.7 & & N & II & 52 & 7275.28 & & S1 & I \\
\hline $7189 \cdot 17$ & & Fo & I & 463 & 7277.67 & & Br & II \\
\hline 7189.57 & & od & II & 138 & 7278.48 & $\mathbf{P}$ & $\mathrm{Fe}$ & I \\
\hline 7189.89 & & $T 1$ & I & 285 & 7278.72 & & $\mathrm{Br}$ & II \\
\hline 7190.12 & $\mathbf{P}$ & $\mathrm{Fe}$ & I & 463 & 7280.298 & & $\mathrm{Ba}$ & I \\
\hline 7181.66 & & $\mathrm{Fe}$ & I & 1274 & 7281.349 & & He & I \\
\hline 7193.20 & $\mathbf{P}$ & 48 & I & 31 & 7282.36 & & La & II \\
\hline 7193.23 & & $\mathrm{Fe}$ & II & 187 & 7282.39 & & $F_{a}$ & I \\
\hline 7193.56 & & $\mathbf{s 1}$ & & 25 & 7283.80 & & $M n$ & I \\
\hline
\end{tabular}




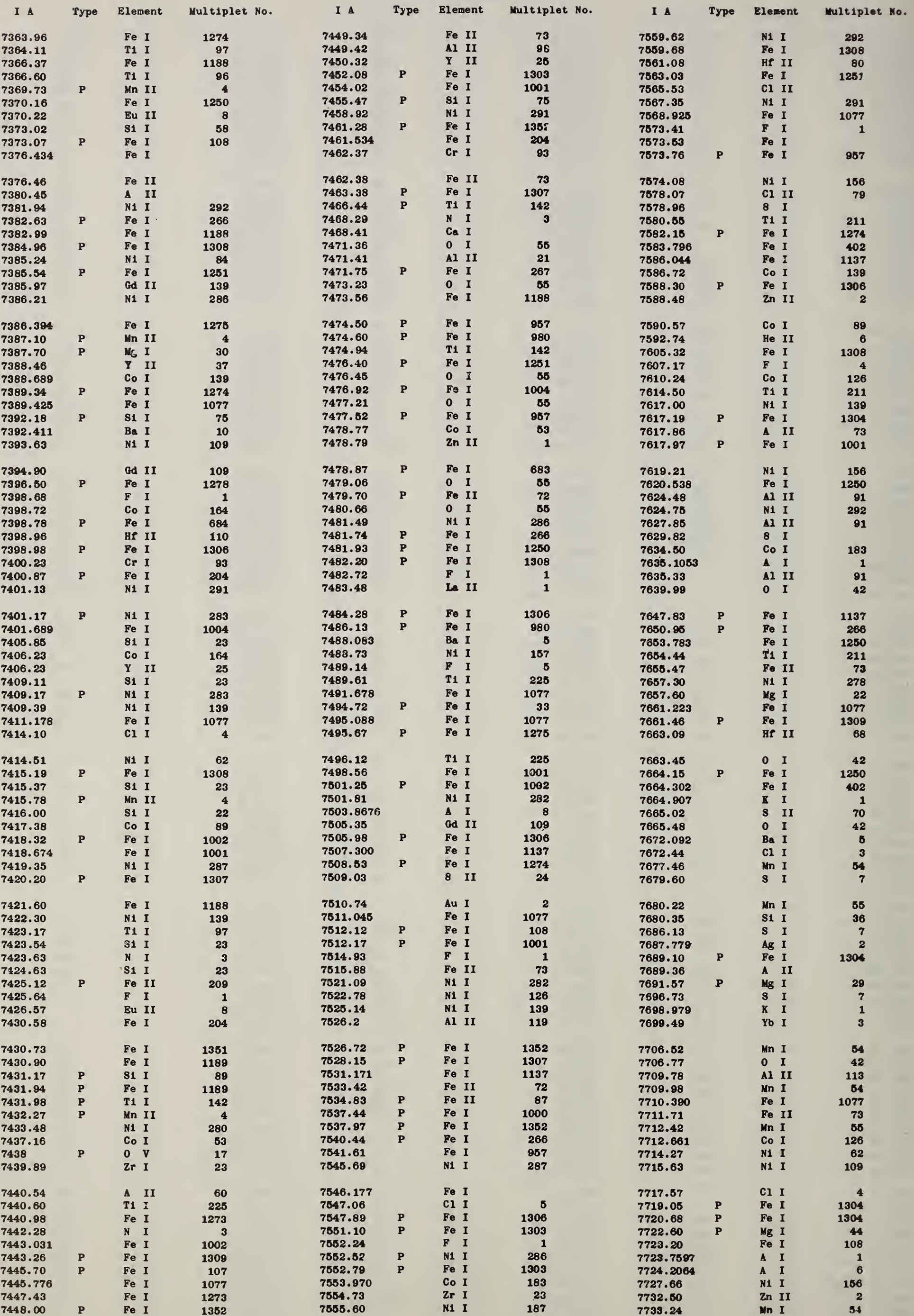


I A Type Element Multiplet No.

I A Type Element Multiplet No.

I A Type Element

Multiplet No.

\begin{tabular}{|c|c|c|c|c|c|c|c|c|}
\hline 7733.50 & & Gd & I & 1 & 7909.60 & $\mathbf{P}$ & $\mathrm{Fe}$ & I \\
\hline 7733.68 & $\mathbf{P}$ & $\mathbf{F e}$ & I & 1306 & 7910.50 & & $\mathrm{Cr}$ & $\mathbf{I}$ \\
\hline 7734.43 & & Mn & I & 55 & 7911.338 & & $B a$ & I \\
\hline 7735.99 & & N1 & I & 281 & 7912.55 & & s1 & I \\
\hline 7737.67 & $\mathbf{P}$ & $\mathrm{Fe}$ & I & 1137 & 7912.866 & & $\mathrm{Fe}$ & I \\
\hline 7742.7 & & S1 & $\mathbf{I}$ & & 7913.47 & & $\mathbf{S}_{1}$ & I \\
\hline 7742.71 & & $\mathrm{Fe}$ & I & 1306 & 7917.48 & & N1 & I \\
\hline 7743.2 & & S1 & I & & 7917.85 & & Cr & I \\
\hline 7743.27 & & Co & I & 183 & $7918 \cdot 38$ & & S1 & $\mathbf{I}$ \\
\hline 7744.94 & & $\mathrm{Cl}$ & I & 5 & 7923.95 & & $\mathbf{s}$ & I \\
\hline 7745.05 & & $\mathbf{s}$ & I I & 70 & 7924.14 & $\mathbf{P}$ & $\mathrm{Fe}$ & I \\
\hline 7745.48 & $\mathbf{P}$ & $\mathrm{Fe}$ & I & 1305 & 7924.62 & & $\mathrm{Cl}$ & I \\
\hline 7746.56 & $\mathbf{P}$ & $\mathrm{Fe}$ & I & 1309 & 7926.37 & & Ti & I \\
\hline 7748.281 & & $\mathrm{Fe}$ & I & 402 & 7928.14 & & Sm & II \\
\hline 7748.37 & & Gd & II & 142 & 7928.84 & & $\mathbf{s}$ & I \\
\hline 7748.93 & & N1 & I & 156 & 7930.25 & & od & II \\
\hline 7751.18 & & $\mathbf{F e}$ & $\mathbf{I}$ & 1304 & 7930.33 & & $\mathbf{s}$ & $\mathbf{I}$ \\
\hline 7754.70 & & $\mathbf{F}$ & I & 4 & 7830.83 & $\mathbf{P}$ & $\mathrm{Mg}$ & I \\
\hline 7755.15 & & Mn & I & 55 & $7931 \cdot 70$ & & $\mathbf{s}$ & I \\
\hline 7757.89 & & ня & II & 66 & 7932.20 & & S1 & I \\
\hline 7764.72 & & Mn & $\mathbf{I}$ & 54 & 7933.130 & & $\mathrm{Cu}$ & I \\
\hline 7766.72 & $\mathbf{P}$ & $\mathbf{F e}$ & I & 857 & 7937.166 & & $\mathrm{Fe}$ & I \\
\hline 7771.74 & & $\mathrm{Cr}$ & I & & 7938.53 & $\mathbf{P}$ & T1 & I \\
\hline 7771.96 & & 0 & I & 1 & 7939.49 & & 0 & I \\
\hline 7774.18 & & 0 & I & 1 & 7841.09 & & $\mathrm{Fe}$ & I \\
\hline 7775.40 & & 0 & I & 1 & 7941.84 & $\mathbf{P}$ & $\mathrm{Fe}$ & I \\
\hline 7780.479 & & $\mathrm{Ba}$ & I & 5 & 7942.02 & & $\mathrm{Cr}$ & I \\
\hline 7780.586 & & $\mathrm{Fe}$ & I & 1154 & 7942.81 & & Mn & I \\
\hline 7788.95 & & N1 & I & 62 & 7943.16 & & 0 & I \\
\hline 7797.62 & & N1 & I & 201 & 7943.1802 & & $\mathrm{Ne}$ & I \\
\hline 7798.90 & $\mathbf{P}$ & $\mathrm{Fe}$ & I & 403 & 7943.93 & & T1 & I \\
\hline 7800.0 & & 81 & I & 81 & 7943.94 & & 81 & I \\
\hline 7800.22 & & $\mathbf{F}$ & I & 4 & 7944.65 & & $\mathrm{Zr}$ & I \\
\hline 7800.227 & & $\mathbf{R b}$ & I & 1 & 7945.878 & & $\mathrm{Fe}$ & I \\
\hline 7802.49 & $\mathbf{P}$ & Fe & I & 1303 & 7845.98 & $\mathbf{P}$ & $\mathrm{Fe}$ & I \\
\hline 7807.87 & $\mathbf{P}$ & $\mathrm{Fe}$ & I & 1303 & 7947.204 & & 0 & I \\
\hline 7808.04 & & $\mathbf{F e}$ & $I$ & & 7947.56 & & 0 & I \\
\hline 7809.24 & & Co & I & 79 & 7847.60 & & $\mathbf{R b}$ & I \\
\hline 7809.4 & & $\mathrm{Na}$ & I & 20 & $7948 \cdot 1754$ & & $\mathbf{A}$ & I \\
\hline 7810.81 & $\mathbf{P}$ & $\mathbf{F e}$ & I & 1303 & 7948.17 & & $\mathbf{T 1}$ & I \\
\hline 7811.14 & $\mathbf{P}$ & Mg & I & 43 & 7850.83 & & 0 & I \\
\hline 7812.31 & & $\Delta 1$ & II & 90 & 7952.18 & & 0 & I \\
\hline 7813.62 & $\mathbf{P}$ & $\mathbf{F e}$ & I & 1305 & 7953.11 & & N1 & I \\
\hline 7815.83 & & Al & I I & 80 & 7954.84 & $\mathbf{P}$ & $\mathrm{Fe}$ & I \\
\hline 7816.16 & & He & I & 69 & 7955.81 & & $\mathrm{Fe}$ & I \\
\hline 7820.80 & $\mathbf{P}$ & $\mathrm{Fe}$ & I & 1118 & 7956.69 & & $\mathbf{Z r}$ & I \\
\hline 7821.47 & & 8 & II & 31 & 7959.21 & & $\mathrm{Fe}$ & I \\
\hline 7823.72 & & $\mathbf{A l}$ & II & 90 & 7861.58 & & $\mathrm{~T} 1$ & I \\
\hline 7826.81 & & N1 & I & 109 & 7963.25 & & od & II \\
\hline 7832.224 & & Fe & I & 1164 & 7964.93 & $\mathbf{P}$ & $\mathbf{F e}$ & I \\
\hline 7835.08 & & $8 m$ & II & 69 & 7865.52 & $\mathbf{P}$ & $\mathrm{Fe}$ & I \\
\hline 7835.33 & & Al & I & 10 & 7967.03 & $\mathbf{P}$ & $\mathrm{Fe}$ & I \\
\hline 7836.15 & & $\mathbf{A 1}$ & I & 10 & 7867.43 & & $\mathbf{s}$ & II \\
\hline 7837.27 & & Sm & II & 64 & 7970.26 & & s1 & $\mathbf{I}$ \\
\hline 7838.09 & $\mathbf{P}$ & $\mathrm{Fe}$ & II & 87 & 7978.88 & & $\mathbf{T} 1$ & I \\
\hline 7841.40 & $\mathbf{P}$ & Fe & I I & 72 & 7980.04 & $\mathbf{P}$ & $\mathrm{Fe}$ & I \\
\hline 7844.55 & $\mathbf{P}$ & $\mathrm{Fe}$ & I & 1250 & 7880.58 & & CI & I \\
\hline 7844.87 & & ad & II & & 7881.97 & & 0 & $I$ \\
\hline 7846.35 & & od & II & & 7882.41 & & 0 & I \\
\hline 7846.47 & $\mathbf{P}$ & $\mathrm{Fe}$ & I & 1323 & 7883.66 & & HF & II \\
\hline 7849.38 & & $\mathbf{z r}$ & I & 40 & 7987.00 & & 0 & I \\
\hline 7850 & & C & I & 32 & 7987.34 & & 0 & I \\
\hline 7850.5 & & 81 & I & 81 & 7987.36 & & Co & I \\
\hline 7852.74 & $\mathbf{P}$ & T1 & I & 34 & 7889.38 & & $\mathbf{C r}$ & $\mathbf{I}$ \\
\hline 7855.12 & & N1 & I & 267 & 7994.473 & & Fe & I \\
\hline 7855.41 & $\mathbf{P}$ & $\mathrm{Fe}$ & I & 1305 & 7895.00 & $\mathbf{P}$ & 81 & I \\
\hline $7861 \cdot 10$ & & N1 & $\mathbf{I}$ & 156 & 7985.12 & & 0 & $\mathbf{I}$ \\
\hline 7861.22 & & HP & II & 66 & 7996.53 & & $\mathbf{T} 1$ & I \\
\hline 7863.79 & & N1 & I & 268 & 7896.80 & & Co & I \\
\hline 7869.65 & & $\mathrm{Fe}$ & I & 1137 & 7997.80 & & Cl & I \\
\hline 7869.868 & & Co & I & 189 & 7997.85 & & 8 & II \\
\hline 7870.00 & & $\mathbf{2 r}$ & I & 41 & 7998.972 & & Fe & I \\
\hline 7871.370 & & Co & I & 188 & 8002.55 & $\mathbf{P}$ & Fo & I \\
\hline 7877.13 & & $\mathrm{~kg}$ & II & 8 & 8005.24 & & 8 & II \\
\hline 7878.22 & & Cl & I & 3 & 8006.1566 & & $\mathbf{A}$ & I \\
\hline 7879.75 & $\mathbf{P}$ & Fe & I & 1306 & 8009.38 & $\mathbf{P}$ & 81 & I \\
\hline 7881.90 & & $\mathbf{I}$ & II & 32 & 8014.7856 & & 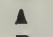 & I \\
\hline 7886.00 & $\mathbf{p}$ & $\mathbf{T} 1$ & I & 34 & 8018.51 & $\mathbf{P}$ & Fo & I \\
\hline 7885.26 & & 8 & II & 68 & 8018 & & C & I \\
\hline 7886.31 & & 0 & I & 64 & 8018.04 & & $\mathrm{Cr}_{\mathrm{r}}$ & I \\
\hline 7880.22 & & N1 & I & 200,267 & 8018.70 & & 8 & II \\
\hline 7895.50 & $\mathbf{P}$ & $\mathbf{T 1}$ & $I$ & 34 & 8024.50 & & Fo & $\mathbf{I}$ \\
\hline 7896.37 & & $\mathrm{~kg}$ & II & 8 & 8024.84 & & $\mathbf{T} \mathbf{1}$ & I \\
\hline 7898.38 & $\mathbf{P}$ & 81 & $I$ & 69 & $8025 \cdot 12$ & & 8 & II \\
\hline 7904.12 & $\mathbf{P}$ & $\mathrm{Fe}$ & I & 403 & 8026.32 & & 8. & II \\
\hline 7905.751 & & Ba & I & 10 & 8027.36 & & $\mathbf{v}$ & $I$ \\
\hline 7908.06 & & Od & II & 120 & 8027.96 & $\mathbf{P}$ & Fo & I \\
\hline 7808.30 & & $\mathbf{C r}$ & I & 316 & 8028.341 & & F• & I \\
\hline 7908.679 & & Co & I & 189 & 8032.03 & & 8 & II \\
\hline & $\mathbf{p}$ & & & & 8034.66 & & & \\
\hline
\end{tabular}


I A Type Element Multiplet No.

\begin{tabular}{|c|c|c|c|}
\hline $\begin{array}{l}8203.2 \\
8203.572\end{array}$ & $\mathbf{P}$ & $\begin{array}{l}\mathrm{Ca} \\
\mathrm{H}\end{array}$ & I I \\
\hline 8204.10 & $\mathbf{P}$ & Fe & I \\
\hline 8204.83 & $\mathbf{P}$ & Fo & I \\
\hline 8207.767 & & Fe & I \\
\hline 8208.57 & & Co & I \\
\hline 8210.64 & & $\mathbf{N}$ & I \\
\hline 8211.48 & & $\mathbf{s 1}$ & I \\
\hline 8212.00 & & Cl & I \\
\hline 8212.43 & & Mn & I \\
\hline 8212.59 & & $\mathbf{Z r}$ & I \\
\hline 8213.02 & $\mathbf{P}$ & $\mathrm{Mg}$ & I \\
\hline 8216.28 & & $\mathrm{Cr}$ & I \\
\hline 8216.28 & & $\mathbf{N}$ & I \\
\hline 8217.8 & $\mathbf{P}$ & $\mathrm{Mg}$ & II \\
\hline 8220.40 & & Cl & I \\
\hline 8220.406 & & Fe & I \\
\hline 8221.63 & & $\mathbf{s}$ & II \\
\hline 8221.73 & & Cl & I \\
\hline 8221.84 & & 0 & I \\
\hline 8222.15 & & $\mathbf{s}$ & II \\
\hline 8223.07 & & $\mathbf{N}$ & I \\
\hline 8223.16 & & $\mathbf{s}$ & II \\
\hline 8224.08 & & $\mathrm{Cr}$ & I \\
\hline 8225.15 & & 8 & II \\
\hline 8225.67 & & $\mathrm{Cr}$ & I \\
\hline 8227.64 & & 0 & I \\
\hline 8230.01 & & 0 & I \\
\hline 8230.67 & & S1 & I \\
\hline 8232.347 & & $\mathrm{Fe}$ & I \\
\hline
\end{tabular}

8233. 30

8235.31

8235.88

8236.13

8236.77

8238. 2

8238.4

8238.130

13
14
12
12
1136
183
2
19
2

40
28

28

2

3
136

31

34

68

2

98

68

99

34

34
19

18

$\begin{array}{llll}8242.34 & & \text { N I } & \\ 8248.151 & & \text { Fe I } & 1138 \\ 8250.2 & \text { P } & \text { Ca II } & 13 \\ 8253.51 & & \text { V I } & \\ 8253.78 & \text { P } & \text { Fe I } & 121 \\ 8254.10 & & \text { Be I } & \\ 8254.34 & \text { P } & \text { Fe I } & 508 \\ 8255.153 & & \text { H } & \\ 8255.80 & & \text { V I } & \\ 8256.1 & \text { P } & \text { Ca II } & \\ 8257.859 & & \text { H } & \end{array}$

8257.858
8258.27
8260.838
8261.95
8263.86
8264.27
8264.288
8264.5209
8266.076
8267.041

\begin{tabular}{|c|c|c|c|}
\hline 8268.66 & $\mathbf{P}$ & $\mathrm{Fe}$ & I \\
\hline 8271.834 & & H & \\
\hline 8273.46 & & 8 & I I \\
\hline 8273.518 & & 48 & I \\
\hline 8274.28 & & $\mathrm{Fe}$ & I \\
\hline 8276.81 & & $\mathrm{Fe}$ & I \\
\hline 8276.310 & & H & \\
\hline 8281.128 & & B & \\
\hline 8286.434 & & H & \\
\hline 8287.38 & & $\mathrm{Cr}$ & I \\
\hline 8290.62 & & $\mathrm{Cr}$ & I \\
\hline 8282.309 & & H & \\
\hline 8283.627 & & $\mathrm{Fe}$ & I \\
\hline 8286.90 & & $\mathrm{Cr}$ & I \\
\hline 8297.58 & & $\mathrm{Cr}$ & I \\
\hline 8298.837 & & H & \\
\hline 8300.01 & $\mathbf{P}$ & $\mathrm{Fe}$ & I \\
\hline 8300.3258 & & Ne & I \\
\hline 8303.11 & $\mathbf{P}$ & $\mathrm{Fe}$ & I \\
\hline 8303.18 & & $\mathrm{Cr}$ & I \\
\hline 8305.62 & & As & I \\
\hline 8305.78 & & $8 m$ & II \\
\hline 8305.94 & & $\mathbf{Z r}$ & I \\
\hline 8306.115 & & H & \\
\hline $8306: 80$ & & 81 & I \\
\hline 8307.41 & & T1 & I \\
\hline 2907.61 & $\mathbf{P}$ & $\mathrm{Fe}$ & I \\
\hline 9210.88 & $\mathbf{P}$ & $\mathrm{Fe}$ & I \\
\hline 8314.262 & & 月 & \\
\hline 8314.73 & & 8 & I I \\
\hline
\end{tabular}

$\begin{array}{ll}0 & 1 \\ 8 & \end{array}$

0 I

Cr I

He II

He II

Cr I

Mg II

$\begin{array}{ll}\mathrm{V} & I \\ & \end{array}$

34

34

65

6

7

30

2

16

2
08

508
14

H 14

\begin{tabular}{rrr}
14 & 14 \\
\hline & 1 &
\end{tabular}

27
13

18

8
2
2

13
13
13

8

298
12
823
57
87
12
31
12
165
57
5
68
40
12
19
33
12
12
12
12

2
7
7
2
12
12
57
12
5
68
40
12
19
33
12
12
12
12

\begin{abstract}
I A Type Eloment Multiplet No.
\end{abstract}

I A Type Element Multiplet No.

$\begin{array}{cc}\text { I A } & \text { Type } \\ 8428.342 & \\ 8428.94 & \\ 8429.128 & \\ 8428.36 & \\ 8431.20 & \\ 8434.51 & P \\ 8434.98 & \\ 8435.28 & P \\ 8435.68 & \\ 8437.858 & \\ & \\ 8438.93 & \\ 8439.603 & \\ 8442.58 & \\ 8442.98 & \\ 8444.00 & \\ 8444.48 & \\ 8446.35 & \\ 8446.42 & P \\ 8446.56 & P \\ 8446.76 & \\ & \end{array}$

$\begin{array}{llr}0 & \text { I } & 54 \\ \text { As } & \text { I } & 4 \\ 0 & \text { I } & 54 \\ \text { Y } & \text { II } & \\ \text { Mn } & \text { I } & 53 \\ \text { Fe } & \text { I } & 1270 \\ \text { T1 } & \text { I } & 33 \\ \text { S1 } & \text { I } & 8 \\ \text { T1 } & \text { I } & 33 \\ \text { H } & & 10\end{array}$

\begin{tabular}{|c|c|c|c|c|}
\hline 8316.38 & & Gd & II & \\
\hline 8317.45 & & 81 & I & 18 \\
\hline 8322.06 & & $\mathrm{Cr}$ & I & 298 \\
\hline 8323.428 & & H & & 12 \\
\hline 8323.44 & & $\mathrm{Cr}$ & I & 288 \\
\hline 8327.063 & & Fe & I & 60 \\
\hline 8331.941 & & $\mathrm{Fe}$ & I & 1153 \\
\hline 8333.29 & & $\mathrm{Cl}$ & I & 2 \\
\hline 8333.785 & & H & & 11 \\
\hline 8334.37 & & T1 & I & 33 \\
\hline 8335.18 & & C & I & 10 \\
\hline 8338.43 & & S1 & I & 33 \\
\hline 8338.83 & & $\mathrm{Cr}_{\mathrm{r}}$ & I & 298 \\
\hline 8339.431 & & $\mathrm{Fe}$ & I & 1153 \\
\hline 8342.21 & $\mathbf{P}$ & Fo & I & 401 \\
\hline 8342.85 & & $\mathrm{Fe}$ & I & 1270 \\
\hline 8345.20 & $\mathbf{P}$ & $\mathrm{Fe}$ & I & 265 \\
\hline 8345.553 & & H & & 11 \\
\hline 8346.13 & $\mathbf{P}$ & $\mathrm{Mg}$ & I & 40 \\
\hline 8348.28 & & $\mathrm{Cr}$ & I & 56 \\
\hline 8348.68 & & Sm & I I & 64 \\
\hline 8349.06 & $\mathbf{P}$ & Fe & I & 12 \\
\hline 8353.15 & & T1 & I & 33 \\
\hline 8354.35 & & Al & I I & 40 \\
\hline $8355 \cdot 16$ & $\mathbf{P}$ & $\mathrm{Fe}$ & I & 1050 \\
\hline 8356.07 & $\mathbf{P}$ & Fo & I & 1117 \\
\hline 8358.53 & $\mathbf{P}$ & Fe & I & 401 \\
\hline 8358.006 & & H & & 11 \\
\hline 8358.23 & & Al & II & 40 \\
\hline 8359.57 & & Al & II & 40 \\
\hline
\end{tabular}

$\begin{array}{rllr}8447.41 & P & \text { Fe I } & 1266 \\ 8447.63 & P & \text { Fe I } & 12\end{array}$

$8448.54 \quad S$ S $~ 14$

$\begin{array}{rrr}8450.26 & \text { Cr I } & 68 \\ 8450.88 & \text { T1 } & \text { I }\end{array}$

$\begin{array}{lll}8451.05 & S \text { I } & 14\end{array}$

$\begin{array}{llll}8462.14 & 8 & 1 & 14\end{array}$

$\begin{array}{rrr}8455.24 & \text { Cr I } & 56 \\ 8457.10 & \text { T1 I } & 141\end{array}$

$\begin{array}{rrrr}8459.01 & P & \text { Fe } I & 1270\end{array}$

8360.63 Cl II

$\begin{array}{lrr}8361.77 & \text { Fe I } & 1153 \\ 8362 & \text { He I } & 68\end{array}$

$\begin{array}{llll}8361.85 & 8 & \text { II } & 31\end{array}$

8363.30 Al II 40

$\begin{array}{rllr}8363.52 & & \text { Al I I } & 40 \\ 8363.58 & \text { P } & \text { TI I } & 182\end{array}$

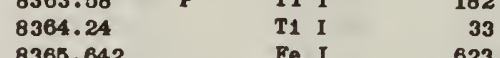

$\begin{array}{lllr}8365.642 & & F \theta_{1} & 623 \\ 8369.87 & P & F e I & 1271\end{array}$

$\begin{array}{lll}8370.21 & \text { Zr I } & 40\end{array}$

$\begin{array}{llr}8372.79 & \text { Co I } & 40 \\ 8374.478 & \text { H } & 193\end{array}$

$\begin{array}{llr}8374.478 & \text { CI I } & 11 \\ 8375.95 & \text { No I } & 12\end{array}$

$\begin{array}{lll}8376.41 & \text { Ne I } & 12 \\ 8377.6068 & \text { Ne I } & 12\end{array}$

$\begin{array}{lll}8377.79 & \text { S II } & 31\end{array}$

$\begin{array}{rrr}8377.90 & \text { T1 I } & 33 \\ 8379.44 & \text { Co I } & 193\end{array}$

$\begin{array}{ll}8379.44 & \text { Co I } \\ 8380.77 & \text { Mn I }\end{array}$

$\begin{array}{lllr}8382.23 & \text { P } & \text { Fe I } & 12 \\ 8382.54 & & \text { T1 I } & 33 \\ 8382.82 & & \text { Ti I } & 33 \\ 8386.24 & \text { P } & \text { TI I } & 182 \\ 8387.781 & & \text { Fe I } & 60 \\ 8389.42 & & \text { Zr I } & 40 \\ 8389.48 & & \text { T. I } & 182 \\ 8392.400 & & \text { H } & 11 \\ 8395.87 & & \text { Mn I } & 53 \\ 8396.93 & & \text { T1 I } & 33\end{array}$

$\begin{array}{llll}8461.41 & P & \text { Fe I } & 814\end{array}$

$\begin{array}{rrrr}8461.41 & P & \text { Fe } & 814 \\ 8464.02 & P & F e & 1\end{array}$

$\begin{array}{lllr}8464.65 & & \mathrm{Zr} I & 10 \\ 8485.23 & P & \mathrm{Fe} I & 1270\end{array}$

$\begin{array}{llll}8466.10 & P & \text { Fe I } & 1260\end{array}$

$\begin{array}{lllr}8466.54 & P & F e I & 999\end{array}$

$\begin{array}{llll}8467.15 & P & \text { TI I } & 989 \\ 8467.256 & & H & 182\end{array}$

$\begin{array}{lll}8487.256 & \text { H } & 10 \\ 8468.413 & \mathrm{Fe} \mathrm{I} & 60\end{array}$

$\begin{array}{lll}8468.46 & \text { Ti } & 1\end{array}$

$8471.75 \quad$ Fe I 1270

$\begin{array}{rlrr}8480.63 & \text { P } & \text { Fe I } & 1272 \\ 8481.96 & \text { P } & \text { Fe I } & 890\end{array}$

$\begin{array}{lll}8483.16 & \text { TI I } & 150\end{array}$

$\begin{array}{rllr}8485.98 & & 8 m \text { II } & 66 \\ 8493.78 & P & \text { Fo I } & 1268\end{array}$

$\begin{array}{rrrr}8493.78 & P & \text { Fe I } & 1268 \\ 8494.42 & & \text { T1 I } & 141\end{array}$

$8496.3600 \quad$ Ne $I \quad 18$

$\begin{array}{llc}8495.51 & \text { T1 I } & 210 \\ 8496.03 & \text { T1 I } & 209,313\end{array}$

$\begin{array}{llll}8486.51 & P & F e & I\end{array}$

$8487.00 \quad$ Fe I 1172

8498.018 Ca II 2

$\begin{array}{lll}8498.44 & \text { Zr I } & 40 \\ 8501.50 & \text { S1 I } & 47\end{array}$

$\begin{array}{rrr}8501.81 & \text { N1 } ~ & 47 \\ 8502.38 & \text { BI } & 186\end{array}$

$\begin{array}{lll}8502.38 & 81 \text { I } & 46 \\ 8502.487 & \text { H } & 10\end{array}$

$\begin{array}{lllr}8503.17 & & \text { S1 I } & \\ 8509.63 & \text { P } & \text { Fe I } & 1136\end{array}$

$\begin{array}{lll}8397.04 & \mathrm{Cr} I & 57\end{array}$

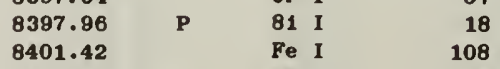

$\begin{array}{rrrr}8401.68 & P & \text { Fe } I & 108 \\ 8 & & 1136\end{array}$

$\begin{array}{llr}8402.54 & \text { T1 } I & 224\end{array}$

8408.208 A I

$\begin{array}{ll}8408.88 & \text { Mn } 1 \\ 8412.36 & \text { T1 I }\end{array}$

$\begin{array}{lll}8412.36 & \text { TI I } & 33 \\ 8413.321 & \text { H } & 10 \\ 8414.00 & \text { Zr I } & 40\end{array}$

$\begin{array}{llll}8414.08 & P & \text { Fe } I & 1154\end{array}$

$\begin{array}{rrrr}8414.08 & \text { P } & \text { Fe I } & 1164 \\ 8416.97 & & \text { T1 I } & 224 \\ 8417.24 & & \text { N1 I } & 156\end{array}$

$\begin{array}{lll}8417.24 & \text { N1 I } & 156 \\ 8417.54 & \text { T1 I } & 182\end{array}$

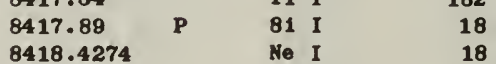

$\begin{array}{ll}8418.4274 & \text { Ne } I \\ 8418.70 & \text { Ti I }\end{array}$

$\begin{array}{llll}8420.968 & 0 & \text { I } & 54 \\ 8422.38 & 8 & \text { II } & 37\end{array}$

$\begin{array}{rlr}8422.95 & \text { Fo I } & 989\end{array}$

$8423.10 \quad$ T1 I $\quad 160$

$\begin{array}{rrr}8424.14 & \text { Fe I } & 1272 \\ 8424.41 & \text { T1 I } & 182\end{array}$

$\begin{array}{llr}8424.647 & \text { A I } & 182 \\ 8424.780 & \end{array}$

$8424.780 \quad 0 \quad I$

8425.37 P 8 II

$\begin{array}{lll}8425.89 & P & \text { Fo } I \\ 8426.326 & & 0\end{array}$

$8426.50 \quad$ T1 I

8428.25

$\begin{array}{ll}\text { T1 } & 1 \\ \text { C1 } & 1\end{array}$

\begin{tabular}{|c|c|c|c|c|}
\hline 8510.90 & & $8 \mathrm{~m}$ & II & 64 \\
\hline 8512.85 & $\mathbf{P}$ & $\mathrm{Fe}$ & I & 462 \\
\hline 8514.075 & & Fe & I & 60 \\
\hline 8514.64 & $\mathbf{P}$ & 81 & I & 18 \\
\hline 8515.08 & & Fe & I & 401 \\
\hline 8515.48 & & 8 & II & 37 \\
\hline 8518.05 & & T1 & I & 182 \\
\hline 8518.37 & & T1 & I & 150 \\
\hline 8519.05 & $\mathbf{P}$ & $\mathrm{Fe}$ & I & 1267 \\
\hline 8520.23 & & $\mathbf{s}$ & II & 34 \\
\hline 8621.10 & & Cs & I & 1 \\
\hline 8521.4407 & & A & I & 8 \\
\hline 8522.64 & & $\mathbf{s}$ & II & 62 \\
\hline 8525.04 & $\mathbf{P}$ & Fe & I & 1215 \\
\hline 8526.685 & & Fe & I & 1270 \\
\hline 8327.32 & $\mathbf{P}$ & s1 & I & 18 \\
\hline 8527.88 & $\mathbf{P}$ & $\mathrm{Fe}$ & I & 1270 \\
\hline 8531.36 & & T1 & I & 141 \\
\hline 8523.38 & & S1 & I & 80 \\
\hline $8538^{\circ} .02$ & $\mathbf{P}$ & Fe & I & 1266 \\
\hline 8539.36 & & T1 & I & 200 \\
\hline 8541.65 & & As & I & 3 \\
\hline 8542.089 & & Ce & II & 2 \\
\hline 8543.72 & & $\mathrm{Cr}$ & I & 36 \\
\hline 8545.384 & & H & & 10 \\
\hline 8548.07 & & T1 & I & 150 \\
\hline 8548.83 & & $\mathrm{Cr}$ & I & 56 \\
\hline 8550.34 & $\mathbf{P}$ & s1 & I & 88 \\
\hline 8550.46 & & Cl & I & 13 \\
\hline 8650.84 & & T1 & I & 141 \\
\hline
\end{tabular}


11

Type Blement Multiplet No.

56
45
1321
1153
3
141
8
1268
209
1272

2
87

1215

141
56

401

2

$\begin{array}{llllr}8584.82 & \text { P } & \text { Fe I } & 1270 \\ 8585.60 & & \text { S I } & \\ 8585.96 & & \text { CI I } & 2\end{array}$

$\begin{array}{llll}8586.20 & P & \text { N1 } ~ & 296\end{array}$

$\begin{array}{lllr}8586.20 & \text { P } & \text { N1 I } & 296 \\ 8589.78 & & \text { Co I } & 193 \\ 8591.2584 & & \text { Ne I } & 30 \\ 8582.10 & \text { P } & \text { Fe I } & 1269 \\ 8592.97 & & \text { Fe I } & 1267 \\ 8594.01 & & \text { N I } & 8 \\ 8596.02 & \text { P } & \text { S1 I } & 80 \\ 8597.00 & & \text { S1 I } & 80 \\ 8598.18 & & \text { T1 I } & 236 \\ 8598.394 & & \text { H } & 9 \\ 8598.79 & & \text { Fe I } & 1153 \\ 8600.98 & & \text { T1 I } & 141,209 \\ 8606.43 & & \text { S1 I } & 55 \\ 8606.45 & & \text { N1 I } & 275 \\ 8607.08 & \text { P } & \text { Fe I } & 1272 \\ 8610.62 & \text { P } & \text { Fe I } & 1153 \\ 8611.807 & & \text { Fe I } & 339 \\ 8612.91 & & \text { T1 I } & 141 \\ 8613.93 & \text { P } & \text { Fe I } & 1272 \\ 8616.27 & \text { P } & \text { Fe I } & 1266 \\ & & & \end{array}$

$\begin{array}{lll}8618.44 & \text { T1 } I & 209\end{array}$

$8621.612 \quad \mathrm{Fe} \mathrm{I}$

$8621.612 \quad$ Fo I

8629.24 N I

$\begin{array}{llll}8632.42 & \text { P } & \text { Fe } I \\ 8634.6480 & & \text { Ne I }\end{array}$

$8636.26 \quad$ Cr I

$8637.04 \quad$ N1 I

8640.70 AI II

$\begin{array}{ll}8641.47 & \text { T1 I } \\ 8643.03 & \text { CF I }\end{array}$

401

1050

23

186
4

$\begin{array}{llll}8643.29 & P & \text { Fe } I & 1261\end{array}$

8647.05 Ne I

8648.89 S1 I

$8648.6 \quad \mathrm{Na} I$

$\begin{array}{lll}8652.50 & P & \text { Fe I } \\ 8654.16 & & \text { As I }\end{array}$

8654.16 As I

$\begin{array}{llll}8654.3835 & & \text { Ne I } \\ 8654.40 & P & \text { Pe I }\end{array}$

8654.51 Ne I

8654.63 Wn I

19
1050

1050
3

33
623

33

$\begin{array}{lllr}8655.88 & & \text { N I } & 8 \\ 8656.67 & \text { P } & \text { Pe I } & 1269 \\ 8659.38 & & \text { Mn I } & 59 \\ 8661.908 & & \text { Fe I } & 60 \\ 8662.140 & & \text { Ca II } & 2 \\ 8683.73 & \text { P } & \text { Fe I } & 1270 \\ 8665.021 & & \text { H } & 9 \\ 8667.37 & \text { P } & \text { Fe I } & 166 \\ 8667.40 & \text { P } & \text { S1 I } & 55\end{array}$

59

8
1269

59
60

2
270

$8667.9430 \quad A$ I

8670.19

s I

8670.65

8670.92

8671.28

8871.37

8671.86

8672.06

8873.97

8674.751
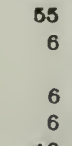

M I

Al II

A1 II

8 I

P

8674.92

8675.28

8675.38

8679.00

8679.491

8678.61

8679.70

8680.24

8680.31

Fe I

Mn I

Yn I

AI II

A1 II

T1 I

S I

Ne I

Fe I

8 I

N I

Al II

$8 \mathrm{~s}$

112
I A Type Element Multiplet No.

$\begin{array}{llrrr}8680.77 & \text { P } & \text { Fe I } & 999 \\ 8681.920 & & \text { Ne I } & 23 \\ 8682.99 & & \text { TI I } & 68 \\ 8683.38 & & \text { N I } & 1 \\ 8686.13 & & \text { N I I } & 1 \\ 8686.28 & & \text { CI I } & 14 \\ 8686.77 & \text { P } & \text { Fe I } & 1269 \\ 8686.79 & \text { P } & \text { Fe I } & 956 \\ 8688.633 & & \text { Fe I } & 60 \\ 8689.71 & \text { P } & \text { Fe I } & 507\end{array}$

I A Ţpe Element Multiplet No.

$\begin{array}{llll}8689.83 & P & \text { Fe } I & 1330\end{array}$

$\begin{array}{lllr}8689.83 & \text { P } & \text { Fe I } & 1330 \\ 8692.34 & & \text { TI I } & 68 \\ 8693.24 & & \text { S I } & 6\end{array}$

8693.98 S I

$\begin{array}{llll}8694.70 & & S & I \\ 8698.71 & P & \text { Fe I }\end{array}$

$8699.13 \quad M n ~ I \quad 49$

$\begin{array}{llll}8699.43 & & \text { Fe I } & 1267 \\ 8700.34 & \text { P } & \text { Fe I } & 1266\end{array}$

$\begin{array}{rrr}8701.05 & \text { Mn I } & 49\end{array}$

$8702.49 \quad$ N1 I 83

8703.24

8703.76

8704.15

8707.42

8707.95
8710.29

8710.82

8711.69

N I

Mn I

Cr I

36

$\begin{array}{rr}\text { Cr } & 296 \\ \text { Pe } & 1\end{array}$

Ba II

$\begin{array}{ll}\mathrm{N} & \mathrm{I} \\ \mathrm{Fe} & \mathrm{I}\end{array}$

8718.70

8718.82

8719.56

$8727.10 \quad P \quad$ Fe I

8728.38

8728.88

$8729.02 \quad$ N

$8729.12 \quad P \quad F$

8732.17

Cr I

400,1267

$\begin{array}{ll}8734.60 & \text { Mn I } \\ 8734.70 & \text { T1 I }\end{array}$

$\begin{array}{llll}8736.0 & P & \text { Mg I } \\ 8737.32 & & \text { Mn I }\end{array}$

$\begin{array}{ll}8737.32 & \text { Mn I } \\ 8737.74 & \text { Ba II }\end{array}$

8740.93 Mn I

$8742.60 \quad$ S1

$\begin{array}{ll}8747.32 & \text { Fe I } \\ 8747.35 & \text { N }\end{array}$

8750.13 Co I

8750.475 H

8751.18 P S1

$\begin{array}{ll}8752.17 & \text { S1 } \\ 8757.192 & \text { Fe I }\end{array}$

$8757.192 \quad \mathrm{Fe}$

$8764.000 \quad \mathrm{Fe}$

8766.64 T1

$\begin{array}{lll}8766.68 & & \text { S1 I } \\ 8767.65 & P & \text { Fe I }\end{array}$

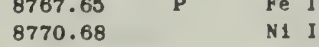

8771.70 Ne I

$\begin{array}{ll}8772.88 & \text { Al I } \\ 8773.56 & \text { Cr I }\end{array}$

$8773.91 \quad A 1$

8778.66 TI

$\begin{array}{llll}8779.12 & P & F e & I \\ 8780.6223 & & \text { Ne }\end{array}$

8783.755 Ne

$8784.44 \quad F e$

8786.28 P Cr I

8786.96

8790.62

8790.88

8791.28

8793.376

8796

8796.42

$8798.05 \quad P \quad P$ I 1260

$\begin{array}{llll}8801.78 & P & \text { Fe I } & 856\end{array}$

8804.624

286
1
140
139
999
79
1
79
713
296

$\begin{array}{ll}8819.42 & \mathrm{P} \\ 8819.48 & \mathrm{P} \\ 8820.45 & \\ 8821.14 & \\ 8821.76 & \\ 8824.227 & \\ 8828.08 & \mathrm{P} \\ 8828.91 & \\ 8834.04 & \mathrm{P} \\ 8835.67 & \\ & \\ 8835.85 & \\ 8838.433 & \\ 8841.26 & \\ 8846.82 & \\ 8848.25 & \mathrm{P} \\ 8848.46 & \mathrm{P} \\ 8852.30 & \mathrm{P} \\ 8853.867 & \\ 8858.39 & \\ 8858.77 & \\ 8862.59 & \end{array}$

Fe I 1266

Fe I 1269

T1 I 139

$\begin{array}{rrr}\text { As } & 1 & 139 \\ \text { As } & 3\end{array}$

Fe $1 \quad 60$

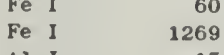

Al I 15

$\begin{array}{lr}\text { Cr I } & 142\end{array}$

8882.787

8863.64

8865.759

8866.961

8868.42

8869.68

8874.53

8876.13

Y II 30

Fe I 339

$\begin{array}{lr}\text { Al } & \text { Fe I } \\ \text { Fe } & 1267\end{array}$

$\mathrm{Fe} I \quad 1153$

$\begin{array}{lll} & 1214\end{array}$

Ne $I \quad 27$

$\begin{array}{ll}\text { Al II } & 115 \\ \text { Al II } & 115\end{array}$

8877.07

N1 I 214

$\begin{array}{llr}\text { Fe I } & 1283 \\ \text { Ne } & I & 8\end{array}$

Fe I 1172

Fe I 400

$\begin{array}{lr}\text { As } 1 & 4 \\ \text { S } & 1\end{array}$

$\begin{array}{llr}\text { S } & I & 21 \\ \text { Fe } & I & 1267\end{array}$

$8878.26 \quad P \quad F e I$

$\begin{array}{rrrr}8878.76 & P & \text { Fe I } & 401 \\ 8 & & \text { S I } & \end{array}$

$8880.70 \quad$ S I 21

S $1 \quad 21$

$\begin{array}{lll}8883.84 & \text { S1 I } & 54 \\ 8884.23 & \text { S I } & 21\end{array}$

$\begin{array}{llll}8887.10 & P & \text { Fe I } & 1265\end{array}$

$\begin{array}{llll}8892.13 & P & F e & 1302\end{array}$

$\begin{array}{rrrr}8892.97 & & \text { S1 I } & 54 \\ 8886.00 & P & \text { Fe } & \text { I }\end{array}$

$\begin{array}{lll}8898.97 & \text { S1 I } & 79,86\end{array}$

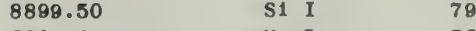

$8901.0 \quad M n I \quad 56$

$\begin{array}{llll}8902.94 & \text { P } & \text { Fe I } & 1266 \\ 8905.98 & \text { P } & \text { Fe I } & 1302\end{array}$

$8912.88 \quad A 1$ I 14

$\begin{array}{lll}8812.88 & \text { Fe I } & 32\end{array}$

$\begin{array}{llr}8916.26 & \text { Fe I } & 32 \\ 8918.88 & \text { Se I } & 1 \\ 8919.50 & \text { Ne I } & 27\end{array}$

8918.95 Fe I 1301

$\begin{array}{lllll}8920.02 & P & F e & 1261\end{array}$

$\begin{array}{llll}8922.66 & P & \text { Pe I } & 1298\end{array}$

$8923.56 \quad$ Al I $\quad 14$

$8925.55 \quad$ S1 I 54

8925.75 Cr I 142

$\begin{array}{rrr}8926.06 & \text { Mn I } & 56 \\ 8929.04 & \text { Fe I } & 1301\end{array}$

8929.72 Mn I 56

$\begin{array}{llll}8931.78 & P & \text { Fe } I & 507\end{array}$

8943.00 Fe I 338

8943.50 Cs I 1

8945.204 Pe I 1301

8946.25 Fe I 338

$\begin{array}{lll}8947.20 & \text { Cr I } & 142\end{array}$

$\begin{array}{rrr}8948.01 & \text { C1 } 1 & 1 \\ 8949.33 & \text { S1 } & 54\end{array}$

8950.20 P Fe I $\quad 1050$

8954.65

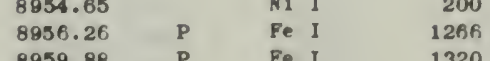

$\begin{array}{llll}9959.88 & P & \text { Fe I } & 1320\end{array}$

8965.84 N1 I 225

$8967.53 \quad P \quad$ Fe $I$ 1 1286

$\begin{array}{lll}8988.20 & \text { N1 } & 284 \\ 8975.408 & \text { F. I } & 400\end{array}$

8976. B8 $P$ CF 1 142

$\begin{array}{rrrr}8978.04 & \text { P } & \text { Pe I } & 1266 \\ 8878.17 & \text { P } & \text { Pe I } & 713\end{array}$

$\begin{array}{llll}8805.21 & P & \text { Fe I } & 1265\end{array}$

$8806.7032 \quad \mathrm{Pg}$

$8806.7358 \quad$ Mg I

$8806.7678 \quad \mathrm{Mg}_{\mathrm{B}}$

8808.17

8809.47

$8814.50 \quad P \quad \mathrm{Fe}$

$8816.86 \quad P \quad P e$

8819. 11 
I A Type Element Multiplet No.

9009.0

9009.95

9010.55

9012.098

9013.90

9014.911

9015.16
9017.10

9019.84

9021.69

9024.47

$\begin{array}{rlrr}9024.78 & \text { P } & \text { Fe I } & 1297 \\ 9027.32 & & \text { TI I } & 138\end{array}$

$\begin{array}{lll}9027.90 \quad P & \text { TI II } & 138\end{array}$

9028.9

9030.67

9035.86

9035.92

N I

Fe I

$\begin{array}{ll}\text { Cr } & \text { I } \\ S & I\end{array}$

S I

9036.73

$$
\text { S I }
$$

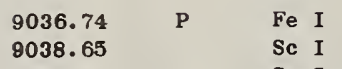

$\begin{array}{lll}9038.65 & & \text { Sc I } \\ 9038.72 & & \text { S I } \\ 9038.84 & \text { P } & \text { Fe I }\end{array}$

$\begin{array}{llll}9038.84 & \mathrm{P} & \mathrm{Fe} & \mathrm{I} \\ 9039.27 & & \mathrm{~S} & \mathrm{I} \\ & & \mathrm{Cl} & \mathrm{I}\end{array}$

$9045.40 \quad \mathrm{Cl}$ I

$\begin{array}{lll}9052.56 & P & \text { Fe } I \\ 9059.74 & & \text { Cr I }\end{array}$

$\begin{array}{llll}9060.6 & & \text { N } & \text { I } \\ 9061.33 & \text { P } & \text { Fe } & \text { II }\end{array}$

9061.48

9062.24

9062.53

9063.40

9070.42

9073.15

9078.32

9079.599

8080.48

9084.20

9084.29

9088.57

9089.413

9090.70

9094.89

9103.37

9103.64

\section{I}

Fe I

He I

S1 I

$\mathrm{Fe}$

Cl I

c

Ee I

Fe

9106.40

9111.85

9112.25

9112.95

9114.02

9116.89

9117.10

9118.888
9121.10

9122.9660

$\mathrm{Fe}$

Fe I

C I

Fe I

T1 I

C I

$\begin{array}{ll}\mathrm{Fe} & \text { I } \\ \text { S1 } & \text { I }\end{array}$

Fe I

100
15

338
142

13

13

1269

1
13

400
13

13
1342

1342

15
71

$$
3
$$

1301

3
77
91

1076,1300

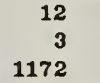

1265,1298

1076

339

400

138
3

1264

66
1076

Ni I

Fe I

Fe II

Mn I

Fe I

$\mathrm{Fe} \mathrm{I}$

$\mathrm{Fe} \mathrm{I}$

Cl I
A I

9124.27

9140.15 Al II

9146.11 $\mathrm{P} \mathrm{Fe}$

$\begin{array}{ll}9147.800 & \mathrm{Fe} \\ 9148.45 & \mathrm{Cr}\end{array}$

9148.68

9154.?

$9155.67 \quad \mathrm{Na}$

$\begin{array}{lll}9156.23 & P & \mathrm{Fe}\end{array}$

$\begin{array}{llll}9157.07 & P & F e & \end{array}$

$\begin{array}{llll}9157.08 & P & \text { Fe } & \\ 91 & 1261\end{array}$

9164.51 $\mathrm{Fe}$

9172.09

$9173.20 \quad P \quad \mathrm{Fe}$

$9173.63 \quad \mathrm{P} \quad \mathrm{Fe}$

$9173.83 \quad \mathrm{Fe}$

9175.85

9178.57

9191.67

$$
\text { Fe } \text { I }
$$

9197.49

9199.52

9201.76

9203.10 $\mathrm{P} \quad \mathrm{Ne}$

9208.29 $\mathrm{Cr}$

9208.55

9210.030

9210.28

9212.91

289
3
1297
71
46
1265
338
338
1
1

108

622
202

1301

30

25

1301

400

1261
1263

46

203
1300

622

1262

$\begin{array}{ll}\text { Cl I } & 1\end{array}$

14
1298

30

1298

165

66
338
83

He

S I

I A Type Element Multiplet No.

I $\mathbf{A}$

Type Element

Multiplet No.

$\begin{array}{lll}9214.85 & \text { P } & \text { Fe II } \\ 9217.4 & \text { P } & \text { Mg II } \\ 9217.54 & & \text { Fe I } \\ 9220.05 & & \text { Ne I } \\ 9221.59 & & \mathrm{Ne} \text { I } \\ 9221.498 & & \mathrm{~A} \text { I } \\ 9225.55 & & \text { Fe I } \\ 9226.67 & & \text { Ne I } \\ 9228.11 & & \text { S I } \\ 9229.017 & & \text { H }\end{array}$

71
1
1298
33
33
8
1213
30
1
8

$\begin{array}{ll}9413.46 & \\ 9413.59 & \\ 9414.14 & \\ 9415.04 & P \\ 9415.5 & \\ 9421.82 & \\ 9421.93 & \\ 9423.07 & P \\ 9425.38 & \\ 9429.58 & \end{array}$

\begin{tabular}{|c|c|c|c|}
\hline 9233.15 & $\mathbf{P}$ & $\mathrm{Fe}$ & I \\
\hline 9237.49 & & $\mathbf{s}$ & I \\
\hline 9238.60 & & S1 & I \\
\hline 9242.32 & & $\mathrm{Fe}$ & I \\
\hline 9243.29 & & Mn & I \\
\hline 9213.4 & $\mathbf{P}$ & $\mathrm{Mg}$ & II \\
\hline 9246.51 & & $\mathrm{Fe}$ & I \\
\hline 9248.13 & $\mathbf{P}$ & $\mathrm{Fe}$ & I \\
\hline 9248.80 & $\mathbf{P}$ & $\mathrm{Fe}$ & I \\
\hline 9249.41 & & Al & I I \\
\hline 9252.67 & $\mathbf{P}$ & T1 & II \\
\hline 9253.72 & $\mathbf{P}$ & $\mathrm{Fe}$ & I \\
\hline 9254.59 & & S1 & I \\
\hline 9256.0 & & $\mathrm{Mg}$ & I \\
\hline 9258.30 & & $\mathrm{Fe}$ & I \\
\hline 9259.05 & & $\mathrm{Fe}$ & I \\
\hline 9260.88 & & 0 & I \\
\hline 9262.73 & & 0 & I \\
\hline 9263.97 & & $\mathrm{Cr}_{\mathrm{r}}$ & I \\
\hline 9265.99 & & 0 & I \\
\hline
\end{tabular}

$\begin{array}{ll}\text { S } & \text { I } \\ \text { S1 } & \text { I } \\ \text { Fe } & \text { I } \\ \text { Fe } & \text { I } \\ \text { Mg } & \text { I } \\ \text { S1 } & \text { I } \\ \text { S } & \text { I } \\ \text { Fe } & \text { I } \\ \text { Ne } & \text { I } \\ \text { Mn } & \text { I }\end{array}$

18
14
1298
1297
38
72
18
1300
36
57

9437.11

9437.91

9443.98

9444.36

$\mathbf{8 4 4 7 . 0 0}$

9452.06

9452. 45

9454.24

$\mathrm{Fe} \mathrm{I}$

Fe I 129

9459.21

9460.66

9463.57

9466.0

9476.57

9482.8

$P \quad \mathrm{Fe}$

Fe I

9487.49

9267.29 As I 3

$\begin{array}{lll}9276.89 & \text { Zr I } & 39 \\ 9286.578 & \text { AI I I } & 64\end{array}$

$9286.794 \quad$ Al II

9288.145 Al II

9288.550

Al II

9288.82

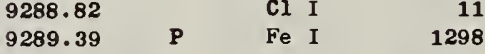

$\begin{array}{llr}9290.44 & \text { Cr I } & 29 \\ 9290.619 & \text { AI II } & 64\end{array}$

9290.747 Al II $\quad 64$

$\begin{array}{rrr}9294.17 & \text { Cr I } & 29 \\ 9294.66 & \text { Fe I } & 1301\end{array}$

$\begin{array}{llll}9297.14 & P & \mathrm{Fe} I & 1247\end{array}$

$\begin{array}{llll}9297.14 & \text { P } & \text { Fe I } & 1247 \\ 9298.05 & \text { P } & \text { Fe I } & 1262\end{array}$

$\begin{array}{rrr}9300.62 & \text { As I } & 5 \\ 9300.85 & & \text { Ne I }\end{array}$

$\begin{array}{rlr}9300.85 & \text { Ne I } & 33 \\ 9304.88 & \text { P } & \text { I }\end{array}$

$9307.94 \quad$ Fe I $\quad 1297$

9313.55

Cr I

1297
80

$9313.98 \quad \mathrm{Ne}$

Fe I 1263

9318.24 Si I 66

9324.07 Fe I 1300

$\begin{array}{rlllr}9326.52 & & \text { Ne } & \text { I } & 36 \\ 9328.64 & P & \text { Fe } & \text { I } & 1261\end{array}$

$9331.546 \quad$ Al II $\quad 56$

0331.979 Al II

$\begin{array}{llr}9333.94 & \text { Fe I } & 1297\end{array}$

$\begin{array}{lllll}9335.27 & P & \text { Fe } & \text { I } & 1338\end{array}$

$\begin{array}{lll}3336.47 & \text { Mn I }\end{array}$

$9343.40 \quad$ Fe I 1300

9344.93

9346. 69

9350.46

9354.218

9359.420

9362.06

9362.370

9370.5

9372.900

9373.28

$\begin{array}{rlrr}9375.14 & \text { P } & \text { Fe I } & 400 \\ 9382.93 & \text { P } & \text { Fe I } & 1284\end{array}$

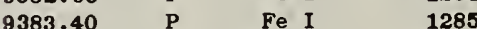

$9385.62 \quad$ N1 I 225

9386.79

9388.28

$\begin{array}{llll}9392.77 & & \mathrm{Fe} \mathrm{I} & 1263 \\ & \mathrm{Fe} \mathrm{I} & 1262\end{array}$

$\begin{array}{rrr}9392.80 & \text { N I } & 7\end{array}$

$\begin{array}{lll}9393.40 & \text { S1 I } & 72 \\ 9393.81 & \text { CI I } & \end{array}$

9394.71 Fe I 1264

0396.57

9403.36

9404. 80

$9409.55 \quad P$

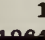

1

$\mathrm{Fe}$
$\mathrm{Ne}$

9498.0

9499. 39

9502.12

9505.28

9505.67

9506.04

9508.49

9510.81

9511.55

9511.80

9513.24

9516.51

9516.66

9520.06

9522. 01

9525.78

9526.17

9527.73

9529. 27

Fe I

$\mathrm{Fe} I$

Cr I

Cr I

$\mathrm{Fe} I$

Fe I

18
1171
1298
29
29
13
1263,1292
1298

9529. 3

9534. 17

9535.72

9543.376

9545. 974

9546.07

9547. 26

9547.40

9550.90

9556.56

9563.45

9568.58

9569.960

9570.08 
FINDING LIST

I A Type Element Multiplet No. $9626.562 \quad$ Fe I 1296

\begin{tabular}{|c|c|c|c|c|}
\hline & & & & \\
\hline 8632.37 & & Cl & I & 12 \\
\hline 8633.0 & $\mathbf{P}$ & $\mathrm{Mg}$ & II & 15 \\
\hline 9633.02 & & Mn & I & 58 \\
\hline 9633.78 & & $\mathbf{s}$ & I & 17 \\
\hline 8634.22 & & $\mathrm{Fe}$ & I & 1296 \\
\hline 9636.69 & & $\mathrm{Fe}$ & I & 1212 \\
\hline 9637.55 & & $\mathrm{Fe}$ & I & \\
\hline 9638.28 & & T1 & I & 32 \\
\hline 9639.40 & $\mathbf{P}$ & $\mathrm{Ca}$ & I & 55 \\
\hline 8647.40 & & Ti & I & 32 \\
\hline 9649.94 & & $\mathbf{S}$ & I & 17 \\
\hline 9653.143 & & $\mathrm{Fe}$ & I & 1247 \\
\hline 2657.00 & & La & I I & 60 \\
\hline 9657.30 & & $\mathrm{Fe}$ & I & 1296 \\
\hline 9657.7841 & & $\mathbf{A}$ & I & 3 \\
\hline 9658.49 & & c & I & 2 \\
\hline 9658.94 & & $\mathrm{Fe}$ & I & 1292 \\
\hline 9661.42 & & $\mathbf{T} \mathbf{1}$ & I & 194 \\
\hline 9663.58 & $\mathbf{P}$ & $\mathrm{Ca}$ & I & 55 \\
\hline 9664.29 & $\mathbf{P}$ & $\mathrm{Ca}$ & I & 55 \\
\hline 9665.426 & & $\mathrm{Ne}$ & I & 8 \\
\hline 9666.59 & & $\mathrm{Fe}$ & I & \\
\hline 9667.20 & & $\mathrm{Cr}$ & I & 29 \\
\hline 9668.9 & & v & I & 106 \\
\hline 9670.48 & & $\mathrm{Cr}$ & I & 28 \\
\hline 9672.34 & & $\mathbf{s}$ & I & 17 \\
\hline 9673.16 & & $\mathrm{Fe}$ & I & 580 \\
\hline 9675.55 & & T1 & I & 32 \\
\hline 9676.25 & $\mathbf{P}$ & $\mathrm{Ca}$ & I & 55 \\
\hline 9676.42 & & $\mathrm{Fe}$ & I & 1345 \\
\hline 9676.50 & & $\mathrm{Mn}$ & I & 60 \\
\hline 9677.41 & & 0 & I & 58 \\
\hline 9680.80 & & $\mathbf{S}$ & I & 17 \\
\hline 9683.57 & & $\mathrm{Fe}$ & I & 1337 \\
\hline 9684.9 & & Mn & I & 60 \\
\hline 9686.3 & & Mn & I & \\
\hline 8688.60 & $\mathbf{P}$ & $\mathrm{Ca}$ & I & 55 \\
\hline 9688.86 & & T1 & I & 32 \\
\hline 8689.35 & & N1 & I & 285 \\
\hline 9689.41 & & S1 & I & 65 \\
\hline 9690.62 & & T1 & I & 194 \\
\hline 9691.58 & & v & I & 108 \\
\hline 9693.68 & & $\mathbf{S}$ & I & 20 \\
\hline 9693.69 & & $\mathrm{Fe}$ & I & 1282 \\
\hline 9697.33 & & $\mathbf{s}$ & I & 17 \\
\hline 9699.70 & & $\mathrm{Fe}$ & I & 1292,1299 \\
\hline 9701.81 & $\mathbf{P}$ & $\mathrm{Ca}$ & I & 55 \\
\hline 9702.35 & & Cl & I & 1 \\
\hline 9702.66 & & He & I & 75 \\
\hline 9702.86 & & $\mathbf{T} 1$ & I & 248 \\
\hline 9705.64 & & T1 & I & 32 \\
\hline 9710.21 & $\mathbf{P}$ & N1 & I & 285 \\
\hline 9717.00 & & $\mathbf{T} \pm$ & I & 248 \\
\hline 9718.96 & & T1 & I & 124 \\
\hline 9722.88 & & $\mathrm{Te}$ & I & 1 \\
\hline 9728.36 & & T1 & I & 32 \\
\hline 9730.32 & & $\mathrm{Cr}$ & I & 226 \\
\hline 9734.52 & & $\mathrm{Cr}$ & I & 29 \\
\hline 0734.74 & & $P$ & I & 2 \\
\hline 9738.50 & & V & I & 106 \\
\hline 9738.60 & & S1 & I & \\
\hline 9738.624 & & $\mathrm{Fe}$ & I & 1296 \\
\hline 9739.74 & & $\mathbf{s}$ & I & 20 \\
\hline 9741.49 & & 0 & I & 57 \\
\hline 9741.93 & & $\mathbf{s}$ & I & 20 \\
\hline 9742.28 & & He & II & 66 \\
\hline 9743.60 & & T1 & I & 32 \\
\hline 9744,33 & & $\mathrm{Cl}$ & I & 10 \\
\hline 9746.86 & & T1 & I & 248 \\
\hline $9747 \cdot 24$ & & $\mathrm{Fe}$ & I & 1209 \\
\hline 9750.73 & & $\mathbf{P}$ & I & 2 \\
\hline 9752.84 & & $\mathrm{Cr}$ & I & 80 \\
\hline 9753.129 & & Fe & I & 1247 \\
\hline 6758.08 & & s1 & I & 65 \\
\hline 9760.65 & & 0 & I & 56 \\
\hline 9763.450 & & $\mathrm{Fe}$ & I & 1296 \\
\hline 9763.913 & & $\mathrm{Fe}$ & I & 1292 \\
\hline 8764.40 & $\mathbf{P}$ & $\mathrm{Fe}$ & I & 1348 \\
\hline 9768.27 & & s1 & I & 7 \\
\hline 9770.10 & & S1 & I & \\
\hline 9770.28 & & T1 & I & 32 \\
\hline 9771.06 & $\mathbf{P}$ & $\mathrm{Fe}$ & I & 1211 \\
\hline 9783.30 & & T1 & I & 32 \\
\hline 9783.58 & & T1 & I & 32 \\
\hline 9783.98 & & $\mathrm{Fe}$ & I & 1295 \\
\hline 8784.5010 & & $A$ & I & 8 \\
\hline 8786.62 & & 80 & I & 1171 \\
\hline 9787.67 & & T1 & I & 32 \\
\hline & & & & 65 \\
\hline
\end{tabular}

I A Type Element Nultiplet No.

I

4
2
1296
1292
1285
106

$9790.08 \quad$ P I $\quad 10061.29 \quad$ N1 I

$9800.335 \quad$ Fe I 1296

$\begin{array}{llll}9800.79 & P & \mathrm{Fe} I & 1292 \\ 9811.36 & & \mathrm{Fe} I & 1285\end{array}$

$\begin{array}{lllr}9811.36 & & \text { Fe } & \\ 9820.24 & P & \text { Fe I } & 106\end{array}$

\begin{tabular}{lll}
9821.8 & N I & 19 \\
\hline & Zr I & 39
\end{tabular}

$\begin{array}{lll}9822.30 & \text { Zr I } & 39 \\ 9826.69 & \text { AS I }\end{array}$

9832.15

T1 I

149

9833.76

8834.04
9839.38

$\begin{array}{lllr}9856.7 & \text { P } & \text { Ca II } & 12 \\ 9861.793 & & \text { Fe I } & 1296\end{array}$

9862.5

9865.44

9868.09

9875.95

$\begin{array}{ll}\text { N } & \text { I } \\ \text { V } & \text { I }\end{array}$

Fe I

19

282,1289

Cl I $\quad 11$

$9878.18 \quad P \quad \mathrm{Fe}$

$\begin{array}{lll}9881.51 & P & \text { TI I } \\ 9886.92 & \text { Fe I }\end{array}$

9886.92

$9889.082 \quad$ Fe I $\quad 1296$

9881.90

9898.90

9900.87

9903.74

9913.16

S1 I

Ni I

Cr I

$\begin{array}{ll}\text { P } & \text { I } \\ \text { S1 } & \text { I }\end{array}$

71

243
80

4
65

9913.19 P Fe I

$9917.93 \quad$ Fe I 1317

9920.46 P $\quad$ Pe I 1292

$\begin{array}{rrrr}9923.03 & & \text { As I } & 2 \\ 9924.35 & P & \mathrm{Fe} \mathrm{I} & 737\end{array}$

$\begin{array}{lll}9927.35 & \text { T1 I } & 149\end{array}$

$9932.26 \quad$ S I 16

$\begin{array}{lllll}9933.3 & P & C a & I I & 12\end{array}$

$9937.10 \quad \mathrm{P} \quad \mathrm{Fe} \mathrm{I} \quad 1210$

9941.33

9944.13

9948.98

99.06

8950.5

9950.70

9851.15

9953.45

9955.2

9955.85

Fe I

T1 I

S I

1285
193

226

8

1209
1346

1346
1346

1346

1211

9958.90 S I

$9959.18 \quad \mathrm{P} \quad \mathrm{Fe} I$

998

$9961.0 \quad \mathrm{Na} \mathrm{I}$

9967.32 P Fe I

9967.46 P $\quad \mathrm{St} I$

9970.26 P Fe I

9976.65

9977.52

9980.55

9981.16

Fe I

T1 I

988
23
1293

1293
64

461

1293

1293
1295

$\begin{array}{llll}9987.0 & & \mathrm{Mg} I & 36 \\ 9987.88 & \mathrm{P} & \mathrm{Fe} \mathrm{I} & 59 \\ 9993.7 & & \mathrm{Mg} \text { I } & 36\end{array}$

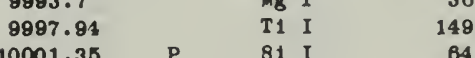

$\begin{array}{rrrrr}10001.35 & P & 81 & I & \\ 10003.02 & & \text { T1 I } & 193\end{array}$

$\begin{array}{rrrr}10011.72 & \text { TI I } & 193 \\ 10012.15 & \text { Fe I } & 1336\end{array}$

$\begin{array}{lllll}10012.15 & P & \text { Fe I } & 1336 \\ 10015.33 & & \text { S1 I } & \end{array}$

$\begin{array}{llll}10016.67 & P & F e & 1\end{array}$

$\begin{array}{llll}10018.77 & P & \text { Fe } I & 1348\end{array}$

10020.16 S1 I $\quad$ S

$\begin{array}{llll}10022.34 & P & \text { Fe } I & 1345\end{array}$

$10025.80 \quad P \quad 81$ I

$\begin{array}{llll}10026.10 & \text { P } & \text { Fo I } & 1211\end{array}$

10027.73

10032.84 P He I

10036.658

10046.64

10048.60

10048.78

10049.38

10051.55

10057.64

10057.69

10068.28

10058.87
10066.47

10067.84

10070.58

10072.10

10076.29

10077.32

10077.53

T1 I

Fe I

Al II

Al II

Al II

CrI

$\begin{array}{llll}10080.44 & P & F e & I\end{array}$

$\begin{array}{llll}10081.40 & P & \text { Fe I } & 106\end{array}$

$\begin{array}{llll}10084.42 & P & F e & 1\end{array}$

$10084.70 \quad \mathrm{Zr} \mathrm{I}$

$10086.27 \quad P \quad$ Fe I $\quad 399$

10091.12 Te I

10107.19 Al II

10108.01

Al II

10108.37

10113.4

10113.86

10117.8

10120.90

10122.50

10123.61

10137.06

10138.50

Al II

N I

18
264

$\mathrm{Fe} \mathrm{I} 264$

Fe I 1295

TI I

Al II

He II

$\mathrm{Fe} I$

1294
88

$10142.82 \quad$ Fe I $\quad 129$

$\begin{array}{llll}10143.59 & P & \text { Fe } & I\end{array}$

$10145.00 \quad P \quad$ Fe I $\quad 621$

10145.37 N1 I 243

10145.48 T1 1

10147.08

10147.3

10149.08

Fe

T1

$\begin{array}{ll}\mathrm{Fe} & \mathrm{I} \\ \mathrm{SI} & \mathrm{I}\end{array}$

1247
315
18

$\begin{array}{lll}10153.13 & P & S 1\end{array}$

$10153.30 \quad \mathrm{P}-\mathrm{F}_{0} \mathrm{I}$

$10155.88 \quad$ S1 I 64

$\begin{array}{llll}10156.50 & P & F e & 1\end{array}$

$10164.5 \quad$ N I 18

$10167.4 \quad$ Fe I

$10170.60 \quad$ T1 I $~ 95$

$\begin{array}{llll}10191.51 & P & F e & 149\end{array}$

$10193.25 \quad$ N1 I 213

10193.66

10185.11

10197.05

10203.45

10204.72

10216.351

10218.36

10262.49

10265.23

$\mathrm{VI}$

Fe I

$\mathrm{Cr}$

$\begin{array}{ll}V & I \\ \text { P } & \text { I }\end{array}$

Fe I

Fe

S1

10283.87 P $\quad \mathrm{Fe}$

Fe I

10288.83 S1

10285.05

10307.48

10307.60

10311.18

10311.37

10311.88

N1 I

N1 I

Fe I

He I

He I

Fe I

76

264

80

1247

461

63
59

1346

Fo 1 I
S1 I 193

$\begin{array}{rlrrr}10155.18 & \mathrm{P} & \mathrm{Fe} & \mathrm{I} & 1348\end{array}$

10189.26 T1 I 95
Fe $I \quad 1348$

10327.30

10327.314

10330.23

10332.33

10333.24

10340.77

10343.85

10348.16

10353.85

10362.73

10364.13

10371.23

10378.62

10379.01

10386.45

10388.73

10392.45

10395.75

10396.86

10401.72

Se I

Sr II

N1 I

Fe I

Fe I

Ca I

$\begin{array}{ll}\text { Fe I } & 1347 \\ \text { Fe I } & 1346\end{array}$

Fe I 1347

$\begin{array}{lll}\text { S1 } & \text { I } & \text { N1 }\end{array}$

Fe $1 \quad 58$

Se I

$\begin{array}{lr}\text { Fe I } & 1346 \\ \text { Cl I } & 10\end{array}$

T1 I

Fi I 
I A

11564.8

$\mathrm{Ca} \mathrm{I} \quad 56$

10838.77

10844.02
10844.54

10847.72

10849.68

10861.51

10863.60

10863.72

10869.37

T1 I $\quad 223$

10459.46

10469.59

A I

10486.05

10496.14

10506.5

10509. 96

10511.45

10529.45

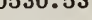

10532.21

10539.0

10548.0

10548.0

10553.02

10562.43

10565.97

10577.14

10581.52

10582.66

10584. 66

10585.12

10596.92

10603.38

10607.78

10616.75

10627.81

10647.66

10653.6

10660.98

10661. 61

Cr I

N I

Cr I

$\begin{array}{ll}\text { P } & \text { I } \\ \text { P } & \text { I }\end{array}$

N1 I

$\begin{array}{ll}\mathrm{Fe} & \mathrm{I} \\ \mathrm{N} & \mathrm{I}\end{array}$

6
118

10869.54

10872.47
10875.00

10879.78

10881.65

10882. 66

10884.30

10885.16

10890.13

10891.21

10891.25

10893.72

10896.10

10896.30

10912.92

10914.2

10914.877

10916.98

10925.80

10929.90

10935.11

10938.09

10947.51

10949.4

10957.19

10961.2

10966. 1

10979.27

10979.87

10982.28

10984.27

10987.22
10996.55

10996.55
11012.97

11013.27

11015.63

11018.00

11022.3

11044.64

11044.95

11119.80

11125.28

11130.37

11149.34

11157.03

11187.74

11196.70

11202.02

11225.83

11227.5

11230.91

S1 I

$\begin{array}{ll}\mathrm{Ne} & \mathrm{I} \\ \mathrm{T} 1 & \mathrm{I}\end{array}$

Fe I

Ca I

$\mathrm{Fe}$ I

$\begin{array}{ll}\mathrm{Ca} & \mathrm{I} \\ \mathrm{Ca} & \mathrm{I}\end{array}$

S1 I

56
31
26
31
1352
56
1246
56
56
13

Al I

$\begin{array}{ll}\mathrm{Fe} & \mathrm{I} \\ \mathrm{Ca} & \mathrm{I}\end{array}$

Fe I

S1 I

$\mathrm{Fe}$ I

S1 I

Fe I

A1 I

12
1316

56

336

53
979

77
1341

12

S1 I

224

T1 I

78
310

$\begin{array}{ll} & 461\end{array}$

He I

Mg II

Sr II

He I

$\mathrm{Fe} I$

Cr I

H

Ge I

$\begin{array}{ll}\text { Ge } & \text { I } \\ \mathrm{Mg} & \text { II }\end{array}$

Cr I

Mg I

$\mathrm{Mg} \mathrm{I}$

S1 I

N1 I

S1 I
S1. I

79
3

3
2
84

1350

118
2

Fe I

$\begin{array}{ll}\mathrm{He} & \text { I } \\ \mathrm{He} & \mathrm{I}\end{array}$

$\mathrm{He} I$

Cr I

S1 I

K I

Cr I

He I

$\mathrm{Fe}$

Ge I

S1 I

$\mathrm{Fe}$ I

Cr I

S1 I

10729.59

10734.14

10745.9
10748.7

Ge I

$\mathrm{Na} I$

S1 I

11251.09

11253.81

11255.69

11290.01

11294.0

11294.97

11297.54

11298.83

11302.22

11308.45

S1 I

He I

N I

T1 I

8

3
118

118
35

35

242

77
77

337

78
70

1246

221

39
9

118
88

337

6
77
336

221

76
81
76

76

17

Fe I

Al I

Al I

S1 I

$\begin{array}{ll}N & \text { I } \\ \text { o } & \text { I }\end{array}$

o I

$\mathrm{Fe}$ I

0

11313.8

11329.0

11330.36

11355.97

11374.02

11381.21

11381.53

11403.55

11403.89

11422.30

337

337
8
8
76
17
7
7
337
7
76

\begin{tabular}{ll}
$N$ & I \\
N & I \\
\hline & I
\end{tabular}

Fe I

$\mathrm{Fe}$.I

Ne I

Na

T1 I

$\begin{array}{ll}\mathrm{Na} & \mathrm{I} \\ \mathrm{T} 1 \mathrm{I}\end{array}$

Fe I

11439.06

11453

11464

11468.54

11472

11479.87

11485.68

11502.94

11539.50

11542. 96

$\begin{array}{ll}\mathrm{Fe} & \mathrm{I} \\ \mathrm{S} & \mathrm{I}\end{array}$

s I

S1 I

$S$ I

Fe I

Fe I

S1 I

T1 I

Fe I

11667.1

11863.0

11880.4

11882.80

11894.

11905.83

11927.89

11947.0

11969.07

11973.01

11984.20

11991.57

11997.9

12031.49

12074 . 1

12083.79

12103.46

12107.4

12128.6

12186. 9

12203.4

Type Element

Mult1plet Mo.

$\begin{array}{llll}11564.8 & \text { N } & \text { I } & 12\end{array}$

$11588.73 \quad P \quad$ N1 I $\quad 242$

$\begin{array}{lll}11591.98 & \text { S1 I } & 90 \\ 11593.55 & \text { Fe I } & 58\end{array}$

$11602.94 \quad P \quad C \quad I \quad 25$

$\begin{array}{lll}11607.42 & \text { S1 I } & 82 \\ 11607.57 & \text { Fe I } & 58\end{array}$

$\begin{array}{lllll}11609.41 & & \text { T1 } & & \\ 11609.91 & P & { }^{\circ} C & I & 25\end{array}$

$\begin{array}{lll}11611.49 & \text { S1 I } & 90\end{array}$

$11619.0 \quad \mathrm{C}$

$\begin{array}{ll}11626.40 & \text { He I } \\ 11628.0 & \text { N I I }\end{array}$

11631.59 P $\quad C$ I

$11638.25 \quad$ Fe I

$\begin{array}{lll}11638.60 & P & \text { C } \\ 11640.58 & & \text { S1 }\end{array}$

$11656.0 \quad$ C I

$\begin{array}{ll}\mathrm{N} & \mathrm{I} \\ \mathrm{C} & \mathrm{I}\end{array}$

25,29

$\begin{array}{llllr}11676.99 & \text { P } & \text { C } & \text { I } & 25 \\ 11689.76 & & \text { K } & \text { I } & 6 \\ 11689.98 & & \text { Fe } & \text { I } & 58 \\ 11747.5 & & \text { C } & \text { I } & 24 \\ 11754.0 & & \text { C } & \text { I } & 24 \\ 11769.41 & & \text { K } & \text { I } & 6 \\ 11772.66 & & \text { K } & \text { I } & 6 \\ 11783.28 & & \text { Fe } & \text { I } & 337 \\ 11801.8 & & \text { C } & \text { I } & 24 \\ 11828.8 & & \text { Mg I } & 6\end{array}$

$11836.4 \quad P \quad C a$ II

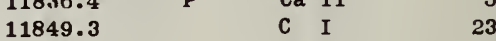

$11890.44 \quad P \quad S 1$ I 
FINDING LIST

Forbldden Lines

I A

Element

Multiplet No.

2972

2972. 3

2990.4

3000.6

3006.1

3008.4

3026.4

3035.3

3042.44

3042.61

048

3049.05

3063.0

3086.28

3068.8

3070.8

3071.0

3074.0

3074.11

3075.6

3081.6

3089.76

3093.4

3098.6

3088.7

3106.0

3106.1

3109.0

3117.1

3124.18

3158.5

3162.21

3165.4

3168.2

3170.55

3175.38

3181.05

3186.01

3188.79

3188.1

3190.76

3181.2

3181.3

3202.25

3203.3

3207.46

3207.6

3208.3

3209.94

3212.75

3214.5

3214.5

3214.67

3216.32

3224.54

3226.7

3226.89

3228.2

3230.17

3236.7

3238.07

3239.7

3239.8

3243.2

3244.18

3254.24

3254.7

3256.31

3256.73

261.7

3263.1

3264.84

3272.8

3273.6

3274.7

3275.02

3277.12

3277.3

3277.55

3283.1

3285.6

3286.2

3287.35

3289.46

3289.89

3295.4

$\begin{array}{llll}\text { Ne V } & 2 & F \\ 0 & \text { I } & 2 & F \\ \text { N1 VII } & 1 & F \\ \text { N1 VII } & 3 & F \\ \text { A III } & 2 & \text { F } \\ \text { T1 III } & 6 & \text { F } \\ \text { N1 VIII } & 4 & F \\ \text { N1 VIII } & 3 & F \\ \text { N1 VII } & 1 & F \\ \text { N1 VII } & 3 & F\end{array}$

Mn II

Mn II

N1 VII

Mn II

N II

Cr II

Al VII

$\begin{array}{ll}\text { Co } & \text { VII }\end{array}$

AI VII

N1 VIII

N1 VII

Cr II

AI VII

Al VII

NI VII

A III

N1 VII

Cl IV

Fe II

Fe II

NI VII

Co VII

Cr II

Fe II

Fe II

Cr II
Co VII

Fe II

N1 VII

N1 VII

Cr I

$\begin{array}{ll}\text { Cl } & \text { IV } \\ \text { Cr } & \text { II }\end{array}$

T1 III

Fe II $12 \mathrm{~F}$

Cr II 6 F

N VII $1 \mathrm{~F}$

TI III $\quad \begin{array}{ll}7 & F \\ \text { Fe } & \end{array}$

Cr II 6 F

$\begin{array}{llll}\text { Fe II } & 11 & \mathrm{~F} \\ \text { T1 III } & 7 & \mathrm{~F}\end{array}$

Fe II $11 \mathrm{~F}$

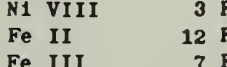

Fe III 7

Fe III 6

$\begin{array}{ll}\text { Co VII } & 3 \mathrm{~F} \\ \text { N1 VII } & \text { I F }\end{array}$

$\mathrm{Fe}$ II

$11 \mathrm{~F}$

Fe III

$\begin{array}{ll}\text { Fe } & \text { II } \\ \text { Fe } & \text { II }\end{array}$

Co VII

T1 VII

Fe II

Co vI

Cr IX

Fe II

Fe II

Co VI

Fe II

Co III

Pe III

Fe II

Fe II

Fe II

Co VI
298.61

3298.61

3289.8

3300.5

3301.6

3307.0

3316.1

3318.38

3319.3

3322.54

33.8

3333.8

3334.8

3336.9

3337.7

3337.77

3338.5

3339.14

3340.7

3341.38

3341.5

3342.7

3342.8

3344.72

3315.9

3353.4

3356.5

3356.6

3361.?

3362.5

3363.2

3366.2

367.3

3367.5

3368.8

3371.4

3374.6

3376.20

3378.4

3378.55

3378.7

3380.95

3387.10

3387.7

3388.2

3396.7

3388.6

3400.3

3402.50

3403.3

3403.65

3408.39

3406.2

3406. 6

3413.3

3425.8

3428.8

3430.3

3438.29

3440.3

3440.89

3444.1

3445.4

3445.8

3452.30

3452.54

3454.34

3455 . 11

3457.3

3458.73

3460 . 20

3461.42

3463.4

3465. 7

3470.0

3476.5

3481.5

3484.01

3485.5

3486.6

3487.2

3488.1

3489.07

3480.98

Element Multiplet No.

$\begin{array}{lr}\text { Cr II } & 5 \\ \text { N1 VII } & 1 \\ \text { Co VI } & 1 \\ \text { Fe III } & 6 \\ \text { Fo III } & 6 \\ \text { Co VI } & 2 \\ \text { Fe III } & 7 \\ \text { Fe II } & 27 \\ \text { Fe III } & 6 \\ \text { Na IV } & 1 \\ \text { Fe III } & 5 \\ \text { Co VI } & 2 \\ \text { Ca XII } & 1 \\ \text { Fe III } & 6 \\ \text { Fe III } & 6 \\ \text { Co VI } & 2 \\ \text { TI III } & 4 \\ \text { Cr II } & 5 \\ \text { Mn II } & 3 \\ \text { Co VII } & 3\end{array}$

Fe II $27 \mathrm{~F}$

Fe III $6 \mathrm{~F}$

Yn II 3 F

Cl III

Ne II I

In II

Ne $V$

Fe III

Fe III

Co VII

Fe $V$

T1 III

Fe III

Fe III

Co VI

Fe V

$\mathrm{Fe}$ III
$\mathrm{Fe} \mathrm{V}$

$F$
$F$
$F$
$F$
$F$
$F$
$F$
$F$
$F$
$F$

I A

Element

Multiplet No.

3193.55

3494 . 7

3500.4

3501.62

3503.5

3503.8

3504.02
3504.51

3505.81

3509. 78

3511.6

3511.64

3512.8

3516.17

3522.76

3524.38

3527. 33

3528.28

3536.25

3538.69

3538.8

3539.19

3543.5

3558.1

3559.8

3569.0
3575.6 
Forbidden Lines

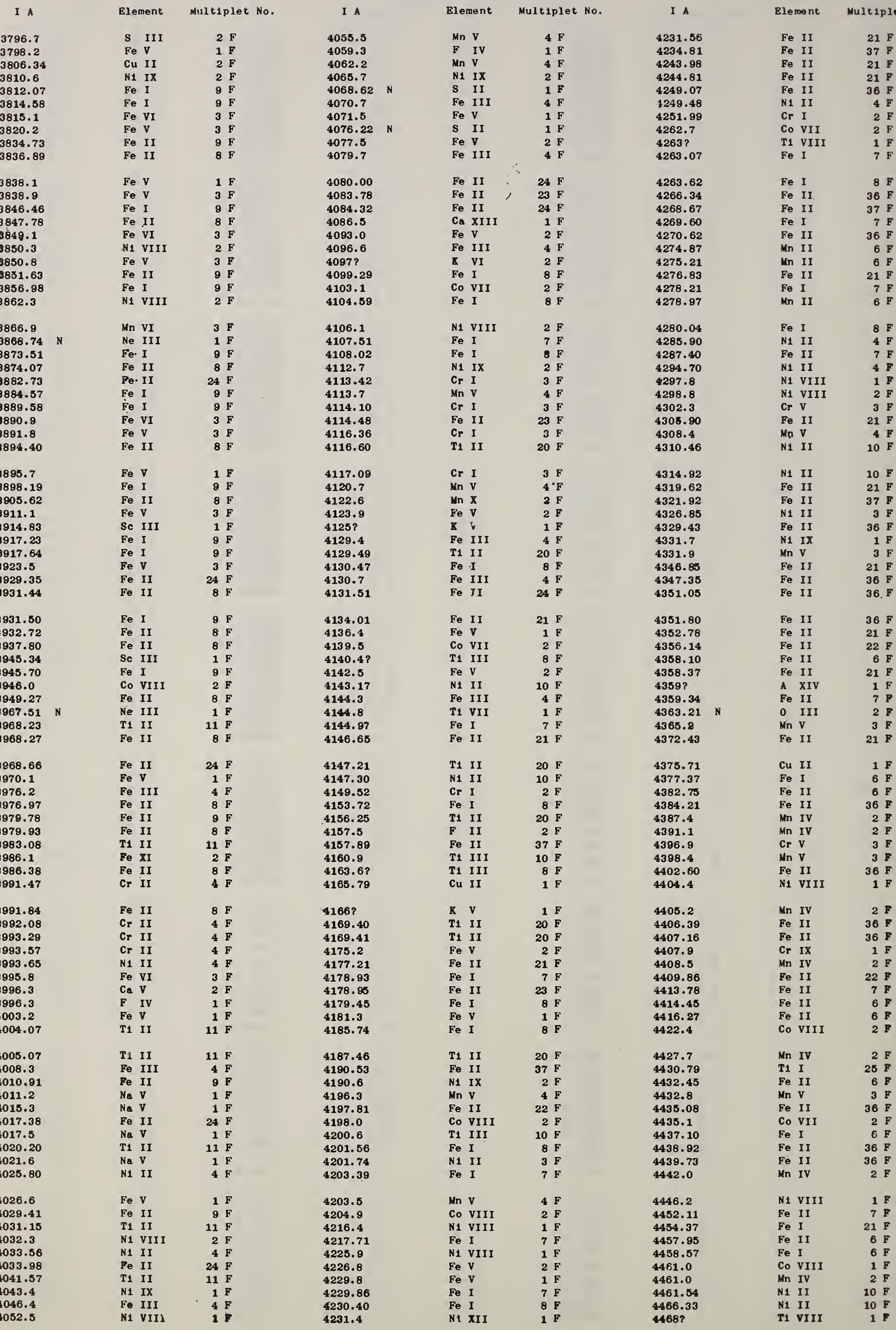


Forbidden Lines

I $\Lambda$

Element Nultiplet No.

470.28

472.37

4473.46

447.81

4477.81

4478.8

$\$ 480.6$

484.84
485.87

486.35

4488.75

4488.76

492.3

4492.64

493.23

493.3

494.57

4495.3

4496.21

4497.23

4487.4

4498.90

4500.00

4501.36

4504.71

4505.8

4508.52

4509.61

4509.85

4510.63

4511.0

4514.90

4515.52

4516.60

4517.36

4521.76

4523.6

4528.55

4528.3

4528.38

4528.7

4532.08

4533.00

4535.7

4544 . 36

4545 ?

4545.20

4548.3

4548.32

4548.5

4550.48

4550.64

4551.98

4554.48

4555.01

4563.7

4564.7

4573.23

4573.45

4573.9

4573.93

4575.46

4575.84

4577.32

4578.83

4580.88

4581.18

4589.0

4591.4

4598.07

4603.66

4604.48

4607.0

4610 ?

4618.97

4621.5

4622. 19

627.3

4630.06

4631.93

632.27

4638.68

4640.05

4644.2

4652 .

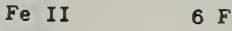

$\begin{array}{lrl}\text { Fe II } & 6 \mathrm{~F} \\ \text { T1 I } & 19 \mathrm{~F} \\ \text { Fe I } & 6 \mathrm{~F} \\ \text { Fe II } & 7 \mathrm{~F} \\ \text { Co VII } & 2 & \mathrm{~F} \\ \text { T1 I } & 15 \mathrm{~F} \\ \text { Nn IV } & 1 \mathrm{~F} \\ \text { Mn IV } & 2 \mathrm{~F} \\ \text { I1 I } & 19 \mathrm{~F} \\ \text { N1 II } & 3 \mathrm{~F}\end{array}$

T1 I $19 \mathrm{~F}$

T1 I $\quad 6$ F

Co VIII

$\mathrm{Fe}$ II $6 \mathrm{P}$

N1 VIII $\quad 1 \mathrm{~F}$

$\begin{array}{ll}\text { Fe I } & 6 \text { F } \\ \text { Mn IV } & 2 \text { F }\end{array}$

T1 I

$19 \mathrm{~F}$

TI I

Mn IV

T1

T1 I

T1 I

Co VII

T1 I

Fe II

$\begin{array}{rl}19 & \mathrm{~F} \\ 1 & \mathrm{~F} \\ 19 & \mathrm{~F} \\ 18 & \mathrm{~F} \\ 18 & \mathrm{~F} \\ 18 & \mathrm{~F} \\ 1 & \mathrm{~F} \\ 2 & \mathrm{~F} \\ 19 & \mathrm{~F} \\ 6 & \mathrm{~F}\end{array}$

\section{I1 I}

I IV

Fe II
T1 I

T1 I

T1 I

T1 I

$\begin{array}{ll}\mathrm{N} 1 & \mathrm{~J} \\ \mathrm{Cr} & \mathrm{V}\end{array}$

\section{I1 I}

Fe IV

Fe II

Fe I

Fe II

Mn IV

$\begin{array}{ll}\text { T1 } & I \\ \text { Fe } & I\end{array}$

II VIII

$19 \mathrm{~F}$

$\begin{array}{ll}6 & F \\ 2 & F \\ 6 & F\end{array}$

$6 \mathrm{~F}$
$19 \mathrm{~F}$

$6 \mathrm{~F}$

$18 \mathrm{~F}$

$19 \mathrm{~F}$

Be I 21

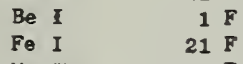

Mn IV $2 \mathrm{~F}$

$\begin{array}{lll}\mathrm{Fe} \text { II } & 6 \mathrm{~F} \\ \mathrm{Fe} & \mathrm{G} & 6 \mathrm{~F}\end{array}$

Fe I

Fe II

Fe II

$\begin{array}{ll}6 & F \\ 6 & F \\ 6 & F\end{array}$

$\begin{array}{ll}6 & F \\ 6 & F \\ 2 & F\end{array}$

Co VIII $2 \mathrm{~F}$

Fe I $\quad 6 \mathrm{~F}$

Fe III $3 \mathrm{~F}$

Cr I $1 \mathrm{~F}$

$\begin{array}{lll}\mathrm{Ca} & \mathrm{I} & 2 \mathrm{~F} \\ \mathrm{Cr} & \mathrm{I} & 1 \mathrm{~F}\end{array}$

Cr I

Fo I

Cr II $3 \mathrm{~F}$

Cr II $3 F$

$\begin{array}{ll}\mathrm{Cr} \text { II } & 3 \mathrm{~F}\end{array}$

In IV

Fe II $4 \mathrm{~F}$

$\begin{array}{lll}\mathrm{Fe} & \mathrm{I} & 5 \mathrm{~F} \\ \mathrm{Fe} & \text { II } & 5 \mathrm{~F}\end{array}$

Fe III $3 \mathrm{~F}$

$\triangle \mathrm{V} \quad 2 \mathrm{~F}$

C I

$\begin{array}{ll}\mathrm{Fe} & \mathrm{I} \\ \mathrm{C} & \mathrm{I}\end{array}$

Fe I

Fe I

Fe II

$\begin{array}{ll}\text { Fe } & \text { II } \\ r_{0} & \text { I }\end{array}$

N1 VIII

Co VII

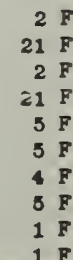

$\mathrm{Fe} \mathrm{I} \quad 5 \mathrm{~F}$

Fe I 21

I A

4658.1

4662.7

4664.97

4665.5

4665.65

4667.0

4672.2

4674.64

4677.94

4685.93

4687.56

4892.6

4683.56

4699.0

4699.3

4701.5

4711.4
4711.86

4714 ?

4715.21

$4716 ?$

4716. 36

4717 ?

4719.7

4720 ?
4723.38

4728.07

4733.8

4734

4738.6

4738.9

4740.3

4745.48

4750.57
4751.75

4754.7

4761.9

4768.4
4771.54

4772.07

4772.4

4774.74

4777.7

4778 ?

4785.21

4789.19

4789.5

4783.03

798.28

4789.31

4798.4

4799.5

4806.83

4807.5

4813.27

4813.8

4814.0

4814.85

4823.44

4824 ?

4824.1

4837.42

4838.7

4842.4

4843.1

4843.34

4843.81

4847.01

4847.58

4850.9

4851.6

4852.73

4857.50

4858.4

4858.87

4861.41
4862.80

4863.8

486.3

4870.8

4871.43

4872.80

4873.4

Element vultiplet No.

$\begin{array}{ll}\text { Fe III } & 3 \mathrm{~F} \\ \text { Mn IV } & \text { I F }\end{array}$

Fe II $\quad$ I F

$\begin{array}{ll}\text { Fe II } & 5 \mathrm{~F} \\ \text { Co VII } & 2 \mathrm{~F}\end{array}$

Fe II 4 F

$P$ II $2 \mathrm{~F}$

$\begin{array}{lll}\text { Se VI } & 1 & F \\ \text { Fe I } & 21 & F\end{array}$

Fe I $21 \mathrm{~F}$

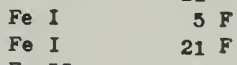

$\begin{array}{lll}\text { Fe II } & 21 & \text { F } \\ \text { Co } & \text { III } & \text { I F }\end{array}$

$\begin{array}{ll}\text { Co VII } & 1 \mathrm{~F} \\ \mathrm{Fe} \mathrm{I} & 5 \mathrm{~F}\end{array}$

Fe I $5 \mathrm{~F}$

$\begin{array}{ll}\text { Fe VII } & 2 F \\ \text { Mn IV } & \text { I F }\end{array}$

Fe III

$\begin{array}{ll}1 & F \\ 3 & F\end{array}$

$\triangle$ IV $1 \mathrm{~F}$

Ne IV $\quad 21 \mathrm{~F}$

$\begin{array}{lll}\text { Fe I } & 21 \mathrm{~F} \\ \text { Ne IV } & \text { I }\end{array}$

Fe II $5 \mathrm{~F}$

Ne IV $I F$

$\begin{array}{lll}\text { No IV } & \text { I F } \\ \text { Ne IV } & \text { I F }\end{array}$

$\begin{array}{lr}\text { Ne IV } & 1 \mathrm{~F} \\ \text { Fe } & 21 \mathrm{~F}\end{array}$

Fe II $4 \mathrm{~F}$

$\begin{array}{ll}\text { Fe III } & 3 \mathrm{~F} \\ V \text { VIII } & 1 \mathrm{~F}\end{array}$

P II 2 F

$\triangle$ IV $I F$

Fe II $20 \mathrm{~F}$

$\begin{array}{ll}\mathrm{Fe} \text { II } & 5 \mathrm{~F} \\ \mathrm{Fe} I & 4 \mathrm{~F}\end{array}$

Fe III $\quad 3 \mathrm{~F}$

NI IV $1 \mathrm{~F}$

$\begin{array}{lr}\text { Fe III } & 3 \mathrm{~F} \\ \text { I1 II } & 10 \mathrm{~F}\end{array}$

Fe II $4 \mathrm{~F}$

N1 VIII 1 P

Fe II 20

$\begin{array}{lll}\text { Fe III } & 3 \text { F } \\ \text { II VIII } & 1\end{array}$

II II $\quad 10 \mathrm{~F}$

Fe I 4 F

F II 1 II

Fe II $4 \mathrm{~F}$

Fo II $4 \mathrm{~F}$

$\begin{array}{ll}\text { CP IV } & 5 \mathrm{~F} \\ \text { Fe III } & 3 \mathrm{~F}\end{array}$

I1 II $10 \mathrm{~F}$

$\begin{array}{ll}\text { Fe VI } & 2 \text { F } \\ \text { N1 I } & 3 \text { F }\end{array}$

Fe III 3 F

Fe II $20 \mathrm{~F}$

Mn IV $1 F$ 
I A

Element Multiplet No.

5032.7

5034.05

5035.50

5036.55

5039.10

5043.53

5045 ?

5047.81

5049. 29

5049.73

5056.5

5060.3

5063.7

5065.43

5071.6

5072.40

5074.90

5076.3

5076.57

5080.84

5082.54

5083.72

5084.8

5092.60

5092.87

5098.44

5100.4

5105.16

5107.95

5108.53
5108.57

5108.57
5111.63

$5116.03 \mathrm{C}$

5116. 3

5118.07

5119.47

5124.41

5126.25

5127.09

5134.16

5136.3

5144.39

5145.5

5143.55

5146.8

5147.16

5150.07

5151.9

5157.28

$\mathbf{5 1 5 8 . 0 0}$

5158.3

5158.81

5162.53

5163.94

5165.88

Fe III

Cr II

Fe II

Fe II

T1 I

Fe II

Sc VII

Fe 1 II

Cr II

N1 IX
Fe II I

Fe III

T1 II

Fe II

Fe I

Co VII

Fe II

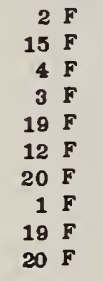

I A

Element Multiplet No.

I A

Element Multiplet No.

T1 II $19 \mathrm{~F}$

$\begin{array}{lll}\text { Cr I } & 16 \mathrm{~F} \\ \text { Fe II } & 35 \mathrm{~F}\end{array}$

Fe III $\quad 1 \mathrm{~F}$

Cr II $2 \mathrm{~F}$

Cr I $8 \mathrm{~F}$

CrI $\quad 16 \mathrm{~F}$

$\begin{array}{lll}\text { Fe VI } & 1 \mathrm{~F} \\ \text { T1 VII } & 1 \mathrm{~F}\end{array}$

Cr I $8 \mathrm{~F}$

$\begin{array}{lrl}\text { Fe II } & 18 \mathrm{~F} \\ \text { Cr I } & 8 \mathrm{~F}\end{array}$

Cr II $\quad 14 \mathrm{~F}$

Fe II $19 \mathrm{~F}$

N1 XIII $1 \mathrm{~F}$

$\begin{array}{ll}\text { N1 XIII } & 1 \mathrm{~F} \\ \mathrm{Z} \text { III } & 1 \mathrm{~F}\end{array}$

(r) III

Cr II

$\begin{array}{ll}2 & F \\ 7 & F\end{array}$

Cr I $16 \mathrm{~F}$

Cr I $16 \mathrm{~F}$

Cr II $14 \mathrm{~F}$

Cr I $15 \mathrm{~F}$

$\begin{array}{lll}\text { Co VII } & \text { I F } \\ \text { Zr II } & 7 & \text { F }\end{array}$

$\begin{array}{ll}\text { Zr II } & 7 \text { F } \\ \text { Cr IV } & 3 \text { F }\end{array}$

Cr I $\quad 15 \mathrm{~F}$

$\begin{array}{lr}\text { Fe I } & 2 \mathrm{~F}\end{array}$

Cr I $\quad 15 \mathrm{~F}$

Fe III $\quad 1 \mathrm{~F}$

Cr II 14 F

Fe II $18 \mathrm{~F}$

$\begin{array}{lr}\text { Fe VII } & 2 \mathrm{~F} \\ \mathrm{Fe} \text { II } & 19 \mathrm{~F}\end{array}$

Cr I $15 \mathrm{~F}$

Fe II $35 \mathrm{~F}$

T I $15 \mathrm{~F}$

5170.84
5174.95

5177.0

5180.78

5181.21

6181. 97

5182.71

5184.80

5185

5191.4 N

Fe I

Cr II

Fe I

Cr I

Fe II

Cr I

Fe II

Co XI

$\begin{array}{rl}3 & \mathrm{~F} \\ 14 & \mathrm{~F} \\ 2 & \mathrm{~F} \\ 20 & \mathrm{~F} \\ 15 & \mathrm{~F} \\ 18 & \mathrm{~F} \\ 16 & \mathrm{~F} \\ 19 & \mathrm{~F} \\ 1 & \mathrm{~F} \\ 3 & \mathrm{~F}\end{array}$

5193.13

5193.82

5197.31

5198.5

5199.18

5200.7

3206.02

5206.84

5209.1

5212.95

5216.07

5219.02

5220.06

5220.56

5224 ?

5224.15

525.00

5226.64

Fe I $19 \mathrm{~F}$

Cr I $\quad 15$ F

$\mathrm{Fe} \mathrm{I} \quad 20 \mathrm{~F}$

Cr I $15 \mathrm{~F}$

N I $\quad 1 \mathrm{~F}$

$\begin{array}{llrl}\text { Fe II } & 35 & \mathrm{~F} \\ \mathrm{~N} & \mathrm{I} & 1 & \mathrm{~F}\end{array}$

Cr II $14 \mathrm{~F}$

$\begin{array}{lll}\text { Zr II } & 7 & \text { F } \\ \text { Cr IV } & 3 & \text { F }\end{array}$

Fe I $\quad 19 \mathrm{~F}$

Cr II $13 \mathrm{~F}$

Fe II $19 \mathrm{~F}$

$\begin{array}{lll}\mathrm{Fe} I & 3 \mathrm{~F} \\ \mathrm{Sc} \text { VII } & 1 \mathrm{~F}\end{array}$

Fe I

Cr II

$\checkmark$ I I

$\begin{array}{rr}3 & \mathrm{~F} \\ 13 & \mathrm{~F}\end{array}$

Cr I
5227.25

6228. 44

5229.06
5235.07

5236.6

5237.7

5238.35

5238.47

5242.00
5245.25

5247.84

5248.64

5254.49

5255.97

5261.61

5268.82

5268.88

5269.16

5270.19

5270.4

5273.38

5274.27

5275.83

5276.1

5278.39

5279.2

5279.80

5280.25
5281.46

5282.88

3283.11

5285.21

5285.34

5286.31

5288.83

5289.66

5290.75

5295.70

5286.3

5296.84

5299.42
5302.86

5303.37

5303.6

5303.99

5304.06

5308.68

5308.9

5310. 36

5312.52

5313.88

5316.87

5323.64

5326.5

5331.46

5332.4

5333.66

5334. 30

5336.4

5338.65

5341.39

5347.67

5352. 29

5354.10

5354.76

5355.9

5356.32

5358.79

5362.06

5363.81

3368. 91

5370.5

5374.6

5376.47

3382.26

5386.27

5394. 78

5396. 71

5404.80

5412.0

5412.64

5412.97

5413. 34

5415.04

3418.0

5427.17

$\begin{array}{llr}\text { V II } & 6 \\ \text { Cr II } & 13 \\ \text { Zr II } & 7 \\ \text { V II } & 6 \\ \text { Fe VI } & 1 \\ \text { V IV } & 3 \\ \text { Cr II } & 13 \\ \text { Cr I } & 15 \\ \text { Cr II } & 13 \\ \text { V II } & 6\end{array}$

$\begin{array}{rl}6 & F \\ 13 & F \\ 7 & F \\ 6 & F \\ 1 & F \\ 3 & F \\ 13 & F \\ 15 & F \\ 13 & F \\ 6 & F\end{array}$

Cr II

$\mathrm{Cr}$ I

Cr II

Co VIII

$\mathrm{Fe} I$

Fe II

Cr II

$\begin{array}{rl}13 & \mathrm{~F} \\ 14 & \mathrm{~F} \\ 6 & \mathrm{~F} \\ 13 & \mathrm{~F} \\ 19 & \mathrm{~F} \\ 1 & \mathrm{~F} \\ 18 & \mathrm{~F} \\ 18 & \mathrm{~F} \\ 14 & \mathrm{~F} \\ 13 & \mathrm{~F}\end{array}$

Fe III

Fe II

N1 II

NI II

Fe VII

Fe VI

Cr II

Fe II

$\begin{array}{rl}1 & F \\ 18 & F \\ 8 & F\end{array}$

$14 \mathrm{~F}$
$2 \mathrm{~F}$

$35 \mathrm{~F}$

$1 \mathrm{~F}$

$13 \mathrm{~F}$

V II

Fe II

Cr II

Cr I

Cr II

$\mathrm{Fe} I$

$\mathrm{Fe} I$

Fe II

B F

$\begin{array}{ll}35 & \mathrm{~F} \\ 13 & \mathrm{~F}\end{array}$

$15 \mathrm{~F}$

$10 \mathrm{~F}$

$12 \mathrm{~F}$

$19 \mathrm{~F}$

$17 \mathrm{~F}$

Fe II $19 \mathrm{~F}$

$\begin{array}{ll}\text { Cr II } & 13 \mathrm{~F}\end{array}$

$\mathrm{Zr}$ III

Fe XIV 1 F

Fe I

Fe I

Cr II 
Forbldden Lines

I A Element Multiplet No.

6588.16

5581.3

6581.9

5685.31

6600.1

5600.66

5601.

5603.2

5600.36

6609.27

6611.94

6613.81

5614.62

6615.18

6615.8

5618.9

6625.0

6630.85

6631.0

5631.6

5634.78

5639.56

6643.02

6643.44

6648 ?

5649.67

6850.38

6660.84

5654.85

B656.39

6659.83

5662.62

6664.02

6669.88

5678.0

6678.0
6679.3

6683.86

5689.3

6684 .

6696.36

6699.5

6703.3

6703.64
6708.96

5711.46

6712.7

6713.35

5715.84

6720.8

5721.2

6721.35

6724.62

6726.9

6733.0

5741.11

5742.07

5746.48

5745.70

5746. 98

5750.80

6753.8

6754.8

6755.38

5756.60

5766.4

6773.51

6778.35

6778.97

6780.28

6783.
6785

5704.16

6795.58

5796.28

$6799.5:$

6804.4

5809.4 :

6812.5

5815.5:

6815.7

5819.5

6828.12

\begin{tabular}{|c|c|c|c|c|}
\hline $\mathrm{Fe}$ & II & & $\mathbf{F}$ & 5832.40 \\
\hline $\mathrm{Cr}$ & III & 2 & $\mathbf{F}$ & 5834.64 \\
\hline Mn & v & 2 & $F$ & 6836.21 \\
\hline $\mathrm{T} 1$ & I & 8 & $\mathbf{F}$ & 5843.6 \\
\hline$C_{r}$ & III & 1 & $F$ & 5843.90 \\
\hline $\mathbf{P e}$ & II & 33 & $\mathbf{F}$ & 5846.3 \\
\hline Mn & VI & 2 & $\mathbf{F}$ & 6862.48 \\
\hline $\mathbf{x}$ & VI & 1 & $\mathbf{F}$ & 5852.8 \\
\hline v & II & 14 & $\mathbf{F}$ & 5855.37 \\
\hline $\mathbf{F e}$ & I & 31 & $\boldsymbol{F}$ & 5863.1 \\
\hline
\end{tabular}

5867.17

5868.3

5872.77

5876.23

5876.92

5884.9

5888.0

6893. 89

5888.30

5801.26

5902.64

6907. 1

5913.34

6829.20

6929.31

5931.19

5932.88

6034.41

5934.73

5936.99

594.

5846.87

5848.99

5851.24

6952.21

5855.61

5968.87

6971.33

6972. 59

5975.39

5882.55

5883.89

6988.76

5990.31

6991.0

5892.16

6899.99

6007.34

6010.53

6013.28

6015.26

6016.15

6018.64

6018.63

6026.18

6029.7

6040.31

6040.84

6044.94

6046.80

6047.46

6048.37

8053.14

6059.21

6061.50

8065.2

6065.34

6067.88

6069.2

6071.36

6077.80

6083.2

6085.5

6085.8

6087.77

6093.32

6094.65

6095.96

6096.3

6088.1

6098.31
Element Multiplet No.

I A

6100.26

6101.1

6104.8

6106.17

6111.14

6113.40

6113.97

6114.52

6114.66

6117.60

6124.57

6140.20

6147.13

6151.82

6152.9

6159.3

6150.3

6160.1

Fe I $\quad 2$ F

Fe II 34 F

Fe I $18 \mathrm{~F}$

Un VI $2 \mathrm{~F}$

Cr I $13 \mathrm{~F}$

2r II $12 \mathrm{~F}$

$\begin{array}{lr}\text { Bu II } & 2 \mathrm{~F} \\ \mathrm{Fe} \mathrm{I} & 17 \mathrm{~F}\end{array}$

Zr II 6 F

Mn VI 1 F

Fe I $2 \mathrm{~F}$

Cr I $14 \mathrm{~F}$

Fe I $2 \mathrm{~F}$

2 F

$\mathrm{Fe} \mathrm{I} 30 \mathrm{~F}$

$\begin{array}{lll}\text { Cr I } & 13 \mathrm{~F} \\ \text { Cr I } & 14 \mathrm{~F}\end{array}$

F* I $30 \mathrm{~F}$

Fe I $\quad 30 \mathrm{~F}$

$\begin{array}{lll}\mathrm{Fe} I & 2 \mathrm{~F} \\ \mathrm{Fe} & \mathrm{I} & 17 \mathrm{~F}\end{array}$

Co II 2 P

Cr I $13 \mathrm{~F}$

Cr I $13 \mathrm{~F}$

Cr I $7 \mathrm{~F}$

$\begin{array}{ll}\text { Cr I } & 7 \mathrm{~F}\end{array}$

Cr I 7 F

Mn $V \quad 1 \mathrm{~F}$

Cr

Fe I

N1 II

Cr I

T1 II

Zr II

Fe I

Fe

Cr

un $\mathrm{V}$

$\begin{array}{ll}\mathrm{Cr} & \mathrm{II}\end{array}$

Zr II

Cr I

TI II

Cr I

TII

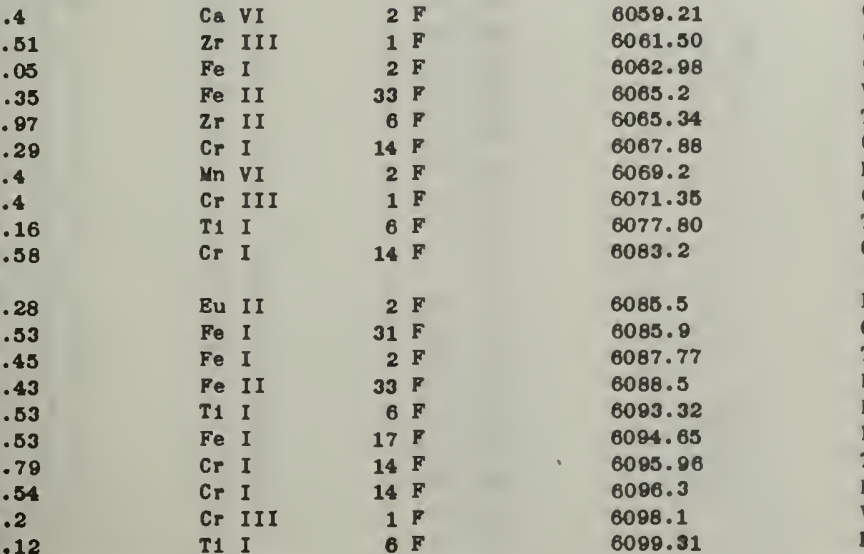

6164.64

6167.7

6167.84

6169.37

6172.91

6174.44

6176.08

6177.21

6178.35

6180.8

6184.51

6188.56

6193.7

6196.53

6186.75

6215.6

6220.7

6223.4

6226.64

6227.19

6229.2

6230.4

6231.27

6233.8

6249.35

6249.75

6250.51

6251.33

6258.22

6277.3

6280.22

6310.2

6314.58

6316.6

6317.64

6328.46

6333.46

6339.70

6342.88

6344.56

6346.2

6349.5

6360.66

6365.52

6367.28

6372.11

6372.9

6376.6

6377.58

6377.83

6381.13

6382.03

6391.51

6393.72

6396.2

6396.30

6404.46

0406.27

6405.67

6408.5

6409.46

6414.83

6415,69

6418. 86

6420.88

$6300.23 \mathrm{~L}$

6363.88 L

$6374.51 \mathrm{C}$ 
Forbidden Lines

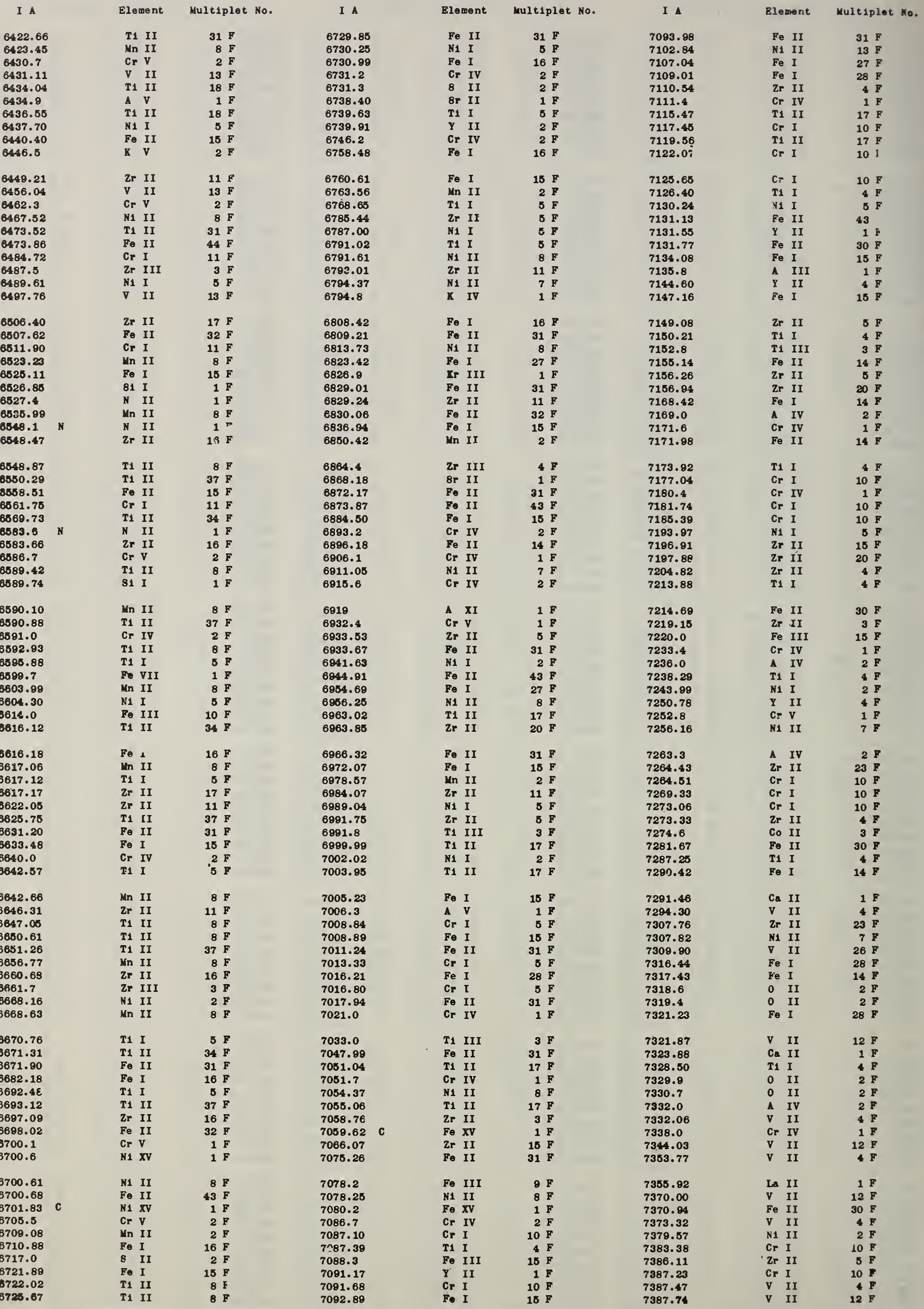


Forbldden Lines

I A

7388.16

7390.6

7393.71

7395.79

7398.95

7404.36

7406.61

411.90

7413.33

7418.75

7418.42

7421.5

7430.26

7431.08

7431.2

7432.23

7438.58

7440.62

7444.80

7445.63

7448.45

7452.50

7.154 .82

7457.80

7459.30

7460.57

7460.93

7464.38

7467.0

7468.52

7469.44

7470.10

7475.84

7477.26

7478.78

7489.15

7487.68

7507.44

7510.54

7515.13

7518.35

7518.81

7523.27

7526.46

7526.94

7530.8

7533.84

7536

7536.83

7539.67

7540.1

7540.54

7540.74

7641.42

7541.95

7544.00

7547.77

7551.8

7556.03

7567.6

7571.69

7586.23

7604.53

7611.2

7612.96

7013.15

7623.44

7626.54

7637.52

7640.39

7642.3

7642.61

7647.06

7658.84

7658.92

7682.36

7686.28

7673.74

7674.06

7681.88

7684.16

7685.58

7686.18

7686.90

7687.94

7688.04
Element Multiplet No.

I $\mathbf{A}$

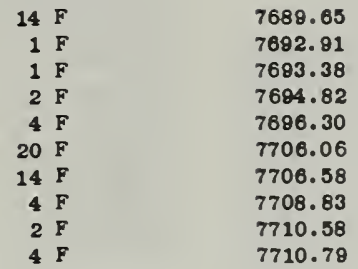

7717.28

7724.7
7733.12

7740.11

7741.96

7750.56

7751.0

7752.86

7757.43

7758.47

7750.25
7764.68

7768.35

7773.91

$\mathbf{7 7 8 6 . 0 3}$

7787.00

7793.8

7803.90

7805.47

7805.66

7806.22

7806.88

7835.98

7845.41

7847.76

7849.08

7853.51

7867.83

7869.5

7874.23

7876.34

7878.32

7888.6

7889.15

7893.57

7894.10

7899.63

7904.04

7906.95

7908.30

7816.25

7916.88

7917.03

7926.90

7828.70

7838.32

7838.41

7940.71

7045.02

7947.28

7854.24

7954.76

7856.80

7958.50

7859.00

7960.85

7964.27

7965.96

7966.36

7974.31

7975.58

7876.96

7878.7
7999.47

8000.12

8009.53

8012.08

8022.25

8022.63

8024

8024.21

8028.94

8033.86

8037.28
Element Vultiplet fio.

$\begin{array}{rl}11 & \mathrm{~F} \\ 7 & \mathrm{~F} \\ 7 & \mathrm{~F} \\ 7 & \mathrm{~F} \\ 7 & \mathrm{~F} \\ 3 & \mathrm{~F} \\ 20 & \mathrm{~F} \\ 1 & \mathrm{~F} \\ 23 & \mathrm{~F} \\ 30 & \mathrm{~F}\end{array}$

I1 I $F$

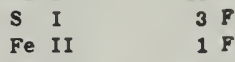

Fe II $29 \mathrm{~F}$

$\mathrm{Fe} \mathrm{I} \quad 14 \mathrm{~F}$

Cr II $11 F$

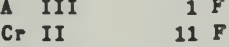

Fe I $14 \mathrm{~F}$

Cr II 11

$\begin{array}{lll}\text { Fe I } & 14 \mathrm{~F} \\ \text { Fe II } & 30 \mathrm{~F}\end{array}$

ZI II $4 \mathrm{~F}$

Fe I $13 \mathrm{~F}$

$\mathrm{Zr}$ II $3 \mathrm{~F}$

$\begin{array}{ll}I & 3 \mathrm{~F}\end{array}$

Co II 3 F

Fo II 1

Nn II

$\begin{array}{ll}\text { T1 } & \text { I } \\ \text { Mn } & \text { II }\end{array}$

Fe II

$\begin{array}{rl}7 & F \\ 23 & F \\ 7 & \end{array}$

Cr II

T1 II

Cr II

Cr II

Zr II

Zr III

Cr

Fe I

P II

Fo II

Fe I

Yn II

Fo XI

Zr II

\section{Zr II}

$\begin{array}{ll}\text { T1 II } & 6 \mathrm{~F} \\ \mathrm{Fe} \mathrm{I} & 14 \mathrm{~F}\end{array}$

I II $3 \mathrm{~F}$

ZF II $22 \mathrm{~F}$

N1 I $\quad 4 \mathrm{~F}$

Fe II $29 \mathrm{~F}$

$\begin{array}{lr}\text { T1 II } & 20 \mathrm{~F} \\ \text { Fo II } & \text { I F }\end{array}$

N1 I $8 \mathrm{~F}$

Fe I $26 \mathrm{~F}$

Cr I $8 \mathrm{P}$

$\begin{array}{lll}C \text { C I } & 8 & F \\ \text { TI II } & 7 & \text { F }\end{array}$

Cr II $20 \mathrm{~F}$

$1113 \mathrm{~F}$

$\begin{array}{lll}\text { ZF II } & 25 & \mathrm{~F} \\ \text { T1 II } & 38 \mathrm{~F}\end{array}$

Fo II $\quad 29 \mathrm{~F}$

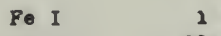

Cr II $20 \mathrm{~F}$

Fo I $1 \mathrm{~F}$

$\begin{array}{lr}\text { Cr II } & 11 \mathrm{~F} \\ \text { T1 II } & 7 \mathrm{P}\end{array}$

Cr II

T1 II

T1 II

Mn IX
Fe II

Cr II

P. II

Fe II

Fo I

N1 $X V$

N1 XV

II II

N1 II
Fo II

I $\mathrm{A}$

Blement

Multiplet No.

8038.68

8043.80

8045.57

8046.1

8047.93

80.8

8074.28

8076.58

8085.17

8086.73

8081.87

8098.70

8101.03

8106.38

8106.

8118.16

8119.46

8125.50

8137.88

8138.58

8138.62

8148.37

8151.33

8153.46

8160.66

8164.85

8166.83

8183.68

8185.52

8189.44

8104.57

8201.77

8220.64

8225.25

8228.81

8229.81

8231.57

8235.69

8245.12

8249.61

8251.14

8252.38

8261.21

8261.58

8268.36

8271.32

8272.21

8275.57

8278.88

8284.1

8289.45

8303.23

8307.67

8308.68

8315.71

8321.51 
FINDING LIST

Forbidden Lines

I A

8433. 7

8436.37

8437.9

8441.27

8444. 83

8445.28

8446.11

8456.74

8457.2

8466. 38

8466. 95

8469. 75

8471.07

8481.6

8485.90

8490.18

8490.34

840.44

8491.16

8483.1

8501.8

8518.20

8520.22

8521.66

8525.41

8529.50

8530.15

8532.12

8644.49

8545.12

8553.73

8553.87

8561.42

8664.56

8565.94

8567.60

86.75 .4

8579.15

8579.5

8582.52

8585.04

8585.14

8588.84

8596.27

8698.3

8599.1

8612.91

8613.35

8615.4

8621.67

8623.51

8625. 26

8625.8

8625.93

8626.86

8627.35

8640.22

8640.27

8643.14

8645.95

8647. 89

8648.72

8649. 11

8649.72

8651.14

8652.17

8653.20

8658.20

8661.20

8661.96

8605.66

1050.28

0974.27

8082.13

8683.4

8689.73

8691.53

8698.18

8698.69
Blement Multiplet No.

I A

Element

Multiplet No.

I A

8105.8

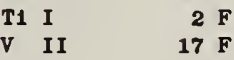

24. $\mathrm{F}$

$\mathrm{Fe} \mathrm{I} \quad 25 \mathrm{~F}$

$\checkmark$ II $\quad 11 \mathrm{~F}$

T1 II $27 \mathrm{~F}$

$\begin{array}{llll}V & \text { III } & \text { I F } \\ \text { Cl III } & 3 & F\end{array}$

$\mathrm{V}$ II $11 \mathrm{~F}$

$\begin{array}{rr}\text { Sc II } & 2 \mathrm{~F} \\ \text { Cr II } & 19 \mathrm{~F}\end{array}$

T1 I

$\begin{array}{rl}19 & \mathrm{~F} \\ 3 & \mathrm{~F}\end{array}$

Zr II $\quad 10 \mathrm{~F}$

Cr II $19 \mathrm{~F}$

$\begin{array}{llll}\text { T1 } & \text { I } & 30 & \mathrm{~F} \\ V & \text { II } & 28 & \mathrm{~F}\end{array}$

$\checkmark$ II $2 \mathrm{~F}$

T1 II $\quad 16 \mathrm{~F}$

$\begin{array}{llrl}\text { Cl } & \text { III } & 3 & \mathrm{~F} \\ \text { T1 } & \text { II } & 16 & \mathrm{~F}\end{array}$

$\begin{array}{ll}\mathrm{V} \text { II } & 16 \mathrm{~F}\end{array}$

Zr II 26 F

$\mathrm{Fe} \mathrm{I} \quad 1 \mathrm{~F}$

T1 II $15 \mathrm{~F}$

$\begin{array}{lll}\text { Sc II } & 2 & \text { F } \\ V & \text { IV } & \text { I }\end{array}$

T1 I $22 \mathrm{~F}$

$\checkmark$ II $11 \mathrm{~F}$

$\begin{array}{llrl}C & \text { II } & 1 & \mathrm{~F} \\ V & \text { II } & 28 & \mathrm{~F}\end{array}$

T1 II

$15 \mathrm{~F}$

$\mathrm{Fe} \mathrm{I} \quad 33 \mathrm{~F}$

$\begin{array}{llr}\text { T1 I } & 2 & \text { F } \\ \text { Fe I } & 33 & \text { F }\end{array}$

$\checkmark$ III $2 \mathrm{~F}$

T1 I $\quad 29 \mathrm{~F}$

T1 I $30 \mathrm{~F}$

T1 $12 \mathrm{~F}$

$\begin{array}{llrl}\text { V } & \text { III } & 2 & \mathrm{~F} \\ \text { Fe } & \text { II } & 13 & \text { F }\end{array}$

Zr II

$\mathrm{Fe} \mathrm{I} \quad 33 \mathrm{~F}$

$\begin{array}{ll}\mathrm{Zr} \text { II } & 8 \mathrm{~F} \\ \mathrm{~V} \text { III } & \text { I F }\end{array}$

T1 II $16 \mathrm{~F}$

T1 I $16 \mathrm{~F}$

$\begin{array}{llll}V & \text { II } & 11 & \mathrm{~F} \\ \text { T1 } & \text { I } & 22 & \mathrm{~F}\end{array}$

T1 I $29 \mathrm{~F}$

T1 I 22 F

$\begin{array}{ll}\text { Fe } & \text { I } \\ \text { T1 } & \text { II }\end{array}$

Sc II

Fe I

T1 II

$\begin{array}{ll}\text { Cr } & \text { II } \\ \text { Cr } & \text { II }\end{array}$

T1 I

T1 II

$\begin{array}{ll}\text { T1 } & \text { II } \\ Y & \text { II }\end{array}$

T1 I

V II

V III

T1 I

Zr II

V II

$\begin{array}{rl}1 & \mathrm{~F} \\ 16 & \mathrm{~F} \\ 2 & \mathrm{~F} \\ 24 & \mathrm{~F} \\ 29 & \mathrm{~F} \\ 18 & \mathrm{~F} \\ 19 & \mathrm{~F} \\ 16 & \mathrm{~F} \\ 15 & \mathrm{~F}\end{array}$

$\begin{array}{rl}15 & \mathrm{~F} \\ 6 & \mathrm{~F} \\ 2 & \mathrm{~F}\end{array}$

$2 \mathrm{~F}$
$17 \mathrm{~F}$

$2 \mathrm{~F}$

$1 \mathrm{~F}$
$16 \mathrm{~F}$

$19 \mathrm{~F}$

$11 \mathrm{~F}$
8702.70
8703.03

8703.79

8704.24

8705.08

8706.79

8708.23

8715.84

8716.24

8719.70

8721.54

8723.13

8727.4

8728.09

8730.02

8731.38

8735.0

$\begin{array}{llrl}\text { Zr II } & 9 & \mathrm{~F} \\ \text { T1 } & \text { II } & 33 & \mathrm{~F} \\ \text { Cr II } & 18 & \mathrm{~F} \\ \text { N1 } & \text { II } & 12 & \mathrm{~F} \\ \text { T1 } & \text { I } & 16 & \mathrm{~F} \\ \text { Fe } & \text { II } & 52 & \mathrm{~F} \\ \text { T1 } & \text { I } & 17 & \mathrm{~F} \\ \text { V } & \text { II } & 2 & \mathrm{~F} \\ \text { Fe } & \text { II } & 42 & \mathrm{~F} \\ \text { T1 } & \text { I } & 2 & \mathrm{~F}\end{array}$

T1 II 36

T1 I $16 \mathrm{~F}$

T1 II $16 \mathrm{~F}$

T1 I $29 \mathrm{~F}$

C I $3 \mathrm{~F}$

$\begin{array}{lrl}\text { Fe III } & 8 & \text { F } \\ \text { Cr II } & 18 & \text { F }\end{array}$

$\begin{array}{llll}\text { Cr II } & 18 & \text { F } \\ \text { T1 } & \text { I } & 17 & \text { F }\end{array}$

$\checkmark$ III $1 \mathrm{~F}$

8739. 71

8740.05

8743.65

8743.66

8745.0

8746.99

8761.8

8763.28

8763.95

8770.71

8771.24

8774.69

8775.19

8777.26

8782.6

8787.6

8787.81

8789.70
8792.09

8792.49

8794.80

8798. 79

8798.82

8799.09

8799.1

8808.47

8815.9

8826.02

8830.3

8830.7

8831.94

8832.31

8838.2

8843.42

8848.50

8850.73

8851.13

8851.45

8858.94

8862.47

8868.91

8872.37

8878.98

8884.12

8885.66

8891. 88

8899.71

8909.40

8921.0

8929. 91

8930.70

8931.47

8854.34

8969.06

8970.23

8970.56

8983.71

8012.04

9033.73

9043.52

9051.92

9069.4

9071.07
9072.86

9089.24

9093.67

9096.76

$\begin{array}{llrl}\text { T1 } & \text { I } & 16 & \mathrm{~F} \\ \text { T1 } & \text { I } & 17 & \mathrm{~F} \\ \text { Zr } & \text { II } & 10 & \mathrm{~F} \\ \text { T1 } & \text { II } & 29 & \mathrm{~F} \\ \text { V } & \text { III } & 1 & \mathrm{~F} \\ \text { Fe } & \text { I } & 33 & \mathrm{~F} \\ \text { N1 } & \text { VIII } & 5 & \mathrm{~F} \\ \text { V } & \text { II } & 2 & \mathrm{~F} \\ \text { T1 } & \text { II } & 36 & \mathrm{~F} \\ \text { Zr } & \text { II } & 10 & \mathrm{~F}\end{array}$

$\begin{array}{llrl}\text { T1 } & \text { I } & 17 & \mathrm{~F} \\ \text { Fe I } & 12 & \mathrm{~F} \\ \text { V } & \text { II } & 11 & \mathrm{~F} \\ \text { Fe I } & 33 & \mathrm{~F} \\ \text { T1 } & \text { I } & 2 & \mathrm{~F} \\ \text { V } & \text { III } & 2 & \mathrm{~F} \\ \text { P } & \text { I } & 1 & \mathrm{~F} \\ \text { T1 I } & 17 & \mathrm{~F} \\ \text { T1 II } & 29 & \mathrm{~F} \\ \text { Cr II } & 18 & \mathrm{~F}\end{array}$

$\begin{array}{llrl}\text { Fe I } & 24 & \text { F } \\ \text { Fe I } & 25 & \text { F } \\ \text { T1 II } & 33 & \text { F } \\ \text { Zr II } & 26 & \text { F } \\ \text { T1 I } & 2 & \text { F } \\ \text { P I } & 1 & \text { F } \\ \text { T1 I } & 17 & \text { F } \\ \text { V } & \text { IV } & 1 & \text { F } \\ \text { Cr II } & 19 & \text { F } \\ \text { Co II } & 1 & \text { F }\end{array}$

Fe III $8 \mathrm{~F}$

Cr II $18 \mathrm{~F}$

N1 I $7 \mathrm{~F}$

Fe III $8 \mathrm{~F}$

N1 I $1 \mathrm{~F}$

T1 I $17 \mathrm{~F}$

$\mathrm{Zr}$ II $9 \mathrm{~F}$

$\begin{array}{lll}\text { Fe II } & 52 & \text { F } \\ \text { T1 I } & 17 & \text { F }\end{array}$

$\begin{array}{llll}\text { T1 I } & 17 & \text { F } \\ \text { Cr II } & 18 & \text { F }\end{array}$

Zr II 26

Fo

Zr II

I I

$\begin{array}{ll}1 & \mathrm{~F} \\ 8 & \mathrm{~F}\end{array}$

Pe II $42 \mathrm{~F}$

Fe II $13 \mathrm{~F}$

Cr II $16 \mathrm{~F}$

$\begin{array}{lll}\text { Zr II } & 2 & \text { F } \\ \text { Zr III } & 5 & \text { F }\end{array}$

Cr II $\quad 18$ F

T1 I $\quad 17 \mathrm{~F}$

$\begin{array}{lrl}\text { Fe II } & 49 \mathrm{~F} \\ \mathrm{Z} & \text { II } & 9 \mathrm{~F}\end{array}$

Zr II $22 \mathrm{~F}$

T1 I $2 \mathrm{~F}$

Cr II $\quad 18 \mathrm{~F}$

$\begin{array}{lr}\text { Eu II } & 1 \mathrm{~F} \\ \text { Cr II } & 18 \mathrm{~F}\end{array}$

Fe II $13 \mathrm{~F}$

Cr II $\quad 16$ F

$\begin{array}{lll}V & \text { II } & 10 \mathrm{~F} \\ \mathrm{Fe} & \text { II } & 13 \mathrm{~F}\end{array}$

Zr II $2 \mathrm{~F}$

5 III 1 F

T1 II $\quad 35 \mathrm{~F}$

$\begin{array}{lll}\text { Cr II } & 24 & \mathrm{~F} \\ \text { Zr II } & 10 \mathrm{~F}\end{array}$

$\begin{array}{llll}\text { Zr } & \text { II } & 10 & \mathrm{~F} \\ \text { Fe } & \text { I } & 36 & \text { F } \\ \text { V } & \text { II } & 10 & \text { F }\end{array}$ 
FINDING LIST

Forbidden Lines

I A Element Multiplet No.

9512.58

8513.87

$\$ 517.76$

9522.24

9632.1

8034.75

9543.3

9544.00

9658.6

9565.8

8670.24

9682.55

9800.9

0594.5

9883.12

9085.8

9608.6

8619.74

9622.68

9635.8

9639.4

9642.42

9644. 96

9648.8

9652.70

8670.04

9670.87

8671.2

9674.66

9681.8

9682.13

8686.7

9694.0

9687.4

9701.3

9704.1

9720.20

9731.40

9733.52

9765.81

9774.53

9775.8

9778.67

9778.70

9795.21

9806.20

9808.9

9822.50

9823.4

9826.83

9831.2

9862.2

9866.49

9870.08

8884.28

9885.7

9886.8

8887.1

8902.2

9903.31

9916.3

8917.8

9926.0

9837.20

9837.27

8941.20

8942.2

9947. 19

9949.32

8953.5

8957.23

8957.44

9960.0

8988.6

9872.58

9974.41

8977.1

8982.17

8986.60

8997.3

9898.31

10013.8

$\begin{array}{lr}\text { Zr II } & 27 \\ \text { Cr II } & 16 \\ \text { Pe II } & 41 \\ \text { Fe II } & 52 \\ \text { V II } & 32 \\ \text { S III } & 1 \\ \text { Zr II } & 14 \\ \mathrm{Zr} \text { III } & 5 \\ \text { TI I } & 28 \\ \text { Co VII } & 5\end{array}$

N1 VIII $5 \mathrm{~F}$

$\checkmark$ II $9 \mathrm{~F}$

$\mathrm{Zr} I I \quad 8 \mathrm{~F}$

T1 III 2 F

T1 I $28 \mathrm{~F}$

$\checkmark$ II $32 \mathrm{~F}$

$\begin{array}{lrl}\text { Zr II } & 8 \mathrm{~F} \\ \text { Fe III } & 12 \mathrm{~F}\end{array}$

$\begin{array}{ll}\text { Fo I } & 12 \mathrm{~F} \\ & 12 \mathrm{~F}\end{array}$

T1 $1 \quad 28$

Co II $\quad 4$

T1 II

$\checkmark$ II $25 \mathrm{~F}$

T1 II $21 \mathrm{~F}$

Cr II $17 \mathrm{~F}$

Zr II $24 \mathrm{~F}$

Zr II

Zr III

T1 I

Pe II

Cr II

Bu II

T1 I

Fe III

TI III

$27 \mathrm{~F}$

$5 \mathrm{~F}$

$28 \mathrm{~F}$

$41 \mathrm{~F}$

$16 \mathrm{~F}$

$1 \mathrm{~F}$

$28 \mathrm{~F}$

$11 \mathrm{~F}$
$9 \mathrm{~F}$

T1 I

Fe I

Co VII

Fe II

Pr II

I1 I

$12 \mathrm{~F}$

Fe II $28 \mathrm{~F}$

Cr II $17 \mathrm{~F}$

C I $1 \mathrm{~F}$

Fe I $39 \mathrm{~F}$

$\begin{array}{lll}C & I & 1 \\ \text { Fe I } & 11 \mathrm{~F}\end{array}$

I1 I $20 \mathrm{~F}$

Fe II $40 \mathrm{~F}$

Cr II $17 \mathrm{~F}$

$\mathrm{ZF}$ II $8 \mathrm{~F}$

T1 I $20 \mathrm{~F}$

$\mathrm{Zr} I \mathrm{2I} \mathrm{F}$

N1 I $6 \mathrm{~F}$

$\mathrm{Kr} I I I \quad 1 \mathrm{~F}$

La II 1 F

$S$ VIII $1 \mathrm{~F}$

Fe II $31 \mathrm{~F}$

ZF III 5 F

$\mathrm{Zr} \mathrm{II} 27 \mathrm{~F}$

Fe II $51 \mathrm{~F}$

Fe III $11 \mathrm{~F}$

$\mathrm{Zr}$ II $14 \mathrm{~F}$

Co VII $\mathrm{F}$

N1 II $12 \mathrm{~F}$

Fe III 8 F

Fe III $8 \mathrm{~F}$

T1 II $\quad 5 \mathrm{~F}$

$\begin{array}{llrl}\text { Fe I } & 23 & \mathrm{~F} \\ \text { N1 VIII } & 5 & \mathrm{~F}\end{array}$

V II $16 \mathrm{~F}$

$39 \mathrm{~F}$

Mn $X$

Fe I

$11 \mathrm{~F}$
$11 \mathrm{~F}$
Blement Multiplet No.

$\begin{array}{rl}4 & F \\ 28 & F \\ 1 & F \\ 5 & F \\ 40 & F \\ 48 & F \\ 11 & F \\ 5 & F \\ 6 & F \\ 1 & F\end{array}$

\begin{tabular}{|c|c|c|}
\hline $\mathrm{Pe}$ & & 38 \\
\hline $\mathrm{Zr}$ & I I & 13 \\
\hline $\mathrm{Cr}$ & VIII & 1 \\
\hline T1 & II I & 4 \\
\hline $\mathrm{Cr}_{\mathrm{r}}$ & II & 22 \\
\hline $\mathbf{Z r}$ & II & 24 \\
\hline T1 & II & 5 \\
\hline $\mathrm{Z}_{\mathbf{r}}$ & II & 27 \\
\hline $\mathrm{Cr}$ & I I & 10 \\
\hline $\mathrm{Cr}_{\mathrm{r}}$ & II & 10 \\
\hline
\end{tabular}

Cr II

T1 II

Fe I

Co II

$\mathrm{Fe} I$

T1 II

Zr II

N1 II

Cr II

$\begin{array}{ll}\text { Cr } & \text { II } \\ \text { Cr II } & \text { II }\end{array}$

Cr II

Cr II

TI II

N1 VIII

Fe I

$\mathrm{Fe} I$

Co II

$\begin{array}{ll}\text { Zr } & \text { II } \\ \mathrm{Fe} & \text { I } \\ \mathrm{Fe} & \text { I }\end{array}$

Co II

S II

$\checkmark$ II

Cr II

Ti I

Cr II

Cr II

Cr II

TI II

Cr II

Fe I

S II

Fe I

Fe II

Fe II

Cr II

Cr II

Cr II

7r I

Zr II

V II

T1 I

Cr II

$S$ I

10366.28

10372.30

Cr II

Cr II

TI II

Cr II

TI I

Cr II

Cr V

N I

10394.3

10395.4

10400.63

10404 . 1

10431.10

10432.60

10443.85

10447.44

10452.56

10458.8

I A

10461.85

10464.94

10475.86

10486.87

10481.98

10494.00

10500.68

10502.67

10503.47

10504.3

10508.07

10810.26

10518.77

10553.58

10561.05

10568.84

10588.44

10676.88

10582.32

10601.80

10603.65

10608.1

10608.18

10627.5

10640.18

10640.4

10642.86

10660.35

10671.7

10676.61

10686.87

10718.16

10718.84

$10746.80 \mathrm{C}$

10747.64

10749.7

10758.91

10758.04

10758.32

10770.38

10771.88

10780.17

10784.80

10786.00

10796.2

10796.48

10797.66

10797.95

10798.14

10800.75

10807.8

10818.8

10860.44

10872.05

10882.6

10880.02

10901.70

10908.34

10812.8

10916.5

10916.64

10821.07 
11123.63 11132.24

11151.54

11173.94

11178.94

11185.14

11185.70

11191.43

11193.04

11202.11

11203.92

11228.14

11233.80

11237.04

11242.12

11246.87

11261.79

11272.6

11280.5

11284.9

11305.8

11315.62

11324.18

11332.50

11347.6

11359.87

11368.21

11396.50

11402.87

11414.22

11432.93

11444.61

11444.66

11450.66

11458.27

11471.69.

11477.29

11478.82

11479.61

11483.2

11495.96

11509.6

11518.28

11520.46

11521.31

11524.46

11537.68

11557.08

11568.38

11580.17

$\begin{array}{lll}\text { T1 I } & 26 \mathrm{~F} & 11595.50 \\ \text { Zr II } & 21 \mathrm{~F} & 11602.41 \\ \text { T1 II } & 14 \mathrm{~F} & 11606.00 \\ \text { T1 II } & 14 \mathrm{~F} & 11611.10 \\ \text { T1 II } & 13 \mathrm{~F} & 11616.88 \\ \text { T1 I } & 26 \mathrm{~F} & 11618.68 \\ \text { T1 II } & 14 \mathrm{~F} & 11619.10 \\ \text { T1 I } & 26 \mathrm{~F} & 11621.54 \\ \text { T1 I } & 26 \mathrm{~F} & 11658.88 \\ \text { Fe I } & 41 \mathrm{~F} & 11659.62\end{array}$

11665.66

Zr II 13 F

T1 II

Fe I

$\mathrm{Fe} I$

T1 II

V II

T1 I

Fe III

Co II

Fe III

$\begin{array}{rl}2 & \mathrm{~F} \\ 22 & \mathrm{~F} \\ 32 & \mathrm{~F} \\ 2 & \mathrm{~F} \\ 1 & \mathrm{~F} \\ 26 & \mathrm{~F} \\ 13 & \mathrm{~F} \\ 1 & \mathrm{~F} \\ 13 & \mathrm{~F}\end{array}$

$\begin{array}{ll}S & I \\ V & I\end{array}$

V II

T1 I

Co VII

N1 II

V II

T1 II

$\begin{array}{ll}\text { T1 } & \text { I } \\ \text { V } & \text { II }\end{array}$

T1 II

$\begin{array}{ll}\text { V } & \text { II } \\ \text { V } & \text { II }\end{array}$

Fe I

T1 II

$\checkmark$ II

T1 II

T1 II

V II

II

Fe I

N1 VIII

Fe I

T1 I

T1 1

$\begin{array}{ll}\text { T1 } & \text { I } \\ \text { Fo I }\end{array}$

$\mathrm{Fe} I$

T1 II

$\begin{array}{ll}\text { V } & \text { II } \\ \text { V } & \text { II }\end{array}$

11748.60

11764.23

11765.16

11767.30

11771.95

11778.39

11782.27

11782.63

11784.62

11785.17

11786.08

11789.27

11790.50

11791.90

11792.65

11799.5

11823.03

11836.06

11849.83

11852.48

11856.02

11857.28

11867.96

11881.68

11884.67

11896.48

11898.2

11918.75

11933.60

11943.76

11960.77
Element Multiplet No.

$\begin{array}{ll}\text { Zr } & \text { II } \\ \text { T1 } & \text { II } \\ \text { V } & \text { II } \\ \text { T1 } & \text { II } \\ \text { N1 } & \text { II } \\ \text { T1 } & \text { II } \\ \text { V } & \text { II } \\ \text { T1 } & \text { I } \\ \text { V } & \text { II } \\ \text { Zr } & \text { II }\end{array}$

$\begin{array}{rl}18 & \mathrm{~F} \\ 1 & \mathrm{~F} \\ 23 & \mathrm{~F} \\ 12 & \mathrm{~F} \\ 1 & \mathrm{~F} \\ 2 & \mathrm{~F} \\ 1 & \mathrm{~F} \\ 1 & \mathrm{~F} \\ 22 & \mathrm{~F} \\ 18 & \mathrm{~F}\end{array}$

T1 I

T1 1

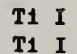

Zr II

T1 II

$\begin{array}{ll}\text { T } & \text { II } \\ \text { II }\end{array}$

T1 I

V II

Fe

Fe I

T1 I

T1 I

T1 II

T1 II

Cr II

Cr II

Cr II

Fe I

Cr II

Fe I

Fe I

T1 I

T1 III

TI II

T1 I

T1 I

V II

T1 I

V II

T1 II

T1 I

TI II

Sc II

$P$ II

V II

T1 I

Cr II

TI I
I A

11961.78

11871.26

11897.42

12012.60

12019.17

12024.89

12025.23

12061.0

12072.48

12094.78

12095.67

12168.18

12168.8

12168.80

12170.50

12178.83

12209.6

12211.22

12219.66

Blement

Mult1plet No.

12323.27

12372.65

12387.48

12417.8

12460.65

12463.08

12471.70

12645.23
Cr II

T1 II

Fe I

T1 I

Fe I

T1 I

Fe I

T1 III

$\mathrm{Fe} I$

Zr II

T1 I

Cr II

Co II

T1 I

Cr. II

Cr II

Co VII

Zr II

$\checkmark$ II

Cr II

Cr II

N1 II

Fe I

Fe I

T1 III

Cr II

Cr II

Cr II

Fe I

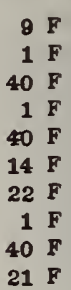

$14 \mathrm{~F}$

$8 \mathrm{~F}$

$1 \mathrm{~F}$

1

$9 \mathrm{~F}$

$6 \mathrm{~F}$

$18 \mathrm{~F}$

(2)

$26 \mathrm{~F}$

$11 \mathrm{~F}$

$22 \mathrm{~F}$

$22 \mathrm{~F}$

$\begin{array}{ll}1 & \mathrm{~F} \\ 9 & \mathrm{~F}\end{array}$

$9 \mathrm{~F}$

(

$22 \mathrm{~F}$ 


\section{Publications in the National Standard Reference Data Series National Bureau of Standards}

You may use this listing as your order form by checking the proper box of the publication(s) you desire or by providing the full identification of the publication you wish to purchase. The full letter symbols with each publication number and full title of the publication and author must be given in your order, e.g. NSRDS-NBS-21, Kinetic Data on Gas Phase Unimolecular Reactions, by S. W. Benson and H. E. O'Neal.

Pay for publications by check, money order, or Superintendent of Documents coupons or deposit account. Make checks and money orders payable to Superintendent of Documents. Foreign remittances should be made either

NSRDS-NBS 1, National Standard Reference Data SystemPlan of Operation, by E. L. Brady and M. B. Wallenstein, 1964 (15 cents), SD Catalog No. C13.48:1.

NSRDS-NBS 2, Thermal Properties of Aqueous Uni-univalent Electrolytes, by V. B. Parker, 1965 (45 cents), SD Catalog No. C13.48:2.

NSRDS-NBS 3, Sec. 1, Selected Tables of Atomic Spectra, Atomic Energy Levels and Multiplet Tables, Si II, Si III, Si IV, by C. E. Moore, 1965 (35 cents), SD Catalog No. C13.48:3/Sec.1.

NSRDS-NBS 3, Sec. 2, Selected Tables of Atomic Spectra, Atomic Energy Levels and Multiplet Tables, Si I, by C. E. Moore, 1967 (20 cents), SD Catalog No. C13.48:3/Sec.2.

NSRDS-NBS 3, Sec. 3, Selected Tables of Atomic Spectra, Atomic Energy Levels and Multiplet Tables, C I, C II, C III, C Iv, C v, C VI, by C. E. Moore, 1970 (\$1), SD Catalog No. C13.48:3/ $\mathrm{Sec} .3$.

$\square$ NSRDS-NBS 3, Sec. 4, Selected Tables of Atomic Spectra, Atomic Energy Levels and Multiplet Tables, N Iv, N v, N vi, N viI, by C. E. Moore, 1971 (55 cents), SD Catalog No. C13.48:3/Sec. 4.

$\square$ NSRDS-NBS 4, Atomic Transition Probabilities, Vol. I, Hydrogen Through Neon, by W. L. Wiese, M. W. Smith, and B. M. Glennon, 1966 ( $\$ 2.50)$, SD Catalog No. C13.48:4/Vol.I.

NSRDS-NBS 5, The Band Spectrum of Carbon Morioxide, by P. H. Krupenie, 1966 (70 cents), SD Catalog No. C13.48:5

$\square$ NSRDS-NBS 6 , Tables of Molecular Vibrational Frequencies, Part 1, by T. Shimanouchi, 1967 (40 cents), SD Catalog No. C13.48:6/Pt.1.

NSRDS-NBS 7, High Temperature Properties and Decomposition of Inorganic Salts, Part 1. Sulfates, by K. H. Stern and E. L. Weise, 1966 (35 cents), SD Catalog No. C13.48:7/Pt.1.

$\square$ NSRDS-NBS 8, Thermal Conductivity of Selected Materials, by R. W. Powell, C. Y. Ho, and P. E. Liley, 1966 (\$3). PB189698 NSRDS-NBS 9, Tables of Bimolecular Gas Reactions, by A. F. Trotman-Dickenson and G. S. Milne, 1967 (\$2), SD Catalog No. C13.48:9.

NSRDS-NBS 10, Selected Values of Electric Dipole Moments for Molecules in the Gas Phase, by R. D. Nelson, Jr., D. R. Lide, Jr., and A. A. Maryott, 1967 (40 cents), SD Catalog No. C13.48:10.

NSRDS-NBS 11, Tables of Molecular Vibrational Frequencies, Part 2, by T. Shimanouchi, 1967 (30 cents), SD Catalog No. C13.48:11/Pt.2.

$\square$ NSRDS-NBS 12, Tables for the Rigid Asymmetric Rotor: Trans formation Coefficients from Symmetric to Asymmetric Bases and Expectation Values of $\mathbf{P}_{2}^{2}, \mathbf{P}_{z}^{4}$, and $\mathbf{P}_{z}^{6}$, by $R$. $H$. Schwendeman, 1968 (60 cents), SD Catalog No. C13.48:12

NSRDS-NBS 13, Hydrogenation of Ethylene on Metallic Catalysts, by J. Horiuti and K. Miyahara, 1968 (\$1), SD Catalog No. C13.48:13.

$\square$ NSRDS-NBS 14, X-Ray Wavelengths and X-Ray Atomic Energy Levels, by J. A. Bearden, 1967 (40 cents), SD Catalog No. C13.48:14.

* Available from National Technical Information Service, Springfield, Virginia 22151 by international money order or draft on an American bank. Postage stamps are not acceptable.

No charge is made for postage to destinations in the United States and possessions, Canada, Mexico, and certain Central and South American countries. To other countries, payments for documents must cover postage. Therefore, one-fourth of the price of the publication should be added for postage.

Send your order together with remittance to Superin tendent of Documents, Government Printing Office, Washington, D.C. 20402.

NSRDS-NBS 15, Molten Salts: Vol. 1, Electrical Conductance, Density, and Viscosity Data, by G. J. Janz, F. W. Dampier, G. R. Lakshminarayanan, P. K. Lorenz, and R. P. T. Tomkins, 1968 (\$3), SD Catalog No. C13.48:15/Vol.1.

$\square$ NSRDS-NBS 16, Thermal Conductivity of Selected Materials, Part 2, by C. Y. Ho, R. W. Powell, and P. E. Liley, 1968 (\$2), SD Catalog No. C13.48:16/Pt.2.

NSRDS-NBS 17, Tables of Molecular Vibrational Frequencies, Part 3, by T. Shimanouchi, 1968 (30 cents), SD Catalog No. C13.48:17/Pt.3.

$\square$ NSRDS-NBS 18, Critical Analysis of the Heat-Capacity Dats of the Literature and Evaluation of Thermodynamic Properties of Copper, Silver, and Gold From 0 to $300^{\circ} \mathrm{K}$, by G. T. Furukawa, W. G. Saba, and M. L. Reilly, 1968 (40 cents), SD Catalog No. C13.48:18

$\square$ NSRDS-NBS 19, Thermodynamic Properties of Ammonia an Ideal Gas, by L. Haar, 1968 (20 cents), SD Catalog No. C13.48:19.

NSRDS-NBS 20, Gas Phase Reaction Kinetics of Neutral Oxygen Species, by H. S. Johnston, 1968 (45 cents), SD Cata $\log$ No. C13.48:20.

$\square$ NSRDS-NBS 21, Kinetic Data on Gas Phase Unimolecular Reactions, by S. W. Benson and H. E. O'Neal, 1970 (\$7), SD Catalog No. C13.48:21.

$\square$ NSRDS-NBS 22, Atomic Transition Probabilities, Vol. II, Sodium Through Calcium, A Critical Data Compilation, by W. L. Wiese, M. W. Smith, and B. M. Miles, 1969 (\$4.50), SD Catalog No. C13.48:22/Vol.II.

NSRDS-NBS 23, Partial Grotrian Dlagrams of Astrophysical Interest, by C. E. Moore and P. W. Merrill, 1968 (55 cents), SD Catalog No. C13.48:23.

$\square$ NSRDS-NBS 24, Theoretical Mean Activity Coefficlents of Strong Electrolytes in Aqueous Solutions from 0 to $100^{\circ} \mathrm{C}$, by Walter J. Hamer, 1968 (\$4.25), SD Catalog No. C13.48:24.

$\square$ NSRDS-NBS 25, Electron Impact Excitation of Atoms, by B. L. Moiseiwitsch and S. J. Smith, 1968 (\$2), SD Catalog No. C13.48:25.

NSRDS-NBS 26, Ionization Potentials, Appearance Potentlals, and Heats of Formation of Gaseous Positive lons, by J. L. Franklin, J. G. Dillard, H. M. Rosenstock, J. T. Herron, K. Draxl, and F. H. Field, 1969 (\$4), SD Catalog No. C13.48:26.

NSRDS-NBS 27, Thermodynamic Propertles of Argon from the Triple Point to $300 \mathrm{~K}$ at Pressures to 1000 Atmospheres, by A. L. Gosman, R. D. McCarty, and J. G. Hust, 1969 (\$1.25), SD Catalog No. C13.48:27.

$\square$ NSRDS.NBS 28, Molten Salts: Vol. 2, Section 1, Electrochemistry of Molten Salts: Gibbs Free Energies and Excess Free Energies From Equilibrium-Type Cells, by G. J. Janz and C. G. M. Dijkhuis. Section 2, Surface Tension Data, by G. J. Janz, G. R. Lakshminarayanan, R. P. T. Tomkins, and J. Wong, 1969 (\$2.75), SD Catalog No. C13.48:28/Vol.2.

$\square$ NSRDS-NBS 29, Photon Cross Sections, Attenuation Coefficients, and Energy Absorption Coefficients From $10 \mathrm{keV}$ to $100 \mathrm{GeV}$, by J. H. Hubbell, 1969 (75 cents), SD Catalog No. C13.48:29. 
$\square$ NSRDS-NBS 30, High Temperature Properties and Decomposition of Inorganic Salts, Part 2, Carbonates, by K. H. Stern and E. L. Weise, 1969 (45 cents). SD Catalog No. C13.48:30/Pt. 2

$\square$ NSRDS-NBS 31, Bond Discrimination Energies in Simple Molecules, by B. deB. Darwent, 1970 (55 cents). SD Catalog No. C13.48:31.

$\square$ NSRDS-NBS 32, Phase Behavior in Binary Multicomponent Systems at Elevated Pressures: $\eta$-Pentane and Methane- $\eta$-Pentane, by V. M. Berry and B. H. Sage, 1970 (70 cents). SD Catalog No. C13.48.32.

$\square$ NSRDS-NBS 33, Electrolytic and Conductance and the Conductance of the Acids in Water, by W. J. Hamer and H. J. DeWane, 1970 (50 cents). SD Catalog No. C13.48:34.

$\square$ NSRDS-NBS 34, Ionization Potentials and Ionization Limits Derived from the Analyses of Optical Spectra, by C. E. Moore, 1970 (75 cents). SD Catalog No. C13.48:33.

$\square$ NSRDS-NBS 35, Atomic Energy Levels, Vol. I 1 H to $23 \mathrm{~V}$; Vol. II ${ }^{24} \mathrm{Cr}$ to $41 \mathrm{Nb}$; $\mathrm{Vol}$ III $22 \mathrm{Mo}$ to $57 \mathrm{La}-72 \mathrm{Hf}$ to ${ }^{89} \mathrm{Ac}$, by C. E.
Moore, 1971 (In press). SD Catalog No. C13.48:35/Vols. I, II, and III.

NSRDS-NBS 36, Micelle Concentrations of Aqueous Surfactant Systems, by P. Mukerjee and K. J. Mysels, 1971 (In press). SD Catalog No. C13.48:36

$\square$ NSRDS-NBS 37, JANAF Thermochemical Tables, 2d Edition, by D. R. Stull, H. Prophet, et al., 1971 (in press), SD Catalog No. C13.48:37.

$\square$ NSRDS-NBS 38, Critical Review of Ultraviolet Photoabsorption Cross Sections for Molecules of Astrophysical and Aeronomic Interest, by R. D. Hudson, 1971 (in press), SD Catalog No. C13.48:38.

$\square$ NSRDS-NBS 39, Tables of Molecular Vibrational Frequencies, Consolidated Volume I, by T. Shimanouchi, 1971 (in press). SD Catalog No. C13.48:39.

$\square$ NSRDS-NBS 40, A Multiplet Table of Astrophysical Interest, by C. E. Moore, 1971 ( $\$$ ). SD Catalog No. C13.48:40. 


\section{Announcement of New Publications in National Standard Reference Data Series}

Superintendent of Documents, Government Printing Office, Washington, D.C. 20402

Dear Sir:

Please add my name to the announcement list of new publications to be issued in the series: National Standard Reference Data Series-National Bureau of Standards.

Name....

Company.

Address

City.

State

Zip Code.

(Notification key $\mathrm{N}-337$ ) 



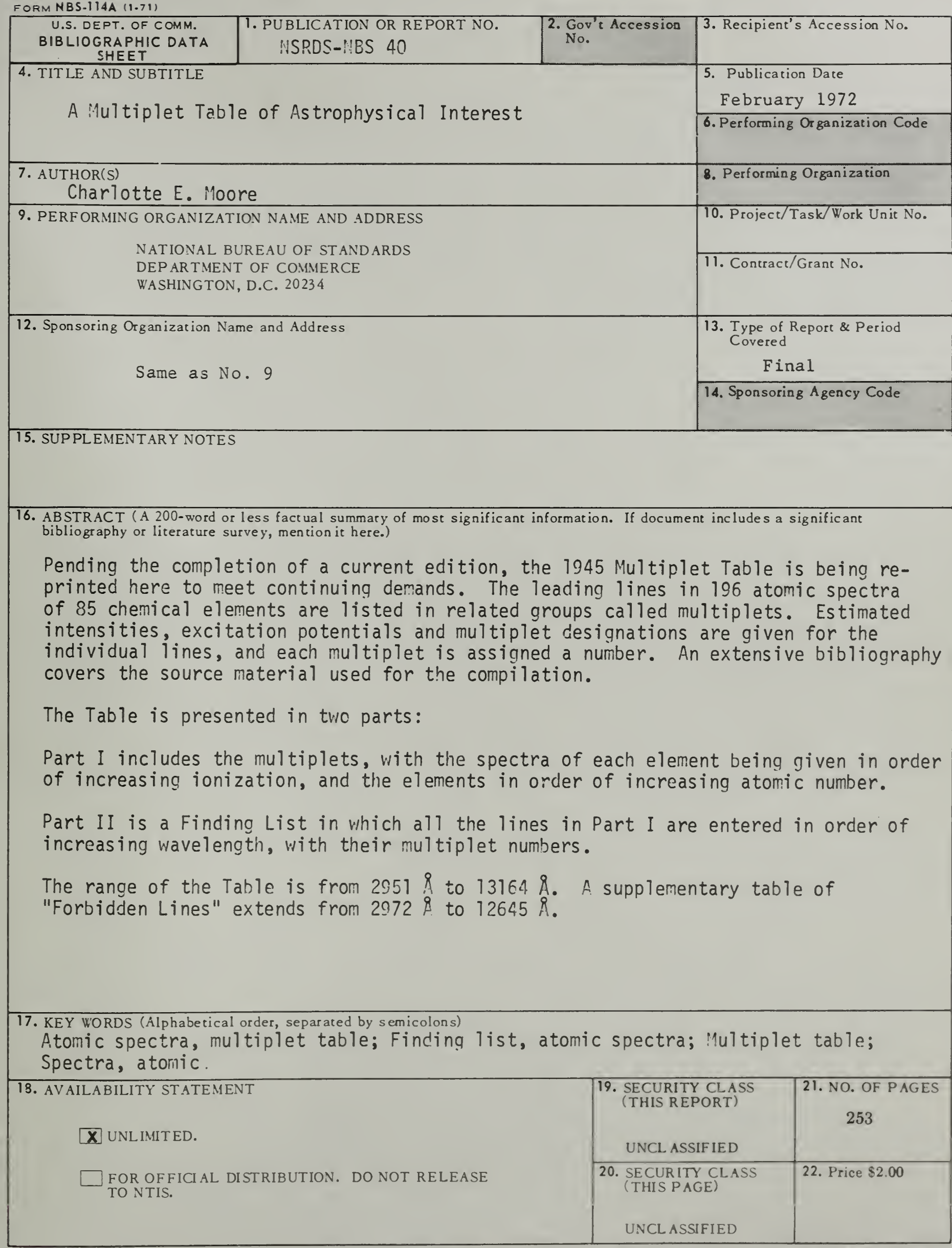





\section{NBS TECHNICAL PUBLICATIONS}

\section{PERIODICALS}

JOURNAL OF RESEARCH reports National Bureau of Standards research and development in pliysics, mathematics, chemistry, and engineering. Comprehensive scientific papers give complete details of the work. including laboratory data, experimental procedures, and theoretical and mathernatical analyses. Illustrated with photographs, drawings, and charts.

Published in three sections, available separately:

\section{- Physics and Chemistry}

Papers of interest primarily to scientists working in these fields. This section covers a broad range of physical and chemical research, with major emphasis on standards of physical measurement, fundamental constants, and properties of matter. Issued six times a year. Annual subscription: Domestic, \$9.50; \$2.25 additional for foreign mailing.

\section{- Mathematical Sciences}

Studies and compilations designed mainly for the mathematician and theoretical physicist. Topics in mathernatical statistics, theory of experiment design, numerical analysis, theoretical physics and chemisty, logical design and programming of computers and computer systems. Short numerical tables. Issued quarterly. Annual subscription: Domestic, \$5.00; $\$ 1.25$ additional for foreign mailing.

\section{- Engineering and Instrumentation}

Reporting results of interest chiefly to the engineer and the applied scientist. This section includes many of the new developments in instrumentation resulting from the Bureau's work in physical measurement, data processing, and development of test methods. It will also cover some of the work in acoustics, applied mechanics, building research, and cryogenic engineering. Issued quarterly. Annual subscription: Domestic, $\$ 5.00 ; \$ 1.25$ additional for foreign mailing.

\section{TECHNICAL NEWS BULLETIN}

The best single source of information concerning the Bureau's research. developmental, cooperative, and publication activities, this monthly publication is designed for the industry-oriented individual whore daily work involves intinate contact with science and terhnology- for enginerers, chemists, phuicists, research managers, froduct-dectepment manager, and compan) (xicutizes. Innunl subscription. Donestif $\$ 3.00 ; \$ 1.00$ additional for foresen manling

\section{NONPERIODICALS}

Applied Mathematics Series. Mathematical tables manuals, and studies.

Building Science Series. Research results, test methods, and performance criteria of building materials, components, systems, and structures.

Handbooks. Recommended codes of engineering and industrial practice (including safety codes) developed in cooperation with interested industries, professional organizations, and regulatory bodies.

Special Publications. Proceedings of NBS conferences, bibliographies, annual reports, wall charts, pamphlets, etc.

Monographs. Major contributions to the technical literature on various subjects related to the Bureau's scientific and technical activities.

National Standard Reference Data Series. NSRDS provides quantitative data on the physical and chemical properties of materials, compiled from the world's literature and critically evaluated.

Product Standards. Provide requirements for sizes, types, quality, and methods for testing various industrial products. These standards are developed cooperatively with interested Government and industry groups and provide the basis for common understanding of product characteristics for both buyers and sellers. Their use is voluntary.

Technical Notes. This series consists of communications and reports (covering both other agency and NBS-sponsored work) of limited or transitory interest.

Federal Information Processing Standards Publications. This series is the official publication within the Federal Government for information on standards adopted and promulgated under the Public Law 89-306, and Bureau of the Budget Circular A-86 entitled, Standardization of Data Elements and Codes in Data Systems

Consumer Information Series. Practical information, based on NBS research and experience, coveriur areas of interest to the consume. Easily understandable languase and illustrations provide Lseful backsround knowledge for shoppine in todavs te hnolo: ic al marketplace.

NBS Special Iuhlication :30.5, Supplement 1, I'ublications of the NBS, 1968-1969. Wh norcer-

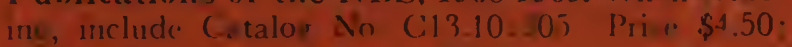
S1 25 wdditional for foreiv $n$ mesluty 
